LOCKHEED MATTIN

ENVIRONMENTAL RESTORATION

PROGRAM
RET EIVED

MAY 281996

OSTI

\title{
Preliminary Remediation Goals for Use at the U.S. Department of Energy Oak Ridge Operations Office
}


This report has been reproduced directly from the best available copy.

Available to DOE and DOE contractors from the Office of Scientific and Technical Information, P.O. Box 62, Oak Ridge, TN 37831; prices available from 615-576-8401 (fax 423-576-2865).

Available to the public from the National Technical Information Service, U.S. Department of Commerce, 5285 Port Royal Rd., Springfield, VA 22161. 


\title{
Preliminary Remediation Goals for Use at the U.S. Department of Energy Oak Ridge Operations Office
}

\author{
Environmental Restoration Division \\ P.O. Box 2003 \\ Oak Ridge, Tennessee 37831-7298
}

Date Issued-May 1996

\section{Prepared for}

U.S. Department of Energy

Office of Environmental Management

under budget and reporting code EW 20

LOCKHEED MARTIN ENERGY SYSTEMS, INC.

managing the

Environmental Management Activities at

Oak Ridge K-25 Site Paducah Gaseous Diffusion Plant

Oak Ridge Y-12 Plant Portsmouth Gaseous Diffusion Plant

Oak Ridge National Laboratory

under contract DE-AC05-84OR21400

for the

U.S. DEPARTMENT OF ENERGY 



\section{DISCLAIMER}

This report was prepared as an account of work sponsored by an agency of the United States Government. Neither the United States. Government nor any agency thereof, nor any of their employees, makes any warranty, express or implied, or assumes any legal liability or responsibility for the accuracy, completeness, or usefulness of any information, apparatus, product, or process disclosed, or represents that its use would not infringe privately owned rights. Reference herein to any specific commercial product, process, or service by trade name, trademark, manufacturer, or otherwise does not necessarily constitute or imply its endorsement, recommendation, or favoring by the United States Government or' any agency thereof. The views and opinions of authors expressed herein do not necessarily state or reflect those of the United States Government or any agency thereof.

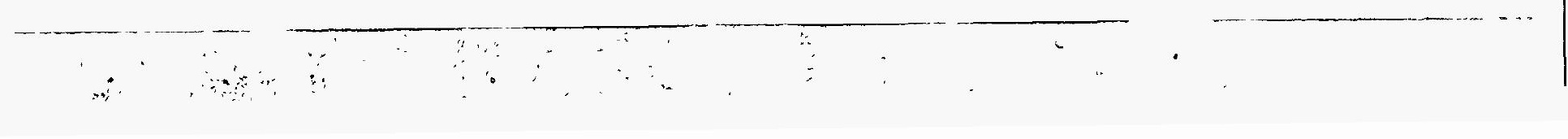




\section{PREFACE}

This technical memorandum presents Preliminary Remediation Goals (PRGs) for use in human health risk assessment efforts under the United States Department of Energy, Oak Ridge Operations Office Environmental Restoration (ER) Division. This work was performed by the ER Risk Assessment Program under Work Breakdown Structure 1.4.12.2.3.04.07.01 entitled Risk Assessment Review, Planning, and Coordination and within the Activity Data Sheet 8304, Technical Integration. This document provides the ER Division with standardized PRGs which are integral to the Remedial Investigation/Feasibility Study process. They are used during project scoping (Data Quality Objectives development), in screening level risk assessments to support early action or No Further Investigation decisions, and in the baseline risk assessment where they are employed in the selection of chemicals of potential concern. The primary objective of this document is to standardize these values and eliminate any duplication of effort by providing PRGs to all contractors involved in risk activities. In addition, by managing the assumptions and systems used in PRG derivation, the ER Risk Assessment Program will be able to control the level of quality assurance associated with these risk-based guideline values. 
, 


\section{ACKNOWLEDGMENTS}

The Risk Assessment Program would like to acknowledge Leslie D. Bloom for her assistance and dedication to the preparation of this technical memorandum. 


\section{CONTENTS}

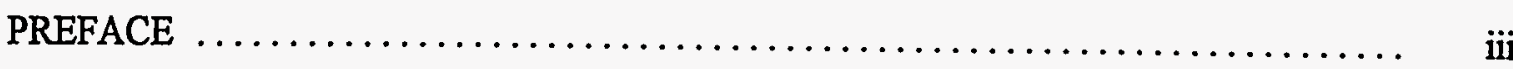

ACKNOWLEDGMENTS $\ldots \ldots \ldots \ldots \ldots \ldots \ldots \ldots \ldots \ldots \ldots \ldots \ldots \ldots \ldots \ldots \ldots \ldots \ldots$

ABBREVIATIONS $\ldots \ldots \ldots \ldots \ldots \ldots \ldots \ldots \ldots \ldots \ldots \ldots \ldots \ldots \ldots \ldots \ldots \ldots \ldots \ldots \ldots \ldots \ldots$

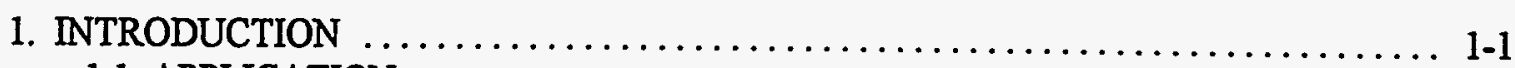

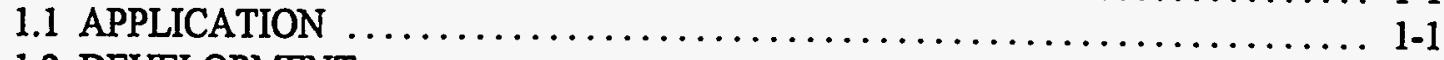

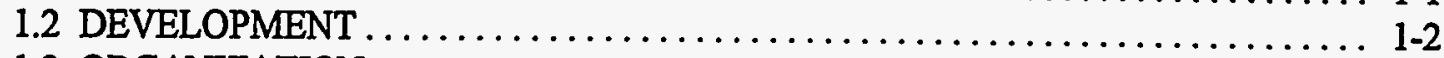

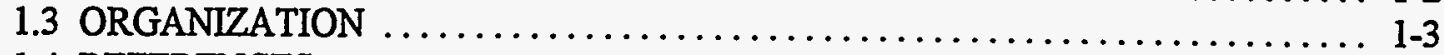

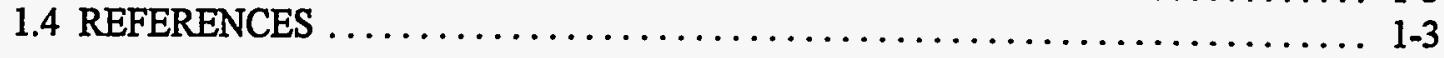

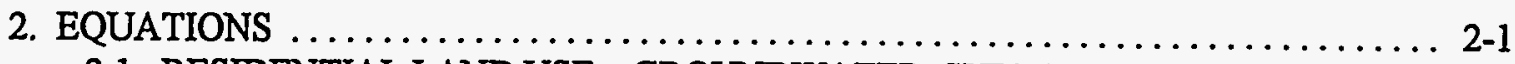

2.1 RESIDENTIAL LAND USE-GROUNDWATER, SURFACE WATER, SOIL,

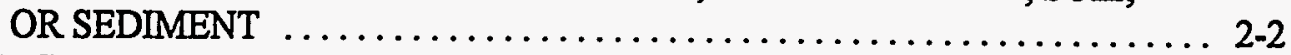

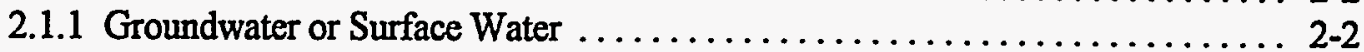

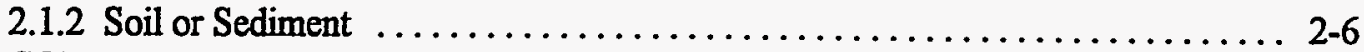

2.2 COMMERCIAL/INDUSTRIAL LAND USE-GROUNDWATER, SURFACE WATER,

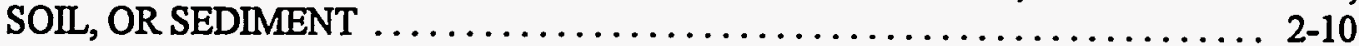

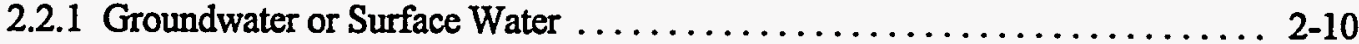

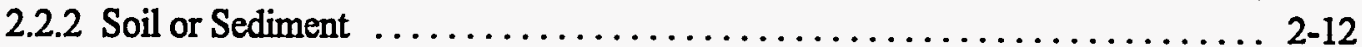

2.3 AGRICULTURAL LAND USE-PRODUCE, WHOLE MILK, AND BEEF . . . . 2-20

2.3.1 Nonradionuclide Contaminant ......................... 2-20

2.3.2 Radionuclide Contaminant ......................... 2-24

2.3.3 Back-Calculated Concentrations for Groundwater and Soil ........... 2-25

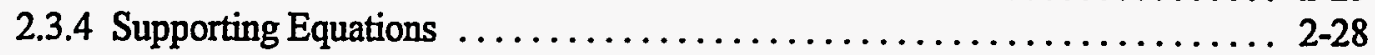

2.4 RECREATIONAL LAND USE_SURFACE WATER, SOIL, OR SEDIMENT . . . 2-32

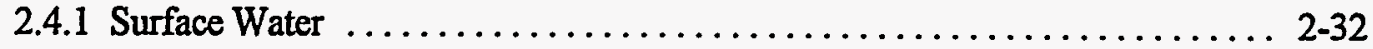

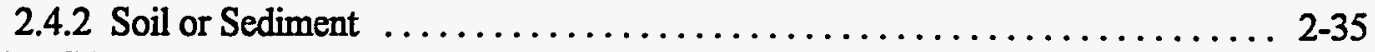

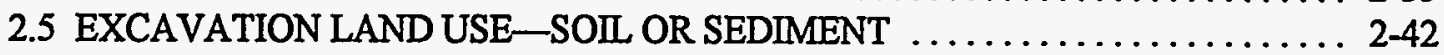

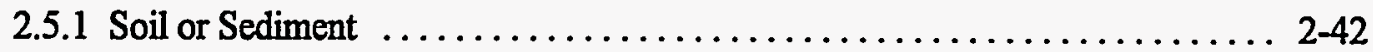

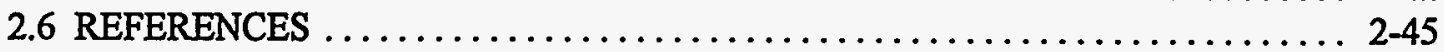

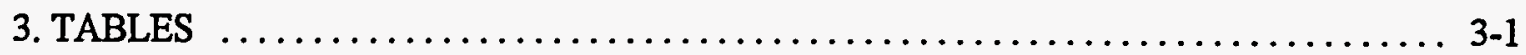

3.1 RESIDENTIAL LAND USE

3.1a Risk-based Preliminary Remediation Goals for nonradionuclides

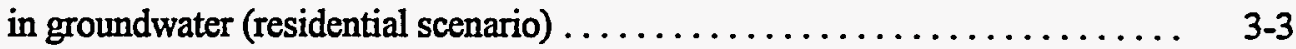

3.1b Risk-based Preliminary Remediation Goals for radionuclides in groundwater (residential scenario) . . . . . . . . . . . 39.........

3.1c Risk-based Preliminary Remediation Goals for nonradionuclides

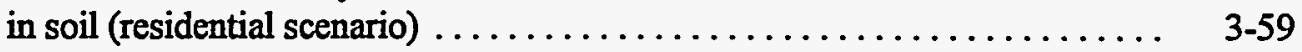

3.1d Risk-based Preliminary Remediation Goals for radionuclides in soil (residential scenario) $\ldots \ldots \ldots \ldots \ldots \ldots \ldots \ldots \ldots \ldots \ldots$ 3-101

3.1e Risk-based Preliminary Remediation Goals for nonradionuclides in fish (residential scenario) 
3.1f Risk-based Preliminary Remediation Goals for radionuclides in fish (residential scenario)

3.1g Risk-based Preliminary Remediation Goals for water based on ingestion

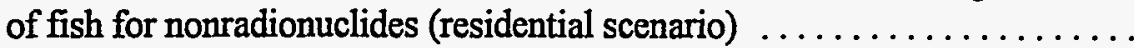

3.1h Risk-based Preliminary Remediation Goals for water based on ingestion of fish for radionuclides (residential scenario) $\ldots \ldots \ldots \ldots \ldots \ldots \ldots$

3.2 INDUSTRIAL LAND USE

3.2a Risk-based Preliminary Remediation Goals for nonradionuclides in groundwater (industrial scenario) $\ldots \ldots \ldots \ldots \ldots \ldots \ldots \ldots \ldots . . . \ldots \ldots$

3.2b Risk-based Preliminary Remediation Goals for radionuclides in groundwater (industrial scenario) $\ldots \ldots \ldots \ldots \ldots \ldots \ldots \ldots \ldots \ldots$

3.2c Risk-based Preliminary Remediation Goals for nonradionuclides in soil (industrial scenario)

3.2d Risk-based Preliminary Remediation Goals for radionuclides in soil (industrial scenario)

3.3 AGRICULTURAL LAND USE

3.3a Risk-based Preliminary Remediation Goals for nonradionuclides in water (agricultural scenario) . . . . . . . . . . . . . . . . . .

3.3b Risk-based Preliminary Remediation Goals for radionuclides

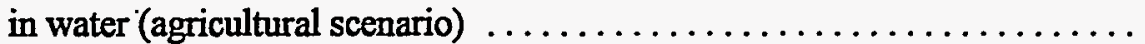

3.3c Risk-based Preliminary Remediation Goals for nonradionuclides

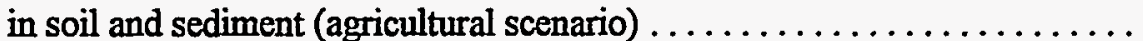

3.3d Risk-based Preliminary Remediation Goals for radionuclides

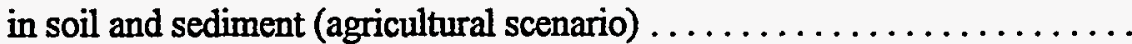

3.3e Risk-based Preliminary Remediation Goals for nonradionuclides in water and soil (agricultural scenario) $\ldots \ldots \ldots \ldots \ldots \ldots \ldots \ldots$

3.3f Risk-based Preliminary Remediation Goals for radionuclides in water and soil (agricultural scenario)

3.4 RECREATIONAL LAND USE

3.4a Risk-based Preliminary Remediation Goals for nonradionuclides in groundwater (recreational scenario)

3.4b Risk-based Preliminary Remediation Goals for radionuclides in groundwater (recreational scenario) $\ldots \ldots \ldots \ldots \ldots \ldots \ldots \ldots \ldots$

3.4c Risk-based Preliminary Remediation Goals for nonradionuclides in soil (recreational scenario) . . . . . . . . . . . . . . . . . . . .

3.4d Risk-based Preliminary Remediation Goals for radionuclides in soil (recreational scenario) . . . . . . . . . . . . . . . . . . . .

3.4e Risk-based Preliminary Remediation Goals for nonradionuclides in fish (recreational scenario)

3.4f Risk-based Preliminary Remediation Goals for radionuclides in fish (recreational scenario)

3.4g Risk-based Preliminary Remediation Goals for water based on ingestion

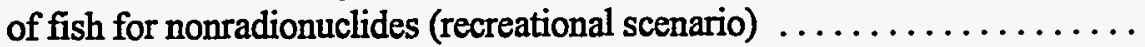

3.4h Risk-based Preliminary Remediation Goals for water based on ingestion of fish for radionuclides (recreational scenario) $\ldots \ldots \ldots \ldots \ldots \ldots$

\subsection{EXCAVATION LAND USE}

3.5a Risk-based Preliminary Remediation Goals for nonradionuclides

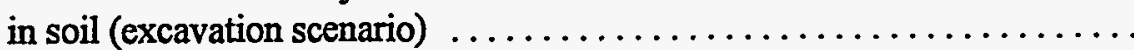

3.5b Risk-based Preliminary Remediation Goals for radionuclides

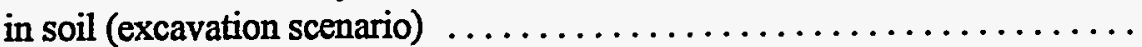




\subsection{CHEMICAL-SPECIFIC PARAMETERS}

3.6a Toxicity values used in calculating risk-based

Preliminary Remediation Goals (nonradionuclides)

3.6b Chemical-specific parameters used in calculating risk-based

Preliminary Remediation Goals (nonradionuclides)

3.6c Chemical-specific parameters used in calculating risk-based

Preliminary Remediation Goals (radionuclides)

\section{FIGURES}

1. Land uses and exposure routes considered in the development of PRGs $\ldots \ldots \ldots \ldots 1-5$

2. Combination PRGs for bis(2-ethylhexyl)phthalate $\ldots \ldots \ldots \ldots \ldots \ldots \ldots \ldots \ldots .2-22$ 
. 


\section{ABBREVIATIONS}

$\begin{array}{ll}\text { ARARs } & \text { Applicable or Relevant and Applicable Requirements } \\ \text { CASRN } & \text { chemical abstract registration number } \\ \text { COPCs } & \text { contaminants of potential concern } \\ \text { DOE-OR/ERD } & \text { Department of Energy-Oak Ridge/Environmental Restoration Division } \\ \text { EPA } & \text { United States Environmental Protection Agency } \\ \text { HEAST } & \text { Health Effects Assessment Summary Tables } \\ \text { HQ } & \text { hazard quotient } \\ \text { IRIS } & \text { Integrated Risk Information System } \\ \text { NCP } & \text { National Contingency Plan } \\ \text { NPL } & \text { National Priorities List } \\ \text { ORR } & \text { Oak Ridge Reservation } \\ \text { PRGs } & \text { Preliminary Remediation Goals } \\ \text { RAGS } & \text { Risk Assessment Guidance for Superfund } \\ \text { RIFS } & \text { Remedial Investigation/Feasibility Study } \\ \text { ROD } & \text { Record of Decision }\end{array}$




\section{INTRODUCTION}

Chemical-specific preliminary remediation goals (PRGs) are concentration goals for individual chemicals for specific medium and land use combinations. The PRGs provided in this report are referred to as risk-based because they have been calculated using risk assessment procedures. Risk-based calculations set concentration limits using both carcinogenic or noncarcinogenic toxicity values under specific exposure pathways. The PRG is a concentration that is derived from a specified excess cancer risk level (carcinogenic PRG) or hazard quotient (HQ) (noncarcinogenic PRG).

Risk Assessment Guidance for Superfund: Volume I-Human Health Evaluation Manual (Part B) or RAGS, Part B [1], was prepared for risk assessors, remedial project managers, and others to assist them in developing PRGs for National Priorities List (NPL) sites. RAGS, Part B, provides guidance on using United States Environmental Protection Agency (EPA) toxicity values and exposure information to derive risk-based PRGs [1]. The Risk Assessment Program for Environmental Restoration is providing these values to all organizations involved in risk assessment activities to ensure consistent development and application of PRGs for Oak Ridge Operations.

\subsection{APPLICATION}

The National Contingency Plan (NCP) preamble indicates that PRGs are developed typically during the project scoping phase or concurrently with initial Remedial Investigation and Feasibility Study (RI/FS) activities (i.e., prior to completion of the baseline risk assessment). This early determination of PRGs facilitates development of appropriate detection limits and remedial alternatives.

Preliminary remediation goals can also be used as screening tools. Screening against PRGs and other limiting criteria is discussed in the Remedial Site Evaluation Report as a preliminary step in the RI/FS process. Comparisons can be used to focus concern on a specific medium or chemical of potential concern and support "no further action" recommendations. In addition, PRG screens can be used in place of most toxicity screens for baseline risk assessments. The toxicity screen is a tool used to identify contaminants of potential concern (COPCs) in baseline risk assessments. The objective is to eliminate chemicals that pose little or no risk at a site by comparing relative toxicities and identifying those chemicals contributing less than $1 \%$ of the total risk. The PRG screen is a more useful tool because each chemical is weighed on the basis of its toxicity rather than as a fraction of total risk.

The reader should note that risk-based PRGs (either during scoping or later) are initial guidelines (protective of human health and the environment) that are based on readily available information and comply with applicable or relevant and appropriate requirements (ARARs). They do not establish that cleanup to meet these goals is warranted. Preliminary remediation goals developed at the scoping phase using readily available information are generally modified based on site-specific data gathered during the RI/FS. Design staff may be able to streamline the consideration of remedialalternatives by developing PRGs early in the decision-making process (before the RI/FS and the baseline risk assessment are completed). A risk-based concentration will be considered a final remediation level only after appropriate analysis in the RI/FS and Record of Decision (ROD) [1]. 


\subsection{DEVELOPMENT}

The general equations used to calculate the PRGs were obtained from RAGS, Part B [1], where available; however, the remaining equations, such as concentrations for agricultural products, were developed using sources specific to the study of agricultural consumption by humans. The toxicity values used in the development of these PRGs were prepared by the Chemical Hazard Evaluation Group of the Biomedical and Environmental Information Analysis Section of the Health Sciences Research Division at the Oak Ridge National Laboratory. These values were compiled from EPA's Integrated Risk Information System (IRIS) [2] and Health Effects Assessment Summary Tables (HEAST) [3]. These values have been approved for use in risk assessments at all Department of Energy-Oak Ridge/Environmental Restoration Division (DOE-OR/ERD) sites.

Carcinogenic toxicity values are referred to as slope factors. Different slope factor values for ingestion, inhalation, and external exposure are provided. In addition, slope factors for dermal exposure (absorbed dose) are calculated by using the gastrointestinal absorption factor to adjust the oral slope factor [4]. In addition, skin absorption factors are used in the equations to adjust for dermal exposure. Noncarcinogenic toxicity values are referred to as reference doses (oral) and reference concentrations (inhalation). Both are subdivided into chronic and subchronic levels. For PRG calculations, only the chronic values are used because these are available for all chemicals with reference doses and are more conservative relative to human health. The inhalation reference concentration has been converted to a dose by the units conversion factor recommended in HEAST. Reference doses for dermal exposure (absorbed dose) are also calculated by using the gastrointestinal absorption factor to adjust the oral chronic reference dose.

The IRIS/HEAST databases contain toxicity values for more than 600 nonradioactive chemicals and more than 300 radioactive chemicals. However, toxicity values are not available for many chemicals. As the IRIS/HEAST databases are updated with toxicity changes and additions, this PRGs report will be updated also.

Preliminary remediation goals have been calculated for five land use scenarios that may be considered reasonable for future use of some areas of the Oak Ridge Reservation (ORR). The residential and industrial scenarios are recommended in RAGS, Part B [1]. In addition, recreation, agriculture, and excavation scenarios are included. The recreation and agriculture scenarios are mentioned in RAGS, Part A [5], for baseline risk assessments. The excavation scenario is included also because it is a likely industrial occurrence at ORR. Equation default parameters from RAGS have been used when available; others were obtained from documents pertaining to the particular land use scenario. The media evaluated are groundwater/surface water and soil/sediment.

The exposure routes considered are the same for groundwater and surface water; therefore, a single set of equations is used to represent the "water" exposure pathway. Likewise, the PRGs for soil are representative of sediment as well. For the resident, the routes of exposure are ingestion, inhalation of volatiles, and dermal contact with groundwater or surface water and ingestion, inhalation, dermal contact, and external exposure to soil or sediment. For the industrial worker, the routes of exposure are ingestion of groundwater or surface water and ingestion, inhalation, dermal contact, and external exposure to soil or sediment. 
Introduction

Organization/References

The agricultural scenario evaluates ingestion of produce, beef, and milk grown on the ORR using groundwater, surface water, soil, and sediment. The underlying assumptions for agricultural product ingestion are that the produce would be irrigated with either groundwater or surface water during the growing season and the pasture the cattle graze on would not be irrigated because of the sufficient average amount of rainfall for the region. Both vegetation and pasture would grow on existing soil and sediment around the ORR.

The exposure routes for the recreational visitor are ingestion and dermal contact with surface water; ingestion of fish caught in surface water; and ingestion, inhalation, dermal contact, and external exposure with soil or sediment or both. The exposure routes for the excavation worker are ingestion, inhalation, dermal contact, and external exposure to soil or sediment or both. These scenarios are demonstrated in Fig. 1.

The default parameters specified in RAGS, Parts A [5] and B [1], were used to develop the PRGS in this document. Defaults not available in RAGS, Parts $A$ and $B$, were obtained from land use- and pathway-specific literature searches. Media-specific information such as soil moisture content $(\theta$ and $\theta_{m}$ page 2-15) has been changed due to climatic conditions indigenous to the ORR.

\subsection{ORGANIZATION}

The remainder of this document is organized by land use scenario. In Chapter 2, each scenario is described, and likely exposure routes are represented. Each equation is numbered sequentially with the parameters and defaults listed immediately afterwards. The tables (Chapter 3 ) are arranged by scenario in the following order: 1) Residential, 2) Industrial, 3) Agriculture, 4) Recreation, and 5) Excavation. Each table contains the chemical name, its corresponding chemical abstract registration number (CASRN), and the appropriate PRG for each pathway at risk levels of 1.0E-04 and 1.0E-06 and an HI level of 1 . The tables are differentiated based on the medium (water or soil) and the chemical type (nonradionuclide or radionuclide). For each scenario, risk-based PRGs for nonradionuclides in water are followed by risk-based PRGs for radionuclides in water, nonradionuclides in soil, and radionuclides in soil.

\subsection{REFERENCES}

[1] United States Environmental Protection Agency (EPA). 1991. Risk Assessment Guidance for Superfund: Volume I - Human Health Evaluation Manual (Part B, Development of Risk-based Preliminary Remediation Goals). Office of Emergency and Remedial Response, U.S. Environmental Protection Agency, Washington, D.C.

[2] United States Environmental Protection Agency (EPA). 1994. Integrated Risk Information System (IRIS). Environmental Criteria and Assessment Office. Cincinnati, $\mathrm{OH}$.

[3] United States Environmental Protection Agency (EPA). 1993. Health Effects Assessment Summary Tables. Annual FY-1993. OERR 9200.6-303-(93-1). PB93-921199. March And Supplement No. 1, OERR 9200.6-303 (93-2). PB93-921101. July. 
Introduction

References

[4] United States Environmental Protection Agency (EPA). 1992. Dermal Exposure Assessment: Principles and Application. Interim Report. EPA/600/8-91/011B. Office of Research and Development, Washington, D.C.

[5] United States Environmental Protection Agency (EPA). 1989. Risk Assessment Guidance for Superfund: Volume I-Human Health Evaluation Manual. Office of Emergency and Remedial Response, U.S. Environmental Protection Agency, Washington, D.C. 


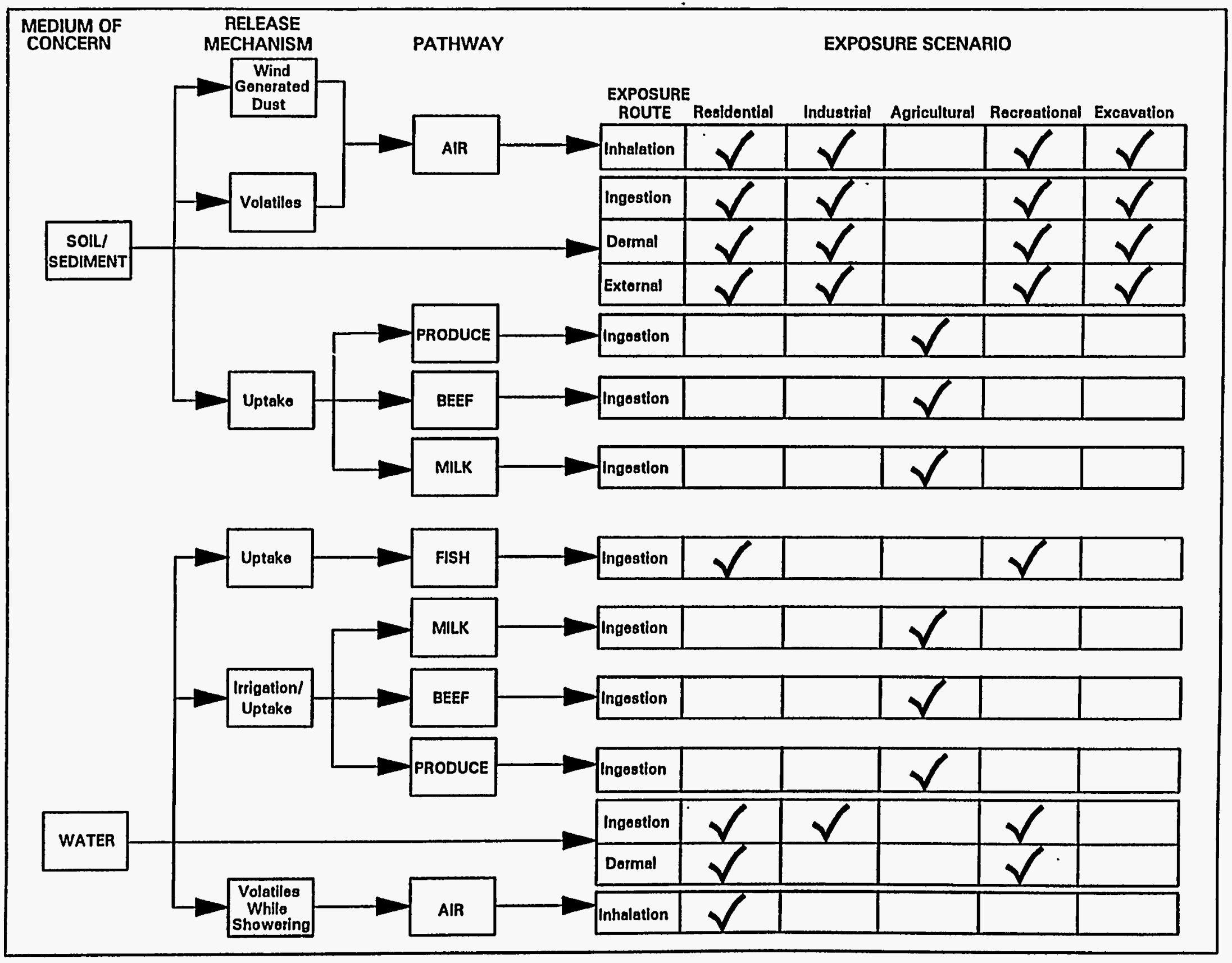

Fig. 1. Land uses and exposure routes considered in the development of PRGs. 


\section{EQUATIONS}

The reader should note that for purposes of readability and convenience, the equations have been placed as close as possible to their respective parameters. To ensure this accessibility for the reader, the chapter has been formatted with each equation followed immediately by its parameters. Blank pages have been inserted where necessary to separate groups of equations and keep this order. 
Residential Land Use

\subsection{RESIDENTIAL LAND USE—GROUNDWATER, SURFACE WATER, SOIL, OR SEDIMENT}

Under residential land use, residents are expected to be in frequent, repeated contact with contaminated media. The contamination may be on the site itself or may have migrated from it. The assumptions in this case account for daily exposure over the long term and generally result in the highest potential exposures and risk. Risk from groundwater or surface water contaminants is assumed to be primarily from direct ingestion, inhalation of volatiles from household water use, and dermal contact while showering. Risk from soil or sediment is assumed to be from direct ingestion, inhalation of dust and particulates, dermal exposure from chemicals, and external exposure from radionuclides. Risks from two or more exposure pathways are combined where available, and the risk-based concentration is derived to be protective for exposure from all included pathways.

\subsubsection{Groundwater or Surface Water}

\subsubsection{Nonradionuclide contaminant}

1. Ingestion only

$$
C=\frac{T \times B W \times A T}{T V_{0} \times I R_{w} \times E F \times E D}
$$

2. Inhalation of vapor-phase chemicals (volatile organics only)

$$
C=\frac{T \times B W \times A T}{T V_{i} \times K \times I R_{a} \times E F \times E D}
$$

3. Dermal contact only

$$
C=\frac{T \times B W \times A T}{T V_{a d} \times S A \times K_{p} \times E T \times E F \times E D \times C F_{1}}
$$

4. Ingestion and inhalation and dermal contact

$$
C=\frac{T \times B W \times A T}{E F \times E D \times\left[\left(T V_{o} \times I R_{w}\right)+\left(T V_{i} \times K \times I R_{a}\right)+\left(T V_{a d} \times S A \times K_{p} \times E T \times C F_{1}\right)\right]}
$$

5. Ingestion of fish-concentration in water

$$
C=\frac{T \times B W \times A T}{T V_{0} \times I R_{f} \times F T \times E F \times E D \times B F}
$$


Equations

Residential Land Use

6. Ingestion of fish-concentration in fish

$$
C_{f}=\frac{T \times B W \times A T}{T V_{o} \times I R_{f} \times F I \times E F \times E D}
$$

where

\begin{tabular}{|c|c|c|}
\hline Parameters & Definition (units) & Default Value \\
\hline AT & averaging time $(\mathrm{yr} \times \mathrm{day} / \mathrm{yr})$ & $\begin{array}{l}70 \times 365 \text { (carcinogen) [1] } \\
\text { ED } \times 365 \\
\text { (noncarcinogen) }[1]\end{array}$ \\
\hline $\mathrm{BF}$ & bioaccumulation factor $(\mathrm{L} / \mathrm{kg})$ & chemical-specific \\
\hline BW & adult body weight $(\mathbf{k g})$ & $70[1]$ \\
\hline C & chemical PRG in water $(\mathrm{mg} / \mathrm{L})$ & - \\
\hline $\mathrm{C}_{\mathrm{f}}$ & chemical PRG in fish $(\mathrm{mg} / \mathrm{kg})$ & - \\
\hline $\mathrm{CF}_{1}$ & units conversion factor $(\mathrm{L}-\mathrm{m}) /\left(\mathrm{cm}-\mathrm{m}^{3}\right)$ & 10 \\
\hline $\mathrm{ED}$ & exposure duration (yr) & $30[1]$ \\
\hline $\mathrm{EF}$ & exposure frequency (day/yr) & $350[1]$ \\
\hline ET & exposure time (hr/day) & $0.25[4]$ \\
\hline FI & fraction ingested (unitless) & $1[12]$ \\
\hline $\mathbb{I R}_{\mathrm{a}}$ & inhalation rate $\left(\mathrm{m}^{3} /\right.$ day $)$ & $20[28]$ \\
\hline $\mathbb{I R}_{\mathbf{f}}$ & fish ingestion rate (kg/day) & 0.054 [9] \\
\hline $\mathbb{I R}_{w}$ & water ingestion rate (L/day) & $2[1]$ \\
\hline $\mathrm{K}$ & volatilization factor of Andelman (1990) $\left(\mathrm{L} / \mathrm{m}^{3}\right)$ & $0.0005 \times 10^{3}[2]$ \\
\hline $\mathrm{K}_{\mathrm{p}}$ & permeability constant $(\mathbf{c m} / \mathbf{h r})$ & chemical-specific \\
\hline $\mathrm{RfD}_{\mathrm{ad}}$ & absorbed chronic reference dose (mg/kg-day) & chemical-specific [3] \\
\hline $\mathrm{RfD}_{\mathrm{i}}$ & inhalation chronic reference dose (mg/kg-day) & chemical-specific [3] \\
\hline $\mathrm{RfD}_{0}$ & oral chronic reference dose (mg/kg-day) & chemical-specific [3] \\
\hline SA & adult total body surface area $\left(\mathbf{m}^{2}\right)$ & $1.94[4]$ \\
\hline $\mathrm{SF}_{\mathrm{ad}}$ & absorbed dose slope factor $\left((\mathrm{mg} / \mathrm{kg}-\mathrm{day})^{-1}\right)$ & chemical-specific [3] \\
\hline $\mathrm{SF}_{\mathrm{i}}$ & inhalation slope factor $\left((\mathrm{mg} / \mathrm{kg}-\mathrm{day})^{-1}\right)$ & chemical-specific [3] \\
\hline $\mathrm{SF}_{\mathrm{o}}$ & oral slope factor $\left((\mathrm{mg} / \mathrm{kg}-\mathrm{day})^{-1}\right)$ & chemical-specific [3] \\
\hline $\mathrm{T}$ & target (unitless) & $\begin{array}{l}\text { TR (carcinogen) } \\
\text { THI (noncarcinogen) }\end{array}$ \\
\hline THI & target hazard index (unitless) & 1 and 0.1 \\
\hline TR & target excess individual lifetime cancer risk (unitless) & $10^{-6}$ and $10^{-4}$ \\
\hline $\mathrm{TV}_{\mathrm{ad}}$ & absorbed toxicity value & $\begin{array}{l}\mathrm{SF}_{\mathrm{ad}} \text { (carcinogen) } \\
\mathrm{l} / \mathrm{RfD}_{\mathrm{ad}} \text { (noncarcinogen) }\end{array}$ \\
\hline $\mathrm{TV}_{\mathrm{i}}$ & inhalation toxicity value & $\begin{array}{l}\mathrm{SF}_{\mathrm{i}}(\text { carcinogen) } \\
1 / \mathrm{RfD}_{\mathrm{i}} \text { (noncarcinogen) }\end{array}$ \\
\hline $\mathrm{TV}_{\mathrm{o}}$ & oral toxicity value & $\begin{array}{l}\mathrm{SF}_{\circ}(\text { carcinogen }) \\
\mathrm{I}_{\mathrm{RfD}} \text { (noncarcinogen) }\end{array}$ \\
\hline
\end{tabular}


Residential Land Use

\subsubsection{Radionuclide contaminants}

Since most radionuclides are not considered to be volatile, the inhalation pathway is not usually considered for exposure through groundwater or surface water; the exceptions are tritium and radon.* Tritium easily volatilizes in water because it acts like hydrogen. Groundwater that is in contact with rock or soil containing radium will pick up radon-222 and release it to the atmosphere when the water comes to the surface. With a contribution of $5 \times 10^{8} \mathrm{Ci}$ of radon-222 per year, groundwater is considered the second largest source of environmental radon [15].

1. Ingestion only

$$
C=\frac{T R}{S F_{o} \times I R_{w} \times E F \times E D}
$$

2. Inhalation only (tritium, radon-220, and radon-222)*

$$
C=\frac{T R}{S F_{i} \times I R_{a i r} \times E F \times E D \times C F_{1} \times I E F}
$$

3. Ingestion and inhalation (volatiles only)

$$
C=\frac{T R}{E F \times E D \times\left[\left(S F_{o} \times I R_{w}\right)+\left(S F_{i} \times I R_{a i r} \times C F_{1} \times I E F\right)\right]}
$$

4. Ingestion of fish - concentration in water

$$
C=\frac{T R}{S F_{o} \times I R_{f} \times F I \times E F \times E D \times B F}
$$

5. Ingestion of fish - concentration in fish

\begin{tabular}{|c|c|c|}
\hline Parameters & Definition (units) & Default Value \\
\hline $\mathrm{BF}$ & bioaccumulation factor $(\mathrm{L} / \mathrm{kg})$ & radionuclide-specific \\
\hline $\mathrm{C}$ & radionuclide $P R G$ in water $(\mathbf{p C i} / \mathrm{L})$ & - \\
\hline $\mathrm{C}_{\mathrm{f}}$ & radionuclide $P R G$ in fish $(\mathrm{pCi} / \mathrm{g})$ & - \\
\hline $\mathrm{CF}_{1}$ & units conversion factor (day/hr) & $1 / 24$ \\
\hline $\mathrm{CF}_{2}$ & units conversion factor $(\mathrm{g} / \mathrm{kg})$ & 1000 \\
\hline $\mathrm{ED}$ & exposure duration (yr) & $30[1]$ \\
\hline $\mathrm{EF}$ & exposure frequency (day/yr) & $350[1]$ \\
\hline FI & fraction ingested (unitless) & $1[12]$ \\
\hline IEF & inhalation exposure factor $(\mathbf{L}-\mathrm{hr}) /\left(\mathrm{m}^{3}\right.$-day $)$ & $\begin{array}{l}0.2802 \text { (tritium) [14] } \\
7.6030 \text { (radon) [14] }\end{array}$ \\
\hline
\end{tabular}

$$
C_{f}=\frac{T R}{S F_{o} \times I R_{f} \times C F_{2} \times F I \times E F \times E D}
$$

where 
$\mathrm{IR}_{\mathrm{air}} \quad$ inhalation rate $\left(\mathrm{m}^{3} /\right.$ day)

$\operatorname{IR}_{\mathbf{f}}$

$\mathbb{I R}_{w}$

$\mathrm{SF}_{\mathrm{i}}$

$\mathrm{SF}_{\mathrm{o}}$

TR fish ingestion rate (kg/day)

water ingestion rate (L/day)

inhalation slope factor (risk/pCi)

oral slope factor (risk/pCi)

target excess individual lifetime cancer risk (unitless)
20 [28]

0.054 [9]

2 [1]

radionuclide-specific[3] radionuclide-specific[3] $10^{-6}$ and $10^{-4}$ 
Residential Land Use

\subsubsection{Soil or Sediment}

Under residential land use, risk of contamination from soil is caused by direct ingestion, inhalation of dust and particulates, dermal exposure to chemicals, and external exposure to radionuclides. Because the soil ingestion rate is different for children and adults, the carcinogenic risk due to direct ingestion of soil is calculated using an age-adjusted ingestion factor. This takes into account the differences in daily soil ingestion rates, body weights, and exposure durations for the two exposure groups. Exposure frequency is assumed to be the same for the two groups. Calculated in this manner, the factor leads to a more protective risk-based concentration compared to an adult-only assumption. Due to differences in averaging times for carcinogens and noncarcinogens, the noncarcinogenic hazard is calculated separately for adults and children. This procedure will give a more protective concentration than the adult-only assumption.

\subsubsection{Nonradionuclide contaminant}

1. Ingestion only

a. Carcinogen

$$
C=\frac{T \times A T}{T V_{0} \times C F \times E F \times I F_{\text {soilladij }} \times F I}
$$

b. Noncarcinogenic (adult and child calculated separately)

$$
C=\frac{T \times B W_{n} \times A T_{n}}{T V_{0} \times C F \times E F \times E D_{n} \times I R_{n} \times F I}
$$

2. Inhalation only

$$
C=\frac{T \times B W \times A T}{T V_{i} \times E F \times E D \times\left(\frac{1}{V F}+\frac{1}{P E F}\right) \times I R_{\text {air }}}
$$

3. Dermal contact only

$$
C=\frac{T \times B W \times A T}{T V_{a d} \times C F_{d} \times S A \times A F \times A B S \times E F \times E D}
$$

4. Ingestion and inhalation and dermal contact (adult only)

$$
C=\frac{T \times B W_{a} \times A T}{E F \times E D\left[\left(T V_{o} \times C F \times I R_{a} \times F T\right)+\left(T V_{i} \times\left(\frac{1}{V F}+\frac{1}{P E F}\right) \times I R_{a i r}\right)+\left(T V_{a d} \times C F_{d} \times S A \times A F \times A B S\right)\right]}
$$


Equations

Residential Land Use

where

Parameters

ABS

$\mathrm{AF}$

AT

$\mathrm{AT}_{\mathrm{n}}$

BW

$\mathrm{BW}_{\mathrm{n}}$

C

$\mathrm{CF}$

$\mathrm{CF}_{\mathrm{d}}$

ED

$\mathrm{ED}_{\mathrm{n}}$

$\mathrm{EF}$

FI

$\mathrm{IF}_{\text {roil/edj }}$

$\mathbb{I R}_{\mathrm{a}}$

$\mathrm{IR}_{\mathrm{air}}$

$\mathrm{IR}_{\mathfrak{n}}$

PEF

$\mathrm{RfD}_{\mathrm{ad}}$

$\mathrm{RfD}_{i}$

$\mathrm{RfD}_{\text {。 }}$

SA

$\mathrm{SF}_{\mathrm{ad}}$

$\mathrm{SF}_{\mathrm{i}}$

$\mathrm{SF}_{\text {。 }}$

$\mathrm{T}$

THI

TR

$\mathrm{TV}_{\mathrm{gd}}$

TV

. TV。

VF
Definition (units)

absorption factor (unitless)

adherence factor $\left(\mathrm{mg} / \mathrm{cm}^{2}\right)$

averaging time $(\mathrm{yr} \times$ day $/ \mathrm{yr})$

averaging time - noncarcinogenic ingestion only

$(\mathrm{yr} \times \mathrm{day} / \mathrm{yr})$

adult body weight $(\mathrm{kg})$

body weight - noncarcinogenic ingestion only (kg)

chemical PRG in soil (mg/kg)

units conversion factor $(\mathrm{kg} / \mathrm{mg})$

units conversion factor - dermal $\left(\mathrm{kg}-\mathrm{cm}^{2}\right) /\left(\mathbf{m g}-\mathrm{m}^{2}\right)$

exposure duration (yr)

exposure duration - noncarcinogenic ingestion

only (yr)

exposure frequency (day/yr)

fraction ingested (unitless)

age-adjusted soil ingestion factor (mg-yr/kg-day)

soil ingestion rate for adult (mg/day)

total inhalation rate $\left(\mathrm{m}^{3} / \mathrm{day}\right)$

soil ingestion rate - noncarcinogenic (mg/day)

particulate emission factor $\left(\mathrm{m}^{3} / \mathrm{kg}\right)$

absorbed chronic reference dose (mg/kg-day)

inhalation chronic reference dose ( $\mathrm{mg} / \mathrm{kg}$-day)

oral chronic reference dose ( $\mathrm{mg} / \mathrm{kg}$-day)

adult surface area (head, hands, forearms, lower legs)

$\left(\mathrm{m}^{2} /\right.$ day)

absorbed dose slope factor ((mg/kg-day $\left.)^{-1}\right)$

inhalation slope factor $\left((\mathrm{mg} / \mathrm{kg}-\mathrm{day})^{-1}\right)$

oral slope factor $\left(\left(\mathrm{mg} / \mathrm{kg}^{-d a y}\right)^{-1}\right)$

target (unitless)

target hazard index (unitless)

target excess individual lifetime cancer risk (unitless)

absorbed toxicity value

inhalation toxicity value

oral toxicity value

volatilization factor (volatile organics only) $\left(\mathrm{m}^{3} / \mathrm{kg}\right)$
Default Value

chemical-specific [4]

$1[11]$

$70 \times 365$ (carcinogen)[1]

$\mathrm{ED} \times 365$

(noncarcinogen)[1]

$\mathrm{ED}_{\mathrm{n}} \times 365[1]$

70 [1]

70 (adult) [1]

15 (child) [1]

-

$10^{-6}$

0.01

30 [1]

24 (adult) [1]

6 (child) [1]

350 [1]

1 [12]

$\sim 114$ (see Eq. 21) [1]

100 [1]

20 [9]

100 (adult) [1]

200 (child) [1]

$4.28 \times 10^{9}$ (see Eq. 40)[5]

chemical-specific [3]

chemical-specific [3]

chemical-specific [3]

0.53 [4]

chemical-specific [3]

chemical-specific [3]

chemical-specific [3]

TR (carcinogen)

THI (noncarcinogen)

1 and 0.1

$10^{-6}$ and $10^{-4}$

$\mathrm{SF}_{\mathrm{ad}}$ (carcinogen)

$1 / \mathrm{RfD}_{\mathrm{ad}}$ (noncarcinogen)

$\mathrm{SF}_{\mathrm{i}}$ (carcinogen)

$1 / \mathrm{RfD}_{\mathrm{i}}$ (noncarcinogen)

$\mathrm{SF}_{0}$ (carcinogen)

1/RfD。 (noncarcinogen)

chemical-specific

(see Eqs. 34-39) [5] 
Equations

Residential Land Use

\subsubsection{Radionuclide contaminant}

1. Ingestion only

$$
C=\frac{T R}{S F_{o} \times C F \times E F \times I F_{\text {soilladj }} \times F I}
$$

2. External radiation only

$$
C=\frac{T R}{S F_{x} \times E D \times E F_{x} \times\left(1-S_{e}\right) \times T_{e}}
$$

3. Inhalation only

$$
C=\frac{T R}{S F_{i} \times E D \times C F_{i} \times E F \times\left(\frac{1}{V F}+\frac{1}{P E F}\right) \times I R_{\text {air }}}
$$

4. Ingestion and external radiation and inhalation

$$
C=\frac{T R}{\left(S F_{0} \times C F \times E F \times E D \times I R_{a} \times F T\right)+\left(S F_{x} \times E D \times E F_{x} \times\left(1-S_{e}\right) \times T_{e}\right)+\left(S F_{l} \times E D \times C F_{i} \times E F \times\left(\frac{1}{V F}+\frac{1}{P E F}\right) \times I R_{a i r}\right)}
$$

\begin{tabular}{|c|c|c|}
\hline Parameters & Definition (units) & Default Value \\
\hline $\mathrm{C}$ & radionuclide $\mathrm{PRG}$ in soil $(\mathrm{pCi} / \mathrm{g})$ & - \\
\hline $\mathrm{CF}$ & units conversion factor (g/mg) & $10^{-3}$ \\
\hline $\mathrm{CF}_{\mathrm{i}}$ & units conversion factor -inhalation $(\mathrm{g} / \mathrm{kg})$ & $10^{3}$ \\
\hline $\mathrm{ED}$ & exposure duration (yr) & $30[1]$ \\
\hline $\mathrm{EF}$ & exposure frequency (day/yr) & $350[1]$ \\
\hline $\mathrm{EF}_{\mathrm{x}}$ & exposure frequency - external (day/day) & $350 / 365[1]$ \\
\hline FI & fraction ingested (unitless) & $1[12]$ \\
\hline $\mathrm{IF}_{\text {soil/adj }}$ & age-adjusted soil ingestion factor (mg-yr/day) & 3600 (see Eq. 22) [6] \\
\hline $\mathbb{R}_{\mathbf{a}}$ & soil ingestion rate for adult (mg/day) & $100[1]$ \\
\hline $\mathbb{I R}_{\text {air }}$ & total inhalation rate $\left(\mathrm{m}^{3} /\right.$ day $)$ & $20[9]$ \\
\hline PEF & particulate emission factor $\left(\mathrm{m}^{3} / \mathrm{kg}\right)$ & $4.28 \times 10^{9}$ (see Eq. 40)[5] \\
\hline$S_{c}$ & gamma shielding factor (unitless) & $0.2[1]$ \\
\hline $\mathrm{SF}_{\mathbf{i}}$ & inhalation slope factor (risk/pCi) & radionuclide-specific [3] \\
\hline $\mathrm{SF}_{\mathrm{o}}$ & oral slope factor (risk/pCi) & radionuclide-specific [3] \\
\hline $\mathrm{SF}_{\mathrm{x}}$ & external exposure slope factor ((risk-g)/(pCi-yr)) & radionuclide-specific [3] \\
\hline $\mathrm{T}_{\mathrm{c}}$ & gamma exposure time factor $(\mathbf{h r} / \mathbf{h r})$ & $24 / 24[1]$ \\
\hline TR & target excess individual lifetime cancer risk (unitless) & $10^{-6}$ and $10^{-4}$ \\
\hline VF & volatilization factor (volatiles only) $\left(\mathrm{m}^{3} / \mathrm{kg}\right)$ & radionuclide-specific [5] \\
\hline
\end{tabular}

where 
Equations

Residential Land Use

\subsubsection{Supporting equations}

1. Age-adjusted soil ingestion factor for nonradionuclide (carcinogenic risk only)

$$
I F_{\text {soilladj }}=\frac{I R_{\text {soillage 1-6 }} \times E D_{\text {age 1-6 }}}{B W_{\text {age 1-6 }}}+\frac{I R_{\text {soillage 7-31 }} \times E D_{\text {age 7-31 }}}{B W_{\text {age 7-31 }}}
$$

where

\begin{tabular}{|c|c|c|}
\hline Parameters & Definition (units) & Default Value \\
\hline$\overline{B W_{\text {age 1-6 }}}$ & average body weight from ages 1-6 (kg) & $15[1]$ \\
\hline $\mathrm{BW}_{\text {ago 7-31 }}$ & average body weight from ages $7-31$ (kg) & $70[1]$ \\
\hline $\mathrm{ED}_{\text {age 1-6 }}$ & exposure duration during ages $1-6(y r)$ & $6[1]$ \\
\hline $\mathrm{ED}_{\text {age } 7-31}$ & exposure duration during ages $7-31$ (yr) & $24[1]$ \\
\hline $\mathrm{IF}_{\text {soil/adj }}$ & age-adjusted soil ingestion factor (mg-yr/kg-day) & $\sim 114[1]$ \\
\hline $\operatorname{IR}_{\text {soil/age 1-6 }}$ & ingestion rate of soil ages 1 to 6 (mg/day) & $200[1]$ \\
\hline $\mathrm{IR}_{\text {zoil/ago 7-31 }}$ & ingestion rate of soil ages 7 to 31 (mg/day) & $100[1]$ \\
\hline
\end{tabular}

2. Age-adjusted soil ingestion factor for radionuclide

$$
I F_{\text {soilladif }}=\left(I R_{\text {soillage 1-6 }} \times E D_{\text {age 1-6 }}\right)+\left(I R_{\text {soillage 7-31 }} \times E D_{\text {age 7-31 }}\right)
$$

\begin{tabular}{|c|c|c|}
\hline Parameters & Definition_units) & Default Value \\
\hline $\mathrm{ED}_{\mathrm{age} \mathrm{1-6}}$ & exposure duration during ages 1-6 (yr) & $6[1]$ \\
\hline $\mathrm{ED}_{2 \mathrm{ges}} 7-31$ & exposure duration during ages 7-31 (yr) & $24[1]$ \\
\hline $\mathrm{IF}_{\text {soil/adj }}$ & age-adjusted soil ingestion factor (mg-yr/day) & $3600[1]$ \\
\hline $\mathbb{I R}_{\text {zoil/age 1-6 }}$ & ingestion rate of soil ages $1-6$ (mg/day) & $200[1]$ \\
\hline $\operatorname{IR}_{20 \mathrm{il} / \mathrm{gge}} 7-31$ & ingestion rate of soil ages $7-31$ (mg/day) & $100[1]$ \\
\hline
\end{tabular}

where 
Commercial/Industrial Land Use

\subsection{COMMERCIAL/INDUSTRIAL LAND USE-GROUNDWATER, SURFACE WATER, SOIL, OR SEDIMENT}

Under commercial/industrial land use, workers are exposed to contaminants within a commercial area or industrial site. Workers are expected to be routinely exposed to contaminated media. Risk of exposure to the contaminant from groundwater or surface water is assumed to be from incidental ingestion only. Risk of contamination from soil or sediment is assumed to be from direct ingestion, inhalation of dust and particulates, dermal exposure to chemicals, and external exposure from radionuclides. It is assumed that there is potential for use of heavy equipment and related traffic in and around contaminated soils and therefore a potential for soils to be disturbed and produce particulate and volatile emissions.

\subsubsection{Groundwater or Surface Water}

\subsubsection{Nonradionuclide contaminant}

Ingestion only

$$
C=\frac{T \times B W \times A T}{T V_{o} \times I R_{w} \times E F \times E D}
$$

where

\begin{tabular}{|c|c|c|}
\hline \multirow{2}{*}{$\frac{\text { Parameters }}{\mathrm{AT}}$} & Definition (units) & Default Value \\
\hline & averaging time $(\mathrm{yr} \times \mathrm{day} / \mathrm{yr})$ & $\begin{array}{l}70 \times 365 \text { (carcinogen) }[1] \\
\mathrm{ED} \times 365 \\
(\text { noncarcinogen) }[1]\end{array}$ \\
\hline BW & adult body weight (kg) & $70[1]$ \\
\hline $\mathrm{C}$ & chemical PRG in water $(\mathrm{mg} / \mathrm{L})$ & - \\
\hline ED & exposure duration (yr) & $25[1]$ \\
\hline EF & exposure frequency (day/yr) & $250[1]$ \\
\hline $\mathbb{R}_{\mathrm{w}}$ & water ingestion rate (L/day) & $1[9]$ \\
\hline $\mathrm{RfD}_{0}$ & oral chronic reference dose (mg/kg-day) & chemical-specific [3] \\
\hline $\mathrm{SF}_{0}$ & oral slope factor $\left((\mathrm{mg} / \mathrm{kg}-\mathrm{day})^{-1}\right)$ & chemical-specific [3] \\
\hline \multirow[t]{2}{*}{$\mathrm{T}$} & \multirow{2}{*}{ target (unitless) } & TR (carcinogen) \\
\hline & & THI (noncarcinogen) \\
\hline THI & target hazard index (unitless) & 1 and 0.1 \\
\hline TR & target excess individual lifetime cancer risk (unitless) & $10^{-6}$ and $10^{-4}$ \\
\hline \multirow[t]{2}{*}{$\mathrm{TV}_{\mathrm{o}}$} & oral toxicity value & $\mathrm{SF}_{\mathrm{o}}$ (carcinogen) \\
\hline & & $1 / \mathrm{RfD}_{\mathrm{o}}$ (noncarcinogen) \\
\hline
\end{tabular}


Equations

Commercial/Industrial Land Use

\subsubsection{Radionuclide contaminant}

Ingestion only

$$
C=\frac{T R}{S F_{o} \times I R_{w} \times E F \times E D}
$$

where

Parameters

C

ED

$\mathrm{EF}$

$\mathrm{IR}_{\mathrm{w}}$

$\mathrm{SF}_{\mathrm{o}}$

TR

\section{Definition (units)}

radionuclide $P R G$ in water $(\mathrm{pCi} / \mathrm{L})$

exposure duration (yr)

exposure frequency (day/yr)

water ingestion rate $(\mathrm{L} / \mathrm{day})$

oral slope factor (risk/pCi)

target excess individual lifetime cancer risk (unitless)
Default Value

$25[1]$

$250[1]$

1 [9]

radionuclide-specific [3]

$10^{-6}$ and $10^{-4}$ 
Commercial/Industrial Land Use

\subsubsection{Soil or Sediment}

\subsubsection{Nonradionuclide contaminant}

1. Ingestion only

$$
C=\frac{T \times B W \times A T}{T V_{0} \times C F \times E F \times E D \times I R_{s}}
$$

2. Inhalation only

$$
C=\frac{T \times B W \times A T}{T V_{i} \times E F \times E D \times\left(\frac{1}{V F}+\frac{1}{P E F}\right) \times I R_{a}}
$$

3. Dermal contact only

$$
C=\frac{T \times B W \times A T}{T V_{a d} \times C F_{d} \times S A \times A F \times A B S \times E F \times E D}
$$

4. Ingestion and inhalation and dermal contact

$$
C=\frac{T \times B W \times A T}{E F \times E D \times\left[\left(T V_{o} \times C F \times I R_{s}\right)+\left(T V_{i} \times\left(\frac{1}{V F}+\frac{1}{P E F}\right) \times I R_{a}\right)+\left(T V_{a d} \times C F_{d} \times S A \times A F \times A B S\right)\right]}
$$


Equations

Commercial/Industrial Land Use

where

\begin{tabular}{|c|c|c|}
\hline Parameters & Definition (units) & Default Value \\
\hline ABS & absorption factor (unitless) & chemical-specific [4] \\
\hline $\mathrm{AF}$ & adherence factor $\left(\mathrm{mg} / \mathrm{cm}^{2}\right)$ & $1[11]$ \\
\hline AT & averaging time $(\mathrm{yr} \times \mathrm{day} / \mathrm{yr})$ & $\begin{array}{l}70 \times 365 \text { (carcinogen) [1] } \\
\text { ED } \times 365 \\
(\text { noncarcinogen) }[1]\end{array}$ \\
\hline BW & adult body weight $(\mathrm{kg})$ & $70[1]$ \\
\hline C. & chemical PRG in soil $(\mathrm{mg} / \mathrm{kg})$ & - \\
\hline $\mathrm{CF}$ & units conversion factor $(\mathrm{kg} / \mathrm{mg})$ & $10^{-6}$ \\
\hline $\mathrm{CF}_{\mathrm{d}}$ & units conversion factor - dermal $\left(\mathrm{kg}-\mathrm{cm}^{2}\right) /\left(\mathbf{m g}-\mathrm{m}^{2}\right)$ & 0.01 \\
\hline $\mathrm{ED}$ & exposure duration (yr) & $25[1]$ \\
\hline $\mathrm{EF}$ & exposure frequency (day/yr) & $250[1]$ \\
\hline $\mathrm{IR}_{\mathrm{a}}$ & total inhalation rate $\left(\mathrm{m}^{3} /\right.$ day $)$ & $20[1]$ \\
\hline $\mathbb{R}_{\mathbf{R}}$ & soil ingestion rate (mg/day) & $50[1]$ \\
\hline PEF & particulate emission factor $\left(\mathrm{m}^{3} / \mathrm{kg}\right)$ & $4.28 \times 10^{9}$ (see Eq. 40$)[5]$ \\
\hline $\mathrm{RfD}_{\mathrm{ad}}$ & absorbed chronic reference dose (mg/kg-day) & chemical-specific [3] \\
\hline $\mathrm{RfD}_{\mathrm{i}}$ & inhalation chronic reference dose (mg/kg-day) & chemical-specific [3] \\
\hline $\mathrm{RfD}_{0}$ & oral chronic reference dose (mg/kg-day) & chemical-specific [3] \\
\hline SA & adult surface area (head, hands, forearms) ( $\mathrm{m}^{2} /$ day) & $0.316[4]$ \\
\hline $\mathrm{SF}_{\mathrm{ad}}$ & absorbed dose slope factor $\left((\mathrm{mg} / \mathrm{kg}-\mathrm{day})^{-1}\right)$ & chemical-specific [3] \\
\hline $\mathrm{SF}_{\mathrm{i}}$ & inhalation slope factor $\left((\mathrm{mg} / \mathrm{kg}-\mathrm{day})^{-1}\right)$ & chemical-specific [3] \\
\hline $\mathrm{SF}_{\mathrm{o}}$ & oral slope factor $\left((\mathrm{mg} / \mathrm{kg}-\mathrm{day})^{-1}\right)$ & chemical-specific [3] \\
\hline $\mathrm{T}$ & target (unitless) & $\begin{array}{l}\text { TR (carcinogen) [3] } \\
\text { THI (noncarcinogen) }\end{array}$ \\
\hline THI & target hazard index (unitless) & 1 and 0.1 \\
\hline TR & target excess individual lifetime cancer risk (unitless) & $10^{-6}$ and $10^{-4}$ \\
\hline $\mathrm{TV}_{\mathrm{ad}}$ & absorbed toxicity value & $\begin{array}{l}\mathrm{SF}_{\mathrm{ad}} \text { (carcinogen) } \\
1 / \mathrm{RfD}_{\mathrm{ad}} \text { (noncarcinogen) }\end{array}$ \\
\hline $\mathrm{TV}_{\mathrm{i}}$ & inhalation toxicity value & $\begin{array}{l}\mathrm{SF}_{\mathrm{i}}(\text { carcinogen) } \\
1 / \mathrm{RfD}_{\mathrm{i}} \text { (noncarcinogen) }\end{array}$ \\
\hline $\mathrm{TV}_{\mathrm{o}}$ & oral toxicity value & $\begin{array}{l}\mathrm{SF}_{\circ} \text { (carcinogen) } \\
1 / \mathrm{RfD}_{\mathrm{o}} \text { (noncarcinogen) }\end{array}$ \\
\hline VF & volatilization factor $\left(\mathrm{m}^{3} / \mathrm{kg}\right)$ & $\begin{array}{l}\text { chemical-specific } \\
\text { (see Eqs. 34-39)[5] }\end{array}$ \\
\hline
\end{tabular}




\subsubsection{Radionuclide contaminant}

1. Ingestion only

$$
C=\frac{T R}{S F_{0} \times E D \times C F \times E F \times I R_{s}}
$$

2. Inhalation only

$$
C=\frac{T R}{S F_{i} \times E D \times C F_{i} \times E F \times\left(\frac{1}{V F}+\frac{1}{P E F}\right) \times I R_{a}}
$$

3. External radiation only

$$
C=\frac{T R}{S F_{x} \times E D \times E F_{x} \times\left(1-S_{e}\right) \times T_{e}}
$$

4. Ingestion and inhalation and external radiation

$$
C=\frac{T R}{E D \times\left[\left(S F_{0} \times C F \times E F \times I R_{s}\right)+\left(S F_{i} \times C F_{i} \times E F \times\left(\frac{1}{V F}+\frac{1}{P E F}\right) \times I R_{a}\right)+\left(S F_{x} \times E F_{x} \times\left(1-S_{e}\right) \times T_{e}\right)\right]}
$$

\begin{tabular}{|c|c|c|}
\hline Parameters & Definition (units) & Default Value \\
\hline $\mathrm{C}$ & radionuclide $\mathrm{PRG}$ in soil (pCi/g) & - \\
\hline $\mathrm{CF}$ & units conversion factor $(\mathrm{g} / \mathrm{mg})$ & $10^{-3}$ \\
\hline $\mathrm{CF}_{\mathrm{i}}$ & units conversion factor - inhalation $(\mathrm{g} / \mathrm{kg})$ & $10^{3}$ \\
\hline ED & exposure duration (yr) & $25[1]$ \\
\hline EF & exposure frequency (day/yr) & $250[1]$ \\
\hline $\mathrm{EF}_{\mathrm{x}}$ & exposure frequency - external (day/day) & $250 / 365[1]$ \\
\hline $\mathrm{IR}_{\mathrm{a}}$ & total inhalation rate $\left(\mathrm{m}^{3} /\right.$ day $)$ & $20[1]$ \\
\hline $\mathrm{IR}_{\mathrm{s}}$ & soil ingestion rate (mg/day) & $50[1]$ \\
\hline PEF & particulate emission factor $\left(\mathrm{m}^{3} / \mathrm{kg}\right)$ & $4.28 \times 10^{9}$ (see Eq. 40$)[5]$ \\
\hline$S_{e}$ & gamma shielding factor (unitless) & $0.2[1]$ \\
\hline $\mathrm{SF}_{\mathrm{i}}$ & inhalation slope factor (risk/pCi) & radionuclide-specific [3] \\
\hline $\mathrm{SF}_{\mathrm{o}}$ & oral slope factor (risk/pCi) & radionuclide-specific [3] \\
\hline $\mathrm{SF}_{\mathrm{x}}$ & external exposure slope factor ((risk-g)/(pCi-yr)) & radionuclide-specific [3] \\
\hline $\mathrm{T}_{\mathrm{c}}$ & gamma exposure factor $(\mathrm{hr} / \mathrm{hr})$ & $8 / 24[6]$ \\
\hline TR & target excess individual lifetime cancer risk (unitless) & $10^{-6}$ and $10^{-4}$ \\
\hline VF & volatilization factor $\left(\mathrm{m}^{3} / \mathrm{kg}\right)$ & radionuclide-specific [5] \\
\hline
\end{tabular}

where

Most radionuclides are heavy metal elements and are nonvolatile under normal, ambient conditions. However, some radionuclides are volatile under certain conditions (i.e., carbon-14, tritium, phosphorus-32, sulfur-35) and still others undergo radioactive decay to yield gaseous daughter isotopes 
Equations

Supporting Equations

(i.e., radium-226 and radium-224 decay to form radon-222 and radon-220, respectively). The United States Environmental Protection Agency [1] describes how to calculate volatilization factors (VF) for these exceptions.

\subsubsection{Supporting equations}

The volatilization factor, VF, defines the relationship between the concentration of contaminants in soil and the volatilization of contaminants in air. The chemical concentration in soil (C) calculated using the VF must be compared with the soil saturation concentration $\left(C_{\text {sat }}\right)$. If $C$ is greater than $C_{\text {sat }}$ then the $P R G$ is set equal to $C_{\text {zat }}[1]$.

1. Soil saturation concentration

$$
C_{\text {sat }}=\frac{\left(K_{d} \times C_{w} \times \beta\right)+\left(C_{w} \times P_{w}\right)+\left(C_{w} \times H^{\prime} \times P_{a}\right)}{\beta}
$$

where

Parameters Definition (units)

$\mathrm{C}_{\text {2at }} \quad$ soil saturation concentration $(\mathrm{mg} / \mathrm{kg})$

$\mathrm{C}_{\mathrm{w}}$

$\mathrm{H}$

$\mathrm{H}^{\prime}$

$K_{d}$

$\mathrm{K}_{\infty}$

$\mathrm{OC}$

$P_{a}$

$P_{t}$

$P_{\text {w }}$

$\mathbf{s}$

$\beta$

$\theta$

$\theta_{\mathrm{m}}$

$\rho_{3}$ upper limit of free moisture in soil (mg/L-water)

Henry's Law constant (atm-m ${ }^{3} / \mathrm{mol}$ )

Henry's Law constant (unitless)

soil-water partition coefficient $(\mathrm{L} / \mathrm{kg})$

organic carbon partition coefficient $(\mathrm{L} / \mathrm{kg})$

organic carbon content of soil (fraction)

air-filled soil porosity (unitless)

total soil porosity (unitless)

water filled soil porosity (unitless)

solubility in water ( $\mathrm{mg} / \mathrm{L}$-water)

soil bulk density $(\mathrm{kg} / \mathrm{L})$

soil moisture content (L-water/kg-soil)

soil moisture content (kg-water/kg-soil)

true soil density or particle density $(\mathrm{kg} / \mathrm{L})$
Default Value

$-$

$\mathrm{s} \times \theta_{\mathrm{m}}[6]$

chemical-specific [6]

$\mathrm{H} \times 41$, where 41 is a conversion factor [6]

chemical-specific, or

$\mathrm{K}_{\mathrm{oc}} \times \mathrm{OC}[6]$

chemical-specific [6]

site-specific, or 0.02 [6]

$\mathrm{P}_{\mathrm{t}}-\theta \beta[6]$

$1-\left(\beta / p_{2}\right)[6]$

$P_{t}-P_{a}$, or $\theta \beta[6]$

chemical-specific [6]

1.5 [6]

site-specific, or $0.15[6]$

site-specific, or $0.15[6]$

2.65 [6]

Please note that the equation presented here for $\mathrm{C}_{2 a t}$ is also a modification of the equation presented in RAGS, Part B [1] guidance. This equation also considers the amount of contaminant in vapor phase in the pore spaces of the soil. 
Equations

Supporting Equations

The VF assumes that the contaminant concentration in the soil is homogeneous from the soil surface to the depth of concern and that the contaminated material is not covered by contaminant-free soil material. For the purpose of calculating the VF, depth of concern is defined as the depth at which a near impenetrable layer or the permanent groundwater level is reached. The following equation represents revisions to RAGS, Part B, Sect. 3.3.1 [1] incorporating the 95\% upper confidence limit of the mean normalized concentration as determined by regression analysis of the modeled data. This methodology uses the most conservative results of the three source shapes modeled (1:3 aspect ratio rectangle).

2. Soil-to-air volatilization factor

$$
V F=Q / C \times \frac{(3.1416 \times \alpha \times T)^{\frac{1}{2}}}{\left(2 \times D_{e i} \times P_{a} \times K_{a s}\right)} \times 10^{-4} \mathrm{~m}^{2} / \mathrm{cm}^{2}
$$

3. $\alpha$

$$
\alpha=\frac{\left(D_{e i} \times P_{a}\right)}{P_{a}+\left(\rho_{s}\right)\left(1-P_{a}\right) / K_{a s}}
$$

4. $\mathrm{Q} / \mathrm{C}$

$$
Q / C=\left(\exp \left[\hat{Y}_{h}+2.92 s\left(\hat{Y}_{h}\right)\right]\right)^{-1}
$$

5. $s\left(\hat{Y}_{\mathrm{b}}\right)$

$$
s\left(\hat{Y}_{h}\right)=0.02685\left[0.25+\frac{\left(X_{h}-11.0509\right)^{2}}{26.3608}\right]
$$

6. $Y_{h}$

$$
\hat{Y}_{h}=0.1004 X_{h}-5.3466
$$


where

\begin{tabular}{|c|c|c|}
\hline Parameters & Definition (units) & Default Value \\
\hline $\mathrm{D}_{\mathrm{ei}}$ & effective diffusivity $\left(\mathrm{cm}^{2} / \mathrm{s}\right)$ & $\mathrm{D}_{\mathrm{i}}\left(\mathrm{P}_{\mathrm{a}}^{3.3} / \mathrm{P}_{\mathrm{t}}^{2}\right)[5]$ \\
\hline$D_{i}$ & molecular diffusivity $\left(\mathrm{cm}^{2} / \mathrm{s}\right)$ & chemical-specific \\
\hline $\mathrm{H}$ & Henry's law constant (atm-m $\left.\mathrm{m}^{3} / \mathrm{mol}\right)$ & chemical-specific \\
\hline $\mathrm{K}_{\mathrm{as}}$ & soil/air partition coefficient (g-soil/ $\mathrm{cm}^{3}$-air) & $\begin{array}{l}\left(\mathrm{H} / \mathrm{K}_{\mathrm{d}}\right) \times 41, \text { where } 41 \\
\text { is a units conversion } \\
\text { factor [5] }\end{array}$ \\
\hline $\mathrm{K}_{\mathrm{d}}$ & soil-water partition coefficient $\left(\mathrm{cm}^{3} / \mathrm{g}\right)$ & $\begin{array}{l}\text { chemical-specific, or } \\
\mathrm{K}_{\mathrm{oc}} \times \mathrm{OC}[5]\end{array}$ \\
\hline $\mathrm{K}_{o c}$ & organic carbon partition coefficient $\left(\mathrm{cm}^{3} / \mathrm{g}\right)$ & chemical-specific [5] \\
\hline $\mathrm{OC}$ & organic carbon content of soil (fraction) & site-specific or $0.02[5]$ \\
\hline $\mathrm{P}_{\mathrm{a}}$ & air-filled soil porosity (unitless) & $P_{t}-\theta \beta[5]$ \\
\hline$P_{t}$ & total soil porosity (unitless) & $1-\left(\beta / \rho_{s}\right)[5]$ \\
\hline $\mathrm{Q} / \mathrm{C}$ & $\begin{array}{l}\text { inverse of the normalized concentration from EQ } \\
1993 \text { and represents the emission flux }\left(\mathrm{g} / \mathrm{m}^{2}-\mathrm{s}\right) \text { per } \\
\text { unit concentration }\left(\mathrm{kg} / \mathrm{m}^{3}\right)\end{array}$ & $92.5247[5]$ \\
\hline$s\left(\hat{Y}_{h}\right)$ & $\begin{array}{l}\text { UCL for a } 95 \% \text { confidence interval on the mean } \\
\text { response at the point } x=x_{0}\end{array}$ & $0.0187[5]$ \\
\hline $\mathrm{T}$ & exposure interval (s) & $7.9 \times 10^{8}[5]$ \\
\hline VF & volatilization factor $\left(\mathrm{m}^{3} / \mathrm{kg}\right)$ & - \\
\hline $\mathrm{X}$ & contiguous area of contamination $\left(\mathbf{m}^{2}\right)$ & 2025 [5] \\
\hline$X_{h}$ & $\begin{array}{l}\text { natural logarithm of the contiguous area of } \\
\text { contamination }\left(\mathrm{m}^{2}\right)\end{array}$ & $\ln (X)[5]$ \\
\hline$Y_{h}$ & linear regression line & - \\
\hline$\beta$ & soil bulk density $(\mathrm{kg} / \mathrm{L})$ & $1.5[5]$ \\
\hline$\theta$ & soil moisture content (L-water/kg-soil) & site-specific or 0.15 [5] \\
\hline$\rho_{s}$ & true soil density or particulate density $\left(\mathrm{g} / \mathrm{cm}^{3}\right)$ & $2.65[5]$ \\
\hline
\end{tabular}


Equations

Supporting Equations

7. Molecular diffusivity (used in calculating VF)

$$
D_{i}=\frac{0.001 T^{1.75} \sqrt{\frac{1}{M W_{i}}+\frac{1}{M W_{a}}}}{P_{a}\left[\left(\Sigma V_{1}\right)^{1 / 3}+\left(\Sigma V_{a}\right)^{1 / 3}\right]^{2}}
$$

where

Parameters Definition (units)

$\mathrm{D}_{\mathrm{i}}$

$\mathrm{MW}_{\mathrm{a}}$

$\mathrm{MW}_{\mathrm{i}}$

$\mathbf{P}_{\mathbf{a}}$

T average temperature for year expressed as absolute

$\Sigma V_{1} \quad$ molecular diffusion volume of toxic substance (g/mole); This is the sum of the atomic diffusion volumes of the

$\Sigma \mathrm{V}_{\mathrm{a}}$ temperature $\left({ }^{\circ} \mathrm{K}\right)$ compound components $\left(\mathrm{cm}^{3} / \mathrm{mole}\right)$.

molecular diffusivity $\left(\mathrm{cm}^{2} / \mathrm{s}\right)$

molecular weight of air (g/mole)

molecular weight of toxic substance (g/mole)

absolute pressure (atm)

molecular diffusion volume for air $\left(\mathrm{cm}^{3} / \mathrm{mole}\right)$
Default Value

28.8

chemical-specific

site-specific or 0.9825

(estimated)

site-specific or

$284.82^{\circ} \mathrm{K}=53^{\circ} \mathrm{F}$

(estimated)

chemical-specific

20.1

To estimate short-term (maximum) release rates, use a value for the temperature that reflects the expected summer maximum temperatures. Annual average temperatures are used here to estimate longterm (average) release rates.

Relevant atomic diffusion volume coefficients for use in estimating $D_{i}$ are [16]:

$\begin{array}{lll}\mathrm{C} & = & 16.5 \\ \mathrm{H} & = & 1.98 \\ \mathrm{O} & = & 5.48 \\ \mathrm{~N} & = & 5.69 \\ \mathrm{Cl} & = & 19.5 \\ \mathrm{Br} & = & 35.5 \\ \mathrm{~F} & = & 25.0[18] \\ \mathrm{S} & = & 17.0 \\ \text { Aromatic ring } & = & -20.2 \\ \text { Heterocyclic ring } & = & -20.2\end{array}$

The United States Environmental Protection Agency [17] provides appropriate diffusion coefficients. The diffusion coefficient $\left(D_{i}\right)$ can also be calculated by Fuller's method [16]. 
8. Particulate emission factor

$$
P E F=Q / C \times \frac{3600 s / h}{R S F \times(1-G) \times\left(U_{m} / U_{t}\right)^{3} \times F(x)}
$$

where

Parameters

Definition (units)

function dependent on $U_{m} / U_{t}$ (unitless) fraction of vegetative cover (unitless) particulate emission factor $\left(\mathrm{m}^{3} / \mathrm{kg}\right)$ inverse of the normalized concentration from $E Q$ 1993 and represents the emission flux $\left(\mathrm{g} / \mathrm{m}^{2}-\mathrm{s}\right)$ per unit concentration $\left(\mathrm{kg} / \mathrm{m}^{3}\right)$

RSF

$\mathrm{U}_{\mathrm{m}}$

$\mathrm{U}_{\mathrm{t}}$ respirable fraction $\left(\mathrm{g} / \mathrm{m}^{2}-\mathrm{hr}\right)$ mean annual wind speed $(\mathrm{m} / \mathrm{s})$ equivalent threshold value of wind speed at $10 \mathrm{~m}(\mathrm{~m} / \mathrm{s})$
Default Value

$0.0497[19]$

0 [5]

$4.28 \times 10^{9}[5]$

$92.5247[5]$

$0.036[5]$

$4.5[5]$

12.8 [5] 
Equations

Agricultural Land Use

\subsection{AGRICULTURAL LAND USE_PRODUCE, WHOLE MILK, AND BEEF}

Miller et al. [20] state that all sites at the Oak Ridge Reservation (ORR) undergoing a remedial investigation will include a comparison of the site data to agricultural PRGs. The agricultural pathways addressed for the ORR in this document include ingestion of produce, milk, and beef. The agricultural land use scenario integrates exposure from two different exposure media (soil and water) and integrates the results into a single risk number for a given food chain pathway. This presents unique problems for the calculation of media-based PRGs since a PRG based on a risk level for a given medium is not necessarily protective of the pathway. Therefore, the calculations presented here provide the slopes and y-intercepts for an algebraic equation that shows the linear relationship between the PRGs for water and soil with respect to a given risk level when contamination from both soil and water is present. Figure 2 demonstrates the use of this equation. The three graphs represent the relationship between the soil and water at different risk and HI levels. Please note that the change in scale in each graph should be used to interpret PRG level. The $x$ - and y-intercepts show the PRGs for soil and water, respectively, in the absence of contamination in the other media. Every point on the line represents a combination of contamination levels in soil and water that yield a risk equivalent to the target risk level. If a plotted point for a site falls above this line based on the representative soil and water concentrations for a particular contaminant, then the site has exceeded the combined PRG for that contaminant and pathway at the target risk level.

Because the milk ingestion rate is different for children and adults, the carcinogenic risk due to ingestion of milk is calculated using an age-adjusted ingestion factor. This takes into account the differences in daily milk ingestion rates, body weights, and exposure durations for the two exposure groups. Exposure frequency is assumed to be the same for the two groups. Calculated in this manner, the factor leads to a more protective risk-based concentration compared to an adult-only assumption. Due to differences in averaging times for carcinogens and noncarcinogens, the noncarcinogenic hazard is calculated separately for adults and children. This procedure will give a more protective concentration than the adult-only assumption.

\subsubsection{Nonradionuclide Contaminant}

\subsubsection{Ingestion of vegetable only}

$$
C_{v}=\frac{T \times B W \times A T}{T V_{o} \times I R_{v} \times F I_{v} \times E F \times E D}
$$

\subsubsection{Ingestion of whole milk only}

1. Carcinogen

$$
C_{m}=\frac{T \times A T}{T V_{0} \times F I \times E F \times I F_{\text {milddactj }}}
$$


Equations

Agricultural Land Use

2. Noncarcinogen (adult and child calculated separately)

$$
C_{m}=\frac{T \times B W_{n} \times A T_{n}}{T V_{0} \times I R_{m} \times F I \times E F \times E D_{n}}
$$

\subsubsection{Ingestion of beef only}

$$
C_{b}=\frac{T \times B W \times A T}{T V_{o} \times I R_{b} \times F I \times E F \times E D}
$$

\begin{tabular}{|c|c|c|}
\hline Parameters & Definition (units) & Default Value \\
\hline AT & averaging time $(\mathrm{yr} \times \mathrm{day} / \mathrm{yr})$ & $\begin{array}{l}70 \times 365 \text { (carcinogen) }[1 \\
\text { ED } \times 365 \\
(\text { noncarcinogen) }[1]\end{array}$ \\
\hline $\mathrm{AT}_{\mathrm{n}}$ & $\begin{array}{l}\text { averaging time - noncarcinogenic ingestion of } \\
\text { milk only (yr } \times \text { day/yr) }\end{array}$ & $\mathrm{ED}_{\mathrm{n}} \times 365[1]$ \\
\hline BW & adult body weight $(\mathrm{kg})$ & $70[1]$ \\
\hline $\mathrm{BW}_{\mathrm{n}}$ & $\begin{array}{l}\text { body weight - noncarcinogenic ingestion } \\
\text { of milk only (kg) }\end{array}$ & $\begin{array}{l}70 \text { (adult) [1] } \\
15 \text { (child) [1] }\end{array}$ \\
\hline $\mathrm{C}_{\mathrm{b}}$ & chemical concentration in beef $(\mathrm{mg} / \mathrm{kg})$ & - \\
\hline $\mathrm{C}_{\mathrm{m}}$ & chemical concentration in milk (mg/kg) & - \\
\hline$C_{\mathrm{v}}$ & chemical concentration in vegetable $(\mathrm{mg} / \mathrm{kg})$ & - \\
\hline ED & exposure duration (yr) & $30[1]$ \\
\hline $\mathrm{ED}_{\mathrm{n}}$ & $\begin{array}{l}\text { exposure duration - noncarcinogenic ingestion } \\
\text { of milk only (yr) }\end{array}$ & 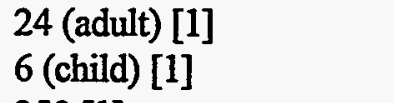 \\
\hline $\mathrm{EF}$ & exposure frequency (day/yr) & $350[1]$ \\
\hline FI & fraction ingested (unitless) & $1[12]$ \\
\hline $\mathrm{FL}_{\mathrm{v}}$ & fraction ingested - vegetable (unitless) & $0.4[10]$ \\
\hline $\mathrm{IF}_{\text {milleadj }}$ & age-adjusted milk ingestion factor ( $\mathrm{kg}-\mathrm{yr} / \mathrm{kg}$-day) & $\sim 0.308$ (see Eq. 66) \\
\hline $\mathrm{IR}_{\mathrm{b}}$ & ingestion rate - beef (kg/day) & $0.075[10]$ \\
\hline $\mathbf{I R}_{\mathbf{v}}$ & ingestion rate - vegetable (kg/day) & $0.2[10]$ \\
\hline $\mathrm{IR}_{\mathrm{m}}$ & $\begin{array}{l}\text { ingestion rate - milk } \\
\text { (noncarcinogenic ingestion only) (kg/day) }\end{array}$ & $\begin{array}{l}0.305 \text { (adult) [10] } \\
0.509 \text { (child) [21] }\end{array}$ \\
\hline $\mathrm{RfD}_{0}$ & oral chronic reference dose ( $\mathrm{mg} / \mathrm{kg}$-day) & chemical-specific [3] \\
\hline $\mathrm{SF}_{\mathrm{o}}$ & oral slope factor $\left((\mathrm{mg} / \mathrm{kg}-\mathrm{day})^{-1}\right)$ & chemical-specific [3] \\
\hline $\mathrm{T}$ & target (unitless) & $\begin{array}{l}\text { TR (carcinogen) } \\
\text { THI (noncarcinogen) }\end{array}$ \\
\hline THI & target hazard index (unitless) & 1 and 0.1 \\
\hline TR & target excess individual lifetime cancer risk (unitless) & $10^{-6}$ and $10^{-4}$ \\
\hline $\mathrm{TV}_{0}$ & oral toxicity value & $\begin{array}{l}\mathrm{SF}_{\circ} \text { (carcinogen) } \\
1 / \mathrm{RfD}_{\circ} \text { (noncarcinogen) }\end{array}$ \\
\hline
\end{tabular}

where 
Equations

Agricultural Land Use

Bis(2-ethylhexyl)phthalate *

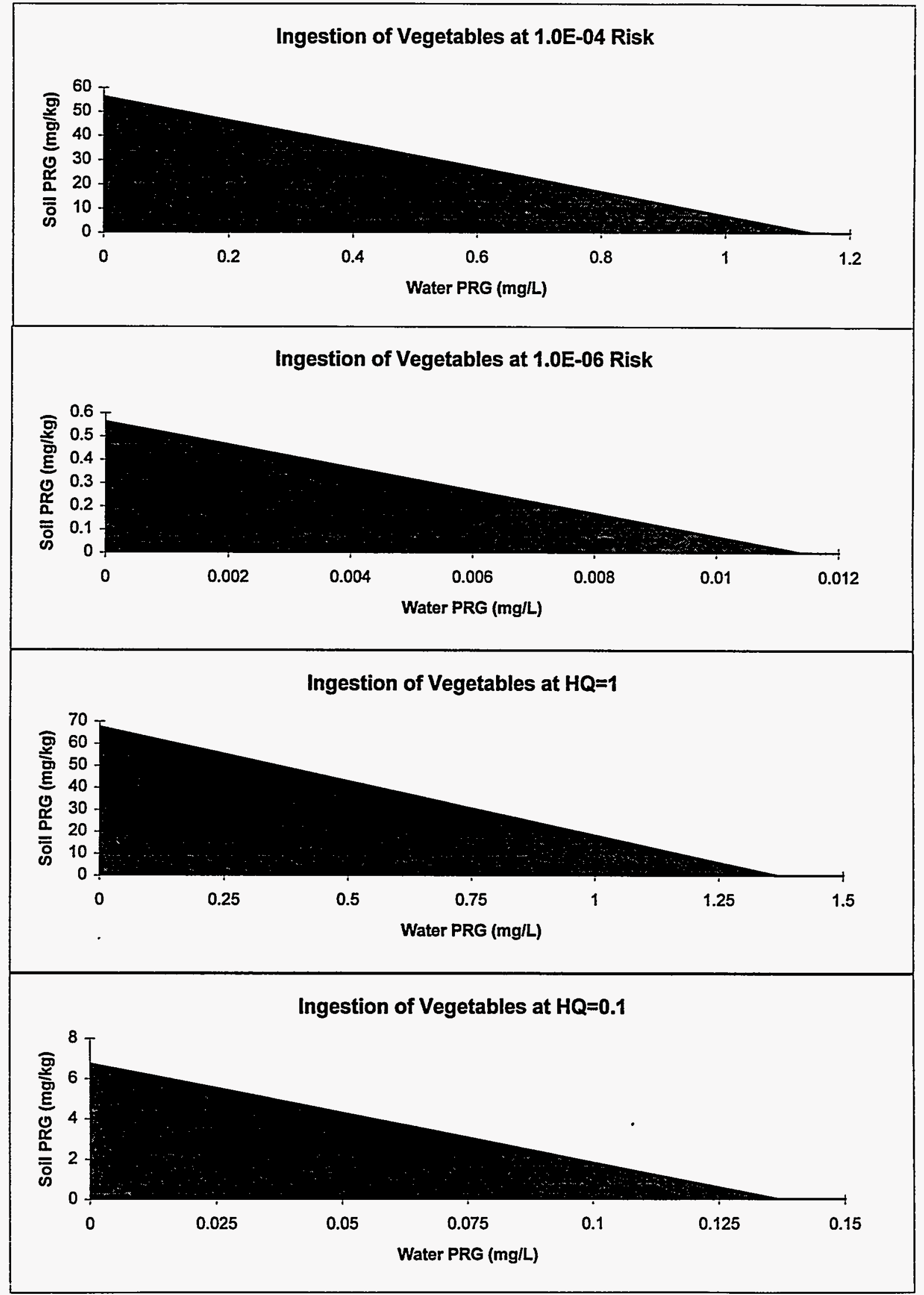

- Please note different scale for each plot

Fig. 2. Combination PRGs for bis(2-ethylhexvl)phthalate. 

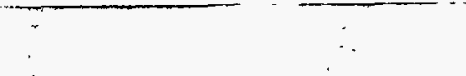
Equations

Agricultural Land Use

\subsubsection{Radionuclide Contaminant}

2.3.2.1 Ingestion of vegetables only

$$
C_{v}=\frac{T R}{S F_{o} \times I R_{v} \times F I_{v} \times E F \times E D \times C F}
$$

2.3.2.2 Ingestion of whole milk only

$$
C_{m}=\frac{T R}{S F_{o} \times I F_{\text {mildadi }} \times F I \times E F \times C F}
$$

2.3.2.3 Ingestion of beef only

$$
C_{b}=\frac{T R}{S F_{o} \times I R_{b} \times F I \times E F \times E D \times C F}
$$

where

\section{Parameters}

$\mathrm{C}_{\mathrm{b}}$

CF

$\mathrm{C}_{\mathrm{m}}$

$\mathrm{C}_{\mathrm{v}}$

ED

$\mathrm{EF}$

FI

$\mathrm{FI}_{\mathrm{v}}$

IF $_{\text {milk/adj }}$

$\mathrm{IR}_{\mathrm{b}}$

$\mathrm{IR}_{\mathrm{v}}$

$\mathrm{SF}_{\mathrm{o}}$

TR
Definition_(units)

radionuclide concentration in beef ( $\mathrm{pCi} / \mathrm{g}$ )

units conversion factor ( $\mathrm{g} / \mathrm{kg}$ )

radionuclide concentration in milk ( $p$ Ci/g)

radionuclide concentration in vegetable ( $\mathrm{pCi} / \mathrm{g}$ )

exposure duration (yr)

exposure frequency (day/yr)

fraction ingested (unitless)

fraction ingested - vegetable (unitless)

age-adjusted milk ingestion factor (kg-yr/day)

ingestion rate - beef ( $\mathrm{kg} /$ day)

ingestion rate - vegetable (kg/day)

oral slope factor (risk/pCi)

target excess individual lifetime

cancer risk (unitless)
Default Value

1000

-

30 [1]

$350[1]$

$1[12]$

$0.4[10]$

$\sim 10.374$ (see Eq. 67)

$0.075[10]$

$0.2[10]$

chemical-specific

$10^{-6}$ and $10^{-4}$ 
Equations

Agricultural Land Use

\subsubsection{Back-Calculated Concentrations for Groundwater and Soil}

Root uptake - leafy vegetables [38]

$$
R_{\mathrm{upv}}=B v_{\text {wet }}
$$

Root uptake - grazing pasture [38]

$$
R_{u p p}=B v_{d r y}
$$

Resuspension [32]

$$
R_{e s}=M L F
$$

Root uptake from irrigation [37], [39], [27]

$$
I r r_{n p p}=\frac{I r \times F \times B v_{\text {wet }} \times\left[1-\exp \left(-\lambda_{B} \times t_{b}\right)\right]}{\mathrm{P} \times \lambda_{B}}
$$

Resuspension from irrigation [32], [27]

$$
I r r_{\text {res }}=\frac{I r \times F \times M L F \times\left[1-\exp \left(-\lambda_{B} \times t_{b}\right)\right]}{\mathrm{P} \times \lambda_{B}}
$$

Aerial deposition from irrigation [37], [27]

$$
I r r_{\text {dep }}=\frac{I r \times F \times I_{f} \times T \times\left[1-\exp \left(-\lambda_{E} \times t_{v}\right)\right]}{Y_{v} \times \lambda_{E}}
$$

\subsubsection{Water contamination only}

1. Vegetable

$$
C W=\frac{C_{v}}{C F^{*} \times\left(I r r_{n p p}+I r r_{r e s}+I r r_{d e p}\right)}
$$


Equations

Agricultural Land Use

2. Whole Milk

$$
C W=\frac{C_{m}}{F_{m} \times Q_{w} \times C F^{*}}
$$

3. Beef

$$
C W=\frac{C_{b}}{F_{b} \times Q_{w} \times C F^{*}}
$$

\subsubsection{Soil contamination only}

1. Vegetable

$$
C S=\frac{C_{v}}{R_{u p v}+R_{e s}}
$$

2. Whole milk

$$
C S=\frac{C_{m}}{F_{m} \times\left[\left(Q_{p} \times f_{p} \times f_{s} \times\left(R_{u p p}+R_{e s}\right)\right)+\left(Q_{s} \times f_{p}\right)\right]}
$$

3. Beef

$$
C S=\frac{C_{b}}{F_{b} \times\left[\left(Q_{p} \times f_{p} \times f_{s} \times\left(R_{u p p}+R_{e s}\right)\right)+\left(Q_{s} \times f_{p}\right)\right]}
$$

\subsubsection{Water and soil contamination}

1. Vegetable

$$
\begin{gathered}
I N T C P T=\frac{C_{v}}{R_{u p v}+R_{e s}} \\
S L O P E=\frac{-\left(I r r_{r u p}+I r r_{r e s}+I r r_{d e p}\right)}{R_{u p v}+R_{e s}}
\end{gathered}
$$

* This conversion factor is for radionuclides only. 
2. Whole milk

$$
\begin{gathered}
I N T C P T=\frac{C_{m}}{F_{m} \times\left[\left(Q_{p} \times f_{p} \times f_{s} \times\left(R_{u p p}+R_{e s}\right)\right)+\left(Q_{s} \times f_{p}\right)\right]} \\
S L O P E=\frac{-Q_{w}}{\left(Q_{p} \times f_{p} \times f_{s} \times\left(R_{u p p}+R_{e s}\right)\right)+\left(Q_{s} \times f_{p}\right)}
\end{gathered}
$$

3. Beef

$$
\begin{gathered}
I N T C P T=\frac{C_{b}}{F_{b} \times\left[\left(Q_{p} \times f_{p} \times f_{s} \times\left(R_{u p p}+R_{e s}\right)\right)+\left(Q_{s} \times f_{p}\right)\right]} \\
S L O P E=\frac{-Q_{w}}{\left(Q_{p} \times f_{p} \times f_{s} \times\left(R_{u p p}+R_{e s}\right)\right)+\left(Q_{s} \times f_{p}\right)}
\end{gathered}
$$

\begin{tabular}{|c|c|}
\hline Parameters & Definition (units) \\
\hline $\mathrm{Bv}_{\text {wet }}$ & soil to plant uptake wet weight (kg/kg) \\
\hline$B v_{d r y}$ & soil to plant uptake dry weight (kg/kg) \\
\hline $\mathrm{C}_{\mathrm{b}}$ & $\mathrm{PRG}$ in beef $(\mathrm{mg} / \mathrm{kg}$ or $\mathrm{pCi} / \mathrm{g})$ \\
\hline CF & units conversion factor $(\mathrm{kg} / \mathrm{g})$ \\
\hline $\mathrm{C}_{\mathrm{m}}$ & $\mathrm{PRG}$ in milk (mg/kg or $\mathrm{pCi} / \mathrm{g})$ \\
\hline CS & PRG in soil (mg/kg or pCi/g) \\
\hline $\mathrm{C}_{\mathrm{v}}$ & $\mathrm{PRG}$ in vegetable (mg/kg or $\mathrm{pCi} / \mathrm{g}$ ) \\
\hline $\mathrm{CW}$ & PRG in water $(\mathrm{mg} / \mathrm{L}$ or $\mathrm{pCi} / \mathrm{L})$ \\
\hline $\begin{array}{l}f_{p} \\
f_{z}\end{array}$ & $\begin{array}{l}\text { fraction of year animal is on site (unitless) } \\
\text { fraction of animal's food is from site (unitless) }\end{array}$ \\
\hline $\mathrm{F}$ & irrigation period (unitless) \\
\hline$F_{b}$ & transfer coefficient - beef (day/kg) \\
\hline $\mathrm{F}_{\mathrm{m}}$ & transfer coefficient - milk (day/kg) \\
\hline$Y_{v}$ & plant yield (wet) $\left(\mathrm{kg} / \mathrm{m}^{2}\right)$ \\
\hline$I_{f}$ & interception fraction (unitless) \\
\hline $\operatorname{Irr}_{\text {rup }}$ & root uptake from irrigation multiplier (L/kg) \\
\hline
\end{tabular}

where

Default Value chemical-specific, or $7.7 \times \mathrm{K}_{\text {ow }}^{-0.58}$ [29] chemical-specific, or $38 \times \mathrm{K}_{\text {ow }}{ }^{-0.58}$ [29]

$1 / 1000$

$-$

-

-

$-$

1.0

0.90 (beef cattle) [23]

0.60 (dairy cattle) [23]

0.25 (based on 3 months per year) [23] chemical-specific chemical-specific 2 [22]

0.42 [30] 
Equations

Agricultural Land Use

\begin{tabular}{|c|c|c|}
\hline $\operatorname{Irr}_{\text {res }}$ & resuspension from irrigation multiplier (L/kg) & - \\
\hline $\operatorname{Irr}_{\text {dep }}$ & aerial deposition from irrigation multiplier $(\mathbf{L} / \mathrm{kg})$ & - \\
\hline INTCPT & equation intercept & - \\
\hline Ir & irrigation rate $\left(\mathbf{L} / \mathbf{m}^{2}\right.$-day) & $3.62[23]$ \\
\hline MLF & plant mass loading factor (unitless) & $\begin{array}{l}0.26 \text { (vegetables) [31] } \\
0.25 \text { (pasture) [32] }\end{array}$ \\
\hline P & area density for root zone $\left(\mathrm{kg} / \mathrm{m}^{2}\right)$ & $240[33],[34],[35]$ \\
\hline $\mathrm{Q}_{\mathbf{P}}$ & quantity of pasture ingested ( $\mathrm{kg} /$ day) & $\begin{array}{l}7.2 \text { (beef cattle) [36] } \\
16.1 \text { (dairy cattle) [36] }\end{array}$ \\
\hline$Q_{s}$ & quantity of soil ingested (kg/day) & $1[26]$ \\
\hline $\mathrm{Q}_{\mathrm{W}}$ & quantity of water ingested (L/day) & $\begin{array}{l}50 \text { (beef cattle) [36] } \\
75 \text { (dairy cattle) [36] }\end{array}$ \\
\hline$R_{\text {upv }}$ & wet root uptake for vegetables multiplier (unitless) & - \\
\hline$R_{\text {upp }}$ & dry root uptake for pasture multiplier (unitless) & - \\
\hline $\mathrm{R}_{\mathrm{g}}$ & resuspension multiplier (unitless) & - \\
\hline SLOPE & equation slope & - \\
\hline $\mathrm{T}$ & translocation factor (unitless) & $1[37]$ \\
\hline$t_{b}$ & long term deposition and buildup (day) & $10950[22]$ \\
\hline & half-life (day) & chemical-specific \\
\hline$t_{v}$ & above ground exposure time (day) & 60 (vegetable) [22] \\
\hline$t_{w}$ & weathering half-life (day) & $14[22]$ \\
\hline$\lambda_{\mathrm{B}}$ & effective rate for removal (1/day) & $\lambda_{\mathrm{i}}+\lambda_{\mathrm{FL}}[8]$ \\
\hline$\lambda_{\mathrm{E}}$ & decay for removal on produce (1/day) & $\lambda_{i}+\left(0.693 / t_{w}\right)[8]$ \\
\hline$\lambda_{\mathrm{HL}}$ & soil leaching rate $(1 /$ day) & $0.000027[8]$ \\
\hline$\lambda_{i}$ & decay (1/day) & $0.693 / \mathrm{T}_{\mathrm{R}}[8]$ \\
\hline
\end{tabular}

\subsubsection{Supporting Equations}

2.3.4.1 Age-adjusted milk ingestion factor for nonradionuclides (carcinogenic risk only)

$$
I F_{\text {milldadj }}=\frac{I R_{\text {mildeage 1-6 }} \times E D_{\text {age 1-6 }}}{B W_{\text {age 1-6 }}}+\frac{I R_{\text {milldage 7-31 }} \times E D_{\text {age 7-31 }}}{B W_{\text {age 7-31 }}}
$$

where

\begin{tabular}{lll} 
Parameters & Definition (units) & Default Value \\
\hline $\mathrm{BW}_{\text {age 1-6 }}$ & average body weight from ages 1-6 (kg) & $15[1]$ \\
$\mathrm{BW}_{\text {age 7-31 }}$ & average body weight from ages 7-31 (kg) & $70[1]$ \\
$\mathrm{ED}_{\text {age 1-6 }}$ & exposure duration during ages 1-6 (yr) & $6[1]$ \\
$\mathrm{ED}_{\text {age 7-31 }}$ & exposure duration during ages 7-31 (yr) & $24[1]$ \\
$\mathrm{IF}_{\text {milk/adj }}$ & age-adjusted milk ingestion factor (kg-yr/kg-day) & $\sim 0.308[10]$ \\
$\mathrm{IR}_{\text {milk/age 1-6 }}$ & ingestion rate of milk for ages 1-6 (kg/day) & $0.509[21]$ \\
$\mathbb{R}_{\text {mill/age 7-31 }}$ & ingestion rate of milk for ages 7-31 (kg/day) & $0.305[10]$
\end{tabular}


Equations

Agricultural Land Use

2.3.4.2 Age-adjusted milk ingestion factor for radionuclides

$$
I F_{\text {mildtadj }}=\left(I R_{\text {mildage 1-6 }} \times E D_{\text {age 1-6 }}\right)+\left(I R_{\text {milldage 7-31 }} \times E D_{\text {age 7-31) }}\right)
$$

where

Parameters Definition (units) Default Value

$\mathrm{ED}_{\mathrm{ege} \mathrm{1-6}}$ exposure duration during ages 1-6 (yr)

$\mathrm{ED}_{\mathrm{age}} 7-31 \quad$ exposure duration during ages 7-31 (yr)

EDFe 7-31 24 [1]

$\mathrm{IF}_{\text {milkzadj }} \quad$ age-adjusted milk ingestion factor (kg-yr/day) 10.374

$\mathrm{IR}_{\text {milk/age 1-6 }} \quad$ ingestion rate of milk for ages 1-6 (kg/day) $0.509[21]$

$\mathbb{R}_{\text {millkage }}$ 7-31 ingestion rate of milk for ages 7-31 (kg/day) 0.305 [10]

2.3.4.3 Chemical concentration in vegetables

$$
C_{v}=\left(C_{w} \times I r r_{r q p} \times C F^{*}\right)+\left(C_{s} \times R_{v p}\right)+\left(C_{w} \times I r r_{r e s} \times C F^{\prime \prime}\right)+\left(C_{s} \times R_{e s}\right)+\left(C_{w} \times I r r_{d p p} \times C F^{*}\right)
$$

2.3.4.4 Chemical concentration in pasture (assuming no irrigation)

$$
C_{p}=\left(C_{s} \times R_{u p p}\right)+\left(C_{s} \times R_{e s}\right)
$$

2.3.4.5 Chemical concentration in beef

$$
C_{b}=F_{b} \times\left[\left(C_{p} \times Q_{p} \times f_{p} \times f_{s}\right)+\left(C_{s} \times Q_{s} \times f_{p}\right)+\left(C_{w} \times C F^{*} \times Q_{w}\right)\right]
$$

2.3.4.6 Chemical concentration in milk

$$
C_{m}=F_{m} \times\left[\left(C_{p} \times Q_{p} \times f_{p} \times f_{s}\right)+\left(C_{s} \times Q_{s} \times f_{p}\right)+\left(C_{w} \times C F^{*} \times Q_{w}\right)\right]
$$

where

$\begin{array}{lll}\frac{\text { Parameters }}{\mathrm{Bv}_{\text {wet }}} & \begin{array}{l}\text { Definition (units) } \\ \text { soil to plant uptake wet weight }(\mathrm{kg} / \mathrm{kg})\end{array} & \begin{array}{l}\text { Default Value } \\ \text { chemical-specific, }\end{array} \\ \mathrm{Bv}_{\text {dry }} & \text { soil to plant uptake dry weight }(\mathrm{kg} / \mathrm{kg}) & \begin{array}{l}\text { or } 7.7 \times \mathrm{K}_{\text {ow }}^{-0.58}[29] \\ \text { chemical-specific, }\end{array} \\ \mathrm{C} & \text { or } 38 \times \mathrm{K}_{\text {ow }}^{-0.58}[29] \\ \mathrm{CF} & \mathrm{PRG} \text { in beef }(\mathrm{mg} / \mathrm{kg} \text { or } \mathrm{pCi} / \mathrm{g}) & - \\ & \text { units conversion factor }(\mathrm{kg} / \mathrm{g}) & 1 / 1000\end{array}$

* This conversion factor is for radionuclides only. 
Equations

Agricultural Land Use

$\mathrm{C}_{\mathrm{m}}$
$\mathrm{CS}$
$\mathrm{C}_{\mathrm{v}}$
$\mathrm{CW}$
$\mathrm{f}_{\mathrm{p}}$
$\mathrm{f}_{\mathrm{s}}$

F irrigation period (unitless)

per

$F_{b}$

$F_{m}$

$\mathrm{Y}_{\mathrm{v}}$

$I_{8}$

$\mathrm{Irr}_{\text {rup }}$

$\mathrm{Irr}_{\text {res }}$

$\mathrm{IrT}_{\text {dep }}$

INTCPT

Ir

MLF

P

$Q_{P}$

$\mathrm{Q}_{\mathrm{s}}$

$\mathrm{Q}_{\mathrm{w}}$

$R_{\text {upv }}$

$\mathrm{R}_{\text {upp }}$

$\mathrm{R}_{\mathrm{c}}$

SLOPE

$T$

$\mathrm{t}_{\mathrm{b}}$

$T_{R}$

t.

$\mathrm{t}_{\mathrm{w}}$

$\lambda_{\mathrm{B}}$

$\lambda_{\mathrm{E}}$

$\lambda_{\mathrm{HL}}$

$\lambda_{i}$

plant yield (wet) $\left(\mathrm{kg} / \mathrm{m}^{2}\right)$

equation intercept

irrigation rate $\left(\mathrm{L} / \mathrm{m}^{2}\right.$-day $)$

equation slope

half-life (day)

weathering half-life (day)

soil leaching rate (1/day)

decay $(1 /$ day $)$
PRG in milk (mg/kg or $\mathrm{pCi} / \mathrm{g}$ )

PRG in soil (mg/kg or pCi/g)

$\mathrm{PRG}$ in vegetable (mg/kg or $\mathrm{pCi} / \mathrm{g})$

$\mathrm{PRG}$ in water $(\mathrm{mg} / \mathrm{L}$ or $\mathrm{pCi} / \mathrm{L})$

fraction of year animal is on site (unitless)

fraction of animal's food is from site (unitless)

transfer coefficient - beef (day/kg)

transfer coefficient - milk (day/kg)

interception fraction (unitless)

root uptake from irrigation multiplier (L/kg)

resuspension from irrigation multiplier $(\mathrm{L} / \mathrm{kg})$

aerial deposition from irrigation multiplier $(\mathrm{L} / \mathrm{kg})$

plant mass loading factor (unitless)

area density for root zone $\left(\mathrm{kg} / \mathrm{m}^{2}\right)$

quantity of pasture ingested $(\mathrm{kg} / \mathrm{day})$

quantity of soil ingested (kg/day)

quantity of water ingested (L/day)

wet root uptake for vegetables multiplier (unitless)

dry root uptake for pasture multiplier (unitless)

resuspension multiplier (unitless)

translocation factor (unitless)

long term deposition and buildup (day)

above ground exposure time (day)

effective rate for removal (1/day)

decay for removal on produce (1/day)
$-$

1.0

0.90 (beef cattle) [23]

0.60 (dairy cattle) [23]

0.25 (based on 3 months

year) [23]

chemical-specific, or $2.5 \times 10^{-8} \mathrm{~K}_{\text {ow }}$ [35]

chemical-specific, or $7.9 \times 10^{-9} \mathrm{~K}_{\text {ow }}$ [35]

2 [22]

$0.42[30]$

$-$

$-$

$-$

3.62 [23]

0.26 (vegetables) [31]

0.25 (pasture) [32]

240 [33], [34], [35]

7.2 (beef cattle) [36]

16.1 (dairy cattle) [36]

1 [29]

50 (beef cattle) [36]

75 (dairy cattle) [36]

-

$-$

$-$

1 [29]

10950 [22]

chemical-specific

60 (vegetable) [22]

14 [22]

$\lambda_{\mathrm{i}}+\lambda_{\text {II }}[8]$

$\lambda_{\mathrm{i}}+\left(0.693 / \mathrm{t}_{\mathrm{w}}\right)[8]$

0.000027 [8]

$0.693 / \mathrm{T}_{\mathrm{R}}[8]$ 


\subsection{RECREATIONAL LAND USE—SURFACE WATER, SOIL, OR SEDIMENT}

The recreational land use scenario addresses exposure to people who spend a limited amount of time at or near a site while playing, fishing, hunting, hiking, or engaging in other outdoor activities. This includes what is often described as the "trespasser" or "site visitor" scenario. Risk of contamination from surface water is assumed to be primarily due to ingestion and dermal contact with chemicals while swimming and ingestion of fish. Risk of contamination from soil or sediment is assumed to be from direct ingestion, inhalation of dust and particulates, dermal contact with chemicals in the soil, and extemal exposure from radionuclides. Because the soil ingestion rate is different for children and adults, the carcinogenic risk due to direct ingestion of soil is calculated using an age-adjusted ingestion factor. This takes into account the differences in daily soil ingestion rates, body weights, and exposure durations for the two exposure groups. Exposure frequency is assumed to be the same for the two groups. Calculated in this manner, the factor leads to a more protective risk-based concentration compared to an adult-only assumption. Due to differences in averaging times for carcinogens and noncarcinogens, the noncarcinogenic hazard is calculated separately for adults and children. This procedure will give a more protective concentration than the adult-only assumption.

\subsubsection{Surface Water}

\subsubsection{Nonradionuclide contaminant}

1. Ingestion only

$$
C=\frac{T \times B W \times A T}{T V_{0} \times I R_{w} \times E F \times E D \times E T}
$$

2. Dermal contact only

$$
C=\frac{T \times B W \times A T}{T V_{a d} \times S A \times K_{p} \times E T \times E F \times E D \times C F_{1}}
$$

3. Ingestion and dermal contact

$$
C=\frac{T \times B W \times A T}{E F \times E D \times\left[\left(T V_{o} \times I R_{w} \times E T\right)+\left(T V_{a d} \times S A \times K_{p} \times E T \times C F_{\mathfrak{l}}\right)\right]}
$$

4. Ingestion of fish only - concentration in water

$$
C=\frac{T \times B W \times A T}{T V_{o} \times I R_{f} \times F I \times E F_{f} \times E D \times B F}
$$


Equations

Recreational Land Use

5. Ingestion of fish only - concentration in fish

$$
C_{f}=\frac{T \times B W \times A T}{T V_{o} \times I R_{f} \times F I \times E F_{f} \times E D}
$$

where

Parameters Definition (units)

AT

BF

BW

C

$\mathrm{C}_{\mathrm{f}}$

$\mathrm{CF}_{1}$

ED

$\mathrm{EF}$

$\mathrm{EF}_{\mathrm{f}}$

ET

FI

$\mathrm{IR}_{\mathrm{f}}$

$\mathrm{IR}_{\mathrm{w}}$

$\mathrm{K}_{\mathrm{p}}$

$\mathrm{RfD}_{\mathrm{ad}}$

$\mathrm{RfD}_{0}$

SA

$\mathrm{SF}_{\text {ad }}$

$\mathrm{SF}_{\text {。 }}$

$\mathrm{T}$

THI

TR

$\mathrm{TV}_{\mathrm{ad}}$

$\mathrm{TV}_{\mathrm{o}}$

target (unitless)

oral toxicity value averaging time (yr $\times$ day/yr)

bioaccumulation factor $(\mathrm{L} / \mathrm{kg})$

adult body weight (kg)

chemical PRG in water $(\mathrm{mg} / \mathrm{L})$

chemical PRG in fish $(\mathrm{mg} / \mathrm{kg})$

units conversion factor $\left(\mathrm{L} / \mathrm{cm}-\mathrm{m}^{2}\right)$

exposure duration (yr)

exposure frequency (day/yr)

exposure frequency fishing (day/yr)

exposure time (hr/day)

fraction ingested (unitless)

ingestion rate - fish ( $\mathrm{kg} /$ day)

water ingestion rate $(\mathrm{L} / \mathrm{hr})$

permeability constant $(\mathbf{c m} / \mathbf{h r})$

absorbed chronic reference dose (mg/kg-day)

oral chronic reference dose ( $\mathrm{mg} / \mathbf{k g}$-day)

adult surface area $\left(\mathrm{m}^{2}\right)$

absorbed dose slope factor ((mg/kg-day $\left.)^{-1}\right)$

oral slope factor $\left((\mathrm{mg} / \mathrm{kg}-\mathrm{day})^{-1}\right)$

target hazard index (unitless)

target excess individual lifetime cancer risk (unitless)

absorbed toxicity value
Default Value

$70 \times 365$ (carcinogen) [1]

$\mathrm{ED} \times 365$

(noncarcinogen) [1]

chemical-specific

70 [1]

$-$

$-$

10

30 [1]

$45[28]$

48 [13]

1 [4]

$1[12]$

0.054 [9]

0.05 [28]

chemical-specific

chemical-specific [3]

chemical-specific [3]

1.94 [4]

chemical-specific [3]

chemical-specific [3]

TR (carcinogen)

THI (noncarcinogen)

1 and 0.1

$10^{-6}$ and $10^{-4}$

$\mathrm{SF}_{\mathrm{ad}}$ (carcinogen)

$1 / \mathrm{RfD}_{\mathrm{ad}}$ (noncarcinogen)

$\mathrm{SF}_{\mathrm{o}}$ (carcinogen)

$1 / \mathrm{RfD}_{\mathrm{o}}$ (noncarcinogen) 
Recreational Land Use

\subsubsection{Radionuclide contaminant}

1. Ingestion only

$$
C=\frac{T R}{S F_{o} \times I R_{w} \times E F \times E D \times E T}
$$

2. Ingestion of fish only - concentration in water

$$
C=\frac{T R}{S F_{o} \times I R_{f} \times E F_{f} \times F I \times E D \times B F}
$$

3. Ingestion of fish only - concentration in fish

$$
C_{f}=\frac{T R}{S F_{o} \times I R_{f} \times C F \times E F_{f} \times F I \times E D}
$$

where

Parameters
$\mathrm{BF}$
$\mathrm{C}$
$\mathrm{C}_{\mathrm{f}}$
$\mathrm{CF}$
$\mathrm{ED}$
$\mathrm{EF}$
$\mathrm{EF}$
$\mathrm{ET}$
$\mathrm{FI}$
$\mathrm{IR}_{\mathrm{f}}$
$\mathrm{IR}_{\mathrm{w}}$
$\mathrm{SF}_{\mathrm{o}}$
$\mathrm{TR}$

Definition (units)

bioaccumulation factor $(\mathrm{L} / \mathrm{kg})$

radionuclide $P R G$ in water $(\mathrm{pCi} / \mathrm{L})$

radionuclide $P R G$ in fish (pCi/g)

units conversion factor $(\mathrm{g} / \mathrm{kg})$

exposure duration (yr)

exposure frequency (day/yr)

exposure frequency fishing (day/yr)

exposure time (hr/day)

fraction ingested (unitless)

ingestion rate - fish (kg/day)

water ingestion rate $(\mathrm{L} / \mathrm{hr})$

oral slope factor (risk/pCi)

target excess individual lifetime cancer risk (unitless)
Default Value

radionuclide-specific

-

$-$

1000

30 [1]

$45[28]$

48 [13]

1 [4]

1 [12]

0.054 [9]

0.05 [28]

radionuclide-specific

$10^{-6}$ and $10^{-4}$ 


\subsubsection{Soil or Sediment}

\subsubsection{Nonradionuclide contaminant}

1. Ingestion only

a. Carcinogen

$$
C=\frac{T \times A T}{T V_{0} \times C F \times E F \times F I \times I F_{\text {soilladj }} \times E T \times C F_{2}}
$$

b. Noncarcinogen (adult and child calculated separately)

$$
C=\frac{T \times B W_{n} \times A T_{n}}{T V_{0} \times C F \times E F \times E D_{n} \times I R_{n} \times F T \times E T \times C F_{2}}
$$

2. Inhalation only

$$
C=\frac{T \times B W \times A T}{T V_{i} \times E F \times E D \times\left(\frac{1}{V F}+\frac{1}{P E F}\right) \times I R_{a i r} \times E T \times C F_{2}}
$$

3. Dermal contact only

$$
C=\frac{T \times B W \times A T}{T V_{a d} \times C F_{d} \times S A \times A F \times A B S \times E F \times E D}
$$

4. Ingestion and inhalation and dermal contact

$$
C=\frac{T \times B W \times A T}{E F \times E D\left[\left(T V_{0} \times C F \times F I \times I R_{a} \times E T \times C F_{2}\right)+\left(T V_{1} \times\left(\frac{1}{V F}+\frac{1}{P E F}\right) \times I R_{a t r} \times E T \times C F_{2}\right)+\left(T V_{a d} \times C F_{d} \times S A \times A F \times A B S\right)\right]}
$$

where

Parameters

ABS

$\mathrm{AF}$

AT

$\mathrm{AT}_{\mathrm{n}}$

BW

\section{Definition_(units)} absorption factor (unitless) adherence factor $\left(\mathrm{mg} / \mathrm{cm}^{2}\right)$ averaging time (yr $\times$ day/yr)

averaging time - noncarcinogenic ingestion only (yr $\times$ day $/ y r)$

adult body weight (kg)
Default Value chemical-specific [4]

1 [11] $70 \times 365$ (carcinogen) [1] $\mathrm{ED} \times 365$ (noncarcinogen) [1] $\mathrm{ED}_{\mathrm{n}} \times 365[1]$

$70[1]$ 


\begin{tabular}{|c|c|c|}
\hline \multicolumn{3}{|c|}{ Recreational Land Use } \\
\hline $\mathrm{BW}_{\mathrm{n}}$ & body weight - noncarcinogenic ingestion only (kg) & $\begin{array}{l}70 \text { (adult) [1] } \\
15 \text { (child) [1] }\end{array}$ \\
\hline $\mathrm{C}$ & chemical PRG in soil (mg/kg) & - \\
\hline $\mathrm{CF}$ & units conversion factor $(\mathrm{kg} / \mathrm{mg})$ & $10^{-6}$ \\
\hline $\mathrm{CF}_{2}$ & units conversion factor (day/hr) & $1 / 24$ \\
\hline $\mathrm{CF}_{\mathrm{d}}$ & units conversion factor - dermal $\left(\mathrm{kg}-\mathrm{cm}^{2}\right) /\left(\mathrm{mg}-\mathrm{m}^{2}\right)$ & 0.01 \\
\hline $\mathrm{ED}$ & exposure duration (yr) & $30[1]$ \\
\hline $\mathrm{ED}_{\mathrm{n}}$ & $\begin{array}{l}\text { exposure duration - noncarcinogenic ingestion } \\
\text { only (yr) }\end{array}$ & $\begin{array}{l}24 \text { (adult) [1] } \\
6 \text { (child) [1] }\end{array}$ \\
\hline $\mathrm{EF}$ & exposure frequency (day/yr) & $75[4]$ \\
\hline ET & exposure time (hr/day) & $1[4]$ \\
\hline FI & fraction ingested (unitless) & $1[12]$ \\
\hline $\mathrm{IF}_{\text {soil/adj }}$ & age-adjusted soil ingestion factor (mg-yr/kg-day) & $\sim 114$ (see Eq. 89) [1] \\
\hline $\mathbb{I R}_{\mathbf{a}}$ & ingestion rate for adult (mg/day) & $100[1]$ \\
\hline $\mathrm{IR}_{\mathrm{ait}}$ & total inhalation rate $\left(\mathrm{m}^{3} / \mathrm{day}\right)$ & $20[9]$ \\
\hline $\mathbb{I R}_{\mathbf{n}}$ & $\begin{array}{l}\text { ingestion rate - noncarcinogenic ingestion only } \\
\text { (mg/day) }\end{array}$ & $\begin{array}{l}100 \text { (adult) [1] } \\
200 \text { (child) [1] }\end{array}$ \\
\hline PEF & particulate emission factor $\left(\mathrm{m}^{3} / \mathrm{kg}\right)$ & $4.28 \times 10^{9}$ (see Eq. 40$)[5]$ \\
\hline $\mathrm{RfD}_{\mathrm{ad}}$ & absorbed chronic reference dose (mg/kg-day) & chemical-specific [3] \\
\hline $\mathrm{RfD}_{\mathrm{i}}$ & inhalation chronic reference dose (mg/kg-day) & chemical-specific [3] \\
\hline $\mathrm{RfD}_{0}$ & oral chronic reference dose (mg/kg-day) & chemical-specific [3] \\
\hline $\mathrm{SA}$ & adult surface area $\left(\mathrm{m}^{2} /\right.$ day $)$ & $0.53[4]$ \\
\hline $\mathrm{SF}_{\mathrm{ad}}$ & absorbed dose slope factor ((mg/kg-day $\left.)^{-1}\right)$ & chemical-specific [3] \\
\hline $\mathrm{SF}_{\mathrm{i}}$ & inhalation slope factor $\left((\mathrm{mg} / \mathrm{kg}-\mathrm{day})^{-1}\right)$ & chemical-specific [3] \\
\hline $\mathrm{SF}_{\mathrm{o}}$ & oral slope factor ((mg/kg-day $\left.)^{-1}\right)$ & chemical-specific [3] \\
\hline $\mathrm{T}$ & target (unitless) & $\begin{array}{l}\text { TR (carcinogen) } \\
\text { THI (noncarcinogen) }\end{array}$ \\
\hline THI & target hazard index (unitless) & 1 and 0.1 \\
\hline TR & target excess individual lifetime cancer risk (unitless) & $10^{-6}$ and $10^{-4}$ \\
\hline $\mathrm{TV}_{\mathrm{ad}}$ & absorbed toxicity value & 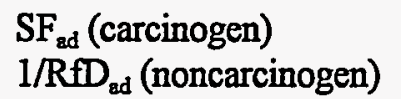 \\
\hline $\mathrm{TV}_{\mathrm{i}}$ & inhalation toxicity value & $\begin{array}{l}\mathrm{SF}_{\mathrm{i}}(\text { carcinogen } \\
1 / \mathrm{RfD}_{\mathrm{i}} \text { (noncarcinogen) }\end{array}$ \\
\hline $\mathrm{TV}_{0}$ & oral toxicity value & $\begin{array}{l}\mathrm{SF}_{\mathrm{o}} \text { (carcinogen) } \\
1 / \mathrm{RfD}_{\mathrm{o}} \text { (noncarcinogen) }\end{array}$ \\
\hline VF & volatilization factor $\left(\mathrm{m}^{3} / \mathrm{kg}\right)$ & $\begin{array}{l}\text { chemical-specific } \\
\text { (see Eqs. 34-39)[5] }\end{array}$ \\
\hline
\end{tabular}


THIS PAGE LEFT BLANK 


\section{Equations}

Recreational Land Use

\subsubsection{Radionuclide contaminant}

1. Ingestion only

$$
C=\frac{T R}{S F_{o} \times C F \times E F \times I F_{\text {soillatj }} \times E T \times C F_{2}}
$$

2. External radiation only

$$
C=\frac{T R}{S F_{x} \times E D \times E F_{x} \times\left(1-S_{e}\right) \times T_{e}}
$$

3. Inhalation only

$$
C=\frac{T R}{S F_{i} \times E D \times C F_{i} \times E F \times\left(\frac{1}{V F}+\frac{1}{P E F}\right) \times I R_{a i r} \times E T \times C F_{2}}
$$

4. Ingestion and external radiation and inhalation (adult only)

$$
C=\frac{T R}{E D \times\left[\left(S F_{0} \times C F \times E F \times I R_{a} \times E T \times C F_{2}\right)+\left(S F_{x} \times E F_{x} \times\left(1-S_{e}\right) \times T_{e}\right)+\left(S F_{t} \times C F_{1} \times E F \times\left(\frac{1}{V F}+\frac{1}{P E F}\right) \times I R_{d t r} \times E T \times C F_{2}\right)\right]}
$$


Equations

Recreational Land Use

where

\begin{tabular}{|c|c|c|}
\hline Parameters & Definition (units) & Default Value \\
\hline $\mathrm{C}$ & radionuclide $\mathrm{PRG}$ in soil (pCi/g) & - \\
\hline CF & units conversion factor $(\mathrm{g} / \mathrm{mg})$ & $10^{-3}$ \\
\hline $\mathrm{CF}_{2}$ & units conversion factor (day/hr) & $1 / 24$ \\
\hline $\mathrm{CF}_{\mathrm{i}}$ & units conversion factor - inhalation $(\mathrm{g} / \mathrm{kg})$ & $10^{3}$ \\
\hline ED & exposure duration (yr) & $30[1]$ \\
\hline $\mathrm{EF}$ & exposure frequency (day/yr) & $75[4]$ \\
\hline $\mathrm{EF}_{\mathrm{x}}$ & exposure frequency - external (day/day) & $75 / 365$ \\
\hline ET & exposure time (hr/day) & $1[4]$ \\
\hline $\mathrm{IF}_{\mathrm{xoi} / \mathrm{ddj}}$ & age-adjusted soil ingestion factor (mg-yr/day) & 3600 (see Eq. 90) [6] \\
\hline $\mathbb{I R}_{\mathrm{a}}$ & adult ingestion rate (mg/day) & $100[1]$ \\
\hline $\mathrm{IR}_{\text {air }}$ & total inhalation rate $\left(\mathrm{m}^{3} / \mathrm{day}\right)$ & $20[9]$ \\
\hline PEF & particulate emission factor $\left(\mathrm{m}^{3} / \mathrm{kg}\right)$ & $\begin{array}{l}\left.4.28 \times 10^{9} \text { (see Eq. } 40\right) \\
{[5]}\end{array}$ \\
\hline $\mathbf{S}_{\mathrm{c}}$ & gamma shielding factor (unitless) & 0.2 (see EPA, 1991) [1] \\
\hline $\mathrm{SF}_{\mathrm{i}}$ & inhalation slope factor (risk/pCi) & radionuclide-specific [3] \\
\hline $\mathrm{SF}_{\mathrm{o}}$ & oral slope factor (risk/pCi) & radionuclide-specific [3] \\
\hline $\mathrm{SF}_{\mathrm{x}}$ & external exposure slope factor ((risk-g)/(pCi-yr)) & radionuclide-specific [3] \\
\hline $\mathrm{T}_{\mathrm{e}}$ & gamma exposure time factor $(\mathrm{hr} / \mathrm{hr})$ & $1 / 24$ \\
\hline TR & target excess individual lifetime cancer risk (unitless) & $10^{-6}$ and $10^{-4}$ \\
\hline VF & volatilization factor $\left(\mathrm{m}^{3} / \mathrm{kg}\right)$ & radionuclide-specific[5] \\
\hline
\end{tabular}

\subsubsection{Supporting equations}

1. Age-adjusted Soil Ingestion Factor for Nonradionuclides (carcinogenic risk only)

$$
I F_{\text {soillauf }}=\frac{I R_{\text {soillage 1-6 }} \times E D_{\text {age 1-6 }}}{B W_{\text {age 1-6 }}}+\frac{I R_{\text {soillage 7-31 }} \times E D_{\text {age 7-31 }}}{B W_{\text {age 7-31 }}}
$$

where

\begin{tabular}{|c|c|c|}
\hline Parameters & Definition (units) & Default Value \\
\hline $\mathrm{BW}_{\mathrm{age} 1.6}$ & average body weight from ages $1-6(\mathrm{~kg})$ & $15[1]$ \\
\hline $\mathrm{BW}_{\mathrm{age}}$ 7-31 & average body weight from ages $7-31(\mathrm{~kg})$ & $70[1]$ \\
\hline $\mathrm{ED}_{\text {ape } 1-6}$ & exposure duration during ages $1-6$ (yr) & $6[1]$ \\
\hline $\mathrm{ED}_{\mathrm{age}} 7-31$ & exposure duration during ages $7-31$ (yr) & $24[1]$ \\
\hline$I_{\text {soil/adj }}$ & age-adjusted soil ingestion factor (mg-yr/kg-day) & $\sim 114[1]$ \\
\hline $\operatorname{IR}_{\text {LoiVkge 1-6 }}$ & ingestion rate of soil for ages $1-6$ (mg/day) & $200[1]$ \\
\hline $\mathbb{I R}_{\text {pil/gre }} 7.31$ & ingestion rate of soil for ages 7-31 (mg/day) & $100[1]$ \\
\hline
\end{tabular}


Recreational Land Use

2. Age-adjusted soil ingestion factor for radionuclides

$$
I F_{\text {soilladi }}=\left(I R_{\text {soillage 1-6 }} \times E D_{\text {age 1-6 }}\right)+\left(I R_{\text {soillage 7-31 }} \times E D_{\text {age 7-31 }}\right)
$$

where

Parameters

$\mathrm{ED}_{\text {age 1-6 }}$

$\mathrm{ED}_{\text {age } 7-31}$

IF $_{\text {soil/adj }}$

$\mathbb{R}_{\text {soil/age 1-6 }}$

$\mathrm{IR}_{\text {soivage }}$ 7-31
Definition (units)

exposure duration during ages 1-6 (yr) exposure duration during ages 7-31 (yr) age-adjusted soil ingestion factor (mg-yr/day) ingestion rate of soil for ages 1-6 (mg/day) ingestion rate of soil for ages 7-31 (mg/day)
Default Value

$6[1]$

24 [1]

3600 [1]

$200[1]$

100 [1] 
Equations

Excavation Land Use

\subsection{EXCAVATION LAND USE_SOIL OR SEDIMENT}

The excavation land use scenario is assumed to be an infrequent and short-lived occurrence. Due to weather considerations for this scenario, exposure frequency is assumed to be one month of the worker year, or approximately 20 days per year. In addition, excavation is assumed to be a rare occurrence, so the exposure duration for any one worker is assumed to be one year. Risk of contamination is assumed to be due to direct ingestion, inhalation of dust and particulates, dermal contact with chemicals, and external exposure to radionuclides.

\subsubsection{Soil or Sediment}

\subsubsection{Nonradionuclide contaminant}

1. Ingestion only

$$
C=\frac{T \times B W \times A T}{T V_{o} \times C F \times E F \times E D \times I R_{s}}
$$

2. Inhalation only

$$
C=\frac{T \times B W \times A T}{T V_{i} \times E F \times E D \times\left(\frac{1}{V F}+\frac{1}{P E F}\right) \times I R_{a}}
$$

3. Dermal contact only

$$
C=\frac{T \times B W \times A T}{T V_{a d} \times C F_{d} \times S A \times A F \times A B S \times E F \times E D}
$$

4. Ingestion and inhalation and dermal contact

$$
C=\frac{T \times B W \times A T}{E F \times E D \times\left[\left(T V_{o} \times C F \times I R_{s}\right)+\left(T V_{i} \times\left(\frac{1}{V F}+\frac{1}{P E F}\right) \times I R_{a}\right)+\left(T V_{a d} \times C F_{d} \times S A \times A F \times A B S\right)\right]}
$$


Equations

Excavation Land Use

where

\begin{tabular}{|c|c|c|}
\hline Parameters & Definition (units) & Default Value \\
\hline ABS & absorption factor (unitless) & chemical-specific [4] \\
\hline $\mathrm{AF}$ & adherence factor $\left(\mathrm{mg} / \mathrm{cm}^{2}\right)$ & $1[11]$ \\
\hline AT & averaging time $(\mathrm{yr} \times$ day/yr) & $\begin{array}{l}70 \times 365 \text { (carcinogen) [1] } \\
\text { ED } \times 365 \\
(\text { noncarcinogen) }[1]\end{array}$ \\
\hline BW & adult body weight $(\mathrm{kg})$ & $70[1]$ \\
\hline C & chemical PRG in soil (mg/kg) & - \\
\hline CF & units conversion factor ( $\mathrm{kg} / \mathrm{mg}$ ) & $10^{-6}$ \\
\hline $\mathrm{CF}_{\mathrm{d}}$ & $\begin{array}{l}\text { units conversion factor - dermal } \\
\left(\mathrm{kg}-\mathrm{cm}^{2}\right) /\left(\mathrm{mg}-\mathrm{m}^{2}\right)\end{array}$ & 0.01 \\
\hline ED & exposure duration (yr) & 1 \\
\hline $\mathrm{EF}$ & exposure frequency (day/yr) & $20(\sim 1$ worker month $)$ \\
\hline $\mathbb{I R}_{\mathbf{z}}$ & total inhalation rate $\left(\mathrm{m}^{3} / \mathrm{day}\right)$ & $20[9]$ \\
\hline $\mathbb{R}$ & soil ingestion rate (mg/day) & $480[6]$ \\
\hline $\mathrm{PEF}$ & particulate emission factor $\left(\mathrm{m}^{3} / \mathrm{kg}\right)$ & $4.28 \times 10^{9}$ (see Eq. 40$)[5]$ \\
\hline $\mathrm{RfD}_{\mathrm{ad}}$ & absorbed subchronic reference dose (mg/kg-day) & chemical-specific [3] \\
\hline $\mathrm{RfD}_{\mathrm{i}}$ & inhalation subchronic reference dose (mg/kg-day) & chemical-specific [3] \\
\hline $\mathrm{RfD}_{0}$ & oral subchronic reference dose (mg/kg-day) & chemical-specific [3] \\
\hline SA & adult surface area $\left(\mathrm{m}^{2} /\right.$ day $)$ & $0.316[4]$ \\
\hline $\mathrm{SF}_{\mathrm{ad}}$ & absorbed dose slope factor $\left((\mathrm{mg} / \mathrm{kg}-\mathrm{day})^{-1}\right)$ & chemical-specific [3] \\
\hline $\mathrm{SF}_{\mathrm{i}}$ & inhalation slope factor $\left((\mathrm{mg} / \mathrm{kg}-\mathrm{day})^{-1}\right)$ & chemical-specific [3] \\
\hline $\mathbf{S F}_{\mathrm{o}}$ & oral slope factor $\left((\mathrm{mg} / \mathrm{kg}-\mathrm{day})^{-1}\right)$ & chemical-specific [3] \\
\hline $\mathrm{T}$ & target (unitless) & $\begin{array}{l}\text { TR (carcinogen) } \\
\text { THI (noncarcinogen) }\end{array}$ \\
\hline THI & target hazard index (unitless) & 1 and 0.1 \\
\hline TR & target excess individual lifetime cancer risk (unitless) & $10^{-6}$ and $10^{-4}$ \\
\hline $\mathrm{TV}_{\mathrm{ed}}$ & absorbed toxicity value & $\begin{array}{l}\mathrm{SF}_{\mathrm{ad}} \text { (carcinogen) } \\
\mathrm{LRfD}_{\mathrm{ad}} \text { (noncarcinogen) }\end{array}$ \\
\hline $\mathrm{TV}_{\mathrm{i}}$ & inhalation toxicity value & $\begin{array}{l}\mathrm{SF}_{\mathrm{i}} \text { (carcinogen) } \\
1 / \mathrm{RfD}_{\mathrm{i}} \text { (noncarcinogen) }\end{array}$ \\
\hline $\mathrm{TV}_{0}$ & oral toxicity value & $\begin{array}{l}\mathrm{SF}_{\circ} \text { (carcinogen) } \\
1 / \mathrm{RfD}_{0} \text { (noncarcinogen) }\end{array}$ \\
\hline VF & volatilization factor $\left(\mathrm{m}^{3} / \mathrm{kg}\right)$ & $\begin{array}{l}\text { chemical-specific } \\
\text { (see Eqs. 34-39)[5] }\end{array}$ \\
\hline
\end{tabular}


Excovation Land Use

\subsubsection{Radionuclide contaminant}

1. Ingestion only

$$
C=\frac{T R}{S F_{o} \times E D \times C F \times E F \times I R_{s}}
$$

2. Inhalation only

$$
C=\frac{T R}{S F_{i} \times E D \times C F_{i} \times E F \times\left(\frac{1}{V F}+\frac{1}{P E F}\right) \times I R_{a}}
$$

3. External radiation only

$$
C=\frac{T R}{S F_{x} \times E D \times E F_{x} \times\left(1-S_{e}\right) \times T_{e}}
$$

4. Ingestion and inhalation and external radiation

$$
C=\frac{T R}{E D \times\left[\left(S F_{o} \times C F \times E F \times I R_{s}\right)+\left(S F_{i} \times C F_{i} \times E F \times\left(\frac{1}{V F}+\frac{1}{P E F}\right) \times I R_{a}\right)+\left(S F_{x} \times E F_{x} \times\left(1-S_{e}\right) \times T_{e}\right)\right]}
$$

where

Parameters

C

CF

$\mathrm{CF}_{\mathrm{i}}$

ED

EF

$\mathrm{EF}_{\mathrm{x}}$

$\mathrm{IR}_{\mathrm{a}}$

$\mathrm{IR}_{3}$

PEF

$\mathrm{S}_{\mathrm{e}}$

$\mathrm{SF}_{\mathbf{i}}$

SF。

$\mathrm{SF}_{\mathrm{x}}$

$T_{e}$

TR

VF
Definition (units)

radionuclide $P R G$ in soil (pCi/g)

units conversion factor (g/mg)

units conversion factor - inhalation (g/kg)

exposure duration (yr)

exposure frequency (day/yr)

exposure frequency - external (day/day)

total inhalation rate $\left(\mathrm{m}^{3} /\right.$ day $)$

soil ingestion rate (mg/day)

particulate emission factor $\left(\mathrm{m}^{3} / \mathrm{kg}\right)$

gamma shielding factor (unitless)

inhalation slope factor (risk/pCi)

oral slope factor (risk/pCi)

external exposure slope factor ((risk-g)/(pCi-yr))

gamma exposure factor (hr/hr)

target excess individual lifetime cancer risk (unitless)

volatilization factor $\left(\mathrm{m}^{3} / \mathrm{kg}\right)$
Default Value

$-$

$10^{-3}$

$10^{3}$

1

20 ( one worker month)

20/365

20 [9]

480 [6]

$4.28 \times 10^{9}$ (see Eq. 40)[5]

0.2 [1]

radionuclide-specific [3]

radionuclide-specific [3]

radionuclide-specific [3]

$8 / 24$ [6]

$10^{-6}$ and $10^{-4}$

radionuclide-specific [5] 
Equations

References

\subsection{REFERENCES}

[1] United States Environmental Protection Agency (EPA). 1991. Risk Assessment Guidance for Superfund: Volume I - Humain Health Evaluation Manual (Part B, Development of Risk-based Preliminary Remediation Goals). Office of Emergency and Remedial Response, U.S. Environmental Protection Agency, Washington, D.C.

[2] Andelman, J.B. 1990. Total Exposure to Volatile Organic Chemicals in Potable Water. N.M. Ram, R.F. Christman, K.P. Cantor (eds.). Lewis Publishers.

[3] Oak Ridge National Laboratory (ORNL). 1994. Toxicity Values for Use in Hazardous Waste Risk Assessment and Remediation. Vol. I. ES/ER/TM-76. Biomedical and Environmental Analysis Section. Health Sciences Research Division.

[4] United States Environmental Protection Agency (EPA). 1992. Dermal Exposure Assessment: Principles and Application. Interim Report. EPA/600/8-91/011B. Office of Research and Development, Washington, D.C.

[5] Appendix D of Risk Assessment Guidance for Superfund: Volume I - Human Health Evaluation Manual (Part B, Development of Risk-based Preliminary Remediation Goals). 1991. Office of Emergency and Remedial Response, U.S. Environmental Protection Agency, Washington, D.C.

[6] United States Environmental Protection Agency (EPA). 1991. Risk Assessment Guidance for Superfund. Volume I, Human Health Evaluation Manual (Part B, Development of Risk -based Preliminary Remediation Goals). OSWER Directive 9285.7-01B. Office of Emergency and Remedial Response, Washington, D.C.

[7] International Atomic Energy Agency (IAEA). 1982. Generic Models Parameters for Assessing the Environmental Transfer of Radionuclides from Routine Releases: Exposures of Critical Groups.

[8] National Council on Radiation Protection Measurement (NCRP). January 1989. Screening Techniques for Determining Compliance with Environmental Standards. Releases of Radionuclides to the Atmosphere. Bethesda, Maryland.

[9] United States Environmental Protection Agency (EPA). 1991. Risk Assessment Guidance for Superfund Volume I: Human Health Evaluation Manual Supplemental Guidance Standard Default Exposure Factors. OSWER Directive 9285.6-03. Office of Emergency and Remedial Response. Toxics Integration Branch.

[10] United States Environmental Protection Agency (EPA). 1989. Exposure Factors Handbook. EPA/600/8-89/043. Office of Health and Environmental Assessment, Washington, D.C.

[11] United States Environmental Protection Agency (EPA) Region IV. February 11, 1992. New Interim Region IV Guidance Memorandum from Region IV, Atlanta, Georgia.

[12] Maximum value used; equivalent to $100 \%$ 
Equations

References

[13] United States Environmental Protection Agency (EPA). 1989. Risk Assessment Guidance for Superfund: Volume I-Human Health Evaluation Manual. Office of Emergency and Remedial Response, U.S. Environmental Protection Agency, Washington, D.C.

[14] McKone, T. E. 1987. Human Exposure to Volatile Organic Compounds in Household Tap Water: The Indoor Inhalation Pathway. Environ. Sci. Technol. 21:1194-1201

[15] Cockerham, L. G. and B. S. Shane [eds]. 1994. Basic Environmental Toxicology. CRC Press, Inc.

[16] Perry, R. and C. Chilton. 1973 Chemical Engineers Handbook. 5th edition. New York: McGraw Hill.

[17] United States Environmental Protection Agency (EPA). 1988. Superfund Exposure Assessment Manual. Washington, D.C., U.S. Environmental Protection Agency Office of Remedial Response. EPA/540/1-88/001 OSWER directive 9285.5-1

[18] Shen, T. 1981. "Estimating hazardous air emissions from disposal sites." Pollution Engineering. 13(8):31-34.

[19] Cowherd, C., G. Muleski, P. Engelhart, and D. Gillete. 1985. Rapid Assessment of Exposure to Particulate Emissions from Surface Contamination. Prepared for EPA Office of Health and Environmental Assessment. EPA/600/8-85/002.

[20] Miller, P. D., C. W. McGinn, S. T. Purucker, and R. K. White. 1995. Defining the Role of Risk Assessment in the Comprehensive Environmental Response, Compensation, and Liability Act (CERCLA) Remedial Investigation Process at the DOE-OR. ES/ER/TM-58.

[21] Pao, E. M., K. H. Fleming, P. M. Gueuther, and S. J. Mickle. 1982. Studies of ingestion dose pathways from the nuclear fuel services fuel reprocessing plant. U.S. Department of Agriculture.

[22] National Council on Radiation Protection and Measurements (NCRP). 1985. Radiological Assessment: Predicting the Transport, Bioaccumulation, and Uptake by Man of Radionuclides Released to the Environment. NCRP Report No. 76.

[23] Personal communication with the Roane County, Tennessee, Extension Agent.

[24] Till, J. E. and H. R. Meyer. Radiological Assessment: A Textbook on Environmental Dose Analysis. ORNL-5968. U.S. Department of Commerce, National Technical Information Service.

[25] Nuclear. Regulatory Commission (NRC). 1977. Regulatory Guide 1.109: Calculation of Annual Doses to Man from Routine Releases of Reactor Effluents for the Purpose of Evaluating Compliance with 10 CFR Part 50, Appendix 1.

[26] Darwin, R. 1990. Soil ingestion by dairy cattle. Pacific Northwest Laboratory. Richland, Washington. 
[27] United States Department of Energy (DOE). 1987. Remedial Actions Priority Systems (RAPS) Mathematical Formulations. DOE/RLL/87-06.

[28] United States Environmental Protection Agency (EPA). 1995. Supplemental Guidance to RAGS: Region 4 Bulletins, Human Health Risk Assessment (Interim Guidance). Waste Management Division, Office of Health Assessment.

[29] McKone, T. E. 1994. Uncertainty and variability in human exposures to soil contaminants through home-grown food: a Monte Carlo assessment. Risk Anal. 14(4):449-463. .

[30] Miller, C. W. 1980. An analysis of measured values for the fraction of a radioactive aerosol intercepted by vegetation. Health Phys. 38:705-712.

[31] Pinder, J. E. ,. I., and K. W. McLeod. 1989. Mass loading of soil particles on plant surfaces. Health Phys. 57:935-942.

[32] Hinton, T. G. 1992. Contamination of plants by resuspension: a review, with critique of measurement methods. Sci. Total Environ. 121:177-193.

[33] Hoffman, F. O., R. H. Gardner, and K. F. Eckerman. 1982. Variability in dose estimates associated with the food chain transport and ingestion of selected radionuclides. NUREG/CR-2612. Oak Ridge National Laboratory, Oak Ridge, TN.

[34] Peterson, H. T. .. Jr.. 1983. Terrestrial and aquatic food chain pathways, pp. 5-1 to 5-156 in J. E. Till, and H. R. Meyer, eds. Radiological Assessment: a textbook on environmental dose analysis. U.S. Nuclear Regulatory Commission, Washington, D.C.

[35] McKone, T. E. 1994. Uncertainty and variability in human exposures to soil contaminants through home-grown food: a Monte Carlo assessment. Risk Anal. 14(4):449-463.

[36] IAEA. 1994. Handbook of parameter values for the prediction of radionuclide transfer in temperate environment. Tech. Rep. Ser. No. 364, Vienna, Austria.

[37] NCRP (National Council on Radiation Protection and Measurements). 1984. Radiological assessment: predicting the transport, bioaccumulation, and uptake by man of radionuclides released to the environment. NCRP Rep. No. 76, Bethesda, MD.

[38] EPA. 1989. Risk Assessment Guidance for Superfund: Volume 1-Human Health Evaluation Manual (Part A). EPA/540/1-89/002. Office of Emergency and Remedial Response, Washington, D.C. 
Equations

References

[39] Whelan, G., D. L. Strenge, J. G. ,. Jr. Droppo, B. L. Steelman, and J. W. Buck. 1987. The remedial action priority system (RAPS): mathematical formulations. DOE/RLL/87-09. Pacific Northwest Laboratory, Richland, WA. 


\section{TABLES}

The previous equations and parameters were used to generate the following 23 tables which are arranged by scenario in the following order: (1) Residential, (2) Industrial, (3) Agriculture, (4) Recreation, and (5) Excavation. Each table contains the chemical name, its corresponding chemical abstract registration number (CASRN), and the appropriate $\mathrm{PRG}$ for each pathway at risk levels of 1.0E04 and 1.0E-06 and an HI level of 1. The tables are differentiated based on the medium (water or soil) and the chemical type (nonradionuclide or radionuclide). For each scenario, risk-based PRGs for nonradionuclides in water are followed by risk-based PRGs for radionuclides in water, nonradionuclides in soil, and radionuclides in soil. 
Table 3.1a. Risk-based Preliminary Remediation Goals for nonradionuclides in groundwater (residential scenario)

\begin{tabular}{|c|c|c|c|c|c|c|c|c|c|c|c|c|c|c|c|c|c|}
\hline \multirow[t]{2}{*}{ Chemical } & \multirow[t]{2}{*}{ CASRN } & \multicolumn{4}{|c|}{ Ingestion (mg/L)" } & \multicolumn{4}{|c|}{$\operatorname{Dermal}(\mathrm{mg} / \mathrm{L})^{*}$} & \multicolumn{4}{|c|}{ Inhalation $(\mathbf{m g} / \mathbf{L})^{\dagger *}$} & \multicolumn{4}{|c|}{$\begin{array}{l}\text { Ingestion + Dermal + } \\
\text { Inhalation }(\mathrm{mg} / \mathrm{L})^{\dagger+*}\end{array}$} \\
\hline & & $10^{-4}$ & $10^{5}$ & $\mathbf{H Q}=\mathbf{1}$ & $H Q=0.1$ & $10^{-4}$ & $10^{4}$ & $H Q=1$ & $\mathrm{HQ}=0.1$ & $-10^{-4}$ & $10^{5}$ & $H Q=1$ & $\mathrm{HQ}=0.1$ & $10^{-4}$ & $10^{4}$ & $H I=1$ & $H I=0.1$ \\
\hline ALAR & $001596-84-5$ & & & $5.5 \mathrm{E}+00$ & $5.5 \mathrm{E}-01$ & & & & & & & & & & & & \\
\hline Acenaphthene & $000083-32-9$ & & & $2.2 E+00$ & $2.2 \mathrm{E}-01$ & & & $1.1 \mathrm{E}+00$ & $1.1 \mathrm{E}-01$ & & & & & & & $7.5 \mathrm{E}-01$ & $7.5 \mathrm{E}-02$ \\
\hline Acenaphthylene & $000208-96-8$ & & & & & & & & & & & & & & & & \\
\hline Acephate & $030560-19-1$ & $9.8 \mathrm{E}-01$ & $9.8 \mathrm{E}-03$ & $1.5 \mathrm{E}-01$ & $1.5 \mathrm{E}-02$ & $5.6 \mathrm{E}+03$ & $5.6 \mathrm{E}+01$ & $8.3 \mathrm{E}+02$ & $8.3 \mathrm{E}+01$ & & & & & $9.8 \mathrm{E}-01$ & $9.8 \mathrm{E}-03$ & $1.5 \mathrm{E}-01$ & $1.5 \mathrm{E}-02$ \\
\hline Acetaldehyde & $000075-07-0$ & & & & & & & & & $2.2 \mathrm{E}-01$ & $2.2 \mathrm{E}-03$ & $1.9 \mathrm{E}-02$ & $1.9 \mathrm{E}-03$ & & & & \\
\hline Acetochlor & $034256-82-1$ & & & 7.3E-01 & $7.3 \mathrm{E}-02$ & & & & & & & & & & & & \\
\hline Acetone & $000067-64-1$ & & & $3.7 \mathrm{E}+00$ & $3.7 \mathrm{E}-01$ & & & $2.2 \mathrm{E}+03$ & $2.2 \mathrm{E}+02$ & & & & & & & $3.6 \mathrm{E}+00$ & $3.6 \mathrm{E}-01$ \\
\hline Acetone Cyanohydrin & $000075-86-5$ & & & $2.9 \mathrm{E}-02$ & $2.9 \mathrm{E}-03$ & & & & & & & & & & & & \\
\hline Acetonitrile & $000075-05-8$ & & & $2.2 \mathrm{E}-01$ & $2.2 \mathrm{E}-02$ & & & $1.2 \mathrm{E}+02$ & $1.2 \mathrm{E}+01$ & & & $1.0 \mathrm{E}-01$ & $1.0 \mathrm{E}-02$ & & & $7.1 \mathrm{E}-02$ & $7.1 \mathrm{E}-03$ \\
\hline Acetophenone $e^{(s)}$ & $000098-86-2$ & & & 3.7E+00 & $3.7 \mathrm{E}-01$ & & & $2.1 \mathrm{E}+02$ & $2.1 \mathrm{E}+01$ & & & $4.2 \mathrm{E}-05$ & 4.2E-06 & & & $4.2 \mathrm{E}-05$ & 4.2E-06 \\
\hline Acrolein ${ }^{(6)}$ & $000107-02-8$ & & & 7.3E-01 & 7.3E-02 & & & $3.3 \mathrm{E}+02$ & $3.3 \mathrm{E}+01$ & & & 4.2E-05 & 4.2E-06 & & & $4.2 \mathrm{E}-05$ & 4.2E-06 \\
\hline Actylamide & $000079-06-1$ & $1.9 \mathrm{E}-03$ & $1.9 \mathrm{E}-05$ & $7.3 \mathrm{E}-03$ & 7.3E-04 & $1.6 \mathrm{E}+00$ & $1.6 \mathrm{E}-02$ & $6.3 \mathrm{E}+00$ & $6.3 \mathrm{E}-01$ & & & & & $1.9 \mathrm{E}-03$ & $1.9 \mathrm{E}-05$ & 7.3E-03 & 7.3E-04 \\
\hline Acrylic Acid & $000079-10-7$ & & & $1.8 \mathrm{E}+01$ & $1.8 \mathrm{E}+00$ & & & $4.2 E+03$ & $4.2 \mathrm{E}+02$ & & & & & & & $1.8 \mathrm{E}+01$ & $1.8 \mathrm{E}+00$ \\
\hline Acrylonitrile & $000107-13-1$ & $1.6 \mathrm{E}-02$ & 1.6E-04 & $3.7 \mathrm{E}-02$ & $3.7 \mathrm{E}-03$ & $3.7 \mathrm{E}+00$ & 3.7E-02 & $8.6 \mathrm{E}+00$ & $8.6 \mathrm{E}-01$ & $7.1 \mathrm{E}-03$ & $7.1 \mathrm{E}-05$ & 4.2E-03 & 4.2E-04 & $4.9 \mathrm{E}-03$ & $4.9 \mathrm{E}-05$ & $3.7 \mathrm{E}-03$ & 3.7E-04 \\
\hline Alachlor & $015972-60-8$ & $1.1 \mathrm{E}-01$ & $1.1 \mathrm{E}-03$ & 3.7E-01 & 3.7E-02 & $4.4 \mathrm{E}+00$ & $4.4 \mathrm{E}-02$ & $1.5 \mathrm{E}+01$ & $1.5 \mathrm{E}+00$ & & & & & $1.0 \mathrm{E}-01$ & $1.0 \mathrm{E}-03$ & $3.6 \mathrm{E}-01$ & $3.6 \mathrm{E}-02$ \\
\hline Aldicarb & $000116-06-3$ & & & $3.7 \mathrm{E}-02$ & $3.7 \mathrm{E}-03$ & & & $9.0 \mathrm{E}+00$ & $9.0 \mathrm{E}-01$ & & & & & & & $3.6 \mathrm{E}-02$ & $3.6 \mathrm{E}-03$ \\
\hline Aldicarb Sulfone & $001646-88-4$ & & & $3.7 \mathrm{E}-02$ & $3.7 \mathrm{E}-03$ & & & & & & & & & & & & \\
\hline Aldrin & $000309-00-2$ & $5.0 \mathrm{E}-04$ & $5.0 \mathrm{E}-06$ & $1.1 \mathrm{E}-03$ & $1.1 \mathrm{E}-04$ & $6.5 \mathrm{E}-02$ & $6.5 \mathrm{E}-04$ & $1.4 \mathrm{E}-01$ & $1.4 \mathrm{E}-02$ & & & & & $5.0 \mathrm{E}-04$ & $5.0 \mathrm{E}-06$ & $1.1 \mathrm{E}-03$ & $1.1 \mathrm{E}-04$ \\
\hline Ally & $074223-64-6$ & & & $9.1 \mathrm{E}+00$ & 9.1E-01 & & & $2.0 \mathrm{E}+05$ & $2.0 \mathrm{E}+04$ & & & & & & & $9.1 \mathrm{E}+00$ & $9.1 \mathrm{E}-01$ \\
\hline Allyl Alcoho! & $000107-18-6$ & & & $1.8 \mathrm{E}-01$ & $1.8 \mathrm{E}-02$ & & & $1.0 \mathrm{E}+02$ & $1.0 \mathrm{E}+01$ & & & & & & & $1.8 \mathrm{E}-01$ & $1.8 \mathrm{E}-02$ \\
\hline Allyl Chloride ${ }^{(3)}$ & $000107-05-1$ & & & $1.8 \mathrm{E}+00$ & $1.8 \mathrm{E}-01$ & & & $8.6 \mathrm{E}+01$ & $8.6 \mathrm{E}+00$ & & & 2.1E-03 & 2.1E-04 & & & 2.1E-03 & 2.1E-04 \\
\hline
\end{tabular}

Nonradionuclides/groundwater/residential

Page 1 of 36 
Table 3.1a. (continued)

\begin{tabular}{|c|c|c|c|c|c|c|c|c|c|c|c|c|c|c|c|c|c|}
\hline \multirow[t]{2}{*}{ Chemical } & \multirow[t]{2}{*}{ CASRN } & \multicolumn{4}{|c|}{ Ingestion (mg/L)" } & \multicolumn{4}{|c|}{ Dermal (mg/L)" } & \multicolumn{4}{|c|}{ Inhalation (mg/L) $)^{\dagger \oplus}$} & \multicolumn{4}{|c|}{$\begin{array}{l}\text { Ingestion + Dermal + } \\
\text { Inhalation }(\mathrm{mg} / \mathrm{L})^{\mathrm{H}^{+}}\end{array}$} \\
\hline & & $10^{-4}$ & $10^{-5}$ & $\mathrm{HQ}=1$ & $\mathrm{HQ}=0.1$ & $10^{-4}$ & $10^{5}$ & $H Q=1$ & $\mathrm{HQ}=0.1$ & $10^{-4}$ & $10^{4}$ & $\mathrm{HQ}=\mathbf{1}$ & $\mathrm{HQ}=0.1$ & $10^{-4}$ & $10^{5}$ & $\mathrm{HI}=\mathbf{1}$ & $\mathrm{HI}=0.1$ \\
\hline Aluminum & $007429-90-5$ & & & & & & & & & & & & & & & & \\
\hline Aluminum Phosphide & $020859-73-8$ & & & $1.5 \mathrm{E}-02$ & $1.5 \mathrm{E}-03$ & & & $1.2 \mathrm{E}+00$ & $1.2 \mathrm{E}-01$ & & & & & & & $1.4 \mathrm{E}-02$ & $1.4 \mathrm{E}-03$ \\
\hline Amdro & $067485-29-4$ & & & $1.1 \mathrm{E}-02$ & $1.1 \mathrm{E}-03$ & & & & & & & & & & & & \\
\hline Ametryn & $000834-12-8$ & & & 3.3E-01 & $3.3 \mathrm{E}-02$ & & & $9.2 \mathrm{E}+01$ & $9.2 \mathrm{E}+00$ & & & & & & & $3.3 \mathrm{E}-01$ & 3.3E-02 \\
\hline Aminophenol, $\mathrm{m}$ - & $000591-27-5$ & & & $2.6 \mathrm{E}+00$ & $2.6 \mathrm{E}-01$ & & & $9.1 \mathrm{E}+02$ & $9.1 \mathrm{E}+01$ & & & & & & & $2.5 \mathrm{E}+00$ & 2.5E-01 \\
\hline Aminopyridine, 4- & $000504-24-5$ & & & $7.3 \mathrm{E}-04$ & $7.3 \mathrm{E}-05$ & & & $1.9 \mathrm{E}-01$ & $1.9 \mathrm{E}-02$ & & & & & & & 7.3E-04 & 7.3E-05 \\
\hline Amitraz & 033089-61-1 & & & $9.1 E-02$ & $9.1 \mathrm{E}-03$ & & & & & & & & & & & & \\
\hline Ammonia & $007664-41-7$ & & & & & & & & & & & & & & & & \\
\hline Ammonium Sulfamate & $007773-06-0$ & & & $7.3 \mathrm{E}+00$ & $7.3 \mathrm{E}-01$ & & & $6.0 \mathrm{E}+02$ & $6.0 \mathrm{E}+01$ & & & & & & & $7.2 \mathrm{E}+00$ & 7.2E-01 \\
\hline Aniline & $000062-53-3$ & $1.5 \mathrm{E}+00$ & $1.5 \mathrm{E}-02$ & & & $7.5 \mathrm{E}+00$ & $7.5 \mathrm{E}-02$ & & & & & & & $1.2 \mathrm{E}+00$ & $1.2 \mathrm{E}-02$ & & \\
\hline Anthracene & $000120-12-7$ & & & $1.1 \mathrm{E}+01$ & $1.1 \mathrm{E}+00$ & & & $1.5 \mathrm{E}+01$ & $1.5 \mathrm{E}+00$ & & & & & & & $6.4 \mathrm{E}+00$ & $6.4 \mathrm{E}-01$ \\
\hline Antimony (metallic) & $007440-36-0$ & & & $1.5 \mathrm{E}-02$ & $1.5 \mathrm{E}-03$ & & & $1.2 \mathrm{E}-01$ & $1.2 \mathrm{E}-02$ & & & & & & & $1.3 \mathrm{E}-02$ & $1.3 \mathrm{E}-03$ \\
\hline Antimony Pentoxide & $001314-60-9$ & & & $1.8 \mathrm{E}-02$ & $1.8 \mathrm{E}-03$ & & & $1.5 \mathrm{E}+00$ & $1.5 \mathrm{E}-01$ & & & & & & & $1.8 \mathrm{E}-02$ & $1.8 \mathrm{E}-03$ \\
\hline $\begin{array}{l}\text { Antimony Potassium } \\
\text { Tartrate }\end{array}$ & $000304-61-0$ & & & $3.3 \mathrm{E}-02$ & 3.3E-03 & & & $2.7 \mathrm{E}+00$ & $2.7 E-01$ & & & & & & & $3.2 \mathrm{E}-02$ & $3.2 \mathrm{E}-03$ \\
\hline Antimony Tetroxide & $001332-81-6$ & & & $1.5 \mathrm{E}-02$ & $1.5 \mathrm{E}-03$ & & & $1.2 \mathrm{E}+00$ & $1.2 \mathrm{E}-01$ & & & & & & & $1.4 E-02$ & $1.4 \mathrm{E}-03$ \\
\hline Antimony Trioxide & $001309-64-4$ & & & $1.5 \mathrm{E}-02$ & $1.5 \mathrm{E}-03$ & & & $1.2 E+00$ & $1.2 \mathrm{E}-01$ & & & & & & & $1.4 \mathrm{E}-02$ & $1.4 \mathrm{E}-03$ \\
\hline Apollo & 074115-24-5 & & & $4.7 \mathrm{E}-01$ & $4.7 \mathrm{E}-02$ & & & & & & & & & & & & \\
\hline Aramite & $000140-57-8$ & $3.4 \mathrm{E}-01$ & $3.4 \mathrm{E}-03$ & $1.8 \mathrm{E}+00$ & $1.8 \mathrm{E}-01$ & & & & & & & & & & & & \\
\hline Aroclor 1016 & $012674-11-2$ & & & $2.6 \mathrm{E}-03$ & $2.6 \mathrm{E}-04$ & & & $1.2 \mathrm{E}-03$ & $1.2 \mathrm{E}-04$ & & & & & & & $8.2 \mathrm{E}-04$ & $8.2 E-05$ \\
\hline Aroclor $122 \mathrm{I}^{(1)}$ & $011104-28-2$ & $1.1 \mathrm{E}-03$ & $1.1 \mathrm{E}-05$ & & & $4.5 \mathrm{E}-03$ & 4.5E-05 & & & & & & & $8.9 E-04$ & $8.9 \mathrm{E}-06$ & & \\
\hline Aroclor $1232^{(1)}$ & $011141-16.5$ & $1.1 \mathrm{E}-03$ & $1.1 \mathrm{EE}-05$ & & & $3.0 \mathrm{E}-02$ & $3.0 \mathrm{E}-04$ & & & & & & & $1.1 \mathrm{E}-03$ & 1.1E- -05 & & \\
\hline
\end{tabular}


Table 3.1a. (continued)

\begin{tabular}{|c|c|c|c|c|c|c|c|c|c|c|c|c|c|c|c|c|c|}
\hline \multirow[t]{2}{*}{ Chemical } & \multirow[t]{2}{*}{ CASRN } & \multicolumn{4}{|c|}{ Ingestion (mg/L)" } & \multicolumn{4}{|c|}{ Dermal (mg/L)" } & \multicolumn{4}{|c|}{ Inhalation $(\mathrm{mg} / \mathrm{L})^{t^{*}}$} & \multicolumn{4}{|c|}{$\begin{array}{l}\text { Ingestion + Dermal + } \\
\text { Inhalation }(\mathrm{mg} / \mathrm{L})^{\mathrm{tt*}^{*}}\end{array}$} \\
\hline & & $10^{4}$ & $10^{4}$ & $H Q=1$ & $\mathrm{HQ}=0.1$ & $10^{-4}$ & $10^{6}$ & $\mathrm{HQ}=1$ & $\mathrm{HQ}=0.1$ & $10^{-4}$ & $10^{4}$ & $H Q=1$ & $\mathrm{HQ}=0.1$ & $10^{-4}$ & $10^{5}$ & $\mathrm{HI}=\mathbf{I}$ & $H I=0.1$ \\
\hline Aroclor $1242^{(1)}$ & $053469-21-9$ & $1.1 \mathrm{E}-03$ & L.1E-05 & & & $1.1 \mathrm{E}-02$ & 1.1E-04 & & & & & & & $1.0 \mathrm{E}-03$ & $1.0 \mathrm{E}-05$ & & \\
\hline Aroclor $1248^{(1)}$ & $012672-29-6$ & 1.1E-03 & 1.IE-05 & & & $1.1 \mathrm{E}-03$ & $1.1 \mathrm{E}-05$ & & & & & & & $5.5 \mathrm{E}-04$ & $5.5 \mathrm{E}-06$ & & \\
\hline Aroclor 1254 & 01 1097-69-1 & & & 7.3E-04 & 7.3E-05 & & & $7.8 \mathrm{E}-04$ & 7.8E-05 & & & & & & & $3.8 \mathrm{E}-04$ & $3.8 \mathrm{E}-05$ \\
\hline Aroclor $1260^{(1)}$ & $011096-82-5$ & 1.1E-03 & $1.1 \mathrm{E}-05$ & & & $3.9 \mathrm{E}-04$ & $3.9 \mathrm{E}-06$ & & & & & & & $2.9 \mathrm{E}-04$ & $2.9 \mathrm{E}-06$ & & \\
\hline Arsenic Salts & NA & & & & & & & & & & & & & & & & \\
\hline Arsenic, Inorganic & $007440-38-2$ & 5.7E-03 & $5.7 \mathrm{E}-05$ & $1.1 \mathrm{E}-02$ & $1.1 \mathrm{E}-03$ & $9.6 \mathrm{E}-01$ & $9.6 \mathrm{E}-03$ & $1.9 \mathrm{E}+00$ & $1.9 \mathrm{E}-0 \mathrm{i}$ & & & & & $5.6 \mathrm{E}-03$ & $5.6 \mathrm{E}-05$ & $1.1 \mathrm{E}-02$ & $1.1 \mathrm{E}-03$ \\
\hline Arsine & $007784-42-1$ & & & & & & & & & & & & & & & & \\
\hline Asbestos & $001332-21-4$ & & & & & & & & & & & & & & & & \\
\hline Assure & $076578-14-8$ & & & $3.3 \mathrm{E}-01$ & $3.3 \mathrm{E}-02$ & & & & & & & & & & & & \\
\hline Asulam & $003337-71-1$ & & & $1.8 \mathrm{E}+00$ & $1.8 \mathrm{E}-01$ & & & & & & & & & & & & \\
\hline Atrazine & $001912-24-9$ & $3.8 \mathrm{E}-02$ & $3.8 \mathrm{E}-04$ & $1.3 \mathrm{E}+00$ & $1.3 \mathrm{E}-01$ & $1.0 \mathrm{E}+00$ & $1.0 \mathrm{E}-02$ & $3.5 \mathrm{E}+01$ & $3.5 \mathrm{E}+00$ & & & & & $3.7 \mathrm{E}-02$ & 3.7E-04 & $1.2 \mathrm{E}+00$ & $1.2 \mathrm{E}-01$ \\
\hline Avermectin B1 & $065195-55-3$ & & & $1.5 \mathrm{E}-02$ & $1.5 \mathrm{E}-03$ & & & & & & & & & & & & \\
\hline Azobenzene & $000103-33-3$ & 7.7E-02 & 7.7E-04 & & & $2.2 \mathrm{E}-01$ & $2.2 \mathrm{E}-03$ & & & & & & & $5.7 \mathrm{E}-02$ & $5.7 \mathrm{E}-04$ & & \\
\hline Barium & $007440-39-3$ & & & $2.6 \mathrm{E}+00$ & $2.6 \mathrm{E}-01$ & & & 7.4E+01 & $7.4 \mathrm{E}+00$ & & & & & & & $2.5 \mathrm{E}+00$ & $2.5 \mathrm{E}-01$ \\
\hline Barium Cyanide & $000542-62-1$ & & & $3.7 \mathrm{E}+00$ & $3.7 \mathrm{E}-01$ & & & $3.0 \mathrm{E}+02$ & $3.0 \mathrm{E}+01$ & & & & & & & $3.6 \mathrm{E}+00$ & $3.6 \mathrm{E}-01$ \\
\hline Baygon & $000114-26-1$ & & & $1.5 \mathrm{E}-01$ & $1.5 \mathrm{E}-02$ & & & $2.2 \mathrm{E}+01$ & $2.2 \mathrm{E}+00$ & & & & & & & $1.5 \mathrm{E}-01$ & $1.5 \mathrm{E}-02$ \\
\hline Bayleton & $043121-43-3$ & & & $1.1 \mathrm{E}+00$ & $1.1 \mathrm{E}-01$ & & & $1.7 \mathrm{E}+02$ & $1.7 \mathrm{E}+01$ & & & & & & & $1.1 \mathrm{E}+00$ & $1.1 \mathrm{E}-01$ \\
\hline Baythroid & $068359-37-5$ & & & $9.1 \mathrm{E}-01$ & $9.1 \mathrm{E}-02$ & & & & & & & & & & & & \\
\hline Benefin & $001861-40-1$ & & & $1.1 \mathrm{E}+01$ & $1.1 \mathrm{E}+00$ & & & $7.9 \mathrm{E}+01$ & $7.9 \mathrm{E}+00$ & & & & & & & $9.6 \mathrm{E}+00$ & $9.6 \mathrm{E}-01$ \\
\hline Benomyl & $017804-35-2$ & & & $1.8 \mathrm{E}+00$ & $1.8 \mathrm{E}-01$ & & & $2.7 \mathrm{E}+02$ & $2.7 \mathrm{E}+01$ & & - & & & & & $1.8 \mathrm{E}+00$ & $1.8 \mathrm{E}-01$ \\
\hline Bentazon & $025057-89-0$ & & & 9.1E-02 & $9.1 \mathrm{E}-03$ & & & $4.8 \mathrm{E}+01$ & $4.8 \mathrm{E}+00$ & & & & & & & 9.1E-02 & $9.1 \mathrm{E}-03$ \\
\hline
\end{tabular}


Table 3.1a. (continued)

\begin{tabular}{|c|c|c|c|c|c|c|c|c|c|c|c|c|c|c|c|c|c|}
\hline \multirow[t]{2}{*}{ Chemical } & \multirow[t]{2}{*}{ CASRN } & \multicolumn{4}{|c|}{ Ingestion (mg/L) } & \multicolumn{4}{|c|}{ Dermal (mg/L)" } & \multicolumn{4}{|c|}{ Inhalation (mg/L) ${ }^{\dagger *}$} & \multicolumn{4}{|c|}{$\begin{array}{c}\text { Ingestion + Dermal + } \\
\text { Inhalation }(\mathrm{mg} / \mathrm{L})^{+{ }^{+*}}\end{array}$} \\
\hline & & $10^{-1}$ & $10^{4}$ & $\mathrm{HQ}=\mathbf{1}$ & $\mathrm{HQ}=0.1$ & $10^{-4}$ & $10^{6}$ & $\mathrm{HQ}=1$ & $H Q=0.1$ & $10^{-4}$ & $10^{4}$ & $H Q=1$ & $\mathrm{HQ}=0.1$ & $10^{-4}$ & $10^{6}$ & $\mathrm{HI}=1$ & $\mathrm{HI}=0.1$ \\
\hline Benz[a]anthracene ${ }^{(2)}$ & $000056-55-3$ & $1.2 \mathrm{E}-02$ & $1.2 \mathrm{E}-04$ & & & $1.8 \mathrm{E}-03$ & $1.8 \mathrm{E}-05$ & & & & & & & $1.6 \mathrm{E}-03$ & $1.6 \mathrm{E}-05$ & & \\
\hline Benzaldehyde & $000100-52-7$ & & & $3.7 \mathrm{E}+00$ & $3.7 \mathrm{E}-01$ & & & $6.5 \mathrm{E}+01$ & $6.5 \mathrm{E}+00$ & & & & & & & $3.5 \mathrm{E}+00$ & $3.5 \mathrm{E}-01$ \\
\hline Benzene & $000071-43-2$ & $2.9 \mathrm{E}-01$ & $2.9 \mathrm{E}-03$ & & & $5.6 \mathrm{E}+00$ & $5.6 \mathrm{E}-02$ & & & $5.9 \mathrm{E}-02$ & $5.9 \mathrm{E}-04$ & & & $4.9 \mathrm{E}-02$ & $4.9 \mathrm{E}-04$ & & \\
\hline Benzene Hexachloride & NA & & & & & & & & & & & & & & & & \\
\hline Benzene, Ethyldimethy! & NA & & & & & & & & & & & & & & & & \\
\hline Benzene, Ethyimethyl & NA & & & & & & & & & & & & & & & & \\
\hline $\begin{array}{l}\text { Benzene, } \\
\text { Methylpropenyl }\end{array}$ & NA & & & & & & & & & & & & & & & & \\
\hline Benzene, Methylpropyl & NA & & & & & & & $\cdot$ & & & & & & & & & \\
\hline Benzene, Trimethyl & $025551-13-7$ & & & & & & & & & & & & & & & & \\
\hline Benzenethiol & $000108-98-5$ & & & 3.7E-04 & 3.7E-05 & & & 3.1E-03 & $3.1 \mathrm{E}-04$ & & & & & & & 3.3E-04 & 3.3E-05 \\
\hline Benzidine & $000092-87-5$ & 3.7E-05 & $3.7 \mathrm{E}-07$ & $1.1 \mathrm{E}-01$ & 1.1E-02 & 9.4E-03 & $9.4 \mathrm{E}-05$ & $2.8 \mathrm{E}+01$ & $2.8 \mathrm{E}+00$ & & & & & $3.7 \mathrm{E}-05$ & 3.7E-07 & $1.1 \mathrm{E}-01$ & 1.1E-02 \\
\hline Benzo[a]pyrene $e^{(2)}$ & $000050-32-8$ & $1.2 \mathrm{E}-03$ & $1.2 \mathrm{E}-05$ & & & $1.2 \mathrm{E}-04$ & $1.2 \mathrm{E}-06$ & & & & & & & $1.1 \mathrm{E}-04$ & $1.1 \mathrm{E}-06$ & & \\
\hline Benzo[b]fluoranthene ${ }^{(2)}$ & $000205-99-2$ & $1.2 \mathrm{E}-02$ & $1.2 \mathrm{E}-04$ & & & $1.2 \mathrm{E}-03$ & $1.2 \mathrm{E}-05$ & & & & & & & $1.1 \mathrm{E}-03$ & $1.1 \mathrm{E}-05$ & & \\
\hline Benzo[g,h,i]perylene & $000191-24-2$ & & & & & & & & & & & & & & & & \\
\hline Benzo[k]fluoranthene ${ }^{(z)}$ & $000207-08-9$ & $1.2 \mathrm{E}-01$ & $1.2 \mathrm{E}-03$ & & & $2.5 \mathrm{E}-02$ & $2.5 \mathrm{E}-04$ & & & & & & & $2.0 \mathrm{E}-02$ & $2.0 \mathrm{E}-04$ & & \\
\hline Benzoic Acid & $000065-85-0$ & & & $1.5 E+02$ & $1.5 \mathrm{E}+01$ & & & $8.2 \mathrm{E}+03$ & $8.2 \mathrm{E}+02$ & & & & · & & & $1.4 \mathrm{E}+02$ & $1.4 \mathrm{E}+01$ \\
\hline Benzotrichloride & $000098-07-7$ & $6.6 \mathrm{E}-04$ & $6.6 \mathrm{E}-06$ & & & $9.0 \mathrm{E}-03$ & $9.0 \mathrm{E}-05$ & & & & & & & $6.1 \mathrm{E}-04$ & $6.1 \mathrm{E}-06$ & & \\
\hline Benzyl Alcohol & $000100-51-6$ & & & $1.1 \mathrm{E}+01$ & $1.1 \mathrm{E}+00$ & & & $5.0 \mathrm{E}+02$ & $5.0 \mathrm{E}+01$ & & & & & & & $1.1 E+01$ & $1.1 \mathrm{E}+00$ \\
\hline Benzyl Chloride & $000100-44-7$ & $5.0 \mathrm{E}-02$ & $5.0 \mathrm{E}-04$ & & & $1.2 \mathrm{E}+00$ & $1.2 \mathrm{E}-02$ & & & & & & & $4.8 \mathrm{E}-02$ & $4.8 \mathrm{E}-04$ & & \\
\hline Beryllium & $007440-41-7$ & $2.0 \mathrm{E}-03$ & $2.0 \mathrm{E}-05$ & $1.8 \mathrm{E}-01$ & $1.8 \mathrm{E}-02$ & 8.2E-03 & $8.2 \mathrm{E}-05$ & 7.5E-01 & $7.5 \mathrm{E}-02$ & & & & & $1.6 \mathrm{E}-03$ & $1.6 \mathrm{E}-05$ & $1.5 \mathrm{E}-01$ & $1.5 \mathrm{E}-02$ \\
\hline Bidrin & $000141-66-2$ & & & $3.7 \mathrm{E}-03$ & 3.7E-04 & & & $3.4 \mathrm{E}+01$ & $3.4 \mathrm{E}+00$ & & & & & & & $3.6 \mathrm{E}-03$ & $3.6 \mathrm{E}-04$ \\
\hline
\end{tabular}


Table 3.1a. (continued)

\begin{tabular}{|c|c|c|c|c|c|c|c|c|c|c|c|c|c|c|c|c|c|}
\hline \multirow[t]{2}{*}{ Chemical } & \multirow[t]{2}{*}{ CASRN } & \multicolumn{4}{|c|}{ Ingestion (mg/L)" } & \multicolumn{4}{|c|}{ Dermal (mg/L)" } & \multicolumn{4}{|c|}{ Inhalation $(\mathrm{mg} / \mathrm{L})^{t^{*}}$} & \multicolumn{4}{|c|}{$\begin{array}{l}\text { Ingestion + Dermal + } \\
\text { Inhalation }(\mathrm{mg} / \mathrm{L})^{t^{*}}\end{array}$} \\
\hline & & $10^{-4}$ & $10^{4}$ & $\mathrm{HQ}=1$ & $\mathrm{HQ}=0.1$ & $10^{-4}$ & $10^{-4}$ & HQ=1 & $\mathrm{HQ}=0.1$ & $10^{-4}$ & $10^{4}$ & $\mathbf{H Q}=\mathbf{l}$ & $\mathrm{HQ}=0.1$ & $10^{4}$ & $10^{\circ}$ & $H I=1$ & $H I=0.1$ \\
\hline Biphenthrin & $082657-04-3$ & & & $5.5 \mathrm{E}-01$ & $5.5 \mathrm{E}-02$ & & & & & & & & & & & & \\
\hline Biphenyl, 1,1'- & $000092-52-4$ & & & $1.8 \mathrm{E}+00$ & $1.8 \mathrm{E}-01$ & & & $2.1 \mathrm{E}+00$ & $2.1 \mathrm{E}-01$ & & & & & & & $9.8 \mathrm{E}-01$ & $9.8 \mathrm{E}-02$ \\
\hline $\begin{array}{l}\text { Bis(2-chloro-1- } \\
\text { methylethyl)ether } \\
\text { (Technical) }\end{array}$ & $000108-60-1$ & $1.2 \mathrm{E}-01$ & $1.2 \mathrm{E}-03$ & & & $3.4 \mathrm{E}+00$ & $3.4 \mathrm{E}-02$ & & & $4.9 \mathrm{E}-02$ & $4.9 \mathrm{E}-04$ & & & $3.4 \mathrm{E}-02$ & $3.4 \mathrm{E}-04$ & & \\
\hline $\begin{array}{l}\text { Bis(2- } \\
\text { chloroethoxy)methane }\end{array}$ & $000111-91-1$ & & & & & & & & & & & & & & & & \\
\hline Bis(2-chloroethyl)ether & $000111-44-4$ & 7.7E-03 & $7.7 \mathrm{E}-05$ & & & $1.2 \mathrm{E}+00$ & $1.2 \mathrm{E}-02$ & & & $1.5 \mathrm{E}-03$ & $1.5 \mathrm{E}-05$ & & & $1.3 \mathrm{E}-03$ & $1.3 \mathrm{E}-05$ & & \\
\hline $\begin{array}{l}\text { Bis(2- } \\
\text { chloroisopropyl)ether }\end{array}$ & $039638-32-9$ & & & $1.5 \mathrm{E}+00$ & $1.5 \mathrm{E}-01$ & & & $9.0 \mathrm{E}+01$ & $9.0 \mathrm{E}+00$ & & & & & & & $1.4 E+00$ & $1.4 \mathrm{E}-01$ \\
\hline $\begin{array}{l}\text { Bis(2- } \\
\text { ethylhexyl)phthalate }\end{array}$ & $000117-81-7$ & $6.1 \mathrm{E}-01$ & 6.IE-03 & $7.3 \mathrm{E}-01$ & $7.3 \mathrm{E}-02$ & $2.0 \mathrm{E}+00$ & $2.0 \mathrm{E}-02$ & $2.4 \mathrm{E}+00$ & $2.4 \mathrm{E}-01$ & & & & & $4.7 \mathrm{E}-01$ & $4.7 \mathrm{E}-03$ & $5.6 \mathrm{E}-01$ & $5.6 \mathrm{E}-02$ \\
\hline Bis(chloromethyl)ether & $000542-88-1$ & $3.9 \mathrm{E}-05$ & $3.9 \mathrm{E}-07$ & & & $1.8 \mathrm{E}-02$ & $1.8 \mathrm{E}-04$ & & & 7.7E-06 & 7.7E-08 & & & $6.4 \mathrm{E}-06$ & $6.4 \mathrm{E}-08$ & & \\
\hline Bisphenol A & $000080-05-7$ & & & $1.8 \mathrm{E}+00$ & $1.8 \mathrm{E}-01$ & & & $1.4 \mathrm{E}+01$ & $1.4 \mathrm{E}+00$ & & & & & & & $1.6 \mathrm{E}+00$ & $1.6 \mathrm{E}-01$ \\
\hline Boron And Borates Only & $007440-42-8$ & & & $3.3 \mathrm{E}+00$ & $3.3 \mathrm{E}-01$ & & & $1.2 \mathrm{E}+03$ & $1.2 \mathrm{E}+02$ & & & & & & & $3.3 \mathrm{E}+00$ & 3.3E-01 \\
\hline Boron Trifluoride & $007637-07-2$ & & & & & & & & & & & & & & & & \\
\hline Bromochloromethane & $000074-97-5$ & & & & & & & & & & & & & & & & \\
\hline Bromodichloromethane & $000075-27-4$ & $1.4 \mathrm{E}-01$ & $1.4 \mathrm{E}-03$ & 7.3E-01 & 7.3E-02 & $9.6 \mathrm{E}+00$ & $9.6 \mathrm{E}-02$ & $5.1 \mathrm{E}+01$ & $5.1 E+00$ & & & & & $1.4 E-01$ & $1.4 \mathrm{E}-03$ & $7.2 \mathrm{E}-01$ & $7.2 \mathrm{E}-02$ \\
\hline Bromodiphenyl Ether, p- & $000101-55-3$ & & & & & & & & & & & & & & & & \\
\hline Bromoform & $000075-25-2$ & $1.1 E+00$ & $1.1 \mathrm{E}-02$ & $7.3 \mathrm{E}-01$ & $7.3 \mathrm{E}-02$ & $1.0 \mathrm{E}+02$ & $1.0 \mathrm{E}+00$ & $6.9 \mathrm{E}+01$ & $6.9 \mathrm{E}+00$ & 4.4E-01 & $4.4 \mathrm{E}-03$ & & & $3.1 \mathrm{E}-01$ & $3.1 \mathrm{E}-03$ & $7.2 \mathrm{E}-01$ & $7.2 \mathrm{E}-02$ \\
\hline Bromomethane ${ }^{(6)}$ & $000074-83-9$ & & & $5.1 \mathrm{E}-02$ & $5.1 \mathrm{E}-03$ & & & $4.8 \mathrm{E}+00$ & $4.8 \mathrm{E}-01$ & & & $1.0 \mathrm{E}-02$ & $1.0 \mathrm{E}-03$ & & & $8.6 \mathrm{E}-03$ & $8.6 \mathrm{E}-04$ \\
\hline Bromophos & $002104-96-3$ & & & $1.8 \mathrm{E}-01$ & $1.8 \mathrm{E}-02$ & & & & & & & & & & & & \\
\hline Bromotrichloromethane & $000075-62-7$ & & & & & & & & & & & & & & & & \\
\hline Bromoxynil & $001689-84-5$ & & & $7.3 \mathrm{E}-01$ & 7.3E-02 & & & $1.7 \mathrm{E}+03$ & $1.7 E+02$ & & & & & & & $7.3 \mathrm{E}-01$ & $7.3 \mathrm{E}-02$ \\
\hline
\end{tabular}


Table 3.1a. (continued)

\begin{tabular}{|c|c|c|c|c|c|c|c|c|c|c|c|c|c|c|c|c|c|}
\hline \multirow[t]{2}{*}{ Chemical } & \multirow[t]{2}{*}{ CASRN } & \multicolumn{4}{|c|}{ Ingestion (mg/L)" } & \multicolumn{4}{|c|}{ Dermal (mg/L)" } & \multicolumn{4}{|c|}{ Inhalation $(\mathrm{mg} / \mathrm{L})^{\dagger^{\star}}$} & \multicolumn{4}{|c|}{$\begin{array}{l}\text { Ingestion + Dermal + } \\
\text { Inhalation }(\mathrm{mg} / \mathrm{L})^{\dagger{ }^{*}}\end{array}$} \\
\hline & & $10^{-1}$ & $10^{6}$ & $H Q=1$ & $\mathrm{HQ}=0.1$ & $10^{-4}$ & $10^{6}$ & $H Q=1$ & $\mathrm{HQ}=0.1$ & $10^{-4}$ & $10^{4}$ & $H Q=1$ & $H Q=0.1$ & $10^{4}$ & $10^{4}$ & $\mathrm{HI}=1$ & $H \mathrm{I}=0.1$ \\
\hline Bromoxynil Octanoate & $001689-99-2$ & & & $7.3 \mathrm{E}-01$ & 7.3E-02 & & & & & & & & & & & & \\
\hline Butadiene, 1,3- & $000106-99-0$ & & & & & & & & & $9.5 \mathrm{E}-04$ & $9.5 E-06$ & & & & & & \\
\hline Butanol, N- & $000071-36-3$ & & & $3.7 \mathrm{E}+00$ & $3.7 \mathrm{E}-01$ & & & $3.0 \mathrm{E}+02$ & $3.0 \mathrm{E}+01$ & & & & & & & $3.6 \mathrm{E}+00$ & $3.6 \mathrm{E}-01$ \\
\hline $\begin{array}{l}\text { Butanone-2, 4-chloro- } \\
\text { 4,4-difluoro }\end{array}$ & NA & & & & & & & & & & & & & & & & \\
\hline Butyl Benzyl Phthlate & $000085-68-7$ & & & $7.3 E+00$ & 7.3E-01 & & & $2.6 \mathrm{E}+01$ & $2.6 \mathrm{E}+00$ & & & & & & & $5.7 \mathrm{E}+00$ & $5.7 \mathrm{E}-01$ \\
\hline Butylate & $002008-41-5$ & & & $1.8 \mathrm{E}+00$ & $1.8 \mathrm{E}-01$ & & & & . & & & & & & & & \\
\hline Butylchloride, t- & $000507-20-0$ & & & & & & & & & & & & & & & & \\
\hline $\begin{array}{l}\text { Butylphthalyl } \\
\text { Butylglycolate }\end{array}$ & $000085-70-1$ & & & $3.7 \mathrm{E}+01$ & $3.7 \mathrm{E}+00$ & & & & & & & & & & & & \\
\hline Cacodylic Acid & $000075-60-5$ & & & $1.1 \mathrm{E}-01$ & $1.1 \mathrm{E}-02$ & & & $8.2 \mathrm{E}+01$ & $8.2 \mathrm{E}+00$ & & & & & & & $1.1 \mathrm{E}-01$ & $1.1 \mathrm{E}-02$ \\
\hline Cadmium (Water). & $007440-43-9$ & & & $1.8 \mathrm{E}-02$ & $1.8 \mathrm{E}-03$ & & & 7.5E-02 & $7.5 \mathrm{E}-03$ & & & & & & & $1.5 \mathrm{E}-02$ & $1.5 \mathrm{E}-03$ \\
\hline Calcium Cyanide & $000592-01-8$ & & & $1.5 \mathrm{E}+00$ & $1.5 \mathrm{E}-01$ & & & $1.2 \mathrm{E}+02$ & $1.2 \mathrm{E}+01$ & & & & & & & $1.4 \mathrm{E}+00$ & $1.4 \mathrm{E}-01$ \\
\hline Caprolactam & $000105-60-2$ & & & $1.8 \mathrm{E}+01$ & $1.8 \mathrm{E}+00$ & & & $1.3 \mathrm{E}+04$ & $1.3 \mathrm{E}+03$ & & & & & & & $1.8 \mathrm{E}+01$ & $1.8 \mathrm{E}+00$ \\
\hline Captafol & $002425-06-1$ & $9.9 \mathrm{E}-01$ & $9.9 \mathrm{E}-03$ & 7.3E-02 & 7.3E-03 & $2.4 \mathrm{E}+02$ & $2.4 E+00$ & $1.8 \mathrm{E}+01$ & $1.8 \mathrm{E}+00$ & & & & & $9.9 \mathrm{E}-01$ & $9.9 \mathrm{E}-03$ & 7.3E-02 & 7.3E-03 \\
\hline Captan & $000133-06-2$ & $2.4 \mathrm{E}+00$ & $2.4 \mathrm{E}-02$ & $4.7 \mathrm{E}+00$ & 4.7E-01 & $3.9 \mathrm{E}+02$ & $3.9 \mathrm{E}+00$ & $7.5 \mathrm{E}+02$ & $7.5 \mathrm{E}+01$ & & & & & $2.4 \mathrm{E}+00$ & $2.4 \mathrm{E}-02$ & $4.7 \mathrm{E}+00$ & 4.7E-01 \\
\hline Carbaryl & $000063-25-2$ & & & $3.7 \mathrm{E}+00$ & $3.7 \mathrm{E}-01$ & & & $1.6 \mathrm{E}+02$ & $1.6 \mathrm{E}+01$ & & & & & & & $3.6 \mathrm{E}+00$ & $3.6 \mathrm{E}-01$ \\
\hline Carbazole & $000086-74-8$ & $4.3 \mathrm{E}-01$ & 4.3E-03 & & & $1.4 \mathrm{E}+00$ & $1.4 \mathrm{E}-02$ & & & & & & & $3.2 \mathrm{E}-01$ & $3.2 \mathrm{E}-03$ & & \\
\hline Carbofuran & $001563-66-2$ & & & $1.8 \mathrm{E}-01$ & $1.8 \mathrm{E}-02$ & & & $3.2 \mathrm{E}+01$ & $3.2 \mathrm{E}+00$ & & & & & & & $1.8 \mathrm{E}-01$ & $1.8 \mathrm{E}-02$ \\
\hline Carbon Disulfide & $000075 \cdot 15-0$ & & & $3.7 \mathrm{E}+00$ & $3.7 \mathrm{E}-01$ & & & $4.0 \mathrm{E}+01$ & $4.0 \mathrm{E}+00$ & & & $\begin{array}{l}1.5 \mathrm{E}+0 \\
0\end{array}$ & $1.5 \mathrm{E}-01$ & & & $1.0 \mathrm{E}+00$ & $1.0 \mathrm{E}-01$ \\
\hline Carbon Tetrachloride ${ }^{(6)}$ & $000056-23-5$ & $6.6 \mathrm{E}-02$ & $6.6 \mathrm{E}-04$ & 2.6E-02 & 2.6E-03 & $8.0 \mathrm{E}-01$ & $8.0 \mathrm{E}-03$ & $3.1 \mathrm{E}-01$ & 3.1E-02 & $3.2 \mathrm{E}-02$ & 3.2E-04 & & & 2.1E-02 & 2.1E-04 & $2.4 \mathrm{E}-02$ & $2.4 \mathrm{E}-03$ \\
\hline Carbosulfan & $055285-14-8$ & & & 3.7E-01 & 3.7E-02 & & & & & & & & & & & & \\
\hline
\end{tabular}


Table 3.1a. (continued)

\begin{tabular}{|c|c|c|c|c|c|c|c|c|c|c|c|c|c|c|c|c|c|}
\hline \multirow[t]{2}{*}{ Chemical } & \multirow[t]{2}{*}{ CASRN } & \multicolumn{4}{|c|}{ Ingestion (mg/L) } & \multicolumn{4}{|c|}{ Dermal (mg/L)* } & \multicolumn{4}{|c|}{ Inhalation $(\mathrm{mg} / \mathrm{L})^{t^{*}}$} & \multicolumn{4}{|c|}{$\begin{array}{l}\text { Ingestion + Dermal + } \\
\text { Inhalation }(\mathrm{mg} / \mathrm{L})^{t^{*}}\end{array}$} \\
\hline & & $10^{-1}$ & $10^{4}$ & $H Q=1$ & $\mathrm{HQ}=0.1$ & $10^{-4}$ & $10^{4}$ & $H Q=1$ & $\mathrm{HQ}=0.1$ & $10^{-1}$ & $10^{-6}$ & $\mathrm{HQ}=1$ & HQ $=0.1$ & $10^{-4}$ & $10^{5}$ & $\mathrm{HI}=1$ & $\mathrm{HI}=0.1$ \\
\hline Carboxin & $005234-68-4$ & & r & $3.7 E+00$ & $3.7 \mathrm{E}-01$ & & & $3.5 E+02$ & $3.5 \mathrm{E}+01$ & & & & & & & $3.6 \mathrm{E}+00$ & $3.6 \mathrm{E}-01$ \\
\hline Chloral & $000075-87-6$ & & & $7.3 \mathrm{E}-02$ & $7.3 \mathrm{E}-03$ & & & & & & & & & & & & \\
\hline Chloramben & $000133-90-4$ & & & 5.5E-01 & $5.5 \mathrm{E}-02$ & & & & & & & & & & & & \\
\hline Chloranil & $000118-75-2$ & 2.1E-02 & $2.1 E-04$ & & & & & & & & & & & & & & \\
\hline Chlordane $^{(6)}$ & $000057-74-9$ & $6.6 \mathrm{E}-03$ & $6.6 \mathrm{E}-05$ & $2.2 \mathrm{E}-03$ & $2.2 \mathrm{E}-04$ & $2.6 \mathrm{E}-02$ & $2.6 \mathrm{E}-04$ & 8.7E-03 & 8.7E-04 & & & & & $5.2 \mathrm{E}-03$ & $5.2 \mathrm{E}-05$ & $1.7 \mathrm{E}-03$ & $1.7 \mathrm{E}-04$ \\
\hline Chloride & $016887-00-6$ & & & & & & & & & & & & & & & & \\
\hline Chlorimuron, Ethyl- & $090982-32-4$ & & & $7.3 \mathrm{E}-01$ & $7.3 \mathrm{E}-02$ & & & & & & & & & & & & \\
\hline Chlorine & $007782-50-5$ & & & $3.7 \mathrm{E}+00$ & $3.7 \mathrm{E}-01$ & & & $3.0 \mathrm{E}+02$ & $3.0 \mathrm{E}+01$ & & & & & & & $3.6 \mathrm{E}+00$ & $3.6 \mathrm{E}-01$ \\
\hline Chlorine Dioxide & $010049-04-4$ & & & & & & & & & & & & & & & & \\
\hline Chiorite (Sodium Salt) & $007758-19-2$ & & & & & & & & & & & & & & & & \\
\hline $\begin{array}{l}\text { Chloro-1,1- } \\
\text { difluorocthane, 1- }\end{array}$ & $000075-68-3$ & & & & & & & & & & & $2.0 E+0$ & $1.0 \mathrm{E}+01$ & & & & \\
\hline Chloro-1,3-butadiene, 2- & $000126-99-8$ & & & $7.3 \mathrm{E}-01$ & $7.3 \mathrm{E}-02$ & & & $2.3 \mathrm{E}+01$ & 2.3E+00 & & & $1.5 \mathrm{E}-02$ & $1.5 \mathrm{E}-03$ & & & $1.4 \mathrm{E}-02$ & $1.4 \mathrm{E}-03$ \\
\hline $\begin{array}{l}\text { Chloro-2-methylaniline } \\
\mathrm{HCl} \text { 4- }\end{array}$ & $003165-93-3$ & $1.9 E-02$ & $1.9 \mathrm{E}-04$ & & & & & & & & & & & & & & \\
\hline $\begin{array}{l}\text { Chloro-2-methylaniline, } \\
\text { 4- }\end{array}$ & $000095-69-2$ & $1.5 \mathrm{E}-02$ & $1.5 \mathrm{E}-04$ & & & & & & & & & & & & & & \\
\hline Chloroacetic Acid & $000079-11-8$ & & & 7.3E-02 & $7.3 \mathrm{E}-03$ & & & $4.2 \mathrm{E}+00$ & 4.2E-01 & & & & & & & $7.2 \mathrm{E}-02$ & $7.2 \mathrm{E}-03$ \\
\hline Chloroacetophenone, 2 - & $000532-27-4$ & & & & & & & & & & & & & & & & \\
\hline Chloroaniline, p- & $000106-47-8$ & & & $1.5 \mathrm{E}-01$ & $1.5 \mathrm{E}-02$ & & & $9.7 \mathrm{E}-01$ & $9.7 \mathrm{E}-02$ & & & & & & & $1.3 \mathrm{E}-01$ & $1.3 \mathrm{E}-02$ \\
\hline Chlorobenzene ${ }^{(5)}$ & $000108-90-7$ & & & $7.3 \mathrm{E}-01$ & $7.3 E-02$ & & & $2.3 \mathrm{E}+00$ & $2.3 \mathrm{E}-01$ & & & 4.2E-02 & $4.2 \mathrm{E}-03$ & & & $3.9 \mathrm{E}-02$ & $3.9 \mathrm{E}-03$ \\
\hline Chlorobenzilate & $000510-15.6$ & $3.2 \mathrm{E}-02$ & $3.2 \mathrm{E}-04$ & $7.3 \mathrm{E}-01$ & $7.3 E-02$ & $2.1 \mathrm{E}-01$ & 2.1E-03 & $4.8 \mathrm{E}+00$ & $4.8 \mathrm{E}-01$ & & & & & $2.7 \mathrm{E}-02$ & 2.7E-04 & $6.3 \mathrm{E}-01$ & $6.3 \mathrm{E}-02$ \\
\hline Chlorobenzoic Acid, p- & $000074-11-3$ & & & $7.3 E+00$ & 7.3E-01 & & & $8.6 \mathrm{E}+01$ & $8.6 \mathrm{E}+00$ & & & & & & & $6.7 E+00$ & $6.7 \mathrm{E}-01$ \\
\hline
\end{tabular}


Table 3.1a. (continued)

\begin{tabular}{|c|c|c|c|c|c|c|c|c|c|c|c|c|c|c|c|c|c|}
\hline \multirow[t]{2}{*}{ Chemical } & \multirow[t]{2}{*}{ CASRN } & \multicolumn{4}{|c|}{ Ingestion (mg/L)" } & \multicolumn{4}{|c|}{ Dermal (mg/L)" } & \multicolumn{4}{|c|}{ Inhalation (mg/L) ${ }^{t^{*}}$} & \multicolumn{4}{|c|}{$\begin{array}{l}\text { Ingestion + Dermal + } \\
\text { Inhalation }(\mathrm{mg} / \mathrm{L})^{\dagger+^{n}}\end{array}$} \\
\hline & & $10^{-4}$ & $10^{6}$ & $\mathrm{HQ}=1$ & $\mathrm{HQ}=0.1$ & $10^{-4}$ & $10^{4}$ & $H Q=1$ & $\mathrm{HQ}=0.1$ & $10^{-1}$ & $10^{6}$ & $H Q=1$ & $\mathrm{HQ}=0.1$ & $10^{-4}$ & $10^{-6}$ & $H I=1$ & $\mathrm{HI}=0.1$ \\
\hline $\begin{array}{l}\text { Chlorobenzotrifluoride, } \\
\text { 4- }\end{array}$ & $000098-56-6$ & & & 7.3E-01 & $7.3 \mathrm{E}-02$ & & & $2.2 \mathrm{E}+00$ & $2.2 \mathrm{E}-01$ & . & & & & & & $5.5 \mathrm{E}-01$ & $5.5 \mathrm{E}-02$ \\
\hline Chlorobutane, 1- & $000109-69-3$ & & & $1.5 \mathrm{E}+01$ & $1.5 \mathrm{E}+00$ & & & $1.3 \mathrm{E}+02$ & $1.3 E+01$ & & & & & & & $1.3 \mathrm{E}+01$ & $1.3 E+00$ \\
\hline Chlorobutane, 2- & $000078-86-4$ & & & & & & & & & & & & & & & & \\
\hline Chlorocyclopentadiene & $041851-50-7$ & & & & & & & & & & & & & & & & \\
\hline Chlorodibromoethane & $073506-94-2$ & $1.0 \mathrm{E}-01$ & $1.0 \mathrm{E}-03$ & & & & & & & & & & & & & & \\
\hline Chlorodifluoromethane & $000075-45-6$ & & & & & & & & & & & $2^{1.0 \mathrm{E}+0}$ & $1.0 \mathrm{E}+01$ & & & & \\
\hline Chloroform ${ }^{(6)}$ & $000067-66-3$ & $1.4 \mathrm{E}+00$ & $1.4 \mathrm{E}-02$ & $3.7 \mathrm{E}-01$ & $3.7 \mathrm{E}-02$ & $1.3 \mathrm{E}+01$ & $1.3 \mathrm{E}-01$ & $3.4 \mathrm{E}+00$ & $3.4 \mathrm{E}-01$ & 2.1E-02 & 2.1E-04 & & & 2.1E-02 & 2.1E-04 & 3.3E-01 & $3.3 \mathrm{E}-02$ \\
\hline Chloromethane $\mathrm{e}^{(6)}$ & $000074-87-3$ & $6.6 \mathrm{E}-01$ & $6.6 \mathrm{E}-03$ & & & $5.1 \mathrm{E}+01$ & 5.1E-01 & & & 2.7E-01 & $2.7 \mathrm{E}-03$ & & & $1.9 \mathrm{E}-01$ & $1.9 \mathrm{E}-03$ & & \\
\hline $\begin{array}{l}\text { Chloromethyl Methyl } \\
\text { Ether }\end{array}$ & $000107-30-2$ & & & & & & & & & & & & & & & & \\
\hline $\begin{array}{l}\text { Chloronaphthalene, } \\
\text { Beta- }\end{array}$ & $000091-58-7$ & & & $2.9 \mathrm{E}+00$ & $2.9 \mathrm{E}-01$ & & & $6.1 \mathrm{E}+00$ & $6.1 \mathrm{E}-01$ & & & & & & & $2.0 \mathrm{E}+00$ & 2.0E-01 \\
\hline Chloronitrobenzene, $0-$ & $000088-73-3$ & $3.4 \mathrm{E}-01$ & $3.4 \mathrm{E}-03$ & & & $1.5 \mathrm{E}+0 \mathrm{t}$ & $1.5 \mathrm{E}-01$ & & & & & & & $3.3 \mathrm{E}-01$ & 3.3E-03 & & \\
\hline Chloronitrobenzene, $\mathrm{p}$ - & $000121-73-3$ & $4.7 \mathrm{E}-01$ & 4.7E-03 & & & $1.5 \mathrm{E}+01$ & $1.5 \mathrm{E}-01$ & & & & & & & $4.6 \mathrm{E}-01$ & 4.6E-03 & & \\
\hline Chlorophenol, 2- & $000095.57-8$ & & & $1.8 \mathrm{E}-01$ & $1.8 \mathrm{E}-02$ & & & $1.8 \mathrm{E}+00$ & $1.8 \mathrm{E}-01$ & & & & & & & $1.7 \mathrm{E}-01$ & $1.7 \mathrm{E}-02$ \\
\hline $\begin{array}{l}\text { Chlorophenyl Methyl } \\
\text { Sulfide, p- }\end{array}$ & $000123-09-1$ & & & & & & & & & & & & & & & & \\
\hline $\begin{array}{l}\text { Chlorophenyl Methyl } \\
\text { Sulfoxide }\end{array}$ & $000934-73-6$ & & & & & & & & & & & & & & & & \\
\hline Chloropropane, 2- & $000075-29-6$ & & & & & & & & & & & 2.1E-01 & 2.1E-02 & & & & \\
\hline Chlorothalonil & $001897-45-6$ & $7.7 \mathrm{E}-01$ & 7.7E-03 & $5.5 \mathrm{E}-01$ & $5.5 \mathrm{E}-02$ & $6.4 \mathrm{E}+00$ & $6.4 \mathrm{E}-02$ & 4.5E +00 & 4.5E-01 & & & & & $6.9 \mathrm{E}-01$ & $6.9 \mathrm{E}-03$ & $4.9 \mathrm{E}-01$ & $4.9 \mathrm{E}-02$ \\
\hline Chlorotoluene, 0 - & $000095-49-8$ & & & 7.3E-01 & 7.3E-02 & & & $2.9 \mathrm{E}+00$ & $2.9 \mathrm{E}-01$ & & & & & & & $5.8 \mathrm{E}-01$ & $5.8 \mathrm{E}-02$ \\
\hline
\end{tabular}


Table 3.1a. (continued)

\begin{tabular}{|c|c|c|c|c|c|c|c|c|c|c|c|c|c|c|c|c|c|}
\hline \multirow[t]{2}{*}{ Chemical } & \multirow[t]{2}{*}{ CASRN } & \multicolumn{4}{|c|}{ Ingestion (mg/L)" } & \multicolumn{4}{|c|}{ Dermal (mg/L)" } & \multicolumn{4}{|c|}{ Inhalation $(\mathrm{mg} / \mathrm{L})^{\dagger *}$} & \multicolumn{4}{|c|}{$\begin{array}{l}\text { Ingestion + Dermal + } \\
\text { Inhalation }(\mathrm{mg} / \mathrm{L})^{++{ }^{+*}}\end{array}$} \\
\hline & & $10^{-4}$ & $10^{6}$ & HQ=1 & $\mathrm{HQ}=0.1$ & $10^{-4}$ & $10^{5}$ & $\mathrm{HQ}=1$ & HQ $=0.1$ & $10^{-4}$ & $10^{5}$ & $\mathrm{HQ}=\mathbf{1}$ & $H Q=0.1$ & $10^{-4}$ & $10^{4}$ & $\mathrm{HI}=1$ & $\mathrm{HI}=0.1$ \\
\hline Chlorpropham & $000101-21-3$ & & & $7.3 \mathrm{E}+00$ & 7.3E-01 & & & $1.4 \mathrm{E}+02$ & $1.4 E+01$ & & & & & & & $6.9 \mathrm{E}+00$ & $6.9 \mathrm{E}-01$ \\
\hline Chlorpyrifos & $002921-88-2$ & & & $1.1 \mathrm{E}-01$ & $1.1 \mathrm{E}-02$ & & & 2.8E-01 & $2.8 E-02$ & & & & & & & $7.9 \mathrm{E}-02$ & 7.9E-03 \\
\hline Chlopyrifos Methyl & $005598-13-0$ & & & $3.7 \mathrm{E}-01$ & $3.7 \mathrm{E}-02$ & & & $5.9 \mathrm{E}+00$ & $5.9 \mathrm{E}-01$ & & & & & & & $3.4 \mathrm{E}-01$ & $3.4 \mathrm{E}-02$ \\
\hline Chlorsulfuron & $064902-72-3$ & & & $1.8 \mathrm{E}+00$ & $1.8 \mathrm{E}-01$ & & & $2.7 \mathrm{E}+05$ & $2.7 \mathrm{E}+04$ & & & & & & & $1.8 \mathrm{E}+00$ & $1.8 \mathrm{E}-01$ \\
\hline Chlorthiophos & $060238-56-4$ & & & $2.9 E-02$ & $2.9 \mathrm{E}-03$ & & & & & & & & & & & & \\
\hline $\begin{array}{l}\text { Chromium (III) } \\
\text { (Insoluble Salts) }\end{array}$ & 016065-83-1 & & & 3.7E+01 & $3.7 \mathrm{E}+00$ & & & $7.5 \mathrm{E}+01$ & $7.5 \mathrm{E}+00$ & & & & & & & $2.5 E+01$ & $2.5 \mathrm{E}+00$ \\
\hline Chromium (V) & $018540-29-9$ & & & $1.8 \mathrm{E}-01$ & $1.8 \mathrm{E}-02$ & & & $1.5 \mathrm{E}+00$ & $1.5 \mathrm{E}-01$ & & & & & & & $1.6 \mathrm{E}-01$ & $1.6 \mathrm{E}-02$ \\
\hline Chromium Salts & NA & & & & & & & & & & & & & & & & \\
\hline Chrysene $e^{(2)}$ & $000218-01-9$ & $1.2 \mathrm{E}+00$ & $1.2 \mathrm{E}-02$ & & & $1.8 \mathrm{E}-01$ & $1.8 \mathrm{E}-03$ & & & & & & & $1.6 \mathrm{E}-01$ & $1.6 \mathrm{E}-03$ & & \\
\hline Cobalt & $007440-48-4$ & & & & & & & & & & & & & & & & \\
\hline Coke Oven Emissions & $008007-45-2$ & & & & & & & & & & & & & & & & \\
\hline Copper & $007440-50-8$ & & & & & & & & & & & & & & & & \\
\hline Copper Cyanide & $000544-92-3$ & & & $1.8 \mathrm{E}-01$ & $1.8 \mathrm{E}-02$ & & & $1.5 \mathrm{E}+01$ & $1.5 \mathrm{E}+00$ & & & & & & & $1.8 \mathrm{E}-01$ & $1.8 \mathrm{E}-02$ \\
\hline Creosote & $008001-58-9$ & & & & & & & & & & & & & & & & \\
\hline Cresol, m- & $000108-39-4$ & & & $1.8 \mathrm{E}+00$ & $1.8 \mathrm{E}-01$ & & & $2.5 \mathrm{E}+01$ & $2.5 \mathrm{E}+00$ & & & & & & & $1.7 \mathrm{E}+00$ & $1.7 \mathrm{E}-01$ \\
\hline Cresol, o- & $000095-48-7$ & & & $1.8 \mathrm{E}+00$ & $1.8 \mathrm{E}-01$ & & & $2.4 \mathrm{E}+0 \mathrm{I}$ & $2.4 \mathrm{E}+00$ & & & & & & & $1.7 E+00$ & $1.7 \mathrm{E}-01$ \\
\hline Cresol, p- & $000106-44-5$ & & & $1.8 \mathrm{E}-01$ & $1.8 \mathrm{E}-02$ & & & $2.7 \mathrm{E}+00$ & $2.7 \mathrm{E}-01$ & & & & & & & $1.7 \mathrm{E}-01$ & $1.7 \mathrm{E}-02$ \\
\hline Crotonaldehyde & $000123-73-9$ & 4.5E-03 & $4.5 \mathrm{E}-05$ & & & $6.8 \mathrm{E}-02$ & $6.8 \mathrm{E}-04$ & & & & & & & $4.2 \mathrm{E}-03$ & $4.2 \mathrm{E}-05$ & & \\
\hline Cumene & $000098-82-8$ & & & $1.5 \mathrm{E}+00$ & $1.5 \mathrm{E}-01$ & & & 4.5E+00 & 4.5E-01 & & & $1.9 \mathrm{E}-02$ & $1.9 \mathrm{E}-03$ & & & $1.8 \mathrm{E}-02$ & $1.8 \mathrm{E}-03$ \\
\hline Cyanazine & $021725-46-2$ & $1.0 \mathrm{E}-02$ & $1.0 \mathrm{E}-04$ & 7.3E-02 & 7.3E-03 & $8.6 \mathrm{E}-01$ & $8.6 \mathrm{E}-03$ & $6.2 \mathrm{E}+00$ & $6.2 \mathrm{E}-01$ & & & & & $1.0 \mathrm{E}-02$ & $1.0 E-04$ & $7.2 \mathrm{E}-02$ & $7.2 \mathrm{E}-03$ \\
\hline Cyanide (CN-) & $000057-12-5$ & & & 7.3E- 01 & 7.3E-02 & & & $6.8 \mathrm{E}+00$ & $6.8 \mathrm{E}-01$ & & & & & & & $6.6 \mathrm{E}-01$ & $6.6 \mathrm{E}-02$ \\
\hline
\end{tabular}


Table 3.1a. (continued)

\begin{tabular}{|c|c|c|c|c|c|c|c|c|c|c|c|c|c|c|c|c|c|}
\hline \multirow[t]{2}{*}{ Chemical } & \multirow[t]{2}{*}{ CASRN } & \multicolumn{4}{|c|}{ Ingestion (mg/L)" } & \multicolumn{4}{|c|}{ Dermal (mg/L)" } & \multicolumn{4}{|c|}{ Inhalation $(\mathrm{mg} / \mathrm{L})^{\dagger *}$} & \multicolumn{4}{|c|}{$\begin{array}{l}\text { Ingestion + Dermal + } \\
\text { Inhalation }(\mathrm{mg} / \mathrm{L})^{\mathrm{ft}{ }^{*}}\end{array}$} \\
\hline & & $10^{-4}$ & $10^{4}$ & $\mathrm{HQ}=\mathbf{1}$ & $\mathrm{HQ}=0.1$ & $10^{-1}$ & $10^{-5}$ & $\mathrm{HQ}=1$ & $\mathrm{HQ}=0.1$ & $10^{-4}$ & $10^{5}$ & $\mathrm{HQ}=1$ & $\mathrm{HQ}=0.1$ & $10^{-4}$ & $10^{-4}$ & $\mathrm{HI}=\mathbf{1}$ & $\mathrm{HI}=0.1$ \\
\hline Cyanogen & $000460-19-5$ & & & $1.5 \mathrm{E}+00$ & $1.5 \mathrm{E}-01$ & & & & & & & & & & & & \\
\hline Cyanogen Bromide & $000506-68-3$ & & & $3.3 \mathrm{E}+00$ & $3.3 \mathrm{E}-01$ & & & & & & & & & & & & \\
\hline Cyanogen Chloride & $000506-77-4$ & & & $1.8 \mathrm{E}+00$ & $1.8 \mathrm{E}-01$ & & & $5.4 \mathrm{E}+02$ & $5.4 \mathrm{E}+01$ & & & & & & & $1.8 \mathrm{E}+00$ & $1.8 \mathrm{E}-01$ \\
\hline $\begin{array}{l}\text { Cyclohexane, 1,2,3,4,5- } \\
\text { pentabromo-6-chloro- }\end{array}$ & $000087-84-3$ & $3.7 \mathrm{E}-01$ & 3.7E-03 & & & & & & & & & & & & & & \\
\hline Cyclohexanone & 000108-94-1 & & & $1.8 \mathrm{E}+02$ & $1.8 \mathrm{E}+01$ & & & $3.3 \mathrm{E}+04$ & $3.3 \mathrm{E}+03$ & & & & & & & $1.8 \mathrm{E}+02$ & $1.8 \mathrm{E}+01$ \\
\hline Cyclohexylamine & $000108-91-8$ & & & $7.3 \mathrm{E}+00$ & $7.3 \mathrm{E}-01$ & & & $2.7 \mathrm{E}+02$ & $2.7 \mathrm{E}+01$ & & & & & & & $7.1 \mathrm{E}+00$ & $7.1 \mathrm{E}-01$ \\
\hline Cyclopentadiene & $000542-92-7$ & & & & & & & & & & & & & & & & \\
\hline Cyhalothrin/karate & $068085-85-8$ & & & $1.8 \mathrm{E}-01$ & $1.8 \mathrm{E}-02$ & & & $1.4 \mathrm{E}-01$ & $1.4 \mathrm{E}-02$ & & & & & & & 7.9E-02 & 7.9E-03 \\
\hline Cypermethrin & $052315-07-8$ & & & $3.7 \mathrm{E}-01$ & $3.7 E-02$ & & & & & & & & & & & & \\
\hline Cyromazine & $066215-27-8$ & & & $2.7 \mathrm{E}-01$ & $2.7 \mathrm{E}-02$ & & & & & & & & & & & & \\
\hline DDD & $000072-54-8$ & $3.5 \mathrm{E}-02$ & 3.5E-04 & & & $3.7 \mathrm{E}-02$ & 3.7E-04 & & & & & & & $1.8 \mathrm{E}-02$ & $1.8 \mathrm{E}-04$ & & \\
\hline DDE & $000072-55-9$ & $2.5 \mathrm{E}-02$ & 2.5E-04 & & & $3.0 \mathrm{E}-02$ & $3.0 \mathrm{E}-04$ & & & & & & & $1.4 \mathrm{E}-02$ & $1.4 \mathrm{E}-04$ & & \\
\hline DDT & $000050-29-3$ & $2.5 \mathrm{E}-02$ & $2.5 \mathrm{E}-04$ & $1.8 \mathrm{E}-02$ & $1.8 \mathrm{E}-03$ & $1.7 \mathrm{E}-02$ & $1.7 \mathrm{E}-04$ & $1.2 \mathrm{E}-02$ & $1.2 \mathrm{E}-03$ & & & & & $1.0 \mathrm{E}-02$ & $1.0 \mathrm{E}-04$ & 7.3E-03 & 7.3E-04 \\
\hline Dacthal & 001861-32-1 & & & $3.7 \mathrm{E}-01$ & $3.7 \mathrm{E}-02$ & & & & & & & & & & & & \\
\hline Dalapon & $000075-99-0$ & & & $1.1 \mathrm{E}+00$ & $1.1 \mathrm{E}-01$ & & & $2.5 \mathrm{E}+02$ & $2.5 \mathrm{E}+01$ & & & & & & & $1.1 \mathrm{E}+00$ & $1.1 \mathrm{E}-01$ \\
\hline $\begin{array}{l}\text { Decabromodiphenyl } \\
\text { Ether }\end{array}$ & $001163-19-5$ & & & $3.7 \mathrm{E}-01$ & $3.7 \mathrm{E}-02$ & & & $8.5 E+03$ & $8.5 \mathrm{E}+02$ & & & & & & & 3.6E-01 & $3.6 \mathrm{E}-02$ \\
\hline Demeton & $008065-48-3$ & & & $1.5 \mathrm{E}-03$ & $1.5 \mathrm{E}-04$ & & & & & & & & & & & & \\
\hline Di(2-ethylhexyl)adipate & $000103-23-1$ & $7.1 \mathrm{E}+00$ & 7.1E-02 & $2.2 \mathrm{E}+01$ & $2.2 E+00$ & 4.1E-01 & 4.1E- 03 & $1.3 \mathrm{E}+00$ & $1.3 \mathrm{E}-01$ & & & & & $3.8 \mathrm{E}-01$ & $3.8 E-03$ & $1.2 \mathrm{E}+00$ & $1.2 \mathrm{E}-01$ \\
\hline Diallate & $002303-16-4$ & $1.4 \mathrm{E}-01$ & $1.4 \mathrm{E}-03$ & & & $3.3 \mathrm{E}+02$ & $3.3 \mathrm{E}+00$ & & & & & & & $1.4 \mathrm{E}-01$ & $1.4 \mathrm{E}-03$ & & \\
\hline Diazinon & $000333-41-5$ & & & $3.3 \mathrm{E}-02$ & 3.3E-03 & & & 5.IE-01 & $5.1 \mathrm{E}-02$ & & & & & & & $3.1 E-02$ & $3.1 \mathrm{E}-03$ \\
\hline
\end{tabular}


Table 3.1a. (continued)

\begin{tabular}{|c|c|c|c|c|c|c|c|c|c|c|c|c|c|c|c|c|c|}
\hline \multirow[t]{2}{*}{ Chemical } & \multirow[t]{2}{*}{ CASRN } & \multicolumn{4}{|c|}{ Ingestion (mg/L)* } & \multicolumn{4}{|c|}{ Dermal (mg/L)" } & \multicolumn{4}{|c|}{ Inhalation $(\mathrm{mg} / \mathrm{L})^{\dagger^{\star}}$} & \multicolumn{4}{|c|}{$\begin{array}{l}\text { Ingestion + Dermal + } \\
\text { Inhalation }(\mathrm{mg} / \mathrm{L})^{\dagger t^{*}}\end{array}$} \\
\hline & & $10^{-4}$ & $10^{+5}$ & $\mathrm{HQ}=1$ & $\mathrm{HQ}=0.1$ & $10^{-4}$ & $10^{+5}$ & $H Q=1$ & $\mathrm{HQ}=0.1$ & $10^{-4}$ & $10^{4}$ & $H Q=1$ & $\mathrm{HQ}=0.1$ & $10^{-4}$ & $10^{-6}$ & $\mathrm{HI}=1$ & $\mathrm{HI}=0.1$ \\
\hline Dibenz[a,h]anthracene $e^{(n)}$ & $000053-70-3$ & $1.2 \mathrm{E}-03$ & $1.2 \mathrm{E}-05$ & & & $5.5 \mathrm{E}-05$ & $5.5 \mathrm{E}-07$ & & & & & & & $5.3 \mathrm{E}-05$ & $5.3 \mathrm{E}-07$ & & \\
\hline $\begin{array}{l}\text { Dibromo-3- } \\
\text { chloropropane, 1,2- }\end{array}$ & $000096-12-8$ & $6.1 \mathrm{E}-03$ & 6.1E-05 & & & $4.2 \mathrm{E}-01$ & $4.2 \mathrm{E}-03$ & & & $7.1 \mathrm{E}-01$ & 7.1E-03 & $4.2 \mathrm{E}-04$ & $4.2 \mathrm{E}-05$ & $5.9 \mathrm{E}-03$ & $5.9 \mathrm{E}-05$ & & \\
\hline Dibromobenzene, 1,4- & $000106-37-6$ & & & 3.7E-01 & $3.7 \mathrm{E}-02$ & & & $3.5 \mathrm{E}+00$ & 3.5E-01 & & & & & & & $3.3 \mathrm{E}-01$ & 3.3E-02 \\
\hline Dibromochloromethane & $000124-48-1$ & $1.0 \mathrm{E}-01$ & $1.0 \mathrm{E}-03$ & $7.3 \mathrm{E}-01$ & $7.3 \mathrm{E}-02$ & $6.4 \mathrm{E}+00$ & $6.4 \mathrm{E}-02$ & $4.6 \mathrm{E}+01$ & $4.6 \mathrm{E}+00$ & & & & & $1.0 \mathrm{E}-01$ & $1.0 \mathrm{E}-03$ & $7.2 \mathrm{E}-01$ & $7.2 \mathrm{E}-02$ \\
\hline $\begin{array}{l}\text { Dibromodichloromethan } \\
\text { e }\end{array}$ & $000594-18-3$ & & & & & & & & & & & & & & & & \\
\hline $\begin{array}{l}\text { Dibromodiphenyl Ether, } \\
\text { p,p- }\end{array}$ & $002050-47-7$ & & & & & & & & & & & & & & & & \\
\hline Dibromoethane, 1,2- & $000106-93-4$ & $1.0 \mathrm{E}-04$ & $1.0 \mathrm{E}-06$ & & & $1.0 \mathrm{E}-02$ & $1.0 \mathrm{E}-04$ & & & $2.2 \mathrm{E}-03$ & $2.2 \mathrm{E}-05$ & $4.2 \mathrm{E}-04$ & 4.2E-05 & $9.5 \mathrm{E}-05$ & $9.5 \mathrm{E}-07$ & & \\
\hline $\begin{array}{l}\text { Dibromomethane } \\
\text { (Methylene Bromide) }\end{array}$ & $000074-95-3$ & & & 3.7E-01 & $3.7 \mathrm{E}-02$ & & & $1.0 \mathrm{E}+02$ & $1.0 \mathrm{E}+01$ & & & & & & & $3.6 \mathrm{E}-01$ & $3.6 \mathrm{E}-02$ \\
\hline Dibutyl Phthalate & $000084-74-2$ & & & $3.7 \mathrm{E}+00$ & $3.7 \mathrm{E}-01$ & & & $1.3 \mathrm{E}+01$ & $1.3 \mathrm{E}+00$ & & & & & & & $2.9 \mathrm{E}+00$ & $2.9 \mathrm{E}-01$ \\
\hline Dicamba & $001918-00-9$ & & & $1.1 \mathrm{E}+00$ & $1.1 \mathrm{E}-01$ & & & $2.0 \mathrm{E}+01$ & $2.0 \mathrm{E}+00$ & & & & & & & $1.0 \mathrm{E}+00$ & $1.0 \mathrm{E}-01$ \\
\hline Dichloro-2-butene, 1,4- & $000764-41-0$ & & & & & & & & & $1.8 \mathrm{E}-04$ & $1.8 \mathrm{E}-06$ & & & & & & \\
\hline \multicolumn{18}{|l|}{ Dichloroacetic Acid } \\
\hline Dichlorobenzene, $1,2^{(3)}$ & $000095-50-1$ & & & $3.3 \mathrm{E}+00$ & $3.3 \mathrm{E}-01$ & & & $1.8 \mathrm{E}+01$ & $1.8 \mathrm{E}+00$ & & & $4.2 \mathrm{E}-01$ & $4.2 \mathrm{E}-02$ & - & & $3.6 \mathrm{E}-01$ & $3.6 \mathrm{E}-02$ \\
\hline Dichlorobenzene, 1,3- & $000541-73-1$ & & & & & & & & & & & & & & & & \\
\hline Dichlorobenzene, 1,4- & $000106-46-7$ & $3.5 \mathrm{E}-01$ & $3.5 \mathrm{E}-03$ & & & $2.1 \mathrm{E}+00$ & 2.1E-02 & & & & & $\begin{array}{l}1.7 E+0 \\
0\end{array}$ & $1.7 \mathrm{E}-01$ & $3.0 \mathrm{E}-01$ & $3.0 \mathrm{E}-03$ & & \\
\hline Dichlorobenzidine, 3,3'- & $000091-94-1$ & $1.9 \mathrm{E}-02$ & $1.9 \mathrm{E}-04$ & & & 2.3E-01 & $2.3 \mathrm{E}-03$ & & & & & & & $1.7 \mathrm{E}-02$ & $1.7 \mathrm{E}-04$ & & \\
\hline Dichlorodifluoromethane & $000075-71-8$ & & & $7.3 \mathrm{E}+00$ & 7.3E-01 & & & $5.8 \mathrm{E}+01$ & $5.8 E+00$ & & & $4.2 \mathrm{E}-01$ & 4.2E-02 & & & $3.9 E-01$ & $3.9 \mathrm{E}-02$ \\
\hline Dichloroethane, 1,1- & $000075-34-3$ & & & $3.7 E+00$ & $3.7 \mathrm{E}-01$ & & & $1.7 E+02$ & $1.7 \mathrm{E}+01$ & & & $\begin{array}{l}1.0 \mathrm{E}+0 \\
0\end{array}$ & $1.0 \mathrm{E}-01$ & & & $8.1 \mathrm{E}-01$ & $8.1 \mathrm{E}-02$ \\
\hline
\end{tabular}


Table 3.1a. (continued)

\begin{tabular}{|c|c|c|c|c|c|c|c|c|c|c|c|c|c|c|c|c|c|}
\hline \multirow[t]{2}{*}{ Chemical } & \multirow[t]{2}{*}{ CASRN } & \multicolumn{4}{|c|}{ Ingestion (mg/L)" } & \multicolumn{4}{|c|}{ Dermal (mg/L)" } & \multicolumn{4}{|c|}{ Inhalation $(\mathrm{mg} / \mathrm{L})^{\dagger *}$} & \multicolumn{4}{|c|}{$\begin{array}{l}\text { Ingestion + Dermal + } \\
\text { Inhalation }(\mathrm{mg} / \mathrm{L})^{\dagger+*}\end{array}$} \\
\hline & & $10^{-4}$ & $10^{4}$ & $H Q=1$ & $H Q=0.1$ & $10^{-4}$ & $10^{4}$ & $H Q=1$ & $\mathrm{HQ}=0.1$ & $10^{-4}$ & $10^{6}$ & $H Q=1$ & $\mathrm{HQ}=0.1$ & $10^{-4}$ & $10^{6}$ & HI=1 & $\mathrm{HI}=0.1$ \\
\hline Dichloroethane, 1,2- & $000107-06-2$ & $9.4 \mathrm{E}-02$ & $9.4 \mathrm{E}-04$ & & & $7.3 \mathrm{E}+00$ & $7.3 \mathrm{E}-02$ & & & $1.9 \mathrm{E}-02$ & $1.9 \mathrm{E}-04$ & & & $1.6 \mathrm{E}-02$ & $1.6 \mathrm{E}-04$ & & \\
\hline Dichloroethylene, 1,1- & $000075-35-4$ & $1.4 \mathrm{E}-02$ & $1.4 \mathrm{E}-04$ & $3.3 \mathrm{E}-01$ & $3.3 \mathrm{E}-02$ & $6.6 \mathrm{E}-01$ & $6.6 \mathrm{E}-03$ & $1.5 \mathrm{E}+01$ & $1.5 \mathrm{E}+00$ & $1.4 \mathrm{E}-03$ & $1.4 \mathrm{E}-05$ & & & $1.3 \mathrm{E}-03$ & 1.3E-05 & $3.2 \mathrm{E}-01$ & $3.2 \mathrm{E}-02$ \\
\hline $\begin{array}{l}\text { Dichloroethylene, 1,2- } \\
\text { (Mixed Isomers) }\end{array}$ & $000540-59-0$ & & & $3.3 \mathrm{E}-01$ & $3.3 \mathrm{E}-02$ & & & $1.0 \mathrm{E}+02$ & $1.0 E+01$ & & & & & & & 3.3E-01 & $3.3 \mathrm{E}-02$ \\
\hline $\begin{array}{l}\text { Dichloroethylene, 1,2- } \\
\text { cis- }\end{array}$ & $000156-59-2$ & & & 3.7E-01 & 3.7E-02 & & & $1.5 \mathrm{E}+01$ & $1.5 \mathrm{E}+00$ & & & & & & & $3.6 \mathrm{E}-01$ & $3.6 \mathrm{E}-02$ \\
\hline $\begin{array}{l}\text { Dichloroethylene, 1,2- } \\
\text { trans- }\end{array}$ & $000156-60-5$ & & & $7.3 \mathrm{E}-01$ & 7.3E-02 & & & $2.8 \mathrm{E}+02$ & $2.8 \mathrm{E}+01$ & & & & & & & 7.3E-01 & 7.3E-02 \\
\hline Dichlorophenol, 2,4- & $000120-83-2$ & & & $1.1 \mathrm{E}-01$ & $1.1 \mathrm{E}-02$ & & & $6.2 \mathrm{E}-01$ & $6.2 \mathrm{E}-02$ & & & & & & & $9.3 \mathrm{E}-02$ & $9.3 \mathrm{E}-03$ \\
\hline $\begin{array}{l}\text { Dichlorophenoxy Acetic } \\
\text { Acid, 2,4- }\end{array}$ & $000094-75-7$ & & & 3.7E-01 & 3.7E-02 & & & $2.4 \mathrm{E}+01$ & $2.4 \mathrm{E}+00$ & & & & & & & $3.6 \mathrm{E}-01$ & $3.6 \mathrm{E}-02$ \\
\hline $\begin{array}{l}\text { Dichlorophenoxy)butyric } \\
\text { Acid, 4-(2,4- }\end{array}$ & $000094-82-6$ & & & $2.9 \mathrm{E}-01$ & $2.9 \mathrm{E}-02$ & & & $1.3 \mathrm{E}+01$ & $1.3 \mathrm{E}+00$ & & & & & & & $2.9 \mathrm{E}-01$ & $2.9 \mathrm{E}-02$ \\
\hline Dichloropropane, 1,2- & $000078-87-5$ & $1.3 \mathrm{E}-01$ & $1.3 \mathrm{E}-03$ & & & $3.8 \mathrm{E}+00$ & $3.8 \mathrm{E}-02$ & & & & & $8.3 \mathrm{E}-03$ & $8.3 \mathrm{E}-04$ & $1.2 \mathrm{E}-01$ & $1.2 \mathrm{E}-03$ & & \\
\hline Dichloropropanol, 2,3- & $000616-23-9$ & & & $1.1 \mathrm{E}-01$ & $1.1 \mathrm{E}-02$ & & & & & & & & & & & & \\
\hline Dichloropropene, 1,3- & $000542-75-6$ & 4.7E-02 & 4.7E-04 & $1.1 \mathrm{E}-02$ & $1.1 \mathrm{E}-03$ & $2.0 \mathrm{E}+00$ & $2.0 \mathrm{E}-02$ & 4.5E-01 & $4.5 \mathrm{E}-02$ & $1.3 \mathrm{E}-02$ & $1.3 \mathrm{E}-04$ & $4.2 \mathrm{E}-02$ & $4.2 \mathrm{E}-03$ & $1.0 \mathrm{E}-02$ & $1.0 \mathrm{E}-04$ & $8.5 \mathrm{E}-03$ & $8.5 \mathrm{E}-04$ \\
\hline Dichlorvos & $000062-73-7$ & $2.9 \mathrm{E}-02$ & $2.9 \mathrm{E}-04$ & $1.8 \mathrm{E}-02$ & $1.8 \mathrm{E}-03$ & $6.4 \mathrm{E}+00$ & $6.4 \mathrm{E}-02$ & $4.0 \mathrm{E}+00$ & $4.0 \mathrm{E}-01$ & & & & & $2.9 \mathrm{E}-02$ & $2.9 \mathrm{E}-04$ & $1.8 \mathrm{E}-02$ & $1.8 \mathrm{E}-03$ \\
\hline Dicofol & $000115-32-2$ & & & & & & & & & & & & & & & & \\
\hline Dicyclopentadiene & $000077-73-6$ & & & $1.1 E+00$ & $1.1 \mathrm{E}-01$ & & & $1.1 E+01$ & $1.1 \mathrm{E}+\infty 0$ & & & $4.2 \mathrm{E}-04$ & 4.2E-05 & & & $4.2 \mathrm{E}-04$ & 4.2E-05 \\
\hline Dieldrin & $000060-57-1$ & $5.3 \mathrm{E}-04$ & $5.3 \mathrm{E}-06$ & $1.8 \mathrm{E}-03$ & $1.8 \mathrm{E}-04$ & $6.9 \mathrm{E}-03$ & $6.9 \mathrm{E}-05$ & $2.4 \mathrm{E}-02$ & $2.4 \mathrm{E}-03$ & & & & & $4.9 \mathrm{E}-04$ & $4.9 \mathrm{E}-06$ & $1.7 \mathrm{E}-03$ & 1.7E-04 \\
\hline Diethyl Phthalate & $000084-66-2$ & & & $2.9 E+01$ & $2.9 \mathrm{E}+00$ & & & $2.3 \mathrm{E}+03$ & $2.3 \mathrm{E}+02$ & & & & & & & $2.9 \mathrm{E}+01$ & $2.9 E+00$ \\
\hline $\begin{array}{l}\text { Diethyl-p- } \\
\text { nitrophenylphosphate }\end{array}$ & $000311-45-5$ & & & & & & & & & & & & & & & & \\
\hline $\begin{array}{l}\text { Diethylene Glycol } \\
\text { Dinitrate (DEGDN) }\end{array}$ & $000693-21-0$ & & & & & & & & & & & & & & & & \\
\hline
\end{tabular}


Table 3.1a. (continued)

\begin{tabular}{|c|c|c|c|c|c|c|c|c|c|c|c|c|c|c|c|c|c|}
\hline \multirow[t]{2}{*}{ Chemical } & \multirow[t]{2}{*}{ CASRN } & \multicolumn{4}{|c|}{ Ingestion (mg/L)" } & \multicolumn{4}{|c|}{ Dermal (mg/L)" } & \multicolumn{4}{|c|}{ Inhalation $(\mathrm{mg} / \mathrm{L})^{\dagger *}$} & \multicolumn{4}{|c|}{$\begin{array}{l}\text { Ingestion + Dermal + } \\
\text { Inhalation }(\mathbf{m g} / \mathbf{L})^{\text {t+ }^{+}}\end{array}$} \\
\hline & & $10^{-4}$ & $10^{5}$ & $\mathrm{HO}=1$ & $\mathrm{HQ}=0.1$ & $10^{-1}$ & $10^{4}$ & HQ=1 & $\mathrm{HQ}=0.1$ & $10^{-4}$ & $10^{5}$ & $\mathrm{HQ}=1$ & $\mathrm{HQ}=0.1$ & $10^{-4}$ & $10^{8}$ & $\mathrm{HI}=\mathbf{1}$ & $\mathrm{HI}=0.1$ \\
\hline $\begin{array}{l}\text { Diethylene Glycol } \\
\text { Monobutyl Ether }\end{array}$ & $000112-34-5$ & & & & & & & & & & & & & & & & \\
\hline $\begin{array}{l}\text { Diethylene Glycol } \\
\text { Monocthyl Ether }\end{array}$ & $000111-90-0$ & & & $7.3 \mathrm{E}+01$ & $7.3 E+00$ & & & $6.0 \mathrm{E}+04$ & $6.0 \mathrm{E}+03$ & & & & & & & $7.3 E+01$ & $7.3 E+00$ \\
\hline Diethylformamide & $000617-84-5$ & & & $4.0 \mathrm{E}-01$ & $4.0 \mathrm{E}-02$ & & & & & & & & & & & & \\
\hline Diethylstibesterol & $000056-53-1$ & $1.8 \mathrm{E}-06$ & $1.8 \mathrm{E}-08$ & & & 1.1.E-06 & 1.1E- -08 & & & & & & & $6.7 \mathrm{E}-07$ & $6.7 \mathrm{E}-09$ & & \\
\hline Difenzoquat & $043222-48-6$ & & & $2.9 \mathrm{E}+00$ & $2.9 \mathrm{E}-01$ & & & & & & & & & & & & \\
\hline Diflubenzuron & 035367-38-5 & & & 7.3E-01 & 7.3E-02 & & & & & & & & & & & & \\
\hline Difluoroethane, $1,1-$ & $000075-37-6$ & & & & & & & & & & & $i^{8.3 \mathrm{E}+0}$ & $8.3 E+00$ & & & & \\
\hline $\begin{array}{l}\text { Diisopropyl } \\
\text { Methylphosphonate }\end{array}$ & $001445-75-6$ & & & $2.9 \mathrm{E}+00$ & $2.9 \mathrm{E}-01$ & & & $7.4 \mathrm{E}+02$ & $7.4 \mathrm{E}+01$ & & & & & & & $2.9 \mathrm{E}+00$ & $2.9 \mathrm{E}-01$ \\
\hline Dimethipin & $055290-64-7$ & & & $7.3 \mathrm{E}-01$ & 7.3E-02 & & & & & & & & & & & & \\
\hline Dimethoate & $000060-51-5$ & & & 7.3E-03 & 7.3E-04 & & & $2.4 \mathrm{E}-01$ & $2.4 \mathrm{E}-02$ & & & & & & & 7.1E-03 & 7.1E-04 \\
\hline $\begin{array}{l}\text { Dimethoxybenzidine, } \\
3,33^{-}\end{array}$ & $000119-90-4$ & $6.1 \mathrm{E}-01$ & $6.1 \mathrm{E}-03$ & & & $1.3 \mathrm{E}+02$ & $1.3 \mathrm{E}+00$ & & & & & & & $6.1 \mathrm{E}-01$ & $6.1 \mathrm{E}-03$ & & \\
\hline Dimethyl Sulfate & $000077-78-1$ & & & & & & & & & & & & & & & & \\
\hline $\begin{array}{l}\text { Dimethylaniline } \mathrm{HCl}, \\
2,4 \cdot\end{array}$ & $021436-96-4$ & $1.5 \mathrm{E}-02$ & $1.5 \mathrm{E}-04$ & & & & & & & & & & & & & & \\
\hline Dimethylaniline, $2,4-$ & $000095-68.1$ & $1.1 \mathrm{E}-02$ & $1.1 \mathrm{E}-04$ & & & & & & & & & & & & & & \\
\hline Dimethylaniline, N,N- & $000121-69-7$ & & & 7.3E-02 & 7.3E-03 & & & $6.2 \mathrm{E}-01$ & $6.2 \mathrm{E}-02$ & & & & & & & $6.5 \mathrm{E}-02$ & $6.5 \mathrm{E}-03$ \\
\hline Dimethylbenzidine, 3,3'- & $000119-93-7$ & $9.3 \mathrm{E}-04$ & 9.3E-06 & & & 4.3E-02 & 4.3E-04 & & & & & & & 9.1E-04 & 9.1E-06 & & \\
\hline Dimethylethyl Lead & $107584-40-7$ & & & & & & & & & & & & & & & & \\
\hline Dimethylformanide & $000068-12-2$ & & & $3.7 \mathrm{E}+00$ & $3.7 \mathrm{E}-01$ & & & $5.7 E+03$ & $5.7 \mathrm{E}+02$ & & & & & & & $3.6 \mathrm{E}+00$ & $3.6 \mathrm{E}-01$ \\
\hline
\end{tabular}


Table 3.1a. (continued)

\begin{tabular}{|c|c|c|c|c|c|c|c|c|c|c|c|c|c|c|c|c|c|}
\hline \multirow[t]{2}{*}{ Chemical } & \multirow[t]{2}{*}{ CASRN } & \multicolumn{4}{|c|}{ Ingestion (mg/L)" } & \multicolumn{4}{|c|}{ Dermal (mg/L)" } & \multicolumn{4}{|c|}{ Inhalation $(\mathrm{mg} / \mathrm{L})^{\dagger \dagger}$} & \multicolumn{4}{|c|}{$\begin{array}{l}\text { Ingestion + Dermal + } \\
\text { Inhalation }(\mathrm{mg} / \mathrm{L})^{1 \mathrm{ta}^{\circ}}\end{array}$} \\
\hline & & $10^{-4}$ & $10^{4}$ & $\dot{H} Q=1$ & $\mathrm{HQ}=0.1$ & $10^{-1}$ & $10^{6}$ & $\mathrm{HQ}=1$ & $\mathrm{HQ}=0.1$ & $10^{-4}$ & $10^{4}$ & $\mathrm{HQ}=1$ & $\mathrm{HQ}=0.1$ & $10^{-4}$ & $10^{4}$ & $\mathrm{HI}=\mathbf{1}$ & $\mathrm{HI}=0.1$ \\
\hline Bimethylhydrazine, 1,1- & $000057-14-7$ & $3.3 \mathrm{E}-03$ & 3.3E-05 & & & & & & & & & & & & & & \\
\hline Dimethylhydrazine, 1,2- & 000540-73-8 & & & & & & & & & & & & & & & & \\
\hline Dimethylphenol, 2,4- & $000105-67-9$ & & & $7.3 \mathrm{E}-01$ & $.7 .3 \mathrm{E}-02$ & & & $2.2 \mathrm{E}+00^{\circ}$ & $2.2 E-01$ & & & & & & & $5.5 \mathrm{E}-01$ & $5.5 \mathrm{E}-02$ \\
\hline Dimethylphenol, 2,6- & $000576-26-1$ & & & $2.2 \mathrm{E}-02$ & $2.2 \mathrm{E}-03$ & & & $2.8 \mathrm{E}-01$ & $2.8 \mathrm{E}-02$ & & & & & & & $2.0 \mathrm{E}-02$ & $2.0 \mathrm{E}-03$ \\
\hline Dimethylphenol, 3,4- & $000095-65-8$ & & & $3.7 \mathrm{E}-02$ & $3.7 \mathrm{E}-03$ & & & $1.9 \mathrm{E}-01$ & $1.9 \mathrm{E}-02$ & & & & & & & $3.1 \mathrm{E}-02$ & $3.1 \mathrm{E}-03$ \\
\hline Dimethylphthalate & $000131-11-3$ & & & $3.7 \mathrm{E}+02$ & $3.7 \mathrm{E}+01$ & & & $8.5 \mathrm{E}+04$ & $8.5 E+03$ & & & & & & & $3.6 \mathrm{E}+02$ & $3.6 \mathrm{E}+01$ \\
\hline Dimethylterephthalate & $000120-61-6$ & & & $3.7 \mathrm{E}+00$ & $3.7 \mathrm{E}-01$ & & & & & & & & & & & & \\
\hline Dinitro-o-cresol, 4,6- & 000534-52-1 & & & & & & & & & & & & & & & & \\
\hline $\begin{array}{l}\text { Dinitro-o-cyclohexyl } \\
\text { Phenol, 4,6- }\end{array}$ & 000131-89-5 & & & $7.3 \mathrm{E}-02$ & $7.3 \mathrm{E}-03$ & & & $2.7 \mathrm{E}+04$ & $2.7 \mathrm{E}+03$ & & & & & & & $7.3 \mathrm{E}-02$ & 7.3E-03 \\
\hline Dinitrobenzene, 1,2- & $000528-29-0$ & & & $1.5 \mathrm{E}-02$ & $1.5 \mathrm{E}-03$ & & & $2.3 \mathrm{E}+00$ & $2.3 \mathrm{E}-01$ & & & & & & & $1.5 \mathrm{E}-02$ & $1.5 \mathrm{E}-03$ \\
\hline Dinitrobenzene, 1,3- & $000099-65-0$ & & & $3.7 \mathrm{E}-03$ & $3.7 \mathrm{E}-04$ & & & 4.1E-01 & $4.1 \mathrm{E}-02$ & & & & & & & $3.6 \mathrm{E}-03$ & 3.6E-04 \\
\hline Dinitrobenzene, 1,4- & $000100-25-4$ & & & $1.5 \mathrm{E}-02$ & $1.5 \mathrm{E}-03$ & & & $1.4 \mathrm{E}+00$ & $1.4 \mathrm{E}-01$ & & & & & & & $1.4 \mathrm{E}-02$ & $1.4 \mathrm{E}-03$ \\
\hline Dinitrophenol, 2,4- & $000051-28-5$ & & & $7.3 \mathrm{E}-02$ & $7.3 \mathrm{E}-03$ & & & $9.4 E+00$ & $9.4 \mathrm{E}-01$ & & & & & & & 7.2E-02 & $7.2 \mathrm{E}-03$ \\
\hline Dinitrotoluene, 2,4- & $000121-14-2$ & $1.3 \mathrm{E}-02$ & $1.3 \mathrm{E}-04$ & $7.3 \mathrm{E}-02$ & $7.3 \mathrm{E}-03$ & $1.2 E+00$ & $1.2 \mathrm{E}-02$ & $6.7 \mathrm{E}+00$ & $6.7 \mathrm{E}-01$ & & & & & $1.2 \mathrm{E}-02$ & $1.2 \mathrm{E}-04$ & $7.2 \mathrm{E}-02$ & $7.2 \mathrm{E}-03$ \\
\hline Dinitrotoluene, 2,6- & $000606-20-2$ & $1.3 \mathrm{E}-02$ & $1.3 \mathrm{E}-04$ & $3.7 \mathrm{E}-02$ & 3.7E-03 & $1.8 \mathrm{E}+00$ & $1.8 \mathrm{E}-02$ & $5.1 \mathrm{E}+00$ & $5.1 \mathrm{E}-01$ & & & & & $1.2 \mathrm{E}-02$ & $1.2 \mathrm{E}-04$ & $3.6 \mathrm{E}-02$ & $3.6 \mathrm{E}-03$ \\
\hline $\begin{array}{l}\text { Dinitrotoluene, 2-Amino- } \\
\text { 4,6- }\end{array}$ & 035572-78-2 & & & & & & & & & & & & & & & & \\
\hline Dinoseb & 000088-85-7 & & & $3.7 \mathrm{E}-02$ & $3.7 \mathrm{E}-03$ & & & $2.7 \mathrm{E}-01$ & $2.7 \mathrm{E}-02$ & & & & & & & $3.2 \mathrm{E}-02$ & $3.2 \mathrm{E}-03$ \\
\hline Dioxane, 1,4- & 000123-91-1 & 7.7E-01 & 7.7E-03 & & & $6.4 E+02$ & $6.4 \mathrm{E}+00$ & & & & & & & $7.7 \mathrm{E}-01$ & $7.7 \mathrm{E}-03$ & & \\
\hline Diphenamid & $000957-51-7$ & & & $1.1 \mathrm{E}+00$ & $1.1 \mathrm{E}-01$ & & & $9.8 \mathrm{E}+01$ & $9.8 \mathrm{E}+00$ & & & & & & & $1.1 \mathrm{E}+00$ & $1.1 \mathrm{E}-01$ \\
\hline Diphenylamine & $000122-39-4$ & & & $9.1 \mathrm{E}-01$ & $9.1 E-02$ & & & $3.5 \mathrm{E}+00$ & $3.5 \mathrm{E}-01$ & & & & & & & $7.2 \mathrm{E}-01$ & $7.2 E-02$ \\
\hline
\end{tabular}


Table 3.1a. (continued)

\begin{tabular}{|c|c|c|c|c|c|c|c|c|c|c|c|c|c|c|c|c|c|}
\hline \multirow[t]{2}{*}{ Chemical } & \multirow[t]{2}{*}{ CASRN } & \multicolumn{4}{|c|}{ Ingestion (mg/L)" } & \multicolumn{4}{|c|}{ Dermal (mg/L)" } & \multicolumn{4}{|c|}{ Inhalation (mg/L) ${ }^{\dagger *}$} & \multicolumn{4}{|c|}{$\begin{array}{l}\text { Ingestion + Dermal + } \\
\text { Inhalation }(\mathrm{mg} / \mathbf{L})^{++{ }^{+*}}\end{array}$} \\
\hline & & $10^{-4}$ & $10^{4}$ & $\mathrm{HQ}=1$ & $\mathrm{HQ}=0.1$ & $10^{-1}$ & $10^{6}$ & $\mathrm{HQ}=1$ & $\mathrm{HQ}=0.1$ & $10^{-4}$ & $10^{4}$ & $\mathrm{HQ}=1$ & $\mathrm{HQ}=0.1$ & $10^{-4}$ & $10^{4}$ & $\mathrm{HI}=\mathbf{1}$ & $\mathrm{Hl}=0.1$ \\
\hline Diphenylhydrazine, 1,2- & $000122-66-7$ & $1.1 E-02$ & $1.1 E-04$ & & & $1.2 E-01$ & $1.2 \mathrm{E}-03$ & & & & & & & $9.8 \mathrm{E}-03$ & $9.8 \mathrm{E}-05$ & & \\
\hline Diquat & $000085-00-7$ & & & $8.0 E-02$ & $8.0 E-03$ & & & $1.6 \mathrm{E}+05$ & $1.6 \mathrm{E}+04$ & & & & & & & $8.0 \mathrm{E}-02$ & $8.0 \mathrm{E}-03$ \\
\hline Direct Black 38 & 001937-37-7 & $9.9 E-04$ & $9.9 \mathrm{E}-06$ & & & & & & & & & & & & & & \\
\hline Direct Blue 6 & $002602-46-2$ & $1.1 \mathrm{E}-03$ & $1.1 \mathrm{E}-05$ & & & & & & & & & & & & & & \\
\hline Direct Brown 95 & $016071-86-6$ & $9.2 \mathrm{E}-04$ & $9.2 \mathrm{E}-06$ & & & & & & & & & & & & & & \\
\hline Direct Sky Blue & $002610-05-1$ & & & & & & & & & & & & & & & & \\
\hline Disulfoton & $000298-04-4$ & & & $1.5 \mathrm{E}-03$ & 1.5E-04 & & & $1.1 \mathrm{E}-02$ & $1.1 \mathrm{E}-03$ & & & & & & & $1.3 \mathrm{E}-03$ & $1.3 \mathrm{E}-04$ \\
\hline Diuron & $000330-54-1$ & & & $7.3 \mathrm{E}-02$ & $7.3 \mathrm{E}-03$ & & & $2.5 \mathrm{E}+00$ & $2.5 \mathrm{E}-01$ & & & & & & & $7.1 \mathrm{E}-02$ & $7.1 \mathrm{E}-03$ \\
\hline Dodine & $002439-10-3$ & & & $1.5 \mathrm{E}-01$ & $1.5 \mathrm{E}-02$ & & & & & & & & & & & & \\
\hline EPTC & $000759-94-4$ & & & $9.1 E-01$ & $9.1 \mathrm{E}-02$ & & & & & & & & & & & & \\
\hline Endosuffan & 000115-29.7 & & & $2.2 \mathrm{E}-01$ & $2.2 \mathrm{E}-02$ & & & & & & & & & & & & \\
\hline Endothall & 000145-73-3 & & & $7.3 \mathrm{E}-01$ & 7.3E-02 & & & & & & & & & & & & \\
\hline Endrin & $000072-20-8$ & & & $1.1 \mathrm{E}-02$ & 1.1E--03 & & & $5.6 \mathrm{E}-03$ & $5.6 \mathrm{E}-04$ & & & & & & & 3.7E-03 & $3.7 \mathrm{E}-04$ \\
\hline Epichlorohydrin & $000106-89-8$ & $8.6 \mathrm{E}-01$ & $8.6 \mathrm{E}-03$ & $7.3 \mathrm{E}-02$ & $7.3 \mathrm{E}-03$ & $7.7 E+02$ & $7.7 \mathrm{E}+00$ & $6.5 \mathrm{E}+01$ & $6.5 \mathrm{E}+00$ & 4.1E- 01 & $4.1 \mathrm{E}-03$ & $2.1 \mathrm{E}-03$ & 2.1E-04 & $2.8 \mathrm{E}-01$ & $2.8 \mathrm{E}-03$ & $2.0 \mathrm{E}-03$ & $2.0 \mathrm{E}-04$ \\
\hline Epoxybutane, 1,2- & $000106-88-7$ & & & & & & & & & & & & & & & & \\
\hline Ethephon & $016672-87-0$ & & & $1.8 \mathrm{E}-01$ & $1.8 \mathrm{E}-02$ & & & & & & & & & & & & \\
\hline Ethion & $000563-12-2$ & & & $1.8 \mathrm{E}-02$ & $1.8 \mathrm{E}-03$ & & & $1.1 \mathrm{E}-01$ & 1.1EE-02 & & & & & & & $1.6 \mathrm{E}-02$ & $1.6 \mathrm{E}-03$ \\
\hline $\begin{array}{l}\text { Ethoxyethanol Acctate, } \\
2 \text { - }\end{array}$ & $000111-15-9$ & & & $1.1 E+01$ & $1.1 \mathrm{E}+00$ & & & $2.6 \mathrm{E}+03$ & $2.6 \mathrm{E}+02$ & & & & & & & $1.1 E+01$ & $1.1 \mathrm{E}+00$ \\
\hline Ethoxyethanol, 2- & $000110-80-5$ & & & $1.5 \mathrm{E}+01$ & $1.5 \mathrm{E}+00$ & & & $1.0 E+04$ & $1.0 \mathrm{E}+03$ & & & & & & & $1.5 \mathrm{E}+01$ & $1.5 \mathrm{E}+00$ \\
\hline Ethyl Acelate & $000141-78-6$ & & & $3.3 E+01$ & $3.3 E+00$ & & & $5.2 \mathrm{E}+03$ & $5.2 E+02$ & & & & & & & $3.3 \mathrm{E}+01$ & $3.3 \mathrm{E}+00$ \\
\hline Ethyl Acrylate & $000140-88-5$ & $1.8 \mathrm{E}-01$ & $1.8 \mathrm{E}-03$ & & & $9.1 E+00$ & $9.1 \mathrm{E}-02$ & & & & & & & $1.7 \mathrm{E}-01$ & $1.7 \mathrm{E}-03$ & & \\
\hline
\end{tabular}


Table 3.1a. (continued)

\begin{tabular}{|c|c|c|c|c|c|c|c|c|c|c|c|c|c|c|c|c|c|}
\hline \multirow[t]{2}{*}{ Chemical } & \multirow[t]{2}{*}{ CASRN } & \multicolumn{4}{|c|}{ Ingestion $(\mathrm{mg} / \mathrm{L})^{*}$} & \multicolumn{4}{|c|}{ Dermal (mg/L)" } & \multicolumn{4}{|c|}{ Inhalation $(\mathrm{mg} / \mathrm{L})^{\dagger^{\star}}$} & \multicolumn{4}{|c|}{$\begin{array}{c}\text { Ingestion + Dermal + } \\
\text { Inhalation }(\mathrm{mg} / \mathrm{L})^{\dagger+{ }^{\star}}\end{array}$} \\
\hline & & $10^{-4}$ & $10^{4}$ & HQ $=1$ & $\mathrm{HQ}=0.1$ & $10^{-4}$ & $10^{6}$ & $\mathrm{HQ}=1$ & $\mathrm{HQ}=0.1$ & $10^{4}$ & $10^{4}$ & $\mathrm{HQ}=1$ & $\mathrm{HQ}=0.1$ & $10^{-t}$ & $10^{4}$ & Hl=1 & $\mathrm{HI}=0.1$ \\
\hline Ethyl Chloride & $000075-00-3$ & & & & & & & & & & & $\begin{array}{l}2.1 \mathrm{E}+0 \\
1\end{array}$ & $2.1 \mathrm{E}+00$ & & & & \\
\hline Ethyl Ether & $000060-29-7$ & & & $7.3 \mathrm{E}+00$ & 7.3E- 01 & & & $1.4 \mathrm{E}+02$ & $1.4 \mathrm{E}+01$ & & & & & & & $6.9 \mathrm{E}+00$ & $6.9 \mathrm{E}-01$ \\
\hline Ethyl Methacrylate & $000097-63-2$ & & & $3.3 \mathrm{E}+00$ & 3.3E-01 & & . & $1.3 \mathrm{E}+02$ & $1.3 \mathrm{E}+01$ & & & & & & & $3.2 \mathrm{E}+00$ & $3.2 \mathrm{E}-01$ \\
\hline $\begin{array}{l}\text { Ethyl-p-nitrophenyl } \\
\text { Phosphonate }\end{array}$ & 002104-64-5 & & & 3.7E-04 & $3.7 \mathrm{E}-05$ & & & $7.4 \mathrm{E}-03$ & 7.4E-04 & & & & & & & $3.5 E-04$ & $3.5 \mathrm{E}-05$ \\
\hline Ethylbenzene $e^{(s)}$ & $000100-41-4$ & & & $3.7 \mathrm{E}+00$ & 3.7E-01 & & & $2.0 \mathrm{E}+01$ & $2.0 \mathrm{E}+00$ & & & $\begin{array}{l}2.1 E+0 \\
0\end{array}$ & $2.1 \mathrm{E}-01$ & & & $1.2 \mathrm{E}+00$ & $1.2 \mathrm{E}-01$ \\
\hline Ethylene Cyanohydrin & $000109-78-4$ & & & $1.1 \mathrm{E}+01$ & $1.1 \mathrm{E}+00$ & & & $3.7 E+04$ & $3.7 \mathrm{E}+03$ & & & & & & & $1.1 \mathrm{E}+01$ & $1.1 \mathrm{E}+00$ \\
\hline Euhylene Diamine & $000107-15-3$ & & & $7.3 \mathrm{E}-01$ & $7.3 \mathrm{E}-02$ & & & $1.7 \mathrm{E}+02$ & $1.7 \mathrm{E}+01$ & & & & & & & $7.3 \mathrm{E}-01$ & $7.3 \mathrm{E}-02$ \\
\hline Ethylene Glycol & $000107-21-1$ & & & $7.3 \mathrm{E}+01$ & $7.3 \mathrm{E}+00$ & & & $4.2 E+05$ & $4.2 \mathrm{E}+04$ & & & & & & & $7.3 \mathrm{E}+01$ & 7.3E+00 \\
\hline $\begin{array}{l}\text { Ethylene Glycol } \\
\text { Monobutyly Ether }\end{array}$ & $000111-76-2$ & & & & & & & & & & & & & & & & \\
\hline Ethylene Oxide & $000075-21-8$ & $8.3 \mathrm{E}-03$ & $8.3 \mathrm{E}-05$ & & & $4.4 \mathrm{E}+00$ & $4.4 \mathrm{E}-02$ & & & $4.9 \mathrm{E}-03$ & $4.9 \mathrm{E}-05$ & & & $3.1 \mathrm{E}-03$ & 3.1E-05 & & \\
\hline Ethylene Thiourea & $000096-45-7$ & 7.7E-02 & $7.7 \mathrm{E}-04$ & $2.9 \mathrm{E}-03$ & $2.9 \mathrm{E}-04$ & $9.4 \mathrm{E}+01$ & $9.4 \mathrm{E}-01$ & $3.5 E+00$ & $3.5 \mathrm{E}-01$ & & & & & $7.7 \mathrm{E}-02$ & 7.7E-04 & $2.9 \mathrm{E}-03$ & $2.9 \mathrm{E}-04$ \\
\hline $\begin{array}{l}\text { Ethylphthalyl Ethyl } \\
\text { Glycolate }\end{array}$ & $000084-72-0$ & & & $1.1 E+02$ & $1.1 \mathrm{E}+01$ & & & & & & & & & & & & \\
\hline Express & $101200-48-0$ & & & 2.9E-01 & $2.9 \mathrm{E}-02$ & & & & & & & & & & & & \\
\hline Fenamiphos & $022224-92-6$ & & & 9.1E-03 & $9.1 \mathrm{E}-04$ & & & & & & & & & & & & \\
\hline Fenpropathrin & 039515-41-8 & & & 9.1E-01 & $9.1 \mathrm{E}-02$ & & & & & & & & & & & & \\
\hline Fluometuron & $002164-17-2$ & & & $4.7 \mathrm{E}-01$ & 4.7E-02 & & & $2.6 \mathrm{E}+01$ & $2.6 \mathrm{E}+00$ & & & & & & & $4.7 \mathrm{E}-01$ & $4.7 \mathrm{E}-02$ \\
\hline Fluoranthene & $000206-44-0$ & & & $1.5 \mathrm{E}+00$ & 1.SE-01 & & & $5.2 \mathrm{E}-01$ & $5.2 E-02$ & & & & & & & $3.8 \mathrm{E}-01$ & $3.8 \mathrm{E}-02$ \\
\hline Fluorene & $000086-73-7$ & & & $1.5 \mathrm{E}+00$ & $1.5 \mathrm{E}-01$ & & & $2.0 \mathrm{E}+00$ & $2.0 E-01$ & & & & & & & $8.4 \mathrm{E}-01$ & $8.4 \mathrm{E}-02$ \\
\hline Fluoride & $007782-41-4$ & & & $2.2 \mathrm{E}+00$ & $2.2 \mathrm{E}-01$ & & & $8.8 \mathrm{E}+02$ & $8.8 \mathrm{E}+01$ & & & & & & & $2.2 \mathrm{E}+00$ & $2.2 \mathrm{E}-01$ \\
\hline
\end{tabular}


Table 3.1a. (continued)

\begin{tabular}{|c|c|c|c|c|c|c|c|c|c|c|c|c|c|c|c|c|c|}
\hline \multirow[t]{2}{*}{ Chemical } & \multirow[t]{2}{*}{ CASRN } & \multicolumn{4}{|c|}{ Ingestion (mg/L)" } & \multicolumn{4}{|c|}{ Dermal (mg/L)* } & \multicolumn{4}{|c|}{ Inhalation $(\mathrm{mg} / \mathbf{L})^{t^{*}}$} & \multicolumn{4}{|c|}{$\begin{array}{l}\text { Ingestion + Dermal + } \\
\text { Inhalation }(\mathrm{mg} / \mathrm{L})^{+t^{*}}\end{array}$} \\
\hline & & $10^{-4}$ & $10^{6}$ & $\mathrm{HQ}=1$ & $\mathrm{HQ}=0.1$ & $10^{-4}$ & $10^{4}$ & $H Q=1$ & $\mathrm{HQ}=0.1$ & $10^{-4}$ & $10^{4}$ & $\mathrm{HQ}=1$ & $H Q=0.1$ & $10^{4}$ & $10^{6}$ & $H I=1$ & $\mathrm{HI}=0.1$ \\
\hline Fluridone & $059756-60-4$ & & & $2.9 \mathrm{E}+00$ & $2.9 \mathrm{E}-01$ & & & & & & & & & & & & \\
\hline Flurprimidol & $056425-91-3$ & & & 7.3E-01 & $7.3 \mathrm{E}-02$ & & & & & & & & & & & & \\
\hline Flutolanil & $066332-96-5$ & & & $2.2 \mathrm{E}+00$ & $2.2 \mathrm{E}-01$ & & & & & & & & & & & & \\
\hline Folpet & $000133-07-3$ & $2.4 \mathrm{E}+00$ & $2.4 \mathrm{E}-02$ & $3.7 \mathrm{E}+00$ & 3.7E-01 & $1.1 \mathrm{E}+02$ & $1.1 \mathrm{E}+00$ & $1.6 \mathrm{E}+02$ & $1.6 \mathrm{E}+01$ & & & & & $2.4 \mathrm{E}+00$ & $2.4 \mathrm{E}-02$ & $3.6 \mathrm{E}+00$ & $3.6 \mathrm{E}-01$ \\
\hline Fomesafen & $072178-02-0$ & $4.5 \mathrm{E}-02$ & 4.5E-04 & & & & & & & & & & & & & & \\
\hline Fonofos & $000944-22-9$ & & & 7.3E-02 & 7.3E-03 & & & 4.0E-0! & $4.0 \mathrm{E}-02$ & & & & & & & $6.2 \mathrm{E}-02$ & $6.2 \mathrm{E}-03$ \\
\hline Formaldehyde & $000050-00-0$ & & & $7.3 \mathrm{E}+00$ & $7,3 \mathrm{E}-01$ & & & $1.1 \mathrm{E}+03$ & $1.1 \mathrm{E}+02$ & $3.8 \mathrm{E}-02$ & $3.8 \mathrm{E}-04$ & & & & & $7.3 E+00$ & $7.3 \mathrm{E}-01$ \\
\hline Formic Acid & $000064-18-6$ & & & $7.3 \mathrm{E}+01$ & $7.3 \mathrm{E}+00$ & & & $3.6 \mathrm{E}+04$ & $3.6 \mathrm{E}+03$ & & & & & & & $7.3 \mathrm{E}+01$ & $7.3 \mathrm{E}+00$ \\
\hline Furan & $000110-00-9$ & & & $3.7 \mathrm{E}-02$ & $3.7 \mathrm{E}-03$ & & & $2.0 \mathrm{E}+00$ & $2.0 \mathrm{E}-01$ & & & & & & & $3.6 \mathrm{E}-02$ & $3.6 \mathrm{E}-03$ \\
\hline Furazolidone & $000067-45-8$ & $2.2 \mathrm{E}-03$ & $2.2 \mathrm{E}-05$ & & & $1.9 E+00$ & $1.9 \mathrm{E}-02$ & & & & & & & $2.2 \mathrm{E}-03$ & $2.2 \mathrm{E}-05$ & & \\
\hline Furfural & $000098-01-1$ & & & 1.IE-01 & 1.1E-02 & & & $2.1 \mathrm{E}-01$ & 2.1E-02 & & & & & & & $7.2 \mathrm{E}-02$ & $7.2 \mathrm{E}-03$ \\
\hline Furium & $000531-82-8$ & $1.7 \mathrm{E}-04$ & $1.7 \mathrm{E}-06$ & & & & & & & & & & & & & & \\
\hline Furmecyclox & $060568-05-0$ & $2.8 \mathrm{E}-01$ & $2.8 \mathrm{E}-03$ & & & & & & & & & & & & & & \\
\hline Glufosinate, Ammonium & $077182-82-2$ & & & $1.5 \mathrm{E}-02$ & $1.5 \mathrm{E}-03$ & & & & & & & & & & & & \\
\hline Glycidyl & $000765-34-4$ & & & $1.5 \mathrm{E}-02$ & $1.5 \mathrm{E}-03$ & & & $1.4 \mathrm{E}+01$ & $1.4 \mathrm{E}+00$ & & & & & & & $1.5 \mathrm{E}-02$ & $1.5 \mathrm{E}-03$ \\
\hline Glyphosate & $001071-83-6$ & & & $3.7 \mathrm{E}+00$ & $3.7 \mathrm{E}-01$ & & & $5.7 \mathrm{E}+05$ & $5.7 E+04$ & & & & & & & $3.6 E+00$ & $3.6 \mathrm{E}-01$ \\
\hline Goal & 042874-03-3 & & & $1.1 \mathrm{E}-01$ & $1.1 \mathrm{E}-02$ & & & & & & & & & & & & \\
\hline Haloxyfop, Methyl & $069806-40-2$ & & & $1.8 \mathrm{E}-03$ & $1.8 \mathrm{E}-04$ & & & & & & & & & & & & \\
\hline Harmony & $079277-27-3$ & & & 4.7E-01 & 4.7E-02 & & & & & & & & & & & & \\
\hline
\end{tabular}


Table 3.1a. (continued)

\begin{tabular}{|c|c|c|c|c|c|c|c|c|c|c|c|c|c|c|c|c|c|}
\hline \multirow[t]{2}{*}{ Chemical } & \multirow[t]{2}{*}{ CASRN } & \multicolumn{4}{|c|}{ Ingestion (mg/L)" } & \multicolumn{4}{|c|}{ Dermal (mg/L)" } & \multicolumn{4}{|c|}{ Inhalation $(\mathbf{m g} / \mathbf{L})^{t^{\star}}$} & \multicolumn{4}{|c|}{$\begin{array}{l}\text { Ingestion + Dermal + } \\
\text { Inhalation }(\mathrm{mg} / \mathrm{L})^{+t^{*}}\end{array}$} \\
\hline & & $10^{-4}$ & $10^{6}$ & HQ=1 & $\mathrm{HQ}=0.1$ & $10^{-4}$ & $10^{-4}$ & $\mathrm{HQ}=\mathbf{1}$ & $\mathrm{HQ}=0.1$ & $10^{-4}$ & $10^{5}$ & $\mathrm{HQ}=1$ & $H Q=0.1$ & $10^{-1}$ & $10^{4}$ & $\mathbf{H I}=\mathbf{1}$ & $\mathrm{HI}=0.1$ \\
\hline Heptachlor & $000076-44-8$ & $1.9 \mathrm{E}-03$ & $1.9 \mathrm{E}-05$ & $1.8 \mathrm{E}-02$ & $1.8 \mathrm{E}-03$ & 5.1E-02 & $5.1 \mathrm{E}-04$ & $4.9 \mathrm{E}-01$ & $4.9 \mathrm{E}-02$ & & & & & $1.8 \mathrm{E}-03$ & $1.8 \mathrm{E}-05$ & $1.8 \mathrm{E}-02$ & $1.8 \mathrm{E}-03$ \\
\hline Heptachlor Epoxide & $001024-57-3$ & $9.4 \mathrm{E}-04$ & 9.4E-06 & 4.7E-04 & 4.7E-05 & 5.1E-03 & $5.1 \mathrm{E}-05$ & $2.6 \mathrm{E}-03$ & $2.6 \mathrm{E}-04$ & & & & & $7.9 \mathrm{E}-04$ & $7.9 \mathrm{E}-06$ & $4.0 \mathrm{E}-04$ & $4.0 \mathrm{E}-05$ \\
\hline Heptane, N- & $000142-82-5$ & & & & & & & & & & & & & & & & \\
\hline Hexabromobenzene & $000087-82-1$ & & & 7.3E-02 & $7.3 \mathrm{E}-03$ & & & $1.4 \mathrm{E}+00$ & $1.4 \mathrm{E}-01$ & & & & & & & $6.9 \mathrm{E}-02$ & $6.9 \mathrm{E}-03$ \\
\hline Hexachlorobenzene $e^{(6)}$ & $000118-74-1$ & $5.3 \mathrm{E}-03$ & 5.3E-05 & $2.9 \mathrm{E}-02$ & $2.9 \mathrm{E}-03$ & $8.4 \mathrm{E}-03$ & $8.4 \mathrm{E}-05$ & $4.6 \mathrm{E}-02$ & $4.6 \mathrm{E}-03$ & $1.1 \mathrm{E}-03$ & $1.1 \mathrm{E}-05$ & & & $8.0 \mathrm{E}-04$ & $8.0 \mathrm{E}-06$ & $1.8 \mathrm{E}-02$ & $1.8 \mathrm{E}-03$ \\
\hline Hexachlorobutadiene ${ }^{(6)}$ & $000087-68-3$ & $1.1 \mathrm{E}-01$ & $1.1 \mathrm{E}-03$ & 7.3E-03 & 7.3E-04 & $3.0 \mathrm{E}-01$ & $3.0 \mathrm{E}-03$ & $2.0 \mathrm{E}-02$ & $2.0 E-03$ & $2.2 \mathrm{E}-02$ & $2.2 \mathrm{E}-04$ & & & $1.7 \mathrm{E}-02$ & $1.7 \mathrm{E}-04$ & $5.4 \mathrm{E}-03$ & $5.4 \mathrm{E}-04$ \\
\hline $\begin{array}{l}\text { Hexachlorocyclohexane, } \\
\text { Alpha- }\end{array}$ & $000319-84-6$ & $1.4 \mathrm{E}-03$ & $1.4 \mathrm{E}-0 \mathrm{~s}$ & & & $2.9 \mathrm{E}-02$ & $2.9 \mathrm{E}-04$ & & & & & & & $1.3 \mathrm{E}-03$ & $1.3 \mathrm{E}-05$ & & \\
\hline $\begin{array}{l}\text { Hexachlorocyclohexane, } \\
\text { Beta- }\end{array}$ & $000319-85-7$ & $4.7 \mathrm{E}-03$ & $4.7 \mathrm{E}-05$ & & & $8.0 E-02$ & $8.0 E-04$ & & & & & & & $4.5 \mathrm{E}-03$ & 4.5E-05 & & \\
\hline $\begin{array}{l}\text { Hexachlorocyclohexane, } \\
\text { Delta- }\end{array}$ & $000319-86-8$ & & & & & & & & & & & & & & & & \\
\hline $\begin{array}{l}\text { Hexachlorocyclohexane, } \\
\text { Epsilon }\end{array}$ & 006108-10-7 & & & & & & & & & & & & & & & & \\
\hline $\begin{array}{l}\text { Hexachlorocyclohexane, } \\
\text { Gamma- }\end{array}$ & 000058-89-9 & $6.6 \mathrm{E}-03$ & $6.6 \mathrm{E}-05$ & $1.1 \mathrm{E}-02$ & $1.1 \mathrm{E}-03$ & $1.9 \mathrm{E}-01$ & $1.9 \mathrm{E}-03$ & 3.1E-01 & 3.1E-02 & & & & & $6.3 \mathrm{E}-03$ & $6.3 \mathrm{E}-05$ & $1.1 \mathrm{E}-02$ & $1.1 \mathrm{E}-03$ \\
\hline $\begin{array}{l}\text { Hexachlorocyclohexane, } \\
\text { Technical }\end{array}$ & 000608-73-1 & $4.7 \mathrm{E}-03$ & $4.7 \mathrm{E}-05$ & & & & & & & & & & & & & & \\
\hline $\begin{array}{l}\text { Hexachlorocyclopentadie } \\
\text { ne }\end{array}$ & $000077-47-4$ & & & $2.6 \mathrm{E}-01$ & $2.6 \mathrm{E}-02$ & & & $2.9 \mathrm{E}+00$ & $2.9 \mathrm{E}-01$ & & & $1.5 \mathrm{E}-04$ & $1.5 \mathrm{E}-05$ & & & $1.5 \mathrm{E}-04$ & $1.5 \mathrm{E}-05$ \\
\hline $\begin{array}{l}\text { Hexachlorodibenzo-p- } \\
\text { dioxin, Mixture }\end{array}$ & 019408-74-3 & $1.4 \mathrm{E}-06$ & $1.4 \mathrm{E}-08$ & & & $6.3 \mathrm{E}-07$ & $6.3 \mathrm{E}-09$ & & & & & & & $4.3 E-07$ & $4.3 \mathrm{E}-09$ & & \\
\hline Hexachloroethane & $000067-72-1$ & $6.1 \mathrm{E}-01$ & $6.1 \mathrm{E}-03$ & $3.7 \mathrm{E}-02$ & $3.7 \mathrm{E}-03$ & $4.8 \mathrm{E}+00$ & $4.8 \mathrm{E}-02$ & $2.9 \mathrm{E}-01$ & $2.9 \mathrm{E}-02$ & $1.2 \mathrm{E}-01$ & $1.2 E-03$ & & & $9.9 \mathrm{E}-02$ & $9.9 \mathrm{E}-04$ & $3.2 \mathrm{E}-02$ & $3.2 \mathrm{E}-03$ \\
\hline Hexachlorophene & $000070-30-4$ & & & $1.1 E-02$ & $1.1 \mathrm{E}-03$ & & & $1.7 \mathrm{E}-03$ & $1.7 \mathrm{E}-04$ & & & & & & & $1.5 \mathrm{E}-03$ & $1.5 \mathrm{E}-04$ \\
\hline $\begin{array}{l}\text { Hexahydro-1,3,5-trinitro- } \\
1,3,5 \text {-triazine (RDX) }\end{array}$ & $000121-82-4$ & $7.7 \mathrm{E}-02$ & $7.7 \mathrm{E}-04$ & 1.IE-01 & $1.1 \mathrm{E}-02$ & $1.7 E+00$ & $1.7 \mathrm{E}-02$ & $2.4 E+00$ & $2.4 \mathrm{E}-01$ & & & & & $7.4 \mathrm{E}-02$ & $7.4 \mathrm{E}-04$ & $1.0 \mathrm{E}-01$ & $1.0 \mathrm{E}-02$ \\
\hline
\end{tabular}


Table 3.1a. (continued)

\begin{tabular}{|c|c|c|c|c|c|c|c|c|c|c|c|c|c|c|c|c|c|}
\hline \multirow[t]{2}{*}{ Chemical } & \multirow[t]{2}{*}{ CASRN } & \multicolumn{4}{|c|}{ Ingestion (mg/L)* } & \multicolumn{4}{|c|}{ Dermal (mg/L)" } & \multicolumn{4}{|c|}{ Inhalation $(\mathrm{mg} / \mathbf{L})^{\dagger^{*}}$} & \multicolumn{4}{|c|}{$\begin{array}{l}\text { Ingestion + Dermal + } \\
\text { Inhalation (mg/L) }\end{array}$} \\
\hline & & $10^{-4}$ & $10^{-4}$ & $\mathrm{HQ}=1$ & $\mathrm{HQ}=0.1$ & $10^{-1}$ & $10^{-5}$ & HQ=1 & $\mathrm{HQ}=0.1$ & $10^{-4}$ & $10^{5}$ & $\mathrm{HQ}=1$ & $\mathrm{HQ}=0.1$ & $10^{-4}$ & $10^{4}$ & HI=1 & $\mathrm{Hl}=0.1$ \\
\hline $\begin{array}{l}\text { Hexamethylene } \\
\text { Diisocyanate, 1,6- }\end{array}$ & $000822-06-0$ & & & & & & & & & & & & & & & & \\
\hline Hexane, N- & $000110-54-3$ & & & $2.2 \mathrm{E}+00$ & $2.2 \mathrm{E}-01$ & & & $2.2 \mathrm{E}+00$ & $2.2 \mathrm{E}-01$ & & & $4.2 \mathrm{E}-01$ & $4.2 \mathrm{E}-02$ & & & $3.0 \mathrm{E}-01$ & $3.0 \mathrm{E}-02$ \\
\hline Hexanone, 2- & $000591-78-6$ & & & & & & & & & & & & & & & & \\
\hline Hexazinone & $051235-04-2$ & $\cdot$ & & $1.2 \mathrm{E}+00$ & $1.2 \mathrm{E}-01$ & & & $1.0 \mathrm{E}+06$ & $6.0 \mathrm{E}+05$ & & & & & & & $1.2 \mathrm{E}+00$ & $1.2 \mathrm{E}-01$ \\
\hline $\mathrm{HpCDD}, 2,3,7,8=(1)$ & $037871-00-4$ & $5.7 \mathrm{E}-06$ & $5.7 E-08$ & & & & & & & & & & & & & & \\
\hline $\mathrm{HpCDF}, 2,3,7,88^{(4)}$ & 038998-75-3 & 5.7E-06 & 5.7E-08 & & & & & & & & & & & & & & \\
\hline $\mathrm{HxCDD}, 2,3,7,8^{(1)}$ & $034465-46-8$ & $5.7 \mathrm{E}-07$ & $5.7 \mathrm{E}-09$ & & & & & & & & & & & & & & \\
\hline $\mathrm{HxCDF}, 2,3,7,8^{-(4)}$ & 055684-94-1 & $5.7 E-07$ & $5.7 \mathrm{E}-09$ & & & & & & & & & & & & & & \\
\hline Hydrazine & $000302-01-2$ & $2.8 \mathrm{E}-03$ & 2.8E-05 & & & $1.4 \mathrm{E}+01$ & $1.4 \mathrm{E}-01$ & & & & & & & $2.8 \mathrm{E}-03$ & $2.8 \mathrm{E}-05$ & & \\
\hline Hydrazine Sulfate & $010034-93-2$ & $2.8 \mathrm{E}-03$ & $2.8 \mathrm{E}-05$ & & & $1.4 \mathrm{E}+01$ & $1.4 \mathrm{E}-01$ & & & & & & & $2.8 \mathrm{E}-03$ & $2.8 \mathrm{E}-05$ & & \\
\hline Hydrogen Chloride & $007647-01-0$ & & & & & & & & & & & & & & & & \\
\hline Hydrogen Cyanide & $000074-90-8$ & & & $7.3 \mathrm{E}-01$ & $7.3 \mathrm{E}-02$ & & & $2.1 E+01$ & $2.1 \mathrm{E}+00$ & & & & & & & 7.1E-01 & 7.1E-02 \\
\hline Hydrogen Sulfide & 007783-06-4 & & & $1.1 \mathrm{E}-01$ & $1.1 \mathrm{E}-02$ & & & $1.6 \mathrm{E}+00$ & $1.6 \mathrm{E}-01$ & & & & & & & $1.0 \mathrm{E}-01$ & $1.0 \mathrm{E}-02$ \\
\hline Hydroquinone & $000123-31-9$ & & & $1.5 \mathrm{E}+00$ & $1.5 \mathrm{E}-01$ & & & $3.0 \mathrm{E}+02$ & $3.0 \mathrm{E}+01$ & & & & & & & $1.5 \mathrm{E}+00$ & $1.5 \mathrm{E}-01$ \\
\hline Imazalil & $035554-44-0$ & & & $4.7 \mathrm{E}-01$ & $4.7 \mathrm{E}-02$ & & & & & & & & & & & & \\
\hline Imazaquin & 081335-37-7 & & & $9.1 \mathrm{E}+00$ & 9.1E-01 & & & & & & & & & & & & \\
\hline Indeno $\left[1,2,3-c\right.$-cd pyrene $e^{(2)}$ & $000193-39-5$ & $1.2 \mathrm{E}-02$ & $1.2 \mathrm{E}-04$ & & & $7.8 \mathrm{E}-04$ & $7.8 \mathrm{E}-06$ & . & & & & & & 7.4E-04 & $7,4 \mathrm{E}-06$ & & \\
\hline Iprodione & $036734-19-7$ & & & $1.5 \mathrm{E}+00$ & $1.5 \mathrm{E}-01$ & & & & & & & & & & & & \\
\hline Iron & $007439-89-6$ & & & & & & & & & & & & & & & & \\
\hline Isobutyl Alcohol & 000078-83-1 & & & $1.1 \mathrm{E}+01$ & $1.1 \mathrm{E}+00$ & & & $1.4 E+03$ & $1.4 \mathrm{E}+02$ & & & & & & & $1.1 \mathrm{E}+01$ & $1.1 \mathrm{E}+00$ \\
\hline Isophorone & $000078-59-1$ & $9.0 \mathrm{E}+00$ & $9.0 \mathrm{E}-02$ & $7.3 \mathrm{E}+00$ & 7.3E-01 & $4.2 \mathrm{E}+02$ & $4.2 E+00$ & $3.4 E+02$ & $3.4 \mathrm{E}+01$ & & & & & $8.8 \mathrm{E}+00$ & $8.8 \mathrm{E}-02$ & 7.1E+00 & 7.1E-01 \\
\hline
\end{tabular}




\begin{tabular}{|c|c|c|c|c|c|c|c|c|c|c|c|c|c|c|c|c|c|}
\hline & & & & & & & & & & & & I0-3I'I & $00+9[1]$ & & & L-10-810800 & qวzosure $N$ \\
\hline $50-\pi E L$ & $B 0-a E L$ & & & & & & & $20-30^{\circ} \varepsilon$ & $10-\Xi 0^{\circ} \varepsilon$ & & & $50-\exists E L$ & DOE-EEL & & & $\varepsilon-L L-601000$ & 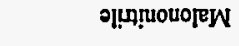 \\
\hline \multirow[t]{2}{*}{$00+981$} & $10+98^{\prime} 1$ & & & & & & & $\varepsilon 0+98^{\circ} \mathrm{I}$ & $50+981$ & & & $00+98 \%$ & $10+98^{\prime 1}$ & & & $1-\varepsilon \varepsilon-\varepsilon z 1000$ & 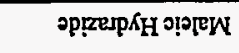 \\
\hline & & & & & & & & & & & & $10-\exists<\mathcal{E} \varepsilon$ & $00+\exists L \cdot E$ & & & $9-1 \varepsilon-801000$ & 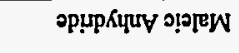 \\
\hline \multirow[t]{2}{*}{$20 \cdot \operatorname{ar} 2$} & $10-a \tau^{\prime}<$ & & & & & & & $00+9126$ & $10+a ! c$ & & & $20-9 \varepsilon .6$ & $10-3 E^{\prime} L$ & & & S-SL-IZ1000 & 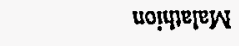 \\
\hline & & & & & & & & & & & & & & & & $\leftarrow S 6-6 \varepsilon \forall<00$ & um!sousew \\
\hline \multirow[t]{2}{*}{$\varepsilon 0-\overline{E S} \mathcal{\varepsilon}$} & 20-as' $\varepsilon$ & & & & & & & I0-ain' & $00+a 1 \cdot 1$ & & & $\mathcal{E} 0 \cdot a^{\prime} \mathcal{E}$ & $20-\exists L \cdot \varepsilon$ & & & $\tau-\varsigma 9-\varepsilon 60000$ & ddכW \\
\hline & & & & & & & & & & & & $20-\mathrm{a} L \mathcal{L} \mathrm{E}$ & $10-\Xi L \mathcal{E}$ & & & $s=18-760000$ & gdכพ \\
\hline \multirow[t]{12}{*}{$\varepsilon 0-78$ 't } & $20-38^{\prime \prime 1}$ & & & & & & & $20 \cdot 3 i 2$ & $10-a C L$ & & & $\varepsilon 0-\mathrm{As}^{\prime} \mathrm{I}$ & $20-581$ & & & $9-t<-t 60000$ & VdכW \\
\hline & & & & & & & & & & & & $10-3 E^{\circ} \mathrm{C}$ & $00+\exists \varepsilon^{\prime} L$ & & & $9-66-\varsigma 50 \varepsilon 80$ & хериот \\
\hline & & & & & & & & & & & & & & & & $\tau-\varepsilon 6-6 \varepsilon \nabla<00$ & นีท!ฺ!? \\
\hline & & & & & & & & & & & & $E 0-E^{\prime} L$ & $20-\bar{g} E L$ & & & Z-Sร-0દE000 & นomuฺ 1 \\
\hline & & & & & & & & & & & & $20-\mathrm{aS} I$ & $20-95$ & & & I-Z6-6£\$LOO & spunoduro $\mathrm{J}$ pur per \\
\hline & & & & & & & & & & & & & & & & $\mathrm{VN}$ & s[N]IV peə] \\
\hline & & & & & & & & & & & & $\varepsilon 0-a E^{\prime} L$ & $20-a E^{\prime} L$ & & & TEE-IOSLLO & นวग010อ7 \\
\hline & & & & & & & & & & & & $10-3 L 2$ & $00+\pi \iota z$ & & & $\varsigma-85-0 \varsigma 6 \varepsilon z 0$ & qיX \\
\hline & & & & & & & & & & & & $20-981$ & $10-38^{\prime} \mathrm{I}$ & & & $9-80-59 p 160$ & भाष्ट्र \\
\hline & & & & & & & & & & & & $10-98^{\prime \prime}$ & $00+781$ & & & $L-0 S-8 S S Z 80$ & usqexosI \\
\hline & & & & & & & & & & & & $10 \cdot \exists \iota^{\prime} \varepsilon$ & $00+\exists L^{\prime} \varepsilon$ & & & $8 * t s-2 \varepsilon 8100$ & 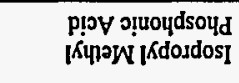 \\
\hline & & & & & & & & & & & & & & & & $0-\varepsilon 9-\angle 90000$ & poredodosi \\
\hline . & & & & & & & & & & & & $20-35 \%$ & 10-as's & & & 0 - $\varepsilon S-0 z 8 \varepsilon \varepsilon 0$ & u|̣pedosdosI \\
\hline$I^{\prime} 0=\mathrm{IH}$ & $\mathrm{I}=\mathrm{IH}$ & , or & rol & $I^{\prime} 0=\partial H$ & $I=O H$ & ,01 & rot & $I^{\circ} 0=0 \mathrm{H}$ & $I=0 H$ &, $0 \mathrm{I}$ & roI & $r^{\prime} 0=\partial H$ & $I=\partial H$ &, 01 & roI & & \\
\hline \multicolumn{4}{|c|}{ 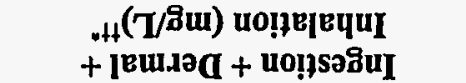 } & \multicolumn{4}{|c|}{ 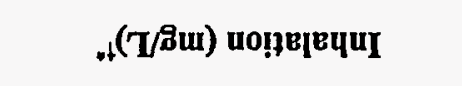 } & \multicolumn{4}{|c|}{ 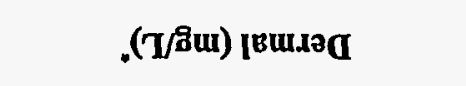 } & \multicolumn{4}{|c|}{.(T/8ีu) uo!̣sว8ิuI } & Nysvว & |вอุุเนวน \\
\hline
\end{tabular}


Table 3.1a. (continued)

\begin{tabular}{|c|c|c|c|c|c|c|c|c|c|c|c|c|c|c|c|c|c|}
\hline \multirow[t]{2}{*}{ Chemical } & \multirow[t]{2}{*}{ CASRN } & \multicolumn{4}{|c|}{ Ingestion (mg/L) } & \multicolumn{4}{|c|}{ Dermal (mg/L)" } & \multicolumn{4}{|c|}{ Inhalation (mg/L) ${ }^{t^{*}}$} & \multicolumn{4}{|c|}{$\begin{array}{l}\text { Ingestion + Dermal + } \\
\text { Inhalation }(\mathrm{mg} / \mathrm{L})^{t^{*}}\end{array}$} \\
\hline & & $10^{-4}$ & $10^{5}$ & $H Q=1$ & $\mathrm{HQ}=0.1$ & $10^{4}$ & $10^{4}$ & $H Q=1$ & $H Q=0.1$ & $10^{-4}$ & $10^{4}$ & $H Q=1$ & $H Q=0.1$ & $10^{-4}$ & $10^{6}$ & $\mathrm{HI}=1$ & $H I=0.1$ \\
\hline Maneb & $012427-38-2$ & & & $1.8 \mathrm{E}-01$ & $1.8 \mathrm{E}-02$ & & & $8.2 \mathrm{E}+02$ & $8.2 E+01$ & & & & & & & $1.8 \mathrm{E}-01$ & $1.8 \mathrm{E}-02$ \\
\hline Manganese (Water) & $007439-96-5$ & & & $1.7 \mathrm{E}+00$ & $1.7 \mathrm{E}-01$ & & & $2.8 \mathrm{E}+01$ & $2.8 \mathrm{E}+00$ & & & & & & & $1.6 \mathrm{E}+00$ & $1.6 \mathrm{E}-01$ \\
\hline Mephosfolan & $000950-10-7$ & & & $3.3 \mathrm{E}-03$ & 3.3E-04 & & & & & & & & & & & & \\
\hline Mepiquat Chloride & $024307-26-4$ & & & $1.1 \mathrm{E}+00$ & $1.1 \mathrm{E}-01$ & & & & & & & & & & & & \\
\hline Mercuric Chloride & $007487-94-7$ & & & $1.1 \mathrm{E}-02$ & $1.1 \mathrm{E}-03$ & & & $9.0 \mathrm{E}-01$ & $9.0 \mathrm{E}-02$ & & & & & & & $1.1 \mathrm{E}-02$ & $1.1 \mathrm{E}-03$ \\
\hline Mercury (elemental) & $007439-97-6$ & & & $1.1 \mathrm{E}-02$ & $1.1 \mathrm{E}-03$ & & & 4.5E-04 & 4.5E-05 & & & & & & & $4.3 \mathrm{E}-04$ & $4.3 \mathrm{E}-05$ \\
\hline Mercury, Inorganic Salt & $007439-97-6$ & & & $1.1 \mathrm{E}-02$ & $1.1 \mathrm{E}-03$ & & & $3.2 \mathrm{E}-01$ & $3.2 \mathrm{E}-02$ & & & & & & & $1.1 \mathrm{E}-02$ & $1.1 \mathrm{E}-03$ \\
\hline Merphos & 000150.50 .5 & & & $1.1 \mathrm{E}-03$ & $1.1 \mathrm{E}-04$ & & & & & & & & & & & & \\
\hline Merphos Oxide & $000078-48-8$ & & & $1.1 \mathrm{E}-03$ & $1.1 \mathrm{E}-04$ & & & $8.8 \mathrm{E}-04$. & $8.8 \mathrm{E}-05$ & & & & & & . & $4.9 \mathrm{E}-04$ & $4.9 \mathrm{E}-05$ \\
\hline Metalaxyl & $057837-19-1$ & & & $2.2 E+00$ & $2.2 \mathrm{E}-01$ & & & & & & & & & & & & \\
\hline Methacrylonitrile & $000126-98-7$ & & & $3.7 \mathrm{E}-03$ & 3.7E-04 & & & $6.7 \mathrm{E}-01$ & $6.7 \mathrm{E}-02$ & & & $1.5 \mathrm{E}-03$ & $1.5 \mathrm{E}-04$ & & & $1.0 \mathrm{E}-03$ & $1.0 \mathrm{E}-04$ \\
\hline Methamidophos & $010265-92-6$ & & & $1.8 \mathrm{E}-03$ & $1.8 \mathrm{E}-04$ & & & $4.2 \mathrm{E}+00$ & $4.2 \mathrm{E}-01$ & & & & & & & $1.8 \mathrm{E}-03$ & $1.8 \mathrm{E}-04$ \\
\hline Methanol & $000067-56-1$ & & & $1.8 \mathrm{E}+01$ & $1.8 \mathrm{E}+00$ & & & $3.8 \mathrm{E}+03$ & $3.8 \mathrm{E}+02$ & & & & & & & $1.8 \mathrm{E}+01$ & $1.8 \mathrm{E}+00$ \\
\hline Methidathion & $000950-37-8$ & & & $3.7 \mathrm{E}-02$ & $3.7 \mathrm{E}-03$ & & & & & & & & & & & & \\
\hline Methomyl & 016752.77 .5 & & & $9.1 \mathrm{E}-01$ & $9.1 \mathrm{E}-02$ & & & $3.6 \mathrm{E}+02$ & $3.6 \mathrm{E}+01$ & & & & & & & $9.1 \mathrm{E}-01$ & $9.1 \mathrm{E}-02$ \\
\hline $\begin{array}{l}\text { Methoxy-5-nitroaniline, } \\
\text { 2- }\end{array}$ & $000099.59-2$ & $1.9 \mathrm{E}-01$ & $1.9 \mathrm{E}-03$ & & & $1.5 \mathrm{E}+01$ & $1.5 \mathrm{E}-01$ & & & & & & & $1.8 \mathrm{E}-01$ & $1.8 \mathrm{E}-03$ & & \\
\hline Methoxychlor & $000072-43-5$ & & & $1.8 \mathrm{E}-01$ & $1.8 \mathrm{E}-02$ & & & $1.9 \mathrm{E}+00$ & $1.9 \mathrm{E}-01$ & & & & & & & $1.7 \mathrm{E}-01$ & $1.7 \mathrm{E}-02$ \\
\hline $\begin{array}{l}\text { Methoxyethanol Acetate, } \\
2-\end{array}$ & $000110-49-6$ & & & $7.3 \mathrm{E}-02$ & 7.3E-03 & & & & & & & & & & & & \\
\hline Methoxyethanol, 2- & $000109-86-4$ & & & 3.7E-02 & $3.7 \mathrm{E}-03$ & & & $4.0 E+01$ & $4.0 E+00$ & & & & & & & 3.6E-02 & $3.6 \mathrm{E}-03$ \\
\hline Methyl Acetate & $000079-20-9$ & & & $3.7 \mathrm{E}+01$ & $3.7 \mathrm{E}+00$ & & & $8.3 \mathrm{E}+03$ & $8.3 \mathrm{E}+02$ & & & & & & & $3.6 \mathrm{E}+01$ & $3.6 \mathrm{E}+00$ \\
\hline
\end{tabular}


Table 3.1a. (continued)

\begin{tabular}{|c|c|c|c|c|c|c|c|c|c|c|c|c|c|c|c|c|c|}
\hline \multirow[t]{2}{*}{ Chemical } & \multirow[t]{2}{*}{ CASRN } & \multicolumn{4}{|c|}{ Ingestion (mg/L)" } & \multicolumn{4}{|c|}{ Dermal (mg/L) } & \multicolumn{4}{|c|}{ Inhalation $(\mathrm{mg} / \mathrm{L})^{\dagger \bullet}$} & \multicolumn{4}{|c|}{$\begin{array}{l}\text { Ingestion + Dermal + } \\
\text { Inhalation }(\mathrm{mg} / \mathrm{L})^{1+{ }^{\star *}}\end{array}$} \\
\hline & & $10^{-4}$ & $10^{6}$ & $H Q=1$ & $\mathrm{HQ}=0.1$ & $10^{-4}$ & $10^{6}$ & $H Q=1$ & $H Q=0.1$ & $10^{-4}$ & $10^{4}$ & $H Q=1$ & $\mathrm{HQ}=0.1$ & $10^{-4}$ & $10^{6}$ & $H I=1$ & $\mathrm{HI}=0.1$ \\
\hline Methyl Acrylate & $000096-33-3$ & & & $1.1 \mathrm{E}+00$ & $1.1 \mathrm{E}-01$ & & & $1.3 \mathrm{E}+02$ & $1.3 \mathrm{E}+01$ & & & & & & & $1.1 \mathrm{E}+00$ & 1.IE-0I \\
\hline Methyl Ethyl Ketone & $000078-93-3$ & & & $2.2 \mathrm{E}+01$ & $2.2 \mathrm{E}+00$ & & & $6.6 \mathrm{E}+03$ & $6.6 \mathrm{E}+02$ & & & $\begin{array}{l}2.1 E+0 \\
0\end{array}$ & $2.1 \mathrm{E}-01$ & & & $1.9 \mathrm{E}+00$ & $1.9 \mathrm{E}-01$ \\
\hline Methyl Isobutyl Ketone & $000108-10-1$ & & & $2.9 \mathrm{E}+00$ & $2.9 \mathrm{E}-01$ & & & $2.9 \mathrm{E}+02$ & $2.9 \mathrm{E}+01$ & & & $1.7 \mathrm{E}-01$ & $1.7 \mathrm{E}-02$ & & & $1.6 \mathrm{E}-01$ & $1.6 \mathrm{E}-02$ \\
\hline Methyl Mercury & $022967-92-6$ & & & $3.7 \mathrm{E}-03$ & 3.7E-04 & & & & & & & & & & & & \\
\hline Methyl Methacrylate & $000080-62-6$ & & & $2.9 \mathrm{E}+00$ & $2.9 \mathrm{E}-01$ & & & $2.5 \mathrm{E}+02$ & $2.5 \mathrm{E}+01$ & & & & & & & $2.9 \mathrm{E}+00$ & $2.9 \mathrm{E}-01$ \\
\hline Methyl Parathion & $000298-00-0$ & & & $9.1 \mathrm{E}-03$ & $9.1 \mathrm{E}-04$ & & & $1.8 \mathrm{E}+00$ & $1.8 \mathrm{E}-01$ & & & & & & & $9.1 \mathrm{E}-03$ & $9.1 \mathrm{E}-04$ \\
\hline $\begin{array}{l}\text { Methyl Styrene (Mixed } \\
\text { Isomers) }\end{array}$ & $025013-15-4$ & & & $2.2 \mathrm{E}-01$ & $2.2 \mathrm{E}-02$ & & & $8.3 E-01$ & $8.3 \mathrm{E}-02$ & & & 8.3E-02 & $8.3 \mathrm{E}-03$ & & & $5.6 \mathrm{E}-02$ & $5.6 \mathrm{E}-03$ \\
\hline Methyl hydrazine $e^{(3)}$ & $000060-34-4$ & $7.7 \mathrm{E}-03$ & 7.7E-05 & & & & & & & & & & & & & & \\
\hline $\begin{array}{l}\text { Methyl tert-Butyl Ether } \\
\text { (MTBE) }\end{array}$ & 001634-04-4 & & & & & & & & & & & $\begin{array}{l}6.3 \mathrm{E}+0 \\
0\end{array}$ & $6.3 \mathrm{E}-01$ & & & & \\
\hline Methyl-5-Nitroaniline, 2- & $000099-55-8$ & $2.6 \mathrm{E}-01$ & $2.6 \mathrm{E}-03$ & & & $9.0 \mathrm{E}+00$ & $9.0 \mathrm{E}-02$ & & & & & & & 2.5E-01 & $2.5 \mathrm{E}-03$ & & \\
\hline $\begin{array}{l}\text { Methylaniline } \\
\text { Hydrochloride, 2- }\end{array}$ & $000636-21-5$ & $4.7 \mathrm{E}-02$ & $4.7 \mathrm{E}-04$ & & & $4.6 \mathrm{E}+00$ & $4.6 \mathrm{E}-02$ & & & & & & & $4.7 \mathrm{E}-02$ & 4.7E-04 & & \\
\hline Methylcyclohexane & $000108-87-2$ & & & & & & & & & & & $\begin{array}{l}6.3 \mathrm{E}+0 \\
0\end{array}$ & $6.3 \mathrm{E}-01$ & & & & \\
\hline Methylene Chloride & $000075-09-2$ & $1.1 E+00$ & 1.JE-02 & $2.2 \mathrm{E}+00$ & 2.2E-01 & $9.9 \mathrm{E}+01$ & $9.9 \mathrm{E}-01$ & $1.9 \mathrm{E}+02$ & $1.9 \mathrm{E}+01$ & $\begin{array}{l}1.0 \mathrm{E}+0 \\
0\end{array}$ & $1.0 \mathrm{E}-02$ & $\begin{array}{l}6.3 \mathrm{E}+0 \\
0\end{array}$ & $6.3 \mathrm{E}-01$ & $5.4 \mathrm{E}-01$ & $5.4 \mathrm{E}-03$ & $1.6 \mathrm{E}+00$ & $1.6 \mathrm{E}-01$ \\
\hline $\begin{array}{l}\text { Methylene-bis(2- } \\
\text { chloroaniline), 4,4'- }\end{array}$ & $000101-14-4$ & $6.6 \mathrm{E}-02$ & $6.6 \mathrm{E}-04$ & $2.6 \mathrm{E}-02$ & $2.6 \mathrm{E}-03$ & $4.8 \mathrm{E}-01$ & $4.8 \mathrm{E}-03$ & $1.9 \mathrm{E}-01$ & $1.9 \mathrm{E}-02$ & & & & & $5.8 \mathrm{E}-02$ & $5.8 \mathrm{E}-04$ & $2.2 \mathrm{E}-02$ & $2.2 \mathrm{E}-03$ \\
\hline $\begin{array}{l}\text { Methylene-bis(N,N- } \\
\text { dimethyl) Aniline, 4,4- }\end{array}$ & $000101-61-1$ & $1.9 \mathrm{E}-01$ & $1.9 \mathrm{E}-03$ & & & $2.9 \mathrm{E}-01$ & $2.9 \mathrm{E}-03$ & & & & & & & $1.1 \mathrm{E}-01$ & $1.1 \mathrm{E}-03$ & & \\
\hline $\begin{array}{l}\text { Methylenebisbenzenami } \\
\text { ne, } 4,4^{\prime}\left({ }^{(5)}\right.\end{array}$ & $000101-77-9$ & $3.4 \mathrm{E}-02$ & $3.4 \mathrm{E}-04$ & & & $4.4 \mathrm{E}+00$ & $4.4 \mathrm{E}-02$ & & & & & & & $3.4 \mathrm{E}-02$ & $3.4 \mathrm{E}-04$ & & \\
\hline
\end{tabular}


Table 3.1a. (continued)

\begin{tabular}{|c|c|c|c|c|c|c|c|c|c|c|c|c|c|c|c|c|c|}
\hline \multirow[t]{2}{*}{ Chemical } & \multirow[t]{2}{*}{ CASRN } & \multicolumn{4}{|c|}{ Ingestion (mg/L)" } & \multicolumn{4}{|c|}{ Dermal (mg/L) } & \multicolumn{4}{|c|}{ Inhalation $(\mathrm{mg} / \mathrm{L})^{\dagger *}$} & \multicolumn{4}{|c|}{$\begin{array}{l}\text { Ingestion + Dermal + } \\
\text { Inhalation }(\mathrm{mg} / \mathrm{L})^{1 t^{*}}\end{array}$} \\
\hline & & $10^{-5}$ & $10^{+}$ & $\mathrm{HQ}=1$ & $\mathrm{HQ}=0.1$ & $10^{-1}$ & $10^{4}$ & $\mathrm{HQ}=\mathbf{1}$ & $\mathrm{HQ}=0.1$ & $10^{-4}$ & $10^{4}$ & $\mathrm{HQ}=1$ & $\mathrm{HQ}=0.1$ & $10^{-4}$ & $10^{5}$ & $\mathrm{HI}=1$ & $\mathrm{HI}=0.1$ \\
\hline $\begin{array}{l}\text { Methylenediphenyl } \\
\text { Isocyanate, 4,4- }\end{array}$ & 000101-68-8 & & & & & & & & & & & $4.2 \mathrm{E}-05$ & $4.2 \mathrm{E}-06$ & & & & \\
\hline Methylstyrene, Alpha- & 000098-83-9 & & & $2.6 \mathrm{E}+00$ & $2.6 \mathrm{E}-01$ & & & $9.7 \mathrm{E}+00$. & $9.7 \mathrm{E}-01$ & & & & & & & $2.0 E+00$ & $2.0 \mathrm{E}-01$ \\
\hline Methyltriethyl Lead & $001762-28-3$ & & & & & & & & & & & & & & & & \\
\hline Metolachlor & $051218-45-2$ & & & $5.5 \mathrm{E}+00$ & $5.5 \mathrm{E}-01$ & & & & & & & & & & & & \\
\hline Metribuzin's) & $021087-64-9$ & & & $9.1 \mathrm{E}-01$ & $9.1 E-02$ & & & $1.2 \mathrm{E}+02$ & $1.2 \mathrm{E}+01$ & & & & & & & $9.1 \mathrm{E}-01$ & $9.1 \mathrm{E}-02$ \\
\hline Mirex & $002385-85-5$ & 4.7E- -03 & 4.7E-05 & 7.3E-03 & 7.3E-04 & $1.9 \mathrm{E}-01$ & $1.9 \mathrm{E}-03$. & $3.0 \mathrm{E}-01$ & $3.0 \mathrm{E}-02$ & & & & & $4.6 \mathrm{E}-03$ & $4.6 \mathrm{E}-05$ & 7.1E-03 & 7.1E-04 \\
\hline Molinate & $002212-67-1$ & & & $7.3 \mathrm{E}-02$ & $7.3 \mathrm{E}-03$ & & & $3.4 \mathrm{E}-01$ & $3.4 \mathrm{E}-02$ & & & & & & & $6.0 \mathrm{E}-02$ & $6.0 \mathrm{E}-03$ \\
\hline Molybdenum & 007439-98-7 & & & $1.8 \mathrm{E}-01$ & $1.8 \mathrm{E}-02$ & & & $2.9 E+01$ & $2.9 E+00$ & & & & & & & $1.8 \mathrm{E}-01$ & $1.8 \mathrm{E}-02$ \\
\hline Monochloramine & 010599-90-3 & & & $3.7 E+00$ & $3.7 \mathrm{E}-01$ & & & & & & & & & & & & \\
\hline Monochlorobutanes & $025154-42-1$ & & & $1.5 \mathrm{E}+01$ & $1.5 \mathrm{E}+00$ & & & & & & & & & & & & \\
\hline Naled & $000300-76-5$ & & & $7.3 \mathrm{E}-02$ & $7.3 \mathrm{E}-03$ & & & $1.7 \mathrm{E}+01$ & $1.7 \mathrm{E}+00$ & & & & & & & $7.3 \mathrm{E}-02$ & 7.3E-03 \\
\hline Naphthalene ${ }^{(6)}$ & $000091-20-3$ & & & $1.3 E+00$ & $1.3 \mathrm{E}-01$ & & & $6.2 E+00$ & $6.2 \mathrm{E}-01$ & & & & & & & $1.1 \mathrm{E}+00$ & $1.1 \mathrm{E}-01$ \\
\hline Naphthalene, 1-Methyl & $000090-12-0$ & & & & & & & & & & & & & & & & \\
\hline Naphthalene, 2-Methyl & $000091-57-6$ & & & & & & & & & & & & & & & & \\
\hline Napropamide & 015299-99-7 & & & $3.7 \mathrm{E}+00$ & $3.7 \mathrm{E}-01$ & & & $6.1 E+03$ & $6.1 E+02$ & & & & & & & $3.6 \mathrm{E}+00$ & $3.6 \mathrm{E}-01$ \\
\hline Niagara Blue 4B & $002429-74-5$ & & & & & & & & & & & & & & & & \\
\hline Nickel Carbonyl & $013463-39-3$ & & & & & & & & & & & & & & & & \\
\hline Nickel Refinery Dust & $\mathrm{NA}$ & & & & & & & & & & & & & & & & \\
\hline Nickel Soluble Salts & $007440-02-0$ & & & 7.3E-01 & $7.3 \mathrm{E}-02$ & & & $8.1 \mathrm{E}+01$ & $8.1 \mathrm{E}+00$ & & & & & & & $7.2 \mathrm{E}-01$ & $7.2 \mathrm{E}-02$ \\
\hline Nickel Subsulfide & $012035-72-2$ & & & & & & & & & & & & & & & & \\
\hline Nitrapyrin't) & $001929-82-4$ & & & $5.5 \mathrm{E}-02$ & $5.5 \mathrm{E}-03$ & & & $1.1 \mathrm{E}+00$ & $1.1 \mathrm{E}-01$ & & & & & & & $5.2 \mathrm{E}-02$ & $5.2 \mathrm{E}-03$ \\
\hline
\end{tabular}


Table 3.1a. (continued)

\begin{tabular}{|c|c|c|c|c|c|c|c|c|c|c|c|c|c|c|c|c|c|}
\hline \multirow[t]{2}{*}{ Chemical } & \multirow[t]{2}{*}{ CASRN } & \multicolumn{4}{|c|}{ Ingestion (mg/L)" } & \multicolumn{4}{|c|}{ Dermal (mg/L)" } & \multicolumn{4}{|c|}{ Inhalation $(\mathrm{mg} / \mathrm{L})^{\dagger^{\star}}$} & \multicolumn{4}{|c|}{$\begin{array}{l}\text { Ingestion + Dermal + } \\
\text { Inhalation }(\mathbf{m g} / \mathbf{L})^{++^{\star}}\end{array}$} \\
\hline & & $10^{-4}$ & $10^{-5}$ & $\mathrm{HQ}=1$ & $\mathrm{HQ}=0.1$ & $10^{-4}$ & $10^{6}$ & $\mathrm{HQ}=1$ & $\mathrm{HQ}=0.1$ & $10^{4}$ & $10^{6}$ & $\mathrm{HQ}=1$ & $\mathrm{HQ}=0.1$ & $10^{-4}$ & $10^{4}$ & $H I=1$ & $\mathrm{HI}=0.1$ \\
\hline Nitrate & $014797-55-8$ & & & $5.8 \mathrm{E}+01$ & $5.8 \mathrm{E}+00$ & & & $1.2 \mathrm{E}+04$ & $1.2 \mathrm{E}+03$ & & & & & & & $5.8 \mathrm{E}+01$ & $5.8 \mathrm{E}+00$ \\
\hline Nitric Oxide & $010102-43-9$ & & & $3.7 \mathrm{E}+00$ & $3.7 \mathrm{E}-01$ & & & $3.0 \mathrm{E}+02$ & $3.0 \mathrm{E}+01$ & & & & & & & $3.6 \mathrm{E}+00$ & $3.6 \mathrm{E}-01$ \\
\hline Nitrite & $014797-65-0$ & & & $3.7 \mathrm{E}+00$ & $3.7 \mathrm{E}-01$ & & & $7.5 \mathrm{E}+02$ & $7.5 \mathrm{E}+01$ & & & & & & & $3.6 \mathrm{E}+00$ & $3.6 \mathrm{E}-01$ \\
\hline Nitroaniline, 2- & $000088-74-4$ & & & & & & & & & & & 4.2E-04 & $4.2 \mathrm{E}-05$ & & & & \\
\hline Nitrobenzene & 000098-95-3 & & & $1.8 \mathrm{E}-02$ & $1.8 \mathrm{E}-03$ & & & $1.1 \mathrm{E}+00$ & $1.1 \mathrm{E}-01$ & & & $4.2 \mathrm{E}-03$ & $4.2 \mathrm{E}-04$ & & & $3.4 \mathrm{E}-03$ & 3.4E-04 \\
\hline Nitrofurantoin & $000067-20-9$ & & & $2.6 \mathrm{E}+00$ & $2,6 \mathrm{E}-01$ & & & & & & & & & & & & \\
\hline Nitrofurazone & $000059-87-0$ & $5.7 \mathrm{E}-03$ & 5.7E-05 & & & & & & & & & & & & & & \\
\hline Nitrogen Dioxide & $010102-44-0$ & & & $3.7 \mathrm{E}+01$ & $3.7 \mathrm{E}+00$ & & & $1.2 \mathrm{E}+04$ & $1.2 E+03$ & & & & & & & $3.6 \mathrm{E}+01$ & $3.6 \mathrm{E}+00$ \\
\hline Nitroguanidine & $000556-88-7$ & & & $3.7 \mathrm{E}+00$ & $3.7 \mathrm{E}-01$ & & & & & & & & & & & & \\
\hline Nitrophenol, 4- & $000100-02-7$ & & & & & & & & & & & & & & & & \\
\hline Nitropropane, 2-(3) & $000079-46-9$ & $9.0 \mathrm{E}-04$ & $9.0 E-06$ & & & $3.0 \mathrm{E}-01$ & $3.0 \mathrm{E}-03$ & & & $1.8 \mathrm{E}-04$ & $1.8 \mathrm{E}-06$ & $4.2 \mathrm{E}-02$ & $4.2 E-03$ & $1.5 \mathrm{E}-04$ & $1.5 \mathrm{E}-06$ & & \\
\hline Nitroso-N-ethylurea, N- & $000759-73-9$ & $6.1 \mathrm{E}-05$ & $6.1 \mathrm{E}-07$ & & & $2.3 \mathrm{E}-02$ & $2.3 \mathrm{E}-04$ & & & & & & & $6.1 \mathrm{E}-05$ & $6.1 \mathrm{E}-07$ & & \\
\hline $\begin{array}{l}\text { Nitroso-N-methylurea, } \\
\mathrm{N} \text { - }\end{array}$ & $000684-93-5$ & & & & & & & & & & & & & & & & \\
\hline $\begin{array}{l}\text { Nitroso-di-N-butylamine, } \\
\text { N- }\end{array}$ & $000924-16-3$ & $1.6 \mathrm{E}-03$ & $1.6 \mathrm{E}-05$ & & & $1.1 \mathrm{E}-01$ & $1.1 \mathrm{E}-03$ & & & $3.2 \mathrm{E}-04$ & $3.2 \mathrm{E}-06$ & & & $2.6 \mathrm{E}-04$ & $2.6 \mathrm{E}-06$ & & \\
\hline $\begin{array}{l}\begin{array}{l}\text { Nitroso-di-N- } \\
\text { propylamine, } \mathrm{N}-\end{array} \\
\end{array}$ & $000621-64-7$ & $1.2 \mathrm{E}-03$ & $1.2 \mathrm{E}-05$ & & & $4.5 \mathrm{E}-02$ & $4.5 \mathrm{E}-04$ & & & & & & & $1.2 \mathrm{E}-03$ & $1.2 \mathrm{E}-05$ & & \\
\hline $\begin{array}{l}\text { Nitrosodiethanolamine, } \\
\mathrm{N}-\end{array}$ & 001116-54-7 & $3.0 \mathrm{E}-03$ & $3.0 \mathrm{E}-05$ & & & $1.3 \mathrm{E}+02$ & $1.3 \mathrm{E}+00$ & & & & & & & $3.0 \mathrm{E}-03$ & $3.0 \mathrm{E}-05$ & & \\
\hline Nitrosodiethylanine, $\mathrm{N}$ - & $000055-18.5$ & 5.7E-05 & 5.7E-07' & & & $9.8 \mathrm{E}-03$ & $9.8 \mathrm{E}-05$ & & & & & & & $5.6 \mathrm{E}-05$ & $5.6 \mathrm{E}-07$ & & \\
\hline $\begin{array}{l}\text { Nitrosodimethylamine, } \\
\text { N- }\end{array}$ & $000062-75-9$ & $1.7 \mathrm{E}-04$ & $1.7 \mathrm{E}-06$ & & & $1.3 \mathrm{E}-01$ & $1.3 \mathrm{E}-03$ & & & & & & & $1.7 \mathrm{E}-04$ & $1.7 \mathrm{E}-06$ & & \\
\hline
\end{tabular}


Table 3.1a. (continued)

\begin{tabular}{|c|c|c|c|c|c|c|c|c|c|c|c|c|c|c|c|c|c|}
\hline \multirow[t]{2}{*}{ Chemical } & \multirow[t]{2}{*}{ CASRN } & \multicolumn{4}{|c|}{ Ingestion (mg/L)" } & \multicolumn{4}{|c|}{ Dermal (mg/L)" } & \multicolumn{4}{|c|}{ Inhalation $(\mathrm{mg} / \mathrm{L})^{\dagger^{\star}}$} & \multicolumn{4}{|c|}{$\begin{array}{l}\text { Ingestion + Dermal + } \\
\text { Inhalation }(\mathbf{m g} / \mathbf{L})^{t^{*}}\end{array}$} \\
\hline & & $10^{-1}$ & $10^{5}$ & $\mathrm{HQ}=1$ & $\mathrm{HQ}=0.1$ & $10^{-4}$ & $10^{5}$ & HQ=1 & $\mathrm{HQ}=0.1$ & $10^{-4}$ & $10^{6}$ & $\mathrm{HQ}=1$ & $\mathrm{HQ}=0.1$ & $10^{-4}$ & $10^{4}$ & $\mathrm{HII}=1$ & $\mathrm{HI}=0.1$ \\
\hline $\begin{array}{l}\text { Nitrosodiphenylamine, } \\
\mathrm{N} \text { - }\end{array}$ & $000086-30-6$ & $1.7 \mathrm{E}+00$ & $1.7 E-02$ & & & $9.2 \mathrm{E}+00$ & $9.2 \mathrm{E}-02$ & & & & & & & $1.5 \mathrm{E}+00$ & $1.5 \mathrm{E}-02$ & & \\
\hline $\begin{array}{l}\text { Nitrosomethylethylamine } \\
\text {, N- }\end{array}$ & $010595-95-6$ & $3.9 \mathrm{E}-04$ & $3.9 E-06$ & & & & & & & & & & & & & & \\
\hline $\begin{array}{l}\text { Nitrosomethylvinylamine } \\
\text {. N- }\end{array}$ & $004549-40-0$ & & & & & & & & & & & & & & & & \\
\hline Nitrosopyrolidine, $\mathrm{N}$ - & $000930-55-2$ & 4.IE-03 & 4.1E-05 & & & $1.1 \mathrm{E}+01$ & $1.1 \mathrm{E}-01$ & & & & & & & 4.1E-03 & 4.1E-05 & & \\
\hline $\begin{array}{l}\text { Nitrotoluene, 4-Amino- } \\
\text { 2- }\end{array}$ & $000119-32-4$ & & & & & & & & & & & & & & & & \\
\hline Nitrotoluene, $m$ - & $000099-08-1$ & & & $3.7 \mathrm{E}-01$ & $3.7 \mathrm{E}-02$ & & & $8.6 \mathrm{E}+00$ & $8.6 \mathrm{E}-01$ & & & & & & & $3.5 \mathrm{E}-01$ & $3.5 \mathrm{E}-02$ \\
\hline Nitrololuene, o- & $000088-72-2$ & & & $3.7 \mathrm{E}-01$ & $3.7 \mathrm{E}-02$ & & & $1.0 E+01$ & $1.0 \mathrm{E}+00$ & & & & & & & $3.5 \mathrm{E}-01$ & $3.5 \mathrm{E}-02$ \\
\hline Nitrotoluene, p- & $000099-99-0$ & & & $3.7 \mathrm{E}-01$ & $3.7 \mathrm{E}-02$ & & & $8.6 \mathrm{E}+00$ & $8.6 \mathrm{E}-01$ & & & & & & & 3.5E-01 & 3.5E-02 \\
\hline Norflurazon & $027314-13-2$ & & & $1.5 \mathrm{E}+00$ & $1.5 \mathrm{E}-01$ & & & & & & & & & & & & \\
\hline Nustar & 085509-19-9 & & & $2.6 \mathrm{E}-02$ & $2.6 \mathrm{E}-03$ & & & & & & & & & & & & \\
\hline $\mathrm{OCDD}^{(4)}$ & $003268-87-9$ & $5.7 \mathrm{E}-0 \mathrm{~S}$ & 5.7E-07 & & & & & & & & & & & & & & \\
\hline$O C D F^{\prime \prime \prime}$ & $039001-02-0$ & $5.7 \mathrm{E}-05$ & 5.7E-07 & & & & & & & & & & & & & & \\
\hline $\begin{array}{l}\text { Octabromodiphenyl } \\
\text { Ether }\end{array}$ & $032536-52-0$ & & & $1.1 \mathrm{E}-01$ & $1.1 \mathrm{E}-02$ & & & & & & & & & & & & \\
\hline $\begin{array}{l}\text { Octahydro-1,3,5,7- } \\
\text { tetranitro-1,3,5,7-tetra } \\
\text { (HMX) }\end{array}$ & $002691-41-0$ & & & $1.8 \mathrm{E}+00$ & $1.8 \mathrm{E}-01$ & & & $1.4 E+03$ & $1.4 \mathrm{E}+02$ & & & & & & & $1.8 \mathrm{E}+00$ & $1.8 \mathrm{E}-01$ \\
\hline $\begin{array}{l}\text { Octamethylpyrophosphor } \\
\text { amide }\end{array}$ & $000152-16-9$ & & & $7.3 \mathrm{E}-02$ & $7.3 \mathrm{E}-03$ & & & & & & & & & & & & \\
\hline Octyl Phthalate, di-N- & $000117-84-0$ & & & $7.3 \mathrm{E}-01$ & $7.3 \mathrm{E}-02$ & & & $1.0 \mathrm{E}-02$ & $1.0 \mathrm{E}-03$ & & & & & & & $9.9 E-03$ & $9.9 \mathrm{E}-04$ \\
\hline Oryzalin & 019044-88-3 & & & $1.8 \mathrm{E}+00$ & $1.8 \mathrm{E}-01$ & & & & & & & & & & & & \\
\hline
\end{tabular}


Table 3.1a. (continued)

\begin{tabular}{|c|c|c|c|c|c|c|c|c|c|c|c|c|c|c|c|c|c|}
\hline \multirow[t]{2}{*}{ Chemical } & \multirow[t]{2}{*}{ CASRN } & \multicolumn{4}{|c|}{ Ingestion (mg/L)" } & \multicolumn{4}{|c|}{ Dermal (mg/L)" } & \multicolumn{4}{|c|}{ Inhalation $(\mathbf{m g} / \mathbf{L})^{\dagger^{\star}}$} & \multicolumn{4}{|c|}{$\begin{array}{l}\text { Ingestion + Dermal + } \\
\text { Inhalation }(\mathrm{mg} / \mathrm{L})^{\mathrm{t+}^{\star}}\end{array}$} \\
\hline & & $10^{-4}$ & $10^{6}$ & $\mathrm{HQ}=1$ & $\mathrm{HQ}=0.1$ & $10^{-4}$ & $10^{-5}$ & $\mathrm{HQ}=1$ & $\mathrm{HQ}=0.1$ & $10^{-4}$ & $10^{4}$ & $\mathrm{HQ}=1$ & $\mathrm{HQ}=0.1$ & $10^{-4}$ & $10^{4}$ & $\mathrm{HI}=1$ & $\mathrm{HI}=0.1$ \\
\hline Oxadiazon & 019666-30-9 & & & $1.8 \mathrm{E}-01$ & $1.8 \mathrm{E}-02$ & & & & & & & & & & & & \\
\hline Oxamyl & $023135-22-0$ & & & $9.1 \mathrm{E}-01$ & $9.1 \mathrm{E}-02$ & & & & & & & & & & & & \\
\hline Paclobutrazol & $076738-62-0$ & & & $4.7 \mathrm{E}-01$ & $4.7 \mathrm{E}-02$ & & & & & & & & & & & & \\
\hline Paraquat & $001910-42-5$ & & & $1.6 \mathrm{E}-01$ & $1.6 \mathrm{E}-02$ & & & $2.4 \mathrm{E}+05$ & $2.4 \mathrm{E}+04$ & & & & & & & $1.6 \mathrm{E}-01$ & $1.6 \mathrm{E}-02$ \\
\hline Parathion & $000056-38-2$ & & & $2.2 \mathrm{E}-01$ & $2.2 \mathrm{E}-02$ & & & $2.7 \mathrm{E}+00$ & $2.7 \mathrm{E}-01$ & & & & & & & $2.0 E-01$ & $2.0 \mathrm{E}-02$ \\
\hline PeCDD, 2,3,7,8-(4) & $036088-22-9$ & $1.1 \mathrm{E}-07$ & $1.1 \mathrm{E}-09$ & & & & & & & & & & & & & & \\
\hline $\mathrm{PeCDF}, 1,2,3,7,8$-(4) & $057117-41-6$ & $1.1 E-07$ & $1.1 \mathrm{E}-09$ & & & & & & & & & & & & & & \\
\hline PeCDF, 2,3,4,7,8-(4) & $057117.31-4$ & $1.1 \mathrm{E}-06$ & $1.1 E-08$ & & & & & & & & & & & & & & \\
\hline Pebulate & $001114-71-2$ & & & $1.8 E+00$ & $1.8 \mathrm{E}-01$ & & & & & & & & & & & & \\
\hline Pendimethalin & $040487-42-1$ & & & $1.5 \mathrm{E}+00$ & $1.5 E-01$ & & & & & & & & & & & & \\
\hline $\begin{array}{l}\text { Pentabromodiphenyl } \\
\text { Ether }\end{array}$ & $032534-81-9$ & & & $7.3 \mathrm{E}-02$ & $7.3 \mathrm{E}-03$ & & & & & & & & & & & & \\
\hline Pentachlorobenzene & 000608-93-5 & & & $2.9 \mathrm{E}-02$ & $2.9 \mathrm{E}-03$ & & & $3.5 \mathrm{E}-02$ & 3.5E-03 & & & & & & & $1.6 \mathrm{E}-02$ & $1.6 \mathrm{E}-03$ \\
\hline $\begin{array}{l}\text { Pentachlorocyclopentadi } \\
\text { ene }\end{array}$ & 025329-35-5 & & & & & & & & & & & & & & & & \\
\hline Pentachloronitrobenzene & 000082-68-8 & $3.3 \mathrm{E}-02$ & $3.3 \mathrm{E}-04$ & $1.1 \mathrm{E}-01$ & $1.1 \mathrm{E}-02$ & $1.8 \mathrm{E}-01$ & $1.8 \mathrm{E}-03$ & $6.1 \mathrm{E}-01$ & $6.1 \mathrm{E}-02$ & & & & & $2.8 \mathrm{E}-02$ & $2.8 \mathrm{E}-04$ & $9.3 \mathrm{E}-02$ & $9.3 \mathrm{E}-03$ \\
\hline Pentachlorophenol & $000087-86-5$ & $7.1 \mathrm{E}-02$ & $7.1 \mathrm{E}-04$ & $1.1 \mathrm{E}+00$ & 1.1E-01 & $4.5 \mathrm{E}-02$ & $4.5 \mathrm{E}-04$ & $6.9 \mathrm{E}-01$ & $6.9 \mathrm{E}-02$ & & & & & $2.8 \mathrm{E}-02$ & $2.8 \mathrm{E}-04$ & $4.3 \mathrm{E}-01$ & 4.3E-02 \\
\hline Pentyl Alcohol, N- & $000071-41-0$ & & & & & & & & & & & & & & & & \\
\hline Permethrin & $052645-53-1$ & & & $1.8 \mathrm{E}+00$ & $1.8 \mathrm{E}-01$ & & & $1.6 \mathrm{E}+02$ & $1.6 \mathrm{E}+01$ & & & & & & & $1.8 \mathrm{E}+00$ & $1.8 \mathrm{E}-01$ \\
\hline Phenanthrene & $000085-01-8$ & & & & & & & & & & & & & & & & \\
\hline Phenmedipham & $013684-63-4$ & & & $9.1 \mathrm{E}+00$ & $9.1 \mathrm{E}-01$ & & & $5.6 \mathrm{E}+0.5$ & $5.6 \mathrm{E}+04$ & & & & & & & $9.1 \mathrm{E}+00$ & $9.1 \mathrm{E}-01$ \\
\hline Phenol & $000108-95-2$ & & & $2.2 E+01$ & $2.2 \mathrm{E}+00$ & & & $1.5 \mathrm{E}+03$ & $1.5 \mathrm{E}+02$ & & & & & & & $2.2 \mathrm{E}+01$ & $2.2 \mathrm{E}+00$ \\
\hline
\end{tabular}




\begin{tabular}{|c|c|c|c|c|c|c|c|c|c|c|c|c|c|c|c|c|c|}
\hline $20 \cdot-37 \div s$ & $10-a b^{\prime} s$ & & & & & & & $00+\pi \varepsilon \varepsilon$ & $10+[\varepsilon \varepsilon$ & & & $20-35 \cdot s$ & $10-95 ' s$ & & & $0-81-019100$ & 40156104d \\
\hline & & & & $\cdot$ & & & & & & & & $20-9 z \tau$ & $10-a z z$ & & & $0-9 \varepsilon \cdot 66 \varepsilon 970$ & uppenjosd \\
\hline & & & & & & & & & & & & $20-a \varepsilon \varepsilon$ & $10-a \varepsilon \varepsilon$ & $60-3 a \cdot s$ & $20-a L \cdot 5$ & $s=60-\angle P L L 90$ & zesolpo01d \\
\hline $10-a \tau^{\prime} L$. & $00+a z L$ & & & & & & & $10+909$ & $20+30.9$ & & & $10-3 E<L$ & $00+\pi \varepsilon L$ & & & $9-19-905000$ & $\begin{array}{r}\text { oppruero } \\
\text { sal!S un!sseiod }\end{array}$ \\
\hline \multirow[t]{4}{*}{$10-98 \mathrm{t}$} & $00+381$ & & & & & & & $20+321$ & $\varepsilon 0+\nexists Z \mathbf{I}$ & & & $10-38.1$ & $00+9811$ & & & $8-05-151000$ & opptrreßo um!sstod \\
\hline & & $90-a / s$ & $50-a / 2 \cdot s$ & & & & & & & so:3z'I & $\varepsilon 0-\mathrm{sz} \mathfrak{\tau}$ & & & so-ar't & Eo-aI' I & $\varepsilon-9 \varepsilon-9 \varepsilon \varepsilon 100$ & 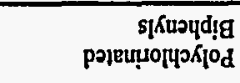 \\
\hline & & & & & & & & & & & & $50-39 \cdot z$ & to-a9:z & $90-39 \cdot 6$ & $50-99 \% 6$ & YN & 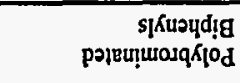 \\
\hline & & & & & & & & & & & & $20-a L^{\prime} \varepsilon$ & $10-a c^{\cdot} \varepsilon$ & & & 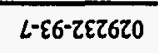 & 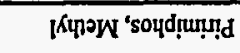 \\
\hline 10-ast $z$ & $00+3 s^{2} \tau$ & & & & & & & $10+38 z$ & $20+382$ & & & $10-39 \cdot z$ & $00+997$ & & & $1-20-816100$ & uresop!̣ d \\
\hline $00+3 \varepsilon L$ & $10+a E E^{\prime} L$ & & & & & & & $20+3 L T$ & $s_{0}+3 L^{\prime} t$ & & & $00+\nexists E L L$ & $10+\pi \varepsilon L$ & & & $6-t+580000$ & 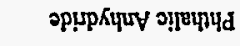 \\
\hline \multirow[t]{2}{*}{$00+39^{\circ} \varepsilon$} & $10+39 \varepsilon$ & & & & & & & $20+\pi 5 \cdot z$ & $\varepsilon 0+3 \xi z$ & & & $00+a l^{\prime} \varepsilon$ & $10+9 L \cdot \varepsilon$ & & & $0-12-001000$ & -d 'p!०७ ग़ाष्यापd \\
\hline & & & & & & & & & & & & & & & & $\tau-8 \varepsilon-699<00$ & p!oy orougdsold \\
\hline$\varepsilon 0-a ! \cdot$ & zo-ar't & & & & & & & $20-30 \cdot 6$ & $10-90 \% 6$ & & & E0-aI' & $20-9 I^{\prime \prime 1}$ & & & $\tau-15-\varepsilon 08<00$ & aụydsoyd \\
\hline $20 \cdot a z L$ & $10-9 z<2$ & & & & & & & $00+30^{\circ} \mathrm{L}$ & $10+30^{\circ} \mathrm{L}$ & & & $20-a \varepsilon L$ & $10-a E L$ & & & 9-II-ZEL000 & fowsoyd \\
\hline \multirow[t]{3}{*}{$50-a \varepsilon<$} & $\varepsilon 0-a \varepsilon<$ & & & & & & & $10-96 \cdot \mathrm{t}$ & $00+96^{\circ} \mathrm{I}$ & & & $50-a \varepsilon L$ & $\varepsilon 0-a \varepsilon L$ & & & $z-20-86 z 000$ & गुस०णपd \\
\hline & & $20-\Xi 6 \cdot \varepsilon$ & $00+\Xi 66^{\prime} \varepsilon$ & & & & & & & I0-a $\varepsilon \varepsilon$ & $10+\exists E E$ & & & $20-a b^{2} t$ & $00+3 p+b$ & L-Et-060000 & 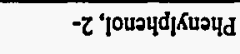 \\
\hline & & & & & & & & & & & & $10-36 \mathrm{z}$ & $\varepsilon 0^{-a 6} \tau$ & & & $58 \varepsilon-290000$ & 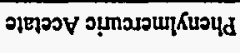 \\
\hline \multirow[t]{2}{*}{$10-969$} & $00+969$ & & & & & & & $\varepsilon 0+a b z$ & $60+3 t z$ & & & $10-369$ & $00+969$ & & & $\varepsilon-05-901000$ & 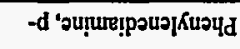 \\
\hline & & $\varepsilon 0-381$ & $10-38 \cdot 1$ & & & & & & & 10-3t5 & $10+a b r$ & & & $\varepsilon 0-38^{\circ} \mathrm{I}$ & $10-38^{\prime \prime} \mathrm{I}$ & $s-t s-s 60000$ & 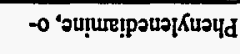 \\
\hline $20-a z z$ & $10-\exists z z$ & & & & & & & $10+a c^{\prime} \varepsilon$ & $20+\nexists 2 \varepsilon \varepsilon$ & & & $20-3 z z$ & $10-3 z z$ & & & $\tau-5 t-801000$ & 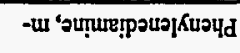 \\
\hline$I^{r} 0=1 \mathrm{H}$ & $\mathbf{I}=\mathbf{I H}$ & ,0I & roI & $I^{\circ} 0=O H$ & $I=O H$ & ,0I & .OI & $I^{\prime} \mathrm{O}=\partial \mathrm{H}$ & $I=\partial H$ & $.0 \mathrm{I}$ & .0I & $I^{\circ} 0=\mathrm{OH}$ & $\mathrm{I}=\mathrm{OH}$ & ,0I & .0I & & \\
\hline \multicolumn{4}{|c|}{ 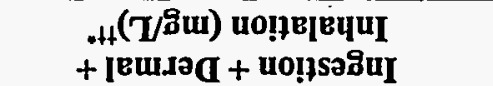 } & \multicolumn{4}{|c|}{ 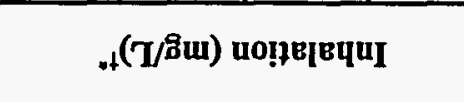 } & \multicolumn{4}{|c|}{ 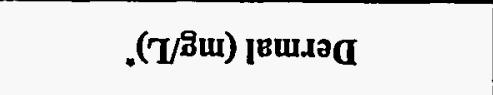 } & \multicolumn{4}{|c|}{ 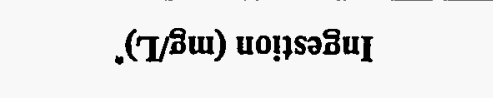 } & NQSVD & 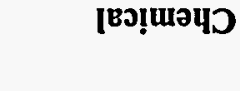 \\
\hline
\end{tabular}


Table 3.1a. (continued)

\begin{tabular}{|c|c|c|c|c|c|c|c|c|c|c|c|c|c|c|c|c|c|}
\hline \multirow[t]{2}{*}{ Chemical } & \multirow[t]{2}{*}{ CASRN } & \multicolumn{4}{|c|}{ Ingestion (mg/L)* } & \multicolumn{4}{|c|}{ Dermal (mg/L)" } & \multicolumn{4}{|c|}{ Inhalation $(\mathrm{mg} / \mathbf{L})^{\dagger *}$} & \multicolumn{4}{|c|}{$\begin{array}{l}\text { Ingestion + Dermal + } \\
\text { Inhalation }(\mathrm{mg} / \mathrm{L})^{+t^{*}}\end{array}$} \\
\hline & & $10^{-4}$ & $10^{-6}$ & $\mathrm{HQ}=1$ & $\mathrm{HQ}=0.1$ & $10^{-4}$ & $10^{-5}$ & $H Q=1$ & $\mathrm{HQ}=0.1$ & $10^{-4}$ & $10^{6}$ & $\mathrm{HQ}=\mathbf{I}$ & $\mathrm{HQ}=0.1$ & $10^{-4}$ & $10^{6}$ & $\mathrm{HI}=\mathbf{1}$ & $\mathrm{HI}=0.1$ \\
\hline Prometryn & $007287-19-6$ & & & $1.5 \mathrm{E}-01$ & $1.5 \mathrm{E}-02$ & & & $7.9 \mathrm{E}+00$ & $7.9 \mathrm{E}-01$ & & & & & & & $1.4 \mathrm{E}-01$ & $1.4 \mathrm{E}-02$ \\
\hline Propachlor & 001918-16-7 & & & $4.7 \mathrm{E}-01$ & $4.7 \mathrm{E}-02$ & & & & & & & & & & & & \\
\hline Propanil & 000709-98-8 & & & $1.8 \mathrm{E}-01$ & $1.8 \mathrm{E}-02$ & & & $2.6 \mathrm{E}+00$ & $2.6 \mathrm{E}-01$ & & & & & & & $1.7 \mathrm{E}-01$ & $1.7 \mathrm{E}-02$ \\
\hline Propargite & $002312-35-8$ & & & 7.3E-01 & 7.3E-02 & & & & & & & & & & & & \\
\hline Propargyl Alcohol & $000107-19-7$ & & & 7.3E-02 & 7.3E-03 & & & & & & & & & & & & \\
\hline Propazine & $000139-40-2$ & & & 7.3E-01 & 7.3E-02 & & & $4.0 \mathrm{E}+01$ & $4.0 \mathrm{E}+00$ & & & & & & & $7.2 \mathrm{E}-01$ & $7.2 \mathrm{E}-02$ \\
\hline Propham & $000122-42-9$ & & & 7.3E-01 & $7.3 \mathrm{E}-02$ & & & $5.2 \mathrm{E}+00$ & $5.2 \mathrm{E}-01$ & & & & & & & $6.4 \mathrm{E}-01$ & $6.4 \mathrm{E}-02$ \\
\hline Propiconazole & $060207-90-1$ & & & 4.7E-01 & $4.7 \mathrm{E}-02$ & & & & & & & & & & & & \\
\hline Propylene Glycol & $000057-55-6$ & & & $7.3 \mathrm{E}+02$ & $7.3 \mathrm{E}+01$ & & & $1.0 \mathrm{E}+06$ & $3.7 \mathrm{E}+05$ & & & & & & & $7.3 \mathrm{E}+02$ & $7.3 E+01$ \\
\hline $\begin{array}{l}\text { Propylene Glycol } \\
\text { Monoethyl Ether }\end{array}$ & $001569.02-4$ & & & $2.6 \mathrm{E}+01$ & $2.6 \mathrm{E}+00$ & & & & & & & & & & & & \\
\hline $\begin{array}{l}\text { Propylene Glycol } \\
\text { Monomethyl Ether }\end{array}$ & $000107-98-2$ & & & $2.6 \mathrm{E}+01$ & $2.6 \mathrm{E}+00$ & & & $1.3 \mathrm{E}+04$ & $1.3 \mathrm{E}+03$ & & & & & & & $2.6 \mathrm{E}+01$ & $2.6 \mathrm{E}+00$ \\
\hline Propylene Oxide & $000075-56-9$ & 3.5E-02 & 3.5E-04 & & & $1.3 \mathrm{E}+01$ & $1.3 \mathrm{E}-01$ & & & $1.3 \mathrm{E}-01$ & $1.3 \mathrm{E}-03$ & $6.3 \mathrm{E}-02$ & $6.3 \mathrm{E}-03$ & $2.8 \mathrm{E}-02$ & $2.8 \mathrm{E}-04$ & & \\
\hline Pursuit & $081335-77.5$ & & & $9.1 \mathrm{E}+00$ & 9.1E-01 & & & & & & & & & & & & \\
\hline Pydrin & 051630-58-1 & & & 9.1E- 01 & $9.1 \mathrm{E}-02$ & & & $2.6 \mathrm{E}+01$ & $2.6 \mathrm{E}+00$ & & & & & & & $8.8 \mathrm{E}-01$ & $8.8 \mathrm{E}-02$ \\
\hline Pyrene & $000129-00-0$ & & & $1.1 \mathrm{E}+00$ & $1.1 \mathrm{E}-01$ & & & $4.3 \mathrm{E}-01$ & $4.3 \mathrm{E}-02$ & & & & & & & $3.1 \mathrm{E}-01$ & 3.1E- -02 \\
\hline Pyridine & 000110-86-1 & & & $3.7 \mathrm{E}-02$ & $3.7 \mathrm{E}-03$ & & & $1.4 \mathrm{E}+00$ & $1.4 \mathrm{E}-01$ & & & & & & & 3.6E-02 & $3.6 \mathrm{E}-03$ \\
\hline Quinalphos & $013593-03-8$ & & & $1.8 \mathrm{E}-02$ & $1.8 \mathrm{E}-03$ & & & & & & & & & & & & \\
\hline Quinoline & $000091-22-5$ & 7.1E-04 & 7.1E-06 & & & $1.5 \mathrm{E}-02$ & $1.5 \mathrm{E}-04$ & & & & & & & $6.8 \mathrm{E}-04$ & $6.8 \mathrm{E}-06$ & & \\
\hline $\begin{array}{l}\text { Refractory Ceramic } \\
\text { Fibers }\end{array}$ & NA & & & & & & & & & & & & & & & & \\
\hline Resmethrin & $010453-86-8$ & & & $1.1 \mathrm{E}+00$ & $1.1 \mathrm{E}-01$ & & & & & & & & & & & & \\
\hline
\end{tabular}


Table 3.1a. (continued)

\begin{tabular}{|c|c|c|c|c|c|c|c|c|c|c|c|c|c|c|c|c|c|}
\hline \multirow[t]{2}{*}{ Chemical } & \multirow[t]{2}{*}{ CASRN } & \multicolumn{4}{|c|}{ Ingestion (mg/L)" } & \multicolumn{4}{|c|}{ Dermal (mg/L) } & \multicolumn{4}{|c|}{ Inhalation $(\mathrm{mg} / \mathrm{L})^{\dagger^{\star}}$} & \multicolumn{4}{|c|}{$\begin{array}{l}\text { Ingestion + Dermal + } \\
\text { Inhalation }(\mathrm{mg} / \mathrm{L})^{\mathrm{t+}}\end{array}$} \\
\hline & & $10^{-1}$ & $10^{-4}$ & $H Q=1$ & $H Q=0.1$ & $10^{-4}$ & $10^{4}$ & $H Q=1$ & $\mathrm{HQ}=0.1$ & $10^{-4}$ & $10^{-6}$ & $\mathrm{HQ}=1$ & $\mathrm{HQ}=0.1$ & $10^{4}$ & $10^{6}$ & $H I=I$ & $\mathrm{HI}=0.1$ \\
\hline Ronnel & $000299-84-3$ & & & $1.8 \mathrm{E}+00$ & $1.8 \mathrm{E}-01$ & & & $4.3 E+00$ & 4.3E-01 & & & & & & & $1.3 \mathrm{E}+00$ & $1.3 E-01$ \\
\hline Rotenone & $000083-79-4$ & & & $1.5 \mathrm{E}-01$ & $1.5 \mathrm{E}-02$ & & & $4.9 \mathrm{E}+00$ & $4.9 \mathrm{E}-01$ & & & & & & & $1.4 \mathrm{E}-01$ & $1.4 \mathrm{E}-02$ \\
\hline Savey & $078587-05-0$ & & & $9.1 \mathrm{E}-01$ & 9.1E-02 & & & & & & & & & & & & \\
\hline Selenious Acid & $007783-00-8$ & & & $1.8 \mathrm{E}-01$ & $1.8 \mathrm{E}-02$ & & & & & & & & & & & & \\
\hline Selenite & $014124-67-5$ & & & & & & & & & & & & & & & & \\
\hline Selenium & $007782-49-2$ & & & $1.8 \mathrm{E}-01$ & $1.8 \mathrm{E}-02$ & & & $3.3 \mathrm{E}+01$ & $3.3 \mathrm{E}+00$ & & & & & & & $1.8 \mathrm{E}-01$ & $1.8 \mathrm{E}-02$ \\
\hline Selenium Sulfide & $007446-34-6$ & & & & & & & & & & & & & & & & \\
\hline Selenourea & $000630-10-4$ & & & $1.8 \mathrm{E}-01$ & $1.8 \mathrm{E}-02$ & & & & & & & & & & & & \\
\hline Sethoxydim & $074051-80-2$ & & & $3.3 \mathrm{E}+00$ & $3.3 \mathrm{E}-01$ & & & & & & & & & & & & \\
\hline Silver & $007440-22-4$ & & & $1.8 \mathrm{E}-01$ & $1.8 \mathrm{E}-02$ & & & $1.4 \mathrm{E}+01$ & $1.4 \mathrm{E}+00$ & & & & & & & $1.8 \mathrm{E}-01$ & $1.8 \mathrm{E}-02$ \\
\hline Silver Cyanide & $000506-64-9$ & & & $3.7 \mathrm{E}+00$ & $3.7 \mathrm{E}-01$ & & & $3.0 \mathrm{E}+02$ & $3.0 \mathrm{E}+01$ & & & & & & & $3.6 \mathrm{E}+00$ & $3.6 \mathrm{E}-01$ \\
\hline Simazine & $000122-34-9$ & 7.1E-02 & 7.1E-04 & $1.8 \mathrm{E}-01$ & $1.8 \mathrm{E}-02$ & $3.6 \mathrm{E}+00$ & $3.6 \mathrm{E}-02$ & $9.2 \mathrm{E}+00$ & $9.2 \mathrm{E}-01$ & & & & & $7.0 \mathrm{E}-02$ & $7.0 \mathrm{E}-04$ & $1.8 \mathrm{E}-01$ & $1.8 \mathrm{E}-02$ \\
\hline Sodium & $007440-23-5$ & & & & & & & & & & & & & & & & \\
\hline Sodium Acifluorfen & $062476-59-9$ & & & 4.7E-0l & 4.7E-02 & & & & & & & & & & & & \\
\hline Sodium Azide & $026628-22-8$ & & & $1.5 \mathrm{E}-01$ & $1.5 \mathrm{E}-02$ & & & $1.2 \mathrm{E}+0 \mathrm{t}$ & $1.2 E+00$ & & & & & & & $1.4 \mathrm{E}-01$ & $1.4 \mathrm{E}-02$ \\
\hline Sodium Cyanide & $000143-33-9$ & & & $1.5 \mathrm{E}+00$ & $1.5 \mathrm{E}-01$ & & & $1.9 \mathrm{E}+03$ & $1.9 \mathrm{E}+02$ & & & & & & & $1.5 \mathrm{E}+00$ & $1.5 \mathrm{E}-01$ \\
\hline $\begin{array}{l}\text { Sodium } \\
\text { Diethyldithiocarbamate }\end{array}$ & $000148-18-5$ & $3.2 \mathrm{E}-02$ & $3.2 \mathrm{E}-04$ & $1.1 \mathrm{E}+00$ & $1.1 \mathrm{E}-01$ & & & & & & & & & & & & \\
\hline Sodium Fluoroacetate & $000062-74-8$ & & & $7.3 \mathrm{E}-04$ & 7.3E-05 & & & & & & & & & & & & \\
\hline Sodium Metavanadate & $013718-26-8$ & & & $3,7 \mathrm{E}-02$ & $3.7 \mathrm{E}-03$ & & & $3.0 \mathrm{E}+00$ & $3.0 \mathrm{E}-01$ & & & & & & & $3.6 \mathrm{E}-02$ & $3.6 \mathrm{E}-03$ \\
\hline $\begin{array}{l}\text { Stirofos } \\
\text { (Tetrachlorovinphos) }\end{array}$ & $000961-11-5$ & $3.5 \mathrm{E}-01$ & $3.5 \mathrm{E}-03$ & $1.1 \mathrm{E}+00$ & $1.1 \mathrm{E}-01$ & & & & & & & & & & & & \\
\hline
\end{tabular}


Table 3.1a. (continued)

\begin{tabular}{|c|c|c|c|c|c|c|c|c|c|c|c|c|c|c|c|c|c|}
\hline \multirow[t]{2}{*}{ Chemical } & \multirow[t]{2}{*}{ CASRN } & \multicolumn{4}{|c|}{ Ingestion (mg/L)" } & \multicolumn{4}{|c|}{ Dermal (mg/L)" } & \multicolumn{4}{|c|}{ Inhalation $(\mathrm{mg} / \mathrm{L})^{\dagger *}$} & \multicolumn{4}{|c|}{$\begin{array}{l}\text { Ingestion + Dermal + } \\
\text { Inhalation }(\mathrm{mg} / \mathbf{L})^{1 t^{*}}\end{array}$} \\
\hline & & $10^{-1}$ & $10^{6}$ & $\mathrm{HQ}=1$ & $\mathrm{HQ}=0.1$ & $10^{-1}$ & $10^{-5}$ & $\mathrm{HQ}=1$ & $\mathrm{HQ}=0.1$ & $10^{-1}$ & $10^{5}$ & $\mathrm{HQ}=1$ & $\mathrm{HQ}=0.1$ & $10^{-4}$ & $10^{5}$ & $\mathrm{HI}=1$ & $\mathrm{HI}=0.1$ \\
\hline Strontium, Stable & $007440-24-6$ & & & $2.2 \mathrm{E}+01$ & $2.2 E+00$ & & & $1.8 \mathrm{E}+03$ & $1.8 \mathrm{E}+02$ & & & & & & & $2.2 \mathrm{E}+01$ & $2.2 \mathrm{E}+00$ \\
\hline Strychnine & $000057-24-9$ & & & $1.1 E-02$ & $1.1 \mathrm{E}-03$ & & & $5.8 \mathrm{E}+00$ & $5.8 \mathrm{E}-01$ & & & & & & & $1.1 \mathrm{E}-02$ & $1.1 \mathrm{E}-03$ \\
\hline Styrene ${ }^{(6)}$ & $000100-42-5$ & & & $7.3 \mathrm{E}+00$ & 7.3E-01 & & & $3.6 \mathrm{E}+00$ & $3.6 \mathrm{E}-01$ & & & $\begin{array}{l}2.1 \mathrm{E}+0 \\
0\end{array}$ & $2.1 E-01$ & & & $1.1 \mathrm{E}+00$ & $1.1 \mathrm{E}-01$ \\
\hline Sulfate & 014808-79-8 & & & & & & & & & & & & & & & & \\
\hline Systhane & $088671-89-0$ & & & $9.1 \mathrm{E}-01$ & $9.1 \mathrm{E}-02$ & & & & & & & & & & & & \\
\hline TCDD, 2,3,7,8-(4) & $001746-01-6$ & $5.7 \mathrm{E}-08$ & $5.7 \mathrm{E}-10$ & & & $8.4 \mathrm{E}-09$ & $8.4 E-11$ & & & & & & & 7.3E-09 & $7.3 \mathrm{E}-11$ & & \\
\hline TCDF, 2,3,7,8.(1) & $051207-31-9$ & $5.7 \mathrm{E}-07$ & $5.7 \mathrm{E}-09$ & & & & & & & & & & & & & & \\
\hline TCMTB & $021564-17-0$ & & & $1.1 \mathrm{E}+00$ & $1.1 \mathrm{E}-01$ & & & & & & & & & & & & \\
\hline Tebuthiuron & 034014-18-1 & & & $2.6 \mathrm{E}+00$ & $2.6 \mathrm{E}-01$ & & & $5.3 \mathrm{E}+03$ & $5.3 \mathrm{E}+02$ & & & & & & & $2.6 \mathrm{E}+00$ & 2.6E-01 \\
\hline Temephos & 003383-96-8 & & & $7.3 \mathrm{E}-01$ & $7.3 \mathrm{E}-02$ & & & & & & & & & & & & \\
\hline Terbacil & $005902-51-2$ & & & $4.7 \mathrm{E}-01$ & $4.7 \mathrm{E}-02$ & & & & & & & & & & & & \\
\hline Terbufos & $013071-79-9$ & & & $9.1 \mathrm{E}-04$ & $9.1 \mathrm{E}-05$ & & & $1.4 \mathrm{E}-02$ & $1.4 \mathrm{E}-03$ & & & & & & & $8.6 \mathrm{E}-04$ & 8.6E-05 \\
\hline Terbutryn & $000886-50-0$ & & & $3.7 E-02$ & $3.7 \mathrm{E}-03$ & & & $2.6 \mathrm{E}-01$ & $2.6 \mathrm{E}-02$ & & & & & & & $3.2 \mathrm{E}-02$ & $3.2 \mathrm{E}-03$ \\
\hline Tetrabutyl Lead & $001920-90-7$ & & & & & & & & & & & & & & & & \\
\hline $\begin{array}{l}\text { Tetrachlorobenzene, } \\
1,2,4,5-\end{array}$ & $000095-94-3$ & & & $1.1 \mathrm{E}-02$ & $1.1 \mathrm{E}-03$ & & & 2.1E-02 & $2.1 \mathrm{E}-03$ & & & & & & & 7.2E- 03 & 7.2E-04 \\
\hline $\begin{array}{l}\text { Tetrachloroethane, } \\
1,1,1,2-\end{array}$ & $000630-20.6$ & 3.3E-01 & 3.3E-03 & $1.1 \mathrm{E}+00$ & $1 . \mathrm{IE}-01$ & $4.5 \mathrm{E}+00$ & $4.5 \mathrm{E}-02$ & $1.5 \mathrm{E}+01$ & $1.5 \mathrm{E}+00$ & $6.6 \mathrm{E}-02$ & $6.6 \mathrm{E}-04$ & & & $5.4 \mathrm{E}-02$ & $5.4 \mathrm{E}-04$ & $1.0 \mathrm{E}+00$ & $1.0 \mathrm{E}-01$ \\
\hline $\begin{array}{l}\text { Tetrachloroethane, } \\
1,1,2,2-\end{array}$ & $000079-34-5$ & 4.3E-02 & 4.3E-04 & & & $1.4 \mathrm{E}+00$ & $1.4 \mathrm{E}-02$ & & & $8.5 \mathrm{E}-03$ & $8.5 \mathrm{E}-05$ & & & 7.1E-03 & 7.1E-05 & & \\
\hline Tetrachtoroethylene ${ }^{(s)}$ & $000127-18-4$ & $1,6 \mathrm{E}-01$ & $1.6 \mathrm{E}-03$ & 3.7E-01 & $3.7 \mathrm{E}-02$ & $1.8 \mathrm{E}-01$ & $1.8 \mathrm{E}-03$ & 4.1E-01. & $4.1 E-02$ & $8.5 \mathrm{E}-01$ & $8.5 E-03$ & & & $7.8 \mathrm{E}-02$ & $7.8 \mathrm{E}-04$ & $1.9 \mathrm{E}-01$ & $1.9 \mathrm{E}-02$ \\
\hline $\begin{array}{l}\text { Tetrachlorophenol, } \\
2,3,4,6-\end{array}$ & $000058-90-2$ & & & $1.1 \mathrm{E}+00$ & $1.1 \mathrm{E}-01$ & & & $3.8 \mathrm{E}+00$ & $3.8 \mathrm{E}-01$ & & & & & & & $8.5 \mathrm{E}-01$ & $8.5 \mathrm{E}-02$ \\
\hline
\end{tabular}


Table 3.1a. (continued)

\begin{tabular}{|c|c|c|c|c|c|c|c|c|c|c|c|c|c|c|c|c|c|}
\hline \multirow[t]{2}{*}{ Chemical } & \multirow[t]{2}{*}{ CASRN } & \multicolumn{4}{|c|}{ Ingestion (mg/L)" } & \multicolumn{4}{|c|}{ Dermal (mg/L)" } & \multicolumn{4}{|c|}{ Inhalation $(\mathrm{mg} / \mathrm{L})^{\dagger \oplus}$} & \multicolumn{4}{|c|}{$\begin{array}{l}\text { Ingestion + Dermal + } \\
\text { Inhalation }(\mathrm{mg} / \mathrm{L})^{++^{+}}\end{array}$} \\
\hline & & $10^{-4}$ & $10^{4}$ & $H Q=1$ & $\mathrm{HQ}=0.1$ & $10^{-1}$ & $10^{-5}$ & $\mathrm{HQ}=1$ & $\mathrm{HQ}=0.1$ & $10^{4}$ & $10^{4}$ & $\mathrm{HQ}=1$ & $\mathrm{HQ}=0.1$ & $10^{-4}$ & $10^{4}$ & $\mathrm{HI}=1$ & $\mathrm{HI}=0.1$ \\
\hline $\begin{array}{l}\text { Tetrachlorotoluene, p- } \\
\text { alpha, alpha, alpha- }\end{array}$ & $005216-25-1$ & $4.3 \mathrm{E}-04$ & $4.3 \mathrm{E}-06$ & & . & & & & & & & & & ' & & & \\
\hline $\begin{array}{l}\text { Tetraethyl } \\
\text { Dithiopyrophosphate }\end{array}$ & $003689-24-5$ & & & $1.8 \mathrm{E}-02$ & $1.8 \mathrm{E}-03$ & & & $1.4 E+00$ & $1.4 \mathrm{E}-01$ & & & & & & & $1.8 \mathrm{E}-02$ & $1.8 \mathrm{E}-03$ \\
\hline Tetraethyl Lead & $000078-00-2$ & & & & & & & & & & & & & & & & \\
\hline $\begin{array}{l}\text { Tetrafluoroethane, } \\
1,1,1,2-\end{array}$ & $000811-97-2$ & & & & & & & & & & & $\begin{array}{l}1.7 \mathrm{E}+0 \\
2^{2} \\
\end{array}$ & $1.7 \mathrm{E}+01$ & & & & \\
\hline Tetramethyl Lead & $000075-74-1$ & & & & & & & & & & & & & & & & \\
\hline Tetrapropyl Lead & $003440-75-3$ & & & & & & & & & & & & & & & & \\
\hline Thallic Oxide & $001314-32-5$ & & & $2.6 \mathrm{E}-03$ & $2.6 \mathrm{E}-04$ & & & & & & & & & & & & \\
\hline Thallium (I) Nitrate & $010102-45-1$ & & & 3.3E-03 & $3.3 \mathrm{E}-04$ & & & $2.7 \mathrm{E}-01$ & $2.7 \mathrm{E}-02$ & & & & & & & $3.2 \mathrm{E}-03$ & $3.2 \mathrm{E}-04$ \\
\hline Thallium (Soluble Salts) & $007440-28-0$ & & & & & & & & & & & & & & & & \\
\hline Thallium Acelate & $000563-68-8$ & & & $3.3 \mathrm{E}-03$ & $3.3 \mathrm{E}-04$ & & & & & & & & & & & & \\
\hline Thallium Carbonate & 006533.73 .9 & & & $2.9 \mathrm{E}-03$ & $2.9 \mathrm{E}-04$ & & & & & & & & & & & & \\
\hline Thallium Chloride & $007791-12-0$ & & & $2.9 \mathrm{E}-03$ & $2.9 E-04$ & & & $2.4 E-01$ & $2.4 \mathrm{E}-02$ & & & & & & & $2.9 E-03$ & $2.9 \mathrm{E}-04$ \\
\hline Thallium Selenite & $012039-52-0$ & & & $3.3 \mathrm{E}-03$ & $3.3 \mathrm{E}-04$ & & & $2.7 \mathrm{E}-01$ & $2.7 \mathrm{E}-02$ & & & & & & & $3.2 \mathrm{E}-03$ & $3.2 \mathrm{E}-04$ \\
\hline Thallium Sulfate & $007446-18-6$ & & & $2.9 \mathrm{E}-03$ & $2.9 E-04$ & & & $2.4 \mathrm{E}-01$ & $2.4 \mathrm{E}-02$ & & & & & & & $2.9 \mathrm{E}-03$ & $2.9 \mathrm{E}-04$ \\
\hline Thiobencarb & $028249-77-6$ & & & $3.7 \mathrm{E}-01$ & $3.7 \mathrm{E}-02$ & & & & & & & & & & & & \\
\hline Thiofanox & 039196-18-4 & & & 1.1.E-02 & $1 . \mathrm{IE}-03$ & & & $3.9 \mathrm{E}+02$ & $3.9 \mathrm{E}+01$ & & & & & & & $1.1 \mathrm{E}-02$ & $1.1 \mathrm{E}-03$ \\
\hline Thiophanate, Methyl & 023564-05-8 & & & $2.9 \mathrm{E}+00$ & $2.9 \mathrm{E}-01$ & & & & & & & & & & & & \\
\hline Thiram & $000137-26.8$ & & & 1.8E-01 & $1.8 \mathrm{E}-02$ & & & & & & & & & & & & \\
\hline Thonium & $007440-29-1$ & & & & & & & & & & & & & & & & \\
\hline Tin & 007440-31-5 & & & $2.2 \mathrm{E}+01$ & $2.2 E+00$ & & & $9.0 \mathrm{E}+02$ & $9.0 E+01$ & & & & & & & $2.1 E+01$ & 2.1E +00 \\
\hline
\end{tabular}


Table 3.1a. (continued)

\begin{tabular}{|c|c|c|c|c|c|c|c|c|c|c|c|c|c|c|c|c|c|}
\hline \multirow[t]{2}{*}{ Chemical } & \multirow[t]{2}{*}{ CASRN } & \multicolumn{4}{|c|}{ Ingestion (mg/L) } & \multicolumn{4}{|c|}{ Dermal (mg/L)" } & \multicolumn{4}{|c|}{ Inhalation (mg/L) ${ }^{t^{n}}$} & \multicolumn{4}{|c|}{$\begin{array}{l}\text { Ingestion + Dermal + } \\
\text { Inhalation }(\mathrm{mg} / \mathrm{L})^{++^{\star}}\end{array}$} \\
\hline & & $10^{-4}$ & $10^{4}$ & $\mathrm{HQ}=\mathbf{I}$ & $\mathrm{HQ}=0.1$ & $10^{-1}$ & $10^{4}$ & $\mathrm{HQ}=\mathbf{I}$ & $\mathrm{HQ}=0.1$ & $10^{-4}$ & $10^{5}$ & $H Q=1$ & $\mathrm{HQ}=0.1$ & $10^{4}$ & $10^{6}$ & $\mathrm{HI}=\mathbf{1}$ & $\mathrm{HI}=0.1$ \\
\hline Titanium & $007440-32-6$ & & & & & & & & & & & & & & & & \\
\hline Toluene $e^{(6)}$ & $000108-88-3$ & & & $7.3 \mathrm{E}+00$ & $7.3 \mathrm{E}-01$ & & & $5.4 \mathrm{E}+01$ & $5.4 \mathrm{E}+00$ & & & $8.3 \mathrm{E}-01$ & $8.3 \mathrm{E}-02$ & & & $7.4 \mathrm{E}-01$ & 7.4E-02 \\
\hline $\begin{array}{l}\text { Toluene diisocyanate } \\
\text { mixture (TDI) }\end{array}$ & $026471-62-5$ & & & & & & & & & & & $1.5 \mathrm{E}-04$ & $1.5 \mathrm{E}-05$ & & & & \\
\hline Toluene-2,4-diamine & $000095-80.7$ & $2.7 \mathrm{E}-03$ & $2.7 \mathrm{E}-05$ & & . & $9.1 \mathrm{E}-01$ & 9.1E-03 & & & & & & & $2.7 \mathrm{E}-03$ & $2.7 \mathrm{E}-05$ & & \\
\hline Toluene-2,5-diamine & $000095-70-5$ & & & $2.2 \mathrm{E}+01$ & $2.2 \mathrm{E}+00$ & & & & & & & & & & & & \\
\hline Toluene-2,6-diamine & $000823-40-5$ & & & $7.3 \mathrm{E}+00$ & $7.3 \mathrm{E}-01$ & & & & & & & & & & & & \\
\hline $\begin{array}{l}\text { Toluidine, o- } \\
\text { (Methylariline, 2-) }\end{array}$ & $000095-53-4$ & $3.5 \mathrm{E}-02$ & 3.5E-04 & & & $2.0 \mathrm{E}+00$ & $2.0 E-02$ & & & & & & & $3.5 \mathrm{E}-02$ & 3.5E-04 & & \\
\hline Toluidine, p- & $000106-49-0$ & 4.5E-02 & $4.5 \mathrm{E}-04$ & & & $8.3 \mathrm{E}-01$ & $8.3 E-03$ & & & & & & & $4.3 \mathrm{E}-02$ & 4.3E-04 & & \\
\hline Toxaphene & 008001-35-2 & 7.7E-03 & $7.7 \mathrm{E}-05$ & & & $1.1 \mathrm{E}-01$ & $1.1 E-03$ & & & & & & & 7.2E- -03 & $7.2 \mathrm{E}-05$ & & \\
\hline Tralomethrin & $066841-25-6$ & & & $2.7 \mathrm{E}-01$ & $2.7 \mathrm{E}-02$ & & & & & & & & & & & & \\
\hline Triallate & 002303-17-5 & & & $4.7 \mathrm{E}-01$ & $4.7 \mathrm{E}-02$ & & & $5.5 \mathrm{E}+00$ & $5.5 \mathrm{E}-01$ & & & & & & & $4.4 \mathrm{E}-01$ & $4.4 \mathrm{E}-02$ \\
\hline Triasulfuron & 082097-50-5 & & & $3.7 \mathrm{E}-01$ & $3.7 \mathrm{E}-02$ & & & & & & & & & & & & \\
\hline Tribromobenzene, 1,2,4- & $000615-54-3$ & & & $1.8 \mathrm{E}-01$ & $1.8 \mathrm{E}-02$ & & & & & & & & & & & & \\
\hline Tribrombchloromethane & $000594-15-0$ & & & & & & & & & & & & & & & & \\
\hline Tribromodiphenyl Ether & 049690-944-0 & & & & & & & & & & & & & & & & \\
\hline Tributyltin Oxide & 000056-35-9 & & & $1.1 \mathrm{E}-03$ & $1.1 \mathrm{E}-04$ & & & & & & & & & & & & \\
\hline $\begin{array}{l}\text { Trichloro-1,2,2- } \\
\text { trifluoroethane, 1,1,2- }\end{array}$ & 000076-13-1 & & & $1.1 \mathrm{E}+03$ & $1.1 \mathrm{E}+02$ & & & $3.8 \mathrm{E}+04$ & $3.8 \mathrm{E}+03$ & & & $\begin{array}{l}6_{1}^{6.3 \mathrm{E}+0} \\
1^{-1}\end{array}$ & $6.3 \mathrm{E}+00$ & & & $5.9 \mathrm{E}+01$ & $5.9 \mathrm{E}+00$ \\
\hline $\begin{array}{l}\text { Trichloro-2'- } \\
\text { hydroxydiphenylether }\end{array}$ & 003380-34-5 & & & & & & & & & & & & & & & & \\
\hline Trichloroacetic Acid & & & & & & & & & & & & & & & & & \\
\hline
\end{tabular}


Table 3.1a. (continued)

\begin{tabular}{|c|c|c|c|c|c|c|c|c|c|c|c|c|c|c|c|c|c|}
\hline \multirow[t]{2}{*}{ Chemical } & \multirow[t]{2}{*}{ CASRN } & \multicolumn{4}{|c|}{ Ingestion (mg/L) } & \multicolumn{4}{|c|}{ Dermal (mg/L)" } & \multicolumn{4}{|c|}{ Inhalation $(\mathrm{mg} / \mathrm{L})^{\dagger^{*}}$} & \multicolumn{4}{|c|}{$\begin{array}{l}\text { Ingestion + Dermal + } \\
\text { Inhalation }(\mathrm{mg} / \mathrm{L})^{++^{*}}\end{array}$} \\
\hline & & $10^{-4}$ & $10^{4}$ & $\mathrm{HQ}=1$ & $\mathrm{HQ}=0.1$ & $10^{-4}$ & $10^{-4}$ & $H Q=1$ & $\mathrm{HQ}=0.1$ & $10^{-4}$ & $10^{4}$ & $\mathbf{H Q}=\mathbf{I}$ & $\mathrm{HQ}=0.1$ & $10^{-4}$ & $10^{+}$ & HI=1 & $\mathrm{HI}=0.1$ \\
\hline $\begin{array}{l}\text { Trichloroaniline } \mathrm{HCl} \text {, } \\
2,4,6-\end{array}$ & $033663-50-2$ & $2.9 \mathrm{E}-01$ & $2.9 \mathrm{E}-03$ & & & & & & & & & & & & & & \\
\hline Trichloroaniline, 2,4,6- & $000634-93-5$ & $2.5 \mathrm{E}-01$ & $2.5 \mathrm{E}-03$ & & & & & & & & & & & & & & \\
\hline Trichlorobenzene, 1,2,4- & $000120-82-1$ & & & $3.7 \mathrm{E}-01$ & $3.7 \mathrm{E}-02$ & & & $1.5 \mathrm{E}+00$ & $1.5 \mathrm{E}-01$ & & & $4.2 \mathrm{E}-01$ & $4.2 \mathrm{E}-02$ & & & $1.7 \mathrm{E}-01$ & $1.7 \mathrm{E}-02$ \\
\hline Trichloroethane, $1,1,1^{(3)}$ & $000071-55-6$ & & & $3.3 \mathrm{E}+00$ & $3.3 \mathrm{E}-01$ & & & $7.2 \mathrm{E}+01$ & $7.2 \mathrm{E}+00$ & & & $\begin{array}{l}2.1 E+0 \\
0\end{array}$ & $2.1 \mathrm{E}-01$ & & & $1.3 E+00$ & $1.3 \mathrm{E}-01$ \\
\hline Trichloroethane, 1,1,2- & $000079-00-5$ & $1.5 \mathrm{E}-01$ & $1.5 \mathrm{E}-03$ & $1.5 \mathrm{E}-01$ & $1.5 \mathrm{E}-02$ & $5.9 \mathrm{E}+00$ & $5.9 \mathrm{E}-02$ & $5.8 \mathrm{E}+00$ & $5.8 \mathrm{E}-01$ & $3.0 \mathrm{E}-02$ & $3.0 \mathrm{E}-04$ & & & 2.5E-02 & 2.5E-04 & $1.4 \mathrm{E}-01$ & $1.4 \mathrm{E}-02$ \\
\hline Trichloroethylene $^{(\mathfrak{)})}$ & $000079-01-6$ & 7.7E-01 & 7.7E- 03 & & & $3.0 \mathrm{E}+00$ & $3.0 \mathrm{E}-02$ & & & $2.8 \mathrm{E}-01$ & $2.8 \mathrm{E}-03$ & & & $1.9 \mathrm{E}-01$ & $1.9 \mathrm{E}-03$ & & \\
\hline Trichlorofluoromethane & $000075-69-4$ & & & $1.1 \mathrm{E}+01$ & $1.1 \mathrm{E}+00$ & & & $6.1 \mathrm{E}+01$ & $6.1 E+00$ & & & $\begin{array}{l}1.5 \mathrm{E}+0 \\
0\end{array}$ & $1.5 \mathrm{E}-01$ & & & $1.3 \mathrm{E}+00$ & $1.3 \mathrm{E}-01$ \\
\hline Trichlorophenol, 2,4,5- & $000095-95-4$ & & & $3.7 \mathrm{E}+00$ & 3.7E-01 & & & $2.0 \mathrm{E}+01$ & $2.0 \mathrm{E}+00$ & & & & & & & $3.1 E+00$ & $3.1 \mathrm{E}-01$ \\
\hline Trichlorophenol, 2,4,6- & $000088-06-2$ & $7.7 \mathrm{E}-01$ & 7.7E-03 & & & $2.7 \mathrm{E}+00$ & $2.7 \mathrm{E}-02$ & & & & & & & $6.0 \mathrm{E}-01$ & $6.0 \mathrm{E}-03$ & & \\
\hline $\begin{array}{l}\text { Trichlorophenoxy) } \\
\text { Propionic Acid, 2(2,4,5- }\end{array}$ & $000093-72-1$ & & & $2.9 \mathrm{E}-01$ & $2.9 \mathrm{E}-02$ & & & $2.4 \mathrm{E}+00$ & $2.4 \mathrm{E}-01$ & & & & & & & $2.6 \mathrm{E}-01$ & $2.6 \mathrm{E}-02$ \\
\hline $\begin{array}{l}\text { Trichlorophenoxyacetic } \\
\text { Acid, 2,4,5- }\end{array}$ & 000093-76-5 & & & $3.7 \mathrm{E}-01$ & $3.7 \mathrm{E}-02$ & , & & $2.1 \mathrm{E}+00$ & 2.1E-01 & & & & & & & $3.1 \mathrm{E}-01$ & $3.1 \mathrm{E}-02$ \\
\hline Trichloropropane, 1,1,2- & $000598.77-6$ & & & $1.8 \mathrm{E}-01$ & $1.8 \mathrm{E}-02$ & & & & & & & & & & & & \\
\hline Trichloropropane, 1,2,3- & $000096-18-4$ & $1.2 \mathrm{E}-03$ & $1.2 \mathrm{E}-05$ & $2.2 \mathrm{E}-01$ & $2.2 \mathrm{E}-02$ & $9.1 \mathrm{E}-04$ & $9.1 \mathrm{E}-06$ & $1.6 \mathrm{E}-01$ & $1.6 \mathrm{E}-02$ & & & & & $5.2 \mathrm{E}-04$ & $5.2 \mathrm{E}-06$ & 9.3E-02 & $9.3 \mathrm{E}-03$ \\
\hline Trichloropropene, 1,2,3- & $000096 \cdot 19-5$ & & & $1.8 \mathrm{E}-01$ & $1.8 \mathrm{E}-02$ & & & & & & & & & & & & \\
\hline Trichlorotoluene, 2,3,6- & $002077-46-5$ & & & & & & & & & & & & & & & & \\
\hline $\begin{array}{l}\text { Trichlorotoluene, alpha } \\
2,6 \text { - }\end{array}$ & $002014-83-7$ & & & & & & & & & & & & & & & & \\
\hline Tridiphane & $058138-08-2$ & & & $1.1 \mathrm{E}-01$ & $1.1 \mathrm{E}-02$ & & & & & & & & & & & & \\
\hline Triethyl Lead & $005224-23-7$ & & & & & & & & & & & & & & & & \\
\hline
\end{tabular}


Table 3.1a. (continued)

\begin{tabular}{|c|c|c|c|c|c|c|c|c|c|c|c|c|c|c|c|c|c|}
\hline \multirow[t]{2}{*}{ Chemical } & \multirow[t]{2}{*}{ CASRN } & \multicolumn{4}{|c|}{ Ingestion (mg/L) } & \multicolumn{4}{|c|}{ Dermal (mg/L)" } & \multicolumn{4}{|c|}{ Inhalation (mg/L) ${ }^{{ }^{\wedge}}$} & \multicolumn{4}{|c|}{$\begin{array}{l}\text { Ingestion + Dermal + } \\
\text { Inhalation }(\mathrm{mg} / \mathrm{L})^{\dagger+*}\end{array}$} \\
\hline & & $10^{-4}$ & $10^{4}$ & $H Q=1$ & $\mathrm{HQ}=0.1$ & $10^{-1}$ & $10^{6}$ & HQ=1 & $\mathrm{HQ}=0.1$ & $10^{-4}$ & $10^{5}$ & $\mathrm{HQ}=\mathbf{1}$ & $H Q=0.1$ & $10^{-4}$ & $10^{4}$ & Hl=1 & $\mathrm{HI}=0.1$ \\
\hline Triethylamine & $000121-44-8$ & & & & & & & & & & & 1.5E-02 & 1.5E-03 & & & & \\
\hline Trifluralin & $001582-09-8$ & $1.1 \mathrm{E}+00$ & $1.1 \mathrm{E}-02$ & 2.7E-01 & $2.7 \mathrm{E}-02$ & $2.3 \mathrm{E}+00$ & 2.3E-02 & $5.7 \mathrm{E}-01$ & $5.7 \mathrm{E}-02$ & & & & & $7.5 \mathrm{E}-01$ & 7.5E-03 & $1.8 \mathrm{E}-01$ & $1.8 \mathrm{E}-02$ \\
\hline Trimethyl Lead & 007442-13-9 & & & & & & & & & & & & & & & & \\
\hline Trimethyl Phosphate & $000512-56-1$ & $2.3 \mathrm{E}-01$ & 2.3E-03 & & & & & & & & & & & & & & \\
\hline Trimethylethyl Lead & $001762-26-1$ & & & & & & & & & & & & & & & & \\
\hline Trinitrobenzene, 1,3,5- & $000099-35-4$ & & & $1.8 \mathrm{E}-03$ & $1.8 \mathrm{E}-04$ & & & $7.2 \mathrm{E}-01$ & 7.2E-02 & & & & & & & $1.8 \mathrm{E}-03$ & $1.8 \mathrm{E}-04$ \\
\hline $\begin{array}{l}\text { Trinitrophenylmethylnitr } \\
\text { amine }\end{array}$ & $000479-45-8$ & & & $3.7 E-01$ & $3.7 \mathrm{E}-02$ & & & $8.5 \mathrm{E}+01$ & $8.5 \mathrm{E}+00$ & & & & & & & $3.6 \mathrm{E}-01$ & $3.6 \mathrm{E}-02$ \\
\hline Trinitrotoluene, $2,4,6$. & $000118-96-7$ & $2.8 \mathrm{E}-01$ & $2.8 \mathrm{E}-03$ & $1.8 \mathrm{E}-02$ & $1.8 \mathrm{E}-03$ & $2.1 \mathrm{E}+01$ & 2.1E-01 & $1.3 \mathrm{E}+00$ & $1.3 \mathrm{E}-01$ & & & & & $2.8 \mathrm{E}-01$ & $2.8 \mathrm{E}-03$ & $1.8 \mathrm{E}-02$ & $1.8 \mathrm{E}-03$ \\
\hline Tripropyl Lead & 006618-03-7 & & & & & & & & & & & & & & & & \\
\hline Uranium (Soluble Salts) & $\mathrm{NA}$ & & & $1.1 \mathrm{E}-01$ & $1.1 \mathrm{E}-02$ & & & $3.8 \mathrm{E}+01$ & $3.8 \mathrm{E}+00$ & & & . & & & & $1.1 E-01$ & $1.1 \mathrm{E}-02$ \\
\hline Vanadium Pentoxide & $001314-62-1$ & & & 3.3E-01 & $3.3 \mathrm{E}-02$ & & & $2.7 \mathrm{E}+01$ & $2.7 \mathrm{E}+00$ & & & & & & & $3.2 \mathrm{E}-01$ & $3.2 \mathrm{E}-02$ \\
\hline Vanadium Sulfate & $036907-42-3$ & & & $7.3 \mathrm{E}-01$ & $7.3 \mathrm{E}-02$ & & & $6.0 \mathrm{E}+01$ & $6.0 \mathrm{E}+00$ & & & & & & & $7.2 \mathrm{E}-01$ & $7.2 \mathrm{E}-02$ \\
\hline Vanadium, Metallic & $007440-62-2$ & & & $2.6 \mathrm{E}-01$ & $2.6 \mathrm{E}-02$ & & & $1.1 \mathrm{E}+00$ & $1.1 \mathrm{E}-01$ & & & & & & & $2.1 \mathrm{E}-01$ & $2.1 \mathrm{E}-02$ \\
\hline Vanadyl Sulfate & $027774-13-6$ & & & 7.3E- 01 & $7.3 \mathrm{E}-02$ & & & $6.0 \mathrm{E}+01$ & $6.0 \mathrm{E}+00$ & & $\cdot$ & & & & & $7.2 \mathrm{E}-01$ & 7.2E-02 \\
\hline Vemolate & $001929-77-7$ & & & $3.7 \mathrm{E}-02$ & $3.7 \mathrm{E}-03$ & & & & & & & & & & & & \\
\hline Vinclozolin & $050471-44-8$ & & & $9.1 \mathrm{E}-01$ & $9.1 \mathrm{E}-02$ & & & & & & & & & & & & \\
\hline Vinyl Acetate & $000108-05-4$ & & & $3.7 E+01$ & $3.7 \mathrm{E}+00$ & & & $8.4 \mathrm{E}+02$ & $8.4 \mathrm{E}+01$ & & & $4.2 \mathrm{E}-01$ & $4.2 \mathrm{E}-02$ & & & $4.1 E-01$ & $4.1 \mathrm{E}-02$ \\
\hline Vinyl Bromide & $000593-60-2$ & & & & & & & & & $1.5 \mathrm{E}-02$ & $1.5 \mathrm{E}-04$ & $6.3 \mathrm{E}-03$ & $6.3 \mathrm{E}-04$ & & & & \\
\hline Vinyl Chloride ${ }^{(6)}$ & $000075-01-4$ & $4.5 \mathrm{E}-03$ & $4.5 \mathrm{E}-05$ & & & $2.5 \mathrm{E}-01$ & $2.5 \mathrm{E}-03$ & & & $5.7 \mathrm{E}-03$ & $5.7 E-05$ & & & $2.5 \mathrm{E}-03$ & $2.5 \mathrm{E}-05$ & & \\
\hline Warfarin & $000081-81-2$ & & & $1.1 \mathrm{E}-02$ & $1.1 \mathrm{E}-03$ & & & $4.8 \mathrm{E}-01$ & $4.8 \mathrm{E}-02$ & & & & & & & $1.1 \mathrm{E}-02$ & $1.1 \mathrm{E}-03$ \\
\hline White Phosphorus & $007223-14-0$ & & & $7.3 \mathrm{E}-04$ & 7.3E-05 & & & $6.0 \mathrm{E}-02$ & $6.0 \mathrm{E}-03$ & & & & & & & 7.2E-04 & $7.2 \mathrm{E}-05$ \\
\hline
\end{tabular}


Table 3.1a. (continued)

\begin{tabular}{|c|c|c|c|c|c|c|c|c|c|c|c|c|c|c|c|c|c|}
\hline \multirow[t]{2}{*}{ Chemical } & \multirow[t]{2}{*}{ CASRN } & \multicolumn{4}{|c|}{ Ingestion (mg/L)" } & \multicolumn{4}{|c|}{ Dermal (mg/L)" } & \multicolumn{4}{|c|}{ Inhalation (mg/L) ${ }^{\dagger^{\star}}$} & \multicolumn{4}{|c|}{$\begin{array}{l}\text { Ingestion + Dermal + } \\
\text { Inhalation }(\mathrm{mg} / \mathrm{L})^{+t^{*}}\end{array}$} \\
\hline & & $10^{-4}$ & $10^{6}$ & $H Q=1$ & $\mathrm{HQ}=0.1$ & $10^{-4}$ & $10^{6}$ & $\mathrm{HQ}=\mathbf{1}$ & $\mathrm{HQ}=0.1$ & $10^{-4}$ & $10^{5}$ & $\mathrm{HQ}=\mathbf{1}$ & $\mathrm{HQ}=0.1$ & $10^{-4}$ & $10^{4}$ & $\mathrm{HI}=\mathrm{I}$ & $\mathrm{HI}=0.1$ \\
\hline Xylene, Mixture & $001330-20-7$ & & & $7.3 \mathrm{E}+01$ & $7.3 \mathrm{E}+00$ & & & $2.9 E+02$ & $2.9 E+01$ & & & & & & & $5.8 \mathrm{E}+01$ & $5.8 \mathrm{E}+00$ \\
\hline Xylene, $P_{-}(6)$ & $000106-42-3$ & & & & & & & & & & & & & & & & \\
\hline Xylene, $m-(1)$ & 000108-38-3 & & & $7.3 \mathrm{E}+01$ & $7.3 \mathrm{E}+00$ & & & $3.0 \mathrm{E}+02$ & $3.0 E+01$ & & & & & & & $5.9 \mathrm{E}+01$ & $5.9 \mathrm{E}+00$ \\
\hline Xylene, o.(6) & $000095-47-6$ & & & $7.3 \mathrm{E}+01$ & $7.3 \mathrm{E}+00$ & & & $3.0 \mathrm{E}+02$ & $3.0 E+01$ & & & & & & & $5.9 \mathrm{E}+01$ & $5.9 \mathrm{E}+00$ \\
\hline Zinc (Metallic) & $007440-66-6$ & & & $1.1 \mathrm{E}+01$ & $1.1 \mathrm{E}+00$ & & & $9.0 \mathrm{E}+02$ & $9.0 \mathrm{E}+01$ & & & & & & & $1.1 \mathrm{E}+01$ & $1.1 \mathrm{E}+00$ \\
\hline Zinc Cyanide & $000557-21-1$ & & & $1.8 \mathrm{E}+00$ & $1.8 \mathrm{E}-01$ & & & $1.5 \mathrm{E}+02$ & $1.5 \mathrm{E}+01$ & & & & & & & $1.8 \mathrm{E}+00$ & $1.8 \mathrm{E}-01$ \\
\hline Zinc Phosphide & $001314-84-7$ & & & $1.1 \mathrm{E}-02$ & $1.1 \mathrm{E}-03$ & & & $9.0 \mathrm{E}-01$ & $9.0 \mathrm{E}-02$ & & & & & & & $1.1 \mathrm{E}-02$ & $1.1 \mathrm{E}-03$ \\
\hline Zineb & $012122-67-7$ & & & $1.8 \mathrm{E}+00$ & $1.8 \mathrm{E}-01$ & & & & & & & & & & & & \\
\hline Zirconium & $007440-67-7$ & & & & & & & & & & & & . & & & & \\
\hline
\end{tabular}

Notes:

$\dagger \quad$ Although an inhalation RfD could be calculated or an inhalation slope factor was available, the risk from indoor inhalation of volatiles was assumed to be relevant for only those chemicals that volatilize easily. The risk equation incorporates a water-air concentration relationship that is applicable only to chemicals with a Henry's Law Constant of greater that $1 \times 10^{-5} \mathrm{~atm}-\mathrm{m}^{3} / \mathrm{mole}$ and a molecular weight of less than $200 \mathrm{~g} /$ mole. These criteria were used to identify those chemicals that generally should be considered for the inhalation pathway when developing risk-based PRGs. However, chemicals that do not meet these criteria may pose significant site risks (and require risk-based goals) through inhalation of volatiles. The ultimate decision regarding which contaminants should be considered in the FS must be made on a site-specific basis following completion of the baseline risk assessment.

i† If slope factors and/or reference doses were available for only one pathway (i.e., ingestion, dermal, OR inhalation), the total for ingestion plus dermal plus inhalation was not calculated.

* By definition, $1 \mathrm{~L}$ (water) $=1.0 \mathrm{E}+06 \mathrm{mg}$ (water). Therefore, a limit of $1.0 \mathrm{E}+06 \mathrm{mg} / \mathrm{L}$ was used for any PRG that was calculated to be above this limit.

(1) The same oral slope factor as used for polychlorinated biphenyls.

(2) The PRG was calculated considering Supplemental Guidance from RAGS: Region 4 Bulletins, Human Health Risk Assessment (Interim Guidance) from EPA (received February 11, 1992). The slope factors for chemicals so marked indicate that a toxicity equivalency factor (TEF) method was used for carcinogenic PAHs based on each compound's relative potency to the potency of benzo[a]pyrene. The following TEFs were used to convert each PAH slope factor to an equivalent slope factor of benzo[a]pyrene [that has a new cancer slope factor of $7.3(\mathrm{mg} / \mathrm{kg} / \text { day) })^{-1}$ identified by EPA's CRAVE workgroup]: (1) benzo[a]pyrene, TEF $=1.0 ;(2)$ benzo[a]anthracene, 
Table 3.1a. (continued)

$\mathrm{TEF}=0.1 ;(3)$ benzo[b]fluoranthene, TEF $=0.1 ;(4)$ benzo[k]fluoranthene, TEF $=0.01 ;(5)$ chrysene, $\mathrm{TEF}=0.001 ;(6)$ dibenz[a,h]anthracene, TEF $=1.0 ;$ and $(7)$ indeno[1,2,3-c,d]pyrene, $\mathrm{TEF}=0.1$.

(3) The toxicity values for trichloroethylene have been withdrawn from IRIS and HEAST pending further investigation. However, the most recent toxicity values have been used to calculate the PRG.

(4) The PRG was calculated considering Supplemental Guidance from RAGS: Region 4 Bulletins, Human Health Risk Assessment (Interim Guidance) from EPA (November 1995). The slope factors for chemicals so marked indicate that a TEF methodology was used for chlorinated dioxin and furan congeners based on each compound's relative potency to the potency of 2,3,7,8-tetrachlorodibenzodioxin (TCDD). The following TEFs were used to convert each slope factor to an equivalent slope factor of 2,3,7,8TCDD: (1) 2,3,7,8-TCDD, TEF = 1.0; (2) 2,3,7,8-PeCDD, TEF =0.5; (3) 2,3,7,8-HxCDD, TEF =0.1; (4) 2,3,7,8-HpCDD, TEF =0.01; (5) OCDD, TEF = 0.001; (6) $2,3,7,8-\mathrm{TCDF}, \mathrm{TEF}=0.1 ;(7) 1,2,3,7,8-\mathrm{PeCDF}, \mathrm{TEF}=0.5 ;(8) 2,3,4,7,8-\mathrm{PeCDF}, \mathrm{TEF}=0.05 ;(9) 2,3,7,8-\mathrm{HxCDF}, \mathrm{TEF}=0.1 ;(10) 2,3,7,8-\mathrm{HpCDF}, \mathrm{TEF}=0.01 ;$ and (11) $\mathrm{OCDF}, \mathrm{TEF}=0.001$.

(5) Toxicity values for these chemicals have been withdrawn from IRIS and HEAST pending further investigation. However, the most recent toxicity values have been used to calculate the PRG.

(6) Toxicity values for these chemicals were obtained from the Superfund Health Risk Technical Support Center. 
Table 3.1b. Risk-based Preliminary Remediation Goals for radionuclides in groundwater (residential scenario)

\begin{tabular}{|c|c|c|c|c|c|c|c|}
\hline \multirow[t]{2}{*}{ Chemical $^{\dagger}$} & \multirow[t]{2}{*}{ CASRN } & \multicolumn{2}{|c|}{ Ingestion (pCi/L) } & \multicolumn{2}{|c|}{ Inhalation ( $\mathrm{pCi} / \mathrm{L})$} & \multicolumn{2}{|c|}{$\begin{array}{c}\text { Ingestion + Inhalation } \\
(\mathrm{pCi} / \mathbf{L})^{+t}\end{array}$} \\
\hline & & $10^{-4}$ & $10^{-5}$ & $10^{-4}$ & $10^{-6}$ & $10^{-4}$ & $10^{-6}$ \\
\hline Ac-225 & $014265-85-1$ & $3.4 \mathrm{E}+01$ & 3.4E-01 & & & & \\
\hline Ac-227 & $014952-40-0$ & $1.4 \mathrm{E}+01$ & $1.4 \mathrm{E}-01$ & & & & \\
\hline$A C-227+D$ & $014952-40-0$ & $7.6 \mathrm{E}+00$ & $7.6 \mathrm{E}-02$ & & & & \\
\hline Ac-228 & $014331-83-0$ & $2.9 \mathrm{E}+03$ & $2.9 \mathrm{E}+01$ & & & & \\
\hline $\mathrm{Ag}-105$ & $014928-14-4$ & $2.9 \mathrm{E}+03$ & $2.9 \mathrm{E}+01$ & & & & \\
\hline Ag-108 & 014391-65-2 & $6.9 E+05$ & $6.9 \mathrm{E}+03$ & & & & \\
\hline $\mathrm{Ag}-108 \mathrm{~m}$ & $014391-65-2$ & $7.9 \mathrm{E}+02$ & $7.9 \mathrm{E}+00$ & & & & \\
\hline$A g-108 m+D$ & $014391-65-2$ & $7.9 \mathrm{E}+02$ & $7.9 \mathrm{E}+00$ & & & & \\
\hline Ag- $109 m$ & $014378-38-2$ & $1.8 \mathrm{E}+07$ & $1.8 \mathrm{E}+05$ & & & & \\
\hline $\mathrm{Ag}-110$ & 014391-76-5 & $2.0 \mathrm{E}+06$ & $2.0 \mathrm{E}+04$ & & & & \\
\hline $\mathrm{Ag}-110 \mathrm{~m}$ & 014391-76-5 & $5.6 \mathrm{E}+02$ & $5.6 \mathrm{E}+00$ & & & & \\
\hline Ag-111 & $015760-04-0$ & $7.0 \mathrm{E}+02$ & $7.0 \mathrm{E}+00$ & & & & \\
\hline Am-241 & 014596-10-2 & $1.5 \mathrm{E}+01$ & $1.5 \mathrm{E}-01$ & & & & \\
\hline Am-242 & 013981-54-9 & $3.2 \mathrm{E}+03$ & $3.2 \mathrm{E}+01$ & & & & \\
\hline$A m-242 m$ & 013981-54-9 & $1.6 \mathrm{E}+01$ & $1.6 \mathrm{E}-01$ & & & & \\
\hline Am-243 & 014993-75-0 & $1.5 \mathrm{E}+01$ & $1.5 \mathrm{E}-01$ & & & & \\
\hline Am-243+D & 014993-75-0 & $1.4 \mathrm{E}+01$ & $1.4 \mathrm{E}-01$ & & & & \\
\hline
\end{tabular}


Table 3.1b. (continued)

\begin{tabular}{|c|c|c|c|c|c|c|c|}
\hline \multirow[t]{2}{*}{ Chemical $^{\dagger}$} & \multirow[t]{2}{*}{ CASRN } & \multicolumn{2}{|c|}{ Ingestion $(\mathrm{pCi} / \mathrm{L})$} & \multicolumn{2}{|c|}{ Inhalation (pCi/L) } & \multicolumn{2}{|c|}{$\begin{array}{c}\text { Ingestion + Inhalation } \\
(\mathrm{pCi} / \mathrm{L})^{\dagger+}\end{array}$} \\
\hline & & $10^{-4}$ & $10^{-6}$ & $10^{-4}$ & $10^{-6}$ & $10^{-4}$ & $10^{-6}$ \\
\hline $\operatorname{Ar}-41$ & 014163-25-8 & & & & & & \\
\hline At-217 & $017239-90-6$ & $5.3 \mathrm{E}+08$ & $5.3 \mathrm{E}+06$ & & & & \\
\hline $\mathrm{Au}-196$ & 014914-16-0 & $3.7 \mathrm{E}+03$ & $3.7 \mathrm{E}+01$ & & & & \\
\hline Au-198 & 010043-49-0 & $9.0 \mathrm{E}+02$ & $9.0 \mathrm{E}+00$ & & & & \\
\hline Ba-131 & $014914-75-1$ & $2.8 \mathrm{E}+03$ & $2.8 \mathrm{E}+01$ & & & & \\
\hline $\mathrm{Ba}-133$ & $013981-41-4$ & $1.8 \mathrm{E}+03$ & $1.8 \mathrm{E}+01$ & & & & \\
\hline $\mathrm{Ba}-133 \mathrm{~m}$ & $013981-41-4$ & $1.7 \mathrm{E}+03$ & $1.7 \mathrm{E}+01$ & & & & \\
\hline $\mathrm{Ba}-137 \mathrm{~m}$ & 013981-97-0 & $2.0 \mathrm{E}+06$ & $2.0 \mathrm{E}+04$ & & & & \\
\hline Ba-139 & $014378-25-7$ & $1.6 \mathrm{E}+04$ & $1.6 \mathrm{E}+02$ & & & & \\
\hline $\mathrm{Ba}-140$ & $014798-08-4$ & $4.0 \mathrm{E}+02$ & $4.0 \mathrm{E}+00$ & & & & \\
\hline $\mathrm{Be}-7$ & $013966-02-4$ & $5.5 \mathrm{E}+04$ & $5.5 \mathrm{E}+02$ & & & & \\
\hline $\mathrm{Bi}-206$ & 015776-19-9 & $6.7 \mathrm{E}+02$ & $6.7 \mathrm{E}+00$ & & & & \\
\hline $\mathrm{Bi}-207$ & $013982-38-2$ & $9.4 \mathrm{E}+02$ & $9.4 \mathrm{E}+00$ & & & & \\
\hline $\mathrm{Bi}-210$ & $014331-79-4$ & $6.5 E+02$ & $6.5 \mathrm{E}+00$ & & & & \\
\hline Bi-211 & 015229-37-5 & $2.6 \mathrm{E}+05$ & $2.6 \mathrm{E}+03$ & & & & \\
\hline $\mathrm{Bi}-212$ & 014913-49-6 & $7.7 \mathrm{E}+03$ & $7.7 \mathrm{E}+01$ & & & & \\
\hline $\mathrm{Bi}-213$ & $015776-20-2$ & $1.1 \mathrm{E}+04$ & $1.1 \mathrm{E}+02$ & & & & \\
\hline
\end{tabular}


Table 3.1b. (continued)

\begin{tabular}{|c|c|c|c|c|c|c|c|}
\hline \multirow[t]{2}{*}{ Chemical $^{\dagger}$} & \multirow[t]{2}{*}{ CASRN } & \multicolumn{2}{|c|}{ Ingestion (pCi/L) } & \multicolumn{2}{|c|}{ Inhalation (pCi/L) } & \multicolumn{2}{|c|}{$\begin{array}{l}\text { Ingestion + Inhalation } \\
(\mathrm{pCi} / \mathrm{L})^{t+}\end{array}$} \\
\hline & & $10^{-4}$ & $10^{-6}$ & $10^{-4}$ & $10^{-6}$ & $10^{-4}$ & $10^{-6}$ \\
\hline $\mathrm{Bi}-214$ & 014733-03-0 & $2.4 \mathrm{E}+04$ & $2.4 \mathrm{E}+02$ & & & & \\
\hline $\mathrm{Br}-82$ & $014686-69-2$ & $3.4 \mathrm{E}+03$ & $3.4 \mathrm{E}+01$ & & & & \\
\hline$C-11$ & 014333-33-6 & $1.1 \mathrm{E}+05$ & $1.1 \mathrm{E}+03$ & & & & \\
\hline C-14 & $014762-75-5$ & $4.6 \mathrm{E}+03$ & $4.6 \mathrm{E}+01$ & & & & \\
\hline C-15 & $015929-23-4$ & $7.2 \mathrm{E}+06$ & $7.2 \mathrm{E}+04$ & & & & \\
\hline $\mathrm{Ca}-45$ & 013966-05-7 & $2.4 \mathrm{E}+03$ & $2.4 \mathrm{E}+01$ & & & & \\
\hline $\mathrm{Ca}-47$ & $001439-99-2$ & $7.2 \mathrm{E}+02$ & $7.2 \mathrm{E}+00$ & & & & \\
\hline Cd-109 & $014109-32-1$ & $5.9 \mathrm{E}+02$ & $5.9 \mathrm{E}+00$ & & & & \\
\hline $\mathrm{Cd}-115$ & $014336-68-6$ & $6.5 \mathrm{E}+02$ & $6.5 \mathrm{E}+00$ & & & & \\
\hline $\mathrm{Cd}-115 \mathrm{~m}$ & $014336-68-6$ & $3.4 \mathrm{E}+02$ & $3.4 \mathrm{E}+00$ & - & & & \\
\hline Ce-141 & $013967-74-3$ & $1.2 \mathrm{E}+03$ & $1.2 \mathrm{E}+01$ & & & & \\
\hline Ce-143 & 014119-19-8 & $8.1 \mathrm{E}+02$ & $8.1 \mathrm{E}+00$ & & & & \\
\hline Ce-144 & $014762-78-8$ & $1.6 \mathrm{E}+02$ & $1.6 \mathrm{E}+00$ & & & & \\
\hline Ce-144+D & 014762-78-8 & $1.6 \mathrm{E}+02$ & $1.6 \mathrm{E}+00$ & & & & \\
\hline $\mathrm{Cl}-36$ & $013981-43-6$ & $2.1 \mathrm{E}+03$ & $2.1 \mathrm{E}+01$ & & & & \\
\hline $\mathrm{Cl}-38$ & 014158-34-0 & $2.3 \mathrm{E}+04$ & $2.3 \mathrm{E}+02$ & & & & \\
\hline $\mathrm{Cm}-242$ & $015510-73-3$ & $1.2 \mathrm{E}+02$ & $1.2 \mathrm{E}+00$ & & & & \\
\hline
\end{tabular}


Table 3.1b. (continued)

\begin{tabular}{|c|c|c|c|c|c|c|c|}
\hline \multirow[t]{2}{*}{ Chemical $^{\dagger}$} & \multirow[t]{2}{*}{ CASRN } & \multicolumn{2}{|c|}{ Ingestion $(\mathrm{pCi} / \mathrm{L})$} & \multicolumn{2}{|c|}{ Inhalation (pCi/L) } & \multicolumn{2}{|c|}{$\begin{array}{l}\text { Ingestion + Inhalation } \\
(\mathrm{pCi} / \mathrm{L})^{t+}\end{array}$} \\
\hline & & $10^{-4}$ & $10^{-6}$ & $10^{-4}$ & $10^{-6}$ & $10^{-4}$ & $10^{-6}$ \\
\hline $\mathrm{Cm}-243$ & $015757-87-6$ & $1.9 \mathrm{E}+01$ & $1.9 \mathrm{E}-01$ & & & & \\
\hline $\mathrm{Cm}-243+\mathrm{D}$ & $015757-87-6$ & $1.9 \mathrm{E}+01$ & $1.9 \mathrm{E}-01$ & & & & \\
\hline $\mathrm{Cm}-244$ & $013981-15-2$ & $2.3 \mathrm{E}+01$ & $2.3 \mathrm{E}-01$ & & & & \\
\hline $\mathrm{Cm}-245$ & $015621-76-8$ & $1.4 \mathrm{E}+01$ & $1.4 \mathrm{E}-01$ & & & & \\
\hline $\mathrm{Cm}-246$ & $015757-90-1$ & $1.4 \mathrm{E}+01$ & $1.4 \mathrm{E}-01$ & & & & \\
\hline $\mathrm{Cm}-247$ & $015758-32-4$ & $1.5 \mathrm{E}+01$ & $1.5 \mathrm{E}-01$ & & & & \\
\hline $\mathrm{Cm}-248$ & $015758-33-5$ & $3.6 \mathrm{E}+00$ & $3.6 \mathrm{E}-02$ & & & & \\
\hline Co-57 & 013981-50-5 & $4.9 \mathrm{E}+03$ & $4.9 \mathrm{E}+01$ & & & & \\
\hline Co-58 & 013981-38-9 & $1.7 \mathrm{E}+03$ & $1.7 \mathrm{E}+01$ & & & & \\
\hline Co-58m & 013981-38-9 & $5.0 \mathrm{E}+04$ & $5.0 \mathrm{E}+02$ & & & & \\
\hline Co-60 & $010198-40-0$ & $2.5 \mathrm{E}+02$ & $2.5 \mathrm{E}+00$ & & & & \\
\hline $\mathrm{Cr}-51$ & 014392-02-0 & $3.5 \mathrm{E}+04$ & $3.5 \mathrm{E}+02$ & & & & \\
\hline Cs-131 & $014914-76-2$ & $2.6 \mathrm{E}+04$ & $2.6 \mathrm{E}+02$ & & & & \\
\hline Cs-134 & 013967-70-9 & $1.0 \mathrm{E}+02$ & $1.0 \mathrm{E}+00$ & & & & \\
\hline Cs- $134 \mathrm{~m}$ & 013967-70-9 & $1.0 \mathrm{E}+05$ & $1.0 \mathrm{E}+03$ & & & & \\
\hline Cs-135 & $015726-30-4$ & $1.1 \mathrm{E}+03$ & $1.1 \mathrm{E}+01$ & & & & \\
\hline Cs-136 & $014234-29-8$ & $6.2 \mathrm{E}+02$ & $6.2 \mathrm{E}+00$ & & & & \\
\hline
\end{tabular}


Table 3.1b. (continued)

\begin{tabular}{|c|c|c|c|c|c|c|c|}
\hline \multirow[t]{2}{*}{ Chemical $^{\dagger}$} & \multirow[t]{2}{*}{ CASRN } & \multicolumn{2}{|c|}{ Ingestion (pCi/L) } & \multicolumn{2}{|c|}{ Inhalation (pCi/L) } & \multicolumn{2}{|c|}{$\begin{array}{c}\text { Ingestion + Inhalation } \\
(\mathrm{pCi} / \mathrm{L})^{+\dagger}\end{array}$} \\
\hline & & $10^{-4}$ & $10^{-6}$ & $10^{-4}$ & $10^{-6}$ & $10^{-4}$ & $10^{-6}$ \\
\hline Cs-137 & $010045-97-3$ & $1.5 \mathrm{E}+02$ & $1.5 \mathrm{E}+00^{\circ}$ & & & & \\
\hline Cs-137+D & $010045-97-3$ & $1.5 \mathrm{E}+02$ & $1.5 \mathrm{E}+00$ & & & & \\
\hline Cs-138 & 015758-29-9 & $2.7 \mathrm{E}+04$ & $2.7 \mathrm{E}+02$ & & & & \\
\hline Cu-64 & $013981-25-4$ & $9.1 \mathrm{E}+03$ & $9.1 \mathrm{E}+01$ & & & & \\
\hline Dy-165 & 013967-64-1 & $1.5 E+04$ & $1.5 \mathrm{E}+02$ & & & & \\
\hline Dy-166 & $015840-01-4$ & $5.1 \mathrm{E}+02$ & $5.1 \mathrm{E}+00$ & & & & \\
\hline Er-169 & $015840-13-8$ & $2.2 \mathrm{E}+03$ & $2.2 \mathrm{E}+01$ & & & & \\
\hline Er-171 & $014391-45-8$ & $2.9 \mathrm{E}+03$ & $2.9 \mathrm{E}+01$ & & & & \\
\hline Eu-152 & 014683-23-9 & $8.3 E+02$ & $8.3 E+00$ & & & & \\
\hline Eu-154 & $015585-10-1$ & $5.1 \mathrm{E}+02$ & $5.1 E+00$ & & & & \\
\hline Eu-155 & $014391-16-3$ & $2.9 \mathrm{E}+03$ & $2.9 \mathrm{E}+01$ & & & & \\
\hline Eu-156 & $014280-35-4$ & $4.4 \mathrm{E}+02$ & $4.4 \mathrm{E}+00$ & & & & \\
\hline F-18 & $013981-56-1$ & $4.4 \mathrm{E}+04$ & $4.4 \mathrm{E}+02$ & & & & \\
\hline Fe-55 & $014681-59-5$ & $1.4 \mathrm{E}+04$ & $1.4 \mathrm{E}+02$ & & & & \\
\hline $\mathrm{Fe}-59$ & 014596-12-4 & $8.1 E+02$ & $8.1 E+00$ & & & & \\
\hline Fr-221 & $015756-41-9$ & $3.3 E+04$ & $3.3 E+02$ & & & & \\
\hline Fr-223 & 015756-98-6 & $1.1 \mathrm{E}+04$ & $1.1 \mathrm{E}+02$ & & & & \\
\hline
\end{tabular}


Table 3.1b. (continued)

\begin{tabular}{|c|c|c|c|c|c|c|c|}
\hline \multirow{2}{*}{ Chemical $^{\dagger}$} & \multirow{2}{*}{ CASRN } & \multicolumn{2}{|c|}{ Ingestion (pCi/L) } & \multicolumn{2}{|c|}{ Inhalation $(\mathrm{pCi} / \mathrm{L})$} & \multicolumn{2}{|c|}{$\begin{array}{c}\text { Ingestion + Inhalation } \\
(\mathrm{pCi} / \mathrm{L})^{+\dagger}\end{array}$} \\
\hline & & $10^{-4}$ & $10^{-6}$ & $10^{-4}$ & $10^{-6}$ & $10^{-4}$ & $10^{-6}$ \\
\hline Ga-67 & $014119-09-6$ & $5.7 E+03$ & $5.7 \mathrm{E}+01$ & & & & \\
\hline Ga-72 & $013982-22-4$ & $1.0 \mathrm{E}+03$ & $1.0 \mathrm{E}+01$ & & & & \\
\hline Gd-153 & $014276-65-4$ & $3.6 \mathrm{E}+03$ & $3.6 \mathrm{E}+01$ & & & & \\
\hline Gd-159 & $014041-42-0$ & $1.8 \mathrm{E}+03$ & $1.8 \mathrm{E}+01$ & & & & \\
\hline Ge-71 & $014374-81-3$ & $4.0 \mathrm{E}+05$ & $4.0 \mathrm{E}+03$ & & & & \\
\hline $\mathrm{H}-3$ & $010028-17-8$ & $6.7 \mathrm{E}+04$ & $6.7 \mathrm{E}+02$ & $4.3 E+05$ & $4.3 \mathrm{E}+03$ & $5.8 \mathrm{E}+04$ & $5.8 \mathrm{E}+02$ \\
\hline $\mathrm{Hg}-197$ & $013981-51-6$ & $4.0 \mathrm{E}+03$ & $4.0 \mathrm{E}+01$ & & & & \\
\hline $\mathrm{Hg}-203$ & 013982-78-0 & $1.8 \mathrm{E}+03$ & $1.8 \mathrm{E}+01$ & & & & \\
\hline Ho-166 & $013967-65-2$ & $6.3 \mathrm{E}+02$ & $6.3 \mathrm{E}+00$ & & & & \\
\hline$[-122$ & $018287-75-7$ & $2.2 \mathrm{E}+05$ & $2.2 \mathrm{E}+03$ & & & & \\
\hline I-123 & 015715-08-9 & $8.8 \mathrm{E}+03$ & $8.8 \mathrm{E}+01$ & & & & \\
\hline $\mathrm{I}-125$ & 014158-31-7 & $1.8 \mathrm{E}+02$ & $1.8 \mathrm{E}+00$ & & & & \\
\hline $\mathrm{I}-126$ & 014158-32-8 & $9.9 \mathrm{E}+01$ & $9.9 \mathrm{E}-01$ & & & & \\
\hline $\mathrm{I}-129$ & $015046-84-1$ & $2.6 \mathrm{E}+01$ & $2.6 \mathrm{E}-01$ & & & & \\
\hline $\mathrm{I}-130$ & $014914-02-4$ & $9.8 \mathrm{E}+02$ & $9.8 \mathrm{E}+00$ & & & & \\
\hline $\mathrm{I}-131$ & 010043-66-0 & $1.3 \mathrm{E}+02$ & $1.3 \mathrm{E}+00$ & & & & \\
\hline $\mathrm{I}-132$ & $014683-16-0$ & $7.2 \mathrm{E}+03$ & $7.2 \mathrm{E}+01$ & & & & \\
\hline
\end{tabular}


Table 3.1b. (continued)

\begin{tabular}{|c|c|c|c|c|c|c|c|}
\hline \multirow[t]{2}{*}{ Chemical $^{\dagger}$} & \multirow[t]{2}{*}{ CASRN } & \multicolumn{2}{|c|}{ Ingestion $(\mathrm{pCi} / \mathrm{L})$} & \multicolumn{2}{|c|}{ Inhalation (pCi/L) } & \multicolumn{2}{|c|}{$\begin{array}{c}\text { Ingestion + Inhalation } \\
(\mathrm{pCi} / \mathrm{L})^{+t}\end{array}$} \\
\hline & & $10^{-4}$ & $10^{-6}$ & $10^{-4}$ & $10^{-6}$ & $10^{-4}$ & $10^{-6}$ \\
\hline I-133 & $014834-67-4$ & $4.5 \mathrm{E}+02$ & $4.5 \mathrm{E}+00$ & & & & \\
\hline I-134 & $014914-27-3$ & $2.1 \mathrm{E}+04$ & $2.1 \mathrm{E}+02$ & & & & \\
\hline $\mathrm{I}-135$ & $014834-68-5$ & $2.1 \mathrm{E}+03$ & 2.1E+01 & & & & \\
\hline In-113m & $014885-78-0$ & $5.7 E+04$ & $5.7 \mathrm{E}+02$ & & & & \\
\hline In-114 & $013981-55-0$ & $1.1 \mathrm{E}+06$ & $1.1 \mathrm{E}+04$ & & & & \\
\hline In-114m & $013981-55-0$ & $2.3 \mathrm{E}+02$ & $2.3 \mathrm{E}+00$ & & & & \\
\hline In-115 & $014191-71-0$ & $1.4 E+02$ & $1.4 \mathrm{E}+00$ & & & & \\
\hline In-115m & $014191-71-0$ & $1.4 \mathrm{E}+04$ & $1.4 \mathrm{E}+02$ & & & & \\
\hline Ir-190 & 014981-91-0 & $9.6 \mathrm{E}+02$ & $9.6 \mathrm{E}+00$ & & & & \\
\hline Ir-194 & $014158-35-1$ & $6.8 \mathrm{E}+02$ & $6.8 \mathrm{E}+00$ & & & & \\
\hline $\mathrm{K}-40$ & $013966-00-2$ & $3.8 \mathrm{E}+02$ & $3.8 \mathrm{E}+00$ & & & & \\
\hline $\mathrm{K}-42$ & $014378-21-3$ & $3.7 \mathrm{E}+03$ & $3.7 \mathrm{E}+01$ & & & & \\
\hline $\mathrm{Kr}-83 \mathrm{~m}$ & 013965-98-5 & & & & & & \\
\hline $\mathrm{Kr}-85$ & $013983-27-2$ & & & & & & \\
\hline $\mathrm{Kr}-85 \mathrm{~m}$ & $013983-27-2$ & & & & & & \\
\hline $\mathrm{Kr}-87$ & $014809-68-8$ & & & & & & \\
\hline $\mathrm{Kr}-88$ & 014995-61-0 & & & & & & \\
\hline
\end{tabular}


Table 3.1b. (continued)

\begin{tabular}{|c|c|c|c|c|c|c|c|}
\hline \multirow[t]{2}{*}{ Chemical $^{\dagger}$} & \multirow[t]{2}{*}{ CASRN } & \multicolumn{2}{|c|}{ Ingestion (pCi/L) } & \multicolumn{2}{|c|}{ Inhalation $(\mathrm{pCi} / \mathrm{L})$} & \multicolumn{2}{|c|}{$\begin{array}{c}\text { Ingestion + Inhalation } \\
(\mathrm{pCi} / \mathrm{L})^{\dagger+}\end{array}$} \\
\hline & & $10^{-4}$ & $10^{-6}$ & $10^{-4}$ & $10^{\cdot 6}$ & $10^{-4}$ & $10^{-6}$ \\
\hline $\mathrm{Kr}-89$ & $016316-03-3$ & & & & & & \\
\hline $\mathrm{Kr}-90$ & $015741-13-6$ & & & & & & \\
\hline La-140 & $013981-28-7$ & $5.0 \mathrm{E}+02$ & $5.0 \mathrm{E}+00$ & & & & \\
\hline Lu-177 & $014265-75-9$ & $1.6 \mathrm{E}+03$ & $1.6 \mathrm{E}+01$ & & & & \\
\hline $\mathrm{Mn}-52$ & $014092-99-0$ & $7.9 \mathrm{E}+02$ & $7.9 \mathrm{E}+00$ & & & & \\
\hline $\mathrm{Mn}-54$ & $013966-31-9$ & $2.4 \mathrm{E}+03$ & $2.4 \mathrm{E}+01$ & & & & \\
\hline Mn-56 & $014681-52-8$ & $5.6 \mathrm{E}+03$ & $5.6 \mathrm{E}+01$ & & & & \\
\hline Mo-99 & $014119-15-4$ & $2.1 \mathrm{E}+03$ & $2.1 \mathrm{E}+01$ & & & & \\
\hline $\mathrm{Na}-22$ & $013966-32-0$ & $5.9 \mathrm{E}+02$ & $5.9 \mathrm{E}+00$ & & & & \\
\hline $\mathrm{Na}-24$ & $013982-04-2$ & $3.5 \mathrm{E}+03$ & $3.5 \mathrm{E}+01$ & & & & \\
\hline $\mathrm{Nb}-93 \mathrm{~m}$ & $007440-03-1$ & $7.2 \mathrm{E}+03$ & $7.2 \mathrm{E}+01$ & & & & \\
\hline Nb-94 & $014681-63-1$ & $6.9 \mathrm{E}+02$ & $6.9 \mathrm{E}+00$ & & & & \\
\hline $\mathrm{Nb}-95$ & $013967-76-5$ & $2.1 \mathrm{E}+03$ & $2.1 \mathrm{E}+01$ & & & & \\
\hline $\mathrm{Nb}-95 \mathrm{~m}$ & $013967-76-5$ & $1.6 \mathrm{E}+03$ & $1.6 \mathrm{E}+01$ & & & & \\
\hline $\mathrm{Nb}-97$ & $018496-04-3$ & $2.7 \mathrm{E}+04$ & $2.7 \mathrm{E}+02$ & & & & \\
\hline $\mathrm{Nb}-97 \mathrm{~m}$ & $018496-04-3$ & $1.5 \mathrm{E}+06$ & $1.5 \mathrm{E}+04$ & & & & \\
\hline Nd-147 & $014269-74-0$ & $8.1 \mathrm{E}+02$ & $8.1 \mathrm{E}+00$ & & & & \\
\hline
\end{tabular}


Table 3.1b. (continued)

\begin{tabular}{|c|c|c|c|c|c|c|c|}
\hline \multirow[t]{2}{*}{ Chemical $^{\dagger}$} & \multirow[t]{2}{*}{ CASRN } & \multicolumn{2}{|c|}{ Ingestion (pCi/L) } & \multicolumn{2}{|c|}{ Inhalation (pCi/L) } & \multicolumn{2}{|c|}{$\begin{array}{c}\text { Ingestion + Inhalation } \\
(\mathrm{pCi} / \mathrm{L})^{+t}\end{array}$} \\
\hline & & $10^{-4}$ & $10^{-6}$ & $10^{-4}$ & $10^{-6}$ & $10^{-4}$ & $10^{-6}$ \\
\hline Nd-149 & $015749-81-2$ & $1.0 \mathrm{E}+04$ & $1.0 \mathrm{E}+02$ & & & & \\
\hline $\mathrm{Ni}-59$ & 014336-70-0 & $2.6 \mathrm{E}+04$ & $2.6 \mathrm{E}+02$ & & & & \\
\hline $\mathrm{Ni}-63$ & $013981-37-8$ & $8.7 \mathrm{E}+03$ & $8.7 \mathrm{E}+01$ & & & & \\
\hline $\mathrm{Ni}-65$ & $014833-49-9$ & $8.5 \mathrm{E}+03$ & $8.5 \mathrm{E}+01$ & & & & \\
\hline Np-236 & $015700-36-4$ & $5.1 \mathrm{E}+03$ & $5.1 E+01$ & & & & \\
\hline Np-237 & $013994-20-2$ & $1.6 \mathrm{E}+01$ & $1.6 \mathrm{E}-01$ & & & & \\
\hline $\mathrm{Np}-237+\mathrm{D}$ & 013994-20-2 & $1.6 \mathrm{E}+01$ & $1.6 \mathrm{E}-01$ & & & & \\
\hline $\mathrm{Np}-238$ & $015766-25-3$ & $1.0 \mathrm{E}+03$ & $1.0 \mathrm{E}+01$ & & & & \\
\hline Np-239 & $013968-59-7$ & $1.1 \mathrm{E}+03$ & $1.1 E+01$ & & & & \\
\hline $\mathrm{Np}-240$ & $015690-84-3$ & $2.7 \mathrm{E}+04$ & $2.7 \mathrm{E}+02$ & & & & \\
\hline $\mathrm{Np}-240 \mathrm{~m}$ & $015690-84-3$ & $2.0 \mathrm{E}+05$ & $2.0 \mathrm{E}+03$ & & & & \\
\hline Os-185 & $015766-50-4$ & $2.6 \mathrm{E}+03$ & $2.6 \mathrm{E}+01$ & & & & \\
\hline Os-191 & 014119-24-5 & $1.6 \mathrm{E}+03$ & $1.6 \mathrm{E}+01$ & & & & \\
\hline Os-191m & 014119-24-5 & $9.6 \mathrm{E}+03$ & $9.6 \mathrm{E}+01$ & & & & \\
\hline Os-193 & $016057-77-5$ & $1.1 \mathrm{E}+03$ & $1.1 \mathrm{E}+01$ & & & & \\
\hline $\mathrm{P}-32$ & $014596-37-3$ & $7.8 \mathrm{E}+02$ & $7.8 \mathrm{E}+00$ & & & & \\
\hline P-33 & 015749-66-3 & $6.1 E+03$ & $6.1 \mathrm{E}+01$ & & & & \\
\hline
\end{tabular}


Table 3.1b. (continued)

\begin{tabular}{|c|c|c|c|c|c|c|c|}
\hline \multirow[t]{2}{*}{ Chemical $^{\dagger}$} & \multirow{2}{*}{ CASRN } & \multicolumn{2}{|c|}{ Ingestion (pCi/L) } & \multicolumn{2}{|c|}{ Inhalation (pCi/L) } & \multicolumn{2}{|c|}{$\begin{array}{c}\text { Ingestion + Inhalation } \\
(\mathrm{pCi} / \mathrm{L})^{\dagger+}\end{array}$} \\
\hline & & $10^{-4}$ & $10^{-6}$ & $10^{-4}$ & $10^{-6}$ & $10^{-4}$ & $10^{-6}$ \\
\hline $\mathrm{Pa}-231$ & 014331-85-2 & $3.2 \mathrm{E}+01$ & $3.2 \mathrm{E}-01$ & & & & \\
\hline $\mathrm{Pa}-233$ & 013981-14-1 & $1.0 \mathrm{E}+03$ & $1.0 \mathrm{E}+01$ & & & & \\
\hline $\mathrm{Pa}-234$ & $015100-28-4$ & $2.2 \mathrm{E}+03$ & $2.2 \mathrm{E}+01$ & & & & \\
\hline $\mathrm{Pa}-234 \mathrm{~m}$ & $015100-28-4$ & $1.0 \mathrm{E}+06$ & $1.0 \mathrm{E}+04$ & & & & \\
\hline $\mathrm{Pb}-203$ & $014687-25-3$ & $4.6 \mathrm{E}+03$ & $4.6 \mathrm{E}+01$ & & & & \\
\hline $\mathrm{Pb}-209$ & 014119-30-3 & $2.3 E+04$ & 2.3E+02 & & & & \\
\hline $\mathrm{Pb}-210$ & 014255-04-0 & $7.1 E+00$ & $7.1 \mathrm{E}-02$ & & & & \\
\hline $\mathrm{Pb}-210+\mathrm{D}$ & 014255-04-0 & 4.7E+00 & 4.7E-02 & & & & \\
\hline $\mathrm{Pb}-211$ & $015816-77-0$ & $1.4 \mathrm{E}+04$ & $1.4 \mathrm{E}+02$ & & & & \\
\hline $\mathrm{Pb}-212$ & $015092-94-1$ & $2.6 \mathrm{E}+02$ & $2.6 \mathrm{E}+00$ & & & & \\
\hline $\mathrm{Pb}-214$ & $015067-28-4$ & $1.6 \mathrm{E}+04$ & $1.6 \mathrm{E}+02$ & & & & \\
\hline Pd-100 & $015690-69-4$ & $1.3 \mathrm{E}+03$ & $1.3 \mathrm{E}+01$ & & & & \\
\hline Pd-101 & 015749-54-9 & $1.3 \mathrm{E}+04$ & $1.3 \mathrm{E}+02$ & & & & \\
\hline Pd-103 & $014967-68-1$ & $4.5 \mathrm{E}+03$ & $4.5 \mathrm{E}+01$ & & & & \\
\hline Pd-107 & 017637-99-9 & $2.3 \mathrm{E}+04$ & $2.3 \mathrm{E}+02$ & & & & \\
\hline Pd-109 & $014981-64-7$ & $1.4 \mathrm{E}+03$ & $1.4 \mathrm{E}+01$ & & & & \\
\hline Pm-147 & $014380-75-7$ & $3.4 \mathrm{E}+03$ & $3.4 \mathrm{E}+01$ & & & & \\
\hline
\end{tabular}


Table 3.1b. (continued)

\begin{tabular}{|c|c|c|c|c|c|c|c|}
\hline \multirow[t]{2}{*}{ Chemical $^{\dagger}$} & \multirow[t]{2}{*}{ CASRN } & \multicolumn{2}{|c|}{ Ingestion ( $\mathrm{pCi} / \mathrm{L}$ ) } & \multicolumn{2}{|c|}{ Inhalation (pCi/L) } & \multicolumn{2}{|c|}{$\begin{array}{c}\text { Ingestion + Inhalation } \\
(\mathrm{pCi} / \mathrm{L})^{+t}\end{array}$} \\
\hline & & $10^{-4}$ & $10^{-6}$ & $10^{-4}$ & $10^{-6}$ & $10^{-4}$ & $10^{-6}$ \\
\hline Pm-148 & 014683-19-3 & $3.3 E+02$ & $3.3 \mathrm{E}+00$ & & & & \\
\hline Pm-148m & 014683-19-3 & $4.8 E+02$ & $4.8 \mathrm{E}+00$ & & & & \\
\hline Pm-149 & 015765-31-8 & $8.6 \mathrm{E}+02$ & $8.6 \mathrm{E}+00$ & & & & \\
\hline Po-210 & 013981-52-7 & $1.5 \mathrm{E}+01$ & $1.5 \mathrm{E}-01$ & & & & \\
\hline Po-212 & $015389-34-1$ & $1.1 \mathrm{E}+14$ & $1.1 \mathrm{E}+12$ & & & & \\
\hline Po-213 & $015756-57-7$ & $7.1 E+12$ & $7.1 \mathrm{E}+10$ & & & & \\
\hline Po-214 & $015735-67-8$ & $2.2 E+11$ & $2.2 \mathrm{E}+09$ & & & & \\
\hline Po-215 & $015706-52-2$ & $9.5 \mathrm{E}+09$ & $9.5 \mathrm{E}+07$ & & & & \\
\hline Po-216 & 015756-58-8 & $5.4 \mathrm{E}+07$ & $5.4 \mathrm{E}+05$ & & & & \\
\hline Po-218 & $015422-24-9$ & $9.4 \mathrm{E}+04$ & $9.4 \mathrm{E}+02$ & & & & \\
\hline Pr-142 & 014191-64-1 & $6.8 \mathrm{E}+02$ & $6.8 \mathrm{E}+00$ & & & & \\
\hline $\operatorname{Pr}-143$ & $014981-79-4$ & $7.2 E+02$ & $7.2 \mathrm{E}+00$ & & & & \\
\hline Pr-144 & 014119-05-2 & $5.9 \mathrm{E}+04$ & $5.9 \mathrm{E}+02$ & & & & \\
\hline Pr-144m & 014119-05-2 & $1.5 \mathrm{E}+05$ & $1.5 \mathrm{E}+03$ & & & & \\
\hline Pt-191 & $015706-36-2$ & $3.2 \mathrm{E}+03$ & $3.2 \mathrm{E}+01$ & & & & \\
\hline Pt-193 & $015735-70-3$ & $2.9 \mathrm{E}+04$ & $2.9 \mathrm{E}+02$ & & & & \\
\hline Pt-193m & 015735-70-3 & $1.9 \mathrm{E}+03$ & $1.9 \mathrm{E}+01$ & & & & \\
\hline
\end{tabular}


Table 3.1b. (continued)

\begin{tabular}{|c|c|c|c|c|c|c|c|}
\hline \multirow[t]{2}{*}{ Chemical $^{\dagger}$} & \multirow[t]{2}{*}{ CASRN } & \multicolumn{2}{|c|}{ Ingestion (pCi/L) } & \multicolumn{2}{|c|}{ Inhalation (pCi/L) } & \multicolumn{2}{|c|}{$\begin{array}{c}\text { Ingestion + Inhalation } \\
(\mathrm{pCi} / \mathrm{L})^{\dagger}\end{array}$} \\
\hline & & $10^{-4}$ & $10^{-6}$ & $10^{-4}$ & $10^{-6}$ & $10^{-4}$ & $10^{-6}$ \\
\hline Pt-197 & $015735-74-7$ & $2.2 \mathrm{E}+03$ & $2.2 \mathrm{E}+01$ & & & & \\
\hline Pt-197m & $015735-74-7$ & $1.5 \mathrm{E}+04$ & $1.5 \mathrm{E}+02$ & & & & \\
\hline Pu-236 & $015411-92-4$ & $6.2 \mathrm{E}+01$ & $6.2 \mathrm{E}-01$ & & & & \\
\hline $\mathrm{Pu}-238$ & 013981-16-3 & $1.6 \mathrm{E}+01$ & $1.6 \mathrm{E}-01$ & & & & \\
\hline Pu-239 & $015117-48-3$ & $1.5 \mathrm{E}+01$ & $1.5 \mathrm{E}-01$ & & & & \\
\hline $\mathrm{Pu}-240$ & $014119-33-6$ & $1.5 \mathrm{E}+01$ & $1.5 \mathrm{E}-01$ & & & & \\
\hline $\mathrm{Pu}-241$ & 014119-32-5 & $9.2 \mathrm{E}+02$ & $9.2 \mathrm{E}+00$ & & & & \\
\hline Pu-241+D & 014119-32-5 & $1.4 \mathrm{E}+01$ & $1.4 \mathrm{E}-01$ & & & & \\
\hline $\mathrm{Pu}-242$ & 013982-10-0 & $1.6 \mathrm{E}+01$ & $1.6 \mathrm{E}-01$ & & & & \\
\hline $\mathrm{Pu}-243$ & $015706-37-3$ & $1.3 \mathrm{E}+04$ & $1.3 E+02$ & & & & \\
\hline Pu-244 & 014119-34-7 & $1.5 \mathrm{E}+01$ & $1.5 \mathrm{E}-01$ & & & & \\
\hline $\mathrm{Pu}-244+\mathrm{D}$ & 014119-34-7 & $1.5 \mathrm{E}+01$ & $1.5 \mathrm{E}-01$ & & & & \\
\hline $\mathrm{Ra}-223$ & $015623-45-7$ & $2.0 \mathrm{E}+01$ & $2.0 \mathrm{E}-01$ & & & & \\
\hline $\mathrm{Ra}-224$ & $013233-32-4$ & $3.2 \mathrm{E}+01$ & $3.2 \mathrm{E}-01$ & & & & \\
\hline $\mathrm{Ra}-225$ & 013981-53-8 & $3.0 \mathrm{E}+01$ & $3.0 \mathrm{E}-01$ & & & & \\
\hline $\mathrm{Ra}-226$ & 013982-63-3 & $1.6 \mathrm{E}+01$ & $1.6 \mathrm{E}-01$ & & & & \\
\hline $\mathrm{Ra}-226+\mathrm{D}$ & 013982-63-3 & $1.6 \mathrm{E}+01$ & $1.6 \mathrm{E}-01$ & & & & \\
\hline
\end{tabular}


Table 3.1b. (continued)

\begin{tabular}{|c|c|c|c|c|c|c|c|}
\hline \multirow[t]{2}{*}{ Chemical $^{\dagger}$} & \multirow[t]{2}{*}{ CASRN } & \multicolumn{2}{|c|}{ Ingestion (pCi/L) } & \multicolumn{2}{|c|}{ Inhalation (pCi/L) } & \multicolumn{2}{|c|}{$\begin{array}{c}\text { Ingestion + Inhalation } \\
(\mathrm{pCi} / \mathrm{L})^{\dagger+}\end{array}$} \\
\hline & & $10^{-4}$ & $10^{-6}$ & $10^{-4}$ & $10^{-6}$ & $10^{-4}$ & $10^{-6}$ \\
\hline Ra-228 & $015262-20-1$ & $1.9 \mathrm{E}+01$ & $1.9 \mathrm{E}-01$ & & & & \\
\hline $\mathrm{Ra}-228+\mathrm{D}$ & $015262-20-1$ & $1.9 \mathrm{E}+01$ & $1.9 \mathrm{E}-01$ & & & & \\
\hline $\mathrm{Rb}-82$ & 014391-63-0 & $4.5 \mathrm{E}+05$ & $4.5 E+03$ & & & & \\
\hline $\mathrm{Rb}-86$ & 014932-53-7 & $6.7 \mathrm{E}+02$ & $6.7 \mathrm{E}+00$ & & & & \\
\hline $\mathrm{Rb}-87$ & 013982-13-3 & $1.3 \mathrm{E}+03$ & $1.3 \mathrm{E}+01$ & & & & \\
\hline $\mathrm{Rb}-88$ & 014928-36-0 & $3.3 \mathrm{E}+04$ & $3.3 \mathrm{E}+02$ & . & & & \\
\hline $\mathrm{Rb}-89$ & $014191-65-2$ & $5.5 \mathrm{E}+04$ & $5.5 \mathrm{E}+02$ & & & & \\
\hline $\mathrm{Rh}-103 \mathrm{~m}$ & $007440-16-6$ & $5.8 \mathrm{E}+05$ & $5.8 \mathrm{E}+03$ & & & & \\
\hline $\mathrm{Rh}-105$ & 014913-89-4 & $2.5 \mathrm{E}+03$ & $2.5 \mathrm{E}+01$ & & & & \\
\hline Rh-105m & $014913-89-4$ & $4.4 \mathrm{E}+06$ & $4.4 \mathrm{E}+04$ & & & & \\
\hline Rh-106 & $014234-34-5$ & $1.3 \mathrm{E}+06$ & $1.3 E+04$ & & & & \\
\hline $\mathrm{Rn}-219$ & $014835-02-0$ & & & & & & \\
\hline $\mathrm{Rn}-220$ & $022481-48-7$ & & & $7.8 \mathrm{E}+03$ & $7.8 \mathrm{E}+01$ & & \\
\hline $\mathrm{Rn}-222+\mathrm{D}$ & $014859-67-7$ & & & $2.0 \mathrm{E}+02$ & $2.0 \mathrm{E}+00$ & & \\
\hline $\mathrm{Ru}-103$ & 013968-53-1 & $1.4 \mathrm{E}+03$ & $1.4 \mathrm{E}+01$ & & & & \\
\hline Ru-105 & $014331-95-4$ & $4.1 \mathrm{E}+03$ & $4.1 \mathrm{E}+01$ & & & & \\
\hline Ru-106 & $013967-48-1$ & $1.4 \mathrm{E}+02$ & $1.4 \mathrm{E}+00$ & & & & \\
\hline
\end{tabular}


Table 3.1b. (continued)

\begin{tabular}{|c|c|c|c|c|c|c|c|}
\hline \multirow[t]{2}{*}{ Chemical $^{\dagger}$} & \multirow[t]{2}{*}{ CASRN } & \multicolumn{2}{|c|}{ Ingestion (pCi/L) } & \multicolumn{2}{|c|}{ Inhalation (pCi/L) } & \multicolumn{2}{|c|}{$\begin{array}{c}\text { Ingestion + Inhalation } \\
(\mathrm{pCi} / \mathrm{L})^{\dagger t}\end{array}$} \\
\hline & & $10^{-4}$ & $10^{-6}$ & $10^{-4}$ & $10^{-6}$ & $10^{-4}$ & $10^{-6}$ \\
\hline $\mathrm{Ru}-106+\mathrm{D}$ & $013967-48-1$ & $1.4 \mathrm{E}+02$ & $1.4 \mathrm{E}+00$ & & & & \\
\hline Ru-97 & $015758-35-7$ & $8.1 \mathrm{E}+03$ & $8.1 E+01$ & & & & \\
\hline $\mathrm{S}-35$ & $015117-53-0$ & $1.1 \mathrm{E}+04$ & $1.1 \mathrm{E}+02$ & & & & \\
\hline Sb-122 & 014374-79-9 & $5.4 \mathrm{E}+02$ & $5.4 \mathrm{E}+00$ & & & & \\
\hline Sb-124 & $014683-10-4$ & $4.5 \mathrm{E}+02$ & $4.5 \mathrm{E}+00$ & & & & \\
\hline Sb-125 & 014234-35-6 & $1.6 \mathrm{E}+03$ & $1.6 \mathrm{E}+01$ & & & & \\
\hline $\mathrm{Sb}-125+\mathrm{D}$ & 014234-35-6 & $1.3 \mathrm{E}+03$ & $1.3 \mathrm{E}+01$ & & & & \\
\hline $\mathrm{Sb}-126$ & 015756-32-8 & $4.9 \mathrm{E}+02$ & $4.9 E+00$ & & & & \\
\hline $\mathrm{Sb}-126 \mathrm{~m}$ & 015756-32-8 & $6.5 \mathrm{E}+04$ & $6.5 \mathrm{E}+02$ & & & & \\
\hline Sb-127 & 013968-50-8 & $5.6 \mathrm{E}+02$ & $5.6 \mathrm{E}+00$ & & & & \\
\hline Sb-129 & 014331-88-5 & $2.6 \mathrm{E}+03$ & $2.6 \mathrm{E}+01$ & & & & \\
\hline Sc-46 & 013967-63-0 & $8.3 E+02$ & $8.3 E+00$ & & & & \\
\hline $\mathrm{Sc}-47$ & 014391-96-9 & $1.6 \mathrm{E}+03$ & $1.6 \mathrm{E}+01$ & & & & \\
\hline Sc-48 & 014391-86-7 & $7.2 \mathrm{E}+02$ & $7.2 \mathrm{E}+00$ & & & & \\
\hline Se-75 & $014265-71-5$ & $7.3 E+02$ & $7.3 \mathrm{E}+00$ & & & & \\
\hline Si-31 & $014276-49-4$ & $9.4 \mathrm{E}+03$ & $9.4 \mathrm{E}+01$ & & & & \\
\hline Sm-147 & $014392-33-7$ & $1.9 E+02$ & $1.9 \mathrm{E}+00$ & & & & \\
\hline
\end{tabular}


Table 3.1b. (continued)

\begin{tabular}{|c|c|c|c|c|c|c|c|}
\hline \multirow[t]{2}{*}{ Chemical $^{\dagger}$} & \multirow[t]{2}{*}{ CASRN } & \multicolumn{2}{|c|}{ Ingestion $(\mathrm{pCi} / \mathrm{L})$} & \multicolumn{2}{|c|}{ Inhalation (pCi/L) } & \multicolumn{2}{|c|}{$\begin{array}{c}\text { Ingestion + Inhalation } \\
(\mathrm{pCi} / \mathbf{L})^{+t}\end{array}$} \\
\hline & & $10^{-4}$ & $10^{-6}$ & $10^{-4}$ & $10^{-6}$ & $10^{-4}$ & $10^{-6}$ \\
\hline Sm-151 & $015715-94-3$ & $1.0 \mathrm{E}+04$ & $1.0 \mathrm{E}+02$ & & & & \\
\hline Sm-153 & $015766-00-4$ & $1.2 \mathrm{E}+03$ & $1.2 \mathrm{E}+01$ & & & & \\
\hline Sn-113 & 013966-06-8 & $1.3 \mathrm{E}+03$ & $1.3 \mathrm{E}+01$ & & & & \\
\hline Sn-121 & $014683-06-8$ & $3.9 \mathrm{E}+03$ & $3.9 \mathrm{E}+01$ & & & & \\
\hline $\mathrm{Sn}-121 \mathrm{~m}$ & $014683-06-8$ & $2.4 \mathrm{E}+03$ & $2.4 \mathrm{E}+01$ & & & & \\
\hline $\mathrm{Sn}-125$ & $014683-08-0$ & $2.8 \mathrm{E}+02$ & $2.8 \mathrm{E}+00$ & & & & \\
\hline Sn-126 & $015832-50-5$ & $2.2 \mathrm{E}+02$ & $2.2 \mathrm{E}+00$ & & & & \\
\hline $\mathrm{Sr}-82$ & $014809-50-8$ & $1.8 \mathrm{E}+02$ & $1.8 \mathrm{E}+00$ & & & & \\
\hline Sr-85 & $013967-73-2$ & $3.4 \mathrm{E}+03$ & $3.4 \mathrm{E}+01$ & & & & \\
\hline $\mathrm{Sr}-85 \mathrm{~m}$ & $013967-73-2$ & $2.6 \mathrm{E}+05$ & $2.6 \mathrm{E}+03$ & & & & \\
\hline $\mathrm{Sr}-89$ & $014158-27-1$ & $4.6 \mathrm{E}+02$ & $4.6 \mathrm{E}+00$ & & & & \\
\hline Sr-90 & $010098-97-2$ & $1.2 \mathrm{E}+02$ & $1.2 \mathrm{E}+00$ & & & & \\
\hline Sr-90+D & $010098-97-2$ & $8.5 \mathrm{E}+01$ & $8.5 \mathrm{E}-01$ & & & & \\
\hline Sr-91 & 014331-91-0 & $1.7 \mathrm{E}+03$ & $1.7 \mathrm{E}+01$ & & & & \\
\hline Sr-92 & $014928-29-1$ & $2.3 \mathrm{E}+03$ & $2.3 E+01$ & & & & \\
\hline Ta-182 & 013982-00-8 & $6.8 \mathrm{E}+02$ & $6.8 \mathrm{E}+00$ & & & & \\
\hline Tb-158 & $015759-55-4$ & $1.1 \mathrm{E}+03$ & $1.1 \mathrm{E}+01$ & & & & \\
\hline
\end{tabular}


Table 3.1b. (continued)

\begin{tabular}{|c|c|c|c|c|c|c|c|}
\hline \multirow[t]{2}{*}{ Chemical $^{\dagger}$} & \multirow[t]{2}{*}{ CASRN } & \multicolumn{2}{|c|}{ Ingestion $(\mathrm{pCi} / \mathrm{L})$} & \multicolumn{2}{|c|}{ Inhalation (pCi/L) } & \multicolumn{2}{|c|}{$\begin{array}{c}\text { Ingestion + Inhalation } \\
(\mathrm{pCi} / \mathrm{L})^{+t}\end{array}$} \\
\hline & & $10^{-4}$ & $10^{-6}$ & $10^{-4}$ & $10^{-6}$ & $10^{-4}$ & $10^{-6}$ \\
\hline $\mathrm{Tb}-160^{\circ}$ & 013981-29-8 & $6.2 \mathrm{E}+02$ & $6.2 \mathrm{E}+00$ & & & & \\
\hline Tc-95 & $014809-56-4$ & $7.0 \mathrm{E}+04$ & $7.0 \mathrm{E}+02$ & & & & \\
\hline Tc-95m & $014809-56-4$ & $3.8 \mathrm{E}+03$ & $3.8 \mathrm{E}+01$ & & & & \\
\hline Tc-96 & $014808-44-7$ & $2.1 E+03$ & $2.1 \mathrm{E}+01$ & & & & \\
\hline Tc-96m & 014808-44-7 & $1.8 \mathrm{E}+05$ & $1.8 \mathrm{E}+03$ & & & & \\
\hline Tc-97 & 015759-35-0 & $3.0 \mathrm{E}+04$ & $3.0 \mathrm{E}+02$ & & & & \\
\hline Tc-97m & 015759-35-0 & $4.0 \mathrm{E}+03$ & $4.0 \mathrm{E}+01$ & & & & \\
\hline Tc-99 & 014133-76-7 & $3.4 \mathrm{E}+03$ & $3.4 \mathrm{E}+01$ & & & & \\
\hline Tc-99m & 014133-76-7 & $8.5 E+04$ & $8.5 \mathrm{E}+02$ & & & & \\
\hline $\mathrm{Te}-125 \mathrm{~m}$ & 014390-73-9 & $1.9 \mathrm{E}+03$ & $1.9 \mathrm{E}+01$ & & & & \\
\hline $\mathrm{Te}-127$ & 013981-49-2 & $5.6 \mathrm{E}+03$ & $5.6 \mathrm{E}+01$ & & & & \\
\hline $\mathrm{Te}-127 \mathrm{~m}$ & $013981-49-2$ & $7.9 \mathrm{E}+02$ & $7.9 \mathrm{E}+00$ & & & & \\
\hline Te-129 & $014269-71-7$ & $3.2 \mathrm{E}+04$ & $3.2 \mathrm{E}+02$ & & & & \\
\hline $\mathrm{Te}-129 \mathrm{~m}$ & 014269-71-7 & 4.1E+02 & $4.1 \mathrm{E}+00$ & & & & \\
\hline $\mathrm{Te}-131$ & 014683-12-6 & $1.2 \mathrm{E}+04$ & $1.2 E+02$ & & & & \\
\hline $\mathrm{Te}-131 \mathrm{~m}$ & 014683-12-6 & $5.4 \mathrm{E}+02$ & $5.4 \mathrm{E}+00$ & & & & \\
\hline Te-132 & $014234-28-7$ & $3.9 \mathrm{E}+02$ & $3.9 \mathrm{E}+00$ & & & & \\
\hline
\end{tabular}


Table 3.1b. (continued)

\begin{tabular}{|c|c|c|c|c|c|c|c|}
\hline \multirow[t]{2}{*}{ Chemical $^{\dagger}$} & \multirow[t]{2}{*}{ CASRN } & \multicolumn{2}{|c|}{ Ingestion (pCi/L) } & \multicolumn{2}{|c|}{ Inhalation (pCi/L) } & \multicolumn{2}{|c|}{$\begin{array}{c}\text { Ingestion + Inhalation } \\
(\mathrm{pCi} / \mathbf{L})^{+t}\end{array}$} \\
\hline & & $10^{-4}$ & $10^{-6}$ & $10^{-4}$ & $10^{-6}$ & $10^{-4}$ & $10^{-6}$ \\
\hline Th-227 & $015623-47-9$ & $1.2 \mathrm{E}+02$ & $1.2 \mathrm{E}+00$ & & & & \\
\hline Th-228 & $014274-82-9$ & $7.6 \mathrm{E}+01$ & 7.6E-01 & & - & & \\
\hline Th-228+D & $014274-82-9$ & $2.1 \mathrm{E}+01$ & 2.1E-01 & & & & \\
\hline Th-229 & $015594-54-4$ & $8.4 \mathrm{E}+01$ & 8.4E-01 & & & & \\
\hline Th-229+D & $015594-54-4$ & $1.3 \mathrm{E}+01$ & $1.3 \mathrm{E}-01$ & & & & \\
\hline Th-230 & $014269-63-7$ & $1.3 \mathrm{E}+02$ & $1.3 \mathrm{E}+00$ & & & & \\
\hline Th-231 & $014932-40-2$ & $2.7 \mathrm{E}+03$ & $2.7 \mathrm{E}+01$ & & & & \\
\hline Th-232 & $007440-29-1$ & $1.5 \mathrm{E}+02$ & $1.5 \mathrm{E}+00$ & & & & \\
\hline Th-234 & $015065-10-8$ & $2.5 \mathrm{E}+02$ & $2.5 \mathrm{E}+00$ & & & & \\
\hline Tl-202 & $015720-57-7$ & $4.7 \mathrm{E}+03$ & $4.7 \mathrm{E}+01$ & & & & \\
\hline Tl-204 & $013968-51-9$ & $2.4 \mathrm{E}+03$ & $2.4 \mathrm{E}+01$ & & & & \\
\hline Tl-208 & 014913-50-9 & $2.7 \mathrm{E}+05$ & $2.7 \mathrm{E}+03$ & & & & \\
\hline Tl-209 & $015690-73-0$ & $3.4 \mathrm{E}+05$ & $3.4 \mathrm{E}+03$ & & & & \\
\hline Tm-170 & $013981-30-1$ & $6.3 E+02$ & $6.3 \mathrm{E}+00$ & & & & \\
\hline Tm-171 & $014333-45-0$ & 8.1E+03 & $8.1 \mathrm{E}+01$ & & & & \\
\hline U-232 & $014158-29-3$ & $5.9 \mathrm{E}+01$ & $5.9 \mathrm{E}-01$ & & & & \\
\hline U-233 & 013968-55-3 & $1.1 \mathrm{E}+02$ & $1.1 \mathrm{E}+00$ & & & & \\
\hline
\end{tabular}


Table 3.1b. (continued)

\begin{tabular}{|c|c|c|c|c|c|c|c|}
\hline \multirow[t]{2}{*}{ Chemical $^{\dagger}$} & \multirow[t]{2}{*}{ CASRN } & \multicolumn{2}{|c|}{ Ingestion (pCi/L) } & \multicolumn{2}{|c|}{ Inhalation (pCi/L) } & \multicolumn{2}{|c|}{$\begin{array}{c}\text { Ingestion + Inhalation } \\
(\mathrm{pCi} / \mathrm{L})^{\mathrm{tt}}\end{array}$} \\
\hline & & $10^{-4}$ & $10^{-6}$ & $10^{-4}$ & $10^{-6}$ & $10^{-4}$ & $10^{-6}$ \\
\hline $\mathrm{U}-234$ & 013966-29-5 & $1.1 \mathrm{E}+02$ & $1.1 \mathrm{E}+00$ & & & & \\
\hline $\mathrm{U}-235$ & 015117-96-1 & $1.1 \mathrm{E}+02$ & $1.1 \mathrm{E}+00$ & & & & \\
\hline $\mathrm{U}-235+\mathrm{D}$ & 015117-96-1 & $1.0 \mathrm{E}+02$ & $1.0 \mathrm{E}+00$ & & & & \\
\hline U-236 & $013982-70-2$ & $1.1 \mathrm{E}+02$ & $1.1 \mathrm{E}+00$ & & & & \\
\hline U-237 & $014269-75-1$ & $1.2 \mathrm{E}+03$ & $1.2 \mathrm{E}+01$ & & & & \\
\hline U-238 & $007440-61-1$ & $1.1 \mathrm{E}+02$ & $1.1 \mathrm{E}+00$ & & & & \\
\hline$U-238+D$ & $007440-61-1$ & $7.7 \mathrm{E}+01$ & 7.7E-01 & & & & \\
\hline $\mathrm{U}-240$ & $015687-53-3$ & $8.7 \mathrm{E}+02$ & $8.7 \mathrm{E}+00$ & & & & \\
\hline V-48 & 014331-97-6 & $6.3 \mathrm{E}+02$ & $6.3 \mathrm{E}+00$ & & & & \\
\hline$W-181$ & $015749-46-9$ & $1.8 \mathrm{E}+04$ & $1.8 \mathrm{E}+02$ & & & & \\
\hline W-185 & $014932-41-3$ & $2.3 \mathrm{E}+03$ & $2.3 \mathrm{E}+01$ & & & & \\
\hline W-187 & 014983-48-3 & $1.9 \mathrm{E}+03$ & $1.9 \mathrm{E}+01$ & & & & \\
\hline $\mathrm{Xe}-122$ & $015151-09-4$ & & & & & & \\
\hline $\mathrm{Xe}-123$ & $015700-10-4$ & & & & & & \\
\hline $\mathrm{Xe}-125$ & 013994-18-8 & & & & & & \\
\hline $\mathrm{Xe}-127$ & 013994-19-9 & & & & & & \\
\hline $\mathrm{Xe}-129 \mathrm{~m}$ & $013965-99-6$ & & & & & & \\
\hline
\end{tabular}


Table 3.1b. (continued)

\begin{tabular}{|c|c|c|c|c|c|c|c|}
\hline \multirow[t]{2}{*}{ Chemical $^{t}$} & \multirow[t]{2}{*}{ CASRN } & \multicolumn{2}{|c|}{ Ingestion (pCi/L) } & \multicolumn{2}{|c|}{ Inhalation $(\mathrm{pCi} / \mathrm{L})$} & \multicolumn{2}{|c|}{$\begin{array}{c}\text { Ingestion + Inhalation } \\
(\mathrm{pCi} / \mathrm{L})^{+t}\end{array}$} \\
\hline & & $10^{-4}$ & $10^{-6}$ & $10^{-4}$ & $10^{-6}$ & $10^{-4}$ & $10^{-6}$ \\
\hline $\mathrm{Xe}-131 \mathrm{~m}$ & $014683-11-5$ & & & & & & \\
\hline Xe-133 & $014932-42-4$ & & & & & & \\
\hline $\mathrm{Xe}-133 \mathrm{~m}$ & $014932-42-4$ & & & & & & \\
\hline Xe-135 & $014995-62-1$ & & & & & & \\
\hline $\mathrm{Xe}-135 \mathrm{~m}$ & $014995-62-1$ & & & & & & \\
\hline Xe-137 & $014835-21-3$ & $\cdot$ & & & & & \\
\hline Xe-138 & 015751-81-2 & & & & & & \\
\hline$Y-90$ & $010098-91-6$ & $3.2 \mathrm{E}+02$ & $3.2 \mathrm{E}+00$ & & & & \\
\hline$Y-91$ & $014234-24-3$ & $3.5 \mathrm{E}+02$ & $3.5 \mathrm{E}+00$ & & & & \\
\hline Y-91m & $014234-24-3$ & $1.3 \mathrm{E}+05$ & $1.3 \mathrm{E}+03$ & & & & \\
\hline Y-92 & $015751-59-4$ & $2.4 \mathrm{E}+03$ & $2.4 \mathrm{E}+01$ & & & & \\
\hline$Y-93$ & $014981-70-5$ & $8.3 \mathrm{E}+02$ & $8.3 \mathrm{E}+00$ & & & & \\
\hline $\mathrm{Zn}-65$ & 013982-39-3 & $4.8 \mathrm{E}+02$ & $4.8 \mathrm{E}+00$ & & & & \\
\hline $\mathrm{Zn}-69$ & $013982-23-5$ & $7.7 \mathrm{E}+04$ & $7.7 E+02$ & & & & \\
\hline $\mathrm{Zn}-69 \mathrm{~m}$ & $013982-23-5$ & $3.1 \mathrm{E}+03$ & $3.1 \mathrm{E}+01$ & & & & \\
\hline $\mathrm{Zr}-93$ & $015751-77-6$ & $9.1 \mathrm{E}+03$ & $9.1 \mathrm{E}+01$ & & & & \\
\hline $\mathrm{Zr}-95$ & $013967-71-0$ & $1.2 \mathrm{E}+03$ & $1.2 \mathrm{E}+01$ & & & & \\
\hline
\end{tabular}


Table 3.1b. (continued)

\begin{tabular}{|l|l|c|c|c|c|c|c|}
\hline \multirow{2}{*}{ Chemical } & \multirow{2}{*}{ CASRN } & \multicolumn{2}{|c|}{ Ingestion (pCi/L) } & \multicolumn{2}{c|}{ Inhalation (pCi/L) } & \multicolumn{2}{|c|}{$\begin{array}{c}\text { Ingestion + Inhalation } \\
(\mathrm{pCi} / \mathrm{L})^{+\dagger}\end{array}$} \\
\cline { 3 - 8 } & & $10^{-4}$ & $10^{-6}$ & $10^{-4}$ & $10^{-6}$ & \multicolumn{1}{c|}{$10^{-4}$} & $10^{-6}$ \\
\hline $\mathrm{Zr}-97$ & $014928-30-4$ & $4.6 \mathrm{E}+02$ & $4.6 \mathrm{E}+00$ & & & & \\
\hline
\end{tabular}

Notes:

$\dagger \quad$ Isotopes designated with an " $m$ " indicate that the isotope is metastable; isotopes designated with a " $\mathrm{D}$ " indicate that the radionuclide slope factors include contributions from daughter products.

i† The total PRG for ingestion plus inhalation is not equal to the sum of the individual pathway PRGs. If slope factors were available for only one pathway (i.e., ingestion $O R$ inhalation), the total for ingestion plus inhalation was not calculated. 
Table 3.1c. Risk-based Preliminary Remediation Goals for nonradionuclides in soil (residential scenario)

\begin{tabular}{|c|c|c|c|c|c|c|c|c|c|c|c|c|c|c|c|c|c|c|c|}
\hline \multirow{2}{*}{ Chemical } & \multirow{2}{*}{ CASRN } & \multicolumn{6}{|c|}{ Ingestion (mg/kg) } & \multicolumn{4}{|c|}{ Dermal (mg/kg) } & \multicolumn{4}{|c|}{ Inhalation (mg/kg) } & \multicolumn{4}{|c|}{$\begin{aligned} & \text { Ingestion + Dermal } \\
+ & \text { Inhalation }(\mathrm{mg} / \mathrm{kg})^{+\bullet \gamma}\end{aligned}$} \\
\hline & & $10^{-4}$ & $10^{6}$ & $\begin{array}{l}\mathrm{HQ}=1 \\
\text { Adult }\end{array}$ & $\begin{array}{l}\text { HQ=1 } \\
\text { Child }\end{array}$ & $\begin{array}{c}\mathrm{HQ}=0.1 \\
\text { Adult }\end{array}$ & $\begin{array}{c}\text { HQ }=0.1 \\
\text { Child }\end{array}$ & $10^{-1}$ & $10^{5}$ & $\mathrm{HQ}=1$ & $H Q=0.1$ & $10^{4}$ & $10^{6}$ & $H Q=1$ & $\mathrm{HQ}=0.1$ & $10^{-4}$ & $10^{4}$ & $\mathrm{HI}=1$ & $\mathrm{HI}=0.1$ \\
\hline ALAR & $001596-84.5$ & & & $1.1 \mathrm{E}+05$ & $1.2 \mathrm{E}+04$ & $1.1 E+04$ & $1.2 E+03$ & & & $1.0 \mathrm{E}+05$ & $1.0 \mathrm{E}+04$ & & & & & & & $5.3 \mathrm{E}+04$ & $5.3 E+03$ \\
\hline Acenaphthene & $000083-32-9$ & & & $4.4 \mathrm{E}+04$ & 4.7E+03 & $4.4 E+03$ & $4.7 \mathrm{E}+02$ & & & $2.6 \mathrm{E}+04$ & $2.6 \mathrm{E}+03$ & & & & & & & $1.6 \mathrm{E}+04$ & $1.6 \mathrm{E}+03$ \\
\hline $\begin{array}{l}\text { Acenuphthy. } \\
\text { lene }\end{array}$ & $000208-96-8$ & & & & & & & & & & & & & & & & & & \\
\hline Acephate & $030560-19-1$ & $7.3 \mathrm{E}+03$ & $7.3 \mathrm{E}+01$ & $2.9 \mathrm{E}+03$ & $3.1 \mathrm{E}+02$ & $2.9 \mathrm{E}+02$ & $3.1 \mathrm{E}+01$ & $1.8 \mathrm{E}+04$ & $1.8 \mathrm{E}+02$ & $2.8 \mathrm{E}+03$ & $2.8 \mathrm{E}+02$ & & & & & $9.5 \mathrm{E}+03$ & $9.5 E+01$ & $1.4 \mathrm{E}+03$ & $1.4 \mathrm{E}+02$ \\
\hline Acetaldehyde & $000075 \cdot 07-0$ & & & & & & & & & & & $6.6 \mathrm{E}+03$ & $6.6 \mathrm{E}+01$ & $5.6 \mathrm{E}+02$ & $5.6 \mathrm{E}+01$ & & & & \\
\hline Acetochlor & $034256-82-1$ & & & $1.5 \mathrm{E}+04$ & $1.6 \mathrm{E}+03$ & $1.5 \mathrm{E}+03$ & $1.6 \mathrm{E}+02$ & & & $1.4 E+04$ & $1.4 E+03$ & & & & & & & $7.1 E+03$ & $7.1 \mathrm{E}+02$ \\
\hline Acetone & $000067-64-1$ & & & $7.3 \mathrm{E}+04$ & $7,8 \mathrm{E}+03$ & 7.3E+03 & $7.8 \mathrm{E}+02$ & & " & $1.1 \mathrm{E}+05$ & $1.1 \mathrm{E}+04$ & & & & & & & $4.5 E+04$ & $4.5 \mathrm{E}+03$ \\
\hline $\begin{array}{l}\text { Acelone Cya. } \\
\text { nohydrin }\end{array}$ & $000075-86-5$ & & & $5.8 \mathrm{E}+02$ & $6.3 \mathrm{E}+01$ & $5.8 \mathrm{E}+01$ & $6.3 \mathrm{E}+00$ & & & S.SE+02 & $5.5 \mathrm{E}+01$ & & & $1.0 \mathrm{E}+06$ & $1.0 \mathrm{E}+06$ & & & $2.8 \mathrm{E}+02$ & $2.8 \mathrm{E}+01$ \\
\hline Acetonitrile & $000075-05-8$ & & & $4.4 E+03$ & $4.7 \mathrm{E}+02$ & $4,4 E+02$ & $4.7 \mathrm{E}+01$ & & & $6.6 \mathrm{E}+03$ & $6.6 \mathrm{E}+02$ & & & $1.0 \mathrm{E}+06$ & $1.0 \mathrm{E}+06$ & & & $2.6 \mathrm{E}+03$ & $2.6 \mathrm{E}+02$ \\
\hline $\begin{array}{l}\text { Acetophe- } \\
\text { nones }\end{array}$ & $000098.86-2$ & & & $7.3 E+04$ & $7.8 \mathrm{E}+03$ & $7.3 \mathrm{E}+03$ & $7.8 \mathrm{E}+02$ & & & $1.1 \mathrm{E}+05$ & 1.IE+04 & & & $8.9 \mathrm{E}+04$ & $8.9 \mathrm{E}+03$ & & & $2.9 \mathrm{E}+04$ & $2.9 \mathrm{E}+03$ \\
\hline Acrolein ${ }^{(1)}$ & $000107-02-8$ & & & $1.5 \mathrm{E}+04$ & $1.6 \mathrm{E}+03$ & $1.5 \mathrm{E}+03$ & $1.6 \mathrm{E}+02$ & & & $2.2 \mathrm{E}+04$ & $2.2 \mathrm{E}+03$ & & & $2.8 \mathrm{E}-01$ & $2.8 \mathrm{E}-02$ & & & $2.8 \mathrm{E}-01$ & $28 \mathrm{E}-02$ \\
\hline Acrylamide & $000079-06-1$ & $1.4 \mathrm{E}+01$ & $1.4 \mathrm{E}-01$ & 1.5E+02 & $1.6 \mathrm{E}+01$ & $1.5 \mathrm{E}+01$ & $1.6 \mathrm{E}+00$ & $3.6 \mathrm{E}+01$ & $3.6 \mathrm{E}-01$ & $1.4 \mathrm{E}+02$ & $1.4 \mathrm{E}+01$ & 8.1E+05 & $8.1 \mathrm{E}+03$ & & & $1.8 \mathrm{E}+01$ & $1.8 \mathrm{E}-01$ & 7.1E+01 & $7.1 E+\infty 0$ \\
\hline Acrylic Acid & $000079-10-7$ & & & $3.7 \mathrm{E}+05$ & $3.9 E+04$ & $3.7 \mathrm{E}+04$ & $3.9 E+03$ & & & $3.4 \mathrm{E}+05$ & $3.4 E+04$ & & & $1.0 \mathrm{E}+06$ & 4.5E+05 & & & $1.7 \mathrm{E}+0 \mathrm{~S}$ & $1.7 E+04$ \\
\hline Acrylonitrile & $000107-13-1$ & $1,2 E+02$ & $1.2 \mathrm{E}+\infty$ & $7.3 \mathrm{E}+02$ & $7.8 \mathrm{E}+01$ & $7.3 E+01$ & $7.8 \mathrm{E}+00$ & $4.8 \mathrm{E}+02$ & $4.8 \mathrm{E}+\infty 0$ & $1.1 \mathrm{E}+03$ & $1.1 \mathrm{E}+02$ & $1.3 \mathrm{E}+01$ & $1.3 \mathrm{E}-01$ & $7.6 \mathrm{E}+00$ & $7.6 \mathrm{E}-0 \mathrm{t}$ & $1.2 \mathrm{E}+01$ & $1.2 \mathrm{E}-01$ & $7.4 E+\infty 0$ & $7.4 \mathrm{E}-01$ \\
\hline Alachlor & 015972.60 .8 & $8.0 \mathrm{E}+02$ & $8.0 \mathrm{E}+00$ & $7.3 E+03$ & $7.8 \mathrm{E}+02$ & $7.3 \mathrm{E}+02$ & $7.8 \mathrm{E}+0 \mathrm{t}$ & $2.0 \mathrm{E}+03$ & $2.0 \mathrm{E}+01$ & $6.9 \mathrm{E}+03$ & $6.9 \mathrm{E}+02$ & & & & & $1.0 E+03$ & $1.0 \mathrm{E}+01$ & $3.5 \mathrm{E}+03$ & $3.5 E+02$ \\
\hline Aldicarb & $000116-06-3$ & & & $7.3 \mathrm{E}+02$ & $7.8 \mathrm{E}+01$ & $7.3 E+01$ & $7.8 \mathrm{E}+00$ & & & $6.9 \mathrm{E}+02$ & $6.9 E+01$ & & & & & & & $3.5 \mathrm{E}+02$ & $3.5 \mathrm{E}+01$ \\
\hline $\begin{array}{l}\text { Aldicarb Sul- } \\
\text { fone }\end{array}$ & $001646.88-4$ & & & $7.3 \mathrm{E}+02$ & $7.8 \mathrm{E}+01$ & $7.3 E+01$ & $7.8 \mathrm{E}+00$ & & & $6.9 \mathrm{E}+02$ & $6.9 \mathrm{E}+01$ & & & & & & & $3.5 E+02$ & $3.5 \mathrm{E}+01$ \\
\hline Addrin & $000309-00-2$ & $3.8 \mathrm{E}+00$ & $3.8 \mathrm{E}-02$ & $2.2 \mathrm{E}+01$ & $2.3 \mathrm{E}+\infty 0$ & $2.2 \mathrm{E}+00$ & 2.3E-01 & $9.5 E+\infty 0$ & 9.5E-02 & $2.1 E+01$ & $2.1 \mathrm{E}+00$ & $2.1 \mathrm{E}+05$ & $2.1 E+03$ & & & $4.9 E+\infty$ & 4.9E-02 & $1.1 E+01$ & $1.1 \mathrm{E}+00$ \\
\hline Ally & $074223-64-6$ & & & $1.8 \mathrm{E}+05$ & $2.0 \mathrm{E}+04$ & $1.8 E+04$ & $2.0 \mathrm{E}+03$ & & & $1.7 \mathrm{E}+05$ & $1.7 \mathrm{E}+04$ & & & & & & & $8.9 E+04$ & $8.9 E+03$ \\
\hline Allyl Alcohol & $000107-18-6$ & & & $3.7 \mathrm{E}+03$ & $3.9 E+02$ & $3.7 E+02$ & $3.9 E+01$ & & & $5.5 \mathrm{E}+03$ & $5.5 E+02$ & & & & & & & $2.2 \mathrm{E}+03$ & $2.2 \mathrm{E}+02$ \\
\hline
\end{tabular}

Nonradionuclides/soil/residential

Page 1 of 41 
Table 3.1c. (continued)

\begin{tabular}{|c|c|c|c|c|c|c|c|c|c|c|c|c|c|c|c|c|c|c|c|}
\hline \multirow{2}{*}{ Chemical } & \multirow{2}{*}{ CASRN } & \multicolumn{6}{|c|}{ Ingestion (mg/kg) } & \multicolumn{4}{|c|}{ Dermal (mg/kg) } & \multicolumn{4}{|c|}{ Inhalation $(\mathrm{mg} / \mathrm{kg})^{\wedge v}$} & \multicolumn{4}{|c|}{$\begin{array}{c}\text { Ingestion + Dermal } \\
+ \text { Inhalation }(\mathrm{mg} / \mathrm{kg})^{\dagger \cdot v} \\
\end{array}$} \\
\hline & & $10^{-4}$ & $10^{6}$ & $\begin{array}{l}\mathrm{HQ}=1 \\
\text { Adult }\end{array}$ & $\begin{array}{l}\mathrm{HQ}=1 \\
\text { Child }\end{array}$ & $\begin{array}{c}\mathrm{HQ}=0.1 \\
\text { Adult }\end{array}$ & $\begin{array}{c}\text { HQ }=0.1 \\
\text { Child }\end{array}$ & $10^{-4}$ & $10^{4}$ & $H Q=1$ & $\mathrm{HQ}=0.1$ & $10^{-4}$ & $10^{4}$ & $H Q=1$ & $\mathrm{HQ}=0.1$ & $10^{-4}$ & $10^{6}$ & $H I=1$ & $\mathrm{HI}=0.1$ \\
\hline $\begin{array}{l}\text { Allyl Chlo- } \\
\text { ride }\end{array}$ & $000107-05-1$ & & & $3.7 \mathrm{E}+04$ & $3.9 E+03$ & $3.7 \mathrm{E}+03$ & $3.9 \mathrm{E}+02$ & & & $5.5 \mathrm{E}+04$ & $5.5 \mathrm{E}+03$ & & & $1.0 \mathrm{E}+01$ & $1,0 \mathrm{E}+00$ & & & $1.0 \mathrm{E}+01$ & $1.0 E+00$ \\
\hline Aluminum & $007429-90-5$ & & & & & & & & & & & & & & & & & & \\
\hline $\begin{array}{l}\text { Aluminum } \\
\text { Phosphide }\end{array}$ & 020859-73-8 & & & $2.9 \mathrm{E}+02$ & $3.1 E+01$ & $2.9 \mathrm{E}+01$ & $3.1 \mathrm{E}+\infty$ & & & $1.1 \mathrm{E}+03$ & 1. $.1 E+02$ & & & & & & & $2.3 \mathrm{E}+02$ & $2.3 E+01$ \\
\hline Amdro & $067485-29-4$ & & & $2.2 \mathrm{E}+02$ & $2.3 \mathrm{E}+01$ & $2.2 \mathrm{E}+01$ & $2.3 E+\infty 0$ & & & $2.1 E+02$ & 2.IE+01 & & & & & & & $1.1 \mathrm{E}+02$ & 1. $1 \mathrm{E}+01$ \\
\hline Ametryn & $000834-12-8$ & & & $6.6 \mathrm{E}+03$ & $7.0 \mathrm{E}+02$ & $6.6 \mathrm{E}+02$ & $7.0 E+01$ & & & $6.2 \mathrm{E}+03$ & $6.2 \mathrm{E}+02$ & & & & & & & $3.2 \mathrm{E}+03$ & $3.2 \mathrm{E}+02$ \\
\hline $\begin{array}{l}\text { Aminophenol, } \\
\mathrm{m} \text { - }\end{array}$ & $000591-27-5$ & & & $5.1 \mathrm{E}+04$ & $5.5 \mathrm{E}+03$ & $5.1 \mathrm{E}+03$ & $5.5 E+02$ & & & $4.8 E+04$ & $4.8 E+03$ & & & & & & & $2.5 \mathrm{E}+04$ & $2.5 \mathrm{E}+03$ \\
\hline $\begin{array}{l}\text { Aminopyri- } \\
\text { dine, 4- }\end{array}$ & $000504-24-5$ & & & $1.5 \mathrm{E}+01$ & $1.6 \mathrm{E}+\infty$ & $1.5 \mathrm{E}+00$ & $1.6 \mathrm{E}-0 \mathrm{I}$ & & & $1.4 E+01$ & $1.4 \mathrm{E}+00$ & & & & & & & $7.1 \mathrm{E}+\infty$ & $7.1 \mathrm{E}-01$ \\
\hline Amitraz & $033089-61-1$ & & & $1.8 \mathrm{E}+03$ & $2.0 \mathrm{E}+02$ & $1.8 \mathrm{E}+02$ & $2.0 \mathrm{E}+01$ & & & $1.7 \mathrm{E}+03$ & $1.7 E+02$ & & & & & & & $8.9 E+02$ & $8.9 E+01$ \\
\hline Ammonia & $007664-41-7$ & & & & & & & & & & & & & $1.0 \mathrm{E}+06$ & $1.0 E+06$ & & & & \\
\hline $\begin{array}{l}\text { Ammonium } \\
\text { Sulfamate }\end{array}$ & $007773.06 \cdot 0$ & & & $1.5 E+05$ & $1.6 \mathrm{E}+04$ & $1.5 \mathrm{E}+04$ & $1.6 \mathrm{E}+03$ & & & $5.5 E+05$ & $5.5 \mathrm{E}+04$ & . & & & & & & $1.2 \mathrm{E}+05$ & $1.2 \mathrm{E}+04$ \\
\hline Aniline & 000062.53 .3 & $1.1 \mathrm{E}+04$ & $1.1 \mathrm{E}+02$ & & & & & $2.8 \mathrm{E}+04$ & $2.8 \mathrm{E}+02$ & & & & & $1.0 \mathrm{E}+06$ & 4.5E+05 & $1.5 E+04$ & $1.5 \mathrm{E}+02$ & & \\
\hline Anthracene & $000120-12-7$ & & & $2.2 \mathrm{E}+0 \mathrm{~S}$ & $2.3 \mathrm{E}+04$ & $2.2 \mathrm{E}+04$ & $2.3 \mathrm{E}+03$ & & & $3.1 E+05$ & $3.1 \mathrm{E}+04$ & & & & & & & $1.3 \mathrm{E}+0 \mathrm{~S}$ & $1.3 E+04$ \\
\hline $\begin{array}{l}\text { Antimony } \\
\text { (metallic) }\end{array}$ & $007440-36.0$ & & & $2.9 \mathrm{E}+02$ & $3.1 E+01$ & $2.9 \mathrm{E}+01$ & $3.1 \mathrm{E}+00$ & & & $1.1 \mathrm{E}+02$ & $1.1 \mathrm{E}+01$ & & & & & & & $8.0 \mathrm{E}+01$ & $8.0 \mathrm{E}+\infty 0$ \\
\hline $\begin{array}{l}\text { Antimony } \\
\text { Pentoxide }\end{array}$ & $001314-60-9$ & & & $3.7 \mathrm{E}+02$ & $3.9 E+01$ & $3.7 \mathrm{E}+01$ & $3.9 \mathrm{E}+00$ & & & $1.4 \mathrm{E}+03$ & $1.4 \mathrm{E}+02$ & & & & & & & $2.9 E+02$ & $2.9 \mathrm{E}+01$ \\
\hline $\begin{array}{l}\text { Antimony Po- } \\
\text { tassium Tar- } \\
\text { trate }\end{array}$ & $000304-61-0$ & & & $6.6 \mathrm{E}+02$ & $7.0 E+01$ & $6.6 \mathrm{E}+01$ & $7.0 \mathrm{E}+00$ & & & $2.5 \mathrm{E}+03$ & $2.5 \mathrm{E}+02$ & & & $\cdot$ & & & & $5.2 \mathrm{E}+02$ & $5.2 \mathrm{E}+01$ \\
\hline $\begin{array}{l}\text { Antimony Te- } \\
\text { troxide }\end{array}$ & 001332.81 .6 & & & $2.9 E+02$ & $3.1 E+01$ & $2.9 \mathrm{E}+01$ & $3.1 E+00$ & & & $1.1 E+03$ & $1.1 E+02$ & & & & & & & $2.3 \mathrm{E}+02$ & $2.3 E+01$ \\
\hline $\begin{array}{l}\text { Antimony Tri- } \\
\text { oxide }\end{array}$ & $001309-64-4$ & & & $2.9 \mathrm{E}+02$ & $3.1 E+01$ & $2.9 E+01$ & $3.1 \mathrm{E}+\infty$ & & & $1.1 \mathrm{E}+03$ & $1.1 \mathrm{E}+02$ & & & $8.9 \mathrm{E}+05$ & $8.9 \mathrm{E}+04$ & & & $2.3 E+02$ & $2.3 E+01$ \\
\hline Apollo & $074115-24-5$ & & & $9.5 \mathrm{E}+03$ & $1.0 \mathrm{E}+03$ & $9.5 E+02$ & $1.0 \mathrm{E}+02$ & & & $9.0 E+03$ & $9.0 \mathrm{E}+02$ & & & & & & & $4.6 \mathrm{E}+03$ & $4.6 \mathrm{E}+02$ \\
\hline
\end{tabular}

Nonradionuclides/soil/residential

Page 2 of 41 
Table 3.1c. (continued)

\begin{tabular}{|c|c|c|c|c|c|c|c|c|c|c|c|c|c|c|c|c|c|c|c|}
\hline \multirow{2}{*}{ Chemical } & \multirow{2}{*}{ CASRN } & \multicolumn{6}{|c|}{ Ingestion (mg/kg) } & \multicolumn{4}{|c|}{ Dermal (mg/kg) } & \multicolumn{4}{|c|}{ Inhalation (mg/kg) } & \multicolumn{4}{|c|}{$\begin{array}{c}\text { Ingestion + Dermal } \\
+ \text { Inhalation }(\mathrm{mg} / \mathrm{kg})^{\dagger \cdot \mathrm{x}}\end{array}$} \\
\hline & & $10^{-4}$ & $10^{4}$ & $\begin{array}{l}\text { HQ=1 } \\
\text { Adult }\end{array}$ & $\begin{array}{l}\text { HQ=1 } \\
\text { Child }\end{array}$ & $\begin{array}{c}\text { HQ }=0.1 \\
\text { Adult }\end{array}$ & $\begin{array}{c}\text { HQ }=0.1 \\
\text { Child }\end{array}$ & $10^{-4}$ & $10^{6}$ & $\mathrm{HQ}=1$ & $H Q=0.1$ & $10^{-4}$ & $10^{4}$ & $H Q=1$ & $\mathrm{HQ}=0.1$ & $10^{4}$ & $10^{4}$ & $H I=1$ & $\mathrm{HI}=0.1$ \\
\hline Asamite & $000140-57-8$ & $2.6 \mathrm{E}+03$ & $2.6 \mathrm{E}+01$ & 3.TE+04 & $3.9 \mathrm{E}+03$ & 3.TE+03 & $3.9 \mathrm{E}+02$ & $6.4 E+03$ & $6.4 E+01$ & $3.4 E+04$ & $3.4 E+03$ & $1.0 E+06$ & $1.0 \mathrm{E}+06$ & & & $3.3 \mathrm{E}+03$ & $3.3 \mathrm{E}+01$ & $1.8 \mathrm{E}+04$ & $1.8 \mathrm{E}+03$ \\
\hline Aroclor 1016 & $012674-11-2$ & & & $5.1 \mathrm{E}+01$ & $5.5 \mathrm{E}+00$ & $5.1 \mathrm{E}+\infty 0$ & $5.5 \mathrm{E}-01$ & & $\cdot$ & $1.4 \mathrm{E}+01$ & $1.4 \mathrm{E}+00$ & & & & & & & $1.1 E+01$ & $1.1 E+\infty$ \\
\hline $\begin{array}{l}\text { Aroclor } \\
1221^{101}\end{array}$ & $011104-28-2$ & $8.3 E+00$ & $8.3 \mathrm{E}-02$ & & & & & $6.3 \mathrm{E}+00$ & $6.3 \mathrm{E}-02$ & & & & & & & $4.9 E+00$ & $4.9 \mathrm{E} \cdot 02$ & & \\
\hline $\begin{array}{l}\text { Aroclor } \\
1232^{(1)}\end{array}$ & $011141-16-5$ & $8.3 E+00$ & $8.3 \mathrm{E}-02$ & & & & & $6.3 \mathrm{E}+00$ & $6.3 \mathrm{E}-02$ & & & & & & & $4.9 \mathrm{E}+00$ & $4.9 \mathrm{E}-02$ & & \\
\hline $\begin{array}{l}\text { Aroclor } \\
1242^{111}\end{array}$ & $053469-21-9$ & $8.3 E+\infty 0$ & $8.3 \mathrm{E}-02$ & & & & & $6.3 \mathrm{E}+00$ & $6.3 \mathrm{E}-02$ & & & & & & & $4.9 \mathrm{E}+00$ & $4.9 \mathrm{E}-02$ & & \\
\hline $\begin{array}{l}\text { Aroclor } \\
1248^{(11}\end{array}$ & $012672-29-6$ & $8.3 \mathrm{E}+\infty 0$ & $8.3 \mathrm{E}-02$ & & & & & $6,3 \mathrm{E}+00$ & $6.3 \mathrm{E}-02$ & & & & & & & $4.9 \mathrm{E}+00$ & 4.9E-02 & & \\
\hline Aroclor 1254 & $011097-69-1$ & & & $1.5 \mathrm{E}+01$ & $1.6 \mathrm{E}+\infty$ & $1,5 \mathrm{E}+00$ & $1.6 \mathrm{E}-01$ & & & 4. $1 E+\infty$ & 4.1E-01 & & & & & & & $3.2 E+\infty$ & $3.2 \mathrm{E}-01$ \\
\hline $\begin{array}{l}\text { Aroclor } \\
1260^{11}\end{array}$ & $011096.82-5$ & $8.3 E+00$ & $8.3 \mathrm{E}-02$ & & & & & $6.3 \mathrm{E}+10$ & $6.3 \mathrm{E}-02$ & & & & & & & $4.9 \mathrm{E}+00$ & $4.9 \mathrm{E}-02$ & & \\
\hline Arsenic Salts & $\mathrm{NA}$ & & & & & & & & & & & & & & & & & & \\
\hline $\begin{array}{l}\text { Arsenic, Inor- } \\
\text { ganic }\end{array}$ & $007440-38-2$ & $4.3 \mathrm{E}+01$ & 4.3E-01 & $2.2 \mathrm{E}+02$ & $2.3 \mathrm{E}+01$ & $2.2 E+01$ & $2.3 \mathrm{E}+\infty 0$ & $8.8 \mathrm{E}+02$ & $8.8 E+\infty$ & $1.7 E+03$ & $1.7 \mathrm{E}+02$ & $7.3 E+04$ & $7.3 \mathrm{E}+02$ & & & $1.0 \mathrm{E}+02$ & $1.0 \mathrm{E}+\infty 0$ & $1.9 \mathrm{E}+02$ & $1.9 \mathrm{E}+01$ \\
\hline Arsine & $007784-42-1$ & & & & & & & & & & & & & $2.2 E+05$ & $2.2 \mathrm{E}+04$ & & & & \\
\hline Asbestos & $001332-21-4$ & & & & & & & & & & & & & & & & & & \\
\hline Assure & $076578-14-8$ & & & $6.6 \mathrm{E}+03$ & $7,0 \mathrm{E}+02$ & $6.6 \mathrm{E}+02$ & $7.0 \mathrm{E}+01$ & & & $6.2 \mathrm{E}+03$ & $6.2 E+02$ & & & & & & & $3.2 E+03$ & $3.2 E+02$ \\
\hline Asulam & $003337-71-1$ & & & $3.7 \mathrm{E}+04$ & $3.9 E+03$ & $3.7 E+03$ & $3.9 E+02$ & & & $3.4 \mathrm{E}+04$ & $3.4 \mathrm{E}+03$ & & & & & & & $1.8 \mathrm{E}+04$ & $1.8 \mathrm{E}+03$ \\
\hline Atravine & $001912-24-9$ & $2.9 \mathrm{E}+02$ & $2.9 \mathrm{E}+\infty 0$ & $2.6 \mathrm{E}+04$ & $2.7 \mathrm{E}+03$ & $2.6 \mathrm{E}+03$ & $2.7 \mathrm{E}+02$ & $7.2 \mathrm{E}+02$ & $7.2 E+\infty$ & $2.4 \mathrm{E}+04$ & $2.4 E+03$ & & & & & $3.7 \mathrm{E}+02$ & $3.7 E+00$ & $1.2 E+04$ & $1.2 \mathrm{E}+03$ \\
\hline $\begin{array}{l}\text { Avermectin } \\
\text { B1 }\end{array}$ & $065195-55-3$ & & & $2.9 \mathrm{E}+02$ & $3.1 \mathrm{E}+01$ & $2.9 \mathrm{E}+01$ & $3.1 E+\infty$ & & & $2.8 \mathrm{E}+02$ & $2.8 \mathrm{E}+01$ & & & & & & & $1.4 \mathrm{E}+02$ & $1.4 \mathrm{E}+01$ \\
\hline Azobenzene & $000103-33-3$ & $5.8 \mathrm{E}+02$ & $5.8 \mathrm{E}+\infty$ & & & & & $1.5 \mathrm{E}+03$ & $1.5 E+01$ & & & $1.0 \mathrm{E}+06$ & $3.3 E+05$ & & & 7.5E+02 & 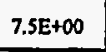 & & \\
\hline Barium & $007440-39-3$ & & & $5.1 \mathrm{E}+04$ & $5.5 \mathrm{E}+03$ & $5.1 \mathrm{E}+03$ & $5.5 \mathrm{E}+02$ & & & $6.7 E+04$ & $6.7 \mathrm{E}+03$ & & & $1.0 \mathrm{E}+06$ & $2.2 \mathrm{E}+0 \mathrm{~S}$ & & & $2.9 \mathrm{E}+04$ & $2.9 E+03$ \\
\hline $\begin{array}{l}\text { Barium Cya- } \\
\text { nide }\end{array}$ & $000542-62-1$ & & & $7.3 E+04$ & $7.8 \mathrm{E}+03$ & $7.3 \mathrm{E}+03$ & $7.8 \mathrm{E}+02$ & & & $2.8 \mathrm{E}+05$ & $2.8 \mathrm{E}+04$ & & & & & & & $5.8 \mathrm{E}+04$ & $5.8 \mathrm{E}+03$ \\
\hline
\end{tabular}

Nonradionuclides/soil/residential

Page 3 of 41 
Table 3.1c. (continued)

\begin{tabular}{|c|c|c|c|c|c|c|c|c|c|c|c|c|c|c|c|c|c|c|c|}
\hline \multirow{2}{*}{ Chemical } & \multirow{2}{*}{ CASRN } & \multicolumn{6}{|c|}{ Ingestion (mg/kg) } & \multicolumn{4}{|c|}{ Dermal (mg/kg) } & \multicolumn{4}{|c|}{ Inhalation (mg/kg) } & \multicolumn{4}{|c|}{$\begin{array}{c}\text { Ingestion + Dermal } \\
+ \text { Inhalation }(\mathrm{mg} / \mathrm{kg})^{+*} \\
\end{array}$} \\
\hline & & $10^{-4}$ & $10^{4}$ & $\begin{array}{l}H Q=1 \\
\text { Adult }\end{array}$ & $\begin{array}{l}\text { HQ=1 } \\
\text { Child }\end{array}$ & $\begin{array}{c}\text { HQ }=0.1 \\
\text { Adult }\end{array}$ & $\begin{array}{l}\text { HQ }=0.1 \\
\text { Child }\end{array}$ & $10^{-4}$ & $10^{-6}$ & $\mathrm{HQ}=1$ & $\mathrm{HQ}=0.1$ & $10^{-4}$ & $10^{6}$ & $H Q=1$ & $\mathrm{HQ}=0.1$ & $10^{-4}$ & $10^{4}$ & $\mathrm{HI}=1$ & $\mathrm{HI}=0.1$ \\
\hline Baygon & $000114-26-1$ & & & $2.9 \mathrm{E}+03$ & $3.1 \mathrm{E}+02$ & $2.9 \mathrm{E}+02$ & $3.1 \mathrm{E}+01$ & & & $2.8 \mathrm{E}+03$ & $2.8 \mathrm{E}+02$ & & & & & & & $1.4 \mathrm{E}+03$ & $1.4 \mathrm{E}+02$ \\
\hline Bayleton & $043121-43-3$ & & & $2.2 E+04$ & $2.3 \mathrm{E}+03$ & $2.2 \mathrm{E}+03$ & 2.3E+02 & & & 2.1E+04 & $2.1 \mathrm{E}+03$ & & & & & & & $1.1 \mathrm{E}+04$ & $1.1 \mathrm{E}+03$ \\
\hline Baythroid & $068359-37.5$ & & & $1.8 \mathrm{E}+04$ & $2.0 \mathrm{E}+03$ & $1.8 \mathrm{E}+03$ & $2.0 E+02$ & & & $1.7 \mathrm{E}+04$ & $1.7 \mathrm{E}+03$ & & & & & & & $8.9 \mathrm{E}+03$ & $8.9 \mathrm{E}+02$ \\
\hline Benefin & $001861-40-1$ & & & $2.2 \mathrm{E}+05$ & $2.3 \mathrm{E}+04$ & $2.2 \mathrm{E}+04$ & $2.3 \mathrm{E}+03$ & & & $2.1 \mathrm{E}+05$ & $2.1 \mathrm{E}+04$ & & & & & & & $1.1 E+05$ & $1.1 \mathrm{E}+04$ \\
\hline Benomyl & $017804-35-2$ & & & 3.7E+04 & $3.9 \mathrm{E}+03$ & $3.7 \mathrm{E}+03$ & $3.9 \mathrm{E}+02$ & & & $3.4 E+04$ & $3.4 E+03$ & & & & & & & $1,8 \mathrm{E}+04$ & $1.8 \mathrm{E}+03$ \\
\hline Bentazon & $025057-89-0$ & & & $1,8 \mathrm{E}+03$ & $2.0 \mathrm{E}+02$ & $1.8 \mathrm{E}+02$ & $2.0 \mathrm{E}+01$ & & & $1.7 \mathrm{E}+03$ & $1.7 \mathrm{E}+02$ & & & & & & & $8.9 \mathrm{E}+02$ & $8.9 E+01$ \\
\hline $\begin{array}{l}\text { Benz[a]an- } \\
\text { thracene }{ }^{(2)}\end{array}$ & $000056.55-3$ & $8.8 \mathrm{E}+01$ & $8.8 \mathrm{E}-01$ & & & & & $1.4 \mathrm{E}+02$ & $1.4 \mathrm{E}+\infty$ & & & $1.0 \mathrm{E}+06$ & $1.2 \mathrm{E}+05$ & & & $8.6 \mathrm{E}+01$ & $8.6 \mathrm{E}-01$ & & \\
\hline Benzaldehyde & $000100-52-7$ & & & $7.3 \mathrm{E}+04$ & $7.8 \mathrm{E}+03$ & $7.3 E+03$ & $7.8 \mathrm{E}+02$ & & & $1.1 \mathrm{E}+05$ & $1.1 \mathrm{E}+04$ & & & & & & & 4.4E+04 & $4.4 \mathrm{E}+03$ \\
\hline Benzene & $000071-43-2$ & $2.2 \mathrm{E}+03$ & $2.2 E+01$ & & & & . & $1.1 \mathrm{E}+04$ & $1.1 E+02$ & & & $5.0 \mathrm{E}+02$ & $5.5 \mathrm{E}+00$ & & & $4.8 \mathrm{E}+02$ & $4.8 \mathrm{E}+00$ & & \\
\hline $\begin{array}{l}\text { Benzene } \\
\text { Hexachloride }\end{array}$ & NA & & & & & & & & & & & & & & & & & & \\
\hline $\begin{array}{l}\text { Benzene, } \\
\text { Ethyldimethyl }\end{array}$ & NA & & & & & & & & & & & & & & & & & & \\
\hline $\begin{array}{l}\text { Benzene, } \\
\text { Ethylmethyl }\end{array}$ & NA & & & & & & & & & & & & & & & & & & \\
\hline $\begin{array}{l}\text { Benzene, } \\
\text { Methylpro- } \\
\text { penyl }\end{array}$ & NA & & & & & & & & & & & & & & & & & & \\
\hline $\begin{array}{l}\text { Benzene, } \\
\text { Methylpropyl }\end{array}$ & NA & & & & & & & & & & & & & & & & & & \\
\hline $\begin{array}{l}\text { Berzene, } \\
\text { Trimethyl }\end{array}$ & $025551-13-7$ & & & & & & & & & & & & & & & & & & \\
\hline Benzenethiol & 000108.98 .5 & & & $7.3 \mathrm{E}+00$ & $7.8 \mathrm{E}-01$ & 7.3E-01 & $7.8 \mathrm{E}-02$ & & & $6.9 \mathrm{E}+00$ & $6.9 \mathrm{E}-01$ & & & & & & & $3.5 E+00$ & $3.5 \mathrm{E}-01$ \\
\hline Benzidine & 000092.87 .5 & $2.8 \mathrm{E}-01$ & $2.8 \mathrm{E}-03$ & $2.2 E+03$ & $2.3 \mathrm{E}+02$ & $2.2 \mathrm{E}+02$ & $2.3 \mathrm{E}+01$ & $1.1 \mathrm{E}+\infty$ & $1.1 \mathrm{E}-02$ & $3.3 \mathrm{E}+03$ & $3.3 \mathrm{E}+02$ & $1.6 \mathrm{E}+04$ & $1.6 \mathrm{E}+02$ & & & 4.5E-01 & 4.5E-03 & $1.3 \mathrm{E}+03$ & $1.3 E+02$ \\
\hline $\begin{array}{l}\text { Benzo[a]py- } \\
\text { rene }\end{array}$ & 000050.32 .8 & $8.8 \mathrm{E}+\infty$ & $8.8 \mathrm{E}-02$ & & & & & $1.4 E+01$ & $1.4 \mathrm{E}-01$ & & & $1.0 \mathrm{E}+06$ & $1.2 E+04$ & & & $8.6 \mathrm{E}+00$ & $8.6 \mathrm{E}-02$ & & \\
\hline
\end{tabular}


Table 3.1c. (continued)

\begin{tabular}{|c|c|c|c|c|c|c|c|c|c|c|c|c|c|c|c|c|c|c|c|}
\hline \multirow{2}{*}{ Chemical } & \multirow{2}{*}{ CASRN } & \multicolumn{6}{|c|}{ Ingestion (mg/kg) } & \multicolumn{4}{|c|}{ Dermal (mg/kg) } & \multicolumn{4}{|c|}{ Inhalation (mg/kg) } & \multicolumn{4}{|c|}{$\begin{array}{c}\text { Ingestion + Dermal } \\
+ \text { Inhalation (mg/kg) }\end{array}$} \\
\hline & & $10^{-4}$ & $10^{4}$ & $\begin{array}{l}\text { HQ=1 } \\
\text { Adult }\end{array}$ & $\begin{array}{l}\mathrm{HQ}=1 \\
\text { Child }\end{array}$ & $\begin{array}{c}\mathrm{HQ}=0.1 \\
\text { Adult }\end{array}$ & $\begin{array}{c}H Q=0.1 \\
\text { Child }\end{array}$ & $10^{-4}$ & $10^{5}$ & $H Q=1$ & $\mathrm{HQ}=0.1$ & $10^{-4}$ & $10^{4}$ & $H Q=1$ & $\mathrm{HQ}=0.1$ & $10^{-4}$ & $10^{4}$ & $H I=1$ & $\mathrm{HI}=0.1$ \\
\hline $\begin{array}{l}\text { Benzo[b]- } \\
\text { Aluoranthene }\end{array}$ & $000205-99-2$ & $8.8 \mathrm{E}+01$ & $8.8 \mathrm{E}-01$ & & & & & $1.4 \mathrm{E}+02$ & $1.4 E+\infty$ & & & t.0E+06 & $1.2 \mathrm{E}+05$ & & & $8.6 \mathrm{E}+01$ & $8.6 \mathrm{E}-01$ & & \\
\hline $\begin{array}{l}\text { Benzo[g,h,i]- } \\
\text { perylene }\end{array}$ & $000191-24-2$ & & & & & & & & & & & & & & & & & . & \\
\hline $\begin{array}{l}\text { Benzo[kj]- } \\
\text { fluoranthene }\end{array}$ & $000207-08-9$ & $8.8 \mathrm{E}+02$ & $8.8 \mathrm{E}+00$ & & & & & $1.4 \mathrm{E}+03$ & $1.4 \mathrm{E}+01$ & & & $1.0 \mathrm{E}+06$ & $1.0 E+06$ & & & $8.6 \mathrm{E}+02$ & $8.6 \mathrm{E}+00$ & & \\
\hline Benzoic Acid & $000065-85-0$ & & & $1.0 E+06$ & $3.1 E+05$ & $2.9 E+05$ & $3.1 E+04$ & & & $1.0 \mathrm{E}+06$ & $5.5 E+05$ & & & & & & & $1.0 \mathrm{E}+06$ & $1.9 E+05$ \\
\hline $\begin{array}{l}\text { Benzotrichlo- } \\
\text { ride }\end{array}$ & $000098-07-7$ & $4.9 E+\infty 0$ & $4.9 \mathrm{E}-02$ & & & & & $1.2 \mathrm{E}+01$ & $1.2 \mathrm{E}-01$ & & & & & & & $6.4 \mathrm{E}+00$ & 6.4E-02 & & \\
\hline $\begin{array}{l}\text { Benzyl Alco- } \\
\text { hol }\end{array}$ & $000100-51-6$ & & & 2.2E+05 & $2.3 \mathrm{E}+04$ & $2.2 E+04$ & $2.3 \mathrm{E}+03$ & & & $2.7 \mathrm{E}+05$ & 2.7E+04 & & & & & & & $1.2 E+05$ & $1.2 \mathrm{E}+04$ \\
\hline $\begin{array}{l}\text { Benzyl Chlo- } \\
\text { ride }\end{array}$ & $000100-44-7$ & $3.8 \mathrm{E}+02$ & $3.8 \mathrm{E}+00$ & & & & & $1.5 \mathrm{E}+03$ & $1.5 E+01$ & & & & & & & $6.0 \mathrm{E}+02$ & $6.0 \mathrm{E}+00$ & & \\
\hline Beryllium & $007440-41-7$ & $1.5 \mathrm{E}+01$ & 1.5E-01 & 3.7E+03 & $3.9 \mathrm{E}+02$ & $3.7 \mathrm{E}+02$ & $3.9 \mathrm{E}+01$ & $7.5 \mathrm{E}+00$ & 7.5E-02 & $6.9 \mathrm{E}+02$ & $6.9 \mathrm{E}+01$ & $4.3 \mathrm{E}+05$ & $4.3 E+03$ & & & $6.3 \mathrm{E}+00$ & $6.3 \mathrm{E}-02$ & $5.8 E+02$ & $5.8 \mathrm{E}+01$ \\
\hline Bidrin & $000141.66-2$ & & & $7.3 \mathrm{E}+01$ & $7.8 \mathrm{E}+\infty 0$ & $7.3 E+00$ & $7.8 \mathrm{E}-01$ & & & $6.9 \mathrm{E}+01$ & $6.9 \mathrm{E}+00$ & & & & & & & $3.5 \mathrm{E}+01$ & 3.5E+00 \\
\hline Biphenthrin & $082657-04-3$ & & & $1.1 E+04$ & $1.2 \mathrm{E}+03$ & $1.1 E+03$ & $1.2 \mathrm{E}+02$ & & & $1.0 E+04$ & $1.0 E+03$ & & & & & & & $5.3 \mathrm{E}+03$ & $5.3 \mathrm{E}+02$ \\
\hline Biphenyl, 1,1:- & $000092-52-4$ & & & $3.7 E+04$ & $3.9 \mathrm{E}+03$ & $3.7 E+03$ & $3.9 E+02$ & & & $3.4 E+04$ & $3.4 \mathrm{E}+03$ & & & & & & & $1.8 \mathrm{E}+04$ & $1.8 \mathrm{E}+03$ \\
\hline $\begin{array}{l}\text { Bis(2-chloro- } \\
\text { 1-methyl- } \\
\text { ethyl)ether } \\
\text { (Technical) }\end{array}$ & $000108-60-1$ & $9.1 E+02$ & $9.1 E+\infty 0$ & & & & & $3.7 \mathrm{E}+03$ & $3.7 E+01$ & & & $3.5 \mathrm{E}+02$ & $3.4 E+01$ & & & $3.5 \mathrm{E}+02$ & $1.0 E+01$ & & \\
\hline $\begin{array}{l}\text { Bis(2- } \\
\text { chloroethoxy) } \\
\text { methane }\end{array}$ & $000111-91-1$ & & & & & & & & & & & & & & & & & & \\
\hline $\begin{array}{l}\text { Bis(2- } \\
\text { chloroethyl)- } \\
\text { ether }\end{array}$ & $000111-44-4$ & $5.8 \mathrm{E}+01$ & $5.8 \mathrm{E}-01$ & & & & & $2.3 E+02$ & $2.3 \mathrm{E}+\infty$ & & & $1.4 E+02$ & $1.4 \mathrm{E}+\infty 0$ & & & $5.6 \mathrm{E}+01$ & $5.6 E-01$ & & \\
\hline $\begin{array}{l}\text { Bis(2. } \\
\text { chloroisopro- } \\
\text { pyl)ether }\end{array}$ & $039638-32-9$ & & & $2.9 \mathrm{E}+04$ & $3.1 E+03$ & $2.9 \mathrm{E}+03$ & $3.1 \mathrm{E}+02$ & & & $4.4 E+04$ & $4.4 E+03$ & & & & & & & $1.8 \mathrm{E}+04$ & $1.8 E+03$ \\
\hline
\end{tabular}


Table 3.1c. (continued)

\begin{tabular}{|c|c|c|c|c|c|c|c|c|c|c|c|c|c|c|c|c|c|c|c|}
\hline \multirow{2}{*}{ Chemical } & \multirow{2}{*}{ CASRN } & \multicolumn{6}{|c|}{ Ingestion (mg/kg)" } & \multicolumn{4}{|c|}{ Dermal (mg/kg) } & \multicolumn{4}{|c|}{ Inhalation (mg/kg) } & \multicolumn{4}{|c|}{$\begin{aligned} & \text { Ingestion + Dermal } \\
+ & \text { Inhalation (mg/kg) }\end{aligned}$} \\
\hline & & $10^{-4}$ & $10^{4}$ & $\begin{array}{l}\mathrm{HQ}=1 \\
\text { Adult }\end{array}$ & $\begin{array}{l}\text { HQ }=1 \\
\text { Child }\end{array}$ & $\begin{array}{c}\text { HQ }=0.1 \\
\text { Adult }\end{array}$ & $\begin{array}{c}\text { HQ }=0.1 \\
\text { Child }\end{array}$ & $10^{-1}$ & $10^{6}$ & $\mathrm{HQ}=1$ & $\mathrm{HQ}=0.1$ & $10^{-4}$ & $10^{4}$ & $H Q=1$ & $\mathrm{HQ}=0.1$ & $10^{-4}$ & $10^{6}$ & $\mathrm{HI}=\mathbf{I}$ & $\mathrm{HI}=0.1$ \\
\hline $\begin{array}{l}\text { Bis(2- } \\
\text { ethylhexyly)- } \\
\text { phthasalete }\end{array}$ & 000117.81 .7 & $4.6 \mathrm{E}+03$ & $4.6 E+01$ & 1.5E+04 & $1.6 \mathrm{E}+03$ & $1.5 \mathrm{E}+03$ & $1.6 \mathrm{E}+02$ & $4.4 \mathrm{E}+03$ & $4.4 E+01$ & $5.2 E+03$ & $5.2 E+02$ & & & & & $3.2 E+03$ & $3.2 \mathrm{E}+01$ & $3.9 E+03$ & $3.9 \mathrm{E}+02$ \\
\hline $\begin{array}{l}\text { Bis(chloro- } \\
\text { methyl)ether }\end{array}$ & $000542-88-1$ & $2.9 \mathrm{E}-01$ & $2.9 \mathrm{E}-03$ & & & & & $1.2 \mathrm{E}+00$ & $1.2 \mathrm{E}-02$ & & & 4.4E-01 & $4.4 \mathrm{E} \cdot 03$ & & & $2.3 \mathrm{E}-01$ & $2.3 \mathrm{E}-03$ & & \\
\hline Bisphenol A & $000080.05-7$ & & & $3.7 \mathrm{E}+04$ & $3.9 E+03$ & $3.7 E+03$ & $3.9 \mathrm{E}+02$ & & & $3.4 \mathrm{E}+04$ & $3.4 E+03$ & & & & & & & $1.8 \mathrm{E}+04$ & $1.8 \mathrm{E}+03$ \\
\hline $\begin{array}{l}\text { Boron And } \\
\text { Borates Only }\end{array}$ & $007440-42-8$ & & & $6.6 \mathrm{E}+04$ & $7.0 \mathrm{E}+03$ & $6.6 \mathrm{E}+03$ & $7.0 \mathrm{E}+02$ & & & $1.0 \mathrm{E}+06$ & $1.1 \mathrm{E}+05$ & & & $1.0 \mathrm{E}+06$ & $1.0 \mathrm{E}+06$ & & & $6.2 \mathrm{E}+04$ & $6.2 \mathrm{E}+03$ \\
\hline $\begin{array}{l}\text { Boron Tri- } \\
\text { fluoride }\end{array}$ & $007637.07-2$ & & & & & & & & & & & & & $1.0 \mathrm{E}+06$ & $3.1 E+05$ & & & & \\
\hline $\begin{array}{l}\text { Bromochloro- } \\
\text { methane }\end{array}$ & $000074-97-5$ & & & & & & & & & & & & & & & & & & \\
\hline $\begin{array}{l}\text { Bromodichlo- } \\
\text { romethiane }\end{array}$ & $000075-27-4$ & $1.0 E+03$ & $1.0 \mathrm{E}+01$ & $1.5 \mathrm{E}+04$ & $1.6 \mathrm{E}+03$ & $1.5 E+03$ & $1.6 \mathrm{E}+02$ & $5.1 \mathrm{E}+03$ & $5.1 E+01$ & $2.7 E+04$ & $2.7 \mathrm{E}+03$ & & & & & $1.8 \mathrm{E}+03$ & $1.8 \mathrm{E}+01$ & $9.5 \mathrm{E}+03$ & $9.5 \mathrm{E}+02$ \\
\hline $\begin{array}{l}\text { Bromodi-phe- } \\
\text { nyl Ether, p- }\end{array}$ & $000101-55-3$ & & & & & & & & & & & & & & & & & & \\
\hline Bromoform & $000075-25-2$ & 8.1E+03 & $8.1 E+01$ & $1.5 \mathrm{E}+04$ & $1.6 \mathrm{E}+03$ & $1.5 \mathrm{E}+03$ & $1.6 \mathrm{E}+02$ & $2.4 E+04$ & $2.4 E+02$ & $1.7 \mathrm{E}+04$ & $1.7 \mathrm{E}+03$ & $1.0 \mathrm{E}+06$ & $1.0 \mathrm{E}+06$ & & & $1.1 \mathrm{E}+04$ & $1.1 E+02$ & $7.8 \mathrm{E}+03$ & $7.8 \mathrm{E}+02$ \\
\hline $\begin{array}{l}\text { Bromometh- } \\
\text { ane }\end{array}$ & $000074-83-9$ & & & $1.0 \mathrm{E}+03$ & $1.1 E+02$ & $1.0 \mathrm{E}+02$ & $1.1 \mathrm{E}+01$ & & & $1.5 \mathrm{E}+03$ & $1.5 \mathrm{E}+02$ & & & $1.7 E+01$ & $1.7 \mathrm{E}+\infty$ & & & $1.7 \mathrm{E}+01$ & $1.7 E+00$ \\
\hline Bromophos & $002104-96-3$ & & & $3.7 \mathrm{E}+03$ & $3.9 \mathrm{E}+02$ & $3.7 \mathrm{E}+02$ & $3.9 \mathrm{E}+01$ & & & $3.4 E+03$ & $3.4 \mathrm{E}+02$ & & & & & & & $1.8 \mathrm{E}+03$ & $1.8 \mathrm{E}+02$ \\
\hline $\begin{array}{l}\text { Bromotri- } \\
\text { chloromethane }\end{array}$ & $000075.62-7$ & & & & & & & & & & & & & & & & & & \\
\hline Bromoxynil & $001689-84-5$ & & & $1.5 \mathrm{E}+04$ & $1.6 \mathrm{E}+03$ & $1.5 \mathrm{E}+03$ & $1.6 \mathrm{E}+02$ & & & $1.4 E+04$ & $1.4 \mathrm{E}+03$ & & & & & & & $7.1 E+03$ & $7.1 E+02$ \\
\hline $\begin{array}{l}\text { Bromoxynil } \\
\text { Octanoate }\end{array}$ & $001689-99-2$ & & & $1.5 \mathrm{E}+04$ & $1.6 \mathrm{E}+03$ & $1.5 \mathrm{E}+03$ & $1.6 \mathrm{E}+02$ & & & $1.4 E+04$ & $1.4 \mathrm{E}+03$ & & & & & & & 7.1E+03 & $7.1 E+02$ \\
\hline $\begin{array}{l}\text { Butadiene, } \\
\text { 1,3. }\end{array}$ & $000106-99-0$ & & & & & & & & & & & $2.9 E+\infty$ & $2.9 \mathrm{E} \cdot 02$ & & & & & & \\
\hline Butanol, N- & $000071-36-3$ & & & $7.3 E+04$ & $7.8 \mathrm{E}+03$ & $7.3 E+03$ & $7.8 \mathrm{E}+02$ & & & $6.9 E+04$ & $6.9 \mathrm{E}+03$ & & & & & & & $3.5 E+04$ & $3.5 E+03$ \\
\hline
\end{tabular}


Table 3.1c. (continued)

\begin{tabular}{|c|c|c|c|c|c|c|c|c|c|c|c|c|c|c|c|c|c|c|c|}
\hline \multirow{2}{*}{ Chemical } & \multirow{2}{*}{ CASRN } & \multicolumn{6}{|c|}{ Ingestion (mg/kg) } & \multicolumn{4}{|c|}{ Dermal (mg/kg)" } & \multicolumn{4}{|c|}{ Inhalation $(\mathrm{mg} / \mathrm{kg})^{* \boldsymbol{\gamma}}$} & \multicolumn{4}{|c|}{$\begin{aligned} & \text { Ingestion + Dermal } \\
+ & \text { Inhalation }\left(\mathrm{mg} / \mathrm{kg}^{)^{\cdot \cdot}}\right.\end{aligned}$} \\
\hline & & $10^{-1}$ & $10^{4}$ & $\begin{array}{l}\text { HQ=1 } \\
\text { Adult }\end{array}$ & $\begin{array}{l}\mathrm{HQ}=1 \\
\text { Child }\end{array}$ & $\begin{array}{c}\mathrm{HQ}=0.1 \\
\text { Adult }\end{array}$ & $\begin{array}{l}\text { HQ=0.1 } \\
\text { Child }\end{array}$ & $10^{-4}$ & $10^{5}$ & $H Q=1$ & $\mathrm{HQ}=0.1$ & $10^{-4}$ & $10^{4}$ & $H Q=1$ & $\mathrm{HQ}=0.1$ & $10^{-4}$ & $10^{5}$ & $\mathrm{HI}=1$ & $\mathrm{HI}=0.1$ \\
\hline $\begin{array}{l}\text { Butanone-2, } \\
\text { 4-chloro-4,4- } \\
\text { difluoro }\end{array}$ & NA & & & & & & & & & & & & & & & & & & \\
\hline $\begin{array}{l}\text { Butyl Benzyl } \\
\text { Phthlate }\end{array}$ & $000085-68-7$ & & & $1.5 \mathrm{E}+05$ & $1.6 \mathrm{E}+04$ & $1.5 \mathrm{E}+04$ & $1.6 \mathrm{E}+03$ & & & $1.7 E+05$ & $1.7 \mathrm{E}+04$ & & & & & & & $7.8 \mathrm{E}+04$ & $7.8 \mathrm{E}+03$ \\
\hline Butylate & $002008-41-5$ & & & $3.7 \mathrm{E}+04$ & $3.9 E+03$ & $3.7 \mathrm{E}+03$ & $3.9 E+02$ & & & $3.4 \mathrm{E}+04$ & $3.4 E+03$ & & & & & & & $1.8 E+04$ & $1.8 \mathrm{E}+03$ \\
\hline $\begin{array}{l}\text { Butylchloride } \\
\text { to }\end{array}$ & $000507-20.0$ & & & & & & & & & & & & & & & & & & \\
\hline $\begin{array}{l}\text { Butylphthalyl } \\
\text { Butylyglycolate }\end{array}$ & $000085-70-1$ & & & $7.3 \mathrm{E}+05$ & $7.8 \mathrm{E}+04$ & $7.3 \mathrm{E}+04$ & $7.8 \mathrm{E}+03$ & & & $6.9 \mathrm{E}+05$ & $6.9 E+04$ & & & & & & & $3.5 \mathrm{E}+05$ & $3.5 \mathrm{E}+04$ \\
\hline $\begin{array}{l}\text { Cacodylic } \\
\text { Acid }\end{array}$ & $000075-60-5$ & & & $2.2 \mathrm{E}+03$ & $2.3 E+02$ & $2.2 \mathrm{E}+02$ & 2.3E+01 & & & 2.IE+03 & $2.1 E+02$ & & & & & & & $1.1 \mathrm{E}+03$ & $1.1 \mathrm{E}+02$ \\
\hline $\begin{array}{l}\text { Cadmium } \\
\text { (Dite) }\end{array}$ & $007440-43.9$ & & & $7.3 \mathrm{E}+02$ & $7.8 \mathrm{E}+01$ & 7.3E+01 & $7.8 \mathrm{E}+00$ & & & $1.4 \mathrm{E}+01$ & $1.4 \mathrm{E}+\infty 0$ & $6.0 \mathrm{E}+05$ & $6.0 E+03$ & & & & & $1.4 \mathrm{E}+0 \mathrm{I}$ & $1.4 \mathrm{E}+00$ \\
\hline $\begin{array}{l}\text { Calcium Cya- } \\
\text { nide }\end{array}$ & $000592-01-8$ & & & $2.9 E+04$ & $3.1 \mathrm{E}+03$ & $2.9 \mathrm{E}+03$ & $3.1 E+02$ & & & $1.1 \mathrm{E}+0 \mathrm{~S}$ & L.1E+04 & & & & & & & $2.3 E+04$ & $2.3 \mathrm{E}+03$ \\
\hline Caprolactam & $000105-60-2$ & & & $3.7 \mathrm{E}+05$ & $3.9 E+04$ & 3.7E+04 & $3.9 E+03$ & & & $3.4 \mathrm{E}+05$ & $3.4 \mathrm{E}+04$ & & & & & & & $1.8 \mathrm{E}+05$ & $1.8 \mathrm{E}+04$ \\
\hline Captafol & $002425-06-1$ & $7.4 \mathrm{E}+03$ & $7,4 \mathrm{E}+01$ & $1.5 \mathrm{E}+03$ & $1.6 \mathrm{E}+02$ & 1.SE+02 & $1.6 \mathrm{E}+01$ & $1.9 \mathrm{E}+04$ & $1.9 E+02$ & $1.4 \mathrm{E}+03$ & $1.4 E+02$ & & & & & $9.6 \mathrm{E}+03$ & $9.6 \mathrm{E}+01$ & $7.1 \mathrm{E}+02$ & $7.1 E+01$ \\
\hline Captan & $000133-06-2$ & $1.8 \mathrm{E}+04$ & $1.8 \mathrm{E}+02$ & $9.5 \mathrm{E}+04$ & $1.0 \mathrm{E}+04$ & $9.5 \mathrm{E}+03$ & $1.0 \mathrm{E}+03$ & $4.6 \mathrm{E}+04$ & $4.6 \mathrm{E}+02$ & $9.0 \mathrm{E}+04$ & $9.0 \mathrm{E}+03$ & & & & & $2.4 \mathrm{E}+04$ & $2,4 \mathrm{E}+02$ & $4.6 \mathrm{E}+04$ & $4,6 \mathrm{E}+03$ \\
\hline Carbary! & $000063 \cdot 25.2$ & & & $7.3 E+04$ & $7.8 \mathrm{E}+03$ & 7.3E+03 & $7.8 \mathrm{E}+02$ & & & $6.9 \mathrm{E}+04$ & $6.9 \mathrm{E}+03$ & & & & & & & $3.5 \mathrm{E}+04$ & $3.5 \mathrm{E}+03$ \\
\hline Carbazole & $000086-74-8$ & $3.2 E+03$ & $3.2 \mathrm{E}+01$ & & & & & $1.1 \mathrm{E}+04$ & $1.1 E+02$ & & & & & & & $4.8 \mathrm{E}+03$ & $4.8 \mathrm{E}+01$ & & \\
\hline Carbofunan & $001563-66-2$ & & & $3.7 E+03$ & $3.9 E+02$ & $3.7 E+02$ & $3.9 E+01$ & & & $3.4 E+03$ & $3.4 \mathrm{E}+02$ & & & & & & & $1.8 \mathrm{E}+03$ & $1.8 \mathrm{E}+02$ \\
\hline $\begin{array}{l}\text { Carbon Disul- } \\
\text { fide }\end{array}$ & 000075.15 .0 & & & $7.3 \mathrm{E}+04$ & $7.8 \mathrm{E}+03$ & $7.3 \mathrm{E}+03$ & $7.8 E+02$ & & & $3.5 \mathrm{E}+03$ & $3.5 \mathrm{E}+02$ & & & $1.7 E+03$ & $1.4 E+03$ & & & $1.7 \mathrm{E}+03$ & $2.7 E+02$ \\
\hline $\begin{array}{l}\text { Carbon Tetra. } \\
\text { chloride }\end{array}$ & $000056-23-5$ & $4.9 E+02$ & $4.9 \mathrm{E}+00$ & $5.1 E+02$ & $5.5 E+01$ & $5.1 E+01$ & $5.5 \mathrm{E}+\infty$ & $1.6 \mathrm{E}+03$ & $1.6 \mathrm{E}+01$ & $6.3 \mathrm{E}+02$ & $6.3 \mathrm{E}+01$ & $3.1 \mathrm{E}+02$ & $3.1 \mathrm{E}+\infty$ & & & $2.1 E+02$ & $2.1 E+\infty 0$ & $2.8 \mathrm{E}+02$ & $28 \mathrm{E}+01$ \\
\hline Carbosulfan & $055285-14.8$ & & & 7.3E+03 & $7.8 E+02$ & $7.3 E+02$ & $7.8 \mathrm{E}+01$ & & & $6.9 \mathrm{E}+03$ & $6.9 E+02$ & & & & & & & $3.5 \mathrm{E}+03$ & $3.5 \mathrm{E}+02$ \\
\hline Carboxin & $005234-68-4$ & & & $7.3 E+04$ & $7.8 E+03$ & $7.3 \mathrm{E}+03$ & $7.8 E+02$ & & & $6.9 E+04$ & $6.9 \mathrm{E}+03$ & & & & & & & $3.5 E+04$ & 3.5E+03 \\
\hline
\end{tabular}

Nonradionuclides/soil/residential

Page 7 of 41 
Table 3.1c. (continued)

\begin{tabular}{|c|c|c|c|c|c|c|c|c|c|c|c|c|c|c|c|c|c|c|c|}
\hline \multirow{2}{*}{ Chemical } & \multirow{2}{*}{ CASRN } & \multicolumn{6}{|c|}{ Ingestion (mg/kg) } & \multicolumn{4}{|c|}{ Dermal (mg/kg) } & \multicolumn{4}{|c|}{ Inhalation $(\mathrm{mg} / \mathrm{kg})^{\circ i}$} & \multicolumn{4}{|c|}{$\begin{aligned} & \text { Ingestion + Dermal } \\
&+ \text { Inhalation (mg/kg) } \\
&\end{aligned}$} \\
\hline & & $10^{-4}$ & $10^{4}$ & $\begin{array}{l}\text { HQ=1 } \\
\text { Adult }\end{array}$ & $\begin{array}{l}\text { HQ=1 } \\
\text { Child }\end{array}$ & $\begin{array}{c}\mathrm{HQ}=0.1 \\
\text { Adult }\end{array}$ & $\begin{array}{c}\mathrm{HQ}=0.1 \\
\text { Child }\end{array}$ & $10^{-4}$ & $10^{-4}$ & $H Q=1$ & $\mathrm{HQ}=0.1$ & $10^{-4}$ & $10^{-6}$ & $H Q=1$ & $\mathrm{HQ}=0.1$ & $10^{-4}$ & $10^{4}$ & $\mathrm{HI}=1$ & $\mathrm{HI}=0.1$ \\
\hline Chloral & $000075 \cdot 87.6$ & & & $1.5 \mathrm{E}+03$ & $1,6 \mathrm{E}+02$ & $1.5 \mathrm{E}+02$ & $1.6 \mathrm{E}+01$ & & & $1.4 \mathrm{E}+03$ & $1.4 E+02$ & & & & & & & 7.1E+02 & 7.1E+01 \\
\hline Chloramben & $000133-90-4$ & & & $1.1 \mathrm{E}+04$ & $1.2 \mathrm{E}+03$ & $1.1 \mathrm{E}+03$ & $1.2 E+02$ & & & $1.0 \mathrm{E}+04$ & $1.0 \mathrm{E}+03$ & & & & & & & $5.3 E+03$ & $5.3 \mathrm{E}+02$ \\
\hline Chloranil & $000118-75-2$ & $1.6 \mathrm{E}+02$ & $1.6 \mathrm{E}+00$ & & & & & $4.0 \mathrm{E}+02$ & $4.0 E+\infty 0$ & & & & & & & $2.1 E+02$ & $2.1 \mathrm{E}+00$ & & \\
\hline Chlordane $^{(1)}$ & $000057-74.9$ & $4.9 \mathrm{E}+01$ & $4.9 \mathrm{E}-01$ & 4.4E+01 & $4.7 \mathrm{E}+00$ & $4.4 \mathrm{E}+00$ & 4.7E-01 & $1.2 \mathrm{E}+02$ & $1.2 \mathrm{E}+00$ & $4.1 \mathrm{E}+01$ & $4.1 \mathrm{E}+00$ & $1.0 \mathrm{E}+06$ & $2.8 \mathrm{E}+04$ & & & $6.4 E+01$ & $6.4 \mathrm{E}-01$ & $2.1 \mathrm{E}+01$ & $2.1 \mathrm{E}+\infty 0$ \\
\hline Chloride & $016887.00-6$ & & & & & & & & & $\therefore$ & & & & & & & & & \\
\hline $\begin{array}{l}\text { Chlorimuron, } \\
\text { Ethyl- }\end{array}$ & $090982-32-4$ & & & $1.5 \mathrm{E}+04$ & $1.6 \mathrm{E}+03$ & $1.5 \mathrm{E}+03$ & $1.6 \mathrm{E}+02$ & & & $1.4 E+04$ & $1.4 E+03$ & & & & & & & $7.1 \mathrm{E}+03$ & $7.1 \mathrm{E}+02$ \\
\hline Chlorine & 007782.50 .5 & & & 7.3E+04 & $7.8 \mathrm{E}+03$ & $7.3 E+03$ & $7.8 \mathrm{E}+02$ & & & $2.8 \mathrm{E}+05$ & $2.8 \mathrm{E}+04$ & & & & & & & $5.8 \mathrm{E}+04$ & $5.8 \mathrm{E}+03$ \\
\hline $\begin{array}{l}\text { Chlorine Diox- } \\
\text { ide }\end{array}$ & $010049-04-4$ & & & & & & & & & & & & & $8.9 \mathrm{E}+05$ & $8.9 \mathrm{E}+04$ & & & & \\
\hline $\begin{array}{l}\text { Chlorite (So- } \\
\text { dium Salt) }\end{array}$ & $007758-19-2$ & & & & & & & & & & & & & & & & & & \\
\hline $\begin{array}{l}\text { Chloro-1,1- } \\
\text { difluoroethane, } \\
\text { 1- }\end{array}$ & $000075-68-3$ & - & & & & & & & & & & & & $1.0 \mathrm{E}+06$ & $1.0 \mathrm{E}+06$ & & & & \\
\hline $\begin{array}{l}\text { Chloro-1,3- } \\
\text { butadiene, 2- }\end{array}$ & $000126-99-8$ & & & $1.5 E+04$ & $1.6 \mathrm{E}+03$ & $1.5 \mathrm{E}+03$ & $1.6 \mathrm{E}+02$ & & & $2.2 E+04$ & $2.2 \mathrm{E}+03$ & & & 3.1E+02 & $3.1 E+01$ & & & $3.0 \mathrm{E}+02$ & $3.0 \mathrm{E}+01$ \\
\hline $\begin{array}{l}\text { Chloro-2- } \\
\text { methylaniline } \\
\mathrm{HCl}_{4} 4-\end{array}$ & $003165-93-3$ & $1.4 \mathrm{E}+02$ & $1.4 \mathrm{E}+00$ & & & & & $3.5 E+02$ & $3.5 \mathrm{E}+00$ & & & & & & & $1.8 \mathrm{E}+02$ & $1.8 \mathrm{E}+\infty$ & & \\
\hline $\begin{array}{l}\text { Chloro-2:- } \\
\text { methylaniline, } \\
\text { 4- }\end{array}$ & $000095-69-2$ & $1.1 \mathrm{E}+02$ & $1.1 \mathrm{E}+00$ & & & & & $2.8 \mathrm{E}+02$ & $2.8 E+\infty 0$ & & & & & & & $1.4 E+02$ & $1.4 E+\infty$ & & \\
\hline $\begin{array}{l}\text { Chloroacetic } \\
\text { Acid }\end{array}$ & $000079-11-8$ & & & $1.5 E+03$ & $1.6 \mathrm{E}+02$ & $1.5 E+02$ & $1.6 \mathrm{E}+01$ & & & $1.4 \mathrm{E}+03$ & $1.4 \mathrm{E}+02$ & & & & & & & $7.1 \mathrm{E}+02$ & $7.1 E+01$ \\
\hline $\begin{array}{l}\text { Chloroaceto- } \\
\text { phenone, 2- }\end{array}$ & $000532-27-4$ & & & & & & & & & & & & & $1.3 \mathrm{E}+05$ & $1.3 \mathrm{E}+04$ & & & & \\
\hline $\begin{array}{l}\text { Chloroaniline, } \\
\text { p- }\end{array}$ & $000106-47-8$ & & & $2.9 E+03$ & $3.1 E+02$ & $2.9 \mathrm{E}+02$ & $3.1 \mathrm{E}+01$ & & & $2.8 \mathrm{E}+03$ & $2.8 \mathrm{E}+02$ & & & & & & & $1.4 E+03$ & $1.4 E+02$ \\
\hline
\end{tabular}


Table 3.1c. (continued)

\begin{tabular}{|c|c|c|c|c|c|c|c|c|c|c|c|c|c|c|c|c|c|c|c|}
\hline \multirow{2}{*}{ Chemical } & \multirow{2}{*}{ CASRN } & \multicolumn{6}{|c|}{ Ingestion (mg/kg) } & \multicolumn{4}{|c|}{ Dermal (mg/kg) } & \multicolumn{4}{|c|}{ Inhalation (mg/kg) } & \multicolumn{4}{|c|}{$\begin{array}{l}\text { Ingestion + Dermal } \\
+ \text { Inhalation }(\mathrm{mg} / \mathrm{kg})^{+\bullet \varepsilon}\end{array}$} \\
\hline & & $10^{-4}$ & $10^{4}$ & $\begin{array}{l}\text { HQ=1 } \\
\text { Adult }\end{array}$ & $\begin{array}{l}H Q=1 \\
\text { Child }\end{array}$ & $\begin{array}{c}\mathrm{HQ}=0.1 \\
\text { Adult }\end{array}$ & $\begin{array}{l}H Q=0.1 \\
\text { Child }\end{array}$ & $10^{-4}$ & $10^{4}$ & $H Q=1$ & $\mathrm{HQ}=0.1$ & $10^{-4}$ & $10^{4}$ & $H Q=1$ & $\mathrm{HQ}=0.1$ & $10^{-1}$ & $10^{6}$ & $H I=1$ & $\mathrm{HI}=0.1$ \\
\hline $\begin{array}{l}\text { Chloroben- } \\
\text { zenee }\end{array}$ & $000108-90-7$ & & & 1.5E+04 & $1.6 \mathrm{E}+03$ & $1.5 \mathrm{E}+03$ & $1.6 \mathrm{E}+02$ & & & $8.5 E+03$ & $8.5 \mathrm{E}+02$ & - & & $5.1 E+02$ & $1.0 \mathrm{E}+02$ & & & $5.1 \mathrm{E}+02$ & $86 E+01$ \\
\hline $\begin{array}{l}\text { Chlorobenzil- } \\
\text { ate }\end{array}$ & $000510-15-6$ & $2.4 \mathrm{E}+02$ & $2.4 E+00$ & $1.5 \mathrm{E}+04$ & $1.6 \mathrm{E}+03$ & $1.5 \mathrm{E}+03$ & $1.6 \mathrm{E}+02$ & $6.0 E+02$ & $6.0 \mathrm{E}+00$ & $1.4 E+04$ & $1.4 \mathrm{E}+03$ & $1.0 \mathrm{E}+06$ & $1.4 E+05$ & & & $3.1 E+02$ & $3.1 E+\infty$ & $7.1 E+03$ & $7.1 E+02$ \\
\hline $\begin{array}{l}\text { Chlorobenzoic } \\
\text { Acid, p. }\end{array}$ & $000074 \cdot 11-3$ & & & $1.5 E+05$ & $1.6 \mathrm{E}+04$ & $1.5 \mathrm{E}+04$ & $1.6 \mathrm{E}+03$ & & & $1.4 \mathrm{E}+05$ & $1.4 \mathrm{E}+04$ & & & & & & & 7.1E+04 & 7.1E+03 \\
\hline $\begin{array}{l}\text { Chlorobenzo- } \\
\text { trifluoride, } 4 \text { - }\end{array}$ & $000098.56-6$ & & & $1.5 E+04$ & $1.6 E+03$ & $1.5 \mathrm{E}+03$ & $1.6 E+02$ & & & $1.4 \mathrm{E}+04$ & $1.4 E+03$ & & & & & & & 7.1E+03 & $7.1 \mathrm{E}+02$ \\
\hline $\begin{array}{l}\text { Chlorobutane, } \\
\text { 1- }\end{array}$ & 000109.69 .3 & & & $2.9 E+05$ & $3.1 E+04$ & $2.9 \mathrm{E}+04$ & $3.1 \mathrm{E}+03$ & & & $4.4 E+05$ & $4.4 E+04$ & & & & & & & $1.8 \mathrm{E}+05$ & $1.8 \mathrm{E}+04$ \\
\hline $\begin{array}{l}\text { Chlorobutane, } \\
2-\end{array}$ & $000078-86-4$ & & & & & & & & & & & & & & & & & & \\
\hline $\begin{array}{l}\text { Chlorocyclo- } \\
\text { pentadiene }\end{array}$ & $041851.50-7$ & & & & & & & & & & & & & & & & & & \\
\hline $\begin{array}{l}\text { Chlorodibro- } \\
\text { moethane }\end{array}$ & $073506-94-2$ & $7.6 \mathrm{E}+02$ & $7.6 \mathrm{E}+\infty$ & & & & & $3.1 \mathrm{E}+03$ & $3.1 E+01$ & & & & & & & $1.2 \mathrm{E}+03$ & $1.2 \mathrm{E}+01$ & & \\
\hline $\begin{array}{l}\text { Chlorodi- } \\
\text { fluoromethane }\end{array}$ & $000075-45-6$ & & & & & & & & & & & & & $1.0 \mathrm{E}+06$ & $1.0 \mathrm{E}+06$ & & & & \\
\hline Chloroform ${ }^{(1)}$ & $000067-66-3$ & $1.0 E+04$ & $1.0 \mathrm{E}+02$ & $7.3 \mathrm{E}+03$ & $7,8 \mathrm{E}+02$ & $7.3 E+02$ & $7.8 \mathrm{E}+01$ & $1.1 \mathrm{E}+04$ & $1.1 \mathrm{E}+02$ & $2.8 \mathrm{E}+03$ & $2.8 \mathrm{E}+02$ & $1.9 E+02$ & $1.9 \mathrm{E}+00$ & & & $1.8 \mathrm{E}+02$ & $1.8 \mathrm{E}+\infty 0$ & $1.5 \mathrm{E}+03$ & $2.0 \mathrm{E}+02$ \\
\hline $\begin{array}{l}\text { Chlorometh- } \\
\text { ande }\end{array}$ & $000074-87-3$ & $4.9 E+03$ & $4.9 \mathrm{E}+01$ & & & & & $2.0 \mathrm{E}+04$ & $2.0 \mathrm{E}+02$ & & & $4.4 \mathrm{E}+02$ & $4.4 \mathrm{E}+00$ & & & $4.2 \mathrm{E}+02$ & $4.2 \mathrm{E}+\infty$ & & \\
\hline $\begin{array}{l}\text { Chloromethy! } \\
\text { Methyl Ether }\end{array}$ & $000107 \cdot 30-2$ & & & & & & & & & & & & & & & & & & \\
\hline $\begin{array}{l}\text { Chlorora- } \\
\text { phthalene, } \\
\text { Betz- }\end{array}$ & $000091-58-7$ & & & $5.8 \mathrm{E}+04$ & $6.3 \mathrm{E}+03$ & $5.8 \mathrm{E}+03$ & $6.3 \mathrm{E}+02$ & & & $8.8 \mathrm{E}+04$ & $8.8 E+03$ & & & & & & & $3.5 E+04$ & $3.5 \mathrm{E}+03$ \\
\hline $\begin{array}{l}\text { Chloronitro- } \\
\text { benzene, o- }\end{array}$ & $000088-73-3$ & $2.6 \mathrm{E}+03$ & $2.6 \mathrm{E}+01$ & & & & & $1.0 \mathrm{E}+04$ & $1.0 \mathrm{E}+02$ & & & & & & & $4.1 E+03$ & $4.1 \mathrm{E}+01$ & & \\
\hline $\begin{array}{l}\text { Chloronitro- } \\
\text { benzenc, p- }\end{array}$ & $000121-73-3$ & $3.5 \mathrm{E}+03$ & $3.5 E+01$ & & & & & $1.4 E+04$ & $1.4 \mathrm{E}+02$ & & & & & & & $5.7 E+03$ & $5.7 E+01$ & & \\
\hline
\end{tabular}


Table 3.1c. (continued)

\begin{tabular}{|c|c|c|c|c|c|c|c|c|c|c|c|c|c|c|c|c|c|c|c|}
\hline \multirow{2}{*}{ Chemical } & \multirow{2}{*}{ CASRN } & \multicolumn{6}{|c|}{ Ingestion (mg/kg) } & \multicolumn{4}{|c|}{ Dermal (mg/kg) } & \multicolumn{4}{|c|}{ Inhalation (mg/kg) } & \multicolumn{4}{|c|}{$\begin{array}{r}\text { Ingestion + Dermal } \\
+ \text { Inhalation (mg/kg) } \\
\end{array}$} \\
\hline & & $10^{-4}$ & $10^{6}$ & $\begin{array}{l}\text { HQ=1 } \\
\text { Adult }\end{array}$ & $\begin{array}{l}\mathrm{HQ}=1 \\
\text { Child }\end{array}$ & $\begin{array}{c}\mathrm{HQ}=0.1 \\
\text { Adult }\end{array}$ & $\begin{array}{c}\text { HQ }=0.1 \\
\text { Child }\end{array}$ & $10^{-4}$ & $10^{5}$ & $\mathrm{HQ}=1$ & $\mathrm{HQ}=0.1$ & $10^{-4}$ & $10^{4}$ & HQ=1 & $\mathrm{HQ}=0.1$ & $10^{-4}$ & $10^{4}$ & $\mathrm{HI}=1$ & $\mathrm{HI}=0.1$ \\
\hline $\begin{array}{l}\text { Chlorophenol, } \\
2 .\end{array}$ & $000095-57-8$ & & & $3.7 \mathrm{E}+03$ & $3.9 E+02$ & $3.7 E+02$ & $3.9 \mathrm{E}+01$ & & & $5.5 \mathrm{E}+03$ & $5.5 E+02$ & & & & & & & $2.2 \mathrm{E}+03$ & $2.2 E+02$ \\
\hline $\begin{array}{l}\text { Chlorophenyl } \\
\text { Methyl Sul- } \\
\text { fide, p- }\end{array}$ & $000123-09-1$ & & & & & & & & & & & & & & & & & & \\
\hline $\begin{array}{l}\text { Chlorophenyl } \\
\text { Methyl Sulfox. } \\
\text { ide }\end{array}$ & $000934-73-6$ & & & & & & & & & & & & & & & & & & \\
\hline $\begin{array}{l}\text { Chloropro- } \\
\text { pane, 2- }\end{array}$ & $000075-29-6$ & & & & & & & & & & & & & $1.5 E+03$ & $1.5 E+02$ & & & & \\
\hline Chlorothelonil & $001897-45-6$ & $5.8 E+03$ & $5.8 \mathrm{E}+01$ & $1.1 E+04$ & $1.2 E+03$ & $1.1 \mathrm{E}+03$ & $1.2 E+02$ & $1.5 \mathrm{E}+04$ & $1.5 E+02$ & $1.0 E+04$ & $1.0 \mathrm{E}+03$ & & & & & $7.5 E+03$ & 7.5E+01 & $5.3 \mathrm{E}+03$ & $5.3 \mathrm{E}+02$ \\
\hline $\begin{array}{l}\text { Chlorotoluene, } \\
\text { o. }\end{array}$ & $000095-49.8$ & & . & $1.5 E+04$ & $1.6 \mathrm{E}+03$ & $1.5 \mathrm{E}+03$ & $1.6 \mathrm{E}+02$ & & & $2.2 \mathrm{E}+04$ & $2.2 \mathrm{E}+03$ & & & & & & & $8.8 \mathrm{E}+03$ & $8.8 \mathrm{E}+02$ \\
\hline Chloppropham & $000101-21-3$ & & & $1.5 E+05$ & $1.6 \mathrm{E}+04$ & $1.5 E+04$ & $1.6 \mathrm{E}+03$ & & & $1.4 E+05$ & $1.4 E+04$ & & & & & & & $7.1 \mathrm{E}+04$ & 7. $. \mathrm{E}+03$ \\
\hline Chlorpyrifos & $002921-88-2$ & & & $2.2 E+03$ & $2.3 E+02$ & $2.2 E+02$ & $2.3 E+01$ & & & $2.1 E+03$ & $2.1 E+02$ & & & & & & & $1.1 \mathrm{E}+03$ & $1.1 \mathrm{E}+02$ \\
\hline $\begin{array}{l}\text { CMloppyrifos } \\
\text { Mehyl }\end{array}$ & $005598-13-0$ & & & $7.3 E+03$ & $7.8 \mathrm{E}+02$ & $7.3 \mathrm{E}+02$ & $7.8 E+01$ & & & $6.9 E+03$ & $6.9 E+02$ & & & & & & & $3.5 E+03$ & -3.5E+02 \\
\hline Chlorsulfuron & $064902-72-3$ & & & $3.7 E+04$ & $3.9 E+03$ & 3. $\pi+03$ & $3.9 E+02$ & & & $3.4 E+04$ & $3.4 E+03$ & & & & & & & $1.8 \mathrm{E}+04$ & $1.8 \mathrm{E}+03$ \\
\hline Chlorthiophos & $050238-56-4$ & & & $5.8 \mathrm{E}+02$ & $6.3 \mathrm{E}+01$ & $5.8 \mathrm{E}+01$ & $6.3 \mathrm{E}+00$ & & & $5.5 E+02$ & $5.5 E+01$ & & & & & & & $2.8 \mathrm{E}+02$ & $2.8 \mathrm{E}+01$ \\
\hline $\begin{array}{l}\text { Chromium } \\
\text { (IIII) (nsoluble } \\
\text { Salts) }\end{array}$ & 016065-83-1 & & & $7.3 \mathrm{E}+05$ & $7.8 E+04$ & $7.3 E+04$ & $7.8 \mathrm{E}+03$ & & & $6.9 \mathrm{E}+04$ & $6.9 \mathrm{E}+03$ & & & & & & & $6.3 \mathrm{E}+04$ & $6.3 \mathrm{E}+03$ \\
\hline $\begin{array}{l}\text { Chromium } \\
\left(\text { (VI) }^{(1)}\right.\end{array}$ & $018540-29.9$ & & & $3.7 \mathrm{E}+03$ & $3.9 \mathrm{E}+02$ & $3.7 \mathrm{E}+02$ & $3.9 E+01$ & & & $1.4 \mathrm{E}+03$ & $1.4 E+02$ & $8.9 E+04$ & $8.9 E+02$ & & & & & $1.0 \mathrm{E}+03$ & $1.0 E+02$ \\
\hline $\begin{array}{l}\text { Chromium } \\
\text { Salts }\end{array}$ & $\mathrm{NA}$ & & & & & & & & & & & & & & & & & & \\
\hline Chrysene ${ }^{m}$ & $000218-01-9$ & $8.8 E+03$ & $8.8 \mathrm{E}+01$ & & & & & $1.4 E+04$ & $1.4 E+02$ & & & $1.0 \mathrm{E}+06$ & $1.0 E+06$ & & & $8.6 \mathrm{E}+03$ & $8.6 \mathrm{E}+01$ & & \\
\hline Cobalt & $007440-48-4$ & & & & & & & & & & & & & & & & & & \\
\hline $\begin{array}{l}\text { Coke Oven } \\
\text { Emissions }\end{array}$ & $008007-45 \cdot 2$ & & & & & & & & & & & $1.0 E+06$ & $1.7 \mathrm{E}+04$ & & & & & & \\
\hline
\end{tabular}


Table 3.1c. (continued)

\begin{tabular}{|c|c|c|c|c|c|c|c|c|c|c|c|c|c|c|c|c|c|c|c|}
\hline \multirow{2}{*}{ Chemical } & \multirow{2}{*}{ CASRN } & \multicolumn{6}{|c|}{ Ingestion (mg/kg) } & \multicolumn{4}{|c|}{ Dermal (mg/kg) } & \multicolumn{4}{|c|}{ Inhalation (mg/kg) } & \multicolumn{4}{|c|}{$\begin{aligned} & \text { Ingestion + Dermal } \\
&+ \text { Inhalation (mg } / \mathbf{k g})^{\dagger \cdot v}\end{aligned}$} \\
\hline & & $10^{-4}$ & $10^{4}$ & $\begin{array}{l}\text { HQ=1 } \\
\text { Adult }\end{array}$ & $\begin{array}{l}\text { HQ }=1 \\
\text { Child }\end{array}$ & $\begin{array}{c}\text { HQ }=0.1 \\
\text { Adult }\end{array}$ & $\begin{array}{c}\mathrm{HQ}=0.1 \\
\text { Child }\end{array}$ & $10^{4}$ & $10^{4}$ & $H Q=1$ & $\mathrm{HQ}=0.1$ & $10^{4}$ & $10^{4}$ & $H Q=1$ & $\mathrm{HQ}=0.1$ & $10^{4}$ & $10^{6}$ & $H I=1$ & $\mathrm{HI}=0.1$ \\
\hline Copper & $007440-50-8$ & & & & & & & & & & & & & & & & & & \\
\hline $\begin{array}{l}\text { Copper Cya- } \\
\text { nide }\end{array}$ & $000544-92-3$ & & & $3.7 E+03$ & $3.9 E+02$ & $3.7 \mathrm{E}+02$ & $3.9 E+01$ & & & $1.4 E+04$ & $1.4 \mathrm{E}+03$ & & & & & & & $2.9 E+03$ & $2.9 E+02$ \\
\hline Creosote & $008001-58-9$ & & & & & & & & & & & & & & & & & & \\
\hline Cresol, m- & $000108-39-4$ & & & $3.7 E+04$ & $3.9 E+03$ & $3.7 E+03$ & $3.9 E+02$ & & & $3.4 E+04$ & $3,4 E+03$ & & & & & & & $1.8 \mathrm{E}+04$ & $1.8 \mathrm{E}+03$ \\
\hline Cresol, o- & $000095-48-7$ & & & $3.7 \mathrm{E}+04$ & $3.9 \mathrm{E}+03$ & $3.7 \mathrm{E}+03$ & $3.9 E+02$ & & & $3.4 E+04$ & $3.4 \mathrm{E}+03$ & & & & & & & $1.8 \mathrm{E}+04$ & $1.8 \mathrm{E}+03$ \\
\hline Cresol, p- & $000106-44-5$ & & & $3.7 E+03$ & $3.9 E+02$ & $3.7 \mathrm{E}+02$ & $3.9 \mathrm{E}+01$ & & & $4.5 \mathrm{E}+03$ & $4.5 \mathrm{E}+02$ & & & & & & & $2.0 \mathrm{E}+03$ & $2.0 \mathrm{E}+02$ \\
\hline $\begin{array}{l}\text { Crotonalde- } \\
\text { hyde }\end{array}$ & $000123-73.9$ & $3.4 E+01$ & $3.4 \mathrm{E}-01$ & & & & & $8.5 E+01$ & $8.5 \mathrm{E}-01$ & & & & & & & $4.4 E+01$ & $4.4 \mathrm{E}-01$ & & \\
\hline Cumene & $000098-82-8$ & & & $2.9 E+04$ & $3.1 E+03$ & $2.9 \mathrm{E}+03$ & $3.1 \mathrm{E}+02$ & & & 4.4E+04 & $4.4 E+03$ & & & $3.5 E+02$ & $6.6 \mathrm{E}+01$ & & & $3.5 \mathrm{E}+02$ & $6.4 E+01$ \\
\hline Cyanazine & $021725-46-2$ & $7.6 \mathrm{E}+01$ & $7.6 \mathrm{E}-01$ & $1.5 \mathrm{E}+03$ & $1.6 \mathrm{E}+02$ & $1.5 \mathrm{E}+02$ & $1.6 \mathrm{E}+01$ & $1.9 \mathrm{E}+02$ & $1.9 \mathrm{E}+\infty$ & $1.4 E+03$ & $1.4 \mathrm{E}+02$ & & & & & $9.8 \mathrm{E}+01$ & $9.8 \mathrm{E}-01$ & 7.1E+02 & $7.1 \mathrm{E}+01$ \\
\hline Cyanide (CN-) & $000057-12-5$ & & & $1.5 \mathrm{E}+04$ & $1.6 \mathrm{E}+03$ & 1.5E+03 & $1.6 \mathrm{E}+02$ & & & $4.7 E+03$ & 4.7E+02 & & & & & & & $3.5 \mathrm{E}+03$ & $3.5 \mathrm{E}+02$ \\
\hline Cyanogen & $000460-19-5$ & & & $2.9 \mathrm{E}+04$ & $3.1 E+03$ & $2.9 E+03$ & $3.1 E+02$ & & & $4.4 \mathrm{E}+04$ & $4.4 \mathrm{E}+03$ & & & & & & & $1.8 \mathrm{E}+04$ & $1.8 \mathrm{E}+03$ \\
\hline $\begin{array}{l}\text { Cyanogen } \\
\text { Bromide }\end{array}$ & $000506-68-3$ & & & $6.6 \mathrm{E}+04$ & $7.0 E+03$ & $6.6 \mathrm{E}+03$ & $7.0 E+02$ & & & $9.9 \mathrm{E}+04$ & $9.9 \mathrm{E}+03$ & & & & & & & $4.0 \mathrm{E}+04$ & $4.0 \mathrm{E}+03$ \\
\hline $\begin{array}{l}\text { Cyanogen } \\
\text { Chloride }\end{array}$ & $000506-77-4$ & & & $3.7 \mathrm{E}+04$ & $3.9 E+03$ & $3.7 E+03$ & $3.9 \mathrm{E}+02$ & & & $5.5 \mathrm{E}+04$ & $5.5 \mathrm{E}+03$ & & & & & & & $2.2 E+04$ & $2.2 E+03$ \\
\hline $\begin{array}{l}\text { Cyclohexane, } \\
1,2,3,4,5 \text {. } \\
\text { pentabromo-6- } \\
\text { chloro- }\end{array}$ & $000087-84-3$ & $2.8 \mathrm{E}+03$ & $2.8 \mathrm{E}+01$ & & & & & $7.0 \mathrm{E}+03$ & $7.0 \mathrm{E}+01$ & & & & & & & $3.6 \mathrm{E}+03$ & $3.6 \mathrm{E}+01$ & & \\
\hline $\begin{array}{l}\text { Cyclohexa- } \\
\text { none }\end{array}$ & $000108-94-1$ & & & $1.0 E+06$ & $3.9 \mathrm{E}+05$ & 3.7E+0S & $3.9 \mathrm{E}+04$ & & & $1.0 \mathrm{E}+06$ & $5.5 E+05$ & & & & & & & $1.0 \mathrm{E}+06$ & $2.2 \mathrm{E}+05$ \\
\hline $\begin{array}{l}\text { Cyclohexyla- } \\
\text { mine }\end{array}$ & $000108-91-8$ & & & $1.5 E+05$ & $1.6 \mathrm{E}+04$ & $1.5 \mathrm{E}+04$ & $1.6 \mathrm{E}+03$ & & & $1.4 \mathrm{E}+05$ & $1.4 E+04$ & & & & & & & $7.1 E+04$ & $7.1 E+03$ \\
\hline $\begin{array}{l}\text { Cyclopenta- } \\
\text { diene }\end{array}$ & 000542.92 .7 & & & & & & & & & & & & & & & & & & \\
\hline $\begin{array}{l}\text { Cyhalothring } \\
\text { karate }\end{array}$ & $068085-85-8$ & & & $3.7 \mathrm{E}+03$ & $3.9 E+02$ & $3.7 \mathrm{E}+02$ & $3.9 \mathrm{E}+01$ & & & $3.4 E+03$ & $3.4 E+02$ & & & & & & & $1.8 \mathrm{E}+03$ & $1.8 \mathrm{E}+02$ \\
\hline
\end{tabular}

Nonradionuclides/soil/residential

Page 11 of 41 
Table 3.1c. (continued)

\begin{tabular}{|c|c|c|c|c|c|c|c|c|c|c|c|c|c|c|c|c|c|c|c|}
\hline \multirow{2}{*}{ Chemical } & \multirow{2}{*}{ CASRN } & \multicolumn{6}{|c|}{ Ingestion (mg/kg) } & \multicolumn{4}{|c|}{ Dermal (mg/kg) } & \multicolumn{4}{|c|}{ Inhalation (mg/kg) } & \multicolumn{4}{|c|}{$\begin{array}{c}\text { Ingestion + Dermal } \\
+ \text { Inhalation }(\mathrm{mg} / \mathrm{kg})^{+\bullet v} \\
\end{array}$} \\
\hline & & $10^{-1}$ & $10^{4}$ & $\begin{array}{l}\mathrm{HQ}=1 \\
\text { Adult }\end{array}$ & $\begin{array}{l}\text { HQ=1 } \\
\text { Child }\end{array}$ & $\begin{array}{c}\mathrm{HQ}=0.1 \\
\text { Adult }\end{array}$ & $\begin{array}{c}\text { HQ }=0.1 \\
\text { Child }\end{array}$ & $10^{-4}$ & $10^{4}$ & $\mathrm{HQ}=1$ & $H Q=0.1$ & $10^{-4}$ & $10^{4}$ & $\mathrm{HQ}=1$ & $\mathrm{HQ}=0.1$ & $10^{-4}$ & $10^{5}$ & HI=I & $\mathrm{HI}=0.1$ \\
\hline Cypermethrin & 052315.07 .8 & & & $7.3 \mathrm{E}+03$ & $7.8 E+02$ & $7.3 \mathrm{E}+02$ & $7.8 \mathrm{E}+01$ & & & $6.9 E+03$ & $6.9 E+02$ & & & & & & & 3.5E+03 & $3.5 \mathrm{E}+02$ \\
\hline Cyromazine & $066215-27.8$ & & & $5.5 \mathrm{E}+03$ & $5.9 E+02$ & $5.5 \mathrm{E}+02$ & $5.9 \mathrm{E}+01$ & & & $5.2 \mathrm{E}+03$ & $5.2 \mathrm{E}+02$ & & & & & & & $2.7 \mathrm{E}+03$ & $2.7 \mathrm{E}+02$ \\
\hline DDD & $000072-54-8$ & $2.7 E+02$ & $2.7 \mathrm{E}+00$ & & & & & $9.4 \mathrm{E}+02$ & $9.4 E+00$ & & & & & & & $4.0 E+02$ & $4.0 E+00$ & & \\
\hline DDE & $000072-55.9$ & $1.9 \mathrm{E}+02$ & $1.9 \mathrm{E}+00$ & & & & & $6.6 \mathrm{E}+02$ & $6.6 \mathrm{E}+00$ & & & & & & & $2.9 E+02$ & $2.9 \mathrm{E}+00$ & & \\
\hline DDT & $000050-29-3$ & $1.9 E+02$ & $1.9 \mathrm{E}+00$ & $3.7 \mathrm{E}+02$ & $3.9 \mathrm{E}+01$ & 3.7E+01 & $3.9 E+00$ & $6.6 \mathrm{E}+02$ & $6.6 \mathrm{E}+00$ & $4.8 \mathrm{E}+02$ & $4.8 \mathrm{E}+01$ & $1.0 E+06$ & L.1E+0S & & & $2.9 \mathrm{E}+02$ & $2.9 E++0$ & $2.1 E+02$ & 2.1E+01 \\
\hline Dacthal & $001861-32-1$ & & & $7.3 \mathrm{E}+03$ & $7.8 E+02$ & $7.3 \mathrm{E}+02$ & $7.8 \mathrm{E}+01$ & & & $6.9 E+03$ & $6.9 \mathrm{E}+02$ & & & & & & & $3.5 \mathrm{E}+03$ & $3.5 \mathrm{E}+02$ \\
\hline Dalapon & $000075-99.0$ & & & $2.2 \mathrm{E}+04$ & $2.3 E+03$ & $2.2 E+03$ & $2.3 \mathrm{E}+02$ & & & 2.1E+04 & $2.1 \mathrm{E}+03$ & & & & & & & 1.1.E+04 & I. $. \mathrm{E}+03$ \\
\hline $\begin{array}{l}\begin{array}{l}\text { Decabromodi- } \\
\text { phenyl Ether }\end{array} \\
\end{array}$ & $001163-19-5$ & & & $7.3 E+03$ & $7.8 \mathrm{E}+02$ & $7.3 \mathrm{E}+02$ & $7.8 E+01$ & & & 1.1.E+04 & $1.1 E+03$ & & & & & & & $4.4 E+03$ & $4.4 \mathrm{E}+02$ \\
\hline Demetion & $0080055-48-3$ & & & $2.9 \mathrm{E}+01$ & $3.1 \mathrm{E}+00$ & $2.9 \mathrm{E}+00$ & $3.1 \mathrm{E}-01$ & & & $2.8 \mathrm{E}+01$ & $2.8 E+\infty 0$ & & & & & & & $1.4 \mathrm{E}+01$ & $1.4 E++00$ \\
\hline $\begin{array}{l}\text { Di(2- } \\
\text { eltyyhhexyl)- } \\
\text { adipate }\end{array}$ & $000103-23-1$ & $5.3 E+04$ & $5.3 \mathrm{E}+02$ & $4.4 \mathrm{E}+05$ & $4.7 \mathrm{E}+04$ & $4.4 E+04$ & $4.7 \mathrm{E}+03$ & $1.3 \mathrm{E}+05$ & 1. $3 E+03$ & $4.1 E+05$ & 4.1E+04 & & & & & $6.9 E+04$ & $6.9 \mathrm{E}+02$ & $2.1 \mathrm{E}+05$ & 2.1E+04 \\
\hline Diallate & $002303-16-4$ & $1.0 \mathrm{E}+03$ & $1.0 \mathrm{E}+01$ & & & & & $4.2 \mathrm{E}+03$ & $4.2 E+01$ & & & & & & & $1.7 E+03$ & $1.7 \mathrm{E}+01$ & & \\
\hline Diazinon & $000333-41-5$ & & & $6.6 \mathrm{E}+02$ & $7.0 \mathrm{E}+01$ & $6.6 \mathrm{E}+01$ & $7.0 E+00$ & & & $6.2 \mathrm{E}+02$ & $6.2 E+01$ & & & & & & & $3.2 \mathrm{E}+02$ & $3.2 E+01$ \\
\hline $\begin{array}{l}\text { Dibenz[a,h]- } \\
\text { anthracene }\end{array}$ & $000053-70.3$ & $8.8 E+00$ & $8.8 \mathrm{E}-02$ & & & & & $1.4 \mathrm{E}+01$ & $1.4 \mathrm{E}-01$ & & & $1.0 \mathrm{E}+06$ & $1.2 E+04$ & & & $8.6 \mathrm{E}+00$ & $8.6 \mathrm{E}-02$ & & \\
\hline $\begin{array}{l}\text { Dibromo-3. } \\
\text { chloroporo- } \\
\text { pane, i,2. }\end{array}$ & $000096-12.8$ & $4.6 \mathrm{E}+01$ & $4.6 \mathrm{E}-01$ & & & & & $1.8 \mathrm{E}+02$ & $1.8 \mathrm{E}+00$ & & & $1.0 E+06$ & $1.0 \mathrm{E}+06$ & $8.9 E+05$ & $8.9 \mathrm{E}+04$ & $7.3 E+01$ & 7.3E-01 & & \\
\hline $\begin{array}{l}\text { Dibroma-ben- } \\
\text { zene, 1,4- }\end{array}$ & $000006-37-6$ & & & $7.3 E+03$ & $7.8 E+02$ & $7.3 E+02$ & $7.8 E \div 01$ & & & $1.1 E+04$ & $1.1 \mathrm{E}+03$ & & & & & & & $4.4 E+03$ & $4.4 \mathrm{E}+02$ \\
\hline $\begin{array}{l}\begin{array}{l}\text { Dibromochlo- } \\
\text { romethane }\end{array} \\
\end{array}$ & $000124-48-1$ & $7.6 \mathrm{E}+02$ & $7.6 \mathrm{E}++00$ & $1.5 E+04$ & $1.6 \mathrm{E}+03$ & $1.5 E+03$ & $1.6 \mathrm{E}+02$ & $2.3 \mathrm{E}+03$ & $2.3 \mathrm{E}+01$ & $1.7 E+04$ & $1.7 E+03$ & & & & & 1.1.E+03 & $1.1 \mathrm{E}+01$ & $7.8 E+03$ & $7.8 E+02$ \\
\hline $\begin{array}{l}\text { Dibromodi- } \\
\text { chloromethane }\end{array}$ & $000594-18-3$ & & & & & & & & & & & & & & & & & & \\
\hline $\begin{array}{l}\text { Dibromodi. } \\
\text { phenyl Ether, } \\
\text { p.p.t. }\end{array}$ & $002050-47.7$ & & & & & & & & & & & & & & & & & & \\
\hline
\end{tabular}


Table 3.1c. (continued)

\begin{tabular}{|c|c|c|c|c|c|c|c|c|c|c|c|c|c|c|c|c|c|c|c|}
\hline \multirow{2}{*}{ Chemical } & \multirow{2}{*}{ CASRN } & \multicolumn{6}{|c|}{ Ingestion (mg/kg) } & \multicolumn{4}{|c|}{ Dermal (mg/kg) } & \multicolumn{4}{|c|}{ Inhalation (mg/kg) } & \multicolumn{4}{|c|}{$\begin{aligned} & \text { Ingestion + Dermal } \\
+ & \text { Inhalation }\left(\mathrm{mg} / \mathrm{kg}^{)^{+\bullet}}\right.\end{aligned}$} \\
\hline & & $10^{-4}$ & $10^{4}$ & $\begin{array}{l}\mathrm{HQ}=1 \\
\text { Adult }\end{array}$ & $\begin{array}{l}\mathrm{HQ}=1 \\
\text { Child }\end{array}$ & $\begin{array}{c}H Q=0.1 \\
\text { Adult }\end{array}$ & $\begin{array}{c}\mathrm{HQ}=0.1 \\
\text { Child }\end{array}$ & $10^{-4}$ & $10^{-4}$ & $\mathrm{HQ}=1$ & $H Q=0.1$ & $10^{-1}$ & $10^{4}$ & $H Q=1$ & $\mathrm{HQ}=0.1$ & $10^{-4}$ & $10^{5}$ & $H I=I$ & $\mathrm{HI}=0.1$ \\
\hline $\begin{array}{l}\text { Dibromoeth- } \\
\text { nne, 1,2- }\end{array}$ & $000106-93-4$ & $7.5 \mathrm{E}-01$ & $7.5 \mathrm{E}-03$ & & & & & $3.0 E+\infty 0$ & $3.0 \mathrm{E}-02$ & & & $1.5 E+02$ & $1.5 E+\infty$ & $2.8 \mathrm{E}+01$ & $2.8 \mathrm{E}+00$ & $1.2 \mathrm{E}+00$ & $1.2 \mathrm{E}-02$ & & \\
\hline $\begin{array}{l}\text { Dibromom- } \\
\text { ethane (Meth- } \\
\text { ylene Bro- } \\
\text { mide) }\end{array}$ & $000074.95-3$ & & & $7.3 \mathrm{E}+03$ & $7.8 \mathrm{E}+02$ & $7.3 E+02$ & $7.8 \mathrm{E}+01$ & & & $1.1 \mathrm{E}+04$ & $1.1 \mathrm{E}+03$ & & & & & & & $4.4 E+03$ & $4.4 E+02$ \\
\hline $\begin{array}{l}\text { Dibutyl } \\
\text { Phthaslate }\end{array}$ & $000084-74-2$ & & & $7.3 E+04$ & $7.8 \mathrm{E}+03$ & $7.3 E+03$ & $7.8 \mathrm{E}+02$ & & & $1.4 \mathrm{E}+05$ & $1.4 \mathrm{E}+04$ & & & & & & & $4.8 \mathrm{E}+04$ & $4.8 \mathrm{E}+03$ \\
\hline Dicamba & $001918-00.9$ & & & $2.2 E+04$ & $2.3 \mathrm{E}+03$ & $2.2 \mathrm{E}+03$ & $2.3 \mathrm{E}+02$ & & & $2.1 E+04$ & $2.1 \mathrm{E}+03$ & & & & & & & $1.1 \mathrm{E}+04$ & $1.1 E+03$ \\
\hline $\begin{array}{l}\text { Dichloro-2- } \\
\text { butene, } 1,4-\end{array}$ & $000764-41-0$ & & & & & & & & & & & $1.2 \mathrm{E}+01$ & $1.2 \mathrm{E}-01$ & & & & & & \\
\hline \multicolumn{20}{|l|}{$\begin{array}{l}\text { Dichloroacetic } \\
\text { Acid }\end{array}$} \\
\hline $\begin{array}{l}\text { Dichloroben- } \\
\text { zene, } 1,2^{(3)}\end{array}$ & $000095-50-1$ & & & $6.6 \mathrm{E}+04$ & $7.0 \mathrm{E}+03$ & $6.6 \mathrm{E}+03$ & $7.0 \mathrm{E}+02$ & & & $9.9 E+04$ & $9.9 E+03$ & & & $5.1 E+02$ & $5.1 E+02$ & & & $5.1 E+02$ & $5.1 E+02$ \\
\hline $\begin{array}{l}\text { Dichloroben- } \\
\text { zene, 1,3- }\end{array}$ & $000541-73-1$ & & & & & & & & & & & & & & & & & & \\
\hline $\begin{array}{l}\text { Dichloroben- } \\
\text { zene, 1,4e }\end{array}$ & $000106-46-7$ & $2.7 \mathrm{E}+03$ & $2.7 E+01$ & & & & & $1.2 \mathrm{E}+04$ & $1.2 \mathrm{E}+02$ & & & & & $3.2 \mathrm{E}+01$ & $3.2 \mathrm{E}+0 \mathrm{t}$ & $3.2 \mathrm{E}+01$ & $3.2 \mathrm{E}+01$ & & \\
\hline $\begin{array}{l}\text { Dichlorobenz- } \\
\text { idine, 3,3- }\end{array}$ & $000091-94-1$ & $1.4 \mathrm{E}+02$ & $1.4 \mathrm{E}+00$ & & & & & $3.6 \mathrm{E}+02$ & $3.6 \mathrm{E}+\infty 0$ & & & & & & & $1.8 \mathrm{E}+02$ & $1.8 \mathrm{E}+00$ & & \\
\hline $\begin{array}{l}\text { Dichlorodinu- } \\
\text { oromethane }\end{array}$ & $000075-71-8$ & & & $1.5 \mathrm{E}+05$ & $1.6 \mathrm{E}+04$ & $1.5 \mathrm{E}+04$ & $1.6 \mathrm{E}+03$ & & & $6.3 \mathrm{E}+04$ & $6.3 \mathrm{E}+03$ & & & $2.3 \mathrm{E}+02$ & $2.3 E+01$ & & & $2.3 E+02$ & $2.3 \mathrm{E}+01$ \\
\hline $\begin{array}{l}\text { Dichloroeth- } \\
\text { ane, 1,1- }\end{array}$ & $000075-34-3$ & & & $7.3 \mathrm{E}+04$ & $7.8 \mathrm{E}+03$ & $7.3 E+03$ & $7.8 \mathrm{E}+02$ & & & $1.4 \mathrm{E}+05$ & $1.4 \mathrm{E}+04$ & & & $5.6 \mathrm{E}+00$ & $5.6 \mathrm{E}-01$ & & & $5.6 \mathrm{E}+00$ & $5.6 \mathrm{E}-01$ \\
\hline $\begin{array}{l}\text { Dichloroeth- } \\
\text { ane, 1,2. }\end{array}$ & $000107.06-2$ & $7.0 \mathrm{E}+02$ & $7.0 \mathrm{E}+00$ & & & & & $3.5 \mathrm{E}+03$ & $3.5 \mathrm{E}+01$ & & & $2.0 \mathrm{E}+02$ & $2.0 \mathrm{E}+00$ & & & $1.7 E+02$ & $1.7 E+\infty$ & & \\
\hline $\begin{array}{l}\text { Dichloroethyl- } \\
\text { ene, 1,1- }\end{array}$ & $000075-35-4$ & $1.1 \mathrm{E}+02$ & $1.1 \mathrm{E}+00$ & $6.6 \mathrm{E}+03$ & $7.0 \mathrm{E}+02$ & $6.6 \mathrm{E}+02$ & $7.0 \mathrm{E}+01$ & $5.4 E+02$ & $5.4 E+\infty$ & $1.2 E+04$ & $1.2 \mathrm{E}+03$ & 4.5E+00 & 4.5E-02 & & & $4.4 \mathrm{E}+00$ & $4.4 \mathrm{E}-02$ & $1.2 \mathrm{E}+03$ & $4.3 \mathrm{E}+02$ \\
\hline
\end{tabular}


Table 3.1c. (continued)

\begin{tabular}{|c|c|c|c|c|c|c|c|c|c|c|c|c|c|c|c|c|c|c|c|}
\hline \multirow{2}{*}{ Chemical } & \multirow{2}{*}{ CASRN } & \multicolumn{6}{|c|}{ Ingestion $(\mathrm{mg} / \mathrm{kg})^{\circ}$} & \multicolumn{4}{|c|}{ Dermal (mg/kg)' } & \multicolumn{4}{|c|}{ Inhalation (mg/kg) } & \multicolumn{4}{|c|}{$\begin{array}{c}\text { Ingestion + Dermal } \\
+ \text { Inhalation }(\mathrm{mg} / \mathrm{kg})^{+* v}\end{array}$} \\
\hline & & $10^{-4}$ & $10^{6}$ & $\begin{array}{l}\text { HQ=1 } \\
\text { Adult }\end{array}$ & $\begin{array}{l}\text { HQ=1 } \\
\text { Child }\end{array}$ & $\begin{array}{c}\mathrm{HQ}=0.1 \\
\text { Adult }\end{array}$ & $\begin{array}{c}\text { HQ }=0.1 \\
\text { Child }\end{array}$ & $10^{-4}$ & $10^{6}$ & $H Q=1$ & HQ $=0.1$ & $10^{-4}$ & $10^{4}$ & $H Q=1$ & $\mathrm{HQ}=0.1$ & $10^{-4}$ & $10^{6}$ & $H I=1$ & $\mathrm{HI}=0.1$ \\
\hline $\begin{array}{l}\text { Dichloroethyl- } \\
\text { ene, 1,2- } \\
\text { (Mixed Iso- } \\
\text { mers) }\end{array}$ & $000540-59.0$ & & & $6.6 \mathrm{E}+03$ & $7.0 \mathrm{E}+02$ & $6.6 \mathrm{E}+02$ & $7.0 \mathrm{E}+01$ & & & $9.9 \mathrm{E}+03$ & $9.9 E+02$ & & & & & & & $4.0 E+03$ & $4.0 \mathrm{E}+02$ \\
\hline $\begin{array}{l}\text { Dichloroethyl- } \\
\text { ene, 1,2-cis- }\end{array}$ & $000156-59-2$ & & & $7.3 E+03$ & $7.8 \mathrm{E}+02$ & $7.3 \mathrm{E}+02$ & $7.8 \mathrm{E}+01$ & & & $1.4 \mathrm{E}+04$ & $1.4 E+03$ & & & & & & & $4.8 \mathrm{E}+03$ & $4.8 \mathrm{E}+02$ \\
\hline $\begin{array}{l}\text { Dichloroethyl- } \\
\text { ene, 1,2-trans- }\end{array}$ & 000156.60 .5 & & & $1.5 E+04$ & $1.6 \mathrm{E}+03$ & $1.5 \mathrm{E}+03$ & $1.6 \mathrm{E}+02$ & & & $2.8 E+04$ & $2.8 \mathrm{E}+03$ & & & & & & & $9.5 \mathrm{E}+03$ & $9.5 \mathrm{E}+02$ \\
\hline $\begin{array}{l}\text { Dichloro-phe- } \\
\text { nol, 2,4- }\end{array}$ & 000120.83 .2 & & & $2.2 \mathrm{E}+03$ & 2.3E+02 & $2.2 \mathrm{E}+02$ & $2.3 \mathrm{E}+01$ & & & $3.4 E+03$ & $3.4 E+02$ & & & & & & & $1.3 \mathrm{E}+03$ & $1.3 E+02$ \\
\hline $\begin{array}{l}\text { Dichlora-phe- } \\
\text { noxy Acetic } \\
\text { Acid, 2,4- }\end{array}$ & $000094-75-7$ & & & 7.3E+03 & $7.8 \mathrm{E}+02$ & $7.3 \mathrm{E}+02$ & $7.8 \mathrm{E}+01$ & & & $1.1 \mathrm{E}+04$ & $1.1 \mathrm{E}+03$ & & & & & & & $4.4 \mathrm{E}+03$ & $4.4 \mathrm{E}+02$ \\
\hline $\begin{array}{l}\text { Dichlora-phe- } \\
\text { noxy)butyric } \\
\text { Acid, 4-(2,4- }\end{array}$ & $000094-82-6$ & & & $5.8 \mathrm{E}+03$ & $6.3 \mathrm{E}+02$ & $5.8 \mathrm{E}+02$ & $6.3 \mathrm{E}+01$ & & & $5.5 \mathrm{E}+03$ & $5.5 \mathrm{E}+02$ & & & & & & & $2.8 \mathrm{E}+03$ & $2.8 \mathrm{E}+02$ \\
\hline $\begin{array}{l}\text { Dichloropro- } \\
\text { pane, 1,2- }\end{array}$ & $000078-87.5$ & $9.4 \mathrm{E}+02$ & $9.4 \mathrm{E}+00$ & & & & & $3.5 \mathrm{E}+03$ & $3.5 \mathrm{E}+01$ & & & & & $1.0 \mathrm{E}+02$ & $1.0 \mathrm{E}+01$ & $4.8 E+02$ & $1.5 \mathrm{E}+01$ & & \\
\hline $\begin{array}{l}\text { Dichloropro- } \\
\text { panol, 2,3-. }\end{array}$ & $000616-23.9$ & & & $2.2 E+03$ & $2.3 \mathrm{E}+02$ & $2.2 \mathrm{E}+02$ & $2.3 \mathrm{E}+01$ & & & $2.1 \mathrm{E}+03$ & $2.1 E+02$ & & & & & & & $1.1 E+03$ & $1.1 \mathrm{E}+02$ \\
\hline $\begin{array}{l}\text { Dichloropro- } \\
\text { pene, 1,3- }\end{array}$ & $000542-75-6$ & $3.5 \mathrm{E}+02$ & $3.5 \mathrm{E}+\infty$ & $2.2 \mathrm{E}+02$ & $2.3 \mathrm{E}+01$ & $2.2 \mathrm{E}+01$ & $2.3 \mathrm{E}+00$ & $9.8 \mathrm{E}+02$ & $9.8 \mathrm{E}+\infty$ & $2.3 \mathrm{E}+02$ & $2.3 E+01$ & $5.4 \mathrm{E}+02$ & $5.4 E+00$ & $1.7 E+03$ & $1.7 \mathrm{E}+02$ & $2.5 \mathrm{E}+02$ & $2.5 \mathrm{E}+\infty 0$ & $1.0 \mathrm{E}+02$ & $1.0 \mathrm{E}+01$ \\
\hline Dichlorvos & $000062-73-7$ & $2.2 \mathrm{E}+02$ & $2.2 \mathrm{E}+00$ & $3.7 \mathrm{E}+02$ & $3.9 \mathrm{E}+01$ & $3.7 E+01$ & $3.9 \mathrm{E}+00$ & $5.5 E+02$ & $5.5 \mathrm{E}+\infty$ & $3.4 E+02$ & $3.4 E+01$ & & & $1.0 \mathrm{E}+06$ & $2.2 E+05$ & $2.9 \mathrm{E}+02$ & $2.9 \mathrm{E}+\infty$ & $1.8 \mathrm{E}+02$ & $1.8 \mathrm{E}+01$ \\
\hline Dicofol & $000115-32-2$ & & & & & & & & & & & & & & & & & & \\
\hline $\begin{array}{l}\text { Dicyclopenta- } \\
\text { diene }\end{array}$ & $000077-73.6$ & & & $2.2 E+04$ & $2.3 E+03$ & $2.2 \mathrm{E}+03$ & $2.3 \mathrm{E}+02$ & & & $3.3 \mathrm{E}+04$ & $3.3 \mathrm{E}+03$ & & & $3.0 \mathrm{E}+01$ & $3.0 E+00$ & & & $3.0 \mathrm{E}+01$ & $3.0 E+\infty 0$ \\
\hline Dieldrin & $000060-57-1$ & $4.0 \mathrm{E}+00$ & $4.0 \mathrm{E}-02$ & $3.7 E+01$ & $3.9 E+00$ & $3.7 E+00$ & $3.9 \mathrm{E}-01$ & $1.0 \mathrm{E}+01$ & $1.0 \mathrm{E}-01$ & $3.4 E+01$ & $3.4 \mathrm{E}+00$ & 2.3E+05 & $2.3 \mathrm{E}+03$ & & & $5.2 \mathrm{E}+\infty$ & $5.2 \mathrm{E}-02$ & $1.8 \mathrm{E}+01$ & $1.8 \mathrm{E}+\infty$ \\
\hline $\begin{array}{l}\text { Diethyl Phthal- } \\
\text { ate }\end{array}$ & $000084-66-2$ & & & $5.8 \mathrm{E}+05$ & $6.3 E+04$ & $5.8 \mathrm{E}+04$ & $6.3 \mathrm{E}+03$ & & & $9.9 E+0 S$ & $9.9 \mathrm{E}+04$ & & & & & & & 3.7E+05 & $3.7 \mathrm{E}+04$ \\
\hline $\begin{array}{l}\text { Diethyl-p- } \\
\text { nitrophenyl- } \\
\text { phosphate }\end{array}$ & $000311-45-5$ & & & & & & & & & & & & & & & & & & \\
\hline
\end{tabular}


Table 3.1c. (continued)

\begin{tabular}{|c|c|c|c|c|c|c|c|c|c|c|c|c|c|c|c|c|c|c|c|}
\hline \multirow{2}{*}{ Chemical } & \multirow{2}{*}{ CASRN } & \multicolumn{6}{|c|}{ Ingestion (mg/kg) } & \multicolumn{4}{|c|}{ Dermal (mg/kg) } & \multicolumn{4}{|c|}{ Inhalation (mg/kg) ${ }^{-\mathbf{T}}$} & \multicolumn{4}{|c|}{$\begin{array}{r}\text { Ingestion + Dermal } \\
+ \text { Inhalation }(\mathrm{mg} / \mathrm{kg})^{+* v}\end{array}$} \\
\hline & & $10^{-4}$ & $10^{6}$ & $\begin{array}{l}\text { HQ=1 } \\
\text { Adult }\end{array}$ & $\begin{array}{l}\text { HQ=1 } \\
\text { Child }\end{array}$ & $\begin{array}{c}\mathrm{HQ}=0.1 \\
\text { Adult }\end{array}$ & $\begin{array}{l}\mathrm{HQ}=0.1 \\
\text { Child }\end{array}$ & $10^{-1}$ & $10^{4}$ & $\mathrm{HQ}=1$ & $\mathrm{HQ}=0.1$ & $10^{-4}$ & $10^{4}$ & $\mathrm{HQ}=1$ & $\mathrm{HQ}=0.1$ & $10^{-4}$ & $10^{4}$ & HI=I & $\mathrm{HI}=0.1$ \\
\hline $\begin{array}{l}\text { Diethylene } \\
\text { Glyyol } \\
\text { Dinitrate } \\
\text { (DEGDM) }\end{array}$ & $0006933-21-0$ & & & & & & & & & & & & & & & & & . & \\
\hline $\begin{array}{l}\text { Diethylene } \\
\text { Glycol } \\
\text { Monobutyl } \\
\text { Ether }\end{array}$ & $000112-34-5$ & & & & & & & & & & & & & $1.0 E+06$ & $1.0 E+06$ & & & & \\
\hline $\begin{array}{l}\text { Ditcthylene } \\
\text { Glyeol } \\
\text { Monoethyl } \\
\text { Ether }\end{array}$ & $000111-90-0$ & & & $1.0 \mathrm{E}+06$ & $1.6 \mathrm{E}+05$ & $1.5 \mathrm{E}+0.5$ & $1.6 E+04$ & & & $1.0 E+06$ & $1.4 E+05$ & & & & & & & $7.1 E+05$ & $7.1 E+04$ \\
\hline $\begin{array}{l}\text { Diechylformas- } \\
\text { mide }\end{array}$ & $000617-84-5$ & & & $8.0 E+03$ & $8.6 \mathrm{E}+02$ & $8.0 E+02$ & $8.6 \mathrm{E}+01$ & & & $7.6 \mathrm{E}+03$ & $7.6 \mathrm{E}+02$ & & & & & & & $3.9 E+03$ & $3.9 \mathrm{E}+02$ \\
\hline $\begin{array}{l}\text { Diethystil- } \\
\text { besterol }\end{array}$ & $000056-53-1$ & L.AE-02 & $1.4 E-04$ & & & & & 3.4E- 02 & $3.4 \mathrm{E}-04$ & & & $7.4 E+03$ & $7.4 \mathrm{E}+01$ & & & $1.8 \mathrm{E}-02$ & $1.8 \mathrm{E}-04$ & & \\
\hline Difenzoquat & $043222-48-6$ & & & $5.8 \mathrm{E}+04$ & $6.3 \mathrm{E}+03$ & $5.8 E+03$ & $6.3 \mathrm{E}+02$ & & & $5.5 E+04$ & $5.5 E+03$ & & & & & & & $2.8 \mathrm{E}+04$ & $2.8 \mathrm{E}+03$ \\
\hline Diflubenzaron & $033367-38-5$ & & & $1.5 \mathrm{E}+04$ & $1.6 \mathrm{E}+03$ & $1.5 \mathrm{E}+03$ & $1.6 \mathrm{E}+02$ & & & $1.4 E+04$ & $1.4 \mathrm{E}+03$ & & & & & & & $7.1 E+03$ & $7.1 E+02$ \\
\hline $\begin{array}{l}\text { Difluoroeth- } \\
\text { anc, 1,1- }\end{array}$ & $000075-37-6$ & & & & & & & & & & & & & $1.0 \mathrm{E}+06$ & $1.0 E+06$ & & & & \\
\hline $\begin{array}{l}\text { Ditsopropyl } \\
\text { Methylphos. } \\
\text { phonate }\end{array}$ & $001445-75-6$ & & & $5.8 E+04$ & $6.3 \mathrm{E}+03$ & $5.8 E+03$ & $6.3 \mathrm{E}+02$ & & & $5.5 E+04$ & $5.5 E+03$ & & & & & & & $2.8 \mathrm{E}+04$ & $2.8 \mathrm{E}+03$ \\
\hline Dimethipin & $055290-64-7$ & & & $1.5 E+04$ & $1.6 \mathrm{E}+03$ & $1.5 \mathrm{E}+03$ & $1.6 \mathrm{E}+02$ & & & $1.4 E+04$ & $1.4 E+03$ & & & & & & & $7.1 E+03$ & $7.1 E+02$ \\
\hline Dimethoate & $000060.51-5$ & & & $1.5 \mathrm{E}+02$ & $1.6 \mathrm{E}+01$ & $1.5 E+01$ & $1.6 \mathrm{E}+\infty 0$ & & & $1.4 E+02$ & $1.4 \mathrm{E}+01$ & & & & & & & $7.1 E+01$ & $7.1 E+00$ \\
\hline $\begin{array}{l}\text { Dimethoxy- } \\
\text { bentidine, } \\
\text { 3,3!- }\end{array}$ & $000119.90-4$ & $4.6 \mathrm{E}+03$ & $4.6 \mathrm{E}+01$ & & & & & $1.1 \mathrm{E}+04$ & 1.1.EF+02 & & & & & & & $5.9 E+03$ & $5.9 \mathrm{E}+01$ & & \\
\hline $\begin{array}{l}\text { Dimethyl Sul- } \\
\text { fate }\end{array}$ & $000077-78-1$ & & & & & & & & & & & & & & & & & & \\
\hline $\begin{array}{l}\text { Dimethylanit- } \\
\text { ine } \mathrm{HCl}, 2,4-\end{array}$ & $021436-96-4$ & $1.1 E+02$ & 1.1EE+100 & & & . & & $2.8 \mathrm{E}+02$ & $2.8 \mathrm{E}+\infty 0$ & & & & & & & $1.4 E+02$ & $1.4 E+00$ & & \\
\hline
\end{tabular}

Nonradionuclides/soil/residential

Page 15 of 41 
Table 3.1c. (continued)

\begin{tabular}{|c|c|c|c|c|c|c|c|c|c|c|c|c|c|c|c|c|c|c|c|}
\hline \multirow{2}{*}{ Chemical } & \multirow{2}{*}{ CASRN } & \multicolumn{6}{|c|}{ Ingestion (mg/kg) } & \multicolumn{4}{|c|}{ Dermal (mg/kg) } & \multicolumn{4}{|c|}{ Inhalation (mg/kg) } & \multicolumn{4}{|c|}{$\begin{array}{l}\text { Ingestion + Dermal } \\
+ \text { Inhalation (mg/kg) }\end{array}$} \\
\hline & & $10^{-4}$ & $10^{-6}$ & $\begin{array}{l}\text { HQ }=1 \\
\text { Adult }\end{array}$ & $\begin{array}{l}H Q=1 \\
\text { Child }\end{array}$ & $\begin{array}{c}\mathrm{HQ}=0.1 \\
\text { Adult }\end{array}$ & $\begin{array}{l}\text { HQ }=0.1 \\
\text { Child }\end{array}$ & $10^{-4}$ & $10^{5}$ & HQ=1 & $\mathrm{HQ}=0.1$ & $10^{-4}$ & $10^{6}$ & $H Q=1$ & $\mathrm{HQ}=0.1$ & $10^{-4}$ & $10^{5}$ & $\mathrm{HI}=1$ & $\mathrm{HI}=0.1$ \\
\hline $\begin{array}{l}\text { Dimethylanil- } \\
\text { ine, 2,4- }\end{array}$ & $000095-68-1$ & $8.5 \mathrm{E}+01$ & $8.5 \mathrm{E}-01$ & & & & & $2.1 E+02$ & $2.1 E+\infty$ & & & & & & & $1.1 \mathrm{E}+02$ & $1.1 \mathrm{E}+\infty 0$ & & \\
\hline $\begin{array}{l}\text { Dimethylanil- } \\
\text { ine, } N, N \text { - }\end{array}$ & $000121-69-7$ & & & $1.5 \mathrm{E}+03$ & $1.6 \mathrm{E}+02$ & $1.5 E+02$ & $1.6 \mathrm{E}+01$ & & & $1.4 \mathrm{E}+03$ & $1.4 \mathrm{E}+02$ & & & & & & & $7.1 \mathrm{E}+02$ & $7.1 E+01$ \\
\hline $\begin{array}{l}\text { Dimethylbenz- } \\
\text { idine, 3,3:- }\end{array}$ & 000119.93 .7 & $6.9 \mathrm{E}+00$ & $6.9 \mathrm{E}-02$ & & & & & $1.7 E+01$ & $1.7 E-01$ & & & & & & & $9.0 \mathrm{E}+\infty 0$ & $9.0 \mathrm{E}-02$ & & \\
\hline $\begin{array}{l}\text { Dimethylethyl } \\
\text { Lead }\end{array}$ & $107584-40-7$ & & & & & & & & & & & & & & & & & & \\
\hline $\begin{array}{l}\text { Dimethylform- } \\
\text { amide }\end{array}$ & $000068-12-2$ & & & 7.3E+04 & $7.8 \mathrm{E}+03$ & $7.3 \mathrm{E}+03$ & $7.8 \mathrm{E}+02$ & & & $6.9 \mathrm{E}+04$ & $6.9 E+03$ & & & $1.0 \mathrm{E}+06$ & $1.0 E+06$ & & & $3.5 \mathrm{E}+04$ & $3.5 \mathrm{E}+03$ \\
\hline $\begin{array}{l}\text { Dimethylhy- } \\
\text { drazing, 1,1.(4) }\end{array}$ & $000057-14-7$ & $2.5 \mathrm{E}+01$ & $2.5 \mathrm{E}-01$ & & & & & $6.2 \mathrm{E}+01$ & $6.2 \mathrm{E}-01$ & & & $1.0 \mathrm{E}+06$ & $1.0 \mathrm{E}+04$ & & & $3.2 \mathrm{E}+01$ & $3.2 \mathrm{E}-01$ & & \\
\hline $\begin{array}{l}\text { Dimethylhy- } \\
\text { drazine, 1,2- }\end{array}$ & $000540-73.8$ & & & & & & & & & & & & & & & & & & \\
\hline $\begin{array}{l}\text { Dimethylphe- } \\
\text { nol, 2,4- }\end{array}$ & 000105.67 .9 & & & $1.5 \mathrm{E}+04$ & $1.6 \mathrm{E}+03$ & 1.5E+03 & $1.6 \mathrm{E}+02$ & & & $2.2 \mathrm{E}+04$ & $2.2 \mathrm{E}+03$ & & & & & & & $8.8 \mathrm{E}+03$ & $8.8 E+02$ \\
\hline $\begin{array}{l}\text { Dimethylphe- } \\
\text { nol, 2,6- }\end{array}$ & $000576-26-1$ & & & $4.4 \mathrm{E}+02$ & $4.7 E+01$ & $4.4 \mathrm{E}+0 \mathrm{I}$ & $4.7 E+\infty$ & & & $4.1 E+02$ & $4.1 \mathrm{E}+01$ & & & & & & & $2.1 \mathrm{E}+02$ & $2.1 E+01$ \\
\hline $\begin{array}{l}\text { Dimethylphe- } \\
\text { nol, 3,4- }\end{array}$ & $000095-65-8$ & & & $7.3 \mathrm{E}+02$ & $7.8 \mathrm{E}+01$ & $7.3 \mathrm{E}+01$ & $7.8 \mathrm{E}+00$ & & & $6.9 E+02$ & $6.9 \mathrm{E}+01$ & & & & & & & $3.5 E+02$ & $3.5 \mathrm{E}+01$ \\
\hline $\begin{array}{l}\text { Dimethyl- } \\
\text { phthalate(t) }\end{array}$ & $000131-11-3$ & & & $1.0 \mathrm{E}+06$ & $7.8 E+05$ & $7.3 \mathrm{E}+05$ & $7.8 E+04$ & & & $1.0 \mathrm{E}+06$ & $1.0 E+06$ & & & & & & & $1.0 \mathrm{E}+06$ & $4.6 \mathrm{E}+05$ \\
\hline $\begin{array}{l}\text { Dimethylte- } \\
\text { rephthalate }\end{array}$ & $000120.61-6$ & & & $7.3 E+04$ & $7.8 E+03$ & $7.3 E+03$ & $7.8 E+02$ & & & $6.9 \mathrm{E}+04$ & $6.9 \mathrm{E}+03$ & & & & & & & $3.5 \mathrm{E}+04$ & $3.5 E+03$ \\
\hline $\begin{array}{l}\text { Dinitro-0-cre- } \\
\text { sol, 4,6- }\end{array}$ & $000534-52-1$ & & & & & & & & & & & & & & & & & & \\
\hline $\begin{array}{l}\text { Dinitro-o- } \\
\text { cyclohexyl } \\
\text { Phenol, 4,6- }\end{array}$ & $000131-89-5$ & & & $1.5 E+03$ & $1.6 \mathrm{E}+02$ & $1.5 \mathrm{E}+02$ & $1.6 \mathrm{E}+01$ & & & $1.4 \mathrm{E}+03$ & $1.4 \mathrm{E}+02$ & & & & & & & $7.1 E+02$ & $7.1 E+01$ \\
\hline $\begin{array}{l}\text { Dinitroben- } \\
\text { zene, 1,2- }\end{array}$ & $000528-29-0$ & & & $2.9 \mathrm{E}+02$ & $3.1 E+01$ & $2.9 E+01$ & $3.1 E+\infty$ & & & $5.1 E+02$ & $5.1 E+01$ & & & & & & & $1.9 \mathrm{E}+02$ & $1.9 E+01$ \\
\hline
\end{tabular}


Table 3.1c. (continued)

\begin{tabular}{|c|c|c|c|c|c|c|c|c|c|c|c|c|c|c|c|c|c|c|c|}
\hline \multirow{2}{*}{ Chemical } & \multirow{2}{*}{ CASRN } & \multicolumn{6}{|c|}{ Ingestion (mg/kg) } & \multicolumn{4}{|c|}{ Dermal (mg/kg) } & \multicolumn{4}{|c|}{ Inhalation (mg/kg) } & \multicolumn{4}{|c|}{$\begin{aligned} & \text { Ingestion + Dermal } \\
&+ \text { Inhalation }(\mathrm{mg} / \mathrm{kg})^{+\bullet v} \\
&\end{aligned}$} \\
\hline & & $10^{-4}$ & $10^{4}$ & $\begin{array}{l}\text { HQ=1 } \\
\text { Adult }\end{array}$ & $\begin{array}{l}\mathrm{HQ}=1 \\
\text { Child }\end{array}$ & $\begin{array}{c}\text { HQ }=0.1 \\
\text { Adult }\end{array}$ & $\begin{array}{c}\text { HQ }=0.1 \\
\text { Child }\end{array}$ & $10^{-4}$ & $10^{4}$ & $\mathrm{HQ}=1$ & $\mathrm{HQ}=0.1$ & $10^{-4}$ & $10^{6}$ & $H Q=1$ & $\mathrm{HQ}=0.1$ & $10^{-4}$ & $10^{4}$ & $H I=1$ & $\mathrm{HI}=0.1$ \\
\hline $\begin{array}{l}\text { Dinitroben- } \\
\text { zene, 1,3- }\end{array}$ & $000099-65-0$ & & & $7.3 \mathrm{E}+01$ & $7.8 \mathrm{E}+00$ & $7.3 E+\infty$ & $7.8 \mathrm{E}-01$ & & . & $9.0 E+01$ & $9.0 \mathrm{E}+00$ & & & & & & & $4.0 E+01$ & $4.0 \mathrm{E}+\infty 0$ \\
\hline $\begin{array}{l}\text { Dinitroben- } \\
\text { zene, 1,4- }\end{array}$ & $000100-25-4$ & & & $2.9 E+02$ & $3.1 \mathrm{E}+01$ & $2.9 E+01$ & $3.1 E+\infty$ & & & $2.8 \mathrm{E}+02$ & $2.8 \mathrm{E}+01$ & & & & & & & $1.4 E+02$ & $1.4 \mathrm{E}+0 \mathrm{I}$ \\
\hline $\begin{array}{l}\text { Dinitrophenol, } \\
2,4-\end{array}$ & $000051-28.5$ & & & $1.5 E+03$ & $1.6 \mathrm{E}+02$ & $1.5 \mathrm{E}+02$ & $1.6 \mathrm{E}+01$ & & & $2.8 E+03$ & $2.8 \mathrm{E}+02$ & & & & & & & $9.5 \mathrm{E}+02$ & $9.5 \mathrm{E}+01$ \\
\hline $\begin{array}{l}\text { Dinitrotolu- } \\
\text { ene, 2,4- }\end{array}$ & $000121-14-2$ & $9.4 \mathrm{E}+01$ & $9.4 \mathrm{E}-01$ & $1.5 E+03$ & $1.6 E+02$ & $1,5 E+02$ & $1.6 \mathrm{E}+01$ & $4.08+02$ & $4.0 \mathrm{E}+\infty 0$ & $2.3 \mathrm{E}+03$ & $2.3 \mathrm{E}+02$ & & & & & $1.5 \mathrm{E}+02$ & $1.5 E+00$ & $9.0 E+02$ & $9.0 E+01$ \\
\hline $\begin{array}{l}\text { Dinitrotolu- } \\
\text { ene, 2,6- }\end{array}$ & $000506-20-2$ & $9.4 \mathrm{E}+01$ & 9.4E-01 & $7.3 E+02$ & $7.8 \mathrm{E}+01$ & $7.3 E+01$ & $7.8 \mathrm{E}+00$ & $4.0 \mathrm{E}+02$ & $4.0 \mathrm{E}+00$ & $1.2 \mathrm{E}+03$ & $1.2 \mathrm{E}+02$ & & & & & $1.5 E+02$ & $1.5 \mathrm{E}+\infty$ & $4.5 E+02$ & $4.5 \mathrm{E}+01$ \\
\hline $\begin{array}{l}\text { Dinitrotolu- } \\
\text { ene, 2-Amino- } \\
4,6 \text { - }\end{array}$ & $035572.78-2$ & & & & & & & & & & & & & & & & & & \\
\hline Dinoseb & $000088-85.7$ & & & $7.3 \mathrm{E}+02$ & $7.8 \mathrm{E}+01$ & 7.3E+01 & $7.8 \mathrm{E}+\infty$ & & & $6.9 \mathrm{E}+02$ & $6.9 \mathrm{E}+01$ & & & & & & & $3.5 E+02$ & $3.5 \mathrm{E}+01$ \\
\hline Dioxane, 1,4- & $000123-91-1$ & $5.8 \mathrm{E}+03$ & $5.8 \mathrm{E}+01$ & & & & & $2.3 E+04$ & $2.3 \mathrm{E}+02$ & & & & & & & $9.3 \mathrm{E}+03$ & $9.3 \mathrm{E}+01$ & & \\
\hline Diphensmid & 000957.51 .7 & & & $2.2 \mathrm{E}+04$ & $2.3 \mathrm{E}+03$ & $2.2 E+03$ & $2.3 E+02$ & & & $2.1 E+04$ & $2.1 \mathrm{E}+03$ & & & & & & & $1.1 \mathrm{E}+04$ & $1.1 \mathrm{E}+03$ \\
\hline Diphenylamine & $000122-39-4$ & & & $1.8 \mathrm{E}+04$ & $2.0 E+03$ & $1.8 \mathrm{E}+03$ & $2.0 \mathrm{E}+02$ & & & $1.7 E+04$ & $1.7 E+03$ & & & & & & & $8.9 E+03$ & $8.9 \mathrm{E}+02$ \\
\hline $\begin{array}{l}\text { Diphenylhy- } \\
\text { drazine, 1,2. }\end{array}$ & $000122.66-7$ & $8.0 E+01$ & $8.0 E-01$ & & & & & $2.0 \mathrm{E}+02$ & $2.0 \mathrm{E}+00$ & & & $1.0 E+06$ & $4.6 \mathrm{E}+04$ & & & $1.0 \mathrm{E}+02$ & $1.0 \mathrm{E}+00$ & & \\
\hline Diquat & $000085-00-7$ & & & $1.6 \mathrm{E}+03$ & $1.7 \mathrm{E}+02$ & $1.6 \mathrm{E}+02$ & $1.7 \mathrm{E}+01$ & & & $1.5 E+03$ & 1. $.5 \mathrm{E}+02$ & & & & & & & $7.8 \mathrm{E}+02$ & $7.8 \mathrm{E}+01$ \\
\hline $\begin{array}{l}\text { Direct } \\
\text { Black } 38\end{array}$ & 001937.37 .7 & $7.4 \mathrm{E}+00$ & $7.4 \mathrm{E}-02$ & & & & & $1.9 \mathrm{E}+01$ & $1.9 \mathrm{E}-01$ & & & & & & & $9.6 \mathrm{E}+00$ & $9.6 \mathrm{E}-02$ & & \\
\hline Direet Blue 6 & $002602-46-2$ & $7.9 E+00$ & 7.9E-02 & & & & & $2.0 E+01$ & $2.0 \mathrm{E}-01$ & & & & & & & $1,0 \mathrm{E}+01$ & $1,0 \mathrm{E}-01$ & & \\
\hline $\begin{array}{l}\text { Direct } \\
\text { Brown 95 }\end{array}$ & $016071-86-6$ & $6.9 E+00$ & $6.9 \mathrm{E}-02$ & & & & & $1.7 E+01$ & $1.7 \mathrm{E}-01$ & & & & & & & $8.9 E+00$ & $8.9 E-02$ & & \\
\hline $\begin{array}{l}\text { Direct Sky } \\
\text { Blue }\end{array}$ & $002610-05-1$ & & & & & & & & & & & & & & & & & & \\
\hline Disulfoton & $000298-04-4$ & & & $2.9 \mathrm{E}+01$ & $3.1 \mathrm{E}+\infty$ & $2.9 E+\infty$ & $3.1 \mathrm{E}-01$ & & & $2.8 E+01$ & $2.8 E+\infty 0$ & & & & & & & $1.4 E+01$ & $1.4 \mathrm{E}+00$ \\
\hline Diuron & $000330-54-1$ & & & $1.5 \mathrm{E}+03$ & $1.6 \mathrm{E}+02$ & $1.5 E+02$ & $1.6 \mathrm{E}+01$ & & & $1.4 E+03$ & $1.4 E+02$ & & & & & & & $7.1 \varepsilon+02$ & 7.1E+01 \\
\hline
\end{tabular}

Nonradionuclides/soil/residential

Page 17 of 41 
Table 3.1c. (continued)

\begin{tabular}{|c|c|c|c|c|c|c|c|c|c|c|c|c|c|c|c|c|c|c|c|}
\hline \multirow{2}{*}{ Chemical } & \multirow{2}{*}{ CASRN } & \multicolumn{6}{|c|}{ Ingestion (mg/kg) } & \multicolumn{4}{|c|}{ Dermal (mg/kg) } & \multicolumn{4}{|c|}{ Inhalation $(\mathrm{mg} / \mathrm{kg})^{* v}$} & \multicolumn{4}{|c|}{$\begin{array}{c}\text { Ingestion + Dermal } \\
+ \text { Inhalation }(\mathrm{mg} / \mathrm{kg})^{)^{-\gamma}}\end{array}$} \\
\hline & & $10^{-4}$ & $10^{6}$ & $\begin{array}{l}\mathrm{HQ}=1 \\
\text { Adult }\end{array}$ & $\begin{array}{l}H Q=1 \\
\text { Child }\end{array}$ & $\begin{array}{l}\text { HQ }=0.1 \\
\text { Adult }\end{array}$ & $\begin{array}{l}\text { HQ }=0.1 \\
\text { Child . }\end{array}$ & $10^{-4}$ & $10^{6}$ & $H Q=1$ & $\mathrm{HQ}=0.1$ & $10^{-4}$ & $10^{6}$ & $H Q=1$ & $\mathrm{HQ}=0.1$ & $10^{-4}$ & $10^{5}$ & $\mathrm{HI}=1$ & $\mathrm{HI}=0.1$ \\
\hline Dodine & $002439-10-3$ & & & $2.9 \mathrm{E}+03$ & $3.1 \mathrm{E}+02$ & $2.9 \mathrm{E}+02$ & 3.1E+01 & & & $2.8 \mathrm{E}+03$ & $2,8 \mathrm{E}+02$ & & & & & & & $1.4 \mathrm{E}+03$ & $1.4 \mathrm{E}+02$ \\
\hline EPTC & $000759-94-4$ & & & $1.8 \mathrm{E}+04$ & $2.0 E+03$ & $1.8 \mathrm{E}+03$ & $2.0 \mathrm{E}+02$ & & & $1.7 \mathrm{E}+04$ & $1.7 \mathrm{E}+03$ & & & & & & & $8.9 E+03$ & $8.9 \mathrm{E}+02$ \\
\hline Endosulfan & $000115-29-7$ & & & $4.4 \mathrm{E}+03$ & $4.7 \mathrm{E}+02$ & $4.4 E+02$ & 4.7E+01 & & & $4.1 E+03$ & 4.IE+02 & & & & & & & $2.1 \mathrm{E}+03$ & $2.1 E+02$ \\
\hline Endothall & $000145-73-3$ & & & $1.5 \mathrm{E}+04$ & $1.6 \mathrm{E}+03$ & $1.5 \mathrm{E}+03$ & $1.6 \mathrm{E}+02$ & & & $1.4 \mathrm{E}+0.4$ & $1.4 \mathrm{E}+03$ & & & & & & & $7,1 \mathrm{E}+03$ & $7.1 \mathrm{E}+02$ \\
\hline Endrin & $000072-20.8$ & & & $2.2 \mathrm{E}+02$ & $2.3 \mathrm{E}+01$ & $2.2 \mathrm{E}+01$ & $2.3 \mathrm{E}+\infty$ & & & $8.3 \mathrm{E}+\infty$ & $8.3 \mathrm{E}-01$ & & & & & & & $8.0 \mathrm{E}+00$ & $8.0 \mathrm{E}-01$ \\
\hline $\begin{array}{l}\text { Epichlorohy- } \\
\text { drin }\end{array}$ & $000106 \cdot 89.8$ & $6.5 \mathrm{E}+03$ & $6.5 \mathrm{E}+01$ & $1.5 \mathrm{E}+03$ & $1.6 \mathrm{E}+02$ & $1.5 E+02$ & $1.6 \mathrm{E}+01$ & $2.6 \mathrm{E}+04$ & $2.6 \mathrm{E}+02$ & $2.2 \mathrm{E}+03$ & $2.2 \mathrm{E}+02$ & $3.2 \mathrm{E}+03$ & $1.9 \mathrm{E}+02$ & $9.9 \mathrm{E}+01$ & $9.9 E+\infty 0$ & $3.2 \mathrm{E}+03$ & $6.7 \mathrm{E}+01$ & $8.9 \mathrm{E}+01$ & $8.9 \mathrm{E}+\infty 0$ \\
\hline $\begin{array}{l}\text { Epoxybutane, } \\
\text { 1,2. }\end{array}$ & $000106-88-7$ & & & & & & & & & & & & & $1.0 \mathrm{E}+06$ & $1.0 \mathrm{E}+06$ & & & & \\
\hline Ethephon & $016672-87.0$ & & & $3.7 \mathrm{E}+03$ & $3.9 E+02$ & $3.7 \mathrm{E}+02$ & $3.9 \mathrm{E}+01$ & & & $3.4 \mathrm{E}+03$ & $3.4 E+02$ & & & & & & & $1.8 \mathrm{E}+03$ & $1.8 E+02$ \\
\hline Ethion & $000563-12-2$ & & & $3.7 \mathrm{E}+02$ & $3.9 \mathrm{E}+01$ & $3.7 E+01$ & $3.9 \mathrm{E}+00$ & & & $3.4 E+02$ & $3.4 \mathrm{E}+01$ & & & & & & & $1.8 \mathrm{E}+02$ & $1.8 \mathrm{E}+01$ \\
\hline $\begin{array}{l}\text { Ethoxyethanol } \\
\text { Acetate, } 2 \text {. }\end{array}$ & $000111-15.9$ & & & $2.2 E+05$ & $2.3 \mathrm{E}+04$ & $2.2 \mathrm{E}+04$ & $2.3 \mathrm{E}+03$ & & & 2. $\mathrm{tE}+05$ & $2.1 E+04$ & & & & & & & $1.1 E+05$ & $1.1 \mathrm{E}+04$ \\
\hline $\begin{array}{l}\text { Ethoxyetha- } \\
\text { nol, 2- }\end{array}$ & $000110-80-5$ & & & $2.9 \mathrm{E}+05$ & $3.1 E+04$ & $2.9 E+04$ & $3.1 \mathrm{E}+03$ & & & $2.8 \mathrm{E}+05$ & $2.8 \mathrm{E}+04$ & & & $1.0 \mathrm{E}+06$ & $1.0 \mathrm{E}+06$ & & & $1.4 \mathrm{E}+05$ & $1.4 \mathrm{E}+04$ \\
\hline Ethyl Acetate & $000141-78.6$ & & & $6.6 \mathrm{E}+0 \mathrm{~s}$ & $7.0 \mathrm{E}+04$ & $6.6 \mathrm{E}+04$ & $7.0 \mathrm{E}+03$ & & & $9.9 \mathrm{E}+05$ & $9.9 \mathrm{E}+04$ & & & & & & & $4.0 \mathrm{E}+05$ & $4.0 \mathrm{E}+04$ \\
\hline Ethyl Acrylate & $000140-88-5$ & $1.3 \mathrm{E}+03$ & $1.3 \mathrm{E}+01$ & & & & & $3.3 \mathrm{E}+03$ & $3.3 \mathrm{E}+01$ & & & & & & & $1.7 E+03$ & $1.7 \mathrm{E}+01$ & & \\
\hline Ethyl Chloride & $000075-00-3$ & & & & & & & & & & & & & $1.5 E+02$ & 1.5E+02 & & & & \\
\hline Ethyl Elher & $000060-29-7$ & & & $1.5 \mathrm{E}+05$ & $1.6 \mathrm{E}+04$ & $1.5 \mathrm{E}+04$ & $1.6 \mathrm{E}+03$ & & & $2.2 \mathrm{E}+05$ & $2.2 \mathrm{E}+04$ & & & & & & & $8.8 \mathrm{E}+04$ & $8.8 E+03$ \\
\hline $\begin{array}{l}\text { Ethy! Methac- } \\
\text { rylate }\end{array}$ & $000097-63.2$ & & & $6.6 \mathrm{E}+04$ & $7.0 E+03$ & $6.6 \mathrm{E}+03$ & $7.0 \mathrm{E}+02$ & & & $9.9 \mathrm{E}+04$ & $9.9 \mathrm{E}+03$ & & & & & & & $4.0 \mathrm{E}+04$ & $4.0 E+03$ \\
\hline $\begin{array}{l}\text { Ethyl-p- } \\
\text { nitrophenyl } \\
\text { Phosphonste }\end{array}$ & $002104-64-5$ & & & $7.3 E+\infty$ & $7.8 \mathrm{E}-01$ & 7.3E-01 & $7.8 \mathrm{E}-02$ & & & $6.9 \mathrm{E}+\infty$ & $6.9 \mathrm{E}-01$ & & & & & & & $3.5 \mathrm{E}+00$ & $3.5 E-01$ \\
\hline Ethylberzene (i) & $000100-41-4$ & & & $7.3 \mathrm{E}+04$ & $7.8 \mathrm{E}+03$ & $7.3 E+03$ & $7.8 \mathrm{E}+02$ & & & $1.3 \mathrm{E}+05$ & $1.3 \mathrm{E}+04$ & & & $4.7 \mathrm{E}-01$ & 4.7E-01 & & & $4.7 \mathrm{E}-01$ & $4.7 \mathrm{E}-01$ \\
\hline $\begin{array}{l}\text { Ethylene Cyz- } \\
\text { nohydrin }\end{array}$ & $000109-78-4$ & & & $2.2 \mathrm{E}+05$ & $2.3 \mathrm{E}+04$ & 2.2E+04 & $2.3 \mathrm{E}+03$ & & & 2.1E+0S & $2.1 E+04$ & & & & & & & $1.1 E+05$ & $1.1 E+04$ \\
\hline
\end{tabular}


Table 3.1c. (continued)

\begin{tabular}{|c|c|c|c|c|c|c|c|c|c|c|c|c|c|c|c|c|c|c|c|}
\hline \multirow{2}{*}{ Chemical } & \multirow{2}{*}{ CASRN } & \multicolumn{6}{|c|}{ Ingestion (mg/kg) } & \multicolumn{4}{|c|}{ Dermal (mg/kg)" } & \multicolumn{4}{|c|}{ Inhalation (mg/kg) } & \multicolumn{4}{|c|}{$\begin{array}{c}\text { Ingestion + Dermal } \\
+ \text { Inhalation (mg/kg) }\end{array}$} \\
\hline & & $10^{-4}$ & $10^{6}$ & $\begin{array}{l}\text { HQ }=1 \\
\text { Adult }\end{array}$ & $\begin{array}{l}\text { HQ }=1 \\
\text { Child }\end{array}$ & $\begin{array}{c}\text { HQ }=0.1 \\
\text { Adult }\end{array}$ & $\begin{array}{l}\mathrm{HQ}=0.1 \\
\text { Child }\end{array}$ & $10^{4}$ & $10^{6}$ & $H Q=1$ & $\mathrm{HQ}=0.1$ & $10^{-4}$ & $10^{5}$ & $H Q=1$ & $H Q=0.1$ & $10^{-4}$ & $10^{4}$ & $H I=1$ & $H I=0.1$ \\
\hline $\begin{array}{l}\text { Elhylene Dia- } \\
\text { mine }\end{array}$ & $000107-15-3$ & & & $1.5 \mathrm{E}+04$ & $1.6 \mathrm{E}+03$ & $1.5 \mathrm{E}+03$ & $1.6 \mathrm{E}+02$ & & & $1.4 E+04$ & $1.4 \mathrm{E}+03$ & & & & & & & $7.1 E+03$ & 7.1E+02 \\
\hline $\begin{array}{l}\text { Ethylene Gly. } \\
\text { col }\end{array}$ & $000107-21-1$ & & & $1.0 \mathrm{E}+06$ & $1.6 \mathrm{E}+05$ & $1.5 \mathrm{E}+05$ & $1.6 E+04$ & & & $1.0 E+06$ & $1.4 E+05$ & & & & & & & $7.1 E+05$ & $7.1 E+04$ \\
\hline $\begin{array}{l}\text { Eithylene Gly- } \\
\text { col Monobutyl } \\
\text { Ether }\end{array}$ & 000111.76 .2 & & & & & & & & & & & & & $1.0 \mathrm{E}+06$ & $1.0 \mathrm{E}+06$ & & & & \\
\hline $\begin{array}{l}\text { Eithylene Ox- } \\
\text { ide }\end{array}$ & $000075-21.8$ & $6.3 \mathrm{E}+01$ & $6.3 \mathrm{E}-01$ & & & & & $2.5 \mathrm{E}+02$ & $2.5 E+00$ & & & $4.9 \mathrm{E}+01$ & $4.9 \mathrm{E}-01$ & & & $3.3 \mathrm{E}+01$ & $3,3 \mathrm{E}-01$ & & \\
\hline $\begin{array}{l}\text { Ethylene Thio- } \\
\text { urea }\end{array}$ & $000096-45-7$ & $5.8 E+02$ & $5.8 E+\infty$ & $5.8 E+01$ & $6.3 E+\infty$ & $5.8 E+\infty$ & $6.3 \mathrm{E}-01$ & $1.5 \mathrm{E}+03$ & $1.5 \mathrm{E}+0 \mathrm{I}$ & $5.5 E+01$ & $5.5 E+\infty$ & & & & & $7.5 \mathrm{E}+02$ & $7.5 E+00$ & $2.8 \mathrm{E}+01$ & $2.8 \mathrm{E}+00$ \\
\hline $\begin{array}{l}\text { Ethylphthalyl } \\
\text { Ethyl Glyco- } \\
\text { late }\end{array}$ & $000084-72-0$ & & & $1.0 \mathrm{E}+06$ & $2.3 E+05$ & $2.2 \mathrm{E}+05$ & $2.3 \mathrm{E}+04$ & & & $1.0 \mathrm{E}+06$ & 2.1E+05 & & & & & & & $1,0 E+06$ & $1.1 \mathrm{E}+05$ \\
\hline Express & $101200-48-0$ & & & $5.8 \mathrm{E}+03$ & $6.3 \mathrm{E}+02$ & $5.8 E+02$ & $6.3 \mathrm{E}+01$ & & & $5.5 \mathrm{E}+03$ & $5.5 \mathrm{E}+02$ & & & & & & & $2,8 \mathrm{E}+03$ & $2.8 \mathrm{E}+02$ \\
\hline Fenamiphos & $022224-92-6$ & & & $1.8 \mathrm{E}+02$ & $2.0 E+01$ & $1.8 \mathrm{E}+01$ & $2.0 \mathrm{E}+\infty$ & & & $1.7 E+02$ & $1.7 E+01$ & & & & & & & $8.9 E+01$ & $8.9 \mathrm{E}+00$ \\
\hline Fenpropathrin & $039515-41-8$ & & & $1.8 \mathrm{E}+0.4$ & $2.0 \mathrm{E}+03$ & $1.8 \mathrm{E}+03$ & $2.0 \mathrm{E}+02$ & & & $1.7 E+04$ & $1.7 \mathrm{E}+03$ & & & & & & & $8.9 E+03$ & $8.9 \mathrm{E}+02$ \\
\hline Fluometuron & $002164-17-2$ & & & $9.5 \mathrm{E}+03$ & $1.0 \mathrm{E}+03$ & $9.5 \mathrm{E}+02$ & $1.0 \mathrm{E}+02$ & & & $9.0 E+03$ & $9.0 \mathrm{E}+02$ & & & & & & & $4.6 \mathrm{E}+03$ & $4.6 \mathrm{E}+02$ \\
\hline Fluoranthene & $000206-44-0$ & & & $2.9 \mathrm{E}+04$ & $3.1 \mathrm{E}+03$ & $2.9 E+03$ & $3.1 E+02$ & & & $1.7 \mathrm{E}+04$ & $1.7 \mathrm{E}+03$ & & & & & & & $1.1 \mathrm{E}+04$ & $1.1 \mathrm{E}+03$ \\
\hline Fluorene & $000086-73-7$ & & & $2.9 \mathrm{E}+04$ & $3.1 \mathrm{E}+03$ & $2.9 E+03$ & $3.1 E+02$ & & & 4.4E+04 & $4.4 \mathrm{E}+03$ & & & & & & & $1.8 \mathrm{E}+04$ & $1.8 \mathrm{E}+03$ \\
\hline Fluoride & $007782-41-4$ & & & 4.4E 404 & $4.7 \mathrm{E}+03$ & $4.4 E+03$ & 4.7E+02 & & & $8.0 E+05$ & $8.0 E+04$ & & & & & & & $4.2 E+04$ & $4.2 \mathrm{E}+03$ \\
\hline Fluridone & $059756.60-4$ & & & $5.8 \mathrm{E}+04$ & $6.3 E+03$ & $5.8 E+03$ & $6.3 \mathrm{E}+02$ & & & $5.5 E+04$ & $5.5 E+03$ & & & & & & & $2.8 E+04$ & $2.8 \mathrm{E}+03$ \\
\hline Flurprimidol & $056425-91-3$ & & & $1.5 \mathrm{E}+04$ & $1.6 \mathrm{E}+03$ & $1.5 \mathrm{E}+03$ & $1.6 \mathrm{E}+02$ & & & $1.4 E+04$ & $1.4 E+03$ & & & & & & & 7.1E+03 & $7.1 E+02$ \\
\hline Flutolenil & $086332-96-5$ & & & $4,4 E+04$ & $4.7 \mathrm{E}+03$ & 4.4E+03 & $4.7 E+02$ & & & 4.1E+04 & $4.1 E+03$ & & & & & & & $2.1 E+04$ & $2.1 E+03$ \\
\hline Fluvalinate & 069409.94 .5 & & & $7.3 \mathrm{E}+03$ & $7.8 \mathrm{E}+02$ & $7.3 E+02$ & $7.8 \mathrm{E}+01$ & & & $6.9 \mathrm{E}+03$ & $6.9 \mathrm{E}+02$ & & & & & & & $3.5 \mathrm{E}+03$ & $3.5 E+02$ \\
\hline Folpet & $000133-07-3$ & $1.8 \mathrm{E}+04$ & $1.8 \mathrm{E}+02$ & $7.3 \mathrm{E}+04$ & $7.8 \mathrm{E}+03$ & 7.3E+03 & $7.8 \mathrm{E}+02$ & 4.6E+04 & $4.6 \mathrm{E}+02$ & $6.9 \mathrm{E}+04$ & $6.9 E+03$ & & & & & $2.4 E+04$ & $2.4 E+02$ & $3.5 \mathrm{E}+04$ & $3.5 E+03$ \\
\hline Fomessiren & $072178.02-0$ & $3.4 E+02$ & $3.4 \mathrm{E}+00$ & & & & & $8.5 \mathrm{E}+02$ & $8.5 E+\infty 0$ & & & & & & & $4.4 E+02$ & $4.4 E+\infty 0$ & & \\
\hline
\end{tabular}


Table 3.1c. (continued)

\begin{tabular}{|c|c|c|c|c|c|c|c|c|c|c|c|c|c|c|c|c|c|c|c|}
\hline \multirow{2}{*}{ Chemical } & \multirow{2}{*}{ CASRN } & \multicolumn{6}{|c|}{ Ingestion (mg/kg) } & \multicolumn{4}{|c|}{ Dermal (mg/kg) } & \multicolumn{4}{|c|}{ Inhalation $(\mathrm{mg} / \mathrm{kg})^{-6}$} & \multicolumn{4}{|c|}{$\begin{array}{c}\text { Ingestion + Dermal } \\
+ \text { + Inhalation (mg/kg) }\end{array}$} \\
\hline & & $10^{-4}$ & $10^{6}$ & $\begin{array}{l}\text { HQ=1 } \\
\text { Adult }\end{array}$ & $\begin{array}{l}H Q=1 \\
\text { Child }\end{array}$ & $\begin{array}{c}\mathrm{HQ}=0.1 \\
\text { Adult }\end{array}$ & $\begin{array}{c}\text { HQ }=0.1 \\
\text { Child }\end{array}$ & $10^{-4}$ & $10^{6}$ & $\mathrm{HQ}=1$ & $\mathrm{HQ}=0.1$ & $10^{-4}$ & $10^{6}$ & $H Q=1$ & $\mathrm{HQ}=0.1$ & $10^{-4}$ & $10^{5}$ & $H I=1$ & $\mathrm{HI}=0.1$ \\
\hline Fonofos & $000944-22-9$ & & & $1.5 E+03$ & $1.6 \mathrm{E}+02$ & $1.5 \mathrm{E}+02$ & $1,6 \mathrm{E}+01$ & & & $1.4 \mathrm{E}+03$ & $1.4 E+02$ & & & & & & & $7.1 E+02$ & $7.1 E+01$ \\
\hline Formaldehyde & $000050-00-0$ & & & $1.5 E+05$ & $1.6 \mathrm{E}+04$ & $1.5 E+04$ & $1.6 \mathrm{E}+03$ & & & $2.2 \mathrm{E}+05$ & $2.2 \mathrm{E}+04$ & $1.0 \mathrm{E}+06$ & 8. $1 \mathrm{E}+05$ & & & & & $8.8 E+04$ & $8.8 \mathrm{E}+03$ \\
\hline Formic Acid & $000064-18-6$ & & & $1.0 \mathrm{E}+06$ & $1.6 \mathrm{E}+05$ & $1.5 \mathrm{E}+05$ & $1.6 \mathrm{E}+04$ & & & $1.0 \mathrm{E}+06$ & $1.4 E+05$ & & & & & & & $7.3 E+05$ & $7.1 E+04$ \\
\hline Fosetyl-AL & $039148-24-8$ & & & $1.0 \mathrm{E}+06$ & $2.3 \mathrm{E}+05$ & $2.2 \mathrm{E}+05$ & $2.3 \mathrm{E}+04$ & & & $1.0 \mathrm{E}+06$ & 2.1E+05 & & & & & & & $1.0 \mathrm{E}+06$ & $1.1 \mathrm{E}+05$ \\
\hline Furan & 000110.00 .9 & & & $7.3 E+02$ & 7.8E+01 & $7.3 \mathrm{E}+01$ & $7.8 \mathrm{E}+00$ & & & $1.1 \mathrm{E}+03$ & $1.1 \mathrm{E}+02$ & & & & · & & & $4.4 \mathrm{E}+02$ & $4.4 \mathrm{E}+01$ \\
\hline Furazolidone & $000067-45-8$ & $1.7 \mathrm{E}+01$ & $1.7 \mathrm{E}-01$ & & & & & $4.2 \mathrm{E}+01$ & $4.2 \mathrm{E}-01$ & & & & & & & $2.2 \mathrm{E}+01$ & 2.2E-01 & & \\
\hline Furfiural & $000098-01-1$ & & & $2.2 \mathrm{E}+03$ & $2.3 \mathrm{E}+02$ & $2.2 \mathrm{E}+02$ & $2.3 E+01$ & & & $2.1 \mathrm{E}+03$ & $2.1 \mathrm{E}+02$ & & & $1.0 \mathrm{E}+06$ & $1.0 \mathrm{E}+06$ & & & $1.1 E+03$ & $1.1 \mathrm{E}+02$ \\
\hline Furium & 000531.82 .8 & $1.3 \mathrm{E}+\infty$ & $1.3 \mathrm{E}-02$ & & & & & $3.2 \mathrm{E}+\infty$ & $3.2 \mathrm{E}-02$ & & & & & & & $1.7 E+00$ & $1.7 \mathrm{E}-02$ & & \\
\hline Furmecyciox & $060568-05-0$ & $2.1 E+03$ & $2.1 \mathrm{E}+01$ & & & & & $5.4 \mathrm{E}+03$ & $5.4 E+01$ & & & & & & & $2.8 \mathrm{E}+03$ & $2.8 \mathrm{E}+01$ & & \\
\hline $\begin{array}{l}\text { Glufosinate, } \\
\text { Ammonium }\end{array}$ & $077182-82-2$ & & & $2.9 \mathrm{E}+02$ & $3.1 \mathrm{E}+01$ & $2.9 \mathrm{E}+01$ & $3.1 E+\infty$ & & & $2.8 \mathrm{E}+02$ & $2.8 \mathrm{E}+01$ & & & & & & & $1.4 E+02$ & $1.4 E+01$ \\
\hline Glycidyl & $000765-34-4$ & & & $2.9 \mathrm{E}+02$ & $3.1 E+01$ & $2.9 E+01$ & $3.1 \mathrm{E}+\infty$ & & & $2.8 \mathrm{E}+02$ & $2.8 \mathrm{E}+01$ & & & $1.0 E+06$ & 4.5E+05 & & & $1.4 E+02$ & $1.4 \mathrm{E}+01$ \\
\hline Glyphosate & $001071-83.6$ & & & $7.3 \mathrm{E}+04$ & $7.8 \mathrm{E}+03$ & $7.3 E+03$ & $7.8 \mathrm{E}+02$ & & & $6.9 \mathrm{E}+04$ & $6.9 \mathrm{E}+03$ & & & & & & & $3.5 \mathrm{E}+04$ & $3.5 \mathrm{E}+03$ \\
\hline Goal & $042874-03-3$ & & & $2.2 \mathrm{E}+03$ & $2.3 \mathrm{E}+02$ & $2.2 \mathrm{E}+02$ & $2.3 \mathrm{E}+01$ & & & $2.1 \mathrm{E}+03$ & $2.1 E+02$ & & & & & & & $1.1 \mathrm{E}+03$ & $1.1 \mathrm{E}+02$ \\
\hline $\begin{array}{l}\text { Maloxyfop, } \\
\text { Methyl }\end{array}$ & $069806-40-2$ & & & $3.7 E+01$ & $3.9 \mathrm{E}+00$ & $3.7 \mathrm{E}+00$ & $3.9 \mathrm{E}-01$ & & & $3.4 \mathrm{E}+01$ & $3.4 E+\infty 0$ & & & & & & & $1.8 \mathrm{E}+01$ & $1.8 \mathrm{E}+00$ \\
\hline Harmony & $079277-27-3$ & & & $9.5 \mathrm{E}+03$ & $1.0 \mathrm{E}+03$ & $9.5 \mathrm{E}+02$ & $1.0 \mathrm{E}+02$ & & & $9.0 \mathrm{E}+03$ & $9.0 \mathrm{E}+02$ & & & & & & & $4.6 \mathrm{E}+03$ & $4.6 \mathrm{E}+02$ \\
\hline Heptachlor & $000076-44-8$ & $1.4 \mathrm{E}+01$ & $1.4 \mathrm{E}-01$ & $3.7 \mathrm{E}+02$ & $3.9 \mathrm{E}+01$ & $3.7 E+01$ & $3.9 E+\infty 0$ & $5.1 E+01$ & 5.1E-01 & $5.0 \mathrm{E}+02$ & $5.0 \mathrm{E}+01$ & $8.1 \mathrm{E}+05$ & $8.1 \mathrm{E}+03$ & & & $2.2 \mathrm{E}+01$ & $2.2 \mathrm{E}-01$ & $2.1 E+02$ & $2.1 E+01$ \\
\hline $\begin{array}{l}\text { Heptachlor } \\
\text { Epoxide }\end{array}$ & $001024-57-3$ & $7.0 \mathrm{E}+00$ & $7.0 \mathrm{E}-02$ & $9.5 \mathrm{E}+00$ & $1.0 \mathrm{E}+00$ & $9.5 \mathrm{E}-01$ & $1.0 \mathrm{E}-01$ & $2.6 \mathrm{E}+0 \mathrm{I}$ & $2.6 \mathrm{E}-01$ & $1.3 \mathrm{E}+01$ & $1.3 \mathrm{E}+\infty$ & $4.0 \mathrm{E}+05$ & $4.0 \mathrm{E}+03$ & & & $1.1 E+01$ & $1.1 E-01$ & $5.5 \mathrm{E}+00$ & $5.5 \mathrm{E}-01$ \\
\hline Heptene, N- & 000142.82 .5 & & & & & & & & & & & & & & & & & & \\
\hline $\begin{array}{l}\text { Hexabromobe } \\
\text { nzene }\end{array}$ & $000087-82-1$ & & & $1.5 \mathrm{E}+03$ & $1.6 \mathrm{E}+02$ & $1.5 \mathrm{E}+02$ & $1.6 \mathrm{E}+01$ & & & $2.2 E+03$ & $2.2 E+02$ & & & & & & & $8.8 \mathrm{E}+02$ & $8.8 \mathrm{E}+01$ \\
\hline $\begin{array}{l}\text { Hexschloro- } \\
\text { benzene }\end{array}$ & $000118-74-1$ & $4.0 \mathrm{E}+01$ & $4.0 \mathrm{E}-01$ & $5.8 \mathrm{E}+02$ & $6.3 E+01$ & $5.8 \mathrm{E}+01$ & $6.3 E+\infty$ & $1.6 \mathrm{E}+02$ & $1.6 \mathrm{E}+\infty 0$ & $8.8 E+02$ & $8.8 E+01$ & $1.0 \mathrm{E}+06$ & $2.3 E+04$ & & & $6.4 E+01$ & $6.4 \mathrm{E}-01$ & $3.5 E+02$ & $3.5 E+01$ \\
\hline
\end{tabular}


Table 3.1c. (continued)

\begin{tabular}{|c|c|c|c|c|c|c|c|c|c|c|c|c|c|c|c|c|c|c|c|}
\hline \multirow{2}{*}{ Chemical } & \multirow{2}{*}{ CASRN } & \multicolumn{6}{|c|}{ Ingestion (mg/kg) } & \multicolumn{4}{|c|}{ Dermal (mg/kg) } & \multicolumn{4}{|c|}{ Inhalation (mg/kg) } & \multicolumn{4}{|c|}{$\begin{array}{c}\text { Ingestion + Dermal } \\
+ \text { Inhalation (mg/kg) } \\
\end{array}$} \\
\hline & & $10^{-4}$ & $10^{-6}$ & $\begin{array}{l}\mathrm{HQ}=1 \\
\text { Adult }\end{array}$ & $\begin{array}{l}\mathrm{HQ}=1 \\
\text { Child }\end{array}$ & $\begin{array}{c}\mathrm{HQ}=0.1 \\
\text { Adult }\end{array}$ & $\begin{array}{c}\text { HQ }=0.1 \\
\text { Child }\end{array}$ & $10^{-4}$ & $10^{4}$ & $H Q=1$ & $H Q=0.1$ & $10^{-4}$ & $10^{6}$ & $H Q=1$ & $H Q=0.1$ & $10^{-4}$ & $10^{6}$ & $\mathbf{H I}=\mathbf{1}$ & $\mathrm{HI}=0.1$ \\
\hline $\begin{array}{l}\text { Hexachloro- } \\
\text { butadienef(i) }\end{array}$ & $000087-68-3$ & $8.2 \mathrm{E}+02$ & $8.2 E+\infty 0$ & $1.5 \mathrm{E}+02$ & $1.6 \mathrm{E}+01$ & I. $.5 E+01$ & $1.6 \mathrm{E}+00$ & $3.3 E+03$ & $3.3 E+01$ & $2.2 \mathrm{E}+02$ & $2.2 E+01$ & $1.0 E+06$ & 4.7E+05 & & & $1.3 \mathrm{E}+03$ & $1.3 \mathrm{E}+01$ & $8.8 E+01$ & $8.8 \mathrm{E}+00$ \\
\hline $\begin{array}{l}\text { Hexachlorocy- } \\
\text { clohexane, } \\
\text { Apha- }\end{array}$ & $000319-84-6$ & $1.0 E+01$ & $1.0 \mathrm{E}-01$ & & & & * & $5.0 E+01$ & $5.0 \mathrm{E}-01$ & & & $5.8 \mathrm{E}+05$ & $5.8 E+03$ & & & $1.7 E+01$ & $1.7 \mathrm{E}-01$ & & \\
\hline $\begin{array}{l}\text { Hexachlorocy- } \\
\text { clohexsene, } \\
\text { Beta*- }\end{array}$ & $000319-85-7$ & $3.5 E+01$ & 3.SE-0I & & & & & $1.6 \mathrm{E}+02$ & $1.6 \mathrm{E}+\infty$ & & & $1.0 \mathrm{E}+06$ & $2.0 \mathrm{E}+04$ & & & $6.0 E+01$ & $6.0 \mathrm{E}-01$ & & \\
\hline $\begin{array}{l}\text { Hexachlorocy- } \\
\text { clohexane, } \\
\text { Delta- }\end{array}$ & $000319-86.8$ & & & & & & & & & & & & & & & & & & \\
\hline $\begin{array}{l}\text { Hexachlorocy- } \\
\text { clohexane, } \\
\text { Epsilon }\end{array}$ & $006108-10-7$ & & & & & & & & & & & & & & & & & & \\
\hline $\begin{array}{l}\text { Hexachlorocy- } \\
\text { clohexane, } \\
\text { Gamma. }\end{array}$ & $000058-89-9$ & $4.9 \mathrm{E}+01$ & $4.9 \mathrm{E}-01$ & $2.2 E+02$ & $2.3 \mathrm{E}+01$ & $2.2 \mathrm{E}+01$ & $2.3 \mathrm{E}+00$ & $2.4 \mathrm{E}+02$ & $2.4 E+\infty 0$ & $4.0 \mathrm{E}+02$ & $4.0 \mathrm{E}+01$ & & & & & 8.5E+01 & 8.5E-01 & $1.4 \mathrm{E}+02$ & $1.4 \mathrm{E}+01$ \\
\hline $\begin{array}{l}\text { Hexachlorocy- } \\
\text { clohexane, } \\
\text { Technical }\end{array}$ & $000608-73-1$ & 3.SE+01 & $3.5 \mathrm{E}-01$ & & & & & $8.9 E+01$ & $8.9 \mathrm{E}-01$ & & & $1.0 \mathrm{E}+06$ & $2.0 \mathrm{E}+04$ & & & $4.6 \mathrm{E}+01$ & $4.6 \mathrm{E}-01$ & & \\
\hline $\begin{array}{l}\text { Hexuchlorocy- } \\
\text { clopentediene }\end{array}$ & $000077-47-4$ & & & $5.1 E+03$ & $5.5 E+02$ & $5.1 E+02$ & $5.5 E+01$ & & & $7.7 \mathrm{E}+03$ & $7.7 E+02$ & & & $3.1 \mathrm{E}+05$ & $3.1 \mathrm{E}+04$ & & & $3.0 \mathrm{E}+03$ & $3.0 \mathrm{E}+02$ \\
\hline $\begin{array}{l}\text { Hexachlorodi- } \\
\text { benzo-p-di- } \\
\text { oxin, Mixture }\end{array}$ & $019408-74-3$ & $1.0 \mathrm{E}-02$ & $1.0 \mathrm{E}-04$ & & & & & $2.6 \mathrm{E}-02$ & $2.6 \mathrm{E}-04$ & & & $8.0 \mathrm{E}+02$ & $8.0 E+00$ & & & $1.3 \mathrm{E}-02$ & $1.3 \mathrm{E}-04$ & & \\
\hline $\begin{array}{l}\text { Hexachloro. } \\
\text { ethane }\end{array}$ & $000067-72-1$ & $4.6 E+03$ & $4.6 \mathrm{E}+01$ & $7.3 \mathrm{E}+02$ & $7.8 \mathrm{E}+01$ & $7.3 \mathrm{E}+01$ & $7.8 \mathrm{E}+00$ & $1.8 \mathrm{E}+04$ & $1.8 \mathrm{E}+02$ & $1.1 \mathrm{E}+03$ & $1.1 \mathrm{E}+02$ & $1.0 \mathrm{E}+06$ & $1.0 \mathrm{E}+06$ & & & $7.3 E+03$ & $7.3 \mathrm{E}+01$ & $4.4 E+02$ & $4.4 E+01$ \\
\hline $\begin{array}{l}\text { Hexsechloro. } \\
\text { phene }\end{array}$ & $000070-30-4$ & & & $2.2 \mathrm{E}+02$ & $2.3 E+01$ & $2.2 \mathrm{E}+01$ & $2.3 \mathrm{E}+\infty 0$ & & & $2.1 E+02$ & 2.1E+01 & & & & & & & $1.1 \mathrm{E}+02$ & $1.1 \mathrm{E}+01$ \\
\hline $\begin{array}{l}\text { Hexahydro- } \\
1,3,5 \text {-trinitro- } \\
1,3,5 \text {-triazine } \\
\text { (RDX) }\end{array}$ & $000121-82-4$ & $5.8 \mathrm{E}+02$ & $5.8 \mathrm{E}+00$ & $2.2 \mathrm{E}+03$ & $2.3 E+02$ & $2.2 E+02$ & $2.3 E+01$ & $2.9 \mathrm{E}+03$ & $2.9 \mathrm{E}+01$ & $4.1 E+03$ & $4.1 \mathrm{E}+02$ & & & & & $1.0 \mathrm{E}+03$ & $1.0 E+01$ & $1.4 \mathrm{E}+03$ & $1.4 E+02$ \\
\hline
\end{tabular}


Table 3.1c. (continued)

\begin{tabular}{|c|c|c|c|c|c|c|c|c|c|c|c|c|c|c|c|c|c|c|c|}
\hline \multirow{2}{*}{ Chemical } & \multirow{2}{*}{ CASRN } & \multicolumn{6}{|c|}{ Ingestion (mg/kg) } & \multicolumn{4}{|c|}{ Dermal (mg/kg) } & \multicolumn{4}{|c|}{ Inhalation (mg/kg) } & \multicolumn{4}{|c|}{$\begin{array}{l}\text { Ingestion + Dermal } \\
+ \text { Inhalation (mg/kg) }\end{array}$} \\
\hline & & $10^{-4}$ & $10^{4}$ & $\begin{array}{l}\text { HQ }=1 \\
\text { Adult }\end{array}$ & $\begin{array}{l}\mathrm{HQ}=1 \\
\text { Child }\end{array}$ & $\begin{array}{c}H Q=0.1 \\
\text { Adult }\end{array}$ & $\begin{array}{c}\mathrm{HQ}=0.1 \\
\text { Child }\end{array}$ & $10^{-4}$ & $10^{6}$ & $\mathrm{HQ}=1$ & $\mathrm{HQ}=0.1$ & $10^{-4}$ & $10^{6}$ & $H Q=1$ & $\mathrm{HQ}=0.1$ & $10^{-4}$ & $10^{5}$ & $H I=1$ & $\mathrm{HI}=0.1$ \\
\hline $\begin{array}{l}\text { Hexamethyl- } \\
\text { ene Di- } \\
\text { isocyanate, } \\
1,6 \text { - }\end{array}$ & $000822-06.0$ & & & & & & & & & & & & & 4.5E+04 & $4.5 \mathrm{E}+03$ & & & & \\
\hline Hexane, N- & $000110-54-3$ & & & 4.4E+04 & $4.7 E+03$ & $4.4 E+03$ & 4.7E+02 & & & $6.6 \mathrm{E}+04$ & $6.6 \mathrm{E}+03$ & & & 9. $I E+01$ & $9.1 E+01$ & & & $9.1 E+01$ & $9.1 E+01$ \\
\hline Hexanone, 2- & $000591-78-6$ & & & & & & & & & & & & & & & & & & \\
\hline Hexazinone & $051235.04-2$ & & & $2.4 \mathrm{E}+04$ & $2.6 \mathrm{E}+03$ & $2.4 E+03$ & $2.6 \mathrm{E}+02$ & & & $2.3 E+04$ & $2.3 E+03$ & & & & & & & $1.2 \mathrm{E}+04$ & $1.2 E+03$ \\
\hline $\begin{array}{l}\mathrm{HpCDD}_{2,} \\
2,3,7,8^{(i)}\end{array}$ & $037871-00-4$ & $4.3 \mathrm{E}-02$ & 4.3E-04 & & & & & $3.6 \mathrm{E}-02$ & $3.6 \mathrm{E}-04$ & & & $2.4 E+03$ & $2.4 E+01$ & & & $2.7 \mathrm{E}-02$ & 2.7E-04 & & \\
\hline $\begin{array}{l}\mathrm{HpCDF}_{2,3,7, \mathbf{f}^{(1)}}\end{array}$ & $038998-75-3$ & 4.3E-02 & 4.3E-04 & & & & & $1.1 \mathrm{E}-01$ & $1.1 \mathrm{E}-03$ & & & $2.4 E+03$ & $2.4 \mathrm{E}+01$ & & & S.SE-02 & $5.5 \mathrm{E}-04$ & & \\
\hline $\begin{array}{l}\mathrm{HxCDD} \\
2,3,7,8^{(i)}\end{array}$ & $034465-46-8$ & 4.3E-03 & 4.3E-05 & & & & & 3.6E-03 & $3.6 \mathrm{E}-05$ & & & $2.4 E+02$ & $2.4 \mathrm{E}+00$ & & & $2.7 \mathrm{E}-03$ & 2.7E-05 & & \\
\hline $\begin{array}{l}\mathrm{HxCDF} \\
2,3,7,8=1,1\end{array}$ & $055684-94-1$ & 4.3E-03 & 4.3E-0S & & & & & $1.1 \mathrm{E}-02$ & $1.1 \mathrm{E}-04$ & & & $2.4 E+02$ & $2.4 E+\infty 0$ & & & $5.5 \mathrm{E}-03$ & S.5E.05 & & \\
\hline Hydrazine & $000302.01-2$ & $2.1 E+01$ & 2.1E.01 & & & & & $5.4 \mathrm{E}+01$ & $5.4 \mathrm{E}-01$ & & & $2.1 E+05$ & $2.1 E+03$ & & & $2.8 \mathrm{E}+01$ & $2.8 \mathrm{E} \cdot 01$ & & \\
\hline $\begin{array}{l}\text { Hydrazine Sul- } \\
\text { fate }\end{array}$ & $010034-93-2$ & $2.1 \mathrm{E}+01$ & 2.1E-01 & & & & & $5.4 E+01$ & $5.4 \mathrm{E}-01$ & & & $2.1 E+05$ & $2.1 E+03$ & & & $2.8 \mathrm{E}+01$ & $2.8 \mathrm{E}-01$ & & \\
\hline $\begin{array}{l}\text { Hydrogen } \\
\text { Chloride }\end{array}$ & $007647-01-0$ & & & & & & & & & & & & & $1.0 \mathrm{E}+06$ & $1.0 \mathrm{E}+06$ & & & & \\
\hline $\begin{array}{l}\text { Hydrogen } \mathrm{Cy}- \\
\text { anide }\end{array}$ & $000074-90.8$ & & & $1.5 E+04$ & $1.6 \mathrm{E}+03$ & $1.5 \mathrm{E}+03$ & $1.6 \mathrm{E}+02$ & & & $4.7 E+04$ & $4.7 E+03$ & & & $1.0 \mathrm{E}+06$ & $1.0 \mathrm{E}+06$ & & & $1.1 \mathrm{E}+04$ & $1.1 \mathrm{E}+03$ \\
\hline $\begin{array}{l}\text { Hydrogen Sul- } \\
\text { fide }\end{array}$ & $007783-06-4$ & & & $2.2 E+03$ & $2.3 E+02$ & $2.2 \mathrm{E}+02$ & $2.3 E+01$ & & & $8.3 E+03$ & $8.3 E+02$ & & & $1.0 \mathrm{E}+06$ & 4.5E+05 & & & $1.7 \mathrm{E}+03$ & $1.7 \mathrm{E}+02$ \\
\hline Hydroquinone & $000123-31.9$ & & & $2.9 \mathrm{E}+04$ & $3.1 E+03$ & $2.9 E+03$ & $3.1 \mathrm{E}+02$ & & & $2.8 \mathrm{E}+04$ & $2.8 E+03$ & & & & & & & $1.4 \mathrm{E}+04$ & $1.4 \mathrm{E}+03$ \\
\hline Imazalit & $035554-44.0$ & & & $9.5 \mathrm{E}+03$ & $1.0 E+03$ & $9.5 \mathrm{E}+02$ & $1.0 \mathrm{E}+02$ & & & $9.0 E+03$ & $9.0 E+02$ & & & & & & & $4.6 \mathrm{E}+03$ & $4.6 \mathrm{E}+02$ \\
\hline Imazaquin & $081335-37-7$ & & & $1.8 \mathrm{E}+0 \mathrm{~S}$ & $2.0 E+04$ & $1.8 E+04$ & $2.0 E+03$ & & & $1.7 \mathrm{E}+0 \mathrm{~S}$ & $1.7 \mathrm{E}+04$ & & & & & & & $8.9 E+04$ & $8.9 E+03$ \\
\hline $\begin{array}{l}\text { Indeno[1,2,3- } \\
\text { ed]pyrene }\end{array}$ & $000193-39-5$ & $8.8 E+01$ & $8.8 \mathrm{E}-01$ & & & & & $1.4 E+02$ & $1.4 \mathrm{E}+\infty 0$ & & & $1.0 \mathrm{E}+06$ & $1.2 E+05$ & & & $8.6 E+01$ & $8.6 \mathrm{E}-01$ & & \\
\hline
\end{tabular}


Table 3.1c. (continued)

\begin{tabular}{|c|c|c|c|c|c|c|c|c|c|c|c|c|c|c|c|c|c|c|c|}
\hline \multirow{2}{*}{ Chemical } & \multirow{2}{*}{ CASRN } & \multicolumn{6}{|c|}{ Ingestion (mg/kg) } & \multicolumn{4}{|c|}{ Dermal (mg/kg)" } & \multicolumn{4}{|c|}{ Inhalation (mg/kg) } & \multicolumn{4}{|c|}{$\begin{aligned} & \text { Ingestion + Dermal } \\
+ & \text { Inhalation }(\mathrm{mg} / \mathrm{kg})^{+* v}\end{aligned}$} \\
\hline & & $10^{-4}$ & $10^{4}$ & $\begin{array}{l}\text { HQ=1 } \\
\text { Adult }\end{array}$ & $\begin{array}{l}\text { HQ=1 } \\
\text { Child }\end{array}$ & $\begin{array}{c}\text { HQ }=0.1 \\
\text { Adult }\end{array}$ & $\begin{array}{c}\mathrm{HQ}=0,1 \\
\text { Child }\end{array}$ & $10^{-4}$ & $10^{6}$ & $H Q=1$ & $\mathrm{HQ}=0.1$ & $10^{-4}$ & $10^{4}$ & $H Q=1$ & $\mathrm{HQ}=0.1$ & $10^{-1}$ & $10^{4}$ & $H I=I$ & $\mathrm{HI}=0.1$ \\
\hline Iprodione & $036734-19-7$ & & & $2.9 E+04$ & $3.1 E+03$ & $2.9 \mathrm{E}+03$ & $3.1 \mathrm{E}+02$ & & & $2.8 \mathrm{E}+04$ & $2.8 E+03$ & & & & & & & $1.4 E+04$ & $1.4 \mathrm{E}+03$ \\
\hline Iron & $007439.89-6$ & & & & & & & & & & & & & & & & & & \\
\hline $\begin{array}{l}\text { Isobutyl Alco- } \\
\text { hol }\end{array}$ & $000078-83-1$ & & & $2.2 E+05$ & $2.3 \mathrm{E}+04$ & $2.2 E^{\circ}+04$ & $2.3 \mathrm{E}+03$ & & & 3.3E+05 & $3.3 \mathrm{E}+04$ & & & & & & & $1.3 E+05$ & $1.3 E+04$ \\
\hline Isophorone & $000078-59-1$ & $6.7 E+04$ & $6.7 \mathrm{E}+02$ & $1.5 \mathrm{E}+05$ & $1.6 \mathrm{E}+04$ & $1.5 \mathrm{E}+04$ & $1.6 \mathrm{E}+03$ & $1.7 E+05$ & $1.7 \mathrm{E}+03$ & $1.4 \mathrm{E}+05$ & $1.4 \mathrm{E}+04$ & & & & & $8.7 E+04$ & $8.7 \mathrm{E}+02$ & $7.1 E+04$ & $7.1 E+03$ \\
\hline Isopropalin & 033820.53 .0 & & & $1.1 E+04$ & $1.2 \mathrm{E}+03$ & $1.1 \mathrm{E}+03$ & $1.2 \mathrm{E}+02$ & & & $1.0 \mathrm{E}+04$ & $1.0 \mathrm{E}+03$ & & & & & & & $5.3 \mathrm{E}+03$ & $5.3 E+02$ \\
\hline Isopropenol & $000067-63-0$ & & & & & & & & & & & & & & & & & & \\
\hline $\begin{array}{l}\text { Isopropyl } \\
\text { Methyl } \\
\text { Phosphonic } \\
\text { Acid }\end{array}$ & $001832-54-8$ & & & $7.3 \mathrm{E}+04$ & $7.8 \mathrm{E}+03$ & $7.3 \mathrm{E}+03$ & $7.8 \mathrm{E}+02$ & & & $\begin{array}{l}6.9 \mathrm{E}+04 \\
.\end{array}$ & $6.9 \mathrm{E}+03$ & & & & & & & $3.5 \mathrm{E}+04$ & $3.5 \mathrm{E}+03$ \\
\hline Isoxaben & 082558.50 .7 & & & $3.7 \mathrm{E}+04$ & $3.9 \mathrm{E}+03$ & $3.7 \mathrm{E}+03$ & $3.9 \mathrm{E}+02$ & & & $3.4 E+04$ & $3.4 E+03$ & & & & & & & $1.8 \mathrm{E}+04$ & $1.8 \mathrm{E}+03$ \\
\hline Karate & $091465-08-6$ & & & $3.7 \mathrm{E}+03$ & $3.9 E+02$ & $3.7 \mathrm{E}+02$ & $3.9 E+01$ & & & $3.4 \mathrm{E}+03$ & $3.4 \mathrm{E}+02$ & & & & & & & $1.8 \mathrm{E}+03$ & $1.8 \mathrm{E}+02$ \\
\hline Kerb & 023950.58 .5 & & & $5.5 \mathrm{E}+04$ & $5.9 E+03$ & $5.5 \mathrm{E}+03$ & $5.9 \mathrm{E}+02$ & & & $5.2 E+04$ & $5.2 E+03$ & & & & & & & $2.7 E+04$ & $2.7 E+03$ \\
\hline Lactofen & $077501-63-4$ & & & $1.5 E+03$ & $1.6 \mathrm{E}+02$ & $1.5 \mathrm{E}+02$ & $1.6 \mathrm{E}+01$ & & & $1.4 \mathrm{E}+03$ & $1.4 \mathrm{E}+02$ & & & & & & & $7.1 E+02$ & $7.1 \mathrm{E}+01$ \\
\hline Lead Alkyls & NA & & & & & & & & & & & & & & & & & & \\
\hline $\begin{array}{l}\text { Lead And } \\
\text { Compounds }\end{array}$ & $007439.92-1$ & & & $4.0 \mathrm{E}+02$ & $4.0 E+02$ & $4.0 \mathrm{E}+02$ & $4.0 E+02$ & & & & & & & & & & & & \\
\hline Linuron & $000330.55-2$ & & & $1.5 \mathrm{E}+03$ & $1.6 \mathrm{E}+02$ & $1.5 \mathrm{E}+02$ & $1.6 \mathrm{E}+01$ & & & $1.4 E+03$ & $1.4 \mathrm{E}+02$ & & & & & & & $7.1 E+02$ & $7.1 E+01$ \\
\hline Lithium & 007439.93 .2 & & & & & & & & & & & & & & & & & & \\
\hline Londax & $083055-99.6$ & & & $1.5 \mathrm{E}+05$ & $1.6 \mathrm{E}+04$ & $1.5 \mathrm{E}+04$ & $1.6 \mathrm{E}+03$ & & & $1.4 \mathrm{E}+0 \mathrm{~S}$ & $1.4 \mathrm{E}+04$ & & & & & & & $7.1 \mathrm{E}+04$ & $7.1 \mathrm{E}+03$ \\
\hline MCPA & $000094-74-6$ & & & $3.7 \mathrm{E}+02$ & $3.9 \mathrm{E}+01$ & $3.7 \mathrm{E}+01$ & $3.9 E+\infty 0$ & & & $3.4 E+02$ & $3,4 E+01$ & & & & & & & $1.8 \mathrm{E}+02$ & $1.8 \mathrm{E}+01$ \\
\hline MCPB & $000094-81-5$ & & & $7.3 E+03$ & $7.8 \mathrm{E}+02$ & $7.3 \mathrm{E}+02$ & $7.8 \mathrm{E}+01$ & & & $6.9 \mathrm{E}+03$ & $6.9 \mathrm{E}+02$ & & & & & & & 3.5E+03 & $3.5 E+02$ \\
\hline MCPP & 000093.65 .2 & & & 7.3E+02 & $7.8 \mathrm{E}+01$ & 7.3E+01 & $7.8 E+\infty 0$ & & & $6.9 \mathrm{E}+02$ & $6.9 \mathrm{E}+01$ & & & & & & & $3.5 E+02$ & $3.5 E+01$ \\
\hline Magnesium & $007439-95-4$ & & & & & & & & & & & & & & & & & & \\
\hline
\end{tabular}


Table 3.1c. (continued)

\begin{tabular}{|c|c|c|c|c|c|c|c|c|c|c|c|c|c|c|c|c|c|c|c|}
\hline \multirow{2}{*}{ Chemical } & \multirow{2}{*}{ CASRN } & \multicolumn{6}{|c|}{ Ingestion (mg/kg) } & \multicolumn{4}{|c|}{ Dermal (mg/kg) } & \multicolumn{4}{|c|}{ Inhalation $(\mathrm{mg} / \mathrm{kg})^{*}$} & \multicolumn{4}{|c|}{$\begin{aligned} & \text { Ingestion + Dermal } \\
&+ \text { Inhalation }\left(\mathrm{mg} / \mathrm{kg}^{+* *}\right. \\
&\end{aligned}$} \\
\hline & & $10^{-4}$ & $10^{-5}$ & $\begin{array}{l}\text { HQ=1 } \\
\text { Adult }\end{array}$ & $\begin{array}{l}\mathrm{HQ}=1 \\
\text { Child }\end{array}$ & $\begin{array}{c}\mathrm{HQ}=0.1 \\
\text { Adult }\end{array}$ & $\begin{array}{c}\mathrm{HQ}=0.1 \\
\text { Child }\end{array}$ & $10^{-4}$ & $10^{6}$ & $\mathrm{HQ}=1$ & $H Q=0.1$ & $10^{-4}$ & $10^{6}$ & $H Q=1$ & $\mathrm{HQ}=0.1$ & $10^{-4}$ & $10^{4}$ & $\mathrm{HI}=1$ & $\mathrm{HI}=0.1$ \\
\hline Malathion & $000121-75.5$ & & & $1.5 \mathrm{E}+04$ & $1.6 \mathrm{E}+03$ & $1.5 \mathrm{E}+03$ & $1.6 \mathrm{E}+02$ & & & $1.4 E+04$ & $1.4 E+03$ & & & & & & & $7.1 \mathrm{E}+03$ & $7.1 E+02$ \\
\hline $\begin{array}{l}\text { Maleic Anhy- } \\
\text { dride }\end{array}$ & $000108-31-6$ & & & $7.3 \mathrm{E}+04$ & $7.8 \mathrm{E}+03$ & $7.3 \mathrm{E}+03$ & $7.8 \mathrm{E}+02$ & & & $6.9 \mathrm{E}+04$ & $6.9 E+03$ & & & & & & & $3.5 \mathrm{E}+04$ & $3.5 \mathrm{E}+03$ \\
\hline $\begin{array}{l}\text { Maleie Hydra- } \\
\text { zide }\end{array}$ & $000123-33-1$ & & & 3.7E+05 & $3.9 E+04$ & $3.7 \mathrm{E}+04$ & $3.9 \mathrm{E}+03$ & & & $3.4 \mathrm{E}+05$ & $3.4 E+04$ & & & & & & & $1.8 \mathrm{E}+05$ & $1.8 \mathrm{E}+04$ \\
\hline Malononitrile & $000109-77-3$ & & & $1.5 \mathrm{E}+01$ & $1,6 \mathrm{E}+00$ & $1.5 \mathrm{E}+00$ & $1,6 \mathrm{E}-01$ & & & $2.2 \mathrm{E}+01$ & $2.2 E+\infty 0$ & & & & & & & $8.8 \mathrm{E}+00$ & $8.8 \mathrm{E}-01$ \\
\hline Mancozeb & $008018.01-7$ & & & $2.2 \mathrm{E}+04$ & $2.3 E+03$ & $2.2 \mathrm{E}+03$ & $2.3 \mathrm{E}+02$ & & & $2.1 \mathrm{E}+04$ & 2. $1 E+03$ & & & & & & & $1.1 \mathrm{E}+04$ & $1.1 \mathrm{E}+03$ \\
\hline Maneb & $012427-38-2$ & & & $3.7 \mathrm{E}+03$ & $3.9 E+02$ & $3.7 \mathrm{E}+02$ & $3.9 \mathrm{E}+01$ & & & $3.4 E+03$ & $3.4 \mathrm{E}+02$ & & & & & & & $1.8 \mathrm{E}+03$ & $1.8 \mathrm{E}+02$ \\
\hline $\begin{array}{l}\text { Manganese } \\
\text { (Water) }\end{array}$ & $007439-96-5$ & & & $3.4 E+04$ & $3.7 E+03$ & $3.4 E+03$ & $3.7 E+02$ & & & $2.6 \mathrm{E}+04$ & $2.6 \mathrm{E}+03$ & & & $2.2 \mathrm{E}+05$ & $2.2 E+04$ & & & $1.4 \mathrm{E}+04$ & $1.4 \mathrm{E}+03$ \\
\hline Mephosfolan & $000950-10-7$ & & & $6.6 \mathrm{E}+01$ & $7.0 E+\infty$ & $6.6 \mathrm{E}+\infty 0$ & $7.0 \mathrm{E}-01$ & & & $6.2 \mathrm{E}+01$ & $6.2 \mathrm{E}+00$ & & & & & & & $3.2 \mathrm{E}+01$ & $3.2 \mathrm{E}+00$ \\
\hline $\begin{array}{l}\text { Mepiquat } \\
\text { Chloride }\end{array}$ & $024307-26-4$ & & & $2.2 E+04$ & $2.3 \mathrm{E}+03$ & $2.2 \mathrm{E}+03$ & $2.3 \mathrm{E}+02$ & & & $2.1 E+04$ & $2.1 E+03$ & & & & & & & 1. $1 E+04$ & $1.1 \mathrm{E}+03$ \\
\hline $\begin{array}{l}\text { Mercuric } \\
\text { Chloride }\end{array}$ & $007487-94-7$ & & & $2.2 \mathrm{E}+02$ & $2.3 E+01$ & $2.2 \mathrm{E}+01$ & $2.3 \mathrm{E}+\infty$ & & & 8.3E+02 & $8.3 E+01$ & & & & & & & $1.7 E+02$ & $1.7 \mathrm{E}+01$ \\
\hline $\begin{array}{l}\text { Mercury (cle- } \\
\text { mental) }\end{array}$ & $007439-97-6$ & & & $2.2 \mathrm{E}+02$ & $2.3 \mathrm{E}+01$ & $2.2 \mathrm{E}+01$ & $2.3 E+00$ & & & 4.1E-01 & $4.1 \mathrm{E}-02$ & & & $1.0 \mathrm{E}+06$ & $1.3 \mathrm{E}+05$ & & & $4.1 \mathrm{E}-01$ & $4.1 \mathrm{E}-02$ \\
\hline $\begin{array}{l}\text { Mercury, Inor- } \\
\text { ganic Salt }\end{array}$ & $007439-97-6$ & & & $2.2 \mathrm{E}+02$ & $2.3 \mathrm{E}+01$ & $2.2 \mathrm{E}+01$ & $2.3 E+\infty$ & & & $2.9 E+02$ & $2.9 \mathrm{E}+01$ & & & $1.0 \mathrm{E}+06$ & $1.3 E+05$ & & & $1.2 E+02$ & $1.2 \mathrm{E}+01$ \\
\hline Merphos & 000150.50 .5 & & & $2.2 \mathrm{E}+0 \mathrm{I}$ & $2.3 E+\infty$ & $2.2 E+\infty 0$ & $2.3 \mathrm{E}-01$ & & & $2.1 E+01$ & $2.1 E+\infty$ & & & & & & & $1.1 E+01$ & $1.1 E+\infty$ \\
\hline $\begin{array}{l}\text { Merphos Ox- } \\
\text { ide }\end{array}$ & $000078-48-8$ & & & $2.2 \mathrm{E}+01$ & $2.3 E+\infty$ & $2.2 \mathrm{E}+00$ & $2.3 \mathrm{E}-01$ & & & $2.1 \mathrm{E}+01$ & $2.1 E+\infty$ & & & & & & & $1.1 E+01$ & $1.1 E+\infty$ \\
\hline Met2alaxyl & $057837-19-1$ & & & $4.4 \mathrm{E}+04$ & $4.7 E+03$ & $4.4 \mathrm{E}+03$ & $4.7 \mathrm{E}+02$ & & & 4.1E+04 & $4.1 E+03$ & & & & & & & $2.1 E+04$ & $2.1 \mathrm{E}+03$ \\
\hline $\begin{array}{l}\text { Methacrylo- } \\
\text { nitrile }\end{array}$ & $000126-98-7$ & & & $7.3 \mathrm{E}+01$ & $7.8 \mathrm{E}+\infty$ & $7.3 E+\infty$ & $7.8 \mathrm{E}-01$ & & & $1.1 E+02$ & $1.18+01$ & & & $8.3 \mathrm{E}+01$ & $8.3 E+\infty$ & & & $2.9 E+01$ & $2.9 E+\infty$ \\
\hline $\begin{array}{l}\text { Methamido- } \\
\text { phos }\end{array}$ & $010265-92-6$ & & & 3.7E+01 & $3.9 E+\infty 0$ & $3.7 E+00$ & $3.9 \mathrm{E}-01$ & & & $3.4 E+01$ & $3.4 E+\infty$ & & & & & & & $1.8 \mathrm{E}+01$ & $1.8 \mathrm{E}+00$ \\
\hline Methanol & $000067-56-1$ & & & $3.7 E+05$ & $3.9 E+04$ & $3.7 E+04$ & $3.9 E+03$ & & & $5.5 \mathrm{E}+05$ & $5.5 E+04$ & & & & & & & $2.2 E+0 S$ & $2.2 E+04$ \\
\hline
\end{tabular}


Table 3.1c. (continued)

\begin{tabular}{|c|c|c|c|c|c|c|c|c|c|c|c|c|c|c|c|c|c|c|c|}
\hline \multirow{2}{*}{ Chemical } & \multirow{2}{*}{ CASRN } & \multicolumn{6}{|c|}{ Ingestion (mg/kg) } & \multicolumn{4}{|c|}{ Dermal (mg/kg) } & \multicolumn{4}{|c|}{ Inhalation (mg/kg) } & \multicolumn{4}{|c|}{$\begin{aligned} & \text { Ingestion + Dermal } \\
+ & \text { Inhalation (mg/kg) }\end{aligned}$} \\
\hline & & $10^{4}$ & $10^{4}$ & $\begin{array}{l}\text { HQ=1 } \\
\text { Adult }\end{array}$ & $\begin{array}{l}\mathrm{HQ}=1 \\
\text { Child }\end{array}$ & $\begin{array}{c}\text { HQ=0.1 } \\
\text { Adult }\end{array}$ & $\begin{array}{l}\text { HQ }=0.1 \\
\text { Child }\end{array}$ & $10^{-4}$ & $10^{4}$ & $H Q=1$ & $\mathrm{HQ}=0.1$ & $10^{-4}$ & $10^{4}$ & $H Q=1$ & $H Q=0.1$ & $10^{-4}$ & $10^{4}$ & $H I=1$ & $H I=0.1$ \\
\hline Methidathion & 000950.37 .8 & & & $7.3 E+02$ & $7.8 \mathrm{E}+01$ & $7.3 \mathrm{E}+01$ & $7.8 \mathrm{E}+00$ & & & $6.9 \mathrm{E}+02$ & $6.9 \mathrm{E}+01$ & & & & & & & $3.5 E+02$ & $3.5 \mathrm{E}+01$ \\
\hline Methomyl & $016752-77.5$ & & & $1.8 \mathrm{E}+04$ & $2.0 E+03$ & $1.8 \mathrm{E}+03$ & $2.0 \mathrm{E}+02$ & & & $1.7 \mathrm{E}+04$ & $1.7 E+03$ & & & & & & & $8.9 E+03$ & $8.9 E+02$ \\
\hline $\begin{array}{l}\text { Methoxy-5- } \\
\text { nitroasiline, } 2 \text { - }\end{array}$ & $000099-59-2$ & $1.4 \mathrm{E}+03$ & $1.4 E+01$ & & & & & $3.5 \mathrm{E}+03$ & $3.5 \mathrm{E}+01$ & & & & & & & $1.8 \mathrm{E}+03$ & $1.8 \mathrm{E}+01$ & & \\
\hline Methoxychlor & $000072-43-5$ & & & $3.7 \mathrm{E}+03$ & $3.9 \mathrm{E}+02$ & 3.7E+02 & $3.9 E+01$ & & & $3.4 \mathrm{E}+03$ & $3.4 E+02$ & & & & & & & $1.8 \mathrm{E}+03$ & $1.8 \mathrm{E}+02$ \\
\hline $\begin{array}{l}\text { Methoxyeth- } \\
\text { anol Acetate, } \\
\text { 2- }\end{array}$ & $000110-49-6$ & & & $1.5 E+03$ & $1.6 \mathrm{E}+02$ & $1.5 \mathrm{E}+02$ & $1.6 \mathrm{E}+01$ & & & $1.4 E+03$ & $1.4 E+02$ & & & & & & & $7.1 E+02$ & $7.1 \mathrm{E}+01$ \\
\hline $\begin{array}{l}\text { Methoxyeth- } \\
\text { anol, 2- }\end{array}$ & $000109-86-4$ & & & $7.3 E+02$ & $7.8 \mathrm{E}+01$ & $7.3 E+01$ & $7.8 \mathrm{E}+00$ & & & $6.9 E+02$ & $6.9 \mathrm{E}+01$ & & & $1,0 \mathrm{E}+06$ & $1.0 \mathrm{E}+06$ & & & $3.5 \mathrm{E}+02$ & $3.5 E+01$ \\
\hline $\begin{array}{l}\text { Methyl Ace- } \\
\text { tate }\end{array}$ & $000079-20.9$ & & & $7.3 E+05$ & $7.8 \mathrm{E}+04$ & $7.3 \mathrm{E}+04$ & $7.8 \mathrm{E}+03$ & & & $6.9 \mathrm{E}+05$ & $6.9 E+04$ & & & & & & & 3.5E+05 & $3.5 \mathrm{E}+04$ \\
\hline $\begin{array}{l}\text { Methyl Acry- } \\
\text { late }\end{array}$ & $000096-33-3$ & & & $2.2 E+04$ & $2.3 \mathrm{E}+03$ & $2.2 \mathrm{E}+03$ & $2.3 \mathrm{E}+02$ & & & $2.1 \mathrm{E}+04$ & $2.1 \mathrm{E}+03$ & & & & & & & $1.1 E+04$ & $1.1 E+03$ \\
\hline $\begin{array}{l}\text { Methyl Ethyl } \\
\text { Ketore }\end{array}$ & $000078-93.3$ & & & $4.4 E+05$ & 4.7E+04 & $4.4 E+04$ & $4.7 \mathrm{E}+03$ & & & $6.6 \mathrm{E}+05$ & $6.6 \mathrm{E}+04$ & & & $9.2 \mathrm{E}+03$ & $2.6 E+03$ & & & $9.2 \mathrm{E}+03$ & $2.4 E+03$ \\
\hline $\begin{array}{l}\text { Methyl Isobu. } \\
\text { tyl Ketone }\end{array}$ & $000108-10-1$ & & & $5.8 E+04$ & $6.3 \mathrm{E}+03$ & $5.8 E+03$ & $6.3 \mathrm{E}+02$ & & & $8.8 \mathrm{E}+04$ & $8.8 \mathrm{E}+03$ & & & $7.0 \mathrm{E}+02$ & $5.4 \mathrm{E}+02$ & & & $7.0 E+02$ & $4.7 E+02$ \\
\hline $\begin{array}{l}\text { Methyl Mer- } \\
\text { cury }\end{array}$ & $022967-92-6$ & & & $7.3 E+01$ & $7.8 E+\infty 0$ & $7.3 E+\infty$ & $7.8 \mathrm{E}-01$ & & & $1.2 \mathrm{E}+02$ & $1.2 \mathrm{E}+01$ & & & & & & & $4.6 \mathrm{E}+01$ & $4.6 \mathrm{E}+00$ \\
\hline $\begin{array}{l}\text { Methyl Meth- } \\
\text { acrylate . }\end{array}$ & $000080-62-6$ & & & $5.8 E+04$ & $6.3 \mathrm{E}+03$ & $5.8 \mathrm{E}+03$ & $6.3 \mathrm{E}+02$ & & & $8.8 \mathrm{E}+04$ & $8.8 \mathrm{E}+03$ & & & & & & & $3.5 \mathrm{E}+04$ & $3.5 \mathrm{E}+03$ \\
\hline $\begin{array}{l}\text { Methyl Parn. } \\
\text { thion }\end{array}$ & $000298-00-0$ & & & $1.8 \mathrm{E}+02$ & $2.0 \mathrm{E}+01$ & $1.8 \mathrm{E}+01$ & $2.0 \mathrm{E}+00$ & & & $1.7 E+02$ & $1.7 \mathrm{E}+01$ & & & & & & & $8.9 E+01$ & $8.9 E+\infty 0$ \\
\hline $\begin{array}{l}\text { Methyl Sty- } \\
\text { rene (Mixed } \\
\text { isomers) }\end{array}$ & $025013-15-4$ & & & $4.4 E+03$ & $4.7 \mathrm{E}+02$ & $4.4 \mathrm{E}+02$ & $4.7 \mathrm{E}+01$ & & & $6.6 \mathrm{E}+03$ & $6.6 \mathrm{E}+02$ & & & $1.0 \mathrm{E}+06$ & $1.0 E+06$ & & & $2.6 \mathrm{E}+03$ & $2.6 \mathrm{E}+02$ \\
\hline $\begin{array}{l}\text { Methyl hydra- } \\
\text { zine }\end{array}$ & $000060-34-4$ & $5.8 E+01$ & $5.8 \mathrm{E}-01$ & & & & & 1. $.5 E+02$ & $1.5 E+\infty 0$ & & & & & & & 7.5E+01 & $7.5 \mathrm{E}-01$ & & \\
\hline
\end{tabular}


Table 3.1c. (continued)

\begin{tabular}{|c|c|c|c|c|c|c|c|c|c|c|c|c|c|c|c|c|c|c|c|}
\hline \multirow{2}{*}{ Chemical } & \multirow{2}{*}{ CASRN } & \multicolumn{6}{|c|}{ Ingestion (mg/kg) } & \multicolumn{4}{|c|}{ Dermal (mg/kg) } & \multicolumn{4}{|c|}{ Inhalation $(\mathbf{m g} / \mathbf{k g})^{-v}$} & \multicolumn{4}{|c|}{$\begin{aligned} & \text { Ingestion + Dermal } \\
+ & \text { Inhalation (mg/kg) }\end{aligned}$} \\
\hline & & $10^{-4}$ & $10^{4}$ & $\begin{array}{l}\mathrm{HQ}=1 \\
\text { Adult }\end{array}$ & $\begin{array}{l}\text { HQ }=1 \\
\text { Child }\end{array}$ & $\begin{array}{c}\text { HQ }=0.1 \\
\text { Adult }\end{array}$ & $\begin{array}{c}\mathrm{HQ}=0.1 \\
\text { Child }\end{array}$ & $10^{-4}$ & $10^{6}$ & $H Q=1$. & $\mathrm{HQ}=0.1$ & $10^{-4}$ & $10^{4}$ & $\mathbf{H Q}=1$ & $H Q=0.1$ & $10^{-4}$ & $10^{6}$ & $\mathrm{HI}=1$ & $\mathrm{HI}=0.1$ \\
\hline $\begin{array}{l}\text { Methyl tert- } \\
\text { Butyl Ether } \\
\text { (MTBE) }\end{array}$ & $001634.04-4$ & & & & & & & & & & & & & $1.0 \mathrm{E}+06$ & $1.0 \mathrm{E}+06$ & & & & \\
\hline $\begin{array}{l}\text { Methyl-5- } \\
\text { Nitroaniline, } \\
2-\end{array}$ & $000099-55.8$ & $1.9 \mathrm{E}+03$ & $1.9 \mathrm{E}+01$ & & & & & $4.9 \mathrm{E}+03$ & $4.9 \mathrm{E}+01$ & & & & & & & $2.5 \mathrm{E}+03$ & $2.5 E+01$ & & \\
\hline $\begin{array}{l}\text { Methylaniline } \\
\text { Hydrochloride, } \\
\text { 2. }\end{array}$ & $000636-21-5$ & $3.5 \mathrm{E}+02$ & $3.5 \mathrm{E}+00$ & & & & & $8.9 E+02$ & $8.9 E+00$ & & & & & & & $4.6 \mathrm{E}+02$ & $4.6 \mathrm{E}+00$ & & \\
\hline $\begin{array}{l}\text { Methylcyclo- } \\
\text { hexane }\end{array}$ & $000108.87-2$ & & & & & & & & & & & & & $2.4 \mathrm{E}+02$ & $2.4 \mathrm{E}+02$ & & & & \\
\hline $\begin{array}{l}\text { Methylene } \\
\text { Chloride }\end{array}$ & $000075-09-2$ & $8.5 E+03$ & $8.5 E+01$ & $4,4 E+04$ & $4.7 \mathrm{E}+03$ & $4.4 \mathrm{E}+03$ & $4.7 \mathrm{E}+02$ & $4.1 \mathrm{E}+04$ & 4.1E+02 & $7.9 \mathrm{E}+04$ & $7.9 E+03$ & $1.0 E+03$ & $4.8 \mathrm{E}+0 !$ & $1.0 \mathrm{E}+03$ & $1.0 \mathrm{E}+03$ & $1.0 \mathrm{E}+03$ & $3.6 \mathrm{E}+01$ & $1.0 \mathrm{E}+03$ & $1.0 E+03$ \\
\hline $\begin{array}{l}\text { Methylene- } \\
\text { bis(2- } \\
\text { chloroaniline), } \\
4,4^{\prime-}\end{array}$ & $000101-14-4$ & $4.9 E+02$ & $4.9 \mathrm{E}+00$ & $5.1 E+02$ & S.5E+01 & $5.1 \mathrm{E}+01$ & $5.5 \mathrm{E}+\infty 0$ & $1.2 \mathrm{E}+03$ & $1.2 \mathrm{E}+0 \mathrm{I}$ & $4.8 \mathrm{E}+02$ & $4.8 \mathrm{E}+0 \mathrm{t}$ & $1.0 \mathrm{E}+06$ & $2.8 \mathrm{E}+05$ & & & $6.4 \mathrm{E}+02$ & $6.4 \mathrm{E}+00$ & $2,5 \mathrm{E}+02$ & $2.5 E+01$ \\
\hline $\begin{array}{l}\text { Methylene- } \\
\text { bis(Ni,N-di- } \\
\text { methyl) Ani- } \\
\text { line, 4,4-- }\end{array}$ & $000101-61-1$ & $1.4 \mathrm{E}+03$ & $1.4 \mathrm{E}+01$ & & & & & $3.5 \mathrm{E}+03$ & $3.5 E+01$ & & & & & & & $1.8 \mathrm{E}+03$ & $1.8 \mathrm{E}+01$ & & \\
\hline $\begin{array}{l}\text { Methylenebis- } \\
\text { benzenamine, } \\
4,4^{\prime}(-)\end{array}$ & $000101-77-9$ & $2.6 \mathrm{E}+02$ & $2.6 \mathrm{E}+00$ & & & & & $6.4 \mathrm{E}+02$ & $6.4 \mathrm{E}+00$ & & & & & & & $3.3 \mathrm{E}+02$ & $3.3 \mathrm{E}+00$ & & \\
\hline $\begin{array}{l}\text { Methylenedi- } \\
\text { phenyl } \\
\text { Isocyanate, } \\
\text { 4,4- }\end{array}$ & $000101-68-8$ & & & & & & & & & & & & & $8.9 E+04$ & $8.9 E+03$ & & & & \\
\hline $\begin{array}{l}\text { Methylstyrene, } \\
\text { Alphs- }\end{array}$ & $000098-83-9$ & & & $5.1 E+04$ & $5.5 \mathrm{E}+03$ & $5.1 E+03$ & $5.5 \mathrm{E}+02$ & & & $7.7 E+04$ & $7.7 E+03$ & & & & & & & $3.1 E+04$ & $3.1 E+03$ \\
\hline $\begin{array}{l}\text { Methyltriethyl } \\
\text { Lead }\end{array}$ & $001762-28-3$ & & & & & & & & & & & & & & & & & & \\
\hline Metolachlor & $051218-45-2$ & & & $1.1 \mathrm{E}+05$ & $1.2 \mathrm{E}+04$ & $1.1 E+04$ & $1.2 \mathrm{E}+03$ & & & $1.0 \mathrm{E}+0 \mathrm{~s}$ & $1.0 \mathrm{E}+04$ & & & & & & & $5.3 \mathrm{E}+04$ & $5.3 E+03$ \\
\hline Metribuzining) & $021087-64-9$ & & & $1.8 \mathrm{E}+04$ & $2.0 \mathrm{E}+03$ & $1.8 \mathrm{E}+03$ & $2.0 E+02$ & & & $1.7 \mathrm{E}+04$ & $1.7 E+03$ & & & & & & & $8.9 E+03$ & $8.9 E+02$ \\
\hline
\end{tabular}


Table 3.1c. (continued)

\begin{tabular}{|c|c|c|c|c|c|c|c|c|c|c|c|c|c|c|c|c|c|c|c|}
\hline \multirow{2}{*}{ Chemical } & \multirow{2}{*}{ CASRN } & \multicolumn{6}{|c|}{ Ingestion (mg/kg) } & \multicolumn{4}{|c|}{ Dermal (mg/kg) } & \multicolumn{4}{|c|}{ Inhalation (mg/kg) } & \multicolumn{4}{|c|}{$\begin{aligned} & \text { Ingestion + Dermal } \\
&+ \text { Inhalation }(\mathrm{mg} / \mathrm{kg})^{1 \cdot v}\end{aligned}$} \\
\hline & & $10^{-4}$ & $10^{6}$ & $\begin{array}{l}\text { HQ=1 } \\
\text { Adult }\end{array}$ & $\begin{array}{l}\mathrm{HQ}=1 \\
\text { Child }\end{array}$ & $\begin{array}{c}\mathrm{HQ}=0.1 \\
\text { Adult }\end{array}$ & $\begin{array}{c}H Q=0.1 \\
\text { Child }\end{array}$ & $10^{-4}$ & $10^{4}$ & $H Q=1$ & $\mathrm{HQ}=0.1$ & $10^{-4}$ & $10^{4}$ & $H Q=1$ & $\mathrm{HQ}=0.1$ & $10^{-4}$ & $10^{4}$ & $H I=1$ & $\mathrm{HI}=0.1$ \\
\hline Mirex & $002385-85-5$ & 3.5E+01 & $3.5 \mathrm{E}-01$ & $1.5 E+02$ & $1.6 \mathrm{E}+01$ & $1.5 \mathrm{E}+01$ & $1.6 \mathrm{E}+\infty$ & $8.9 \mathrm{E}+01$ & $8.9 \mathrm{E}-01$ & $1.4 E+02$ & $1.4 E+01$ & & & & & $4.6 \mathrm{E}+01$ & $4,6 \mathrm{E}-01$ & $7.1 \mathrm{E}+01$ & $7.1 E+\infty 00$ \\
\hline Molinate & $002212-67.1$ & & & $1.5 \mathrm{E}+03$ & $1.6 \mathrm{E}+02$ & $1.5 E+02$ & $1.6 \mathrm{E}+01$ & & & $1.4 E+03$ & $1.4 \mathrm{E}+02$ & & & & & & & $7.1 E+02$ & $7.1 E+01$ \\
\hline Molybdenum & $007439-98-7$ & & & $3.7 E+03$ & $3.9 \mathrm{E}+02$ & $3.7 \mathrm{E}+02$ & $3.9 \mathrm{E}+01$ & & & $2.6 \mathrm{E}+04$ & $2.6 \mathrm{E}+03$ & & & & & & & $3.2 \mathrm{E}+03$ & $3.2 E+02$ \\
\hline $\begin{array}{l}\text { Monochlor. } \\
\text { amine }\end{array}$ & $010599-90-3$ & & & 7.3E+04 & $7.8 \mathrm{E}+03$ & $7.3 \mathrm{E}+03$ & $7.8 \mathrm{E}+02$ & & & $6.9 \mathrm{E}+04$ & $6.9 \mathrm{E}+03$ & & & & & & & $3.5 \mathrm{E}+04$ & $3.5 \mathrm{E}+03$ \\
\hline $\begin{array}{l}\text { Monochloro- } \\
\text { butanes }\end{array}$ & $025154-42-1$ & & & $2.9 E+05$ & $3.1 E+04$ & $2.9 E+04$ & $3.1 \mathrm{E}+03$ & & & $2.8 \mathrm{E}+05$ & $2.8 \mathrm{E}+04$ & & & & & & & $1.4 \mathrm{E}+05$ & $1.4 \mathrm{E}+04$ \\
\hline Naled & $000300-76.5$ & & & $1.5 E+03$ & $1.6 \mathrm{E}+02$ & $1.5 \mathrm{E}+02$ & $1.6 \mathrm{E}+01$ & & & $1.4 E+03$ & $1.4 \mathrm{E}+02$ & & & & & & & $7.1 E+02$ & $7.1 E+01$ \\
\hline Naphthalene $e^{(0)}$ & $000091-20-3$ & & & $2.6 \mathrm{E}+04$ & $2.8 \mathrm{E}+03$ & $2,6 \mathrm{E}+03$ & $2.8 \mathrm{E}+02$ & & & $3.9 \mathrm{E}+04$ & $3.9 \mathrm{E}+03$ & & & & & & & $1.6 \mathrm{E}+04$ & $1.6 \mathrm{E}+03$ \\
\hline $\begin{array}{l}\text { Naphthalene, } \\
\text { 1-Methyl }\end{array}$ & $000090-12.0$ & & & & & & & & & & & & & & & & & & \\
\hline $\begin{array}{l}\text { Naphthalene, } \\
\text { 2-Methyl }\end{array}$ & 000091.57 .6 & & & & & & & & & & & & & & & & & & \\
\hline Napropanide & 015299-99-7 & & & $7.3 \mathrm{E}+04$ & $7.8 \mathrm{E}+03$ & 7.3E+03 & $7.8 \mathrm{E}+02$ & & & $6.9 E+04$ & $6.9 \mathrm{E}+03$ & & & & & & & $3.5 \mathrm{E}+04$ & $3.5 \mathrm{E}+03$ \\
\hline $\begin{array}{l}\text { Niagara Blue } \\
4 \mathrm{~B}\end{array}$ & $002429-74-5$ & & & & & & & & & & & & & & & & & & \\
\hline $\begin{array}{l}\text { Nickel Car- } \\
\text { bonyl }\end{array}$ & $013463-39-3$ & & & & & & & & & & & & & & & & & & \\
\hline $\begin{array}{l}\text { Nickel Refin- } \\
\text { ery Dust }\end{array}$ & NA & & & & & & & & & & & $1.0 \mathrm{E}+08$ & $4.3 \mathrm{E}+04$ & & & & & & \\
\hline $\begin{array}{l}\text { Nickel Soluble } \\
\text { Salts }\end{array}$ & $0.07440-02.0$ & & & 1.5E+04 & $1.6 \mathrm{E}+03$ & $1.5 \mathrm{E}+03$ & $1.6 \mathrm{E}+02$ & & & 7.4E+04 & $7.4 \mathrm{E}+03$ & & & & & & & $1.2 E+04$ & $1.2 \mathrm{E}+03$ \\
\hline $\begin{array}{l}\text { Nickel } \\
\text { Subsulfide }\end{array}$ & $012035-72-2$ & & & & & & & & & & & $1.0 E+06$ & 2.1E+04 & & & & & & \\
\hline Nitrapyrin & $001929-82-4$ & & & $1.1 E+03$ & $1.2 \mathrm{E}+02$ & $1.1 \mathrm{E}+02$ & $1.2 \mathrm{E}+0 !$ & & & $1.0 \mathrm{E}+03$ & $1.0 \mathrm{E}+02$ & & & & & & & $5.3 E+02$ & $5.3 E+01$ \\
\hline Nitrate & $014797-55.8$ & & & $1.0 E+06$ & $1.3 E+05$ & $1.2 \mathrm{E}+05$ & $1.3 E+04$ & & & $1.0 \mathrm{E}+06$ & $1.0 \mathrm{E}+06$ & & & & & & & $1.0 E+06$ & $1.1 E+05$ \\
\hline Nitric Oxide() & $010102-43-9$ & & & 7,3E+04 & $7.8 \mathrm{E}+03$ & $7,3 E+03$ & $7.8 \mathrm{E}+02$ & & & $2.8 \mathrm{E}+05$ & $2.8 \mathrm{E}+04$ & & & & & & & $5.8 E+04$ & $5.8 \mathrm{E}+03$ \\
\hline
\end{tabular}


Table 3.1c. (continued)

\begin{tabular}{|c|c|c|c|c|c|c|c|c|c|c|c|c|c|c|c|c|c|c|c|}
\hline \multirow{2}{*}{ Chemical } & \multirow{2}{*}{ CASRN } & \multicolumn{6}{|c|}{ Ingestion (mg/kg) } & \multicolumn{4}{|c|}{ Dermal (mg/kg)" } & \multicolumn{4}{|c|}{ Inhalation (mg/kg) } & \multicolumn{4}{|c|}{$\begin{array}{l}\text { Ingestion + Dermal } \\
+ \text { Inhalation }(\mathrm{mg} / \mathrm{kg})^{1 \cdot \gamma}\end{array}$} \\
\hline & & $10^{-4}$ & $10^{6}$ & $\begin{array}{l}\text { HQ=1 } \\
\text { Adult }\end{array}$ & $\begin{array}{l}\text { HQ }=1 \\
\text { Child }\end{array}$ & $\begin{array}{c}\text { HQ }=0.1 \\
\text { Adult }\end{array}$ & $\begin{array}{l}\text { HQ }=0.1 \\
\text { Child }\end{array}$ & $10^{-4}$ & $10^{4}$ & $H Q=1$ & $\mathrm{HQ}=0.1$ & $10^{-4}$ & $10^{6}$ & $H Q=1$ & $\mathrm{HQ}=0.1$ & $10^{-4}$ & $10^{6}$ & $H I=1$ & $\mathrm{HI}=0.1$ \\
\hline Nitrite & 014797.65 .0 & & & $7.3 E+04$ & $7.8 \mathrm{E}+03$ & $7.3 \mathrm{E}+03$ & $7.8 E+02$ & & & $6.9 \mathrm{E}+05$ & $6.9 E+04$ & & & & & & & $6.6 \mathrm{E}+04$ & $6.6 \mathrm{E}+03$ \\
\hline $\begin{array}{l}\text { Nitroaniline, } \\
2-\end{array}$ & $000088-74-4$ & & & & & . & . & & & & & & & $1.5 \mathrm{E}+01$ & $1.5 \mathrm{E}+00$ & & & & \\
\hline Nitrobenzene & $000098-95-3$ & & & $3.7 \mathrm{E}+02$ & $3.9 \mathrm{E}+01$ & $3.7 E+01$ & $3.9 \mathrm{E}+00$ & & & $6.7 \mathrm{E}+02$ & $6.7 \mathrm{E}+01$ & & & $8.6 \mathrm{E}+\infty$ & $8.6 \mathrm{E}-01$ & & & $8.3 \mathrm{E}+00$ & $8,3 \mathrm{E}-01$ \\
\hline Nitrofurantoin & $000067-20-9$ & & & $5.1 \mathrm{E}+04$ & $5.5 E+03$ & $5.1 E+03$ & $5.5 E+02$ & & & $4.8 \mathrm{E}+04$ & $4.8 \mathrm{E}+03$ & & & & & & & $2.5 \mathrm{E}+04$ & $2.5 \mathrm{E}+03$ \\
\hline Nitrofurazone & 000059.87 .0 & 4.3E+01 & $4.3 \mathrm{E}-01$ & & & & & $1.1 \mathrm{E}+02$ & $1.1 \mathrm{E}+\infty$ & & & & & & & $5.5 E+01$ & 5.SE-01 & & \\
\hline $\begin{array}{l}\text { Nitrogen Di- } \\
\text { oxide } e^{(3)}\end{array}$ & $010102-44-0$ & & & $7.3 \mathrm{E}+05$ & $7.8 \mathrm{E}+04$ & $7.3 \mathrm{E}+04$ & $7.8 \mathrm{E}+03$ & & & $1.0 \mathrm{E}+06$ & $1.0 \mathrm{E}+06$ & & & & & & & $6.8 \mathrm{E}+05$ & $6.8 \mathrm{E}+04$ \\
\hline Nitroguaridine & $000556-88-7$ & & & 7.3E+04 & $7.8 \mathrm{E}+03$ & $7.3 \mathrm{E}+03$ & $7.8 \mathrm{E}+02$ & & & $6.9 E+04$ & $6.9 E+03$ & & & & & & & $3.5 E+04$ & $3.5 \mathrm{E}+03$ \\
\hline $\begin{array}{l}\text { Nitrophenol, } \\
\text { 4- }\end{array}$ & $000100-02-7$ & & & & & & & & & & & & & & & & & & \\
\hline $\begin{array}{l}\text { Nitropropane, } \\
2_{-(3)}\end{array}$ & $000079-46-9$ & $6.7 E+00$ & $6.7 \mathrm{E}-02$ & & & & & 2.7E+01 & $2.7 E-01$ & & & $8.4 \mathrm{E}+00$ & $8.4 \mathrm{E}-02$ & $1.9 E+03$ & $1.9 E+02$ & 4.7E+00 & 4.7E-02 & & \\
\hline $\begin{array}{l}\text { Nitroso-N- } \\
\text { ethylures, N- }\end{array}$ & 000759.73 .9 & $4.6 \mathrm{E}-01$ & $4.6 \mathrm{E}-03$ & & & & & $1.1 E+\infty$ & $1.1 \mathrm{E}-02$ & & & & & & & $5.9 \mathrm{E}-01$ & S.9E.03 & & \\
\hline $\begin{array}{l}\text { Nitroso-N- } \\
\text { methylures, N. }\end{array}$ & $000684-93-5$ & & & & & & & & & & & & & & & & & & \\
\hline $\begin{array}{l}\text { Nitroso-di-N. } \\
\text { butylamine, N- }\end{array}$ & $000924-16-3$ & $1.2 \mathrm{E}+01$ & $1.2 \mathrm{E}-01$ & & & & & $4.8 \mathrm{E}+01$ & $4.8 \mathrm{E}-01$ & & & $6.1 \mathrm{E}+0 !$ & 6.IE-01 & & & $1.4 \mathrm{E}+01$ & $1.4 \mathrm{E}-01$ & & \\
\hline $\begin{array}{l}\text { Nitroso-di-N- } \\
\text { propylamine, } \\
\text { N- }\end{array}$ & 000621.64 .7 & $9.1 \mathrm{E}+\infty$ & $9.1 \mathrm{E}-02$ & & & & & $1.1 E+01$ & $1.1 \mathrm{E}-01$ & & & & & & & $7.8 \mathrm{E}+00$ & $7.8 \mathrm{E}-02$ & & \\
\hline $\begin{array}{l}\text { Nitrosodi-eth- } \\
\text { anolamine, N- }\end{array}$ & $001116-54-7$ & $2.3 \mathrm{E}+01$ & $2.3 \mathrm{E}-01$ & & & & & $5.7 \mathrm{E}+01$ & $5.7 \mathrm{E}-01$ & & & & & & & $3.0 \mathrm{E}+01$ & $3,0 \mathrm{E}-01$ & & \\
\hline $\begin{array}{l}\text { Nitrosodi- } \\
\text { ethylamine, N- }\end{array}$ & $000055-18-5$ & $4.3 \mathrm{E}-01$ & 4.3E-03 & & & & & $1.1 \mathrm{E}+\infty$ & $1.1 \mathrm{E}-02$ & & & $2.4 E+04$ & $2.4 \mathrm{E}+02$ & & & $5.5 \mathrm{E}-01$ & $5.5 \dot{\mathrm{E}}-03$ & & \\
\hline $\begin{array}{l}\text { Nitrosodi-me- } \\
\text { thylamine, N- }\end{array}$ & $000062-75.9$ & $1.3 \mathrm{E}+00$ & $1.3 \mathrm{E}-02$ & & & & & $3.2 \mathrm{E}+00$ & $3.2 \mathrm{E}-02$ & & & $7.2 E+04$ & $7.2 \mathrm{E}+02$ & & & $1.6 \mathrm{E}+00$ & $1.6 \mathrm{E}-02$ & & \\
\hline
\end{tabular}


Table 3.1c. (continued)

\begin{tabular}{|c|c|c|c|c|c|c|c|c|c|c|c|c|c|c|c|c|c|c|c|}
\hline \multirow{2}{*}{ Chemical } & \multirow{2}{*}{ CASRN } & \multicolumn{6}{|c|}{ Ingestion (mg/kg) } & \multicolumn{4}{|c|}{ Dermal (mg/kg) } & \multicolumn{4}{|c|}{ Inhalation (mg/kg) } & \multicolumn{4}{|c|}{$\begin{aligned} & \text { Ingestion + Dermal } \\
&+ \text { Inhalation }(\mathrm{mg} / \mathrm{kg})^{\bullet \cdot v}\end{aligned}$} \\
\hline & & $10^{-4}$ & $10^{6}$ & $\begin{array}{l}H Q=1 \\
\text { Adult }\end{array}$ & $\begin{array}{l}\mathrm{HQ}=1 \\
\text { Child }\end{array}$ & $\begin{array}{c}H Q=0.1 \\
\text { Adult }\end{array}$ & $\begin{array}{c}\mathrm{HQ}=0.1 \\
\text { Child }\end{array}$ & $10^{-4}$ & $10^{4}$ & $\mathrm{HQ}=1$ & $H Q=0.1$ & $10^{-4}$ & $10^{4}$ & $H Q=1$ & $\mathrm{HQ}=0.1$ & $10^{+}$ & $10^{6}$ & $H I=1$ & $H I=0.1$ \\
\hline $\begin{array}{l}\text { Nitrosodi- } \\
\text { phenylamine, } \\
\text { N- }\end{array}$ & $000086-30-6$ & $1.3 \mathrm{E}+04$ & $1.3 \mathrm{E}+02$ & & & & & $1.6 \mathrm{E}+04$ & $1.6 \mathrm{E}+02$ & & & & & & & $1.1 E+04$ & $1.1 \mathrm{E}+02$ & & \\
\hline $\begin{array}{l}\text { Nitrosomethyl } \\
\text { ethylamine, } N \text {. }\end{array}$ & $010595-95-6$ & $2.9 \mathrm{E}+00$ & $2.9 E-02$ & & & & & $7.3 \mathrm{E}+00$ & 7.3E-02 & & & & & & & $3.8 \mathrm{E}+00$ & $3.8 \mathrm{E}-02$ & & \\
\hline $\begin{array}{l}\text { Nitrosomethyl } \\
\text { vinylamine, N- }\end{array}$ & $004549-40-0$ & & & & & & & & & & & & & & & & & & \\
\hline $\begin{array}{l}\text { Nitrosopyr- } \\
\text { rolldine, N. }\end{array}$ & $000930-55-2$ & $3.0 \mathrm{E}+01$ & $3.0 \mathrm{E}-01$ & & & & & $7.7 \mathrm{E}+01$ & 7.7E-01 & & & $1.0 \mathrm{E}+06$ & $1.7 \mathrm{E}+04$ & & & $3.9 \mathrm{E}+01$ & $3.9 \mathrm{E}-01$ & & \\
\hline $\begin{array}{l}\text { Nitrololuene, } \\
\text { 4-Amino-2. }\end{array}$ & $000119.32-4$ & & & & & & & & & & & & & & & & & & \\
\hline $\begin{array}{l}\text { Nitrotoluene, } \\
\text { m- }\end{array}$ & $000099-08-1$ & & & $7.3 \mathrm{E}+03$ & $7.8 E+02$ & $7.3 \mathrm{E}+02$ & $7.8 E+01$ & & & $1.1 E+04$ & $1.1 \mathrm{E}+03$ & & & & & & & $4.4 E+03$ & $4.4 \mathrm{E}+02$ \\
\hline $\begin{array}{l}\text { Nitrotoluene, } \\
\text { o- }\end{array}$ & $000088-72-2$ & & & $7.3 E+03$ & $7.8 \mathrm{E}+02$ & $7.3 \mathrm{E}+02$ & $7.8 E+01$ & & & $1.1 E+04$ & $1.1 E+03$ & & & & & & & $4.4 E+03$ & $4.4 E+02$ \\
\hline $\begin{array}{l}\text { Nitrotoluene, } \\
\text { p- }\end{array}$ & $000099.99-0$ & & & $7.3 E+03$ & $7.8 \mathrm{E}+02$ & $7.3 \mathrm{E}+02$ & $7.8 \mathrm{E}+01$ & & & 1.tE+04 & $1.1 E+03$ & & & & & & & $4.4 \mathrm{E}+03$ & $4.4 \mathrm{E}+02$ \\
\hline Norflurazon & $027314-13-2$ & & & $2.9 E+04$ & $3.1 \mathrm{E}+03$ & $2.9 E+03$ & $3.1 \mathrm{E}+02$ & & & $2.8 \mathrm{E}+04$ & $2.8 \mathrm{E}+03$ & & & & & & & $1.4 E+04$ & $1.4 E+03$ \\
\hline Nustar & $085509-19-9$ & & & $5.1 E+02$ & $5.5 \mathrm{E}+01$ & S.1E+01 & $5.5 E+00$ & & & $4.8 \mathrm{E}+02$ & $4.8 \mathrm{E}+01$ & & & & & & & $2.5 E+02$ & $2.5 E+01$ \\
\hline $\mathrm{OCDD}^{(4)}$ & $003268-87.9$ & $4.3 \mathrm{E}-01$ & $4.3 \mathrm{E}-03$ & & & & & 3.6E-01 & $3.6 \mathrm{E}-03$ & & & $2.4 E+04$ & $2.4 E+02$ & & & $2.7 \mathrm{E}-0 !$ & 2.7E-03 & & \\
\hline$O C D F^{(\prime)}$ & $039001-02-0$ & $4.3 \mathrm{E}-01$ & $4.3 E-03$ & & & & & $1.1 \mathrm{E}+00$ & 1.1E-02 & & & $2.4 E+04$ & $2.4 \mathrm{E}+02$ & & & $5.5 E-01$ & $5.5 \mathrm{E}-03$ & & \\
\hline $\begin{array}{l}\text { Octibromo. } \\
\text { diphenyl Ether }\end{array}$ & $032536-52.0$ & . & & $2.2 \mathrm{E}+03$ & $2.3 E+02$ & $2.2 E+02$ & $2.3 E+01$ & & & $2.1 E+03$ & 2.IE+02 & & & & & & & 1.1E+03 & $1.1 E+02$ \\
\hline $\begin{array}{l}\text { Octahydro- } \\
1,3,5,7- \\
\text { tetranitro- } \\
1,3,5,7 \text {-tetra } \\
\text { (HMX) }\end{array}$ & $002691-41-0$ & & & $3.7 E+04$ & $3.9 E+03$ & $3.7 E+03$ & $3.9 E+02$ & & & $1.0 E+04$ & $1.0 \mathrm{E}+03$ & & & & & & & $8.1 \mathrm{E}+03$ & $8.1 E+02$ \\
\hline $\begin{array}{l}\text { Octamethyl. } \\
\text { pyrophospho. } \\
\text { ramide }\end{array}$ & $000152-16.9$ & & & $1.5 E+03$ & $1.6 \mathrm{E}+02$ & $1.5 \mathrm{E}+02$ & $1.6 \mathrm{E}+01$ & & & $1.4 \mathrm{E}+03$ & $1.4 E+02$ & & & & & & & $7.1 E+02$ & 7.1E+01 \\
\hline
\end{tabular}


Table 3.1c. (continued)

\begin{tabular}{|c|c|c|c|c|c|c|c|c|c|c|c|c|c|c|c|c|c|c|c|}
\hline \multirow{2}{*}{ Chemical } & \multirow{2}{*}{ CASRN } & \multicolumn{6}{|c|}{ Ingestion (mg/kg) } & \multicolumn{4}{|c|}{ Dermal (mg/kg) } & \multicolumn{4}{|c|}{ Inhalation (mg/kg) } & \multicolumn{4}{|c|}{$\begin{array}{c}\text { Ingestion + Dermal } \\
\text { + Inhalation }(\mathrm{mg} / \mathrm{kg})^{\mid \cdot *}\end{array}$} \\
\hline & & $10^{-4}$ & $10^{5}$ & $\begin{array}{l}\mathrm{HQ}=1 \\
\text { Adult }\end{array}$ & $\begin{array}{l}\mathrm{HQ}=1 \\
\text { Child }\end{array}$ & $\begin{array}{c}\mathrm{HQ}=0.1 \\
\text { Adult }\end{array}$ & $\begin{array}{c}\mathrm{HQ}=0.1 \\
\text { Child }\end{array}$ & $10^{-4}$ & $10^{4}$ & $\mathrm{HQ}=1$ & $\mathrm{HQ}=0.1$ & $10^{-4}$ & $10^{4}$ & $H Q=1$ & $\mathrm{HQ}=0.1$ & $10^{-4}$ & $10^{4}$ & HI $=1$ & $\mathrm{HI}=0.1$ \\
\hline $\begin{array}{l}\text { Octy Phthal- } \\
\text { ate, di-N- }\end{array}$ & $000117-84-0$ & & & $1,5 \mathrm{E}+04$ & $1.6 \mathrm{E}+03$ & $1.5 \mathrm{E}+03$ & $1.6 \mathrm{E}+02$ & & & $2.5 E+04$ & $2.5 \mathrm{E}+03$ & & & & & & & $9.2 E+03$ & $9.2 \mathrm{E}+02$ \\
\hline Oryzalin & $019044-88-3$ & & & $3.7 \mathrm{E}+04$ & $3.9 E+03$ & $3.7 \mathrm{E}+03$ & $3.9 E+02$ & & & $3.4 \mathrm{E}+04$ & $3.4 \mathrm{E}+03$ & & & & & & & $1.8 \mathrm{E}+04$ & $1.8 E+03$ \\
\hline Oxadiazon & $019666-30-9$ & & & $3.7 E+03$ & $3.9 \mathrm{E}+02$ & $3.7 \mathrm{E}+02$ & $3.9 E+01$ & & & $3.4 E+03$ & $3.4 \mathrm{E}+02$ & & & & & & & $1.8 \mathrm{E}+03$ & $1.8 \mathrm{E}+02$ \\
\hline Oxamyl & $023135-22-0$ & & & $1.8 \mathrm{E}+04$ & $2.0 \mathrm{E}+03$ & $1.8 \mathrm{E}+03$ & $2.0 E+02$ & & & $1.7 \mathrm{E}+04$ & $1.7 \mathrm{E}+03$ & & & & & & & $8.9 E+03$ & $8.9 E+02$ \\
\hline Paclobutrazol & $0767388-62.0$ & & & $9.5 \mathrm{E}+03$ & $1.0 E+03$ & $9.5 E+02$ & $1.0 E+02$ & & & $9.0 E+03$ & $9.0 E+02$ & & & & & & & $4.6 \mathrm{E}+03$ & $4.6 \mathrm{E}+02$ \\
\hline Paraquat & $001910-42-5$ & & & $3.3 E+03$ & $3.5 \mathrm{E}+02$ & $3.3 \mathrm{E}+02$ & $3.5 \mathrm{E}+01$ & & & $3.1 \mathrm{E}+03$ & 3.IE+02 & & & & & & & $1.6 \mathrm{E}+03$ & $1.6 \mathrm{E}+02$ \\
\hline Parathion & $000056-38-2$ & & & $4.4 E+03$ & $4.7 E+02$ & $4.4 E+02$ & $4.7 E+01$ & & & $4.1 E+03$ & $4.1 \mathrm{E}+02$ & & & & & & & $2.1 E+03$ & $2.1 E+02$ \\
\hline $\begin{array}{l}P_{2,3,7,8.14} \\
\text { PeCDD }\end{array}$ & $036088-22-9$ & $8.5 E-04$ & 8.5E-06 & & & & & 7.1E-04 & 7.1E- -06 & & & $4.9 \mathrm{E}+01$ & 4.9E-01 & & & $5.4 \mathrm{E}-04$ & $5.4 \mathrm{E}-06$ & & \\
\hline $\begin{array}{l}\mathrm{PeCDF}, \\
1,2,3,7,8(1)\end{array}$ & $057117-41-6$ & 8.5E-04 & $8.5 \mathrm{E}-06$ & & & & & 2.1E-03 & 2.1E-05 & & & $4.9 \mathrm{E}+01$ & $4.9 \mathrm{E}-01$ & & & $1.1 \mathrm{E}-03$ & t.1E-05 & & \\
\hline $\begin{array}{l}\text { PeCDF, } \\
\left.2,3,4,7,8_{-1}\right)\end{array}$ & 057117-31-4 & 8.5E-03 & 8.5E- 05 & & & & & 2.1E-02 & 2.IE-04 & & & $4.9 \mathrm{E}+02$ & $4.9 \mathrm{E}+\infty$ & & & 1.1EE-02 & 1.1E-04 & & \\
\hline Pebulate & $0011144-71-2$ & & & $3.7 \mathrm{E}+04$ & $3.9 E+03$ & $3.7 \mathrm{E}+03$ & $3.9 E+02$ & & & $3.4 E+04$ & $3.4 E+03$ & & & & & & & $1.8 \mathrm{E}+04$ & $1.8 \mathrm{E}+03$ \\
\hline Pendimethalin & $040487-42-1$ & & & $2.9 E+04$ & $3.1 \mathrm{E}+03$ & $2.9 E+03$ & $3.1 E+02$ & & & $2.8 \mathrm{E}+04$ & $2.8 E+03$ & & & & & & & $1.4 \mathrm{E}+04$ & $1.4 E+03$ \\
\hline $\begin{array}{l}\text { Penatabomo- } \\
\text { diphenyl Ether }\end{array}$ & 032534-81-9 & & & $1.5 \mathrm{E}+03$ & $1.6 \mathrm{E}+02$ & $1.5 E+02$ & $1.6 \mathrm{E}+01$ & & & $1.4 E+03$ & $1.4 E+02$ & & & & & & & $7.1 \mathrm{E}+02$ & $7.1 E+01$ \\
\hline 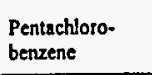 & $000608-93.5$ & & & $5.8 \mathrm{E}+02$ & $6.3 E+01$ & $5.8 E+01$ & $6.3 \mathrm{E}+00$ & & & $8.8 \mathrm{E}+02$ & $8.8 \mathrm{E}+01$ & & & & & & & $3.5 \mathrm{E}+02$ & 3.5E+01 \\
\hline $\begin{array}{l}\text { Pentachloro- } \\
\text { cyclopenta- } \\
\text { dienc }\end{array}$ & $025329-35$-5 & & & & & & & & & & & & & & & & & & \\
\hline 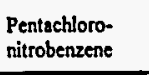 & $000082-68-8$ & $2.5 \mathrm{E}+02$ & $2.5 \mathrm{E}+00$ & $2.2 \mathrm{E}+03$ & $2.3 E+02$ & $2.2 E+02$ & $2.3 \mathrm{E}+01$ & $9.9 E+02$ & $9.9 E++0$ & $3.3 \mathrm{E}+03$ & 3.3E+02 & & & & & $3.9 E+02$ & $3.9 E+00$ & $1.3 \mathrm{E}+03$ & $1.3 \mathrm{E}+02$ \\
\hline $\begin{array}{l}\text { Pentuchloro- } \\
\text { phenol }\end{array}$ & $000087-86-5$ & $5.3 E+02$ & $5.3 \mathrm{E}+00$ & $22 \mathrm{E}+04$ & $2.3 \mathrm{E}+03$ & $2.2 \mathrm{E}+03$ & $2.3 \mathrm{E}+02$ & $2.7 \mathrm{E}+\overline{03}$ & $2.7 \mathrm{E}+01$ & $4.1 \mathrm{E}+04$ & 4.1E+03 & & & & & $9.3 \mathrm{E}+02$ & $9.3 \mathrm{E}+00$ & $1.4 E+04$ & 1.4E+03 \\
\hline $\begin{array}{l}\text { Pentyl Aleo- } \\
\text { hol, N. }\end{array}$ & $000071-41-0$ & & & & & & & & & & & & & & & & & & \\
\hline
\end{tabular}

Nonradionuclides/soil/residential

Page 30 of 41 
Table 3.1c. (continued)

\begin{tabular}{|c|c|c|c|c|c|c|c|c|c|c|c|c|c|c|c|c|c|c|c|}
\hline \multirow{2}{*}{ Chemical } & \multirow{2}{*}{ CASRN } & \multicolumn{6}{|c|}{ Ingestion (mg/kg) } & \multicolumn{4}{|c|}{ Dermal (mg/kg) } & \multicolumn{4}{|c|}{ Inhalation (mg/kg) } & \multicolumn{4}{|c|}{$\begin{array}{c}\text { Ingestion + Dermal } \\
+ \text { Inhalation (mg/kg) }\end{array}$} \\
\hline & & $10^{-4}$ & $10^{6}$ & $\begin{array}{l}\text { HQ=1 } \\
\text { Adult }\end{array}$ & $\begin{array}{l}\mathrm{HQ}=1 \\
\text { Child }\end{array}$ & $\begin{array}{c}H Q=0.1 \\
\text { Adult }\end{array}$ & $\begin{array}{l}\mathrm{HQ}=0.1 \\
\text { Child }\end{array}$ & $10^{-4}$ & $10^{4}$ & $H Q=1$ & $H Q=0.1$ & $10^{-1}$ & $10^{4}$ & HQ $=1$ & $\mathrm{HQ}=0.1$ & $10^{-4}$ & $10^{4}$ & $H I=1$ & $H I=0.1$ \\
\hline Permethrin & $052645-53.1$ & & & 3.7E+04 & $3.9 E+03$ & $3.7 E+03$ & $3.9 E+02$ & & & $3.4 E+04$ & $3.4 E+03$ & & & & & & & $1.8 \mathrm{E}+04$ & $1.8 \mathrm{E}+03$ \\
\hline Phenanthrene & $000085-01-8$ & & & & & & & & & & & & & & & & & & \\
\hline $\begin{array}{l}\text { Phenmedi- } \\
\text { pham }\end{array}$ & $013684-63-4$ & & & $1.8 \mathrm{E}+05$ & $2.0 \mathrm{E}+04$ & $1.8 \mathrm{E}+04$ & $2.0 E+03$ & & & $1.7 \mathrm{E}+05$ & $1.7 E+04$ & & & & & & & $8.9 E+04$ & $8.9 E+03$ \\
\hline Phenot & $000108-95-2$ & & & $4.4 \mathrm{E}+05$ & 4.7E+04 & 4.4E+04 & $4.7 \mathrm{E}+03$ & & & $7.4 \mathrm{E}+05$ & $7.4 \mathrm{E}+04$ & & & & & & & $2.8 \mathrm{E}+05$ & $2.8 E+04$ \\
\hline $\begin{array}{l}\text { Phenylenedi- } \\
\text { amine, m- }\end{array}$ & $000108-45-2$ & & & $4.4 E+03$ & $4.7 \mathrm{E}+02$ & $4.4 E+02$ & $4.7 E+01$ & & & 4.1E+03 & $4.1 E+02$ & & & & & & & $2.1 \mathrm{E}+03$ & $2.1 E+02$ \\
\hline $\begin{array}{l}\text { Phenylenedi- } \\
\text { amine, o- }\end{array}$ & $000095-54-5$ & $1.4 \mathrm{E}+03$ & $.1 .4 E+01$ & & & & & $3.4 \mathrm{E}+03$ & $3.4 E+01$ & & & & & & & $1.8 \mathrm{E}+03$ & $1,8 \mathrm{E}+0 \mathrm{t}$ & & \\
\hline $\begin{array}{l}\text { Phenyienedi- } \\
\text { amine, p- }\end{array}$ & $000106-50-3$ & & & $1.4 \mathrm{E}+05$ & $1.5 E+04$ & $1.4 E+04$ & $1.5 E+03$ & & & $1.3 \mathrm{E}+05$ & $1.3 \mathrm{E}+04$ & & & & & & & $6.7 E+04$ & $6.7 \mathrm{E}+03$ \\
\hline $\begin{array}{l}\text { Phenylmerctu- } \\
\text { ric Acetate }\end{array}$ & $000062-38-4$ & & & $5.8 \mathrm{E}+01$ & $6.3 \mathrm{E}+00$ & $5.8 \mathrm{E}+00$ & 6.3E-01 & & & S.SE+0I & $5.5 \mathrm{E}+00$ & & & & & & & $2.8 \mathrm{E}+01$ & $2,8 \mathrm{E}+\infty$ \\
\hline $\begin{array}{l}\text { Phenylphenol, } \\
2 .\end{array}$ & $000090-43.7$ & $3.3 E+04$ & $3.3 \mathrm{E}+02$ & & & & & $8.3 E+04$ & 8.3E+02 & & & & & & & $4.3 E+04$ & $4.3 E+02$ & & \\
\hline Phorate & $000298-02-2$ & & & $1.5 \mathrm{E}+02$ & $1.6 \mathrm{E}+01$ & $1.5 \mathrm{E}+01$ & $1.6 \mathrm{E}+\infty 0$ & & & $1.4 \mathrm{E}+02$ & $1.4 \mathrm{E}+01$ & & & & & & & $7.1 \mathrm{E}+01$ & $7.1 E+\infty 0$ \\
\hline Phosmet & $000732-11-6$ & & & $1.5 \mathrm{E}+0.4$ & $1.6 \mathrm{E}+03$ & $1.5 \mathrm{E}+03$ & $1.6 \mathrm{E}+02$ & & & $1.4 \mathrm{E}+04$ & $1.4 \mathrm{E}+03$ & & & & & & & $7.1 \mathrm{E}+03$ & 7. $1 \mathrm{E}+02$ \\
\hline Phosphine & $007803-51.2$ & & & $2.2 \mathrm{E}+02$ & 2.3E+01 & $2.2 \mathrm{E}+01$ & $2.3 \mathrm{E}+\infty 0$ & & & $8.3 \mathrm{E}+02$ & $8.3 E+01$ & & & $1.0 \mathrm{E}+06$ & $1.3 \mathrm{E}+05$ & & & $1.7 \mathrm{E}+02$ & $1.7 E+01$ \\
\hline $\begin{array}{l}\text { Phosphoric } \\
\text { Acid }\end{array}$ & $007664-38-2$ & & & & & & & & & & & & & $1.0 \mathrm{E}+06$ & $1.0 \mathrm{E}+06$ & & & & \\
\hline $\begin{array}{l}\text { Phthalic Acid, } \\
\text { P- }\end{array}$ & $000100-21-0$ & & & $7.3 E+05$ & $7.8 E+04$ & 7.3E+04 & $7.8 \mathrm{E}+03$ & & & $6.9 E+0 S$ & $6.9 E+04$ & & & & & & & $3.5 E+05$ & $3.5 E+04$ \\
\hline $\begin{array}{l}\text { Phthalic Anthy- } \\
\text { dride }\end{array}$ & $000085-44-9$ & & & $1.0 \mathrm{E}+06$ & $1.6 \mathrm{E}+05$ & $1.5 \mathrm{E}+05$ & $1.6 \mathrm{E}+04$ & & & $1.0 \mathrm{E}+06$ & $1.4 \mathrm{E}+05$ & & & $1.0 \mathrm{E}+06$ & $1.0 \mathrm{E}+06$ & & & $7.1 \mathrm{E}+05$ & $7.1 E+04$ \\
\hline Picloram & $001918-02-1$ & & & $5.1 \mathrm{E}+04$ & $5.5 \mathrm{E}+03$ & $5.1 \mathrm{E}+03$ & $5.5 \mathrm{E}+02$ & & & $4.8 E+04$ & $4.8 \mathrm{E}+03$ & & & & & & & $2.5 E+04$ & $25 E+03$ \\
\hline $\begin{array}{l}\text { Pirimiphos, } \\
\text { Methyl }\end{array}$ & $029232-93-7$ & & & $7.3 \mathrm{E}+03$ & $7.8 \mathrm{E}+02$ & $7.3 E+02$ & $7.8 E+01$ & & & $6.9 \mathrm{E}+03$ & $6.9 \mathrm{E}+02$ & & & & & & & $3.5 \mathrm{E}+03$ & $3.5 \mathrm{E}+02$ \\
\hline $\begin{array}{l}\text { Polybrominate } \\
\text { d Biphenyls }\end{array}$ & NA & $7.2 E+\infty 0$ & 7.2E-.02 & $5.1 E+00$ & 5.5E-01 & $5.1 \mathrm{E}-01$ & $5.5 \mathrm{E}-02$ & $3.4 E+01$ & $3.4 \mathrm{E}-01$ & $9.0 E+\infty 0$ & $9.0 \mathrm{E}-01$ & & & & & $1.2 \mathrm{E}+01$ & $1.2 \mathrm{E}-01$ & $3.3 E+\infty$ & 3.3E-01 \\
\hline
\end{tabular}

Nonradionuclides/soil/residential

Page 31 of 41 
Table 3.1c. (continued)

\begin{tabular}{|c|c|c|c|c|c|c|c|c|c|c|c|c|c|c|c|c|c|c|c|}
\hline \multirow{2}{*}{ Chemical } & \multirow{2}{*}{ CASRN } & \multicolumn{6}{|c|}{ Ingestion (mg/kg) } & \multicolumn{4}{|c|}{ Dermal (mg/kg) } & \multicolumn{4}{|c|}{ Inhalation (mg/kg) } & \multicolumn{4}{|c|}{$\begin{array}{c}\text { Ingestion + Dermal } \\
+ \text { Inhalation (mg/kg) }{ }^{+*} \\
\end{array}$} \\
\hline & & $10^{-1}$ & $10^{4}$ & $\begin{array}{l}H Q=1 \\
\text { Adult }\end{array}$ & $\begin{array}{l}\mathrm{HQ}=1 \\
\text { Child }\end{array}$ & $\begin{array}{c}\mathrm{HQ}=0.1 \\
\text { Adult }\end{array}$ & $\begin{array}{c}\text { HQ=0.1 } \\
\text { Child }\end{array}$ & $10^{-4}$ & $10^{4}$ & HQ=1 & $\mathrm{HQ}=0.1$ & $10^{-4}$ & $10^{4}$ & $\mathrm{HQ}=1$ & $H Q=0.1$ & $10^{-4}$ & $10^{-5}$ & $\mathrm{HI}=1$ & $\mathrm{HI}=0.1$ \\
\hline $\begin{array}{l}\text { Polychlorinate } \\
\text { dBiphenyls }\end{array}$ & $001336-36-3$ & $8.3 \mathrm{E}+00$ & $8.3 \mathrm{E}-02$ & & & & & $6.3 E+00$ & $6.3 \mathrm{E}-02$ & & & & & & & $4.9 E+00$ & $4.9 E-02$ & & \\
\hline $\begin{array}{l}\text { Potassium Cy- } \\
\text { anide }\end{array}$ & $000151-50.8$ & & & $3.7 \mathrm{E}+04$ & $3.9 E+03$ & $3.7 \mathrm{E}+03$ & $3.9 E+02$ & & & $5.5 \mathrm{E}+04$ & $5.5 E+03$ & & & & & & & $2.2 \mathrm{E}+04$ & $2.2 \mathrm{E}+03$ \\
\hline $\begin{array}{l}\text { Potassium Sil- } \\
\text { ver Cyanide }\end{array}$ & $0005066-61-6$ & & & $1.5 \mathrm{E}+05$ & $1.6 \mathrm{E}+04$ & $1.5 \mathrm{E}+04$ & $1.6 \mathrm{E}+03$ & & & $5.5 E+05$ & $5.5 E+04$ & & & & & & & $1.2 \mathrm{E}+0 \mathrm{~s}$ & $1.2 \mathrm{E}+04$ \\
\hline Prochloraz & $067747-09 \cdot 5$ & $4.3 \mathrm{E}+02$ & $4.3 E+00$ & $6.6 \mathrm{E}+03$ & $7.0 \mathrm{E}+02$ & $6.6 \mathrm{E}+02$ & $7.0 \mathrm{E}+01$ & 1.1.E+ +03 & $1.1 \mathrm{E}+01$ & $6.2 \mathrm{E}+03$ & $6.2 E+02$ & & & & & $5.5 \mathrm{E}+02$ & $5.5 E+\infty 0$ & $3.2 \mathrm{E}+03$ & $3.2 \mathrm{E}+02$ \\
\hline Profluralin & $0263999-36-0$ & & & $4.4 E+03$ & $4.7 E+02$ & $4.4 \mathrm{E}+02$ & $4.7 \mathrm{E}+01$ & & & $4.1 E+03$ & $4.1 E+02$ & & & & & & & 2. $.1 \mathrm{E}+03$ & $2.1 \mathrm{E}+02$ \\
\hline Prometon & $001610-18-0$ & & & $1.1 E+04$ & $1.2 E+03$ & $1.1 \mathrm{E}+03$ & $1.2 E+02$ & & & $1.0 \mathrm{E}+04$ & $1.0 E+03$ & & & & & & & $5.3 \mathrm{E}+03$ & $5.3 \mathrm{E}+02$ \\
\hline Prometryn & $007287-19-6$ & & & $2.9 E+03$ & 3.IE+02 & $2.9 E+02$ & 3.1E+01 & & & $2.8 \mathrm{E}+03$ & $2.8 \mathrm{E}+02$ & & & & & & & $1.4 E+03$ & $1.4 \mathrm{E}+02$ \\
\hline Propachlor & $001918-16-7$ & & & $9.5 \mathrm{E}+03$ & $1.0 E+03$ & $9.5 \mathrm{E}+02$ & $1.0 E+02$ & & & $9.0 E+03$ & $9.0 E+02$ & & & & & & & $4.6 \mathrm{E}+03$ & $4.6 \mathrm{E}+02$ \\
\hline Propanil & $000709-98-8$ & & & $3.7 E+03$ & $3.9 E+02$ & $3 . \pi \mathrm{E}+02$ & $3.9 \mathrm{E}+01$ & & & $3.4 E+03$ & $3.4 \mathrm{E}+02$ & & & & & & & $1.8 \mathrm{E}+03$ & $1.8 \mathrm{E}+02$ \\
\hline Propargite & $002312-35-8$ & & & $1.5 E+04$ & $1.6 \mathrm{E}+03$ & $1.5 \mathrm{E}+03$ & $1.6 \mathrm{E}+02$ & & & $1.4 \mathrm{E}+04$ & $1.4 \mathrm{E}+03$ & & & & & & & 7. $1 \mathrm{E}+03$ & 7. $1 \mathrm{E}+02$ \\
\hline 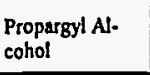 & $000107-19-7$ & & & $1.5 E+03$ & $1.6 \mathrm{E}+02$ & $1.5 \mathrm{E}+02$ & $1.6 \mathrm{E}+01$ & & & $2.2 \mathrm{E}+03$ & $2.2 E+02$ & & & & & & & $8.8 \mathrm{E}+02$ & $8.8 \mathrm{E}+01$ \\
\hline Propazine & $000139-40 \cdot 2$ & & & $1.5 E+04$ & $1.6 \mathrm{E}+03$ & $1.5 \mathrm{E}+03$ & $1.6 \mathrm{E}+02$ & & & $1.4 E+04$ & $1.4 E+03$ & & & & & & & $7.1 E+03$ & $7.1 \mathrm{E}+02$ \\
\hline Propham & $000122-42.9$ & & & $1.5 \mathrm{E}+04$ & $1.6 \mathrm{E}+03$ & $1.5 E+03$ & $1.6 \mathrm{E}+02$ & & & $1.4 E+04$ & $1.4 E+03$ & & & & & & & $7.1 E+03$ & $7.1 E+02$ \\
\hline Propiconazole & 060207-90-1 & & & 9.5E+03 & $1.0 \mathrm{E}+03$ & $9.5 \mathrm{E}+02$ & $1.0 \mathrm{E}+02$ & & & $9.0 \mathrm{E}+03$ & $9.0 \mathrm{E}+02$ & & & & & & & $4.6 \mathrm{E}+03$ & $4.6 \mathrm{E}+02$ \\
\hline $\begin{array}{l}\text { Propylene Gly- } \\
\text { col }\end{array}$ & $000057-55.6$ & & & $1.0 E+06$ & $1.0 E+06$ & $1.0 E+06$ & $1.6 \mathrm{E}+05$ & & & $1.0 E+06$ & $1.0 \mathrm{E}+06$ & & & & & & & $1.0 E+06$ & $7.1 E+05$ \\
\hline $\begin{array}{l}\text { Propylenc Gly- } \\
\text { col Menoethyl } \\
\text { Elther }\end{array}$ & $001569-02-4$ & & & S.IEE+OS & $5.5 \mathrm{E}+04$ & $5.1 E+04$ & $5.5 E+03$ & & & $4.8 \mathrm{E}+0 \mathrm{~s}$ & $4.8 E+04$ & & & & & & & $2.5 \mathrm{E}+0 \mathrm{~S}$ & 2.5E+04 \\
\hline $\begin{array}{l}\text { Propylene Gly- } \\
\text { col } \\
\text { Monomethyl } \\
\text { Ether }\end{array}$ & 000107-98-2 & & & S.1E+05 & $5.5 \mathrm{E}+04$ & S.1E+04 & $5.5 E+03$ & & & $4.8 E+05$ & $4.8 E+04$ & & & $1.0 \mathrm{E}+06$ & $1.0 \mathrm{E}+06$ & & & $2.5 \mathrm{E}+0.5$ & $2.5 E+04$ \\
\hline $\begin{array}{l}\text { Propylene Ox- } \\
\text { ide }\end{array}$ & $000075-56-9$ & $2.7 \mathrm{E}+02$ & $2.7 \mathrm{E}+\infty 0$ & & & & & 1. $1 E+03$ & 1.1.E+01 & & & $4.2 E+03$ & $4.2 \mathrm{E}+01$ & $2.0 \mathrm{E}+03$ & $2.0 E+02$ & $3.9 E+02$ & $3.9 E+\infty 0$ & & \\
\hline
\end{tabular}


Table 3.1c. (continued)

\begin{tabular}{|c|c|c|c|c|c|c|c|c|c|c|c|c|c|c|c|c|c|c|c|}
\hline \multirow{2}{*}{ Chemical } & \multirow{2}{*}{ CASRN } & \multicolumn{6}{|c|}{ Ingestion (mg/kg) } & \multicolumn{4}{|c|}{ Dermal (mg/kg) } & \multicolumn{4}{|c|}{ Inhalation (mg/kg) } & \multicolumn{4}{|c|}{$\begin{array}{c}\text { Ingestion + Dermal } \\
+ \text { Inhalation (mg/kg) }\end{array}$} \\
\hline & & $10^{-4}$ & $10^{4}$ & $\begin{array}{l}\text { HQ=1 } \\
\text { Adult }\end{array}$ & $\begin{array}{l}\mathrm{HQ}=1 \\
\text { Child }\end{array}$ & $\begin{array}{l}\text { HQ }=0.1 \\
\text { Adult }\end{array}$ & $\begin{array}{l}\text { HQ }=0.1 \\
\text { Child }\end{array}$ & $10^{-4}$ & $10^{-6}$ & $\mathrm{HQ}=1$ & $H Q=0.1$ & $10^{-4}$ & $10^{5}$ & $H Q=1$ & $\mathrm{HQ}=0.1$ & $10^{-4}$ & $10^{6}$ & $H I=1$ & $\mathrm{HI}=0.1$ \\
\hline Pursuit & $081335-77.5$ & & & $1.8 E+05$ & $2.0 \mathrm{E}+04$ & $1.8 \mathrm{E}+04$ & $2.0 \mathrm{E}+03$ & & & $1.7 E+05$ & $1.7 E+04$ & & & & & & & $8.9 E+04$ & $8.9 \mathrm{E}+03$ \\
\hline Pydrin & $051630-58-1$ & & & $1.8 \mathrm{E}+04$ & $2.0 \mathrm{E}+03$ & $1.8 \mathrm{E}+03$ & $2.0 \mathrm{E}+02$ & & & $1.7 E+04$ & $1.7 \mathrm{E}+03$ & & & & & & & $8.9 E+03$ & $8.9 E+02$ \\
\hline Pyrene & $000129-00-0$ & & & $2.2 \mathrm{E}+04$ & $2.3 \mathrm{E}+03$ & $2.2 \mathrm{E}+03$ & $2.3 \mathrm{E}+02$ & & & $1.3 E+04$ & $1.3 \mathrm{E}+03$ & & & & & & & $8.1 \mathrm{E}+03$ & 8.IE+02 \\
\hline Pyridine & 000110.86 .1 & & & $7.3 \mathrm{E}+02$ & $7.8 \mathrm{E}+01$ & $7.3 E+01$ & $7.8 \mathrm{E}+\infty$ & & & $6.9 E+02$ & $6.9 \mathrm{E}+01$ & & & & & & & $3.5 \mathrm{E}+02$ & $3.5 \mathrm{E}+01$ \\
\hline Quinalphos & 013593.03 .8 & & & $3.7 \mathrm{E}+02$ & $3.9 \mathrm{E}+01$ & $3.7 E+01$ & $3.9 E+\infty 0$ & & & $3.4 \mathrm{E}+02$ & $3,4 E+01$ & & & & & & & $1.8 \mathrm{E}+02$ & $1.8 \mathrm{E}+01$ \\
\hline Quinoline & $000091-22-5$ & $5.3 \mathrm{E}+00$ & $5.3 \mathrm{E}-02$ & & & & & $1.3 \mathrm{E}+01$ & 1.3E-01 & & & & & & & $6.9 \mathrm{E}+\infty$ & $6.9 \mathrm{E}-02$ & & \\
\hline $\begin{array}{l}\text { Refractory } \\
\text { Ceramic Fibers }\end{array}$ & NA & & & & & & & & & & & & & & & & & & \\
\hline Resmethrin & $010453-86.8$ & & & $2.2 E+04$ & $2.3 \mathrm{E}+03$ & $2.2 E+03$ & $2.3 \mathrm{E}+02$ & & & $2.1 \mathrm{E}+04$ & $2.1 \mathrm{E}+03$ & & & & & & & $1.1 \mathrm{E}+04$ & $1.1 \mathrm{E}+03$ \\
\hline Ronnel & $000299-84-3$ & & & $3.7 \mathrm{E}+04$ & $3.9 \mathrm{E}+03$ & $3.7 E+03$ & $3.9 E+02$ & & & 3.4E+04 & $3.4 E+03$ & & & & & & & $1.8 E+04$ & $1.8 \mathrm{E}+03$ \\
\hline Rotenone & $000083-79-4$ & & & $2.9 E+03$ & $3.1 E+02$ & $2.9 \mathrm{E}+02$ & $3.1 E+01$ & & & $2.8 \mathrm{E}+03$ & $2.8 \mathrm{E}+02$ & & & & & & & $1.4 \mathrm{E}+03$ & $1.4 E+02$ \\
\hline Savey & $078587-05-0$ & & & $1.8 \mathrm{E}+04$ & $2.0 E+03$ & $1.8 \mathrm{E}+03$ & $2.0 \mathrm{E}+02$ & & & $1.7 \mathrm{E}+04$ & $1.7 \mathrm{E}+03$ & & & & & & & $8.9 \mathrm{E}+03$ & $8.9 E+02$ \\
\hline Selenious Acid & 007783.00 .8 & & & $3.7 \mathrm{E}+03$ & $3.9 \mathrm{E}+02$ & $3.7 \mathrm{E}+02$ & $3.9 \mathrm{E}+01$ & & & $6.0 \mathrm{E}+03$ & $6.0 \mathrm{E}+02$ & & & & & & & $2.3 E+03$ & $2.3 \mathrm{E}+02$ \\
\hline Selenile & $014124-67.5$ & & & & & & & & & & & & & & & & & & \\
\hline Selenium & $007782-49-2$ & & & $3.7 \mathrm{E}+03$ & $3.9 E+02$ & 3. $\mathrm{TE}+02$ & $3.9 \mathrm{E}+01$ & & & $3.0 \mathrm{E}+04$ & $3.0 \mathrm{E}+03$ & & & & & & & $3.3 \mathrm{E}+03$ & $3.3 \mathrm{E}+02$ \\
\hline $\begin{array}{l}\text { Selenium Sul- } \\
\text { fide }\end{array}$ & $007446-34-6$ & & & & & & & & & & & & & & & & & & \\
\hline Selenourea & $000630.10-4$ & & & $3.7 \mathrm{E}+03$ & $3.9 E+02$ & 3.7E+02 & $3.9 E+01$ & & & 3.4E+03 & $3.4 \mathrm{E}+02$ & & & & & & & $1.8 E+03$ & $1.8 \mathrm{E}+02$ \\
\hline Sethoxydim & 074051.80 .2 & & & $6.6 \mathrm{E}+04$ & $7.0 E+03$ & $6.6 \mathrm{E}+03$ & $7.0 E+02$ & & & $6.2 \mathrm{E}+04$ & $6.2 \mathrm{E}+03$ & & & & & & & $3.2 \mathrm{E}+04$ & $3.2 \mathrm{E}+03$ \\
\hline Silver & $007440-22-4$ & & & $3.7 E+03$ & $3.9 E+02$ & $3.7 \mathrm{E}+02$ & $3.9 \mathrm{E}+01$ & & & $1.2 \mathrm{E}+04$ & $1.2 \mathrm{E}+03$ & & & & & & & $2.8 \mathrm{E}+03$ & $2.8 \mathrm{E}+02$ \\
\hline Silver Cyanide & $000506-64.9$ & & & $7.3 \mathrm{E}+04$ & $7.8 \mathrm{E}+03$ & $7.3 E+03$ & $7.8 \mathrm{E}+02$ & & & $2.8 \mathrm{E}+05$ & $2.8 \mathrm{E}+04$ & & & & & & & $5.8 E+04$ & $5.8 \mathrm{E}+03$ \\
\hline Simazine & $000122-34-9$ & $5.3 \mathrm{E}+02$ & $5.3 \mathrm{E}+00$ & $3.7 \mathrm{E}+03$ & $3.9 E+02$ & $3.7 \mathrm{E}+02$ & $3.9 \mathrm{E}+01$ & $1.3 \mathrm{E}+03$ & $1.3 \mathrm{E}+01$ & $3.4 E+03$ & $3.4 \mathrm{E}+02$ & & & & & $6.9 \mathrm{E}+02$ & $6.9 E+00$ & $1.8 E+03$ & $1.8 \mathrm{E}+02$ \\
\hline Sodium & $007440-23-5$ & & & & & & & & & & & & & & & & & & \\
\hline
\end{tabular}


Table 3.1c. (continued)

\begin{tabular}{|c|c|c|c|c|c|c|c|c|c|c|c|c|c|c|c|c|c|c|c|}
\hline \multirow{2}{*}{ Chemical } & \multirow{2}{*}{ CASRN } & \multicolumn{6}{|c|}{ Ingestion (mg/kg) } & \multicolumn{4}{|c|}{ Dermal (mg/kg) } & \multicolumn{4}{|c|}{ Inhalation $(\mathrm{mg} / \mathrm{kg})^{\cdot v}$} & \multicolumn{4}{|c|}{$\begin{aligned} & \text { Ingestion + Dermal } \\
+ & \text { Inhalation }(\mathrm{mg} / \mathrm{kg})^{+\cdot v}\end{aligned}$} \\
\hline & & $10^{-4}$ & $10^{4}$ & $\begin{array}{l}H Q=1 \\
\text { Adult }\end{array}$ & $\begin{array}{l}\text { HQ=1 } \\
\text { Child }\end{array}$ & $\begin{array}{c}H Q=0.1 \\
\text { Adult }\end{array}$ & $\begin{array}{c}\mathrm{HQ}=0.1 \\
\text { Child }\end{array}$ & $10^{-4}$ & $10^{4}$ & $H Q=1$ & $H Q=0.1$ & $10^{-4}$ & $10^{6}$ & $H Q=1$ & $H Q=0.1$ & $10^{-1}$ & $10^{4}$ & $\mathrm{HI}=1$ & $H \mathrm{I}=0.1$ \\
\hline $\begin{array}{l}\text { Sodium } \\
\text { Acifluoren }\end{array}$ & 062476.59 .9 & & & $9.5 \mathrm{E}+03$ & $1.0 \mathrm{E}+03$ & $9.5 E+02$ & $1.0 \mathrm{E}+02$ & & & $9.0 \mathrm{E}+03$ & $9.0 \mathrm{E}+02$ & & & & & & & $4.6 \mathrm{E}+03$ & $4.6 \mathrm{E}+02$ \\
\hline Sodium Azide & $026628-22.8$ & & & $2.9 \mathrm{E}+03$ & 3.IE+02 & $2.9 \mathrm{E}+02$ & $3.1 \mathrm{E}+01$ & & & $1.1 E+04$ & $1.1 \mathrm{E}+03$ & & & & & & & $2.3 \mathrm{E}+03$ & $2.3 \mathrm{E}+02$ \\
\hline $\begin{array}{l}\text { Sodium Cya- } \\
\text { nide }\end{array}$ & $000143-33-9$ & & & $2.9 \mathrm{E}+04$ & $3.1 E+03$ & $2.9 \mathrm{E}+03$ & $3.1 \mathrm{E}+02$ & & & $4.4 E+04$ & $4.4 \mathrm{E}+03$ & & & & & & & $1.8 \mathrm{E}+04$ & $1.8 \mathrm{E}+03$ \\
\hline $\begin{array}{l}\text { Sodium } \\
\text { Diethyldithioc } \\
\text { arbamate }\end{array}$ & $000148-18-5$ & $2.4 \mathrm{E}+02$ & $2.4 \mathrm{E}+00$ & 2.2E+04 & $2.3 \mathrm{E}+03$ & $2.2 E+03$ & 2.3E+02 & $6.0 \mathrm{E}+02$ & $6.0 \mathrm{E}+00$ & $2.1 \mathrm{E}+04$ & $2.1 E+03$ & & & & & $3.1 \mathrm{E}+02$ & $3.1 \mathrm{E}+\infty$ & 1. $1 \mathrm{E}+04$ & $1.1 E+03$ \\
\hline $\begin{array}{l}\text { Sodium } \\
\text { Fluoroacetate }\end{array}$ & $000062-74-8$ & & & $1.5 \mathrm{E}+0 \mathrm{t}$ & $1.6 \mathrm{E}+00$ & $1.5 \mathrm{E}+00$ & $1.6 \mathrm{E}-01$ & & & $1.4 \mathrm{E}+01$ & $1.4 \mathrm{E}+00$ & & & & & & & $7.1 E+\infty$ & $7.1 \mathrm{E}-01$ \\
\hline $\begin{array}{l}\text { Sodium } \\
\text { Metavanadate }\end{array}$ & $013718-26-8$ & & & $7.3 \mathrm{E}+02$ & $7.8 \mathrm{E}+01$ & $7,3 \mathrm{E}+01$ & $7.8 E+00$ & & & $2.8 \mathrm{E}+03$ & $2.8 \mathrm{E}+02$ & & & & & & & $5.8 E+02$ & $5.8 E+01$ \\
\hline $\begin{array}{l}\text { Stirofos } \\
\text { (Tetrachloro- } \\
\text { vinphos) }\end{array}$ & $000961-11-5$ & $2.7 E+03$ & $2.7 \mathrm{E}+01$ & $2.2 \mathrm{E}+04$ & $2.3 \mathrm{E}+03$ & $2.2 \mathrm{E}+03$ & $2.3 \mathrm{E}+02$ & $6.7 E+03$ & $6.7 \mathrm{E}+01$ & $2.1 E+04$ & $2.1 \mathrm{E}+03$ & & & & & $3.4 \mathrm{E}+03$ & $3.4 E+01$ & $1.1 \mathrm{E}+04$ & $1.1 \mathrm{E}+03$ \\
\hline $\begin{array}{l}\text { Strontium, } \\
\text { Stable }\end{array}$ & $007440-24-6$ & & & 4. $4 E+05$ & 4.7E+04 & $4.4 \mathrm{E}+04$ & 4.7E+03 & & & $1.0 \mathrm{E}+06$ & $1.7 \mathrm{E}+0 \mathrm{~S}$ & & & & & & & $3.5 \mathrm{E}+05$ & $3.5 \mathrm{E}+04$ \\
\hline Strychnine & $000057 \cdot 24.9$ & & & $2.2 \mathrm{E}+02$ & $2.3 \mathrm{E}+01$ & $2.2 \mathrm{E}+01$ & $2.3 \mathrm{E}+00$ & & & $2.1 \mathrm{E}+02$ & $2.1 \mathrm{E}+0 \mathrm{I}$ & & & & & & & $1.1 \mathrm{E}+02$ & 1. $1 E+01$ \\
\hline Styrene ${ }^{(4)}$ & $000100-42-5$ & & & $1.5 E+05$ & $1.6 E+04$ & $1.5 \mathrm{E}+04$ & $1.6 \mathrm{E}+03$ & & & $2.2 \mathrm{E}+05$ & $2.2 E+04$ & & & $6.7 E+02$ & $6.7 \mathrm{E}+02$ & & & $6.7 \mathrm{E}+02$ & $6.7 E+02$ \\
\hline Sulfate & $014808-79-8$ & & & & & & & & & & & & & & & & & & \\
\hline Systhane & $088671-89.0$ & & & $1.8 \mathrm{E}+04$ & $2.0 E+03$ & $1.8 \mathrm{E}+03$ & $2.0 E+02$ & & & $1.7 E+04$ & $1.7 \mathrm{E}+03$ & & & & & & & $8.9 \mathrm{E}+03$ & $8.9 E+02$ \\
\hline $\begin{array}{l}\mathrm{TCDD} \\
2,3,7,8 .(1)\end{array}$ & 001746.01 .6 & 4.3E-04 & $4.3 E-06$ & & & & & 3.6E-04 & $3.6 \mathrm{E}-06$ & & & $2.4 E+01$ & $2.4 \mathrm{E}-0 \mathrm{I}$ & & & $2.7 \mathrm{E}-04$ & 2.7E-06 & & \\
\hline $\begin{array}{l}\mathrm{TCDF} \\
2,3,7,8^{(1)}\end{array}$ & $051207-31-9$ & $4.3 \mathrm{E}-03$ & 4.3E-05 & & & & & $1.1 \mathrm{E}-02$ & $1.1 \mathrm{E}-04$ & & & $2.4 E+02$ & $2.4 \mathrm{E}+00$ & & & $5.5 \mathrm{E}-03$ & $5.5 \mathrm{E}-05$ & & \\
\hline TCMTB & $021564-17.0$ & & & $2.2 E+04$ & $2.3 \mathrm{E}+03$ & $2.2 \mathrm{E}+03$ & $2.3 \mathrm{E}+02$ & & & 2.IE+04 & $2.1 E+03$ & & & & & & & $1.1 E+04$ & 1. $I E+03$ \\
\hline Tebuthiuron & 034014-18-1 & & & $5.1 \mathrm{E}+04$ & $5.5 \mathrm{E}+03$ & $5.1 E+03$ & $5.5 \mathrm{E}+02$ & & & $4.8 \mathrm{E}+04$ & $4.8 \mathrm{E}+03$ & & & & & & & $2.5 E+04$ & $2.5 \mathrm{E}+03$ \\
\hline Temephos & $003383.96-8$ & & & $1.5 \mathrm{E}+04$ & $1.6 \mathrm{E}+03$ & $1.5 E+03$ & $1.6 \mathrm{E}+02$ & & & $1.4 \mathrm{E}+04$ & $1.4 \mathrm{E}+03$ & & & & & & & $7.1 \mathrm{E}+03$ & $7.1 E+02$ \\
\hline Terbacil & $005902-51-2$ & & & $9.5 E+03$ & $1.0 E+03$ & $9.5 \mathrm{E}+02$ & $1.0 E+02$ & & & $9.0 \mathrm{E}+03$ & $9.0 E+02$ & & & & & & & $4.6 \mathrm{E}+03$ & $4.6 \mathrm{E}+02$ \\
\hline
\end{tabular}

Nonradionuclides/soil/residential

Page 34 of 41 
Table 3.1c. (continued)

\begin{tabular}{|c|c|c|c|c|c|c|c|c|c|c|c|c|c|c|c|c|c|c|c|}
\hline \multirow{2}{*}{ Chemical } & \multirow{2}{*}{ CASRN } & \multicolumn{6}{|c|}{ Ingestion (mg/kg) } & \multicolumn{4}{|c|}{ Dermal (mg/kg) } & \multicolumn{4}{|c|}{ Inhalation (mg/kg) } & \multicolumn{4}{|c|}{$\begin{array}{c}\text { Ingestion + Dermal } \\
+ \text { Inhalation (mg/kg) } \\
\end{array}$} \\
\hline & & $10^{-4}$ & $10^{5}$ & $\begin{array}{l}\text { HQ=1 } \\
\text { Adult }\end{array}$ & $\begin{array}{l}\text { HQ=1 } \\
\text { Child }\end{array}$ & $\begin{array}{c}\mathrm{HQ}=0.1 \\
\text { Adult }\end{array}$ & $\begin{array}{c}H Q=0.1 \\
\text { Child }\end{array}$ & $10^{-4}$ & $10^{5}$ & $\mathrm{HQ}=1$ & $\mathrm{HQ}=0.1$ & $10^{-4}$ & $10^{4}$ & $H Q=1$ & $\mathrm{HQ}=0.1$ & $10^{-4}$ & $10^{5}$ & $\mathbf{H I}=\mathbf{1}$ & $\mathrm{HI}=0.1$ \\
\hline Terbufos & 013071.79 .9 & & & $1.8 \mathrm{E}+01$ & $2.0 E+00$ & $1.8 \mathrm{E}+\infty 0$ & $2.0 \mathrm{E}-01$ & & & $1.7 \mathrm{E}+01$ & $1.7 \mathrm{E}+\infty 0$ & & & & & & & $8.9 \mathrm{E}+00$ & $8.9 E-01$ \\
\hline Terbutryn & $000886-50-0$ & & & $7.3 \mathrm{E}+02$ & $7.8 \mathrm{E}+01$ & $7.3 E+01$ & $7.8 \mathrm{E}+\infty$ & & & $6.9 \mathrm{E}+02$ & $6.9 \mathrm{E}+01$ & & & & & & & $3.5 \mathrm{E}+02$ & $3.5 \mathrm{E}+01$ \\
\hline $\begin{array}{l}\text { Tetrabutyl } \\
\text { Lead }\end{array}$ & $001920-90-7$ & 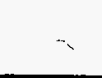 & & & & & & & & & & & & & & & & & \\
\hline $\begin{array}{l}\text { Tetrachloro- } \\
\text { benzene, } \\
1,2,4,5-\end{array}$ & $000095-94-3$ & & & $2.2 \mathrm{E}+02$ & $2.3 E+01$ & $2.2 E+01$ & $2.3 \mathrm{E}+\infty 0$ & & & $3.3 \mathrm{E}+02$ & $3.3 \mathrm{E}+01$ & & & & & & & $1.3 E+02$ & $1.3 \mathrm{E}+01$ \\
\hline $\begin{array}{l}\text { Tetrachloro- } \\
\text { ethane, } \\
1,1,1,2 .\end{array}$ & $000630-20-6$ & $2.5 E+03$ & $2.5 E+01$ & $2.2 E+04$ & $2.3 E+03$ & $2.2 \mathrm{E}+03$ & 2.3E+02 & $9.9 \mathrm{E}+03$ & $9.9 \mathrm{E}+01$ & $3.3 E+04$ & $3.3 \mathrm{E}+03$ & $7.7 E+02$ & $1.7 E+01$ & & & $7.7 \mathrm{E}+02$ & $1.2 \mathrm{E}+01$ & $7.7 E+02$ & $7.7 E+02$ \\
\hline $\begin{array}{l}\text { Tetrachloro- } \\
\text { ethane, } \\
1, \mathbf{1}, \mathbf{2 , 2} \text {. }\end{array}$ & $000079-34-5$ & $3.2 \mathrm{E}+02$ & $3.2 E+\infty 0$ & & & & & $1.1 E+03$ & $1.1 E+01$ & & & $4.0 E+02$ & $4.0 E+\infty$ & & & $2.2 \mathrm{E}+02$ & $2.2 E+00$ & & \\
\hline $\begin{array}{l}\text { Tetrachioro- } \\
\text { ethylene } e^{(1)}\end{array}$ & $000127 \cdot 18-4$ & $1.2 \mathrm{E}+03$ & $1.2 \mathrm{E}+01$ & $7.3 E+03$ & $7.8 E+02$ & $7.3 \mathrm{E}+02$ & $7.8 \mathrm{E}+01$ & $6.2 \mathrm{E}+03$ & $6.2 \mathrm{E}+01$ & $1.4 E+04$ & $1.4 \mathrm{E}+03$ & $1.2 \mathrm{E}+02$ & $9.4 E+01$ & & & $1.2 \mathrm{E}+02$ & $1.7 \mathrm{E}+01$ & $1.2 \mathrm{E}+02$ & $1.2 E+02$ \\
\hline $\begin{array}{l}\text { Tetrachioro- } \\
\text { phenol, } \\
2,3,4,6-\end{array}$ & $000058.90-2$ & & & $2.2 \mathrm{E}+04$ & $2.3 \mathrm{E}+03$ & $2.2 \mathrm{E}+03$ & $2.3 \mathrm{E}+02$ & & & 2.1E+04 & $2.1 E+03$ & & & & & & & $1.1 E+04$ & $1.1 E+03$ \\
\hline $\begin{array}{l}\text { Tetrachloro- } \\
\text { toluene, p- } \\
\text { alpha, alpha, } \\
\text { alpha- }\end{array}$ & $005216-25-1$ & $3.2 \mathrm{E}+00$ & $3.2 \mathrm{E}-02$ & & & & & $1.3 \mathrm{E}+01$ & $1.3 \mathrm{E}-01$ & & & & & & & $5.1 \mathrm{E}+00$ & $5.1 \mathrm{E}-02$ & & \\
\hline $\begin{array}{l}\text { Tetraethyl } \\
\text { Dithiopyro- } \\
\text { phosphate }\end{array}$ & $003689-24-5$ & & & $3.7 E+02$ & $3.9 E+01$ & $3.7 \mathrm{E}+01$ & $3.9 \mathrm{E}+00$ & & & $3.4 \mathrm{E}+02$ & $3.4 \mathrm{E}+01$ & & & & & & & $1.8 \mathrm{E}+02$ & $1.8 \mathrm{E}+01$ \\
\hline $\begin{array}{l}\text { Tetracthyl } \\
\text { Lead }\end{array}$ & $000078-00-2$ & & & & & & & & & & & & & & & & & & \\
\hline $\begin{array}{l}\text { Tetrafluoro- } \\
\text { ethane, } \\
1,1,1,2 .\end{array}$ & $000811-97-2$ & & & & & & & & & & & & & $1.1 \mathrm{E}+02$ & $1.1 \mathrm{E}+02$ & & & & \\
\hline $\begin{array}{l}\text { Tetramethyl } \\
\text { Lead }\end{array}$ & $000075-74-1$ & & & & & & & & & & & & & & & & & & \\
\hline $\begin{array}{l}\text { Tetrapropyl } \\
\text { Lead }\end{array}$ & $003440-75-3$ & & & & & & & & & & & & & & & & & & \\
\hline
\end{tabular}


Table 3.1c. (continued)

\begin{tabular}{|c|c|c|c|c|c|c|c|c|c|c|c|c|c|c|c|c|c|c|c|}
\hline \multirow{2}{*}{ Chemical } & \multirow{2}{*}{ CASRN } & \multicolumn{6}{|c|}{ Ingestion (mg/kg) } & \multicolumn{4}{|c|}{ Dermal (mg/kg) } & \multicolumn{4}{|c|}{ Inhalation (mg/kg) } & \multicolumn{4}{|c|}{$\begin{array}{l}\text { Ingestion + Dermal } \\
+ \text { Inhalation }\left(\mathrm{mg} / \mathrm{kg}^{\mathbf{1}^{\bullet *}}\right.\end{array}$} \\
\hline & & $10^{-4}$ & $10^{4}$ & $\begin{array}{l}\text { HQ=1 } \\
\text { Adult }\end{array}$ & $\begin{array}{l}\text { HQ=1 } \\
\text { Child }\end{array}$ & $\begin{array}{l}\text { HQ }=0.1 \\
\text { Adult }\end{array}$ & $\begin{array}{c}\text { HQ }=0.1 \\
\text { Child }\end{array}$ & $10^{-4}$ & $10^{5}$ & $\mathrm{HQ}=1$ & $\mathrm{HQ}=0.1$ & $10^{4}$ & $10^{4}$ & $H Q=1$ & $\mathrm{HQ}=0.1$ & $10^{-4}$ & $10^{6}$ & $H I=1$ & $\mathrm{HI}=0.1$ \\
\hline $\begin{array}{l}\text { Thallic Ox- } \\
\text { ide }\end{array}$ & $001314-32-5$ & & & S.1E+01 & $5.5 E+00$ & $5.1 E+00$ & $5.5 \mathrm{E}-01$ & & & $4.8 \mathrm{E}+01$ & $4.8 \mathrm{E}+00$ & & & & & & & $2.5 \mathrm{E}+01$ & $2.5 \mathrm{E}+00$ \\
\hline $\begin{array}{l}\text { Thallium (l) } \\
\text { Nitrate }\end{array}$ & $010102-45-1$ & & & $6.6 \mathrm{E}+01$ & $7.0 \mathrm{E}+00$ & $6.6 \mathrm{E}+\infty$ & $7.0 \mathrm{E}-01$ & & & $2.5 \mathrm{E}+02$ & $2.5 E+01$ & & & & & & & $5.2 \mathrm{E}+01$ & $5.2 \mathrm{E}+00$ \\
\hline $\begin{array}{l}\text { Thallium (Sol- } \\
\text { uble Salts) }\end{array}$ & $007440-28-0$ & & & & & & & & & & & & & & & & & & \\
\hline $\begin{array}{l}\text { Thallium } \\
\text { Acetate }\end{array}$ & $000563-68-8$ & & & $6.6 \mathrm{E}+01$ & $7.0 \mathrm{E}+00$ & $.6 .6 \mathrm{E}+\infty$ & $7.0 \mathrm{E}-01$ & & & $6.2 \mathrm{E}+01$ & $6.2 \mathrm{E}+00$ & & & & & & & $3.2 \mathrm{E}+01$ & $3.2 \mathrm{E}+00$ \\
\hline $\begin{array}{l}\text { Thallium } \\
\text { Carbonate }\end{array}$ & $006533-73-9$ & & & $5.8 \mathrm{E}+01$ & $6.3 \mathrm{E}+00$ & $5.8 \mathrm{E}+00$ & $6.3 \mathrm{E}-01$ & & & $5.5 \mathrm{E}+01$ & $5.5 \mathrm{E}+\infty$ & & & & & & & $2.8 \mathrm{E}+01$ & $2.8 \mathrm{E}+00$ \\
\hline $\begin{array}{l}\text { Thallium } \\
\text { Chloride }\end{array}$ & $007791-12.0$ & & & $5,8 \mathrm{E}+01$ & $6.3 \mathrm{E}+00$ & $5.8 \mathrm{E}+\infty 0$ & $6.3 \mathrm{E}-01$ & & & $2.2 \mathrm{E}+02$ & $2.2 \mathrm{E}+01$ & & & & & & & $4.6 \mathrm{E}+01$ & $4.6 \mathrm{E}+00$ \\
\hline $\begin{array}{l}\text { Thallium } \\
\text { Selenite" }\end{array}$ & $012039-52.0$ & & & $6.6 \mathrm{E}+01$ & $7.0 \mathrm{E}+00$ & $6.6 \mathrm{E}+\infty$ & $7.0 \mathrm{E}-01$ & & & $2.5 \mathrm{E}+02$ & $2.5 \mathrm{E}+01$ & & & & & & & $5.2 \mathrm{E}+01$ & $5.2 \mathrm{E}+00$ \\
\hline $\begin{array}{l}\text { Thallium } \\
\text { Sulfate }\end{array}$ & $007446-18-6$ & & & S.8E+01 & $6.3 \mathrm{E}+00$ & $5.8 \mathrm{E}+\infty$ & $6.3 \mathrm{E}-01$ & & & $2.2 \mathrm{E}+02$ & $2.2 \mathrm{E}+01$ & & & & & & & $4.6 \mathrm{E}+01$ & $4.6 \mathrm{E}+00$ \\
\hline Thiobencarb & $028249-77.6$ & & & $7.3 \mathrm{E}+03$ & $7.8 \mathrm{E}+02$ & $7.3 \mathrm{E}+02$ & $7.8 \mathrm{E}+01$ & & & $6.9 \mathrm{E}+03$ & $6.9 \mathrm{E}+02$ & & & & & & & $3.5 \mathrm{E}+03$ & $3.5 \mathrm{E}+02$ \\
\hline Thiofanox & $039196-18-4$ & & & $2.2 \mathrm{E}+02$ & $2.3 \mathrm{E}+01$ & $2.2 \mathrm{E}+01$ & $2.3 \mathrm{E}+00$ & & & $2.1 \mathrm{E}+02$ & $2.1 \mathrm{E}+01$ & & & & & & & $1.1 \mathrm{E}+02$ & $1.1 \mathrm{E}+01$ \\
\hline $\begin{array}{l}\text { Thiophanale, } \\
\text { Methyl }\end{array}$ & $023564-05.8$ & & & $5.8 \mathrm{E}+04$ & $6.3 \mathrm{E}+03$ & $5.8 \mathrm{E}+03$ & $6.3 \mathrm{E}+02$ & & & $5.5 \mathrm{E}+04$ & $5.5 \mathrm{E}+03$ & & & & & & & $2.8 \mathrm{E}+04$ & $2.8 \mathrm{E}+03$ \\
\hline Thiram & 000137.26 .8 & & & $3.7 \mathrm{E}+03$ & $3.9 \mathrm{E}+02$ & $3.7 \mathrm{E}+02$ & $3.9 \mathrm{E}+01$ & & & $3.4 \mathrm{E}+03$ & $3.4 \mathrm{E}+02$ & & & & & & & $1.8 E+03$ & $1.8 \mathrm{E}+02$ \\
\hline Thorium & $007440-29-1$ & & & & & & & & & & & & & & & & & & \\
\hline Tin & $007440-31-5$ & & & $4.4 \mathrm{E}+05$ & 4.7E+04 & $4.4 E+04$ & $4.7 E+03$ & & & $8.3 \mathrm{E}+05$ & $8.3 \mathrm{E}+04$ & & & & & & & $2.9 E+05$ & $2.9 E+04$ \\
\hline Titanium & $007440-32-6$ & & & & & & & & & & & & & & & & & & \\
\hline Toluene $^{(6)}$ & $000108-88-3$ & & & $1.5 E+05$ & $1.6 \mathrm{E}+04$ & $1.5 \mathrm{E}+04$ & $1.6 \mathrm{E}+03$ & & & 2.2E+0S & $2.2 \mathrm{E}+04$ & & & $2.5 E+02$ & $2.5 \mathrm{E}+02$ & & & $2.5 \mathrm{E}+02$ & $2.5 \mathrm{E}+02$ \\
\hline $\begin{array}{l}\text { Toluene } \\
\text { diisocyanate } \\
\text { mixture (TDl) }\end{array}$ & $026471-62-5$ & & & & & & & & $\cdot$ & & & & & $3.1 \mathrm{E}+05$ & 3.1E+04 & & & & \\
\hline
\end{tabular}


Table 3.1c. (continued)

\begin{tabular}{|c|c|c|c|c|c|c|c|c|c|c|c|c|c|c|c|c|c|c|c|}
\hline \multirow{2}{*}{ Chemical } & \multirow{2}{*}{ CASRN } & \multicolumn{6}{|c|}{ Ingestion (mg/kg) } & \multicolumn{4}{|c|}{ Dermal (mg/kg) } & \multicolumn{4}{|c|}{ Inhalation $(\mathrm{mg} / \mathrm{kg})^{*}$} & \multicolumn{4}{|c|}{$\begin{array}{c}\text { Ingestion + Dermal } \\
+ \text { Inhalation }(\mathrm{mg} / \mathrm{kg})^{\wedge * \boldsymbol{v}}\end{array}$} \\
\hline & & $10^{-4}$ & $10^{5}$ & $\begin{array}{l}\text { HQ=1 } \\
\text { Adult }\end{array}$ & $\begin{array}{l}H Q=1 \\
\text { Child }\end{array}$ & $\begin{array}{c}H Q=0.1 \\
\text { Adult }\end{array}$ & $\begin{array}{c}\text { HQ }=0.1 \\
\text { Child }\end{array}$ & $10^{-4}$ & $10^{4}$ & $H Q=1$ & $\mathrm{HQ}=0.1$ & $10^{-4}$ & $10^{5}$ & HQ=1 & $\mathrm{HQ}=0.1$ & $10^{-1}$ & $10^{4}$ & $\mathrm{HI}=1$ & $\mathrm{Hl}=0.1$ \\
\hline $\begin{array}{l}\text { Toluene-2,4- } \\
\text { diamine- }\end{array}$ & $000095-80-7$ & $2.0 E+01$ & $2.0 \mathrm{E}-01$ & & & & & $5.0 E+01$ & $5.0 E-01$ & & & & & & & $2.6 \mathrm{E}+08$ & $2.6 \mathrm{E}-01$ & & \\
\hline $\begin{array}{l}\text { Toluene-2,s. } \\
\text { diamine }\end{array}$ & $000095-70-5$ & & & $4.4 E+05$ & $4.7 E+04$ & $4.4 E+04$ & 4. $7 E+03$ & & & $4.1 E+05$ & 4.1E+04 & & & & & & & 2. $\mathrm{tE}+05$ & $2.1 E+04$ \\
\hline $\begin{array}{l}\text { Toluene-2,6- } \\
\text { diamine }\end{array}$ & $0008233-40.5$ & & & $1.5 \mathrm{E}+05$ & $1.6 \mathrm{E}+04$ & $1.5 E+04$ & $1.6 \mathrm{E}+03$ & & & $1.4 E+05$ & $1.4 E+04$ & & & & & & & $7.1 \mathrm{E}+04$ & $7.1 E+03$ \\
\hline $\begin{array}{l}\text { Toluididine, o- } \\
\text { Methylaniline, } \\
\text { 2.) }\end{array}$ & $0000955.53-4$ & $2.7 E+02$ & $2.7 E+\infty 0$ & & & & & $6.7 \mathrm{E}+02$ & $6.7 E+\infty 0$ & & & & & & & $3.4 \mathrm{E}+02$ & $3.4 \mathrm{E}+00$ & & \\
\hline Toluidine, $p$. & $000106-49-0$ & $3.4 E+02$ & $3.4 \mathrm{E}+\infty 0$ & & & & & $8.5 \mathrm{E}+02$ & $8.5 \mathrm{E}+00$ & & & & & & & $4.4 \mathrm{E}+02$ & $4.4 \mathrm{E}+00$ & & \\
\hline Toxaphene & $008001-35-2$ & $5.8 \mathrm{E}+01$ & $5.8 \mathrm{E}-01$ & & & & & $1.5 \mathrm{E}+02$ & $1.5 \mathrm{E}+\infty 0$ & & & $1.0 E+06$ & $3.3 \mathrm{E}+04$ & & & $7.5 \mathrm{E}+01$ & 7.5E-01 & & \\
\hline Tralomethrin & $066841-25-6$ & & & $5.5 \mathrm{E}+03$ & $5.9 \mathrm{E}+02$ & $5.5 E+02$ & $5.9 \mathrm{E}+01$ & & & $5.2 E+03$ & $5.2 \mathrm{E}+02$ & & & & & & & $2.7 \mathrm{E}+03$ & $2.7 E+02$ \\
\hline Triallate & $002303-17-5$ & & & $9.5 \mathrm{E}+03$ & $1.0 \mathrm{E}+03$ & $9.5 \mathrm{E}+02$ & $1.0 \mathrm{E}+02$ & & & $9.0 \mathrm{E}+03$ & $9.0 \mathrm{E}+02$ & & & & & & & $4.6 \mathrm{E}+03$ & $4.6 \mathrm{E}+02$ \\
\hline Triasulfuron & 082097.50 .5 & & & $7.3 \mathrm{E}+03$ & $7.8 \mathrm{E}+02$ & $7.3 \mathrm{E}+02$ & $7.8 \mathrm{E}+01$ & & & $6.9 \mathrm{E}+03$ & $6.9 \mathrm{E}+02$ & & & & & & & 3.5E+03 & $3.5 \mathrm{E}+02$ \\
\hline $\begin{array}{l}\text { Tribromoben. } \\
\text { zenre, 1,2,4. }\end{array}$ & $000615-54-3$ & & & $3.7 \mathrm{E}+03$ & $3.9 \mathrm{E}+02$ & $3.7 \mathrm{E}+02$ & $3.9 \mathrm{E}+01$ & & & $5.5 E+03$ & $5.5 \mathrm{E}+02$ & & & & & & & $2.2 E+03$ & $2.2 E+02$ \\
\hline $\begin{array}{l}\text { Tribroma- } \\
\text { chloromethane }\end{array}$ & $000594-15-0$ & & & & & & & & & & & & & & & & & & \\
\hline $\begin{array}{l}\text { Tribromodi- } \\
\text { phenyl Eher }\end{array}$ & 049690-94-0 & & & & & & & & & & & & & & & & & & \\
\hline $\begin{array}{l}\text { Tributyltin } \\
\text { Oxide }\end{array}$ & $000056-35.9$ & & & $2.2 \mathrm{E}+01$ & $2.3 \mathrm{E}+00$ & $2.2 \mathrm{E}+00$ & $2.3 \mathrm{E}-01$ & & & $2.1 \mathrm{E}+01$ & $2.1 E+\infty 0$ & & & & & & & 1.1E+01 & $1.1 E+00$ \\
\hline $\begin{array}{l}\text { Trichloro- } \\
1,2,2,2- \\
\text { tifluoroeth- } \\
\text { ane, 1,1,2- }\end{array}$ & $000076-13-1$ & & & $1.0 E+06$ & $1.0 E+06$ & $1.0 \mathrm{E}+06$ & $2.3 \mathrm{E}+05$ & & & $1.0 E+06$ & $1.0 E+06$ & & & $2.1 \mathrm{E}+02$ & $2.1 E+02$ & & & 2.1E+02 & 2.IE+02 \\
\hline $\begin{array}{l}\text { Trichloro-2- } \\
\text { hydroxydi- } \\
\text { phenylether }\end{array}$ & $003380-34.5$ & & & & & & & & & & & & & & & & & & \\
\hline $\begin{array}{l}\text { Trichloroace. } \\
\text { tic Acid }\end{array}$ & & & & & & & & & & & & & & & & & & & \\
\hline
\end{tabular}


Table 3.1c. (continued)

\begin{tabular}{|c|c|c|c|c|c|c|c|c|c|c|c|c|c|c|c|c|c|c|c|}
\hline \multirow{2}{*}{ Chemical } & \multirow{2}{*}{ CASRN } & \multicolumn{6}{|c|}{ Ingestion (mg/kg) } & \multicolumn{4}{|c|}{ Dermal (mg/kg) } & \multicolumn{4}{|c|}{ Inhalation $(\mathrm{mg} / \mathrm{kg})^{-\mathbf{r}}$} & \multicolumn{4}{|c|}{$\begin{array}{l}\text { Ingestion + Dermal } \\
+ \text { Inhalation (mg/kg) }\end{array}$} \\
\hline & & $10^{-4}$ & $10^{-6}$ & $\begin{array}{l}H Q=1 \\
\text { Adult }\end{array}$ & $\begin{array}{l}\text { HQ=1 } \\
\text { Child }\end{array}$ & $\begin{array}{c}\mathrm{HQ}=0.1 \\
\text { Adult }\end{array}$ & $\begin{array}{l}\text { HQ=0.1 } \\
\text { Child }\end{array}$ & $10^{-4}$ & $10^{4}$ & $H Q=1$ & $\mathrm{HQ}=0.1$ & $10^{-4}$ & $10^{6}$ & $\mathrm{HQ}=1$ & $\mathrm{HQ}=0.1$ & $10^{-4}$ & $10^{4}$ & $\mathrm{HI}=1$ & $\mathrm{HI}=0.1$ \\
\hline $\begin{array}{l}\text { Trichloroanil- } \\
\text { ine } \mathrm{HCl}, 2,4,6-\end{array}$ & $033663-50-2$ & $2.2 E+03$ & $2.2 \mathrm{E}+01$ & & & & & $5.5 E+03$ & $5.5 \mathrm{E}+01$ & & & & & & & $2.9 E+03$ & $2.9 \mathrm{E}+01$ & & \\
\hline $\begin{array}{l}\text { Trichloroanil- } \\
\text { ine, 2,4,6- }\end{array}$ & 000634.93 .5 & $1.9 \mathrm{E}+03$ & $1.9 \mathrm{E}+01$ & & & & & $4.7 \mathrm{E}+03$ & 4.7E+01 & & & & & & & $2.4 \mathrm{E}+03$ & $2.4 \mathrm{E}+01$ & & \\
\hline $\begin{array}{l}\text { Trichloroben- } \\
\text { zene, 1,2,4- }\end{array}$ & $000120-82-1$ & & & $7.3 \mathrm{E}+03$ & $7.8 \mathrm{E}+02$ & $7.3 E+02$ & $7.8 \mathrm{E}+01$ & & & $1.3 \mathrm{E}+04$ & $1.3 \mathrm{E}+03$ & & & $8.3 \mathrm{E}+02$ & 8.3E+02 & & & $8.3 E+02$ & $4.4 E+02$ \\
\hline $\begin{array}{l}\text { Trichloroeth- } \\
\text { ane, } 1,1,1_{1}^{13}\end{array}$ & $000071-55-6$ & & & $6.6 \mathrm{E}+04$ & $7.0 \mathrm{E}+03$ & $6.6 \mathrm{E}+03$ & $7.0 \mathrm{E}+02$ & & & 1.1E+05 & $1.1 \mathrm{E}+04$ & & & $7.3 \mathrm{E}+02$ & $7.3 \mathrm{E}+02$ & & & $7.3 \mathrm{E}+02$ & $7.3 \mathrm{E}+02$ \\
\hline $\begin{array}{l}\text { Trichloroeth- } \\
\text { ane, 1,1,2- }\end{array}$ & $000079-00-5$ & $1.1 \mathrm{E}+03$ & $1.1 \mathrm{E}+01$ & $2.9 \mathrm{E}+03$ & $3.1 E+02$ & $2.9 \mathrm{E}+02$ & $3.1 \mathrm{E}+01$ & $4.6 \mathrm{E}+03$ & $4.6 \mathrm{E}+01$ & $4.5 E+03$ & $4.5 \mathrm{E}+02$ & $5.3 \mathrm{E}+02$ & $5.3 \mathrm{E}+\infty$ & & & 4.1E+02 & $4.1 \mathrm{E}+00$ & 8.5E+02 & $1.8 \mathrm{E}+02$ \\
\hline $\begin{array}{l}\text { Trichloroethyl- } \\
\text { ene }\end{array}$ & $000079-01-6$ & $5.8 \mathrm{E}+03$ & $5.8 \mathrm{E}+01$ & & & & & $4.4 E+03$ & $4.4 E+01$ & & & $4.5 \mathrm{E}+02$ & $2.7 \mathrm{E}+01$ & & & 4.5E+02 & $1.5 \mathrm{E}+01$ & & \\
\hline $\begin{array}{l}\text { Trichloro- } \\
\text { fluoromethane }\end{array}$ & $000075-69-4$ & & & $2.2 \mathrm{E}+05$ & $2.3 \mathrm{E}+04$ & $2.2 \mathrm{E}+04$ & $2.3 \mathrm{E}+03$ & & & $9.5 \mathrm{E}+0.4$ & $9.5 \mathrm{E}+03$ & & & 7.1E+02 & $4.3 \mathrm{E}+02$ & & & $7.1 \mathrm{E}+02$ & $4.0 \mathrm{E}+02$ \\
\hline $\begin{array}{l}\text { Trichlorophe- } \\
\text { nol, 2,4,5- }\end{array}$ & $000095-95-4$ & & & $7.3 \mathrm{E}+04$ & $7.8 \mathrm{E}+03$ & $7.3 E+03$ & $7.8 E+02$ & & & $1.1 E+05$ & 1.1E+04 & & & & & & & $4.4 \mathrm{E}+04$ & $4.4 E+03$ \\
\hline $\begin{array}{l}\text { Trichlorophe- } \\
\text { nol, 2,4,6- }\end{array}$ & $000088-06-2$ & $5.8 \mathrm{E}+03$ & $5.8 \mathrm{E}+01$ & & & & & $1.5 \mathrm{E}+04$ & $1.5 \mathrm{E}+02$ & & & $1.0 \mathrm{E}+06$ & $1.0 \mathrm{E}+06$ & & & $7.5 \mathrm{E}+03$ & $7.5 \mathrm{E}+01$ & & \\
\hline $\begin{array}{l}\text { Trichlorophe- } \\
\text { noxy) Propi- } \\
\text { onic Acid, } \\
\text { 2(2,4,5- }\end{array}$ & $000093-72-1$ & & & $5.8 \mathrm{E}+03$ & $6.3 \mathrm{E}+02$ & $5.8 \mathrm{E}+02$ & $6.3 \mathrm{E}+01$ & & & $5.5 E+03$ & $5.5 \mathrm{E}+02$ & & & & & & & $2.8 \mathrm{E}+03$ & $2.8 \mathrm{E}+02$ \\
\hline $\begin{array}{l}\text { Trichlorophe- } \\
\text { noxyacetic } \\
\text { Acid, 2,4,5- }\end{array}$ & $000093-76.5$ & & & $7.3 E+03$ & $7.8 \mathrm{E}+02$ & 7.3E+02 & $7.8 \mathrm{E}+01$ & & & $6.9 E+03$ & $6.9 \mathrm{E}+02$ & & & & & & & $3.5 E+03$ & $3.5 \mathrm{E}+02$ \\
\hline $\begin{array}{l}\text { Trichloropro- } \\
\text { pane, 1,1,2- }\end{array}$ & $000598-77-6$ & & & $3.7 \mathrm{E}+03$ & $3.9 \mathrm{E}+02$ & $3.7 E+02$ & $3.9 E+01$ & & & $5.5 \mathrm{E}+03$ & $5.5 \mathrm{E}+02$ & & & & & & & $2.2 E+03$ & $2.2 \mathrm{E}+02$ \\
\hline $\begin{array}{l}\text { Trichloropro- } \\
\text { pane, 1,2,3- }\end{array}$ & $000096-18-4$ & $9.1 E+00$ & $9.1 \mathrm{E}-02$ & $4.4 E+03$ & 4.7E+02 & $4.4 \mathrm{E}+02$ & 4.7E+01 & $3.7 E+01$ & $3.7 E-01$ & $6.6 \mathrm{E}+03$ & $6.6 \mathrm{E}+02$ & & & & & $1.5 E+01$ & $1.5 \mathrm{E}-01$ & $2.6 \mathrm{E}+03$ & $2.6 \mathrm{E}+02$ \\
\hline $\begin{array}{l}\text { Trichloropro- } \\
\text { pere, 1,2,3. }\end{array}$ & $000096-19-5$ & & & $3.7 E+03$ & $3.9 E+02$ & $3.7 E+02$ & $3.9 E+01$ & & & $5.5 \mathrm{E}+03$ & $5.5 E+02$ & & & & & & & $2.2 E+03$ & $2.2 E+02$ \\
\hline
\end{tabular}


Table 3.1c. (continued)

\begin{tabular}{|c|c|c|c|c|c|c|c|c|c|c|c|c|c|c|c|c|c|c|c|}
\hline \multirow{2}{*}{ Chemical } & \multirow{2}{*}{ CASRN } & \multicolumn{6}{|c|}{ Ingestion (mg/kg) } & \multicolumn{4}{|c|}{ Dermal (mg/kg) } & \multicolumn{4}{|c|}{ Inhalation (mg/kg) } & \multicolumn{4}{|c|}{$\begin{array}{c}\text { Ingestion + Dermal } \\
+ \text { Inhalation (mg/kg) }{ }^{+*} \\
\end{array}$} \\
\hline & & $10^{-1}$ & $10^{4}$ & $\begin{array}{l}\text { HQ=1 } \\
\text { Adult }\end{array}$ & $\begin{array}{l}\text { HQ=1 } \\
\text { Child }\end{array}$ & $\begin{array}{c}\mathrm{HQ}=0.1 \\
\text { Adult }\end{array}$ & $\begin{array}{c}\mathrm{HQ}=0.1 \\
\text { Child }\end{array}$ & $10^{-4}$ & $10^{4}$ & $\mathrm{HQ}=1$ & $\mathrm{HQ}=0.1$ & $10^{4}$ & $10^{4}$ & $H Q=1$ & $\mathrm{HQ}=0.1$ & $10^{-4}$ & $10^{6}$ & $\mathrm{HI}=1$ & $\mathrm{HI}=0.1$ \\
\hline $\begin{array}{l}\text { Trichhorotolulu- } \\
\text { ene, 2,3,6- }\end{array}$ & $002077-46-5$ & & & & & & & & & & & & & & & & & & \\
\hline $\begin{array}{l}\text { Trichlorotolu- } \\
\text { ene, alpha 2,6- }\end{array}$ & $002014-83-7$ & & & & - & & & & & & & & & & & & & & \\
\hline Tridiphane & $058138-08-2$ & $\cdot$ & & $2.2 \mathrm{E}+03$ & $2.3 \mathrm{E}+02$ & $2.2 E+02$ & 2.3E+01 & & & $2.1 E+03$ & $2.1 \mathrm{E}+02$ & & & & & & & $1.1 E+03$ & $1.1 E+02$ \\
\hline Triethyl Lead & $005224-23-7$ & & & & & & & & & & & & & & & & & & \\
\hline Triethylamine & $000121-44.8$ & & & & & & & & & & & & & $1.2 \mathrm{E}+03$ & $1.2 E+02$ & & & & \\
\hline Trifluralin & $001582-09-8$ & $8.3 \mathrm{E}+03$ & 8.3E+01 & $5.5 \mathrm{E}+03$ & $5.9 \mathrm{E}+02$ & $5.5 \mathrm{E}+02$ & $5.9 \mathrm{E}+01$ & $2.1 \mathrm{E}+04$ & 2.LE+02 & $5,2 \mathrm{E}+03$ & $5.2 \mathrm{E}+02$ & & & & & $1.1 \mathrm{E}+04$ & $1.1 \mathrm{E}+02$ & $2.7 E+03$ & $2.7 E+02$ \\
\hline $\begin{array}{l}\text { Trimethyl } \\
\text { Lead }\end{array}$ & $007442 \cdot 13.9$ & & & & & & & & & & & & & & & & & & \\
\hline $\begin{array}{l}\text { Trimethyl } \\
\text { Phosphate }\end{array}$ & $000512-56-1$ & $1.7 E+03$ & $1.7 E+01$ & & & & & $4.3 \mathrm{E}+03$ & $4.3 \mathrm{E}+01$ & & & & & & & $2.2 \mathrm{E}+03$ & $2.2 \mathrm{E}+01$ & & \\
\hline $\begin{array}{l}\text { Trimethylethyl } \\
\text { Lead }\end{array}$ & $001762-26-1$ & & & & & & & & & & & & & & & & & & \\
\hline $\begin{array}{l}\text { Trinitroben- } \\
\text { zene, 1,3,5- }\end{array}$ & $000099-35-4$ & & & $3.7 \mathrm{E}+01$ & $3.9 \mathrm{E}+\infty 0$ & $3.7 E+\infty 0$ & $3.9 \mathrm{E}-01$ & & & 4.5E+01 & $4.5 \mathrm{E}+00$ & & & & & & & $2.0 \mathrm{E}+01$ & $2.0 \mathrm{E}+00$ \\
\hline $\begin{array}{l}\text { Trinitrophenyl } \\
\text { methylnitr- } \\
\text { amine }\end{array}$ & $000479-45-8$ & & & $7.3 E+03$ & $7.8 \mathrm{E}+02$ & $7.3 \mathrm{E}+02$ & $7.8 \mathrm{E}+01$ & & & $6.9 \mathrm{E}+03$ & $6.9 \mathrm{E}+02$ & & & & & & & $3.5 E+03$ & $3.5 \mathrm{E}+02$ \\
\hline $\begin{array}{l}\text { Trinitrotolu- } \\
\text { ene, 2,4,6- }\end{array}$ & $000118-96-7$ & $2.1 E+03$ & $2.1 \mathrm{E}+01$ & $3.7 \mathrm{E}+02$ & $3.9 \mathrm{E}+01$ & $3.7 E+01$ & $3.9 E+\infty 0$ & $6.4 \mathrm{E}+03$ & $6.4 \mathrm{E}+01$ & $4.1 \mathrm{E}+02$ & 4.1E+01 & & & & & $3.0 E+03$ & $3.0 \mathrm{E}+01$ & $1.9 \mathrm{E}+02$ & $1.9 \mathrm{E}+01$ \\
\hline Tripropyl Lead & $006618-03-7$ & & & & & & & & & & & & & & & & & & \\
\hline $\begin{array}{l}\text { Unanium (Sol- } \\
\text { uble Salts) }\end{array}$ & $\mathrm{NA}$ & & & $2.2 E+03$ & $2.3 \mathrm{E}+02$ & $2.2 \mathrm{E}+02$ & $2.3 \mathrm{E}+01$ & & & 3.5E+04 & $3.5 \mathrm{E}+03$ & & & & & & & 2. $1 \mathrm{E}+03$ & $2.1 \mathrm{E}+02$ \\
\hline $\begin{array}{l}\text { Varadium } \\
\text { Pentoxide }\end{array}$ & $001314-62-1$ & & & $6.6 \mathrm{E}+03$ & $7.0 \mathrm{E}+02$ & $6.6 \mathrm{E}+02$ & $7.0 \mathrm{E}+01$ & & & $2.5 \mathrm{E}+04$ & 2.5E+03 & & & & & & & $5.2 \mathrm{E}+03$ & $5.2 E+02$ \\
\hline $\begin{array}{l}\text { Vanadium Sul- } \\
\text { fate }\end{array}$ & $036907-42-3$ & & & $1.5 \mathrm{E}+04$ & $1.6 \mathrm{E}+03$ & $1.5 \mathrm{E}+03$ & $1.6 \mathrm{E}+02$ & & & 5.5E+04 & $5.5 \mathrm{E}+03$ & & & & & & & $1.2 \mathrm{E}+04$ & $1.2 E+03$ \\
\hline $\begin{array}{l}\text { Vanadium, } \\
\text { Metallic }\end{array}$ & $007440-62-2$ & & & $5.1 E+03$ & $5.5 E+02$ & $5.1 E+02$ & $5.5 \mathrm{E}+01$ & & & $9.6 \mathrm{E}+02$ & $9.6 \mathrm{E}+01$ & & & & & & & $8.1 E+02$ & $8.1 E+01$ \\
\hline
\end{tabular}

Nonradionuclides/soil/residential

Page 39 of 41 
Table 3.1c. (continued)

\begin{tabular}{|c|c|c|c|c|c|c|c|c|c|c|c|c|c|c|c|c|c|c|c|}
\hline \multirow{2}{*}{ Chemical } & \multirow{2}{*}{ CASRN } & \multicolumn{6}{|c|}{ Ingestion (mg/kg) } & \multicolumn{4}{|c|}{ Dermal (mg/kg) } & \multicolumn{4}{|c|}{ Inhalation $(\mathrm{mg} / \mathrm{kg})^{* v}$} & \multicolumn{4}{|c|}{$\begin{array}{l}\text { Ingestion + Dermal } \\
+ \text { Inhalation (mg/kg) }\end{array}$} \\
\hline & & $10^{-4}$ & $10^{4}$ & $\begin{array}{l}\text { HQ=1 } \\
\text { Adult }\end{array}$ & $\begin{array}{l}\mathrm{HQ}=1 \\
\text { Child }\end{array}$ & $\begin{array}{c}H Q=0.1 \\
\text { Adult }\end{array}$ & $\begin{array}{c}\text { HQ }=0.1 \\
\text { Child }\end{array}$ & $10^{-4}$ & $10^{-6}$ & $\mathrm{HQ}=1$ & $\mathrm{HQ}=0.1$ & $10^{-4}$ & $10^{4}$ & $H Q=1$ & $\mathrm{HQ}=0.1$ & $10^{-4}$ & $10^{4}$ & $\mathrm{HI}=1$ & $H I=0.1$ \\
\hline $\begin{array}{l}\text { Vanadyl Sul- } \\
\text { fate }\end{array}$ & $027774-13-6$ & . & & $1.5 \mathrm{E}+04$ & $1.6 \mathrm{E}+03$ & $1.5 \mathrm{E}+03$ & $1.6 \mathrm{E}+02$ & & & S.SE+04 & $5.5 E+03$ & & & & & & & $1.2 \mathrm{E}+04$ & $1.2 \mathrm{E}+03$ \\
\hline Vemolate & $001929-77.7$ & & & $7.3 E+02$ & $7.8 \mathrm{E}+01$ & $7.3 E+01$ & $7.8 \mathrm{E}+00$ & & & $6.9 E+02$ & $6.9 E+01$ & & & & & & & $3.5 \mathrm{E}+02$ & $3.5 \mathrm{E}+01$ \\
\hline Vinclozolin & $050471-44-8$ & & & $1.8 \mathrm{E}+04$ & $2.0 \mathrm{E}+03$ & $1.8 \mathrm{E}+03$ & $2.0 \mathrm{E}+02$ & & & $1.7 \mathrm{E}+04$ & $1.7 E+03$ & & & & & & & $8.9 E+03$ & $8.9 E+02$ \\
\hline Vinyl Acetate & $000108.05-4$ & & & 7.3E+05 & $7.8 \mathrm{E}+04$ & $7.3 E+04$ & $7.8 \mathrm{E}+03$ & & & $9.0 \mathrm{E}+05$ & $9.0 \mathrm{E}+04$ & & & $7.8 \mathrm{E}+02$ & $2.8 \mathrm{E}+02$ & & & $7.8 \mathrm{E}+02$ & $2.7 \mathrm{E}+02$ \\
\hline Vinyl Bromide & $000593-60-2$ & & & & & & & & & & & $1.1 \mathrm{E}+02$ & $1.1 \mathrm{E}+\infty$ & $4.4 E+01$ & $4.4 E+\infty 0$ & & & & \\
\hline $\begin{array}{l}\text { Viny! Chlo- } \\
\text { ride }\end{array}$ & $000075.01-4$ & $3.4 E+01$ & $3.4 \mathrm{E}-01$ & & & & & $1.7 E+02$ & $1.7 \mathrm{E}+\infty$ & & & 2.5E-02 & 2.5E-04 & & & $2.4 \mathrm{E}-02$ & 2.4E-04 & & \\
\hline Warferin & $000081-81-2$ & & & $2.2 E+02$ & $2.3 \mathrm{E}+01$ & $2.2 E+01$ & $2.3 \mathrm{E}+00$ & & & $2.1 \mathrm{E}+02$ & $2.1 E+01$ & & & & & & & $1.1 \mathrm{E}+02$ & $1.1 \mathrm{E}+01$ \\
\hline $\begin{array}{l}\text { White Phos- } \\
\text { phorus }\end{array}$ & $007723-14-0$ & & & $1.5 \mathrm{E}+01$ & $1.6 \mathrm{E}+00$ & $1.5 \mathrm{E}+\infty$ & $1.6 \mathrm{E}-01$ & & & $5.5 \mathrm{E}+01$ & $5.5 \mathrm{E}+00$ & & & & & & & $1.2 \mathrm{E}+01$ & $1.2 \mathrm{E}+\infty$ \\
\hline $\begin{array}{l}\text { Xylence, Mix- } \\
\text { ture }\end{array}$ & $001330-20-7$ & & & $1.0 \mathrm{E}+06$ & $1.6 \mathrm{E}+05$ & $1.5 \mathrm{E}+05$ & $1.6 \mathrm{E}+04$ & & & $1.0 \mathrm{E}+06$ & $2.5 E+05$ & & & & & & & $9.3 \mathrm{E}+05$ & $9.3 \mathrm{E}+04$ \\
\hline Xylene, $P_{-(n)}$ & $000106-42-3$ & & & & & & & & & & & & & & & & & & \\
\hline Xylene, m-(i) & $000108-38-3$ & & & $1.0 \mathrm{E}+06$ & $1.6 \mathrm{E}+05$ & $1.5 \mathrm{E}+05$ & $1.6 \mathrm{E}+04$ & & & $1.0 \mathrm{E}+06$ & $2.2 E+05$ & & & & & & & $8.8 \mathrm{E}+05$ & $8.8 \mathrm{E}+04$ \\
\hline Xylene, $0 .(n)$ & $000095-47.6$ & & & $1.0 \mathrm{E}+06$ & $1.6 \mathrm{E}+05$ & $1.5 E+05$ & $1.6 \mathrm{E}+04$ & & & $1.0 \mathrm{E}+06$ & $2.2 E+05$ & & & & & & & $8.8 \mathrm{E}+05$ & $8.8 E+04$ \\
\hline Zinc (Metallic) & 007440.66 .6 & & & $2.2 E+05$ & $2.3 \mathrm{E}+04$ & $2.2 E+04$ & $2.3 \mathrm{E}+03$ & & & $8.3 E+05$ & $8.3 E+04$ & & & & & & & $1.7 E+05$ & $1.7 \mathrm{E}+04$ \\
\hline Zinc Cyanide & $000557-21-1$ & & & 3.7E+04 & $3.9 \mathrm{E}+03$ & $3.7 E+03$ & $3.9 E+02$ & & & $1.4 E+05$ & $1.4 E+04$ & & & & & & & $2.9 E+04$ & $2.9 \mathrm{E}+03$ \\
\hline $\begin{array}{l}\text { Zinc Phos- } \\
\text { phide }\end{array}$ & $001314-84-7$ & & & $2.2 \mathrm{E}+02$ & $2.3 E+0 !$ & $2.2 \mathrm{E}+01$ & $2.3 \mathrm{E}+\infty$ & & & $8.3 E+02$ & $8.3 E+01$ & & & & & & & $1.7 \mathrm{E}+02$ & $1.7 \mathrm{E}+01$ \\
\hline Zineb & $012122.67-7$ & & & 3.7E+04 & $3.9 E+03$ & $3.7 \mathrm{E}+03$ & $3.9 E+02$ & & & $3.4 E+04$ & $3.4 E+03$ & & & & & & & $1.8 E+04$ & $1.8 \mathrm{E}+03$ \\
\hline Zirconium & $007440-67-7$ & & & & & & & & & & & & & & & & & & \\
\hline
\end{tabular}

Notes:

* By definition, $1 \mathrm{~kg}=1.0 \mathrm{E}+06 \mathrm{mg}$. Therefore, a limit of $1.0 \mathrm{E}+06 \mathrm{mg} / \mathrm{kg}$ was used for any PRG that was calculated to be above this limit. 


\section{Table 3.1c. (continued)}

$\dagger \quad$ If slope factors and/or reference doses were available for only one pathway (i.e., ingestion, dermal, OR inhalation), the total for ingestion plus inhalation plus dermal was not calculated.

¥ The volatilization factor defines the relationship between the concentration of contaminants in soil and the volatilization of contaminants in air. The chemical concentration in soil $(C)$ calculated using the volatilization factor must be compared with the soil saturation concentration $\left(C_{s a t}\right)$. If $C$ is greater than $C_{\text {sat }}$, then the PRG is set equal to $\mathrm{C}_{\mathrm{sat}}$

(1) The same oral slope factor as used for polychlorinated biphenyls.

(2) The PRG was calculated considering Supplemental Guidance from RAGS: Region 4 Bulletins, Human Health Risk Assessment (Interim Guidance) from EPA (received February 11, 1992). The slope factors for chemicals so marked indicate that a toxicity equivalency factor (TEF) method was used for carcinogenic PAHs based on each compound's relative potency to the potency of benzo[a]pyrene. The following TEFs were used to convert each PAH slope factor to an equivalent slope factor of benzo[a]pyrene [that has a new cancer slope factor of $7.3(\mathrm{mg} / \mathrm{kg} / \mathrm{day})^{-1}$ identified by EPA's CRAVE workgroup]: (1) benzo[a]pyrene, TEF $=1.0 ;(2)$ benzo[a]anthracene, TEF $=0.1$; (3) benzo[b]fluoranthene, TEF $=0.1$; (4) benzo[k]fluoranthene, TEF $=0.01 ;(5)$ chrysene, TEF $=0.001 ;(6)$ dibenz $[\mathrm{a}, \mathrm{h}]$ anthracene, TEF $=1.0$; and $(7)$ indeno[ $[1,2,3-c, d]$ pyrene, $T E F=0.1$.

(3) The toxicity values for trichloroethylene have been withdrawn from IRIS and HEAST pending further investigation. However, the most recent toxicity values have been used to calculate the PRG.

(4) The PRG was calculated considering Supplemental Guidance from RAGS: Region 4 Bulletins, Human Health Risk Assessment (Interim Guidance) from EPA (November 1995). The slope factors for chemicals so marked indicate that a TEF methodology was used for chlorinated dioxin and furan congeners based on each compound's relative potency to the potency of 2,3,7,8-tetrachlorodibenzodioxin (TCDD). The following TEFs were used to convert each slope factor to an equivalent slope factor of 2,3,7,8TCDD: (1) 2,3,7,8-TCDD, TEF = 1.0; (2) 2,3,7,8-PeCDD, TEF = 0.5; (3) 2,3,7,8-HxCDD, TEF = 0.1; (4) 2,3,7,8-HpCDD, TEF =0.01; (5) OCDD, TEF = 0.001; (6) $2,3,7,8-\mathrm{TCDF}, \mathrm{TEF}=0.1 ;$ (7) $1,2,3,7,8-\mathrm{PeCDF}, \mathrm{TEF}=0.5 ;(8)$ 2,3,4,7,8-PeCDF, TEF $=0.05 ;(9) 2,3,7,8-\mathrm{HxCDF}, \mathrm{TEF}=0.1 ;(10) 2,3,7,8-\mathrm{HpCDF}, \mathrm{TEF}=0.01$; and (11) OCDF, TEF $=0.001$.

(5) Toxicity values for these chemicals have been withdrawn from IRIS and HEAST pending further investigation. However, the most recent toxicity values have been used to calculate the PRG.

(6) Toxicity values for these chemicals were obtained from the Superfund Health Risk Technical Support Center. 
เ 
Table 3.1d. Risk-based Preliminary Remediation Goals for radionuclides in soil (residential scenario)

\begin{tabular}{|c|c|c|c|c|c|c|c|c|c|}
\hline \multirow[t]{2}{*}{ Chemical $^{t}$} & \multirow[t]{2}{*}{ CASRN } & \multicolumn{2}{|c|}{$\begin{array}{c}\text { Ingestion } \\
\text { (pCi/g) }\end{array}$} & \multicolumn{2}{|c|}{$\begin{array}{c}\text { Inhalation } \\
\text { (pCi/g) }\end{array}$} & \multicolumn{2}{|c|}{$\begin{array}{l}\text { External Exposure } \\
(\mathrm{pCi} / \mathrm{g})^{t+}\end{array}$} & \multicolumn{2}{|c|}{$\begin{array}{l}\text { Ingestion + External + } \\
\text { Inhalation (pCi/g) }\end{array}$} \\
\hline & & $10^{-4}$ & $10^{-6}$ & $10^{-4}$ & $10^{-6}$ & $10^{-4}$ & $10^{-6}$ & $10^{-4}$ & $10^{-6}$ \\
\hline Ac-225 & $014265-85-1$ & $5.6 \mathrm{E}+02$ & $5.6 \mathrm{E}+00$ & $4.9 \mathrm{E}+05$ & $4.9 \mathrm{E}+03$ & $5.6 \mathrm{E}+02$ & $5.6 \mathrm{E}+00$ & $3.0 \mathrm{E}+02$ & $3.0 \mathrm{E}+00$ \\
\hline Ac-227 & $014952-40-0$ & $2.3 \mathrm{E}+02$ & $2.3 \mathrm{E}+00$ & $2.9 \mathrm{E}+04$ & $2.9 \mathrm{E}+02$ & $1.8 \mathrm{E}+05$ & $1.8 \mathrm{E}+03$ & $2.7 E+02$ & $2.7 \mathrm{E}+00$ \\
\hline Ac-227+D & $014952-40-0$ & $1.3 \mathrm{E}+02$ & $1.3 \mathrm{E}+00$ & $2.6 \mathrm{E}+04$ & $2.6 \mathrm{E}+02$ & $7.3 E+00$ & 7.3E-02 & $6.9 E+00$ & $6.9 \mathrm{E}-02$ \\
\hline Ac- 228 & 014331-83-0 & $4.9 E+04$ & $4.9 \mathrm{E}+02$ & $6.2 \mathrm{E}+07$ & $6.2 \mathrm{E}+05$ & $1.3 \mathrm{E}+00$ & $1.3 \mathrm{E}-02$ & $1.3 \mathrm{E}+00$ & $1.3 \mathrm{E}-02$ \\
\hline $\mathrm{Ag}-105$ & 014928-14-4 & $4.9 \mathrm{E}+04$ & $4.9 E+02$ & $8.8 \mathrm{E}+08$ & $8.8 \mathrm{E}+06$ & & & $5.8 \mathrm{E}+04$ & $5.8 \mathrm{E}+02$ \\
\hline $\mathrm{Ag}-108$ & 014391-65-2 & $1.1 E+07$ & $1.1 E+05$ & $2.2 \mathrm{E}+11$ & $2.2 \mathrm{E}+09$ & $7.5 \mathrm{E}+01$ & 7.5E-01 & $7.5 \mathrm{E}+01$ & $7.5 \mathrm{E}-01$ \\
\hline Ag-108m & $014391-65-2$ & $1.3 E+04$ & $1.3 \mathrm{E}+02$ & $2.9 \mathrm{E}+07$ & $2.9 E+05$ & 7.7E-01 & 7.7E-03 & $7.7 \mathrm{E}-01$ & 7.7E-03 \\
\hline$A g-108 m+D$ & $014391-65-2$ & $1.3 \mathrm{E}+04$ & $1.3 \mathrm{E}+02$ & $2.9 \mathrm{E}+07$ & $2.9 \mathrm{E}+05$ & 7.7E-01 & 7.7E-03 & 7.7E-01 & 7.7E-03 \\
\hline $\mathrm{Ag}-109 \mathrm{~m}$ & $014378-38-2$ & $2.9 E+08$ & $2.9 E+06$ & $5.9 \mathrm{E}+12$ & $5.9 \mathrm{E}+10$ & $3.7 \mathrm{E}+03$ & $3.7 \mathrm{E}+01$ & $3.7 \mathrm{E}+03$ & $3.7 \mathrm{E}+01$ \\
\hline Ag-110 & 014391-76-5 & $3.3 \mathrm{E}+07$ & 3.3E+05 & $6.5 \mathrm{E}+11$ & $6.5 E+09$ & $3.8 \mathrm{E}+01$ & $3.8 \mathrm{E}-01$ & $3.8 E+01$ & $3.8 \mathrm{E}-01$ \\
\hline $\mathrm{Ag}-110 \mathrm{~m}$ & $015760-04-0$ & $9.4 \mathrm{E}+03$ & $9.4 \mathrm{E}+01$ & $6.4 \mathrm{E}+07$ & $6.4 \mathrm{E}+05$ & $4.1 \mathrm{E}-01$ & 4.1E-03 & $4.1 \mathrm{E}-01$ & $4.1 \mathrm{E}-03$ \\
\hline $\mathrm{Ag}-111$ & $157690-04-0$ & $1.2 \mathrm{E}+04$ & $1.2 \mathrm{E}+02$ & $3.9 \mathrm{E}+08$ & $3.9 \mathrm{E}+06$ & $5.1 \mathrm{E}+01$ & $5.1 \mathrm{E}-01$ & $5.1 \mathrm{E}+01$ & $5.1 \mathrm{E}-01$ \\
\hline Am-241 & $014596-10-2$ & $2.4 \mathrm{E}+02$ & $2.4 \mathrm{E}+00$ & $5.3 \mathrm{E}+04$ & $5.3 \mathrm{E}+02$ & $9.5 \mathrm{E}+02$ & $9.5 \mathrm{E}+00$ & $2.2 \mathrm{E}+02$ & $2.2 \mathrm{E}+00$ \\
\hline Am-242 & 013981-54-9 & $5.4 E+04$ & $5.4 \mathrm{E}+02$ & $2.0 \mathrm{E}+08$ & $2.0 \mathrm{E}+06$ & $7.5 E+02$ & $7.5 \mathrm{E}+00$ & $7.5 \mathrm{E}+02$ & $7.5 \mathrm{E}+00$ \\
\hline $\mathrm{Am}-242 \mathrm{~m}$ & 013981-54-9 & $2.7 E+02$ & $2.7 \mathrm{E}+00$ & $5.8 \mathrm{E}+04$ & $5.8 \mathrm{E}+02$ & $5.0 \mathrm{E}+04$ & $5.0 \mathrm{E}+02$ & $3.2 \mathrm{E}+02$ & $3.2 \mathrm{E}+00$ \\
\hline Am-243 & 014993-75-0 & $2.4 \mathrm{E}+02$ & $2.4 \mathrm{E}+00$ & $5.3 \mathrm{E}+04$ & $5.3 E+02$ & $1.8 \mathrm{E}+02$ & $1.8 \mathrm{E}+00$ & $1.1 \mathrm{E}+02$ & $1.1 \mathrm{E}+00$ \\
\hline$A m-243+D$ & 014993-75-0 & $2.4 \mathrm{E}+02$ & $2.4 \mathrm{E}+00$ & $5.3 \mathrm{E}+04$ & $5.3 \mathrm{E}+02$ & $1.6 \mathrm{E}+01$ & $1.6 \mathrm{E}-01$ & $1.5 \mathrm{E}+01$ & $1.5 \mathrm{E}-01$ \\
\hline
\end{tabular}


Table 3.1d. (continued)

\begin{tabular}{|c|c|c|c|c|c|c|c|c|c|}
\hline \multirow[t]{2}{*}{ Chemical ${ }^{\dagger}$} & \multirow[t]{2}{*}{ CASRN } & \multicolumn{2}{|c|}{$\begin{array}{c}\text { Ingestion } \\
\text { (pCi/g) }\end{array}$} & \multicolumn{2}{|c|}{$\begin{array}{c}\text { Inhalation } \\
\text { (pCi/g) }\end{array}$} & \multicolumn{2}{|c|}{$\begin{array}{c}\text { External Exposure } \\
(\mathrm{pCi} / \mathrm{g})^{\dagger \dagger}\end{array}$} & \multicolumn{2}{|c|}{$\begin{array}{c}\text { Ingestion + External + } \\
\text { Inhalation (pCi/g) }\end{array}$} \\
\hline & & $10^{-4}$ & $10^{-6}$ & $10^{-4}$ & $10^{-6}$ & $10^{-4}$ & $10^{-6}$ & $10^{-4}$ & $10^{-6}$ \\
\hline Ar-41 & $014163-25-8$ & & & $4.3 E+12$ & $4.3 \mathrm{E}+10$ & & & & \\
\hline At-217 & $017239-90-6$ & $8.8 \mathrm{E}+09$ & $8.8 \mathrm{E}+07$ & $4.0 \mathrm{E}+12$ & $4.0 \mathrm{E}+10$ & $5.0 \mathrm{E}+03$ & $5.0 \mathrm{E}+01$ & $5.0 \mathrm{E}+03$ & $5.0 \mathrm{E}+01$ \\
\hline$A u-196$ & 014914-16-0 & $6.1 E+04$ & $6.1 \mathrm{E}+02$ & $2.0 \mathrm{E}+09$ & $2.0 \mathrm{E}+07$ & $3.1 \mathrm{E}+00$ & $3.1 \mathrm{E}-02$ & $3.1 \mathrm{E}+00$ & $3.1 \mathrm{E}-02$ \\
\hline Au-198 & 010043-49-0 & $1.5 \mathrm{E}+04$ & $1.5 \mathrm{E}+02$ & $5.6 \mathrm{E}+08$ & $5.6 \mathrm{E}+06$ & $3.2 \mathrm{E}+00$ & $3.2 \mathrm{E}-02$ & $3.2 \mathrm{E}+00$ & $3.2 \mathrm{E}-02$ \\
\hline $\mathrm{Ba}-131$ & $014914-75-1$ & $4.7 \mathrm{E}+04$ & $4.7 \mathrm{E}+02$ & $4.3 \mathrm{E}+09$ & $4.3 E+07$ & $3.4 \mathrm{E}+00$ & $3.4 \mathrm{E}-02$ & $3.4 \mathrm{E}+00$ & $3.4 \mathrm{E}-02$ \\
\hline Ba-133 & $013981-41-4$ & $2.9 \mathrm{E}+04$ & $2.9 \mathrm{E}+02$ & $5.1 \mathrm{E}+08$ & $5.1 \mathrm{E}+06$ & $4.7 \mathrm{E}+00$ & $4.7 \mathrm{E}-02$ & $4.7 \mathrm{E}+00$ & 4.7E-02 \\
\hline Ba-133m & $013981-41-4$ & $2.9 \mathrm{E}+04$ & $2.9 \mathrm{E}+02$ & $3.6 \mathrm{E}+09$ & $3.6 \mathrm{E}+07$ & $4.3 \mathrm{E}+01$ & $4.3 \mathrm{E}-01$ & $4.3 \mathrm{E}+01$ & $4.3 \mathrm{E}-01$ \\
\hline $\mathrm{Ba}-137 \mathrm{~m}$ & $013981-97-0$ & $3.3 \mathrm{E}+07$ & $3.3 E+05$ & $1.3 \mathrm{E}+12$ & $1.3 \mathrm{E}+10$ & $2.0 \mathrm{E}+00$ & $2.0 \mathrm{E}-02$ & $2.0 \mathrm{E}+00$ & $2.0 \mathrm{E}-02$ \\
\hline Ba-139 & $014378-25-7$ & $2.6 \mathrm{E}+05$ & $2.6 \mathrm{E}+03$ & $1.3 \mathrm{E}+10$ & $1.3 E+08$ & $5.2 \mathrm{E}+01$ & $5.2 \mathrm{E}-01$ & $5.2 \mathrm{E}+01$ & $5.2 \mathrm{E}-01$ \\
\hline $\mathrm{Ba}-140$ & $014798-08-4$ & $6.7 \mathrm{E}+03$ & $6.7 \mathrm{E}+01$ & $6.4 \mathrm{E}+08$ & $6.4 \mathrm{E}+06$ & $7.2 \mathrm{E}+00$ & $7.2 \mathrm{E}-02$ & $7.2 \mathrm{E}+00$ & $7.2 \mathrm{E}-02$ \\
\hline $\mathrm{Be}-7$ & $013966-02-4$ & $9.2 \mathrm{E}+05$ & $9.2 \mathrm{E}+03$ & $1.1 \mathrm{E}+10$ & $1.1 \mathrm{E}+08$ & $2.5 \mathrm{E}+01$ & $2.5 \mathrm{E}-01$ & $2.5 \mathrm{E}+01$ & $2.5 \mathrm{E}-01$ \\
\hline Bi-206 & 015776-19-9 & $1.1 \mathrm{E}+04$ & $1.1 \mathrm{E}+02$ & $4.0 \mathrm{E}+08$ & $4.0 \mathrm{E}+06$ & $3.6 \mathrm{E}-01$ & $3.6 \mathrm{E}-03$ & $3.6 \mathrm{E}-01$ & $3.6 \mathrm{E}-03$ \\
\hline Bi-207 & $013982-38-2$ & $1.6 \mathrm{E}+04$ & $1.6 \mathrm{E}+02$ & $2.2 \mathrm{E}+08$ & $2.2 E+06$ & $7.9 \mathrm{E}-01$ & $7.9 \mathrm{E}-03$ & $7.9 \mathrm{E}-01$ & $7.9 \mathrm{E}-03$ \\
\hline $\mathrm{Bi}-210$ & $014331-79-4$ & $1.1 \mathrm{E}+04$ & $1.1 \mathrm{E}+02$ & $4.0 \mathrm{E}+07$ & $4.0 \mathrm{E}+05$ & & & $1.3 E+04$ & $1.3 \mathrm{E}+02$ \\
\hline Bi-211 & 015229-37-5 & $4.4 \mathrm{E}+06$ & $4.4 \mathrm{E}+04$ & $1.2 \mathrm{E}+09$ & $1.2 \mathrm{E}+07$ & $2.9 \mathrm{E}+01$ & $2.9 \mathrm{E}-01$ & $2.9 \mathrm{E}+01$ & $2.9 \mathrm{E}-01$ \\
\hline $\mathrm{Bi}-212$ & $014913-49-6$ & $1.3 \mathrm{E}+05$ & $1.3 \mathrm{E}+03$ & $5.6 \mathrm{E}+07$ & $5.6 \mathrm{E}+05$ & $6.5 \mathrm{E}+00$ & $6.5 \mathrm{E}-02$ & $6.5 \mathrm{E}+00$ & $6.5 \mathrm{E}-02$ \\
\hline $\mathrm{Bi}-213$ & $015776-20-2$ & $1.8 \mathrm{E}+05$ & $1.8 \mathrm{E}+03$ & $6.6 \mathrm{E}+07$ & $6.6 \mathrm{E}+05$ & $9.4 \mathrm{E}+00$ & $9.4 \mathrm{E}-02$ & $9.4 \mathrm{E}+00$ & 9.4E-02 \\
\hline
\end{tabular}


Table 3.1d. (continued)

\begin{tabular}{|c|c|c|c|c|c|c|c|c|c|}
\hline \multirow[t]{2}{*}{ Chemical' ${ }^{\dagger}$} & \multirow[t]{2}{*}{ CASRN } & \multicolumn{2}{|c|}{$\begin{array}{c}\text { Ingestion } \\
\text { (pCi/g) }\end{array}$} & \multicolumn{2}{|c|}{$\begin{array}{c}\text { Inhalation } \\
\text { (pCi/g) }\end{array}$} & \multicolumn{2}{|c|}{$\begin{array}{l}\text { External Exposure } \\
(\mathrm{pCi} / \mathrm{g})^{\dagger t}\end{array}$} & \multicolumn{2}{|c|}{$\begin{array}{c}\text { Ingestion + External + } \\
\text { Inhalation (pCi/g) }\end{array}$} \\
\hline & & $10^{-4}$ & $10^{-5}$ & $10^{-4}$ & $10^{-6}$ & $10^{-4}$ & $10^{-6}$ & $10^{-4}$ & $10^{-6}$ \\
\hline $\mathrm{Bi}-214$ & 014733-03-0 & $4.1 \mathrm{E}+05$ & $4.1 \mathrm{E}+03$ & $1.4 \mathrm{E}+08$ & $1.4 \mathrm{E}+06$ & $7.2 \mathrm{E}-01$ & $7.2 \mathrm{E}-03$ & $7.2 \mathrm{E}-01$ & $7.2 \mathrm{E}-03$ \\
\hline $\mathrm{Br}-82$ & $014686-69-2$ & $5.6 \mathrm{E}+04$ & $5.6 \mathrm{E}+02$ & $2.6 \mathrm{E}+09$ & $2.6 \mathrm{E}+07$ & $4.3 \mathrm{E}-01$ & $4.3 \mathrm{E}-03$ & $4.3 \mathrm{E}-01$ & $4.3 \mathrm{E}-03$ \\
\hline C-11 & 014333-33-6 & $1.8 \mathrm{E}+06$ & $1.8 \mathrm{E}+04$ & $6.0 \mathrm{E}+10$ & $6.0 \mathrm{E}+08$ & $1.2 \mathrm{E}+00$ & $1.2 \mathrm{E}-02$ & $1.2 \mathrm{E}+00$ & $1.2 \mathrm{E}-02$ \\
\hline C-14 & $014762-75-5$ & $7.7 \mathrm{E}+04$ & $7.7 \mathrm{E}+02$ & $2.9 \mathrm{E}+11$ & $2.9 E+09$ & & & $9.2 \mathrm{E}+04$ & $9.2 \mathrm{E}+02$ \\
\hline $\mathrm{C}-15$ & 015929-23-4 & $1.2 \mathrm{E}+08$ & $1.2 \mathrm{E}+06$ & $2.5 \mathrm{E}+12$ & $2.5 \mathrm{E}+10$ & & & $1.4 \mathrm{E}+08$ & $1.4 \mathrm{E}+06$ \\
\hline $\mathrm{Ca}-45$ & 013966-05-7 & $3.9 E+04$ & $3.9 \mathrm{E}+02$ & 8.1E+08 & $8.1 E+06$ & $1.1 \mathrm{E}+12$ & $1.1 \mathrm{E}+10$ & 4.7E+04 & $4.7 \mathrm{E}+02$ \\
\hline $\mathrm{Ca}-47$ & 001439-99-2 & $1.2 \mathrm{E}+04$ & $1.2 \mathrm{E}+02$ & $3.9 \mathrm{E}+08$ & $3.9 \mathrm{E}+06$ & $1.1 \mathrm{E}+00$ & $1.1 \mathrm{E}-02$ & $1.1 \mathrm{E}+00$ & $1.1 \mathrm{E}-02$ \\
\hline Cd-109 & 014109-32-1 & $9.9 \mathrm{E}+03$ & $9.9 \mathrm{E}+01$ & $1.1 \mathrm{E}+08$ & $1.1 E+06$ & $7.7 \mathrm{E}+03$ & $7.7 \mathrm{E}+01$ & $4.7 \mathrm{E}+03$ & $4.7 \mathrm{E}+01$ \\
\hline Cd-115 & $014336-68-6$ & $1.1 \mathrm{E}+04$ & $1.1 \mathrm{E}+02$ & $4.1 \mathrm{E}+08$ & $4.1 E+06$ & $6.2 \mathrm{E}+00$ & $6.2 \mathrm{E}-02$ & $6.2 \mathrm{E}+00$ & $6.2 \mathrm{E}-02$ \\
\hline $\mathrm{Cd}-115 \mathrm{~m}$ & 014336-68-6 & $5.6 \mathrm{E}+03$ & $5.6 \mathrm{E}+01$ & $1.2 \mathrm{E}+08$ & $1.2 \mathrm{E}+06$ & $5.1 \mathrm{E}+01$ & $5.1 \mathrm{E}-01$ & $5.0 \mathrm{E}+01$ & $5.0 \mathrm{E}-01$ \\
\hline Ce-141 & $013967-74-3$ & $2.0 \mathrm{E}+04$ & $2.0 \mathrm{E}+02$ & $4.7 \mathrm{E}+08$ & $4.7 \mathrm{E}+06$ & $3.1 \mathrm{E}+01$ & $3.1 \mathrm{E}-01$ & $3.1 \mathrm{E}+01$ & $3.1 \mathrm{E}-01$ \\
\hline Ce-143 & 014119-19-8 & $1.3 \mathrm{E}+04$ & $1.3 E+02$ & $5.3 \mathrm{E}+08$ & $5.3 \mathrm{E}+06$ & $5.9 \mathrm{E}+00$ & $5.9 \mathrm{E}-02$ & $5.9 E+00$ & $5.9 \mathrm{E}-02$ \\
\hline Ce-144 & $014762-78-8$ & $2.7 \mathrm{E}+03$ & 2.7E+01 & $1.9 \mathrm{E}+07$ & $1.9 \mathrm{E}+05$ & $1.7 \mathrm{E}+02$ & $1.7 \mathrm{E}+00$ & $1.6 \mathrm{E}+02$ & $1.6 \mathrm{E}+00$ \\
\hline Ce-144+D & $014762-78-8$ & $2.7 \mathrm{E}+03$ & $2.7 \mathrm{E}+01$ & $1.9 \mathrm{E}+07$ & $1.9 E+05$ & $2.8 \mathrm{E}+01$ & $2.8 \mathrm{E}-01$ & $2.8 \mathrm{E}+01$ & 2.8E-01 \\
\hline $\mathrm{Cl}-36$ & 013981-43-6 & $3.6 E+04$ & $3.6 \mathrm{E}+02$ & $1.6 \mathrm{E}+09$ & $1.6 \mathrm{E}+07$ & & & $4.3 E+04$ & $4.3 E+02$ \\
\hline $\mathrm{Cl}-38$ & 014158-34-0 & $3.8 \mathrm{E}+05$ & $3.8 \mathrm{E}+03$ & $1.3 E+10$ & $1.3 E+08$ & $6.7 \mathrm{E}-01$ & $6.7 \mathrm{E}-03$ & $6.7 \mathrm{E}-01$ & $6.7 \mathrm{E}-03$ \\
\hline $\mathrm{Cm}-242$ & $015510-73-3$ & $2.1 E+03$ & $2.1 \mathrm{E}+01$ & $6.5 \mathrm{E}+05$ & $6.5 E+03$ & $1.9 \mathrm{E}+05$ & $1.9 \mathrm{E}+03$ & $2.4 \mathrm{E}+03$ & $2.4 \mathrm{E}+01$ \\
\hline
\end{tabular}




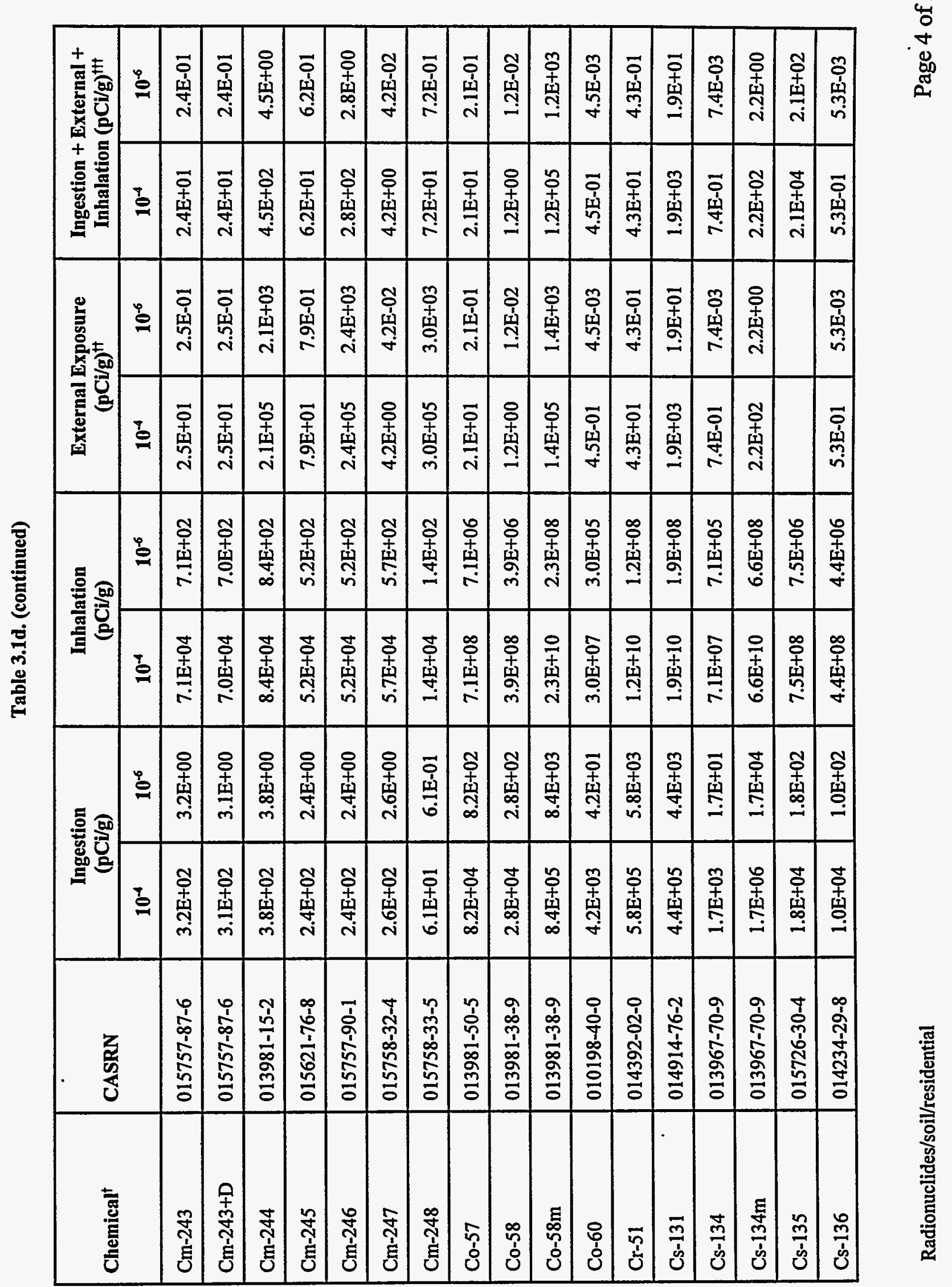


Table 3.1d. (continued)

\begin{tabular}{|c|c|c|c|c|c|c|c|c|c|}
\hline \multirow[t]{2}{*}{ Chemical $^{\dagger}$} & \multirow[t]{2}{*}{ CASRN } & \multicolumn{2}{|c|}{$\begin{array}{l}\text { Ingestion } \\
(\mathrm{pCi} / \mathrm{g})\end{array}$} & \multicolumn{2}{|c|}{$\begin{array}{c}\text { Inhalation } \\
\text { (pCi/g) }\end{array}$} & \multicolumn{2}{|c|}{$\begin{array}{c}\text { External Exposure } \\
(\mathrm{pCi} / \mathrm{g})^{\dagger+}\end{array}$} & \multicolumn{2}{|c|}{$\begin{array}{l}\text { Ingestion + External + } \\
\text { Inhalation }(\mathrm{pCi} / \mathrm{g})^{1+1}\end{array}$} \\
\hline & & $10^{-4}$ & $10^{-6}$ & $10^{-4}$ & $10^{-6}$ & $10^{-4}$ & $10^{-6}$ & $10^{-4}$ & $10^{-6}$ \\
\hline Cs-137 & $010045-97-3$ & $2.5 E+03$ & $2.5 \mathrm{E}+01$ & $1.1 \mathrm{E}+08$ & $1.1 E+06$ & & & $3.0 \mathrm{E}+03$ & $3.0 \mathrm{E}+01$ \\
\hline Cs-137+D & 010045-97-3 & $2.5 E+03$ & $2.5 \mathrm{E}+01$ & $1.1 \mathrm{E}+08$ & $1.1 \mathrm{E}+06$ & $2.1 E+00$ & $2.1 \mathrm{E}-02$ & $2.1 \mathrm{E}+00$ & 2.1E-02 \\
\hline Cs-138 & $015758-29-9$ & $4.5 E+05$ & $4.5 \mathrm{E}+03$ & $1.6 \mathrm{E}+10$ & $1.6 \mathrm{E}+08$ & $4.6 \mathrm{E}-01$ & $4.6 \mathrm{E}-03$ & $4.6 \mathrm{E}-01$ & $4.6 \mathrm{E}-03$ \\
\hline $\mathrm{Cu}-64$ & $013981-25-4$ & $1.5 \mathrm{E}+05$ & $1.5 \mathrm{E}+03$ & $4.9 \mathrm{E}+09$ & $4.9 E+07$ & $6.5 \mathrm{E}+00$ & $6.5 \mathrm{E}-02$ & $6.5 \mathrm{E}+00$ & $6.5 \mathrm{E}-02$ \\
\hline Dy-165 & $013967-64-1$ & $2.4 \mathrm{E}+05$ & $2.4 E+03$ & $9.1 \mathrm{E}+09$ & $9.1 E+07$ & $7.0 \mathrm{E}+01$ & $7.0 \mathrm{E}-01$ & $7.0 \mathrm{E}+01$ & $7.0 \mathrm{E}-01$ \\
\hline Dy-166 & $015840-01-4$ & $8.4 E+03$ & $8.4 \mathrm{E}+01$ & $2.6 E+08$ & $2.6 \mathrm{E}+06$ & $1.6 \mathrm{E}+02$ & $1.6 \mathrm{E}+00$ & $1.6 \mathrm{E}+02$ & $1.6 \mathrm{E}+00$ \\
\hline Er-169 & 015840-13-8 & 3.7E+04 & $3.7 \mathrm{E}+02$ & $1.4 \mathrm{E}+09$ & $1.4 \mathrm{E}+07$ & $6.7 \mathrm{E}+05$ & $6.7 \mathrm{E}+03$ & $4.2 \mathrm{E}+04$ & $4.2 \mathrm{E}+02$ \\
\hline Er-171 & $014391-45-8$ & $4.9 E+04$ & $4.9 E+02$ & $2.7 \mathrm{E}+09$ & $2.7 \mathrm{E}+07$ & $4.2 \mathrm{E}+00$ & $4.2 \mathrm{E}-02$ & $4.2 \mathrm{E}+00$ & $4.2 \mathrm{E}-02$ \\
\hline Eu-152 & $014683-23-9$ & $1.4 \mathrm{E}+04$ & $1.4 E+02$ & $2.6 \mathrm{E}+07$ & $2.6 \mathrm{E}+05$ & $1.1 \mathrm{E}+00$ & $1.1 \mathrm{E}-02$ & $1.1 \mathrm{E}+00$ & $1.1 \mathrm{E}-02$ \\
\hline Eu-154 & $015585-10-1$ & $8.5 \mathrm{E}+03$ & $8.5 E+01$ & $2.2 \mathrm{E}+07$ & $2.2 \mathrm{E}+05$ & 9.3E-01 & $9.3 \mathrm{E}-03$ & 9.3E-01 & $9.3 \mathrm{E}-03$ \\
\hline Eu-155 & 014391-16-3 & $4.8 E+04$ & $4.8 \mathrm{E}+02$ & $2.1 E+08$ & $2.1 E+06$ & $7.1 \mathrm{E}+01$ & 7.1E-01 & $7.1 \mathrm{E}+01$ & $.7 .1 \mathrm{E}-01$ \\
\hline Eu-156 & $014280-35-4$ & $7.3 \mathrm{E}+03$ & 7.3E+01 & $2.2 E+08$ & $2.2 \mathrm{E}+06$ & $8.0 \mathrm{E}-01$ & $8.0 \mathrm{E}-03$ & $8.0 \mathrm{E}-01$ & $8.0 \mathrm{E}-03$ \\
\hline $\mathrm{F}-18$ & $013981-56-1$ & $7.3 \mathrm{E}+05$ & $7.3 \mathrm{E}+03$ & $3.1 E+10$ & $3.1 \mathrm{E}+08$ & $1.2 \mathrm{E}+00$ & $1.2 \mathrm{E}-02$ & $1.2 \mathrm{E}+00$ & $1.2 \mathrm{E}-02$ \\
\hline Fe-55 & 014681-59-5 & $2.3 \mathrm{E}+05$ & $2.3 \mathrm{E}+03$ & $3.6 \mathrm{E}+09$ & $3.6 \mathrm{E}+07$ & & & $2.7 \mathrm{E}+05$ & $2.7 \mathrm{E}+03$ \\
\hline Fe-59 & $014596-12-4$ & $1.4 \mathrm{E}+04$ & $1.4 \mathrm{E}+02$ & $2.9 \mathrm{E}+08$ & $2.9 \mathrm{E}+06$ & $9.4 \mathrm{E}-01$ & $9.4 \mathrm{E}-03$ & $9.4 \mathrm{E}-01$ & $9.4 \mathrm{E}-03$ \\
\hline Fr-221 & $015756-41-9$ & $5.5 \mathrm{E}+05$ & $5.5 \mathrm{E}+03$ & $2.5 \mathrm{E}+08$ & $2.5 \mathrm{E}+06$ & $6.4 \mathrm{E}+01$ & $6.4 \mathrm{E}-01$ & $6.4 \mathrm{E}+01$ & $6.4 \mathrm{E}-01$ \\
\hline Fr-223 & $015756-98-6$ & $1.8 \mathrm{E}+05$ & $1.8 \mathrm{E}+03$ & $3.5 \mathrm{E}+09$ & $3.5 \mathrm{E}+07$ & $1.0 \mathrm{E}+02$ & $1.0 \mathrm{E}+00$ & $1.0 \mathrm{E}+02$ & $1.0 \mathrm{E}+00$ \\
\hline
\end{tabular}


Table 3.1d. (continued)

\begin{tabular}{|c|c|c|c|c|c|c|c|c|c|}
\hline \multirow{2}{*}{ Chemical $^{\dagger}$} & \multirow{2}{*}{ CASRN } & \multicolumn{2}{|c|}{$\begin{array}{c}\text { Ingestion } \\
\text { (pCi/g) }\end{array}$} & \multicolumn{2}{|c|}{$\begin{array}{c}\text { Inhalation } \\
\text { (pCi/g) }\end{array}$} & \multicolumn{2}{|c|}{$\begin{array}{c}\text { External Exposure } \\
(\mathrm{pCi} / \mathrm{g})^{\dagger t}\end{array}$} & \multicolumn{2}{|c|}{$\begin{array}{c}\text { Ingestion + External + } \\
\text { Inhalation (pCi/g) }\end{array}$} \\
\hline & & $10^{-4}$ & $10^{-6}$ & $10^{-4}$ & $10^{-6}$ & $10^{-4}$ & $10^{-6}$ & $10^{-4}$ & $10^{-6}$ \\
\hline Ga-67 & 014119-09-6 & $9.5 \mathrm{E}+04$ & $9.5 \mathrm{E}+02$ & $4.0 \mathrm{E}+09$ & $4.0 \mathrm{E}+07$ & $1.2 \mathrm{E}+01$ & $1.2 \mathrm{E}-01$ & $1.2 \mathrm{E}+01$ & $1.2 \mathrm{E}-01$ \\
\hline $\mathrm{Ga}-72$ & $013982-22-4$ & $1.7 \mathrm{E}+04$ & $1.7 \mathrm{E}+02$ & $9.4 \mathrm{E}+08$ & $9.4 \mathrm{E}+06$ & $3.9 \mathrm{E}-01$ & $3.9 \mathrm{E}-03$ & $3.9 \mathrm{E}-01$ & $3.9 \mathrm{E}-03$ \\
\hline Gd-153 & $014276-65-4$ & $6.0 \mathrm{E}+04$ & $6.0 \mathrm{E}+02$ & $6.4 \mathrm{E}+08$ & $6.4 E+06$ & $6.0 \mathrm{E}+01$ & $6.0 \mathrm{E}-01$ & $6.0 \mathrm{E}+01$ & $6.0 \mathrm{E}-01$ \\
\hline Gd-159 & $014041-42-0$ & $3.1 \mathrm{E}+04$ & $3.1 \mathrm{E}+02$ & $1.6 \mathrm{E}+09$ & $1.6 \mathrm{E}+07$ & $4.5 \mathrm{E}+01$ & $4.5 \mathrm{E}-01$ & $4.5 \mathrm{E}+01$ & $4.5 \mathrm{E}-01$ \\
\hline Ge-71 & $014374-81-3$ & $6.7 \mathrm{E}+06$ & $6.7 E+04$ & $3.5 \mathrm{E}+10$ & $3.5 \mathrm{E}+08$ & $2.8 \mathrm{E}+05$ & $2.8 \mathrm{E}+03$ & $2.7 \mathrm{E}+05$ & $2.7 \mathrm{E}+03$ \\
\hline $\mathrm{H}-3$ & $010028-17-8$ & $1.1 E+06$ & $1.1 \mathrm{E}+04$ & $2.1 E+10$ & $2.1 \mathrm{E}+08$ & & & $1.3 \mathrm{E}+06$ & $1.3 E+04$ \\
\hline $\mathrm{Hg}-197$ & $013981-51-6$ & $6.7 \mathrm{E}+04$ & $6.7 \mathrm{E}+02$ & $2.9 \mathrm{E}+0.9$ & $2.9 \mathrm{E}+07$ & $7.9 \mathrm{E}+01$ & $7.9 \mathrm{E}-01$ & $7.9 \mathrm{E}+01$ & $7.9 \mathrm{E}-01$ \\
\hline $\mathrm{Hg}-203$ & 013982-78-0 & $3.0 \mathrm{E}+04$ & $3.0 \mathrm{E}+02$ & $6.7 E+08$ & $6.7 \mathrm{E}+06$ & $6.9 \mathrm{E}+00$ & $6.9 \mathrm{E}-02$ & $6.9 \mathrm{E}+00$ & $6.9 \mathrm{E}-02$ \\
\hline Ho-166 & $013967-65-2$ & $1.0 \mathrm{E}+04$ & $1.0 \mathrm{E}+02$ & $5.0 \mathrm{E}+08$ & $5.0 \mathrm{E}+06$ & $6.2 \mathrm{E}+01$ & $6.2 \mathrm{E}-01$ & $6.2 \mathrm{E}+01$ & $6.2 \mathrm{E}-01$ \\
\hline I -122 & $018287-75-7$ & $3.7 \mathrm{E}+06$ & $3.7 \mathrm{E}+04$ & $9.1 \mathrm{E}+10$ & $9.1 \mathrm{E}+08$ & $1.3 \mathrm{E}+00$ & $1.3 \mathrm{E}-02$ & $1.3 \mathrm{E}+00$ & $1.3 \mathrm{E}-02$ \\
\hline I-123 & 015715-08-9 & $1.5 \mathrm{E}+05$ & $1.5 \mathrm{E}+03$ & $6.9 E+09$ & $6.9 \mathrm{E}+07$ & $1.7 \mathrm{E}+01$ & $1.7 \mathrm{E}-01$ & $1.7 \mathrm{E}+01$ & $1.7 \mathrm{E}-01$ \\
\hline I -125 & 014158-31-7 & $3.1 \mathrm{E}+03$ & $3.1 \mathrm{E}+01$ & $1.2 \mathrm{E}+08$ & $1.2 \mathrm{E}+06$ & $1.8 \mathrm{E}+03$ & $1.8 \mathrm{E}+01$ & $1.2 \mathrm{E}+03$ & $1.2 \mathrm{E}+01$ \\
\hline $\mathrm{I}-126$ & 014158-32-8 & $1.6 \mathrm{E}+03$ & $1.6 \mathrm{E}+01$ & $6.5 \mathrm{E}+07$ & $6.5 \mathrm{E}+05$ & $2.9 \mathrm{E}+00$ & $2.9 \mathrm{E}-02$ & $2.9 \mathrm{E}+00$ & $2.9 \mathrm{E}-02$ \\
\hline I-129 & $015046-84-1$ & $4.3 \mathrm{E}+02$ & $4.3 \mathrm{E}+00$ & $1.7 \mathrm{E}+07$ & $1.7 \mathrm{E}+05$ & $1.6 \mathrm{E}+03$ & $1.6 \mathrm{E}+01$ & $3.9 \mathrm{E}+02$ & $3.9 \mathrm{E}+00$ \\
\hline $1-130$ & $014914-02-4$ & $1.6 \mathrm{E}+04$ & $1.6 \mathrm{E}+02$ & $7.8 \mathrm{E}+08$ & $7.8 \mathrm{E}+06$ & $5.5 \mathrm{E}-01$ & $5.5 \mathrm{E}-03$ & $5.5 \mathrm{E}-01$ & $5.5 \mathrm{E}-03$ \\
\hline $\mathrm{I}-131$ & 010043-66-0 & $2.2 \mathrm{E}+03$ & $2.2 \mathrm{E}+01$ & $8.8 \mathrm{E}+07$ & $8.8 \mathrm{E}+05$ & $3.5 \mathrm{E}+00$ & $3.5 \mathrm{E}-02$ & $3.5 \mathrm{E}+00$ & 3.5E-02 \\
\hline $1-132$ & 014683-16-0 & $1.2 E+05$ & $1.2 \mathrm{E}+03$ & $5.8 \mathrm{E}+09$ & $5.8 \mathrm{E}+07$ & $5.0 \mathrm{E}-01$ & $5.0 \mathrm{E}-03$ & $5.0 \mathrm{E}-01$ & $5.0 \mathrm{E}-03$ \\
\hline
\end{tabular}


Table 3.1d. (continued)

\begin{tabular}{|c|c|c|c|c|c|c|c|c|c|}
\hline \multirow[t]{2}{*}{ Chemical $^{t}$} & \multirow{2}{*}{$\begin{array}{c}\text { CASRN } \\
- \\
\end{array}$} & \multicolumn{2}{|c|}{$\begin{array}{c}\text { Ingestion } \\
\text { (pCi/g) }\end{array}$} & \multicolumn{2}{|c|}{$\begin{array}{c}\text { Inhalation } \\
\text { (pCi/g) }\end{array}$} & \multicolumn{2}{|c|}{$\begin{array}{c}\text { External Exposure } \\
(\mathrm{pCi} / \mathrm{g})^{H}\end{array}$} & \multicolumn{2}{|c|}{$\begin{array}{l}\text { Ingestion + External + } \\
\text { Inhalation }(\mathrm{pCi} / \mathrm{g})^{\mathrm{Ht}}\end{array}$} \\
\hline & & $10^{-4}$ & $10^{-6}$ & $10^{-4}$ & $10^{-6}$ & $10^{-4}$ & $10^{-6}$ & $10^{-4}$ & $10^{-6}$ \\
\hline I-133 & $014834-67-4$ & $7.5 E+03$ & $7.5 \mathrm{E}+01$ & $3.4 \mathrm{E}+08$ & $3.4 \mathrm{E}+06$ & $2.0 \mathrm{E}+00$ & $2.0 \mathrm{E}-02$ & $2.0 \mathrm{E}+00$ & $2.0 \mathrm{E}-02$ \\
\hline I-134 & $014914-27-3$ & $3.4 \mathrm{E}+05$ & $3.4 \mathrm{E}+03$ & $1.5 \mathrm{E}+10$ & $1.5 E+08$ & $4.3 \mathrm{E}-01$ & $4.3 \mathrm{E}-03$ & 4.3E-01 & $4.3 \mathrm{E}-03$ \\
\hline I-135 & $014834-68-5$ & $3.5 \mathrm{E}+04$ & $3.5 \mathrm{E}+02$ & $1.7 \mathrm{E}+09$ & $1.7 \mathrm{E}+07$ & $7.0 \mathrm{E}-01$ & $7.0 \mathrm{E}-03$ & $7.0 \mathrm{E}-01$ & $7.0 \mathrm{E}-03$ \\
\hline In-113m & $014885-78-0$ & $9.6 E+05$ & $9.6 \mathrm{E}+03$ & $3.5 \mathrm{E}+10$ & $3.5 \mathrm{E}+08$ & $5.6 \mathrm{E}+00$ & $5.6 \mathrm{E}-02$ & $5.6 \mathrm{E}+00$ & $5.6 \mathrm{E}-02$ \\
\hline In-114 & $013981-55-0$ & $1.8 \mathrm{E}+07$ & $1.8 \mathrm{E}+05$ & $3.5 \mathrm{E}+11$ & $3.5 \mathrm{E}+09$ & $3.8 \mathrm{E}+01$ & $3.8 \mathrm{E}-01$ & $3.8 \mathrm{E}+01$ & $3.8 \mathrm{E}-01$ \\
\hline In-114m & $013981-55-0$ & $3.9 \mathrm{E}+03$ & $3.9 \mathrm{E}+01$ & $8.1 \mathrm{E}+07$ & 8.1E+05 & $2.2 \mathrm{E}+01$ & $2.2 \mathrm{E}-01$ & $2.2 \mathrm{E}+01$ & $2.2 \mathrm{E}-01$ \\
\hline In-115 & $014191-71-0$ & $2.3 E+03$ & $2.3 E+01$ & $9.9 \mathrm{E}+06$ & $9.9 \mathrm{E}+04$ & & & $2.7 \mathrm{E}+03$ & $2.7 \mathrm{E}+01$ \\
\hline In-115m & $014191-71-0$ & $2.3 E+05$ & $2.3 E+03$ & $1.2 \mathrm{E}+10$ & $1.2 \mathrm{E}+08$ & $1.0 \mathrm{E}+01$ & $1.0 \mathrm{E}-01$ & $1.0 \mathrm{E}+01$ & $1.0 \mathrm{E}-01$ \\
\hline Ir-190 & 014981-91-0 & $1.6 \mathrm{E}+04$ & $1.6 \mathrm{E}+02$ & $4.5 \mathrm{E}+08$ & $4.5 \mathrm{E}+06$ & $9.3 \mathrm{E}-01$ & $9.3 \mathrm{E}-03$ & $9.3 \mathrm{E}-01$ & 9.3E-03 \\
\hline Ir-194 & $014158-35-1$ & $1.1 \mathrm{E}+04$ & $1.1 \mathrm{E}+02$ & $4.9 \mathrm{E}+08$ & $4.9 \mathrm{E}+06$ & $1.4 \mathrm{E}+01$ & $1.4 \mathrm{E}-01$ & $1.4 \mathrm{E}+01$ & $1.4 \mathrm{E}-01$ \\
\hline $\mathrm{K}-40$ & $013966-00-2$ & $6.3 \mathrm{E}+03$ & $6.3 \mathrm{E}+01$ & $2.7 \mathrm{E}+08$ & $2.7 \mathrm{E}+06$ & $7.1 \mathrm{E}+00$ & 7.1E-02 & $7.1 E+00$ & 7.1E-02 \\
\hline $\mathrm{K}-42$ & $014378-21-3$ & $6.2 E+04$ & $6.2 \mathrm{E}+02$ & $2.7 E+09$ & $2.7 \mathrm{E}+07$ & $4.0 \mathrm{E}+00$ & $4.0 \mathrm{E}-02$ & $4.0 \mathrm{E}+00$ & 4.0E-02 \\
\hline $\mathrm{Kr}-83 \mathrm{~m}$ & $013965-98-5$ & & & $5.9 \mathrm{E}+13$ & $5.9 \mathrm{E}+11$ & & & & \\
\hline $\mathrm{Kr}-85$ & $013983-27-2$ & & & $7.1 \mathrm{E}+12$ & $7.1 \mathrm{E}+10$ & & & & \\
\hline $\mathrm{Kr}-85 \mathrm{~m}$ & $013983-27-2$ & & & $7.4 \mathrm{E}+12$ & $7.4 \mathrm{E}+10$ & & & & \\
\hline $\mathrm{Kr}-87$ & 014809-68-8 & & & $1.7 \mathrm{E}+12$ & $1.7 \mathrm{E}+10$ & & & & \\
\hline $\mathrm{Kr}-88$ & $014995-61-0$ & & & $9.3 \mathrm{E}+11$ & $9.3 \mathrm{E}+09$ & & & & \\
\hline
\end{tabular}


Table 3.1d. (continued)

\begin{tabular}{|c|c|c|c|c|c|c|c|c|c|}
\hline \multirow[t]{2}{*}{ Chemical ${ }^{\dagger}$} & \multirow[t]{2}{*}{ CASRN } & \multicolumn{2}{|c|}{$\begin{array}{c}\text { Ingestion } \\
\text { (pCi/g) }\end{array}$} & \multicolumn{2}{|c|}{$\begin{array}{c}\text { Inhalation } \\
\text { (pCi/g) }\end{array}$} & \multicolumn{2}{|c|}{$\begin{array}{l}\text { External Exposure } \\
(\mathrm{pCi} / \mathrm{g})^{\dagger+}\end{array}$} & \multicolumn{2}{|c|}{$\begin{array}{c}\text { Ingestion + External + } \\
\text { Inhalation }(\mathrm{pCi} / \mathrm{g})^{\mathrm{tt}}\end{array}$} \\
\hline & & $10^{-4}$ & $10^{-6}$ & $10^{-4}$ & $10^{-6}$ & $10^{-4}$ & $10^{-6}$ & $10^{-4}$ & $10^{-6}$ \\
\hline $\mathrm{Kr}-89$ & 016316-03-3 & & & $1.3 \mathrm{E}+12$ & $1.3 \mathrm{E}+10$ & & & & \\
\hline $\mathrm{Kr}-90$ & 015741-13-6 & & & $1.3 \mathrm{E}+12$ & $1.3 E+10$ & & & & \\
\hline La-140 & $013981-28-7$ & $8.4 \mathrm{E}+03$ & $8.4 \mathrm{E}+01$ & $4.0 \mathrm{E}+08$ & $4.0 \mathrm{E}+06$ & $4.8 \mathrm{E}-01$ & $4.8 \mathrm{E}-03$ & $4.8 \mathrm{E}-01$ & $4.8 \mathrm{E}-03$ \\
\hline Lu-177 & 014265-75-9 & $2.7 \mathrm{E}+04$ & $2.7 \mathrm{E}+02$ & $9.3 \mathrm{E}+08$ & $9.3 E+06$ & $6.0 \mathrm{E}+01$ & $6.0 \mathrm{E}-01$ & $6.0 \mathrm{E}+01$ & $6.0 \mathrm{E}-01$ \\
\hline $\mathrm{Mn}-52$ & 014092-99-0 & $1.3 \mathrm{E}+04$ & $1.3 \mathrm{E}+02$ & $4.6 E+08$ & $4.6 E+06$ & $3.2 \mathrm{E}-01$ & $3.2 \mathrm{E}-03$ & $3.2 \mathrm{E}-01$ & $3.2 \mathrm{E}-03$ \\
\hline $\mathrm{Mn}-54$ & 013966-31-9 & $4.0 \mathrm{E}+04$ & $4.0 \mathrm{E}+02$ & $5.5 \mathrm{E}+08$ & $5.5 \mathrm{E}+06$ & $1.3 \mathrm{E}+00$ & $1.3 \mathrm{E}-02$ & $1.3 \mathrm{E}+00$ & $1.3 \mathrm{E}-02$ \\
\hline $\mathrm{Mn}-56$ & $014681-52-8$ & $9.3 E+04$ & $9.3 E+02$ & $3.9 \mathrm{E}+09$ & $3.9 E+07$ & $6.3 \mathrm{E}-01$ & $6.3 \mathrm{E}-03$ & $6.3 \mathrm{E}-01$ & $6.3 \mathrm{E}-03$ \\
\hline Mo-99 & 014119-15-4 & $3.5 \mathrm{E}+04$ & $3.5 \mathrm{E}+02$ & $4.6 \mathrm{E}+08$ & $4.6 \mathrm{E}+06$ & $8.0 \mathrm{E}+00$ & $8.0 \mathrm{E}-02$ & $8.0 \mathrm{E}+00$ & $8.0 \mathrm{E}-02$ \\
\hline $\mathrm{Na}-22$ & 013966-32-0 & $9.9 \mathrm{E}+03$ & $9.9 \mathrm{E}+01$ & $4.2 E+08$ & $4.2 E+06$ & $5.3 \mathrm{E}-01$ & $5.3 \mathrm{E}-03$ & $5.3 \mathrm{E}-01$ & $5.3 \mathrm{E}-03$ \\
\hline $\mathrm{Na}-24$ & $013982-04-2$ & $5.8 \mathrm{E}+04$ & $5.8 \mathrm{E}+02$ & $2.7 \mathrm{E}+09$ & $2.7 \mathrm{E}+07$ & $2.5 \mathrm{E}-01$ & $2.5 \mathrm{E}-03$ & $2.5 \mathrm{E}-01$ & $2.5 \mathrm{E}-03$ \\
\hline $\mathrm{Nb}-93 \mathrm{~m}$ & 007440-03-1 & $1.2 \mathrm{E}+05$ & $1.2 \mathrm{E}+03$ & $4.7 \mathrm{E}+08$ & $4.7 \mathrm{E}+06$ & $1.2 \mathrm{E}+05$ & $1.2 \mathrm{E}+03$ & $6.5 \mathrm{E}+04$ & $6.5 \mathrm{E}+02$ \\
\hline $\mathrm{Nb}-94$ & $014681-63-1$ & $1.1 E+04$ & $1.1 \mathrm{E}+02$ & $2.5 E+07$ & $2.5 \mathrm{E}+05$ & 7.1E-01 & 7.1E-03 & $7.1 \mathrm{E}-01$ & 7.1E-03 \\
\hline $\mathrm{Nb}-95$ & $013967-76-5$ & $3.5 \mathrm{E}+04$ & $3.5 \mathrm{E}+02$ & $6.6 \mathrm{E}+08$ & $6.6 \mathrm{E}+06$ & $1.5 \mathrm{E}+00$ & $1.5 \mathrm{E}-02$ & $1.5 \mathrm{E}+00$ & $1.5 \mathrm{E}-02$ \\
\hline $\mathrm{Nb}-95 \mathrm{~m}$ & $013967-76-5$ & $2.6 \mathrm{E}+04$ & $2.6 \mathrm{E}+02$ & $9.1 E+08$ & $9.1 \mathrm{E}+06$ & $5.0 \mathrm{E}+01$ & $5.0 \mathrm{E}-01$ & $5.0 \mathrm{E}+01$ & $5.0 \mathrm{E}-01$ \\
\hline $\mathrm{Nb}-97$ & $018496-04-3$ & $4.5 \mathrm{E}+05$ & $4.5 \mathrm{E}+03$ & $9.6 \mathrm{E}+09$ & $9.6 \mathrm{E}+07$ & $1.7 \mathrm{E}+00$ & $1.7 \mathrm{E}-02$ & $1.7 \mathrm{E}+00$ & $1.7 \mathrm{E}-02$ \\
\hline $\mathrm{Nb}-97 \mathrm{~m}$ & 018496-04-3 & $2.4 \mathrm{E}+07$ & $2.4 \mathrm{E}+05$ & $6.1 \mathrm{E}+11$ & $6.1 E+09$ & $1.6 \mathrm{E}+00$ & $1.6 \mathrm{E}-02$ & $1.6 \mathrm{E}+00$ & $1.6 \mathrm{E}-02$ \\
\hline Nd-147 & 014269-74-0 & $1.3 E+04$ & $1.3 \mathrm{E}+02$ & $4.2 E+08$ & $4.2 E+06$ & $1.3 \mathrm{E}+01$ & $1.3 \mathrm{E}-01$ & $1.3 E+01$ & $1.3 \mathrm{E}-01$ \\
\hline
\end{tabular}


Table 3.1d. (continued)

\begin{tabular}{|c|c|c|c|c|c|c|c|c|c|}
\hline \multirow[t]{2}{*}{ Chemical $^{\dagger}$} & \multirow[t]{2}{*}{ CASRN } & \multicolumn{2}{|c|}{$\begin{array}{c}\text { Ingestion } \\
\text { (pCi/g) }\end{array}$} & \multicolumn{2}{|c|}{$\begin{array}{l}\text { Inhalation } \\
\text { (pCi/g) }\end{array}$} & \multicolumn{2}{|c|}{$\begin{array}{l}\text { External Exposure } \\
(\mathrm{pCi} / \mathrm{g})^{\dagger t}\end{array}$} & \multicolumn{2}{|c|}{$\begin{array}{l}\text { Ingestion + External + } \\
\text { Inhalation (pCi/g) }\end{array}$} \\
\hline & & $10^{-4}$ & $10^{-6}$ & $10^{-4}$ & $10^{-5}$ & $10^{-4}$ & $10^{-6}$ & $10^{-4}$ & $10^{-6}$ \\
\hline Nd-149 & $015749-81-2$ & $1.7 \mathrm{E}+05$ & $1.7 \mathrm{E}+03$ & $4.8 \mathrm{E}+09$ & $4.8 \mathrm{E}+07$ & $4.0 \mathrm{E}+00$ & $4.0 \mathrm{E}-02$ & $4.0 \mathrm{E}+00$ & $4.0 \mathrm{E}-02$ \\
\hline Ni-59 & 014336-70-0 & $4.3 \mathrm{E}+05$ & $4.3 \mathrm{E}+03$ & $5.1 \mathrm{E}+09$ & $5.1 \mathrm{E}+07$ & & & $5.1 E+05$ & $5.1 \mathrm{E}+03$ \\
\hline $\mathrm{Ni}-63$ & $013981-37-8$ & $1.4 \mathrm{E}+05$ & $1.4 \mathrm{E}+03$ & $2.0 \mathrm{E}+09$ & $2.0 \mathrm{E}+07$ & & & $1.7 \mathrm{E}+05$ & $1.7 \mathrm{E}+03$ \\
\hline $\mathrm{Ni}-65$ & 014833-49-9 & $1.4 \mathrm{E}+05$ & $1.4 \mathrm{E}+03$ & $5.7 \mathrm{E}+09$ & $5.7 \mathrm{E}+07$ & $2.0 \mathrm{E}+00$ & $2.0 \mathrm{E}-02$ & $2.0 \mathrm{E}+00$ & $2.0 \mathrm{E}-02$ \\
\hline Np-236 & $015700-36-4$ & $8.5 \mathrm{E}+04$ & $8.5 E+02$ & $5.3 \mathrm{E}+08$ & $5.3 \mathrm{E}+06$ & $4.7 \mathrm{E}+01$ & 4.7E-01 & $4.7 \mathrm{E}+01$ & 4.7E-01 \\
\hline Np-237 & $013994-20-2$ & $2.7 \mathrm{E}+02$ & $2.7 \mathrm{E}+00$ & $5.9 \mathrm{E}+04$ & $5.9 \mathrm{E}+02$ & $5.7 \mathrm{E}+02$ & $5.7 \mathrm{E}+00$ & $2.1 E+02$ & $2.1 E+00$ \\
\hline $\mathrm{Np}-237+\mathrm{D}$ & 013994-20-2 & $2.6 \mathrm{E}+02$ & $2.6 \mathrm{E}+00$ & $5.9 \mathrm{E}+04$ & $5.9 \mathrm{E}+02$ & $9.4 \mathrm{E}+00$ & $9.4 \mathrm{E}-02$ & $9.1 \mathrm{E}+00$ & $9.1 \mathrm{E}-02$ \\
\hline Np-238 & $015766-25-3$ & $1.7 \mathrm{E}+04$ & $1.7 \mathrm{E}+02$ & $4.4 \mathrm{E}+08$ & $4.4 \mathrm{E}+06$ & $2.2 \mathrm{E}+00$ & $2.2 \mathrm{E}-02$ & $2.2 \mathrm{E}+00$ & $2.2 \mathrm{E}-02$ \\
\hline $\mathrm{Np}-239$ & 013968-59-7 & $1.9 \mathrm{E}+04$ & $1.9 \mathrm{E}+02$ & $8.5 E+08$ & $8.5 \mathrm{E}+06$ & $1.8 \mathrm{E}+01$ & $1.8 \mathrm{E}-01$ & $1.8 \mathrm{E}+01$ & $1.8 \mathrm{E}-01$ \\
\hline $\mathrm{Np}-240$ & $015690-84-3$ & $4.5 \mathrm{E}+05$ & $4.5 E+03$ & $1.6 \mathrm{E}+10$ & $1.6 \mathrm{E}+08$ & $1.2 \mathrm{E}+00$ & $1.2 \mathrm{E}-02$ & $1.2 \mathrm{E}+00$ & $1.2 \mathrm{E}-02$ \\
\hline $\mathrm{Np}-240 \mathrm{~m}$ & $015690-84-3$ & $3.3 \mathrm{E}+06$ & $3.3 E+04$ & $7.2 \mathrm{E}+10$ & $7.2 \mathrm{E}+08$ & $4.1 E+00$ & 4.1E-02 & 4.1E+00 & $4.1 \mathrm{E}-02$ \\
\hline Os-185 & $015766-50-4$ & $4.4 \mathrm{E}+04$ & $4.4 \mathrm{E}+02$ & $4.4 \mathrm{E}+08$ & $4.4 \mathrm{E}+06$ & $1.8 \mathrm{E}+00$ & $1.8 \mathrm{E}-02$ & $1.8 \mathrm{E}+00$ & $1.8 \mathrm{E}-02$ \\
\hline Os-191 & $014119-24-5$ & $2.6 \mathrm{E}+04$ & $2.6 \mathrm{E}+02$ & $7.6 \mathrm{E}+08$ & $7.6 \mathrm{E}+06$ & $5.0 \mathrm{E}+01$ & $5.0 \mathrm{E}-01$ & $5.0 \mathrm{E}+01$ & $5.0 \mathrm{E}-01$ \\
\hline Os-191m & $014119-24-5$ & $1.6 \mathrm{E}+05$ & $1.6 \mathrm{E}+03$ & $6.1 \mathrm{E}+09$ & $6.1 \mathrm{E}+07$ & $1.3 E+03$ & $1.3 E+01$ & $1.3 E+03$ & $1.3 \mathrm{E}+01$ \\
\hline Os-193 & $016057-77-5$ & $1.8 \mathrm{E}+04$ & $1.8 \mathrm{E}+02$ & $7.6 \mathrm{E}+08$ & $7.6 \mathrm{E}+06$ & $2.4 \mathrm{E}+01$ & $2.4 \mathrm{E}-01$ & $2.4 \mathrm{E}+01$ & $2.4 \mathrm{E}-01$ \\
\hline P-32 & $014596-37-3$ & $1.3 E+04$ & $1.3 E+02$ & $7.0 \mathrm{E}+08$ & $7.0 \mathrm{E}+06$ & & & $1.6 E+04$ & $1.6 \mathrm{E}+02$ \\
\hline P-33 & $015749-66-3$ & $1.0 \mathrm{E}+05$ & $1.0 \mathrm{E}+03$ & $5.2 \mathrm{E}+09$ & $5.2 E+07$ & & & $1.2 \mathrm{E}+05$ & $1.2 \mathrm{E}+03$ \\
\hline
\end{tabular}


Table 3.1d. (continued)

\begin{tabular}{|c|c|c|c|c|c|c|c|c|c|}
\hline \multirow{2}{*}{ Chemical $^{\dagger}$} & \multirow{2}{*}{ CASRN } & \multicolumn{2}{|c|}{$\begin{array}{c}\text { Ingestion } \\
\text { (pCi/g) }\end{array}$} & \multicolumn{2}{|c|}{$\begin{array}{l}\text { Inhalation } \\
\text { (pCi/g) }\end{array}$} & \multicolumn{2}{|c|}{$\begin{array}{l}\text { External Exposure } \\
(\mathrm{pCi} / \mathrm{g})^{\dagger+}\end{array}$} & \multicolumn{2}{|c|}{$\begin{array}{c}\text { Ingestion + External + } \\
\text { Inhalation (pCi/g) }\end{array}$} \\
\hline & & $10^{-4}$ & $10^{-6}$ & $10^{-4}$ & $10^{-6}$ & $10^{-4}$ & $10^{-6}$ & $10^{-4}$ & $10^{-6}$ \\
\hline $\mathrm{Pa}-231$ & $014331-85-2$ & $5.3 \mathrm{E}+02$ & $5.3 \mathrm{E}+00$ & $8.4 E+04$ & $8.4 \mathrm{E}+02$ & $1.6 \mathrm{E}+02$ & $1.6 \mathrm{E}+00$ & $1.3 \mathrm{E}+02$ & $1.3 \mathrm{E}+00$ \\
\hline $\mathrm{Pa}-233$ & 013981-14-1 & $1.7 \mathrm{E}+04$ & $1.7 \mathrm{E}+02$ & $4.1 E+08$ & $4.1 \mathrm{E}+06$ & $9.6 \mathrm{E}+00$ & $9.6 \mathrm{E}-02$ & $9.6 \mathrm{E}+00$ & $9.6 \mathrm{E}-02$ \\
\hline $\mathrm{Pa}-234$ & $015100-28-4$ & $3.7 \mathrm{E}+04$ & $3.7 \mathrm{E}+02$ & $1.6 \mathrm{E}+09$ & $1.6 \mathrm{E}+07$ & $6.6 \mathrm{E}-01$ & $6.6 \mathrm{E}-03$ & $6.6 \mathrm{E}-01$ & $6.6 \mathrm{E}-03$ \\
\hline $\mathrm{Pa}-234 \mathrm{~m}$ & $015100-28-4$ & $1.7 \mathrm{E}+07$ & $1.7 \mathrm{E}+05$ & $3.3 \mathrm{E}+11$ & $3.3 \mathrm{E}+09$ & $1.1 \mathrm{E}+02$ & $1.1 \mathrm{E}+00$ & $1.1 \mathrm{E}+02$ & $1.1 \mathrm{E}+00$ \\
\hline $\mathrm{Pb}-203$ & 014687-25-3 & 7.7E+04 & $7.7 \mathrm{E}+02$ & $6.6 \mathrm{E}+09$ & $6.6 \mathrm{E}+07$ & $6.8 \mathrm{E}+00$ & $6.8 \mathrm{E}-02$ & $6.8 \mathrm{E}+00$ & $6.8 \mathrm{E}-02$ \\
\hline $\mathrm{Pb}-209$ & 014119-30-3 & $3.8 \mathrm{E}+05$ & $3.8 \mathrm{E}+03$ & $3.0 \mathrm{E}+10$ & $3.0 \mathrm{E}+08$ & & & 4.6E+05 & $4.6 E+03$ \\
\hline $\mathrm{Pb}-210$ & 014255-04-0 & $1.2 \mathrm{E}+02$ & $1.2 \mathrm{E}+00$ & $1.2 \mathrm{E}+06$ & $1.2 \mathrm{E}+04$ & $3.9 \mathrm{E}+04$ & $3.9 \mathrm{E}+02$ & $1.4 \mathrm{E}+02$ & $1.4 \mathrm{E}+00$ \\
\hline $\mathrm{Pb}-210+\mathrm{D}$ & $014255-04-0$ & $7.9 E+01$ & $7.9 \mathrm{E}-01$ & $5.3 \mathrm{E}+05$ & $5.3 \mathrm{E}+03$ & $3.0 \mathrm{E}+04$ & $3.0 \mathrm{E}+02$ & $9.4 \mathrm{E}+01$ & 9.4E-01 \\
\hline $\mathrm{Pb}-211$ & 015816-77-0 & $2.3 \mathrm{E}+05$ & $2.3 \mathrm{E}+03$ & $2.0 \mathrm{E}+08$ & $2.0 \mathrm{E}+06$ & $2.3 E+01$ & $2.3 \mathrm{E}-01$ & $2.3 E+01$ & $2.3 \mathrm{E}-01$ \\
\hline $\mathrm{Pb}-212$ & 015092-94-1 & $4.4 \mathrm{E}+03$ & $4.4 \mathrm{E}+01$ & $5.3 \mathrm{E}+07$ & $5.3 \mathrm{E}+05$ & $1.4 \mathrm{E}+01$ & $1.4 \mathrm{E}-01$ & $1.4 \mathrm{E}+01$ & $1.4 \mathrm{E}-01$ \\
\hline $\mathrm{Pb}-214$ & $015067-28-4$ & $2.7 \mathrm{E}+05$ & 2.7E+03 & $3.3 E+08$ & $3.3 \mathrm{E}+06$ & $6.1 E+00$ & $6.1 \mathrm{E}-02$ & $6.1 \mathrm{E}+00$ & $6.1 \mathrm{E}-02$ \\
\hline Pd-100 & $015690-69-4$ & $2.1 \mathrm{E}+04$ & $2.1 \mathrm{E}+02$ & $5.7 \mathrm{E}+08$ & $5.7 \mathrm{E}+06$ & & & $2.5 \mathrm{E}+04$ & $2.5 \mathrm{E}+02$ \\
\hline Pd-101 & 015749-54-9 & $2.1 \mathrm{E}+05$ & $2.1 \mathrm{E}+03$ & $8.9 E+09$ & $8.9 \mathrm{E}+07$ & & & $2.5 \mathrm{E}+05$ & $2.5 \mathrm{E}+03$ \\
\hline Pd-103 & $014967-68-1$ & $7.6 \mathrm{E}+04$ & $7.6 \mathrm{E}+02$ & $1.9 \mathrm{E}+09$ & $1.9 \mathrm{E}+07$ & $8.1 E+03$ & $8.1 \mathrm{E}+01$ & $7.4 \mathrm{E}+03$ & $7.4 \mathrm{E}+01$ \\
\hline Pd-107 & 017637-99-9 & $3.8 \mathrm{E}+05$ & $3.8 \mathrm{E}+03$ & $1.4 \mathrm{E}+09$ & $1.4 \mathrm{E}+07$ & & & $4.6 \mathrm{E}+05$ & $4.6 \mathrm{E}+03$ \\
\hline Pd-109 & 014981-64-7 & $2.4 \mathrm{E}+04$ & $2.4 \mathrm{E}+02$ & $1.0 \mathrm{E}+09$ & $1.0 \mathrm{E}+07$ & $1.8 \mathrm{E}+03$ & $1.8 \mathrm{E}+01$ & $1.7 \mathrm{E}+03$ & $1.7 \mathrm{E}+01$ \\
\hline Pm-147 & $014380-75-7$ & $5.6 \mathrm{E}+04$ & $5.6 \mathrm{E}+02$ & $2.7 \mathrm{E}+08$ & $2.7 \mathrm{E}+06$ & $6.8 \mathrm{E}+05$ & $6.8 \mathrm{E}+03$ & $6.1 E+04$ & $6.1 \mathrm{E}+02$ \\
\hline
\end{tabular}


Table 3.1d. (continued)

\begin{tabular}{|c|c|c|c|c|c|c|c|c|c|}
\hline \multirow{2}{*}{ Chemical $^{\dagger}$} & \multirow{2}{*}{ CASRN } & \multicolumn{2}{|c|}{$\begin{array}{c}\text { Ingestion } \\
\text { (pCi/g) }\end{array}$} & \multicolumn{2}{|c|}{$\begin{array}{c}\text { Inhalation } \\
\text { (pCi/g) }\end{array}$} & \multicolumn{2}{|c|}{$\begin{array}{c}\text { External Exposure } \\
(\mathrm{pCi} / \mathrm{g})^{+t}\end{array}$} & \multicolumn{2}{|c|}{$\begin{array}{l}\text { Ingestion + External + } \\
\text { Inhalation (pCi/g) }\end{array}$} \\
\hline & & $10^{-4}$ & $10^{-6}$ & $10^{-4}$ & $10^{-6}$ & $10^{-4}$ & $10^{-6}$ & $10^{-4}$ & $10^{-6}$ \\
\hline Pm-148 & 014683-19-3 & $5.5 \mathrm{E}+03$ & $5.5 \mathrm{E}+01$ & $1.9 \mathrm{E}+08$ & $1.9 \mathrm{E}+06$ & $2.0 \mathrm{E}+00$ & $2.0 \mathrm{E}-02$ & $2.0 \mathrm{E}+00$ & $2.0 \mathrm{E}-02$ \\
\hline Pm-148m & 014683-19-3 & $8.0 \mathrm{E}+03$ & $8.0 \mathrm{E}+01$ & $6.9 E+07$ & $6.9 \mathrm{E}+05$ & $5.9 \mathrm{E}-01$ & $5.9 \mathrm{E}-03$. & $5.9 \mathrm{E}-01$ & $5.9 \mathrm{E}-03$ \\
\hline Pm-149 & $015765-31-8$ & $1.4 \mathrm{E}+04$ & $1.4 \mathrm{E}+02$ & $5.7 \mathrm{E}+08$ & $5.7 \mathrm{E}+06$ & $1.2 \mathrm{E}+02$ & $1.2 \mathrm{E}+00$ & $1.2 \mathrm{E}+02$ & $1.2 \mathrm{E}+00$ \\
\hline Po-210 & $013981-52-7$ & $2.4 \mathrm{E}+02$ & $2.4 \mathrm{E}+00$ & $9.5 E+05$ & $9.5 E+03$ & $1.3 \mathrm{E}+05$ & $1.3 E+03$ & $2.9 \mathrm{E}+02$ & $2.9 \mathrm{E}+00$ \\
\hline Po-212 & $015389-34-1$ & $1.8 \mathrm{E}+15$ & $1.8 \mathrm{E}+13$ & $3.4 \mathrm{E}+17$ & $3.4 \mathrm{E}+15$ & & & $2.1 \mathrm{E}+15$ & $2.1 E+13$ \\
\hline Po-213 & 015756-57-7 & $1.2 \mathrm{E}+14$ & $1.2 \mathrm{E}+12$ & $2.6 \mathrm{E}+16$ & $2.6 \mathrm{E}+14$ & $3.7 \mathrm{E}+04$ & $3.7 \mathrm{E}+02$ & 3.7E+04 & $3.7 \mathrm{E}+02$ \\
\hline Po-214 & 015735-67-8 & 3.7E+12 & $3.7 \mathrm{E}+10$ & $7.4 \mathrm{E}+14$ & $7.4 \mathrm{E}+12$ & $1.3 \mathrm{E}+04$ & $1.3 \mathrm{E}+02$ & $1.3 E+04$ & $1.3 \mathrm{E}+02$ \\
\hline Po-215 & $015706-52-2$ & $1.6 \mathrm{E}+11$ & $1.6 \mathrm{E}+09$ & $4.6 \mathrm{E}+13$ & $4.6 \mathrm{E}+11$ & $8.5 E+03$ & $8.5 E+01$ & $8.5 \mathrm{E}+03$ & $8.5 \mathrm{E}+01$ \\
\hline Po-216 & 015756-58-8 & $9.0 \mathrm{E}+08$ & $9.0 \mathrm{E}+06$ & $6.9 \mathrm{E}+11$ & $6.9 \mathrm{E}+09$ & $7.7 \mathrm{E}+04$ & $7.7 \mathrm{E}+02$ & $7.7 \mathrm{E}+04$ & $7.7 \mathrm{E}+02$ \\
\hline Po-218 & $015422-24-9$ & $1.6 \mathrm{E}+06$ & $1.6 \mathrm{E}+04$ & $5.5 \mathrm{E}+08$ & $5.5 \mathrm{E}+06$ & & & $1.9 E+06$ & $1.9 \mathrm{E}+04$ \\
\hline Pr-142 & 014191-64-1 & $1.1 \mathrm{E}+04$ & $1.1 \mathrm{E}+02$ & $4.9 E+08$ & $4.9 E+06$ & $1.9 \mathrm{E}+01$ & $1.9 \mathrm{E}-01$ & $1.9 \mathrm{E}+01$ & $1.9 \mathrm{E}-01$ \\
\hline Pr-143 & $014981-79-4$ & $1.2 \mathrm{E}+04$ & $1.2 E+02$ & $3.6 \mathrm{E}+08$ & $3.6 E+06$ & $1.3 E+08$ & $1.3 E+06$ & $1.4 \mathrm{E}+04$ & $1.4 \mathrm{E}+02$ \\
\hline Pr-144 & $014119-05-2$ & $9.8 \mathrm{E}+05$ & $9.8 \mathrm{E}+03$ & $1.6 \mathrm{E}+10$ & $1.6 \mathrm{E}+08$ & $3.3 \mathrm{E}+01$ & $3.3 \mathrm{E}-01$ & $3.3 \mathrm{E}+01$ & $3.3 \mathrm{E}-01$ \\
\hline Pr-144m & 014119-05-2 & $2.5 \mathrm{E}+06$ & $2.5 \mathrm{E}+04$ & $3.6 \mathrm{E}+10$ & $3.6 \mathrm{E}+08$ & $2.3 E+03$ & $2.3 \mathrm{E}+01$ & $2.3 E+03$ & $2.3 \mathrm{E}+01$ \\
\hline Pt-191 & $015706-36-2$ & $5.3 \mathrm{E}+04$ & $5.3 \mathrm{E}+02$ & $4.9 \mathrm{E}+09$ & $4.9 \mathrm{E}+07$ & $6.4 \mathrm{E}+00$ & $6.4 \mathrm{E}-02$ & $6.4 \mathrm{E}+00$ & $6.4 \mathrm{E}-02$ \\
\hline Pt-193 & $015735-70-3$ & $4.9 \mathrm{E}+05$ & $4.9 \mathrm{E}+03$ & $2.6 \mathrm{E}+10$ & $2.6 \mathrm{E}+08$ & & & $5.9 \mathrm{E}+05$ & $5.9 \mathrm{E}+03$ \\
\hline Pt-193m & $015735-70-3$ & $3.2 \mathrm{E}+04$ & $3.2 \mathrm{E}+02$ & $3.5 \mathrm{E}+09$ & $3.5 \mathrm{E}+07$ & $5.8 \mathrm{E}+02$ & $5.8 \mathrm{E}+00$ & $5.8 \mathrm{E}+02$ & $5.8 \mathrm{E}+00$ \\
\hline
\end{tabular}


Table 3.1d. (continued)

\begin{tabular}{|c|c|c|c|c|c|c|c|c|c|}
\hline \multirow[t]{2}{*}{ Chemical $^{\dagger}$} & \multirow[t]{2}{*}{ CASRN } & \multicolumn{2}{|c|}{$\begin{array}{l}\text { Ingestion } \\
\text { (pCi/g) }\end{array}$} & \multicolumn{2}{|c|}{$\begin{array}{c}\text { Inhalation } \\
\text { (pCi/g) }\end{array}$} & \multicolumn{2}{|c|}{$\begin{array}{c}\text { External Exposure } \\
(\mathrm{pCi} / \mathrm{g})^{\dagger+}\end{array}$} & \multicolumn{2}{|c|}{$\begin{array}{c}\text { Ingestion + External + } \\
\text { Inhalation (pCi/g) }{ }^{+t+}\end{array}$} \\
\hline & & $10^{-4}$ & $10^{-6}$ & $10^{-4}$ & $10^{-6}$ & $10^{-4}$ & $10^{-6}$ & $10^{-4}$ & $10^{-6}$ \\
\hline Pt-197 & 015735-74-7 & $3.7 E+04$ & $3.7 \mathrm{E}+02$ & $4.5 E+09$ & 4.5E+07 & $1.4 \mathrm{E}+02$ & $1.4 \mathrm{E}+00$ & $1.4 \mathrm{E}+02$ & $1.4 \mathrm{E}+00$ \\
\hline Pt-197m & 015735-74-7 & $2.4 E+05$ & $2.4 \mathrm{E}+03$ & $2.0 \mathrm{E}+10$ & $2.0 \mathrm{E}+08$ & $2.6 \mathrm{E}+01$ & $2.6 \mathrm{E}-01$ & $2.6 \mathrm{E}+01$ & $2.6 \mathrm{E}-01$ \\
\hline $\mathrm{Pu}-236$ & $015411-92-4$ & $1.0 \mathrm{E}+03$ & $1.0 \mathrm{E}+01$ & $1.5 \mathrm{E}+05$ & $1.5 \mathrm{E}+03$ & $1.9 \mathrm{E}+05$ & $1.9 \mathrm{E}+03$ & $1.2 E+03$ & $1.2 \mathrm{E}+01$ \\
\hline Pu-238 & 013981-16-3 & $2.7 \mathrm{E}+02$ & $2.7 \mathrm{E}+00$ & $7.4 \mathrm{E}+04$ & $7.4 \mathrm{E}+02$ & $2.2 \mathrm{E}+05$ & $2.2 \mathrm{E}+03$ & $3.2 \mathrm{E}+02$ & $3.2 \mathrm{E}+00$ \\
\hline Pu-239 & $015117-48-3$ & $2.5 \mathrm{E}+02$ & $2.5 \mathrm{E}+00$ & $7.3 E+04$ & $7.3 \mathrm{E}+02$ & $3.4 \mathrm{E}+05$ & $3.4 \mathrm{E}+03$ & $3.0 \mathrm{E}+02$ & $3.0 \mathrm{E}+00$ \\
\hline $\mathrm{Pu}-240$ & 014119-33-6 & $2.5 \mathrm{E}+02$ & $2.5 \mathrm{E}+00$ & $7.3 \mathrm{E}+04$ & $7.3 E+02$ & $2.3 E+05$ & $2.3 E+03$ & $3.0 \mathrm{E}+02$ & $3.0 \mathrm{E}+00$ \\
\hline $\mathrm{Pu}-241$ & $014119-32-5$ & $1.5 \mathrm{E}+04$ & $\cdot 1.5 \mathrm{E}+02$ & $7.3 E+06$ & $7.3 \mathrm{E}+04$ & & & $1.8 \mathrm{E}+04$ & $1.8 \mathrm{E}+02$ \\
\hline $\mathrm{Pu}-241+\mathrm{D}$ & $014119-32-5$ & $2.4 \mathrm{E}+02$ & $2.4 \mathrm{E}+00$ & $5.3 \mathrm{E}+04$ & $5.3 \mathrm{E}+02$ & $9.5 \mathrm{E}+02$ & $9.5 \mathrm{E}+00$ & $2.2 \mathrm{E}+02$ & $2.2 \mathrm{E}+00$ \\
\hline $\mathrm{Pu}-242$ & $013982-10-0$ & $2.6 \mathrm{E}+02$ & $2.6 \mathrm{E}+00$ & $7.7 \mathrm{E}+04$ & $7.7 \mathrm{E}+02$ & $2.8 \mathrm{E}+05$ & $2.8 E+03$ & $3.2 \mathrm{E}+02$ & $3.2 \mathrm{E}+00$ \\
\hline $\mathrm{Pu}-243$ & $015706-37-3$ & $2.2 \mathrm{E}+05$ & $2.2 \mathrm{E}+03$ & $7.6 \mathrm{E}+09$ & $7.6 \mathrm{E}+07$ & $2.3 E+02$ & $2.3 \mathrm{E}+00$ & $2.3 E+02$ & $2.3 \mathrm{E}+00$ \\
\hline $\mathrm{Pu}-244$ & 014119-34-7 & $2.5 E+02$ & $2.5 \mathrm{E}+00$ & $7.6 \mathrm{E}+04$ & $7.6 \mathrm{E}+02$ & $3.4 \mathrm{E}+05$ & $3.4 \mathrm{E}+03$ & $3.0 \mathrm{E}+02$ & $3.0 \mathrm{E}+00$ \\
\hline $\mathrm{Pu}-244+\mathrm{D}$ & 014119-34-7 & $2.5 \mathrm{E}+02$ & $2.5 \mathrm{E}+00$ & $7.6 \mathrm{E}+04$ & $7.6 \mathrm{E}+02$ & $1.2 \mathrm{E}+00$ & $1.2 \mathrm{E}-02$ & $1.2 \mathrm{E}+00$ & $1.2 \mathrm{E}-02$ \\
\hline $\mathrm{Ra}-223$ & 015623-45-7 & $3.4 \mathrm{E}+02$ & $3.4 \mathrm{E}+00$ & $5.7 \mathrm{E}+05$ & $5.7 \mathrm{E}+03$ & $1.8 \mathrm{E}+01$ & $1.8 \mathrm{E}-01$ & $1.7 \mathrm{E}+01$ & $1.7 \mathrm{E}-01$ \\
\hline Ra-224 & 013233-32-4 & $5.3 E+02$ & $5.3 \mathrm{E}+00$ & $9.1 \mathrm{E}+05$ & $9.1 \mathrm{E}+03$ & $1.8 \mathrm{E}+02$ & $1.8 \mathrm{E}+00$ & $1.4 \mathrm{E}+02$ & $1.4 \mathrm{E}+00$ \\
\hline $\mathrm{Ra}-224^{(1)}$ & $013233-32-4$ & $5.3 \mathrm{E}+02$ & $5.3 E+00$ & $7.9 \mathrm{E}+02$ & $7.9 E+00$ & $1.8 \mathrm{E}+02$ & $1.8 \mathrm{E}+00$ & $1.2 \mathrm{E}+02$ & $1.2 \mathrm{E}+00$ \\
\hline $\mathrm{Ra}-225$ & 013981-53-8 & $5.1 \mathrm{E}+02$ & $5.1 E+00$ & $8.6 \mathrm{E}+05$ & $8.6 \mathrm{E}+03$ & $2.5 \mathrm{E}+03$ & $2.5 \mathrm{E}+01$ & $4.9 E+02$ & $4.9 \mathrm{E}+00$ \\
\hline Ra-226 & 013982-63-3 & $2.7 \mathrm{E}+02$ & $2.7 E+00$ & $7.5 \mathrm{E}+05$ & $7.5 \mathrm{E}+03$ & $3.3 \mathrm{E}+02$ & $3.3 E+00$ & $1.6 \mathrm{E}+02$ & $1.6 \mathrm{E}+00$ \\
\hline
\end{tabular}


Table 3.1d. (continued)

\begin{tabular}{|c|c|c|c|c|c|c|c|c|c|}
\hline \multirow[t]{2}{*}{ Chemical $^{\dagger}$} & \multirow[t]{2}{*}{ CASRN } & \multicolumn{2}{|c|}{$\begin{array}{c}\text { Ingestion } \\
\text { (pCi/g) }\end{array}$} & \multicolumn{2}{|c|}{$\begin{array}{c}\text { Inhalation } \\
\text { (pCi/g) }\end{array}$} & \multicolumn{2}{|c|}{$\begin{array}{c}\text { External Exposure } \\
(\mathrm{pCi} / \mathrm{g})^{t+}\end{array}$} & \multicolumn{2}{|c|}{$\begin{array}{l}\text { Ingestion + External + } \\
\text { Inhalation (pCi/g) }{ }^{\mathrm{ft+}}\end{array}$} \\
\hline & & $10^{-4}$ & $10^{-6}$ & $10^{-4}$ & $10^{-6}$ & $10^{-4}$ & $10^{-6}$ & $10^{-4}$ & $10^{-6}$ \\
\hline $\mathrm{Ra}-226^{(2)}$ & $013982-63-3$ & $2.7 \mathrm{E}+02$ & $2.7 \mathrm{E}+00$ & $4.9 \mathrm{E}-01$ & $4.9 \mathrm{E}-03$ & $3.3 \mathrm{E}+02$ & $3.3 \mathrm{E}+00$ & $4.9 \mathrm{E}-01$ & $4.9 \mathrm{E}-03$ \\
\hline $\mathrm{Ra}-226+\mathrm{D}$ & $013982-63-3$ & $2.7 \mathrm{E}+02$ & $2.7 \mathrm{E}+00$ & $7.4 \mathrm{E}+05$ & $7.4 \mathrm{E}+03$ & $6.4 \mathrm{E}-01$ & $6.4 \mathrm{E}-03$ & $6.4 \mathrm{E}-01$ & $6.4 \mathrm{E}-03$ \\
\hline $\mathrm{Ra}-226+\mathrm{D}^{(2)}$ & $013982-63-3$ & $2.7 \mathrm{E}+02$ & $2.7 \mathrm{E}+00$ & $4.9 \mathrm{E}-01$ & $4.9 \mathrm{E}-03$ & $6.4 \mathrm{E}-01$ & $6.4 \mathrm{E}-03$ & $2.8 \mathrm{E}-01$ & $2.8 \mathrm{E}-03$ \\
\hline $\mathrm{Ra}-228$ & $015262-20-1$ & $3.2 E+02$ & $3.2 \mathrm{E}+00$ & $2.1 \mathrm{E}+06$ & $2.1 E+04$ & & & $3.9 E+02$ & $3.9 \mathrm{E}+00$ \\
\hline $\mathrm{Ra}-228+\mathrm{D}$ & $015262-20-1$ & $3.2 \mathrm{E}+02$ & $3.2 \mathrm{E}+00$ & $2.1 \mathrm{E}+06$ & $2.1 \mathrm{E}+04$ & $1.3 \mathrm{E}+00$ & $1.3 \mathrm{E}-02$ & $1.3 E+00$ & $1.3 \mathrm{E}-02$ \\
\hline $\mathrm{Rb}-82$ & 014391-63-0 & $7.6 \mathrm{E}+06$ & $7.6 \mathrm{E}+04$ & $1.7 \mathrm{E}+11$ & $1.7 \mathrm{E}+09$ & $1.1 \mathrm{E}+00$ & $1.1 \mathrm{E}-02$ & $1.1 \mathrm{E}+00$ & $1.1 \mathrm{E}-02$ \\
\hline $\mathrm{Rb}-86$ & $014932-53-7$ & $1.1 E+04$ & $1.1 \mathrm{E}+02$ & $4.8 \mathrm{E}+08$ & $4.8 \mathrm{E}+06$ & $1.2 \mathrm{E}+01$ & $1.2 \mathrm{E}-01$ & $1.2 \mathrm{E}+01$ & $1.2 \mathrm{E}-01$ \\
\hline $\mathrm{Rb}-87$ & 013982-13-3 & $2.2 E+04$ & $2.2 \mathrm{E}+02$ & $9.0 \mathrm{E}+08$ & $9.0 \mathrm{E}+06$ & & & $2.6 \mathrm{E}+04$ & $2.6 \mathrm{E}+02$ \\
\hline $\mathrm{Rb}-88$ & 014928-36-0 & $5.4 \mathrm{E}+05$ & $5.4 \mathrm{E}+03$ & $1.5 \mathrm{E}+10$ & $1.5 \mathrm{E}+08$ & $1.6 \mathrm{E}+00$ & $1.6 \mathrm{E}-02$ & $1.6 \mathrm{E}+00$ & $1.6 \mathrm{E}-02$ \\
\hline $\mathrm{Rb}-89$ & $014191-65-2$ & $9.2 \mathrm{E}+05$ & $9.2 \mathrm{E}+03$ & $2.9 \mathrm{E}+10$ & $2.9 \mathrm{E}+08$ & - $5.1 \mathrm{E}-01$ & $5.1 \mathrm{E}-03$ & $5.1 \mathrm{E}-01$ & $5.1 \mathrm{E}-03$ \\
\hline $\mathrm{Rh}-103 \mathrm{~m}$ & 007440-16-6 & $9.7 \mathrm{E}+06$ & $9.7 \mathrm{E}+04$ & $1.6 \mathrm{E}+11$ & $1.6 \mathrm{E}+09$ & $7.4 \mathrm{E}+04$ & $7.4 \mathrm{E}+02$ & $7.4 \mathrm{E}+04$ & $7.4 \mathrm{E}+02$ \\
\hline Rh-105 & 014913-89-4 & $4.1 E+04$ & $4.1 \mathrm{E}+02$ & $1.7 \mathrm{E}+09$ & $1.7 \mathrm{E}+07$ & $1.7 \mathrm{E}+01$ & $1.7 \mathrm{E}-01$ & $1.7 \mathrm{E}+01$ & $1.7 \mathrm{E}-01$ \\
\hline Rh-105m & $014913-89-4$ & 7.3E+07 & $7.3 \mathrm{E}+05$ & $2.2 \mathrm{E}+12$ & $2.2 E+10$ & $1.9 \mathrm{E}+02$ & $1.9 \mathrm{E}+00$ & $1.9 \mathrm{E}+02$ & $1.9 \mathrm{E}+00$ \\
\hline Rh-106 & 014234-34-5 & $2.2 \mathrm{E}+07$ & $2.2 E+05$ & $4.4 \mathrm{E}+11$ & $4.4 \mathrm{E}+09$ & $5.7 E+00$ & $5.7 \mathrm{E}-02$ & $5.7 \mathrm{E}+00$ & $5.7 \mathrm{E}-02$ \\
\hline Rn-219 & 014835-02-0 & & & $3.0 \mathrm{E}+10$ & $3.0 \mathrm{E}+08$ & $2.5 \mathrm{E}+01$ & $2.5 \mathrm{E}-01$ & $2.5 \mathrm{E}+01$ & $2.5 \mathrm{E}-01$ \\
\hline $\mathrm{Rn}-220$ & 022481-48-7 & & & $1.1 \mathrm{E}+10$ & $1.1 \mathrm{E}+08$ & $2.3 E+03$ & $2.3 \mathrm{E}+01$ & $2.3 E+03$ & $2.3 \mathrm{E}+01$ \\
\hline $\mathrm{Rn}-222+\mathrm{D}$ & 014859-67-7 & & & $2.7 \mathrm{E}+08$ & $2.7 E+06$ & & & & \\
\hline
\end{tabular}


Table 3.1d. (continued)

\begin{tabular}{|c|c|c|c|c|c|c|c|c|c|}
\hline \multirow[t]{2}{*}{ Chemical $^{\dagger}$} & \multirow[t]{2}{*}{ CASRN } & \multicolumn{2}{|c|}{$\begin{array}{c}\text { Ingestion } \\
\text { (pCi/g) }\end{array}$} & \multicolumn{2}{|c|}{$\begin{array}{c}\text { Inhalation } \\
\text { (pCi/g) }\end{array}$} & \multicolumn{2}{|c|}{$\begin{array}{l}\text { External Exposure } \\
(\mathrm{pCi} / \mathrm{g})^{+t}\end{array}$} & \multicolumn{2}{|c|}{$\begin{array}{c}\text { Ingestion + External + } \\
\text { Inhalation (pCi/g) }{ }^{+t+}\end{array}$} \\
\hline & & $10^{-4}$ & $10^{-6}$ & $10^{-4}$ & $10^{-6}$ & $10^{-4}$ & $10^{-6}$ & $10^{-4}$ & $10^{-6}$ \\
\hline Ru-103 & 013968-53-1 & $2.4 \mathrm{E}+04$ & $2.4 \mathrm{E}+02$ & $4.4 \mathrm{E}+08$ & $4.4 \mathrm{E}+06$ & $2.6 \mathrm{E}+00$ & 2.6E-02 & $2.6 \mathrm{E}+00$ & $2.6 \mathrm{E}-02$ \\
\hline Ru-105 & 014331-95-4 & $6.9 E+04$ & $6.9 \mathrm{E}+02$ & $2.5 \mathrm{E}+09$ & $2.5 \mathrm{E}+07$ & $1.5 \mathrm{E}+00$ & $1.5 \mathrm{E}-02$ & $1.5 \mathrm{E}+00$ & $1.5 \mathrm{E}-02$ \\
\hline Ru-106 & 013967-48-1 & $2.3 E+03$ & $2.3 E+01$ & $1.8 \mathrm{E}+07$ & $1.8 \mathrm{E}+05$ & & & $2.8 \mathrm{E}+03$ & $2.8 \mathrm{E}+01$ \\
\hline Ru-106+D & $013967-48-1$ & $2.3 \mathrm{E}+03$ & $2.3 \mathrm{E}+01$ & $1.8 \mathrm{E}+07$ & $1.8 \mathrm{E}+05$ & $5.7 \mathrm{E}+00$ & $5.7 \mathrm{E}-02$ & $5.7 \mathrm{E}+00$ & $5.7 \mathrm{E}-02$ \\
\hline Ru-97 & 015758-35-7 & $1.3 \mathrm{E}+05$ & $1.3 \mathrm{E}+03$ & $5.0 \mathrm{E}+09$ & $5.0 \mathrm{E}+07$ & $9.6 \mathrm{E}+00$ & $9.6 \mathrm{E}-02$ & $9.6 \mathrm{E}+00$ & $9.6 \mathrm{E}-02$ \\
\hline$S-35$ & $015117-53-0$ & $1.9 \mathrm{E}+05$ & $1.9 E+03$ & $1.1 \mathrm{E}+10$ & $1.1 \mathrm{E}+08$ & & & $2.3 \mathrm{E}+05$ & $2.3 \mathrm{E}+03$ \\
\hline Sb-122 & 014374-79-9 & $9.0 \mathrm{E}+03$ & $9.0 \mathrm{E}+01$ & $3.7 \mathrm{E}+08$ & $3.7 \mathrm{E}+06$ & $2.7 \mathrm{E}+00$ & 2.7E-02 & $2.7 \mathrm{E}+00$ & 2.7E-02 \\
\hline Sb-124 & 014683-10-4 & $7.4 \mathrm{E}+03$ & $7.4 \mathrm{E}+01$ & $1.5 \mathrm{E}+08$ & $1.5 \mathrm{E}+06$ & $5.9 \mathrm{E}-01$ & $5.9 \mathrm{E}-03$ & $5.9 \mathrm{E}-01$ & $5.9 \mathrm{E}-03$ \\
\hline $\mathrm{Sb}-125$ & 014234-35-6 & $2.7 \mathrm{E}+04$ & $2.7 \mathrm{E}+02$ & $3.9 E+08$ & $3.9 \mathrm{E}+06$ & $3.2 \mathrm{E}+00$ & $3.2 \mathrm{E}-02$ & $3.2 \mathrm{E}+00$ & $3.2 \mathrm{E}-02$ \\
\hline Sb-125+D & 014234-35-6 & $2.2 \mathrm{E}+04$ & $2.2 \mathrm{E}+02$ & $3.5 E+08$ & $3.5 \mathrm{E}+06$ & $3.2 \mathrm{E}+00$ & 3.2E-02 & $3.2 \mathrm{E}+00$ & $3.2 \mathrm{E}-02$ \\
\hline Sb-126 & 015756-32-8 & $8.2 \mathrm{E}+03$ & $8.2 \mathrm{E}+01$ & $2.4 \mathrm{E}+08$ & $2.4 \mathrm{E}+06$ & 4.2E-01 & $4.2 \mathrm{E}-03$ & 4.2E-01 & 4.2E-03 \\
\hline $\mathrm{Sb}-126 \mathrm{~m}$ & $015756-32-8$ & $1.1 \mathrm{E}+06$ & $1.1 \mathrm{E}+04$ & $3.2 \mathrm{E}+10$ & $3.2 \mathrm{E}+08$ & $7.5 \mathrm{E}-01$ & 7.5E-03 & $7.5 \mathrm{E}-01$ & 7.5E-03 \\
\hline Sb-127 & 013968-50-8 & $9.4 \mathrm{E}+03$ & $9.4 \mathrm{E}+01$ & $3.4 \mathrm{E}+08$ & $3.4 \mathrm{E}+06$ & $1.8 \mathrm{E}+00$ & $1.8 \mathrm{E}-02$ & $1.8 \mathrm{E}+00$ & $1.8 \mathrm{E}-02$ \\
\hline Sb-129 & 014331-88-5 & $4.3 \mathrm{E}+04$ & 4.3E+02 & $2.4 \mathrm{E}+09$ & $2.4 \mathrm{E}+07$ & $7.8 \mathrm{E}-01$ & $7.8 \mathrm{E}-03$ & $7.8 \mathrm{E}-01$ & $7.8 \mathrm{E}-03$ \\
\hline Sc-46 & 013967-63-0 & $1.4 \mathrm{E}+04$ & $1.4 \mathrm{E}+02$ & $1.6 \mathrm{E}+08$ & $1.6 \mathrm{E}+06$ & $5.5 \mathrm{E}-01$ & $5.5 \mathrm{E}-03$ & $5.5 \mathrm{E}-01$ & $5.5 \mathrm{E}-03$ \\
\hline Sc-47 & 014391-96-9 & 2.7E+04 & $2.7 E+02$ & $1.0 \mathrm{E}+09$ & $1.0 \mathrm{E}+07$ & $1.7 \mathrm{E}+01$ & $1.7 \mathrm{E}-01$ & $1.7 \mathrm{E}+01$ & $1.7 \mathrm{E}-01$ \\
\hline Sc-48 & $014391-86-7$ & $1.2 \mathrm{E}+04$ & $1.2 \mathrm{E}+02$ & $4.9 E+08$ & $4.9 E+06$ & $3.3 \mathrm{E}-01$ & $3.3 \mathrm{E}-03$ & $3.3 \mathrm{E}-01$ & 3.3E-03 \\
\hline
\end{tabular}

Radionuclides/soil/residential

Page 14 of 20 
Table 3.1d. (continued)

\begin{tabular}{|c|c|c|c|c|c|c|c|c|c|}
\hline \multirow[t]{2}{*}{ Chemical $^{\dagger}$} & \multirow[t]{2}{*}{ CASRN } & \multicolumn{2}{|c|}{$\begin{array}{l}\text { Ingestion } \\
\text { (pCi/g) }\end{array}$} & \multicolumn{2}{|c|}{$\begin{array}{c}\text { Inhalation } \\
\text { (pCi/g) }\end{array}$} & \multicolumn{2}{|c|}{$\begin{array}{c}\text { External Exposure } \\
(\mathrm{pCi} / \mathrm{g})^{\dagger t}\end{array}$} & \multicolumn{2}{|c|}{$\begin{array}{c}\text { Ingestion + External + } \\
\text { Inhalation }(\mathrm{pCi} / \mathrm{g})^{\mathrm{tt+}}\end{array}$} \\
\hline & & $10^{-4}$ & $10^{-6}$ & $10^{-4}$ & $10^{-6}$ & $10^{-4}$ & $10^{-6}$ & $10^{-4}$ & $10^{-6}$ \\
\hline Se-75 & $014265-71-5$ & $1.2 \mathrm{E}+04$ & $1.2 \mathrm{E}+02$ & $4.1 \mathrm{E}+08$ & $4.1 E+06$ & $4.9 \mathrm{E}+00$ & $4.9 \mathrm{E}-02$ & $4.9 \mathrm{E}+00$ & $4.9 \mathrm{E}-02$ \\
\hline Si-31 & $014276-49-4$ & $1.6 \mathrm{E}+05$ & $1.6 \mathrm{E}+03$ & $6.2 \mathrm{E}+09$ & $6.2 \mathrm{E}+07$ & $1.3 \mathrm{E}+03$ & $1.3 E+01$ & $1.3 \mathrm{E}+03$ & $1.3 \mathrm{E}+01$ \\
\hline $\mathrm{Sm}-147$ & 014392-33-7 & $3.2 \mathrm{E}+03$ & $3.2 \mathrm{E}+01$ & $2.9 \mathrm{E}+05$ & $2.9 \mathrm{E}+03$ & & & $3.7 \mathrm{E}+03$ & $3.7 \mathrm{E}+01$ \\
\hline $\mathrm{Sm}-151$ & $015715-94-3$ & $1.7 \mathrm{E}+05$ & $1.7 \mathrm{E}+03$ & $4.4 \mathrm{E}+08$ & $4.4 \mathrm{E}+06$ & $1.5 \mathrm{E}+07$ & $1.5 \mathrm{E}+05$ & $2.0 \mathrm{E}+05$ & $2.0 \mathrm{E}+03$ \\
\hline Sm-153 & $015766-00-4$ & $2.0 \mathrm{E}+04$ & $2.0 \mathrm{E}+02$ & $9.4 \mathrm{E}+08$ & $9.4 \mathrm{E}+06$ & $9.3 \mathrm{E}+01$ & 9.3E-01 & $9.3 \mathrm{E}+01$ & $9.3 \mathrm{E}-01$ \\
\hline Sn-113 & $013966-06-8$ & $2.1 \mathrm{E}+04$ & $2.1 E+02$ & $3.1 \mathrm{E}+08$ & $3.1 \mathrm{E}+06$ & $1.5 \mathrm{E}+03$ & $1.5 \mathrm{E}+01$ & $1.4 \mathrm{E}+03$ & $1.4 \mathrm{E}+01$ \\
\hline $\mathrm{Sn}-121$ & 014683-06-8 & $6.5 \mathrm{E}+04$ & $6.5 E+02$ & $3.3 E+09$ & $3.3 \mathrm{E}+07$ & & & $7.8 \mathrm{E}+04$ & $7.8 \mathrm{E}+02$ \\
\hline Sn-121m & 014683-06-8 & $4.0 \mathrm{E}+04$ & $4.0 \mathrm{E}+02$ & $2.7 \mathrm{E}+08$ & $2.7 \mathrm{E}+06$ & & & $4.8 \mathrm{E}+04$ & $4.8 \mathrm{E}+02$ \\
\hline Sn-125 & $014683-08-0$ & $4.7 \mathrm{E}+03$ & $4.7 \mathrm{E}+01$ & $1.7 \mathrm{E}+08$ & $1.7 \mathrm{E}+06$ & $3.6 \mathrm{E}+00$ & $3.6 \mathrm{E}-02$ & $3.6 \mathrm{E}+00$ & $3.6 \mathrm{E}-02$ \\
\hline Sn-126 & $015832-50-5$ & $3.7 \mathrm{E}+03$ & $3.7 \mathrm{E}+01$ & $4.8 \mathrm{E}+07$ & $4.8 \mathrm{E}+05$ & $1.3 \mathrm{E}+02$ & $1.3 \mathrm{E}+00$ & $1.3 \mathrm{E}+02$ & $1.3 \mathrm{E}+00$ \\
\hline $\mathrm{Sr}-82$ & 014809-50-8 & $3.1 \mathrm{E}+03$ & $3.1 E+01$ & $2.3 E+08$ & $2.3 E+06$ & $4.8 \mathrm{E}+04$ & $4.8 \mathrm{E}+02$ & $3.4 \mathrm{E}+03$ & $3.4 \mathrm{E}+01$ \\
\hline $\mathrm{Sr}-85$ & $013967-73-2$ & $5.7 \mathrm{E}+04$ & $5.7 \mathrm{E}+02$ & $1.8 \mathrm{E}+09$ & $1.8 \mathrm{E}+07$ & $2.8 \mathrm{E}+00$ & $2.8 \mathrm{E}-02$ & $2.8 \mathrm{E}+00$ & $2.8 \mathrm{E}-02$ \\
\hline $\mathrm{Sr}-85 \mathrm{~m}$ & 013967-73-2 & $4.4 \mathrm{E}+06$ & $4.4 \mathrm{E}+04$ & $2.9 \mathrm{E}+11$ & $2.9 \mathrm{E}+09$ & $8.3 \mathrm{E}+00$ & $8.3 \mathrm{E}-02$ & $8.3 E+00$ & $8.3 \mathrm{E}-02$ \\
\hline Sr-89 & 014158-27-1 & $7.7 \mathrm{E}+03$ & $7.7 \mathrm{E}+01$ & $5.5 \mathrm{E}+08$ & $5.5 \mathrm{E}+06$ & $8.1 E+03$ & $8.1 E+01$ & $4.3 E+03$ & $4.3 \mathrm{E}+01$ \\
\hline Sr-90 & $010098-97-2$ & $1.9 \mathrm{E}+03$ & $1.9 \mathrm{E}+01$ & $3.4 \mathrm{E}+07$ & $3.4 \mathrm{E}+05$ & & & $2.3 E+03$ & $2.3 \mathrm{E}+01$ \\
\hline Sr-90+D & $010098-97-2$ & $1.4 \mathrm{E}+03$ & $1.4 \mathrm{E}+01$ & $2.9 \mathrm{E}+07$ & $2.9 \mathrm{E}+05$ & & & $1.7 \mathrm{E}+03$ & $1.7 \mathrm{E}+01$ \\
\hline Sr-91 & 014331-91-0 & $2.8 \mathrm{E}+04$ & $2.8 \mathrm{E}+02$ & $2.6 \mathrm{E}+09$ & $2.6 \mathrm{E}+07$ & $1.6 \mathrm{E}+00$ & $1.6 \mathrm{E}-02$ & $1.6 \mathrm{E}+00$ & $1.6 \mathrm{E}-02$ \\
\hline
\end{tabular}


Table 3.1d. (continued)

\begin{tabular}{|c|c|c|c|c|c|c|c|c|c|}
\hline \multirow[t]{2}{*}{ Chemical $^{\dagger}$} & \multirow[t]{2}{*}{ CASRN } & \multicolumn{2}{|c|}{$\begin{array}{c}\text { Ingestion } \\
\text { (pCi/g) }\end{array}$} & \multicolumn{2}{|c|}{$\begin{array}{c}\text { Inhalation } \\
(\mathrm{pCi} / \mathrm{g})\end{array}$} & \multicolumn{2}{|c|}{$\begin{array}{c}\text { External Exposure } \\
(\mathrm{pCi} / \mathrm{g})^{\dagger+}\end{array}$} & \multicolumn{2}{|c|}{$\begin{array}{c}\text { Ingestion + External + } \\
\text { Inhalation (pCi/g) }\end{array}$} \\
\hline & & $10^{-4}$ & $10^{-6}$ & $10^{-4}$ & $10^{-6}$ & $10^{-4}$ & $10^{-6}$ & $10^{-4}$ & $10^{-6}$ \\
\hline Sr-92 & 014928-29-1 & $3.9 \mathrm{E}+04$ & $3.9 \mathrm{E}+02$ & $4.3 E+09$ & $4.3 \mathrm{E}+07$ & $8.4 \mathrm{E}-01$ & $8.4 \mathrm{E}-03$ & $8.4 \mathrm{E}-01$ & $8.4 \mathrm{E}-03$ \\
\hline Ta-182 & $013982-00-8$ & $1.1 E+04$ & $1.1 \mathrm{E}+02$ & $1.2 \mathrm{E}+08$ & $1.2 \mathrm{E}+06$ & $9.3 \mathrm{E}-01$ & 9.3E-03 & 9.3E-01 & $9.3 \mathrm{E}-03$ \\
\hline Tb-158 & 015759-55-4 & $1.9 E+04$ & $1.9 \mathrm{E}+02$ & $2.9 \mathrm{E}+07$ & $2.9 \mathrm{E}+05$ & & & $2.3 E+04$ & $2.3 \mathrm{E}+02$ \\
\hline Tb-160 & 013981-29-8 & $1.0 \mathrm{E}+04$ & $1.0 \mathrm{E}+02$ & $1.8 \mathrm{E}+08$ & $1.8 \mathrm{E}+06$ & $1.1 \mathrm{E}+00$ & $1.1 \mathrm{E}-02$ & $1.1 \mathrm{E}+00$ & $1.1 \mathrm{E}-02$ \\
\hline Tc-95 & 014809-56-4 & $1.2 E+06$ & $1.2 \mathrm{E}+04$ & $6.0 \mathrm{E}+10$ & $6.0 \mathrm{E}+08$ & $1.6 \mathrm{E}+00$ & $1.6 \mathrm{E}-02$ & $1.6 \mathrm{E}+00$ & $1.6 \mathrm{E}-02$ \\
\hline Tc-95m & 014809-56-4 & $6.4 \mathrm{E}+04$ & $6.4 \mathrm{E}+02$ & $9.7 E+08$ & $9.7 \mathrm{E}+06$ & $2.1 E+00$ & 2.1E-02 & $2.1 \mathrm{E}+00$ & $2.1 \mathrm{E}-02$ \\
\hline Tc-96 & $014808-44-7$ & $3.5 E+04$ & $3.5 E+02$ & $1.1 \mathrm{E}+09$ & $1.1 \mathrm{E}+07$ & $4.6 \mathrm{E}-01$ & $4.6 \mathrm{E}-03$ & $4.6 \mathrm{E}-01$ & $4.6 \mathrm{E}-03$ \\
\hline Tc-96m & 014808-44-7 & $3.0 \mathrm{E}+06$ & $3.0 \mathrm{E}+04$ & $9.0 \mathrm{E}+10$ & $9.0 \mathrm{E}+08$ & $5.6 \mathrm{E}+01$ & $5.6 \mathrm{E}-01$ & $5.6 \mathrm{E}+01$ & $5.6 \mathrm{E}-01$ \\
\hline Tc-97 & 015759-35-0 & $5.0 \mathrm{E}+05$ & $5.0 \mathrm{E}+03$ & $5.9 \mathrm{E}+09$ & $5.9 \mathrm{E}+07$ & $1.7 \mathrm{E}+04$ & $1.7 \mathrm{E}+02$ & $1.7 \mathrm{E}+04$ & $1.7 \mathrm{E}+02$ \\
\hline Tc-97m & 015759-35-0 & $6.6 \mathrm{E}+04$ & $6.6 \mathrm{E}+02$ & $1.0 \mathrm{E}+09$ & $1.0 \mathrm{E}+07$ & $1.6 \mathrm{E}+04$ & $1.6 \mathrm{E}+02$ & $1.4 \mathrm{E}+04$ & $1.4 \mathrm{E}+02$ \\
\hline Tc-99 & $014133-76-7$ & $5.7 \mathrm{E}+04$ & $5.7 \mathrm{E}+02$ & $7.1 \mathrm{E}+08$ & $7.1 \mathrm{E}+06$ & $7.0 \mathrm{E}+06$ & $7.0 \mathrm{E}+04$ & $6.7 E+04$ & $6.7 E+02$ \\
\hline Tc-99m & 014133-76-7 & $1.4 \mathrm{E}+06$ & $1.4 \mathrm{E}+04$ & $5.8 \mathrm{E}+10$ & $5.8 \mathrm{E}+08$ & $1.7 \mathrm{E}+01$ & $1.7 \mathrm{E}-01$ & $1.7 \mathrm{E}+01$ & $1.7 \mathrm{E}-01$ \\
\hline $\mathrm{Te}-125 \mathrm{~m}$ & 014390-73-9 & $3.2 E+04$ & $3.2 \mathrm{E}+02$ & $7.2 \mathrm{E}+08$ & $7.2 \mathrm{E}+06$ & $2.0 \mathrm{E}+03$ & $2.0 \mathrm{E}+01$ & $1.9 \mathrm{E}+03$ & $1.9 \mathrm{E}+01$ \\
\hline Te-127 & $013981-49-2$ & $9.3 \mathrm{E}+04$ & $9.3 \mathrm{E}+02$ & $4.7 \mathrm{E}+09$ & $4.7 \mathrm{E}+07$ & $2.7 E+02$ & $2.7 \mathrm{E}+00$ & $2.7 \mathrm{E}+02$ & $2.7 \mathrm{E}+00$ \\
\hline Te-127m & $013981-49-2$ & $1.3 E+04$ & $1.3 \mathrm{E}+02$ & $1.6 \mathrm{E}+08$ & $1.6 \mathrm{E}+06$ & $6.1 E+03$ & $6.1 \mathrm{E}+01$ & $4.4 \mathrm{E}+03$ & $4.4 \mathrm{E}+01$ \\
\hline Te-129 & $014269-71-7$ & $5.4 \mathrm{E}+05$ & $5.4 \mathrm{E}+03$ & $1.4 \mathrm{E}+10$ & $1.4 \mathrm{E}+08$ & $3.0 \mathrm{E}+01$ & $3.0 \mathrm{E}-01$ & $3.0 \mathrm{E}+01$ & $3.0 \mathrm{E}-01$ \\
\hline $\mathrm{Te}-129 \mathrm{~m}$ & 014269-71-7 & $6.8 \mathrm{E}+03$ & $6.8 \mathrm{E}+01$ & $1.5 \mathrm{E}+08$ & $1.5 \mathrm{E}+06$ & $6.3 \mathrm{E}+01$ & $6.3 \mathrm{E}-01$ & $6.2 \mathrm{E}+01$ & $6.2 \mathrm{E}-01$ \\
\hline
\end{tabular}


Table 3.1d. (continued)

\begin{tabular}{|c|c|c|c|c|c|c|c|c|c|}
\hline \multirow[t]{2}{*}{ Chemical $^{\dagger}$} & \multirow[t]{2}{*}{ CASRN } & \multicolumn{2}{|c|}{$\begin{array}{c}\text { Ingestion } \\
\text { (pCi/g) }\end{array}$} & \multicolumn{2}{|c|}{$\begin{array}{c}\text { Inhalation } \\
\text { (pCi/g) }\end{array}$} & \multicolumn{2}{|c|}{$\begin{array}{c}\text { External Exposure } \\
(\mathrm{pCi} / \mathrm{g})^{H+}\end{array}$} & \multicolumn{2}{|c|}{$\begin{array}{c}\text { Ingestion + External + } \\
\text { Inhalation (pCi/g) }\end{array}$} \\
\hline & & $10^{-4}$ & $10^{-6}$ & $10^{-4}$ & $10^{-6}$ & $10^{-4}$ & $10^{-6}$ & $10^{-4}$ & $10^{-6}$ \\
\hline Te-131 & $014683-12-6$ & $2.0 \mathrm{E}+05$ & $2.0 \mathrm{E}+03$ & $8.2 E+09$ & $8.2 E+07$ & $3.2 \mathrm{E}+00$ & $3.2 \mathrm{E}-02$ & $3.2 \mathrm{E}+00$ & $3.2 \mathrm{E}-02$ \\
\hline $\mathrm{Te}-131 \mathrm{~m}$ & $014683-12-6$ & $9.0 \mathrm{E}+03$ & $9.0 \mathrm{E}+01$ & $2.4 \mathrm{E}+08$ & $2.4 \mathrm{E}+06$ & $8.2 \mathrm{E}-01$ & $8.2 \mathrm{E}-03$ & $8.2 \mathrm{E}-01$ & $8.2 \mathrm{E}-03$ \\
\hline Te-132 & $014234-28-7$ & $6.5 \mathrm{E}+03$ & $6.5 \mathrm{E}+01$ & $2.4 \mathrm{E}+08$ & $2.4 \mathrm{E}+06$ & $1.0 \mathrm{E}+01$ & $1.0 \mathrm{E}-01$ & $1.0 \mathrm{E}+01$ & $1.0 \mathrm{E}-01$ \\
\hline Th-227 & $015623-47-9$ & $2.0 \mathrm{E}+03$ & $2.0 \mathrm{E}+01$ & $4.7 \mathrm{E}+05$ & 4.7E+03 & $2.5 \mathrm{E}+01$ & $2.5 \mathrm{E}-01$ & $2.5 \mathrm{E}+01$ & $2.5 \mathrm{E}-01$ \\
\hline Th-228 & $014274-82-9$ & $1.3 \mathrm{E}+03$ & $1.3 \mathrm{E}+01$ & $2.2 \mathrm{E}+04$ & $2.2 \mathrm{E}+02$ & $8.2 E+03$ & $8.2 \mathrm{E}+01$ & $1.2 \mathrm{E}+03$ & $1.2 \mathrm{E}+01$ \\
\hline Th-228+D & $014274-82-9$ & $3.4 \mathrm{E}+02$ & $3.4 \mathrm{E}+00$ & $2.1 \mathrm{E}+04$ & $2.1 \mathrm{E}+02$ & $4.4 \mathrm{E}+00$ & $4.4 \mathrm{E}-02$ & $4.3 \mathrm{E}+00$ & 4.3E-02 \\
\hline Th-229 & 015594-54-4 & $1.4 \mathrm{E}+03$ & $1.4 \mathrm{E}+01$ & $2.7 E+04$ & $2.7 \mathrm{E}+02$ & $7.3 \mathrm{E}+01$ & 7.3E-01 & $7.0 \mathrm{E}+01$ & $7.0 \mathrm{E}-01$ \\
\hline Th-229+D & $015594-54-4$ & $2.2 E+02$ & $2.2 \mathrm{E}+00$ & $2.5 \mathrm{E}+04$ & $2.5 \mathrm{E}+02$ & $7.3 E+00$ & 7.3E-02 & $7.1 \mathrm{E}+00$ & 7.1E-02 \\
\hline Th-230 & $014269-63-7$ & $2.1 \mathrm{E}+03$ & $2.1 E+01$ & $1.2 \mathrm{E}+05$ & $1.2 \mathrm{E}+03$ & $9.9 E+04$ & $9.9 \mathrm{E}+02$ & $2.4 \mathrm{E}+03$ & $2.4 \mathrm{E}+01$ \\
\hline Th-231 & $014932-40-2$ & $4.4 \mathrm{E}+04$ & $4.4 \mathrm{E}+02$ & $1.9 \mathrm{E}+09$ & $1.9 \mathrm{E}+07$ & $2.1 E+03$ & $2.1 E+01$ & $2.0 \mathrm{E}+03$ & $2.0 \mathrm{E}+01$ \\
\hline Th-232 & $007440-29-1$ & $2.4 E+03$ & $2.4 \mathrm{E}+01$ & $1.1 \mathrm{E}+05$ & $1.1 \mathrm{E}+03$ & $2.2 E+05$ & $2.2 E+03$ & $2.8 \mathrm{E}+03$ & $2.8 \mathrm{E}+01$ \\
\hline Th-234 & $015065-10-8$ & $4.1 \mathrm{E}+03$ & 4. $1 \mathrm{E}+01$ & $1.1 \mathrm{E}+08$ & $1.1 \mathrm{E}+06$ & $1.2 E+03$ & $1.2 \mathrm{E}+01$ & $9.9 \mathrm{E}+02$ & $9.9 \mathrm{E}+00$ \\
\hline Tl-202 & $015720-57-7$ & $7.9 \mathrm{E}+04$ & $7.9 \mathrm{E}+02$ & $3.4 \mathrm{E}+09$ & $3.4 \mathrm{E}+07$ & $3.1 \mathrm{E}+00$ & $3.1 \mathrm{E}-02$ & $3.1 \mathrm{E}+00$ & $3.1 \mathrm{E}-02$ \\
\hline Tl-204 & $013968-51-9$ & $4.0 E+04$ & $4.0 \mathrm{E}+02$ & $1.8 \mathrm{E}+09$ & $1.8 \mathrm{E}+07$ & $5.0 \mathrm{E}+03$ & $5.0 \mathrm{E}+01$ & $4.5 \mathrm{E}+03$ & $4.5 \mathrm{E}+01$ \\
\hline Tl-208 & 014913-50-9 & $4.5 \mathrm{E}+06$ & $4.5 E+04$ & $1.5 \mathrm{E}+11$ & $1.5 E+09$ & $3.0 \mathrm{E}-01$ & $3.0 \mathrm{E}-03$ & $3.0 \mathrm{E}-01$ & $3.0 \mathrm{E}-03$ \\
\hline Tl-209 & 015690-73-0 & $5.7 \mathrm{E}+06$ & $5.7 \mathrm{E}+04$ & $1.8 \mathrm{E}+11$ & $1.8 \mathrm{E}+09$ & $5.5 \mathrm{E}-01$ & $5.5 \mathrm{E}-03$ & $5.5 \mathrm{E}-01$ & $5.5 \mathrm{E}-03$ \\
\hline $\mathrm{Tm}-170$ & 013981-30-1 & $1.1 \mathrm{E}+04$ & $1.1 \mathrm{E}+02$ & $1.9 \mathrm{E}+08$ & $1.9 \mathrm{E}+06$ & $1.1 E+03$ & $1.1 \mathrm{E}+01$ & $1.0 \mathrm{E}+03$ & $1.0 \mathrm{E}+01$ \\
\hline
\end{tabular}


Table 3.1d. (continued)

\begin{tabular}{|c|c|c|c|c|c|c|c|c|c|}
\hline \multirow[t]{2}{*}{ Chemical $^{\dagger}$} & \multirow[t]{2}{*}{ CASRN } & \multicolumn{2}{|c|}{$\begin{array}{c}\text { Ingestion } \\
\text { (pCi/g) }\end{array}$} & \multicolumn{2}{|c|}{$\begin{array}{l}\text { Inhalation } \\
\text { (pCi/g) }\end{array}$} & \multicolumn{2}{|c|}{$\begin{array}{l}\text { External Exposure } \\
(\mathrm{pCi} / \mathrm{g})^{\dagger+}\end{array}$} & \multicolumn{2}{|c|}{$\begin{array}{l}\text { Ingestion + External + } \\
\text { Inhalation }(\mathrm{pCi} / \mathrm{g})^{t+1}\end{array}$} \\
\hline & & $10^{-4}$ & $10^{-6}$ & $10^{-4}$ & $10^{-6}$ & $10^{-4}$ & $10^{-6}$ & $10^{-4}$ & $10^{-6}$ \\
\hline Tm-171 & $014333-45-0$ & $1.4 E+05$ & $1.4 \mathrm{E}+03$ & $1.1 \mathrm{E}+09$ & $1.1 \mathrm{E}+07$ & $1.4 \mathrm{E}+04$ & $1.4 \mathrm{E}+02$ & $1.3 \mathrm{E}+04$ & $1.3 E+02$ \\
\hline U-232 & $014158-29-3$ & $9.8 \mathrm{E}+02$ & $9.8 \mathrm{E}+00$ & $3.9 \mathrm{E}+04$ & $3.9 \mathrm{E}+02$ & $1.3 \mathrm{E}+05$ & $1.3 \mathrm{E}+03$ & $1.1 \mathrm{E}+03$ & $1.1 \mathrm{E}+01$ \\
\hline U-233 & $013968-55-3$ & $1.8 \mathrm{E}+03$ & $1.8 \mathrm{E}+01$ & $1.4 \mathrm{E}+05$ & $1.4 \mathrm{E}+03$ & $1.2 \mathrm{E}+05$ & $1.2 \mathrm{E}+03$ & $2.1 E+03$ & $2.1 \mathrm{E}+01$ \\
\hline U-234 & 013966-29-5 & $1.8 \mathrm{E}+03$ & $1.8 \mathrm{E}+01$ & $1.5 \mathrm{E}+05$ & $1.5 \mathrm{E}+03$ & $2.0 \mathrm{E}+05$ & $2.0 \mathrm{E}+03$ & $2.1 \mathrm{E}+03$ & $2.1 \mathrm{E}+01$ \\
\hline $\mathrm{U}-235$ & 015117-96-1 & $1.8 \mathrm{E}+03$ & $1.8 \mathrm{E}+01$ & $1.6 \mathrm{E}+05$ & $1.6 \mathrm{E}+03$ & $1.7 \mathrm{E}+01$ & $1.7 \mathrm{E}-01$ & $1.6 \mathrm{E}+01$ & $1.6 \mathrm{E}-01$ \\
\hline U-235+D & 015117-96-1 & $1.7 \mathrm{E}+03$ & $1.7 \mathrm{E}+01$ & $1.6 \mathrm{E}+05$ & $1.6 \mathrm{E}+03$ & $1.6 \mathrm{E}+01$ & $1.6 \mathrm{E}-01$ & $1.6 \mathrm{E}+01$ & $1.6 \mathrm{E}-01$ \\
\hline U-236 & $013982-70-2$ & $1.9 \mathrm{E}+03$ & $1.9 \mathrm{E}+01$ & $1.5 \mathrm{E}+05$ & $1.5 \mathrm{E}+03$ & $2.5 \mathrm{E}+05$ & $2.5 \mathrm{E}+03$ & $2.2 \mathrm{E}+03$ & $2.2 \mathrm{E}+01$ \\
\hline U-237 & $014269-75-1$ & $2.0 \mathrm{E}+04$ & $2.0 \mathrm{E}+02$ & $6.5 \mathrm{E}+08$ & $6.5 E+06$ & $3.2 \mathrm{E}+01$ & $3.2 \mathrm{E}-01$ & $3.2 \mathrm{E}+01$ & $3.2 \mathrm{E}-01$ \\
\hline U-238 & 007440-61-1 & $1.9 \mathrm{E}+03$ & $1.9 \mathrm{E}+01$ & $1.6 \mathrm{E}+05$ & $1.6 \mathrm{E}+03$ & $2.9 \mathrm{E}+05$ & $2.9 \mathrm{E}+03$ & $2.2 \mathrm{E}+03$ & $2.2 \mathrm{E}+01$ \\
\hline$U-238+D$ & 007440-61-1 & $1.3 \mathrm{E}+03$ & $1.3 \mathrm{E}+01$ & $1.6 \mathrm{E}+05$ & $1.6 \mathrm{E}+03$ & $8.3 E+01$ & $8.3 \mathrm{E}-01$ & $7.8 \mathrm{E}+01$ & $7.8 \mathrm{E}-01$ \\
\hline U-240 & $015687-53-3$ & $1.5 \mathrm{E}+04$ & $1.5 \mathrm{E}+02$ & $6.1 \mathrm{E}+08$ & $6.1 \mathrm{E}+06$ & $4.0 \mathrm{E}+04$ & $4.0 \mathrm{E}+02$ & $1.2 \mathrm{E}+04$ & $1.2 \mathrm{E}+02$ \\
\hline$V-48$ & $014331-97-6$ & $1.0 \mathrm{E}+04$ & $1.0 \mathrm{E}+02$ & $3.0 \mathrm{E}+08$ & $3.0 \mathrm{E}+06$ & $3.9 \mathrm{E}-01$ & $3.9 \mathrm{E}-03$ & $3.9 \mathrm{E}-01$ & $3.9 \mathrm{E}-03$ \\
\hline W-181 & 015749-46-9 & $2.9 E+05$ & $2.9 \mathrm{E}+03$ & $2.5 \mathrm{E}+10$ & $2.5 \mathrm{E}+08$ & $2.1 \mathrm{E}+02$ & $2.1 \mathrm{E}+00$ & $2.1 \mathrm{E}+02$ & $2.1 E+00$ \\
\hline W-185 & $014932-41-3$ & $3.9 \mathrm{E}+04$ & $3.9 \mathrm{E}+02$ & $4.8 \mathrm{E}+09$ & $4.8 \mathrm{E}+07$ & $8.6 \mathrm{E}+04$ & $8.6 \mathrm{E}+02$ & $3.0 \mathrm{E}+04$ & $3.0 \mathrm{E}+02$ \\
\hline W-187 & $014983-48-3$ & $3.2 \mathrm{E}+04$ & $3.2 \mathrm{E}+02$ & $3.9 \mathrm{E}+09$ & $3.9 \mathrm{E}+07$ & $2.7 \mathrm{E}+00$ & $2.7 \mathrm{E}-02$ & $2.7 \mathrm{E}+00$ & 2.7E-02 \\
\hline $\mathrm{Xe}-122$ & $015151-09-4$ & & & $6.6 \mathrm{E}+11$ & $6.6 \mathrm{E}+09$ & & & & \\
\hline $\mathrm{Xe}-123$ & $015700-10-4$ & & & $2.3 \mathrm{E}+12$ & $2.3 \mathrm{E}+10$ & & & & \\
\hline
\end{tabular}


Table 3.1d. (continued)

\begin{tabular}{|c|c|c|c|c|c|c|c|c|c|}
\hline \multirow[t]{2}{*}{ Chemical $^{\dagger}$} & \multirow[t]{2}{*}{ CASRN } & \multicolumn{2}{|c|}{$\begin{array}{c}\text { Ingestion } \\
(\mathrm{pCi} / \mathrm{g})\end{array}$} & \multicolumn{2}{|c|}{$\begin{array}{c}\text { Inhalation } \\
\text { (pCi/g) }\end{array}$} & \multicolumn{2}{|c|}{$\begin{array}{c}\text { External Exposure } \\
(\mathrm{pCi} / \mathrm{g})^{\dagger \dagger}\end{array}$} & \multicolumn{2}{|c|}{$\begin{array}{l}\text { Ingestion + External + } \\
\text { Inhalation (pCi/g) }\end{array}$} \\
\hline & & $10^{-4}$ & $10^{-6}$ & $10^{-4}$ & $10^{-6}$ & $10^{-4}$ & $10^{-6}$ & $10^{-4}$ & $10^{-6}$ \\
\hline $\mathrm{Xe}-125$ & 013994-18-8 & & & $1.7 \mathrm{E}+12$ & $1.7 \mathrm{E}+10$ & & & & \\
\hline $\mathrm{Xe}-127$ & 013994-19-9 & & & $5.0 \mathrm{E}+12$ & $5.0 \mathrm{E}+10$ & & & & \\
\hline $\mathrm{Xe}-129 \mathrm{~m}$ & 013965-99-6 & & & $3.6 \mathrm{E}+12$ & $3.6 \mathrm{E}+10$ & & & & \\
\hline $\mathrm{Xe}-131 \mathrm{~m}$ & 014683-11-5 & & & $4.9 \mathrm{E}+12$ & $4.9 \mathrm{E}+10$ & & & & \\
\hline $\mathrm{Xe}-133$ & $014932-42-4$ & & & $4.9 \mathrm{E}+12$ & $4.9 E+10$ & & & & \\
\hline $\mathrm{Xe}-133 \mathrm{~m}$ & $014932-42-4$ & & & $4.0 \mathrm{E}+12$ & $4.0 \mathrm{E}+10$ & & & & \\
\hline $\mathrm{Xe}-135$ & $014995-62-1$ & & & $2.7 E+12$ & $2.7 \mathrm{E}+10$ & & & & \\
\hline $\mathrm{Xe}-135 \mathrm{~m}$ & $014995-62-1$ & & & $1.1 \mathrm{E}+13$ & $1.1 \mathrm{E}+11$ & & & & \\
\hline Xe-137 & $014835-21-3$ & & & $1.5 \mathrm{E}+12$ & $1.5 \mathrm{E}+10$ & & & & \\
\hline $\mathrm{Xe}-138$ & $015751-81-2$ & & & $9.9 \mathrm{E}+11$ & $9.9 \mathrm{E}+09$ & & & & \\
\hline$Y-90$ & 010098-91-6 & $5.3 \mathrm{E}+03$ & $5.3 \mathrm{E}+01$ & $2.1 \mathrm{E}+08$ & $2.1 E+06$ & & & $6.3 E+03$ & $6.3 \mathrm{E}+01$ \\
\hline$Y-91$ & $014234-24-3$ & $5.9 \mathrm{E}+03$ & $5.9 E+01$ & $1.1 \mathrm{E}+08$ & $1.1 E+06$ & $3.1 \mathrm{E}+02$ & $3.1 E+00$ & $3.0 \mathrm{E}+02$ & $3.0 \mathrm{E}+00$ \\
\hline$Y-91 \mathrm{~m}$ & $014234-24-3$ & $2.2 \mathrm{E}+06$ & $2.2 \mathrm{E}+04$ & $6.8 \mathrm{E}+10$ & $6.8 \mathrm{E}+08$ & $2.3 \mathrm{E}+00$ & $2.3 \mathrm{E}-02$ & $2.3 E+00$ & 2.3E-02 \\
\hline$Y-92$ & 015751-59-4 & 4.1E+04 & $4.1 \mathrm{E}+02$ & $1.3 \mathrm{E}+09$ & $1.3 E+07$ & $4.4 \mathrm{E}+00$ & $4.4 \mathrm{E}-02$ & $4.4 \mathrm{E}+00$ & $4.4 \mathrm{E}-02$ \\
\hline Y-93 & 014981-70-5 & $1.4 \mathrm{E}+04$ & $1.4 \mathrm{E}+02$ & $5.9 \mathrm{E}+08$ & $5.9 \mathrm{E}+06$ & $1.3 \mathrm{E}+01$ & $1.3 \mathrm{E}-01$ & $1.3 \mathrm{E}+01$ & $1.3 \mathrm{E}-01$ \\
\hline $\mathrm{Zn}-65$ & 013982-39-3 & $8.0 \mathrm{E}+03$ & $8.0 \mathrm{E}+01$ & $2.0 \mathrm{E}+08$ & $2.0 \mathrm{E}+06$ & $1.9 \mathrm{E}+00$ & $1.9 \mathrm{E}-02$ & $1.9 \mathrm{E}+00$ & $1.9 \mathrm{E}-02$ \\
\hline Zn-69 & $013982-23-5$ & $1.3 E+06$ & $1.3 E+04$ & $2.0 \mathrm{E}+10$ & $2.0 \mathrm{E}+08$ & $2.1 \mathrm{E}+05$ & $2.1 E+03$ & $1.9 E+05$ & $1.9 \mathrm{E}+03$ \\
\hline
\end{tabular}


Table 3.1d. (continued)

\begin{tabular}{|l|l|c|c|c|c|c|c|c|c|}
\hline \multirow{2}{*}{ Chemical } & \multirow{2}{*}{} & \multirow{2}{*}{ CASRN } & \multicolumn{2}{|c|}{$\begin{array}{c}\text { Ingestion } \\
(\mathrm{pCi} / \mathrm{g})\end{array}$} & \multicolumn{2}{c|}{$\begin{array}{c}\text { Inhalation } \\
(\mathrm{pCi} / \mathrm{g})\end{array}$} & \multicolumn{2}{c|}{$\begin{array}{c}\text { External Exposure } \\
(\mathrm{pCi} / \mathrm{g})^{\dagger \dagger}\end{array}$} & \multicolumn{2}{|c|}{$\begin{array}{c}\text { Ingestion + External + } \\
\text { Inhalation (pCi/g) }\end{array}$} \\
\cline { 3 - 10 } & & $10^{-4}$ & $10^{-6}$ & $10^{-4}$ & $10^{-6}$ & $10^{-4}$ & $10^{-6}$ & $10^{-4}$ & $10^{-6}$ \\
\hline $\mathrm{Zn}-69 \mathrm{~m}$ & $013982-23-5$ & $5.2 \mathrm{E}+04$ & $5.2 \mathrm{E}+02$ & $1.7 \mathrm{E}+09$ & $1.7 \mathrm{E}+07$ & $3.0 \mathrm{E}+00$ & $3.0 \mathrm{E}-02$ & $3.0 \mathrm{E}+00$ & $3.0 \mathrm{E}-02$ \\
\hline $\mathrm{Zr}-93$ & $015751-77-6$ & $1.5 \mathrm{E}+05$ & $1.5 \mathrm{E}+03$ & $3.9 \mathrm{E}+08$ & $3.9 \mathrm{E}+06$ & & & $1.8 \mathrm{E}+05$ & $1.8 \mathrm{E}+03$ \\
\hline $\mathrm{Zr}-95$ & $013967-71-0$ & $2.0 \mathrm{E}+04$ & $2.0 \mathrm{E}+02$ & $3.1 \mathrm{E}+08$ & $3.1 \mathrm{E}+06$ & $1.5 \mathrm{E}+00$ & $1.5 \mathrm{E}-02$ & $1.5 \mathrm{E}+00$ & $1.5 \mathrm{E}-02$ \\
\hline $\mathrm{Zr}-97$ & $014928-30-4$ & $7.6 \mathrm{E}+03$ & $7.6 \mathrm{E}+01$ & $4.3 \mathrm{E}+08$ & $4.3 \mathrm{E}+06$ & $6.3 \mathrm{E}+00$ & $6.3 \mathrm{E}-02$ & $6.3 \mathrm{E}+00$ & $6.3 \mathrm{E}-02$ \\
\hline
\end{tabular}

\section{Notes:}

$+\quad$ Isotopes designated with an " $\mathrm{m}$ " indicate that the isotope is metastable; isotopes designated with a " $+\mathrm{D}$ " indicate that the radionuclide slope factors include contributions from daughter products.

i† If no PRG for external exposure was calculated, then no external exposure slope factors were available or the external exposure slope factor was equal to $0.00 \mathrm{E}+00$ (resulting in an undefined PRG for the external exposure pathway for soil under residential land use).

tit If slope factors and/or reference doses were available for only one pathway (i.e., ingestion, inhalation, OR external exposure), the total for ingestion plus inhalation plus external exposure was not calculated.

(1) The default volatilization factor of 200 for Ra-224 was calculated as the ratio of the average Ra-224 background concentration in soil to the average $\mathrm{Rn}-220$ background concentration in air. Inhalation slope factor values are for $\mathrm{Rn}-222$ plus decay products (i.e., $\mathrm{Rn}-222+\mathrm{D}$ ) formed from the radioactive decay of Ra-226 and for Rn-220+D from the decay of Ra-224. Slope factor values were taken from Table 4a of EPA's Health Effects Assessment Summary Tables (HEAST 1992).

(2) The default volatilization factor of 8 for Ra-226 was calculated as the ratio of the average natural background concentration of Ra-226 in soil to the corresponding average natural background concentration of $\mathrm{Rn}-222$ in air. Inhalation slope factor values are for $\mathrm{Rn}-222$ plus decay products (i.e., $\mathrm{Rn}$ $222+\mathrm{D}$ ) formed from the radioactive decay of Ra-226 and for $\mathrm{Rn}-220+\mathrm{D}$ from the decay of Ra-224. Slope factor values were taken from Table $4 \mathrm{a}$ of EPA's Health Effects Assessment Summary Tables (HEAST 1992). 
Table 3.1e. Risk-based Preliminary Remediation Goals for nonradionuclides in fish (residential scenario)

\begin{tabular}{|l|l|l|c|c|c|}
\hline \multirow{2}{*}{ Chemical } & \multirow{2}{*}{ CASRN } & \multicolumn{4}{|c|}{ Ingestion of fish (mg/kg) } \\
\cline { 4 - 6 } & & $10^{-4}$ & $10^{-6}$ & HQ=1 & HQ=0.1 \\
\hline ALAR & $001596-84-5$ & & & $2.0 \mathrm{E}+02$ & $2.0 \mathrm{E}+01$ \\
\hline Acenaphthene & $000083-32-9$ & & & $8.1 \mathrm{E}+01$ & $8.1 \mathrm{E}+00$ \\
\hline Acenaphthylene & $000208-96-8$ & & & & \\
\hline Acephate & $030560-19-1$ & $3.6 \mathrm{E}+01$ & $3.6 \mathrm{E}-01$ & $5.4 \mathrm{E}+00$ & $5.4 \mathrm{E}-01$ \\
\hline Acetaldehyde & $000075-07-0$ & & & & \\
\hline Acetochlor & $034256-82-1$ & & & $2.7 \mathrm{E}+01$ & $2.7 \mathrm{E}+00$ \\
\hline Acetone & $000067-64-1$ & & & $1.4 \mathrm{E}+02$ & $1.4 \mathrm{E}+01$ \\
\hline Acetone Cyanohydrin & $000075-86-5$ & & & $1.1 \mathrm{E}+00$ & $1.1 \mathrm{E}-01$ \\
\hline Acetonitrile & $000075-05-8$ & & & $8.1 \mathrm{E}+00$ & $8.1 \mathrm{E}-01$ \\
\hline Acetophenone ${ }^{(5)}$ & $000098-86-2$ & & & $1.4 \mathrm{E}+02$ & $1.4 \mathrm{E}+01$ \\
\hline Acrolein ${ }^{(6)}$ & $000107-02-8$ & & & $2.7 \mathrm{E}+01$ & $2.7 \mathrm{E}+00$ \\
\hline Acrylamide & $000079-06-1$ & $7.0 \mathrm{E}-02$ & $7.0 \mathrm{E}-04$ & $2.7 \mathrm{E}-01$ & $2.7 \mathrm{E}-02$ \\
\hline Acrylic Acid & $000079-10-7$ & & & $6.8 \mathrm{E}+02$ & $6.8 \mathrm{E}+01$ \\
\hline Acrylonitrile & $000107-13-1$ & $5.8 \mathrm{E}-01$ & $5.8 \mathrm{E}-03$ & $1.4 \mathrm{E}+00$ & $1.4 \mathrm{E}-01$ \\
\hline Alachlor & $015972-60-8$ & $3.9 \mathrm{E}+00$ & $3.9 \mathrm{E}-02$ & $1.4 \mathrm{E}+01$ & $1.4 \mathrm{E}+00$ \\
\hline Aldicarb & $000116-06-3$ & & & $1.4 \mathrm{E}+00$ & $1.4 \mathrm{E}-01$ \\
\hline Aldicarb Sulfone & $001646-88-4$ & & & $1.4 \mathrm{E}+00$ & $1.4 \mathrm{E}-01$ \\
\hline Aldrin & $000309-00-2$ & $1.9 \mathrm{E}-02$ & $1.9 \mathrm{E}-04$ & $4.1 \mathrm{E}-02$ & $4.1 \mathrm{E}-03$ \\
\hline
\end{tabular}


Table 3.1e. (continued)

\begin{tabular}{|c|c|c|c|c|c|}
\hline \multirow{2}{*}{ Chemical } & \multirow{2}{*}{ CASRN } & \multicolumn{4}{|c|}{ Ingestion of fish (mg/kg)" } \\
\hline & & $10^{-4}$ & $10^{-6}$ & $H Q=1$ & $\mathrm{HQ}=0.1$ \\
\hline Ally & $074223-64-6$ & & & $3.4 \mathrm{E}+02$ & $3.4 \mathrm{E}+01$ \\
\hline Allyl Alcohol & $000107-18-6$ & & & $6.8 \mathrm{E}+00$ & $6.8 \mathrm{E}-01$ \\
\hline Allyl Chloride ${ }^{(5)}$ & $000107-05-1$ & & & $6.8 \mathrm{E}+01$ & $6.8 \mathrm{E}+00$ \\
\hline Aluminum & 007429-90-5 & & & & \\
\hline Aluminum Phosphide & 020859-73-8 & & & $5.4 \mathrm{E}-01$ & $5.4 \mathrm{E}-02$ \\
\hline Amdro & 067485-29-4 & & & $4.1 \mathrm{E}-01$ & 4.1E-02 \\
\hline Ametryn & 000834-12-8 & & & $1.2 \mathrm{E}+01$ & $1.2 \mathrm{E}+00$ \\
\hline Aminophenol, m- & 000591-27-5 & & & $9.5 \mathrm{E}+01$ & $9.5 \mathrm{E}+00$ \\
\hline Aminopyridine, 4- & $000504-24-5$ & & & $2.7 \mathrm{E}-02$ & $2.7 \mathrm{E}-03$ \\
\hline Amitraz & 033089-61-1 & & & $3.4 \mathrm{E}+00$ & $3.4 \mathrm{E}-01$ \\
\hline Ammonia & $007664-41-7$ & & & & \\
\hline Ammonium Sulfamate & 007773-06-0 & & & $2.7 \mathrm{E}+02$ & $2.7 \mathrm{E}+01$ \\
\hline Aniline & 000062-53-3 & $5.5 \mathrm{E}+01$ & $5.5 \mathrm{E}-01$ & & \\
\hline Anthracene & 000120-12-7 & & & $4.1 \mathrm{E}+02$ & $4.1 \mathrm{E}+01$ \\
\hline Antimony (metallic) & 007440-36-0 & & & $5.4 \mathrm{E}-01$ & $5.4 \mathrm{E}-02$ \\
\hline Antimony Pentoxide & $001314-60-9$ & & & $6.8 \mathrm{E}-01$ & $6.8 \mathrm{E}-02$ \\
\hline Antimony Potassium Tartrate & 000304-61-0 & & & $1.2 \mathrm{E}+00$ & $1.2 \mathrm{E}-01$ \\
\hline Antimony Tetroxide & 001332-81-6 & & & $5.4 \mathrm{E}-01$ & $5.4 \mathrm{E}-02$ \\
\hline
\end{tabular}


Table 3.1e. (continued)

\begin{tabular}{|l|l|l|c|c|c|}
\hline \multirow{2}{*}{ Chemical } & \multirow{2}{*}{ CASRN } & \multicolumn{4}{|c|}{ Ingestion of fish (mg/kg) } \\
\cline { 4 - 6 } & & $10^{-4}$ & $10^{-6}$ & HQ=1 & HQ=0.1 \\
\hline Antimony Trioxide & $001309-64-4$ & & & $5.4 \mathrm{E}-01$ & $5.4 \mathrm{E}-02$ \\
\hline Apollo & $074115-24-5$ & & & $1.8 \mathrm{E}+01$ & $1.8 \mathrm{E}+00$ \\
\hline Aramite & $000140-57-8$ & $1.3 \mathrm{E}+01$ & $1.3 \mathrm{E}-01$ & $6.8 \mathrm{E}+01$ & $6.8 \mathrm{E}+00$ \\
\hline Aroclor 1016 & $012674-11-2$ & & & $9.5 \mathrm{E}-02$ & $9.5 \mathrm{E}-03$ \\
\hline Aroclor 1221() & $011104-28-2$ & $4.1 \mathrm{E}-02$ & $4.1 \mathrm{E}-04$ & & \\
\hline Aroclor 1232(1) & $011141-16-5$ & $4.1 \mathrm{E}-02$ & $4.1 \mathrm{E}-04$ & & \\
\hline Aroclor 1242(1) & $053469-21-9$ & $4.1 \mathrm{E}-02$ & $4.1 \mathrm{E}-04$ & & \\
\hline Aroclor 1248(1) & $012672-29-6$ & $4.1 \mathrm{E}-02$ & $4.1 \mathrm{E}-04$ & & \\
\hline Aroclor 1254 & $011097-69-1$ & & & $2.7 \mathrm{E}-02$ & $2.7 \mathrm{E}-03$ \\
\hline Aroclor 1260 (1) & $011096-82-5$ & $4.1 \mathrm{E}-02$ & $4.1 \mathrm{E}-04$ & & \\
\hline Arsenic Salts & $\mathrm{NA}$ & & & & \\
\hline Arsenic, Inorganic & $007440-38-2$ & $2.1 \mathrm{E}-01$ & $2.1 \mathrm{E}-03$ & $4.1 \mathrm{E}-01$ & $4.1 \mathrm{E}-02$ \\
\hline Arsine & $007784-42-1$ & & & & \\
\hline Asbestos & $001332-21-4$ & & & & \\
\hline Assure & $076578-14-8$ & & & $1.2 \mathrm{E}+01$ & $1.2 \mathrm{E}+00$ \\
\hline Asulam & $003337-71-1$ & & & $6.8 \mathrm{E}+01$ & $6.8 \mathrm{E}+00$ \\
\hline Atrazine & $001912-24-9$ & $1.4 \mathrm{E}+00$ & $1.4 \mathrm{E}-02$ & $4.7 \mathrm{E}+01$ & $4.7 \mathrm{E}+00$ \\
\hline Avermectin B1 & $065195-55-3$ & & & $5.4 \mathrm{E}-01$ & $5.4 \mathrm{E}-02$ \\
\hline
\end{tabular}


Table 3.1e. (continued)

\begin{tabular}{|c|c|c|c|c|c|}
\hline \multirow{2}{*}{ Chemical } & \multirow{2}{*}{ CASRN } & \multicolumn{4}{|c|}{ Ingestion of fish (mg/kg) } \\
\hline & & $10^{-4}$ & $10^{-6}$ & $\mathbf{H Q}=\mathbf{1}$ & $\mathrm{HQ}=0.1$ \\
\hline Azobenzene & 000103-33-3 & $2.9 \mathrm{E}+00$ & $2.9 \mathrm{E}-02$ & & \\
\hline Barium & 007440-39-3 & & & $9.5 \mathrm{E}+01$ & $9.5 \mathrm{E}+00$ \\
\hline Barium Cyanide & $000542-62-1$ & & & $1.4 \mathrm{E}+02$ & $1.4 \mathrm{E}+01$ \\
\hline Baygon & 000114-26-1 & & & $5.4 \mathrm{E}+00$ & $5.4 \mathrm{E}-01$ \\
\hline Bayleton & $043121-43-3$ & & & $4.1 \mathrm{E}+01$ & 4.1E+00 \\
\hline Baythroid & 068359-37-5 & & & $3.4 \mathrm{E}+01$ & $3.4 \mathrm{E}+00$ \\
\hline Benefin & $001861-40-1$ & & & $4.1 \mathrm{E}+02$ & 4.1E+01 \\
\hline Benomyl & $017804-35-2$ & & & $6.8 \mathrm{E}+01$ & $6.8 \mathrm{E}+00$ \\
\hline Bentazon & 025057-89-0 & & & $3.4 \mathrm{E}+00$ & $3.4 \mathrm{E}-01$ \\
\hline Benz[a]anthracene $e^{(2)}$ & 000056-55-3 & 4.3E-01 & 4.3E-03 & & \\
\hline Benzaldehyde & $000100-52-7$ & & & $1.4 \mathrm{E}+02$ & $1.4 \mathrm{E}+01$ \\
\hline Benzene & $000071-43-2$ & $1.1 \mathrm{E}+01$ & $1.1 \mathrm{E}-01$ & & \\
\hline Benzene Hexachloride & NA & & & & \\
\hline Benzene, Ethyldimethyl & NA & & & & \\
\hline Benzene, Ethylmethyl & NA & & & & \\
\hline Benzene, Methylpropenyl & NA & & & & \\
\hline Benzene, Methylpropyl & NA & & & & \\
\hline Benzene, Trimethyl & 025551-13-7 & & & & \\
\hline
\end{tabular}


Table 3.1e. (continued)

\begin{tabular}{|l|l|l|l|l|l|}
\hline \multirow{2}{*}{ Chemical } & \multirow{2}{*}{ CASRN } & \multicolumn{3}{|c|}{ Ingestion of fish (mg/kg) } \\
\cline { 4 - 6 } & & $10^{-4}$ & $10^{-5}$ & HQ=1 & HQ=0.1 \\
\hline Benzenethiol & $000108-98-5$ & & & $1.4 \mathrm{E}-02$ & $1.4 \mathrm{E}-03$ \\
\hline Benzidine & $000092-87-5$ & $1.4 \mathrm{E}-03$ & $1.4 \mathrm{E}-05$ & $4.1 \mathrm{E}+00$ & $4.1 \mathrm{E}-01$ \\
\hline Benzo[a]pyrene ${ }^{(2)}$ & $000050-32-8$ & $4.3 \mathrm{E}-02$ & $4.3 \mathrm{E}-04$ & & \\
\hline Benzo[b]fluoranthene(2) & $000205-99-2$ & $4.3 \mathrm{E}-01$ & $4.3 \mathrm{E}-03$ & & \\
\hline Benzo[g,h,i]perylene & $000191-24-2$ & & & & \\
\hline Benzo[k]fluoranthene(2) & $000207-08-9$ & $4.3 \mathrm{E}+00$ & $4.3 \mathrm{E}-02$ & & \\
\hline Benzoic Acid & $000065-85-0$ & & & $5.4 \mathrm{E}+03$ & $5.4 \mathrm{E}+02$ \\
\hline Benzotrichloride & $000098-07-7$ & $2.4 \mathrm{E}-02$ & $2.4 \mathrm{E}-04$ & & \\
\hline Benzyl Alcohol & $000100-51-6$ & & & $4.1 \mathrm{E}+02$ & $4.1 \mathrm{E}+01$ \\
\hline Benzyl Chloride & $000100-44-7$ & $1.9 \mathrm{E}+00$ & $1.9 \mathrm{E}-02$ & & \\
\hline Beryllium & $007440-41-7$ & $7.3 \mathrm{E}-02$ & $7.3 \mathrm{E}-04$ & $6.8 \mathrm{E}+00$ & $6.8 \mathrm{E}-01$ \\
\hline Bidrin & $000141-66-2$ & & & $1.4 \mathrm{E}-01$ & $1.4 \mathrm{E}-02$ \\
\hline Biphenthrin & $082657-04-3$ & & & $2.0 \mathrm{E}+01$ & $2.0 \mathrm{E}+00$ \\
\hline Biphenyl, 1,1'- & $000092-52-4$ & & & $6.8 \mathrm{E}+01$ & $6.8 \mathrm{E}+00$ \\
\hline $\begin{array}{l}\text { Bis(2-chloro-1-methylethyl)ether } \\
\text { (Technical) }\end{array}$ & $000108-60-1$ & $4.5 \mathrm{E}+00$ & $4.5 \mathrm{E}-02$ & & \\
\hline Bis(2-chloroethoxy)methane & $000111-91-1$ & & & & \\
\hline Bis(2-chloroethyl)ether & $000111-44-4$ & $2.9 \mathrm{E}-01$ & $2.9 \mathrm{E}-03$ & & \\
\hline
\end{tabular}




\begin{tabular}{|c|c|c|c|c|c|}
\hline$I 0+B t$ & $20+960 . I$ & & & $\varepsilon-9 \varepsilon-I<0000$ & $-N^{c}$ joure, \\
\hline & & & & $0-66-901000$ & -દ'I 'วuə̣pe]ng \\
\hline $00+\theta L \cdot z$ & $10+\exists L \cdot \tau$ & & & $z-66-689100$ & 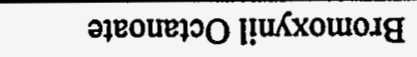 \\
\hline \multirow[t]{2}{*}{$00+\exists L \cdot \tau$} & $10+B L^{\prime} Z$ & & & $s-t 8-689100$ & 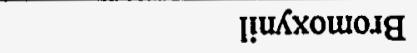 \\
\hline & & & & $L-Z 9-\varsigma \angle 0000$ & 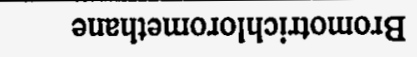 \\
\hline $10-78^{\circ} 9$ & $00+38^{\circ} 9$ & & & $\varepsilon-96-\not 0 I Z 00$ & soųdouro.rg \\
\hline$I 0^{-3}-\mathrm{6} \cdot \mathrm{I}$ & $00+36 . I$ & & & $6-\varepsilon 8-\downarrow L 0000$ & $(\text { g) })^{\text {วurqpowowosg }}$ \\
\hline \multirow[t]{2}{*}{$00+B L \cdot Z$} & $10+3 L \cdot Z$ & $10-30^{\circ} \mathrm{t}$ & $10+30^{\circ} t$ & $Z-\varsigma Z-\varsigma \angle 0000$ & wrojourosg \\
\hline & & & & $\varepsilon$-ss-โ01000 & 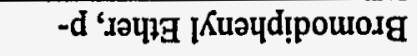 \\
\hline \multirow[t]{3}{*}{$00+B L \cdot Z$} & $10+9 L Z$ & 20-BI's & $00+B I S S$ & $\nabla-L Z-S \angle 0000$ & 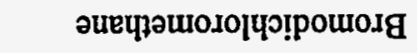 \\
\hline & & & & $s-\angle 6-\downarrow \angle 0000$ & әиечрәитологчроштолg \\
\hline & & & & $\tau=\angle 0-\angle E 9 L 00$ & 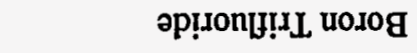 \\
\hline$I 0+3 Z^{\prime} I$ & $Z 0+a Z Z^{\prime} I$ & & & $8-2 t-0 t b<00$ & КโuO sәредog pư uodog \\
\hline \multirow[t]{2}{*}{$00+38.9$} & $10+38 \cdot 9$ & & & $L-S 0-080000$ & V louəyds!g \\
\hline & & S0-ヨt'I & $\varepsilon 0-\exists \dagger^{\top} I$ & {$[-88-\tau+\subseteq \varsigma 000$} & 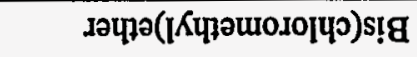 \\
\hline $00+9 L 2$ & $10+B L 2 Z$ & $10-\exists E \cdot Z$ & $10+3 \varepsilon^{\prime} Z$ & $L-I 8-L I I 000$ & 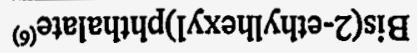 \\
\hline $00+3 b 5$ & $10+\mathrm{A} t \mathrm{t} \mathrm{S}$ & & & 6-ZЕ-8Е96Еะ0 & 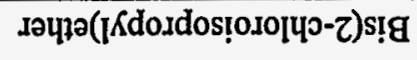 \\
\hline$I^{\circ} 0=\partial H$ & $\mathbf{I}=\mathbf{O H}$ & $9.0 I$ & rOI & \multirow{2}{*}{ N\&SVD } & \multirow{2}{*}{ ןвอ|นวนว } \\
\hline & (8y/giur) & 10!psə8ีuI & & & \\
\hline
\end{tabular}

(pənụ̣uoo) 'əI'E ว 
Table 3.1e. (continued)

\begin{tabular}{|l|l|l|c|c|c|}
\hline \multirow{2}{*}{ Chemical } & \multirow{2}{*}{ CASRN } & \multicolumn{4}{|c|}{ Ingestion of fish (mg/kg) } \\
\cline { 4 - 6 } & & $10^{-4}$ & $10^{-6}$ & HQ=1 & HQ=0.1 \\
\hline $\begin{array}{l}\text { Butanone-2, 4-chloro-4,4- } \\
\text { difluoro }\end{array}$ & NA & & & & \\
\hline Butyl Benzyl Phthlate & $000085-68-7$ & & & $2.7 \mathrm{E}+02$ & $2.7 \mathrm{E}+01$ \\
\hline Butylate & $002008-41-5$ & & & $6.8 \mathrm{E}+01$ & $6.8 \mathrm{E}+00$ \\
\hline Butylchloride, t- & $000507-20-0$ & & & \\
\hline Butylphthalyl Butylglycolate & $000085-70-1$ & & & $1.4 \mathrm{E}+03$ & $1.4 \mathrm{E}+02$ \\
\hline Cacodylic Acid & $000075-60-5$ & & & $4.1 \mathrm{E}+00$ & $4.1 \mathrm{E}-01$ \\
\hline Cadmium (Diet) & $007440-43-9$ & & & $1.4 \mathrm{E}+00$ & $1.4 \mathrm{E}-01$ \\
\hline Calcium Cyanide & $000592-01-8$ & & & $5.4 \mathrm{E}+01$ & $5.4 \mathrm{E}+00$ \\
\hline Caprolactam & $000105-60-2$ & & & $6.8 \mathrm{E}+02$ & $6.8 \mathrm{E}+01$ \\
\hline Captafol & $002425-06-1$ & $3.7 \mathrm{E}+01$ & $3.7 \mathrm{E}-01$ & $2.7 \mathrm{E}+00$ & $2.7 \mathrm{E}-01$ \\
\hline Captan & $000133-06-2$ & $9.0 \mathrm{E}+01$ & $9.0 \mathrm{E}-01$ & $1.8 \mathrm{E}+02$ & $1.8 \mathrm{E}+01$ \\
\hline Carbaryl & $000063-25-2$ & & & $1.4 \mathrm{E}+02$ & $1.4 \mathrm{E}+01$ \\
\hline Carbazole & $000086-74-8$ & $1.6 \mathrm{E}+01$ & $1.6 \mathrm{E}-01$ & & \\
\hline Carbofuran & $001563-66-2$ & & & $6.8 \mathrm{E}+00$ & $6.8 \mathrm{E}-01$ \\
\hline Carbon Disulfide & $000075-15-0$ & & & $1.4 \mathrm{E}+02$ & $1.4 \mathrm{E}+01$ \\
\hline Carbon Tetrachloride ${ }^{(6)}$ & $000056-23-5$ & $2.4 \mathrm{E}+00$ & $2.4 \mathrm{E}-02$ & $9.5 \mathrm{E}-01$ & $9.5 \mathrm{E}-02$ \\
\hline Carbosulfan & $055285-14-8$ & & & $1.4 \mathrm{E}+01$ & $1.4 \mathrm{E}+00$ \\
\hline
\end{tabular}




\begin{tabular}{|c|c|c|c|c|c|}
\hline $00+\exists L \cdot \tau$ & $10+g \angle Z$ & & & $L-06-80 I 000$ & 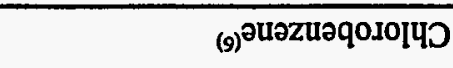 \\
\hline \multirow[t]{2}{*}{$10-3 t 5$} & $00+365$ & & & $8-\angle t-901000$ & -d 'วu!!!นеодоцว \\
\hline & & & & $\downarrow-L Z-Z \varepsilon \varsigma \subseteq 000$ & 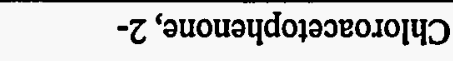 \\
\hline \multirow[t]{3}{*}{$10-\exists L \cdot Z$} & $00+B L \cdot Z$ & & & 8-โI-6L0000 & 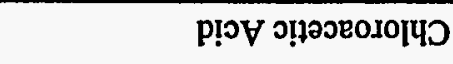 \\
\hline & & $\varepsilon 0-\exists b \cdot \varsigma$ & I0-at's & $z-69-\$ 60000$ & 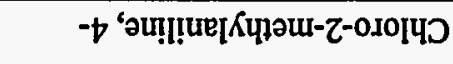 \\
\hline & & $\varepsilon 0-36^{\circ} 9$ & $10-36.9$ & $\varepsilon-\varepsilon 6-\varsigma 9$ I $\varepsilon 00$ & 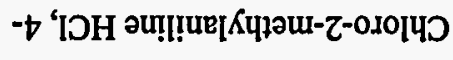 \\
\hline \multirow[t]{4}{*}{$00+\exists L \cdot \tau$} & $10+B L \cdot Z$ & & & 8-66-9Z1000 & 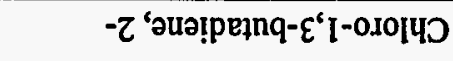 \\
\hline & & & & $\varepsilon-89-\varsigma<0000$ & 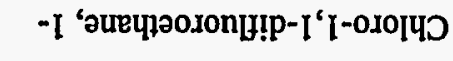 \\
\hline & & & & $z-6 I-8 S L L 00$ & 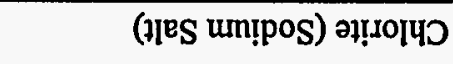 \\
\hline & & & & $t-b 0-6 t 0010$ & 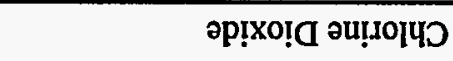 \\
\hline$I 0+3 b^{\circ} I$ & $20+96 \cdot 1$ & & & S-0S-Z8LL00 & 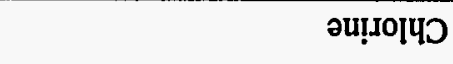 \\
\hline \multirow[t]{2}{*}{$00+B L \cdot \tau$} & $10+B L \cdot Z$ & & & $\downarrow-Z \varepsilon-286060$ & 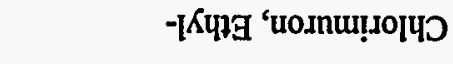 \\
\hline & & & & $9-00-\angle 889 I 0$ & әр!ฺำ५ \\
\hline \multirow[t]{2}{*}{$\varepsilon 0-\bar{g} I^{\circ} 8$} & $20-9 I^{\prime} 8$ & $\varepsilon 0-\exists b+2$ & $10-\exists b^{2} Z$ & $6-t L-L S 0000$ & 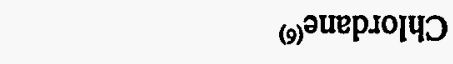 \\
\hline & & $\varepsilon 0-38^{\circ} \mathrm{L}$ & $10-98 . \mathrm{L}$ & $\tau-S L-8 I I 000$ & โ!นะ.ภำว \\
\hline $00+B 0^{\circ} Z$ & $10+B 0 \tau$ & & & t-06-EعI000 & นəqureso|ఘว \\
\hline $10-\exists L \iota^{\prime} Z$ & $00+G L \cdot Z$ & & & $9-\angle 8-S \angle 0000$ & ןело|Чว \\
\hline$I 0+36 \cdot I$ & $20+\not b \cdot I$ & & & $\downarrow-89-\downarrow \varepsilon Z \$ 00$ & u!xoqIeว \\
\hline$I^{*} 0=\partial H$ & $I=\partial H$ & $9.0 I$ & rOI & \multirow{2}{*}{ NASVD } & \multirow{2}{*}{ โвวุแวนว } \\
\hline & 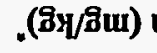 & 10!̣sอ8ิuI & & & \\
\hline
\end{tabular}

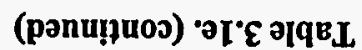


Table 3.1e. (continued)

\begin{tabular}{|l|l|l|c|c|c|}
\hline \multirow{2}{*}{ Chemical } & \multirow{2}{*}{ CASRN } & \multicolumn{4}{|c|}{ Ingestion of fish (mg/kg) } \\
\cline { 3 - 6 } & & $10^{-4}$ & $10^{-6}$ & HQ=1 & HQ=0.1 \\
\hline Chlorobenzilate & $000510-15-6$ & $1.2 \mathrm{E}+00$ & $1.2 \mathrm{E}-02$ & $2.7 \mathrm{E}+01$ & $2.7 \mathrm{E}+00$ \\
\hline Chlorobenzoic Acid, p- & $000074-11-3$ & & & $2.7 \mathrm{E}+02$ & $2.7 \mathrm{E}+01$ \\
\hline Chlorobenzotrifluoride, 4- & $000098-56-6$ & & & $2.7 \mathrm{E}+01$ & $2.7 \mathrm{E}+00$ \\
\hline Chlorobutane, 1- & $000109-69-3$ & & & $5.4 \mathrm{E}+02$ & $5.4 \mathrm{E}+01$ \\
\hline Chlorobutane, 2- & $000078-86-4$ & & & \\
\hline Chlorocyclopentadiene & $041851-50-7$ & & & & \\
\hline Chlorodibromoethane & $073506-94-2$ & $3.8 \mathrm{E}+00$ & $3.8 \mathrm{E}-02$ & & \\
\hline Chlorodifluoromethane & $000075-45-6$ & & & & \\
\hline Chloroform ${ }^{(6)}$ & $000067-66-3$ & $5.2 \mathrm{E}+01$ & $5.2 \mathrm{E}-01$ & $1.4 \mathrm{E}+01$ & $1.4 \mathrm{E}+00$ \\
\hline Chloromethane ${ }^{(6)}$ & $000074-87-3$ & $2.4 \mathrm{E}+01$ & $2.4 \mathrm{E}-01$ & & \\
\hline Chloromethyl Methyl Ether & $000107-30-2$ & & & & \\
\hline Chloronaphthalene, Beta- & $000091-58-7$ & & & $1.1 \mathrm{E}+02$ & $1.1 \mathrm{E}+01$ \\
\hline Chloronitrobenzene, 0- & $000088-73-3$ & $1.3 \mathrm{E}+01$ & $1.3 \mathrm{E}-01$ & & \\
\hline Chloronitrobenzene, p- & $000121-73-3$ & $1.8 \mathrm{E}+01$ & $1.8 \mathrm{E}-01$ & & \\
\hline Chlorophenol, 2- & $000095-57-8$ & & & $6.8 \mathrm{E}+00$ & $6.8 \mathrm{E}-01$ \\
\hline Chlorophenyl Methyl Sulfide, p- & $000123-09-1$ & & & & \\
\hline Chlorophenyl Methyl Sulfoxide & $000934-73-6$ & & & & \\
\hline Chloropropane, 2- & $000075-29-6$ & & & & \\
\hline
\end{tabular}




\begin{tabular}{|c|c|c|c|c|c|}
\hline $00+389$ & $10+3899$ & & & $L-8 b-\$ 60000$ & -0 tosajo \\
\hline \multirow[t]{2}{*}{$00+38^{\prime} 9$} & $10+389$ & & & $t-6 \varepsilon-80 I 000$ & 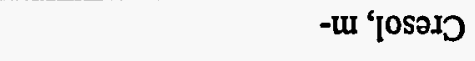 \\
\hline & & & & 6-8S-I00800 & 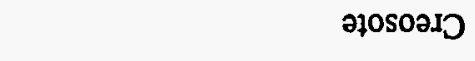 \\
\hline \multirow[t]{6}{*}{ I0-78.9 } & $00+98 \cdot 9$ & & & $\varepsilon-Z 6 ־ t \downarrow S 000$ & 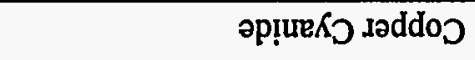 \\
\hline & & & & $8-0 S-0 b t<00$ & Iəddo \\
\hline & & & & $\tau-S_{t}-\angle 00800$ & 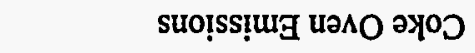 \\
\hline & & & & $t-8 t-0 t t<00$ & HeqOJ \\
\hline & & {$\left[0-\exists \varepsilon^{*} \downarrow\right.$} & $10+B \varepsilon \cdot t$ & $6-10-8 I Z 000$ & 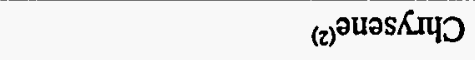 \\
\hline & & & & $\mathrm{VN}$ & słןes unṭurory \\
\hline $10-98^{\circ} 9$ & $00+38^{\circ} 9$ & & & $6-6 z-0+5810$ & 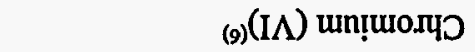 \\
\hline $20+3 t I$ & $\varepsilon 0+B \nabla^{\circ} I$ & & & I-£8-§909I0 & 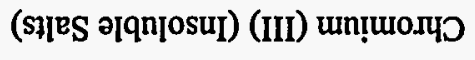 \\
\hline I0-G['] & $00+A I^{*} I$ & & & $t-9 S-8 \varepsilon Z 090$ & 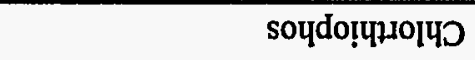 \\
\hline $00+38 \cdot 9$ & $10+98^{\circ} 9$ & & & $\varepsilon-z L-z 06+90$ & 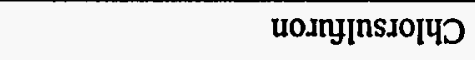 \\
\hline $00+3 b^{\circ} I$ & $I 0+\exists b^{\circ} I$ & & & $0-\varepsilon[-86 \varsigma \$ 00$ & 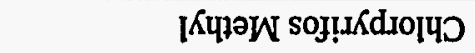 \\
\hline I0-GI't & $00+B I^{\circ} \mathrm{t}$ & & & $\tau-88-I Z 6 Z 00$ & 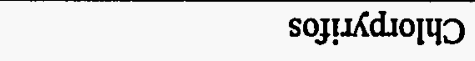 \\
\hline $10+B L Z$ & $Z 0+\exists L Z$ & & & $\varepsilon-I Z-I 01000$ & ureq̨dordıo[૫ \\
\hline $00+\xi L \cdot \tau$ & $10+g L Z$ & & & $8-6 t-\$ 60000$ & 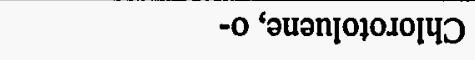 \\
\hline $00+30^{\circ} z$ & $10+\exists 0^{\circ} Z$ & $10-967$ & $10+36 \cdot \tau$ & 9-St-L68I00 & 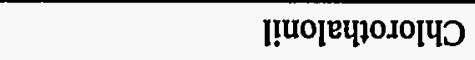 \\
\hline$I^{\prime} \mathbf{0}=\partial \mathrm{H}$ & $\mathbf{I}=\mathbf{O H}$ & $9.0 I$ & rOI & Prayn & 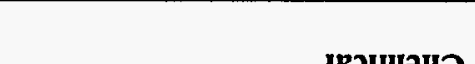 \\
\hline \multicolumn{4}{|c|}{ 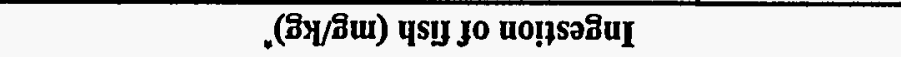 } & tres & The \\
\hline
\end{tabular}

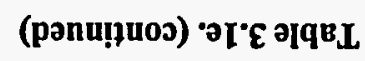


Table 3.1e. (continued)

\begin{tabular}{|c|c|c|c|c|c|}
\hline \multirow{2}{*}{ Chemical } & \multirow{2}{*}{ CASRN } & \multicolumn{4}{|c|}{ Ingestion of fish (mg/kg) } \\
\hline & & $10^{-4}$ & $10^{-6}$ & $\mathbf{H Q}=\mathbf{1}$ & $\mathrm{HQ}=0.1$ \\
\hline Cresol, p- & 000106-44-5 & & & $6.8 \mathrm{E}+00$ & $6.8 \mathrm{E}-01$ \\
\hline Crotonaldehyde & 000123-73-9 & $1.7 \mathrm{E}-01$ & $1.7 \mathrm{E}-03$ & & \\
\hline Cumene & 000098-82-8 & & & $5.4 \mathrm{E}+01$ & $5.4 \mathrm{E}+00$ \\
\hline Cyanazine & $021725-46-2$ & $3.8 \mathrm{E}-01$ & $3.8 \mathrm{E}-03$ & $2.7 \mathrm{E}+00$ & 2.7E-01 \\
\hline Cyanide (CN-) & $000057-12-5$ & & & $2.7 \mathrm{E}+01$ & $2.7 \mathrm{E}+00$ \\
\hline Cyanogen & $000460-19-5$ & & & $5.4 \mathrm{E}+01$ & $5.4 \mathrm{E}+00$ \\
\hline Cyanogen Bromide & 000506-68-3 & & & $1.2 \mathrm{E}+02$ & $1.2 \mathrm{E}+01$ \\
\hline Cyanogen Chloride & $000506-77-4$ & & & $6.8 \mathrm{E}+01$ & $6.8 \mathrm{E}+00$ \\
\hline $\begin{array}{l}\text { Cyclohexane, } 1,2,3,4,5- \\
\text { pentabromo-6-chloro- }\end{array}$ & 000087-84-3 & $1.4 \mathrm{E}+01$ & $1.4 \mathrm{E}-01$ & & \\
\hline Cyclohexanone & 000108-94-1 & & & $6.8 \mathrm{E}+03$ & $6.8 \mathrm{E}+02$ \\
\hline Cyclohexylamine & 000108-91-8 & & & $2.7 \mathrm{E}+02$ & $2.7 \mathrm{E}+01$ \\
\hline Cyclopentadiene & 000542-92-7 & & & & \\
\hline Cyhalothrin/karate & 068085-85-8 & & & $6.8 \mathrm{E}+00$ & $6.8 \mathrm{E}-01$ \\
\hline Cypermethrin & 052315-07-8 & & & $1.4 \mathrm{E}+01$ & $1.4 \mathrm{E}+00$ \\
\hline Cyromazine & 066215-27-8 & & & $1.0 \mathrm{E}+01$ & $1.0 \mathrm{E}+00$ \\
\hline DDD & $000072-54-8$ & $1.3 \mathrm{E}+00$ & $1.3 \mathrm{E}-02$ & & \\
\hline DDE & 000072-55-9 & $9.3 \mathrm{E}-01$ & 9.3E-03 & & \\
\hline
\end{tabular}


Table 3.1e. (continued)

\begin{tabular}{|c|c|c|c|c|c|}
\hline \multirow{2}{*}{ Chemical } & \multirow{2}{*}{ CASRN } & \multicolumn{4}{|c|}{ Ingestion of fish (mg/kg)" } \\
\hline & & $10^{-4}$ & $10^{-6}$ & $\mathbf{H Q}=\mathbf{1}$ & $\mathrm{HQ}=0.1$ \\
\hline DDT & $000050-29-3$ & 9.3E-01 & $9.3 \mathrm{E}-03$ & $6.8 \mathrm{E}-01$ & $6.8 \mathrm{E}-02$ \\
\hline Dacthal & $001861-32-1$ & & & $1.4 \mathrm{E}+01$ & $1.4 \mathrm{E}+00$ \\
\hline Dalapon & 000075-99-0 & & & $4.1 \mathrm{E}+01$ & $4.1 \mathrm{E}+00$ \\
\hline Decabromodiphenyl Ether & 001163-19-5 & & & $1.4 \mathrm{E}+01$ & $1.4 \mathrm{E}+00$ \\
\hline Demeton & 008065-48-3 & & & $5.4 \mathrm{E}-02$ & $5.4 \mathrm{E}-03$ \\
\hline Di(2-ethylhexyl)adipate & 000103-23-1 & $2.6 \mathrm{E}+02$ & $2.6 \mathrm{E}+00$ & 8.1E+02 & $8.1 \mathrm{E}+01$ \\
\hline Diallate & 002303-16-4 & $5.2 \mathrm{E}+00$ & $5.2 \mathrm{E}-02$ & & \\
\hline Diazinon & $000333-41-5$ & & & $1.2 \mathrm{E}+00$ & $1.2 \mathrm{E}-01$ \\
\hline Dibenz[a,h]anthracene ${ }^{(2)}$ & 000053-70-3 & $4.3 \mathrm{E}-02$ & 4.3E-04 & & \\
\hline Dibromo-3-chloropropane, 1,2- & 000096-12-8 & 2.3E-01 & $2.3 \mathrm{E}-03$ & & \\
\hline Dibromobenzene, 1,4- & 000106-37-6 & & & $1.4 \mathrm{E}+01$ & $1.4 \mathrm{E}+00$ \\
\hline Dibromochloromethane & 000124-48-1 & $3.8 \mathrm{E}+00$ & $3.8 \mathrm{E}-02$ & $2.7 \mathrm{E}+01$ & $2.7 \mathrm{E}+00$ \\
\hline Dibromodichloromethane & 000594-18-3 & & & & \\
\hline Dibromodiphenyl Ether, p,p'- & $002050-47-7$ & & & & \\
\hline Dibromoethane, 1,2- & 000106-93-4 & 3.7E-03 & 3.7E-05 & & \\
\hline $\begin{array}{l}\text { Dibromomethane (Methylene } \\
\text { Bromide) }\end{array}$ & 000074-95-3 & & & $1.4 \mathrm{E}+01$ & $1.4 \mathrm{E}+00$ \\
\hline Dibutyl Phthalate & 000084-74-2 & & & $1.4 \mathrm{E}+02$ & $1.4 \mathrm{E}+01$ \\
\hline
\end{tabular}


Table 3.1e. (continued)

\begin{tabular}{|c|c|c|c|c|c|}
\hline \multirow{2}{*}{ Chemical } & \multirow{2}{*}{ CASRN } & \multicolumn{4}{|c|}{ Ingestion of fish (mg/kg)" } \\
\hline & & $10^{-4}$ & $10^{-6}$ & $H Q=1$ & $H Q=0.1$ \\
\hline Dicamba & 001918-00-9 & & & $4.1 \mathrm{E}+01$ & $4.1 \mathrm{E}+00$ \\
\hline Dichloro-2-butene, 1,4- & $000764-41-0$ & & & & \\
\hline \multicolumn{6}{|l|}{ Dichloroacetic Acid } \\
\hline Dichlorobenzene, 1,2-(3) & $000095-50-1$ & & & $1.2 \mathrm{E}+02$ & $1.2 \mathrm{E}+01$ \\
\hline Dichlorobenzene, 1,3- & $000541-73-1$ & & & & \\
\hline Dichlorobenzene, 1,4- & $000106-46-7$ & $1.3 \mathrm{E}+01$ & $1.3 \mathrm{E}-01$ & & \\
\hline Dichlorobenzidine, 3,3'- & $000091-94-1$ & $7.0 \mathrm{E}-01$ & $7.0 \mathrm{E}-03$ & & \\
\hline Dichlorodifluoromethane & $000075-71-8$ & & & $2.7 E+02$ & $2.7 E+01$ \\
\hline Dichloroethane, 1,1- & $000075-34-3$ & & & $1.4 \mathrm{E}+02$ & $1.4 \mathrm{E}+01$ \\
\hline Dichloroethane, 1,2- & $000107-06-2$ & $3.5 \mathrm{E}+00$ & $3.5 \mathrm{E}-02$ & & \\
\hline Dichloroethylene, 1,1- & $000075-35-4$ & $5.3 \mathrm{E}-01$ & $5.3 \mathrm{E}-03$ & $1.2 \mathrm{E}+01$ & $1.2 \mathrm{E}+00$ \\
\hline $\begin{array}{l}\text { Dichloroethylene, 1,2- (Mixed } \\
\text { Isomers) }\end{array}$ & 000540-59-0 & & & $1.2 \mathrm{E}+01$ & $1.2 \mathrm{E}+00$ \\
\hline Dichloroethylene, 1,2-cis- & $000156-59-2$ & & & $1.4 \mathrm{E}+01$ & $1.4 \mathrm{E}+00$ \\
\hline Dichloroethylene, 1,2-trans- & $000156-60-5$ & & & $2.7 \mathrm{E}+01$ & $2.7 \mathrm{E}+00$ \\
\hline Dichlorophenol, 2,4- & $000120-83-2$ & & & $4.1 E+00$ & 4.1E-01 \\
\hline $\begin{array}{l}\text { Dichlorophenoxy Acetic Acid, } \\
2,4-\end{array}$ & $000094-75-7$ & & & $1.4 \mathrm{E}+01$ & $1.4 \mathrm{E}+00$ \\
\hline
\end{tabular}


Table 3.1e. (continued)

\begin{tabular}{|c|c|c|c|c|c|}
\hline \multirow{2}{*}{ Chemical } & \multirow{2}{*}{ CASRN } & \multicolumn{4}{|c|}{ Ingestion of fish (mg/kg) } \\
\hline & & $10^{-4}$ & $10^{-6}$ & $\mathbf{H Q}=\mathbf{1}$ & HQ $=0.1$ \\
\hline $\begin{array}{l}\text { Dichlorophenoxy)butyric Acid, } \\
\text { 4-(2,4- }\end{array}$ & 000094-82-6 & & & $1.1 \mathrm{E}+01$ & $1.1 \mathrm{E}+00$ \\
\hline Dichloropropane, 1,2- & 000078-87-5 & $4.6 \mathrm{E}+00$ & $4.6 \mathrm{E}-02$ & & \\
\hline Dichloropropanol, 2,3- & 000616-23-9 & & & 4.1E+00 & 4.1E-01 \\
\hline Dichloropropene, 1,3- & 000542-75-6 & $1.8 \mathrm{E}+00$ & $1.8 \mathrm{E}-02$ & 4.1E-01 & $4.1 \mathrm{E}-02$ \\
\hline Dichlorvos & 000062-73-7 & $1.1 E+00$ & $1.1 \mathrm{E}-02$ & $6.8 \mathrm{E}-01$ & $6.8 \mathrm{E}-02$ \\
\hline Dicofol & $000115-32-2$ & & & & \\
\hline Dicyclopentadiene & $000077-73-6$ & & & $4.1 \mathrm{E}+01$ & $4.1 \mathrm{E}+00$ \\
\hline Dieldrin & $000060-57-1$ & $2.0 \mathrm{E}-02$ & $2.0 \mathrm{E}-04$ & $6.8 \mathrm{E}-02$ & $6.8 \mathrm{E}-03$ \\
\hline Diethyl Phthalate & $000084-66-2$ & & & $1.1 \mathrm{E}+03$ & $1.1 \mathrm{E}+02$ \\
\hline Diethyl-p-nitrophenylphosphate & 000311-45-5 & & & & \\
\hline $\begin{array}{l}\text { Diethylene Glycol Dinitrate } \\
\text { (DEGDN) }\end{array}$ & 000693-21-0 & & & & \\
\hline $\begin{array}{l}\text { Diethylene Glycol Monobutyl } \\
\text { Ether }\end{array}$ & $000112-34-5$ & & & & \\
\hline $\begin{array}{l}\text { Diethylene Glycol Monoethyl } \\
\text { Ether }\end{array}$ & $000111-90-0$ & & & $2.7 \mathrm{E}+03$ & 2.7E+02 \\
\hline Diethylformamide & 000617-84-5 & & & $1.5 \mathrm{E}+01$ & $1.5 \mathrm{E}+00$ \\
\hline Diethylstilbesterol & $000056-53-1$ & $6.7 \mathrm{E}-05$ & $6.7 \mathrm{E}-07$ & & \\
\hline
\end{tabular}




\begin{tabular}{|c|c|c|c|c|c|}
\hline $20-91 \cdot 8$ & $10-9 I^{\circ} 8$ & & & $I-9 Z-9 L \$ 000$ & $-9^{6} Z^{\prime}$ ' \\
\hline \multirow[t]{3}{*}{$00+B<\cdot z$} & $10+B L \cdot \tau$ & & & $6-\angle 9-\varsigma 0 I 000$ & 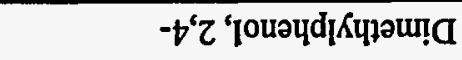 \\
\hline & & & & $8-\varepsilon L-0 b S 000$ & 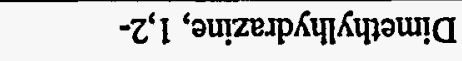 \\
\hline & & $\varepsilon 0-\mathrm{BZ} \cdot \mathrm{I}$ & $I 0-\exists Z^{\prime} I$ & $L-b I-L S 0000$ & 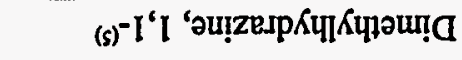 \\
\hline \multirow[t]{3}{*}{$10+3 t^{\circ} I$} & $20+a+1$ & & & $\tau-\tau I-890000$ & 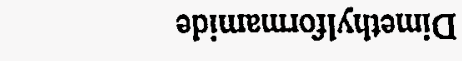 \\
\hline & & & & $L-0 t-\downarrow 8 S L 0 I$ & 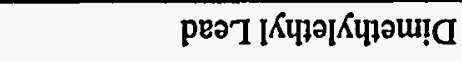 \\
\hline & & 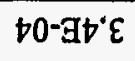 & $20-\exists b^{\circ} \varepsilon$ & L-E6-6I I000 & 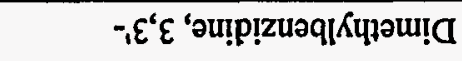 \\
\hline \multirow[t]{5}{*}{$10-\exists L L^{\prime} Z$} & $00+a L Z \tau$ & & & $L-69-I Z I 000$ & 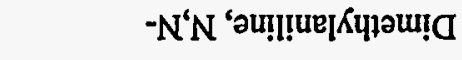 \\
\hline & & $\varepsilon 0-\exists Z^{*} \downarrow$ & I0-gz"b & $I-89-\$ 60000$ & 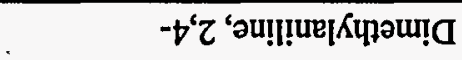 \\
\hline & & $\varepsilon 0-\exists b^{\prime} S$ & I0-ats & t-96-9عtIZ0 & 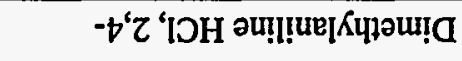 \\
\hline & & & & $I-8 L-L L 0000$ & 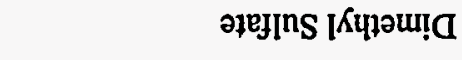 \\
\hline & & I0-ヨE'ح & $10+\exists \varepsilon z$ & $b-06-611000$ & 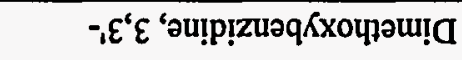 \\
\hline $20-\exists L \cdot \tau$ & $10-\exists L L^{\prime} Z$ & & & S-IS-090000 & әјеочрәи! \\
\hline $00+B L \cdot Z$ & $10+B L \cdot Z$ & & & L-t9-06Zsऽ0 & 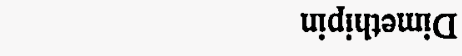 \\
\hline \multirow[t]{2}{*}{$I 0+B I \cdot I$} & $20+B I^{-I}$ & & & $9-\varsigma L-S t b I 00$ & 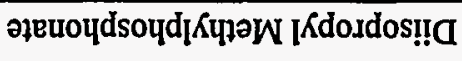 \\
\hline & & & & $9-L \varepsilon-\varsigma \angle 0000$ & 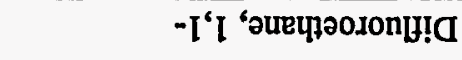 \\
\hline $00+g L \tau$ & $10+\exists L Z$ & & & ऽ-8E-L9ESE0 & uomzuaqnu!! \\
\hline$I 0+B I^{*} I$ & $20+B I \cdot I$ & & & $9-8 \triangleright-\tau Z Z \varepsilon \vdash 0$ & Ienbozuəy!a \\
\hline$I^{\circ} 0=\partial H$ & $\mathbf{I}=\mathbf{O H}$ & $9-0 \mathrm{I}$ & -OI & \multirow{2}{*}{ N\&SVD } & \multirow{2}{*}{ |вว|ุนวนว } \\
\hline & 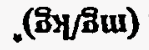 & 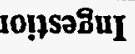 & & & \\
\hline
\end{tabular}




\begin{tabular}{|c|c|c|c|c|c|}
\hline & & $\varepsilon 0-\bar{G} 6^{\circ} \varepsilon$ & {$\left[0-96^{\circ} \varepsilon\right.$} & L-99-ZZ1000 & 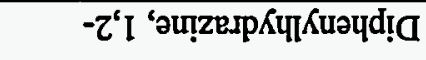 \\
\hline $00+3 t^{\circ} \varepsilon$ & $10+\pi t^{\circ} \varepsilon$ & & & $\downarrow-6 \varepsilon-2 Z 1000$ & 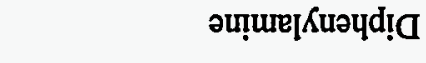 \\
\hline \multirow[t]{2}{*}{$00+3 I^{\circ}$} & $10+B I^{\circ} t$ & & & $L-I S-L S 6000$ & p!̣uruaนd! $ם$ \\
\hline & & $10-367$ & $10+36 \tau$ & I-I6-EZI000 & $-\nabla^{\prime} I$ 'auexo!d \\
\hline \multirow[t]{2}{*}{$I 0-9 b \cdot I$} & $00+\exists b I$ & & & L-\$8-880000 & qəosou!a \\
\hline & & & & Z-8L-ZLSSE0 & 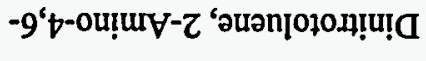 \\
\hline$I 0-\exists b^{I} I$ & $00+B b^{\circ} I$ & $\varepsilon 0-39^{\circ} \downarrow$ & $10-39^{\circ}$ & $z=0 z-909000$ & 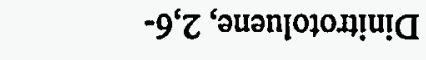 \\
\hline $10-\exists L^{\prime} Z$ & $00+B L \cdot Z$ & $\varepsilon 0-39^{\circ} t$ & $10-399^{\circ}$ & $Z-b I-I Z I 000$ & 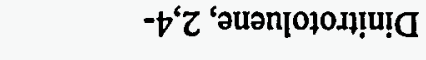 \\
\hline $10-\exists L L^{\prime} Z$ & $00+3 L^{\prime} Z$ & & & S-8Z-I IS0000 & $-t^{\prime} Z$ 'jouəudon!u!a \\
\hline $20-\exists t \cdot S$ & $10-\exists t^{\circ} S$ & & & $t-\varsigma Z-001000$ & - †'l 'әuәzuәqoमn!u! \\
\hline $20-\exists b I$ & $10-\exists b^{\prime} I$ & & - & $0-\$ 9-660000$ & 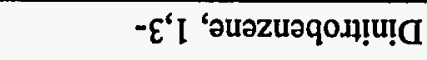 \\
\hline $20-9 b \cdot s$ & $10-a t \cdot S$ & & & $0-6 z-8 z \varsigma 000$ & 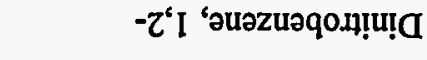 \\
\hline \multirow[t]{2}{*}{$10-\exists L L^{\prime} Z$} & $00+\exists L \cdot Z$ & & & S-68-IEI000 & 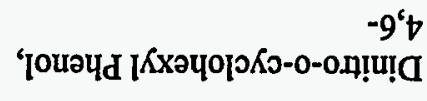 \\
\hline & & & & $I-\tau \varsigma-\downarrow \varepsilon \varsigma 000$ & 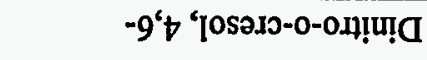 \\
\hline $10+3 b^{\prime} I$ & $20+3 t I$ & & & 9-โ9-0ZІ000 & 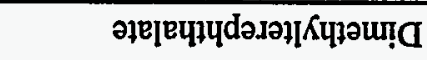 \\
\hline$\varepsilon 0+3 t^{\circ} I$ & †0+ロヤ゙I & & & $\varepsilon-I I-I \varepsilon I 000$ & 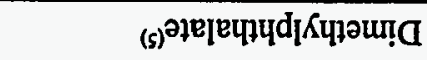 \\
\hline I0-at'I & $00+3 t$ & & & $8-\varsigma 9-\varsigma 60000$ & 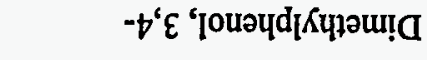 \\
\hline$I^{\prime} 0=\partial H$ & $I=\mathbf{O H}$ & $9-0 I$ & rOI & & 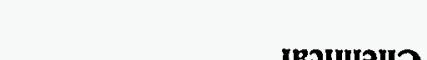 \\
\hline \multicolumn{4}{|c|}{ 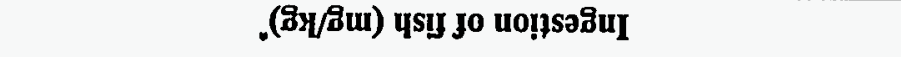 } & 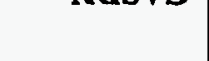 & \\
\hline
\end{tabular}

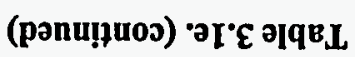


Table 3.1e. (continued)

\begin{tabular}{|c|c|c|c|c|c|}
\hline \multirow{2}{*}{ Chemical } & \multirow{2}{*}{ CASRN } & \multicolumn{4}{|c|}{ Ingestion of fish (mg/kg) } \\
\hline & & $10^{-4}$ & $10^{-6}$ & $\mathbf{H Q}=\mathbf{1}$ & $\mathrm{HQ}=0.1$ \\
\hline Diquat & 000085-00-7 & & & $3.0 \mathrm{E}+00$ & $3.0 \mathrm{E}-01$ \\
\hline Direct Black 38 & 001937-37-7 & $3.7 \mathrm{E}-02$ & 3.7E-04 & & \\
\hline Direct Blue 6 & $002602-46-2$ & $3.9 \mathrm{E}-02$ & $3.9 \mathrm{E}-04$ & & \\
\hline Direct Brown 95 & $016071-86-6$ & $3.4 \mathrm{E}-02$ & 3.4E-04 & & \\
\hline Direct Sky Blue & 002610-05-1 & & & & \\
\hline Disulfoton & $000298-04-4$ & & & $5.4 \mathrm{E}-02$ & $5.4 \mathrm{E}-03$ \\
\hline Diuron & 000330-54-1 & & & $2.7 \mathrm{E}+00$ & 2.7E-01 \\
\hline Dodine & 002439-10-3 & & & $5.4 \mathrm{E}+00$ & $5.4 \mathrm{E}-01$ \\
\hline EPTC & 000759-94-4 & & & $3.4 \mathrm{E}+01$ & $3.4 \mathrm{E}+00$ \\
\hline Endosulfan & 000115-29-7 & & & $8.1 \mathrm{E}+00$ & $8.1 \mathrm{E}-01$ \\
\hline Endothall & 000145-73-3 & & & $2.7 \mathrm{E}+01$ & $2.7 \mathrm{E}+00$ \\
\hline Endrin & 000072-20-8 & & & 4.1E-01 & 4.1E-02 \\
\hline Epichlorohydrin & 000106-89-8 & $3.2 \mathrm{E}+01$ & $3.2 \mathrm{E}-01$ & $2.7 \mathrm{E}+00$ & 2.7E-01 \\
\hline Epoxybutane, 1,2- & 000106-88-7 & & & & \\
\hline Ethephon & $016672-87-0$ & & & $6.8 \mathrm{E}+00$ & $6.8 \mathrm{E}-01$ \\
\hline Ethion & $000563-12-2$ & & & $6.8 \mathrm{E}-01$ & $6.8 \mathrm{E}-02$ \\
\hline Ethoxyethanol Acetate, 2- & 000111-15-9 & & & $4.1 \mathrm{E}+02$ & $4.1 \mathrm{E}+01$ \\
\hline Ethoxyethanol, 2- & $000110-80-5$ & & & $5.4 \mathrm{E}+02$ & $5.4 \mathrm{E}+01$ \\
\hline
\end{tabular}


Table 3.1e. (continued)

\begin{tabular}{|c|c|c|c|c|c|}
\hline \multirow{2}{*}{ Chemical } & \multirow{2}{*}{ CASRN } & \multicolumn{4}{|c|}{ Ingestion of fish (mg/kg) } \\
\hline & & $10^{-4}$ & $10^{-6}$ & $H Q=1$ & $\mathrm{HQ}=0.1$ \\
\hline Ethyl Acetate & $000141-78-6$ & & & $1.2 \mathrm{E}+03$ & $1.2 \mathrm{E}+02$ \\
\hline Ethyl Acrylate & 000140-88-5 & $6.6 \mathrm{E}+00$ & $6.6 \mathrm{E}-02$ & & \\
\hline Ethyl Chloride & $000075-00-3$ & & & & \\
\hline Ethyl Ether & 000060-29-7 & & & $2.7 \mathrm{E}+02$ & 2.7E+01 \\
\hline Ethyl Methacrylate & 000097-63-2 & & & $1.2 \mathrm{E}+02$ & $1.2 \mathrm{E}+01$ \\
\hline Ethyl-p-nitrophenyl Phosphonate & 002104-64-5 & & & $1.4 \mathrm{E}-02$ & $1.4 \mathrm{E}-03$ \\
\hline Ethylbenzene $^{(6)}$ & $000100-41-4$ & & & $1.4 \mathrm{E}+02$ & $1.4 \mathrm{E}+01$ \\
\hline Ethylene Cyanohydrin & $000109-78-4$ & & & $4.1 \mathrm{E}+02$ & $4.1 \mathrm{E}+01$ \\
\hline Ethylene Diamine & 000107-15-3 & & & $2.7 \mathrm{E}+01$ & $2.7 \mathrm{E}+00$ \\
\hline Ethylene Glycol & 000107-21-1 & & & $2.7 \mathrm{E}+03$ & $2.7 \mathrm{E}+02$ \\
\hline $\begin{array}{l}\text { Ethylene Glycol Monobutyl } \\
\text { Ether }\end{array}$ & $000111-76-2$ & & & . & \\
\hline Ethylene Oxide & $000075-21-8$ & $3.1 \mathrm{E}-01$ & $3.1 \mathrm{E}-03$ & & \\
\hline Ethylene Thiourea & 000096-45-7 & $2.9 \mathrm{E}+00$ & $2.9 \mathrm{E}-02$ & $1.1 \mathrm{E}-01$ & $1.1 \mathrm{E}-02$ \\
\hline Ethylphthalyl Ethyl Glycolate & $000084-72-0$ & & & 4.1E+03 & 4.1E+02 \\
\hline Express & $101200-48-0$ & & & $1.1 \mathrm{E}+01$ & $1.1 \mathrm{E}+00$ \\
\hline Fenamiphos & 022224-92-6 & & & $3.4 \mathrm{E}-01$ & $3.4 \mathrm{E}-02$ \\
\hline Fenpropathrin & $039515-41-8$ & & & $3.4 \mathrm{E}+01$ & $3.4 \mathrm{E}+00$ \\
\hline
\end{tabular}


Table 3.1e. (continued)

\begin{tabular}{|l|l|l|c|c|c|}
\hline \multirow{2}{*}{ Chemical } & \multirow{2}{*}{ CASRN } & \multicolumn{4}{|c|}{ Ingestion of fish (mg/kg) } \\
\cline { 4 - 6 } & & $10^{-4}$ & $10^{-6}$ & HQ=1 & HQ=0.1 \\
\hline Fluometuron & $002164-17-2$ & & & $1.8 \mathrm{E}+01$ & $1.8 \mathrm{E}+00$ \\
\hline Fluoranthene & $000206-44-0$ & & & $5.4 \mathrm{E}+01$ & $5.4 \mathrm{E}+00$ \\
\hline Fluorene & $000086-73-7$ & & & $5.4 \mathrm{E}+01$ & $5.4 \mathrm{E}+00$ \\
\hline Fluoride & $007782-41-4$ & & & $8.1 \mathrm{E}+01$ & $8.1 \mathrm{E}+00$ \\
\hline Fluridone & $059756-60-4$ & & & $1.1 \mathrm{E}+02$ & $1.1 \mathrm{E}+01$ \\
\hline Flurprimidol & $056425-91-3$ & & & $2.7 \mathrm{E}+01$ & $2.7 \mathrm{E}+00$ \\
\hline Flutolanil & $066332-96-5$ & & & $8.1 \mathrm{E}+01$ & $8.1 \mathrm{E}+00$ \\
\hline Fluvalinate & $069409-94-5$ & & & $1.4 \mathrm{E}+01$ & $1.4 \mathrm{E}+00$ \\
\hline Folpet & $000133-07-3$ & $9.0 \mathrm{E}+01$ & $9.0 \mathrm{E}-01$ & $1.4 \mathrm{E}+02$ & $1.4 \mathrm{E}+01$ \\
\hline Fomesafen & $072178-02-0$ & $1.7 \mathrm{E}+00$ & $1.7 \mathrm{E}-02$ & & \\
\hline Fonofos & $000944-22-9$ & & & $2.7 \mathrm{E}+00$ & $2.7 \mathrm{E}-01$ \\
\hline Formaldehyde & $000050-00-0$ & & & $2.7 \mathrm{E}+02$ & $2.7 \mathrm{E}+01$ \\
\hline Formic Acid & $000064-18-6$ & & & $2.7 \mathrm{E}+03$ & $2.7 \mathrm{E}+02$ \\
\hline Fosetyl-AL & $039148-24-8$ & & & $4.1 \mathrm{E}+03$ & $4.1 \mathrm{E}+02$ \\
\hline Furan & $000110-00-9$ & & & $1.4 \mathrm{E}+00$ & $1.4 \mathrm{E}-01$ \\
\hline Furazolidone & $000067-45-8$ & $8.3 \mathrm{E}-02$ & $8.3 \mathrm{E}-04$ & & \\
\hline Furfural & $000098-01-1$ & & & $4.1 \mathrm{E}+00$ & $4.1 \mathrm{E}-01$ \\
\hline Furium & $000531-82-8$ & $6.3 \mathrm{E}-03$ & $6.3 \mathrm{E}-05$ & & \\
\hline
\end{tabular}


Table 3.1e. (continued)

\begin{tabular}{|c|c|c|c|c|c|}
\hline \multirow{2}{*}{ Chemical } & \multirow{2}{*}{ CASRN } & \multicolumn{4}{|c|}{ Ingestion of fish (mg/kg)" } \\
\hline & & $10^{-4}$ & $10^{-6}$ & $H Q=1$ & $\mathrm{HQ}=0.1$ \\
\hline Furmecyclox & 060568-05-0 & $1.1 \mathrm{E}+01$ & $1.1 \mathrm{E}-01$ & & \\
\hline Glufosinate, Ammonium & 077182-82-2 & & & $5.4 \mathrm{E}-01$ & $5.4 \mathrm{E}-02$ \\
\hline Glycidyl & 000765-34-4 & & & $5.4 \mathrm{E}-01$ & $5.4 \mathrm{E}-02$ \\
\hline Glyphosate & 001071-83-6 & & & $1.4 \mathrm{E}+02$ & $1.4 \mathrm{E}+01$ \\
\hline Goal & 042874-03-3 & & & 4.1E+00 & 4.1E-01 \\
\hline Haloxyfop, Methyl & 069806-40-2 & & & $6.8 \mathrm{E}-02$ & $6.8 \mathrm{E}-03$ \\
\hline Harmony & 079277-27-3 & & & $1.8 \mathrm{E}+01$ & $1.8 \mathrm{E}+00$ \\
\hline Heptachlor & 000076-44-8 & $7.0 \mathrm{E}-02$ & 7.0E-04 & $6.8 \mathrm{E}-01$ & $6.8 \mathrm{E}-02$ \\
\hline Heptachlor Epoxide & 001024-57-3 & $3.5 \mathrm{E}-02$ & $3.5 \mathrm{E}-04$ & $1.8 \mathrm{E}-02$ & $1.8 \mathrm{E}-03$ \\
\hline Heptane, N- & $000142-82-5$ & & & & \\
\hline Hexabromobenzene & 000087-82-1 & & & $2.7 \mathrm{E}+00$ & 2.7E-01 \\
\hline Hexachlorobenzene ${ }^{(6)}$ & 000118-74-1 & 2.0E-01 & $2.0 \mathrm{E}-03$ & $1.1 \mathrm{E}+00$ & $1.1 \mathrm{E}-01$ \\
\hline Hexachlorobutadiene $e^{(6)}$ & $000087-68-3$ & $4.0 \mathrm{E}+00$ & 4.0E-02 & $2.7 \mathrm{E}-01$ & $2.7 \mathrm{E}-02$ \\
\hline Hexachlorocyclohexane, Alpha- & 000319-84-6 & $5.0 \mathrm{E}-02$ & $5.0 \mathrm{E}-04$ & & \\
\hline Hexachlorocyclohexane, Beta- & $000319-85-7$ & $1.8 \mathrm{E}-01$ & $1.8 \mathrm{E}-03$ & & \\
\hline Hexachlorocyclohexane, Delta- & 000319-86-8 & & & & \\
\hline Hexachlorocyclohexane, Epsilon & 006108-10-7 & & & & \\
\hline
\end{tabular}


Table 3.1e. (continued)

\begin{tabular}{|l|l|l|c|c|c|}
\hline \multirow{2}{*}{ Chemical } & \multirow{2}{*}{ CASRN } & \multicolumn{4}{|c|}{ Ingestion of fish (mg/kg) $^{*}$} \\
\cline { 3 - 6 } & & $10^{-4}$ & $10^{-6}$ & HQ=1 & HQ=0.1 \\
\hline $\begin{array}{l}\text { Hexachlorocyclohexane, } \\
\text { Gamma- }\end{array}$ & $000058-89-9$ & $2.4 \mathrm{E}-01$ & $2.4 \mathrm{E}-03$ & $4.1 \mathrm{E}-01$ & $4.1 \mathrm{E}-02$ \\
\hline $\begin{array}{l}\text { Hexachlorocyclohexane, } \\
\text { Technical }\end{array}$ & $000608-73-1$ & $1.8 \mathrm{E}-01$ & $1.8 \mathrm{E}-03$ & & \\
\hline Hexachlorocyclopentadiene & $000077-47-4$ & & & $9.5 \mathrm{E}+00$ & $9.5 \mathrm{E}-01$ \\
\hline $\begin{array}{l}\text { Hexachlorodibenzo-p-dioxin, } \\
\text { Mixture }\end{array}$ & $019408-74-3$ & $5.1 \mathrm{E}-05$ & $5.1 \mathrm{E}-07$ & & \\
\hline Hexachloroethane & $000067-72-1$ & $2.3 \mathrm{E}+01$ & $2.3 \mathrm{E}-01$ & $1.4 \mathrm{E}+00$ & $1.4 \mathrm{E}-01$ \\
\hline Hexachlorophene & $000070-30-4$ & & & $4.1 \mathrm{E}-01$ & $4.1 \mathrm{E}-02$ \\
\hline $\begin{array}{l}\text { Hexahydro-1,3,5-trinitro-1,3,5- } \\
\text { triazine (RDX) }\end{array}$ & $000121-82-4$ & $2.9 \mathrm{E}+00$ & $2.9 \mathrm{E}-02$ & $4.1 \mathrm{E}+00$ & $4.1 \mathrm{E}-01$ \\
\hline $\begin{array}{l}\text { Hexamethylene Diisocyanate, } \\
\text { 1,6- }\end{array}$ & $000822-06-0$ & & & & \\
\hline Hexane, N- & $000110-54-3$ & & & & \\
\hline Hexanone, 2- & $000591-78-6$ & & & & \\
\hline Hexazinone & $051235-04-2$ & & & & \\
\hline HpCDD, 2,3,7,8-(4) & $037871-00-4$ & $2.1 \mathrm{E}-04$ & $2.1 \mathrm{E}-06$ & & \\
\hline HpCDF, 2,3,7,8-(4) & $038998-75-3$ & $2.1 \mathrm{E}-04$ & $2.1 \mathrm{E}-06$ & & \\
\hline HxCDD, 2,3,7,8-(4) & $034465-46-8$ & $2.1 \mathrm{E}-05$ & $2.1 \mathrm{E}-07$ & & \\
\hline HxCDF, 2,3,7,8-(4) & $055684-94-1$ & $2.1 \mathrm{E}-05$ & $2.1 \mathrm{E}-07$ & & \\
\hline
\end{tabular}


Table 3.1e. (continued)

\begin{tabular}{|c|c|c|c|c|c|}
\hline \multirow{2}{*}{ Chemical } & \multirow{2}{*}{ CASRN } & \multicolumn{4}{|c|}{ Ingestion of fish (mg/kg)" } \\
\hline & & $10^{-4}$ & $10^{-6}$ & $\mathbf{H Q}=\mathbf{1}$ & $\mathrm{HQ}=0.1$ \\
\hline Hydrazine & $000302-01-2$ & $1.1 \mathrm{E}-01$ & $1.1 \mathrm{E}-03$ & & \\
\hline Hydrazine Sulfate & 010034-93-2 & $1.1 \mathrm{E}-01$ & $1.1 \mathrm{E}-03$ & & \\
\hline Hydrogen Chloride & 007647-01-0 & & & & \\
\hline Hydrogen Cyanide & 000074-90-8 & & & $2.7 \mathrm{E}+01$ & $2.7 \mathrm{E}+00$ \\
\hline Hydrogen Sulfide & 007783-06-4 & & & $4.1 \mathrm{E}+00$ & $4.1 \mathrm{E}-01$ \\
\hline Hydroquinone & 000123-31-9 & & & $5.4 \mathrm{E}+01$ & $5.4 \mathrm{E}+00$ \\
\hline Imazalil & 035554-44-0 & & & $1.8 \mathrm{E}+01$ & $1.8 \mathrm{E}+00$ \\
\hline Imazaquin & 081335-37-7 & & & $3.4 \mathrm{E}+02$ & $3.4 \mathrm{E}+01$ \\
\hline Indeno $[1,2,3-c d]$ pyrene ${ }^{(2)}$ & 000193-39-5 & 4.3E-01 & $4.3 \mathrm{E}-03$ & & \\
\hline Iprodione & $036734-19-7$ & & & $5.4 \mathrm{E}+01$ & $5.4 \mathrm{E}+00$ \\
\hline Iron & 007439-89-6 & & & & \\
\hline Isobutyl Alcohol & 000078-83-1 & & & $4.1 \mathrm{E}+02$ & 4.1E+01 \\
\hline Isophorone & 000078-59-1 & $3.3 \mathrm{E}+02$ & $3.3 \mathrm{E}+00$ & $2.7 \mathrm{E}+02$ & $2.7 \mathrm{E}+01$ \\
\hline Isopropalin & 033820-53-0 & & & $2.0 \mathrm{E}+01$ & $2.0 \mathrm{E}+00$ \\
\hline Isopropanol & 000067-63-0 & & & & \\
\hline $\begin{array}{l}\text { Isopropyl Methyl Phosphonic } \\
\text { Acid }\end{array}$ & $001832-54-8$ & & & $1.4 \mathrm{E}+02$ & $1.4 \mathrm{E}+01$ \\
\hline Isoxaben & 082558-50-7 & & & $6.8 \mathrm{E}+01$ & $6.8 \mathrm{E}+00$ \\
\hline
\end{tabular}


Table 3.1e. (continued)

\begin{tabular}{|c|c|c|c|c|c|}
\hline \multirow{2}{*}{ Chemical } & \multirow{2}{*}{ CASRN } & \multicolumn{4}{|c|}{ Ingestion of fish (mg/kg) } \\
\hline & & $10^{-4}$ & $10^{-6}$ & $\mathbf{H Q}=\mathbf{1}$ & $H Q=0.1$ \\
\hline Karate & 091465-08-6 & & & $6.8 \mathrm{E}+00$ & $6.8 \mathrm{E}-01$ \\
\hline Kerb & $023950-58-5$ & & & $1.0 \mathrm{E}+02$ & $1.0 \mathrm{E}+01$ \\
\hline Lactofen & $077501-63-4$ & & & $2.7 \mathrm{E}+00$ & $2.7 \mathrm{E}-01$ \\
\hline Lead Alkyls & NA & & & & \\
\hline Lead And Compounds & $007439-92-1$ & & & & \\
\hline Linuron & $000330-55-2$ & & & $2.7 \mathrm{E}+00$ & 2.7E-01 \\
\hline Lithium & 007439-93-2 & & & & \\
\hline Londax & 083055-99-6 & & & $2.7 \mathrm{E}+02$ & $2.7 \mathrm{E}+01$ \\
\hline MCPA & $000094-74-6$ & & & $6.8 \mathrm{E}-01$ & $6.8 \mathrm{E}-02$ \\
\hline $\mathrm{MCPB}$ & $000094-81-5$ & & & $1.4 \mathrm{E}+01$ & $1.4 \mathrm{E}+00$ \\
\hline MCPP & $000093-65-2$ & & & $1.4 \mathrm{E}+00$ & $1.4 \mathrm{E}-01$ \\
\hline Magnesium & 007439-95-4 & & & & \\
\hline Malathion & $000121-75-5$ & & & $2.7 \mathrm{E}+01$ & $2.7 \mathrm{E}+00$ \\
\hline Maleic Anhydride & $000108-31-6$ & & & $1.4 \mathrm{E}+02$ & $1.4 \mathrm{E}+01$ \\
\hline Maleic Hydrazide & 000123-33-1 & & & $6.8 \mathrm{E}+02$ & $6.8 \mathrm{E}+01$ \\
\hline Malononitrile & $000109-77-3$ & & & 2.7E-02 & $2.7 \mathrm{E}-03$ \\
\hline Mancozeb & 008018-01-7 & & & $4.1 \mathrm{E}+01$ & 4.1E+00 \\
\hline Maneb & $012427-38-2$ & & & $6.8 \mathrm{E}+00$ & $6.8 \mathrm{E}-01$ \\
\hline
\end{tabular}


Table 3.1e. (continued)

\begin{tabular}{|c|c|c|c|c|c|}
\hline \multirow{2}{*}{ Chemical } & \multirow{2}{*}{ CASRN } & \multicolumn{4}{|c|}{ Ingestion of fish (mg/kg)" } \\
\hline & & $10^{-4}$ & $10^{-6}$ & $\mathbf{H Q}=\mathbf{1}$ & $H Q=0.1$ \\
\hline Manganese (Diet) & 007439-96-5 & & & $1.9 \mathrm{E}+02$ & $1.9 \mathrm{E}+01$ \\
\hline Mephosfolan & 000950-10-7 & & & $1.2 \mathrm{E}-01$ & $1.2 \mathrm{E}-02$ \\
\hline Mepiquat Chloride & $024307-26-4$ & & & $4.1 \mathrm{E}+01$ & 4.1E+00 \\
\hline Mercuric Chloride & $007487-94-7$ & & & 4.1E-01 & 4:1E-02 \\
\hline Mercury (elemental) & 007439-97-6 & & & 4.1E-01 & $4.1 \mathrm{E}-02$ \\
\hline Mercury, Inorganic Salt & $007439-97-6$ & & & 4.1E-01 & 4.1E-02 \\
\hline Merphos & 000150-50-5 & & & 4.1E-02 & 4.1E-03 \\
\hline Merphos Oxide & $000078-48-8$ & & & 4.1E-02 & $4.1 \mathrm{E}-03$ \\
\hline Metalaxyl & $057837-19-1$ & & & 8.1E+01 & $8.1 \mathrm{E}+00$ \\
\hline Methacrylonitrile & 000126-98-7 & & & $1.4 \mathrm{E}-01$ & $1.4 \mathrm{E}-02$ \\
\hline Methamidophos & $010265-92-6$ & & & $6.8 \mathrm{E}-02$ & $6.8 \mathrm{E}-03$ \\
\hline Methanol & $000067-56-1$ & & & $6.8 \mathrm{E}+02$ & $6.8 \mathrm{E}+01$ \\
\hline Methidathion & 000950-37-8 & & & $1.4 \mathrm{E}+00$ & $1.4 \mathrm{E}-01$ \\
\hline Methomyl & $016752-77-5$ & & & $3.4 \mathrm{E}+01$ & $3.4 \mathrm{E}+00$ \\
\hline Methoxy-5-nitroaniline, 2- & $000099-59-2$ & $6.9 \mathrm{E}+00$ & $6.9 \mathrm{E}-02$ & & \\
\hline Methoxychlor & $000072-43-5$ & & & $6.8 \mathrm{E}+00$ & $6.8 \mathrm{E}-01$ \\
\hline Methoxyethanol Acetate, 2- & $000110-49-6$ & & & $2.7 \mathrm{E}+00$ & $2.7 \mathrm{E}-01$ \\
\hline Methoxyethanol, 2- & $000109-86-4$ & & & $1.4 \mathrm{E}+00$ & $1.4 \mathrm{E}-01$ \\
\hline
\end{tabular}


Table 3.1e. (continued)

\begin{tabular}{|c|c|c|c|c|c|}
\hline \multirow{2}{*}{ Chemical } & \multirow{2}{*}{ CASRN } & \multicolumn{4}{|c|}{ Ingestion of fish (mg/kg)* } \\
\hline & & $10^{-4}$ & $10^{-6}$ & $\mathbf{H Q}=\mathbf{1}$ & $H Q=0.1$ \\
\hline Methyl Acetate & $000079-20-9$ & & & $1.4 \mathrm{E}+03$ & $1.4 \mathrm{E}+02$ \\
\hline Methyl Acrylate & 000096-33-3 & & & $4.1 E+01$ & 4.1E+00 \\
\hline Methyl Ethyl Ketone & 000078-93-3 & & & 8.1E+02 & $8.1 \mathrm{E}+01$ \\
\hline Methyl Isobutyl Ketone & $000108-10-1$ & & & $1.1 \mathrm{E}+02$ & $1.1 \mathrm{E}+01$ \\
\hline Methyl Mercury & $022967-92-6$ & & & $1.4 \mathrm{E}-01$ & 1.4E-02 \\
\hline Methyl Methacrylate & $000080-62-6$ & & & $1.1 \mathrm{E}+02$ & $1.1 \mathrm{E}+01$ \\
\hline Methyl Parathion & 000298-00-0 & & & $3.4 \mathrm{E}-01$ & $3.4 \mathrm{E}-02$ \\
\hline Methyl Styrene (Mixed Isomers) & $025013-15-4$ & & & $8.1 \mathrm{E}+00$ & 8.1E-01 \\
\hline Methyl hydrazine $^{(s)}$ & $000060-34-4$ & $2.9 \mathrm{E}-01$ & $2.9 \mathrm{E}-03$ & & \\
\hline Methyl tert-Butyl Ether (MTBE) & $001634-04-4$ & & & & \\
\hline Methyl-5-Nitroaniline, 2- & 000099-55-8 & $9.6 \mathrm{E}+00$ & $9.6 \mathrm{E}-02$ & & \\
\hline Methylaniline Hydrochloride, 2- & $000636-21-5$ & $1.8 \mathrm{E}+00$ & $1.8 \mathrm{E}-02$ & & \\
\hline Methylcyclohexane & 000108-87-2 & & & & \\
\hline Methylene Chloride & $000075-09-2$ & $4.2 E+01$ & 4.2E-01 & $8.1 E+01$ & $8.1 \mathrm{E}+00$ \\
\hline $\begin{array}{l}\text { Methylene-bis(2-chloroaniline), } \\
4,4^{\prime} \text { - }\end{array}$ & $000101-14-4$ & $2.4 \mathrm{E}+00$ & 2.4E-02 & $9.5 \mathrm{E}-01$ & $9.5 \mathrm{E}-02$ \\
\hline $\begin{array}{l}\text { Methylene-bis(N,N-dimethyl) } \\
\text { Aniline, 4,4-- }\end{array}$ & $000101-61-1$ & $6.9 \mathrm{E}+00$ & $6.9 \mathrm{E}-02$ & & \\
\hline
\end{tabular}


Table 3.1e. (continued)

\begin{tabular}{|c|c|c|c|c|c|}
\hline \multirow{2}{*}{ Chemical } & \multirow{2}{*}{ CASRN } & \multicolumn{4}{|c|}{ Ingestion of fish (mg/kg) } \\
\hline & & $10^{-4}$ & $10^{-6}$ & $\mathbf{H Q}=\mathbf{1}$ & $\mathrm{HQ}=0.1$ \\
\hline Methylenebisbenzenamine, 4,4'- & 000101-77-9 & $1.3 \mathrm{E}+00$ & $1.3 \mathrm{E}-02$ & & \\
\hline $\begin{array}{l}\text { Methylenediphenyl Isocyanate, } \\
\text { 4,4'- }\end{array}$ & 000101-68-8 & & & & \\
\hline Methylstyrene, Alpha- & 000098-83-9 & & & $9.5 \mathrm{E}+01$ & $9.5 \mathrm{E}+00$ \\
\hline Methyltriethyl Lead & 001762-28-3 & & & & \\
\hline Metolachlor & $051218-45-2$ & & & $2.0 \mathrm{E}+02$ & $2.0 \mathrm{E}+01$ \\
\hline Metribuzin $^{(5)}$ & 021087-64-9 & & & $3.4 \mathrm{E}+01$ & $3.4 \mathrm{E}+00$ \\
\hline $\operatorname{Mirex}^{(5)}$ & 002385-85-5 & $1.8 \mathrm{E}-01$ & $1.8 \mathrm{E}-03$ & $2.7 \mathrm{E}-01$ & 2.7E-02 \\
\hline Molinate & $002212-67-1$ & & & $2.7 \mathrm{E}+00$ & 2.7E-01 \\
\hline Molybdenum & 007439-98-7 & & & $6.8 \mathrm{E}+00$ & $6.8 \mathrm{E}-01$ \\
\hline Monochloramine & 010599-90-3 & & & $1.4 \mathrm{E}+02$ & $1.4 \mathrm{E}+01$ \\
\hline Monochlorobutanes & $025154-42-1$ & & & $5.4 \mathrm{E}+02$ & $5.4 \mathrm{E}+01$ \\
\hline Naled & $000300-76-5$ & & & $2.7 \mathrm{E}+00$ & 2.7E-01 \\
\hline Naphthalene ${ }^{(6)}$ & 000091-20-3 & & & $4.8 \mathrm{E}+01$ & $4.8 \mathrm{E}+00$ \\
\hline Naphthalene, 1-Methyl & 000090-12-0 & & & & \\
\hline Naphthalene, 2-Methyl & 000091-57-6 & & & & \\
\hline Napropamide & 015299-99-7 & & & $1.4 \mathrm{E}+02$ & $1.4 \mathrm{E}+01$ \\
\hline Niagara Blue 4B & 002429-74-5 & & & & \\
\hline
\end{tabular}


Table 3.1e. (continued)

\begin{tabular}{|c|c|c|c|c|c|}
\hline \multirow{2}{*}{ Chemical } & \multirow{2}{*}{ CASRN } & \multicolumn{4}{|c|}{ Ingestion of fish (mg/kg) } \\
\hline & & $10^{-4}$ & $10^{-6}$ & $\mathbf{H Q}=\mathbf{1}$ & $H Q=0.1$ \\
\hline Nickel Carbonyl & $013463-39-3$ & & & & \\
\hline Nickel Refinery Dust & NA & & & & \\
\hline Nickel Soluble Salts & $007440-02-0$ & & & 2.7E+01 & $2.7 \mathrm{E}+00$ \\
\hline Nickel Subsulfide & $012035-72-2$ & & & & \\
\hline Nitrapyrin $^{(5)}$ & $001929-82-4$ & & & $2.0 \mathrm{E}+00$ & $2.0 \mathrm{E}-01$ \\
\hline Nitrate & $014797-55-8$ & & & $2.2 \mathrm{E}+03$ & $2.2 \mathrm{E}+02$ \\
\hline Nitric Oxide ${ }^{(3)}$ & $010102-43-9$ & & & $1.4 \mathrm{E}+02$ & $1.4 \mathrm{E}+01$ \\
\hline Nitrite & $014797-65-0$ & & & $1.4 \mathrm{E}+02$ & $1.4 \mathrm{E}+01$ \\
\hline Nitroaniline, 2- & $000088-74-4$ & & & & \\
\hline Nitrobenzene & $000098-95-3$ & & & $6.8 \mathrm{E}-01$ & $6.8 \mathrm{E}-02$ \\
\hline Nitrofurantoin & $000067-20-9$ & & & $9.5 \mathrm{E}+01$ & $9.5 \mathrm{E}+00$ \\
\hline Nitrofurazone & $000059-87-0$ & $2.1 \mathrm{E}-01$ & 2.1E-03 & & \\
\hline Nitrogen Dioxide ${ }^{(3)}$ & $010102-44-0$ & & & $1.4 \mathrm{E}+03$ & $1.4 \mathrm{E}+02$ \\
\hline Nitroguanidine & 000556-88-7 & & & $1.4 \mathrm{E}+02$ & $1.4 \mathrm{E}+01$ \\
\hline Nitrophenol, 4- & $000100-02-7$ & & & & \\
\hline Nitropropane, 2-(5) & $000079-46-9$ & 3.3E-02 & $3.3 \mathrm{E}-04$ & & \\
\hline Nitroso-N-ethylurea, N- & 000759-73-9 & $2.3 \mathrm{E}-03$ & 2.3E-05 & & \\
\hline Nitroso-N-methylurea, N- & $000684-93-5$ & & & & \\
\hline
\end{tabular}


Table 3.1e. (continued)

\begin{tabular}{|l|l|l|l|l|l|}
\hline \multirow{2}{*}{ Chemical } & \multirow{2}{*}{ CASRN } & \multicolumn{3}{|c|}{ Ingestion of fish (mg/kg) } \\
\cline { 3 - 6 } & & $10^{-4}$ & $10^{-6}$ & HQ=1 & HQ=0.1 \\
\hline Nitroso-di-N-butylamine, N- & $000924-16-3$ & $5.8 \mathrm{E}-02$ & $5.8 \mathrm{E}-04$ & & \\
\hline Nitroso-di-N-propylamine, N- & $000621-64-7$ & $4.5 \mathrm{E}-02$ & $4.5 \mathrm{E}-04$ & & \\
\hline Nitrosodiethanolamine, N- & $001116-54-7$ & $1.1 \mathrm{E}-01$ & $1.1 \mathrm{E}-03$ & & \\
\hline Nitrosodiethylamine, N- & $000055-18-5$ & $2.1 \mathrm{E}-03$ & $2.1 \mathrm{E}-05$ & & \\
\hline Nitrosodimethylamine, N- & $000062-75-9$ & $6.2 \mathrm{E}-03$ & $6.2 \mathrm{E}-05$ & & \\
\hline Nitrosodiphenylamine, N- & $000086-30-6$ & $6.4 \mathrm{E}+01$ & $6.4 \mathrm{E}-01$ & & \\
\hline Nitrosomethylethylamine, N- & $010595-95-6$ & $1.4 \mathrm{E}-02$ & $1.4 \mathrm{E}-04$ & & \\
\hline Nitrosomethylvinylamine, N- & $004549-40-0$ & & & & \\
\hline Nitrosopyrrolidine, N- & $000930-55-2$ & $1.5 \mathrm{E}-01$ & $1.5 \mathrm{E}-03$ & & \\
\hline Nitrotoluene, 4-Amino-2- & $000119-32-4$ & & & & \\
\hline Nitrotoluene, m- & $000099-08-1$ & & & $1.4 \mathrm{E}+00$ \\
\hline Nitrotoluene, o- & $000088-72-2$ & & & $1.4 \mathrm{E}+01$ & $1.4 \mathrm{E}+00$ \\
\hline Nitrotoluene, $\mathrm{p}-$ & $000099-99-0$ & & & $1.4 \mathrm{E}+01$ & $1.4 \mathrm{E}+00$ \\
\hline Norflurazon & $027314-13-2$ & & & $5.4 \mathrm{E}+01$ & $5.4 \mathrm{E}+00$ \\
\hline Nustar & $085509-19-9$ & & & $9.5 \mathrm{E}-01$ & $9.5 \mathrm{E}-02$ \\
\hline OCDD(4) & $003268-87-9$ & $2.1 \mathrm{E}-03$ & $2.1 \mathrm{E}-05$ & & \\
\hline OCDF(4) & $039001-02-0$ & $2.1 \mathrm{E}-03$ & $2.1 \mathrm{E}-05$ & & \\
\hline Octabromodiphenyl Ether & $032536-52-0$ & & & $4.1 \mathrm{E}+00$ & $4.1 \mathrm{E}-01$ \\
\hline
\end{tabular}


Table 3.1e. (continued)

\begin{tabular}{|c|c|c|c|c|c|}
\hline \multirow{2}{*}{ Chemical } & \multirow{2}{*}{ CASRN } & \multicolumn{4}{|c|}{ Ingestion of fish (mg/kg) } \\
\hline & & $10^{-4}$ & $10^{-6}$ & $H Q=1$ & $\mathrm{HQ}=0.1$ \\
\hline $\begin{array}{l}\text { Octahydro-1,3,5,7-tetranitro- } \\
\text { 1,3,5,7-tetra (HMX) }\end{array}$ & $002691-41-0$ & & & $6.8 \mathrm{E}+01$ & $6.8 \mathrm{E}+00$ \\
\hline Octamethylpyrophosphoramide & 000152-16-9 & & & $2.7 \mathrm{E}+00$ & 2.7E-01 \\
\hline Octyl Phthalate, di-N- & $000117-84-0$ & & & $2.7 \mathrm{E}+01$ & 2.7E+00 \\
\hline Oryzalin & 019044-88-3 & & & $6.8 \mathrm{E}+01$ & $6.8 \mathrm{E}+00$ \\
\hline Oxadiazon & 019666-30-9 & & & $6.8 \mathrm{E}+00$ & $6.8 \mathrm{E}-01$ \\
\hline Oxamyl & $023135-22-0$ & & & $3.4 \mathrm{E}+01$ & $3.4 \mathrm{E}+00$ \\
\hline Paclobutrazol & $076738-62-0$ & & & $1.8 \mathrm{E}+01$ & $1.8 \mathrm{E}+00$ \\
\hline Paraquat & $001910-42-5$ & & & $6.1 \mathrm{E}+00$ & $6.1 \mathrm{E}-01$ \\
\hline Parathion & 000056-38-2 & & & 8.1E+00 & 8.1E-01 \\
\hline PeCDD, 2,3,7,8-(4) & 036088-22-9 & $4.2 \mathrm{E}-06$ & $4.2 \mathrm{E}-08$ & & \\
\hline PeCDF, $1,2,3,7,8$-(4) $^{(1)}$ & $057117-41-6$ & $4.2 \mathrm{E}-06$ & $4.2 \mathrm{E}-08$ & & \\
\hline PeCDF, 2,3,4,7,8-(4) & $057117-31-4$ & 4.2E-05 & $4.2 \mathrm{E}-07$ & & \\
\hline Pebulate & $001114-71-2$ & & & $6.8 \mathrm{E}+01$ & $6.8 \mathrm{E}+00$ \\
\hline Pendimethalin & $040487-42-1$ & & & $5.4 \mathrm{E}+01$ & $5.4 \mathrm{E}+00$ \\
\hline Pentabromodiphenyl Ether & 032534-81-9 & & & $2.7 \mathrm{E}+00$ & $2.7 \mathrm{E}-01$ \\
\hline Pentachlorobenzene & 000608-93-5 & & & $1.1 \mathrm{E}+00$ & $1.1 \mathrm{E}-01$ \\
\hline Pentachlorocyclopentadiene & 025329-35-5 & & & & \\
\hline
\end{tabular}




\begin{tabular}{|c|c|c|c|c|c|}
\hline$z 0+\theta L \cdot z$ & $\varepsilon 0+g<{ }^{\prime} \tau$ & & & $6-t b-\varsigma 80000$ & 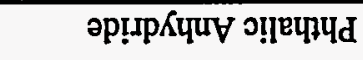 \\
\hline \multirow[t]{2}{*}{$z 0+96 \cdot I$} & $\varepsilon 0+\exists b^{\circ} l$ & & & $0-12-001000$ & 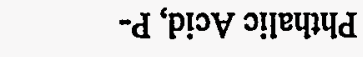 \\
\hline & & & & $z-8 \varepsilon-\dagger 99<00$ & p!৩V ग!ıoųdsoपd \\
\hline zo-gl'b & $I 0-9 I^{\prime} t$ & & & $\tau-I S-\varepsilon 08<00$ & әu!̣dsoपd \\
\hline $00+B L \cdot \tau$ & $10+\exists L \cdot 2$ & & & 9-II-ZEL000 & fousoyd \\
\hline \multirow[t]{2}{*}{$20-\mathrm{a} L^{\prime} Z$} & $10-3 L^{\prime} \tau$ & & & $z-z 0-86 z 000$ & गұЕ.0Чd \\
\hline & & $00+39 \cdot I$ & $20+39^{\circ} I$ & $L-\varepsilon t-060000$ & 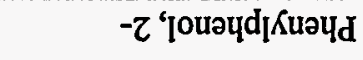 \\
\hline Z0-घI'I & I0-BI'I & & & 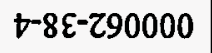 & 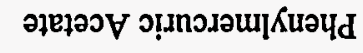 \\
\hline \multirow[t]{2}{*}{$10+39^{\circ} z$} & $20+39 \cdot 2$ & & & $\varepsilon-0 S-901000$ & 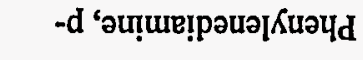 \\
\hline & & $20-\exists L^{\prime} 9$ & $00+\mathrm{B}<\cdot 9$ & $s-t s-s 60000$ & -o 'әu!̣uе!̣рәиәןКиәчd \\
\hline I0-91'8 & $00+9 I^{\circ} 8$ & & & $\tau-s t-801000$ & -ш ‘әu!̣uе!рәиәкиәчd \\
\hline $10+3 I^{\prime} 8$ & $20+91 \cdot 8$ & & & $\tau-\varsigma 6-801000$ & ןouər|d \\
\hline \multirow[t]{2}{*}{$10+\exists b^{\bullet} \varepsilon$} & $20+\theta t \varepsilon$ & & & 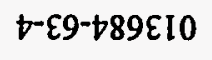 & ureud!pәuшuәчd \\
\hline & & & & $8-10-\varsigma 80000$ & әนอгqрนеนวЧ \\
\hline \multirow[t]{2}{*}{$00+788^{\circ} 9$} & $10+38^{\circ} 9$ & & & {$[-\varepsilon \varsigma-\varsigma \$ 9 Z S 0$} & 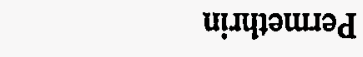 \\
\hline & & & & $0-I t-I L 0000$ & 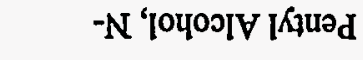 \\
\hline $00+3 I^{\circ} t$ & $I 0+I^{\circ} \mathrm{t}$ & $20-39^{\circ} \tau$ & $00+39 \cdot 2$ & $\varsigma-98-\angle 80000$ & 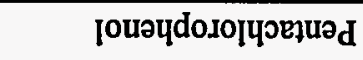 \\
\hline I0-EI't & $00+3 I^{\prime} t$ & $20-\exists Z^{\prime} I$ & $00+A Z Z^{\prime I}$ & $8-89-280000$ & 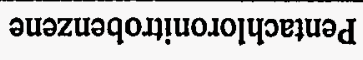 \\
\hline$I^{*} 0=\partial H$ & $\mathrm{I}=\mathbf{O H}$ & $9.0 I$ & mOI & \multirow{2}{*}{ N\&SVD } & \multirow{2}{*}{ 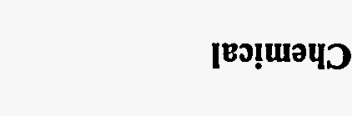 } \\
\hline & 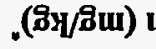 & 10!!sอ8ิ4I & & & \\
\hline
\end{tabular}

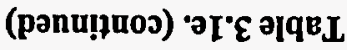




\begin{tabular}{|c|c|c|c|c|c|}
\hline$\varepsilon 0+\exists L\urcorner$ & $70+B L^{\circ} Z$ & & & 9-SS-LS0000 & 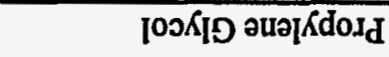 \\
\hline $00+988^{\circ}$ & $10+38^{\circ} I$ & & & I-06-L0Z090 & शpozeuoo!̣doxd \\
\hline $00+\exists L Z$ & $10+\exists L \cdot Z$ & & & $6-2 t-2 z 1000$ & ureqdord \\
\hline $00+\theta \angle \cdot Z$ & $10+B L \tau$ & & & $z-0 t-6 \varepsilon 1000$ & วụzedoId \\
\hline $\mathfrak{I} 0-\exists L \cdot Z$ & $00+\theta \angle \cdot Z$ & & & $L-6 \mathrm{I}-\angle 0 \mathrm{I} 000$ & ןочоगV !Кร.sedoxd \\
\hline $00+\exists \angle L^{\circ} \tau$ & $10+B L \cdot Z$ & & & 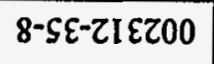 & ว!lठf.sedoxd \\
\hline $10-98 \cdot 9$ & $00+98.9$ & & & $8-86-60<000$ & I!uedord \\
\hline $00+78^{\circ} I$ & $I 0+98^{\circ} I$ & & & $L-9 I-816100$ & 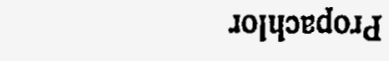 \\
\hline $10-9 t \cdot s$ & $00+a t \cdot s$ & & & $9-6 \mathrm{I}-\angle 8 Z \angle 00$ & uKnouro. d \\
\hline $00+30^{\circ} \tau$ & $10+30^{\circ} \tau$ & & & $0-8 I-0 I 9 I 00$ & 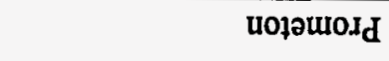 \\
\hline I0-9I'8 & $00+9 I^{\bullet} 8$ & & & $0-9 \varepsilon-66 £ 9 Z 0$ & u!jexn|Joxd \\
\hline $00+a Z \cdot I$ & 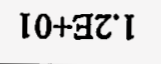 & $20-\mathrm{gl} \cdot \mathrm{Z}$ & $00+\mathrm{Al} Z$ & $s-60-\angle b L \angle 90$ & zeso]4001 \\
\hline$I 0+G \angle \cdot Z$ & $Z 0+B L \cdot \tau$ & & & 9-โ9-90\$000 & 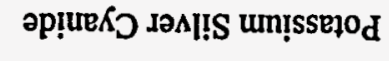 \\
\hline \multirow[t]{2}{*}{$00+98^{\circ} 9$} & $10+98^{\circ} 9$ & & & 8-0S-ISI000 & 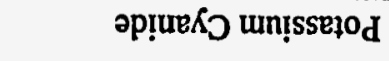 \\
\hline & & $t 0-9 I \%$ & $20-9 I^{\circ} \mathrm{t}$ & $\varepsilon-9 \varepsilon-9 \varepsilon \varepsilon 100$ & s]Кนәцd! \\
\hline t0-as.6 & $\varepsilon 0-\exists S^{\prime} 6$ & $t 0-a \varsigma^{\bullet} \varepsilon$ & $20-\exists S^{\circ} \mathcal{E}$ & $\mathrm{VN}$ & 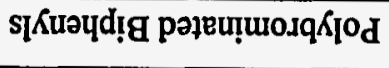 \\
\hline $00+3 t I$ & $I 0+B t$ & & & $L-\varepsilon 6-\succsim \varepsilon z \measuredangle \zeta Z 0$ & 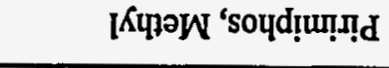 \\
\hline $00+3 \varsigma^{\circ} 6$ & $10+3 s^{\circ} 6$ & & & $\mathrm{I}-20-8 \mathrm{I} 6 \mathrm{I00}$ & urexopग!d \\
\hline$I 0=\partial \mathrm{H}$ & $\mathbf{I}=\mathbf{O H}$ & $9.0 \mathrm{I}$ & mOI & מסyey & 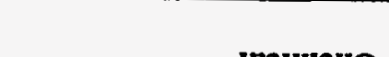 \\
\hline \multicolumn{4}{|c|}{ 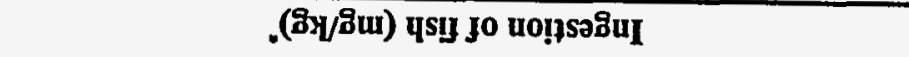 } & Nastrs & S \\
\hline
\end{tabular}

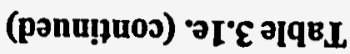


Table 3.1e. (continued)

\begin{tabular}{|c|c|c|c|c|c|}
\hline \multirow{2}{*}{ Chemical } & \multirow{2}{*}{ CASRN } & \multicolumn{4}{|c|}{ Ingestion of fish (mg/kg) } \\
\hline & & $10^{-4}$ & $10^{-6}$ & $\mathbf{H Q}=\mathbf{1}$ & $\mathrm{HQ}=0.1$ \\
\hline $\begin{array}{l}\text { Propylene Glycol Monoethyl } \\
\text { Ether }\end{array}$ & $001569-02-4$ & & & $9.5 \mathrm{E}+02$ & $9.5 \mathrm{E}+01$ \\
\hline $\begin{array}{l}\text { Propylene Glycol Monomethyl } \\
\text { Ether }\end{array}$ & $000107-98-2$ & & & $9.5 \mathrm{E}+02$ & $9.5 \mathrm{E}+01$ \\
\hline Propylene Oxide & $000075-56-9$ & $1.3 \mathrm{E}+00$ & $1.3 \mathrm{E}-02$ & & \\
\hline Pursuit & 081335-77-5 & & & $3.4 \mathrm{E}+02$ & $3.4 \mathrm{E}+01$ \\
\hline Pydrin & $051630-58-1$ & & & $3.4 \mathrm{E}+01$ & $3.4 \mathrm{E}+00$ \\
\hline Pyrene & 000129-00-0 & & & $4.1 \mathrm{E}+01$ & 4.1E+00 \\
\hline Pyridine & $000110-86-1$ & & & $1.4 \mathrm{E}+00$ & $1.4 \mathrm{E}-01$ \\
\hline Quinalphos & 013593-03-8 & & & $6.8 \mathrm{E}-01$ & $6.8 \mathrm{E}-02$ \\
\hline Quinoline & $000091-22-5$ & $2.6 \mathrm{E}-02$ & $2.6 \mathrm{E}-04$ & & \\
\hline Refractory Ceramic Fibers & NA & & & & \\
\hline Resmethrin & $010453-86-8$ & & & 4.1E+01 & $4.1 \mathrm{E}+00$ \\
\hline Ronnel & $000299-84-3$ & & & $6.8 \mathrm{E}+01$ & $6.8 \mathrm{E}+00$ \\
\hline Rotenone & $000083-79-4$ & & & $5.4 \mathrm{E}+00$ & $5.4 \mathrm{E}-01$ \\
\hline Savey & $078587-05-0$ & & & $3.4 \mathrm{E}+01$ & $3.4 \mathrm{E}+00$ \\
\hline Selenious Acid & 007783-00-8 & & & $6.8 \mathrm{E}+00$ & $6.8 \mathrm{E}-01$ \\
\hline Selenite & $014124-67-5$ & & & & \\
\hline Selenium & $007782-49-2$ & & & $6.8 \mathrm{E}+00$ & $6.8 \mathrm{E}-01$ \\
\hline
\end{tabular}


Table 3.1e. (continued)

\begin{tabular}{|l|l|l|l|l|l|}
\hline \multirow{2}{*}{ Chemical } & \multirow{2}{*}{ CASRN } & \multicolumn{4}{|c|}{ Ingestion of fish (mg/kg) $^{\circ}$} \\
\cline { 4 - 6 } & & $10^{-1}$ & $10^{-6}$ & HQ=1 & HQ=0.1 \\
\hline Selenium Sulfide & $007446-34-6$ & & & & \\
\hline Selenourea & $000630-10-4$ & & & $6.8 \mathrm{E}+00$ & $6.8 \mathrm{E}-01$ \\
\hline Sethoxydim & $074051-80-2$ & & & $1.2 \mathrm{E}+02$ & $1.2 \mathrm{E}+01$ \\
\hline Silver & $007440-22-4$ & & & $6.8 \mathrm{E}+00$ & $6.8 \mathrm{E}-01$ \\
\hline Silver Cyanide & $000506-64-9$ & & & $1.4 \mathrm{E}+02$ & $1.4 \mathrm{E}+01$ \\
\hline Simazine & $000122-34-9$ & $2.6 \mathrm{E}+00$ & $2.6 \mathrm{E}-02$ & $6.8 \mathrm{E}+00$ & $6.8 \mathrm{E}-01$ \\
\hline Sodium & $007440-23-5$ & & & & \\
\hline Sodium Acifluorfen & $062476-59-9$ & & & $1.8 \mathrm{E}+01$ & $1.8 \mathrm{E}+00$ \\
\hline Sodium Azide & $026628-22-8$ & & & $5.4 \mathrm{E}+00$ & $5.4 \mathrm{E}-01$ \\
\hline Sodium Cyanide & $000143-33-9$ & & & $5.4 \mathrm{E}+01$ & $5.4 \mathrm{E}+00$ \\
\hline Sodium Diethyldithiocarbamate & $000148-18-5$ & $1.2 \mathrm{E}+00$ & $1.2 \mathrm{E}-02$ & $4.1 \mathrm{E}+01$ & $4.1 \mathrm{E}+00$ \\
\hline Sodium Fluoroacetate & $000062-74-8$ & & & $2.7 \mathrm{E}-02$ & $2.7 \mathrm{E}-03$ \\
\hline Sodium Metavanadate & $013718-26-8$ & & & $1.4 \mathrm{E}+00$ & $1.4 \mathrm{E}-01$ \\
\hline Stirofos (Tetrachlorovinphos) & $000961-11-5$ & $1.3 \mathrm{E}+01$ & $1.3 \mathrm{E}-01$ & $4.1 \mathrm{E}+01$ & $4.1 \mathrm{E}+00$ \\
\hline Strontium, Stable & $007440-24-6$ & & & $8.1 \mathrm{E}+02$ & $8.1 \mathrm{E}+01$ \\
\hline Strychnine & $000057-24-9$ & & & $4.1 \mathrm{E}-01$ & $4.1 \mathrm{E}-02$ \\
\hline Styrene ${ }^{(6)}$ & $000100-42-5$ & & & $2.7 \mathrm{E}+02$ & $2.7 \mathrm{E}+01$ \\
\hline Sulfate & $014808-79-8$ & & & \\
\hline
\end{tabular}


Table 3.1e. (continued)

\begin{tabular}{|l|l|l|c|c|c|}
\hline \multirow{2}{*}{ Chemical } & \multirow{2}{*}{ CASRN } & \multicolumn{4}{|c|}{ Ingestion of fish (mg/kg) } \\
\cline { 3 - 6 } & & $10^{-4}$ & $10^{-6}$ & HQ=1 & HQ=0.1 \\
\hline Systhane & $088671-89-0$ & & & $3.4 \mathrm{E}+01$ & $3.4 \mathrm{E}+00$ \\
\hline TCDD, 2,3,7,8-(4) & $001746-01-6$ & $2.1 \mathrm{E}-06$ & $2.1 \mathrm{E}-08$ & & \\
\hline TCDF, 2,3,7,8-(4) & $051207-31-9$ & $2.1 \mathrm{E}-05$ & $2.1 \mathrm{E}-07$ & & \\
\hline TCMTB & $021564-17-0$ & & & $4.1 \mathrm{E}+01$ & $4.1 \mathrm{E}+00$ \\
\hline Tebuthiuron & $034014-18-1$ & & & $9.5 \mathrm{E}+01$ & $9.5 \mathrm{E}+00$ \\
\hline Temephos & $003383-96-8$ & & & $2.7 \mathrm{E}+01$ & $2.7 \mathrm{E}+00$ \\
\hline Terbacil & $005902-51-2$ & & & $1.8 \mathrm{E}+01$ & $1.8 \mathrm{E}+00$ \\
\hline Terbufos & $013071-79-9$ & & & $3.4 \mathrm{E}-02$ & $3.4 \mathrm{E}-03$ \\
\hline Terbutryn & $000886-50-0$ & & & $1.4 \mathrm{E}+00$ & $1.4 \mathrm{E}-01$ \\
\hline Tetrabutyl Lead & $001920-90-7$ & & & & \\
\hline Tetrachlorobenzene, 1,2,4,5- & $000095-94-3$ & & & & \\
\hline Tetrachloroethane, 1,1,1,2- & $000630-20-6$ & $1.2 \mathrm{E}+01$ & $1.2 \mathrm{E}-01$ & $4.1 \mathrm{E}+01$ & $4.1 \mathrm{E}+00$ \\
\hline Tetrachloroethane, 1,1,2,2- & $000079-34-5$ & $1.6 \mathrm{E}+00$ & $1.6 \mathrm{E}-02$ & & \\
\hline Tetrachloroethylene(6) & $000127-18-4$ & $6.1 \mathrm{E}+00$ & $6.1 \mathrm{E}-02$ & $1.4 \mathrm{E}+01$ & $1.4 \mathrm{E}+00$ \\
\hline Tetrachlorophenol, 2,3,4,6- & $000058-90-2$ & & & $4.1 \mathrm{E}+01$ & $4.1 \mathrm{E}+00$ \\
\hline $\begin{array}{l}\text { Tetrachlorotoluene, p- alpha, } \\
\text { alpha, alpha- }\end{array}$ & $005216-25-1$ & $1.6 \mathrm{E}-02$ & $1.6 \mathrm{E}-04$ & & \\
\hline Tetraethyl Dithiopyrophosphate & $003689-24-5$ & & & $6.8 \mathrm{E}-01$ & $6.8 \mathrm{E}-02$ \\
\hline
\end{tabular}


Table 3.1e. (continued)

\begin{tabular}{|c|c|c|c|c|c|}
\hline \multirow{2}{*}{ Chemical } & \multirow{2}{*}{ CASRN } & \multicolumn{4}{|c|}{ Ingestion of fish (mg/kg)" } \\
\hline & & $10^{-4}$ & $10^{-6}$ & $\mathbf{H Q}=\mathbf{1}$ & $\mathrm{HQ}=0.1$ \\
\hline Tetraethyl Lead & $000078-00-2$ & & & & \\
\hline Tetrafluoroethane, $1,1,1,2-$ & $000811-97-2$ & & & & \\
\hline Tetramethyl Lead & $000075-74-1$ & & & & \\
\hline Tetrapropyl Lead & 003440-75-3 & & & & \\
\hline Thallic Oxide $^{(5)}$ & 001314-32-5 & & & $9.5 \mathrm{E}-02$ & $9.5 \mathrm{E}-03$ \\
\hline Thallium (I) Nitrate & $010102-45-1$ & & & $1.2 \mathrm{E}-01$ & $1.2 \mathrm{E}-02$ \\
\hline Thallium (Soluble Salts) & $007440-28-0$ & & & & \\
\hline Thallium Acetate & $000563-68-8$ & & & $1.2 \mathrm{E}-01$ & $1.2 \mathrm{E}-02$ \\
\hline Thallium Carbonate & 006533-73-9 & & & $1.1 \mathrm{E}-01$ & 1.1E-02 \\
\hline Thallium Chloride & $007791-12-0$ & & & $1.1 \mathrm{E}-01$ & $1.1 \mathrm{E}-02$ \\
\hline Thallium Selenite $^{(s)}$ & $012039-52-0$ & & & $1.2 \mathrm{E}-01$ & $1.2 \mathrm{E}-02$ \\
\hline Thallium Sulfate & 007446-18-6 & & & $1.1 \mathrm{E}-01$ & $1.1 \mathrm{E}-02$ \\
\hline Thiobencarb & $028249-77-6$ & & & $1.4 \mathrm{E}+01$ & $1.4 \mathrm{E}+00$ \\
\hline Thiofanox & 039196-18-4 & & & 4.1E-01 & $4.1 \mathrm{E}-02$ \\
\hline Thiophanate, Methyl & 023564-05-8 & & & $1.1 \mathrm{E}+02$ & $1.1 \mathrm{E}+01$ \\
\hline Thiram & 000137-26-8 & & & $6.8 \mathrm{E}+00$ & $6.8 \mathrm{E}-01$ \\
\hline Thorium & 007440-29-1 & & & & \\
\hline Tin & $007440-31-5$ & & & $8.1 \mathrm{E}+02$ & $8.1 \mathrm{E}+01$ \\
\hline
\end{tabular}


Table 3.1e. (continued)

\begin{tabular}{|c|c|c|c|c|c|}
\hline \multirow{2}{*}{ Chemical } & \multirow{2}{*}{ CASRN } & \multicolumn{4}{|c|}{ Ingestion of fish (mg/kg) } \\
\hline & & $10^{-4}$ & $10^{-6}$ & $H Q=1$ & $H Q=0.1$ \\
\hline Titanium & $007440-32-6$ & & & & . \\
\hline Toluene $^{(6)}$ & 000108-88-3 & & & $2.7 \mathrm{E}+02$ & $2.7 \mathrm{E}+01$ \\
\hline $\begin{array}{l}\text { Toluene diisocyanate mixture } \\
\text { (TDI) }\end{array}$ & $026471-62-5$ & & & & \\
\hline Toluene-2,4-diamine & $000095-80-7$ & $9.9 \mathrm{E}-02$ & 9.9E-04 & & \\
\hline Toluene-2,5-diamine & $000095-70-5$ & & & $8.1 \mathrm{E}+02$ & 8.1E+01 \\
\hline Toluene-2,6-diamine & $000823-40-5$ & & & $2.7 \mathrm{E}+02$ & 2.7E+01 \\
\hline Toluidine, 0 - (Methylaniline, 2-) & $000095-53-4$ & $1.3 \mathrm{E}+00$ & $1.3 \mathrm{E}-02$ & & \\
\hline Toluidine, $\mathrm{p}$ - & $000106-49-0$ & $1.7 \mathrm{E}+00$ & $1.7 \mathrm{E}-02$ & & \\
\hline Toxaphene & $008001-35-2$ & $2.9 \mathrm{E}-01$ & $2.9 \mathrm{E}-03$ & & \\
\hline Tralomethrin & $066841-25-6$ & & & $1.0 \mathrm{E}+01$ & $1.0 \mathrm{E}+00$ \\
\hline Triallate & $002303-17-5$ & & & $1.8 \mathrm{E}+01$ & $1.8 \mathrm{E}+00$ \\
\hline Triasulfuron & 082097-50-5 & & & $1.4 \mathrm{E}+01$ & $1.4 \mathrm{E}+00$ \\
\hline Tribromobenzene, 1,2,4- & 000615-54-3 & & & $6.8 \mathrm{E}+00$ & $6.8 \mathrm{E}-01$ \\
\hline Tribromochloromethane & 000594-15-0 & & & & \\
\hline Tribromodiphenyl Ether & 049690-94-0 & & & & \\
\hline Tributyltin Oxide & 000056-35-9 & & & $4.1 \mathrm{E}-02$ & 4.1E-03 \\
\hline $\begin{array}{l}\text { Trichloro-1,2,2-trifluoroethane, } \\
\text { 1,1,2- }\end{array}$ & $000076-13-1$ & & & $4.1 \mathrm{E}+04$ & $4.1 \mathrm{E}+03$ \\
\hline
\end{tabular}


Table 3.1e. (continued)

\begin{tabular}{|c|c|c|c|c|c|}
\hline \multirow{2}{*}{ Chemical } & \multirow{2}{*}{ CASRN } & \multicolumn{4}{|c|}{ Ingestion of fish (mg/kg)" } \\
\hline & & $10^{-4}$ & $10^{-6}$ & $H Q=1$ & $H Q=0.1$ \\
\hline $\begin{array}{l}\text { Trichloro-2'- } \\
\text { hydroxydiphenylether }\end{array}$ & $003380-34-5$ & & & & \\
\hline \multicolumn{6}{|l|}{ Trichloroacetic Acid } \\
\hline Trichloroaniline $\mathrm{HCl}, 2,4,6-$ & 033663-50-2 & $1.1 \mathrm{E}+01$ & $1.1 \mathrm{E}-01$ & & \\
\hline Trichloroaniline, 2,4,6- & 000634-93-5 & $9.3 \mathrm{E}+00$ & 9.3E-02 & & \\
\hline Trichlorobenzene, 1,2,4- & $000120-82-1$ & & & $1.4 \mathrm{E}+01$ & $1.4 \mathrm{E}+00$ \\
\hline Trichloroethane, $1,1,1-^{(3)}$ & $000071-55-6$ & & & $1.2 \mathrm{E}+02$ & $1.2 \mathrm{E}+01$ \\
\hline Trichloroethane, 1,1,2- & $000079-00-5$ & $5.5 \mathrm{E}+00$ & $5.5 \mathrm{E}-02$ & $5.4 \mathrm{E}+00$ & $5.4 \mathrm{E}-01$ \\
\hline Trichloroethylene $^{(3)}$ & $000079-01-6$ & $2.9 \mathrm{E}+01$ & $2.9 \mathrm{E}-01$ & & \\
\hline Trichlorofluoromethane & $000075-69-4$ & & & $4.1 E+02$ & 4.1E+01 \\
\hline Trichlorophenol, 2,4,5- & $000095-95-4$ & & & $1.4 \mathrm{E}+02$ & $1.4 \mathrm{E}+01$ \\
\hline Trichlorophenol, 2,4,6- & $000088-06-2$ & $2.9 \mathrm{E}+01$ & $2.9 \mathrm{E}-01$ & & \\
\hline $\begin{array}{l}\text { Trichlorophenoxy) Propionic } \\
\text { Acid, 2(2,4,5- }\end{array}$ & $000093-72-1$ & & & $1.1 E+01$ & $1.1 \mathrm{E}+00$ \\
\hline $\begin{array}{l}\text { Trichlorophenoxyacetic Acid, } \\
\text { 2,4,5- }\end{array}$ & $000093-76-5$ & & & $1.4 \mathrm{E}+01$ & $1.4 \mathrm{E}+00$ \\
\hline Trichloropropane, 1,1,2- & $000598-77-6$ & & & $6.8 \mathrm{E}+00$ & $6.8 \mathrm{E}-01$ \\
\hline Trichloropropane, 1,2,3- & 000096-18-4 & $4.5 \mathrm{E}-02$ & 4.5E-04 & 8.1E+00 & 8.1E-01 \\
\hline Trichloropropene, 1,2,3- & $000096-19-5$ & & & $6.8 \mathrm{E}+00$ & $6.8 \mathrm{E}-01$ \\
\hline
\end{tabular}


Table 3.1e. (continued)

\begin{tabular}{|l|l|l|l|l|l|}
\hline \multirow{2}{*}{ Chemical } & \multirow{2}{*}{ CASRN } & \multicolumn{4}{|c|}{ Ingestion of fish (mg/kg) } \\
\cline { 4 - 6 } & & $10^{-4}$ & $10^{-6}$ & HQ=1 & HQ=0.1 \\
\hline Trichlorotoluene, 2,3,6- & $002077-46-5$ & & & & \\
\hline Trichlorotoluene, alpha 2,6- & $002014-83-7$ & & & & \\
\hline Tridiphane & $058138-08-2$ & & & $4.1 \mathrm{E}+00$ & $4.1 \mathrm{E}-01$ \\
\hline Triethyl Lead & $005224-23-7$ & & & \\
\hline Triethylamine & $000121-44-8$ & & & & \\
\hline Trifluralin & $001582-09-8$ & $4.1 \mathrm{E}+01$ & $4.1 \mathrm{E}-01$ & $1.0 \mathrm{E}+01$ & $1.0 \mathrm{E}+00$ \\
\hline Trimethyl Lead & $007442-13-9$ & & & & \\
\hline Trimethyl Phosphate & $000512-56-1$ & $8.5 \mathrm{E}+00$ & $8.5 \mathrm{E}-02$ & & \\
\hline Trimethylethyl Lead & $001762-26-1$ & & & & \\
\hline Trinitrobenzene, 1,3,5- & $000099-35-4$ & & & $6.8 \mathrm{E}-02$ & $6.8 \mathrm{E}-03$ \\
\hline Trinitrophenylmethylnitramine & $000479-45-8$ & & & $1.4 \mathrm{E}+01$ & $1.4 \mathrm{E}+00$ \\
\hline Trinitrotoluene, 2,4,6- & $000118-96-7$ & $1.1 \mathrm{E}+01$ & $1.1 \mathrm{E}-01$ & $6.8 \mathrm{E}-01$ & $6.8 \mathrm{E}-02$ \\
\hline Tripropyl Lead & $006618-03-7$ & & & & \\
\hline Uranium (Soluble Salts) & NA & & & $4.1 \mathrm{E}+00$ & $4.1 \mathrm{E}-01$ \\
\hline Vanadium Pentoxide & $001314-62-1$ & & & $1.2 \mathrm{E}+01$ & $1.2 \mathrm{E}+00$ \\
\hline Vanadium Sulfate & $036907-42-3$ & & & $2.7 \mathrm{E}+01$ & $2.7 \mathrm{E}+00$ \\
\hline Vanadium, Metallic & $007440-62-2$ & & & $9.5 \mathrm{E}+00$ & $9.5 \mathrm{E}-01$ \\
\hline Vanadyl Sulfate & $027774-13-6$ & & & $2.7 \mathrm{E}+01$ & $2.7 \mathrm{E}+00$ \\
\hline
\end{tabular}


Table 3.1e. (continued)

\begin{tabular}{|c|c|c|c|c|c|}
\hline \multirow{2}{*}{ Chemical } & \multirow{2}{*}{ CASRN } & \multicolumn{4}{|c|}{ Ingestion of fish $(\mathrm{mg} / \mathrm{kg})^{*}$} \\
\hline & & $10^{-4}$ & $10^{-6}$ & $\mathbf{H Q}=\mathbf{1}$ & $\mathrm{HQ}=0.1$ \\
\hline Vernolate & $001929-77-7$ & & & $1.4 \mathrm{E}+00$ & $1.4 \mathrm{E}-01$ \\
\hline Vinclozolin & $050471-44-8$ & & & $3.4 \mathrm{E}+01$ & $3.4 \mathrm{E}+00$ \\
\hline Vinyl Acetate & $000108-05-4$ & & & $1.4 \mathrm{E}+03$ & $1.4 \mathrm{E}+02$ \\
\hline Vinyl Bromide & $000593-60-2$ & & & & \\
\hline Vinyl Chloride ${ }^{(6)}$ & $000075-01-4$ & $1.7 \mathrm{E}-01$ & $1.7 \mathrm{E}-03$ & & \\
\hline Warfarin & $000081-81-2$ & & & 4.1E-01 & 4.1E-02 \\
\hline White Phosphorus & $007723-14-0$ & & & $2.7 \mathrm{E}-02$ & $2.7 \mathrm{E}-03$ \\
\hline Xylene, Mixture $^{(6)}$ & $001330-20-7$ & & & $2.7 \mathrm{E}+03$ & $2.7 \mathrm{E}+02$ \\
\hline Xylene, $P_{-}^{(6)}$ & $000106-42-3$ & & & & \\
\hline Xylene, $m_{-(6)}^{(6)}$ & 000108-38-3 & & & $2.7 \mathrm{E}+03$ & $2.7 \mathrm{E}+02$ \\
\hline Xylene, $0^{((s)}$ & $000095-47-6$ & & & $2.7 \mathrm{E}+03$ & $2.7 \mathrm{E}+02$ \\
\hline Zinc (Metallic) & $007440-66-6$ & & & $4.1 \mathrm{E}+02$ & 4.1E+01 \\
\hline Zinc Cyanide & $000557-21-1$ & & & $6.8 \mathrm{E}+01$ & $6.8 \mathrm{E}+00$ \\
\hline Zinc Phosphide & $001314-84-7$ & & & 4.1E-01 & 4.1E-02 \\
\hline Zineb & $012122-67-7$ & & & $6.8 \mathrm{E}+01$ & $6.8 \mathrm{E}+00$ \\
\hline Zirconium & 007440-67-7 & & & & \\
\hline
\end{tabular}


Table 3.1e. (continued)

Notes:

* By definition, $1 \mathrm{~kg}=1.0 \mathrm{E}+06 \mathrm{mg}$. Therefore, a limit of $1.0 \mathrm{E}+06 \mathrm{mg} / \mathrm{kg}$ was used for any PRG that was calculated to be above this limit.

(1) The same oral slope factor as used for polychlorinated biphenyls.

(2) The PRG was calculated considering Supplemental Guidance from RAGS: Region 4 Bulletins, Human Health Risk Assessment (Interim Guidance) from EPA (received February 11, 1992). The slope factors for chemicals so marked indicate that a toxicity equivalency factor (TEF) method was used for carcinogenic PAHs based on each compound's relative potency to the potency of benzo[a]pyrene. The following TEFs were used to convert each PAH slope factor to an equivalent slope factor of benzo[a]pyrene [that has a new cancer slope factor of $7.3(\mathrm{mg} / \mathrm{kg} / \text { day) })^{-1}$ identified by EPA's CRAVE workgroup]: (1) benzo[a]pyrene, TEF $=1.0$; (2) benzo[a]anthracene, TEF $=0.1$; (3) benzo[b]fluoranthene, TEF $=0.1$; (4) benzo[k]fluoranthene, TEF $=0.01 ;(5)$ chrysene, TEF $=0.001 ;(6)$ dibenz $[\mathrm{a}, \mathrm{h}]$ anthracene, $\mathrm{TEF}=1.0$; and (7) indeno $[1,2,3-\mathrm{c}, \mathrm{d}]$ pyrene, $\mathrm{TEF}=0.1$.

(3) The toxicity values for trichloroethylene have been withdrawn from IRIS and HEAST pending further investigation. However, the most recent toxicity values have been used to calculate the PRG.

(4) The PRG was calculated considering Supplemental Guidance from RAGS: Region 4 Bulletins, Human Health Risk Assessment (Interim Guidance) from EPA (November 1995). The slope factors for chemicals so marked indicate that a TEF methodology was used for chlorinated dioxin and furan congeners based on each compound's relative potency to the potency of 2,3,7,8-tetrachlorodibenzodioxin (TCDD). The following TEFs were used to convert each slope factor to an equivalent slope factor of 2,3,7,8-TCDD: (1) 2,3,7,8-TCDD, TEF $=1.0 ;(2) 2,3,7,8-\mathrm{PeCDD}, \mathrm{TEF}=0.5 ;$; (3) $2,3,7,8-$ HxCDD, TEF $=0.1$; (4) 2,3,7,8-HpCDD, TEF $=0.01$; (5) OCDD, TEF $=0.001 ;(6) 2,3,7,8-\mathrm{TCDF}, \mathrm{TEF}=0.1 ;(7) 1,2,3,7,8-\mathrm{PeCDF}, \mathrm{TEF}=0.5 ;(8)$ $2,3,4,7,8-\mathrm{PeCDF}, \mathrm{TEF}=0.05 ;(9) 2,3,7,8-\mathrm{HxCDF}, \mathrm{TEF}=0.1 ;(10) 2,3,7,8-\mathrm{HpCDF}, \mathrm{TEF}=0.01$; and (11) OCDF, $\mathrm{TEF}=0.001$

(5) Toxicity values for these chemicals have been withdrawn from IRIS and HEAST pending further investigation. However, the most recent toxicity values have been used to calculate the PRG.

(6) Toxicity values for these chemicals were obtained from the Superfund Health Risk Technical Support Center. 
Table 3.1f. Risk-based Preliminary Remediation Goals for radionuclides in fish (residential scenario)

\begin{tabular}{|c|c|c|c|}
\hline \multirow{2}{*}{ Chemical $^{\dagger}$} & \multirow{2}{*}{ CASRN } & \multicolumn{2}{|c|}{ Ingestion of fish (pCi/g) } \\
\hline & & $10^{-4}$ & $10^{-6}$ \\
\hline Ac-225 & $014265-85-1$ & $1.2 E+00$ & $1.2 \mathrm{E}-02$ \\
\hline Ac-227 & $014952-40-0$ & $5.0 \mathrm{E}-01$ & $5.0 \mathrm{E}-03$ \\
\hline Ac- $227+D$ & $014952-40-0$ & $2.8 \mathrm{E}-01$ & $2.8 \mathrm{E}-03$ \\
\hline Ac-228 & $014331-83-0$ & $1.1 E+02$ & $1.1 \mathrm{E}+00$ \\
\hline Ag-105 & $014928-14-4$ & $1.1 \mathrm{E}+02$ & $1.1 \mathrm{E}+00$ \\
\hline Ag-108 & $014391-65-2$ & $2.5 E+04$ & $2.5 \mathrm{E}+02$ \\
\hline Ag-108m & $014391-65-2$ & $2.9 \mathrm{E}+01$ & $2.9 \mathrm{E}-01$ \\
\hline $\mathrm{Ag}-108 \mathrm{~m}+\mathrm{D}$ & $014391-65-2$ & $2.9 E+01$ & $2.9 \mathrm{E}-01$ \\
\hline Ag-109m & $014378-38-2$ & $6.5 E+05$ & $6.5 \mathrm{E}+03$ \\
\hline $\mathrm{Ag}-110$ & $014391-76-5$ & $7.2 E+04$ & $7.2 E+02$ \\
\hline $\mathrm{Ag}-110 \mathrm{~m}$ & $015760-04-0$ & $2.1 \mathrm{E}+01$ & $2.1 \mathrm{E}-01$ \\
\hline $\mathrm{Ag}-111$ & $157690-04-0$ & $2.6 \mathrm{E}+01$ & $2.6 \mathrm{E}-01$ \\
\hline Am-241 & $014596-10-2$ & $5.4 \mathrm{E}-01$ & $5.4 \mathrm{E}-03$ \\
\hline Am-242 & $013981-54-9$ & $1.2 \mathrm{E}+02$ & $1.2 \mathrm{E}+00$ \\
\hline$A m-242 m$ & $013981-54-9$ & $6.0 \mathrm{E}-01$ & $6.0 \mathrm{E}-03$ \\
\hline Am-243 & 014993-75-0 & $5.4 \mathrm{E}-01$ & $5.4 \mathrm{E}-03$ \\
\hline$A m-243+D$ & 014993-75-0 & $5.3 \mathrm{E}-01$ & 5.3E-03 \\
\hline
\end{tabular}


Table 3.1f. (continued)

\begin{tabular}{|l|l|c|c|}
\hline \multirow{2}{*}{ Chemical $^{\dagger}$} & \multirow{2}{*}{ CASRN } & \multicolumn{2}{|c|}{ Ingestion of fish (pCi/g) } \\
\cline { 3 - 4 } & & $10^{-4}$ & $10^{-6}$ \\
\hline Ar-41 & $014163-25-8$ & & \\
\hline At-217 & $017239-90-6$ & $2.0 \mathrm{E}+07$ & $2.0 \mathrm{E}+05$ \\
\hline Au-196 & $014914-16-0$ & $1.4 \mathrm{E}+02$ & $1.4 \mathrm{E}+00$ \\
\hline Au-198 & $010043-49-0$ & $3.3 \mathrm{E}+01$ & $3.3 \mathrm{E}-01$ \\
\hline Ba-131 & $014914-75-1$ & $1.0 \mathrm{E}+02$ & $1.0 \mathrm{E}+00$ \\
\hline Ba-133 & $013981-41-4$ & $6.5 \mathrm{E}+01$ & $6.5 \mathrm{E}-01$ \\
\hline Ba-133m & $013981-41-4$ & $6.4 \mathrm{E}+01$ & $6.4 \mathrm{E}-01$ \\
\hline Ba-137m & $013981-97-0$ & $7.3 \mathrm{E}+04$ & $7.3 \mathrm{E}+02$ \\
\hline Ba-139 & $014378-25-7$ & $5.8 \mathrm{E}+02$ & $5.8 \mathrm{E}+00$ \\
\hline Ba-140 & $014798-08-4$ & $1.5 \mathrm{E}+01$ & $1.5 \mathrm{E}-01$ \\
\hline Be-7 & $013966-02-4$ & $2.0 \mathrm{E}+03$ & $2.0 \mathrm{E}+01$ \\
\hline Bi-206 & $015776-19-9$ & $2.5 \mathrm{E}+01$ & $2.5 \mathrm{E}-01$ \\
\hline Bi-207 & $013982-38-2$ & $3.5 \mathrm{E}+01$ & $3.5 \mathrm{E}-01$ \\
\hline Bi-210 & $014331-79-4$ & $2.4 \mathrm{E}+01$ & $2.4 \mathrm{E}-01$ \\
\hline Bi-211 & $015229-37-5$ & $9.7 \mathrm{E}+03$ & $9.7 \mathrm{E}+01$ \\
\hline Bi-212 & $014913-49-6$ & $2.8 \mathrm{E}+02$ & $2.8 \mathrm{E}+00$ \\
\hline Bi-213 & $015776-20-2$ & $4.0 \mathrm{E}+02$ & $4.0 \mathrm{E}+00$ \\
\hline Bi-214 & $014733-03-0$ & $9.0 \mathrm{E}+02$ & $9.0 \mathrm{E}+00$ \\
\hline & & & \\
\hline
\end{tabular}


Table 3.1f. (continued)

\begin{tabular}{|l|l|c|c|}
\hline \multirow{2}{*}{ Chemical } & \multirow{2}{*}{ CASRN } & \multicolumn{2}{|c|}{ Ingestion of fish (pCi/g) } \\
\cline { 2 - 4 } & & $10^{-4}$ & $10^{-6}$ \\
\hline Br-82 & $014686-69-2$ & $1.2 \mathrm{E}+02$ & $1.2 \mathrm{E}+00$ \\
\hline C-11 & $014333-33-6$ & $3.9 \mathrm{E}+03$ & $3.9 \mathrm{E}+01$ \\
\hline C-14 & $014762-75-5$ & $1.7 \mathrm{E}+02$ & $1.7 \mathrm{E}+00$ \\
\hline C-15 & $015929-23-4$ & $2.7 \mathrm{E}+05$ & $2.7 \mathrm{E}+03$ \\
\hline Ca-45 & $013966-05-7$ & $8.7 \mathrm{E}+01$ & $8.7 \mathrm{E}-01$ \\
\hline Ca-47 & $001439-99-2$ & $2.6 \mathrm{E}+01$ & $2.6 \mathrm{E}-01$ \\
\hline Cd-109 & $014109-32-1$ & $2.2 \mathrm{E}+01$ & $2.2 \mathrm{E}-01$ \\
\hline Cd-115 & $014336-68-6$ & $2.4 \mathrm{E}+01$ & $2.4 \mathrm{E}-01$ \\
\hline Cd-115m & $014336-68-6$ & $1.2 \mathrm{E}+01$ & $1.2 \mathrm{E}-01$ \\
\hline Ce-141 & $013967-74-3$ & $4.5 \mathrm{E}+01$ & $4.5 \mathrm{E}-01$ \\
\hline Ce-143 & $014119-19-8$ & $3.0 \mathrm{E}+01$ & $3.0 \mathrm{E}-01$ \\
\hline Ce-144 & $014762-78-8$ & $6.0 \mathrm{E}+00$ & $6.0 \mathrm{E}-02$ \\
\hline Ce-144+D & $014762-78-8$ & $5.9 \mathrm{E}+00$ & $5.9 \mathrm{E}-02$ \\
\hline Cl-36 & $013981-43-6$ & $7.9 \mathrm{E}+01$ & $7.9 \mathrm{E}-01$ \\
\hline Cl-38 & $014158-34-0$ & $8.5 \mathrm{E}+02$ & $8.5 \mathrm{E}+00$ \\
\hline Cm-242 & $015510-73-3$ & $4.6 \mathrm{E}+00$ & $4.6 \mathrm{E}-02$ \\
\hline Cm-243 & $015757-87-6$ & $7.0 \mathrm{E}-01$ & $7.0 \mathrm{E}-03$ \\
\hline Cm-243+D & $015757-87-6$ & $7.0 \mathrm{E}-01$ & $7.0 \mathrm{E}-03$ \\
\hline
\end{tabular}


Table 3.1f. (continued)

\begin{tabular}{|l|l|c|c|}
\hline \multirow{2}{*}{ Chemical $^{\dagger}$} & \multirow{2}{*}{ CASRN } & \multicolumn{2}{|c|}{ Ingestion of fish (pCi/g) } \\
\cline { 2 - 4 } & & $10^{-4}$ & $10^{-6}$ \\
\hline Cm-244 & $013981-15-2$ & $8.4 \mathrm{E}-01$ & $8.4 \mathrm{E}-03$ \\
\hline Cm-245 & $015621-76-8$ & $5.3 \mathrm{E}-01$ & $5.3 \mathrm{E}-03$ \\
\hline Cm-246 & $015757-90-1$ & $5.3 \mathrm{E}-01$ & $5.3 \mathrm{E}-03$ \\
\hline Cm-247 & $015758-32-4$ & $5.7 \mathrm{E}-01$ & $5.7 \mathrm{E}-03$ \\
\hline Cm-248 & $015758-33-5$ & $1.3 \mathrm{E}-01$ & $1.3 \mathrm{E}-03$ \\
\hline Co-57 & $013981-50-5$ & $1.8 \mathrm{E}+02$ & $1.8 \mathrm{E}+00$ \\
\hline Co-58 & $013981-38-9$ & $6.3 \mathrm{E}+01$ & $6.3 \mathrm{E}-01$ \\
\hline Co-58m & $013981-38-9$ & $1.9 \mathrm{E}+03$ & $1.9 \mathrm{E}+01$ \\
\hline Co-60 & $010198-40-0$ & $9.3 \mathrm{E}+00$ & $9.3 \mathrm{E}-02$ \\
\hline Cr-51 & $014392-02-0$ & $1.3 \mathrm{E}+03$ & $1.3 \mathrm{E}+01$ \\
\hline Cs-131 & $014914-76-2$ & $9.8 \mathrm{E}+02$ & $9.8 \mathrm{E}+00$ \\
\hline Cs-134 & $013967-70-9$ & $3.7 \mathrm{E}+00$ & $3.7 \mathrm{E}-02$ \\
\hline Cs-134m & $013967-70-9$ & $3.9 \mathrm{E}+03$ & $3.9 \mathrm{E}+01$ \\
\hline Cs-135 & $015726-30-4$ & $3.9 \mathrm{E}+01$ & $3.9 \mathrm{E}-01$ \\
\hline Cs-136 & $014234-29-8$ & $2.3 \mathrm{E}+01$ & $2.3 \mathrm{E}-01$ \\
\hline Cs-137 & $010045-97-3$ & $5.6 \mathrm{E}+00$ & $5.6 \mathrm{E}-02$ \\
\hline Cs-137+D & $010045-97-3$ & $5.6 \mathrm{E}+00$ & $5.6 \mathrm{E}-02$ \\
\hline Cs-138 & $015758-29-9$ & $1.0 \mathrm{E}+03$ & $1.0 \mathrm{E}+01$ \\
\hline
\end{tabular}


Table 3.1f. (continued)

\begin{tabular}{|l|l|c|c|}
\hline \multirow{2}{*}{ Chemical $^{\dagger}$} & \multirow{2}{*}{ CASRN } & \multicolumn{2}{|c|}{ Ingestion of fish (pCi/g) } \\
\cline { 3 - 4 } & & $10^{-4}$ & $10^{-6}$ \\
\hline Cu-64 & $013981-25-4$ & $3.4 \mathrm{E}+02$ & $3.4 \mathrm{E}+00$ \\
\hline Dy-165 & $013967-64-1$ & $5.4 \mathrm{E}+02$ & $5.4 \mathrm{E}+00$ \\
\hline Dy-166 & $015840-01-4$ & $1.9 \mathrm{E}+01$ & $1.9 \mathrm{E}-01$ \\
\hline Er-169 & $015840-13-8$ & $8.3 \mathrm{E}+01$ & $8.3 \mathrm{E}-01$ \\
\hline Er-171 & $014391-45-8$ & $1.1 \mathrm{E}+02$ & $1.1 \mathrm{E}+00$ \\
\hline Eu-152 & $014683-23-9$ & $3.1 \mathrm{E}+01$ & $3.1 \mathrm{E}-01$ \\
\hline Eu-154 & $015585-10-1$ & $1.9 \mathrm{E}+01$ & $1.9 \mathrm{E}-01$ \\
\hline Eu-155 & $014391-16-3$ & $1.1 \mathrm{E}+02$ & $1.1 \mathrm{E}+00$ \\
\hline Eu-156 & $014280-35-4$ & $1.6 \mathrm{E}+01$ & $1.6 \mathrm{E}-01$ \\
\hline F-18 & $013981-56-1$ & $1.6 \mathrm{E}+03$ & $1.6 \mathrm{E}+01$ \\
\hline Fe-55 & $014681-59-5$ & $5.0 \mathrm{E}+02$ & $5.0 \mathrm{E}+00$ \\
\hline Fe-59 & $014596-12-4$ & $3.0 \mathrm{E}+01$ & $3.0 \mathrm{E}-01$ \\
\hline Fr-221 & $015756-41-9$ & $1.2 \mathrm{E}+03$ & $1.2 \mathrm{E}+01$ \\
\hline Fr-223 & $015756-98-6$ & $4.0 \mathrm{E}+02$ & $4.0 \mathrm{E}+00$ \\
\hline Ga-67 & $014119-09-6$ & $2.1 \mathrm{E}+02$ & $2.1 \mathrm{E}+00$ \\
\hline Ga-72 & $013982-22-4$ & $3.7 \mathrm{E}+01$ & $3.7 \mathrm{E}-01$ \\
\hline Gd-153 & $014276-65-4$ & $1.3 \mathrm{E}+02$ & $1.3 \mathrm{E}+00$ \\
\hline Gd-159 & $014041-42-0$ & $6.8 \mathrm{E}+01$ & $6.8 \mathrm{E}-01$ \\
\hline
\end{tabular}


Table 3.1f. (continued)

\begin{tabular}{|l|l|c|c|}
\hline \multirow{2}{*}{ Chemical } & \multirow{2}{*}{ CASRN } & \multicolumn{2}{|c|}{ Ingestion of fish (pCi/g) } \\
\cline { 2 - 4 } & & $10^{-4}$ & $10^{-6}$ \\
\hline Ge-71 & $014374-81-3$ & $1.5 \mathrm{E}+04$ & $1.5 \mathrm{E}+02$ \\
\hline H-3 & $010028-17-8$ & $2.5 \mathrm{E}+03$ & $2.5 \mathrm{E}+01$ \\
\hline Hg-197 & $013981-51-6$ & $1.5 \mathrm{E}+02$ & $1.5 \mathrm{E}+00$ \\
\hline Hg-203 & $013982-78-0$ & $6.7 \mathrm{E}+01$ & $6.7 \mathrm{E}-01$ \\
\hline Ho-166 & $013967-65-2$ & $2.3 \mathrm{E}+01$ & $2.3 \mathrm{E}-01$ \\
\hline I-122 & $018287-75-7$ & $8.2 \mathrm{E}+03$ & $8.2 \mathrm{E}+01$ \\
\hline I-123 & $015715-08-9$ & $3.3 \mathrm{E}+02$ & $3.3 \mathrm{E}+00$ \\
\hline I-125 & $014158-31-7$ & $6.8 \mathrm{E}+00$ & $6.8 \mathrm{E}-02$ \\
\hline I-126 & $014158-32-8$ & $3.7 \mathrm{E}+00$ & $3.7 \mathrm{E}-02$ \\
\hline I-129 & $015046-84-1$ & $9.6 \mathrm{E}-01$ & $9.6 \mathrm{E}-03$ \\
\hline I-130 & $014914-02-4$ & $3.6 \mathrm{E}+01$ & $3.6 \mathrm{E}-01$ \\
\hline I-131 & $010043-66-0$ & $4.9 \mathrm{E}+00$ & $4.9 \mathrm{E}-02$ \\
\hline I-132 & $014683-16-0$ & $2.7 \mathrm{E}+02$ & $2.7 \mathrm{E}+00$ \\
\hline I-133 & $014834-67-4$ & $1.7 \mathrm{E}+01$ & $1.7 \mathrm{E}-01$ \\
\hline I-134 & $014914-27-3$ & $7.6 \mathrm{E}+02$ & $7.6 \mathrm{E}+00$ \\
\hline I-135 & $014834-68-5$ & $7.8 \mathrm{E}+01$ & $7.8 \mathrm{E}-01$ \\
\hline In-113m & $014885-78-0$ & $2.1 \mathrm{E}+03$ & $2.1 \mathrm{E}+01$ \\
\hline In-114 & $013981-55-0$ & $3.9 \mathrm{E}+04$ & $3.9 \mathrm{E}+02$ \\
\hline & & & \\
\hline
\end{tabular}


Table 3.1f. (continued)

\begin{tabular}{|c|c|c|c|}
\hline \multirow{2}{*}{ Chemical $^{\dagger}$} & \multirow{2}{*}{ CASRN } & \multicolumn{2}{|c|}{ Ingestion of fish (pCi/g) } \\
\hline & & $10^{-4}$ & $10^{-6}$ \\
\hline In-114m & 013981-55-0 & $8.6 \mathrm{E}+00$ & $8.6 \mathrm{E}-02$ \\
\hline $\operatorname{In}-115$ & $014191-71-0$ & $5.1 \mathrm{E}+00$ & $5.1 \mathrm{E}-02$ \\
\hline In-115m & 014191-71-0 & $5.2 \mathrm{E}+02$ & $5.2 \mathrm{E}+00$ \\
\hline Ir-190 & 014981-91-0 & $3.6 \mathrm{E}+01$ & $3.6 \mathrm{E}-01$ \\
\hline Ir-194 & 014158-35-1 & $2.5 \mathrm{E}+01$ & $2.5 \mathrm{E}-01$ \\
\hline $\mathrm{K}-40$ & $013966-00-2$ & $1.4 \mathrm{E}+01$ & $1.4 \mathrm{E}-01$ \\
\hline $\mathrm{K}-42$ & 014378-21-3 & $1.4 \mathrm{E}+02$ & $1.4 \mathrm{E}+00$ \\
\hline $\mathrm{Kr}-83 \mathrm{~m}$ & 013965-98-5 & & \\
\hline $\mathrm{Kr}-85$ & 013983-27-2 & & \\
\hline $\mathrm{Kr}-85 \mathrm{~m}$ & 013983-27-2 & & \\
\hline $\mathrm{Kr}-87$ & $014809-68-8$ & & \\
\hline $\mathrm{Kr}-88$ & 014995-61-0 & & \\
\hline $\mathrm{Kr}-89$ & $016316-03-3$ & & \\
\hline $\mathrm{Kr}-90$ & 015741-13-6 & & \\
\hline La-140 & 013981-28-7 & $1.9 \mathrm{E}+01$ & $1.9 \mathrm{E}-01$ \\
\hline Lu-177 & 014265-75-9 & $6.0 \mathrm{E}+01$ & $6.0 \mathrm{E}-01$ \\
\hline $\mathrm{Mn}-52$ & 014092-99-0 & $2.9 \mathrm{E}+01$ & $2.9 \mathrm{E}-01$ \\
\hline $\mathrm{Mn}-54$ & 013966-31-9 & $9.0 \mathrm{E}+01$ & $9.0 \mathrm{E}-01$ \\
\hline
\end{tabular}


Table 3.1f. (continued)

\begin{tabular}{|l|l|c|c|}
\hline \multirow{2}{*}{ Chemical } & \multirow{2}{*}{ CASRN } & \multicolumn{2}{|c|}{ Ingestion of fish (pCi/g) } \\
\cline { 2 - 4 } & & $10^{-4}$ & $10^{-6}$ \\
\hline Mn-56 & $014681-52-8$ & $2.1 \mathrm{E}+02$ & $2.1 \mathrm{E}+00$ \\
\hline Mo-99 & $014119-15-4$ & $7.8 \mathrm{E}+01$ & $7.8 \mathrm{E}-01$ \\
\hline Na-22 & $013966-32-0$ & $2.2 \mathrm{E}+01$ & $2.2 \mathrm{E}-01$ \\
\hline Na-24 & $013982-04-2$ & $1.3 \mathrm{E}+02$ & $1.3 \mathrm{E}+00$ \\
\hline Nb-93m & $007440-03-1$ & $2.7 \mathrm{E}+02$ & $2.7 \mathrm{E}+00$ \\
\hline Nb-94 & $014681-63-1$ & $2.6 \mathrm{E}+01$ & $2.6 \mathrm{E}-01$ \\
\hline Nb-95 & $013967-76-5$ & $7.8 \mathrm{E}+01$ & $7.8 \mathrm{E}-01$ \\
\hline Nb-95m & $013967-76-5$ & $5.8 \mathrm{E}+01$ & $5.8 \mathrm{E}-01$ \\
\hline Nb-97 & $018496-04-3$ & $1.0 \mathrm{E}+03$ & $1.0 \mathrm{E}+01$ \\
\hline Nb-97m & $018496-04-3$ & $5.4 \mathrm{E}+04$ & $5.4 \mathrm{E}+02$ \\
\hline Nd-147 & $014269-74-0$ & $3.0 \mathrm{E}+01$ & $3.0 \mathrm{E}-01$ \\
\hline Nd-149 & $015749-81-2$ & $3.9 \mathrm{E}+02$ & $3.9 \mathrm{E}+00$ \\
\hline Ni-59 & $014336-70-0$ & $9.5 \mathrm{E}+02$ & $9.5 \mathrm{E}+00$ \\
\hline Ni-63 & $013981-37-8$ & $3.2 \mathrm{E}+02$ & $3.2 \mathrm{E}+00$ \\
\hline Ni-65 & $014833-49-9$ & $3.1 \mathrm{E}+02$ & $3.1 \mathrm{E}+00$ \\
\hline Np-236 & $015700-36-4$ & $1.9 \mathrm{E}+02$ & $1.9 \mathrm{E}+00$ \\
\hline Np-237 & $013994-20-2$ & $6.0 \mathrm{E}-01$ & $6.0 \mathrm{E}-03$ \\
\hline Np-237+D & $013994-20-2$ & $5.9 \mathrm{E}-01$ & $5.9 \mathrm{E}-03$ \\
\hline
\end{tabular}


Table 3.1f. (continued)

\begin{tabular}{|l|l|c|c|}
\hline \multirow{2}{*}{ Chemical } & \multirow{2}{*}{ CASRN } & \multicolumn{2}{|c|}{ Ingestion of fish (pCi/g) } \\
\cline { 2 - 4 } & & $10^{-4}$ & $10^{-6}$ \\
\hline $\mathrm{Np}-238$ & $015766-25-3$ & $3.9 \mathrm{E}+01$ & $3.9 \mathrm{E}-01$ \\
\hline Np-239 & $013968-59-7$ & $4.1 \mathrm{E}+01$ & $4.1 \mathrm{E}-01$ \\
\hline Np-240 & $015690-84-3$ & $1.0 \mathrm{E}+03$ & $1.0 \mathrm{E}+01$ \\
\hline Np-240m & $015690-84-3$ & $7.3 \mathrm{E}+03$ & $7.3 \mathrm{E}+01$ \\
\hline Os-185 & $015766-50-4$ & $9.8 \mathrm{E}+01$ & $9.8 \mathrm{E}-01$ \\
\hline Os-191 & $014119-24-5$ & $5.8 \mathrm{E}+01$ & $5.8 \mathrm{E}-01$ \\
\hline Os-191m & $014119-24-5$ & $3.6 \mathrm{E}+02$ & $3.6 \mathrm{E}+00$ \\
\hline Os-193 & $016057-77-5$ & $4.0 \mathrm{E}+01$ & $4.0 \mathrm{E}-01$ \\
\hline $\mathrm{P}-32$ & $014596-37-3$ & $2.9 \mathrm{E}+01$ & $2.9 \mathrm{E}-01$ \\
\hline $\mathrm{P}-33$ & $015749-66-3$ & $2.3 \mathrm{E}+02$ & $2.3 \mathrm{E}+00$ \\
\hline $\mathrm{Pa}-231$ & $014331-85-2$ & $1.2 \mathrm{E}+00$ & $1.2 \mathrm{E}-02$ \\
\hline $\mathrm{Pa}-233$ & $013981-14-1$ & $3.8 \mathrm{E}+01$ & $3.8 \mathrm{E}-01$ \\
\hline $\mathrm{Pa}-234$ & $015100-28-4$ & $8.3 \mathrm{E}+01$ & $8.3 \mathrm{E}-01$ \\
\hline $\mathrm{Pa}-234 \mathrm{~m}$ & $015100-28-4$ & $3.7 \mathrm{E}+04$ & $3.7 \mathrm{E}+02$ \\
\hline $\mathrm{Pb}-203$ & $014687-25-3$ & $1.7 \mathrm{E}+02$ & $1.7 \mathrm{E}+00$ \\
\hline $\mathrm{Pb}-209$ & $014119-30-3$ & $8.4 \mathrm{E}+02$ & $8.4 \mathrm{E}+00$ \\
\hline $\mathrm{Pb}-210$ & $014255-04-0$ & $2.6 \mathrm{E}-01$ & $2.6 \mathrm{E}-03$ \\
\hline $\mathrm{Pb}-210+\mathrm{D}$ & $014255-04-0$ & $1.7 \mathrm{E}-01$ & $1.7 \mathrm{E}-03$ \\
\hline
\end{tabular}


Table 3.1f. (continued)

\begin{tabular}{|l|l|c|c|}
\hline \multirow{2}{*}{ Chemical } & \multirow{2}{*}{ CASRN } & \multicolumn{2}{|c|}{ Ingestion of fish (pCi/g) } \\
\cline { 2 - 4 } & & $10^{-4}$ & $10^{-6}$ \\
\hline Pb-211 & $015816-77-0$ & $5.2 \mathrm{E}+02$ & $5.2 \mathrm{E}+00$ \\
\hline Pb-212 & $015092-94-1$ & $9.8 \mathrm{E}+00$ & $9.8 \mathrm{E}-02$ \\
\hline Pb-214 & $015067-28-4$ & $6.0 \mathrm{E}+02$ & $6.0 \mathrm{E}+00$ \\
\hline Pd-100 & $015690-69-4$ & $4.7 \mathrm{E}+01$ & $4.7 \mathrm{E}-01$ \\
\hline Pd-101 & $015749-54-9$ & $4.7 \mathrm{E}+02$ & $4.7 \mathrm{E}+00$ \\
\hline Pd-103 & $014967-68-1$ & $1.7 \mathrm{E}+02$ & $1.7 \mathrm{E}+00$ \\
\hline Pd-107 & $017637-99-9$ & $8.4 \mathrm{E}+02$ & $8.4 \mathrm{E}+00$ \\
\hline Pd-109 & $014981-64-7$ & $5.3 \mathrm{E}+01$ & $5.3 \mathrm{E}-01$ \\
\hline Pm-147 & $014380-75-7$ & $1.3 \mathrm{E}+02$ & $1.3 \mathrm{E}+00$ \\
\hline Pm-148 & $014683-19-3$ & $1.2 \mathrm{E}+01$ & $1.2 \mathrm{E}-01$ \\
\hline Pm-148m & $014683-19-3$ & $1.8 \mathrm{E}+01$ & $1.8 \mathrm{E}-01$ \\
\hline Pm-149 & $015765-31-8$ & $3.2 \mathrm{E}+01$ & $3.2 \mathrm{E}-01$ \\
\hline Po-210 & $013981-52-7$ & $5.4 \mathrm{E}-01$ & $5.4 \mathrm{E}-03$ \\
\hline Po-212 & $015389-34-1$ & $3.9 \mathrm{E}+12$ & $3.9 \mathrm{E}+10$ \\
\hline Po-213 & $015756-57-7$ & $2.6 \mathrm{E}+11$ & $2.6 \mathrm{E}+09$ \\
\hline Po-214 & $015735-67-8$ & $8.3 \mathrm{E}+09$ & $8.3 \mathrm{E}+07$ \\
\hline Po-215 & $015706-52-2$ & $3.5 \mathrm{E}+08$ & $3.5 \mathrm{E}+06$ \\
\hline Po-216 & $015756-58-8$ & $2.0 \mathrm{E}+06$ & $2.0 \mathrm{E}+04$ \\
\hline
\end{tabular}


Table 3.1f. (continued)

\begin{tabular}{|l|l|c|c|}
\hline \multirow{2}{*}{ Chemical $^{\dagger}$} & \multirow{2}{*}{ CASRN } & \multicolumn{2}{|c|}{ Ingestion of fish (pCi/g) } \\
\cline { 2 - 4 } & & $10^{-4}$ & $10^{-5}$ \\
\hline Po-218 & $015422-24-9$ & $3.5 \mathrm{E}+03$ & $3.5 \mathrm{E}+01$ \\
\hline Pr-142 & $014191-64-1$ & $2.5 \mathrm{E}+01$ & $2.5 \mathrm{E}-01$ \\
\hline Pr-143 & $014981-79-4$ & $2.7 \mathrm{E}+01$ & $2.7 \mathrm{E}-01$ \\
\hline Pr-144 & $014119-05-2$ & $2.2 \mathrm{E}+03$ & $2.2 \mathrm{E}+01$ \\
\hline Pr-144m & $014119-05-2$ & $5.5 \mathrm{E}+03$ & $5.5 \mathrm{E}+01$ \\
\hline Pt-191 & $015706-36-2$ & $1.2 \mathrm{E}+02$ & $1.2 \mathrm{E}+00$ \\
\hline Pt-193 & $015735-70-3$ & $1.1 \mathrm{E}+03$ & $1.1 \mathrm{E}+01$ \\
\hline Pt-193m & $015735-70-3$ & $7.0 \mathrm{E}+01$ & $7.0 \mathrm{E}-01$ \\
\hline Pt-197 & $015735-74-7$ & $8.3 \mathrm{E}+01$ & $8.3 \mathrm{E}-01$ \\
\hline Pt-197m & $015735-74-7$ & $5.4 \mathrm{E}+02$ & $5.4 \mathrm{E}+00$ \\
\hline Pu-236 & $015411-92-4$ & $2.3 \mathrm{E}+00$ & $2.3 \mathrm{E}-02$ \\
\hline Pu-238 & $013981-16-3$ & $6.0 \mathrm{E}-01$ & $6.0 \mathrm{E}-03$ \\
\hline Pu-239 & $015117-48-3$ & $5.6 \mathrm{E}-01$ & $5.6 \mathrm{E}-03$ \\
\hline Pu-240 & $014119-33-6$ & $5.6 \mathrm{E}-01$ & $5.6 \mathrm{E}-03$ \\
\hline Pu-241 & $014119-32-5$ & $3.4 \mathrm{E}+01$ & $3.4 \mathrm{E}-01$ \\
\hline Pu-241+D & $014119-32-5$ & $5.3 \mathrm{E}-01$ & $5.3 \mathrm{E}-03$ \\
\hline Pu-242 & $013982-10-0$ & $5.9 \mathrm{E}-01$ & $5.9 \mathrm{E}-03$ \\
\hline Pu-243 & $015706-37-3$ & $4.8 \mathrm{E}+02$ & $4.8 \mathrm{E}+00$ \\
\hline
\end{tabular}


Table 3.1f. (continued)

\begin{tabular}{|l|l|c|c|}
\hline \multirow{2}{*}{ Chemical $^{\dagger}$} & \multirow{2}{*}{ CASRN } & \multicolumn{2}{|c|}{ Ingestion of fish (pCi/g) } \\
\cline { 2 - 4 } & & $10^{-4}$ & $10^{-6}$ \\
\hline Pu-244 & $014119-34-7$ & $5.6 \mathrm{E}-01$ & $5.6 \mathrm{E}-03$ \\
\hline Pu-244+D & $014119-34-7$ & $5.5 \mathrm{E}-01$ & $5.5 \mathrm{E}-03$ \\
\hline Ra-223 & $015623-45-7$ & $7.5 \mathrm{E}-01$ & $7.5 \mathrm{E}-03$ \\
\hline Ra-224 & $013233-32-4$ & $1.2 \mathrm{E}+00$ & $1.2 \mathrm{E}-02$ \\
\hline Ra-225 & $013981-53-8$ & $1.1 \mathrm{E}+00$ & $1.1 \mathrm{E}-02$ \\
\hline Ra-226 & $013982-63-3$ & $6.0 \mathrm{E}-01$ & $6.0 \mathrm{E}-03$ \\
\hline Ra-226+D & $013982-63-3$ & $6.0 \mathrm{E}-01$ & $6.0 \mathrm{E}-03$ \\
\hline Ra-228 & $015262-20-1$ & $7.2 \mathrm{E}-01$ & $7.2 \mathrm{E}-03$ \\
\hline Ra-228+D & $015262-20-1$ & $7.1 \mathrm{E}-01$ & $7.1 \mathrm{E}-03$ \\
\hline Rb-82 & $014391-63-0$ & $1.7 \mathrm{E}+04$ & $1.7 \mathrm{E}+02$ \\
\hline Rb-86 & $014932-53-7$ & $2.5 \mathrm{E}+01$ & $2.5 \mathrm{E}-01$ \\
\hline Rb-87 & $013982-13-3$ & $4.8 \mathrm{E}+01$ & $4.8 \mathrm{E}-01$ \\
\hline Rb-88 & $014928-36-0$ & $1.2 \mathrm{E}+03$ & $1.2 \mathrm{E}+01$ \\
\hline Rb-89 & $014191-65-2$ & $2.0 \mathrm{E}+03$ & $2.0 \mathrm{E}+01$ \\
\hline Rh-103m & $007440-16-6$ & $2.2 \mathrm{E}+04$ & $2.2 \mathrm{E}+02$ \\
\hline Rh-105 & $014913-89-4$ & $9.1 \mathrm{E}+01$ & $9.1 \mathrm{E}-01$ \\
\hline Rh-105m & $014913-89-4$ & $1.6 \mathrm{E}+05$ & $1.6 \mathrm{E}+03$ \\
\hline Rh-106 & $014234-34-5$ & $4.9 \mathrm{E}+04$ & $4.9 \mathrm{E}+02$ \\
\hline
\end{tabular}


Table 3.1f. (continued)

\begin{tabular}{|l|l|c|c|}
\hline \multirow{2}{*}{ Chemical } & \multirow{2}{*}{ CASRN } & \multicolumn{2}{|c|}{ Ingestion of fish (pCi/g) } \\
\cline { 3 - 4 } & & $10^{-4}$ & $10^{-6}$ \\
\hline Rn-219 & $014835-02-0$ & & \\
\hline Rn-220 & $022481-48-7$ & & \\
\hline Rn-222+D & $014859-67-7$ & & \\
\hline Ru-103 & $013968-53-1$ & $5.3 \mathrm{E}+01$ & $5.3 \mathrm{E}-01$ \\
\hline Ru-105 & $014331-95-4$ & $1.5 \mathrm{E}+02$ & $1.5 \mathrm{E}+00$ \\
\hline Ru-106 & $013967-48-1$ & $5.1 \mathrm{E}+00$ & $5.1 \mathrm{E}-02$ \\
\hline Ru-106+D & $013967-48-1$ & $5.1 \mathrm{E}+00$ & $5.1 \mathrm{E}-02$ \\
\hline Ru-97 & $015758-35-7$ & $3.0 \mathrm{E}+02$ & $3.0 \mathrm{E}+00$ \\
\hline S-35 & $015117-53-0$ & $4.2 \mathrm{E}+02$ & $4.2 \mathrm{E}+00$ \\
\hline Sb-122 & $014374-79-9$ & $2.0 \mathrm{E}+01$ & $2.0 \mathrm{E}-01$ \\
\hline Sb-124 & $014683-10-4$ & $1.6 \mathrm{E}+01$ & $1.6 \mathrm{E}-01$ \\
\hline Sb-125 & $014234-35-6$ & $5.9 \mathrm{E}+01$ & $5.9 \mathrm{E}-01$ \\
\hline Sb-125+D & $014234-35-6$ & $5.0 \mathrm{E}+01$ & $5.0 \mathrm{E}-01$ \\
\hline Sb-126 & $015756-32-8$ & $1.8 \mathrm{E}+01$ & $1.8 \mathrm{E}-01$ \\
\hline Sb-126m & $015756-32-8$ & $2.4 \mathrm{E}+03$ & $2.4 \mathrm{E}+01$ \\
\hline Sb-127 & $013968-50-8$ & $2.1 \mathrm{E}+01$ & $2.1 \mathrm{E}-01$ \\
\hline Sb-129 & $014331-88-5$ & $9.5 \mathrm{E}+01$ & $9.5 \mathrm{E}-01$ \\
\hline Sc-46 & $013967-63-0$ & $3.1 \mathrm{E}+01$ & $3.1 \mathrm{E}-01$ \\
\hline
\end{tabular}


Table 3.1f. (continued)

\begin{tabular}{|l|l|c|c|}
\hline \multirow{2}{*}{ Chemical $^{\dagger}$} & \multirow{2}{*}{ CASRN } & \multicolumn{2}{|c|}{ Ingestion of fish (pCi/g) } \\
\cline { 3 - 4 } & & $10^{-4}$ & $10^{-6}$ \\
\hline Sc-47 & $014391-96-9$ & $6.0 \mathrm{E}+01$ & $6.0 \mathrm{E}-01$ \\
\hline Sc-48 & $014391-86-7$ & $2.7 \mathrm{E}+01$ & $2.7 \mathrm{E}-01$ \\
\hline Se-75 & $014265-71-5$ & $2.7 \mathrm{E}+01$ & $2.7 \mathrm{E}-01$ \\
\hline Si-31 & $014276-49-4$ & $3.5 \mathrm{E}+02$ & $3.5 \mathrm{E}+00$ \\
\hline Sm-147 & $014392-33-7$ & $7.0 \mathrm{E}+00$ & $7.0 \mathrm{E}-02$ \\
\hline Sm-151 & $015715-94-3$ & $3.8 \mathrm{E}+02$ & $3.8 \mathrm{E}+00$ \\
\hline Sm-153 & $015766-00-4$ & $4.4 \mathrm{E}+01$ & $4.4 \mathrm{E}-01$ \\
\hline Sn-113 & $013966-06-8$ & $4.7 \mathrm{E}+01$ & $4.7 \mathrm{E}-01$ \\
\hline Sn-121 & $014683-06-8$ & $1.4 \mathrm{E}+02$ & $1.4 \mathrm{E}+00$ \\
\hline Sn-121m & $014683-06-8$ & $8.8 \mathrm{E}+01$ & $8.8 \mathrm{E}-01$ \\
\hline Sn-125 & $014683-08-0$ & $1.0 \mathrm{E}+01$ & $1.0 \mathrm{E}-01$ \\
\hline Sn-126 & $015832-50-5$ & $8.3 \mathrm{E}+00$ & $8.3 \mathrm{E}-02$ \\
\hline Sr-82 & $014809-50-8$ & $6.8 \mathrm{E}+00$ & $6.8 \mathrm{E}-02$ \\
\hline Sr-85 & $013967-73-2$ & $1.3 \mathrm{E}+02$ & $1.3 \mathrm{E}+00$ \\
\hline Sr-85m & $013967-73-2$ & $9.8 \mathrm{E}+03$ & $9.8 \mathrm{E}+01$ \\
\hline Sr-89 & $014158-27-1$ & $1.7 \mathrm{E}+01$ & $1.7 \mathrm{E}-01$ \\
\hline Sr-90 & $010098-97-2$ & $4.3 \mathrm{E}+00$ & $4.3 \mathrm{E}-02$ \\
\hline Sr-90+D & $010098-97-2$ & $3.2 \mathrm{E}+00$ & $3.2 \mathrm{E}-02$ \\
\hline & & & \\
\hline
\end{tabular}


Table 3.1f. (continued)

\begin{tabular}{|l|l|c|c|}
\hline \multirow{2}{*}{ Chemical } & \multirow{2}{*}{ CASRN } & \multicolumn{2}{|c|}{ Ingestion of fish (pCi/g) } \\
\cline { 2 - 4 } & & $10^{-4}$ & $10^{-6}$ \\
\hline Sr-91 & $014331-91-0$ & $6.3 \mathrm{E}+01$ & $6.3 \mathrm{E}-01$ \\
\hline Sr-92 & $014928-29-1$ & $8.7 \mathrm{E}+01$ & $8.7 \mathrm{E}-01$ \\
\hline Ta-182 & $013982-00-8$ & $2.5 \mathrm{E}+01$ & $2.5 \mathrm{E}-01$ \\
\hline Tb-158 & $015759-55-4$ & $4.2 \mathrm{E}+01$ & $4.2 \mathrm{E}-01$ \\
\hline Tb-160 & $013981-29-8$ & $2.3 \mathrm{E}+01$ & $2.3 \mathrm{E}-01$ \\
\hline Tc-95 & $014809-56-4$ & $2.6 \mathrm{E}+03$ & $2.6 \mathrm{E}+01$ \\
\hline Tc-95m & $014809-56-4$ & $1.4 \mathrm{E}+02$ & $1.4 \mathrm{E}+00$ \\
\hline Tc-96 & $014808-44-7$ & $7.7 \mathrm{E}+01$ & $7.7 \mathrm{E}-01$ \\
\hline Tc-96m & $014808-44-7$ & $6.8 \mathrm{E}+03$ & $6.8 \mathrm{E}+01$ \\
\hline Tc-97 & $015759-35-0$ & $1.1 \mathrm{E}+03$ & $1.1 \mathrm{E}+01$ \\
\hline Tc-97m & $015759-35-0$ & $1.5 \mathrm{E}+02$ & $1.5 \mathrm{E}+00$ \\
\hline Tc-99 & $014133-76-7$ & $1.3 \mathrm{E}+02$ & $1.3 \mathrm{E}+00$ \\
\hline Tc-99m & $014133-76-7$ & $3.2 \mathrm{E}+03$ & $3.2 \mathrm{E}+01$ \\
\hline Te-125m & $014390-73-9$ & $7.0 \mathrm{E}+01$ & $7.0 \mathrm{E}-01$ \\
\hline Te-127 & $013981-49-2$ & $2.1 \mathrm{E}+02$ & $2.1 \mathrm{E}+00$ \\
\hline Te-127m & $013981-49-2$ & $2.9 \mathrm{E}+01$ & $2.9 \mathrm{E}-01$ \\
\hline Te-129 & $014269-71-7$ & $1.2 \mathrm{E}+03$ & $1.2 \mathrm{E}+01$ \\
\hline Te-129m & $014269-71-7$ & $1.5 \mathrm{E}+01$ & $1.5 \mathrm{E}-01$ \\
\hline
\end{tabular}


Table 3.1f. (continued)

\begin{tabular}{|l|l|c|c|}
\hline \multirow{2}{*}{ Chemical $^{\dagger}$} & \multirow{2}{*}{ CASRN } & \multicolumn{2}{|c|}{ Ingestion of fish (pCi/g) } \\
\cline { 2 - 4 } & & $10^{-4}$ & $10^{-6}$ \\
\hline Te-131 & $014683-12-6$ & $4.5 \mathrm{E}+02$ & $4.5 \mathrm{E}+00$ \\
\hline Te-131m & $014683-12-6$ & $2.0 \mathrm{E}+01$ & $2.0 \mathrm{E}-01$ \\
\hline Te-132 & $014234-28-7$ & $1.4 \mathrm{E}+01$ & $1.4 \mathrm{E}-01$ \\
\hline Th-227 & $015623-47-9$ & $4.4 \mathrm{E}+00$ & $4.4 \mathrm{E}-02$ \\
\hline Th-228 & $014274-82-9$ & $2.8 \mathrm{E}+00$ & $2.8 \mathrm{E}-02$ \\
\hline Th-228+D & $014274-82-9$ & $7.6 \mathrm{E}-01$ & $7.6 \mathrm{E}-03$ \\
\hline Th-229 & $015594-54-4$ & $3.1 \mathrm{E}+00$ & $3.1 \mathrm{E}-02$ \\
\hline Th-229+D & $015594-54-4$ & $5.0 \mathrm{E}-01$ & $5.0 \mathrm{E}-03$ \\
\hline Th-230 & $014269-63-7$ & $4.7 \mathrm{E}+00$ & $4.7 \mathrm{E}-02$ \\
\hline Th-231 & $014932-40-2$ & $9.9 \mathrm{E}+01$ & $9.9 \mathrm{E}-01$ \\
\hline Th-232 & $007440-29-1$ & $5.4 \mathrm{E}+00$ & $5.4 \mathrm{E}-02$ \\
\hline Th-234 & $015065-10-8$ & $9.1 \mathrm{E}+00$ & $9.1 \mathrm{E}-02$ \\
\hline Tl-202 & $015720-57-7$ & $1.7 \mathrm{E}+02$ & $1.7 \mathrm{E}+00$ \\
\hline Tl-204 & $013968-51-9$ & $9.0 \mathrm{E}+01$ & $9.0 \mathrm{E}-01$ \\
\hline Tl-208 & $014913-50-9$ & $1.0 \mathrm{E}+04$ & $1.0 \mathrm{E}+02$ \\
\hline Tl-209 & $015690-73-0$ & $1.3 \mathrm{E}+04$ & $1.3 \mathrm{E}+02$ \\
\hline Tm-170 & $013981-30-1$ & $2.4 \mathrm{E}+01$ & $2.4 \mathrm{E}-01$ \\
\hline Tm-171 & $014333-45-0$ & $3.0 \mathrm{E}+02$ & $3.0 \mathrm{E}+00$ \\
\hline
\end{tabular}


Table 3.1f. (continued)

\begin{tabular}{|l|l|c|c|}
\hline \multirow{2}{*}{ Chemical } & \multirow{2}{*}{ CASRN } & \multicolumn{2}{|c|}{ Ingestion of fish (pCi/g) } \\
\cline { 2 - 4 } & & $10^{-4}$ & $10^{-6}$ \\
\hline U-232 & $014158-29-3$ & $2.2 \mathrm{E}+00$ & $2.2 \mathrm{E}-02$ \\
\hline U-233 & $013968-55-3$ & $3.9 \mathrm{E}+00$ & $3.9 \mathrm{E}-02$ \\
\hline U-234 & $013966-29-5$ & $4.0 \mathrm{E}+00$ & $4.0 \mathrm{E}-02$ \\
\hline U-235 & $015117-96-1$ & $3.9 \mathrm{E}+00$ & $3.9 \mathrm{E}-02$ \\
\hline U-235+D & $015117-96-1$ & $3.8 \mathrm{E}+00$ & $3.8 \mathrm{E}-02$ \\
\hline U-236 & $013982-70-2$ & $4.2 \mathrm{E}+00$ & $4.2 \mathrm{E}-02$ \\
\hline U-237 & $014269-75-1$ & $4.4 \mathrm{E}+01$ & $4.4 \mathrm{E}-01$ \\
\hline U-238 & $007440-61-1$ & $4.1 \mathrm{E}+00$ & $4.1 \mathrm{E}-02$ \\
\hline U-238+D & $007440-61-1$ & $2.8 \mathrm{E}+00$ & $2.8 \mathrm{E}-02$ \\
\hline U-240 & $015687-53-3$ & $3.2 \mathrm{E}+01$ & $3.2 \mathrm{E}-01$ \\
\hline V-48 & $014331-97-6$ & $2.3 \mathrm{E}+01$ & $2.3 \mathrm{E}-01$ \\
\hline W-181 & $015749-46-9$ & $6.5 \mathrm{E}+02$ & $6.5 \mathrm{E}+00$ \\
\hline W-185 & $014932-41-3$ & $8.6 \mathrm{E}+01$ & $8.6 \mathrm{E}-01$ \\
\hline W-187 & $014983-48-3$ & $7.2 \mathrm{E}+01$ & $7.2 \mathrm{E}-01$ \\
\hline Xe-122 & $015151-09-4$ & & \\
\hline Xe-123 & $015700-10-4$ & & \\
\hline Xe-125 & $013994-18-8$ & & \\
\hline Xe-127 & $013994-19-9$ & & \\
\hline & & & \\
\hline
\end{tabular}


Table 3.1f. (continued)

\begin{tabular}{|l|l|c|c|}
\hline \multirow{2}{*}{ Chemical $^{\dagger}$} & \multirow{2}{*}{ CASRN } & \multicolumn{2}{|c|}{ Ingestion of fish (pCi/g) } \\
\cline { 3 - 4 } & & $10^{-4}$ & $10^{-6}$ \\
\hline Xe-129m & $013965-99-6$ & & \\
\hline Xe-131m & $014683-11-5$ & & \\
\hline Xe-133 & $014932-42-4$ & & \\
\hline Xe-133m & $014932-42-4$ & & \\
\hline Xe-135 & $014995-62-1$ & & \\
\hline$X e-135 m$ & $014995-62-1$ & & \\
\hline$X e-137$ & $014835-21-3$ & & \\
\hline$X e-138$ & $015751-81-2$ & & $1.2 \mathrm{E}-01$ \\
\hline $\mathrm{Y}-90$ & $010098-91-6$ & & $1.3 \mathrm{E}-01$ \\
\hline $\mathrm{Y}-91$ & $014234-24-3$ & $1.2 \mathrm{E}+01$ & $4.8 \mathrm{E}+01$ \\
\hline $\mathrm{Y}-91 \mathrm{~m}$ & $014234-24-3$ & $1.3 \mathrm{E}+01$ & $9.0 \mathrm{E}-01$ \\
\hline $\mathrm{Y}-92$ & $015751-59-4$ & $4.8 \mathrm{E}+03$ & $3.1 \mathrm{E}-01$ \\
\hline $\mathrm{Y}-93$ & $014981-70-5$ & $9.0 \mathrm{E}+01$ & $1.8 \mathrm{E}-01$ \\
\hline $\mathrm{Zn}-65$ & $013982-39-3$ & $3.1 \mathrm{E}+01$ & $2.8 \mathrm{E}+01$ \\
\hline $\mathrm{Zn}-69$ & $013982-23-5$ & $1.8 \mathrm{E}+01$ & $1.2 \mathrm{E}+00$ \\
\hline $\mathrm{Zn}-69 \mathrm{~m}$ & $013982-23-5$ & $2.8 \mathrm{E}+03$ & $3.4 \mathrm{E}+00$ \\
\hline $\mathrm{Zr}-93$ & $015751-77-6$ & $1.2 \mathrm{E}+02$ & $4.5 \mathrm{E}-01$ \\
\hline $\mathrm{Zr}-95$ & $013967-71-0$ & & \\
\hline & & & \\
\hline
\end{tabular}


Table 3.1f. (continued)

\begin{tabular}{|l|l|c|c|}
\hline \multirow{2}{*}{ Chemical $^{\dagger}$} & \multirow{2}{*}{ CASRN } & \multicolumn{2}{|c|}{ Ingestion of fish (pCi/g) } \\
\cline { 3 - 4 } & & $10^{-4}$ & $10^{-6}$ \\
\hline Zr-97 & $014928-30-4$ & $1.7 \mathrm{E}+01$ & $1.7 \mathrm{E}-01$ \\
\hline
\end{tabular}

Note:

$\dagger$ Isotopes designated with an " $m$ " indicate that the isotope is metastable; isotopes designated with a " $+D$ " indicate that the radionuclide slope factors include contributions from daughter products. 
$\checkmark$ 
Table 3.1g. Risk-based Preliminary Remediation Goals for water based on ingestion of fish for nonradionuclides (residential scenario)

\begin{tabular}{|c|c|c|c|c|c|}
\hline \multirow{2}{*}{ Chemical } & \multirow{2}{*}{ CASRN } & \multicolumn{4}{|c|}{ Ingestion of fish (concentration in water) (mg/L)* } \\
\hline & & $10^{-4}$ & $10^{-6}$ & $\mathbf{H Q}=\mathbf{1}$ & $H Q=0.1$ \\
\hline ALAR & 001596-84-5 & & & & \\
\hline Acenaphthene & 000083-32-9 & & & 7.4E-02 & 7.4E-03 \\
\hline Acenaphthylene & 000208-96-8 & & & & \\
\hline Acephate & 030560-19-1 & $2.7 \mathrm{E}+02$ & $2.7 \mathrm{E}+00$ & 4.1E+01 & $4.1 \mathrm{E}+00$ \\
\hline Acetaldehyde & $000075-07-0$ & & & & \\
\hline Acetochlor & 034256-82-1 & & & & \\
\hline Acetone & $000067-64-1$ & & & $3.5 \mathrm{E}+02$ & $3.5 \mathrm{E}+01$ \\
\hline Acetone Cyanohydrin & $000075-86-5$ & & & & \\
\hline Acetonitrile & 000075-05-8 & & & $2.5 \mathrm{E}+01$ & $2.5 \mathrm{E}+00$ \\
\hline Acetophenone $\mathrm{s}^{(5)}$ & 000098-86-2 & & & $1.2 \mathrm{E}+01$ & $1.2 \mathrm{E}+00$ \\
\hline Acrolein $^{(6)}$ & $000107-02-8$ & & & $5.5 \mathrm{E}+01$ & $5.5 \mathrm{E}+00$ \\
\hline Acrylamide & $000079-06-1$ & $3.8 \mathrm{E}-01$ & $3.8 \mathrm{E}-03$ & $1.5 \mathrm{E}+00$ & $1.5 \mathrm{E}-01$ \\
\hline Acrylic Acid & 000079-10-7 & & & $8.7 E+02$ & $8.7 \mathrm{E}+01$ \\
\hline Acrylonitrile & $000107-13-1$ & $6.4 \mathrm{E}-01$ & $6.4 \mathrm{E}-03$ & $1.5 \mathrm{E}+00$ & $1.5 \mathrm{E}-01$ \\
\hline Alachlor & $015972-60-8$ & 4.2E-02 & $4.2 \mathrm{E}-04$ & $1.4 \mathrm{E}-01$ & $1.4 \mathrm{E}-02$ \\
\hline Aldicarb & $000116-06-3$ & & & $3.2 \mathrm{E}-01$ & $3.2 \mathrm{E}-02$ \\
\hline Aldicarb Sulfone & 001646-88-4 & & & & \\
\hline Aldrin & $000309-00-2$ & $1.7 \mathrm{E}-04$ & $1.7 \mathrm{E}-06$ & $3.6 \mathrm{E}-04$ & $3.6 \mathrm{E}-05$ \\
\hline
\end{tabular}


Table 3.1g. (continued)

\begin{tabular}{|c|c|c|c|c|c|}
\hline \multirow{2}{*}{ Chemical } & \multirow{2}{*}{ CASRN } & \multicolumn{4}{|c|}{ Ingestion of fish (concentration in water) $(\mathrm{mg} / \mathrm{L})^{*}$} \\
\hline & & $10^{-4}$ & $10^{-6}$ & $\mathbf{H Q}=\mathbf{1}$ & $H Q=0.1$ \\
\hline Ally & 074223-64-6 & & & $5.6 \mathrm{E}+02$ & $5.6 \mathrm{E}+01$ \\
\hline Allyl Alcohol & 000107-18-6 & & & $1.7 \mathrm{E}+01$ & $1.7 \mathrm{E}+00$ \\
\hline Allyl Chloride ${ }^{(5)}$ & 000107-05-1 & & & $9.9 \mathrm{E}+00$ & $9.9 \mathrm{E}-01$ \\
\hline Aluminum & 007429-90-5 & & & & \\
\hline Aluminum Phosphide & 020859-73-8 & & & & \\
\hline Amdro & 067485-29-4 & & & & \\
\hline Ametryn & 000834-12-8 & & & $8.9 \mathrm{E}-01$ & $8.9 \mathrm{E}-02$ \\
\hline Aminophenol, m- & 000591-27-5 & & & $1.1 \mathrm{E}+02$ & $1.1 \mathrm{E}+01$ \\
\hline Aminopyridine, 4- & 000504-24-5 & & & $2.8 \mathrm{E}-02$ & $2.8 \mathrm{E}-03$ \\
\hline Amitraz & $033089-61-1$ & & & & \\
\hline Ammonia & $007664-41-7$ & & & & \\
\hline Ammonium Sulfamate & 007773-06-0 & & & & \\
\hline Aniline & $000062-53-3$ & $1.9 \mathrm{E}+01$ & $1.9 \mathrm{E}-01$ & & \\
\hline Anthracene & 000120-12-7 & & & 3.1E-01 & $3.1 \mathrm{E}-02$ \\
\hline Antimony (metallic) & $007440-36-0$ & & & $5.4 \mathrm{E}-03$ & $5.4 \mathrm{E}-04$ \\
\hline Antimony Pentoxide & $001314-60-9$ & & & & \\
\hline Antimony Potassium Tartrate & $000304-61-0$ & & & & \\
\hline Antimony Tetroxide & $001332-81-6$ & & & & \\
\hline
\end{tabular}


Table 3.1g. (continued)

\begin{tabular}{|c|c|c|c|c|c|}
\hline \multirow{2}{*}{ Chemical } & \multirow{2}{*}{ CASRN } & \multicolumn{4}{|c|}{ Ingestion of fish (concentration in water) (mg/L)* } \\
\hline & & $10^{-4}$ & $10^{-6}$ & $\mathbf{H Q}=\mathbf{1}$ & $\mathrm{HQ}=0.1$ \\
\hline Antimony Trioxide & 001309-64-4 & & & & \\
\hline Apollo & 074115-24-5 & & & & \\
\hline Aramite & 000140-57-8 & & & & \\
\hline Aroclor 1016 & 012674-11-2 & & & $5.3 \mathrm{E}-06$ & 5.3E-07 \\
\hline Aroclor $1221^{(1)}$ & 011104-28-2 & 5.3E-05 & 5.3E-07 & & \\
\hline Aroclor $1232^{(1)}$ & 011141-16-5 & 2.6E-04 & 2.6E-06 & & \\
\hline Aroclor $1242^{(1)}$ & $053469-21-9$ & $5.3 \mathrm{E}-05$ & $5.3 \mathrm{E}-07$ & & \\
\hline Aroclor $1248^{(1)}$ & $012672-29-6$ & 2.7E-06 & 2.7E-08 & & \\
\hline Aroclor 1254 & $011097-69-1$ & & & $1.3 \mathrm{E}-06$ & $1.3 \mathrm{E}-07$ \\
\hline Aroclor $1260^{(1)}$ & 011096-82-5 & $2.8 \mathrm{E}-07$ & $2.8 \mathrm{E}-09$ & & \\
\hline Arsenic Salts & NA & & & & \\
\hline Arsenic, Inorganic & 007440-38-2 & & & & \\
\hline Arsine & $007784-42-1$ & & & & \\
\hline Asbestos & 001332-21-4 & & & & \\
\hline Assure & 076578-14-8 & & & & \\
\hline Asulam & 003337-71-1 & & & & \\
\hline Atrazine & 001912-24-9 & $2.1 \mathrm{E}-02$ & $2.1 \mathrm{E}-04$ & $7.1 \mathrm{E}-01$ & $7.1 \mathrm{E}-02$ \\
\hline Avermectin B1 & 065195-55-3 & & & & \\
\hline
\end{tabular}


Table 3.1g. (continued)

\begin{tabular}{|c|c|c|c|c|c|}
\hline \multirow{2}{*}{ Chemical } & \multirow{2}{*}{ CASRN } & \multicolumn{4}{|c|}{ Ingestion of fish (concentration in water) $(\mathrm{mg} / \mathrm{L})^{*}$} \\
\hline & & $10^{-4}$ & $10^{-6}$ & $\mathrm{HQ}=\mathbf{1}$ & $\mathrm{HQ}=0.1$ \\
\hline Azobenzene & 000103-33-3 & $6.3 \mathrm{E}-03$ & $6.3 \mathrm{E}-05$ & & \\
\hline Barium & 007440-39-3 & & & $2.4 \mathrm{E}+01$ & $2.4 \mathrm{E}+00$ \\
\hline Barium Cyanide & $000542-62-1$ & & & & \\
\hline Baygon & $000114-26-1$ & & & $5.6 \mathrm{E}-01$ & $5.6 \mathrm{E}-02$ \\
\hline Bayleton & 043121-43-3 & & & $1.2 \mathrm{E}+00$ & $1.2 \mathrm{E}-01$ \\
\hline Baythroid & 068359-37-5 & & & & \\
\hline Benefin & 001861-40-1 & & & $2.4 \mathrm{E}-01$ & $2.4 \mathrm{E}-02$ \\
\hline Benomyl & $017804-35-2$ & & & $2.1 \mathrm{E}+00$ & 2.1E-01 \\
\hline Bentazon & $025057-89-0$ & & & 8.4E-01 & $8.4 \mathrm{E}-02$ \\
\hline Benz[a]anthracene ${ }^{(2)}$ & $000056-55-3$ & $3.4 \mathrm{E}-05$ & 3.4E-07 & & \\
\hline Benzaldehyde & $000100-52-7$ & & & $4.1 \mathrm{E}+00$ & 4.1E-01 \\
\hline Benzene & $000071-43-2$ & 4.7E-01 & 4.7E-03 & & \\
\hline Benzene Hexachloride & NA & & & & \\
\hline Benzene, Ethyldimethyl & NA & & & & \\
\hline Benzene, Ethylmethyl & NA & & & & \\
\hline Benzene, Methylpropenyl & NA & & & & \\
\hline Benzene, Methylpropyl & NA & & & & \\
\hline Benzene, Trimethyl & $025551-13-7$ & & & & \\
\hline
\end{tabular}


Table 3.1g. (continued)

\begin{tabular}{|c|c|c|c|c|c|}
\hline \multirow{2}{*}{ Chemical } & \multirow{2}{*}{ CASRN } & \multicolumn{4}{|c|}{ Ingestion of fish (concentration in water) (mg/L)" } \\
\hline & & $10^{-4}$ & $10^{-6}$ & $\mathbf{H Q}=\mathbf{1}$ & $\mathrm{HQ}=0.1$ \\
\hline Benzenethiol & 000108-98-5 & & & $2.9 \mathrm{E}-04$ & 2.9E-05 \\
\hline Benzidine & 000092-87-5 & 2.4E-04 & $2.4 \mathrm{E}-06$ & 7.1E-01 & 7.1E-02 \\
\hline Benzo[a]pyrene ${ }^{(2)}$ & $000050-32-8$ & $1.7 \mathrm{E}-06$ & $1.7 \mathrm{E}-08$ & & \\
\hline Benzo[b]fluoranthene ${ }^{(2)}$ & 000205-99-2 & $1.7 \mathrm{E}-05$ & $1.7 \mathrm{E}-07$ & & \\
\hline Benzo[g,h,i]perylene & 000191-24-2 & & & & \\
\hline Benzo[k]fluoranthene ${ }^{(2)}$ & 000207-08-9 & $5.0 \mathrm{E}-05$ & $5.0 \mathrm{E}-07$ & & \\
\hline Benzoic Acid & 000065-85-0 & & & $3.3 \mathrm{E}+02$ & $3.3 \mathrm{E}+01$ \\
\hline Benzotrichloride & 000098-07-7 & $2.6 \mathrm{E}-04$ & $2.6 \mathrm{E}-06$ & & \\
\hline Benzyl Alcohol & $000100-51-6$ & & & $1.0 \mathrm{E}+02$ & $1.0 \mathrm{E}+01$ \\
\hline Benzyl Chloride & $000100-44-7$ & $5.6 \mathrm{E}-02$ & $5.6 \mathrm{E}-04$ & & \\
\hline Beryllium & $007440-41-7$ & 7.3E-04 & 7.3E-06 & $6.8 \mathrm{E}-02$ & $6.8 \mathrm{E}-03$ \\
\hline Bidrin & 000141-66-2 & & & 7.5E-01 & 7.5E-02 \\
\hline Biphenthrin & 082657-04-3 & & & & \\
\hline Biphenyl, 1,1'- & $000092-52-4$ & & & $8.8 \mathrm{E}-02$ & $8.8 \mathrm{E}-03$ \\
\hline $\begin{array}{l}\text { Bis(2-chloro-1-methylethyl)ether } \\
\text { (Technical) }\end{array}$ & $000108-60-1$ & 8.4E-02 & 8.4E-04 & & \\
\hline Bis(2-chloroethoxy)methane & $000111-91-1$ & & & & \\
\hline Bis(2-chloroethyl)ether & $000111-44-4$ & $5.0 \mathrm{E}-02$ & $5.0 \mathrm{E}-04$ & & \\
\hline
\end{tabular}




\begin{tabular}{|c|c|c|c|c|c|}
\hline & & & & $\mathrm{VN}$ & 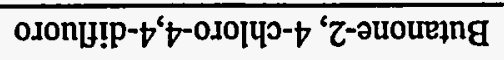 \\
\hline \multirow[t]{3}{*}{$00+B t^{\circ} L$} & $10+3+2 L$ & & & $\varepsilon-9 \varepsilon-I L 0000$ & $-\mathrm{N}^{\prime}$ 'ouręng \\
\hline & & & & $0-66-901000$ & $-\varepsilon^{6} I$ 'əuə̣p \\
\hline & & & & $\tau-66-689100$ & 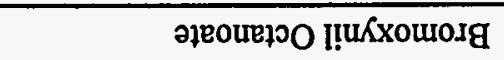 \\
\hline \multirow[t]{3}{*}{$00+36 . I$} & $10+96^{\circ} \mathrm{I}$ & & & $s-\diamond 8-689100$ & I!UKXourodg \\
\hline & & & & $\angle-Z 9-\varsigma \angle 0000$ & әиечрәшолорчр!дошо.дg \\
\hline & & & & $\varepsilon-96-\not 0 I Z 00$ & soydourag \\
\hline $20^{-}-96^{\circ} \varepsilon$ & $10-76^{\circ} \varepsilon$ & & & $6-\varepsilon 8-\downarrow \angle 0000$ & 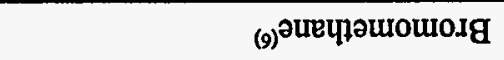 \\
\hline \multirow[t]{2}{*}{$20-86^{\circ} 9$} & $10-36.9$ & $20-50^{\circ} \mathrm{I}$ & $00+30^{\circ} I$ & $\tau-\varsigma \tau-\varsigma \angle 0000$ & whojourosg \\
\hline & & & & $\varepsilon-S S-I 0 I 000$ & 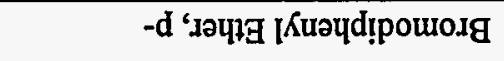 \\
\hline \multirow[t]{4}{*}{$I 0-\exists Z^{\prime} I$} & $00+g z \cdot I$ & $\varepsilon 0-z^{\prime} \tau^{\prime} Z$ & I0-घZZ & $t-L Z-\varsigma L 0000$ & 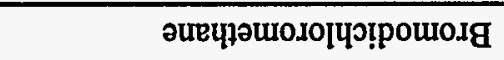 \\
\hline & & & & $s-\angle 6-t \angle 0000$ & 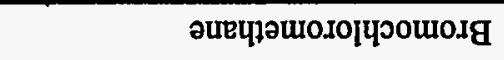 \\
\hline & & & & $Z-\angle 0-\angle E 9 L O O$ & әp!IonIJ! \\
\hline & & & & $8-Z t-0 b t<00$ & КโuO sejeIog pur uojog \\
\hline \multirow[t]{2}{*}{$z 0-\mathrm{gI} z$} & I0-GI'Z & & & L-S0-080000 & V Iouəuds!g \\
\hline & & SO-تีદ I & $\varepsilon 0-\Xi \varepsilon \varepsilon^{\prime} I$ & {$[-88-z b \varsigma 000$} & 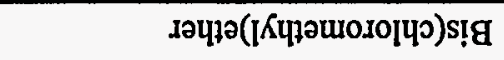 \\
\hline t0-3L'8 & $\varepsilon 0-\exists L^{\prime} 8$ & SO- $3 Z: L$ & $\varepsilon 0-\exists Z^{\prime} L$ & $L-I 8-L I I 000$ & 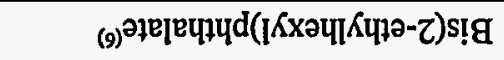 \\
\hline I0-gॄ'乙 & $00+\exists \varepsilon \tau$ & & & $6-Z \varepsilon-8 \varepsilon 96 \varepsilon 0$ & 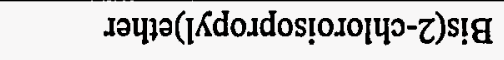 \\
\hline$I^{\circ} 0=\partial H$ & $\mathbf{I}=\mathbf{O H}$ & $9-0 I$ & r.0I & \multirow{2}{*}{ N\&SYD } & \multirow{2}{*}{ |вอุแวนว } \\
\hline & дәрім घ! u & ग) ysı jo & & & \\
\hline
\end{tabular}


Table 3.1g. (continued)

\begin{tabular}{|c|c|c|c|c|c|}
\hline \multirow{2}{*}{ Chemical } & \multirow{2}{*}{ CASRN } & \multicolumn{4}{|c|}{ Ingestion of fish (concentration in water) $(\mathrm{mg} / \mathrm{L})^{*}$} \\
\hline & & $10^{-4}$ & $10^{-5}$ & $H Q=1$ & $\mathrm{HQ}=0.1$ \\
\hline Butyl Benzyl Phthlate & 000085-68-7 & & & 8.7E-02 & 8.7E-03 \\
\hline Butylate & $002008-41-5$ & & & & \\
\hline Butylchloride, $t-$ & 000507-20-0 & & & & \\
\hline Butylphthalyl Butylglycolate & 000085-70-1 & & & & \\
\hline Cacodylic Acid & $000075-60-5$ & & & $6.9 \mathrm{E}+00$ & $6.9 \mathrm{E}-01$ \\
\hline Cadmium (Diet) & $007440-43-9$ & & & $6.8 \mathrm{E}-03$ & $6.8 \mathrm{E}-04$ \\
\hline Calcium Cyanide & 000592-01-8 & & & & \\
\hline Caprolactam & 000105-60-2 & & & $1.6 \mathrm{E}+03$ & $1.6 \mathrm{E}+02$ \\
\hline Captafol & $002425-06-1$ & 7.7E-01 & 7.7E-03 & $5.7 \mathrm{E}-02$ & $5.7 \mathrm{E}-03$ \\
\hline Captan & 000133-06-2 & $2.7 \mathrm{E}+00$ & 2.7E-02 & $5.3 \mathrm{E}+00$ & $5.3 \mathrm{E}-01$ \\
\hline Carbaryl & $000063-25-2$ & & & $4.1 E+00$ & $4.1 \mathrm{E}-01$ \\
\hline Carbazole & 000086-74-8 & $3.5 \mathrm{E}-02$ & $3.5 \mathrm{E}-04$ & & \\
\hline Carbofuran & 001563-66-2 & & & $7.0 \mathrm{E}-01$ & 7.0E-02 \\
\hline Carbon Disulfide & 000075-15-0 & & & $4.9 \mathrm{E}+00$ & $4.9 \mathrm{E}-01$ \\
\hline Carbon Tetrachloride ${ }^{(6)}$ & 000056-23-5 & $3.1 \mathrm{E}-02$ & $3.1 \mathrm{E}-04$ & $1.2 \mathrm{E}-02$ & $1.2 \mathrm{E}-03$ \\
\hline Carbosulfan & $055285-14-8$ & & & & - \\
\hline Carboxin & $005234-68-4$ & & & $5.8 \mathrm{E}+00$ & $5.8 \mathrm{E}-01$ \\
\hline Chloral & $000075-87-6$ & & & & \\
\hline
\end{tabular}


Table 3.1g. (continued)

\begin{tabular}{|c|c|c|c|c|c|}
\hline \multirow{2}{*}{ Chemical } & \multirow{2}{*}{ CASRN } & \multicolumn{4}{|c|}{ Ingestion of fish (concentration in water) (mg/L)* } \\
\hline & & $10^{-4}$ & $10^{-6}$ & $\mathbf{H Q}=\mathbf{1}$ & $\mathrm{HQ}=0.1$ \\
\hline Chloramben & 000133-90-4 & & & & \\
\hline Chloranil & 000118-75-2 & & & & \\
\hline Chlordane $^{(6)}$ & $000057-74-9$ & 2.7E-05 & 2.7E-07 & $9.1 \mathrm{E}-06$ & 9.1E-07 \\
\hline Chloride & $016887-00-6$ & & & & \\
\hline Chlorimuron, Ethyl- & 090982-32-4 & & & & \\
\hline Chlorine & 007782-50-5 & & & & \\
\hline Chlorine Dioxide & 010049-04-4 & & & & \\
\hline Chlorite (Sodium Salt) & 007758-19-2 & & & & \\
\hline Chloro-1,1-difluoroethane, 1- & 000075-68-3 & & $\cdot$ & & \\
\hline Chloro-1,3-butadiene, 2- & 000126-99-8 & & & $2.0 \mathrm{E}+00$ & $2.0 \mathrm{E}-01$ \\
\hline Chloro-2-methylaniline $\mathrm{HCl}, 4-$ & 003165-93-3 & & & & \\
\hline Chloro-2-methylaniline, 4- & 000095-69-2 & & & & \\
\hline Chloroacetic Acid & $000079-11-8$ & & & $5.6 \mathrm{E}-01$ & $5.6 \mathrm{E}-02$ \\
\hline Chloroacetophenone, 2- & $000532-27-4$ & & & & \\
\hline Chloroaniline, p- & $000106-47-8$ & & & $6.8 \mathrm{E}-02$ & $6.8 \mathrm{E}-03$ \\
\hline Chlorobenzene ${ }^{(6)}$ & $000108-90-7$ & & & $3.4 \mathrm{E}-01$ & $3.4 \mathrm{E}-02$ \\
\hline Chlorobenzilate & $000510-15-6$ & 7.5E-04 & $7.5 \mathrm{E}-06$ & $1.7 \mathrm{E}-02$ & $1.7 \mathrm{E}-03$ \\
\hline Chlorobenzoic Acid, p- & $000074-11-3$ & & & $4.1 \mathrm{E}+00$ & 4.1E-01 \\
\hline
\end{tabular}


Table 3.1g. (continued)

\begin{tabular}{|c|c|c|c|c|c|}
\hline \multirow{2}{*}{ Chemical } & \multirow{2}{*}{ CASRN } & \multicolumn{4}{|c|}{ Ingestion of fish (concentration in water) (mg/L)" } \\
\hline & & $10^{-4}$ & $10^{-6}$ & $\mathbf{H Q}=\mathbf{1}$ & $\mathrm{HQ}=0.1$ \\
\hline Chlorobenzotrifluoride, 4- & $000098-56-6$ & & & $6.6 \mathrm{E}-02$ & $6.6 \mathrm{E}-03$ \\
\hline Chlorobutane, 1- & 000109-69-3 & & & $9.7 \mathrm{E}+00$ & 9.7E-01 \\
\hline Chlorobutane, 2- & $000078-86-4$ & & & & \\
\hline Chlorocyclopentadiene & $041851-50-7$ & & & & \\
\hline Chlorodibromoethane & 073506-94-2 & & & & \\
\hline Chlorodifluoromethane & 000075-45-6 & & & & \\
\hline Chloroform $^{(6)}$ & $000067-66-3$ & $2.7 \mathrm{E}+00$ & $2.7 \mathrm{E}-02$ & $6.9 \mathrm{E}-01$ & $6.9 \mathrm{E}-02$ \\
\hline Chloromethane $^{(6)}$ & $000074-87-3$ & $8.4 E+00$ & $8.4 \mathrm{E}-02$ & & \\
\hline Chloromethyl Methyl Ether & $000107-30-2$ & & & & \\
\hline Chloronaphthalene, Beta- & 000091-58-7 & & & $1.4 \mathrm{E}-01$ & $1.4 \mathrm{E}-02$ \\
\hline Chloronitrobenzene, o- & 000088-73-3 & $4.6 \mathrm{E}-01$ & $4.6 \mathrm{E}-03$ & & \\
\hline Chloronitrobenzene, $\mathrm{p}$ - & $000121-73-3$ & $4.5 \mathrm{E}-01$ & 4.5E-03 & & \\
\hline Chlorophenol, 2- & 000095-57-8 & & & $2.4 \mathrm{E}-01$ & $2.4 \mathrm{E}-02$ \\
\hline Chlorophenyl Methyl Sulfide, p- & 000123-09-1 & & & & \\
\hline Chlorophenyl Methyl Sulfoxide & $000934-73-6$ & & & & \\
\hline Chloropropane, 2- & $000075-29-6$ & & & & \\
\hline Chlorothalonil & $001897-45-6$ & $5.3 \mathrm{E}-02$ & $5.3 \mathrm{E}-04$ & 3.7E-02 & 3.7E-03 \\
\hline Chlorotoluene, o- & 000095-49-8 & & & $1.2 \mathrm{E}-01$ & $1.2 \mathrm{E}-02$ \\
\hline
\end{tabular}


Table 3.1g. (continued)

\begin{tabular}{|c|c|c|c|c|c|}
\hline \multirow{2}{*}{ Chemical } & \multirow{2}{*}{ CASRN } & \multicolumn{4}{|c|}{ Ingestion of fish (concentration in water) $(\mathrm{mg} / \mathrm{L})^{*}$} \\
\hline & & $10^{-4}$ & $10^{-6}$ & $H Q=1$ & $H Q=0.1$ \\
\hline Chlorpropham & $000101-21-3$ & & & $2.9 \mathrm{E}+00$ & $2.9 \mathrm{E}-01$ \\
\hline Chlorpyrifos & 002921-88-2 & & & $6.5 \mathrm{E}-04$ & $6.5 \mathrm{E}-05$ \\
\hline Chlorpyrifos Methyl & 005598-13-0 & & & $1.2 \mathrm{E}-02$ & $1.2 \mathrm{E}-03$ \\
\hline Chlorsulfuron & $064902-72-3$ & & & $1.2 \mathrm{E}+03$ & $1.2 \mathrm{E}+02$ \\
\hline Chlorthiophos & 060238-56-4 & & & & \\
\hline Chromium (III) (Insoluble Salts) & 016065-83-1 & & & $6.8 \mathrm{E}+00$ & $6.8 \mathrm{E}-01$ \\
\hline Chromium (VI) ${ }^{(6)}$ & $018540-29-9$ & & & 3.4E-02 & 3.4E-03 \\
\hline Chromium Salts & NA & & & & \\
\hline Chrysene $^{(2)}$ & 000218-01-9 & 3.4E-03 & 3.4E-05 & & \\
\hline Cobalt & $007440-48-4$ & & & & \\
\hline Coke Oven Emissions & $008007-45-2$ & & & & \\
\hline Copper & 007440-50-8 & & & & \\
\hline Copper Cyanide & $000544-92-3$ & & & & \\
\hline Creosote & 008001-58-9 & & & & \\
\hline Cresol, m- & $000108-39-4$ & & & $3.5 \mathrm{E}+00$ & $3.5 \mathrm{E}-01$ \\
\hline Cresol, o- & $000095-48-7$ & & & $4.1 \mathrm{E}+00$ & 4.1E-01 \\
\hline Cresol, p- & $000106-44-5$ & & & 4.1E-01 & 4.1E-02 \\
\hline Crotonaldehyde & $000123-73-9$ & $1.2 \mathrm{E}-02$ & $1.2 \mathrm{E}-04$ & & \\
\hline
\end{tabular}


Table 3.1g. (continued)

\begin{tabular}{|c|c|c|c|c|c|}
\hline \multirow{2}{*}{ Chemical } & \multirow{2}{*}{ CASRN } & \multicolumn{4}{|c|}{ Ingestion of fish (concentration in water) $(\mathrm{mg} / \mathrm{L})^{*}$} \\
\hline & & $10^{-4}$ & $10^{-6}$ & $\mathbf{H Q}=\mathbf{1}$ & $\mathrm{HQ}=\mathbf{0 . 1}$ \\
\hline Cumene & 000098-82-8 & & & $2.0 \mathrm{E}-01$ & $2.0 \mathrm{E}-02$ \\
\hline Cyanazine & $021725-46-2$ & $1.3 \mathrm{E}-02$ & $1.3 \mathrm{E}-04$ & $9.4 \mathrm{E}-02$ & $9.4 \mathrm{E}-03$ \\
\hline Cyanide (CN-) & $000057-12-5$ & & & $6.7 \mathrm{E}+00$ & 6.7E-01 \\
\hline Cyanogen & $000460-19-5$ & & & & \\
\hline Cyanogen Bromide & 000506-68-3 & & & & \\
\hline Cyanogen Chloride & $000506-77-4$ & & & $8.1 \mathrm{E}+01$ & $8.1 \mathrm{E}+00$ \\
\hline $\begin{array}{l}\text { Cyclohexane, 1,2,3,4,5-pentabromo-6- } \\
\text { chloro- }\end{array}$ & 000087-84-3 & & & & \\
\hline Cyclohexanone & 000108-94-1 & & & $2.8 \mathrm{E}+03$ & $2.8 \mathrm{E}+02$ \\
\hline Cyclohexylamine & 000108-91-8 & & & $3.3 \mathrm{E}+01$ & $3.3 \mathrm{E}+00$ \\
\hline Cyclopentadiene & $000542-92-7$ & & & & \\
\hline Cyhalothrin/karate & 068085-85-8 & & & $6.5 \mathrm{E}-05$ & $6.5 \mathrm{E}-06$ \\
\hline Cypermethrin & $052315-07-8$ & & & & \\
\hline Cyromazine & $066215-27-8$ & & & & \\
\hline DDD & $000072-54-8$ & 8.7E-05 & 8.7E-07 & & \\
\hline $\mathrm{DDE}$ & $000072-55-9$ & 7.3E-05 & 7.3E-07 & & \\
\hline DDT & $000050-29-3$ & $2.3 \mathrm{E}-05$ & $2.3 \mathrm{E}-07$ & $1.7 \mathrm{E}-05$ & $1.7 \mathrm{E}-06$ \\
\hline Dacthal & $001861-32-1$ & & & & \\
\hline
\end{tabular}


Table 3.1g. (continued)

\begin{tabular}{|c|c|c|c|c|c|}
\hline \multirow{2}{*}{ Chemical } & \multirow{2}{*}{ CASRN } & \multicolumn{4}{|c|}{ Ingestion of fish (concentration in water) (mg/L)" } \\
\hline & & $10^{-4}$ & $10^{-6}$ & $\mathbf{H Q}=\mathbf{1}$ & $\mathrm{HQ}=0.1$ \\
\hline Dalapon & 000075-99-0 & & & $1.8 \mathrm{E}+01$ & $1.8 \mathrm{E}+00$ \\
\hline Decabromodiphenyl Ether & 001163-19-5 & & & $2.4 \mathrm{E}-03$ & 2.4E-04 \\
\hline Demeton & 008065-48-3 & & & & \\
\hline Di(2-ethylhexyl)adipate & 000103-23-1 & 5.3E-04 & $5.3 \mathrm{E}-06$ & $1.6 \mathrm{E}-03$ & $1.6 \mathrm{E}-04$ \\
\hline Diallate & 002303-16-4 & $2.4 \mathrm{E}+00$ & $2.4 \mathrm{E}-02$ & & \\
\hline Diazinon & 000333-41-5 & & & $2.7 \mathrm{E}-03$ & 2.7E-04 \\
\hline Dibenz[a,h]anthracene ${ }^{(2)}$ & 000053-70-3 & $5.0 \mathrm{E}-07$ & $5.0 \mathrm{E}-09$ & & \\
\hline Dibromo-3-chloropropane, 1,2- & 000096-12-8 & $4.0 \mathrm{E}-03$ & 4.0E-05 & & \\
\hline Dibromobenzene, 1,4- & 000106-37-6 & & & $3.0 \mathrm{E}-02$ & $3.0 \mathrm{E}-03$ \\
\hline Dibromochloromethane & 000124-48-1 & $1.4 \mathrm{E}-01$ & $1.4 \mathrm{E}-03$ & $9.8 \mathrm{E}-01$ & $9.8 \mathrm{E}-02$ \\
\hline Dibromodichloromethane & 000594-18-3 & & & & \\
\hline Dibromodiphenyl Ether, p,p'- & $002050-47-7$ & & & & \\
\hline Dibromoethane, 1,2- & 000106-93-4 & $1.9 \mathrm{E}-04$ & $1.9 \mathrm{E}-06$ & & \\
\hline $\begin{array}{l}\text { Dibromomethane (Methylene } \\
\text { Bromide) }\end{array}$ & 000074-95-3 & & & $2.8 \mathrm{E}+00$ & $2.8 \mathrm{E}-01$ \\
\hline Dibutyl Phthalate & 000084-74-2 & & & $4.3 \mathrm{E}-02$ & 4.3E-03 \\
\hline Dicamba & 001918-00-9 & & & $3.6 \mathrm{E}-01$ & $3.6 \mathrm{E}-02$ \\
\hline Dichloro-2-butene, 1,4- & $000764-41-0$ & & & & \\
\hline
\end{tabular}


Table 3.1g. (continued)

\begin{tabular}{|c|c|c|c|c|c|}
\hline \multirow{2}{*}{ Chemical } & \multirow{2}{*}{ CASRN } & \multicolumn{4}{|c|}{ Ingestion of fish (concentration in water) $(\mathrm{mg} / \mathrm{L})$} \\
\hline & & $10^{-4}$ & $10^{-6}$ & $\mathbf{H Q}=\mathbf{1}$ & $H Q=0.1$ \\
\hline \multicolumn{6}{|l|}{ Dichloroacetic Acid } \\
\hline Dichlorobenzene, $1,2^{-(5)}$ & 000095-50-1 & & & $5.4 \mathrm{E}-01$ & $5.4 \mathrm{E}-02$ \\
\hline Dichlorobenzene, 1,3- & 000541-73-1 & & & & \\
\hline Dichlorobenzene, 1,4- & 000106-46-7 & $5.8 \mathrm{E}-02$ & $5.8 \mathrm{E}-04$ & & \\
\hline Dichlorobenzidine, 3,3'- & 000091-94-1 & $2.6 \mathrm{E}-03$ & 2.6E-05 & & \\
\hline Dichlorodifluoromethane & 000075-71-8 & & & $9.8 \mathrm{E}+00$ & $9.8 \mathrm{E}-01$ \\
\hline Dichloroethane, 1,1- & 000075-34-3 & & & $9.8 \mathrm{E}+00$ & 9.8E-01 \\
\hline Dichloroethane, 1,2- & 000107-06-2 & $4.3 \mathrm{E}-01$ & 4.3E-03 & & \\
\hline Dichloroethylene, 1,1- & $000075-35-4$ & $3.8 \mathrm{E}-02$ & 3.8E-04 & $8.9 \mathrm{E}-01$ & $8.9 \mathrm{E}-02$ \\
\hline $\begin{array}{l}\text { Dichloroethylene, 1,2-(Mixed } \\
\text { Isomers) }\end{array}$ & 000540-59-0 & & & $8.9 \mathrm{E}+00$ & $8.9 \mathrm{E}-01$ \\
\hline Dichloroethylene, 1,2-cis- & 000156-59-2 & & & $8.3 \mathrm{E}-01$ & 8.3E-02 \\
\hline Dichloroethylene, 1,2-trans- & $000156-60-5$ & & & $2.0 \mathrm{E}+01$ & $2.0 \mathrm{E}+00$ \\
\hline Dichlorophenol, 2,4- & 000120-83-2 & & & $4.3 \mathrm{E}-02$ & $4.3 \mathrm{E}-03$ \\
\hline Dichlorophenoxy Acetic Acid, 2,4- & 000094-75-7 & & & $2.9 \mathrm{E}-01$ & $2.9 \mathrm{E}-02$ \\
\hline Dichlorophenoxy)butyric Acid, 4-(2,4- & 000094-82-6 & & & $3.8 \mathrm{E}-02$ & $3.8 \mathrm{E}-03$ \\
\hline Dichloropropane, 1,2- & 000078-87-5 & $2.4 \mathrm{E}-01$ & $2.4 \mathrm{E}-03$ & & \\
\hline Dichloropropanol, 2,3- & 000616-23-9 & & & & \\
\hline
\end{tabular}


Table 3.1g. (continued)

\begin{tabular}{|c|c|c|c|c|c|}
\hline \multirow{2}{*}{ Chemical } & \multirow{2}{*}{ CASRN } & \multicolumn{4}{|c|}{ Ingestion of fish (concentration in water) (mg/L)" } \\
\hline & & $10^{-4}$ & $10^{-6}$ & $\mathbf{H Q}=\mathbf{1}$ & $\mathrm{HQ}=0.1$ \\
\hline Dichloropropene, 1,3- & $000542-75-6$ & $1.8 \mathrm{E}-01$ & $1.8 \mathrm{E}-03$ & $4.2 \mathrm{E}-02$ & 4.2E-03 \\
\hline Dichlorvos & $000062-73-7$ & $5.3 \mathrm{E}-02$ & $5.3 \mathrm{E}-04$ & 3.3E-02 & 3.3E-03 \\
\hline Dicofol & 000115-32-2 & & & & \\
\hline Dicyclopentadiene & 000077-73-6 & & & $4.4 \mathrm{E}-01$ & 4.4E-02 \\
\hline Dieldrin & $000060-57-1$ & $1.1 \mathrm{E}-05$ & 1.1E-07 & 3.7E-05 & 3.7E-06 \\
\hline Diethyl Phthalate & $000084-66-2$ & & & $2.3 \mathrm{E}+01$ & $2.3 \mathrm{E}+00$ \\
\hline Diethyl-p-nitrophenylphosphate & $000311-45-5$ & & & & \\
\hline Diethylene Glycol Dinitrate (DEGDN) & 000693-21-0 & & & & \\
\hline Diethylene Glycol Monobutyl Ether & $000112-34-5$ & & & & \\
\hline Diethylene Glycol Monoethyl Ether & 000111-90-0 & & & $5.3 \mathrm{E}+03$ & $5.3 \mathrm{E}+02$ \\
\hline Diethylformamide & $000617-84-5$ & & & & \\
\hline Diethylstilbesterol & $000056-53-1$ & 7.5E-09 & 7.5E-11 & & \\
\hline Difenzoquat & $043222-48-6$ & & & & \\
\hline Diflubenzuron & $035367-38-5$ & & & & \\
\hline Difluoroethane, 1,1- & $000075-37-6$ & & & & \\
\hline Diisopropyl Methylphosphonate & 001445-75-6 & & & $3.0 \mathrm{E}+01$ & $3.0 \mathrm{E}+00$ \\
\hline Dimethipin & $055290-64-7$ & & & & \\
\hline Dimethoate & $000060-51-5$ & & & 4.1E-03 & 4.1E-04 \\
\hline
\end{tabular}


Table 3.1g. (continued)

\begin{tabular}{|c|c|c|c|c|c|}
\hline \multirow{2}{*}{ Chemical } & \multirow{2}{*}{ CASRN } & \multicolumn{4}{|c|}{ Ingestion of fish (concentration in water) $(\mathrm{mg} / \mathrm{L})^{*}$} \\
\hline & & $10^{-4}$ & $10^{-6}$ & $\mathbf{H Q}=\mathbf{1}$ & $\mathrm{HQ}=\mathbf{0 . 1}$ \\
\hline Dimethoxybenzidine, 3,3'- & $000119-90-4$ & $1.6 \mathrm{E}+00$ & $1.6 \mathrm{E}-02$ & & \\
\hline Dimethyl Sulfate & 000077-78-1 & & & & \\
\hline Dimethylaniline $\mathrm{HCl}, 2,4-$ & 021436-96-4 & & & & \\
\hline Dimethylaniline, 2,4- & 000095-68-1 & & & & \\
\hline Dimethylaniline, $\mathrm{N}, \mathrm{N}-$ & $000121-69-7$ & & & $4.9 \mathrm{E}-02$ & $4.9 \mathrm{E}-03$ \\
\hline Dimethylbenzidine, 3,3'- & 000119-93-7 & $1.0 \mathrm{E}-03$ & $1.0 \mathrm{E}-05$ & & \\
\hline Dimethylethyl Lead & $107584-40-7$ & & & & \\
\hline Dimethylformamide & $000068-12-2$ & & & $1.3 \mathrm{E}+03$ & $1.3 \mathrm{E}+02$ \\
\hline Dimethylhydrazine, $1,1-^{(3)}$ & 000057-14-7 & & & & \\
\hline Dimethylhydrazine, 1,2- & 000540-73-8 & & & & \\
\hline Dimethylphenol, 2,4- & $000105-67-9$ & & & $8.2 \mathrm{E}-01$ & $8.2 \mathrm{E}-02$ \\
\hline Dimethylphenol, 2,6- & 000576-26-1 & & & $2.2 \mathrm{E}-02$ & $2.2 \mathrm{E}-03$ \\
\hline Dimethylphenol, 3,4- & 000095-65-8 & & & $4.9 \mathrm{E}-02$ & 4.9E-03 \\
\hline Dimethylphthalate ${ }^{(5)}$ & $000131-11-3$ & & & $1.4 \mathrm{E}+03$ & $1.4 \mathrm{E}+02$ \\
\hline Dimethylterephthalate & 000120-61-6 & & & $1.2 \mathrm{E}+00$ & $1.2 \mathrm{E}-01$ \\
\hline Dinitro-0-cresol, 4,6- & $000534-52-1$ & & & & \\
\hline Dinitro-0-cyclohexyl Phenol, 4,6- & 000131-89-5 & & & $5.2 \mathrm{E}+02$ & $5.2 \mathrm{E}+01$ \\
\hline Dinitrobenzene, 1,2- & $000528-29-0$ & & & $5.6 \mathrm{E}-02$ & $5.6 \mathrm{E}-03$ \\
\hline
\end{tabular}


Table 3.1g. (continued)

\begin{tabular}{|c|c|c|c|c|c|}
\hline \multirow{2}{*}{ Chemical } & \multirow{2}{*}{ CASRN } & \multicolumn{4}{|c|}{ Ingestion of fish (concentration in water) $(\mathrm{mg} / \mathrm{L})$} \\
\hline & & $10^{-4}$ & $10^{-6}$ & $\mathbf{H Q}=\mathbf{1}$ & $\mathrm{HQ}=0.1$ \\
\hline Dinitrobenzene, 1,3- & $000099-65-0$ & & & $1.4 \mathrm{E}-02$ & $1.4 \mathrm{E}-03$ \\
\hline Dinitrobenzene, 1,4- & $000100-25-4$ & & & $6.7 \mathrm{E}-02$ & $6.7 \mathrm{E}-03$ \\
\hline Dinitrophenol, 2,4- & 000051-28-5 & & & $3.3 \mathrm{E}-01$ & 3.3E-02 \\
\hline Dinitrotoluene, 2,4- & $000121-14-2$ & 2.4E-02 & $2.4 \mathrm{E}-04$ & $1.4 \mathrm{E}-01$ & 1.4E-02 \\
\hline Dinitrotoluene, 2,6- & $000606-20-2$ & $4.0 \mathrm{E}-02$ & 4.0E-04 & $1.2 \mathrm{E}-01$ & $1.2 \mathrm{E}-02$ \\
\hline Dinitrotoluene, 2-Amino-4,6- & $035572-78-2$ & & & & \\
\hline Dinoseb & 000088-85-7 & & & $3.5 \mathrm{E}-03$ & 3.5E-04 \\
\hline Dioxane, 1,4- & $000123-91-1$ & $7.8 \mathrm{E}+01$ & 7.8E-01 & & \\
\hline Diphenamid & $000957-51-7$ & & & $1.5 \mathrm{E}+00$ & $1.5 \mathrm{E}-01$ \\
\hline Diphenylamine & $000122-39-4$ & & & $1.3 \mathrm{E}-01$ & $1.3 \mathrm{E}-02$ \\
\hline Diphenylhydrazine, 1,2- & $000122-66-7$ & $4.2 \mathrm{E}-03$ & $4.2 \mathrm{E}-05$ & & \\
\hline Diquat & $000085-00-7$ & & & $1.1 \mathrm{E}+03$ & $1.1 \mathrm{E}+02$ \\
\hline Direct Black 38 & $001937-37-7$ & & & & \\
\hline Direct Blue 6 & $002602-46-2$ & & & & \\
\hline Direct Brown 95 & $016071-86-6$ & & & & \\
\hline Direct Sky Blue & $002610-05-1$ & & & & \\
\hline Disulfoton & 000298-04-4 & & & 8.4E-05 & $8.4 \mathrm{E}-06$ \\
\hline Diuron & 000330-54-1 & & & 4.1E-02 & 4.1E-03 \\
\hline
\end{tabular}


Table 3.1g. (continued)

\begin{tabular}{|c|c|c|c|c|c|}
\hline \multirow{2}{*}{ Chemical } & \multirow{2}{*}{ CASRN } & \multicolumn{4}{|c|}{ Ingestion of fish (concentration in water) $(\mathrm{mg} / \mathrm{L})^{*}$} \\
\hline & & $10^{-4}$ & $10^{-6}$ & $\mathbf{H Q}=\mathbf{1}$ & $\mathrm{HQ}=0.1$ \\
\hline Dodine & $002439-10-3$ & & & & \\
\hline EPTC & 000759-94-4 & & & & \\
\hline Endosulfan & 000115-29-7 & & & & \\
\hline Endothall & 000145-73-3 & & & & \\
\hline Endrin & $000072-20-8$ & & & $2.2 \mathrm{E}-04$ & 2.2E-05 \\
\hline Epichlorohydrin & 000106-89-8 & $7.8 E+01$ & $7.8 \mathrm{E}-01$ & $6.6 \mathrm{E}+00$ & $6.6 \mathrm{E}-01$ \\
\hline Epoxybutane, 1,2- & 000106-88-7 & & & & \\
\hline Ethephon & $016672-87-0$ & & & & \\
\hline Ethion & 000563-12-2 & & & $1.6 \mathrm{E}-04$ & $1.6 \mathrm{E}-05$ \\
\hline Ethoxyethanol Acetate, 2- & 000111-15-9 & & & $2.2 E+02$ & $2.2 \mathrm{E}+01$ \\
\hline Ethoxyethanol, 2- & $000110-80-5$ & & & $1.1 \mathrm{E}+03$ & $1.1 \mathrm{E}+02$ \\
\hline Ethyl Acetate & 000141-78-6 & & & $5.0 \mathrm{E}+02$ & $5.0 \mathrm{E}+01$ \\
\hline Ethyl Acrylate & 000140-88-5 & $1.1 E+00$ & $1.1 \mathrm{E}-02$ & & \\
\hline Ethyl Chloride & $000075-00-3$ & & & & \\
\hline Ethyl Ether & 000060-29-7 & & & $9.7 \mathrm{E}+01$ & $9.7 \mathrm{E}+00$ \\
\hline Ethyl Methacrylate & 000097-63-2 & & & $7.4 \mathrm{E}+00$ & 7.4E-01 \\
\hline Ethyl-p-nitrophenyl Phosphonate & 002104-64-5 & & & 3.0E-05 & 3.0E-06 \\
\hline Ethylbenzene ${ }^{(6)}$ & $000100-41-4$ & & & $1.0 \mathrm{E}+00$ & $1.0 \mathrm{E}-01$ \\
\hline
\end{tabular}


Table 3.1g. (continued)

\begin{tabular}{|c|c|c|c|c|c|}
\hline \multirow{2}{*}{ Chemical } & \multirow{2}{*}{ CASRN } & \multicolumn{4}{|c|}{ Ingestion of fish (concentration in water) $(\mathrm{mg} / \mathrm{L}){ }^{n}$} \\
\hline & & $10^{-4}$ & $10^{-6}$ & $\mathbf{H Q}=\mathbf{1}$ & $H Q=0.1$ \\
\hline Ethylene Cyanohydrin & 000109-78-4 & & & $9.5 \mathrm{E}+03$ & $9.5 \mathrm{E}+02$ \\
\hline Ethylene Diamine & 000107-15-3 & & & $4.2 \mathrm{E}+01$ & $4.2 \mathrm{E}+00$ \\
\hline Ethylene Glycol & 000107-21-1 & & & $1.3 E+05$ & $1.3 \mathrm{E}+04$ \\
\hline Ethylene Glycol Monobutyl Ether & 000111-76-2 & & & & \\
\hline Ethylene Oxide & $000075-21-8$ & $8.9 \mathrm{E}-01$ & $8.9 \mathrm{E}-03$ & & \\
\hline Ethylene Thiourea & 000096-45-7 & $1.5 \mathrm{E}+01$ & $1.5 \mathrm{E}-01$ & $5.8 \mathrm{E}-01$ & $5.8 \mathrm{E}-02$ \\
\hline Ethylphthalyl Ethyl Glycolate & $000084-72-0$ & & & & \\
\hline Express & $101200-48-0$ & & & & \\
\hline Fenamiphos & $022224-92-6$ & & & & \\
\hline Fenpropathrin & 039515-41-8 & & & & . \\
\hline Fluometuron & 002164-17-2 & & & $4.5 \mathrm{E}-01$ & $4.5 \mathrm{E}-02$ \\
\hline Fluoranthene & 000206-44-0 & & & $1.7 \mathrm{E}-02$ & $1.7 \mathrm{E}-03$ \\
\hline Fluorene & $000086-73-7$ & & & $4.2 \mathrm{E}-02$ & $4.2 \mathrm{E}-03$ \\
\hline Fluoride & $007782-41-4$ & & & & \\
\hline Fluridone & $059756-60-4$ & & & & \\
\hline Flurprimidol & $056425-91-3$ & & & & \\
\hline Flutolanil & $066332-96-5$ & & & & \\
\hline Fluvalinate & $069409-94-5$ & & & & \\
\hline
\end{tabular}


Table 3.1g. (continued)

\begin{tabular}{|c|c|c|c|c|c|}
\hline \multirow{2}{*}{ Chemical } & \multirow{2}{*}{ CASRN } & \multicolumn{4}{|c|}{ Ingestion of fish (concentration in water) (mg/L)" } \\
\hline & & $10^{-4}$ & $10^{-6}$ & $\mathbf{H Q}=\mathbf{1}$ & $H Q=0.1$ \\
\hline Folpet & 000133-07-3 & $6.7 \mathrm{E}-01$ & $6.7 \mathrm{E}-03$ & $1.0 \mathrm{E}+00$ & $1.0 \mathrm{E}-01$ \\
\hline Fomesafen & 072178-02-0 & & & & \\
\hline Fonofos & 000944-22-9 & & & 4.7E-03 & 4.7E-04 \\
\hline Formaldehyde & 000050-00-0 & & & $4.6 \mathrm{E}+02$ & $4.6 \mathrm{E}+01$ \\
\hline Formic Acid & 000064-18-6 & & & $1.2 \mathrm{E}+04$ & $1.2 \mathrm{E}+03$ \\
\hline Fosetyl-AL & 039148-24-8 & & & & \\
\hline Furan & $000110-00-9$ & & & $2.4 \mathrm{E}-01$ & $2.4 \mathrm{E}-02$ \\
\hline Furazolidone & 000067-45-8 & 4.3E-02 & 4.3E-04 & & \\
\hline Furfural & 000098-01-1 & & & $1.5 \mathrm{E}-02$ & $1.5 \mathrm{E}-03$ \\
\hline Furium & $000531-82-8$ & & & & \\
\hline Furmecyclox & 060568-05-0 & & & & \\
\hline Glufosinate, Ammonium & $077182-82-2$ & & & & \\
\hline Glycidyl & 000765-34-4 & & & $3.3 \mathrm{E}+00$ & 3.3E-01 \\
\hline Glyphosate & $001071-83-6$ & & & $4.4 \mathrm{E}+04$ & $4.4 \mathrm{E}+03$ \\
\hline Goal & $042874-03-3$ & & & & \\
\hline Haloxyfop, Methyl & $069806-40-2$ & & & & \\
\hline Harmony & $079277-27-3$ & & & & \\
\hline Heptachlor & $000076-44-8$ & $6.4 \mathrm{E}-05$ & $6.4 \mathrm{E}-07$ & $6.2 \mathrm{E}-04$ & $6.2 \mathrm{E}-05$ \\
\hline
\end{tabular}


Table 3.1g. (continued)

\begin{tabular}{|c|c|c|c|c|c|}
\hline \multirow{2}{*}{ Chemical } & \multirow{2}{*}{ CASRN } & \multicolumn{4}{|c|}{ Ingestion of fish (concentration in water) (mg/L)" } \\
\hline & & $10^{-4}$ & $10^{-6}$ & $\mathbf{H Q}=\mathbf{1}$ & $\mathrm{HQ}=0.1$ \\
\hline Heptachlor Epoxide & 001024-57-3 & $4.6 \mathrm{E}-06$ & $4.6 \mathrm{E}-08$ & $2.3 \mathrm{E}-06$ & 2.3E-07 \\
\hline Heptane, N- & $000142-82-5$ & & & & \\
\hline Hexabromobenzene & $000087-82-1$ & & & $1.1 \mathrm{E}-04$ & $1.1 \mathrm{E}-05$ \\
\hline Hexachlorobenzene ${ }^{(6)}$ & 000118-74-1 & 3.1E-05 & 3.1E-07 & $1.7 \mathrm{E}-04$ & 1.7E-05 \\
\hline Hexachlorobutadiene ${ }^{(6)}$ & 000087-68-3 & $1.5 \mathrm{E}-03$ & $1.5 \mathrm{E}-05$ & $1.0 \mathrm{E}-04$ & $1.0 \mathrm{E}-05$ \\
\hline Hexachlorocyclohexane, Alpha- & 000319-84-6 & 9.2E-05 & $9.2 \mathrm{E}-07$ & & \\
\hline Hexachlorócyclohexane, Beta- & 000319-85-7 & 2.7E-04 & 2.7E-06 & & \\
\hline Hexachlorocyclohexáne, Delta- & 000319-86-8 & & & & \\
\hline Hexachlorocyclohexane, Epsilon & 006108-10-7 & & & & \\
\hline Hexachlorocyclohexane, Gamma- & 000058-89-9 & $6.4 \mathrm{E}-04$ & $6.4 \mathrm{E}-06$ & $1.1 \mathrm{E}-03$ & $1.1 \mathrm{E}-04$ \\
\hline Hexachlorocyclohexane, Technical & 000608-73-1 & & & & \\
\hline Hexachlorocyclopentadiene & $000077-47-4$ & & & $1.5 \mathrm{E}-02$ & $1.5 \mathrm{E}-03$ \\
\hline Hexachlorodibenzo-p-dioxin, Mixture & 019408-74-3 & $7.0 \mathrm{E}-10$ & $7.0 \mathrm{E}-12$ & & \\
\hline Hexachloroethane & $000067-72-1$ & $4.2 \mathrm{E}-02$ & $4.2 \mathrm{E}-04$ & $2.5 \mathrm{E}-03$ & $2.5 \mathrm{E}-04$ \\
\hline Hexachlorophene & $000070-30-4$ & & & $1.4 \mathrm{E}-06$ & $1.4 \mathrm{E}-07$ \\
\hline $\begin{array}{l}\text { Hexahydro-1,3,5-trinitro-1,3,5-triazine } \\
\text { (RDX) }\end{array}$ & $000121-82-4$ & $1.5 \mathrm{E}-02$ & $1.5 \mathrm{E}-04$ & $2.1 \mathrm{E}-02$ & $2.1 \mathrm{E}-03$ \\
\hline Hexamethylene Diisocyanate, 1,6- & $000822-06-0$ & & & & \\
\hline
\end{tabular}


Table 3.1g. (continued)

\begin{tabular}{|c|c|c|c|c|c|}
\hline \multirow{2}{*}{ Chemical } & \multirow{2}{*}{ CASRN } & \multicolumn{4}{|c|}{ Ingestion of fish (concentration in water) (mg/L)" } \\
\hline & & $10^{-4}$ & $10^{-6}$ & $\mathbf{H Q}=\mathbf{1}$ & $\mathrm{HQ}=0.1$ \\
\hline Hexane, $N-$ & $000110-54-3$ & & & $1.5 \mathrm{E}-01$ & $1.5 \mathrm{E}-02$ \\
\hline Hexanone, 2- & 000591-78-6 & & & & \\
\hline Hexazinone & 051235-04-2 & & & $1.7 \mathrm{E}+05$ & $1.7 \mathrm{E}+04$ \\
\hline $\mathrm{HpCDD}, 2,3,7,8-^{(4)}$ & $037871-00-4$ & & & & \\
\hline $\mathrm{HpCDF}, 2,3,7,8$-(4) $^{-1}$ & 038998-75-3 & & & & \\
\hline HxCDD, 2,3,7,8-(4) & $034465-46-8$ & & & & \\
\hline $\mathrm{HxCDF}, 2,3,7,8-(4)$ & $055684-94-1$ & & & & \\
\hline Hydrazine & $000302-01-2$ & $7.0 \mathrm{E}+00$ & 7.0E-02 & & \\
\hline Hydrazine Sulfate & $010034-93-2$ & $7.0 \mathrm{E}+00$ & 7.0E-02 & & \\
\hline Hydrogen Chloride & $007647-01-0$ & & & & \\
\hline Hydrogen Cyanide & $000074-90-8$ & & & $2.4 \mathrm{E}+01$ & $2.4 \mathrm{E}+00$ \\
\hline Hydrogen Sulfide & 007783-06-4 & & & $1.3 \mathrm{E}+00$ & $1.3 \mathrm{E}-01$ \\
\hline Hydroquinone & 000123-31-9 & & & $3.5 \mathrm{E}+01$ & $3.5 \mathrm{E}+00$ \\
\hline Imazalil & 035554-44-0 & & & & \\
\hline Imazaquin & 081335-37-7 & & & & \\
\hline Indeno $[1,2,3-c d]$ pyrene $e^{(2)}$ & 000193-39-5 & 7.1E-06 & 7.1E-08 & & \\
\hline Iprodione & $036734-19-7$ & & & & \\
\hline Iron & 007439-89-6 & & & & \\
\hline
\end{tabular}


Table 3.1g. (continued)

\begin{tabular}{|c|c|c|c|c|c|}
\hline \multirow{2}{*}{ Chemical } & \multirow{2}{*}{ CASRN } & \multicolumn{4}{|c|}{ Ingestion of fish (concentration in water) $(\mathrm{mg} / \mathrm{L}){ }^{*}$} \\
\hline & & $10^{-4}$ & $10^{-6}$ & $\mathbf{H Q}=\mathbf{1}$ & $\mathrm{HQ}=0.1$ \\
\hline Isobutyl Alcohol & 000078-83-1 & & & $1.6 \mathrm{E}+02$ & $1.6 \mathrm{E}+01$ \\
\hline Isophorone & 000078-59-1 & $2.9 \mathrm{E}+01$ & 2.9E-01 & 2.3E+01 & $2.3 \mathrm{E}+00$ \\
\hline Isopropalin & $033820-53-0$ & & & & \\
\hline Isopropanol & 000067-63-0 & & & & \\
\hline Isopropyl Methyl Phosphonic Acid & 001832-54-8 & & $\cdot$ & & \\
\hline Isoxaben & 082558-50-7 & & & & \\
\hline Karate & 091465-08-6 & & & & \\
\hline Kerb & $023950-58-5$ & & & & \\
\hline Lactofen & $077501-63-4$ & & & & \\
\hline Lead Alkyls & NA & & & & \\
\hline Lead And Compounds & 007439-92-1 & & & & \\
\hline Linuron & $000330-55-2$ & & & & \\
\hline Lithium & 007439-93-2 & & & & \\
\hline Londax & 083055-99-6 & & & & \\
\hline MCPA & 000094-74-6 & & & 2.1E-02 & $2.1 \mathrm{E}-03$ \\
\hline MCPB & $000094-81-5$ & & & & \\
\hline MCPP & 000093-65-2 & & & $2.4 \mathrm{E}-02$ & $2.4 \mathrm{E}-03$ \\
\hline Magnesium & 007439-95-4 & & & & \\
\hline
\end{tabular}


Table 3.1g. (continued)

\begin{tabular}{|c|c|c|c|c|c|}
\hline \multirow{2}{*}{ Chemical } & \multirow{2}{*}{ CASRN } & \multicolumn{4}{|c|}{ Ingestion of fish (concentration in water) (mg/L) } \\
\hline & & $10^{-4}$ & $10^{-6}$ & $\mathbf{H Q}=\mathbf{1}$ & $\mathrm{HQ}=\mathbf{0 . 1}$ \\
\hline Malathion & $000121-75-5$ & & & $2.9 \mathrm{E}-01$ & $2.9 \mathrm{E}-02$ \\
\hline Maleic Anhydride & $000108-31-6$ & & & & \\
\hline Maleic Hydrazide & $000123-33-1$ & & & $2.3 \mathrm{E}+03$ & $2.3 \mathrm{E}+02$ \\
\hline Malononitrile & 000109-77-3 & & & 4.3E-02 & $4.3 \mathrm{E}-03$ \\
\hline Mancozeb & 008018-01-7 & & & & \\
\hline Maneb & $012427-38-2$ & & & $1.1 E+01$ & $1.1 \mathrm{E}+00$ \\
\hline Manganese (Diet) & 007439-96-5 & & & 4.7E-01 & $4.7 \mathrm{E}-02$ \\
\hline Mephosfolan & $000950-10-7$ & & & & \\
\hline Mepiquat Chloride & $024307-26-4$ & & & & \\
\hline Mercuric Chloride & $007487-94-7$ & & & & \\
\hline Mercury (elemental) & 007439-97-6 & & & 4.1E-04 & 4.1E-05 \\
\hline Mercury, Inorganic Salt & $007439-97-6$ & & & 4.1E-04 & 4.1E-05 \\
\hline Merphos & 000150-50-5 & & & & \\
\hline Merphos Oxide & $000078-48-8$ & & & $3.2 \mathrm{E}-06$ & $3.2 \mathrm{E}-07$ \\
\hline Metalaxyl & $057837-19-1$ & & & & \\
\hline Methacrylonitrile & $000126-98-7$ & & & $8.9 \mathrm{E}-02$ & $8.9 \mathrm{E}-03$ \\
\hline Methamidophos & $010265-92-6$ & & & $3.6 \mathrm{E}-01$ & $3.6 \mathrm{E}-02$ \\
\hline Methanol & $000067-56-1$ & & & $4.4 E+03$ & $4.4 E+02$ \\
\hline
\end{tabular}




\begin{tabular}{|c|c|c|c|c|c|}
\hline & & $20-\exists 0^{\circ} \varepsilon$ & $00+30^{\circ} \varepsilon$ & $s-I \tau-9 \varepsilon 9000$ & 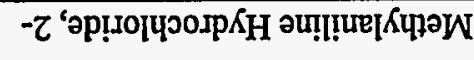 \\
\hline & & $\varepsilon 0-96^{\circ} t$ & $10-96 . t$ & $8-\varsigma \varsigma-660000$ & 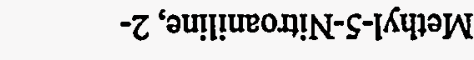 \\
\hline & & & & $\downarrow \vdash 0-\downarrow \varepsilon 9100$ & 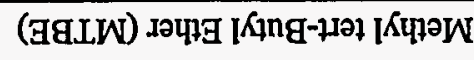 \\
\hline & & & & $\downarrow-t \varepsilon-090000$ & 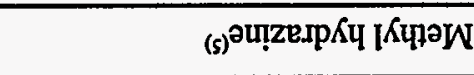 \\
\hline$\varepsilon 0^{-96} \varepsilon$ & $20-\exists 6^{\circ} \varepsilon$ & & & $\downarrow-S I-\varepsilon I 0 S Z O$ & 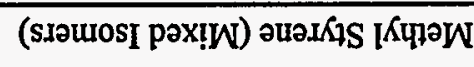 \\
\hline$\varepsilon 0-$ Gl' $Z$ & Z0-ヨI'Z & & & $0-00-862000$ & 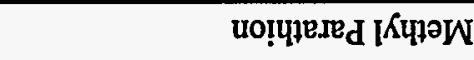 \\
\hline \multirow[t]{2}{*}{$00+36^{\circ} I$} & $\mathrm{I} 0+\mathrm{B} 6^{\circ} \mathrm{I}$ & & & $9-Z 9-080000$ & 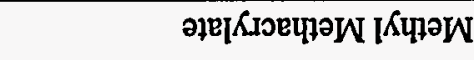 \\
\hline & & & & $9-Z 6-\angle 96 Z Z 0$ & KInગ.əW IКц1әW \\
\hline $00+\exists z \cdot z$ & $10+\theta z \cdot \tau$ & & & {$[-0 I-80 I 000$} & 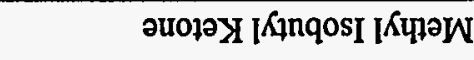 \\
\hline$[0+39 \cdot 8$ & $20+39 \cdot 8$ & & & $\varepsilon-\varepsilon 6-8<0000$ & 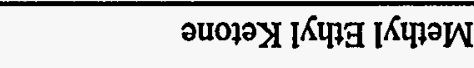 \\
\hline $00+3 I \tau$ & $I 0+3 I^{\circ} Z$ & & & $\varepsilon-\varepsilon \varepsilon-960000$ & 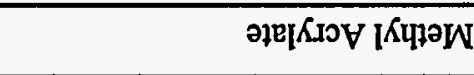 \\
\hline $20+B L \cdot I$ & $\varepsilon 0+\exists L \cdot I$ & & & 6-0z-6L0000 & 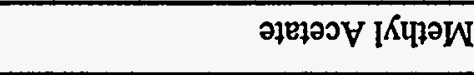 \\
\hline \multirow[t]{2}{*}{$10-98 \cdot 8$} & $00+788^{\circ} 8$ & & & $t-98-601000$ & 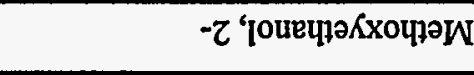 \\
\hline & & & & $9-6 t-0$ I L000 & 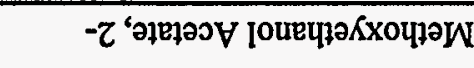 \\
\hline \multirow[t]{2}{*}{$t 0-a z \cdot S$} & $\varepsilon 0-a z \cdot s$ & & & $s-\varepsilon t-Z<0000$ & ХоІчОКхочю्) \\
\hline & & $\varepsilon 0-9 t^{\circ} 8$ & $10-9 t^{\prime} 8$ & $z-6 S-660000$ & 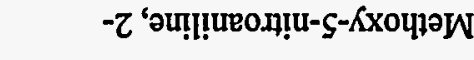 \\
\hline \multirow[t]{2}{*}{$00+30 \% 2$} & $10+30 \cdot z$ & & & $S-L L-Z S \angle 910$ & ІКшочрәЈ \\
\hline & & & & $8-L E-0 S 6000$ & 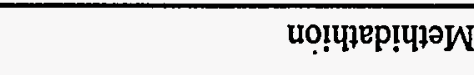 \\
\hline$I^{*} 0=\mathbf{O H}$ & $\mathrm{I}=\mathbf{O} \mathbf{H}$ & $9-01$ & r.OI & \multirow{2}{*}{ N\&SVD } & \multirow{2}{*}{ [षวุயวนุด } \\
\hline & Iอ|вM แ! u & ICU & & & \\
\hline
\end{tabular}


Table 3.1g. (continued)

\begin{tabular}{|c|c|c|c|c|c|}
\hline \multirow{2}{*}{ Chemical } & \multirow{2}{*}{ CASRN } & \multicolumn{4}{|c|}{ Ingestion of fish (concentration in water) (mg/L)" } \\
\hline & & $10^{-4}$ & $10^{-6}$ & $\mathbf{H Q}=\mathbf{1}$ & $\mathrm{HQ}=0.1$ \\
\hline Methylcyclohexane & 000108-87-2 & & & & \\
\hline Methylene Chloride & 000075-09-2 & $7.3 E+00$ & 7.3E-02 & $1.4 \mathrm{E}+01$ & $1.4 \mathrm{E}+00$ \\
\hline Methylene-bis(2-chloroaniline), 4,4'- & $000101-14-4$ & $4.5 \mathrm{E}-03$ & $4.5 \mathrm{E}-05$ & $1.7 \mathrm{E}-03$ & 1.7E-04 \\
\hline $\begin{array}{l}\text { Methylene-bis(N,N-dimethyl) Aniline, } \\
4,4^{\prime} \text { - }\end{array}$ & $000101-61-1$ & $2.6 \mathrm{E}-03$ & $2.6 \mathrm{E}-05$ & & \\
\hline Methylenebisbenzenamine, $4,4^{\prime}\left({ }^{(3)}\right.$ & $000101-77-9$ & $1.3 \mathrm{E}-01$ & $1.3 \mathrm{E}-03$ & & \\
\hline Methylenediphenyl Isocyanate, 4,4'- & 000101-68-8 & & & & \\
\hline Methylstyrene, Alpha- & 000098-83-9 & & & $4.6 \mathrm{E}-01$ & $4.6 \mathrm{E}-02$ \\
\hline Methyltriethyl Lead & 001762-28-3 & & & & \\
\hline Metolachlor & $051218-45-2$ & & & & \\
\hline Metribuzin $^{(3)}$ & 021087-64-9 & & & $2.9 \mathrm{E}+00$ & $2.9 \mathrm{E}-01$ \\
\hline Mirex ${ }^{(3)}$ & 002385-85-5 & $2.9 \mathrm{E}-05$ & $2.9 \mathrm{E}-07$ & $4.5 \mathrm{E}-05$ & $4.5 \mathrm{E}-06$ \\
\hline Molinate & $002212-67-1$ & & & $9.5 \mathrm{E}-03$ & $9.5 \mathrm{E}-04$ \\
\hline Molybdenum & 007439-98-7 & & & $6.8 \mathrm{E}-01$ & $6.8 \mathrm{E}-02$ \\
\hline Monochloramine & 010599-90-3 & & & & \\
\hline Monochlorobutanes & $025154-42-1$ & & & & \\
\hline Naled & $000300-76-5$ & & & $3.4 \mathrm{E}-02$ & $3.4 \mathrm{E}-03$ \\
\hline Naphthalene ${ }^{(6)}$ & 000091-20-3 & & & $2.5 \mathrm{E}-01$ & $2.5 \mathrm{E}-02$ \\
\hline
\end{tabular}


Table 3.1g. (continued)

\begin{tabular}{|c|c|c|c|c|c|}
\hline \multirow{2}{*}{ Chemical } & \multirow{2}{*}{ CASRN } & \multicolumn{4}{|c|}{ Ingestion of fish (concentration in water) (mg/L)" } \\
\hline & & $10^{-4}$ & $10^{-6}$ & $\mathbf{H Q}=\mathbf{1}$ & $\mathrm{HQ}=0.1$ \\
\hline Naphthalene, 1-Methyl & $000090-12-0$ & & & & \\
\hline Naphthalene, 2-Methyl & $000091-57-6$ & & & & \\
\hline Napropamide & 015299-99-7 & & & $7.2 E+01$ & $7.2 \mathrm{E}+00$ \\
\hline Niagara Blue 4B & 002429-74-5 & & & & \\
\hline Nickel Carbonyl & 013463-39-3 & & & & \\
\hline Nickel Refinery Dust & NA & & & & \\
\hline Nickel Soluble Salts & $007440-02-0$ & & & $2.7 \mathrm{E}-01$ & 2.7E-02 \\
\hline Nickel Subsulfide & $012035-72-2$ & & & & \\
\hline Nitrapyrin $^{(5)}$ & 001929-82-4 & & & $1.8 \mathrm{E}-02$ & $1.8 \mathrm{E}-03$ \\
\hline Nitrate & 014797-55-8 & & & & \\
\hline Nitric Oxide ${ }^{(5)}$ & 010102-43-9 & & & & \\
\hline Nitrite & $014797-65-0$ & & & & \\
\hline Nitroaniline, 2- & 000088-74-4 & & & & \\
\hline Nitrobenzene & 000098-95-3 & & & $4.9 \mathrm{E}-02$ & $4.9 \mathrm{E}-03$ \\
\hline Nitrofurantoin & 000067-20-9 & & & & \\
\hline Nitrofurazone & 000059-87-0 & & & & \\
\hline Nitrogen Dioxide ${ }^{(5)}$ & 010102-44-0 & & & & \\
\hline Nitroguanidine & 000556-88-7 & & & & \\
\hline
\end{tabular}




\begin{tabular}{|c|c|c|c|c|c|}
\hline & & & & $\tau-\varepsilon I-\downarrow I \varepsilon L Z O$ & LOZEInJION \\
\hline$z 0-\exists t^{\prime} \varepsilon$ & $I 0-\exists t^{\circ} \mathcal{E}$ & & & $0-66-660000$ & 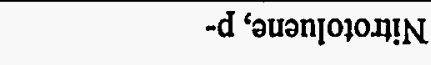 \\
\hline $20-3 I^{\prime} t$ & $I 0-9 I^{\prime} t$ & & & $\tau-Z L-880000$ & 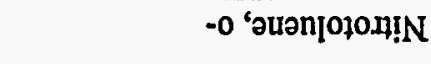 \\
\hline \multirow[t]{15}{*}{$z 0-\exists b^{\circ} \varepsilon$. } & 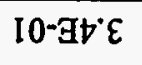 & & & {$[-80-660000$} & -u 'әuวก|одод!N \\
\hline & & & & t-ZE-6II000 & 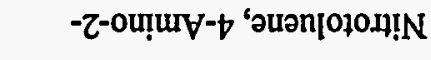 \\
\hline & & $20-3 L \cdot I$ & $00+\theta L \cdot I$ & $\tau-\mathcal{S S}-0 \varepsilon 6000$ & -N 'ou!p!|OLuKdoson! $N$ \\
\hline & & & & $0-0 b-6 t s+00$ & 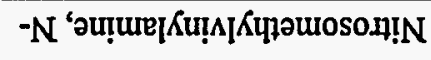 \\
\hline & & & & $9-\varsigma 6-\varsigma 6 \varsigma 0 I 0$ & 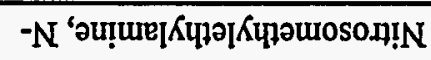 \\
\hline & & $\varepsilon 0-988^{\circ} \downarrow$ & $10-98^{\circ} t$ & $9-0 \varepsilon-980000$ & -N “әu!ure]кuәчd!poso.p!N \\
\hline & & $t 0-988^{\prime} z$ & $20-38 \%$ & 6-SL-Z90000 & -N 'әи!ure[Кцрәш!роsод!N \\
\hline & & SO-GS'I & $\varepsilon 0-\exists S^{\prime} I$ & $\varsigma-8[-\varsigma \varsigma 0000$ & 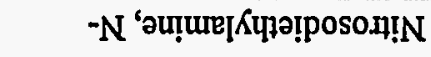 \\
\hline & & $20-\exists I^{\circ} \varepsilon$ & $00+\exists I^{\circ} \mathcal{E}$ & L-tS-9III00 & 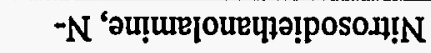 \\
\hline & & S0-99.9 & E0-39.9 & L-t9-IZ9000 & -N 'әu!ure[Sdodd-N-!p-oson!! \\
\hline & & S0- $39^{\circ} \varepsilon$ & $\varepsilon 0-39^{\circ} \varepsilon$ & $\varepsilon-9[-\downarrow Z 6000$ & 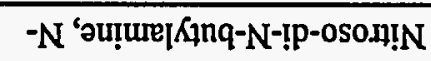 \\
\hline & & & & $\varsigma-\varepsilon 6-\downarrow 89000$ & 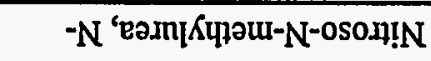 \\
\hline & & $s 0-39^{\circ} \tau$ & $\varepsilon 0-899^{\prime} 乙$ & $6-\varepsilon L-6 S L 000$ & 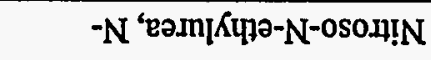 \\
\hline & & $t 0-a z \cdot z$ & Z0-घ乙乙乙 & $6-9 b-6<0000$ & $(s))^{Z}$ 'vurdosdon!N \\
\hline & & & & $L-20-00 I 000$ & - ' 'ouəudon!N \\
\hline$I^{\prime} 0=\partial H$ & $\mathbf{I}=\mathbf{O H}$ & $9.0 \mathrm{I}$ & rOI & \multirow{2}{*}{ N\&SVכ } & \multirow{2}{*}{ |вगุนวุว } \\
\hline \multicolumn{4}{|c|}{ 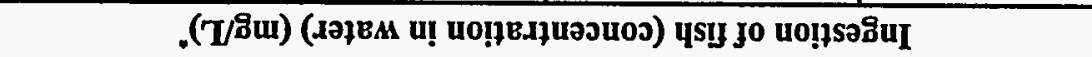 } & & \\
\hline
\end{tabular}


Table 3.1g. (continued)

\begin{tabular}{|c|c|c|c|c|c|}
\hline \multirow{2}{*}{ Chemical } & \multirow{2}{*}{ CASRN } & \multicolumn{4}{|c|}{ Ingestion of fish (concentration in water) $(\mathrm{mg} / \mathrm{L})^{*}$} \\
\hline & & $10^{-4}$ & $10^{-6}$ & $\mathbf{H Q}=\mathbf{1}$ & $\mathrm{HQ}=0.1$ \\
\hline Nustar & 085509-19-9 & & & & \\
\hline $\mathrm{OCDD}^{(4)}$ & 003268-87-9 & $7.6 \mathrm{E}-10$ & $7.6 \mathrm{E}-12$ & & \\
\hline $\mathrm{OCDF}^{(4)}$ & 039001-02-0 & & & & \\
\hline Octabromodiphenyl Ether & 032536-52-0 & & & & \\
\hline $\begin{array}{l}\text { Octahydro-1,3,5,7-tetranitro-1,3,5,7- } \\
\text { tetra (HMX) }\end{array}$ & $002691-41-0$ & & & 4.1E+01 & $4.1 \mathrm{E}+00$ \\
\hline Octamethylpyrophosphoramide & 000152-16-9 & & & & \\
\hline Octyl Phthalate, di-N- & $000117-84-0$ & & & 4.7E-06 & 4.7E-07 \\
\hline Oryzalin & 019044-88-3 & & & & \\
\hline Oxadiazon & 019666-30-9 & & & & \\
\hline Oxamyl & 023135-22-0 & & & & \\
\hline Paclobutrazol & 076738-62-0 & & & & \\
\hline Paraquat & $001910-42-5$ & & & $1.7 E+04$ & $1.7 \mathrm{E}+03$ \\
\hline Parathion & 000056-38-2 & & & $1.8 \mathrm{E}-02$ & $1.8 \mathrm{E}-03$ \\
\hline PeCDD, $2,3,7,8-^{-(4)}$ & 036088-22-9 & 8.2E-11 & $8.2 \mathrm{E}-13$ & & \\
\hline PeCDF, $1,2,3,7,8-^{(4)}$ & $057117-41-6$ & & & & \\
\hline PeCDF, $2,3,4,7,8-(4)$ & $057117-31-4$ & & & & \\
\hline Pebulate & $001114-71-2$ & & & & \\
\hline
\end{tabular}




\begin{tabular}{|c|c|c|c|c|c|}
\hline$z 0-\exists t^{\circ} \varepsilon$ & {$\left[0-\exists t^{\circ} \varepsilon\right.$} & & & $9-I I-Z E<000$ & zausoपd \\
\hline \multirow[t]{3}{*}{$\varepsilon 0-\exists \varepsilon z$} & Z0-ヨE'乙 & & & $z-20-86 z 000$ & 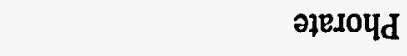 \\
\hline & & $z 0-g Z^{\prime} I$ & $00+a z I$ & $L-\varepsilon t-060000$ & -Z' 'ouəud] אuәपd \\
\hline & & & & t-8ع-290000 & 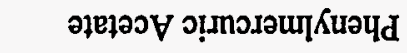 \\
\hline \multirow[t]{2}{*}{$20+39^{\circ} \varepsilon$} & $\varepsilon 0+39^{\circ} \varepsilon$ & & & $\varepsilon-0 S-901000$ & 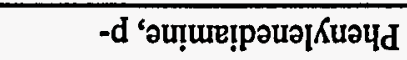 \\
\hline & & $z 0-\exists \varepsilon^{\circ} \varsigma$ & $00+B \varepsilon^{*} S$ & $s-t \varsigma-\varsigma 60000$ & 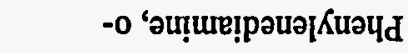 \\
\hline $00+B I^{\circ} S$ & $I 0+B I \cdot S$ & & & $\tau-\varsigma t-801000$ & -ш 'әи!ше!рәиә[Киәчd \\
\hline$\left[0+30^{\circ}\right]$ & $20+30^{\circ} I$ & & & $z-\varsigma 6-801000$ & jouəपd \\
\hline \multirow[t]{2}{*}{$20+39^{\circ} \mathrm{s}$} & $\varepsilon 0+39^{\circ} S$ & & & 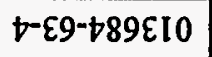 & ureчd!̣рәитиәчd \\
\hline & & & & 8- $10-\$ 80000$ & әшәгциненчд \\
\hline \multirow[t]{2}{*}{ 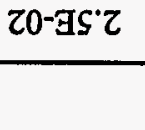 } & I0-as’z & & & $\mathfrak{I}-\varepsilon \varsigma-\varsigma$ †9ZS0 & น!ฺपрәшนә \\
\hline & & & & $0-I t-I L 0000$ & 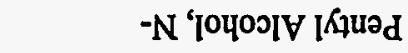 \\
\hline 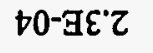 & $\varepsilon 0-\exists \varepsilon \cdot \tau$ & $90-\exists S^{\prime} I$ & $t 0-3 S I$ & $S-98-\angle 80000$ & 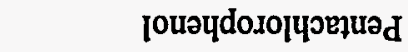 \\
\hline \multirow[t]{2}{*}{$\forall 0-\exists z^{\prime} z$} & $\varepsilon 0-\exists Z \cdot Z$ & $90-39 \cdot 9$ & t0-39・9 & $8-89-280000$ & 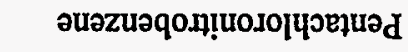 \\
\hline & & & & ৎ-ৎE-6ZEৎZO & 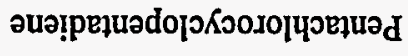 \\
\hline \multirow[t]{3}{*}{$s_{0}-\exists I \cdot z$} & $t 0-\exists\left[I^{\circ} Z\right.$ & & & s-६6-809000 & 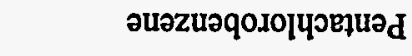 \\
\hline & & & & 6-I8-tESZE0 & 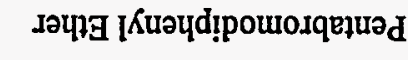 \\
\hline & & & & $I-Z t-\angle 8 t 0 t 0$ & 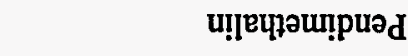 \\
\hline$I^{*} 0=\partial H$ & $\mathbf{I}=\mathbf{O H}$ & $9-0 I$ & HOI & \multirow{2}{*}{ N\&SYO } & \multirow{2}{*}{ |вว!ุயวนว } \\
\hline & OfeM u! u & b) पsIJ jo & & & \\
\hline
\end{tabular}

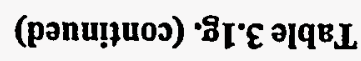


Table 3.1g. (continued)

\begin{tabular}{|c|c|c|c|c|c|}
\hline \multirow{2}{*}{ Chemical } & \multirow{2}{*}{ CASRN } & \multicolumn{4}{|c|}{ Ingestion of fish (concentration in water) $(\mathrm{mg} / \mathrm{L})^{*}$} \\
\hline & & $10^{-4}$ & $10^{-6}$ & $\mathrm{HQ}=1$ & $H Q=0.1$ \\
\hline Phosphine & 007803-51-2 & & & & \\
\hline Phosphoric Acid & 007664-38-2 & & & & \\
\hline Phthalic Acid, P- & 000100-21-0 & & & $1.2 \mathrm{E}+02$ & $1.2 \mathrm{E}+01$ \\
\hline Phthalic Anhydride & 000085-44-9 & & & $1.4 \mathrm{E}+04$ & $1.4 \mathrm{E}+03$ \\
\hline Picloram & 001918-02-1 & & & $2.4 \mathrm{E}+00$ & $2.4 \mathrm{E}-01$ \\
\hline Pirimiphos, Methyl & 029232-93-7 & & & & \\
\hline Polybrominated Biphenyls & NA & & & & \\
\hline Polychlorinated Biphenyls & 001336-36-3 & $1.9 \mathrm{E}-06$ & $1.9 \mathrm{E}-08$ & & \\
\hline Potassium Cyanide & 000151-50-8 & & & $1.8 \mathrm{E}+02$ & $1.8 \mathrm{E}+01$ \\
\hline Potassium Silver Cyanide & 000506-61-6 & & & & \\
\hline Prochloraz & 067747-09-5 & & & & \\
\hline Profluralin & 026399-36-0 & & & & \\
\hline Prometon & 001610-18-0 & & & $6.2 \mathrm{E}-01$ & $6.2 \mathrm{E}-02$ \\
\hline Prometryn & 007287-19-6 & & & $1.2 \mathrm{E}-01$ & $1.2 \mathrm{E}-02$ \\
\hline Propachlor & 001918-16-7 & & & & \\
\hline Propanil & 000709-98-8 & & & $4.9 \mathrm{E}-02$ & $4.9 \mathrm{E}-03$ \\
\hline Propargite & $002312-35-8$ & & & & \\
\hline Propargyl Alcohol & 000107-19-7 & & & & \\
\hline
\end{tabular}


Table 3.1g. (continued)

\begin{tabular}{|c|c|c|c|c|c|}
\hline \multirow{2}{*}{ Chemical } & \multirow{2}{*}{ CASRN } & \multicolumn{4}{|c|}{ Ingestion of fish (concentration in water) (mg/L)" } \\
\hline & & $10^{-4}$ & $10^{-6}$ & $\mathbf{H Q}=\mathbf{1}$ & $H Q=0.1$ \\
\hline Propazine & 000139-40-2 & & & $6.9 \mathrm{E}-01$ & $6.9 \mathrm{E}-02$ \\
\hline Propham & $000122-42-9$ & & & $1.7 \mathrm{E}-01$ & $1.7 \mathrm{E}-02$ \\
\hline Propiconazole & 060207-90-1 & & & & \\
\hline Propylene Glycol & $000057-55-6$ & & & $9.0 \mathrm{E}+05$ & $9.0 \mathrm{E}+04$ \\
\hline Propylene Glycol Monoethyl Ether & $001569-02-4$ & & & & \\
\hline Propylene Glycol Monomethyl Ether & 000107-98-2 & & & $2.2 \mathrm{E}+03$ & $2.2 \mathrm{E}+02$ \\
\hline Propylene Oxide & $000075-56-9$ & 2.1E+00 & 2.1E-02 & & \\
\hline Pursuit & 081335-77-5 & & & & \\
\hline Pydrin & 051630-58-1 & & & 2.5E-02 & $2.5 \mathrm{E}-03$ \\
\hline Pyrene & $000129-00-0$ & & & $1.3 \mathrm{E}-02^{\circ}$ & $1.3 \mathrm{E}-03$ \\
\hline Pyridine & 000110-86-1 & & & 2.4E-01 & $2.4 \mathrm{E}-02$ \\
\hline Quinalphos & 013593-03-8 & & & & \\
\hline Quinoline & 000091-22-5 & $1.1 \mathrm{E}-03$ & $1.1 \mathrm{E}-05$ & & \\
\hline Refractory Ceramic Fibers & NA & & & & \\
\hline Resmethrin & 010453-86-8 & & & & \\
\hline Ronnel & 000299-84-3 & & & $1.5 \mathrm{E}-02$ & $1.5 \mathrm{E}-03$ \\
\hline Rotenone & $000083-79-4$ & & & $7.0 \mathrm{E}-03$ & 7.0E-04 \\
\hline Savey & 078587-05-0 & & & & \\
\hline
\end{tabular}


Table 3.1g. (continued)

\begin{tabular}{|c|c|c|c|c|c|}
\hline \multirow{2}{*}{ Chemical } & \multirow{2}{*}{ CASRN } & \multicolumn{4}{|c|}{ Ingestion of fish (concentration in water) (mg/L) } \\
\hline & & $10^{-4}$ & $10^{-6}$ & $\mathbf{H Q}=\mathbf{1}$ & $\mathrm{HQ}=0.1$ \\
\hline Selenious Acid & 007783-00-8 & & & & \\
\hline Selenite & $014124-67-5$ & & & & \\
\hline Selenium & $007782-49-2$ & & & & \\
\hline Selenium Sulfide & $007446-34-6$ & & & & \\
\hline Selenourea & 000630-10-4 & & & & \\
\hline Sethoxydim & 074051-80-2 & & & & \\
\hline Silver & 007440-22-4 & & & $1.4 \mathrm{E}+00$ & $1.4 \mathrm{E}-01$ \\
\hline Silver Cyanide & 000506-64-9 & & & & \\
\hline Simazine & 000122-34-9 & $9.5 \mathrm{E}-02$ & $9.5 \mathrm{E}-04$ & $2.4 \mathrm{E}-01$ & 2.4E-02 \\
\hline Sodium & 007440-23-5 & & & & \\
\hline Sodium Acifluorfen & 062476-59-9 & & & & \\
\hline Sodium Azide & 026628-22-8 & & & & \\
\hline Sodium Cyanide & 000143-33-9 & & & $3.9 \mathrm{E}+02$ & $3.9 \mathrm{E}+01$ \\
\hline Sodium Diethyldithiocarbamate & 000148-18-5 & & & & \\
\hline Sodium Fluoroacetate & $000062-74-8$ & & & & \\
\hline Sodium Metavanadate & $013718-26-8$ & & & & \\
\hline Stirofos (Tetrachlorovinphos) & $000961-11-5$ & & & & \\
\hline Strontium, Stable & 007440-24-6 & & & $1.4 \mathrm{E}+01$ & $1.4 \mathrm{E}+00$ \\
\hline
\end{tabular}


Table 3.1g. (continued)

\begin{tabular}{|c|c|c|c|c|c|}
\hline \multirow{2}{*}{ Chemical } & \multirow{2}{*}{ CASRN } & \multicolumn{4}{|c|}{ Ingestion of fish (concentration in water) (mg/L)" } \\
\hline & & $10^{-4}$ & $10^{-6}$ & $\mathbf{H Q}=\mathbf{1}$ & $\mathrm{HQ}=\mathbf{0 . 1}$ \\
\hline Strychnine & 000057-24-9 & & & $2.5 \mathrm{E}-02$ & $2.5 \mathrm{E}-03$ \\
\hline Styrene ${ }^{(())}$ & $000100-42-5$ & & & $2.9 \mathrm{E}+00$ & $2.9 \mathrm{E}-01$ \\
\hline Sulfate & 014808-79-8 & & & & \\
\hline Systhane & 088671-89-0 & & & & \\
\hline TCDD, 2,3,7,8-(4) & 001746-01-6 & $2.4 \mathrm{E}-11$ & 2.4E-13 & & \\
\hline TCDF, $2,3,7,8^{-(4)}$ & 051207-31-9 & $1.3 \mathrm{E}-09$ & $1.3 \mathrm{E}-11$ & & \\
\hline TCMTB & $021564-17-0$ & & & & \\
\hline Tebuthiuron & 034014-18-1 & & & $1.2 \mathrm{E}+02$ & $1.2 \mathrm{E}+01$ \\
\hline Temephos & 003383-96-8 & & & & \\
\hline Terbacil & 005902-51-2 & & & & \\
\hline Terbufos & $013071-79-9$ & & & $9.2 \mathrm{E}-05$ & $9.2 \mathrm{E}-06$ \\
\hline Terbutryn & 000886-50-0 & & & 3.3E-03 & $3.3 \mathrm{E}-04$ \\
\hline Tetrabutyl Lead & 001920-90-7 & & & & \\
\hline Tetrachlorobenzene, $1,2,4,5-$ & 000095-94-3 & & & $2.2 \mathrm{E}-04$ & $2.2 \mathrm{E}-05$ \\
\hline Tetrachloroethane, 1,1,1,2- & 000630-20-6 & $1.1 \mathrm{E}-01$ & $1.1 \mathrm{E}-03$ & $3.6 \mathrm{E}-01$ & $3.6 \mathrm{E}-02$ \\
\hline Tetrachloroethane, 1,1,2,2- & $000079-34-5$ & $4.0 \mathrm{E}-02$ & $4.0 \mathrm{E}-04$ & & \\
\hline Tetrachloroethylene ${ }^{(6)}$ & $000127-18-4$ & $1.1 \mathrm{E}-01$ & $1.1 \mathrm{E}-03$ & $2.4 \mathrm{E}-01$ & $2.4 \mathrm{E}-02$ \\
\hline Tetrachlorophenol, 2,3,4,6- & 000058-90-2 & & & $5.3 \mathrm{E}-02$ & $5.3 \mathrm{E}-03$ \\
\hline
\end{tabular}


Table 3.1g. (continued)

\begin{tabular}{|c|c|c|c|c|c|}
\hline \multirow{2}{*}{ Chemical } & \multirow{2}{*}{ CASRN } & \multicolumn{4}{|c|}{ Ingestion of fish (concentration in water) $(\mathrm{mg} / \mathrm{L}){ }^{*}$} \\
\hline & & $10^{-4}$ & $10^{-6}$ & $\mathbf{H Q}=\mathbf{1}$ & HQ $=0.1$ \\
\hline $\begin{array}{l}\text { Tetrachlorotoluene, p- alpha, alpha, } \\
\text { alpha- }\end{array}$ & 005216-25-1 & & & & \\
\hline Tetraethyl Dithiopyrophosphate & $003689-24-5$ & & & $6.0 \mathrm{E}-03$ & $6.0 \mathrm{E}-04$ \\
\hline Tetraethyl Lead & 000078-00-2 & & & & \\
\hline Tetrafluoroethane, 1,1,1,2- & $000811-97-2$ & & & & \\
\hline Tetramethyl Lead & $000075-74-1$ & & & & \\
\hline Tetrapropyl Lead & 003440-75-3 & & & & \\
\hline Thallic Oxide ${ }^{(5)}$ & 001314-32-5 & & & & \\
\hline Thallium (I) Nitrate & $010102-45-1$ & & & & \\
\hline Thallium (Soluble Salts) & 007440-28-0 & & & & \\
\hline Thallium Acetate & 000563-68-8 & & & & \\
\hline Thallium Carbonate & 006533-73-9 & & & & \\
\hline Thallium Chloride & 007791-12-0 & & & & \\
\hline Thallium Selenite $^{(s)}$ & 012039-52-0 & & & & \\
\hline Thallium Sulfate & 007446-18-6 & & & & \\
\hline Thiobencarb & $028249-77-6$ & & & & \\
\hline Thiofanox & 039196-18-4 & & & $1.3 \mathrm{E}+01$ & $1.3 \mathrm{E}+00$ \\
\hline Thiophanate, Methyl & 023564-05-8 & & & & \\
\hline
\end{tabular}


Table 3.1g. (continued)

\begin{tabular}{|c|c|c|c|c|c|}
\hline \multirow{2}{*}{ Chemical } & \multirow{2}{*}{ CASRN } & \multicolumn{4}{|c|}{ Ingestion of fish (concentration in water) (mg/L)" } \\
\hline & & $10^{-4}$ & $10^{-6}$ & $\mathbf{H Q}=1$ & $\mathrm{HQ}=0.1$ \\
\hline Thiram & $000137-26-8$ & & & & \\
\hline Thorium & $007440-29-1$ & & & & \\
\hline Tin & 007440-31-5 & & & 2.7E-01 & 2.7E-02 \\
\hline Titanium & $007440-32-6$ & & & & \\
\hline Toluene ${ }^{(6)}$ & 000108-88-3 & & & 4.1E+00 & 4.1E-01 \\
\hline Toluene diisocyanate mixture (TDI) & $026471-62-5$ & & & & \\
\hline Toluene-2,4-diamine & 000095-80-7 & 9.2E-02 & 9.2E-04 & & \\
\hline Toluene-2,5-diamine & 000095-70-5 & & & & \\
\hline Toluene-2,6-diamine & $000823-40-5$ & & & & \\
\hline Toluidine, o- (Methylaniline, 2-) & 000095-53-4 & $2.3 \mathrm{E}-01$ & $2.3 \mathrm{E}-03$ & & \\
\hline Toluidine, $\mathrm{p}$ - & $000106-49-0$ & $8.5 \mathrm{E}-02$ & 8.5E-04 & & \\
\hline Toxaphene & $008001-35-2$ & $1.1 \mathrm{E}-04$ & $1.1 E-06$ & & \\
\hline Tralomethrin & $066841-25-6$ & & & & \\
\hline Triallate & $002303-17-5$ & & & $2.8 \mathrm{E}-02$ & $2.8 \mathrm{E}-03$ \\
\hline Triasulfuron & 082097-50-5 & & & & \\
\hline Tribromobenzene, 1,2,4- & 000615-54-3 & & & & \\
\hline Tribromochloromethane & $000594-15-0$ & & & & \\
\hline Tribromodiphenyl Ether & $049690-94-0$ & & & & \\
\hline
\end{tabular}


Table 3.1g. (continued)

\begin{tabular}{|c|c|c|c|c|c|}
\hline \multirow{2}{*}{ Chemical } & \multirow{2}{*}{ CASRN } & \multicolumn{4}{|c|}{ Ingestion of fish (concentration in water) $(\mathrm{mg} / \mathrm{L})^{n}$} \\
\hline & & $10^{-4}$ & $10^{-6}$ & $\mathbf{H Q}=\mathbf{1}$ & $\mathrm{HQ}=0.1$ \\
\hline Tributyltin Oxide & 000056-35-9 & & & & \\
\hline Trichloro-1,2,2-trifluoroethane, 1,1,2- & 000076-13-1 & & & $7.3 \mathrm{E}+02$ & 7.3E+01 \\
\hline Trichloro-2'-hydroxydiphenylether & 003380-34-5 & & & & \\
\hline \multicolumn{6}{|l|}{ Trichloroacetic Acid } \\
\hline Trichloroaniline $\mathrm{HCl}, 2,4,6-$ & 033663-50-2 & & & & \\
\hline Trichloroaniline, 2,4,6- & 000634-93-5 & & & & \\
\hline Trichlorobenzene, 1,2,4- & 000120-82-1 & & & $2.1 \mathrm{E}-02$ & 2.1E-03 \\
\hline Trichloroethane, 1,1,1-(5) & 000071-55-6 & & & $2.6 \mathrm{E}+00$ & $2.6 \mathrm{E}-01$ \\
\hline Trichloroethane, 1,1,2- & 000079-00-5 & $2.8 \mathrm{E}-01$ & 2.8E-03 & $2.8 \mathrm{E}-01$ & $2.8 \mathrm{E}-02$ \\
\hline Trichloroethylene $^{(3)}$ & 000079-01-6 & 7.3E-01 & 7.3E-03 & & \\
\hline Trichlorofluoromethane & $000075-69-4$ & & & $8.7 \mathrm{E}+00$ & 8.7E-01 \\
\hline Trichlorophenol, 2,4,5- & 000095-95-4 & & & $3.0 \mathrm{E}-01$ & $3.0 \mathrm{E}-02$ \\
\hline Trichlorophenol, 2,4,6- & 000088-06-2 & 7.5E-02 & 7.5E-04 & & \\
\hline $\begin{array}{l}\text { Trichlorophenoxy) Propionic Acid, } \\
\text { 2(2,4,5- }\end{array}$ & 000093-72-1 & & & $2.0 \mathrm{E}-02$ & $2.0 \mathrm{E}-03$ \\
\hline Trichlorophenoxyacetic Acid, 2,4,5- & 000093-76-5 & & & $2.1 \mathrm{E}-02$ & $2.1 \mathrm{E}-03$ \\
\hline Trichloropropane, 1,1,2- & 000598-77-6 & & & & \\
\hline Trichloropropane, 1,2,3- & 000096-18-4 & $2.4 \mathrm{E}-05$ & $2.4 \mathrm{E}-07$ & $4.4 \mathrm{E}-03$ & 4.4E-04 \\
\hline
\end{tabular}




\begin{tabular}{|c|c|c|c|c|c|}
\hline $20-\not S^{\circ} 6$ & I0-9S"6 & & & $z-Z 9-0 t+\angle 00$ & 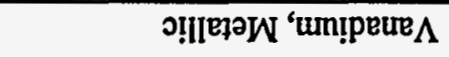 \\
\hline & & & & $\varepsilon-\tau ち-\angle 069 \varepsilon 0$ & әұвJInS un!̣peue $\Lambda$ \\
\hline & & & & $I-Z 9-\dagger l \varepsilon 100$ & 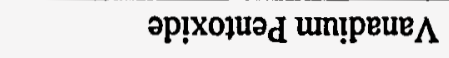 \\
\hline \multirow[t]{2}{*}{$20-31 \%$} & $[0-8]]^{\circ} t$ & & & $\mathrm{VN}$ & (słes ગqnıos) um!̣uex $\Omega$ \\
\hline & & & & L-E0-8I9900 & pea T IKdoJd!̣L \\
\hline$\varepsilon 0-\exists I I^{\circ} Z$ & $20-\exists[\tau$ & $\varepsilon 0-\exists \tau^{*} \varepsilon$ & {$\left[0-a z^{*} \varepsilon\right.$} & $L-96-811000$ & 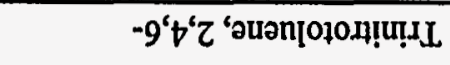 \\
\hline $20-96.9$ & $10-36^{* 9}$ & & & $8-S t-6<t 000$ & 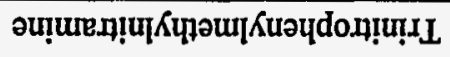 \\
\hline \multirow[t]{4}{*}{$\varepsilon 0-\exists t^{\prime} I$} & $20-a t i$ & & & $b-\varsigma \varepsilon-660000$ & 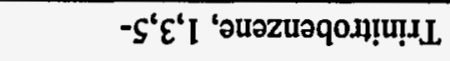 \\
\hline & & & & I-9z-Z9L100 & 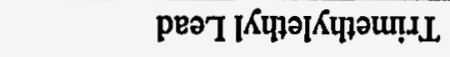 \\
\hline & & & & I-9S-ZIS000 & 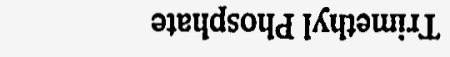 \\
\hline & & & & $6-\varepsilon l-z \not \downarrow \vdash 00$ & 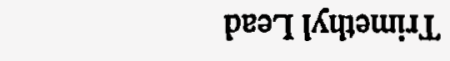 \\
\hline \multirow[t]{7}{*}{ t0-999.I } & $\varepsilon 0-\exists 9^{\circ} I$ & S0-AS'9 & $\varepsilon 0-\not \varsigma^{\prime} 9$ & $8-60-28 S I 00$ & u!̣em|!LL \\
\hline & & & & $8-t t-I Z 1000$ & 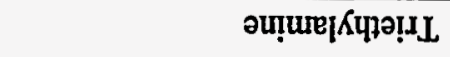 \\
\hline & & & & $L-\varepsilon Z-\downarrow Z Z S 00$ & 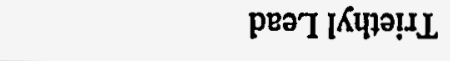 \\
\hline & & & & $\tau-80-8 \varepsilon I 8 S 0$ & әueud!̣!!II \\
\hline & & & & 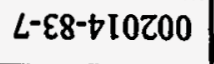 & 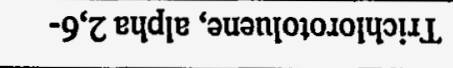 \\
\hline & & & & $s-9 r<<0 z 00$ & 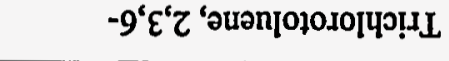 \\
\hline & & & & s-6I-960000 & 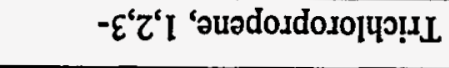 \\
\hline$I^{\prime} 0=\partial H$ & $I=\mathbf{O H}$ & $9-0 I$ & rOI & \multirow{2}{*}{ NYSVD } & \multirow{2}{*}{ [вง!ุนวัว } \\
\hline & g'M u! u & )) YSIJ Jo & & & \\
\hline
\end{tabular}

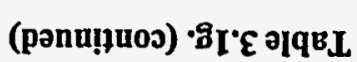


Table 3.1g. (continued)

\begin{tabular}{|c|c|c|c|c|c|}
\hline \multirow{2}{*}{ Chemical } & \multirow{2}{*}{ CASRN } & \multicolumn{4}{|c|}{ Ingestion of fish (concentration in water) (mg/L)" } \\
\hline & & $10^{-4}$ & $10^{-6}$ & $\mathbf{H Q}=\mathbf{1}$ & $\mathrm{HQ}=0.1$ \\
\hline Vanadyl Sülfate & $027774-13-6$ & & & & \\
\hline Vernolate & 001929-77-7 & & & & \\
\hline Vinclozolin & 050471-44-8 & & & & \\
\hline Vinyl Acetate & 000108-05-4 & & & $6.4 \mathrm{E}+02$ & $6.4 \mathrm{E}+01$ \\
\hline Vinyl Bromide & $000593-60-2$ & & & & \\
\hline Vinyl Chloride ${ }^{(6)}$ & 000075-01-4 & 2.4E-02 & 2.4E-04 & & \\
\hline Warfarin & 000081-81-2 & & & $2.5 \mathrm{E}-03$ & $2.5 \mathrm{E}-04$ \\
\hline White Phosphorus & 007723-14-0 & & & & \\
\hline Xylene, Mixture ${ }^{(6)}$ & 001330-20-7 & & & $1.4 \mathrm{E}+01$ & $1.4 \mathrm{E}+00$ \\
\hline Xylene, P-(6) & $000106-42-3$ & & & & \\
\hline Xylene, $\mathrm{m}^{-(6)}$ & 000108-38-3 & & & $1.7 \mathrm{E}+01$ & $1.7 \mathrm{E}+00$ \\
\hline Xylene, $0^{(6)}$ & 000095-47-6 & & & $1.7 \mathrm{E}+01$ & $1.7 \mathrm{E}+00$ \\
\hline Zinc (Metallic) & $007440-66-6$ & & & 4.1E-01 & 4.1E-02 \\
\hline Zinc Cyanide & $000557-21-1$ & & & & \\
\hline Zinc Phosphide & $001314-84-7$ & & & & \\
\hline Zineb & $012122-67-7$ & & & & \\
\hline Zirconium & $007440-67-7$ & & & & \\
\hline
\end{tabular}

Notes: By definition, $1 \mathrm{~L}$ (water) $=1.0 \mathrm{E}+06 \mathrm{mg}$ (water). Therefore, a limit of $1.0 \mathrm{E}+06 \mathrm{mg} / \mathrm{L}$ was used for any PRG that was calculated to be above this limit. 


\section{Table 3.1g. (continued)}

(1) The same oral slope factor as used for polychlorinated biphenyls.

(2) The PRG was calculated considering Supplemental Guidance from RAGS: Region 4 Bulletins, Human Health Risk Assessment (Interim Guidance) from EPA (received February 11, 1992). The slope factors for chemicals so marked indicate that a toxicity equivalency factor (TEF) method was used for carcinogenic PAHs based on each compound's relative potency to the potency of benzo[a]pyrene. The following TEFs were used to convert each PAH slope factor to an equivalent slope factor of benzo[a]pyrene [that has a new cancer slope factor of 7.3 (mg/kg/day) $)^{-1}$ identified by EPA's CRAVE workgroup]: (1) benzo[a]pyrene, TEF $=1.0 ;$ (2) benzo[a]anthracene, TEF $=0.1 ;(3)$ benzo[b]fluoranthene, TEF $=0.1 ;(4)$ benzo[k]fluoranthene, TEF $=0.01 ;(5)$ chrysene, TEF $=0.001 ;(6)$ dibenz $[\mathrm{a}, \mathrm{h}]$ anthracene, $\mathrm{TEF}=1.0 ;$ and $(7)$ indeno[1,2,3-c,d]pyrene, TEF $=0.1$.

(3) The toxicity values for trichloroethylene have been withdrawn from IRIS and HEAST pending further investigation. However, the most recent toxicity values have been used to calculate the PRG.

(4) The PRG was calculated considering Supplemental Guidance from RAGS: Region 4 Bulletins, Human Health Risk Assessment (Interim Guidance) from EPA (November 1995). The slope factors for chemicals so marked indicate that a TEF methodology was used for chlorinated dioxin and furan congeners based on each compound's relative potency to the potency of 2,3,7,8-tetrachlorodibenzodioxin (TCDD). The following TEFs were used to convert each slope factor to an equivalent slope factor of 2,3,7,8-TCDD: (1) $2,3,7,8-\mathrm{TCDD}, \mathrm{TEF}=1.0 ;$ (2) 2,3,7,8-PeCDD, TEF $=0.5 ;(3) 2,3,7,8-\mathrm{HxCDD}, \mathrm{TEF}=0.1 ;(4) 2,3,7,8-\mathrm{HpCDD}, \mathrm{TEF}=0.01 ;$ (5) OCDD, TEF = 0.001; (6) 2,3,7,8-TCDF, $\mathrm{TEF}=0.1$; (7) $1,2,3,7,8-\mathrm{PeCDF}, \mathrm{TEF}=0.5 ;(8)$ 2,3,4,7,8-PeCDF, TEF $=0.05 ;(9) \quad 2,3,7,8-\mathrm{HxCDF}, \mathrm{TEF}=0.1 ;(10) 2,3,7,8-\mathrm{HpCDF}$, TEF $=0.01$; and $(11)$ OCDF, $\mathrm{TEF}=0.001$.

(5) Toxicity values for these chemicals have been withdrawn from IRIS and HEAST pending further investigation. However, the most recent toxicity values have been used to calculate the PRG.

(6) Toxicity values for these chemicals were obtained from the Superfund Health Risk Technical Support Center. 
Table 3.1h. Risk-based Preliminary Remediation Goals for water based on ingestion of fish for radionuclides (residential scenario)

\begin{tabular}{|c|c|c|c|}
\hline \multirow[t]{2}{*}{ Chemical $^{\dagger}$} & \multirow[t]{2}{*}{ CASRN } & \multicolumn{2}{|c|}{$\begin{array}{l}\text { Ingestion of fish (concentration in water) } \\
(\mathrm{pCi} / \mathrm{L})\end{array}$} \\
\hline & & $10^{-4}$ & $10^{-6}$ \\
\hline Ac-225 & $014265-85-1$ & $5.0 \mathrm{E}+01$ & 5.0E-01 \\
\hline Ac-227 & $014952-40-0$ & $2.0 \mathrm{E}+01$ & $2.0 \mathrm{E}-01$ \\
\hline$A c-227+D$ & $014952-40-0$ & $5.8 \mathrm{E}-01$ & 5.8E-03 \\
\hline Ac-228 & $014331-83-0$ & $4.4 E+03$ & $4.4 E+01$ \\
\hline Ag-105 & $014928-14-4$ & $2.2 \mathrm{E}+04$ & $2.2 \mathrm{E}+02$ \\
\hline Ag-108 & $014391-65-2$ & $5.1 \mathrm{E}+06$ & $5.1 \mathrm{E}+04$ \\
\hline $\mathrm{Ag}-108 \mathrm{~m}$ & $014391-65-2$ & $5.8 \mathrm{E}+03$ & $5.8 \mathrm{E}+01$ \\
\hline $\mathrm{Ag}-108 \mathrm{~m}+\mathrm{D}$ & $014391-65-2$ & $5.8 \mathrm{E}+03$ & $5.8 \mathrm{E}+01$ \\
\hline Ag-109m & $014378-38-2$ & $1.3 \mathrm{E}+08$ & $1.3 E+06$ \\
\hline $\mathrm{Ag}-110$ & $014391-76-5$ & $1.4 E+07$ & $1.4 \mathrm{E}+05$ \\
\hline $\mathrm{Ag}-110 \mathrm{~m}$ & $014391-76-5$ & $4.2 \mathrm{E}+03$ & $4.2 \mathrm{E}+01$ \\
\hline Ag-111 & $015760-04-0$ & $5.2 \mathrm{E}+03$ & $5.2 \mathrm{E}+01$ \\
\hline Am-241 & $014596-10-2$ & $1.8 \mathrm{E}+01$ & $1.8 \mathrm{E}-01$ \\
\hline Am-242 & 013981-54-9 & $4.0 \mathrm{E}+03$ & $4.0 \mathrm{E}+01$ \\
\hline$A m-242 m$ & 013981-54-9 & $2.0 \mathrm{E}+01$ & $2.0 \mathrm{E}-01$ \\
\hline$A m-243$ & 014993-75-0 & $1.8 \mathrm{E}+01$ & $1.8 \mathrm{E}-01$ \\
\hline $\mathrm{Am}-243+\mathrm{D}$ & 014993-75-0 & $8.9 E+00$ & $8.9 \mathrm{E}-02$ \\
\hline
\end{tabular}


Table 3.1h. (continued)

\begin{tabular}{|l|l|c|c|}
\hline \multirow{2}{*}{ Chemical } & \multirow{2}{*}{ CASRN } & \multicolumn{2}{|c|}{$\begin{array}{c}\text { Ingestion of fish (concentration in water) } \\
\text { (pCi/L) }\end{array}$} \\
\cline { 3 - 4 } & & $10^{-4}$ & $10^{-6}$ \\
\hline Ar-41 & $014163-25-8$ & & \\
\hline At-217 & $017239-90-6$ & & \\
\hline Au-196 & $014914-16-0$ & & \\
\hline Au-198 & $010043-49-0$ & & $2.6 \mathrm{E}+02$ \\
\hline Ba-131 & $014914-75-1$ & $2.6 \mathrm{E}+04$ & $1.6 \mathrm{E}+02$ \\
\hline Ba-133 & $013981-41-4$ & $1.6 \mathrm{E}+04$ & $1.6 \mathrm{E}+02$ \\
\hline Ba-133m & $013981-41-4$ & $1.6 \mathrm{E}+04$ & $1.8 \mathrm{E}+05$ \\
\hline Ba-137m & $013981-97-0$ & $1.8 \mathrm{E}+07$ & $1.5 \mathrm{E}+03$ \\
\hline Ba-139 & $014378-25-7$ & $1.5 \mathrm{E}+05$ & $3.7 \mathrm{E}+01$ \\
\hline Ba-140 & $014798-08-4$ & $3.7 \mathrm{E}+03$ & $2.0 \mathrm{E}+02$ \\
\hline Be-7 & $013966-02-4$ & $2.0 \mathrm{E}+04$ & $2.5 \mathrm{E}+01$ \\
\hline Bi-206 & $015776-19-9$ & $2.5 \mathrm{E}+03$ & $3.5 \mathrm{E}+01$ \\
\hline Bi-207 & $013982-38-2$ & $3.5 \mathrm{E}+03$ & $2.4 \mathrm{E}+01$ \\
\hline Bi-210 & $014331-79-4$ & $2.4 \mathrm{E}+03$ & $9.7 \mathrm{E}+03$ \\
\hline Bi-211 & $015229-37-5$ & $9.7 \mathrm{E}+05$ & $2.8 \mathrm{E}+02$ \\
\hline Bi-212 & $014913-49-6$ & $2.8 \mathrm{E}+04$ & $4.0 \mathrm{E}+02$ \\
\hline Bi-213 & $015776-20-2$ & $4.0 \mathrm{E}+04$ & \\
\hline & & & \\
\hline & & & \\
\hline
\end{tabular}


Table 3.1h. (continued)

\begin{tabular}{|l|l|c|c|}
\hline \multirow{2}{*}{ Chemical $^{\dagger}$} & \multirow{2}{*}{ CASRN } & \multicolumn{2}{|c|}{$\begin{array}{c}\text { Ingestion of fish (concentration in water) } \\
\text { (pCi/L) }\end{array}$} \\
\cline { 3 - 4 } & & $10^{-1}$ & $10^{-6}$ \\
\hline Bi-214 & $014733-03-0$ & $9.0 \mathrm{E}+04$ & $9.0 \mathrm{E}+02$ \\
\hline Br-82 & $014686-69-2$ & $3.1 \mathrm{E}+02$ & $3.1 \mathrm{E}+00$ \\
\hline $\mathrm{C}-11$ & $014333-33-6$ & & \\
\hline $\mathrm{C}-15$ & $014762-75-5$ & & \\
\hline Ca-45 & $015929-23-4$ & & \\
\hline Ca-47 & $013966-05-7$ & & \\
\hline Cd-109 & $001439-99-2$ & & $1.1 \mathrm{E}+00$ \\
\hline Cd-115 & $014109-32-1$ & $1.1 \mathrm{E}+02$ & $1.2 \mathrm{E}+00$ \\
\hline Cd-115m & $014336-68-6$ & $1.2 \mathrm{E}+02$ & $6.2 \mathrm{E}-01$ \\
\hline Ce-141 & $014336-68-6$ & $6.2 \mathrm{E}+01$ & $1.5 \mathrm{E}+01$ \\
\hline Ce-143 & $013967-74-3$ & $1.5 \mathrm{E}+03$ & $9.9 \mathrm{E}+00$ \\
\hline Ce-144 & $014119-19-8$ & $9.9 \mathrm{E}+02$ & $2.0 \mathrm{E}+00$ \\
\hline Ce-144+D & $014762-78-8$ & $2.0 \mathrm{E}+02$ & $2.6 \mathrm{E}-01$ \\
\hline Cl-36 & $014762-78-8$ & $2.6 \mathrm{E}+01$ & $1.5 \mathrm{E}+00$ \\
\hline Cl-38 & $013981-43-6$ & & \\
\hline Cm-242 & $014158-34-0$ & & \\
\hline & $015510-73-3$ & $1.5 \mathrm{E}+02$ & \\
\hline
\end{tabular}


Table 3.1h. (continued)

\begin{tabular}{|l|l|c|c|}
\hline \multirow{2}{*}{ Chemical $^{\dagger}$} & \multirow{2}{*}{ CASRN } & \multicolumn{2}{|c|}{$\begin{array}{c}\text { Ingestion of fish (concentration in water) } \\
\text { (pCiL) }\end{array}$} \\
\cline { 3 - 4 } & & $10^{-4}$ & $10^{-6}$ \\
\hline Cm-243 & $015757-87-6$ & $2.3 \mathrm{E}+01$ & $2.3 \mathrm{E}-01$ \\
\hline Cm-243+D & $015757-87-6$ & $7.1 \mathrm{E}-01$ & $7.1 \mathrm{E}-03$ \\
\hline Cm-244 & $013981-15-2$ & $2.8 \mathrm{E}+01$ & $2.8 \mathrm{E}-01$ \\
\hline Cm-245 & $015621-76-8$ & $1.8 \mathrm{E}+01$ & $1.8 \mathrm{E}-01$ \\
\hline Cm-246 & $015757-90-1$ & $1.8 \mathrm{E}+01$ & $1.8 \mathrm{E}-01$ \\
\hline Cm-247 & $015758-32-4$ & $1.9 \mathrm{E}+01$ & $1.9 \mathrm{E}-01$ \\
\hline Cm-248 & $015758-33-5$ & $4.5 \mathrm{E}+00$ & $4.5 \mathrm{E}-02$ \\
\hline Co-57 & $013981-50-5$ & $6.1 \mathrm{E}+02$ & $6.1 \mathrm{E}+00$ \\
\hline Co-58 & $013981-38-9$ & $2.1 \mathrm{E}+02$ & $2.1 \mathrm{E}+00$ \\
\hline Co-58m & $013981-38-9$ & $6.2 \mathrm{E}+03$ & $6.2 \mathrm{E}+01$ \\
\hline Co-60 & $010198-40-0$ & $3.1 \mathrm{E}+01$ & $3.1 \mathrm{E}-01$ \\
\hline Cr-51 & $014392-02-0$ & $6.4 \mathrm{E}+03$ & $6.4 \mathrm{E}+01$ \\
\hline Cs-131 & $014914-76-2$ & $4.9 \mathrm{E}+02$ & $4.9 \mathrm{E}+00$ \\
\hline Cs-134 & $013967-70-9$ & $1.9 \mathrm{E}+00$ & $1.9 \mathrm{E}-02$ \\
\hline Cs-134m & $013967-70-9$ & $1.9 \mathrm{E}+03$ & $1.9 \mathrm{E}+01$ \\
\hline Cs-135 & $015726-30-4$ & $1.9 \mathrm{E}+01$ & $1.9 \mathrm{E}-01$ \\
\hline Cs-136 & $1.1 \mathrm{E}+01$ & $1.1 \mathrm{E}-01$ \\
\hline
\end{tabular}


Table 3.1h. (continued)

\begin{tabular}{|l|l|c|c|}
\hline \multirow{2}{*}{ Chemical } & \multirow{2}{*}{ CASRN } & \multicolumn{2}{|c|}{$\begin{array}{c}\text { Ingestion of fish (concentration in water) } \\
\text { (pCi/L) }\end{array}$} \\
\cline { 2 - 4 } & & $10^{-4}$ & $10^{-6}$ \\
\hline Cs-137 & $010045-97-3$ & $2.8 \mathrm{E}+00$ & $2.8 \mathrm{E}-02$ \\
\hline Cs-137+D & $010045-97-3$ & $2.8 \mathrm{E}+00$ & $2.8 \mathrm{E}-02$ \\
\hline Cs-138 & $015758-29-9$ & $5.0 \mathrm{E}+02$ & $5.0 \mathrm{E}+00$ \\
\hline Cu-64 & $013981-25-4$ & $1.7 \mathrm{E}+03$ & $1.7 \mathrm{E}+01$ \\
\hline Dy-165 & $013967-64-1$ & & \\
\hline Dy-166 & $015840-01-4$ & & \\
\hline Er-169 & $015840-13-8$ & & \\
\hline Er-171 & $014391-45-8$ & & $6.2 \mathrm{E}+00$ \\
\hline Eu-152 & $014683-23-9$ & $6.2 \mathrm{E}+02$ & $3.8 \mathrm{E}+00$ \\
\hline Eu-154 & $015585-10-1$ & $3.8 \mathrm{E}+02$ & $2.1 \mathrm{E}+01$ \\
\hline Eu-155 & $014391-16-3$ & $2.1 \mathrm{E}+03$ & $3.2 \mathrm{E}+00$ \\
\hline Eu-156 & $014280-35-4$ & $3.2 \mathrm{E}+02$ & \\
\hline F-18 & $013981-56-1$ & & $2.5 \mathrm{E}+01$ \\
\hline Fe-55 & $014681-59-5$ & $2.5 \mathrm{E}+03$ & $1.5 \mathrm{E}+00$ \\
\hline Fe-59 & $014596-12-4$ & $1.5 \mathrm{E}+02$ & \\
\hline Fr-221 & $015756-41-9$ & & \\
\hline Fr-223 & $015756-98-6$ & & \\
\hline & & & \\
\hline
\end{tabular}


Table 3.1h. (continued)

\begin{tabular}{|l|l|c|c|}
\hline \multirow{2}{*}{ Chemical } & \multirow{2}{*}{ CASRN } & \multicolumn{2}{|c|}{$\begin{array}{c}\text { Ingestion of fish (concentration in water) } \\
\text { (pCi/L) }\end{array}$} \\
\cline { 3 - 4 } & & $10^{-4}$ & $10^{-6}$ \\
\hline Ga-67 & $014119-09-6$ & & \\
\hline Ga-72 & $013982-22-4$ & & \\
\hline Gd-153 & $014276-65-4$ & & \\
\hline Ge-71 & $014041-42-0$ & & \\
\hline H-3 & $014374-81-3$ & & $2.5 \mathrm{E}+04$ \\
\hline Hg-197 & $010028-17-8$ & & $1.5 \mathrm{E}+00$ \\
\hline Hg-203 & $013981-51-6$ & $2.5 \mathrm{E}+06$ & $6.7 \mathrm{E}-01$ \\
\hline Ho-166 & $013982-78-0$ & $1.5 \mathrm{E}+02$ & \\
\hline I-122 & $013967-65-2$ & $6.7 \mathrm{E}+01$ & \\
\hline I-123 & $018287-75-7$ & & $2.0 \mathrm{E}+03$ \\
\hline I-125 & $015715-08-9$ & $8.1 \mathrm{E}+03$ & $8.1 \mathrm{E}+01$ \\
\hline I-126 & $014158-31-7$ & $1.7 \mathrm{E}+02$ & $1.7 \mathrm{E}+00$ \\
\hline I-129 & $014158-32-8$ & $9.1 \mathrm{E}+01$ & $9.1 \mathrm{E}-01$ \\
\hline I-130 & $015046-84-1$ & $2.4 \mathrm{E}+01$ & $2.4 \mathrm{E}-01$ \\
\hline I-131 & $014914-02-4$ & $9.1 \mathrm{E}+02$ & $9.1 \mathrm{E}+00$ \\
\hline I-132 & $010043-66-0$ & $1.2 \mathrm{E}+02$ & $1.2 \mathrm{E}+00$ \\
\hline & $014683-16-0$ & $6.7 \mathrm{E}+03$ & $6.7 \mathrm{E}+01$ \\
\hline
\end{tabular}


Table 3.1h. (continued)

\begin{tabular}{|l|l|c|c|}
\hline \multirow{2}{*}{ Chemical $^{\dagger}$} & \multirow{2}{*}{ CASRN } & \multicolumn{2}{|c|}{$\begin{array}{c}\text { Ingestion of fish (concentration in water) } \\
\text { (pCi/L) }\end{array}$} \\
\cline { 3 - 4 } & & $10^{-4}$ & $10^{-6}$ \\
\hline $\mathrm{I}-133$ & $014834-67-4$ & $4.2 \mathrm{E}+02$ & $4.2 \mathrm{E}+00$ \\
\hline $\mathrm{I}-134$ & $014914-27-3$ & $1.9 \mathrm{E}+04$ & $1.9 \mathrm{E}+02$ \\
\hline $\mathrm{I}-135$ & $014834-68-5$ & $1.9 \mathrm{E}+03$ & $1.9 \mathrm{E}+01$ \\
\hline In-113m & $014885-78-0$ & & \\
\hline In-114 & $013981-55-0$ & & \\
\hline In-114m & $013981-55-0$ & & \\
\hline In-115 & $014191-71-0$ & & \\
\hline In-115m & $014191-71-0$ & & \\
\hline Ir-190 & $014981-91-0$ & & \\
\hline Ir-194 & $014158-35-1$ & & \\
\hline $\mathrm{K}-40$ & $013966-00-2$ & & \\
\hline $\mathrm{K}-42$ & $014378-21-3$ & & \\
\hline $\mathrm{Kr}-83 \mathrm{~m}$ & $013965-98-5$ & & \\
\hline $\mathrm{Kr}-85$ & $013983-27-2$ & & \\
\hline $\mathrm{Kr}-85 \mathrm{~m}$ & $013983-27-2$ & & \\
\hline $\mathrm{Kr}-87$ & $014809-68-8$ & & \\
\hline $\mathrm{Kr}-88$ & $014995-61-0$ & & \\
\hline & & & \\
\hline
\end{tabular}


Table 3.1h. (continued)

\begin{tabular}{|l|l|c|c|}
\hline \multirow{2}{*}{ Chemical } & \multirow{2}{*}{ CASRN } & \multicolumn{2}{|c|}{$\begin{array}{c}\text { Ingestion of fish (concentration in water) } \\
\text { (pCi/L) }\end{array}$} \\
\cline { 3 - 4 } & & $10^{-4}$ & $10^{-6}$ \\
\hline Kr-89 & $016316-03-3$ & & \\
\hline Kr-90 & $015741-13-6$ & & \\
\hline La-140 & $013981-28-7$ & $6.2 \mathrm{E}+02$ & $6.2 \mathrm{E}+00$ \\
\hline Lu-177 & $014265-75-9$ & & $7.3 \mathrm{E}-01$ \\
\hline $\mathrm{Mn}-52$ & $014092-99-0$ & $7.3 \mathrm{E}+01$ & $2.2 \mathrm{E}+00$ \\
\hline $\mathrm{Mn}-54$ & $013966-31-9$ & $2.2 \mathrm{E}+02$ & $5.1 \mathrm{E}+00$ \\
\hline $\mathrm{Mn}-56$ & $014681-52-8$ & $5.1 \mathrm{E}+02$ & $7.8 \mathrm{E}+01$ \\
\hline $\mathrm{Mo}-99$ & $014119-15-4$ & $7.8 \mathrm{E}+03$ & $1.1 \mathrm{E}+01$ \\
\hline $\mathrm{Na}-22$ & $013966-32-0$ & $1.1 \mathrm{E}+03$ & $6.4 \mathrm{E}+01$ \\
\hline $\mathrm{Na}-24$ & $013982-04-2$ & $6.4 \mathrm{E}+03$ & $8.9 \mathrm{E}+00$ \\
\hline $\mathrm{Nb}-93 \mathrm{~m}$ & $007440-03-1$ & $8.9 \mathrm{E}+02$ & $8.5 \mathrm{E}-01$ \\
\hline $\mathrm{Nb}-94$ & $014681-63-1$ & $8.5 \mathrm{E}+01$ & $2.6 \mathrm{E}+00$ \\
\hline $\mathrm{Nb}-95$ & $013967-76-5$ & $2.6 \mathrm{E}+02$ & $1.9 \mathrm{E}+00$ \\
\hline $\mathrm{Nb}-95 \mathrm{~m}$ & $013967-76-5$ & $1.9 \mathrm{E}+02$ & $3.4 \mathrm{E}+01$ \\
\hline $\mathrm{Nb}-97$ & $018496-04-3$ & $3.4 \mathrm{E}+03$ & $1.8 \mathrm{E}+03$ \\
\hline $\mathrm{Nb}-97 \mathrm{~m}$ & $018496-04-3$ & $1.8 \mathrm{E}+05$ & $3.0 \mathrm{E}+00$ \\
\hline $\mathrm{Nd}-147$ & $014269-74-0$ & $3.0 \mathrm{E}+02$ & \\
\hline & & & \\
\hline
\end{tabular}


Table 3.1h. (continued)

\begin{tabular}{|l|l|c|c|}
\hline \multirow{2}{*}{ Chemical } & \multirow{2}{*}{ CASRN } & \multicolumn{2}{|c|}{$\begin{array}{c}\text { Ingestion of fish (concentration in water) } \\
\text { (pCi/L) }\end{array}$} \\
\cline { 2 - 4 } & & $10^{-4}$ & $10^{-6}$ \\
\hline Nd-149 & $015749-81-2$ & $3.9 \mathrm{E}+03$ & $3.9 \mathrm{E}+01$ \\
\hline Ni-59 & $014336-70-0$ & $9.5 \mathrm{E}+03$ & $9.5 \mathrm{E}+01$ \\
\hline Ni-63 & $013981-37-8$ & $3.2 \mathrm{E}+03$ & $3.2 \mathrm{E}+01$ \\
\hline Ni-65 & $014833-49-9$ & $3.1 \mathrm{E}+03$ & $3.1 \mathrm{E}+01$ \\
\hline Np-236 & $015700-36-4$ & $6.3 \mathrm{E}+03$ & $6.3 \mathrm{E}+01$ \\
\hline Np-237 & $013994-20-2$ & $2.0 \mathrm{E}+01$ & $2.0 \mathrm{E}-01$ \\
\hline Np-237+D & $013994-20-2$ & $1.5 \mathrm{E}+01$ & $1.5 \mathrm{E}-01$ \\
\hline Np-238 & $015766-25-3$ & $1.3 \mathrm{E}+03$ & $1.3 \mathrm{E}+01$ \\
\hline Np-239 & $013968-59-7$ & $1.4 \mathrm{E}+03$ & $1.4 \mathrm{E}+01$ \\
\hline Np-240 & $015690-84-3$ & $3.3 \mathrm{E}+04$ & $3.3 \mathrm{E}+02$ \\
\hline Np-240m & $015690-84-3$ & $2.4 \mathrm{E}+05$ & $2.4 \mathrm{E}+03$ \\
\hline Os-185 & $015766-50-4$ & & $4.5 \mathrm{E}-02$ \\
\hline Os-191 & $014119-24-5$ & & \\
\hline Os-191m & $014119-24-5$ & & \\
\hline Os-193 & $016057-77-5$ & & \\
\hline P-32 & $014596-37-3$ & $5.8 \mathrm{E}-01$ & \\
\hline P-33 & $015749-66-3$ & $4.5 \mathrm{E}+00$ & \\
\hline
\end{tabular}


Table 3.1h. (continued)

\begin{tabular}{|l|l|c|c|}
\hline \multirow{2}{*}{ Chemical $^{\dagger}$} & \multirow{2}{*}{ CASRN } & \multicolumn{2}{|c|}{$\begin{array}{c}\text { Ingestion of fish (concentration in water) } \\
\text { (pCiL) }\end{array}$} \\
\cline { 2 - 4 } & & $10^{-4}$ & $10^{-6}$ \\
\hline $\mathrm{Pa}-231$ & $014331-85-2$ & $1.2 \mathrm{E}+02$ & $1.2 \mathrm{E}+00$ \\
\hline $\mathrm{Pa}-233$ & $013981-14-1$ & $3.8 \mathrm{E}+03$ & $3.8 \mathrm{E}+01$ \\
\hline $\mathrm{Pa}-234$ & $015100-28-4$ & $8.3 \mathrm{E}+03$ & $8.3 \mathrm{E}+01$ \\
\hline $\mathrm{Pa}-234 \mathrm{~m}$ & $015100-28-4$ & $3.7 \mathrm{E}+06$ & $3.7 \mathrm{E}+04$ \\
\hline $\mathrm{Pb}-203$ & $014687-25-3$ & $5.7 \mathrm{E}+02$ & $5.7 \mathrm{E}+00$ \\
\hline $\mathrm{Pb}-209$ & $014119-30-3$ & $2.8 \mathrm{E}+03$ & $2.8 \mathrm{E}+01$ \\
\hline $\mathrm{Pb}-210$ & $014255-04-0$ & $8.7 \mathrm{E}-01$ & $8.7 \mathrm{E}-03$ \\
\hline $\mathrm{Pb}-210+\mathrm{D}$ & $014255-04-0$ & $4.9 \mathrm{E}-01$ & $4.9 \mathrm{E}-03$ \\
\hline $\mathrm{Pb}-211$ & $015816-77-0$ & $1.7 \mathrm{E}+03$ & $1.7 \mathrm{E}+01$ \\
\hline $\mathrm{Pb}-212$ & $015092-94-1$ & $3.3 \mathrm{E}+01$ & $3.3 \mathrm{E}-01$ \\
\hline $\mathrm{Pb}-214$ & $015067-28-4$ & $2.0 \mathrm{E}+03$ & $2.0 \mathrm{E}+01$ \\
\hline $\mathrm{Pd}-100$ & $015690-69-4$ & & $4.2 \mathrm{E}+01$ \\
\hline $\mathrm{Pd}-101$ & $015749-54-9$ & & \\
\hline $\mathrm{Pd}-103$ & $014967-68-1$ & & \\
\hline $\mathrm{Pd}-107$ & $017637-99-9$ & & \\
\hline $\mathrm{Pd}-109$ & $014981-64-7$ & & \\
\hline $\mathrm{Pm}-147$ & $014380-75-7$ & & \\
\hline & & & \\
\hline & & & \\
\hline
\end{tabular}


Table 3.1h. (continued)

\begin{tabular}{|l|l|c|c|}
\hline \multirow{2}{*}{ Chemical $^{\dagger}$} & \multirow{2}{*}{ CASRN } & \multicolumn{2}{|c|}{$\begin{array}{c}\text { Ingestion of fish (concentration in water) } \\
\text { (pCi/L) }\end{array}$} \\
\cline { 2 - 4 } & & $10^{-4}$ & $10^{-6}$ \\
\hline Pm-148 & $014683-19-3$ & $4.1 \mathrm{E}+02$ & $4.1 \mathrm{E}+00$ \\
\hline Pm-148m & $014683-19-3$ & $5.9 \mathrm{E}+02$ & $5.9 \mathrm{E}+00$ \\
\hline Pm-149 & $015765-31-8$ & $1.1 \mathrm{E}+03$ & $1.1 \mathrm{E}+01$ \\
\hline Po-210 & $013981-52-7$ & $1.1 \mathrm{E}+01$ & $1.1 \mathrm{E}-01$ \\
\hline Po-212 & $015389-34-1$ & $7.8 \mathrm{E}+13$ & $7.8 \mathrm{E}+11$ \\
\hline Po-213 & $015756-57-7$ & $5.3 \mathrm{E}+12$ & $5.3 \mathrm{E}+10$ \\
\hline Po-214 & $015735-67-8$ & $1.7 \mathrm{E}+11$ & $1.7 \mathrm{E}+09$ \\
\hline Po-215 & $015706-52-2$ & $7.1 \mathrm{E}+09$ & $7.1 \mathrm{E}+07$ \\
\hline Po-216 & $015756-58-8$ & $4.0 \mathrm{E}+07$ & $4.0 \mathrm{E}+05$ \\
\hline Po-218 & $015422-24-9$ & $6.9 \mathrm{E}+04$ & $6.9 \mathrm{E}+02$ \\
\hline Pr-142 & $014191-64-1$ & $2.5 \mathrm{E}+02$ & $2.5 \mathrm{E}+00$ \\
\hline Pr-143 & $014981-79-4$ & $2.7 \mathrm{E}+02$ & $2.7 \mathrm{E}+00$ \\
\hline Pr-144 & $014119-05-2$ & $2.2 \mathrm{E}+04$ & $2.2 \mathrm{E}+02$ \\
\hline Pr-144m & $014119-05-2$ & $5.5 \mathrm{E}+04$ & $5.5 \mathrm{E}+02$ \\
\hline Pt-191 & $015706-36-2$ & & \\
\hline Pt-193 & $015735-70-3$ & & \\
\hline Pt-193m & $015735-70-3$ & & \\
\hline & & & \\
\hline
\end{tabular}


Table 3.1h. (continued)

\begin{tabular}{|l|l|c|c|}
\hline \multirow{2}{*}{ Chemical } & \multirow{2}{*}{ CASRN } & \multicolumn{2}{|c|}{$\begin{array}{l}\text { Ingestion of fish (concentration in water) } \\
\text { (pCi/L) }\end{array}$} \\
\cline { 3 - 4 } & & $10^{-4}$ & $10^{-6}$ \\
\hline Pt-197 & $015735-74-7$ & & \\
\hline Pt-197m & $015735-74-7$ & & \\
\hline Pu-236 & $015411-92-4$ & $7.7 \mathrm{E}+01$ & $7.7 \mathrm{E}-01$ \\
\hline Pu-238 & $013981-16-3$ & $2.0 \mathrm{E}+01$ & $2.0 \mathrm{E}-01$ \\
\hline Pu-239 & $015117-48-3$ & $1.9 \mathrm{E}+01$ & $1.9 \mathrm{E}-01$ \\
\hline Pu-240 & $014119-33-6$ & $1.9 \mathrm{E}+01$ & $1.9 \mathrm{E}-01$ \\
\hline Pu-241 & $014119-32-5$ & $1.1 \mathrm{E}+03$ & $1.1 \mathrm{E}+01$ \\
\hline Pu-241+D & $014119-32-5$ & $7.6 \mathrm{E}+00$ & $7.6 \mathrm{E}-02$ \\
\hline Pu-242 & $013982-10-0$ & $2.0 \mathrm{E}+01$ & $2.0 \mathrm{E}-01$ \\
\hline Pu-243 & $015706-37-3$ & $1.6 \mathrm{E}+04$ & $1.6 \mathrm{E}+02$ \\
\hline Pu-244 & $014119-34-7$ & $1.9 \mathrm{E}+01$ & $1.9 \mathrm{E}-01$ \\
\hline Pu-244+D & $014119-34-7$ & $7.9 \mathrm{E}+00$ & $7.9 \mathrm{E}-02$ \\
\hline Ra-223 & $015623-45-7$ & $1.5 \mathrm{E}+01$ & $1.5 \mathrm{E}-01$ \\
\hline Ra-224 & $013233-32-4$ & $2.4 \mathrm{E}+01$ & $2.4 \mathrm{E}-01$ \\
\hline Ra-225 & $013981-53-8$ & $2.2 \mathrm{E}+01$ & $2.2 \mathrm{E}-01$ \\
\hline Ra-226 & $013982-63-3$ & $1.2 \mathrm{E}+01$ & $1.2 \mathrm{E}-01$ \\
\hline Ra-226+D & $013982-63-3$ & $1.3 \mathrm{E}+00$ & $1.3 \mathrm{E}-02$ \\
\hline
\end{tabular}


Table 3.1h. (continued)

\begin{tabular}{|l|l|c|c|}
\hline \multirow{2}{*}{ Chemical $^{\dagger}$} & \multirow{2}{*}{ CASRN } & \multicolumn{2}{|c|}{$\begin{array}{c}\text { Ingestion of fish (concentration in water) } \\
\text { (pCi/L) }\end{array}$} \\
\cline { 3 - 4 } & & $10^{-4}$ & $10^{-6}$ \\
\hline Ra-228 & $015262-20-1$ & $1.4 \mathrm{E}+01$ & $1.4 \mathrm{E}-01$ \\
\hline Ra-228+D & $015262-20-1$ & $1.4 \mathrm{E}+01$ & $1.4 \mathrm{E}-01$ \\
\hline Rb-82 & $014391-63-0$ & $8.4 \mathrm{E}+03$ & $8.4 \mathrm{E}+01$ \\
\hline Rb-86 & $014932-53-7$ & $1.2 \mathrm{E}+01$ & $1.2 \mathrm{E}-01$ \\
\hline Rb-87 & $013982-13-3$ & $2.4 \mathrm{E}+01$ & $2.4 \mathrm{E}-01$ \\
\hline Rb-88 & $014928-36-0$ & $6.0 \mathrm{E}+02$ & $6.0 \mathrm{E}+00$ \\
\hline Rb-89 & $014191-65-2$ & $1.0 \mathrm{E}+03$ & $1.0 \mathrm{E}+01$ \\
\hline Rh-103m & $007440-16-6$ & $2.2 \mathrm{E}+06$ & $2.2 \mathrm{E}+04$ \\
\hline Rh-105 & $014913-89-4$ & $9.1 \mathrm{E}+03$ & $9.1 \mathrm{E}+01$ \\
\hline Rh-105m & $014913-89-4$ & $1.6 \mathrm{E}+07$ & $1.6 \mathrm{E}+05$ \\
\hline Rh-106 & $014234-34-5$ & $4.9 \mathrm{E}+06$ & $4.9 \mathrm{E}+04$ \\
\hline Rn-219 & $014835-02-0$ & & \\
\hline Rn-220 & $022481-48-7$ & & $5.1 \mathrm{E}+00$ \\
\hline Rn-222+D & $014859-67-7$ & & $5.5 \mathrm{E}+01$ \\
\hline Ru-103 & $013968-53-1$ & $5.3 \mathrm{E}+03$ & \\
\hline Ru-105 & $014331-95-4$ & $1.5 \mathrm{E}+04$ & \\
\hline Ru-106 & $013967-48-1$ & $5.1 \mathrm{E}+02$ & \\
\hline
\end{tabular}


Table 3.1h. (continued)

\begin{tabular}{|l|l|c|c|}
\hline \multirow{2}{*}{ Chemical } & \multirow{2}{*}{ CASRN } & \multicolumn{2}{|c|}{$\begin{array}{c}\text { Ingestion of fish (concentration in water) } \\
\text { (pCi/L) }\end{array}$} \\
\cline { 3 - 4 } & & $10^{-4}$ & $10^{-6}$ \\
\hline Ru-106+D & $013967-48-1$ & $2.6 \mathrm{E}+02$ & $2.6 \mathrm{E}+00$ \\
\hline Ru-97 & $015758-35-7$ & $3.0 \mathrm{E}+04$ & $3.0 \mathrm{E}+02$ \\
\hline S-35 & $015117-53-0$ & $5.3 \mathrm{E}+02$ & $5.3 \mathrm{E}+00$ \\
\hline Sb-122 & $014374-79-9$ & $2.0 \mathrm{E}+02$ & $2.0 \mathrm{E}+00$ \\
\hline Sb-124 & $014683-10-4$ & $1.6 \mathrm{E}+02$ & $1.6 \mathrm{E}+00$ \\
\hline Sb-125 & $014234-35-6$ & $5.9 \mathrm{E}+02$ & $5.9 \mathrm{E}+00$ \\
\hline Sb-125+D & $014234-35-6$ & $1.0 \mathrm{E}+02$ & $1.0 \mathrm{E}+00$ \\
\hline Sb-126 & $015756-32-8$ & $1.8 \mathrm{E}+02$ & $1.8 \mathrm{E}+00$ \\
\hline Sb-126m & $015756-32-8$ & $2.4 \mathrm{E}+04$ & $2.4 \mathrm{E}+02$ \\
\hline Sb-127 & $013968-50-8$ & $2.1 \mathrm{E}+02$ & $2.1 \mathrm{E}+00$ \\
\hline Sb-129 & $014331-88-5$ & $9.5 \mathrm{E}+02$ & $9.5 \mathrm{E}+00$ \\
\hline Sc-46 & $013967-63-0$ & $3.1 \mathrm{E}+02$ & $3.1 \mathrm{E}+00$ \\
\hline Sc-47 & $014391-96-9$ & $6.0 \mathrm{E}+02$ & $6.0 \mathrm{E}+00$ \\
\hline Sc-48 & $014391-86-7$ & $2.7 \mathrm{E}+02$ & $2.7 \mathrm{E}+00$ \\
\hline Se-75 & $014265-71-5$ & & \\
\hline Si-31 & $014276-49-4$ & & \\
\hline Sm-147 & $014392-33-7$ & & \\
\hline & & & \\
\hline
\end{tabular}


Table 3.1h. (continued)

\begin{tabular}{|l|l|c|c|}
\hline \multirow{2}{*}{ Chemical } & \multirow{2}{*}{ CASRN } & \multicolumn{2}{|c|}{$\begin{array}{c}\text { Ingestion of fish (concentration in water) } \\
\text { (pCi/L) }\end{array}$} \\
\cline { 3 - 4 } & & $10^{-4}$ & $10^{-6}$ \\
\hline Sm-151 & $015715-94-3$ & & \\
\hline Sm-153 & $015766-00-4$ & & \\
\hline Sn-113 & $013966-06-8$ & $1.6 \mathrm{E}+01$ & $1.6 \mathrm{E}-01$ \\
\hline Sn-121 & $014683-06-8$ & $4.8 \mathrm{E}+01$ & $4.8 \mathrm{E}-01$ \\
\hline Sn-121m & $014683-06-8$ & $2.9 \mathrm{E}+01$ & $2.9 \mathrm{E}-01$ \\
\hline Sn-125 & $014683-08-0$ & $3.5 \mathrm{E}+00$ & $3.5 \mathrm{E}-02$ \\
\hline Sn-126 & $015832-50-5$ & $2.8 \mathrm{E}+00$ & $2.8 \mathrm{E}-02$ \\
\hline Sr-82 & $014809-50-8$ & $1.1 \mathrm{E}+02$ & $1.1 \mathrm{E}+00$ \\
\hline Sr-85 & $013967-73-2$ & $2.1 \mathrm{E}+03$ & $2.1 \mathrm{E}+01$ \\
\hline Sr-85m & $013967-73-2$ & $1.6 \mathrm{E}+05$ & $1.6 \mathrm{E}+03$ \\
\hline Sr-89 & $014158-27-1$ & $2.9 \mathrm{E}+02$ & $2.9 \mathrm{E}+00$ \\
\hline Sr-90 & $010098-97-2$ & $7.2 \mathrm{E}+01$ & $7.2 \mathrm{E}-01$ \\
\hline Sr-90+D & $010098-97-2$ & $3.5 \mathrm{E}+01$ & $3.5 \mathrm{E}-01$ \\
\hline Sr-91 & $014331-91-0$ & $1.0 \mathrm{E}+03$ & $1.0 \mathrm{E}+01$ \\
\hline Sr-92 & $014928-29-1$ & $1.4 \mathrm{E}+03$ & $1.4 \mathrm{E}+01$ \\
\hline Ta-182 & $013982-00-8$ & $2.5 \mathrm{E}+02$ & $2.5 \mathrm{E}+00$ \\
\hline Tb-158 & $015759-55-4$ & & \\
\hline & & & \\
\hline
\end{tabular}


Table 3.1h. (continued)

\begin{tabular}{|l|l|c|c|}
\hline \multirow{2}{*}{ Chemical } & \multirow{2}{*}{ CASRN } & \multicolumn{2}{|c|}{$\begin{array}{c}\text { Ingestion of fish (concentration in water) } \\
\text { (pCi/L) }\end{array}$} \\
\cline { 3 - 4 } & & $10^{-4}$ & $10^{-6}$ \\
\hline Tb-160 & $013981-29-8$ & & \\
\hline Tc-95 & $014809-56-4$ & $1.3 \mathrm{E}+05$ & $1.3 \mathrm{E}+03$ \\
\hline Tc-95m & $014809-56-4$ & $7.1 \mathrm{E}+03$ & $7.1 \mathrm{E}+01$ \\
\hline Tc-96 & $014808-44-7$ & $3.9 \mathrm{E}+03$ & $3.9 \mathrm{E}+01$ \\
\hline Tc-96m & $014808-44-7$ & $3.4 \mathrm{E}+05$ & $3.4 \mathrm{E}+03$ \\
\hline Tc-97 & $015759-35-0$ & $5.6 \mathrm{E}+04$ & $5.6 \mathrm{E}+02$ \\
\hline Tc-97m & $015759-35-0$ & $7.3 \mathrm{E}+03$ & $7.3 \mathrm{E}+01$ \\
\hline Tc-99 & $014133-76-7$ & $6.3 \mathrm{E}+03$ & $6.3 \mathrm{E}+01$ \\
\hline Tc-99m & $014133-76-7$ & $1.6 \mathrm{E}+05$ & $1.6 \mathrm{E}+03$ \\
\hline Te-125m & $014390-73-9$ & $1.8 \mathrm{E}+02$ & $1.8 \mathrm{E}+00$ \\
\hline Te-127 & $013981-49-2$ & $5.2 \mathrm{E}+02$ & $5.2 \mathrm{E}+00$ \\
\hline Te-127m & $013981-49-2$ & $7.3 \mathrm{E}+01$ & $7.3 \mathrm{E}-01$ \\
\hline Te-129 & $014269-71-7$ & $3.0 \mathrm{E}+03$ & $3.0 \mathrm{E}+01$ \\
\hline Te-129m & $014269-71-7$ & $3.8 \mathrm{E}+01$ & $3.8 \mathrm{E}-01$ \\
\hline Te-131 & $014683-12-6$ & $1.1 \mathrm{E}+03$ & $1.1 \mathrm{E}+01$ \\
\hline Te-131m & $014683-12-6$ & $5.0 \mathrm{E}+01$ & $5.0 \mathrm{E}-01$ \\
\hline Te-132 & $014234-28-7$ & $3.6 \mathrm{E}+01$ & $3.6 \mathrm{E}-01$ \\
\hline & & & \\
\hline
\end{tabular}


Table 3.1h. (continued)

\begin{tabular}{|l|l|c|c|}
\hline \multirow{2}{*}{ Chemical } & \multirow{2}{*}{ CASRN } & \multicolumn{2}{|c|}{$\begin{array}{c}\text { Ingestion of fish (concentration in water) } \\
\text { (pCi/L) }\end{array}$} \\
\cline { 3 - 4 } & & $10^{-4}$ & $10^{-6}$ \\
\hline Th-227 & $015623-47-9$ & $4.4 \mathrm{E}+01$ & $4.4 \mathrm{E}-01$ \\
\hline Th-228 & $014274-82-9$ & $2.8 \mathrm{E}+01$ & $2.8 \mathrm{E}-01$ \\
\hline Th-228+D & $014274-82-9$ & $1.4 \mathrm{E}+00$ & $1.4 \mathrm{E}-02$ \\
\hline Th-229 & $015594-54-4$ & $3.1 \mathrm{E}+01$ & $3.1 \mathrm{E}-01$ \\
\hline Th-229+D & $015594-54-4$ & $9.5 \mathrm{E}-01$ & $9.5 \mathrm{E}-03$ \\
\hline Th-230 & $014269-63-7$ & $4.7 \mathrm{E}+01$ & $4.7 \mathrm{E}-01$ \\
\hline Th-231 & $014932-40-2$ & $9.9 \mathrm{E}+02$ & $9.9 \mathrm{E}+00$ \\
\hline Th-232 & $007440-29-1$ & $5.4 \mathrm{E}+01$ & $5.4 \mathrm{E}-01$ \\
\hline Th-234 & $015065-10-8$ & $9.1 \mathrm{E}+01$ & $9.1 \mathrm{E}-01$ \\
\hline Tl-202 & $015720-57-7$ & & \\
\hline Tl-204 & $013968-51-9$ & & $3.9 \mathrm{E}+00$ \\
\hline Tl-208 & $014913-50-9$ & & \\
\hline Tl-209 & $015690-73-0$ & & \\
\hline Tm-170 & $013981-30-1$ & & \\
\hline Tm-171 & $014333-45-0$ & & \\
\hline U-232 & $014158-29-3$ & & \\
\hline U-233 & $013968-55-3$ & & \\
\hline & & & \\
\hline
\end{tabular}


Table 3.1h. (continued)

\begin{tabular}{|l|l|c|c|}
\hline \multirow{2}{*}{ Chemical $^{\dagger}$} & \multirow{2}{*}{ CASRN } & \multicolumn{2}{|c|}{$\begin{array}{c}\text { Ingestion of fish (concentration in water) } \\
\text { (pCi/L) }\end{array}$} \\
\cline { 3 - 4 } & & $10^{-4}$ & $10^{-6}$ \\
\hline U-234 & $013966-29-5$ & $4.0 \mathrm{E}+02$ & $4.0 \mathrm{E}+00$ \\
\hline U-235 & $015117-96-1$ & $3.9 \mathrm{E}+02$ & $3.9 \mathrm{E}+00$ \\
\hline U-235+D & $015117-96-1$ & $3.4 \mathrm{E}+01$ & $3.4 \mathrm{E}-01$ \\
\hline U-236 & $013982-70-2$ & $4.2 \mathrm{E}+02$ & $4.2 \mathrm{E}+00$ \\
\hline U-237 & $014269-75-1$ & $4.4 \mathrm{E}+03$ & $4.4 \mathrm{E}+01$ \\
\hline U-238 & $007440-61-1$ & $4.1 \mathrm{E}+02$ & $4.1 \mathrm{E}+00$ \\
\hline U-238+D & $007440-61-1$ & $2.2 \mathrm{E}+01$ & $2.2 \mathrm{E}-01$ \\
\hline U-240 & $015687-53-3$ & $3.2 \mathrm{E}+03$ & $3.2 \mathrm{E}+01$ \\
\hline V-48 & $014331-97-6$ & $2.3 \mathrm{E}+03$ & $2.3 \mathrm{E}+01$ \\
\hline W-181 & $015749-46-9$ & $6.5 \mathrm{E}+04$ & $6.5 \mathrm{E}+02$ \\
\hline W-185 & $014932-41-3$ & $8.6 \mathrm{E}+03$ & $8.6 \mathrm{E}+01$ \\
\hline W-187 & $014983-48-3$ & $7.2 \mathrm{E}+03$ & $7.2 \mathrm{E}+01$ \\
\hline Xe-122 & $015151-09-4$ & & \\
\hline Xe-123 & $015700-10-4$ & & \\
\hline Xe-125 & $013994-18-8$ & & \\
\hline Xe-127 & $013994-19-9$ & & \\
\hline Xe-129m & $013965-99-6$ & & \\
\hline
\end{tabular}


Table 3.1h. (continued)

\begin{tabular}{|c|c|c|c|}
\hline \multirow[t]{2}{*}{ Chemical $^{\dagger}$} & \multirow[t]{2}{*}{ CASRN } & \multicolumn{2}{|c|}{$\begin{array}{l}\text { Ingestion of fish (concentration in water) } \\
\qquad(\mathrm{pCi} / \mathrm{L})\end{array}$} \\
\hline & & $10^{-4}$ & $10^{-6}$ \\
\hline $\mathrm{Xe}-13 \mathrm{~lm}$ & $014683-11-5$ & & \\
\hline $\mathrm{Xe}-133$ & $014932-42-4$ & & \\
\hline $\mathrm{Xe}-133 \mathrm{~m}$ & $014932-42-4$ & & \\
\hline $\mathrm{Xe}-135$ & 014995-62-1 & & \\
\hline $\mathrm{Xe}-135 \mathrm{~m}$ & 014995-62-1 & & \\
\hline $\mathrm{Xe}-137$ & 014835-21-3 & & \\
\hline $\mathrm{Xe}-138$ & $015751-81-2$ & & \\
\hline$Y-90$ & 010098-91-6 & $3.9 \mathrm{E}+02$ & $3.9 \mathrm{E}+00$ \\
\hline Y-91 & $014234-24-3$ & $4.4 \mathrm{E}+02$ & $4.4 \mathrm{E}+00$ \\
\hline Y-91m & $014234-24-3$ & $1.6 \mathrm{E}+05$ & $1.6 \mathrm{E}+03$ \\
\hline Y-92 & $015751-59-4$ & $3.0 \mathrm{E}+03$ & $3.0 \mathrm{E}+01$ \\
\hline Y-93 & 014981-70-5 & $1.0 \mathrm{E}+03$ & $1.0 \mathrm{E}+01$ \\
\hline $\mathrm{Zn}-65$ & 013982-39-3 & $1.8 \mathrm{E}+01$ & $1.8 \mathrm{E}-01$ \\
\hline $\mathrm{Zn}-69$ & 013982-23-5 & $2.8 \mathrm{E}+03$ & $2.8 \mathrm{E}+01$ \\
\hline $\mathrm{Zn}-69 \mathrm{~m}$ & 013982-23-5 & $1.2 \mathrm{E}+02$ & $1.2 \mathrm{E}+00$ \\
\hline Zr-93 & 015751-77-6 & $1.1 \mathrm{E}+03$ & $1.1 \mathrm{E}+01$ \\
\hline Zr-95 & 013967-71-0 & $1.5 \mathrm{E}+02$ & $1.5 \mathrm{E}+00$ \\
\hline
\end{tabular}


Table 3.1h. (continued)

\begin{tabular}{|l|l|c|c|}
\hline \multirow{2}{*}{ Chemical $^{\dagger}$} & \multirow{2}{*}{ CASRN } & \multicolumn{2}{|c|}{ Ingestion of fish (concentration in water) } \\
& & $10^{-4}$ & $10^{-6}$ \\
\cline { 3 - 4 } & & $5.7 \mathrm{E}+01$ & $5.7 \mathrm{E}-01$ \\
\hline $\mathrm{Zr}-97$ & $014928-30-4$ & & \\
\hline
\end{tabular}

Note:

$\dagger \quad$ Isotopes designated with an " $\mathrm{m}$ " indicate that the isotope is metastable; isotopes designated with a " $+D$ " indicate that the radionuclide slope factors include contributions from daughter products. 
Table 3.2a. Risk-based Preliminary Remediation Goals for nonradionuclides in groundwater (industrial scenario)

\begin{tabular}{|c|c|c|c|c|c|}
\hline \multirow{2}{*}{ Chemical } & \multirow{2}{*}{ CASRN } & \multicolumn{4}{|c|}{ Ingestion (mg/L)" } \\
\hline & & $10^{-4}$ & $10^{-6}$ & $H Q=1$ & $\mathrm{HQ}=0.1$ \\
\hline ALAR & 001596-84-5 & & & $1.5 \mathrm{E}+01$ & $1.5 \mathrm{E}+00$ \\
\hline Acenaphthene & 000083-32-9 & & & $6.1 \mathrm{E}+00$ & 6.1E-01 \\
\hline Acenaphthylene & $000208-96-8$ & & & & \\
\hline Acephate & $030560-19-1$ & $3.3 \mathrm{E}+00$ & 3.3E-02 & 4.1E-01 & 4.1E-02 \\
\hline Acetaldehyde & 000075-07-0 & & & & \\
\hline Acetochlor & $034256-82-1$ & & & $2.0 \mathrm{E}+00$ & $2.0 \mathrm{E}-01$ \\
\hline Acetone & $000067-64-1$ & & & $1.0 E+01$ & $1.0 \mathrm{E}+00$ \\
\hline Acetone Cyanohydrin & $000075-86-5$ & & & $8.2 \mathrm{E}-02$ & 8.2E-03 \\
\hline Acetonitrile & 000075-05-8 & & & $6.1 \mathrm{E}-01$ & $6.1 \mathrm{E}-02$ \\
\hline Acetophenone $\mathrm{e}^{(\mathrm{s})}$ & 000098-86-2 & & & $1.0 \mathrm{E}+01$ & $1.0 \mathrm{E}+00$ \\
\hline Acrolein $^{(6)}$ & $000107-02-8$ & & & $2.0 \mathrm{E}+00$ & $2.0 \mathrm{E}-01$ \\
\hline Acrylamide & $000079-06-1$ & $6.4 \mathrm{E}-03$ & $6.4 \mathrm{E}-05$ & $2.0 \mathrm{E}-02$ & $2.0 \mathrm{E}-03$ \\
\hline Acrylic Acid & $000079-10-7$ & & & $5.1 \mathrm{E}+01$ & $5.1 \mathrm{E}+00$ \\
\hline Acrylonitrile & $000107-13-1$ & $5.3 \mathrm{E}-02$ & $5.3 \mathrm{E}-04$ & $1.0 \mathrm{E}-01$ & $1.0 \mathrm{E}-02$ \\
\hline Alachlor & $015972-60-8$ & $3.6 \mathrm{E}-01$ & $3.6 \mathrm{E}-03$ & $1.0 \mathrm{E}+00$ & $1.0 \mathrm{E}-01$ \\
\hline Aldicarb & $000116-06-3$ & & & $1.0 \mathrm{E}-01$ & $1.0 \mathrm{E}-02$ \\
\hline Aldicarb Sulfone & 001646-88-4 & & & $1.0 \mathrm{E}-01$ & $1.0 \mathrm{E}-02$ \\
\hline Aldrin & 000309-00-2 & $1.7 \mathrm{E}-03$ & $1.7 \mathrm{E}-05$ & 3.1E-03 & $3.1 \mathrm{E}-04$ \\
\hline
\end{tabular}


Table 3.2a. (continued)

\begin{tabular}{|c|c|c|c|c|c|}
\hline \multirow{2}{*}{ Chemical } & \multirow{2}{*}{ CASRN } & \multicolumn{4}{|c|}{ Ingestion (mg/L) } \\
\hline & & $10^{-4}$ & $10^{-6}$ & $\mathbf{H Q}=\mathbf{1}$ & $H Q=0.1$ \\
\hline Ally & $074223-64-6$ & & & $2.6 \mathrm{E}+01$ & $2.6 \mathrm{E}+00$ \\
\hline Allyl Alcohol & $000107-18-6$ & & & $5.1 \mathrm{E}-01$ & $5.1 \mathrm{E}-02$ \\
\hline Allyl Chloride ${ }^{(5)}$ & $000107-05-1$ & & & $5.1 \mathrm{E}+00$ & $5.1 \mathrm{E}-01$ \\
\hline Aluminum & $007429-90-5$ & & & & \\
\hline Aluminum Phosphide & 020859-73-8 & & & $4.1 \mathrm{E}-02$ & $4.1 \mathrm{E}-03$ \\
\hline Amdro & $067485-29-4$ & & & 3.1E-02 & $3.1 \mathrm{E}-03$ \\
\hline Ametryn & $000834-12-8$ & & & $9.2 \mathrm{E}-01$ & $9.2 \mathrm{E}-02$ \\
\hline Aminophenol, m- & $000591-27-5$ & & & $7.2 \mathrm{E}+00$ & 7.2E-01 \\
\hline Aminopyridine, 4- & 000504-24-5 & & & $2.0 \mathrm{E}-03$ & $2.0 \mathrm{E}-04$ \\
\hline Amitraz & 033089-61-1 & & & $2.6 \mathrm{E}-01$ & 2.6E-02 \\
\hline Ammonia & $007664-41-7$ & & & & \\
\hline Ammonium Sulfamate & 007773-06-0 & & & $2.0 \mathrm{E}+01$ & $2.0 \mathrm{E}+00$ \\
\hline Aniline & $000062-53-3$ & $5.0 \mathrm{E}+00$ & $5.0 \mathrm{E}-02$ & & \\
\hline Anthracene & $000120-12-7$ & & & $3.1 \mathrm{E}+01$ & $3.1 \mathrm{E}+00$ \\
\hline Antimony (metallic) & $007440-36-0$ & & & $4.1 \mathrm{E}-02$ & 4.1E-03 \\
\hline Antimony Pentoxide & $001314-60-9$ & & & $5.1 \mathrm{E}-02$ & $5.1 \mathrm{E}-03$ \\
\hline Antimony Potassium Tartrate & $000304-61-0$ & & & $9.2 \mathrm{E}-02$ & $9.2 \mathrm{E}-03$ \\
\hline Antimony Tetroxide & $001332-81-6$ & & & 4.1E-02 & 4.1E-03 \\
\hline
\end{tabular}


Table 3.2a. (continued)

\begin{tabular}{|l|l|l|l|c|c|}
\hline \multirow{2}{*}{ Chemical } & \multirow{2}{*}{ CASRN } & \multicolumn{3}{|c|}{ Ingestion (mg/L)* } \\
\cline { 4 - 6 } & & $10^{-4}$ & $10^{-6}$ & HQ=1 & HQ=0.1 \\
\hline Antimony Trioxide & $001309-64-4$ & & & $4.1 \mathrm{E}-02$ & $4.1 \mathrm{E}-03$ \\
\hline Apollo & $074115-24-5$ & & & $1.3 \mathrm{E}+00$ & $1.3 \mathrm{E}-01$ \\
\hline Aramite & $000140-57-8$ & $1.1 \mathrm{E}+00$ & $1.1 \mathrm{E}-02$ & $5.1 \mathrm{E}+00$ & $5.1 \mathrm{E}-01$ \\
\hline Aroclor 1016 & $012674-11-2$ & & & $7.2 \mathrm{E}-03$ & $7.2 \mathrm{E}-04$ \\
\hline Aroclor 1221(1) & $011104-28-2$ & $3.7 \mathrm{E}-03$ & $3.7 \mathrm{E}-05$ & & \\
\hline Aroclor 1232(1) & $011141-16-5$ & $3.7 \mathrm{E}-03$ & $3.7 \mathrm{E}-05$ & & \\
\hline Aroclor 1242(1) & $053469-21-9$ & $3.7 \mathrm{E}-03$ & $3.7 \mathrm{E}-05$ & & \\
\hline Aroclor 1248(1) & $012672-29-6$ & $3.7 \mathrm{E}-03$ & $3.7 \mathrm{E}-05$ & & \\
\hline Aroclor 1254 & $011097-69-1$ & & & $2.0 \mathrm{E}-03$ & $2.0 \mathrm{E}-04$ \\
\hline Aroclor 1260 & & & \\
\hline Arsenic Salts & $011096-82-5$ & $3.7 \mathrm{E}-03$ & $3.7 \mathrm{E}-05$ & & \\
\hline Arsenic, Inorganic & NA & & & & \\
\hline Arsine & $007440-38-2$ & $1.9 \mathrm{E}-02$ & $1.9 \mathrm{E}-04$ & $3.1 \mathrm{E}-02$ & $3.1 \mathrm{E}-03$ \\
\hline Asbestos & $007784-42-1$ & & & & \\
\hline Assure & $001332-21-4$ & & & & \\
\hline Asulam & $076578-14-8$ & & & $9.2 \mathrm{E}-01$ & $9.2 \mathrm{E}-02$ \\
\hline Atrazine & $003337-71-1$ & & & $5.1 \mathrm{E}+00$ & $5.1 \mathrm{E}-01$ \\
\hline Avermectin B1 & $001912-24-9$ & $1.3 \mathrm{E}-01$ & $1.3 \mathrm{E}-03$ & $3.6 \mathrm{E}+00$ & $3.6 \mathrm{E}-01$ \\
\hline
\end{tabular}


Table 3.2a. (continued)

\begin{tabular}{|l|l|l|l|l|l|}
\hline \multirow{2}{*}{ Chemical } & \multirow{2}{*}{ CASRN } & \multicolumn{4}{|c|}{ Ingestion (mg/L) } \\
\cline { 3 - 6 } & & $10^{-4}$ & $10^{-6}$ & HQ=1 & HQ=0.1 \\
\hline Azzobenzene & $000103-33-3$ & $2.6 \mathrm{E}-01$ & $2.6 \mathrm{E}-03$ & & \\
\hline Barium & $007440-39-3$ & & & $7.2 \mathrm{E}+00$ & $7.2 \mathrm{E}-01$ \\
\hline Barium Cyanide & $000542-62-1$ & & & $1.0 \mathrm{E}+01$ & $1.0 \mathrm{E}+00$ \\
\hline Baygon & $000114-26-1$ & & & $4.1 \mathrm{E}-01$ & $4.1 \mathrm{E}-02$ \\
\hline Bayleton & $043121-43-3$ & & & $3.1 \mathrm{E}+00$ & $3.1 \mathrm{E}-01$ \\
\hline Baythroid & $068359-37-5$ & & & $2.6 \mathrm{E}+00$ & $2.6 \mathrm{E}-01$ \\
\hline Benefin & $001861-40-1$ & & & $3.1 \mathrm{E}+01$ & $3.1 \mathrm{E}+00$ \\
\hline Benomyl & $017804-35-2$ & & & $5.1 \mathrm{E}+00$ & $5.1 \mathrm{E}-01$ \\
\hline Bentazon & $025057-89-0$ & & & $2.6 \mathrm{E}-01$ & $2.6 \mathrm{E}-02$ \\
\hline Benz[a]anthracene ${ }^{(2)}$ & $000056-55-3$ & $3.9 \mathrm{E}-02$ & $3.9 \mathrm{E}-04$ & & \\
\hline Benzaldehyde & $000100-52-7$ & & & $1.0 \mathrm{E}+01$ & $1.0 \mathrm{E}+00$ \\
\hline Benzene & $000071-43-2$ & $9.9 \mathrm{E}-01$ & $9.9 \mathrm{E}-03$ & & \\
\hline Benzene Hexachloride & NA & & & & \\
\hline Benzene, Ethyldimethyl & NA & & & & \\
\hline Benzene, Ethylmethyl & NA & & & & \\
\hline Benzene, Methylpropenyl & NA & & & & \\
\hline Benzene, Methylpropyl & NA & & & & \\
\hline Benzene, Trimethyl & $025551-13-7$ & & & & \\
\hline
\end{tabular}


Table 3.2a. (continued)

\begin{tabular}{|l|l|l|l|l|l|}
\hline \multirow{2}{*}{ Chemical } & \multirow{2}{*}{ CASRN } & \multicolumn{4}{|c|}{ Ingestion (mg/L) } \\
\cline { 4 - 6 } & & $10^{-4}$ & $10^{-6}$ & HQ=1 & HQ=0.1 \\
\hline Benzenethiol & $000108-98-5$ & & & $1.0 \mathrm{E}-03$ & $1.0 \mathrm{E}-04$ \\
\hline Benzidine & $000092-87-5$ & $1.2 \mathrm{E}-04$ & $1.2 \mathrm{E}-06$ & $3.1 \mathrm{E}-01$ & $3.1 \mathrm{E}-02$ \\
\hline Benzo[a]pyrene ${ }^{(2)}$ & $000050-32-8$ & $3.9 \mathrm{E}-03$ & $3.9 \mathrm{E}-05$ & & \\
\hline Benzo[b]fluoranthene(2) & $000205-99-2$ & $3.9 \mathrm{E}-02$ & $3.9 \mathrm{E}-04$ & & \\
\hline Benzo[g,h,i]perylene & $000191-24-2$ & & & & \\
\hline Benzo[k]fluoranthene(2) & $000207-08-9$ & $3.9 \mathrm{E}-01$ & $3.9 \mathrm{E}-03$ & & \\
\hline Benzoic Acid & $000065-85-0$ & & & $4.1 \mathrm{E}+02$ & $4.1 \mathrm{E}+01$ \\
\hline Benzotrichloride & $000098-07-7$ & $2.2 \mathrm{E}-03$ & $2.2 \mathrm{E}-05$ & & \\
\hline Benzyl Alcohol & $000100-51-6$ & & & $3.1 \mathrm{E}+01$ & $3.1 \mathrm{E}+00$ \\
\hline Benzyl Chloride & $000100-44-7$ & $1.7 \mathrm{E}-01$ & $1.7 \mathrm{E}-03$ & & \\
\hline Beryllium & $007440-41-7$ & $6.7 \mathrm{E}-03$ & $6.7 \mathrm{E}-05$ & $5.1 \mathrm{E}-01$ & $5.1 \mathrm{E}-02$ \\
\hline Bidrin & $000141-66-2$ & & & $1.0 \mathrm{E}-02$ & $1.0 \mathrm{E}-03$ \\
\hline Biphenthrin & $082657-04-3$ & & & $1.5 \mathrm{E}+00$ & $1.5 \mathrm{E}-01$ \\
\hline Biphenyl, 1,1'- & $000092-52-4$ & & & $5.1 \mathrm{E}+00$ & $5.1 \mathrm{E}-01$ \\
\hline $\begin{array}{l}\text { Bis(2-chloro-1-methylethyl)ether } \\
\text { (Technical) }\end{array}$ & $000108-60-1$ & $4.1 \mathrm{E}-01$ & $4.1 \mathrm{E}-03$ & & \\
\hline Bis(2-chloroethoxy)methane & $000111-91-1$ & & & & \\
\hline Bis(2-chloroethyl)ether & $000111-44-4$ & $2.6 \mathrm{E}-02$ & $2.6 \mathrm{E}-04$ & & \\
\hline & & & & & \\
\hline
\end{tabular}


Table 3.2a. (continued)

\begin{tabular}{|c|c|c|c|c|c|}
\hline \multirow{2}{*}{ Chemical } & \multirow{2}{*}{ CASRN } & \multicolumn{4}{|c|}{ Ingestion (mg/L) } \\
\hline & & $10^{-4}$ & $10^{-6}$ & $\mathbf{H Q}=\mathbf{1}$ & $H Q=0.1$ \\
\hline Bis(2-chloroisopropyl)ether & 039638-32-9 & & & $4.1 \mathrm{E}+00$ & 4.1E-01 \\
\hline Bis(2-ethylhexyl)phthalate ${ }^{(6)}$ & $000117-81-7$ & $2.0 \mathrm{E}+00$ & $2.0 \mathrm{E}-02$ & $2.0 \mathrm{E}+00$ & $2.0 \mathrm{E}-01$ \\
\hline Bis(chloromethyl)ether & $000542-88-1$ & $1.3 \mathrm{E}-04$ & $1.3 \mathrm{E}-06$ & & \\
\hline Bisphenol A & $000080-05-7$ & & & $5.1 \mathrm{E}+00$ & $5.1 \mathrm{E}-01$ \\
\hline Boron And Borates Only & $007440-42-8$ & & & $9.2 \mathrm{E}+00$ & $9.2 \mathrm{E}-01$ \\
\hline Boron Trifluoride & $007637-07-2$ & & & & \\
\hline Bromochloromethane & $000074-97-5$ & & & & \\
\hline Bromodichloromethane & $000075-27-4$ & 4.6E-01 & $4.6 \mathrm{E}-03$ & $2.0 \mathrm{E}+00$ & $2.0 \mathrm{E}-01$ \\
\hline Bromodiphenyl Ether, p- & $000101-55-3$ & & & & \\
\hline Bromoform & $000075-25-2$ & $3.6 \mathrm{E}+00$ & $3.6 \mathrm{E}-02$ & $2.0 \mathrm{E}+00$ & $2.0 \mathrm{E}-01$ \\
\hline Bromomethane ${ }^{(6)}$ & 000074-83-9 & & & $1.4 \mathrm{E}-01$ & $1.4 \mathrm{E}-02$ \\
\hline Bromophos & 002104-96-3 & & & $5.1 \mathrm{E}-01$ & $5.1 \mathrm{E}-02$ \\
\hline Bromotrichloromethane & $000075-62-7$ & & & & \\
\hline Bromoxynil & $001689-84-5$ & & & $2.0 \mathrm{E}+00$ & $2.0 \mathrm{E}-01$ \\
\hline Bromoxynil Octanoate & $001689-99-2$ & & & $2.0 \mathrm{E}+00$ & $2.0 \mathrm{E}-01$ \\
\hline Butadiene, 1,3- & 000106-99-0 & & & & \\
\hline Butanol, N- & $000071-36-3$ & & & $1.0 \mathrm{E}+01$ & $1.0 \mathrm{E}+00$ \\
\hline Butanone-2, 4-chloro-4,4-difluoro & NA & & & & \\
\hline
\end{tabular}


Table 3.2a. (continued)

\begin{tabular}{|c|c|c|c|c|c|}
\hline \multirow{2}{*}{ Chemical } & \multirow{2}{*}{ CASRN } & \multicolumn{4}{|c|}{ Ingestion (mg/L)" } \\
\hline & & $10^{-4}$ & $10^{-6}$ & $\mathbf{H Q}=\mathbf{1}$ & $\mathrm{HQ}=0.1$ \\
\hline Butyl Benzyl Phthlate & 000085-68-7 & & & $2.0 \mathrm{E}+01$ & $2.0 \mathrm{E}+00$ \\
\hline Butylate & $002008-41-5$ & & & $5.1 \mathrm{E}+00$ & $5.1 \mathrm{E}-01$ \\
\hline Butylchloride, t- & $000507-20-0$ & & & & \\
\hline Butylphthalyl Butylglycolate & 000085-70-1 & & & $1.0 \mathrm{E}+02$ & $1.0 \mathrm{E}+01$ \\
\hline Cacodylic Acid & $000075-60-5$ & & & $3.1 \mathrm{E}-01$ & $3.1 \mathrm{E}-02$ \\
\hline Cadmium (Water) & $007440-43-9$ & & & $5.1 \mathrm{E}-02$ & $5.1 \mathrm{E}-03$ \\
\hline Calcium Cyanide & 000592-01-8 & & & $4.1 E+00$ & $4.1 \mathrm{E}-01$ \\
\hline Caprolactam & 000105-60-2 & & & $5.1 \mathrm{E}+01$ & $5.1 \mathrm{E}+00$ \\
\hline Captafol & 002425-06-1 & $3.3 \mathrm{E}+00$ & 3.3E-02 & $2.0 \mathrm{E}-01$ & $2.0 \mathrm{E}-02$ \\
\hline Captan & $000133-06-2$ & $8.2 \mathrm{E}+00$ & $8.2 \mathrm{E}-02$ & $1.3 \mathrm{E}+01$ & $1.3 \mathrm{E}+00$ \\
\hline Carbaryl & 000063-25-2 & & & $1.0 \mathrm{E}+01$ & $1.0 \mathrm{E}+00$ \\
\hline Carbazole & $000086-74-8$ & $1.4 \mathrm{E}+00$ & $1.4 \mathrm{E}-02$ & & \\
\hline Carbofuran & $001563-66-2$ & & & $5.1 \mathrm{E}-01$ & $5.1 \mathrm{E}-02$ \\
\hline Carbon Disulfide & $000075-15-0$ & & & $1.0 \mathrm{E}+01$ & $1.0 \mathrm{E}+00$ \\
\hline Carbon Tetrachloride ${ }^{(\sigma)}$ & 000056-23-5 & $2.2 \mathrm{E}-01$ & $2.2 \mathrm{E}-03$ & $7.2 \mathrm{E}-02$ & $7.2 \mathrm{E}-03$ \\
\hline Carbosulfan & 055285-14-8 & & & $1.0 \mathrm{E}+00$ & $1.0 \mathrm{E}-01$ \\
\hline Carboxin & 005234-68-4 & & & $1.0 \mathrm{E}+01$ & $1.0 \mathrm{E}+00$ \\
\hline Chloral & 000075-87-6 & & & $2.0 \mathrm{E}-01$ & $2.0 \mathrm{E}-02$ \\
\hline
\end{tabular}


Table 3.2a. (continued)

\begin{tabular}{|c|c|c|c|c|c|}
\hline \multirow{2}{*}{ Chemical } & \multirow{2}{*}{ CASRN } & \multicolumn{4}{|c|}{ Ingestion (mg/L)" } \\
\hline & & $10^{-4}$ & $10^{-6}$ & $H Q=1$ & $\mathrm{HQ}=0.1$ \\
\hline Chloramben & 000133-90-4 & & & $1.5 \mathrm{E}+00$ & $1.5 \mathrm{E}-01$ \\
\hline Chloranil & 000118-75-2 & $7.1 \mathrm{E}-02$ & 7.1E-04 & & \\
\hline Chlordane $^{(6)}$ & $000057-74-9$ & $2.2 \mathrm{E}-02$ & $2.2 \mathrm{E}-04$ & $6.1 \mathrm{E}-03$ & $6.1 \mathrm{E}-04$ \\
\hline Chloride & $.016887-00-6$ & & & & \\
\hline Chlorimuron, Ethyl- & 090982-32-4. & & & $2.0 \mathrm{E}+00$ & $2.0 \mathrm{E}-01$ \\
\hline Chlorine & 007782-50-5 & & & $1.0 \mathrm{E}+01$ & $1.0 \mathrm{E}+00$ \\
\hline Chlorine Dioxide & 010049-04-4 & & & & \\
\hline Chlorite (Sodium Salt) & 007758-19-2 & & $=$ & & \\
\hline Chloro-1,1-difluoroethane, 1- & 000075-68-3 & & & & \\
\hline Chloro-1,3-butadiene, 2- & 000126-99-8 & & & $2.0 \mathrm{E}+00$ & $2.0 \mathrm{E}-01$ \\
\hline Chloro-2-methylaniline $\mathrm{HCl}$, 4- & 003165-93-3 & $6.2 \mathrm{E}-02$ & $6.2 \mathrm{E}-04$ & & \\
\hline Chloro-2-methylaniline, 4- & 000095-69-2 & $4.9 \mathrm{E}-02$ & $4.9 \mathrm{E}-04$ & & \\
\hline Chloroacetic Acid & 000079-11-8 & & & $2.0 \mathrm{E}-01$ & $2.0 \mathrm{E}-02$ \\
\hline Chloroacetophenone, 2- & $000532-27-4$ & & & & \\
\hline Chloroaniline, $\mathrm{p}$ - & $000106-47-8$ & & & 4.1E-01 & 4.1E-02 \\
\hline Chlorobenzene ${ }^{(6)}$ & 000108-90-7 & & & $2.0 \mathrm{E}+00$ & $2.0 \mathrm{E}-01$ \\
\hline Chlorobenzilate & 000510-15-6 & $1.1 \mathrm{E}-01$ & $1.1 \mathrm{E}-03$ & $2.0 \mathrm{E}+00$ & $2.0 \mathrm{E}-01$ \\
\hline Chlorobenzoic Acid, p- & $000074-11-3$ & & & $2.0 \mathrm{E}+01$ & $2.0 \mathrm{E}+00$ \\
\hline
\end{tabular}


Table 3.2a. (continued)

\begin{tabular}{|l|l|l|l|l|l|}
\hline \multirow{2}{*}{ Chemical } & \multirow{2}{*}{ CASRN } & \multicolumn{4}{|c|}{ Ingestion (mg/L) } \\
\cline { 4 - 6 } & & $10^{-4}$ & $10^{-6}$ & HQ=1 & HQ=0.1 \\
\hline Chlorobenzotrifluoride, 4- & $000098-56-6$ & & & $2.0 \mathrm{E}+00$ & $2.0 \mathrm{E}-01$ \\
\hline Chlorobutane, 1- & $000109-69-3$ & & & $4.1 \mathrm{E}+01$ & $4.1 \mathrm{E}+00$ \\
\hline Chlorobutane, 2- & $000078-86-4$ & & & & \\
\hline Chlorocyclopentadiene & $041851-50-7$ & & & & \\
\hline Chlorodibromoethane & $073506-94-2$ & $3.4 \mathrm{E}-01$ & $3.4 \mathrm{E}-03$ & & \\
\hline Chlorodifluoromethane & $000075-45-6$ & & & & \\
\hline Chloroform ${ }^{(6)}$ & $000067-66-3$ & $4.7 \mathrm{E}+00$ & $4.7 \mathrm{E}-02$ & $1.0 \mathrm{E}+00$ & $1.0 \mathrm{E}-01$ \\
\hline Chloromethane(6) & $000074-87-3$ & $2.2 \mathrm{E}+00$ & $2.2 \mathrm{E}-02$ & & \\
\hline Chloromethyl Methyl Ether & $000107-30-2$ & & & & \\
\hline Chloronaphthalene, Beta- & $000091-58-7$ & & & $8.2 \mathrm{E}+00$ & $8.2 \mathrm{E}-01$ \\
\hline Chloronitrobenzene, 0- & $000088-73-3$ & $1.1 \mathrm{E}+00$ & $1.1 \mathrm{E}-02$ & & \\
\hline Chloronitrobenzene, p- & $000121-73-3$ & $1.6 \mathrm{E}+00$ & $1.6 \mathrm{E}-02$ & & \\
\hline Chlorophenol, 2- & $000095-57-8$ & & & $5.1 \mathrm{E}-01$ & $5.1 \mathrm{E}-02$ \\
\hline Chlorophenyl Methyl Sulfide, p- & $000123-09-1$ & & & & \\
\hline Chlorophenyl Methyl Sulfoxide & $000934-73-6$ & & & & \\
\hline Chloropropane, 2- & $000075-29-6$ & & & & \\
\hline Chlorothalonil & $001897-45-6$ & $2.6 \mathrm{E}+00$ & $2.6 \mathrm{E}-02$ & $1.5 \mathrm{E}+00$ & $1.5 \mathrm{E}-01$ \\
\hline Chlorotoluene, 0- & $000095-49-8$ & & & $2.0 \mathrm{E}+00$ & $2.0 \mathrm{E}-01$ \\
\hline
\end{tabular}


Table 3.2a. (continued)

\begin{tabular}{|c|c|c|c|c|c|}
\hline \multirow{2}{*}{ Chemical } & \multirow{2}{*}{ CASRN } & \multicolumn{4}{|c|}{ Ingestion (mg/L)* } \\
\hline & & $10^{-4}$ & $10^{-6}$ & $\mathbf{H Q}=\mathbf{1}$ & $\mathrm{HQ}=0.1$ \\
\hline Chlorpropham & $000101-21-3$ & & & $2.0 \mathrm{E}+01$ & $2.0 \mathrm{E}+00$ \\
\hline Chlorpyrifos & 002921-88-2 & & & 3.1E-01 & $3.1 \mathrm{E}-02$ \\
\hline Chlorpyrifos Methyl & 005598-13-0 & & & $1.0 \mathrm{E}+00$ & $1.0 \mathrm{E}-01$ \\
\hline Chlorsulfuron & $064902-72-3$ & & & $5.1 \mathrm{E}+00$ & $5.1 \mathrm{E}-01$ \\
\hline Chlorthiophos & $060238-56-4$ & & & $8.2 \mathrm{E}-02$ & $8.2 \mathrm{E}-03$ \\
\hline Chromium (III) (Insoluble Salts) & 016065-83-1 & & & $1.0 \mathrm{E}+02$ & $1.0 \mathrm{E}+01$ \\
\hline Chromium (VI) ${ }^{(6)}$ & 018540-29-9 & & & $5.1 \mathrm{E}-01$ & $5.1 \mathrm{E}-02$ \\
\hline Chromium Salts & NA & & & & \\
\hline Chrysene $^{(2)}$ & 000218-01-9 & $3.9 \mathrm{E}+00$ & $3.9 \mathrm{E}-02$ & & \\
\hline Cobalt & $007440-48-4$ & & & & \\
\hline Coke Oven Emissions & $008007-45-2$ & & & & \\
\hline Copper & 007440-50-8 & & & & \\
\hline Copper Cyanide & 000544-92-3 & & & $5.1 \mathrm{E}-01$ & $5.1 \mathrm{E}-02$ \\
\hline Creosote & 008001-58-9 & & & & \\
\hline Cresol, m- & 000108-39-4 & & & $5.1 E+00$ & $5.1 \mathrm{E}-01$ \\
\hline Cresol, o- & 000095-48-7 & & & $5.1 \mathrm{E}+00$ & $5.1 \mathrm{E}-01$ \\
\hline Cresol, p- & $000106-44-5$ & & & 5.1E-01 & $5.1 \mathrm{E}-02$ \\
\hline Crotonaldehyde & 000123-73-9 & $1.5 \mathrm{E}-02$ & $1.5 \mathrm{E}-04$ & & \\
\hline
\end{tabular}


Table 3.2a. (continued)

\begin{tabular}{|c|c|c|c|c|c|}
\hline \multirow{2}{*}{ Chemical } & \multirow{2}{*}{ CASRN } & \multicolumn{4}{|c|}{ Ingestion (mg/L)" } \\
\hline & & $10^{-4}$ & $10^{-6}$ & $\mathbf{H Q}=\mathbf{1}$ & $\mathrm{HQ}=0.1$ \\
\hline Cumene & $000098-82-8$ & & & 4.1E+00 & 4.1E-01 \\
\hline Cyanazine & $021725-46-2$ & 3.4E-02 & 3.4E-04 & $2.0 \mathrm{E}-01$ & $2.0 \mathrm{E}-02$ \\
\hline Cyanide (CN-) & $000057-12-5$ & & & $2.0 \mathrm{E}+00$ & $2.0 \mathrm{E}-01$ \\
\hline Cyanogen & 000460-19-5 & & & $4.1 \mathrm{E}+00$ & 4.1E-01 \\
\hline Cyanogen Bromide & 000506-68-3 & & & $9.2 E+00$ & $9.2 \mathrm{E}-01$ \\
\hline Cyanogen Chloride & $000506-77-4$ & & & $5.1 \mathrm{E}+00$ & $5.1 \mathrm{E}-01$ \\
\hline $\begin{array}{l}\text { Cyclohexane, 1,2,3,4,5-pentabromo-6- } \\
\text { chloro- }\end{array}$ & $000087-84-3$ & $1.2 \mathrm{E}+00$ & $1.2 \mathrm{E}-02$ & & \\
\hline Cyclohexanone & 000108-94-1 & & & $5.1 E+02$ & $5.1 \mathrm{E}+01$ \\
\hline Cyclohexylamine & 000108-91-8 & & & $2.0 \mathrm{E}+01$ & $2.0 \mathrm{E}+00$ \\
\hline Cyclopentadiene & $000542-92-7$ & & & & \\
\hline Cyhalothrin/karate & $068085-85-8$ & & & $5.1 \mathrm{E}-01$ & $5.1 \mathrm{E}-02$ \\
\hline Cypermethrin & 052315-07-8 & & & $1.0 \mathrm{E}+00$ & $1.0 \mathrm{E}-01$ \\
\hline Cyromazine & $066215-27-8$ & & & 7.7E-01 & 7.7E-02 \\
\hline DDD & $000072-54-8$ & $1.2 \mathrm{E}-01$ & $1.2 \mathrm{E}-03$ & & \\
\hline DDE & $000072-55-9$ & $8.4 \mathrm{E}-02$ & $8.4 \mathrm{E}-04$ & & \\
\hline DDT & 000050-29-3 & $8.4 \mathrm{E}-02$ & 8.4E-04 & $5.1 \mathrm{E}-02$ & $5.1 \mathrm{E}-03$ \\
\hline Dacthal & 001861-32-1 & & & $1.0 \mathrm{E}+00$ & $1.0 \mathrm{E}-01$ \\
\hline
\end{tabular}


Table 3.2a. (continued)

\begin{tabular}{|l|l|l|c|c|c|}
\hline \multirow{2}{*}{ Chemical } & \multirow{2}{*}{ CASRN } & \multicolumn{3}{|c|}{ Ingestion (mg/L)" } \\
\cline { 4 - 6 } & & $10^{-4}$ & $10^{-6}$ & HQ=1 & HQ=0.1 \\
\hline Dalapon & $000075-99-0$ & & & $3.1 \mathrm{E}+00$ & $3.1 \mathrm{E}-01$ \\
\hline Decabromodiphenyl Ether & $001163-19-5$ & & & $1.0 \mathrm{E}+00$ & $1.0 \mathrm{E}-01$ \\
\hline Demeton & $008065-48-3$ & & & $4.1 \mathrm{E}-03$ & $4.1 \mathrm{E}-04$ \\
\hline Di(2-ethylhexyl)adipate & $000103-23-1$ & $2.4 \mathrm{E}+01$ & $2.4 \mathrm{E}-01$ & $6.1 \mathrm{E}+01$ & $6.1 \mathrm{E}+00$ \\
\hline Diallate & $002303-16-4$ & $4.7 \mathrm{E}-01$ & $4.7 \mathrm{E}-03$ & & \\
\hline Diazinon & $000333-41-5$ & & & $9.2 \mathrm{E}-02$ & $9.2 \mathrm{E}-03$ \\
\hline Dibenz[a,h]anthracene ${ }^{(2)}$ & $000053-70-3$ & $3.9 \mathrm{E}-03$ & $3.9 \mathrm{E}-05$ & & \\
\hline Dibromo-3-chloropropane, 1,2- & $000096-12-8$ & $2.0 \mathrm{E}-02$ & $2.0 \mathrm{E}-04$ & & \\
\hline Dibromobenzene, 1,4- & $000106-37-6$ & & & $1.0 \mathrm{E}+00$ & $1.0 \mathrm{E}-01$ \\
\hline Dibromochloromethane & $000124-48-1$ & $3.4 \mathrm{E}-01$ & $3.4 \mathrm{E}-03$ & $2.0 \mathrm{E}+00$ & $2.0 \mathrm{E}-01$ \\
\hline Dibromodichloromethane & $000594-18-3$ & & & & \\
\hline Dibromodiphenyl Ether, p,p'- & $002050-47-7$ & & & & \\
\hline Dibromoethane, 1,2- & $000106-93-4$ & $3.4 \mathrm{E}-04$ & $3.4 \mathrm{E}-06$ & & \\
\hline $\begin{array}{l}\text { Dibromomethane (Methylene } \\
\text { Bromide) }\end{array}$ & $000074-95-3$ & & & $1.0 \mathrm{E}+00$ & $1.0 \mathrm{E}-01$ \\
\hline Dibutyl Phthalate & $000084-74-2$ & & & $1.0 \mathrm{E}+01$ & $1.0 \mathrm{E}+00$ \\
\hline Dicamba & $001918-00-9$ & & & $3.1 \mathrm{E}+00$ & $3.1 \mathrm{E}-01$ \\
\hline Dichloro-2-butene, 1,4- & $000764-41-0$ & & & & \\
\hline
\end{tabular}


Table 3.2a. (continued)

\begin{tabular}{|c|c|c|c|c|c|}
\hline \multirow{2}{*}{ Chemical } & \multirow{2}{*}{ CASRN } & \multicolumn{4}{|c|}{ Ingestion (mg/L)" } \\
\hline & & $10^{-4}$ & $10^{-6}$ & $\mathbf{H Q}=\mathbf{1}$ & $\mathrm{HQ}=0.1$ \\
\hline \multicolumn{6}{|l|}{ Dichloroacetic Acid } \\
\hline Dichlorobenzene, $1,2^{(5)}$ & 000095-50-1 & & & $9.2 \mathrm{E}+00$ & $9.2 \mathrm{E}-01$ \\
\hline Dichlorobenzene, 1,3- & 000541-73-1 & & & & \\
\hline Dichlorobenzene, 1,4- & 000106-46-7 & $1.2 \mathrm{E}+00$ & $1.2 \mathrm{E}-02$ & & \\
\hline Dichlorobenzidine, 3,3'- & 000091-94-1 & $6.4 \mathrm{E}-02$ & $6.4 \mathrm{E}-04$ & & \\
\hline Dichlorodifluoromethane & 000075-71-8 & & & $2.0 \mathrm{E}+01$ & $2.0 \mathrm{E}+00$ \\
\hline Dichloroethane, 1,1- & 000075-34-3 & & & $1.0 \mathrm{E}+01$ & $1.0 \mathrm{E}+00$ \\
\hline Dichloroethane, 1,2- & 000107-06-2 & 3.1E-01 & $3.1 \mathrm{E}-03$ & & \\
\hline Dichloroethylene, 1,1- & 000075-35-4 & $4.8 \mathrm{E}-02$ & $4.8 \mathrm{E}-04$ & $9.2 \mathrm{E}-01$ & $9.2 \mathrm{E}-02$ \\
\hline $\begin{array}{l}\text { Dichloroethylene, 1,2-(Mixed } \\
\text { Isomers) }\end{array}$ & 000540-59-0 & & & $9.2 \mathrm{E}-01$ & $9.2 \mathrm{E}-02$ \\
\hline Dichloroethylene, 1,2-cis- & $000156-59-2$ & & & $1.0 \mathrm{E}+00$ & $1.0 \mathrm{E}-01$ \\
\hline Dichloroethylene, 1,2-trans- & 000156-60-5 & & & $2.0 \mathrm{E}+00$ & 2.0E-01 \\
\hline Dichlorophenol, 2,4- & 000120-83-2 & & & 3.1E-01 & 3.1E-02 \\
\hline Dichlorophenoxy Acetic Acid, 2,4- & 000094-75-7 & & & $1.0 \mathrm{E}+00$ & $1.0 \mathrm{E}-01$ \\
\hline Dichlorophenoxy)butyric Acid, 4-(2,4- & 000094-82-6 & & & $8.2 \mathrm{E}-01$ & $8.2 \mathrm{E}-02$ \\
\hline Dichloropropane, 1,2- & $000078-87-5$ & $4.2 \mathrm{E}-01$ & $4.2 \mathrm{E}-03$ & & \\
\hline Dichloropropanol, 2,3- & 000616-23-9 & & & $3.1 \mathrm{E}-01$ & 3.1E-02 \\
\hline
\end{tabular}




\begin{tabular}{|c|c|c|c|c|c|}
\hline$\varepsilon 0-\exists 0^{\circ} z$ & $20-80^{\circ} z$ & & & S-IS-090000 & әңвочрәน!ฺ \\
\hline $10-80^{\circ} z$ & $00+\mathrm{B} 00^{\circ} \mathrm{Z}$ & & & $\left\llcorner-t 9-06 Z \varsigma \varsigma_{0}\right.$ & u!̣d!̣|әш!̣ \\
\hline \multirow[t]{2}{*}{ I0-3z'8 } & $00+3 z \cdot 8$ & & & 9-SL-StDI00 & 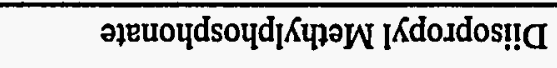 \\
\hline & & & & $9-\angle \varepsilon-\varsigma L 0000$ & 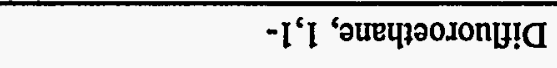 \\
\hline I0-ت10 $Z$ & $00+80 \%$ & & & S-8E-L9ESEO & uodnzueqni!! \\
\hline \multirow[t]{2}{*}{ [0-تZ'8 } & $00+928$ & & & $9-8 t-z Z \tau \varepsilon+0$ & penbozuəy!d \\
\hline & & $80-\mathrm{Gl} 99$ & $90-9 I^{\circ} 9$ & {$[-\varepsilon \varsigma-9 \$ 0000$} & 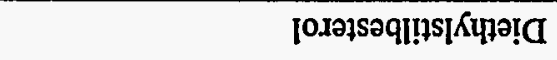 \\
\hline$I 0-3 I \cdot I$ & $00+9 I^{\circ} \mathrm{I}$ & & & $s-t 8-\angle 19000$ & әр!шешшоу Кцрә! \\
\hline \multirow[t]{4}{*}{$10+30^{\circ} \tau$} & $20+90 \cdot \tau$ & & & 0-06-III000 & 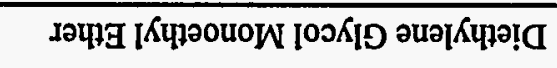 \\
\hline & & - & & $s-\downarrow \varepsilon-Z I I 000$ & 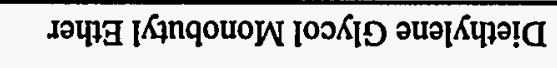 \\
\hline & & & & $0-I Z-\varepsilon 69000$ & 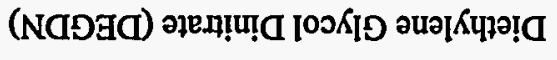 \\
\hline & & & & S-St-II $\varepsilon 000$ & 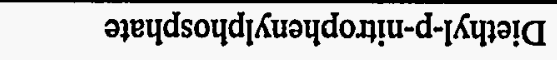 \\
\hline $00+\exists Z^{\circ} 8$ & $\mathrm{I} 0+\mathrm{BZ} \cdot 8$ & & & $\tau-99-\not 80000$ & 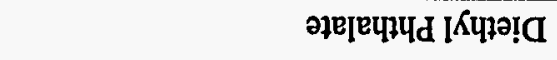 \\
\hline$t 0-\exists I \cdot S$ & $\varepsilon 0-\exists l \cdot s$ & S0-38. I & $\varepsilon 0^{-38^{*} I}$ & $I-\angle S-090000$ & น!ррә!व \\
\hline \multirow[t]{2}{*}{$10-3 I^{\circ} \varepsilon$} & $00+\theta I^{\circ} \varepsilon$ & & & $9-\varepsilon L-L L 0000$ & 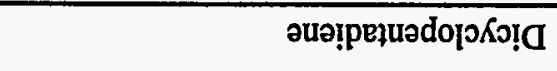 \\
\hline & & & & $\tau-\tau \varepsilon-S I I 000$ & [оjоэ! \\
\hline$\varepsilon 0-\mathrm{Gl} \cdot \mathrm{s}$ & $20-\mathrm{Al}^{\prime} \mathrm{s}$ & $t 0-96.6$ & $20-36.6$ & $L-\varepsilon L-Z 90000$ & SOАІоүчग! \\
\hline$\varepsilon 0-\exists I \cdot \varepsilon$ & Z0-घI’ $\varepsilon$ & $\varepsilon 0-39 \cdot I$ & $10-89^{\circ} I$ & $9-\varsigma L-\tau \pitchfork S 000$ & 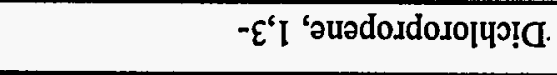 \\
\hline$I^{\circ} 0=\partial H$ & $I=\partial \mathbf{H}$ & $9-0 I$ & rOI & \multirow{2}{*}{ NySV } & \multirow{2}{*}{ |вวุฒอนว } \\
\hline &.$(T / 8 ิ \mathrm{~m}$ & วิชีนI & & & \\
\hline
\end{tabular}

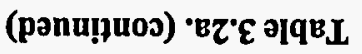


Table 3.2a. (continued)

\begin{tabular}{|c|c|c|c|c|c|}
\hline \multirow{2}{*}{ Chemical } & \multirow{2}{*}{ CASRN } & \multicolumn{4}{|c|}{ Ingestion (mg/L)* } \\
\hline & & $10^{-4}$ & $10^{-6}$ & $H Q=1$ & $\mathrm{HQ}=0.1$ \\
\hline Dimethoxybenzidine, 3,3'- & 000119-90-4 & $2.0 \mathrm{E}+00$ & 2.0E-02 & & \\
\hline Dimethyl Sulfate & $000077-78-1$ & & & & \\
\hline Dimethylaniline $\mathrm{HCl}, 2,4-$ & $021436-96-4$ & $4.9 \mathrm{E}-02$ & $4.9 \mathrm{E}-04$ & & \\
\hline Dimethylaniline, 2,4- & 000095-68-1 & $3.8 \mathrm{E}-02$ & 3.8E-04 & & \\
\hline Dimethylaniline, N,N- & $000121-69-7$ & & & 2.0E-01 & $2.0 \mathrm{E}-02$ \\
\hline Dimethylbenzidine, 3,3'- & 000119-93-7 & $3.1 \mathrm{E}-03$ & 3.1E-05 & & \\
\hline Dimethylethyl Lead & $107584-40-7$ & & & & \\
\hline Dimethylformamide & $000068-12-2$ & & & $1.0 \mathrm{E}+01$ & $1.0 \mathrm{E}+00$ \\
\hline Dimethylhydrazine, 1,1-(3) & $000057-14-7$ & $1.1 \mathrm{E}-02$ & $1.1 \mathrm{E}-04$ & & \\
\hline Dimethylhydrazine, 1,2- & 000540-73-8 & & & & \\
\hline Dimethylphenol, 2,4- & 000105-67-9 & & & $2.0 \mathrm{E}+00$ & $2.0 \mathrm{E}-01$ \\
\hline Dimethylphenol, 2,6- & 000576-26-1 & & & $6.1 \mathrm{E}-02$ & $6.1 \mathrm{E}-03$ \\
\hline Dimethylphenol, 3,4- & 000095-65-8 & & & $1.0 \mathrm{E}-01$ & $1.0 \mathrm{E}-02$ \\
\hline Dimethylphthalate ${ }^{(5)}$ & $000131-11-3$ & & & $1.0 \mathrm{E}+03$ & $1.0 \mathrm{E}+02$ \\
\hline Dimethylterephthalate & $000120-61-6$ & & & $1.0 \mathrm{E}+01$ & $1.0 \mathrm{E}+00$ \\
\hline Dinitro-o-cresol, 4,6- & $000534-52-1$ & & & & \\
\hline Dinitro-o-cyclohexyl Phenol, 4,6- & 000131-89-5 & & & $2.0 \mathrm{E}-01$ & $2.0 \mathrm{E}-02$ \\
\hline Dinitrobenzene, 1,2- & 000528-29-0 & & & 4.1E-02 & 4.1E-03 \\
\hline
\end{tabular}


Table 3.2a. (continued)

\begin{tabular}{|c|c|c|c|c|c|}
\hline \multirow{2}{*}{ Chemical } & \multirow{2}{*}{ CASRN } & \multicolumn{4}{|c|}{ Ingestion (mg/L)* } \\
\hline & & $10^{-4}$ & $10^{-6}$ & $\mathbf{H Q}=\mathbf{1}$ & $\mathbf{H Q}=0.1$ \\
\hline Dinitrobenzene, 1,3- & $000099-65-0$ & & & $1.0 \mathrm{E}-02$ & $1.0 \mathrm{E}-03$ \\
\hline Dinitrobenzene, 1,4- & $000100-25-4$ & & & 4.1E-02 & 4.1E-03 \\
\hline Dinitrophenol, 2,4- & $000051-28-5$ & & & $2.0 \mathrm{E}-01$ & $2.0 \mathrm{E}-02$ \\
\hline Dinitrotoluene, 2,4- & $000121-14-2$ & 4.2E-02 & 4.2E-04 & $2.0 \mathrm{E}-01$ & $2.0 \mathrm{E}-02$ \\
\hline Dinitrotoluene, 2,6- & $000606-20-2$ & 4.2E-02 & 4.2E-04 & $1.0 \mathrm{E}-01$ & $1.0 \mathrm{E}-02$ \\
\hline Dinitrotoluene, 2-Amino-4,6- & $035572-78-2$ & & & & \\
\hline Dinoseb & $000088-85-7$ & & & $1.0 \mathrm{E}-01$ & $1.0 \mathrm{E}-02$ \\
\hline Dioxane, 1,4- & $000123-91-1$ & $2.6 \mathrm{E}+00$ & $2.6 \mathrm{E}-02$ & & \\
\hline Diphenamid & 000957-51-7 & & & $3.1 \mathrm{E}+00$ & 3.1E-01 \\
\hline Diphenylamine & $000122-39-4$ & & & $2.6 \mathrm{E}+00$ & 2.6E-01 \\
\hline Diphenylhydrazine, 1,2- & $000122-66-7$ & $3.6 \mathrm{E}-02$ & $3.6 \mathrm{E}-04$ & & \\
\hline Diquat & $000085-00-7$ & & & $2.2 \mathrm{E}-01$ & 2.2E-02 \\
\hline Direct Black 38 & $001937-37-7$ & 3.3E-03 & $3.3 \mathrm{E}-05$ & & \\
\hline Direct Blue 6 & $002602-46-2$ & $3.5 \mathrm{E}-03$ & $3.5 \mathrm{E}-05$ & & \\
\hline Direct Brown 95 & $016071-86-6$ & $3.1 E-03$ & $3.1 \mathrm{E}-05$ & & \\
\hline Direct Sky Blue & $002610-05-1$ & & & & \\
\hline Disulfoton & $000298-04-4$ & & & 4.1E-03 & 4.1E-04 \\
\hline Diuron & $000330-54-1$ & & & $2.0 \mathrm{E}-01$ & $2.0 \mathrm{E}-02$ \\
\hline
\end{tabular}


Table 3.2a. (continued)

\begin{tabular}{|c|c|c|c|c|c|}
\hline \multirow{2}{*}{ Chemical } & \multirow{2}{*}{ CASRN } & \multicolumn{4}{|c|}{ Ingestion (mg/L)" } \\
\hline & & $10^{-4}$ & $10^{-6}$ & $\mathbf{H Q}=\mathbf{1}$ & $\mathrm{HQ}=0.1$ \\
\hline Dodine & $002439-10-3$ & & & 4.1E-01 & 4.1E-02 \\
\hline EPTC & $000759-94-4$ & & & $2.6 \mathrm{E}+00$ & 2.6E-01 \\
\hline Endosulfan & $000115-29-7$ & & & $6.1 \mathrm{E}-01$ & $6.1 \mathrm{E}-02$ \\
\hline Endothall & $000145-73-3$ & & & $2.0 \mathrm{E}+00$ & $2.0 \mathrm{E}-01$ \\
\hline Endrin & $000072-20-8$ & & & $3.1 \mathrm{E}-02$ & $3.1 \mathrm{E}-03$ \\
\hline Epichlorohydrin & $000106-89-8$ & $2.9 \mathrm{E}+00$ & $2.9 \mathrm{E}-02$ & 2.0E-01 & $2.0 \mathrm{E}-02$ \\
\hline Epoxybutane, 1,2- & 000106-88-7 & & & & \\
\hline Ethephon & $016672-87-0$ & & & $5.1 \mathrm{E}-01$ & $5.1 \mathrm{E}-02$ \\
\hline Ethion & $000563-12-2$ & & & $5.1 \mathrm{E}-02$ & $5.1 \mathrm{E}-03$ \\
\hline Ethoxyethanol Acetate, 2- & $000111-15-9$ & & & $3.1 \mathrm{E}+01$ & $3.1 \mathrm{E}+00$ \\
\hline Ethoxyethanol, 2- & $000110-80-5$ & & & $4.1 \mathrm{E}+01$ & $4.1 \mathrm{E}+00$ \\
\hline Ethyl Acetate & $000141-78-6$ & & & $9.2 \mathrm{E}+01$ & $9.2 \mathrm{E}+00$ \\
\hline Ethyl Acrylate & 000140-88-5 & $6.0 \mathrm{E}-01$ & $6.0 \mathrm{E}-03$ & & \\
\hline Ethyl Chloride & $000075-00-3$ & & & & \\
\hline Ethyl Ether & $000060-29-7$ & & & $2.0 \mathrm{E}+01$ & $2.0 \mathrm{E}+00$ \\
\hline Ethyl Methacrylate & $000097-63-2$ & & & $9.2 \mathrm{E}+00$ & $9.2 \mathrm{E}-01$ \\
\hline Ethyl-p-nitrophenyl Phosphonate & $002104-64-5$ & & & $1.0 \mathrm{E}-03$ & $1.0 \mathrm{E}-04$ \\
\hline Ethylbenzene $e^{(6)}$ & $000100-41-4$ & & & $1.0 \mathrm{E}+01$ & $1.0 \mathrm{E}+00$ \\
\hline
\end{tabular}




\begin{tabular}{|c|c|c|c|c|c|}
\hline $\mathrm{I0}-\mathrm{E} 0^{\circ} \mathrm{I}$ & $00+30^{\circ} I$ & & & $s-\nabla 6-60 t 690$ & 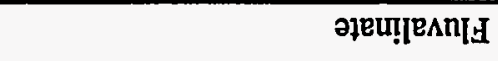 \\
\hline I0-GI'9 & $00+9 l^{\circ} 9$ & & & S-96-Z££990 & 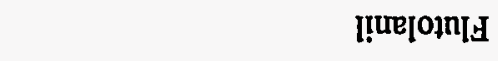 \\
\hline 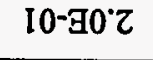 & $00+30 \%$ & & & $\varepsilon-I 6-\varsigma Z \pitchfork 9 S 0$ & 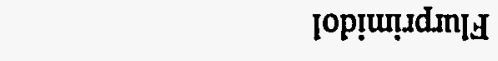 \\
\hline [0-az’8 & $00+9 Z 8$ & & & $t-09-9 S \angle 6 S 0$ & әuop!̣nIA \\
\hline I0-GI'9 & $00+31 \cdot 9$ & & & $t-I t-z 8<L 00$ & әp!̣on广E \\
\hline $10-9 I^{\prime} \mathrm{t}$ & $00+9 I^{\circ}$ & & & $L-\varepsilon L-980000$ & әuə.jonj] \\
\hline$I 0-B I+t$ & $00+g I^{\circ} b$ & & & $0-t t-90 z 000$ & 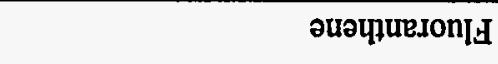 \\
\hline $\mathfrak{I 0}-\exists \varepsilon^{\prime} I$ & $00+B \varepsilon^{\cdot} I$ & & & $Z-L I-t 9 I Z 00$ & uompawon!H \\
\hline I0- $-89^{\circ} Z$ & $00+897$ & & & 8-It-SIS6E0 & u!лиреdosduä \\
\hline$\varepsilon 0-89^{\circ} z$ & $20-\exists 9^{\circ} Z$ & & & 9-Z6-๖zZzZ๐ & soudịureuəd \\
\hline $20-a z \cdot 8$ & $10-9 Z^{\prime} 8$ & & & $0-8 t-00 Z I 0 I$ & sserdxg \\
\hline$\left[0+9 I^{\bullet} \varepsilon\right.$ & $\tau 0+B I \cdot \varepsilon$ & & & $0-\tau L-\downarrow 80000$ & 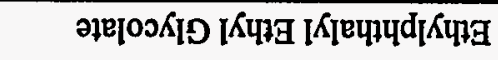 \\
\hline t0-az'8 & $\varepsilon 0-\exists Z^{\prime} 8$ & $\varepsilon 0-89 \cdot z$ & I0- & L-St-960000 & 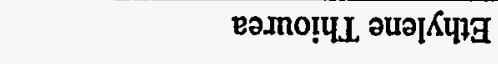 \\
\hline & & t0- $-98^{\circ} Z$ & $20-98^{\circ} z$ & 8-IZ-SL0000 & әр!̣ \\
\hline & & & & $\tau-9 L-I I I 000$ & 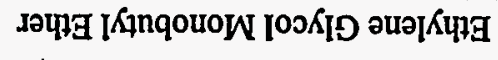 \\
\hline $10+90^{\circ} Z$ & $20+90^{\circ} z$ & & & $I-I Z-L 01000$ & [00К[D әนәโКนุ] \\
\hline $10-80 \cdot 2$ & $00+90^{\circ} z$ & & & $\varepsilon-\varsigma I-\angle 0 I 000$ & 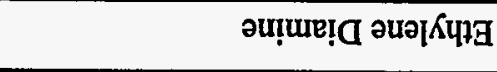 \\
\hline $00+B I^{\circ} \varepsilon$ & $I 0+B I^{\circ} \varepsilon$ & & & $t-8 L-601000$ & 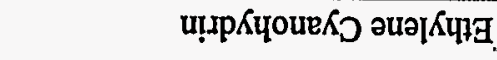 \\
\hline$I^{\prime} 0=\partial H$ & $\mathbf{I}=\mathbf{O} \mathbf{H}$ & $9-0 \mathrm{I}$ & $r \mathrm{OI}$ & \multirow{2}{*}{ N\&SYD } & \multirow{2}{*}{ [вगุщนวцว } \\
\hline \multicolumn{4}{|c|}{.(T/1ిu) uo!̣sว8ిuI } & & \\
\hline
\end{tabular}


Table 3.2a. (continued)

\begin{tabular}{|l|l|l|c|c|c|}
\hline \multirow{2}{*}{ Chemical } & \multirow{2}{*}{ CASRN } & \multicolumn{4}{|c|}{ Ingestion (mg/L) } \\
\cline { 3 - 6 } & & $10^{-4}$ & $10^{-5}$ & HQ=1 & HQ=0.1 \\
\hline Folpet & $000133-07-3$ & $8.2 \mathrm{E}+00$ & $8.2 \mathrm{E}-02$ & $1.0 \mathrm{E}+01$ & $1.0 \mathrm{E}+00$ \\
\hline Fomesafen & $072178-02-0$ & $1.5 \mathrm{E}-01$ & $1.5 \mathrm{E}-03$ & & \\
\hline Fonofos & $000944-22-9$ & & & $2.0 \mathrm{E}-01$ & $2.0 \mathrm{E}-02$ \\
\hline Formaldehyde & $000050-00-0$ & & & $2.0 \mathrm{E}+01$ & $2.0 \mathrm{E}+00$ \\
\hline Formic Acid & $000064-18-6$ & & & $2.0 \mathrm{E}+02$ & $2.0 \mathrm{E}+01$ \\
\hline Fosetyl-AL & $039148-24-8$ & & & $3.1 \mathrm{E}+02$ & $3.1 \mathrm{E}+01$ \\
\hline Furan & $000110-00-9$ & & & $1.0 \mathrm{E}-01$ & $1.0 \mathrm{E}-02$ \\
\hline Furazolidone & $000067-45-8$ & $7.5 \mathrm{E}-03$ & $7.5 \mathrm{E}-05$ & & \\
\hline Furfural & $000098-01-1$ & & & $3.1 \mathrm{E}-01$ & $3.1 \mathrm{E}-02$ \\
\hline Furium & $000531-82-8$ & $5.7 \mathrm{E}-04$ & $5.7 \mathrm{E}-06$ & & \\
\hline Furmecyclox & $060568-05-0$ & $9.5 \mathrm{E}-01$ & $9.5 \mathrm{E}-03$ & & \\
\hline Glufosinate, Ammonium & $077182-82-2$ & & & $4.1 \mathrm{E}-02$ & $4.1 \mathrm{E}-03$ \\
\hline Glycidyl & $000765-34-4$ & & & $4.1 \mathrm{E}-02$ & $4.1 \mathrm{E}-03$ \\
\hline Glyphosate & $001071-83-6$ & & & $1.0 \mathrm{E}+01$ & $1.0 \mathrm{E}+00$ \\
\hline Goal & $042874-03-3$ & & & $3.1 \mathrm{E}-01$ & $3.1 \mathrm{E}-02$ \\
\hline Haloxyfop, Methyl & $069806-40-2$ & & & $5.1 \mathrm{E}-03$ & $5.1 \mathrm{E}-04$ \\
\hline Harmony & $079277-27-3$ & & & $1.3 \mathrm{E}+00$ & $1.3 \mathrm{E}-01$ \\
\hline Heptachlor & $000076-44-8$ & $6.4 \mathrm{E}-03$ & $6.4 \mathrm{E}-05$ & $5.1 \mathrm{E}-02$ & $5.1 \mathrm{E}-03$ \\
\hline
\end{tabular}


Table 3.2a. (continued)

\begin{tabular}{|c|c|c|c|c|c|}
\hline \multirow{2}{*}{ Chemical } & \multirow{2}{*}{ CASRN } & \multicolumn{4}{|c|}{ Ingestion $(\mathrm{mg} / \mathrm{L})^{n}$} \\
\hline & & $10^{-4}$ & $10^{-6}$ & $\mathbf{H Q}=\mathbf{1}$ & $\mathrm{HQ}=\mathbf{0 . 1}$ \\
\hline Heptachlor Epoxide & $001024-57-3$ & $3.1 \mathrm{E}-03$ & $3.1 \mathrm{E}-05$ & $1.3 \mathrm{E}-03$ & $1.3 \mathrm{E}-04$ \\
\hline Heptane, N- & $000142-82-5$ & & & & \\
\hline Hexabromobenzene & $000087-82-1$ & & & $2.0 \mathrm{E}-01$ & $2.0 \mathrm{E}-02$ \\
\hline Hexachlorobenzene ${ }^{(6)}$ & $000118-74-1$ & $1.8 \mathrm{E}-02$ & $1.8 \mathrm{E}-04$ & $8.2 \mathrm{E}-02$ & 8.2E-03 \\
\hline Hexachlorobutadiene ${ }^{(6)}$ & $000087-68-3$ & 3.7E-01 & 3.7E-03 & $2.0 \mathrm{E}-02$ & $2.0 \mathrm{E}-03$ \\
\hline Hexachlorocyclohexane, Alpha- & 000319-84-6 & $4.5 \mathrm{E}-03$ & 4.5E-05 & & \\
\hline Hexachlorocyclohexane, Beta- & 000319-85-7 & $1.6 \mathrm{E}-02$ & $1.6 \mathrm{E}-04$ & & \\
\hline Hexachlorocyclohexane, Delta- & 000319-86-8 & & & & \\
\hline Hexachlorocyclohexane, Epsilon & 006108-10-7 & & & & \\
\hline Hexachlorocyclohexane, Gamma- & 000058-89-9 & $2.2 \mathrm{E}-02$ & $2.2 \mathrm{E}-04$ & $3.1 \mathrm{E}-02$ & 3.1E-03 \\
\hline Hexachlorocyclohexane, Technical & 000608-73-1 & $1.6 \mathrm{E}-02$ & $1.6 \mathrm{E}-04$ & & \\
\hline Hexachlorocyclopentadiene & $000077-47-4$ & & & 7.2E-01 & 7.2E-02 \\
\hline Hexachlorodibenzo-p-dioxin, Mixture & 019408-74-3 & $4.6 \mathrm{E}-06$ & $4.6 \mathrm{E}-08$ & & \\
\hline Hexachloroethane & $000067-72-1$ & $2.0 \mathrm{E}+00$ & $2.0 \mathrm{E}-02$ & $1.0 \mathrm{E}-01$ & $1.0 \mathrm{E}-02$ \\
\hline Hexachlorophene & $000070-30-4$ & & & $3.1 \mathrm{E}-02$ & $3.1 \mathrm{E}-03$ \\
\hline $\begin{array}{l}\text { Hexahydro-1,3,5-trinitro-1,3,5-triazine } \\
\text { (RDX) }\end{array}$ & $000121-82-4$ & $2.6 \mathrm{E}-01$ & $2.6 \mathrm{E}-03$ & $3.1 \mathrm{E}-01$ & $3.1 \mathrm{E}-02$ \\
\hline Hexamethylene Diisocyanate, 1,6- & $000822-06-0$ & & & & \\
\hline
\end{tabular}


Table 3.2a. (continued)

\begin{tabular}{|c|c|c|c|c|c|}
\hline \multirow{2}{*}{ Chemical } & \multirow{2}{*}{ CASRN } & \multicolumn{4}{|c|}{ Ingestion (mg/L)" } \\
\hline & & $10^{-4}$ & $10^{-6}$ & $H Q=1$ & $\mathrm{HQ}=0.1$ \\
\hline Hexane, N- & $000110-54-3$ & & & $6.1 E+00$ & $6.1 \mathrm{E}-01$ \\
\hline Hexanone, 2- & 000591-78-6 & & & & \\
\hline Hexazinone & $051235-04-2$ & & & $3.4 \mathrm{E}+00$ & $3.4 \mathrm{E}-01$ \\
\hline $\mathrm{HpCDD}, 2,3,7,8-{ }^{-(4)}$ & $037871-00-4$ & $1.9 \mathrm{E}-05$ & $1.9 \mathrm{E}-07$ & & \\
\hline HpCDF, 2,3,7,8-(4) & 038998-75-3 & $1.9 \mathrm{E}-05$ & $1.9 \mathrm{E}-07$ & & \\
\hline $\mathrm{HxCDD}, 2,3,7,8-^{(4)}$ & $034465-46-8$ & $1.9 \mathrm{E}-06$ & $1.9 \mathrm{E}-08$ & & \\
\hline $\mathrm{HxCDF}, 2,3,7,8^{-(4)}$ & 055684-94-1 & $1.9 \mathrm{E}-06$ & $1.9 \mathrm{E}-08$ & & \\
\hline Hydrazine & $000302-01-2$ & $9.5 \mathrm{E}-03$ & 9.5E-05 & & \\
\hline Hydrazine Sulfate & $010034-93-2$ & $9.5 \mathrm{E}-03$ & $9.5 \mathrm{E}-05$ & & \\
\hline Hydrogen Chloride & $007647-01-0$ & & & & \\
\hline Hydrogen Cyanide & $000074-90-8$ & & & $2.0 \mathrm{E}+00$ & $2.0 \mathrm{E}-01$ \\
\hline Hydrogen Sulfide & 007783-06-4 & & & $3.1 \mathrm{E}-01$ & 3.1E-02 \\
\hline Hydroquinone & 000123-31-9 & & & $4.1 \mathrm{E}+00$ & 4.1E-01 \\
\hline Imazalil & $035554-44-0$ & & & $1.3 E+00$ & $1.3 \mathrm{E}-01$ \\
\hline Imazaquin & 081335-37-7 & & & $2.6 \mathrm{E}+01$ & $2.6 \mathrm{E}+00$ \\
\hline Indeno[ $[1,2,3-c d]$ pyrene ${ }^{(2)}$ & 000193-39-5 & $3.9 \mathrm{E}-02$ & $3.9 \mathrm{E}-04$ & & \\
\hline Iprodione & 036734-19-7 & & & $4.1 \mathrm{E}+00$ & 4.1E-01 \\
\hline Iron & 007439-89-6 & & & & \\
\hline
\end{tabular}


Table 3.2a. (continued)

\begin{tabular}{|c|c|c|c|c|c|}
\hline \multirow{2}{*}{ Chemical } & \multirow{2}{*}{ CASRN } & \multicolumn{4}{|c|}{ Ingestion (mg/L)" } \\
\hline & & $10^{-4}$ & $10^{-6}$ & $H Q=1$ & $\mathrm{HQ}=0.1$ \\
\hline Isobutyl Alcohol & 000078-83-1 & & & $3.1 \mathrm{E}+01$ & $3.1 \mathrm{E}+00$ \\
\hline Isophorone & 000078-59-1 & $3.0 \mathrm{E}+01$ & $3.0 \mathrm{E}-01$ & $2.0 \mathrm{E}+01$ & $2.0 \mathrm{E}+00$ \\
\hline Isopropalin & 033820-53-0 & & & $1.5 \mathrm{E}+00$ & $1.5 \mathrm{E}-01$ \\
\hline Isopropanol & 000067-63-0 & & & & \\
\hline Isopropyl Methyl Phosphonic Acid & 001832-54-8 & & & $1.0 \mathrm{E}+01$ & $1.0 \mathrm{E}+00$ \\
\hline Isoxaben & 082558-50-7 & & & $5.1 \mathrm{E}+00$ & $5.1 \mathrm{E}-01$ \\
\hline Karate & 091465-08-6 & & & $5.1 \mathrm{E}-01$ & 5.1E-02 \\
\hline Kerb & 023950-58-5 & & & $7.7 \mathrm{E}+00$ & 7.7E-01 \\
\hline Lactofen & $077501-63-4$ & & & $2.0 \mathrm{E}-01$ & 2.0E-02 \\
\hline Lead Alkyls & NA & & & & \\
\hline Lead And Compounds & 007439-92-1 & & & & \\
\hline Linuron & $000330-55-2$ & & & $2.0 \mathrm{E}-01$ & $2.0 \mathrm{E}-02$ \\
\hline Lithium & 007439-93-2 & & & & \\
\hline Londax & 083055-99-6 & & & $2.0 \mathrm{E}+01$ & $2.0 \mathrm{E}+00$ \\
\hline MCPA & 000094-74-6 & & & $5.1 \mathrm{E}-02$ & $5.1 \mathrm{E}-03$ \\
\hline MCPB & 000094-81-5 & & & $1.0 \mathrm{E}+00$ & $1.0 \mathrm{E}-01$ \\
\hline MCPP & 000093-65-2 & & & $1.0 \mathrm{E}-01$ & $1.0 \mathrm{E}-02$ \\
\hline Magnesium & 007439-95-4 & & & & \\
\hline
\end{tabular}


Table 3.2a. (continued)

\begin{tabular}{|c|c|c|c|c|c|}
\hline \multirow{2}{*}{ Chemical } & \multirow{2}{*}{ CASRN } & \multicolumn{4}{|c|}{ Ingestion (mg/L)" } \\
\hline & & $10^{-4}$ & $10^{-6}$ & $\mathbf{H Q}=1$ & $\mathrm{HQ}=0.1$ \\
\hline Malathion & $000121-75-5$ & & & $2.0 \mathrm{E}+00$ & $2.0 \mathrm{E}-01$ \\
\hline Maleic Anhydride & $000108-31-6$ & & & $1.0 \mathrm{E}+01$ & $1.0 \mathrm{E}+00$ \\
\hline Maleic Hydrazide & 000123-33-1 & & & $5.1 \mathrm{E}+01$ & $5.1 \mathrm{E}+00$ \\
\hline Malononitrile & $000109-77-3$ & & & $2.0 \mathrm{E}-03$ & $2.0 \mathrm{E}-04$ \\
\hline Mancozeb & 008018-01-7 & & & $3.1 \mathrm{E}+00$ & $3.1 \mathrm{E}-01$ \\
\hline Maneb & $012427-38-2$ & & & $5.1 \mathrm{E}-01$ & $5.1 \mathrm{E}-02$ \\
\hline Manganese (Water) & $007439-96-5$ & & & $4.8 \mathrm{E}+00$ & $4.8 \mathrm{E}-01$ \\
\hline Mephosfolan & 000950-10-7 & & & $9.2 \mathrm{E}-03$ & $9.2 \mathrm{E}-04$ \\
\hline Mepiquat Chloride & $024307-26-4$ & & & $3.1 \mathrm{E}+00$ & $3.1 \mathrm{E}-01$ \\
\hline Mercuric Chloride & $007487-94-7$ & & & $3.1 \mathrm{E}-02$ & $3.1 \mathrm{E}-03$ \\
\hline Mercury (elemental) & 007439-97-6 & & & $3.1 \mathrm{E}-02$ & $3.1 \mathrm{E}-03$ \\
\hline Mercury, Inorganic Salt & $007439-97-6$ & & & 3.1E-02 & $3.1 \mathrm{E}-03$ \\
\hline Merphos & 000150-50-5 & & & $3.1 \mathrm{E}-03$ & $3.1 \mathrm{E}-04$ \\
\hline Merphos Oxide & $000078-48-8$ & & & $3.1 \mathrm{E}-03$ & $3.1 \mathrm{E}-04$ \\
\hline Metalaxyl & 057837-19-1 & & & $6.1 \mathrm{E}+00$ & $6.1 \mathrm{E}-01$ \\
\hline Methacrylonitrile & 000126-98-7 & & & $1.0 \mathrm{E}-02$ & $1.0 \mathrm{E}-03$ \\
\hline Methamidophos & $010265-92-6$ & & & $5.1 \mathrm{E}-03$ & 5.1E-04 \\
\hline Methanol & $000067-56-1$ & & & $5.1 \mathrm{E}+01$ & $5.1 \mathrm{E}+00$ \\
\hline
\end{tabular}




\begin{tabular}{|c|c|c|c|c|c|}
\hline & & $\varepsilon 0^{-39} 9^{\circ}$ & $I 0-39 \cdot I$ & s-IZ-9E9000 & 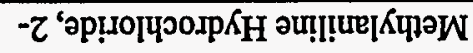 \\
\hline & & $\varepsilon 0-g L \cdot 8$ & $10-\exists L \cdot 8$ & $8-\varsigma \varsigma-660000$ & 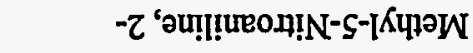 \\
\hline & & & & $\downarrow \rightarrow \downarrow 0-\downarrow \varepsilon 9 I 00$ & 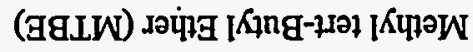 \\
\hline & & t0-घ9' & $20-399^{2} z$ & $\downarrow-\downarrow \varepsilon-090000$ & 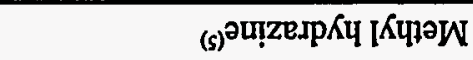 \\
\hline $20-9 I^{\circ} 9$ & I0-GI'9 & & & † I-EI0SZ0 & 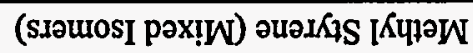 \\
\hline$\varepsilon 0-899^{\circ} 乙$ & $20-39^{\circ} \tau$ & & & $0-00-862000$ & 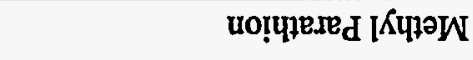 \\
\hline I0-az'8 & $00+3 Z^{\prime} 8$ & & & $9-29-080000$ & 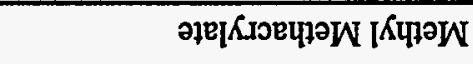 \\
\hline$\varepsilon 0-\exists 0^{\circ} I$ & $Z 0^{-G 0^{\circ} I}$ & & & 9-Z6-८96ZZ0 & SmOدәW I КчрәЈ \\
\hline $10-3 z^{\prime} 8$ & $00+92 \cdot 8$ & & & $\mathfrak{I}-0 \mathfrak{l}-80 \mathfrak{l} 000$ & 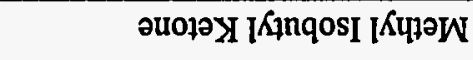 \\
\hline $00+3[9$ & {$[0+9]^{\circ} 9$} & & & $\varepsilon-\varepsilon 6-8\llcorner 0000$ & 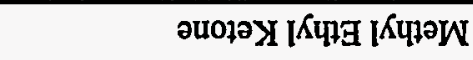 \\
\hline I0-AI' $\mathcal{E}$ & $00+B I^{\cdot} \varepsilon$ & & & $\varepsilon-\varepsilon \varepsilon-960000$ & 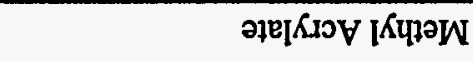 \\
\hline $10+30.1$ & $20+30^{\circ} I$ & & & $6-0 z-6 L 0000$ & 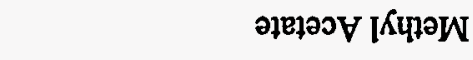 \\
\hline 20-G0.I & $10-30^{\circ} 1$ & & & $t-98-601000$ & 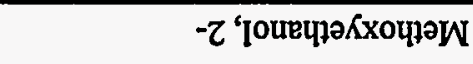 \\
\hline 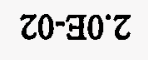 & $10-80 \cdot z$ & & & $9-6 \mathrm{t}-0 \mathrm{II} 1000$ & 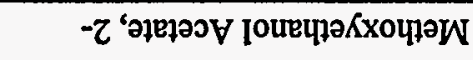 \\
\hline zo-gl's & $10-\mathrm{aI} \cdot \mathrm{S}$ & & & $s-\varepsilon \forall-Z L 0000$ & ХОГЧОКхочрәЖ \\
\hline & & $\varepsilon 0-\exists \tau^{\prime} 9$ & $10-\exists \tau^{\prime} 9$ & $\tau-6 \varsigma-660000$ & 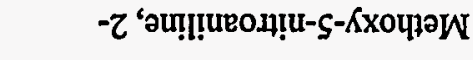 \\
\hline I0-39-Z & $00+39 \cdot \tau$ & & & $S-L L-Z S L 9 I 0$ & [КயочрәШ \\
\hline $20-90^{\circ} I$ & I0- $100^{\circ} \mathrm{I}$ & & & 8-LE-0S6000 & นо!ฺчıер!чюәW \\
\hline$I^{\prime} 0=\partial H$ & $\mathbf{I}=\mathbf{O H}$ & $9.0 I$ & rOI & \multirow{2}{*}{ N\&SYD } & \multirow{2}{*}{ 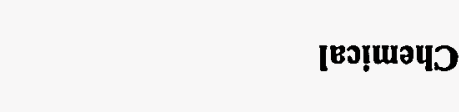 } \\
\hline \multicolumn{4}{|c|}{ 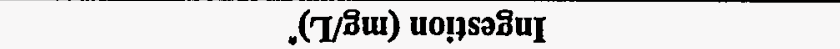 } & & \\
\hline
\end{tabular}

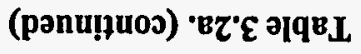


Table 3.2a. (continued)

\begin{tabular}{|l|l|l|l|l|l|}
\hline \multirow{2}{*}{ Chemical } & \multirow{2}{*}{ CASRN } & \multicolumn{4}{|c|}{ Ingestion (mg/L)* } \\
\cline { 4 - 6 } & & $10^{-4}$ & $10^{-6}$ & HQ=1 & HQ=0.1 \\
\hline Methylcyclohexane & $000108-87-2$ & & & & \\
\hline Methylene Chloride & $000075-09-2$ & $3.8 \mathrm{E}+00$ & $3.8 \mathrm{E}-02$ & $6.1 \mathrm{E}+00$ & $6.1 \mathrm{E}-01$ \\
\hline Methylene-bis(2-chloroaniline), 4,4'- & $000101-14-4$ & $2.2 \mathrm{E}-01$ & $2.2 \mathrm{E}-03$ & $7.2 \mathrm{E}-02$ & $7.2 \mathrm{E}-03$ \\
\hline $\begin{array}{l}\text { Methylene-bis(N,N-dimethyl) Aniline, } \\
\text { 4,4'- }\end{array}$ & $000101-61-1$ & $6.2 \mathrm{E}-01$ & $6.2 \mathrm{E}-03$ & & \\
\hline Methylenebisbenzenamine, 4,4'-(5) & $000101-77-9$ & $1.1 \mathrm{E}-01$ & $1.1 \mathrm{E}-03$ & & \\
\hline Methylenediphenyl Isocyanate, 4,4'- & $000101-68-8$ & & & & \\
\hline Methylstyrene, Alpha- & $000098-83-9$ & & & $7.2 \mathrm{E}+00$ & $7.2 \mathrm{E}-01$ \\
\hline Methyltriethyl Lead & $001762-28-3$ & & & & \\
\hline Metolachlor & $051218-45-2$ & & & $1.5 \mathrm{E}+01$ & $1.5 \mathrm{E}+00$ \\
\hline Metribuzin (3) & $021087-64-9$ & & & $2.6 \mathrm{E}+00$ & $2.6 \mathrm{E}-01$ \\
\hline Mirex ${ }^{(5)}$ & $002385-85-5$ & $1.6 \mathrm{E}-02$ & $1.6 \mathrm{E}-04$ & $2.0 \mathrm{E}-02$ & $2.0 \mathrm{E}-03$ \\
\hline Molinate & $002212-67-1$ & & & $2.0 \mathrm{E}-01$ & $2.0 \mathrm{E}-02$ \\
\hline Molybdenum & $007439-98-7$ & & & $5.1 \mathrm{E}-01$ & $5.1 \mathrm{E}-02$ \\
\hline Monochloramine & $010599-90-3$ & & & $1.0 \mathrm{E}+01$ & $1.0 \mathrm{E}+00$ \\
\hline Monochlorobutanes & $025154-42-1$ & & & $4.1 \mathrm{E}+01$ & $4.1 \mathrm{E}+00$ \\
\hline Naled & $000300-76-5$ & & & $2.0 \mathrm{E}-01$ & $2.0 \mathrm{E}-02$ \\
\hline Naphthalene $^{(6)}$ & $000091-20-3$ & & & $3.6 \mathrm{E}+00$ & $3.6 \mathrm{E}-01$ \\
\hline
\end{tabular}


Table 3.2a. (continued)

\begin{tabular}{|c|c|c|c|c|c|}
\hline \multirow{2}{*}{ Chemical } & \multirow{2}{*}{ CASRN } & \multicolumn{4}{|c|}{ Ingestion (mg/L)" } \\
\hline & & $10^{-4}$ & $10^{-6}$ & $H Q=1$ & $\mathrm{HQ}=0.1$ \\
\hline Naphthalene, 1-Methyl & $000090-12-0$ & & & & \\
\hline Naphthalene, 2-Methyl & $000091-57-6$ & & & & \\
\hline Napropamide & 015299-99-7 & & & $1.0 \mathrm{E}+01$ & $1.0 \mathrm{E}+00$ \\
\hline Niagara Blue 4B & 002429-74-5 & & & & \\
\hline Nickel Carbonyl & 013463-39-3 & & & & \\
\hline Nickel Refinery Dust & NA & & & & \\
\hline Nickel Soluble Salts & $007440-02-0$ & & & $2.0 \mathrm{E}+00$ & $2.0 \mathrm{E}-01$ \\
\hline Nickel Subsulfide & $012035-72-2$ & & & & \\
\hline Nitrapyrin $^{(5)}$ & 001929-82-4 & & & $1.5 \mathrm{E}-01$ & $1.5 \mathrm{E}-02$ \\
\hline Nitrate & $014797-55-8$ & & & $1.6 \mathrm{E}+02$ & $1.6 \mathrm{E}+01$ \\
\hline Nitric Oxide ${ }^{(5)}$ & 010102-43-9 & & & $1.0 \mathrm{E}+01$ & $1.0 \mathrm{E}+00$ \\
\hline Nitrite & 014797-65-0 & & & $1.0 \mathrm{E}+01$ & $1.0 \mathrm{E}+00$ \\
\hline Nitroaniline, 2- & $000088-74-4$ & & & & \\
\hline Nitrobenzene & 000098-95-3 & & & $5.1 \mathrm{E}-02$ & $5.1 \mathrm{E}-03$ \\
\hline Nitrofurantoin & 000067-20-9 & & & $7.2 \mathrm{E}+00$ & 7.2E-01 \\
\hline Nitrofurazone & 000059-87-0 & $1.9 \mathrm{E}-02$ & $1.9 \mathrm{E}-04$ & & \\
\hline Nitrogen Dioxide ${ }^{(s)}$ & $010102-44-0$ & & & $1.0 \mathrm{E}+02$ & $1.0 \mathrm{E}+01$ \\
\hline Nitroguanidine & 000556-88-7 & & & $1.0 \mathrm{E}+01$ & $1.0 \mathrm{E}+00$ \\
\hline
\end{tabular}


Table 3.2a. (continued)

\begin{tabular}{|c|c|c|c|c|c|}
\hline \multirow{2}{*}{ Chemical } & \multirow{2}{*}{ CASRN } & \multicolumn{4}{|c|}{ Ingestion $(\mathrm{mg} / \mathrm{L})^{\circ}$} \\
\hline & & $10^{-4}$ & $10^{-6}$ & $H Q=1$ & $H Q=0.1$ \\
\hline Nitrophenol, 4- & $000100-02-7$ & & & & \\
\hline Nitropropane, $2^{-(5)}$ & $000079-46-9$ & $3.0 \mathrm{E}-03$ & $3.0 \mathrm{E}-05$ & & \\
\hline Nitroso-N-ethylurea, N- & 000759-73-9 & $2.0 \mathrm{E}-04$ & $2.0 \mathrm{E}-06$ & & \\
\hline Nitroso-N-methylurea, N- & $000684-93-5$ & & & & \\
\hline Nitroso-di-N-butylamine, $\mathrm{N}$ - & $000924-16-3$ & $5.3 \mathrm{E}-03$ & $5.3 \mathrm{E}-05$ & & \\
\hline Nitroso-di-N-propylamine, N- & $000621-64-7$ & $4.1 \mathrm{E}-03$ & 4.1E-05 & & \\
\hline Nitrosodiethanolamine, $\mathrm{N}-$ & $001116-54-7$ & $1.0 \mathrm{E}-02$ & $1.0 \mathrm{E}-04$ & & \\
\hline Nitrosodiethylamine, N- & $000055-18-5$ & $1.9 \mathrm{E}-04$ & $1.9 \mathrm{E}-06$ & & \\
\hline Nitrosodimethylamine, N- & $000062-75-9$ & $5.6 \mathrm{E}-04$ & $5.6 \mathrm{E}-06$ & & \\
\hline Nitrosodiphenylamine, $\mathrm{N}$ - & 000086-30-6 & $5.8 \mathrm{E}+00$ & $5.8 \mathrm{E}-02$ & & \\
\hline Nitrosomethylethylamine, N- & 010595-95-6 & $1.3 \mathrm{E}-03$ & $1.3 \mathrm{E}-05$ & & \\
\hline Nitrosomethylvinylamine, $\mathrm{N}$ - & $004549-40-0$ & & & & \\
\hline Nitrosopyrrolidine, N- & 000930-55-2 & $1.4 \mathrm{E}-02$ & $1.4 \mathrm{E}-04$ & & \\
\hline Nitrotoluene, 4-Amino-2- & $000119-32-4$ & & & & \\
\hline Nitrotoluene, m- & 000099-08-1 & & & $1.0 \mathrm{E}+00$ & $1.0 \mathrm{E}-01$ \\
\hline Nitrotoluene, o- & $000088-72-2$ & & & $1.0 \mathrm{E}+00$ & $1.0 \mathrm{E}-01$ \\
\hline Nitrotoluene, p- & 000099-99-0 & & & $1.0 \mathrm{E}+00$ & $1.0 \mathrm{E}-01$ \\
\hline Norflurazon & 027314-13-2 & & & $4.1 \mathrm{E}+00$ & 4.1E-01 \\
\hline
\end{tabular}


Table 3.2a. (continued)

\begin{tabular}{|c|c|c|c|c|c|}
\hline \multirow{2}{*}{ Chemical } & \multirow{2}{*}{ CASRN } & \multicolumn{4}{|c|}{ Ingestion $(\mathrm{mg} / \mathrm{L})^{n}$} \\
\hline & & $10^{-4}$ & $10^{-6}$ & $\mathbf{H Q}=\mathbf{1}$ & $\mathrm{HQ}=0.1$ \\
\hline Nustar & 085509-19-9 & & & $7.2 \mathrm{E}-02$ & $7.2 \mathrm{E}-03$ \\
\hline $\mathrm{OCDD}^{(4)}$ & 003268-87-9 & $1.9 \mathrm{E}-04$ & $1.9 \mathrm{E}-06$ & & \\
\hline $\mathrm{OCDF}^{(4)}$ & 039001-02-0 & $1.9 \mathrm{E}-04$ & $1.9 \mathrm{E}-06$ & & \\
\hline Octabromodiphenyl Ether & $032536-52-0$ & & & $3.1 \mathrm{E}-01$ & $3.1 \mathrm{E}-02$ \\
\hline $\begin{array}{l}\text { Octahydro-1,3,5,7-tetranitro-1,3,5,7- } \\
\text { tetra (HMX) }\end{array}$ & $002691-41-0$ & & & $5.1 \mathrm{E}+00$ & $5.1 \mathrm{E}-01$ \\
\hline Octamethylpyrophosphoramide & 000152-16-9 & & & $2.0 \mathrm{E}-01$ & $2.0 \mathrm{E}-02$ \\
\hline Octyl Phthalate, di-N- & 000117-84-0 & & & $2.0 \mathrm{E}+00$ & $2.0 \mathrm{E}-01$ \\
\hline Oryzalin & 019044-88-3 & & & $5.1 \mathrm{E}+00$ & 5.1E-01 \\
\hline Oxadiazon & 019666-30-9 & & & $5.1 \mathrm{E}-01$ & $5.1 \mathrm{E}-02$ \\
\hline Oxamyl & $023135-22-0$ & & & $2.6 \mathrm{E}+00$ & $2.6 \mathrm{E}-01$ \\
\hline Paclobutrazol & $076738-62-0$ & & & $1.3 \mathrm{E}+00$ & $1.3 \mathrm{E}-01$ \\
\hline Paraquat & 001910-42-5 & & & 4.6E-01 & $4.6 \mathrm{E}-02$ \\
\hline Parathion & $000056-38-2$ & & & $6.1 \mathrm{E}-01$ & $6.1 \mathrm{E}-02$ \\
\hline PeCDD, 2,3,7,8-(4) & 036088-22-9 & $3.8 \mathrm{E}-07$ & $3.8 \mathrm{E}-09$ & & \\
\hline PeCDF, $1,2,3,7,8-_{-(4)}$ & $057117-41-6$ & 3.8E-07 & $3.8 \mathrm{E}-09$ & & \\
\hline PeCDF, 2,3,4,7,8-(4) & $057117-31-4$ & $3.8 \mathrm{E}-06$ & $3.8 \mathrm{E}-08$ & & \\
\hline Pebulate & 001114-71-2 & & & $5.1 \mathrm{E}+00$ & $5.1 \mathrm{E}-01$ \\
\hline
\end{tabular}


Table 3.2a. (continued)

\begin{tabular}{|l|l|l|l|l|l|}
\hline \multirow{2}{*}{ Chemical } & \multirow{2}{*}{ CASRN } & \multicolumn{4}{|c|}{ Ingestion (mg/L) } \\
\cline { 4 - 6 } & & $10^{-4}$ & $10^{-5}$ & HQ=1 & HQ=0.1 \\
\hline Pendimethalin & $040487-42-1$ & & & $4.1 \mathrm{E}+00$ & $4.1 \mathrm{E}-01$ \\
\hline Pentabromodiphenyl Ether & $032534-81-9$ & & & $2.0 \mathrm{E}-01$ & $2.0 \mathrm{E}-02$ \\
\hline Pentachlorobenzene & $000608-93-5$ & & & $8.2 \mathrm{E}-02$ & $8.2 \mathrm{E}-03$ \\
\hline Pentachlorocyclopentadiene & $025329-35-5$ & & & & \\
\hline Pentachloronitrobenzene & $000082-68-8$ & $1.1 \mathrm{E}-01$ & $1.1 \mathrm{E}-03$ & $3.1 \mathrm{E}-01$ & $3.1 \mathrm{E}-02$ \\
\hline Pentachlorophenol & $000087-86-5$ & $2.4 \mathrm{E}-01$ & $2.4 \mathrm{E}-03$ & $3.1 \mathrm{E}+00$ & $3.1 \mathrm{E}-01$ \\
\hline Pentyl Alcohol, N- & $000071-41-0$ & & & & \\
\hline Permethrin & $052645-53-1$ & & & $5.1 \mathrm{E}+00$ & $5.1 \mathrm{E}-01$ \\
\hline Phenanthrene & $000085-01-8$ & & & & \\
\hline Phenmedipham & $013684-63-4$ & & & $2.6 \mathrm{E}+01$ & $2.6 \mathrm{E}+00$ \\
\hline Phenol & $000108-95-2$ & & & $6.1 \mathrm{E}+01$ & $6.1 \mathrm{E}+00$ \\
\hline Phenylenediamine, m- & $000108-45-2$ & & & $6.1 \mathrm{E}-01$ & $6.1 \mathrm{E}-02$ \\
\hline Phenylenediamine, o- & $000095-54-5$ & $6.1 \mathrm{E}-01$ & $6.1 \mathrm{E}-03$ & & \\
\hline Phenylenediamine, p- & $000106-50-3$ & & & $1.9 \mathrm{E}+01$ & $1.9 \mathrm{E}+00$ \\
\hline Phenylmercuric Acetate & $000062-38-4$ & & & $8.2 \mathrm{E}-03$ & $8.2 \mathrm{E}-04$ \\
\hline Phenylphenol, 2- & $000090-43-7$ & $1.5 \mathrm{E}+01$ & $1.5 \mathrm{E}-01$ & & \\
\hline Phorate & $000298-02-2$ & & & $2.0 \mathrm{E}-02$ & $2.0 \mathrm{E}-03$ \\
\hline Phosmet & $000732-11-6$ & & & $2.0 \mathrm{E}+00$ & $2.0 \mathrm{E}-01$ \\
\hline
\end{tabular}


Table 3.2a. (continued)

\begin{tabular}{|l|l|l|l|l|l|}
\hline \multirow{2}{*}{ Chemical } & \multirow{2}{*}{ CASRN } & \multicolumn{4}{|c|}{ Ingestion (mg/L) } \\
\cline { 4 - 6 } & & $10^{-4}$ & $10^{-6}$ & HQ=1 & HQ=0.1 \\
\hline Phosphine & $007803-51-2$ & & & $3.1 \mathrm{E}-02$ & $3.1 \mathrm{E}-03$ \\
\hline Phosphoric Acid & $007664-38-2$ & & & & \\
\hline Phthalic Acid, P- & $000100-21-0$ & & & $1.0 \mathrm{E}+02$ & $1.0 \mathrm{E}+01$ \\
\hline Phthalic Anhydride & $000085-44-9$ & & & $2.0 \mathrm{E}+02$ & $2.0 \mathrm{E}+01$ \\
\hline Picloram & $001918-02-1$ & & & $7.2 \mathrm{E}+00$ & $7.2 \mathrm{E}-01$ \\
\hline Pirimiphos, Methyl & $029232-93-7$ & & & $1.0 \mathrm{E}+00$ & $1.0 \mathrm{E}-01$ \\
\hline Polybrominated Biphenyls & NA & $3.2 \mathrm{E}-03$ & $3.2 \mathrm{E}-05$ & $7.2 \mathrm{E}-04$ & $7.2 \mathrm{E}-05$ \\
\hline Polychlorinated Biphenyls & $001336-36-3$ & $3.7 \mathrm{E}-03$ & $3.7 \mathrm{E}-05$ & & \\
\hline Potassium Cyanide & $000151-50-8$ & & & $5.1 \mathrm{E}+00$ & $5.1 \mathrm{E}-01$ \\
\hline Potassium Silver Cyanide & $000506-61-6$ & & & $2.0 \mathrm{E}+01$ & $2.0 \mathrm{E}+00$ \\
\hline Prochloraz & $067747-09-5$ & $1.9 \mathrm{E}-01$ & $1.9 \mathrm{E}-03$ & $9.2 \mathrm{E}-01$ & $9.2 \mathrm{E}-02$ \\
\hline Profluralin & $026399-36-0$ & & & $6.1 \mathrm{E}-01$ & $6.1 \mathrm{E}-02$ \\
\hline Prometon & $001610-18-0$ & & & $1.5 \mathrm{E}+00$ & $1.5 \mathrm{E}-01$ \\
\hline Prometryn & $007287-19-6$ & & & $4.1 \mathrm{E}-01$ & $4.1 \mathrm{E}-02$ \\
\hline Propachlor & $001918-16-7$ & & & $1.3 \mathrm{E}+00$ & $1.3 \mathrm{E}-01$ \\
\hline Propanil & $000709-98-8$ & & & $5.1 \mathrm{E}-01$ & $5.1 \mathrm{E}-02$ \\
\hline Propargite & $002312-35-8$ & & & $2.0 \mathrm{E}+00$ & $2.0 \mathrm{E}-01$ \\
\hline Propargyl Alcohol & $000107-19-7$ & & & $2.0 \mathrm{E}-01$ & $2.0 \mathrm{E}-02$ \\
\hline
\end{tabular}


Table 3.2a. (continued)

\begin{tabular}{|c|c|c|c|c|c|}
\hline \multirow{2}{*}{ Chemical } & \multirow{2}{*}{ CASRN } & \multicolumn{4}{|c|}{ Ingestion (mg/L)" } \\
\hline & & $10^{-4}$ & $10^{-6}$ & $\mathbf{H Q}=\mathbf{1}$ & $\mathrm{HQ}=0.1$ \\
\hline Propazine & $000139-40-2$ & & & $2.0 \mathrm{E}+00$ & $2.0 \mathrm{E}-01$ \\
\hline Propham & $000122-42-9$ & & & $2.0 \mathrm{E}+00$ & $2.0 \mathrm{E}-01$ \\
\hline Propiconazole & 060207-90-1 & & & $1.3 \mathrm{E}+00$ & $1.3 \mathrm{E}-01$ \\
\hline Propylene Glycol & 000057-55-6 & & & $2.0 \mathrm{E}+03$ & $2.0 \mathrm{E}+02$ \\
\hline Propylene Glycol Monoethyl Ether & 001569-02-4 & & & $7.2 E+01$ & $7.2 \mathrm{E}+00$ \\
\hline Propylene Glycol Monomethyl Ether & 000107-98-2 & & & $7.2 \mathrm{E}+01$ & $7.2 \mathrm{E}+00$ \\
\hline Propylene Oxide & 000075-56-9 & $1.2 \mathrm{E}-01$ & $1.2 \mathrm{E}-03$ & & \\
\hline Pursuit & $081335-77-5$ & & & $2.6 \mathrm{E}+01$ & $2.6 \mathrm{E}+00$ \\
\hline Pydrin & 051630-58-1 & & & $2.6 \mathrm{E}+00$ & $2.6 \mathrm{E}-01$ \\
\hline Pyrene & $000129-00-0$ & & & 3.1E+00 & $3.1 \mathrm{E}-01$ \\
\hline Pyridine & 000110-86-1 & & & $1.0 \mathrm{E}-01$ & $1.0 \mathrm{E}-02$ \\
\hline Quinalphos & 013593-03-8 & & & $5.1 \mathrm{E}-02$ & $5.1 \mathrm{E}-03$ \\
\hline Quinoline & 000091-22-5 & $2.4 \mathrm{E}-03$ & $2.4 \mathrm{E}-05$ & & \\
\hline Refractory Ceramic Fibers & NA & & & & \\
\hline Resmethrin & 010453-86-8 & & & $3.1 \mathrm{E}+00$ & $3.1 \mathrm{E}-01$ \\
\hline Ronnel & 000299-84-3 & & & $5.1 E+00$ & $5.1 \mathrm{E}-01$ \\
\hline Rotenone & 000083-79-4 & & & 4.1E-01 & $4.1 \mathrm{E}-02$ \\
\hline Savey & 078587-05-0 & & & $2.6 \mathrm{E}+00$ & $2.6 \mathrm{E}-01$ \\
\hline
\end{tabular}


Table 3.2a. (continued)

\begin{tabular}{|l|l|l|l|l|l|}
\hline \multirow{2}{*}{ Chemical } & \multirow{2}{*}{ CASRN } & \multicolumn{4}{|c|}{ Ingestion (mg/L) } \\
\cline { 4 - 6 } & & $10^{-4}$ & $10^{-6}$ & HQ=1 & HQ=0.1 \\
\hline Selenious Acid & $007783-00-8$ & & & $5.1 \mathrm{E}-01$ & $5.1 \mathrm{E}-02$ \\
\hline Selenite & $014124-67-5$ & & & & \\
\hline Selenium & $007782-49-2$ & & & $5.1 \mathrm{E}-01$ & $5.1 \mathrm{E}-02$ \\
\hline Selenium Sulfide & $007446-34-6$ & & & & \\
\hline Selenourea & $000630-10-4$ & & & $5.1 \mathrm{E}-01$ & $5.1 \mathrm{E}-02$ \\
\hline Sethoxydim & $074051-80-2$ & & & $9.2 \mathrm{E}+00$ & $9.2 \mathrm{E}-01$ \\
\hline Silver & $007440-22-4$ & & & $5.1 \mathrm{E}-01$ & $5.1 \mathrm{E}-02$ \\
\hline Silver Cyanide & $000506-64-9$ & & & $1.0 \mathrm{E}+01$ & $1.0 \mathrm{E}+00$ \\
\hline Simazine & $000122-34-9$ & $2.4 \mathrm{E}-01$ & $2.4 \mathrm{E}-03$ & $5.1 \mathrm{E}-01$ & $5.1 \mathrm{E}-02$ \\
\hline Sodium & $007440-23-5$ & & & & \\
\hline Sodium Acifluorfen & $062476-59-9$ & & & $1.3 \mathrm{E}+00$ & $1.3 \mathrm{E}-01$ \\
\hline Sodium Azide & $026628-22-8$ & & & $4.1 \mathrm{E}-01$ & $4.1 \mathrm{E}-02$ \\
\hline Sodium Cyanide & $000143-33-9$ & & & $4.1 \mathrm{E}+00$ & $4.1 \mathrm{E}-01$ \\
\hline Sodium Diethyldithiocarbamate & $000148-18-5$ & $1.1 \mathrm{E}-01$ & $1.1 \mathrm{E}-03$ & $3.1 \mathrm{E}+00$ & $3.1 \mathrm{E}-01$ \\
\hline Sodium Fluoroacetate & $000062-74-8$ & & & $2.0 \mathrm{E}-03$ & $2.0 \mathrm{E}-04$ \\
\hline Sodium Metavanadate & $013718-26-8$ & & & $1.0 \mathrm{E}-01$ & $1.0 \mathrm{E}-02$ \\
\hline Stirofos (Tetrachlorovinphos) & $000961-11-5$ & $1.2 \mathrm{E}+00$ & $1.2 \mathrm{E}-02$ & $3.1 \mathrm{E}+00$ & $3.1 \mathrm{E}-01$ \\
\hline Strontium, Stable & $007440-24-6$ & & & $6.1 \mathrm{E}+01$ & $6.1 \mathrm{E}+00$ \\
\hline
\end{tabular}


Table 3.2a. (continued)

\begin{tabular}{|l|l|l|l|l|l|}
\hline \multirow{2}{*}{ Chemical } & \multirow{2}{*}{ CASRN } & \multicolumn{4}{|c|}{ Ingestion (mg/L) } \\
\cline { 4 - 6 } & & $10^{-4}$ & $10^{-6}$ & HQ=1 & HQ=0.1 \\
\hline Strychnine & $000057-24-9$ & & & $3.1 \mathrm{E}-02$ & $3.1 \mathrm{E}-03$ \\
\hline Styrene(6) & $000100-42-5$ & & & $2.0 \mathrm{E}+01$ & $2.0 \mathrm{E}+00$ \\
\hline Sulfate & $014808-79-8$ & & & & \\
\hline Systhane & $088671-89-0$ & & & $2.6 \mathrm{E}+00$ & $2.6 \mathrm{E}-01$ \\
\hline TCDD, 2,3,7,8-(4) & $001746-01-6$ & $1.9 \mathrm{E}-07$ & $1.9 \mathrm{E}-09$ & & \\
\hline TCDF, 2,3,7,8-(4) & $051207-31-9$ & $1.9 \mathrm{E}-06$ & $1.9 \mathrm{E}-08$ & & \\
\hline TCMTB & $021564-17-0$ & & & $3.1 \mathrm{E}+00$ & $3.1 \mathrm{E}-01$ \\
\hline Tebuthiuron & $034014-18-1$ & & & $7.2 \mathrm{E}+00$ & $7.2 \mathrm{E}-01$ \\
\hline Temephos & $003383-96-8$ & & & $2.0 \mathrm{E}+00$ & $2.0 \mathrm{E}-01$ \\
\hline Terbacil & $005902-51-2$ & & & $1.3 \mathrm{E}+00$ & $1.3 \mathrm{E}-01$ \\
\hline Terbufos & $013071-79-9$ & & & $2.6 \mathrm{E}-03$ & $2.6 \mathrm{E}-04$ \\
\hline Terbutryn & $000886-50-0$ & & & $1.0 \mathrm{E}-01$ & $1.0 \mathrm{E}-02$ \\
\hline Tetrabutyl Lead & $001920-90-7$ & & & & \\
\hline Tetrachlorobenzene, 1,2,4,5- & $000095-94-3$ & & & $3.1 \mathrm{E}-02$ & $3.1 \mathrm{E}-03$ \\
\hline Tetrachloroethane, 1,1,1,2- & $000630-20-6$ & $1.1 \mathrm{E}+00$ & $1.1 \mathrm{E}-02$ & $3.1 \mathrm{E}+00$ & $3.1 \mathrm{E}-01$ \\
\hline Tetrachloroethane, 1,1,2,2- & $000079-34-5$ & $1.4 \mathrm{E}-01$ & $1.4 \mathrm{E}-03$ & & \\
\hline Tetrachloroethylene & & & & \\
\hline Tetrachlorophenol, 2,3,4,6- & $000127-18-4$ & $5.5 \mathrm{E}-01$ & $5.5 \mathrm{E}-03$ & $1.0 \mathrm{E}+00$ & $1.0 \mathrm{E}-01$ \\
\hline & $000058-90-2$ & & & $3.1 \mathrm{E}+00$ & $3.1 \mathrm{E}-01$ \\
\hline
\end{tabular}


Table 3.2a. (continued)

\begin{tabular}{|c|c|c|c|c|c|}
\hline \multirow{2}{*}{ Chemical } & \multirow{2}{*}{ CASRN } & \multicolumn{4}{|c|}{ Ingestion (mg/L) } \\
\hline & & $10^{-4}$ & $10^{-6}$ & - $\mathbf{H Q}=\mathbf{1}$ & $\mathrm{HQ}=\mathbf{0 . 1}$ \\
\hline $\begin{array}{l}\text { Tetrachlorotoluene, p- alpha, alpha, } \\
\text { alpha- }\end{array}$ & $005216-25-1$ & $1.4 \mathrm{E}-03$ & $1.4 \mathrm{E}-05$ & & \\
\hline Tetraethyl Dithiopyrophosphate & 003689-24-5 & & & $5.1 \mathrm{E}-02$ & $5.1 \mathrm{E}-03$ \\
\hline Tetraethyl Lead & 000078-00-2 & & & & \\
\hline Tetrafluoroethane, 1,1,1,2- & $000811-97-2$ & & & & \\
\hline Tetramethyl Lead & $000075-74-1$ & & & & \\
\hline Tetrapropyl Lead & $003440-75-3$ & & & & \\
\hline Thallic Oxide ${ }^{(5)}$ & $001314-32-5$ & & & $7.2 \mathrm{E}-03$ & 7.2E-04 \\
\hline Thallium (I) Nitrate & $010102-45-1$ & & & $9.2 \mathrm{E}-03$ & $9.2 \mathrm{E}-04$ \\
\hline Thallium (Soluble Salts) & $007440-28-0$ & & & & \\
\hline Thallium Acetate & 000563-68-8 & & & $9.2 \mathrm{E}-03$ & $9.2 \mathrm{E}-04$ \\
\hline Thallium Carbonate & $006533-73-9$ & & & $8.2 \mathrm{E}-03$ & 8.2E-04 \\
\hline Thallium Chloride & $007791-12-0$ & & & $8.2 \mathrm{E}-03$ & $8.2 \mathrm{E}-04$ \\
\hline Thallium Selenite $^{(5)}$ & $012039-52-0$ & & & $9.2 \mathrm{E}-03$ & $9.2 \mathrm{E}-04$ \\
\hline Thallium Sulfate & 007446-18-6 & & & $8.2 \mathrm{E}-03$ & 8.2E-04 \\
\hline Thiobencarb & $028249-77-6$ & & & $1.0 \mathrm{E}+00$ & $1.0 \mathrm{E}-01$ \\
\hline Thiofanox & 039196-18-4 & & & $3.1 \mathrm{E}-02$ & $3.1 \mathrm{E}-03$ \\
\hline Thiophanate, Methyl & 023564-05-8 & & & $8.2 \mathrm{E}+00$ & 8.2E-01 \\
\hline
\end{tabular}


Table 3.2a. (continued)

\begin{tabular}{|c|c|c|c|c|c|}
\hline \multirow{2}{*}{ Chemical } & \multirow{2}{*}{ CASRN } & \multicolumn{4}{|c|}{ Ingestion $(\mathbf{m g} / \mathbf{L})^{*}$} \\
\hline & & $10^{-4}$ & $10^{-6}$ & $\mathbf{H Q}=\mathbf{1}$ & $\mathrm{HQ}=0.1$ \\
\hline Thiram & $000137-26-8$ & & & $5.1 \mathrm{E}-01$ & 5.1E-02 \\
\hline Thorium & $007440-29-1$ & & & & \\
\hline Tin & $007440-31-5$ & & & $6.1 E+01$ & $6.1 \mathrm{E}+00$ \\
\hline Titanium & 007440-32-6 & & & & \\
\hline Toluene $^{(6)}$ & 000108-88-3 & & & $2.0 \mathrm{E}+01$. & $2.0 \mathrm{E}+00$ \\
\hline Toluene diisocyanate mixture (TDI) & $026471-62-5$ & & & & \\
\hline Toluene-2,4-diamine & 000095-80-7 & $8.9 \mathrm{E}-03$ & $8.9 \mathrm{E}-05$ & & \\
\hline Toluene-2,5-diamine & $000095-70-5$ & & & $6.1 \mathrm{E}+01$ & $6.1 \mathrm{E}+00$ \\
\hline Toluene-2,6-diamine & $000823-40-5$ & & & $2.0 \mathrm{E}+01$ & $2.0 \mathrm{E}+00$ \\
\hline Toluidine, o- (Methylaniline, 2-) & $000095-53-4$ & $1.2 \mathrm{E}-01$ & $1.2 \mathrm{E}-03$ & & \\
\hline Toluidine, $\mathrm{p}$ - & 000106-49-0 & $1.5 \mathrm{E}-01$ & $1.5 \mathrm{E}-03$ & & \\
\hline Toxaphene & $008001-35-2$ & $2.6 \mathrm{E}-02$ & $2.6 \mathrm{E}-04$ & & \\
\hline Tralomethrin & $066841-25-6$ & & & 7.7E-01 & 7.7E-02 \\
\hline Triallate & $002303-17-5$ & & & $1.3 \mathrm{E}+00$ & $1.3 \mathrm{E}-01$ \\
\hline Triasulfuron & $082097-50-5$ & & & $1.0 \mathrm{E}+00$ & $1.0 \mathrm{E}-01$ \\
\hline Tribromobenzene, 1,2,4- & 000615-54-3 & & & $5.1 \mathrm{E}-01$ & $5.1 \mathrm{E}-02$ \\
\hline Tribromochloromethane & 000594-15-0 & & & & \\
\hline Tribromodiphenyl Ether & 049690-94-0 & & & & \\
\hline
\end{tabular}


Table 3.2a. (continued)

\begin{tabular}{|c|c|c|c|c|c|}
\hline \multirow{2}{*}{ Chemical } & \multirow{2}{*}{ CASRN } & \multicolumn{4}{|c|}{ Ingestion (mg/L)* } \\
\hline & & $10^{-4}$ & $10^{-6}$ & $\mathbf{H Q}=\mathbf{1}$ & $\mathrm{HQ}=0.1$ \\
\hline Tributyltin Oxide & $000056-35-9$ & & & $3.1 E-03$ & 3.1E-04 \\
\hline Trichloro-1,2,2-trifluoroethane, 1,1,2- & $000076-13-1$ & & & $3.1 \mathrm{E}+03$ & $3.1 \mathrm{E}+02$ \\
\hline Trichloro-2'-hydroxydiphenylether & $003380-34-5$ & & & & \\
\hline \multicolumn{6}{|l|}{ Trichloroacetic Acid } \\
\hline Trichloroaniline $\mathrm{HCl}, 2,4,6-$ & $033663-50-2$ & $9.9 \mathrm{E}-01$ & 9.9E-03 & & \\
\hline Trichloroaniline, 2,4,6- & $000634-93-5$ & $8.4 \mathrm{E}-01$ & 8.4E-03 & & \\
\hline Trichlorobenzene, $1,2,4-$ & $000120-82-1$ & & & $1.0 \mathrm{E}+00$ & $1.0 \mathrm{E}-01$ \\
\hline Trichloroethane, $1,1,1^{(3)}$ & $000071-55-6$ & & & $9.2 \mathrm{E}+00$ & $9.2 \mathrm{E}-01$ \\
\hline Trichloroethane, 1,1,2- & $000079-00-5$ & $5.0 \mathrm{E}-01$ & $5.0 \mathrm{E}-03$ & 4.1E-01 & 4.1E-02 \\
\hline Trichloroethylene $e^{(3)}$ & $000079-01-6$ & $2.6 \mathrm{E}+00$ & $2.6 \mathrm{E}-02$ & & \\
\hline Trichlorofluoromethane & $000075-69-4$ & & & $3.1 E+01$ & $3.1 \mathrm{E}+00$ \\
\hline Trichlorophenol, 2,4,5- & $000095-95-4$ & & & $1.0 \mathrm{E}+01$ & $1.0 \mathrm{E}+00$ \\
\hline Trichlorophenol, 2,4,6- & 000088-06-2 & $2.6 \mathrm{E}+00$ & $2.6 \mathrm{E}-02$ & & \\
\hline $\begin{array}{l}\text { Trichlorophenoxy) Propionic Acid, } \\
2(2,4,5 \text { - }\end{array}$ & $000093-72-1$ & & & 8.2E-01 & 8.2E-02 \\
\hline Trichlorophenoxyacetic Acid, 2,4,5- & $000093-76-5$ & & & $1.0 \mathrm{E}+00$ & $1.0 \mathrm{E}-01$ \\
\hline Trichloropropane, 1,1,2- & $000598-77-6$ & & & $5.1 \mathrm{E}-01$ & $5.1 \mathrm{E}-02$ \\
\hline Trichloropropane, 1,2,3- & 000096-18-4 & 4.1E-03 & 4.1E-05 & $6.1 \mathrm{E}-01$ & $6.1 \mathrm{E}-02$ \\
\hline
\end{tabular}


Table 3.2a. (continued)

\begin{tabular}{|l|l|l|l|l|l|}
\hline \multirow{2}{*}{ Chemical } & \multirow{2}{*}{ CASRN } & \multicolumn{4}{|c|}{ Ingestion (mg/L)" } \\
\cline { 4 - 6 } & & $10^{-4}$ & $10^{-6}$ & HQ=1 & HQ=0.1 \\
\hline Trichloropropene, 1,2,3- & $000096-19-5$ & & & $5.1 \mathrm{E}-01$ & $5.1 \mathrm{E}-02$ \\
\hline Trichlorotoluene, 2,3,6- & $002077-46-5$ & & & & \\
\hline Trichlorotoluene, alpha 2,6- & $002014-83-7$ & & & & \\
\hline Tridiphane & $058138-08-2$ & & & $3.1 \mathrm{E}-01$ & $3.1 \mathrm{E}-02$ \\
\hline Triethyl Lead & $005224-23-7$ & & & & \\
\hline Triethylamine & $000121-44-8$ & & & & \\
\hline Trifluralin & $001582-09-8$ & $3.7 \mathrm{E}+00$ & $3.7 \mathrm{E}-02$ & $7.7 \mathrm{E}-01$ & $7.7 \mathrm{E}-02$ \\
\hline Trimethyl Lead & $007442-13-9$ & & & & \\
\hline Trimethyl Phosphate & $000512-56-1$ & $7.7 \mathrm{E}-01$ & $7.7 \mathrm{E}-03$ & & \\
\hline Trimethylethyl Lead & $001762-26-1$ & & & & \\
\hline Trinitrobenzene, 1,3,5- & $000099-35-4$ & & & $5.1 \mathrm{E}-03$ & $5.1 \mathrm{E}-04$ \\
\hline Trinitrophenylmethylnitramine & $000479-45-8$ & & & $1.0 \mathrm{E}+00$ & $1.0 \mathrm{E}-01$ \\
\hline Trinitrotoluene, 2,4,6- & $000118-96-7$ & $9.5 \mathrm{E}-01$ & $9.5 \mathrm{E}-03$ & $5.1 \mathrm{E}-02$ & $5.1 \mathrm{E}-03$ \\
\hline Tripropyl Lead & $006618-03-7$ & & & & \\
\hline Uranium (Soluble Salts) & NA & & & $3.1 \mathrm{E}-01$ & $3.1 \mathrm{E}-02$ \\
\hline Vanadium Pentoxide & $001314-62-1$ & & & $9.2 \mathrm{E}-01$ & $9.2 \mathrm{E}-02$ \\
\hline Vanadium Sulfate & $036907-42-3$ & & & $2.0 \mathrm{E}+00$ & $2.0 \mathrm{E}-01$ \\
\hline Vanadium, Metallic & $007440-62-2$ & & & $7.2 \mathrm{E}-01$ & $7.2 \mathrm{E}-02$ \\
\hline
\end{tabular}


Table 3.2a. (continued)

\begin{tabular}{|l|l|l|l|l|l|}
\hline \multirow{2}{*}{ Chemical } & \multirow{2}{*}{ CASRN } & \multicolumn{3}{|c|}{ Ingestion (mg/L) $^{*}$} \\
\cline { 4 - 6 } & & $10^{-4}$ & $10^{-6}$ & HQ=1 & HQ=0.1 \\
\hline Vanadyl Sulfate & $027774-13-6$ & & & $2.0 \mathrm{E}+00$ & $2.0 \mathrm{E}-01$ \\
\hline Vernolate & $001929-77-7$ & & & $1.0 \mathrm{E}-01$ & $1.0 \mathrm{E}-02$ \\
\hline Vinclozolin & $050471-44-8$ & & & $2.6 \mathrm{E}+00$ & $2.6 \mathrm{E}-01$ \\
\hline Vinyl Acetate & $000108-05-4$ & & & $1.0 \mathrm{E}+02$ & $1.0 \mathrm{E}+01$ \\
\hline Vinyl Bromide & $000593-60-2$ & & & & \\
\hline Vinyl Chloride ${ }^{(6)}$ & $000075-01-4$ & $1.5 \mathrm{E}-02$ & $1.5 \mathrm{E}-04$ & & \\
\hline Warfarin & $000081-81-2$ & & & $3.1 \mathrm{E}-02$ & $3.1 \mathrm{E}-03$ \\
\hline White Phosphorus & $007723-14-0$ & & & $2.0 \mathrm{E}-03$ & $2.0 \mathrm{E}-04$ \\
\hline Xylene, Mixture ${ }^{(6)}$ & $001330-20-7$ & & & $2.0 \mathrm{E}+02$ & $2.0 \mathrm{E}+01$ \\
\hline Xylene, P-(6) & $000106-42-3$ & & & & \\
\hline Xylene, $\mathrm{m}^{(6)}$ & $000108-38-3$ & & & $2.0 \mathrm{E}+02$ & $2.0 \mathrm{E}+01$ \\
\hline Xylene, 0-(6) & $000095-47-6$ & & & $2.0 \mathrm{E}+02$ & $2.0 \mathrm{E}+01$ \\
\hline Zinc (Metallic) & $007440-66-6$ & & & $3.1 \mathrm{E}+01$ & $3.1 \mathrm{E}+00$ \\
\hline Zinc Cyanide & $000557-21-1$ & & & $5.1 \mathrm{E}+00$ & $5.1 \mathrm{E}-01$ \\
\hline Zinc Phosphide & $001314-84-7$ & & & $3.1 \mathrm{E}-02$ & $3.1 \mathrm{E}-03$ \\
\hline Zineb & $012122-67-7$ & & & $5.1 \mathrm{E}+00$ & $5.1 \mathrm{E}-01$ \\
\hline Zirconium & $007440-67-7$ & & & & \\
\hline
\end{tabular}


Notes:

* By definition, $1 \mathrm{~L}$ (water) $=1.0 \mathrm{E}+06 \mathrm{mg}$ (water). Therefore, a limit of $1.0 \mathrm{E}+06 \mathrm{mg} / \mathrm{L}$ was used for any PRG that was calculated to be above this limit.

(1) The same oral slope factor as used for polychlorinated biphenyls.

(2) The PRG was calculated considering Supplemental Guidance from RAGS: Region 4 Bulletins, Human Health Risk Assessment (Interim Guidance) from EPA (received February 11, 1992). The slope factors for chemicals so marked indicate that a toxicity equivalency factor (TEF) method was used for carcinogenic PAHs based on each compound's relative potency to the potency of benzo[a]pyrene. The following TEFs were used to convert each PAH slope factor to an equivalent slope factor of benzo[a]pyrene [that has a new cancer slope factor of $7.3\left(\mathrm{mg} / \mathrm{kg} /\right.$ day) ${ }^{-1}$ identified by EPA's CRAVE workgroup]: (1) benzo[a]pyrene, TEF $=1.0 ;(2)$ benzo[a] anthracene, TEF $=0.1$; (3) benzo[b]fluoranthene, TEF $=0.1$; (4) benzo[k]fluoranthene, TEF $=0.01$; (5) chrysene, TEF $=0.001$; (6) dibenz $[\mathrm{a}$, h] anthracene, TEF $=1.0 ;$ and $(7)$ indeno[1,2,3-c,d]pyrene, $\mathrm{TEF}=0.1$.

(3) The toxicity values for trichloroethylene have been withdrawn from IRIS and HEAST pending further investigation. However, the most recent toxicity values have been used to calculate the PRG.

(4) The PRG was calculated considering Supplemental Guidance from RAGS: Region 4 Bulletins, Human Health Risk Assessment (Interim Guidance) from EPA (November 1995). The slope factors for chemicals so marked indicate that a TEF methodology was used for chlorinated dioxin and furan congeners based on each compound's relative potency to the potency of 2,3,7,8-tetrachlorodibenzodioxin (TCDD). The following TEFs were used to convert each slope factor to an equivalent slope factor of 2,3,7,8-TCDD: (1) 2,3,7,8-TCDD, $\mathrm{TEF}=1.0$; (2) 2,3,7,8-PeCDD, TEF $=0.5 ;$ (3) 2,3,7,8-HxCDD, TEF $=0.1$; (4) 2,3,7,8-HpCDD, TEF $=0.01$; (5) OCDD, TEF $=0.001$; (6) 2,3,7,8-TCDF, TEF =0.1; (7) 1,2,3,7,8-PeCDF, TEF $=0.5$; (8) 2,3,4,7,8-PeCDF, TEF $=0.05 ;(9) 2,3,7,8-\mathrm{HxCDF}, \mathrm{TEF}=0.1$; (10) $2,3,7,8-\mathrm{HpCDF}, \mathrm{TEF}=0.01 ;$ and $(11) \mathrm{OCDF}, \mathrm{TEF}=0.001$.

(5) Toxicity values for these chemicals have been withdrawn from IRIS and HEAST pending further investigation. However, the most recent toxicity values have been used to calculate the PRG.

(6) Toxicity values for these chemicals were obtained from the Superfund Health Risk Technical Support Center. 
Table 3.2b. Risk-based Preliminary Remediation Goals for radionuclides in ground water (industrial scenario)

\begin{tabular}{|l|l|c|c|}
\hline \multirow{2}{*}{ Chemical } & \multirow{2}{*}{ CASRN } & \multicolumn{2}{|c|}{ Ingestion (pCi/L) } \\
\cline { 3 - 4 } & & $10^{-4}$ & $10^{-6}$ \\
\hline Ac-225 & $014265-85-1$ & $1.1 \mathrm{E}+02$ & $1.1 \mathrm{E}+00$ \\
\hline Ac-227 & $014952-40-0$ & $4.5 \mathrm{E}+01$ & $4.5 \mathrm{E}-01$ \\
\hline Ac-227+D & $014952-40-0$ & $2.6 \mathrm{E}+01$ & $2.6 \mathrm{E}-01$ \\
\hline Ac-228 & $014331-83-0$ & $9.9 \mathrm{E}+03$ & $9.9 \mathrm{E}+01$ \\
\hline Ag-105 & $014928-14-4$ & $9.8 \mathrm{E}+03$ & $9.8 \mathrm{E}+01$ \\
\hline Ag-108 & $014391-65-2$ & $2.3 \mathrm{E}+06$ & $2.3 \mathrm{E}+04$ \\
\hline Ag-108m & $014391-65-2$ & $2.6 \mathrm{E}+03$ & $2.6 \mathrm{E}+01$ \\
\hline Ag-108m+D & $014391-65-2$ & $2.6 \mathrm{E}+03$ & $2.6 \mathrm{E}+01$ \\
\hline Ag-109m & $014378-38-2$ & $5.9 \mathrm{E}+07$ & $5.9 \mathrm{E}+05$ \\
\hline Ag-110 & $014391-76-5$ & $6.6 \mathrm{E}+06$ & $6.6 \mathrm{E}+04$ \\
\hline Ag-110m & $014391-76-5$ & $1.9 \mathrm{E}+03$ & $1.9 \mathrm{E}+01$ \\
\hline Ag-111 & $015760-04-0$ & $2.3 \mathrm{E}+03$ & $2.3 \mathrm{E}+01$ \\
\hline Am-241 & $014596-10-2$ & $4.9 \mathrm{E}+01$ & $4.9 \mathrm{E}-01$ \\
\hline Am-242 & $013981-54-9$ & $1.1 \mathrm{E}+04$ & $1.1 \mathrm{E}+02$ \\
\hline Am-242m & $013981-54-9$ & $5.5 \mathrm{E}+01$ & $5.5 \mathrm{E}-01$ \\
\hline Am-243 & $014993-75-0$ & $4.9 \mathrm{E}+01$ & $4.9 \mathrm{E}-01$ \\
\hline Am-243+D & $014993-75-0$ & $4.8 \mathrm{E}+01$ & $4.8 \mathrm{E}-01$ \\
\hline
\end{tabular}


Table 3.2b. (continued)

\begin{tabular}{|c|c|c|c|}
\hline \multirow{2}{*}{ Chemical $^{\dagger}$} & \multirow{2}{*}{ CASRN } & \multicolumn{2}{|c|}{ Ingestion ( $(\mathrm{C} \mathbf{i} / \mathbf{L})$} \\
\hline & & $10^{-4}$ & $10^{-6}$ \\
\hline Ar-41 & $014163-25-8$ & & \\
\hline At -217 & $017239-90-6$ & $1.8 \mathrm{E}+09$ & $1.8 \mathrm{E}+07$ \\
\hline Au-196 & $014914-16-0$ & $1.2 E+04$ & $1.2 \mathrm{E}+02$ \\
\hline Au-198 & $010043-49-0$ & $3.0 \mathrm{E}+03$ & $3.0 \mathrm{E}+01$ \\
\hline Ba-131 & 014914-75-1 & $9.4 \mathrm{E}+03$ & $9.4 \mathrm{E}+01$ \\
\hline $\mathrm{Ba}-133$ & $013981-41-4$ & $5.9 \mathrm{E}+03$ & $5.9 \mathrm{E}+01$ \\
\hline $\mathrm{Ba}-133 \mathrm{~m}$ & $013981-41-4$ & $5.8 \mathrm{E}+03$ & $5.8 \mathrm{E}+01$ \\
\hline $\mathrm{Ba}-137 \mathrm{~m}$ & $013981-97-0$ & $6.6 \mathrm{E}+06$ & $6.6 \mathrm{E}+04$ \\
\hline Ba-139 & $014378-25-7$ & $5.3 E+04$ & $5.3 E+02$ \\
\hline $\mathrm{Ba}-140$ & $014798-08-4$ & $1.4 \mathrm{E}+03$ & $1.4 \mathrm{E}+01$ \\
\hline $\mathrm{Be}-7$ & $013966-02-4$ & $1.9 \mathrm{E}+05$ & $1.9 \mathrm{E}+03$ \\
\hline $\mathrm{Bi}-206$ & $015776-19-9$ & $2.3 \mathrm{E}+03$ & $2.3 \mathrm{E}+01$ \\
\hline Bi-207 & $013982-38-2$ & $3.2 E+03$ & $3.2 \mathrm{E}+01$ \\
\hline Bi-210 & $014331-79-4$ & $2.2 \mathrm{E}+03$ & $2.2 \mathrm{E}+01$ \\
\hline $\mathrm{Bi}-211$ & $015229-37-5$ & $8.8 E+05$ & $8.8 \mathrm{E}+03$ \\
\hline Bi-212 & $014913-49-6$ & $2.6 \mathrm{E}+04$ & $2.6 \mathrm{E}+02$ \\
\hline $\mathrm{Bi}-213$ & $015776-20-2$ & $3.6 \mathrm{E}+04$ & $3.6 \mathrm{E}+02$ \\
\hline Bi-214 & $014733-03-0$ & $8.2 E+04$ & $8.2 E+02$ \\
\hline
\end{tabular}


Table 3.2b. (continued)

\begin{tabular}{|l|l|c|c|}
\hline \multirow{2}{*}{ Chemical } & \multirow{2}{*}{ CASRN } & \multicolumn{2}{|c|}{ Ingestion (pCi/L) } \\
\cline { 2 - 4 } & & $10^{-4}$ & $10^{-6}$ \\
\hline $\mathrm{Br}-82$ & $014686-69-2$ & $1.1 \mathrm{E}+04$ & $1.1 \mathrm{E}+02$ \\
\hline $\mathrm{C}-11$ & $014333-33-6$ & $3.6 \mathrm{E}+05$ & $3.6 \mathrm{E}+03$ \\
\hline $\mathrm{C}-14$ & $014762-75-5$ & $1.6 \mathrm{E}+04$ & $1.6 \mathrm{E}+02$ \\
\hline $\mathrm{C}-15$ & $015929-23-4$ & $2.4 \mathrm{E}+07$ & $2.4 \mathrm{E}+05$ \\
\hline $\mathrm{Ca}-45$ & $013966-05-7$ & $7.9 \mathrm{E}+03$ & $7.9 \mathrm{E}+01$ \\
\hline $\mathrm{Ca}-47$ & $001439-99-2$ & $2.4 \mathrm{E}+03$ & $2.4 \mathrm{E}+01$ \\
\hline $\mathrm{Cd}-109$ & $014109-32-1$ & $2.0 \mathrm{E}+03$ & $2.0 \mathrm{E}+01$ \\
\hline $\mathrm{Cd}-115$ & $014336-68-6$ & $2.2 \mathrm{E}+03$ & $2.2 \mathrm{E}+01$ \\
\hline $\mathrm{Cd}-115 \mathrm{~m}$ & $014336-68-6$ & $1.1 \mathrm{E}+03$ & $1.1 \mathrm{E}+01$ \\
\hline $\mathrm{Ce}-141$ & $013967-74-3$ & $4.1 \mathrm{E}+03$ & $4.1 \mathrm{E}+01$ \\
\hline Ce-143 & $014119-19-8$ & $2.7 \mathrm{E}+03$ & $2.7 \mathrm{E}+01$ \\
\hline $\mathrm{Ce}-144$ & $014762-78-8$ & $5.4 \mathrm{E}+02$ & $5.4 \mathrm{E}+00$ \\
\hline $\mathrm{Ce}-144+\mathrm{D}$ & $014762-78-8$ & $5.4 \mathrm{E}+02$ & $5.4 \mathrm{E}+00$ \\
\hline $\mathrm{Cl}-36$ & $013981-43-6$ & $7.2 \mathrm{E}+03$ & $7.2 \mathrm{E}+01$ \\
\hline $\mathrm{Cl}-38$ & $014158-34-0$ & $7.7 \mathrm{E}+04$ & $7.7 \mathrm{E}+02$ \\
\hline $\mathrm{Cm}-242$ & $015510-73-3$ & $4.2 \mathrm{E}+02$ & $4.2 \mathrm{E}+00$ \\
\hline $\mathrm{Cm}-243$ & $015757-87-6$ & $6.4 \mathrm{E}+01$ & $6.4 \mathrm{E}-01$ \\
\hline $\mathrm{Cm}-243+\mathrm{D}$ & $015757-87-6$ & $6.3 \mathrm{E}+01$ & $6.3 \mathrm{E}-01$ \\
\hline
\end{tabular}


Table 3.2b. (continued)

\begin{tabular}{|l|l|c|c|}
\hline \multirow{2}{*}{ Chemical $^{\dagger}$} & \multirow{2}{*}{ CASRN } & \multicolumn{2}{|c|}{ Ingestion (pCi/L) } \\
\cline { 3 - 4 } & & $10^{-4}$ & $10^{-6}$ \\
\hline Cm-244 & $013981-15-2$ & $7.6 \mathrm{E}+01$ & $7.6 \mathrm{E}-01$ \\
\hline Cm-245 & $015621-76-8$ & $4.8 \mathrm{E}+01$ & $4.8 \mathrm{E}-01$ \\
\hline Cm-246 & $015757-90-1$ & $4.8 \mathrm{E}+01$ & $4.8 \mathrm{E}-01$ \\
\hline Cm-247 & $015758-32-4$ & $5.2 \mathrm{E}+01$ & $5.2 \mathrm{E}-01$ \\
\hline Cm-248 & $015758-33-5$ & $1.2 \mathrm{E}+01$ & $1.2 \mathrm{E}-01$ \\
\hline Co-57 & $013981-50-5$ & $1.6 \mathrm{E}+04$ & $1.6 \mathrm{E}+02$ \\
\hline Co-58 & $013981-38-9$ & $5.7 \mathrm{E}+03$ & $5.7 \mathrm{E}+01$ \\
\hline Co-58m & $013981-38-9$ & $1.7 \mathrm{E}+05$ & $1.7 \mathrm{E}+03$ \\
\hline Co-60 & $010198-40-0$ & $8.5 \mathrm{E}+02$ & $8.5 \mathrm{E}+00$ \\
\hline Cr-51 & $014392-02-0$ & $1.2 \mathrm{E}+05$ & $1.2 \mathrm{E}+03$ \\
\hline Cs-131 & $014914-76-2$ & $8.9 \mathrm{E}+04$ & $8.9 \mathrm{E}+02$ \\
\hline Cs-134 & $013967-70-9$ & $3.4 \mathrm{E}+02$ & $3.4 \mathrm{E}+00$ \\
\hline Cs-134m & $013967-70-9$ & $3.5 \mathrm{E}+05$ & $3.5 \mathrm{E}+03$ \\
\hline Cs-135 & $015726-30-4$ & $3.5 \mathrm{E}+03$ & $3.5 \mathrm{E}+01$ \\
\hline Cs-136 & $014234-29-8$ & $2.1 \mathrm{E}+03$ & $2.1 \mathrm{E}+01$ \\
\hline Cs-137 & $010045-97-3$ & $5.1 \mathrm{E}+02$ & $5.1 \mathrm{E}+00$ \\
\hline Cs-137+D & $010045-97-3$ & $5.1 \mathrm{E}+02$ & $5.1 \mathrm{E}+00$ \\
\hline Cs-138 & $015758-29-9$ & $9.1 \mathrm{E}+04$ & $9.1 \mathrm{E}+02$ \\
\hline
\end{tabular}


Table 3.2b. (continued)

\begin{tabular}{|l|l|c|c|}
\hline \multirow{2}{*}{ Chemical $^{\dagger}$} & \multirow{2}{*}{ CASRN } & \multicolumn{2}{|c|}{ Ingestion (pCi/L) } \\
\cline { 3 - 4 } & \multicolumn{1}{|c|}{$10^{-4}$} & $10^{-6}$ \\
\hline Cu-64 & $013981-25-4$ & $3.0 \mathrm{E}+04$ & $3.0 \mathrm{E}+02$ \\
\hline Dy-165 & $013967-64-1$ & $4.9 \mathrm{E}+04$ & $4.9 \mathrm{E}+02$ \\
\hline Dy-166 & $015840-01-4$ & $1.7 \mathrm{E}+03$ & $1.7 \mathrm{E}+01$ \\
\hline Er-169 & $015840-13-8$ & $7.5 \mathrm{E}+03$ & $7.5 \mathrm{E}+01$ \\
\hline Er-171 & $014391-45-8$ & $9.8 \mathrm{E}+03$ & $9.8 \mathrm{E}+01$ \\
\hline Eu-152 & $014683-23-9$ & $2.8 \mathrm{E}+03$ & $2.8 \mathrm{E}+01$ \\
\hline Eu-154 & $015585-10-1$ & $1.7 \mathrm{E}+03$ & $1.7 \mathrm{E}+01$ \\
\hline Eu-155 & $014391-16-3$ & $9.7 \mathrm{E}+03$ & $9.7 \mathrm{E}+01$ \\
\hline Eu-156 & $014280-35-4$ & $1.5 \mathrm{E}+03$ & $1.5 \mathrm{E}+01$ \\
\hline F-18 & $013981-56-1$ & $1.5 \mathrm{E}+05$ & $1.5 \mathrm{E}+03$ \\
\hline Fe-55 & $014681-59-5$ & $4.6 \mathrm{E}+04$ & $4.6 \mathrm{E}+02$ \\
\hline Fe-59 & $014596-12-4$ & $2.7 \mathrm{E}+03$ & $2.7 \mathrm{E}+01$ \\
\hline Fr-221 & $015756-41-9$ & $1.1 \mathrm{E}+05$ & $1.1 \mathrm{E}+03$ \\
\hline Fr-223 & $015756-98-6$ & $3.6 \mathrm{E}+04$ & $3.6 \mathrm{E}+02$ \\
\hline Ga-67 & $014119-09-6$ & $1.9 \mathrm{E}+04$ & $1.9 \mathrm{E}+02$ \\
\hline Ga-72 & $013982-22-4$ & $3.4 \mathrm{E}+03$ & $3.4 \mathrm{E}+01$ \\
\hline Gd-153 & $014276-65-4$ & $1.2 \mathrm{E}+04$ & $1.2 \mathrm{E}+02$ \\
\hline Gd-159 & $014041-42-0$ & $6.2 \mathrm{E}+03$ & $6.2 \mathrm{E}+01$ \\
\hline
\end{tabular}


Table 3.2b. (continued)

\begin{tabular}{|l|l|c|c|}
\hline \multirow{2}{*}{ Chemical $^{\dagger}$} & \multirow{2}{*}{ CASRN } & \multicolumn{2}{|c|}{ Ingestion (pCi/L) } \\
\cline { 3 - 4 } & & $10^{-4}$ & $10^{-6}$ \\
\hline Ge-71 & $014374-81-3$ & $1.4 \mathrm{E}+06$ & $1.4 \mathrm{E}+04$ \\
\hline H-3 & $010028-17-8$ & $2.2 \mathrm{E}+05$ & $2.2 \mathrm{E}+03$ \\
\hline Hg-197 & $013981-51-6$ & $1.4 \mathrm{E}+04$ & $1.4 \mathrm{E}+02$ \\
\hline Hg-203 & $013982-78-0$ & $6.1 \mathrm{E}+03$ & $6.1 \mathrm{E}+01$ \\
\hline Ho-166 & $013967-65-2$ & $2.1 \mathrm{E}+03$ & $2.1 \mathrm{E}+01$ \\
\hline I-122 & $018287-75-7$ & $7.4 \mathrm{E}+05$ & $7.4 \mathrm{E}+03$ \\
\hline I-123 & $015715-08-9$ & $3.0 \mathrm{E}+04$ & $3.0 \mathrm{E}+02$ \\
\hline I-125 & $014158-31-7$ & $6.2 \mathrm{E}+02$ & $6.2 \mathrm{E}+00$ \\
\hline I-126 & $014158-32-8$ & $3.3 \mathrm{E}+02$ & $3.3 \mathrm{E}+00$ \\
\hline I-129 & $015046-84-1$ & $8.7 \mathrm{E}+01$ & $8.7 \mathrm{E}-01$ \\
\hline I-130 & $014914-02-4$ & $3.3 \mathrm{E}+03$ & $3.3 \mathrm{E}+01$ \\
\hline I-131 & $010043-66-0$ & $4.4 \mathrm{E}+02$ & $4.4 \mathrm{E}+00$ \\
\hline I-132 & $014683-16-0$ & $2.4 \mathrm{E}+04$ & $2.4 \mathrm{E}+02$ \\
\hline I-133 & $014834-67-4$ & $1.5 \mathrm{E}+03$ & $1.5 \mathrm{E}+01$ \\
\hline I-134 & $014914-27-3$ & $6.9 \mathrm{E}+04$ & $6.9 \mathrm{E}+02$ \\
\hline I-135 & $014834-68-5$ & $7.0 \mathrm{E}+03$ & $7.0 \mathrm{E}+01$ \\
\hline In-113m & $014885-78-0$ & $1.9 \mathrm{E}+05$ & $1.9 \mathrm{E}+03$ \\
\hline In-114 & $013981-55-0$ & $3.5 \mathrm{E}+06$ & $3.5 \mathrm{E}+04$ \\
\hline
\end{tabular}


Table 3.2b. (continued)

\begin{tabular}{|l|l|c|c|}
\hline \multirow{2}{*}{ Chemical } & \multirow{2}{*}{ CASRN } & \multicolumn{2}{|c|}{ Ingestion (pCi/L) } \\
\cline { 3 - 4 } & & $10^{-4}$ & $10^{-6}$ \\
\hline In-114m & $013981-55-0$ & $7.8 \mathrm{E}+02$ & $7.8 \mathrm{E}+00$ \\
\hline In-115 & $014191-71-0$ & $4.6 \mathrm{E}+02$ & $4.6 \mathrm{E}+00$ \\
\hline In-115m & $014191-71-0$ & $4.7 \mathrm{E}+04$ & $4.7 \mathrm{E}+02$ \\
\hline Ir-190 & $014981-91-0$ & $3.2 \mathrm{E}+03$ & $3.2 \mathrm{E}+01$ \\
\hline Ir-194 & $014158-35-1$ & $2.3 \mathrm{E}+03$ & $2.3 \mathrm{E}+01$ \\
\hline $\mathrm{K}-40$ & $013966-00-2$ & $1.3 \mathrm{E}+03$ & $1.3 \mathrm{E}+01$ \\
\hline $\mathrm{K}-42$ & $014378-21-3$ & $1.2 \mathrm{E}+04$ & $1.2 \mathrm{E}+02$ \\
\hline Kr-83m & $013965-98-5$ & & \\
\hline Kr-85 & $013983-27-2$ & & \\
\hline Kr-85m & $013983-27-2$ & & \\
\hline Kr-87 & $014809-68-8$ & & \\
\hline Kr-88 & $014995-61-0$ & & \\
\hline Kr-89 & $016316-03-3$ & & \\
\hline Kr-90 & $015741-13-6$ & & \\
\hline La-140 & $013981-28-7$ & $1.7 \mathrm{E}+03$ & $1.7 \mathrm{E}+01$ \\
\hline Lu-177 & $014265-75-9$ & $5.4 \mathrm{E}+03$ & $5.4 \mathrm{E}+01$ \\
\hline Mn-52 & $014092-99-0$ & $2.7 \mathrm{E}+03$ & \\
\hline Mr-54 & $013966-31-9$ & $8.2 \mathrm{E}+03$ & \\
\hline
\end{tabular}


Table 3.2b. (continued)

\begin{tabular}{|c|c|c|c|}
\hline \multirow{2}{*}{ Chemical $^{\dagger}$} & \multirow{2}{*}{ CASRN } & \multicolumn{2}{|c|}{ Ingestion (pCi/L) } \\
\hline & & $10^{-4}$ & $10^{-6}$ \\
\hline $\mathrm{Mn}-56$ & $014681-52-8$ & $1.9 E+04$ & $1.9 \mathrm{E}+02$ \\
\hline Mo-99 & $014119-15-4$ & $7.0 \mathrm{E}+03$ & $7.0 \mathrm{E}+01$ \\
\hline $\mathrm{Na}-22$ & $013966-32-0$ & $2.0 \mathrm{E}+03$ & $2.0 \mathrm{E}+01$ \\
\hline $\mathrm{Na}-24$ & 013982-04-2 & $1.2 \mathrm{E}+04$ & $1.2 E+02$ \\
\hline $\mathrm{Nb}-93 \mathrm{~m}$ & $007440-03-1$ & $2.4 \mathrm{E}+04$ & $2.4 \mathrm{E}+02$ \\
\hline $\mathrm{Nb}-94$ & $014681-63-1$ & $2.3 E+03$ & $2.3 \mathrm{E}+01$ \\
\hline $\mathrm{Nb}-95$ & $013967-76-5$ & $7.1 \mathrm{E}+03$ & $7.1 \mathrm{E}+01$ \\
\hline $\mathrm{Nb}-95 \mathrm{~m}$ & $013967-76-5$ & $5.2 \mathrm{E}+03$ & $5.2 \mathrm{E}+01$ \\
\hline $\mathrm{Nb}-97$ & 018496-04-3 & $9.1 E+04$ & $9.1 \mathrm{E}+02$ \\
\hline $\mathrm{Nb}-97 \mathrm{~m}$ & $018496-04-3$ & $4.9 E+06$ & $4.9 E+04$ \\
\hline Nd-147 & $014269-74-0$ & $2.7 \mathrm{E}+03$ & 2.7E+01 \\
\hline Nd-149 & $015749-81-2$ & $3.5 E+04$ & $3.5 \mathrm{E}+02$ \\
\hline $\mathrm{Ni}-59$ & $014336-70-0$ & $8.6 E+04$ & $8.6 \mathrm{E}+02$ \\
\hline $\mathrm{Ni}-63$ & $013981-37-8$ & $2.9 \mathrm{E}+04$ & $2.9 \mathrm{E}+02$ \\
\hline $\mathrm{Ni}-65$ & $014833-49-9$ & $2.8 \mathrm{E}+04$ & $2.8 \mathrm{E}+02$ \\
\hline $\mathrm{Np}-236$ & $015700-36-4$ & $1.7 \mathrm{E}+04$ & $1.7 E+02$ \\
\hline Np-237 & $013994-20-2$ & $5.4 \mathrm{E}+01$ & $5.4 \mathrm{E}-01$ \\
\hline $\mathrm{Np}-237+\mathrm{D}$ & 013994-20-2 & $5.3 \mathrm{E}+01$ & 5.3E-01 \\
\hline
\end{tabular}


Table 3.2b. (continued)

\begin{tabular}{|l|l|c|c|}
\hline \multirow{2}{*}{ Chemical } & \multirow{2}{*}{ CASRN } & \multicolumn{2}{|c|}{ Ingestion (pCi/L) } \\
\cline { 3 - 4 } & & $10^{-4}$ & $10^{-6}$ \\
\hline Np-238 & $015766-25-3$ & $3.5 \mathrm{E}+03$ & $3.5 \mathrm{E}+01$ \\
\hline Np-239 & $013968-59-7$ & $3.7 \mathrm{E}+03$ & $3.7 \mathrm{E}+01$ \\
\hline Np-240 & $015690-84-3$ & $9.0 \mathrm{E}+04$ & $9.0 \mathrm{E}+02$ \\
\hline $\mathrm{Np}-240 \mathrm{~m}$ & $015690-84-3$ & $6.6 \mathrm{E}+05$ & $6.6 \mathrm{E}+03$ \\
\hline Os-185 & $015766-50-4$ & $8.9 \mathrm{E}+03$ & $8.9 \mathrm{E}+01$ \\
\hline Os-191 & $014119-24-5$ & $5.3 \mathrm{E}+03$ & $5.3 \mathrm{E}+01$ \\
\hline $\mathrm{Os}-191 \mathrm{~m}$ & $014119-24-5$ & $3.2 \mathrm{E}+04$ & $3.2 \mathrm{E}+02$ \\
\hline $\mathrm{Os}-193$ & $016057-77-5$ & $3.7 \mathrm{E}+03$ & $3.7 \mathrm{E}+01$ \\
\hline $\mathrm{P}-32$ & $014596-37-3$ & $2.6 \mathrm{E}+03$ & $2.6 \mathrm{E}+01$ \\
\hline $\mathrm{P}-33$ & $015749-66-3$ & $2.0 \mathrm{E}+04$ & $2.0 \mathrm{E}+02$ \\
\hline $\mathrm{Pa}-231$ & $014331-85-2$ & $1.1 \mathrm{E}+02$ & $1.1 \mathrm{E}+00$ \\
\hline $\mathrm{Pa}-233$ & $013981-14-1$ & $3.4 \mathrm{E}+03$ & $3.4 \mathrm{E}+01$ \\
\hline $\mathrm{Pa}-234$ & $015100-28-4$ & $7.5 \mathrm{E}+03$ & $7.5 \mathrm{E}+01$ \\
\hline $\mathrm{Pa}-234 \mathrm{~m}$ & $015100-28-4$ & $3.4 \mathrm{E}+06$ & $3.4 \mathrm{E}+04$ \\
\hline $\mathrm{Pb}-203$ & $014687-25-3$ & $1.6 \mathrm{E}+04$ & $1.6 \mathrm{E}+02$ \\
\hline $\mathrm{Pb}-209$ & $014119-30-3$ & $7.7 \mathrm{E}+04$ & $7.7 \mathrm{E}+02$ \\
\hline $\mathrm{Pb}-210$ & $014255-04-0$ & $2.4 \mathrm{E}+01$ & $2.4 \mathrm{E}-01$ \\
\hline $\mathrm{Pb}-210+\mathrm{D}$ & $014255-04-0$ & $1.6 \mathrm{E}+01$ & $1.6 \mathrm{E}-01$ \\
\hline
\end{tabular}


Table 3.2b. (continued)

\begin{tabular}{|c|c|c|c|}
\hline \multirow{2}{*}{ Chemical $^{\dagger}$} & \multirow{2}{*}{ CASRN } & \multicolumn{2}{|c|}{ Ingestion (pCi/L) } \\
\hline & & $10^{-4}$ & $10^{-6}$ \\
\hline $\mathrm{Pb}-211$ & $015816-77-0$ & 4.7E+04 & 4.7E+02 \\
\hline $\mathrm{Pb}-212$ & $015092-94-1$ & $8.9 E+02$ & 8.9E+00 \\
\hline $\mathrm{Pb}-214$ & $015067-28-4$ & $5.4 E+04$ & $5.4 \mathrm{E}+02$ \\
\hline $\mathrm{Pd}-100$ & $015 \underline{690}-69-4$ & $4.3 \mathrm{E}+03$ & $4.3 E+01$ \\
\hline $\mathrm{Pd}-101$ & $015749-54-9$ & $4.3 E+04$ & 4.3E+02 \\
\hline $\mathrm{Pd}-103$ & $014967-68-1$ & $1.5 \mathrm{E}+04$ & $1.5 E+02$ \\
\hline $\mathrm{Pd}-107$ & $017637-99-9$ & 7.7E+04 & $7.7 \mathrm{E}+02$ \\
\hline Pd-109 & $014981-64-7$ & $4.8 \mathrm{E}+03$ & $4.8 \mathrm{E}+01$ \\
\hline Pm-147 & $014380-75-7$ & $1.1 E+04$ & $1.1 E+02$ \\
\hline Pm-148 & $014683-19-3$ & $1.1 E+03$ & $1.1 \mathrm{E}+01$ \\
\hline Pm-148m & $014683-19-3$ & $1.6 E+03$ & $1.6 \mathrm{E}+01$ \\
\hline Pm-149 & $015765-31-8$ & $2.9 \mathrm{E}+03$ & $2.9 \mathrm{E}+01$ \\
\hline Po-210 & $013981-52-7$ & $4,9 \mathrm{E}+01$ & $4.9 \mathrm{E}-01$ \\
\hline Po-212 & $015389-34-1$ & $3.5 \mathrm{E}+14$ & $3.5 \mathrm{E}+12$ \\
\hline Po-213 & $015756-57-7$ & $2.4 \mathrm{E}+13$ & $2.4 \mathrm{E}+11$ \\
\hline Po-214 & $015735-67-8$ & $7.5 \mathrm{E}+11$ & $7.5 \mathrm{E}+09$ \\
\hline Po-215 & $015706-52-2$ & $3.2 E+10$ & $3.2 E+08$ \\
\hline Po-216 & $015756-58-8$ & $1.8 \mathrm{E}+08$ & $1.8 \mathrm{E}+06$ \\
\hline
\end{tabular}


Table 3.2b. (continued)

\begin{tabular}{|l|l|c|c|}
\hline \multirow{2}{*}{ Chemical } & \multirow{2}{*}{ CASRN } & \multicolumn{2}{|c|}{ Ingestion (pCi/L) } \\
\cline { 3 - 4 } & & $10^{-4}$ & $10^{-6}$ \\
\hline Po-218 & $015422-24-9$ & $3.1 \mathrm{E}+05$ & $3.1 \mathrm{E}+03$ \\
\hline Pr-142 & $014191-64-1$ & $2.3 \mathrm{E}+03$ & $2.3 \mathrm{E}+01$ \\
\hline Pr-143 & $014981-79-4$ & $2.4 \mathrm{E}+03$ & $2.4 \mathrm{E}+01$ \\
\hline Pr-144 & $014119-05-2$ & $2.0 \mathrm{E}+05$ & $2.0 \mathrm{E}+03$ \\
\hline Pr-144m & $014119-05-2$ & $5.0 \mathrm{E}+05$ & $5.0 \mathrm{E}+03$ \\
\hline Pt-191 & $015706-36-2$ & $1.1 \mathrm{E}+04$ & $1.1 \mathrm{E}+02$ \\
\hline Pt-193 & $015735-70-3$ & $9.9 \mathrm{E}+04$ & $9.9 \mathrm{E}+02$ \\
\hline Pt-193m & $015735-70-3$ & $6.4 \mathrm{E}+03$ & $6.4 \mathrm{E}+01$ \\
\hline Pt-197 & $015735-74-7$ & $7.5 \mathrm{E}+03$ & $7.5 \mathrm{E}+01$ \\
\hline Pt-197m & $015735-74-7$ & $4.9 \mathrm{E}+04$ & $4.9 \mathrm{E}+02$ \\
\hline Pu-236 & $015411-92-4$ & $2.1 \mathrm{E}+02$ & $2.1 \mathrm{E}+00$ \\
\hline Pu-238 & $013981-16-3$ & $5.4 \mathrm{E}+01$ & $5.4 \mathrm{E}-01$ \\
\hline Pu-239 & $015117-48-3$ & $5.1 \mathrm{E}+01$ & $5.1 \mathrm{E}-01$ \\
\hline Pu-240 & $014119-33-6$ & $5.1 \mathrm{E}+01$ & $5.1 \mathrm{E}-01$ \\
\hline Pu-241 & $014119-32-5$ & $3.1 \mathrm{E}+03$ & $3.1 \mathrm{E}+01$ \\
\hline Pu-241+D & $014119-32-5$ & $4.8 \mathrm{E}+01$ & $4.8 \mathrm{E}-01$ \\
\hline Pu-242 & $013982-10-0$ & $5.3 \mathrm{E}+01$ & $5.3 \mathrm{E}-01$ \\
\hline Pu-243 & $015706-37-3$ & $4.3 \mathrm{E}+04$ & $4.3 \mathrm{E}+02$ \\
\hline
\end{tabular}


Table 3.2b. (continued)

\begin{tabular}{|l|l|c|c|}
\hline \multirow{2}{*}{ Chemical } & \multirow{2}{*}{ CASRN } & \multicolumn{2}{|c|}{ Ingestion (pCi/L) } \\
\cline { 3 - 4 } & & $10^{-4}$ & $10^{-6}$ \\
\hline Pu-244 & $014119-34-7$ & $5.1 \mathrm{E}+01$ & $5.1 \mathrm{E}-01$ \\
\hline Pu-244+D & $014119-34-7$ & $5.0 \mathrm{E}+01$ & $5.0 \mathrm{E}-01$ \\
\hline Ra-223 & $015623-45-7$ & $6.8 \mathrm{E}+01$ & $6.8 \mathrm{E}-01$ \\
\hline Ra-224 & $013233-32-4$ & $1.1 \mathrm{E}+02$ & $1.1 \mathrm{E}+00$ \\
\hline Ra-225 & $013981-53-8$ & $1.0 \mathrm{E}+02$ & $1.0 \mathrm{E}+00$ \\
\hline Ra-226 & $013982-63-3$ & $5.4 \mathrm{E}+01$ & $5.4 \mathrm{E}-01$ \\
\hline Ra-226+D & $013982-63-3$ & $5.4 \mathrm{E}+01$ & $5.4 \mathrm{E}-01$ \\
\hline Ra-228 & $015262-20-1$ & $6.5 \mathrm{E}+01$ & $6.5 \mathrm{E}-01$ \\
\hline Ra-228+D & $015262-20-1$ & $6.5 \mathrm{E}+01$ & $6.5 \mathrm{E}-01$ \\
\hline Rb-82 & $014391-63-0$ & $1.5 \mathrm{E}+06$ & $1.5 \mathrm{E}+04$ \\
\hline Rb-86 & $014932-53-7$ & $2.2 \mathrm{E}+03$ & $2.2 \mathrm{E}+01$ \\
\hline Rb-87 & $013982-13-3$ & $4.3 \mathrm{E}+03$ & $4.3 \mathrm{E}+01$ \\
\hline Rb-88 & $014928-36-0$ & $1.1 \mathrm{E}+05$ & $1.1 \mathrm{E}+03$ \\
\hline Rb-89 & $014191-65-2$ & $1.8 \mathrm{E}+05$ & $1.8 \mathrm{E}+03$ \\
\hline Rh-103m & $007440-16-6$ & $2.0 \mathrm{E}+06$ & $2.0 \mathrm{E}+04$ \\
\hline Rh-105 & $014913-89-4$ & $8.3 \mathrm{E}+03$ & $8.3 \mathrm{E}+01$ \\
\hline Rh-105m & $014913-89-4$ & $1.5 \mathrm{E}+07$ & $1.5 \mathrm{E}+05$ \\
\hline Rh-106 & $014234-34-5$ & $4.4 \mathrm{E}+06$ & $4.4 \mathrm{E}+04$ \\
\hline
\end{tabular}


Table 3.2b. (continued)

\begin{tabular}{|l|l|c|c|}
\hline \multirow{2}{*}{ Chemical } & \multirow{2}{*}{ CASRN } & \multicolumn{2}{|c|}{ Ingestion (pCi/L) } \\
\cline { 3 - 4 } & & $10^{-4}$ & $10^{-6}$ \\
\hline Rn-219 & $014835-02-0$ & & \\
\hline Rn-220 & $022481-48-7$ & & \\
\hline Rn-222+D & $014859-67-7$ & & \\
\hline Ru-103 & $013968-53-1$ & $4.8 \mathrm{E}+03$ & $4.8 \mathrm{E}+01$ \\
\hline Ru-105 & $014331-95-4$ & $1.4 \mathrm{E}+04$ & $1.4 \mathrm{E}+02$ \\
\hline Ru-106 & $013967-48-1$ & $4.6 \mathrm{E}+02$ & $4.6 \mathrm{E}+00$ \\
\hline Ru-106+D & $013967-48-1$ & $4.6 \mathrm{E}+02$ & $4.6 \mathrm{E}+00$ \\
\hline Ru-97 & $015758-35-7$ & $2.7 \mathrm{E}+04$ & $2.7 \mathrm{E}+02$ \\
\hline S-35 & $015117-53-0$ & $3.8 \mathrm{E}+04$ & $3.8 \mathrm{E}+02$ \\
\hline Sb-122 & $014374-79-9$ & $1.8 \mathrm{E}+03$ & $1.8 \mathrm{E}+01$ \\
\hline Sb-124 & $014683-10-4$ & $1.5 \mathrm{E}+03$ & $1.5 \mathrm{E}+01$ \\
\hline Sb-125 & $014234-35-6$ & $5.4 \mathrm{E}+03$ & $5.4 \mathrm{E}+01$ \\
\hline Sb-125+D & $014234-35-6$ & $4.5 \mathrm{E}+03$ & $4.5 \mathrm{E}+01$ \\
\hline Sb-126 & $015756-32-8$ & $1.6 \mathrm{E}+03$ & $1.6 \mathrm{E}+01$ \\
\hline Sb-126m & $015756-32-8$ & $2.2 \mathrm{E}+05$ & $2.2 \mathrm{E}+03$ \\
\hline Sb-127 & $013968-50-8$ & $1.9 \mathrm{E}+03$ & $1.9 \mathrm{E}+01$ \\
\hline Sb-129 & $014331-88-5$ & $8.6 \mathrm{E}+03$ & $8.6 \mathrm{E}+01$ \\
\hline Sc-46 & $013967-63-0$ & $2.8 \mathrm{E}+03$ & $2.8 \mathrm{E}+01$ \\
\hline
\end{tabular}


Table 3.2b. (continued)

\begin{tabular}{|l|l|c|c|}
\hline \multirow{2}{*}{ Chemical $^{\dagger}$} & \multirow{2}{*}{ CASRN } & \multicolumn{2}{|c|}{ Ingestion (pCi/L) } \\
\cline { 3 - 4 } & & $10^{-4}$ & $10^{-6}$ \\
\hline Sc-47 & $014391-96-9$ & $5.4 \mathrm{E}+03$ & $5.4 \mathrm{E}+01$ \\
\hline Sc-48 & $014391-86-7$ & $2.4 \mathrm{E}+03$ & $2.4 \mathrm{E}+01$ \\
\hline Se-75 & $014265-71-5$ & $2.5 \mathrm{E}+03$ & $2.5 \mathrm{E}+01$ \\
\hline Si-31 & $014276-49-4$ & $3.2 \mathrm{E}+04$ & $3.2 \mathrm{E}+02$ \\
\hline Sm-147 & $014392-33-7$ & $6.4 \mathrm{E}+02$ & $6.4 \mathrm{E}+00$ \\
\hline Sm-151 & $015715-94-3$ & $3.5 \mathrm{E}+04$ & $3.5 \mathrm{E}+02$ \\
\hline Sm-153 & $015766-00-4$ & $4.0 \mathrm{E}+03$ & $4.0 \mathrm{E}+01$ \\
\hline Sn-113 & $013966-06-8$ & $4.3 \mathrm{E}+03$ & $4.3 \mathrm{E}+01$ \\
\hline Sn-121 & $014683-06-8$ & $1.3 \mathrm{E}+04$ & $1.3 \mathrm{E}+02$ \\
\hline Sn-121m & $014683-06-8$ & $8.0 \mathrm{E}+03$ & $8.0 \mathrm{E}+01$ \\
\hline Sn-125 & $014683-08-0$ & $9.5 \mathrm{E}+02$ & $9.5 \mathrm{E}+00$ \\
\hline Sn-126 & $015832-50-5$ & $7.5 \mathrm{E}+02$ & $7.5 \mathrm{E}+00$ \\
\hline Sr-82 & $014809-50-8$ & $6.2 \mathrm{E}+02$ & $6.2 \mathrm{E}+00$ \\
\hline Sr-85 & $013967-73-2$ & $1.1 \mathrm{E}+04$ & $1.1 \mathrm{E}+02$ \\
\hline Sr-85m & $013967-73-2$ & $8.9 \mathrm{E}+05$ & $8.9 \mathrm{E}+03$ \\
\hline Sr-89 & $014158-27-1$ & $1.6 \mathrm{E}+03$ & $1.6 \mathrm{E}+01$ \\
\hline Sr-90 & $010098-97-2$ & $3.9 \mathrm{E}+02$ & $3.9 \mathrm{E}+00$ \\
\hline Sr-90+D & $010098-97-2$ & $2.9 \mathrm{E}+02$ & $2.9 \mathrm{E}+00$ \\
\hline
\end{tabular}


Table 3.2b. (continued)

\begin{tabular}{|l|l|c|c|}
\hline \multirow{2}{*}{ Chemical } & \multirow{2}{*}{ CASRN } & \multicolumn{2}{|c|}{ Ingestion (pCi/L) } \\
\cline { 3 - 4 } & & $10^{-4}$ & $10^{-5}$ \\
\hline Sr-91 & $014331-91-0$ & $5.7 \mathrm{E}+03$ & $5.7 \mathrm{E}+01$ \\
\hline Sr-92 & $014928-29-1$ & $7.9 \mathrm{E}+03$ & $7.9 \mathrm{E}+01$ \\
\hline Ta-182 & $013982-00-8$ & $2.3 \mathrm{E}+03$ & $2.3 \mathrm{E}+01$ \\
\hline Tb-158 & $015759-55-4$ & $3.8 \mathrm{E}+03$ & $3.8 \mathrm{E}+01$ \\
\hline Tb-160 & $013981-29-8$ & $2.1 \mathrm{E}+03$ & $2.1 \mathrm{E}+01$ \\
\hline Tc-95 & $014809-56-4$ & $2.3 \mathrm{E}+05$ & $2.3 \mathrm{E}+03$ \\
\hline Tc-95m & $014809-56-4$ & $1.3 \mathrm{E}+04$ & $1.3 \mathrm{E}+02$ \\
\hline Tc-96 & $014808-44-7$ & $7.0 \mathrm{E}+03$ & $7.0 \mathrm{E}+01$ \\
\hline Tc-96m & $014808-44-7$ & $6.1 \mathrm{E}+05$ & $6.1 \mathrm{E}+03$ \\
\hline Tc-97 & $015759-35-0$ & $1.0 \mathrm{E}+05$ & $1.0 \mathrm{E}+03$ \\
\hline Tc-97m & $015759-35-0$ & $1.3 \mathrm{E}+04$ & $1.3 \mathrm{E}+02$ \\
\hline Tc-99 & $014133-76-7$ & $1.1 \mathrm{E}+04$ & $1.1 \mathrm{E}+02$ \\
\hline Tc-99m & $014133-76-7$ & $2.9 \mathrm{E}+05$ & $2.9 \mathrm{E}+03$ \\
\hline Te-125m & $014390-73-9$ & $6.4 \mathrm{E}+03$ & $6.4 \mathrm{E}+01$ \\
\hline Te-127 & $013981-49-2$ & $1.9 \mathrm{E}+04$ & $1.9 \mathrm{E}+02$ \\
\hline Te-127m & $013981-49-2$ & $2.7 \mathrm{E}+03$ & $2.7 \mathrm{E}+01$ \\
\hline Te-129 & $014269-71-7$ & $1.1 \mathrm{E}+05$ & $1.1 \mathrm{E}+03$ \\
\hline Te-129m & $014269-71-7$ & $1.4 \mathrm{E}+03$ & $1.4 \mathrm{E}+01$ \\
\hline
\end{tabular}


Table 3.2b. (continued)

\begin{tabular}{|l|l|c|c|}
\hline \multirow{2}{*}{ Chemical } & \multirow{2}{*}{ CASRN } & \multicolumn{2}{|c|}{ Ingestion (pCi/L) } \\
\cline { 3 - 4 } & & $10^{-4}$ & $10^{-6}$ \\
\hline Te-131 & $014683-12-6$ & $4.1 \mathrm{E}+04$ & $4.1 \mathrm{E}+02$ \\
\hline Te-131m & $014683-12-6$ & $1.8 \mathrm{E}+03$ & $1.8 \mathrm{E}+01$ \\
\hline Te-132 & $014234-28-7$ & $1.3 \mathrm{E}+03$ & $1.3 \mathrm{E}+01$ \\
\hline Th-227. & $015623-47-9$ & $4.0 \mathrm{E}+02$ & $4.0 \mathrm{E}+00$ \\
\hline Th-228 & $014274-82-9$ & $2.5 \mathrm{E}+02$ & $2.5 \mathrm{E}+00$ \\
\hline Th-228+D & $014274-82-9$ & $6.9 \mathrm{E}+01$ & $6.9 \mathrm{E}-01$ \\
\hline Th-229 & $015594-54-4$ & $2.8 \mathrm{E}+02$ & $2.8 \mathrm{E}+00$ \\
\hline Th-229+D & $015594-54-4$ & $4.5 \mathrm{E}+01$ & $4.5 \mathrm{E}-01$ \\
\hline Th-230 & $014269-63-7$ & $4.3 \mathrm{E}+02$ & $4.3 \mathrm{E}+00$ \\
\hline Th-231 & $014932-40-2$ & $8.9 \mathrm{E}+03$ & $8.9 \mathrm{E}+01$ \\
\hline Th-232 & $007440-29-1$ & $4.9 \mathrm{E}+02$ & $4.9 \mathrm{E}+00$ \\
\hline Th-234 & $015065-10-8$ & $8.3 \mathrm{E}+02$ & $8.3 \mathrm{E}+00$ \\
\hline Tl-202 & $015720-57-7$ & $1.6 \mathrm{E}+04$ & $1.6 \mathrm{E}+02$ \\
\hline Tl-204 & $013968-51-9$ & $8.1 \mathrm{E}+03$ & $8.1 \mathrm{E}+01$ \\
\hline Tl-208 & $014913-50-9$ & $9.1 \mathrm{E}+05$ & $9.1 \mathrm{E}+03$ \\
\hline Tl-209 & $015690-73-0$ & $1.1 \mathrm{E}+06$ & $1.1 \mathrm{E}+04$ \\
\hline Tm-170 & $013981-30-1$ & $2.1 \mathrm{E}+03$ & $2.1 \mathrm{E}+01$ \\
\hline Tm-171 & $014333-45-0$ & $2.7 \mathrm{E}+04$ & $2.7 \mathrm{E}+02$ \\
\hline
\end{tabular}


Table 3.2b. (continued)

\begin{tabular}{|c|c|c|c|}
\hline \multirow{2}{*}{ Chemical $^{\dagger}$} & \multirow{2}{*}{ CASRN } & \multicolumn{2}{|c|}{ Ingestion (pCi/L) } \\
\hline & & $10^{-4}$ & $10^{-6}$ \\
\hline $\mathrm{U}-232$ & $014158-29-3$ & $2.0 \mathrm{E}+02$ & $2.0 \mathrm{E}+00$ \\
\hline U-233 & 013968-55-3 & $3.6 \mathrm{E}+02$ & $3.6 \mathrm{E}+00$ \\
\hline U-234 & 013966-29-5 & $3.6 \mathrm{E}+02$ & $3.6 \mathrm{E}+00$ \\
\hline U-235 & $015117-96-1$ & $3.5 \mathrm{E}+02$ & $3.5 \mathrm{E}+00$ \\
\hline$U-235+D$ & $015117-96-1$ & $3.4 \mathrm{E}+02$ & $3.4 \mathrm{E}+00$ \\
\hline U-236 & 013982-70-2 & $3.8 \mathrm{E}+02$ & $3.8 \mathrm{E}+00$ \\
\hline U-237 & $014269-75-1$ & $4.0 \mathrm{E}+03$ & $4.0 \mathrm{E}+01$ \\
\hline U-238 & $007440-61-1$ & $3.7 \mathrm{E}+02$ & $3.7 \mathrm{E}+00$ \\
\hline$U-238+D$ & 007440-61-1 & $2.6 \mathrm{E}+02$ & $2.6 \mathrm{E}+00$ \\
\hline U-240 & 015687-53-3 & $2.9 \mathrm{E}+03$ & $2.9 \mathrm{E}+01$ \\
\hline$V-48$ & $014331-97-6$ & $2.1 \mathrm{E}+03$ & $2.1 \mathrm{E}+01$ \\
\hline$W-181$ & $015749-46-9$ & $5.9 \mathrm{E}+04$ & $5.9 \mathrm{E}+02$ \\
\hline W-185 & $014932-41-3$ & $7.8 \mathrm{E}+03$ & $7.8 \mathrm{E}+01$ \\
\hline$W-187$ & 014983-48-3 & $6.5 E+03$ & $6.5 \mathrm{E}+01$ \\
\hline $\mathrm{Xe}-122$ & $015151-09-4$ & & \\
\hline $\mathrm{Xe}-123$ & $015700-10-4$ & & \\
\hline $\mathrm{Xe}-125$ & 013994-18-8 & & \\
\hline $\mathrm{Xe}-127$ & 013994-19-9 & & \\
\hline
\end{tabular}


Table 3.2b. (continued)

\begin{tabular}{|c|c|c|c|}
\hline \multirow{2}{*}{ Chemical $^{\dagger}$} & \multirow{2}{*}{ CASRN } & \multicolumn{2}{|c|}{ Ingestion $(\mathrm{pCi} / \mathrm{L})$} \\
\hline & & $10^{-4}$ & $10^{-6}$ \\
\hline $\mathrm{Xe}-129 \mathrm{~m}$ & 013965-99-6 & & \\
\hline $\mathrm{Xe}-131 \mathrm{~m}$ & 014683-11-5 & & \\
\hline $\mathrm{Xe}-133$ & $014932-42-4$ & & \\
\hline $\mathrm{Xe}-133 \mathrm{~m}$ & $014932-42-4$ & & \\
\hline $\mathrm{Xe}-135$ & 014995-62-1 & & \\
\hline $\mathrm{Xe}-135 \mathrm{~m}$ & 014995-62-1 & & \\
\hline $\mathrm{Xe}-137$ & $014835-21=3$ & & \\
\hline $\mathrm{Xe}-138$ & $015751-81-2$ & & \\
\hline Y-90 & 010098-91-6 & $1.1 \mathrm{E}+03$ & $1.1 \mathrm{E}+01$ \\
\hline Y-91 & 014234-24-3 & $1.2 \mathrm{E}+03$ & $1.2 E+01$ \\
\hline $\mathrm{Y}-91 \mathrm{~m}$ & $014234-24-3$ & $4.3 \mathrm{E}+05$ & $4.3 \mathrm{E}+03$ \\
\hline Y-92 & 015751-59-4 & $8.2 \mathrm{E}+03$ & $8.2 \mathrm{E}+01$ \\
\hline Y-93 & 014981-70-5 & $2.8 \mathrm{E}+03$ & $2.8 \mathrm{E}+01$ \\
\hline $\mathrm{Zn}-65$ & 013982-39-3 & $1.6 \mathrm{E}+03$ & $1.6 \mathrm{E}+01$ \\
\hline $\mathrm{Zn}-69$ & $013982-23-5$ & $2.6 \mathrm{E}+05$ & $2.6 \mathrm{E}+03$ \\
\hline $\mathrm{Zn}-69 \mathrm{~m}$ & 013982-23-5 & $1.1 \mathrm{E}+04$ & $1.1 \mathrm{E}+02$ \\
\hline $\mathrm{Zr}-93$ & 015751-77-6 & $3.1 E+04$ & $3.1 \mathrm{E}+02$ \\
\hline Zr-95 & 013967-71-0 & $4.1 E+03$ & $4.1 \mathrm{E}+01$ \\
\hline
\end{tabular}


Table 3.2b. (continued)

\begin{tabular}{|l|l|c|c|}
\hline \multirow{2}{*}{ Chemical $^{\dagger}$} & \multirow{2}{*}{ CASRN } & \multicolumn{2}{|c|}{ Ingestion (pCi/L) } \\
\cline { 3 - 4 } & & $10^{-4}$ & $10^{-6}$ \\
\hline Zr-97 & $014928-30-4$ & $1.5 \mathrm{E}+03$ & $1.5 \mathrm{E}+01$ \\
\hline
\end{tabular}

Note:

$\dagger$ Isotopes designated with an " $\mathrm{m}$ " indicate that the isotope is metastable; isotopes designated with $a$ " $+D$ " indicate that the radionuclide slope factors include contributions from daughter products. 
Table 3.2c. Risk-based Preliminary Remediation Goals for nonradionuclides in soil (industrial scenario)

\begin{tabular}{|c|c|c|c|c|c|c|c|c|c|c|c|c|c|c|c|c|c|}
\hline \multirow[t]{2}{*}{ Chemical } & \multirow[t]{2}{*}{ CASRN } & \multicolumn{4}{|c|}{ Ingestion (mg/kg) } & \multicolumn{4}{|c|}{ Inhalation (mg/kg) } & \multicolumn{4}{|c|}{ Dermal (mg/kg) } & \multicolumn{4}{|c|}{$\begin{array}{c}\text { Ingestion + Inhalation } \\
+ \text { Dermal (mg/kg) } \\
\end{array}$} \\
\hline & & $10^{-4}$ & $10^{4}$ & $H Q=1$ & $\mathrm{HQ}=0.1$ & $10^{-4}$ & $10^{6}$ & $H Q=1$ & $\mathrm{HQ}=0.1$ & $10^{-4}$ & $10^{5}$ & $H Q=1$ & $\mathrm{HQ}=0.1$ & $10^{-4}$ & $10^{4}$ & $\mathrm{HI}=1$ & $\mathrm{HI}=0 . \mathrm{I}$ \\
\hline ALAR & $001596-84-5$ & & & $3.1 \mathrm{E}+05$ & $3.1 E+04$ & & & & & & & $2.4 E+05$ & $2.4 E+04$ & & & $1.4 \mathrm{E}+05$ & $1.4 \mathrm{E}+04$ \\
\hline Acenaphthene & $000083-32-9$ & & & $1.2 \mathrm{E}+05$ & $1.2 E+04$ & & & & & & & $6.0 \mathrm{E}+04$ & $6.0 E+03$ & & & $4.0 \mathrm{E}+04$ & $4.0 \mathrm{E}+03$ \\
\hline Acenaphthylene & $000208-96-8$ & & & & & & & & & & & & & & & & \\
\hline Acephate & $030560-19-1$ & $6.6 \mathrm{E}+04$ & $6.6 \mathrm{E}+02$ & $8.2 \mathrm{E}+03$ & $8.2 E+02$ & & & & & $5.2 \mathrm{E}+04$ & $5.2 \mathrm{E}+02$ & $6.5 \mathrm{E}+03$ & $6.5 \mathrm{E}+02$ & $2.9 \mathrm{E}+04$ & $2.9 \mathrm{E}+02$ & $3.6 \mathrm{E}+03$ & $3.6 \mathrm{E}+02$ \\
\hline Acetaidehyde & $000075-07-0$ & & & & & $1.1 \mathrm{E}+04$ & $1.1 \mathrm{E}+02$ & $7.9 \mathrm{E}+02$ & $7.9 \mathrm{E}+01$ & & & & & & & & \\
\hline Acetochlor & $034256-82-1$ & & & 4.1E+04 & $4.1 \mathrm{E}+03$ & & & & & & & $3.2 \mathrm{E}+04$ & $3.2 \mathrm{E}+03$ & & & $1.8 \mathrm{E}+04$ & $1.8 \mathrm{E}+03$ \\
\hline Acetone & $000067-64-1$ & & & $2.0 \mathrm{E}+05$ & $2.0 \mathrm{E}+04$ & & & & & & & $2.7 E+05$ & $2.7 \mathrm{E}+04$ & & & $1.2 \mathrm{E}+05$ & $1.2 \mathrm{E}+04$ \\
\hline Acetone Cyanohydrin & $000075-86-5$ & & & $1.6 \mathrm{E}+03$ & $1.6 \mathrm{E}+02$ & & & $1.0 \mathrm{E}+06$ & $1.0 \mathrm{E}+06$ & & & $1.3 \mathrm{E}+03$ & $1.3 \mathrm{E}+02$ & & & $7.2 \mathrm{E}+02$ & $7.2 E+01$ \\
\hline Acetonitrile & $000075-05-8$ & & & $1.2 \mathrm{E}+04$ & $1.2 \mathrm{E}+03$ & & & $1.0 \mathrm{E}+06$ & $1.0 \mathrm{E}+06$ & & & $1.6 \mathrm{E}+04$ & $1.6 \mathrm{E}+03$ & & & $6.9 \mathrm{E}+03$ & $6.9 \mathrm{E}+02$ \\
\hline Acetophenone $e^{(3)}$ & $000098-86-2$ & & & $2.0 \mathrm{E}+05$ & $2.0 \mathrm{E}+04$ & & & $1.3 \mathrm{E}+05$ & $1.3 \mathrm{E}+04$ & & & $2.6 \mathrm{E}+05$ & $2.6 \mathrm{E}+04$ & & & $6.0 \mathrm{E}+04$ & $6.0 \mathrm{E}+03$ \\
\hline Acrolein ${ }^{(6)}$ & $000107-02-8$ & & & $4.1 \mathrm{E}+04$ & $4.1 \mathrm{E}+03$ & & & $3.9 \mathrm{E}-01$ & $3.9 \mathrm{E}-02$ & & & $5.2 \mathrm{E}+04$ & $5.2 \mathrm{E}+03$ & & & $3.9 \mathrm{E}-01$ & $3.9 \mathrm{E}-02$ \\
\hline Acrylamide & $000079-06-1$ & $1.3 \mathrm{E}+02$ & $1.3 \mathrm{E}+00$ & $4.1 \mathrm{E}+02$ & $4.1 \mathrm{E}+01$ & $1.0 \mathrm{E}+06$ & $1.4 \mathrm{E}+04$ & & & $1.0 \mathrm{E}+02$ & $1.0 \mathrm{E}+00$ & $3.2 \mathrm{E}+02$ & $3.2 \mathrm{E}+01$ & $5.6 \mathrm{E}+01$ & $5.6 \mathrm{E}-01$ & $1.8 \mathrm{E}+02$ & $1.8 \mathrm{E}+01$ \\
\hline Acrylic Acid & $000079-10-7$ & & & $1.0 \mathrm{E}+06$ & $1.0 \mathrm{E}+05$ & & & $1.0 \mathrm{E}+06$ & $6.3 \mathrm{E}+05$ & & & $8.1 \mathrm{E}+05$ & $8.1 \mathrm{E}+04$ & & & $4.2 \mathrm{E}+05$ & $4.2 \mathrm{E}+04$ \\
\hline Acrylonitrile & $000107-13-1$ & $1.1 \mathrm{E}+03$ & $1.1 \mathrm{E}+01$ & $2.0 \mathrm{E}+03$ & $2.0 \mathrm{E}+02$ & $2.2 \mathrm{E}+01$ & 2.2E-01 & $1.1 \mathrm{E}+01$ & $1.1 \mathrm{E}+00$ & $1.3 E+03$ & $1.3 E+01$ & $2.6 \mathrm{E}+03$ & $2.6 \mathrm{E}+02$ & $2.1 \mathrm{E}+01$ & 2.1E-01 & $1.1 \mathrm{E}+01$ & $1.1 \mathrm{E}+00$ \\
\hline Alachlor & $015972-60-8$ & $7.2 \mathrm{E}+03$ & $7.2 \mathrm{E}+01$ & $2.0 \mathrm{E}+04$ & $2.0 \mathrm{E}+03$ & & & & & $5.7 \mathrm{E}+03$ & $5.7 \mathrm{E}+01$ & $1.6 \mathrm{E}+04$ & $1.6 \mathrm{E}+03$ & $3.2 \mathrm{E}+03$ & $3.2 \mathrm{E}+01$ & $9.0 \mathrm{E}+03$ & $9.0 \mathrm{E}+02$ \\
\hline Aldicarb & $000116 \cdot 06-3$ & & & $2.0 \mathrm{E}+03$ & $2.0 \mathrm{E}+02$ & & & & & & & $1.6 \mathrm{E}+03$ & $1.6 \mathrm{E}+02$ & & & $9.0 \mathrm{E}+02$ & $9.0 \mathrm{E}+01$ \\
\hline Aldicarb Sulfone & $001646-88-4$ & & & $2.0 \mathrm{E}+03$ & $2.0 \mathrm{E}+02$ & & & & & & & $1.6 \mathrm{E}+03$ & $1.6 \mathrm{E}+02$ & & & $9.0 \mathrm{E}+02$ & $9.0 E+01$ \\
\hline Aldrin & $000309-00-2$ & $3.4 E+01$ & $3.4 \mathrm{E}-01$ & $6.1 \mathrm{E}+01$ & $6.1 \mathrm{E}+00$ & $3.6 \mathrm{E}+05$ & $3.6 \mathrm{E}+03$ & & & $2.7 \mathrm{E}+01$ & 2.7E-01 & $4.9 \mathrm{E}+01$ & $4.9 \mathrm{E}+00$ & $1.5 \mathrm{E}+01$ & $1.5 \mathrm{E}-0 \mathrm{t}$ & $2.7 \mathrm{E}+01$ & $2.7 E+00$ \\
\hline Ally & $074223-64-6$ & & & $5.1 \mathrm{E}+05$ & $5.1 \mathrm{E}+04$ & & & & & & & $4.0 \mathrm{E}+05$ & $4.0 \mathrm{E}+04$ & & & $2.3 \mathrm{E}+05$ & $2.3 \mathrm{E}+04$ \\
\hline Allyl Alcohol & $000107-18-6$ & & & $1.0 \mathrm{E}+04$ & $1.0 \mathrm{E}+03$ & & & & & & & $1.3 E+04$ & $1.3 \mathrm{E}+03$ & & & $5.7 \mathrm{E}+03$ & $5.7 \mathrm{E}+02$ \\
\hline Allyl Chloride $e^{(3)}$ & $000107-05-1$ & & & $1.0 E+05$ & $1.0 \mathrm{E}+04$ & & & $1.4 \mathrm{E}+01$ & $1.4 \mathrm{E}+00$ & & & $1.3 \mathrm{E}+05$ & $1.3 \mathrm{E}+04$ & & & $1.4 \mathrm{E}+01$ & $1.4 \mathrm{E}+00$ \\
\hline Aluminum & $007429-90-5$ & & & & & & & & & & & & & & & & \\
\hline Aluminum Phosphide & $020859-73-8$ & & & $8.2 E+02$ & $8.2 \mathrm{E}+01$ & & & & & & & $2.6 \mathrm{E}+03$ & $2.6 \mathrm{E}+02$ & & & $6.2 \mathrm{E}+02$ & $6.2 \mathrm{E}+01$ \\
\hline
\end{tabular}


Table 3.2c. (continued)

\begin{tabular}{|c|c|c|c|c|c|c|c|c|c|c|c|c|c|c|c|c|c|}
\hline \multirow{2}{*}{ Chemical } & \multirow{2}{*}{ CASRN } & \multicolumn{4}{|c|}{ Ingestion (mg/kg) } & \multicolumn{4}{|c|}{ Inhalation $(\mathrm{mg} / \mathrm{kg})^{\bullet v}$} & \multicolumn{4}{|c|}{ Dermal (mg/kg) } & \multicolumn{4}{|c|}{$\begin{array}{l}\text { Ingestion + Inhalation } \\
+ \text { Dermal }(\mathrm{mg} / \mathrm{kg})^{+\vee}\end{array}$} \\
\hline & & $10^{-4}$ & $10^{4}$ & $\mathrm{HQ}=1$ & $\mathrm{HQ}=0.1$ & $10^{-4}$ & $10^{5}$ & $H Q=1$ & $\mathrm{HQ}=0.1$ & $10^{-4}$ & $10^{4}$ & $H Q=1$ & $\mathrm{HQ}=0.1$ & $10^{-1}$ & $10^{4}$ & $H I=1$ & $\mathrm{HI}=0.1$ \\
\hline Ametryn & $000834-12-8$ & & & $1.8 \mathrm{E}+04$ & $1.8 \mathrm{E}+03$ & & & & & & & $1.5 \mathrm{E}+04$ & $1.5 \mathrm{E}+03$ & & & $8.1 E+03$ & $8.1 \mathrm{E}+02$ \\
\hline Aminophenol, m- & $000591-27-5$ & & & $1.4 \mathrm{E}+05$ & $1.4 \mathrm{E}+04$ & & & & & & & $1.1 \mathrm{E}+05$ & $1.1 \mathrm{E}+04$ & & & $6.3 \mathrm{E}+04$ & $6.3 \mathrm{E}+03$ \\
\hline Amitraz & $033089-61-1$ & & & $5.1 \mathrm{E}+03$ & $5.1 \mathrm{E}+02$ & & & & & & & $4.0 \mathrm{E}+03$ & $4.0 \mathrm{E}+02$ & & & $2.3 \mathrm{E}+03$ & $2.3 \mathrm{E}+02$ \\
\hline Ammonia & $007664-41-7$ & & & & & & & $1.0 \mathrm{E}+06$ & $1.0 \mathrm{E}+06$ & & & & & & & & \\
\hline Ammonium Sulfamate & 007773.06 .0 & & & 4.1E+05 & $4.1 E+04$ & & & & & & & $1.0 \mathrm{E}+06$ & $1.3 \mathrm{E}+05$ & & & $3.1 \mathrm{E}+05$ & $3.1 \mathrm{E}+04$ \\
\hline Aniline & $000062-53-3$ & $1.0 \mathrm{E}+05$ & $1.0 \mathrm{E}+03$ & & & & & $1.0 \mathrm{E}+06$ & $6.3 \mathrm{E}+05$ & $7.9 \mathrm{E}+04$ & $7.9 E+02$ & & & $4.4 \mathrm{E}+04$ & $4.4 \mathrm{E}+02$ & & \\
\hline Anthracene & $000120-12-7$ & & & $6.1 \mathrm{E}+05$ & $6.1 \mathrm{E}+04$ & & & & & & & 7.4E+05 & $7.4 \mathrm{E}+04$ & & & $3.3 \mathrm{E}+05$ & $3.3 \mathrm{E}+04$ \\
\hline Antimony Pentoxide & $001314-60-9$ & & & $1.0 \mathrm{E}+03$ & $1.0 \mathrm{E}+02$ & & & & & & & $3.2 \mathrm{E}+03$ & $3.2 \mathrm{E}+02$ & & & $7.8 \mathrm{E}+02$ & $7.8 \mathrm{E}+01$ \\
\hline $\begin{array}{l}\text { Antimony Potassium } \\
\text { Tartrate }\end{array}$ & $000304-61-0$ & & & $1.8 \mathrm{E}+03$ & $1.8 \mathrm{E}+02$ & & & & & & & $5.8 \mathrm{E}+03$ & $5.8 \mathrm{E}+02$ & & & $1.4 \mathrm{E}+03$ & $1.4 \mathrm{E}+02$ \\
\hline Antimony Tetroxide & $001332-81-6$ & & & $8.2 \mathrm{E}+02$ & $8.2 \mathrm{E}+01$ & & & & & & & $2.6 \mathrm{E}+03$ & $2.6 \mathrm{E}+02$ & & & $6.2 \mathrm{E}+02$ & $6.2 \mathrm{E}+01$ \\
\hline Antimony Trioxide & $001309-64-4$ & & & $8.2 \mathrm{E}+02$ & $8.2 \mathrm{E}+01$ & & & $1.0 \mathrm{E}+06$ & $1.3 \mathrm{E}+05$ & & & $2.6 \mathrm{E}+03$ & $2.6 \mathrm{E}+02$ & & & $6.2 \mathrm{E}+02$ & $6.2 \mathrm{E}+01$ \\
\hline Apollo & $074115-24-5$ & & & $2.7 \mathrm{E}+04$ & $2.7 \mathrm{E}+03$ & & & & & & & $2.1 \mathrm{E}+04$ & $2.1 E+03$ & & & $1.2 \mathrm{E}+04$ & $1.2 \mathrm{E}+03$ \\
\hline Aramite & $000140-57-8$ & $2.3 \mathrm{E}+04$ & $2.3 E+02$ & $1.0 \mathrm{E}+05$ & $1.0 \mathrm{E}+04$ & $1.0 \mathrm{E}+06$ & $1.0 \mathrm{E}+06$ & & & $1.8 \mathrm{E}+04$ & $1.8 \mathrm{E}+02$ & $8.1 \mathrm{E}+04$ & $8.1 E+03$ & $1.0 \mathrm{E}+04$ & $1.0 E+02$ & $4.5 \mathrm{E}+04$ & $4.5 \mathrm{E}+03$ \\
\hline Aroclor 1016 & $012674-11-2$ & & & $1.4 \mathrm{E}+02$ & $1.4 \mathrm{E}+01$ & & & & & & & $3.4 \mathrm{E}+01$ & $3.4 \mathrm{E}+00$ & & & $2.7 \mathrm{E}+01$ & $2.7 E+00$ \\
\hline Aroclor $1221^{(1)}$ & $011104-28-2$ & 7.4E+01 & $7.4 \mathrm{E}-01$ & & & & & & & $1.8 \mathrm{E}+01$ & $1.8 \mathrm{E}-01$ & & & $1.4 \mathrm{E}+01$ & $1.4 \mathrm{E}-01$ & & \\
\hline Aroclor $1232^{(1)}$ & $011141-16.5$ & $7.4 \mathrm{E}+01$ & $7.4 \mathrm{E}-01$ & & & & & & & $1.8 \mathrm{E}+01$ & $1.8 \mathrm{E}-01$ & & & $1.4 \mathrm{E}+01$ & $1.4 \mathrm{E}-01$ & & \\
\hline Aroclor $1242^{(1)}$ & $053469-21-9$ & $7.4 \mathrm{E}+01$ & $7.4 \mathrm{E}-01$ & & & & & & & $1.8 \mathrm{E}+01$ & $1.8 \mathrm{E}-01$ & & & $1.4 \mathrm{E}+01$ & $1.4 \mathrm{E}-01$ & & \\
\hline Aroclor $1248^{(1)}$ & $012672-29-6$ & $7.4 \mathrm{E}+01$ & $7.4 \mathrm{E}-01$ & & & & & & & $1.8 \mathrm{E}+01$ & $1.8 \mathrm{E}-01$ & & & $1.4 \mathrm{E}+01$ & $1.4 \mathrm{E}-01$ & & \\
\hline Aroclor $1260^{(1)}$ & $011096-82-5$ & $7.4 E+01$ & $7.4 \mathrm{E}-01$ & & & & & & & $1.8 \mathrm{E}+01$ & $1.8 \mathrm{E}-01$ & & & $1.4 \mathrm{E}+01$ & $1.4 \mathrm{E}-01$ & & \\
\hline
\end{tabular}

Nonradionuclides/soil/industrial

Page 2 of 32 
Table 3.2c. (continued)

\begin{tabular}{|c|c|c|c|c|c|c|c|c|c|c|c|c|c|c|c|c|c|}
\hline \multirow[t]{2}{*}{ Chemical } & \multirow[t]{2}{*}{ CASRN } & \multicolumn{4}{|c|}{$\begin{array}{c}\text { Ingestion (mg/kg) } \\
.\end{array}$} & \multicolumn{4}{|c|}{ Inhalation (mg/kg) } & \multicolumn{4}{|c|}{ Dermal (mg/kg) } & \multicolumn{4}{|c|}{$\begin{array}{l}\text { Ingestion + Inhalation } \\
+ \text { Dermal (mg/kg) }\end{array}$} \\
\hline & & $10^{-1}$ & $10^{5}$ & $H Q=1$ & $\mathrm{HQ}=0.1$ & $10^{-4}$ & $10^{4}$ & $H Q=1$ & $\mathrm{HQ}=0.1$ & $10^{-4}$ & $10^{4}$ & $H Q=1$ & $\mathrm{HQ}=0.1$ & $20^{-4}$ & $10^{6}$ & MI=I & $\mathrm{HI}=0.1$ \\
\hline Arsenic Salts & NA & & & & & & & & & & & & & & & & \\
\hline Arsenic, Inorganic & $007440-38-2$ & $3.8 E+02$ & $3.8 \mathrm{E}+00$ & $6.1 \mathrm{E}+02$ & $6.1 \mathrm{E}+01$ & $1.2 \mathrm{E}+05$ & $1.2 \mathrm{E}+03$ & & & $2.5 E+03$ & $2.5 \mathrm{E}+01$ & $4.0 \mathrm{E}+03$ & $4.0 \mathrm{E}+02$ & $3.3 \mathrm{E}+02$ & $3.3 E+00$ & $5.3 \mathrm{E}+02$ & $5.3 \mathrm{E}+01$ \\
\hline Arsine & $007784-42-1$ & & & & & & & $3.1 E+05$ & $3.1 E+04$ & & & & & & & & \\
\hline Asbestos & $001332-21-4$ & & & & & & & & & & & & & & & & \\
\hline Assure & $076578-14-8$ & & & $1.8 \mathrm{E}+04$ & $1.8 \mathrm{E}+03$ & & & & & & & $1.5 \mathrm{E}+04$ & $1.5 \mathrm{E}+03$ & & & $8.1 E+03$ & 8.1E+02 \\
\hline Asulam & $003337-71-1$ & & & $1.0 E+05$ & $1.0 \mathrm{E}+04$ & & & & & & & $8.1 E+04$ & $8.1 E+03$ & & & $4.5 \mathrm{E}+04$ & $4.5 \mathrm{E}+03$ \\
\hline Atrazine & $001912-24-9$ & $2.6 \mathrm{E}+03$ & $2.6 \mathrm{E}+01$ & $7.2 \mathrm{E}+04$ & $7.2 \mathrm{E}+03$ & & & & & $2.0 \mathrm{E}+03$ & $2.0 \mathrm{E}+01$ & $5.7 E+04$ & $5.7 \mathrm{E}+03$ & $1.1 E+03$ & $1.1 \mathrm{E}+01$ & $3.2 \mathrm{E}+04$ & $3.2 \mathrm{E}+03$ \\
\hline Avermectin Bl & $065195-55-3$ & & & $8.2 \mathrm{E}+02$ & $8.2 \mathrm{E}+01$ & & & & & & & $6.5 \mathrm{E}+02$ & $6.5 \mathrm{E}+01$ & & & $3.6 \mathrm{E}+02$ & $3.6 \mathrm{E}+01$ \\
\hline Azobenzene & $000103-33-3$ & $5.2 \mathrm{E}+03$ & $5.2 \mathrm{E}+01$ & & & $1.0 \mathrm{E}+06$ & $5.6 \mathrm{E}+05$ & & & $4.1 \mathrm{E}+03$ & $4.1 \mathrm{E}+01$ & & & $2.3 \mathrm{E}+03$ & $2.3 \mathrm{E}+01$ & $\cdot$ & \\
\hline Barium & $007440-39.3$ & & & $1.4 \mathrm{E}+05$ & $1.4 \mathrm{E}+04$ & & & $1.0 \mathrm{E}+06$ & $3.1 \mathrm{E}+05$ & & & $1.6 \mathrm{E}+05$ & $1.6 \mathrm{E}+04$ & & & $7.3 \mathrm{E}+04$ & 7.3E+03 \\
\hline Barium Cyanide & $000542-62-1$ & & & $2.0 \mathrm{E}+05$ & $2.0 \mathrm{E}+04$ & & & & & & & $6.5 \mathrm{E}+05$ & $6.5 \mathrm{E}+04$ & & & $1.6 \mathrm{E}+05$ & $1.6 \mathrm{E}+04$ \\
\hline Baygon & $000114-26-1$ & & & $8.2 \mathrm{E}+03$ & $8.2 \mathrm{E}+02$ & & & & & & & $6.5 \mathrm{E}+03$ & $6.5 \mathrm{E}+02$ & & & $3.6 \mathrm{E}+03$ & $3.6 \mathrm{E}+02$ \\
\hline Bayleton & $043121-43-3$ & & & $6.1 \mathrm{E}+04$ & $6.1 \mathrm{E}+03$ & & & & & & & $4.9 \mathrm{E}+04$ & $4.9 \mathrm{E}+03$ & & & $2.7 \mathrm{E}+04$ & $2.7 \mathrm{E}+03$ \\
\hline Baythroid & 068359-37-5 & & & $5.1 \mathrm{E}+04$ & $5.1 \mathrm{E}+03$ & & & & & & & $4.0 \mathrm{E}+04$ & $4.0 \mathrm{E}+03$ & & & $2.3 \mathrm{E}+04$ & $2.3 \mathrm{E}+03$ \\
\hline Benefin & $001861-40.1$ & & & $6.1 \mathrm{E}+05$ & $6.1 \mathrm{E}+04$ & & & & & & & $4.9 \mathrm{E}+05$ & $4.9 \mathrm{E}+04$ & & & $2.7 \mathrm{E}+05$ & $2.7 E+04$ \\
\hline Benomyl & $017804-35-2$ & & & $1.0 \mathrm{E}+05$ & $1.0 \mathrm{E}+04$ & & & & & & & $8.1 \mathrm{E}+04$ & $8.1 E+03$ & & & $4.5 \mathrm{E}+04$ & $4.5 \mathrm{E}+03$ \\
\hline Bentazon & $025057-89-0$ & & & $5.1 \mathrm{E}+03$ & $5.1 \mathrm{E}+02$ & & & & & & & $4.0 \mathrm{E}+03$ & $4.0 \mathrm{E}+02$ & & & $2.3 E+03$ & $2.3 E+02$ \\
\hline Benz[a]anthracene ${ }^{(z)}$ & $000056-55-3$ & $7.8 \mathrm{E}+02$ & $7.8 \mathrm{E}+00$ & & & $1.0 \mathrm{E}+06$ & $2.0 \mathrm{E}+05$ & & & $3.8 \mathrm{E}+02$ & $3.8 \mathrm{E}+00$ & & & $2.6 \mathrm{E}+02$ & $2.6 \mathrm{E}+00$ & & \\
\hline Benzaldehyde & $000100-52-7$ & & & $2.0 \mathrm{E}+05$ & $2.0 \mathrm{E}+04$ & & & & & & & $2.6 \mathrm{E}+05$ & $2.6 \mathrm{E}+04$ & & & $1.1 E+05$ & $1.1 E+04$ \\
\hline Benzene & $000071-43-2$ & $2.0 \mathrm{E}+04$ & $2.0 \mathrm{E}+02$ & & & $5.0 \mathrm{E}+02$ & $9.3 \mathrm{E}+00$ & & & $3.0 \mathrm{E}+04$ & $3.0 \mathrm{E}+02$ & & & $5.0 \mathrm{E}+02$ & $8.6 \mathrm{E}+00$ & & \\
\hline Benzene Hexachloride & NA & & & & & & & & & & & & & & & & \\
\hline Benzene, Ethyldimethyl & NA & & & & & & & & & & & & & & & & \\
\hline Benzene, Ethylmethyl & NA & & & & & & & & & & & & & & & & \\
\hline
\end{tabular}


Table 3.2c. (continued)

\begin{tabular}{|c|c|c|c|c|c|c|c|c|c|c|c|c|c|c|c|c|c|}
\hline \multirow[t]{2}{*}{ Chemical } & \multirow[t]{2}{*}{ CASRN } & \multicolumn{4}{|c|}{ Ingestion (mg/kg) } & \multicolumn{4}{|c|}{ Inhalation (mg/kg) } & \multicolumn{4}{|c|}{ Dermal (mg/kg) } & \multicolumn{4}{|c|}{$\begin{array}{l}\text { Ingestion + Inhalation } \\
+ \text { Dermal }(\mathrm{mg} / \mathrm{kg})^{\dagger \cdot \bullet}\end{array}$} \\
\hline & & $10^{-4}$ & $10^{6}$ & $H Q=1$ & $H Q=0.1$ & $10^{-4}$ & $10^{4}$ & $H Q=1$ & $\mathrm{HQ}=0.1$ & $10^{-4}$ & $10^{5}$ & $H Q=1$ & $\mathrm{HQ}=0.1$ & $10^{-4}$ & $10^{6}$ & $\mathrm{HI}=1$ & $\mathrm{HI}=0.1$ \\
\hline Benzene, Methylpropenyl & NA & & & & & & & & & & & & & & & & \\
\hline Benzene, Methylpropyl & NA & & & & & & & & & & & & & & & & \\
\hline Benzene, Trimethyl & $025551-13-7$ & & & & & & & & & & & & & & & & \\
\hline Benzenethiol & $000108-98-5$ & & & $2.0 \mathrm{E}+01$ & $2.0 \mathrm{E}+00$ & & & & & & & $1.6 \mathrm{E}+01$ & $1.6 \mathrm{E}+00$ & & & $9.0 \mathrm{E}+00$ & $9.0 \mathrm{E}-01$ \\
\hline Benzidine & $000092-87-5$ & $2.5 \mathrm{E}+00$ & $2.5 \mathrm{E}-02$ & $6.1 E+03$ & $6.1 \mathrm{E}+02$ & $2.7 \mathrm{E}+04$ & $2.7 \mathrm{E}+02$ & & & $3.1 \mathrm{E}+00$ & $3.1 \mathrm{E}-02$ & $7.8 \mathrm{E}+03$ & $7.8 \mathrm{E}+02$ & $1.4 \mathrm{E}+00$ & $1.4 \mathrm{E}-02$ & $3.4 \mathrm{E}+03$ & $3.4 \mathrm{E}+02$ \\
\hline Benzo[a]pyrene $e^{(2)}$ & $000050-32-8$ & $7.8 \mathrm{E}+01$ & $7.8 \mathrm{E}-01$ & & & $1.0 \mathrm{E}+06$ & $2.0 \mathrm{E}+04$ & & & $3.9 \mathrm{E}+01$ & $3.9 \mathrm{E}-01$ & & & $2.6 \mathrm{E}+01$ & $2.6 \mathrm{E}-01$ & & \\
\hline Benzo[b]fluoranthene $e^{(2)}$ & $000205-99-2$ & $7.8 \mathrm{E}+02$ & $7.8 \mathrm{E}+00$ & & & $1.0 \mathrm{E}+06$ & $2.0 \mathrm{E}+05$ & & & $3.8 \mathrm{E}+02$ & $3.8 \mathrm{E}+00$ & & & $2.6 \mathrm{E}+02$ & $2.6 \mathrm{E}+00$ & & \\
\hline Benzo $[\mathrm{g}, \mathrm{h}, \mathrm{i}]$ perylene & $000191-24-2$ & & & & & & & & & & & & & & & & \\
\hline Benzo[k]fiuoranthene $e^{(2)}$ & $000207-08-9$ & $7.8 \mathrm{E}+03$ & $7.8 \mathrm{E}+01$ & & & $1.0 \mathrm{E}+06$ & $1.0 \mathrm{E}+06$ & & & $3.8 \mathrm{E}+03$ & $3.8 \mathrm{E}+01$ & & & $2.6 \mathrm{E}+03$ & $2.6 \mathrm{E}+01$ & & \\
\hline Benzoic Acid & $000065-85-0$ & & & $1.0 \mathrm{E}+06$ & $8.2 \mathrm{E}+05$ & & & & & & & $1.0 \mathrm{E}+06$ & $1.0 \mathrm{E}+06$ & & & $1.0 \mathrm{E}+06$ & $5.0 \mathrm{E}+05$ \\
\hline Benzotrichloride & $000098-07-7$ & $4.4 \mathrm{E}+01$ & 4.4E-01 & & & & & & & $3.5 \mathrm{E}+01$ & $3.5 \mathrm{E}-01$ & & & $1.9 \mathrm{E}+01$ & $1.9 \mathrm{E}-01$ & & \\
\hline Benzyl Alcohol & $000100-51-6$ & & & $6.1 E+05$ & $6.1 \mathrm{E}+04$ & & . & & & & & $6.4 \mathrm{E}+05$ & $6.4 \mathrm{E}+04$ & & & $3.1 \mathrm{E}+05$ & $3.1 \mathrm{E}+04$ \\
\hline Benzyl Chloride & $000100-44-7$ & $3.4 \mathrm{E}+03$ & $3.4 \mathrm{E}+01$ & & & & & & & $4.3 \mathrm{E}+03$ & $4.3 \mathrm{E}+01$ & & & $1.9 \mathrm{E}+03$ & $1.9 \mathrm{E}+01$ & & \\
\hline Beryllium & $007440-41-7$ & $1.3 \mathrm{E}+02$ & $1.3 \mathrm{E}+00$ & $1.0 \mathrm{E}+04$ & $1.0 \mathrm{E}+03$ & $7.3 \mathrm{E}+05$ & $7.3 \mathrm{E}+03$ & & & $2.1 \mathrm{E}+01$ & 2.1E-01 & $1.6 \mathrm{E}+03$ & $1.6 \mathrm{E}+02$ & $1.8 \mathrm{E}+01$ & $1.8 \mathrm{E}-01$ & $1.4 \mathrm{E}+03$ & $1.4 \mathrm{E}+02$ \\
\hline Bidrin & $000141-66-2$ & & & $2.0 \mathrm{E}+02$ & $2.0 \mathrm{E}+01$ & & & & & & & $1.6 \mathrm{E}+02$ & $1.6 \mathrm{E}+01$ & & & $9.0 \mathrm{E}+01$ & $9.0 \mathrm{E}+00$ \\
\hline Biphenthrin & $082657-04-3$ & & & $3.1 \mathrm{E}+04$ & $3.1 \mathrm{E}+03$ & & & & & & & $2.4 \mathrm{E}+04$ & $2.4 \mathrm{E}+03$ & & & $1.4 \mathrm{E}+04$ & $1.4 \mathrm{E}+03$ \\
\hline Biphenyl, 1,1'- & $000092-52-4$ & & & $1.0 \mathrm{E}+05$ & $1.0 \mathrm{E}+04$ & & & & & & & $8.1 \mathrm{E}+04$ & $8.1 E+03$ & & & $4.5 E+04$ & $4.5 \mathrm{E}+03$ \\
\hline $\begin{array}{l}\text { Bis(2-chloro-1- } \\
\text { methylethyl)ether } \\
\text { (Technical) }\end{array}$ & $000108-60-1$ & $8.2 \mathrm{E}+03$ & $8.2 \mathrm{E}+01$ & & & $3.5 \mathrm{E}+02$ & $5.7 \mathrm{E}+01$ & & & $1.0 \mathrm{E}+04$ & $1.0 \mathrm{E}+02$ & & & $3.5 \mathrm{E}+02$ & . $2.5 \mathrm{E}+01$ & & \\
\hline $\begin{array}{l}\text { Bis(2-chloro- } \\
\text { ethoxy)methane }\end{array}$ & $000111-91-1$ & & & & & & & & & & & & & & & & \\
\hline Bis(2-chloroethyl)ether & $000111-44-4$ & $5.2 \mathrm{E}+02$ & $5.2 \mathrm{E}+00$ & & & $2.3 \mathrm{E}+02$ & $2.3 \mathrm{E}+00$ & & & $6.6 \mathrm{E}+02$ & $6.6 \mathrm{E}+00$ & & & $1.3 \mathrm{E}+02$ & $1.3 \mathrm{E}+00$ & & \\
\hline $\begin{array}{l}\text { Bis(2-chloroiso- } \\
\text { propyl)ether }\end{array}$ & $039638-32-9$ & & & $8.2 \mathrm{E}+04$ & $8.2 \mathrm{E}+03$ & & & & & & & $1.0 \mathrm{E}+05$ & $1.0 \mathrm{E}+04$ & & & $4.6 \mathrm{E}+04$ & $4.6 \mathrm{E}+03$ \\
\hline
\end{tabular}


Table 3.2c. (continued)

\begin{tabular}{|c|c|c|c|c|c|c|c|c|c|c|c|c|c|c|c|c|c|}
\hline \multirow{2}{*}{ Chemical } & \multirow[t]{2}{*}{ CASRN } & \multicolumn{4}{|c|}{ Ingestion (mg/kg) } & \multicolumn{4}{|c|}{ Inhalation (mg/kg) } & \multicolumn{4}{|c|}{ Dermal (mg/kg) } & \multicolumn{4}{|c|}{$\begin{array}{c}\text { Ingestion + Inhalation } \\
\text { + Dermal (mg/kg) }\end{array}$} \\
\hline & & $10^{-1}$ & $10^{5}$ & $\mathrm{HQ}=1$ & $\mathrm{HQ}=0.1$ & $10^{-4}$ & $10^{6}$ & $\mathrm{HQ}=\mathbf{I}$ & $\mathrm{HQ}=0.1$ & $10^{-4}$ & $10^{5}$ & $\mathrm{HQ}=1$ & $\mathrm{HQ}=0.1$ & $10^{-4}$ & $10^{4}$ & $\mathrm{HI}=\mathbf{1}$ & $\mathrm{HI}=0.1$ \\
\hline $\begin{array}{l}\text { Bis(2-ethyl- } \\
\text { hexyl)phthalate }\end{array}$ & $000117-81-7$ & $4.1 E+04$ & $4.1 E+02$ & $4.1 E+04$ & $4.1 E+03$ & & & & & $1.2 \mathrm{E}+04$ & $1.2 \mathrm{E}+02$ & $1.2 E+04$ & $1.2 \mathrm{E}+03$ & $9.4 \mathrm{E}+03$ & $9.4 E+01$ & $9.4 \mathrm{E}+03$ & $9.4 E+02$ \\
\hline Bis(chloromethyl)ether & $000542-88-1$ & $2.6 \mathrm{E}+00$ & $2.6 \mathrm{E}-02$ & & & 7.4E-01 & $7.4 \mathrm{E}-03$ & & & $3.3 \mathrm{E}+00$ & 3.3E-02 & & & $4.9 \mathrm{E}-01$ & 4.9E-03 & & \\
\hline Bisphenol A & $000080-05-7$ & & & $1.0 \mathrm{E}+05$ & $1.0 \mathrm{E}+04$ & & & & & & & $8.1 E+04$ & $8.1 E+03$ & & & $4.5 \mathrm{E}+04$ & $4.5 \mathrm{E}+03$ \\
\hline Boron And Borates Only & $007440-42-8$ & & & $1.8 \mathrm{E}+05$ & $1.8 \mathrm{E}+04$ & & & $1.0 \mathrm{E}+06$ & $1.0 \mathrm{E}+06$ & & & $1.0 E+06$ & $2.6 \mathrm{E}+05$ & & & $1.7 \mathrm{E}+05$ & $1.7 \mathrm{E}+04$ \\
\hline Boron Trifluoride & $007637-07-2$ & & & & & & & $1.0 \mathrm{E}+06$ & $4.4 E+05$ & & & & & & & & \\
\hline Bromochloromethane & $000074-97-5$ & & & & & & & & & & & & & & & & \\
\hline Bromodichloromethane & $000075-27-4$ & $9.2 \mathrm{E}+03$ & $9.2 \mathrm{E}+01$ & $4.1 E+04$ & $4.1 E+03$ & & & & & $1.4 E+04$ & $1.4 E+02$ & $6.3 \mathrm{E}+04$ & $6.3 \mathrm{E}+03$ & $5.6 \mathrm{E}+03$ & $5.6 \mathrm{E}+01$ & $2.5 \mathrm{E}+04$ & $2.5 \mathrm{E}+03$ \\
\hline Bromodiphenyl Ether, p- & $000101-55-3$ & & & & & & & & & & & & & & & & \\
\hline Bromoform & $000075-25-2$ & $7.2 \mathrm{E}+04$ & $7.2 \mathrm{E}+02$ & $4.1 \mathrm{E}+04$ & 4.1E+03 & $1.0 \mathrm{E}+06$ & $1.0 E+06$ & & & $6.9 E+04$ & $6.9 \mathrm{E}+02$ & $3.9 E+04$ & $3.9 E+03$ & $3.5 \mathrm{E}+04$ & $3.5 \mathrm{E}+02$ & $2.0 \mathrm{E}+04$ & $2.0 \mathrm{E}+03$ \\
\hline Bromomethane $e^{(6)}$ & 000074-83-9 & & & $2.9 \mathrm{E}+03$ & $2.9 \mathrm{E}+02$ & & & $2.4 \mathrm{E}+01$ & $2.4 \mathrm{E}+00$ & & & $3.6 \mathrm{E}+03$ & $3.6 \mathrm{E}+02$ & & & $2.4 \mathrm{E}+01$ & $2.4 \mathrm{E}+00$ \\
\hline Bromophos & 002104-96-3 & & & $1.0 \mathrm{E}+04$ & $1.0 \mathrm{E}+03$ & & & & & & & $8.1 E+03$ & $8.1 E+02$ & & & $4.5 \mathrm{E}+03$ & $4.5 \mathrm{E}+02$ \\
\hline Bromotrichloromethane & $000075-62-7$ & & & & & & & & & & & & & & & & \\
\hline Bromoxynil & $001689.84-5$ & & & 4.1E+04 & $4.1 \mathrm{E}+03$ & & & & & & & $3.2 \mathrm{E}+04$ & $3.2 \mathrm{E}+03$ & & & $1.8 \mathrm{E}+04$ & $1.8 \mathrm{E}+03$ \\
\hline Bromoxynil Octanoate & $001689-99-2$ & & & $4.1 E+04$ & $4.1 E+03$ & & & & & & & $3.2 \mathrm{E}+04$ & $3.2 E+03$ & & & $1.8 \mathrm{E}+04$ & $1.8 \mathrm{E}+03$ \\
\hline Butadiene, 1,3- & $000106-99-0$ & & & & & $4.8 \mathrm{E}+00$ & $4.8 \mathrm{E}-02$ & & & & & & & & & & \\
\hline Butanol, N- & $000071-36-3$ & & & $2.0 \mathrm{E}+05$ & $2.0 \mathrm{E}+04$ & & & & & & & $1.6 \mathrm{E}+05$ & $1.6 \mathrm{E}+04$ & & & $9.0 \mathrm{E}+04$ & $9.0 \mathrm{E}+03$ \\
\hline $\begin{array}{l}\text { Butanone-2, 4-chloro-4,4- } \\
\text { difluoro }\end{array}$ & $\mathrm{NA}$ & & & & & & & & & & & & & & & & \\
\hline Butyl Benzyl Phthlate & 000085-68-7 & & & $4.1 \mathrm{E}+05$ & $4.1 \mathrm{E}+04$ & & & & & & & $3.9 \mathrm{E}+05$ & $3.9 \mathrm{E}+04$ & & & $2.0 \mathrm{E}+05$ & $2.0 \mathrm{E}+04$ \\
\hline Butylate & 002008-41-5 & & & $1.0 \mathrm{E}+05$ & $1.0 \mathrm{E}+04$ & & & & & & & $8.1 E+04$ & $8.1 \mathrm{E}+03$ & & & $4.5 \mathrm{E}+04$ & $4.5 \mathrm{E}+03$ \\
\hline Butylchloride, t- & $000507-20-0$ & & & & & & & & & & & & & & & & \\
\hline $\begin{array}{l}\text { Butylyphthalyl } \\
\text { Butylglycolate }\end{array}$ & 000085-70-1 & & & $1.0 \mathrm{E}+06$ & $2.0 \mathrm{E}+05$ & & & & & & & $1.0 \mathrm{E}+06$ & $1.6 \mathrm{E}+05$ & & & $9.0 \mathrm{E}+05$ & $9.0 \mathrm{E}+04$ \\
\hline Cacodylic Acid & $000075-60-5$ & & & $6.1 \mathrm{E}+03$ & $6.1 \mathrm{E}+02$ & & & & & & & $4.9 \mathrm{E}+03$ & $4.9 \mathrm{E}+02$ & & & $2.7 E+03$ & $2.7 E+02$ \\
\hline
\end{tabular}

Nonradionuclides/soil/industrial

Page 5 of 32 


\begin{tabular}{|c|c|c|c|c|c|c|c|c|c|c|c|c|c|c|c|c|c|c|c|c|c|c|c|c|}
\hline \multirow{4}{*}{ 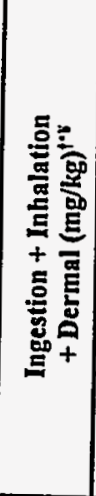 } & 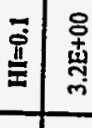 & 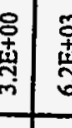 & 怘 & \begin{tabular}{l}
$\stackrel{+}{+}$ \\
萦 \\
\multirow{\sigma}{*}{}
\end{tabular} & 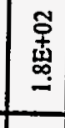 & 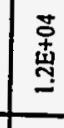 & 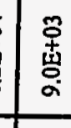 & & 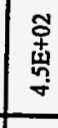 & \begin{tabular}{|c} 
草 \\
总 \\
n.
\end{tabular} & 罍 & 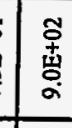 & \begin{tabular}{|l} 
学 \\
产 \\
व.
\end{tabular} & 茧 & 空 & & 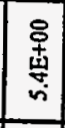 & & 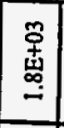 & 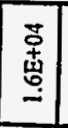 & & & & 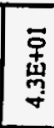 \\
\hline & 预 & 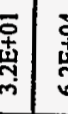 & 岽 & 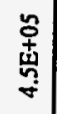 & 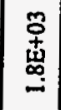 & 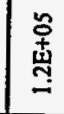 & 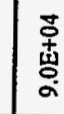 & & $\begin{array}{l}\stackrel{0}{0} \\
\stackrel{4}{4} \\
\stackrel{4}{*}\end{array}$ & 空 & 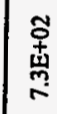 & 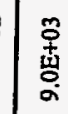 & 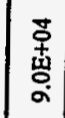 & 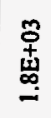 & $\begin{array}{l}\text { 莒 } \\
\text { 索 } \\
=\end{array}$ & & 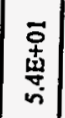 & & 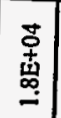 & 草 & & & & 恿 \\
\hline & $\stackrel{\dot{g}}{\mathrm{~g}}$ & & & & 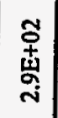 & 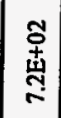 & & 草 & & & 罍 & & & & & 号 & 辛 & & & & & & & \\
\hline & 름 & & & & $\begin{array}{l}\text { 茣 } \\
\text { 䓌 } \\
\stackrel{\sim}{*}\end{array}$ & 草 & & 䔅 & & & \begin{tabular}{l}
$\underset{+}{4}$ \\
\multirow{4}{*}{} \\
\multirow{\sigma}{*}{}
\end{tabular} & & & & & 草 & 容 & & & & & & & \\
\hline \multirow{4}{*}{ 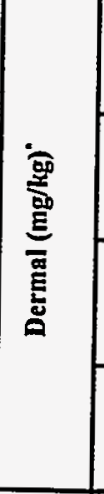 } & 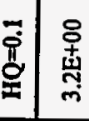 & 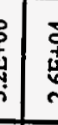 & 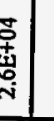 & 虽 & 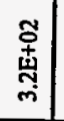 & 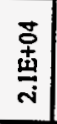 & 㤐 & & 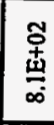 & 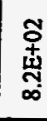 & 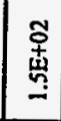 & 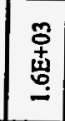 & 䓌 & 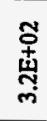 & 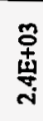 & & 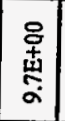 & & 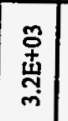 & 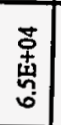 & & & & 密 \\
\hline & 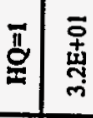 & 焉 & . & 突 & $\begin{array}{c}\stackrel{p}{9} \\
\stackrel{4}{4} \\
\end{array}$ & 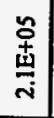 & 总 & & 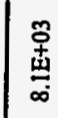 & 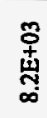 & 亳 & $\mid \begin{array}{l}\text { 葍 } \\
\text { 晋 }\end{array}$ & 惹 & 旁 & $\begin{array}{l}\stackrel{+}{0} \\
\text { 壱 } \\
\text { i }\end{array}$ & & 虽 & & 龺 & $\begin{array}{l}\text { 号 } \\
\text { 岕 } \\
\text {. }\end{array}$ & & & & 孛 \\
\hline & $\dot{\ddot{g}}$ & & & & 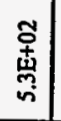 & 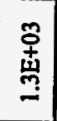 & & $\begin{array}{l}\text { 莒 } \\
\text { 尊 }\end{array}$ & & & 京 & & & & & 哭 & 总 & & & & & & & \\
\hline & : & & & & 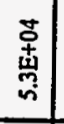 & 㠇 & & 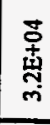 & & & 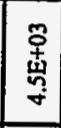 & & & & & 骞 & 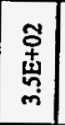 & & & & & & & \\
\hline \multirow{4}{*}{ 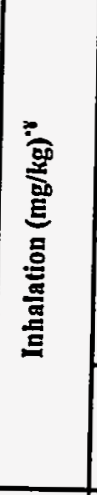 } & 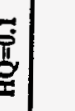 & & & & & & & & & 恿 & & & & & & & & & & & $\begin{array}{c}\text { 畐 } \\
\text { 界 }\end{array}$ & & $\begin{array}{l}\text { 古 } \\
\text { 莺 }\end{array}$ & $\begin{array}{l}\overline{\mathbf{q}} \\
\text { 孛 }\end{array}$ \\
\hline & $\stackrel{\pi}{\pi}$ & & & & & & & & & 亳 & & & & & & & & & & & $\begin{array}{l}\stackrel{0}{9} \\
\text { 索 } \\
-\end{array}$ & & 哭 & 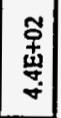 \\
\hline & 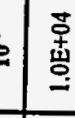 & & & & & & & & & & $\begin{array}{c}\stackrel{8}{+} \\
\stackrel{4}{4} \\
\text { s }\end{array}$ & & & & & & 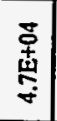 & & & & & & & \\
\hline & 草 & & & & & & & & & & 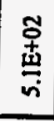 & & & & & & 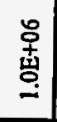 & & & & & & & \\
\hline \multirow{4}{*}{ 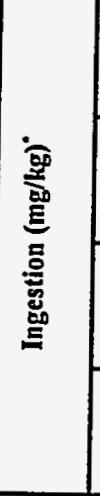 } & 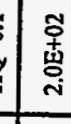 & 章 & & 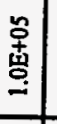 & 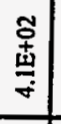 & 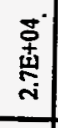 & $\begin{array}{l}\text { 草 } \\
\text { 兽 } \\
\end{array}$ & & 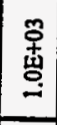 & $\begin{array}{l}\text { 員 } \\
\text { 总 } \\
\text { in }\end{array}$ & $\begin{array}{l}\text { 号 } \\
\text { 率 } \\
-\end{array}$ & 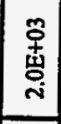 & 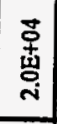 & $\begin{array}{l}\text { 号 } \\
\text { 學 } \\
\end{array}$ & 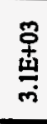 & & $\begin{array}{c}\overline{\mathbf{q}} \\
\underset{\mathrm{W}}{\mathrm{W}} \\
-\end{array}$ & & 总 & 蛄 & & & & 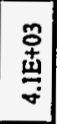 \\
\hline & $\begin{array}{l}\stackrel{0}{+} \\
\text { 畜 } \\
\text { }\end{array}$ & 尔 & 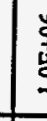 & 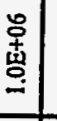 & 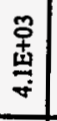 & 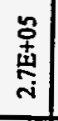 & $\begin{array}{l}\text { 呆 } \\
\text { 营 } \\
\text { N }\end{array}$ & & $\begin{array}{l}\text { 草 } \\
\text { 索 }\end{array}$ & 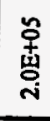 & 总 & $\begin{array}{l}\text { 草 } \\
\text { 怘 } \\
\text { i }\end{array}$ & 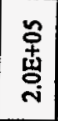 & 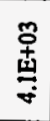 & 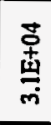 & & 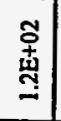 & & 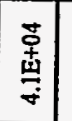 & 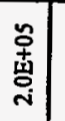 & & & & 㽞 \\
\hline & & & & & 客 & 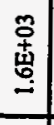 & & 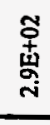 & & & 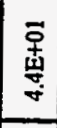 & & & & & $\begin{array}{l}\text { 古 } \\
\text { 索 }\end{array}$ & 号 & & & & & & & \\
\hline & & & & & 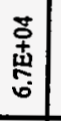 & 亳 & & 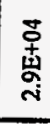 & & & $\begin{array}{l}\text { 卓 } \\
\mathbf{9} \\
\stackrel{5}{+}\end{array}$ & & & & & 辛 & $\begin{array}{l}\text { 草 } \\
\text { 索 } \\
\end{array}$ & & & & & & & \\
\hline 蛋 & 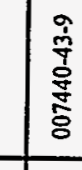 & 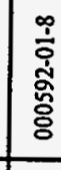 & ह & ริ & 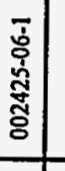 & 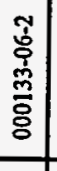 & 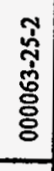 & 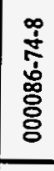 & 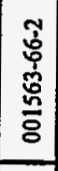 & 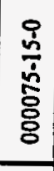 & 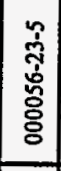 & 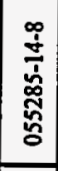 & 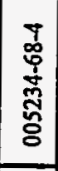 & 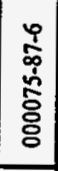 & 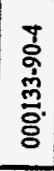 & 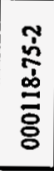 & $\begin{array}{l}\frac{9}{5} \\
\frac{1}{3} \\
\frac{\mathbf{s}}{8} \\
\end{array}$ & 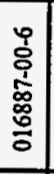 & 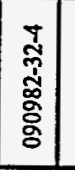 & 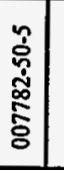 & 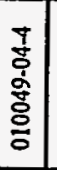 & 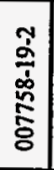 & 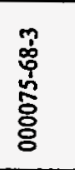 & 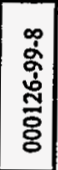 \\
\hline 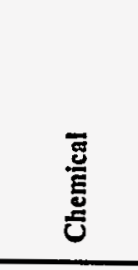 & 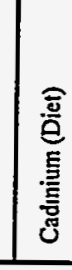 & 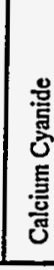 & 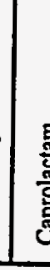 & 密 & 홇 & 혛 & 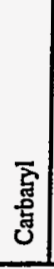 & 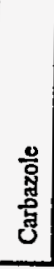 & $\begin{array}{l}\text { 열 } \\
\text { 영 }\end{array}$ & 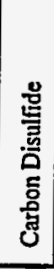 & 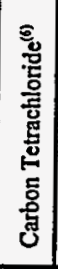 & 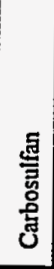 & 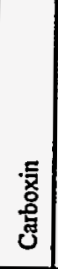 & $\begin{array}{l}\overrightarrow{\mathrm{g}} \\
\mathrm{d} \\
\overline{\mathrm{g}} \\
\mathrm{s}\end{array}$ & 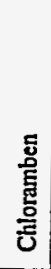 & $\begin{array}{l}\text { 鱿 } \\
\frac{\partial}{\partial} \\
\end{array}$ & 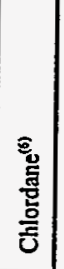 & $\begin{array}{l}\stackrel{0}{\circ} \\
\text { 흔 }\end{array}$ & 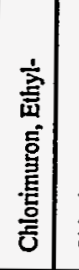 & 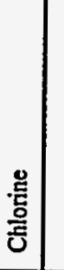 & 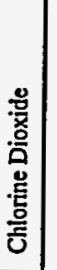 & 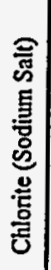 & 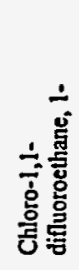 & 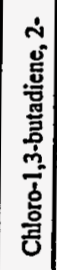 \\
\hline
\end{tabular}

N
4
0
0
0
0
0
0
0

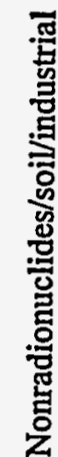


Table 3.2c. (continued)

\begin{tabular}{|c|c|c|c|c|c|c|c|c|c|c|c|c|c|c|c|c|c|}
\hline \multirow[t]{2}{*}{ Chemical } & \multirow[t]{2}{*}{ CASRN } & \multicolumn{4}{|c|}{ Ingestion (mg/kg) } & \multicolumn{4}{|c|}{ Inhalation (mg/kg) } & \multicolumn{4}{|c|}{ Dermal (mg/kg) } & \multicolumn{4}{|c|}{$\begin{array}{l}\text { Ingestion + Inhalation } \\
+ \text { Dermal (mg/kg) }\end{array}$} \\
\hline & & $10^{-4}$ & $10^{5}$ & $\mathrm{HQ}=1$ & $H Q=0.1$ & $10^{-4}$ & $10^{4}$ & $H Q=1$ & $\mathrm{HQ}=0.1$ & $10^{-4}$ & $10^{6}$ & $H Q=1$ & $\mathrm{HQ}=0.1$ & $10^{-4}$ & $10^{4}$ & $H I=1$ & $\mathrm{HI}=0.1$ \\
\hline $\begin{array}{l}\text { Chloro-2-methylaniline } \\
\mathrm{HCl}, 4-\end{array}$ & $003165-93-3$ & $1.2 \mathrm{E}+03$ & $1.2 E+01$ & & & & & & & $9.8 \mathrm{E}+02$ & $9.8 \mathrm{E}+00$ & & & $5.5 \mathrm{E}+02$ & $5.5 \mathrm{E}+00$ & & \\
\hline $\begin{array}{l}\text { Chloro-2-methylaniline, } \\
\text { 4- }\end{array}$ & $000095-69-2$ & $9.9 E+02$ & $9.9 E+00$ & & & & & & & $7.8 \mathrm{E}+02$ & $7.8 \mathrm{E}+00$ & & & $4.4 \mathrm{E}+02$ & $4.4 \mathrm{E}+00$ & & \\
\hline Chloroacetic Acid & $000079-11-8$ & & & $4.1 \mathrm{E}+03$ & $4.1 \mathrm{E}+02$ & & & & & & & $3.2 \mathrm{E}+03$ & $3.2 E+02$ & & & $1.8 \mathrm{E}+03$ & $1.8 \mathrm{E}+02$ \\
\hline Chloroacetophenone, 2- & $000532-27-4$ & & & & & & & $1.9 E+05$ & $1.9 \mathrm{E}+04$ & & & & & & & & \\
\hline Chloroaniline, p- & $000106-47-8$ & & & $8.2 \mathrm{E}+03$ & $8.2 E+02$ & & & & & & & $6.5 \mathrm{E}+03$ & $6.5 \mathrm{E}+02$ & & & $3.6 \mathrm{E}+03$ & $3.6 \mathrm{E}+02$ \\
\hline Chlorobenzene $e^{(6)}$ & $000108-90-7$ & & & 4.1E+04 & $4.1 \mathrm{E}+03$ & & & $5.1 \mathrm{E}+02$ & $1.4 \mathrm{E}+02$ & & & $2.0 \mathrm{E}+04$ & $2.0 \mathrm{E}+03$ & & & $5.1 \mathrm{E}+02$ & $1.3 E+02$ \\
\hline Chlorobenzilate & $000510-15-6$ & $2.1 E+03$ & $2.1 \mathrm{E}+01$ & $4.1 \mathrm{E}+04$ & $4.1 \mathrm{E}+03$ & $1.0 \mathrm{E}+06$ & $2.3 \mathrm{E}+05$ & & & $1.7 \mathrm{E}+03$ & $1.7 E+01$ & $3.2 \mathrm{E}+04$ & $3.2 \mathrm{E}+03$ & $9.4 \mathrm{E}+02$ & $9.4 \mathrm{E}+00$ & $1.8 \mathrm{E}+04$ & $1.8 \mathrm{E}+03$ \\
\hline Chlorobenzoic Acid, p- & $000074-11-3$ & & & $4.1 \mathrm{E}+05$ & $4.1 \mathrm{E}+04$ & & & & & & & $3.2 \mathrm{E}+05$ & $3.2 \mathrm{E}+04$ & & & $1.8 \mathrm{E}+05$ & $1.8 \mathrm{E}+04$ \\
\hline $\begin{array}{l}\text { Chlorobenzotrifluoride, } \\
\text { 4- }\end{array}$ & $000098-56-6$ & & & $4.1 \mathrm{E}+04$ & $4.1 \mathrm{E}+03$ & & & & & & " & $3.2 \mathrm{E}+04$ & $3.2 \mathrm{E}+03$ & & & $1.8 \mathrm{E}+04$ & $1.8 \mathrm{E}+03$ \\
\hline Chlorobutane, 1- & $000109-69-3$ & & & $8.2 \mathrm{E}+05$ & $8.2 E+04$ & & & & & & & $1.0 \mathrm{E}+06$ & $1.0 \mathrm{E}+05$ & & & $4.6 \mathrm{E}+0 \mathrm{~S}$ & $4.6 \mathrm{E}+04$ \\
\hline Chlorobutane, 2- & $000078-86-4$ & & & & & & & & & & & & & & & & \\
\hline Chlorocyclopentadiene & $041851 \cdot 50-7$ & & & & & & & & & & & & & & & & \\
\hline Chlorodibromoethane & $073506-94-2$ & $6.8 \mathrm{E}+03$ & $6.8 \mathrm{E}+01$ & & & & & & & $8.6 \mathrm{E}+03$ & $8.6 \mathrm{E}+01$ & & & $3.8 \mathrm{E}+03$ & $3.8 \mathrm{E}+01$ & & \\
\hline Chlorodifluoromethane & $000075-45-6$ & & & & & & & $1.0 \mathrm{E}+06$ & $1.0 \mathrm{E}+06$ & & & & & & & & \\
\hline Chloroform ${ }^{(6)}$ & $000067-66-3$ & $9.4 \mathrm{E}+04$ & $9.4 \mathrm{E}+02$ & $2.0 \mathrm{E}+04$ & $2.0 \mathrm{E}+03$ & $3.1 \mathrm{E}+02$ & $3.1 \mathrm{E}+00$ & & & $3.0 \mathrm{E}+04$ & $3.0 \mathrm{E}+02$ & $6.5 \mathrm{E}+03$ & $6.5 \mathrm{E}+02$ & $3.1 \mathrm{E}+02$ & $3.1 \mathrm{E}+00$ & $1.5 \mathrm{E}+03$ & $4.9 \mathrm{E}+02$ \\
\hline Chloromethane $^{(5)}$ & $000074-87-3$ & $4.4 E+04$ & $4.4 \mathrm{E}+02$ & & & $7.4 \mathrm{E}+02$ & $7.4 \mathrm{E}+00$ & & & $5.6 \mathrm{E}+04$ & $5.6 \mathrm{E}+02$ & & & $7.2 \mathrm{E}+02$ & $7.2 \mathrm{E}+00$ & & \\
\hline $\begin{array}{l}\text { Chloromethyl Methyl } \\
\text { Ether }\end{array}$ & $000107-30-2$ & & & & & & & & & & & & & & & & \\
\hline Chloronaphthalene, Beta- & $000091-58-7$ & & & $1.6 \mathrm{E}+05$ & $1.6 \mathrm{E}+04$ & & & & & & & $2.1 \mathrm{E}+05$ & $2.1 \mathrm{E}+04$ & & & $9.1 \mathrm{E}+04$ & $9.1 E+03$ \\
\hline Chloronitrobenzene, o- & $000088-73-3$ & $2.3 E+04$ & $2.3 \mathrm{E}+02$ & & & & & & & $2.9 E+04$ & $2.9 \mathrm{E}+02$ & & & $1.3 \mathrm{E}+04$ & $1.3 \mathrm{E}+02$ & & \\
\hline Chloronitrobenzene, p- & $000121-73-3$ & $3.2 \mathrm{E}+04$ & 3.2E+02 & & & & & & & $4.0 E+04$ & $4.0 \mathrm{E}+02$ & & & $1.8 \mathrm{E}+04$ & $1.8 \mathrm{E}+02$ & & \\
\hline Chlorophenol, 2- & $000095-57-8$ & & & $1.0 \mathrm{E}+04$ & $1.0 \mathrm{E}+03$ & & & & & & & $1.3 \mathrm{E}+04$ & $1.3 \mathrm{E}+03$ & & & $5.7 \mathrm{E}+03$ & $5.7 \mathrm{E}+02$ \\
\hline
\end{tabular}

Nonradionuclides/soil/industrial

Page 7 of 32 
Table 3.2c. (continued)

\begin{tabular}{|c|c|c|c|c|c|c|c|c|c|c|c|c|c|c|c|c|c|}
\hline \multirow{2}{*}{ Chemical } & \multirow[t]{2}{*}{ CASRN } & \multicolumn{4}{|c|}{ Ingestion (mg/kg) } & \multicolumn{4}{|c|}{ Inhalation (mg/kg) } & \multicolumn{4}{|c|}{ Dermal (mg/kg)" } & \multicolumn{4}{|c|}{$\begin{array}{l}\text { Ingestion + Inhalation } \\
+ \text { Dermal (mg/kg) }\end{array}$} \\
\hline & & $10^{-4}$ & $10^{4}$ & $H Q=1$ & $\mathrm{HQ}=0.1$ & $10^{4}$ & $10^{6}$ & $H Q=1$ & $\mathrm{HQ}=0.1$ & $10^{-1}$ & $10^{-4}$ & $H Q=1$ & $H Q=0.1$ & $10^{-4}$ & $10^{6}$ & $H I=1$ & $\mathrm{HI}=0.1$ \\
\hline $\begin{array}{l}\text { Chlorophenyl Methyl } \\
\text { Sulfide, p- }\end{array}$ & $000123-09-1$ & & & & & & & & & & & & & & & & \\
\hline $\begin{array}{l}\text { Chlorophenyl Methyl } \\
\text { Sulfoxide }\end{array}$ & $000934-73-6$ & & & & & & & & & & & & & & & & \\
\hline Chloropropane, 2- & $000075-29-6$ & & & & & & & $1.6 \mathrm{E}+03$ & $2.2 \mathrm{E}+02$ & & & & & & & & \\
\hline Chlorothalonil & $001897-45-6$ & $5.2 \mathrm{E}+04$ & $5.2 \mathrm{E}+02$ & $3.1 \mathrm{E}+04$ & $3.1 E+03$ & & & & & 4.1E+04 & $4.1 \mathrm{E}+02$ & $2.4 \mathrm{E}+04$ & $2.4 \mathrm{E}+03$ & $2.3 \mathrm{E}+04$ & $2.3 \mathrm{E}+02$ & $1.4 \mathrm{E}+04$ & $1.4 \mathrm{E}+03$ \\
\hline Chlorotoluene, 0 - & $000095-49-8$ & & & 4.1E+04 & $4.1 \mathrm{E}+03$ & & & & & & & $5.2 \mathrm{E}+04$ & $5.2 \mathrm{E}+03$ & & & $2.3 E+04$ & $2.3 \mathrm{E}+03$ \\
\hline Chlorpropham & $000101-21-3$ & & & $4.1 \mathrm{E}+05$ & $4.1 \mathrm{E}+04$ & & & & & & & $3.2 \mathrm{E}+05$ & $3.2 \mathrm{E}+04$ & & & $1.8 \mathrm{E}+05$ & $1.8 \mathrm{E}+04$ \\
\hline Chlorpyrifos & $002921-88-2$ & & & $6.1 E+03$ & $6.1 E+02$ & & & & & & & $4.9 \mathrm{E}+03$ & $4.9 \mathrm{E}+02$ & & & $2.7 \mathrm{E}+03$ & $2.7 \mathrm{E}+02$ \\
\hline Chlorpyrifos Methyl & $005598-13-0$ & & & $2.0 \mathrm{E}+04$ & $2.0 \mathrm{E}+03$ & & & & & & & $1.6 \mathrm{E}+04$ & $1.6 \mathrm{E}+03$ & & & $9.0 \mathrm{E}+03$ & $9.0 \mathrm{E}+02$ \\
\hline Chlorsulfuron & $064902-72-3$ & & & $1.0 \mathrm{E}+05$ & $1.0 \mathrm{E}+04$ & & & & & & & $8.1 \mathrm{E}+04$ & $8.1 \mathrm{E}+03$ & & & $4.5 \mathrm{E}+04$ & $4.5 \mathrm{E}+03$ \\
\hline Chlorthiophos & $060238-56-4$ & & & $1.6 \mathrm{E}+03$ & $1.6 \mathrm{E}+02$ & & & & & & & $1.3 \mathrm{E}+03$ & $1.3 \mathrm{E}+02$ & & & $7.2 \mathrm{E}+02$ & $7.2 \mathrm{E}+01$ \\
\hline $\begin{array}{l}\text { Chromium (III) (Insoluble } \\
\text { Salts) }\end{array}$ & $016065-83-1$ & & & $1.0 E+06$ & $2.0 \mathrm{E}+05$ & & & & & & & $1.6 \mathrm{E}+05$ & $1.6 \mathrm{E}+04$ & & & $1.5 \mathrm{E}+05$ & $1.5 \mathrm{E}+04$ \\
\hline Chromium $(\mathrm{VI})^{(6)}$ & $018540-29-9$ & & & $1.0 \mathrm{E}+04$ & $1.0 \mathrm{E}+03$ & $1.5 \mathrm{E}+05$ & $1.5 \mathrm{E}+03$ & & & & . & $3.2 \mathrm{E}+03$ & $3.2 \mathrm{E}+02$ & & & $2.5 \mathrm{E}+03$ & $2.5 E+02$ \\
\hline Chromium Salts & NA & & & & & & & & & & & & & & & & \\
\hline Chrysene $^{(2)}$ & $000218-01-9$ & $7.8 \mathrm{E}+04$ & $7.8 \mathrm{E}+02$ & & & $1.0 \mathrm{E}+06$ & $1.0 \mathrm{E}+06$ & & & $3.8 \mathrm{E}+04$ & $3.8 \mathrm{E}+02$ & & & $2.6 \mathrm{E}+04$ & $2.6 E+02$ & & \\
\hline Cobalt & $007440-48-4$ & & & & & & & & & & & & & & & & \\
\hline Coke Oven Emissions & $008007-45-2$ & & & & & $1.0 \mathrm{E}+06$ & $2.8 \mathrm{E}+04$ & & & & & & & & & & \\
\hline Copper & $007440-50-8$ & & & & & & & & & & & & & & & & \\
\hline Copper Cyanide & $000544-92-3$ & & & $1.0 \mathrm{E}+04$ & $1.0 \mathrm{E}+03$ & & & & & & & $3.2 \mathrm{E}+04$ & $3.2 \mathrm{E}+03$ & & & $7.8 \mathrm{E}+03$ & $7.8 \mathrm{E}+02$ \\
\hline Creosote & $008001-58-9$ & & & & & & & & & & & & & & & & \\
\hline Cresol, m- & $000108-39-4$ & & & $1.0 \mathrm{E}+05$ & $1.0 \mathrm{E}+04$ & & & & & & & $8.1 \mathrm{E}+04$ & $8.1 \mathrm{E}+03$ & & & $4.5 \mathrm{E}+04$ & $4.5 \mathrm{E}+03$ \\
\hline Cresol, o- & $000095-48-7$ & & & $1.0 \mathrm{E}+05$ & $1.0 \mathrm{E}+04$ & & & & & & & $8.1 \mathrm{E}+04$ & $8.1 \mathrm{E}+03$ & & & $4.5 E+04$ & $4.5 \mathrm{E}+03$ \\
\hline Cresol, p- & $000106-44-5$ & & & $1.0 \mathrm{E}+04$ & $1.0 \mathrm{E}+03$ & & & & & & & $1.1 \mathrm{E}+04$ & $1.1 \mathrm{E}+03$ & & & $5.2 \mathrm{E}+03$ & $5.2 \mathrm{E}+02$ \\
\hline
\end{tabular}


Table 3.2c. (continued)

\begin{tabular}{|c|c|c|c|c|c|c|c|c|c|c|c|c|c|c|c|c|c|}
\hline \multirow{2}{*}{ Chemical } & \multirow{2}{*}{ CASRN } & \multicolumn{4}{|c|}{ Ingestion (mg/kg) } & \multicolumn{4}{|c|}{ Inhalation $(\mathrm{mg} / \mathrm{kg})^{-\gamma}$} & \multicolumn{4}{|c|}{ Dermal (mg/kg)' } & \multicolumn{4}{|c|}{$\begin{array}{l}\text { Ingestion + Inhalation } \\
+ \text { Dermal }(\mathrm{mg} / \mathrm{kg})^{+* \bullet} \\
\end{array}$} \\
\hline & & $10^{-4}$ & $10^{4}$ & $\mathrm{HQ}=1$ & $\mathrm{HQ}=0.1$ & $10^{-4}$ & $10^{4}$ & $H Q=1$ & $\mathrm{HQ}=0.1$ & $10^{-4}$ & $10^{6}$ & $H Q=1$ & $\mathrm{HQ}=0.1$ & $10^{-4}$ & $10^{5}$ & $H I=1$ & $\mathrm{HI}=0.1$ \\
\hline Crotonaldehyde & $000123-73-9$ & $3.0 \mathrm{E}+02$ & $3.0 \mathrm{E}+00$ & & & & & & & $2.4 \mathrm{E}+02$ & $2.4 \mathrm{E}+00$ & & & $1.3 \mathrm{E}+02$ & $1.3 \mathrm{E}+00$ & & \\
\hline Cumene & $000098-82-8$ & & & $8.2 E+04$ & $8.2 \mathrm{E}+03$ & & & $3.5 \mathrm{E}+02$ & $9.3 \mathrm{E}+01$ & & & $1.0 \mathrm{E}+05$ & $1.0 \mathrm{E}+04$ & & & $3.5 \mathrm{E}+02$ & $9.1 \mathrm{E}+01$ \\
\hline Cyanazine & $021725-46-2$ & $6.8 \mathrm{E}+02$ & $6.8 \mathrm{E}+00$ & 4.1E+03 & 4.1E+02 & & & & & $5.4 \mathrm{E}+02$ & $5.4 \mathrm{E}+00$ & $3.2 \mathrm{E}+03$ & $3.2 E+02$ & $3.0 \mathrm{E}+02$ & $3.0 \mathrm{E}+00$ & $1.8 \mathrm{E}+03$ & $1.8 \mathrm{E}+02$ \\
\hline Cyanide (CN-) & $000057-12.5$ & & & 4.1E+04 & $4.1 E+03$ & & & & & & & $1.1 \mathrm{E}+04$ & $1.1 \mathrm{E}+03$ & & & $8.7 \mathrm{E}+03$ & $8.7 \mathrm{E}+02$ \\
\hline Cyanogen & $000460-19.5$ & & & $8.2 E+04$ & $8.2 E+03$ & & & & & & & $1.0 \mathrm{E}+05$ & $1.0 \mathrm{E}+04$ & & & $4.6 \mathrm{E}+04$ & $4.6 \mathrm{E}+03$ \\
\hline Cyanogen Bromide & $000506-68-3$ & & & $1.8 \mathrm{E}+05$ & $1.8 \mathrm{E}+04$ & & & & . & & & $2.3 \mathrm{E}+05$ & $2.3 \mathrm{E}+04$ & & & $1.0 \mathrm{E}+05$ & $1.0 \mathrm{E}+04$ \\
\hline Cyanogen Chloride & $000506 \cdot 77-4$ & & & $1.0 \mathrm{E}+05$ & $1.0 \mathrm{E}+04$ & & & & & & & $1.3 \mathrm{E}+05$ & $1.3 E+04$ & & & $5.7 E+04$ & $5.7 \mathrm{E}+03$ \\
\hline $\begin{array}{l}\text { Cyclohexane, 1,2,3,4,5- } \\
\text { pentabromo-6-chloro- }\end{array}$ & $000087-84-3$ & $2.5 \mathrm{E}+04$ & $2.5 \mathrm{E}+02$ & & & & & & & $2.0 \mathrm{E}+04$ & $2.0 \mathrm{E}+02$ & & & $1.1 \mathrm{E}+04$ & $1.1 \mathrm{E}+02$ & & \\
\hline Cyclohexanone & $000108-94-1$ & & $\cdot$ & $1.0 \mathrm{E}+06$ & $1.0 \mathrm{E}+06$ & & & & & & & $1.0 \mathrm{E}+06$ & $1.0 \mathrm{E}+06$ & & & $1.0 \mathrm{E}+06$ & $5.7 \mathrm{E}+05$ \\
\hline Cyclohexylamine & $000108-91-8$ & & & 4.1E+05 & 4.1E+04 & & & & & & & $3.2 \mathrm{E}+05$ & $3.2 E+04$ & & & $1.8 \mathrm{E}+05$ & $1.8 \mathrm{E}+04$ \\
\hline Cyclopentadiene & $000542-92-7$ & & & & & & & & & & & & & & & & \\
\hline Cyhalothrin/karate & $068085-85-8$ & & & $1.0 \mathrm{E}+04$ & $1.0 \mathrm{E}+03$ & & & & & & & $8.1 \mathrm{E}+03$ & $8.1 \mathrm{E}+02$ & & & 4.5E+03 & $4.5 \mathrm{E}+02$ \\
\hline Cypermethrin & $052315-07-8$ & & & $2.0 \mathrm{E}+04$ & $2.0 \mathrm{E}+03$ & & & & & & & $1.6 \mathrm{E}+04$ & $1.6 \mathrm{E}+03$ & & & $9.0 \mathrm{E}+03$ & $9.0 \mathrm{E}+02$ \\
\hline Cyromazine & $066215-27-8$ & & & $1.5 \mathrm{E}+04$ & $1.5 \mathrm{E}+03$ & & & & & & & $1.2 \mathrm{E}+04$ & $1.2 \mathrm{E}+03$ & & & $6.8 \mathrm{E}+03$ & $6.8 \mathrm{E}+02$ \\
\hline DDD & $000072-54-8$ & $2.4 \mathrm{E}+03$ & $2.4 \mathrm{E}+01$ & & & & & & & $2.6 \mathrm{E}+03$ & $2.6 \mathrm{E}+01$ & & & $1.3 \mathrm{E}+03$ & $1.3 \mathrm{E}+01$ & & \\
\hline $\mathrm{DDE}$ & $000072-55-9$ & $1.7 \mathrm{E}+03$ & $1.7 \mathrm{E}+01$ & & & & & & & $1.9 \mathrm{E}+03$ & $1.9 \mathrm{E}+01$ & & & $8.8 \mathrm{E}+02$ & $8.8 \mathrm{E}+00$ & & \\
\hline $\mathrm{DDT}$ & $000050-29-3$ & $1.7 \mathrm{E}+03$ & $1.7 \mathrm{E}+01$ & $1.0 \mathrm{E}+03$ & $1.0 \mathrm{E}+02$ & $1.0 \mathrm{E}+06$ & $1,8 \mathrm{E}+05$ & & & $1.9 \mathrm{E}+03$ & $1.9 \mathrm{E}+01$ & $1.1 \mathrm{E}+03$ & $1.1 \mathrm{E}+02$ & $8.8 \mathrm{E}+02$ & $8.8 \mathrm{E}+00$ & $5.4 \mathrm{E}+02$ & $5.4 \mathrm{E}+01$ \\
\hline Dacthal & $001861-32-1$ & & & $2.0 \mathrm{E}+04$ & $2.0 \mathrm{E}+03$ & & & & & & & $1.6 \mathrm{E}+04$ & $1.6 \mathrm{E}+03$ & & & $9.0 E+03$ & $9.0 \mathrm{E}+02$ \\
\hline Dalapon & $000075-99.0$ & & & $6.1 \mathrm{E}+04$ & $6.1 \mathrm{E}+03$ & & & & & & & $4.9 \mathrm{E}+04$ & $4.9 \mathrm{E}+03$ & & & 2.7E+04 & $2.7 \mathrm{E}+03$ \\
\hline $\begin{array}{l}\text { Decabromodiphenyl } \\
\text { Ether }\end{array}$ & $001163-19-5$ & & & $2.0 \mathrm{E}+04$ & $2.0 \mathrm{E}+03$ & & & & & & & $2.6 \mathrm{E}+04$ & $2.6 \mathrm{E}+03$ & & & $1.1 \mathrm{E}+04$ & $1.1 \mathrm{E}+03$ \\
\hline Demeton & $008065-48-3$ & & & $8.2 \mathrm{E}+01$ & $8.2 \mathrm{E}+00$ & & & & & & & $6.5 \mathrm{E}+01$ & $6.5 \mathrm{E}+00$ & & & $3.6 \mathrm{E}+01$ & $3.6 \mathrm{E}+00$ \\
\hline Di(2-ethylhexyl)adipate & $000103-23-1$ & $4.8 \mathrm{E}+05$ & $4.8 \mathrm{E}+03$ & $1.0 \mathrm{E}+06$ & $1.2 \mathrm{E}+05$ & & & & & $3.8 \mathrm{E}+05$ & $3.8 \mathrm{E}+03$ & $9.7 \mathrm{E}+05$ & $9.7 E+04$ & $2.1 \mathrm{E}+05$ & $2.1 \mathrm{E}+03$ & $5.4 \mathrm{E}+05$ & $5.4 E+04$ \\
\hline
\end{tabular}

Nonradionuclides/soil/industrial

Page 9 of 32 
Table 3.2c. (continued)

\begin{tabular}{|c|c|c|c|c|c|c|c|c|c|c|c|c|c|c|c|c|c|}
\hline \multirow[t]{2}{*}{ Chemical } & \multirow[t]{2}{*}{ CASRN } & \multicolumn{4}{|c|}{ Ingestion (mg/kg) } & \multicolumn{4}{|c|}{ Inhalation (mg/kg) } & \multicolumn{4}{|c|}{ Dermal (mg/kg)" } & \multicolumn{4}{|c|}{$\begin{array}{c}\text { Ingestion + Inhalation } \\
+ \text { Dermal (mg/kg) }\end{array}$} \\
\hline & & $10^{-4}$ & $10^{5}$ & $H Q=1$ & $\mathrm{HQ}=0.1$ & $10^{-4}$ & $10^{4}$ & $\mathrm{HQ}=1$ & $\mathrm{HQ}=0.1$ & $10^{-4}$ & $10^{6}$ & $\mathrm{HQ}=1$ & $\mathrm{HQ}=0.1$ & $10^{-4}$ & $10^{6}$ & HI $=1$ & $H \mathrm{l}=0.1$ \\
\hline Diallate & $002303-16-4$ & $9.4 \mathrm{E}+03$ & $9.4 \mathrm{E}+01$ & & & & & & & $1.2 \mathrm{E}+04$ & $1.2 \mathrm{E}+02$ & & & $5.2 \mathrm{E}+03$ & $5.2 \mathrm{E}+01$ & & \\
\hline Diazinon & $000333-41-5$ & & & $1.8 \mathrm{E}+03$ & $1.8 \mathrm{E}+02$ & & & & & & & $1.5 \mathrm{E}+03$ & $1.5 \mathrm{E}+02$ & & & $8.1 \mathrm{E}+02$ & $8.1 E+01$ \\
\hline Dibenz[a,h]anthracene $e^{(3)}$ & $000053-70-3$ & $7.8 \mathrm{E}+01$ & $7.8 \mathrm{E}-01$ & & & $1.0 \mathrm{E}+06$ & $2.0 \mathrm{E}+04$ & & & $3.8 \mathrm{E}+01$ & $3.8 \mathrm{E}-01$ & & & $2.6 \mathrm{E}+01$ & $2.6 \mathrm{E}-01$ & & \\
\hline $\begin{array}{l}\text { Dibromo-3- } \\
\text { chloropropane, 1,2- }\end{array}$ & $000096-12.8$ & $4.1 \mathrm{E}+02$ & $4.1 \mathrm{E}+00$ & & & $1.0 \mathrm{E}+06$ & $1.0 \mathrm{E}+06$ & $1.0 \mathrm{E}+06$ & $1.3 E+05$ & $5.2 \mathrm{E}+02$ & $5.2 \mathrm{E}+00$ & & & $2.3 \mathrm{E}+02$ & $2.3 \mathrm{E}+00$ & & \\
\hline Dibromobenzene, 1,4- & $000106-37-6$ & & & $2.0 \mathrm{E}+04$ & $2.0 \mathrm{E}+03$ & & & & & & & $2.6 \mathrm{E}+04$ & $2.6 \mathrm{E}+03$ & & & $1.1 E+04$ & $1.1 \mathrm{E}+03$ \\
\hline Dibromochloromethane & $000124-48-1$ & $6.8 \mathrm{E}+03$ & $6.8 \mathrm{E}+01$ & $4.1 \mathrm{E}+04$ & $4.1 \mathrm{E}+03^{\circ}$ & & & & & $6.5 \mathrm{E}+03$ & $6.5 \mathrm{E}+01$ & $3.9 \mathrm{E}+04$ & $3.9 \mathrm{E}+03$ & $3.3 \mathrm{E}+03$ & $3.3 \mathrm{E}+01$ & $2.0 \mathrm{E}+04$ & $2.0 \mathrm{E}+03$ \\
\hline Dibromodichloromethane & $000594-18-3$ & & & & & & & & & & & & & & & & \\
\hline $\begin{array}{l}\text { Dibromodiphenyl Ether, } \\
\text { p,p'- }\end{array}$ & $002050-47-7$ & & & & & & & & & & & & & & & & \\
\hline Dibromoethane, 1,2- & $000106-93-4$ & $6.7 \mathrm{E}+00$ & $6.7 \mathrm{E}-02$ & & & $2.5 \mathrm{E}+02$ & $2.5 \mathrm{E}+00$ & $3.9 \mathrm{E}+01$ & $3.9 \mathrm{E}+00$ & $8.5 \mathrm{E}+00$ & 8.5E-02 & & & $3.7 \mathrm{E}+00$ & $3.7 \mathrm{E}-02$ & & \\
\hline $\begin{array}{l}\text { Dibromomethane } \\
\text { (Methylene Bromide) }\end{array}$ & $000074-95-3$ & & & $2.0 \mathrm{E}+04$ & $2.0 \mathrm{E}+03$ & & & & & & & $2.6 \mathrm{E}+04$ & $2.6 \mathrm{E}+03$ & & & $1.1 \mathrm{E}+04$ & $1.1 \mathrm{E}+03$ \\
\hline Dibutyl Phthalate & $000084-74-2$ & & & $2.0 \mathrm{E}+05$ & $2.0 \mathrm{E}+04$ & & & & & & & $3.2 E+05$ & $3.2 \mathrm{E}+04$ & & & $1.3 \mathrm{E}+05$ & $1.3 \mathrm{E}+04$ \\
\hline Dicamba & $001918-00-9$ & & & $6.1 \mathrm{E}+04$ & $6.1 \mathrm{E}+03$ & & & & & & & $4.9 \mathrm{E}+04$ & $4.9 \mathrm{E}+03$ & & & $2.7 \mathrm{E}+04$ & $2.7 \mathrm{E}+03$ \\
\hline Dichloro-2-butene, 1,4- & $000764-41-0$ & & & & & $2.0 \mathrm{E}+01$ & $2.0 \mathrm{E}-01$ & & & & & & & & & & \\
\hline \multicolumn{18}{|l|}{ Dichloroacetic Acid } \\
\hline Dichlorobenzene, 1,2-(3) & $000095-50-1$ & & & $1.8 \mathrm{E}+05$ & $1.8 \mathrm{E}+04$ & & & $5.1 \mathrm{E}+02$ & $5.1 \mathrm{E}+02$ & & & $2.3 E+05$ & $2.3 E+04$ & & & $5.1 E+02$ & $5.1 \mathrm{E}+02$ \\
\hline Dichlorobenzene, 1,3- & $000541-73-1$ & & & & & & & & & & & & & & & & \\
\hline Dichlorobenzene, 1,4- & $000106-46-7$ & $2.4 \mathrm{E}+04$ & $2.4 \mathrm{E}+02$ & & & & & $3.2 \mathrm{E}+01$ & $3.2 \mathrm{E}+01$ & $3.4 \mathrm{E}+04$ & $3.4 \mathrm{E}+02$ & & & $3.2 \mathrm{E}+01$ & $3.2 \mathrm{E}+01$ & & \\
\hline Dichlorobenzidine, 3,3'- & $000091-94-1$ & $1.3 \mathrm{E}+03$ & $1.3 \mathrm{E}+01$ & & & & & & & $1.0 \mathrm{E}+03$ & $1.0 \mathrm{E}+01$ & & & $5.6 \mathrm{E}+02$ & $5.6 \mathrm{E}+00$ & & \\
\hline Dichlorodifluoromethane & $000075-71-8$ & & & $4.1 \mathrm{E}+05$ & 4.1E+04 & & & $3.2 E+02$ & $3.2 \mathrm{E}+01$ & & & $1.5 \mathrm{E}+05$ & $1.5 \mathrm{E}+04$ & & & $3.2 \mathrm{E}+02$ & $3.2 E+01$ \\
\hline Dichloroethane, 1,1- & $000075-34-3$ & & & $2.0 \mathrm{E}+05$ & $2.0 \mathrm{E}+04$ & & & $7.8 \mathrm{E}+00$ & $7.8 \mathrm{E}-01$ & & & $3.2 \mathrm{E}+05$ & $3.2 \mathrm{E}+04$ & & & $7.8 \mathrm{E}+00$ & $7.8 \mathrm{E}-01$ \\
\hline Dichloroethane, 1,2- & $000107-06-2$ & $6.3 \mathrm{E}+03$ & $6.3 \mathrm{E}+01$ & & & $3.4 \mathrm{E}+02$ & $3.4 \mathrm{E}+00$ & & & $1.0 \mathrm{E}+04$ & $1.0 \mathrm{E}+02$ & & & $3.1 \mathrm{E}+02$ & $3.1 \mathrm{E}+00$ & & \\
\hline Dichloroethylene, 1,1- & $000075-35-4$ & $9.5 E+02$ & $9.5 \mathrm{E}+00$ & $1.8 \mathrm{E}+04$ & $1.8 \mathrm{E}+03$ & $7.5 \mathrm{E}+00$ & 7.5E-02 & & & $1.5 \mathrm{E}+03$ & $1.5 \mathrm{E}+01$ & $2.9 \mathrm{E}+04$ & $2.9 \mathrm{E}+03$ & $7.4 \mathrm{E}+00$ & 7.4E-02 & $1.2 \mathrm{E}+03$ & $1.1 \mathrm{E}+03$ \\
\hline
\end{tabular}


Table 3.2c. (continued)

\begin{tabular}{|c|c|c|c|c|c|c|c|c|c|c|c|c|c|c|c|c|c|}
\hline \multirow{2}{*}{ Chemical } & \multirow{2}{*}{ CASRN } & \multicolumn{4}{|c|}{ Ingestion (mg/kg) } & \multicolumn{4}{|c|}{ Inhalation (mg/kg) } & \multicolumn{4}{|c|}{ Dermal (mg/kg) } & \multicolumn{4}{|c|}{$\begin{array}{l}\text { Ingestion + Inhalation } \\
+ \text { Dermal (mg/kg) }\end{array}$} \\
\hline & & $10^{-4}$ & $10^{4}$ & $\mathrm{HQ}=\mathbf{I}$ & $H Q=0.1$ & $10^{4}$ & $10^{5}$ & $H Q=1$ & $\mathrm{HQ}=0.1$ & $10^{-4}$ & $10^{4}$ & $H Q=1$ & $\mathrm{HQ}=0.1$ & $10^{-4}$ & $10^{4}$ & $\mathrm{HI}=1$ & $H I=0.1$ \\
\hline $\begin{array}{l}\text { Dichloroethylene, 1,2- } \\
\text { (Mixed Isomers) }\end{array}$ & $000540-59-0$ & & & $1.8 \mathrm{E}+04$ & $1.8 \mathrm{E}+03$ & & & & & & & $2.3 \mathrm{E}+04$ & $2.3 \mathrm{E}+03$ & & & $1.0 \mathrm{E}+04$ & $1.0 E+03$ \\
\hline Dichloroethylene, 1,2-cis- & $000156-59-2$ & & & $2.0 \mathrm{E}+04$ & $2.0 \mathrm{E}+03$ & & & & & & & $3.2 \mathrm{E}+04$ & $3.2 \mathrm{E}+03$ & & & $1.3 E+04$ & $1.3 \mathrm{E}+03$ \\
\hline $\begin{array}{l}\text { Dichloroethylene, 1,2- } \\
\text { trans- }\end{array}$ & $000156-60-5$ & & & $4.1 \mathrm{E}+04$ & $4.1 \mathrm{E}+03$ & & & & & & & $6.5 \mathrm{E}+04$ & $6.5 \mathrm{E}+03$ & & & $2.5 \mathrm{E}+04$ & $2.5 \mathrm{E}+03$ \\
\hline Dichlorophenol, 2,4- & $000120-83-2$ & & & $6.1 \mathrm{E}+03$ & $6.1 \mathrm{E}+02$ & & & & & & & $8.0 \mathrm{E}+03$ & $8.0 E+02$ & & & $3.5 \mathrm{E}+03$ & $3.5 \mathrm{E}+02$ \\
\hline $\begin{array}{l}\text { Dichlorophenoxy Acetic } \\
\text { Acid, 2,4- }\end{array}$ & $000094-75-7$ & & & $2.0 E+04$ & $2.0 E+03$ & & & & & & & $2.6 \mathrm{E}+04$ & $2.6 \mathrm{E}+03$ & & & $1.1 \mathrm{E}+04$ & $1.1 \mathrm{E}+03$ \\
\hline $\begin{array}{l}\text { Dichlorophenoxy)butyric } \\
\text { Acid, } 4-(2,4-\end{array}$ & $000094-82-6$ & & & $1.6 \mathrm{E}+04$ & $1.6 \mathrm{E}+03$ & & & & & & & $1.3 \mathrm{E}+04$ & $1.3 \mathrm{E}+03$ & & & $7.2 \mathrm{E}+03$ & $7.2 \mathrm{E}+02$ \\
\hline Dichloropropane, 1,2- & $000078-87-5$ & $8.4 \mathrm{E}+03$ & $8.4 \mathrm{E}+01$ & & & & & $1.4 \mathrm{E}+02$ & $1.4 \mathrm{E}+01$ & $9.9 \mathrm{E}+03$ & $9.9 E+01$ & & & $4.8 \mathrm{E}+02$ & $4.5 \mathrm{E}+01$ & & \\
\hline Dichloropropanol, 2,3- & $000616-23.9$ & & & $6.1 \mathrm{E}+03$ & $6.1 \mathrm{E}+02$ & & & & & & & $4.9 \mathrm{E}+03$ & $4.9 \mathrm{E}+02$ & & & $2.7 \mathrm{E}+03$ & $2.7 \mathrm{E}+02$ \\
\hline Dichloropropene, 1,3- & $000542-75-6$ & $3.2 \mathrm{E}+03$ & $3.2 \mathrm{E}+01$ & $6.1 \mathrm{E}+02$ & $6.1 \mathrm{E}+01$ & $9.0 \mathrm{E}+02$ & $9.0 \mathrm{E}+00$ & $2.4 \mathrm{E}+03$ & $2.4 \mathrm{E}+02$ & $2.8 \mathrm{E}+03$ & $2.8 E+01$ & $5.3 \mathrm{E}+02$ & $5.3 E+01$ & $5.6 \mathrm{E}+02$ & $5.6 \mathrm{E}+00$ & $2.5 E+02$ & $2.5 E+01$ \\
\hline Dichlorvos & $000062-73-7$ & $2.0 \mathrm{E}+03$ & $2.0 \mathrm{E}+01$ & $1.0 \mathrm{E}+03$ & $1.0 \mathrm{E}+02$ & & & $1.0 \mathrm{E}+06$ & $3.1 \mathrm{E}+05$ & $1.6 \mathrm{E}+03$ & $1.6 \mathrm{E}+01$ & $8.1 \mathrm{E}+02$ & $8.1 E+01$ & $8.7 E+02$ & $8.7 E+00$ & $4.5 \mathrm{E}+02$ & $4.5 \mathrm{E}+01$ \\
\hline Dicofol & $000115-32-2$ & & & & & & & & & & & & & & & & \\
\hline Dicyclopentadiene & $000077-73-6$ & & & $6.1 E+04$ & $6.1 \mathrm{E}+03$ & & & $4.2 \mathrm{E}+01$ & $4.2 \mathrm{E}+00$ & & & $7.8 \mathrm{E}+04$ & $7.8 \mathrm{E}+03$ & & & $4.2 \mathrm{E}+01$ & $4.2 \mathrm{E}+00$ \\
\hline Dieldrin & $000050-57-1$ & $3.6 \mathrm{E}+01$ & $3.6 \mathrm{E}-01$ & $1.0 \mathrm{E}+02$ & $1.0 \mathrm{E}+01$ & $3.8 \mathrm{E}+05$ & $3.8 \mathrm{E}+03$ & & & $2.8 \mathrm{E}+01$ & $2.8 \mathrm{E}-01$ & $8.1 \mathrm{E}+01$ & $8.1 \mathrm{E}+00$ & $1.6 \mathrm{E}+01$ & $1.6 \mathrm{E}-01$ & $4.5 \mathrm{E}+01$ & $4.5 E+00$ \\
\hline Diethyl Phthalate & $000084-66-2$ & & & $1.0 \mathrm{E}+06$ & $1.6 \mathrm{E}+05$ & & & & & & & $1.0 \mathrm{E}+06$ & $2.3 \mathrm{E}+05$ & & & $9.6 \mathrm{E}+05$ & $9.6 E+04$ \\
\hline $\begin{array}{l}\text { Diethyl-p- } \\
\text { nitrophenylphosphate }\end{array}$ & $000311-45-5$ & & & & & & & & & & & & & & & & \\
\hline $\begin{array}{l}\text { Diethylene Glycol } \\
\text { Dinitrate (DEGDN) }\end{array}$ & $000693-21-0$ & & & & & & & & & & & & & & & & \\
\hline $\begin{array}{l}\text { Diethylene Glycol } \\
\text { Monobutyl Ether }\end{array}$ & $000112-34-5$ & & & & & & & $1.0 E+06$ & $1.0 \mathrm{E}+06$ & & & & & & & & \\
\hline $\begin{array}{l}\text { Diethylene Glycol } \\
\text { Monoethyl Ether }\end{array}$ & $000111-90-0$ & & & $1.0 \mathrm{E}+06$ & 4.1E+05 & & & & & & & $1.0 \mathrm{E}+06$ & $3.2 \mathrm{E}+0 \mathrm{~s}$ & & & $1.0 E+06$ & $1.8 \mathrm{E}+05$ \\
\hline Diethylformamide & $000617-84-5$ & & & $2.2 \mathrm{E}+04$ & $2.2 \mathrm{E}+03$ & & & & & & & $1.8 \mathrm{E}+04$ & $1.8 \mathrm{E}+03$ & & & $9.9 \mathrm{E}+03$ & $9.9 \mathrm{E}+02$ \\
\hline Diethylstilbesterol & $000056-53-1$ & $1.2 \mathrm{E}-01$ & $1.2 \mathrm{E}-03$ & & & $1.3 E+04$ & $1.3 \mathrm{E}+02$ & & & $9.6 \mathrm{E}-02$ & $9.6 \mathrm{E}-04$ & & & $5.4 \mathrm{E}-02$ & 5.4E-04 & & \\
\hline
\end{tabular}

Nonradionuclides/soil/industrial

Page 11 of 32 
Table 3.2c. (continued)

\begin{tabular}{|c|c|c|c|c|c|c|c|c|c|c|c|c|c|c|c|c|c|}
\hline \multirow[t]{2}{*}{ Chemical } & \multirow[t]{2}{*}{ CASRN } & \multicolumn{4}{|c|}{ Ingestion (mg/kg) } & \multicolumn{4}{|c|}{ Inhalation (mg/kg) } & \multicolumn{4}{|c|}{ Dermal (mg/kg) } & \multicolumn{4}{|c|}{$\begin{array}{l}\text { Ingestion + Inhalation } \\
+ \text { Dermal (mg/kg) }{ }^{+\vee y}\end{array}$} \\
\hline & & $10^{-4}$ & $10^{-6}$ & $H Q=1$ & $\mathrm{HQ}=0.1$ & $10^{-4}$ & $10^{4}$ & $H Q=1$ & $\mathrm{HQ}=0.1$ & $10^{-4}$ & $10^{4}$ & $H Q=1$ & $H Q=0.1$ & $10^{-4}$ & $10^{6}$ & HI $=1$ & $H I=0.1$ \\
\hline Difenzoquat & $043222-48-6$ & & & $1.6 \mathrm{E}+05$ & $1.6 \mathrm{E}+04$ & & & & & & & $1.3 \mathrm{E}+05$ & $1.3 \mathrm{E}+04$ & & & $7.2 E+04$ & $7.2 \mathrm{E}+03$ \\
\hline Diflubenzuron & $035367-38-5$ & & & $4.1 \mathrm{E}+04$ & $4.1 \mathrm{E}+03$ & & & & & & & $3.2 \mathrm{E}+04$ & $3.2 \mathrm{E}+03$ & & & $1.8 \mathrm{E}+04$ & $1.8 \mathrm{E}+03$ \\
\hline Difluoroethane, 1,1- & $000075-37-6$ & & & & & & & $1.0 \mathrm{E}+06$ & $1.0 \mathrm{E}+06$ & & & & & & & & \\
\hline $\begin{array}{l}\text { Diisopropyl } \\
\text { Methylphosphonate }\end{array}$ & $001445-75-6$ & & & $1.6 \mathrm{E}+05$ & $1.6 \mathrm{E}+04$ & & & & & & & $1.3 \mathrm{E}+05$ & $1.3 \mathrm{E}+04$ & & & $7.2 E+04$ & $7.2 \mathrm{E}+03$ \\
\hline Dimethipin & $055290-64-7$ & & & 4.1E+04 & $4.1 \mathrm{E}+03$ & & & & & & & $3.2 \mathrm{E}+04$ & $3.2 E+03$ & & & $1.8 \mathrm{E}+04$ & $1.8 \mathrm{E}+03$ \\
\hline Dimethoate & $000060-51-5$ & & & $4.1 \mathrm{E}+02$ & 4.IE+01 & & & & & & & $3.2 \mathrm{E}+02$ & $3.2 \mathrm{E}+01$ & & & $1.8 \mathrm{E}+02$ & $1.8 \mathrm{E}+01$ \\
\hline $\begin{array}{l}\text { Dimethoxybenzidine, } \\
\text { 3,3'- }\end{array}$ & $000119-90-4$ & $4.1 \mathrm{E}+04$ & 4.1E+02 & & & & & & & $3.2 \mathrm{E}+04$ & $3.2 \mathrm{E}+02$ & & & $1.8 \mathrm{E}+04$ & $1.8 \mathrm{E}+02$ & & \\
\hline Dimethyl Sulfate & $000077-78-1$ & & & & & & & & & & & & & & & & \\
\hline Dimethylaniline $\mathrm{HCl}, 2,4-$ & $021436-96-4$ & $9.9 \mathrm{E}+02$ & $9.9 \mathrm{E}+00$ & & & & & & & $7.8 \mathrm{E}+02$ & $7.8 \mathrm{E}+00$ & & & $4.4 \mathrm{E}+02$ & $4.4 \mathrm{E}+00$ & & \\
\hline Dimethylaniline, 2,4- & $000095-68-1$ & $7.6 \mathrm{E}+02$ & $7.6 \mathrm{E}+00$ & & & & & & & $6.0 \mathrm{E}+02$ & $6.0 \mathrm{E}+00$ & & & $3.4 \mathrm{E}+02$ & $3.4 E+00$ & & \\
\hline Dimethylaniline, $\mathrm{N}, \mathrm{N}-$ & $000121-69-7$ & & & $4.1 \mathrm{E}+03$ & 4.1E+02 & & & & & & & $3.2 \mathrm{E}+03$ & $3.2 \mathrm{E}+02$ & & & $1.8 \mathrm{E}+03$ & $1.8 \mathrm{E}+02$ \\
\hline Dimethylbenzidine, 3,3'- & $000119-93-7$ & $6.2 \mathrm{E}+01$ & $6.2 \mathrm{E}-01$ & & & & & & & $4.9 \mathrm{E}+01$ & $4.9 \mathrm{E}-01$ & & & $2.7 \mathrm{E}+01$ & $2.7 \mathrm{E}-01$ & & \\
\hline Dimethylethyl Lead & $107584-40-7$ & & & & & & & & & & & & & & & & \\
\hline Dimethylformamide & $000068-12-2$ & & & $2.0 \mathrm{E}+05$ & $2.0 \mathrm{E}+04$ & & & $1.0 \mathrm{E}+06$ & $1.0 \mathrm{E}+06$ & & & $1.6 \mathrm{E}+05$ & $1.6 \mathrm{E}+04$ & & & $9.0 \mathrm{E}+04$ & $9.0 \mathrm{E}+03$ \\
\hline Dimethylhydrazine, $1,1{ }^{(3)}$ & $000057-14-7$ & $2.2 \mathrm{E}+02$ & $2.2 \mathrm{E}+00$ & & & $1.0 \mathrm{E}+06$ & $1.8 \mathrm{E}+04$ & & & $1.7 \mathrm{E}+02$ & $1.7 \mathrm{E}+00$ & & & $9.7 \mathrm{E}+01$ & $9.7 \mathrm{E}-01$ & & \\
\hline Dimethylhydrazine, 1,2- & $000540-73-8$ & & & & & & & & & & & & & & & & \\
\hline Dimethylphenol, 2,4- & $000105-67-9$ & & & $4.1 \mathrm{E}+04$ & 4.IE+03 & & & & & & & $5.2 \mathrm{E}+04$ & $5.2 \mathrm{E}+03$ & & & $2.3 \mathrm{E}+04$ & $2.3 \mathrm{E}+03$ \\
\hline Dimethylphenol, 2,6- & $000576-26-1$ & & & $1.2 \mathrm{E}+03$ & $1.2 \mathrm{E}+02$ & & & & & & & $9.7 \mathrm{E}+02$ & $9.7 \mathrm{E}+01$ & & & $5.4 \mathrm{E}+02$ & $5.4 \mathrm{E}+01$ \\
\hline Dimethylphenol, 3,4- & $000095-65-8$ & & & $2.0 \mathrm{E}+03$ & $2.0 \mathrm{E}+02$ & & & & & & & $1.6 \mathrm{E}+03$ & $1.6 \mathrm{E}+02$ & & & $9.0 \mathrm{E}+02$ & $9.0 \mathrm{E}+01$ \\
\hline Dimethylphthalate ${ }^{(3)}$ & $000131-11-3$ & & & $1.0 \mathrm{E}+06$ & $1.0 \mathrm{E}+06$ & & & & & & & $1.0 \mathrm{E}+06$ & $1.0 \mathrm{E}+06$ & & & $1.0 \mathrm{E}+06$ & $1.0 \mathrm{E}+06$ \\
\hline Dimethyiterephthalate & $000120-61-6$ & & & $2.0 \mathrm{E}+05$ & $2.0 \mathrm{E}+04$ & & & & & & & $1.6 \mathrm{E}+05$ & $1.6 \mathrm{E}+04$ & & & $9.0 \mathrm{E}+04$ & $9.0 \mathrm{E}+03$ \\
\hline Dinitro-a-cresol, 4,6- & $000534-52-1$ & & & & & & & & & & & & & & & & \\
\hline
\end{tabular}


Table 3.2c. (continued)

\begin{tabular}{|c|c|c|c|c|c|c|c|c|c|c|c|c|c|c|c|c|c|}
\hline \multirow[t]{2}{*}{ Chemical } & \multirow[t]{2}{*}{ CASRN } & \multicolumn{4}{|c|}{ Ingestion (mg/kg) } & \multicolumn{4}{|c|}{ Inhalation (mg/kg) } & \multicolumn{4}{|c|}{ Dermal (mg/kg) } & \multicolumn{4}{|c|}{$\begin{array}{l}\text { Ingestion + Inhalation } \\
+ \text { Dermal (mg/kg) }\end{array}$} \\
\hline & & $10^{-4}$ & $10^{6}$ & $\mathrm{HQ}=1$ & $\mathrm{HQ}=0.1$ & $10^{-1}$ & $10^{5}$ & $\mathrm{HQ}=1$ & $\mathrm{HQ}=0.1$ & $10^{-4}$ & $10^{4}$ & $H Q=1$ & $\mathrm{HQ}=0.1$ & $10^{-4}$ & $10^{6}$ & $H I=1$ & $\mathrm{HI}=0.1$ \\
\hline $\begin{array}{l}\text { Dinitro-0-cyclohexyl } \\
\text { Phenol, 4,6- }\end{array}$ & $000131-89-5$ & & & $4.1 \mathrm{E}+03$ & $4.1 \mathrm{E}+02$ & & & & & & & $3.2 \mathrm{E}+03$ & $3.2 \mathrm{E}+02$ & & & $1.8 \mathrm{E}+03$ & $1.8 \mathrm{E}+02$ \\
\hline Dinitrobenzene, 1,2- & $000528-29-0$ & & & $8.2 \mathrm{E}+02$ & $8.2 \mathrm{E}+01$ & & & & & & & $1.2 \mathrm{E}+03$ & $1.2 \mathrm{E}+02$ & & & $4.9 \mathrm{E}+02$ & $4.9 \mathrm{E}+01$ \\
\hline Dinitrobenzene, 1,3- & $000099-65-0$ & & & $2.0 \mathrm{E}+02$ & $2.0 \mathrm{E}+01$ & & & & & & & $2.1 \mathrm{E}+02$ & $2.1 \mathrm{E}+01$ & & & $1.0 \mathrm{E}+02$ & $1.0 \mathrm{E}+01$ \\
\hline Dinitrobenzene, 1,4- & $000100-25-4$ & & & $8.2 E+02$ & $8.2 \mathrm{E}+01$ & & & & & & & $6.5 \mathrm{E}+02$ & $6.5 \mathrm{E}+01$ & & & $3.6 \mathrm{E}+02$ & $3.6 \mathrm{E}+01$ \\
\hline Dinitrophenol, 2,4- & $000051-28-5$ & & & 4.1E+03 & 4.1E+02 & & & & & & & $6.5 \mathrm{E}+03$ & $6.5 \mathrm{E}+02$ & & & $2.5 \mathrm{E}+03$ & $2.5 E+02$ \\
\hline Dinitrotoluene, 2,4- & $000121-14-2$ & $8.4 E+02$ & $8.4 \mathrm{E}+00$ & $4.1 \mathrm{E}+03$ & $4.1 \mathrm{E}+02$ & & & & & $1.1 E+03$ & $1.1 \mathrm{E}+01$ & $5.5 \mathrm{E}+03$ & $5.5 \mathrm{E}+02$ & $4.8 \mathrm{E}+02$ & $4.8 \mathrm{E}+00$ & $2.3 E+03$ & $2.3 \mathrm{E}+02$ \\
\hline Dinitrotoluene, 2,6- & $000606-20-2$ & $8.4 E+02$ & $8.4 E+00$ & $2.0 E+03$ & $2.0 \mathrm{E}+02$ & & & & & $1.1 \mathrm{E}+03$ & $1.1 \mathrm{E}+01$ & $2.7 \mathrm{E}+03$ & $2.7 \mathrm{E}+02$ & $4.8 \mathrm{E}+02$ & $4.8 \mathrm{E}+00$ & $1.2 \mathrm{E}+03$ & $1.2 \mathrm{E}+02$ \\
\hline $\begin{array}{l}\text { Dinitrotoluene, 2-Amino- } \\
\text { 4,6- }\end{array}$ & $035572-78-2$ & & & & & & & & & & & & & & & & \\
\hline Dinoseb & $000088-85-7$ & & & $2.0 \mathrm{E}+03$ & $2.0 \mathrm{E}+02$ & & & & & & & $1.6 \mathrm{E}+03$ & $1.6 \mathrm{E}+02$ & & & $9.0 E+02$ & $9.0 E+01$ \\
\hline Dioxane, 1,4- & $000123-91-1$ & $5.2 E+04$ & $5.2 \mathrm{E}+02$ & & & & & & & $6.6 \mathrm{E}+04$ & $6.6 \mathrm{E}+02$ & & & $2.9 E+04$ & $2.9 E+02$ & & \\
\hline Diphenamid & $000957-51-7$ & & & $6.1 \mathrm{E}+04$ & $6.1 \mathrm{E}+03$ & & & & & & & $4.9 \mathrm{E}+04$ & $4.9 E+03$ & & & 2.7E+04 & $2.7 \mathrm{E}+03$ \\
\hline Diphenylamine & $000122-39-4$ & & & $5.1 \mathrm{E}+04$ & $5.1 \mathrm{E}+03$ & & & . & & & & $4.0 \mathrm{E}+04$ & $4.0 \mathrm{E}+03$ & & & 2.3E+04 & $2.3 E+03$ \\
\hline Diphenylhydrazine, 1,2- & $000122-66-7$ & $7.2 \mathrm{E}+02$ & $7.2 \mathrm{E}+00$ & & & $1.0 \mathrm{E}+06$ & $7.7 \mathrm{E}+04$ & & & $5.7 \mathrm{E}+02$ & $5.7 \mathrm{E}+00$ & & & $3.2 \mathrm{E}+02$ & $3.2 \mathrm{E}+00$ & & \\
\hline Diquat & $000085-00-7$ & & & $4.5 \mathrm{E}+03$ & $4.5 \mathrm{E}+02$ & & & & & & & $3.6 \mathrm{E}+03$ & $3.6 \mathrm{E}+02$ & & & $2.0 E+03$ & $2.0 E+02$ \\
\hline Direct Black 38 & $001937-37-7$ & $6.7 \mathrm{E}+01$ & $6.7 \mathrm{E}-01$ & & & & & & & $5.3 \mathrm{E}+01$ & $5.3 \mathrm{E}-01$ & & & $2.9 \mathrm{E}+01$ & $2.9 \mathrm{E}-01$ & & \\
\hline Direct Blue 6 & $002602-46-2$ & $7.1 \mathrm{E}+01$ & 7.1E-01 & & & & & & & $5.6 \mathrm{E}+01$ & $5.6 \mathrm{E}-01$ & & & $3.1 \mathrm{E}+01$ & $3.1 \mathrm{E}-01$ & & \\
\hline Direct Brown 95 & $016071-86-6$ & $6.2 \mathrm{E}+01$ & $6.2 \mathrm{E}-01$ & & & & & & & $4.9 \mathrm{E}+01$ & $4.9 \mathrm{E}-01$ & & & $2.7 \mathrm{E}+01$ & 2.7E-01 & & \\
\hline Direct Sky Blue & $002610-05-1$ & & & & & & & & & & & & & & & & \\
\hline Disulfoton & $000298-04-4$ & & & $8.2 \mathrm{E}+01$ & $8.2 \mathrm{E}+00$ & & & & & & & $6.5 E+01$ & $6.5 \mathrm{E}+00$ & & & $3.6 \mathrm{E}+01$ & $3.6 \mathrm{E}+00$ \\
\hline Diuron & $000330-54-1$ & & & 4.1E+03 & 4.1E+02 & & & & & & & $3.2 \mathrm{E}+03$ & $3.2 \mathrm{E}+02$ & & & $1.8 \mathrm{E}+03$ & $1.8 \mathrm{E}+02$ \\
\hline Dodine & $002439-10-3$ & & & $8.2 \mathrm{E}+03$ & $8.2 \mathrm{E}+02$ & & & & & & & $6.5 \mathrm{E}+03$ & $6.5 \mathrm{E}+02$ & & & $3.6 \mathrm{E}+03$ & $3.6 \mathrm{E}+02$ \\
\hline EPTC & $000759-94-4$ & & & $5.1 \mathrm{E}+04$ & $5.1 E+03$ & & & & & & & $4.0 E+04$ & $4.0 \mathrm{E}+03$ & & . & $2.3 \mathrm{E}+04$ & $2.3 E+03$ \\
\hline
\end{tabular}


Table 3.2c. (continued)

\begin{tabular}{|c|c|c|c|c|c|c|c|c|c|c|c|c|c|c|c|c|c|}
\hline \multirow{2}{*}{ Chemical } & \multirow{2}{*}{ CASRN } & \multicolumn{4}{|c|}{ Ingestion (mg/kg) } & \multicolumn{4}{|c|}{ Inhalation (mg/kg) } & \multicolumn{4}{|c|}{ Dermal (mg/kg) } & \multicolumn{4}{|c|}{$\begin{array}{l}\text { Ingestion + Inhalation } \\
+ \text { Dermal }(\mathrm{mg} / \mathrm{kg})^{1 * \vartheta}\end{array}$} \\
\hline & & $10^{-4}$ & $10^{4}$ & $H Q=1$ & $\mathrm{HQ}=0.1$ & $10^{-4}$ & $10^{4}$ & HQ=1 & $\mathrm{HQ}=0.1$ & $10^{-4}$ & $10^{6}$ & $H Q=1$ & $\mathrm{HQ}=0.1$ & $10^{-4}$ & $10^{4}$ & $H I=1$ & $H I=0.1$ \\
\hline Endosulfan & $000115-29-7$ & & & $1.2 \mathrm{E}+04$ & $1.2 \mathrm{E}+03$ & & & & & & & $9.7 \mathrm{E}+03$ & $9.7 \mathrm{E}+02$ & & & $5.4 \mathrm{E}+03$ & $5.4 \mathrm{E}+02$ \\
\hline Endothall & $000145-73-3$ & & & 4.1E+04 & $4.1 \mathrm{E}+03$ & & & & & & & $3.2 \mathrm{E}+04$ & $3.2 \mathrm{E}+03$ & & & $1.8 \mathrm{E}+04$ & $1.8 E+03$ \\
\hline Endrin & $000072-20-8$ & & & $6.1 E+02$ & $6.1 \mathrm{E}+01$ & & & & & & & $1.9 \mathrm{E}+01$ & $1.9 \mathrm{E}+00$ & & & $1.9 \mathrm{E}+01$ & $1.9 E+00$ \\
\hline Epichlorohydrin & $000106-89-8$ & $5.8 \mathrm{E}+04$ & $5.8 \mathrm{E}+02$ & $4.1 \mathrm{E}+03$ & $4.1 \mathrm{E}+02$ & $3.2 \mathrm{E}+03$ & 3.2E+02 & $1.4 \mathrm{E}+02$ & $1.4 \mathrm{E}+01$ & $7.3 \mathrm{E}+04$ & $7.3 \mathrm{E}+02$ & $5.2 \mathrm{E}+03$ & $5.2 E+02$ & $3.2 \mathrm{E}+03$ & $1.6 \mathrm{E}+02$ & $1.3 \mathrm{E}+02$ & $1.3 \mathrm{E}+01$ \\
\hline Epoxybutane, 1,2- & $000106-88-7$ & & & & & & & $1.0 \mathrm{E}+06$ & $1.0 \mathrm{E}+06$ & & & & & & & & \\
\hline Ethephon & $016672-87-0$ & & & $1.0 \mathrm{E}+04$ & $1.0 \mathrm{E}+03$ & & & & & & & $8.1 E+03$ & $8.1 E+02$ & & & $4.5 \mathrm{E}+03$ & $4.5 \mathrm{E}+02$ \\
\hline Ethion & $000563-12-2$ & & & $1.0 \mathrm{E}+03$ & $1.0 \mathrm{E}+02$ & & & & & & & $8.1 \mathrm{E}+02$ & $8.1 E+01$ & & & $4.5 \mathrm{E}+02$ & $4.5 \mathrm{E}+01$ \\
\hline Ethoxyethanol Acetate, 2- & $000111-15-9$ & & & $6.1 \mathrm{E}+05$ & $6.1 \mathrm{E}+04$ & & & & & & & $4.9 \mathrm{E}+05$ & $4.9 \mathrm{E}+04$ & & & $2.7 \mathrm{E}+05$ & $2.7 \mathrm{E}+04$ \\
\hline Ethoxyethanol, 2- & $000110-80-5$ & & & $8.2 \mathrm{E}+05$ & $8.2 \mathrm{E}+04$ & & & $1.0 \mathrm{E}+06$ & $1.0 \mathrm{E}+06$ & & & $6.5 \mathrm{E}+05$ & $6.5 \mathrm{E}+04$ & & & $3.6 \mathrm{E}+05$ & $3.6 \mathrm{E}+04$ \\
\hline Ethyl Acetate & $000141-78-6$ & & & $1.0 \mathrm{E}+06$ & $1.8 \mathrm{E}+05$ & & & & & & & $1.0 \mathrm{E}+06$ & $2.3 \mathrm{E}+05$ & & & $1.0 \mathrm{E}+06$ & $1.0 \mathrm{E}+05$ \\
\hline Ethyl Acrylate & $000140-88-5$ & $1.2 \mathrm{E}+04$ & $1.2 \mathrm{E}+02$ & & & & & & & $9.4 \mathrm{E}+03$ & $9.4 \mathrm{E}+01$ & & & $5,3 \mathrm{E}+03$ & $5.3 \mathrm{E}+01$ & & \\
\hline Ethyl Chloride & $000075-00-3$ & & & & & & & $1.5 \mathrm{E}+02$ & $1.5 \mathrm{E}+02$ & & & & & & & & \\
\hline Ethyl Ether & $000060-29-7$ & & & $4.1 \mathrm{E}+05$ & $4.1 \mathrm{E}+04$ & & & & & & & $5.2 \mathrm{E}+05$ & $5.2 \mathrm{E}+04$ & & & $2.3 \mathrm{E}+05$ & $2.3 \mathrm{E}+04$ \\
\hline Ethyl Methacrylate & $000097-63-2$ & & & $1.8 \mathrm{E}+05$ & $1.8 \mathrm{E}+04$ & & & & & & & $2.3 \mathrm{E}+05$ & $2.3 \mathrm{E}+04$ & & & $1.0 \mathrm{E}+05$ & $1.0 \mathrm{E}+04$ \\
\hline $\begin{array}{l}\text { Ethyl-p-nitrophenyl } \\
\text { Phosphonate }\end{array}$ & $002104-64-5$ & & & $2.0 \mathrm{E}+01$ & $2.0 \mathrm{E}+00$ & & & & & & & $1.6 \mathrm{E}+01$ & $1.6 \mathrm{E}+00$ & & & $9.0 E+00$ & $9.0 \mathrm{E}-01$ \\
\hline Ethylbenzene ${ }^{(6)}$ & $000100-41-4$ & & & $2.0 \mathrm{E}+05$ & $2.0 \mathrm{E}+04$ & & & 4.7E-01 & 4.7E-01 & & & $3.1 \mathrm{E}+05$ & $3.1 \mathrm{E}+04$ & & & 4.7E-01 & $4.7 \mathrm{E}-01$ \\
\hline Ethylene Cyanohydrin & $000109-78-4$ & & & $6.1 E+05$ & $6.1 \mathrm{E}+04$ & & & & & & & $4.9 \mathrm{E}+05$ & $4.9 \mathrm{E}+04$ & & & $2.7 \mathrm{E}+05$ & $2.7 \mathrm{E}+04$ \\
\hline Ethylene Diamine & $000107-15-3$ & & & $4.1 \mathrm{E}+04$ & $4.1 \mathrm{E}+03$ & & & & & & & $3.2 \mathrm{E}+04$ & $3.2 \mathrm{E}+03$ & & & $1.8 \mathrm{E}+04$ & $1.8 \mathrm{E}+03$ \\
\hline Ethylene Glycol & $000107-21-1$ & & & $1.0 \mathrm{E}+06$ & $4.1 \mathrm{E}+05$ & & & & & & & $1.0 \mathrm{E}+06$ & $3.2 \mathrm{E}+05$ & & & $1.0 \mathrm{E}+06$ & $1.8 E+05$ \\
\hline $\begin{array}{l}\text { Ethylene Glycol } \\
\text { Monobutyl Ether }\end{array}$ & $000111-76-2$ & & & & & & & $1.0 \mathrm{E}+06$ & $1.0 \mathrm{E}+06$ & & & & & & & & \\
\hline Ethylene Oxide & $000075-21-8$ & $5.6 \mathrm{E}+02$ & $5.6 \mathrm{E}+00$ & & & $8.2 E+01$ & $8.2 \mathrm{E}-01$ & & & $7.1 E+02$ & $7.1 E+00$ & & & $6.5 \mathrm{E}+01$ & $6.5 \mathrm{E}-01$ & & \\
\hline Ethylene Thiourea & $000096-45-7$ & $5.2 \mathrm{E}+03$ & $5.2 \mathrm{E}+01$ & $1.6 \mathrm{E}+02$ & $1.6 \mathrm{E}+01$ & & & & & $4.1 \mathrm{E}+03$ & 4.1E+01 & $1.3 E+02$ & $1.3 \mathrm{E}+01$ & $2.3 \mathrm{E}+03$ & $2.3 \mathrm{E}+01$ & $7.2 E+01$ & $7.2 E+00$ \\
\hline
\end{tabular}


Table 3.2c. (continued)

\begin{tabular}{|c|c|c|c|c|c|c|c|c|c|c|c|c|c|c|c|c|c|}
\hline \multirow[t]{2}{*}{ Chemical } & \multirow[t]{2}{*}{ CASRN } & \multicolumn{4}{|c|}{ Ingestion (mg/kg) } & \multicolumn{4}{|c|}{ Inhalation (mg/kg) } & \multicolumn{4}{|c|}{ Dermal (mg/kg) } & \multicolumn{4}{|c|}{$\begin{array}{l}\text { Ingestion + Inhalation } \\
+ \text { Dermal }(\mathrm{mg} / \mathrm{kg})^{\varphi^{-3}}\end{array}$} \\
\hline & & $10^{-4}$ & $10^{4}$ & $\mathrm{HQ}=1$ & $\mathrm{HQ}=0.1$ & $10^{-4}$ & $10^{4}$ & $\mathrm{HQ}=1$ & $\mathrm{HQ}=0.1$ & $10^{-4}$ & $10^{6}$ & $\mathbf{H Q}=1$ & $\mathrm{HQ}=0.1$ & $10^{-4}$ & $10^{6}$ & $H I=1$ & $\mathrm{HI}=0.1$ \\
\hline $\begin{array}{l}\text { Ethylphthalyl Ethyl } \\
\text { Glycolate }\end{array}$ & $000084-72-0$ & & & $1.0 \mathrm{E}+06$ & $6.1 \mathrm{E}+05$ & & & & & & & $1.0 \mathrm{E}+06$ & $4.9 \mathrm{E}+05$ & & & $1.0 \mathrm{E}+06$ & $2.7 \mathrm{E}+05$ \\
\hline Express & $101200-48-0$ & & & $1.6 \mathrm{E}+04$ & $1.6 \mathrm{E}+03$ & • & & & & & & $1.3 E+04$ & $1.3 \mathrm{E}+03$ & & & $7.2 \mathrm{E}+03$ & $7.2 E+02$ \\
\hline Fenamiphos & $022224-92-6$ & & & $5.1 E+02$ & $5.1 \mathrm{E}+01$ & & & & & & & $4.0 \mathrm{E}+02$ & $4.0 \mathrm{E}+01$ & & & $2.3 \mathrm{E}+02$ & $2.3 \mathrm{E}+0 \mathrm{t}$ \\
\hline Fenpropathrin & $039515-41-8$ & & & $5.1 E+04$ & $5.1 E+03$ & & & & & & & $4.0 \mathrm{E}+04$ & $4.0 E+03$ & & & $2.3 \mathrm{E}+04$ & $2.3 \mathrm{E}+03$ \\
\hline Fluometuron & $002164-17-2$ & & & $2.7 \mathrm{E}+04$ & $2.7 \mathrm{E}+03$ & & & & & & & 2.1E+04 & $2.1 \mathrm{E}+03$ & & & $1.2 E+04$ & $1.2 \mathrm{E}+03$ \\
\hline Fluoranthene & $000206-44-0$ & & & $8.2 E+04$ & $8.2 \mathrm{E}+03$ & & & & & & & $4.0 \mathrm{E}+04$ & $4.0 \mathrm{E}+03$ & & & $2.7 E+04$ & $2.7 \mathrm{E}+03$ \\
\hline Fluorene & $000086-73-7$ & & & $8.2 E+04$ & $8.2 \mathrm{E}+03$ & & & & & & & $1.0 \mathrm{E}+05$ & $1.0 \mathrm{E}+04$ & & & $4.6 \mathrm{E}+04$ & $4.6 \mathrm{E}+03$ \\
\hline Fluoride & $007782-41-4$ & & & $1.2 \mathrm{E}+05$ & $1.2 \mathrm{E}+04$ & & & & & & & $1.0 \mathrm{E}+06$ & $1.9 \mathrm{E}+05$ & & & $1.2 \mathrm{E}+05$ & $1.2 \mathrm{E}+04$ \\
\hline Fluridone & $059756-60-4$ & & & $1.6 \mathrm{E}+05$ & $1.6 \mathrm{E}+04$ & & & & & & & $1.3 \mathrm{E}+05$ & $1.3 E+04$ & & & $7.2 \mathrm{E}+04$ & $7.2 \mathrm{E}+03$ \\
\hline Flurprimidol & $056425-91-3$ & & & $4.1 \mathrm{E}+04$ & $4.1 \mathrm{E}+03$ & & & & & & & $3.2 \mathrm{E}+04$ & $3.2 \mathrm{E}+03$ & & & $1.8 \mathrm{E}+04$ & $1.8 \mathrm{E}+03$ \\
\hline Flutolanil & $066332-96-5$ & & & $1.2 \mathrm{E}+05$ & $1.2 \mathrm{E}+04$ & & & & & & & $9.7 E+04$ & $9.7 E+03$ & & & $5.4 \mathrm{E}+04$ & $5.4 E+03$ \\
\hline Fluvalinate & 069409-94-5 & & & $2.0 \mathrm{E}+04$ & $2.0 \mathrm{E}+03$ & & & & & & & $1.6 \mathrm{E}+04$ & $1.6 \mathrm{E}+03$ & & & $9.0 \mathrm{E}+03$ & $9.0 \mathrm{E}+02$ \\
\hline Folpet & $000133-07-3$ & $1.6 \mathrm{E}+05$ & $1.6 \mathrm{E}+03$ & $2.0 \mathrm{E}+05$ & $2.0 \mathrm{E}+04$ & & & & & $1.3 \mathrm{E}+05$ & $1.3 \mathrm{E}+03$ & $1.6 \mathrm{E}+05$ & $1.6 \mathrm{E}+04$ & $7.2 E+04$ & $7.2 \mathrm{E}+02$ & $9.0 E+04$ & $9.0 \mathrm{E}+03$ \\
\hline Fomesafen & $072178-02-0$ & $3.0 \mathrm{E}+03$ & $3.0 \mathrm{E}+01$ & & & & & & & $2.4 \mathrm{E}+03$ & $2.4 \mathrm{E}+01$ & & & $1.3 \mathrm{E}+03$ & $1.3 \mathrm{E}+01$ & & \\
\hline Fonofos & $000944-22-9$ & & & $4.1 \mathrm{E}+03$ & $4.1 E+02$ & & & & & & & $3.2 \mathrm{E}+03$ & $3.2 \mathrm{E}+02$ & & & $1.8 \mathrm{E}+03$ & $1.8 \mathrm{E}+02$ \\
\hline Formaldehyde & $000050-00-0$ & & & $4.1 \mathrm{E}+05$ & $4.1 \mathrm{E}+04$ & $1.0 \mathrm{E}+06$ & $1.0 \mathrm{E}+06$ & & & & & $5.2 \mathrm{E}+05$ & $5.2 E+04$ & & & $2.3 \mathrm{E}+05$ & $2.3 \mathrm{E}+04$ \\
\hline Formic Acid & $000064-18-6$ & & & $1.0 \mathrm{E}+06$ & $4.1 \mathrm{E}+05$ & & & & & & & $1.0 \mathrm{E}+06$ & $3.2 \mathrm{E}+05$ & & & $1.0 \mathrm{E}+06$ & $1.8 \mathrm{E}+05$ \\
\hline Fosetyl-AL & $039148-24-8$ & & & $1.0 \mathrm{E}+06$ & $6.1 \mathrm{E}+05$ & & & & & & & $1.0 \mathrm{E}+06$ & $4.9 \mathrm{E}+05$ & & & $1.0 \mathrm{E}+06$ & 2.7E+05 \\
\hline Furan & $000110-00.9$ & & & $2.0 \mathrm{E}+03$ & $2.0 E+02$ & & & & & & & $2.6 \mathrm{E}+03$ & $2.6 \mathrm{E}+02$ & & & $1.1 E+03$ & $1.1 \mathrm{E}+02$ \\
\hline Furazolidone & $000067-45-8$ & $1.5 \mathrm{E}+02$ & $1.5 \mathrm{E}+00$ & & & & & & & $1.2 \mathrm{E}+02$ & $1.2 \mathrm{E}+00$ & & & $6.7 \mathrm{E}+01$ & $6.7 \mathrm{E}-01$ & & \\
\hline Furfural & $000098-01-1$ & & & $6.1 \mathrm{E}+03$ & $6.1 \mathrm{E}+02$ & & & $1.0 \mathrm{E}+06$ & $1.0 \mathrm{E}+06$ & & & $4.9 \mathrm{E}+03$ & $4.9 E+02$ & & & $2.7 \mathrm{E}+03$ & $2.7 \mathrm{E}+02$ \\
\hline Furium & $000531-82-8$ & $1.1 \mathrm{E}+01$ & $1.1 \mathrm{E}-01$ & & & & & & & $9.1 \mathrm{E}+00$ & $9.1 \mathrm{E}-02$ & & & $5.1 \mathrm{E}+00$ & $5.1 \mathrm{E}-02$ & & \\
\hline Furmecyclox & $060568-05-0$ & $1.9 \mathrm{E}+04$ & $1.9 E+02$ & & & & & & & $1.5 E+04$ & $1.5 \mathrm{E}+02$ & & & $8.4 E+03$ & $8.4 E+01$ & & \\
\hline
\end{tabular}

Nonradionuclides/soil/industrial

Page 15 of 32 
Table 3.2c. (continued)

\begin{tabular}{|c|c|c|c|c|c|c|c|c|c|c|c|c|c|c|c|c|c|}
\hline \multirow{2}{*}{ Chemical } & \multirow{2}{*}{ CAȘRN } & \multicolumn{4}{|c|}{ Ingestion (mg/kg)* } & \multicolumn{4}{|c|}{ Inhalation $(\mathrm{mg} / \mathrm{kg})^{\cdot \gamma}$} & \multicolumn{4}{|c|}{ Dermal (mg/kg) } & \multicolumn{4}{|c|}{$\begin{array}{l}\text { Ingestion + Inhalation } \\
+ \text { Dermal (mg/kg) }\end{array}$} \\
\hline & & $10^{-4}$ & $10^{-5}$ & $H Q=1$ & $\mathrm{HQ}=0.1$ & $10^{-4}$ & $10^{4}$ & $\mathrm{HQ}=1$ & $\mathrm{HQ}=0.1$ & $10^{-4}$ & $10^{4}$ & $H Q=1$ & $H Q=0.1$ & $10^{-1}$ & $10^{6}$ & $H I=1$ & $\mathrm{HI}=0.1$ \\
\hline Glufosinate, Ammonium & $077182-82-2$ & & & $8.2 \mathrm{E}+02$ & $8.2 \mathrm{E}+01$ & & & & & & & $6.5 \mathrm{E}+02$ & $6.5 \mathrm{E}+01$ & & & $3.6 \mathrm{E}+02$ & $3.6 \mathrm{E}+01$ \\
\hline Glycidyl & $000765-34-4$ & & & $8.2 E+02$ & $8.2 \mathrm{E}+01$ & & & $1.0 \mathrm{E}+06$ & $6.3 \mathrm{E}+05$ & & & $6.5 \mathrm{E}+02$ & $6.5 \mathrm{E}+01$ & & & $3.6 \mathrm{E}+02$ & $3.6 \mathrm{E}+01$ \\
\hline Glyphosate & $001071-83-6$ & & & $2.0 \mathrm{E}+05$ & $2.0 \mathrm{E}+04$ & & & & & & & $1.6 \mathrm{E}+05$ & $1.6 \mathrm{E}+04$ & & & $9.0 \mathrm{E}+04$ & $9.0 \mathrm{E}+03$ \\
\hline Goal & $042874-03-3$ & & & $6.1 E+03$ & $6.1 \mathrm{E}+02$ & & & & & & & $4.9 \mathrm{E}+03$ & $4.9 \mathrm{E}+02$ & & & $2.7 \mathrm{E}+03$ & $2.7 E+02$ \\
\hline Haloxyfop, Methyl & $069806-40-2$ & & & $1.0 \mathrm{E}+02$ & $1.0 \mathrm{E}+01$ & & & & & & & $8.1 \mathrm{E}+01$ & $8.1 E+00$ & & & $4.5 \mathrm{E}+01$ & $4.5 \mathrm{E}+00$ \\
\hline Harmony & $079277-27-3$ & & & $2.7 \mathrm{E}+04$ & $2.7 \mathrm{E}+03$ & & & & & & & $2.1 \mathrm{E}+04$ & $2.1 E+03$ & & & $1.2 \mathrm{E}+04$ & $1.2 \mathrm{E}+03$ \\
\hline Heptachlor & $000076-44-8$ & $1.3 \mathrm{E}+02$ & $1.3 \mathrm{E}+00$ & $1.0 \mathrm{E}+03$ & $1.0 \mathrm{E}+02$ & $1.0 \mathrm{E}+06$ & $1.4 \mathrm{E}+04$ & & & $1.4 \mathrm{E}+02$ & $1.4 \mathrm{E}+00$ & $1.2 \mathrm{E}+03$ & $1.2 \mathrm{E}+02$ & $6.8 \mathrm{E}+01$ & $6.8 \mathrm{E}-01$ & $5.4 \mathrm{E}+02$ & $5.4 \mathrm{E}+01$ \\
\hline Heptachlor Epoxide & $001024-57-3$ & $6.3 \mathrm{E}+01$ & $6.3 \mathrm{E}-01$ & $2.7 \mathrm{E}+01$ & $2.7 \mathrm{E}+00$ & $6.7 \mathrm{E}+05$ & $6.7 \mathrm{E}+03$ & & & $7.2 \mathrm{E}+01$ & $7.2 \mathrm{E}-01$ & $3.0 \mathrm{E}+01$ & $3.0 \mathrm{E}+00$ & $3.4 \mathrm{E}+01$ & $3.4 \mathrm{E}-01$ & $1.4 \mathrm{E}+01$ & $1.4 \mathrm{E}+00$ \\
\hline Heptane, N. & $000142-82-5$ & & & & & & & & & & & & & & & & \\
\hline Hexabromobenzene & $000087-82-1$ & & & $4.1 \mathrm{E}+03$ & 4.1E+02 & & & & & & & $5.2 \mathrm{E}+03$ & $5.2 \mathrm{E}+02$ & & & $2.3 \mathrm{E}+03$ & $2.3 \mathrm{E}+02$ \\
\hline Hexachlorobenzene $e^{(6)}$ & $000118-74-1$ & $3.6 \mathrm{E}+02$ & $3.6 \mathrm{E}+00$ & $1.6 \mathrm{E}+03$ & $1.6 \mathrm{E}+02$ & $1.0 \mathrm{E}+06$ & $3.8 \mathrm{E}+04$ & & & 4.5E+02 & $4.5 \mathrm{E}+00$ & $2.1 \mathrm{E}+03$ & $2.1 \mathrm{E}+02$ & $2.0 \mathrm{E}+02$ & $2.0 \mathrm{E}+00$ & $9.1 \mathrm{E}+02$ & $9.1 \mathrm{E}+01$ \\
\hline Hexachlorobutadiene ${ }^{(s)}$ & $000087-68-3$ & $7.3 \mathrm{E}+03$ & $7.3 \mathrm{E}+01$ & $4.1 \mathrm{E}+02$ & 4.1E+01 & $1.0 \mathrm{E}+06$ & $7.9 E+05$ & & & $9.3 E+03$ & $9.3 \mathrm{E}+01$ & $5.2 \mathrm{E}+02$ & $5.2 \mathrm{E}+01$ & 4.1E+03 & 4.1E+01 & $2.3 \mathrm{E}+02$ & $2.3 \mathrm{E}+01$ \\
\hline $\begin{array}{l}\text { Hexachlorocyclohexane, } \\
\text { Alpha- }\end{array}$ & $000319-84-6$ & $9.1 \mathrm{E}+01$ & $9.1 \mathrm{E}-01$ & & & $9.7 \mathrm{E}+05$ & $9.7 \mathrm{E}+03$ & & & $1.4 \mathrm{E}+02$ & $1.4 \mathrm{E}+00$ & & & $5.5 \mathrm{E}+01$ & $5.5 \mathrm{E}-01$ & & \\
\hline $\begin{array}{l}\text { Hexachlorocyclohexane, } \\
\text { Beta- }\end{array}$ & $000319-85-7$ & $3.2 \mathrm{E}+02$ & $3.2 \mathrm{E}+00$ & & & $1.0 \mathrm{E}+06$ & $3.4 \mathrm{E}+04$ & & & $4.6 \mathrm{E}+02$ & $4.6 \mathrm{E}+00$ & & & $1.9 \mathrm{E}+02$ & $1.9 \mathrm{E}+00$ & & \\
\hline $\begin{array}{l}\text { Hexachlorocyclohexane, } \\
\text { Della- }\end{array}$ & $000319-86-8$ & & & & & & & & & & & & & & & & \\
\hline $\begin{array}{l}\text { Hexachlorocyclohexane, } \\
\text { Epsilon }\end{array}$ & $006108-10-7$ & & & & & & & & & & & & & & & & \\
\hline $\begin{array}{l}\text { Hexachlorocyclohexane, } \\
\text { Gamma- }\end{array}$ & $000058-89-9$ & $4.4 \mathrm{E}+02$ & $4.4 E+00$ & $6.1 \mathrm{E}+02$ & $6.1 \mathrm{E}+01$ & & & & & $6.8 \mathrm{E}+02$ & $6.8 \mathrm{E}+00$ & $9.4 \mathrm{E}+02$ & $9.4 \mathrm{E}+01$ & $2.7 \mathrm{E}+02$ & $2.7 \mathrm{E}+00$ & $3.7 E+02$ & $3.7 \mathrm{E}+01$ \\
\hline $\begin{array}{l}\text { Hexachlorocyclohexane, } \\
\text { Technical }\end{array}$ & 000608-73-1 & $3.2 \mathrm{E}+02$ & $3.2 \mathrm{E}+00$ & & & $1.0 \mathrm{E}+06$ & $3.4 \mathrm{E}+04$ & & & $2.5 \mathrm{E}+02$ & $2.5 \mathrm{E}+00$ & & & $1.4 \mathrm{E}+02$ & $1.4 \mathrm{E}+00$ & & \\
\hline $\begin{array}{l}\text { Hexachlorocyclopenta- } \\
\text { diene }\end{array}$ & $000077-47-4$ & & & $1.4 \mathrm{E}+04$ & $1.4 E+03$ & & & $4.4 E+05$ & $4.4 E+04$ & & & $1.8 \mathrm{E}+04$ & $1.8 \mathrm{E}+03$ & & & $7.8 \mathrm{E}+03$ & $7.8 E+02$ \\
\hline $\begin{array}{l}\text { Hexachlorodibenzo-p- } \\
\text { dioxin, Mixture }\end{array}$ & $019408-74-3$ & $9.2 \mathrm{E}-02$ & $9.2 \mathrm{E}-04$ & & & $1.3 \mathrm{E}+03$ & $1.3 \mathrm{E}+01$ & & & 7.3E-02 & 7.3E-04 & & & 4.1E-02 & 4.1E-04 & & \\
\hline
\end{tabular}

Nonradionuclides/soil/industrial

Page 16 of 32 
Table 3.2c. (continued)

\begin{tabular}{|c|c|c|c|c|c|c|c|c|c|c|c|c|c|c|c|c|c|}
\hline \multirow[t]{2}{*}{ Chemical } & \multirow[t]{2}{*}{ CASRN } & \multicolumn{4}{|c|}{ Ingestion (mg/kg) } & \multicolumn{4}{|c|}{ Inbalation (mg/kg) } & \multicolumn{4}{|c|}{ Dermal (mg/kg) } & \multicolumn{4}{|c|}{$\begin{array}{l}\text { Ingestion + Inhalation } \\
+ \text { Dermal (mg/kg) }\end{array}$} \\
\hline & & $10^{-4}$ & $10^{4}$ & $H Q=1$ & $H Q=0.1$ & $10^{-4}$ & $10^{s}$ & $\mathrm{HQ}=1$ & $\mathrm{HQ}=0.1$ & $10^{-4}$ & $10^{4}$ & $H Q=1$ & $\mathrm{HQ}=0.1$ & $10^{-4}$ & $10^{6}$ & $H I=1$ & $\mathrm{HI}=0.1$ \\
\hline Hexachloroethane & $000067-72-1$ & $4.1 E+04$ & 4.1E+02 & $2.0 E+03$ & $2.0 \mathrm{E}+02$ & $1.0 \mathrm{E}+06$ & $1.0 \mathrm{E}+06$ & & & $5.2 \mathrm{E}+04$ & $5.2 E+02$ & $2.6 \mathrm{E}+03$ & $2.6 \mathrm{E}+02$ & $2.3 \mathrm{E}+04$ & $2.3 \mathrm{E}+02$ & 1:1E+03 & $1.1 E+02$ \\
\hline Hexachlorophene & $000070-30-4$ & & & $6.1 E+02$ & $6.1 \mathrm{E}+01$ & & & & & & & $4.9 \mathrm{E}+02$ & $4.9 E+01$ & & & $2.7 \mathrm{E}+02$ & $2.7 \mathrm{E}+01$ \\
\hline $\begin{array}{l}\text { Hexahydro-1,3,5-trinitro- } \\
\text { 1,3,5-triazine (RDX) }\end{array}$ & $000121-82-4$ & $5.2 \mathrm{E}+03$ & $5.2 \mathrm{E}+01$ & $6.1 E+03$ & $6.1 \mathrm{E}+02$ & & & & & $8.2 E+03$ & $8.2 E+01$ & $9.7 \mathrm{E}+03$ & $9.7 \mathrm{E}+02$ & $3.2 \mathrm{E}+03$ & $3.2 \mathrm{E}+01$ & $3.8 \mathrm{E}+03$ & $3.8 \mathrm{E}+02$ \\
\hline $\begin{array}{l}\text { Hexamethylene } \\
\text { Diisocyanate, 1,6. }\end{array}$ & $000822-06-0$ & & & & & & & $6.3 \mathrm{E}+04$ & $6.3 \mathrm{E}+03$ & & & & & & & & \\
\hline Hexane, N- & $000110-54-3$ & & & $1.2 \mathrm{E}+05$ & $1.2 E+04$ & & & $9.1 \mathrm{E}+01$ & $9.1 \mathrm{E}+01$ & & & $1.6 \mathrm{E}+05$ & $1.6 \mathrm{E}+04$ & & & $9.1 E+01$ & $9.1 E+01$ \\
\hline Hexanone, 2- & $000591-78-6$ & & & & & & & & & & & & & & & & \\
\hline Hexazinone & $051235-04-2$ & & & $6.7 E+04$ & $6.7 E+03$ & & & & & & & $5.3 \mathrm{E}+04$ & $5.3 \mathrm{E}+03$ & & & $3.0 \mathrm{E}+04$ & $3.0 \mathrm{E}+03$ \\
\hline $\mathrm{HpCDD}, 2,3,7,8{ }^{\left({ }^{(4)}\right.}$ & $037871-00-4$ & $3.8 \mathrm{E}-01$ & $3.8 \mathrm{E}-03$ & & & $4.1 E+03$ & $4.1 \mathrm{E}+01$ & & & $1.0 \mathrm{E}-01$ & $1.0 \mathrm{E}-03$ & & & $8.0 \mathrm{E}-02$ & $8.0 \mathrm{E}-04$ & & \\
\hline HpCDF, 2,3,7,8-(1) & 038998-75-3 & $3.8 \mathrm{E}-01$ & $3.8 \mathrm{E}-03$ & & & 4.1E+03 & $4.1 \mathrm{E}+01$ & & & $3.0 \mathrm{E}-01$ & $3.0 \mathrm{E}-03$ & & & $1.7 \mathrm{E}-01$ & $1.7 \mathrm{E}-03$ & & \\
\hline $\mathrm{HxCDD}, 2,3,7,8-^{(4)}$ & $034465-46-8$ & $3.8 \mathrm{E}-02$ & $3.8 \mathrm{E}-04$ & & & 4.1E+02 & 4.1E+00 & & & $1.0 E-02$ & $1.0 \mathrm{E}-04$ & & & $8.0 \mathrm{E}-03$ & $8.0 E-05$ & & \\
\hline $\mathrm{HxCDF}, 2,3,7,8-(1)$ & 055684-94-1 & $3.8 \mathrm{E}-02$ & $3.8 \mathrm{E}-04$ & & & $4.1 E+02$ & $4.1 \mathrm{E}+00$ & & & $3.0 \mathrm{E}-02$ & $3.0 \mathrm{E}-04$ & & & $1.7 \mathrm{E}-02$ & $1.7 \mathrm{E}-04$ & & \\
\hline Hydrazine & $000302-01-2$ & $1.9 \mathrm{E}+02$ & $1.9 \mathrm{E}+00$ & & & $3.6 \mathrm{E}+05$ & $3.6 \mathrm{E}+03$ & & & $1.5 E+02$ & $1.5 \mathrm{E}+00$ & & & $8.4 \mathrm{E}+01$ & $8.4 \mathrm{E}-01$ & & \\
\hline Hydrazine Sulfate & 010034.93 .2 & $1.9 \mathrm{E}+02$ & $1.9 \mathrm{E}+00$ & & & $3.6 \mathrm{E}+05$ & $3.6 \mathrm{E}+03$ & & & $1.5 \mathrm{E}+02$ & $1.5 \mathrm{E}+00$ & & & $8.4 \mathrm{E}+01$ & $8.4 \mathrm{E}-01$ & & \\
\hline Hydrogen Chloride & $007647-01-0$ & & & & & & & $1.0 \mathrm{E}+06$ & $1.0 \mathrm{E}+06$ & & & & & & & & \\
\hline Hydrogen Cyanide & $000074-90-8$ & & & 4.1E+04 & $4.1 \mathrm{E}+03$ & & & $1.0 \mathrm{E}+06$ & $1.0 \mathrm{E}+06$ & & & $1.1 \mathrm{E}+05$ & $1.1 \mathrm{E}+04$ & & & $3.0 \mathrm{E}+04$ & $3.0 \mathrm{E}+03$ \\
\hline Hydrogen Sulfide & $007783-06-4$ & & & $6.1 E+03$ & $6.1 \mathrm{E}+02$ & & & $1.0 \mathrm{E}+06$ & $6.3 \mathrm{E}+05$ & & & $1.9 \mathrm{E}+04$ & $1.9 \mathrm{E}+03$ & & & $4.7 \mathrm{E}+03$ & $4.7 \mathrm{E}+02$ \\
\hline Hydroquinone & $000123-31-9$ & & & $8.2 E+04$ & $8.2 \mathrm{E}+03$ & & & & & & & $6.5 \mathrm{E}+04$ & $6.5 \mathrm{E}+03$ & & & $3.6 \mathrm{E}+04$ & $3.6 \mathrm{E}+03$ \\
\hline Imazalil & $035554-44-0$ & & & $2.7 \mathrm{E}+04$ & $2.7 \mathrm{E}+03$ & & & & & & & $2.1 \mathrm{E}+04$ & $2.1 \mathrm{E}+03$ & & & $1.2 \mathrm{E}+04$ & $1.2 \mathrm{E}+03$ \\
\hline Imazaquin & $081335-37-7$ & & & $5.1 E+05$ & $5.1 E+04$ & & & & & & & $4.0 \mathrm{E}+05$ & $4.0 E+04$ & & & $2.3 \mathrm{E}+05$ & $2.3 \mathrm{E}+04$ \\
\hline Indeno[1,2,3-cd]pyrene ${ }^{(2)}$ & $000193-39-5$ & $7.8 \mathrm{E}+02$ & $7.8 \mathrm{E}+00$ & & & $1.0 \mathrm{E}+06$ & $2.0 \mathrm{E}+05$ & & & $3.8 \mathrm{E}+02$ & $3.8 \mathrm{E}+00$ & & & $2.6 \mathrm{E}+02$ & $2.6 \mathrm{E}+00$ & & \\
\hline Iprodione & $036734-19-7$ & & & $8.2 \mathrm{E}+04$ & $8.2 E+03$ & & & & & & & $6.5 \mathrm{E}+04$ & $6.5 \mathrm{E}+03$ & & & $3.6 \mathrm{E}+04$ & $3.6 \mathrm{E}+03$ \\
\hline Iron & $007439-89-6$ & & & & & & & & & & & & & & & & \\
\hline
\end{tabular}


Table 3.2c. (continued)

\begin{tabular}{|c|c|c|c|c|c|c|c|c|c|c|c|c|c|c|c|c|c|}
\hline \multirow[t]{2}{*}{ Chemical } & \multirow[t]{2}{*}{ CASRN } & \multicolumn{4}{|c|}{ Ingestion (mg/kg) } & \multicolumn{4}{|c|}{ Inhalation $(\mathrm{mg} / \mathrm{kg})^{-8}$} & \multicolumn{4}{|c|}{ Dermal (mg/kg) } & \multicolumn{4}{|c|}{$\begin{array}{l}\text { Ingestion + Inhalation } \\
+ \text { Dermal }(\mathrm{mg} / \mathrm{kg})^{1 \cdot v}\end{array}$} \\
\hline & & $10^{-4}$ & $10^{4}$ & $H Q=1$ & $\mathrm{HQ}=0.1$ & $10^{-4}$ & $10^{-4}$ & $\mathrm{HQ}=1$ & $\mathrm{HQ}=0.1$ & $10^{4}$ & $10^{4}$ & $H Q=1$ & $H Q=0.1$ & $10^{-4}$ & $10^{4}$ & $\mathrm{HI}=1$ & $H I=0.1$ \\
\hline Isobutyl Alcohol & $000078-83-1$ & & & $6.1 \mathrm{E}+05$ & $6.1 \mathrm{E}+04$ & & & & & & & $7.8 \mathrm{E}+05$ & $7.8 \mathrm{E}+04$ & & & $3.4 \mathrm{E}+05$ & $3.4 \mathrm{E}+04$ \\
\hline Isophorone & $000078-59-1$ & $6.0 \mathrm{E}+05$ & $6.0 \mathrm{E}+03$ & 4.1E+05 & $4.1 \mathrm{E}+04$ & & & & & $4.8 \mathrm{E}+05$ & $4.8 \mathrm{E}+03$ & $3.2 \mathrm{E}+05$ & $3.2 \mathrm{E}+04$ & $2.7 \mathrm{E}+05$ & $2.7 \mathrm{E}+03$ & $1.8 \mathrm{E}+05$ & $1.8 \mathrm{E}+04$ \\
\hline Isopropalin & $033820-53-0$ & & & $3.1 \mathrm{E}+04$ & $3.1 \mathrm{E}+03$ & & & & & & & $2.4 \mathrm{E}+04$ & $2.4 \mathrm{E}+03$ & & & $1.4 \mathrm{E}+04$ & $1.4 \mathrm{E}+03$ \\
\hline Isopropanol & $000067-63-0$ & & & & & & & & & & & & & & & & \\
\hline $\begin{array}{l}\text { Isopropyl Methyl } \\
\text { Phosphonic Acid }\end{array}$ & $001832-54-8$ & & & $2.0 \mathrm{E}+05$ & $2.0 \mathrm{E}+04$ & & & & & & & $1.6 \mathrm{E}+05$ & $1.6 \mathrm{E}+04$ & & & $9.0 \mathrm{E}+04$ & $9.0 \mathrm{E}+03$ \\
\hline Isoxaben & $082558-50-7$ & & & $1.0 \mathrm{E}+05$ & $1.0 \mathrm{E}+04$ & & & & & & & $8.1 E+04$ & $8.1 E+03$ & & & $4.5 \mathrm{E}+04$ & $4.5 \mathrm{E}+03$ \\
\hline Karate & $091465-08-6$ & & & $1.0 \mathrm{E}+04$ & $1.0 \mathrm{E}+03$ & & & & & & & $8.1 E+03$ & $8.1 \mathrm{E}+02$ & & & $4.5 \mathrm{E}+03$ & $4.5 \mathrm{E}+02$ \\
\hline Kerb & $023950-58-5$ & & & $1.5 \mathrm{E}+05$ & $1.5 \mathrm{E}+04$ & & & & & & & $1.2 \mathrm{E}+05$ & $1.2 \mathrm{E}+04$ & & & $6.8 \mathrm{E}+04$ & $6.8 \mathrm{E}+03$ \\
\hline Lactofen & $077501-63-4$ & & & $4.1 \mathrm{E}+03$ & $4.1 \mathrm{E}+02$ & & & & & & & $3.2 \mathrm{E}+03$ & $3.2 \mathrm{E}+02$ & & & $1.8 \mathrm{E}+03$ & $1.8 \mathrm{E}+02$ \\
\hline Lead Alkyls & NA & & & & & & & & & & & & & & & & \\
\hline Lead And Compounds & $007439-92-1$ & & & & & & & & & & & & & & & & \\
\hline Linuron & $000330-55-2$ & . & & $4.1 \mathrm{E}+03$ & $4.1 \mathrm{E}+02$ & & & & & & & $3.2 \mathrm{E}+03$ & $3.2 \mathrm{E}+02$ & & & $1.8 \mathrm{E}+03$ & $1.8 E+02$ \\
\hline Lithium & $007439-93-2$ & & & & & & & & & & & & & & & & \\
\hline Londax & 083055-99-6 & & & $4.1 \mathrm{E}+05$ & $4.1 \mathrm{E}+04$ & & & & & & & $3.2 \mathrm{E}+05$ & $3.2 \mathrm{E}+04$ & & & $1.8 \mathrm{E}+05$ & $1.8 \mathrm{E}+04$ \\
\hline $\mathrm{MCPA}$ & $000094-74-6$ & & & $1.0 \mathrm{E}+03$ & $1.0 \mathrm{E}+02$ & & & & & & & $8.1 \mathrm{E}+02$ & $8.1 E+01$ & & & $4.5 \mathrm{E}+02$ & $4.5 \mathrm{E}+01$ \\
\hline MCPB & $000094-81-5$ & & & $2.0 \mathrm{E}+04$ & $2.0 \mathrm{E}+03$ & & & & & & & $1.6 \mathrm{E}+04$ & $1.6 \mathrm{E}+03$ & & & $9.0 E+03$ & $9.0 \mathrm{E}+02$ \\
\hline MCPP & $000093-65-2$ & & & $2.0 \mathrm{E}+03$ & $2.0 \mathrm{E}+02$ & & & & & & & $1.6 \mathrm{E}+03$ & $1.6 \mathrm{E}+02$ & & & $9.0 \mathrm{E}+02$ & $9.0 \mathrm{E}+01$ \\
\hline Magnesium & $007439-95-4$ & & & $\cdot$ & & & & & & & & & & & & & \\
\hline Malathion & $000121-75-5$ & & & $4.1 \mathrm{E}+04$ & $4.1 \mathrm{E}+03$ & & & & & & & $3.2 \mathrm{E}+04$ & $3.2 \mathrm{E}+03$ & & & $1.8 \mathrm{E}+04$ & $1.8 \mathrm{E}+03$ \\
\hline Maleic Anhydride & $000108-31-6$ & & & $2.0 \mathrm{E}+05$ & $2.0 \mathrm{E}+04$ & & & & & & & $1.6 \mathrm{E}+05$ & $1.6 \mathrm{E}+04$ & & & $9.0 \mathrm{E}+04$ & $9.0 \mathrm{E}+03$ \\
\hline Maleic Hydrazide & $000123-33-1$ & & & $1.0 \mathrm{E}+06$ & $1.0 \mathrm{E}+05$ & & & & & & & $8.1 \mathrm{E}+05$ & $8.1 \mathrm{E}+04$ & & & $4.5 \mathrm{E}+05$ & 4.5E+04 \\
\hline Malononitrile & $000109-77-3$ & & & $4.1 \mathrm{E}+01$ & $4.1 \mathrm{E}+00$ & & & & & & & $5.2 \mathrm{E}+01$ & $5.2 \mathrm{E}+00$ & & & $2.3 \mathrm{E}+01$ & $2.3 \mathrm{E}+00$ \\
\hline Mancozeb & $008018-01-7$ & & & $6.1 \mathrm{E}+04$ & $6.1 \mathrm{E}+03$ & & & & & & & $4.9 E+04$ & $4.9 E+03$ & & & $2.7 E+04$ & 2.7E+03 \\
\hline
\end{tabular}


Table 3.2c. (continued)

\begin{tabular}{|c|c|c|c|c|c|c|c|c|c|c|c|c|c|c|c|c|c|}
\hline \multirow[t]{2}{*}{ Chemical } & \multirow{2}{*}{ CASRN } & \multicolumn{4}{|c|}{ Ingestion (mg/kg) } & \multicolumn{4}{|c|}{ Inhalation (mg/kg) } & \multicolumn{4}{|c|}{ Dermal (mg/kg) } & \multicolumn{4}{|c|}{$\begin{array}{l}\text { Ingestion + Inhalation } \\
+ \text { Dermal }(\mathrm{mg} / \mathrm{kg})^{+-\boldsymbol{r}}\end{array}$} \\
\hline & & $10^{-4}$ & $10^{6}$ & $\mathrm{HQ}=1$ & $\mathrm{HQ}=0.1$ & $10^{-4}$ & $10^{6}$ & $\mathrm{HQ}=1$ & $\mathrm{HQ}=0.1$ & $10^{-4}$ & $10^{5}$ & $\mathrm{HQ}=1$ & $\mathrm{HQ}=0.1$ & $10^{-4}$ & $10^{6}$ & $\mathrm{HI}=1$ & $\mathrm{HI}=0.1$ \\
\hline Maneb & $012427-38-2$ & & & $1.0 \mathrm{E}+04$ & $1.0 \mathrm{E}+03$ & & & & & & & 8.1E+03 & $8.1 E+02$ & & & $4.5 \mathrm{E}+03$ & $4.5 \mathrm{E}+02$ \\
\hline Manganese (Water) & $007439-96-5$ & & & $9.5 \mathrm{E}+04$ & $9.5 \mathrm{E}+03$ & & & $3.1 \mathrm{E}+05$ & $3.1 \mathrm{E}+04$ & & & $6.0 \mathrm{E}+04$ & $6.0 \mathrm{E}+03$ & & & $3.3 E+04$ & $3.3 \mathrm{E}+03$ \\
\hline Mephosfolan & $000950-10-7$ & & & $1.8 \mathrm{E}+02$ & $1.8 \mathrm{E}+01$ & & & & & & & $1.5 \mathrm{E}+02$ & $1.5 \mathrm{E}+01$ & & & $8.1 E+01$ & $8.1 E+00$ \\
\hline Mepiquat Chloride & $024307-26-4$ & & & $6.1 E+04$ & $6.1 \mathrm{E}+03$ & & & & & & & $4.9 \mathrm{E}+04$ & $4.9 \mathrm{E}+03$ & & & $2.7 E+04$ & $2.7 \mathrm{E}+03$ \\
\hline Mercuric Chloride & $007487-94-7$ & & & $6.1 \mathrm{E}+02$ & $6.1 \mathrm{E}+01$ & & & & & & & $1.9 \mathrm{E}+03$ & $1.9 \mathrm{E}+02$ & & & $4.7 E+02$ & $4.7 \mathrm{E}+01$ \\
\hline Mereury (elemental) & $007439-97-6$ & & & $6.1 E+02$ & $6.1 \mathrm{E}+01$ & & & $1.0 \mathrm{E}+06$ & $1.9 \mathrm{E}+0 \mathrm{~S}$ & & & 9.7E-01 & $9.7 \mathrm{E}-02$ & & & 9.7E-01 & $9.7 \mathrm{E}-02$ \\
\hline Mercury, Inorganic Salt & $007439-97-6$ & & & $6.1 \mathrm{E}+02$ & $6.1 \mathrm{E}+01$ & & & $1.0 \mathrm{E}+06$ & $1.9 \mathrm{E}+05$ & & & $6.8 \mathrm{E}+02$ & $6.8 \mathrm{E}+01$ & & & $3.2 \mathrm{E}+02$ & $3.2 \mathrm{E}+01$ \\
\hline Merphos & $000150-50-5$ & & & $6.1 \mathrm{E}+01$ & $6.1 E+00$ & & & & & & & $4.9 \mathrm{E}+01$ & $4.9 \mathrm{E}+00$ & & & $2.7 \mathrm{E}+01$ & $2.7 \mathrm{E}+00$ \\
\hline Merphos Oxide & $000078-48-8$ & & & $6.1 \mathrm{E}+01$ & $6.1 \mathrm{E}+00$ & & & & & & & $4.9 E+01$ & $4.9 \mathrm{E}+00$ & & & $2.7 E+01$ & $2.7 \mathrm{E}+00$ \\
\hline Metalaxyl & $057837-19-1$ & & & $1.2 E+05$ & $1.2 E+04$ & & & & & & & $9.7 \mathrm{E}+04$ & $9.7 \mathrm{E}+03$ & & & $5.4 \mathrm{E}+04$ & $5.4 \mathrm{E}+03$ \\
\hline Methacrylonitrile & $000126-98-7$ & & & $2.0 \mathrm{E}+02$ & $2.0 \mathrm{E}+01$ & & & $1.2 \mathrm{E}+02$ & $1.2 \mathrm{E}+01$ & & & $2.6 \mathrm{E}+02$ & $2.6 \mathrm{E}+01$ & & & $5.8 \mathrm{E}+01$ & $5.8 \mathrm{E}+00$ \\
\hline Methamidophos & $010265-92-6$ & & & $1.0 \mathrm{E}+02$ & $1.0 \mathrm{E}+01$ & & & & & & & $8.1 E+01$ & $8.1 E+00$ & & & $4.5 \mathrm{E}+01$ & $4.5 \mathrm{E}+00$ \\
\hline Methanol & $000067-56-1$ & & & $1.0 \mathrm{E}+06$ & $1.0 \mathrm{E}+05$ & & & & & & & $1.0 \mathrm{E}+06$ & $1.3 E+05$ & & & $5.7 \mathrm{E}+05$ & $5.7 \mathrm{E}+04$ \\
\hline Methidathion & $000950-37-8$ & & & $2.0 \mathrm{E}+03$ & $2.0 \mathrm{E}+02$ & & & & & & & $1.6 \mathrm{E}+03$ & $1.6 \mathrm{E}+02$ & & & $9.0 \mathrm{E}+02$ & $9.0 \mathrm{E}+0 \mathrm{I}$ \\
\hline Methomyl & $016752-77-5$ & & & $5.1 E+04$ & $5.1 \mathrm{E}+03$ & & & & & & & $4.0 \mathrm{E}+04$ & $4.0 \mathrm{E}+03$ & & & $2.3 \mathrm{E}+04$ & $2.3 \mathrm{E}+03$ \\
\hline $\begin{array}{l}\text { Methoxy-5-nitroaniline, } \\
2 \text { - }\end{array}$ & $000099-59-2$ & $1.2 \mathrm{E}+04$ & $1.2 \mathrm{E}+02$ & & & & & & & $9.8 \mathrm{E}+03$ & $9.8 E+01$ & & & $5.5 \mathrm{E}+03$ & $5.5 \mathrm{E}+01$ & & \\
\hline Methoxychlor & $000072-43-5$ & & & $1.0 \mathrm{E}+04$ & $1.0 \mathrm{E}+03$ & & & & & & & $8.1 E+03$ & $8.1 E+02$ & & & $4.5 \mathrm{E}+03$ & $4.5 \mathrm{E}+02$ \\
\hline $\begin{array}{l}\text { Methoxyethanol Acetate, } \\
2-\end{array}$ & $000110-49-6$ & & & $4.1 \mathrm{E}+03$ & $4.1 \mathrm{E}+02$ & & & & & 1 & & $3.2 \mathrm{E}+03$ & $3.2 \mathrm{E}+02$ & & & $1.8 \mathrm{E}+03$ & $1.8 \mathrm{E}+02$ \\
\hline Methoxyethanol, 2 . & $000109-86-4$ & & & $2.0 \mathrm{E}+03$ & $2.0 \mathrm{E}+02$ & & & $1.0 \mathrm{E}+06$ & $1.0 \mathrm{E}+06$ & & & $1.6 \mathrm{E}+03$ & $1.6 \mathrm{E}+02$ & & & $9.0 \mathrm{E}+02$ & $9.0 \mathrm{E}+01$ \\
\hline Methyl Acetate & $000079-20-9$ & & & $1.0 \mathrm{E}+06$ & $2.0 E+05$ & & & & & & & $1.0 \mathrm{E}+06$ & $1.6 \mathrm{E}+05$ & & & $9.0 \mathrm{E}+05$ & $9.0 \mathrm{E}+04$ \\
\hline Methyl Acrylate & $000096-33-3$ & & & $6.1 \mathrm{E}+04$ & $6.1 \mathrm{E}+03$ & & & & & & & $4.9 \mathrm{E}+04$ & $4.9 \mathrm{E}+03$ & & & $2.7 \mathrm{E}+04$ & $2.7 \mathrm{E}+03$ \\
\hline Methyl Ethyl Ketone & $000078-93-3$ & & & $1.0 \mathrm{E}+06$ & $1.2 \mathrm{E}+05$ & & & $9.2 E+03$ & $3.6 \mathrm{E}+03$ & & & $1.0 \mathrm{E}+06$ & $1.6 \mathrm{E}+05$ & & & $9.2 \mathrm{E}+03$ & $3.5 E+03$ \\
\hline
\end{tabular}

Nonradionuclides/soil/industrial

Page 19 of 32 
Table 3.2c. (continued)

\begin{tabular}{|c|c|c|c|c|c|c|c|c|c|c|c|c|c|c|c|c|c|}
\hline \multirow{2}{*}{ Chemical } & \multirow[t]{2}{*}{ CASRN } & \multicolumn{4}{|c|}{ Ingestion (mg/kg) } & \multicolumn{4}{|c|}{ Inhalation $(\mathrm{mg} / \mathrm{kg})^{\cdot \mathbf{y}}$} & \multicolumn{4}{|c|}{ Dermal (mg/kg) } & \multicolumn{4}{|c|}{$\begin{array}{l}\text { Ingestion + Inhalation } \\
+ \text { Dermal }(\mathrm{mg} / \mathrm{kg})^{+* \vartheta}\end{array}$} \\
\hline & & $10^{-4}$ & $10^{6}$ & $H Q=1$ & $\mathrm{HQ}=0.1$ & $10^{-1}$ & $10^{-4}$ & $H Q=1$ & $\mathrm{HQ}=0.1$ & $10^{-4}$ & $10^{5}$ & $H Q=1$ & $\mathrm{HQ}=0.1$ & $10^{-4}$ & $10^{6}$ & $H I=1$ & $H I=0.1$ \\
\hline Methyl Isobutyl Ketone & $000108-10-1$ & & & $1.6 \mathrm{E}+05$ & $1.6 \mathrm{E}+04$ & & & $7.0 \mathrm{E}+02$ & $7.0 \mathrm{E}+02$ & & & $2.1 \mathrm{E}+05$ & $2.1 \mathrm{E}+04$ & & & $7.0 \mathrm{E}+02$ & $7.0 E+02$ \\
\hline Methyl Mercury & $022967-92-6$ & & & $2.0 \mathrm{E}+02$ & $2.0 \mathrm{E}+01$ & & & & & & & $2.9 \mathrm{E}+02$ & $2.9 \mathrm{E}+01$ & & & $1.2 \mathrm{E}+02$ & $1.2 \mathrm{E}+01$ \\
\hline Methyl Methacrylate & $000080.62-6$ & & & $1.6 \mathrm{E}+05$ & $1.6 \mathrm{E}+04$ & & & & & & & $2.1 \mathrm{E}+05$ & $2.1 \mathrm{E}+04$ & & & $9.1 \mathrm{E}+04$ & $9.1 E+03$ \\
\hline Methyl Parathion & $000298-00-0$ & & & $5.1 \mathrm{E}+02$ & $5.1 \mathrm{E}+01$ & & & & & & & $4.0 E+02$ & $4.0 \mathrm{E}+01$ & & & $2.3 \mathrm{E}+02$ & $2.3 \mathrm{E}+01$ \\
\hline $\begin{array}{l}\text { Methyl Styrene (Mixed } \\
\text { Isomers) }\end{array}$ & $025013-15-4$ & & & $1.2 \mathrm{E}+04$ & $1.2 \mathrm{E}+03$ & & & $1.0 \mathrm{E}+06$ & $1.0 \mathrm{E}+06$ & & & $1.6 \mathrm{E}+04$ & $1.6 \mathrm{E}+03$ & & & $6.9 \mathrm{E}+03$ & $6.9 \mathrm{E}+02$ \\
\hline Methyl hydrazine $e^{(3)}$ & $000060-34-4$ & $5.2 \mathrm{E}+02$ & $5.2 \mathrm{E}+00$ & & & & & & & $4.1 \mathrm{E}+02$ & $4.1 \mathrm{E}+00$ & & & $2.3 \mathrm{E}+02$ & $2.3 \mathrm{E}+00$ & & \\
\hline $\begin{array}{l}\text { Methyl tert-Butyl Ether } \\
\text { (MTBE) }\end{array}$ & $001634-04-4$ & & & & & & & $1.0 \mathrm{E}+06$ & $1.0 \mathrm{E}+06$ & & & & & & & & \\
\hline Methyl-5-Nitroaniline, 2- & $000099-55-8$ & $1.7 \mathrm{E}+04$ & $1.7 \mathrm{E}+02$ & & & & & & & $1.4 \mathrm{E}+04$ & $1.4 \mathrm{E}+02$ & & & $7.7 \mathrm{E}+03$ & $7.7 E+01$ & & \\
\hline $\begin{array}{l}\text { Methylaniline } \\
\text { Hydrochloride, 2- }\end{array}$ & $000636-21-5$ & $3.2 \mathrm{E}+03$ & $3.2 \mathrm{E}+01$ & & & & & & & $2.5 \mathrm{E}+03$ & $2.5 \mathrm{E}+0 \mathrm{t}$ & & & $1.4 \mathrm{E}+03$ & $1.4 \mathrm{E}+01$ & & \\
\hline Methylcyclohexane & $000108-87-2$ & & & & & & & $2.4 \mathrm{E}+02$ & $2.4 \mathrm{E}+02$ & & & & & & & & \\
\hline Methylene Chloride & $000075-09-2$ & $7.6 \mathrm{E}+04$ & $7.6 \mathrm{E}+02$ & $1.2 \mathrm{E}+05$ & $1.2 \mathrm{E}+04$ & $1.0 \mathrm{E}+03$ & $8.1 \mathrm{E}+01$ & $1.0 \mathrm{E}+03$ & $1.0 \mathrm{E}+03$ & $1.1 \mathrm{E}+05$ & $1.1 \mathrm{E}+03$ & $1.8 \mathrm{E}+05$ & $1.8 \mathrm{E}+04$ & $1.0 \mathrm{E}+03$ & $6.9 \mathrm{E}+01$ & $1.0 E+03$ & $1.0 \mathrm{E}+03$ \\
\hline $\begin{array}{l}\text { Methylene-bis(2- } \\
\text { chloroaniline), 4,4- }\end{array}$ & $000101-14-4$ & $4.4 \mathrm{E}+03$ & $4.4 \mathrm{E}+01$ & $1.4 \mathrm{E}+03$ & $1.4 \mathrm{E}+02$ & $1.0 \mathrm{E}+06$ & $4.7 \mathrm{E}+05$ & & & $3.5 \mathrm{E}+03$ & $3.5 \mathrm{E}+01$ & $1.1 \mathrm{E}+03$ & $1.1 \mathrm{E}+02$ & $1.9 \mathrm{E}+03$ & $1.9 \mathrm{E}+01$ & $6.3 E+02$ & $6.3 \mathrm{E}+01$ \\
\hline $\begin{array}{l}\text { Methylene-bis(N,N- } \\
\text { dimethyl) Aniline, 4,4- }\end{array}$ & $000101-61-1$ & $1.2 \mathrm{E}+04$ & $1.2 \mathrm{E}+02$ & & & & & & & $9.8 \mathrm{E}+03$ & $9.8 \mathrm{E}+01$ & & & $5.5 \mathrm{E}+03$ & $5.5 \mathrm{E}+01$ & & \\
\hline $\begin{array}{l}\text { Methylenebisbenzen- } \\
\text { amine, } 4,4^{-1(3)}\end{array}$ & $000101-77-9$ & $2.3 \mathrm{E}+03$ & $2.3 \mathrm{E}+01$ & & & & & & & $1.8 \mathrm{E}+03$ & $1.8 \mathrm{E}+01$ & & & $1.0 \mathrm{E}+03$ & $1.0 \mathrm{E}+01$ & & \\
\hline $\begin{array}{l}\text { Methylenediphenyl } \\
\text { Isocyanate, 4,4:- }\end{array}$ & $000101-68-8$ & & & & & & & $1.3 \mathrm{E}+05$ & $1.3 E+04$ & & & & & & & & \\
\hline Methylstyrene, Alpha- & 000098-83-9 & & & $1.4 \mathrm{E}+05$ & $1.4 \mathrm{E}+04$ & & & & & & & $1.8 \mathrm{E}+05$ & $1.8 \mathrm{E}+04$ & & & $8.0 E+04$ & $8.0 \mathrm{E}+03$ \\
\hline Methyltriethyl Lead & $001762-28-3$ & & & & & & & & & & & & & & & & \\
\hline Metolachlor & $051218-45-2$ & & & $3.1 \mathrm{E}+05$ & $3.1 \mathrm{E}+04$ & & & & & & & $2.4 \mathrm{E}+05$ & $2.4 \mathrm{E}+04$ & & & $1.4 \mathrm{E}+05$ & $1.4 \mathrm{E}+04$ \\
\hline Metribuzin ${ }^{(3)}$ & $021087-64-9$ & & & $5.1 \mathrm{E}+04$ & $5.1 \mathrm{E}+03$ & & & & & & & $4.0 E+04$ & $4.0 \mathrm{E}+03$ & & & $2.3 \mathrm{E}+04$ & $2.3 \mathrm{E}+03$ \\
\hline Mirex & $002385-85.5$ & $3.2 \mathrm{E}+02$ & $3.2 \mathrm{E}+00$ & $4.1 \mathrm{E}+02$ & $4.1 \mathrm{E}+01$ & & & & & $2.5 \mathrm{E}+02$ & $2.5 \mathrm{E}+00$ & $3.2 \mathrm{E}+02$ & $3.2 \mathrm{E}+01$ & $1.4 \mathrm{E}+02$ & $1.4 \mathrm{E}+00$ & $1.8 \mathrm{E}+02$ & $1.8 \mathrm{E}+01$ \\
\hline
\end{tabular}


Table 3.2c. (continued)

\begin{tabular}{|c|c|c|c|c|c|c|c|c|c|c|c|c|c|c|c|c|c|}
\hline \multirow[t]{2}{*}{ Chemical } & \multirow[t]{2}{*}{ CASRN } & \multicolumn{4}{|c|}{ Ingestion $(\mathrm{mg} / \mathrm{kg})^{\circ}$} & \multicolumn{4}{|c|}{ Inhalation (mg/kg) } & \multicolumn{4}{|c|}{ Dermal (mg/kg) } & \multicolumn{4}{|c|}{$\begin{array}{l}\text { Ingestion + Inhalation } \\
+ \text { Dermal (mg/kg) }\end{array}$} \\
\hline & & $10^{-4}$ & $10^{4}$ & $\mathrm{HQ}=1$ & $\mathrm{HQ}=0.1$ & $10^{-4}$ & $10^{4}$ & $\mathrm{HQ}=1$ & $\mathrm{HQ}=0.1$ & $10^{-4}$ & $10^{4}$ & $\mathrm{HQ}=1$ & $\mathrm{HQ}=0.1$ & $10^{-4}$ & $10^{5}$ & $H I=1$ & $\mathrm{HI}=0.1$ \\
\hline Molinate & $002212-67-1$ & & & 4.1E+03 & $4.1 \mathrm{E}+02$ & & & & & & & $3.2 \mathrm{E}+03$ & $3.2 E+02$ & & & $1.8 \mathrm{E}+03$ & $1.8 \mathrm{E}+02$ \\
\hline Molybdenum & $007439-98-7$ & & & $1.0 \mathrm{E}+04$ & $1.0 \mathrm{E}+03$ & & & & & & & $6.1 E+04$ & $6.1 \mathrm{E}+03$ & & & $8.8 \mathrm{E}+03$ & $8.8 \mathrm{E}+02$ \\
\hline Monochloramine & $010599-90-3$ & & & $2.0 \mathrm{E}+05$ & $2.0 \mathrm{E}+04$ & & & & & & & $1.6 \mathrm{E}+05$ & $1.6 \mathrm{E}+04$ & & & $9.0 \mathrm{E}+04$ & $9.0 \mathrm{E}+03$ \\
\hline Monochlorobutanes & $025154-42-1$ & & & $8.2 \mathrm{E}+05$ & $8.2 \mathrm{E}+04$ & & & & & & & $6.5 \mathrm{E}+05$ & $6.5 \mathrm{E}+04$ & & & $3.6 \mathrm{E}+05$ & $3.6 \mathrm{E}+04$ \\
\hline Naled & $000300-76-5$ & & & $4.1 E+03$ & $4.1 \mathrm{E}+02$ & & & & & & & $3.2 \mathrm{E}+03$ & $3.2 E+02$ & & & $1.8 \mathrm{E}+03$ & $1.8 \mathrm{E}+02$ \\
\hline Naphthalene $e^{(0)}$ & $000091-20-3$ & & & $7.3 \mathrm{E}+04$ & $7.3 \mathrm{E}+03$ & & & & & & & $9.2 \mathrm{E}+04$ & $9.2 \mathrm{E}+03$ & & & $4.1 E+04$ & $4.1 \mathrm{E}+03$ \\
\hline Naphthalene, 1-Methyl & $000090-12-0$ & & & & & & & & & & & & & & & & \\
\hline Naphthalene, 2-Methyl & $000091-57.6$ & & & & & & & & & & & & & & & . & \\
\hline Napropamide & $015299-99.7$ & & & $2.0 \mathrm{E}+05$ & $2.0 \mathrm{E}+04$ & & & & & & & $1.6 \mathrm{E}+05$ & $1.6 \mathrm{E}+04$ & & & $9.0 \mathrm{E}+04$ & $9.0 \mathrm{E}+03$ \\
\hline Niagara Blue 4B & $002429-74-5$ & & & & & & & & & & & & & & & & \\
\hline Nickel Carbonyl & 013463-39-3 & & & & & & & & & & & & & & & & \\
\hline Nickel Refinery Dust & NA & & & & & $1.0 \mathrm{E}+06$ & $7.3 \mathrm{E}+04$ & & & & & & & & & & \\
\hline Nickel Soluble Salts & $007440-02-0$ & & & 4.1E+04 & 4.1E+03 & & & & & & & $1.7 \mathrm{E}+05$ & $1.7 \mathrm{E}+04$ & & & $3.3 \mathrm{E}+04$ & $3.3 \mathrm{E}+03$ \\
\hline Nickel Subsulfide & $012035-72-2$ & & & & & $1.0 \mathrm{E}+06$ & $3.6 \mathrm{E}+04$ & & & & & & & & & & \\
\hline Nitrapyrin ${ }^{(3)}$ & $001929-82-4$ & & & $3.1 \mathrm{E}+03$ & $3.1 \mathrm{E}+02$ & & & & & & & $2.4 \mathrm{E}+03$ & $2.4 \mathrm{E}+02$ & & & $1.4 E+03$ & $1.4 \mathrm{E}+02$ \\
\hline Nitrate & $014797-55-8$ & & & $1.0 \mathrm{E}+06$ & $3.3 \mathrm{E}+05$ & & & & & & & $1.0 \mathrm{E}+06$ & $1.0 \mathrm{E}+06$ & & & $1.0 \mathrm{E}+06$ & $2.9 \mathrm{E}+05$ \\
\hline Nitric Oxide ${ }^{(3)}$ & $010102-43-9$ & & & $2.0 \mathrm{E}+0 \mathrm{~S}$ & $2.0 \mathrm{E}+04$ & & & & & & & $6.5 \mathrm{E}+05$ & $6.5 \mathrm{E}+04$ & & & $1.6 \mathrm{E}+05$ & $1.6 \mathrm{E}+04$ \\
\hline Nitrite & $014797-65-0$ & & & $2.0 \mathrm{E}+05$ & $2.0 \mathrm{E}+04$ & & & & & & & $1.0 \mathrm{E}+06$ & $1.6 \mathrm{E}+05$ & & & $1.8 \mathrm{E}+05$ & $1.8 \mathrm{E}+04$ \\
\hline Nitroaniline, 2- & $000088-74-4$ & & & & & & & $2.1 \mathrm{E}+01$ & $2.1 \mathrm{E}+00$ & & & & & & & & \\
\hline Nitrobenzene & $000098-95-3$ & & & $1.0 \mathrm{E}+03$ & $1.0 \mathrm{E}+02$ & & & $1.2 \mathrm{E}+01$ & $1.2 \mathrm{E}+00$ & & & $1.6 \mathrm{E}+03$ & $1.6 \mathrm{E}+02$ & & & $1.2 \mathrm{E}+01$ & $1.2 \mathrm{E}+00$ \\
\hline Nitrofurantoin & $000067-20-9$ & & & $1.4 E+05$ & $1.4 \mathrm{E}+04$ & & & & & & & $1.1 \mathrm{E}+0 \mathrm{~s}$ & $1.1 \mathrm{E}+04$ & & & $6.3 \mathrm{E}+04$ & $6.3 \mathrm{E}+03$ \\
\hline Nitrofurazone & $000059-87.0$ & $3.8 \mathrm{E}+02$ & $3.8 \mathrm{E}+00$ & & & & & & & $3.0 \mathrm{E}+02$ & $3.0 \mathrm{E}+00$ & & & $1.7 E+02$ & $1.7 \mathrm{E}+00$ & & \\
\hline Nitrogen Dioxide & $010102-44-0$ & & & $1.0 \mathrm{E}+06$ & $2.0 \mathrm{E}+05$ & & & & & & & $1.0 \mathrm{E}+06$ & $1.0 \mathrm{E}+06$ & & & $1.0 \mathrm{E}+06$ & $1.9 \mathrm{E}+05$ \\
\hline
\end{tabular}


Table 3.2c. (continued)

\begin{tabular}{|c|c|c|c|c|c|c|c|c|c|c|c|c|c|c|c|c|c|}
\hline \multirow{2}{*}{ Chemical } & \multirow[t]{2}{*}{ CASRN } & \multicolumn{4}{|c|}{ Ingestion (mg/kg) } & \multicolumn{4}{|c|}{ Inhalation $(\mathrm{mg} / \mathrm{kg})^{\circ \mathrm{r}}$} & \multicolumn{4}{|c|}{ Dermal (mg/kg) } & \multicolumn{4}{|c|}{$\begin{array}{l}\text { Ingestion + Inhalation } \\
+ \text { Dermal (mg/kg) }\end{array}$} \\
\hline & & $10^{-4}$ & $10^{-4}$ & $H Q=1$ & $\mathrm{HQ}=0.1$ & $10^{-4}$ & $10^{6}$ & $H Q=1$ & $H Q=0.1$ & $10^{-4}$ & $10^{5}$ & $H Q=1$ & $\mathrm{HQ}=0.1$ & $10^{-4}$ & $10^{5}$ & $H I=1$ & $\mathrm{HI}=0.1$ \\
\hline Nitroguanidine & $000556-88-7$ & & & $2.0 \mathrm{E}+05$ & $2.0 \mathrm{E}+04$ & & & & & & & $1.6 \mathrm{E}+05$ & $1.6 \mathrm{E}+04$ & & & $9.0 \mathrm{E}+04$ & $9.0 \mathrm{E}+03$ \\
\hline Nitrophenol, $4-$ & $000100-02-7$ & & & & & & & & & & & & & & & & \\
\hline Nitropropane, 2-(s) & $000079-46-9$ & $6.0 \mathrm{E}+01$ & $6,0 \mathrm{E}-01$ & & & $1.4 \mathrm{E}+01$ & $1.4 \mathrm{E}-01$ & $2.7 \mathrm{E}+03$ & $2.7 \mathrm{E}+02$ & $7.6 \mathrm{E}+01$ & $7.6 \mathrm{E}-01$ & & & $9.9 E+00$ & $9.9 \mathrm{E}-02$ & & \\
\hline Nitroso-N-ethylurea, N- & $000759-73-9$ & $4.1 \mathrm{E}+00$ & $4,1 \mathrm{E}-02$ & & & & & & & $3.2 \mathrm{E}+00$ & $3.2 \mathrm{E}-02$ & & & $1.8 E+00$ & $1.8 \mathrm{E}-02$ & & \\
\hline Nitroso-N-methylurea, N- & $000684-93-5$ & & & & & & & & & & & & & & & & \\
\hline $\begin{array}{l}\text { Nitroso-di-N-butylamine, } \\
\text { N- }\end{array}$ & $000924-16-3$ & $1.1 \mathrm{E}+02$ & $1.1 \mathrm{E}+00$ & & & $1.0 \mathrm{E}+02$ & $1.0 \mathrm{E}+00$ & & & $1.3 \mathrm{E}+02$ & $1.3 \mathrm{E}+00$ & & & $3.8 \mathrm{E}+01$ & $3.8 \mathrm{E}-01$ & & \\
\hline $\begin{array}{l}\text { Nitroso-di-N- } \\
\text { propylamine, N- }\end{array}$ & $000621-64-7$ & $8.2 E+01$ & 8.2E-01 & & & & & & & $3.2 \mathrm{E}+01$ & $3.2 \mathrm{E}-01$ & & & $2.3 \mathrm{E}+01$ & 2.3E-01 & & \\
\hline $\begin{array}{l}\text { Nitrosodiethanolamine, } \\
\text { N- }\end{array}$ & $001116-54-7$ & $2.0 \mathrm{E}+02$ & $=2.0 \mathrm{E}+00$ & & & & & & & $1.6 \mathrm{E}+02$ & $1.6 \mathrm{E}+00$ & & & $9.0 \mathrm{E}+01$ & $9.0 \mathrm{E}-01$ & & \\
\hline Nitrosodiethylamine, N- & $000055-18-5$ & $3.8 \mathrm{E}+00$ & 3.8E-02 & & & $4.1 \mathrm{E}+04$ & $4.1 \mathrm{E}+02$ & & & $3.0 \mathrm{E}+00$ & $3.0 \mathrm{E}-02$ & & & $1.7 \mathrm{E}+00$ & $1.7 \mathrm{E}-02$ & & \\
\hline Nitrosodimethylamine, N- & $000062-75-9$ & $1.1 E+01$ & $1.1 \mathrm{E}-01$ & & & $1.2 \mathrm{E}+05$ & $1.2 \mathrm{E}+03$ & & & $8.9 \mathrm{E}+00$ & $8.9 \mathrm{E}-02$ & & & $5.0 \mathrm{E}+00$ & $5.0 \mathrm{E}-02$ & & \\
\hline Nitrosodiphenylamine, $\mathrm{N}$ - & $000086-30-6$ & $1.2 \mathrm{E}+05$ & $1.2 E+03$ & & & & & & & $4.6 \mathrm{E}+04$ & $4.6 \mathrm{E}+02$ & & & $3.3 \mathrm{E}+04$ & $3.3 \mathrm{E}+02$ & & \\
\hline $\begin{array}{l}\text { Nitrosomethylethylamine, } \\
\text { N- }\end{array}$ & $010595-95-6$ & $2.6 \mathrm{E}+01$ & $2.6 \mathrm{E}-01$ & & & & & & & $2.1 E+01$ & 2.1E-01 & & & $1.1 \mathrm{E}+01$ & $1.1 \mathrm{E}-01$ & & \\
\hline $\begin{array}{l}\text { Nitrosomethylvinylamine, } \\
\text { N- }\end{array}$ & $004549-40-0$ & & & & & & & & & & & & & & & & \\
\hline Nitrosopymolidine, N- & $000930-55-2$ & $2.7 \mathrm{E}+02$ & $2.7 \mathrm{E}+00$ & & & $1.0 \mathrm{E}+06$ & $2.9 \mathrm{E}+04$ & & & $2.2 \mathrm{E}+02$ & $2.2 \mathrm{E}+00$ & & & $1.2 \mathrm{E}+02$ & $1.2 \mathrm{E}+00$ & & \\
\hline Nitrotoluene, 4-Amino-2- & $000119-32-4$ & & & & & & & & & & & & & & & & \\
\hline Nitrotoluene, m- & $000099-08-1$ & & & $2.0 \mathrm{E}+04$ & $2.0 \mathrm{E}+03$ & & & & & & & $2.6 \mathrm{E}+04$ & $2.6 \mathrm{E}+03$ & & & $1.1 \mathrm{E}+04$ & $1.1 \mathrm{E}+03$ \\
\hline Nitrotoluene, 0 - & $000088-72-2$ & & & $2.0 \mathrm{E}+04$ & $2.0 \mathrm{E}+03$ & & & & & & & $2.6 \mathrm{E}+04$ & $2.6 \mathrm{E}+03$ & & & $1.1 \mathrm{E}+04$ & $1.1 \mathrm{E}+03$ \\
\hline Nitrotoluene, $\mathrm{p}$ - & $000099.99-0$ & & & $2.0 \mathrm{E}+04$ & $2.0 \mathrm{E}+03$ & & & & & & & $2.6 \mathrm{E}+04$ & $2.6 \mathrm{E}+03$ & & & $1.1 \mathrm{E}+04$ & $1.1 \mathrm{E}+03$ \\
\hline Norflurazon & $027314-13-2$ & & & $8.2 \mathrm{E}+04$ & $8.2 \mathrm{E}+03$ & & & & & & & $6.5 \mathrm{E}+04$ & $6.5 \mathrm{E}+03$ & & & $3.6 \mathrm{E}+04$ & $3.6 \mathrm{E}+03$ \\
\hline Nustar & $085509-19-9$ & & & $1.4 \mathrm{E}+03$ & $1.4 \mathrm{E}+02$ & & & & & & & $1.1 \mathrm{E}+03$ & $1.1 \mathrm{E}+02$ & & & $6.3 \mathrm{E}+02$ & $6.3 \mathrm{E}+01$ \\
\hline $\mathrm{OCDD}^{(4)}$ & $003268-87-9$ & $3.8 \mathrm{E}+00$ & $3.8 \mathrm{E}-02$ & & & 4.1E+04 & 4.1E+02 & & & $1.0 \mathrm{E}+00$ & $1.0 \mathrm{E}-02$ & & & $8.0 \mathrm{E}-01$ & $8.0 \mathrm{E}-03$ & & \\
\hline
\end{tabular}

Nonradionuclides/soil/industrial

Page 22 of 32 
Table 3.2c. (continued)

\begin{tabular}{|c|c|c|c|c|c|c|c|c|c|c|c|c|c|c|c|c|c|}
\hline \multirow{2}{*}{ Chemical } & \multirow{2}{*}{ CASRN } & \multicolumn{4}{|c|}{ Ingestion (mg/kg) } & \multicolumn{4}{|c|}{ Inhalation (mg/kg) ${ }^{-y}$} & \multicolumn{4}{|c|}{ Dermal (mg/kg) } & \multicolumn{4}{|c|}{$\begin{array}{l}\text { Ingestion + Inhalation } \\
+ \text { Dermal }(\mathrm{mg} / \mathrm{kg})^{\prime \cdot v}\end{array}$} \\
\hline & & $10^{-4}$ & $10^{6}$ & $\mathrm{HQ}=1$ & $\mathrm{HQ}=0.1$ & $10^{-5}$ & $10^{6}$ & $\mathrm{HQ}=1$ & $\mathrm{HQ}=0.1$ & $10^{-4}$ & $10^{-5}$ & $\mathrm{HQ}=1$ & $\mathrm{HQ}=0.1$ & $10^{-4}$ & $10^{4}$ & $H I=1$ & $\mathrm{HI}=0.1$ \\
\hline $\mathrm{OCDF}^{(4)}$ & $039001-02-0$ & $3.8 E+00$ & $3.8 \mathrm{E}-02$ & & & 4.1E+04 & 4.1E+02 & & & $3.0 \mathrm{E}+00$ & $3.0 \mathrm{E}-02$ & & & $1.7 \mathrm{E}+00$ & $1.7 \mathrm{E}-02$ & & \\
\hline Octabromodiphenyl Ether & $032536-52-0$ & & & $6.1 \mathrm{E}+03$ & $6.1 \mathrm{E}+02$ & & & $\cdot$ & & & & $4.9 E+03$ & $4.9 \mathrm{E}+02$ & & & $2.7 \mathrm{E}+03$ & $2.7 \mathrm{E}+02$ \\
\hline $\begin{array}{l}\text { Octahydro-1,3,5,7- } \\
\text { tetranitro-1,3,5,7-tetra } \\
\text { (FMX) }\end{array}$ & $002691-41-0$ & & & $1.0 \mathrm{E}+05$ & $1.0 \mathrm{E}+04$ & & & & & & & $2.4 E+04$ & $2.4 \mathrm{E}+03$ & & & $2.0 \mathrm{E}+04$ & $2.0 \mathrm{E}+03$ \\
\hline $\begin{array}{l}\text { Octamethylpyrophosph- } \\
\text { oramide }\end{array}$ & $000152-16-9$ & & & $4.1 \mathrm{E}+03$ & $4.1 E+02$ & & & & & & & $3.2 \mathrm{E}+03$ & $3.2 \mathrm{E}+02$ & & & $1.8 \mathrm{E}+03$ & $1.8 E+02$ \\
\hline Octyl Phthalate, di-N. & $000117-84-0$ & & & 4.1E+04 & 4.1E+03 & & & & & & & $5.8 \mathrm{E}+04$ & $5.8 \mathrm{E}+03$ & & & $2.4 \mathrm{E}+04$ & $2.4 \mathrm{E}+03$ \\
\hline Oryzalin & $019044-88-3$ & & & $1,0 \mathrm{E}+05$ & $1.0 \mathrm{E}+04$ & & & & & & & $8.1 E+04$ & $8.1 E+03$ & & & $4.5 \mathrm{E}+04$ & $4.5 \mathrm{E}+03$ \\
\hline Oxadiazon & $019666-30-9$ & & & $1.0 \mathrm{E}+04$ & $1.0 \mathrm{E}+03$ & & & & & & & $8.1 E+03$ & $8.1 \mathrm{E}+02$ & & & $4.5 \mathrm{E}+03$ & $4.5 \mathrm{E}+02$ \\
\hline Oxamyl & $023135-22-0$ & & & $5.1 \mathrm{E}+04$ & $5.1 \mathrm{E}+03$ & & & & & & & $4.0 E+04$ & $4.0 \mathrm{E}+03$ & & & $2.3 \mathrm{E}+04$ & $2.3 E+03$ \\
\hline Paclobutrazol & $076738-62-0$ & & & $2.7 \mathrm{E}+04$ & $2.7 \mathrm{E}+03$ & & & & & & & $2.1 \dot{E}+04$ & $2.1 \mathrm{E}+03$ & & & $1.2 \mathrm{E}+04$ & $1.2 \mathrm{E}+03$ \\
\hline Paraquat & $001910-42-5$ & & & $9.2 \mathrm{E}+03$ & $9.2 \mathrm{E}+02$ & & & & & & & $7.3 \mathrm{E}+03$ & $7.3 \mathrm{E}+02$ & & & 4.1E+03 & $4.1 E+02$ \\
\hline Parathion & $000056-38-2$ & & & $1.2 \mathrm{E}+04$ & $1.2 \mathrm{E}+03$ & & & & & & & $9.7 \mathrm{E}+03$ & $9.7 \mathrm{E}+02$ & & & $5.4 \mathrm{E}+03$ & $5.4 \mathrm{E}+02$ \\
\hline $\mathrm{PeCDD}, 2,3,7,8$ ( $^{(4)}$ & $036088-22-9$ & 7.6E-03 & $7.6 \mathrm{E}-05$ & & & $8.2 \mathrm{E}+01$ & $8.2 \mathrm{E}-01$ & & & $2.0 \mathrm{E}-03$ & $2.0 \mathrm{E}-05$ & & & $1.6 \mathrm{E}-03$ & $1.6 \mathrm{E}-05$ & & \\
\hline PeCDF, 1,2,3,7,8.(1) & $057117-41-6$ & $7.6 \mathrm{E}-03$ & 7.6E-05 & & & $8.2 E+01$ & $8.2 \mathrm{E}-01$ & & & $6.0 \mathrm{E}-03$ & $6.0 \mathrm{E}-05$ & & & $3.4 \mathrm{E}-03$ & $3.4 \mathrm{E}-05$ & & \\
\hline $\mathrm{PeCDF}, 2,3,4,7,8^{-(1)}$ & $057117-31-4$ & $7.6 \mathrm{E}-02$ & $7.6 \mathrm{E}-04$ & & & $8.2 E+02$ & $8.2 E+00$ & & & $6.0 \mathrm{E}-02$ & $6.0 \mathrm{E}-04$ & & & $3.4 \mathrm{E}-02$ & $3.4 \mathrm{E}-04$ & & \\
\hline Pebulate & $001114-71-2$ & & & $1.0 \mathrm{E}+05$ & $1.0 E+04$ & & & & & & & $8.1 \mathrm{E}+04$ & $8.1 E+03$ & & & $4.5 \mathrm{E}+04$ & $4.5 \mathrm{E}+03$ \\
\hline Pendimethalin & $040487-42-1$ & & & $8.2 \mathrm{E}+04$ & $8.2 \mathrm{E}+03$ & & & & & & & $6.5 \mathrm{E}+04$ & $6.5 \mathrm{E}+03$ & & & $3,6 \mathrm{E}+04$ & $3.6 \mathrm{E}+03$ \\
\hline $\begin{array}{l}\text { Pentabromodiphenyl } \\
\text { Ether }\end{array}$ & $032534-81-9$ & & & 4.1E+03 & 4.1E+02 & & & & & & & $3.2 \mathrm{E}+03$ & $3.2 E+02$ & & & $1.8 \mathrm{E}+03$ & $1.8 \mathrm{E}+02$ \\
\hline Pentachlorobenzene & $000608-93.5$ & & & $1.6 \mathrm{E}+03$ & $1.6 \mathrm{E}+02$ & & & & & & & $2.1 \mathrm{E}+03$ & $2.1 \mathrm{E}+02$ & & & $9.1 \mathrm{E}+02$ & $9.1 \mathrm{E}+01$ \\
\hline $\begin{array}{l}\text { Pentachlorocyclopenta- } \\
\text { diene }\end{array}$ & $025329-35-5$ & & & & & & & & & & & & & & & & \\
\hline Pentachloronitrobenzene & $000082-68-8$ & $2.2 \mathrm{E}+03$ & $2.2 E+01$ & $6.1 \mathrm{E}+03$ & $6.1 \mathrm{E}+02$ & & & & & $2.8 \mathrm{E}+03$ & $2.8 \mathrm{E}+01$ & $7.8 \mathrm{E}+03$ & $7.8 \mathrm{E}+02$ & $1.2 \mathrm{E}+03$ & $1,2 \mathrm{E}+01$ & $3.4 \mathrm{E}+03$ & $3.4 \mathrm{E}+02$ \\
\hline Pentachlorophenol & $000087-86-5$ & $4.8 \mathrm{E}+03$ & $4.8 \mathrm{E}+01$ & $6.1 \mathrm{E}+04$ & $6.1 \mathrm{E}+03$ & & & & & $7.5 \mathrm{E}+03$ & 7.5E+01 & $9.7 \mathrm{E}+04$ & $9.7 \mathrm{E}+03$ & $2.9 \mathrm{E}+03$ & $2.9 E+01$ & $3.8 \mathrm{E}+04$ & $3.8 \mathrm{E}+03$ \\
\hline
\end{tabular}

Nonradionuclides/soil/industrial

Page 23 of 32 
Table 3.2c. (continued)

\begin{tabular}{|c|c|c|c|c|c|c|c|c|c|c|c|c|c|c|c|c|c|}
\hline \multirow{2}{*}{ Chemical } & \multirow[t]{2}{*}{ CASRN } & \multicolumn{4}{|c|}{ Ingestion (mg/kg) } & \multicolumn{4}{|c|}{ Inhalation (mg/kg) } & \multicolumn{4}{|c|}{ Dermal (mg/kg) } & \multicolumn{4}{|c|}{$\begin{array}{l}\text { Ingestion + Inhalation } \\
+ \text { Dermal }(\mathrm{mg} / \mathrm{kg})^{\dagger \vee v}\end{array}$} \\
\hline & & $10^{-4}$ & $10^{-4}$ & $H Q=1$ & $\mathrm{HQ}=0.1$ & $10^{-4}$ & $10^{-6}$ & $\mathrm{HQ}=1$ & $\mathrm{HQ}=0.1$ & $10^{-4}$ & $10^{6}$ & $H Q=1$ & $\mathrm{HQ}=0.1$ & $10^{-4}$ & $10^{6}$ & $\mathrm{H} \mathbf{I}=\mathbf{1}$ & $\mathrm{HI}=0.1$ \\
\hline Pentyl Alcohol, N. & $000071-41-0$ & & & & & & & & & & & & & & & & \\
\hline Permethrin & $052645-53-1$ & & & $1.0 \mathrm{E}+05$ & $1.0 \mathrm{E}+04$ & & & & & & & $8.1 E+04$ & $8.1 E+03$ & & & $4.5 \mathrm{E}+04$ & $4.5 \mathrm{E}+03$ \\
\hline Phenanthrene & $000085-01-8$ & & & & & & & & & & & & & & & & \\
\hline Phenmedipham & $013684-63-4$ & & & $5.1 \mathrm{E}+05$ & $5.1 \mathrm{E}+04$ & & & & & & & $4.0 \mathrm{E}+05$ & $4.0 \mathrm{E}+04$ & & & $2.3 \mathrm{E}+05$ & $2.3 E+04$ \\
\hline Phenol & $000108-95-2$ & & & $1.0 \mathrm{E}+06$ & $1.2 \mathrm{E}+05$ & & & & & & & $1.0 \mathrm{E}+06$ & $1.7 \mathrm{E}+05$ & & & $7.2 \mathrm{E}+05$ & $7.2 \mathrm{E}+0.4$ \\
\hline Phenylenediamine, m- & $000108-45-2$ & & & $1.2 \mathrm{E}+04$ & $1.2 E+03$ & & & & & & & $9.7 E+03$ & $9.7 \mathrm{E}+02$ & & & $5.4 \mathrm{E}+03$ & $5.4 \mathrm{E}+02$ \\
\hline Phenylenediamine, o- & $000095-54-5$ & $1.2 E+04$ & $1.2 \mathrm{E}+02$ & & & & & & & $9.6 \mathrm{E}+03$ & $9.6 \mathrm{E}+01$ & & & $5.4 \mathrm{E}+03$ & $5.4 \mathrm{E}+01$ & & \\
\hline Phenylenediamine, $\mathrm{p}$ - & $000106-50-3$ & & & $3.9 E+05$ & $3.9 E+04$ & & $\cdot$ & & & & & $3.1 \mathrm{E}+05$ & 3.1E+04 & & & $1.7 \mathrm{E}+05$ & $1.7 E+04$ \\
\hline Phenylmercuric Acetate & $000062-38-4$ & & & $1.6 \mathrm{E}+02$ & $1.6 \mathrm{E}+01$ & & & & & & & $1.3 \mathrm{E}+02$ & $1.3 \mathrm{E}+01$ & & & $7.2 \mathrm{E}+01$ & $7.2 \mathrm{E}+00$ \\
\hline Phenylphenol, 2 - & $000090-43-7$ & $3.0 \mathrm{E}+05$ & $3.0 \mathrm{E}+03$ & & & & & & & $2.3 E+05$ & $2.3 \mathrm{E}+03$ & & & $1.3 \mathrm{E}+05$ & $1.3 \mathrm{E}+03$ & & \\
\hline Phorate & $000298-02-2$ & & & $4.1 E+02$ & 4.1E+01 & & & & & & & $3.2 \mathrm{E}+02$ & $3.2 \mathrm{E}+01$ & & & $1.8 \mathrm{E}+02$ & $1.8 \mathrm{E}+01$ \\
\hline Phosmet & $000732-11-6$ & & & 4.1E+04 & 4.1E+03 & & & & & & & $3.2 \mathrm{E}+04$ & $3.2 \mathrm{E}+03$ & & & $1.8 \mathrm{E}+04$ & $1.8 \mathrm{E}+03$ \\
\hline Phosphine & $007803-51-2$ & & & $6.1 \mathrm{E}+02$ & $6.1 \mathrm{E}+01$ & & & $1.0 \mathrm{E}+06$ & $1.9 \mathrm{E}+05$ & & & $1.9 \mathrm{E}+03$ & $1.9 \mathrm{E}+02$ & & & $4.7 \mathrm{E}+02$ & $4.7 \mathrm{E}+01$ \\
\hline Phosphoric Acid & $007664-38-2$ & & & & & & & $1.0 \mathrm{E}+06$ & $1.0 \mathrm{E}+06$ & & & & & & & & \\
\hline Phthalic Acid, P. & $000100-21-0$ & & & $1.0 \mathrm{E}+06$ & $2.0 \mathrm{E}+05$ & & & & & & & $1.0 \mathrm{E}+06$ & $1.6 \mathrm{E}+05$ & & & $9.0 \mathrm{E}+05$ & $9.0 \mathrm{E}+04$ \\
\hline Phthalic Anhydride & $000085-44-9$ & & & $1.0 E+06$ & $4.1 \mathrm{E}+05$ & & & $1.0 \mathrm{E}+06$ & $1.0 \mathrm{E}+06$ & & & $1.0 \mathrm{E}+06$ & $3.2 \mathrm{E}+05$ & & & $1.0 \mathrm{E}+06$ & $1.8 \mathrm{E}+05$ \\
\hline Picloram & $001918-02-1$ & & & $1.4 \mathrm{E}+05$ & $1.4 \mathrm{E}+04$ & & & & & & & $1.1 \mathrm{E}+05$ & $1.1 \mathrm{E}+04$ & & & $6.3 E+04$ & $6.3 \mathrm{E}+03$ \\
\hline Pirimiphos, Methyl & 029232-93-7 & & & $2.0 \mathrm{E}+04$ & $2.0 \mathrm{E}+03$ & & & & & & & $1.6 \mathrm{E}+04$ & $1.6 \mathrm{E}+03$ & & & $9.0 \mathrm{E}+03$ & $9.0 \mathrm{E}+02$ \\
\hline $\begin{array}{l}\text { Polybrominated } \\
\text { Biphenyls }\end{array}$ & NA & $6.4 E+01$ & $6.4 \mathrm{E}-01$ & $1.4 E+01$ & $1.4 \mathrm{E}+00$ & & & & & $9.5 \mathrm{E}+01$ & $9.5 \mathrm{E}-01$ & $2.1 E+01$ & $2.1 \mathrm{E}+00$ & $3.8 \mathrm{E}+01$ & $3.8 \mathrm{E}-01$ & $8.5 \mathrm{E}+00$ & $8.5 \mathrm{E}-01$ \\
\hline $\begin{array}{l}\text { Polychlorinated } \\
\text { Biphenyls }\end{array}$ & $001336-36-3$ & $7.4 \mathrm{E}+01$ & $7.4 \mathrm{E}-01$ & & & & & & & $1.8 \mathrm{E}+01$ & $1.8 \mathrm{E}-01$ & & & $1.4 \mathrm{E}+01$ & $1.4 \mathrm{E}-01$ & & \\
\hline Potassium Cyanide & $000151 \cdot 50-8$ & & & $1.0 E+05$ & $1.0 \mathrm{E}+04$ & & & & & & & $1.3 \mathrm{E}+05$ & $1.3 \mathrm{E}+04$ & & & $5.7 \mathrm{E}+04$ & $5.7 \mathrm{E}+03$ \\
\hline Potassium Silver Cyanide & $000506-61-6$ & & & 4.1E+05 & $4.1 \mathrm{E}+04$ & & & & & & & $1.0 \mathrm{E}+06$ & $1.3 \mathrm{E}+05$ & & & $3.1 E+05$ & $3.1 \mathrm{E}+04$ \\
\hline
\end{tabular}


Table 3.2c. (continued)

\begin{tabular}{|c|c|c|c|c|c|c|c|c|c|c|c|c|c|c|c|c|c|}
\hline \multirow[t]{2}{*}{ Chemical } & \multirow[t]{2}{*}{ CASRN } & \multicolumn{4}{|c|}{ Ingestion (mg/kg) } & \multicolumn{4}{|c|}{ Inhalation (mg/kg) } & \multicolumn{4}{|c|}{ Dermal (mg/kg) } & \multicolumn{4}{|c|}{$\begin{array}{l}\text { Ingestion + Inhalation } \\
+ \text { Dermal }(\mathrm{mg} / \mathrm{kg})^{+* \mathrm{~b}}\end{array}$} \\
\hline & & $10^{-1}$ & $10^{4}$ & $\mathrm{HQ}=1$ & $\mathrm{HQ}=0.1$ & $10^{-4}$ & $10^{4}$ & $\mathrm{HQ}=1$ & $\mathrm{HQ}=0.1$ & $10^{-4}$ & $10^{5}$ & $H Q=1$ & $\mathrm{HQ}=0.1$ & $10^{-4}$ & $10^{6}$ & $\mathrm{HI}=1$ & $\mathrm{HI}=0.1$ \\
\hline Prochloraz & $067747-09-5$ & $3.8 \mathrm{E}+03$ & $3.8 \mathrm{E}+01$ & $1.8 E+04$ & $1.8 \mathrm{E}+03$ & & & & & $3.0 E+03$ & $3.0 \mathrm{E}+01$ & $1.5 \mathrm{E}+04$ & $1.5 \mathrm{E}+03$ & $1.7 E+03$ & $1.7 \mathrm{E}+01$ & $8.1 E+03$ & $8.1 \mathrm{E}+02$ \\
\hline Profluralin & $026399-36-0$ & & & $1.2 \mathrm{E}+04$ & $1.2 \mathrm{E}+03$ & & & & & & & $9.7 \mathrm{E}+03$ & $9.7 \mathrm{E}+02$ & & & $5.4 \mathrm{E}+03$ & $5.4 \mathrm{E}+02$ \\
\hline Prometon & $001610-18-0$ & & & $3.1 \mathrm{E}+04$ & $3.1 E+03$ & & & & & & & $2.4 \mathrm{E}+04$ & $2.4 \mathrm{E}+03$ & & & $1.4 \mathrm{E}+04$ & $1.4 \mathrm{E}+03$ \\
\hline Prometryn & $007287-19-6$ & & & $8.2 \mathrm{E}+03$ & $8.2 \mathrm{E}+02$ & & & & & & & $6.5 \mathrm{E}+03$ & $6.5 \mathrm{E}+02$ & & & $3.6 \mathrm{E}+03$ & $3.6 \mathrm{E}+02$ \\
\hline Propachlor & $001918-16-7$ & & & $2.7 \mathrm{E}+04$ & $2.7 \mathrm{E}+03$ & & & & & & & $2.1 \mathrm{E}+04$ & $2.1 \mathrm{E}+03$ & & & $1.2 E+04$ & $1.2 \mathrm{E}+03$ \\
\hline Propanil & $000709-98-8$ & & & $1.0 \mathrm{E}+04$ & $1.0 \mathrm{E}+03$ & & & & & & & $8.1 E+03$ & $8.1 \mathrm{E}+02$ & & & $4.5 \mathrm{E}+03$ & $4.5 \mathrm{E}+02$ \\
\hline Propargite & $002312-35-8$ & & & $4.1 \mathrm{E}+04$ & $4.1 \mathrm{E}+03$ & & & & & & & $3.2 E+04$ & $3.2 \mathrm{E}+03$ & & & $1.8 \mathrm{E}+04$ & $1.8 \mathrm{E}+03$ \\
\hline Propargyl Alcohol & $000107-19-7$ & & & $4.1 E+03$ & $4.1 E+02$ & & & & & & & $5.2 \mathrm{E}+03$ & $5.2 \mathrm{E}+02$ & & & $2.3 \mathrm{E}+03$ & $2.3 \mathrm{E}+02$ \\
\hline Propazine & $000139-40-2$ & & & $4.1 \mathrm{E}+04$ & $4.1 \mathrm{E}+03$ & & & & & & & $3.2 \mathrm{E}+04$ & $3.2 \mathrm{E}+03$ & & & $1.8 \mathrm{E}+04$ & $1.8 \mathrm{E}+03$ \\
\hline Propham & $000122-42-9$ & & & $4.1 \mathrm{E}+04$ & $4.1 E+03$ & & & & & & & $3.2 \mathrm{E}+04$ & $3.2 \mathrm{E}+03$ & & & $1.8 \mathrm{E}+04$ & $1.8 \mathrm{E}+03$ \\
\hline Propiconazole & $060207-90.1$ & & & $2.7 \mathrm{E}+04$ & $2.7 \mathrm{E}+03$ & & & & & & & $2.1 E+04$ & $2.1 \mathrm{E}+03$ & & & $1.2 \mathrm{E}+04$ & $1.2 \mathrm{E}+03$ \\
\hline Propylene Glycol & $000057-55-6$ & & & $1.0 \mathrm{E}+06$ & $1.0 E+06$ & & & & & & & $1.0 \mathrm{E}+06$ & $1.0 \mathrm{E}+06$ & & & $1.0 \mathrm{E}+06$ & $1.0 \mathrm{E}+06$ \\
\hline $\begin{array}{l}\text { Propylene Glycol } \\
\text { Monoethyl Ether }\end{array}$ & $001569-02-4$ & & & $1.0 E+06$ & $1.4 E+05$ & & & & & & & $1.0 \mathrm{E}+06$ & $1.1 \mathrm{E}+05$ & & & $6.3 \mathrm{E}+05$ & $6.3 \mathrm{E}+04$ \\
\hline $\begin{array}{l}\text { Propylene Glycol } \\
\text { Monomethyl Ether }\end{array}$ & $000107-98-2$ & & & $1.0 \mathrm{E}+06$ & $1.4 \mathrm{E}+05$ & & & $1.0 \mathrm{E}+06$ & $1.0 \mathrm{E}+06$ & & & $1.0 \mathrm{E}+06$ & $1.1 \mathrm{E}+05$ & & & $6.3 \mathrm{E}+05$ & $6.3 E+04$ \\
\hline Propylene Oxide & $000075-56-9$ & $2.4 \mathrm{E}+03$ & $2.4 \mathrm{E}+01$ & & & $7.0 \mathrm{E}+03$ & $7.0 \mathrm{E}+01$ & $2.8 \mathrm{E}+03$ & $2.8 \mathrm{E}+02$ & $3.0 \mathrm{E}+03$ & $3.0 E+01$ & & & $1.1 E+03$ & $1.1 \mathrm{E}+01$ & & \\
\hline Pursuit & $081335-77-5$ & & & $5.1 \mathrm{E}+05$ & $5.1 \mathrm{E}+04$ & & & & & & & $4.0 \mathrm{E}+05$ & $4.0 \mathrm{E}+04$ & & & $2.3 \mathrm{E}+05$ & $2,3 \mathrm{E}+04$ \\
\hline Pydrin & $051630-58-1$ & & & $5.1 \mathrm{E}+04$ & $5.1 E+03$ & & & & & & & $4.0 \mathrm{E}+04$ & $4.0 \mathrm{E}+03$ & & & $2.3 \mathrm{E}+04$ & $2.3 \mathrm{E}+03$ \\
\hline Pyrene & $000129-00-0$ & & & $6.1 E+04$ & $6.1 \mathrm{E}+03$ & & & & & & & $3.0 \mathrm{E}+04$ & $3.0 \mathrm{E}+03$ & & & $2.0 \mathrm{E}+04$ & $2.0 \mathrm{E}+03$ \\
\hline Pyridine & $000110-86-1$ & & & $2.0 E+03$ & $2.0 E+02$ & & & & & & & $1.6 \mathrm{E}+03$ & $1.6 \mathrm{E}+02$ & & & $9.0 \mathrm{E}+02$ & $9.0 \mathrm{E}+01$ \\
\hline Quinalphos & $013593-03.8$ & & & $1.0 \mathrm{E}+03$ & $1.0 \mathrm{E}+02$ & & & & & & & $8.1 E+02$ & $8.1 E+01$ & & & $4.5 \mathrm{E}+02$ & $4.5 \mathrm{E}+01$ \\
\hline Quinoline & $000091-22-5$ & $4.8 \mathrm{E}+01$ & $4.8 \mathrm{E}-01$ & & & & & & & $3.8 \mathrm{E}+01$ & $3.8 \mathrm{E}-01$ & & & $2.1 E+01$ & $2.1 \mathrm{E}-01$ & & \\
\hline $\begin{array}{l}\text { Refractory Ceramic } \\
\text { Fibers }\end{array}$ & $\mathrm{NA}$ & & & & & & & & & & & & & & & & \\
\hline
\end{tabular}


Table 3.2c. (continued)

\begin{tabular}{|c|c|c|c|c|c|c|c|c|c|c|c|c|c|c|c|c|c|}
\hline \multirow{2}{*}{ Chemical } & \multirow{2}{*}{ CASRN } & \multicolumn{4}{|c|}{ Ingestion (mg/kg) } & \multicolumn{4}{|c|}{ Inhalation $(\mathrm{mg} / \mathrm{kg})^{-\mathrm{s}}$} & \multicolumn{4}{|c|}{ Dermal (mg/kg) } & \multicolumn{4}{|c|}{$\begin{array}{l}\text { Ingestion + Inhalation } \\
+ \text { Dermal }(\mathrm{mg} / \mathrm{kg})^{+\vee *}\end{array}$} \\
\hline & & $10^{-4}$ & $10^{-6}$ & $H Q=1$ & $\mathrm{HQ}=0.1$ & $10^{-4}$ & $10^{4}$ & $H Q=1$ & $H Q=0.1$ & $10^{-4}$ & $10^{4}$ & $\mathrm{HQ}=1$ & $\mathrm{HQ}=0.1$ & $10^{4}$ & $10^{6}$ & $\mathrm{HI}=1$ & $\mathrm{HI}=0.1$ \\
\hline Resmethrin & $010453-86-8$ & & & $6.1 \mathrm{E}+04$ & $6.1 \mathrm{E}+03$ & & & & & & & $4.9 \mathrm{E}+04$ & $4.9 \mathrm{E}+03$ & & & $2.7 \mathrm{E}+04$ & $2.7 \mathrm{E}+03$ \\
\hline Ronnel & $000299-84-3$ & & & $1.0 \mathrm{E}+05$ & $1.0 \mathrm{E}+04$ & & & & & & & $8.1 \mathrm{E}+04$ & $8.1 E+03$ & & & $4.5 \mathrm{E}+04$ & $4.5 E+03$ \\
\hline Rotenone & $000083-79-4$ & & & $8.2 E+03$ & $8.2 \mathrm{E}+02$ & & & & & & & $6.5 \mathrm{E}+03$ & $6.5 \mathrm{E}+02$ & & & $3.6 \mathrm{E}+03$ & $3.6 \mathrm{E}+02$ \\
\hline Savey & $078587-05-0$ & & & $5.1 \mathrm{E}+04$ & $5.1 \mathrm{E}+03$ & & & & & & & $4.0 \mathrm{E}+04$ & $4.0 \mathrm{E}+03$ & & & $2.3 \mathrm{E}+04$ & $2.3 \mathrm{E}+03$ \\
\hline Selenious Acid & $007783-00-8$ & & & $1.0 E+04$ & $1.0 \mathrm{E}+03$ & & & & & & & $1.4 E+04$ & $1.4 \mathrm{E}+03$ & & & $5.9 E+03$ & $5.9 \mathrm{E}+02$ \\
\hline Selenite & $014124-67-5$ & & & & & & & & & & & & & & & & \\
\hline Selenium & $007782-49-2$ & & & $1.0 \mathrm{E}+04$ & $1.0 \mathrm{E}+03$ & & & & & & & $7.1 \mathrm{E}+04$ & $7.1 \mathrm{E}+03$ & & & $8.9 E+03$ & $8.9 \mathrm{E}+02$ \\
\hline Selenium Sulfide & $007446-34-6$ & & & & & & & & & & & & & & & & \\
\hline Selenourea & $000630-10-4$ & & & $1.0 \mathrm{E}+04$ & $1.0 \mathrm{E}+03$ & & & & & & & 8.1E+03 & $8.1 E+02$ & & & $4.5 \mathrm{E}+03$ & $4.5 \mathrm{E}+02$ \\
\hline Sethoxydim & $074051-80-2$ & & & $1.8 \mathrm{E}+05$ & $1.8 \mathrm{E}+04$ & & & & & & & $1.5 \mathrm{E}+05$ & $1.5 \mathrm{E}+04$ & & & $8.1 \mathrm{E}+04$ & $8.1 E+03$ \\
\hline Silver & $007440-22-4$ & & & $1.0 \mathrm{E}+04$ & $1.0 E+03$ & & & & & & & $2.9 \mathrm{E}+04$ & $2.9 E+03$ & & & $7.6 \mathrm{E}+03$ & $7.6 \mathrm{E}+02$ \\
\hline Silver Cyanide & $000506-64-9$ & & & $2.0 \mathrm{E}+05$ & $2.0 \mathrm{E}+04$ & & & & & & & $6.5 \mathrm{E}+05$ & $6.5 \mathrm{E}+04$ & & & $1.6 \mathrm{E}+05$ & $1.6 \mathrm{E}+04$ \\
\hline Simazine & $000122-34-9$ & $4.8 \mathrm{E}+03$ & $4.8 \mathrm{E}+01$ & $1.0 \mathrm{E}+04$ & $1.0 \mathrm{E}+03$ & & & & & $3.8 \mathrm{E}+03$ & $3.8 \mathrm{E}+01$ & $8.1 \mathrm{E}+03$ & $8.1 \mathrm{E}+02$ & $2.1 E+03$ & $2.1 E+01$ & $4.5 \mathrm{E}+03$ & $4.5 \mathrm{E}+02$ \\
\hline Sodium & $007440-23-5$ & & & & & & & & & & & & & & & & \\
\hline Sodium Acifluorfen & $062476-59-9$ & & & $2.7 \mathrm{E}+04$ & $2.7 \mathrm{E}+03$ & & & & & & & $2.1 \mathrm{E}+04$ & $2.1 \mathrm{E}+03$ & & & $1.2 \mathrm{E}+04$ & $1.2 \mathrm{E}+03$ \\
\hline Sodium Azide & $026628-22-8$ & & & $8.2 \mathrm{E}+03$ & $8.2 \mathrm{E}+02$ & & & & & & & $2.6 \mathrm{E}+04$ & $2.6 \mathrm{E}+03$ & & & $6.2 \mathrm{E}+03$ & $6.2 \mathrm{E}+02$ \\
\hline Sodium Cyanide & $000143-33-9$ & & & $8.2 \mathrm{E}+04$ & $8.2 \mathrm{E}+03$ & & & & & & & $1.0 \mathrm{E}+05$ & $1.0 \mathrm{E}+04$ & & & $4.6 E+04$ & $4.6 \mathrm{E}+03$ \\
\hline $\begin{array}{l}\text { Sodium } \\
\text { Diethyldithiocarbarnate }\end{array}$ & $000148-18-5$ & $2.1 E+03$ & $2.1 E+01$ & $6.1 E+04$ & $6.1 E+03$ & & & & & $1.7 \mathrm{E}+03$ & $1.7 \mathrm{E}+01$ & $4.9 E+04$ & $4.9 E+03$ & $9.4 \mathrm{E}+02$ & $9.4 \mathrm{E}+00$ & $2.7 E+04$ & $2.7 \mathrm{E}+03$ \\
\hline Sodium Fluoroacetate & $000062-74-8$ & & & $4.1 \mathrm{E}+01$ & $4.1 \mathrm{E}+00$ & & & & & & & $3.2 \mathrm{E}+01$ & $3.2 \mathrm{E}+00$ & & & $1.8 \mathrm{E}+01$ & $1.8 \mathrm{E}+00$ \\
\hline Sodium Metavanadate & $013718-26-8$ & & & $2.0 \mathrm{E}+03$ & $2.0 \mathrm{E}+02$ & & & & & & & $6.5 \mathrm{E}+03$ & $6.5 \mathrm{E}+02$ & & & $1.6 \mathrm{E}+03$ & $1.6 \mathrm{E}+02$ \\
\hline $\begin{array}{l}\text { Stirofos } \\
\text { (Tetrachlorovinphos) }\end{array}$ & $000961-11-5$ & $2.4 E+04$ & $2.4 E+02$ & $6.1 \mathrm{E}+04$ & $6.1 E+03$ & & & & & $1.9 \mathrm{E}+04$ & $1.9 \mathrm{E}+02$ & $4.9 \mathrm{E}+04$ & $4.9 \mathrm{E}+03$ & $1.1 E+04$ & $1.1 \mathrm{E}+02$ & $2.7 \mathrm{E}+04$ & $2.7 \mathrm{E}+03$ \\
\hline Strontium, Stable & $007440-24-6$ & & & $1.0 \mathrm{E}+06$ & $1.2 \mathrm{E}+05$ & & & & & & & $1.0 \mathrm{E}+06$ & $3.9 \mathrm{E}+05$ & & & $9.3 \mathrm{E}+05$ & $9.3 \mathrm{E}+04$ \\
\hline
\end{tabular}


Table 3.2c. (continued)

\begin{tabular}{|c|c|c|c|c|c|c|c|c|c|c|c|c|c|c|c|c|c|}
\hline \multirow[t]{2}{*}{ Chemical } & \multirow[t]{2}{*}{ CASRN } & \multicolumn{4}{|c|}{ Ingestion (mg/kg) } & \multicolumn{4}{|c|}{ Inhalation (mg/kg) } & \multicolumn{4}{|c|}{$\operatorname{Dermal}(\mathrm{mg} / \mathrm{kg})^{\circ}$} & \multicolumn{4}{|c|}{$\begin{array}{l}\text { Ingestion + Inhalation } \\
\text { + Dermal (mg/kg) }\end{array}$} \\
\hline & & $10^{-4}$ & $10^{4}$ & $H Q=1$ & $\mathrm{HQ}=0.1$ & $10^{-4}$ & $10^{4}$ & $H Q=1$ & $\mathrm{HQ}=0.1$ & $10^{-4}$ & $10^{5}$ & $\mathrm{HQ}=1$ & $\mathrm{HQ}=0.1$ & $10^{-4}$ & $10^{6}$ & $H I=1$ & $\mathrm{HI}=0.1$ \\
\hline Strychnine & $000057-24-9$ & & & $6.1 E+02$ & $6.1 \mathrm{E}+01$ & & & & & & & $4.9 \mathrm{E}+02$ & $4.9 \mathrm{E}+01$ & & & $2.7 \mathrm{E}+02$ & $2.7 \mathrm{E}+01$ \\
\hline Styrene $e^{(6)}$ & $000100-42-5$ & & & 4.1E+05 & $4.1 \mathrm{E}+04$ & & & $6.7 \mathrm{E}+02$ & $6.7 E+02$ & & & $5.2 \mathrm{E}+05$ & $5.2 \mathrm{E}+04$ & & & $6.7 E+02$ & $6.7 \mathrm{E}+02$ \\
\hline Sulfate & $014808-79-8$ & & & & & & & & & & & & & & & & \\
\hline Systhane & $088671-89-0$ & & & $5.1 \mathrm{E}+04$ & $5.1 \mathrm{E}+03$ & & & & & & & $4.0 \mathrm{E}+04$ & $4.0 \mathrm{E}+03$ & & & $2.3 \mathrm{E}+04$ & $2.3 \mathrm{E}+03$ \\
\hline TCDD, 2,3,7,8-(1) & $001746-01-6$ & $3.8 \mathrm{E}-03$ & $3.8 \mathrm{E}-05$ & & $\cdot$ & $4.1 \mathrm{E}+01$ & 4.1E-01 & & & $1.0 \mathrm{E}-03$ & $1.0 \mathrm{E}-05$ & & & $8.0 \mathrm{E}-04$ & $8.0 \mathrm{E}-06$ & & \\
\hline TCDF, 2,3,7,8-(1) & $051207-31-9$ & $3.8 \mathrm{E}-02$ & $3.8 \mathrm{E}-04$ & & & 4.1E+02 & $4.1 \mathrm{E}+00$ & & & $3.0 \mathrm{E}-02$ & $3.0 \mathrm{E}-04$ & & & $1.7 \mathrm{E} \cdot 02$ & $1.7 \mathrm{E}-04$ & & \\
\hline TCMTB & $021564-17-0$ & & & $6.1 \mathrm{E}+04$ & $6.1 \mathrm{E}+03$ & & & & & & & $4.9 \mathrm{E}+04$ & $4.9 \mathrm{E}+03$ & & & $2.7 \mathrm{E}+04$ & $2.7 \mathrm{E}+03$ \\
\hline Tebuthiuron & $034014-18-1$ & & & $1.4 \mathrm{E}+05$ & $1.4 E+04$ & & & & & & & $1.1 \mathrm{E}+05$ & $1.1 \mathrm{E}+04$ & & & $6.3 E+04$ & $6.3 \mathrm{E}+03$ \\
\hline Temephos & $003383-96-8$ & & & 4.1E+04 & $4.1 E+03$ & & & & & & & $3.2 \mathrm{E}+04$ & $3.2 \mathrm{E}+03$ & & & $1.8 \mathrm{E}+04$ & $1.8 \mathrm{E}+03$ \\
\hline Terbacil & $005902-51-2$ & & & $2.7 \mathrm{E}+04$ & $2.7 \mathrm{E}+03$ & & & & & & & $2.1 \mathrm{E}+04$ & $2.1 \mathrm{E}+03$ & & & $1.2 \mathrm{E}+04$ & $1.2 \mathrm{E}+03$ \\
\hline Terbufos & $013071-79-9$ & & & $5.1 E+01$ & $5.1 \mathrm{E}+00$ & & & & & & & $4.0 \mathrm{E}+01$ & $4.0 E+00$ & & & $2.3 E+01$ & $2.3 \mathrm{E}+00$ \\
\hline Terbutryn & $000886-50-0$ & & & $2.0 \mathrm{E}+03$ & $2.0 \mathrm{E}+02$ & & & & & & & $1.6 \mathrm{E}+03$ & $1.6 \mathrm{E}+02$ & & & $9.0 E+02$ & $9.0 \mathrm{E}+01$ \\
\hline Tetrabutyl Lead & $001920-90-7$ & & & & & & & & & & & & & & & & \\
\hline $\begin{array}{l}\text { Tetrachlorobenzene, } \\
1,2,4,5 \text { - }\end{array}$ & $000095-94-3$ & & & $6.1 E+02$ & $6.1 \mathrm{E}+01$ & & & & . & & & $7.8 \mathrm{E}+02$ & $7.8 \mathrm{E}+01$ & & & $3.4 E+02$ & $3.4 \mathrm{E}+01$ \\
\hline $\begin{array}{l}\text { Tetrachloroethane, } \\
1,1,1,2-\end{array}$ & $000630-20-6$ & $2.2 \mathrm{E}+04$ & $2.2 \mathrm{E}+02$ & $6.1 \mathrm{E}+04$ & $6.1 \mathrm{E}+03$ & $7.7 \mathrm{E}+02$ & $2.9 \mathrm{E}+01$ & & & $2.8 \mathrm{E}+04$ & $2.8 \mathrm{E}+02$ & $7.8 \mathrm{E}+04$ & $7.8 \mathrm{E}+03$ & $7.7 E+02$ & $2.4 E+01$ & $7.7 \mathrm{E}+02$ & $7.7 \mathrm{E}+02$ \\
\hline $\begin{array}{l}\text { Tetrachloroethane, } \\
1,1,2,2-\end{array}$ & $000079-34-5$ & $2.9 \mathrm{E}+03$ & $2.9 \mathrm{E}+01$ & & & $6.8 \mathrm{E}+02$ & $6.8 \mathrm{E}+00$ & & & $3.2 \mathrm{E}+03$ & $3.2 \mathrm{E}+01$ & & & $4.7 E+02$ & $4.7 \mathrm{E}+00$ & & \\
\hline Tetrachloroethylene & $000127-18-4$ & $1.1 \mathrm{E}+04$ & $1.1 \mathrm{E}+02$ & $2.0 \mathrm{E}+04$ & $2.0 \mathrm{E}+03$ & $1.2 \mathrm{E}+02$ & $1.2 \mathrm{E}+02$ & & & $1.7 \mathrm{E}+04$ & $1.7 \mathrm{E}+02$ & $3.2 \mathrm{E}+04$ & $3.2 \mathrm{E}+03$ & $1.2 \mathrm{E}+02$ & $4.7 \mathrm{E}+01$ & $1.2 \mathrm{E}+02$ & $1.2 \mathrm{E}+02$ \\
\hline $\begin{array}{l}\text { Tetrachlorophenol, } \\
2,3,4,6-\end{array}$ & $000058-90-2$ & & & $6.1 \mathrm{E}+04$ & $6.1 \mathrm{E}+03$ & & & & & & & $4.9 E+04$ & $4.9 \mathrm{E}+03$ & & & $2.7 \mathrm{E}+04$ & $2.7 \mathrm{E}+03$ \\
\hline $\begin{array}{l}\text { Tetrachlorotoluene, p- } \\
\text { alpha, alpha, alpha- }\end{array}$ & $005216-25-1$ & $2.9 \mathrm{E}+01$ & $2.9 \mathrm{E}-01$ & & & & & & & $3.6 \mathrm{E}+01$ & $3.6 \mathrm{E}-01$ & & & $1.6 \mathrm{E}+01$ & $1.6 \mathrm{E}-01$ & & \\
\hline $\begin{array}{l}\text { Tetraethyl } \\
\text { Dithiopyrophosphate }\end{array}$ & $003689-24-5$ & & & $1.0 \mathrm{E}+03$ & $1.0 E+02$ & & & & & & & $8.1 E+02$ & $8.1 E+01$ & & & $4.5 E+02$ & $4.5 \mathrm{E}+01$ \\
\hline
\end{tabular}


Table 3.2c. (continued)

\begin{tabular}{|c|c|c|c|c|c|c|c|c|c|c|c|c|c|c|c|c|c|}
\hline \multirow[t]{2}{*}{ Chemical } & \multirow[t]{2}{*}{ CASRN } & \multicolumn{4}{|c|}{ Ingestion (mg/kg) } & \multicolumn{4}{|c|}{ Inhalation $(\mathrm{mg} / \mathrm{kg})^{-\boldsymbol{x}}$} & \multicolumn{4}{|c|}{ Dermal (mg/kg) } & \multicolumn{4}{|c|}{$\begin{array}{l}\text { Ingestion + Inhalation } \\
+ \text { Dermal (mg/kg) }\end{array}$} \\
\hline & & $10^{-4}$ & $10^{4}$ & $H Q=1$ & $\mathrm{HQ}=0.1$ & $10^{-4}$ & $10^{4}$ & $H Q=1$ & $\mathrm{HQ}=0.1$ & $10^{-4}$ & $10^{4}$ & $\mathrm{HQ}=1$ & $\mathrm{HQ}=0.1$ & $10^{-4}$ & $10^{6}$ & $\mathrm{HI}=1$ & $H I=0.1$ \\
\hline Tetraethyl Lead & $000078-00-2$ & & & & & & & & & & & & & & & & \\
\hline $\begin{array}{l}\text { Tetrafluoroethane, } \\
1,1,1,2 \text { - }\end{array}$ & $000811-97-2$ & & & & & & & $1.1 \mathrm{E}+02$ & $1.1 \mathrm{E}+02$ & & & & & & & & \\
\hline Tetramethyl Lead & $000075-74-1$ & & & & & & & & & & & & & & & & \\
\hline Tetrapropyl Lead & $003440-75-3$ & & & & & & & & & & & & & & & & \\
\hline Thallic Oxide ${ }^{(3)}$ & $001314-32-5$ & & & $1.4 \mathrm{E}+02$ & $1.4 \mathrm{E}+01$ & & & & & & & $1.1 \mathrm{E}+02$ & $1.1 \mathrm{E}+01$ & & & $6.3 \mathrm{E}+01$ & $6.3 \mathrm{E}+00$ \\
\hline Thallium (I) Nitrate & $010102-45-1$ & & & $1.8 \mathrm{E}+02$ & $1.8 \mathrm{E}+01$. & & & & & & & $5.8 \mathrm{E}+02$ & $5.8 \mathrm{E}+01$ & & & $1.4 \mathrm{E}+02$ & $1.4 \mathrm{E}+01$ \\
\hline Thallium (Soluble Salts) & $007440-28-0$ & & & & & & & & & & & & & & & & \\
\hline Thallium Acetate & $000563-68-8$ & & & $1.8 \mathrm{E}+02$ & $1.8 \mathrm{E}+01$ & & & & & & & $1.5 \mathrm{E}+02$ & $1.5 \mathrm{E}+01$ & & & $8.1 E+01$ & $8.1 \mathrm{E}+00$ \\
\hline Thallium Carbonate & $006533-73-9$ & & & $1.6 \mathrm{E}+02$ & $1.6 \mathrm{E}+01$ & & & & & & & $1.3 \mathrm{E}+02$ & $1.3 \mathrm{E}+01$ & & & $7.2 \mathrm{E}+01$ & $7.2 \mathrm{E}+00$ \\
\hline Thallium Chloride & $007791-12-0$ & & & $1.6 \mathrm{E}+02$ & $1.6 \mathrm{E}+01$ & & & & & & & $5.2 \mathrm{E}+02$ & $5.2 \mathrm{E}+01$ & & & $1.2 \mathrm{E}+02$ & $1.2 \mathrm{E}+01$ \\
\hline Thallium Selenite ${ }^{(3)}$ & $012039-52-0$ & & & $1.8 \mathrm{E}+02$ & $1.8 \mathrm{E}+01$ & & & & & & & $5.8 \mathrm{E}+02$ & $5.8 \mathrm{E}+01$ & & & $1.4 \mathrm{E}+02$ & $1.4 \mathrm{E}+01$ \\
\hline Thallium Sulfate & $007446-18-6$ & & & $1.6 \mathrm{E}+02$ & $1.6 \mathrm{E}+01$ & & & & & & & $5.2 \mathrm{E}+02$ & $5.2 \mathrm{E}+01$ & & & $1.2 \mathrm{E}+02$ & $1.2 \mathrm{E}+01$ \\
\hline Thiobencarb & $028249-77-6$ & & & $2.0 \mathrm{E}+04$ & $2.0 \mathrm{E}+03$ & & & & & & & $1.6 \mathrm{E}+04$ & $1.6 \mathrm{E}+03$ & & & $9.0 \mathrm{E}+03$ & $9.0 \mathrm{E}+02$ \\
\hline Thiofanox & $039196-18-4$ & & & $6.1 \mathrm{E}+02$ & $6.1 \mathrm{E}+01$ & & & & & & & $4.9 \mathrm{E}+02$ & $4.9 \mathrm{E}+01$ & & & $2.7 \mathrm{E}+02$ & $2.7 \mathrm{E}+01$ \\
\hline Thiophanate, Methyl & $023564-05-8$ & & & $1.6 \mathrm{E}+05$ & $1.6 \mathrm{E}+04$ & & & & & & & $1.3 \mathrm{E}+05$ & $1.3 \mathrm{E}+04$ & & & $7.2 \mathrm{E}+04$ & $7.2 \mathrm{E}+03$ \\
\hline Thiram & $000137-26-8$ & & & $1.0 \mathrm{E}+04$ & $1.0 \mathrm{E}+03$ & & & & & & & $8.1 \mathrm{E}+03$ & $8.1 \mathrm{E}+02$ & & & $4.5 \mathrm{E}+03$ & $4.5 \mathrm{E}+02$ \\
\hline Thorium & $007440-29-1$ & & & & & & & & & & & & & & & & \\
\hline Tin & $007440-31-5$ & & & $1.0 \mathrm{E}+06$ & $1.2 \mathrm{E}+05$ & & & & & & & $1.0 \mathrm{E}+06$ & $1.9 \mathrm{E}+05$ & & & $7.5 E+05$ & $7.5 \mathrm{E}+04$ \\
\hline Titanium & $007440-32-6$ & & & & & & & & & & & & & & & & \\
\hline Toluene $^{(6)}$ & $000108-88-3$ & & & $4.1 \mathrm{E}+05$ & $4.1 \mathrm{E}+04$ & & & $2.5 \mathrm{E}+02$ & $2.5 E+02$ & & & $5.2 \mathrm{E}+05$ & $5.2 \mathrm{E}+04$ & & & $2.5 \mathrm{E}+02$ & $2.5 \mathrm{E}+02$ \\
\hline $\begin{array}{l}\text { Toluene diisocyanate } \\
\text { mixture (IDI) }\end{array}$ & $026471-62-5$ & & & & & & & $4.4 E+05$ & $4.4 \mathrm{E}+04$ & & & & & & & & \\
\hline Toluene-2,4-diamine & $000095-80-7$ & $1.8 \mathrm{E}+02$ & $1.8 \mathrm{E}+00$ & & & & & & & $1.4 \mathrm{E}+02$ & $1.4 \mathrm{E}+00$ & & & $7.9 \mathrm{E}+01$ & $7.9 \mathrm{E}-01$ & & \\
\hline
\end{tabular}


Table 3.2c. (continued)

\begin{tabular}{|c|c|c|c|c|c|c|c|c|c|c|c|c|c|c|c|c|c|}
\hline \multirow[t]{2}{*}{ Chemical } & \multirow[t]{2}{*}{ CASRN } & \multicolumn{4}{|c|}{ Ingestion (mg/kg) } & \multicolumn{4}{|c|}{ Inhalation $(\mathrm{mg} / \mathrm{kg})^{\cdot \boldsymbol{x}}$} & \multicolumn{4}{|c|}{ Dermal (mg/kg) } & \multicolumn{4}{|c|}{$\begin{array}{l}\text { Ingestion + Inhalation } \\
+ \text { Dermal }(\mathrm{mg} / \mathrm{kg})^{1^{-\gamma}}\end{array}$} \\
\hline & & $10^{4}$ & $10^{6}$ & $H Q=1$ & $\mathrm{HQ}=0.1$ & $10^{-4}$ & $10^{6}$ & $H Q=1$ & $\mathrm{HQ}=0.1$ & $10^{-4}$ & $10^{5}$ & $H Q=1$ & $\mathrm{HQ}=0.1$ & $10^{-4}$ & $10^{4}$ & $\mathrm{HI}=\mathbf{1}$ & $\mathrm{HI}=0.1$ \\
\hline Toluene-2,5-diamine & $000095-70-5$ & & & $1.0 \mathrm{E}+06$ & $1.2 \mathrm{E}+05$ & & & & & & & $9.7 \mathrm{E}+05$ & $9.7 \mathrm{E}+04$ & & & $5.4 \mathrm{E}+05$ & $5.4 \mathrm{E}+04$ \\
\hline Toluene-2,6-diamine & $000823-40-5$ & & & 4.1E+05 & $4.1 \mathrm{E}+04$ & & & & & & & $3.2 E+05$ & $3.2 \mathrm{E}+04$ & & & $1.8 \mathrm{E}+05$ & $1.8 \mathrm{E}+04$ \\
\hline $\begin{array}{l}\text { Toluidine, o- } \\
\text { (Methylaniline, 2-) }\end{array}$ & $000095-53-4$ & $2.4 \mathrm{E}+03$ & $2.4 \mathrm{E}+01$ & & & & & & & $1.9 \mathrm{E}+03$ & $1.9 \mathrm{E}+01$ & & & $1.1 \mathrm{E}+03$ & $1.1 \mathrm{E}+01$ & & \\
\hline Toluidine, p- & $000106-49-0$ & $3.0 \mathrm{E}+03$ & $3.0 \mathrm{E}+01$ & & & & & & & $2.4 \mathrm{E}+03$ & $2.4 \mathrm{E}+01$ & & & $1.3 \mathrm{E}+03$ & $1.3 E+01$ & & \\
\hline Toxaphene & $008001-35-2$ & $5.2 \mathrm{E}+02$ & $5.2 \mathrm{E}+00$ & & & $1.0 \mathrm{E}+06$ & $5.6 \mathrm{E}+04$ & & & $4.1 E+02$ & 4. $. \mathrm{IE}+00$ & & & $2.3 E+02$ & $2.3 E+\infty 0$ & & \\
\hline Tralomethrin & $066841-25-6$ & & & $1.5 \mathrm{E}+04$ & $1.5 \mathrm{E}+03$ & & & & & & & $1.2 \mathrm{E}+04$ & $1.2 \mathrm{E}+03$ & & & $6.8 \mathrm{E}+03$ & $6.8 \mathrm{E}+02$ \\
\hline Triallate & $002303-17-5$ & & & $2.7 \mathrm{E}+04$ & $2.7 \mathrm{E}+03$ & & & & & & & $2.1 \mathrm{E}+04$ & $2.1 E+03$ & & & $1.2 \mathrm{E}+04$ & $1.2 \mathrm{E}+03$ \\
\hline Triasulfuron & $082097-50-5$ & & & $2.0 E+04$ & $2.0 \mathrm{E}+03$ & & & & & & & $1.6 \mathrm{E}+04$ & $1.6 \mathrm{E}+03$ & & & $9.0 \mathrm{E}+03$ & $9.0 \mathrm{E}+02$ \\
\hline Tribromobenzene, 1,2,4- & $000615-54-3$ & & & $1.0 \mathrm{E}+04$ & $1.0 \mathrm{E}+03$ & & & & & & & $1.3 \mathrm{E}+04$ & $1.3 \mathrm{E}+03$ & & & $5.7 \mathrm{E}+03$ & $5.7 \mathrm{E}+02$ \\
\hline Tribromochloromethane & $000594-15-0$ & & & & & & & & & & & & & & & & \\
\hline Tribromodiphenyl Ether & $049690-94-0$ & & & & & & & & & & & & & & & & \\
\hline Tributyltin Oxide & $000056-35.9$ & & & $6.1 \mathrm{E}+01$ & $6.1 \mathrm{E}+00$ & & & & & & & $4.9 E+01$ & $4.9 \mathrm{E}+00$ & & & 2.7E+01 & $2.7 \mathrm{E}+00$ \\
\hline $\begin{array}{l}\text { Trichloro-1,2,2- } \\
\text { trifluoroethane, 1,1,2- }\end{array}$ & $000076-13-1$ & & & $1.0 \mathrm{E}+06$ & $1.0 \mathrm{E}+06$ & & & $2.1 \mathrm{E}+02$ & $2.1 \mathrm{E}+02$ & & & $1.0 \mathrm{E}+06$ & $1.0 \mathrm{E}+06$ & & & $2.1 \mathrm{E}+02$ & $2.1 \mathrm{E}+02$ \\
\hline $\begin{array}{l}\text { Trichloro-2- } \\
\text { hydroxydiphenylether }\end{array}$ & $003380-34-5$ & & & & & & & & & & & & & & & & \\
\hline \multicolumn{18}{|l|}{ Trichloroacetic Acid } \\
\hline $\begin{array}{l}\text { Trichloroaniline } \mathrm{HCl} \text {, } \\
2,4,6 \cdot\end{array}$ & $033663-50-2$ & $2.0 \mathrm{E}+04$ & $2.0 \mathrm{E}+02$ & & & & & & & $1.6 \mathrm{E}+04$ & $1.6 \mathrm{E}+02$ & & & $\begin{array}{c}8.7 \mathrm{E}+03 \\
.\end{array}$ & $8.7 \mathrm{E}+01$ & & \\
\hline Trichloroaniline, 2,4,6- & $000634-93-5$ & $1.7 \mathrm{E}+04$ & $1.7 \mathrm{E}+02$ & & & & & & & $1.3 \mathrm{E}+04$ & $1.3 \mathrm{E}+02$ & & & $7.4 \mathrm{E}+03$ & $7.4 \mathrm{E}+01$ & & \\
\hline Trichlorobenzene, 1,2,4- & $000120-82-1$ & & & $2.0 \mathrm{E}+04$ & $2.0 \mathrm{E}+03$ & & & $8.3 \mathrm{E}+02$ & $8.3 \mathrm{E}+02$ & & & $3.1 E+04$ & $3.1 \mathrm{E}+03$ & $\cdot$ & & $8.3 \mathrm{E}+02$ & $8.3 \mathrm{E}+02$ \\
\hline Trichloroethane, $1,1,1_{-}^{(3)}$ & $000071-55-6$ & & & $1.8 \mathrm{E}+05$ & $1.8 \mathrm{E}+04$ & & & $7.3 \mathrm{E}+02$ & $7.3 \mathrm{E}+02$ & & & $2.6 \mathrm{E}+05$ & $2.6 \mathrm{E}+04$ & & & $7.3 \mathrm{E}+02$ & $7.3 \mathrm{E}+02$ \\
\hline Trichlorocthane, 1,1,2- & $000079-00-5$ & $1.0 \mathrm{E}+04$ & $1.0 \mathrm{E}+02$ & $8.2 E+03$ & $8.2 \mathrm{E}+02$ & $8.5 E+02$ & $8.8 \mathrm{E}+00$ & & & $1.3 \mathrm{E}+04$ & $1.3 \mathrm{E}+02$ & $1.0 \mathrm{E}+04$ & $1.0 \mathrm{E}+03$ & $7.6 \mathrm{E}+02$ & $7.6 \mathrm{E}+00$ & $8.5 E+02$ & $4.6 \mathrm{E}+02$ \\
\hline Trichloroethylene ${ }^{(0)}$ & $000079-01-6$ & $5.2 \mathrm{E}+04$ & $5.2 \mathrm{E}+02$ & & & $4.5 \mathrm{E}+02$ & $4.5 \mathrm{E}+01$ & & & $1.2 \mathrm{E}+04$ & $1.2 \mathrm{E}+02$ & & & $4.5 E+02$ & $3.1 E+01$ & & \\
\hline
\end{tabular}

Nonradionuclides/soil/industrial

Page 29 of 32 
Table 3.2c. (continued)

\begin{tabular}{|c|c|c|c|c|c|c|c|c|c|c|c|c|c|c|c|c|c|}
\hline \multirow[t]{2}{*}{ Chemical } & \multirow[t]{2}{*}{ CASRN } & \multicolumn{4}{|c|}{ Ingestion (mg/kg) } & \multicolumn{4}{|c|}{ Inhalation $(\mathrm{mg} / \mathrm{kg})^{\circ z}$} & \multicolumn{4}{|c|}{ Dermal (mg/kg) } & \multicolumn{4}{|c|}{$\begin{array}{l}\text { Ingestion + Inhalation } \\
+ \text { Dermal (mg/kg) }\end{array}$} \\
\hline & & $10^{-4}$ & $10^{4}$ & $H Q=1$ & $H Q=0.1$ & $10^{-4}$ & $10^{4}$ & $H Q=1$ & $\mathrm{HQ}=0.1$ & $10^{-4}$ & $10^{6}$ & $\mathrm{HQ}=1$ & $\mathrm{HQ}=0.1$ & $10^{-4}$ & $10^{4}$ & $H \mathrm{I}=1$ & $\mathrm{HI}=0.1$ \\
\hline Trichlorofluoromethane & $000075-69-4$ & & & $6.1 \mathrm{E}+05$ & $6.1 \mathrm{E}+04$ & & & $7.1 \mathrm{E}+02$ & $6.0 E+02$ & & & $2.2 \mathrm{E}+05$ & $2.2 \mathrm{E}+04$ & & & $7.1 \mathrm{E}+02$ & $5.7 \mathrm{E}+02$ \\
\hline Trichlorophenol, 2,4,5- & $000095-95-4$ & & & $2.0 \mathrm{E}+05$ & $2.0 \mathrm{E}+04$ & & & & & & & $2.6 \mathrm{E}+05$ & $2.6 \mathrm{E}+04$ & & & $1.1 \mathrm{E}+05$ & $1.1 \mathrm{E}+04$ \\
\hline Trichlorophenol, 2,4,6- & $000088-06-2$ & $5.2 \mathrm{E}+04$ & $5.2 \mathrm{E}+02$ & & & $1.0 \mathrm{E}+06$ & $1.0 \mathrm{E}+06$ & & & 4.1E+04 & 4.1E+02 & & & $2.3 E+04$ & $2.3 \mathrm{E}+02$ & & \\
\hline $\begin{array}{l}\text { Trichlorophenoxyacetic } \\
\text { Acid, 2,4,5- }\end{array}$ & $000093-76-5$ & & & $2.0 E+04$ & $2.0 \mathrm{E}+03$ & & & & & & & $1.6 \mathrm{E}+04$ & $1.6 \mathrm{E}+03$ & & & $9.0 \mathrm{E}+03$ & $9.0 \mathrm{E}+02$ \\
\hline Trichloropropane, 1,1,2- & $000598-77-6$ & & & $1.0 \mathrm{E}+04$ & $1.0 \mathrm{E}+03$ & & & & & & & $1.3 \mathrm{E}+04$ & $1.3 \mathrm{E}+03$ & & & $5.7 \mathrm{E}+03$ & $5.7 \mathrm{E}+02$ \\
\hline Trichloropropane, 1,2,3- & $000096-18-4$ & $8.2 \mathrm{E}+01$ & $8.2 \mathrm{E}-01$ & $1.2 \mathrm{E}+04$ & $1.2 E+03$ & & & & & $1.0 \mathrm{E}+02$ & $1.0 \mathrm{E}+00$ & $1.6 \mathrm{E}+04$ & $1.6 \mathrm{E}+03$ & $4.6 \mathrm{E}+01$ & $4.6 \mathrm{E}-01$ & $6.9 \mathrm{E}+03$ & $6.9 \mathrm{E}+02$ \\
\hline Trichloropropene, 1,2,3- & $000096-19-5$ & & & $1.0 \mathrm{E}+04$ & $1.0 \mathrm{E}+03$ & & & & & & & $1.3 \mathrm{E}+04$ & $1.3 \mathrm{E}+03$ & & & $5.7 \mathrm{E}+03$ & $5.7 \mathrm{E}+02$ \\
\hline $\begin{array}{l}\text { Trichlorotoluene, alpha } \\
\text { 2,6- }\end{array}$ & $002014-83-7$ & & & & & & & & & & & & & & & & \\
\hline Tridiphane & $058138-08-2$ & & & $6.1 \mathrm{E}+03$ & $6.1 \mathrm{E}+02$ & & & & & & & $4.9 \mathrm{E}+03$ & $4.9 E+02$ & & & $2.7 \mathrm{E}+03$ & $2.7 \mathrm{E}+02$ \\
\hline Triethyl Lead & $005224-23-7$ & & & & & & & & & & & & & & & & \\
\hline Triethylamine & $000121-44-8$ & & & & & & & $1.7 \mathrm{E}+03$ & $1.7 \mathrm{E}+02$ & & & & & & & & \\
\hline Trifluralin & $001582-09-8$ & $7.4 \mathrm{E}+04$ & $7.4 E+02$ & $1.5 \mathrm{E}+04$ & $1.5 \mathrm{E}+03$ & & & & & $5.9 \mathrm{E}+04$ & $5.9 \mathrm{E}+02$ & $1.2 \mathrm{E}+04$ & $1.2 \mathrm{E}+03$ & $3.3 E+04$ & $3.3 \mathrm{E}+02$ & $6.8 \mathrm{E}+03$ & $6.8 \mathrm{E}+02$ \\
\hline Trimethyl Lead & $007442-13-9$ & & & & & & & & & & & & & & & & \\
\hline Trimethyl Phosphate & $000512-56-1$ & $1.5 \mathrm{E}+04$ & $1.5 \mathrm{E}+02$ & & & & & & & $1.2 \mathrm{E}+04$ & $1.2 \mathrm{E}+02$ & & & $6.8 \mathrm{E}+03$ & $6.8 \mathrm{E}+0 \mathrm{I}$ & & \\
\hline Trimethylethyl Lead & $001762-26-1$ & & & & & & & & & & & & & & & & \\
\hline Trinitrobenzene, 1,3,5- & $000099-35-4$ & & & $1.0 \mathrm{E}+02$ & $1.0 \mathrm{E}+01$ & & & & & & & $1.1 \mathrm{E}+02$ & $1.1 \mathrm{E}+01$ & & & $5.2 \mathrm{E}+01$ & $5.2 \mathrm{E}+00$ \\
\hline $\begin{array}{l}\text { Trinitrophenylmethyl- } \\
\text { nitramine }\end{array}$ & $000479-45-8$ & & & $2.0 \mathrm{E}+04$ & $2.0 \mathrm{E}+03$ & & & & & & & $1.6 \mathrm{E}+04$ & $1.6 \mathrm{E}+03$ & & & $9.0 \mathrm{E}+03$ & $9.0 \mathrm{E}+02$ \\
\hline Trinitrotoluene, 2,4,6- & $000118-96-7$ & $1.9 \mathrm{E}+04$ & $1.9 \mathrm{E}+02$ & $1.0 \mathrm{E}+03$ & $1.0 \mathrm{E}+02$ & & & & & $1.8 \mathrm{E}+04$ & $1.8 \mathrm{E}+02$ & $9.7 \mathrm{E}+02$ & $9.7 \mathrm{E}+01$ & $9.3 \mathrm{E}+03$ & $9.3 \mathrm{E}+01$ & $5.0 \mathrm{E}+02$ & $5.0 \mathrm{E}+01$ \\
\hline Tripropyl Lead & $006618-03-7$ & & & & & & & & & & & & & & & & \\
\hline
\end{tabular}


Table 3.2c. (continued)

\begin{tabular}{|c|c|c|c|c|c|c|c|c|c|c|c|c|c|c|c|c|c|}
\hline \multirow[t]{2}{*}{ Chemical } & \multirow[t]{2}{*}{ CASRN } & \multicolumn{4}{|c|}{ Ingestion (mg/kg) } & \multicolumn{4}{|c|}{ Inhalation (mg/kg) } & \multicolumn{4}{|c|}{ Dermal (mg/kg)" } & \multicolumn{4}{|c|}{$\begin{array}{l}\text { Ingestion + Inhalation } \\
+ \text { Dermal (mg/kg) }\end{array}$} \\
\hline & & $10^{-4}$ & $10^{4}$ & $\mathrm{HQ}=1$ & $\mathrm{HQ}=0.1$ & $10^{-4}$ & $10^{4}$ & $\mathrm{HQ}=1$ & $\mathrm{HQ}=0.1$ & $10^{-4}$ & $10^{6}$ & $H Q=1$ & $\mathrm{HQ}=0.1$ & $10^{-4}$ & $10^{5}$ & $\mathbf{H I}=1$ & $\mathrm{HI}=0.1$ \\
\hline Uranium (Soluble Salts) & NA & & & $6.1 \mathrm{E}+03$ & $6.1 E+02$ & & & & & & & $8.2 E+04$ & $8.2 \mathrm{E}+03$ & & & $5.7 \mathrm{E}+03$ & $5.7 \mathrm{E}+02$ \\
\hline Vanadium Pentoxide & $001314-62-1$ & & & $1.8 \mathrm{E}+04$ & $1.8 \mathrm{E}+03$ & & & & & & & $5.8 \mathrm{E}+04$ & $5.8 \mathrm{E}+03$ & & & $1.4 \mathrm{E}+04$ & $1.4 \mathrm{E}+03$ \\
\hline Vanadium Sulfate & $036907-42-3$ & & & $4.1 \mathrm{E}+04$ & $4.1 E+03$ & & & & & & & $1.3 \mathrm{E}+05$ & $1.3 E+04$ & & & $3.1 E+04$ & $3.1 \mathrm{E}+03$ \\
\hline Vanadium, Metallic & $007440-62-2$ & & & $1.4 \mathrm{E}+04$ & $1.4 E+03$ & & & & & & & $2.3 E+03$ & $2.3 \mathrm{E}+02$ & & & $2.0 \mathrm{E}+03$ & $2.0 \mathrm{E}+02$ \\
\hline Vanadyl Sulfate & $027774-13-6$ & & & $4.1 \mathrm{E}+04$ & 4.1E+03 & & & & & & & $1.3 \mathrm{E}+05$ & $1.3 \mathrm{E}+04$ & & & $3.1 E+04$ & $3.1 E+03$ \\
\hline Vemolate & $001929-77-7$ & & & $2.0 \mathrm{E}+03$ & $2.0 \mathrm{E}+02$ & & & & & & & $1.6 \mathrm{E}+03$ & $1.6 \mathrm{E}+02$ & & & $9.0 \mathrm{E}+02$ & $9.0 \mathrm{E}+01$ \\
\hline Vinclozolin & $050471-44-8$ & $\cdot$ & & $5.1 \mathrm{E}+04$ & $5.1 \mathrm{E}+03$ & & & & & & & $4.0 \mathrm{E}+04$ & $4.0 \mathrm{E}+03$ & & & $2.3 E+04$ & $2.3 E+03$ \\
\hline Vinyl Acetate & $000108-05-4$ & & & $1.0 \mathrm{E}+06$ & $2.0 \mathrm{E}+05$ & & & $7.8 \mathrm{E}+02$ & $3.9 \mathrm{E}+02$ & & & $1.0 \mathrm{E}+06$ & $2.1 E+05$ & & & $7.8 \mathrm{E}+02$ & $3.9 \mathrm{E}+02$ \\
\hline Vinyl Bromide & $000593-60-2$ & & & & & $1.8 \mathrm{E}+02$ & $1.8 \mathrm{E}+00$ & $6.2 \mathrm{E}+01$ & $6.2 \mathrm{E}+00$ & & & & & & & & \\
\hline Vinyl Chloride(6) & $000075-01-4$ & $3.0 \mathrm{E}+02$ & $3.0 \mathrm{E}+00$ & & & $4.1 \mathrm{E}-02$ & $4.1 \mathrm{E}-04$ & & & $4.8 \mathrm{E}+02$ & $4.8 \mathrm{E}+00$ & & & 4.1E-02 & 4.1E-04 & & \\
\hline Warfarin & $000081-81-2$ & & & $6.1 E+02$ & $6.1 \mathrm{E}+01$ & & & & & & & $4.9 \mathrm{E}+02$ & $4.9 \mathrm{E}+01$ & & & $2.7 E+02$ & $2.7 E+01$ \\
\hline White Phosphorus & $007723-14-0$ & & & 4.1E+01 & $4.1 \mathrm{E}+00$ & & & & & & & $1.3 \mathrm{E}+02$ & $1.3 \mathrm{E}+01$ & & & $3.1 E+01$ & $3.1 \mathrm{E}+00$ \\
\hline Xylene, Mixture $e^{(6)}$ & $001330-20-7$ & & & $1.0 \mathrm{E}+06$ & 4.1E+05 & & & & & & & $1.0 \mathrm{E}+06$ & $6.0 \mathrm{E}+05$ & & & $1.0 \mathrm{E}+06$ & $2.4 \mathrm{E}+05$ \\
\hline Xylene, $P_{-}^{(6)}$ & $000106-42-3$ & & & & & & & & & & & & & & & & \\
\hline Xylene, m.(6) & $000108-38-3$ & & & $1.0 \mathrm{E}+06$ & $4.1 \mathrm{E}+05$ & & & & & & & $1.0 \mathrm{E}+06$ & $5.2 \mathrm{E}+05$ & & & $1.0 \mathrm{E}+06$ & $2.3 E+05$ \\
\hline Xylene, $0 .(6)$ & $000095-47-6$ & & & $1.0 \mathrm{E}+06$ & $4.1 \mathrm{E}+05$ & & & & & & & $1.0 \mathrm{E}+06$ & $5.2 \mathrm{E}+05$ & & & $1.0 \mathrm{E}+06$ & 2.3E+05 \\
\hline Zinc (Metallic) & $007440-66-6$ & & & $6.1 \mathrm{E}+05$ & $6.1 \mathrm{E}+04$ & & & & & & & $1.0 \mathrm{E}+06$ & $1.9 \mathrm{E}+05$ & & & $4.7 \mathrm{E}+05$ & $4.7 \mathrm{E}+04$ \\
\hline Zinc Cyanide & $000557-21-1$ & & & $1.0 \mathrm{E}+05$ & $1.0 \mathrm{E}+04$ & & & & & & & $3.2 \mathrm{E}+05$ & $3.2 \mathrm{E}+04$ & & & $7.8 \mathrm{E}+04$ & $7.8 \mathrm{E}+03$ \\
\hline Zinc Phosphide & $001314-84-7$ & & & $6.1 \mathrm{E}+02$ & $6.1 \mathrm{E}+01$ & & & & & & & $1.9 \mathrm{E}+03$ & $1.9 \mathrm{E}+02$ & & & $4.7 \mathrm{E}+02$ & $4.7 \mathrm{E}+01$ \\
\hline
\end{tabular}


Table 3.2c. (continued)

\begin{tabular}{|c|c|c|c|c|c|c|c|c|c|c|c|c|c|c|c|c|c|}
\hline \multirow{2}{*}{ Chemical } & \multirow[t]{2}{*}{ CASRN } & \multicolumn{4}{|c|}{ Ingestion (mg/kg) } & \multicolumn{4}{|c|}{ Inhalation (mg/kg) } & \multicolumn{4}{|c|}{ Dermal (mg/kg) } & \multicolumn{4}{|c|}{$\begin{array}{c}\text { Ingestion + Inhalation } \\
+ \text { Dermal }(\mathrm{mg} / \mathrm{kg})^{\dagger+v}\end{array}$} \\
\hline & & $10^{-4}$ & $10^{4}$ & $\mathrm{HQ}=1$ & $H Q=0.1$ & $10^{-1}$ & $10^{4}$ & $H Q=1$ & $\mathrm{HQ}=0.1$ & $10^{-t}$ & $10^{6}$ & $\mathrm{HQ}=1$ & $\mathrm{HQ}=0.1$ & $10^{-4}$ & $10^{6}$ & $\mathrm{HI}=1$ & $\mathrm{HI}=0.1$ \\
\hline Zineb & $012122-67-7$ & & & $1.0 \mathrm{E}+05$ & $1.0 E+04$ & & & & & & & 8. $1 E+04$ & $8.1 E+03$ & & & $4.5 \mathrm{E}+04$ & $4.5 \mathrm{E}+03$ \\
\hline Zirconium & $007440-67-7$ & & & & & & & & & & & & & & & & \\
\hline
\end{tabular}

Notes:

* By definition, $1 \mathrm{~kg}=1.0 \mathrm{E}+06_{\mathrm{mg}}$. Therefore, a limit of $1.0 \mathrm{E}+06 \mathrm{mg} / \mathrm{kg}$ was used for any PRG that was calculated to be above this limit.

$\dagger$ If slope factors and/or reference doses were available for only one pathway (i.e., ingestion, dermal, OR inhalation), the total for ingestion plus inhalation plus dermal was not calculated.

¥ The volatilization factor defines the relationship between the concentration of contaminants in soil and the volatilization of contaminants in air. The chemical concentration in soil (C) calculated using the volatilization factor must be compared with the soil saturation concentration $\left(C_{s t a t}\right)$. If $C$ is greater than $C_{s a t}$ then the PRG is set equal to $C_{s a t}$.

(1) The same oral slope factor as used for polychlorinated biphenyls.

(2) The PRG was calculated considering Supplemental Guidance from RAGS: Region 4 Bulletins, Human Health Risk Assessment (Interim Guidance) from EPA (received February 11, 1992). The slope factors for chemicals so marked indicate that a toxicity equivalency factor (TEF) method was used for carcinogenic PAHs based on each compound's relative potency to the potency of benzo[a]pyrene. The following TEFs were used to convert each PAH slope factor to an equivalent slope factor of benzo[a]pyrene [that has a new cancer slope factor of $7.3(\mathrm{mg} / \mathrm{kg} / \mathrm{day})^{-1}$ identified by EPA's CRAVE workgroup]: (1) benzo[a]pyrene, TEF $=1.0$; (2) benzo[a]anthracene, TEF $=0.1 ;$ (3) benzo[b]fluoranthene, TEF $=0.1 ;$ (4) benzo[k]fluoranthene, TEF $=0.01$; (5) chrysene, $\mathrm{TEF}=0.001 ;(6)$ dibenz $[\mathrm{a}, \mathrm{h}]$ anthracene, $\mathrm{TEF}=1.0$; and $(7)$ indeno[1,2,3-c,d]pyrene, $\mathrm{TEF}=0.1$.

(3) The toxicity values for trichloroethylene have been withdrawn from IRIS and HEAST pending further investigation. However, the most recent toxicity values have been used to calculate the PRG.

(4) The PRG was calculated considering Supplemental Guidance from RAGS: Region 4 Bulletins, Human Health Risk Assessment (Interim Guidance) from EPA (November 1995). The slope factors for chemicals so marked indicate that a TEF methodology was used for chlorinated dioxin and furan congeners based on each compound's relative potency to the potency of 2,3,7,8tetrachlorodibenzodioxin (TCDD). The following TEFs were used to convert each slope factor to an equivalent slope factor of 2,3,7,8-TCDD: (1) 2,3,7,8-TCDD, TEF = 1.0; (2) 2,3,7,8$\mathrm{PeCDD}, \mathrm{TEF}=0.5 ;$ (3) 2,3,7,8-HxCDD, TEF $=0.1 ;(4) 2,3,7,8-\mathrm{HpCDD}, \mathrm{TEF}=0.01 ;(5) \mathrm{OCDD}, \mathrm{TEF}=0.001 ;(6) 2,3,7,8-\mathrm{TCDF}, \mathrm{TEF}=0.1 ;(7) 1,2,3,7,8-\mathrm{PeCDF}, \mathrm{TEF}=0.5 ;(8) 2,3,4,7,8-$ $\mathrm{PeCDF}, \mathrm{TEF}=0.05 ;(9) 2,3,7,8-\mathrm{HxCDF}, \mathrm{TEF}=0.1 ;(10) 2,3,7,8-\mathrm{HpCDF}, \mathrm{TEF}=0.01$; and $(11) \mathrm{OCDF}, \mathrm{TEF}=0.001$.

(5) Toxicity values for these chemicals have been withdrawn from IRIS and HEAST pending further investigation. However, the most recent toxicity values have been used to calculate the PRG.

(6) Toxicity values for these chemicals were obtained from the Superfund Health Risk Technical Support Center. 
Table 3.2d. Risk-based Preliminary Remediation Goals for radionuclides in soil (industrial scenario)

\begin{tabular}{|c|c|c|c|c|c|c|c|c|c|}
\hline \multirow[t]{2}{*}{ Chemical $^{\dagger}$} & \multirow[t]{2}{*}{ CASRN } & \multicolumn{2}{|c|}{ Ingestion (pCi/g) } & \multicolumn{2}{|c|}{ Inhalation (pCi/g) } & \multicolumn{2}{|c|}{$\begin{array}{l}\text { External Exposure } \\
(\mathrm{pCi} / \mathrm{g})^{+t}\end{array}$} & \multicolumn{2}{|c|}{$\begin{array}{c}\text { Ingestion + Inhalation + } \\
\text { External Exposure } \\
(\mathrm{pCi} / \mathrm{g})^{\mathrm{ft \prime}}\end{array}$} \\
\hline & & $10^{-4}$ & $10^{-6}$ & $10^{-4}$ & $10^{-6}$ & $10^{-4}$ & $10^{-6}$ & $10^{-4}$ & $10^{-6}$ \\
\hline Ac-225 & $014265-85-1$ & $2.3 \mathrm{E}+03$ & $2.3 \mathrm{E}+01$ & $8.2 E+05$ & $8.2 E+03$ & $2.8 \mathrm{E}+03$ & $2.8 \mathrm{E}+01$ & $1.2 \mathrm{E}+03$ & $1.2 \mathrm{E}+01$ \\
\hline Ac-227 & $014952-40-0$ & $9.1 \mathrm{E}+02$ & $9.1 \mathrm{E}+00$ & $4.8 \mathrm{E}+04$ & $4.8 \mathrm{E}+02$ & $9.3 \mathrm{E}+05$ & $9.3 E+03$ & $8.9 \mathrm{E}+02$ & $8.9 \mathrm{E}+00$ \\
\hline$A C-227+D$ & $014952-40-0$ & $5.1 \mathrm{E}+02$ & $5.1 \mathrm{E}+00$ & $4.4 \mathrm{E}+04$ & $4.4 \mathrm{E}+02$ & $3.7 \mathrm{E}+01$ & 3.7E-01 & $3.4 \mathrm{E}+01$ & $3.4 \mathrm{E}-01$ \\
\hline Ac-228 & $014331-83-0$ & $2.0 \mathrm{E}+05$ & $2.0 \mathrm{E}+03$ & $1.0 \mathrm{E}+08$ & $1.0 \mathrm{E}+06$ & $6.7 \mathrm{E}+00$ & $6.7 \mathrm{E}-02$ & $6.7 E+00$ & $6.7 \mathrm{E}-02$ \\
\hline Ag-105 & $014928-14-4$ & $2.0 \mathrm{E}+05$ & $2.0 \mathrm{E}+03$ & $1.5 \mathrm{E}+09$ & $1.5 \mathrm{E}+07$ & & & $2.0 \mathrm{E}+05$ & $2.0 \mathrm{E}+03$ \\
\hline $\mathrm{Ag}-108$ & $014391-65-2$ & $4.6 E+07$ & $4.6 \mathrm{E}+05$ & $3.6 \mathrm{E}+11$ & $3.6 \mathrm{E}+09$ & $3.8 \mathrm{E}+02$ & $3.8 \mathrm{E}+00$ & $3.8 \mathrm{E}+02$ & $3.8 \mathrm{E}+00$ \\
\hline $\mathrm{Ag}-108 \mathrm{~m}$ & $014391-65-2$ & $5.3 \mathrm{E}+04$ & $5.3 \mathrm{E}+02$ & $4.9 \mathrm{E}+07$ & $4.9 \mathrm{E}+05$ & $3.9 \mathrm{E}+00$ & $3.9 \mathrm{E}-02$ & $3.9 \mathrm{E}+00$ & $3.9 \mathrm{E}-02$ \\
\hline $\mathrm{Ag}-108 \mathrm{~m}+\mathrm{D}$ & $014391-65-2$ & $5.3 \mathrm{E}+04$ & $5.3 \mathrm{E}+02$ & $4.9 \mathrm{E}+07$ & $4.9 \mathrm{E}+05$ & $3.9 \mathrm{E}+00$ & $3.9 \mathrm{E}-02$ & $3.9 \mathrm{E}+00$ & $3.9 \mathrm{E}-02$ \\
\hline $\mathrm{Ag}-109 \mathrm{~m}$ & $014378-38-2$ & $1.2 \mathrm{E}+09$ & $1.2 \mathrm{E}+07$ & $9.9 E+12$ & $9.9 \mathrm{E}+10$ & $1.9 \mathrm{E}+04$ & $1.9 \mathrm{E}+02$ & $1.9 \mathrm{E}+04$ & $1.9 \mathrm{E}+02$ \\
\hline Ag-110 & $014391-76-5$ & $1.3 E+08$ & $1.3 \mathrm{E}+06$ & $1.1 \mathrm{E}+12$ & $1.1 \mathrm{E}+10$ & $1.9 \mathrm{E}+02$ & $1.9 \mathrm{E}+00$ & $1.9 \mathrm{E}+02$ & $1.9 \mathrm{E}+00$ \\
\hline $\mathrm{Ag}-110 \mathrm{~m}$ & $014391-76-5$ & $3.8 \mathrm{E}+04$ & $3.8 \mathrm{E}+02$ & $1.1 \mathrm{E}+08$ & $1.1 \mathrm{E}+06$ & $2.1 \mathrm{E}+00$ & 2.1E-02 & $2.1 \mathrm{E}+00$ & $2.1 \mathrm{E}-02$ \\
\hline $\mathrm{Ag}-111$ & $015760-04-0$ & $4.7 \mathrm{E}+04$ & $4.7 \mathrm{E}+02$ & $6.5 \mathrm{E}+08$ & $6.5 \mathrm{E}+06$ & $2.6 \mathrm{E}+02$ & $2.6 \mathrm{E}+00$ & $2.6 E+02$ & $2.6 \mathrm{E}+00$ \\
\hline Am-241 & $014596-10-2$ & $9.8 \mathrm{E}+02$ & $9.8 \mathrm{E}+00$ & $8.9 E+04$ & $8.9 \mathrm{E}+02$ & $4.8 E+03$ & $4.8 \mathrm{E}+01$ & $8.0 \mathrm{E}+02$ & $8.0 \mathrm{E}+00$ \\
\hline Am-242 & $013981-54-9$ & $2.2 E+05$ & $2.2 \mathrm{E}+03$ & $3.3 E+08$ & $3.3 \mathrm{E}+06$ & $3.8 \mathrm{E}+03$ & $3.8 \mathrm{E}+01$ & $3.7 \mathrm{E}+03$ & $3.7 \mathrm{E}+01$ \\
\hline Am-242m & $013981-54-9$ & $1.1 \mathrm{E}+03$ & $1.1 \mathrm{E}+01$ & $9.8 \mathrm{E}+04$ & $9.8 \mathrm{E}+02$ & $2.5 \mathrm{E}+05$ & $2.5 E+03$ & $1.1 \mathrm{E}+03$ & $1.1 E+01$ \\
\hline Am-243 & $014993-75-0$ & $9.8 \mathrm{E}+02$ & $9.8 \mathrm{E}+00$ & $9.0 \mathrm{E}+04$ & $9.0 \mathrm{E}+02$ & $9.0 \mathrm{E}+02$ & $9.0 \mathrm{E}+00$ & $4.7 \mathrm{E}+02$ & $4.7 \mathrm{E}+00$ \\
\hline$A m-243+D$ & 014993-75-0 & $9.7 \mathrm{E}+02$ & $9.7 \mathrm{E}+00$ & $9.0 \mathrm{E}+04$ & $9.0 \mathrm{E}+02$ & $8.2 \mathrm{E}+01$ & $8.2 \mathrm{E}-01$ & $7.6 \mathrm{E}+01$ & $7.6 \mathrm{E}-01$ \\
\hline
\end{tabular}

Radionuclides/soil/industrial

Page 1 of 20 


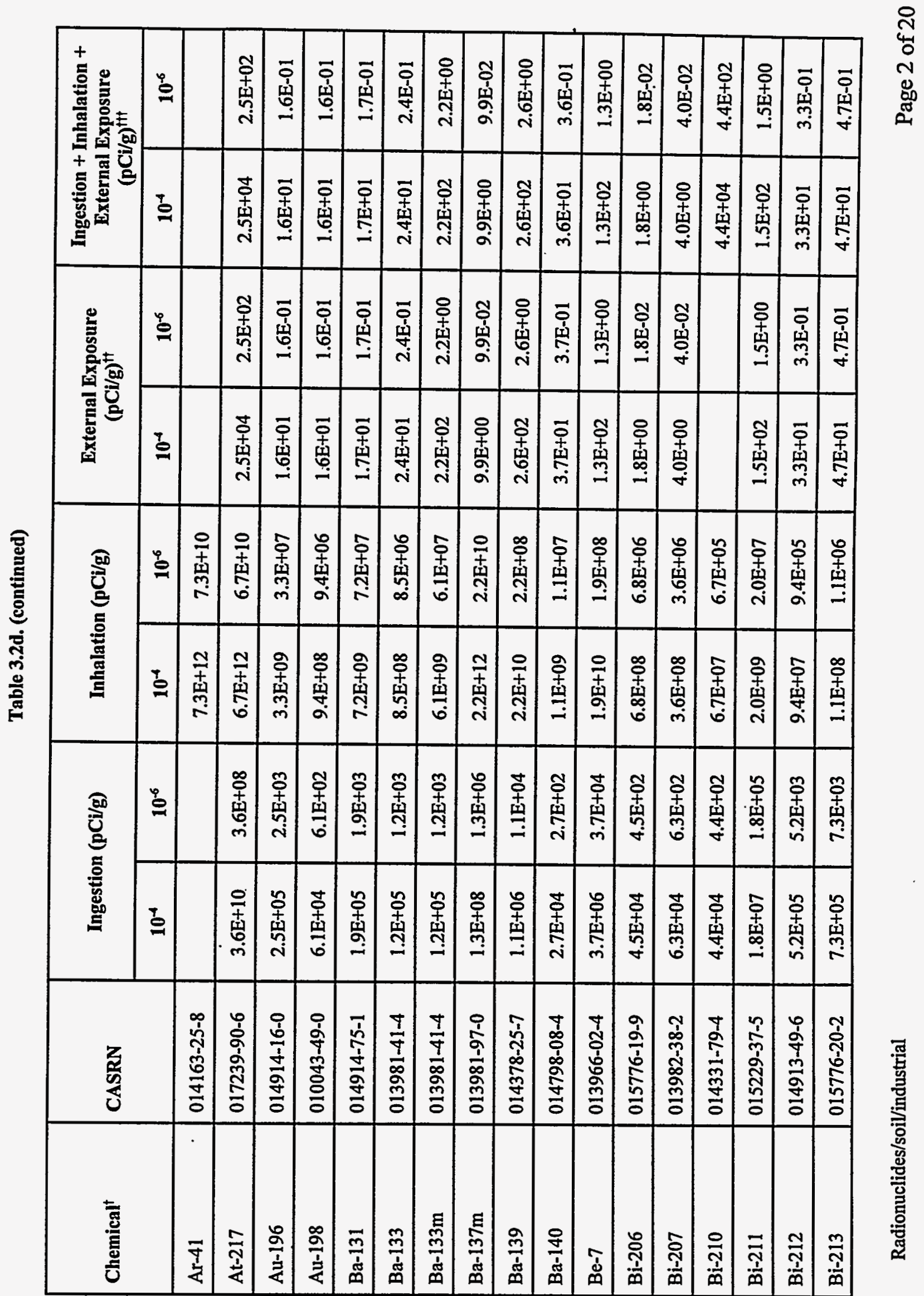


Table 3.2d. (continued)

\begin{tabular}{|c|c|c|c|c|c|c|c|c|c|}
\hline \multirow[t]{2}{*}{ Chemical $^{\dagger}$} & \multirow[t]{2}{*}{ CASRN } & \multicolumn{2}{|c|}{ Ingestion (pCi/g) } & \multicolumn{2}{|c|}{ Inhalation (pCi/g) } & \multicolumn{2}{|c|}{$\begin{array}{l}\text { External Exposure } \\
(\mathrm{pCi} / \mathrm{g})^{++}\end{array}$} & \multicolumn{2}{|c|}{$\begin{array}{c}\text { Ingestion + Inhalation + } \\
\text { External Exposure } \\
(\mathrm{pCi} / \mathrm{g})^{\mathrm{ft}}\end{array}$} \\
\hline & & $10^{-4}$ & $10^{-6}$ & $10^{-4}$ & $10^{-6}$ & $10^{-4}$ & $10^{-6}$ & $10^{-4}$ & $10^{-6}$ \\
\hline $\mathrm{Bi}-214$ & 014733-03-0 & $1.6 \mathrm{E}+06$ & $1.6 \mathrm{E}+04$ & $2.3 \mathrm{E}+08$ & $2.3 E+06$ & $3.6 \mathrm{E}+00$ & $3.6 \mathrm{E}-02$ & $3.6 \mathrm{E}+00$ & $3.6 \mathrm{E}-02$ \\
\hline $\mathrm{Br}-82$ & $014686-69-2$ & $2.3 \mathrm{E}+05$ & $2.3 \mathrm{E}+03$ & $4.4 \mathrm{E}+09$ & $4.4 \mathrm{E}+07$ & $2.2 \mathrm{E}+00$ & $2.2 \mathrm{E}-02$ & $2.2 \mathrm{E}+00$ & 2.2E-02 \\
\hline C-11 & 014333-33-6 & $7.1 E+06$ & $7.1 \mathrm{E}+04$ & $1.0 \mathrm{E}+11$ & $1.0 \mathrm{E}+09$ & $6.1 \mathrm{E}+00$ & $6.1 \mathrm{E}-02$ & $6.1 \mathrm{E}+00$ & $6.1 \mathrm{E}-02$ \\
\hline C-14 & $014762-75-5$ & $3.1 \mathrm{E}+05$ & $3.1 \mathrm{E}+03$ & $4.9 \mathrm{E}+11$ & $4.9 \mathrm{E}+09$ & & & $3.1 E+05$ & $3.1 \mathrm{E}+03$ \\
\hline C-15 & $015929-23-4$ & $4.8 \mathrm{E}+08$ & $4.8 \mathrm{E}+06$ & $4.3 \mathrm{E}+12$ & $4.3 E+10$ & & & $4.8 \mathrm{E}+08$ & $4.8 \mathrm{E}+06$ \\
\hline $\mathrm{Ca}-45$ & 013966-05-7 & $1.6 \mathrm{E}+05$ & $1.6 \mathrm{E}+03$ & $1.4 \mathrm{E}+09$ & $1.4 \mathrm{E}+07$ & $5.6 \mathrm{E}+12$ & $5.6 \mathrm{E}+10$ & $1.6 \mathrm{E}+05$ & $1.6 \mathrm{E}+03$ \\
\hline $\mathrm{Ca}-47$ & 001439-99-2 & $4.8 \mathrm{E}+04$ & $4.8 \mathrm{E}+02$ & $6.6 \mathrm{E}+08$ & $6.6 \mathrm{E}+06$ & $5.3 \mathrm{E}+00$ & $5.3 \mathrm{E}-02$ & $5.3 \mathrm{E}+00$ & 5.3E-02 \\
\hline Cd-109 & $014109-32-1$ & $4.0 \mathrm{E}+04$ & $4.0 \mathrm{E}+02$ & $1.9 \mathrm{E}+08$ & $1.9 E+06$ & $3.9 \mathrm{E}+04$ & $3.9 E+02$ & $2.0 \mathrm{E}+04$ & $2.0 \mathrm{E}+02$ \\
\hline $\mathrm{Cd}-115$ & 014336-68-6 & $4.4 \mathrm{E}+04$ & $4.4 \mathrm{E}+02$ & $7.0 \mathrm{E}+08$ & $7.0 \mathrm{E}+06$ & $3.1 \mathrm{E}+01$ & $3.1 \mathrm{E}-01$ & $3.1 E+01$ & $3.1 \mathrm{E}-01$ \\
\hline $\mathrm{Cd}-115 \mathrm{~m}$ & 014336-68-6 & $2.3 E+04$ & $2.3 E+02$ & $2.0 \mathrm{E}+08$ & $2.0 \mathrm{E}+06$ & $2.6 \mathrm{E}+02$ & $2.6 \mathrm{E}+00$ & $2.5 \mathrm{E}+02$ & $2.5 \mathrm{E}+00$ \\
\hline Ce-141 & 013967-74-3 & $8.2 E+04$ & $8.2 E+02$ & $7.9 \mathrm{E}+08$ & $7.9 E+06$ & $1.6 \mathrm{E}+02$ & $1.6 \mathrm{E}+00$ & $1.6 \mathrm{E}+02$ & $1.6 \mathrm{E}+00$ \\
\hline Ce-143 & 014119-19-8 & $5.4 \mathrm{E}+04$ & $5.4 \mathrm{E}+02$ & $8.9 \mathrm{E}+08$ & $8.9 E+06$ & $3.0 \mathrm{E}+01$ & $3.0 \mathrm{E}-01$ & $3.0 \mathrm{E}+01$ & $3.0 \mathrm{E}-01$ \\
\hline Ce-144 & $014762-78-8$ & $1.1 \mathrm{E}+04$ & $1.1 E+02$ & $3.2 E+07$ & $3.2 E+05$ & $8.5 \mathrm{E}+02$ & $8.5 \mathrm{E}+00$ & $7.9 \mathrm{E}+02$ & $7.9 E+00$ \\
\hline Ce-144+D & $014762-78-8$ & $1.1 E+04$ & $1.1 \mathrm{E}+02$ & $3.2 E+07$ & $3.2 \mathrm{E}+05$ & $1.4 \mathrm{E}+02$ & $1.4 \mathrm{E}+00$ & $1.4 \mathrm{E}+02$ & $1.4 \mathrm{E}+00$ \\
\hline $\mathrm{Cl}-36$ & $013981-43-6$ & $1.4 \mathrm{E}+05$ & $1.4 \mathrm{E}+03$ & $2.6 \mathrm{E}+09$ & $2.6 \mathrm{E}+07$ & & & $1.4 E+05$ & $1.4 \mathrm{E}+03$ \\
\hline Cl-38 & 014158-34-0 & $1.5 \mathrm{E}+06$ & $1.5 \mathrm{E}+04$ & $2.1 \mathrm{E}+10$ & $2.1 E+08$ & $3.4 \mathrm{E}+00$ & $3.4 \mathrm{E}-02$ & $3.4 \mathrm{E}+00$ & $3.4 \mathrm{E}-02$ \\
\hline $\mathrm{Cm}-242$ & 015510-73-3 & $8.4 \mathrm{E}+03$ & $8.4 \mathrm{E}+01$ & $1.1 \mathrm{E}+06$ & $1.1 \mathrm{E}+04$ & $9.4 \mathrm{E}+05$ & $9.4 E+03$ & $8.2 \mathrm{E}+03$ & $8.2 \mathrm{E}+01$ \\
\hline
\end{tabular}


Table 3.2d. (continued)

\begin{tabular}{|c|c|c|c|c|c|c|c|c|c|}
\hline \multirow[t]{2}{*}{ Chemical $^{\dagger}$} & \multirow[t]{2}{*}{ CASRN } & \multicolumn{2}{|c|}{ Ingestion (pCi/g) } & \multicolumn{2}{|c|}{ Inhalation (pCi/g) } & \multicolumn{2}{|c|}{$\begin{array}{l}\text { External Exposure } \\
(\mathrm{pCi} / \mathrm{g})^{H+}\end{array}$} & \multicolumn{2}{|c|}{$\begin{array}{c}\text { Ingestion + Inhalation + } \\
\text { External Exposure } \\
(\mathrm{pCi} / \mathrm{g})^{++1}\end{array}$} \\
\hline & & $10^{-4}$ & $10^{-6}$ & $10^{-4}$ & $10^{-6}$ & $10^{-4}$ & $10^{-6}$ & $10^{-4}$ & $10^{-6}$ \\
\hline $\mathrm{Cm}-243$ & $015757-87-6$ & $1.3 \mathrm{E}+03$ & $1.3 \mathrm{E}+01$ & $1.2 \mathrm{E}+05$ & $1.2 \mathrm{E}+03$ & $1.3 \mathrm{E}+02$ & $1.3 \mathrm{E}+00$ & $1.2 \mathrm{E}+02$ & $1.2 \mathrm{E}+00$ \\
\hline $\mathrm{Cm}-243+\mathrm{D}$ & $015757-87-6$ & $1.3 \mathrm{E}+03$ & $1.3 \mathrm{E}+01$ & $1.2 \mathrm{E}+05$ & $1.2 \mathrm{E}+03$ & $1.3 \mathrm{E}+02$ & $1.3 \mathrm{E}+00$ & $1.2 \mathrm{E}+02$ & $1.2 \mathrm{E}+00$ \\
\hline $\mathrm{Cm}-244$ & $013981-15-2$ & $1.5 \mathrm{E}+03$ & $1.5 \mathrm{E}+01$ & $1.4 \mathrm{E}+05$ & $1.4 \mathrm{E}+03$ & $1.1 \mathrm{E}+06$ & $1.1 \mathrm{E}+04$ & $1.5 \mathrm{E}+03$ & $1.5 \mathrm{E}+01$ \\
\hline $\mathrm{Cm}-245$ & $015621-76-8$ & $9.6 \mathrm{E}+02$ & $9.6 \mathrm{E}+00$ & $8.7 \mathrm{E}+04$ & 8.7E+02 & $4.0 \mathrm{E}+02$ & $4.0 \mathrm{E}+00$ & $2.8 \mathrm{E}+02$ & $2.8 \mathrm{E}+00$ \\
\hline $\mathrm{Cm}-246$ & $015757-90-1$ & $9.6 \mathrm{E}+02$ & $9.6 \mathrm{E}+00$ & $8.8 \mathrm{E}+04$ & $8.8 \mathrm{E}+02$ & $1.2 \mathrm{E}+06$ & $1.2 \mathrm{E}+04$ & $9.5 \mathrm{E}+02$ & $9.5 \mathrm{E}+00$ \\
\hline $\mathrm{Cm}-247$ & $015758-32-4$ & $1.0 \mathrm{E}+03$ & $1.0 \mathrm{E}+01$ & $9.6 \mathrm{E}+04$ & $9.6 \mathrm{E}+02$ & $2.1 \mathrm{E}+01$ & $2.1 \mathrm{E}-01$ & $2.1 \mathrm{E}+01$ & $2.1 \mathrm{E}-01$ \\
\hline $\mathrm{Cm}-248$ & 015758-33-5 & $2.4 \mathrm{E}+02$ & $2.4 \mathrm{E}+00$ & $2.3 E+04$ & $2.3 \mathrm{E}+02$ & $1.5 \mathrm{E}+06$ & $1.5 \mathrm{E}+04$ & $2.4 \mathrm{E}+02$ & $2.4 \mathrm{E}+00$ \\
\hline Co-57 & $013981-50-5$ & $3.3 \mathrm{E}+05$ & $3.3 \mathrm{E}+03$ & $1.2 \mathrm{E}+09$ & $1.2 \mathrm{E}+07$ & $1.1 \mathrm{E}+02$ & $1.1 \mathrm{E}+00$ & $1.1 \mathrm{E}+02$ & $1.1 \mathrm{E}+00$ \\
\hline $\mathrm{Co}-58$ & 013981-38-9 & $1.1 \mathrm{E}+05$ & $1.1 \mathrm{E}+03$ & $6.6 \mathrm{E}+08$ & $6.6 \mathrm{E}+06$ & $5.9 \mathrm{E}+00$ & $5.9 \mathrm{E}-02$ & $5.9 \mathrm{E}+00$ & $5.9 \mathrm{E}-02$ \\
\hline Co-58m & 013981-38-9 & $3.4 \mathrm{E}+06$ & $3.4 \mathrm{E}+04$ & $3.9 \mathrm{E}+10$ & $3.9 \mathrm{E}+08$ & $6.8 \mathrm{E}+05$ & $6.8 \mathrm{E}+03$ & $5.7 \mathrm{E}+05$ & $5.7 \mathrm{E}+03$ \\
\hline Co-60 & 010198-40-0 & $1.7 \mathrm{E}+04$ & $1.7 E+02$ & $5.0 \mathrm{E}+07$ & $5.0 \mathrm{E}+05$ & $2.2 \mathrm{E}+00$ & $2.2 \mathrm{E}-02$ & $2.2 \mathrm{E}+00$ & 2.2E-02 \\
\hline $\mathrm{Cr}-51$ & 014392-02-0 & $2.3 \mathrm{E}+06$ & $2.3 E+04$ & $2.0 \mathrm{E}+10$ & $2.0 \mathrm{E}+08$ & $2.1 \mathrm{E}+02$ & $2.1 \mathrm{E}+00$ & $2.1 \mathrm{E}+02$ & $2.1 \mathrm{E}+00$ \\
\hline Cs-131 & 014914-76-2 & $1.8 \mathrm{E}+06$ & $1.8 \mathrm{E}+04$ & $3.2 \mathrm{E}+10$ & $3.2 \mathrm{E}+08$ & $9.4 \mathrm{E}+03$ & $9.4 \mathrm{E}+01$ & $9.3 \mathrm{E}+03$ & $9.3 \mathrm{E}+01$ \\
\hline Cs-134 & 013967-70-9 & $6.8 \mathrm{E}+03$ & $6.8 \mathrm{E}+01$ & $1.2 \mathrm{E}+08$ & $1.2 \mathrm{E}+06$ & $3.7 \mathrm{E}+00$ & 3.7E-02 & $3.7 \mathrm{E}+00$ & $3.7 \mathrm{E}-02$ \\
\hline Cs-134m & $013967-70-9$ & $7.0 \mathrm{E}+06$ & $7.0 \mathrm{E}+04$ & $1.1 \mathrm{E}+11$ & $1.1 \mathrm{E}+09$ & $1.1 \mathrm{E}+03$ & $1.1 \mathrm{E}+01$ & $1.1 \mathrm{E}+03$ & $1.1 \mathrm{E}+01$ \\
\hline Cs-135 & $015726-30-4$ & $7.1 \mathrm{E}+04$ & $7.1 \mathrm{E}+02$ & $1.3 E+09$ & $1.3 E+07$ & & & $7.1 \mathrm{E}+04$ & $7.1 \mathrm{E}+02$ \\
\hline Cs-136 & 014234-29-8 & $4.1 \mathrm{E}+04$ & 4.1E+02 & $7.4 \mathrm{E}+08$ & $7.4 E+06$ & $2.7 \mathrm{E}+00$ & $2.7 \mathrm{E}-02$ & $2.7 \mathrm{E}+00$ & 2.7E-02 \\
\hline
\end{tabular}

Radionuclides/soil/industrial

Page 4 of 20 
Table 3.2d. (continued)

\begin{tabular}{|c|c|c|c|c|c|c|c|c|c|}
\hline \multirow[t]{2}{*}{ Chemical $^{+}$} & \multirow[t]{2}{*}{ CASRN } & \multicolumn{2}{|c|}{ Ingestion (pCi/g) } & \multicolumn{2}{|c|}{ Inhalation (pCi/g) } & \multicolumn{2}{|c|}{$\begin{array}{l}\text { External Exposure } \\
(\mathrm{pCi} / \mathrm{g})^{\dagger+}\end{array}$} & \multicolumn{2}{|c|}{$\begin{array}{c}\text { Ingestion + Inhalation + } \\
\text { External Exposure } \\
(\mathrm{pCi} / \mathrm{g})^{\mathrm{ft}}\end{array}$} \\
\hline & & $10^{-4}$ & $10^{-6}$ & $10^{-4}$ & $10^{-6}$ & $10^{-4}$ & $10^{-6}$ & $10^{-4}$ & $10^{-6}$ \\
\hline Cs-137 & $010045-97-3$ & $1.0 \mathrm{E}+04$ & $1.0 \mathrm{E}+02$ & $1.8 \mathrm{E}+08$ & $1.8 \mathrm{E}+06$ & & & $1.0 \mathrm{E}+04$ & $1.0 \mathrm{E}+02$ \\
\hline Cs-137+D & $010045-97-3$ & $1.0 \mathrm{E}+04$ & $1.0 \mathrm{E}+02$ & $1.8 \mathrm{E}+08$ & $1.8 \mathrm{E}+06$ & $1.0 \mathrm{E}+01$ & $1.0 \mathrm{E}-01$ & $1.0 \mathrm{E}+01$ & $1.0 \mathrm{E}-01$ \\
\hline Cs-138 & 015758-29-9 & $1.8 \mathrm{E}+06$ & $1.8 \mathrm{E}+04$ & $2.6 \mathrm{E}+10$ & $2.6 \mathrm{E}+08$ & $2.3 \mathrm{E}+00$ & $2.3 \mathrm{E}-02$ & $2.3 \mathrm{E}+00$ & $2.3 \mathrm{E}-02$ \\
\hline $\mathrm{Cu}-64$ & $013981-25-4$ & $6.1 \mathrm{E}+05$ & $6.1 E+03$ & $8.2 \mathrm{E}+09$ & $8.2 E+07$ & $3.3 E+01$ & 3.3E-01 & $3.3 \mathrm{E}+01$ & 3.3E-01 \\
\hline Dy-165 & $013967-64-1$ & $9.8 \mathrm{E}+05$ & $9.8 \mathrm{E}+03$ & $1.5 \mathrm{E}+10$ & $1.5 \mathrm{E}+08$ & $3.5 \mathrm{E}+02$ & $3.5 \mathrm{E}+00$ & $3.5 \mathrm{E}+02$ & $3.5 \mathrm{E}+00$ \\
\hline Dy-166 & $015840-01-4$ & $3.4 \mathrm{E}+04$ & $3.4 \mathrm{E}+02$ & $4.4 \mathrm{E}+08$ & $4.4 \mathrm{E}+06$ & 8.1E+02 & $8.1 E+00$ & $7.9 E+02$ & $7.9 \mathrm{E}+00$ \\
\hline Er-169 & $015840-13-8$ & $1.5 \mathrm{E}+05$ & $1.5 \mathrm{E}+03$ & $2.3 E+09$ & $2.3 \mathrm{E}+07$ & $3.4 \mathrm{E}+06$ & $3.4 \mathrm{E}+04$ & $1.4 \mathrm{E}+05$ & $1.4 \mathrm{E}+03$ \\
\hline Er-171 & $014391-45-8$ & $2.0 \mathrm{E}+05$ & $2.0 \mathrm{E}+03$ & $4.6 \mathrm{E}+09$ & $4.6 \mathrm{E}+07$ & $2.1 \mathrm{E}+01$ & $2.1 \mathrm{E}-01$ & $2.1 \mathrm{E}+01$ & $2.1 \mathrm{E}-01$ \\
\hline Eu-152 & 014683-23-9 & $5.6 \mathrm{E}+04$ & $5.6 \mathrm{E}+02$ & $4.3 \mathrm{E}+07$ & $4.3 \mathrm{E}+05$ & $5.4 \mathrm{E}+00$ & $5.4 \mathrm{E}-02$ & $5.4 \mathrm{E}+00$ & $5.4 \mathrm{E}-02$ \\
\hline Eu-154 & $015585-10-1$ & $3.4 \mathrm{E}+04$ & $3.4 \mathrm{E}+02$ & $3.7 \mathrm{E}+07$ & 3.7E+05 & 4.7E+00 & $4.7 \mathrm{E}-02$ & $4.7 E+00$ & 4.7E-02 \\
\hline Eu-155 & $014391-16-3$ & $1.9 \mathrm{E}+05$ & $1.9 \mathrm{E}+03$ & $3.6 \mathrm{E}+08$ & $3.6 \mathrm{E}+06$ & $3.6 \mathrm{E}+02$ & $3.6 \mathrm{E}+00$ & $3.6 \mathrm{E}+02$ & $3.6 \mathrm{E}+00$ \\
\hline Eu-156 & $014280-35-4$ & $2.9 \mathrm{E}+04$ & $2.9 E+02$ & $3.7 \mathrm{E}+08$ & $3.7 E+06$ & 4.1E+00 & $4.1 \mathrm{E}-02$ & $4.1 E+00$ & $4.1 \mathrm{E}-02$ \\
\hline $\mathrm{F}-18$ & 013981-56-1 & $2.9 \mathrm{E}+06$ & $2.9 \mathrm{E}+04$ & $5.2 \mathrm{E}+10$ & $5.2 \mathrm{E}+08$ & $6.3 \mathrm{E}+00$ & $6.3 \mathrm{E}-02$ & $6.3 \mathrm{E}+00$ & $6.3 \mathrm{E}-02$ \\
\hline $\mathrm{Fe}-55$ & 014681-59-5 & $9.1 \mathrm{E}+05$ & $9.1 \mathrm{E}+03$ & $6.1 E+09$ & $6.1 E+07$ & & & $9.1 \mathrm{E}+05$ & $9.1 E+03$ \\
\hline Fe-59 & $014596-12-4$ & $5.5 \mathrm{E}+04$ & $5.5 \mathrm{E}+02$ & $4.8 \mathrm{E}+08$ & $4.8 E+06$ & $4.7 \mathrm{E}+00$ & $4.7 \mathrm{E}-02$ & 4.7E+00 & 4.7E-02 \\
\hline Fr-221 & 015756-41-9 & $2.2 \mathrm{E}+06$ & $2.2 \mathrm{E}+04$ & $4.3 E+08$ & $4.3 E+06$ & $3.2 E+02$ & $3.2 E+00$ & $3.2 \mathrm{E}+02$ & $3.2 \mathrm{E}+00$ \\
\hline Fr-223 & 015756-98-6 & $7.2 \mathrm{E}+05$ & $7.2 \mathrm{E}+03$ & $5.8 \mathrm{E}+09$ & $5.8 \mathrm{E}+07$ & $5.3 \mathrm{E}+02$ & $5.3 \mathrm{E}+00$ & $5.2 \mathrm{E}+02$ & $5.2 \mathrm{E}+00$ \\
\hline
\end{tabular}




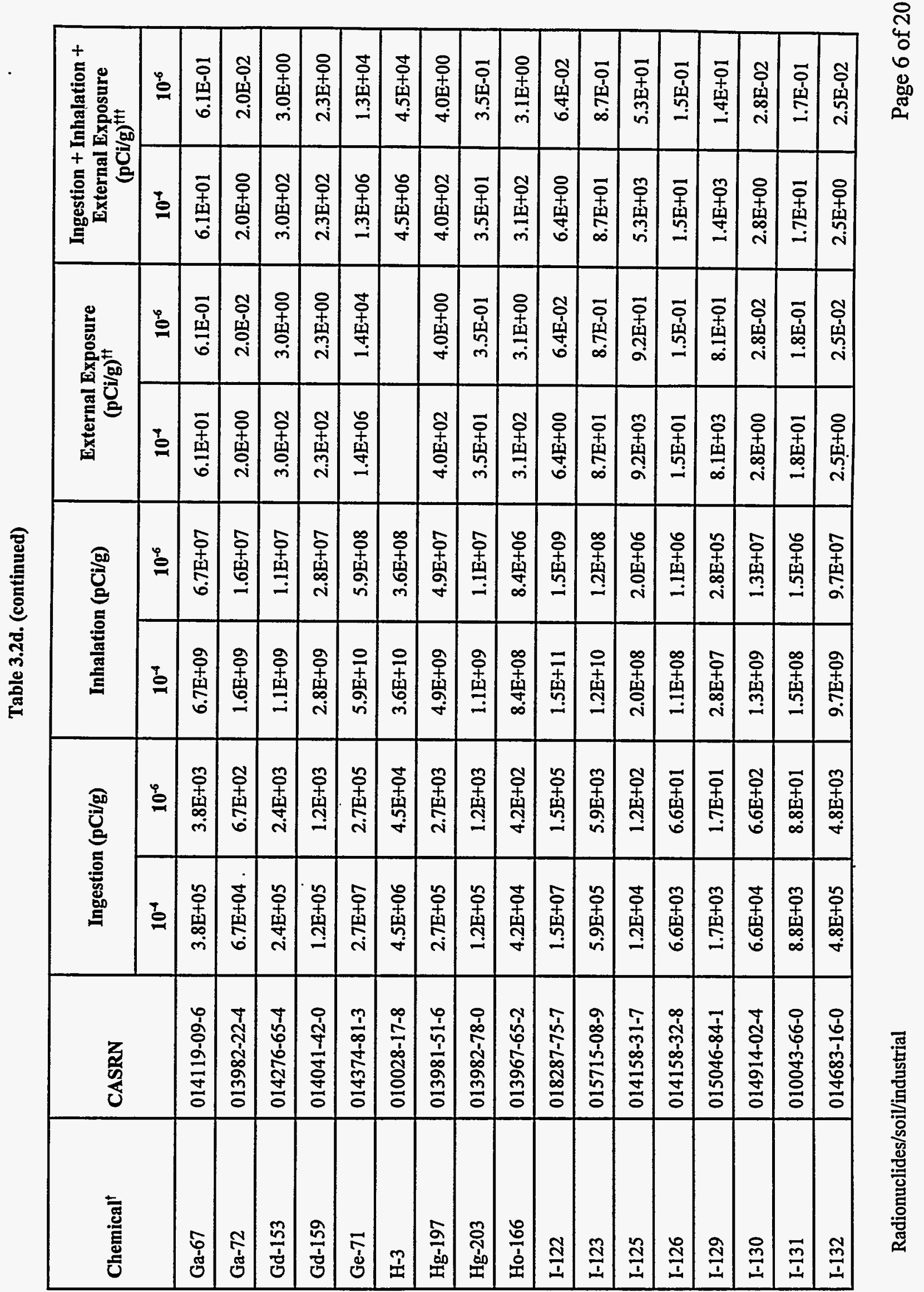


Table 3.2d. (continued)

\begin{tabular}{|c|c|c|c|c|c|c|c|c|c|}
\hline \multirow[t]{2}{*}{ Chemical $^{\dagger}$} & \multirow[t]{2}{*}{ CASRN } & \multicolumn{2}{|c|}{ Ingestion (pCi/g) } & \multicolumn{2}{|c|}{ Inhalation (pCi/g) } & \multicolumn{2}{|c|}{$\begin{array}{c}\text { External Exposure } \\
(\mathrm{pCi} / \mathrm{g})^{+t}\end{array}$} & \multicolumn{2}{|c|}{$\begin{array}{c}\text { Ingestion + Inhalation + } \\
\text { External Exposure } \\
(\mathrm{pCi} / \mathrm{g})^{\mathrm{tt}}\end{array}$} \\
\hline & & $10^{-4}$ & $10^{-6}$ & $10^{-4}$ & $10^{-6}$ & $10^{-4}$ & $10^{-5}$ & $10^{-4}$ & $10^{-6}$ \\
\hline I-133 & $014834-67-4$ & $3.0 \mathrm{E}+04$ & $3.0 \mathrm{E}+02$ & $5.7 \mathrm{E}+08$ & $5.7 \mathrm{E}+06$ & $1.0 \mathrm{E}+01$ & $1.0 \mathrm{E}-01$ & $1.0 \mathrm{E}+01$ & $1.0 \mathrm{E}-01$ \\
\hline I-134 & $014914-27-3$ & $1.4 \mathrm{E}+06$ & $1.4 \mathrm{E}+04$ & $2.5 \mathrm{E}+10$ & $2.5 E+08$ & $2.1 E+00$ & $2.1 \mathrm{E}-02$ & $2.1 \mathrm{E}+00$ & $2.1 \mathrm{E}-02$ \\
\hline I-135 & $014834-68-5$ & $1.4 \mathrm{E}+05$ & $1.4 \mathrm{E}+03$ & $2.9 \mathrm{E}+09$ & $2.9 \mathrm{E}+07$ & $3.5 \mathrm{E}+00$ & $3.5 \mathrm{E}-02$ & $3.5 \mathrm{E}+00$ & $3.5 \mathrm{E}-02$ \\
\hline In-113m & $014885-78-0$ & $3.9 \mathrm{E}+06$ & $3.9 \mathrm{E}+04$ & $5.9 \mathrm{E}+10$ & $5.9 \mathrm{E}+08$ & $2.8 \mathrm{E}+01$ & $2.8 \mathrm{E}-01$ & $2.8 \mathrm{E}+01$ & $2.8 \mathrm{E}-01$ \\
\hline In-114 & 013981-55-0 & $7.1 \mathrm{E}+07$ & $7.1 \mathrm{E}+05$ & $5.9 E+11$ & $5.9 \mathrm{E}+09$ & $1.9 \mathrm{E}+02$ & $1.9 \mathrm{E}+00$ & $1.9 \mathrm{E}+02$ & $1.9 \mathrm{E}+00$ \\
\hline In-114m & $013981-55-0$ & $1.6 \mathrm{E}+04$ & $1.6 \mathrm{E}+02$ & $1.4 \mathrm{E}+08$ & $1.4 \mathrm{E}+06$ & $1.1 E+02$ & $1.1 \mathrm{E}+00$ & $1.1 \mathrm{E}+02$ & $1.1 \mathrm{E}+00$ \\
\hline In-115 & 014191-71-0 & $9.2 \mathrm{E}+03$ & $9.2 \mathrm{E}+01$ & $1.7 \mathrm{E}+07$ & $1.7 \mathrm{E}+05$ & & & $9.2 \mathrm{E}+03$ & $9.2 \mathrm{E}+01$ \\
\hline In-115m & 014191-71-0 & $9.4 \mathrm{E}+05$ & $9.4 \mathrm{E}+03$ & $2.0 \mathrm{E}+10$ & $2.0 \mathrm{E}+08$ & $5.1 \mathrm{E}+01$ & $5.1 \mathrm{E}-01$ & $5.1 \mathrm{E}+01$ & $5.1 \mathrm{E}-01$ \\
\hline Ir-190 & $014981-91-0$ & $6.5 \mathrm{E}+04$ & $6.5 \mathrm{E}+02$ & $7.6 \mathrm{E}+08$ & $7.6 \mathrm{E}+06$ & $4.7 \mathrm{E}+00$ & $4.7 \mathrm{E}-02$ & $4.7 \mathrm{E}+00$ & 4.7E-02 \\
\hline Ir-194 & $014158-35-1$ & $4.6 \mathrm{E}+04$ & 4.6E+02 & $8.2 E+08$ & $8.2 E+06$ & $6.9 \mathrm{E}+01$ & $6.9 \mathrm{E}-01$ & $6.9 E+01$ & $6.9 \mathrm{E}-01$ \\
\hline $\mathrm{K}-40$ & $013966-00-2$ & $2.6 \mathrm{E}+04$ & $2.6 \mathrm{E}+02$ & $4.6 \mathrm{E}+08$ & $4.6 \mathrm{E}+06$ & $3.6 \mathrm{E}+01$ & $3.6 \mathrm{E}-01$ & $3.6 \mathrm{E}+01$ & $3.6 \mathrm{E}-01$ \\
\hline $\mathrm{K}-42$ & $014378-21-3$ & $2.5 \mathrm{E}+05$ & $2.5 \mathrm{E}+03$ & $4.5 \mathrm{E}+09$ & $4.5 \mathrm{E}+07$ & $2.0 \mathrm{E}+01$ & $2.0 \mathrm{E}-01$ & $2.0 \mathrm{E}+01$ & $2.0 \mathrm{E}-01$ \\
\hline $\mathrm{Kr}-83 \mathrm{~m}$ & $013965-98-5$ & & & $9.8 E+13$ & $9.8 \mathrm{E}+11$ & & & & \\
\hline $\mathrm{Kr}-85$ & $013983-27-2$ & & & $1.2 \mathrm{E}+13$ & $1.2 \mathrm{E}+11$ & & & & \\
\hline $\mathrm{Kr}-85 \mathrm{~m}$ & $013983-27-2$ & & & $1.2 \mathrm{E}+13$ & $1.2 \mathrm{E}+11$ & & & & \\
\hline $\mathrm{Kr}-87$ & $014809-68-8$ & & & $2.9 \mathrm{E}+12$ & $2.9 E+10$ & & & & \\
\hline $\mathrm{Kr}-88$ & 014995-61-0 & & & $1.6 \mathrm{E}+12$ & $1.6 \mathrm{E}+10$ & & & & \\
\hline
\end{tabular}


Table 3.2d. (continued)

\begin{tabular}{|c|c|c|c|c|c|c|c|c|c|}
\hline \multirow[t]{2}{*}{ Chemical $^{\dagger}$} & \multirow[t]{2}{*}{ CASRN } & \multicolumn{2}{|c|}{ Ingestion (pCi/g) } & \multicolumn{2}{|c|}{ Inhalation (pCi/g) } & \multicolumn{2}{|c|}{$\begin{array}{l}\text { External Exposure } \\
(\mathrm{pCi} / \mathrm{g})^{\mathrm{tt}}\end{array}$} & \multicolumn{2}{|c|}{$\begin{array}{c}\text { Ingestion + Inhalation }+ \\
\text { External Exposure } \\
(\mathrm{pCi} / \mathrm{g})^{\mathrm{ftt}}\end{array}$} \\
\hline & & $10^{-4}$ & $10^{-6}$ & $10^{-4}$ & $10^{-6}$ & $10^{-4}$ & $10^{-6}$ & $10^{-4}$ & $10^{-6}$ \\
\hline $\mathrm{Kr}-89$ & 016316-03-3 & & & $2.1 \mathrm{E}+12$ & $2.1 E+10$ & & & & \\
\hline $\mathrm{Kr}-90$ & 015741-13-6 & & & $2.1 \mathrm{E}+12$ & $2.1 \mathrm{E}+10$ & & & & \\
\hline La-140 & $013981-28-7$ & $3.4 E+04$ & $3.4 \mathrm{E}+02$ & $6.7 \mathrm{E}+08$ & $6.7 \mathrm{E}+06$ & $2.4 \mathrm{E}+00$ & $2.4 \mathrm{E}-02$ & $2.4 \mathrm{E}+00$ & 2.4E-02 \\
\hline Lu-177 & $014265-75-9$ & $1.1 \mathrm{E}+05$ & $1.1 \mathrm{E}+03$ & $1.6 \mathrm{E}+09$ & $1.6 \mathrm{E}+07$ & $3.0 \mathrm{E}+02$ & $3.0 \mathrm{E}+00$ & $3.0 \mathrm{E}+02$ & $3.0 \mathrm{E}+00$ \\
\hline $\mathrm{Mn}-52$ & $014092-99-0$ & $5.3 \mathrm{E}+04$ & $5.3 \mathrm{E}+02$ & $7.8 \mathrm{E}+08$ & $7.8 \mathrm{E}+06$ & $1.6 \mathrm{E}+00$ & $1.6 \mathrm{E}-02$ & $1.6 \mathrm{E}+00$ & $1.6 \mathrm{E}-02$ \\
\hline $\mathrm{Mn}-54$ & 013966-31-9 & $1.6 \mathrm{E}+05$ & $1.6 \mathrm{E}+03$ & $9.3 \mathrm{E}+08$ & $9.3 \mathrm{E}+06$ & $6.7 \mathrm{E}+00$ & $6.7 \mathrm{E}-02$ & $6.7 \mathrm{E}+00$ & $6.7 \mathrm{E}-02$ \\
\hline $\mathrm{Mn}-56$ & 014681-52-8 & $3.7 \mathrm{E}+05$ & $3.7 E+03$ & $6.6 E+09$ & $6.6 \mathrm{E}+07$ & $3.2 \mathrm{E}+00$ & $3.2 \mathrm{E}-02$ & $3.2 \mathrm{E}+00$ & $3.2 \mathrm{E}-02$ \\
\hline Mo-99 & $014119-15-4$ & $1.4 \mathrm{E}+05$ & $1.4 \mathrm{E}+03$ & $7.7 \mathrm{E}+08$ & $7.7 E+06$ & $4.0 \mathrm{E}+01$ & $4.0 \mathrm{E}-01$ & $4.0 \mathrm{E}+01$ & $4.0 \mathrm{E}-01$ \\
\hline $\mathrm{Na}-22$ & 013966-32-0 & $4.0 \mathrm{E}+04$ & $4.0 \mathrm{E}+02$ & $7.0 \mathrm{E}+08$ & $7.0 \mathrm{E}+06$ & $2.7 \mathrm{E}+00$ & 2.7E-02 & $2.7 \mathrm{E}+00$ & 2.7E-02 \\
\hline $\mathrm{Na}-24$ & 013982-04-2 & $2.3 \mathrm{E}+05$ & $2.3 \mathrm{E}+03$ & $4.6 \mathrm{E}+09$ & $4.6 \mathrm{E}+07$ & $1.2 \mathrm{E}+00$ & $1.2 \mathrm{E}-02$ & $1.2 \mathrm{E}+00$ & $1.2 \mathrm{E}-02$ \\
\hline $\mathrm{Nb}-93 \mathrm{~m}$ & $007440-03-1$ & $4.8 \mathrm{E}+05$ & $4.8 \mathrm{E}+03$ & $7.9 \mathrm{E}+08$ & $7.9 \mathrm{E}+06$ & $6.0 \mathrm{E}+05$ & $6.0 \mathrm{E}+03$ & $2.7 \mathrm{E}+05$ & $2.7 \mathrm{E}+03$ \\
\hline $\mathrm{Nb}-94$ & $014681-63-1$ & $4.6 \mathrm{E}+04$ & $4.6 \mathrm{E}+02$ & $4.2 E+07$ & $4.2 \mathrm{E}+05$ & $3.6 \mathrm{E}+00$ & $3.6 \mathrm{E}-02$ & $3.6 \mathrm{E}+00$ & $3.6 \mathrm{E}-02$ \\
\hline $\mathrm{Nb}-95$ & $013967-76-5$ & $1.4 \mathrm{E}+05$ & $1.4 \mathrm{E}+03$ & $1.1 \mathrm{E}+09$ & $1.1 \mathrm{E}+07$ & $7.4 \mathrm{E}+00$ & 7.4E-02 & $7.4 \mathrm{E}+00$ & $7.4 \mathrm{E}-02$ \\
\hline $\mathrm{Nb}-95 \mathrm{~m}$ & $013967-76-5$ & $1.0 \mathrm{E}+05$ & $1.0 \mathrm{E}+03$ & $1.5 \mathrm{E}+09$ & $1.5 \mathrm{E}+07$ & $2.5 \mathrm{E}+02$ & $2.5 \mathrm{E}+00$ & $2.5 \mathrm{E}+02$ & $2.5 \mathrm{E}+00$ \\
\hline $\mathrm{Nb}-97$ & $018496-04-3$ & $1.8 \mathrm{E}+06$ & $1.8 \mathrm{E}+04$ & $1.6 \mathrm{E}+10$ & $1.6 \mathrm{E}+08$ & $8.8 \mathrm{E}+00$ & $8.8 \mathrm{E}-02$ & $8.8 \mathrm{E}+00$ & $8.8 \mathrm{E}-02$ \\
\hline $\mathrm{Nb}-97 \mathrm{~m}$ & 018496-04-3 & $9.8 \mathrm{E}+07$ & $9.8 \mathrm{E}+05$ & $1.0 \mathrm{E}+12$ & $1.0 \mathrm{E}+10$ & $7.9 \mathrm{E}+00$ & $7.9 \mathrm{E}-02$ & $7.9 \mathrm{E}+00$ & $7.9 \mathrm{E}-02$ \\
\hline Nd-147 & 014269-74-0 & $5.4 \mathrm{E}+04$ & $5.4 \mathrm{E}+02$ & $7.1 \mathrm{E}+08$ & $7.1 \mathrm{E}+06$ & $6.8 \mathrm{E}+01$ & $6.8 \mathrm{E}-01$ & $6.8 \mathrm{E}+01$ & $6.8 \mathrm{E}-01$ \\
\hline
\end{tabular}

Radionuclides/soil/industrial

Page 8 of 20 
Table 3.2d. (continued)

\begin{tabular}{|c|c|c|c|c|c|c|c|c|c|}
\hline \multirow[t]{2}{*}{ Chemical $^{\dagger}$} & \multirow[t]{2}{*}{ CASRN } & \multicolumn{2}{|c|}{ Ingestion (pCi/g) } & \multicolumn{2}{|c|}{ Inhalation (pCi/g) } & \multicolumn{2}{|c|}{$\begin{array}{c}\text { External Exposure } \\
(\mathrm{pCi} / \mathrm{g})^{+t}\end{array}$} & \multicolumn{2}{|c|}{$\begin{array}{c}\text { Ingestion + Inhalation + } \\
\text { External Exposure } \\
(\mathrm{pCi} / \mathrm{g})^{\mathrm{ft}}\end{array}$} \\
\hline & & $10^{-4}$ & $10^{-6}$ & $10^{-4}$ & $10^{-6}$ & $10^{-4}$ & $10^{-6}$ & $10^{-4}$ & $10^{-6}$ \\
\hline $\mathrm{Nd}-149$ & $015749-81-2$ & $7.0 \mathrm{E}+05$ & $7.0 \mathrm{E}+03$ & $8.1 E+09$ & 8.1E+07 & $2.0 \mathrm{E}+01$ & $2.0 \mathrm{E}-01$ & $2.0 \mathrm{E}+01$ & $2.0 \mathrm{E}-01$ \\
\hline $\mathrm{Ni}-59$ & 014336-70-0 & $1.7 \mathrm{E}+06$ & $1.7 \mathrm{E}+04$ & $8.5 E+09$ & $8.5 \mathrm{E}+07$ & & & $1.7 \mathrm{E}+06$ & $1.7 \mathrm{E}+04$ \\
\hline $\mathrm{Ni}-63$ & $013981-37-8$ & $5.8 \mathrm{E}+05$ & $5.8 \mathrm{E}+03$ & $3.4 \mathrm{E}+09$ & $3.4 \mathrm{E}+07$ & & & $5.8 \mathrm{E}+05$ & $5.8 \mathrm{E}+03$ \\
\hline $\mathrm{Ni}-65$ & 014833-49-9 & $5.7 \mathrm{E}+05$ & $5.7 \mathrm{E}+03$ & $9.5 \mathrm{E}+09$ & $9.5 \mathrm{E}+07$ & $1.0 \mathrm{E}+01$ & $1.0 \mathrm{E}-01$ & $1.0 \mathrm{E}+01$ & $1.0 \mathrm{E}-01$ \\
\hline $\mathrm{Np}-236$ & $015700-36-4$ & $3.4 \mathrm{E}+05$ & $3.4 \mathrm{E}+03$ & $8.9 E+08$ & $8.9 \mathrm{E}+06$ & $2.4 \mathrm{E}+02$ & $2.4 \mathrm{E}+00$ & $2.4 \mathrm{E}+02$ & $2.4 \mathrm{E}+00$ \\
\hline $\mathrm{Np}-237$ & 013994-20-2 & $1.1 \mathrm{E}+03$ & $1.1 \mathrm{E}+01$ & $9.9 \mathrm{E}+04$ & $9.9 \mathrm{E}+02$ & $2.9 E+03$ & $2.9 \mathrm{E}+01$ & $7.8 \mathrm{E}+02$ & $7.8 \mathrm{E}+00$ \\
\hline $\mathrm{Np}-237+\mathrm{D}$ & 013994-20-2 & $1.1 \mathrm{E}+03$ & $1.1 \mathrm{E}+01$ & $9.9 \mathrm{E}+04$ & $9.9 \mathrm{E}+02$ & $4.7 \mathrm{E}+01$ & 4.7E-01 & $4.5 \mathrm{E}+01$ & $4.5 \mathrm{E}-01$ \\
\hline $\mathrm{Np}-238$ & $015766-25-3$ & $7.0 \mathrm{E}+04$ & $7.0 \mathrm{E}+02$ & $7.3 \mathrm{E}+08$ & $7.3 \mathrm{E}+06$ & $1.1 \mathrm{E}+01$ & $1.1 \mathrm{E}-01$ & $1.1 \mathrm{E}+01$ & $1.1 \mathrm{E}-01$ \\
\hline $\mathrm{Np}-239$ & $013968-59-7$ & $7.5 E+04$ & $7.5 \mathrm{E}+02$ & $1.4 \mathrm{E}+09$ & $1.4 \mathrm{E}+07$ & $9.0 \mathrm{E}+01$ & $9.0 \mathrm{E}-01$ & $9.0 \mathrm{E}+01$ & $9.0 \mathrm{E}-01$ \\
\hline $\mathrm{Np}-240$ & 015690-84-3 & $1.8 E+06$ & $1.8 \mathrm{E}+04$ & $2.6 \mathrm{E}+10$ & $2.6 \mathrm{E}+08$ & $6.0 \mathrm{E}+00$ & $6.0 \mathrm{E}-02$ & $6.0 \mathrm{E}+00$ & $6.0 \mathrm{E}-02$ \\
\hline $\mathrm{Np}-240 \mathrm{~m}$ & $015690-84-3$ & $1.3 E+07$ & $1.3 \mathrm{E}+05$ & $1.2 \mathrm{E}+11$ & $1.2 \mathrm{E}+09$ & $2.1 E+01$ & 2.1E-01 & $2.1 \mathrm{E}+01$ & 2.1E-01 \\
\hline Os-185 & $015766-50-4$ & $1.8 \mathrm{E}+05$ & $1.8 \mathrm{E}+03$ & $7.4 E+08$ & $7.4 \mathrm{E}+06$ & $8.9 \mathrm{E}+00$ & $8.9 \mathrm{E}-02$ & $8.9 \mathrm{E}+00$ & 8.9E-02 \\
\hline Os-191 & 014119-24-5 & $1.1 \mathrm{E}+05$ & $1.1 \mathrm{E}+03$ & $1.3 E+09$ & $1.3 \mathrm{E}+07$ & $2.5 \mathrm{E}+02$ & $2.5 \mathrm{E}+00$ & $2.5 \mathrm{E}+02$ & $2.5 \mathrm{E}+00$ \\
\hline Os-191m & 014119-24-5 & $6.5 \mathrm{E}+05$ & $6.5 \mathrm{E}+03$ & $1.0 \mathrm{E}+10$ & $1.0 \mathrm{E}+08$ & $6.8 \mathrm{E}+03$ & $6.8 \mathrm{E}+01$ & $6.7 \mathrm{E}+03$ & $6.7 E+01$ \\
\hline Os-193 & $016057-77-5$ & $7.3 \mathrm{E}+04$ & $7.3 \mathrm{E}+02$ & $1.3 E+09$ & $1.3 \mathrm{E}+07$ & $1.2 \mathrm{E}+02$ & $1.2 \mathrm{E}+00$ & $1.2 \mathrm{E}+02$ & $1.2 \mathrm{E}+00$ \\
\hline P-32 & $014596-37-3$ & $5.2 \mathrm{E}+04$ & $5.2 \mathrm{E}+02$ & $1.2 \mathrm{E}+09$ & $1.2 \mathrm{E}+07$ & & & $5.2 E+04$ & $5.2 \mathrm{E}+02$ \\
\hline P-33 & $015749-66-3$ & $4.1 \mathrm{E}+05$ & $4.1 \mathrm{E}+03$ & $8.7 \mathrm{E}+09$ & $8.7 \mathrm{E}+07$ & & & $4.1 E+05$ & $4.1 \mathrm{E}+03$ \\
\hline
\end{tabular}

Radionuclides/soil/industrial

Page 9 of 20 
Table 3.2d. (continued)

\begin{tabular}{|c|c|c|c|c|c|c|c|c|c|}
\hline \multirow[t]{2}{*}{ Chemical $^{\dagger}$} & \multirow[t]{2}{*}{ CASRN } & \multicolumn{2}{|c|}{ Ingestion (pCi/g) } & \multicolumn{2}{|c|}{ Inhalation (pCi/g) } & \multicolumn{2}{|c|}{$\begin{array}{l}\text { External Exposure } \\
(\text { pCi/g) })^{+t}\end{array}$} & \multicolumn{2}{|c|}{$\begin{array}{c}\text { Ingestion }+ \text { Inhalation }+ \\
\text { External Exposure } \\
(\mathrm{pCi} / \mathrm{g})^{\dagger+t}\end{array}$} \\
\hline & & $10^{-4}$ & $10^{-6}$ & $10^{-4}$ & $10^{-6}$ & $10^{-4}$ & $10^{-6}$ & $10^{-4}$ & $10^{-6}$ \\
\hline $\mathrm{Pa}-231$ & $014331-85-2$ & $2.1 \mathrm{E}+03$. & $2.1 \mathrm{E}+01$ & $1.4 \mathrm{E}+05$ & $1.4 \mathrm{E}+03$ & $8.1 \mathrm{E}+02$ & $8.1 \mathrm{E}+00$ & $5.8 \mathrm{E}+02$ & $5.8 \mathrm{E}+00$ \\
\hline $\mathrm{Pa}-233$ & 013981-14-1 & $6.8 \mathrm{E}+04$ & $6.8 \mathrm{E}+02$ & $7.0 \mathrm{E}+08$ & $7.0 \mathrm{E}+06$ & $4.8 \mathrm{E}+01$ & $4.8 \mathrm{E}-01$ & $4.8 \mathrm{E}+01$ & $4.8 \mathrm{E}-01$ \\
\hline $\mathrm{Pa}-234$ & $015100-28-4$ & $1.5 \mathrm{E}+05$ & $1.5 \mathrm{E}+03$ & $2.6 \mathrm{E}+09$ & $2.6 \mathrm{E}+07$ & $3.3 \mathrm{E}+00$ & 3.3E-02 & $3.3 \mathrm{E}+00$ & 3.3E-02 \\
\hline $\mathrm{Pa}-234 \mathrm{~m}$ & $015100-28-4$ & $6.7 \mathrm{E}+07$ & $6.7 \mathrm{E}+05$ & $5.5 \mathrm{E}+11$ & $5.5 \mathrm{E}+09$ & $5.4 \mathrm{E}+02$ & $5.4 \mathrm{E}+00$ & $5.4 \mathrm{E}+02$ & $5.4 \mathrm{E}+00$ \\
\hline $\mathrm{Pb}-203$ & $014687-25-3$ & $3.1 \mathrm{E}+05$ & $3.1 \mathrm{E}+03$ & $1.1 \mathrm{E}+10$ & $1.1 \mathrm{E}+08$ & $3.4 \mathrm{E}+01$ & $3.4 \mathrm{E}-01$ & $3.4 \mathrm{E}+01$ & 3.4E-01 \\
\hline Pb-209 & $014119-30-3$ & $1.5 \mathrm{E}+06$ & $1.5 \mathrm{E}+04$ & $5.0 \mathrm{E}+10$ & $5.0 \mathrm{E}+08$ & & & $1.5 \mathrm{E}+06$ & $1.5 E+04$ \\
\hline $\mathrm{Pb}-210$ & $014255-04-0$ & $4.7 \mathrm{E}+02$ & $4.7 \mathrm{E}+00$ & $2.1 \mathrm{E}+06$ & $2.1 \mathrm{E}+04$ & $2.0 \mathrm{E}+05$ & $2.0 \mathrm{E}+03$ & $4.7 \mathrm{E}+02$ & $4.7 \mathrm{E}+00$ \\
\hline $\mathrm{Pb}-210+\mathrm{D}$ & 014255-04-0 & $3.2 \mathrm{E}+02$ & $3.2 \mathrm{E}+00$ & $8.9 E+05$ & $8.9 \mathrm{E}+03$ & $1.5 \mathrm{E}+05$ & $1.5 \mathrm{E}+03$ & $3.2 \mathrm{E}+02$ & $3.2 \mathrm{E}+00$ \\
\hline $\mathrm{Pb}-211$ & $015816-77-0$ & $9.5 \mathrm{E}+05$ & $9.5 \mathrm{E}+03$ & $3.3 \mathrm{E}+08$ & $3.3 \mathrm{E}+06$ & $1.2 \mathrm{E}+02$ & $1.2 \mathrm{E}+00$ & $1.2 \mathrm{E}+02$ & $1.2 \mathrm{E}+00$ \\
\hline $\mathrm{Pb}-212$ & 015092-94-1 & $1.8 \mathrm{E}+04$ & $1.8 \mathrm{E}+02$ & $8.9 \mathrm{E}+07$ & $8.9 \mathrm{E}+05$ & 7.3E+01 & 7.3E-01 & $7.3 \mathrm{E}+01$ & 7.3E-01 \\
\hline $\mathrm{Pb}-214$ & $015067-28-4$ & $1.1 \mathrm{E}+06$ & $1.1 \mathrm{E}+04$ & $5.5 \mathrm{E}+08$ & $5.5 \mathrm{E}+06$ & $3.1 \mathrm{E}+01$ & $3.1 \mathrm{E}-01$ & $3.1 \mathrm{E}+01$ & $3.1 \mathrm{E}-01$ \\
\hline $\mathrm{Pd}-100$ & $015690-69-4$ & $8.6 \mathrm{E}+04$ & $8.6 \mathrm{E}+02$ & $9.7 \mathrm{E}+08$ & $9.7 \mathrm{E}+06$ & & & $8.6 E+04$ & $8.6 \mathrm{E}+02$ \\
\hline Pd-101 & $015749-54-9$ & $8.6 \mathrm{E}+05$ & $8.6 \mathrm{E}+03$. & $1.5 \mathrm{E}+10$ & $1.5 \mathrm{E}+08$ & & & $8.6 \mathrm{E}+05$ & $8.6 \mathrm{E}+03$ \\
\hline $\mathrm{Pd}-103$ & $014967-68-1$ & $3.0 \mathrm{E}+05$ & $3.0 \mathrm{E}+03$ & $3.2 \mathrm{E}+09$ & $3.2 \mathrm{E}+07$ & $4.1 E+04$ & $4.1 \mathrm{E}+02$ & $3.6 \mathrm{E}+04$ & $3.6 \mathrm{E}+02$ \\
\hline Pd-107 & $017637-99-9$ & $1.5 \mathrm{E}+06$ & $1.5 \mathrm{E}+04$ & $2.3 E+09$ & $2.3 \mathrm{E}+07$ & & & $1.5 \mathrm{E}+06$ & $1.5 \mathrm{E}+04$ \\
\hline Pd-109 & $014981-64-7$ & $9.6 \mathrm{E}+04$ & $9.6 \mathrm{E}+02$ & $1.7 \mathrm{E}+09$ & $1.7 \mathrm{E}+07$ & $9.0 \mathrm{E}+03$ & $9.0 \mathrm{E}+01$ & $8.2 E+03$ & $8.2 \mathrm{E}+01$ \\
\hline Pm-147 & $014380-75-7$ & $2.3 E+05$ & $2.3 \mathrm{E}+03$ & $4.6 \mathrm{E}+08$ & $4.6 \mathrm{E}+06$ & $3.4 \mathrm{E}+06$ & $3.4 \mathrm{E}+04$ & $2.1 \mathrm{E}+05$ & $2.1 \mathrm{E}+03$ \\
\hline
\end{tabular}

Radionuclides/soil/industrial

Page 10 of 20 
Table 3.2d. (continued)

\begin{tabular}{|c|c|c|c|c|c|c|c|c|c|}
\hline \multirow[t]{2}{*}{ Chemical $^{\dagger}$} & \multirow[t]{2}{*}{ CASRN } & \multicolumn{2}{|c|}{ Ingestion (pCi/g) } & \multicolumn{2}{|c|}{ Inhalation (pCi/g) } & \multicolumn{2}{|c|}{$\begin{array}{l}\text { External Exposure } \\
(\text { (pCi/g })^{\dagger+}\end{array}$} & \multicolumn{2}{|c|}{$\begin{array}{c}\text { Ingestion + Inhalation + } \\
\text { External Exposure } \\
(\mathrm{pCi} / \mathrm{g})^{\mathrm{ftt}}\end{array}$} \\
\hline & & $10^{-4}$ & $10^{-6}$ & $10^{-4}$ & $10^{-6}$ & $10^{-4}$ & $10^{-6}$ & $10^{-4}$ & $10^{-6}$ \\
\hline Pm-148 & 014683-19-3 & $2.2 E+04$ & $2.2 \mathrm{E}+02$ & $3.3 \mathrm{E}+08$ & $3.3 \mathrm{E}+06$ & $9.9 \mathrm{E}+00$ & $9.9 \mathrm{E}-02$ & $9.9 \mathrm{E}+00$ & $9.9 \mathrm{E}-02$ \\
\hline Pm-148m & $014683-19-3$ & $3.2 E+04$ & $3.2 \mathrm{E}+02$ & $1.2 \mathrm{E}+08$ & $1.2 \mathrm{E}+06$ & $3.0 \mathrm{E}+00$ & $3.0 \mathrm{E}-02$ & $3.0 \mathrm{E}+00$ & $3.0 \mathrm{E}-02$ \\
\hline Pm-149 & $015765-31-8$ & $5.8 \mathrm{E}+04$ & $5.8 \mathrm{E}+02$ & $9.6 \mathrm{E}+08$ & $9.6 \mathrm{E}+06$ & $6.0 \mathrm{E}+02$ & $6.0 \mathrm{E}+00$ & $5.9 \mathrm{E}+02$ & $5.9 \mathrm{E}+00$ \\
\hline Po-210 & $013981-52-7$ & $9.8 \mathrm{E}+02$ & $9.8 \mathrm{E}+00$ & $1.6 \mathrm{E}+06$ & $1.6 \mathrm{E}+04$ & $6.6 \mathrm{E}+05$ & $6.6 \mathrm{E}+03$ & $9.8 \mathrm{E}+02$ & $9.8 \mathrm{E}+00$ \\
\hline Po-212 & 015389-34-1 & $7.1 \mathrm{E}+15$ & $7.1 \mathrm{E}+13$ & $5.8 \mathrm{E}+17$ & $5.8 \mathrm{E}+15$ & & & $7.0 \mathrm{E}+15$ & $7.0 \mathrm{E}+13$ \\
\hline Po-213 & $015756-57-7$ & $4.8 \mathrm{E}+14$ & $4.8 \mathrm{E}+12$ & $4.4 \mathrm{E}+16$ & $4.4 \mathrm{E}+14$ & $1.9 \mathrm{E}+05$ & $1.9 \mathrm{E}+03$ & $1.9 \mathrm{E}+05$ & $1.9 \mathrm{E}+03$ \\
\hline Po-214 & $015735-67-8$ & $1.5 \mathrm{E}+13$ & $1.5 \mathrm{E}+11$ & $1.2 \mathrm{E}+15$ & $1.2 \mathrm{E}+13$ & $6.8 \mathrm{E}+04$ & $6.8 \mathrm{E}+02$ & $6.8 \mathrm{E}+04$ & $6.8 \mathrm{E}+02$ \\
\hline Po-215 & $015706-52-2$ & $6.4 \mathrm{E}+11$ & $6.4 \mathrm{E}+09$ & $7.7 \mathrm{E}+13$ & $7.7 \mathrm{E}+11$ & $4.3 \mathrm{E}+04$ & $4.3 \mathrm{E}+02$ & $4.3 E+04$ & $4.3 \mathrm{E}+02$ \\
\hline Po-216 & 015756-58-8 & $3.6 \mathrm{E}+09$ & $3.6 \mathrm{E}+07$ & $1.2 \mathrm{E}+12$ & $1.2 \mathrm{E}+10$ & $3.9 \mathrm{E}+05$ & $3.9 E+03$ & $3.9 \mathrm{E}+05$ & $3.9 \mathrm{E}+03$ \\
\hline Po-218 & 015422-24-9 & $6.3 E+06$ & $6.3 E+04$ & $9.3 E+08$ & $9.3 E+06$ & & & $6.3 E+06$ & $6.3 E+04$ \\
\hline $\operatorname{Pr}-142$ & 014191-64-1 & $4.6 \mathrm{E}+04$ & $4.6 \mathrm{E}+02$ & $8.2 E+08$ & $8.2 \mathrm{E}+06$ & $9.4 \mathrm{E}+01$ & $9.4 \mathrm{E}-01$ & $9.3 \mathrm{E}+01$ & 9.3E-01 \\
\hline Pr-143 & 014981-79-4 & $4.8 \mathrm{E}+04$ & $4.8 \mathrm{E}+02$ & $6.1 \mathrm{E}+08$ & $6.1 \mathrm{E}+06$ & $6.4 \mathrm{E}+08$ & $6.4 \mathrm{E}+06$ & $4.8 \mathrm{E}+04$ & $4.8 \mathrm{E}+02$ \\
\hline Pr-144 & $014119-05-2$ & $4.0 \mathrm{E}+06$ & $4.0 \mathrm{E}+04$ & $2.6 \mathrm{E}+10$ & $2.6 \mathrm{E}+08$ & $1.6 \mathrm{E}+02$ & $1.6 \mathrm{E}+00$ & $1.6 \mathrm{E}+02$ & $1.6 \mathrm{E}+00$ \\
\hline Pr- $144 m$ & 014119-05-2 & $9.9 E+06$ & $9.9 E+04$ & $6.1 \mathrm{E}+10$ & $6.1 \mathrm{E}+08$ & $1.2 E+04$ & $1.2 \mathrm{E}+02$ & $1.2 \mathrm{E}+04$ & $1.2 \mathrm{E}+02$ \\
\hline Pt-191 & $015706-36-2$ & $2.1 E+05$ & $2.1 \mathrm{E}+03$ & $8.3 \mathrm{E}+09$ & $8.3 \mathrm{E}+07$ & $3.2 \mathrm{E}+01$ & $3.2 \mathrm{E}-01$ & $3.2 \mathrm{E}+01$ & $3.2 \mathrm{E}-01$ \\
\hline Pt-193 & 015735-70-3 & $2.0 \mathrm{E}+06$ & $2.0 \mathrm{E}+04$ & $4.3 \mathrm{E}+10$ & $4.3 \mathrm{E}+08$ & & & $2.0 \mathrm{E}+06$ & $2.0 \mathrm{E}+04$ \\
\hline Pt-193m & 015735-70-3 & $1.3 \mathrm{E}+05$ & $1.3 \mathrm{E}+03$ & $6.0 \mathrm{E}+09$ & $6.0 \mathrm{E}+07$ & $2.9 \mathrm{E}+03$ & $2.9 \mathrm{E}+01$ & $2.9 \mathrm{E}+03$ & $2.9 \mathrm{E}+01$ \\
\hline
\end{tabular}

Radionuclides/soil/industrial

Page 11 of 20 


\begin{tabular}{|c|c|c|c|c|c|c|c|c|c|c|c|c|c|c|c|c|c|c|}
\hline 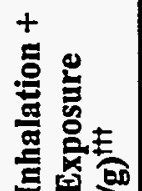 & $\stackrel{\mathscr{Q}}{\mathcal{E}}$ & 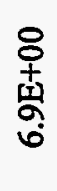 & 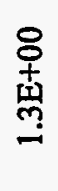 & $\begin{array}{l} \\
\text { 畐 } \\
\text { + }\end{array}$ & 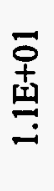 & 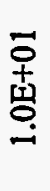 & $\begin{array}{l}\overline{0} \\
\text { 离 } \\
\text {. }\end{array}$ & 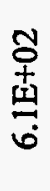 & $\begin{array}{l}8 \\
\text { 市 } \\
\text { 帘 } \\
\end{array}$ & 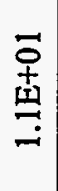 & 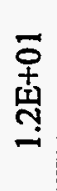 & $\begin{array}{l}\overline{0} \\
\text { 龺 } \\
\text { - }\end{array}$ & 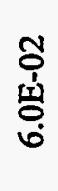 & 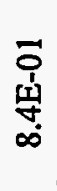 & $\begin{array}{l}8 \\
\text { †े } \\
\text { બ్ } \\
\text { M. } \\
0\end{array}$ & \begin{tabular}{l}
8 \\
† \\
I⿱ \\
m \\
\multirow{\forall}{*}{}
\end{tabular} & 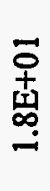 & 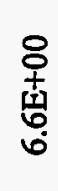 \\
\hline 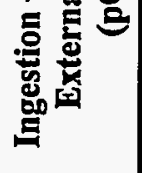 & I & 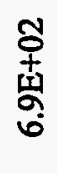 & 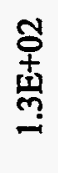 & 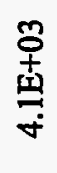 & 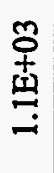 & 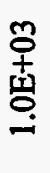 & 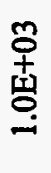 & 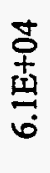 & 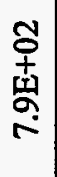 & 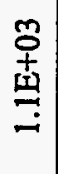 & 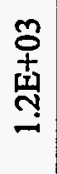 & 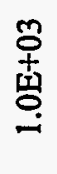 & $\begin{array}{l}8 \\
\text { +⿱ } \\
\text { 秀 } \\
\text { ○े } \\
0\end{array}$ & 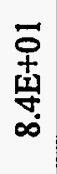 & 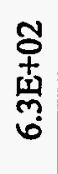 & 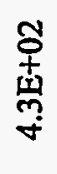 & $\begin{array}{l}\stackrel{8}{+} \\
\frac{1}{0} \\
\stackrel{\infty}{0}\end{array}$ & 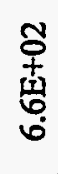 \\
\hline
\end{tabular}

兽

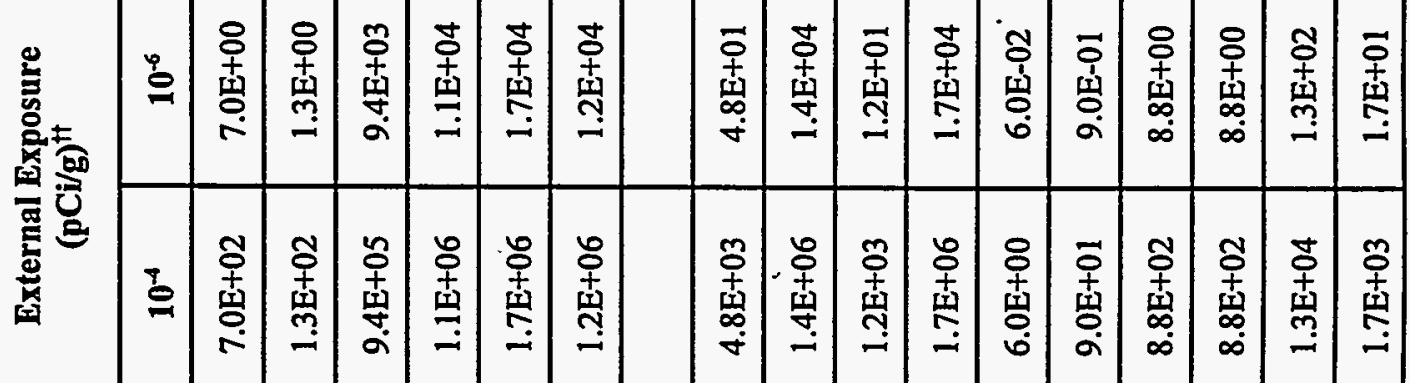

\begin{tabular}{|c|c|c|c|c|c|c|c|c|c|c|c|c|c|c|c|c|}
\hline 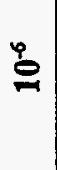 & $\begin{array}{l}\text { ô } \\
\text { 离 } \\
\text { مَ }\end{array}$ & 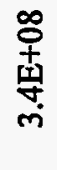 & 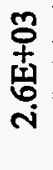 & 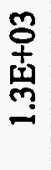 & 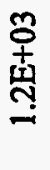 & 等 & 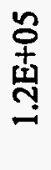 & 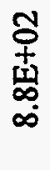 & 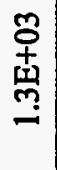 & 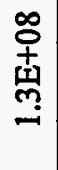 & 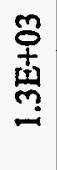 & $\begin{array}{l}\text { o. } \\
\text { 怘 } \\
\text { ma }\end{array}$ & $\begin{array}{l}\text { o } \\
\text { 离 } \\
\text { ă }\end{array}$ & 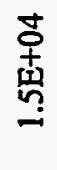 & 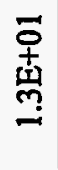 & 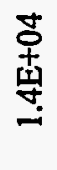 \\
\hline 了] & $\begin{array}{l}\text { oे } \\
\text { 4 } \\
\text { م }\end{array}$ & 章 & 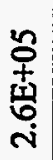 & 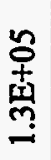 & 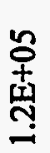 & 草 & $\begin{array}{l}\text { 京 } \\
\text { 祀 }\end{array}$ & $\begin{array}{l}\mathbf{0} \\
+ \\
\text { ț } \\
\infty \\
\infty \\
\infty\end{array}$ & 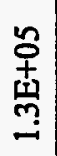 & $\begin{array}{l}\frac{9}{7} \\
\underset{m}{m}\end{array}$ & 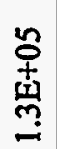 & 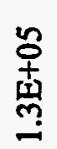 & 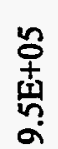 & 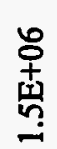 & 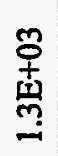 & 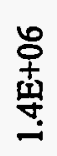 \\
\hline
\end{tabular}

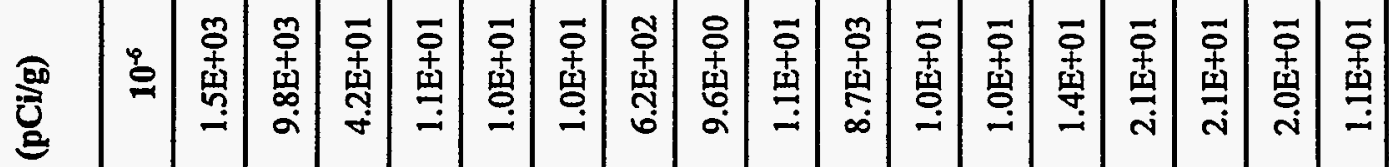

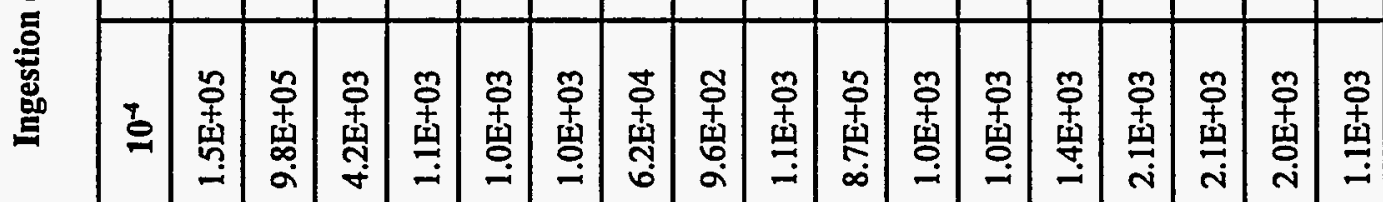

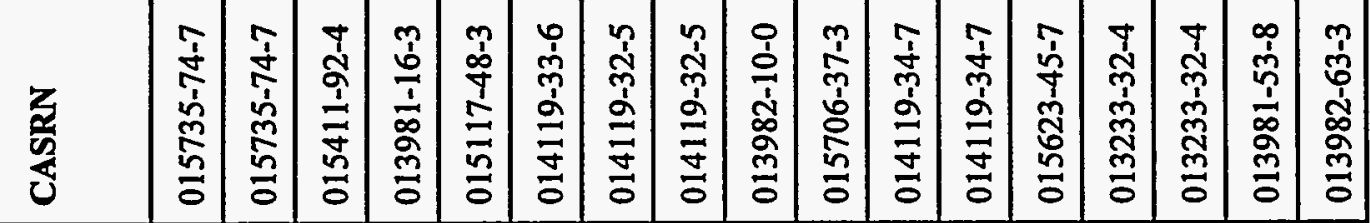

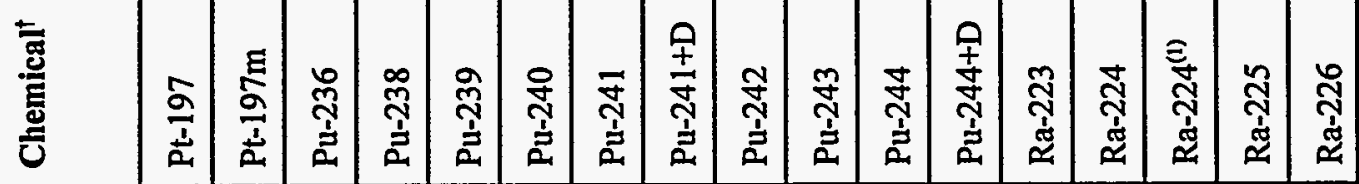


Table 3.2d. (continued)

\begin{tabular}{|c|c|c|c|c|c|c|c|c|c|}
\hline \multirow[t]{2}{*}{ Chemical $^{\dagger}$} & \multirow[t]{2}{*}{ CASRN } & \multicolumn{2}{|c|}{ Ingestion (pCi/g) } & \multicolumn{2}{|c|}{ Inhalation (pCi/g) } & \multicolumn{2}{|c|}{$\begin{array}{l}\text { External Exposure } \\
(\mathrm{pCi} / \mathrm{g})^{\mathrm{tt}}\end{array}$} & \multicolumn{2}{|c|}{$\begin{array}{c}\text { Ingestion + Inhalation + } \\
\text { External Exposure } \\
(\mathrm{pCi} / \mathrm{g})^{\mathrm{ft}}\end{array}$} \\
\hline & & $10^{-4}$ & $10^{-6}$ & $10^{-4}$ & $10^{-6}$ & $10^{-4}$ & $10^{-6}$ & $10^{-4}$ & $10^{-6}$ \\
\hline $\mathrm{Ra}-226^{(2)}$ & $013982-63-3$ & $1.1 \mathrm{E}+03$ & $1.1 \mathrm{E}+01$ & 8.3E-01 & 8.3E-03 & $1.7 \mathrm{E}+03$ & $1.7 \mathrm{E}+01$ & 8.3E-01 & 8.3E-03 \\
\hline $\mathrm{Ra}-226+\mathrm{D}$ & 013982-63-3 & $1.1 \mathrm{E}+03$ & $1.1 \mathrm{E}+01$ & $1.2 \mathrm{E}+06$ & $1.2 \mathrm{E}+04$ & $3.2 \mathrm{E}+00$ & $3.2 \mathrm{E}-02$ & $3.2 \mathrm{E}+00$ & $3.2 \mathrm{E}-02$ \\
\hline $\mathrm{Ra}-226+\mathrm{D}^{(2)}$ & $013982-63-3$ & $1.1 \mathrm{E}+03$ & $1.1 \mathrm{E}+01$ & $8.3 \mathrm{E}-01$ & $8.3 \mathrm{E}-03$ & $3.2 \mathrm{E}+00$ & $3.2 \mathrm{E}-02$ & $6.6 \mathrm{E}-01$ & $6.6 \mathrm{E}-03$ \\
\hline $\mathrm{Ra}-228$ & $015262-20-1$ & $1.3 \mathrm{E}+03$ & $1.3 \mathrm{E}+01$ & $3.6 \mathrm{E}+06$ & $3.6 \mathrm{E}+04$ & & & $1.3 E+03$ & $1.3 E+01$ \\
\hline $\mathrm{Ra}-228+\mathrm{D}$ & $015262-20-1$ & $1.3 \mathrm{E}+03$ & $1.3 E+01$ & $3.4 \mathrm{E}+06$ & $3.4 \mathrm{E}+04$ & $6.7 \mathrm{E}+00$ & $6.7 \mathrm{E}-02$ & $6.6 \mathrm{E}+00$ & $6.6 \mathrm{E}-02$ \\
\hline $\mathrm{Rb}-82$ & 014391-63-0 & $3.0 \mathrm{E}+07$ & $3.0 \mathrm{E}+05$ & $2.9 \mathrm{E}+11$ & $2.9 E+09$ & $5.6 \mathrm{E}+00$ & $5.6 \mathrm{E}-02$ & $5.6 \mathrm{E}+00$ & $5.6 \mathrm{E}-02$ \\
\hline $\mathrm{Rb}-86$ & 014932-53-7 & $4.5 \mathrm{E}+04$ & $4.5 \mathrm{E}+02$ & $8.1 E+08$ & $8.1 \mathrm{E}+06$ & $5.9 \mathrm{E}+01$ & $5.9 \mathrm{E}-01$ & $5.9 \mathrm{E}+01$ & $5.9 \mathrm{E}-01$ \\
\hline $\mathrm{Rb}-87$ & $013982-13-3$ & $8.7 \mathrm{E}+04$ & $8.7 E+02$ & $1.5 \mathrm{E}+09$ & $1.5 \mathrm{E}+07$ & & & $8.7 \mathrm{E}+04$ & $8.7 \mathrm{E}+02$ \\
\hline $\mathrm{Rb}-88$ & 014928-36-0 & $2.2 \mathrm{E}+06$ & $2.2 \mathrm{E}+04$ & $2.5 \mathrm{E}+10$ & $2.5 \mathrm{E}+08$ & $8.2 \mathrm{E}+00$ & $8.2 \mathrm{E}-02$ & $8.2 E+00$ & 8.2E-02 \\
\hline $\mathrm{Rb}-89$ & 014191-65-2 & $3.7 \mathrm{E}+06$ & $3.7 \mathrm{E}+04$ & $5.0 \mathrm{E}+10$ & $5.0 \mathrm{E}+08$ & $2.6 \mathrm{E}+00$ & $2.6 \mathrm{E}-02$ & $2.6 \mathrm{E}+00$ & $2.6 \mathrm{E}-02$ \\
\hline $\mathrm{Rh}-103 \mathrm{~m}$ & $007440-16-6$ & $3.9 \mathrm{E}+07$ & $3.9 \mathrm{E}+05$ & $2.7 \mathrm{E}+11$ & $2.7 E+09$ & $3.7 \mathrm{E}+05$ & $3.7 \mathrm{E}+03$ & $3.7 E+05$ & $3.7 \mathrm{E}+03$ \\
\hline Rh-105 & 014913-89-4 & $1.7 \mathrm{E}+05$ & $1.7 \mathrm{E}+03$ & $2.8 \mathrm{E}+09$ & $2.8 \mathrm{E}+07$ & $8.8 \mathrm{E}+01$ & $8.8 \mathrm{E}-01$ & $8.8 \mathrm{E}+01$ & $8.8 \mathrm{E}-01$ \\
\hline $\mathrm{Rh}-105 \mathrm{~m}$ & $014913-89-4$ & $3.0 \mathrm{E}+08$ & $3.0 \mathrm{E}+06$ & $3.7 \mathrm{E}+12$ & $3.7 \mathrm{E}+10$ & $9.6 \mathrm{E}+02$ & $9.6 \mathrm{E}+00$ & $9.6 \mathrm{E}+02$ & $9.6 \mathrm{E}+00$ \\
\hline $\mathrm{Rh}-106$ & 014234-34-5 & $8.8 E+07$ & $8.8 \mathrm{E}+05$ & $7.4 \mathrm{E}+11$ & $7.4 \mathrm{E}+09$ & $2.9 \mathrm{E}+01$ & $2.9 \mathrm{E}-01$ & $2.9 \mathrm{E}+01$ & $2.9 \mathrm{E}-01$ \\
\hline $\mathrm{Rn}-219$ & $014835-02-0$ & & & $5.0 \mathrm{E}+10$ & $5.0 \mathrm{E}+08$ & $1.3 \mathrm{E}+02$ & $1.3 \mathrm{E}+00$ & $1.3 E+02$ & $1.3 E+00$ \\
\hline $\mathrm{Rn}-220$ & $022481-48-7$ & & & $1.8 \mathrm{E}+10$ & $1.8 \mathrm{E}+08$ & $1.2 \mathrm{E}+04$ & $1.2 \mathrm{E}+02$ & $1.2 \mathrm{E}+04$ & $1.2 \mathrm{E}+02$ \\
\hline$R n-222+D$ & $014859-67-7$ & & & $4.5 \mathrm{E}+08$ & $4.5 \mathrm{E}+06$ & & & & \\
\hline
\end{tabular}


'Table 3.2d. (continued)

\begin{tabular}{|c|c|c|c|c|c|c|c|c|c|}
\hline \multirow[t]{2}{*}{ Chemical $^{\dagger}$} & \multirow[t]{2}{*}{ CASRN } & \multicolumn{2}{|c|}{ Ingestion (pCi/g) } & \multicolumn{2}{|c|}{ Inhalation (pCi/g) } & \multicolumn{2}{|c|}{$\begin{array}{l}\text { External Exposure } \\
(\mathrm{pCi} / \mathrm{g})^{\dagger \dagger}\end{array}$} & \multicolumn{2}{|c|}{$\begin{array}{c}\text { Ingestion + Inhalation + } \\
\text { External Exposure } \\
(\mathbf{p C i} / \mathbf{g})^{\dagger+t}\end{array}$} \\
\hline & & $10^{-4}$ & $10^{-6}$ & $10^{-4}$ & $10^{-6}$ & $10^{-4}$ & $10^{-6}$ & $10^{-4}$ & $10^{-6}$ \\
\hline Ru-103 & $013968-53-1$ & $9.6 \mathrm{E}+04$ & $9.6 \mathrm{E}+02$ & $7.5 \mathrm{E}+08$ & 7.5E+06 & $1.3 \mathrm{E}+01$ & $1.3 \mathrm{E}-01$ & $1.3 \mathrm{E}+01$ & $1.3 \mathrm{E}-01$ \\
\hline Ru-105 & $014331-95-4$ & $2.8 \mathrm{E}+05$ & $2.8 \mathrm{E}+03$ & $4.3 E+09$ & $4.3 \mathrm{E}+07$ & $7.6 \mathrm{E}+00$ & $7.6 \mathrm{E}-02$ & $7.6 \mathrm{E}+00$ & 7.6E-02 \\
\hline Ru-106 & $013967-48-1$ & $9.3 \mathrm{E}+03$ & $9.3 \mathrm{E}+01$ & $3.0 \mathrm{E}+07$ & $3.0 \mathrm{E}+05$ & & & $9.3 \mathrm{E}+03$ & $9.3 E+01^{\circ}$ \\
\hline Ru-106+D & 013967-48-1 & $9.3 \mathrm{E}+03$ & $9.3 \mathrm{E}+01$ & $3.0 \mathrm{E}+07$ & $3.0 \mathrm{E}+05$ & $2.9 \mathrm{E}+01$ & $2.9 \mathrm{E}-01$ & $2.9 \mathrm{E}+01$ & $2.9 \mathrm{E}-01$ \\
\hline Ru-97 & $015758-35-7$ & $5.4 \mathrm{E}+05$ & $5.4 \mathrm{E}+03$ & $8.4 \mathrm{E}+09$ & $8.4 \mathrm{E}+07$ & $4.8 \mathrm{E}+01$ & $4.8 \mathrm{E}-01$ & $4.8 \mathrm{E}+01$ & $4.8 \mathrm{E}-01$ \\
\hline S-35 & 015117-53-0 & $7.7 \mathrm{E}+05$ & $7.7 \mathrm{E}+03$ & $1.9 \mathrm{E}+10$ & $1.9 \mathrm{E}+08$ & & & $7.7 \mathrm{E}+05$ & $7.7 \mathrm{E}+03$ \\
\hline $\mathrm{Sb}-122$ & 014374-79-9 & $3.6 \mathrm{E}+04$ & $3.6 \mathrm{E}+02$ & $6.3 \mathrm{E}+08$ & $6.3 \mathrm{E}+06$ & $1.4 \mathrm{E}+01$ & $1.4 \mathrm{E}-01$ & $1.4 \mathrm{E}+01$ & $1.4 \mathrm{E}-01$ \\
\hline $\mathrm{Sb}-124$ & $014683-10-4$ & $3.0 \mathrm{E}+04$ & $3.0 \mathrm{E}+02$ & $2.6 \mathrm{E}+08$ & $2.6 \mathrm{E}+06$ & $3.0 \mathrm{E}+00$ & $3.0 \mathrm{E}-02$ & $3.0 \mathrm{E}+00$ & $3.0 \mathrm{E}-02$ \\
\hline $\mathrm{Sb}-125$ & 014234-35-6 & $1.1 \mathrm{E}+05$ & $1.1 \mathrm{E}+03$ & $6.6 \mathrm{E}+08$ & $6.6 \mathrm{E}+06$ & $1.6 \mathrm{E}+01$ & $1.6 \mathrm{E}-01$ & $1.6 \mathrm{E}+01$ & $1.6 \mathrm{E}-01$ \\
\hline $\mathrm{Sb}-125+\mathrm{D}$ & 014234-35-6 & $9.0 \mathrm{E}+04$ & $9.0 \mathrm{E}+02$ & $5.9 \mathrm{E}+08$ & $5.9 \mathrm{E}+06$ & $1.6 \mathrm{E}+01$ & $1.6 \mathrm{E}-01$ & $1.6 \mathrm{E}+01$ & $1.6 \mathrm{E}-01$ \\
\hline Sb-126 & 015756-32-8 & $3.3 \mathrm{E}+04$ & $3.3 \mathrm{E}+02$ & $4.1 \mathrm{E}+08$ & $4.1 \mathrm{E}+06$ & $2.1 E+00$ & $2.1 \mathrm{E}-02$ & $2.1 \mathrm{E}+00$ & $2.1 \mathrm{E}-02$ \\
\hline Sb-126m & 015756-32-8 & $4.4 \mathrm{E}+06$ & $4.4 \mathrm{E}+04$ & $5.3 \mathrm{E}+10$ & $5.3 \mathrm{E}+08$ & $3.8 \mathrm{E}+00$ & $3.8 \mathrm{E}-02$ & $3.8 \mathrm{E}+00$ & $3.8 \mathrm{E}-02$ \\
\hline Sb-127 & 013968-50-8 & $3.8 \mathrm{E}+04$ & $3.8 \mathrm{E}+02$ & $5.7 \mathrm{E}+08$ & $5.7 \mathrm{E}+06$ & $9.1 \mathrm{E}+00$ & $9.1 \mathrm{E}-02$ & $9.1 \mathrm{E}+00$ & $9.1 \mathrm{E}-02$ \\
\hline Sb-129 & 014331-88-5 & $1.7 \mathrm{E}+05$ & $1.7 \mathrm{E}+03$ & $4.0 \mathrm{E}+09$ & $4.0 \mathrm{E}+07$ & $3.9 E+00$ & $3.9 \mathrm{E}-02$ & $3.9 \mathrm{E}+00$ & $3.9 \mathrm{E}-02$ \\
\hline Sc-46 & $013967-63-0$ & $5.6 \mathrm{E}+04$ & $5.6 \mathrm{E}+02$ & $2.6 \mathrm{E}+08$ & $2.6 \mathrm{E}+06$ & $2.8 \mathrm{E}+00$ & $2.8 \mathrm{E}-02$ & $2.8 \mathrm{E}+00$ & $2.8 \mathrm{E}-02$ \\
\hline Sc-47 & 014391-96-9 & $1.1 \mathrm{E}+05$ & $1.1 \mathrm{E}+03$ & $1.7 \mathrm{E}+09$ & $1.7 \mathrm{E}+07$ & $8.8 E+01$ & $8.8 \mathrm{E}-01$ & $8.8 \mathrm{E}+01$ & $8.8 \mathrm{E}-01$ \\
\hline Sc-48 & 014391-86-7 & $4.8 \mathrm{E}+04$ & $4.8 \mathrm{E}+02$ & $8.2 E+08$ & $8.2 E+06$ & $1.7 \mathrm{E}+00$ & $1.7 \mathrm{E}-02$ & $1.7 \mathrm{E}+00$ & $1.7 \mathrm{E}-02$ \\
\hline
\end{tabular}

Radionuclides/soil/industrial

Page 14 of 20 
Table 3.2d. (continued)

\begin{tabular}{|c|c|c|c|c|c|c|c|c|c|}
\hline \multirow[t]{2}{*}{ Chemical } & \multirow[t]{2}{*}{ CASRN } & \multicolumn{2}{|c|}{ Ingestion (pCi/g) } & \multicolumn{2}{|c|}{ Inhalation (pCi/g) } & \multicolumn{2}{|c|}{$\begin{array}{c}\text { External Exposure } \\
(\mathrm{pCi} / \mathrm{g})^{\dagger+}\end{array}$} & \multicolumn{2}{|c|}{$\begin{array}{c}\text { Ingestion + Inhalation }+ \\
\text { External Exposure } \\
(\mathrm{pCi} / \mathrm{g})^{\mathrm{tt}}\end{array}$} \\
\hline & & $10^{-4}$ & $10^{-6}$ & $10^{-4}$ & $10^{-6}$ & $10^{-4}$ & $10^{-6}$ & $10^{-4}$ & $10^{-6}$ \\
\hline Se-75 & $014265-71-5$ & $4.9 \mathrm{E}+04$ & $4.9 \mathrm{E}+02$ & $7.0 \mathrm{E}+08$ & $7.0 \mathrm{E}+06$ & $2.5 \mathrm{E}+01$ & 2.5E-01 & $2.5 \mathrm{E}+01$ & $2.5 \mathrm{E}-01$ \\
\hline Si-31 & $014276-49-4$ & $6.3 E+05$ & $6.3 E+03$ & $1.0 \mathrm{E}+10$ & $1.0 \mathrm{E}+08$ & $6.3 \mathrm{E}+03$ & $6.3 \mathrm{E}+01$ & $6.3 \mathrm{E}+03$ & $6.3 E+01$ \\
\hline Sm-147 & $014392-33-7$ & $1.3 \mathrm{E}+04$ & $1.3 \mathrm{E}+02$ & $4.9 \mathrm{E}+05$ & $4.9 \mathrm{E}+03$ & & & $1.2 \mathrm{E}+04$ & $1.2 \mathrm{E}+02$ \\
\hline Sm-151 & $015715-94-3$ & $7.0 \mathrm{E}+05$ & $7.0 \mathrm{E}+03$ & $7.4 \mathrm{E}+08$ & $7.4 \mathrm{E}+06$ & $7.5 \mathrm{E}+07$ & $7.5 \mathrm{E}+05$ & $6.9 E+05$ & $6.9 \mathrm{E}+03$ \\
\hline Sm-153 & $015766-00-4$ & $8.0 \mathrm{E}+04$ & $8.0 \mathrm{E}+02$ & $1.6 \mathrm{E}+09$ & $1.6 \mathrm{E}+07$ & $4.7 \mathrm{E}+02$ & $4.7 E+00$ & 4.7E+02 & $4.7 \mathrm{E}+00$ \\
\hline Sn-113 & 013966-06-8 & $8.6 E+04$ & $8.6 \mathrm{E}+02$ & $5.2 E+08$ & $5.2 \mathrm{E}+06$ & $7.4 \mathrm{E}+03$ & $7.4 \mathrm{E}+01$ & $6.8 E+03$ & $6.8 \mathrm{E}+01$ \\
\hline Sn-121 & 014683-06-8 & $2.6 \mathrm{E}+05$ & $2.6 \mathrm{E}+03$ & $5.6 \mathrm{E}+09$ & $5.6 \mathrm{E}+07$ & & & $2.6 \mathrm{E}+05$ & $2.6 \mathrm{E}+03$ \\
\hline $\mathrm{Sn}-121 \mathrm{~m}$ & 014683-06-8 & $1.6 \mathrm{E}+05$ & $1.6 \mathrm{E}+03$ & $4.6 \mathrm{E}+08$ & $4.6 E+06$ & & & $1.6 \mathrm{E}+05$ & $1.6 E+03$ \\
\hline $\mathrm{Sn}-125$ & $014683-08-0$ & $1.9 \mathrm{E}+04$ & $1.9 \mathrm{E}+02$ & $2.9 \mathrm{E}+08$ & $2.9 \mathrm{E}+06$ & $1.8 \mathrm{E}+01$ & $1.8 \mathrm{E}-01$ & $1.8 \mathrm{E}+01$ & $1.8 \mathrm{E}-01$ \\
\hline Sn-126 & $015832-50-5$ & $1.5 \mathrm{E}+04$ & $1.5 \mathrm{E}+02$ & $8.0 \mathrm{E}+07$ & $8.0 E+05$ & $6.6 E+02$ & $6.6 \mathrm{E}+00$ & $6.3 E+02$ & $6.3 \mathrm{E}+00$ \\
\hline Sr-82 & 014809-50-8 & $1.2 \mathrm{E}+04$ & $1.2 \mathrm{E}+02$ & $3.9 E+08$ & $3.9 E+06$ & $2.4 \mathrm{E}+05$ & $2.4 \mathrm{E}+03$ & $1.2 \mathrm{E}+04$ & $1.2 \mathrm{E}+02$ \\
\hline Sr-85 & 013967-73-2 & $2.3 \mathrm{E}+05$ & $2.3 E+03$ & $3.0 \mathrm{E}+09$ & $3.0 \mathrm{E}+07$ & $1.4 \mathrm{E}+01$ & $1.4 \mathrm{E}-01$ & $1.4 \mathrm{E}+01$ & $1.4 \mathrm{E}-01$ \\
\hline $\mathrm{Sr}-85 \mathrm{~m}$ & 013967-73-2 & $1.8 \mathrm{E}+07$ & $1.8 \mathrm{E}+05$ & $4.8 \mathrm{E}+11$ & $4.8 \mathrm{E}+09$ & $4.2 \mathrm{E}+01$ & 4.2E-01 & $4.2 \mathrm{E}+01$ & $4.2 \mathrm{E}-01$ \\
\hline Sr-89 & $014158-27-1$ & $3.1 E+04$ & $3.1 E+02$ & $9.3 \mathrm{E}+08$ & $9.3 \mathrm{E}+06$ & $4.1 \mathrm{E}+04$ & 4.1E+02 & $1.8 \mathrm{E}+04$ & $1.8 \mathrm{E}+02$ \\
\hline Sr-90 & 010098-97-2 & $7.8 E+03$ & $7.8 \mathrm{E}+01$ & $5.8 \mathrm{E}+07$ & $5.8 \mathrm{E}+05$ & & & $7.8 \mathrm{E}+03$ & $7.8 \mathrm{E}+01$ \\
\hline Sr-90+D & $010098-97-2$ & $5.7 \mathrm{E}+03$ & $5.7 \mathrm{E}+01$ & $4.9 \mathrm{E}+07$ & $4.9 \mathrm{E}+05$ & & & $5.7 \mathrm{E}+03$ & $5.7 \mathrm{E}+01$ \\
\hline Sr-91 & $014331-91-0$ & $1.1 \mathrm{E}+05$ & $1.1 \mathrm{E}+03$ & $4.4 \mathrm{E}+09$ & $4.4 \mathrm{E}+07$ & $8.2 \mathrm{E}+00$ & $8.2 \mathrm{E}-02$ & $8.2 E+00$ & $8.2 \mathrm{E}-02$ \\
\hline
\end{tabular}

Radionuclides/soil/industrial

Page 15 of 20 
Table 3.2d. (continued)

\begin{tabular}{|c|c|c|c|c|c|c|c|c|c|}
\hline \multirow[t]{2}{*}{ Chemical $^{\dagger}$} & \multirow[t]{2}{*}{ CASRN } & \multicolumn{2}{|c|}{ Ingestion (pCi/g) } & \multicolumn{2}{|c|}{ Inhalation ( $\mathrm{pCi} / \mathrm{g}$ ) } & \multicolumn{2}{|c|}{$\begin{array}{l}\text { External Exposure } \\
(\mathrm{pCi} / \mathrm{g})^{\mathrm{tt}}\end{array}$} & \multicolumn{2}{|c|}{$\begin{array}{c}\text { Ingestion + Inhalation + } \\
\text { External Exposure } \\
(\mathrm{pCi} / \mathrm{g})^{\mathrm{tt+}}\end{array}$} \\
\hline & & $10^{-4}$ & $10^{-6}$ & $10^{-4}$ & $10^{-6}$ & $10^{-4}$ & $10^{-6}$ & $10^{-4}$ & $10^{-6}$ \\
\hline Sr-92 & 014928-29-1 & $1.6 \mathrm{E}+05$ & $1.6 \mathrm{E}+03$ & $7.3 \mathrm{E}+09$ & 7.3E+07 & $4.2 \mathrm{E}+00$ & 4.2E-02 & $4.2 \mathrm{E}+00$ & $4.2 \mathrm{E}-02$ \\
\hline Ta-182 & $013982-00-8$ & $4.6 \mathrm{E}+04$ & $4.6 \mathrm{E}+02$ & 2.1E+08 & $2.1 \mathrm{E}+06$ & $4.7 \mathrm{E}+00$ & $4.7 \mathrm{E}-02$ & $4.7 \mathrm{E}+00$ & 4.7E-02 \\
\hline $\mathrm{Tb}-158$ & $015759-55-4$ & $7.6 E+04$ & $7.6 \mathrm{E}+02$ & $4.9 \mathrm{E}+07$ & $4.9 \mathrm{E}+05$ & & & $7.6 \mathrm{E}+04$ & $7.6 \mathrm{E}+02$ \\
\hline Tb-160 & 013981-29-8 & $4.2 E+04$ & $4.2 \mathrm{E}+02$ & $3.0 \mathrm{E}+08$ & $3.0 \mathrm{E}+06$ & $5.4 \mathrm{E}+00$ & $5.4 \mathrm{E}-02$ & $5.4 \mathrm{E}+00$ & $5.4 \mathrm{E}-02$ \\
\hline Tc-95 & $014809-56-4$ & $4.7 \mathrm{E}+06$ & $4.7 \mathrm{E}+04$ & $1.0 \mathrm{E}+11$ & $1.0 \mathrm{E}+09$ & $8.1 \mathrm{E}+00$ & 8.1E-02 & $8.1 E+00$ & 8.1E-02 \\
\hline Tc-95m & $014809-56-4$ & $2.6 E+05$ & $2.6 \mathrm{E}+03$ & $1.6 \mathrm{E}+09$ & $1.6 \mathrm{E}+07$ & $1.1 \mathrm{E}+01$ & $1.1 \mathrm{E}-01$ & $1.1 \mathrm{E}+01$ & $1.1 \mathrm{E}-01$ \\
\hline Tc-96 & $014808-44-7$ & $1.4 \mathrm{E}+05$ & $1.4 \mathrm{E}+03$ & $1.8 \mathrm{E}+09$ & $1.8 \mathrm{E}+07$ & $2.3 \mathrm{E}+00$ & 2.3E-02 & $2.3 \mathrm{E}+00$ & $2.3 \mathrm{E}-02$ \\
\hline Tc-96m & $014808-44-7$ & $1.2 E+07$ & $1.2 \mathrm{E}+05$ & $1.5 \mathrm{E}+11$ & $1.5 \mathrm{E}+09$ & $2.8 \mathrm{E}+02$ & $2.8 \mathrm{E}+00$ & $2.8 \mathrm{E}+02$ & $2.8 \mathrm{E}+00$ \\
\hline Tc-97 & $015759-35-0$ & $2.0 \mathrm{E}+06$ & $2.0 \mathrm{E}+04$ & $1.0 \mathrm{E}+10$ & $1.0 \mathrm{E}+08$ & $8.8 \mathrm{E}+04$ & $8.8 \mathrm{E}+02$ & $8.4 \mathrm{E}+04$ & $8.4 \mathrm{E}+02$ \\
\hline Tc-97m & 015759-35-0 & $2.7 \mathrm{E}+05$ & $2.7 \mathrm{E}+03$ & $1.7 \mathrm{E}+09$ & $1.7 \mathrm{E}+07$ & $8.2 E+04$ & $8.2 E+02$ & $6.3 E+04$ & $6.3 \mathrm{E}+02$ \\
\hline Tc-99 & 014133-76-7 & $2.3 E+05$ & $2.3 \mathrm{E}+03$ & $1.2 \mathrm{E}+09$ & $1.2 \mathrm{E}+07$ & $3.5 \mathrm{E}+07$ & $3.5 \mathrm{E}+05$ & $2.3 E+05$ & $2.3 \mathrm{E}+03$ \\
\hline Tc-99m & 014133-76-7 & $5.7 \mathrm{E}+06$ & $5.7 \mathrm{E}+04$ & $9.8 \mathrm{E}+10$ & $9.8 \mathrm{E}+08$ & 8.7E+01 & 8.7E-01 & $8.7 \mathrm{E}+01$ & $8.7 \mathrm{E}-01$ \\
\hline $\mathrm{Te}-125 \mathrm{~m}$ & 014390-73-9 & $1.3 \mathrm{E}+05$ & $1.3 \mathrm{E}+03$ & $1.2 \mathrm{E}+09$ & $1.2 \mathrm{E}+07$ & $1.0 \mathrm{E}+04$ & $1.0 \mathrm{E}+02$ & $9.4 \mathrm{E}+03$ & $9.4 \mathrm{E}+01$ \\
\hline $\mathrm{Te}-127$ & $013981-49-2$ & $3.7 \mathrm{E}+05$ & $3.7 \mathrm{E}+03$ & $7.9 \mathrm{E}+09$ & $7.9 \mathrm{E}+07$ & $1.4 \mathrm{E}+03$ & $1.4 \mathrm{E}+01$ & $1.3 E+03$ & $1.3 E+01$ \\
\hline Te-127m & $013981-49-2$ & $5.3 E+04$ & $5.3 \mathrm{E}+02$ & $2.6 \mathrm{E}+08$ & $2.6 \mathrm{E}+06$ & $3.1 E+04$ & $3.1 E+02$ & $2.0 \mathrm{E}+04$ & $2.0 \mathrm{E}+02$ \\
\hline $\mathrm{Te}-129$ & 014269-71-7 & $2.2 \mathrm{E}+06$ & $2.2 \mathrm{E}+04$ & $2.3 E+10$ & $2.3 E+08$ & $1.5 \mathrm{E}+02$ & $1.5 \mathrm{E}+00$ & $1.5 E+02$ & $1.5 \mathrm{E}+00$ \\
\hline Te-129m & $014269-71-7$ & $2.7 E+04$ & $2.7 \mathrm{E}+02$ & $2.6 \mathrm{E}+08$ & $2.6 \mathrm{E}+06$ & $3.2 \mathrm{E}+02$ & $3.2 \mathrm{E}+00$ & $3.1 \mathrm{E}+02$ & $3.1 E+00$ \\
\hline
\end{tabular}

Radionuclides/soil/industrial

Page 16 of 20 


\begin{tabular}{|c|c|c|c|c|c|c|c|c|c|c|c|c|c|c|c|c|c|c|}
\hline 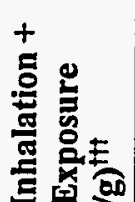 & $\stackrel{\varrho}{\varrho}$ & 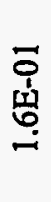 & 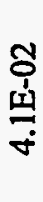 & 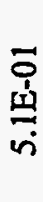 & 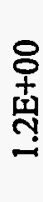 & $\begin{array}{l}\overline{0} \\
+ \\
\text { 索 } \\
\dot{\forall}\end{array}$ & 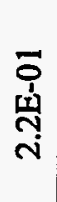 & $\begin{array}{l}8 \\
+ \\
\text { 跻 }\end{array}$ & 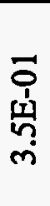 & 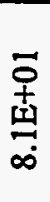 & $\begin{array}{l}\overline{0} \\
+ \\
\text { 产 } \\
\text { à }\end{array}$ & 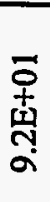 & 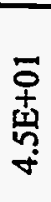 & $\begin{array}{l}\overline{0} \\
\text { 㟧 } \\
\text { - }\end{array}$ & 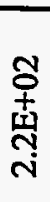 & 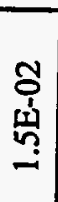 & 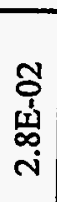 & 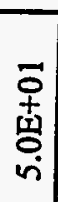 \\
\hline 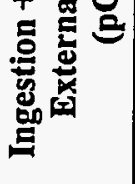 & ?ב & 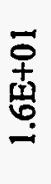 & $\begin{array}{l}8 \\
+ \\
\text { 至 } \\
\dot{8}\end{array}$ & $\begin{array}{l}\text { 辛 } \\
\text { 出 } \\
\text { v }\end{array}$ & 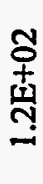 & $\begin{array}{l}m \\
\text { o } \\
\text { 章 } \\
\dot{q}\end{array}$ & $\begin{array}{l}\overline{0} \\
+ \\
\text { స్ } \\
\text { N }\end{array}$ & 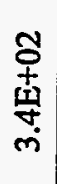 & 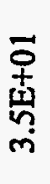 & 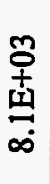 & 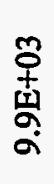 & 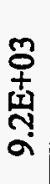 & 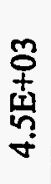 & 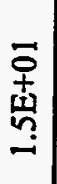 & 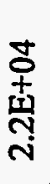 & 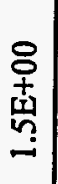 & $\begin{array}{l}8 \\
+ \\
+1 \\
\text { ஸे } \\
\text { i }\end{array}$ & 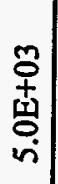 \\
\hline
\end{tabular}

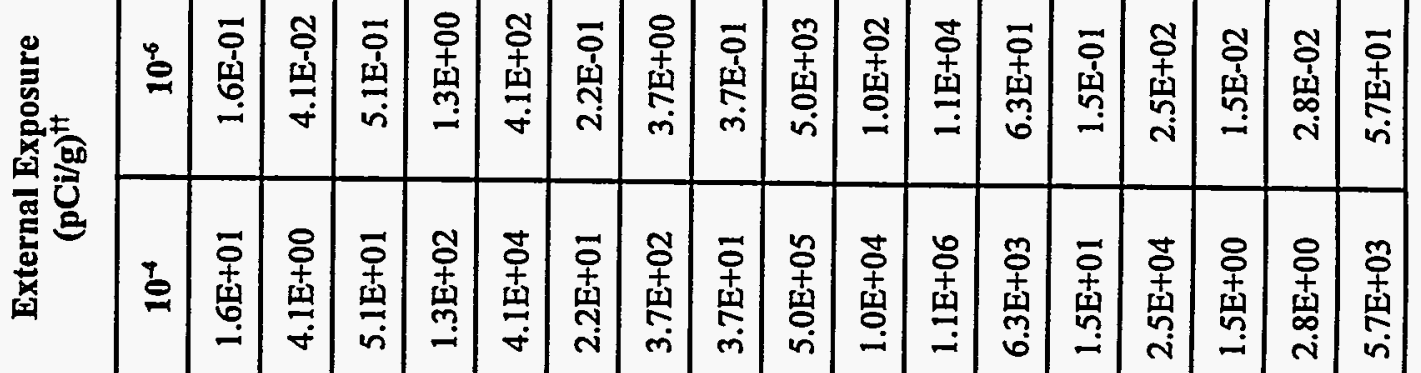

苞

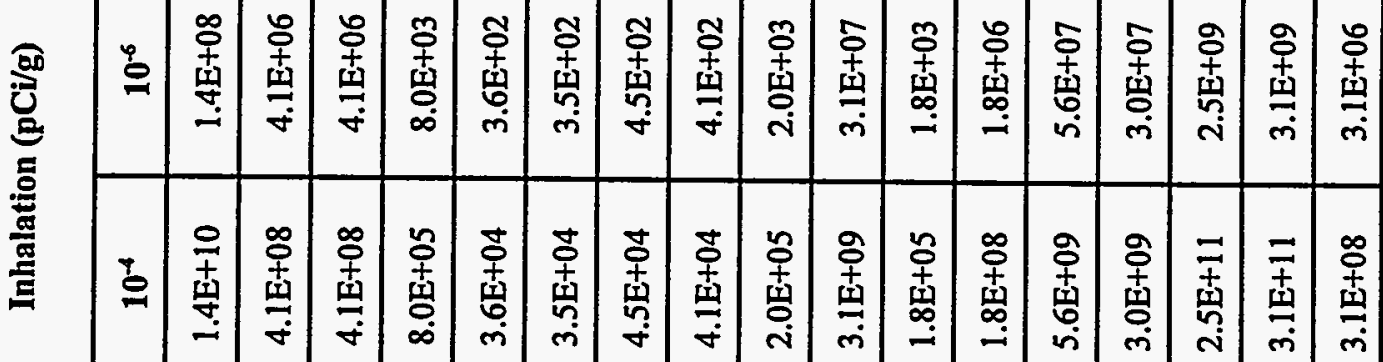

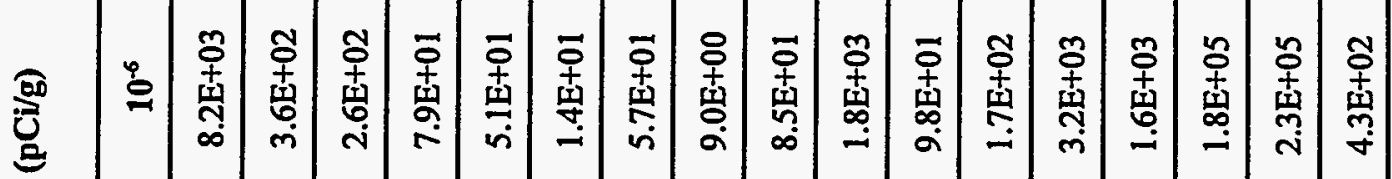

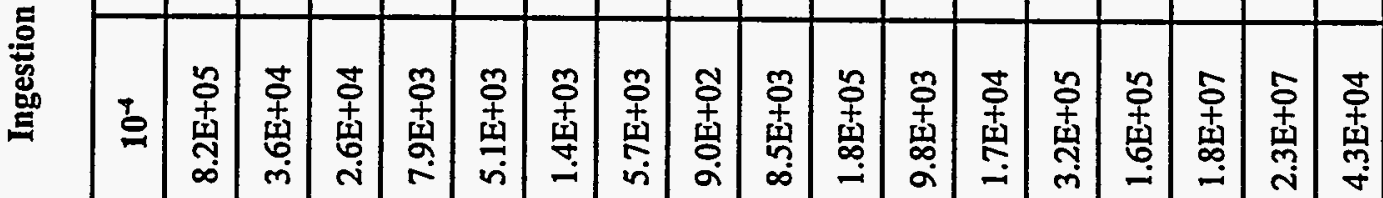

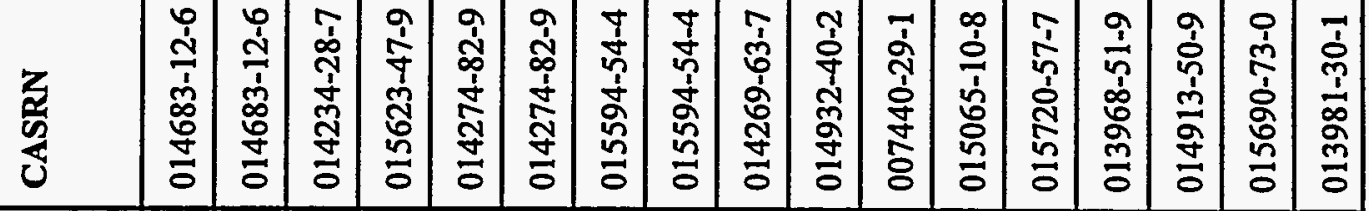

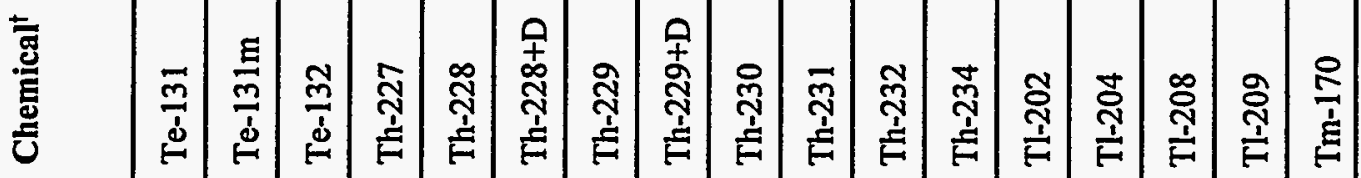

푤 
Table 3.2d. (continued)

\begin{tabular}{|c|c|c|c|c|c|c|c|c|c|}
\hline \multirow[t]{2}{*}{ Chemical $^{\dagger}$} & \multirow[t]{2}{*}{ CASRN } & \multicolumn{2}{|c|}{ Ingestion (pCi/g) } & \multicolumn{2}{|c|}{ Inhalation (pCi/g) } & \multicolumn{2}{|c|}{$\begin{array}{l}\text { External Exposure } \\
\qquad(\mathrm{pCi} / \mathrm{g})^{\dagger t}\end{array}$} & \multicolumn{2}{|c|}{$\begin{array}{c}\text { Ingestion + Inhalation }+ \\
\text { External Exposure } \\
(p C i / g)^{\dagger t t}\end{array}$} \\
\hline & & $10^{-4}$ & $10^{-6}$ & $10^{-4}$ & $10^{-6}$ & $10^{-4}$ & $10^{-6}$ & $10^{-4}$ & $10^{-6}$ \\
\hline Tm-171 & $014333-45-0$ & $5.5 E+05$ & $5.5 E+03$ & $1.9 \mathrm{E}+09$ & $1.9 E+07$ & $7.0 \mathrm{E}+04$ & $7.0 \mathrm{E}+02$ & $6.2 \mathrm{E}+04$ & $6.2 E+02$ \\
\hline $\mathrm{U}-232$ & $014158-29-3$ & $3.9 \mathrm{E}+03$ & $3.9 \mathrm{E}+01$ & $6.5 E+04$ & $6.5 E+02$ & $6.4 \mathrm{E}+05$ & $6.4 \mathrm{E}+03$ & $3.7 E+03$ & $3.7 \mathrm{E}+01$ \\
\hline $\mathrm{U}-233$ & $013968-55-3$ & $7.1 \mathrm{E}+03$ & $7.1 \mathrm{E}+01$ & $2.4 E+05$ & $2.4 E+03$ & $6.2 E+05$ & $6.2 E+03$ & $6.9 \mathrm{E}+03$ & $6.9 \mathrm{E}+01$ \\
\hline $\mathrm{U}-234$ & $013966-29-5$ & $7.2 E+03$ & $7.2 \mathrm{E}+01$ & $2.4 \mathrm{E}+05$ & $2.4 \mathrm{E}+03$ & $1.0 \mathrm{E}+06$ & $1.0 E+04$ & $7.0 \mathrm{E}+03$ & $7.0 \mathrm{E}+01$ \\
\hline U-235 & $015117-96-1$ & $7.1 E+03$ & $7.1 \mathrm{E}+01$ & $2.6 \mathrm{E}+05$ & $2.6 \mathrm{E}+03$ & $8.3 \mathrm{E}+01$ & 8.3E-01 & $8.2 E+01$ & $8.2 E-01$ \\
\hline $\mathrm{U}-235+\mathrm{D}$ & $015117-96-1$ & $6.8 \mathrm{E}+03$ & $6.8 \mathrm{E}+01$ & $2.6 \mathrm{E}+05$ & $2.6 \mathrm{E}+03$ & $8.3 \mathrm{E}+01$ & 8.3E-01 & $8.2 E+01$ & $8.2 \mathrm{E}-01$ \\
\hline $\mathrm{U}-236$ & $013982-70-2$ & $7.6 \mathrm{E}+03$ & $7.6 \mathrm{E}+01$ & $2.6 \mathrm{E}+05$ & $2.6 E+03$ & $1.3 \mathrm{E}+06$ & $1.3 E+04$ & $7.3 E+03$ & $7.3 \mathrm{E}+01$ \\
\hline U-237 & $014269-75-1$ & $8.0 \mathrm{E}+04$ & $8.0 \mathrm{E}+02$ & $1.1 E+09$ & $1.1 \mathrm{E}+07$ & $1.6 \mathrm{E}+02$ & $1.6 \mathrm{E}+00$ & $1.6 \mathrm{E}+02$ & $1.6 \mathrm{E}+00$ \\
\hline $\mathrm{U}-238$ & $007440-61-1$ & $7.5 \mathrm{E}+03$ & $7.5 \mathrm{E}+01$ & $2.8 \mathrm{E}+05$ & $2.8 E+03$ & $1.5 E+06$ & $1.5 \mathrm{E}+04$ & $7.3 \mathrm{E}+03$ & $7.3 \mathrm{E}+01$ \\
\hline $\mathrm{U}-238+\mathrm{D}$ & $007440-61-1$ & $5.2 E+03$ & $5.2 E+01$ & $2.8 \mathrm{E}+05$ & $2.8 \mathrm{E}+03$ & $4.2 E+02$ & $4.2 \mathrm{E}+00$ & $3.9 \mathrm{E}+02$ & $3.9 \mathrm{E}+00$ \\
\hline $\mathrm{U}-240$ & $015687-53-3$ & $5.9 \mathrm{E}+04$ & $5.9 E+02$ & $1.0 \mathrm{E}+09$ & $1.0 \mathrm{E}+07$ & $2.0 \mathrm{E}+05$ & $2.0 \mathrm{E}+03$ & $4.5 E+04$ & $4.5 \mathrm{E}+02$ \\
\hline$V-48$ & $014331-97-6$ & $4.2 E+04$ & $4.2 \mathrm{E}+02$ & $5.0 \mathrm{E}+08$ & $5.0 \mathrm{E}+06$ & $2.0 \mathrm{E}+00$ & $2.0 \mathrm{E}-02$ & $2.0 \mathrm{E}+00$ & $2.0 \mathrm{E}-02$ \\
\hline W-181 & $015749-46-9$ & $1.2 E+06$ & $1.2 \mathrm{E}+04$ & $4.3 \mathrm{E}+10$ & $4.3 E+08$ & $1.0 \mathrm{E}+03$ & $1.0 \mathrm{E}+01$ & $1.0 \mathrm{E}+03$ & $1.0 \mathrm{E}+01$ \\
\hline W-185 & $014932-41-3$ & $1.6 \mathrm{E}+05$ & $1.6 \mathrm{E}+03$ & $8.0 E+09$ & $8.0 \mathrm{E}+07$ & $4.4 \mathrm{E}+05$ & $4.4 E+03$ & $1.2 E+05$ & $1.2 E+03$ \\
\hline W-187 & $014983-48-3$ & $1.3 E+05$ & $1.3 E+03$ & $6.5 E+09$ & $6.5 E+07$ & $1.3 \mathrm{E}+01$ & $1.3 \mathrm{E}-01$ & $1.3 \mathrm{E}+01$ & $1.3 \mathrm{E}-01$ \\
\hline $\mathrm{Xe}-122$ & $015151-09-4$ & & & $1.1 \mathrm{E}+12$ & $1.1 \mathrm{E}+10$ & & & & \\
\hline $\mathrm{Xe}-123$ & $015700-10-4$ & & & $3.8 \mathrm{E}+12$ & $3.8 \mathrm{E}+10$ & & & & \\
\hline
\end{tabular}

Radionuclides/soil/industrial

Page 18 of 20 
Table 3.2d. (continued)

\begin{tabular}{|c|c|c|c|c|c|c|c|c|c|}
\hline \multirow[t]{2}{*}{ Chemical $^{\dagger}$} & \multirow[t]{2}{*}{ CASRN } & \multicolumn{2}{|c|}{ Ingestion (pCi/g) } & \multicolumn{2}{|c|}{ Inhalation (pCi/g) } & \multicolumn{2}{|c|}{$\begin{array}{l}\text { External Exposure } \\
\text { (pCi/g) }\end{array}$} & \multicolumn{2}{|c|}{$\begin{array}{c}\text { Ingestion + Inhalation + } \\
\text { External Exposure } \\
(\text { pCi/g) })^{f+t}\end{array}$} \\
\hline & & $10^{-4}$ & $10^{-6}$ & $10^{-4}$ & $10^{-6}$ & $10^{-4}$ & $10^{-6}$ & $10^{-4}$ & $10^{-6}$ \\
\hline $\mathrm{Xe}-125$ & 013994-18-8 & & & $2.9 \mathrm{E}+12$ & $2.9 \mathrm{E}+10$ & & & & \\
\hline $\mathrm{Xe}-127$ & 013994-19-9 & & & $8.4 \mathrm{E}+12$ & $8.4 \mathrm{E}+10$ & & & & \\
\hline $\mathrm{Xe}-129 \mathrm{~m}$ & 013965-99-6 & & & $6.0 \mathrm{E}+12$ & $6.0 \mathrm{E}+10$ & & & & \\
\hline $\mathrm{Xe}-131 \mathrm{~m}$ & $014683-11-5$ & & & $8.3 E+12$ & $8.3 E+10$ & & & & \\
\hline $\mathrm{Xe}-133$ & $014932-42-4$ & & & $8.3 E+12$ & $8.3 E+10$ & & & & \\
\hline $\mathrm{Xe}-133 \mathrm{~m}$ & $014932-42-4$ & & & $6.7 \mathrm{E}+12$ & $6.7 \mathrm{E}+10$ & & & & \\
\hline $\mathrm{Xe}-135$ & $014995-62-1$ & & & $4.6 \mathrm{E}+12$ & $4.6 \mathrm{E}+10$ & & & & \\
\hline $\mathrm{Xe}-135 \mathrm{~m}$ & $014995-62-1$ & & & $1.8 \mathrm{E}+13$ & $1.8 \mathrm{E}+11$ & & & & \\
\hline $\mathrm{Xe}-137$ & $014835-21-3$ & & & $2.5 \mathrm{E}+12$ & $2.5 \mathrm{E}+10$ & & & & \\
\hline $\mathrm{Xe}-138$ & $015751-81-2$ & & & $1.7 \mathrm{E}+12$ & $1.7 \mathrm{E}+10$ & & & & \\
\hline Y-90 & $010098-91-6$ & $2.1 E+04$ & $2.1 \mathrm{E}+02$ & $3.5 \mathrm{E}+08$ & $3.5 \mathrm{E}+06$ & & & $2.1 \mathrm{E}+04$ & $2.1 \mathrm{E}+02$ \\
\hline$Y-91$ & $014234-24-3$ & $2.4 \mathrm{E}+04$ & $2.4 \mathrm{E}+02$ & $1.9 \mathrm{E}+08$ & $1.9 \mathrm{E}+06$ & $1.6 \mathrm{E}+03$ & $1.6 \mathrm{E}+01$ & $1.5 E+03$ & $1.5 \mathrm{E}+01$ \\
\hline Y-91m & $014234-24-3$ & $8.7 \mathrm{E}+06$ & $8.7 E+04$ & $1.1 \mathrm{E}+11$ & $1.1 \mathrm{E}+09$ & $1.2 \mathrm{E}+01$ & $1.2 \mathrm{E}-01$ & $1.2 \mathrm{E}+01$ & $1.2 \mathrm{E}-01$ \\
\hline$Y-92$ & $015751-59-4$ & $1.6 \mathrm{E}+05$ & $1.6 \mathrm{E}+03$ & $2.1 \mathrm{E}+09$ & $2.1 \mathrm{E}+07$ & $2.2 \mathrm{E}+01$ & $2.2 \mathrm{E}-01$ & $2.2 \mathrm{E}+01$ & $2.2 \mathrm{E}-01$ \\
\hline Y-93 & $014981-70-5$ & $5.6 \mathrm{E}+04$ & $5.6 \mathrm{E}+02$ & $9.8 \mathrm{E}+08$ & $9.8 \mathrm{E}+06$ & $6.4 \mathrm{E}+01$ & $6.4 \mathrm{E}-01$ & $6.4 \mathrm{E}+01$ & $6.4 \mathrm{E}-01$ \\
\hline $\mathrm{Zn}-65$ & 013982-39-3 & $3.2 E+04$ & $3.2 \mathrm{E}+02$ & $3.4 \mathrm{E}+08$ & $3.4 \mathrm{E}+06$ & $9.6 \mathrm{E}+00$ & $9.6 \mathrm{E}-02$ & $9.6 \mathrm{E}+00$ & $9.6 \mathrm{E}-02$ \\
\hline $\mathrm{Zn}-69$ & 013982-23-5 & $5.2 \mathrm{E}+06$ & $5.2 \mathrm{E}+04$ & $3.3 \mathrm{E}+10$ & $3.3 E+08$ & $1.1 \mathrm{E}+06$ & $1.1 \mathrm{E}+04$ & $8.9 E+05$ & $8.9 E+03$ \\
\hline
\end{tabular}


Table 3.2d. (continued)

\begin{tabular}{|c|c|c|c|c|c|c|c|c|c|}
\hline \multirow[t]{2}{*}{ Chemical $^{\dagger}$} & \multirow[t]{2}{*}{ CASRN } & \multicolumn{2}{|c|}{ Ingestion (pCi/g) } & \multicolumn{2}{|c|}{ Inhalation (pCi/g) } & \multicolumn{2}{|c|}{$\begin{array}{l}\text { External Exposure } \\
(\mathrm{pCi} / \mathrm{g})^{H}\end{array}$} & \multicolumn{2}{|c|}{$\begin{array}{c}\text { Ingestion + Inhalation + } \\
\text { External Exposure } \\
(\mathrm{pCi} / \mathrm{g})^{\mathrm{ftt}}\end{array}$} \\
\hline & & $10^{-4}$ & $10^{-6}$ & $10^{-4}$ & $10^{-6}$ & $10^{-4}$ & $10^{-6}$ & $10^{-4}$ & $10^{-6}$ \\
\hline $\mathrm{Zn}-69 \mathrm{~m}$ & $013982-23-5$ & $2.1 \mathrm{E}+05$ & $2.1 E+03$ & $2.9 E+09$ & $2.9 \mathrm{E}+07$ & $1.5 \mathrm{E}+01$ & $1.5 \mathrm{E}-01$ & $1.5 \mathrm{E}+01$ & $1.5 \mathrm{E}-01$ \\
\hline $\mathrm{Zr}-93$ & $015751-77-6$ & $6.1 \mathrm{E}+05$ & $6.1 E+03$ & $6.5 \mathrm{E}+08$ & $6.5 \mathrm{E}+06$ & & & $6.1 \mathrm{E}+05$ & $6.1 \mathrm{E}+03$ \\
\hline $\mathrm{Zr}-95$ & $013967-71-0$ & $8.2 E+04$ & $8.2 \mathrm{E}+02$ & $5.3 \mathrm{E}+08$ & $5.3 \mathrm{E}+06$ & $7.8 \mathrm{E}+00$ & 7.8E-02 & $7.8 \mathrm{E}+00$ & $7.8 \mathrm{E}-02$ \\
\hline $\mathrm{Zr}-97$ & $014928-30-4$ & $3.1 \mathrm{E}+04$ & $3.1 \mathrm{E}+02$ & $7.2 E+08$ & $7.2 \mathrm{E}+06$ & $3.2 \mathrm{E}+01$ & $3.2 \mathrm{E}-01$ & $3.2 \mathrm{E}+01$ & $3.2 \mathrm{E}-01$ \\
\hline
\end{tabular}

Notes:

$\dagger \quad$ Isotopes designated with an " $m$ " indicate that the isotope is metastable; isotopes designated with a " $+\mathrm{D}$ " indicate that the radionuclide slope factors include contributions from daughter products.

†† If no PRG for external exposure was calculated, then no external exposure slope factors were available or the external exposure slope factor was equal to $0.00 \mathrm{E}+00$ (resulting in an undefined PRG for the external exposure pathway for soil under industrial land use).

$\dagger \dagger \dagger$ If slope factors and/or reference doses were available for only one pathway (i.e., ingestion, inhalation, OR external exposure), the total for ingestion plus inhalation plus external exposure was not calculated.

(1) The default volatilization factor of 200 for Ra-224 was calculated as the ratio of the average Ra-224 background concentration in soil to the average Rn-220 background concentration in air. Inhalation slope factor values are for Rn-222 plus decay products (i.e., Rn-222+D) formed from the radioactive decay of Ra-226 and for Rn-220+D from the decay of Ra-224. Slope factor values were taken from Table 4a of EPA's Health Effects Assessment Summary Tables (HEAST 1992).

(2) The default volatilization factor of 8 for Ra-226 was calculated as the ratio of the average natural background concentration of Ra-226 in soil to the corresponding average natural background concentration of Rn-222 in air. Inhalation slope factor values are for $\mathrm{Rn}-222$ plus decay products (i.e., $\mathrm{Rn}-222+\mathrm{D}$ ) formed from the radioactive decay of Ra-226 and for Rn-220+D from the decay of Ra-224. Slope factor values were taken from Table 4a of EPA's Health Effects Assessment Summary Tables (HEAST 1992). 
Table 3.3a. Risk-based Preliminary Remediation Goals for nonradionuclides in water (agricultural scenario)

\begin{tabular}{|c|c|c|c|c|c|c|c|c|c|c|c|c|c|c|c|}
\hline \multirow{2}{*}{ Chemical } & \multirow{2}{*}{ CASRN } & \multicolumn{4}{|c|}{$\begin{array}{c}\text { Ingestion of Vegetables } \\
(\mathrm{mg} / \mathrm{L})^{*}\end{array}$} & \multicolumn{4}{|c|}{$\begin{array}{c}\text { Ingestion of Beef } \\
(\mathrm{mg} / \mathrm{L})^{*}\end{array}$} & \multicolumn{6}{|c|}{$\begin{array}{c}\text { Ingestion of Milk } \\
(\mathrm{mg} / \mathrm{L})^{*}\end{array}$} \\
\hline & & $10^{4}$ & $10^{5}$ & $H Q=1$ & $\mathrm{HQ}=0.1$ & $10^{-4}$ & $10^{5}$ & нQ-1 & $\mathrm{HQ}=0.1$ & $10^{-4}$ & $10^{6}$ & $\begin{array}{l}\text { HQ }=1 \\
\text { Adult }\end{array}$ & $\begin{array}{l}\text { HQ=1 } \\
\text { Child }\end{array}$ & $\begin{array}{c}\text { HQ }=0.1 \\
\text { Adult }\end{array}$ & $\begin{array}{c}\text { HQ }=0.1 \\
\text { Child }\end{array}$ \\
\hline ALAR & $001596-84-5$ & & & & & & & & & & & & & & \\
\hline Acenaphthene & $000083-32-9$ & & & $4.0 \mathrm{E}+\infty 0$ & 4.0E-01 & & & $2.3 E+03$ & $2.3 \mathrm{E}+02$ & & & $1.2 \mathrm{E}+03$ & $1.6 \mathrm{E}+02$ & $1.2 \mathrm{E}+02$ & $1.6 \mathrm{E}+01$ \\
\hline Acenaphthylene & $000208-96-8$ & & & & $\cdot$ & & & & & & & & & & \\
\hline Acephate & $030560-19-1$ & $2.8 \mathrm{E}-02$ & $2,8 \mathrm{E}-04$ & $4.2 \mathrm{E}-03$ & 4.2E-04 & $1.0 \mathrm{E}+06$ & $1.0 \mathrm{E}+06$ & $1.0 \mathrm{E}+06$ & $1.0 \mathrm{E}+06$ & $1.0 \mathrm{E}+06$ & $3.3 \mathrm{E}+05$ & $1.0 \mathrm{E}+06$ & $1.0 \mathrm{E}+06$ & $1.0 \mathrm{E}+06$ & $1.5 \mathrm{E}+05$ \\
\hline Acetaldehyde & $000075-07-0$ & & & & & & & & & & & & & & \\
\hline Acetochlor & $034256-82-1$ & & & & & & & & & & & & & & $\cdot$ \\
\hline Acetone & $000067-64-1$ & & & $2.3 \mathrm{E}-01$ & 2.3E-02 & & & $1.0 \mathrm{E}+06$ & $1.0 \mathrm{E}+06$ & & & $1.0 \mathrm{E}+06$ & $1.0 \mathrm{E}+06$ & $1.0 E+06$ & $9.0 \mathrm{E}+05$ \\
\hline Acetone Cyanohydrin & $000075-86-5$ & & & & & & & & & & & & & & \\
\hline Acetonitrile & $000075-05-8$ & & & $1.2 \mathrm{E}-02$ & $1.2 \mathrm{E}-03$ & & & $1.0 \mathrm{E}+06$ & $1.0 \mathrm{E}+06$ & & & $1.0 \mathrm{E}+06$ & $6.8 \mathrm{E}+05$ & $5.3 E+05$ & $6.8 \mathrm{E}+04$ \\
\hline Acetophenone $e^{(3)}$ & $000098-86-2$ & & & $2.2 E+00$ & $2.2 \mathrm{E}-01$ & & & $1.0 \mathrm{E}+06$ & $1.6 \mathrm{E}+05$ & & & $8.1 E+05$ & $1.0 \mathrm{E}+05$ & $8.1 \mathrm{E}+04$ & $1.0 \mathrm{E}+04$ \\
\hline Acrolein $^{(6)}$ & $000107-02-8$ & & & $5.6 \mathrm{E}-02$ & $5.6 \mathrm{E}-03$ & & & $1.0 \mathrm{E}+06$ & $1.0 \mathrm{E}+06$ & & & $1.0 \mathrm{E}+06$ & $1.0 \mathrm{E}+06$ & $1.0 \mathrm{E}+06$ & $1.3 \mathrm{E}+05$ \\
\hline Acrylamide & $000079-06-1$ & $6.9 \mathrm{E}-05$ & $6.9 \mathrm{E}-07$ & 2.7E-04 & 2.7E-05 & $1.9 \mathrm{E}+05$ & $1.9 \mathrm{E}+03$ & 7.3E+0S & $7.3 \mathrm{E}+04$ & $4.2 \mathrm{E}+04$ & $4.2 \mathrm{E}+02$ & $3.8 \mathrm{E}+05$ & $4.9 \mathrm{E}+04$ & $3.8 \mathrm{E}+04$ & $4.9 E+03$ \\
\hline Acrylic Acid & $000079 \cdot 10-7$ & & & $1.9 \mathrm{E}+00$ & $1.9 \mathrm{E}-01$ & & & $1.0 \mathrm{E}+06$ & $1.0 \mathrm{E}+06$ & & & $1.0 \mathrm{E}+06$ & $1.0 \mathrm{E}+06$ & $1.0 \mathrm{E}+06$ & $1.0 \mathrm{E}+06$ \\
\hline Acrylonitrile & $000107-13-1$ & $1.9 \mathrm{E}-03$ & $1.9 \mathrm{E}_{-05}$ & 4.3E-03 & 4.3E-04 & $1.9 \mathrm{E}+05$ & $1.9 \mathrm{E}+03$ & $4.4 \mathrm{E}+05$ & $4.4 \mathrm{E}+04$ & $4.2 \mathrm{E}+04$ & $4.2 \mathrm{E}+02$ & $2.3 \mathrm{E}+05$ & $2.9 \mathrm{E}+04$ & $2.3 \mathrm{E}+04$ & $2.9 \mathrm{E}+03$ \\
\hline Alachlor & $015972-60-8$ & $1.4 \mathrm{E}-01$ & $1.4 \mathrm{E}-03$ & $4.9 \mathrm{E}-01$ & $4.9 \mathrm{E}-02$ & $2.9 \mathrm{E}+03$ & $2.9 \mathrm{E}+01$ & $9.8 \mathrm{E}+03$ & $9.8 \mathrm{E}+02$ & $6,3 \mathrm{E}+02$ & $6.3 \mathrm{E}+00$ & $5.1 \mathrm{E}+03$ & $6.5 \mathrm{E}+02$ & $5.1 \mathrm{E}+02$ & $6.5 \mathrm{E}+01$ \\
\hline Aldicarb & $000116-06-3$ & & & $1.2 \mathrm{E}-02$ & $1.2 \mathrm{E}-03$ & & & $5.8 \mathrm{E}+04$ & $5.8 \mathrm{E}+03$ & & & $3.0 \mathrm{E}+04$ & $3.8 \mathrm{E}+03$ & $3.0 \mathrm{E}+03$ & $3.8 \mathrm{E}+02$ \\
\hline Aldicarb Sulfone & $001646-88-4$ & & & & & & & & & & & & & & \\
\hline Aldrin & $000309-00-2$ & 7.0E-04 & $7.0 \mathrm{E}-06$ & $1.5 \mathrm{E}-03$ & $1.5 \mathrm{E}-04$ & $1.1 \mathrm{E}+01$ & $1.1 \mathrm{E}-01$ & $2.3 \mathrm{E}+01$ & $2.3 \mathrm{E}+00$ & $2.4 \mathrm{E}+00$ & $2.4 \mathrm{E}-02$ & $1.2 \mathrm{E}+01$ & $1.6 \mathrm{E}+00$ & $1.2 \mathrm{E}+00$ & $1.6 \mathrm{E}-01$ \\
\hline Ally & $074223-64-6$ & & & 8.1E-01 & $8.1 \mathrm{E}-02$ & & & $1.0 \mathrm{E}+06$ & $1.0 \mathrm{E}+06$ & & & $1.0 \mathrm{E}+06$ & $1.0 \mathrm{E}+06$ & $1.0 \mathrm{E}+06$ & $1.0 \mathrm{E}+06$ \\
\hline Allyl Ảlcohol & $000107-18-6$ & & & $1.2 \mathrm{E}-02$ & $1.2 \mathrm{E}-03$ & & & $1.0 \mathrm{E}+06$ & $6.5 \mathrm{E}+05$ & & & $1.0 \mathrm{E}+06$ & $4.3 \mathrm{E}+05$ & $3.4 E+05$ & $4.3 \mathrm{E}+04$ \\
\hline Allyl Chloride & $000107-05-1$ & & & 8.2E-01 & 8.2E-02 & & & $1.0 \mathrm{E}+06$ & $1.5 \mathrm{E}+05$ & & & $8.0 E+05$ & $1.0 \mathrm{E}+05$ & $8.0 E+04$ & $1.0 \mathrm{E}+04$ \\
\hline
\end{tabular}


Table 3.3a. (continued)

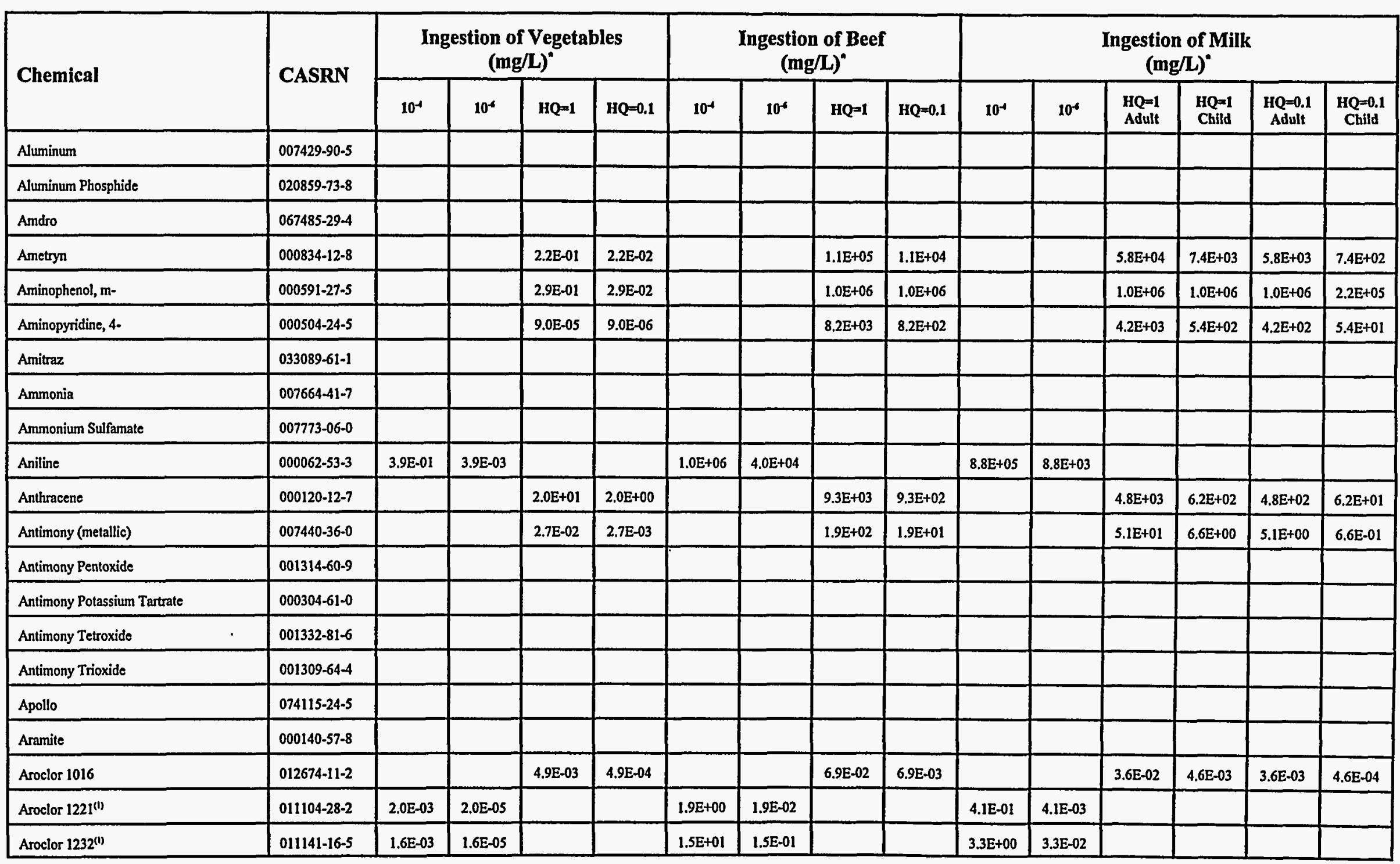


Table 3.3a. (continued)

\begin{tabular}{|c|c|c|c|c|c|c|c|c|c|c|c|c|c|c|c|}
\hline \multirow{2}{*}{ Chemical } & \multirow{2}{*}{ CASRN } & \multicolumn{4}{|c|}{$\begin{array}{l}\text { Ingestion of Vegetables } \\
\text { (mg/L) }\end{array}$} & \multicolumn{4}{|c|}{$\begin{array}{c}\text { Ingestion of Beef } \\
(\mathrm{mg} / \mathrm{L})^{*}\end{array}$} & \multicolumn{6}{|c|}{$\begin{array}{c}\text { Ingestion of Milk } \\
(\mathrm{mg} / \mathrm{L})^{*}\end{array}$} \\
\hline & & $10^{-4}$ & $10^{6}$ & $H Q=1$ & $\mathrm{HQ}=0.1$ & $10^{-4}$ & $10^{6}$ & $H Q=1$ & $\mathrm{HQ}=0.1$ & $10^{-4}$ & $10^{6}$ & $\begin{array}{l}\text { HQ=1 } \\
\text { Adult }\end{array}$ & $\begin{array}{l}\text { HQ=1 } \\
\text { Child }\end{array}$ & $\begin{array}{c}\mathrm{HQ}=0.1 \\
\text { Adult }\end{array}$ & $\begin{array}{c}\text { HQ }=0.1 \\
\text { Child }\end{array}$ \\
\hline Aroclor $1242^{(1)}$ & 053469-21-9 & $2.0 \mathrm{E}-03$ & $2.0 \mathrm{E}-05$ & & & $1.9 \mathrm{E}+00$ & $1.9 \mathrm{E}-02$ & & & 4.1E-01 & 4.1E-03 & & & & \\
\hline Aroclor $1248^{(1)}$ & $012672-29-6$ & 2.1E-03 & 2.1E-05 & . & & 3.7E-02 & 3.7E-04 & & & $8.2 \mathrm{E}-03$ & $8.2 \mathrm{E}-05$ & & & & \\
\hline Aroclor 1254 & $011097-69-1$ & & & $1.4 \mathrm{E}-03$ & $1.4 \mathrm{E}-04$ & & & $1.6 \mathrm{E}-02$ & $1.6 \mathrm{E}-03$ & & & 8.1E-03 & $1.0 \mathrm{E}-03$ & 8.1E-04 & $1.0 \mathrm{E}-04$ \\
\hline Aroclor 1260(1) & $011096-82-5$ & 2.1E-03 & 2.1E-05 & & & $1.9 \mathrm{E}-03$ & $1.9 \mathrm{E}-05$ & & & 4.1E-04 & 4.1E-06 & & & & \\
\hline Arsenic Salts & NA & & & & & & & & & & & & & & \\
\hline Arsenic, Inorganic & $007440-38-2$ & $1.1 \mathrm{E}-02$ & $1.1 \mathrm{E}-04$ & $2.1 \mathrm{E}-02$ & 2.1E-03 & $1.5 \mathrm{E}+00$ & $1.5 \mathrm{E}-02$ & $2.9 \mathrm{E}+00$ & $2.9 \mathrm{E}-01$ & $3.5 \mathrm{E}+00$ & $3.5 \mathrm{E}-02$ & $1.6 \mathrm{E}+01$ & $2.0 \mathrm{E}+00$ & $1.6 \mathrm{E}+00$ & $2.0 \mathrm{E}-01$ \\
\hline Arsine & $007784-42-1$ & & & & & & & & & & & & & & \\
\hline Asbestos & $001332-21-4$ & & & & & & & & & & & & & & \\
\hline Assure & $076578-14-8$ & & & & & & & & & & & & & & \\
\hline Asulam & $003337-71-1$ & & & & & & & & & & & & & & \\
\hline Atrazine & $001912-24-9$ & $4.7 \mathrm{E}-02$ & $4.7 \mathrm{E}-04$ & $1.6 \mathrm{E}+00$ & $1.6 \mathrm{E}-01$ & $1.6 \mathrm{E}+03$ & $1.6 \mathrm{E}+01$ & $5.4 \mathrm{E}+04$ & $5.4 \mathrm{E}+03$ & $3.6 \mathrm{E}+02$ & $3.6 \mathrm{E}+00$ & $2.8 \mathrm{E}+04$ & $3.6 \mathrm{E}+03$ & $2.8 \mathrm{E}+03$ & $3.6 \mathrm{E}+02$ \\
\hline Avermectin B1 & $065195-55-3$ & & & & & & & & & & & & & & \\
\hline Azobenzene & $000103-33-3$ & $1.3 \mathrm{E}-01$ & $1.3 \mathrm{E}-03$ & & & $2.6 \mathrm{E}+02$ & $2.6 \mathrm{E}+00$ & & & $5.8 \mathrm{E}+01$ & $5.8 \mathrm{E}-01$ & & & & \\
\hline Barium & $007440-39-3$ & & & $4.9 \mathrm{E}+00$ & $4.9 \mathrm{E}-01$ & & & $6.8 \mathrm{E}+03$ & $6.8 \mathrm{E}+02$ & & & $4.7 \mathrm{E}+02$ & $6.0 \mathrm{E}+01$ & $4.7 \mathrm{E}+01$ & $6.0 \mathrm{E}+00$ \\
\hline Barium Cyanide & $000542-62-1$ & & & & & & & & & & & & & & 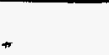 \\
\hline Baygon & $000114-26-1$ & & & $8.0 \mathrm{E}-02$ & $8.0 \mathrm{E}-03$ & & & $7.8 \mathrm{E}+04$ & $7.8 \mathrm{E}+03$ & & & 4.1E+04 & $5.2 \mathrm{E}+03$ & $4.1 E+03$ & $5.2 \mathrm{E}+02$ \\
\hline Bayleton & $043121-43-3$ & & & $1.1 \mathrm{E}+00$ & $1.1 \mathrm{E}-01$ & & & $1.2 \mathrm{E}+05$ & $1.2 \mathrm{E}+04$ & & & $6.1 E+04$ & $7.8 \mathrm{E}+03$ & $6.1 \mathrm{E}+03$ & $7.8 \mathrm{E}+02$ \\
\hline Baythroid & 068359-37-5 & & & & & & & & & & & & & & \\
\hline Benefin & $001861-40-1$ & & & $2.0 \mathrm{E}+01$ & $2.0 \mathrm{E}+00$ & & & $6.7 \mathrm{E}+03$ & $6.7 \mathrm{E}+02$ & & & $3.5 \mathrm{E}+03$ & $4.5 \mathrm{E}+02$ & $3.5 \mathrm{E}+02$ & $4.5 \mathrm{E}+01$ \\
\hline Benomyl & $017804-35-2$ & & & $1.8 \mathrm{E}+\infty 0$ & $1.8 \mathrm{E}-01$ & & & $2.0 \mathrm{E}+05$ & $2.0 \mathrm{E}+04$ & & & $1.0 \mathrm{E}+05$ & $1.3 E+04$ & $1.0 E+04$ & $1.3 E+03$ \\
\hline Bentazon & $025057-89-0$ & & & $3.0 \mathrm{E}-02$ & $3.0 \mathrm{E}-03$ & & & $1.5 E+05$ & $1.5 \mathrm{E}+04$ & & & $8.0 \mathrm{E}+04$ & $1.0 \mathrm{E}+04$ & $8.0 E+03$ & $1.0 \mathrm{E}+03$ \\
\hline
\end{tabular}


Table 3.3a. (continued)

\begin{tabular}{|c|c|c|c|c|c|c|c|c|c|c|c|c|c|c|c|}
\hline \multirow{2}{*}{ Chemical } & \multirow{2}{*}{ CASRN } & \multicolumn{4}{|c|}{$\begin{array}{c}\text { Ingestion of Vegetables } \\
(\mathrm{mg} / \mathrm{L})^{\circ}\end{array}$} & \multicolumn{4}{|c|}{$\begin{array}{c}\text { Ingestion of Beef } \\
(\mathrm{mg} / \mathrm{L})^{*}\end{array}$} & \multicolumn{6}{|c|}{$\begin{array}{l}\text { Ingestion of Milk } \\
(\mathrm{mg} / \mathrm{L})^{*}\end{array}$} \\
\hline & & $10^{-4}$ & $10^{6}$ & $\mathrm{HO}=1$ & $\mathrm{HQ}=0.1$ & $10^{-1}$ & $10^{5}$ & HQ=1 & $\mathrm{HQ}=0.1$ & $10^{-1}$ & $10^{4}$ & $\begin{array}{l}\text { HQ=1 } \\
\text { Adult }\end{array}$ & $\begin{array}{l}\mathrm{HO}=\mathbf{1} \\
\text { Chill }\end{array}$ & $\begin{array}{c}\text { HQ }=0.1 \\
\text { Adult }\end{array}$ & $\begin{array}{l}\text { HO=0.1 } \\
\text { Child }\end{array}$ \\
\hline Benzaldehyde & $000100-52-7$ & & & $3.6 \mathrm{E}+00$ & $3.6 \mathrm{E}-01$ & & & $3.9 \mathrm{E}+05$ & $3.9 E+04$ & & & $2.0 E+05$ & $2.6 \mathrm{E}+04$ & $2.0 E+04$ & $2.6 \mathrm{E}+03$ \\
\hline Benzene & $000071-43-2$ & 2.5E-01 & $2.5 \mathrm{E}-03$ & & & $5.0 \mathrm{E}+04$ & $5.0 \mathrm{E}+02$ & & & $1.1 E+04$ & $1.1 E+02$ & & & & \\
\hline Benzene, Ethylmethyl & NA & & & 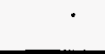 & & & & & & & & & & & \\
\hline Benzene, MethylpropenyI & $\mathrm{NA}$ & & & & & & & & & & . & & & & \\
\hline Benzene, Methylpropyl & $\mathrm{NA}$ & & & & & & & & & & & & & & \\
\hline Benzene, Trimethyl & 025551-13-7 & & & & & & & & & & & & & & \\
\hline Benzo[g,h,i]perylene & $000191-24-2$ & & & & & & & & & & & & & & \\
\hline Benzo[k] fluoranthene $e^{(2)}$ & $000207-08-9$ & $2.2 \mathrm{E}-01$ & $2.2 \mathrm{E}-03$ & & & $3.9 \mathrm{E}-01$ & $3.9 E-03$ & & & $8.7 \mathrm{E}-02$ & $8.7 \mathrm{E}-04$ & & & & \\
\hline Benzoic Acid & $000065-85-0$ & & & $1.1 \mathrm{E}+02$ & $1.1 \mathrm{E}+01$ & & & $1.0 E+06$ & $1.0 \mathrm{E}+06$ & & & $1.0 E+06$ & $1.0 E+06$ & $1.0 \mathrm{E}+06$ & 2.6E+05 \\
\hline Benzotrichloride & $000098-07-7$ & $8.8 \mathrm{E}-04$ & $8.8 \mathrm{E}-06$ & & & $1.8 \mathrm{E}+01$ & $1.8 \mathrm{E}-01$ & & & $3.9 \mathrm{E}+00$ & $3.9 \mathrm{E}-02$ & & & & \\
\hline Benzyl Alcohol & $000100-51-6$ & & & $3.6 \mathrm{E}+00$ & $3.6 \mathrm{E}-01$ & & & $1.0 \mathrm{E}+06$ & $1.0 E+06$ & & & $1.0 E+06$ & $1.0 E+06$ & $9.6 \mathrm{E}+05$ & $1.2 \mathrm{E}+05$ \\
\hline Benzyl Chloride & $000100-44-7$ & $4.9 \mathrm{E}-02$ & $4.9 \mathrm{E}-04$ & & & $5.4 \mathrm{E}+03$ & $5.4 \mathrm{E}+01$ & & & $1.2 \mathrm{E}+03$ & $1.2 \mathrm{E}+01$ & & & & \\
\hline Beryllium & $007440-41-7$ & $3.8 \mathrm{E}-03$ & $3.8 \mathrm{E}-05$ & $3.5 \mathrm{E}-01$ & 3.SE-02 & $1.1 \mathrm{E}+00$ & $1.1 E-02$ & $9.7 E+01$ & $9.7 E+00$ & $8.2 \mathrm{E}+01$ & $8.2 \mathrm{E}-01$ & $1.8 \mathrm{E}+04$ & $2.3 \mathrm{E}+03$ & $1.8 E+03$ & $2.3 \mathrm{E}+02$ \\
\hline Bidrin & $000141-66-2$ & & & $1.3 \mathrm{E}-04$ & $1.3 \mathrm{E}-05$ & & & $3.7 E+05$ & $3.7 E+04$ & & & $1.9 \mathrm{E}+05$ & $2.5 E+04$ & $1.9 \mathrm{E}+04$ & $2.5 \mathrm{E}+03$ \\
\hline
\end{tabular}


Table 3.3a. (continued)

\begin{tabular}{|c|c|c|c|c|c|c|c|c|c|c|c|c|c|c|c|}
\hline \multirow{2}{*}{ Chemical } & \multirow{2}{*}{ CASRN } & \multicolumn{4}{|c|}{$\begin{array}{c}\text { Ingestion of Vegetables } \\
(\mathrm{mg} / \mathrm{L})^{*}\end{array}$} & \multicolumn{4}{|c|}{$\begin{array}{c}\text { Ingestion of Beef } \\
(\mathrm{mg} / \mathrm{L})^{*}\end{array}$} & \multicolumn{6}{|c|}{$\begin{array}{l}\text { Ingestion of Milk } \\
(\mathbf{m g} / \mathbf{L})^{*}\end{array}$} \\
\hline & & $10^{4}$ & $10^{4}$ & $H Q=1$ & $\mathrm{HQ}=0.1$ & $10^{-4}$ & $10^{4}$ & $H Q=1$ & $\mathrm{HQ}=0.1$ & $10^{-4}$ & $10^{6}$ & $\begin{array}{l}\text { HQ=1 } \\
\text { Adult }\end{array}$ & $\begin{array}{l}\text { HQ=1 } \\
\text { Child }\end{array}$ & $\begin{array}{c}\text { HQ }=0.1 \\
\text { Adult }\end{array}$ & $\begin{array}{c}\text { HQ }=0.1 \\
\text { Child }\end{array}$ \\
\hline Biphenthrin & $082657-04-3$ & & & & & & & & & & & & & & $\ldots$ \\
\hline Biphenyl, 1,1'- & $000092-52-4$ & & & $3.2 \mathrm{E}+00$ & $3.2 \mathrm{E}-01$ & & & $3.1 \mathrm{E}+03$ & $3.1 \mathrm{E}+02$ & & & $1.6 \mathrm{E}+03$ & $2.1 E+02$ & $1.6 \mathrm{E}+02$ & $2.1 E+01$ \\
\hline $\begin{array}{l}\text { Bis(2-chloro-1-methylethyl)ether } \\
\text { (Technical) }\end{array}$ & $000108-60-1$ & $1.4 \mathrm{E}-01$ & $1.4 \mathrm{E}-03$ & & & $6.8 \mathrm{E}+03$ & $6.8 \mathrm{E}+01$ & & & $1.5 \mathrm{E}+03$ & $1.5 \mathrm{E}+01$ & & & & \\
\hline Bis(2-chloroethoxy)methane & $000111-91-1$ & & & & & & & & & & & & & & \\
\hline Bis(2-chloroethyl)ether & $000111-44-4$ & $3.2 \mathrm{E}-03$ & $3.2 \mathrm{E}-05$ & & & $8.3 \mathrm{E}+03$ & $8.3 \mathrm{E}+01$ & & & $1.8 \mathrm{E}+03$ & $1.8 \mathrm{E}+01$ & & & & \\
\hline Bis(2-chloroisopropyl)ether & $039638-32-9$ & & & $1.2 \mathrm{E}+00$ & $1.2 \mathrm{E}-01$ & & & $2.5 \mathrm{E}+05$ & $2.5 \mathrm{E}+04$ & & & $1.3 \mathrm{E}+05$ & $1.6 \mathrm{E}+04$ & 1.3E+04 & $1.6 \mathrm{E}+03$ \\
\hline Bis(2-ethylhexyl)phthalate $e^{(6)}$ & $000117-81-7$ & $1.1 \mathrm{E}+00$ & $1.1 \mathrm{E}-02$ & $1.4 \mathrm{E}+00$ & $1.4 \mathrm{E}-01$ & $1.6 \mathrm{E}+02$ & $1.6 \mathrm{E}+00$ & $2.0 \mathrm{E}+02$ & $2.0 \mathrm{E}+01$ & $3.6 \mathrm{E}+01$ & $3.6 \mathrm{E}-01$ & $1.0 \mathrm{E}+02$ & $1.3 \mathrm{E}+01$ & $1.0 \mathrm{E}+01$ & $1.3 \mathrm{E}+00$ \\
\hline Bis(chloromethyl)ether & $000542-88-1$ & $5.4 E-06$ & $5.4 \mathrm{E}-08$ & & & $3.4 E+02$ & $3.4 \mathrm{E}+00$ & & & $7.6 \mathrm{E}+01$ & $7.6 \mathrm{E}-01$ & & & & \\
\hline Bisphenol A & $000080-05-7$ & & & $3.0 \mathrm{E}+00$ & $3.0 \mathrm{E}-01$ & & & $9.8 \mathrm{E}+03$ & $9.8 \mathrm{E}+02$ & & & $5.1 \mathrm{E}+03$ & $6.5 \mathrm{E}+02$ & $5.1 \mathrm{E}+02$ & $6.5 \mathrm{E}+01$ \\
\hline Boron And Borates Only & $007440-42-8$ & & & $1.7 \mathrm{E}+00$ & $1.7 E-01$ & & & $2.2 \mathrm{E}+03$ & $2.2 \mathrm{E}+02$ & & & $1.9 \mathrm{E}+02$ & $2.5 \mathrm{E}+01$ & $1.9 \mathrm{E}+01$ & $2.5 \mathrm{E}+00$ \\
\hline Boron Trifluoride & $007637-07-2$ & & & & & & & & & & & & & & \\
\hline Bromochloromethane & $000074-97-5$ & & & & & & & & & & & & & & \\
\hline Bromodichloromethane & $000075-27-4$ & $1.2 \mathrm{E}-01$ & $1.2 \mathrm{E}-03$ & $6.2 \mathrm{E}-01$ & $6.2 E-02$ & $2.3 E+04$ & $2.3 \mathrm{E}+02$ & $1.2 \mathrm{E}+05$ & $1.2 \mathrm{E}+04$ & $5.1 E+03$ & $5.1 E+01$ & $6.4 \mathrm{E}+04$ & $8.2 E+03$ & $6.4 \mathrm{E}+03$ & $8.2 \mathrm{E}+02$ \\
\hline Bromodiphenyl Ether, p- & $000101-55-3$ & & & & & & & & & & & & & & \\
\hline Bromoform & $000075-25-2$ & $1.1 \mathrm{E}+00$ & $1.1 \mathrm{E}-02$ & $7.6 \mathrm{E}-01$ & $7.6 \mathrm{E}-02$ & $9.2 \mathrm{E}+04$ & $9.2 \mathrm{E}+02$ & $6.2 \mathrm{E}+04$ & $6.2 \mathrm{E}+03$ & $2.0 \mathrm{E}+04$ & $2.0 \mathrm{E}+02$ & $3.2 \mathrm{E}+04$ & $4.1 E+03$ & $3.2 \mathrm{E}+03$ & $4.1 \mathrm{E}+02$ \\
\hline Bromomethane $e^{(b)}$ & $000074-83-9$ & & & $1.9 \mathrm{E}-02$ & $1.9 \mathrm{E}-03$ & & & $6.9 E+04$ & $6.9 \mathrm{E}+03$ & & & $3.6 \mathrm{E}+04$ & $4.6 \mathrm{E}+03$ & $3.6 \mathrm{E}+03$ & $4.6 \mathrm{E}+02$ \\
\hline Bromophos & $002104-96-3$ & & & & & & & & & & & & & & \\
\hline Bromotrichloromethane & $000075-62-7$ & & & & & & & & & & . & & & & \\
\hline Bromoxynil & $001689-84-5$ & & & $1.2 \mathrm{E}-01$ & $1.2 \mathrm{E}-02$ & & & $1.0 \mathrm{E}+06$ & $5.0 \mathrm{E}+05$ & & & $1.0 \mathrm{E}+06$ & $3.4 \mathrm{E}+05$ & $2.6 \mathrm{E}+05$ & $3.4 E+04$ \\
\hline Bromoxynil Octanoate & 001689-99-2 & & & & & & & & & & & & & & \\
\hline
\end{tabular}


Table 3.3a. (continued)

\begin{tabular}{|c|c|c|c|c|c|c|c|c|c|c|c|c|c|c|c|}
\hline \multirow{2}{*}{ Chemical } & \multirow{2}{*}{ CASRN } & \multicolumn{4}{|c|}{$\begin{array}{l}\text { Ingestion of Vegetables } \\
(\mathrm{mg} / \mathrm{L})^{n}\end{array}$} & \multicolumn{4}{|c|}{$\begin{array}{c}\text { Ingestion of Beef } \\
(\mathrm{mg} / \mathrm{L})^{*}\end{array}$} & \multicolumn{6}{|c|}{$\begin{array}{c}\text { Ingestion of Milk } \\
(\mathrm{mg} / \mathrm{L})^{n}\end{array}$} \\
\hline & & $10^{-4}$ & $10^{4}$ & $\mathrm{HQ}=1$ & $\mathrm{HQ}=0.1$ & $10^{-4}$ & $10^{4}$ & $\mathrm{HQ}=1$ & $\mathrm{HQ}=0.1$ & $10^{-4}$ & $10^{6}$ & $\begin{array}{l}H Q=1 \\
\text { Adult }\end{array}$ & $\begin{array}{l}H Q=1 \\
\text { Child }\end{array}$ & $\begin{array}{c}H Q=0.1 \\
\text { Adult }\end{array}$ & $\begin{array}{c}\text { HQ }=0.1 \\
\text { Child }\end{array}$ \\
\hline Butadiene, 1,3- & $000106-99-0$ & & & & & & & & & & & & & & \\
\hline Butanol, N- & $000071-36-3$ & & & 7.1E-01 & 7.1E-02 & & & $1.0 \mathrm{E}+06$ & $1.0 \mathrm{E}+06$ & & & $1.0 \mathrm{E}+06$ & $1.0 E+06$ & $9.0 \mathrm{E}+05$ & $1.2 \mathrm{E}+05$ \\
\hline Butanone-2, 4-chloro-4,4-difluoro & NA & & & & & & & & & & & & & & \\
\hline Butyl Benzyl Phthlate & $000085-68-7$ & & & $1.4 \mathrm{E}+01$ & $1.4 \mathrm{E}+00$ & & & $2.0 \mathrm{E}+03$ & $2.0 \mathrm{E}+02$ & & & $1.0 \mathrm{E}+03$ & $1.3 E+02$ & $1.0 \mathrm{E}+02$ & $1.3 \mathrm{E}+01$ \\
\hline Butylate & $002008-41-5$ & & & & & & & & & & & & & & \\
\hline Butylchloride, t- & $000507-20-0$ & & & & & & & & & & & & & & \\
\hline Butylphthalyl Butylglycolate & $000085-70-1$ & & & & & & - & & & & & & & & \\
\hline Cacodylic Acid & $000075-60-5$ & & & 9.5E-03 & $9.5 \mathrm{E}-04$ & & & $1.0 \mathrm{E}+06$ & $2.3 \mathrm{E}+05$ & & & $1.0 \mathrm{E}+06$ & $1.6 \mathrm{E}+05$ & $1.2 \mathrm{E}+05$ & $1.6 \mathrm{E}+04$ \\
\hline Cadmium (Diet) & $007440-43.9$ & & & $5.1 \mathrm{E}-02$ & 5.1E-03 & & & $4.9 \mathrm{E}+01$ & $4.9 \mathrm{E}+00$ & & & $3.2 \mathrm{E}+00$ & 4.1E-01 & $3.2 \mathrm{E}-01$ & 4.1E-02 \\
\hline Calcium Cyanide & $000592-01-8$ & & & & & & & & & & & & & & \\
\hline Caprolactam & $000105-60-2$ & & & $1.2 \mathrm{E}+00$ & $1.2 \mathrm{E}-01$ & & & $1.0 \mathrm{E}+06$ & $1.0 \mathrm{E}+06$ & & & $1.0 \mathrm{E}+06$ & $1.0 \mathrm{E}+06$ & $1.0 \mathrm{E}+06$ & $1.0 \mathrm{E}+06$ \\
\hline Captafol & $002425-06-1$ & $1.1 \mathrm{E}+00$ & $1.1 \mathrm{E}-02$ & 8.1E-02 & 8.1E-03 & $6.5 \mathrm{E}+04$ & $6.5 \mathrm{E}+02$ & $4.8 \mathrm{E}+03$ & $4.8 \mathrm{E}+02$ & $1.4 \mathrm{E}+04$ & $1.4 \mathrm{E}+02$ & $2.5 \mathrm{E}+03$ & $3.2 \mathrm{E}+02$ & $2.5 \mathrm{E}+02$ & $3.2 \mathrm{E}+01$ \\
\hline Captan & $000133-06-2$ & $2.4 E+00$ & 2.4E-02 & $4.6 \mathrm{E}+00$ & $4.6 \mathrm{E}-01$ & $2.6 \mathrm{E}+05$ & $2.6 \mathrm{E}+03$ & $5.1 \mathrm{E}+05$ & $5.1 \mathrm{E}+04$ & $5.7 \mathrm{E}+04$ & $5.7 \mathrm{E}+02$ & $2.6 \mathrm{E}+05$ & $3.4 \mathrm{E}+04$ & $2.6 E+04$ & $3.4 \mathrm{E}+03$ \\
\hline Carbaryl & $000063-25-2$ & & & $3.6 \mathrm{E}+00$ & $3.6 \mathrm{E}-01$ & & & $3.9 E+05$ & $3.9 E+04$ & & & $2.0 \mathrm{E}+05$ & $2.6 \mathrm{E}+04$ & $2.0 \mathrm{E}+04$ & $2.6 \mathrm{E}+03$ \\
\hline Carbazole & $000086-74-8$ & 7.3E-01 & 7.3E-03 & & & $1.4 \mathrm{E}+03$ & $1.4 \mathrm{E}+01$ & & & $3.2 \mathrm{E}+02$ & $3.2 \mathrm{E}+00$ & & & & \\
\hline Carbofuran & $001563-66-2$ & & & $1.0 \mathrm{E}-01$ & $1.0 \mathrm{E}-02$ & & & $9.8 \mathrm{E}+04$ & $9.8 \mathrm{E}+03$ & & & $5.1 E+04$ & $6.5 \mathrm{E}+03$ & $5.1 E+03$ & $6.5 E+02$ \\
\hline Carbon Disulfide & $000075-15-0$ & & & $3.3 \mathrm{E}+00$ & $3,3 \mathrm{E}-01$ & & & $4.9 E+05$ & $4.9 E+04$ & & & 2.5E+05 & $3.3 \mathrm{E}+04$ & $2.5 \mathrm{E}+04$ & $3.3 \mathrm{E}+03$ \\
\hline Carbon Tetrachloride ${ }^{(6)}$ & $000056-23-5$ & 8.4E-02 & $8.4 E-04$ & $3.3 \mathrm{E}-02$ & 3.3E-03 & $2.2 \mathrm{E}+03$ & $2.2 \mathrm{E}+01$ & $8.6 \mathrm{E}+02$ & $8.6 \mathrm{E}+01$ & $4.9 E+02$ & $4.9 E+00$ & $4.5 \mathrm{E}+02$ & $5.8 \mathrm{E}+01$ & $4.5 \mathrm{E}+01$ & $5.8 \mathrm{E}+00$ \\
\hline Carbosulfan & $055285-14-8$ & & & & & & & & & & & & & & \\
\hline Carboxin & $005234-68-4$ & & & $3.1 \mathrm{E}+00$ & 3.1E-01 & & & $6.2 \mathrm{E}+05$ & $6.2 \mathrm{E}+04$ & & & $3.2 E+05$ & 4.1E+04 & 3.2E+04 & $4.1 E+03$ \\
\hline Chloral & $000075-87-6$ & & & & & & & & & & & & & & \\
\hline
\end{tabular}

Nonradionuclides/water/agricultural

Page 6 of 33 
Table 3.3a. (continued)

\begin{tabular}{|c|c|c|c|c|c|c|c|c|c|c|c|c|c|c|c|}
\hline \multirow[t]{2}{*}{ Chemical } & \multirow[t]{2}{*}{ CASRN } & \multicolumn{4}{|c|}{$\begin{array}{l}\text { Ingestion of Vegetables } \\
(\mathrm{mg} / \mathrm{L})^{*}\end{array}$} & \multicolumn{4}{|c|}{$\begin{array}{c}\text { Ingestion of Beef } \\
(\mathrm{mg} / \mathrm{L})^{*}\end{array}$} & \multicolumn{6}{|c|}{$\begin{array}{l}\text { Ingestion of Milk } \\
\text { (mg/L) }\end{array}$} \\
\hline & & $10^{-4}$ & $10^{4}$ & $H Q=1$ & HQ $=0.1$ & $10^{-4}$ & $10^{4}$ & $\mathrm{HQ}=1$ & $H Q=0.1$ & $10^{-4}$ & $10^{6}$ & $\begin{array}{l}\text { HQ=1 } \\
\text { Adult }\end{array}$ & $\begin{array}{l}\text { HQ }=1 \\
\text { Child }\end{array}$ & $\begin{array}{c}\text { HQ }=0.1 \\
\text { Adult }\end{array}$ & $\begin{array}{c}\text { HQ }=0.1 \\
\text { Child }\end{array}$ \\
\hline Chloramben & $000133.90-4$ & & & & & & & & & & & & & & \\
\hline Chloranil & $000118-75-2$ & & & & & & & & & & & & & & \\
\hline Chlordane $e^{(6)}$ & $000057-74-9$ & $1.2 \mathrm{E}-02$ & $1.2 \mathrm{E}-04$ & $4.2 \mathrm{E}-03$ & $4.2 \mathrm{E}-04$ & $4.4 \mathrm{E}-01$ & 4.4E-03 & $1.5 \mathrm{E}-01$ & $1.5 \mathrm{E}-02$ & 9.7E-02 & 9.7E-04 & 7.7E-02 & $9.8 \mathrm{E}-03$ & 7.7E-03 & $9.8 \mathrm{E}-04$ \\
\hline Chloride & $016887-00-6$ & & & & & & & & & & & & & & \\
\hline Chlorimuron, Ethyl- & $090982-32-4$ & & & & & & & & & & & & & & \\
\hline Chlorine & $007782-50-5$ & & & $1.4 \mathrm{E}-01$ & $1.4 \mathrm{E}-02$ & & & $9.7 \mathrm{E}+01$ & $9.7 \mathrm{E}+00$ & & & $1.9 \mathrm{E}+0 \mathrm{I}$ & $2.4 \mathrm{E}+00$ & $1.9 \mathrm{E}+00$ & $2.4 \mathrm{E}-01$ \\
\hline Chlorine Dioxide & $010049-04-4$ & & & & & & & & & & & & & & \\
\hline Chlorite (Sodium Salt) & $007758-19-2$ & & & & & & & & & & & & & & \\
\hline Chloro-1,1-difluoroethane, 1- & $000075-68-3$ & & & & & & & & & & & & & & \\
\hline Chloro-1,3-butadiene, 2- & $000126-99-8$ & & & $4.8 \mathrm{E}-01$ & $4.8 \mathrm{E}-02$ & & & $2.5 \mathrm{E}+05$ & $2.5 \mathrm{E}+04$ & & & $1.3 E+05$ & $1.6 \mathrm{E}+04$ & $1.3 \mathrm{E}+04$ & $1.6 \mathrm{E}+03$ \\
\hline Chloro-2-methylaniline $\mathrm{HCl}$, 4- & $003165-93-3$ & & & & & & & & & & & & & & \\
\hline Chloro-2-methylaniline, 4- & $000095-69-2$ & & & & & & & & & & & & & & \\
\hline Chloroacetic Acid & $000079-11-8$ & & & 2.7E-02 & $2.7 \mathrm{E}-03$ & & & $9.8 \mathrm{E}+04$ & $9.8 \mathrm{E}+03$ & & & $5.1 \mathrm{E}+04$ & $6.5 \mathrm{E}+03$ & $5.1 \mathrm{E}+03$ & $6.5 \mathrm{E}+02$ \\
\hline Chloroacetophenone, 2- & $000532-27-4$ & & & & & & & & & & & & & & \\
\hline Chloroaniline, p- & $000106-47-8$ & & & $1.9 \mathrm{E}-01$ & $1.9 \mathrm{E}-02$ & & & $4.9 \mathrm{E}+03$ & $4.9 \mathrm{E}+02$ & & & $2.6 \mathrm{E}+03$ & $3.3 \mathrm{E}+02$ & $2.6 \mathrm{E}+02$ & $3.3 \mathrm{E}+01$ \\
\hline Chlorobenzene $e^{(1)}$ & $000108-90-7$ & & & $9.4 E-01$ & $9.4 \mathrm{E}-02$ & & & $2.5 \mathrm{E}+04$ & $2.5 \mathrm{E}+03$ & & & $1.3 E+04$ & $1.6 \mathrm{E}+03$ & $1.3 E+03$ & $1.6 \mathrm{E}+02$ \\
\hline Chlorobenzilate & $000510-15-6$ & 5.8E-02 & $5.8 \mathrm{E}-04$ & $1.3 \mathrm{E}+00$ & $1.3 \mathrm{E}-01$ & $2.1 \mathrm{E}+01$ & $2.1 \mathrm{E}-01$ & $4.9 \mathrm{E}+02$ & $4.9 E+01$ & $4.7 \mathrm{E}+00$ & 4.7E-02 & $2.6 \mathrm{E}+02$ & $3.3 \mathrm{E}+01$ & $2.6 \mathrm{E}+01$ & $3.3 \mathrm{E}+00$ \\
\hline Chlorobenzoic Acid, p- & $000074-11-3$ & & & $8.9 E+\infty$ & $8.9 \mathrm{E}-01$ & & & $3.1 \mathrm{E}+05$ & $3.1 \mathrm{E}+04$ & & & $1.6 \mathrm{E}+05$ & 2.1E+04 & $1.6 \mathrm{E}+04$ & $2.1 \mathrm{E}+03$ \\
\hline Chlorobenzotrifluoride, 4- & $000098-56-6$ & & & $1.2 \mathrm{E}+00$ & $1.2 \mathrm{E}-01$ & & & $2.8 \mathrm{E}+03$ & $2.8 \mathrm{E}+02$ & & & $1.5 E+03$ & $1.9 \mathrm{E}+02$ & $1.5 \mathrm{E}+02$ & $1.9 E+01$ \\
\hline Chlorobutare, 1- & $000109-69-3$ & & & $1.7 \mathrm{E}+01$ & $1.7 \mathrm{E}+00$ & & & $7.8 \mathrm{E}+05$ & $7.8 \mathrm{E}+04$ & & & 4.1E+05 & $5.2 \mathrm{E}+04$ & $4.1 \mathrm{E}+04$ & $5.2 \mathrm{E}+03$ \\
\hline Chlorobutane, 2 - & $000078-86-4$ & & & & & & & & & & & & & & \\
\hline
\end{tabular}

Nonradionuclides/water/agricultural

Page 7 of 33 
Table 3.3a. (continued)

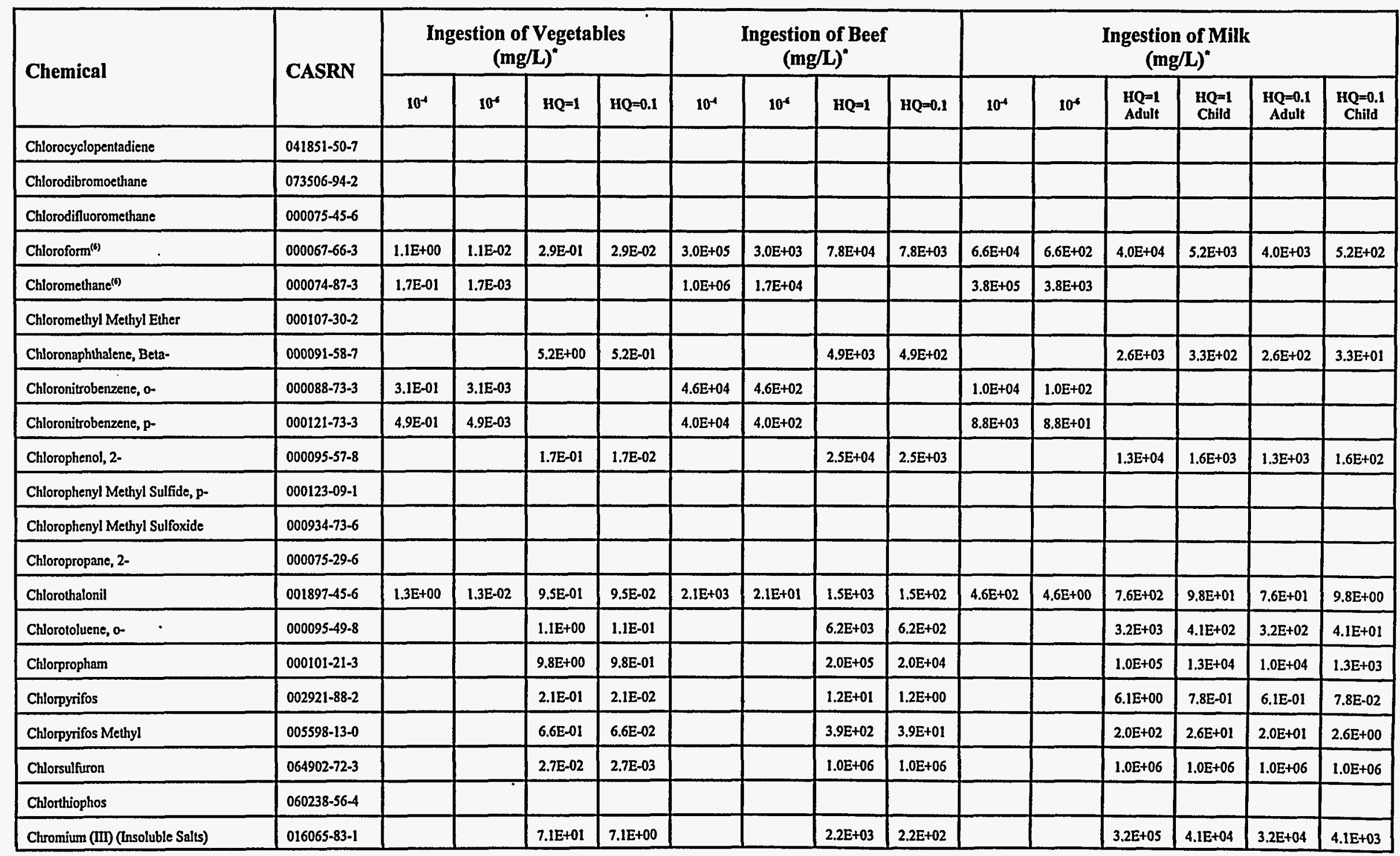

Nonradionuclides/water/agricultural

Page 8 of 33 


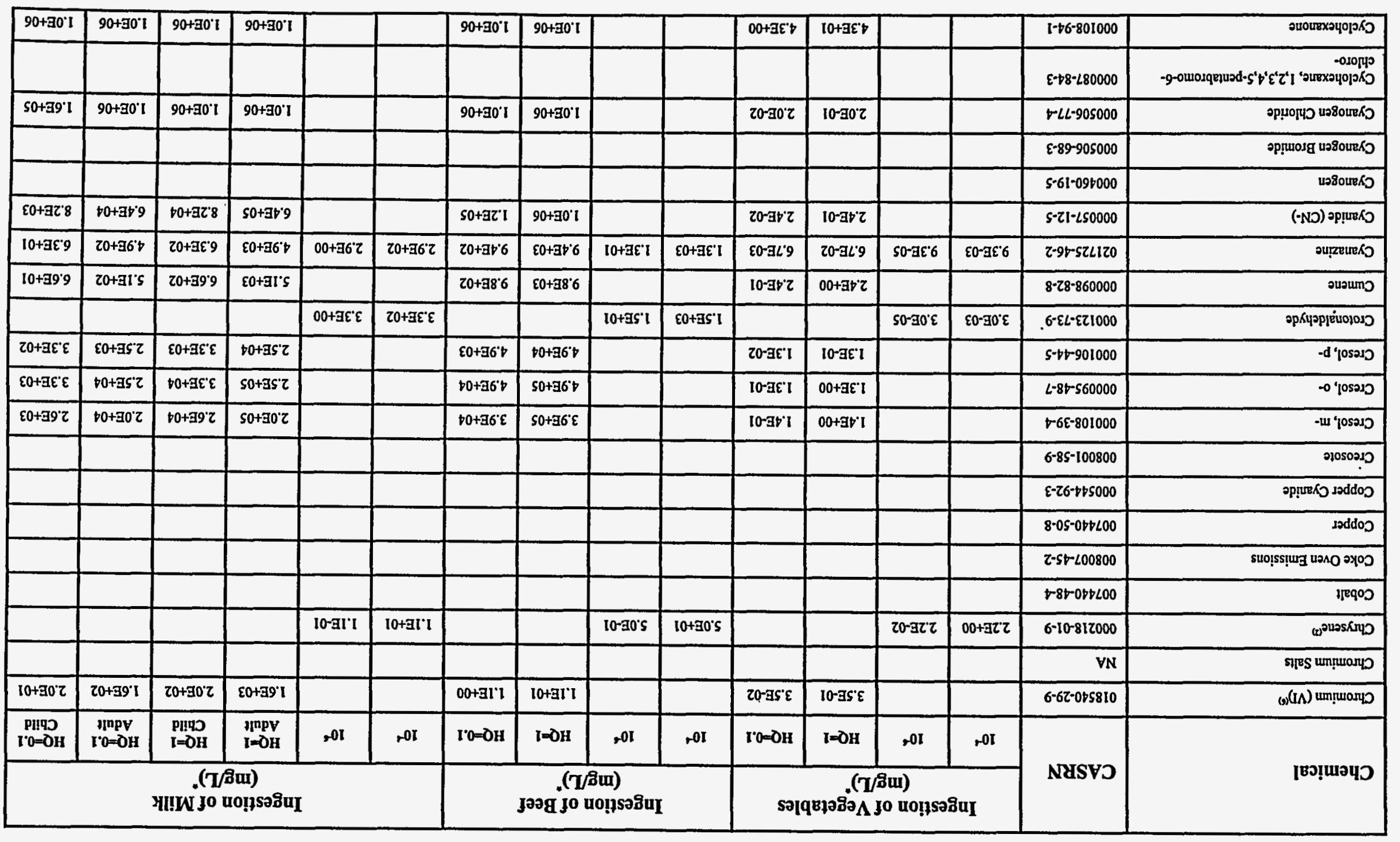

(pənu!วน0ว) 'вє' 
Table 3.3a. (continued)

\begin{tabular}{|c|c|c|c|c|c|c|c|c|c|c|c|c|c|c|c|}
\hline \multirow{2}{*}{ Chemical } & \multirow{2}{*}{ CASRN } & \multicolumn{4}{|c|}{$\begin{array}{l}\text { Ingestion of Vegetables } \\
(\mathrm{mg} / \mathrm{L})^{n}\end{array}$} & \multicolumn{4}{|c|}{$\begin{array}{c}\text { Ingestion of Beef } \\
(\mathrm{mg} / \mathrm{L})^{n}\end{array}$} & \multicolumn{6}{|c|}{$\begin{array}{l}\text { Ingestion of Milk } \\
(\mathrm{mg} / \mathrm{L})^{*}\end{array}$} \\
\hline & & $10^{-4}$ & $10^{4}$ & $H Q=1$ & $\mathrm{HQ}=0.1$ & $10^{-4}$ & $10^{6}$ & $\mathrm{HQ}=1$ & $\mathrm{HQ}=0.1$ & $10^{-4}$ & $10^{5}$ & $\begin{array}{l}\text { HQ=1 } \\
\text { Adult }\end{array}$ & $\begin{array}{l}\text { HQ=1 } \\
\text { Child }\end{array}$ & $\begin{array}{c}\text { HQ }=0.1 \\
\text { Adult }\end{array}$ & $\begin{array}{c}\text { HQ }=0.1 \\
\text { Child }\end{array}$ \\
\hline Cyclohexylamine & $000108-91-8$ & & & $3.6 \mathrm{E}+00$ & $3.6 \mathrm{E}-01$ & & & $1.0 \mathrm{E}+06$ & 4.9E+05 & & & $1.0 \mathrm{E}+06$ & $3.3 E+05$ & $2.6 \mathrm{E}+05$ & $3.3 \mathrm{E}+04$ \\
\hline Cyclopentadiene & $000542-92-7$ & & & & & & & & & & & & & & \\
\hline Cyhalothrin/karate & $068085-85-8$ & & & $3.5 \mathrm{E}-01$ & $3.5 \mathrm{E}-02$ & & & $4.9 \mathrm{E}-01$ & $4.9 \mathrm{E}-02$ & & & $2.5 \mathrm{E}-01$ & 3.3E-02 & $2.5 \mathrm{E}-02$ & 3.3E-03 \\
\hline Cypermethrin & $052315-07-8$ & & & & & & & & & & & & & & \\
\hline Cyromazine & $066215-27-8$ & & & & & & & & & & & & & & \\
\hline DDD & $000072-54-8$ & $6.8 \mathrm{E}-02$ & $6.8 \mathrm{E}-04$ & & & $1.2 \mathrm{E}+00$ & $1.2 \mathrm{E}-02$ & & & $2.6 \mathrm{E}-01$ & $2.6 \mathrm{E}-03$ & & & & \\
\hline DDE & $000072-55-9$ & 4.8E- 02 & $4.8 \mathrm{E}-04$ & & & $1.1 \mathrm{E}+00$ & $1.1 \mathrm{E}-02$ & & & $2.3 \mathrm{E}-01$ & $2.3 \mathrm{E}-03$ & & & & \\
\hline DDT & $000050-29-3$ & $4.8 \mathrm{E}-02$ & $4.8 \mathrm{E}-04$ & $3.5 \mathrm{E}-02$ & $3.5 \mathrm{E}-03$ & $2.3 \mathrm{E}-01$ & 2.3E-03 & $1.7 \mathrm{E}-01$ & $1.7 \mathrm{E}-02$ & $5.1 E-02$ & $5.1 \mathrm{E}-04$ & $8.8 \mathrm{E}-02$ & $1.1 \mathrm{E}-02$ & $8.8 \mathrm{E}-03$ & 1.1E-03 \\
\hline Dacthal & $001861-32-1$ & & & & & & & & & & & & & & \\
\hline Dalapon & $000075-99-0$ & & & 2.5E-01 & $2.5 \mathrm{E}-02$ & & & $1.0 \mathrm{E}+06$ & $3.9 \mathrm{E}+05$ & & & $1.0 \mathrm{E}+06$ & $2.6 \mathrm{E}+05$ & $2.0 \mathrm{E}+05$ & $2.6 \mathrm{E}+04$ \\
\hline Decabromodiphenyl Ether & $001163-19-5$ & & & $6.9 \mathrm{E}-01$ & $6.9 \mathrm{E}-02$ & & & $4.5 \mathrm{E}+01$ & $4.5 \mathrm{E}+00$ & & & $2.3 E+01$ & $3.0 \mathrm{E}+00$ & $2.3 E+00$ & $3.0 \mathrm{E}-01$ \\
\hline Demeton & $008065-48-3$ & & & & & & & & & & & & & & \\
\hline Di(2-ethylhexyl)adipate & $000103-23-1$ & $1.4 \mathrm{E}+01$ & $1.4 E-01$ & $4.2 \mathrm{E}+01$ & $4.2 \mathrm{E}+00$ & $2.4 \mathrm{E}+00$ & $2.4 \mathrm{E}-02$ & $7.4 \mathrm{E}+00$ & 7.4E-01 & 5.3E-01 & $5.3 \mathrm{E}-03$ & $3.8 \mathrm{E}+00$ & $4.9 \mathrm{E}-01$ & $3.8 \mathrm{E}-01$ & $4.9 \mathrm{E}-02$ \\
\hline Diallate & $002303-16-4$ & $3.0 \mathrm{E}-02$ & $3.0 \mathrm{E}-04$ & & & $5.5 \mathrm{E}+0 \mathrm{~S}$ & $5.5 \mathrm{E}+03$ & & & $1.2 \mathrm{E}+05$ & $1.2 \mathrm{E}+03$ & & & & \\
\hline Diazinon & $000333-41-5$ & & & $5.6 \mathrm{E}-02$ & $5.6 \mathrm{E}-03$ & & & $1.1 \mathrm{E}+02$ & $1.1 \mathrm{E}+01$ & & & $5.8 \mathrm{E}+01$ & $7.4 \mathrm{E}+00$ & $5.8 \mathrm{E}+00$ & 7.4E-01 \\
\hline Dibenz $[a, h]$ anthracene $e^{(x)}$ & $000053-70-3$ & $2.2 \mathrm{E}-03$ & $2.2 \mathrm{E}-05$ & & & $3.9 \mathrm{E}-03$ & $3.9 \mathrm{E}-05$ & & & 8.7E-04 & 8.7E-06 & & & & \\
\hline Dibromo-3-chloropropane, 1,2- & $000096-12-8$ & 7.1E-03 & 7.1E-05 & & & $3.3 \mathrm{E}+02$ & $3.3 \mathrm{E}+00$ & & & $7.2 \mathrm{E}+01$ & 7.2E-01 & & & & \\
\hline Dibromobenzene, 1,4- & $000106 \cdot 37-6$ & & & $6.2 \mathrm{E}-01$ & $6.2 \mathrm{E}-02$ & & & $1.2 \mathrm{E}+03$ & $1.2 \mathrm{E}+02$ & & & $6.4 \mathrm{E}+02$ & $8.2 E+01$ & $6.4 E+01$ & $8.2 E+00$ \\
\hline Dibromochloromethane & $000124-48-1$ & $9.2 \mathrm{E}-02$ & $9.2 \mathrm{E}-04$ & $6.6 \mathrm{E}-01$ & $6.6 \mathrm{E}-02$ & $1.4 \mathrm{E}+04$ & $1.4 \mathrm{E}+02$ & $9.8 E+04$ & $9.8 \mathrm{E}+03$ & $3.0 \mathrm{E}+03$ & $3.0 \mathrm{E}+01$ & $5.1 E+04$ & $6.5 \mathrm{E}+03$ & $5.1 \mathrm{E}+03$ & $6.5 \mathrm{E}+02$ \\
\hline Dibromodichloromethane & $000594-18-3$ & & & & & & & & & & & & & & \\
\hline Dibromodiphenyl Ether, p.p'- & $002050-47-7$ & & & & & & & & & & & & & & \\
\hline
\end{tabular}

Nonradionuclides/water/agricultural

Page 10 of 33 
Table 3.3a. (continued)

\begin{tabular}{|c|c|c|c|c|c|c|c|c|c|c|c|c|c|c|c|}
\hline \multirow{2}{*}{ Chemical } & \multirow{2}{*}{ CASRN } & \multicolumn{4}{|c|}{$\begin{array}{l}\text { Ingestion of Vegetables } \\
\text { (mg/L) }\end{array}$} & \multicolumn{4}{|c|}{$\begin{array}{c}\text { Ingestion of Beef } \\
(\mathrm{mg} / \mathrm{L})^{*}\end{array}$} & \multicolumn{6}{|c|}{$\begin{array}{c}\text { Ingestion of Milk } \\
\text { (mg/L) }\end{array}$} \\
\hline & & $10^{-4}$ & $10^{5}$ & $H Q=1$ & $H Q=0.1$ & $10^{-4}$ & $10^{5}$ & $H Q=1$ & $\mathrm{HQ}=0.1$ & $10^{-4}$ & $10^{6}$ & $\begin{array}{l}\text { HQ=1 } \\
\text { Adult }\end{array}$ & $\begin{array}{l}\text { HQ=1 } \\
\text { Child }\end{array}$ & $\begin{array}{c}H Q=0.1 \\
\text { Adult }\end{array}$ & $\begin{array}{c}\mathrm{HQ}=0.1 \\
\text { Child }\end{array}$ \\
\hline Dibromoethane, 1,2- & $000106-93-4$ & $7.8 \mathrm{E}-05$ & $7.8 \mathrm{E}-07$ & & & $2.1 \mathrm{E}+01$ & 2.1E-01 & & & 4.7E+co & 4.7E-02 & & & & \\
\hline Dibromomethane (Methylene Bromide) & $000074-95-3$ & & & $1.3 \mathrm{E}-01$ & $1.3 \mathrm{E}-02$ & & & $4.9 \mathrm{E}+05$ & $4.9 \mathrm{E}+04$ & & & $2.5 \mathrm{E}+05$ & 3.3E+04 & $2.5 \mathrm{E}+04$ & 3.3E+03 \\
\hline Dibutyl Phthalate & $000084-74-2$ & & & $6.8 \mathrm{E}+00$ & $6.8 \mathrm{E}-01$ & & & $9.8 \mathrm{E}+02$ & $9.8 \mathrm{E}+01$ & & & $5.1 \mathrm{E}+02$ & $6.5 \mathrm{E}+01$ & $5.1 \mathrm{E}+01$ & $6.5 \mathrm{E}+00$ \\
\hline Dicamba & $001918-00.9$ & & & $1.5 E+00$ & $1.5 \mathrm{E}-01$ & & & $2.3 \mathrm{E}+04$ & $2.3 \mathrm{E}+03$ & & . & $1.2 \mathrm{E}+04$ & $1.6 \mathrm{E}+03$ & $1.2 \mathrm{E}+03$ & $1.6 \mathrm{E}+02$ \\
\hline Dichloro-2-butene, 1,4- & $000764-41-0$ & & & & & & & & & & & & & & \\
\hline \multicolumn{16}{|l|}{ Dichloroacetic Acid } \\
\hline Dichlorobenzene, 1,2.(5) & $000095-50-1$ & & & $5.2 \mathrm{E}+00$ & $5.2 \mathrm{E}-01$ & & & $2.8 \mathrm{E}+04$ & $2.8 \mathrm{E}+03$ & & & $1.4 \mathrm{E}+04$ & $1.9 \mathrm{E}+03$ & $1.4 \mathrm{E}+03$ & $1.9 \mathrm{E}+02$ \\
\hline Dichlorobenzene, 1,3- & 000541-73-1 & & & & & & & & & & & & & & \\
\hline Dichlorobenzene, 1,4- & $000106-46-7$ & $5.6 \mathrm{E}-01$ & $5.6 \mathrm{E}-03$ & & & $3.0 \mathrm{E}+03$ & $3.0 \mathrm{E}+01$ & & & $6.6 \mathrm{E}+02$ & $6.6 \mathrm{E}+00$ & & & & \\
\hline Dichlorobenzidine, 3,3'- & $000091-94-1$ & $3.1 \mathrm{E}-02$ & $3.1 \mathrm{E}-04$ & & & $1.3 \mathrm{E}+02$ & $1.3 \mathrm{E}+00$ & & & $2.8 \mathrm{E}+01$ & $2.8 \mathrm{E}-01$ & & & & \\
\hline Dichlorodifluoromethane & $000075-71-8$ & & & $6.6 \mathrm{E}+00$ & $6.6 \mathrm{E}-01$ & & & $9.8 \mathrm{E}+05$ & $9.8 \mathrm{E}+04$ & & & $5.1 \mathrm{E}+05$ & $6.5 \mathrm{E}+04$ & $5.1 \mathrm{E}+04$ & $6.5 \mathrm{E}+03$ \\
\hline Dichloroethane, 1,1- & $000075-34-3$ & & & $2.4 \mathrm{E}+00$ & $2.4 \mathrm{E}-01$ & & & $1.0 \mathrm{E}+06$ & $1.2 \mathrm{E}+05$ & & & $6.4 \mathrm{E}+05$ & $8.2 \mathrm{E}+04$ & $6.4 \mathrm{E}+04$ & $8.2 E+03$ \\
\hline Dichloroethane, 1,2- & $000107-06-2$ & 4.7E-02 & 4.7E-04 & & & $6.3 \mathrm{E}+04$ & $6.3 \mathrm{E}+02$ & & & $1.4 \mathrm{E}+04$ & $1.4 \mathrm{E}+02$ & & & & \\
\hline Dichloroethylene, 1,1- & $000075-35-4$ & $9.4 \mathrm{E}-03$ & 9.4E-05 & $2.2 \mathrm{E}-01$ & $2.2 \mathrm{E}-02$ & $4.8 \mathrm{E}+03$ & $4.8 \mathrm{E}+01$ & $1.1 E+05$ & $1.1 \mathrm{E}+04$ & $1.1 \mathrm{E}+03$ & 1.1E+01 & $5.8 \mathrm{E}+04$ & $7.4 \mathrm{E}+03$ & $5.8 \mathrm{E}+03$ & $7.4 \mathrm{E}+02$ \\
\hline Dichloroethylene, 1,2-(Mixed Isomers) & $000540.59-0$ & & & $5.2 \mathrm{E}-02$ & $5.2 \mathrm{E}-03$ & & & $1.0 \mathrm{E}+06$ & $2.3 \mathrm{E}+05$ & & & $1.0 \mathrm{E}+06$ & $1.5 \mathrm{E}+05$ & $1.2 \mathrm{E}+05$ & $1.5 \mathrm{E}+04$ \\
\hline Dichloroethylene, 1,2-cis- & $000156.59-2$ & & & 2.6E-01 & $2.6 \mathrm{E}-02$ & & & $9.8 \mathrm{E}+04$ & $9.8 \mathrm{E}+03$ & & & $5.1 \mathrm{E}+04$ & $6.5 \mathrm{E}+03$ & $5.1 E+03$ & $6.5 \mathrm{E}+02$ \\
\hline Dichloroethylene, 1,2-trans- & $000156-60-5$ & & & $1.2 \mathrm{E}-01$ & $1.2 \mathrm{E}-02$ & & & $1.0 \mathrm{E}+06$ & $5.2 \mathrm{E}+05$ & & & $1.0 \mathrm{E}+06$ & $3.4 \mathrm{E}+05$ & 2.7E+05 & $3.4 \mathrm{E}+04$ \\
\hline Dichlorophenol, 2,4- & $000120-83-2$ & & & $1.5 \mathrm{E}-01$ & $1.5 \mathrm{E}-02$ & & & $2.9 E+03$ & $2.9 \mathrm{E}+02$ & & & $1.5 \mathrm{E}+03$ & $2.0 \mathrm{E}+02$ & $1.5 \mathrm{E}+02$ & $2.0 E+01$ \\
\hline Dichlorophenoxy Acetic Acid, 2,4- & $000094-75-7$ & & & 4.0E-01 & 4.0E-02 & & & $2.5 \mathrm{E}+04$ & $2.5 \mathrm{E}+03$ & & & $1.3 \mathrm{E}+04$ & $1.6 \mathrm{E}+03$ & $1.3 \mathrm{E}+03$ & $1.6 \mathrm{E}+02$ \\
\hline Dichlorophenoxy)butyric Acid, 4-(2,4- & $000094-82-6$ & & & 4.7E-01 & 4.7E-02 & & & $1.8 \mathrm{E}+03$ & $1.8 \mathrm{E}+02$ & & & $9.5 \mathrm{E}+02$ & $1.2 \mathrm{E}+02$ & 9.5E+01 & $1.2 \mathrm{E}+01$ \\
\hline Dichloropropane, 1,2- & $000078-87-5$ & $9.8 \mathrm{E}-02$ & $9.8 \mathrm{E}-04$ & & & $2.7 \mathrm{E}+04$ & $2.7 \mathrm{E}+02$ & & & $5.9 \mathrm{E}+03$ & $5.9 \mathrm{E}+0 \mathrm{l}$ & & & & \\
\hline
\end{tabular}

Nonradionuclides/water/agricultural

Page 11 of 33 
Table 3.3a. (continued)

\begin{tabular}{|c|c|c|c|c|c|c|c|c|c|c|c|c|c|c|c|}
\hline \multirow{2}{*}{ Chemical } & \multirow{2}{*}{ CASRN } & \multicolumn{4}{|c|}{$\begin{array}{l}\text { Ingestion of Vegetables } \\
(\mathrm{mg} / \mathrm{L})^{*}\end{array}$} & \multicolumn{4}{|c|}{$\begin{array}{l}\text { Ingestion of Beef } \\
(\mathrm{mg} / \mathrm{L})^{*}\end{array}$} & \multicolumn{6}{|c|}{$\begin{array}{l}\text { Ingestion of Milk } \\
(\mathrm{mg} / \mathrm{L})^{*}\end{array}$} \\
\hline & & $10^{-1}$ & $10^{4}$ & $H Q=1$ & $\mathrm{HQ}=0.1$ & $10^{-4}$ & $10^{4}$ & $H Q=1$ & $\mathrm{HQ}=0.1$ & $10^{-4}$ & $10^{4}$ & $\begin{array}{l}H Q=1 \\
\text { Adult }\end{array}$ & $\begin{array}{l}\text { HQ=1 } \\
\text { Child }\end{array}$ & $\begin{array}{c}\text { HQ }=0.1 \\
\text { Adult }\end{array}$ & $\begin{array}{c}\text { HQ }=0.1 \\
\text { Child }\end{array}$ \\
\hline Dichloropropanol, 2,3- & $000616-23-9$ & & & & & & & & & & & & & & \\
\hline Dichloropropene, 1,3- & $000542-75-6$ & $2.6 \mathrm{E}-02$ & 2.6E-04 & $6.0 \mathrm{E}-03$ & $6.0 \mathrm{E}-04$ & $2.5 \mathrm{E}+04$ & $2.5 \mathrm{E}+02$ & $5.9 \mathrm{E}+03$ & $5.9 \mathrm{E}+02$ & $5.6 \mathrm{E}+03$ & $5.6 \mathrm{E}+01$ & $3.0 \mathrm{E}+03$ & $3.9 \mathrm{E}+02$ & $3.0 \mathrm{E}+02$ & $3.9 E+01$ \\
\hline Dichlorvos & $000062-73-7$ & $2.4 \mathrm{E}-02$ & $2.4 \mathrm{E}-04$ & $1.5 \mathrm{E}-02$ & $1.5 \mathrm{E}-03$ & $5.8 \mathrm{E}+03$ & $5.8 \mathrm{E}+01$ & $3.6 \mathrm{E}+03$ & $3.6 \mathrm{E}+02$ & $1.3 \mathrm{E}+03$ & $1.3 \mathrm{E}+01$ & $1.9 \mathrm{E}+03$ & $2.4 \mathrm{E}+02$ & $1.9 \mathrm{E}+02$ & $2.4 \mathrm{E}+01$ \\
\hline Dicofol & $000115-32-2$ & & & & & & & & & & & & & & \\
\hline Dicyclopentadiene & $000077-73-6$ & & & $1.5 \mathrm{E}+00$ & $1.5 \mathrm{E}-01$ & & & $3.0 \mathrm{E}+04$ & $3.0 \mathrm{E}+03$ & & & $1.6 \mathrm{E}+04$ & $2.0 \mathrm{E}+03$ & $1.6 \mathrm{E}+03$ & $2.0 \mathrm{E}+02$ \\
\hline Dieldrin & $000060-57-1$ & $9.8 \mathrm{E}-04$ & $9.8 \mathrm{E}-06$ & $3.4 \mathrm{E}-03$ & $3.4 \mathrm{E}-04$ & $2.9 \mathrm{E}-01$ & $2.9 \mathrm{E}-03$ & $9.8 \mathrm{E}-01$ & $9.8 \mathrm{E}-02$ & $6.3 \mathrm{E}-02$ & $6.3 \mathrm{E}-04$ & 5.1E-01 & $6.5 \mathrm{E}-02$ & $5.1 \mathrm{E}-02$ & $6.5 \mathrm{E}-03$ \\
\hline Diethyl Phthalate & $000084-66-2$ & & & $3.2 \mathrm{E}+01$ & $3.2 \mathrm{E}+00$ & & & $1.0 \mathrm{E}+06$ & $2.0 \mathrm{E}+05$ & & & $1.0 \mathrm{E}+06$ & $1.3 \mathrm{E}+05$ & $1.0 \mathrm{E}+05$ & $1.3 \mathrm{E}+04$ \\
\hline Diethyl-p-nitrophenylphosphate & $000311-45-5$ & & & & & & & & & & & & & & \\
\hline Diethylene Glycol Dinitrate (DEGDN) & $000693-21-0$ & & & & & & & & & & & & & & \\
\hline Diethylene Glycol Monobutyl Ether & $000112-34-5$ & & & & & & & & & & & & & & \\
\hline Diethylene Glycol Monoethyl Ether & $000111-90-0$ & & & $5.7 \mathrm{E}+00$ & $5.7 \mathrm{E}-01$ & & & $1.0 \mathrm{E}+06$ & $1.0 \mathrm{E}+06$ & & & $1.0 \mathrm{E}+06$ & $1.0 \mathrm{E}+06$ & $1.0 \mathrm{E}+06$ & $1.0 \mathrm{E}+06$ \\
\hline Diethylformamide & $000617-84-5$ & & & & & & & & & & & & & & \\
\hline Diethylstilbesterol & $000056-53-1$ & 3.5E-06 & $3.5 \mathrm{E}-08$ & & & $1.2 \mathrm{E}-04$ & $1.2 \mathrm{E}-06$ & & & 2.7E-05 & 2.7E-07 & & & & \\
\hline Difenzoquat & $043222-48-6$ & & & & & & & & & & & & & & \\
\hline Diflubenzuron & 035367-38-5 & & & & & & & & & & & & & & \\
\hline Difluoroethane, 1,1- & $000075-37-6$ & & & & & & & & & & & & & & \\
\hline Diisopropyl Methylphosphonate & $001445-75-6$ & & & $8.8 \mathrm{E}-01$ & $8.8 \mathrm{E}-02$ & & & $1.0 \mathrm{E}+06$ & $5.8 \mathrm{E}+05$ & & & $1.0 \mathrm{E}+06$ & $3.9 \mathrm{E}+05$ & $3.0 \mathrm{E}+05$ & $3.9 \mathrm{E}+04$ \\
\hline Dimethipin & $055290-64-7$ & & & & & & & & & & & & & & \\
\hline Dimethoate & $000060-51-5$ & & & $8.9 \mathrm{E}-03$ & $8.9 \mathrm{E}-04$ & & & $3.1 \mathrm{E}+02$ & $3.1 \mathrm{E}+01$ & & & $1.6 \mathrm{E}+02$ & 2.1E+01 & $1.6 \mathrm{E}+01$ & $2.1 E+00$ \\
\hline Dimethoxybenzidine, 3,3'- & $000119-90-4$ & 4.0E-01 & $4.0 \mathrm{E}-03$ & & & 2.1E+0S & $2.1 \mathrm{E}+03$ & & & $4.5 \mathrm{E}+04$ & $4.5 \mathrm{E}+02$ & & & & \\
\hline Dimethyl Sulfate & $000077-78-1$ & & & & & & & & & & & & & & \\
\hline
\end{tabular}


Table 3.3a. (continued)

\begin{tabular}{|c|c|c|c|c|c|c|c|c|c|c|c|c|c|c|c|}
\hline \multirow{2}{*}{ Chemical } & \multirow{2}{*}{ CASRN } & \multicolumn{4}{|c|}{$\begin{array}{c}\text { Ingestion of Vegetables } \\
\text { (mg/L) }\end{array}$} & \multicolumn{4}{|c|}{$\begin{array}{c}\text { Ingestion of Beef } \\
(\mathrm{mg} / \mathrm{L})^{\circ}\end{array}$} & \multicolumn{6}{|c|}{$\begin{array}{l}\text { Ingestion of Milk } \\
(\mathrm{mg} / \mathrm{L})^{*}\end{array}$} \\
\hline & & $10^{-4}$ & $10^{4}$ & $H Q=1$ & $H Q=0.1$ & $10^{-4}$ & $10^{4}$ & $H Q=1$ & $\mathrm{HQ}=0.1$ & $10^{-4}$ & $10^{5}$ & $\begin{array}{l}\text { HQ=1 } \\
\text { Adult }\end{array}$ & $\begin{array}{l}\mathrm{HQ}=1 \\
\text { Child }\end{array}$ & $\begin{array}{c}\text { HQ }=0.1 \\
\text { Adult }\end{array}$ & $\begin{array}{c}\text { HQ }=0.1 \\
\text { Child }\end{array}$ \\
\hline Dimethylaniline $\mathrm{HCl}, 2,4-$ & $021436-96-4$ & & & & & & & & & & & & & & \\
\hline Dimethylaniline, 2,4- & $000095-68-1$ & & & & & & & & & & & & & & \\
\hline Dimethylaniline, N,N- & $000121-69-7$ & & & 8.5E-02 & 8.5E-03 & & & $3.9 E+03$ & $3.9 \mathrm{E}+02$ & & & $2.0 \mathrm{E}+03$ & $2.6 \mathrm{E}+02$ & $2.0 \mathrm{E}+02$ & $2.6 \mathrm{E}+01$ \\
\hline Dimethylbenzidine, 3,3'- & $000119-93-7$ & $9.0 \mathrm{E}-04$ & $9.0 \mathrm{E}-06$ & & & $9.9 \mathrm{E}+01$ & $9.9 \mathrm{E}-01$ & & & $2.2 E+01$ & $2.2 \mathrm{E}-01$ & & & & \\
\hline Dimethylethyl Lead & $107584-40.7$ & & & & & & & & & & & & & & \\
\hline Dimethylformamide & $000068-12-2$ & & & $8.6 \mathrm{E}-02$ & $8.6 \mathrm{E}-03$ & & & $1.0 \mathrm{E}+06$ & $1.0 \mathrm{E}+06$ & & & $1.0 E+06$ & $1.0 \mathrm{E}+06$ & $1.0 \mathrm{E}+06$ & $1.0 \mathrm{E}+06$ \\
\hline Dimethylhydrazine, 1,1-(3) & $000057-14-7$ & & & & & & & & & & & & & & \\
\hline Dimethylhydrazine, 1,2- & $000540-73-8$ & & & & & & & & & & & & & & \\
\hline Dimethyiphenol, 2,4- & $000105-67-9$ & & & $7.1 \mathrm{E}-01$ & 7.1E-02 & & & $7.8 \mathrm{E}+04$ & $7.8 \mathrm{E}+03$ & & & $4.0 \mathrm{E}+04$ & $5.2 \mathrm{E}+03$ & $4.0 \mathrm{E}+03$ & $5.2 \mathrm{E}+02$ \\
\hline Dimethylphenol, 2,6- & $000576-26-1$ & & & $2.2 \mathrm{E}-02$ & $2.2 \mathrm{E}-03$ & & & $2.0 \mathrm{E}+03$ & $2.0 \mathrm{E}+02$ & & & $1.1 \mathrm{E}+03$ & $1.4 \mathrm{E}+02$ & $1.1 E+02$ & $1.4 \mathrm{E}+01$ \\
\hline Dimethyiphenol, 3,4- & $000095-65.8$ & & & 3.3E-02 & $3.3 \mathrm{E}-03$ & & & $4.9 \mathrm{E}+03$ & $4.9 \mathrm{E}+02$ & & & $2.5 E+03$ & $3.3 \mathrm{E}+02$ & $2.5 E+02$ & $3.3 \mathrm{E}+01$ \\
\hline Dimethylphthalate ${ }^{(3)}$ & $000131-11-3$ & & & $2.0 \mathrm{E}+02$ & $2.0 \mathrm{E}+01$ & & & $1.0 \mathrm{E}+06$ & $1.0 \mathrm{E}+06$ & & & $1.0 \mathrm{E}+06$ & $1.0 \mathrm{E}+06$ & $1.0 \mathrm{E}+06$ & $1.0 \mathrm{E}+06$ \\
\hline Dimethylterephthalate & $000120-61-6$ & & & $5.1 \mathrm{E}+00$ & S.1E-01 & & & $7.8 \mathrm{E}+04$ & $7.8 \mathrm{E}+03$ & & & $4.0 \mathrm{E}+04$ & $5.2 \mathrm{E}+03$ & $4.0 \mathrm{E}+03$ & $5.2 \mathrm{E}+02$ \\
\hline Dinitro-a-cresol, 4,6- & $000534-52-1$ & & & & & & & & & & & & & & \\
\hline Dinitro-o-cyclohexyl Phenol, 4,6. & $000131-89-5$ & & & $1.8 \mathrm{E}-04$ & $1.8 \mathrm{E}-05$ & & & $1.0 \mathrm{E}+06$ & $1.0 \mathrm{E}+06$ & & & $1.0 \mathrm{E}+06$ & $1.0 \mathrm{E}+06$ & $1.0 \mathrm{E}+06$ & $1.0 \mathrm{E}+06$ \\
\hline Dinitrobenzene, 1,2- & $000528-29-0$ & & & $8.0 \mathrm{E}-03$ & $8.0 E-04$ & & & $7.8 \mathrm{E}+03$ & $7.8 \mathrm{E}+02$ & & & 4.1E+03 & $5.2 \mathrm{E}+02$ & $4.1 \mathrm{E}+02$ & $5.2 \mathrm{E}+01$ \\
\hline Dinitrobenzene, 1,3- & $000099-65-0$ & & & $2.0 \mathrm{E}-03$ & $2.0 \mathrm{E}-04$ & & & $2.0 \mathrm{E}+03$ & $2.0 \mathrm{E}+02$ & & & $1.0 \mathrm{E}+03$ & $1.3 E+02$ & $1.0 \mathrm{E}+02$ & $1,3 \mathrm{E}+01$ \\
\hline Dinitrobenzene, 1,4- & $000100-25-4$ & & & 7.3E-03 & 7.3E-04 & & & $9.8 \mathrm{E}+03$ & $9.8 \mathrm{E}+02$ & & & $5.1 \mathrm{E}+03$ & $6.6 \mathrm{E}+02$ & $5.1 \mathrm{E}+02$ & $6.6 \mathrm{E}+01$ \\
\hline Dinitrophenol, 2,4- & $000051-28.5$ & & & $3.6 \mathrm{E}-02$ & $3.6 \mathrm{E}-03$ & & & $4.9 \mathrm{E}+04$ & $4.9 E+03$ & & & $2.6 \mathrm{E}+04$ & $3.3 \mathrm{E}+03$ & $2.6 \mathrm{E}+03$ & 3.3E+02 \\
\hline Dinitrotoluene, 2,4- & $000121-14-2$ & $9.8 \mathrm{E}-03$ & 9.8E-05 & 5.7E-02 & 5.7E-03 & $2.7 \mathrm{E}+03$ & $2.7 \mathrm{E}+01$ & $1.6 \mathrm{E}+04$ & $1.6 \mathrm{E}+03$ & $5.9 E+02$ & $5.9 \mathrm{E}+00$ & 8.1E+03 & $1.0 E+03$ & $8.1 E+02$ & $1.0 \mathrm{E}+02$ \\
\hline Dinitrotoluene, 2,6- & $000606-20-2$ & $7.6 \mathrm{E}-03$ & 7.6E-05 & $2.2 \mathrm{E}-02$ & $2.2 \mathrm{E}-03$ & $5.3 \mathrm{E}+03$ & $5.3 \mathrm{E}+01$ & $1.6 \mathrm{E}+04$ & $1.6 \mathrm{E}+03$ & $1.2 \mathrm{E}+03$ & $1.2 \mathrm{E}+01$ & $8.1 E+03$ & $1.0 \mathrm{E}+03$ & $8.1 \mathrm{E}+02$ & $1.0 \mathrm{E}+02$ \\
\hline
\end{tabular}

Nonradionuclides/water/agricultural

Page 13 of 33 
Table 3.3a. (continued)

\begin{tabular}{|c|c|c|c|c|c|c|c|c|c|c|c|c|c|c|c|}
\hline \multirow{2}{*}{ Chemical } & \multirow{2}{*}{ CASRN } & \multicolumn{4}{|c|}{$\begin{array}{c}\text { Ingestion of Vegetables } \\
(\mathrm{mg} / \mathrm{L})^{*}\end{array}$} & \multicolumn{4}{|c|}{$\begin{array}{c}\text { Ingestion of Beef } \\
(\mathrm{mg} / \mathrm{L})^{*}\end{array}$} & \multicolumn{6}{|c|}{$\begin{array}{l}\text { Ingestion of Milk } \\
(\mathrm{mg} / \mathrm{L})^{i}\end{array}$} \\
\hline & & $10^{-4}$ & $10^{4}$ & $H Q=1$ & $\mathrm{HQ}=0.1$ & $10^{-4}$ & $10^{6}$ & $\mathrm{HQ}=1$ & $H Q=0.1$ & $10^{-4}$ & $10^{4}$ & $\begin{array}{l}\text { HQ=1 } \\
\text { Adult }\end{array}$ & $\begin{array}{l}\text { HQ=1 } \\
\text { Child }\end{array}$ & $\begin{array}{c}H Q=0.1 \\
\text { Adult }\end{array}$ & $\begin{array}{c}\text { HQ }=0.1 \\
\text { Child }\end{array}$ \\
\hline Dinitrotoluene, 2-Amino-4,6- & $035572-78-2$ & & & & & & & & & & & & & & \\
\hline Dinoseb & $000088-85-7$ & & & $6.1 \mathrm{E}-02$ & $6.1 \mathrm{E}-03$ & & & $1.6 \mathrm{E}+02$ & $1.6 \mathrm{E}+01$ & & & $8.1 E+01$ & $1.0 \mathrm{E}+01$ & $8.1 E+00$ & $1.0 \mathrm{E}+00$ \\
\hline Dioxane, 1,4- & $000123-91-1$ & 4.7E-02 & 4.7E-04 & & & $1.0 \mathrm{E}+06$ & $3.1 \mathrm{E}+05$ & & & $1.0 \mathrm{E}+06$ & $6.8 \mathrm{E}+04$ & & & & \\
\hline Diphenamid & $000957-51-7$ & & & 9.7E-01 & 9.7E-02 & & & $1.6 \mathrm{E}+05$ & $1.6 \mathrm{E}+04$ & & & $8.2 E+04$ & $1.1 \mathrm{E}+04$ & $8.2 \mathrm{E}+03$ & $1.1 \mathrm{E}+03$ \\
\hline Diphenylamine & $000122-39-4$ & & & $1.5 \mathrm{E}+00$ & $1.5 \mathrm{E}-01$ & & & $6.2 \mathrm{E}+03$ & $6.2 \mathrm{E}+02$ & & & $3.2 \mathrm{E}+03$ & 4.1E+02 & $3.2 \mathrm{E}+02$ & $4.1 \mathrm{E}+01$ \\
\hline Diphenylhydrazine, 1,2- & $000122-66-7$ & $1.4 \mathrm{E}-02$ & $1.4 \mathrm{E}-04$ & & & $2.9 \mathrm{E}+02$ & $2.9 \mathrm{E}+00$ & & & $6.3 \mathrm{E}+01$ & $6.3 \mathrm{E}-01$ & & & & \\
\hline Diquat & $000085-00-7$ & & & $1.2 \mathrm{E}-04$ & $1.2 \mathrm{E}-05$ & & & $1.0 \mathrm{E}+06$ & $1.0 \mathrm{E}+06$ & & & $1.0 \mathrm{E}+06$ & $1.0 \mathrm{E}+06$ & $1.0 \mathrm{E}+06$ & $1.0 \mathrm{E}+06$ \\
\hline Direct Black 38 & $001937-37-7$ & & & & & & & & & & & & & & \\
\hline Direct Blue 6 & $002602-46-2$ & & & & & & & & & & & & & & \\
\hline Direct Brown 95 & $016071-86-6$ & & & & & & & & & & & & & & \\
\hline Direct Sky Blue & $002610-05-1$ & & & & & & & & & & & & & & \\
\hline Disulfoton & $000298-04-4$ & & & $2.6 \mathrm{E}-03$ & 2.6E-04 & & & $3.1 \mathrm{E}+00$ & $3.1 \mathrm{E}-01$ & & & $1.6 \mathrm{E}+00$ & $2.1 \mathrm{E}-01$ & $1.6 \mathrm{E}-01$ & $2.1 \mathrm{E}-02$ \\
\hline Diuron & $000330-54-1$ & & & $8.9 \mathrm{E}-02$ & $8.9 \mathrm{E}-03$ & & & $3.1 \mathrm{E}+03$ & $3.1 \mathrm{E}+02$ & & & $1.6 \mathrm{E}+03$ & $2.1 \mathrm{E}+02$ & $1.6 \mathrm{E}+02$ & $2.1 \mathrm{E}+01$ \\
\hline Dodine & $002439-10-3$ & & & & & & & & & & & & & & \\
\hline EPTC & $000759-94-4$ & & & & & & & & & & & & & & \\
\hline Endosulfan & $000115-29-7$ & & & & & & & & & & & & & & \\
\hline Endothall & $000145-73-3$ & & & & & & & & & & & & & & \\
\hline Endrin & $000072-20-8$ & & & $2.0 \mathrm{E}-02$ & $2.0 \mathrm{E}-03$ & & & $5.9 \mathrm{E}+00$ & $5.9 \mathrm{E}-01$ & & & $3.0 \mathrm{E}+00$ & $3.9 \mathrm{E}-01$ & $3.0 \mathrm{E}-01$ & $3.9 \mathrm{E}-02$ \\
\hline Epichlorohydrin & $000106-89-8$ & 5.7E-02 & $5.7 \mathrm{E}-04$ & $4.8 \mathrm{E}-03$ & 4.8E-04 & $1.0 \mathrm{E}+06$ & $3.0 \mathrm{E}+05$ & $1.0 \mathrm{E}+06$ & $2.5 \mathrm{E}+05$ & $1.0 \mathrm{E}+06$ & $6.5 E+04$ & $1.0 \mathrm{E}+06$ & $1.7 \mathrm{E}+05$ & $1.3 \mathrm{E}+05$ & $1.7 E+04$ \\
\hline Epoxybutane, 1,2- & $000106-88-7$ & & & & & & & & & & & & & & \\
\hline Ethephon & $016672-87-0$ & & & & & & & & & & & & & & \\
\hline
\end{tabular}

Nonradionuclides/water/agricultural

Page 14 of 33 
Table 3.3a. (continued)

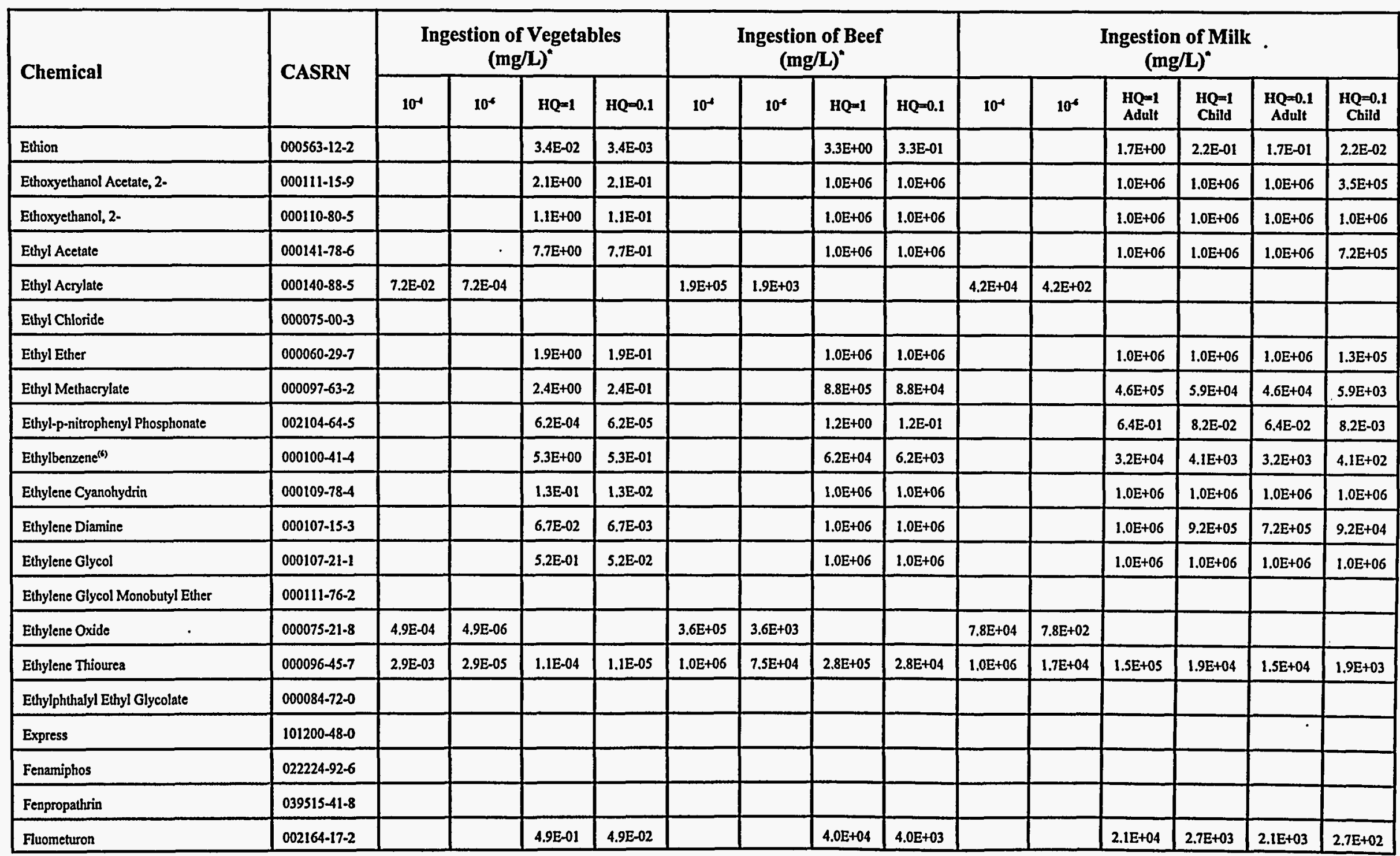


Table 3.3a. (continued)

\begin{tabular}{|c|c|c|c|c|c|c|c|c|c|c|c|c|c|c|c|}
\hline \multirow{2}{*}{ Chemical } & \multirow{2}{*}{ CASRN } & \multicolumn{4}{|c|}{$\begin{array}{l}\text { Ingestion of Vegetables } \\
(\mathrm{mg} / \mathrm{L})^{n}\end{array}$} & \multicolumn{4}{|c|}{$\begin{array}{c}\text { Ingestion of Beef } \\
(\mathrm{mg} / \mathrm{L})^{*}\end{array}$} & \multicolumn{6}{|c|}{$\begin{array}{c}\text { Ingestion of Milk } \\
(\mathrm{mg} / \mathrm{L})^{*}\end{array}$} \\
\hline & & $10^{-4}$ & $10^{6}$ & $H Q=1$ & $H Q=0.1$ & $10^{-4}$ & $10^{4}$ & $H Q=1$ & $\mathrm{HQ}=0.1$ & $10^{-4}$ & $10^{6}$ & $\begin{array}{l}\text { HQ=1 } \\
\text { Adult }\end{array}$ & $\begin{array}{l}H Q=1 \\
\text { Child }\end{array}$ & $\begin{array}{c}\text { HQ }=0.1 \\
\text { Adult }\end{array}$ & $\begin{array}{c}H Q=0.1 \\
\text { Child }\end{array}$ \\
\hline Fluoranthene & $000206-44-0$ & & & $2.7 \mathrm{E}+00$ & 2.7E-01 & & & $3.9 \mathrm{E}+02$ & $3.9 E+01$ & & & $2.0 \mathrm{E}+02$ & $2.6 \mathrm{E}+01$ & $2.0 \mathrm{E}+01$ & $2.6 \mathrm{E}+00$ \\
\hline Fluorene & $000086-73-7$ & & & $2.7 \mathrm{E}+00$ & 2.7E-01 & & & $1.2 \mathrm{E}+03$ & $1.2 \mathrm{E}+02$ & & & $6.4 \mathrm{E}+02$ & $8.3 \mathrm{E}+01$ & $6.4 \mathrm{E}+01$ & $8.3 \mathrm{E}+00$ \\
\hline Fluoride & $007782-41-4$ & & & & & & & & & & & & & & \\
\hline Fluridone & $059756-60-4$ & & & & & & & & & & & & & & \\
\hline Flurprimidol & $056425-91-3$ & & & & & & & & & & & & & & \\
\hline Flutolanil & $066332-96-5$ & & & & & & & & & & & & & & \\
\hline Fluvalinate & $069409-94-5$ & & & & & & & & & & & & & & \\
\hline Folpet & $000133-07-3$ & $3.5 \mathrm{E}+00$ & $3.5 \mathrm{E}-02$ & $5.3 E+00$ & $5.3 \mathrm{E}-01$ & $4.1 \mathrm{E}+04$ & 4.1E+02 & $6.2 \mathrm{E}+04$ & $6.2 \mathrm{E}+03$ & $9.1 E+03$ & 9.1E+01 & $3.2 \mathrm{E}+04$ & $4.1 \mathrm{E}+03$ & $3.2 \mathrm{E}+03$ & $4.1 \mathrm{E}+02$ \\
\hline Fomesafen & $072178-02-0$ & & & & & & & & & & & & & & \\
\hline Fonofos & $000944-22-9$ & & & $1.3 \mathrm{E}-01$ & $1.3 \mathrm{E}-02$ & & & $1.8 \mathrm{E}+02$ & $1.8 \mathrm{E}+01$ & & & $9.3 \mathrm{E}+01$ & $1.2 \mathrm{E}+01$ & $9.3 \mathrm{E}+00$ & $1.2 \mathrm{E}+00$ \\
\hline Fornaldehyde & $000050-00-0$ & & & $6.3 \mathrm{E}-01$ & $6.3 \mathrm{E}-02$ & & & $1.0 \mathrm{E}+06$ & $1.0 \mathrm{E}+06$ & & & $1.0 \mathrm{E}+06$ & $1.0 \mathrm{E}+06$ & $1.0 \mathrm{E}+06$ & $1.0 \mathrm{E}+06$ \\
\hline Formic Acid & $000064-18-6$ & & & $3.2 \mathrm{E}+00$ & $3.2 \mathrm{E}-01$ & & & $1.0 \mathrm{E}+06$ & $1.0 \mathrm{E}+06$ & & & $1.0 \mathrm{E}+06$ & $1.0 \mathrm{E}+06$ & $1.0 \mathrm{E}+06$ & $1.0 \mathrm{E}+06$ \\
\hline Fosetyl-AL & $039148-24-8$ & & & & & & & & & & & & & & \\
\hline Furan & $000110-00-9$ & & & $1.5 \mathrm{E}-02$ & $1.5 \mathrm{E}-03$ & & & $3.9 E+04$ & $3.9 \mathrm{E}+03$ & & & $2.0 \mathrm{E}+04$ & $2.6 \mathrm{E}+03$ & $2.0 \mathrm{E}+03$ & $2.6 \mathrm{E}+02$ \\
\hline Furazolidone & $000067-45-8$ & 4.5E-04 & 4.5E-06 & & & $1.0 \mathrm{E}+04$ & $1.0 \mathrm{E}+02$ & & & $2.2 \mathrm{E}+03$ & $2.2 E+01$ & & & & \\
\hline Furfural & $000098-01-1$ & & & $1.8 \mathrm{E}-01$ & $1.8 \mathrm{E}-02$ & & & 7.4E+02 & $7.4 E+01$ & & & $3.8 \mathrm{E}+02$ & $4.9 \mathrm{E}+01$ & $3.8 \mathrm{E}+01$ & $4.9 \mathrm{E}+00$ \\
\hline Furium & $000531-82-8$ & & & & & & & & & & & & & & \\
\hline Furmecyclox & $060568-05-0$ & & & & & & & & & & & & & & \\
\hline Glufosinate, Ammonium & $077182-82-2$ & & & & & & & & & & & & & & \\
\hline Glycidyl & $000765-34-4$ & & & $4.9 \mathrm{E}-04$ & $4.9 \mathrm{E}-05$ & & & $1.0 \mathrm{E}+06$ & $1.7 \mathrm{E}+05$ & & & $8.7 \mathrm{E}+05$ & $1.1 \mathrm{E}+05$ & $8.7 \mathrm{E}+04$ & $1.1 \mathrm{E}+04$ \\
\hline Glyphosate & $001071-83-6$ & & & $6.0 \mathrm{E}-03$ & $6.0 \mathrm{E}-04$ & & & $1.0 \mathrm{E}+06$ & $1.0 \mathrm{E}+06$ & & & $1.0 E+06$ & $1.0 \mathrm{E}+06$ & $1.0 \mathrm{E}+06$ & $1.0 \mathrm{E}+06$ \\
\hline
\end{tabular}

Nonradionuclides/water/agricultural

Page 16 of 33 
Table 3.3a. (continued)

\begin{tabular}{|c|c|c|c|c|c|c|c|c|c|c|c|c|c|c|c|}
\hline \multirow{2}{*}{ Chemical } & \multirow{2}{*}{ CASRN } & \multicolumn{4}{|c|}{$\begin{array}{l}\text { Ingestion of Vegetables } \\
(\mathrm{mg} / \mathrm{L})^{*}\end{array}$} & \multicolumn{4}{|c|}{$\begin{array}{l}\text { Ingestion of Beef } \\
(\mathrm{mg} / \mathrm{L})^{*}\end{array}$} & \multicolumn{6}{|c|}{$\begin{array}{c}\text { Ingestion of Milk } \\
(\mathrm{mg} / \mathrm{L})^{*}\end{array}$} \\
\hline & & $10^{-4}$ & $10^{5}$ & HQ-1 & $H Q=0.1$ & $10^{-1}$ & $10^{6}$ & HQ $=1$ & $\mathrm{HQ}=0.1$ & $10^{-4}$ & $10^{5}$ & $\begin{array}{l}\text { HQ }=1 \\
\text { Adult }\end{array}$ & $\begin{array}{l}\text { HQ=1 } \\
\text { Child }\end{array}$ & $\begin{array}{c}\mathrm{HQ}=0.1 \\
\text { Adult }\end{array}$ & $\begin{array}{c}\text { HQ }=0.1 \\
\text { Child }\end{array}$ \\
\hline Goal & $042874-03-3$ & & , & & & & & & & & & & & & \\
\hline Haloxyfop, Methyl & $069806-40-2$ & & & . & . & & & & & & & & & & \\
\hline Harmony & $079277-27-3$ & & & & & & & & & & & & & & \\
\hline Heptachlor & $000076-44-8$ & $3.4 \mathrm{E}-03$ & $3.4 \mathrm{E}-05$ & $3.3 \mathrm{E}-02$ & $3.3 \mathrm{E}-03$ & $2.0 E+00$ & $2.0 \mathrm{E}-02$ & $2.0 \mathrm{E}+01$ & $2.0 E+00$ & $4.5 \mathrm{E}-01$ & 4.5E-03 & $1.0 E+01$ & $1.3 \mathrm{E}+00$ & $1.0 \mathrm{E}+00$ & $1.3 \mathrm{E}-01$ \\
\hline Heptachlor Epoxide & $001024-57-3$ & $1.8 \mathrm{E}-03$ & $1.8 \mathrm{E}-05$ & $9.0 \mathrm{E}-04$ & $9.0 \mathrm{E}-05$ & $7.9 \mathrm{E}-02$ & $7.9 \mathrm{E}-04$ & $4.0 \mathrm{E}-02$ & $4.0 \mathrm{E}-03$ & $1.7 \mathrm{E}-02$ & $1.7 E-04$ & 2.1E-02 & $2.7 \mathrm{E}-03$ & 2.1E-03 & $-2.7 \mathrm{E}-04$ \\
\hline Heptane, N- & $000142-82-5$ & & & & & & & & & & & & & &. \\
\hline Hexabromobenzene & $000087-82-1$ & & & $1.4 \mathrm{E}-01$ & $1.4 \mathrm{E}-02$ & & & $1.3 \mathrm{E}+00$ & $1.3 \mathrm{E}-01$ & & , & $6.9 \mathrm{E}-01$ & $8.8 \mathrm{E}-02$ & $6.9 \mathrm{E}-02$ & $8.8 \mathrm{E}-03$ \\
\hline Hexachlorobenzene ${ }^{(1)}$ & $000118-74-1$ & $1.0 \mathrm{E}-02$ & $1.0 \mathrm{E}-04$ & $5.5 \mathrm{E}-02$ & $5.5 \mathrm{E}-03$ & $5.7 \mathrm{E}-01$ & $5.7 \mathrm{E}-03$ & $3.1 \mathrm{E}+\infty$ & $3.1 \mathrm{E}-01$ & $1.3 \mathrm{E}-01$ & $1.3 \mathrm{E}-03$ & $1.6 \mathrm{E}+00$ & 2.1E-01 & $1.6 \mathrm{E}-01$ & $2.1 E-02$ \\
\hline Hexachlorobutadiene $e^{(b)}$ & $000087-68-3$ & $2.0 \mathrm{E}-01$ & $2.0 \mathrm{E}-03$ & $1.4 \mathrm{E}-02$ & $1.4 \mathrm{E}-03$ & $3.7 \mathrm{E}+01$ & 3.7E-01 & $2.5 \mathrm{E}+00$ & $2.5 E-01$ & $8.1 E+00$ & $8.1 \mathrm{E}-02$ & $1.3 \mathrm{E}+00$ & $1.6 \mathrm{E}-01$ & $1.3 \mathrm{E}-01$ & $1.6 \mathrm{E}-02$ \\
\hline Hexachlorocyclohexane, Alpha. & $000319-84-6$ & $2.3 \mathrm{E}-03$ & 2.3E-05 & & & $3.6 \mathrm{E}+00$ & $3.6 \mathrm{E}-02$ & & & $8.0 \mathrm{E}-01$ & $8.0 \mathrm{E}-03$ & & & & \\
\hline Hexachlorocyclohexane, Beta. & $000319-85-7$ & 8.3E-03 & 8.3E-05 & & & $1.0 \mathrm{E}+01$ & $1.0 \mathrm{E}-01$ & & & $2.2 \mathrm{E}+00$ & $2.2 \mathrm{E}-02$ & & & & \\
\hline Hexachlorocyclohexane, Delta. & $000319-86.8$ & & & & & & & & & & & & & & \\
\hline Hexachlorocyclohexane, Epsiton & $006108-10-7$ & & & & & & & & & & & & & & \\
\hline Hexachlorocyclohexane, Gammz- & $000058-89-9$ & 1.1E-02 & 1.1E-04 & $1.8 \mathrm{E}-02$ & $1.8 \mathrm{E}-03$ & $2.8 \mathrm{E}+01$ & $2.8 \mathrm{E}-01$ & $4.7 \mathrm{E}+01$ & $4.7 \mathrm{E}+00$ & $6.1 \mathrm{E}+00$ & $6.1 \mathrm{E}-02$ & $2.4 E+01$ & $3.1 \mathrm{E}+00$ & $2.4 E+00$ & $3.1 \mathrm{E}-01$ \\
\hline Hexachlorocyclohexane, Technical & 000608-73-1 & & & & & & & & & & & & & & . \\
\hline Hexachlorocyclopentadiene & $000077-47-4$ & & & $4.5 \mathrm{E}-01$ & $4.5 \mathrm{E}-02$ & & & $5.5 \mathrm{E}+02$ & $5.5 \mathrm{E}+01$ & & & $2.8 \mathrm{E}+02$ & $3.6 \mathrm{E}+01$ & $2.8 \mathrm{E}+01$ & $3.6 \mathrm{E}+00$ \\
\hline Hexachlorodibenzo-p-dioxin, Mixture & $019408-74-3$ & 2.6E-06 & $2.6 \mathrm{E}-08$ & & & $5.8 \mathrm{E}-06$ & $5.8 \mathrm{E}-08$ & & & $1.3 \mathrm{E}-06$ & $1.3 \mathrm{E}-08$ & & & & \\
\hline Hexachloroethane & $000067-72-1$ & $1.1 E+00$ & $1.1 \mathrm{E}-02$ & $6.3 \mathrm{E}-02$ & $6.3 \mathrm{E}-03$ & $1.6 \mathrm{E}+03$ & $1.6 \mathrm{E}+01$ & $9.8 \mathrm{E}+01$ & $9.8 \mathrm{E}+00$ & $3.6 \mathrm{E}+02$ & $3.6 \mathrm{E}+00$ & $5.1 \mathrm{E}+01$ & $6.5 \mathrm{E}+00$ & $5.1 \mathrm{E}+00$ & $6.5 \mathrm{E}-01$ \\
\hline Hexachlorophene & $000070-30-4$ & & & $2.1 \mathrm{E}-02$ & 2.1E-03 & & & 7.4E-03 & 7.4E-04 & & & $3.8 \mathrm{E}-03$ & 4.9E-04 & $3.8 \mathrm{E}-04$ & $4.9 E-05$ \\
\hline $\begin{array}{l}\text { Hexahydro-1,3,5-trinitro-1,3,5-triazine } \\
\text { (RDX) }\end{array}$ & $000121-82-4$ & $1.2 \mathrm{E}-01$ & $1.2 \mathrm{E}-03$ & $1.7 \mathrm{E}-01$ & $1.7 \mathrm{E}-02$ & $8.3 \mathrm{E}+02$ & $8.3 E+00$ & $1.2 \mathrm{E}+03$ & $1.2 \mathrm{E}+02$ & $1.8 \mathrm{E}+02$ & $1.8 \mathrm{E}+00$ & $6.1 \mathrm{E}+02$ & $7.8 \mathrm{E}+01$ & $6.1 E+01$ & $7.8 \mathrm{E}+00$ \\
\hline
\end{tabular}


Table 3.3a. (continued)

\begin{tabular}{|c|c|c|c|c|c|c|c|c|c|c|c|c|c|c|c|}
\hline \multirow{2}{*}{ Chemical } & \multirow{2}{*}{ CASRN } & \multicolumn{4}{|c|}{$\begin{array}{l}\text { Ingestion of Vegetables } \\
(\mathrm{mg} / \mathrm{L})^{*}\end{array}$} & \multicolumn{4}{|c|}{$\begin{array}{l}\text { Ingestion of Beef } \\
(\mathrm{mg} / \mathrm{L})^{*}\end{array}$} & \multicolumn{6}{|c|}{$\begin{array}{l}\text { Ingestion of Milk } \\
(\mathrm{mg} / \mathrm{L})^{*}\end{array}$} \\
\hline & & $10^{-4}$ & $10^{4}$ & $H Q=1$ & $\mathrm{HQ}=0.1$ & $10^{-4}$ & $10^{5}$ & $\mathrm{HQ}=1$ & $\mathrm{HQ}=0.1$ & $10^{-4}$ & $10^{-6}$ & $\begin{array}{l}\text { HQ=1 } \\
\text { Adult }\end{array}$ & $\begin{array}{l}\text { HQ=1 } \\
\text { Child }\end{array}$ & $\begin{array}{c}\mathrm{HQ}=0.1 \\
\text { Adult }\end{array}$ & $\begin{array}{c}\text { HQ }=0.1 \\
\text { Child }\end{array}$ \\
\hline Hexamethyleme Diisocyanate, 1,6- & $000822-06-0$ & & & & & & & & & & & & & & \\
\hline Hexane, N- & $000110-54-3$ & & & $3.8 \mathrm{E}+00$ & $3.8 \mathrm{E}-01$ & & & $5.9 \mathrm{E}+03$ & $5.9 \mathrm{E}+02$ & & & $3.1 E+03$ & $3.9 \mathrm{E}+02$ & $3.1 \mathrm{E}+02$ & $3.9 \mathrm{E}+01$ \\
\hline Hexanone, 2- & $000591-78-6$ & & & & & & & & & & & & & & \\
\hline Hexazinone & $051235-04-2$ & & & 3.1E-04 & 3.1E-05 & & & $1.0 \mathrm{E}+06$ & $1.0 E+06$ & & & $1.0 \mathrm{E}+06$ & $1.0 \mathrm{E}+06$ & $1.0 \mathrm{E}+06$ & $1.0 \mathrm{E}+06$ \\
\hline $\mathrm{HpCDD}, 2,3,7,8^{(1)}$ & $037871-00-4$ & & & & & & & & & & & & & & \\
\hline $\mathrm{HpCDF}, 2,3,7,8^{(4)}$ & 038998-75-3 & & & & & & & & & & & & & & \\
\hline $\mathrm{HxCDD}, 2,3,7,8_{-(4)}^{(4)}$ & $034465-46-8$ & & & & & & & & & & & & & & \\
\hline $\mathrm{HxCDF}, 2,3,7,8-(4)$ & $055684-94-1$ & & & & & & & & & & & & & & \\
\hline Hydrazine & $000302-01-2$ & $1.6 \mathrm{E}-05$ & $1.6 \mathrm{E}-07$ & & & $1.0 \mathrm{E}+06$ & $7.6 \mathrm{E}+04$ & & & $1.0 \mathrm{E}+06$ & $1.7 \mathrm{E}+04$ & & & & \\
\hline Hydrazine Sulfate & $010034-93-2$ & $1.6 \mathrm{E}-05$ & $1.6 \mathrm{E}-07$ & & & $1.0 \mathrm{E}+06$ & $7.6 \mathrm{E}+04$ & & & $1.0 \mathrm{E}+06$ & $1.7 E+04$ & & & & \\
\hline Hydrogen Chloride & $007647-01-0$ & & & & & & & & & & & & & & \\
\hline Hydrogen Cyanide & $000074-90-8$ & & & $1.0 \mathrm{E}-01$ & $1.0 \mathrm{E}-02$ & & & $1.0 \mathrm{E}+06$ & $6.5 \mathrm{E}+05$ & & & $1.0 \mathrm{E}+06$ & $4.3 \mathrm{E}+05$ & $3.4 \mathrm{E}+05$ & $4.3 \mathrm{E}+04$ \\
\hline Hydrogen Sulfide & $007783-06-4$ & & & $3.0 \mathrm{E}-02$ & $3.0 \mathrm{E}-03$ & & & $2.6 \mathrm{E}+05$ & $2.6 \mathrm{E}+04$ & & & $1.4 \mathrm{E}+05$ & $1.7 \mathrm{E}+04$ & $1.4 \mathrm{E}+04$ & $1.7 \mathrm{E}+03$ \\
\hline Hydroquinone & $000123-31-9$ & & & $2.5 \mathrm{E}-01$ & $2.5 \mathrm{E}-02$ & & & $1.0 \mathrm{E}+06$ & $8.8 \mathrm{E}+05$ & & & $1.0 \mathrm{E}+06$ & $5.8 \mathrm{E}+05$ & $4.6 \mathrm{E}+05$ & $5.8 \mathrm{E}+04$ \\
\hline Imazalil & $035554-44-0$ & & & & & & & & & & & & & & \\
\hline Imazaquin & $081335-37-7$ & & $\cdot$ & & & & & & & & & & & & \\
\hline Indeno[1,2,3-cd]pyrene ${ }^{(2)}$ & $000193-39-5$ & $2.2 \mathrm{E}-02$ & $2.2 \mathrm{E}-04$ & & & $6.3 \mathrm{E}-02$ & $6.3 \mathrm{E}-04$ & & & $1.4 \mathrm{E}-02$ & $1.4 \mathrm{E}-04$ & & & & \\
\hline Iprodione & 036734-19-7 & & & & & & & & & & & & & & \\
\hline Iron & $007439-89-6$ & & & & & & & & & & & & & & \\
\hline Isobutyl Alcohol & $000078-83-1$ & & & $2.6 \mathrm{E}+00$ & 2.6E-01 & & & $1.0 E+06$ & $1.0 \mathrm{E}+06$ & & & $1.0 \mathrm{E}+06$ & $1.0 \mathrm{E}+06$ & $1.0 \mathrm{E}+06$ & $2.3 \mathrm{E}+05$ \\
\hline Isophorone & $000078-59-1$ & $5.4 \mathrm{E}+00$ & $5.4 \mathrm{E}-02$ & $4.4 \mathrm{E}+00$ & 4.4E-01 & $1.0 E+06$ & $3.8 \mathrm{E}+04$ & $1.0 \mathrm{E}+06$ & $3.1 \mathrm{E}+05$ & $8.4 \mathrm{E}+05$ & $8.4 \mathrm{E}+03$ & $1.0 \mathrm{E}+06$ & $2.1 E+05$ & $1.6 \mathrm{E}+05$ & $2.1 E+04$ \\
\hline
\end{tabular}

Nonradionuclides/water/agricultural

Page 18 of 33 


\begin{tabular}{|c|c|c|c|c|c|c|c|c|c|c|c|c|c|c|c|}
\hline & & & & & & & & & & & & & & $L-10-810800$ & q9zostrew \\
\hline $10+9 s^{\prime} 6$ & $20+a b i c$ & $20+956$ & $\varepsilon 0+\exists \not \subset l<$ & & & $\varepsilon_{0}+I_{b} \mathbf{t}$ & $60+361$ & & & $90-\mathrm{a} \iota^{\prime} 9$ & so-al'9 & & & $\varepsilon=\angle L-601000$ & 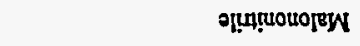 \\
\hline $90+30^{\prime} \mathrm{I}$ & $90+30^{\circ} 1$ & $90+30^{\circ} \mathrm{t}$ & $90+30^{\circ} \mathrm{I}$ & & & $90+30^{\circ} \mathrm{t}$ & $90+3011$ & & & $20-a s^{\prime} 6$ & $10-a s^{\prime} 6$ & & & I- $\varepsilon \varepsilon-\varepsilon \tau 1000$ & 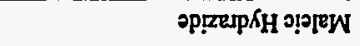 \\
\hline & & & & & & & & & & & & & & $9-[\varepsilon-801000$ & 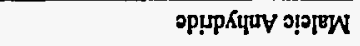 \\
\hline $20+9 E=1$ & $\varepsilon 0+30^{\circ} \mathrm{t}$ & $\varepsilon 0+\{E 1$ & $50+30^{\circ} \mathrm{I}$ & & & $\varepsilon 0+\pi 0^{\circ} z$ & $60+700 \tau$ & & & $20 \cdot 38 \cdot 6$ & $10-386$ & & & S-SL-IZ1000 & uọpperew \\
\hline & & & & & & & & & & & & & & $+56.65 t<00$ & um!ssor88 \\
\hline $10+a \varepsilon^{\prime} 1$ & $20+300^{\circ} \mathrm{I}$ & $20+3 \varepsilon^{\prime} t$ & $\varepsilon 0+90^{\circ} 1$ & & & $20+ \pm 0^{\circ} \tau$ & $\varepsilon 0+30^{\circ} z$ & & & E0-az' & $20-3 z^{\prime}$ & & & $z-\$ 9-\varepsilon 60000$ & ddכW \\
\hline & & & & & & & & & & & & & & $s-18-660000$ & gdכw \\
\hline $10+\exists E^{\prime} \tau$ & $20+30.1$ & $20+a \varepsilon^{\prime} l$ & $\varepsilon 0+30^{\circ} \mathrm{I}$ & & & $20+70^{\circ} z$ & $\varepsilon 0+\nexists 0^{\circ} z$ & & & E0-ت78' I & $20-3811$ & & & $9-b l-b 60000$ & $\forall d \supset \mathcal{W}$ \\
\hline & & & & & & & & & & & & & & $9-66-5 \varsigma 0 \varepsilon 80$ & xерuот \\
\hline & & & & & & & & & & & & & & $\tau-\varepsilon 6-6 \varepsilon \downarrow<00$ & wn!m!!7 \\
\hline & & & & & & & & & & & & & & Z-Ss-0E£000 & นоли! 1 \\
\hline & & & & & & & & & & & & & & $1-26-65 \div<00$ & sptmoduloo ptry per7 \\
\hline & & & & & & & & & & & & & & $\mathrm{VN}$ & STSIIV PBDT \\
\hline & & & & & & & & & & & & & & $T E 9-10 S \angle L O$ & แวзорอา \\
\hline & & & & & & & & & & & & & & $5-85-056 \varepsilon 20$ & q1יX \\
\hline & & & & & & & & & & & & & & $9-80-596160$ & शвमт \\
\hline & & & & & & & & & & & & & & $\iota-0 S-85 S 280$ & แəqеxоs! \\
\hline & & & & & & & & & & & & & & $8-t s-2 E 8100$ & 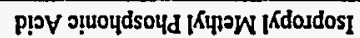 \\
\hline & & & & & & & & & & & & & & $0-\varepsilon 9-\angle 90000$ & porrdadosI \\
\hline & & & & & & & & & & & & & & 0 - $\varepsilon S-0 z 8 \varepsilon \varepsilon 0$ & ụpedoudos! \\
\hline 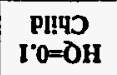 & 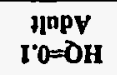 & $\stackrel{P I ! q O}{I=O H}$ & $\prod_{l=O H}^{n p y}$ & , or & .0I & $1^{1} \mathrm{O}=\mathrm{OH}$ & $\mathfrak{l}=\mathbf{O H}$ & .01 & rOI & $\mathrm{I}^{\mathrm{O}}=\mathrm{OH}$ & $I=\partial H$ & ,0I & rOI & \multirow[b]{2}{*}{ N\&SYD } & \multirow[b]{2}{*}{ [вว!ณวนว } \\
\hline \multicolumn{6}{|c|}{ 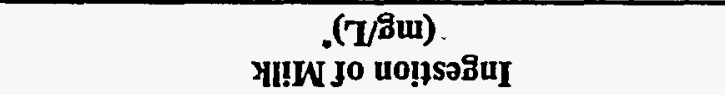 } & \multicolumn{4}{|c|}{ 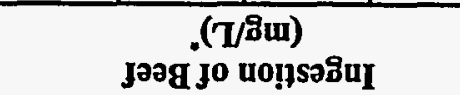 } & \multicolumn{4}{|c|}{ 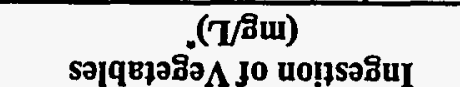 } & & \\
\hline
\end{tabular}


Table 3.3a. (continued)

\begin{tabular}{|c|c|c|c|c|c|c|c|c|c|c|c|c|c|c|c|}
\hline \multirow{2}{*}{ Chemical } & \multirow{2}{*}{ CASRN } & \multicolumn{4}{|c|}{$\begin{array}{l}\text { Ingestion of Vegetables } \\
(\mathrm{mg} / \mathrm{L})^{*}\end{array}$} & \multicolumn{4}{|c|}{$\begin{array}{l}\text { Ingestion of Beef } \\
(\mathrm{mg} / \mathrm{L})^{*}\end{array}$} & \multicolumn{6}{|c|}{$\begin{array}{l}\text { Ingestion of Milk } \\
\text { (mg/L)* }\end{array}$} \\
\hline & & $10^{-4}$ & $10^{4}$ & $\mathrm{HQ}=1$ & $\mathrm{HQ}=0.1$ & $10^{-1}$ & $10^{5}$ & HQ=1 & $\mathrm{HQ}=0.1$ & $10^{-4}$ & $10^{4}$ & $\begin{array}{l}\text { HQ=1 } \\
\text { Adult }\end{array}$ & $\begin{array}{l}\text { HQ=1 } \\
\text { Child }\end{array}$ & $\begin{array}{c}\text { HQ }=0.1 \\
\text { Adult }\end{array}$ & $\begin{array}{c}\text { HQ }=0.1 \\
\text { Child }\end{array}$ \\
\hline Manganese (Diet) & $007439-96-5$ & & & $8.3 \mathrm{E}+00$ & $8.3 \mathrm{E}-01$ & & & $5.5 \mathrm{E}+03$ & $5.5 \mathrm{E}+02$ & & & $1.5 E+04$ & $1.9 \mathrm{E}+03$ & $1.5 \mathrm{E}+03$ & $1.9 E+02$ \\
\hline Mephosfolan & $000950-10-7$ & & & & & & & & & & & & & & \\
\hline Mercury (elemental) & $007439-97-6$ & & & $1.2 \mathrm{E}-02$ & $1.2 \mathrm{E}-03$ & & & $5.8 \mathrm{E}-01$ & $5.8 \mathrm{E}-02$ & & & $2.0 \mathrm{E}+00$ & $2.6 \mathrm{E}-01$ & $2.0 \mathrm{E}-01$ & $2.6 \mathrm{E}-02$ \\
\hline Mercury, Inorganic Salt & $007439-97-6$ & & & $1.2 \mathrm{E}-02$ & $1.2 \mathrm{E}-03$ & & & $5.8 \mathrm{E}-01$ & $5.8 \mathrm{E}-02$ & & & $2.0 \mathrm{E}+00$ & $2.6 \mathrm{E}-01$ & $2.0 \mathrm{E}-01$ & $2.6 \mathrm{E}-02$ \\
\hline Merphos & $000150-50-5$ & & & & & & & & & & & & & & \\
\hline Merphos Oxide & $000078-48-8$ & & & $2.1 \mathrm{E}-03$ & $2.1 \mathrm{E}-04$ & & & $4.7 \mathrm{E}-02$. & $4.7 \mathrm{E}-03$ & & & $2.4 \mathrm{E}-02$ & $3.1 \mathrm{E}-03$ & $2.4 \mathrm{E}-03$ & 3.1E-04 \\
\hline Methidathion & $000950-37-8$ & & & & & & & & & & & & & & \\
\hline Methomyl & $016752-77-5$ & & & $1.7 \mathrm{E}-01$ & $1.7 E-02$ & & & $1.0 \mathrm{E}+06$ & $4.9 \mathrm{E}+05$ & & & $1.0 \mathrm{E}+06$ & $3.3 \mathrm{E}+05$ & $2.5 \mathrm{E}+05$ & $3.3 E+04$ \\
\hline Methoxy-S-nitroaniline, 2- & $000099-59-2$ & $9.2 \mathrm{E}-02$ & $9.2 E-04$ & & & $1.2 E+05$ & $1.2 \mathrm{E}+03$ & & & $2.7 \mathrm{E}+04$ & $2.7 \mathrm{E}+02$ & & & & \\
\hline Methoxychlor & $000072-43-5$ & & & $3.3 \mathrm{E}-01$ & $3.3 \mathrm{E}-02$ & & & $1.5 \mathrm{E}+02$ & $1.5 \mathrm{E}+01$ & & & $8.0 E+01$ & $1.0 \mathrm{E}+01$ & $8.0 \mathrm{E}+00$ & $1.0 \mathrm{E}+00$ \\
\hline Methoxyethanol Acetate, 2- & $000110-49-6$ & & & & & & & & & & & & & & \\
\hline Methoxyethanol, 2- & $000109-86-4$ & & & $1.2 E-03$ & $1.2 E-04$ & & & $1.0 \mathrm{E}+06$ & $4.6 \mathrm{E}+05$ & & & $1.0 \mathrm{E}+06$ & $3.1 \mathrm{E}+05$ & $2.4 E+05$ & $3.1 \mathrm{E}+04$ \\
\hline Methyl Acetate & $000079-20-9$ & & & $4.0 E+00$ & $4.0 E-01$ & & & $1.0 \mathrm{E}+06$ & $1.0 \mathrm{E}+06$ & & & $1.0 E+06$ & $1.0 \mathrm{E}+06$ & $1.0 E+06$ & $1.0 E+06$ \\
\hline Methyl Acrylate & $000096-33-3$ & & & $2.2 \mathrm{E}-01$ & $2.2 \mathrm{E}-02$ & & & $1.0 \mathrm{E}+06$ & $5.0 E+05$ & & & $1.0 E+06$ & $3.3 \mathrm{E}+05$ & $2.6 \mathrm{E}+05$ & $3.3 \mathrm{E}+04$ \\
\hline
\end{tabular}

Nonradionuclides/water/agricultural

Page 20 of 33 
Table 3.3a. (continued)

\begin{tabular}{|c|c|c|c|c|c|c|c|c|c|c|c|c|c|c|c|}
\hline \multirow{2}{*}{ Chemical } & \multirow{2}{*}{ CASRN } & \multicolumn{4}{|c|}{$\begin{array}{c}\text { Ingestion of Vegetables } \\
(\mathrm{mg} / \mathrm{L})^{*}\end{array}$} & \multicolumn{4}{|c|}{$\begin{array}{c}\text { Ingestion of Beef } \\
(\mathrm{mg} / \mathrm{L})^{*}\end{array}$} & \multicolumn{6}{|c|}{$\begin{array}{c}\text { Ingestion of Milk } \\
\text { (mg/L) }\end{array}$} \\
\hline & & $10^{-4}$ & $10^{6}$ & $H Q=1$ & $\mathrm{HQ}=0.1$ & $10^{-4}$ & $10^{\circ}$ & $\mathrm{HQ}=\mathbf{I}$ & $H Q=0.1$ & $10^{-4}$ & $10^{4}$ & $\begin{array}{l}\text { HQ=1 } \\
\text { Adult }\end{array}$ & $\begin{array}{l}\text { HQ=1 } \\
\text { Child }\end{array}$ & $\begin{array}{c}\text { HQ }=0.1 \\
\text { Adult }\end{array}$ & $\begin{array}{c}\mathrm{HQ}=0.1 \\
\text { Child }\end{array}$ \\
\hline Methyl Ethyl Ketone & $000078-93-3$ & & & 2.7E+00 & 2.7E-01 & & & $1.0 \mathrm{E}+06$ & $1.0 \mathrm{E}+06$ & & & $1.0 \mathrm{E}+06$ & $1.0 \mathrm{E}+06$ & $1.0 \mathrm{E}+06$ & $1.0 \mathrm{E}+06$ \\
\hline Methyl lsobutyl Ketone & $000108-10-1$ & & & $1.1 \mathrm{E}+00$ & $1.1 \mathrm{E}-01$ & & & $1.0 \mathrm{E}+06$ & $3.9 E+05$ & & & $1.0 \mathrm{E}+06$ & $2.6 \mathrm{E}+05$ & $2.0 \mathrm{E}+05$ & $2.6 \mathrm{E}+04$ \\
\hline Methyl Mercury & $022967-92-6$ & & & & & & & & & & & & & & \\
\hline Methyl Methacrylate & $000080-62-6$ & & & $1.2 \mathrm{E}+00$ & $1.2 \mathrm{E}-01$ & & & $1.0 \mathrm{E}+06$ & $3.1 \mathrm{E}+05$ & & & $1.0 \mathrm{E}+06$ & $2.1 \mathrm{E}+05$ & $1.6 \mathrm{E}+05$ & $2.1 E+04$ \\
\hline Methyl Parathion & $000298-00-0$ & & & $6.6 \mathrm{E}-03$ & $6.6 \mathrm{E}-04$ & & & $2.5 \mathrm{E}+03$ & $2.5 \mathrm{E}+02$ & & & $1.3 \mathrm{E}+03$ & $1.6 \mathrm{E}+02$ & $1.3 \mathrm{E}+02$ & $1.6 \mathrm{E}+01$ \\
\hline Methyl Styrene (Mixed Isomers) & $025013-15-4$ & & & $3.4 \mathrm{E}-01$ & $3.4 \mathrm{E}-02$ & & & $2.1 \mathrm{E}+03$ & $2.1 \mathrm{E}+02$ & & & $1.1 \mathrm{E}+03$ & $1.4 \mathrm{E}+02$ & $1.1 \mathrm{E}+02$ & $1.4 E+01$ \\
\hline Methyl hydrazine $e^{(s)}$. & $000060-34-4$ & & & & & & & & & & & & & & \\
\hline Methyl tert-Butyl Ether (MTBE) & $001634-04-4$ & & & & & & & & & & & & & & \\
\hline Methyl-5-Nitroaniline, 2 - & $000099-55-8$ & $2.0 \mathrm{E}-01$ & $2.0 \mathrm{E}-03$ & & & $5.5 \mathrm{E}+04$ & $5.5 \mathrm{E}+02$ & & & $1.2 \mathrm{E}+04$ & $1.2 \mathrm{E}+02$ & & & & \\
\hline Methylaniline Hydrochloride, 2- & $000636-21-5$ & 4.1E-03 & 4.1E-05 & & & $1.0 \mathrm{E}+06$ & $1.0 \mathrm{E}+04$ & & & $2.2 \mathrm{E}+05$ & $2.2 E+03$ & & & & \\
\hline Methylcyclohexane & $000108-87-2$ & & & & & & & & & & & & & & \\
\hline Methylene Chloride & $000075-09.2$ & $4.6 \mathrm{E}-01$ & $4.6 \mathrm{E}-03$ & $8.9 \mathrm{E}-01$ & $8.9 \mathrm{E}-02$ & $1.0 \mathrm{E}+06$ & $1.2 \mathrm{E}+04$ & $1.0 \mathrm{E}+06$ & $2.3 \mathrm{E}+05$ & $2.7 E+05$ & $2.7 \mathrm{E}+03$ & $1.0 \mathrm{E}+06$ & $1.6 \mathrm{E}+05$ & $1.2 \mathrm{E}+05$ & $1.6 \mathrm{E}+04$ \\
\hline Methylene-bis(2-chloroaniline), 4,4- & $000101-14-4$ & $1.1 \mathrm{E}-01$ & $1.1 \mathrm{E}-03$ & $4.4 \mathrm{E}-02$ & $4.4 \mathrm{E}-03$ & $1.8 \mathrm{E}+02$ & $1.8 E+00$ & $6.9 E+01$ & $6.9 \mathrm{E}+00$ & $3.9 \mathrm{E}+01$ & $3.9 \mathrm{E}-01$ & $3.6 \mathrm{E}+01$ & $4.6 \mathrm{E}+00$ & $3.6 \mathrm{E}+00$ & $4.6 \mathrm{E}-01$ \\
\hline $\begin{array}{l}\text { Methylene-bis(N,N-dimethyl) Aniline, } \\
\text { 4,4-' }\end{array}$ & $000101-61-1$ & $3.5 \mathrm{E}-01$ & $3.5 \mathrm{E}-03$ & & & $6.3 \mathrm{E}+01$ & $6.3 \mathrm{E}-01$ & & & $1.4 \mathrm{E}+01$ & $1.4 \mathrm{E}-01$ & & & & \\
\hline Methylenebisbenzenamine, $4,4^{\prime} \cdot(3)$ & $000101-77-9$ & $1.9 \mathrm{E}-02$ & $1.9 \mathrm{E}-04$ & & & $1.8 \mathrm{E}+04$ & $1.8 \mathrm{E}+02$ & & & $4.0 \mathrm{E}+03$ & $4.0 \mathrm{E}+01$ & & & & \\
\hline Methylenediphenyl Isocyanate, 4,4'- & $000101-68-8$ & & & & & & & & & & & & & & \\
\hline Methylstyrene, Alpha- & $000098-83.9$ & & & $4.0 \mathrm{E}+00$ & 4.0E-01 & & & $2.4 E+04$ & $2.4 \mathrm{E}+03$ & & & $1.3 \mathrm{E}+04$ & $1.6 \mathrm{E}+03$ & $1.3 \mathrm{E}+03$ & $1.6 \mathrm{E}+02$ \\
\hline Methyltriethyl Lead & $001762-28-3$ & & & & " & & & & & & & & & & \\
\hline Metolachlor & $051218-45-2$ & & & $\therefore$ & & & & & & & & & & & \\
\hline Metribuzin & $021087-64-9$ & & & 5.5E-01 & 5.5E-02 & & & $3.9 \mathrm{E}+05$ & $3.9 \mathrm{E}+04$ & & & $2.0 E+05$ & $2.6 \mathrm{E}+04$ & $2.0 \mathrm{E}+04$ & $2.6 \mathrm{E}+03$ \\
\hline
\end{tabular}

Nonradionuclides/water/agricultural

Page 21 of 33 
Table 3.3a. (continued)

\begin{tabular}{|c|c|c|c|c|c|c|c|c|c|c|c|c|c|c|c|}
\hline \multirow{2}{*}{ Chemical } & \multirow{2}{*}{ CASRN } & \multicolumn{4}{|c|}{$\begin{array}{c}\text { Ingestion of Vegetables } \\
(\mathrm{mg} / \mathrm{L})^{n}\end{array}$} & \multicolumn{4}{|c|}{$\begin{array}{c}\text { Ingestion of Beef } \\
(\mathrm{mg} / \mathrm{L})^{n}\end{array}$} & \multicolumn{6}{|c|}{$\begin{array}{l}\text { Ingestion of Milk } \\
(\mathrm{mg} / \mathrm{L})^{*}\end{array}$} \\
\hline & & $10^{-4}$ & $10^{4}$ & $H Q=1$ & $H Q=0.1$ & $10^{-4}$ & $10^{-6}$ & $H Q=1$ & $H Q=0.1$ & $10^{-4}$ & $10^{5}$ & $\begin{array}{l}\text { HQ=1 } \\
\text { Adult }\end{array}$ & $\begin{array}{l}\mathrm{HQ}=1 \\
\text { Child }\end{array}$ & $\begin{array}{c}H Q=0.1 \\
\text { Adult }\end{array}$ & $\begin{array}{c}\mathrm{HQ}=0.1 \\
\text { Child }\end{array}$ \\
\hline Mirex & 002385-85-5 & $9.0 \mathrm{E}-03$ & $9.0 \mathrm{E}-05$ & $1.4 \mathrm{E}-02$ & $1.4 \mathrm{E}-03$ & $5.3 \mathrm{E}-01$ & 5.3E-03 & $8.2 \mathrm{E}-0 \mathrm{I}$ & $8.2 \mathrm{E}-02$ & $1.2 \mathrm{E}-01$ & $1.2 \mathrm{E}-03$ & 4.2E-01 & $5.4 \mathrm{E}-02$ & $4.2 \mathrm{E}-02$ & $5.4 \mathrm{E}-03$ \\
\hline Molinate & $002212-67-1$ & & & $1.2 \mathrm{E}-01$ & $1.2 \mathrm{E}-02$ & & & $4.6 \mathrm{E}+02$ & $4.6 \mathrm{E}+01$ & & & $2.4 \mathrm{E}+02$ & $3.1 \mathrm{E}+01$ & $2.4 E+01$ & $3.1 \mathrm{E}+00$ \\
\hline Molybdenum & 007439-98-7 & & & 2.9E-01 & $2.9 \mathrm{E}-02$ & & & $9.7 \mathrm{E}+01$ & 9.7E+00 & & & $9.4 E+00$ & $1.2 \mathrm{E}+00$ & $9.4 \mathrm{E}-01$ & $1.2 \mathrm{E}-01$ \\
\hline Monochloramine & 010599-90-3 & & & & & & & . & & & & & & & \\
\hline Monochlorobutanes & $025154-42-1$ & & & & & & & & & & & & & & \\
\hline Naled & $000300-76-5$ & & & $9.4 \mathrm{E}-02$ & $9.4 \mathrm{E}-03$ & & & $2.5 \mathrm{E}+03$ & $2.5 \mathrm{E}+02$ & & & $1.3 \mathrm{E}+03$ & $1.6 \mathrm{E}+02$ & $1.3 \mathrm{E}+02$ & $1.6 \mathrm{E}+01$ \\
\hline Naphthalene $e^{(0)}$ & $000091-20-3$ & & & $2.0 \mathrm{E}+00$ & $2.0 \mathrm{E}-01$ & & & $1.4 \mathrm{E}+04$ & $1.4 \mathrm{E}+03$ & & & $7.2 \mathrm{E}+03$ & $9.3 \mathrm{E}+02$ & $7.2 \mathrm{E}+02$ & $9.3 \mathrm{E}+01$ \\
\hline Naphthalene, 1-Methyl & $000090-12-0$ & & & & & & & & & & & & & & \\
\hline Naphthalene, 2-Methyl & $000091-57-6$ & & & & & & & & & & & & & & \\
\hline Napropamide & $015299-99-7$ & & & $7.2 \mathrm{E}-01$ & $7.2 \mathrm{E}-02$ & & & $1.0 \mathrm{E}+06$ & $1.0 \mathrm{E}+06$ & & & $1.0 \mathrm{E}+06$ & $1.0 \mathrm{E}+06$ & $8.8 \mathrm{E}+05$ & $1.1 \mathrm{E}+05$ \\
\hline Niagara Blue 4B & $002429-74-5$ & & & & & & & & & & & & & & \\
\hline Nickel Carbonyl & $013463-39-3$ & & & & & & & & & & & & & & \\
\hline Nickel Refinery Dust & NA & & & & & & & & & & & & & & \\
\hline Nickel Soluble Salts & $007440-02-0$ & & & $1.2 \mathrm{E}+00$ & $1.2 \mathrm{E}-01$ & & & $7.8 \mathrm{E}+01$ & $7.8 \mathrm{E}+00$ & & & $4.0 \mathrm{E}+00$ & $5.1 \mathrm{E}-0 \mathrm{t}$ & $4.0 \mathrm{E}-01$ & 5.1E-02 \\
\hline Nickel Subsulfide & $012035-72-2$ & & & & & & & & & & & & & & \\
\hline Nitrapyrin ${ }^{(3)}$ & $001929-82-4$ & & & $7.6 \mathrm{E}-02$ & $7.6 \mathrm{E}-03$ & & & $1.2 \mathrm{E}+03$ & $1.2 \mathrm{E}+02$ & & & $6.1 \mathrm{E}+02$ & $7.8 \mathrm{E}+01$ & $6.1 E+01$ & $7.8 \mathrm{E}+00$ \\
\hline Nitrate & $014797-55-8$ & & & & & & & & & & & & & & \\
\hline Nitric Oxide & $010102-43-9$ & & & & & & & & & & & & & & \\
\hline Nitrite & $014797-65.0$ & & & & & & & & & & & & & & \\
\hline Nitroaniline, 2- & $000088-74-4$ & & & & & & & & & & & & & & \\
\hline Nitrobenzene & $000098-95-3$ & & & $1.2 \mathrm{E}-02$ & $1.2 \mathrm{E}-03$ & & & $6.2 \mathrm{E}+03$ & $6.2 \mathrm{E}+02$ & & & $3.2 \mathrm{E}+03$ & $4.1 E+02$ & $3.2 \mathrm{E}+02$ & $4.1 E+01$ \\
\hline
\end{tabular}

Nonradionuclides/water/agricultural

Page 22 of 33 
Table 3.3a. (continued)

\begin{tabular}{|c|c|c|c|c|c|c|c|c|c|c|c|c|c|c|c|}
\hline \multirow{2}{*}{ Chemical } & \multirow{2}{*}{ CASRN } & \multicolumn{4}{|c|}{$\begin{array}{c}\text { Ingestion of Vegetables } \\
(\mathrm{mg} / \mathrm{L})^{n}\end{array}$} & \multicolumn{4}{|c|}{$\begin{array}{l}\text { Ingestion of Beef } \\
(\mathrm{mg} / \mathrm{L})^{*}\end{array}$} & \multicolumn{6}{|c|}{$\begin{array}{c}\text { Ingestion of Milk } \\
(\mathrm{mg} / \mathrm{L})^{n}\end{array}$} \\
\hline & & $10^{-4}$ & $10^{5}$ & HQ-1 & $\mathrm{HQ}=0.1$ & $10^{4}$ & $10^{6}$ & $\mathrm{HQ}=1$ & $\mathrm{HQ}=0.1$ & $10^{-4}$ & $10^{6}$ & $\begin{array}{l}\text { HQ=1 } \\
\text { Adult }\end{array}$ & $\begin{array}{l}\text { HQ=1 } \\
\text { Child }\end{array}$ & $\begin{array}{c}\text { HQ }=0.1 \\
\text { Adult }\end{array}$ & $\begin{array}{c}\text { HQ }=0.1 \\
\text { Child }\end{array}$ \\
\hline Nitrofurantoin & $000067-20-9$ & & & & & & & & & & & & & & \\
\hline Nitrofurazone & $000059-87-0$ & & & & & & & & & & & & & & \\
\hline Nitrogen Dioxide $e^{(s)}$ & $010102-44-0$ & & & & & & & & & & & & & & \\
\hline Nitroguanidine & $000556-88-7$ & & & & & & & & & & & & & & \\
\hline Nitrophenol, 4- & $000100-02-7$ & & & & & & & & & & & & & & \\
\hline Nitropropane, 2-(3) & $000079-46-9$ & $1.5 \mathrm{E}-04$ & $1.5 \mathrm{E}-06$ & & & $5.4 \mathrm{E}+03$ & $5.4 \mathrm{E}+01$ & & & $1.2 \mathrm{E}+03$ & $1.2 \mathrm{E}+01$ & & & & \\
\hline Nitroso-N-ethylurea, $\mathrm{N}$ - & $000759-73-9$ & $7,1 \mathrm{E}-06$ & 7.1E-08 & & & $7.6 \mathrm{E}+02$ & $7.6 \mathrm{E}+00$ & & & $1.7 \mathrm{E}+02$ & $1.7 \mathrm{E}+00$ & & & & \\
\hline Nitroso-N-methylurea, $\mathrm{N}$ - & $000684-93-5$ & & & & & & & & & & & & & & \\
\hline Nitroso-di-N-butylamine, N- & $000924-16-3$ & $1.1 \mathrm{E}-03$ & 1.1E-05 & & & $4.2 \mathrm{E}+02$ & $4.2 \mathrm{E}+00$ & & & $9.3 \mathrm{E}+01$ & $9.3 \mathrm{E}-01$ & & & & \\
\hline Nitroso-di-N-propylamine, N- & $000621-64-7$ & $5.5 \mathrm{E}-04$ & 5.5E-06 & & & $1.0 \mathrm{E}+03$ & $1.0 \mathrm{E}+01$ & & & $2.3 \mathrm{E}+02$ & $2.3 E+00$ & & & & \\
\hline Nitrosodiethanolamine, $\mathrm{N}$ - & $001116-54-7$ & $3.2 \mathrm{E}-05$ & 3.2E-07 & & & $1.0 \mathrm{E}+06$ & $2.6 \mathrm{E}+04$ & & & $5.7 \mathrm{E}+05$ & $5.7 \mathrm{E}+03$ & & & & \\
\hline Nitrosodiethylamine, $\mathrm{N}$ - & $000055-18-5$ & $9.0 \mathrm{E}-06$ & 9.0E-08 & & & $4.0 \mathrm{E}+02$ & $4.0 E+00$ & & & $8.8 \mathrm{E}+01$ & $8.8 \mathrm{E}-01$ & & & & \\
\hline Nitrosodimethylamine, N- & $000062-75-9$ & $6.9 \mathrm{E}-06$ & $6.9 E-08$ & & & $1.3 \mathrm{E}+04$ & $1.3 \mathrm{E}+02$ & & & $2.9 \mathrm{E}+03$ & $2.9 \mathrm{E}+01$ & & & & \\
\hline Nitrosodiphenylamine, N- & $000086-30-6$ & $2.5 \mathrm{E}+00$ & 2.5E-02 & & & $2.9 \mathrm{E}+04$ & $2.9 \mathrm{E}+02$ & & & $6.5 \mathrm{E}+03$ & $6.5 \mathrm{E}+01$ & & & & \\
\hline Nitrosomethylethylamine, $\mathrm{N}-$ & $010595-95-6$ & & & & & & & & & & & & & & \\
\hline Nitrosomethylvinylamine, $\mathrm{N}$ - & $004549-40-0$ & & & & & & & & & & & & & & \\
\hline Nitrosopyrnolidine, N- & $000930-55-2$ & $8.4 \mathrm{E}-05$ & 8.4E-07 & & & $1.0 \mathrm{E}+06$ & $1.1 \mathrm{E}+04$ & & & $2.4 E+05$ & $2.4 \mathrm{E}+03$ & & & & \\
\hline Nitrotoluene, 4-Amino-2- & $000119-32-4$ & & & & & & & & & & & & & & \\
\hline Nitrotoluene, m- & $000099-08-1$ & & & 3.8E-01 & $3.8 \mathrm{E}-02$ & & & 3.1E+04 & $3.1 E+03$ & & & $1.6 \mathrm{E}+04$ & $2.1 \mathrm{E}+03$ & $1.6 \mathrm{E}+03$ & $2.1 E+02$ \\
\hline Nitrotoluene, 0 - & $000088-72-2$ & & & $3.6 \mathrm{E}-01$ & $3.6 \mathrm{E}-02$ & & & $3.9 \mathrm{E}+04$ & $3.9 E+03$ & & & $2.0 \mathrm{E}+04$ & $2.6 \mathrm{E}+03$ & $2.0 E+03$ & $2.6 \mathrm{E}+02$ \\
\hline Nitrotoluene, p- & $000099-99-0$ & & & $3.8 \mathrm{E}-01$ & $3.8 \mathrm{E}-02$ & & & 3.1E+04 & $3.1 \mathrm{E}+03$ & & & $1.6 \mathrm{E}+04$ & $2.1 \mathrm{E}+03$ & $1.6 \mathrm{E}+03$ & $2.1 \mathrm{E}+02$ \\
\hline
\end{tabular}

Nonradionuclides/water/agricultural

Page 23 of 33 
Table 3.3a. (continued)

\begin{tabular}{|c|c|c|c|c|c|c|c|c|c|c|c|c|c|c|c|}
\hline \multirow{2}{*}{ Chemical } & \multirow{2}{*}{ CASRN } & \multicolumn{4}{|c|}{$\begin{array}{c}\text { Ingestion of Vegetables } \\
(\mathrm{mg} / \mathrm{L})^{*}\end{array}$} & \multicolumn{4}{|c|}{$\begin{array}{c}\text { Ingestion of Beef } \\
(\mathrm{mg} / \mathrm{L})^{*}\end{array}$} & \multicolumn{6}{|c|}{$\begin{array}{c}\text { Ingestion of Milk } \\
(\mathrm{mg} / \mathrm{L})^{\circ}\end{array}$} \\
\hline & & $10^{-4}$ & $10^{4}$ & $\mathrm{HO}=1$ & $\mathrm{HQ}=0.1$ & $10^{-1}$ & $10^{5}$ & $\mathrm{HQ}=1$ & $\mathrm{HQ}=0.1$ & $10^{-1}$ & $10^{-5}$ & $\begin{array}{l}\mathrm{HQ}=\mathbf{1} \\
\text { Adult }\end{array}$ & $\begin{array}{l}\mathrm{HQ}=\mathbf{1} \\
\text { Child }\end{array}$ & $\begin{array}{c}\text { HQ }=0.1 \\
\text { Adult }\end{array}$ & $\begin{array}{c}\text { HQ=0.1 } \\
\text { Child }\end{array}$ \\
\hline Norflurazon & 027314-13-2 & & & & & & & & & & & & & & \\
\hline Nustar & 085509-19-9 & & & & & & & & & & & & & & \\
\hline$O C D D^{(n)}$ & $003268-87-9$ & 1.1E-04 & $1.1 \mathrm{E}-06$ & & & $2.0 \mathrm{E}-06$ & 2.0E-08 & & & $4.4 \mathrm{E}-07$ & 4.4E-09 & & & & \\
\hline$O C D F^{(4)}$ & $039001-02-0$ & & & & & & & & & & & & & & \\
\hline Octabromodiphenyl Ether & $032536-52-0$ & & & & & & & & & & & & & & \\
\hline $\begin{array}{l}\text { Octahydro-1,3,5,7-tetranitro-1,3,5,7-tetra } \\
\text { (FMMX) }\end{array}$ & $002691-41-0$ & & & $3.3 \mathrm{E}-01$ & $3.3 \mathrm{E}-02$ & & & $1.0 \mathrm{E}+06$ & $1.0 \mathrm{E}+06$ & & & $1.0 E+06$ & $6.7 \mathrm{E}+05$ & $5.2 \mathrm{E}+05$ & $6.7 E+04$ \\
\hline Octamethylpyrophosphoramide & $000152-16-9$ & & & & & & & & & & & & & & \\
\hline Octyl Phthalate, di-N- & $000117-84-0$ & & & $1.4 \mathrm{E}+00$ & $1.4 \mathrm{E}-01$ & & & $9.8 \mathrm{E}-03$ & $9.8 \mathrm{E}-04$ & & & $5.1 \mathrm{E}-03$ & $6.5 \mathrm{E}-04$ & 5.1E-04 & $6.5 \mathrm{E}-05$ \\
\hline Oryzalin & 019044-88-3 & & & & & & & & & & & & & & \\
\hline Oxadiazon & 019666-30-9 & & & & & & & & & & & & & & \\
\hline Oxamyl & $023135-22-0$ & & & & & & & & & & & & & & \\
\hline Paclobutrazol & 076738-62-0 & & & & & & & & & & & & & & \\
\hline Paraquat & $001910-42-5$ & & & $5.3 \mathrm{E}-05$ & $5.3 \mathrm{E}-06$ & & & $1.0 \mathrm{E}+06$ & $1.0 \mathrm{E}+06$ & & & $1.0 \mathrm{E}+06$ & $1.0 \mathrm{E}+06$ & $1.0 \mathrm{E}+06$ & $1.0 \mathrm{E}+06$ \\
\hline Parathion & $000056-38-2$ & & & $3.7 \mathrm{E}-01$ & $3.7 \mathrm{E}-02$ & & & $7.4 \mathrm{E}+02$ & $7.4 \mathrm{E}+01$ & & & $3.8 \mathrm{E}+02$ & $4.9 \mathrm{E}+01$ & $3.8 \mathrm{E}+01$ & $4.9 \mathrm{E}+00$ \\
\hline PeCDD, 2,3,7,8-(1) & $036088-22-9$ & $2.2 \mathrm{E}-07$ & $2.2 \mathrm{E}-09$ & & & $7.7 \mathrm{E}-07$ & $7.7 \mathrm{E}-09$ & & & $1.7 \mathrm{E}-07$ & $1.7 \mathrm{E}-09$ & & & & \\
\hline $\mathrm{PeCDF}, 1,2,3,7,8^{-(4)}$ & $057117-41-6$ & & & & & & & & & & & & & & \\
\hline PeCDF, 2,3,4,7,8, (4) & $057117-31-4$ & & & & . & & & & . & & & & & & \\
\hline Pebulate & $001114-71-2$ & & & & & & & & & & & & & & \\
\hline Pendimethalin & 040487-42-1 & & & & & & & & & & & & & & \\
\hline Pentabromodiphenyl Ether & $032534-81-9$ & & & & & & & & & & & & & & \\
\hline
\end{tabular}

Nonradionuclides/water/agricultural

Page 24 of 33 
Table 3.3a. (continued)

\begin{tabular}{|c|c|c|c|c|c|c|c|c|c|c|c|c|c|c|c|}
\hline \multirow{2}{*}{ Chemical } & \multirow{2}{*}{ CASRN } & \multicolumn{4}{|c|}{$\begin{array}{c}\text { Ingestion of Vegetables } \\
\text { (mg/L) }\end{array}$} & \multicolumn{4}{|c|}{$\begin{array}{c}\text { Ingestion of Beef } \\
(\mathrm{mg} / \mathrm{L})^{*}\end{array}$} & \multicolumn{6}{|c|}{$\begin{array}{c}\text { Ingestion of Milk } \\
(\mathrm{mg} / \mathrm{L})^{*}\end{array}$} \\
\hline & & $10^{-4}$ & $10^{4}$ & $H Q=1$ & $H Q=0.1$ & $10^{+}$ & $10^{6}$ & $H Q=1$ & HQ $=0.1$ & $10^{-4}$ & $10^{4}$ & $\begin{array}{l}\text { HQ=1 } \\
\text { Adult }\end{array}$ & $\begin{array}{l}\text { HQ=1 } \\
\text { Child }\end{array}$ & $\begin{array}{c}H Q=0.1 \\
\text { Adult }\end{array}$ & $\begin{array}{c}\text { HQ }=0.1 \\
\text { Child }\end{array}$ \\
\hline Pentachlorobenzene & 000608-93-5 & & & $5.5 \mathrm{E}-02$ & 5.5E-03 & & & $3.9 \mathrm{E}+00$ & $3.9 \mathrm{E}-01$ & & & $2.0 \mathrm{E}+00$ & $2.6 \mathrm{E}-01$ & $2.0 \mathrm{E}-01$ & 2.6E-02 \\
\hline Pentachlorocyclopentadiene & 025329-35-5 & & & & & & & & & & & & & & \\
\hline Pentachloronitrobenzene & $000082-68-8$ & $6.1 \mathrm{E}-02$ & 6.1E-04 & $2.0 \mathrm{E}-01$ & $2.0 \mathrm{E}-02$ & $1.8 \mathrm{E}+01$ & $1.8 \mathrm{E}-01$ & $5.9 \mathrm{E}+01$ & $5.9 \mathrm{E}+00$ & $3.9 \mathrm{E}+00$ & $3.9 \mathrm{E}-02$ & $3.0 \mathrm{E}+01$ & $3.9 \mathrm{E}+00$ & $3.0 \mathrm{E}+00$ & $3.9 \mathrm{E}-01$ \\
\hline Pentachlorophenol & $000087-86-5$ & $1.4 \mathrm{E}-01$ & $1.4 \mathrm{E}-03$ & $2.1 \mathrm{E}+00$ & $2.1 \mathrm{E}-01$ & $1.9 \mathrm{E}+00$ & $1.9 \mathrm{E}-02$ & $2.9 \mathrm{E}+01$ & $2.9 \mathrm{E}+00$ & $4.2 \mathrm{E}-01$ & 4.2E-03 & $1.5 \mathrm{E}+01$ & $2.0 \mathrm{E}+00$ & $1.5 \mathrm{E}+00$ & 2.0E-01 \\
\hline Pentyl Alcohol, N. & $000071-41-0$ & & & & & & & & & & & & & & \\
\hline Permethrin & $052645-53-1$ & & & $2.9 \mathrm{E}+00$ & $2.9 \mathrm{E}-01$ & & & $1.2 \mathrm{E}+04$ & $1.2 \mathrm{E}+03$ & & & $6.4 E+03$ & $8.2 \mathrm{E}+02$ & $6.4 \mathrm{E}+02$ & $8.2 \mathrm{E}+01$ \\
\hline Phenanthrene & $000085-01-8$ & & & & & & & & & & & & & & \\
\hline Phenmedipham & $013684-63-4$ & & & $1.4 \mathrm{E}-01$ & $1.4 \mathrm{E}-02$ & & & $1.0 \mathrm{E}+06$ & $1.0 \mathrm{E}+06$ & & & $1.0 \mathrm{E}+06$ & $1.0 E+06$ & $1.0 \mathrm{E}+06$ & $1.0 \mathrm{E}+06$ \\
\hline Phenol & $000108-95-2$ & & & $1.1 \mathrm{E}+01$ & $1.1 \mathrm{E}+00$ & & & $1.0 \mathrm{E}+06$ & $1.0 \mathrm{E}+06$ & & & $1.0 \mathrm{E}+06$ & $9.8 \mathrm{E}+05$ & $7.7 \mathrm{E}+05$ & $9.8 \mathrm{E}+04$ \\
\hline Phenylenediamine, $\mathrm{m}$ - & $000108-45-2$ & & & $7.2 \mathrm{E}-03$ & $7.2 \mathrm{E}-04$ & & & $1.0 \mathrm{E}+06$ & $1.0 \mathrm{E}+06$ & & & $1.0 \mathrm{E}+06$ & $1.0 \mathrm{E}+06$ & $1.0 \mathrm{E}+06$ & $1.8 \mathrm{E}+05$ \\
\hline Phenylenediamine, 0 - & $000095-54-5$ & $2.7 \mathrm{E}-02$ & $2.7 \mathrm{E}-04$ & & & $1.0 \mathrm{E}+06$ & $1.4 \mathrm{E}+04$ & & & $3.1 \mathrm{E}+05$ & $3.1 E+03$ & & & & \\
\hline Phenylenediamine, p- & $000106-50-3$ & & & $1.3 \mathrm{E}-01$ & $1.3 \mathrm{E}-02$ & & & $1.0 \mathrm{E}+06$ & $1.0 \mathrm{E}+06$ & & & $1.0 \mathrm{E}+06$ & $1.0 \mathrm{E}+06$ & $1.0 \mathrm{E}+06$ & $1.0 \mathrm{E}+06$ \\
\hline Phenylmercuric Acetate & $000062-38-4$ & & & & & & & & & & & & & & \\
\hline Phenylphenol, 2- & $000090-43-7$ & $6.3 \mathrm{E}+00$ & $6.3 \mathrm{E}-02$ & & & $7.4 E+04$ & $7.4 E+02$ & & & $1.6 E+04$ & $1.6 \mathrm{E}+02$ & & & & \\
\hline Phorate & $000298-02-2$ & & & 4.4E-03 & 4.4E-04 & & & $3.1 \mathrm{E}+03$ & $3.1 \mathrm{E}+02$ & & & $1.6 \mathrm{E}+03$ & $2.1 \mathrm{E}+02$ & $1.6 \mathrm{E}+02$ & $2.1 \mathrm{E}+01$ \\
\hline Phosmet & $000732-11-6$ & & & $9.4 \mathrm{E}-01$ & $9.4 \mathrm{E}-02$ & & & $2.5 \mathrm{E}+04$ & $2.5 \mathrm{E}+03$ & & & $1.3 \mathrm{E}+04$ & $1.6 \mathrm{E}+03$ & $1.3 E+03$ & $1.6 \mathrm{E}+02$ \\
\hline Phosphine & $007803-51-2$ & & & & & & & & & & & & & & \\
\hline Phosphoric Acid & $007664-38-2$ & & & & & & & & & & & & & & \\
\hline Phthalic Acid, P- & $000100-21-0$ & & & $2.2 E+01$ & $2.2 E+\infty 0$ & & & $1.0 E+06$ & $1.0 \mathrm{E}+06$ & & & $1.0 \mathrm{E}+06$ & $1.0 \mathrm{E}+06$ & $8.1 E+05$ & $1.0 \mathrm{E}+05$ \\
\hline Phthalic Anhydride & $000085-44-9$ & & & $2.8 \mathrm{E}+00$ & $2.8 \mathrm{E}-01$ & & & $1.0 \mathrm{E}+06$ & $1.0 \mathrm{E}+06$ & & & $1.0 \mathrm{E}+06$ & $1.0 \mathrm{E}+06$ & $1.0 \mathrm{E}+06$ & $1.0 \mathrm{E}+06$ \\
\hline Picloram & $001918-02-1$ & & & $2.7 \mathrm{E}+00$ & 2.7E-01 & & & $2.2 \mathrm{E}+05$ & $2.2 \mathrm{E}+04$ & & & $1.1 \mathrm{E}+05$ & $1.4 \mathrm{E}+04$ & $1.1 \mathrm{E}+04$ & $1.4 \mathrm{E}+03$ \\
\hline
\end{tabular}

Nonradionuclides/water/agricultural

Page 25 of 33 
Table 3.3a. (continued)

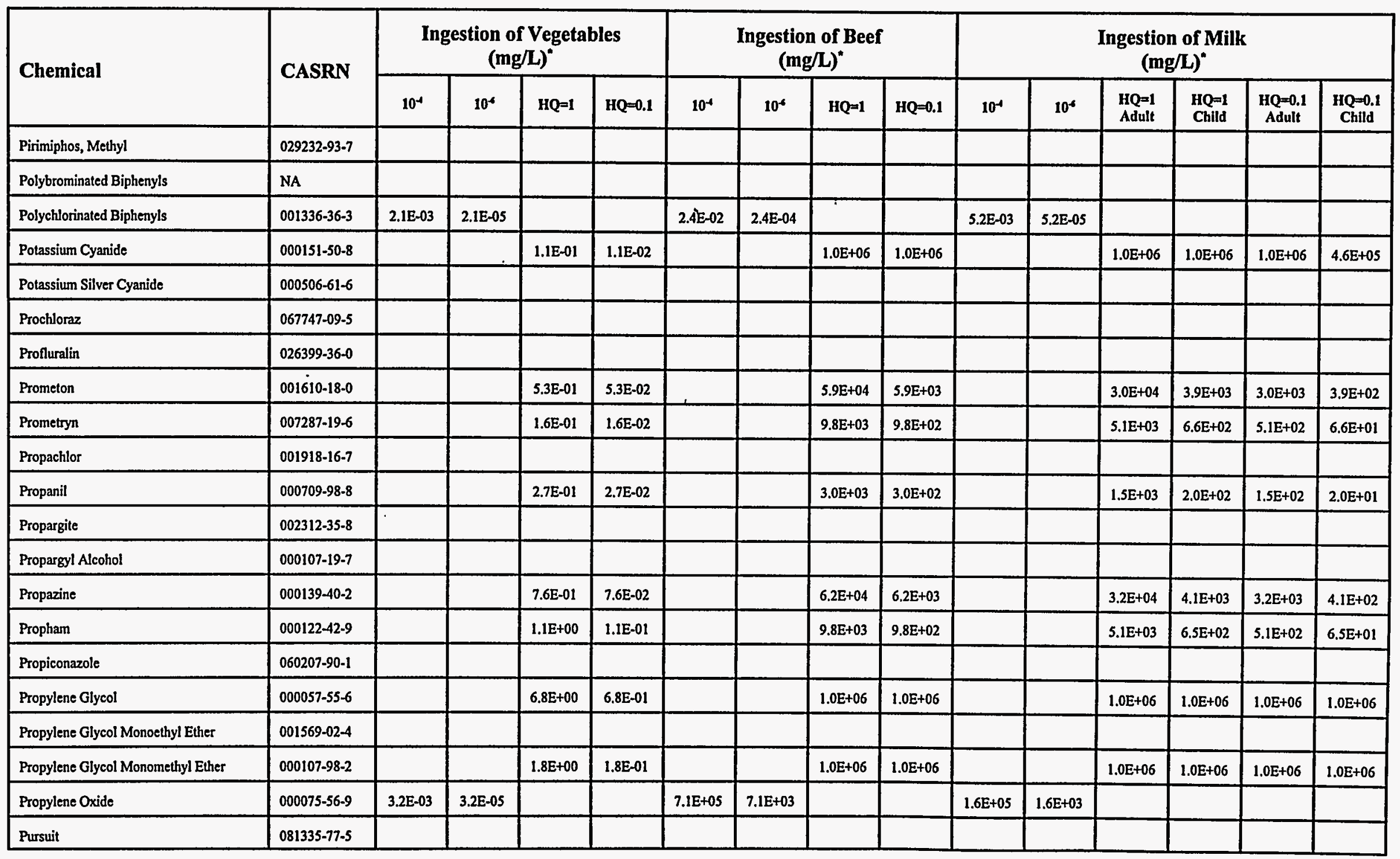

Nonradionuclides/water/agricultural

Page 26 of 33 
Table 3.3a. (continued)

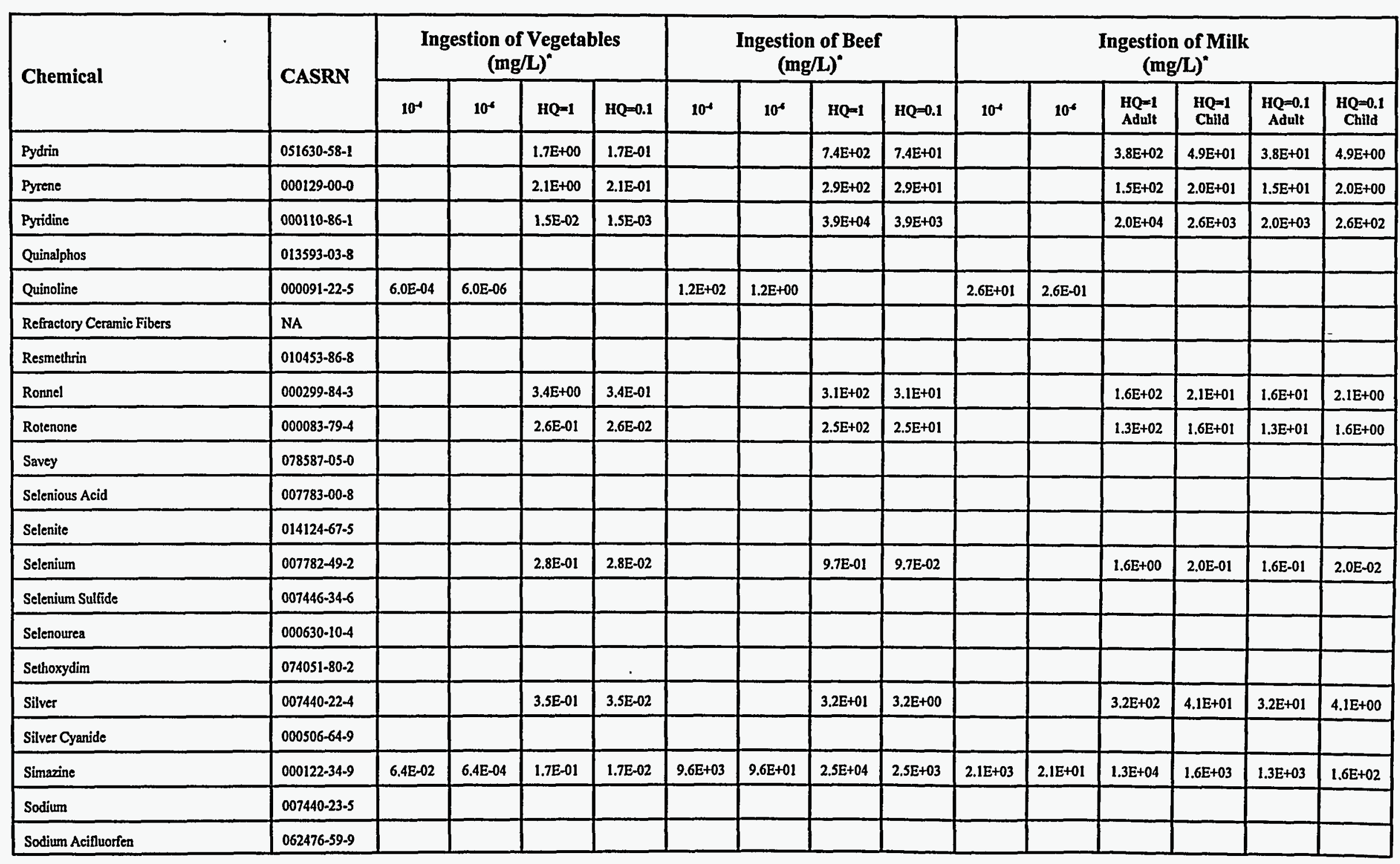




\begin{tabular}{|c|c|c|c|c|c|c|c|c|c|c|c|c|c|c|c|}
\hline $20-\pi \sigma^{*} \varepsilon$ & $\mathbb{1 0 - 3 0} \varepsilon$ & $10-36 \mathfrak{\varepsilon}$ & $00+ \pm 0^{\circ} \varepsilon$ & & & $10-365$ & $00+36 \mathrm{~s}$ & & & $\varepsilon 0-30 \tau$ & $20-302 z$ & & & $\varepsilon=-66-560000$ & 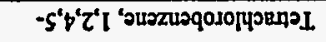 \\
\hline & & & & & & & & & & & & & & $L-06-0 Z 6100$ & 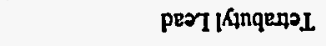 \\
\hline $10-366$ & $00+30 \div 2$ & $00+96 \%$ & $10+\exists i L$ & & & $10+367$ & $20+a b i$ & & & ع0-sz'9 & $z 0-a z^{\prime} 9$ & & & $0-0 S-988000$ & 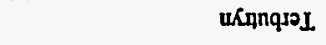 \\
\hline$z 0-3<z$ & $10-a \tau^{\prime} \tau$ & $10-3 c^{\prime} z$ & $00+312$ & & & 10-al't & $00+315$ & & & 60-as't & E0-as'I & & & $6-6 L-1<0 \& 10$ & sognqral \\
\hline & & & & & & & & & & & & & & $z-15-206500$ & !อвчدџ \\
\hline & & & & & & & & & & & & & & 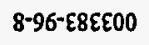 & soldow \\
\hline $50+99 z$ & $90+301$ & $90+90^{\circ} \mathrm{I}$ & $90+30^{\circ} \mathrm{I}$ & & & $90+90 \%$ & $90+30^{\prime} \mathrm{I}$ & & & $z 0-a<\tau$ & $10-3 L \tau$ & & . & 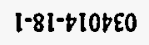 & นоm!ฺqnqวL \\
\hline & & & & & & & & & & & & & & $0-\angle I-\forall 9 s i z 0$ & GIWDI \\
\hline & & & & $80 \cdot 90^{\circ} \mathrm{b}$ & $90-30 . t$ & & & L0- & $50-98^{\prime \prime} \mathrm{I}$ & & & 80-GIT & 90-al't I & 6-IE-LOZISO & $\left(\omega^{-8} 8^{\prime} L^{\prime} \varepsilon^{\prime} \tau^{\prime} \exists a \partial I\right.$ \\
\hline & & & & $01-a^{2} t$ & $80 \cdot a z^{\prime}$ & & & $60-\pi 6^{\prime} \mathrm{I}$ & $\angle 0-36 . \mathrm{I}$ & & & $60-a r \cdot 1$ & LO-GI'I & $9-10-9+<100$ & 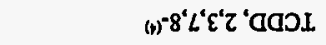 \\
\hline & & & & & & & & & & & & & & $0-68-1<9880$ & 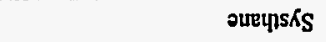 \\
\hline & & & & & & & & & & & & & & $8-6 L-808+10$ & शejns \\
\hline$\varepsilon 0+\Xi \varepsilon: I$ & $60+30^{\circ} \mathrm{I}$ & $p 0+ \pm E E^{\prime} I$ & $50+90^{\circ} I$ & & & $50+907$ & $50+90^{2} z$ & & & $10-986$ & $00+\$ 8 \cdot 6$ & & & $s-z+001000$ & (6) $)^{9} 231 / 4 S$ \\
\hline $10+30 z$ & $20+a s^{\prime} i$ & $20+90 z$ & $\varepsilon 0+\exists S^{\prime} \mathfrak{I}$ & & & $20+96 z$ & $\varepsilon 0+36 z$ & & & $50-96 . \mathrm{L}$ & $\varepsilon 0-36 \% \mathrm{~L}$ & & & $6-\$ z-\angle 50000$ & 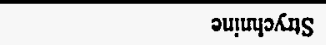 \\
\hline $00+988$ & $10+389$ & $10+988$ & $20+38 \cdot 9$ & & & $20+a s t$ & $\varepsilon 0+3 s: t$ & & & $00+a c z$ & $10+a<z$ & & & $9-t z-0 t \forall<00$ & गqQ1' 'umpurous \\
\hline & & & & & & & & & & & & & & $S \cdot I I-196000$ & 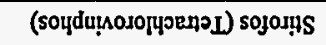 \\
\hline & & & & & & & & & & & & & & 8-9Z-8ILEIO & 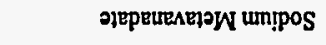 \\
\hline & & & & & & & & & & & & & & $8 \cdot 7 L-290000$ & 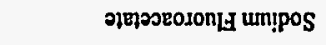 \\
\hline & & & & & & & & & & & & & & $5-81-8+1000$ & 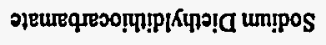 \\
\hline $90+90^{\circ} \mathrm{t}$ & $90+90^{\circ} \mathrm{I}$ & $90+90 \% \mathrm{I}$ & $90+30^{\prime} \mathrm{t}$ & & & $90+30^{\circ} \mathrm{I}$ & $90+90 \% 1$ & & & $\varepsilon 0-a b t$ & $20-3 t 5$ & & & $6-\varepsilon \varepsilon-\varepsilon \not 1000$ & opṭrexo un!pos \\
\hline & & & & & & & & & & & & & & 8-zz-8z99z0 & oppy um!pos \\
\hline $\begin{array}{c}\text { puบว } \\
I^{\prime} 0=\mathrm{OH}\end{array}$ & $\begin{array}{c}\text { InpV } \\
I^{\prime} 0=\partial H\end{array}$ & $\begin{array}{l}P \| 4 J \\
l=O H\end{array}$ & 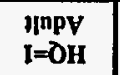 & ,0I & rOI & $I^{\circ} 0=0 H$ & $I=\mathrm{OH}$ & ,0I & rOI & $I^{\prime} 0=\partial H$ & $\mathrm{I}=\mathrm{OH}$ & ,0I & rOI & & \\
\hline & & भा!एW & $\begin{array}{l}\text { Buf) } \\
\text { lọ̣səశి }\end{array}$ & & & & јаәя & $\begin{array}{l}\text { ua) } \\
\text { o!̣sอ8ి }\end{array}$ & & & 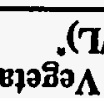 & $\begin{array}{l}\text { w) } \\
\text { uo!! }\end{array}$ & & N\&SVD & |вगุแ०นว \\
\hline
\end{tabular}


Table 3.3a. (continued)

\begin{tabular}{|c|c|c|c|c|c|c|c|c|c|c|c|c|c|c|c|}
\hline \multirow{2}{*}{ Chemical } & \multirow{2}{*}{ CASRN } & \multicolumn{4}{|c|}{$\begin{array}{c}\text { Ingestion of Vegetables } \\
(\mathrm{mg} / \mathrm{L})^{a}\end{array}$} & \multicolumn{4}{|c|}{$\begin{array}{c}\text { Ingestion of Beef } \\
(\mathrm{mg} / \mathrm{L})^{n}\end{array}$} & \multicolumn{6}{|c|}{$\begin{array}{c}\text { Ingestion of Milk } \\
(\mathrm{mg} / \mathrm{L})^{*}\end{array}$} \\
\hline & & $10^{-4}$ & $10^{6}$ & HQ=1 & $\mathrm{HQ}=0.1$ & $10^{-4}$ & $10^{5}$ & $\mathrm{HQ}=1$ & $H Q=0.1$ & $10^{-4}$ & $10^{4}$ & $\begin{array}{l}\text { HQ }=1 \\
\text { Adult }\end{array}$ & $\begin{array}{l}\text { HQ=1 } \\
\text { Child }\end{array}$ & $\begin{array}{l}\mathrm{HQ}=0.1 \\
\text { Adult }\end{array}$ & $\begin{array}{c}\text { HQ }=0.1 \\
\text { Child }\end{array}$ \\
\hline Tetrachloroethane, 1,1,1,2- & $000630-20-6$ & 4.6E-01 & $4.6 \mathrm{E}-03$ & $1.5 \mathrm{E}+00$ & $1.5 \mathrm{E}-01$ & $7.0 \mathrm{E}+03$ & $7.0 \mathrm{E}+01$ & $2.3 E+04$ & $2.3 \mathrm{E}+03$ & $1.5 \mathrm{E}+03$ & $1.5 \mathrm{E}+01$ & $1.2 \mathrm{E}+04$ & $1.6 \mathrm{E}+03$ & $1.2 \mathrm{E}+03$ & $1.6 \mathrm{E}+02$ \\
\hline Tetrachloroethane, 1,1,2,2. & $000079-34-5$ & 4.4E- -02 & 4.4E-04 & & & $3.6 \mathrm{E}+03$ & $3.6 \mathrm{E}+01$ & & & 8.0E+02 & $8.0 \mathrm{E}+00$ & & & & \\
\hline Tetrachloroethylene ${ }^{(6)}$ & $000127-18-4$ & 1.9E-01 & $1.9 \mathrm{E}-03$ & $4.2 \mathrm{E}-01$ & $4.2 \mathrm{E}-02$ & $8.8 \mathrm{E}+03$ & $8.8 \mathrm{E}+01$ & $2.0 \mathrm{E}+04$ & $2.0 \mathrm{E}+03$ & $1.9 \mathrm{E}+03$ & $1.9 \mathrm{E}+01$ & $1.0 \mathrm{E}+04$ & $1.3 \mathrm{E}+03$ & $1.0 \mathrm{E}+03$ & $1.3 E+02$ \\
\hline Tetrachlorophenol, 2,3,4,6- & $000058-90-2$ & & & $1.9 \mathrm{E}+00$ & $1.9 \mathrm{E}-01$ & & & $1.9 \mathrm{E}+03$ & $1.9 \mathrm{E}+02$ & & & $9.6 \mathrm{E}+02$ & $1.2 \mathrm{E}+02$ & $9.6 \mathrm{E}+01$ & $1.2 \mathrm{E}+01$ \\
\hline $\begin{array}{l}\text { Tetrachlorotoluene, p-alpha, alpha, } \\
\text { alpha- }\end{array}$ & $005216-25-1$ & & & & & & & & & & & & & & \\
\hline Tetraethyl Dithiopyrophosphate & $003689-24-5$ & & & $2.5 \mathrm{E}-02$ & $2.5 \mathrm{E}-03$ & & & $3.9 \mathrm{E}+02$ & $3.9 \mathrm{E}+01$ & & & $2.0 \mathrm{E}+02$ & $2.6 \mathrm{E}+01$ & $2.0 E+01$ & $2.6 \mathrm{E}+00$ \\
\hline Tetraethyl Lead & $000078-00-2$ & & & & & & & & & & & & & & \\
\hline Tetrafluoroethane, 1,1,1,2- & $000811-97-2$ & & & & & & & & & & & & & & \\
\hline Tetramethyl Lead & $000075-74-1$ & & & & & & & & & & & & & & \\
\hline Tetrapropyl Lead & $003440-75-3$ & & & & & & & & & & & & & & \\
\hline Thallic Oxide $e^{(3)}$ & $001314-32-5$ & & & & & & & & & & & & & & \\
\hline Thallium (I) Nitrate & $010102-45-1$ & & & & & & & & & & & & & & \\
\hline Thallium (Soluble Salts) & $007440-28.0$ & & & & & & & & & & & & & & \\
\hline Thallium Acetate & $000563-68-8$ & & & & & & & & & & & & & & \\
\hline Thallium Carbonate & $006533-73-9$ & & & & & & & & & & & & & & \\
\hline Thallium Chloride & $007791-12-0$ & & & & & & & & & & & & & & \\
\hline Thallium Selenite & $012039-52-0$ & & & & & & & & & & & & & & \\
\hline Thallium Sulfate & $007446-18-6$ & & & & & & & & & & & & & & \\
\hline Thiobencarb & $028249-77-6$ & & & & & & & & & & & & & & \\
\hline Thiofanox & $039196-18-4$ & & & $1.1 \mathrm{E}-04$ & $1.1 E-05$ & & & $1.0 \mathrm{E}+06$ & $1.0 \mathrm{E}+06$ & & & $1.0 \mathrm{E}+06$ & $7.3 \mathrm{E}+05$ & $5.7 \mathrm{E}+05$ & 7.3E+04 \\
\hline
\end{tabular}


Table 3.3a. (continued)

\begin{tabular}{|c|c|c|c|c|c|c|c|c|c|c|c|c|c|c|c|}
\hline \multirow{2}{*}{ Chemical } & \multirow{2}{*}{ CASRN } & \multicolumn{4}{|c|}{$\begin{array}{c}\text { Ingestion of Vegetables } \\
(\mathrm{mg} / \mathrm{L})^{\prime}\end{array}$} & \multicolumn{4}{|c|}{$\begin{array}{c}\text { Ingestion of Beef } \\
(\mathrm{mg} / \mathrm{L})^{*}\end{array}$} & \multicolumn{6}{|c|}{$\begin{array}{c}\text { Ingestion of Milk } \\
\text { (mg/L) }\end{array}$} \\
\hline & & $10^{-4}$ & $10^{4}$ & $\mathrm{HQ}=1$ & $\mathrm{HQ}=0.1$ & $10^{-4}$ & $10^{4}$ & $\mathrm{HQ}=1$ & $\mathrm{HQ}=0.1$ & $10^{-4}$ & $10^{4}$ & $\begin{array}{l}\text { HQ=1 } \\
\text { Adult }\end{array}$ & $\begin{array}{l}\mathrm{HQ}=1 \\
\text { Child }\end{array}$ & $\begin{array}{c}\mathrm{HQ}=0.1 \\
\text { Adult }\end{array}$ & $\begin{array}{c}H Q=0.1 \\
\text { Child }\end{array}$ \\
\hline Thiophanate, Methyl & $023564-05-8$ & & & & & & & & & & & & & & \\
\hline Thiram & $000137-26.8$ & & & & & & & & & & & & & & \\
\hline Thorium & $007440-29-1$ & & & & & & & & & & & & & & \\
\hline Tin & $007440-31-5$ & & & $2.3 \mathrm{E}+01$ & $2.3 E+00$ & & & $1.2 \mathrm{E}+03$ & $1.2 \mathrm{E}+02$ & $\cdot$ & & $1.9 \mathrm{E}+03$ & $2.5 \mathrm{E}+02$ & $1.9 \mathrm{E}+02$ & $2.5 \mathrm{E}+01$ \\
\hline Titanium & $007440-32-6$ & & & & & & & & & & & & & & \\
\hline Toluene $^{(())}$ & $000108-88-3$ & & & $8.9 \mathrm{E}+00$ & $8.9 \mathrm{E}-01$ & & & $3.1 E+05$ & $3.1 E+04$ & & & $1.6 \mathrm{E}+05$ & $2.1 E+04$ & $1.6 \mathrm{E}+04$ & $2.1 \mathrm{E}+03$ \\
\hline Toluene diisocyanate mixture (TDI) & $026471-62-5$ & & & & & & & & & & & & & & \\
\hline Toluene-2,4-diamine & $000095-80-7$ & 3.5E-04 & $3.5 \mathrm{E}-06$ & & & $2.6 \mathrm{E}+04$ & $2.6 \mathrm{E}+02$ & & & $5.7 \mathrm{E}+03$ & $5.7 \mathrm{E}+01$ & & & & \\
\hline Toluene-2,5-diamine & $000095-70-5$ & & & & & & & & & & & & & & \\
\hline Toluene-2,6-diamine & $000823-40-5$ & & & & & & & & & & & & & & \\
\hline Toluidine, o- (Methylaniline, 2-) & $000095-53-4$ & $1.4 \mathrm{E}-02$ & $1.4 \mathrm{E}-04$ & & & $3.8 \mathrm{E}+04$ & $3.8 \mathrm{E}+02$ & & & $8.3 \mathrm{E}+03$ & $8.3 \mathrm{E}+01$ & & & & \\
\hline Toluidine, p- & $000106-49-0$ & $3.5 \mathrm{E}-02$ & $3.5 \mathrm{E}-04$ & & & $9.6 \mathrm{E}+03$ & $9.6 \mathrm{E}+01$ & & & $2.1 \mathrm{E}+03$ & $2.1 \mathrm{E}+01$ & & & & \\
\hline Toxaphene & $008001-35-2$ & $1.4 \mathrm{E}-02$ & $1.4 \mathrm{E}-04$ & & & $2.6 \mathrm{E}+00$ & $2.6 \mathrm{E}-02$ & & & $5.8 \mathrm{E}-01$ & $5.8 \mathrm{E}-03$ & & & & \\
\hline Tralomethrin & $066841-25-6$ & & & & & & & & & & & & & & \\
\hline Triallate & $002303-17-5$ & & & $8.3 \mathrm{E}-01$ & $8.3 \mathrm{E}-02$ & & & $1.1 \mathrm{E}+03$ & $1.1 \mathrm{E}+02$ & & & $5.5 \mathrm{E}+02$ & $7.1 \mathrm{E}+01$ & $5.5 \mathrm{E}+01$ & $7.1 \mathrm{E}+00$ \\
\hline Triasulfuron & $082097-50-5$ & & & & & & & & & & & & & & \\
\hline Tribromobenzene, 1,2,4- & 000615-54-3 & & & & & & & & & & & & & & \\
\hline Tribromochloromethane & $000594-15-0$ & & & & & & & & & & & & & & \\
\hline Tribromodiphenyl Ether & $049690-94-0$ & & & & & & & & & & & & & & \\
\hline Tributyltin Oxide & $000056-35-9$ & & & & & & & & & & & & & & \\
\hline Trichloro-1,2,2-trifluoroethane, 1,1,2- & $000076-13-1$ & & & $1.3 \mathrm{E}+03$ & $1.3 \mathrm{E}+02$ & & & $1.0 \mathrm{E}+06$ & $1.0 \mathrm{E}+06$ & & & $1.0 E+06$ & $1.0 \mathrm{E}+06$ & $1.0 E+06$ & $3.9 \mathrm{E}+05$ \\
\hline
\end{tabular}

Nonradionuclides/water/agricultural

Page 30 of 33 
Table 3.3a. (continued)

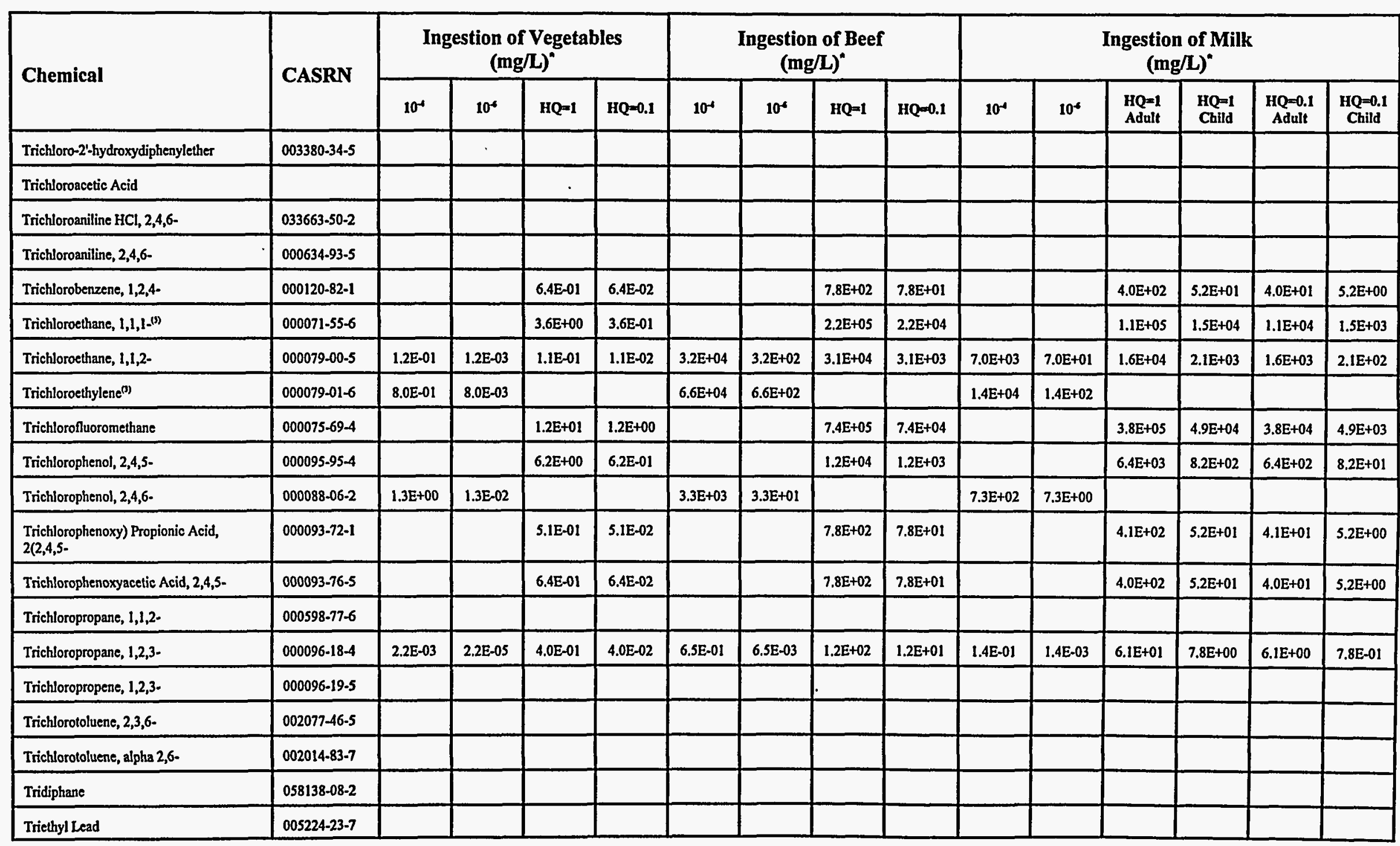


Table 3.3a. (continued)

\begin{tabular}{|c|c|c|c|c|c|c|c|c|c|c|c|c|c|c|c|}
\hline \multirow{2}{*}{ Chemical } & \multirow{2}{*}{ CASRN } & \multicolumn{4}{|c|}{$\begin{array}{l}\text { Ingestion of Vegetables } \\
(\mathrm{mg} / \mathrm{L})^{n}\end{array}$} & \multicolumn{4}{|c|}{$\begin{array}{l}\text { Ingestion of Beef } \\
(\mathrm{mg} / \mathrm{L})^{\circ}\end{array}$} & \multicolumn{6}{|c|}{$\begin{array}{c}\text { Ingestion of Milk } \\
(\mathrm{mg} / \mathrm{L})^{*}\end{array}$} \\
\hline & & $10^{-4}$ & $10^{5}$ & $H Q=1$ & $\mathrm{HQ}=0.1$ & $10^{-4}$ & $10^{4}$ & $\mathrm{HQ}=1$ & $\mathrm{HQ}=0.1$ & $10^{-4}$ & $10^{6}$ & $\begin{array}{l}H Q=1 \\
\text { Adult }\end{array}$ & $\begin{array}{l}\text { HQ=1 } \\
\text { Child }\end{array}$ & $\begin{array}{c}\text { HQ }=0.1 \\
\text { Adult }\end{array}$ & $\begin{array}{c}\mathrm{HQ}=0.1 \\
\text { Child }\end{array}$ \\
\hline Triethylamine & $000121-44-8$ & $\cdot$ & & & & & & & & & & & & & \\
\hline Trifluralin & $001582-09-8$ & $2.1 \mathrm{E}+00$ & 2.1E-02 & $5.2 \mathrm{E}-01$ & $5.2 \mathrm{E}-02$ & $1.2 \mathrm{E}+02$ & $1.2 \mathrm{E}+00$ & $2.9 \mathrm{E}+01$ & $2.9 \mathrm{E}+00$ & $2.6 \mathrm{E}+01$ & $2.6 \mathrm{E}-01$ & $1.5 \mathrm{E}+01$ & $1.9 \mathrm{E}+00$ & $1.5 \mathrm{E}+00$ & $1.9 \mathrm{E}-01$ \\
\hline Trimethyl Lead & $007442-13-9$ & & & & & & & & & & & & & & \\
\hline Trimethyl Phosphate & $000512-56-1$ & & & & & & & & & & & & & & \\
\hline Trimethylethyl Lead & $001762-26-1$ & & & & & & & & & & & & & & \\
\hline Trinitrobenzene, 1,3,5- & $000099-35-4$ & & & 6.7E-04 & $6.7 \mathrm{E}-05$ & & & $2.5 \mathrm{E}+03$ & $2.5 \mathrm{E}+02$ & & & $1.3 \mathrm{E}+03$ & $1.6 \mathrm{E}+02$ & $1.3 \mathrm{E}+02$ & $1.6 \mathrm{E}+01$ \\
\hline Trinitrophenylmethylnitramine & $000479-45-8$ & & & $2.9 \mathrm{E}-01$ & $2.9 \mathrm{E}-02$ & & & $7.8 \mathrm{E}+04$ & $7.8 \mathrm{E}+03$ & & & $4.0 \mathrm{E}+04$ & $5.2 \mathrm{E}+03$ & $4.0 \mathrm{E}+03$ & $5.2 \mathrm{E}+02$ \\
\hline Trinitrotoluene, 2,4,6- & $000118-96-7$ & $2.8 \mathrm{E}-01$ & $2.8 \mathrm{E}-03$ & $1.8 \mathrm{E}-02$ & $1.8 \mathrm{E}-03$ & $3.0 \mathrm{E}+04$ & $3.0 \mathrm{E}+02$ & $2.0 \mathrm{E}+03$ & $2.0 \mathrm{E}+02$ & $6.7 \mathrm{E}+03$ & $6.7 E+01$ & $1.0 \mathrm{E}+03$ & $1.3 \mathrm{E}+02$ & $1.0 \mathrm{E}+02$ & $1.3 \mathrm{E}+01$ \\
\hline Tripropyl Lead & 006618-03-7 & & & & & & & & & & & & & & \\
\hline Uranium (Soluble Salts) & NA & & & 2.1E-01 & 2.1E-02 & & & $1.9 \mathrm{E}+02$ & $1.9 \mathrm{E}+01$ & & & $2.4 \mathrm{E}+01$ & $3.1 \mathrm{E}+00$ & $2.4 E+00$ & $3.1 \mathrm{E}-01$ \\
\hline Vanadium Pentoxide & $001314-62-1$ & & & & & & & & & & & & & & \\
\hline Vanadium Sulfate & $036907-42-3$ & & & & & & & & & & & & & & \\
\hline Vanadium, Metallic & $007440-62-2$ & & & $4.9 \mathrm{E}-01$ & $4.9 \mathrm{E}-02$ & & & $5.5 E+01$ & $5.5 \mathrm{E}+00$ & & & $1.1 \mathrm{E}+03$ & $1.4 E+02$ & $1.1 \mathrm{E}+02$ & $1.4 \mathrm{E}+01$ \\
\hline Vanadyl Sulfate & $027774-13-6$ & & & & & & & & & & & & & & \\
\hline Vernolate & $001929-77.7$ & & & & & & & & & & & & & & \\
\hline Vinclozolin & $050471-44-8$ & & & & & & & & & & & & & & \\
\hline Vinyl Acetate & $000108-05-4$ & & & $7.8 \mathrm{E}+00$ & $7.8 \mathrm{E}-01$ & & & $1.0 \mathrm{E}+06$ & $1.0 \mathrm{E}+06$ & & & $1.0 \mathrm{E}+06$ & $1.0 \mathrm{E}+06$ & $1.0 \mathrm{E}+06$ & $9.7 \mathrm{E}+05$ \\
\hline Vinyl Bromide & $000593-60-2$ & & & & & & & & & & & & & & \\
\hline Vinyl Chloride & $000075-01-4$ & $2.0 \mathrm{E}-03$ & $2.0 \mathrm{E}-05$ & & & $3.8 \mathrm{E}+03$ & $3.8 \mathrm{E}+01$ & & & $8.4 E+02$ & $8.4 \mathrm{E}+00$ & & & & \\
\hline Warfarin & $000081-81-2$ & & & $1.6 \mathrm{E}-02$ & $1.6 \mathrm{E}-03$ & & & $1.5 \mathrm{E}+02$ & $1.5 \mathrm{E}+01$ & & & $7.6 \mathrm{E}+01$ & $9.8 \mathrm{E}+00$ & $7.6 \mathrm{E}+00$ & $9.8 \mathrm{E}-01$ \\
\hline White Phosphorus & 007723-14-0 & & & & & & & & & & & & & & \\
\hline
\end{tabular}


Table 3.3a. (continued)

\begin{tabular}{|c|c|c|c|c|c|c|c|c|c|c|c|c|c|c|c|}
\hline \multirow{2}{*}{ Chemical } & \multirow{2}{*}{ CASRN } & \multicolumn{4}{|c|}{$\begin{array}{l}\text { Ingestion of Vegetables } \\
(\mathrm{mg} / \mathrm{L})^{*}\end{array}$} & \multicolumn{4}{|c|}{$\begin{array}{c}\text { Ingestion of Beef } \\
(\mathrm{mg} / \mathrm{L})^{*}\end{array}$} & \multicolumn{6}{|c|}{$\begin{array}{c}\text { Ingestion of Milk } \\
(\mathrm{mg} / \mathrm{L})^{*}\end{array}$} \\
\hline & & $10^{-4}$ & $10^{6}$ & $\mathrm{HQ}=1$ & $\mathrm{HQ}=0.1$ & $10^{-4}$ & $10^{4}$ & $H Q=1$ & $\mathrm{HQ}=0.1$ & $10^{-4}$ & $10^{4}$ & $\begin{array}{l}\mathrm{HQ}=1 \\
\text { Adult }\end{array}$ & $\begin{array}{l}H Q=1 \\
\text { Child }\end{array}$ & $\begin{array}{c}\text { HQ }=0.1 \\
\text { Adult }\end{array}$ & $\begin{array}{c}\text { HQ }=0.1 \\
\text { Child }\end{array}$ \\
\hline Xylene, Mixture ${ }^{(b)}$ & $001330-20-7$ & & & $1.1 \mathrm{E}+02$ & $1.1 E+01$ & & & $7.8 \mathrm{E}+05$ & $7.8 \mathrm{E}+04$ & & & $4.0 E+05$ & $5.2 \mathrm{E}+04$ & $4.0 \mathrm{E}+04$ & $5.2 \mathrm{E}+03$ \\
\hline Xylene, $P-(0)$ & $000106-42-3$ & & & & & & & & & & & & & & \\
\hline Xylene, $\mathrm{m}_{-}^{(0)}$ & $000108-38-3$ & & & $1.1 \mathrm{E}+02$ & $1.1 \mathrm{E}+01$ & & & $9.8 \mathrm{E}+05$ & $9.8 \mathrm{E}+04$ & & & $5.1 \mathrm{E}+05$ & $6.5 \mathrm{E}+04$ & $5.1 E+04$ & $6.5 \mathrm{E}+03$ \\
\hline Xylene, $0^{(6)}$ & $000095-47-6$ & & & $1.1 \mathrm{E}+02$ & $1.1 \mathrm{E}+01$ & & & $9.8 \mathrm{E}+05$ & $9.8 \mathrm{E}+04$ & & & $5.1 E+05$ & $6.5 \mathrm{E}+04$ & $5.1 \mathrm{E}+04$ & $6.5 \mathrm{E}+03$ \\
\hline Zine (Metallic) & $007440-66-6$ & & & $1.2 \mathrm{E}+01$ & $1.2 \mathrm{E}+00$ & & & $5.8 \mathrm{E}+01$ & $5.8 \mathrm{E}+00$ & & & $9.6 \mathrm{E}+01$ & $1.2 \mathrm{E}+01$ & $9.6 \mathrm{E}+00$ & $1.2 E+00$ \\
\hline Zine Cyanide & $000557-21-1$ & & & & & & & & & & & & & & \\
\hline Zine Phosphide & $001314-84-7$ & & & & & & & & & & & & & & \\
\hline Zineb & $012122-67-7$ & & & & & & & & & & & & & & \\
\hline Zirconium & $007440-67-7$ & & & & & & & & & & & & & & \\
\hline
\end{tabular}

Notes:

* By definition, $1 \mathrm{~L}$ (water) $=1.0 \mathrm{E}+06 \mathrm{mg}$ (water). Therefore, a limit of $1.0 \mathrm{E}+06 \mathrm{mg} / \mathrm{L}$ was used for any PRG that was calculated to be above this limit.

(1) The same oral slope factor as used for polychlorinated biphenyls.

(2) The PRG was calculated considering Supplemental Guidance from RAGS: Region 4 Bulletins, Human Health Risk Assessment (Interim Guidance) from EPA (received February 11 , 1992). The slope factors for chemicals so marked indicate that a toxicity equivalency factor (TEF) method was used for carcinogenic PAHs based on each compound's relative potency to the potency of benzo[a]pyrene. The following TEFs were used to convert each PAH slope factor to an equivalent slope factor of benzo[a]pyrene [that has a new cancer slope factor of 7.3 (mg/kg/day) ${ }^{-1}$ identified by EPA's CRAVE workgroup]: (1) benzo[a]pyrene, TEF $=1.0$; (2) benzo[a]anthracene, TEF $=0.1 ;$ (3) benzo[b]fluoranthene, TEF $=0.1 ;(4)$ benzo[k]fluoranthene, TEF $=0.01 ;(5)$ chrysene, $\mathrm{TEF}=0.001 ;{ }^{(6)}$ dibenz[a,h]anthracene, $\mathrm{TEF}=1.0$; and $(7)$ indeno[1,2,3-c,d]pyrene, $\mathrm{TEF}=0.1$.

(3) The toxicity values for trichloroethylene have been withdrawn from IRIS and HEAST pending further investigation. However, the most recent toxicity values have been used to calculate the PRG.

(4) The PRG was calculated considering Supplemental Guidance from RAGS: Region 4 Bulletins, Human Health Risk Assessment (Interim Guidance) from EPA (November 1995). The slope factors for chemicals so marked indicate that a TEF methodology was used for chlorinated dioxin and furan congeners based on each compound's relative potency to the potency of $2,3,7,8$ tetrachlorodibenzodioxin (TCDD). The following TEFs were used to convert each slope factor to an equivalent slope factor of 2,3,7,8-TCDD: (1) 2,3,7,8-TCDD, TEF = 1.0; (2) 2,3,7,8-PeCDD, $\mathrm{TEF}=0.5 ;(3) 2,3,7,8-\mathrm{HxCDD}$, TEF $=0.1 ;(4) 2,3,7,8-\mathrm{HpCDD}$, TEF $=0.01 ;(5) \mathrm{OCDD}, \mathrm{TEF}=0.001 ;(6) 2,3,7,8-\mathrm{TCDF}, \mathrm{TEF}=0.1 ;(7) 1,2,3,7,8 \mathrm{-PeCDF}, \mathrm{TEF}=0.5 ;(8) 2,3,4,7,8-\mathrm{PeCDF}$, $\mathrm{TEF}=0.05 ;$ (9) $2,3,7,8-\mathrm{HxCDF}, \mathrm{TEF}=0.1 ;(10) 2,3,7,8-\mathrm{HpCDF}, \mathrm{TEF}=0.01$; and $(11) \mathrm{OCDF}, \mathrm{TEF}=0.001$.

(5) Toxicity values for these chemicals have been withdrawn from IRIS and HEAST pending further investigation. However, the most recent toxicity values have been used to calculate the PRG.

(6) Toxicity values for these chemicals were obtained from the Superfund Health Risk Technical Support Center. 
$3-386$

,

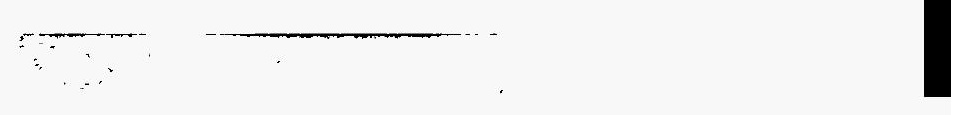




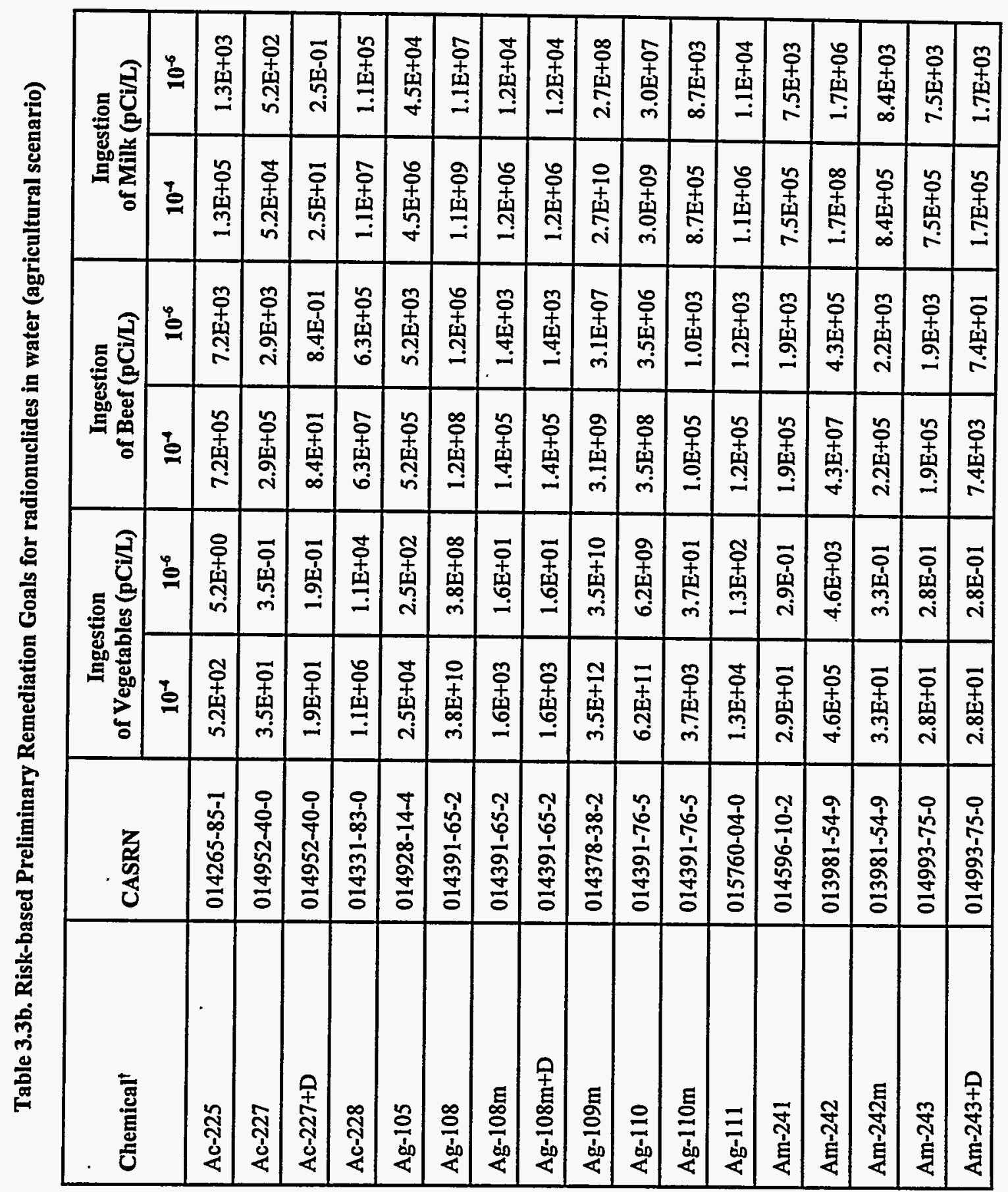


Table 3.3b. (continued)

\begin{tabular}{|c|c|c|c|c|c|c|c|}
\hline \multirow[t]{2}{*}{ Chemical ${ }^{\dagger}$} & \multirow[t]{2}{*}{ CASRN } & \multicolumn{2}{|c|}{$\begin{array}{c}\text { Ingestion } \\
\text { of Vegetables }(\mathrm{pCi} / \mathrm{L})\end{array}$} & \multicolumn{2}{|c|}{$\begin{array}{c}\text { Ingestion } \\
\text { of Beef }(p C i / L)\end{array}$} & \multicolumn{2}{|c|}{$\begin{array}{c}\text { Ingestion } \\
\text { of Milk (pCi/L) }\end{array}$} \\
\hline & & $10^{-4}$ & $10^{-6}$ & $10^{-4}$ & $10^{-6}$ & $10^{-4}$ & $10^{-6}$ \\
\hline $\mathrm{Ar}-41$ & 014163-25-8 & & & & & & \\
\hline At-217 & 017239-90-6 & $1.3 \mathrm{E}+17$ & $1.3 \mathrm{E}+15$ & $2.8 \mathrm{E}+10$ & $2.8 E+08$ & $4.1 E+09$ & 4.1E+07 \\
\hline Au-196 & 014914-16-0 & $7.7 E+04$ & $7.7 \mathrm{E}+02$ & $3.9 \mathrm{E}+05$ & $3.9 \mathrm{E}+03$ & $2.8 \mathrm{E}+07$ & $2.8 \mathrm{E}+05$ \\
\hline $\mathrm{Au}-198$ & 010043-49-0 & $3.6 \mathrm{E}+04$ & $3.6 \mathrm{E}+02$ & $9.6 \mathrm{E}+04$ & $9.6 \mathrm{E}+02$ & $7.0 \mathrm{E}+06$ & $7.0 \mathrm{E}+04$ \\
\hline $\mathrm{Ba}-131$ & 014914-75-1 & $4.0 \mathrm{E}+04$ & $4.0 \mathrm{E}+02$ & $7.5 E+06$ & $7.5 \mathrm{E}+04$ & $4.5 E+05$ & $4.5 \mathrm{E}+03$ \\
\hline Ba-133 & $013981-41-4$ & $5.6 \mathrm{E}+03$ & $5.6 \mathrm{E}+01$ & $4.7 E+06$ & $4.7 \mathrm{E}+04$ & $2.8 E+05$ & $2.8 \mathrm{E}+03$ \\
\hline $\mathrm{Ba}-133 \mathrm{~m}$ & $013981-41-4$ & $1.1 \mathrm{E}+05$ & $1.1 \mathrm{E}+03$ & $4.6 \mathrm{E}+06$ & $4.6 \mathrm{E}+04$ & $2.8 \mathrm{E}+05$ & $2.8 \mathrm{E}+03$ \\
\hline $\mathrm{Ba}-137 \mathrm{~m}$ & $013981-97-0$ & $1.0 \mathrm{E}+11$ & $1.0 \mathrm{E}+09$ & $5.2 \mathrm{E}+09$ & $5.2 \mathrm{E}+07$ & $3.1 \mathrm{E}+08$ & $3.1 \mathrm{E}+06$ \\
\hline Ba-139 & 014378-25-7 & $2.5 \mathrm{E}+07$ & $2.5 \mathrm{E}+05$ & $4.2 \mathrm{E}+07$ & $4.2 \mathrm{E}+05$ & $2.5 \mathrm{E}+06$ & $2.5 \mathrm{E}+04$ \\
\hline $\mathrm{Ba}-140$ & 014798-08-4 & $5.5 \mathrm{E}+03$ & $5.5 \mathrm{E}+01$ & $1.1 \mathrm{E}+06$ & 1.1E+04 & $6.5 \mathrm{E}+04$ & $6.5 \mathrm{E}+02$ \\
\hline $\mathrm{Be}-7$ & $013966-02-4$ & $4.5 \mathrm{E}+05$ & $4.5 E+03$ & $2.9 \mathrm{E}+07$ & $2.9 \mathrm{E}+05$ & 4.7E+09 & $4.7 \mathrm{E}+07$ \\
\hline $\mathrm{Bi}-206$ & 015776-19-9 & $1.4 \mathrm{E}+04$ & $1.4 \mathrm{E}+02$ & $8.9 \mathrm{E}+05$ & $8.9 \mathrm{E}+03$ & $1.0 \mathrm{E}+05$ & $1.0 \mathrm{E}+03$ \\
\hline $\mathrm{Bi}-207$ & 013982-38-2 & $2.2 \mathrm{E}+03$ & $2.2 \mathrm{E}+01$ & $1.3 \mathrm{E}+06$ & $1.3 \mathrm{E}+04$ & $1.5 \mathrm{E}+05$ & $1.5 E+03$ \\
\hline $\mathrm{Bi}-210$ & 014331-79-4 & $1.6 \mathrm{E}+04$ & $1.6 \mathrm{E}+02$ & $8.7 \mathrm{E}+05$ & $8.7 \mathrm{E}+03$ & $1.0 \mathrm{E}+05$ & $1.0 \mathrm{E}+03$ \\
\hline $\mathrm{Bi}-211$ & 015229-37-5 & $1.6 \mathrm{E}+10$ & $1.6 \mathrm{E}+08$ & $3.5 \mathrm{E}+08$ & $3.5 \mathrm{E}+06$ & $4.0 \mathrm{E}+07$ & $4.0 \mathrm{E}+05$ \\
\hline Bi-212 & 014913-49-6 & $1.7 \mathrm{E}+07$ & $1.7 \mathrm{E}+05$ & $1.0 \mathrm{E}+07$ & $1.0 \mathrm{E}+05$ & $1.2 \mathrm{E}+06$ & $1.2 \mathrm{E}+04$ \\
\hline $\mathrm{Bi}-213$ & $015776-20-2$ & $3.1 \mathrm{E}+07$ & $3.1 E+05$ & $1.4 \mathrm{E}+07$ & $1.4 \mathrm{E}+05$ & $1.7 \mathrm{E}+06$ & $1.7 \mathrm{E}+04$ \\
\hline
\end{tabular}


Table 3.3b. (continued)

\begin{tabular}{|l|l|l|c|c|c|c|c|}
\hline \multirow{2}{*}{ Chemical $^{\dagger}$} & \multirow{2}{*}{ CASRN } & \multicolumn{2}{|c|}{$\begin{array}{c}\text { Ingestion } \\
\text { of Vegetables (pCi/L) }\end{array}$} & \multicolumn{2}{c|}{$\begin{array}{c}\text { Ingestion } \\
\text { of Beef (pCi/L) }\end{array}$} & \multicolumn{2}{c|}{$\begin{array}{c}\text { Ingestion } \\
\text { of Milk (pCi/L) }\end{array}$} \\
\cline { 5 - 9 } & & $10^{-4}$ & $10^{-6}$ & $10^{-4}$ & $10^{-6}$ & $10^{-4}$ & $10^{-6}$ \\
\hline Bi-214 & $014733-03-0$ & $1.6 \mathrm{E}+08$ & $1.6 \mathrm{E}+06$ & $3.3 \mathrm{E}+07$ & $3.3 \mathrm{E}+05$ & $3.8 \mathrm{E}+06$ & $3.8 \mathrm{E}+04$ \\
\hline Br-82 & $014686-69-2$ & $2.3 \mathrm{E}+05$ & $2.3 \mathrm{E}+03$ & $7.2 \mathrm{E}+04$ & $7.2 \mathrm{E}+02$ & $1.3 \mathrm{E}+04$ & $1.3 \mathrm{E}+02$ \\
\hline $\mathrm{C}-11$ & $014333-33-6$ & & & & & & \\
\hline $\mathrm{C}-14$ & $014762-75-5$ & & & & & & \\
\hline $\mathrm{C}-15$ & $015929-23-4$ & & & & & & \\
\hline $\mathrm{Ca}-45$ & $013966-05-7$ & $1.3 \mathrm{E}+04$ & $1.3 \mathrm{E}+02$ & $6.3 \mathrm{E}+05$ & $6.3 \mathrm{E}+03$ & $6.1 \mathrm{E}+04$ & $6.1 \mathrm{E}+02$ \\
\hline $\mathrm{Ca}-47$ & $001439-99-2$ & $1.8 \mathrm{E}+04$ & $1.8 \mathrm{E}+02$ & $1.9 \mathrm{E}+05$ & $1.9 \mathrm{E}+03$ & $1.8 \mathrm{E}+04$ & $1.8 \mathrm{E}+02$ \\
\hline $\mathrm{Cd}-109$ & $014109-32-1$ & $3.3 \mathrm{E}+03$ & $3.3 \mathrm{E}+01$ & $7.9 \mathrm{E}+05$ & $7.9 \mathrm{E}+03$ & $4.6 \mathrm{E}+04$ & $4.6 \mathrm{E}+02$ \\
\hline $\mathrm{Cd}-115$ & $014336-68-6$ & $3.1 \mathrm{E}+04$ & $3.1 \mathrm{E}+02$ & $8.7 \mathrm{E}+05$ & $8.7 \mathrm{E}+03$ & $5.0 \mathrm{E}+04$ & $5.0 \mathrm{E}+02$ \\
\hline $\mathrm{Cd}-115 \mathrm{~m}$ & $014336-68-6$ & $2.8 \mathrm{E}+03$ & $2.8 \mathrm{E}+01$ & $4.5 \mathrm{E}+05$ & $4.5 \mathrm{E}+03$ & $2.6 \mathrm{E}+04$ & $2.6 \mathrm{E}+02$ \\
\hline $\mathrm{Ce}-141$ & $013967-74-3$ & $1.1 \mathrm{E}+04$ & $1.1 \mathrm{E}+02$ & $3.2 \mathrm{E}+07$ & $3.2 \mathrm{E}+05$ & $3.1 \mathrm{E}+06$ & $3.1 \mathrm{E}+04$ \\
\hline $\mathrm{Ce}-143$ & $014119-19-8$ & $5.8 \mathrm{E}+04$ & $5.8 \mathrm{E}+02$ & $2.1 \mathrm{E}+07$ & $2.1 \mathrm{E}+05$ & $2.1 \mathrm{E}+06$ & $2.1 \mathrm{E}+04$ \\
\hline $\mathrm{Ce}-144$ & $014762-78-8$ & $1.0 \mathrm{E}+03$ & $1.0 \mathrm{E}+01$ & $4.3 \mathrm{E}+06$ & $4.3 \mathrm{E}+04$ & $4.1 \mathrm{E}+05$ & $4.1 \mathrm{E}+03$ \\
\hline $\mathrm{Ce}-144+\mathrm{D}$ & $014762-78-8$ & $1.0 \mathrm{E}+03$ & $1.0 \mathrm{E}+01$ & $1.4 \mathrm{E}+05$ & $1.4 \mathrm{E}+03$ & $1.8 \mathrm{E}+05$ & $1.8 \mathrm{E}+03$ \\
\hline $\mathrm{Cl}-36$ & $013981-43-6$ & $8.4 \mathrm{E}+01$ & $8.4 \mathrm{E}-01$ & $5.7 \mathrm{E}+04$ & $5.7 \mathrm{E}+02$ & $9.7 \mathrm{E}+03$ & $9.7 \mathrm{E}+01$ \\
\hline $\mathrm{Cl}-38$ & $014158-34-0$ & $6.0 \mathrm{E}+07$ & $6.0 \mathrm{E}+05$ & $6.1 \mathrm{E}+05$ & $6.1 \mathrm{E}+03$ & $1.0 \mathrm{E}+05$ & $1.0 \mathrm{E}+03$ \\
\hline $\mathrm{Cm}-242$ & $015510-73-3$ & $8.6 \mathrm{E}+02$ & $8.6 \mathrm{E}+00$ & $3.3 \mathrm{E}+06$ & $3.3 \mathrm{E}+04$ & $2.4 \mathrm{E}+07$ & $2.4 \mathrm{E}+05$ \\
\hline
\end{tabular}




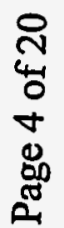

\begin{tabular}{|c|c|c|c|c|c|c|c|c|c|c|c|c|c|c|c|c|c|c|}
\hline : & $\stackrel{\varphi}{g}$ & 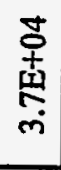 & 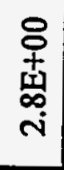 & $\begin{array}{l}\text { 吉 } \\
\text { 蒋 } \\
\text { 子 }\end{array}$ & $\begin{array}{c}\text { 古 } \\
\text { 索 } \\
\text { i }\end{array}$ & 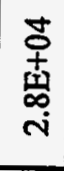 & $\begin{array}{l}\text { 志 } \\
\text { 索 } \\
\dot{m}\end{array}$ & 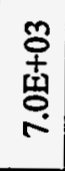 & $\begin{array}{l}\text { 草 } \\
\text { 索 } \\
\text { s. }\end{array}$ & $\begin{array}{l}+ \\
0 \\
+ \\
\text { 壱 } \\
-\end{array}$ & 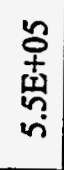 & 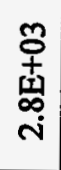 & 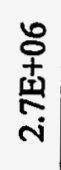 & 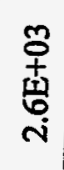 & 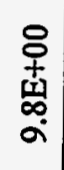 & 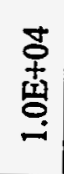 & 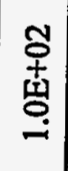 & 宫 \\
\hline 里突 & $\stackrel{7}{0}$ & $\begin{array}{c}8 \\
\stackrel{+}{+} \\
\text { 空 } \\
\text { ஸे }\end{array}$ & 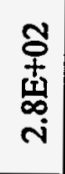 & 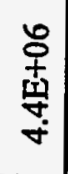 & 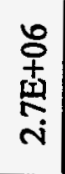 & $\begin{array}{l}0 \\
+ \\
+10 \\
\infty \\
i \\
i\end{array}$ & 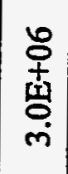 & 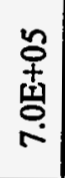 & 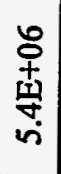 & 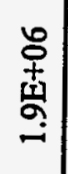 & $\begin{array}{c}5 \\
\vdots \\
\text { 䓌 } \\
\text { vi }\end{array}$ & $\begin{array}{l}n \\
+ \\
\vdots \\
\infty \\
i \\
i\end{array}$ & 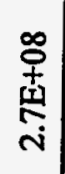 & 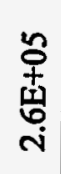 & 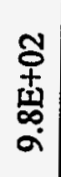 & 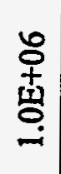 & $\begin{array}{l}\text { 草 } \\
\text { 总 } \\
\text { - }\end{array}$ & $\begin{array}{l}\frac{m}{0} \\
\frac{1}{ \pm 0} \\
0 \\
0\end{array}$ \\
\hline 옳 & $\stackrel{\varphi}{\circ}$ & 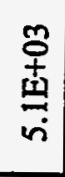 & $\begin{array}{c}8 \\
+ \\
\text { ta } \\
\\
\text { i }\end{array}$ & 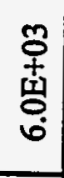 & 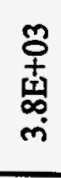 & 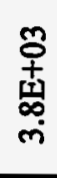 & 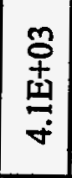 & 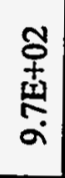 & 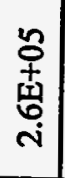 & 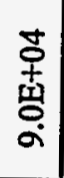 & 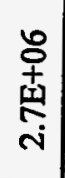 & $\begin{array}{c}\text { 草 } \\
\text { 學 } \\
-\end{array}$ & $\begin{array}{l}\text { 古 } \\
\text { 壱 } \\
\text { i }\end{array}$ & 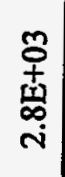 & 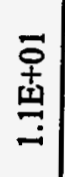 & 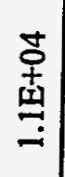 & 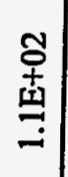 & 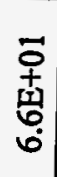 \\
\hline 总离 & తo & 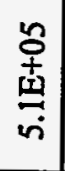 & $\begin{array}{c}\text { O̊ } \\
\text { 豈 } \\
\text { ni }\end{array}$ & 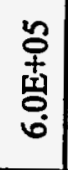 & 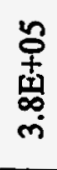 & $\begin{array}{l}n \\
0 \\
+ \\
\text { 亲 } \\
\text { m }\end{array}$ & 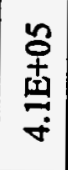 & 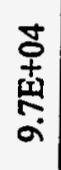 & 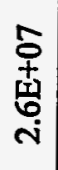 & $\begin{array}{l}\text { oे } \\
\text { 章 } \\
0 \\
0\end{array}$ & 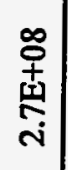 & $\begin{array}{c}\text { o̊ } \\
\text { 罊 } \\
-\end{array}$ & 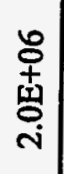 & $\begin{array}{l}n \\
+ \\
+1 \\
\infty \\
i\end{array}$ & 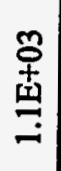 & 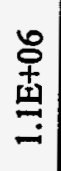 & 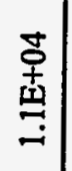 & $\begin{array}{l}\text { m } \\
\text { 吉 } \\
\text { o } \\
0\end{array}$ \\
\hline 莣 & $\stackrel{9}{9}$ & 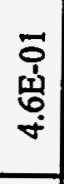 & 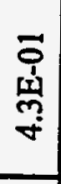 & 㔬 & 离 & 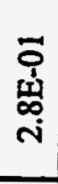 & 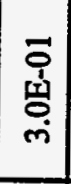 & 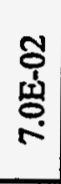 & 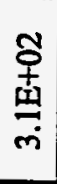 & 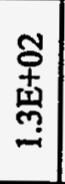 & 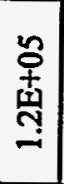 & 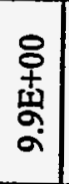 & 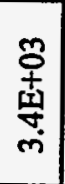 & 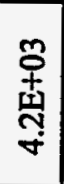 & 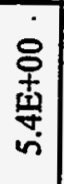 & 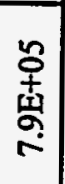 & $\begin{array}{l}\overline{0} \\
\text { 至 } \\
\text { i }\end{array}$ & 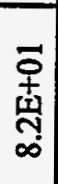 \\
\hline 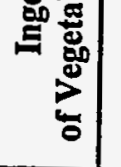 & $\stackrel{7}{\underline{\theta}}$ & 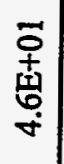 & 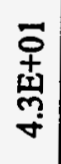 & 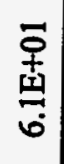 & $\begin{array}{c}\overline{0} \\
\text { 亲 } \\
\text { i }\end{array}$ & $\begin{array}{l}\overline{0} \\
+ \\
10 \\
0 \\
i\end{array}$ & $\begin{array}{l}\bar{\sigma} \\
+ \\
\text { 点 } \\
\dot{m}\end{array}$ & 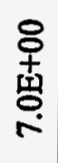 & 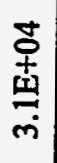 & 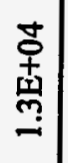 & $\begin{array}{c}\text { S } \\
\text { 壱 } \\
\text { ণ } \\
-\end{array}$ & $\begin{array}{l}\text { ơ } \\
\text { 索 } \\
\text { à }\end{array}$ & 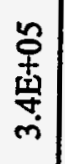 & 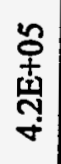 & 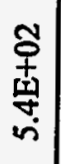 & $\begin{array}{c}5 \\
\text { +⿱ } \\
\text { 高 } \\
\end{array}$ & 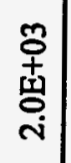 & 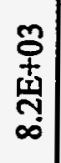 \\
\hline$\frac{Z}{\tilde{z}}$ & & $\begin{array}{l}0 \\
\frac{1}{1} \\
0 \\
\frac{1}{n} \\
\frac{1}{n} \\
0 \\
0\end{array}$ & $\begin{array}{l}0 \\
\frac{1}{0} \\
0 \\
\frac{1}{n} \\
\frac{n}{n} \\
0 \\
0\end{array}$ & 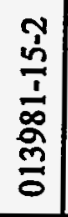 & 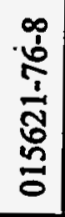 & 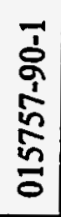 & 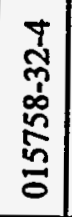 & 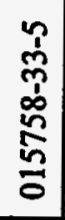 & 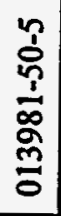 & 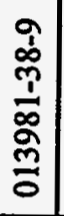 & 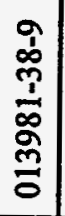 & 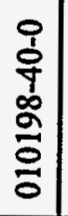 & 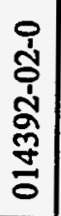 & 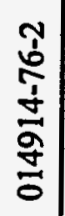 & 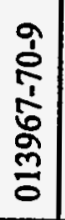 & 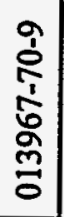 & 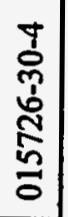 & 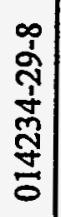 \\
\hline 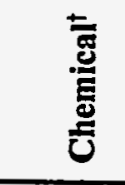 & & $\begin{array}{l}\stackrel{?}{\sim} \\
\stackrel{\mathcal{U}}{U}\end{array}$ & 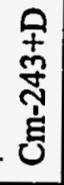 & 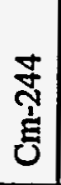 & 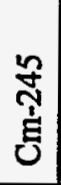 & 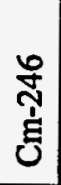 & $\begin{array}{l}\hat{\mathcal{A}} \\
\text { g }\end{array}$ & 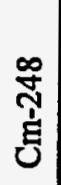 & î́ & i̊ & $\begin{array}{l}\mathbf{g} \\
\infty \\
p \\
\delta \\
ن\end{array}$ & $\begin{array}{l}80 \\
\vdots \\
0\end{array}$ & ปี & $\frac{\bar{m}}{\dot{b}}$ & $\begin{array}{l}\stackrel{ \pm}{7} \\
\stackrel{5}{0}\end{array}$ & $\frac{\text { g }}{\frac{\dot{m}}{\delta}}$ & $\frac{n}{\dot{m}}$ & $\frac{0}{m}$ \\
\hline
\end{tabular}




\begin{tabular}{|c|c|c|c|c|c|c|c|c|c|c|c|c|c|c|c|c|c|c|}
\hline 동월 & $\stackrel{\varphi}{\varrho}$ & 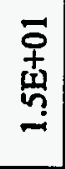 & 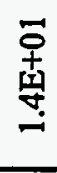 & $\begin{array}{l}8 \\
+ \\
+1 \\
\text { 竞 } \\
\text { ن }\end{array}$ & 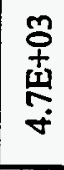 & $\begin{array}{l}2 \\
+ \\
+ \\
\text { 章 } \\
\text { ம் }\end{array}$ & 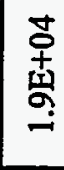 & $\begin{array}{c}\stackrel{+}{+} \\
\underset{\infty}{+} \\
\infty\end{array}$ & 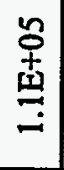 & 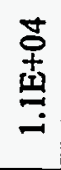 & 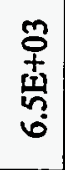 & $\begin{array}{c}\stackrel{+}{0} \\
+ \\
\stackrel{+}{9} \\
\text { ले }\end{array}$ & $\begin{array}{l}\text { o } \\
+ \\
\text { 夏 } \\
\text { ம் }\end{array}$ & 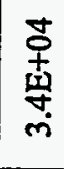 & 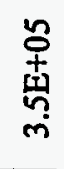 & 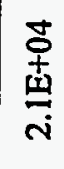 & 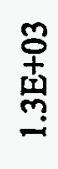 & 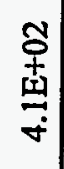 \\
\hline 总空 & $\underline{\underline{g}}$ & 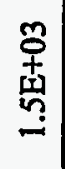 & 总 & 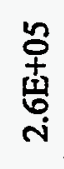 & 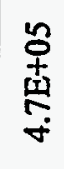 & $\begin{array}{l}\delta \\
\frac{1}{40} \\
\frac{6}{0} \\
\dot{0}\end{array}$ & 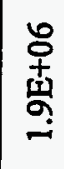 & 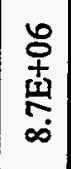 & 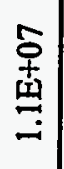 & 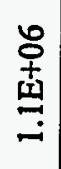 & 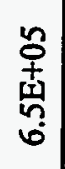 & 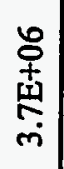 & 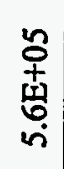 & 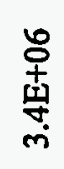 & 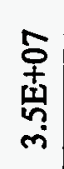 & 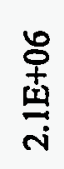 & 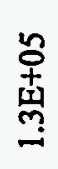 & 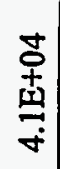 \\
\hline 홍 & $\stackrel{\varphi}{g}$ & 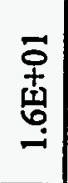 & 审 & 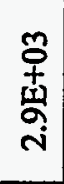 & 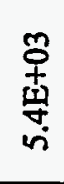 & $\begin{array}{l}\text { 草 } \\
\text { 夏 } \\
\text { - }\end{array}$ & 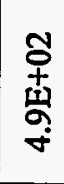 & $\begin{array}{c}m \\
\text { o } \\
\text { 总 } \\
\text { m } \\
m\end{array}$ & 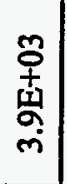 & 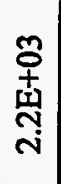 & 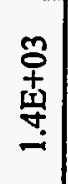 & $\begin{array}{c}\text { o } \\
+ \\
\text { 点 } \\
i\end{array}$ & 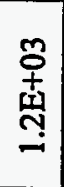 & 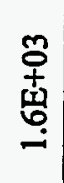 & $\begin{array}{l}\text { o } \\
+ \\
\text { 㟧 } \\
\dot{m}\end{array}$ & 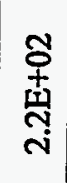 & 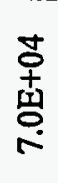 & 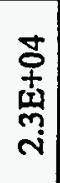 \\
\hline 总总 & 믐 & 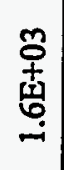 & $\begin{array}{c}m \\
\text { 畐 } \\
\text { 点 } \\
\stackrel{-}{-}\end{array}$ & 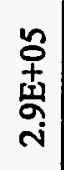 & 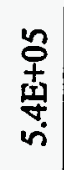 & 号 & 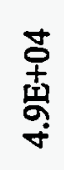 & 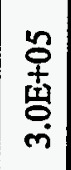 & 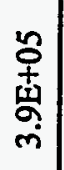 & 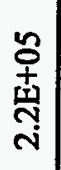 & 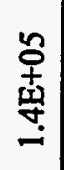 & 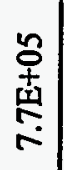 & 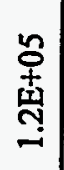 & $\begin{array}{l}\text { 另 } \\
+ \\
\text { 壱 } \\
-1\end{array}$ & 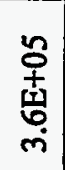 & 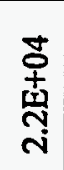 & 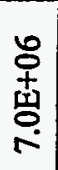 & 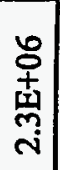 \\
\hline 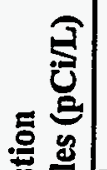 & $\stackrel{\varphi}{\circ}$ & 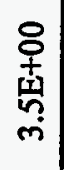 & 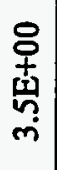 & 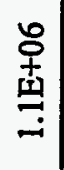 & 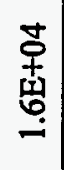 & 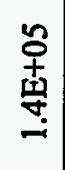 & 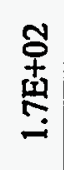 & $\begin{array}{c}\delta \\
\stackrel{+}{0} \\
\text { 兽 } \\
\dot{m}\end{array}$ & 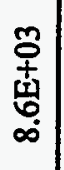 & $\begin{array}{l}\overrightarrow{0} \\
\text { 索 } \\
\text { i }\end{array}$ & 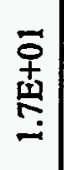 & 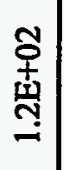 & 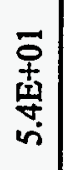 & 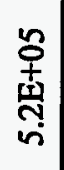 & 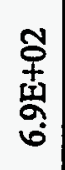 & 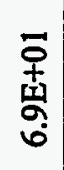 & $\begin{array}{l}\text { o } \\
\text { +⿱ } \\
\text { 究 } \\
\infty\end{array}$ & 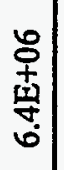 \\
\hline 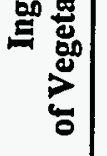 & $\stackrel{Y}{g}$ & 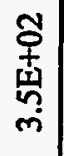 & 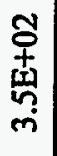 & 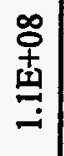 & 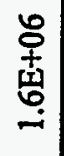 & 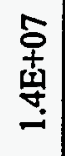 & $\begin{array}{c}\stackrel{+}{0} \\
+ \\
\text { 亲 } \\
-\end{array}$ & 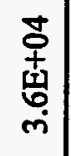 & 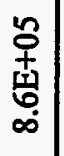 & 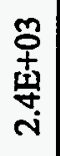 & 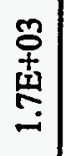 & 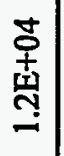 & 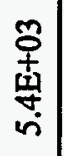 & 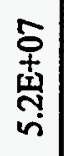 & 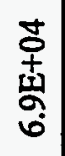 & 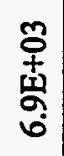 & $\begin{array}{l}\infty \\
0 \\
+ \\
\text { ज్ } \\
\infty \\
\infty\end{array}$ & 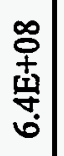 \\
\hline$\frac{z}{0}$ & & $\begin{array}{l}\frac{n}{5} \\
\frac{1}{2} \\
\frac{5}{8} \\
0 \\
0\end{array}$ & $\begin{array}{l}\frac{n}{5} \\
\frac{1}{2} \\
\overleftarrow{8} \\
0 \\
0\end{array}$ & 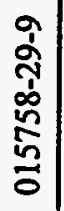 & 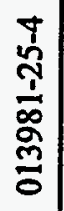 & $\begin{array}{l}\frac{1}{4} \\
\frac{1}{1} \\
\frac{0}{0} \\
\frac{\tilde{m}}{0}\end{array}$ & 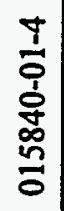 & 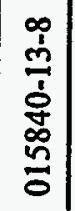 & 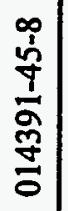 & 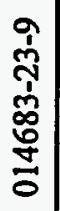 & 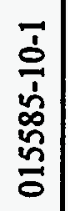 & $\begin{array}{l}\frac{n}{b} \\
\frac{1}{\sigma} \\
\frac{\sigma}{\sigma}\end{array}$ & 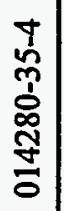 & 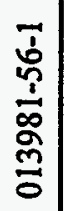 & 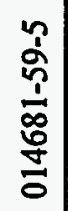 & 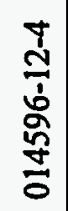 & 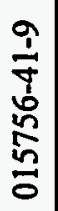 & 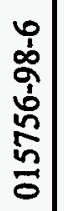 \\
\hline 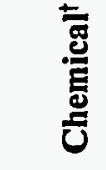 & & $\frac{m}{m}$ & $\begin{array}{l}\frac{p}{+} \\
\frac{m}{2} \\
\frac{w}{3}\end{array}$ & 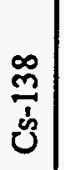 & 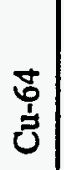 & $\begin{array}{l}n \\
\vdots \\
\vdots \\
\vdots \\
\vdots\end{array}$ & 弚 & $\frac{8}{\frac{1}{1}}$ & $\Xi$ & 告 & 蒙 & $\frac{n}{2}$ & $\frac{\stackrel{0}{7}}{3}$ & $\frac{\infty}{1}$ & గึ & 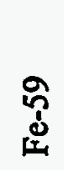 & ્ָષ & ָัָ \\
\hline
\end{tabular}




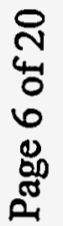

\begin{tabular}{|c|c|c|c|c|c|c|c|c|c|c|c|c|c|c|c|c|c|c|}
\hline 올 & $\stackrel{9}{0}$ & 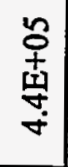 & 龺 & 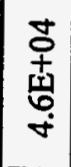 & $\begin{array}{l}\text { t } \\
+ \\
\text { 売 } \\
\text { i }\end{array}$ & 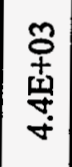 & 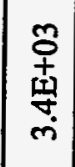 & 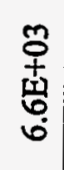 & 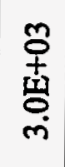 & 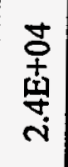 & $\begin{array}{l}\text { 吉 } \\
\text { 索 } \\
-\end{array}$ & 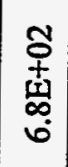 & $\begin{array}{l}\overrightarrow{+} \\
\stackrel{+}{+} \\
\stackrel{+}{+}\end{array}$ & 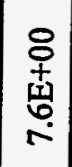 & $\begin{array}{l}\text { 号 } \\
\text { 吉 } \\
\text { i }\end{array}$ & $\begin{array}{l}\overrightarrow{0} \\
\text { 吉 } \\
\stackrel{0}{\circ}\end{array}$ & 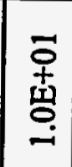 & 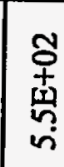 \\
\hline 总壹 & $\stackrel{7}{=}$ & 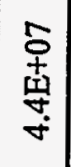 & 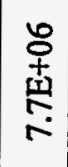 & 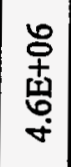 & $\begin{array}{l}\text { o } \\
+ \\
\text { 売 } \\
\text { ণ }\end{array}$ & $\begin{array}{c}\stackrel{0}{+} \\
\stackrel{+}{+1} \\
\stackrel{+}{+} \\
\dot{f}\end{array}$ & 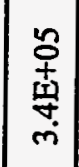 & 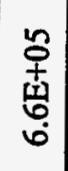 & $\begin{array}{l}\text { on } \\
+ \\
\text { 竞 } \\
\text { m. }\end{array}$ & 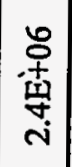 & 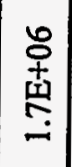 & 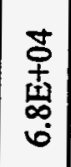 & 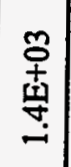 & 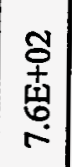 & $\begin{array}{l}\text { 웅 } \\
\text { 咅 } \\
\text { i }\end{array}$ & 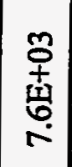 & $\begin{array}{c}\text { @o } \\
\text { 章 } \\
\text { - }\end{array}$ & 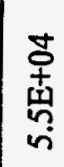 \\
\hline 월 & $\stackrel{\varphi}{\stackrel{\varphi}{0}}$ & 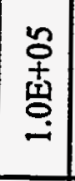 & 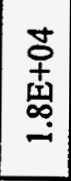 & 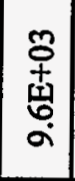 & 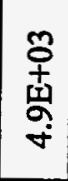 & 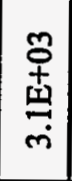 & & 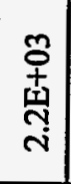 & 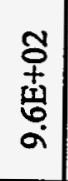 & 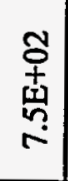 & 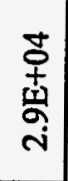 & 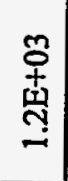 & $\begin{array}{l}\overline{0} \\
+ \\
\text { ज्ञ } \\
\text { i }\end{array}$ & $\begin{array}{c}\overline{0} \\
\stackrel{+}{9} \\
\text { m. }\end{array}$ & $\begin{array}{c}8 \\
8 \\
+ \\
n \\
n \\
m\end{array}$ & 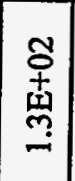 & 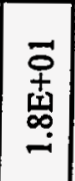 & $\begin{array}{l}\text { ơ } \\
\text { 龺 } \\
\text { a. }\end{array}$ \\
\hline 兽 & $\overline{0}$ & 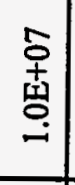 & 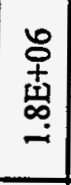 & $\begin{array}{l}\text { n } \\
\text { 点 } \\
\text { 号 } \\
\text { o }\end{array}$ & 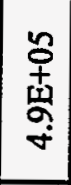 & 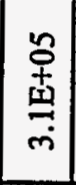 & & 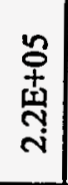 & $\begin{array}{l}\text { 후 } \\
\text { 草 } \\
\sigma\end{array}$ & 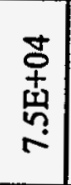 & $\begin{array}{l}\text { ㅇ } \\
\text { 索 } \\
\text { ஸे }\end{array}$ & 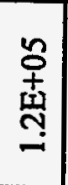 & 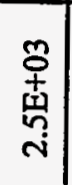 & 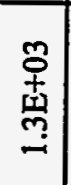 & 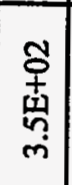 & 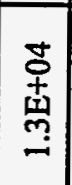 & 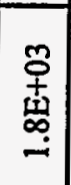 & 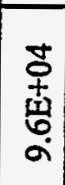 \\
\hline 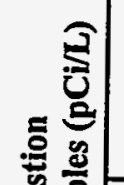 & 을 & 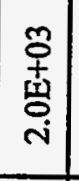 & 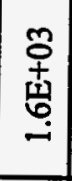 & 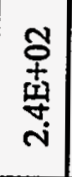 & 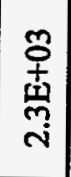 & $\begin{array}{l}\text { 草 } \\
\text { 索 } \\
\text { vi }\end{array}$ & & 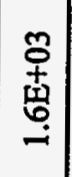 & 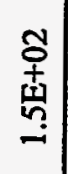 & $\begin{array}{l}\text { o } \\
+ \\
\text { 章 } \\
\text { n. }\end{array}$ & $\begin{array}{c}5 \\
+ \\
+ \\
\text { 章 } \\
\infty\end{array}$ & 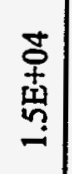 & $\begin{array}{l}\overrightarrow{0} \\
+\frac{1}{n} \\
\text { n- }\end{array}$ & $\begin{array}{l}\overline{0} \\
\text { 离 } \\
\text { m. }\end{array}$ & 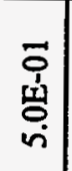 & 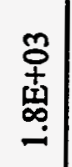 & 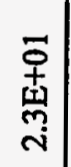 & 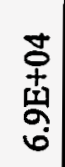 \\
\hline 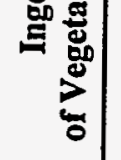 & $\stackrel{\top}{\varrho}$ & 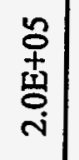 & 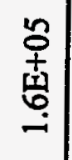 & 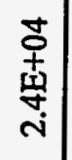 & $\begin{array}{l}\stackrel{n}{0} \\
+ \\
\text { 空 } \\
\text { i }\end{array}$ & 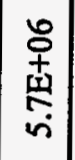 & & 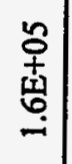 & $\begin{array}{c}\text { ㅎ } \\
+ \\
\text { 됨 } \\
-\end{array}$ & $\begin{array}{l}\text { ț } \\
+ \\
\stackrel{4}{4} \\
n \\
n\end{array}$ & 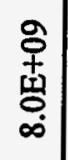 & 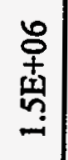 & $\begin{array}{l}m \\
\text { 古 } \\
\text { 孚 } \\
\end{array}$ & 总 & $\begin{array}{l}\overrightarrow{+} \\
\text { 吉 } \\
\text { i }\end{array}$ & 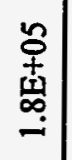 & 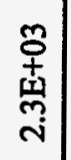 & $\begin{array}{l}\text { 号 } \\
\text { 兽 }\end{array}$ \\
\hline$\frac{Z}{0}$ & & 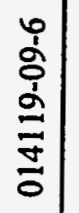 & 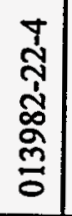 & 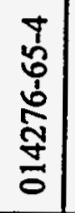 & 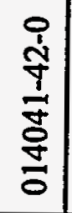 & 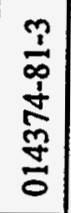 & 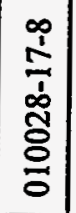 & $\begin{array}{l}\frac{0}{1} \\
\frac{1}{0} \\
\frac{0}{0} \\
\frac{\pi}{0}\end{array}$ & 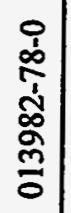 & 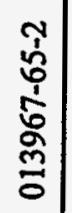 & 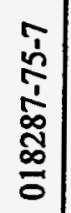 & 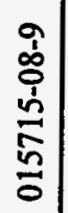 & 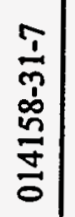 & 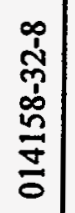 & 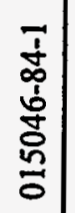 & 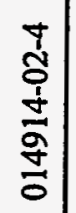 & $\begin{array}{l}0 \\
0 \\
\dot{0} \\
\dot{0} \\
\dot{p} \\
0 \\
0 \\
0\end{array}$ & $\begin{array}{l}0 \\
\dot{0} \\
0 \\
0 \\
0 \\
0 \\
0 \\
0\end{array}$ \\
\hline 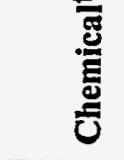 & & $\begin{array}{l}\text { E. } \\
\text { i̊ }\end{array}$ & 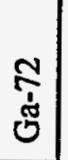 & $\frac{m}{\frac{1}{3}}$ & $\frac{8}{3}$ & $\overline{8}$ & 蛋 & $\frac{5}{5}$ & 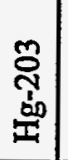 & $\frac{8}{0}$ & $\underset{\Xi}{\Xi}$ & $\underset{\beth}{\stackrel{2}{I}}$ & $\stackrel{\Perp}{\beth}$ & 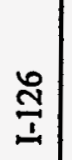 & $\stackrel{\text { త్ }}{\Xi}$ & $\stackrel{\check{m}}{\beth}$ & $\vec{m}$ & $\frac{\tilde{m}}{\mathfrak{I}}$ \\
\hline
\end{tabular}


Table 3.3b. (continued)

\begin{tabular}{|c|c|c|c|c|c|c|c|}
\hline \multirow[t]{2}{*}{ Chemical $^{\dagger}$} & \multirow{2}{*}{ CASRN } & \multicolumn{2}{|c|}{$\begin{array}{c}\text { Ingestion } \\
\text { of Vegetables (pCi/L) }\end{array}$} & \multicolumn{2}{|c|}{$\begin{array}{c}\text { Ingestion } \\
\text { of Beef }(\mathrm{pCi} / \mathrm{L})\end{array}$} & \multicolumn{2}{|c|}{$\begin{array}{c}\text { Ingestion } \\
\text { of Milk (pCi/L) }\end{array}$} \\
\hline & & $10^{-4}$ & $10^{-6}$ & $10^{-4}$ & $10^{-6}$ & $10^{-4}$ & $10^{-6}$ \\
\hline I-133 & $014834-67-4$ & $5.0 \mathrm{E}+04$ & $5.0 \mathrm{E}+02$ & $6.0 \mathrm{E}+03$ & $6.0 \mathrm{E}+01$ & $3.5 \mathrm{E}+03$ & $3.5 \mathrm{E}+01$ \\
\hline I-134 & $014914-27-3$ & $5.1 \mathrm{E}+07$ & $5.1 \mathrm{E}+05$ & $2.7 \mathrm{E}+05$ & $2.7 \mathrm{E}+03$ & $1.6 \mathrm{E}+05$ & $1.6 \mathrm{E}+03$ \\
\hline $\mathrm{I}-135$ & $014834-68-5$ & $7.0 \mathrm{E}+05$ & $7.0 \mathrm{E}+03$ & $2.8 \mathrm{E}+04$ & $2.8 \mathrm{E}+02$ & $1.6 \mathrm{E}+04$ & $1.6 \mathrm{E}+02$ \\
\hline In-113m & 014885-78-0 & $7.6 \mathrm{E}+07$ & $7.6 \mathrm{E}+05$ & $7.6 \mathrm{E}+06$ & $7.6 \mathrm{E}+04$ & $2.2 \mathrm{E}+07$ & $2.2 \mathrm{E}+05$ \\
\hline In-114 & 013981-55-0 & $1.1 \mathrm{E}+11$ & $1.1 \mathrm{E}+09$ & $1.4 \mathrm{E}+08$ & $1.4 \mathrm{E}+06$ & $4.1 \mathrm{E}+08$ & 4.1E+06 \\
\hline In-114m & 013981-55-0 & $1.9 \mathrm{E}+03$ & $1.9 \mathrm{E}+01$ & $3.1 \mathrm{E}+04$ & $3.1 \mathrm{E}+02$ & $8.9 \mathrm{E}+04$ & $8.9 \mathrm{E}+02$ \\
\hline In-115 & 014191-71-0 & $2.6 \mathrm{E}+02$ & $2.6 \mathrm{E}+00$ & $1.8 \mathrm{E}+04$ & $1.8 \mathrm{E}+02$ & $5.3 \mathrm{E}+04$ & $5.3 \mathrm{E}+02$ \\
\hline In-115m & 014191-71-0 & $7.0 \mathrm{E}+06$ & $7.0 \mathrm{E}+04$ & $1.9 \mathrm{E}+06$ & $1.9 \mathrm{E}+04$ & $5.4 \mathrm{E}+06$ & $5.4 \mathrm{E}+04$ \\
\hline Ir-190 & 014981-91-0 & $1.4 \mathrm{E}+04$ & $1.4 \mathrm{E}+02$ & $2.6 \mathrm{E}+05$ & $2.6 \mathrm{E}+03$ & $3.7 \mathrm{E}+07$ & $3.7 \mathrm{E}+05$ \\
\hline Ir-194 & 014158-35-1 & $8.1 E+04$ & $8.1 \mathrm{E}+02$ & $1.8 \mathrm{E}+05$ & $1.8 \mathrm{E}+03$ & $2.6 E+07$ & $2.6 \mathrm{E}+05$ \\
\hline $\mathrm{K}-40$ & $013966-00-2$ & $4.0 \mathrm{E}+02$ & $4.0 \mathrm{E}+00$ & $1.0 \mathrm{E}+04$ & $1.0 \mathrm{E}+02$ & $4.1 E+03$ & $4.1 E+01$ \\
\hline $\mathrm{K}-42$ & $014378-21-3$ & $6.7 \mathrm{E}+05$ & $6.7 E+03$ & $9.8 \mathrm{E}+04$ & $9.8 \mathrm{E}+02$ & $4.0 \mathrm{E}+04$ & $4.0 \mathrm{E}+02$ \\
\hline $\mathrm{Kr}-83 \mathrm{~m}$ & 013965-98-5 & & & & & & \\
\hline $\mathrm{Kr}-85$ & $013983-27-2$ & & & & & & \\
\hline $\mathrm{Kr}-85 \mathrm{~m}$ & $013983-27-2$ & & & & & & \\
\hline $\mathrm{Kr}-87$ & $014809-68-8$ & & & & & & \\
\hline $\mathrm{Kr}-88$ & 014995-61-0 & & & & & & \\
\hline
\end{tabular}


Table 3.3b. (continued)

\begin{tabular}{|l|l|l|c|c|c|c|c|}
\hline \multirow{2}{*}{ Chemical } & \multirow{2}{*}{ CASRN } & \multicolumn{2}{|c|}{$\begin{array}{c}\text { Ingestion } \\
\text { of Vegetables (pCi/L) }\end{array}$} & \multicolumn{2}{c|}{$\begin{array}{c}\text { Ingestion } \\
\text { of Beef (pCi/L) }\end{array}$} & \multicolumn{2}{c|}{$\begin{array}{c}\text { Ingestion } \\
\text { of Milk (pCi/L) }\end{array}$} \\
\cline { 5 - 8 } & & $10^{-4}$ & $10^{-6}$ & $10^{-4}$ & $10^{-6}$ & $10^{-4}$ & $10^{-6}$ \\
\hline Kr-89 & $016316-03-3$ & & & & & & \\
\hline Kr-90 & $015741-13-6$ & & & & & & \\
\hline La-140 & $013981-28-7$ & $3.0 \mathrm{E}+04$ & $3.0 \mathrm{E}+02$ & $8.9 \mathrm{E}+05$ & $8.9 \mathrm{E}+03$ & $1.9 \mathrm{E}+06$ & $1.9 \mathrm{E}+04$ \\
\hline $\mathrm{Lu}-177$ & $014265-75-9$ & $3.2 \mathrm{E}+04$ & $3.2 \mathrm{E}+02$ & $1.9 \mathrm{E}+05$ & $1.9 \mathrm{E}+03$ & $6.2 \mathrm{E}+06$ & $6.2 \mathrm{E}+04$ \\
\hline $\mathrm{Mn}-52$ & $014092-99-0$ & $1.8 \mathrm{E}+04$ & $1.8 \mathrm{E}+02$ & $8.5 \mathrm{E}+05$ & $8.5 \mathrm{E}+03$ & $2.0 \mathrm{E}+06$ & $2.0 \mathrm{E}+04$ \\
\hline $\mathrm{Mn}-54$ & $013966-31-9$ & $1.5 \mathrm{E}+04$ & $1.5 \mathrm{E}+02$ & $2.6 \mathrm{E}+06$ & $2.6 \mathrm{E}+04$ & $6.2 \mathrm{E}+06$ & $6.2 \mathrm{E}+04$ \\
\hline $\mathrm{Mn}-56$ & $014681-52-8$ & $4.7 \mathrm{E}+06$ & $4.7 \mathrm{E}+04$ & $5.9 \mathrm{E}+06$ & $5.9 \mathrm{E}+04$ & $1.4 \mathrm{E}+07$ & $1.4 \mathrm{E}+05$ \\
\hline $\mathrm{Mo}-99$ & $014119-15-4$ & $8.3 \mathrm{E}+04$ & $8.3 \mathrm{E}+02$ & $1.1 \mathrm{E}+06$ & $1.1 \mathrm{E}+04$ & $9.5 \mathrm{E}+04$ & $9.5 \mathrm{E}+02$ \\
\hline $\mathrm{Na}-22$ & $013966-32-0$ & $2.9 \mathrm{E}+03$ & $2.9 \mathrm{E}+01$ & $4.0 \mathrm{E}+03$ & $4.0 \mathrm{E}+01$ & $2.9 \mathrm{E}+03$ & $2.9 \mathrm{E}+01$ \\
\hline $\mathrm{Na}-24$ & $013982-04-2$ & $5.2 \mathrm{E}+05$ & $5.2 \mathrm{E}+03$ & $2.3 \mathrm{E}+04$ & $2.3 \mathrm{E}+02$ & $1.7 \mathrm{E}+04$ & $1.7 \mathrm{E}+02$ \\
\hline $\mathrm{Nb}-93 \mathrm{~m}$ & $007440-03-1$ & $2.0 \mathrm{E}+04$ & $2.0 \mathrm{E}+02$ & $1.3 \mathrm{E}+10$ & $1.3 \mathrm{E}+08$ & $1.3 \mathrm{E}+09$ & $1.3 \mathrm{E}+07$ \\
\hline $\mathrm{Nb}-94$ & $014681-63-1$ & $1.3 \mathrm{E}+03$ & $1.3 \mathrm{E}+01$ & $1.2 \mathrm{E}+09$ & $1.2 \mathrm{E}+07$ & $1.3 \mathrm{E}+08$ & $1.3 \mathrm{E}+06$ \\
\hline $\mathrm{Nb}-95$ & $013967-76-5$ & $1.9 \mathrm{E}+04$ & $1.9 \mathrm{E}+02$ & $3.8 \mathrm{E}+09$ & $3.8 \mathrm{E}+07$ & $4.0 \mathrm{E}+08$ & $4.0 \mathrm{E}+06$ \\
\hline $\mathrm{Nb}-95 \mathrm{~m}$ & $013967-76-5$ & $4.9 \mathrm{E}+04$ & $4.9 \mathrm{E}+02$ & $2.8 \mathrm{E}+09$ & $2.8 \mathrm{E}+07$ & $2.9 \mathrm{E}+08$ & $2.9 \mathrm{E}+06$ \\
\hline $\mathrm{Nb}-97$ & $018496-04-3$ & $4.9 \mathrm{E}+07$ & $4.9 \mathrm{E}+05$ & $4.8 \mathrm{E}+10$ & $4.8 \mathrm{E}+08$ & $5.1 \mathrm{E}+09$ & $5.1 \mathrm{E}+07$ \\
\hline $\mathrm{Nb}-97 \mathrm{~m}$ & $018496-04-3$ & $1.9 \mathrm{E}+11$ & $1.9 \mathrm{E}+09$ & $2.6 \mathrm{E}+12$ & $2.6 \mathrm{E}+10$ & $2.7 \mathrm{E}+11$ & $2.7 \mathrm{E}+09$ \\
\hline $\mathrm{Nd}-147$ & $014269-74-0$ & $1.2 \mathrm{E}+04$ & $1.2 \mathrm{E}+02$ & $1.4 \mathrm{E}+06$ & $1.4 \mathrm{E}+04$ & $3.1 \mathrm{E}+06$ & $3.1 \mathrm{E}+04$ \\
\hline
\end{tabular}




\begin{tabular}{|c|c|c|c|c|c|c|c|c|c|c|c|c|c|c|c|c|c|c|}
\hline : & 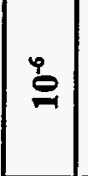 & 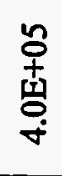 & 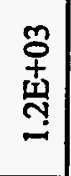 & 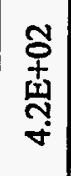 & 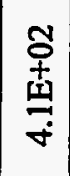 & 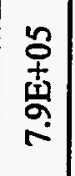 & 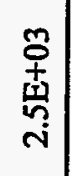 & 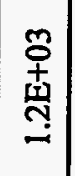 & 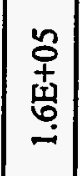 & 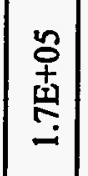 & 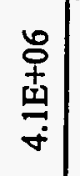 & 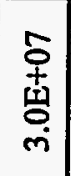 & 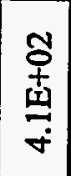 & 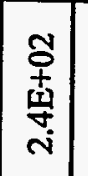 & 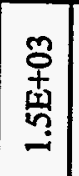 & 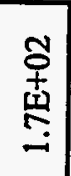 & 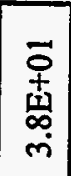 & 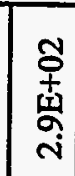 \\
\hline 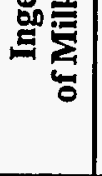 & $\stackrel{T}{\Xi}$ & 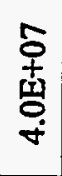 & 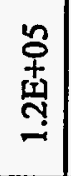 & 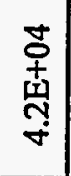 & 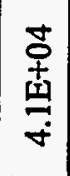 & 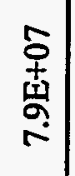 & 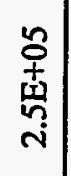 & 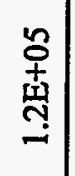 & 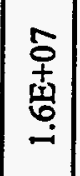 & $\begin{array}{c}0 \\
+ \\
\text { 索 } \\
=\end{array}$ & 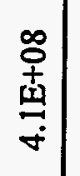 & 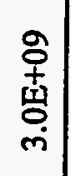 & 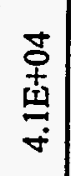 & 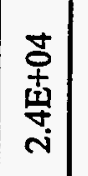 & 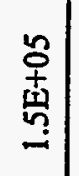 & $\begin{array}{c}\stackrel{+}{+} \\
\text { 索 } \\
=\end{array}$ & 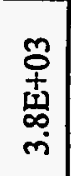 & $\begin{array}{l}\text { 草 } \\
\text { 产 } \\
\text { ì }\end{array}$ \\
\hline 월 & $\stackrel{\varphi}{\varrho}$ & 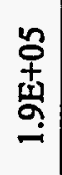 & 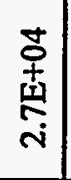 & 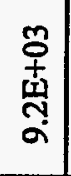 & 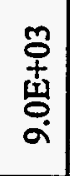 & $\begin{array}{c}\underset{⿱}{+} \\
\text { 壹 } \\
\text { i }\end{array}$ & 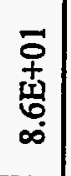 & 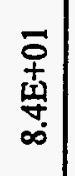 & 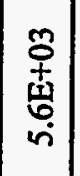 & $\begin{array}{l}\text { oे } \\
\text { 索 } \\
\text { in }\end{array}$ & 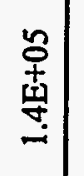 & 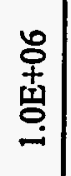 & $\begin{array}{c}\bar{q} \\
\text { 索 } \\
\text { m }\end{array}$ & 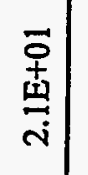 & 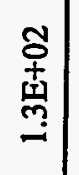 & 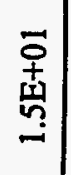 & 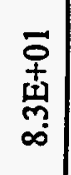 & 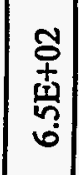 \\
\hline 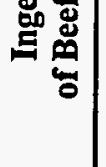 & $\dot{\vec{b}}$ & 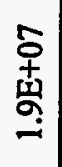 & 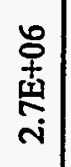 & 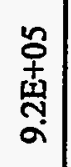 & $\begin{array}{l}0 \\
\vdots \\
+ \\
\vdots \\
\vdots \\
\vdots\end{array}$ & 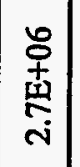 & 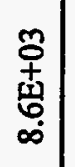 & 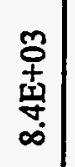 & 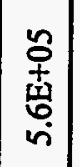 & 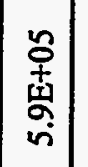 & $\begin{array}{l}\stackrel{0}{+} \\
\text { 孛 } \\
-\end{array}$ & $\begin{array}{c}\text { 吕 } \\
\text { 索 } \\
-\end{array}$ & 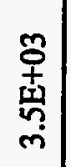 & 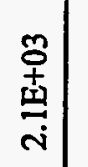 & 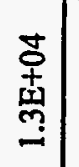 & 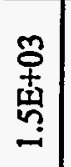 & 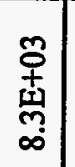 & 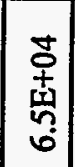 \\
\hline 愿 & $\stackrel{\varphi}{g}$ & 崇 & 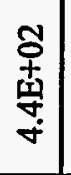 & 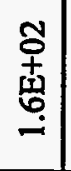 & 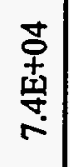 & 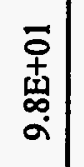 & 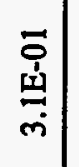 & 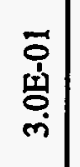 & 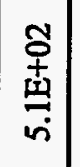 & 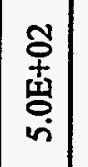 & 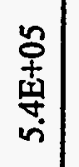 & 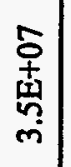 & $\begin{array}{l}\text { 古 } \\
\text { 壱 } \\
\text { is }\end{array}$ & 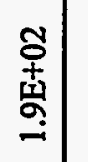 & 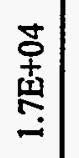 & 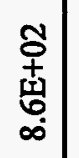 & $\begin{array}{l}\overline{0} \\
\text { 怘 } \\
\text { ه. }\end{array}$ & 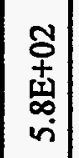 \\
\hline $\mid$ & $\stackrel{+}{=}$ & 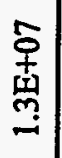 & 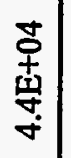 & 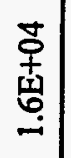 & 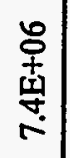 & 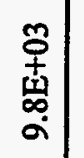 & 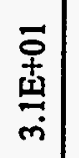 & 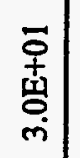 & 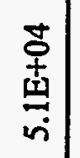 & 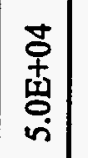 & 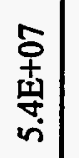 & 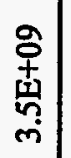 & $\begin{array}{c}\text { 草 } \\
\text { 㟧 } \\
\text { i }\end{array}$ & 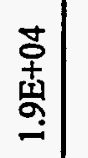 & 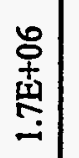 & 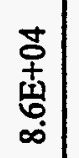 & 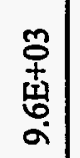 & 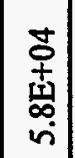 \\
\hline 蛋 & & 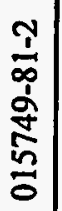 & 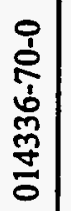 & $\begin{array}{l}\frac{\infty}{1} \\
\frac{1}{9} \\
\frac{1}{\infty} \\
\frac{0}{0} \\
\frac{0}{0}\end{array}$ & 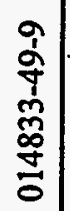 & 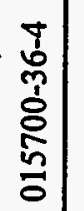 & 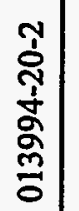 & 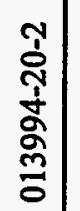 & 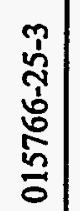 & 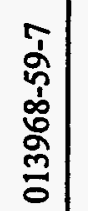 & 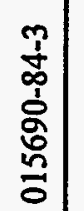 & 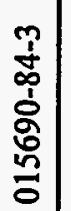 & 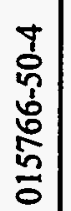 & 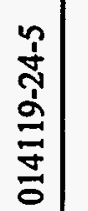 & 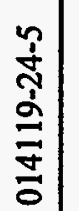 & $\begin{array}{l}? \\
\frac{1}{5} \\
\frac{1}{2} \\
0 \\
0 \\
0\end{array}$ & 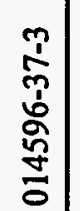 & 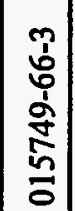 \\
\hline 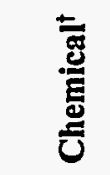 & & $\frac{\frac{g}{1}}{\frac{1}{z}}$ & 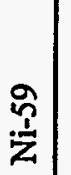 & 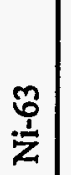 & 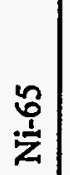 & 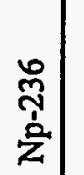 & $\begin{array}{l}\widehat{\hat{T}} \\
\frac{1}{z} \\
\frac{1}{2}\end{array}$ & 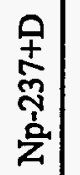 & 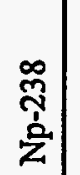 & 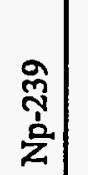 & 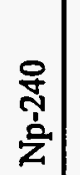 & 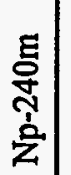 & $\begin{array}{l}\mathscr{\omega} \\
\stackrel{\dot{b}}{0}\end{array}$ & $\frac{\bar{a}}{\dot{b}}$ & $\begin{array}{l}E \\
\frac{E}{9} \\
\dot{b} \\
\dot{0}\end{array}$ & $\begin{array}{l}\text { \%े } \\
\stackrel{3}{b} \\
\text { on }\end{array}$ & న్ & $\stackrel{m}{\stackrel{m}{n}}$ \\
\hline
\end{tabular}




\begin{tabular}{|c|c|c|c|c|c|c|c|c|c|c|c|c|c|c|c|c|c|c|}
\hline 올 & $\stackrel{\varrho}{\varrho}$ & 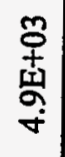 & 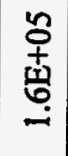 & 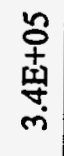 & 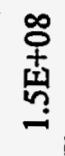 & 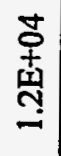 & 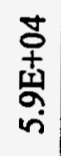 & 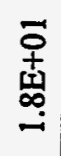 & 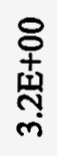 & 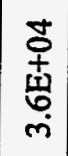 & 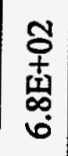 & 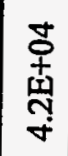 & 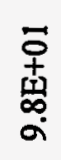 & 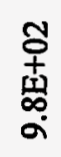 & 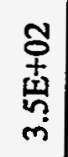 & 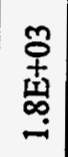 & 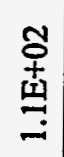 & $\begin{array}{l}n \\
+ \\
\text { qu } \\
\text { m }\end{array}$ \\
\hline 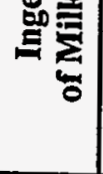 & $\Xi$ & 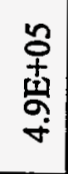 & 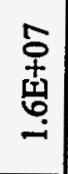 & $\begin{array}{c}5 \\
+ \\
\text { 竎 } \\
\text { ले }\end{array}$ & $\begin{array}{l}\stackrel{0}{7} \\
\frac{1}{5} \\
\end{array}$ & $\begin{array}{l}\text { ๖ } \\
+ \\
\text { 壱 } \\
\stackrel{-}{-}\end{array}$ & 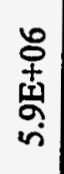 & 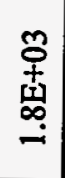 & 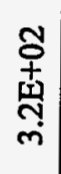 & 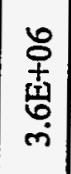 & 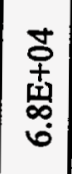 & 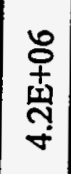 & 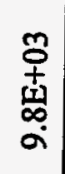 & 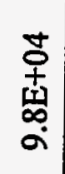 & 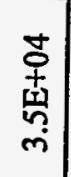 & 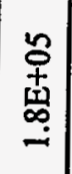 & $\begin{array}{l}\text { 呆 } \\
\text { 章 } \\
-\end{array}$ & 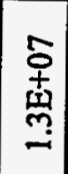 \\
\hline 옹 & 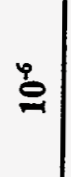 & $\begin{array}{l}\text { 草 } \\
\text { 龺 } \\
\end{array}$ & 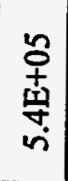 & 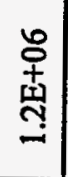 & 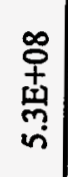 & 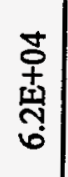 & 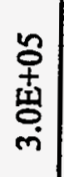 & 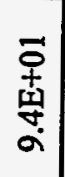 & $\begin{array}{l}\text { 옹 } \\
\text { 壱 } \\
\text { m } \\
+\end{array}$ & 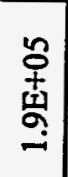 & 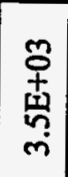 & 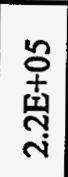 & 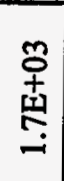 & 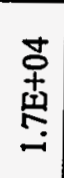 & 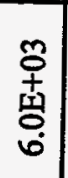 & 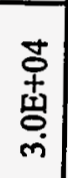 & $\begin{array}{c}\text { m } \\
+ \\
\text { 吉 } \\
\text { - }\end{array}$ & 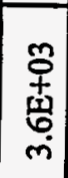 \\
\hline 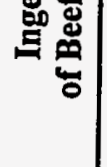 & $\stackrel{ }{0}$ & 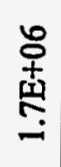 & $\begin{array}{l}5 \\
\text { 웅 } \\
\text { 寽 } \\
\text { ம் }\end{array}$ & 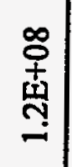 & 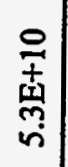 & 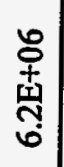 & $\begin{array}{l}\hat{0} \\
\text { +⿱ } \\
\text { 売 } \\
\dot{m}\end{array}$ & $\begin{array}{l}\text { ơ } \\
\text { 龺 } \\
\text { aे }\end{array}$ & 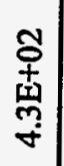 & 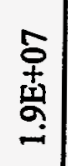 & 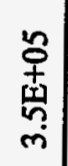 & 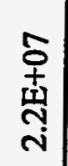 & 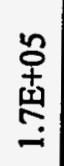 & 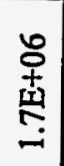 & 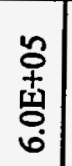 & $\begin{array}{l}\text { o } \\
\text { 吉 } \\
\text { in }\end{array}$ & 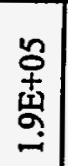 & $\begin{array}{l}\text { 草 } \\
\text { 索 } \\
\dot{m}\end{array}$ \\
\hline 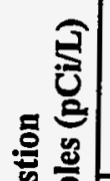 & $\stackrel{\varphi}{\underline{\theta}}$ & 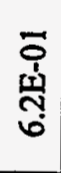 & 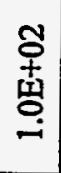 & 年 & $\begin{array}{l}8 \\
0 \\
+ \\
\\
\end{array}$ & 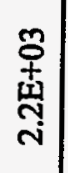 & 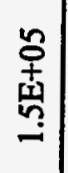 & 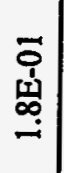 & $\begin{array}{l}\bar{s} \\
\text { जీ } \\
\text { స్ }\end{array}$ & $\begin{array}{l}n \\
+ \\
+9 \\
\text { 省 }\end{array}$ & 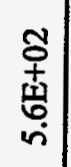 & $\begin{array}{l}2 \\
+ \\
+ \\
\text { વิ } \\
\end{array}$ & 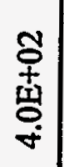 & 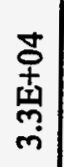 & 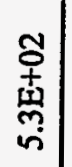 & $\begin{array}{l}\underset{\delta}{+} \\
\text { ț } \\
\stackrel{5}{0} \\
\stackrel{\forall}{*}\end{array}$ & 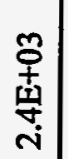 & $\begin{array}{c}\text { 草 } \\
\text { 至 } \\
=\end{array}$ \\
\hline 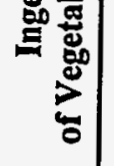 & 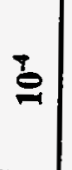 & 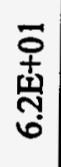 & 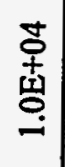 & 告 & $\begin{array}{l}\Xi \\
\stackrel{+}{ \pm} \\
\Xi\end{array}$ & 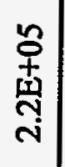 & 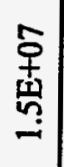 & 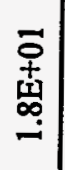 & 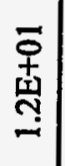 & 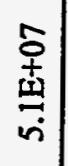 & 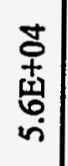 & 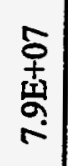 & $\begin{array}{l}\stackrel{+}{0} \\
\text { 索 } \\
\dot{+} \\
\dot{+}\end{array}$ & 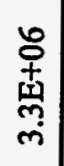 & 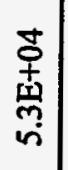 & 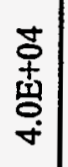 & 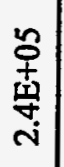 & 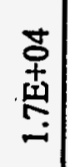 \\
\hline 苞 & & 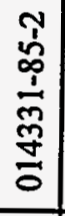 & 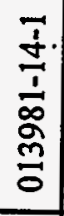 & 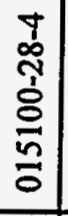 & 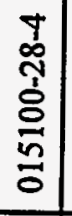 & 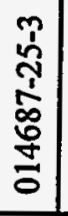 & 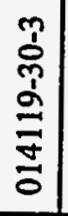 & 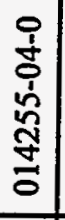 & 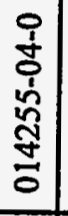 & 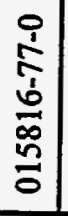 & 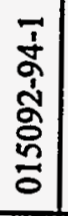 & 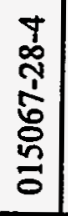 & 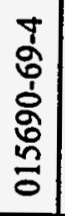 & $\begin{array}{l}\frac{a}{d} \\
\frac{n}{2} \\
\frac{a}{d} \\
\frac{5}{0} \\
\frac{0}{0}\end{array}$ & $\begin{array}{l}\overline{0} \\
0 \\
\frac{1}{0} \\
\stackrel{0}{0} \\
\dot{\sigma} \\
0\end{array}$ & 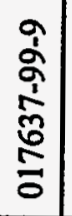 & $\begin{array}{l}\frac{j}{0} \\
\frac{1}{0} \\
\frac{0}{d} \\
0\end{array}$ & 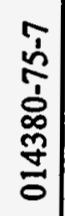 \\
\hline 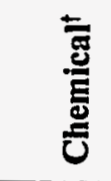 & & 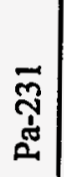 & 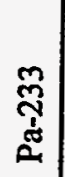 & 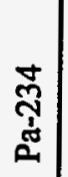 & 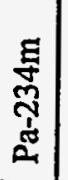 & 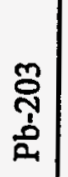 & 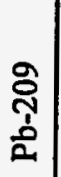 & 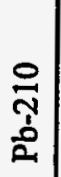 & $\frac{a}{\frac{1}{0}}$ & $\begin{array}{l}\overrightarrow{\vec{T}} \\
\dot{0} \\
\dot{n_{1}}\end{array}$ & $\begin{array}{l}\frac{N}{\pi} \\
\grave{a}\end{array}$ & $\begin{array}{l}\frac{\Delta}{2} \\
\text { م. }\end{array}$ & $\frac{8}{\frac{8}{2}}$ & $\frac{\overline{0}}{\frac{1}{2}}$ & $\frac{m}{0}$ & $\begin{array}{l}\hat{\circ} \\
\frac{1}{2} \\
0\end{array}$ & $\frac{8}{0}$ & $\frac{F}{\dot{E}}$ \\
\hline
\end{tabular}

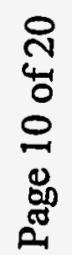


Table 3.3b. (continued)

\begin{tabular}{|l|l|c|c|c|c|c|c|}
\hline \multirow{2}{*}{ Chemical $^{\dagger}$} & \multirow{2}{*}{ CASRN } & \multicolumn{2}{|c|}{$\begin{array}{c}\text { Ingestion } \\
\text { of Vegetables (pCi/L) }\end{array}$} & \multicolumn{2}{c|}{$\begin{array}{c}\text { Ingestion } \\
\text { of Beef (pCi/L) }\end{array}$} & \multicolumn{2}{c|}{$\begin{array}{c}\text { Ingestion } \\
\text { of Milk (pCi/L) }\end{array}$} \\
\cline { 3 - 8 } & & $10^{-4}$ & $10^{-6}$ & $10^{-4}$ & $10^{-6}$ & $10^{-4}$ & $10^{-6}$ \\
\hline Pm-148 & $014683-19-3$ & $7.7 \mathrm{E}+03$ & $7.7 \mathrm{E}+01$ & $3.5 \mathrm{E}+04$ & $3.5 \mathrm{E}+02$ & $1.3 \mathrm{E}+06$ & $1.3 \mathrm{E}+04$ \\
\hline Pm-148m & $014683-19-3$ & $4.2 \mathrm{E}+03$ & $4.2 \mathrm{E}+01$ & $5.1 \mathrm{E}+04$ & $5.1 \mathrm{E}+02$ & $1.8 \mathrm{E}+06$ & $1.8 \mathrm{E}+04$ \\
\hline Pm-149 & $015765-31-8$ & $4.1 \mathrm{E}+04$ & $4.1 \mathrm{E}+02$ & $9.2 \mathrm{E}+04$ & $9.2 \mathrm{E}+02$ & $3.3 \mathrm{E}+06$ & $3.3 \mathrm{E}+04$ \\
\hline Po-210 & $013981-52-7$ & $1.0 \mathrm{E}+02$ & $1.0 \mathrm{E}+00$ & $1.6 \mathrm{E}+03$ & $1.6 \mathrm{E}+01$ & $3.3 \mathrm{E}+03$ & $3.3 \mathrm{E}+01$ \\
\hline Po-212 & $015389-34-1$ & $2.8 \mathrm{E}+27$ & $2.8 \mathrm{E}+25$ & $1.1 \mathrm{E}+16$ & $1.1 \mathrm{E}+14$ & $2.4 \mathrm{E}+16$ & $2.4 \mathrm{E}+14$ \\
\hline Po-213 & $015756-57-7$ & $1.3 \mathrm{E}+25$ & $1.3 \mathrm{E}+23$ & $7.6 \mathrm{E}+14$ & $7.6 \mathrm{E}+12$ & $1.6 \mathrm{E}+15$ & $1.6 \mathrm{E}+13$ \\
\hline Po-214 & $015735-67-8$ & $1.1 \mathrm{E}+22$ & $1.1 \mathrm{E}+20$ & $2.4 \mathrm{E}+13$ & $2.4 \mathrm{E}+11$ & $5.1 \mathrm{E}+13$ & $5.1 \mathrm{E}+11$ \\
\hline Po-215 & $015706-52-2$ & $4.2 \mathrm{E}+19$ & $4.2 \mathrm{E}+17$ & $1.0 \mathrm{E}+12$ & $1.0 \mathrm{E}+10$ & $2.2 \mathrm{E}+12$ & $2.2 \mathrm{E}+10$ \\
\hline Po-216 & $015756-58-8$ & $2.9 \mathrm{E}+15$ & $2.9 \mathrm{E}+13$ & $5.8 \mathrm{E}+09$ & $5.8 \mathrm{E}+07$ & $1.2 \mathrm{E}+10$ & $1.2 \mathrm{E}+08$ \\
\hline Po-218 & $015422-24-9$ & $4.0 \mathrm{E}+09$ & $4.0 \mathrm{E}+07$ & $1.0 \mathrm{E}+07$ & $1.0 \mathrm{E}+05$ & $2.1 \mathrm{E}+07$ & $2.1 \mathrm{E}+05$ \\
\hline Pr-142 & $014191-64-1$ & $8.2 \mathrm{E}+04$ & $8.2 \mathrm{E}+02$ & $1.2 \mathrm{E}+06$ & $1.2 \mathrm{E}+04$ & $2.6 \mathrm{E}+06$ & $2.6 \mathrm{E}+04$ \\
\hline Pr-143 & $014981-79-4$ & $9.5 \mathrm{E}+03$ & $9.5 \mathrm{E}+01$ & $1.3 \mathrm{E}+06$ & $1.3 \mathrm{E}+04$ & $2.8 \mathrm{E}+06$ & $2.8 \mathrm{E}+04$ \\
\hline Pr-144 & $014119-05-2$ & $4.5 \mathrm{E}+08$ & $4.5 \mathrm{E}+06$ & $1.0 \mathrm{E}+08$ & $1.0 \mathrm{E}+06$ & $2.3 \mathrm{E}+08$ & $2.3 \mathrm{E}+06$ \\
\hline Pr-144m & $014119-05-2$ & $2.7 \mathrm{E}+09$ & $2.7 \mathrm{E}+07$ & $2.6 \mathrm{E}+08$ & $2.6 \mathrm{E}+06$ & $5.7 \mathrm{E}+08$ & $5.7 \mathrm{E}+06$ \\
\hline Pt-191 & $015706-36-2$ & $1.3 \mathrm{E}+05$ & $1.3 \mathrm{E}+03$ & $4.2 \mathrm{E}+05$ & $4.2 \mathrm{E}+03$ & $4.9 \mathrm{E}+04$ & $4.9 \mathrm{E}+02$ \\
\hline Pt-193 & $015735-70-3$ & $6.1 \mathrm{E}+04$ & $6.1 \mathrm{E}+02$ & $3.9 \mathrm{E}+06$ & $3.9 \mathrm{E}+04$ & $4.5 \mathrm{E}+05$ & $4.5 \mathrm{E}+03$ \\
\hline Pt-193m & $015735-70-3$ & $5.2 \mathrm{E}+04$ & $5.2 \mathrm{E}+02$ & $2.5 \mathrm{E}+05$ & $2.5 \mathrm{E}+03$ & $2.9 \mathrm{E}+04$ & $2.9 \mathrm{E}+02$ \\
\hline
\end{tabular}


Table 3.3b. (continued)

\begin{tabular}{|l|l|c|c|c|c|c|c|}
\hline \multirow{2}{*}{ Chemical $^{\dagger}$} & \multirow{2}{*}{ CASRN } & \multicolumn{2}{c|}{$\begin{array}{c}\text { Ingestion } \\
\text { of Vegetables (pCi/L) }\end{array}$} & \multicolumn{2}{c|}{$\begin{array}{c}\text { Ingestion } \\
\text { of Beef (pCi/L) }\end{array}$} & \multicolumn{2}{c|}{$\begin{array}{c}\text { Ingestion } \\
\text { of Milk (pCi/L) }\end{array}$} \\
\cline { 3 - 8 } & & $10^{-4}$ & $10^{-6}$ & $10^{-4}$ & $10^{-6}$ & $10^{-4}$ & $10^{-6}$ \\
\hline Pt-197 & $015735-74-7$ & $2.8 \mathrm{E}+05$ & $2.8 \mathrm{E}+03$ & $3.0 \mathrm{E}+05$ & $3.0 \mathrm{E}+03$ & $3.5 \mathrm{E}+04$ & $3.5 \mathrm{E}+02$ \\
\hline Pt-197m & $015735-74-7$ & $2.0 \mathrm{E}+07$ & $2.0 \mathrm{E}+05$ & $2.0 \mathrm{E}+06$ & $2.0 \mathrm{E}+04$ & $2.3 \mathrm{E}+05$ & $2.3 \mathrm{E}+03$ \\
\hline Pu-236 & $015411-92-4$ & $3.1 \mathrm{E}+02$ & $3.1 \mathrm{E}+00$ & $3.3 \mathrm{E}+06$ & $3.3 \mathrm{E}+04$ & $4.3 \mathrm{E}+06$ & $4.3 \mathrm{E}+04$ \\
\hline Pu-238 & $013981-16-3$ & $3.4 \mathrm{E}+01$ & $3.4 \mathrm{E}-01$ & $8.6 \mathrm{E}+05$ & $8.6 \mathrm{E}+03$ & $1.1 \mathrm{E}+06$ & $1.1 \mathrm{E}+04$ \\
\hline Pu-239 & $015117-48-3$ & $2.9 \mathrm{E}+01$ & $2.9 \mathrm{E}-01$ & $8.0 \mathrm{E}+05$ & $8.0 \mathrm{E}+03$ & $1.1 \mathrm{E}+06$ & $1.1 \mathrm{E}+04$ \\
\hline Pu-240 & $014119-33-6$ & $2.9 \mathrm{E}+01$ & $2.9 \mathrm{E}-01$ & $8.1 \mathrm{E}+05$ & $8.1 \mathrm{E}+03$ & $1.1 \mathrm{E}+06$ & $1.1 \mathrm{E}+04$ \\
\hline Pu-241 & $014119-32-5$ & $2.6 \mathrm{E}+03$ & $2.6 \mathrm{E}+01$ & $4.9 \mathrm{E}+07$ & $4.9 \mathrm{E}+05$ & $6.4 \mathrm{E}+07$ & $6.4 \mathrm{E}+05$ \\
\hline Pu-241+D & $014119-32-5$ & $4.1 \mathrm{E}+01$ & $4.1 \mathrm{E}-01$ & $2.2 \mathrm{E}+04$ & $2.2 \mathrm{E}+02$ & $2.7 \mathrm{E}+03$ & $2.7 \mathrm{E}+01$ \\
\hline Pu-242 & $013982-10-0$ & $3.1 \mathrm{E}+01$ & $3.1 \mathrm{E}-01$ & $8.5 \mathrm{E}+05$ & $8.5 \mathrm{E}+03$ & $1.1 \mathrm{E}+06$ & $1.1 \mathrm{E}+04$ \\
\hline Pu-243 & $015706-37-3$ & $5.7 \mathrm{E}+06$ & $5.7 \mathrm{E}+04$ & $6.9 \mathrm{E}+08$ & $6.9 \mathrm{E}+06$ & $9.0 \mathrm{E}+08$ & $9.0 \mathrm{E}+06$ \\
\hline Pu-244 & $014119-34-7$ & $2.9 \mathrm{E}+01$ & $2.9 \mathrm{E}-01$ & $8.1 \mathrm{E}+05$ & $8.1 \mathrm{E}+03$ & $1.1 \mathrm{E}+06$ & $1.1 \mathrm{E}+04$ \\
\hline Pu-244+D & $014119-34-7$ & $2.9 \mathrm{E}+01$ & $2.9 \mathrm{E}-01$ & $6.1 \mathrm{E}+03$ & $6.1 \mathrm{E}+01$ & $2.8 \mathrm{E}+03$ & $2.8 \mathrm{E}+01$ \\
\hline Ra-223 & $015623-45-7$ & $2.9 \mathrm{E}+02$ & $2.9 \mathrm{E}+00$ & $1.2 \mathrm{E}+04$ & $1.2 \mathrm{E}+02$ & $1.2 \mathrm{E}+03$ & $1.2 \mathrm{E}+01$ \\
\hline Ra-224 & $013233-32-4$ & $1.0 \mathrm{E}+03$ & $1.0 \mathrm{E}+01$ & $1.9 \mathrm{E}+04$ & $1.9 \mathrm{E}+02$ & $1.9 \mathrm{E}+03$ & $1.9 \mathrm{E}+01$ \\
\hline Ra-225 & $013981-53-8$ & $3.8 \mathrm{E}+02$ & $3.8 \mathrm{E}+00$ & $1.8 \mathrm{E}+04$ & $1.8 \mathrm{E}+02$ & $1.8 \mathrm{E}+03$ & $1.8 \mathrm{E}+01$ \\
\hline Ra-226 & $013982-63-3$ & $3.1 \mathrm{E}+01$ & $3.1 \mathrm{E}-01$ & $9.6 \mathrm{E}+03$ & $9.6 \mathrm{E}+01$ & $9.6 \mathrm{E}+02$ & $9.6 \mathrm{E}+00$ \\
\hline Ra-226+D & $013982-63-3$ & $3.0 \mathrm{E}+01$ & $3.0 \mathrm{E}-01$ & $7.3 \mathrm{E}+02$ & $7.3 \mathrm{E}+00$ & $4.5 \mathrm{E}+02$ & $4.5 \mathrm{E}+00$ \\
\hline
\end{tabular}


Table 3.3b. (continued)

\begin{tabular}{|l|l|c|c|c|c|c|c|}
\hline \multirow{2}{*}{ Chemical $^{\dagger}$} & \multirow{2}{*}{ CASRN } & \multicolumn{2}{c|}{$\begin{array}{c}\text { Ingestion } \\
\text { of Vegetables (pCi/L) }\end{array}$} & \multicolumn{2}{c|}{$\begin{array}{c}\text { Ingestion } \\
\text { of Beef (pCi/L) }\end{array}$} & \multicolumn{2}{c|}{$\begin{array}{c}\text { Ingestion } \\
\text { of Milk (pCi/L) }\end{array}$} \\
\cline { 3 - 9 } & & $10^{-4}$ & $10^{-6}$ & $10^{-4}$ & $10^{-5}$ & $10^{-4}$ & $10^{-5}$ \\
\hline Ra-228 & $015262-20-1$ & $7.6 \mathrm{E}+01$ & $7.6 \mathrm{E}-01$ & $1.1 \mathrm{E}+04$ & $1.1 \mathrm{E}+02$ & $1.1 \mathrm{E}+03$ & $1.1 \mathrm{E}+01$ \\
\hline Ra-228+D & $015262-20-1$ & $7.5 \mathrm{E}+01$ & $7.5 \mathrm{E}-01$ & $1.1 \mathrm{E}+04$ & $1.1 \mathrm{E}+02$ & $1.1 \mathrm{E}+03$ & $1.1 \mathrm{E}+01$ \\
\hline Rb-82 & $014391-63-0$ & $4.7 \mathrm{E}+10$ & $4.7 \mathrm{E}+08$ & $2.4 \mathrm{E}+07$ & $2.4 \mathrm{E}+05$ & $2.9 \mathrm{E}+06$ & $2.9 \mathrm{E}+04$ \\
\hline Rb-86 & $014932-53-7$ & $7.5 \mathrm{E}+03$ & $7.5 \mathrm{E}+01$ & $3.6 \mathrm{E}+04$ & $3.6 \mathrm{E}+02$ & $4.3 \mathrm{E}+03$ & $4.3 \mathrm{E}+01$ \\
\hline Rb-87 & $013982-13-3$ & $2.0 \mathrm{E}+03$ & $2.0 \mathrm{E}+01$ & $6.9 \mathrm{E}+04$ & $6.9 \mathrm{E}+02$ & $8.3 \mathrm{E}+03$ & $8.3 \mathrm{E}+01$ \\
\hline Rb-88 & $014928-36-0$ & $2.4 \mathrm{E}+08$ & $2.4 \mathrm{E}+06$ & $1.7 \mathrm{E}+06$ & $1.7 \mathrm{E}+04$ & $2.1 \mathrm{E}+05$ & $2.1 \mathrm{E}+03$ \\
\hline Rb-89 & $014191-65-2$ & $4.7 \mathrm{E}+08$ & $4.7 \mathrm{E}+06$ & $2.9 \mathrm{E}+06$ & $2.9 \mathrm{E}+04$ & $3.5 \mathrm{E}+05$ & $3.5 \mathrm{E}+03$ \\
\hline Rh-103m & $007440-16-6$ & $1.4 \mathrm{E}+09$ & $1.4 \mathrm{E}+07$ & $1.6 \mathrm{E}+08$ & $1.6 \mathrm{E}+06$ & $4.5 \mathrm{E}+06$ & $4.5 \mathrm{E}+04$ \\
\hline Rh-105 & $014913-89-4$ & $1.7 \mathrm{E}+05$ & $1.7 \mathrm{E}+03$ & $6.6 \mathrm{E}+05$ & $6.6 \mathrm{E}+03$ & $1.9 \mathrm{E}+04$ & $1.9 \mathrm{E}+02$ \\
\hline Rh-105m & $014913-89-4$ & $7.7 \mathrm{E}+11$ & $7.7 \mathrm{E}+09$ & $1.2 \mathrm{E}+09$ & $1.2 \mathrm{E}+07$ & $3.4 \mathrm{E}+07$ & $3.4 \mathrm{E}+05$ \\
\hline Rh-106 & $014234-34-5$ & $3.4 \mathrm{E}+11$ & $3.4 \mathrm{E}+09$ & $3.5 \mathrm{E}+08$ & $3.5 \mathrm{E}+06$ & $1.0 \mathrm{E}+07$ & $1.0 \mathrm{E}+05$ \\
\hline Rn-219 & $014835-02-0$ & & & & & & \\
\hline Rn-220 & $022481-48-7$ & & & & & & \\
\hline Rn-222+D & $014859-67-7$ & & & & & & \\
\hline Ru-103 & $013968-53-1$ & $1.3 \mathrm{E}+04$ & $1.3 \mathrm{E}+02$ & $1.5 \mathrm{E}+04$ & $1.5 \mathrm{E}+02$ & $3.4 \mathrm{E}+07$ & $3.4 \mathrm{E}+05$ \\
\hline Ru-105 & $014331-95-4$ & $2.1 \mathrm{E}+06$ & $2.1 \mathrm{E}+04$ & $4.3 \mathrm{E}+04$ & $4.3 \mathrm{E}+02$ & $9.7 \mathrm{E}+07$ & $9.7 \mathrm{E}+05$ \\
\hline Ru-106 & $013967-48-1$ & $8.4 \mathrm{E}+02$ & $8.4 \mathrm{E}+00$ & $1.4 \mathrm{E}+03$ & $1.4 \mathrm{E}+01$ & $3.2 \mathrm{E}+06$ & $3.2 \mathrm{E}+04$ \\
\hline
\end{tabular}




\begin{tabular}{|c|c|c|c|c|c|c|c|c|c|c|c|c|c|c|c|c|c|c|}
\hline \multirow{2}{*}{ 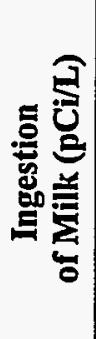 } & $\stackrel{\text { : }}{\circ}$ & 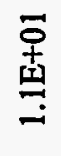 & $\begin{array}{l}\text { ○ั } \\
\text { 茍 }\end{array}$ & $\begin{array}{l}\text { ô } \\
\text { † } \\
\text { 离 } \\
\text { in }\end{array}$ & 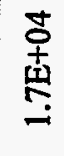 & $\begin{array}{l}\text { 草 } \\
\text { 豈 } \\
\text {. }\end{array}$ & $\begin{array}{l}\text { 号 } \\
\text { 索 } \\
\stackrel{+}{+}\end{array}$ & 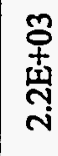 & 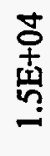 & 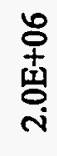 & 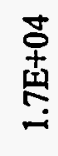 & 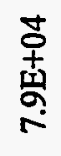 & $\begin{array}{l}\text { 草 } \\
\text { 虫 } \\
\end{array}$ & $\begin{array}{l}\text { 古 } \\
+ \\
\text { 田 } \\
\text { i }\end{array}$ & 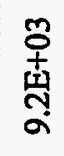 & 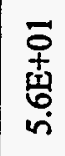 & 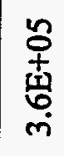 & 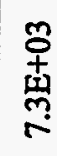 \\
\hline & to & 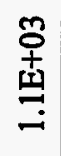 & 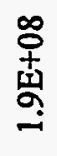 & 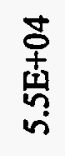 & 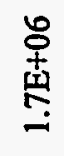 & 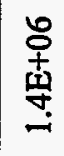 & 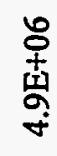 & 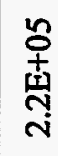 & 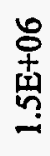 & $\begin{array}{l}\infty \\
0 \\
+1 \\
\text { 㕝 } \\
\text { ㅇ }\end{array}$ & 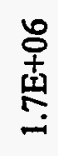 & 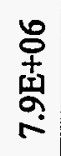 & 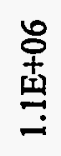 & $\begin{array}{l}\text { o̊ } \\
\text { 晋 } \\
\text { N }\end{array}$ & $\begin{array}{l}\text { o } \\
\text { 怘 } \\
\text { స్ }\end{array}$ & $\begin{array}{l}\text { o } \\
\text { 亲 } \\
\text { in }\end{array}$ & 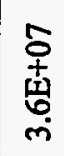 & 号 \\
\hline \multirow{2}{*}{ 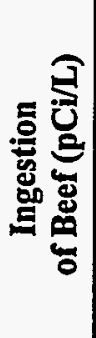 } & 옹 & 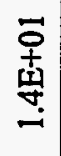 & 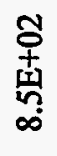 & 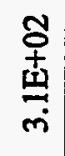 & 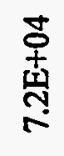 & 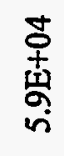 & $\begin{array}{l}\text { o } \\
+ \\
\text { 至 } \\
\text { N }\end{array}$ & 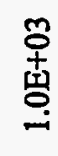 & 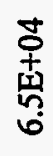 & 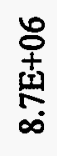 & $\begin{array}{l}\text { 士 } \\
+ \\
\text { 吉 } \\
\text { n }\end{array}$ & 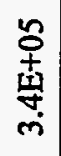 & 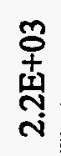 & 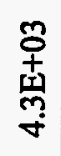 & 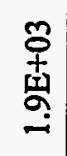 & $\begin{array}{l}\overrightarrow{0} \\
\text { † } \\
\text { 章 } \\
\text { n. }\end{array}$ & 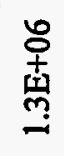 & 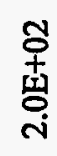 \\
\hline & 흠 & 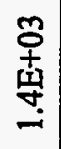 & 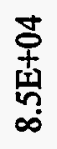 & $\begin{array}{c}\text { 草 } \\
\text { 皇 } \\
\text { en }\end{array}$ & 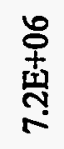 & 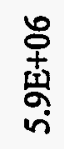 & 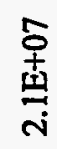 & $\begin{array}{l}\text { n } \\
\text { + } \\
\text { 몽 }\end{array}$ & 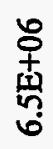 & $\begin{array}{l}\infty \\
\stackrel{+}{+} \\
\underset{\infty}{+} \\
\underset{\infty}{\infty}\end{array}$ & 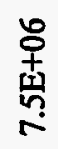 & 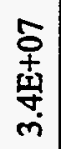 & 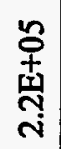 & 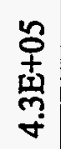 & 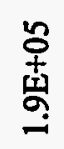 & $\begin{array}{l}m \\
\text { o } \\
\text { 产 } \\
m\end{array}$ & 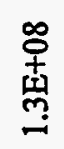 & 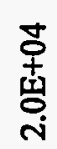 \\
\hline \multirow{2}{*}{ 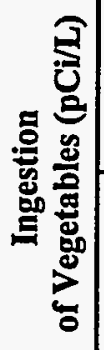 } & $\stackrel{\varphi}{\varrho}$ & 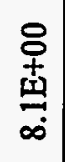 & 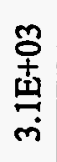 & 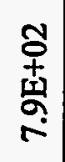 & 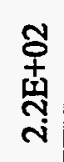 & $\begin{array}{l}\bar{\phi} \\
+ \\
\text { 奌 } \\
\text { m. }\end{array}$ & 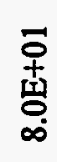 & 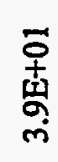 & $\begin{array}{l}\overline{0} \\
\text { 产 } \\
\text { هo }\end{array}$ & 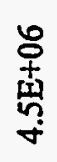 & 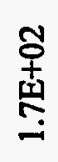 & $\begin{array}{l}\text { 草 } \\
\text { 恶 } \\
\text { - }\end{array}$ & 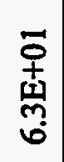 & 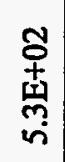 & 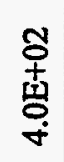 & $\begin{array}{l}\text { 古 } \\
\text { 畐 } \\
\text { n' }\end{array}$ & $\begin{array}{l}\text { 古 } \\
\text { 萦 } \\
\end{array}$ & $\begin{array}{l}8 \\
+ \\
010 \\
\text { ț } \\
\dot{m}\end{array}$ \\
\hline & $\stackrel{t}{g}$ & 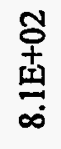 & 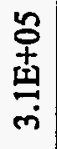 & 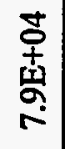 & 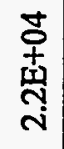 & 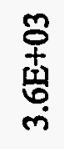 & 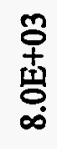 & 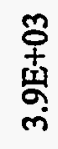 & 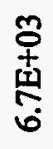 & 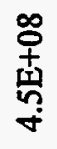 & 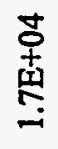 & 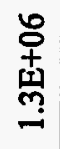 & 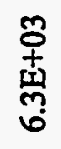 & 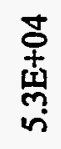 & 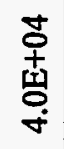 & 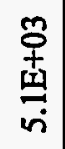 & 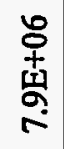 & 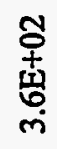 \\
\hline 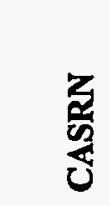 & & 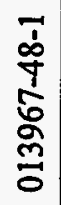 & 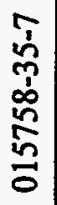 & $\begin{array}{l}o \\
\hat{n} \\
\frac{\hat{D}}{5} \\
\bar{\Xi} \\
0\end{array}$ & $\frac{a}{\frac{a}{T}}$ & 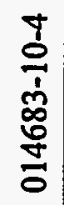 & 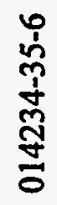 & 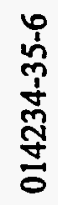 & 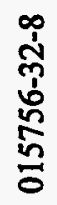 & 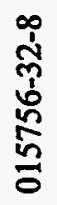 & 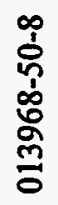 & 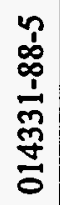 & 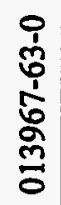 & $\frac{\dot{a}}{\frac{\dot{a}}{a}}$ & 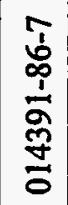 & 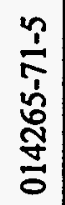 & 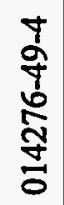 & 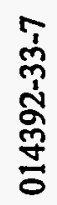 \\
\hline 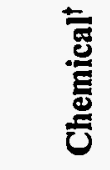 & & 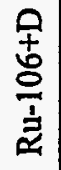 & 氦 & ๓̊ & $\frac{\tilde{d}}{\frac{1}{b}}$ & $\frac{\stackrel{ \pm}{~}}{\dot{b}}$ & $\frac{\check{z}}{\dot{\delta}}$ & $\begin{array}{l}\frac{0}{4} \\
\stackrel{5}{7} \\
\frac{\dot{b}}{b}\end{array}$ & 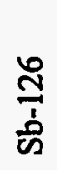 & 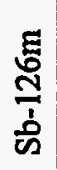 & $\frac{\bar{y}}{\frac{\dot{b}}{b}}$ & $\frac{\text { ̀े }}{\text { हे }}$ & $\begin{array}{l}\text { J̦ } \\
\text { 心 }\end{array}$ & 竎 & 市 & $\frac{n}{3}$ & $\overline{\bar{p}}$ & $\frac{\Xi}{\dot{\Xi}}$ \\
\hline
\end{tabular}




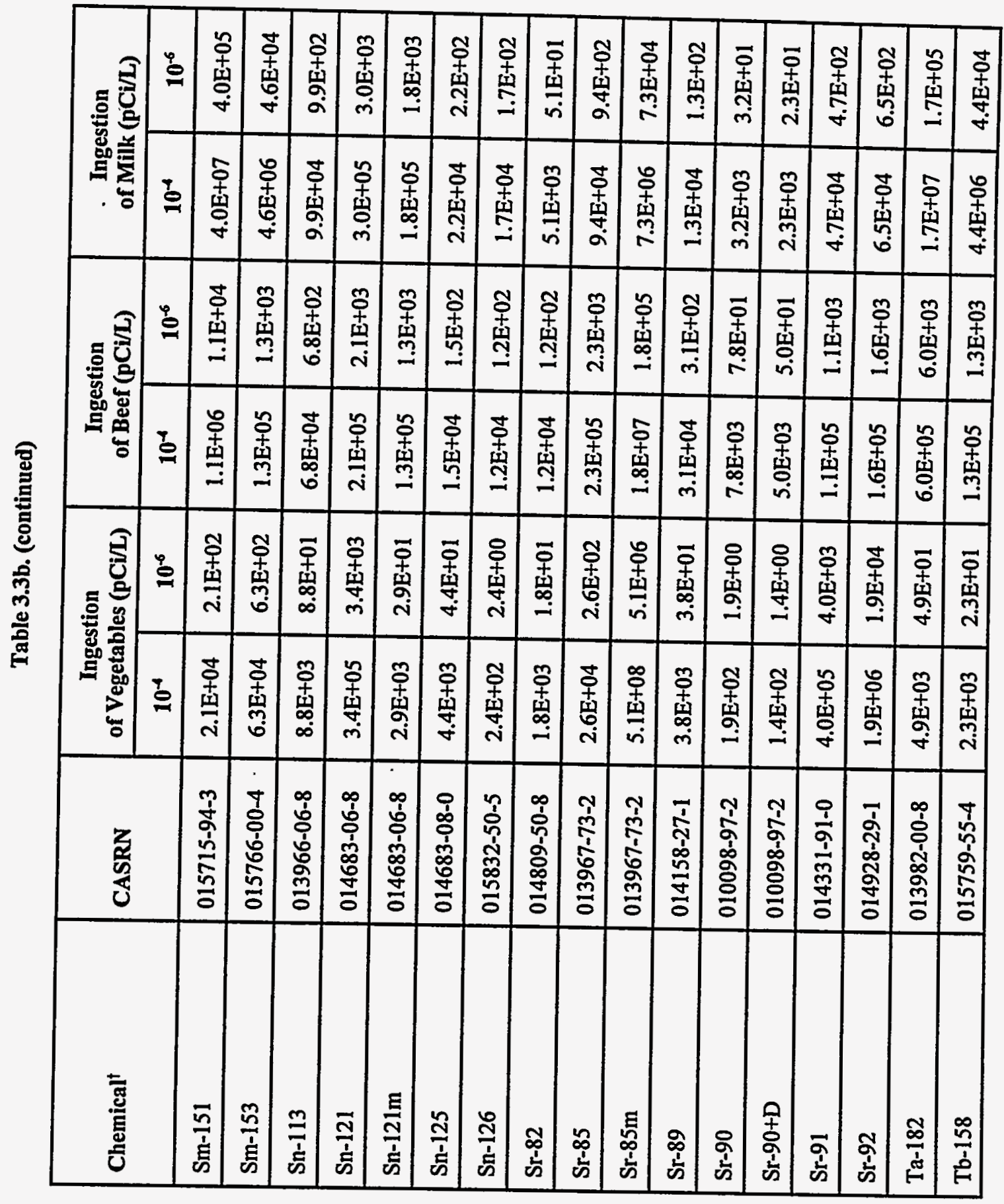


Table 3.3b. (continued)

\begin{tabular}{|l|l|l|c|c|c|c|c|}
\hline \multirow{2}{*}{ Chemical } & \multirow{2}{*}{ CASRN } & \multicolumn{2}{c|}{$\begin{array}{c}\text { Ingestion } \\
\text { of Vegetables (pCi/L) }\end{array}$} & \multicolumn{2}{c|}{$\begin{array}{c}\text { Ingestion } \\
\text { of Beef (pCi/L) }\end{array}$} & \multicolumn{2}{c|}{$\begin{array}{c}\text { Ingestion } \\
\text { of Milk (pCi/L) }\end{array}$} \\
\cline { 3 - 8 } & & $10^{-4}$ & $10^{-6}$ & $10^{-4}$ & $10^{-6}$ & $10^{-4}$ & $10^{-6}$ \\
\hline Tb-160 & $013981-29-8$ & $4.8 \mathrm{E}+03$ & $4.8 \mathrm{E}+01$ & $7.4 \mathrm{E}+04$ & $7.4 \mathrm{E}+02$ & $2.4 \mathrm{E}+06$ & $2.4 \mathrm{E}+04$ \\
\hline Tc-95 & $014809-56-4$ & $1.5 \mathrm{E}+06$ & $1.5 \mathrm{E}+04$ & $3.7 \mathrm{E}+08$ & $3.7 \mathrm{E}+06$ & $3.9 \mathrm{E}+07$ & $3.9 \mathrm{E}+05$ \\
\hline Tc-95m & $014809-56-4$ & $1.3 \mathrm{E}+03$ & $1.3 \mathrm{E}+01$ & $2.0 \mathrm{E}+07$ & $2.0 \mathrm{E}+05$ & $2.1 \mathrm{E}+06$ & $2.1 \mathrm{E}+04$ \\
\hline Tc-96 & $014808-44-7$ & $9.1 \mathrm{E}+03$ & $9.1 \mathrm{E}+01$ & $1.1 \mathrm{E}+07$ & $1.1 \mathrm{E}+05$ & $1.2 \mathrm{E}+06$ & $1.2 \mathrm{E}+04$ \\
\hline Tc-96m & $014808-44-7$ & $9.1 \mathrm{E}+07$ & $9.1 \mathrm{E}+05$ & $9.7 \mathrm{E}+08$ & $9.7 \mathrm{E}+06$ & $1.0 \mathrm{E}+08$ & $1.0 \mathrm{E}+06$ \\
\hline Tc-97 & $015759-35-0$ & $1.0 \mathrm{E}+02$ & $1.0 \mathrm{E}+00$ & $1.6 \mathrm{E}+08$ & $1.6 \mathrm{E}+06$ & $1.7 \mathrm{E}+07$ & $1.7 \mathrm{E}+05$ \\
\hline Tc-97m & $015759-35-0$ & $9.6 \mathrm{E}+02$ & $9.6 \mathrm{E}+00$ & $2.1 \mathrm{E}+07$ & $2.1 \mathrm{E}+05$ & $2.2 \mathrm{E}+06$ & $2.2 \mathrm{E}+04$ \\
\hline Tc-99 & $014133-76-7$ & $1.1 \mathrm{E}+01$ & $1.1 \mathrm{E}-01$ & $1.8 \mathrm{E}+07$ & $1.8 \mathrm{E}+05$ & $1.9 \mathrm{E}+06$ & $1.9 \mathrm{E}+04$ \\
\hline Tc-99m & $014133-76-7$ & $6.1 \mathrm{E}+06$ & $6.1 \mathrm{E}+04$ & $4.6 \mathrm{E}+08$ & $4.6 \mathrm{E}+06$ & $4.7 \mathrm{E}+07$ & $4.7 \mathrm{E}+05$ \\
\hline Te-125m & $014390-73-9$ & $1.4 \mathrm{E}+04$ & $1.4 \mathrm{E}+02$ & $1.4 \mathrm{E}+05$ & $1.4 \mathrm{E}+03$ & $3.3 \mathrm{E}+05$ & $3.3 \mathrm{E}+03$ \\
\hline Te-127 & $013981-49-2$ & $1.3 \mathrm{E}+06$ & $1.3 \mathrm{E}+04$ & $4.2 \mathrm{E}+05$ & $4.2 \mathrm{E}+03$ & $9.5 \mathrm{E}+05$ & $9.5 \mathrm{E}+03$ \\
\hline Te-127m & $013981-49-2$ & $5.1 \mathrm{E}+03$ & $5.1 \mathrm{E}+01$ & $6.0 \mathrm{E}+04$ & $6.0 \mathrm{E}+02$ & $1.4 \mathrm{E}+05$ & $1.4 \mathrm{E}+03$ \\
\hline Te-129 & $014269-71-7$ & $6.0 \mathrm{E}+07$ & $6.0 \mathrm{E}+05$ & $2.5 \mathrm{E}+06$ & $2.5 \mathrm{E}+04$ & $5.5 \mathrm{E}+06$ & $5.5 \mathrm{E}+04$ \\
\hline Te-129m & $014269-71-7$ & $3.6 \mathrm{E}+03$ & $3.6 \mathrm{E}+01$ & $3.1 \mathrm{E}+04$ & $3.1 \mathrm{E}+02$ & $7.0 \mathrm{E}+04$ & $7.0 \mathrm{E}+02$ \\
\hline Te-131 & $014683-12-6$ & $6.3 \mathrm{E}+07$ & $6.3 \mathrm{E}+05$ & $9.3 \mathrm{E}+05$ & $9.3 \mathrm{E}+03$ & $2.1 \mathrm{E}+06$ & $2.1 \mathrm{E}+04$ \\
\hline Te-131m & $014683-12-6$ & $4.2 \mathrm{E}+04$ & $4.2 \mathrm{E}+02$ & $4.1 \mathrm{E}+04$ & $4.1 \mathrm{E}+02$ & $9.3 \mathrm{E}+04$ & $9.3 \mathrm{E}+02$ \\
\hline Te-132 & $014234-28-7$ & $1.3 \mathrm{E}+04$ & $1.3 \mathrm{E}+02$ & $3.0 \mathrm{E}+04$ & $3.0 \mathrm{E}+02$ & $6.7 \mathrm{E}+04$ & $6.7 \mathrm{E}+02$ \\
\hline
\end{tabular}




\begin{tabular}{|c|c|c|c|c|c|c|c|c|c|c|c|c|c|c|c|c|c|c|}
\hline 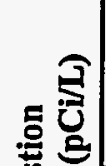 & $\stackrel{\text { ge }}{\varrho}$ & $\begin{array}{l}\text { 古 } \\
\text { 常 } \\
\stackrel{\infty}{-}\end{array}$ & 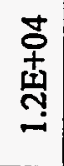 & 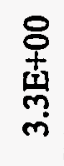 & 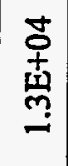 & $\begin{array}{l}\overline{0} \\
\stackrel{\dot{d}}{\dot{m}} \\
\dot{m}\end{array}$ & 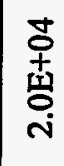 & 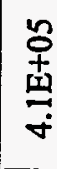 & 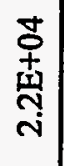 & 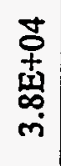 & 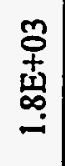 & 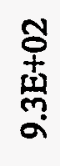 & 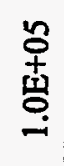 & 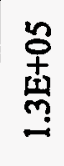 & 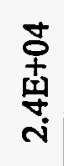 & 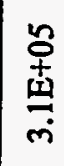 & 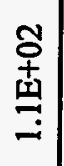 & 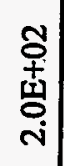 \\
\hline 总壱 & $\stackrel{7}{2}$ & 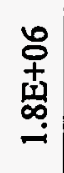 & 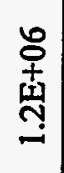 & $\begin{array}{l}\text { Ò } \\
+ \\
\text { I⿱ } \\
m \\
m \\
m\end{array}$ & 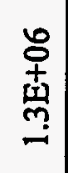 & $\underset{\text { m }}{\stackrel{5}{+}}$ & $\begin{array}{l}\stackrel{0}{+} \\
+ \\
\text { 章 } \\
\text { 시 }\end{array}$ & 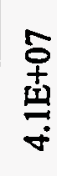 & 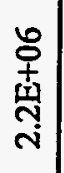 & $\begin{array}{c}0 \\
0 \\
+ \\
\text { 士⿱ } \\
\infty \\
\dot{m} \\
\dot{m}\end{array}$ & 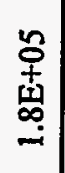 & 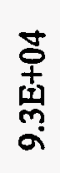 & $\begin{array}{l}5 \\
+ \\
\text { 晋 } \\
-\end{array}$ & 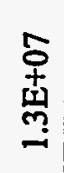 & 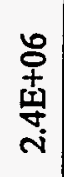 & $\begin{array}{c}5 \\
\frac{1}{9} \\
\text { m. } \\
\text { mi }\end{array}$ & 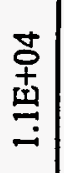 & 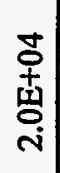 \\
\hline 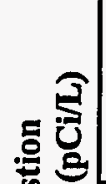 & $\stackrel{\varphi}{\circ}$ & 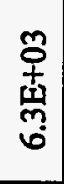 & $\begin{array}{l}\stackrel{0}{0} \\
\text { 要 } \\
\dot{+}\end{array}$ & $\begin{array}{c}8 \\
\stackrel{+}{+} \\
\text { 点 } \\
\text { Ni }\end{array}$ & 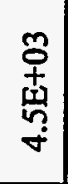 & 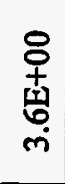 & $\begin{array}{l}\text { no } \\
+ \\
\text { I⿱ } \\
0 \\
0 \\
0\end{array}$ & 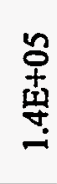 & 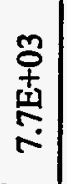 & 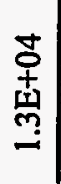 & 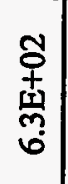 & 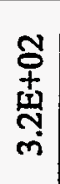 & 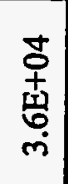 & 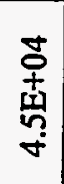 & 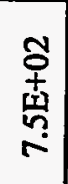 & 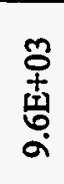 & 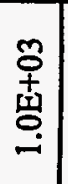 & $\begin{array}{l}\text { o̊ } \\
\text { †े } \\
\text { 空 }\end{array}$ \\
\hline 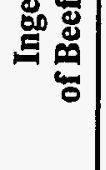 & 3 & 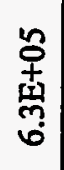 & 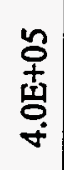 & 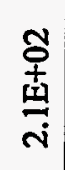 & 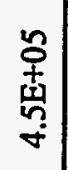 & 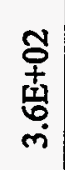 & 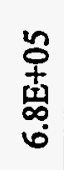 & $\begin{array}{l}5 \\
\text { 孛 } \\
\text { 足 }\end{array}$ & 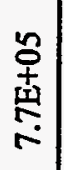 & 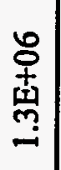 & 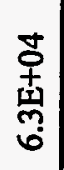 & 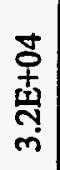 & $\begin{array}{l}\text { ○ } \\
+ \\
\text { 吉 } \\
\dot{m}\end{array}$ & 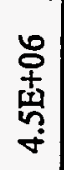 & 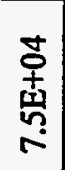 & $\begin{array}{l}\text { 오 } \\
+ \\
\text { 壳 } \\
\alpha\end{array}$ & 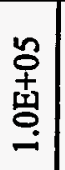 & $\begin{array}{l}n \\
0 \\
+ \\
\text { 言 } \\
\end{array}$ \\
\hline 㺼 & $\stackrel{\varphi}{\varrho}$ & $\begin{array}{l}\overline{0} \\
\text { 索 } \\
\text { ma }\end{array}$ & 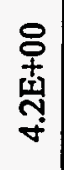 & 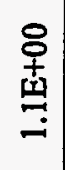 & 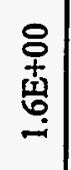 & 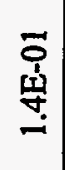 & 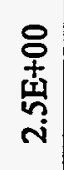 & 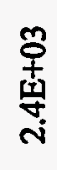 & 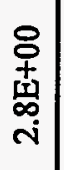 & $\begin{array}{l}\overline{0} \\
\text { 壳 } \\
\text { i }\end{array}$ & 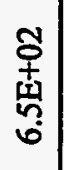 & 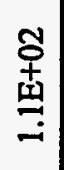 & 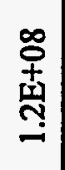 & $\begin{array}{l}\infty \\
\text { o } \\
\text { 吉 } \\
\text { i. }\end{array}$ & 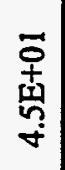 & 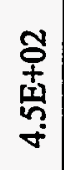 & 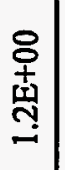 & $\begin{array}{l}\stackrel{8}{+} \\
\text { 至 } \\
\text { is }\end{array}$ \\
\hline 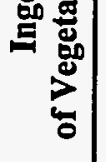 & $\underset{0}{\underline{O}}$ & $\begin{array}{c}m \\
+ \\
\text { 总 } \\
\text { m. } \\
-\end{array}$ & 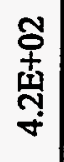 & 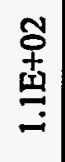 & 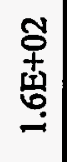 & 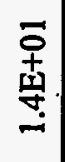 & 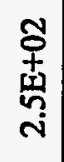 & $\begin{array}{l}n \\
+ \\
\text { 索 } \\
\text { ci }\end{array}$ & 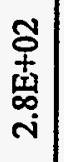 & 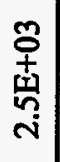 & 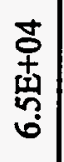 & 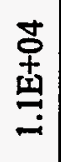 & 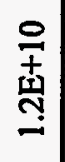 & $\begin{array}{l}\stackrel{0}{+} \\
\text { 壱 } \\
\text { i }\end{array}$ & 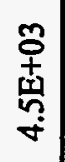 & 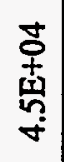 & 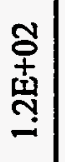 & 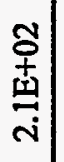 \\
\hline 蛋 & & 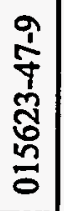 & 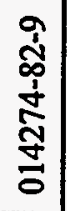 & 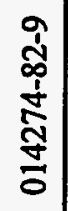 & 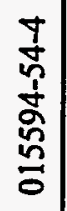 & 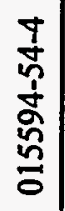 & 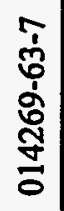 & 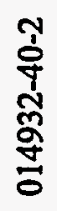 & 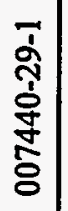 & 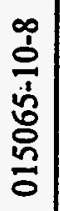 & 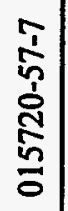 & 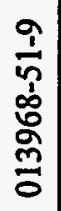 & $\begin{array}{l}9 \\
\dot{b} \\
\frac{m}{2} \\
\frac{9}{0}\end{array}$ & 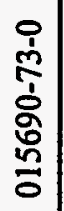 & 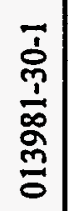 & 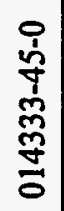 & 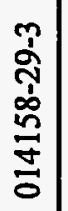 & 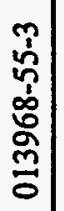 \\
\hline 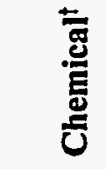 & & 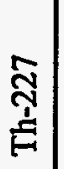 & 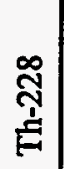 & 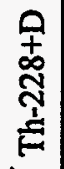 & 突 & 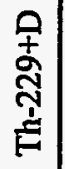 & 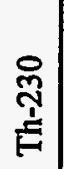 & 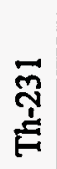 & ָָ̃ & 突 & 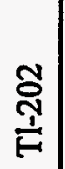 & 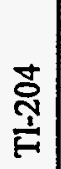 & 究 & 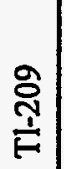 & $\stackrel{?}{\stackrel{B}{g}}$ & $\underset{E}{\vec{D}}$ & $\stackrel{\text { ָ̃ }}{\underset{p}{2}}$ & 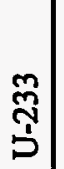 \\
\hline
\end{tabular}


Table 3.3b. (continued)

\begin{tabular}{|c|c|c|c|c|c|c|c|}
\hline \multirow{2}{*}{ Chemical $^{\dagger}$} & \multirow{2}{*}{ CASRN } & \multicolumn{2}{|c|}{$\begin{array}{c}\text { Ingestion } \\
\text { of Vegetables }(\mathrm{pCi} / \mathrm{L})\end{array}$} & \multicolumn{2}{|c|}{$\begin{array}{c}\text { Ingestion } \\
\text { of Beef }(p C i / L)\end{array}$} & \multicolumn{2}{|c|}{$\begin{array}{l}\text { Ingestion } \\
\text { of Milk (pCi/L) }\end{array}$} \\
\hline & & $10^{-4}$ & $10^{-6}$ & $10^{-4}$ & $10^{-6}$ & $10^{-4}$ & $10^{-6}$ \\
\hline $\mathrm{U}-234$ & $013966-29-5$ & $2.1 E+02$ & $2.1 \mathrm{E}+00$ & $1.9 E+05$ & $1.9 E+03$ & $2.1 \mathrm{E}+04$ & $2.1 E+02$ \\
\hline $\mathrm{U}-235$ & $015117-96-1$ & $2.0 \mathrm{E}+02$ & $2.0 \mathrm{E}+00$ & $1.9 E+05$ & $1.9 \mathrm{E}+03$ & $2.0 \mathrm{E}+04$ & $2.0 \mathrm{E}+02$ \\
\hline $\mathrm{U}-235+\mathrm{D}$ & $015117-96-1$ & $2.0 \mathrm{E}+02$ & $2.0 \mathrm{E}+00$ & $1.4 E+05$ & $1.4 \mathrm{E}+03$ & $1.9 \mathrm{E}+04$ & $1.9 \mathrm{E}+02$ \\
\hline $\mathrm{U}-236$ & $013982-70-2$ & $2.2 E+02$ & $2.2 \mathrm{E}+00$ & $2.0 \mathrm{E}+05$ & $2.0 \mathrm{E}+03$ & $2.2 E+04$ & $2.2 \mathrm{E}+02$ \\
\hline U-237 & $014269-75-1$ & $2.4 \mathrm{E}+04$ & $2.4 \mathrm{E}+02$ & $2.1 \mathrm{E}+06$ & $2.1 E+04$ & $2.3 E+05$ & $2.3 \mathrm{E}+03$ \\
\hline U-238 & $007440-61-1$ & $2.2 \mathrm{E}+02$ & $2.2 \mathrm{E}+00$ & $2.0 \mathrm{E}+05$ & $2.0 \mathrm{E}+03$ & $2.1 \mathrm{E}+04$ & $2.1 E+02$ \\
\hline $\mathrm{U}-238+\mathrm{D}$ & $007440-61-1$ & $1.5 \mathrm{E}+02$ & $1.5 \mathrm{E}+00$ & $9.8 E+04$ & $9.8 \mathrm{E}+02$ & $1.4 \mathrm{E}+04$ & $1.4 \mathrm{E}+02$ \\
\hline $\mathrm{U}-240$ & $015687-53-3$ & $1.4 E+05$ & $1.4 \mathrm{E}+03$ & $1.5 E+06$ & $1.5 \mathrm{E}+04$ & $1.7 E+05$ & $1.7 \mathrm{E}+03$ \\
\hline$V-48$ & $014331-97-6$ & $7.6 \mathrm{E}+03$ & $7.6 \mathrm{E}+01$ & $1.3 E+05$ & $1.3 E+03$ & $2.4 \mathrm{E}+06$ & $2.4 \mathrm{E}+04$ \\
\hline W-181 & $015749-46-9$ & $1.3 \mathrm{E}+05$ & $1.3 E+03$ & $2.3 \mathrm{E}+05$ & $2.3 \mathrm{E}+03$ & $4.5 E+06$ & $4.5 E+04$ \\
\hline W-185 & $014932-41-3$ & $1.8 E+04$ & $1.8 E+02$ & $3.1 \mathrm{E}+04$ & $3.1 E+02$ & $6.0 \mathrm{E}+05$ & $6.0 \mathrm{E}+03$ \\
\hline W-187 & $014983-48-3$ & $1.9 \mathrm{E}+05$ & $1.9 E+03$ & $2.6 E+04$ & $2.6 \mathrm{E}+02$ & $5.0 \mathrm{E}+05$ & $5.0 \mathrm{E}+03$ \\
\hline $\mathrm{Xe}-122$ & $015151-09-4$ & & & & & & \\
\hline $\mathrm{Xe}-123$ & $015700-10-4$ & & & & & & \\
\hline $\mathrm{Xe}-125$ & 013994-18-8 & & & & & & \\
\hline $\mathrm{Xe}-127$ & 013994-19-9 & & & & & & \\
\hline $\mathrm{Xe}-129 \mathrm{~m}$ & 013965-99-6 & & & & & & \\
\hline
\end{tabular}


Table 3.3b. (continued)

\begin{tabular}{|l|l|l|c|c|c|c|c|}
\hline \multirow{2}{*}{ Chemical $^{\dagger}$} & \multirow{2}{*}{ CASRN } & \multicolumn{2}{c}{$\begin{array}{c}\text { Ingestion } \\
\text { of Vegetables (pCi/L) }\end{array}$} & \multicolumn{2}{c|}{$\begin{array}{c}\text { Ingestion } \\
\text { of Beef (pCi/L) }\end{array}$} & \multicolumn{2}{c|}{$\begin{array}{c}\text { Ingestion } \\
\text { of Milk (pCi/L) }\end{array}$} \\
\cline { 5 - 9 } & & $10^{-4}$ & $10^{-6}$ & $10^{-4}$ & $10^{-6}$ & $10^{-4}$ & $10^{-6}$ \\
\hline Xe-131m & $014683-11-5$ & & & & & & \\
\hline Xe-133 & $014932-42-4$ & & & & & & \\
\hline Xe-133m & $014932-42-4$ & & & & & & \\
\hline Xe-135 & $014995-62-1$ & & & & & & \\
\hline Xe-135m & $014995-62-1$ & & & & & & \\
\hline Xe-137 & $014835-21-3$ & & & & & & \\
\hline Xe-138 & $015751-81-2$ & & & & & & \\
\hline Y-90 & $010098-91-6$ & $1.3 \mathrm{E}+04$ & $1.3 \mathrm{E}+02$ & $1.7 \mathrm{E}+05$ & $1.7 \mathrm{E}+03$ & $1.2 \mathrm{E}+06$ & $1.2 \mathrm{E}+04$ \\
\hline $\mathrm{Y}-91$ & $014234-24-3$ & $2.8 \mathrm{E}+03$ & $2.8 \mathrm{E}+01$ & $1.9 \mathrm{E}+05$ & $1.9 \mathrm{E}+03$ & $1.4 \mathrm{E}+06$ & $1.4 \mathrm{E}+04$ \\
\hline $\mathrm{Y}-91 \mathrm{~m}$ & $014234-24-3$ & $3.4 \mathrm{E}+08$ & $3.4 \mathrm{E}+06$ & $6.9 \mathrm{E}+07$ & $6.9 \mathrm{E}+05$ & $5.0 \mathrm{E}+08$ & $5.0 \mathrm{E}+06$ \\
\hline $\mathrm{Y}-92$ & $015751-59-4$ & $1.5 \mathrm{E}+06$ & $1.5 \mathrm{E}+04$ & $1.3 \mathrm{E}+06$ & $1.3 \mathrm{E}+04$ & $9.4 \mathrm{E}+06$ & $9.4 \mathrm{E}+04$ \\
\hline $\mathrm{Y}-93$ & $014981-70-5$ & $1.8 \mathrm{E}+05$ & $1.8 \mathrm{E}+03$ & $4.4 \mathrm{E}+05$ & $4.4 \mathrm{E}+03$ & $3.2 \mathrm{E}+06$ & $3.2 \mathrm{E}+04$ \\
\hline $\mathrm{Zn}-65$ & $013982-39-3$ & $2.9 \mathrm{E}+03$ & $2.9 \mathrm{E}+01$ & $2.6 \mathrm{E}+03$ & $2.6 \mathrm{E}+01$ & $3.7 \mathrm{E}+03$ & $3.7 \mathrm{E}+01$ \\
\hline $\mathrm{Zn}-69$ & $013982-23-5$ & $1.8 \mathrm{E}+08$ & $1.8 \mathrm{E}+06$ & $4.1 \mathrm{E}+05$ & $4.1 \mathrm{E}+03$ & $5.9 \mathrm{E}+05$ & $5.9 \mathrm{E}+03$ \\
\hline $\mathrm{Zn}-69 \mathrm{~m}$ & $013982-23-5$ & $5.1 \mathrm{E}+05$ & $5.1 \mathrm{E}+03$ & $1.7 \mathrm{E}+04$ & $1.7 \mathrm{E}+02$ & $2.4 \mathrm{E}+04$ & $2.4 \mathrm{E}+02$ \\
\hline $\mathrm{Zr}-93$ & $015751-77-6$ & $1.8 \mathrm{E}+04$ & $1.8 \mathrm{E}+02$ & $4.9 \mathrm{E}+09$ & $4.9 \mathrm{E}+07$ & $1.3 \mathrm{E}+09$ & $1.3 \mathrm{E}+07$ \\
\hline $\mathrm{Zr}-95$ & $013967-71-0$ & $9.6 \mathrm{E}+03$ & $9.6 \mathrm{E}+01$ & $6.5 \mathrm{E}+08$ & $6.5 \mathrm{E}+06$ & $1.7 \mathrm{E}+08$ & $1.7 \mathrm{E}+06$ \\
\hline
\end{tabular}


Table 3.3b. (continued)

\begin{tabular}{|l|l|c|c|c|c|c|c|}
\hline \multirow{2}{*}{ Chemical $^{\dagger}$} & \multirow{2}{*}{ CASRN } & \multicolumn{2}{|c|}{$\begin{array}{c}\text { Ingestion } \\
\text { of Vegetables (pCi/L) }\end{array}$} & \multicolumn{2}{|c|}{$\begin{array}{c}\text { Ingestion } \\
\text { of Beef (pCi/L) }\end{array}$} & \multicolumn{2}{|c|}{$\begin{array}{c}\text { Ingestion } \\
\text { of Milk (pCi/L) }\end{array}$} \\
\cline { 3 - 8 } & & $10^{-4}$ & $10^{-6}$ & $10^{-4}$ & $10^{-6}$ & $10^{-4}$ & $10^{-6}$ \\
\hline $\mathrm{Zr}-97$ & $014928-30-4$ & $6.2 \mathrm{E}+04$ & $6.2 \mathrm{E}+02$ & $2.4 \mathrm{E}+08$ & $2.4 \mathrm{E}+06$ & $6.4 \mathrm{E}+07$ & $6.4 \mathrm{E}+05$ \\
\hline
\end{tabular}

Note:

$\dagger$ Isotopes designated with an " $m$ " indicate that the isotope is metastable; isotopes designated with a " $D$ " indicate that the radionuclide slope factors include contributions from daughter products. 
Table 3.3c. Risk-based Preliminary Remediation Goals for nonradionuclides in soil or sediment (agricultural scenario)

\begin{tabular}{|c|c|c|c|c|c|c|c|c|c|c|c|c|c|c|c|}
\hline \multirow{2}{*}{ Chemical } & \multirow{2}{*}{ CASRN } & \multicolumn{4}{|c|}{ Ingestion of Vegetables $(\mathrm{mg} / \mathrm{kg})^{*}$} & \multicolumn{4}{|c|}{ Ingestion of Beef ( $\mathrm{mg} / \mathrm{kg})^{\bullet}$} & \multicolumn{6}{|c|}{ Ingestion of Milk (mg/kg)" } \\
\hline & & $10^{-4}$ & $10^{4}$ & $H Q=1$ & $\mathrm{HQ}=0.1$ & $10^{-4}$ & $10^{6}$ & $H Q=1$. & $\mathrm{HQ}=0.1$ & $10^{-4}$ & $10^{6}$ & $\begin{array}{l}\text { HQ=1 } \\
\text { Adult }\end{array}$ & $\begin{array}{l}\text { HQ=1 } \\
\text { Child }\end{array}$ & $\begin{array}{c}H Q=0.1 \\
\text { Adult }\end{array}$ & $\begin{array}{c}\mathrm{HQ}=0.1 \\
\text { Child }\end{array}$ \\
\hline ALAR & $001596-84-5$ & & & & & & & & & & & & & & \\
\hline Acenaphthene & $000083-32-9$ & & & $1.9 E+02$ & $1.9 \mathrm{E}+01$ & & & $3.4 \mathrm{E}+04$ & $3.4 E+03$ & & & $2.0 \mathrm{E}+04$ & $2.5 \mathrm{E}+03$ & $2.0 \mathrm{E}+03$ & $2.5 \mathrm{E}+02$ \\
\hline Acenaphthylene & $000208-96-8$ & & & & & & & & & & & & & & \\
\hline Acephate & $030560-19-1$ & $1.0 \mathrm{E}+00$ & $1.0 \mathrm{E}-02$ & $1.5 \mathrm{E}-01$ & $1.5 \mathrm{E}-02$ & $1.0 \mathrm{E}+06$ & $9.6 \mathrm{E}+04$ & $1.0 \mathrm{E}+06$ & $1.4 \mathrm{E}+05$ & $1.0 \mathrm{E}+06$ & $2.1 \mathrm{E}+04$ & $7.5 \mathrm{E}+05$ & $9.6 \mathrm{E}+04$ & $7.5 \mathrm{E}+04$ & $9.6 \mathrm{E}+03$ \\
\hline Acetaldehyde & $000075-07-0$ & & & & & & & & & & & & & & \\
\hline Acetochlor & $034256-82-1$ & & & & & & & & & & & & $\cdot$ & & \\
\hline Acetone & $000067-64-1$ & & & $8.4 \mathrm{E}+00$ & $8.4 \mathrm{E}-01$ & & & $1.0 \mathrm{E}+06$ & $1.0 \mathrm{E}+06$ & & & $1.0 \mathrm{E}+06$ & $1.0 \mathrm{E}+06$ & $1.0 \mathrm{E}+06$ & $1.3 \mathrm{E}+05$ \\
\hline Acetone Cyanohydrin & $000075-86-5$ & & & & & & & & & & & & & & \\
\hline Acetonitrile & $000075-05-8$ & & & $4.4 \mathrm{E}-01$ & $4.4 \mathrm{E}-02$ & & & $1.0 \mathrm{E}+06$ & $1.3 \mathrm{E}+05$ & & & $6.8 \mathrm{E}+05$ & $8.8 \mathrm{E}+04$ & $6.8 \mathrm{E}+04$ & $8.8 \mathrm{E}+03$ \\
\hline Acetophenone ${ }^{(3)}$ & $000098-86-2$ & & & $8.6 \mathrm{E}+01$ & $8.6 \mathrm{E}+00$ & & & $1.0 \mathrm{E}+06$ & $2.8 \mathrm{E}+05$ & & & $1.0 \mathrm{E}+06$ & $1.9 \mathrm{E}+05$ & $1.5 \mathrm{E}+05$ & $1.9 \mathrm{E}+04$ \\
\hline Acrolein ${ }^{(6)}$ & $000107-02-8$ & & & $2.0 \mathrm{E}+00$ & $2.0 \mathrm{E}-01$ & & & $1.0 \mathrm{E}+06$ & $3.5 \mathrm{E}+05$ & & & $1.0 \mathrm{E}+06$ & 2.3E+05 & $1.8 \mathrm{E}+05$ & $2.3 \mathrm{E}+04$ \\
\hline Acrylamide & $000079-06-1$ & $2.5 E-03$ & 2.5E-05 & $9.6 \mathrm{E}-03$ & $9.6 \mathrm{E}-04$ & $1.6 \mathrm{E}+04$ & $1.6 \mathrm{E}+02$ & $6.0 \mathrm{E}+04$ & $6.0 \mathrm{E}+03$ & $3.5 \mathrm{E}+03$ & $3.5 E+01$ & $3.1 E+04$ & $4.0 \mathrm{E}+03$ & $3.1 \mathrm{E}+03$ & $4.0 \mathrm{E}+02$ \\
\hline Acrylic Acid & $000079-10-7$ & & & $7.0 \mathrm{E}+01$ & $7.0 E+00$ & & & $1.0 \mathrm{E}+06$ & $1.0 \mathrm{E}+06$ & & & $1,0 \mathrm{E}+06$ & $1.0 \mathrm{E}+06$ & $1.0 E+06$ & $4.5 \mathrm{E}+05$ \\
\hline Acrylonitrile & $000107-13-1$ & $6.8 \mathrm{E}-02$ & $6.8 \mathrm{E}-04$ & $1.6 \mathrm{E}-01$ & $1.6 \mathrm{E}-02$ & $5.3 E+04$ & $5.3 \mathrm{E}+02$ & $1.2 E+05$ & $1.2 \mathrm{E}+04$ & $1.2 \mathrm{E}+04$ & $1.2 \mathrm{E}+02$ & $6.4 \mathrm{E}+04$ & $8.2 \mathrm{E}+03$ & $6.4 E+03$ & $8.2 \mathrm{E}+02$ \\
\hline Alachlor & $015972-60-8$ & $6.3 E+00$ & $6.3 \mathrm{E}-02$ & $2.2 \mathrm{E}+01$ & $2.2 \mathrm{E}+00$ & $1.8 \mathrm{E}+04$ & $1.8 \mathrm{E}+02$ & $6.3 E+04$ & $6.3 \mathrm{E}+03$ & $4.3 \mathrm{E}+03$ & $4.3 \mathrm{E}+01$ & $3.5 \mathrm{E}+04$ & $4.4 \mathrm{E}+03$ & $3.5 \mathrm{E}+03$ & $4.4 \mathrm{E}+02$ \\
\hline Aldicarb & $000116-06-3$ & & & $4.6 \mathrm{E}-01$ & 4.6E-02 & & & 5.1E+04 & $5.1 \mathrm{E}+03$ & & & $2.7 \mathrm{E}+04$ & $3.4 \mathrm{E}+03$ & $2.7 E+03$ & $3.4 \mathrm{E}+02$ \\
\hline Aldicarb Sulfone & $001646-88-4$ & & & & & & & & & & & & & & \\
\hline Aldrin & $000309-00-2$ & $3.1 \mathrm{E}-02$ & $3.1 \mathrm{E}-04$ & $6.8 \mathrm{E}-02$ & $6.8 \mathrm{E}-03$ & $7.5 \mathrm{E}+01$ & $7.5 \mathrm{E}-01$ & $1.6 \mathrm{E}+02$ & $1.6 \mathrm{E}+01$ & $1.7 \mathrm{E}+01$ & $1.7 \mathrm{E}-01$ & $9.0 \mathrm{E}+01$ & $1.2 \mathrm{E}+01$ & $9.0 \mathrm{E}+00$ & $1.2 \mathrm{E}+00$ \\
\hline Ally & $074223-64-6$ & & & $2.9 \mathrm{E}+01$ & $2.9 \mathrm{E}+00$ & & & $1.0 \mathrm{E}+06$ & $1.0 \mathrm{E}+06$ & & & $1.0 \mathrm{E}+06$ & $1.0 \mathrm{E}+06$ & $1.0 \mathrm{E}+06$ & 2.6E+0s \\
\hline Allyl Alcohol & $000107-18-6$ & & & 4.3E-01 & $4.3 \mathrm{E}-02$ & & & $9.7 E+05$ & $9.7 \mathrm{E}+04$ & & & $5.1 \mathrm{E}+05$ & $6.5 \mathrm{E}+04$ & $5.1 E+04$ & $6.5 \mathrm{E}+03$ \\
\hline Allyl Chlorideth & $000107-05-1$ & & & $3.2 \mathrm{E}+01$ & $3.2 \mathrm{E}+00$ & & & $1.0 \mathrm{E}+06$ & $1.9 \mathrm{E}+05$ & & & $1.0 \mathrm{E}+06$ & $1.3 \mathrm{E}+05$ & $1.0 \mathrm{E}+05$ & $1.3 \mathrm{E}+04$ \\
\hline
\end{tabular}




\begin{tabular}{|c|c|c|c|c|c|c|c|c|c|c|c|c|c|c|c|}
\hline & & & & $10-362$ & $10+962$ & & & $00+927$ & $20+921$ & & & $50-35 \cdot L$ & $20-35 \cdot L$ & $s-91 \cdot-1$ thIt & (1)ZEZI دорООУ \\
\hline & & & & $20-3 z \cdot 9$ & $00+a z ; 9$ & & & $10-39 \cdot z$ & $10+397$ & & & to-as' 6 & $20-35 \cdot 6$ & $2-82-601110$ & (1)IZZI SOpost \\
\hline$\varepsilon 0-99 \div 6$ & $20-35 \cdot L$ & $20-39^{\circ} 6$ & $10-75 / 2$ & & & $10-\exists \varepsilon^{\prime} \mathrm{I}$ & $00+3 E I$ & & & $20-a+2$ & $10-3 t^{2} z$ & & & $Z=I I+\downarrow L 9 Z 10$ & 9101 sopory \\
\hline & & & & & & & & & & & & & & $8-\angle S-0+1000$ & 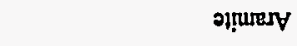 \\
\hline & & & & & & & & & & & & & & $s-\nabla z-s u t<0$ & ollody \\
\hline & & & & & & & & & & & & & & $t+9-60 \varepsilon 100$ & 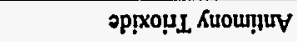 \\
\hline & & & & & & & & & & & & & & 9-18-ZEE100 & oppxonp L Kuounguy \\
\hline & & & & & & & & & & & & & & $0-19-608000$ & opentre L um!sseziod Kuoungury \\
\hline & & & & & & & & & & & & & & 6-09-bIEI00 & әp!xolus d Kuoug̣ury \\
\hline$I 0+\exists E I$ & $10+986$ & $20+a E^{\prime} I$ & $20+386$ & & & $20+a \varepsilon \varepsilon$ & $\varepsilon 0+a \varepsilon \varepsilon$ & & & $10-351$ & $00+a b i$ & & & $0-9 \varepsilon-0+t<00$ & 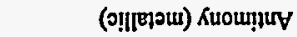 \\
\hline$\varepsilon 0+30 \% 1$ & $\varepsilon 0+31 \cdot 8$ & $50+901$ & $50+71 \cdot 8$ & & & $60+a b ! l$ & $s 0+3 b^{\prime \prime l}$ & & & $10+81 \% 6$ & $20+31 \% 6$ & & & L-zा-0zा000 & әиวэвч⿻ичу \\
\hline & & & & $\varepsilon 0+98$ 's & $50+38.5$ & & & $\$ 0+99 \cdot z$ & $90+90^{\circ} \mathrm{T}$ & & & $10-3 s^{\prime} I$ & $10+3 S^{\prime} t$ & $\varepsilon-\varepsilon s-290000$ & oxpluty \\
\hline & & & & & & & & & & & & & & $0-90-\varepsilon<\angle<00$ & ग)Eurejgns ump̣ounury \\
\hline & & & & & & & & & & & & & & L-It-b99L00 & expourury \\
\hline & & & & & & & & & & & & & & I-19-680££0 & zen!ury \\
\hline $10+99.1$ & $20+92 \cdot 1$ & $20+391$ & $\varepsilon 0+\mathrm{a} z \mathbf{I}$ & & & $20+36 z$ & $E 0+9 t z$ & & & to- $-\mathrm{E} \varepsilon \varepsilon$ & $\varepsilon 0-\exists \varepsilon^{\prime} \varepsilon$ & & & $s-t z-t 0 s 000$ & -t'suppuxdounury \\
\hline $50+30 \% 9$ & $50+3 L b$ & $50+30^{\circ} 9$ & $90+30^{\circ} 1$ & & & $50+968$ & $90+30^{\circ} 1$ & & & $00+9 I^{\prime} I$ & $10+a l^{\prime \prime}$ & & & $s-\angle z-165000$ & -w' "ןourydoụury \\
\hline$\varepsilon 0+9 \varsigma^{\prime} \mathfrak{I}$ & $60+9 z^{\prime} I$ & $50+9 s^{2} 1$ & $50+9 z I$ & & & $00+\exists z z$ & $50+3 z z$ & & & $10-398$ & $00+79.8$ & & & $8-z I-\nabla \varepsilon 8000$ & uќраиу \\
\hline & & & & & & & & & & & & & & $\nabla 6 z-58 \not \angle 90$ & o.pury \\
\hline & & & & & & & & & & & & & & $8-\varepsilon L-6 \varsigma 80 z 0$ & op!̣dsoud unurțumpy \\
\hline & & & & & & & & & & & & & & $s=06-62 \not \angle 00$ & unutumiry \\
\hline $\begin{array}{c}\text { p!!uय } \\
I^{\prime} 0=0 \mathrm{H}\end{array}$ & 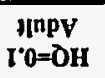 & $\begin{array}{l}\mathrm{P} ! ! q \supset \\
I=\mathrm{OH}\end{array}$ & $\prod_{\mathfrak{l}=\mathrm{OH}}$ & ,0I & rOI & $I^{*} 0=0 H$ & $\mathfrak{I}=\mathbf{O H}$ & .01 & مOI & $I^{n} 0=\mathrm{OH}$ & $I=\mathbf{O H}$ & , ol & rOI & \multirow{2}{*}{ NASVD } & \multirow{2}{*}{ ןยวุแนวนว } \\
\hline \multicolumn{6}{|c|}{ 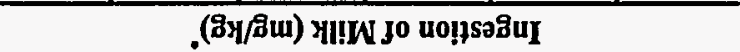 } & \multicolumn{4}{|c|}{ 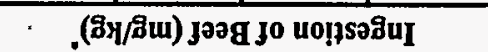 } & \multicolumn{4}{|c|}{ 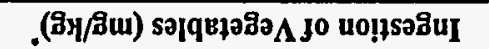 } & & \\
\hline
\end{tabular}

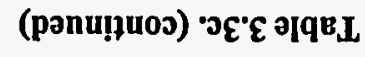


Table 3.3c. (continued)

\begin{tabular}{|c|c|c|c|c|c|c|c|c|c|c|c|c|c|c|c|}
\hline \multirow{2}{*}{ Chemical } & \multirow{2}{*}{ CASRN } & \multicolumn{4}{|c|}{ Ingestion of Vegetables (mg/kg) } & \multicolumn{4}{|c|}{ Ingestion of Beef (mg/kg) } & \multicolumn{6}{|c|}{ Ingestion of Milk (mg/kg) } \\
\hline & & $10^{-4}$ & $10^{5}$ & $H Q=1$ & $\mathrm{HQ}=0.1$ & $10^{-4}$ & $10^{4}$ & $H Q=1$ & $\mathrm{HQ}=0.1$ & $10^{4}$ & $10^{5}$ & $\begin{array}{l}\mathrm{HQ}=1 \\
\text { Adult }\end{array}$ & $\begin{array}{l}\mathrm{HQ}=1 \\
\text { Child }\end{array}$ & $\begin{array}{c}\text { HQ }=0.1 \\
\text { Adult }\end{array}$ & $\begin{array}{c}\mathrm{HQ}=0.1 \\
\text { Child }\end{array}$ \\
\hline Aroclor $1242^{(1)}$ & $053469-21-9$ & $9.5 \mathrm{E}-02$ & $9.5 \mathrm{E}-04$ & & & $2.6 \mathrm{E}+01$ & $2.6 \mathrm{E}-01$ & & & $6.2 \mathrm{E}+00$ & $6.2 \mathrm{E}-02$ & & & & \\
\hline Aroclor $1248^{(1)}$ & $012672-29-6$ & $1.1 \mathrm{E}-01$. & $1.1 \mathrm{E}-03$ & & & $6.9 \mathrm{E}-01$ & $6.9 \mathrm{E}-03$ & & & $1.7 \mathrm{E}-01$ & $1.7 \mathrm{E}-03$ & & & & \\
\hline Aroclor 1254 & $011097-69-1$ & & & $7.0 \mathrm{E}-02$ & $7.0 \mathrm{E}-03$ & & & $2.9 \mathrm{E}-01$ & $2.9 E-02$ & & & $1.7 \mathrm{E}-01$ & $2.2 \mathrm{E}-02$ & $1.7 \mathrm{E}-02$ & $2.2 \mathrm{E}-03$ \\
\hline Aroclor $1260^{(1)}$ & $011096-82-5$ & $1.1 \mathrm{E}-01$ & $1.1 \mathrm{E}-03$ & & & $3.6 \mathrm{E}-02$ & $3.6 \mathrm{E}-04$ & & & $9.0 \mathrm{E}-03$ & $9.0 \mathrm{E}-05$ & & & & \\
\hline Arsenic Salts & NA & & & & & & & & & & & & & & \\
\hline Arsenic, Inorganic & $007440-38-2$ & $5.3 \mathrm{E}-01$ & $5.3 \mathrm{E}-03$ & $1.0 \mathrm{E}+00$ & 1.0E-01 & $2.6 \mathrm{E}+01$ & $2.6 \mathrm{E}-01$ & $5.1 \mathrm{E}+01$ & $5.1 \mathrm{E}+00$ & $6.9 \mathrm{E}+01$ & $6.9 \mathrm{E}-01$ & $3.1 \mathrm{E}+02$ & $4.0 \mathrm{E}+01$ & $3.1 \mathrm{E}+01$ & $4.0 \mathrm{E}+00$ \\
\hline Arsine & $007784-42-1$ & & & & & & & & & & & & & & \\
\hline Asbestos & $001332-21-4$ & & & & & & & & & & & & & & \\
\hline Assure & $076578-14-8$ & & & & & & & & & & & & & & \\
\hline Asulam & $003337-71-1$ & & & & & & & & & & & & & & \\
\hline Atrazine & $001912-24-9$ & $2.0 \mathrm{E}+00$ & $2.0 \mathrm{E}-02$ & $6.8 \mathrm{E}+01$ & $6.8 \mathrm{E}+00$ & $8.8 \mathrm{E}+03$ & $8.8 \mathrm{E}+01$ & $2.9 \mathrm{E}+05$ & $2.9 E+04$ & $2.0 \mathrm{E}+03$ & $2.0 \mathrm{E}+01$ & $1.6 \mathrm{E}+05$ & $2.0 \mathrm{E}+04$ & $1.6 \mathrm{E}+04$ & $2.0 \mathrm{E}+03$ \\
\hline Avermectin B1 & $065195-55-3$ & & & & & & & & & & & & & & \\
\hline Azobenzene & $000103-33-3$ & $6.3 \mathrm{E}+00$ & $6.3 \mathrm{E}-02$ & & & $3.1 \mathrm{E}+03$ & $3.1 \mathrm{E}+01$ & & & 7.6E+02 & $7.6 \mathrm{E}+00$ & & & & \\
\hline Barium & $007440-39-3$ & & & $2.4 E+02$ & $2.4 \mathrm{E}+01$ & & & $1.0 \mathrm{E}+05$ & $1.0 \mathrm{E}+04$ & & & $8.0 \mathrm{E}+03$ & $1.0 \mathrm{E}+03$ & $8.0 \mathrm{E}+02$ & $1.0 \mathrm{E}+02$ \\
\hline Barium Cyanide & $000542-62-1$ & & & & & & & & & & & & & & \\
\hline Baygon & $000114-26-1$ & & & $3.1 \mathrm{E}+00$ & 3.1E-01 & & & $1.2 \mathrm{E}+05$ & $1.2 \mathrm{E}+04$ & & & $6.5 E+04$ & $8.4 E+03$ & $6.5 \mathrm{E}+03$ & $8.4 E+02$ \\
\hline Bayleton & $043121-43-3$ & & & $4.4 E+01$ & $4.4 \mathrm{E}+00$ & & & $4.2 \mathrm{E}+05$ & $4.2 \mathrm{E}+04$ & & & $2.2 \mathrm{E}+05$ & $2.9 \mathrm{E}+04$ & $2.2 \mathrm{E}+04$ & $2.9 \mathrm{E}+03$ \\
\hline Baythroid & $068359-37-5$ & & & & & & & & & & & & & & \\
\hline Benefin & $001861-40-1$ & & & $9.9 E+02$ & $9.9 \mathrm{E}+01$ & & & $1.1 E+05$ & $1.1 \mathrm{E}+04$ & & & $6.1 \mathrm{E}+04$ & $7.9 \mathrm{E}+03$ & $6.1 \mathrm{E}+03$ & $7.9 \mathrm{E}+02$ \\
\hline Benomyl & $017804-35-2$ & & & $7.4 \mathrm{E}+01$ & $7.4 \mathrm{E}+00$ & & & $7.0 \mathrm{E}+05$ & $7.0 \mathrm{E}+04$ & & & 3.7E+05 & $4.8 \mathrm{E}+04$ & $3.7 E+04$ & $4.8 \mathrm{E}+03$ \\
\hline Bentazon & $025057-89-0$ & & & $1.1 \mathrm{E}+00$ & $1.1 \mathrm{E}-01$ & & & $1.3 E+05$ & $1.3 \mathrm{E}+04$ & & & $6.8 \mathrm{E}+04$ & $8.8 \mathrm{E}+03$ & $6.8 \mathrm{E}+03$ & $8.8 \mathrm{E}+02$ \\
\hline
\end{tabular}


Table 3.3c. (continued)

\begin{tabular}{|c|c|c|c|c|c|c|c|c|c|c|c|c|c|c|c|}
\hline \multirow{2}{*}{ Chemical } & \multirow{2}{*}{ CASRN } & \multicolumn{4}{|c|}{ Ingestion of Vegetables ( $\mathrm{mg} / \mathbf{k g})^{*}$} & \multicolumn{4}{|c|}{ Ingestion of Beef (mg/kg)" } & \multicolumn{6}{|c|}{ Ingestion of Milk (mg/kg) } \\
\hline & & $10^{-4}$ & $10^{5}$ & $H Q=1$ & $\mathrm{HQ}=0.1$ & $10^{-4}$ & $10^{-4}$ & $\mathrm{HQ}=1$ & $\mathrm{HQ}=0.1$ & $10^{-4}$ & $10^{-6}$ & $\begin{array}{l}\text { HQ=1 } \\
\text { Adult }\end{array}$ & $\begin{array}{l}\text { HQ=1 } \\
\text { Child }\end{array}$ & $\begin{array}{c}\mathrm{HQ}=0.1 \\
\text { Adult }\end{array}$ & $\begin{array}{c}\mathrm{HQ}=0.1 \\
\text { Child }\end{array}$ \\
\hline Benz[a]anthracene ${ }^{(2)}$ & $000056-55-3$ & $1.1 \mathrm{E}+00$ & $1.1 \mathrm{E}-02$ & & & $9.1 \mathrm{E}+00$ & 9.1E-02 & & & $2.3 \mathrm{E}+00$ & 2.3E-02 & & & & \\
\hline Benzaldehyde & $000100-52-7$ & & & $1.5 \mathrm{E}+02$ & $1.5 \mathrm{E}+01$ & & & $1.0 \mathrm{E}+06$ & $1.4 \mathrm{E}+05$ & & & $7.4 \mathrm{E}+05$ & $9.5 \mathrm{E}+04$ & $7.4 \mathrm{E}+04$ & $9.5 E+03$ \\
\hline Benzene & $000071-43-2$ & $1.0 \mathrm{E}+01$ & $1.0 \mathrm{E}-01$ & & & $1.4 \mathrm{E}+05$ & $1.4 \mathrm{E}+03$ & & & $3.2 \mathrm{E}+04$ & $3.2 \mathrm{E}+02$ & & & & \\
\hline Benzene Hexachloride & NA & & & & & & & & & & & & & & \\
\hline Benzene, Ethyldimethyl & NA & & & & & & & & & & & & & & \\
\hline Benzene, Elhylmethyl & NA & & & & & & & & & & & & & & \\
\hline Benzene, Methylpropenyl & NA & & & & & & & & & & & & & & \\
\hline Benzene, Methylpropyl & NA & & & & & & & & & & & & & & \\
\hline Benzene, Trimethyl & $025551-13-7$ & & & & & & & ' & & & & & & & \\
\hline Benzenethiol & $000108-98-5$ & & & $1.7 \mathrm{E}-02$ & $1.7 \mathrm{E}-03$ & & & $1.1 \mathrm{E}+02$ & $1.1 \mathrm{E}+01$ & & & $5.8 \mathrm{E}+01$ & $7.5 \mathrm{E}+00$ & $5.8 \mathrm{E}+00$ & 7.5E-01 \\
\hline Benzidine & $000092-87-5$ & $5.7 \mathrm{E}-04$ & $5.7 \mathrm{E}-06$ & $1.7 \mathrm{E}+00$ & $1.7 \mathrm{E}-01$ & $4.3 \mathrm{E}+01$ & 4.3E-01 & $1.3 \mathrm{E}+05$ & $1.3 \mathrm{E}+04$ & $9.6 \mathrm{E}+00$ & $9.6 \mathrm{E}-02$ & $6.7 \mathrm{E}+04$ & $8.6 \mathrm{E}+03$ & $6.7 \mathrm{E}+03$ & $8.6 \mathrm{E}+02$ \\
\hline Benzo[a]pyrene $e^{(z)}$ & $000050-32-8$ & $1.1 \mathrm{E}-01$ & 1.1E-03 & & & $3.7 \mathrm{E}-01$ & 3.7E-03 & & & $9.3 \mathrm{E}-02$ & 9.3E-04 & & & & \\
\hline Benzo[b]fluoranthene ${ }^{(z)}$ & $000205-99-2$ & $1.1 \mathrm{E}+00$ & $1.1 \mathrm{E}-02$ & & & $3.7 \mathrm{E}+00$ & $3.7 \mathrm{E}-02$ & & & $9.3 \mathrm{E}-01$ & $9.3 \mathrm{E}-03$ & & & & \\
\hline Benzo[g,h,i]perylene & $000191-24-2$ & & & & & & & & & & & & & & \\
\hline Benzo[k]fluoranthene ${ }^{(z)}$ & $000207-08-9$ & $1.1 \mathrm{E}+01$ & $1.1 \mathrm{E}-01$ & & & $7.4 \mathrm{E}+00$ & 7.4E-02 & & & $1.9 \mathrm{E}+00$ & $1.9 \mathrm{E}-02$ & & & & \\
\hline Benzoic Acid & $000065-85-0$ & & & $4.2 \mathrm{E}+03$ & $4.2 \mathrm{E}+02$ & & & $1.0 \mathrm{E}+06$ & $1.0 \mathrm{E}+06$ & & & $1.0 \mathrm{E}+06$ & $1.0 \mathrm{E}+06$ & $1.0 \mathrm{E}+06$ & $6.0 \mathrm{E}+05$ \\
\hline Benzotrichloride & $000098-07-7$ & $3.9 \mathrm{E}-02$ & $3.9 \mathrm{E}-04$ & & & $1.1 \mathrm{E}+02$ & $1.1 \mathrm{E}+00$ & & & $2.6 \mathrm{E}+01$ & 2.6E-01 & & & & \\
\hline Benzyl Alcohol & $000100-51-6$ & & & $1.3 \mathrm{E}+02$ & $1.3 \mathrm{E}+01$ & & & $1.0 \mathrm{E}+06$ & $1.0 \mathrm{E}+06$ & & & $1.0 \mathrm{E}+06$ & $1.0 \mathrm{E}+06$ & $8.2 \mathrm{E}+05$ & $1.1 \mathrm{E}+05$ \\
\hline Benzyl Chloride & $000100-44-7$ & $2.0 \mathrm{E}+00$ & $2.0 \mathrm{E}-02$ & & & $1.9 E+04$ & $1.9 \mathrm{E}+02$ & & & $4.3 \mathrm{E}+03$ & $4.3 \mathrm{E}+01$ & & & & \\
\hline Beryllium & $007440-41-7$ & $1.9 \mathrm{E}-01$ & $1.9 \mathrm{E}-03$ & $1.7 \mathrm{E}+01$ & $1.7 \mathrm{E}+00$ & $2.0 \mathrm{E}+01$ & $2.0 \mathrm{E}-01$ & $1.8 \mathrm{E}+03$ & $1.8 \mathrm{E}+02$ & $1.7 \mathrm{E}+03$ & $1.7 \mathrm{E}+01$ & $3.8 \mathrm{E}+05$ & $4.9 E+04$ & $3.8 \mathrm{E}+04$ & $4.9 \mathrm{E}+03$ \\
\hline Bidrin & $000141-66-2$ & & & 4.7E-03 & 4.7E-04 & & & $3.0 \mathrm{E}+04$ & $3.0 \mathrm{E}+03$ & & & $1.6 \mathrm{E}+04$ & $2.0 \mathrm{E}+03$ & $1.6 \mathrm{E}+03$ & $2.0 \mathrm{E}+02$ \\
\hline
\end{tabular}


Table 3.3c. (continued)

\begin{tabular}{|c|c|c|c|c|c|c|c|c|c|c|c|c|c|c|c|}
\hline \multirow{2}{*}{ Chemical } & \multirow{2}{*}{ CASRN } & \multicolumn{4}{|c|}{ Ingestion of Vegetables (mg/kg) } & \multicolumn{4}{|c|}{ Ingestion of Beef $(\mathrm{mg} / \mathrm{kg})^{*}$} & \multicolumn{6}{|c|}{ Ingestion of Milk (mg/kg) } \\
\hline & & $10^{-4}$ & $10^{5}$ & $H Q=1$ & $H Q=0.1$ & $10^{-4}$ & $10^{6}$ & HQ=1 & $\mathrm{HQ}=0.1$ & $10^{4}$ & $10^{\circ}$ & $\begin{array}{l}\text { HQ=1 } \\
\text { Adult }\end{array}$ & $\begin{array}{l}\mathrm{HQ}=1 \\
\text { Child }\end{array}$ & $\begin{array}{c}\mathrm{HQ}=0.1 \\
\text { Adult }\end{array}$ & $\begin{array}{c}\text { HQ }=0.1 \\
\text { Child }\end{array}$ \\
\hline Biphenthrin & $082657-04-3$ & & & & & & & & & & & & & & \\
\hline Biphenyl, 1,1'- & $000092-52-4$ & & & $1.6 \mathrm{E}+02$ & $1.6 \mathrm{E}+01$ & & & 4.2E+04 & $4.2 \mathrm{E}+03$ & & & $2.4 \mathrm{E}+04$ & $3.1 E+03$ & $2.4 \mathrm{E}+03$ & $3.1 \mathrm{E}+02$ \\
\hline $\begin{array}{l}\text { Bis(2-chloro-1-methylethyl)ether } \\
\text { (Technical) }\end{array}$ & $000108-60-1$ & $6.0 \mathrm{E}+00$ & $6.0 \mathrm{E}-02$ & ' & & $3.3 E+04$ & $3.3 \mathrm{E}+02$ & & & $7.5 \mathrm{E}+03$ & $7.5 \mathrm{E}+01$ & & & & \\
\hline Bis(2-chloroethoxy)methane & $000111-91-1$ & & & & & & & & & & & & & & \\
\hline Bis(2-chloroethyi)ether & $000111-44-4$ & $1.2 \mathrm{E}-01$ & $1.2 \mathrm{E}-03$ & & & $9.0 \mathrm{E}+03$ & $9.0 \mathrm{E}+01$ & & & $2.0 \mathrm{E}+03$ & $2.0 \mathrm{E}+01$ & & & & \\
\hline Bis(2-chloroisopropyl)ether & $039638-32-9$ & & & $5.0 \mathrm{E}+01$ & $5.0 \mathrm{E}+00$ & & & 7.1E+05 & $7.1 \mathrm{E}+04$ & & & $3.8 \mathrm{E}+05$ & $4.8 \mathrm{E}+04$ & $3.8 \mathrm{E}+04$ & $4.8 \mathrm{E}+03$ \\
\hline Bis(2-ethylhexyl)phthalate() & $000117-81-7$ & $5.6 \mathrm{E}+01$ & $5,6 \mathrm{E}-01$ & $6.7 \mathrm{E}+01$ & $6.7 \mathrm{E}+00$ & 2.7E+03 & $2.7 E+01$ & $3.3 E+03$ & $3.3 \mathrm{E}+02$ & $6.8 \mathrm{E}+02$ & $6.8 \mathrm{E}+00$ & $1.9 E+03$ & $2.5 \mathrm{E}+02$ & $1.9 \mathrm{E}+02$ & $2.5 \mathrm{E}+01$ \\
\hline Bis(chloromethyl)ether & $000542-88-1$ & 2.0E-04 & $2.0 \mathrm{E}-06$ & & & $1.1 \mathrm{E}+02$ & $1.1 \mathrm{E}+00$ & & & $2.5 \mathrm{E}+01$ & $2.5 \mathrm{E}-01$ & & & & \\
\hline Bisphenol A & $000080-05-7$ & & & $1.4 \mathrm{E}+02$ & $1.4 \mathrm{E}+01$ & & & $1.1 E+05$ & 1.1E+04 & & & $5.9 E+04$ & $7.6 \mathrm{E}+03$ & $5.9 \mathrm{E}+03$ & $7.6 \mathrm{E}+02$ \\
\hline Boron And Borates Only & $007440-42-8$ & & & $6.5 \mathrm{E}+0 \mathrm{t}$ & $6.5 \mathrm{E}+00$ & & & $3.8 E+03$ & $3.8 \mathrm{E}+02$ & & & $3.4 E+02$ & $4.4 \mathrm{E}+01$ & $3.4 E+01$ & $4.4 \mathrm{E}+00$ \\
\hline Boron Trifluoride & $007637-07-2$ & & & & & & & & & & & & & & \\
\hline Bromochloromethane & $000074-97-5$ & & & & & & & & & & & & & & \\
\hline Bromodichloromethane & $000075-27-4$ & $4.7 \mathrm{E}+00$ & 4.7E-02 & $2.5 \mathrm{E}+0 \mathrm{t}$ & $2.5 \mathrm{E}+00$ & $6.6 \mathrm{E}+04$ & $6.6 \mathrm{E}+02$ & $3.5 \mathrm{E}+05$ & $3.5 \mathrm{E}+04$ & $1.5 \mathrm{E}+04$ & $1.5 \mathrm{E}+02$ & $1.9 \mathrm{E}+05$ & $2.4 E+04$ & $1.9 \mathrm{E}+04$ & $2.4 \mathrm{E}+03$ \\
\hline Bromodiphenyl Ether, p- & $000101-55-3$ & & & & & & & & & & & & & & \\
\hline Bromoform & $000075-25-2$ & 4.7E+01 & 4.7E-01 & $3.2 \mathrm{E}+01$ & $3.2 \mathrm{E}+00$ & $3.6 \mathrm{E}+05$ & $3.6 \mathrm{E}+03$ & $2.5 \mathrm{E}+05$ & $2.5 \mathrm{E}+04$ & $8.3 \mathrm{E}+04$ & $8.3 \mathrm{E}+02$ & $1.3 E+05$ & $1.7 \mathrm{E}+04$ & $1.3 \mathrm{E}+04$ & $1.7 \mathrm{E}+03$ \\
\hline Bromomethane $($ ()) & $000074-83-9$ & & & 7.1E-01 & 7.1E-02 & & & $6.6 \mathrm{E}+04$ & $6.6 \mathrm{E}+03$ & & & $3.5 \mathrm{E}+04$ & $4.4 \mathrm{E}+03$ & $3.5 \mathrm{E}+03$ & $4.4 E+02$ \\
\hline Bromophos & $002104-96-3$ & & & & & & & & & & & & & & \\
\hline Bromotrichloromethane & $000075-62-7$ & & & & & & & & & & & & & & \\
\hline Bromoxynil & $001689-84-5$ & & & $4.3 E+\infty 0$ & 4.3E-01 & & & $1.0 E+06$ & $1.9 \mathrm{E}+05$ & & & $1.0 \mathrm{E}+06$ & $1.3 \mathrm{E}+05$ & $1.0 \mathrm{E}+05$ & $1.3 \mathrm{E}+04$ \\
\hline Bromoxynil Octanoate & $001689-99-2$ & & & & & & & & & & & & & & \\
\hline Butadiene, 1,3- & $000106-99-0$ & & & & & & & & & & & & & & \\
\hline
\end{tabular}

Nonradionuclides/soil or sediment/agricultural

Page 5 of 33 
Table 3.3c. (continued)

\begin{tabular}{|c|c|c|c|c|c|c|c|c|c|c|c|c|c|c|c|}
\hline \multirow{2}{*}{ Chemical } & \multirow{2}{*}{ CASRN } & \multicolumn{4}{|c|}{ Ingestion of Vegetables (mg/kg)" } & \multicolumn{4}{|c|}{ Ingestion of Beef (mg/kg) } & \multicolumn{6}{|c|}{ Ingestion of Milk (mg/kg) } \\
\hline & & $10^{-4}$ & $10^{5}$ & $H Q=1$ & $\mathrm{HQ}=0.1$ & $10^{-4}$ & $10^{4}$ & $\mathbf{H Q}=\mathbf{1}$ & $\mathrm{HQ}=0.1$ & $10^{-4}$ & $10^{6}$ & $\begin{array}{l}\text { HQ=1 } \\
\text { Adult }\end{array}$ & $\begin{array}{l}\text { HQ=1 } \\
\text { Child }\end{array}$ & $\begin{array}{c}\mathrm{HQ}=0.1 \\
\text { Adult }\end{array}$ & $\begin{array}{c}\text { HQ }=0.1 \\
\text { Child }\end{array}$ \\
\hline Butanol, N- & $000071-36-3$ & & & $2.6 \mathrm{E}+01$ & $2.6 \mathrm{E}+00$ & & & $1.0 \mathrm{E}+06$ & $8.2 \mathrm{E}+05$ & & & $1.0 \mathrm{E}+06$ & $5.5 \mathrm{E}+05$ & $4.3 \mathrm{E}+05$ & $5.5 \mathrm{E}+04$ \\
\hline Butanone-2, 4-chloro-4,4-difluoro & NA & & & & & & & & & & & & & & \\
\hline Butyl Benzyl Phthlate & $000085-68-7$ & & & $6.7 \mathrm{E}+02$ & $6.7 \mathrm{E}+01$ & & & $3.3 \mathrm{E}+04$ & $3.3 \mathrm{E}+03$ & & & $1.9 \mathrm{E}+04$ & $2.5 \mathrm{E}+03$ & $1.9 \mathrm{E}+03$ & $2.5 \mathrm{E}+02$ \\
\hline Butylate & $002008-41-5$ & & & & & & & & & & & & & & \\
\hline Butylchloride, 1- & $000507-20-0^{\circ}$ & & & & & & & & & & & & & & \\
\hline Butylphthalyl Butylglycolate & $000085-70-1$ & & & & & & & & & & & & & & \\
\hline Cacodylic Acid & $000075-60-5$ & & & $3.4 \mathrm{E}-01$ & $3.4 \mathrm{E}-02$ & & & 4.7E+05 & 4.7E+04 & & & $2.5 \mathrm{E}+05$ & $3.1 \mathrm{E}+04$ & $2.5 \mathrm{E}+04$ & $3.1 \mathrm{E}+03$ \\
\hline Cadmium (Diet) & $007440-43-9$ & & & $2.3 \mathrm{E}+00$ & 2.3E-01 & & & $3.9 \mathrm{E}+02$ & $3.9 \mathrm{E}+01$ & & & $2.7 E+01$ & $3.5 \mathrm{E}+00$ & $2.7 \mathrm{E}+00$ & $3.5 \mathrm{E}-01$ \\
\hline Calcium Cyanide & $000592-01-8$ & & & & & & & & & & & & & & \\
\hline Caprolactam & $000105-60-2$ & & & $4.5 \mathrm{E}+01$ & 4.5E+00 & & & $1.0 \mathrm{E}+06$ & $1.0 \mathrm{E}+06$ & & & $1.0 \mathrm{E}+06$ & $1.0 \mathrm{E}+06$ & $1.0 \mathrm{E}+06$ & $6.3 \mathrm{E}+05$ \\
\hline Captafol & $002425-06-1$ & 4.7E+01 & 4.7E-01 & $3.4 \mathrm{E}+00$ & $3.4 \mathrm{E}-01$ & $2.9 \mathrm{E}+05$ & $2.9 \mathrm{E}+03$ & 2.1E+04 & $2.1 \mathrm{E}+03$ & $6.6 \mathrm{E}+04$ & $6.6 \mathrm{E}+02$ & $1.2 \mathrm{E}+04$ & $1.5 \mathrm{E}+03$ & $1.2 \mathrm{E}+03$ & $1.5 \mathrm{E}+02$ \\
\hline Captan & $000133-06-2$ & $9.9 E+01$ & $9.9 \mathrm{E}-01$ & $1.9 \mathrm{E}+02$ & $1.9 \mathrm{E}+01$ & $9.3 \mathrm{E}+05$ & $9.3 \mathrm{E}+03$ & $1.0 \mathrm{E}+06$ & $1.8 \mathrm{E}+05$ & $2.1 \mathrm{E}+05$ & $2.1 \mathrm{E}+03$ & $9.7 E+05$ & $1.2 \mathrm{E}+05$ & $9.7 \mathrm{E}+04$ & $1.2 \mathrm{E}+04$ \\
\hline Carbaryl & $000063-25-2$ & & & $1.5 \mathrm{E}+02$ & $1.5 \mathrm{E}+01$ & & & $1.0 \mathrm{E}+06$ & $1.4 \mathrm{E}+05$ & & & $7.4 E+05$ & $9.5 \mathrm{E}+04$ & $7.4 \mathrm{E}+04$ & $9.5 \mathrm{E}+03$ \\
\hline Carbazole & $000086 \cdot 74-8$ & $3.5 \mathrm{E}+01$ & $3.5 \mathrm{E}-01$ & & & $1.7 \mathrm{E}+04$ & $1.7 \mathrm{E}+02$ & & & $4.2 \mathrm{E}+03$ & $4.2 \mathrm{E}+01$ & & & & \\
\hline Carbofuran & $001563-66-2$ & & & $3.9 E+00$ & $3.9 \mathrm{E}-01$ & & & $1.5 \mathrm{E}+05$ & $1.5 \mathrm{E}+04$ & & & $8.1 \mathrm{E}+04$ & $1.0 \mathrm{E}+04$ & $8.1 \mathrm{E}+03$ & $1.0 \mathrm{E}+03$ \\
\hline Carbon Disulfide & $000075-15-0$ & & & $1.4 E+02$ & $1.4 \mathrm{E}+01$ & & & $1.0 \mathrm{E}+06$ & $1.6 \mathrm{E}+05$ & & & $8.4 E+05$ & $1.1 \mathrm{E}+05$ & $8.4 \mathrm{E}+04$ & $1.1 \mathrm{E}+04$ \\
\hline Carbon Tetrachloride & $000056-23-5$ & $3.7 \mathrm{E}+00$ & 3.7E-02 & $1.4 E+00$ & $1.4 \mathrm{E}-01$ & $1.3 \mathrm{E}+04$ & $1.3 \mathrm{E}+02$ & $5.1 \mathrm{E}+03$ & $5.1 \mathrm{E}+02$ & $3.0 \mathrm{E}+03$ & $3.0 \mathrm{E}+01$ & $2.8 \mathrm{E}+03$ & $3.6 \mathrm{E}+02$ & $2.8 \mathrm{E}+02$ & $3.6 \mathrm{E}+01$ \\
\hline Carbosulfan & $055285-14-8$ & & & & & & & & & & & & & & \\
\hline Carboxin & $005234-68-4$ & & & $1.3 \mathrm{E}+02$ & $1.3 \mathrm{E}+01$ & & & $1.0 \mathrm{E}+06$ & $1.8 \mathrm{E}+05$ & & & $9.4 \mathrm{E}+05$ & $1.2 \mathrm{E}+0 \mathrm{~S}$ & $9.4 E+04$ & $1.2 \mathrm{E}+04$ \\
\hline Chloral & $000075-87-6$ & & & & & & & & & & & & & & \\
\hline Chloramben & $000133-90-4$ & & & & & & & & & & & & & & \\
\hline
\end{tabular}


Table 3.3c. (continued)

\begin{tabular}{|c|c|c|c|c|c|c|c|c|c|c|c|c|c|c|c|}
\hline \multirow{2}{*}{ Chemical } & \multirow{2}{*}{ CASRN } & \multicolumn{4}{|c|}{ Ingestion of Vegetables $(\mathrm{mg} / \mathrm{kg})^{*}$} & \multicolumn{4}{|c|}{ Ingestion of Beef (mg/kg) } & \multicolumn{6}{|c|}{ Ingestion of Milk (mg/kg) } \\
\hline & & $10^{-4}$ & $10^{4}$ & $\mathrm{HQ}=1$ & $\mathrm{HQ}=0.1$ & $10^{-1}$ & $10^{-4}$ & $\mathbf{H Q}=\mathbf{I}$ & $\mathrm{HQ}=0.1$ & $10^{-4}$ & $10^{6}$ & $\begin{array}{l}\text { HQ=1 } \\
\text { Adult }\end{array}$ & $\begin{array}{l}\text { HQ=1 } \\
\text { Child }\end{array}$ & $\begin{array}{c}\text { HQ=0.1 } \\
\text { Adult }\end{array}$ & $\begin{array}{c}\text { HQ }=0.1 \\
\text { Child }\end{array}$ \\
\hline Chloranil & $000118-75-2$ & & & & & & & & & & & & & & \\
\hline Chlordane $e^{(6)}$ & $000057-74-9$ & $6.2 \mathrm{E}-01$ & $6.2 \mathrm{E}-03$ & $2.1 E-01$ & 2.1E-02 & $8.0 \mathrm{E}+00$ & $8.0 \mathrm{E}-02$ & $2.7 E+00$ & 2.7E-01 & $2.0 \mathrm{E}+00$ & $2.0 \mathrm{E}-02$ & $1.6 \mathrm{E}+00$ & $2.0 \mathrm{E}-01$ & $1.6 \mathrm{E}-01$ & $2.0 \mathrm{E}-02$ \\
\hline Chloride & $016887-00-6$ & & & & & & & & & & & & & & \\
\hline Chiorimuron, Ethyl- & $090982-32-4$ & & & & & & & & & & & & & & \\
\hline Chlorine & $007782-50-5$ & & & $5.1 E+00$ & 5.1E-01 & & & $1.1 \mathrm{E}+01$ & $1.1 E+00$ & & & $2.1 E+00$ & $2.7 \mathrm{E}-01$ & $2.1 \mathrm{E}-01$ & $2.7 \mathrm{E}-02$ \\
\hline Chlorine Dioxide & $010049-04-4$ & & & & & & & & & & & & & & \\
\hline Chlorite (Sodium Salt) & $007758-19-2$ & & & & & & & & & & & & & & \\
\hline Chloro-1,1-difluoroethane, 1- & $000075-68-3$ & & & & & & & & & & & & & & \\
\hline Chloro-1,3-butadiene, 2- & $000126-99-8$ & & & $1.9 \mathrm{E}+01$ & $1.9 E+00$ & & & $5.0 \mathrm{E}+05$ & $5.0 \mathrm{E}+04$ & & & $2.6 \mathrm{E}+05$ & $3.4 \mathrm{E}+04$ & $2.6 \mathrm{E}+04$ & $3.4 \mathrm{E}+03$ \\
\hline Chloro-2-methylaniline $\mathrm{HCl}$, 4 - & $003165-93-3$ & & & & & & & & & & & & & & \\
\hline Chloro-2-methylaniline, 4- & $000095-69-2$ & & & & & & & & & & & & & & \\
\hline Chloroacetic Acid & $000079-11-8$ & & & $1.0 \mathrm{E}+00$ & $1.0 \mathrm{E}-01$ & & & $9.4 \mathrm{E}+04$ & $9.4 \mathrm{E}+03$ & & & $4.9 \mathrm{E}+04$ & $6.3 \mathrm{E}+03$ & $4.9 E+03$ & $6.3 \mathrm{E}+02$ \\
\hline Chloroacetophenone, 2 - & $000532-27-4$ & & & & & & & & & & & & & & \\
\hline Chloroaniline, p- & $000106-47-8$ & & & $8.2 \mathrm{E}+00$ & $8.2 \mathrm{E}-01$ & & & $2.9 E+04$ & $2.9 \mathrm{E}+03$ & & & $1.6 \mathrm{E}+04$ & $2.0 \mathrm{E}+03$ & $1.6 \mathrm{E}+03$ & $2.0 \mathrm{E}+02$ \\
\hline Chlorobenzene $^{(6)}$ & $000108-90-7$ & & & 4.1E+01 & $4.1 \mathrm{E}+00$ & & & $1.5 E+05$ & $1.5 \mathrm{E}+04$ & & & $7.9 \mathrm{E}+04$ & $1.0 \mathrm{E}+04$ & $7.9 E+03$ & $1,0 \mathrm{E}+03$ \\
\hline Chlorobenzilate & $000510-15-6$ & $2.8 \mathrm{E}+\dot{00}$ & $2,8 \mathrm{E}-02$ & $6.5 \mathrm{E}+01$ & $6.5 \mathrm{E}+00$ & $3.3 \mathrm{E}+02$ & $3.3 \mathrm{E}+00$ & $7.6 \mathrm{E}+03$ & $7.6 \mathrm{E}+02$ & $8.1 E+01$ & 8.1E-01 & $4.4 \mathrm{E}+03$ & $5.7 \mathrm{E}+02$ & 4.4E+02 & $5.7 \mathrm{E}+01$ \\
\hline Chlorobenzoic Acid, p- & $000074-11-3$ & & & $3.9 \mathrm{E}+02$ & $3.9 \mathrm{E}+01$ & & & $1.0 \mathrm{E}+06$ & $1.7 \mathrm{E}+05$ & & & $9.0 \mathrm{E}+05$ & $1.2 \mathrm{E}+05$ & $9.0 \mathrm{E}+04$ & $1.2 \mathrm{E}+04$ \\
\hline Chlorobenzotrifluoride, 4- & $000098-56-6$ & & & $5.8 \mathrm{E}+01$ & $5.8 \mathrm{E}+00$ & & & $3.3 \mathrm{E}+04$ & $3.3 \mathrm{E}+03$ & & & $1.9 \mathrm{E}+04$ & $2.4 E+03$ & $1.9 \mathrm{E}+03$ & $2.4 \mathrm{E}+02$ \\
\hline Chlorobutane, 1- & $000109-69-3$ & & & $7.3 \mathrm{E}+02$ & $7.3 E+01$ & & & $1.0 \mathrm{E}+06$ & $3.8 \mathrm{E}+05$ & & & $1.0 \mathrm{E}+06$ & $2.6 \mathrm{E}+05$ & $2.1 \mathrm{E}+05$ & $2.6 \mathrm{E}+04$ \\
\hline Chlorobutane, 2- & $000078-86-4$ & & & & & & & & & & & & & & \\
\hline Chlorocyclopentadiene & $041851-50-7$ & & & & & & & & & & & & & & \\
\hline
\end{tabular}


Table 3.3c. (continued)

\begin{tabular}{|c|c|c|c|c|c|c|c|c|c|c|c|c|c|c|c|}
\hline \multirow{2}{*}{ Chemical } & \multirow{2}{*}{ CASRN } & \multicolumn{4}{|c|}{ Ingestion of Vegetables (mg/kg) } & \multicolumn{4}{|c|}{ Ingestion of Beef (mg/kg) } & \multicolumn{6}{|c|}{ Ingestion of Milk (mg/kg) } \\
\hline & & $10^{-4}$ & $10^{4}$ & $H Q=1$ & $\mathrm{HQ}=0.1$ & $10^{-4}$ & $10^{4}$ & HQ=1 & $\mathrm{HQ}=0.1$ & $10^{-4}$ & $10^{4}$ & $\begin{array}{l}\text { HQ=1 } \\
\text { Adult }\end{array}$ & $\begin{array}{l}\text { HQ=1 } \\
\text { Child }\end{array}$ & $\begin{array}{c}\text { HQ }=0.1 \\
\text { Adult }\end{array}$ & $\begin{array}{c}\mathrm{HQ}=0.1 \\
\text { Child }\end{array}$ \\
\hline Chlorodibromoethane & $073506-94-2$ & & & & & & & & & & & & & & \\
\hline Chlorodifluoromethane & $000075-45-6$ & & & & & & & & & & & & & & \\
\hline Chloroform ${ }^{(6)}$ & $000067-66-3$ & $4.4 \mathrm{E}+01$ & 4.4E-01 & $1.2 \mathrm{E}+01$ & $1.2 \mathrm{E}+00$ & $7.6 \mathrm{E}+05$ & $7.6 \mathrm{E}+03$ & $2.0 \mathrm{E}+05$ & $2.0 \mathrm{E}+04$ & $1.7 \mathrm{E}+05$ & $1.7 \mathrm{E}+03$ & $1.1 \mathrm{E}+05$ & $1.4 \mathrm{E}+04$ & $1.1 \mathrm{E}+04$ & $1.4 E+03$ \\
\hline Chloromethane $e^{(6)}$ & $000074-87-3$ & $6.4 \mathrm{E}+00$ & $6.4 \mathrm{E}-02$ & & & $1.0 \mathrm{E}+06$ & $1.1 \mathrm{E}+04$ & & & $2.5 \mathrm{E}+05$ & $2.5 \mathrm{E}+03$ & & & & \\
\hline Chloromethyl Methyl Ether & $000107-30-2$ & & & & & & & & & & & & & & \\
\hline Chloronaphthalene, Beta- & $000091-58-7$ & & & $2.5 \mathrm{E}+02$ & $2.5 \mathrm{E}+01$ & & & $6.8 \mathrm{E}+04$ & $6.8 \mathrm{E}+03$ & & & $3.9 \mathrm{E}+04$ & $5.0 \mathrm{E}+03$ & $3.9 \mathrm{E}+03$ & $5.0 E+02$ \\
\hline Chloronitrobenzene, o- & $000088-73-3$ & $1.3 \mathrm{E}+01$ & $1.3 \mathrm{E}-01$ & & & $1.5 \mathrm{E}+05$ & $1.5 \mathrm{E}+03$ & & & 3.3E+04 & $3.3 \mathrm{E}+02$ & & & & \\
\hline Chloronitrobenzene, p- & $000121-73-3$ & $2.1 \mathrm{E}+01$ & 2.1E-01 & & & $1.6 \mathrm{E}+05$ & $1.6 \mathrm{E}+03$ & & & $3.6 \mathrm{E}+04$ & $3.6 \mathrm{E}+02$ & & & & \\
\hline Chlorophenol, 2- & $000095-57-8$ & & & $6.8 \mathrm{E}+00$ & $6.8 \mathrm{E}-01$ & & & $7.8 \mathrm{E}+04$ & $7.8 \mathrm{E}+03$ & & & $4.2 \mathrm{E}+04$ & $5.4 E+03$ & $4.2 \mathrm{E}+03$ & $5.4 \mathrm{E}+02$ \\
\hline Chlorophenyl Methyl Sulfide, p- & 000123-09-1 & & & & & & & & & & & & & & \\
\hline Chlorophenyl Methyl Sulfoxide & $000934-73-6$ & & & & & & & & & & & & & & \\
\hline Chloropropane, 2- & $000075-29-6$ & & & & & & & & & & & & & & \\
\hline Chlorothalonil & $001897-45-6$ & $6.4 \mathrm{E}+01$ & $6.4 \mathrm{E}-01$ & $4.5 \mathrm{E}+01$ & $4.5 \mathrm{E}+00$ & $2.6 \mathrm{E}+04$ & $2.6 \mathrm{E}+02$ & $1.9 \mathrm{E}+04$ & $1.9 \mathrm{E}+03$ & $6.3 \mathrm{E}+03$ & $6.3 \mathrm{E}+01$ & $1.1 \mathrm{E}+04$ & $1.4 \mathrm{E}+03$ & $1.1 \mathrm{E}+03$ & $1.4 \mathrm{E}+02$ \\
\hline Chlorotoluene, o- & $000095-49-8$ & & & $5.3 \mathrm{E}+01$ & $5.3 \mathrm{E}+00$ & & & $5.9 \mathrm{E}+04$ & $5.9 \mathrm{E}+03$ & & & $3.3 E+04$ & $4.2 \mathrm{E}+03$ & $3.3 \mathrm{E}+03$ & $4.2 \mathrm{E}+02$ \\
\hline Chlorpropham & $000101-21-3$ & & & $4.3 \mathrm{E}+02$ & $4.3 \mathrm{E}+01$ & & & $1.0 \mathrm{E}+06$ & $1.3 \mathrm{E}+05$ & & & $6.9 \mathrm{E}+05$ & $8.9 \mathrm{E}+04$ & $6.9 \mathrm{E}+04$ & $8.9 \mathrm{E}+03$ \\
\hline Chlorpyrifos & 002921-88-2 & & & $1.0 \mathrm{E}+01$ & $1.0 \mathrm{E}+00$ & & & $2.1 \mathrm{E}+02$ & $2.1 \mathrm{E}+01$ & & & $1.2 \mathrm{E}+02$ & $1.6 \mathrm{E}+01$ & $1.2 \mathrm{E}+01$ & $1.6 \mathrm{E}+00$ \\
\hline Chlorpyrifos Methyl & $005598-13-0$ & & & $3.2 \mathrm{E}+01$ & $3.2 \mathrm{E}+00$ & & & $5.7 \mathrm{E}+03$ & $5.7 \mathrm{E}+02$ & & & 3.3E+03 & $4.2 \mathrm{E}+02$ & $3.3 \mathrm{E}+02$ & $4.2 \mathrm{E}+01$ \\
\hline Chlorsulfuron & $064902-72-3$ & & & $9.8 \mathrm{E}-01$ & $9.8 \mathrm{E}-02$ & & & $1.0 \mathrm{E}+06$ & $1.0 \mathrm{E}+06$ & & & $1.0 \mathrm{E}+06$ & $1.0 \mathrm{E}+06$ & $1.0 \mathrm{E}+06$ & $1.9 \mathrm{E}+05$ \\
\hline Chlorthiophos & $060238-56-4$ & & & & & & & & & & & & & & \\
\hline Chromium (III) (Insoluble Salts) & $016065-83-1$ & & & $3.5 \mathrm{E}+03$ & $3.5 \mathrm{E}+02$ & & & $3.8 \mathrm{E}+04$ & $3.8 \mathrm{E}+03$ & & & $1.0 \mathrm{E}+06$ & $8.1 E+05$ & $6.3 \mathrm{E}+05$ & $8.1 \mathrm{E}+04$ \\
\hline Chromium (VI) & $018540-29-9$ & & & $1.8 \mathrm{E}+01$ & $1.8 \mathrm{E}+00$ & & & $1.9 \mathrm{E}+02$ & $1.9 \mathrm{E}+01$ & & & $3.1 \mathrm{E}+04$ & $4.0 E+03$ & $3.1 \mathrm{E}+03$ & $4.0 \mathrm{E}+02$ \\
\hline
\end{tabular}

Nonradionuclides/soil or sediment/agricultural

Page 8 of 33 
Table 3.3c. (continued)

\begin{tabular}{|c|c|c|c|c|c|c|c|c|c|c|c|c|c|c|c|}
\hline \multirow{2}{*}{ Chemical } & \multirow{2}{*}{ CASRN } & \multicolumn{4}{|c|}{ Ingestion of Vegetables (mg/kg)" } & \multicolumn{4}{|c|}{ Ingestion of Beef (mg/kg) } & \multicolumn{6}{|c|}{ Ingestion of Milk (mg/kg) } \\
\hline & & $10^{-4}$ & $10^{5}$ & $H Q=1$ & $\mathrm{HQ}=0.1$ & $10^{-4}$ & $10^{4}$ & $\mathbf{H Q}=\mathbf{1}$ & HQ $=0.1$ & $10^{-4}$ & $10^{5}$ & $\begin{array}{l}\text { HQ }=1 \\
\text { Adult }\end{array}$ & $\begin{array}{l}\text { HQ=1 } \\
\text { Child }\end{array}$ & $\begin{array}{c}\text { HQ }=0.1 \\
\text { Adult }\end{array}$ & $\begin{array}{c}\text { HQ }=0.1 \\
\text { Child }\end{array}$ \\
\hline Chromium Salts & NA & & & & & & & & & & & & & & \\
\hline Chrysene ${ }^{(x)}$ & 000218-01-9 & $1.1 \mathrm{E}+02$ & $1.1 \mathrm{E}+00$ & & & $9.1 E+02$ & $9.1 E+00$ & & & $2.3 E+02$ & $2.3 \mathrm{E}+00$ & & & & \\
\hline Cobalt & $007440-48-4$ & & & & & & & & & & & & & & \\
\hline Coke Oven Emissions & $008007-45 \cdot 2$ & & & & & & & & & & & & & & \\
\hline Copper & $007440-50-8$ & & & & & & & & & & & & & & \\
\hline Copper Cyanide & $000544-92-3$ & & & & & & & & & & & & & & \\
\hline Creosote & $008001-58-9$ & & & & & & & & & & & & & & \\
\hline Cresol, m- & $000108-39-4$ & & & $5.8 \mathrm{E}+01$ & $5.8 \mathrm{E}+00$ & & & $9.9 \mathrm{E}+05$ & $9.9 \mathrm{E}+04$ & & & $5.3 \mathrm{E}+05$ & $6.8 E+04$ & $5.3 \mathrm{E}+04$ & $6.8 \mathrm{E}+03$ \\
\hline Cresol, o- & $000095-48-7$ & & & $5.3 \mathrm{E}+01$ & $5.3 \mathrm{E}+00$ & & & $1.0 \mathrm{E}+06$ & $1.1 \mathrm{E}+05$ & & & $5.9 \mathrm{E}+05$ & $7.5 \mathrm{E}+04$ & $5.9 \mathrm{E}+04$ & $7.5 \mathrm{E}+03$ \\
\hline Cresol, p- & $000106-44-5$ & & & $5.3 \mathrm{E}+00$ & $5.3 \mathrm{E}-01$ & & & $1.1 \mathrm{E}+05$ & $1.1 \mathrm{E}+04$ & & & $5.9 \mathrm{E}+04$ & 7.5E+03 & $5.9 \mathrm{E}+03$ & $7.5 \mathrm{E}+02$ \\
\hline Crotonaldehyde & $000123-73-9$ & $1.2 \mathrm{E}-01$ & $1.2 \mathrm{E}-03$ & & & $3.0 \mathrm{E}+03$ & $3.0 \mathrm{E}+01$ & & & $6.8 \mathrm{E}+02$ & $6.8 \mathrm{E}+00$ & & & & \\
\hline Cumene & $000098-82-8$ & & & $1.1 \mathrm{E}+02$ & $1.1 \mathrm{E}+01$ & & & $1.0 \mathrm{E}+05$ & $1.0 \mathrm{E}+04$ & & & $5.6 E+04$ & $7.2 \mathrm{E}+03$ & $5.6 \mathrm{E}+03$ & $7.2 \mathrm{E}+02$ \\
\hline Cyanazine & $021725-46-2$ & $3.9 \mathrm{E}-01$ & $3.9 \mathrm{E}-03$ & $2.8 \mathrm{E}+00$ & $2.8 \mathrm{E}-01$ & $4.3 \mathrm{E}+03$ & $4.3 \mathrm{E}+01$ & $3.1 \mathrm{E}+04$ & $3.1 \mathrm{E}+03$ & $9.6 \mathrm{E}+02$ & $9.6 \mathrm{E}+00$ & $1.6 \mathrm{E}+04$ & $2.1 E+03$ & $1.6 \mathrm{E}+03$ & 2.1E+02 \\
\hline Cyanide (CN-) & $000057-12-5$ & & & $9.0 \mathrm{E}+00$ & $9.0 \mathrm{E}-01$ & & & $1.0 \mathrm{E}+06$ & $1.0 \mathrm{E}+05$ & & & $5.5 E+05$ & $7.0 E+04$ & $5.5 E+04$ & $7.0 \mathrm{E}+03$ \\
\hline Cyanogen & $000460-19-5$ & & & & & & & & & & & & & & \\
\hline Cyanogen Bromide & $000506-68-3$ & & & & & & & & & & & & & & \\
\hline Cyanogen Chloride & $000506-77-4$ & & & $7.4 \mathrm{E}+00$ & $7.4 \mathrm{E}-01$ & & & $1.0 \mathrm{E}+06$ & $6.4 \mathrm{E}+05$ & & & $1.0 \mathrm{E}+06$ & $4.3 E+05$ & $3.4 E+05$ & $4.3 \mathrm{E}+04$ \\
\hline $\begin{array}{l}\text { Cyclohexane, 1,2,3,4,5-pentabromo-6- } \\
\text { chloro- }\end{array}$ & $000087-84-3$ & & & & & & & & & & & & & & \\
\hline Cyclohexanone & $000108-94-1$ & & & $1.6 \mathrm{E}+03$ & $1.6 \mathrm{E}+02$ & & & $1.0 \mathrm{E}+06$ & $1.0 \mathrm{E}+06$ & & & $1.0 \mathrm{E}+06$ & $1.0 \mathrm{E}+06$ & $1.0 \mathrm{E}+06$ & $1.0 \mathrm{E}+06$ \\
\hline Cyclohexylamine & $000108-91-8$ & & & $1.4 \mathrm{E}+02$ & $1.4 \mathrm{E}+01$ & & & $1.0 \mathrm{E}+06$ & $6.9 \mathrm{E}+05$ & & & $1.0 \mathrm{E}+06$ & $4.6 \mathrm{E}+05$ & $3.6 \mathrm{E}+05$ & $4.6 \mathrm{E}+04$ \\
\hline Cyclopentadiene & $000542-92-7$ & & & & & & & & & & & & & & \\
\hline
\end{tabular}


Table 3.3c. (continued)

\begin{tabular}{|c|c|c|c|c|c|c|c|c|c|c|c|c|c|c|c|}
\hline \multirow{2}{*}{ Chemical } & \multirow{2}{*}{ CASRN } & \multicolumn{4}{|c|}{ Ingestion of Vegetables (mg/kg) } & \multicolumn{4}{|c|}{ Ingestion of Beef (mg/kg) } & \multicolumn{6}{|c|}{ Ingestion of Milk (mg/kg) } \\
\hline & & $10^{-1}$ & $10^{4}$ & $\mathrm{HQ}=1$ & $H Q=0.1$ & $10^{-4}$ & $10^{4}$ & $\mathrm{HQ}=1$ & $\mathrm{HQ}=0.1$ & $10^{-4}$ & $10^{4}$ & $\begin{array}{l}\mathrm{HQ}=1 \\
\text { Adult }\end{array}$ & $\begin{array}{l}H Q=1 \\
\text { Child }\end{array}$ & $\begin{array}{c}H Q=0.1 \\
\text { Adult }\end{array}$ & $\begin{array}{c}\text { HQ }=0.1 \\
\text { Child }\end{array}$ \\
\hline Cyhalothrin/karate & $068085-85-8$ & & & $1.7 \mathrm{E}+01$ & $1.7 \mathrm{E}+00$ & & & $9.3 \mathrm{E}+00$ & $9.3 \mathrm{E}-01$ & & & $5.5 \mathrm{E}+00$ & $7.1 \mathrm{E}-01$ & $5.5 \mathrm{E}-01$ & 7.1E-02 \\
\hline Cypermethrin & $052315-07-8$ & & & & & & & & & & & & & & \\
\hline Cyromazine & $066215-27-8$ & & & & & & & & & & & & & & \\
\hline DDD & $000072-54-8$ & $3.4 \mathrm{E}+00$ & $3.4 \mathrm{E}-02$ & & & $2.2 \mathrm{E}+01$ & $2.2 \mathrm{E}-01$ & & & $5.5 \mathrm{E}+00$ & $5.5 \mathrm{E}-02$ & & & & \\
\hline DDE & $000072-55-9$ & $2.4 \mathrm{E}+00$ & $2.4 \mathrm{E}-02$ & & & $1.9 \mathrm{E}+01$ & $1.9 \mathrm{E}-01$ & & & $4.9 \mathrm{E}+00$ & $4.9 \mathrm{E}-02$ & & & & \\
\hline DDT & $000050-29-3$ & $2.4 \mathrm{E}+00$ & $2.4 \mathrm{E}-02$ & $1.7 \mathrm{E}+00$ & $1.7 \mathrm{E}-01$ & $4.4 \mathrm{E}+00$ & 4.4E-02 & $3.2 \mathrm{E}+00$ & $3.2 \mathrm{E}-01$ & $1.1 \mathrm{E}+00$ & $1.1 \mathrm{E}-02$ & $1.9 \mathrm{E}+00$ & $2.4 \mathrm{E}-01$ & $1.9 \mathrm{E}-01$ & $2.4 \mathrm{E}-02$ \\
\hline Dacthal & $001861-32-1$ & & & & & & & & & & & & & & \\
\hline Dalapon & $000075-99-0$ & & & $9.2 \mathrm{E}+00$ & $9.2 \mathrm{E}-01$ & & & $1.0 \mathrm{E}+06$ & $2.2 \mathrm{E}+05$ & & & $1.0 \mathrm{E}+06$ & $1.5 \mathrm{E}+05$ & $1.1 \mathrm{E}+05$ & $1.5 \mathrm{E}+04$ \\
\hline Decabromodiphenyl Ether & $001163-19-5$ & & & $3.4 \mathrm{E}+01$ & $3.4 \mathrm{E}+00$ & & & $7.9 \mathrm{E}+02$ & $7.9 E+01$ & & & $4.6 \mathrm{E}+02$ & $6.0 \mathrm{E}+01$ & $4.6 \mathrm{E}+01$ & $6.0 \mathrm{E}+00$ \\
\hline Demeton & $008065-48-3$ & & & & & & & & & & & & & & \\
\hline Di(2-ethylhexyl)adipate & $000103-23-1$ & $6.8 \mathrm{E}+02$ & $6.8 \mathrm{E}+00$ & $2.1 \mathrm{E}+03$ & $2.1 \mathrm{E}+02$ & $4.6 \mathrm{E}+01$ & $4.6 \mathrm{E}-01$ & $1.4 \mathrm{E}+02$ & $1.4 \mathrm{E}+01$ & $1.2 \mathrm{E}+01$ & $1.2 \mathrm{E}-01$ & $8.4 \mathrm{E}+01$ & $1.1 \mathrm{E}+01$ & $8.4 \mathrm{E}+00$ & $1.1 \mathrm{E}+00$ \\
\hline Diallate & $002303-16-4$ & $1.1 \mathrm{E}+00$ & 1.1E-02 & & & $2.9 \mathrm{E}+05$ & $2.9 \mathrm{E}+03$ & & & $6.5 \mathrm{E}+04$ & $6.5 \mathrm{E}+02$ & & & & \\
\hline Diazinon & $000333-41-5$ & & & 2.7E+00 & 2.7E-01 & & & $1.3 \mathrm{E}+03$ & $1.3 \mathrm{E}+02$ & & & $7.6 \mathrm{E}+02$ & $9.7 \mathrm{E}+01$ & $7.6 \mathrm{E}+01$ & $9.7 \mathrm{E}+00$ \\
\hline Dibenz $[\mathrm{a}, \mathrm{h}]$ anthracene $e^{(2)}$ & $000053-70-3$ & $1.1 \mathrm{E}-01$ & $1.1 \mathrm{E}-03$ & & & $7.4 \mathrm{E}-02$ & $7.4 \mathrm{E}-04$ & & & $1.9 \mathrm{E}-02$ & $1.9 \mathrm{E}-04$ & & & & \\
\hline Dibromo-3-chloropropane, 1,2- & $000096-12-8$ & $3.0 \mathrm{E}-01$ & $3.0 \mathrm{E}-03$ & & & $1.6 \mathrm{E}+03$ & $1.6 \mathrm{E}+01$ & & & $3.6 \mathrm{E}+02$ & $3.6 \mathrm{E}+00$ & & & & \\
\hline Dibromobenzene, 1,4- & $000106-37-6$ & & & $3.0 \mathrm{E}+01$ & $3.0 \mathrm{E}+00$ & & & $1.5 \mathrm{E}+04$ & $1.5 \mathrm{E}+03$ & & & $8.4 \mathrm{E}+03$ & $1.1 \mathrm{E}+03$ & $8.4 \mathrm{E}+02$ & $1.1 \mathrm{E}+02$ \\
\hline Dibromochloromethane & $000124-48-1$ & $3.8 \mathrm{E}+00$ & $3.8 \mathrm{E}-02$ & 2.7E+01 & $2.7 \mathrm{E}+00$ & $4.4 \mathrm{E}+04$ & $4.4 \mathrm{E}+02$ & 3.1E+05 & $3.1 \mathrm{E}+04$ & $9.9 \mathrm{E}+03$ & $9.9 \mathrm{E}+01$ & $1.7 \mathrm{E}+05$ & $2.1 \mathrm{E}+04$ & $1.7 \mathrm{E}+04$ & $2.1 \mathrm{E}+03$ \\
\hline Dibromodichloromethane & $000594-18-3$ & & & & & & & & & & & & & & \\
\hline Dibromodiphenyl Ether, p,p'- & $002050-47-7$ & & & & & & & & & & & & & & \\
\hline Dibromoethane, 1,2- & $000106-93-4$ & $3.2 \mathrm{E}-03$ & $3.2 \mathrm{E}-05$ & & & $5.4 \mathrm{E}+01$ & $5.4 \mathrm{E}-01$ & & & $1.2 \mathrm{E}+01$ & $1.2 \mathrm{E}-01$ & & & & \\
\hline Dibromomethane (Methylene Bromide) & 000074-95-3 & & & $5.0 \mathrm{E}+00$ & $5.0 \mathrm{E}-01$ & & & 4.7E+05 & $4.7 \mathrm{E}+04$ & & & $2.5 \mathrm{E}+05$ & $3.2 \mathrm{E}+04$ & 2.5E+04 & $3.2 \mathrm{E}+03$ \\
\hline
\end{tabular}


Table 3.3c. (continued)

\begin{tabular}{|c|c|c|c|c|c|c|c|c|c|c|c|c|c|c|c|}
\hline \multirow{2}{*}{ Chemical } & \multirow{2}{*}{ CASRN } & \multicolumn{4}{|c|}{ Ingestion of Vegetables (mg/kg) } & \multicolumn{4}{|c|}{ Ingestion of Beef (mg/kg) } & \multicolumn{6}{|c|}{ Ingestion of Milk (mg/kg) } \\
\hline & & $10^{-1}$ & $10^{6}$ & $\mathrm{HQ}=1$ & $H Q=0.1$ & $10^{-4}$ & $10^{5}$ & $H Q=1$ & $\mathrm{HQ}=0.1$ & $10^{4}$ & $10^{5}$ & $\begin{array}{l}\mathrm{HQ}=\mathbf{1} \\
\text { Adult }\end{array}$ & $\begin{array}{l}\mathrm{HQ}=1 \\
\text { Child }\end{array}$ & $\begin{array}{c}\text { HQ }=0.1 \\
\text { Adult }\end{array}$ & $\begin{array}{c}\text { HQ }=0.1 \\
\text { Child }\end{array}$ \\
\hline Dibutyl Phthalate & $000084-74-2$ & & & $3.4 \mathrm{E}+02$ & $3.4 \mathrm{E}+01$ & & & $1.6 \mathrm{E}+04$ & $1.6 \mathrm{E}+03$ & & & $9.7 \mathrm{E}+03$ & $1.2 \mathrm{E}+03$ & $9.7 \mathrm{E}+02$ & $1.2 \mathrm{E}+02$ \\
\hline Dicamba & $001918-00-9$ & & & $6.8 \mathrm{E}+01$ & $6.8 \mathrm{E}+00$ & & & $1.6 \mathrm{E}+05$ & $1.6 \mathrm{E}+04$ & & & $9.0 \mathrm{E}+04$ & $1.2 \mathrm{E}+04$ & $9.0 \mathrm{E}+03$ & $1.2 \mathrm{E}+03$ \\
\hline Dichloro-2-butene, 1,4- & $000764-41-0$ & & & & & & & & & & & & & & \\
\hline \multicolumn{16}{|l|}{ Dichloroacetic Acid } \\
\hline Dichlorobenzene, 1,2-(s) & $000095-50-1$ & & & $2.4 \mathrm{E}+02$ & $2.4 \mathrm{E}+01$ & & & $2.7 \mathrm{E}+05$ & $2.7 \mathrm{E}+04$ & & & $1.5 \mathrm{E}+05$ & $1.9 \mathrm{E}+04$ & $1.5 \mathrm{E}+04$ & $1.9 \mathrm{E}+03$ \\
\hline Dichlorobenzene, 1,3- & $000541-73-1$ & & & & & & & & & & & & & & \\
\hline Dichlorobenzene, 1,4- & $000106-46-7$ & $2.6 \mathrm{E}+01$ & $2.6 \mathrm{E}-01$ & & & $2.9 E+04$ & $2.9 \mathrm{E}+02$ & & & $6.8 \mathrm{E}+03$ & $6.8 \mathrm{E}+0 \mathrm{t}$ & & & & \\
\hline Dichlorobenzidine, 3,3'- & $000091-94-1$ & $1.4 \mathrm{E}+00$ & $1.4 \mathrm{E}-02$ & & & $1.3 \mathrm{E}+03$ & $1.3 \mathrm{E}+01$ & & & $3.1 \mathrm{E}+02$ & $3.1 \mathrm{E}+00$ & & & & \\
\hline Dichlorodifuoromethane & $000075-71-8$ & & & 2.7E+02 & $2.7 \mathrm{E}+01$ & & & $1.0 E+06$ & $3.1 \mathrm{E}+05$ & & & $1.0 \mathrm{E}+06$ & $2.1 \mathrm{E}+05$ & $1.7 E+05$ & $2.1 E+04$ \\
\hline Dichloroethane, 1,1- & $000075-34-3$ & & & $9.5 \mathrm{E}+01$ & $9.5 \mathrm{E}+00$ & & & $1.0 \mathrm{E}+06$ & $2.5 \mathrm{E}+05$ & & & $1.0 \mathrm{E}+06$ & $1.7 \mathrm{E}+05$ & $1.3 \mathrm{E}+05$ & $1.7 \mathrm{E}+04$ \\
\hline Dichloroethane, 1,2- & $000107-06-2$ & $1.8 \mathrm{E}+00$ & $1.8 \mathrm{E}-02$ & & & $8.8 \mathrm{E}+04$ & $8.8 \mathrm{E}+02$ & & & $2.0 \mathrm{E}+04$ & $2.0 \mathrm{E}+02$ & & & & \\
\hline Dichloroethylene, 1,1- & $000075-35-4$ & $3.7 \mathrm{E}-01$ & 3.7E-03 & $8.6 \mathrm{E}+00$ & $8.6 \mathrm{E}-01$ & $9.6 \mathrm{E}+03$ & $9.6 \mathrm{E}+01$ & $2.2 \mathrm{E}+05$ & $2.2 \mathrm{E}+04$ & $2.2 \mathrm{E}+03$ & $2.2 \mathrm{E}+01$ & $1.2 \mathrm{E}+05$ & $1.5 \mathrm{E}+04$ & $1.2 \mathrm{E}+04$ & $1.5 \mathrm{E}+03$ \\
\hline Dichloroethylene, 1,2-(Mixed Isomers) & $000540-59-0$ & & & $1.9 \mathrm{E}+00$ & $1.9 \mathrm{E}-01$ & & & $8.8 \mathrm{E}+05$ & $8.8 \mathrm{E}+04$ & & & $4.6 \mathrm{E}+05$ & $5.9 \mathrm{E}+04$ & $4.6 \mathrm{E}+04$ & $5.9 \mathrm{E}+03$ \\
\hline Dichloroethylene, 1,2-cis- & $000156-59-2$ & & & $1.1 \mathrm{E}+01$ & $1.1 E+00$ & & & $2.2 \mathrm{E}+05$ & $2.2 \mathrm{E}+04$ & & & $1.2 \mathrm{E}+05$ & $1.5 \mathrm{E}+04$ & $1.2 \mathrm{E}+04$ & $1.5 E+03$ \\
\hline Dichloroethylene, 1,2-trans- & $000156-60-5$ & & & $4.2 E+00$ & $4.2 \mathrm{E}-01$ & & & $1.0 \mathrm{E}+06$ & $1.9 \mathrm{E}+05$ & & & $1.0 \mathrm{E}+06$ & $1.3 \mathrm{E}+05$ & $1.0 \mathrm{E}+05$ & $1.3 E+04$ \\
\hline Dichlorophenol, 2,4- & $000120-83-2$ & & & $6.5 \mathrm{E}+00$ & $6.5 \mathrm{E}-01$ & & & $1.9 \mathrm{E}+04$ & $1.9 \mathrm{E}+03$ & & & $1.0 \mathrm{E}+04$ & $1,3 \mathrm{E}+03$ & $1.0 \mathrm{E}+03$ & $1.3 \mathrm{E}+02$ \\
\hline Dichlorophenoxy Acetic Acid, 2,4- & $000094-75-7$ & & & $1.7 \mathrm{E}+01$ & $1.7 \mathrm{E}+00$ & & & $1.1 \mathrm{E}+05$ & $1.1 \mathrm{E}+04$ & & & $5.8 E+04$ & $7.5 \mathrm{E}+03$ & $5.8 \mathrm{E}+03$ & $7.5 \mathrm{E}+02$ \\
\hline Dichlorophenoxy)butyric Acid, 4-(2,4- & $000094-82-6$ & & & $2.2 \mathrm{E}+01$ & $2.2 \mathrm{E}+00$ & & & $1.9 \mathrm{E}+04$ & $1.9 \mathrm{E}+03$ & & & $1.1 E+04$ & $1.4 \mathrm{E}+03$ & $1.1 \mathrm{E}+03$ & $1.4 \mathrm{E}+02$ \\
\hline Dichloropropane, 1,2- & $000078-87-5$ & $3.9 \mathrm{E}+00$ & $3.9 \mathrm{E}-02$ & & & $6.8 \mathrm{E}+04$ & $6.8 \mathrm{E}+02$ & & & $1.5 \mathrm{E}+04$ & $1.5 E+02$ & & & & \\
\hline Dichloropropanol, 2,3- & $000616-23-9$ & & & & & & & & & & & & & & \\
\hline Dichloropropene, 1,3- & $000542-75-6$ & $1.0 \mathrm{E}+00$ & $1.0 \mathrm{E}-02$ & 2.3E-01 & $2.3 \mathrm{E}-02$ & $4.0 \mathrm{E}+04$ & $4.0 \mathrm{E}+02$ & $9.3 \mathrm{E}+03$ & $9.3 E+02$ & $9.0 \mathrm{E}+03$ & $9.0 \mathrm{E}+01$ & $4.9 \mathrm{E}+03$ & $6.3 \mathrm{E}+02$ & $4.9 \mathrm{E}+02$ & $6.3 \mathrm{E}+01$ \\
\hline
\end{tabular}




\begin{tabular}{|c|c|c|c|c|c|c|c|c|c|c|c|c|c|c|c|}
\hline & & & & & & & & & & & & & & I-89-560000 & 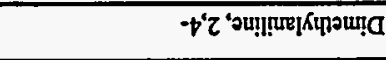 \\
\hline & & & & & & & & & & & & & & $r 96-9 \varepsilon p \mid z 0$ & 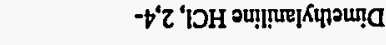 \\
\hline & & & & & & & & & & & & & & $1-8 L-\angle L 0000$ & 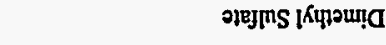 \\
\hline & & & & $20+9 \varepsilon 6$ & $50+3 E 6$ & & & $\varepsilon 0+$ Il't & so+gl't & & & $10 \cdot 99 \cdot 1$ & $10+99 \cdot 1$ & $t-06-611000$ & 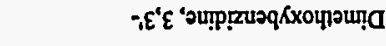 \\
\hline $10+3 z^{2} I$ & $10+30 \%$ & $20+9 z+1$ & $20+30^{\circ} 6$ & & & $20+9 L^{\prime} I$ & $\varepsilon_{0}+a l i$ & & & $20-36^{\prime} \varepsilon$ & $10-36^{\prime} \varepsilon$ & & & S-IS-090000 & ฆвочршта \\
\hline & & & & & & & & & & & & & & $L-b 9-0625 S 0$ & ụd!̣pourạ \\
\hline $00+30^{\circ} \varepsilon$ & $s 0+\exists b z$ & $50+\$ 0^{\circ} \varepsilon$ & $90+80^{\circ} t$ & & & sotast & $90+\$ 011$ & & & $00+\exists \varepsilon \varepsilon$ & $10+9 \varepsilon \varepsilon$ & 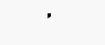 & & 9-SL-Stb100 & 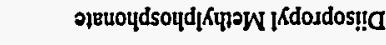 \\
\hline & & & & & & & & & & & & & & 9-LE-SL0000 & 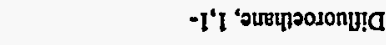 \\
\hline & & & & & & & & & & & & & & S-8E-L9ESE0 & นomzuวqqu!! \\
\hline & & & & & & & & & & & & & & 9-8t-ZzZEห0 & Ienbozus!!a \\
\hline & & & & 90-as's & to-as's & & & $50-\pi z \tau$ & $\varepsilon 0-\exists z ' z$ & & & 90-a $\mathrm{a} \mathrm{L}^{\prime} \mathrm{I}$ & $60-a b \cdot \mathrm{I}$ & I-ES-950000 & 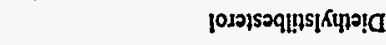 \\
\hline & & & & & & & & & & & & & & $s-\$ 8-\angle I 9000$ & 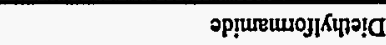 \\
\hline $90+90^{\circ} \mathrm{I}$ & $90+30^{\circ} \mathrm{t}$ & $90+101 \mathrm{t}$ & $90+30^{\prime} \mathrm{T}$ & & & $90+3011$ & $90+300^{\circ}$ & & & $10+31 ' z$ & $z 0+a I^{\prime} z$ & & & 0-06-III000 & 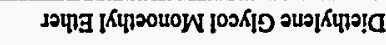 \\
\hline & & & & & & & & & & & & & & $s-t \varepsilon-Z 11000$ & 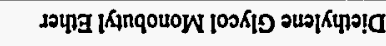 \\
\hline & & & & & & & & & & & & & & $0-1 Z-\varepsilon 69000$ & 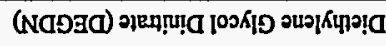 \\
\hline & & & & & & & & & & & & & & S-stIIE000 & 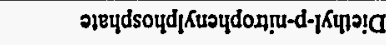 \\
\hline $80+30 \% 9$ & $s 0+a \angle b$ & $50+\pi 0.9$ & $90+200^{\circ} \mathrm{I}$ & & & $50+a<8$ & $90+30^{\circ} \mathrm{t}$ & & & $20+9 y^{\prime} \cdot 1$ & $\varepsilon 0+9 b \cdot 1$ & & & $\tau-99-\$ 80000$ & 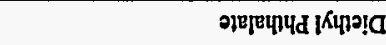 \\
\hline $10-a z i$ & $10-31 \% 6$ & $00+a z-1$ & $00+a 16$ & 20-arit & $00+3 I^{\prime} I$ & $00+39 \cdot \mathrm{I}$ & $10+39^{\circ} \mathrm{l}$ & $20-3 s^{\prime} t$ & $00+3 S^{\prime} t$ & $20-99 \cdot 1$ & $10-39.1$ & 60-38p $p$ & $20-38 \%$ & I-LS-090000 & นب̣pग!̣a \\
\hline$\varepsilon 0+\exists \varepsilon^{\prime \prime} \mathfrak{I}$ & $50+30 \%$ & DO+AET & $50+90^{\circ} \mathrm{I}$ & & & $10+36 . \mathrm{I}$ & $50+36 t$ & & & $00+359$ & $10+759$ & & & $9-\varepsilon L-\angle L 0000$ & 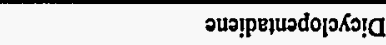 \\
\hline & & & & & & & & & & & & & & $\tau-z \varepsilon-s 11000$ & lojoo! \\
\hline $10+95 \% 9$ & $20+31 \cdot 5$ & $20+359$ & Eo+gI's & $10+3 s^{\prime} \varepsilon$ & $\varepsilon 0+\Xi S \mathcal{\varepsilon}$ & $20+3996$ & $\varepsilon 0+39 \cdot 6$ & $20+3 s^{\prime} i$ & $10+7 S^{\prime} 1$ & $20-96 \div s$ & $10-36 \mathrm{~s}$ & E0-בร' 6 & 10-as'6 & $L-\varepsilon L-Z 90000$ & son:I4ग! \\
\hline $\begin{array}{c}\text { p!!पJ } \\
I^{\prime} 0=0 \mathrm{H}\end{array}$ & 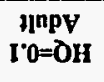 & $\begin{array}{l}\text { P!! } \\
\mathfrak{l}=\mathbf{O H}\end{array}$ & $\begin{array}{l}\Re \text { npy } \\
I=O H\end{array}$ & ,0I & nOI & $I^{\prime} 0=\mathrm{OH}$ & $\mathfrak{I}=\mathrm{OH}$ & .0I & HOI & $I^{\prime} 0=\partial H$ & $I=\partial H$ &, 01 & OI & NYSY? & 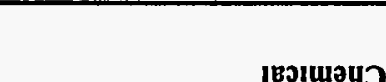 \\
\hline \multicolumn{6}{|c|}{ 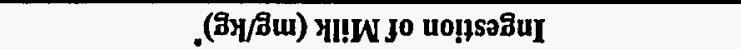 } & \multicolumn{4}{|c|}{ 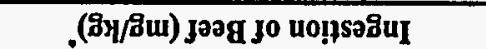 } & \multicolumn{4}{|c|}{ 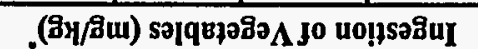 } & & \\
\hline
\end{tabular}

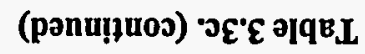


Table 3.3c. (continued)

\begin{tabular}{|c|c|c|c|c|c|c|c|c|c|c|c|c|c|c|c|}
\hline \multirow{2}{*}{ Chemical } & \multirow{2}{*}{ CASRN } & \multicolumn{4}{|c|}{ Ingestion of Vegetables (mg/kg) } & \multicolumn{4}{|c|}{ Ingestion of Beef $(\mathrm{mg} / \mathrm{kg})^{\circ}$} & \multicolumn{6}{|c|}{ Ingestion of Milk (mg/kg) } \\
\hline & & $10^{-4}$ & $10^{6}$ & HQ=1 & $\mathrm{HQ}=0.1$ & $10^{-4}$ & $10^{4}$ & $\mathrm{HQ}=1$ & $\mathrm{HQ}=0.1$ & $10^{-4}$ & $10^{5}$ & $\begin{array}{l}\text { HQ=1 } \\
\text { Adult }\end{array}$ & $\begin{array}{l}\text { HQ }=1 \\
\text { Child }\end{array}$ & $\begin{array}{c}\mathrm{HQ}=0.1 \\
\text { Adult }\end{array}$ & $\begin{array}{c}\text { HQ }=0.1 \\
\text { Child }\end{array}$ \\
\hline Dimethylaniline, $\mathrm{N}, \mathrm{N}-$ & $000121-69-7$ & & & $3.7 \mathrm{E}+00$ & $3.7 E-01$ & & & $1.9 \mathrm{E}+04$ & $1.9 \mathrm{E}+03$ & & & $1.0 \mathrm{E}+04$ & $1.3 \mathrm{E}+03$ & $1.0 \mathrm{E}+03$ & $1.3 \mathrm{E}+02$ \\
\hline Dimethylbenzidine, 3,3'- & $000119-93-7$ & $3.8 \mathrm{E}-02$ & $3.8 \mathrm{E}-04$ & & & 3.5E+02 & $3.5 \mathrm{E}+00$ & & & $8.0 \mathrm{E}+01$ & 8.0E-01 & & & & \\
\hline Dimethylethyl Lead & $107584-40-7$ & & & $\cdot$ & & & & & & & & & & & \\
\hline Dimethylformamide & $000068-12-2$ & & & $3.1 \mathrm{E}+00$ & 3.1E-01 & . & & $1.0 \mathrm{E}+06$ & $1.0 \mathrm{E}+06$ & & & $1.0 \mathrm{E}+06$ & $1.0 \mathrm{E}+06$ & $1.0 \mathrm{E}+06$ & $2.8 \mathrm{E}+05$ \\
\hline Dimethylhydrazine, 1,1-(3) & $000057-14-7$ & & & & & & & & & & & & & & \\
\hline Dimethylhydrazine, 1,2- & $000540-73-8$ & & & & & & & & & & & & & & \\
\hline Dimethylphenol, 2,4- & $000105-67-9$ & & & $3.0 \mathrm{E}+01$ & $3.0 \mathrm{E}+00$ & & & $2.8 \mathrm{E}+05$ & $2.8 \mathrm{E}+04$ & & & $1.5 \mathrm{E}+05$ & $1.9 \mathrm{E}+04$ & $1.5 \mathrm{E}+04$ & $1.9 \mathrm{E}+03$ \\
\hline Dimethylphenol, 2,6- & $000576-26-1$ & & & 9.3E-01 & 9.3E-02 & & & $7.8 \mathrm{E}+03$ & $7.8 \mathrm{E}+02$ & & & $4.2 \mathrm{E}+03$ & $5.3 \mathrm{E}+02$ & $4.2 \mathrm{E}+02$ & $5.3 \mathrm{E}+01$ \\
\hline Dimethylphenol, 3,4- & $000095-65-8$ & & & $1.4 \mathrm{E}+00$ & $1.4 \mathrm{E}-01$ & & & $1.6 \mathrm{E}+04$ & $1.6 \mathrm{E}+03$ & & & $8.4 \mathrm{E}+03$ & $1.1 \mathrm{E}+03$ & $8.4 \mathrm{E}+02$ & $1.1 \mathrm{E}+02$ \\
\hline Dimethylphthalate ${ }^{(3)}$ & $000131-11-3$ & & & $7.8 \mathrm{E}+03$ & $7.8 \mathrm{E}+02$ & & & $1.0 \mathrm{E}+06$ & $1.0 \mathrm{E}+06$ & & & $1.0 \mathrm{E}+06$ & $1.0 \mathrm{E}+06$ & $1.0 \mathrm{E}+06$ & $1.0 \mathrm{E}+06$ \\
\hline Dimethylterephthalate & $000120-61-6$ & & & $2.3 \mathrm{E}+02$ & $2.3 \mathrm{E}+01$ & & & $5.5 \mathrm{E}+05$ & $5.5 \mathrm{E}+04$ & & & $3.0 \mathrm{E}+05$ & $3.9 \mathrm{E}+04$ & $3.0 \mathrm{E}+04$ & $3.9 \mathrm{E}+03$ \\
\hline Dinitro-0-cresol, 4,6- & $000534-52-1$ & & & & & & & & & & & & & & \\
\hline Dinitro-0-cyclohexyl Phenol, 4,6- & $000131-89-5$ & & & $6.4 \mathrm{E}-03$ & 6.4E-04 & & & $1.0 \mathrm{E}+06$ & $4.3 \mathrm{E}+05$ & & & $1.0 \mathrm{E}+06$ & $2.9 \mathrm{E}+05$ & $2.2 E+05$ & $2.9 \mathrm{E}+04$ \\
\hline Dinitrobenzene, 1,2- & $000528-29-0$ & & & 3.1E-01 & $3.1 \mathrm{E}-02$ & & & $1.2 E+04$ & $1.2 \mathrm{E}+03$ & & & $6.5 \mathrm{E}+03$ & $8.4 \mathrm{E}+02$ & $6.5 \mathrm{E}+02$ & $8.4 \mathrm{E}+01$ \\
\hline Dinitrobenzene, 1,3- & $000099-65-0$ & & & 7.8E-02 & $7.8 \mathrm{E}-03$ & & & $3.1 \mathrm{E}+03$ & $3.1 \mathrm{E}+02$ & & & $1.6 \mathrm{E}+03$ & $2.1 \mathrm{E}+02$ & $1.6 \mathrm{E}+02$ & 2.1E+01 \\
\hline Dinitrobenzene, 1,4- & $000100-25-4$ & & & $2.8 \mathrm{E}-01$ & $2.8 \mathrm{E}-02$ & & & $1.4 \mathrm{E}+04$ & $1.4 \mathrm{E}+03$ & & & $7.2 \mathrm{E}+03$ & $9.3 \mathrm{E}+02$ & $7.2 \mathrm{E}+02$ & $9.3 \mathrm{E}+01$ \\
\hline Dinitrophenol, 2,4- & $000051-28-5$ & & & $1.4 \mathrm{E}+00$ & $1.4 \mathrm{E}-01$ & & & $6.9 \mathrm{E}+04$ & $6.9 \mathrm{E}+03$ & & & $3.6 \mathrm{E}+04$ & $4.6 \mathrm{E}+03$ & $3.6 \mathrm{E}+03$ & $4.6 \mathrm{E}+02$ \\
\hline Dinitrotoluene, 2,4- & $000121-14-2$ & $3.9 \mathrm{E}-01$ & $3.9 \mathrm{E}-03$ & $2.3 \mathrm{E}+00$ & $2.3 \mathrm{E}-01$ & $6.8 \mathrm{E}+03$ & $6.8 \mathrm{E}+01$ & $4.0 \mathrm{E}+04$ & $4.0 \mathrm{E}+03$ & $1.5 \mathrm{E}+03$ & $1.5 \mathrm{E}+01$ & $2.1 \mathrm{E}+04$ & $2.7 \mathrm{E}+03$ & $2.1 \mathrm{E}+03$ & $2.7 \mathrm{E}+02$ \\
\hline Dinitrotoluene, 2,6- & $000606-20-2$ & $3.0 \mathrm{E}-01$ & $3.0 \mathrm{E}-03$ & 8.6E-01 & $8.6 \mathrm{E}-02$ & $9.5 \mathrm{E}+03$ & $9.5 E+01$ & $2.8 \mathrm{E}+04$ & $2.8 \mathrm{E}+03$ & $2.1 \mathrm{E}+03$ & $2.1 \mathrm{E}+01$ & $1.5 E+04$ & $1.9 \mathrm{E}+03$ & $1.5 \mathrm{E}+03$ & $1.9 \mathrm{E}+02$ \\
\hline Dinitrotoluene, 2-Amino-4,6- & $035572-78-2$ & & & & & & & & & & & & & & \\
\hline Dinoseb & $000088-85-7$ & & & $2.9 \mathrm{E}+00$ & $2.9 \mathrm{E}-01$ & & & $1.8 \mathrm{E}+03$ & $1.8 \mathrm{E}+02$ & & & $1.0 \mathrm{E}+03$ & $1.3 \mathrm{E}+02$ & $1.0 \mathrm{E}+02$ & $1.3 \mathrm{E}+01$ \\
\hline
\end{tabular}


Table 3.3c. (continued)

\begin{tabular}{|c|c|c|c|c|c|c|c|c|c|c|c|c|c|c|c|}
\hline \multirow{2}{*}{ Chemical } & \multirow{2}{*}{ CASRN } & \multicolumn{4}{|c|}{ Ingestion of Vegetables (mg/kg)" } & \multicolumn{4}{|c|}{ Ingestion of Beef (mg/kg) } & \multicolumn{6}{|c|}{ Ingestion of Milk (mg/kg) } \\
\hline & & $10^{-4}$ & $10^{6}$ & $\mathrm{HQ}=1$ & $\mathrm{HQ}=0.1$ & $10^{-4}$ & $10^{6}$ & $H Q=1$ & $\mathrm{HQ}=0.1$ & $10^{-4}$ & $10^{6}$ & $\begin{array}{c}\text { HQ=1 } \\
\text { Adult }\end{array}$ & $\begin{array}{l}\text { HQ=1 } \\
\text { Child }\end{array}$ & $\begin{array}{c}\text { HQ }=0.1 \\
\text { Adult }\end{array}$ & $\begin{array}{c}\mathrm{HQ}=0.1 \\
\text { Child }\end{array}$ \\
\hline Dioxane, 1,4- & $000123-91-1$ & $1.7 \mathrm{E}+00$ & 1.7E-02 & & & $1.0 \mathrm{E}+06$ & $4.3 \mathrm{E}+04$ & & & $9.6 \mathrm{E}+05$ & $9.6 \mathrm{E}+03$ & & & & \\
\hline Diphenamid & $000957-51-7$ & & & $4.0 \mathrm{E}+01$ & $4.0 \mathrm{E}+00$ & & & $4.9 \mathrm{E}+05$ & $4.9 \mathrm{E}+04$ & & & $2.6 \mathrm{E}+05$ & $3.3 \mathrm{E}+04$ & $2.6 \mathrm{E}+04$ & $3.3 \mathrm{E}+03$ \\
\hline Diphenylamine & $000122-39-4$ & & & $6.9 \mathrm{E}+01$ & $6.9 \mathrm{E}+00$ & & & $6.3 \mathrm{E}+04$ & $6.3 \mathrm{E}+03$ & & & $3.5 \mathrm{E}+04$ & $4.5 \mathrm{E}+03$ & $3.5 \mathrm{E}+03$ & $4.5 \mathrm{E}+02$ \\
\hline Diphenylhydrazine, 1,2- & $000122-66-7$ & $6.3 \mathrm{E}-01$ & 6.3E-03 & & & $1.8 \mathrm{E}+03$ & $1.8 \mathrm{E}+01$ & & & $4.3 \mathrm{E}+02$ & $4.3 \mathrm{E}+00$ & & & & \\
\hline Diquat & $000085-00-7$ & & & $4.4 \mathrm{E}-03$ & 4.4E-04 & & & $1.0 \mathrm{E}+06$ & $6.6 \mathrm{E}+05$ & & & $1.0 \mathrm{E}+06$ & $4.5 \mathrm{E}+05$ & $3.5 \mathrm{E}+05$ & $4.5 \mathrm{E}+04$ \\
\hline Direct Black 38 & $001937-37-7$ & & & & & & & & & & & & & & \\
\hline Direct Blue 6 & $002602-46-2$ & & & & & & & & & & & & & & \\
\hline Direct Brown 95 & $016071-86-6$ & & & & & & & & & & & & & & \\
\hline Direct Sky Blue & $002610-05-1$ & & & & & & & & & & & & & & \\
\hline Disulfoton & $000298-04-4$ & & & $1.2 \mathrm{E}-01$ & $1.2 \mathrm{E}-02$ & & & 4.1E+01 & $4.1 \mathrm{E}+00$ & & & $2.3 \mathrm{E}+01$ & $3.0 \mathrm{E}+00$ & $2.3 \mathrm{E}+00$ & $3.0 \mathrm{E}-01$ \\
\hline Diuron & $000330-54-1$ & & & $3.9 \mathrm{E}+00$ & $3.9 \mathrm{E}-01$ & & & $1.7 \mathrm{E}+04$ & $1.7 \mathrm{E}+03$ & & & $9.0 \mathrm{E}+03$ & $1.2 \mathrm{E}+03$ & $9.0 \mathrm{E}+02$ & $1.2 \mathrm{E}+02$ \\
\hline Dodine & $002439-10-3$ & & & & & & & & & & & & & & \\
\hline EPTC & $000759-94-4$ & & & & & & & & & & & & & & \\
\hline Endosulfan & $000115-29-7$ & & & & & & & & & & & & & & \\
\hline Endothall & $000145-73-3$ & & & & & & & & & & & & & & \\
\hline Endrin & $000072-20-8$ & & & $9.9 \mathrm{E}-01$ & $9.9 \mathrm{E}-02$ & & & $9.3 \mathrm{E}+01$ & $9.3 \mathrm{E}+00$ & & & $5.4 \mathrm{E}+01$ & $7.0 \mathrm{E}+00$ & $5.4 \mathrm{E}+00$ & $7.0 \mathrm{E}-01$ \\
\hline Epichlorohydrin & $000106-89-8$ & $2.1 \mathrm{E}+00$ & 2.1E-02 & $1.7 \mathrm{E}-01$ & 1.7E-02 & $1.0 \mathrm{E}+06$ & $4.5 \mathrm{E}+04$ & $3.8 \mathrm{E}+05$ & $3.8 \mathrm{E}+04$ & $1.0 \mathrm{E}+06$ & $1.0 \mathrm{E}+04$ & $2.0 \mathrm{E}+05$ & $2.6 \mathrm{E}+04$ & $2.0 \mathrm{E}+04$ & $2.6 \mathrm{E}+03$ \\
\hline Epoxybutane, 1,2- & $000106-88-7$ & & & & & & & & & & & & & & \\
\hline Ethephon & $016672-87-0$ & & & & & & & & & & & & & & \\
\hline Ethion & $000563-12-2$ & & & $1.7 E+00$ & $1.7 \mathrm{E}-01$ & & & $5.7 \mathrm{E}+01$ & $5.7 \mathrm{E}+00$ & & & 3.3E+01 & $4.3 \mathrm{E}+00$ & $3.3 \mathrm{E}+00$ & $4.3 \mathrm{E}-01$ \\
\hline Ethoxyethanol Acctate, 2- & $000111-15-9$ & & & $7.8 \mathrm{E}+01$ & $7.8 \mathrm{E}+00$ & & & $1.0 \mathrm{E}+06$ & $1.0 \mathrm{E}+06$ & & & $1.0 \mathrm{E}+06$ & $1.0 \mathrm{E}+06$ & $1.0 \mathrm{E}+06$ & $1.7 \mathrm{E}+05$ \\
\hline
\end{tabular}


Table 3.3c. (continued)

\begin{tabular}{|c|c|c|c|c|c|c|c|c|c|c|c|c|c|c|c|}
\hline \multirow{2}{*}{ Chemical } & \multirow{2}{*}{ CASRN } & \multicolumn{4}{|c|}{ Ingestion of Vegetables (mg/kg)" } & \multicolumn{4}{|c|}{ Ingestion of Beef (mg/kg) } & \multicolumn{6}{|c|}{ Ingestion of Milk (mg/kg) } \\
\hline & & $10^{-4}$ & $10^{4}$ & $\mathrm{HQ}=1$ & $\mathrm{HQ}=0.1$ & $10^{-4}$ & $10^{4}$ & $\mathrm{HQ}=1$ & $\mathrm{HQ}=0.1$ & $10^{-4}$ & $10^{4}$ & $\begin{array}{l}\text { HQ=1 } \\
\text { Adult }\end{array}$ & $\begin{array}{l}\text { HQ=1 } \\
\text { Child }\end{array}$ & $\begin{array}{c}\mathrm{HQ}=0.1 \\
\text { Adult }\end{array}$ & $\begin{array}{c}\mathrm{HQ}=0.1 \\
\text { Child }\end{array}$ \\
\hline Ethoxyethanol, 2- & $000110-80-5$ & & & $4.0 \mathrm{E}+01$ & $4.0 \mathrm{E}+00$ & & & $1.0 \mathrm{E}+06$ & $1.0 \mathrm{E}+06$ & & & $1.0 \mathrm{E}+06$ & $1.0 \mathrm{E}+06$ & $1.0 \mathrm{E}+06$ & $4.6 \mathrm{E}+05$ \\
\hline Ethyl Acetate & $000141-78-6$ & & & $2.9 \mathrm{E}+02$ & $2.9 \mathrm{E}+01$ & & & $1.0 \mathrm{E}+06$ & $1.0 \mathrm{E}+06$ & & & $1.0 \mathrm{E}+06$ & $1.0 \mathrm{E}+06$ & $1.0 \mathrm{E}+06$ & $4.2 \mathrm{E}+05$ \\
\hline Ethyl Acrylate & $000140-88-5$ & $2.7 \mathrm{E}+00$ & 2.7E-02 & & & 2.1E+05 & 2.1E+03 & & & $4.6 \mathrm{E}+04$ & $4.6 \mathrm{E}+02$ & & & & \\
\hline Ethyl Chloride & $000075-00-3$ & & & & & & & & & & & & & & \\
\hline Ethyl Ether & $000060-29-7$ & & & $7.0 \mathrm{E}+01$ & $7.0 \mathrm{E}+00$ & & & $1.0 E+06$ & $1.0 \mathrm{E}+06$ & & & $1.0 \mathrm{E}+06$ & $8.7 \mathrm{E}+05$ & $6.8 \mathrm{E}+05$ & $8.7 E+04$ \\
\hline Ethyl Methacrylate & $000097-63-2$ & & & $9.5 \mathrm{E}+01$ & $9.5 \mathrm{E}+00$ & & & $1.0 \mathrm{E}+06$ & $2.0 \mathrm{E}+05$ & & & $1.0 \mathrm{E}+06$ & $1.4 \mathrm{E}+05$ & $1.1 \mathrm{E}+05$ & $1.4 \mathrm{E}+04$ \\
\hline Ethyl-p-nitrophenyl Phosphonate & $002104-64-5$ & & & $3.0 \mathrm{E}-02$ & $3.0 \mathrm{E}-03$ & & & $1.5 E+01$ & $1.5 \mathrm{E}+00$ & & & $8.4 \mathrm{E}+00$ & $1.1 \mathrm{E}+00$ & $8.4 \mathrm{E}-01$ & 1.1E-01 \\
\hline Ethylbenzene ${ }^{(0)}$ & $000100-41-4$ & & & $2.4 \mathrm{E}+02$ & $2.4 \mathrm{E}+01$ & & & $4.7 \mathrm{E}+05$ & $4.7 \mathrm{E}+04$ & & & 2.6E+05 & $3.3 \mathrm{E}+04$ & $2.6 \mathrm{E}+04$ & $3.3 \mathrm{E}+03$ \\
\hline Ethylene Cyanohydrin & $000109-78-4$ & & & $4.8 \mathrm{E}+00$ & $4.8 \mathrm{E}-01$ & & & $1.0 \mathrm{E}+06$ & $1.0 \mathrm{E}+06$ & & & $1.0 \mathrm{E}+06$ & $1.0 \mathrm{E}+06$ & $1.0 \mathrm{E}+06$ & $1.0 \mathrm{E}+06$ \\
\hline Ethylene Diamine & $000107-15-3$ & & & $2.4 \mathrm{E}+00$ & $2.4 \mathrm{E}-01$ & & & $1.0 \mathrm{E}+06$ & $3.0 \mathrm{E}+05$ & & & $1.0 \mathrm{E}+06$ & $2.0 E+05$ & $1.6 \mathrm{E}+05$ & $2.0 \mathrm{E}+04$ \\
\hline Ethylene Glycol & $000107-21-1$ & & & $1.9 \mathrm{E}+01$ & $1.9 \mathrm{E}+00$ & & & $1.0 \mathrm{E}+06$ & $1.0 \mathrm{E}+06$ & & & $1.0 \mathrm{E}+06$ & $1.0 \mathrm{E}+06$ & $1.0 \mathrm{E}+06$ & $1.0 E+06$ \\
\hline Ethylene Glycol Monobutyl Ether & $000111-76-2$ & & & & & & & & & & & & & & \\
\hline Ethylene Oxide & $000075-21-8$ & $1.8 \mathrm{E}-02$ & $1.8 \mathrm{E}-04$ & & & $4.8 \mathrm{E}+04$ & $4.8 \mathrm{E}+02$ & & & $1.1 \mathrm{E}+04$ & $1.1 \mathrm{E}+02$ & & & & \\
\hline Ethylene Thiourea & $000096-45-7$ & $1.0 \mathrm{E}-01$ & $1.0 \mathrm{E}-03$ & $3.9 \mathrm{E}-03$ & $3.9 \mathrm{E}-04$ & $6.3 \mathrm{E}+05$ & $6.3 \mathrm{E}+03$ & $2.4 \mathrm{E}+04$ & $2.4 \mathrm{E}+03$ & $1.4 \mathrm{E}+05$ & $1.4 \mathrm{E}+03$ & $1.2 \mathrm{E}+04$ & $1.6 \mathrm{E}+03$ & $1.2 \mathrm{E}+03$ & $1.6 \mathrm{E}+02$ \\
\hline Ethylphthalyl Ethyl Glycolate & $000084-72-0$ & & & & & & & & & & & & & & \\
\hline Express & $101200-48-0$ & & & & & & & & & & & & & & \\
\hline Fenamiphos & $022224-92-6$ & & & & & & & & & & & & & & \\
\hline Fenpropathrin & $039515-41-8$ & & & & & & & & & & & & & & \\
\hline Fluometuron & $002164-17-2$ & & & $2.1 \mathrm{E}+01$ & $2.1 \mathrm{E}+00$ & & & $1.6 \mathrm{E}+05$ & $1.6 \mathrm{E}+04$ & & & 8.6E+04 & $1.1 \mathrm{E}+04$ & $8.6 \mathrm{E}+03$ & $1.1 \mathrm{E}+03$ \\
\hline Fluoranthene & $000206-44-0$ & & & $1.3 \mathrm{E}+02$ & $1.3 \mathrm{E}+01$ & & & $6.6 \mathrm{E}+03$ & $6.6 E+02$ & & & $3.9 \mathrm{E}+03$ & $5,0 \mathrm{E}+02$ & $3.9 \mathrm{E}+02$ & $5.0 \mathrm{E}+01$ \\
\hline Fluorene & $000086-73-7$ & & & $1.3 \mathrm{E}+02$ & $1.3 \mathrm{E}+01$ & & & $1.9 \mathrm{E}+04$ & $1.9 \mathrm{E}+03$ & & & $1.1 \mathrm{E}+04$ & $1.4 \mathrm{E}+03$ & $1.1 \mathrm{E}+03$ & $1.4 \mathrm{E}+02$ \\
\hline
\end{tabular}


Table 3.3c. (continued)

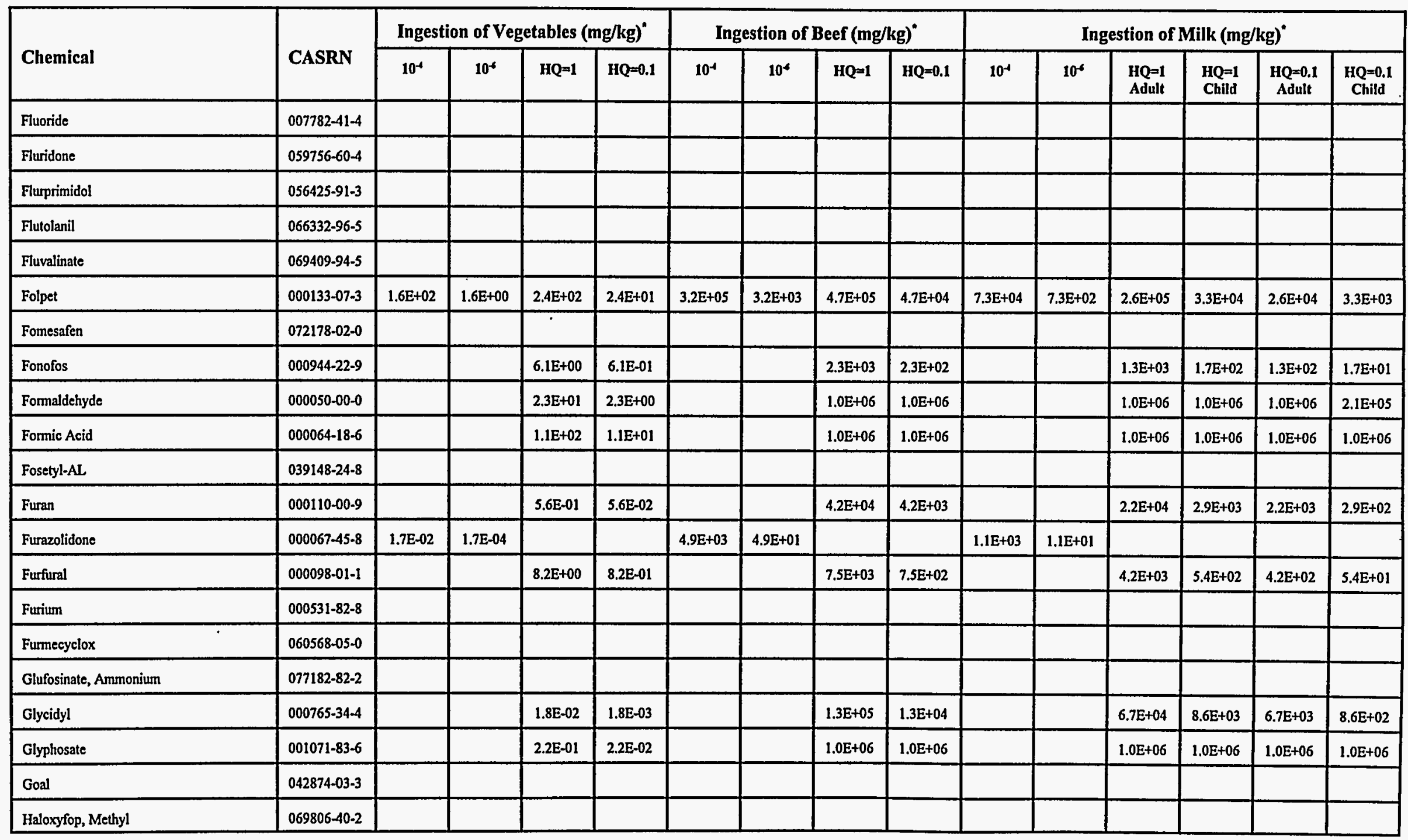

Nonradionuclides/soil or sediment/agricultural

Page 16 of 33 
Table 3.3c. (continued)

\begin{tabular}{|c|c|c|c|c|c|c|c|c|c|c|c|c|c|c|c|}
\hline \multirow{2}{*}{ Chemical } & \multirow{2}{*}{ CASRN } & \multicolumn{4}{|c|}{ Ingestion of Vegetables (mg/kg) } & \multicolumn{4}{|c|}{ Ingestion of Beef (mg/kg) } & \multicolumn{6}{|c|}{ Ingestion of Milk (mg/kg) } \\
\hline & & $10^{4}$ & $10^{4}$ & $H Q=1$ & $\mathrm{HQ}=0.1$ & $10^{-4}$ & $10^{4}$ & $H Q=1$ & $\mathrm{HQ}=0.1$ & $10^{-1}$ & $10^{6}$ & $\begin{array}{l}\mathrm{HQ}=1 \\
\text { Adult }\end{array}$ & $\begin{array}{l}\mathrm{HQ}=1 \\
\text { Child }\end{array}$ & $\begin{array}{c}\text { HQ }=0.1 \\
\text { Adult }\end{array}$ & $\begin{array}{l}\text { HQ }=0.1 \\
\text { Child }\end{array}$ \\
\hline Harmony & $079277-27-3$ & & & & & & & & & & & & & & \\
\hline Heptachlor & $000076-44-8$ & $1.7 \mathrm{E}-01$ & $1.7 \mathrm{E}-03$ & $1.6 \mathrm{E}+00$ & $1.6 \mathrm{E}-01$ & $3.0 \mathrm{E}+01$ & $3.0 \mathrm{E}-01$ & $2.9 \mathrm{E}+02$ & $2.9 \mathrm{E}+01$ & $7.3 \mathrm{E}+00$ & 7.3E-02 & $1.7 \mathrm{E}+02$ & $2.1 \mathrm{E}+01$ & $1.7 \mathrm{E}+01$ & $2.1 \mathrm{E}+00$ \\
\hline Heptachlor Epoxide & $001024-57-3$ & $8.8 \mathrm{E}-02$ & $8.8 \mathrm{E}-04$ & 4.5E-02 & 4.5E-03 & $1.4 \mathrm{E}+00$ & $1.4 \mathrm{E}-02$ & $7.2 \mathrm{E}-01$ & $7.2 \mathrm{E}-02$ & $3.6 \mathrm{E}-01$ & $3.6 \mathrm{E}-03$ & $4.3 \mathrm{E}-01$ & $5.5 \mathrm{E}-02$ & 4.3E-02 & $5.5 \mathrm{E}-03$ \\
\hline Heptane, N- & $000142-82-5$ & & & & & & & & & & & & & & \\
\hline Hexabromobenzene & $000087-82-1$ & & & $7.0 \mathrm{E}+00$ & $7.0 \mathrm{E}-01$ & & & $2.5 \mathrm{E}+01$ & $2.5 \mathrm{E}+00$ & & & $1.5 \mathrm{E}+01$ & $1.9 \mathrm{E}+00$ & $1.5 \mathrm{E}+00$ & $1.9 \mathrm{E}-01$ \\
\hline Hexachlorobenzene $e^{(6)}$ & $000118.74-1$ & $5.0 \mathrm{E}-01$ & $5.0 \mathrm{E}-03$ & $2.7 \mathrm{E}+00$ & $2.7 \mathrm{E}-01$ & $1.0 \mathrm{E}+01$ & $1.0 \mathrm{E}-01$ & $5.5 \mathrm{E}+01$ & $5.5 \mathrm{E}+00$ & $2.5 \mathrm{E}+00$ & $2.5 \mathrm{E}-02$ & $3.3 \mathrm{E}+01$ & $4.2 \mathrm{E}+00$ & $3.3 \mathrm{E}+00$ & $4.2 \mathrm{E}-01$ \\
\hline Hexachlorobutadiene $e^{(6)}$ & $000087-68-3$ & $1.0 \mathrm{E}+01$ & $1.0 \mathrm{E}-01$ & 6.7E-01 & 6.7E-02 & $6.1 \mathrm{E}+02$ & $6.1 E+00$ & 4.1E+01 & $4.1 \mathrm{E}+00$ & $1.5 \mathrm{E}+02$ & $1.5 \mathrm{E}+00$ & $2.4 \mathrm{E}+01$ & $3.1 E+00$ & $2.4 \mathrm{E}+00$ & $3.1 \mathrm{E}-01$ \\
\hline Hexachlorocyclohexane, Alpha- & $000319-84-6$ & $1.1 \mathrm{E}-01$ & $1.1 \mathrm{E}-03$ & & & $4.6 \mathrm{E}+01$ & $4.6 \mathrm{E}-01$ & & & $1.1 \mathrm{E}+01$ & $1.1 \mathrm{E}-01$ & & & & \\
\hline Hexachlorocyclohexane, Beta- & $000319-85-7$ & $4.0 \mathrm{E}-01$ & 4.0E-03 & & & $1.3 \mathrm{E}+02$ & $1.3 E+00$ & & & $3.2 \mathrm{E}+01$ & $3.2 \mathrm{E}-01$ & & & & \\
\hline Hexachlorocyclohexane, Delta* & $000319-86-8$ & & & & & & & & & & & & & & \\
\hline Hexachlorocyclohexane, Epsilon & $006108-10-7$ & & & & & & & & & & & & & & \\
\hline Hexachlorocyclohexane, Gamma- & $000058-89-9$ & $5.2 \mathrm{E}-01$ & $5.2 \mathrm{E}-03$ & 8.7E-01 & 8.7E-02 & $3.2 \mathrm{E}+02$ & $3.2 \mathrm{E}+00$ & $5.3 \mathrm{E}+02$ & $5.3 \mathrm{E}+01$ & $7.6 \mathrm{E}+01$ & $7.6 \mathrm{E}-01$ & $3.0 \mathrm{E}+02$ & $3.9 \mathrm{E}+01$ & $3.0 \mathrm{E}+01$ & $3.9 \mathrm{E}+00$ \\
\hline Hexachlorocyclohexane, Technical & 000608-73-1 & & & & & & & & & & & & & & \\
\hline Hexachlorocyclopentadiene & $000077-47-4$ & & & $2.2 \mathrm{E}+01$ & $2.2 \mathrm{E}+00$ & & & $7.2 E+03$ & $7.2 E+02$ & & & 4.1E+03 & $5.3 \mathrm{E}+02$ & 4.1E+02 & $5.3 \mathrm{E}+01$ \\
\hline Hexachlorodibenzo-p-dioxin, Mixture & $019408-74-3$ & $1.3 \mathrm{E}-04$ & $1.3 \mathrm{E}-06$ & & & 1.1E-04 & $1.1 \mathrm{E}-06$ & & & $2.8 \mathrm{E}-05$ & $2.8 \mathrm{E}-07$ & & & & \\
\hline Hexachloroethane & $000067-72-1$ & $5.0 \mathrm{E}+01$ & $5.0 \mathrm{E}-01$ & $3.0 \mathrm{E}+00$ & $3.0 \mathrm{E}-01$ & $2.1 \mathrm{E}+04$ & $2.1 \mathrm{E}+02$ & $1.2 \mathrm{E}+03$ & $1.2 \mathrm{E}+02$ & $5.0 \mathrm{E}+03$ & $5.0 \mathrm{E}+01$ & $7.0 \mathrm{E}+02$ & $9.0 \mathrm{E}+01$ & $7.0 \mathrm{E}+01$ & $9.0 \mathrm{E}+00$ \\
\hline Hexachlorophene & $000070-30-4$ & & & $1.1 \mathrm{E}+00$ & $1.1 \mathrm{E}-01$ & & & $1.4 \mathrm{E}-01$ & $1.4 \mathrm{E}-02$ & & & $8.4 \mathrm{E}-02$ & $1.1 \mathrm{E}-02$ & $8.4 \mathrm{E}-03$ & $1.1 \mathrm{E}-03$ \\
\hline $\begin{array}{l}\text { Hexahydro-1,3,5-trinitro-1,3,5-triazine } \\
\text { (RDX) }\end{array}$ & $000121-82-4$ & $5.5 E+00$ & $5.5 \mathrm{E}-02$ & $7.7 E+00$ & $7.7 \mathrm{E}-01$ & $7.4 \mathrm{E}+03$ & 7.4E+01 & $1.0 \mathrm{E}+04$ & $1.0 \mathrm{E}+03$ & $1.7 \mathrm{E}+03$ & $1.7 E+01$ & $5.8 \mathrm{E}+03$ & $7.4 \mathrm{E}+02$ & $5.8 \mathrm{E}+02$ & $7.4 \mathrm{E}+01$ \\
\hline Hexamethylene Diisocyanate, 1,6- & $000822-06-0$ & & & & & & & & & & & & & & \\
\hline Hexane, N- & $000110-54-3$ & & & $1.8 \mathrm{E}+02$ & $1.8 \mathrm{E}+01$ & & & $7.4 \mathrm{E}+04$ & $7.4 \mathrm{E}+03$ & & & $4.2 \mathrm{E}+04$ & $5.4 E+03$ & $4.2 \mathrm{E}+03$ & $5.4 \mathrm{E}+02$ \\
\hline Hexanone, 2- & $000591-78-6$ & & & & & & & & & & & & & & \\
\hline
\end{tabular}




\begin{tabular}{|c|c|c|c|c|c|c|c|c|c|c|c|c|c|c|c|}
\hline & & & & & & & & & & & & & & $8-b 5-2 \varepsilon 8100$ & 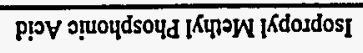 \\
\hline & & & & & & & & & & & & & & $0-\varepsilon 9-\angle 90000$ & porrdardosI \\
\hline & & & & & & & & & & & & & & $0-\varepsilon S-0 z 8 \varepsilon \varepsilon 0$ & u!̣pdodosI \\
\hline $50+38^{\circ} \mathcal{E}$ & $50+96 z$ & $50+98^{\circ} \varepsilon$ & $90+\$ 01 \mathrm{I}$ & $70+a 51$ & $90+30^{\circ} \mathrm{I}$ & $s 0+a s \cdot s$ & $90+90^{\circ} \mathrm{I}$ & $60+389$ & $90+90^{\circ} 1$ & $10+9 L \cdot 1$ & $20+3 L 1$ & $00+2 I \tau$ & $20+912$ & $1-65-820000$ & शuoroydosI \\
\hline $50+\Xi \forall \forall$ & $90+90^{\circ} 1$ & $90+90^{\circ} \mathrm{I}$ & $90+30^{\circ} \mathrm{I}$ & & & $90+90 \%$ & $90+30^{\circ} \mathrm{I}$ & & & $00+986$ & $10+986$ & & & $I-\varepsilon 8-8<0000$ & 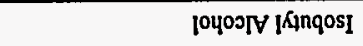 \\
\hline & & & & & & & & & & & & & & $9-68-6 \varepsilon \forall<00$ & นол \\
\hline & & & & & & & & & & & & & & $\iota-61-\nabla \varepsilon<9 \varepsilon 0$ & ouopposdl \\
\hline & & & & $\varepsilon 0^{-190^{\circ} \varepsilon}$ & $10-90^{\circ} \varepsilon$ & & & $20-\pi z t$ & $00+\exists z^{\prime} \mathrm{I}$ & & & $20-a I^{\prime \prime I}$ & $00+$ al'I & $s-6 \varepsilon-\varepsilon 61000$ & (t) \\
\hline & & & & & & & & & & & & & & 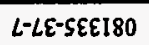 & ựbezeur \\
\hline & & & & & & & & & & & & & & $0-b t-t s s s \varepsilon 0$ & y!lezeur \\
\hline $50+ \pm 02 z$ & $50+96.1$ & $50+\exists t 2$ & $90+30^{\circ} \mathrm{I}$ & & & 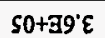 & $90+90^{\circ} \mathrm{I}$ & & & $10-a z ; 6$ & $00+926$ & & & 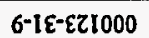 & auou!̣nborp $\mathrm{K}_{\mathrm{H}}$ \\
\hline$\varepsilon 0+\exists z^{\prime} I$ & $\varepsilon 0+396$ & $20+3 z^{\prime} \mathrm{l}$ & $60+7996$ & & & $60+38.1$ & $50+88^{\prime} \mathrm{I}$ & & & $10-3 I^{2} 1$ & $00+a I^{\prime 1} 1$ & & & $1-90-\varepsilon 8 \angle L 00$ & 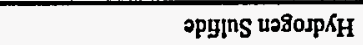 \\
\hline$t 0+a+1$ & so+al' & S0+3bi & $90+30^{\circ} \mathrm{I}$ & & & $50+\mathrm{azz}$ & $90+30^{\circ} \mathrm{I}$ & & & $10-3 L^{\prime} \mathcal{E}$ & $00+g l \cdot \varepsilon$ & & & $8-06-t<0000$ & 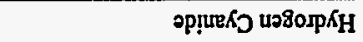 \\
\hline & & & & & & & & & & & & & & $0-10-\angle P 9 L 00$ & 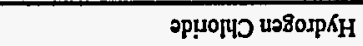 \\
\hline & & & & $20+3 I^{2} z$ & po+al'z & & & $20+a+6$ & $50+356$ & & & $90-39 \cdot 5$ & $60-39 \% 5$ & $z-\varepsilon 6-\downarrow \varepsilon 0010$ & 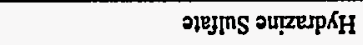 \\
\hline & & & & $20+a 1 z$ & $60+312$ & & & $20+9166$ & $50+3 t^{\prime} 6$ & & & $90-\mathrm{ag} \% \mathrm{~s}$ & $60-39 \% s$ & $z=10-208000$ & गuपzespरH \\
\hline & & & & & & & & & & & & & & $1+66-789550$ & $(1)-8^{-1} L^{\prime} E^{\prime} \tau^{c} \exists G \supset \times H$ \\
\hline & & & & & & & & & & & & & & $8-9 t-59 t p \varepsilon 0$ & 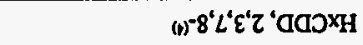 \\
\hline & & & & & & & & & 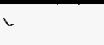 & & & & & $\varepsilon-S L-8668 \varepsilon 0$ & 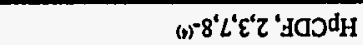 \\
\hline & & & & & & & & & & & & & & T-00-IL8LEO & 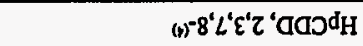 \\
\hline $90+30 \div$ & $90+90^{\circ} I$ & $90+901$ & $90+\mathrm{E0} \mathrm{I}$ & & & $90+30^{\prime} t$ & $90+30^{\circ} \mathrm{I}$ & & & E0-AI' $\mathrm{l}$ & $20-a I^{2} \mathrm{I}$ & & & $\tau-70-$-s\&zISO & энойгехәН \\
\hline $\begin{array}{c}\text { p!!uY } \\
I^{\prime} 0=O H\end{array}$ & 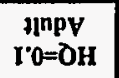 & 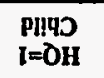 & $\begin{array}{l}\text { unpy } \\
i=0 \mathrm{H}\end{array}$ & ,0I & HOI & $\mathrm{I}^{0} \mathrm{0}=\mathrm{DH}$ & $I=\partial H$ & .01 & ror & $\tau^{0} 0=0 \mathrm{H}$ & $I=\mathrm{OH}$ & ,0I & rol & \multirow{2}{*}{ NQSYD } & \multirow{2}{*}{ ןвэ!щачว } \\
\hline \multicolumn{6}{|c|}{ 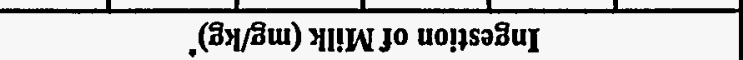 } & \multicolumn{4}{|c|}{ 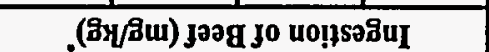 } & \multicolumn{4}{|c|}{ 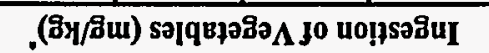 } & & \\
\hline
\end{tabular}

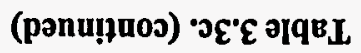


Table 3.3c. (continued)

\begin{tabular}{|c|c|c|c|c|c|c|c|c|c|c|c|c|c|c|c|}
\hline \multirow{2}{*}{ Chemical } & \multirow{2}{*}{ CASRN } & \multicolumn{4}{|c|}{ Ingestion of Vegetables (mg/kg) } & \multicolumn{4}{|c|}{ Ingestion of Beef (mg/kg) } & \multicolumn{6}{|c|}{ Ingestion of Milk (mg/kg) } \\
\hline & & $10^{-4}$ & $10^{5}$ & $H Q=1$ & $\mathrm{HQ}=0.1$ & $10^{-4}$ & $10^{4}$ & $\mathrm{HQ}=1$ & $\mathrm{HQ}=0.1$ & $10^{-4}$ & $10^{6}$ & $\begin{array}{l}\text { HQ=1 } \\
\text { Adult }\end{array}$ & $\begin{array}{l}\text { HQ=1 } \\
\text { Child }\end{array}$ & $\begin{array}{c}\mathrm{HQ}=0.1 \\
\text { Adult }\end{array}$ & $\begin{array}{c}H Q=0.1 \\
\text { Child }\end{array}$ \\
\hline Isoxaben & $082558-50-7$ & & & & & & & & & & & & & & \\
\hline Karate & $091465-08-6$ & & & & & & & & & & & & & & \\
\hline Kerb & $023950-58-5$ & & & & & & & & & & & & & & \\
\hline Lactofen & $077501-63-4$ & & & & & & & & & & & & & & \\
\hline Lead Alkyls & NA & & & & & & & & & & & & & & \\
\hline Lead And Compounds & 007439-92-1 & & & & & & & & & & & & & & \\
\hline Linuron & $000330-55-2$ & & & & & & & & & & & & & & \\
\hline Lithium & 007439-93-2 & & & & & & & & & & & & & & \\
\hline Londax & 083055-99-6 & & & & & & & & & & & & & & \\
\hline MCPA & $000094-74-6$ & & & 7.4E-01 & 7.4E-02 & & & $7.0 \mathrm{E}+03$ & $7.0 \mathrm{E}+02$ & & & $3.7 \mathrm{E}+03$ & $4.8 \mathrm{E}+02$ & $3.7 \mathrm{E}+02$ & $4.8 \mathrm{E}+01$ \\
\hline MCPB & $000094-81-5$ & & & & & & & & & & & & & & \\
\hline MCPP & $000093-65-2$ & & & $1.8 \mathrm{E}+00$ & $1.8 \mathrm{E}-01$ & & & $9.5 \mathrm{E}+03$ & $9.5 \mathrm{E}+02$ & & & $5.1 E+03$ & $6.6 \mathrm{E}+02$ & $5.1 \mathrm{E}+02$ & $6.6 \mathrm{E}+01$ \\
\hline Magnesium & $007439-95-4$ & & & & & & & & & & & & & & \\
\hline Malathion & $000121-75-5$ & & & $4.3 \mathrm{E}+01$ & $4.3 \mathrm{E}+00$ & & & $1.3 \mathrm{E}+05$ & $1.3 E+04$ & & & $6.9 \mathrm{E}+04$ & $8.9 \mathrm{E}+03$ & $6.9 \mathrm{E}+03$ & $8.9 E+02$ \\
\hline Maleic Anhydride & $000108-31-6$ & & & & & & & & & & & & & & \\
\hline Maleic Hydrazide & $000123-33-1$ & & & $3.4 \mathrm{E}+01$ & $3.4 \mathrm{E}+00$ & & & $1.0 \mathrm{E}+06$ & $1.0 \mathrm{E}+06$ & & & $1.0 E+06$ & $1.0 \mathrm{E}+06$ & $1.0 E+06$ & $7.8 \mathrm{E}+05$ \\
\hline Malononitrile & $000109-77-3$ & & ${ }^{\circ}$ & $2.4 \mathrm{E}-03$ & $2.4 \mathrm{E}-04$ & & & $3.0 \mathrm{E}+03$ & $3.0 \mathrm{E}+02$ & & & $1.6 \mathrm{E}+03$ & $2.0 \mathrm{E}+02$ & $1.6 \mathrm{E}+02$ & $2.0 \mathrm{E}+01$ \\
\hline Mancozeb & $008018-01-7$ & & & & & & & & & & & & & & \\
\hline Maneb & $012427-38-2$ & & & $5.7 \mathrm{E}-01$ & $5.7 \mathrm{E}-02$ & & & $7.8 \mathrm{E}+05$ & $7.8 \mathrm{E}+04$ & & & $4.1 E+05$ & $5.2 \mathrm{E}+04$ & $4.1 \mathrm{E}+04$ & $5.2 \mathrm{E}+03$ \\
\hline Manganese (Diet) & $007439-96-5$ & & & $3.9 \mathrm{E}+02$ & $3.9 \mathrm{E}+01$ & & & $3.9 E+04$ & $3.9 \mathrm{E}+03$ & & & $1.1 \mathrm{E}+05$ & $1.4 \mathrm{E}+04$ & $1.1 \mathrm{E}+04$ & $1.4 \mathrm{E}+03$ \\
\hline Mephosfolan & $000950-10-7$ & & & & & & & & & & & & & & \\
\hline
\end{tabular}




\begin{tabular}{|c|c|c|c|c|c|c|c|c|c|c|c|c|c|c|c|}
\hline & & & & & & & & & & & & & & $9-26-\angle 96220$ & 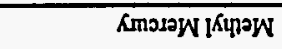 \\
\hline$t 0+3 s z$ & $50+30 \%$ & $s 0+a s z$ & $90+\$ 0^{\circ} \mathrm{I}$ & & & $50+ \pm 8 \cdot \mathcal{E}$ & $90+90 \% 1$ & & & $00+70^{\circ} t$ & $10+30 \%$ & & & $I-0 I-801000$ & 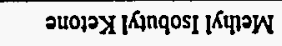 \\
\hline$s 0+\$ 8 b$ & $90+90^{\circ} \mathrm{I}$ & $90+30.1$ & $90+30^{\circ} \mathrm{I}$ & & & $90+901 \mathrm{I}$ & $90+ \pm 0.1$ & & & $00+a L 6$ & $10+3 L \cdot 6$ & & & $\varepsilon-\varepsilon 6-8<0000$ & 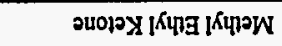 \\
\hline $50+99 \%$ & $50+9 \varepsilon i$ & $50+399^{\circ} \mathrm{I}$ & $90+30^{\circ} 1$ & & & $50+96$ & $90+ \pm 0.1$ & & & $10-90 \% 8$ & $00+30.8$ & & & $\varepsilon-\varepsilon \varepsilon-960000$ & 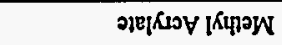 \\
\hline $50+988$ & $90+90^{\circ} \mathrm{I}$ & $90+30^{\circ} \mathrm{I}$ & $90+30^{\circ} 1$ & & & $90+901$ & $90+ \pm 0^{\circ} \mathrm{I}$ & & & $10+a b b^{\prime} I$ & $20+a b t$ & & & $6-0 z-6<0000$ & 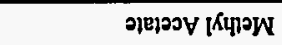 \\
\hline$\varepsilon 0+a z z$ & $50+3<i$ & $50+9 z z$ & $50+a L^{\prime} t$ & & & $50+9 \varepsilon \varepsilon$ & $50+ \pm \varepsilon \varepsilon$ & & & Eo-az' & $20-a z^{\prime}$ & & & $598-601000$ & 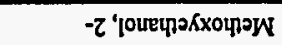 \\
\hline & & & & & & & & & & & & & & $9-6+011000$ & 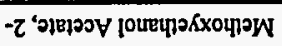 \\
\hline $10+\Xi 2 \cdot 1$ & $20+a b^{\prime} l$ & $20+9 L \cdot 1$ & $\varepsilon 0+\exists \not 1$ & & & $z 0+a \varepsilon z$ & $\varepsilon 0+\nexists \varepsilon z$ & & & $00+39$ & $10+39 \cdot 1$ & & & $s-\varepsilon t-Z<0000$ & 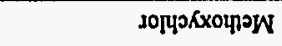 \\
\hline & & & & $20+96 \cdot \varepsilon$ & $20+ \pm 66^{\circ} \varepsilon$ & & & $\varepsilon 0+\mathrm{g}<\cdot \mathrm{I}$ & $50+96 !$ & & & $20-19^{\circ} \varepsilon$ & $00+39^{\circ} \varepsilon$ & $\tau-65-660000$ & 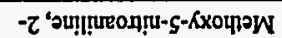 \\
\hline $50+\exists S^{\prime} I$ & SO+Gl'I & $S 0+3 s^{-1}$ & $90+90.1$ & & & $s 0+a z z$ & $90+90^{\circ} \mathrm{I}$ & & & 10-ar'9 & $00+a r ' 9$ & & & $S-L L-Z S L 9 I 0$ & 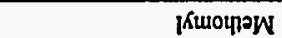 \\
\hline & & & & & & & & & & & & & & $8-\angle \varepsilon-0 S 6000$ & 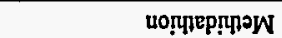 \\
\hline $90+\$ 0^{\circ} \mathrm{I}$ & 90+90 & $90+70^{\circ} \mathrm{I}$ & $90+30^{\circ} \mathrm{I}$ & & & $90+90^{\circ} \mathrm{I}$ & $90+90^{\circ} \mathrm{I}$ & & & $00+912$ & $10+31 z$ & & & I-95- 290000 & joureqpow \\
\hline $20+90 \%$ & $20+98 \cdot \mathrm{L}$ & $\varepsilon 0+30^{\circ} \mathrm{I}$ & $\varepsilon 0+98<L$ & & & $\varepsilon 0+9 S I$ & $60+a S^{\prime} t$ & & & $60-302$ & $\varepsilon 0-a b z$ & & & 9-26-592010 & 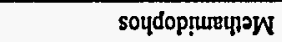 \\
\hline $10+\Xi z^{\prime} 9$ & $20+38^{\circ} \mathrm{t}$ & $20+3 z 9$ & $\varepsilon 0+98^{\circ}$ & & & $20+3 z^{\prime} 6$ & $\varepsilon 0+a z^{\prime} 6$ & & & $\varepsilon 0-\exists \varepsilon z$ & $20-9 \varepsilon z$ & & & $l-86-921000$ & 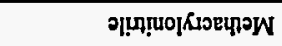 \\
\hline & & & & & & & & & & & & & & I-6I-LE8LS0 & [NXE[E]ON \\
\hline$\varepsilon 0-\bar{z} S^{\prime} 9$ & $20: 30 \div \mathrm{s}$ & $20-a s 9$ & $10-90 \mathrm{~s}$ & & & $20-958$ & $10-358$ & & & $20-30^{\circ} \mathrm{I}$ & 10-90.t & & & $8-8-8 L 0000$ & әp!x O soydrow \\
\hline & & & & & & & & & & & & & & $s=0 s=0 s 1000$ & soqdarw \\
\hline $10-25.1$ & $00+a z i$ & $00+35: 1$ & $10+a z \cdot t$ & & & $10-\mathrm{az} \varepsilon$ & $00+\nexists z \mathcal{E}$ & & & $20-36 t$ & $10-96$ & & & $9-\angle 6-6 \varepsilon \nabla \angle 00$ & 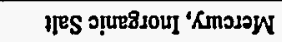 \\
\hline $10-a S^{\prime} \mid$ & $00+\pi z i$ & $00+9 s^{\prime} 1$ & $10+a z i$ & & & 10-az' $\varepsilon$ & $00+9 z \cdot \varepsilon$ & & & $20-96^{\circ} \mathrm{t}$ & $10-36 t$ & & & $9-\angle 6-6 \varepsilon+\angle 00$ & 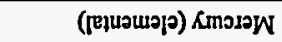 \\
\hline & & & & & & & & & & & & & & $\angle-\downarrow 6-\angle 8+\angle 00$ & 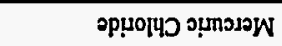 \\
\hline & & & & & & & & & & & & & & F-9Z- LOEマZO & 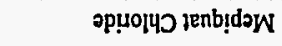 \\
\hline $\begin{array}{c}\text { p!!บ } \\
I^{\prime} 0=0 \mathrm{H}\end{array}$ & 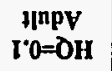 & $\begin{array}{l}\mathrm{P} I ! 4 \mathrm{~J} \\
\mathbf{I}=\mathrm{OH}\end{array}$ & $\begin{array}{l}I_{I} \mathrm{npy} \\
\mathrm{O}=\mathrm{H}\end{array}$ & ,0I & rol & $\mathrm{r}^{\prime} \mathrm{O}=\mathrm{\partial H}$ & $I=O H$ & ,0I & rOI & $\mathrm{I}^{0} \mathrm{O}=\mathrm{\partial H}$ & $I=O H$ & , $0 \mathrm{t}$ & rOI & \multirow{2}{*}{ NQSVD } & \multirow{2}{*}{ ןвว!шәчว } \\
\hline \multicolumn{6}{|c|}{ 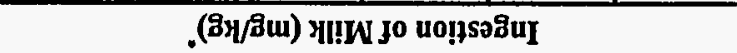 } & \multicolumn{4}{|c|}{ 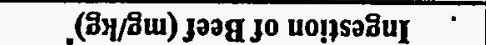 } & \multicolumn{4}{|c|}{ 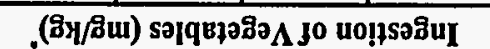 } & & \\
\hline
\end{tabular}




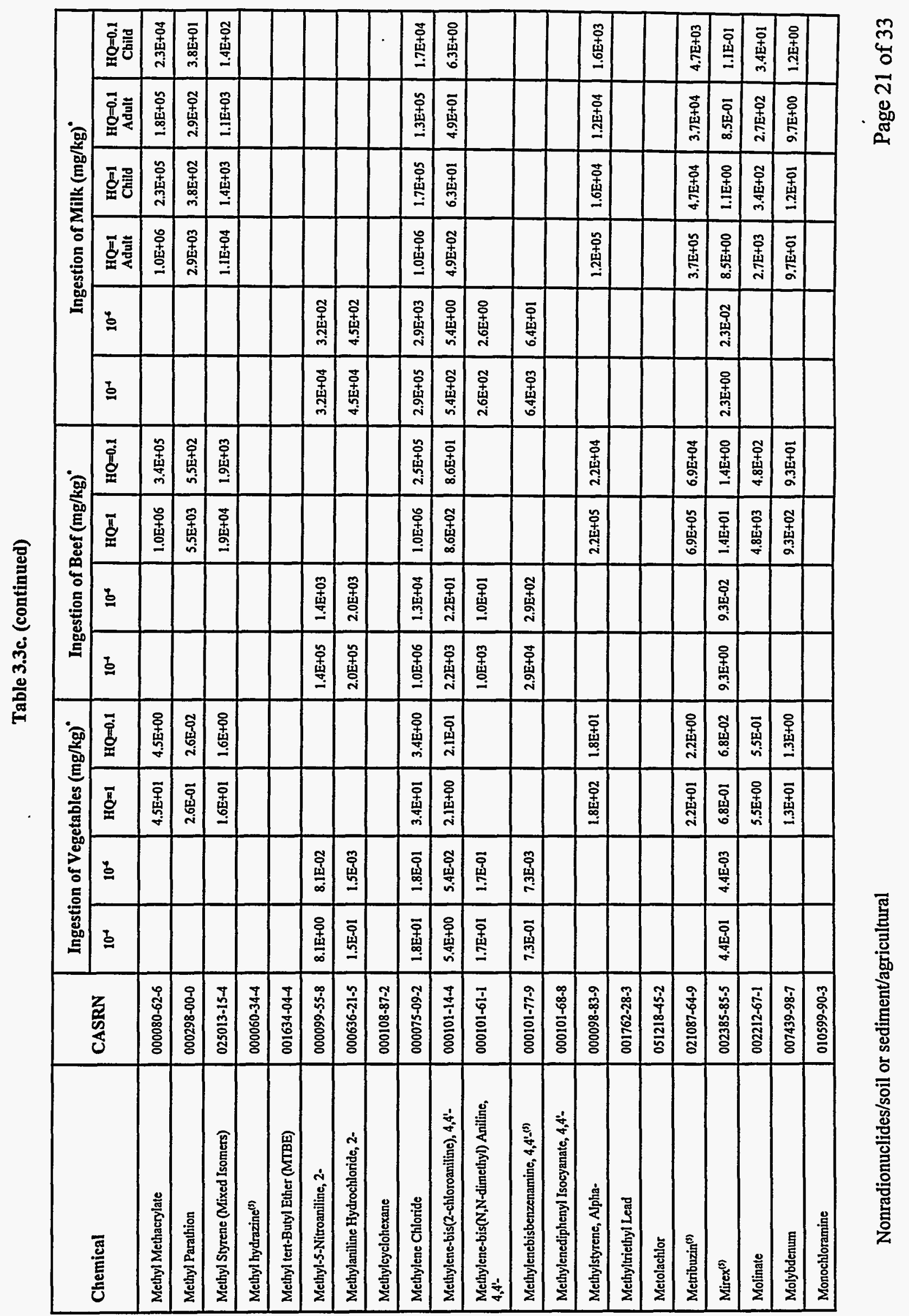


Table 3.3c. (continued)

\begin{tabular}{|c|c|c|c|c|c|c|c|c|c|c|c|c|c|c|c|}
\hline \multirow{2}{*}{ Chemical } & \multirow{2}{*}{ CASRN } & \multicolumn{4}{|c|}{ Ingestion of Vegetables (mg/kg) } & \multicolumn{4}{|c|}{ Ingestion of Beef $(\mathrm{mg} / \mathrm{kg})^{n}$} & \multicolumn{6}{|c|}{ Ingestion of Milk (mg/kg) } \\
\hline & & $10^{-4}$ & $10^{6}$ & $H Q=1$ & $\mathrm{HQ}=0.1$ & $10^{-4}$ & $10^{6}$ & $H Q=1$ & $\mathrm{HQ}=0.1$ & $10^{-4}$ & $10^{6}$ & $\begin{array}{l}\text { HQ=1 } \\
\text { Adult }\end{array}$ & $\begin{array}{l}\text { HQ=1 } \\
\text { Child }\end{array}$ & $\begin{array}{c}H Q=0.1 \\
\text { Adult }\end{array}$ & $\begin{array}{c}\mathrm{HQ}=0.1 \\
\text { Child }\end{array}$ \\
\hline Monochlorobutanes & $025154-42-1$ & & & & & & & & & & & & & & \\
\hline Naled & $000300-76-5$ & & & $4.1 \mathrm{E}+00$ & 4.1E-01 & & & $1.5 \mathrm{E}+04$ & $1.5 \mathrm{E}+03$ & & & $7.9 \mathrm{E}+03$ & $1.0 \mathrm{E}+03$ & $7.9 \mathrm{E}+02$ & $1.0 \mathrm{E}+02$ \\
\hline Naphthalene ${ }^{(6)}$ & $000091-20-3$ & & & $9.2 \mathrm{E}+01$ & $9.2 \mathrm{E}+00$ & & & $1.2 \mathrm{E}+05$ & $1.2 \mathrm{E}+04$ & & & $6.9 \mathrm{E}+04$ & $8.8 \mathrm{E}+03$ & $6.9 \mathrm{E}+03$ & $8.8 \mathrm{E}+02$ \\
\hline Naphthalene, 1-Methyl & $000090-12-0$ & & & & & & & & & & & & & & \\
\hline Naphthalene, 2-Methyl & $000091-57-6$ & & & & & & & & & & & & & & \\
\hline Napropamide & $015299-99-7$ & & & $2.6 \mathrm{E}+01$ & $2.6 \mathrm{E}+00$ & & & $1.0 \mathrm{E}+06$ & 8.1E+05 & & & $1.0 \mathrm{E}+06$ & $5.5 \mathrm{E}+05$ & $4.3 \mathrm{E}+05$ & $5.5 \mathrm{E}+04$ \\
\hline Niagara Blue 4B & $002429-74-5$ & & & & & & & & & & & & & & \\
\hline Nickel Carbonyl & $013463-39-3$ & & & & & & & & & & & & & & \\
\hline Nickel Refinery Dust & NA & & & & & & & & & & & & & & \\
\hline Nickel Soluble Salts & $007440-02-0$ & & & $5.9 \mathrm{E}+01$ & $5.9 \mathrm{E}+00$ & & & $1.0 \mathrm{E}+03$ & $1.0 \mathrm{E}+02$ & & & $5.8 \mathrm{E}+01$ & $7.5 \mathrm{E}+00$ & $5.8 \mathrm{E}+00$ & $7.5 \mathrm{E}-01$ \\
\hline Nickel Subsulfide & $012035-72-2$ & & & & & & & & & & & & & & \\
\hline Nitrapyrin ${ }^{(3)}$ & $001929-82-4$ & & & $3.4 \mathrm{E}+00$ & $3.4 \mathrm{E}-01$ & & & $8.2 E+03$ & $8.2 E+02$ & & & $4.5 \mathrm{E}+03$ & $5.8 \mathrm{E}+02$ & $4.5 \mathrm{E}+02$ & $5.8 \mathrm{E}+01$ \\
\hline Nitrate & $014797-55-8$ & & & & & & & & & & & & & & \\
\hline Nitric Oxide(s) & $010102-43-9$ & & & & & & & & & & & & & & \\
\hline Nitrite & $014797-65-0$ & & & & & & & & & & & & & & \\
\hline Nitroaniline, 2- & $000088-74-4$ & & & & & & & & & & & & & & \\
\hline Nitrobenzene & $000098-95-3$ & & & $4.8 \mathrm{E}-01$ & $4.8 \mathrm{E}-02$ & & & $1.2 \mathrm{E}+04$ & $1.2 \mathrm{E}+03$ & & & $6.6 \mathrm{E}+03$ & $8.4 \mathrm{E}+02$ & $6.6 \mathrm{E}+02$ & $8.4 \mathrm{E}+01$ \\
\hline Nitrofurantoin & $000067-20-9$ & & & & & & & & & & & & & & \\
\hline Nitrofurazone & $000059-87-0$ & & & & & & & & & & & & & & \\
\hline Nitrogen Dioxide ${ }^{(3)}$ & $010102-44-0$ & & & & & & & & & & & & & & \\
\hline Nitroguanidine & $000556-88-7$ & & & & & & & & & & & & & & \\
\hline
\end{tabular}


Table 3.3c. (continued)

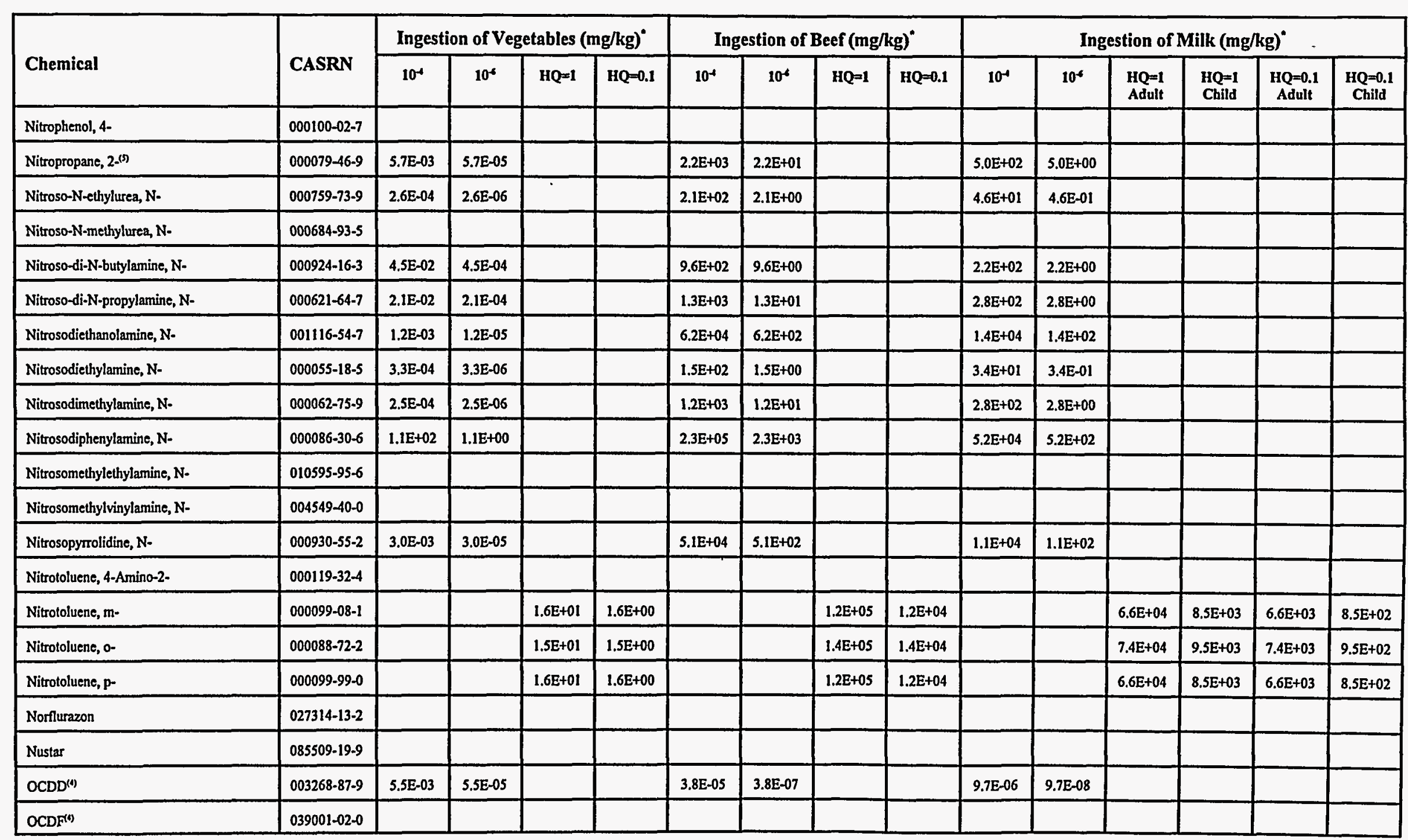


Table 3.3c. (continued)

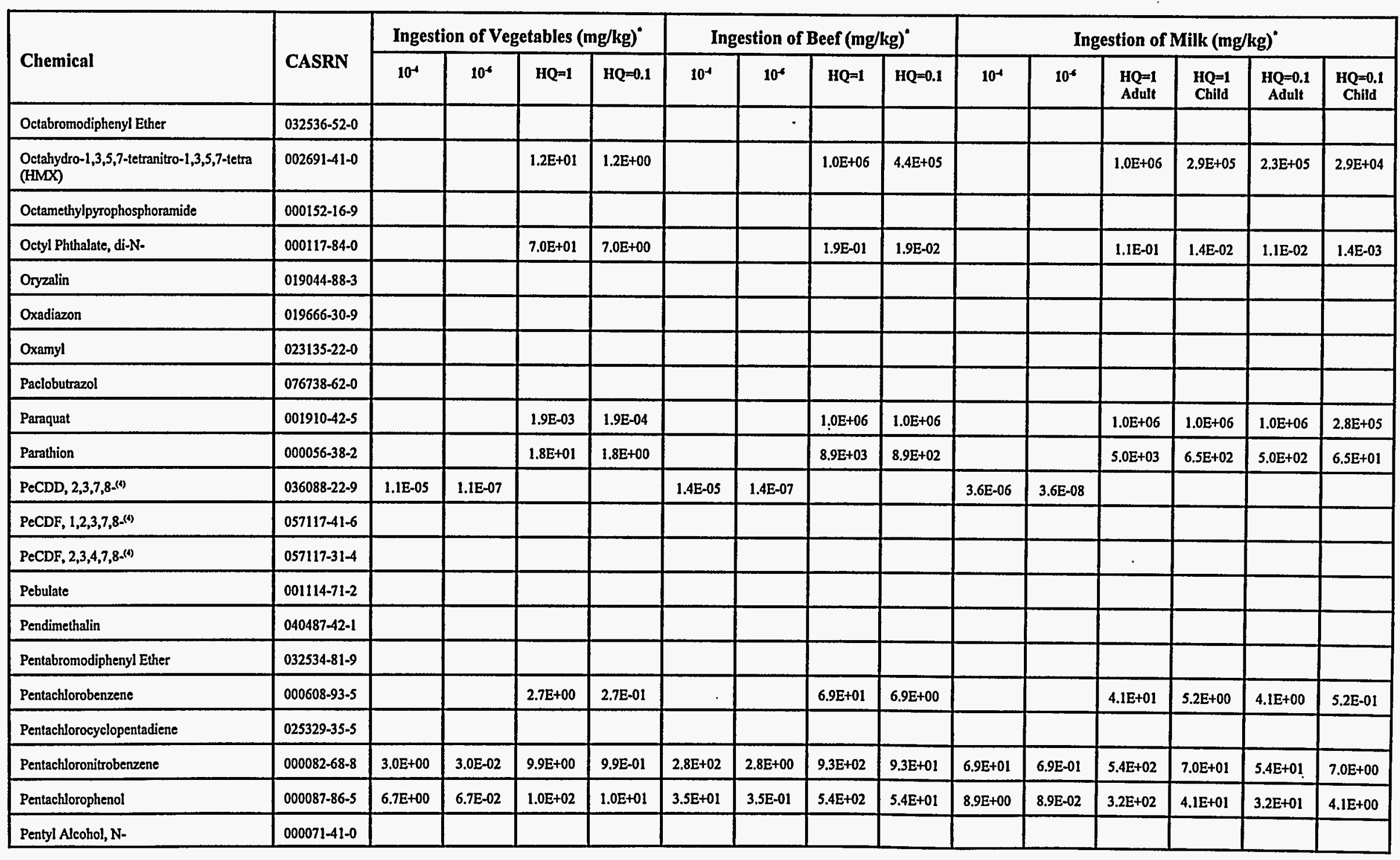


Table 3.3c. (continued)

\begin{tabular}{|c|c|c|c|c|c|c|c|c|c|c|c|c|c|c|c|}
\hline \multirow{2}{*}{ Chemical } & \multirow{2}{*}{ CASRN } & \multicolumn{4}{|c|}{ Ingestion of Vegetables (mg/kg) } & \multicolumn{4}{|c|}{ Ingestion of Beef (mg/kg) } & \multicolumn{6}{|c|}{ Ingestion of Milk (mg/kg) } \\
\hline & & $10^{-4}$ & $10^{6}$ & $\mathrm{HQ}=1$ & $\mathrm{HQ}=0.1$ & $10^{4}$ & $10^{4}$ & $H Q=1$ & $\mathrm{HQ}=0.1$ & $10^{-4}$ & $10^{4}$ & $\begin{array}{l}H Q=1 \\
\text { Adult }\end{array}$ & $\begin{array}{l}H Q=I \\
\text { Child }\end{array}$ & $\begin{array}{c}\mathrm{HQ}=0.1 \\
\text { Adult }\end{array}$ & $\begin{array}{c}\mathrm{HQ}=0.1 \\
\text { Child }\end{array}$ \\
\hline Permethrin & $052645-53-1$ & & & $1.4 \mathrm{E}+02$ & $1.4 \mathrm{E}+01$ & & & $1.3 \mathrm{E}+05$ & $1.3 \mathrm{E}+04$ & & & $7.0 \mathrm{E}+04$ & $9.0 \mathrm{E}+03$ & $7.0 \mathrm{E}+03$ & $9.0 \mathrm{E}+02$ \\
\hline Phenanthrene & $000085-01-8$ & & & & & & & & & & & & & . & \\
\hline Phenmedipham & $013684-63-4$ & & & $5.2 \mathrm{E}+00$ & $5.2 \mathrm{E}-01$ & & & $1.0 \mathrm{E}+06$ & $1.0 \mathrm{E}+06$ & & - & $1.0 \mathrm{E}+06$ & $1.0 \mathrm{E}+06$ & $1.0 \mathrm{E}+06$ & $9.3 \mathrm{E}+05$ \\
\hline Phenol & $000108-95-2$ & & & $4.2 \mathrm{E}+02$ & $4.2 \mathrm{E}+01$ & & & $1.0 \mathrm{E}+06$ & $1.0 \mathrm{E}+06$ & & & $1.0 \mathrm{E}+06$ & $1.0 \mathrm{E}+06$ & $1.0 \mathrm{E}+06$ & $1.4 \mathrm{E}+05$ \\
\hline Phenylenediamine, m- & $000108-45-2$ & & & 2.6E-01 & $2.6 \mathrm{E}-02$ & & & $1.0 \mathrm{E}+06$ & $2.0 \mathrm{E}+05$ & & & $1.0 \mathrm{E}+06$ & $1.3 \mathrm{E}+05$ & $1.0 \mathrm{E}+05$ & $1.3 \mathrm{E}+04$ \\
\hline Phenylenediamine, o- & $000095-54-5$ & $1.0 \mathrm{E}+00$ & $1.0 \mathrm{E}-02$ & & & $5.0 \mathrm{E}+05$ & $5.0 \mathrm{E}+03$ & & & $1.1 \mathrm{E}+05$ & $1.1 \mathrm{E}+03$ & & & & \\
\hline Phenylenediamine, p- & $000106-50-3$ & & & $4.5 \mathrm{E}+00$ & 4.5E-0! & & & $1.0 \mathrm{E}+06$ & $1.0 \mathrm{E}+06$ & & & $1.0 \mathrm{E}+06$ & $1.0 \mathrm{E}+06$ & $1.0 \mathrm{E}+06$ & $6.4 \mathrm{E}+05$ \\
\hline Phenylmercuric Acetate & $000062-38-4$ & & & & & & & & & & & & & & \\
\hline Phenylphenol, 2- & $000090-43-7$ & $2.9 \mathrm{E}+02$ & $2.9 E+00$ & & & $5.7 E+05$ & $5.7 E+03$ & & & $1.3 \mathrm{E}+05$ & $1.3 \mathrm{E}+03$ & & & & \\
\hline Phorate & $000298-02-2$ & & & $1.7 \mathrm{E}-01$ & $1.7 \mathrm{E}-02$ & & & $5.5 \mathrm{E}+03$ & $5.5 \mathrm{E}+02$ & & & $2.9 \mathrm{E}+03$ & $3.8 \mathrm{E}+02$ & $2.9 \mathrm{E}+02$ & $3.8 \mathrm{E}+01$ \\
\hline Phosmet & $000732-11-6$ & & & $4.1 \mathrm{E}+01$ & 4.1E+00 & & & $1.5 \mathrm{E}+05$ & $1.5 \mathrm{E}+04$ & & & $7.9 \mathrm{E}+04$ & $1.0 \mathrm{E}+04$ & $7.9 E+03$ & $1.0 \mathrm{E}+03$ \\
\hline Phosphine & $007803-51-2$ & & & & & & & & & & & & & & \\
\hline Phosphoric Acid & $007664-38-2$ & & & & & & & & & & & & & & \\
\hline Phthalic Acid, P. & $000100-21-0$ & & & $8.6 \mathrm{E}+02$ & $8.6 \mathrm{E}+01$ & & & $1.0 \mathrm{E}+06$ & $1.0 \mathrm{E}+06$ & & & $1.0 \mathrm{E}+06$ & $1.0 \mathrm{E}+06$ & $1.0 \mathrm{E}+06$ & $1.9 \mathrm{E}+05$ \\
\hline Phthalic Anhydride & $000085-44-9$ & & & $1.0 \mathrm{E}+02$ & $1.0 \mathrm{E}+01$ & & & $1.0 \mathrm{E}+06$ & $1.0 \mathrm{E}+06$ & & & $1.0 \mathrm{E}+06$ & $1.0 \mathrm{E}+06$ & $1.0 \mathrm{E}+06$ & $1.0 \mathrm{E}+06$ \\
\hline Picloram & $001918-02-1$ & & & $1.1 \mathrm{E}+02$ & $1.1 \mathrm{E}+01$ & & & $8.6 \mathrm{E}+05$ & $8.6 \mathrm{E}+04$ & & & $4.6 \mathrm{E}+05$ & $5.9 \mathrm{E}+04$ & $4.6 \mathrm{E}+04$ & $5.9 E+03$ \\
\hline Pirimiphos, Methyl & $029232-93-7$ & & & & & & & & & & & & & & \\
\hline Polybrominated Biphenyls & NA & & & & & & & & & & & & & & \\
\hline Polychlorinated Biphenyls & $001336-36-3$ & $1.1 \mathrm{E}-01$ & $1.1 \mathrm{E}-03$ & & & 4.4E-01 & 4.4E-03 & & & $1.1 \mathrm{E}-01$ & 1.1E-03 & & & & \\
\hline Potassium Cyanide & $000151-50-8$ & & & $4.1 \mathrm{E}+00$ & 4.1E-01 & & & $1.0 \mathrm{E}+06$ & $1.0 \mathrm{E}+06$ & & & $1.0 \mathrm{E}+06$ & $6.7 \mathrm{E}+05$ & $5.2 \mathrm{E}+05$ & $6.7 \mathrm{E}+04$ \\
\hline Potassium Silver Cyanide & $000506-61-6$ & & & & & & & & & & & & & & \\
\hline
\end{tabular}


Table 3.3c. (continued)

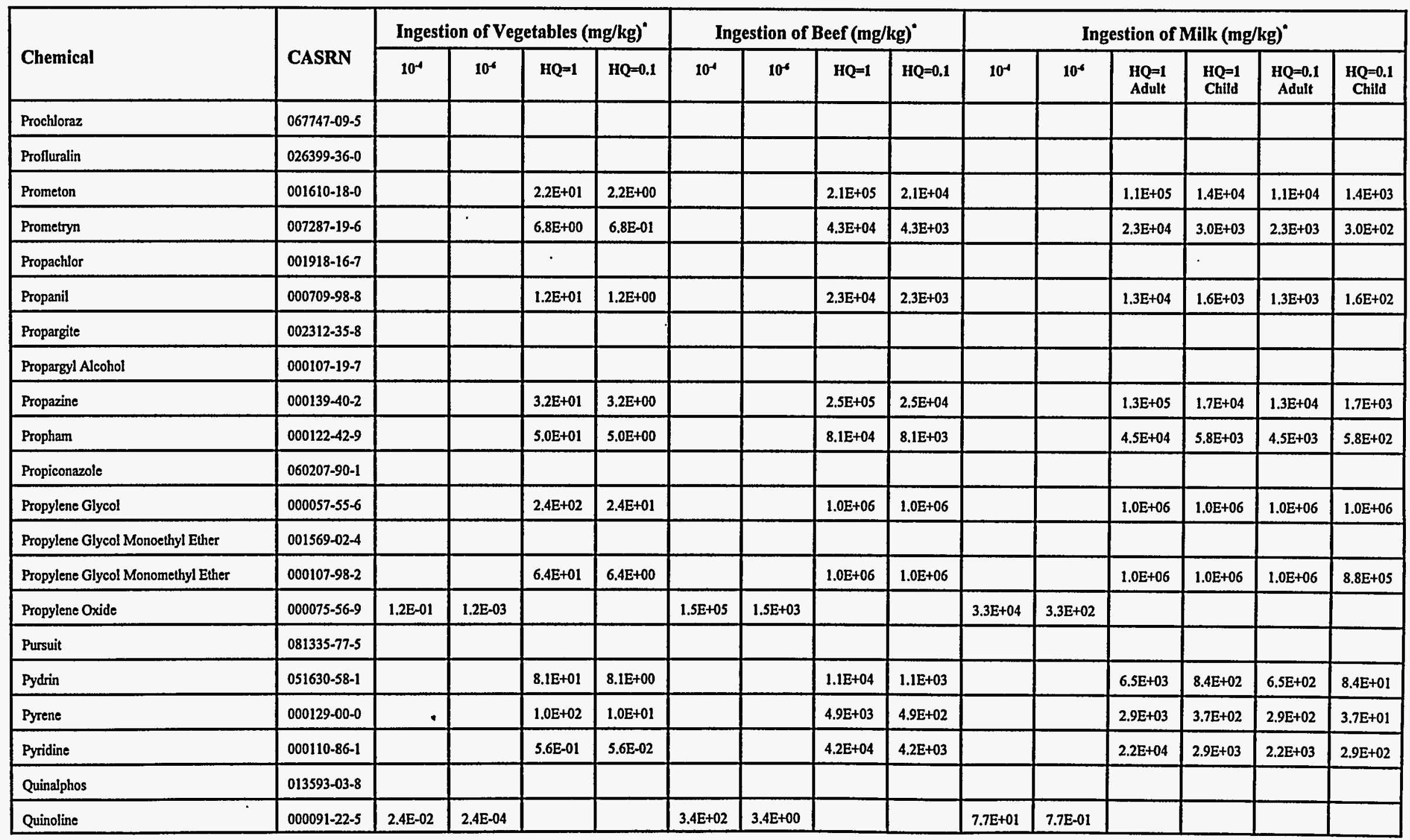


Table 3.3c. (continued)

\begin{tabular}{|c|c|c|c|c|c|c|c|c|c|c|c|c|c|c|c|}
\hline \multirow{2}{*}{ Chemical } & \multirow{2}{*}{ CASRN } & \multicolumn{4}{|c|}{ Ingestion of Vegetables (mg/kg) } & \multicolumn{4}{|c|}{ Ingestion of Beef (mg/kg) } & \multicolumn{6}{|c|}{ Ingestion of Milk (mg/kg)* } \\
\hline & & $10^{-4}$ & $10^{6}$ & $\mathrm{HQ}=1$ & $\mathrm{HQ}=0.1$ & $10^{4}$ & $10^{4}$ & $\mathrm{HQ}=1$ & $\mathrm{HQ}=0.1$ & $10^{-4}$ & $10^{4}$ & $\begin{array}{l}\text { HQ=1 } \\
\text { Adult }\end{array}$ & $\begin{array}{l}\text { HQ }=1 \\
\text { Child }\end{array}$ & $\begin{array}{c}\text { HQ }=0.1 \\
\text { Adult }\end{array}$ & $\begin{array}{c}\text { HQ }=0.1 \\
\text { Child }\end{array}$ \\
\hline Refractory Ceramic Fibers & NA & & & & & & & & & & & & & & \\
\hline Resmethrin & $010453-86-8$ & & & & & & & & & & & & & & \\
\hline Ronnel & $000299-84-3$ & & & $1.7 \mathrm{E}+02$ & $1.7 \mathrm{E}+01$ & & & $5.3 \mathrm{E}+03$ & $5.3 \mathrm{E}+02$ & & & $3.2 \mathrm{E}+03$ & $4.0 \mathrm{E}+02$ & $3.2 \mathrm{E}+02$ & $4.0 \mathrm{E}+01$ \\
\hline Rotenone & $000083-79-4$ & & & $1.2 \mathrm{E}+01$ & $1.2 \mathrm{E}+00$ & & & $3.4 \mathrm{E}+03$ & $3.4 \mathrm{E}+02$ & & & $1.9 \mathrm{E}+03$ & $2.5 \mathrm{E}+02$ & $1.9 \mathrm{E}+02$ & $2.5 E+01$ \\
\hline Savey & $078587-05-0$ & & & & & & & & & & & & & & \\
\hline Selenious Acid & $007783-00-8$ & & & & & & & & & & & & & & \\
\hline Selenite & $014124-67-5$ & & & & & & & & & & & & & & \\
\hline Selenium & $007782-49-2$ & & & $1.3 \mathrm{E}+01$ & $1.3 \mathrm{E}+00$ & & & $8.3 \mathrm{E}+00$ & $8.3 \mathrm{E}-01$ & & & $1.5 \mathrm{E}+01$ & $1.9 \mathrm{E}+00$ & $1.5 \mathrm{E}+00$ & $1.9 \mathrm{E}-01$ \\
\hline Selenium Sulfide & $007446-34-6$ & & & & & & & & & & & & & & \\
\hline Selenourea & $000630-10-4$ & & & & & & & & & & & & & & \\
\hline Sethoxydim & $074051-80-2$ & & & & & & & & & & & & & & \\
\hline Silver & $007440-22-4$ & & & $1.8 \mathrm{E}+01$ & $1.8 \mathrm{E}+00$ & & & $1.8 \mathrm{E}+02$ & $1.8 \mathrm{E}+01$ & & & $1.8 \mathrm{E}+03$ & $2.4 \mathrm{E}+02$ & $1.8 \mathrm{E}+02$ & $2.4 E+01$ \\
\hline Silver Cyanide & $000506-64-9$ & & & & & & & & & & & & & & \\
\hline Simazine & $000122-34-9$ & $2.7 \mathrm{E}+00$ & $2.7 \mathrm{E}-02$ & $6.8 \mathrm{E}+00$ & $6.8 \mathrm{E}-01$ & $3.0 \mathrm{E}+04$ & $3.0 \mathrm{E}+02$ & $7.8 \mathrm{E}+04$ & $7.8 \mathrm{E}+03$ & $6.9 \mathrm{E}+03$ & $6.9 \mathrm{E}+01$ & $4.2 \mathrm{E}+04$ & $5.4 \mathrm{E}+03$ & $4.2 \mathrm{E}+03$ & $5.4 \mathrm{E}+02$ \\
\hline Sodium & $007440-23-5$ & & & & & & & & & & & & & & \\
\hline Sodium Acifluorfen & $062476-59-9$ & & & & & & & & & & & & & & \\
\hline Sodium Azide & $026628-22-8$ & & & & & & & & & & & & & & \\
\hline Sodium Cyanide & $000143-33-9$ & & & $1.6 \mathrm{E}+00$ & $1.6 \mathrm{E}-01$ & & & $1.0 \mathrm{E}+06$ & $1.0 \mathrm{E}+06$ & & & $1.0 \mathrm{E}+06$ & $9.3 \mathrm{E}+05$ & 7.3E+05 & $9.3 \mathrm{E}+04$ \\
\hline Sodium Diethyldihhiocarbamate & $000148-18-5$ & & & & & & & & & & & & & & \\
\hline Sodium Fluoroacetale & $000062-74-8$ & & & & & & & & & & & & & & \\
\hline Sodium Metavanadate & $013718-26-8$ & & & & & & & & & & & & & & \\
\hline
\end{tabular}


Table 3.3c. (continued)

\begin{tabular}{|c|c|c|c|c|c|c|c|c|c|c|c|c|c|c|c|}
\hline \multirow{2}{*}{ Chemical } & \multirow{2}{*}{ CASRN } & \multicolumn{4}{|c|}{ Ingestion of Vegetables (mg/kg) } & \multicolumn{4}{|c|}{ Ingestion of Beef $(\mathrm{mg} / \mathrm{kg})^{*}$} & \multicolumn{6}{|c|}{ Ingestion of Milk (mg/kg) } \\
\hline & & $10^{-1}$ & $10^{6}$ & $\mathbf{H Q}=\mathbf{I}$ & $\mathrm{HQ}=0.1$ & $10^{-4}$ & $10^{6}$ & $\mathbf{H Q}=\mathbf{1}$ & $\mathrm{HQ}=0.1$ & $10^{-4}$ & $10^{6}$ & $\begin{array}{l}\text { HQ=1 } \\
\text { Adult }\end{array}$ & $\begin{array}{l}\text { HQ=1 } \\
\text { Child }\end{array}$ & $\begin{array}{c}\text { HQ }=0.1 \\
\text { Adult }\end{array}$ & $\begin{array}{c}\text { HQ }=0.1 \\
\text { Child }\end{array}$ \\
\hline Stirofos (Tetrachlorovinphos) & $000961-11-5$ & & & & & & & & & & & & & & \\
\hline Strontium, Stable & $007440-24-6$ & & & $1.2 \mathrm{E}+03$ & $1.2 \mathrm{E}+02$ & & & $7.5 \mathrm{E}+03$ & 7.5E+02 & & & $3.7 \mathrm{E}+03$ & $4.7 \mathrm{E}+02$ & $3.7 \mathrm{E}+02$ & $4.7 \mathrm{E}+01$ \\
\hline Strychnine & $000057-24-9$ & & & $3.2 \mathrm{E}-01$ & $3.2 \mathrm{E}-02$ & & & $6.7 \mathrm{E}+03$ & $6.7 E+02$ & & & $3.5 \mathrm{E}+03$ & $4.5 \mathrm{E}+02$ & $3.5 \mathrm{E}+02$ & $4.5 \mathrm{E}+01$ \\
\hline Styrene(6) & $000100-42-5$ & & & $4.3 \mathrm{E}+02$ & $4.3 \mathrm{E}+01$ & & & $1.0 \mathrm{E}+06$ & $1.3 \mathrm{E}+05$ & & & $6.9 \mathrm{E}+05$ & $8.9 E+04$ & $6.9 \mathrm{E}+04$ & $8.9 \mathrm{E}+03$ \\
\hline Sulfate & $014808-79-8$ & & & & & & & & & & & & & & \\
\hline Systhane & $088671-89-0$ & & & & & & & & & & & & & & \\
\hline TCDD, 2,3,7,8-(4) & $001746-01-6$ & 5.4E-06 & 5.4E-08 & & & $3.6 \mathrm{E}-06$ & $3.6 \mathrm{E}-08$ & & & $9.2 \mathrm{E}-07$ & $9.2 \mathrm{E}-09$ & & & & \\
\hline TCDF, 2,3,7,8-(n) & $051207-31-9$ & $5.4 \mathrm{E}-05$ & 5.4E-07 & & & $3.4 \mathrm{E}-04$ & $3.4 \mathrm{E}-06$ & & & 8.5E-05 & $8.5 \mathrm{E}-07$ & & & & \\
\hline TCMTB & $021564-17-0$ & & & & & & & & & & & & & & \\
\hline Tebuthiuron & 034014-18-1 & & & $9.7 E+00$ & $9.7 \mathrm{E}-01$ & & & $1.0 \mathrm{E}+06$ & $9.5 \mathrm{E}+05$ & & & $1.0 \mathrm{E}+06$ & $6.3 \mathrm{E}+05$ & $4.9 \mathrm{E}+05$ & $6.3 \mathrm{E}+04$ \\
\hline Temephos & 003383-96-8 & & & & & & & & & & & & & & \\
\hline Terbacil & $005902-51-2$ & & & & & & & & & & & & & & \\
\hline Terbufos & $013071-79-9$ & & & $7.2 \mathrm{E}-02$ & $7.2 \mathrm{E}-03$ & & & $4.6 \mathrm{E}+01$ & $4.6 \mathrm{E}+00$ & & & $2.6 \mathrm{E}+01$ & $3.3 \mathrm{E}+00$ & $2.6 \mathrm{E}+00$ & 3.3E-01 \\
\hline Terbutryn & $000886-50-0$ & & & $2.9 \mathrm{E}+00$ & $2.9 \mathrm{E}-01$ & & & $1.7 \mathrm{E}+03$ & $1.7 \mathrm{E}+02$ & & & $9.3 \mathrm{E}+02$ & $1.2 \mathrm{E}+02$ & $9.3 \mathrm{E}+01$ & $1.2 \mathrm{E}+01$ \\
\hline Tetrabutyl Lead & 001920-90-7 & & & & & & & & & & & & & & \\
\hline Tetrachlorobenzene, 1,2,4,5- & $000095-94-3$ & & & $9.9 \mathrm{E}-01$ & $9.9 \mathrm{E}-02$ & & & $9.3 \mathrm{E}+01$ & $9.3 \mathrm{E}+00$ & & & $5.4 \mathrm{E}+01$ & $7.0 \mathrm{E}+00$ & $5.4 \mathrm{E}+00$ & 7.0E-01 \\
\hline Tetrachloroethane, 1,1,1,2- & $000630-20-6$ & $2.0 \mathrm{E}+01$ & $2.0 \mathrm{E}-01$ & $6.8 \mathrm{E}+01$ & $6.8 \mathrm{E}+00$ & $4.9 \mathrm{E}+04$ & $4.9 \mathrm{E}+02$ & $1.6 \mathrm{E}+05$ & $1.6 \mathrm{E}+04$ & $1.1 \mathrm{E}+04$ & $1.1 \mathrm{E}+02$ & $9.0 \mathrm{E}+04$ & $1.2 \mathrm{E}+04$ & $9.0 \mathrm{E}+03$ & $1.2 \mathrm{E}+03$ \\
\hline Tetrachloroethane, 1,1,2,2- & $000079-34-5$ & $1.9 \mathrm{E}+00$ & $1.9 \mathrm{E}-02$ & & & $1.4 \mathrm{E}+04$ & $1.4 \mathrm{E}+02$ & & & $3.3 \mathrm{E}+03$ & $3.3 \mathrm{E}+01$ & & & & \\
\hline Tetrachloroethylene ${ }^{(6)}$ & $000127-18-4$ & $8.2 E+00$ & $8.2 \mathrm{E}-02$ & $1.8 \mathrm{E}+01$ & $1.8 \mathrm{E}+00$ & $4.3 \mathrm{E}+04$ & $4.3 \mathrm{E}+02$ & $9.5 \mathrm{E}+04$ & $9.5 \mathrm{E}+03$ & $9.8 \mathrm{E}+03$ & $9.8 \mathrm{E}+01$ & $5.1 \mathrm{E}+04$ & $6.6 \mathrm{E}+03$ & $5.1 \mathrm{E}+03$ & $6.6 \mathrm{E}+02$ \\
\hline Tetrachlorophenol, 2,3,4,6- & $000058-90-2$ & & & $9.4 \mathrm{E}+01$ & $9.4 \mathrm{E}+00$ & & & $2.5 \mathrm{E}+04$ & $2.5 \mathrm{E}+03$ & & & $1.5 \mathrm{E}+04$ & $1.9 \mathrm{E}+03$ & $1.5 \mathrm{E}+03$ & $1.9 \mathrm{E}+02$ \\
\hline Tetrachlorotoluene, p- alpha, alpha, alpha- & $005216-25-1$ & & & & & & & & & & & & & & \\
\hline
\end{tabular}


Table 3.3c. (continued)

\begin{tabular}{|c|c|c|c|c|c|c|c|c|c|c|c|c|c|c|c|}
\hline \multirow{2}{*}{ Chemical } & \multirow{2}{*}{ CASRN } & \multicolumn{4}{|c|}{ Ingestion of Vegetables (mg/kg)" } & \multicolumn{4}{|c|}{ Ingestion of Beef (mg/kg) } & \multicolumn{6}{|c|}{ Ingestion of Milk (mg/kg) } \\
\hline & & $10^{-4}$ & $10^{4}$ & $H Q=1$ & $\mathrm{HQ}=0.1$ & $10^{-4}$ & $10^{8}$ & $H Q=1$ & $\mathrm{HQ}=0.1$ & $10^{-4}$ & $10^{6}$ & $\begin{array}{l}\text { HQ=1 } \\
\text { Adult }\end{array}$ & $\begin{array}{l}\text { HQ=1 } \\
\text { Child }\end{array}$ & $\begin{array}{c}H Q=0.1 \\
\text { Adult }\end{array}$ & $\begin{array}{c}\mathrm{HQ}=0.1 \\
\text { Child }\end{array}$ \\
\hline Tetraethyl Dithiopyrophosphate & $003689-24-5$ & & & $1.1 \mathrm{E}+00$ & $1.1 \mathrm{E}-01$ & & & $2.7 \mathrm{E}+03$ & $2.7 \mathrm{E}+02$ & & & $1.5 \mathrm{E}+03$ & $1.9 \mathrm{E}+02$ & $1.5 \mathrm{E}+02$ & $1.9 \mathrm{E}+01$ \\
\hline Tetraethyl Lead & $000078-00-2$ & & & & & & & & & & & & & & \\
\hline Tetrafluoroethane, 1,1,1,2- & $000811-97-2$ & & & & & & & . & & & & & & & \\
\hline Tetramethyl Lead & $000075-74-1$ & & & & & & & & & & & & & & \\
\hline Tetrapropyl Lead & $003440-75-3$ & & & & & & & & & & & & & & \\
\hline Thallic Oxide(s) & $001314-32-5$ & & & & & & & & & & & & & & \\
\hline Thallium (I) Nitrate & $010102-45-1$ & & & & & & & & & & & & & & \\
\hline Thallium (Solublo Salts) & $007440-28-0$ & & & & & & & & & & & & & & \\
\hline Thallium Acetate & $000563-68-8$ & & & & & & & & & & & & & & \\
\hline Thallium Carbonate & 006533-73-9 & & & & & & & & & & & & & & \\
\hline Thallium Chloride & $007791-12-0$ & & & & & . & & & & & & & & & \\
\hline Thallium Selenite ${ }^{(5)}$ & $012039-52-0$ & & & & & & & & & & & & & & \\
\hline Thallium Sulfate & $007446-18-6$ & & & & & & & & & & & & & & \\
\hline Thiobencarb & $028249-77-6$ & & & & & & & & & & & & & & \\
\hline Thiofanox & $039196-18-4$ & & & $3.8 \mathrm{E}-03$ & $3.8 \mathrm{E}-04$ & & & $2.4 \mathrm{E}+05$ & $2.4 \mathrm{E}+04$ & & & $1.2 \mathrm{E}+05$ & $1.6 \mathrm{E}+04$ & $1.2 \mathrm{E}+04$ & $1.6 \mathrm{E}+03$ \\
\hline Thiophanate, Methyl & $023564-05-8$ & & & & & & & & & & & & & & \\
\hline Thiram & $000137-26-8$ & & & & & & & & & & & & & & \\
\hline Thorium & $007440-29-1$ & & & & & & & & & & & & & & \\
\hline Tin & $007440-31-5$ & & & $9.8 \mathrm{E}+02$ & $9.8 \mathrm{E}+01$ & & & $6.4 \mathrm{E}+03$ & $6.4 \mathrm{E}+02$ & & & $1.1 \mathrm{E}+04$ & $1.4 \mathrm{E}+03$ & $1.1 \mathrm{E}+03$ & $1.4 \mathrm{E}+02$ \\
\hline Titanium & $007440-32-6$ & & & & & & & & & & & & & & \\
\hline Toluene $e^{(6)}$ & $000108-88-3$ & & & $3.9 \mathrm{E}+02$ & $3.9 \mathrm{E}+01$ & & & $1.0 \mathrm{E}+06$ & $1.7 \mathrm{E}+05$ & & & $9.0 \mathrm{E}+05$ & $1.2 \mathrm{E}+05$ & $9.0 E+04$ & $1.2 \mathrm{E}+04$ \\
\hline
\end{tabular}


Table 3.3c. (continued)

\begin{tabular}{|c|c|c|c|c|c|c|c|c|c|c|c|c|c|c|c|}
\hline \multirow{2}{*}{ Chemical } & \multirow{2}{*}{ CASRN } & \multicolumn{4}{|c|}{ Ingestion of Vegetables (mg/kg) } & \multicolumn{4}{|c|}{ Ingestion of Beef (mg/kg) } & \multicolumn{6}{|c|}{ Ingestion of Milk (mg/kg) } \\
\hline & & $10^{-4}$ & $10^{4}$ & $H Q=1$ & $\mathrm{HQ}=0.1$ & $10^{-4}$ & $10^{6}$ & $\mathrm{HQ}=1$ & $\mathrm{HQ}=0.1$ & $10^{-4}$ & $10^{5}$ & $\begin{array}{l}\text { HQ=1 } \\
\text { Adult }\end{array}$ & $\begin{array}{l}\text { HQ=1 } \\
\text { Child }\end{array}$ & $\begin{array}{c}H Q=0.1 \\
\text { Adult }\end{array}$ & $\begin{array}{c}\text { HQ }=0.1 \\
\text { Child }\end{array}$ \\
\hline Toluene diisocyanate mixture (TDI) & $026471-62-5$ & & & & & & & & & & & & & & \\
\hline Toluene-2,4-diamine & $000095-80-7$ & $1.3 \mathrm{E}-02$ & $1.3 \mathrm{E}-04$ & & & $8.2 \mathrm{E}+03$ & $8.2 \mathrm{E}+01$ & & & $1.8 \mathrm{E}+03$ & $1.8 \mathrm{E}+01$ & & & & \\
\hline Toluene-2,5-diamine & $000095-70-5$ & & & & & & & & & & & & & & \\
\hline Toluene-2,6-diamine & $000823-40-5$ & & & & & & & & & & & & & & \\
\hline Toluidine, o-(Methylaniline, 2-) & $000095-53-4$ & 5.5E-01 & $5.5 \mathrm{E}-03$ & & & $4.1 \mathrm{E}+04$ & $4.1 \mathrm{E}+02$ & & & $9.2 \mathrm{E}+03$ & $9.2 \mathrm{E}+01$ & & & & \\
\hline Toluidine, p- & $000106-49-0$ & $1.4 \mathrm{E}+00$ & $1.4 \mathrm{E}-02$ & & & $2.4 \mathrm{E}+0.4$ & $2.4 E+02$ & & & $5.5 \mathrm{E}+03$ & $5.5 \mathrm{E}+01$ & & & & \\
\hline Toxaphene & $008001-35-2$ & 7.1E-01 & 7.1E-03 & & & $4.3 \mathrm{E}+01$ & 4.3E-01 & & & $1.1 \mathrm{E}+01$ & $1.1 \mathrm{E}-01$ & & & & \\
\hline Tralomethrin & $066841-25-6$ & & & & & & & & & & & & & & \\
\hline Triallate & $002303-17-5$ & & & $4.0 \mathrm{E}+01$ & $4.0 \mathrm{E}+00$ & & & $1.4 \mathrm{E}+04$ & $1.4 \mathrm{E}+03$ & & & $7.9 \mathrm{E}+03$ & $1.0 \mathrm{E}+03$ & $7.9 \mathrm{E}+02$ & $1.0 \mathrm{E}+02$ \\
\hline Triasulfuron & $082097-50-5$ & & & & & & & & & & & & & & \\
\hline Tribromobenzene, 1,2,4- & $000615-54-3$ & & & & & & & & & & & & & & \\
\hline Tribromochloromethane & $000594-15-0$ & & & & & & & & & & & & & & \\
\hline Tribromodiphenyl Ether & $049690-94-0$ & & & & & & & & & & & & & & \\
\hline Tributyltin Oxide & $000056-35-9$ & & & & & & & & & & & & & & \\
\hline Trichloro-1,2,2-trifluoroethane, 1,1,2- & 000076-13-1 & & & $5.5 \mathrm{E}+04$ & $5.5 \mathrm{E}+03$ & & & $1.0 \mathrm{E}+06$ & $1.0 \mathrm{E}+06$ & & & $1.0 \mathrm{E}+06$ & $1.0 \mathrm{E}+06$ & $1.0 \mathrm{E}+06$ & $1.0 \mathrm{E}+06$ \\
\hline Trichloro-2'-hydroxydiphenylether & $003380-34-5$ & & & & & & & & & & & & & & \\
\hline \multicolumn{16}{|l|}{ Trichloroacetic Acid } \\
\hline Trichloroaniline $\mathrm{HCl}, 2,4,6-$ & $033663-50-2$ & & & & & & & & & & & & & & \\
\hline Trichloroaniline, 2,4,6. & $000634-93-5$ & & & & & & & & & & & & & & \\
\hline Trichlorobenzene, 1,2,4- & $000120-82-1$ & & & $3.1 \mathrm{E}+01$ & $3.1 \mathrm{E}+00$ & & & $1.0 \mathrm{E}+04$ & $1.0 \mathrm{E}+03$ & & & $5.9 \mathrm{E}+03$ & $7.5 \mathrm{E}+02$ & $5.9 \mathrm{E}+02$ & 7.5E+01 \\
\hline Trichloroethane, $1,1,1-(5)$ & $000071-55-6$ & & & $1.5 \mathrm{E}+02$ & $1.5 \mathrm{E}+01$ & & & $9.8 \mathrm{E}+05$ & $9.8 \mathrm{E}+04$ & & & $5.2 \mathrm{E}+05$ & $6.7 \mathrm{E}+04$ & $5.2 \mathrm{E}+04$ & $6.7 E+03$ \\
\hline
\end{tabular}


Table 3.3c. (continued)

\begin{tabular}{|c|c|c|c|c|c|c|c|c|c|c|c|c|c|c|c|}
\hline \multirow{2}{*}{ Chemical } & \multirow{2}{*}{ CASRN } & \multicolumn{4}{|c|}{ Ingestion of Vegetables (mg/kg) } & \multicolumn{4}{|c|}{ Ingestion of Beef (mg/kg) } & \multicolumn{6}{|c|}{ Ingestion of Milk (mg/kg) } \\
\hline & & $10^{-4}$ & $10^{6}$ & $H Q=1$ & $\mathrm{HQ}=0.1$ & $10^{-4}$ & $10^{5}$ & $H Q=1$ & $\mathrm{HQ}=0.1$ & $10^{-4}$ & $10^{4}$ & $\begin{array}{l}\text { HQ=1 } \\
\text { Adult }\end{array}$ & $\begin{array}{l}\mathrm{HQ}=1 \\
\text { Child }\end{array}$ & $\begin{array}{c}\mathrm{HQ}=0.1 \\
\text { Adult }\end{array}$ & $\begin{array}{l}\text { HQ }=0.1 \\
\text { Child }\end{array}$ \\
\hline Trichloroethane, 1,1,2- & $000079-00-5$ & $4.7 \mathrm{E}+00$ & $4.7 \mathrm{E}-02$ & $4.6 \mathrm{E}+00$ & 4.6E-01 & $8.1 \mathrm{E}+04$ & 8.1E+02 & $7.9 E+04$ & $7.9 \mathrm{E}+03$ & $1.8 \mathrm{E}+04$ & $1.8 \mathrm{E}+02$ & $4.2 E+04$ & $5.4 \mathrm{E}+03$ & $4.2 \mathrm{E}+03$ & $5.4 \mathrm{E}+02$ \\
\hline Trichloroethylene $e^{(0)}$ & $000079-01-6$ & $3.4 \mathrm{E}+01$ & 3.4E-01 & & & $2.6 \mathrm{E}+05$ & $2.6 \mathrm{E}+03$ & & & $5.9 \mathrm{E}+04$ & $5.9 \mathrm{E}+02$ & & & & \\
\hline Trichlorofluoromethane & $000075-69-4$ & & & $5.1 E+02$ & $5.1 \mathrm{E}+01$ & & & $1.0 E+06$ & 3.3E+05 & & & $1.0 \mathrm{E}+06$ & $2.2 E+05$ & $1.7 \mathrm{E}+05$ & $2.2 E+04$ \\
\hline Trichlorophenol, 2,4,5- & $000095-95-4$ & & & $3.0 \mathrm{E}+02$ & $3.0 \mathrm{E}+01$ & & & $1.5 \mathrm{E}+05$ & $1.5 \mathrm{E}+04$ & & & $8.4 \mathrm{E}+04$ & $1.1 \mathrm{E}+04$ & $8.4 E+03$ & $1.1 \mathrm{E}+03$ \\
\hline Trichlorophenol, 2,4,6- & $000088-06-2$ & $6.1 E+01$ & 6.1E-01 & & & $3.8 \mathrm{E}+04$ & $3.8 \mathrm{E}+02$ & & & $9.0 \mathrm{E}+03$ & $9.0 \mathrm{E}+01$ & & & & \\
\hline $\begin{array}{l}\text { Trichlorophenoxy) Propionic Acid, } \\
2(2,4,5-\end{array}$ & $000093-72-1$ & & & $2.4 \mathrm{E}+01$ & $2.4 \mathrm{E}+00$ & & & $9.9 E+03$ & $9.9 \mathrm{E}+02$ & & & $5.6 \mathrm{E}+03$ & $7.2 \mathrm{E}+02$ & $5.6 \mathrm{E}+02$ & $7.2 E+01$ \\
\hline Trichlorophenoxyacetic Acid, 2,4,5. & $000093-76-5$ & & & 3.1E+01 & $3.1 \mathrm{E}+00$ & & & $1.0 E+04$ & $1.0 \mathrm{E}+03$ & & & $5.9 E+03$ & $7.5 \mathrm{E}+02$ & $5.9 \mathrm{E}+02$ & $7.5 \mathrm{E}+01$ \\
\hline Trichloropropane, 1,1,2- & $000598-77-6$ & & & & & & & & & & & & & & \\
\hline Trichloropropane, 1,2,3- & $000096-18-4$ & $1.1 \mathrm{E}-01$ & $1.1 \mathrm{E}-03$ & $2.0 E+01$ & $2.0 \mathrm{E}+00$ & $1.0 \mathrm{E}+01$ & $1.0 \mathrm{E}-01$ & $1.9 \mathrm{E}+03$ & $1.9 \mathrm{E}+02$ & $2.6 \mathrm{E}+00$ & $2.6 \mathrm{E}-02$ & $1.1 \mathrm{E}+03$ & $1.4 \mathrm{E}+02$ & $1.1 E+02$ & $1.4 \mathrm{E}+01$ \\
\hline Trichloropropene, 1,2,3- & $000096-19-5$ & & & & & & & & & & & & & & \\
\hline Trichlorotoluene, 2,3,6- & $002077-46-5$ & & & & & & & & & & & & & & \\
\hline Trichlorotoluene, alpha 2,6- & $002014-83-7$ & & & & & & & & & & & & & & \\
\hline Tridiphane & $058138-08-2$ & & & & & & & & & & & & & & \\
\hline Triethyl Lead & $005224-23-7$ & & & & & & & & & & & & & & \\
\hline Triethylamine & $000121-44-8$ & & & & & & & & & & & & & & \\
\hline Trifluralin & $001582-09-8$ & $1.0 \mathrm{E}+02$ & $1.0 \mathrm{E}+00$ & $2.6 \mathrm{E}+01$ & $2.6 \mathrm{E}+00$ & 2.1E+03 & $2.1 \mathrm{E}+01$ & $5.2 \mathrm{E}+02$ & $5.2 \mathrm{E}+01$ & $5.2 \mathrm{E}+02$ & $5.2 \mathrm{E}+00$ & $3.1 \mathrm{E}+02$ & $3.9 \mathrm{E}+01$ & $3.1 \mathrm{E}+01$ & $3.9 \mathrm{E}+00$ \\
\hline Trimethyl Lead & $007442-13-9$ & & & & & & & & & & & & & & \\
\hline Trimethyl Phosphate & $000512-56-1$ & & & & & & & & & & & & & & \\
\hline Trimethylethyl Lead & $001762-26-1$ & & & & & & & & & & & & & & \\
\hline Trinitrobenzene, 1,3,5- & $000099-35-4$ & & & $2.5 \mathrm{E}-02$ & $2.5 \mathrm{E}-03$ & & & $2.4 \mathrm{E}+03$ & $2.4 \mathrm{E}+02$ & & & $1.2 E+03$ & $1.6 \mathrm{E}+02$ & $1.2 \mathrm{E}+02$ & $1.6 \mathrm{E}+01$ \\
\hline Trinitrophenylmethylnitramine & $000479-45-8$ & & & $1.2 \mathrm{E}+01$ & $1.2 \mathrm{E}+00$ & & & $2.0 \mathrm{E}+05$ & $2.0 \mathrm{E}+04$ & & & $1.1 \mathrm{E}+05$ & $1.4 \mathrm{E}+04$ & $1.1 E+04$ & $1.4 \mathrm{E}+03$ \\
\hline
\end{tabular}

Nonradionuclides/soil or sediment/agricultural

Page 31 of 33 
Table 3.3c. (continued)

\begin{tabular}{|c|c|c|c|c|c|c|c|c|c|c|c|c|c|c|c|}
\hline \multirow{2}{*}{ Chemical } & \multirow{2}{*}{ CASRN } & \multicolumn{4}{|c|}{ Ingestion of Vegetables (mg/kg) } & \multicolumn{4}{|c|}{ Ingestion of Beef (mg/kg) } & \multicolumn{6}{|c|}{ Ingestion of Milk (mg/kg)" } \\
\hline & & $10^{-4}$ & $10^{4}$ & $\mathbf{H Q}=\mathbf{I}$ & $H Q=0.1$ & $10^{-1}$ & $10^{6}$ & $\mathrm{HQ}=\mathbf{I}$ & $\mathbf{H Q}=0.1$ & $10^{-4}$ & $10^{4}$ & $\begin{array}{l}\text { HQ }=1 \\
\text { Adult }\end{array}$ & $\begin{array}{l}\text { HQ=1 } \\
\text { Child }\end{array}$ & $\begin{array}{c}H Q=0.1 \\
\text { Adult }\end{array}$ & $\begin{array}{c}\text { HQ }=0.1 \\
\text { Child }\end{array}$ \\
\hline Trinitrotoluene, 2,4,6- & $000118-96-7$ & $1.2 \mathrm{E}+01$ & $1.2 \mathrm{E}-01$ & $7.4 \mathrm{E}-01$ & $7.4 \mathrm{E}-02$ & $1.1 \mathrm{E}+05$ & $1.1 \mathrm{E}+03$ & $7.0 \mathrm{E}+03$ & $7.0 \mathrm{E}+02$ & $2.5 \mathrm{E}+04$ & $2.5 \mathrm{E}+02$ & $3.7 \mathrm{E}+03$ & $4.8 \mathrm{E}+02$ & $3.7 \mathrm{E}+02$ & $4.8 \mathrm{E}+01$ \\
\hline Tripropyl Lead & 006618-03-7 & & & & & & & & & & & & & & \\
\hline Uranium (Soluble Salts) & NA & & & $1.1 \mathrm{E}+01$ & $1.1 \mathrm{E}+00$ & & & $3.5 \mathrm{E}+03$ & $3.5 \mathrm{E}+02$ & & & $4.9 \mathrm{E}+02$ & $6.3 \mathrm{E}+01$ & $4.9 \mathrm{E}+01$ & $6.3 \mathrm{E}+00$ \\
\hline Vanadium Pentoxide & $001314-62-1$ & & & & & & & & & & & & & & \\
\hline Vanadium Sulfate & $036907-42-3$ & & & & & & & & & & & & & & \\
\hline Vanadium, Metallic & $007440-62-2$ & & & $2.4 E+01$ & $2.4 \mathrm{E}+00$ & & & $1.0 \mathrm{E}+03$ & $1.0 \mathrm{E}+02$ & & & $2.4 \mathrm{E}+04$ & $3.1 \mathrm{E}+03$ & $2.4 \mathrm{E}+03$ & $3.1 E+02$ \\
\hline Vanadyl Sulfate & $027774-13-6$ & & & & & & & & & & & & & & \\
\hline Vemolate & $001929-77-7$ & & & & & & & & & & & & & & \\
\hline Vinclozolin & $050471-44-8$ & & & & & & & & & & & & & & \\
\hline Vinyl Acetate & $000108-05-4$ & & & $2.9 \mathrm{E}+02$ & $2.9 \mathrm{E}+01$ & & & $1.0 \mathrm{E}+06$ & $1.0 \mathrm{E}+06$ & & & $1.0 \mathrm{E}+06$ & $1.0 \mathrm{E}+06$ & $1.0 \mathrm{E}+06$ & $5.1 \mathrm{E}+05$ \\
\hline Vinyl Bromide & $000593-60-2$ & & & & & & & & & & & & & & \\
\hline Vinyl Chloride $e^{(s)}$ & $000075-01-4$ & $7.7 \mathrm{E}-02$ & $7.7 \mathrm{E}-04$ & & & $4.7 \mathrm{E}+03$ & 4.7E+01 & & & $1.0 \mathrm{E}+03$ & $1.0 \mathrm{E}+01$ & & & & \\
\hline Warfarin & $000081-81-2$ & & & 7.5E-01 & $7.5 \mathrm{E}-02$ & & & $1.2 \mathrm{E}+03$ & $1.2 \mathrm{E}+02$ & & & $6.7 \mathrm{E}+02$ & $8.6 \mathrm{E}+01$ & $6.7 \mathrm{E}+01$ & $8.6 \mathrm{E}+00$ \\
\hline White Phosphorus & $007723-14-0$ & & & & & & & & & & & & & & \\
\hline Xylene, Mixture ${ }^{(6)}$ & $001330-20-7$ & & & $5.2 \mathrm{E}+03$ & $5.2 \mathrm{E}+02$ & & & $1.0 \mathrm{E}+06$ & $6.9 E+05$ & & & $1.0 \mathrm{E}+06$ & $4.9 \mathrm{E}+05$ & $3.8 \mathrm{E}+05$ & $4.9 \mathrm{E}+04$ \\
\hline Xylene, P-(b) & $000106-42-3$ & & & & & & & & & & & & & & \\
\hline Xylene, $m_{-(6)}^{(b)}$ & 000108-38-3 & & & $5.0 \mathrm{E}+03$ & $5.0 \mathrm{E}+02$ & & & $1.0 E+06$ & 8.1E+05 & & & $1.0 \mathrm{E}+06$ & $5.8 \mathrm{E}+05$ & 4.5E+05 & $5.8 \mathrm{E}+04$ \\
\hline Xylene, $\sigma^{(5)}$ & $000095-47-6$ & & & $5.0 \mathrm{E}+03$ & $5.0 \mathrm{E}+02$ & & & $1.0 \mathrm{E}+06$ & 8.1E+05 & & & $1.0 \mathrm{E}+06$ & $5.8 \mathrm{E}+05$ & $4.5 \mathrm{E}+05$ & $5.8 \mathrm{E}+04$ \\
\hline Zinc (Metallic) & $007440-66-6$ & & & $5.2 \mathrm{E}+02$ & $5.2 \mathrm{E}+01$ & & & $3.2 \mathrm{E}+02$ & $3.2 \mathrm{E}+01$ & & & $5.5 \mathrm{E}+02$ & 7.1E+01 & $5.5 \mathrm{E}+01$ & $7.1 \mathrm{E}+00$ \\
\hline Zine Cyanide & $000557-21-1$ & & & & & & & & & & & & & & \\
\hline Zinc Phosphide & $001314-84-7$ & & & & & & & & & & & & & & \\
\hline
\end{tabular}


Table 3.3c. (continued)

\begin{tabular}{|c|c|c|c|c|c|c|c|c|c|c|c|c|c|c|c|}
\hline Chemical & CASRN & \multicolumn{4}{|c|}{ Ingestion of Vegetables $(\mathrm{mg} / \mathrm{kg})^{*}$} & \multicolumn{4}{|c|}{ Ingestion of Beef $(\mathrm{mg} / \mathrm{kg})^{\circ}$} & \multicolumn{6}{|c|}{ Ingestion of Milk (mg/kg) } \\
\hline Zineb & $012122-67-7$ & & & & & & & & & & & & & & \\
\hline Zirconium & $007440-67-7$ & & & & & & & & & & & & & & \\
\hline
\end{tabular}

Notes:

* By definition, $1 \mathrm{~kg}=1.0 \mathrm{E}+06 \mathrm{mg}$. Therefore, a limit of $1.0 \mathrm{E}+06 \mathrm{mg} / \mathrm{kg}$ was used for any PRG that was calculated to be above this limit.

(1) The same oral slope factor as used for polychlorinated biphenyls.

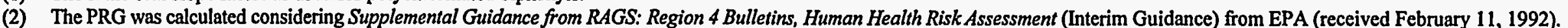
The slope factors for chemicals so marked indicate that a toxicity equivalency factor (TEF) method was used for carcinogenic PAHs based on each compound's relative potency to the potenicy of benzo[a]pyrene. The following TEFs were used to convert each PAH slope factor to an equivalent slope factor of benzo[a]pyrene [that has a new cancer slope factor of 7.3 (mg/kg/day) $)^{-1}$ identified by EPA's CRAVE workgroup]: (1) benzo[a]pyrene, TEF $=1.0 ;(2)$ benzo[a]anthracene, TEF $=0.1 ;(3)$ benzo[b]fluoranthene, TEF $=0.1 ;(4)$ benzo[k]fluoranthene, $\mathrm{TEF}=0.01 ;(5)$ chrysene, $\mathrm{TEF}=0.001 ;(6)$ dibenz $[\mathrm{a}, \mathrm{h}]$ anthracene, $\mathrm{TEF}=1.0 ;$ and $(7)$ indeno $[1,2,3-\mathrm{c}, \mathrm{d}] \mathrm{pyrene}, \mathrm{TEF}=0.1$.

(3) The toxicity values for trichloroethylene have been withdrawn from IRIS and HEAST pending further investigation. However, the most recent toxicity values have been used to calculate the PRG.

(4) The PRG was calculated considering Supplemental Guidance from RAGS: Region 4 Bulletins, Human Health Risk Assessment (Interim Guidance) from EPA (November 1995). The slope factors for chemicals so marked indicate that a TEF methodology was used for chlorinated dioxin and furan congeners based on each compound's relative potency to the potency of $2,3,7,8$ tetrachlorodibenzodioxin (TCDD). The following TEFs were used to convert each slope factor to an equivalent slope factor of $2,3,7,8$-TCDD: $(1) 2,3,7,8-\mathrm{TCDD}, \mathrm{TEF}=1.0 ;(2) 2,3,7,8-$ $\mathrm{PeCDD}, \mathrm{TEF}=0.5 ;$ (3) 2,3,7,8-HxCDD, TEF $=0.1$; (4) $2,3,7,8-\mathrm{HpCDD}, \mathrm{TEF}=0.01 ;(5)$ OCDD, TEF $=0.001 ;(6) 2,3,7,8-\mathrm{TCDF}, \mathrm{TEF}=0.1 ;(7) 1,2,3,7,8-\mathrm{PeCDF}, \mathrm{TEF}=0.5 ;(8) 2,3,4,7,8-$ $\mathrm{PeCDF}, \mathrm{TEF}=0.05 ;(9) 2,3,7,8-\mathrm{HxCDF}, \mathrm{TEF}=0.1 ;(10) 2,3,7,8-\mathrm{HpCDF}, \mathrm{TEF}=0.01$; and $(11) \mathrm{OCDF}, \mathrm{TEF}=0.001$.

(5) Toxicity values for these chemicals have been withdrawn from IRIS and HEAST pending further investigation. However, the most recent toxicity values have been used to calculate the PRG.

(6) Toxicity values for these chemicals were obtained from the Superfund Health Risk Technical Support Center. 
Table 3.3d. Risk-based Preliminary Remediation Goals for radionuclides in soil or sediment (agricultural scenario)

\begin{tabular}{|l|l|l|c|c|c|c|c|}
\hline \multirow{2}{*}{ Chemical } & \multirow{2}{*}{ CASRN } & \multicolumn{2}{|c|}{$\begin{array}{c}\text { Ingestion } \\
\text { of Vegetables (pCi/g) }\end{array}$} & \multicolumn{2}{c|}{$\begin{array}{c}\text { Ingestion } \\
\text { of Beef (pCi/g) }\end{array}$} & \multicolumn{2}{c|}{$\begin{array}{c}\text { Ingestion } \\
\text { of Milk (pCi/g) }\end{array}$} \\
\cline { 3 - 8 } & & $10^{-4}$ & $10^{-6}$ & $10^{-1}$ & $10^{-6}$ & $10^{-4}$ & $10^{-6}$ \\
\hline Ac-225 & $014265-85-1$ & $3.2 \mathrm{E}+00$ & $3.2 \mathrm{E}-02$ & $1.4 \mathrm{E}+04$ & $1.4 \mathrm{E}+02$ & $2.8 \mathrm{E}+03$ & $2.8 \mathrm{E}+01$ \\
\hline Ac-227 & $014952-40-0$ & $1.3 \mathrm{E}+00$ & $1.3 \mathrm{E}-02$ & $5.5 \mathrm{E}+03$ & $5.5 \mathrm{E}+01$ & $1.1 \mathrm{E}+03$ & $1.1 \mathrm{E}+01$ \\
\hline Ac-227+D & $014952-40-0$ & $6.8 \mathrm{E}-01$ & $6.8 \mathrm{E}-03$ & $9.7 \mathrm{E}-01$ & $9.7 \mathrm{E}-03$ & $3.2 \mathrm{E}-01$ & $3.2 \mathrm{E}-03$ \\
\hline $\mathrm{Ac}-228$ & $014331-83-0$ & $2.8 \mathrm{E}+02$ & $2.8 \mathrm{E}+00$ & $1.2 \mathrm{E}+06$ & $1.2 \mathrm{E}+04$ & $2.5 \mathrm{E}+05$ & $2.5 \mathrm{E}+03$ \\
\hline $\mathrm{Ag}-105$ & $014928-14-4$ & $2.8 \mathrm{E}+02$ & $2.8 \mathrm{E}+00$ & $2.9 \mathrm{E}+03$ & $2.9 \mathrm{E}+01$ & $2.6 \mathrm{E}+04$ & $2.6 \mathrm{E}+02$ \\
\hline $\mathrm{Ag}-108$ & $014391-65-2$ & $6.6 \mathrm{E}+04$ & $6.6 \mathrm{E}+02$ & $6.7 \mathrm{E}+05$ & $6.7 \mathrm{E}+03$ & $6.1 \mathrm{E}+06$ & $6.1 \mathrm{E}+04$ \\
\hline $\mathrm{Ag}-108 \mathrm{~m}$ & $014391-65-2$ & $7.6 \mathrm{E}+01$ & $7.6 \mathrm{E}-01$ & $7.7 \mathrm{E}+02$ & $7.7 \mathrm{E}+00$ & $7.0 \mathrm{E}+03$ & $7.0 \mathrm{E}+01$ \\
\hline $\mathrm{Ag}-108 \mathrm{~m}+\mathrm{D}$ & $014391-65-2$ & $7.6 \mathrm{E}+01$ & $7.6 \mathrm{E}-01$ & $7.7 \mathrm{E}+02$ & $7.7 \mathrm{E}+00$ & $7.0 \mathrm{E}+03$ & $7.0 \mathrm{E}+01$ \\
\hline $\mathrm{Ag}-109 \mathrm{~m}$ & $014378-38-2$ & $1.7 \mathrm{E}+06$ & $1.7 \mathrm{E}+04$ & $1.7 \mathrm{E}+07$ & $1.7 \mathrm{E}+05$ & $1.6 \mathrm{E}+08$ & $1.6 \mathrm{E}+06$ \\
\hline $\mathrm{Ag}-110$ & $014391-76-5$ & $1.9 \mathrm{E}+05$ & $1.9 \mathrm{E}+03$ & $1.9 \mathrm{E}+06$ & $1.9 \mathrm{E}+04$ & $1.7 \mathrm{E}+07$ & $1.7 \mathrm{E}+05$ \\
\hline $\mathrm{Ag}-110 \mathrm{~m}$ & $014391-76-5$ & $5.4 \mathrm{E}+01$ & $5.4 \mathrm{E}-01$ & $5.5 \mathrm{E}+02$ & $5.5 \mathrm{E}+00$ & $5.0 \mathrm{E}+03$ & $5.0 \mathrm{E}+01$ \\
\hline $\mathrm{Ag}-111$ & $015760-04-0$ & $6.7 \mathrm{E}+01$ & $6.7 \mathrm{E}-01$ & $6.8 \mathrm{E}+02$ & $6.8 \mathrm{E}+00$ & $6.2 \mathrm{E}+03$ & $6.2 \mathrm{E}+01$ \\
\hline $\mathrm{Am}-241$ & $014596-10-2$ & $1.4 \mathrm{E}+00$ & $1.4 \mathrm{E}-02$ & $3.7 \mathrm{E}+03$ & $3.7 \mathrm{E}+01$ & $1.6 \mathrm{E}+04$ & $1.6 \mathrm{E}+02$ \\
\hline $\mathrm{Am}-242$ & $013981-54-9$ & $3.1 \mathrm{E}+02$ & $3.1 \mathrm{E}+00$ & $8.2 \mathrm{E}+05$ & $8.2 \mathrm{E}+03$ & $3.6 \mathrm{E}+06$ & $3.6 \mathrm{E}+04$ \\
\hline $\mathrm{Am}-242 \mathrm{~m}$ & $013981-54-9$ & $1.6 \mathrm{E}+00$ & $1.6 \mathrm{E}-02$ & $4.1 \mathrm{E}+03$ & $4.1 \mathrm{E}+01$ & $1.8 \mathrm{E}+04$ & $1.8 \mathrm{E}+02$ \\
\hline $\mathrm{Am}-243$ & $014993-75-0$ & $1.4 \mathrm{E}+00$ & $1.4 \mathrm{E}-02$ & $3.7 \mathrm{E}+03$ & $3.7 \mathrm{E}+01$ & $1.6 \mathrm{E}+04$ & $1.6 \mathrm{E}+02$ \\
\hline $\mathrm{Am}-243+\mathrm{D}$ & $014993-75-0$ & $1.4 \mathrm{E}+00$ & $1.4 \mathrm{E}-02$ & $1.2 \mathrm{E}+02$ & $1.2 \mathrm{E}+00$ & $3.1 \mathrm{E}+03$ & $3.1 \mathrm{E}+01$ \\
\hline
\end{tabular}


Table 3.3d. (continued)

\begin{tabular}{|l|l|l|c|c|c|c|c|}
\hline \multirow{2}{*}{ Chemical } & \multirow{2}{*}{ CASRN } & \multicolumn{2}{|c|}{$\begin{array}{c}\text { Ingestion } \\
\text { of Vegetables (pCi/g) }\end{array}$} & \multicolumn{2}{c|}{$\begin{array}{c}\text { Ingestion } \\
\text { of Beef (pCi/g) }\end{array}$} & \multicolumn{2}{c|}{$\begin{array}{c}\text { Ingestion } \\
\text { of Milk (pCi/g) }\end{array}$} \\
\cline { 3 - 8 } & & $10^{-4}$ & $10^{-6}$ & $10^{-4}$ & $10^{-6}$ & $10^{-4}$ & $10^{-6}$ \\
\hline Ar-41 & $014163-25-8$ & & & & & & \\
\hline At-217 & $017239-90-6$ & $2.6 \mathrm{E}+07$ & $2.6 \mathrm{E}+05$ & $1.6 \mathrm{E}+08$ & $1.6 \mathrm{E}+06$ & $2.3 \mathrm{E}+07$ & $2.3 \mathrm{E}+05$ \\
\hline Au-196 & $014914-16-0$ & $2.5 \mathrm{E}+02$ & $2.5 \mathrm{E}+00$ & $3.7 \mathrm{E}+03$ & $3.7 \mathrm{E}+01$ & $2.9 \mathrm{E}+05$ & $2.9 \mathrm{E}+03$ \\
\hline Au-198 & $010043-49-0$ & $6.3 \mathrm{E}+01$ & $6.3 \mathrm{E}-01$ & $9.2 \mathrm{E}+02$ & $9.2 \mathrm{E}+00$ & $7.2 \mathrm{E}+04$ & $7.2 \mathrm{E}+02$ \\
\hline Ba-131 & $014914-75-1$ & $2.7 \mathrm{E}+02$ & $2.7 \mathrm{E}+00$ & $1.1 \mathrm{E}+05$ & $1.1 \mathrm{E}+03$ & $7.7 \mathrm{E}+03$ & $7.7 \mathrm{E}+01$ \\
\hline Ba-133 & $013981-41-4$ & $1.7 \mathrm{E}+02$ & $1.7 \mathrm{E}+00$ & $7.2 \mathrm{E}+04$ & $7.2 \mathrm{E}+02$ & $4.9 \mathrm{E}+03$ & $4.9 \mathrm{E}+01$ \\
\hline Ba-133m & $013981-41-4$ & $1.6 \mathrm{E}+02$ & $1.6 \mathrm{E}+00$ & $7.0 \mathrm{E}+04$ & $7.0 \mathrm{E}+02$ & $4.7 \mathrm{E}+03$ & $4.7 \mathrm{E}+01$ \\
\hline Ba-137m & $013981-97-0$ & $1.9 \mathrm{E}+05$ & $1.9 \mathrm{E}+03$ & $8.0 \mathrm{E}+07$ & $8.0 \mathrm{E}+05$ & $5.4 \mathrm{E}+06$ & $5.4 \mathrm{E}+04$ \\
\hline Ba-139 & $014378-25-7$ & $1.5 \mathrm{E}+03$ & $1.5 \mathrm{E}+01$ & $6.4 \mathrm{E}+05$ & $6.4 \mathrm{E}+03$ & $4.3 \mathrm{E}+04$ & $4.3 \mathrm{E}+02$ \\
\hline Ba-140 & $014798-08-4$ & $3.8 \mathrm{E}+01$ & $3.8 \mathrm{E}-01$ & $1.6 \mathrm{E}+04$ & $1.6 \mathrm{E}+02$ & $1.1 \mathrm{E}+03$ & $1.1 \mathrm{E}+01$ \\
\hline Be-7 & $013966-02-4$ & $5.2 \mathrm{E}+03$ & $5.2 \mathrm{E}+01$ & $5.5 \mathrm{E}+05$ & $5.5 \mathrm{E}+03$ & $1.0 \mathrm{E}+08$ & $1.0 \mathrm{E}+06$ \\
\hline Bi-206 & $015776-19-9$ & $6.2 \mathrm{E}+01$ & $6.2 \mathrm{E}-01$ & $1.6 \mathrm{E}+04$ & $1.6 \mathrm{E}+02$ & $2.1 \mathrm{E}+03$ & $2.1 \mathrm{E}+01$ \\
\hline Bi-207 & $013982-38-2$ & $8.8 \mathrm{E}+01$ & $8.8 \mathrm{E}-01$ & $2.2 \mathrm{E}+04$ & $2.2 \mathrm{E}+02$ & $2.9 \mathrm{E}+03$ & $2.9 \mathrm{E}+01$ \\
\hline Bi-210 & $014331-79-4$ & $6.1 \mathrm{E}+01$ & $6.1 \mathrm{E}-01$ & $1.5 \mathrm{E}+04$ & $1.5 \mathrm{E}+02$ & $2.0 \mathrm{E}+03$ & $2.0 \mathrm{E}+01$ \\
\hline Bi-211 & $015229-37-5$ & $2.4 \mathrm{E}+04$ & $2.4 \mathrm{E}+02$ & $6.1 \mathrm{E}+06$ & $6.1 \mathrm{E}+04$ & $8.1 \mathrm{E}+05$ & $8.1 \mathrm{E}+03$ \\
\hline Bi-212 & $014913-49-6$ & $7.1 \mathrm{E}+02$ & $7.1 \mathrm{E}+00$ & $1.8 \mathrm{E}+05$ & $1.8 \mathrm{E}+03$ & $2.4 \mathrm{E}+04$ & $2.4 \mathrm{E}+02$ \\
\hline Bi-213 & $015776-20-2$ & $1.0 \mathrm{E}+03$ & $1.0 \mathrm{E}+01$ & $2.5 \mathrm{E}+05$ & $2.5 \mathrm{E}+03$ & $3.3 \mathrm{E}+04$ & $3.3 \mathrm{E}+02$ \\
\hline
\end{tabular}


Table 3.3d. (continued)

\begin{tabular}{|c|c|c|c|c|c|c|c|}
\hline \multirow[t]{2}{*}{ Chemical $^{\dagger}$} & \multirow[t]{2}{*}{ CASRN } & \multicolumn{2}{|c|}{$\begin{array}{c}\text { Ingestion } \\
\text { of Vegetables (pCi/g) }\end{array}$} & \multicolumn{2}{|c|}{$\begin{array}{c}\text { Ingestion } \\
\text { of Beef (pCi/g) }\end{array}$} & \multicolumn{2}{|c|}{$\begin{array}{c}\text { Ingestion } \\
\text { of Milk (pCi/g) }\end{array}$} \\
\hline & & $10^{-4}$ & $10^{-5}$ & $10^{-4}$ & $10^{-6}$ & $10^{-4}$ & $10^{-6}$ \\
\hline Bi-214 & 014733-03-0 & $2.3 E+03$ & $2.3 \mathrm{E}+01$ & $5.7 E+05$ & $5.7 E+03$ & $7.5 E+04$ & $7.5 \mathrm{E}+02$ \\
\hline $\mathrm{Br}-82$ & $014686-69-2$ & $1.3 \mathrm{E}+02$ & $1.3 \mathrm{E}+00$ & $2.9 \mathrm{E}+02$ & $2.9 \mathrm{E}+00$ & $5.4 \mathrm{E}+01$ & $5.4 \mathrm{E}-01$ \\
\hline$C-11$ & 014333-33-6 & & & & & & \\
\hline C-14 & $014762-75-5$ & & & & & & \\
\hline$C-15$ & $015929-23-4$ & & & & & & \\
\hline $\mathrm{Ca}-45$ & 013966-05-7 & $5.2 \mathrm{E}+01$ & $5.2 \mathrm{E}-01$ & $1.2 \mathrm{E}+03$ & $1.2 \mathrm{E}+01$ & $1.2 \mathrm{E}+02$ & $1.2 \mathrm{E}+00$ \\
\hline $\mathrm{Ca}-47$ & 001439-99-2 & $1.6 \mathrm{E}+01$ & $1.6 \mathrm{E}-01$ & $3.8 \mathrm{E}+02$ & $3.8 \mathrm{E}+00$ & 3.7E+01 & $3.7 \mathrm{E}-01$ \\
\hline Cd-109 & $014109-32-1$ & $3.7 \mathrm{E}+01$ & $3.7 \mathrm{E}-01$ & $6.4 \mathrm{E}+03$ & $6.4 \mathrm{E}+01$ & $3.9 \mathrm{E}+02$ & $3.9 \mathrm{E}+00$ \\
\hline $\mathrm{Cd}-115$ & $014336-68-6$ & $4.1 \mathrm{E}+01$ & 4.1E-01 & $7.0 \mathrm{E}+03$ & $7.0 \mathrm{E}+01$ & $4.3 E+02$ & $4.3 \mathrm{E}+00$ \\
\hline $\mathrm{Cd}-115 \mathrm{~m}$ & 014336-68-6 & $2.1 \mathrm{E}+01$ & $2.1 \mathrm{E}-01$ & $3.6 \mathrm{E}+03$ & $3.6 \mathrm{E}+01$ & $2.2 \mathrm{E}+02$ & $2.2 \mathrm{E}+00$ \\
\hline Ce-141 & $013967-74-3$ & $1.2 \mathrm{E}+02$ & $1.2 \mathrm{E}+00$ & $5.5 \mathrm{E}+05$ & $5.5 E+03$ & $6.0 \mathrm{E}+04$ & $6.0 \mathrm{E}+02$ \\
\hline Ce-143 & 014119-19-8 & $7.7 \mathrm{E}+01$ & 7.7E-01 & $3.6 \mathrm{E}+05$ & $3.6 \mathrm{E}+03$ & $4.0 \mathrm{E}+04$ & $4.0 \mathrm{E}+02$ \\
\hline Ce-144 & $014762-78-8$ & $1.5 \mathrm{E}+01$ & $1.5 \mathrm{E}-01$ & $7.3 E+04$ & $7.3 E+02$ & $8.0 \mathrm{E}+03$ & $8.0 \mathrm{E}+01$ \\
\hline $\mathrm{Ce}-144+\mathrm{D}$ & $014762-78-8$ & $1.5 \mathrm{E}+01$ & $1.5 \mathrm{E}-01$ & $2.2 \mathrm{E}+03$ & $2.2 \mathrm{E}+01$ & $3.1 \mathrm{E}+03$ & $3.1 E+01$ \\
\hline $\mathrm{Cl}-36$ & $013981-43-6$ & $3.0 \mathrm{E}+00$ & $3.0 \mathrm{E}-02$ & $6.2 \mathrm{E}+00$ & $6.2 \mathrm{E}-02$ & $1.1 \mathrm{E}+00$ & $1.1 \mathrm{E}-02$ \\
\hline $\mathrm{Cl}-38$ & 014158-34-0 & $3.2 \mathrm{E}+01$ & $3.2 \mathrm{E}-01$ & $6.7 \mathrm{E}+01$ & $6.7 \mathrm{E}-01$ & $1.2 \mathrm{E}+01$ & $1.2 \mathrm{E}-01$ \\
\hline $\mathrm{Cm}-242$ & $015510-73-3$ & $1.2 \mathrm{E}+01$ & $1.2 \mathrm{E}-01$ & $6.3 \mathrm{E}+04$ & $6.3 \mathrm{E}+02$ & $5.2 \mathrm{E}+05$ & $5.2 \mathrm{E}+03$ \\
\hline
\end{tabular}


Table 3.3d. (continued)

\begin{tabular}{|l|l|c|c|c|c|c|c|}
\hline \multirow{2}{*}{ Chemical $^{\dagger}$} & \multirow{2}{*}{ CASRN } & \multicolumn{2}{c|}{$\begin{array}{c}\text { Ingestion } \\
\text { of Vegetables (pCi/g) }\end{array}$} & \multicolumn{2}{c|}{$\begin{array}{c}\text { Ingestion } \\
\text { of Beef (pCi/g) }\end{array}$} & \multicolumn{2}{c|}{$\begin{array}{c}\text { Ingestion } \\
\text { of Milk (pCi/g) }\end{array}$} \\
\cline { 3 - 9 } & & $10^{-4}$ & $10^{-6}$ & $10^{-4}$ & $10^{-6}$ & $10^{-4}$ & $10^{-6}$ \\
\hline Cm-243 & $015757-87-6$ & $1.8 \mathrm{E}+00$ & $1.8 \mathrm{E}-02$ & $9.6 \mathrm{E}+03$ & $9.6 \mathrm{E}+01$ & $8.0 \mathrm{E}+04$ & $8.0 \mathrm{E}+02$ \\
\hline Cm-243+D & $015757-87-6$ & $1.7 \mathrm{E}+00$ & $1.7 \mathrm{E}-02$ & $1.9 \mathrm{E}+00$ & $1.9 \mathrm{E}-02$ & $2.7 \mathrm{E}+00$ & $2.7 \mathrm{E}-02$ \\
\hline Cm-244 & $013981-15-2$ & $2.2 \mathrm{E}+00$ & $2.2 \mathrm{E}-02$ & $1.1 \mathrm{E}+04$ & $1.1 \mathrm{E}+02$ & $9.5 \mathrm{E}+04$ & $9.5 \mathrm{E}+02$ \\
\hline Cm-245 & $015621-76-8$ & $1.4 \mathrm{E}+00$ & $1.4 \mathrm{E}-02$ & $7.2 \mathrm{E}+03$ & $7.2 \mathrm{E}+01$ & $6.0 \mathrm{E}+04$ & $6.0 \mathrm{E}+02$ \\
\hline Cm-246 & $015757-90-1$ & $1.4 \mathrm{E}+00$ & $1.4 \mathrm{E}-02$ & $7.3 \mathrm{E}+03$ & $7.3 \mathrm{E}+01$ & $6.1 \mathrm{E}+04$ & $6.1 \mathrm{E}+02$ \\
\hline Cm-247 & $015758-32-4$ & $1.5 \mathrm{E}+00$ & $1.5 \mathrm{E}-02$ & $7.8 \mathrm{E}+03$ & $7.8 \mathrm{E}+01$ & $6.5 \mathrm{E}+04$ & $6.5 \mathrm{E}+02$ \\
\hline Cm-248 & $015758-33-5$ & $3.5 \mathrm{E}-01$ & $3.5 \mathrm{E}-03$ & $1.8 \mathrm{E}+03$ & $1.8 \mathrm{E}+01$ & $1.5 \mathrm{E}+04$ & $1.5 \mathrm{E}+02$ \\
\hline Co-57 & $013981-50-5$ & $4.3 \mathrm{E}+02$ & $4.3 \mathrm{E}+00$ & $4.4 \mathrm{E}+05$ & $4.4 \mathrm{E}+03$ & $1.0 \mathrm{E}+05$ & $1.0 \mathrm{E}+03$ \\
\hline Co-58 & $013981-38-9$ & $1.5 \mathrm{E}+02$ & $1.5 \mathrm{E}+00$ & $1.5 \mathrm{E}+05$ & $1.5 \mathrm{E}+03$ & $3.5 \mathrm{E}+04$ & $3.5 \mathrm{E}+02$ \\
\hline Co-58m & $013981-38-9$ & $4.4 \mathrm{E}+03$ & $4.4 \mathrm{E}+01$ & $4.5 \mathrm{E}+06$ & $4.5 \mathrm{E}+04$ & $1.1 \mathrm{E}+06$ & $1.1 \mathrm{E}+04$ \\
\hline Co-60 & $010198-40-0$ & $2.2 \mathrm{E}+01$ & $2.2 \mathrm{E}-01$ & $2.3 \mathrm{E}+04$ & $2.3 \mathrm{E}+02$ & $5.3 \mathrm{E}+03$ & $5.3 \mathrm{E}+01$ \\
\hline Cr-51 & $014392-02-0$ & $3.3 \mathrm{E}+03$ & $3.3 \mathrm{E}+01$ & $3.6 \mathrm{E}+04$ & $3.6 \mathrm{E}+02$ & $5.3 \mathrm{E}+06$ & $5.3 \mathrm{E}+04$ \\
\hline Cs-131 & $014914-76-2$ & $2.4 \mathrm{E}+03$ & $2.4 \mathrm{E}+01$ & $4.2 \mathrm{E}+03$ & $4.2 \mathrm{E}+01$ & $4.3 \mathrm{E}+03$ & $4.3 \mathrm{E}+01$ \\
\hline Cs-134 & $013967-70-9$ & $9.2 \mathrm{E}+00$ & $9.2 \mathrm{E}-02$ & $1.6 \mathrm{E}+01$ & $1.6 \mathrm{E}-01$ & $1.6 \mathrm{E}+01$ & $1.6 \mathrm{E}-01$ \\
\hline Cs-134m & $013967-70-9$ & $9.6 \mathrm{E}+03$ & $9.6 \mathrm{E}+01$ & $1.7 \mathrm{E}+04$ & $1.7 \mathrm{E}+02$ & $1.7 \mathrm{E}+04$ & $1.7 \mathrm{E}+02$ \\
\hline Cs-135 & $015726-30-4$ & $9.6 \mathrm{E}+01$ & $9.6 \mathrm{E}-01$ & $1.7 \mathrm{E}+02$ & $1.7 \mathrm{E}+00$ & $1.7 \mathrm{E}+02$ & $1.7 \mathrm{E}+00$ \\
\hline Cs-136 & $014234-29-8$ & $5.6 \mathrm{E}+01$ & $5.6 \mathrm{E}-01$ & $9.8 \mathrm{E}+01$ & $9.8 \mathrm{E}-01$ & $1.0 \mathrm{E}+02$ & $1.0 \mathrm{E}+00$ \\
\hline
\end{tabular}


Table 3.3d. (continued)

\begin{tabular}{|l|l|c|c|c|c|c|c|}
\hline \multirow{2}{*}{ Chemical ${ }^{\dagger}$} & \multirow{2}{*}{ CASRN } & \multicolumn{2}{|c|}{$\begin{array}{c}\text { Ingestion } \\
\text { of Vegetables (pCi/g) }\end{array}$} & \multicolumn{2}{c|}{$\begin{array}{c}\text { Ingestion } \\
\text { of Beef (pCi/g) }\end{array}$} & \multicolumn{2}{c|}{$\begin{array}{c}\text { Ingestion } \\
\text { of Milk (pCi/g) }\end{array}$} \\
\cline { 3 - 8 } & & $10^{-4}$ & $10^{-6}$ & $10^{-1}$ & $10^{-6}$ & $10^{-1}$ & $10^{-6}$ \\
\hline Cs-137 & $010045-97-3$ & $1.4 \mathrm{E}+01$ & $1.4 \mathrm{E}-01$ & $2.4 \mathrm{E}+01$ & $2.4 \mathrm{E}-01$ & $2.5 \mathrm{E}+01$ & $2.5 \mathrm{E}-01$ \\
\hline Cs-137+D & $010045-97-3$ & $1.4 \mathrm{E}+01$ & $1.4 \mathrm{E}-01$ & $2.0 \mathrm{E}+01$ & $2.0 \mathrm{E}-01$ & $1.9 \mathrm{E}+01$ & $1.9 \mathrm{E}-01$ \\
\hline Cs-138 & $015758-29-9$ & $2.5 \mathrm{E}+03$ & $2.5 \mathrm{E}+01$ & $4.3 \mathrm{E}+03$ & $4.3 \mathrm{E}+01$ & $4.4 \mathrm{E}+03$ & $4.4 \mathrm{E}+01$ \\
\hline Cu-64 & $013981-25-4$ & $6.7 \mathrm{E}+02$ & $6.7 \mathrm{E}+00$ & $3.4 \mathrm{E}+03$ & $3.4 \mathrm{E}+01$ & $3.1 \mathrm{E}+03$ & $3.1 \mathrm{E}+01$ \\
\hline Dy-165 & $013967-64-1$ & $1.4 \mathrm{E}+03$ & $1.4 \mathrm{E}+01$ & $2.6 \mathrm{E}+04$ & $2.6 \mathrm{E}+02$ & $1.2 \mathrm{E}+06$ & $1.2 \mathrm{E}+04$ \\
\hline Dy-166 & $015840-01-4$ & $4.8 \mathrm{E}+01$ & $4.8 \mathrm{E}-01$ & $9.1 \mathrm{E}+02$ & $9.1 \mathrm{E}+00$ & $4.2 \mathrm{E}+04$ & $4.2 \mathrm{E}+02$ \\
\hline Er-169 & $015840-13-8$ & $2.1 \mathrm{E}+02$ & $2.1 \mathrm{E}+00$ & $5.6 \mathrm{E}+03$ & $5.6 \mathrm{E}+01$ & $1.8 \mathrm{E}+05$ & $1.8 \mathrm{E}+03$ \\
\hline Er-171 & $014391-45-8$ & $2.8 \mathrm{E}+02$ & $2.8 \mathrm{E}+00$ & $7.3 \mathrm{E}+03$ & $7.3 \mathrm{E}+01$ & $2.4 \mathrm{E}+05$ & $2.4 \mathrm{E}+03$ \\
\hline Eu-152 & $014683-23-9$ & $7.9 \mathrm{E}+01$ & $7.9 \mathrm{E}-01$ & $3.8 \mathrm{E}+03$ & $3.8 \mathrm{E}+01$ & $2.1 \mathrm{E}+04$ & $2.1 \mathrm{E}+02$ \\
\hline Eu-154 & $015585-10-1$ & $4.8 \mathrm{E}+01$ & $4.8 \mathrm{E}-01$ & $2.3 \mathrm{E}+03$ & $2.3 \mathrm{E}+01$ & $1.3 \mathrm{E}+04$ & $1.3 \mathrm{E}+02$ \\
\hline Eu-155 & $014391-16-3$ & $2.8 \mathrm{E}+02$ & $2.8 \mathrm{E}+00$ & $1.3 \mathrm{E}+04$ & $1.3 \mathrm{E}+02$ & $7.1 \mathrm{E}+04$ & $7.1 \mathrm{E}+02$ \\
\hline Eu-156 & $014280-35-4$ & $4.2 \mathrm{E}+01$ & $4.2 \mathrm{E}-01$ & $2.0 \mathrm{E}+03$ & $2.0 \mathrm{E}+01$ & $1.1 \mathrm{E}+04$ & $1.1 \mathrm{E}+02$ \\
\hline F-18 & $013981-56-1$ & $4.0 \mathrm{E}+03$ & $4.0 \mathrm{E}+01$ & $2.6 \mathrm{E}+03$ & $2.6 \mathrm{E}+01$ & $6.3 \mathrm{E}+04$ & $6.3 \mathrm{E}+02$ \\
\hline Fe-55 & $014681-59-5$ & $1.3 \mathrm{E}+03$ & $1.3 \mathrm{E}+01$ & $6.7 \mathrm{E}+03$ & $6.7 \mathrm{E}+01$ & $7.4 \mathrm{E}+05$ & $7.4 \mathrm{E}+03$ \\
\hline Fe-59 & $014596-12-4$ & $7.8 \mathrm{E}+01$ & $7.8 \mathrm{E}-01$ & $4.0 \mathrm{E}+02$ & $4.0 \mathrm{E}+00$ & $4.5 \mathrm{E}+04$ & $4.5 \mathrm{E}+02$ \\
\hline Fr-221 & $015756-41-9$ & $3.1 \mathrm{E}+03$ & $3.1 \mathrm{E}+01$ & $1.2 \mathrm{E}+05$ & $1.2 \mathrm{E}+03$ & $2.6 \mathrm{E}+03$ & $2.6 \mathrm{E}+01$ \\
\hline Fr-223 & $015756-98-6$ & $1.0 \mathrm{E}+03$ & $1.0 \mathrm{E}+01$ & $4.0 \mathrm{E}+04$ & $4.0 \mathrm{E}+02$ & $8.3 \mathrm{E}+02$ & $8.3 \mathrm{E}+00$ \\
\hline
\end{tabular}


Table 3.3d. (continued)

\begin{tabular}{|c|c|c|c|c|c|c|c|}
\hline \multirow[t]{2}{*}{ Chemical $^{\dagger}$} & \multirow[t]{2}{*}{ CASRN } & \multicolumn{2}{|c|}{$\begin{array}{c}\text { Ingestion } \\
\text { of Vegetables (pCi/g) }\end{array}$} & \multicolumn{2}{|c|}{$\begin{array}{c}\text { Ingestion } \\
\text { of Beef (pCi/g) }\end{array}$} & \multicolumn{2}{|c|}{$\begin{array}{l}\text { Ingestion } \\
\text { of Milk (pCi/g) }\end{array}$} \\
\hline & & $10^{-4}$ & $10^{-6}$ & $10^{-4}$ & $10^{-6}$ & $10^{-4}$ & $10^{-6}$ \\
\hline Ga-67 & 014119-09-6 & $5.4 \mathrm{E}+02$ & $5.4 \mathrm{E}+00$ & $1.9 \mathrm{E}+05$ & $1.9 \mathrm{E}+03$ & $9.4 \mathrm{E}+05$ & $9.4 \mathrm{E}+03$ \\
\hline Ga-72 & $013982-22-4$ & $9.5 \mathrm{E}+01$ & $9.5 \mathrm{E}-01$ & $3.3 E+04$ & $3.3 \mathrm{E}+02$ & $1.6 \mathrm{E}+05$ & $1.6 \mathrm{E}+03$ \\
\hline Gd-153 & $014276-65-4$ & $3.4 \mathrm{E}+02$ & $3.4 \mathrm{E}+00$ & $1.6 \mathrm{E}+04$ & $1.6 \mathrm{E}+02$ & $8.9 E+04$ & $8.9 E+02$ \\
\hline Gd-159 & $014041-42-0$ & $1.7 \mathrm{E}+02$ & $1.7 \mathrm{E}+00$ & $8.3 E+03$ & $8.3 E+01$ & $4.5 \mathrm{E}+04$ & $4.5 \mathrm{E}+02$ \\
\hline Ge-71 & $014374-81-3$ & $2.8 \mathrm{E}+04$ & $2.8 \mathrm{E}+02$ & $2.9 \mathrm{E}+03$ & $2.9 \mathrm{E}+01$ & $4.6 \mathrm{E}+03$ & $4.6 \mathrm{E}+01$ \\
\hline $\mathrm{H}-3$ & $010028-17-8$ & & & & & & \\
\hline Hg-197 & $013981-51-6$ & $1.8 \mathrm{E}+02$ & $1.8 \mathrm{E}+00$ & $1.2 \mathrm{E}+03$ & $1.2 \mathrm{E}+01$ & $3.8 \mathrm{E}+03$ & $3.8 \mathrm{E}+01$ \\
\hline $\mathrm{Hg}-203$ & 013982-78-0 & $8.1 E+01$ & 8.1E-01 & $5.3 \mathrm{E}+02$ & $5.3 \mathrm{E}+00$ & $1.7 \mathrm{E}+03$ & $1.7 \mathrm{E}+01$ \\
\hline Ho-166 & $013967-65-2$ & $6.0 \mathrm{E}+01$ & $6.0 \mathrm{E}-01$ & $1.4 \mathrm{E}+03$ & $1.4 \mathrm{E}+01$ & $5.2 \mathrm{E}+04$ & $5.2 \mathrm{E}+02$ \\
\hline $\mathrm{I}-122$ & $018287-75-7$ & $2.1 \mathrm{E}+04$ & $2.1 \mathrm{E}+02$ & $5.6 \mathrm{E}+04$ & $5.6 \mathrm{E}+02$ & $3.7 \mathrm{E}+04$ & $3.7 \mathrm{E}+02$ \\
\hline I-123 & 015715-08-9 & $8.4 \mathrm{E}+02$ & $8.4 \mathrm{E}+00$ & $2.2 \mathrm{E}+03$ & $2.2 \mathrm{E}+01$ & $1.5 \mathrm{E}+03$ & $1.5 \mathrm{E}+01$ \\
\hline $\mathrm{I}-125$ & 014158-31-7 & $1.8 \mathrm{E}+01$ & $1.8 \mathrm{E}-01$ & 4.7E+01 & $4.7 \mathrm{E}-01$ & $3.1 \mathrm{E}+01$ & $3.1 \mathrm{E}-01$ \\
\hline I-126 & $014158-32-8$ & $9.4 \mathrm{E}+00$ & 9.4E-02 & $2.5 \mathrm{E}+01$ & $2.5 \mathrm{E}-01$ & $1.7 \mathrm{E}+01$ & $1.7 \mathrm{E}-01$ \\
\hline I-129 & $015046-84-1$ & $2.5 \mathrm{E}+00$ & $2.5 \mathrm{E}-02$ & $6.5 \mathrm{E}+00$ & $6.5 \mathrm{E}-02$ & $4.3 \mathrm{E}+00$ & 4.3E-02 \\
\hline $\mathrm{I}-130$ & $014914-02-4$ & $9.4 \mathrm{E}+01$ & $9.4 \mathrm{E}-01$ & $2.5 E+02$ & $2.5 \mathrm{E}+00$ & $1.6 \mathrm{E}+02$ & $1.6 \mathrm{E}+00$ \\
\hline I-131 & $010043-66-0$ & $1.3 E+01$ & $1.3 \mathrm{E}-01$ & $3.3 E+01$ & $3.3 \mathrm{E}-01$ & $2.2 \mathrm{E}+01$ & $2.2 \mathrm{E}-01$ \\
\hline I-132 & 014683-16-0 & $6.9 \mathrm{E}+02$ & $6.9 \mathrm{E}+00$ & $1.8 \mathrm{E}+03$ & $1.8 \mathrm{E}+01$ & $1.2 \mathrm{E}+03$ & $1.2 \mathrm{E}+01$ \\
\hline
\end{tabular}


Table 3.3d. (continued)

\begin{tabular}{|c|c|c|c|c|c|c|c|}
\hline \multirow[t]{2}{*}{ Chemical $^{\dagger}$} & \multirow[t]{2}{*}{ CASRN } & \multicolumn{2}{|c|}{$\begin{array}{c}\text { Ingestion } \\
\text { of Vegetables (pCi/g) }\end{array}$} & \multicolumn{2}{|c|}{$\begin{array}{c}\text { Ingestion } \\
\text { of Beef (pCi/g) }\end{array}$} & \multicolumn{2}{|c|}{$\begin{array}{c}\text { Ingestion } \\
\text { of Milk ( } \mathrm{pCi} / \mathrm{g})\end{array}$} \\
\hline & & $10^{-4}$ & $10^{-5}$ & $10^{-4}$ & $10^{-6}$ & $10^{-4}$ & $10^{-6}$ \\
\hline I-133 & $014834-67-4$ & $4.3 \mathrm{E}+01$ & 4.3E-01 & $1.1 \mathrm{E}+02$ & $1.1 \mathrm{E}+00$ & $7.5 \mathrm{E}+01$ & $7.5 \mathrm{E}-01$ \\
\hline I-134 & 014914-27-3 & $2.0 \mathrm{E}+03$ & $2.0 \mathrm{E}+01$ & $5.2 \mathrm{E}+03$ & $5.2 \mathrm{E}+01$ & $3.5 \mathrm{E}+03$ & $3.5 \mathrm{E}+01$ \\
\hline I-135 & $014834-68-5$ & $2.0 \mathrm{E}+02$ & $2.0 \mathrm{E}+00$ & $5.3 \mathrm{E}+02$ & $5.3 \mathrm{E}+00$ & $3.5 \mathrm{E}+02$ & $3.5 \mathrm{E}+00$ \\
\hline In-113m & 014885-78-0 & $5.5 \mathrm{E}+03$ & $5.5 \mathrm{E}+01$ & $1.4 \mathrm{E}+05$ & $1.4 \mathrm{E}+03$ & $4.7 E+05$ & $4.7 \mathrm{E}+03$ \\
\hline In-114 & $013981-55-0$ & $1.0 \mathrm{E}+05$ & $1.0 \mathrm{E}+03$ & $2.6 \mathrm{E}+06$ & $2.6 \mathrm{E}+04$ & $8.7 E+06$ & 8.7E+04 \\
\hline In-114m & $013981-55-0$ & $2.2 \mathrm{E}+01$ & $2.2 \mathrm{E}-01$ & $5.7 \mathrm{E}+02$ & $5.7 \mathrm{E}+00$ & $1.9 \mathrm{E}+03$ & $1.9 \mathrm{E}+01$ \\
\hline In-115 & 014191-71-0 & $1.3 \mathrm{E}+01$ & $1.3 \mathrm{E}-01$ & $3.4 \mathrm{E}+02$ & $3.4 \mathrm{E}+00$ & $1.1 \mathrm{E}+03$ & $1.1 \mathrm{E}+01$ \\
\hline In-115m & $014191-71-0$ & $1.3 \mathrm{E}+03$ & $1.3 \mathrm{E}+01$ & $3.5 \mathrm{E}+04$ & $3.5 \mathrm{E}+02$ & $1.1 \mathrm{E}+05$ & $1.1 \mathrm{E}+03$ \\
\hline Ir-190 & 014981-91-0 & $8.3 \mathrm{E}+01$ & $8.3 \mathrm{E}-01$ & $3.3 E+03$ & $3.3 E+01$ & $5.2 \mathrm{E}+05$ & $5.2 \mathrm{E}+03$ \\
\hline Ir-194 & 014158-35-1 & $5.9 \mathrm{E}+01$ & $5.9 \mathrm{E}-01$ & $2.3 E+03$ & $2.3 \mathrm{E}+01$ & $3.7 \mathrm{E}+05$ & $3.7 \mathrm{E}+03$ \\
\hline $\mathrm{K}-40$ & 013966-00-2 & $1.7 \mathrm{E}+01$ & $1.7 \mathrm{E}-01$ & $2.3 \mathrm{E}+01$ & $2.3 \mathrm{E}-01$ & $9.4 \mathrm{E}+00$ & $9.4 \mathrm{E}-02$ \\
\hline $\mathrm{K}-42$ & $014378-21-3$ & $1.6 \mathrm{E}+02$ & $1.6 \mathrm{E}+00$ & $2.2 \mathrm{E}+02$ & $2.2 \mathrm{E}+00$ & $9.2 \mathrm{E}+01$ & $9.2 \mathrm{E}-01$ \\
\hline $\mathrm{Kr}-83 \mathrm{~m}$ & $013965-98-5$ & & & & & & \\
\hline $\mathrm{Kr}-85$ & $013983-27-2$ & & & & & & \\
\hline $\mathrm{Kr}-85 \mathrm{~m}$ & $013983-27-2$ & & & & & & \\
\hline $\mathrm{Kr}-87$ & $014809-68-8$ & & & & & & \\
\hline $\mathrm{Kr}-88$ & 014995-61-0 & & & & & & \\
\hline
\end{tabular}


Table 3.3d. (continued)

\begin{tabular}{|l|l|l|c|c|c|c|c|}
\hline \multirow{2}{*}{ Chemical $^{\dagger}$} & \multirow{2}{*}{ CASRN } & \multicolumn{2}{c|}{$\begin{array}{c}\text { Ingestion } \\
\text { of Vegetables (pCi/g) }\end{array}$} & \multicolumn{2}{c|}{$\begin{array}{c}\text { Ingestion } \\
\text { of Beef (pCi/g) }\end{array}$} & \multicolumn{2}{c|}{$\begin{array}{c}\text { Ingestion } \\
\text { of Milk (pCi/g) }\end{array}$} \\
\cline { 4 - 8 } & & $10^{-4}$ & $10^{-6}$ & $10^{-4}$ & $10^{-6}$ & $10^{-4}$ & $10^{-6}$ \\
\hline $\mathrm{Kr}-89$ & $016316-03-3$ & & & & & & \\
\hline $\mathrm{Kr}-90$ & $015741-13-6$ & & & & & & \\
\hline $\mathrm{La}-140$ & $013981-28-7$ & $4.8 \mathrm{E}+01$ & $4.8 \mathrm{E}-01$ & $1.7 \mathrm{E}+04$ & $1.7 \mathrm{E}+02$ & $4.2 \mathrm{E}+04$ & $4.2 \mathrm{E}+02$ \\
\hline $\mathrm{Lu}-177$ & $014265-75-9$ & $1.5 \mathrm{E}+02$ & $1.5 \mathrm{E}+00$ & $3.6 \mathrm{E}+03$ & $3.6 \mathrm{E}+01$ & $1.3 \mathrm{E}+05$ & $1.3 \mathrm{E}+03$ \\
\hline $\mathrm{Mn}-52$ & $014092-99-0$ & $6.0 \mathrm{E}+01$ & $6.0 \mathrm{E}-01$ & $6.0 \mathrm{E}+03$ & $6.0 \mathrm{E}+01$ & $1.5 \mathrm{E}+04$ & $1.5 \mathrm{E}+02$ \\
\hline $\mathrm{Mn}-54$ & $013966-31-9$ & $1.8 \mathrm{E}+02$ & $1.8 \mathrm{E}+00$ & $1.8 \mathrm{E}+04$ & $1.8 \mathrm{E}+02$ & $4.7 \mathrm{E}+04$ & $4.7 \mathrm{E}+02$ \\
\hline $\mathrm{Mn}-56$ & $014681-52-8$ & $4.2 \mathrm{E}+02$ & $4.2 \mathrm{E}+00$ & $4.2 \mathrm{E}+04$ & $4.2 \mathrm{E}+02$ & $1.1 \mathrm{E}+05$ & $1.1 \mathrm{E}+03$ \\
\hline $\mathrm{Mo}-99$ & $014119-15-4$ & $1.5 \mathrm{E}+02$ & $1.5 \mathrm{E}+00$ & $1.1 \mathrm{E}+04$ & $1.1 \mathrm{E}+02$ & $9.8 \mathrm{E}+02$ & $9.8 \mathrm{E}+00$ \\
\hline $\mathrm{Na}-22$ & $013966-32-0$ & $5.1 \mathrm{E}+01$ & $5.1 \mathrm{E}-01$ & $5.1 \mathrm{E}+01$ & $5.1 \mathrm{E}-01$ & $4.0 \mathrm{E}+01$ & $4.0 \mathrm{E}-01$ \\
\hline $\mathrm{Na}-24$ & $013982-04-2$ & $3.0 \mathrm{E}+02$ & $3.0 \mathrm{E}+00$ & $2.9 \mathrm{E}+02$ & $2.9 \mathrm{E}+00$ & $2.3 \mathrm{E}+02$ & $2.3 \mathrm{E}+00$ \\
\hline $\mathrm{Nb}-93 \mathrm{~m}$ & $007440-03-1$ & $6.8 \mathrm{E}+02$ & $6.8 \mathrm{E}+00$ & $2.0 \mathrm{E}+08$ & $2.0 \mathrm{E}+06$ & $2.3 \mathrm{E}+07$ & $2.3 \mathrm{E}+05$ \\
\hline $\mathrm{Nb}-94$ & $014681-63-1$ & $6.5 \mathrm{E}+01$ & $6.5 \mathrm{E}-01$ & $1.9 \mathrm{E}+07$ & $1.9 \mathrm{E}+05$ & $2.2 \mathrm{E}+06$ & $2.2 \mathrm{E}+04$ \\
\hline $\mathrm{Nb}-95$ & $013967-76-5$ & $2.0 \mathrm{E}+02$ & $2.0 \mathrm{E}+00$ & $5.8 \mathrm{E}+07$ & $5.8 \mathrm{E}+05$ & $6.8 \mathrm{E}+06$ & $6.8 \mathrm{E}+04$ \\
\hline $\mathrm{Nb}-95 \mathrm{~m}$ & $013967-76-5$ & $1.5 \mathrm{E}+02$ & $1.5 \mathrm{E}+00$ & $4.2 \mathrm{E}+07$ & $4.2 \mathrm{E}+05$ & $5.0 \mathrm{E}+06$ & $5.0 \mathrm{E}+04$ \\
\hline $\mathrm{Nb}-97$ & $018496-04-3$ & $2.6 \mathrm{E}+03$ & $2.6 \mathrm{E}+01$ & $7.4 \mathrm{E}+08$ & $7.4 \mathrm{E}+06$ & $8.8 \mathrm{E}+07$ & $8.8 \mathrm{E}+05$ \\
\hline $\mathrm{Nb}-97 \mathrm{~m}$ & $018496-04-3$ & $1.4 \mathrm{E}+05$ & $1.4 \mathrm{E}+03$ & $4.0 \mathrm{E}+10$ & $4.0 \mathrm{E}+08$ & $4.7 \mathrm{E}+09$ & $4.7 \mathrm{E}+07$ \\
\hline $\mathrm{Nd}-147$ & $014269-74-0$ & $7.7 \mathrm{E}+01$ & $7.7 \mathrm{E}-01$ & $2.6 \mathrm{E}+04$ & $2.6 \mathrm{E}+02$ & $6.5 \mathrm{E}+04$ & $6.5 \mathrm{E}+02$ \\
\hline
\end{tabular}


Table 3.3d. (continued)

\begin{tabular}{|l|l|c|c|c|c|c|c|}
\hline \multirow{2}{*}{ Chemical } & \multirow{2}{*}{ CASRN } & \multicolumn{2}{|c|}{$\begin{array}{c}\text { Ingestion } \\
\text { of Vegetables (pCi/g) }\end{array}$} & \multicolumn{2}{c|}{$\begin{array}{c}\text { Ingestion } \\
\text { of Beef (pCi/g) }\end{array}$} & \multicolumn{2}{c|}{$\begin{array}{c}\text { Ingestion } \\
\text { of Milk (pCi/g) }\end{array}$} \\
\cline { 3 - 9 } & & $10^{-4}$ & $10^{-6}$ & $10^{-4}$ & $10^{-6}$ & $10^{-4}$ & $10^{-6}$ \\
\hline Nd-149 & $015749-81-2$ & $1.0 \mathrm{E}+03$ & $1.0 \mathrm{E}+01$ & $3.4 \mathrm{E}+05$ & $3.4 \mathrm{E}+03$ & $8.4 \mathrm{E}+05$ & $8.4 \mathrm{E}+03$ \\
\hline Ni-59 & $014336-70-0$ & $2.1 \mathrm{E}+03$ & $2.1 \mathrm{E}+01$ & $3.6 \mathrm{E}+04$ & $3.6 \mathrm{E}+02$ & $1.8 \mathrm{E}+03$ & $1.8 \mathrm{E}+01$ \\
\hline Ni-63 & $013981-37-8$ & $7.0 \mathrm{E}+02$ & $7.0 \mathrm{E}+00$ & $1.2 \mathrm{E}+04$ & $1.2 \mathrm{E}+02$ & $6.1 \mathrm{E}+02$ & $6.1 \mathrm{E}+00$ \\
\hline Ni-65 & $014833-49-9$ & $6.8 \mathrm{E}+02$ & $6.8 \mathrm{E}+00$ & $1.2 \mathrm{E}+04$ & $1.2 \mathrm{E}+02$ & $5.9 \mathrm{E}+02$ & $5.9 \mathrm{E}+00$ \\
\hline Np-236 & $015700-36-4$ & $4.9 \mathrm{E}+02$ & $4.9 \mathrm{E}+00$ & $4.4 \mathrm{E}+04$ & $4.4 \mathrm{E}+02$ & $1.4 \mathrm{E}+06$ & $1.4 \mathrm{E}+04$ \\
\hline Np-237 & $013994-20-2$ & $1.5 \mathrm{E}+00$ & $1.5 \mathrm{E}-02$ & $1.4 \mathrm{E}+02$ & $1.4 \mathrm{E}+00$ & $4.6 \mathrm{E}+03$ & $4.6 \mathrm{E}+01$ \\
\hline Np-237+D & $013994-20-2$ & $1.5 \mathrm{E}+00$ & $1.5 \mathrm{E}-02$ & $1.4 \mathrm{E}+02$ & $1.4 \mathrm{E}+00$ & $2.2 \mathrm{E}+03$ & $2.2 \mathrm{E}+01$ \\
\hline Np-238 & $015766-25-3$ & $9.9 \mathrm{E}+01$ & $9.9 \mathrm{E}-01$ & $9.1 \mathrm{E}+03$ & $9.1 \mathrm{E}+01$ & $3.0 \mathrm{E}+05$ & $3.0 \mathrm{E}+03$ \\
\hline Np-239 & $013968-59-7$ & $1.1 \mathrm{E}+02$ & $1.1 \mathrm{E}+00$ & $9.7 \mathrm{E}+03$ & $9.7 \mathrm{E}+01$ & $3.2 \mathrm{E}+05$ & $3.2 \mathrm{E}+03$ \\
\hline Np-240 & $015690-84-3$ & $2.6 \mathrm{E}+03$ & $2.6 \mathrm{E}+01$ & $2.3 \mathrm{E}+05$ & $2.3 \mathrm{E}+03$ & $7.6 \mathrm{E}+06$ & $7.6 \mathrm{E}+04$ \\
\hline Np-240m & $015690-84-3$ & $1.9 \mathrm{E}+04$ & $1.9 \mathrm{E}+02$ & $1.7 \mathrm{E}+06$ & $1.7 \mathrm{E}+04$ & $5.6 \mathrm{E}+07$ & $5.6 \mathrm{E}+05$ \\
\hline Os-185 & $015766-50-4$ & $2.5 \mathrm{E}+02$ & $2.5 \mathrm{E}+00$ & $6.5 \mathrm{E}+01$ & $6.5 \mathrm{E}-01$ & $8.6 \mathrm{E}+02$ & $8.6 \mathrm{E}+00$ \\
\hline Os-191 & $014119-24-5$ & $1.5 \mathrm{E}+02$ & $1.5 \mathrm{E}+00$ & $3.8 \mathrm{E}+01$ & $3.8 \mathrm{E}-01$ & $5.1 \mathrm{E}+02$ & $5.1 \mathrm{E}+00$ \\
\hline Os-191m & $014119-24-5$ & $9.1 \mathrm{E}+02$ & $9.1 \mathrm{E}+00$ & $2.4 \mathrm{E}+02$ & $2.4 \mathrm{E}+00$ & $3.1 \mathrm{E}+03$ & $3.1 \mathrm{E}+01$ \\
\hline Os-193 & $016057-77-5$ & $1.0 \mathrm{E}+02$ & $1.0 \mathrm{E}+00$ & $2.7 \mathrm{E}+01$ & $2.7 \mathrm{E}-01$ & $3.5 \mathrm{E}+02$ & $3.5 \mathrm{E}+00$ \\
\hline P-32 & $014596-37-3$ & $1.5 \mathrm{E}+01$ & $1.5 \mathrm{E}-01$ & $1.9 \mathrm{E}+01$ & $1.9 \mathrm{E}-01$ & $8.7 \mathrm{E}+00$ & $8.7 \mathrm{E}-02$ \\
\hline P-33 & $015749-66-3$ & $1.2 \mathrm{E}+02$ & $1.2 \mathrm{E}+00$ & $1.5 \mathrm{E}+02$ & $1.5 \mathrm{E}+00$ & $6.8 \mathrm{E}+01$ & $6.8 \mathrm{E}-01$ \\
\hline
\end{tabular}


일
4
0
0
0
0
0
0

\begin{tabular}{|c|c|c|c|c|c|c|c|c|c|c|c|c|c|c|c|c|c|c|}
\hline. & $\stackrel{9}{=}$ & 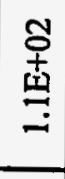 & 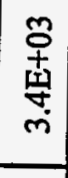 & 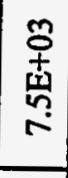 & $\begin{array}{l}\text { o̊ } \\
\text { 孛 } \\
\text { m. }\end{array}$ & 章 & 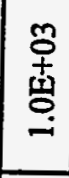 & $\begin{array}{l}\overrightarrow{0} \\
\text { 㟧 } \\
\text { m }\end{array}$ & 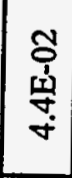 & 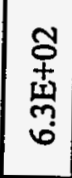 & 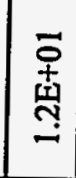 & 客 & 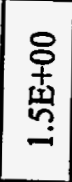 & $\begin{array}{l}\overline{0} \\
+ \\
\text { 㟧 } \\
-\end{array}$ & 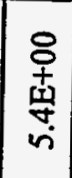 & 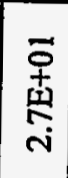 & $\begin{array}{c}8 \\
+ \\
\substack{+1 \\
-1}\end{array}$ & 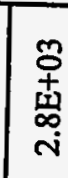 \\
\hline 总空 & $\stackrel{+}{g}$ & 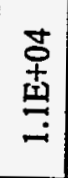 & 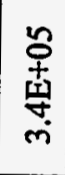 & 号 & 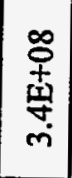 & $\begin{array}{l}\text { t } \\
+ \\
\text { 皿 } \\
\text { i }\end{array}$ & 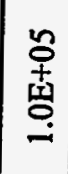 & $\begin{array}{l}\overline{0} \\
\text { 壱 } \\
\text { r }\end{array}$ & $\begin{array}{l}8 \\
\text { ㅁ } \\
\text { 竎 } \\
\forall \\
\forall\end{array}$ & 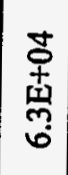 & 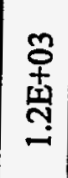 & 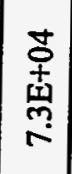 & 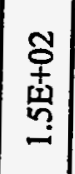 & 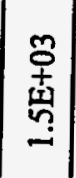 & 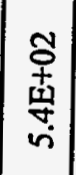 & $\begin{array}{l}\text { 号 } \\
\text { 点 } \\
\text { 今 }\end{array}$ & 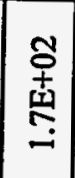 & 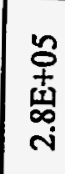 \\
\hline 焉 & $\stackrel{9}{9}$ & 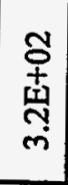 & 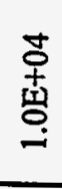 & $\begin{array}{l}\text { J } \\
+ \\
\text { 㟧 } \\
\text { i }\end{array}$ & $\begin{array}{c}5 \\
+ \\
\text { 孛 } \\
-\end{array}$ & 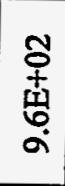 & 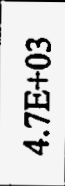 & $\begin{array}{c}8 \\
+ \\
\text { 离 } \\
\end{array}$ & 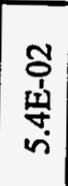 & 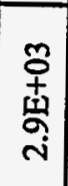 & 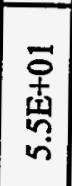 & 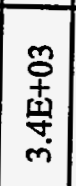 & 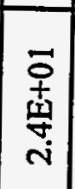 & 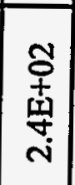 & 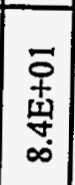 & \begin{tabular}{l}
$\underset{\delta}{+}$ \\
索 \\
\multirow{\forall}{*}{}
\end{tabular} & $\begin{array}{l}\overline{0} \\
\text { 产 } \\
\text { ì }\end{array}$ & 章 \\
\hline 总总 & $\stackrel{\vec{b}}{=}$ & 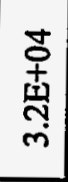 & 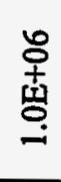 & 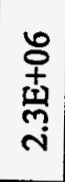 & 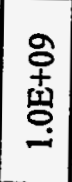 & 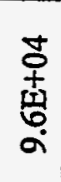 & 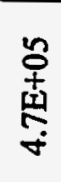 & 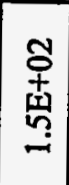 & $\begin{array}{l}8 \\
+ \\
\text { 䒚 } \\
\text { vi }\end{array}$ & $\begin{array}{l}\text { o } \\
\text { 索 } \\
\text { i }\end{array}$ & $\begin{array}{l}\text { mo } \\
\text { 索 } \\
\text { ñ }\end{array}$ & 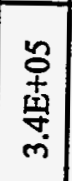 & 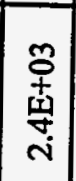 & 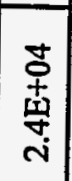 & $\begin{array}{c}m \\
⿱ \\
+ \\
⿱ 亠 䒑 \\
\infty\end{array}$ & 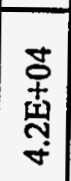 & 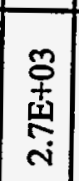 & 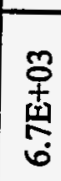 \\
\hline 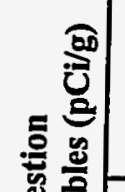 & $\stackrel{\circ}{\varrho}$ & 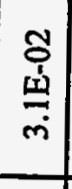 & 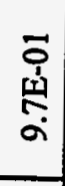 & $\begin{array}{c}8 \\
+ \\
\text { 龺 } \\
\text { i }\end{array}$ & 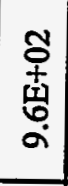 & 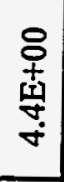 & $\begin{array}{l}\overline{+} \\
\text { ज్ } \\
\text { N }\end{array}$ & 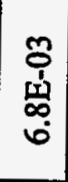 & 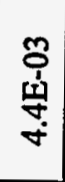 & $\begin{array}{l}\overline{\mathbf{9}} \\
\text { 龺 } \\
-\end{array}$ & 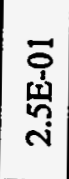 & $\begin{array}{c}\overrightarrow{0} \\
+ \\
\text { 19to } \\
6 \\
-i\end{array}$ & $\begin{array}{l}8 \\
+ \\
\text { 孚 } \\
=\end{array}$ & 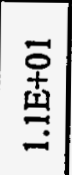 & $\begin{array}{l}8 \\
+ \\
0 \\
\infty \\
\infty \\
\dot{0}\end{array}$ & 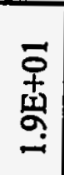 & 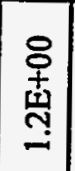 & 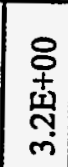 \\
\hline 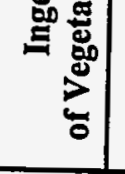 & $\stackrel{1}{0}$ & 辛 & $\begin{array}{c}\overrightarrow{5} \\
+ \\
\frac{1}{2} \\
a\end{array}$ & 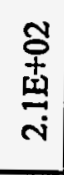 & 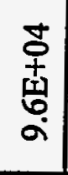 & 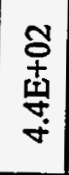 & 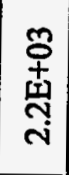 & $\begin{array}{c}\bar{\sigma} \\
\dot{1} \\
0 \\
0 \\
0\end{array}$ & 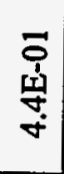 & 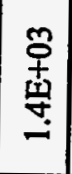 & $\begin{array}{l}\overline{0} \\
+ \\
\text { 站 } \\
\text { in }\end{array}$ & 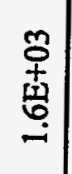 & 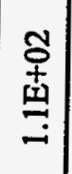 & $\begin{array}{l}\stackrel{9}{+} \\
\text { 豆 } \\
= \\
=\end{array}$ & 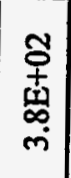 & 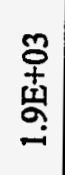 & 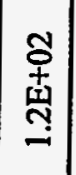 & 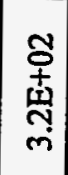 \\
\hline 芯 & & 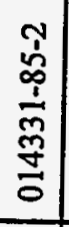 & $\begin{array}{l}\frac{T}{d} \\
\frac{1}{0} \\
\frac{0}{0} \\
\frac{0}{0}\end{array}$ & 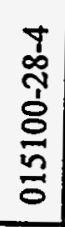 & 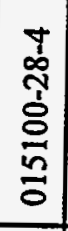 & 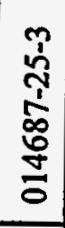 & 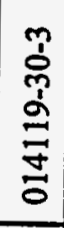 & 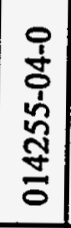 & 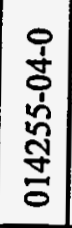 & $\begin{array}{l}\frac{9}{1} \\
\frac{1}{0} \\
0 \\
0 \\
0 \\
0\end{array}$ & 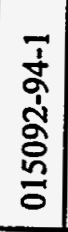 & 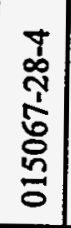 & $\begin{array}{l}7 \\
8 \\
0 \\
0 \\
0 \\
0 \\
0 \\
0\end{array}$ & $\begin{array}{l}\frac{9}{\dot{d}} \\
\frac{\hat{\sigma}}{5} \\
\frac{5}{0}\end{array}$ & 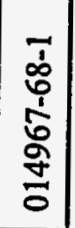 & $\begin{array}{l}\frac{9}{2} \\
\frac{1}{2} \\
\frac{8}{0} \\
\frac{0}{2}\end{array}$ & $\begin{array}{l}\frac{j}{+} \\
\frac{i}{0} \\
\frac{\sigma}{0}\end{array}$ & 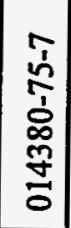 \\
\hline 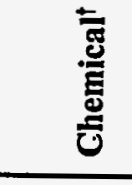 & & 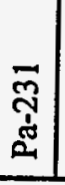 & 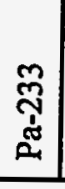 & 壱 & 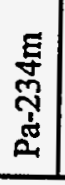 & 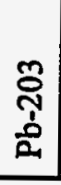 & $\begin{array}{l}\text { के } \\
\text { के }\end{array}$ & $\begin{array}{l}\text { 올 } \\
\text { ฉิ }\end{array}$ & $\begin{array}{l}0 \\
+ \\
0 \\
\\
\hat{2} \\
\text { 2. }\end{array}$ & $\begin{array}{l}\overline{\vec{y}} \\
\dot{b} \\
\dot{0}\end{array}$ & 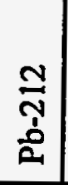 & $\begin{array}{l}\frac{\nabla}{\hat{\gamma}} \\
\text { है }\end{array}$ & $\frac{8}{\frac{8}{2}}$ & $\frac{\overline{0}}{\frac{1}{2}}$ & $\frac{n}{\frac{1}{d}}$ & $\frac{5}{\frac{1}{2}}$ & $\frac{8}{0}$ & $\frac{\tilde{g}}{\dot{g}}$ \\
\hline
\end{tabular}


Table 3.3d. (continued)

\begin{tabular}{|l|l|c|c|c|c|c|c|}
\hline \multirow{2}{*}{ Chemical $^{\dagger}$} & \multirow{2}{*}{ CASRN } & \multicolumn{2}{c|}{$\begin{array}{c}\text { Ingestion } \\
\text { of Vegetables (pCi/g) }\end{array}$} & \multicolumn{2}{c|}{$\begin{array}{c}\text { Ingestion } \\
\text { of Beef (pCi/g) }\end{array}$} & \multicolumn{2}{c|}{$\begin{array}{c}\text { Ingestion } \\
\text { of Milk (pCi/g) }\end{array}$} \\
\cline { 3 - 8 } & & $10^{-4}$ & $10^{-6}$ & $10^{-4}$ & $10^{-6}$ & $10^{-4}$ & $10^{-6}$ \\
\hline Pm-148 & $014683-19-3$ & $3.1 \mathrm{E}+01$ & $3.1 \mathrm{E}-01$ & $6.6 \mathrm{E}+02$ & $6.6 \mathrm{E}+00$ & $2.7 \mathrm{E}+04$ & $2.7 \mathrm{E}+02$ \\
\hline Pm-148m & $014683-19-3$ & $4.6 \mathrm{E}+01$ & $4.6 \mathrm{E}-01$ & $9.5 \mathrm{E}+02$ & $9.5 \mathrm{E}+00$ & $3.9 \mathrm{E}+04$ & $3.9 \mathrm{E}+02$ \\
\hline Pm-149 & $015765-31-8$ & $8.2 \mathrm{E}+01$ & $8.2 \mathrm{E}-01$ & $1.7 \mathrm{E}+03$ & $1.7 \mathrm{E}+01$ & $7.1 \mathrm{E}+04$ & $7.1 \mathrm{E}+02$ \\
\hline Po-210 & $013981-52-7$ & $1.4 \mathrm{E}+00$ & $1.4 \mathrm{E}-02$ & $2.4 \mathrm{E}+01$ & $2.4 \mathrm{E}-01$ & $5.8 \mathrm{E}+01$ & $5.8 \mathrm{E}-01$ \\
\hline Po-212 & $015389-34-1$ & $1.0 \mathrm{E}+13$ & $1.0 \mathrm{E}+11$ & $1.8 \mathrm{E}+14$ & $1.8 \mathrm{E}+12$ & $4.2 \mathrm{E}+14$ & $4.2 \mathrm{E}+12$ \\
\hline Po-213 & $015756-57-7$ & $6.8 \mathrm{E}+11$ & $6.8 \mathrm{E}+09$ & $1.2 \mathrm{E}+13$ & $1.2 \mathrm{E}+11$ & $2.8 \mathrm{E}+13$ & $2.8 \mathrm{E}+11$ \\
\hline Po-214 & $015735-67-8$ & $2.2 \mathrm{E}+10$ & $2.2 \mathrm{E}+08$ & $3.7 \mathrm{E}+11$ & $3.7 \mathrm{E}+09$ & $8.9 \mathrm{E}+11$ & $8.9 \mathrm{E}+09$ \\
\hline Po-215 & $015706-52-2$ & $9.2 \mathrm{E}+08$ & $9.2 \mathrm{E}+06$ & $1.6 \mathrm{E}+10$ & $1.6 \mathrm{E}+08$ & $3.8 \mathrm{E}+10$ & $3.8 \mathrm{E}+08$ \\
\hline Po-216 & $015756-58-8$ & $5.2 \mathrm{E}+06$ & $5.2 \mathrm{E}+04$ & $9.0 \mathrm{E}+07$ & $9.0 \mathrm{E}+05$ & $2.2 \mathrm{E}+08$ & $2.2 \mathrm{E}+06$ \\
\hline Po-218 & $015422-24-9$ & $9.0 \mathrm{E}+03$ & $9.0 \mathrm{E}+01$ & $1.6 \mathrm{E}+05$ & $1.6 \mathrm{E}+03$ & $3.7 \mathrm{E}+05$ & $3.7 \mathrm{E}+03$ \\
\hline Pr-142 & $014191-64-1$ & $6.5 \mathrm{E}+01$ & $6.5 \mathrm{E}-01$ & $2.2 \mathrm{E}+04$ & $2.2 \mathrm{E}+02$ & $5.5 \mathrm{E}+04$ & $5.5 \mathrm{E}+02$ \\
\hline Pr-143 & $014981-79-4$ & $6.9 \mathrm{E}+01$ & $6.9 \mathrm{E}-01$ & $2.3 \mathrm{E}+04$ & $2.3 \mathrm{E}+02$ & $5.8 \mathrm{E}+04$ & $5.8 \mathrm{E}+02$ \\
\hline Pr-144 & $014119-05-2$ & $5.6 \mathrm{E}+03$ & $5.6 \mathrm{E}+01$ & $1.9 \mathrm{E}+06$ & $1.9 \mathrm{E}+04$ & $4.7 \mathrm{E}+06$ & $4.7 \mathrm{E}+04$ \\
\hline Pr-144m & $014119-05-2$ & $1.4 \mathrm{E}+04$ & $1.4 \mathrm{E}+02$ & $4.8 \mathrm{E}+06$ & $4.8 \mathrm{E}+04$ & $1.2 \mathrm{E}+07$ & $1.2 \mathrm{E}+05$ \\
\hline Pt-191 & $015706-36-2$ & $2.8 \mathrm{E}+02$ & $2.8 \mathrm{E}+00$ & $6.5 \mathrm{E}+03$ & $6.5 \mathrm{E}+01$ & $8.5 \mathrm{E}+02$ & $8.5 \mathrm{E}+00$ \\
\hline Pt-193 & $015735-70-3$ & $2.6 \mathrm{E}+03$ & $2.6 \mathrm{E}+01$ & $6.1 \mathrm{E}+04$ & $6.1 \mathrm{E}+02$ & $7.8 \mathrm{E}+03$ & $7.8 \mathrm{E}+01$ \\
\hline Pt-193m & $015735-70-3$ & $1.7 \mathrm{E}+02$ & $1.7 \mathrm{E}+00$ & $3.9 \mathrm{E}+03$ & $3.9 \mathrm{E}+01$ & $5.1 \mathrm{E}+02$ & $5.1 \mathrm{E}+00$ \\
\hline
\end{tabular}


Table 3.3d. (continued)

\begin{tabular}{|l|l|c|c|c|c|c|c|}
\hline \multirow{2}{*}{ Chemical $^{\dagger}$} & \multirow{2}{*}{ CASRN } & \multicolumn{2}{c|}{$\begin{array}{c}\text { Ingestion } \\
\text { of Vegetables (pCi/g) }\end{array}$} & \multicolumn{2}{c|}{$\begin{array}{c}\text { Ingestion } \\
\text { of Beef (pCi/g) }\end{array}$} & \multicolumn{2}{c|}{$\begin{array}{c}\text { Ingestion } \\
\text { of Milk (pCi/g) }\end{array}$} \\
\cline { 3 - 8 } & & $10^{-4}$ & $10^{-6}$ & $10^{-4}$ & $10^{-6}$ & $10^{-4}$ & $10^{-6}$ \\
\hline Pt-197 & $015735-74-7$ & $2.0 \mathrm{E}+02$ & $2.0 \mathrm{E}+00$ & $4.6 \mathrm{E}+03$ & $4.6 \mathrm{E}+01$ & $6.0 \mathrm{E}+02$ & $6.0 \mathrm{E}+00$ \\
\hline Pt-197m & $015735-74-7$ & $1.3 \mathrm{E}+03$ & $1.3 \mathrm{E}+01$ & $3.0 \mathrm{E}+04$ & $3.0 \mathrm{E}+02$ & $3.9 \mathrm{E}+03$ & $3.9 \mathrm{E}+01$ \\
\hline Pu-236 & $015411-92-4$ & $6.0 \mathrm{E}+00$ & $6.0 \mathrm{E}-02$ & $6.3 \mathrm{E}+04$ & $6.3 \mathrm{E}+02$ & $9.5 \mathrm{E}+04$ & $9.5 \mathrm{E}+02$ \\
\hline Pu-238 & $013981-16-3$ & $1.6 \mathrm{E}+00$ & $1.6 \mathrm{E}-02$ & $1.6 \mathrm{E}+04$ & $1.6 \mathrm{E}+02$ & $2.5 \mathrm{E}+04$ & $2.5 \mathrm{E}+02$ \\
\hline Pu-239 & $015117-48-3$ & $1.4 \mathrm{E}+00$ & $1.4 \mathrm{E}-02$ & $1.5 \mathrm{E}+04$ & $1.5 \mathrm{E}+02$ & $2.3 \mathrm{E}+04$ & $2.3 \mathrm{E}+02$ \\
\hline Pu-240 & $014119-33-6$ & $1.5 \mathrm{E}+00$ & $1.5 \mathrm{E}-02$ & $1.5 \mathrm{E}+04$ & $1.5 \mathrm{E}+02$ & $2.3 \mathrm{E}+04$ & $2.3 \mathrm{E}+02$ \\
\hline Pu-241 & $014119-32-5$ & $8.8 \mathrm{E}+01$ & $8.8 \mathrm{E}-01$ & $9.3 \mathrm{E}+05$ & $9.3 \mathrm{E}+03$ & $1.4 \mathrm{E}+06$ & $1.4 \mathrm{E}+04$ \\
\hline Pu-241+D & $014119-32-5$ & $1.4 \mathrm{E}+00$ & $1.4 \mathrm{E}-02$ & $3.9 \mathrm{E}+02$ & $3.9 \mathrm{E}+00$ & $5.6 \mathrm{E}+01$ & $5.6 \mathrm{E}-01$ \\
\hline Pu-242 & $013982-10-0$ & $1.5 \mathrm{E}+00$ & $1.5 \mathrm{E}-02$ & $1.6 \mathrm{E}+04$ & $1.6 \mathrm{E}+02$ & $2.4 \mathrm{E}+04$ & $2.4 \mathrm{E}+02$ \\
\hline Pu-243 & $015706-37-3$ & $1.2 \mathrm{E}+03$ & $1.2 \mathrm{E}+01$ & $1.3 \mathrm{E}+07$ & $1.3 \mathrm{E}+05$ & $2.0 \mathrm{E}+07$ & $2.0 \mathrm{E}+05$ \\
\hline Pu-244 & $014119-34-7$ & $1.5 \mathrm{E}+00$ & $1.5 \mathrm{E}-02$ & $1.5 \mathrm{E}+04$ & $1.5 \mathrm{E}+02$ & $2.3 \mathrm{E}+04$ & $2.3 \mathrm{E}+02$ \\
\hline Pu-244+D & $014119-34-7$ & $1.4 \mathrm{E}+00$ & $1.4 \mathrm{E}-02$ & $9.4 \mathrm{E}+01$ & $9.4 \mathrm{E}-01$ & $4.9 \mathrm{E}+01$ & $4.9 \mathrm{E}-01$ \\
\hline Ra-223 & $015623-45-7$ & $1.9 \mathrm{E}+00$ & $1.9 \mathrm{E}-02$ & $1.9 \mathrm{E}+02$ & $1.9 \mathrm{E}+00$ & $2.2 \mathrm{E}+01$ & $2.2 \mathrm{E}-01$ \\
\hline Ra-224 & $013233-32-4$ & $3.0 \mathrm{E}+00$ & $3.0 \mathrm{E}-02$ & $3.0 \mathrm{E}+02$ & $3.0 \mathrm{E}+00$ & $3.4 \mathrm{E}+01$ & $3.4 \mathrm{E}-01$ \\
\hline Ra-225 & $013981-53-8$ & $2.8 \mathrm{E}+00$ & $2.8 \mathrm{E}-02$ & $2.9 \mathrm{E}+02$ & $2.9 \mathrm{E}+00$ & $3.2 \mathrm{E}+01$ & $3.2 \mathrm{E}-01$ \\
\hline Ra-226 & $013982-63-3$ & $1.5 \mathrm{E}+00$ & $1.5 \mathrm{E}-02$ & $1.5 \mathrm{E}+02$ & $1.5 \mathrm{E}+00$ & $1.7 \mathrm{E}+01$ & $1.7 \mathrm{E}-01$ \\
\hline Ra-226+D & $013982-63-3$ & $1.4 \mathrm{E}+00$ & $1.4 \mathrm{E}-02$ & $7.2 \mathrm{E}+00$ & $7.2 \mathrm{E}-02$ & $4.7 \mathrm{E}+00$ & $4.7 \mathrm{E}-02$ \\
\hline
\end{tabular}


Table 3.3d. (continued)

\begin{tabular}{|c|c|c|c|c|c|c|c|}
\hline \multirow[t]{2}{*}{ Chemical ${ }^{\dagger}$} & \multirow[t]{2}{*}{ CASRN } & \multicolumn{2}{|c|}{$\begin{array}{c}\text { Ingestion } \\
\text { of Vegetables (pCi/g) }\end{array}$} & \multicolumn{2}{|c|}{$\begin{array}{c}\text { Ingestion } \\
\text { of Beef }(\mathrm{pCi} / \mathrm{g})\end{array}$} & \multicolumn{2}{|c|}{$\begin{array}{c}\text { Ingestion } \\
\text { of Milk ( } \mathrm{pCi} / \mathrm{g})\end{array}$} \\
\hline & & $10^{-4}$ & $10^{-6}$ & $10^{-1}$ & $10^{-6}$ & $10^{-4}$ & $10^{-6}$ \\
\hline $\mathrm{Ra}-228$ & $015262-20-1$ & $1.8 \mathrm{E}+00$ & $1.8 \mathrm{E}-02$ & $1.8 \mathrm{E}+02$ & $1.8 \mathrm{E}+00$ & $2.1 \mathrm{E}+01$ & 2.1E-01 \\
\hline $\mathrm{Ra}-228+\mathrm{D}$ & $015262-20-1$ & $1.8 \mathrm{E}+00$ & $1.8 \mathrm{E}-02$ & $1.8 \mathrm{E}+02$ & $1.8 \mathrm{E}+00$ & $2.0 \mathrm{E}+01$ & $2.0 \mathrm{E}-01$ \\
\hline $\mathrm{Rb}-82$ & 014391-63-0 & $3.2 E+04$ & $3.2 \mathrm{E}+02$ & $1.4 \mathrm{E}+05$ & $1.4 \mathrm{E}+03$ & $1.8 \mathrm{E}+04$ & $1.8 \mathrm{E}+02$ \\
\hline $\mathrm{Rb}-86$ & $014932-53-7$ & $4.8 \mathrm{E}+01$ & $4.8 \mathrm{E}-01$ & $2.1 \mathrm{E}+02$ & $2.1 \mathrm{E}+00$ & $2.7 \mathrm{E}+01$ & 2.7E-01 \\
\hline $\mathrm{Rb}-87$ & 013982-13-3 & $9.2 \mathrm{E}+01$ & $9.2 \mathrm{E}-01$ & $4.1 \mathrm{E}+02$ & $4.1 \mathrm{E}+00$ & $5.2 \mathrm{E}+01$ & $5.2 \mathrm{E}-01$ \\
\hline $\mathrm{Rb}-88$ & $014928-36-0$ & $2.3 \mathrm{E}+03$ & $2.3 E+01$ & $1.0 \mathrm{E}+04$ & $1.0 \mathrm{E}+02$ & $1.3 \mathrm{E}+03$ & $1.3 \mathrm{E}+01$ \\
\hline Rb-89 & 014191-65-2 & $3.9 \mathrm{E}+03$ & $3.9 \mathrm{E}+01$ & $1.7 \mathrm{E}+04$ & $1.7 \mathrm{E}+02$ & $2.2 \mathrm{E}+03$ & $2.2 \mathrm{E}+01$ \\
\hline $\mathrm{Rh}-103 \mathrm{~m}$ & $007440-16-6$ & $4.2 \mathrm{E}+04$ & $4.2 \mathrm{E}+02$ & $9.2 \mathrm{E}+05$ & $9.2 \mathrm{E}+03$ & $2.8 \mathrm{E}+04$ & $2.8 \mathrm{E}+02$ \\
\hline Rh-105 & 014913-89-4 & $1.8 \mathrm{E}+02$ & $1.8 \mathrm{E}+00$ & $3.9 \mathrm{E}+03$ & $3.9 \mathrm{E}+01$ & $1.2 \mathrm{E}+02$ & $1.2 \mathrm{E}+00$ \\
\hline $\mathrm{Rh}-105 \mathrm{~m}$ & 014913-89-4 & $3.1 E+05$ & $3.1 \mathrm{E}+03$ & $7.0 \mathrm{E}+06$ & $7.0 \mathrm{E}+04$ & $2.1 \mathrm{E}+05$ & $2.1 E+03$ \\
\hline Rh-106 & $014234-34-5$ & $9.4 \mathrm{E}+04$ & $9.4 \mathrm{E}+02$ & $2.1 \mathrm{E}+06$ & $2.1 E+04$ & $6.3 E+04$ & $6.3 \mathrm{E}+02$ \\
\hline$R n-219$ & $014835-02-0$ & & & & & & \\
\hline $\mathrm{Rn}-220$ & $022481-48-7$ & & & & & & \\
\hline $\mathrm{Rn}-222+\mathrm{D}$ & 014859-67-7 & & & & & & \\
\hline $\mathrm{Ru}-103$ & 013968-53-1 & $1.3 \mathrm{E}+02$ & $1.3 \mathrm{E}+00$ & $1.9 \mathrm{E}+02$ & $1.9 \mathrm{E}+00$ & $4.7 \mathrm{E}+05$ & $4.7 \mathrm{E}+03$ \\
\hline $\mathrm{Ru}-105$ & $014331-95-4$ & $3.6 \mathrm{E}+02$ & $3.6 \mathrm{E}+00$ & $5.5 \mathrm{E}+02$ & $5.5 \mathrm{E}+00$ & $1.4 \mathrm{E}+06$ & $1.4 \mathrm{E}+04$ \\
\hline Ru-106 & 013967-48-1 & $1.2 \mathrm{E}+01$ & $1.2 \mathrm{E}-01$ & $1.8 \mathrm{E}+01$ & $1.8 \mathrm{E}-01$ & $4.5 \mathrm{E}+04$ & $4.5 \mathrm{E}+02$ \\
\hline
\end{tabular}


Table 3.3d. (continued)

\begin{tabular}{|l|l|c|c|c|c|c|c|}
\hline \multirow{2}{*}{ Chemical $^{\dagger}$} & \multirow{2}{*}{ CASRN } & \multicolumn{2}{c}{$\begin{array}{c}\text { Ingestion } \\
\text { of Vegetables (pCi/g) }\end{array}$} & \multicolumn{2}{c|}{$\begin{array}{c}\text { Ingestion } \\
\text { of Beef (pCi/g) }\end{array}$} & \multicolumn{2}{c|}{$\begin{array}{c}\text { Ingestion } \\
\text { of Milk (pCi/g) }\end{array}$} \\
\cline { 3 - 8 } & & $10^{-4}$ & $10^{-6}$ & $10^{-4}$ & $10^{-6}$ & $10^{-4}$ & $10^{-5}$ \\
\hline Ru-106+D & $013967-48-1$ & $9.2 \mathrm{E}+00$ & $9.2 \mathrm{E}-02$ & $7.1 \mathrm{E}+00$ & $7.1 \mathrm{E}-02$ & $5.7 \mathrm{E}+00$ & $5.7 \mathrm{E}-02$ \\
\hline Ru-97 & $015758-35-7$ & $7.1 \mathrm{E}+02$ & $7.1 \mathrm{E}+00$ & $1.1 \mathrm{E}+03$ & $1.1 \mathrm{E}+01$ & $2.7 \mathrm{E}+06$ & $2.7 \mathrm{E}+04$ \\
\hline S-35 & $015117-53-0$ & $3.3 \mathrm{E}+02$ & $3.3 \mathrm{E}+00$ & $9.8 \mathrm{E}+01$ & $9.8 \mathrm{E}-01$ & $1.8 \mathrm{E}+02$ & $1.8 \mathrm{E}+00$ \\
\hline Sb-122 & $014374-79-9$ & $5.0 \mathrm{E}+01$ & $5.0 \mathrm{E}-01$ & $1.2 \mathrm{E}+05$ & $1.2 \mathrm{E}+03$ & $3.2 \mathrm{E}+04$ & $3.2 \mathrm{E}+02$ \\
\hline Sb-124 & $014683-10-4$ & $4.1 \mathrm{E}+01$ & $4.1 \mathrm{E}-01$ & $1.0 \mathrm{E}+05$ & $1.0 \mathrm{E}+03$ & $2.6 \mathrm{E}+04$ & $2.6 \mathrm{E}+02$ \\
\hline Sb-125 & $014234-35-6$ & $1.5 \mathrm{E}+02$ & $1.5 \mathrm{E}+00$ & $3.6 \mathrm{E}+05$ & $3.6 \mathrm{E}+03$ & $9.5 \mathrm{E}+04$ & $9.5 \mathrm{E}+02$ \\
\hline Sb-125+D & $014234-35-6$ & $3.5 \mathrm{E}+01$ & $3.5 \mathrm{E}-01$ & $1.1 \mathrm{E}+02$ & $1.1 \mathrm{E}+00$ & $2.3 \mathrm{E}+02$ & $2.3 \mathrm{E}+00$ \\
\hline Sb-126 & $015756-32-8$ & $4.5 \mathrm{E}+01$ & $4.5 \mathrm{E}-01$ & $1.1 \mathrm{E}+05$ & $1.1 \mathrm{E}+03$ & $2.9 \mathrm{E}+04$ & $2.9 \mathrm{E}+02$ \\
\hline Sb-126m & $015756-32-8$ & $6.1 \mathrm{E}+03$ & $6.1 \mathrm{E}+01$ & $1.5 \mathrm{E}+07$ & $1.5 \mathrm{E}+05$ & $3.9 \mathrm{E}+06$ & $3.9 \mathrm{E}+04$ \\
\hline Sb-127 & $013968-50-8$ & $5.2 \mathrm{E}+01$ & $5.2 \mathrm{E}-01$ & $1.3 \mathrm{E}+05$ & $1.3 \mathrm{E}+03$ & $3.3 \mathrm{E}+04$ & $3.3 \mathrm{E}+02$ \\
\hline Sb-129 & $014331-88-5$ & $2.4 \mathrm{E}+02$ & $2.4 \mathrm{E}+00$ & $5.8 \mathrm{E}+05$ & $5.8 \mathrm{E}+03$ & $1.5 \mathrm{E}+05$ & $1.5 \mathrm{E}+03$ \\
\hline Sc-46 & $013967-63-0$ & $7.9 \mathrm{E}+01$ & $7.9 \mathrm{E}-01$ & $4.1 \mathrm{E}+03$ & $4.1 \mathrm{E}+01$ & $2.3 \mathrm{E}+04$ & $2.3 \mathrm{E}+02$ \\
\hline Sc-47 & $014391-96-9$ & $1.5 \mathrm{E}+02$ & $1.5 \mathrm{E}+00$ & $8.0 \mathrm{E}+03$ & $8.0 \mathrm{E}+01$ & $4.4 \mathrm{E}+04$ & $4.4 \mathrm{E}+02$ \\
\hline Sc-48 & $014391-86-7$ & $6.8 \mathrm{E}+01$ & $6.8 \mathrm{E}-01$ & $3.6 \mathrm{E}+03$ & $3.6 \mathrm{E}+01$ & $2.0 \mathrm{E}+04$ & $2.0 \mathrm{E}+02$ \\
\hline Se-75 & $014265-71-5$ & $5.1 \mathrm{E}+01$ & $5.1 \mathrm{E}-01$ & $3.3 \mathrm{E}+01$ & $3.3 \mathrm{E}-01$ & $5.1 \mathrm{E}+01$ & $5.1 \mathrm{E}-01$ \\
\hline Si-31 & $014276-49-4$ & $6.8 \mathrm{E}+02$ & $6.8 \mathrm{E}+00$ & $1.3 \mathrm{E}+06$ & $1.3 \mathrm{E}+04$ & $4.0 \mathrm{E}+05$ & $4.0 \mathrm{E}+03$ \\
\hline Sm-147 & $014392-33-7$ & $1.8 \mathrm{E}+01$ & $1.8 \mathrm{E}-01$ & $3.8 \mathrm{E}+02$ & $3.8 \mathrm{E}+00$ & $1.6 \mathrm{E}+04$ & $1.6 \mathrm{E}+02$ \\
\hline
\end{tabular}


Table 3.3d. (continued)

\begin{tabular}{|l|l|c|c|c|c|c|c|}
\hline \multirow{2}{*}{ Chemical $^{\dagger}$} & \multirow{2}{*}{ CASRN } & \multicolumn{2}{c|}{$\begin{array}{c}\text { Ingestion } \\
\text { of Vegetables (pCi/g) }\end{array}$} & \multicolumn{2}{c|}{$\begin{array}{c}\text { Ingestion } \\
\text { of Beef (pCi/g) }\end{array}$} & \multicolumn{2}{c|}{$\begin{array}{c}\text { Ingestion } \\
\text { of Milk (pCi/g) }\end{array}$} \\
\cline { 4 - 8 } & & $10^{-4}$ & $10^{-6}$ & $10^{-4}$ & $10^{-6}$ & $10^{-4}$ & $10^{-6}$ \\
\hline Sm-151 & $015715-94-3$ & $9.9 \mathrm{E}+02$ & $9.9 \mathrm{E}+00$ & $2.1 \mathrm{E}+04$ & $2.1 \mathrm{E}+02$ & $8.5 \mathrm{E}+05$ & $8.5 \mathrm{E}+03$ \\
\hline Sm-153 & $015766-00-4$ & $1.1 \mathrm{E}+02$ & $1.1 \mathrm{E}+00$ & $2.4 \mathrm{E}+03$ & $2.4 \mathrm{E}+01$ & $9.8 \mathrm{E}+04$ & $9.8 \mathrm{E}+02$ \\
\hline Sn-113 & $013966-06-8$ & $5.7 \mathrm{E}+01$ & $5.7 \mathrm{E}-01$ & $3.8 \mathrm{E}+02$ & $3.8 \mathrm{E}+00$ & $5.7 \mathrm{E}+02$ & $5.7 \mathrm{E}+00$ \\
\hline Sn-121 & $014683-06-8$ & $1.7 \mathrm{E}+02$ & $1.7 \mathrm{E}+00$ & $1.1 \mathrm{E}+03$ & $1.1 \mathrm{E}+01$ & $1.7 \mathrm{E}+03$ & $1.7 \mathrm{E}+01$ \\
\hline Sn-121m & $014683-06-8$ & $1.1 \mathrm{E}+02$ & $1.1 \mathrm{E}+00$ & $7.0 \mathrm{E}+02$ & $7.0 \mathrm{E}+00$ & $1.1 \mathrm{E}+03$ & $1.1 \mathrm{E}+01$ \\
\hline Sn-125 & $014683-08-0$ & $1.3 \mathrm{E}+01$ & $1.3 \mathrm{E}-01$ & $8.3 \mathrm{E}+01$ & $8.3 \mathrm{E}-01$ & $1.3 \mathrm{E}+02$ & $1.3 \mathrm{E}+00$ \\
\hline Sn-126 & $015832-50-5$ & $1.0 \mathrm{E}+01$ & $1.0 \mathrm{E}-01$ & $6.6 \mathrm{E}+01$ & $6.6 \mathrm{E}-01$ & $9.9 \mathrm{E}+01$ & $9.9 \mathrm{E}-01$ \\
\hline Sr-82 & $014809-50-8$ & $9.9 \mathrm{E}+00$ & $9.9 \mathrm{E}-02$ & $6.3 \mathrm{E}+01$ & $6.3 \mathrm{E}-01$ & $2.7 \mathrm{E}+01$ & $2.7 \mathrm{E}-01$ \\
\hline Sr-85 & $013967-73-2$ & $1.8 \mathrm{E}+02$ & $1.8 \mathrm{E}+00$ & $1.2 \mathrm{E}+03$ & $1.2 \mathrm{E}+01$ & $5.0 \mathrm{E}+02$ & $5.0 \mathrm{E}+00$ \\
\hline Sr-85m & $013967-73-2$ & $1.4 \mathrm{E}+04$ & $1.4 \mathrm{E}+02$ & $9.0 \mathrm{E}+04$ & $9.0 \mathrm{E}+02$ & $3.9 \mathrm{E}+04$ & $3.9 \mathrm{E}+02$ \\
\hline Sr-89 & $014158-27-1$ & $2.5 \mathrm{E}+01$ & $2.5 \mathrm{E}-01$ & $1.6 \mathrm{E}+02$ & $1.6 \mathrm{E}+00$ & $6.8 \mathrm{E}+01$ & $6.8 \mathrm{E}-01$ \\
\hline Sr-90 & $010098-97-2$ & $6.3 \mathrm{E}+00$ & $6.3 \mathrm{E}-02$ & $4.0 \mathrm{E}+01$ & $4.0 \mathrm{E}-01$ & $1.7 \mathrm{E}+01$ & $1.7 \mathrm{E}-01$ \\
\hline Sr-90+D & $010098-97-2$ & $4.6 \mathrm{E}+00$ & $4.6 \mathrm{E}-02$ & $2.6 \mathrm{E}+01$ & $2.6 \mathrm{E}-01$ & $1.2 \mathrm{E}+01$ & $1.2 \mathrm{E}-01$ \\
\hline Sr-91 & $014331-91-0$ & $9.1 \mathrm{E}+01$ & $9.1 \mathrm{E}-01$ & $5.8 \mathrm{E}+02$ & $5.8 \mathrm{E}+00$ & $2.5 \mathrm{E}+02$ & $2.5 \mathrm{E}+00$ \\
\hline Sr-92 & $014928-29-1$ & $1.3 \mathrm{E}+02$ & $1.3 \mathrm{E}+00$ & $8.0 \mathrm{E}+02$ & $8.0 \mathrm{E}+00$ & $3.5 \mathrm{E}+02$ & $3.5 \mathrm{E}+00$ \\
\hline Ta-182 & $013982-00-8$ & $6.5 \mathrm{E}+01$ & $6.5 \mathrm{E}-01$ & $1.1 \mathrm{E}+04$ & $1.1 \mathrm{E}+02$ & $3.7 \mathrm{E}+05$ & $3.7 \mathrm{E}+03$ \\
\hline Tb-158 & $015759-55-4$ & $1.1 \mathrm{E}+02$ & $1.1 \mathrm{E}+00$ & $2.5 \mathrm{E}+03$ & $2.5 \mathrm{E}+01$ & $9.3 \mathrm{E}+04$ & $9.3 \mathrm{E}+02$ \\
\hline
\end{tabular}


Table 3.3d. (continued)

\begin{tabular}{|l|l|c|c|c|c|c|c|}
\hline \multirow{2}{*}{ Chemical } & \multirow{2}{*}{ CASRN } & \multicolumn{2}{|c|}{$\begin{array}{c}\text { Ingestion } \\
\text { of Vegetables (pCi/g) }\end{array}$} & \multicolumn{2}{c|}{$\begin{array}{c}\text { Ingestion } \\
\text { of Beef (pCi/g) }\end{array}$} & \multicolumn{2}{c|}{$\begin{array}{c}\text { Ingestion } \\
\text { of Milk (pCi/g) }\end{array}$} \\
\cline { 3 - 8 } & & $10^{-4}$ & $10^{-6}$ & $10^{-4}$ & $10^{-6}$ & $10^{-4}$ & $10^{-6}$ \\
\hline Tb-160 & $013981-29-8$ & $6.0 \mathrm{E}+01$ & $6.0 \mathrm{E}-01$ & $1.4 \mathrm{E}+03$ & $1.4 \mathrm{E}+01$ & $5.1 \mathrm{E}+04$ & $5.1 \mathrm{E}+02$ \\
\hline Tc-95 & $014809-56-4$ & $8.4 \mathrm{E}+00$ & $8.4 \mathrm{E}-02$ & $3.8 \mathrm{E}+04$ & $3.8 \mathrm{E}+02$ & $3.9 \mathrm{E}+03$ & $3.9 \mathrm{E}+01$ \\
\hline Tc-95m & $014809-56-4$ & $4.6 \mathrm{E}-01$ & $4.6 \mathrm{E}-03$ & $2.1 \mathrm{E}+03$ & $2.1 \mathrm{E}+01$ & $2.2 \mathrm{E}+02$ & $2.2 \mathrm{E}+00$ \\
\hline Tc-96 & $014808-44-7$ & $2.5 \mathrm{E}-01$ & $2.5 \mathrm{E}-03$ & $1.1 \mathrm{E}+03$ & $1.1 \mathrm{E}+01$ & $1.2 \mathrm{E}+02$ & $1.2 \mathrm{E}+00$ \\
\hline Tc-96m & $014808-44-7$ & $2.2 \mathrm{E}+01$ & $2.2 \mathrm{E}-01$ & $9.8 \mathrm{E}+04$ & $9.8 \mathrm{E}+02$ & $1.0 \mathrm{E}+04$ & $1.0 \mathrm{E}+02$ \\
\hline Tc-97 & $015759-35-0$ & $3.6 \mathrm{E}+00$ & $3.6 \mathrm{E}-02$ & $1.6 \mathrm{E}+04$ & $1.6 \mathrm{E}+02$ & $1.7 \mathrm{E}+03$ & $1.7 \mathrm{E}+01$ \\
\hline Tc-97m & $015759-35-0$ & $4.8 \mathrm{E}-01$ & $4.8 \mathrm{E}-03$ & $2.1 \mathrm{E}+03$ & $2.1 \mathrm{E}+01$ & $2.2 \mathrm{E}+02$ & $2.2 \mathrm{E}+00$ \\
\hline Tc-99 & $014133-76-7$ & $4.1 \mathrm{E}-01$ & $4.1 \mathrm{E}-03$ & $1.8 \mathrm{E}+03$ & $1.8 \mathrm{E}+01$ & $1.9 \mathrm{E}+02$ & $1.9 \mathrm{E}+00$ \\
\hline Tc-99m & $014133-76-7$ & $1.0 \mathrm{E}+01$ & $1.0 \mathrm{E}-01$ & $4.6 \mathrm{E}+04$ & $4.6 \mathrm{E}+02$ & $4.8 \mathrm{E}+03$ & $4.8 \mathrm{E}+01$ \\
\hline Te-125m & $014390-73-9$ & $4.9 \mathrm{E}+01$ & $4.9 \mathrm{E}-01$ & $1.5 \mathrm{E}+02$ & $1.5 \mathrm{E}+00$ & $3.4 \mathrm{E}+02$ & $3.4 \mathrm{E}+00$ \\
\hline Te-127 & $013981-49-2$ & $1.5 \mathrm{E}+02$ & $1.5 \mathrm{E}+00$ & $4.4 \mathrm{E}+02$ & $4.4 \mathrm{E}+00$ & $1.0 \mathrm{E}+03$ & $1.0 \mathrm{E}+01$ \\
\hline Te-127m & $013981-49-2$ & $2.1 \mathrm{E}+01$ & $2.1 \mathrm{E}-01$ & $6.3 \mathrm{E}+01$ & $6.3 \mathrm{E}-01$ & $1.4 \mathrm{E}+02$ & $1.4 \mathrm{E}+00$ \\
\hline Te-129 & $014269-71-7$ & $8.4 \mathrm{E}+02$ & $8.4 \mathrm{E}+00$ & $2.6 \mathrm{E}+03$ & $2.6 \mathrm{E}+01$ & $5.8 \mathrm{E}+03$ & $5.8 \mathrm{E}+01$ \\
\hline Te-129m & $014269-71-7$ & $1.1 \mathrm{E}+01$ & $1.1 \mathrm{E}-01$ & $3.2 \mathrm{E}+01$ & $3.2 \mathrm{E}-01$ & $7.4 \mathrm{E}+01$ & $7.4 \mathrm{E}-01$ \\
\hline Te-131 & $014683-12-6$ & $3.2 \mathrm{E}+02$ & $3.2 \mathrm{E}+00$ & $9.7 \mathrm{E}+02$ & $9.7 \mathrm{E}+00$ & $2.2 \mathrm{E}+03$ & $2.2 \mathrm{E}+01$ \\
\hline Te-131m & $014683-12-6$ & $1.4 \mathrm{E}+01$ & $1.4 \mathrm{E}-01$ & $4.3 \mathrm{E}+01$ & $4.3 \mathrm{E}-01$ & $9.8 \mathrm{E}+01$ & $9.8 \mathrm{E}-01$ \\
\hline Te-132 & $014234-28-7$ & $1.0 \mathrm{E}+01$ & $1.0 \mathrm{E}-01$ & $3.1 \mathrm{E}+01$ & $3.1 \mathrm{E}-01$ & $7.1 \mathrm{E}+01$ & $7.1 \mathrm{E}-01$ \\
\hline
\end{tabular}


Table 3.3d. (continued)

\begin{tabular}{|l|l|c|c|c|c|c|c|}
\hline \multirow{2}{*}{ Chemical } & \multirow{2}{*}{ CASRN } & \multicolumn{2}{|c|}{$\begin{array}{c}\text { Ingestion } \\
\text { of Vegetables (pCi/g) }\end{array}$} & \multicolumn{2}{c|}{$\begin{array}{c}\text { Ingestion } \\
\text { of Beef (pCi/g) }\end{array}$} & \multicolumn{2}{c|}{$\begin{array}{c}\text { Ingestion } \\
\text { of Milk (pCi/g) }\end{array}$} \\
\cline { 3 - 8 } & & $10^{-4}$ & $10^{-6}$ & $10^{-4}$ & $10^{-6}$ & $10^{-4}$ & $10^{-5}$ \\
\hline Th-227 & $015623-47-9$ & $1.1 \mathrm{E}+01$ & $1.1 \mathrm{E}-01$ & $1.2 \mathrm{E}+04$ & $1.2 \mathrm{E}+02$ & $3.9 \mathrm{E}+04$ & $3.9 \mathrm{E}+02$ \\
\hline Th-228 & $014274-82-9$ & $7.3 \mathrm{E}+00$ & $7.3 \mathrm{E}-02$ & $7.5 \mathrm{E}+03$ & $7.5 \mathrm{E}+01$ & $2.5 \mathrm{E}+04$ & $2.5 \mathrm{E}+02$ \\
\hline Th-228+D & $014274-82-9$ & $1.8 \mathrm{E}+00$ & $1.8 \mathrm{E}-02$ & $2.0 \mathrm{E}+00$ & $2.0 \mathrm{E}-02$ & $3.4 \mathrm{E}+00$ & $3.4 \mathrm{E}-02$ \\
\hline Th-229 & $015594-54-4$ & $8.1 \mathrm{E}+00$ & $8.1 \mathrm{E}-02$ & $8.4 \mathrm{E}+03$ & $8.4 \mathrm{E}+01$ & $2.8 \mathrm{E}+04$ & $2.8 \mathrm{E}+02$ \\
\hline Th-229+D & $015594-54-4$ & $6.1 \mathrm{E}-01$ & $6.1 \mathrm{E}-03$ & $1.6 \mathrm{E}+00$ & $1.6 \mathrm{E}-02$ & $1.4 \mathrm{E}-01$ & $1.4 \mathrm{E}-03$ \\
\hline Th-230 & $014269-63-7$ & $1.2 \mathrm{E}+01$ & $1.2 \mathrm{E}-01$ & $1.3 \mathrm{E}+04$ & $1.3 \mathrm{E}+02$ & $4.2 \mathrm{E}+04$ & $4.2 \mathrm{E}+02$ \\
\hline Th-231 & $014932-40-2$ & $2.6 \mathrm{E}+02$ & $2.6 \mathrm{E}+00$ & $2.6 \mathrm{E}+05$ & $2.6 \mathrm{E}+03$ & $8.7 \mathrm{E}+05$ & $8.7 \mathrm{E}+03$ \\
\hline Th-232 & $007440-29-1$ & $1.4 \mathrm{E}+01$ & $1.4 \mathrm{E}-01$ & $1.4 \mathrm{E}+04$ & $1.4 \mathrm{E}+02$ & $4.8 \mathrm{E}+04$ & $4.8 \mathrm{E}+02$ \\
\hline Th-234 & $015065-10-8$ & $2.4 \mathrm{E}+01$ & $2.4 \mathrm{E}-01$ & $2.4 \mathrm{E}+04$ & $2.4 \mathrm{E}+02$ & $8.1 \mathrm{E}+04$ & $8.1 \mathrm{E}+02$ \\
\hline Tl-202 & $015720-57-7$ & $4.5 \mathrm{E}+02$ & $4.5 \mathrm{E}+00$ & $1.2 \mathrm{E}+03$ & $1.2 \mathrm{E}+01$ & $3.9 \mathrm{E}+03$ & $3.9 \mathrm{E}+01$ \\
\hline Tl-204 & $013968-51-9$ & $2.3 \mathrm{E}+02$ & $2.3 \mathrm{E}+00$ & $6.1 \mathrm{E}+02$ & $6.1 \mathrm{E}+00$ & $2.0 \mathrm{E}+03$ & $2.0 \mathrm{E}+01$ \\
\hline Tl-208 & $014913-50-9$ & $2.6 \mathrm{E}+04$ & $2.6 \mathrm{E}+02$ & $6.9 \mathrm{E}+04$ & $6.9 \mathrm{E}+02$ & $2.3 \mathrm{E}+05$ & $2.3 \mathrm{E}+03$ \\
\hline Tl-209 & $015690-73-0$ & $3.3 \mathrm{E}+04$ & $3.3 \mathrm{E}+02$ & $8.6 \mathrm{E}+04$ & $8.6 \mathrm{E}+02$ & $2.8 \mathrm{E}+05$ & $2.8 \mathrm{E}+03$ \\
\hline Tm-170 & $013981-30-1$ & $6.0 \mathrm{E}+01$ & $6.0 \mathrm{E}-01$ & $1.4 \mathrm{E}+03$ & $1.4 \mathrm{E}+01$ & $5.2 \mathrm{E}+04$ & $5.2 \mathrm{E}+02$ \\
\hline Tm-171 & $014333-45-0$ & $7.7 \mathrm{E}+02$ & $7.7 \mathrm{E}+00$ & $1.8 \mathrm{E}+04$ & $1.8 \mathrm{E}+02$ & $6.7 \mathrm{E}+05$ & $6.7 \mathrm{E}+03$ \\
\hline U-232 & $014158-29-3$ & $5.6 \mathrm{E}+00$ & $5.6 \mathrm{E}-02$ & $1.9 \mathrm{E}+03$ & $1.9 \mathrm{E}+01$ & $2.3 \mathrm{E}+02$ & $2.3 \mathrm{E}+00$ \\
\hline U-233 & $013968-55-3$ & $1.0 \mathrm{E}+01$ & $1.0 \mathrm{E}-01$ & $3.4 \mathrm{E}+03$ & $3.4 \mathrm{E}+01$ & $4.2 \mathrm{E}+02$ & $4.2 \mathrm{E}+00$ \\
\hline
\end{tabular}




\begin{tabular}{|c|c|c|c|c|c|c|c|c|c|c|c|c|c|c|c|c|c|c|}
\hline 总 & 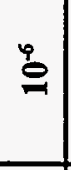 & 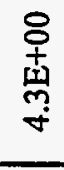 & $\begin{array}{l}\stackrel{8}{+} \\
\text { 章 } \\
\text { ฺ }\end{array}$ & $\begin{array}{l}\text { ᄋ } \\
\text { + } \\
\text { ज్ } \\
\text { ஸे }\end{array}$ & 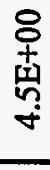 & 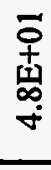 & $\begin{array}{l}8 \\
\text { + } \\
\text { 岁 } \\
\text { 寸 }\end{array}$ & $\begin{array}{l}8 \\
+ \\
+ \\
\text { 离 } \\
\text { i }\end{array}$ & 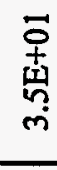 & 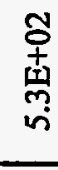 & ণั & 宫 & 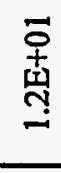 & & & & & \\
\hline 总富 & $\stackrel{ }{\circ}$ & $\begin{array}{l}\text { ơ } \\
+ \\
\text { w } \\
\text { m }\end{array}$ & 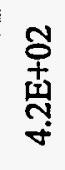 & $\begin{array}{l}\text { 옹 } \\
+ \\
\text { ज్ } \\
\text { ஸे }\end{array}$ & 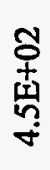 & 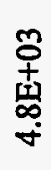 & $\begin{array}{l}\text { O } \\
\text { 章 } \\
\text { 守 }\end{array}$ & 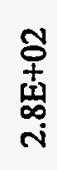 & 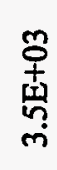 & 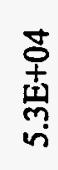 & $\begin{array}{l}\text { ष } \\
+ \\
\text { 명 } \\
\end{array}$ & 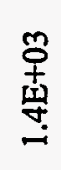 & 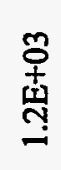 & & & & & \\
\hline : & 边 & 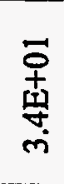 & 章 & 富 & 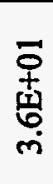 & 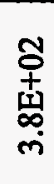 & $\begin{array}{l}\overline{0} \\
+ \\
\text { tọ } \\
\text { r. }\end{array}$ & 畐 & 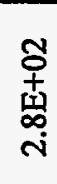 & 㝘 & 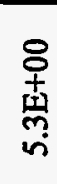 & 审 & 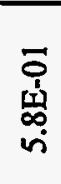 & & & & & \\
\hline 总离 & $\vec{\theta}$ & 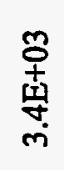 & 号 & 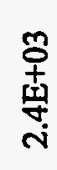 & 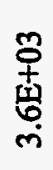 & 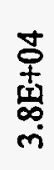 & 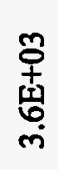 & $\begin{array}{l}\text { 乌 } \\
\text { 龺 } \\
\text { - }\end{array}$ & $\begin{array}{l}\text { J } \\
+ \\
+1 \\
\infty \\
\text { i }\end{array}$ & 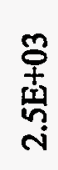 & 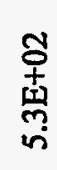 & 亭 & 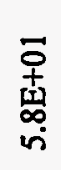 & & & & & \\
\hline 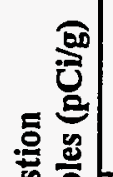 & $\stackrel{\varphi}{\varrho}$ & 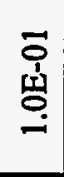 & 㝘 & 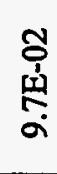 & ت & $\begin{array}{l}8 \\
+ \\
\text { 王 } \\
\end{array}$ & 音 & 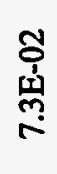 & 宮 & $\begin{array}{l}\overline{0} \\
\text { 山े } \\
\text { ơ }\end{array}$ & 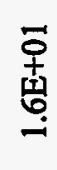 & 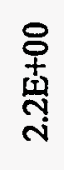 & $\begin{array}{l}8 \\
\text { + } \\
\text { 息 } \\
-\end{array}$ & & & & & \\
\hline 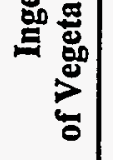 & $\stackrel{T}{b}$ & $\begin{array}{l}\text { 호 } \\
\text { +⿱ } \\
\text { 됭 } \\
\text { - }\end{array}$ & $\begin{array}{c}\text { ㅇ } \\
\text { 晋 } \\
-\end{array}$ & 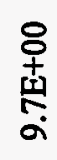 & 훙 & 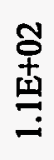 & 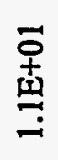 & 号 & 畐 & $\begin{array}{l}\overrightarrow{0} \\
\text { 至 } \\
0 \\
0\end{array}$ & $\begin{array}{l}3 \\
+ \\
+10 \\
0 \\
-1\end{array}$ & 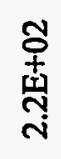 & 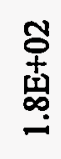 & & & & & \\
\hline 宽 & & 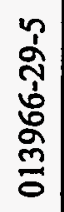 & $\begin{array}{l}\overrightarrow{0} \\
\stackrel{\vdots}{5} \\
\vdots \\
\vdots \\
0\end{array}$ & 嵩 & 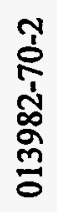 & 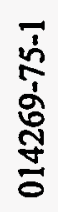 & 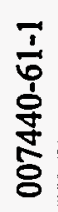 & $\begin{array}{l}\frac{7}{0} \\
0 \\
\frac{8}{8} \\
\frac{5}{8}\end{array}$ & 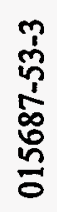 & $\frac{\frac{0}{9}}{\frac{1}{m}}$ & 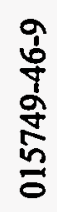 & $\begin{array}{l}\frac{r}{\dot{d}} \\
\text { స్ } \\
\frac{\sigma}{0}\end{array}$ & 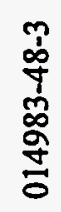 & $\frac{\text { Jे }}{\frac{1}{1}}$ & $\begin{array}{l}\frac{7}{0} \\
\frac{1}{0} \\
\frac{0}{0} \\
\frac{n}{0}\end{array}$ & $\begin{array}{l}\frac{\infty}{0} \\
\frac{1}{7} \\
\frac{5}{5} \\
\frac{\mathrm{m}}{0}\end{array}$ & 产 & 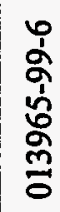 \\
\hline 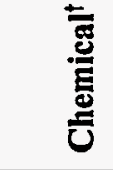 & & 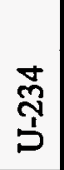 & 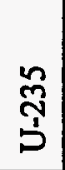 & 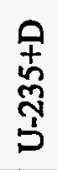 & 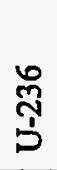 & ஸ̂̀̆ & 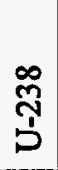 & $\begin{array}{l}\text { 早 } \\
\infty \\
\stackrel{\text { }}{p} \\
\vdots\end{array}$ & 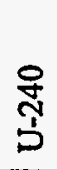 & $\stackrel{\infty}{\dagger}_{>}^{\infty}$ & $\frac{\vec{\infty}}{\frac{1}{3}}$ & $\frac{\infty}{3}$ & $\frac{\infty}{\frac{1}{1}}$ & $\frac{\tilde{y}}{\dot{j}}$ & $\frac{\stackrel{m}{a}}{\dot{d}}$ & 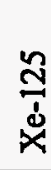 & $\frac{\bar{d}}{\dot{d}}$ & $\frac{\text { g }}{\stackrel{\Xi}{d}}$ \\
\hline
\end{tabular}


Table 3.3d. (continued)

\begin{tabular}{|c|c|c|c|c|c|c|c|}
\hline \multirow[t]{2}{*}{ Chemical $^{\dagger}$} & \multirow[t]{2}{*}{ CASRN } & \multicolumn{2}{|c|}{$\begin{array}{c}\text { Ingestion } \\
\text { of Vegetables (pCi/g) }\end{array}$} & \multicolumn{2}{|c|}{$\begin{array}{c}\text { Ingestion } \\
\text { of Beef (pCi/g) }\end{array}$} & \multicolumn{2}{|c|}{$\begin{array}{c}\text { Ingestion } \\
\text { of Milk (pCi/g) }\end{array}$} \\
\hline & & $10^{-4}$ & $10^{-6}$ & $10^{-4}$ & $10^{-6}$ & $10^{-4}$ & $10^{-6}$ \\
\hline $\mathrm{Xe}-131 \mathrm{~m}$ & 014683-11-5 & & & & & & \\
\hline $\mathrm{Xe}-133$ & $014932-42-4$ & & & & & & \\
\hline $\mathrm{Xe}-133 \mathrm{~m}$ & $014932-42-4$ & & & & & & \\
\hline $\mathrm{Xe}-135$ & 014995-62-1 & & & & & & \\
\hline $\mathrm{Xe}-135 \mathrm{~m}$ & 014995-62-1 & & & & & & \\
\hline Xe-137 & $014835-21-3$ & & & & & & \\
\hline Xe-138 & $015751-81-2$ & & & & & & \\
\hline Y-90 & 010098-91-6 & $3.0 \mathrm{E}+01$ & $3.0 \mathrm{E}-01$ & $3.2 E+03$ & $3.2 \mathrm{E}+01$ & $2.6 \mathrm{E}+04$ & $2.6 \mathrm{E}+02$ \\
\hline$Y-91$ & 014234-24-3 & $3.4 \mathrm{E}+01$ & $3.4 \mathrm{E}-01$ & $3.5 E+03$ & $3.5 \mathrm{E}+01$ & $2.9 E+04$ & $2.9 \mathrm{E}+02$ \\
\hline $\mathrm{Y}-91 \mathrm{~m}$ & 014234-24-3 & $1.2 \mathrm{E}+04$ & $1.2 \mathrm{E}+02$ & $1.3 \mathrm{E}+06$ & $1.3 \mathrm{E}+04$ & $1.1 \mathrm{E}+07$ & $1.1 \mathrm{E}+05$ \\
\hline $\mathrm{Y}-92$ & $015751-59-4$ & $2.3 \mathrm{E}+02$ & $2.3 \mathrm{E}+00$ & $2.4 \mathrm{E}+04$ & $2.4 \mathrm{E}+02$ & $2.0 \mathrm{E}+05$ & $2.0 \mathrm{E}+03$ \\
\hline Y-93 & 014981-70-5 & $7.9 \mathrm{E}+01$ & $7.9 \mathrm{E}-01$ & $8.2 E+03$ & $8.2 E+01$ & $6.8 \mathrm{E}+04$ & $6.8 \mathrm{E}+02$ \\
\hline $\mathrm{Zn}-65$ & 013982-39-3 & $2.3 \mathrm{E}+01$ & 2.3E-01 & $1.4 \mathrm{E}+01$ & $1.4 \mathrm{E}-01$ & $2.1 \mathrm{E}+01$ & $2.1 \mathrm{E}-01$ \\
\hline $\mathrm{Zn}-69$ & 013982-23-5 & $3.7 \mathrm{E}+03$ & $3.7 \mathrm{E}+01$ & $2.3 \mathrm{E}+03$ & $2.3 \mathrm{E}+01$ & $3.4 \mathrm{E}+03$ & $3.4 \mathrm{E}+01$ \\
\hline $\mathrm{Zn}-69 \mathrm{~m}$ & 013982-23-5 & $1.5 \mathrm{E}+02$ & $1.5 \mathrm{E}+00$ & $9.2 \mathrm{E}+01$ & $9.2 \mathrm{E}-01$ & $1.4 \mathrm{E}+02$ & $1.4 \mathrm{E}+00$ \\
\hline Zr-93 & $015751-77-6$ & $8.8 \mathrm{E}+02$ & $8.8 \mathrm{E}+00$ & $9.3 E+07$ & $9.3 E+05$ & $2.8 \mathrm{E}+07$ & $2.8 \mathrm{E}+05$ \\
\hline $\mathrm{Zr}-95$ & $013967-71-0$ & $1.2 \mathrm{E}+02$ & $1.2 \mathrm{E}+00$ & $1.2 \mathrm{E}+07$ & $1.2 \mathrm{E}+05$ & $3.7 \mathrm{E}+06$ & 3.7E+04 \\
\hline
\end{tabular}


Table 3.3d. (continued)

\begin{tabular}{|l|l|c|c|c|c|c|c|}
\hline \multirow{2}{*}{ Chemical $^{\dagger}$} & \multirow{2}{*}{ CASRN } & \multicolumn{2}{c|}{$\begin{array}{c}\text { Ingestion } \\
\text { of Vegetables (pCi/g) }\end{array}$} & \multicolumn{2}{c|}{$\begin{array}{c}\text { Ingestion } \\
\text { of Beef (pCi/g) }\end{array}$} & \multicolumn{2}{c|}{$\begin{array}{c}\text { Ingestion } \\
\text { of Milk (pCi/g) }\end{array}$} \\
\cline { 3 - 8 } & & $10^{-4}$ & $10^{-6}$ & $10^{-4}$ & $10^{-6}$ & $10^{-4}$ & $10^{-6}$ \\
\hline $\mathrm{Zr}-97$ & $014928-30-4$ & $4.4 \mathrm{E}+01$ & $4.4 \mathrm{E}-01$ & $4.6 \mathrm{E}+06$ & $4.6 \mathrm{E}+04$ & $1.4 \mathrm{E}+06$ & $1.4 \mathrm{E}+04$ \\
\hline
\end{tabular}

Note:

$\dagger \quad$ Isotopes designated with an " $\mathrm{m}$ " indicate that the isotope is metastable; isotopes designated with a " $+\mathrm{D}$ " indicate that the radionuclide slope factors include contributions from daughter products. 
Table 3.3e. Risk-based Preliminary Remediation Goals for nonradionuclides in water and soil (agricultural scenario)

\begin{tabular}{|c|c|c|c|c|c|c|c|c|c|c|c|c|c|c|c|c|c|c|}
\hline \multirow{2}{*}{ Chemical } & \multirow{2}{*}{ CASRN } & \multicolumn{4}{|c|}{ Vegetable Intercept (mg/kg) } & \multirow{2}{*}{ Slope } & \multicolumn{4}{|c|}{ Beef Intercept (mg/kg) } & \multirow[b]{2}{*}{ Slope } & \multicolumn{6}{|c|}{ Milk Intercept (mg/kg) } & \multirow[b]{2}{*}{ Slope } \\
\hline & & $10^{-4}$ & $10^{5}$ & $H Q=1$ & $\mathrm{HQ}=0.1$ & & $10^{-4}$ & $10^{6}$ & $H Q=1$ & $\mathrm{HQ}=0.1$ & & $10^{-4}$ & $10^{6}$ & $\begin{array}{l}\text { HQ=1 } \\
\text { Adult }\end{array}$ & $\begin{array}{l}\text { HQ=1 } \\
\text { Child }\end{array}$ & $\begin{array}{c}\mathrm{HQ}=0.1 \\
\text { Adult }\end{array}$ & $\begin{array}{c}\text { HQ }=0.1 \\
\text { Child }\end{array}$ & \\
\hline ALAR & $001596-84.5$ & & & & & & & & & & & & & & & & & \\
\hline Acensphthene & $000083-32-9$ & & & $1.9 E+02$ & $1.9 \mathrm{E}+01$ & $-4.9 \mathrm{E}+01$ & & & $3.4 \mathrm{E}+04$ & $3.4 E+03$ & $-1.5 E+01$ & & & $2.0 \mathrm{E}+04$ & $2.5 E+03$ & $2.0 \mathrm{E}+03$ & $2.5 E+02$ & $-1.6 \mathrm{E}+01$ \\
\hline Acenaphthylene & $000208-96-8$ & & & & & & & & & & & & & & & & & . \\
\hline Acephate & $030560-19-1$ & $1.0 \mathrm{E}+\infty$ & $1.0 \mathrm{E}-02$ & $1.5 \mathrm{E}-01$ & $1.5 \mathrm{E}-02$ & $-3.6 \mathrm{E}+01$ & $1.0 \mathrm{E}+06$ & $9.6 \mathrm{E}+04$ & $1.0 \mathrm{E}+06$ & $1.4 \mathrm{E}+05$ & $-6.5 E-02$ & $1.0 E+06$ & 2.1E+04 & 7.5E+05 & $9.6 \mathrm{E}+04$ & 7.5E+04 & $9.6 \mathrm{E}+03$ & $-6.5 \mathrm{E}-02$ \\
\hline Acetaldehyde & $000075-07-0$ & & & & & & & & & & & & & & & & & \\
\hline Acetochlor & $034256-82-1$ & & & & & & & & & & & & & & & & & \\
\hline Acetone & $000067-64-1$ & & & $8.4 \mathrm{E}+\infty 0$ & $8.4 \mathrm{E}-01$ & $-3.6 \mathrm{E}+01$ & & & $1.0 E+06$ & $1.0 \mathrm{E}+06$ & $-1.5 \mathrm{E}-01$ & & & $1.0 \mathrm{E}+06$ & $1.0 \mathrm{E}+06$ & $1.0 \mathrm{E}+06$ & $1.3 \mathrm{E}+05$ & $-1.5 \mathrm{E}-01$ \\
\hline Acetone Cyanohydrin & 000075.86 .5 & & & & & & & & & & & & & & & & & \\
\hline Acetonitrile & $000075-05-8$ & & & $4.4 \mathrm{E}-01$ & $4,4 \mathrm{E}-02$ & $-3.6 \mathrm{E}+01$ & & & $1.0 \mathrm{E}+06$ & $1.3 \mathrm{E}+05$ & $-1.3 \mathrm{E}-01$ & & & $6.8 \mathrm{E}+05$ & $8.8 \mathrm{E}+04$ & $6.8 \mathrm{E}+04$ & $8.8 E+03$ & $-1.3 \mathrm{E}-01$ \\
\hline Acetophenone ${ }^{17}$ & $000098-86-2$ & & & $8.6 \mathrm{E}+01$ & $8.6 \mathrm{E}+\infty$ & $-3.9 \mathrm{E}+01$ & & & $1.0 \mathrm{E}+06$ & $2.8 \mathrm{E}+05$ & $-1.8 \mathrm{E}+00$ & & & $1.0 \mathrm{E}+06$ & $1.9 \mathrm{E}+05$ & $1.5 \mathrm{E}+05$ & $1.9 \mathrm{E}+04$ & $-1.8 \mathrm{E}+00$ \\
\hline Acrolein & $000107-02-8$ & & & $2.0 E+00$ & $2.0 \mathrm{E}-01$ & $-3.6 \mathrm{E}+01$ & & & $1.0 \mathrm{E}+06$ & $3.5 \mathrm{E}+05$ & $-1.8 \mathrm{E}-01$ & & & $1.0 \mathrm{E}+06$ & 2.3E+05 & $1.8 \mathrm{E}+05$ & $2.3 E+04$ & $-1.8 \mathrm{E}-0 !$ \\
\hline Acrylamide & $000079-06-1$ & $2.5 \mathrm{E}-03$ & 2.5E-05 & $9.6 \mathrm{E}-03$ & $9.6 \mathrm{E}-04$ & $-3.6 \mathrm{E}+01$ & $1.6 \mathrm{E}+04$ & $1.6 \mathrm{E}+02$ & $6.0 \mathrm{E}+04$ & $6.0 E+03$ & $-8.3 \mathrm{E}-02$ & $3.5 \mathrm{E}+03$ & $3.5 \mathrm{E}+01$ & $3.1 \mathrm{E}+04$ & $4.0 \mathrm{E}+03$ & $3.1 \mathrm{E}+03$ & $4.0 \mathrm{E}+02$ & $-8.3 \mathrm{E}-02$ \\
\hline Acrylic Acid & $000079-10-7$ & & & $7.0 \mathrm{E}+01$ & $7.0 \mathrm{E}+\infty$ & $-3.6 \mathrm{E}+01$ & & & $1.0 \mathrm{E}+06$ & $1.0 \mathrm{E}+06$ & $-2.5 \mathrm{E}-01$ & & & $1.0 \mathrm{E}+06$ & $1.0 E+06$ & $1.0 \mathrm{E}+06$ & $4.5 \mathrm{E}+05$ & $-2.5 \mathrm{E}-01$ \\
\hline Acrylonitrile & $000107-13-1$ & $6.8 \mathrm{E}-02$ & $6.8 \mathrm{E}-04$ & $1.6 \mathrm{E}-01$ & $1.6 \mathrm{E}-02$ & $-3.6 \mathrm{E}+01$ & $5.3 \mathrm{E}+04$ & $5.3 \mathrm{E}+02$ & $1.2 \mathrm{E}+05$ & $1.2 \mathrm{E}+04$ & $-2.8 \mathrm{E}-01$ & $1.2 \mathrm{E}+04$ & $1.2 \mathrm{E}+02$ & $6.4 \mathrm{E}+04$ & $8.2 \mathrm{E}+03$ & $6.4 \mathrm{E}+03$ & $8.2 E+02$ & $-2.8 \mathrm{E}-01$ \\
\hline Alachlor & $015972.60-8$ & $6.3 \mathrm{E}+00$ & $6.3 \mathrm{E}-02$ & $2.2 \mathrm{E}+01$ & $2.2 \mathrm{E}+00$ & $-4.4 E+01$ & $1.8 \mathrm{E}+04$ & $1.8 \mathrm{E}+02$ & $6.3 \mathrm{E}+04$ & $6.3 E+03$ & $-6.5 \mathrm{E}+\infty 0$ & $4.3 \mathrm{E}+03$ & $4.3 \mathrm{E}+01$ & $3.5 \mathrm{E}+04$ & $4.4 \mathrm{E}+03$ & $3.5 E+03$ & $4.4 \mathrm{E}+02$ & $-6,8 E+\infty 0$ \\
\hline Addicarb & $000116-06-3$ & & & 4.6E-01 & $4,6 \mathrm{E}-02$ & $-3.8 \mathrm{E}+01$ & & & $5.1 \mathrm{E}+04$ & $5.1 \mathrm{E}+03$ & $-8.8 \mathrm{E}-01$ & & & $2.7 \mathrm{E}+04$ & $3.4 E+03$ & $2.7 \mathrm{E}+03$ & $3.4 E+02$ & $-8,9 \mathrm{E}-01$ \\
\hline Aldicarb Sulfone & $001646-88-4$ & & & & & & & & & & & & & & & & & \\
\hline Addrin & $000309-00.2$ & 3.1E-02 & 3.1E-04 & $6.8 \mathrm{E}-02$ & $6,8 \mathrm{E}-03$ & $-4.5 \mathrm{E}+01$ & 7.5E+01 & $7.5 \mathrm{E}-01$ & $1.6 \mathrm{E}+02$ & $1.6 \mathrm{E}+01$ & $-7.0 E+\infty$ & $1.7 \mathrm{E}+01$ & $1.7 \mathrm{E}-01$ & $9.0 E+01$ & $1.2 \mathrm{E}+01$ & $9.0 \mathrm{E}+00$ & $1.2 \mathrm{E}+\infty$ & $-7.4 E+\infty 0$ \\
\hline Ally & $074223-64-6$ & & & $2.9 \mathrm{E}+01$ & $2.9 \mathrm{E}+\infty 0$ & $-3.6 \mathrm{E}+01$ & & & $1.0 \mathrm{E}+06$ & $1.0 \mathrm{E}+06$ & $-2.0 \mathrm{E}-01$ & & & $1.0 \mathrm{E}+06$ & $1.0 E+06$ & $1.0 \mathrm{E}+06$ & $2.6 \mathrm{E}+05$ & $-2.1 \mathrm{E}-01$ \\
\hline Ally! Alcohol & $000107 \cdot 18-6$ & & & 4.3E-01 & $4.3 \mathrm{E}-02$ & $-3.6 \mathrm{E}+01$ & & & $9.7 \mathrm{E}+05$ & $9.7 \mathrm{E}+04$ & $-1.5 \mathrm{E}-01$ & & & $5.1 \mathrm{E}+05$ & $6.5 \mathrm{E}+04$ & $5.1 \mathrm{E}+04$ & $6.5 \mathrm{E}+03$ & $-1.5 E-01$ \\
\hline Allyl Choride ${ }^{(3)}$ & $000107-05-1$ & & & $3.2 \mathrm{E}+01$ & $3.2 \mathrm{E}+00$ & $-3.8 E+01$ & & & $1.0 E+06$ & $1.9 E+05$ & $-1.2 \mathrm{E}+00$ & & & $1.0 \mathrm{E}+06$ & $1.3 \mathrm{E}+05$ & $1.0 \mathrm{E}+05$ & $1.3 E+04$ & $-1.2 \mathrm{E}+00$ \\
\hline Aluminum & $007429-90-5$ & & & & & & & & & & & & & & & & & \\
\hline Numinum Phosphide & $020859-73-8$ & & & & & & & & & & & & & & & & & \\
\hline
\end{tabular}

Nonradionuclides/water and soil/agricultural

Page 1 of 32 
Table 3.3e. (continued)

\begin{tabular}{|c|c|c|c|c|c|c|c|c|c|c|c|c|c|c|c|c|c|c|}
\hline \multirow[b]{2}{*}{ Chemical } & \multirow[b]{2}{*}{ CASRN } & \multicolumn{4}{|c|}{ Vegetable Intercept (mg/kg) } & \multirow[b]{2}{*}{ Slope } & \multicolumn{4}{|c|}{ Beef Intercept (mg/kg)" } & \multirow[b]{2}{*}{ Slope } & \multicolumn{6}{|c|}{ Milk Intercept (mg/kg)" } & \multirow[b]{2}{*}{ Slope } \\
\hline & & $10^{-1}$ & $10^{-6}$ & $\mathrm{HQ}=1$ & $\mathrm{HQ}=0.1$ & & $10^{-4}$ & $10^{4}$ & $H Q=1$ & $\mathrm{HQ}=0.1$ & & $10^{-4}$ & $10^{6}$ & $\begin{array}{l}\text { HQ=1 } \\
\text { Adult }\end{array}$ & $\begin{array}{l}\text { HQ=1 } \\
\text { Child }\end{array}$ & $\begin{array}{c}\mathrm{HQ}=0.1 \\
\text { Adult }\end{array}$ & $\begin{array}{c}\mathrm{HQ}=0.1 \\
\text { Child }\end{array}$ & \\
\hline Ametryn & $000834-12-8$ & & & $8.6 \mathrm{E}+\infty$ & $8.6 \mathrm{E}-01$ & $-4,0 \mathrm{E}+01$ & & & $2.2 \mathrm{E}+05$ & $2.2 \mathrm{E}+04$ & $-2.0 E+\infty$ & & & $1.2 \mathrm{E}+05$ & $1.5 \mathrm{E}+04$ & $1.2 \mathrm{E}+04$ & $1.5 \mathrm{E}+03$ & $-2.0 \mathrm{E}+\infty 0$ \\
\hline Aminophenol, m- & $000591-27-5$ & & & $1.1 \mathrm{E}+01$ & $1.1 \mathrm{E}+\infty$ & $-3.6 \mathrm{E}+01$ & & & $1.0 \mathrm{E}+06$ & $8.9 E+05$ & $-2.7 \mathrm{E}-01$ & & & $1.0 \mathrm{E}+06$ & $6.0 \mathrm{E}+05$ & $4.7 E+05$ & $6.0 \mathrm{E}+04$ & $-2.7 \mathrm{E}-01$ \\
\hline Aminopyridine, 4- & $000504-24-5$ & & & 3.3E-03 & $3.3 \mathrm{E}-04$ & $-3.6 \mathrm{E}+01$ & & & $2.4 \mathrm{E}+03$ & $2.4 \mathrm{E}+02$ & $-2.9 \mathrm{E}-01$ & & & $1.2 \mathrm{E}+03$ & $1.6 \mathrm{E}+02$ & $1.2 E+02$ & $1.6 \mathrm{E}+01$ & $-2.9 \mathrm{E}-01$ \\
\hline Amitra: & $033089-61-1$ & & & & & & & & & & & & & & & & & \\
\hline Ammoria & $007664-41.7$ & & & & & & & & & & & & & & & & & \\
\hline Ammonium Sulfurmate & $007773-06-0$ & & & & & & & & & & & & & & & & & \\
\hline Aniline & 000062-53-3 & $1.5 \mathrm{E}+01$ & $1.5 \mathrm{E}-01$ & & & $-3.7 \mathrm{E}+01$ & $1.0 \mathrm{E}+\infty 6$ & $2.6 \mathrm{E}+04$ & & & $-6.5 \mathrm{E}-01$ & $5.8 \mathrm{E}+05$ & $5.8 \mathrm{E}+03$ & & & & & $-6.6 \mathrm{E}-01$ \\
\hline Anthracene & $000120-12-7$ & & & $9.7 \mathrm{E}+02$ & $9.7 \mathrm{E}+01$ & $-4.9 \mathrm{E}+01$ & & & $1.4 \mathrm{E}+05$ & $1.4 \mathrm{E}+04$ & $-1.5 E+01$ & & & $8.1 E+04$ & $1.0 \mathrm{E}+04$ & $8.1 \mathrm{E}+03$ & $1.0 \mathrm{E}+03$ & $-1.7 E+01$ \\
\hline Antimony (metallic) & $007440-36-0$ & & & $1.4 \mathrm{E}+00$ & $1.4 \mathrm{E}-01$ & $-4.9 E+01$ & & & 3.3E+03 & $3.3 \mathrm{E}+02$ & $-1.7 \mathrm{E}+01$ & & & $9.8 \mathrm{E}+02$ & $1.3 \mathrm{E}+02$ & $9.8 \mathrm{E}+01$ & $1.3 \mathrm{E}+01$ & $-1.9 E+01$ \\
\hline $\begin{array}{l}\text { Antimony Potassium } \\
\text { Tartrate }\end{array}$ & $000304-61-0$ & & & & & & & & & & & & & & & & & \\
\hline Antimony Tetroxide & $001332-81-6$ & & & & & & & & & & & & & & & & & \\
\hline Antimony Trioxide & $001309-64-4$ & & & & & & & & & & & & & & & & & \\
\hline Apollo & $074115-24-5$ & & & & , & & & & & & & & & & & & & \\
\hline Aramite & 000140.57 .8 & & & & & & & & & & & & & & & & & \\
\hline Aroclor 1016 & $012674-11-2$ & & & $2.4 \mathrm{E}-01$ & 2.4E-02 & $-5.0 E+01$ & & & $1.3 \mathrm{E}+\infty 0$ & $1.3 \mathrm{E}-01$ & $-1.8 E+01$ & & & $7.5 \mathrm{E}-01$ & $9.6 \mathrm{E}-02$ & $7.5 \mathrm{E}-02$ & $9.6 \mathrm{E}-03$ & $-2.1 E+01$ \\
\hline Aroclor $1221^{(1)}$ & $011104-28-2$ & 9.5E-02 & $9.5 \mathrm{E}-04$ & & & $-4.8 E+01$ & $2.6 \mathrm{E}+0 \mathrm{I}$ & $2.6 \mathrm{E}-01$ & & & $-1.4 E+01$ & $6.2 \mathrm{E}+00$ & $6.2 \mathrm{E}-02$ & & & & & $-1.5 E+01$ \\
\hline Aroclor $1232^{(1)}$ & $011141-16-5$ & 7.5E-02 & $7.5 \mathrm{E}-04$ & & & $-4,6 \mathrm{E}+01$ & $1.2 E+02$ & $1.2 E+\infty$ & & & $-8.3 E+\infty$ & $2.9 \mathrm{E}+01$ & $2.9 \mathrm{E}-01$ & & & & & $-8.8 \mathrm{E}+00$ \\
\hline Aroclor 1242(1) & 053469-21-9 & 9.5E-02 & $9.5 \mathrm{E}-04$ & & & $-4.8 \mathrm{E}+01$ & $2.6 \mathrm{E}+01$ & $2.6 \mathrm{E}-01$ & & & $-1.4 E+01$ & $6.2 \mathrm{E}+\infty 0$ & $6.2 \mathrm{E}-02$ & & & & & $-1.5 E+01$ \\
\hline Aroclor 1248(1) & $012672-29-6$ & $1.1 \mathrm{E}-01$ & $1.1 \mathrm{E}-03$ & & & $-5.0 \mathrm{E}+01$ & $6.9 \mathrm{E}-01$ & $6.9 \mathrm{E}-03$ & & & $-1.8 E+01$ & $1.7 \mathrm{E}-01$ & $1.78-03$ & & & & & $-2.1 E+01$ \\
\hline Aroclor 1254 & $011097-69-1$ & & & $7.0 \mathrm{E}-02$ & $7.0 \mathrm{E}-03$ & $-5.0 \mathrm{E}+01$ & & & $2.9 \mathrm{E}-01$ & $2.9 \mathrm{E}-02$ & $-1.9 E+01$ & & & 1.7E-01 & $2.2 E-02$ & $1.7 \mathrm{E}-02$ & 2.2E-03 & $-2.1 E+01$ \\
\hline Aroclor 1260 & $011096-82-5$ & $1.1 E-01$ & $1.1 \mathrm{E}-03$ & & & $-5.0 E+01$ & $3.6 \mathrm{E}-02$ & $3.6 \mathrm{E}-04$ & & & $-1.9 \mathrm{E}+01$ & $9.0 \mathrm{E}-03$ & $9.0 \mathrm{E}-05$ & & & & & $-2.2 E+0 !$ \\
\hline
\end{tabular}

Nonradionuclides/water and soil/agricultural

Page 2 of 32 
Table 3.3e. (continued)

\begin{tabular}{|c|c|c|c|c|c|c|c|c|c|c|c|c|c|c|c|c|c|c|}
\hline \multirow{2}{*}{ Chemical } & \multirow{2}{*}{ CASRN } & \multicolumn{4}{|c|}{ Vegetable Intercept (mg/kg) } & \multirow{2}{*}{ Slope } & \multicolumn{4}{|c|}{ Beef Intercept (mg/kg) } & \multirow{2}{*}{ Slope } & \multicolumn{6}{|c|}{ Milk Intercept (mg/kg)" } & \multirow[b]{2}{*}{ Slope } \\
\hline & & $10^{-4}$ & $10^{4}$ & $H Q=1$ & $\mathrm{HQ}=0.1$ & & $10^{-4}$ & $10^{6}$ & $\mathrm{HQ}=1$ & $\mathrm{HQ}=0.1$ & & $10^{-4}$ & $10^{4}$ & $\begin{array}{l}\text { HQ=1 } \\
\text { Adult }\end{array}$ & $\begin{array}{c}H Q=1 \\
\text { Child }\end{array}$ & $\begin{array}{c}\mathrm{HQ}=0.1 \\
\text { Adult }\end{array}$ & $\begin{array}{c}\text { HQ }=0.1 \\
\text { Child }\end{array}$ & \\
\hline Arsenic, Inorganic & $007440-38-2$ & $5.3 E-01$ & $5.3 \mathrm{E}-03$ & $1.0 E+\infty$ & $1.0 \mathrm{E}-01$ & $-4.9 \mathrm{E}+01$ & $2.6 \mathrm{E}+01$ & $2.6 \mathrm{E}-01$ & S.1E+01 & $5.1 E+\infty$ & $-1.7 E+01$ & $6.9 \mathrm{E}+01$ & $6.9 \mathrm{E}-0 \mathrm{l}$ & $3.1 E+02$ & $4.0 \mathrm{E}+01$ & $3.1 E+01$ & $4.0 \mathrm{E}+00$ & $-2.0 E+01$ \\
\hline Ärsine & $007784-42-1$ & & & & & . & & & & & & & & & & & & \\
\hline Asbestos & $001332-21-4$ & & & & & & & & & & & & & & & & & \\
\hline Assure & $076578-14-8$ & & & & & & & & & & & & & & & & & \\
\hline Asulam & $003337-71-1$ & & & & & & & & & & & & & & & & & \\
\hline Atrazine & $001912-24-9$ & $2.0 E+00$ & $2.0 \mathrm{E}-02$ & $6.8 \mathrm{E}+01$ & $6.8 \mathrm{E}+00$ & $-4.4 E+01$ & $8.8 \mathrm{E}+03$ & $8.8 E+01$ & $2.9 \mathrm{E}+05$ & $2.9 \mathrm{E}+04$ & $-5.4 \mathrm{E}+\infty 0$ & $2.0 E+03$ & $2.0 \mathrm{E}+01$ & $1.6 \mathrm{E}+05$ & $2.0 \mathrm{E}+04$ & $1.6 \mathrm{E}+04$ & $2.0 \mathrm{E}+03$ & $-5.6 \mathrm{E}+00$ \\
\hline Avermectin Bl & 065195.55 .3 & & & & & & & & & & & & & & & & & \\
\hline Azobenzene & $000103-33-3$ & $6.3 \mathrm{E}+00$ & $6.3 \mathrm{E}-02$ & & & $-4.8 \mathrm{E}+01$ & $3.1 \mathrm{E}+03$ & $3.1 E+01$ & & & $-1.2 \mathrm{E}+01$ & $7.6 \mathrm{E}+02$ & $7.6 \mathrm{E}+00$ & & & & & $-1.3 E+01$ \\
\hline Barium & $007440-39-3$ & & & $2.4 E+02$ & $2.4 \mathrm{E}+01$ & $-5.0 E+01$ & & & $1.0 \mathrm{E}+05$ & $1.0 \mathrm{E}+04$ & $-1.5 E+01$ & & & $8.0 E+03$ & $1.0 \mathrm{E}+03$ & $8.0 E+02$ & $1.0 \mathrm{E}+02$ & $-1.7 \mathrm{E}+01$ \\
\hline Baygon & $000114-26-1$ & & & $3.1 E+00$ & $3.1 \mathrm{E}-01$ & $-3.9 \mathrm{E}+01$ & & & $1.2 \mathrm{E}+0 \mathrm{~S}$ & $1.2 E+04$ & $-1.6 \mathrm{E}+00$ & & & $6.5 E+04$ & $8.4 E+03$ & $6.5 \mathrm{E}+03$ & $8.4 E+02$ & $-1.6 \mathrm{E}+00$ \\
\hline Buyleton & $043121-43-3$ & & & $4.4 \mathrm{E}+01$ & $4.4 \mathrm{E}+00$ & $-4.2 \mathrm{E}+01$ & & & $4.2 \mathrm{E}+05$ & 4.2E+04 & $-3.6 E+00$ & & & $2.2 E+05$ & $2.9 \mathrm{E}+04$ & $2.2 E+04$ & $2.9 E+03$ & $-3.7 E+\infty 0$ \\
\hline Baythrold & $068359-37.5$ & & & & & & & & & & & & & & & & & \\
\hline Benefin & $001861-40-1$ & & & $9.9 \mathrm{E}+02$ & $9.9 \mathrm{E}+01$ & $-4.9 \mathrm{E}+01$ & & & $1.1 \mathrm{E}+05$ & $1.1 E+04$ & $-1.6 \mathrm{E}+01$ & & & $6.1 E+04$ & $7.9 E+03$ & $0.1 E+03$ & $7.9 \mathrm{E}+02$ & $-1.8 \mathrm{E}+01$ \\
\hline Benomyl & $017804-35-2$ & & & $7.4 \mathrm{E}+01$ & $7.4 \mathrm{E}+\infty$ & $-4.2 E+01$ & & & $7.0 \mathrm{E}+05$ & $7.0 E+04$ & $-3.6 \mathrm{E}+00$ & & & $3 . \pi+05$ & $4.8 E+04$ & $3.7 E+04$ & $4.8 \mathrm{E}+03$ & $-3.7 E+00$ \\
\hline Bentuzon & $025057-89.0$ & & & 1.1E+00 & 1.IE-01 & $-3.8 E+01$ & & & $1.3 \mathrm{E}+05$ & $1.3 \mathrm{E}+04$ & $-8.4 \mathrm{E}-01$ & & & $6.8 E+04$ & $8.8 E+03$ & $6.8 \mathrm{E}+03$ & $8.8 \mathrm{E}+02$ & $-8.5 \mathrm{E}-01$ \\
\hline Benz[a] anthracene $e^{(m)}$ & $000056.55 .-3$ & $1.1 \mathrm{E}+\infty$ & $1.1 \mathrm{E}-02$ & & & $.5 .0 \mathrm{E}+01$ & $9.1 \mathrm{E}+\infty$ & $9.1 \mathrm{E}-02$ & & & $-1.8 \mathrm{E}+01$ & $2.3 \mathrm{E}+\infty$ & $2.3 \mathrm{E}-02$ & & & & & $-2.1 E+01$ \\
\hline Benzaldehyde & $000100-52-7$ & & & $1.5 \mathrm{E}+02$ & $1.5 \mathrm{E}+01$ & $-4.2 E+01$ & & & $1.0 E+06$ & $1.4 \mathrm{E}+05$ & $-3.6 \mathrm{E}+00$ & & & $7.4 E+05$ & $9.5 \mathrm{E}+04$ & $7.4 E+04$ & $9.5 \mathrm{E}+03$ & $-3.7 \mathrm{E}+00$ \\
\hline Benzene & $000071-43-2$ & $1,0 E+01$ & $1.0 \mathrm{E}-01$ & & & $-4.1 E+01$ & $1.4 \mathrm{E}+05$ & $3.4 \mathrm{E}+03$ & & & $-2.9 E+\infty 0$ & $3.2 \mathrm{E}+04$ & $3.2 \mathrm{E}+02$ & & & & & $-2.9 \mathrm{E}+00$ \\
\hline Benzene Hexachloride & NA & & & & & & & & & & & & & & & & & \\
\hline Benzene, Ethyldimethyl & NA & & & & & & & & & & & & & & & & & \\
\hline Benzene, Ethylmethys & NA & & & & & & & & & & & & & & & & & \\
\hline
\end{tabular}

Nonradionuclides/water and soil/agricultural

Page 3 of 32 
Table 3.3e. (continued)

\begin{tabular}{|c|c|c|c|c|c|c|c|c|c|c|c|c|c|c|c|c|c|c|}
\hline \multirow[b]{2}{*}{ Chemical } & \multirow{2}{*}{ CASRN } & \multicolumn{4}{|c|}{ Vegetable Intercept $(\mathrm{mg} / \mathrm{kg})^{\circ}$} & \multirow[b]{2}{*}{ Slope } & \multicolumn{4}{|c|}{ Beef Intercept (mg/kg) } & \multirow[b]{2}{*}{ Slope } & \multicolumn{6}{|c|}{ Milk Intercept (mg/kg) } & \multirow[b]{2}{*}{ Slope } \\
\hline & & $10^{-4}$ & $10^{4}$ & $H Q=1$ & $\mathrm{HQ}=0.1$ & & $10^{-4}$ & $10^{-6}$ & $\mathrm{HQ}=\mathbf{1}$ & $\mathrm{HQ}=0.1$ & & $10^{-4}$ & $10^{-5}$ & $\begin{array}{l}\text { HQ=1 } \\
\text { Adult }\end{array}$ & $\begin{array}{l}\text { HQ=1 } \\
\text { Child }\end{array}$ & $\begin{array}{c}\mathrm{HQ}=0.1 \\
\text { Adult }\end{array}$ & $\begin{array}{l}\mathrm{HQ}=0.1 \\
\text { Child }\end{array}$ & \\
\hline Benzene, Methylpropyl & NA & & & & & & & & & & & & & & & & & \\
\hline Benzene, Trimethyl & $025551-13-7$ & & & & & & & & & & & & & & & & & \\
\hline Benzenethiol & $000108-98-5$ & & & $1.7 \mathrm{E}-02$ & $1.7 \mathrm{E}-03$ & $-4.3 \mathrm{E}+01$ & & & $1.1 \mathrm{E}+02$ & $1.1 \mathrm{E}+01$ & $-4.4 \mathrm{E}+\infty$ & & & $5.8 \mathrm{E}+01$ & $7.5 E+\infty$ & $5.8 \mathrm{E}+00$ & 7.5E-01 & $-4.6 E+\infty 0$ \\
\hline Benvidine & 000092.87 .5 & 5.7E-04 & 5.TE-06 & $1.7 E+00$ & $1.7 \mathrm{E}-01$ & $-3.8 \mathrm{E}+01$ & 4.3E+01 & 4.3E-01 & $1,3 E+05$ & $1.3 E+04$ & $-1.1 E+\infty$ & $9.6 \mathrm{E}+00$ & $9.6 \mathrm{E}-02$ & $6.7 \mathrm{E}+04$ & $8.6 \mathrm{E}+03$ & $6.7 \mathrm{E}+03$ & $8.6 \mathrm{E}+02$ & $-1.1 \mathrm{E}+\infty$ \\
\hline Benzo[a]pyrene $e^{(2)}$ & $000050-32-8$ & $1.1 \mathrm{E}-01$ & $1.1 \mathrm{E}-03$ & & & $-5.0 E+01$ & 3.7E-01 & $3.7 \mathrm{E}-03$ & & & $-1.9 \mathrm{E}+01$ & $9.3 \mathrm{E}-02$ & $9.3 \mathrm{E}-04$ & & & & & $-2.1 E+01$ \\
\hline Benzo[b]duoranthene $e^{(2)}$ & $000205-99-2$ & $1.1 \mathrm{E}+\infty 0$ & $1.1 \mathrm{E}-02$ & & & $-5.0 \mathrm{E}+01$ & 3.7E+00 & $3.7 \mathrm{E}-02$ & & & $-1.9 \mathrm{E}+01$ & 9.3E-0I & $9.3 \mathrm{E}-03$ & & & & & $-2.1 E+01$ \\
\hline Benzolg.h,i]perylene & $000191-24-2$ & & & & & & & & & & & & & & & & & \\
\hline Benzo[k] luoranthene $e^{(m)}$ & $000207-08-9$ & $1.1 \mathrm{E}+01$ & 1.1E-01 & & & $-5.0 E+01$ & $7.4 E+\infty 0$ & 7.4E-02 & & & $-1.9 \mathrm{E}+01$ & $1.9 \mathrm{E}+00$ & $1.9 \mathrm{E}-02$ & & & & & $-2.2 E+01$ \\
\hline Benzoic Acid & $000065-85-0$ & & & $4.2 E+03$ & 4.2E+02 & $-4.0 \mathrm{E}+01$ & & & $1.0 \mathrm{E}+06$ & $1.0 \mathrm{E}+06$ & $-2.3 \mathrm{E}+00$ & & & $1.0 \mathrm{E}+06$ & $1.0 \mathrm{E}+06$ & $1.0 \mathrm{E}+06$ & $6.0 \mathrm{E}+05$ & $-2.3 \mathrm{E}+\infty 0$ \\
\hline Benzyl Alcohol & $000100-51-6$ & & & $1,3 E+02$ & $1.3 \mathrm{E}+01$ & $-3.8 \mathrm{E}+01$ & & & $1.0 \mathrm{E}+06$ & $1.0 \mathrm{E}+06$ & $-8,4 E-01$ & & & $1.0 \mathrm{E}+06$ & $1.0 \mathrm{E}+06$ & 8.2E+05 & $1.1 E+05$ & $-8.5 E-01$ \\
\hline Benzyl Chloride & $000100-44-7$ & $2.0 \mathrm{E}+00$ & $2.0 \mathrm{E}-02$ & & & $-4.2 \mathrm{E}+01$ & $1.9 \mathrm{E}+04$ & $1.9 \mathrm{E}+02$ & & & $-3.6 \mathrm{E}+00$ & $4.3 \mathrm{E}+03$ & $4.3 \mathrm{E}+01$ & & & & & $-3.7 \mathrm{E}+00$ \\
\hline Beryllium & $007440-41-7$ & $1.9 E-01$ & $1.9 \mathrm{E}-03$ & $1.7 \mathrm{E}+01$ & $1.7 \mathrm{E}+\infty$ & $-5.0 E+01$ & $2.0 \mathrm{E}+01$ & $2.0 \mathrm{E}-01$ & $1.8 E+03$ & $1.8 \mathrm{E}+02$ & $-1.9 \mathrm{E}+01$ & $1.7 \mathrm{E}+03$ & $1.7 \mathrm{E}+01$ & $3.8 \mathrm{E}+05$ & $4.9 \mathrm{E}+04$ & $3.8 \mathrm{E}+04$ & $4.9 E+03$ & $-2.1 \mathrm{E}+01$ \\
\hline Bidrin & $000141-66.2$ & & & 4.TE-03 & 4.7E-04 & $-3.6 \mathrm{E}+01$ & & & $3.0 \mathrm{E}+04$ & $3.0 E+03$ & $-8.2 \mathrm{E}-02$ & & & $1.6 \mathrm{E}+04$ & $2.0 \mathrm{E}+03$ & $1.6 \mathrm{E}+03$ & $2.0 E+02$ & $-8.2 \mathrm{E}-02$ \\
\hline Biphenthrin & $082657-04-3$ & & & & & & & & & & & & & & & & & \\
\hline Biphenyl, 1,1:- & $000092-52-4$ & & & $1.6 \mathrm{E}+02$ & $1.6 \mathrm{E}+01$ & $-4.8 \mathrm{E}+01$ & & & 4.2E+04 & $4.2 \mathrm{E}+03$ & $-1.4 E+01$ & & & $2.4 E+04$ & $3.1 \mathrm{E}+03$ & $2.4 E+03$ & $3.1 \mathrm{E}+02$ & $-1.5 E+01$ \\
\hline $\begin{array}{l}\text { Bis(2-citloro-1- } \\
\text { methylethyl)ether } \\
\text { (Technical) }\end{array}$ & $000108-60-1$ & $6.0 E+00$ & $6.0 \mathrm{E}-02$ & & & $-4.3 E+01$ & $3.3 E+04$ & $3.3 \mathrm{E}+02$ & & & $-4.8 \mathrm{E}+\infty$ & $7.5 E+03$ & 7.5E+01 & & & & & $-5.0 \mathrm{E}+00$ \\
\hline $\begin{array}{l}\text { Bis(2- } \\
\text { chloroethoxy)methane }\end{array}$ & $000111-91-1$ & & & & & & & & & & & & & & & & & \\
\hline Bis(2-ehloroethy) )ether & $000111-44-4$ & $1.2 \mathrm{E}-01$ & $1.2 \mathrm{E}-03$ & & & $-3.8 \mathrm{E}+01$ & $9.0 \mathrm{E}+03$ & $9.0 E+01$ & & & $-1.1 \mathrm{E}+00$ & $2.0 \mathrm{E}+03$ & $2.0 E+01$ & & & & & $-1.1 \mathrm{E}+\infty$ \\
\hline $\begin{array}{l}\text { Bis(2- } \\
\text { chloroisopropyl)ether }\end{array}$ & 039638-32-9 & & & $5.0 \mathrm{E}+01$ & $5.0 \mathrm{E}+00$ & $-4.1 E+01$ & & & 7.1E+05 & $7.1 E+04$ & $-2.9 E+00$ & & & $3.8 \mathrm{E}+05$ & $4.8 \mathrm{E}+04$ & $3.8 \mathrm{E}+04$ & $4.8 E+03$ & $-2.9 \mathrm{E}+00$ \\
\hline
\end{tabular}




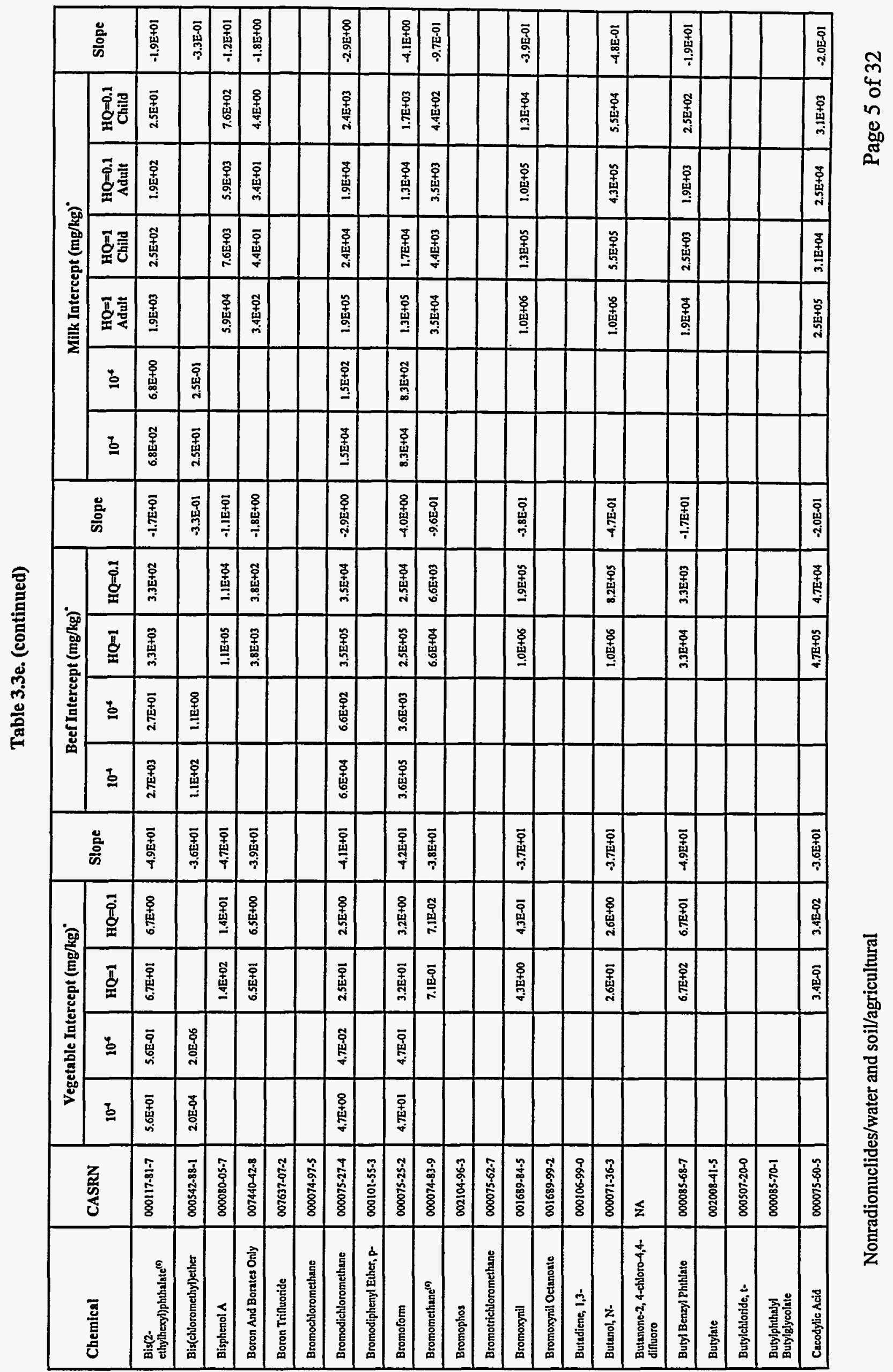


Table 3.3e. (continued)

\begin{tabular}{|c|c|c|c|c|c|c|c|c|c|c|c|c|c|c|c|c|c|c|}
\hline \multirow[b]{2}{*}{ Chemical } & \multirow[b]{2}{*}{ CASRN } & \multicolumn{4}{|c|}{ Vegetable Intercept (mg/kg)" } & \multirow[b]{2}{*}{ Slope } & \multicolumn{4}{|c|}{ Beef Intercept (mg/kg) } & \multirow[b]{2}{*}{ Slope } & \multicolumn{6}{|c|}{ Milk Intercept (mg/kg) } & \multirow[b]{2}{*}{ Slope } \\
\hline & & $10^{-4}$ & $10^{-6}$ & $H Q=1$ & $\mathrm{HQ}=0.1$ & & $10^{-4}$ & $10^{6}$ & $H Q=1$ & $\mathrm{HQ}=0.1$ & & $10^{-4}$ & $10^{4}$ & $\begin{array}{l}\text { HQ=1 } \\
\text { Adult }\end{array}$ & $\begin{array}{l}\text { HQ=1 } \\
\text { Child }\end{array}$ & $\begin{array}{c}H Q=0.1 \\
\text { Adult }\end{array}$ & $\begin{array}{c}\text { HQ }=0.1 \\
\text { Child }\end{array}$ & \\
\hline Calcium Cyanide & $000592-01-8$ & & & & & & & & & & & & & & & & & \\
\hline Caprolectam & $000105-60-2$ & & & $4.5 \mathrm{E}+01$ & $4.5 \mathrm{E}+00$ & $-3.6 \mathrm{E}+01$ & & & $1.0 \mathrm{E}+06$ & $1.0 \mathrm{E}+06$ & $-1.6 \mathrm{E}-01$ & & & $1.0 E+06$ & $1.0 \mathrm{E}+06$ & $1.0 \mathrm{E}+06$ & $6.3 \mathrm{E}+05$ & $-1.6 \mathrm{E}-01$ \\
\hline Captafol & $002425-06-1$ & 4.7E+01 & 4.7E-01 & $3.4 \mathrm{E}+00$ & $3.4 \mathrm{E}-01$ & $-4.3 \mathrm{E}+01$ & $2.9 \mathrm{E}+05$ & $2.9 E+03$ & $2.1 \mathrm{E}+04$ & $2.1 \mathrm{E}+03$ & $-4.4 E+\infty$ & $6.6 \mathrm{E}+04$ & $6.6 \mathrm{E}+02$ & $1.2 \mathrm{E}+04$ & $1.5 E+03$ & $1.2 \mathrm{E}+03$ & $1.5 \mathrm{E}+02$ & $-4.6 \mathrm{E}+\infty 0$ \\
\hline Capten & $000133-06-2$ & $9.9 \mathrm{E}+01$ & $9.9 \mathrm{E}-01$ & $1.9 \mathrm{E}+02$ & $1.9 \mathrm{E}+01$ & $-4.2 \mathrm{E}+01$ & $9.3 \mathrm{E}+05$ & $9.3 \mathrm{E}+03$ & $1.0 \mathrm{E}+06$ & $1.8 \mathrm{E}+05$ & $-3.6 \mathrm{E}+00$ & 2.1E+05 & $2.1 E+03$ & 9.7E+05 & $1.2 E+05$ & $9.7 \mathrm{E}+04$ & $1.2 E+04$ & $-3.7 E+00$ \\
\hline Carbaryl & $000063-25-2$ & & & $1.5 \mathrm{E}+02$ & $1.5 \mathrm{E}+01$ & $-4.2 \mathrm{E}+01$ & & & $1.0 \mathrm{E}+06$ & $1.4 E+05$ & $-3.6 \mathrm{E}+\infty$ & & & 7.4E+05 & $9.5 \mathrm{E}+04$ & $7.4 \mathrm{E}+04$ & $9,5 \mathrm{E}+03$ & $-3.7 E+\infty 0$ \\
\hline Carbazole & $000086-74-8$ & $3.5 \mathrm{E}+01$ & $3.5 \mathrm{E}-01$ & & & $-4.8 \mathrm{E}+01$ & $1.7 \mathrm{E}+04$ & $1.7 E+02$ & & & $-1.2 \mathrm{E}+01$ & $4.2 E+03$ & 4.2E+0I & & & & & $-1.3 E+01$ \\
\hline Carbofuren & $001563-66-2$ & & & $3.9 \mathrm{E}+00$ & $3.9 \mathrm{E}-01$ & $-3.9 E+01$ & & & $1.5 \mathrm{E}+05$ & $1.5 E+04$ & $-1.6 \mathrm{E}+00$ & & & $8.1 E+04$ & $1.0 E+04$ & 8. $1 E+03$ & $1.0 \mathrm{E}+03$ & $-1.6 \mathrm{E}+00$ \\
\hline Carbon Disulfide & $000075-15-0$ & & & $1.4 \mathrm{E}+02$ & $1.4 \mathrm{E}+01$ & $-4.1 E+01$ & & & $1.0 \mathrm{E}+06$ & $1.6 \mathrm{E}+05$ & $-3.2 E+\infty 0$ & & & $8.4 \mathrm{E}+05$ & $1.1 \mathrm{E}+05$ & $8.4 \mathrm{E}+04$ & $1.1 \mathrm{E}+04$ & $-3.3 E+00$ \\
\hline Carbon Tetrachloride' & $000056-23-5$ & $3.7 \mathrm{E}+00$ & 3.7E-02 & $1.4 E+00$ & $1.4 \mathrm{E}-01$ & $-4.4 \mathrm{E}+01$ & $1.3 \mathrm{E}+04$ & $1.3 \mathrm{E}+02$ & $5.1 \mathrm{E}+03$ & $5.1 \mathrm{E}+02$ & $-5.9 E+00$ & $3.0 \mathrm{E}+03$ & $3.0 \mathrm{E}+01$ & $2.8 \mathrm{E}+03$ & $3.6 \mathrm{E}+02$ & $2.8 \mathrm{E}+02$ & $3.6 \mathrm{E}+01$ & $-6.2 \mathrm{E}+00$ \\
\hline Carboxin & $005234-68-4$ & & & $1.3 \mathrm{E}+02$ & $1.3 E+01$ & $-4.1 \mathrm{E}+01$ & & & $1.0 \mathrm{E}+\infty 6$ & $1.8 \mathrm{E}+05$ & $-2.9 E+\infty 0$ & & & $9.4 \mathrm{E}+05$ & $1.2 \mathrm{E}+05$ & $9.4 E+04$ & $1.2 \mathrm{E}+04$ & $-2.9 \mathrm{E}+00$ \\
\hline Chloral & $000075-87-6$ & & & & & & & & & & & & & & & & & \\
\hline Choramben & $000133-90-4$ & & & & & & & & & & & & & & & & & \\
\hline Chloranil & $000118-75-2$ & & & & & & & & & & & & & & & & & \\
\hline Chlordane $e^{(n)}$ & $000057-74-9$ & $6.2 \mathrm{E}-01$ & $6.2 E-03$ & 2.1E-01 & $2.1 \mathrm{E}-02$ & $-4.9 \mathrm{E}+01$ & $8.0 E+\infty$ & $8.0 \mathrm{E}-02$ & 2.7E+00 & $2.7 \mathrm{E}-01$ & $-1.8 E+01$ & $2.0 \mathrm{E}+\infty 0$ & $2.0 \mathrm{E}-02$ & $1.6 \mathrm{E}+\infty$ & $2.0 \mathrm{E}-01$ & $1.6 \mathrm{E}-01$ & $2.0 \mathrm{E}-02$ & $-2.1 \mathrm{E}+01$ \\
\hline Cthoride & $016887-00-6$ & & & & & & & & & & & & & & & & & \\
\hline Chlorimuron, Ethyl- & $090982-32-4$ & & & & & & & & & & & & & & & & & \\
\hline Chlotine & $007782.50-5$ & & & $5.1 \mathrm{E}+00$ & $5.1 \mathrm{E}-01$ & $-3.6 \mathrm{E}+01$ & & & $1.1 E+01$ & $1.1 E+\infty$ & $-1.1 \mathrm{E}-01$ & & & $2.1 \mathrm{E}+00$ & $2.7 \mathrm{E}-01$ & $2.1 E-01$ & 2.7E-02 & $-1 .|\mathrm{E}-0|$ \\
\hline Chlorine Dioxide & $010049-04-4$ & & & & & & & & & & & & & & & & & \\
\hline Chlorite (Sodium Sall) & $007758-19-2$ & & & & · & & & & & & & & & & & & & \\
\hline $\begin{array}{l}\text { Chloro-1,1- } \\
\text { difluoroethene, 1- }\end{array}$ & $000075-68-3$ & & & & & & & & & & & & & & & & & \\
\hline Chloro-1,3-butadiene, 2- & $000126-99-8$ & & & $1.9 \mathrm{E}+01$ & $1.9 \mathrm{E}+\infty 0$ & $-4.0 E+01$ & & & $5.0 \mathrm{E}+05$ & $5.0 E+04$ & $-2.0 E+\infty 0$ & & & $2.6 \mathrm{E}+05$ & $3.4 \mathrm{E}+04$ & $2.6 E+04$ & $3.4 E+03$ & $-2.0 \mathrm{E}+\infty 0$ \\
\hline
\end{tabular}

Nonradionuclides/water and soil/agricultural

Page 6 of 32 
3-467

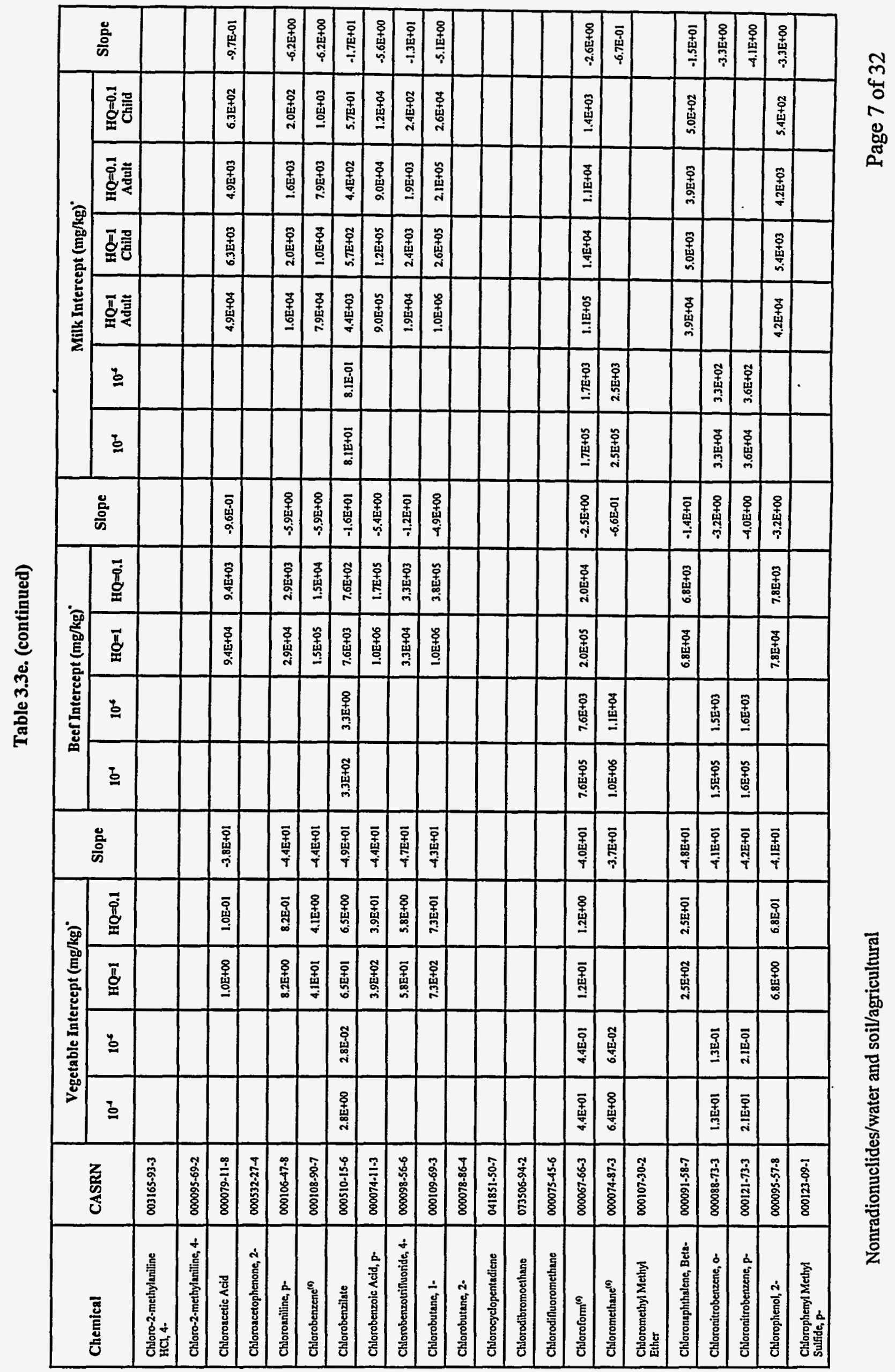


Table 3.3e. (continued)

\begin{tabular}{|c|c|c|c|c|c|c|c|c|c|c|c|c|c|c|c|c|c|c|}
\hline \multirow{2}{*}{ Chemical } & \multirow{2}{*}{ CASRN } & \multicolumn{4}{|c|}{ Vegetable Intercept (mg/kg) } & \multirow[b]{2}{*}{ Slope } & \multicolumn{4}{|c|}{ Beef Intercept (mg/kg) } & \multirow[b]{2}{*}{ Slope } & \multicolumn{6}{|c|}{ Milk Intercept (mg/kg) } & \multirow[b]{2}{*}{ Slope } \\
\hline & & $10^{-4}$ & $10^{6}$ & HQ $=1$ & $H Q=0.1$ & & $10^{-4}$ & $10^{6}$ & $\mathrm{HQ}=1$ & $\mathrm{HQ}=0.1$ & & $10^{-4}$ & $10^{5}$ & $\begin{array}{l}H Q=1 \\
\text { Adult }\end{array}$ & $\begin{array}{l}\text { HQ }=1 \\
\text { Cbild }\end{array}$ & $\begin{array}{c}\mathrm{HQ}=0.1 \\
\text { Adult }\end{array}$ & $\begin{array}{l}\text { HQ }=0.1 \\
\text { Child }\end{array}$ & \\
\hline $\begin{array}{l}\text { Criorophenyl Methyl } \\
\text { Sulfoxide }\end{array}$ & $000934-73-6$ & & & & & & & & & & & & & & & & & \\
\hline Chloropropane, 2 - & $000075-29-6$ & & & & & & & & & & & & & & & & & \\
\hline Chlorothalonil & $001897-45-6$ & $6.4 \mathrm{E}+01$ & $6.4 \mathrm{E}-01$ & 4.5E+01 & $4.5 \mathrm{E}+00$ & $-4.8 E+01$ & 2.6E+04 & $2.6 \mathrm{E}+02$ & $1.9 \mathrm{E}+04$ & $1.9 E+03$ & $-1.3 \mathrm{E}+01$ & $6.3 \mathrm{E}+03$ & $6.3 \mathrm{E}+01$ & $1.1 \mathrm{E}+04$ & $1.4 \mathrm{E}+03$ & $1.1 \mathrm{E}+03$ & $1.4 \mathrm{E}+02$ & $-1.4 E+01$ \\
\hline Chlorotoluene, o- & $000095-49-8$ & & & $5.3 \mathrm{E}+01$ & $5.3 \mathrm{E}+00$ & $-4.6 \mathrm{E}+01$ & & & $5.9 \mathrm{E}+04$ & $5.9 E+03$ & $-9.5 \mathrm{E}+\infty$ & & & $3.3 \mathrm{E}+04$ & $4.2 \mathrm{E}+03$ & $3.3 \mathrm{E}+03$ & $4.2 \mathrm{E}+02$ & $-1.0 \mathrm{E}+01$ \\
\hline Chlorpropham & $000101-21-3$ & & & 4.3E+02 & $4.3 \mathrm{E}+01$ & $-4.4 \mathrm{E}+01$ & & & $1.0 \mathrm{E}+06$ & $1.3 \mathrm{E}+05$ & $-6.5 \mathrm{E}+00$ & & & $6.9 \mathrm{E}+05$ & $8.9 E+04$ & $6.9 \mathrm{E}+04$ & $8.9 E+03$ & $-6.8 \mathrm{E}+\infty$ \\
\hline Chlorpyrifos & $002921.88-2$ & & & $1.0 \mathrm{E}+01$ & $1.0 \mathrm{E}+00$ & $-4.9 E+01$ & & & $2.1 \mathrm{E}+02$ & $2.1 \mathrm{E}+01$ & $-1.8 \mathrm{E}+01$ & & & $1.2 \mathrm{E}+02$ & $1.6 \mathrm{E}+01$ & $1.2 \mathrm{E}+01$ & $1.6 \mathrm{E}+00$ & $-2.0 \mathrm{E}+01$ \\
\hline Chlorpyrifos Methyl & $005598-13-0$ & & & $3.2 \mathrm{E}+01$ & $3.2 \mathrm{E}+00$ & $-4.9 \mathrm{E}+01$ & & & $5.7 \mathrm{E}+03$ & $5.7 \mathrm{E}+02$ & $-1.5 E+01$ & & & $3.3 \mathrm{E}+03$ & $4.2 \mathrm{E}+02$ & $3.3 \mathrm{E}+02$ & $4.2 \mathrm{E}+01$ & $-1.6 \mathrm{E}+01$ \\
\hline Chlorsulturon & $064902-72-3$ & & & $9.8 \mathrm{E}-01$ & $9.8 \mathrm{E}-02$ & $-3.6 \mathrm{E}+01$ & & & $1.0 \mathrm{E}+06$ & $1.0 \mathrm{E}+06$ & $-3.4 \mathrm{E}-02$ & & & $1.0 \mathrm{E}+06$ & $1.0 \mathrm{E}+06$ & $1.0 \mathrm{E}+06$ & $1.9 \mathrm{E}+05$ & $-3,4 \mathrm{E} \cdot 02$ \\
\hline Chiorthiophos & $060238.56-4$ & & & & & & & & & & & & & & & & & \\
\hline $\begin{array}{l}\text { Chromium (III) (Insoluble } \\
\text { Salts) }\end{array}$ & $016065.83-1$ & & & $3.5 E+03$ & $3.5 \mathrm{E}+02$ & $-5.0 E+01$ & & & $3.8 \mathrm{E}+04$ & $3.8 \mathrm{E}+03$ & $-1.7 E+01$ & & & $1.0 \mathrm{E}+06$ & 8.1E+0S & $6.3 \mathrm{E}+05$ & $8.1 \mathrm{E}+04$ & $-2.0 E+01$ \\
\hline Chromium (VI) & $018540-29.9$ & & & $1.8 \mathrm{E}+01$ & $1.8 \mathrm{E}+00$ & $-5.0 \mathrm{E}+01$ & & & $1.9 \mathrm{E}+02$ & $1.9 \mathrm{E}+01$ & $-1.7 E+01$ & & & $3.1 E+04$ & $4.0 \mathrm{E}+03$ & $3.1 E+03$ & $4.0 \mathrm{E}+02$ & $-2,0 \mathrm{E}+01$ \\
\hline Chromium Salts & $\mathrm{NA}$ & & & & & & & & & & & & & & & & & \\
\hline Chrysene $e^{(m)}$ & $000218-01-9$ & $1.1 \mathrm{E}+02$ & $1.1 \mathrm{E}+\infty$ & & & $-5.0 \mathrm{E}+01$ & $9.1 \mathrm{E}+02$ & $9.1 E+\infty$ & & & $-1.8 \mathrm{E}+01$ & $2.3 \mathrm{E}+02$ & $2.3 \mathrm{E}+00$ & & & & & $-2.1 \mathrm{E}+01$ \\
\hline Cobalt & $007440-48-4$ & & & & & & & & & & & & & & & & & \\
\hline Coke Oven Emissions & $008007-45-2$ & & & & & & & & & & & & & $\cdot$ & & & & \\
\hline Copper & $007440-50.8$ & & & & & & & & & & & & & & & & & \\
\hline Copper Cyaride & $000544-92.3$ & & & & & & & & & & & & & & & & & \\
\hline Creosote & $008001-58.9$ & & & & & & & & & & & & & & & & & \\
\hline Cresol, m- & $000108-39-4$ & & & $5.8 \mathrm{E}+01$ & $5.8 \mathrm{E}+00$ & $-4.0 E+01$ & & & $9.9 \mathrm{E}+05$ & $9.9 \mathrm{E}+04$ & $-2.5 \mathrm{E}+00$ & & & $5.3 E+05$ & $6.8 \mathrm{E}+04$ & $5.3 \mathrm{E}+04$ & $6.8 \mathrm{E}+03$ & $-2.6 E+00$ \\
\hline Cresol, O- & $000095-48-7$ & & & $5.3 \mathrm{E}+0 \mathrm{t}$ & $5.3 \mathrm{E}+\infty$ & $-4.0 \mathrm{E}+0 \mathrm{t}$ & & & $1.0 \mathrm{E}+06$ & $1.1 \mathrm{E}+05$ & $-2.3 \mathrm{E}+00$ & & & $5.9 E+05$ & $7.5 E+04$ & $5.9 E+04$ & $7.5 \mathrm{E}+03$ & $-2.3 E+\infty$ \\
\hline Cresol, p- & $000106-44-5$ & & & $5.3 E+\infty$ & $5.3 \mathrm{E}-01$ & $-4.0 \mathrm{E}+01$ & & & 1.1E+05 & 1.1E+04 & $-2.3 \mathrm{E}+\infty 0$ & & & $5.9 E+04$ & $7.5 \mathrm{E}+03$ & $5.9 \mathrm{E}+03$ & $7.5 E+02$ & $-2.3 E+00$ \\
\hline Crotonaldehyde & $000123-73-9$ & $1.2 E-01$ & $1.2 \mathrm{E}-03$ & & & $-4.0 E+01$ & $3.0 \mathrm{E}+03$ & $3.0 \mathrm{E}+01$ & & & $-2.0 \mathrm{E}+00$ & $6.8 \mathrm{E}+02$ & $6.8 E+\infty$ & & & & & $-2.0 E+\infty 0$ \\
\hline
\end{tabular}

Nonradionuclides/water and soil/agricultural

Page 8 of 32 
Table 3.3e. (continued)

\begin{tabular}{|c|c|c|c|c|c|c|c|c|c|c|c|c|c|c|c|c|c|c|}
\hline \multirow[b]{2}{*}{ Chemical } & \multirow[b]{2}{*}{ CASRN } & \multicolumn{4}{|c|}{ Vegetable Intercept (mg/kg) } & \multirow[b]{2}{*}{ Slope } & \multicolumn{4}{|c|}{ Beef Intercept (mg/kg)" } & \multirow[b]{2}{*}{ Slope } & \multicolumn{6}{|c|}{ Milk Intercept (mg/kg) } & \multirow[b]{2}{*}{ Slope } \\
\hline & & $10^{-4}$ & $10^{6}$ & $\mathrm{HQ}=1$ & $\mathrm{HQ}=0.1$ & & $10^{-6}$ & $10^{5}$ & HQ=1 & $\mathrm{HQ}=0.1$ & & $10^{-4}$ & $10^{4}$ & $\begin{array}{l}\text { HQ=1 } \\
\text { Adult }\end{array}$ & $\begin{array}{l}\text { HQ=1 } \\
\text { Child }\end{array}$ & $\begin{array}{c}\text { HQ }=0.1 \\
\text { Adult }\end{array}$ & $\begin{array}{c}\mathrm{HQ}=0.1 \\
\text { Child }\end{array}$ & \\
\hline Cumene & $000098-82-8$ & & & $1.1 E+02$ & $1.1 E+01$ & $-4.7 E+01$ & & & $1.0 \mathrm{E}+05$ & $1.0 \mathrm{E}+04$ & $-1,0 E+01$ & & & $5.6 \mathrm{E}+04$ & $7.2 E+03$ & $5.6 \mathrm{E}+03$ & $7.2 E+02$ & $-1.1 \mathrm{E}+01$ \\
\hline Cyznazine & $021725-46-2$ & 3.9E-01 & $3.9 \mathrm{E}-03$ & $2.8 \mathrm{E}+00$ & 2.8E-01 & $-4.1 E+01$ & $4.3 \mathrm{E}+03$ & $4.3 \mathrm{E}+01$ & 3.1E+04 & $3.1 \mathrm{E}+03$ & $-3.3 E+\infty$ & $9.6 \mathrm{E}+02$ & $9.6 \mathrm{E}+\infty 0$ & $1.6 \mathrm{E}+0.4$ & $2.1 E+03$ & $1.6 \mathrm{E}+03$ & $2.1 E+02$ & $-3.4 E+00$ \\
\hline Cyenide (CN-) & $000057-12.5$ & & & $9.0 \mathrm{E}+\infty 0$ & $9.0 \mathrm{E}-01$ & $-3.8 E+01$ & & & $1.0 \mathrm{E}+06$ & $1.0 \mathrm{E}+05$ & $-8.4 \mathrm{E}-01$ & & & $5.5 \mathrm{E}+05$ & $7.0 E+04$ & $5.5 E+04$ & $7.0 \mathrm{E}+03$ & $-8.5 \mathrm{E}-01$ \\
\hline Cyanogen & $000460-19-5$ & & & & & & & & & & & & & & & & & \\
\hline Cynnogen Bromide & $000506-68-3$ & & & & & & & & & & & & & & & & & \\
\hline Cyenogen Chloride & $000506-77-4$ & & & $7.4 \mathrm{E}+00$ & $7.4 \mathrm{E}-01$ & $-3.6 \mathrm{E}+01$ & & & $1.0 \mathrm{E}+06$ & $6.4 \mathrm{E}+05$ & $-2.6 \mathrm{E}-01$ & & & $1.0 \mathrm{E}+06$ & $4.3 \mathrm{E}+05$ & $3.4 E+05$ & $4.3 E+04$ & $-2.6 \mathrm{E}-01$ \\
\hline $\begin{array}{l}\text { Cyclohexare, 1,2,3,4,5- } \\
\text { pentabromo-6-chloro- }\end{array}$ & $000087-84-3$ & & & & & & & & & & & & & & & & & \\
\hline Cyclohexanone & $000108-94-1$ & & & $1.6 \mathrm{E}+03$ & $1.6 \mathrm{E}+02$ & $-3.7 z+01$ & & & $1.0 \mathrm{E}+06$ & $1.0 \mathrm{E}+06$ & $-5.8 \mathrm{E}-01$ & & & $1.0 \mathrm{E}+06$ & $1.0 \mathrm{E}+06$ & $1.0 \mathrm{E}+06$ & $1.0 \mathrm{E}+06$ & $-5.9 E-01$ \\
\hline Cyclohexylamine & $000108.91-8$ & & & $1.4 \mathrm{E}+02$ & $1.4 E+01$ & $-3.9 \mathrm{E}+01$ & & & $1.0 \mathrm{E}+06$ & $6.9 \mathrm{E}+0 \mathrm{~S}$ & $-1.4 E+\infty 0$ & & & $1.0 \mathrm{E}+06$ & $4.6 \mathrm{E}+05$ & $3.6 \mathrm{E}+05$ & $4.6 \mathrm{E}+04$ & $-1.4 \mathrm{E}+00$ \\
\hline Cyclopentediene & $000542-92-7$ & & & & & & & & & & & & & & & & & \\
\hline Cyhalothrin/karate & 068085.85 .8 & & & $1.7 \mathrm{E}+01$ & $1.7 E+\infty$ & $-5.0 \mathrm{E}+01$ & & & $9.3 \mathrm{E}+00$ & 9.3E-01 & $-1.9 \mathrm{E}+01$ & & & $5.5 \mathrm{E}+\infty 0$ & $7.1 \mathrm{E}-01$ & $5.5 \mathrm{E}-01$ & 7.1E-02 & $-2.2 E+01$ \\
\hline Cypermethrin & $052315-07.8$ & & & & & & & & & & & & & & & & & \\
\hline Cyromaxine & $066215-27-8$ & & & & & & & & & & . & & & & & & & \\
\hline DDD. & $000072-54-8$ & $3.4 \mathrm{E}+\infty 0$ & $3.4 \mathrm{E}-02$ & & & $-5.0 \mathrm{E}+01$ & $2.2 E+01$ & $2.2 \mathrm{E}-01$ & & & $-1.8 \mathrm{E}+01$ & $5.5 E+\infty$ & $5.5 \mathrm{E}-02$ & & & & & $-2.1 E+01$ \\
\hline DDE & $000072-55.9$ & $2.4 E+\infty 0$ & $2.4 \mathrm{E}-02$ & & & $-5.0 \mathrm{E}+01$ & $1.9 \mathrm{E}+01$ & $1.9 \mathrm{E}-01$ & & & $-1.8 \mathrm{E}+01$ & $4.9 \mathrm{E}+00$ & $4.9 \mathrm{E}-02$ & & & & & $-2.1 \mathrm{E}+01$ \\
\hline DDT & $000050-29-3$ & $2.4 \mathrm{E}+\infty$ & $2.4 \mathrm{E}-02$ & $1.7 \mathrm{E}+\infty$ & $1.7 \mathrm{E}-01$ & $-5.0 \mathrm{E}+01$ & $4.4 E+00$ & $4.4 \mathrm{E}-02$ & $3.2 \mathrm{E}+00$ & $3.2 \mathrm{E}-01$ & $-1.9 \mathrm{E}+01$ & $1.1 \mathrm{E}+\infty$ & 1.IE-02 & $1.9 \mathrm{E}+\infty$ & 2.4E-01 & $1.9 \mathrm{E}-01$ & $2.4 \mathrm{E}-02$ & $-2.1 \mathrm{E}+01$ \\
\hline Dacthal & $001861-32-1$ & & & & & & & & & & & & & & & & & \\
\hline Dalapon & 000075.99 .0 & & & $9.2 \mathrm{E}+\infty 0$ & 9.2E- -01 & $-3.7 \mathrm{E}+01$ & & & $1.0 \mathrm{E}+06$ & $2.2 \mathrm{E}+05$ & $-5.6 \mathrm{E}-01$ & & & $1.0 \mathrm{E}+06$ & $1.5 E+05$ & $1.1 \mathrm{E}+05$ & $1.5 \mathrm{E}+04$ & $-5.6 \mathrm{E}-01$ \\
\hline Decabromodiphenyl Ether & $001163-19-5$ & & & $3.4 \mathrm{E}+01$ & $3.4 \mathrm{E}+\infty 0$ & $-4.9 \mathrm{E}+01$ & & & $7.9 \mathrm{E}+02$ & $7.9 \mathrm{E}+01$ & $-1,8 \mathrm{E}+01$ & & & $4.6 \mathrm{E}+02$ & $6.0 \mathrm{E}+01$ & $4.6 \mathrm{E}+01$ & $6.0 \mathrm{E}+\infty 0$ & $-2.0 \mathrm{E}+01$ \\
\hline Demeton & $008065-48-3$ & & & & & & & & & & & & & & & & & \\
\hline Di(2-ethylhexyl)adipate & $000103-23-1$ & $6.8 \mathrm{E}+02$ & $6.8 \mathrm{E}+00$ & 2.1E+03 & $2.1 E+02$ & $-5.0 \mathrm{E}+01$ & $4.6 \mathrm{E}+01$ & $4.6 \mathrm{E}-01$ & $1.4 \mathrm{E}+02$ & $1.4 \mathrm{E}+01$ & $-1.9 \mathrm{E}+01$ & $1.2 \mathrm{E}+01$ & $1.2 \mathrm{E}-01$ & $8.4 E+01$ & $1.1 E+01$ & $8.4 E+00$ & $1.1 \mathrm{E}+\infty 0$ & $-2.2 \mathrm{E}+01$ \\
\hline Diallate & $002303-16-4$ & $1.1 \mathrm{E}+\infty$ & 1.1E-02 & & & $-3.7 E+01$ & $2.9 \mathrm{E}+05$ & $2.9 \mathrm{E}+03$ & & & $-5.2 \mathrm{E}-01$ & $6.5 \mathrm{E}+04$ & $6.5 E+02$ & & & & & $-5.3 \mathrm{E}-01$ \\
\hline Dievinon & $000333-41-5$ & & & $2.7 E+00$ & $2.7 \mathrm{E}-01$ & $-4.8 \mathrm{E}+01$ & & & $1.3 \mathrm{E}+03$ & $1.3 \mathrm{E}+02$ & $-1.2 \mathrm{E}+01$ & & & $7.6 \mathrm{E}+02$ & $9.7 E+01$ & $7.6 \mathrm{E}+01$ & $9.7 E+\infty 0$ & $-1.3 \mathrm{E}+01$ \\
\hline
\end{tabular}

Nonradionuclides/water and soil/agricultural

Page 9 of 32 
$3-470$

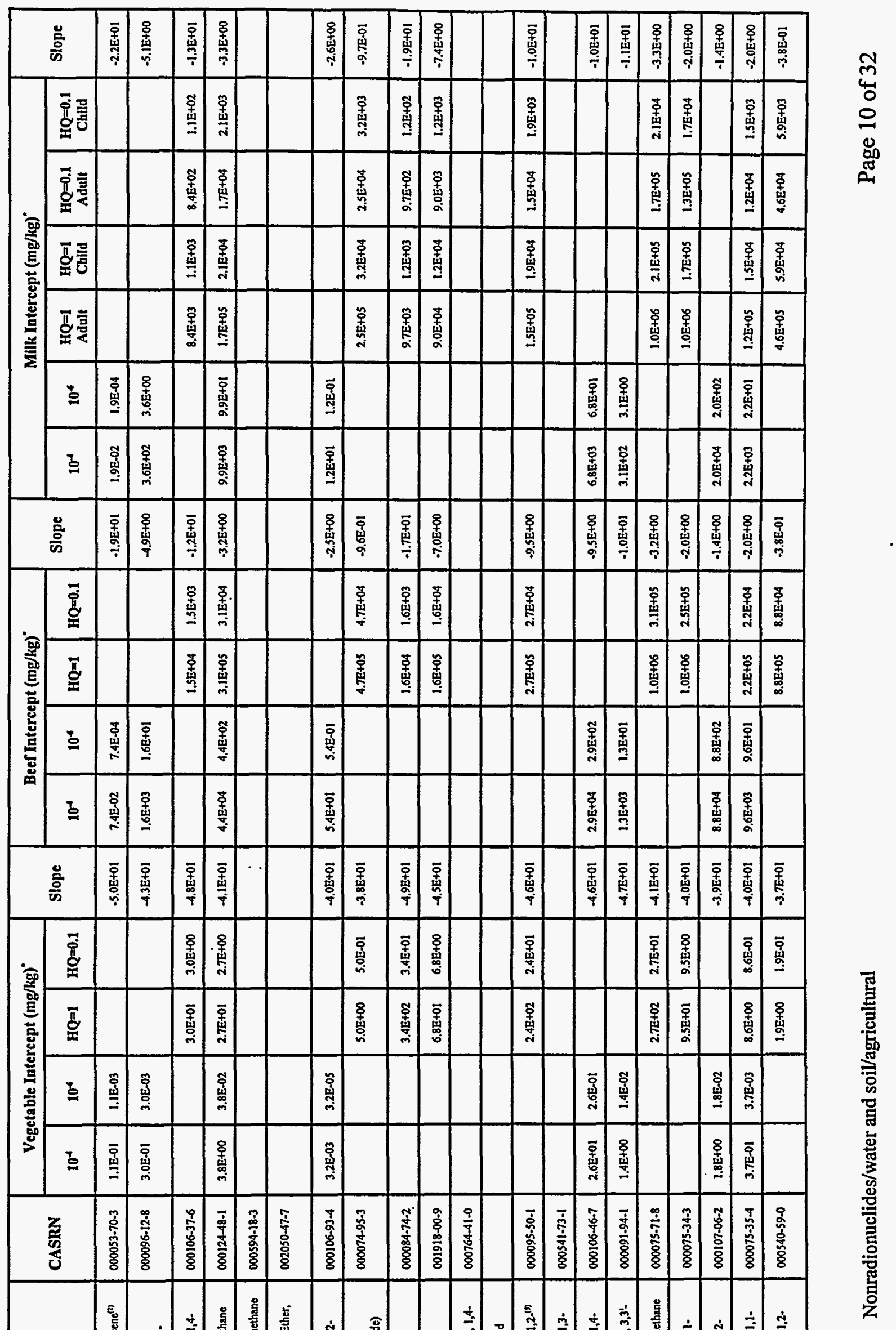




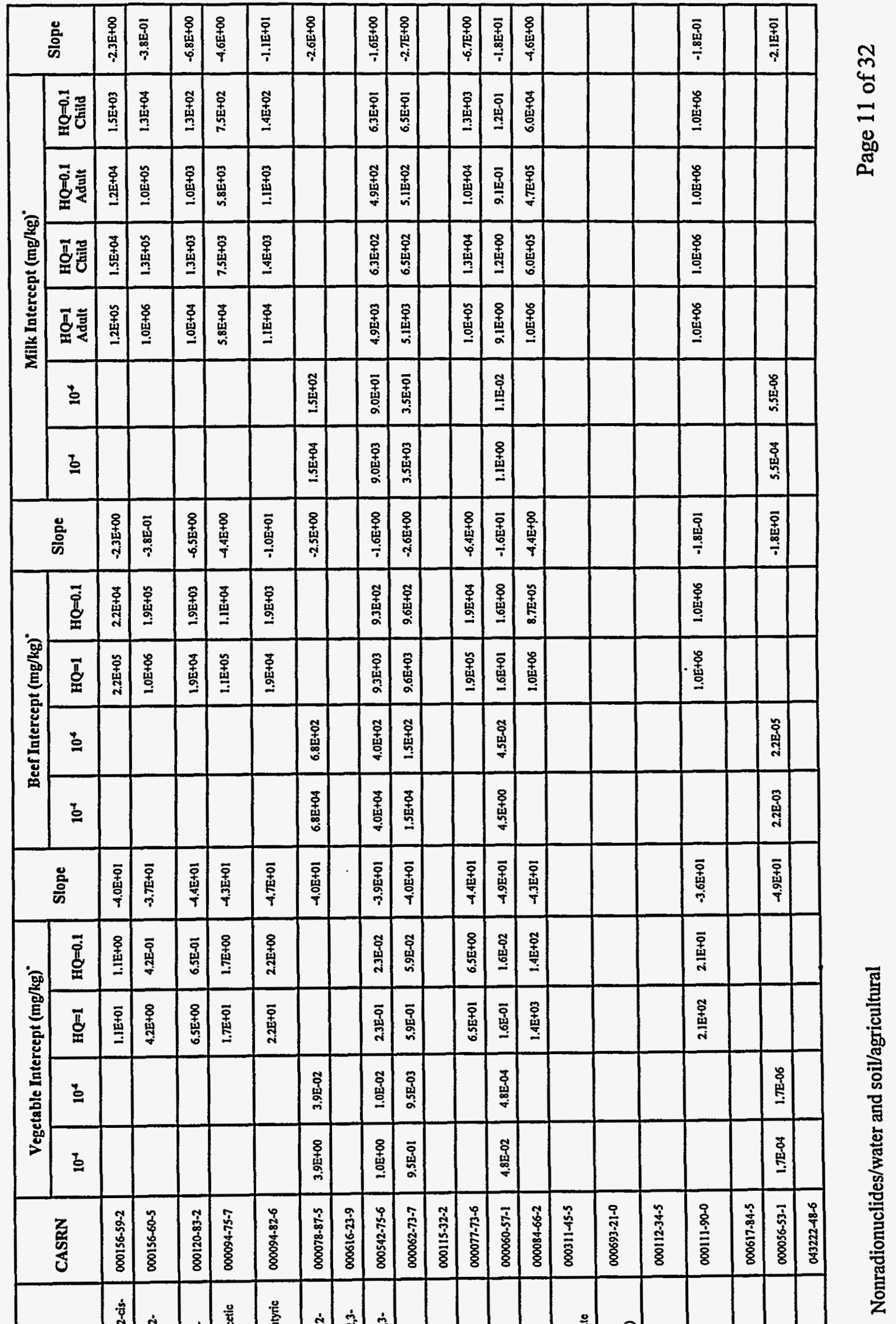

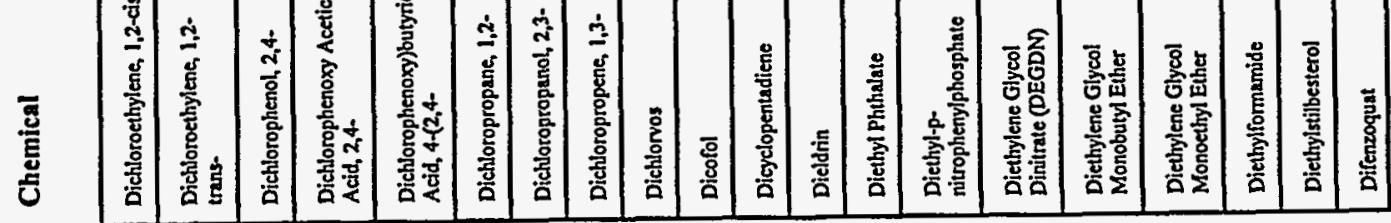


Table 3.3e. (continued)

\begin{tabular}{|c|c|c|c|c|c|c|c|c|c|c|c|c|c|c|c|c|c|c|}
\hline \multirow[b]{2}{*}{ Chemical } & \multirow[b]{2}{*}{ CASRN } & \multicolumn{4}{|c|}{ Vegetable Intercept (mg/kg) } & \multirow[b]{2}{*}{ Slope } & \multicolumn{4}{|c|}{ Beef Intercept (mg/kg) } & \multirow[b]{2}{*}{ Slope } & \multicolumn{6}{|c|}{ Milk Intercept (mg/kg) } & \multirow[b]{2}{*}{ Slope } \\
\hline & & $10^{-4}$ & $10^{4}$ & $\mathbf{H Q}=\mathbf{1}$ & $\mathrm{HQ}=0.1$ & & $10^{-1}$ & $10^{6}$ & $H Q=1$ & $H Q=0.1$ & & $10^{-4}$ & $10^{6}$ & $\begin{array}{l}\text { HQ=1 } \\
\text { Adult }\end{array}$ & $\begin{array}{l}\text { HQ=1 } \\
\text { Child }\end{array}$ & $\begin{array}{c}H Q=0.1 \\
\text { Adult }\end{array}$ & $\begin{array}{c}\text { HQ }=0.1 \\
\text { Child }\end{array}$ & \\
\hline Difluoroethane, 1,1- & $000075-37.6$ & & & & & & & & & & & & & & & & & \\
\hline $\begin{array}{l}\text { Diisopropyl } \\
\text { Methylphosphonate }\end{array}$ & $001445-75-6$ & & & $3,3 \mathrm{E}+01$ & $3.3 \mathrm{E}+00$ & $-3.7 \mathrm{E}+01$ & & & $1.0 \mathrm{E}+06$ & $4.5 \mathrm{E}+05$ & $-7.7 E-01$ & & & $1.0 \mathrm{E}+06$ & $3.0 \mathrm{E}+05$ & $2.4 \mathrm{E}+05$ & $3.0 \mathrm{E}+04$ & $-7.8 \mathrm{E}-01$ \\
\hline Dimethoate & $000060-51-5$ & & & $3.9 \mathrm{E}-01$ & $3.9 \mathrm{E}-02$ & $-4.4 E+01$ & & & $1.7 \mathrm{E}+03$ & $1.7 \mathrm{E}+02$ & $-5.4 \mathrm{E}+\infty 0$ & & & $9.0 \mathrm{E}+02$ & $1.2 \mathrm{E}+02$ & $9.0 \mathrm{E}+01$ & $1.2 \mathrm{E}+01$ & $-5.6 \mathrm{E}+00$ \\
\hline Dimethoxybenzidine, 3,3!- & $000119-90-4$ & $1.6 \mathrm{E}+01$ & $1.6 \mathrm{E}-01$ & & & $-4.0 \mathrm{E}+01$ & $4.1 \mathrm{E}+05$ & $4.1 \mathrm{E}+03$ & & & $-2.0 \mathrm{E}+\infty$ & $9.3 E+04$ & $9.3 E+02$ & & & & & $-2.0 \mathrm{E}+00$ \\
\hline Dimethyl Sulfate & $000077-78-1$ & & & & & & & & & & & & & & & & & \\
\hline Dimethylariline $\mathrm{HCl}, 2,4 \cdot$ & $021436-96-4$ & & & & & & & & & & & & & & & & & \\
\hline Dimethylaniline, 2,4- & $000095-68-1$ & & & & & & & & & & & & & & & & & \\
\hline Dimethylariline, N,N- & $000121-69-7$ & & & $3.7 E+\infty$ & 3.7E-01 & $-4.3 \mathrm{E}+01$ & & & $1.9 \mathrm{E}+04$ & $1.9 \mathrm{E}+03$ & $-4.9 E+\infty$ & & & $1.0 \mathrm{E}+04$ & $1.3 E+03$ & $1.0 \mathrm{E}+03$ & $1.3 \mathrm{E}+02$ & $-5.1 E+\infty$ \\
\hline Dimethylbenzidine, 3,3'- & $000119.93-7$ & $3.8 \mathrm{E} \cdot 02$ & $3.8 \mathrm{E}-04$ & & & $-4.2 \mathrm{E}+01$ & $3.5 \mathrm{E}+02$ & $3.5 \mathrm{E}+\infty 0$ & & & $-3.6 \mathrm{E}+00$ & $8.0 \mathrm{E}+01$ & $8.0 \mathrm{E}-01$ & & & & & $-3.7 \mathrm{E}+00$ \\
\hline Dimethylethyl Lead & $107584-40-7$ & & & & & & & & & & & & & & & & & \\
\hline Dimethylformamide & $000068-12-2$ & & & $3.1 \mathrm{E}+\infty 0$ & $3.1 \mathrm{E}-01$ & $-3.6 \mathrm{E}+01$ & & & $1.0 \mathrm{E}+06$ & $1.0 \mathrm{E}+06$ & $-5.3 \mathrm{E}-02$ & & & $1.0 \mathrm{E}+06$ & $1.0 \mathrm{E}+06$ & $1.0 \mathrm{E}+06$ & $2.8 \mathrm{E}+05$ & $-5.4 \mathrm{E} \cdot 02$ \\
\hline Dimethythydrazine, 1,1.-(n) & $000057 \cdot 14-7$ & & & & & & & & & & & & & & & & & \\
\hline Dimethylhydrazine, 1,2- & $000540.73-8$ & & & & & & & & & & & & & & & & & \\
\hline Dimethylphenol, 2,4- & $000105.67-9$ & & & $3.0 \mathrm{E}+01$ & $3.0 \mathrm{E}+\infty 0$ & $-4.2 \mathrm{E}+0 \mathrm{t}$ & & & $2.8 \mathrm{E}+05$ & $2.8 \mathrm{E}+04$ & $-3.6 \mathrm{E}+00$ & & & $1.5 \mathrm{E}+05$ & $1.9 \mathrm{E}+04$ & $1.5 E+04$ & $1.9 \mathrm{E}+03$ & $-3.7 \mathrm{E}+00$ \\
\hline Dimethylphenol, 2,6- & $000576-26-1$ & & & 9.3E-01 & $9.3 \mathrm{E}-02$ & $-4.2 E+01$ & & & $7.8 \mathrm{E}+03$ & $7.8 \mathrm{E}+02$ & $-3.8 \mathrm{E}+00$ & & & $4.2 E+03$ & $5.3 \mathrm{E}+02$ & $4.2 \mathrm{E}+02$ & $5.3 E+01$ & $-3.9 E+\infty 0$ \\
\hline Dimethylphenol, 3,4- & 000095.65 .8 & & & $1.4 \mathrm{E}+00$ & $1.4 \mathrm{E}-01$ & $-4.1 \mathrm{E}+01$ & & & $1.6 \mathrm{E}+04$ & $1.6 \mathrm{E}+03$ & $-3.2 \mathrm{E}+\infty$ & & & $8.4 E+03$ & $1.1 E+03$ & $8.4 E+02$ & $1.1 E+02$ & $-3.3 \mathrm{E}+00$ \\
\hline Dimethylphthalate & $000131-11-3$ & & & $7.8 \mathrm{E}+03$ & $7.8 \mathrm{E}+02$ & $-3.9 E+01$ & & & $1.0 \mathrm{E}+06$ & $1.0 \mathrm{E}+06$ & $-1.6 \mathrm{E}+00$ & & & $1.0 \mathrm{E}+06$ & $1.0 \mathrm{E}+06$ & $1.0 \mathrm{E}+06$ & $1.0 \mathrm{E}+06$ & $-1.6 \mathrm{E}+00$ \\
\hline Dimethylterephthalate & $000120-61-6$ & & & $2.3 \mathrm{E}+02$ & $2.3 \mathrm{E}+01$ & $-4.5 \mathrm{E}+01$ & & & $5.5 \mathrm{E}+05$ & $5.5 \mathrm{E}+04$ & $-7.0 E+00$ & & & $3.0 \mathrm{E}+05$ & $3.9 E+04$ & $3.0 E+04$ & $3.9 E+03$ & $-7.4 \mathrm{E}+\infty$ \\
\hline Dinitro-0-cresol, 4,6- & $000534-52-1$ & & & & & & & & & & & & & & & & & \\
\hline $\begin{array}{l}\text { Dinitro-o-cyclohexy! } \\
\text { Phenol, 4,6- }\end{array}$ & $000131-89.5$ & & & $6,4 \mathrm{E}-03$ & $6.4 \mathrm{E}-04$ & $-3.6 \mathrm{E}+01$ & & & $1.0 \mathrm{E}+06$ & $4.3 \mathrm{E}+05$ & $-5.5 \mathrm{E}-03$ & & & $1.0 E+06$ & $2.9 \mathrm{E}+05$ & $2.2 \mathrm{E}+05$ & $2.9 \mathrm{E}+04$ & $-5.5 \mathrm{E}-03$ \\
\hline
\end{tabular}

Nonradionuclides/water and soil/agricultural

Page 12 of 32 
Table 3.3e. (continued)

\begin{tabular}{|c|c|c|c|c|c|c|c|c|c|c|c|c|c|c|c|c|c|c|}
\hline \multirow[b]{2}{*}{ Chemical } & \multirow[b]{2}{*}{ CASRN } & \multicolumn{4}{|c|}{ Vegetable Intercept (mg/kg) } & \multirow[b]{2}{*}{ Slope } & \multicolumn{4}{|c|}{ Beef Intercept (mg/kg) } & \multirow[b]{2}{*}{ Slope } & \multicolumn{6}{|c|}{ Milk Intercept (mg/kg) } & \multirow[b]{2}{*}{ Slope } \\
\hline & & $10^{-1}$ & $10^{-5}$ & HQ=1 & $\mathrm{HQ}=0.1$ & & $10^{-4}$ & $10^{5}$ & HQ $=1$ & $\mathrm{HQ}=0.1$ & & $10^{4}$ & $10^{5}$ & $\begin{array}{l}\text { HQ=1 } \\
\text { Adult }\end{array}$ & $\begin{array}{l}\text { HQ=1 } \\
\text { Child }\end{array}$ & $\begin{array}{c}\text { HQ }=0.1 \\
\text { Adult }\end{array}$ & $\begin{array}{c}\mathrm{HQ}=0.1 \\
\text { Child }\end{array}$ & \\
\hline Dinitrobenzene, 1,2- & $000528-29-0$ & & & $3.1 \mathrm{E}-01$ & 3.1EE-02 & $-3.9 E+01$ & & & $1.2 \mathrm{E}+04$ & $1.2 \mathrm{E}+03$ & $-1.6 \mathrm{E}+00$ & & & $6.5 E+03$ & $8.4 E+02$ & $6.5 E+02$ & $8.4 \mathrm{E}+01$ & $-1.6 \mathrm{E}+00$ \\
\hline Dinitrobenzena, 1,3- & $000099-65.0$ & & & $7.8 \mathrm{E}-02$ & $7.8 \mathrm{E}-03$ & $-3.9 E+01$ & & & 3.1E+03 & $3.18+02$ & $-1.6 \mathrm{E}+00$ & & & $1.6 \mathrm{E}+03$ & $2.1 E+02$ & $1.6 E+02$ & $2.1 \mathrm{E}+01$ & $-1.6 \mathrm{E}+00$ \\
\hline Dinitrobenzene, 1,4- & $000100-25-4$ & & & $2.8 \mathrm{E}-01$ & $2.8 \mathrm{E}-02$ & $-3.9 \mathrm{E}+01$ & & & $1.4 \mathrm{EE}+04$ & $1.4 E+03$ & $-1.4 E+00$ & & & $7.2 E+03$ & $9.3 \mathrm{E}+02$ & $7.2 E+02$ & $9.3 E+01$ & $-1.4 E+00$ \\
\hline Dinitrophenol, 2,4. & $000051-28.5$ & & & $1.4 E+00$ & 1.4E-0I & $-3.9 \mathrm{E}+0 !$ & & & $6.9 E+04$ & $6.9 \mathrm{E}+03$ & $-1.4 E+\infty$ & & & $3.6 \mathrm{E}+04$ & $4.6 \mathrm{E}+03$ & $3.6 \mathrm{E}+03$ & $4.6 E+02$ & $-1.4 \mathrm{E}+00$ \\
\hline Dinitrotoluene, 2,4- & $000121-14-2$ & $3.9 \mathrm{E}-01$ & $3.9 \mathrm{E}-03$ & $2.3 \mathrm{E}+\infty 0$ & 2.3E-01 & $-4.0 \mathrm{E}+01$ & $6.8 \mathrm{E}+03$ & $6.8 \mathrm{E}+01$ & $4.0 \mathrm{E}+04$ & $4.0 E+03$ & $-2.5 \mathrm{E}+00$ & $1.5 E+03$ & $1.5 E+01$ & $2.1 E+04$ & $2.7 \mathrm{E}+03$ & $2.1 E+03$ & $2.7 E+02$ & $-2.6 \mathrm{EE}+00$ \\
\hline Dinitrotoluene, 2,6- & $000606-20.2$ & $3.0 \mathrm{E}-0 \mathrm{t}$ & $3.0 E-03$ & $8.6 \mathrm{E}-01$ & 8.6E-02 & $-3.9 E+01$ & $9.5 \mathrm{E}+03$ & $9.5 \mathrm{E}+01$ & $2.88+04$ & $2.8 \mathrm{E}+03$ & $-1.8 \mathrm{E}+\infty 0$ & $2.1 E+03$ & $2.1 E+01$ & $1.5 \mathrm{E}+04$ & $1.9 \mathrm{E}+03$ & $1.5 \mathrm{E}+03$ & $1.9 E+02$ & $-1.8 \mathrm{E}+00$ \\
\hline $\begin{array}{l}\text { Dinitrotoluene, 2-Amino- } \\
\text { 4,6- }\end{array}$ & $035572-78.2$ & & & & & & & - & & & & & & & & & & \\
\hline Dinoseb & $000088-85-7$ & & & $2.9 E+00$ & $2.9 \mathrm{E}-01$ & $-4.7 E+01$ & & & $1.8 \mathrm{E}+03$ & $1.8 \mathrm{E}+02$ & -1.1E+01 & & & $1.0 E+03$ & $1.3 \mathrm{E}+02$ & $1.0 E+02$ & $1.3 \mathrm{E}+01$ & $-1.2 E+01$ \\
\hline Dioxane, 1,4. & $000123-91-1$ & $1.7 \mathrm{E}+\infty 0$ & $1.78-02$ & & & $-3.6 \mathrm{E}+01$ & $1.0 E+06$ & $4.3 \mathrm{E}+04$ & & & $-1.4 \mathrm{E}-01$ & $9.6 \mathrm{E}+05$ & $9.6 \mathrm{E}+03$ & & & & & $-1.4 \mathrm{E}-01$ \\
\hline Diphenamid & $000557.51-7$ & & & $4.0 E+01$ & $4.0 E+\infty 0$ & $-4.1 E+01$ & & & $4.9 E+05$ & $4.9 E+04$ & $-3.1 \mathrm{E}+00$ & & & $2.6 \mathrm{E}+05$ & $3.3 \mathrm{E}+04$ & $2.6 \mathrm{E}+04$ & $3.3 \mathrm{E}+03$ & $-3.2 E+00$ \\
\hline Diphenylamine & $000122-39-4$ & & & $6.9 \mathrm{E}+01$ & $6.9 E+00$ & $-4 . \pi+01$ & & & $6.3 \mathrm{E}+04$ & $6.3 E+03$ & $-1.0 \mathrm{E}+01$ & & & $3.5 E+04$ & $4.5 E+03$ & $3.5 \mathrm{E}+03$ & $4.5 \mathrm{E}+02$ & $-1.1 . \mathrm{E}+01$ \\
\hline Diphenylhydrazine, 1,2- & $000122-66-7$ & $6.3 \mathrm{E}-01$ & $6.3 \mathrm{E}-03$ & & & $-4.4 E+01$ & $1.8 \mathrm{E}+03$ & $1.8 E+01$ & & & $-6.5 \mathrm{E}+00$ & $4.3 \mathrm{E}+02$ & $4.3 \mathrm{E}+00$ & & & & & $-6.8 \mathrm{E}+00$ \\
\hline Diquat & $000085-00-7$ & & & 4.4E-03 & 4.4E-04 & $-3.6 \mathrm{E}+01$ & & & $1.0 E+06$ & $6.6 \mathrm{E}+05$ & $.3 .5 \mathrm{E}-03$ & & & $1.0 E+06$ & 4.5E+0. & $3.5 \mathrm{E}+05$ & 4.5E +04 & $-3.5 E-03$ \\
\hline Direct Black 38 & $001937-37.7$ & & & & & & & & & & & & & & & & & \\
\hline Direct Blue 6 & $002602-46-2$ & & & & & & & & & & & & & & & & & \\
\hline Direct Brown 95 & $016071-86-6$ & & & & & & & & & & & & & & & & & \\
\hline Direet Sky Blue & $002610.05-1$ & & & & & & & & & & & & & & & & & \\
\hline Disulfoton & $000298-04-4$ & & & $1.2 \mathrm{E}-01$ & $1.2 \mathrm{E}-02$ & $-4.8 E+01$ & & & 4.IE+01 & $4.1 E+\infty$ & $-1.3 \mathrm{E}+01$ & & & $2.3 E+01$ & $3.0 E+00$ & $2.3 \mathrm{E}+00$ & $3.0 E-01$ & $-1.5 \mathrm{E}+01$ \\
\hline Diuron & $000330-54-1$ & & & $3.9 \mathrm{E}+\infty 0$ & 3.92-01 & $-4.4 \mathrm{E}+01$ & & & $1.7 z+04$ & $1.7 \mathrm{E}+03$ & $-5,4 \mathrm{E}+\infty 0$ & & & $9.0 E+03$ & $1.2 \mathrm{E}+03$ & $9.0 \mathrm{E}+02$ & $1.2 E+02$ & $-5.6 \mathrm{E}+00$ \\
\hline Dodine & $002439-10-3$ & & & & & & & & & & & & & & & & & \\
\hline EPTC & 000759.94 .4 & & & & & & & & & & & & & & & & & \\
\hline Endosulfin & 000115.29 .7 & & & & & & & & & & & & & & & & & \\
\hline Endothal & $000145-73-3$ & & & & & & & & & & & & & & & & & \\
\hline
\end{tabular}

Nonradionuclides/water and soil/agricultural

Page 13 of 32 
Table 3.3e. (continued)

\begin{tabular}{|c|c|c|c|c|c|c|c|c|c|c|c|c|c|c|c|c|c|c|}
\hline \multirow{2}{*}{ Chemical } & \multirow{2}{*}{ CASRN } & \multicolumn{4}{|c|}{ Vegetable Intercept (mg/kg) } & \multirow{2}{*}{ Slope } & \multicolumn{4}{|c|}{ Beef Intercept (mg/kg) } & \multirow{2}{*}{ Slope } & \multicolumn{6}{|c|}{ Milk Intercept (mg/kg) } & \multirow{2}{*}{ Slope } \\
\hline & & $10^{-4}$ & $10^{-6}$ & $\mathrm{HQ}=1$ & $\mathrm{HQ}=0.1$ & & $10^{-4}$ & $10^{-5}$ & $H Q=1$ & $\mathrm{HQ}=0.1$ & & $10^{-4}$ & $10^{4}$ & $\begin{array}{l}\text { HQ=1 } \\
\text { Adult }\end{array}$ & $\begin{array}{l}\text { HQ=1 } \\
\text { Child }\end{array}$ & $\begin{array}{c}\text { HQ }=0.1 \\
\text { Adult }\end{array}$ & $\begin{array}{c}\mathrm{HQ}=0.1 \\
\text { Child }\end{array}$ & \\
\hline Endrin & $000072-20-8$ & & & $9.9 \mathrm{E} \cdot 01$ & $9.9 \mathrm{E}-02$ & $-4.9 \mathrm{E}+01$ & & & $9.3 \mathrm{E}+01$ & $9.3 \mathrm{E}+00$ & $-1.6 \mathrm{E}+01$ & & & $5.4 \mathrm{E}+01$ & $7.0 \mathrm{E}+00$ & $5.4 \mathrm{E}+00$ & 7.0E-01 & $-1,8 E+01$ \\
\hline Epichlorohydrin & $000106-89-8$ & $2.1 \mathrm{E}+00$ & 2.1E-02 & $1.7 \mathrm{E}-01$ & $1.7 \mathrm{E} \cdot 02$ & $-3.6 \mathrm{E}+01$ & $1.0 \mathrm{E}+06$ & $4.5 \mathrm{E}+04$ & $3.8 \mathrm{E}+05$ & $3.8 \mathrm{E}+04$ & $-1.5 \mathrm{E}-01$ & $1.0 \mathrm{E}+06$ & $1.0 \mathrm{E}+04$ & $2.0 E+05$ & $2.6 \mathrm{E}+04$ & $2.0 \mathrm{E}+04$ & $2.6 \mathrm{E}+03$ & $-1.5 \mathrm{E}-01$ \\
\hline Epoxybutane, 1,2- & $000106-88-7$ & & & & & & & & & & & & & & & & & \\
\hline Ethephon & $016672-87-0$ & & & & & & & & & & & & & & & & & \\
\hline Ethion & $000563-12-2$ & & & $1.7 E+\infty 0$ & $1.7 \mathrm{E}-01$ & $-4.9 \mathrm{E}+01$ & & & $5.7 E+01$ & $5.7 \mathrm{E}+\infty 0$ & $-1.7 \mathrm{E}+01$ & & & $3.3 \mathrm{E}+01$ & $4.3 \mathrm{E}+\infty$ & $3.3 \mathrm{E}+\infty 0$ & 4.3E-01 & $-2.0 \mathrm{E}+01$ \\
\hline Ethoxyethanol Acetate, 2- & $000111-15-9$ & & & $7.8 \mathrm{E}+01$ & $7.8 \mathrm{E}+00$ & $-3.7 E+01$ & & & $1.0 \mathrm{E}+06$ & $1.0 \mathrm{E}+06$ & $-4.7 \mathrm{E}-01$ & & & $1.0 \mathrm{E}+06$ & $1.0 \mathrm{E}+06$ & $1.0 \mathrm{E}+06$ & $1.7 \mathrm{E}+05$ & $-4.8 \mathrm{E}-01$ \\
\hline Ethoxyethanol, 2- & $000110-80.5$ & & & 4.0E+01 & $4.0 \mathrm{E}+00$ & $-3.6 \mathrm{E}+01$ & & & $1.0 \mathrm{E}+06$ & $1.0 \mathrm{E}+06$ & $-1.8 \mathrm{E}-01$ & & & $1.0 \mathrm{E}+06$ & $1.0 \mathrm{E}+06$ & $1.0 \mathrm{E}+06$ & $4.6 \mathrm{E}+05$ & $-1.8 \mathrm{E}-01$ \\
\hline Ethyl Acetale & $000141-78.6$ & & & $2.9 \mathrm{E}+02$ & $2.9 E+01$ & $-3.7 \mathrm{E}+01$ & & & $1.0 \mathrm{E}+06$ & $1.0 \mathrm{E}+06$ & $-5.8 \mathrm{E}-01$ & & & $1.0 \mathrm{E}+06$ & $1.0 \mathrm{E}+06$ & $1.0 \mathrm{E}+06$ & $4.2 \mathrm{E}+05$ & $-5.9 \mathrm{E}-01$ \\
\hline Ethyl Acrylate & $000140-88-5$ & $2.7 \mathrm{E}+00$ & $2.7 \mathrm{E}-02$ & & & $-3.8 \mathrm{E}+01$ & $2.1 \mathrm{E}+05$ & $2.1 \mathrm{E}+03$ & & & $-1.1 \mathrm{E}+\infty 0$ & $4.6 \mathrm{E}+04$ & $4.6 \mathrm{E}+02$ & & & & & $-1.1 E+00$ \\
\hline Ethyl Chloride & $000075-00-3$ & & & & & & & & & & & & & & & & & \\
\hline Ethyl Ether & $000060-29-7$ & & & $7.0 \mathrm{E}+01$ & $7.0 E+00$ & $-3.7 \mathrm{E}+01$ & & & $1.0 \mathrm{E}+06$ & $1.0 \mathrm{E}+06$ & $-6.4 \mathrm{E}-01$ & & & $1.0 \mathrm{E}+06$ & $8.7 E+05$ & $6.8 \mathrm{E}+05$ & $8.7 E+04$ & $-6.5 \mathrm{E}-01$ \\
\hline Ethyl Methacrylate & $000097-63-2$ & & & $9.5 \mathrm{E}+01$ & $9.5 E+00$ & $-4.0 \mathrm{E}+01$ & & & $1.0 \mathrm{E}+06$ & $2.0 E+05$ & $-2.3 \mathrm{E}+00$ & & & $1.0 \mathrm{E}+06$ & $1.4 \mathrm{E}+05$ & I.1E+0s & $1.4 \mathrm{E}+04$ & $-2.3 \mathrm{E}+00$ \\
\hline $\begin{array}{l}\text { Ethyl-p-nitrophenyl } \\
\text { Phosphonate }\end{array}$ & $002104-64-5$ & & & $3.0 \mathrm{E}-02$ & $3.0 \mathrm{E}-03$ & $-4.8 E+01$ & & & $1.5 \mathrm{E}+01$ & $1.5 \mathrm{E}+00$ & $-1.2 E+01$ & & & $8.4 E+\infty 0$ & $1.1 \mathrm{E}+\infty$ & $8.4 \mathrm{E}-01$ & $1.1 \mathrm{E}-01$ & $-1.3 \mathrm{E}+0 \mathrm{t}$ \\
\hline Ethylbenzene ${ }^{(9)}$ & $000100-41-4$ & & & $2.4 \mathrm{E}+02$ & $2.4 \mathrm{E}+01$ & $-4.5 E+01$ & & & 4.7E+0S & 4.TE+04 & $-7.6 \mathrm{E}+00$ & & & $2.6 \mathrm{E}+05$ & $3.3 \mathrm{E}+04$ & $2.6 \mathrm{E}+04$ & $3.3 \mathrm{E}+03$ & $-8.1 E+\infty 0$ \\
\hline Ethylene Cyanohydrin & $000109-78-4$ & & & $4,8 \mathrm{E}+\infty 0$ & $4.8 \mathrm{E}-01$ & $-3,6 \mathrm{E}+01$ & & & $1.0 \mathrm{E}+06$ & $1.0 \mathrm{E}+06$ & $-2.7 \mathrm{E}-02$ & & & $1.0 \mathrm{E}+06$ & $1.0 \mathrm{E}+06$ & $1.0 \mathrm{E}+06$ & $1.0 \mathrm{E}+06$ & $-2.8 \mathrm{E}-02$ \\
\hline Ethylene Dismine & $000107-15-3$ & & & $2.4 \mathrm{E}+00$ & $2.4 \mathrm{E}-01$ & $-3.6 \mathrm{E}+01$ & & & $1.0 \mathrm{E}+06$ & $3.0 \mathrm{E}+05$ & $-2.1 \mathrm{E}-01$ & & & $1.0 \mathrm{E}+06$ & $2.0 E+05$ & $1.6 \mathrm{E}+05$ & $2.0 \mathrm{E}+04$ & $-2.2 \mathrm{E}-01$ \\
\hline Ethylene Glycol & $000107-21-1$ & & & $1.9 \mathrm{E}+01$ & $1.9 \mathrm{E}+00$ & $-3.6 \mathrm{E}+01$ & & & $1.0 \mathrm{E}+06$ & $1.0 \mathrm{E}+06$ & $-1.6 \mathrm{E}-02$ & & & $1.0 \mathrm{E}+06$ & $1.0 E+06$ & $1.0 E+06$ & $1.0 \mathrm{E}+06$ & $-1.6 \mathrm{E} \cdot 02$ \\
\hline $\begin{array}{l}\text { Ethylene Glycol } \\
\text { Monobutyl Ether }\end{array}$ & $000111-76-2$ & & & & & & & & & & & & & & & & & \\
\hline Ethylene Oxide & $000075-21-8$ & $1.8 \mathrm{E}-02$ & $1.8 \mathrm{E}-04$ & & & $-3.6 \mathrm{E}+01$ & $4.8 \mathrm{E}+04$ & $4.8 \mathrm{E}+02$ & & & $-1.4 \mathrm{E}-01$ & $1.1 E+04$ & $1.1 E+02$ & & & & & $-1.4 E-01$ \\
\hline Ethylene Thiourea & $000096-45.7$ & $1.0 \mathrm{E}-01$ & $1.0 \mathrm{E}-03$ & $3.9 E-03$ & $3.9 \mathrm{E}-04$ & $-3.6 \mathrm{E}+01$ & $6.3 \mathrm{E}+05$ & $6.3 \mathrm{E}+03$ & $2.4 \mathrm{E}+04$ & $2.4 \mathrm{E}+03$ & $-8.4 \mathrm{E}-02$ & $1.4 \mathrm{E}+05$ & $1.4 E+03$ & $1.2 \mathrm{E}+04$ & $1.6 \mathrm{E}+03$ & $1.2 E+03$ & $1.6 \mathrm{E}+02$ & $-8.4 \mathrm{E}-02$ \\
\hline $\begin{array}{l}\text { Ethylphthalyl Ethyl } \\
\text { Glycolate }\end{array}$ & $000084-72-0$ & & & & & & & & & & & & & & & & & \\
\hline Express & $101200-48-0$ & & & & & & & & & & & & & & & & & \\
\hline
\end{tabular}

Nonradionuclides/water and soil/agricultural

Page 14 of 32 
Table 3.3e. (continued)

\begin{tabular}{|c|c|c|c|c|c|c|c|c|c|c|c|c|c|c|c|c|c|c|}
\hline \multirow[b]{2}{*}{ Chemical } & \multirow[b]{2}{*}{ CASRN } & \multicolumn{4}{|c|}{ Vegetable Intercept (mg/kg) } & \multirow[b]{2}{*}{ Slope } & \multicolumn{4}{|c|}{ Beef Intercept (mg/kg) } & \multirow[b]{2}{*}{ Slope } & \multicolumn{6}{|c|}{ Milk Intercept (mg/kg) } & \multirow[b]{2}{*}{ Slope } \\
\hline & & $10^{-4}$ & $10^{6}$ & $H Q=1$ & $\mathrm{HQ}=0.1$ & & $10^{-4}$ & $10^{4}$ & $\mathrm{HQ}=1$ & $H Q=0.1$ & & $10^{-4}$ & $10^{-5}$ & $\begin{array}{l}\text { HQ }=1 \\
\text { Adult }\end{array}$ & $\begin{array}{l}\text { HQ=1 } \\
\text { Child }\end{array}$ & $\begin{array}{c}\text { HQ }=0.1 \\
\text { Adult }\end{array}$ & $\begin{array}{c}\text { HQ }=0.1 \\
\text { Child }\end{array}$ & \\
\hline Fenamiphos & $022224-92-6$ & & & & & & & & & & & & & & & & & \\
\hline Fenpropathrin & $039515-41-8$ & & & & & & & & & & & & & & & & & \\
\hline Fluometuron & $002164-17-2$ & & & $2.1 E+01$ & $2.1 E+\infty 0$ & $-4.2 \mathrm{E}+01$ & & & $1.6 E+05$ & $1.6 \mathrm{E}+04$ & $-4.0 E+00$ & & & $8.6 \mathrm{E}+04$ & 1.1E+04 & $8.6 \mathrm{E}+03$ & $1.1 E+03$ & $-4.1 E+\infty$ \\
\hline Fluoranthene & $000206-44-0$ & & & $1.3 \mathrm{E}+02$ & $1.3 \mathrm{E}+0 \mathrm{t}$ & $-4.9 E+01$ & & & $6.6 \mathrm{E}+03$ & $6.6 \mathrm{E}+02$ & $-1.7 E+01$ & & & $3.9 \mathrm{E}+03$ & $5.0 \mathrm{E}+02$ & $3.9 E+02$ & $5.0 \mathrm{E}+01$ & $-1.9 E+01$ \\
\hline Fluorene & $000086-73-7$ & & & $1.3 \mathrm{E}+02$ & $.1 .3 \mathrm{E}+01$ & $-4.9 \mathrm{E}+01$ & & & $1.9 E+04$ & $1.9 \mathrm{E}+03$ & $-1.5 \mathrm{E}+01$ & & & 1.1E+04 & $1.4 \mathrm{E}+03$ & $1.1 \mathrm{E}+03$ & $1,4 \mathrm{E}+02$ & $-1.7 E+01$ \\
\hline Fluoride & $007782-41-4$ & & & & & & & & & & & & & & & & & \\
\hline Furidone & $059756-60-4$ & & & & & & & & & & & & & & & & & \\
\hline Flurprimidol & $056425-91.3$ & & & & & & & & & & & & & & & & & \\
\hline Flutolanil & $066332-96-5$ & & & & & & & & & & & & & & & & & \\
\hline Fuvalinste & $069409-94-5$ & & & & & & & & & & & & & & & & & \\
\hline Folpet & $000133 \cdot 07-3$ & $1.6 \mathrm{E}+02$ & $1.6 \mathrm{E}+\infty$ & $2.4 E+02$ & $2.4 E+01$ & $-4.5 E+01$ & $3.2 E+05$ & $3.2 \mathrm{E}+03$ & 4.7E+05 & $4.7 E+04$ & $-7.6 \mathrm{E}+00$ & $7.3 E+04$ & $7.3 \mathrm{E}+02$ & $2.6 \mathrm{E}+05$ & $3.3 E+04$ & $2.6 \mathrm{E}+04$ & $3.3 E+03$ & $-8,1 E+\infty$ \\
\hline Fomessafen & $072178-02-0$ & & & & & & & & & & & & & & & & & \\
\hline Fonofos & $000944-22-9$ & & & $6.1 \mathrm{E}+\infty$ & $6.1 \mathrm{E}-01$ & $-4.8 \mathrm{E}+01$ & & & $2.3 \mathrm{E}+03$ & $2.3 \mathrm{E}+02$ & $-1.3 E+01$ & & & $1.3 \mathrm{E}+03$ & $1.7 \mathrm{E}+02$ & $1.3 \mathrm{E}+02$ & 1.7E+0I & $-1.4 E+01$ \\
\hline Formaldehyde & $000050-00-0$ & & & $2.3 \mathrm{E}+01$ & $2.3 \mathrm{E}+\infty 0$ & $-3.6 \mathrm{E}+01$ & & & $1.0 \mathrm{E}+06$ & $1,0 \mathrm{E}+06$ & $-2.0 \mathrm{E}-01$ & & & $1.0 \mathrm{E}+06$ & $1.0 \mathrm{E}+06$ & $1.0 \mathrm{E}+06$ & $2.1 \mathrm{E}+05$ & $-2.0 \mathrm{E}-01$ \\
\hline Formic Acid & $000064-18.6$ & & & $1.1 \mathrm{E}+02$ & $1.1 \mathrm{E}+01$ & $-3.6 \mathrm{E}+01$ & & & $1.0 \mathrm{E}+06$ & $1.0 \mathrm{E}+06$ & $-9.8 \mathrm{E}-02$ & & & $1.0 \mathrm{E}+06$ & $1.0 \mathrm{E}+06$ & $1.0 E+06$ & $1.0 \mathrm{E}+06$ & $-9.9 \mathrm{E}-02$ \\
\hline Fosetyl-AL & $039148-24-8$ & & & & & & & & & & & & & & & & & \\
\hline Furan & $000110-00-9$ & & & 5.6E-01 & 5.6E-.02 & $-3,8 \mathrm{E}+01$ & & & $4.2 \mathrm{E}+04$ & $4.2 E+03$ & $-1.1 E+\infty$ & & & $2.2 \mathrm{E}+04$ & $2.9 \mathrm{E}+03$ & $2.2 \mathrm{E}+03$ & $2.9 \mathrm{E}+02$ & $-1.1 \mathrm{E}+00$ \\
\hline Furazolidone & $000067-45.8$ & $1 . \pi E-02$ & $1.7 \mathrm{E}-04$ & & & $-3.7 E+01$ & $4.9 \mathrm{E}+03$ & $4.9 \mathrm{E}+01$ & & & $-4.9 \mathrm{E}-01$ & $1.1 \mathrm{E}+03$ & $1.1 \mathrm{E}+01$ & & & & & $-5.0 \mathrm{E}-01$ \\
\hline Furfural & $000098-01-1$ & & & $8.2 E+00$ & 8.2E-01 & $-4.7 \mathrm{E}+01$ & & & $7.5 \mathrm{E}+03$ & $7.5 \mathrm{E}+02$ & $-1.0 \mathrm{E}+01$ & & & $4.2 \mathrm{E}+03$ & $5.4 \mathrm{E}+02$ & $4.2 \mathrm{E}+02$ & $5.4 E+01$ & $-1.1 E+01$ \\
\hline Furium & $000531-82-8$ & & & & & & & & & & & & & & & & & \\
\hline Furmecyclox & $060568-05-0$ & & & & & & & & & & & & & & & & & \\
\hline Glufosinste, Ammonium & $077182-82-2$ & & & & & & & & & & & & & & & & & \\
\hline Glycidyl & $000765-34-4$ & & & $1.8 \mathrm{E}-02$ & $1.8 \mathrm{E}-03$ & $-3.6 \mathrm{E}+01$ & & & $1.3 \mathrm{E}+05$ & $1.3 \mathrm{E}+04$ & $-7.6 \mathrm{E}-02$ & & & 6.7E+04 & $8.6 \mathrm{E}+03$ & $6.7 E+03$ & $8.6 \mathrm{E}+02$ & $-7.7 E-02$ \\
\hline
\end{tabular}


$3-476$

\begin{tabular}{|c|c|c|c|c|c|c|c|c|c|c|c|c|c|c|c|c|c|c|c|c|}
\hline \multicolumn{2}{|c|}{ 高 } & 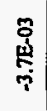 & & & & $\begin{array}{c}\overrightarrow{+} \\
\underset{4}{4} \\
\stackrel{-}{*}\end{array}$ & 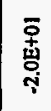 & & 亳 & 帘 & 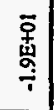 & 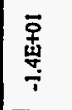 & 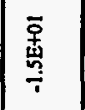 & & & $\begin{array}{l}\text { 㞻 } \\
\stackrel{4}{\rightarrow}\end{array}$ & & 窝 & $\begin{array}{l}\bar{P} \\
\stackrel{4}{4} \\
\text { ᄀ. }\end{array}$ & 韋 \\
\hline \multirow{6}{*}{ 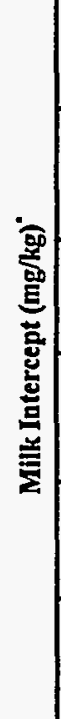 } & 竞总 & 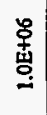 & & & & $\begin{array}{l}\stackrel{8}{4} \\
\text { 索 }\end{array}$ & 总 & & 恿 & 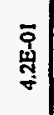 & 离 & & & & & $\begin{array}{l}\text { 古 } \\
\text { 苟 }\end{array}$ & & 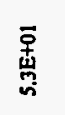 & & $\begin{array}{l}8 \\
\text { 菇 }\end{array}$ \\
\hline & $\begin{array}{l}\text { 苛言 } \\
0\end{array}$ & 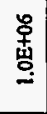 & & & & 哭 & 芦 & & 怘 & 怘 & 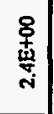 & & & & & 触 & & $\begin{array}{l}\underset{+}{9} \\
\stackrel{4}{*}\end{array}$ & & 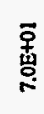 \\
\hline & 몸멸 & 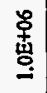 & & & & 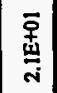 & 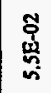 & & $\begin{array}{l}8 \\
\stackrel{8}{4} \\
-9\end{array}$ & 条 & 䤧 & & & & & $\begin{array}{l}\vec{P} \\
\text { 垥 } \\
\dot{m}\end{array}$ & & 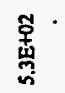 & & 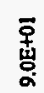 \\
\hline & 兽意 & 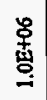 & & & & 恿 & 离 & & 恿 & 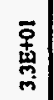 & 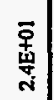 & & & & & 苞 & & 辠 & & 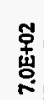 \\
\hline & $\ddot{b}$ & & & & & 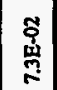 & 总 & & & 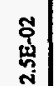 & 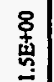 & 兽 & 照 & & & 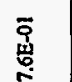 & & & 啇 & $\begin{array}{l}\text { 吴 } \\
\text { 总 }\end{array}$ \\
\hline & $\underline{\underline{z}}$ & & & & & 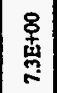 & 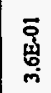 & & & 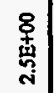 & 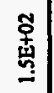 & 量 & 恿 & & & 箇 & & & 芯 & 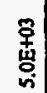 \\
\hline \multicolumn{2}{|c|}{ 高 } & 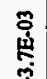 & & & & 㤐 & 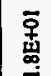 & & 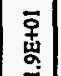 & $\begin{array}{l}\bar{\rho} \\
\text { 畕 } \\
\end{array}$ & 予 & 㞻 & 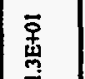 & & & 䂌 & & 宮 & 恿 & 蛋 \\
\hline \multirow{4}{*}{ 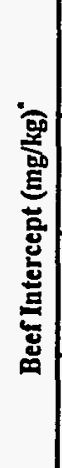 } & $\overline{0}$ & 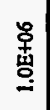 & & & & 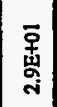 & 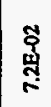 & & $\begin{array}{l}\text { 怘 } \\
\text { â }\end{array}$ & 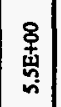 & $\begin{array}{l}\text { 靑 } \\
\text { F }\end{array}$ & & & & & 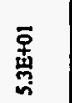 & & 韋 & & 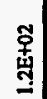 \\
\hline & $\overline{\mathrm{g}}$ & $\begin{array}{l}\text { 怘 } \\
\text { 产 }\end{array}$ & & & & 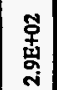 & 京 & & 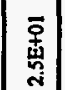 & 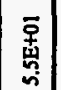 & 親 & & & & & 密 & & 喣 & & 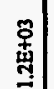 \\
\hline & $\dot{\Xi}$ & & & & & 高 & ఫ్ & & & 离 & 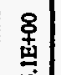 & 宫 & 点 & & & 冬 & & & 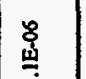 & 糡 \\
\hline & ప & & & & & 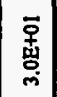 & 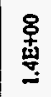 & & & 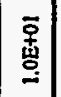 & 章 & $\begin{array}{l}\text { 䓟 } \\
\text { 苦 }\end{array}$ & 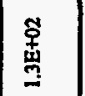 & & & 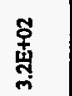 & & & 恚 & $\begin{array}{l}\text { 古 } \\
\text { స. }\end{array}$ \\
\hline \multicolumn{2}{|c|}{ 흠 } & 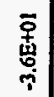 & & & & $\begin{array}{l}\vec{P} \\
\text { 糅 } \\
\end{array}$ & 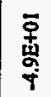 & & 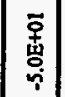 & $\begin{array}{l}\overline{\mathbf{s}} \\
\text { 畺 }\end{array}$ & 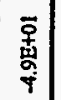 & 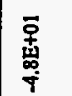 & 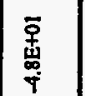 & & & $\begin{array}{c}\bar{P} \\
\substack{\mathbf{H} \\
\dot{y}}\end{array}$ & & 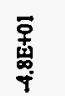 & $\begin{array}{l}\bar{P} \\
\text { 喜 } \\
\stackrel{4}{0}\end{array}$ & 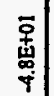 \\
\hline \multirow{4}{*}{ 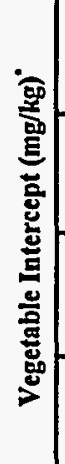 } & $\overline{\underline{0}}$ & 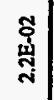 & & & & 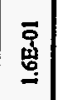 & 总 & & 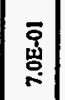 & 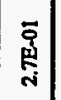 & 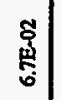 & & & & & 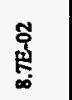 & & $\begin{array}{l}\text { \& } \\
\text { 营 }\end{array}$ & & 寓 \\
\hline & $\underset{\mathrm{g}}{\overrightarrow{\mathrm{g}}}$ & 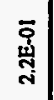 & & & & 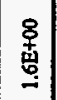 & 离 & & 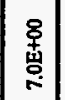 & 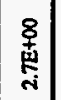 & 䔡 & & & & & 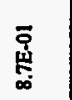 & & 趸 & & $\begin{array}{l}\text { 章 } \\
\text { 兽 }\end{array}$ \\
\hline & $\dot{g}$ & & & & & 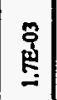 & 志 & & & 兽 & 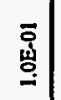 & 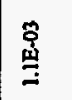 & 总 & & & 旁 & & & $\begin{array}{l}\stackrel{8}{ } \\
\text { 岗 }\end{array}$ & 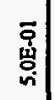 \\
\hline & t) & & & & & 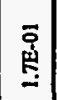 & 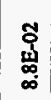 & & & 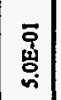 & 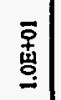 & 离 & $\begin{array}{l}\overrightarrow{0} \\
\text { 产 } \\
\stackrel{4}{*}\end{array}$ & & & 嵹 & & & 莒 & 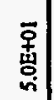 \\
\hline & ż & 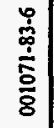 & 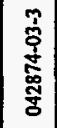 & 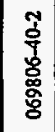 & 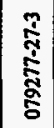 & 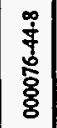 & 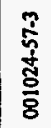 & 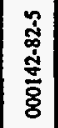 & 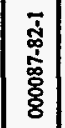 & $\frac{\vec{j}}{\dot{\Phi}}$ & 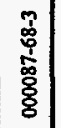 & 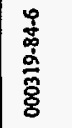 & 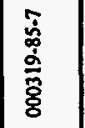 & 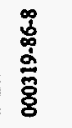 & 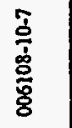 & 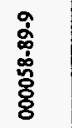 & 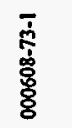 & $\begin{array}{l}\frac{T}{4} \\
\frac{1}{8} \\
8 \\
8\end{array}$ & 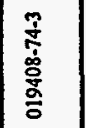 & $\begin{array}{l}\overline{\mathbf{T}} \\
\mathbf{5} \\
\mathbf{8} \\
8\end{array}$ \\
\hline \multicolumn{2}{|c|}{ 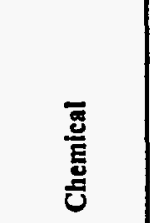 } & 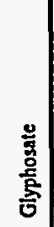 & 总 & 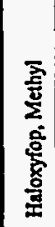 & $\begin{array}{l}\text { 亮 } \\
\text { 总 }\end{array}$ & 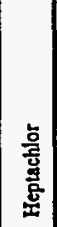 & 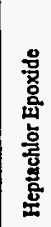 & 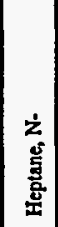 & 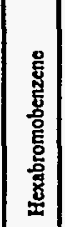 & 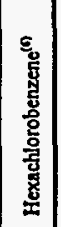 & 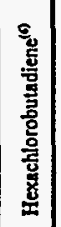 & 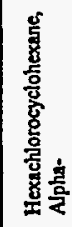 & 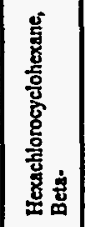 & 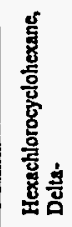 & 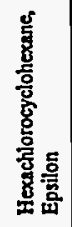 & 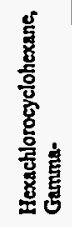 & 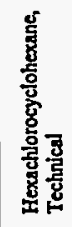 & 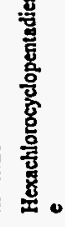 & 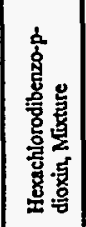 & 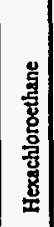 \\
\hline
\end{tabular}

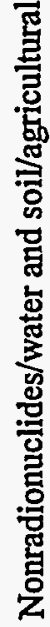


Table 3.3e. (continued)

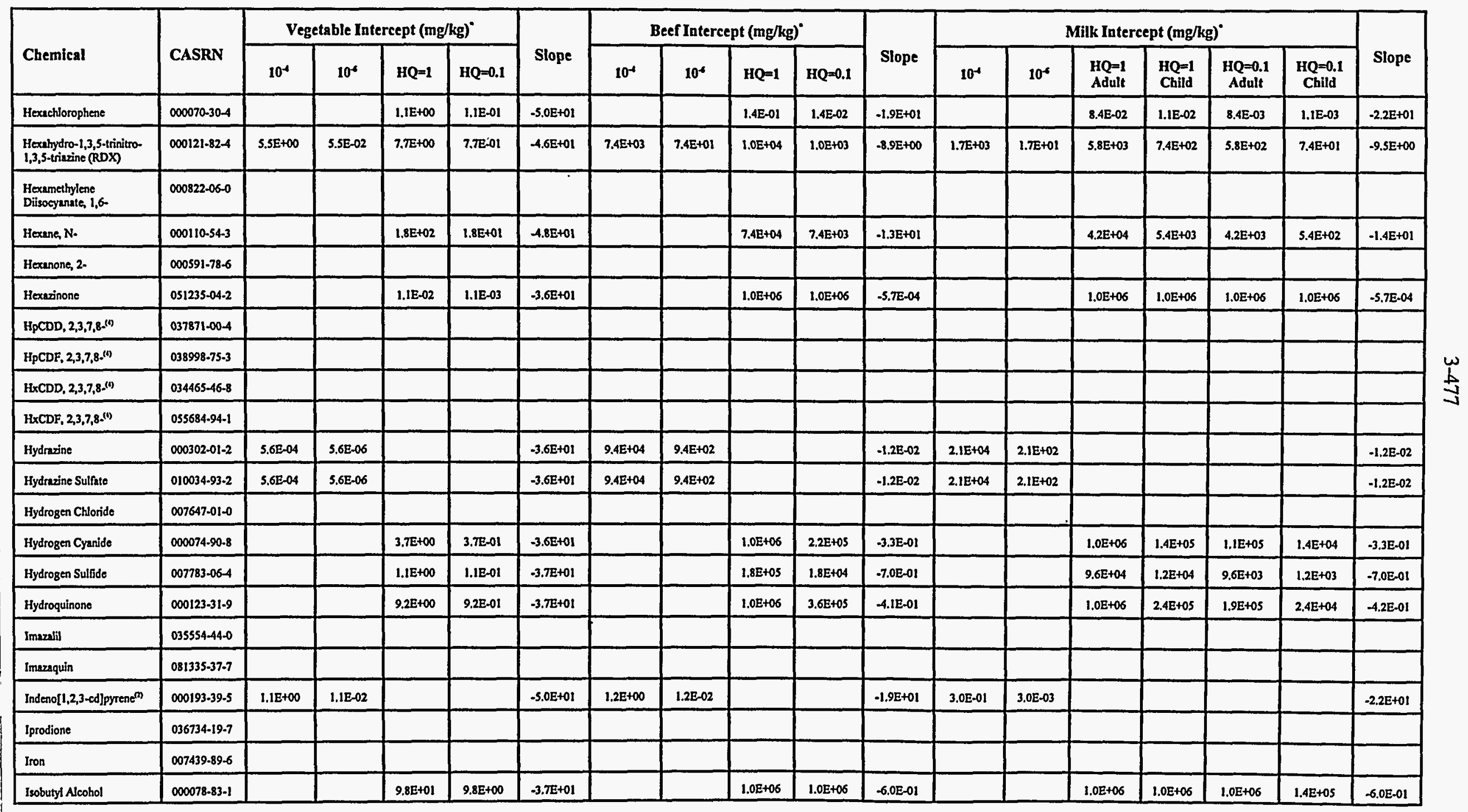

Nonradionuclides/water and soil/agricultural

Page 17 of 32 
乙E ร๐ 8 I วอิอ d

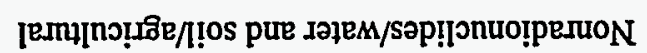

\begin{tabular}{|c|c|c|c|c|c|c|c|c|c|c|c|c|c|c|c|c|c|c|}
\hline $10 \cdot 30 \%$ & $\varepsilon 0+z z ' s$ & $50+316$ & $50+325$ & So+al'b & & & $10-30 z^{-}$ & $50+386$ & $50+38 L$ & & & $10+29 \%-$ & $20-3 / s$ & $10-32$ 's & & & $z-8 \varepsilon-L z b z i 0$ & qurरx \\
\hline & & & & & & & & & & & & & & & & & $L-10-810800$ & $992000 \pi x$ \\
\hline 10-31z & $10+30 z$ & $20+391$ & $20+302$ & $\varepsilon 0+39 \%$ & & & $10-31 z^{-}$ & $20+30 \mathcal{E}$ & $\varepsilon 0+30^{\circ} \varepsilon$ & & & $10+39 \varepsilon^{\circ}$ & $60-3 b^{2} z$ & $\varepsilon 0-3) z$ & & & $\varepsilon-\angle L-601000$ & 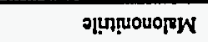 \\
\hline \multirow[t]{2}{*}{ 10-az'1- } & $50+982$ & $90+90.1$ & $90+30^{\circ} \mathrm{t}$ & $90+30.1$ & & & $10-3 z 1$ & $90+90.1$ & $90+90.1$ & & & $10+29 \varepsilon^{-1}$ & $00+a b \varepsilon$ & $10+a b \varepsilon$ & & & $1-\varepsilon \varepsilon-\varepsilon z 1000$ & 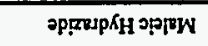 \\
\hline & & & & & & & & & & & & & & & & & 9-[E-801000 & 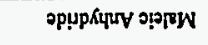 \\
\hline \multirow[t]{2}{*}{$00+38 \%-$} & $20+968$ & $\varepsilon 0+369$ & $\varepsilon 0+968$ & $60+269$ & & & $00+Z S \%$ & $50+9 \varepsilon 1$ & so+aE: & & & $10+a b t$ & $00+a \varepsilon r$ & $10+3 E v$ & & & $S=5 L-1 Z 1000$ & 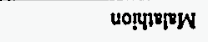 \\
\hline & & & & & & & & & & & & & & & & & FSG-SERLOO & 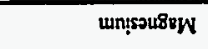 \\
\hline \multirow[t]{2}{*}{$00+$ glis. } & $10+399$ & $20+91 ' s$ & $20+39 \%$ & Eo+al's & & & $00+36 t$ & $20+35 \cdot 6$ & $\varepsilon 0+a 56$ & & & $10+a c t$ & 10-38't & $00+98 \cdot 1$ & & & $\tau-59-[600000$ & ddכW \\
\hline & & & & & & & & & & & & & & & & & $s \cdot 18-660000$ & gdכא \\
\hline \multirow[t]{5}{*}{$00+3 L L^{\prime} \varepsilon-$} & $10+\$ 8 \cdot b$ & $20+\underline{Z} L^{\prime} \varepsilon$ & $20+387$ & $\varepsilon 0+2 L \cdot \varepsilon$ & & & $00+39)^{\prime}$ & $20+30 \% 2$ & $\varepsilon 0+90 \%$ & & & $10+ \pm 2 t$ & $20-a b^{\prime} L$ & $10-3 \times 2$ & & & $9-\nabla L-7600000$ & VdכW \\
\hline & & & & & & & & & & & & & & & & & $9 \cdot 66-550880$ & xppuor \\
\hline & & & & & & & & & & & & & & & & & $\tau-\varepsilon 6-6 \varepsilon \forall \angle 00$ & un!q!? \\
\hline & & & & & & & & & & & & & & & & & $\tau-s 5-0 \varepsilon \varepsilon 000$ & vounu!? \\
\hline & & & & & & & & & & & & & & & & & $1-26-6 \varepsilon \mathrm{ELOO}$ & spunodwo $\mathrm{pury}$ prar \\
\hline & & & & & & & & & & & & & & & & & VN & 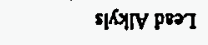 \\
\hline & & & & & & & & & & & & & & & & & FES-10SLLO & 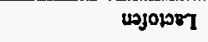 \\
\hline & & & & & & & & & & & & & & & & & $5-85$-056zzo & qक्र \\
\hline & & & & & & & & & & & & & & & & & $9-80-59160$ & ग2erxy \\
\hline & & & & & & & & & & & & & & & & & $L-05-855780$ & แaqqXosi \\
\hline & & & & & & & & & & & & & & & & & $8-t s-288100$ & 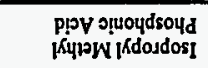 \\
\hline & & & & & & & & & & & & & & & & & $0-\varepsilon 9-\angle 90000$ & |ourdosdos! \\
\hline & & & & & & & & & & & & & & & & & $0-\varepsilon s-0 z 8 \varepsilon \varepsilon 0$ & ugprosdosy \\
\hline $00+381^{-}$ & $80+98 ' \varepsilon$ & $50+962 z$ & $50+98 \cdot \varepsilon$ & $90+90.1$ & $60+3 s^{\prime} t$ & $90+30^{\prime} \mathrm{I}$ & $00+38 t^{\circ}$ & sotas's & $90+30.1$ & $50+989$ & $90+30 \%$ & $10+96 \varepsilon^{-}$ & $10+3 L 1$ & $20+3 L^{\prime} 1$ & $00+31 \cdot z$ & $20+312 z$ & $1-65-810000$ & PuosouddosI \\
\hline \multirow[t]{2}{*}{ odols } & $\begin{array}{c}\text { p!!पว } \\
I^{\prime} 0=\partial \mathrm{H}\end{array}$ & $\begin{array}{l}\text { IInPV } \\
I^{0} 0=\partial H\end{array}$ & $\begin{array}{l}\text { PIIIYJ } \\
I=O H\end{array}$ & $\begin{array}{l}\text { मnpy } \\
I=0 H\end{array}$ & , Or & rOI & \multirow{2}{*}{ Idols } & $r^{0} 0=\partial H$ & $\mathfrak{l}=\mathbf{D H}$ & ,01 & rOI & \multirow{2}{*}{ 2dols } & $I^{\circ} 0=\partial \mathrm{H}$ & $I=\partial \mathrm{H}$ & ,0I & rOI & \multirow{2}{*}{ N\&SVD } & \multirow{2}{*}{ 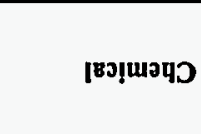 } \\
\hline & & & $y / 2 \mathrm{dw})\} \mathrm{dz}$ & |) भाII & & & & & h/p(bu) \}d & 0 & & & (dy & 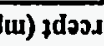 & ग गq & & & \\
\hline
\end{tabular}

(pənu!ฺ 
Table 3.3e. (continued)

\begin{tabular}{|c|c|c|c|c|c|c|c|c|c|c|c|c|c|c|c|c|c|c|}
\hline \multirow[b]{2}{*}{ Chemical } & \multirow[b]{2}{*}{ CASRN } & \multicolumn{4}{|c|}{ Vegetable Intercept (mg/kg) } & \multirow[b]{2}{*}{ Slope } & \multicolumn{4}{|c|}{ Beef Intercept (mg/kg)" } & \multirow[b]{2}{*}{ Slope } & \multicolumn{6}{|c|}{ Milk Intercept (mg/kg)" } & \multirow[b]{2}{*}{ Slope } \\
\hline & & $10^{-4}$ & $10^{4}$ & $\mathbf{H Q}=\mathbf{I}$ & $H Q=0.1$ & & $10^{-4}$ & $10^{4}$ & $\mathrm{HQ}=1$ & $H Q=0.1$ & & $10^{-4}$ & $10^{6}$ & $\begin{array}{l}\text { HQ=1 } \\
\text { Adult }\end{array}$ & $\begin{array}{l}\text { HQ=1 } \\
\text { Child }\end{array}$ & $\begin{array}{c}\mathrm{HQ}=0.1 \\
\text { Adult }\end{array}$ & $\begin{array}{c}\text { HQ }=0.1 \\
\text { Child }\end{array}$ & \\
\hline Manganese (Diet) & $007439-96-5$ & & & $3.9 E+02$ & $3.9 \mathrm{E}+01$ & $-4.7 \mathrm{E}+01$ & & & $3.9 \mathrm{E}+04$ & $3.9 \mathrm{E}+03$ & $-7.1 E+00$ & & & $1.1 E+05$ & $1.4 \mathrm{E}+0.4$ & $1.1 \mathrm{E}+04$ & $1.4 E+03$ & $-7.5 \mathrm{E}+00$ \\
\hline Mephosfolen & $000950-10-7$ & & & & & & & & & & & & & & & & & \\
\hline Mepiquat CHoride & $024307-26-4$ & & & & & & & & & & & & & & & & & \\
\hline Mercuric Chloride & $007487.94-7$ & & & & & & & & & & & & & & & & & \\
\hline Mercury (elementa) & $007439-97-6$ & & & 4.9E-01 & 4.9E-02 & $-4.2 \mathrm{E}+01$ & & & $3.2 \mathrm{E}+\infty$ & 3.2E-01 & $-5.5 \mathrm{E}+\infty 0$ & & & $1.2 E+01$ & $1.5 \mathrm{E}+00$ & $1.2 E+\infty 0$ & $1.5 \mathrm{E}-01$ & $-5.7 \mathrm{E}+00$ \\
\hline Mercury, Inorganic Salt & $007439-97.6$ & & & 4.9E-01 & $4.9 \mathrm{E}-02$ & $-4.2 E+01$ & & & $3.2 \mathrm{E}+00$ & $3.2 \mathrm{E}-01$ & $-5.5 \mathrm{E}+00$ & & & $1.2 E+01$ & 1.5E+00 & $1.2 \mathrm{E}+00$ & $1.5 \mathrm{E}-01$ & $-5.7 E+00$ \\
\hline Merphos & $000150-50-5$ & & & & & & & & & & & & & & & & & \\
\hline Merphos Oxide & $000078-48.8$ & & & $1.0 \mathrm{E}-01$ & $1.0 \mathrm{E}-02$ & $-5.0 \mathrm{E}+01$ & & & 8.5E-01 & 8.5E-02 & $-1.8 \mathrm{E}+01$ & & & $5.0 \mathrm{E}-01$ & $6.5 E-02$ & $5.0 \mathrm{E}-02$ & $6.5 \mathrm{E}-03$ & $-2.1 E+01$ \\
\hline Metalexyl & $057837-19-1$ & & & & & & & & & & & & & & & & & \\
\hline Methacryionitrile & $000126-98-7$ & & & $2.3 \mathrm{E}-02$ & $2.3 \mathrm{E}-03$ & $-3 . \pi \mathrm{E}+01$ & & & $9.2 \mathrm{E}+03$ & $9.2 \mathrm{E}+02$ & $-4.1 \mathrm{E}-01$ & & & $4.8 \mathrm{E}+03$ & $6.2 E+02$ & $4.8 \mathrm{E}+02$ & $6.2 \mathrm{E}+01$ & $-4.1 \mathrm{E}-01$ \\
\hline Methunidophos & $010265.92-6$ & & & $2.4 \mathrm{E}-03$ & $2.4 \mathrm{E}-04$ & $-3.6 \mathrm{E}+01$ & & & $1.5 \mathrm{E}+04$ & $1.5 \mathrm{E}+03$ & $-8.4 \mathrm{E}-02$ & & & $7.8 \mathrm{E}+03$ & $1.0 \mathrm{E}+03$ & $7.8 \mathrm{E}+02$ & $1.0 \mathrm{E}+02$ & $-8.4 \mathrm{E}-02$ \\
\hline Methanol & $000067.56-1$ & & & $2.1 \mathrm{E}+0 \mathrm{t}$ & $2.1 E+\infty$ & $-3.6 \mathrm{E}+01$ & & & $1.0 \mathrm{E}+06$ & $1.0 \mathrm{E}+06$ & $-7.2 \mathrm{E}-02$ & & & $1.0 \mathrm{E}+06$ & $1.0 \mathrm{E}+06$ & $1.0 \mathrm{E}+06$ & $1.0 \mathrm{E}+06$ & $-7.3 E-02$ \\
\hline Methidathion & 000950.37 .8 & & & & & & & & & & & & & & & & & \\
\hline Methomyl & 016752.77 .5 & & & $6.1 \mathrm{E}+\infty$ & 6.IE-01 & $-3.7 \mathrm{E}+01$ & & & $1.0 \mathrm{E}+06$ & $2.2 \mathrm{E}+05$ & $-4,4 \mathrm{E}-01$ & & & $1.08+06$ & $1.5 \mathrm{E}+05$ & $1.1 \mathrm{E}+05$ & $1.5 \mathrm{E}+04$ & $-4.5 \mathrm{E}-01$ \\
\hline Methoxy-5-nitroariline, 2- & $000099-59-2$ & $3.6 \mathrm{E}+00$ & $3.6 \mathrm{E}-02$ & & & $-3.9 E+01$ & $1.7 \mathrm{E}+05$ & $1.7 \mathrm{E}+03$ & & & $-1.4 E+\infty 0$ & $3.9 \mathrm{E}+04$ & $3.9 E+02$ & & & & & $-1.4 E+00$ \\
\hline Methoxychlor & $000072-43.5$ & & & $1.6 \mathrm{E}+01$ & $1.6 \mathrm{E}+00$ & $-4.9 \mathrm{E}+01$ & & & $2.3 \mathrm{E}+03$ & $2.3 \mathrm{E}+02$ & $-1.5 E+01$ & & & $1.4 \mathrm{E}+03$ & $1.7 E+02$ & $1.4 \mathrm{E}+02$ & $1.7 \mathrm{E}+01$ & $-1.7 \mathrm{E}+01$ \\
\hline $\begin{array}{l}\text { Methoxyethanol Acetate, } \\
\text { 2. }\end{array}$ & $000110-49-6$ & & & & & & & & & & & & & & & & $\cdot$ & \\
\hline Methoxyethanol, 2 - & $000109.86-4$ & & & $4.2 \mathrm{E}-02$ & $4.2 \mathrm{E}-03$ & $-3.6 \mathrm{E}+01$ & & & $3.3 \mathrm{E}+05$ & $3.3 \mathrm{E}+04$ & $-7.2 \mathrm{E}-02$ & & & $1.7 \mathrm{E}+05$ & $2.2 \mathrm{E}+04$ & $1.7 E+04$ & $2.2 \mathrm{E}+03$ & $-7.3 \mathrm{E}-02$ \\
\hline Methyl Acetate & $000079-20-9$ & & & $1.4 \mathrm{E}+02$ & $1.4 \mathrm{E}+01$ & $-3.6 \mathrm{E}+01$ & & & $1.0 \mathrm{E}+06$ & $1.0 \mathrm{E}+06$ & $-2.5 \mathrm{E}-01$ & & & $1.0 \mathrm{E}+06$ & $1.0 \mathrm{E}+06$ & $1,0 \mathrm{E}+06$ & $8.8 \mathrm{E}+05$ & $-2.6 \mathrm{E}-01$ \\
\hline Methyl Acrylate & 000096.33 .3 & & & $8.0 \mathrm{E}+00$ & $8.0 \mathrm{~B}-01$ & $-3.7 \mathrm{E}+01$ & & & $1.0 \mathrm{E}+06$ & 2.4E+05 & $-4.8 \mathrm{E}-01$ & & & $1.0 \mathrm{E}+06$ & $1.6 \mathrm{E}+05$ & $1,3 \mathrm{E}+05$ & $1.6 \mathrm{E}+04$ & $-4.9 \mathrm{E}-01$ \\
\hline Methyl Ethyl Ketone & $000078-93-3$ & & & $9.7 E+01$ & $9.7 \mathrm{E}+00$ & $-3.6 \mathrm{E}+01$ & & & $1.0 \mathrm{E}+06$ & $1.0 \mathrm{E}+06$ & $-2.9 \mathrm{E}-01$ & & & $1.0 \mathrm{E}+06$ & $1.0 \mathrm{E}+06$ & $1.0 \mathrm{E}+06$ & $4.8 \mathrm{E}+05$ & $-2.9 \mathrm{E}-01$ \\
\hline Methyl Isobutyl Ketone & $000108-10-1$ & & & $4.0 \mathrm{E}+01$ & $4.0 \mathrm{E}+00$ & $-3.8 \mathrm{E}+01$ & & & $1.0 \mathrm{E}+06$ & $3.8 E+05$ & $-9.6 \mathrm{E}-01$ & & & $1.0 \mathrm{E}+06$ & $2.5 \mathrm{E}+05$ & $2.0 \mathrm{E}+0 \mathrm{~S}$ & $2.5 E+04$ & $-9.7 E-01$ \\
\hline Methyl Mereury & $022967-92-6$ & & & & & & & & & & & & & & & & & \\
\hline
\end{tabular}

Nonradionuclides/water and soil/agricultural

Page 19 of 32 


\begin{tabular}{|c|c|c|c|c|c|c|c|c|c|c|c|c|c|c|c|c|c|c|c|c|c|c|}
\hline & 高 & 隹 & 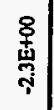 & 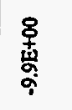 & & & 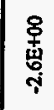 & 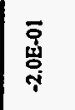 & & 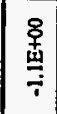 & 哭 & 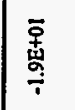 & 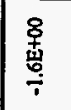 & & 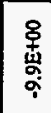 & & & 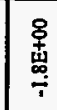 & $\mid \begin{array}{l}\overrightarrow{9} \\
\text { 产 } \\
\text { î }\end{array}$ & 㞻 & $\begin{array}{l}\vec{P} \\
\dot{0} \\
\stackrel{+}{i}\end{array}$ & $\stackrel{\sim}{m}$ \\
\hline & 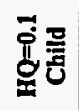 & 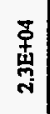 & 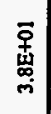 & 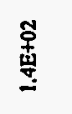 & & & & & & 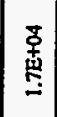 & $\begin{array}{c}\text { 呆 } \\
\text { 点 } \\
0 \\
0\end{array}$ & & & & 总 & & & 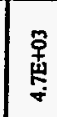 & 离 & 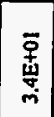 & $\begin{array}{l}8 \\
\text { 怘 } \\
\text { : }\end{array}$ & 잉 \\
\hline & 家言 & 总 & 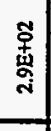 & 总 & & & & & & 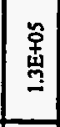 & 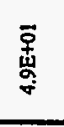 & & & & 菍 & & & $\underset{m}{\stackrel{T}{\mathbf{H}}}$ & 名 & 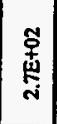 & 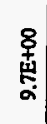 & $A_{r}$ \\
\hline 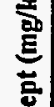 & 预氞 & 旁 & 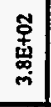 & 骞 & & & & & & 旁 & 帠 & & & & 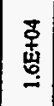 & & & 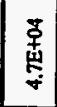 & 点 & 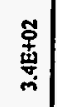 & 㐨 & \\
\hline 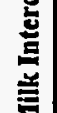 & 要言 & 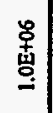 & 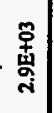 & 莒 & & & & & & 总 & 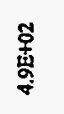 & & & & 亳 & & & 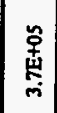 & 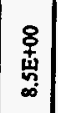 & 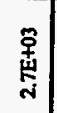 & 京 & \\
\hline & $\ddot{g}$ & & & & & & 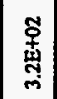 & 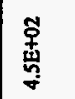 & & 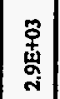 & 竜 & 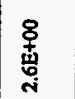 & 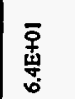 & & & & & & 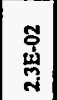 & & & \\
\hline & ] & & & & & & 蒙 & 䓌 & & 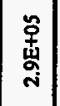 & 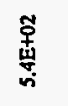 & 䓂 & 害 & & & & & & 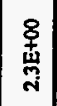 & & & \\
\hline & 흫 & 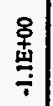 & 各 & 离 & & & 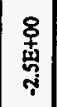 & 率 & & 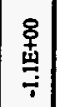 & 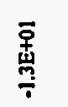 & 恿 & 总 & & 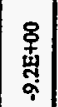 & & & 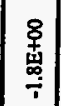 & 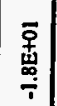 & 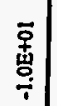 & 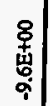 & \\
\hline & 뭄 & 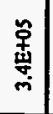 & 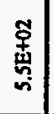 & 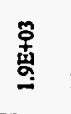 & & & & & & 窝 & 恿 & & & & 䓌 & & & 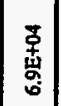 & 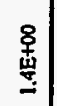 & 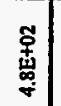 & 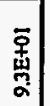 & \\
\hline 兽 & $\overline{\mathrm{g}}$ & 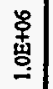 & 旁 & 莒 & & & & & & 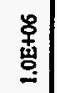 & $\begin{array}{l}\text { 草 } \\
\text { 惫 }\end{array}$ & & & & 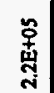 & & & 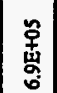 & $\begin{array}{l}\text { 㝵 } \\
\text { 甞 }\end{array}$ & 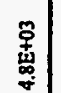 & $\begin{array}{l}\text { 害 } \\
\text { 帘 }\end{array}$ & \\
\hline 竞 & : & & & & & & 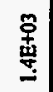 & 总 & & 总 & 名 & 京 & 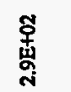 & & & & & & 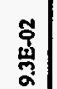 & & & \\
\hline & 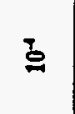 & & & & & & 总 & 藏 & & 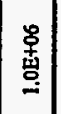 & 䓛 & 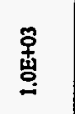 & $\begin{array}{l}\text { 莒 } \\
\text { 兽 }\end{array}$ & & & & & & $\begin{array}{l}\text { \& } \\
\text { 萦 }\end{array}$ & & & \\
\hline & : & 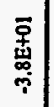 & $\begin{array}{c}\vec{\rho} \\
\mathbf{w} \\
\vec{y} \\
\dot{\gamma}\end{array}$ & 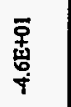 & & & 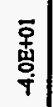 & 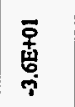 & & 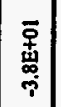 & 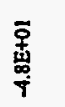 & 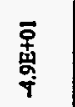 & $\begin{array}{l}\text { 뭉 } \\
\text { 罍 }\end{array}$ & & 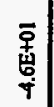 & & & 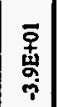 & 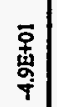 & 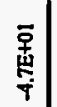 & 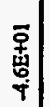 & \\
\hline 可 & 훔 & 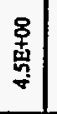 & 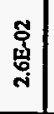 & 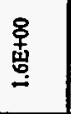 & & & & & & $\begin{array}{l}\frac{8}{4} \\
\text { 索 }\end{array}$ & 离 & & & & 吴 & & & 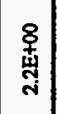 & 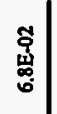 & 离 & 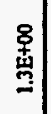 & \\
\hline 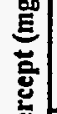 & $\bar{g}$ & 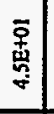 & 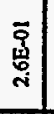 & 窟 & & & & & & 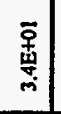 & $\begin{array}{l}\stackrel{8}{4} \\
\underset{\mathbf{w}}{\mathbf{i}}\end{array}$ & & & & 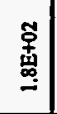 & & & 㤐 & 慝 & 葸 & 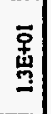 & 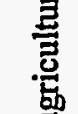 \\
\hline 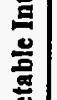 & $\dot{0}$ & & & . & & & 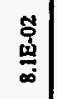 & 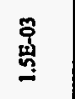 & & 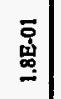 & 岧 & 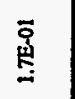 & 䎏 & & & & & & 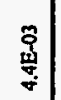 & & & 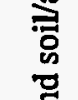 \\
\hline$>$ & $\underline{\sigma}$ & & & & & & $\begin{array}{l}\stackrel{8}{+} \\
\frac{4}{\infty}\end{array}$ & 京 & & 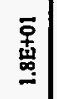 & 量 & 㔬 & 骂 & & & & & & $\begin{array}{l}\overline{\mathbf{s}} \\
\stackrel{4}{+}\end{array}$ & & & 岁 \\
\hline & हु & 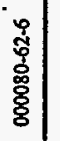 & 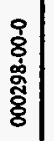 & 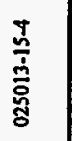 & 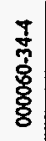 & $\begin{array}{l}\frac{7}{9} \\
\stackrel{3}{8} \\
8\end{array}$ & 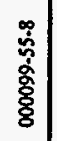 & 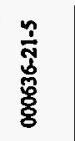 & 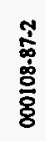 & 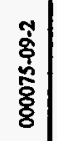 & $\begin{array}{l}\frac{T}{3} \\
\frac{1}{\bar{t}} \\
\frac{8}{8}\end{array}$ & 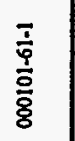 & 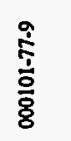 & 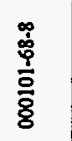 & 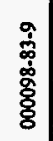 & 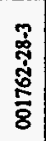 & 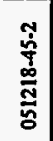 & 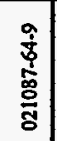 & 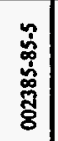 & 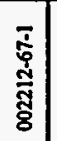 & 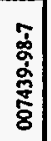 & 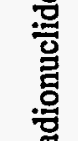 \\
\hline & & 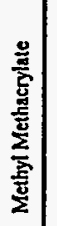 & 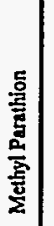 & 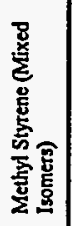 & 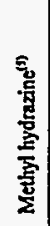 & 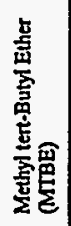 & 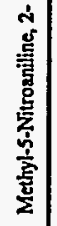 & 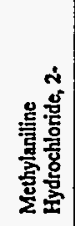 & 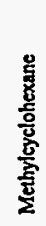 & 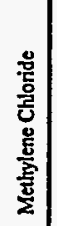 & 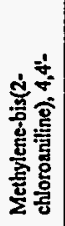 & 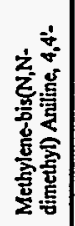 & 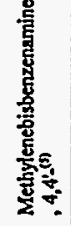 & 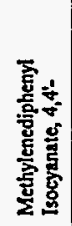 & 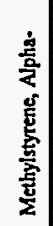 & 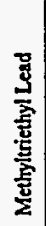 & 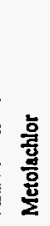 & 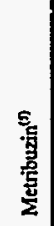 & $\underline{q}$ & $\frac{\mathrm{o}}{\frac{\mathrm{i}}{\mathrm{g}}}$ & 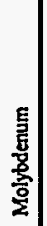 & $\stackrel{0}{Z}$ \\
\hline
\end{tabular}


Table 3.3e. (continued)

\begin{tabular}{|c|c|c|c|c|c|c|c|c|c|c|c|c|c|c|c|c|c|c|}
\hline \multirow{2}{*}{ Chemical } & \multirow{2}{*}{ CASRN } & \multicolumn{4}{|c|}{ Vegetable Intercept (mg/kg) } & \multirow{2}{*}{ Slope } & \multicolumn{4}{|c|}{ Beef Intercept (mg/kg) } & \multirow[b]{2}{*}{ Slope } & \multicolumn{6}{|c|}{ Milk Intercept (mg/kg) } & \multirow[b]{2}{*}{ Slope } \\
\hline & & $10^{-4}$ & $10^{6}$ & $\mathrm{HQ}=1$ & $H Q=0.1$ & & $10^{-4}$ & $10^{5}$ & $\mathbf{H Q}=\mathbf{I}$ & $\mathrm{HQ}=0.1$ & & $10^{-4}$ & $10^{4}$ & $\begin{array}{l}\text { HQ=1 } \\
\text { Adult }\end{array}$ & $\begin{array}{l}\mathrm{HQ}=1 \\
\text { Child }\end{array}$ & $\begin{array}{c}\mathrm{HQ}=0.1 \\
\text { Adult }\end{array}$ & $\begin{array}{c}H Q=0.1 \\
\text { Child }\end{array}$ & \\
\hline Monochloramine & $010599-90-3$ & & & & & & & & & & & & & & & & & \\
\hline Monochlorobutanes & $025154-42-1$ & & & & & & & & & & & & & & & & & \\
\hline Naled & $000300-76-5$ & & & $4.1 \mathrm{E}+\infty$ & 4.1E-01 & $-4.4 E+01$ & & & $1.5 E+04$ & $1.5 \mathrm{E}+03$ & $-5.9 \mathrm{E}+00$ & & & $7.9 \mathrm{E}+03$ & $1.0 \mathrm{E}+03$ & $7.9 E+02$ & $1.0 E+02$ & $-6.2 E+\infty 0$ \\
\hline Naphthalene ${ }^{(9)}$ & $000091-20-3$ & & & $9.2 \mathrm{E}+01$ & $9.2 \mathrm{E}+00$ & $-4.6 \mathrm{E}+01$ & & & $1.2 E+05$ & $1.2 \mathrm{E}+04$ & $-8.9 E+00$ & & & $6.9 \mathrm{E}+04$ & $8.8 E+03$ & $6.9 E+03$ & $8.8 E+02$ & $-9.5 \mathrm{E}+00$ \\
\hline Naphthalene, 1-Methyl & $000090-12-0$ & & & & & & & & & & & & & & & & & \\
\hline Naphthalene, 2-Methyl & $000091-57.6$ & & & & & & & & & & & & & & & & & \\
\hline Napropamide & $015299.99-7$ & & & 2.6E+01 & $2.6 \mathrm{E}+00$ & $-3.7 E+01$ & & & $1.0 \mathrm{E}+06$ & 8.1E+05 & $-4.8 \mathrm{E}-01$ & & & $1.0 \mathrm{E}+06$ & $5.5 \mathrm{E}+05$ & 4.3E+05 & 5.5E+04 & $-4.8 \mathrm{E}-01$ \\
\hline Niagara Blue 4B & $002429-74-5$ & & & & & & & & & & & & & & & & & \\
\hline Nickel Carbonyl & $013463-39-3$ & & & & & & & & & & & & & & & & & \\
\hline Nickel Refinery Dust & NA & & & & & & & & & & & & & & & & & \\
\hline Nickel Soluble Salts & 007440.02 .0 & & & $5.9 \mathrm{E}+01$ & $5.9 \mathrm{E}+00$ & $-4.7 \mathrm{E}+01$ & & & $1.0 \mathrm{E}+03$ & $1.0 \mathrm{E}+02$ & $-1.3 E+01$ & & & $5.8 \mathrm{E}+01$ & $7.5 \mathrm{E}+00$ & $5.8 \mathrm{E}+00$ & $7.5 \mathrm{E}-01$ & $-1.5 E+01$ \\
\hline Nickel Subsutfide & $012035-72-2$ & & & & & & & & & & & & & & & & & \\
\hline Nitrapyrin") & $001929-82-4$ & & & $3.4 \mathrm{E}+00$ & $3,4 \mathrm{E}-01$ & $-4.5 E+01$ & & & $8.2 \mathrm{E}+03$ & $8.2 \mathrm{E}+02$ & $-7.0 E+00$ & & & 4.5E+03 & $5.8 \mathrm{E}+02$ & 4.5E+02 & $5.8 \mathrm{E}+01$ & $-7.4 E+\infty 0$ \\
\hline Nitrate & 014797.55 .8 & & & & & & & & & & & & & & & & & \\
\hline Nitric Oxide & $010102-43-9$ & & & & & & & & & & & & & & & & & \\
\hline Nitrite & $014797-65.0$ & & & & & & & & & & & & & & & & & \\
\hline Nitroaniline, 2-. & $000088-74-4$ & & & & & & & & & & & & & & & & & \\
\hline Nitrobenzene & $000098-95-3$ & & & 4.8E-01 & $4,8 \mathrm{E}-02$ & $-4.0 E+01$ & & & $1.2 \mathrm{E}+04$ & $1.2 E+03$ & $-2.0 E+\infty 0$ & & & $6.6 \mathrm{E}+03$ & $8.4 \mathrm{E}+02$ & $6.6 E+02$ & $8.4 E+01$ & $-2.0 \mathrm{E}+00$ \\
\hline Nitrofurantoin & $000067 \cdot 20-9$ & & & & & & & & & & & & & & & & & \\
\hline Nitrofurazone & $000059-87.0$ & & & & & & & & & & & & & & & & & \\
\hline Nitrogen Dioxide & $010102-44-0$ & & & & & & & & & & & & & & & & & \\
\hline Nitroguanidine & $000556-88-7$ & & & & & & & & & & & & & & & & & \\
\hline Nitrophenol, 4. & $000100-02-7$ & & & & & & & & & & & & & & & & & \\
\hline
\end{tabular}

Nonradionuclides/water and soil/agricultural

Page 21 of 32 
Table 3.3e. (continued)

\begin{tabular}{|c|c|c|c|c|c|c|c|c|c|c|c|c|c|c|c|c|c|c|}
\hline \multirow{2}{*}{ Chemical } & \multirow{2}{*}{ CASRN } & \multicolumn{4}{|c|}{ Vegetable Intercept (mg/kg) } & \multirow{2}{*}{ Slope } & \multicolumn{4}{|c|}{ Beef Intercept (mg/kg) } & \multirow[b]{2}{*}{ Slope } & \multicolumn{6}{|c|}{ Milk Intercept (mg/kg) } & \multirow[b]{2}{*}{ Slope } \\
\hline & & $10^{-4}$ & $10^{4}$ & HQ $=1$ & $\mathrm{HQ}=0.1$ & & $10^{-4}$ & $10^{4}$ & $\mathrm{HQ}=1$ & $H Q=0.1$ & & $10^{-4}$ & $10^{*}$ & $\begin{array}{l}\text { HQ=1 } \\
\text { Adult }\end{array}$ & $\begin{array}{l}\text { HQ=1 } \\
\text { Child }\end{array}$ & $\begin{array}{c}H Q=0.1 \\
\text { Adult }\end{array}$ & $\begin{array}{l}H Q=0.1 \\
\text { Child }\end{array}$ & \\
\hline Nitropropane, 2-(3) & $000079-46-9$ & $5.7 \mathrm{E}-03$ & $5.7 \mathrm{E}-05$ & & & $-3.7 \mathrm{E}+01$ & $2.2 \mathrm{E}+03$ & $2.2 \mathrm{E}+01$ & & & $-4.1 \mathrm{E}-01$ & $5.0 \mathrm{E}+02$ & $5.0 E+\infty 0$ & & & & & $-4.2 \mathrm{E}-01$ \\
\hline Nitroso-N-ethylurea, $\mathrm{N}$ - & $000759-73.9$ & 2.6E-04 & $2.6 \mathrm{E}-06$ & & & $-3.6 \mathrm{E}+01$ & $2.1 \mathrm{E}+02$ & 2.1E+00 & & & $-2.7 \mathrm{E}-01$ & $4.6 \mathrm{E}+01$ & $4.6 \mathrm{E}-01$ & $\cdot$ & & & & $-2.7 \mathrm{E}-01$ \\
\hline Nitroso-N-methylurea, N- & $000684-93-5$ & & & & & & & & & & & & & & & & & \\
\hline $\begin{array}{l}\text { Nitroso-di-N-butylamine, } \\
\text { N- }\end{array}$ & $000924-16-3$ & 4.5E-02 & $4.5 \mathrm{E}-04$ & & & $-4.0 E+01$ & $9.6 \mathrm{E}+02$ & $9.6 \mathrm{E}+00$ & & & $-2.3 \mathrm{E}+00$ & $2.2 E+02$ & $2.2 \mathrm{E}+\infty 0$ & & & & & $-2.3 \mathrm{E}+00$ \\
\hline $\begin{array}{l}\text { Nitroso-di-N. } \\
\text { propylamine, N- }\end{array}$ & $000621-64-7$ & $2.1 \mathrm{E}-02$ & 2.1E-04 & & & $-3.8 \mathrm{E}+01$ & $1.3 \mathrm{E}+03$ & $1.3 \mathrm{E}+01$ & & & $-1.2 \mathrm{E}+00$ & $2.8 \mathrm{E}+02$ & $2.8 \mathrm{E}+00$ & & & & & $-1.2 \mathrm{E}+00$ \\
\hline Nitrosodiethanolamine, N- & $001116-54-7$ & $1.2 \mathrm{E}-03$ & $1.2 \mathrm{E}-05$ & & & $-3.6 \mathrm{E}+01$ & $6.2 \mathrm{E}+04$ & $6.2 \mathrm{E}+02$ & & & $-2.4 \mathrm{E}-02$ & $1.4 \mathrm{E}+04$ & $1.4 \mathrm{E}+02$ & & & & & $-2.4 \mathrm{E}-02$ \\
\hline Nitrosodiethylamine, N. & $000055-18-5$ & 3.3E-04 & 3.3E-06 & & & $-3.7 E+01$ & $1.5 E+02$ & $1.5 \mathrm{E}+\infty 0$ & & & $-3.8 \mathrm{E}-01$ & $3.4 \mathrm{E}+01$ & $3.4 \mathrm{E}-01$ & & & & & $-3.8 \mathrm{E}-01$ \\
\hline Nitrosodimethylarnine, N- & $000062-75-9$ & $2.5 \mathrm{E}-04$ & $2.5 \mathrm{E}-06$ & & & $-3.6 \mathrm{E}+01$ & $1.2 \mathrm{E}+03$ & $1.2 \mathrm{E}+0 \mathrm{I}$ & & & $-9.4 \mathrm{E}-02$ & $2.8 \mathrm{E}+02$ & $2.8 \mathrm{E}+\infty 0$ & & & & & $-9,5 E-02$ \\
\hline Nitrosodiphenylamine, N- & $000086-30-6$ & $1.1 E+02$ & $1.1 \mathrm{E}+\infty$ & & & $-4.5 \mathrm{E}+01$ & $2.3 \mathrm{E}+05$ & $2.3 \mathrm{E}+03$ & & & $-7.6 \mathrm{E}+00$ & $5.2 \mathrm{E}+04$ & $5.2 \mathrm{E}+02$ & & & & & $-8.1 \mathrm{E}+00$ \\
\hline $\begin{array}{l}\text { Nitrosomethylethylamine, } \\
\text { N- }\end{array}$ & $010595-95-6$ & & & & & & & & & & & & & & & & & \\
\hline $\begin{array}{l}\text { Nitrosomethylvinylamine, } \\
\text { N- }\end{array}$ & $004549-40-0$ & & & & & & & & & & & & & & & & & \\
\hline Nitrosopyrrolidine, N- & $000930-55-2$ & $3.0 \mathrm{E}_{-}-03$ & $3.0 \mathrm{E}-05$ & & & $-3.6 \mathrm{E}+01$ & $5.1 \mathrm{E}+04$ & $5.1 \mathrm{E}+02$ & & & $-4.7 \mathrm{E}-02$ & $1.1 \mathrm{E}+04$ & $1.1 \mathrm{E}+02$ & & & & & $-4.7 \mathrm{E}-02$ \\
\hline Nitrotoluene, 4-Amino-2. & $000119-32-4$ & & & & & & & & & & & & & & & & & \\
\hline Nitrotoluene, m- & $000099.08-1$ & & & $1.6 \mathrm{E}+0 \mathrm{I}$ & $1.6 \mathrm{E}+\infty$ & $-4.2 E+01$ & & & $1.2 \mathrm{E}+05$ & $1,2 \mathrm{E}+04$ & $-4.0 E+00$ & & & $6.6 \mathrm{E}+04$ & $8.5 \mathrm{E}+03$ & $6.6 \mathrm{E}+03$ & $8.5 \mathrm{E}+02$ & $-4.1 E+\infty$ \\
\hline Nitrotoluene, o- & $000088-72-2$ & & & $1.5 \mathrm{E}+01$ & $1.5 \mathrm{E}+\infty$ & $-4.2 E+01$ & & & $1.4 \mathrm{E}+05$ & $1.4 \mathrm{E}+04$ & $-3.6 \mathrm{E}+\infty 0$ & & & $7.4 \mathrm{E}+04$ & $9.5 \mathrm{E}+03$ & $7.4 \mathrm{E}+03$ & $9.5 \mathrm{E}+02$ & $-3.7 \mathrm{E}+00$ \\
\hline Nitrotoluene, p- & $000099-99-0$ & & & $1.6 \mathrm{E}+01$ & $1.6 \mathrm{E}+\infty 0$ & $-4.2 \mathrm{E}+01$ & & & $1.2 \mathrm{E}+05$ & $1.2 \mathrm{E}+04$ & $-4.0 E+00$ & & & $6.6 \mathrm{E}+04$ & $8.5 E+03$ & $6.6 \mathrm{E}+03$ & $8.5 \mathrm{E}+02$ & $-4.1 E+\infty 0$ \\
\hline Norflurazon & $027314-13-2$ & & & & & & & & & & & & & & & & & \\
\hline Nustar & $085509-19-9$ & & & & & & & & & & & & & & & & & \\
\hline$O C D D^{(1)}$ & 003268.87 .9 & $5.5 \mathrm{E}-03$ & 5.5E-05 & & & $-5.0 \mathrm{E}+01$ & $3.8 \mathrm{E}-05$ & 3.8E-07 & & & $-1.9 \mathrm{E}+01$ & $9.7 \mathrm{E}-06$ & $9.7 \mathrm{E}-08$ & & & & & $-2.2 \mathrm{E}+01$ \\
\hline$O C D F^{(1)}$ & $039001-02-0$ & & & & & & & & & & & & & & & & & \\
\hline Octabromodiphenyl Ether & $032536-52-0$ & & & & & & & & & & & & & & & & & \\
\hline
\end{tabular}


Table 3.3e. (continued)

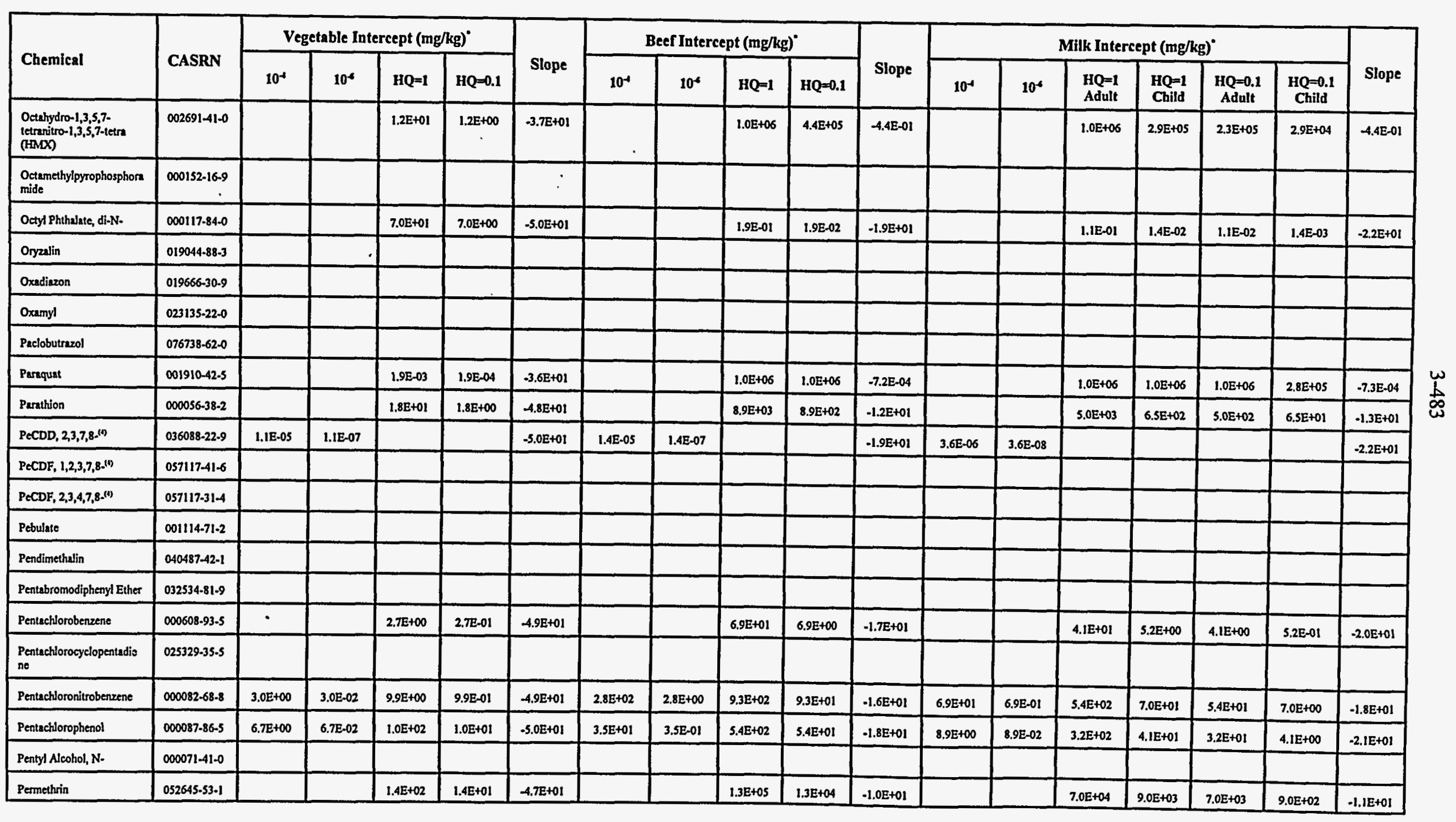

Nonradionuclides/water and soil/agricultural

Page 23 of 32 
Table 3.3e. (continued)

\begin{tabular}{|c|c|c|c|c|c|c|c|c|c|c|c|c|c|c|c|c|c|c|}
\hline \multirow[b]{2}{*}{ Chemical } & \multirow[b]{2}{*}{ CASRN } & \multicolumn{4}{|c|}{ Vegetable Intercept (mg/kg) } & \multirow[b]{2}{*}{ Slope } & \multicolumn{4}{|c|}{ Beef Intercept (mg/kg)' } & \multirow[b]{2}{*}{ Slope } & \multicolumn{6}{|c|}{ Milk Intercept (mg/kg) } & \multirow[b]{2}{*}{ Slope } \\
\hline & & $10^{-4}$ & $10^{6}$ & $\mathrm{HQ}=1$ & $\mathrm{HQ}=0.1$ & & $10^{-4}$ & $10^{6}$ & $H Q=1$ & $\mathrm{HQ}=0.1$ & & $10^{-4}$ & $10^{4}$ & $\begin{array}{l}\text { HQ=1 } \\
\text { Adult }\end{array}$ & $\begin{array}{l}\text { HQ=1 } \\
\text { Child }\end{array}$ & $\begin{array}{c}H Q=0.1 \\
\text { Adult }\end{array}$ & $\begin{array}{c}\text { HQ }=0.1 \\
\text { Child }\end{array}$ & \\
\hline Phenanthrene & $000085-01-8$ & & & & & & & & & & & & & & & & & \\
\hline Phenmedipham & $013684-63-4$ & & & $5.2 \mathrm{E}+00$ & $5.2 \mathrm{E}-01$ & $-3.6 \mathrm{E}+01$ & & & $1.0 \mathrm{E}+06$ & $1.0 \mathrm{E}+06$ & $-3.6 \mathrm{E}-02$ & & & $1.0 E+06$ & $1.0 \mathrm{E}+06$ & $1.0 \mathrm{E}+06$ & $9.3 \mathrm{E}+05$ & $-3.6 \mathrm{E}-02$ \\
\hline Phenol & $000108-95-2$ & & & $4.2 \mathrm{E}+02$ & $4.2 \mathrm{E}+01$ & $-3.9 \mathrm{E}+01$ & & & $1.0 \mathrm{E}+06$ & $1.0 \mathrm{E}+06$ & $-1.4 E+\infty$ & & & $1.0 \mathrm{E}+06$ & $1.0 \mathrm{E}+06$ & $1.0 \mathrm{E}+06$ & $1.4 \mathrm{E}+05$ & $-1.4 \mathrm{E}+00$ \\
\hline Phenylenediamine, m-. & $000108-45-2$ & & & $2.6 \mathrm{E}-0 \mathrm{I}$ & $2.6 \mathrm{E}-02$ & $-3.6 \mathrm{E}+01$ & & & $1.0 \mathrm{E}+06$ & $2.0 \mathrm{E}+05$ & $-7,4 \mathrm{E}-02$ & & & $1.0 \mathrm{E}+06$ & $1.3 \mathrm{E}+05$ & $1.0 \mathrm{E}+05$ & $1.3 \mathrm{E}+04$ & $-7.5 \mathrm{E}-02$ \\
\hline Phenylenedianine, o- & $000095-54-5$ & $1.0 \mathrm{E}+00$ & $1.0 \mathrm{E}-02$ & & & $-3.7 \mathrm{E}+01$ & $5.0 \mathrm{E}+05$ & $5.0 \mathrm{E}+03$ & & & $-3.6 \mathrm{E}-01$ & $1.1 \mathrm{E}+05$ & $1.1 \mathrm{E}+03$ & & & & & $-3.6 \mathrm{E}-01$ \\
\hline Phenylenedianine, p- & $000106-50-3$ & & & $4.5 \mathrm{E}+\infty 0$ & 4.5E-01 & $-3.6 \mathrm{E}+01$ & & & $1.0 \mathrm{E}+06$ & $1.0 \mathrm{E}+06$ & $-4.1 E-02$ & & & $1.0 E+06$ & $1.0 \mathrm{E}+06$ & $1.0 \mathrm{E}+06$ & $6.4 \mathrm{E}+05$ & $-4.1 \mathrm{E} \cdot 02$ \\
\hline Phenylmercuric Acetate & $000062-38-4$ & & & & & & & & & & & & & & & & & \\
\hline Phenylphenol, 2. & $000090-43-7$ & $2.9 \mathrm{E}+02$ & $2.9 \mathrm{E}+\infty 0$ & & & $-4.5 \mathrm{E}+01$ & $5.7 \mathrm{E}+05$ & 5.7E+03 & & & $-7.6 \mathrm{E}+\infty 0$ & $1.3 E+05$ & $1.3 \mathrm{E}+03$ & & & & & $-8.1 E+\infty 0$ \\
\hline Phorate & $000298-02-2$ & & & $1.7 \mathrm{E}-01$ & $1.7 \mathrm{E}-02$ & $-3.9 \mathrm{E}+01$ & & & $5.5 \mathrm{E}+03$ & $5.5 \mathrm{E}+02$ & $-1.8 \mathrm{E}+\infty 0$ & & & $2.9 E+03$ & $3.8 \mathrm{E}+02$ & $2.9 E+02$ & $3.8 \mathrm{E}+01$ & $-1.8 E+00$ \\
\hline Phosmet & $000732-11-6$ & & & $4.1 \mathrm{E}+01$ & $4.1 \mathrm{E}+\infty$ & $-4.4 \mathrm{E}+01$ & & & 1.5E+05 & $1.5 \mathrm{E}+04$ & $-5.9 \mathrm{E}+00$ & & & $7.9 \mathrm{E}+04$ & $1.0 \mathrm{E}+04$ & $7.9 \mathrm{E}+03$ & $1.0 \mathrm{E}+03$ & $-6.2 E+00$ \\
\hline Phosphine & $007803-51-2$ & & & & & & & & & & & & & & & & & \\
\hline Phosphoric Acid & 007664-38-2 & & & & & & & & & & & & & & & & & \\
\hline Phthalic Acid, P- & $000100-21-0$ & & & 8.6E+02 & $8.6 \mathrm{E}+01$ & $-3.9 E+01$ & & & $1.0 \mathrm{E}+06$ & $1.0 E+06$ & $-1.8 E+00$ & & & $1.0 \mathrm{E}+06$ & $1.0 \mathrm{E}+06$ & $1.0 \mathrm{E}+06$ & $1.9 \mathrm{E}+05$ & $-1.8 E+00$ \\
\hline Phthalic Anhydride & $000085-44.9$ & & & $1.0 \mathrm{E}+02$ & $1.0 \mathrm{E}+01$ & $-3.6 \mathrm{E}+01$ & & & $1.0 \mathrm{E}+06$ & $1.0 \mathrm{E}+06$ & $-8.8 \mathrm{E}-02$ & & & $1.0 \mathrm{E}+06$ & $1.0 \mathrm{E}+06$ & $1.0 \mathrm{E}+06$ & $1.0 \mathrm{E}+06$ & $-8.9 \mathrm{E}-02$ \\
\hline Picloram & $001918-02-1$ & & & $1.1 \mathrm{E}+02$ & $1.1 \mathrm{E}+01$ & $-4.2 \mathrm{E}+01$ & & & $8.6 \mathrm{E}+05$ & $8.6 \mathrm{E}+04$ & $-4.0 E+00$ & & & $4.6 E+05$ & $5.9 \mathrm{E}+04$ & $4.6 \mathrm{E}+04$ & $5.9 E+03$ & $-4.1 E+00$ \\
\hline Pirimiphos, Methyl & 029232.93 .7 & & & & & & & & & & & & & & & & & \\
\hline Polybrominated Biphenyls & NA & & & & & & & & & & & & & & & & & \\
\hline Polychlorinated Biphenyls & $001336-36-3$ & $1.1 \mathrm{E}-01$ & $1.1 \mathrm{E}-03$ & & & $-5.0 E+01$ & $4.4 \mathrm{E}-01$ & 4.4E-03 & & & $-1.9 E+01$ & $1.1 \mathrm{E}-01$ & $1.1 \mathrm{E}-03$ & & & & & $-2.1 E+01$ \\
\hline Potassium Cyanide & $000151-50.8$ & & & $4.1 \mathrm{E}+00$ & 4.1E-01 & $-3.6 \mathrm{E}+01$ & & & $1.0 \mathrm{E}+06$ & $1.0 \mathrm{E}+06$ & $-1.4 \mathrm{E}-01$ & & & $1.0 E+06$ & $6.7 \mathrm{E}+05$ & $5.2 \mathrm{E}+05$ & $6.7 \mathrm{E}+04$ & $-1.5 \mathrm{E}-01$ \\
\hline Polassium Siver Cyanide & $000506-61-6$ & & & & & & & & & & & & & & & & & \\
\hline Prochloraz & $067747-09.5$ & & & & & & & & & & & & & & & & & \\
\hline Profluralin & $026399-36-0$ & & & & & & & & & & & & & & & & & \\
\hline Prometon & $001610-18-0$ & & & $2.2 \mathrm{E}+01$ & $2.2 E+\infty 0$ & $-4.2 \mathrm{E}+01$ & & & 2.1E+05 & $2.1 E+04$ & $-3.6 \mathrm{E}+00$ & & & 1.1E+05 & $1.4 E+04$ & $1.1 E+04$ & $1.4 E+03$ & $-3.7 E+\infty 0$ \\
\hline
\end{tabular}

Nonradionuclides/water and soil/agricultural

Page 24 of 32 
Table 3.3e. (continued)

\begin{tabular}{|c|c|c|c|c|c|c|c|c|c|c|c|c|c|c|c|c|c|c|}
\hline \multirow{2}{*}{ Chemical } & \multirow{2}{*}{ CASRN } & \multicolumn{4}{|c|}{ Vegetable Intercept (mg/kg) } & \multirow{2}{*}{ Slope } & \multicolumn{4}{|c|}{ Beef Intercept (mg/kg) } & \multirow[b]{2}{*}{ Slope } & \multicolumn{6}{|c|}{ Milk Intercept (mg/kg) } & \multirow[b]{2}{*}{ Slope } \\
\hline & & $10^{-4}$ & $10^{4}$ & $\mathrm{HQ}=1$ & $\mathrm{HQ}=0.1$ & & $10^{-4}$ & $10^{5}$ & $\mathrm{HQ}=\mathbf{I}$ & $\mathrm{HQ}=0.1$ & & $10^{4}$ & $10^{5}$ & $\begin{array}{l}H Q=1 \\
\text { Adult }\end{array}$ & $\begin{array}{c}\text { HQ }=1 \\
\text { Child }\end{array}$ & $\begin{array}{c}\mathrm{HQ}=0.1 \\
\text { Adult }\end{array}$ & $\begin{array}{c}\mathrm{HQ}=0.1 \\
\text { Child }\end{array}$ & \\
\hline Propachilor & $001918-16-7$ & & & & & & & & & & & & & & & & & \\
\hline Propanil & $000709-98-8$ & & & $1.2 \mathrm{E}+01$ & $1.2 E+\infty$ & $-4.5 \mathrm{E}+0 \mathrm{t}$ & & & $2.3 \mathrm{E}+04$ & $2.3 \mathrm{E}+03$ & $-7.8 E+\infty 0$ & - & & $1.3 E+04$ & $1.6 \mathrm{E}+03$ & $1.3 \mathrm{E}+03$ & $1.6 \mathrm{E}+02$ & $-8.2 \mathrm{E}+00$ \\
\hline Propargite & $002312-35-8$ & & & & & & & & & & & & & & & & & \\
\hline Propargyl Alcohol & $000107-19-7$ & & & & & & & & & & & & & & & & & \\
\hline Propaxine & $000139-40.2$ & & & $3.2 \mathrm{E}+01$ & $3.2 E+\infty$ & $-4.2 E+01$ & & & $2.5 E+05$ & $2.5 E+04$ & $-4.0 E+00$ & & & $1.3 \mathrm{E}+05$ & $1.7 E+04$ & $1.3 \mathrm{E}+04$ & $1.7 \mathrm{E}+03$ & $-4.1 E+\infty$ \\
\hline Propham & $000122-42.9$ & & & $5.0 \mathrm{E}+01$ & $5.0 \mathrm{E}+00$ & $-4.6 \mathrm{E}+01$ & & & $8.1 E+04$ & $8.1 E+03$ & $-8.3 E+\infty$ & & & 4.5E+04 & $5.8 E+03$ & $4.5 \mathrm{E}+03$ & $5.8 \mathrm{E}+02$ & $-8.8 \mathrm{E}+\infty 0$ \\
\hline Propiconazole & $060207-90-1$ & & & & & & & & & & & & & & & & & \\
\hline Propylene Glycol & 000057.55 .6 & & & $2.4 E+02$ & $2.4 \mathrm{E}+01$ & $-3.6 \mathrm{E}+01$ & & & $1.0 E+06$ & $1.0 E+06$ & $-2.1 \mathrm{E}-02$ & & & $1.0 \mathrm{E}+06$ & $1.0 E+06$ & $1.0 \mathrm{E}+06$ & $1.0 E+06$ & $-2.2 \mathrm{E}-02$ \\
\hline $\begin{array}{l}\text { Propylene Glycol } \\
\text { Monoethyl Ether }\end{array}$ & $001569.02-4$ & & & & & & & & & & & & & & & & & \\
\hline Propylene Oxide & $000075-56-9$ & $1.2 \mathrm{E}-01$ & $1.2 \mathrm{E}-03$ & & & $-3.6 \mathrm{E}+01$ & $1.5 \mathrm{E}+05$ & $1.5 \mathrm{E}+03$ & & & $-2.1 E-01$ & $3.3 \mathrm{E}+04$ & $3.3 \mathrm{E}+02$ & & & & & $-2.1 \mathrm{E}-0 \mathrm{I}$ \\
\hline Purssuit & $081335.77-5$ & & & & & & & & & & & & & & & & & \\
\hline Pydrin & 051630-58-1 & & & $8.1 E+01$ & $8.1 E+\infty$ & $-4,9 \mathrm{E}+01$ & & & $1.1 \mathrm{E}+04$ & $1.1 \mathrm{E}+03$ & $-1.5 E+01$ & & & $6.5 E+03$ & $8.4 E+02$ & $6.5 E+02$ & $8.4 E+01$ & $-1.7 \mathrm{E}+0 !$ \\
\hline Pyrene & $000129-00-0$ & & & $1.0 \mathrm{E}+02$ & $1.0 E+01$ & $-4.9 E+01$ & & & $4.9 E+03$ & $4.9 E+02$ & $-1.7 E+01$ & & & $2.9 \mathrm{E}+03$ & $3.7 \mathrm{E}+02$ & $2.9 \mathrm{E}+02$ & $3.7 E+01$ & $-1.9 E+01$ \\
\hline Pyridine & $000110.86-1$ & & & $5.6 \mathrm{E}-01$ & $5.6 \mathrm{E}-02$ & $-3.8 E+01$ & & & 4.2E+04 & $4.2 E+03$ & $-1.1 \mathrm{E}+00$ & & & $2.2 E+04$ & $2.9 \mathrm{E}+03$ & $2.2 E+03$ & $2.9 E+02$ & $-1.1 \mathrm{E}+00$ \\
\hline Quinalphos & $013593-03-8$ & & & & & & & & & & & & & & & & & \\
\hline Quinoline & $000091-22-5$ & 2.4E-02 & $2.4 \mathrm{E}-04$ & & & $-4.1 E+01$ & $3.4 \mathrm{E}+02$ & $3.4 E+\infty 0$ & & & $-2.9 \mathrm{E}+00$ & $7.7 \mathrm{E}+01$ & $7.7 \mathrm{E}-01$ & & & & & $-2.9 \mathrm{E}+00$ \\
\hline Refractory Ceramic Fibers & NA & & & & & & & & & & & & & & & & & \\
\hline Resmethrin & $010453.86-8$ & & & & & & & & & & & & & & & & & \\
\hline Ronnel & $000299-84-3$ & & & $1.7 \mathrm{E}+02$ & $1 . T E+01$ & $-4.9 \mathrm{E}+01$ & & & $5.3 E+03$ & $5.3 E+02$ & $-1.7 \mathrm{E}+01$ & & & $3.2 \mathrm{E}+03$ & $4.0 E+02$ & $3.2 \mathrm{E}+02$ & $4.0 \mathrm{E}+01$ & $-2.0 E+01$ \\
\hline Rotenone & $000083-79-4$ & & & $1.2 E+01$ & $1.2 E+00$ & $-4.8 E+01$ & & & $3.4 E+03$ & $3.4 \mathrm{E}+02$ & $-1.4 E+01$ & & & $1.9 \mathrm{E}+03$ & $2.5 E+02$ & $1.9 \mathrm{E}+02$ & $2.5 \mathrm{E}+01$ & $-1.5 \mathrm{E}+0 \mathrm{I}$ \\
\hline
\end{tabular}

Nonradionuclides/water and soil/agricultural

Page 25 of 32 
Table 3.3e. (continued)

\begin{tabular}{|c|c|c|c|c|c|c|c|c|c|c|c|c|c|c|c|c|c|c|}
\hline \multirow[b]{2}{*}{ Chemical } & \multirow[b]{2}{*}{ CASRN } & \multicolumn{4}{|c|}{ Vegetable Intercept (mg/kg)" } & \multirow[b]{2}{*}{ Slope } & \multicolumn{4}{|c|}{ Beef Intercept (mg/kg) } & \multirow[b]{2}{*}{ Slope } & \multicolumn{6}{|c|}{ Milk Intercept (mg/kg) } & \multirow[b]{2}{*}{ Slope } \\
\hline & & $10^{-4}$ & $10^{4}$ & $H Q=1$ & $\mathrm{HQ}=0.1$ & & $10^{-4}$ & $10^{4}$ & $\mathrm{HQ}=1$ & $\mathrm{HQ}=0.1$ & & $10^{-4}$ & $10^{-6}$ & $\begin{array}{l}H Q=1 \\
\text { Adult }\end{array}$ & $\begin{array}{l}\text { HQ=1 } \\
\text { Child }\end{array}$ & $\begin{array}{c}\text { HQ }=0.1 \\
\text { Adult }\end{array}$ & $\begin{array}{c}\mathrm{HQ}=0.1 \\
\text { Child }\end{array}$ & \\
\hline Savey & $078587-05-0$ & & & & & & & & & & & & & & & & & \\
\hline Selenious Acid & $007783-00-8$ & & & & & & & & & & & & & & & & & \\
\hline Selenite & $014124-67-5$ & & & & & & & & & & & & & & & & & \\
\hline Selenium & $007782-49-2$ & & & $1.3 \mathrm{E}+01$ & $1.3 \mathrm{E}+\infty 0$ & $-4.6 \mathrm{E}+01$ & & & $8.3 \mathrm{E}+\infty$ & $8.3 \mathrm{E}-01$ & $-8.5 E+00$ & & & $1.5 \mathrm{E}+01$ & $1.9 \mathrm{E}+00$ & $1.5 \mathrm{E}+00$ & $1.9 \mathrm{E}-01$ & $-9.1 E+\infty$ \\
\hline Selenium Sulfide & $007446-34-6$ & & & & & & & & & & & & & & & & & \\
\hline Selenourea & $000630-10-4$ & & & & & & & & & & & & & & & & & \\
\hline Sethoxydim & $074051-80-2$ & & & & & & & & & & & & & & & & & \\
\hline Silver & $007440-22-4$ & & & $1.8 \mathrm{E}+01$ & $1.8 \mathrm{E}+\infty 0$ & $-5.0 \mathrm{E}+01$ & & & $1.8 \mathrm{E}+02$ & $1.8 \mathrm{E}+01$ & $-5.5 \mathrm{E}+00$ & & & $1.8 \mathrm{E}+03$ & $2.4 \mathrm{E}+02$ & $1.8 \mathrm{E}+02$ & $2.4 E+01$ & $-5.7 \mathrm{E}+\infty 0$ \\
\hline Silver Cyanide & $000506-64-9$ & & & & & & & & & & & & & & & & & \\
\hline Simuzine & $000122-34-9$ & $2.7 \mathrm{E}+\infty 0$ & $2.7 \mathrm{E}-02$ & $6.8 E+\infty 0$ & $6,8 \mathrm{E}-01$ & $-4.1 E+01$ & $3.0 \mathrm{E}+04$ & $3,0 \mathrm{E}+02$ & $7.8 \mathrm{E}+0.4$ & $7.8 \mathrm{E}+03$ & $-3.2 E+00$ & $6.9 \mathrm{E}+03$ & $6.9 E+01$ & $4.2 \mathrm{E}+04$ & $5.4 \mathrm{E}+03$ & $4.2 E+03$ & $5.4 \mathrm{E}+02$ & $-3.3 \mathrm{E}+\infty 0$ \\
\hline Sodium & $007440-23-5$ & & & & & & & & & & & & & & & & & \\
\hline Sodium Acifluorfen & $062476-59-9$ & & & & & & & & & & & & & & & & & \\
\hline Sodium Azide & $026628-22-8$ & & & & & & & & & & & & & & & & & \\
\hline Sodium Cyanide & $000143-33.9$ & & & $1.6 \mathrm{E}+\infty 0$ & $1,6 \mathrm{E}-01$ & $-3.6 \mathrm{E}+01$ & & & $1.0 E+06$ & $1.0 \mathrm{E}+06$ & $-6,8 \mathrm{E}-02$ & & & $1.0 \mathrm{E}+06$ & $9.3 \mathrm{E}+05$ & $7.3 \mathrm{E}+05$ & $9.3 \mathrm{E}+04$ & $-6.8 \mathrm{E}-02$ \\
\hline $\begin{array}{l}\text { Sodium } \\
\text { Diethyldithiocarbamate }\end{array}$ & $000148-18-5$ & & & & & & & & & & & & & & & & & \\
\hline Sodium Fluoroacetate & $000062-74-8$ & & & & & & & & & & & & & & & & & \\
\hline Sodium Metsvanadate & $013718-26-8$ & & & & & & & & & & & & & & & & & \\
\hline $\begin{array}{l}\text { Stirofos } \\
\text { (Tetrachlorovinphos) }\end{array}$ & $000961-11-5$ & & & & & & & & & & & & & & & & & \\
\hline Strontium, Stable & $007440-24-6$ & & & $1.2 E+03$ & $1.2 \mathrm{E}+02$ & $-4.4 E+01$ & & & 7.5E+03 & $7.5 \mathrm{E}+02$ & $-5.1 E+00$ & & & $3.7 \mathrm{E}+03$ & $4.7 \mathrm{E}+02$ & $3.7 E+02$ & $4.7 E+01$ & $-5.3 E+\infty 0$ \\
\hline Strychnine & $000057-24-9$ & & & $3.2 \mathrm{E}-01$ & $3.2 \mathrm{E}-02$ & $-4.0 E+01$ & & & $6.7 \mathrm{E}+03$ & $6.7 \mathrm{E}+02$ & $-2.3 \mathrm{E}+\infty 0$ & & & $3.5 E+03$ & $4.5 \mathrm{E}+02$ & $3.5 E+02$ & $4.5 E+01$ & $-2.3 E+00$ \\
\hline Styrene & $000100-42-5$ & & & $4.3 E+02$ & $4.3 E+01$ & $-4.4 E+01$ & & & $1.0 \mathrm{E}+06$ & $1.3 \mathrm{E}+05$ & $-6.5 \mathrm{E}+00$ & & & $6.9 \mathrm{E}+0 \mathrm{~S}$ & $8.9 E+04$ & $6.9 \mathrm{E}+04$ & $8.9 E+03$ & $-6.8 E+\infty 0$ \\
\hline Sulfate & $014808-79-8$ & & & & & & & & & & & & & & & & & \\
\hline
\end{tabular}


Table 3.3e. (continued)

\begin{tabular}{|c|c|c|c|c|c|c|c|c|c|c|c|c|c|c|c|c|c|c|}
\hline \multirow[b]{2}{*}{ Chemical } & \multirow[b]{2}{*}{ CASRN } & \multicolumn{4}{|c|}{ Vegetable Intercept (mg/kg) } & \multirow[b]{2}{*}{ Slope } & \multicolumn{4}{|c|}{ Beef Intercept (mg/kg) } & \multirow[b]{2}{*}{ Slope } & \multicolumn{6}{|c|}{ Milk Intercept (mg/kg) } & \multirow[b]{2}{*}{ Slope } \\
\hline & & $10^{-4}$ & $10^{*}$ & $H Q=1$ & $\mathrm{HQ}=0.1$ & & $10^{-4}$ & $10^{4}$ & $H Q=1$ & $\mathrm{HQ}=0.1$ & & $10^{-4}$ & $10^{6}$ & $\begin{array}{l}\text { HQ=1 } \\
\text { Adult }\end{array}$ & $\begin{array}{l}\text { HQ=1 } \\
\text { Child }\end{array}$ & $\begin{array}{l}\text { HQ }=0.1 \\
\text { Adult }\end{array}$ & $\begin{array}{c}\text { HQ }=0.1 \\
\text { Child }\end{array}$ & \\
\hline Systhane & $088671-89-0$ & & & & & & & & & & & & & & & & & \\
\hline TCDD, 2,3,7,8.(1) & $001746-01-6$ & $5.4 \mathrm{E}-06$ & $5.4 \mathrm{E}-08$ & & & $-5.0 \mathrm{E}+01$ & $3.6 \mathrm{E}-06$ & $3.6 \mathrm{E}-08$ & & & $-1.9 E+01$ & $9.2 \mathrm{E}-07$ & $9.2 E-09$ & & & & & $-2.2 \mathrm{E}+01$ \\
\hline TCDF, 2,3,7,8.(1) & $051207-31-9$ & 5.4E-05 & $5.4 \mathrm{E}-07$ & & & $-5.0 \mathrm{E}+01$ & 3.4E-04 & $3.4 \mathrm{E}-06$ & & & $-1.8 E+01$ & 8.5E-05 & 8.5E-07 & & & & & $-2.1 \mathrm{E}+01$ \\
\hline TCMTB & $021564-17-0$ & & & & & & & & & & & & & & & & & \\
\hline Tebuthurun & $034014-18-1$ & & & $9.7 E+\infty 0$ & $9.7 \mathrm{E}-01$ & $-3.6 \mathrm{E}+01$ & & & $1.0 \mathrm{E}+06$ & 9.5E+05 & $-2.4 \mathrm{E}-01$ & & & $1.0 \mathrm{E}+06$ & $6.3 \mathrm{E}+05$ & $4.9 \mathrm{E}+05$ & $6.3 \mathrm{E}+04$ & $-2.5 \mathrm{E}-0 \mathrm{t}$ \\
\hline Temephos & $003383-96-8$ & & & & & & & & & & & & & & & & & \\
\hline Terbacil & 005902.51 .2 & & & & & & & & & & & & & & & & & \\
\hline Terbufos & $013071-79-9$ & & & $7.2 \mathrm{E}-02$ & $7.2 \mathrm{E}-03$ & $-4.7 \mathrm{E}+01$ & & & $4.6 \mathrm{E}+01$ & $4.6 \mathrm{E}+\infty$ & $-1.1 E+01$ & & & $2.6 \mathrm{E}+01$ & $3.3 \mathrm{E}+00$ & $2,6 \mathrm{E}+\infty$ & 3.3E-01 & $-1.2 \mathrm{E}+01$ \\
\hline Terbutryn & $000886-50.0$ & & & $2.9 E+\infty 0$ & $2.9 \mathrm{E}-01$ & $-4.7 \mathrm{E}+01$ & & & $1.7 \mathrm{E}+03$ & $1.7 E+02$ & $-1.2 \mathrm{E}+01$ & & & $9.3 \mathrm{E}+02$ & $1.2 \mathrm{E}+02$ & $9.3 E+01$ & $1.2 \mathrm{E}+0 !$ & $-1.3 E+0 !$ \\
\hline Tetrabutyl Lead & $001920-90-7$ & & & & & & & & & & & & & & & & & \\
\hline $\begin{array}{l}\text { Tetrachlorobenzene, } \\
1,2,4,5 \text {. }\end{array}$ & $000095.94-3$ & & & $9.9 \mathrm{E}-01$ & $9.9 \mathrm{E}-02$ & $-4.9 E+01$ & & & $9.3 \mathrm{E}+01$ & $9.3 \mathrm{E}+00$ & $-1.6 \mathrm{E}+01$ & & & $5.4 E+01$ & $7.0 E+\infty 0$ & $5.4 E+\infty 0$ & $7.0 \mathrm{E}-01$ & $-1,8 E+01$ \\
\hline $\begin{array}{l}\text { Tetrachloroethane, } \\
1,1,1,2^{2}\end{array}$ & $000630-20-6$ & $2.0 \mathrm{E}+01$ & $2.0 \mathrm{E}-01$ & $6.8 \mathrm{E}+01$ & $6.8 \mathrm{E}+\infty 0$ & $-4.5 E+01$ & $4.9 E+04$ & $4.9 \mathrm{E}+02$ & $1.6 \mathrm{E}+05$ & $1.6 \mathrm{E}+04$ & $-7.0 \mathrm{E}+00$ & $1.1 \mathrm{E}+04$ & $1.1 \mathrm{E}+02$ & $9.0 E+04$ & $1.2 \mathrm{E}+04$ & $9.0 E+03$ & $1.2 E+03$ & $-7,4 E+\infty 0$ \\
\hline $\begin{array}{l}\text { Tetrachloroethane, } \\
1,1,2,2-\end{array}$ & $000079-34-5$ & $1.9 \mathrm{E}+\infty 0$ & $1.9 \mathrm{E}-02$ & & & $-4.2 E+01$ & $1.4 \mathrm{E}+04$ & $1.4 \mathrm{E}+02$ & & & $-4.0 \mathrm{E}+00$ & $3.3 E+03$ & $3.3 E+01$ & & & & & $-4.1 E+\infty$ \\
\hline Tetrachloroethylene & $000127-18-4$ & $8.2 \mathrm{E}+\infty 0$ & $8.2 \mathrm{E}-02$ & $1.8 \mathrm{E}+01$ & $1.8 \mathrm{E}+\infty 0$ & $-4.3 E+01$ & $4.3 E+04$ & $4.3 E+02$ & $9.5 E+04$ & $9.5 \mathrm{E}+03$ & $-4.9 \mathrm{E}+00$ & $9.8 \mathrm{E}+03$ & $9.8 \mathrm{E}+01$ & $5.1 E+04$ & $6.6 \mathrm{E}+03$ & $5.1 \mathrm{E}+03$ & $6.6 \mathrm{E}+02$ & $-5.1 E+\infty$ \\
\hline $\begin{array}{l}\text { Tetrachlorophenol, } \\
2,3,4,6 .\end{array}$ & $000058-90-2$ & & & $9.4 E+01$ & $9.4 \mathrm{E}+00$ & $-4.8 E+01$ & & & $2.5 \mathrm{E}+04$ & $2.5 \mathrm{E}+03$ & $-1.4 E+01$ & & & $1.5 \mathrm{E}+04$ & $1.9 E+03$ & $1.5 E+03$ & $1.9 \mathrm{E}+02$ & $-1.5 \mathrm{E}+01$ \\
\hline $\begin{array}{l}\text { Tetrachlorotoluene, p- } \\
\text { alpha, alpha, \&pha- }\end{array}$ & $005216-25-1$ & & & & & & & & & & & & & & & & & \\
\hline $\begin{array}{l}\text { Tetraethyl } \\
\text { Dithiopyrophosphate }\end{array}$ & $003689.24-5$ & & & $1.1 \mathrm{E}+\infty$ & $1.1 \mathrm{E}-01$ & $-4.5 E+01$ & & & $2.7 \mathrm{E}+03$ & 2.7E+02 & $-7.0 \mathrm{E}+\infty 0$ & & & $1.5 \mathrm{E}+03$ & $1.9 \mathrm{E}+02$ & $1.5 \mathrm{E}+02$ & $1.9 \mathrm{E}+01$ & $-7.4 E+00$ \\
\hline Tetraethyt Lead & $000078-00-2$ & & & & & & & & & & & & & & & & & \\
\hline $\begin{array}{l}\text { Tetrafluoroethane, } \\
1,1,1,2-\end{array}$ & $000811-97-2$ & & & & & & & & & & & & & & & & & \\
\hline Tetramethyl Lead & $000075-74-1$ & & & & & & & & & & & & & & & & & \\
\hline
\end{tabular}

Nonradionuclides/water and soil/agricultural

Page 27 of 32 
Table 3.3e. (continued)

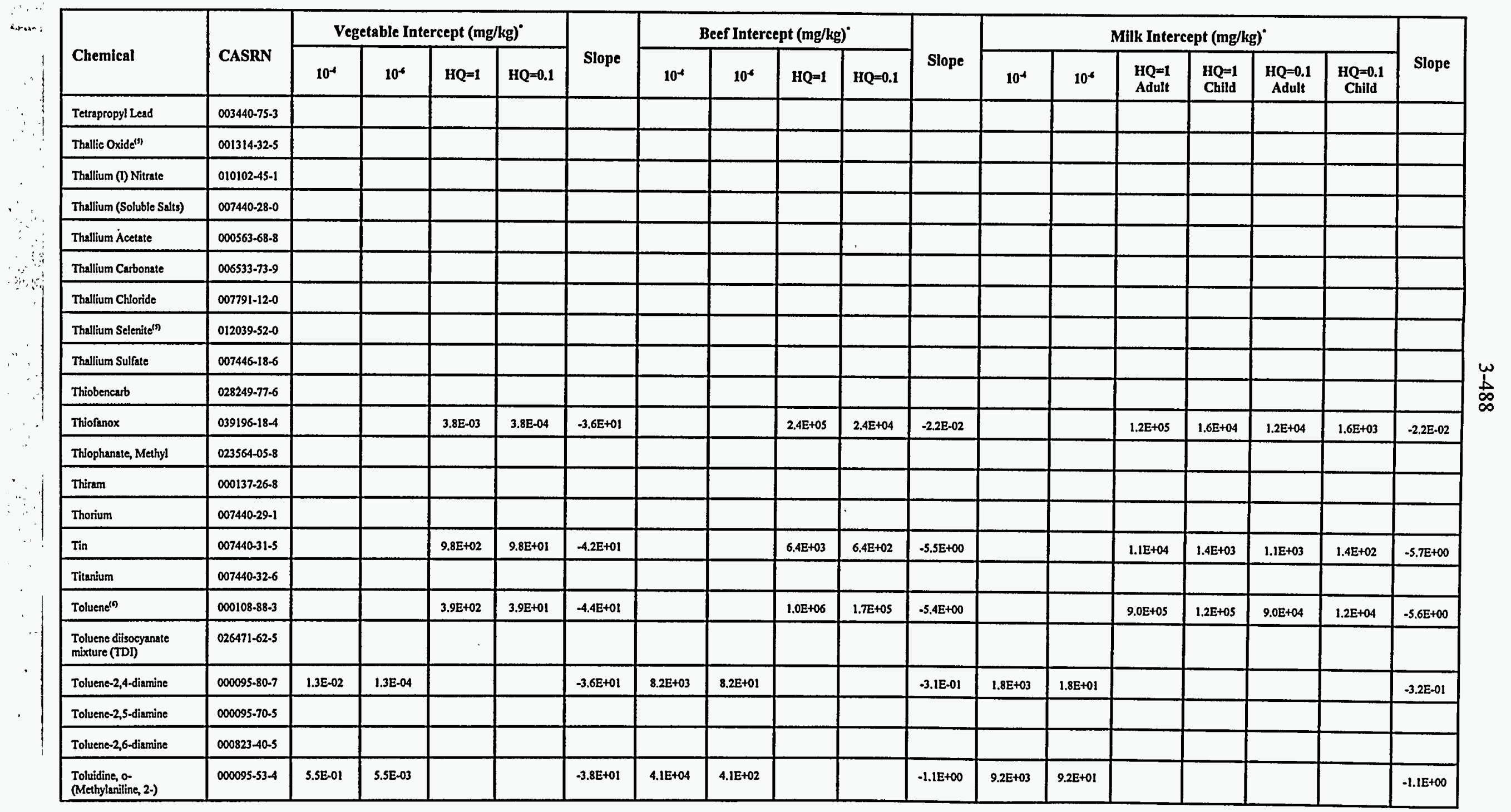


Table 3.3e. (continued)

\begin{tabular}{|c|c|c|c|c|c|c|c|c|c|c|c|c|c|c|c|c|c|c|}
\hline \multirow[b]{2}{*}{ Chemical } & \multirow[b]{2}{*}{ CASRN } & \multicolumn{4}{|c|}{ Vegetable Intercept (mg/kg) } & \multirow[b]{2}{*}{ Slope } & \multicolumn{4}{|c|}{ Beef Intercept (mg/kg)" } & \multirow[b]{2}{*}{ Slope } & \multicolumn{6}{|c|}{ Milk Intercept (mg/kg) } & \multirow[b]{2}{*}{ Slope } \\
\hline & & $10^{-4}$ & $10^{6}$ & $H Q=1$ & $\mathrm{HQ}=0.1$ & & $10^{-4}$ & $10^{6}$ & $H Q=1$ & $\mathrm{HQ}=0.1$ & & $10^{-4}$ & $10^{-4}$ & $\begin{array}{l}H Q=1 \\
\text { Adult }\end{array}$ & $\begin{array}{l}\text { HQ }=1 \\
\text { Child }\end{array}$ & $\begin{array}{c}\mathrm{HQ}=0.1 \\
\text { Adult }\end{array}$ & $\begin{array}{c}\text { HQ }=0.1 \\
\text { Child }\end{array}$ & \\
\hline Toxaphene & $008001-35-2$ & 7.1E-01 & 7.1E-03 & & & $-4.9 E+01$ & 4.3E+01 & 4.3E-01 & & & $-1.7 E+01$ & $1.1 E+01$ & $1.1 \mathrm{E}-01$ & & & & & $-1.9 \mathrm{E}+01$ \\
\hline Tralomethrin & $066841-25-6$ & & & & & & & & & & & & & & & & & \\
\hline Triallate & $002303-17-5$ & & & $4.0 \mathrm{E}+01$ & $4.0 \mathrm{E}+00$ & $-4.8 \mathrm{E}+01$ & & & $1.4 \mathrm{E}+04$ & $1.4 \mathrm{E}+03$ & $-1.3 \mathrm{E}+01$ & & & $7.9 E+03$ & $1.0 \mathrm{E}+03$ & $7.9 \mathrm{E}+02$ & $1.0 E+02$ & $-1,4 E+01$ \\
\hline Triasulfuron & $082097-50-5$ & & & & & & & & & & & & & & & & & \\
\hline Tribromobenzene, 1,2,4. & $000615-54-3$ & & & & & & & & & & & & & & & & & \\
\hline Tribromochloromethane & $000594-15.0$ & & & & & & & & & & & & & & & & & \\
\hline Tribromodiphenyl Ether & $049690-94.0$ & & & & & & & & & & & & & & & & & \\
\hline Tributyltin Oxide & $000056-35-9$ & & & & & & & & & & & & & & & & & \\
\hline $\begin{array}{l}\text { Trichloro-1,2,2. } \\
\text { trilluoroethane, 1,1,2- }\end{array}$ & $000076-13-1$ & & & $5.5 \mathrm{E}+04$ & $5.5 \mathrm{E}+03$ & $-4.3 E+01$ & & & $1.0 \mathrm{E}+06$ & $1.0 \mathrm{E}+06$ & $-4.9 E+\infty 0$ & & & $1.0 \mathrm{E}+06$ & $1.0 E+06$ & $1.0 \mathrm{E}+06$ & $1.0 \mathrm{E}+06$ & $-5.1 \mathrm{E}+\infty 0$ \\
\hline $\begin{array}{l}\text { Trichloro-2'- } \\
\text { hydroxydiphenylether }\end{array}$ & $003380-34-5$ & & & & & & & & & & & & & & & & & \\
\hline \multicolumn{19}{|l|}{ Trichloroncetic Acid } \\
\hline $\begin{array}{l}\text { Trichloroaniline } \mathrm{HCl}, \\
\text { 2,4,6- }\end{array}$ & $033663.50-2$ & & & & & & & & & & & & & & & & & \\
\hline Trichloroaniline, 2,4,6- & $000634-93-5$ & & & & & & & & & & & & & & & & & \\
\hline Trichlorobenzene, 1,2,4- & $000120.82-1$ & & & $3.1 \mathrm{E}+01$ & $3.1 \mathrm{E}+\infty$ & $-4.8 \mathrm{E}+01$ & & & $1.0 \mathrm{E}+04$ & $1.0 \mathrm{E}+03$ & $-1.3 \mathrm{E}+01$ & & & $5.9 \mathrm{E}+03$ & $7.5 \mathrm{E}+02$ & $5.9 \mathrm{E}+02$ & $7.5 \mathrm{E}+01$ & $-1.5 E+01$ \\
\hline Trichlorocthane, 1,1,1,-(s) & $000071-55-6$ & & & $1.5 \mathrm{E}+02$ & $1.5 \mathrm{E}+01$ & $-4.3 \mathrm{E}+01$ & & & $9.8 \mathrm{E}+05$ & $9.8 \mathrm{E}+04$ & $-4.4 \mathrm{E}+\infty$ & & & $5.2 \mathrm{E}+05$ & $6.7 \mathrm{E}+04$ & $5.2 E+04$ & $6.7 \mathrm{E}+03$ & $-4.6 \mathrm{E}+00$ \\
\hline Trichloroethane, 1,1,2- & $000079-00-5$ & $4,7 \mathrm{E}+00$ & 4.TE-02 & $4.6 \mathrm{E}+\infty$ & 4.6E-01 & $-4.0 \mathrm{E}+01$ & $8.1 \mathrm{E}+04$ & $8.1 E+02$ & $7.9 \mathrm{E}+04$ & $7.9 \mathrm{E}+03$ & $-2.5 \mathrm{E}+\infty$ & $1.8 E+04$ & $1.8 \mathrm{E}+02$ & $4.2 E+04$ & $5.4 E+03$ & $4.2 \mathrm{E}+03$ & $5.4 E+02$ & $-2.6 \mathrm{E}+00$ \\
\hline Trichloroethylene $e^{\text {(1) }}$ & $000079-01-6$ & $3.4 \mathrm{E}+01$ & $3,4 \mathrm{E}-01$ & & & $-4.2 \mathrm{E}+01$ & $2.6 \mathrm{E}+05$ & $2.6 \mathrm{E}+03$ & & & $-4.0 E+\infty 0$ & $5.9 \mathrm{E}+04$ & $5.9 \mathrm{E}+02$ & & & & & $-4.1 E+00$ \\
\hline Trichiorofluoromethane & $000075-69-4$ & & & $5.1 \mathrm{E}+02$ & $5.1 \mathrm{E}+01$ & $-4.3 E+01$ & & & $1.0 \mathrm{E}+06$ & $3.3 \mathrm{E}+05$ & $-4.4 \mathrm{E}+00$ & & & $1.0 \mathrm{E}+06$ & $2.2 \mathrm{E}+0 \mathrm{~s}$ & $1.7 E+05$ & $2.2 E+04$ & $-4.6 E+00$ \\
\hline Trictlorophenol, 2,4,5. & $000095.95-4$ & & & $3.0 \mathrm{E}+02$ & $3.0 \mathrm{E}+01$ & $-4.8 E+01$ & & & $1.5 E+05$ & $1.5 E+04$ & $-1.2 \mathrm{E}+01$ & & & $8.4 E+04$ & $1.1 \mathrm{E}+04$ & $8,4 E+03$ & $1.1 \mathrm{E}+03$ & $-1.3 E+01$ \\
\hline Trichlorophenol, 2,4,6- & $000088-06-2$ & $6.1 \mathrm{E}+01$ & $6.1 \mathrm{E}-01$ & & & $-4.7 \mathrm{E}+01$ & $3,8 E+04$ & $3.8 \mathrm{E}+02$ & & & $-1.1 \mathrm{E}+01$ & $9.0 \mathrm{E}+03$ & $9.0 E+01$ & & & & & $-1.2 \mathrm{E}+01$ \\
\hline
\end{tabular}


Table 3.3e. (continued)

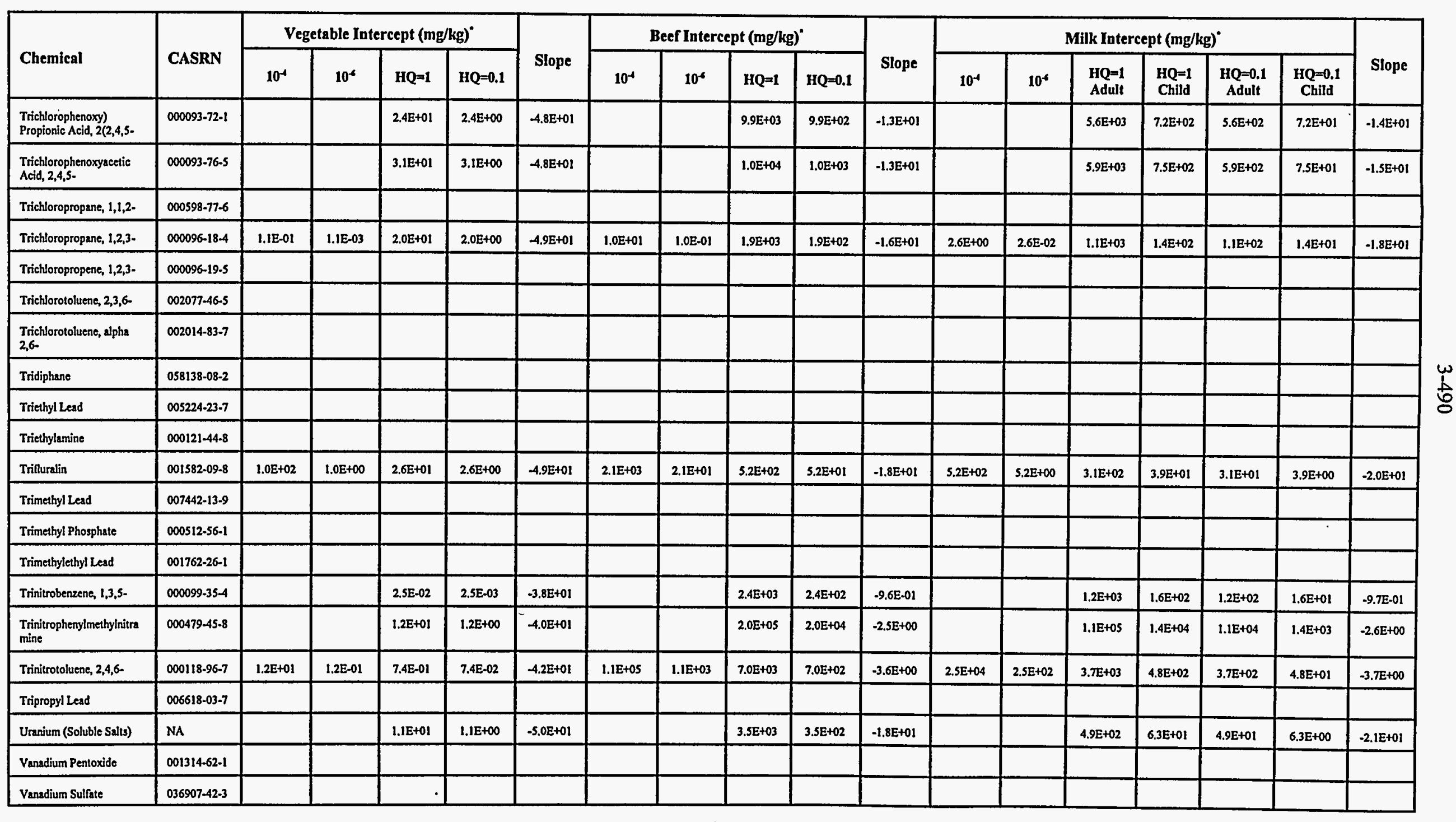


Table 3.3e. (continued)

\begin{tabular}{|c|c|c|c|c|c|c|c|c|c|c|c|c|c|c|c|c|c|c|}
\hline \multirow[b]{2}{*}{ Chemical } & \multirow[b]{2}{*}{ CASRN } & \multicolumn{4}{|c|}{ Vegetable Intercept (mg/kg) } & \multirow[b]{2}{*}{ Slope } & \multicolumn{4}{|c|}{ Beef Intercept (mg/kg) } & \multirow[b]{2}{*}{ Slope } & \multicolumn{6}{|c|}{ Milk Intercept (mg/kg) } & \multirow[b]{2}{*}{$\begin{array}{c}\text { Slope } \\
\text {. }\end{array}$} \\
\hline & & $10^{-4}$ & $10^{6}$ & $H Q=1$ & $\mathrm{HQ}=0.1$ & & $10^{-4}$ & $10^{4}$ & $\mathrm{HQ}=1$ & $\mathrm{HQ}=0.1$ & & $10^{-4}$ & $10^{*}$ & $\begin{array}{l}\text { HQ=1 } \\
\text { Adult }\end{array}$ & $\begin{array}{l}\text { HQ=1 } \\
\text { Child }\end{array}$ & $\begin{array}{c}H Q=0.1 \\
\text { Adult }\end{array}$ & $\begin{array}{c}H Q=0.1 \\
\text { Child }\end{array}$ & \\
\hline Vanadium, Metallic & $007440-62-2$ & & & $2.4 \mathrm{E}+01$ & $2.4 \mathrm{E}+\infty 0$ & $-5.0 \mathrm{E}+01$ & & & $1.0 \mathrm{E}+03$ & $1.0 \mathrm{E}+02$ & $-1.9 E+01$ & & & $2.4 \mathrm{E}+04$ & $3.1 E+03$ & $2.4 E+03$ & $3.1 \mathrm{E}+02$ & $-2.2 E+01$ \\
\hline Vanadyl Sulfate & $027774-13-6$ & & & & & & & & & & & & & & & & & \\
\hline Vernolate & $001929-77-7$ & & & & & & & & & & & & & & & & & \\
\hline Vinclozolin & $050471-44-8$ & & & & & & & & & & & & & & & & & \\
\hline Vinyi Acetate & $000108-05-4$ & & & $2.9 \mathrm{E}+02$ & $2.9 E+01$ & $-3.7 \mathrm{E}+01$ & & & $1.0 E+06$ & $1.0 E+06$ & $-5.2 \mathrm{E}-01$ & & & $1.0 \mathrm{E}+06$ & $1.0 E+06$ & $1.0 E+06$ & $5.1 E+05$ & $-5.3 \mathrm{E}-01$ \\
\hline Vinyl Bromide & $000593-60-2$ & & & & & & & & & & & & & & & & & \\
\hline Vinyl Chloride" & $000075-01-4$ & 7.7E-02 & 7.7E-04 & & & $-3.8 \mathrm{E}+01$ & $4.7 \mathrm{E}+03$ & 4.7E+01 & & & $-1.2 \mathrm{E}+\infty$ & $1.0 \mathrm{E}+03$ & $1.0 \mathrm{E}+01$ & & & & & $-1.2 \mathrm{E}+\infty 0$ \\
\hline Warfasin & $000081-81-2$ & & & 7.5E-01 & 7.5E-02 & $-4.6 \mathrm{E}+01$ & & & $1.2 \mathrm{E}+03^{\circ}$ & $1.2 \mathrm{E}+02$ & $-8.3 \mathrm{E}+00$ & & & $6.7 \mathrm{E}+02$ & $8.6 E+01$ & $6.7 \mathrm{E}+01$ & $8.6 \mathrm{E}+\infty 0$ & $-8.8 \mathrm{E}+00$ \\
\hline White Phosphorus & $007723-14-0$ & & & & & & & & & & & & & & & & & \\
\hline Xylene, Mixture"9 & $001330-20-7$ & & & $5.2 E+03$ & $5.2 E+02$ & $-4.6 \mathrm{E}+01$ & & & $1.0 \mathrm{E}+06$ & $6.9 \mathrm{E}+05$ & $-8.9 \mathrm{E}+\infty 0$ & & & $1.0 \mathrm{E}+06$ & $4.9 E+05$ & $3.8 E+05$ & $4.9 \mathrm{E}+04$ & $-9.5 \mathrm{E}+\infty 0$ \\
\hline Xylene, P-(n) & $000106-42-3$ & & & & & & & & & & & & & & & & & \\
\hline Xylene, $m^{(n)}$ & $000108-38-3$ & & & $5.0 \mathrm{E}+03$ & $5.0 \mathrm{E}+02$ & $-4.6 \mathrm{E}+01$ & & & $1.0 E+06$ & $8.1 E+05$ & $-8.3 \mathrm{E}+\infty 0$ & & & $1.0 \mathrm{E}+06$ & $5.8 \mathrm{E}+05$ & $4.5 E+05$ & $5.8 E+04$ & $-8.8 \mathrm{E}+\infty$ \\
\hline Xylene, $0_{-}^{(n)}$ & $000095-47.6$ & & & $5.0 \mathrm{E}+03$ & $5.0 \mathrm{E}+02$ & $-4.6 \mathrm{E}+01$ & & & $1.0 \mathrm{E}+06$ & $8.1 \mathrm{E}+05$ & $-8.3 \mathrm{E}+\infty 0$ & & & $1.0 \mathrm{E}+06$ & $5.8 E+05$ & 4.5E+05 & $5.8 \mathrm{E}+04$ & $.8 .8 \mathrm{E}+\infty 0$ \\
\hline Zinc (Metallic) & $007440-66-6$ & & & $5.2 \mathrm{E}+02$ & $5.2 \mathrm{E}+01$ & $-4.3 \mathrm{E}+01$ & & & $3.2 \mathrm{E}+02$ & $3.2 \mathrm{E}+01$ & $-5.5 \mathrm{E}+00$ & & & $5.5 \mathrm{E}+02$ & $7.1 E+01$ & $5.5 E+01$ & $7.1 E+\infty 0$ & $-5.8 E+\infty$ \\
\hline Zinc Cyanide & $000557-21-1$ & & & & & & & & & & & & & & & & & \\
\hline Zinc Phosphide & $001314.84-7$ & & & & & & & & & & & & & & & & & \\
\hline Zineb & $012122-67-7$ & & & & & & & & & & & & & & & & & \\
\hline Zireonium & $007440-67-7$ & & & & & & & & & & & & & & & & & \\
\hline
\end{tabular}

Notes:

* By definition, $1 \mathrm{~kg}=1.0 \mathrm{E}+06 \mathrm{mg}$. Therefore, a limit of $1.0 \mathrm{E}+06 \mathrm{mg} / \mathrm{kg}$ was used for any $\mathrm{PRG}$ that was calculated to be above this limit.

(1) The same oral slope factor as used for polychlorinated biphenyls.

(2) The PRG was calculated considering Supplemental Guidance from RAGS: Region 4 Bulletins, Human Health Risk Assessment (Interim Guidance) from EPA (received February 11, 1992).

The slope factors for chemicals so marked indicate that a toxicity equivalency factor (TEF) method was used for carcinogenic PAHs based on each compound's relative potency to the potency

of benzo[a]pyrene. The following TEFs were used to convert each PAH slope factor to an equivalent slope factor of benzo[a]pyrene [that has a new cancer slope factor of 7.3 (mg/kg/day) $)^{-1}$

Nonradionuclides/water and soil/agricultural

Page 31 of 32 


\section{Table 3.3e. (continued)}

identified by EPA's CRAVE workgroup]: (1) benzo[a]pyrene, $\mathrm{TEF}=1.0 ;$ (2) benzo[a]anthracene, $\mathrm{TEF}=0.1 ;(3)$ benzo[b]fluoranthene, $\mathrm{TEF}=0.1 ;$ (4) benzo[k]fluoranthene, $\mathrm{TEF}=0.01$; (5) chrysene, TEF $=0.001$; (6) dibenz[a, h] anthracene, TEF $=1.0$; and (7) indeno[1,2,3-c,d]pyrene, TEF $=0.1$.

(3) The toxicity values for trichloroethylene have been withdrawn from IRIS and HEAST pending further investigation. However, the most recent toxicity values have been used to calculate the PRG.

(4) The PRG was calculated considering Supplemental Guidance from RAGS: Region 4 Bulletins, Human Health Risk Assessment (Interim Guidance) from EPA (November 1995). The slope factors for chemicals so marked indicate that a TEF methodology was used for chlorinated dioxin and furan congeners based on each compound's relative potency to the potency of 2,3,7,8tetrachlorodibenzodioxin (TCDD). The following TEFs were used to convert each slope factor to an equivalent slope factor of 2,3,7,8-TCDD: (1) 2,3,7,8-TCDD, TEF = 1.0; (2) 2,3,7,8PeCDD, TEF $=0.5 ;(3) 2,3,7,8-\mathrm{HxCDD}, \mathrm{TEF}=0.1 ;(4) 2,3,7,8-\mathrm{HpCDD}, \mathrm{TEF}=0.01 ;(5)$ OCDD, TEF $=0.001 ;(6) 2,3,7,8-\mathrm{TCDF}, \mathrm{TEF}=0.1 ;(7) 1,2,3,7,8-\mathrm{PeCDF}, \mathrm{TEF}=0.5 ;(8) 2,3,4,7,8-$ $\mathrm{PeCDF}, \mathrm{TEF}=0.05$; (9) 2,3,7,8-HxCDF, TEF $=0.1$; (10) 2,3,7,8-HpCDF, TEF $=0.01$; and (11) OCDF, $\mathrm{TEF}=0.001$.

(5) Toxicity values for these chemicals have been withdrawn from IRIS and HEAST pending further investigation. However, the most recent toxicity values have been used to calculate the PRG.

(6) Toxicity values for these chemicals were obtained from the Superfund Health Risk Technical Support Center. 
Table 3.3f. Risk-based Preliminary Remediation Goals for radionuclides in water and soil (agricultural scenario)

\begin{tabular}{|c|c|c|c|c|c|c|c|c|c|c|}
\hline \multirow{2}{*}{ Chemical $^{\dagger}$} & \multirow{2}{*}{ CASRN } & \multicolumn{2}{|c|}{$\begin{array}{c}\text { Vegetable Intercept } \\
\text { (pCi/g) }\end{array}$} & \multirow[t]{2}{*}{ Slope } & \multicolumn{2}{|c|}{$\begin{array}{l}\text { Beef Intercept } \\
\text { (pCi/g) }\end{array}$} & \multirow[t]{2}{*}{ Slope } & \multicolumn{2}{|c|}{$\begin{array}{l}\text { Milk Intercept } \\
\text { (pCi/g) }\end{array}$} & \multirow[t]{2}{*}{ Slope } \\
\hline & & $10^{-4}$ & $10^{-6}$ & & $10^{-4}$ & $10^{-6}$ & & $10^{-4}$ & $10^{-6}$ & \\
\hline Ac-225 & $014265-85-1$ & $3.2 \mathrm{E}+00$ & $3.2 \mathrm{E}-02$ & $-6.2 E+00$ & $1.4 \mathrm{E}+04$ & $1.4 \mathrm{E}+02$ & $-1.9 \mathrm{E}+01$ & $2.8 \mathrm{E}+03$ & $2.8 \mathrm{E}+01$ & $-2.2 E+01$ \\
\hline Ac-227 & 014952-40-0 & $1.3 \mathrm{E}+00$ & $1.3 \mathrm{E}-02$ & $-3.8 \mathrm{E}+01$ & $5.5 \mathrm{E}+03$ & $5.5 \mathrm{E}+01$ & $-1.9 \mathrm{E}+01$ & $1.1 \mathrm{E}+03$ & $1.1 \mathrm{E}+01$ & $-2.2 \mathrm{E}+01$ \\
\hline$A c-227+D$ & 014952-40-0 & $6.8 \mathrm{E}-01$ & $6.8 \mathrm{E}-03$ & $-3.7 \mathrm{E}+01$ & $9.7 \mathrm{E}-01$ & $9.7 \mathrm{E}-03$ & $-1.2 \mathrm{E}+01$ & $3.2 \mathrm{E}-01$ & $3.2 \mathrm{E}-03$ & $-1.3 \mathrm{E}+01$ \\
\hline Ac-228 & 014331-83-0 & $2.8 \mathrm{E}+02$ & $2.8 \mathrm{E}+00$ & $-2.7 \mathrm{E}-01$ & $1.2 \mathrm{E}+06$ & $1.2 E+04$ & $-1.9 \mathrm{E}+01$ & $2.5 \mathrm{E}+05$ & $2.5 \mathrm{E}+03$ & $-2.2 \mathrm{E}+01$ \\
\hline $\mathrm{Ag}-105$ & 014928-14-4 & $2.8 \mathrm{E}+02$ & $2.8 \mathrm{E}+00$ & $-1.1 E+01$ & $2.9 \mathrm{E}+03$ & $2.9 \mathrm{E}+01$ & $-5.5 E+00$ & $2.6 E+04$ & $2.6 \mathrm{E}+02$ & $-5.7 \mathrm{E}+00$ \\
\hline Ag-108 & 014391-65-2 & $6.6 E+04$ & $6.6 \mathrm{E}+02$ & $-1.7 \mathrm{E}-03$ & $6.7 \mathrm{E}+05$ & $6.7 \mathrm{E}+03$ & $-5.5 E+00$ & $6.1 E+06$ & $6.1 E+04$ & $-5.7 \mathrm{E}+00$ \\
\hline $\mathrm{Ag}-108 \mathrm{~m}$ & $014391-65-2$ & $7.6 \mathrm{E}+01$ & $7.6 \mathrm{E}-01$ & $-4.7 \mathrm{E}+01$ & $7.7 \mathrm{E}+02$ & $7.7 \mathrm{E}+00$ & $-5.5 E+00$ & $7.0 \mathrm{E}+03$ & $7.0 \mathrm{E}+01$ & $-5.7 \mathrm{E}+00$ \\
\hline Ag-108m+D & $014391-65-2$ & $7.6 \mathrm{E}+01$ & $7.6 \mathrm{E}-01$ & $-4.7 E+01$ & 7.7E+02 & $7.7 E+00$ & $-5.5 \mathrm{E}+00$ & $7.0 \mathrm{E}+03$ & $7.0 \mathrm{E}+01$ & $-5.7 \mathrm{E}+00$ \\
\hline $\mathrm{Ag}-109 \mathrm{~m}$ & 014378-38-2 & $1.7 \mathrm{E}+06$ & $1.7 E+04$ & $-4.9 \mathrm{E}-04$ & $1.7 \mathrm{E}+07$ & $1.7 \mathrm{E}+05$ & $-5.5 \mathrm{E}+00$ & $1.6 \mathrm{E}+08$ & $1.6 \mathrm{E}+06$ & $-5.7 E+00$ \\
\hline $\mathrm{Ag}-110$ & 014391-76-5 & $1.9 \mathrm{E}+05$ & $1.9 \mathrm{E}+03$ & $-3.0 \mathrm{E}-04$ & $1.9 \mathrm{E}+06$ & $1.9 \mathrm{E}+04$ & $-5.5 \mathrm{E}+00$ & $1.7 \mathrm{E}+07$ & $1.7 \mathrm{E}+05$ & $-5.7 E+00$ \\
\hline $\mathrm{Ag}-110 \mathrm{~m}$ & 014391-76-5 & $5.4 \mathrm{E}+01$ & $5.4 \mathrm{E}-01$ & $-1.5 \mathrm{E}+01$ & $5.5 \mathrm{E}+02$ & $5.5 \mathrm{E}+00$ & $-5.5 E+00$ & $5.0 \mathrm{E}+03$ & $5.0 \mathrm{E}+01$ & $-5.7 E+00$ \\
\hline $\mathrm{Ag}-111$ & 015760-04-0 & $6.7 E+01$ & $6.7 \mathrm{E}-01$ & $-5.2 \mathrm{E}+00$ & $6.8 \mathrm{E}+02$ & $6.8 \mathrm{E}+00$ & $-5.5 E+00$ & $6.2 \mathrm{E}+03$ & $6.2 \mathrm{E}+01$ & $-5.7 E+00$ \\
\hline Am-241 & 014596-10-2 & $1.4 \mathrm{E}+00$ & $1.4 \mathrm{E}-02$ & $-4.9 \mathrm{E}+01$ & $3.7 \mathrm{E}+03$ & $3.7 \mathrm{E}+01$ & $-1.9 \mathrm{E}+01$ & $1.6 \mathrm{E}+04$ & $1.6 \mathrm{E}+02$ & $-2.2 \mathrm{E}+01$ \\
\hline Am-242 & 013981-54-9 & $3.1 E+02$ & $3.1 \mathrm{E}+00$ & $-6.7 \mathrm{E}-01$ & $8.2 \mathrm{E}+05$ & $8.2 E+03$ & $-1.9 \mathrm{E}+01$ & $3.6 \mathrm{E}+06$ & $3.6 \mathrm{E}+04$ & $-2.2 \mathrm{E}+01$ \\
\hline Am-242m & $013981-54-9$ & $1.6 \mathrm{E}+00$ & $1.6 \mathrm{E}-02$ & $-4.8 \mathrm{E}+01$ & $\dot{4} .1 \mathrm{E}+03$ & $4.1 E+01$ & $-1.9 \mathrm{E}+01$ & $1.8 \mathrm{E}+04$ & $1.8 \mathrm{E}+02$ & $-2.2 E+01$ \\
\hline $\mathrm{Am}-243$ & 014993-75-0 & $1.4 \mathrm{E}+00$ & $1.4 \mathrm{E}-02$ & $-5.0 \mathrm{E}+01$ & $3.7 \mathrm{E}+03$ & $3.7 \mathrm{E}+01$ & $-1.9 \mathrm{E}+01$ & $1.6 \mathrm{E}+04$ & $1.6 \mathrm{E}+02$ & $-2.2 \mathrm{E}+01$ \\
\hline $\mathrm{Am}-243+\mathrm{D}$ & 014993-75-0 & $1.4 \mathrm{E}+00$ & $1.4 \mathrm{E}-02$ & $-5.0 \mathrm{E}+01$ & $1.2 \mathrm{E}+02$ & $1.2 \mathrm{E}+00$ & $-1.6 \mathrm{E}+01$ & $3.1 \mathrm{E}+03$ & $3.1 \mathrm{E}+01$ & $-1.8 E+01$ \\
\hline
\end{tabular}




\begin{tabular}{|c|c|c|c|c|c|c|c|c|c|c|c|c|c|c|c|c|c|c|}
\hline \multicolumn{2}{|c|}{ 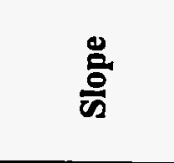 } & & $\begin{array}{l}8 \\
+ \\
\text { 吾 } \\
\text { i }\end{array}$ & $\begin{array}{l}\bar{\delta} \\
\text { 高 } \\
\text { ㅁ }\end{array}$ & 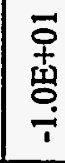 & 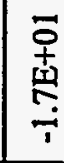 & 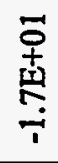 & $\begin{array}{l}\overrightarrow{0} \\
+ \\
\text { 亲 } \\
\dot{7}\end{array}$ & $\begin{array}{l}\overrightarrow{0} \\
\underset{+1}{+} \\
\underset{+}{+}\end{array}$ & 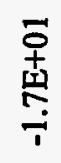 & \begin{tabular}{c}
$\overline{0}$ \\
+ \\
商 \\
\hdashline
\end{tabular} & $\begin{array}{l}\overrightarrow{0} \\
+1 \\
\text { 孚 } \\
\text { ণ }\end{array}$ & 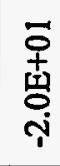 & $\begin{array}{l}\bar{\delta} \\
\text { ㅁ } \\
\text { 夏 } \\
\text { ণ }\end{array}$ & $\begin{array}{l}\text { 우 } \\
\text { 帘 } \\
\text { ㄱ }\end{array}$ & $\begin{array}{l}\text { 여 } \\
\text { 索 } \\
\text { ণ }\end{array}$ & 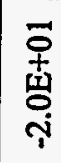 & $\begin{array}{l}\text { 훙 } \\
\text { 㕝 } \\
\text { ஸे }\end{array}$ \\
\hline \multirow{2}{*}{ 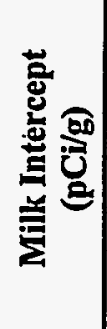 } & $\stackrel{\circ}{\varrho}$ & & $\begin{array}{l}n \\
\text { n } \\
+1 \\
\text { m } \\
\text { ri }\end{array}$ & $\begin{array}{l}\text { 옹 } \\
\text { 壱 } \\
\text { ণ }\end{array}$ & 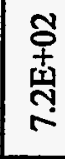 & 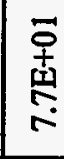 & 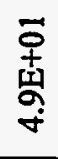 & $\begin{array}{l}\overrightarrow{0} \\
+ \\
\text { 吾 } \\
\text { ㅇ }\end{array}$ & $\begin{array}{l}\text { 草 } \\
+ \\
\text { 竎 } \\
\text { ம் }\end{array}$ & 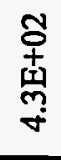 & 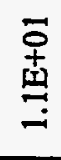 & 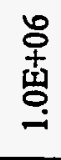 & $\begin{array}{l}\bar{\phi} \\
\text { 豈 } \\
\text { i }\end{array}$ & $\begin{array}{l}\overline{+} \\
\text { 产 } \\
\text { ف }\end{array}$ & $\begin{array}{l}\bar{\phi} \\
\text { 呦 } \\
\text { i }\end{array}$ & 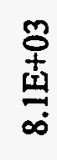 & $\begin{array}{l}\text { ণ } \\
+ \\
\text { 亲 } \\
\text { ণ }\end{array}$ & 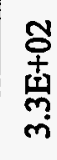 \\
\hline & $\stackrel{1}{0}$ & & $\begin{array}{l}\text { s } \\
+ \\
\text { f1 } \\
\text { ñ } \\
\text { in }\end{array}$ & $\begin{array}{l}\text { n̊ } \\
+ \\
\text { 壳 } \\
\text { ஸे }\end{array}$ & $\begin{array}{l}\text { サ } \\
+ \\
\text { 吉 } \\
\text { N }\end{array}$ & 畩 & $\begin{array}{l}\text { \% } \\
\text { 市 } \\
\text { 章 } \\
\stackrel{+}{+}\end{array}$ & 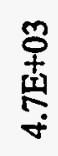 & $\begin{array}{l}\text { o̊ } \\
+ \\
\text { 章 } \\
\text { ம் }\end{array}$ & $\begin{array}{l}\underset{+}{+} \\
\text { 站 } \\
\stackrel{\sim}{\forall}\end{array}$ & 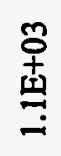 & $\begin{array}{l}\infty \\
\text { o } \\
+ \\
\text { 产 } \\
\end{array}$ & 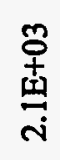 & $\begin{array}{l}\text { ஸे } \\
+ \\
\text { 点 } \\
\text { ஸे }\end{array}$ & $\begin{array}{l}\text { mo } \\
+ \\
\text { + } \\
\text { ஸे } \\
\text { Ni }\end{array}$ & 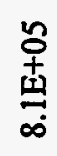 & $\begin{array}{l}\text { 古 } \\
\text { 索 } \\
\text { in }\end{array}$ & $\begin{array}{l}\text { t } \\
\text { +⿱ } \\
\text { m } \\
m \\
m\end{array}$ \\
\hline \multicolumn{2}{|l|}{$\frac{\ddot{2}}{\varpi}$} & & $\begin{array}{l}8 \\
\text { + } \\
\text { 帘 } \\
\text { ஸे }\end{array}$ & 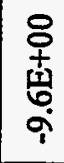 & $\begin{array}{l}\text { 웅 } \\
\text { 点 } \\
\text { ơ }\end{array}$ & 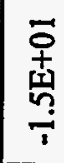 & 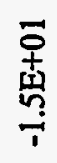 & $\begin{array}{l}\overrightarrow{0} \\
+ \\
\text { 章 } \\
\frac{1}{1}\end{array}$ & \begin{tabular}{l}
$\overline{0}$ \\
+ \\
章 \\
\hdashline
\end{tabular} & 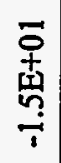 & $\begin{array}{l}\bar{o} \\
\frac{1}{n} \\
\frac{1}{n} \\
\frac{1}{1}\end{array}$ & $\begin{array}{l}\overrightarrow{0} \\
+ \\
\text { ज्ञ } \\
\stackrel{+}{i}\end{array}$ & 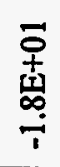 & 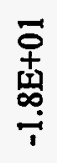 & 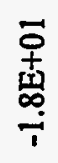 & 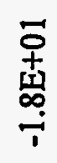 & 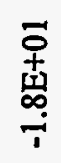 & 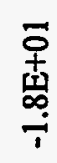 \\
\hline \multirow{2}{*}{ 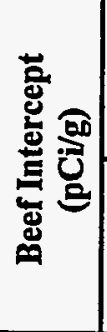 } & $\stackrel{\text { L }}{g}$ & & 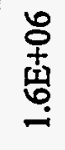 & 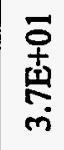 & 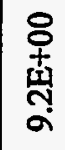 & 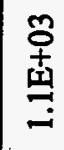 & 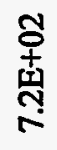 & 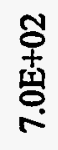 & 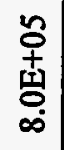 & 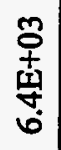 & 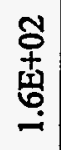 & 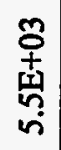 & 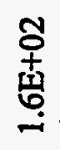 & 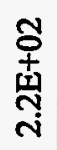 & 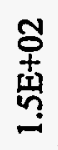 & 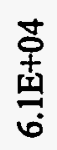 & 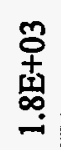 & 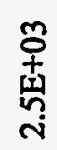 \\
\hline & $\stackrel{t}{\varrho}$ & & 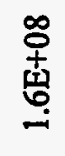 & 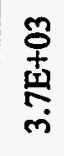 & 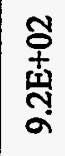 & $\begin{array}{l}\text { 号 } \\
\text { 省 } \\
=\end{array}$ & 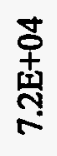 & 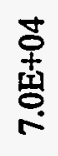 & $\begin{array}{l}0 \\
+ \\
+5 \\
0 \\
\infty \\
\infty\end{array}$ & 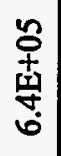 & $\begin{array}{l}\text { 草 } \\
\text { 弆 } \\
\text { - }\end{array}$ & $\begin{array}{l}\text { 号 } \\
\text { 点 } \\
\text { n่ }\end{array}$ & 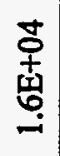 & 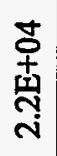 & 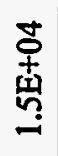 & 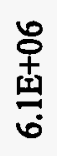 & 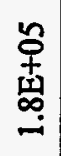 & 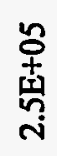 \\
\hline \multicolumn{2}{|l|}{ 음 } & & 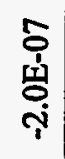 & 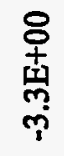 & 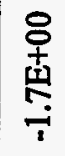 & 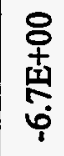 & $\begin{array}{l}\text { 움 } \\
\text { 章 } \\
\text { p் }\end{array}$ & 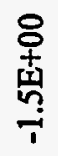 & $\begin{array}{c}\text { \% } \\
\text { 명 } \\
\frac{1}{7}\end{array}$ & $\begin{array}{l}\text { ชै } \\
\text { 떻 } \\
\text { ధํ }\end{array}$ & 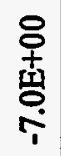 & 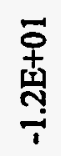 & 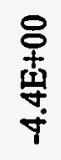 & $\begin{array}{l}\overrightarrow{0} \\
\text { 息 } \\
\text { 安 }\end{array}$ & 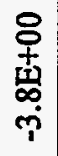 & 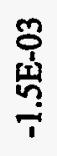 & 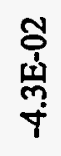 & 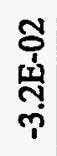 \\
\hline \multirow{2}{*}{ 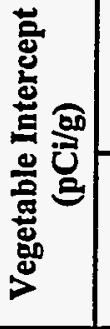 } & $\stackrel{\varphi}{\varrho}$ & & 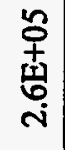 & 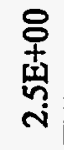 & 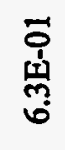 & 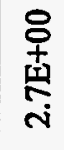 & 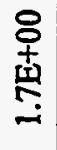 & $\begin{array}{l}8 \\
\text { 市 } \\
\text { 帘 } \\
\stackrel{-}{-}\end{array}$ & 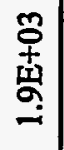 & 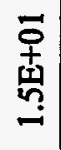 & 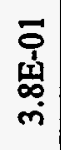 & 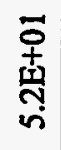 & 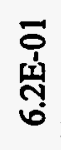 & $\begin{array}{l}\vec{\phi} \\
\text { પ⿱山口 } \\
\infty \\
\infty \\
\infty\end{array}$ & 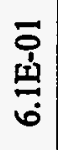 & 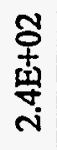 & 章 & $\begin{array}{l}\text { 웅 } \\
\text { 总 }\end{array}$ \\
\hline & $\stackrel{t}{0}$ & & 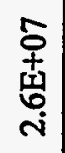 & 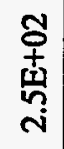 & $\begin{array}{l}\text { 卓 } \\
\text { 孚 } \\
\text { bo }\end{array}$ & $\begin{array}{c}\text { 号 } \\
\text { 点 } \\
\text { ஸ }\end{array}$ & $\begin{array}{l}\text { જ̛ } \\
\text { 章 } \\
\text { I }\end{array}$ & 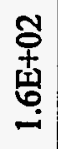 & 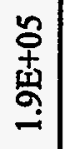 & 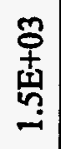 & $\begin{array}{l}\overline{0} \\
\text { 市 } \\
\text { 竞 } \\
\dot{m}\end{array}$ & 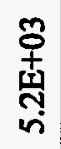 & 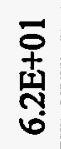 & $\begin{array}{l}\bar{\phi} \\
+ \\
\mathbf{1} \\
\infty \\
\infty\end{array}$ & $\frac{\overrightarrow{0}}{\frac{1}{9}}$ & 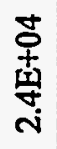 & $\begin{array}{l}\text { 号 } \\
\text { 画 } \\
\text { م }\end{array}$ & $\begin{array}{l}\text { 乌 } \\
+ \\
\text { 됭 } \\
\end{array}$ \\
\hline \multicolumn{2}{|l|}{ 萦 } & 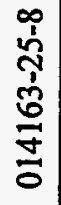 & 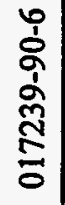 & $\frac{\frac{0}{0}}{\frac{1}{d}}$ & 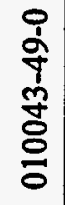 & $\begin{array}{l}\frac{7}{2} \\
\frac{j}{d} \\
\frac{\sigma}{0}\end{array}$ & $\begin{array}{l}\frac{\nabla}{\dot{I}} \\
\frac{1}{0} \\
\frac{m}{0}\end{array}$ & $\begin{array}{l}\frac{I}{\nabla} \\
\frac{\delta}{0} \\
\frac{\tilde{\sigma}}{0}\end{array}$ & $\begin{array}{l}\frac{1}{1} \\
\frac{1}{0} \\
0 \\
\frac{0}{0} \\
0\end{array}$ & 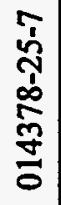 & 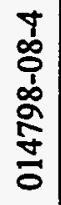 & 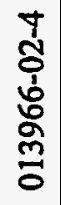 & $\begin{array}{l}\frac{a}{2} \\
\frac{1}{0} \\
\frac{6}{n} \\
0\end{array}$ & 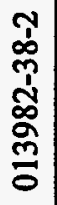 & $\begin{array}{l}\frac{J}{2} \\
\frac{1}{m} \\
\frac{\tilde{g}}{\sigma}\end{array}$ & 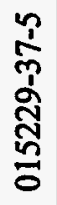 & $\begin{array}{l}\frac{0}{2} \\
\frac{1}{2} \\
\frac{m}{\sigma} \\
\frac{\sigma}{0}\end{array}$ & 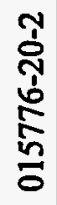 \\
\hline 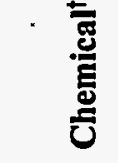 & & 紊 & $\frac{\vec{r}}{\frac{\pi}{\alpha}}$ & $\frac{2}{3}$ & $\frac{\infty}{a}$ & $\frac{\bar{m}}{\dot{m}}$ & $\frac{m}{m}$ & 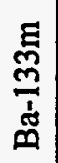 & 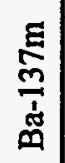 & 突 & $\frac{\stackrel{P}{d}}{\stackrel{d}{\dot{m}}}$ & 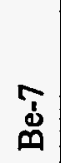 & 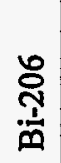 & 突 & $\frac{}{\stackrel{1}{1}}$ & $\overrightarrow{\vec{x}}$ & 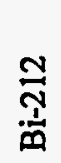 & $\underset{\stackrel{n}{\sim}}{\stackrel{n}{\vec{n}}}$ \\
\hline
\end{tabular}

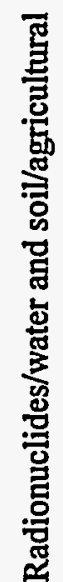


Table 3.3f. (continued)

\begin{tabular}{|c|c|c|c|c|c|c|c|c|c|c|}
\hline \multirow[t]{2}{*}{ Chemical ${ }^{\dagger}$} & \multirow[t]{2}{*}{ CASRN } & \multicolumn{2}{|c|}{$\begin{array}{c}\text { Vegetable Intercept } \\
(\mathrm{pCi} / \mathrm{g})\end{array}$} & \multirow[t]{2}{*}{ Slope } & \multicolumn{2}{|c|}{$\begin{array}{c}\text { Beef Intercept } \\
(\mathrm{pCi} / \mathrm{g})\end{array}$} & \multirow[t]{2}{*}{ Slope } & \multicolumn{2}{|c|}{$\begin{array}{l}\text { Milk Intercept } \\
(\mathrm{pCi} / \mathrm{g})\end{array}$} & \multirow{2}{*}{ Slope } \\
\hline & & $10^{-4}$ & $10^{-6}$ & & $10^{-4}$ & $10^{-6}$ & & $10^{-4}$ & $10^{-6}$ & \\
\hline $\mathrm{Bi}-214$ & 014733-03-0 & $2.3 \mathrm{E}+03$ & $2.3 E+01$ & $-1.4 \mathrm{E}-02$ & $5.7 \mathrm{E}+05$ & $5.7 \mathrm{E}+03$ & $-1.8 \mathrm{E}+01$ & $7.5 \mathrm{E}+04$ & $7.5 \mathrm{E}+02$ & $-2.0 \mathrm{E}+01$ \\
\hline $\mathrm{Br}-82$ & $014686-69-2$ & $1.3 \mathrm{E}+02$ & $1.3 \mathrm{E}+00$ & $-5.8 \mathrm{E}-01$ & $2.9 \mathrm{E}+02$ & $2.9 \mathrm{E}+00$ & $-4.1 E+00$ & $5.4 \mathrm{E}+01$ & $5.4 \mathrm{E}-01$ & $-4.2 \mathrm{E}+00$ \\
\hline$C-11$ & 014333-33-6 & & & & & & & & & \\
\hline$C-14$ & $014762-75-5$ & & & & & & & & $\cdot$ & \\
\hline C-15 & $015929-23-4$ & & & & & & & & & \\
\hline $\mathrm{Ca}-45$ & $013966-05-7$ & $5.2 \mathrm{E}+01$ & $5.2 \mathrm{E}-01$ & $-3.9 \mathrm{E}+00$ & $1.2 \mathrm{E}+03$ & $1.2 \mathrm{E}+01$ & $-2.0 \mathrm{E}+00$ & $1.2 \mathrm{E}+02$ & $1.2 \mathrm{E}+00$ & $-2.0 \mathrm{E}+00$ \\
\hline $\mathrm{Ca}-47$ & $001439-99-2$ & $1.6 \mathrm{E}+01$ & $1.6 \mathrm{E}-01$ & $-8.5 \mathrm{E}-01$ & $3.8 \mathrm{E}+02$ & $3.8 \mathrm{E}+00$ & $-2.0 \mathrm{E}+00$ & $3.7 \mathrm{E}+01$ & 3.7E-01 & $-2.0 \mathrm{E}+00$ \\
\hline Cd-109 & $014109-32-1$ & $3.7 \mathrm{E}+01$ & $3.7 \mathrm{E}-01$ & $-1.1 \mathrm{E}+01$ & $6.4 \mathrm{E}+03$ & $6.4 \mathrm{E}+01$ & $-8.1 E+00$ & $3.9 E+02$ & $3.9 \mathrm{E}+00$ & $-8.6 \mathrm{E}+00$ \\
\hline $\mathrm{Cd}-115$ & $014336-68-6$ & $4.1 \mathrm{E}+01$ & $4.1 \mathrm{E}-01$ & $-1.3 E+00$ & $7.0 \mathrm{E}+03$ & $7.0 \mathrm{E}+01$ & $-8.1 E+00$ & $4.3 \mathrm{E}+02$ & $4.3 E+00$ & $-8.6 \mathrm{E}+00$ \\
\hline Cd-115m & $014336-68-6$ & $2.1 \mathrm{E}+01$ & 2.1E-01 & $-7.4 \mathrm{E}+00$ & $3.6 \mathrm{E}+03$ & $3.6 \mathrm{E}+01$ & $-8.1 E+00$ & $2.2 \mathrm{E}+02$ & $2.2 E+00$ & $-8.6 \mathrm{E}+00$ \\
\hline Ce-141 & $013967-74-3$ & $1.2 \mathrm{E}+02$ & $1.2 \mathrm{E}+00$ & $-1.0 \mathrm{E}+01$ & $5.5 \mathrm{E}+05$ & $5.5 \mathrm{E}+03$ & $-1.7 \mathrm{E}+01$ & $6.0 \mathrm{E}+04$ & $6.0 \mathrm{E}+02$ & $-1.9 \mathrm{E}+01$ \\
\hline Ce-143 & 014119-19-8 & $7.7 \mathrm{E}+01$ & 7.7E-01 & $-1.3 \mathrm{E}+00$ & $3.6 \mathrm{E}+05$ & $3.6 \mathrm{E}+03$ & $-1.7 \mathrm{E}+01$ & $4.0 \mathrm{E}+04$ & $4.0 \mathrm{E}+02$ & $-1.9 \mathrm{E}+01$ \\
\hline Ce-144 & $014762-78-8$ & $1.5 \mathrm{E}+01$ & $1.5 \mathrm{E}-01$ & $-1.5 \mathrm{E}+01$ & $7.3 E+04$ & $7.3 \mathrm{E}+02$ & $-1.7 \mathrm{E}+01$ & $8.0 \mathrm{E}+03$ & $8.0 \mathrm{E}+01$ & $-1.9 \mathrm{E}+01$ \\
\hline Ce-144+D & $014762-78-8$ & $1.5 \mathrm{E}+01$ & $1.5 \mathrm{E}-01$ & $-1.5 \mathrm{E}+01$ & $2.2 E+03$ & $2.2 \mathrm{E}+01$ & $-1.6 \mathrm{E}+01$ & $3.1 E+03$ & $3.1 E+01$ & $-1.8 \mathrm{E}+01$ \\
\hline $\mathrm{Cl}-36$ & $013981-43-6$ & $3.0 \mathrm{E}+00$ & $3.0 \mathrm{E}-02$ & $-3.6 \mathrm{E}+01$ & $6.2 \mathrm{E}+00$ & $6.2 \mathrm{E}-02$ & $-1.1 \mathrm{E}-01$ & $1.1 \mathrm{E}+00$ & $1.1 \mathrm{E}-02$ & $-1.1 \mathrm{E}-01$ \\
\hline $\mathrm{Cl}-38$ & $014158-34-0$ & $3.2 \mathrm{E}+01$ & $3.2 \mathrm{E}-01$ & $-5.4 \mathrm{E}-04$ & $6.7 \mathrm{E}+01$ & $6.7 \mathrm{E}-01$ & $-1.1 \mathrm{E}-01$ & $1.2 \mathrm{E}+01$ & $1.2 \mathrm{E}-01$ & $-1.1 \mathrm{E}-01$ \\
\hline $\mathrm{Cm}-242$ & $015510-73-3$ & $1.2 \mathrm{E}+01$ & $1.2 \mathrm{E}-01$ & $-1.4 \mathrm{E}+01$ & $6.3 \mathrm{E}+04$ & $6.3 E+02$ & $-1.9 \mathrm{E}+01$ & $5.2 \mathrm{E}+05$ & $5.2 \mathrm{E}+03$ & $-2.2 \mathrm{E}+01$ \\
\hline
\end{tabular}


Table 3.3f. (continued)

\begin{tabular}{|c|c|c|c|c|c|c|c|c|c|c|}
\hline \multirow[t]{2}{*}{ Chemical $^{t}$} & \multirow[t]{2}{*}{ CASRN } & \multicolumn{2}{|c|}{$\begin{array}{l}\text { Vegetable Intercept } \\
\text { (pCi/g) }\end{array}$} & \multirow{2}{*}{ Slope } & \multicolumn{2}{|c|}{$\begin{array}{l}\text { Beef Intercept } \\
\text { (pCi/g) }\end{array}$} & \multirow[t]{2}{*}{ Slope } & \multicolumn{2}{|c|}{$\begin{array}{l}\text { Milk Intercept } \\
\text { (pCi/g) }\end{array}$} & \multirow[t]{2}{*}{ Slope } \\
\hline & & $10^{-4}$ & $10^{-6}$ & & $10^{-4}$ & $10^{-6}$ & & $10^{-4}$ & $10^{-6}$ & \\
\hline $\mathrm{Cm}-243$ & $015757-87-6$ & $1.8 \mathrm{E}+00$ & $1.8 \mathrm{E}-02$ & $-4.0 \mathrm{E}+01$ & $9.6 \mathrm{E}+03$ & $9.6 \mathrm{E}+01$ & $-1.9 \mathrm{E}+01$ & 8.0E+04 & $8.0 \mathrm{E}+02$ & $-2.2 E+01$ \\
\hline $\mathrm{Cm}-243+\mathrm{D}$ & $015757-87-6$ & $1.7 \mathrm{E}+00$ & $1.7 \mathrm{E}-02$ & $-3.9 \mathrm{E}+01$ & $1.9 \mathrm{E}+00$ & $1.9 \mathrm{E}-02$ & $-9.1 \mathrm{E}+00$ & $2.7 \mathrm{E}+00$ & 2.7E-02 & $-9.8 \mathrm{E}+00$ \\
\hline $\mathrm{Cm}-244$ & $013981-15-2$ & $2.2 \mathrm{E}+00$ & 2.2E-02 & $-3.6 \mathrm{E}+01$ & $1.1 \mathrm{E}+04$ & $1.1 \mathrm{E}+02$ & $-1.9 \mathrm{E}+01$ & $9.5 \mathrm{E}+04$ & $9.5 \mathrm{E}+02$ & $-2.2 \mathrm{E}+01$ \\
\hline $\mathrm{Cm}-245$ & 015621-76-8 & $1.4 \mathrm{E}+00$ & $1.4 \mathrm{E}-02$ & $-5.0 \mathrm{E}+01$ & $7.2 E+03$ & $7.2 \mathrm{E}+01$ & $-1.9 \mathrm{E}+01$ & $6.0 \mathrm{E}+04$ & $6.0 \mathrm{E}+02$ & $-2.2 \mathrm{E}+01$ \\
\hline $\mathrm{Cm}-246$ & 015757-90-1 & $1.4 \mathrm{E}+00$ & $1.4 \mathrm{E}-02$ & $-5.0 \mathrm{E}+01$ & $7.3 E+03$ & $7.3 \mathrm{E}+01$ & $-1.9 \mathrm{E}+01$ & $6.1 \mathrm{E}+04$ & $6.1 E+02$ & $-2.2 E+01$ \\
\hline $\mathrm{Cm}-247$ & $015758-32-4$ & $1.5 \mathrm{E}+00$ & $1.5 \mathrm{E}-02$ & $-5.0 \mathrm{E}+01$ & $7.8 \mathrm{E}+03$ & $7.8 \mathrm{E}+01$ & $-1.9 \mathrm{E}+01$ & $6.5 \mathrm{E}+04$ & $6.5 \mathrm{E}+02$ & $-2.2 \mathrm{E}+01$ \\
\hline $\mathrm{Cm}-248$ & 015758-33-5 & $3.5 \mathrm{E}-01$ & $3.5 \mathrm{E}-03$ & $-5.0 \mathrm{E}+01$ & $1.8 \mathrm{E}+03$ & $1.8 \mathrm{E}+01$ & $-1.9 \mathrm{E}+01$ & $1.5 \mathrm{E}+04$ & $1.5 \mathrm{E}+02$ & $-2.2 \mathrm{E}+01$ \\
\hline $\mathrm{Co}-57$ & 013981-50-5 & $4.3 \mathrm{E}+02$ & $4.3 \mathrm{E}+00$ & $-1.4 \mathrm{E}+01$ & $4.4 \mathrm{E}+05$ & $4.4 E+03$ & $-1.7 E+01$ & $1.0 \mathrm{E}+05$ & $1.0 \mathrm{E}+03$ & $-1.9 \mathrm{E}+01$ \\
\hline Co-58 & 013981-38-9 & $1.5 E+02$ & $1.5 \mathrm{E}+00$ & $-1.1 \mathrm{E}+01$ & $1.5 \mathrm{E}+05$ & $1.5 E+03$ & $-1.7 \mathrm{E}+01$ & $3.5 \mathrm{E}+04$ & $3.5 E+02$ & $-1.9 \mathrm{E}+01$ \\
\hline Co-58m & 013981-38-9 & $4.4 \mathrm{E}+03$ & $4.4 \mathrm{E}+01$ & $-3.6 \mathrm{E}-01$ & $4.5 \mathrm{E}+06$ & $4.5 E+04$ & $-1.7 \mathrm{E}+01$ & $1.1 \mathrm{E}+06$ & $1.1 \mathrm{E}+04$ & $-1.9 \mathrm{E}+01$ \\
\hline Co- 60 & $010198-40-0$ & $2.2 \mathrm{E}+01$ & 2.2E-01 & $-2.2 \mathrm{E}+01$ & $2.3 E+04$ & $2.3 \mathrm{E}+02$ & $-1.7 \mathrm{E}+01$ & $5.3 \mathrm{E}+03$ & $5.3 \mathrm{E}+01$ & $-1.9 \mathrm{E}+01$ \\
\hline $\mathrm{Cr}-51$ & $014392-02-0$ & $3.3 E+03$ & $3.3 E+01$ & $-9.8 \mathrm{E}+00$ & $3.6 E+04$ & $3.6 \mathrm{E}+02$ & $-1.7 \mathrm{E}+01$ & $5.3 E+06$ & $5.3 E+04$ & $-2.0 \mathrm{E}+01$ \\
\hline Cs-131 & $014914-76-2$ & $2.4 \mathrm{E}+03$ & $2.4 \mathrm{E}+01$ & $-5.8 E+00$ & $4.2 E+03$ & $4.2 \mathrm{E}+01$ & $-1.5 \mathrm{E}+01$ & $4.3 E+03$ & $4.3 \mathrm{E}+01$ & $-1.7 \mathrm{E}+01$ \\
\hline Cs-134 & 013967-70-9 & $9.2 \mathrm{E}+00$ & $9.2 \mathrm{E}-02$ & $-1.7 \mathrm{E}+01$ & $1.6 \mathrm{E}+01$ & $1.6 \mathrm{E}-01$ & $-1.5 E+01$ & $1.6 \mathrm{E}+01$ & $1.6 \mathrm{E}-01$ & $-1.7 \mathrm{E}+01$ \\
\hline Cs-134m & $013967-70-9$ & $9.6 \mathrm{E}+03$ & $9.6 \mathrm{E}+01$ & $-1.2 \mathrm{E}-01$ & $1.7 \mathrm{E}+04$ & $1.7 \mathrm{E}+02$ & $-1.5 \mathrm{E}+01$ & $1.7 \mathrm{E}+04$ & $1.7 \mathrm{E}+02$ & $-1.7 E+01$ \\
\hline Cs-135 & 015726-30-4 & $9.6 \mathrm{E}+01$ & $9.6 \mathrm{E}-01$ & $-4.9 \mathrm{E}+01$ & $1.7 \mathrm{E}+02$ & $1.7 \mathrm{E}+00$ & $-1.5 \mathrm{E}+01$ & $1.7 \mathrm{E}+02$ & $1.7 \mathrm{E}+00$ & $-1.7 \mathrm{E}+01$ \\
\hline Cs-136 & 014234-29-8 & $5.6 \mathrm{E}+01$ & $5.6 \mathrm{E}-01$ & $-6.9 \mathrm{E}+00$ & $9.8 \mathrm{E}+01$ & $9.8 \mathrm{E}-01$ & $-1.5 \mathrm{E}+01$ & $1.0 \mathrm{E}+02$ & $1.0 \mathrm{E}+00$ & $-1.7 E+01$ \\
\hline
\end{tabular}


Table 3.3f. (continued)

\begin{tabular}{|c|c|c|c|c|c|c|c|c|c|c|}
\hline \multirow{2}{*}{ Chemical $^{\dagger}$} & \multirow{2}{*}{ CASRN } & \multicolumn{2}{|c|}{$\begin{array}{l}\text { Vegetable Intercept } \\
\text { (pCi/g) }\end{array}$} & \multirow{2}{*}{ Slope } & \multicolumn{2}{|c|}{$\begin{array}{c}\text { Beef Intercept } \\
\text { (pCi/g) }\end{array}$} & \multirow{2}{*}{ Slope } & \multicolumn{2}{|c|}{$\begin{array}{c}\text { Milk Intercept } \\
\text { (pCi/g) }\end{array}$} & \multirow{2}{*}{ Slope } \\
\hline & & $10^{-4}$ & $10^{-6}$ & & $10^{-4}$ & $10^{-6}$ & & $10^{-4}$ & $10^{-6}$ & \\
\hline Cs-137 & $010045-97-3$ & $1.4 \mathrm{E}+01$ & $1.4 \mathrm{E}-01$ & $-4.0 \mathrm{E}+01$ & $2.4 \mathrm{E}+01$ & $2.4 \mathrm{E}-01$ & $-1.5 \mathrm{E}+01$ & $2.5 \mathrm{E}+01$ & $2.5 \mathrm{E}-01$ & $-1.7 \mathrm{E}+01$ \\
\hline Cs-137+D & $010045-97-3$ & $1.4 \mathrm{E}+01$ & $1.4 \mathrm{E}-01$ & $-3.9 E+01$ & $2.0 \mathrm{E}+01$ & $2.0 \mathrm{E}-01$ & $-1.3 \mathrm{E}+01$ & $1.9 \mathrm{E}+01$ & $1.9 \mathrm{E}-01$ & $-1.4 \mathrm{E}+01$ \\
\hline Cs-138 & 015758-29-9 & $2.5 \mathrm{E}+03$ & $2.5 \mathrm{E}+01$ & $-2.2 \mathrm{E}-02$ & $4.3 E+03$ & $4.3 E+01$ & $-1.5 \mathrm{E}+01$ & $4.4 \mathrm{E}+03$ & $4.4 \mathrm{E}+01$ & $-1.7 \mathrm{E}+01$ \\
\hline $\mathrm{Cu}-64$ & $013981-25-4$ & $6.7 \mathrm{E}+02$ & $6.7 \mathrm{E}+00$ & $-4.1 \mathrm{E}-01$ & $3.4 \mathrm{E}+03$ & $3.4 \mathrm{E}+01$ & $-6.4 \mathrm{E}+00$ & $3.1 E+03$ & $3.1 \mathrm{E}+01$ & $-6.7 E+00$ \\
\hline Dy-165 & $013967-64-1$ & $1.4 \mathrm{E}+03$ & $1.4 \mathrm{E}+01$ & $-1.0 \mathrm{E}-01$ & $2.6 E+04$ & $2.6 \mathrm{E}+02$ & $-1.9 \mathrm{E}+01$ & $1.2 \mathrm{E}+06$ & $1.2 \mathrm{E}+04$ & $-2.1 \mathrm{E}+01$ \\
\hline Dy-166 & $015840-01-4$ & $4.8 \mathrm{E}+01$ & $4.8 \mathrm{E}-01$ & $-2.9 \mathrm{E}+00$ & $9.1 \mathrm{E}+02$ & $9.1 \mathrm{E}+00$ & $-1.9 \mathrm{E}+01$ & $4.2 \mathrm{E}+04$ & $4.2 \mathrm{E}+02$ & $-2.1 \mathrm{E}+01$ \\
\hline Er-169 & $015840-13-8$ & $2.1 \mathrm{E}+02$ & $2.1 \mathrm{E}+00$ & $-5.9 \mathrm{E}+00$ & $5.6 \mathrm{E}+03$ & $5.6 \mathrm{E}+01$ & $-1.9 \mathrm{E}+01$ & $1.8 \mathrm{E}+05$ & $1.8 \mathrm{E}+03$ & $-2.1 E+01$ \\
\hline Er-171 & $014391-45-8$ & $2.8 \mathrm{E}+02$ & $2.8 \mathrm{E}+00$ & $-3.2 \mathrm{E}-01$ & $7.3 E+03$ & 7.3E+01 & $-1.9 \mathrm{E}+01$ & $2.4 \mathrm{E}+05$ & $2.4 \mathrm{E}+03$ & $-2.1 \mathrm{E}+01$ \\
\hline Eu-152 & 014683-23-9 & $7.9 \mathrm{E}+01$ & $7.9 \mathrm{E}-01$ & $-3.3 \mathrm{E}+01$ & $3.8 \mathrm{E}+03$ & $3.8 \mathrm{E}+01$ & $-1.7 \mathrm{E}+01$ & 2.1E+04 & $2.1 \mathrm{E}+02$ & $-1.9 \mathrm{E}+01$ \\
\hline Eu-154 & $015585-10-1$ & $4.8 \mathrm{E}+01$ & $4.8 \mathrm{E}-01$ & $-2.8 \mathrm{E}+01$ & $2.3 E+03$ & $2.3 E+01$ & $-1.7 E+01$ & $1.3 E+04$ & $1.3 \mathrm{E}+02$ & $-1.9 E+01$ \\
\hline Eu-155 & 014391-16-3 & $2.8 \mathrm{E}+02$ & $2.8 \mathrm{E}+00$ & $-2.3 \mathrm{E}+01$ & 1.3E+04 & $1.3 E+02$ & $-1.7 E+01$ & $7.1 \mathrm{E}+04$ & $7.1 \mathrm{E}+02$ & $-1.9 \mathrm{E}+01$ \\
\hline Eu-156 & $014280-35-4$ & $4.2 \mathrm{E}+01$ & $4.2 \mathrm{E}-01$ & $-7.7 \mathrm{E}+00$ & $2.0 \mathrm{E}+03$ & $2.0 \mathrm{E}+01$ & $-1.7 E+01$ & $1.1 \mathrm{E}+04$ & $1.1 \mathrm{E}+02$ & $-1.9 \mathrm{E}+01$ \\
\hline F-18 & $013981-56-1$ & $4.0 \mathrm{E}+03$ & $4.0 \mathrm{E}+01$ & $-7.6 \mathrm{E}-02$ & $2.6 \mathrm{E}+03$ & $2.6 \mathrm{E}+01$ & $-1.7 \mathrm{E}+01$ & $6.3 E+04$ & $6.3 \mathrm{E}+02$ & $-1.9 \mathrm{E}+01$ \\
\hline $\mathrm{Fe}-55$ & 014681-59-5 & $1.3 \mathrm{E}+03$ & $1.3 E+01$ & $-1.9 \mathrm{E}+01$ & $6.7 E+03$ & $6.7 E+01$ & $-1.9 \mathrm{E}+01$ & $7.4 \mathrm{E}+05$ & $7.4 \mathrm{E}+03$ & $-2.1 \mathrm{E}+01$ \\
\hline Fe-59 & 014596-12-4 & $7.8 \mathrm{E}+01$ & $7.8 \mathrm{E}-01$ & $-1.1 \mathrm{E}+01$ & $4.0 \mathrm{E}+02$ & $4.0 \mathrm{E}+00$ & $-1.9 \mathrm{E}+01$ & $4.5 \mathrm{E}+04$ & $4.5 \mathrm{E}+02$ & $-2.1 \mathrm{E}+01$ \\
\hline Fr-221 & $015756-41-9$ & $3.1 \mathrm{E}+03$ & $3.1 \mathrm{E}+01$ & $-3.4 \mathrm{E}-03$ & $1.2 \mathrm{E}+05$ & $1.2 \mathrm{E}+03$ & $-1.8 E+01$ & $2.6 \mathrm{E}+03$ & $2.6 \mathrm{E}+01$ & $-2.0 \mathrm{E}+01$ \\
\hline Fr-223 & 015756-98-6 & $1.0 \mathrm{E}+03$ & $1.0 \mathrm{E}+01$ & $-1.6 \mathrm{E}-03$ & $4.0 \mathrm{E}+04$ & $4.0 \mathrm{E}+02$ & $-1.8 E+01$ & $8.3 E+02$ & $8.3 E+00$ & $-2.0 \mathrm{E}+01$ \\
\hline
\end{tabular}


Table 3.3f. (continued)

\begin{tabular}{|c|c|c|c|c|c|c|c|c|c|c|}
\hline \multirow[t]{2}{*}{ Chemical $^{\dagger}$} & \multirow[t]{2}{*}{ CASRN } & \multicolumn{2}{|c|}{$\begin{array}{c}\text { Vegetable Intercept } \\
(\mathrm{pCi} / \mathrm{g})\end{array}$} & \multirow[t]{2}{*}{ Slope } & \multicolumn{2}{|c|}{$\begin{array}{l}\text { Beef Intercept } \\
\text { (pCi/g) }\end{array}$} & \multirow[t]{2}{*}{ Slope } & \multicolumn{2}{|c|}{$\begin{array}{l}\text { Milk Intercept } \\
\text { (pCi/g) }\end{array}$} & \multirow[t]{2}{*}{ Slope } \\
\hline & & $10^{-4}$ & $10^{-6}$ & & $10^{-4}$ & $10^{-6}$ & & $10^{-4}$ & $10^{-6}$ & \\
\hline $\mathrm{Ga}-67$ & 014119-09-6 & $5.4 \mathrm{E}+02$ & $5.4 \mathrm{E}+00$ & $-2.8 \mathrm{E}+00$ & $1.9 E+05$ & $1.9 \mathrm{E}+03$ & $-1.9 \mathrm{E}+01$ & $9.4 \mathrm{E}+05$ & $9.4 \mathrm{E}+03$ & $-2.1 E+01$ \\
\hline Ga-72 & $013982-22-4$ & $9.5 \mathrm{E}+01$ & $9.5 \mathrm{E}-01$ & $-5.9 \mathrm{E}-01$ & $3.3 E+04$ & $3.3 \mathrm{E}+02$ & $-1.9 \mathrm{E}+01$ & $1.6 \mathrm{E}+05$ & $1.6 \mathrm{E}+03$ & $-2.1 \mathrm{E}+01$ \\
\hline Gd-153 & $014276-65-4$ & $3.4 \mathrm{E}+02$ & $3.4 \mathrm{E}+00$ & $-1.5 \mathrm{E}+01$ & $1.6 \mathrm{E}+04$ & $1.6 \mathrm{E}+02$ & $-1.7 \mathrm{E}+01$ & $8.9 E+04$ & $8.9 E+02$ & $-1.9 \mathrm{E}+01$ \\
\hline Gd-159 & $014041-42-0$ & $1.7 \mathrm{E}+02$ & $1.7 \mathrm{E}+00$ & $-7.7 \mathrm{E}-01$ & $8.3 E+03$ & $8.3 E+01$ & $-1.7 \mathrm{E}+01$ & $4.5 \mathrm{E}+04$ & $4.5 \mathrm{E}+02$ & $-1.9 \mathrm{E}+01$ \\
\hline Ge-71 & $014374-81-3$ & $2.8 \mathrm{E}+04$ & $2.8 \mathrm{E}+02$ & $-4.9 E+00$ & $2.9 \mathrm{E}+03$ & $2.9 \mathrm{E}+01$ & $-9.6 \mathrm{E}+00$ & $4.6 \mathrm{E}+03$ & $4.6 \mathrm{E}+01$ & $-1.0 \mathrm{E}+01$ \\
\hline H-3 & $010028-17-8$ & & & & & & & & & \\
\hline $\mathrm{Hg}-197$ & $013981-51-6$ & $1.8 \mathrm{E}+02$ & $1.8 \mathrm{E}+00$ & $-1.1 \mathrm{E}+00$ & $1.2 \mathrm{E}+03$ & $1.2 \mathrm{E}+01$ & $-5.5 \mathrm{E}+00$ & $3.8 \mathrm{E}+03$ & $3.8 \mathrm{E}+01$ & $-5.7 E+00$ \\
\hline $\mathrm{Hg}-203$ & $013982-78-0$ & 8.1E+01 & 8.1E-01 & $-5.4 \mathrm{E}+00$ & $5.3 \mathrm{E}+02$ & $5.3 \mathrm{E}+00$ & $-5.5 \mathrm{E}+00$ & $1.7 \mathrm{E}+03$ & $1.7 \mathrm{E}+01$ & $-5.7 E+00$ \\
\hline Ho-166 & $013967-65-2$ & $6.0 \mathrm{E}+01$ & $6.0 \mathrm{E}-01$ & $-1.1 E+00$ & $1.4 \mathrm{E}+03$ & $1.4 \mathrm{E}+01$ & $-1.9 \mathrm{E}+01$ & $5.2 E+04$ & $5.2 \mathrm{E}+02$ & $-2.1 \mathrm{E}+01$ \\
\hline I-122 & $018287-75-7$ & $2.1 E+04$ & $2.1 \mathrm{E}+02$ & $-2.6 \mathrm{E}-03$ & $5.6 \mathrm{E}+04$ & $5.6 \mathrm{E}+02$ & $-1.9 \mathrm{E}+01$ & $3.7 \mathrm{E}+04$ & 3.7E+02 & $-2.2 \mathrm{E}+01$ \\
\hline $\mathrm{I}-123$ & $015715-08-9$ & $8.4 \mathrm{E}+02$ & $8.4 \mathrm{E}+00$ & $-5.5 \mathrm{E}-01$ & $2.2 \mathrm{E}+03$ & $2.2 \mathrm{E}+01$ & $-1.9 \mathrm{E}+01$ & $1.5 \mathrm{E}+03$ & $1.5 \mathrm{E}+01$ & $-2.2 \mathrm{E}+01$ \\
\hline I- -125 & $014158-31-7$ & $1.8 \mathrm{E}+01$ & $1.8 \mathrm{E}-01$ & $-1.2 \mathrm{E}+01$ & $4.7 \mathrm{E}+01$ & 4.7E-01 & $-1.9 \mathrm{E}+01$ & $3.1 E+01$ & $3.1 \mathrm{E}-01$ & $-2.2 \mathrm{E}+01$ \\
\hline $\mathrm{I}-126$ & 014158-32-8 & $9.4 \mathrm{E}+00$ & $9.4 \mathrm{E}-02$ & $-7.1 \mathrm{E}+00$ & $2.5 \mathrm{E}+01$ & $2.5 \mathrm{E}-01$ & $-1.9 E+01$ & $1.7 \mathrm{E}+01$ & $1.7 \mathrm{E}-01$ & $-2.2 E+01$ \\
\hline$I-129$ & $015046-84-1$ & $2.5 \mathrm{E}+00$ & $2.5 \mathrm{E}-02$ & $-5.0 \mathrm{E}+01$ & $6.5 \mathrm{E}+00$ & $6.5 \mathrm{E}-02$ & $-1.9 \mathrm{E}+01$ & $4.3 \mathrm{E}+00$ & 4.3E-02 & $-2.2 \mathrm{E}+01$ \\
\hline $1-130$ & $014914-02-4$ & $9.4 \mathrm{E}+01$ & $9.4 \mathrm{E}-01$ & $-5.2 \mathrm{E}-01$ & $2.5 \mathrm{E}+02$ & $2.5 \mathrm{E}+00$ & $-1.9 \mathrm{E}+01$ & $1.6 \mathrm{E}+02$ & $1.6 \mathrm{E}+00$ & $-2.2 \mathrm{E}+01$ \\
\hline $\mathrm{I}-131$ & $010043-66-0$ & $1.3 \mathrm{E}+01$ & $1.3 \mathrm{E}-01$ & $-5.4 \mathrm{E}+00$ & $3.3 \mathrm{E}+01$ & 3.3E-01 & $-1.9 \mathrm{E}+01$ & $2.2 \mathrm{E}+01$ & $2.2 \mathrm{E}-01$ & $-2.2 \mathrm{E}+01$ \\
\hline I-132 & $014683-16-0$ & $6.9 \mathrm{E}+02$ & $6.9 \mathrm{E}+00$ & $-1.0 \mathrm{E}-01$ & $1.8 \mathrm{E}+03$ & $1.8 \mathrm{E}+01$ & $-1.9 \mathrm{E}+01$ & $1.2 \mathrm{E}+03$ & $1.2 \mathrm{E}+01$ & $-2.2 \mathrm{E}+01$ \\
\hline
\end{tabular}


Table 3.3f. (continued)

\begin{tabular}{|c|c|c|c|c|c|c|c|c|c|c|}
\hline \multirow[t]{2}{*}{ Chemical $^{\dagger}$} & \multirow[t]{2}{*}{ CASRN } & \multicolumn{2}{|c|}{$\begin{array}{c}\text { Vegetable Intercept } \\
(\mathrm{pCi} / \mathrm{g})\end{array}$} & \multirow{2}{*}{ Slope } & \multicolumn{2}{|c|}{$\begin{array}{c}\text { Beef Intercept } \\
\text { (pCi/g) }\end{array}$} & \multirow[t]{2}{*}{ Slope } & \multicolumn{2}{|c|}{$\begin{array}{l}\text { Milk Intercept } \\
\text { (pCi/g) }\end{array}$} & \multirow[t]{2}{*}{ Slope } \\
\hline & & $10^{-4}$ & $10^{-5}$ & & $10^{-4}$ & $10^{-6}$ & & $10^{-4}$ & $10^{-6}$ & \\
\hline I-133 & $014834-67-4$ & $4.3 \mathrm{E}+01$ & 4.3E-01 & $-8.6 \mathrm{E}-01$ & $1.1 \mathrm{E}+02$ & $1.1 \mathrm{E}+00$ & $-1.9 E+01$ & $7.5 \mathrm{E}+01$ & 7.5E-01 & $-2.2 E+01$ \\
\hline I-134 & $014914-27-3$ & $2.0 \mathrm{E}+03$ & $2.0 \mathrm{E}+01$ & $-3.8 \mathrm{E}-02$ & $5.2 \mathrm{E}+03$ & $5.2 \mathrm{E}+01$ & $-1.9 E+01$ & $3.5 \mathrm{E}+03$ & $3.5 \mathrm{E}+01$ & $-2.2 E+01$ \\
\hline I-135 & $014834-68-5$ & $2.0 \mathrm{E}+02$ & $2.0 \mathrm{E}+00$ & $-2.8 \mathrm{E}-01$ & $5.3 \mathrm{E}+02$ & $5.3 \mathrm{E}+00$ & $-1.9 \mathrm{E}+01$ & $3.5 \mathrm{E}+02$ & $3.5 \mathrm{E}+00$ & $-2.2 \mathrm{E}+01$ \\
\hline In-113m & 014885-78-0 & $5.5 \mathrm{E}+03$ & $5.5 \mathrm{E}+01$ & $-7.2 \mathrm{E}-02$ & $1.4 \mathrm{E}+05$ & $1.4 \mathrm{E}+03$ & $-1.9 \mathrm{E}+01$ & $4.7 \mathrm{E}+05$ & $4.7 \mathrm{E}+03$ & $-2.1 E+01$ \\
\hline In-114 & 013981-55-0 & $1.0 \mathrm{E}+05$ & $1.0 \mathrm{E}+03$ & $-8.7 \mathrm{E}-04$ & $2.6 \mathrm{E}+06$ & $2.6 E+04$ & $-1.9 \mathrm{E}+01$ & $8.7 \mathrm{E}+06$ & $8.7 E+04$ & $-2.1 E+01$ \\
\hline In-114m & 013981-55-0 & $2.2 E+01$ & $2.2 \mathrm{E}-01$ & $-1.1 \mathrm{E}+01$ & $5.7 \mathrm{E}+02$ & $5.7 \mathrm{E}+00$ & $-1.9 \mathrm{E}+01$ & $1.9 \mathrm{E}+03$ & $1.9 \mathrm{E}+01$ & $-2.1 \mathrm{E}+01$ \\
\hline In-115 & 014191-71-0 & $1.3 E+01$ & $1.3 \mathrm{E}-01$ & $-5.0 \mathrm{E}+01$ & $3.4 \mathrm{E}+02$ & $3.4 \mathrm{E}+00$ & $-1.9 \mathrm{E}+01$ & $1.1 E+03$ & $1.1 \mathrm{E}+01$ & $-2.1 E+01$ \\
\hline In-115m & 014191-71-0 & $1.3 \mathrm{E}+03$ & $1.3 \mathrm{E}+01$ & $-1.9 \mathrm{E}-01$ & $3.5 \mathrm{E}+04$ & $3.5 \mathrm{E}+02$ & $-1.9 \mathrm{E}+01$ & $1.1 E+05$ & $1.1 \mathrm{E}+03$ & $-2.1 \mathrm{E}+01$ \\
\hline Ir- $190^{\circ}$ & 014981-91-0 & $8.3 \mathrm{E}+01$ & $8.3 \mathrm{E}-01$ & $-6.1 E+00$ & $3.3 E+03$ & $3.3 E+01$ & $-1.3 E+01$ & $5.2 \mathrm{E}+05$ & $5.2 \mathrm{E}+03$ & $-1.4 \mathrm{E}+01$ \\
\hline Ir-194 & 014158-35-1 & $5.9 \mathrm{E}+01$ & $5.9 \mathrm{E}-01$ & $-7.2 \mathrm{E}-01$ & $2.3 E+03$ & $2.3 E+01$ & $-1.3 \mathrm{E}+01$ & $3.7 \mathrm{E}+05$ & $3.7 \mathrm{E}+03$ & $-1.4 \mathrm{E}+01$ \\
\hline $\mathrm{K}-40$ & $013966-00-2$ & $1.7 \mathrm{E}+01$ & $1.7 \mathrm{E}-01$ & $-4.2 \mathrm{E}+01$ & $2.3 E+01$ & 2.3E-01 & $-2.3 E+00$ & $9.4 \mathrm{E}+00$ & $9.4 \mathrm{E}-02$ & $-2.3 \mathrm{E}+00$ \\
\hline $\mathrm{K}-42$ & $014378-21-3$ & $1.6 \mathrm{E}+02$ & $1.6 \mathrm{E}+00$ & $-2.5 \mathrm{E}-01$ & $2.2 \mathrm{E}+02$ & $2.2 \mathrm{E}+00$ & $-2.3 \mathrm{E}+00$ & $9.2 \mathrm{E}+01$ & $9.2 \mathrm{E}-01$ & $-2.3 \mathrm{E}+00$ \\
\hline $\mathrm{Kr}-83 \mathrm{~m}$ & $013965-98-5$ & & & & & & & & & \\
\hline $\mathrm{Kr}-85$ & $013983-27-2$ & & & & & & & & & \\
\hline $\mathrm{Kr}-85 \mathrm{~m}$ & $013983-27-2$ & & & & & & & & & \\
\hline $\mathrm{Kr}-87$ & 014809-68-8 & & & & & & & & & \\
\hline $\mathrm{Kr}-88$ & 014995-61-0 & & & & & & & & & \\
\hline
\end{tabular}


Table 3.3f. (continued)

\begin{tabular}{|c|c|c|c|c|c|c|c|c|c|c|}
\hline \multirow[t]{2}{*}{ Chemical ${ }^{\dagger}$} & \multirow[t]{2}{*}{ CASRN } & \multicolumn{2}{|c|}{$\begin{array}{c}\text { Vegetable Intercept } \\
\text { (pCi/g) }\end{array}$} & \multirow[t]{2}{*}{ Slope } & \multicolumn{2}{|c|}{$\begin{array}{l}\text { Beef Intercept } \\
\text { (pCi/g) }\end{array}$} & \multirow[t]{2}{*}{ Slope } & \multicolumn{2}{|c|}{$\begin{array}{l}\text { Milk Intercept } \\
(\mathrm{pCi} / \mathrm{g})\end{array}$} & \multirow{2}{*}{ Slope } \\
\hline & & $10^{-4}$ & $10^{-6}$ & & $10^{-4}$ & $10^{-6}$ & & $10^{-4}$ & $10^{-6}$ & \\
\hline $\mathrm{Kr}-89$ & $016316-03-3$ & & & & & & & & & \\
\hline $\mathrm{Kr}-90$ & 015741-13-6 & & & & & & & & & \\
\hline La-140 & 013981-28-7 & $4.8 \mathrm{E}+01$ & $4.8 \mathrm{E}-01$ & $-1.6 \mathrm{E}+00$ & $1.7 \mathrm{E}+04$ & $1.7 \mathrm{E}+02$ & $-1.9 \mathrm{E}+01$ & $4.2 E+04$ & $4.2 \mathrm{E}+02$ & $-2.2 \mathrm{E}+01$ \\
\hline Lu-177 & 014265-75-9 & $1.5 \mathrm{E}+02$ & $1.5 \mathrm{E}+00$ & $-4.8 \mathrm{E}+00$ & $3.6 \mathrm{E}+03$ & $3.6 \mathrm{E}+01$ & $-1.9 \mathrm{E}+01$ & $1.3 E+05$ & $1.3 \mathrm{E}+03$ & $-2.1 \mathrm{E}+01$ \\
\hline $\mathrm{Mn}-52$ & 014092-99-0 & $6.0 \mathrm{E}+01$ & $6.0 \mathrm{E}-01$ & $-3.4 \mathrm{E}+00$ & $6.0 \mathrm{E}+03$ & $6.0 \mathrm{E}+01$ & $-7.1 \mathrm{E}+00$ & $1.5 \mathrm{E}+04$ & $1.5 \mathrm{E}+02$ & $-7.5 \mathrm{E}+00$ \\
\hline $\mathrm{Mn}-54$ & $013966-31-9$ & $1.8 \mathrm{E}+02$ & $1.8 \mathrm{E}+00$ & $-1.2 \mathrm{E}+01$ & $1.8 E+04$ & $1.8 \mathrm{E}+02$ & $-7.1 \mathrm{E}+00$ & 4.7E+04 & 4.7E+02 & $-7.5 \mathrm{E}+00$ \\
\hline $\mathrm{Mn}-56$ & 014681-52-8 & $4.2 \mathrm{E}+02$ & $4.2 \mathrm{E}+00$ & $-9.0 \mathrm{E}-02$ & $4.2 \mathrm{E}+04$ & $4.2 \mathrm{E}+02$ & $-7.1 \mathrm{E}+00$ & $1.1 \mathrm{E}+05$ & $1.1 \mathrm{E}+03$ & $-7.5 \mathrm{E}+00$ \\
\hline Mo-99 & 014119-15-4 & $1.5 \mathrm{E}+02$ & $1.5 \mathrm{E}+00$ & $-1.9 \mathrm{E}+00$ & $1.1 \mathrm{E}+04$ & $1.1 \mathrm{E}+02$ & $-9.6 \mathrm{E}+00$ & $9.8 \mathrm{E}+02$ & $9.8 \mathrm{E}+00$ & $-1.0 \mathrm{E}+01$ \\
\hline $\mathrm{Na}-22$ & $013966-32-0$ & $5.1 \mathrm{E}+01$ & $5.1 \mathrm{E}-01$ & $-1.7 \mathrm{E}+01$ & $5.1 \mathrm{E}+01$ & $5.1 \mathrm{E}-01$ & $-1.3 E+01$ & $4.0 \mathrm{E}+01$ & $4.0 \mathrm{E}-01$ & $-1.4 \mathrm{E}+01$ \\
\hline $\mathrm{Na}-24$ & 013982-04-2 & $3.0 \mathrm{E}+02$ & $3.0 \mathrm{E}+00$ & $-5.7 \mathrm{E}-01$ & $2.9 \mathrm{E}+02$ & $2.9 E+00$ & $-1.3 E+01$ & $2.3 E+02$ & $2.3 \mathrm{E}+00$ & $-1.4 E+01$ \\
\hline $\mathrm{Nb}-93 \mathrm{~m}$ & $007440-03-1$ & $6.8 \mathrm{E}+02$ & $6.8 \mathrm{E}+00$ & $-3.3 E+01$ & $2.0 \mathrm{E}+08$ & $2.0 \mathrm{E}+06$ & $-1.5 E+01$ & $2.3 E+07$ & $2.3 \mathrm{E}+05$ & $-1.7 \mathrm{E}+01$ \\
\hline $\mathrm{Nb}-94$ & $014681-63-1$ & $6.5 \mathrm{E}+01$ & $6.5 \mathrm{E}-01$ & $-5.0 \mathrm{E}+01$ & $1.9 \mathrm{E}+07$ & $1.9 \mathrm{E}+05$ & $-1.5 \mathrm{E}+01$ & $2.2 \mathrm{E}+06$ & $2.2 \mathrm{E}+04$ & $-1.7 \mathrm{E}+01$ \\
\hline $\mathrm{Nb}-95$ & $013967-76-5$ & $2.0 \mathrm{E}+02$ & $2.0 \mathrm{E}+00$ & $-1.0 \mathrm{E}+01$ & $5.8 \mathrm{E}+07$ & $5.8 \mathrm{E}+05$ & $-1.5 \mathrm{E}+01$ & $6.8 \mathrm{E}+06$ & $6.8 \mathrm{E}+04$ & $-1.7 \mathrm{E}+01$ \\
\hline $\mathrm{Nb}-95 \mathrm{~m}$ & $013967-76-5$ & $1.5 \mathrm{E}+02$ & $1.5 \mathrm{E}+00$ & $-3.0 \mathrm{E}+00$ & $4.2 \mathrm{E}+07$ & $4.2 \mathrm{E}+05$ & $-1.5 \mathrm{E}+01$ & $5.0 \mathrm{E}+06$ & $5.0 \mathrm{E}+04$ & $-1.7 \mathrm{E}+01$ \\
\hline $\mathrm{Nb}-97$ & 018496-04-3 & $2.6 \mathrm{E}+03$ & $2.6 \mathrm{E}+01$ & $-5.2 \mathrm{E}-02$ & $7.4 \mathrm{E}+08$ & $7.4 \mathrm{E}+06$ & $-1.5 \mathrm{E}+01$ & $8.8 \mathrm{E}+07$ & $8.8 \mathrm{E}+05$ & $-1.7 \mathrm{E}+01$ \\
\hline $\mathrm{Nb}-97 \mathrm{~m}$ & $018496-04-3$ & $1.4 \mathrm{E}+05$ & $1.4 \mathrm{E}+03$ & $-7.2 \mathrm{E}-04$ & $4.0 \mathrm{E}+10$ & $4.0 \mathrm{E}+08$ & $-1.5 \mathrm{E}+01$ & $4.7 \mathrm{E}+09$ & $4.7 \mathrm{E}+07$ & $-1.7 \mathrm{E}+01$ \\
\hline Nd-147 & 014269-74-0 & 7.7E+01 & 7.7E-01 & $-6.5 \mathrm{E}+00$ & $2.6 \mathrm{E}+04$ & $2.6 \mathrm{E}+02$ & $-1.8 \mathrm{E}+01$ & $6.5 \mathrm{E}+04$ & $6.5 \mathrm{E}+02$ & $-2.1 \mathrm{E}+01$ \\
\hline
\end{tabular}




\begin{tabular}{|c|c|c|c|c|c|c|c|c|c|c|c|c|c|c|c|c|c|c|}
\hline \multicolumn{2}{|c|}{$\frac{\stackrel{ }{\circ}}{\text { ळ }}$} & 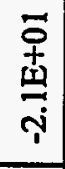 & $\begin{array}{l}\text { ơ } \\
\text { 岃 } \\
\frac{7}{7}\end{array}$ & $\begin{array}{l}\overline{+} \\
+ \\
\text { 章 } \\
7\end{array}$ & $\begin{array}{l}\overrightarrow{0} \\
\text { 故 } \\
\frac{1}{1}\end{array}$ & 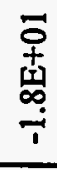 & 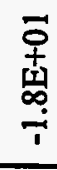 & 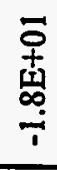 & 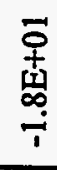 & 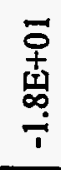 & $\begin{array}{l}\overrightarrow{0} \\
+ \\
+91 \\
\infty \\
+ \\
+\end{array}$ & 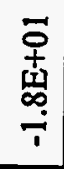 & $\begin{array}{c}\bar{o} \\
\text { 音 } \\
\text { iv }\end{array}$ & 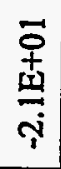 & 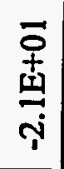 & 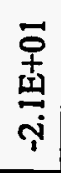 & 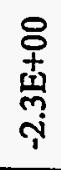 & 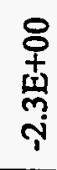 \\
\hline \multirow{2}{*}{ 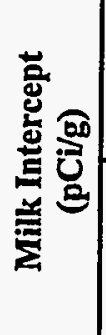 } & $\stackrel{\varphi}{\circ}$ & 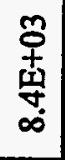 & 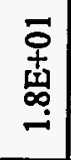 & 辛 & 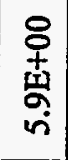 & 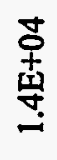 & $\begin{array}{l}\text { 웅 } \\
\text { 点 } \\
\text { f }\end{array}$ & $\begin{array}{l}\bar{\phi} \\
\text { 壱 } \\
\text { ㄱ. }\end{array}$ & $\begin{array}{l}\text { \% } \\
\text { 索 } \\
\text { m }\end{array}$ & $\begin{array}{l}\text { m } \\
\text { +⿱ } \\
\text { 敱 } \\
\text { m }\end{array}$ & $\begin{array}{l}\text { 号 } \\
+ \\
\text { 章 } \\
\sim\end{array}$ & 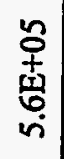 & 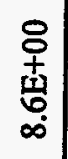 & $\begin{array}{l}8 \\
+ \\
\text { 息 } \\
\text { n் }\end{array}$ & 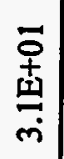 & $\begin{array}{l}8 \\
\text { + } \\
\text { ñ } \\
\text { m }\end{array}$ & 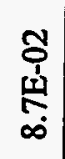 & $\begin{array}{l}\overline{0} \\
\text { 1. } \\
0 \\
0 \\
0\end{array}$ \\
\hline & $\stackrel{7}{\varrho}$ & 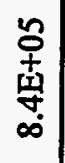 & 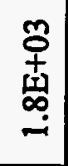 & 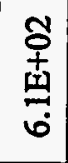 & 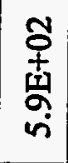 & $\begin{array}{l}\text { 号 } \\
\text { 章 } \\
\text { 足 }\end{array}$ & 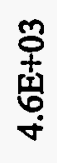 & 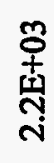 & 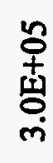 & 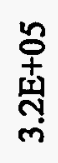 & 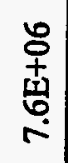 & $\begin{array}{c}\text { so } \\
\text { 盇 } \\
\text { c. }\end{array}$ & $\begin{array}{l}\text { ơ } \\
\text { † } \\
\text { 号 } \\
\infty \\
\infty\end{array}$ & 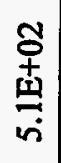 & 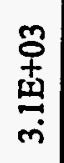 & 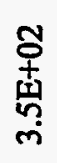 & 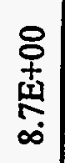 & 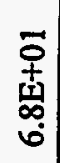 \\
\hline \multicolumn{2}{|c|}{ 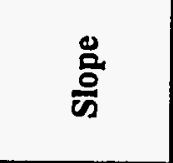 } & 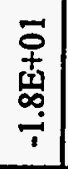 & $\begin{array}{c}\stackrel{0}{+} \\
\text { 孚 } \\
\stackrel{1}{1}\end{array}$ & 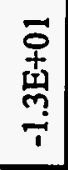 & 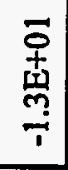 & 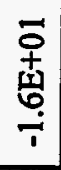 & 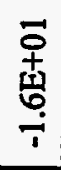 & 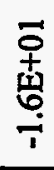 & 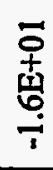 & $\begin{array}{l}\overline{0} \\
\text { 点 } \\
\stackrel{6}{7}\end{array}$ & 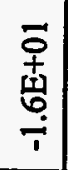 & 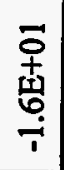 & 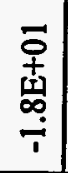 & 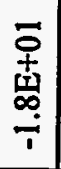 & 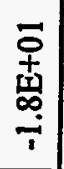 & 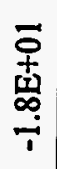 & 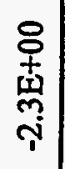 & 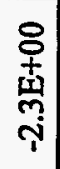 \\
\hline \multirow{2}{*}{ 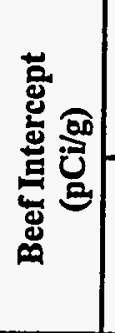 } & $\stackrel{\varphi}{\stackrel{9}{g}}$ & 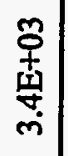 & 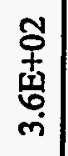 & $\begin{array}{c}\text { 옹 } \\
\text { 岕 } \\
\text { ড̦ }\end{array}$ & 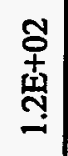 & 尽 & 号 & 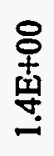 & 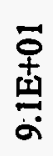 & $\begin{array}{l}\overrightarrow{0} \\
+ \\
\text { 索 } \\
\sigma\end{array}$ & 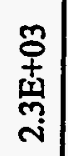 & 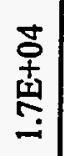 & 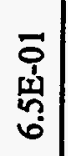 & 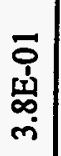 & $\begin{array}{l}\text { 号 } \\
\text { 夏 } \\
\text { in }\end{array}$ & 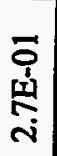 & 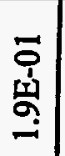 & 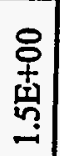 \\
\hline & $\underline{0}$ & $\begin{array}{c}\stackrel{n}{o} \\
\text { 索 } \\
\text { m. }\end{array}$ & 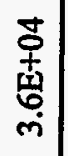 & 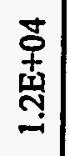 & $\begin{array}{c}\underset{⿱}{+} \\
\text { 壱 } \\
\stackrel{-}{-}\end{array}$ & 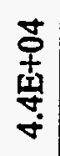 & $\begin{array}{c}\text { S } \\
\text { 龺 } \\
\stackrel{-}{-}\end{array}$ & 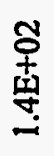 & 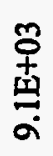 & $\begin{array}{l}\text { o } \\
\text { 章 } \\
\text { å }\end{array}$ & 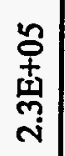 & 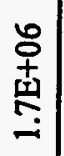 & 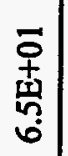 & $\begin{array}{l}\overrightarrow{0} \\
+ \\
\text { 中. } \\
\infty \\
\dot{m}\end{array}$ & $\begin{array}{c}\text { 㟔 } \\
\text { 索 } \\
\text { is }\end{array}$ & $\begin{array}{l}\overrightarrow{+} \\
\text { 商 } \\
\text { i }\end{array}$ & $\begin{array}{l}\overrightarrow{0} \\
+ \\
\text { 离 } \\
\text { a }\end{array}$ & 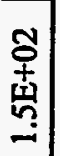 \\
\hline \multicolumn{2}{|c|}{ 응 } & 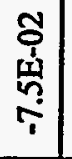 & 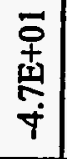 & 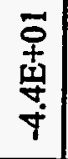 & 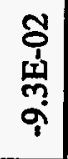 & $\begin{array}{l}\overrightarrow{0} \\
+5 \\
\text { to } \\
\dot{\varphi}\end{array}$ & 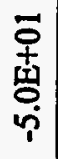 & $\begin{array}{l}\overline{0} \\
\text { 章 } \\
\text { மे }\end{array}$ & 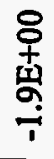 & 용 & 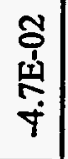 & 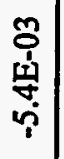 & 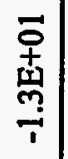 & 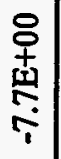 & 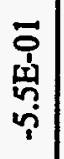 & \begin{tabular}{c}
$\stackrel{8}{0}$ \\
+5 \\
$\stackrel{5}{7}$ \\
\hdashline
\end{tabular} & 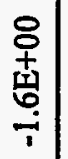 & 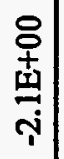 \\
\hline \multirow{2}{*}{ 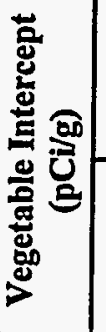 } & $\stackrel{\varphi}{\varrho}$ & 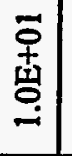 & $\underset{\text { i }}{\stackrel{\overrightarrow{0}}{\mathbf{9}}}$ & 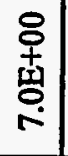 & 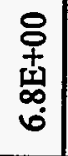 & $\begin{array}{l}8 \\
\text { 市 } \\
\text { 萦 } \\
\text { 子 }\end{array}$ & 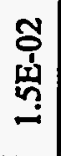 & 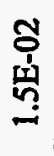 & 호 & $\begin{array}{l}\stackrel{8}{9} \\
\text { 舀 } \\
\end{array}$ & 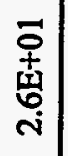 & 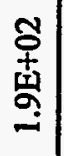 & 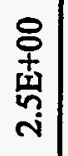 & 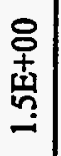 & $\begin{array}{l}\stackrel{8}{+} \\
\text { 至 } \\
\text { ă }\end{array}$ & $\begin{array}{l}\text { 옹 } \\
\text { 南 } \\
.\end{array}$ & 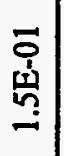 & $\begin{array}{l}\text { oి } \\
\text { 离 } \\
\text { స్ }\end{array}$ \\
\hline & 흠 & 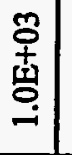 & $\begin{array}{c}\stackrel{3}{0} \\
\text { 点 } \\
\text { ci }\end{array}$ & 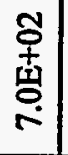 & 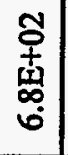 & 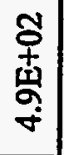 & $\begin{array}{l}8 \\
\stackrel{+}{+} \\
\text { 点 } \\
-\end{array}$ & $\begin{array}{l}8 \\
\frac{1}{4} \\
\text { 窅 } \\
-\end{array}$ & 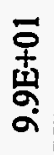 & 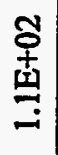 & 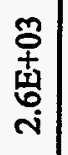 & 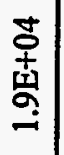 & 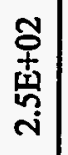 & 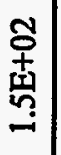 & $\begin{array}{c}\text { 草 } \\
\text { 舁 } \\
\sigma\end{array}$ & $\begin{array}{c}\text { 옹 } \\
\text { 吉 } \\
\text { - }\end{array}$ & 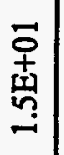 & 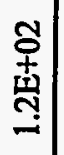 \\
\hline \multicolumn{2}{|l|}{$\frac{z}{\frac{Z}{2}}$} & 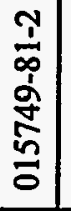 & 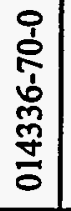 & 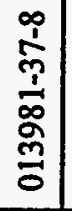 & 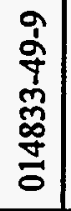 & 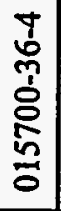 & 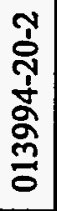 & 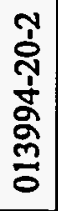 & 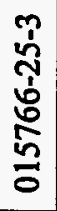 & 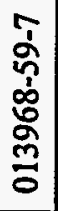 & 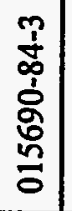 & 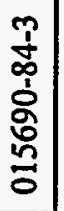 & 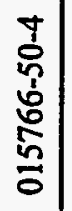 & 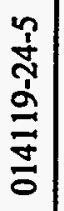 & 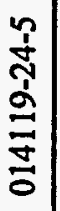 & $\begin{array}{l}n \\
\frac{1}{5} \\
\frac{1}{n} \\
8 \\
0 \\
0\end{array}$ & 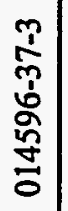 & 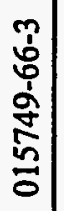 \\
\hline 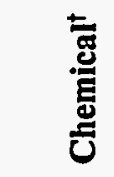 & & $\frac{g}{\frac{\partial}{d}}$ & 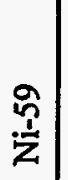 & 兽 & 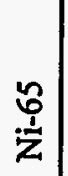 & 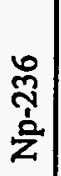 & 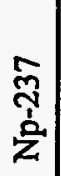 & 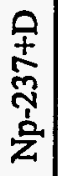 & $\begin{array}{l}\infty \\
\text { స్} \\
\grave{z} \\
z\end{array}$ & $\begin{array}{l}\text { సે } \\
\text { خे. } \\
\text { zे }\end{array}$ & 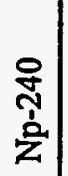 & 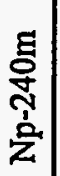 & 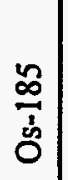 & $\vec{a}$ & $\frac{\underline{E}}{\frac{\sigma}{2}}$ & $\frac{\check{\sigma}}{\dot{\delta}}$ & ָे & $\stackrel{m}{\stackrel{a}{a}}$ \\
\hline
\end{tabular}


Table 3.3f. (continued)

\begin{tabular}{|c|c|c|c|c|c|c|c|c|c|c|}
\hline \multirow[t]{2}{*}{ Chemical $^{\dagger}$} & \multirow[t]{2}{*}{ CASRN } & \multicolumn{2}{|c|}{$\begin{array}{c}\text { Vegetable Intercept } \\
(\mathrm{pCi} / \mathrm{g})\end{array}$} & \multirow{2}{*}{ Slope } & \multicolumn{2}{|c|}{$\begin{array}{l}\text { Beef Intercept } \\
\text { (pCi/g) }\end{array}$} & \multirow[t]{2}{*}{ Slope } & \multicolumn{2}{|c|}{$\begin{array}{l}\text { Milk Intercept } \\
\text { (pCi/g) }\end{array}$} & \multirow[t]{2}{*}{ Slope } \\
\hline & & $10^{-4}$ & $10^{-6}$ & & $10^{-4}$ & $10^{-6}$ & & $10^{-4}$ & $10^{-6}$ & \\
\hline $\mathrm{Pa}-231$ & $014331-85-2$ & $3.1 \mathrm{E}+00$ & 3.1E-02 & $-5.0 \mathrm{E}+01$ & $3.2 \mathrm{E}+04$ & $3.2 \mathrm{E}+02$ & $-1.9 \mathrm{E}+01$ & $1.1 \mathrm{E}+04$ & $1.1 \mathrm{E}+02$ & $-2.2 \mathrm{E}+01$ \\
\hline $\mathrm{Pa}-233$ & 013981-14-1 & $9.7 \mathrm{E}+01$ & $9.7 \mathrm{E}-01$ & $-9.7 \mathrm{E}+00$ & $1.0 \mathrm{E}+06$ & $1.0 \mathrm{E}+04$ & $-1.9 \mathrm{E}+01$ & $3.4 E+05$ & $3.4 \mathrm{E}+03$ & $-2.2 \mathrm{E}+01$ \\
\hline $\mathrm{Pa}-234$ & $015100-28-4$ & $2.1 \mathrm{E}+02$ & $2.1 \mathrm{E}+00$ & $-2.9 \mathrm{E}-01$ & $2.3 E+06$ & $2.3 \mathrm{E}+04$ & $-1.9 \mathrm{E}+01$ & $7.5 \mathrm{E}+05$ & $7.5 \mathrm{E}+03$ & $-2.2 E+01$ \\
\hline $\mathrm{Pa}-234 \mathrm{~m}$ & $015100-28-4$ & $9.6 E+04$ & $9.6 \mathrm{E}+02$ & $-8.6 \mathrm{E}-04$ & $1.0 \mathrm{E}+09$ & $1.0 \mathrm{E}+07$ & $-1.9 \mathrm{E}+01$ & $3.4 \mathrm{E}+08$ & $3.4 \mathrm{E}+06$ & $-2.2 \mathrm{E}+01$ \\
\hline $\mathrm{Pb}-203$ & 014687-25-3 & $4.4 \mathrm{E}+02$ & $4.4 \mathrm{E}+00$ & $-2.0 \mathrm{E}+00$ & $9.6 \mathrm{E}+04$ & $9.6 \mathrm{E}+02$ & $-1.6 \mathrm{E}+01$ & $2.1 \mathrm{E}+04$ & $2.1 \mathrm{E}+02$ & $-1.8 \mathrm{E}+01$ \\
\hline $\mathrm{Pb}-209$ & $014119-30-3$ & $2.2 \mathrm{E}+03$ & $2.2 \mathrm{E}+01$ & $-1.4 \mathrm{E}-01$ & $4.7 E+05$ & $4.7 \mathrm{E}+03$ & $-1.6 \mathrm{E}+01$ & $1.0 \mathrm{E}+05$ & $1.0 \mathrm{E}+03$ & $-1.8 \mathrm{E}+01$ \\
\hline $\mathrm{Pb}-210$ & 014255-04-0 & $6.8 \mathrm{E}-01$ & $6.8 \mathrm{E}-03$ & $-3.8 \mathrm{E}+01$ & $1.5 \mathrm{E}+02$ & $1.5 \mathrm{E}+00$ & $-1.6 \mathrm{E}+01$ & $3.2 \mathrm{E}+01$ & $3.2 \mathrm{E}-01$ & $-1.8 \mathrm{E}+01$ \\
\hline $\mathrm{Pb}-210+\mathrm{D}$ & 014255-04-0 & 4.4E-01 & $4.4 \mathrm{E}-03$ & $-3.7 \mathrm{E}+01$ & $5.4 \mathrm{E}+00$ & $5.4 \mathrm{E}-02$ & $-1.2 \mathrm{E}+01$ & $4.4 \mathrm{E}+00$ & $4.4 \mathrm{E}-02$ & $-1.4 E+01$ \\
\hline $\mathrm{Pb}-211$ & 015816-77-0 & $1.4 \mathrm{E}+03$ & $1.4 \mathrm{E}+01$ & $-2.6 \mathrm{E}-02$ & $2.9 \mathrm{E}+05$ & $2.9 \mathrm{E}+03$ & $-1.6 \mathrm{E}+01$ & $6.3 E+04$ & $6.3 E+02$ & $-1.8 \mathrm{E}+01$ \\
\hline $\mathrm{Pb}-212$ & 015092-94-1 & $2.5 \mathrm{E}+01$ & $2.5 \mathrm{E}-01$ & $-4.5 \mathrm{E}-01$ & $5.5 \mathrm{E}+03$ & $5.5 \mathrm{E}+01$ & $-1.6 \mathrm{E}+01$ & $1.2 \mathrm{E}+03$ & $1.2 \mathrm{E}+01$ & $-1.8 \mathrm{E}+01$ \\
\hline $\mathrm{Pb}-214$ & $015067-28-4$ & $1.6 \mathrm{E}+03$ & $1.6 \mathrm{E}+01$ & $-2.0 \mathrm{E}-02$ & $3.4 \mathrm{E}+05$ & $3.4 \mathrm{E}+03$ & $-1.6 \mathrm{E}+01$ & $7.3 E+04$ & $7.3 \mathrm{E}+02$ & $-1.8 \mathrm{E}+01$ \\
\hline Pd-100 & $015690-69-4$ & $1.1 \mathrm{E}+02$ & $1.1 \mathrm{E}+00$ & $-2.7 \mathrm{E}+00$ & $2.4 \mathrm{E}+03$ & $2.4 \mathrm{E}+01$ & $-1.4 \mathrm{E}+01$ & $1.5 \mathrm{E}+02$ & $1.5 \mathrm{E}+00$ & $-1.5 \mathrm{E}+01$ \\
\hline Pd-101 & 015749-54-9 & $1.1 \mathrm{E}+03$ & $1.1 \mathrm{E}+01$ & $-3.2 \mathrm{E}-01$ & $2.4 \mathrm{E}+04$ & $2.4 \mathrm{E}+02$ & $-1.4 \mathrm{E}+01$ & $1.5 \mathrm{E}+03$ & $1.5 \mathrm{E}+01$ & $-1.5 \mathrm{E}+01$ \\
\hline Pd-103 & 014967-68-1 & $3.8 \mathrm{E}+02$ & $3.8 \mathrm{E}+00$ & $-7.1 \mathrm{E}+00$ & $8.4 \mathrm{E}+03$ & $8.4 \mathrm{E}+01$ & $-1.4 \mathrm{E}+01$ & $5.4 \mathrm{E}+02$ & $5.4 \mathrm{E}+00$ & $-1.5 \mathrm{E}+01$ \\
\hline Pd-107 & 017637-99-9 & $1.9 \mathrm{E}+03$ & $1.9 \mathrm{E}+01$ & $-4.8 \mathrm{E}+01$ & $4.2 \mathrm{E}+04$ & $4.2 \mathrm{E}+02$ & $-1.4 \mathrm{E}+01$ & $2.7 \mathrm{E}+03$ & $2.7 \mathrm{E}+01$ & $-1.5 \mathrm{E}+01$ \\
\hline Pd-109 & 014981-64-7 & $1.2 \mathrm{E}+02$ & $1.2 \mathrm{E}+00$ & $-5.0 \mathrm{E}-01$ & $2.7 \mathrm{E}+03$ & $2.7 \mathrm{E}+01$ & $-1.4 \mathrm{E}+01$ & $1.7 E+02$ & $1.7 \mathrm{E}+00$ & $-1.5 \mathrm{E}+01$ \\
\hline Pm-147 & $014380-75-7$ & $3.2 \mathrm{E}+02$ & $3.2 \mathrm{E}+00$ & $-1.9 \mathrm{E}+01$ & $6.7 \mathrm{E}+03$ & $6.7 E+01$ & $-1.9 \mathrm{E}+01$ & $2.8 E+05$ & $2.8 \mathrm{E}+03$ & $-2.1 E+01$ \\
\hline
\end{tabular}




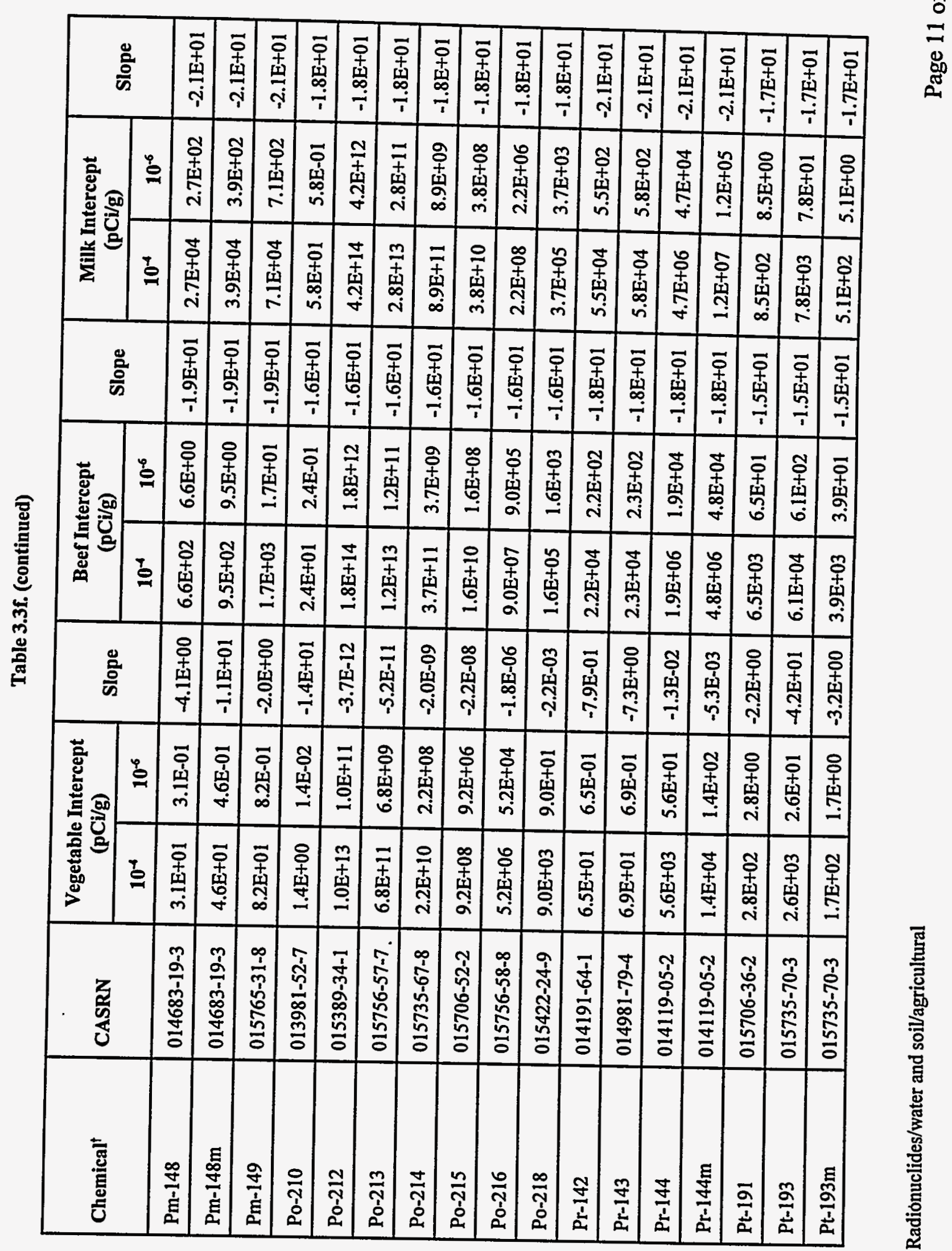




\begin{tabular}{|c|c|c|c|c|c|c|c|c|c|c|c|c|c|c|c|c|c|c|}
\hline \multicolumn{2}{|c|}{ 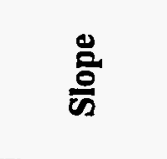 } & $\begin{array}{l}\text { 후 } \\
\text { 龺 } \\
\text { † }\end{array}$ & 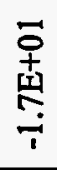 & 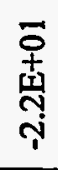 & 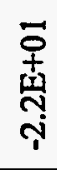 & 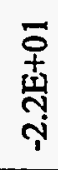 & $\begin{array}{l}\overline{\mathbf{0}} \\
\text { 壳 } \\
\text { ते }\end{array}$ & $\begin{array}{l}\overrightarrow{0} \\
\text { +े } \\
\text { ஸे } \\
\text { †ิ }\end{array}$ & $\begin{array}{c}\overline{0} \\
\stackrel{+}{1} \\
\underset{1}{\mathbf{\gamma}}\end{array}$ & 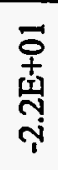 & 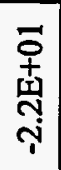 & 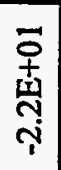 & $\begin{array}{c}\overline{0} \\
\text { 产 } \\
\frac{7}{i}\end{array}$ & $\begin{array}{c}\overrightarrow{0} \\
+1 \\
\frac{1}{0} \\
\stackrel{1}{0}\end{array}$ & 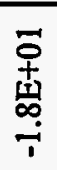 & 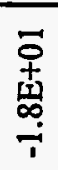 & 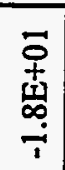 & 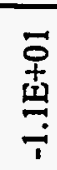 \\
\hline \multirow{2}{*}{ 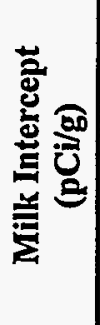 } & $\stackrel{\varphi}{\stackrel{0}{0}}$ & $\begin{array}{l}\text { 옹 } \\
\text { 市 } \\
\text { 우 }\end{array}$ & 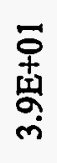 & 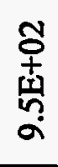 & 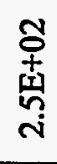 & 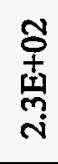 & 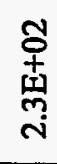 & $\begin{array}{l}\text { 导 } \\
\text { 孛 } \\
\text { 品 }\end{array}$ & 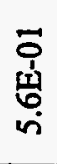 & $\begin{array}{l}\text { Oे } \\
\text { 寽 } \\
\text { ণ }\end{array}$ & $\begin{array}{l}n \\
\text { o } \\
\text { 索 } \\
\text { i }\end{array}$ & 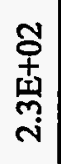 & 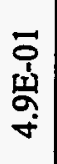 & $\begin{array}{l}\overline{\mathbf{d}} \\
\text { 山్d } \\
\text { స్ }\end{array}$ & $\begin{array}{l}\overrightarrow{0} \\
\text { 峖 } \\
\text { r. }\end{array}$ & 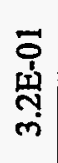 & $\begin{array}{l}\overline{0} \\
\text { 章 } \\
\text { - }\end{array}$ & 客 \\
\hline & 응 & 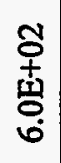 & $\begin{array}{l}m \\
\text { \% } \\
\text { 孚 } \\
\text { m }\end{array}$ & 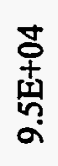 & 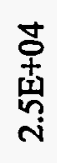 & 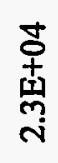 & 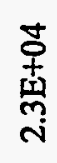 & 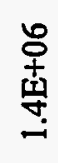 & $\begin{array}{l}\text { 웅 } \\
\text { 章 } \\
\text { in }\end{array}$ & 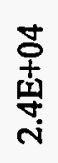 & $\begin{array}{c}\text { o } \\
+ \\
\text { 吉 } \\
\text { i }\end{array}$ & 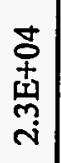 & $\begin{array}{l}\overrightarrow{0} \\
\text { + } \\
\text { 壳 } \\
\dot{+}\end{array}$ & 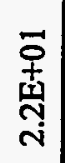 & $\begin{array}{l}\text { D } \\
\text { 湈 } \\
\text { m. }\end{array}$ & $\begin{array}{l}\overline{\text { s }} \\
\text { 离 } \\
\text { ஸे }\end{array}$ & $\begin{array}{c}\overrightarrow{0} \\
\text { 产 } \\
\text { 点 }\end{array}$ & 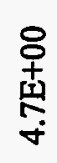 \\
\hline \multicolumn{2}{|c|}{$\frac{\Xi}{\text { 음 }}$} & 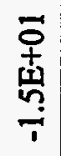 & $\begin{array}{l}\text { o } \\
+ \\
\text { 㟧 } \\
\stackrel{+}{r}\end{array}$ & $\begin{array}{l}\overline{0} \\
\text { 莺 } \\
\stackrel{+}{i}\end{array}$ & \begin{tabular}{l}
$\overrightarrow{0}$ \\
$+\frac{1}{9}$ \\
a \\
\hdashline
\end{tabular} & $\begin{array}{l}\vec{\delta} \\
+ \\
\text { 枽 } \\
\stackrel{i}{7}\end{array}$ & 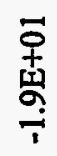 & 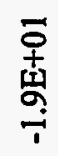 & 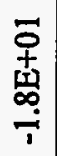 & 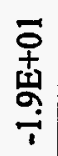 & 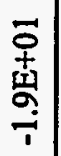 & \begin{tabular}{c}
$\overline{0}$ \\
+ \\
究 \\
\hdashline
\end{tabular} & 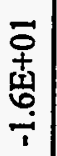 & $\begin{array}{c}\overrightarrow{0} \\
\text { 立 } \\
\text { 号 } \\
\stackrel{i}{1}\end{array}$ & 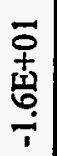 & $\begin{array}{l}\bar{\sigma} \\
+1 \\
\text { 吉 } \\
\frac{1}{1}\end{array}$ & $\begin{array}{c}\overrightarrow{0} \\
\text { 市 } \\
\text { 号 } \\
\stackrel{-}{1}\end{array}$ & $\begin{array}{l}8 \\
\text { ㅇ } \\
\text { In } \\
\infty \\
\text { o }\end{array}$ \\
\hline \multirow{2}{*}{ 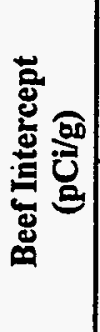 } & $\stackrel{9}{\circ}$ & $\begin{array}{l}\overrightarrow{0} \\
+ \\
\text { 吉 } \\
\dot{+}\end{array}$ & $\begin{array}{l}\text { S } \\
+ \\
\text { 章 } \\
\text { r }\end{array}$ & 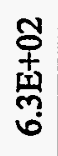 & 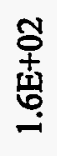 & 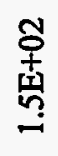 & $\begin{array}{l}\text { ㅇ } \\
\text { 故 } \\
\text { ? }\end{array}$ & 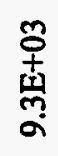 & 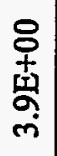 & 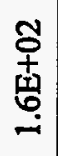 & 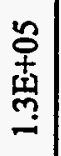 & 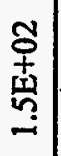 & 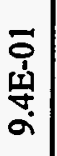 & $\begin{array}{l}8 \\
\text { 怘 } \\
\text { 愛 } \\
-\end{array}$ & 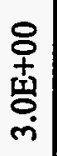 & 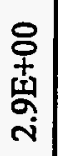 & 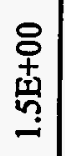 & ণั \\
\hline & $\stackrel{+}{0}$ & $\begin{array}{l}8 \\
0 \\
+ \\
\text { 岁 } \\
\dot{+}\end{array}$ & $\begin{array}{l}\text { 号 } \\
\text { 壱 } \\
\text { r }\end{array}$ & 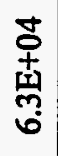 & 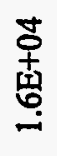 & 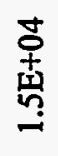 & 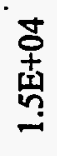 & $\begin{array}{l}\text { o } \\
\text { 离 } \\
\text { ă }\end{array}$ & 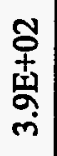 & 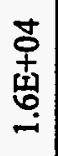 & 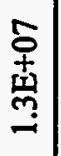 & 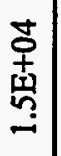 & 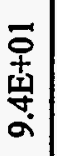 & $\begin{array}{l}\text { ơ } \\
\text { 产 } \\
\text { a }\end{array}$ & $\begin{array}{c}\text { จै } \\
+ \\
\text { 至 } \\
\text { mे }\end{array}$ & 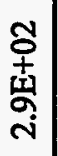 & 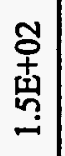 & $\begin{array}{l}\text { 오 } \\
\text { 吉 } \\
\text { ํ. }\end{array}$ \\
\hline \multicolumn{2}{|c|}{ 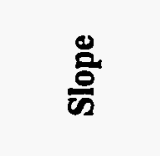 } & 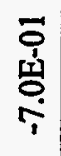 & 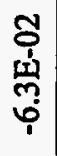 & $\begin{array}{l}\overrightarrow{0} \\
+\frac{1}{\sigma} \\
\text { † }\end{array}$ & $\begin{array}{l}\overline{0} \\
\text { 要 } \\
\text { †े }\end{array}$ & 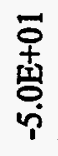 & 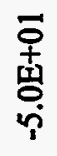 & $\begin{array}{l}\overrightarrow{0} \\
+ \\
\text { 䒚 } \\
\dot{p}\end{array}$ & 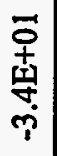 & $\begin{array}{l}\overline{0} \\
\text { 畧 } \\
\text { i }\end{array}$ & 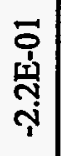 & $\begin{array}{l}\overrightarrow{0} \\
\text { 四 } \\
\text { ن. }\end{array}$ & 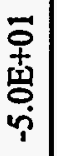 & 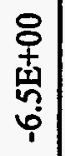 & $\begin{array}{c}\text { 옹 } \\
\text { 商 } \\
\text { ণิ }\end{array}$ & 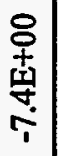 & $\begin{array}{l}\overrightarrow{+} \\
\text { 国 } \\
\text { و } \\
\forall\end{array}$ & 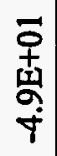 \\
\hline \multirow{2}{*}{ 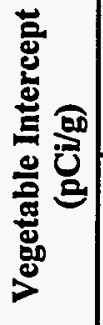 } & 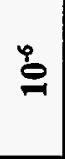 & 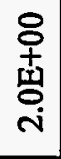 & $\begin{array}{c}\overline{0} \\
\text { 壳 } \\
\stackrel{n}{-}\end{array}$ & 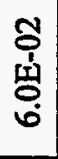 & 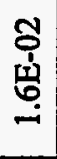 & 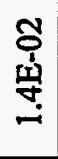 & 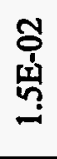 & $\begin{array}{l}\overline{0} \\
\text { 山⿱山口 } \\
\infty \\
\infty \\
\infty\end{array}$ & 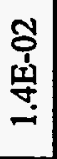 & 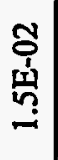 & $\begin{array}{l}\overline{0} \\
\text { 䓌 } \\
\text { స్}\end{array}$ & 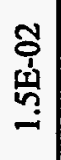 & 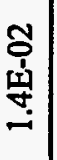 & 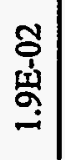 & 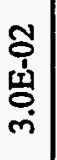 & 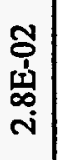 & 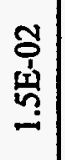 & 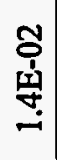 \\
\hline & $\stackrel{T}{\varrho}$ & 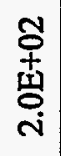 & 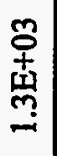 & $\begin{array}{l}8 \\
\text { 立 } \\
\text { 总 } \\
\text { o }\end{array}$ & $\begin{array}{l}\text { 웅 } \\
\text { 章 } \\
\stackrel{-}{-}\end{array}$ & $\begin{array}{l}8 \\
\text { 古 } \\
\text { 孚 } \\
\text { - }\end{array}$ & 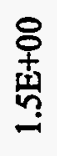 & $\begin{array}{l}\overrightarrow{0} \\
\vdots \\
\text { ț } \\
\infty \\
\infty\end{array}$ & $\begin{array}{l}8 \\
8 \\
\text { 省 } \\
-\end{array}$ & 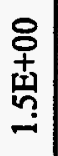 & 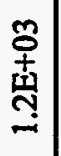 & 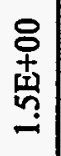 & $\begin{array}{l}8 \\
\text { 古 } \\
\text { 岁 } \\
\stackrel{-}{-}\end{array}$ & $\begin{array}{l}8 \\
\text { 怘 } \\
\text { 高 } \\
-\end{array}$ & 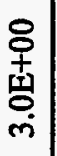 & $\begin{array}{c}8 \\
+ \\
+ \\
\text { 离 } \\
\text { i }\end{array}$ & 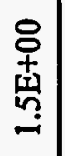 & $\begin{array}{l}\text { 号 } \\
\text { 政 } \\
\text { - }\end{array}$ \\
\hline \multicolumn{2}{|c|}{$\frac{Z}{\text { Zu }}$} & $\begin{array}{l}\frac{1}{5} \\
\frac{1}{n} \\
\frac{n}{n} \\
\frac{n}{0}\end{array}$ & $\begin{array}{l}\frac{r}{d} \\
\frac{1}{n} \\
\frac{n}{n} \\
o \\
0\end{array}$ & 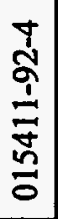 & $\begin{array}{l}\frac{n}{b} \\
\frac{1}{0} \\
\frac{0}{0} \\
\frac{0}{0}\end{array}$ & 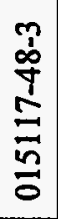 & 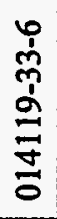 & 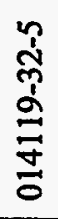 & 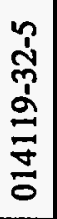 & 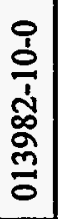 & 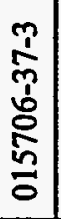 & 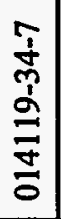 & 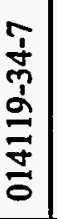 & 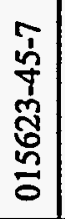 & 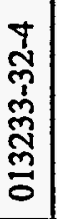 & 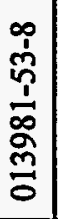 & 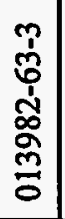 & 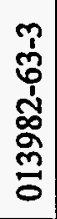 \\
\hline 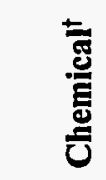 & & $\frac{5}{\vdots}$ & $\begin{array}{l}\frac{\Xi}{5} \\
\frac{a}{ \pm} \\
\frac{\hbar}{2}\end{array}$ & 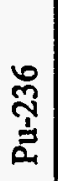 & 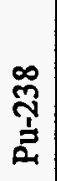 & 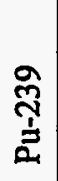 & 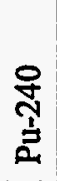 & 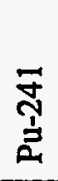 & 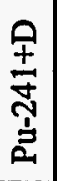 & 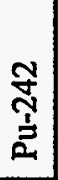 & 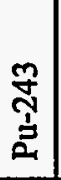 & 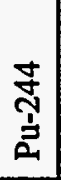 & 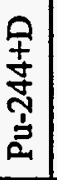 & 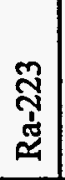 & 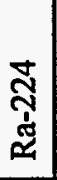 & $\begin{array}{l}\text { సે } \\
\text { 丸ે } \\
\text { జ }\end{array}$ & \begin{tabular}{l} 
స్ \\
\} $\\
{\text { జ }}$ & 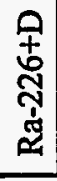 \\
\hline
\end{tabular}
\end{tabular}


Table 3.3f. (continued)

\begin{tabular}{|c|c|c|c|c|c|c|c|c|c|c|}
\hline \multirow[t]{2}{*}{ Chemical $^{t}$} & \multirow[t]{2}{*}{ CASRN } & \multicolumn{2}{|c|}{$\begin{array}{c}\text { Vegetable Intercept } \\
(\mathrm{pCi} / \mathrm{g})\end{array}$} & \multirow[t]{2}{*}{ Slope } & \multicolumn{2}{|c|}{$\begin{array}{l}\text { Beef Intercept } \\
\text { (pCi/g) }\end{array}$} & \multirow[t]{2}{*}{ Slope } & \multicolumn{2}{|c|}{$\begin{array}{l}\text { Milk Intercept } \\
\quad(\mathrm{pCi} / \mathrm{g})\end{array}$} & \multirow[t]{2}{*}{ Slope } \\
\hline & & $10^{-4}$ & $10^{-6}$ & & $10^{-4}$ & $10^{-6}$ & & $10^{-4}$ & $10^{-6}$ & \\
\hline $\mathrm{Ra}-228$ & $015262-20-1$ & $1.8 \mathrm{E}+00$ & $1.8 \mathrm{E}-02$ & $-2.4 \mathrm{E}+01$ & $1.8 \mathrm{E}+02$ & $1.8 \mathrm{E}+00$ & $-1.6 \mathrm{E}+01$ & $2.1 \mathrm{E}+01$ & $2.1 \mathrm{E}-01$ & $-1.8 \mathrm{E}+01$ \\
\hline $\mathrm{Ra}-228+\mathrm{D}$ & $015262-20-1$ & $1.8 \mathrm{E}+00$ & $1.8 \mathrm{E}-02$ & $-2.4 \mathrm{E}+01$ & $1.8 \mathrm{E}+02$ & $1.8 \mathrm{E}+00$ & $-1.6 \mathrm{E}+01$ & $2.0 \mathrm{E}+01$ & $2.0 \mathrm{E}-01$ & $-1.8 \mathrm{E}+01$ \\
\hline $\mathrm{Rb}-82$ & 014391-63-0 & $3.2 E+04$ & $3.2 \mathrm{E}+02$ & $-6.8 \mathrm{E}-04$ & $1.4 \mathrm{E}+05$ & $1.4 \mathrm{E}+03$ & $-5.9 \mathrm{E}+00$ & $1.8 E+04$ & $1.8 \mathrm{E}+02$ & $-6.2 E+00$ \\
\hline $\mathrm{Rb}-86$ & 014932-53-7 & $4.8 \mathrm{E}+01$ & $4.8 \mathrm{E}-01$ & $-6.3 E+00$ & $2.1 E+02$ & $2.1 \mathrm{E}+00$ & $-5.9 \mathrm{E}+00$ & 2.7E+01 & 2.7E-01 & $-6.2 E+00$ \\
\hline $\mathrm{Rb}-87$ & 013982-13-3 & $9.2 \mathrm{E}+01$ & $9.2 \mathrm{E}-01$ & $-4.6 \mathrm{E}+01$ & $4.1 \mathrm{E}+02$ & 4.1E+00 & $-5.9 \mathrm{E}+00$ & $5.2 \mathrm{E}+01$ & $5.2 \mathrm{E}-01$ & $-6.2 \mathrm{E}+00$ \\
\hline $\mathrm{Rb}-88$ & $014928-36-0$ & $2.3 E+03$ & $2.3 \mathrm{E}+01$ & $-9.7 \mathrm{E}-03$ & $1.0 \mathrm{E}+04$ & $1.0 \mathrm{E}+02$ & $-5.9 \mathrm{E}+00$ & $1.3 E+03$ & $1.3 \mathrm{E}+01$ & $-6.2 \mathrm{E}+00$ \\
\hline $\mathrm{Rb}-89$ & 014191-65-2 & $3.9 E+03$ & $3.9 \mathrm{E}+01$ & $-8.4 \mathrm{E}-03$ & $1.7 \mathrm{E}+04$ & $1.7 \mathrm{E}+02$ & $-5.9 \mathrm{E}+00$ & $2.2 \mathrm{E}+03$ & $2.2 \mathrm{E}+01$ & $-6.2 E+00$ \\
\hline $\mathrm{Rh}-103 \mathrm{~m}$ & $007440-16-6$ & $4.2 \mathrm{E}+04$ & $4.2 \mathrm{E}+02$ & $-3.1 \mathrm{E}-02$ & $9.2 \mathrm{E}+05$ & $9.2 \mathrm{E}+03$ & $-5.9 \mathrm{E}+00$ & $2.8 \mathrm{E}+04$ & $2.8 \mathrm{E}+02$ & $-6.2 \mathrm{E}+00$ \\
\hline Rh-105 & 014913-89-4 & $1.8 \mathrm{E}+02$ & $1.8 \mathrm{E}+00$ & $-1.1 \mathrm{E}+00$ & $3.9 \mathrm{E}+03$ & $3.9 \mathrm{E}+01$ & $-5.9 \mathrm{E}+00$ & $1.2 \mathrm{E}+02$ & $1.2 \mathrm{E}+00$ & $-6.2 \mathrm{E}+00$ \\
\hline $\mathrm{Rh}-105 \mathrm{~m}$ & $014913-89-4$ & $3.1 E+05$ & $3.1 \mathrm{E}+03$ & $-4.1 \mathrm{E}-04$ & $7.0 \mathrm{E}+06$ & $7.0 \mathrm{E}+04$ & $-5.9 \mathrm{E}+00$ & $2.1 E+05$ & $2.1 \mathrm{E}+03$ & $-6.2 \mathrm{E}+00$ \\
\hline $\mathrm{Rh}-106$ & $014234-34-5$ & $9.4 \mathrm{E}+04$ & $9.4 \mathrm{E}+02$ & $-2.7 \mathrm{E}-04$ & $2.1 E+06$ & $2.1 \mathrm{E}+04$ & $-5.9 \mathrm{E}+00$ & $6.3 E+04$ & $6.3 \mathrm{E}+02$ & $-6.2 E+00$ \\
\hline $\mathrm{Rn}-219$ & $014835-02-0$ & & & & & & & & & \\
\hline$R n-220$ & $022481-48-7$ & & & & & & & & & \\
\hline $\mathrm{Rn}-222+\mathrm{D}$ & $014859-67-7$ & & & & & & & & & \\
\hline $\mathrm{Ru}-103$ & $013968-53-1$ & $1.3 E+02$ & $1.3 \mathrm{E}+00$ & $-1.0 \mathrm{E}+01$ & $1.9 \mathrm{E}+02$ & $1.9 \mathrm{E}+00$ & $-1.3 \mathrm{E}+01$ & $4.7 \mathrm{E}+05$ & $4.7 E+03$ & $-1.4 E+01$ \\
\hline $\mathrm{Ru}-105$ & $014331-95-4$ & $3.6 \mathrm{E}+02$ & $3.6 \mathrm{E}+00$ & $-1.8 \mathrm{E}-01$ & $5.5 \mathrm{E}+02$ & $5.5 \mathrm{E}+00$ & $-1.3 \mathrm{E}+01$ & $1.4 \mathrm{E}+06$ & $1.4 \mathrm{E}+04$ & $-1.4 \mathrm{E}+01$ \\
\hline $\mathrm{Ru}-106$ & 013967-48-1 & $1.2 \mathrm{E}+01$ & $1.2 \mathrm{E}-01$ & $-1.4 \mathrm{E}+01$ & $1.8 \mathrm{E}+01$ & $1.8 \mathrm{E}-01$ & $-1.3 E+01$ & $4.5 \mathrm{E}+04$ & $4.5 E+02$ & $-1.4 \mathrm{E}+01$ \\
\hline
\end{tabular}


Table 3.3f. (continued)

\begin{tabular}{|c|c|c|c|c|c|c|c|c|c|c|}
\hline \multirow[t]{2}{*}{ Chemical $^{\dagger}$} & \multirow[t]{2}{*}{ CASRN } & \multicolumn{2}{|c|}{$\begin{array}{l}\text { Vegetable Intercept } \\
\text { (pCi/g) }\end{array}$} & \multirow{2}{*}{ Slope } & \multicolumn{2}{|c|}{$\begin{array}{l}\text { Beef Intercept } \\
\text { (pCi/g) }\end{array}$} & \multirow{2}{*}{ Slope } & \multicolumn{2}{|c|}{$\begin{array}{l}\text { Milk Intercept } \\
\quad(\mathrm{pCi} / \mathrm{g})\end{array}$} & \multirow[t]{2}{*}{ Slope } \\
\hline & & $10^{-4}$ & $10^{-6}$ & & $10^{-4}$ & $10^{-6}$ & & $10^{-4}$ & $10^{-6}$ & \\
\hline $\mathrm{Ru}-106+\mathrm{D}$ & 013967-48-1 & $9.2 \mathrm{E}+00$ & $9.2 \mathrm{E}-02$ & $-1.1 \mathrm{E}+01$ & $7.1 \mathrm{E}+00$ & 7.1E-02 & $-5.1 E+00$ & $5.7 \mathrm{E}+00$ & $5.7 \mathrm{E}-02$ & $-5.3 \mathrm{E}+00$ \\
\hline Ru-97 & 015758-35-7 & 7.1E+02 & $7.1 \mathrm{E}+00$ & $-2.3 \mathrm{E}+00$ & $1.1 \mathrm{E}+03$ & $1.1 \mathrm{E}+01$ & $-1.3 \mathrm{E}+01$ & $2.7 \mathrm{E}+06$ & $2.7 \mathrm{E}+04$ & $-1.4 \mathrm{E}+01$ \\
\hline S-35 & $015117-53-0$ & $3.3 \mathrm{E}+02$ & $3.3 \mathrm{E}+00$ & $-4.2 \mathrm{E}+00$ & $9.8 \mathrm{E}+01$ & $9.8 \mathrm{E}-01$ & $-3.2 \mathrm{E}+00$ & $1.8 \mathrm{E}+02$ & $1.8 \mathrm{E}+00$ & $-3.3 E+00$ \\
\hline Sb-122 & $014374-79-9$ & $5.0 \mathrm{E}+01$ & $5.0 \mathrm{E}-01$ & $-2.3 E+00$ & $1.2 \mathrm{E}+05$ & $1.2 \mathrm{E}+03$ & $-1.7 \mathrm{E}+01$ & $3.2 \mathrm{E}+04$ & $3.2 \mathrm{E}+02$ & $-1.9 \mathrm{E}+01$ \\
\hline Sb-124 & $014683-10-4$ & $4.1 \mathrm{E}+01$ & 4.1E-01 & $-1.2 \mathrm{E}+01$ & $1.0 \mathrm{E}+05$ & $1.0 \mathrm{E}+03$ & $-1.7 \mathrm{E}+01$ & $2.6 \mathrm{E}+04$ & $2.6 \mathrm{E}+02$ & $-1.9 \mathrm{E}+01$ \\
\hline Sb-125 & 014234-35-6 & $1.5 \mathrm{E}+02$ & $1.5 \mathrm{E}+00$ & $-1.9 \mathrm{E}+01$ & $3.6 \mathrm{E}+05$ & $3.6 \mathrm{E}+03$ & $-1.7 \mathrm{E}+01$ & $9.5 \mathrm{E}+04$ & $9.5 \mathrm{E}+02$ & $-1.9 \mathrm{E}+01$ \\
\hline$S b-125+D$ & 014234-35-6 & $3.5 \mathrm{E}+01$ & $3.5 \mathrm{E}-01$ & $-9.0 \mathrm{E}+00$ & $1.1 \mathrm{E}+02$ & $1.1 \mathrm{E}+00$ & $-1.0 \mathrm{E}+00$ & $2.3 E+02$ & $2.3 \mathrm{E}+00$ & $-1.0 \mathrm{E}+00$ \\
\hline Sb-126 & $015756-32-8$ & $4.5 \mathrm{E}+01$ & 4.5E-01 & $-6.7 \mathrm{E}+00$ & $1.1 \mathrm{E}+05$ & $1.1 \mathrm{E}+03$ & $-1.7 \mathrm{E}+01$ & $2.9 E+04$ & $2.9 \mathrm{E}+02$ & $-1.9 \mathrm{E}+01$ \\
\hline $\mathrm{Sb}-126 \mathrm{~m}$ & $015756-32-8$ & $6.1 \mathrm{E}+03$ & $6.1 \mathrm{E}+01$ & $-1.3 \mathrm{E}-02$ & $1.5 \mathrm{E}+07$ & $1.5 E+05$ & $-1.7 \mathrm{E}+01$ & $3.9 \mathrm{E}+06$ & $3.9 \mathrm{E}+04$ & $-1.9 \mathrm{E}+01$ \\
\hline Sb-127 & 013968-50-8 & $5.2 \mathrm{E}+01$ & $5.2 \mathrm{E}-01$ & $-3.1 \mathrm{E}+00$ & $1.3 \mathrm{E}+05$ & $1.3 E+03$ & $-1.7 \mathrm{E}+01$ & $3.3 E+04$ & $3.3 E+02$ & $-1.9 E+01$ \\
\hline Sb-129 & $014331-88-5$ & $2.4 \mathrm{E}+02$ & $2.4 \mathrm{E}+00$ & $-1.8 \mathrm{E}-01$ & $5.8 \mathrm{E}+05$ & $5.8 \mathrm{E}+03$ & $-1.7 E+01$ & $1.5 \mathrm{E}+05$ & $1.5 \mathrm{E}+03$ & $-1.9 E+01$ \\
\hline Sc-46 & 013967-63-0 & $7.9 \mathrm{E}+01$ & $7.9 \mathrm{E}-01$ & $-1.3 E+01$ & $4.1 \mathrm{E}+03$ & $4.1 \mathrm{E}+01$ & $-1.9 \mathrm{E}+01$ & $2.3 E+04$ & $2.3 \mathrm{E}+02$ & $-2.1 \mathrm{E}+01$ \\
\hline Sc- 47 & 014391-96-9 & $1.5 \mathrm{E}+02$ & $1.5 \mathrm{E}+00$ & $-2.9 E+00$ & $8.0 \mathrm{E}+03$ & $8.0 \mathrm{E}+01$ & $-1.9 \mathrm{E}+01$ & $4.4 \mathrm{E}+04$ & $4.4 \mathrm{E}+02$ & $-2.1 \mathrm{E}+01$ \\
\hline Sc- 48 & 014391-86-7 & $6.8 \mathrm{E}+01$ & $6.8 \mathrm{E}-01$ & $-1.7 E+00$ & $3.6 \mathrm{E}+03$ & $3.6 \mathrm{E}+01$ & $-1.9 \mathrm{E}+01$ & $2.0 \mathrm{E}+04$ & $2.0 \mathrm{E}+02$ & $-2.1 E+01$ \\
\hline Se-75 & $014265-71-5$ & $5.1 \mathrm{E}+01$ & $5.1 \mathrm{E}-01$ & $-9.9 \mathrm{E}+00$ & $3.3 \mathrm{E}+01$ & $3.3 \mathrm{E}-01$ & $-8.5 \mathrm{E}+00$ & $5.1 \mathrm{E}+01$ & $5.1 \mathrm{E}-01$ & $-9.1 E+00$ \\
\hline Si-31 & $014276-49-4$ & $6.8 \mathrm{E}+02$ & $6.8 \mathrm{E}+00$ & $-8.6 \mathrm{E}-02$ & $1.3 \mathrm{E}+06$ & $1.3 \mathrm{E}+04$ & $-1.0 \mathrm{E}+01$ & $4.0 \mathrm{E}+05$ & $4.0 \mathrm{E}+03$ & $-1.1 \mathrm{E}+01$ \\
\hline Sm-147 & $014392-33-7$ & $1.8 \mathrm{E}+01$ & $1.8 \mathrm{E}-01$ & $-5.0 \mathrm{E}+01$ & $3.8 \mathrm{E}+02$ & $3.8 \mathrm{E}+00$ & $-1.9 \mathrm{E}+01$ & $1.6 \mathrm{E}+04$ & $1.6 \mathrm{E}+02$ & $-2.1 E+01$ \\
\hline
\end{tabular}


Table 3.3f. (continued)

\begin{tabular}{|c|c|c|c|c|c|c|c|c|c|c|}
\hline \multirow[t]{2}{*}{ Chemical $^{\dagger}$} & \multirow{2}{*}{ CASRN } & \multicolumn{2}{|c|}{$\begin{array}{c}\text { Vegetable Intercept } \\
(\mathrm{pCi} / \mathrm{g})\end{array}$} & \multirow{2}{*}{ Slope } & \multicolumn{2}{|c|}{$\begin{array}{c}\text { Beef Intercept } \\
\text { (pCi/g) }\end{array}$} & \multirow{2}{*}{ Slope } & \multicolumn{2}{|c|}{$\begin{array}{l}\text { Milk Intercept } \\
\text { (pCi/g) }\end{array}$} & \multirow{2}{*}{ Slope } \\
\hline & & $10^{-4}$ & $10^{-6}$ & & $10^{-4}$ & $10^{-6}$ & & $10^{-4}$ & $10^{-6}$ & \\
\hline Sm-151 & $015715-94-3$ & $9.9 \mathrm{E}+02$ & $9.9 \mathrm{E}+00$ & $-4.6 \mathrm{E}+01$ & $2.1 \mathrm{E}+04$ & $2.1 E+02$ & $-1.9 \mathrm{E}+01$ & $8.5 E+05$ & $8.5 E+03$ & $-2.1 \mathrm{E}+01$ \\
\hline Sm-153 & $015766-00-4$ & $1.1 \mathrm{E}+02$ & $1.1 \mathrm{E}+00$ & $-1.8 \mathrm{E}+00$ & $2.4 \mathrm{E}+03$ & $2.4 \mathrm{E}+01$ & $-1.9 E+01$ & $9.8 \mathrm{E}+04$ & $9.8 \mathrm{E}+02$ & $-2.1 \mathrm{E}+01$ \\
\hline Sn-113 & $013966-06-8$ & $5.7 \mathrm{E}+01$ & $5.7 \mathrm{E}-01$ & $-6.5 E+00$ & $3.8 \mathrm{E}+02$ & $3.8 \mathrm{E}+00$ & $-5.5 \mathrm{E}+00$ & $5.7 \mathrm{E}+02$ & $5.7 \mathrm{E}+00$ & $-5.7 E+00$ \\
\hline Sn-121 & 014683-06-8 & $1.7 \mathrm{E}+02$ & $1.7 \mathrm{E}+00$ & $-5.2 \mathrm{E}-01$ & $1.1 \mathrm{E}+03$ & $1.1 \mathrm{E}+01$ & $-5.5 \mathrm{E}+00$ & $1.7 \mathrm{E}+03$ & $1.7 \mathrm{E}+01$ & $-5.7 \mathrm{E}+00$ \\
\hline Sn-121m & $014683-06-8$ & $1.1 \mathrm{E}+02$ & $1.1 \mathrm{E}+00$ & $-3.7 \mathrm{E}+01$ & $7.0 \mathrm{E}+02$ & $7.0 \mathrm{E}+00$ & $-5.5 \mathrm{E}+00$ & $1.1 \mathrm{E}+03$ & $1.1 \mathrm{E}+01$ & $-5.7 E+00$ \\
\hline $\mathrm{Sn}-125$ & 014683-08-0 & $1.3 E+01$ & $1.3 \mathrm{E}-01$ & $-2.8 \mathrm{E}+00$ & $8.3 E+01$ & $8.3 \mathrm{E}-01$ & $-5.5 E+00$ & $1.3 \mathrm{E}+02$ & $1.3 \mathrm{E}+00$ & $-5.7 E+00$ \\
\hline $\mathrm{Sn}-126$ & $015832-50-5$ & $1.0 \mathrm{E}+01$ & $1.0 \mathrm{E}-01$ & $-4.2 \mathrm{E}+01$ & $6.6 \mathrm{E}+01$ & $6.6 \mathrm{E}-01$ & $-5.5 \mathrm{E}+00$ & $9.9 \mathrm{E}+01$ & $9.9 \mathrm{E}-01$ & $-5.7 \mathrm{E}+00$ \\
\hline Sr-82 & $014809-50-8$ & $9.9 \mathrm{E}+00$ & $9.9 \mathrm{E}-02$ & $-5.4 \mathrm{E}+00$ & $6.3 E+01$ & $6.3 \mathrm{E}-01$ & $-5.1 E+00$ & $2.7 \mathrm{E}+01$ & 2.7E-01 & $-5.3 E+00$ \\
\hline Sr-85 & $013967-73-2$ & $1.8 \mathrm{E}+02$ & $1.8 \mathrm{E}+00$ & $-7.0 \mathrm{E}+00$ & $1.2 \mathrm{E}+03$ & $1.2 \mathrm{E}+01$ & $-5.1 \mathrm{E}+00$ & $5.0 \mathrm{E}+02$ & $5.0 \mathrm{E}+00$ & $-5.3 E+00$ \\
\hline $\mathrm{Sr}-85 \mathrm{~m}$ & $013967-73-2$ & $1.4 \mathrm{E}+04$ & $1.4 \mathrm{E}+02$ & $-2.8 \mathrm{E}-02$ & $9.0 \mathrm{E}+04$ & $9.0 \mathrm{E}+02$ & $-5.1 E+00$ & $3.9 \mathrm{E}+04$ & $3.9 \mathrm{E}+02$ & $-5.3 \mathrm{E}+00$ \\
\hline Sr-89 & $014158-27-1$ & $2.5 \mathrm{E}+01$ & 2.5E-01 & $-6.6 \mathrm{E}+00$ & $1.6 \mathrm{E}+02$ & $1.6 \mathrm{E}+00$ & $-5.1 \mathrm{E}+00$ & $6.8 \mathrm{E}+01$ & $6.8 \mathrm{E}-01$ & $-5.3 E+00$ \\
\hline Sr -90 & $010098-97-2$ & $6.3 \mathrm{E}+00$ & $6.3 \mathrm{E}-02$ & $-3.4 \mathrm{E}+01$ & $4.0 \mathrm{E}+01$ & $4.0 \mathrm{E}-01$ & $-5.1 E+00$ & $1.7 \mathrm{E}+01$ & $1.7 \mathrm{E}-01$ & $-5.3 E+00$ \\
\hline Sr-90+D & $010098-97-2$ & $4.6 \mathrm{E}+00$ & $4.6 \mathrm{E}-02$ & $-3.4 \mathrm{E}+01$ & $2.6 \mathrm{E}+01$ & $2.6 \mathrm{E}-01$ & $-5.1 E+00$ & $1.2 \mathrm{E}+01$ & $1.2 \mathrm{E}-01$ & $-5.3 E+00$ \\
\hline Sr-91 & $014331-91-0$ & $9.1 \mathrm{E}+01$ & $9.1 \mathrm{E}-01$ & $-2.3 \mathrm{E}-01$ & $5.8 \mathrm{E}+02$ & $5.8 \mathrm{E}+00$ & $-5.1 E+00$ & $2.5 \mathrm{E}+02$ & $2.5 \mathrm{E}+00$ & $-5.3 E+00$ \\
\hline Sr-92 & $014928-29-1$ & $1.3 \mathrm{E}+02$ & $1.3 \mathrm{E}+00$ & $-6.7 \mathrm{E}-02$ & $8.0 \mathrm{E}+02$ & $8.0 \mathrm{E}+00$ & $-5.1 \mathrm{E}+00$ & $3.5 \mathrm{E}+02$ & $3.5 E+00$ & $-5.3 E+00$ \\
\hline Ta-182 & $013982-00-8$ & $6.5 \mathrm{E}+01$ & $6.5 \mathrm{E}-01$ & $-1.3 \mathrm{E}+01$ & $1.1 \mathrm{E}+04$ & $1.1 E+02$ & $-1.9 \mathrm{E}+01$ & $3.7 E+05$ & 3.7E+03 & $-2.1 \mathrm{E}+01$ \\
\hline Tb-158 & $015759-55-4$ & $1.1 \mathrm{E}+02$ & $1.1 \mathrm{E}+00$ & $-4.7 \mathrm{E}+01$ & $2.5 E+03$ & $2.5 \mathrm{E}+01$ & $-1.9 \mathrm{E}+01$ & $9.3 \mathrm{E}+04$ & $9.3 E+02$ & $-2.1 E+01$ \\
\hline
\end{tabular}


Table 3.3f. (continued)

\begin{tabular}{|c|c|c|c|c|c|c|c|c|c|c|}
\hline \multirow[t]{2}{*}{ Chemical $^{\dagger}$} & \multirow[t]{2}{*}{ CASRN } & \multicolumn{2}{|c|}{$\begin{array}{c}\text { Vegetable Intercept } \\
\text { (pCi/g) }\end{array}$} & \multirow[t]{2}{*}{ Slope } & \multicolumn{2}{|c|}{$\begin{array}{l}\text { Beef Intercept } \\
\text { (pCi/g) }\end{array}$} & \multirow[t]{2}{*}{ Slope } & \multicolumn{2}{|c|}{$\begin{array}{l}\text { Milk Intercept } \\
\text { (pCi/g) }\end{array}$} & \multirow[t]{2}{*}{ Slope } \\
\hline & & $10^{-4}$ & $10^{-6}$ & & $10^{-4}$ & $10^{-6}$ & & $10^{-4}$ & $10^{-6}$ & \\
\hline Tb-160 & $013981-29-8$ & $6.0 \mathrm{E}+01$ & $6.0 \mathrm{E}-01$ & $-1.2 \mathrm{E}+01$ & $1.4 \mathrm{E}+03$ & $1.4 \mathrm{E}+01$ & $-1.9 \mathrm{E}+01$ & $5.1 \mathrm{E}+04$ & $5.1 \mathrm{E}+02$ & $-2.1 \mathrm{E}+01$ \\
\hline Tc-95 & $014809-56-4$ & $8.4 E+00$ & $8.4 \mathrm{E}-02$ & $-5.6 \mathrm{E}-03$ & $3.8 \mathrm{E}+04$ & $3.8 \mathrm{E}+02$ & $-1.0 \mathrm{E}-01$ & $3.9 \mathrm{E}+03$ & $3.9 E+01$ & $-1.0 \mathrm{E}-01$ \\
\hline Tc-95m & $014809-56-4$ & $4.6 \mathrm{E}-01$ & $4.6 \mathrm{E}-03$ & $-3.5 \mathrm{E}-01$ & $2.1 \mathrm{E}+03$ & $2.1 \mathrm{E}+01$ & $-1.0 \mathrm{E}-01$ & $2.2 E+02$ & $2.2 \mathrm{E}+00$ & $-1.0 \mathrm{E}-01$ \\
\hline Tc-96 & $014808-44-7$ & $2.5 \mathrm{E}-01$ & $2.5 \mathrm{E}-03$ & $-2.8 \mathrm{E}-02$ & $1.1 \mathrm{E}+03$ & $1.1 \mathrm{E}+01$ & $-1.0 \mathrm{E}-01$ & $1.2 \mathrm{E}+02$ & $1.2 E+00$ & $-1.0 \mathrm{E}-01$ \\
\hline Tc-96m & $014808-44-7$ & $2.2 \mathrm{E}+01$ & 2.2E-01 & $-2.4 \mathrm{E}-04$ & $9.8 \mathrm{E}+04$ & $9.8 \mathrm{E}+02$ & $-1.0 \mathrm{E}-01$ & $1.0 \mathrm{E}+04$ & $1.0 \mathrm{E}+02$ & $-1.0 \mathrm{E}-01$ \\
\hline Tc-97 & $015759-35-0$ & $3.6 \mathrm{E}+00$ & $3.6 \mathrm{E}-02$ & $-3.6 \mathrm{E}+01$ & $1.6 \mathrm{E}+04$ & $1.6 \mathrm{E}+02$ & $-1.0 \mathrm{E}-01$ & $1.7 \mathrm{E}+03$ & $1.7 \mathrm{E}+01$ & $-1.0 \mathrm{E}-01$ \\
\hline Tc-97m & 015759-35-0 & $4.8 \mathrm{E}-01$ & $4.8 \mathrm{E}-03$ & $-5.0 \mathrm{E}-01$ & $2.1 E+03$ & $2.1 \mathrm{E}+01$ & $-1.0 \mathrm{E}-01$ & $2.2 E+02$ & $2.2 E+00$ & $-1.0 \mathrm{E}-01$ \\
\hline Tc-99 & 014133-76-7 & $4.1 \mathrm{E}-01$ & $4.1 \mathrm{E}-03$ & $-3.6 \mathrm{E}+01$ & $1.8 \mathrm{E}+03$ & $1.8 \mathrm{E}+01$ & $-1.0 \mathrm{E}-01$ & $1.9 \mathrm{E}+02$ & $1.9 \mathrm{E}+00$ & $-1.0 \mathrm{E}-01$ \\
\hline Tc-99m & 014133-76-7 & $1.0 \mathrm{E}+01$ & $1.0 \mathrm{E}-01$ & $-1.7 \mathrm{E}-03$ & $4.6 E+04$ & $4.6 \mathrm{E}+02$ & $-1.0 \mathrm{E}-01$ & $4.8 \mathrm{E}+03$ & $4.8 \mathrm{E}+01$ & $-1.0 \mathrm{E}-01$ \\
\hline $\mathrm{Te}-125 \mathrm{~m}$ & 014390-73-9 & $4.9 \mathrm{E}+01$ & $4.9 \mathrm{E}-01$ & $-3.5 E+00$ & $1.5 \mathrm{E}+02$ & $1.5 \mathrm{E}+00$ & $-1.0 \mathrm{E}+00$ & $3.4 \mathrm{E}+02$ & $3.4 \mathrm{E}+00$ & $-1.1 \mathrm{E}+00$ \\
\hline Te-127 & $013981-49-2$ & $1.5 \mathrm{E}+02$ & $1.5 \mathrm{E}+00$ & $-1.1 \mathrm{E}-01$ & $4.4 \mathrm{E}+02$ & $4.4 \mathrm{E}+00$ & $-1.0 \mathrm{E}+00$ & $1.0 \mathrm{E}+03$ & $1.0 \mathrm{E}+01$ & $-1.1 \mathrm{E}+00$ \\
\hline Te-127m & $013981-49-2$ & $2.1 \mathrm{E}+01$ & 2.1E-01 & $-4.0 \mathrm{E}+00$ & $6.3 E+01$ & $6.3 \mathrm{E}-01$ & $-1.0 \mathrm{E}+00$ & $1.4 \mathrm{E}+02$ & $1.4 \mathrm{E}+00$ & $-1.1 \mathrm{E}+00$ \\
\hline Te-129 & $014269-71-7$ & $8.4 \mathrm{E}+02$ & $8.4 \mathrm{E}+00$ & $-1.4 \mathrm{E}-02$ & $2.6 \mathrm{E}+03$ & $2.6 \mathrm{E}+01$ & $-1.0 \mathrm{E}+00$ & $5.8 \mathrm{E}+03$ & $5.8 \mathrm{E}+01$ & $-1.1 E+00$ \\
\hline $\mathrm{Te}-129 \mathrm{~m}$ & $014269-71-7$ & $1.1 \mathrm{E}+01$ & $1.1 \mathrm{E}-01$ & $-3.0 \mathrm{E}+00$ & $3.2 \mathrm{E}+01$ & $3.2 \mathrm{E}-01$ & $-1.0 \mathrm{E}+00$ & $7.4 \mathrm{E}+01$ & $7.4 \mathrm{E}-01$ & $-1.1 E+00$ \\
\hline Te-131 & $014683-12-6$ & $3.2 \mathrm{E}+02$ & $3.2 \mathrm{E}+00$ & $-5.0 \mathrm{E}-03$ & $9.7 \mathrm{E}+02$ & $9.7 \mathrm{E}+00$ & $-1.0 \mathrm{E}+00$ & $2.2 \mathrm{E}+03$ & $2.2 \mathrm{E}+01$ & $-1.1 \mathrm{E}+00$ \\
\hline Te-131m & $014683-12-6$ & $1.4 \mathrm{E}+01$ & $1.4 \mathrm{E}-01$ & $-3.3 \mathrm{E}-01$ & $4.3 \mathrm{E}+01$ & $4.3 \mathrm{E}-01$ & $-1.0 \mathrm{E}+00$ & $9.8 \mathrm{E}+01$ & $9.8 \mathrm{E}-01$ & $-1.1 \mathrm{E}+00$ \\
\hline Te-132 & $014234-28-7$ & $1.0 \mathrm{E}+01$ & $1.0 \mathrm{E}-01$ & $-7.7 \mathrm{E}-01$ & $3.1 \mathrm{E}+01$ & $3.1 \mathrm{E}-01$ & $-1.0 \mathrm{E}+00$ & $7.1 \mathrm{E}+01$ & $7.1 \mathrm{E}-01$ & $-1.1 \mathrm{E}+00$ \\
\hline
\end{tabular}


Table 3.3f. (continued)

\begin{tabular}{|c|c|c|c|c|c|c|c|c|c|c|}
\hline \multirow[t]{2}{*}{ Chemical $^{\dagger}$} & \multirow[t]{2}{*}{ CASRN } & \multicolumn{2}{|c|}{$\begin{array}{c}\text { Vegetable Intercept } \\
(\mathrm{pCi} / \mathrm{g})\end{array}$} & \multirow{2}{*}{ Slope } & \multicolumn{2}{|c|}{$\begin{array}{c}\text { Beef Intercept } \\
\text { (pCi/g) }\end{array}$} & \multirow{2}{*}{ Slope } & \multicolumn{2}{|c|}{$\begin{array}{l}\text { Milk Intercept } \\
\quad \text { (pCi/g) }\end{array}$} & \multirow{2}{*}{ Slope } \\
\hline & & $10^{-4}$ & $10^{-6}$ & & $10^{-4}$ & $10^{-6}$ & & $10^{-4}$ & $10^{-6}$ & \\
\hline Th-227 & $015623-47-9$ & $1.1 \mathrm{E}+01$ & $1.1 \mathrm{E}-01$ & $-8.5 \mathrm{E}+00$ & $1.2 \mathrm{E}+04$ & $1.2 \mathrm{E}+02$ & $-1.9 \mathrm{E}+01$ & $3.9 \mathrm{E}+04$ & $3.9 \mathrm{E}+02$ & $-2.1 E+01$ \\
\hline Th-228 & $014274-82-9$ & $7.3 E+00$ & 7.3E-02 & $-1.7 \mathrm{E}+01$ & $7.5 E+03$ & $7.5 \mathrm{E}+01$ & $-1.9 \mathrm{E}+01$ & $2.5 \mathrm{E}+04$ & $2.5 \mathrm{E}+02$ & $-2.1 E+01$ \\
\hline Th-228+D & $014274-82-9$ & $1.8 \mathrm{E}+00$ & $1.8 \mathrm{E}-02$ & $-1.6 \mathrm{E}+01$ & $2.0 \mathrm{E}+00$ & $2.0 \mathrm{E}-02$ & $-9.6 \mathrm{E}+00$ & $3.4 \mathrm{E}+00$ & $3.4 \mathrm{E}-02$ & $-1.0 \mathrm{E}+01$ \\
\hline Th-229 & 015594-54-4 & $8.1 E+00$ & 8.1E-02 & $-5.0 \mathrm{E}+01$ & $8.4 \mathrm{E}+03$ & $8.4 \mathrm{E}+01$ & $-1.9 \mathrm{E}+01$ & $2.8 \mathrm{E}+04$ & $2.8 \mathrm{E}+02$ & $-2.1 E+01$ \\
\hline Th-229+D & 015594-54-4 & 6.1E-01 & $6.1 \mathrm{E}-03$ & $-4.2 \mathrm{E}+01$ & $1.6 \mathrm{E}+00$ & $1.6 \mathrm{E}-02$ & $-4.3 E+00$ & $1.4 \mathrm{E}-01$ & $1.4 \mathrm{E}-03$ & $-4.5 \mathrm{E}+00$ \\
\hline Th-230 & 014269-63-7 & $1.2 \mathrm{E}+01$ & $1.2 \mathrm{E}-01$ & $-5.0 \mathrm{E}+01$ & $1.3 \mathrm{E}+04$ & $1.3 \mathrm{E}+02$ & $-1.9 \mathrm{E}+01$ & $4.2 E+04$ & $4.2 \mathrm{E}+02$ & $-2.1 \mathrm{E}+01$ \\
\hline Th-231 & 014932-40-2 & $2.6 \mathrm{E}+02$ & $2.6 \mathrm{E}+00$ & $-1.0 \mathrm{E}+00$ & $2.6 \mathrm{E}+05$ & $2.6 \mathrm{E}+03$ & $-1.9 \mathrm{E}+01$ & $8.7 E+05$ & $8.7 E+03$ & $-2.1 E+01$ \\
\hline Th-232 & $007440-29-1$ & $1.4 \mathrm{E}+01$ & $1.4 \mathrm{E}-01$ & $-5.0 \mathrm{E}+01$ & $1.4 \mathrm{E}+04$ & $1.4 \mathrm{E}+02$ & $-1.9 \mathrm{E}+01$ & $4.8 \mathrm{E}+04$ & $4.8 \mathrm{E}+02$ & $-2.1 \mathrm{E}+01$ \\
\hline Th-234 & $015065-10-8$ & $2.4 E+01$ & $2.4 \mathrm{E}-01$ & $-9.4 \mathrm{E}+00$ & $2.4 \mathrm{E}+04$ & $2.4 \mathrm{E}+02$ & $-1.9 \mathrm{E}+01$ & $8.1 E+04$ & $8.1 E+02$ & $-2.1 E+01$ \\
\hline Tl-202 & $015720-57-7$ & $4.5 \mathrm{E}+02$ & $4.5 \mathrm{E}+00$ & $-6.9 \mathrm{E}+00$ & $1.2 E+03$ & $1.2 \mathrm{E}+01$ & $-1.9 \mathrm{E}+01$ & $3.9 \mathrm{E}+03$ & $3.9 \mathrm{E}+01$ & $-2.2 \mathrm{E}+01$ \\
\hline Tl-204 & 013968-51-9 & $2.3 \mathrm{E}+02$ & $2.3 E+00$ & $-2.1 \mathrm{E}+01$ & $6.1 \mathrm{E}+02$ & $6.1 \mathrm{E}+00$ & $-1.9 \mathrm{E}+01$ & $2.0 \mathrm{E}+03$ & $2.0 \mathrm{E}+01$ & $-2.2 \mathrm{E}+01$ \\
\hline Tl-208 & 014913-50-9 & $2.6 \mathrm{E}+04$ & $2.6 \mathrm{E}+02$ & $-2.2 \mathrm{E}-03$ & $6.9 E+04$ & $6.9 \mathrm{E}+02$ & $-1.9 \mathrm{E}+01$ & $2.3 E+05$ & $2.3 \mathrm{E}+03$ & $-2.2 \mathrm{E}+01$ \\
\hline Tl-209 & $015690-73-0$ & $3.3 E+04$ & $3.3 \mathrm{E}+02$ & $-1.6 \mathrm{E}-03$ & $8.6 \mathrm{E}+04$ & $8.6 \mathrm{E}+02$ & $-1.9 \mathrm{E}+01$ & $2.8 \mathrm{E}+05$ & $2.8 \mathrm{E}+03$ & $-2.2 E+01$ \\
\hline Tm-170 & $013981-30-1$ & $6.0 \mathrm{E}+01$ & $6.0 \mathrm{E}-01$ & $-1.3 E+01$ & $1.4 \mathrm{E}+03$ & $1.4 \mathrm{E}+01$ & $-1.9 E+01$ & $5.2 \mathrm{E}+04$ & $5.2 \mathrm{E}+02$ & $-2.1 E+01$ \\
\hline Tm-171 & $014333-45-0$ & 7.7E+02 & $7.7 \mathrm{E}+00$ & $-1.7 \mathrm{E}+01$ & $1.8 \mathrm{E}+04$ & $1.8 \mathrm{E}+02$ & $-1.9 \mathrm{E}+01$ & $6.7 \mathrm{E}+05$ & $6.7 \mathrm{E}+03$ & $-2.1 E+01$ \\
\hline U-232 & 014158-29-3 & $5.6 \mathrm{E}+00$ & $5.6 \mathrm{E}-02$ & $-4.5 \mathrm{E}+01$ & $1.9 E+03$ & $1.9 \mathrm{E}+01$ & $-1.8 \mathrm{E}+01$ & $2.3 \mathrm{E}+02$ & $2.3 \mathrm{E}+00$ & $-2.1 \mathrm{E}+01$ \\
\hline U-233 & 013968-55-3 & $1.0 \mathrm{E}+01$ & $1.0 \mathrm{E}-01$ & $-5.0 \mathrm{E}+01$ & $3.4 \mathrm{E}+03$ & $3.4 \mathrm{E}+01$ & $-1.8 \mathrm{E}+01$ & $4.2 \mathrm{E}+02$ & $4.2 \mathrm{E}+00$ & $-2.1 E+01$ \\
\hline
\end{tabular}




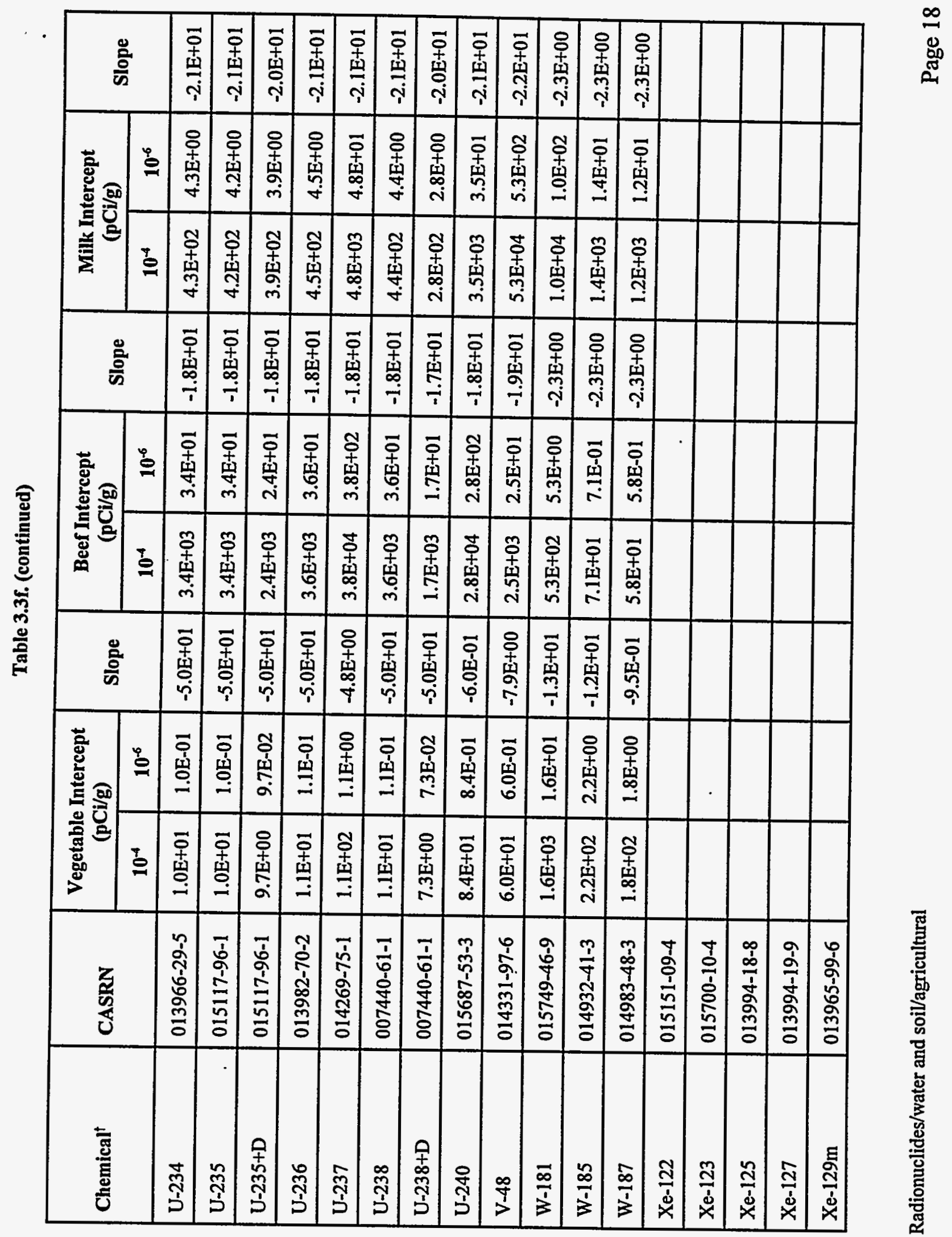


Table 3.3f. (continued)

\begin{tabular}{|c|c|c|c|c|c|c|c|c|c|c|}
\hline \multirow[t]{2}{*}{ Chemical $^{\dagger}$} & \multirow[t]{2}{*}{ CASRN } & \multicolumn{2}{|c|}{$\begin{array}{c}\text { Vegetable Intercept } \\
\text { (pCi/g) }\end{array}$} & \multirow{2}{*}{ Slope } & \multicolumn{2}{|c|}{$\begin{array}{l}\text { Beef Intercept } \\
\text { (pCi/g) }\end{array}$} & \multirow{2}{*}{ Slope } & \multicolumn{2}{|c|}{$\begin{array}{l}\text { Milk Intercept } \\
\text { (pCi/g) }\end{array}$} & \multirow{2}{*}{ Slope } \\
\hline & & $10^{-4}$ & $10^{-6}$ & & $10^{-4}$ & $10^{-6}$ & & $10^{-4}$ & $10^{-6}$ & \\
\hline $\mathrm{Xe}-13 \mathrm{~lm}$ & $014683-11-5$ & . & & & & & & & & \\
\hline $\mathrm{Xe}-133$ & $014932-42-4$ & & . & & & & & & & \\
\hline $\mathrm{Xe-133m}$ & $014932-42-4$ & & & & & & & & & \\
\hline $\mathrm{Xe}-135$ & $014995-62-1$ & & & & & & & & & \\
\hline $\mathrm{Xe}-135 \mathrm{~m}$ & $014995-62-1$ & & & & & & & & & \\
\hline Xe-137 & $014835-21-3$ & & & & & & & & & \\
\hline $\mathrm{Xe}-138$ & $015751-81-2$ & & & & & & & & & \\
\hline$Y-90$ & $010098-91-6$ & $3.0 \mathrm{E}+01$ & $3.0 \mathrm{E}-01$ & $-2.4 \mathrm{E}+00$ & $3.2 \mathrm{E}+03$ & $3.2 \mathrm{E}+01$ & $-1.9 \mathrm{E}+01$ & $2.6 \mathrm{E}+04$ & $2.6 \mathrm{E}+02$ & $-2.1 E+01$ \\
\hline Y-91 & $014234-24-3$ & $3.4 \mathrm{E}+01$ & $3.4 \mathrm{E}-01$ & $-1.2 \mathrm{E}+01$ & $3.5 E+03$ & $3.5 \mathrm{E}+01$ & $-1.9 E+01$ & $2.9 \mathrm{E}+04$ & $2.9 \mathrm{E}+02$ & $-2.1 \mathrm{E}+01$ \\
\hline$Y-91 \mathrm{~m}$ & $014234-24-3$ & $1.2 \mathrm{E}+04$ & $1.2 \mathrm{E}+02$ & $-3.6 \mathrm{E}-02$ & $1.3 E+06$ & $1.3 E+04$ & $-1.9 \mathrm{E}+01$ & $1.1 \mathrm{E}+07$ & $1.1 \mathrm{E}+05$ & $-2.1 \mathrm{E}+01$ \\
\hline$Y-92$ & $015751-59-4$ & $2.3 E+02$ & $2.3 E+00$ & $-1.5 \mathrm{E}-01$ & $2.4 \mathrm{E}+04$ & $2.4 \mathrm{E}+02$ & $-1.9 \mathrm{E}+01$ & $2.0 \mathrm{E}+05$ & $2.0 \mathrm{E}+03$ & $-2.1 \mathrm{E}+01$ \\
\hline$Y-93$ & $014981-70-5$ & $7.9 \mathrm{E}+01$ & $7.9 \mathrm{E}-01$ & $-4.3 \mathrm{E}-01$ & $8.2 \mathrm{E}+03$ & $8.2 \mathrm{E}+01$ & $-1.9 \mathrm{E}+01$ & $6.8 \mathrm{E}+04$ & $6.8 \mathrm{E}+02$ & $-2.1 E+01$ \\
\hline $\mathrm{Zn}-65$ & 013982-39-3 & $2.3 E+01$ & $2.3 \mathrm{E}-01$ & $-7.9 \mathrm{E}+00$ & $1.4 \mathrm{E}+01$ & $1.4 \mathrm{E}-01$ & $-5.5 \mathrm{E}+00$ & $2.1 E+01$ & 2.1E-01 & $-5.8 \mathrm{E}+00$ \\
\hline $\mathrm{Zn}-69$ & $013982-23-5$ & 3.7E+03 & $3.7 E+01$ & $-2.0 \mathrm{E}-02$ & $2.3 E+03$ & $2.3 \mathrm{E}+01$ & $-5.5 \mathrm{E}+00$ & $3.4 \mathrm{E}+03$ & $3.4 \mathrm{E}+01$ & $-5.8 \mathrm{E}+00$ \\
\hline $\mathrm{Zn}-69 \mathrm{~m}$ & $013982-23-5$ & $1.5 \mathrm{E}+02$ & $1.5 \mathrm{E}+00$ & $-2.9 \mathrm{E}-01$ & $9.2 \mathrm{E}+01$ & $9.2 \mathrm{E}-01$ & $-5.5 \mathrm{E}+00$ & $1.4 \mathrm{E}+02$ & $1.4 \mathrm{E}+00$ & $-5.8 E+00$ \\
\hline $\mathrm{Zr}-93$ & $015751-77-6$ & $8.8 \mathrm{E}+02$ & $8.8 E+00$ & $-5.0 \mathrm{E}+01$ & $9.3 E+07$ & $9.3 \mathrm{E}+05$ & $-1.9 \mathrm{E}+01$ & $2.8 E+07$ & $2.8 \mathrm{E}+05$ & $-2.2 \mathrm{E}+01$ \\
\hline Zr-95 & $013967-71-0$ & $1.2 \mathrm{E}+02$ & $1.2 \mathrm{E}+00$ & $-1.2 \mathrm{E}+01$ & $1.2 \mathrm{E}+07$ & $1.2 \mathrm{E}+05$ & $-1.9 \mathrm{E}+01$ & $3.7 \mathrm{E}+06$ & $3.7 E+04$ & $-2.2 E+01$ \\
\hline
\end{tabular}


Table 3.3f. (continued)

\begin{tabular}{|c|c|c|c|c|c|c|c|c|c|c|}
\hline \multirow[t]{2}{*}{ Chemical $^{\dagger}$} & \multirow[t]{2}{*}{ CASRN } & \multicolumn{2}{|c|}{$\begin{array}{c}\text { Vegetable Intercept } \\
(\mathrm{pCi} / \mathrm{g})\end{array}$} & \multirow{2}{*}{ Slope } & \multicolumn{2}{|c|}{$\begin{array}{l}\text { Beef Intercept } \\
\text { (pCi/g) }\end{array}$} & \multirow{2}{*}{ Slope } & \multicolumn{2}{|c|}{$\begin{array}{l}\text { Milk Intercept } \\
\text { (pCi/g) }\end{array}$} & \multirow{2}{*}{ Slope } \\
\hline & & $10^{-4}$ & $10^{-6}$ & & $10^{-4}$ & $10^{-6}$ & & $10^{-4}$ & $10^{-6}$ & \\
\hline Zr-97 & 014928-30-4 & $4.4 \mathrm{E}+01$ & $4.4 \mathrm{E}-01$ & $-7.1 \mathrm{E}-01$ & $4.6 \mathrm{E}+06$ & $4.6 \mathrm{E}+04$ & $-1.9 \mathrm{E}+01$ & $1.4 \mathrm{E}+06$ & $1.4 \mathrm{E}+04$ & $-2.2 \mathrm{E}+01$ \\
\hline
\end{tabular}

Note:

$\dagger$ Isotopes designated with an " $\mathrm{m}$ " indicate that the isotope is metastable; isotopes designated with a " $\mathrm{D}$ " indicate that the radionuclide slope factors include contributions from daughter products. 
Table 3.4a. Risk-based Preliminary Remediation Goals for nonradionuclides in groundwater (recreational scenario)

\begin{tabular}{|c|c|c|c|c|c|c|c|c|c|c|c|c|c|}
\hline Chemical & CASRN & \multicolumn{4}{|c|}{ Ingestion (mg/L)" } & \multicolumn{4}{|c|}{ Dermal (mg/L)" } & \multicolumn{4}{|c|}{ Ingestion + Dermal (mg/L) $)^{+\bullet}$} \\
\hline ALAR & $001596-84-5$ & & & $1.7 \mathrm{E}+03$ & $1.7 E+02$ & . & & & & & & & \\
\hline Acenaphthylene & $000208-96-8$ & & & & & & & & & & & & \\
\hline Acephate & $030560-19-1$ & $3.0 \mathrm{E}+02$ & $3.0 \mathrm{E}+00$ & $4.5 \mathrm{E}+01$ & $4.5 \mathrm{E}+00$ & $1.1 \mathrm{E}+04$ & $1.1 E+02$ & $1.6 \mathrm{E}+03$ & $1.6 \mathrm{E}+02$ & $3.0 \mathrm{E}+02$ & $3.0 \mathrm{E}+00$ & $4.4 \mathrm{E}+01$ & $4.4 \mathrm{E}+00$ \\
\hline Acetochlor & 034256-82-1 & & & $2.3 \mathrm{E}+02$ & 2.3E+01 & & & & & & & & \\
\hline Acetone & $000067-64-1$ & & & $1.1 \mathrm{E}+03$ & $1.1 \mathrm{E}+02$ & & & 4.3E+03 & $4.3 \mathrm{E}+02$ & & & $9.0 \mathrm{E}+02$ & $9.0 \mathrm{E}+01$ \\
\hline Acetone Cyanohydrin & $000075-86-5$ & & & $9.1 \mathrm{E}+00$ & $9.1 \mathrm{E}-01$ & & & & & & & & \\
\hline Acetonitrile & 000075-05-8 & & & $6.8 \mathrm{E}+01$ & $6.8 \mathrm{E}+00$ & & & $2.3 \mathrm{E}+02$ & $2.3 \mathrm{E}+01$ & & & $5.2 \mathrm{E}+01$ & $5.2 \mathrm{E}+00$ \\
\hline Acetophenone $e^{(3)}$ & $000098-86-2$ & & & $1.1 \mathrm{E}+03$ & $1.1 \mathrm{E}+02$ & & & 4.1E+02 & 4.1E+01 & & & $3.0 \mathrm{E}+02$ & $3.0 \mathrm{E}+01$ \\
\hline Acrylonitrile & $000107-13-1$ & $4.9 E+00$ & $4.9 \mathrm{E}-02$ & $1.1 \mathrm{E}+01$ & $1.1 \mathrm{E}+00$ & $7.2 \mathrm{E}+00$ & $7.2 \mathrm{E}-02$ & $1.7 \mathrm{E}+01$ & $1.7 \mathrm{E}+00$ & $2.9 \mathrm{E}+00$ & $2.9 \mathrm{E}-02$ & $6.8 \mathrm{E}+00$ & $6.8 \mathrm{E}-01$ \\
\hline Alachlor & $015972-60-8$ & $3.3 \mathrm{E}+01$ & $3.3 \mathrm{E}-01$ & $1.1 \mathrm{E}+02$ & $1.1 \mathrm{E}+01$ & $8.6 \mathrm{E}+00$ & $8.6 \mathrm{E}-02$ & $3.0 \mathrm{E}+01$ & $3.0 \mathrm{E}+00$ & $6.9 \mathrm{E}+00$ & $6.9 \mathrm{E}-02$ & $2.4 \mathrm{E}+01$ & $2.4 \mathrm{E}+00$ \\
\hline Aldicarb & $000116-06-3$ & & & $1.1 \mathrm{E}+01$ & $1.1 \mathrm{E}+00$ & & & $1.8 \mathrm{E}+01$ & $1.8 E+00$ & & & $6.9 \mathrm{E}+00$ & $6.9 \mathrm{E}-01$ \\
\hline Aldicarb Sulfone & $001646-88-4$ & & & $1.1 E+01$ & $1.1 E+00$ & & . & & & & & & \\
\hline Aldrin & $000309-00-2$ & $1.6 \mathrm{E}-01$ & $1.6 \mathrm{E}-03$ & $3.4 \mathrm{E}-01$ & $3.4 \mathrm{E}-02$ & $1.3 \mathrm{E}-01$ & $1.3 \mathrm{E}-03$ & $2.7 \mathrm{E}-01$ & $2.7 \mathrm{E}-02$ & $7.0 \mathrm{E}-02$ & 7.0E-04 & $1.5 \mathrm{E}-01$ & $1.5 \mathrm{E}-02$ \\
\hline Ally & $074223-64-6$ & & & $2.8 \mathrm{E}+03$ & $2.8 \mathrm{E}+02$ & & & $4.0 \mathrm{E}+05$ & $4.0 \mathrm{E}+04$ & & & $2.8 \mathrm{E}+03$ & $2.8 \mathrm{E}+02$ \\
\hline Allyl Alcohol & $000107-18-6$ & & & $5.7 \mathrm{E}+01$ & 5.7E+00 & & & $2.0 \mathrm{E}+02$ & 2.0E+01 & & & $4.4 \mathrm{E}+01$ & $4.4 E+00$ \\
\hline
\end{tabular}




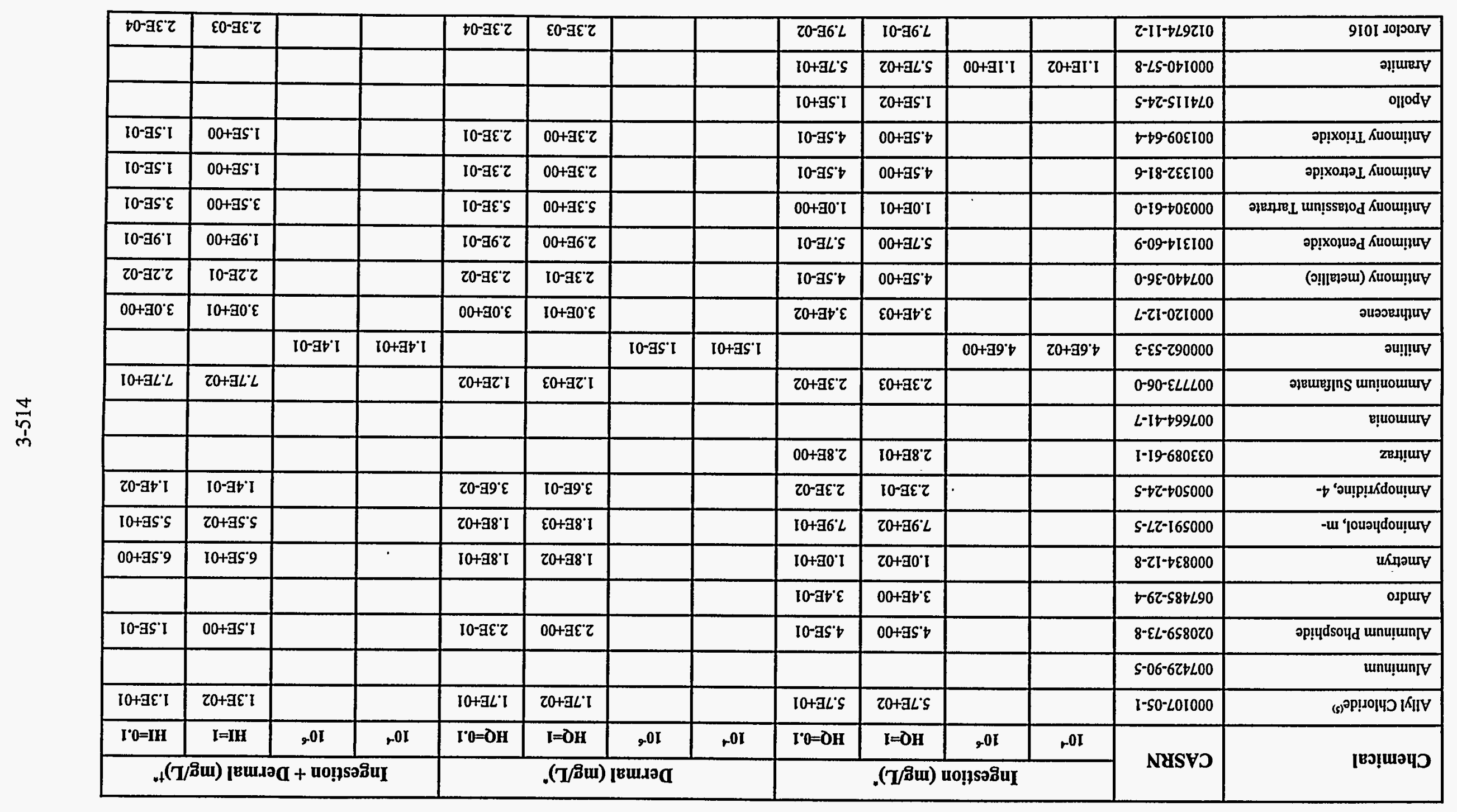

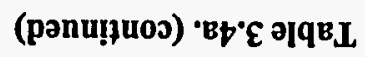


Table 3.4a. (continued)

\begin{tabular}{|c|c|c|c|c|c|c|c|c|c|c|c|c|c|}
\hline Chemical & CASRN & \multicolumn{4}{|c|}{ Ingestion (mg/L) } & \multicolumn{4}{|c|}{ Dermal (mg/L)" } & \multicolumn{4}{|c|}{ Ingestion + Dermal (mg/L) ${ }^{{ }^{\star}}$} \\
\hline Aroclor $1221^{(1)}$ & $011104-28-2$ & $3.4 \mathrm{E}-01$ & $3.4 \mathrm{E}-03$ & & & 8.7E-03 & 8.7E-05 & & & $8.4 \mathrm{E}-03$ & $8.4 \mathrm{E}-05^{\circ}$ & & \\
\hline Aroclor $1242^{(1)}$ & $053469-21-9$ & $3.4 \mathrm{E}-01$ & $3.4 \mathrm{E}-03$ & & & 2.2E-02 & 2.2E-04 & & & 2.1E-02 & 2.1E-04 & & \\
\hline Aroclor $1248^{(1)}$ & $012672-29-6$ & $3.4 \mathrm{E}-01$ & $3.4 \mathrm{E}-03$ & & & $2.2 \mathrm{E}-03$ & $2.2 \mathrm{E}-05$ & & & 2.1E-03 & 2.1E-05 & & \\
\hline Aroclor $1260^{(1)}$ & $011096-82-5$ & $3.4 \mathrm{E}-01$ & $3.4 \mathrm{E}-03$ & & & $7.5 \mathrm{E}-04$ & $7.5 \mathrm{E}-06$ & & & 7.5E-04 & 7.5E-06 & & \\
\hline Arsenic Salts & NA & & & & & & & & & & & & \\
\hline Arsenic, Inorganic & $007440-38-2$ & $1.8 \mathrm{E}+00$ & $1.8 \mathrm{E}-02$ & $3.4 \mathrm{E}+00$ & $3.4 \mathrm{E}-01$ & $1.9 \mathrm{E}+00$ & $1.9 \mathrm{E}-02$ & $3.6 \mathrm{E}+00$ & $3.6 \mathrm{E}-01$ & 9.1E-01 & $9.1 \mathrm{E}-03$ & $1.8 \mathrm{E}+00$ & $1.8 \mathrm{E}-01$ \\
\hline Arsine & $007784-42-1$ & & & & & & & & & & & & \\
\hline Asbestos & $001332-21-4$ & & & & & & & & & & & & \\
\hline Avermectin $\mathrm{BI}$ & $065195-55-3$ & & & $4.5 \mathrm{E}+00$ & 4.5E-01 & & & & & & & & \\
\hline Azobenzene & 000103-33-3 & $2.4 \mathrm{E}+01$ & $2.4 \mathrm{E}-01$ & & & 4.2E-01 & $4.2 \mathrm{E}-03$ & & & 4.1E-01 & $4.1 \mathrm{E}-03$ & & \\
\hline Barium & $007440-39-3$ & & & $7.9 \mathrm{E}+02$ & $7.9 \mathrm{E}+01$ & & & $1.4 \mathrm{E}+02$ & $1.4 \mathrm{E}+01$ & & & $1.2 E+02$ & $1.2 E+01$ \\
\hline Barium Cyanide & $000542-62-1$ & & & $1.1 \mathrm{E}+03$ & $1.1 \mathrm{E}+02$ & & & $5.9 \mathrm{E}+02$ & $5.9 \mathrm{E}+01$ & & & $3.9 \mathrm{E}+02$ & $3.9 \mathrm{E}+01$ \\
\hline Baygon & $000114-26-1$ & & & $4.5 \mathrm{E}+01$ & 4.5E+00 & & & $4.2 E+01$ & $4.2 \mathrm{E}+00$ & & & $2.2 \mathrm{E}+01$ & $2.2 \mathrm{E}+00$ \\
\hline Bayleton & $043121-43-3$ & & & $3.4 \mathrm{E}+02$ & $3.4 \mathrm{E}+01$ & & & $3.3 E+02$ & $3.3 E+01$ & & & $1.7 \mathrm{E}+02$ & $1.7 E+01$ \\
\hline Baythroid & $068359-37-5$ & & & $2.8 \mathrm{E}+02$ & $2.8 \mathrm{E}+01$ & & & & & & & & \\
\hline
\end{tabular}


Table 3.4a. (continued)

\begin{tabular}{|c|c|c|c|c|c|c|c|c|c|c|c|c|c|}
\hline Chemical & CASRN & \multicolumn{4}{|c|}{ Ingestion (mg/L) } & \multicolumn{4}{|c|}{ Dermal (mg/L)" } & \multicolumn{4}{|c|}{ Ingestion + Dermal (mg/L) ${ }^{t^{*}}$} \\
\hline Benefin & $001861-40-1$ & & & $3.4 \mathrm{E}+03$ & $3.4 \mathrm{E}+02$ & & & $1.5 E+02$ & $1.5 \mathrm{E}+01$ & & & $1.5 \mathrm{E}+02$ & $1.5 \mathrm{E}+01$ \\
\hline Bentazon & $025057-89-0$ & & & $2.8 \mathrm{E}+01$ & $2.8 \mathrm{E}+00$ & & & $9.3 E+01$ & $9.3 \mathrm{E}+00$ & & & $2.2 \mathrm{E}+01$ & $2.2 \mathrm{E}+00$ \\
\hline Benz[a]anthracene ${ }^{(2)}$ & $000056-55-3$ & $3.6 \mathrm{E}+00$ & $3.6 \mathrm{E}-02$ & & & $3.6 \mathrm{E}-03$ & $3.6 \mathrm{E}-05$ & & & $3.6 \mathrm{E}-03$ & $3.6 \mathrm{E}-05$ & & \\
\hline Benzene & $000071-43-2$ & $9.1 E+01$ & 9.1E-01 & & & $1.1 \mathrm{E}+01$ & $1.1 \mathrm{E}-01$ & & & $9.7 E+00$ & $9.7 \mathrm{E}-02$ & & \\
\hline Benzene Hexachloride & NA & & & & & & & & & & & & \\
\hline Benzene, Ethyldimethyl & NA & & & & & & & & & & & & \\
\hline Benzene, Ethylmethyl & $\mathrm{NA}$ & & & & & & & & & & & & \\
\hline Benzene, Methylpropenyl & NA & & & & & & & & & & & & \\
\hline Benzidine & $000092-87-5$ & $1.2 \mathrm{E}-02$ & $1.2 \mathrm{E}-04$ & $3.4 \mathrm{E}+01$ & $3.4 \mathrm{E}+00$ & $1.8 \mathrm{E}-02$ & $1.8 \mathrm{E}-04$ & $5.4 \mathrm{E}+01$ & $5.4 \mathrm{E}+00$ & 7.1E-03 & 7.1E-05 & $2.1 E+01$ & $2.1 \mathrm{E}+00$ \\
\hline Benzo[a]pyrene $e^{(2)}$ & $000050-32-8$ & $3.6 \mathrm{E}-01$ & $3.6 \mathrm{E}-03$ & & & $2.4 \mathrm{E}-04$ & $2.4 \mathrm{E}-06$ & & & $2.4 \mathrm{E}-04$ & $2.4 \mathrm{E}-06$ & & \\
\hline Benzo[b]fluoranthene $e^{(2)}$ & $000205-99-2$ & $3.6 \mathrm{E}+00$ & $3.6 \mathrm{E}-02$ & & & $2.4 \mathrm{E}-03$ & $2.4 \mathrm{E}-05$ & & & $2.4 \mathrm{E}-03$ & $2.4 \mathrm{E}-05$ & & \\
\hline Benzo $[\mathrm{g}, \mathrm{h}, \mathrm{i}]$ perylene & $000191-24-2$ & & & & & & & & & & & & \\
\hline Benzo[k]fluoranthene $e^{(2)}$ & $000207-08-9$ & $3.6 \mathrm{E}+01$ & $3.6 \mathrm{E}-01$ & & & $4.8 \mathrm{E}-02$ & $4.8 \mathrm{E}-04$ & & & $4.8 \mathrm{E}-02$ & $4.8 \mathrm{E}-04$ & & \\
\hline Benzoic Acid & $000065-85-0$ & & & 4.5E+04 & $4.5 \mathrm{E}+03$ & & & $1.6 \mathrm{E}+04$ & $1.6 \mathrm{E}+03$ & & & $1.2 \mathrm{E}+04$ & $1.2 \mathrm{E}+03$ \\
\hline Benzotrichloride & $000098-07-7$ & $2.0 \mathrm{E}-01$ & $2.0 \mathrm{E}-03$ & & & $1.8 \mathrm{E}-02$ & $1.8 \mathrm{E}-04$ & & & $1.6 \mathrm{E}-02$ & $1.6 \mathrm{E}-04$ & & \\
\hline
\end{tabular}


Table 3.4a. (continued)

\begin{tabular}{|c|c|c|c|c|c|c|c|c|c|c|c|c|c|}
\hline Chemical & CASRN & \multicolumn{4}{|c|}{ Ingestion (mg/L)" } & \multicolumn{4}{|c|}{ Dermal (mg/L)" } & \multicolumn{4}{|c|}{ Ingestion + Dermal (mg/L) $)^{\dagger *}$} \\
\hline Benzyl Alcohol & $000100-51-6$ & & & $3.4 E+03$ & $3.4 \mathrm{E}+02$ & & & $9.7 \mathrm{E}+02$ & $9.7 E+01$ & & & 7.5E+02 & $7.5 \mathrm{E}+01$ \\
\hline Beryllium & $007440-41-7$ & $6.2 \mathrm{E}-01$ & $6.2 \mathrm{E}-03$ & $5.7 \mathrm{E}+01$ & $5.7 \mathrm{E}+00$ & $1.6 \mathrm{E}-02$ & $1.6 \mathrm{E}-04$ & $1.5 E+00$ & $1.5 \mathrm{E}-01$ & $1.5 \mathrm{E}-02$ & $1.5 \mathrm{E}-04$ & $1.4 \mathrm{E}+00$ & $1.4 \mathrm{E}-01$ \\
\hline Bidrin & $000141-66-2$ & & & $1.1 \mathrm{E}+00$ & $1.1 \mathrm{E}-01$ & & & $6.5 \mathrm{E}+01$ & $6.5 \mathrm{E}+00$ & & & $1.1 \mathrm{E}+00$ & 1.1E-01 \\
\hline Biphenyl, 1,1'- & $000092-52-4$ & & & $5.7 \mathrm{E}+02$ & $5.7 \mathrm{E}+01$ & & & $4.1 \mathrm{E}+00$ & 4.1E-01 & & & $4.1 \mathrm{E}+00$ & 4.1E-01 \\
\hline $\begin{array}{l}\text { Bis(2-chloro-1- } \\
\text { methylethyl)ether (Technical) }\end{array}$ & $000108-60-1$ & $3.8 \mathrm{E}+01$ & $3.8 \mathrm{E}-01$ & & & $6.7 \mathrm{E}+00$ & $6.7 \mathrm{E}-02$ & & & $5.7 \mathrm{E}+00$ & $5.7 \mathrm{E}-02$ & & \\
\hline Bis(2-chloroethoxy)methane & $000111-91-1$ & & & & & & & & & & & & \\
\hline Bis(2-chloroethyl)ether & $000111-44-4$ & $2.4 \mathrm{E}+00$ & $2.4 \mathrm{E}-02$ & & & $2.4 \mathrm{E}+00$ & $2.4 \mathrm{E}-02$ & & & $1.2 \mathrm{E}+00$ & $1.2 \mathrm{E}-02$ & & \\
\hline Bisphenol A & $000080-05-7$ & & & $5.7 \mathrm{E}+02$ & $5.7 \mathrm{E}+01$ & & & $2.6 \mathrm{E}+01$ & $2.6 \mathrm{E}+00$ & & & $2.5 \mathrm{E}+01$ & $2.5 \mathrm{E}+00$ \\
\hline Boron And Borates Only & $007440-42-8$ & & & $1.0 \mathrm{E}+03$ & $1.0 \mathrm{E}+02$ & & & $2.4 \mathrm{E}+03$ & $2.4 \mathrm{E}+02$ & & & $7.1 E+02$ & 7.1E+01 \\
\hline Boron Trifluoride & $007637-07-2$ & & & & & & & & & & & & \\
\hline Bromochloromethane & $000074-97-5$ & & & & & & & & & & & & \\
\hline Bromodichloromethane & $000075-27-4$ & $4.3 \mathrm{E}+01$ & 4.3E-01 & $2.3 \mathrm{E}+02$ & $2.3 E+01$ & $1.9 \mathrm{E}+01$ & $1.9 \mathrm{E}-01$ & $9.9 \mathrm{E}+01$ & $9.9 \mathrm{E}+00$ & $1.3 \mathrm{E}+01$ & $1.3 \mathrm{E}-01$ & $6.9 \mathrm{E}+01$ & $6.9 \mathrm{E}+00$ \\
\hline Bromodiphenyl Ether, p- & $000101-55-3$ & & & & & & & & & & & & \\
\hline Bromoform & 000075-25-2 & $3.4 \mathrm{E}+02$ & $3.4 \mathrm{E}+00$ & $2.3 \mathrm{E}+02$ & $2.3 E+01$ & $2.0 \mathrm{E}+02$ & $2.0 \mathrm{E}+00$ & $1.4 \mathrm{E}+02$ & $1.4 \mathrm{E}+01$ & $1.2 \mathrm{E}+02$ & $1.2 \mathrm{E}+00$ & 8.5E+01 & $8.5 \mathrm{E}+00$ \\
\hline Bromomethane $e^{(\mathfrak{)})}$ & $000074-83-9$ & & & $1.6 \mathrm{E}+01$ & $1.6 \mathrm{E}+00$ & & & $9.4 \mathrm{E}+00$ & $9.4 \mathrm{E}-01$ & & & $5.9 \mathrm{E}+00$ & $5.9 \mathrm{E}-01$ \\
\hline
\end{tabular}

Nonradionuclides/groundwater/recreational

Page 5 of 36 
Table 3.4a. (continued)

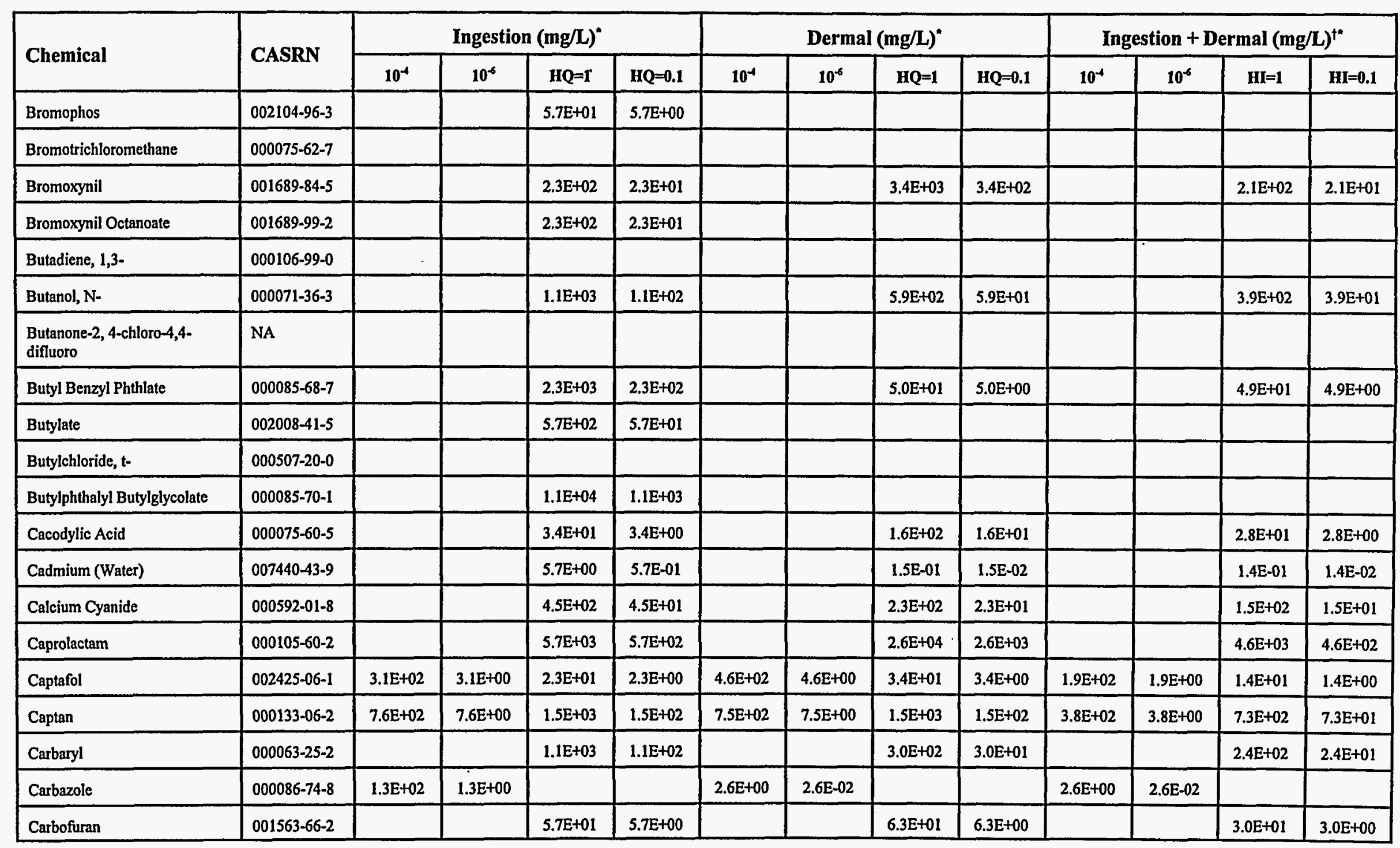


Table 3.4a. (continued)

\begin{tabular}{|c|c|c|c|c|c|c|c|c|c|c|c|c|c|}
\hline Chemical & CASRN & \multicolumn{4}{|c|}{ Ingestion (mg/L)* } & \multicolumn{4}{|c|}{ Dermal (mg/L)* } & \multicolumn{4}{|c|}{ Ingestion + Dermal (mg/L) $)^{\dagger^{*}}$} \\
\hline Carbon Disulfide & $000075-15-0$ & & & $1.1 \mathrm{E}+03$ & $1.1 \mathrm{E}+02$ & & & 7.7E+01 & 7.7E+00 & & & $7.2 \mathrm{E}+01$ & $7.2 \mathrm{E}+00$ \\
\hline Carbosulfan & $055285-14-8$ & & & $1.1 \mathrm{E}+02$ & $1.1 \mathrm{E}+01$ & & & & & & & & \\
\hline Carboxin & $005234-68-4$ & & & $1.1 \mathrm{E}+03$ & $1.1 \mathrm{E}+02$ & & & $6.8 \mathrm{E}+02$ & $6.8 \mathrm{E}+01$ & & & $4.2 \mathrm{E}+02$ & $4.2 \mathrm{E}+01$ \\
\hline Chloramben & $000133-90-4$ & & & $1.7 \mathrm{E}+02$ & $1.7 \mathrm{E}+01$ & & & & & & & & \\
\hline Chloranil & $000118-75-2$ & $6.6 \mathrm{E}+00$ & $6.6 \mathrm{E}-02$ & & & & & & & & & & \\
\hline Chlordane ${ }^{(6)}$ & $000057-74-9$ & $2.0 \mathrm{E}+00$ & $2.0 \mathrm{E}-02$ & $6.8 \mathrm{E}-01$ & $6.8 \mathrm{E}-02$ & $5.1 \mathrm{E}-02$ & $5.1 \mathrm{E}-04$ & $1.7 \mathrm{E}-02$ & $1.7 \mathrm{E}-03$ & $4.9 \mathrm{E}-02$ & $4.9 \mathrm{E}-04$ & $1.6 \mathrm{E}-02$ & $1.6 \mathrm{E}-03$ \\
\hline Chloride & $016887-00-6$ & & & & & & & & & & & & \\
\hline Chlorimuron, Ethyl- & 090982-32-4 & & & $2.3 \mathrm{E}+02$ & $2.3 \mathrm{E}+01$ & & & & & & & & \\
\hline Chloro-1,1-difluoroethane, 1- & $000075-68-3$ & & & & & & & & & & & & \\
\hline Chloro-1,3-butadiene, 2- & 000126-99-8 & & & $2.3 \mathrm{E}+02$ & $2.3 \mathrm{E}+01$ & & & $4.5 \mathrm{E}+01$ & $4.5 \mathrm{E}+00$ & & & $3.8 \mathrm{E}+01$ & $3.8 E+00$ \\
\hline Chloro-2-methylaniline $\mathrm{HCl}, 4$ - & 003165-93-3 & $5.8 \mathrm{E}+00$ & $5.8 \mathrm{E}-02$ & & & & & & & & & & \\
\hline Chloro-2-methylaniline, 4- & $000095-69-2$ & $4.6 \mathrm{E}+00$ & $4.6 \mathrm{E}-02$ & & & & & & & & & & \\
\hline Chloroacetic Acid & $000079-11-8$ & & & $2.3 \mathrm{E}+01$ & $2.3 \mathrm{E}+00$ & & & $8.1 \mathrm{E}+00$ & $8.1 \mathrm{E}-01$ & & & $6.0 \mathrm{E}+00$ & $6.0 \mathrm{E}-01$ \\
\hline Chloroacetophenone, 2- & $000532-27-4$ & & & & & & & & & & & & \\
\hline Chloroaniline, $\mathrm{p}$ - & $000106-47-8$ & & & 4.5E+01 & $4.5 \mathrm{E}+00$ & & & $1.9 \mathrm{E}+00$ & $1.9 \mathrm{E}-01$ & & & $1.8 \mathrm{E}+00$ & $1.8 \mathrm{E}-01$ \\
\hline
\end{tabular}


Table 3.4a. (continued)

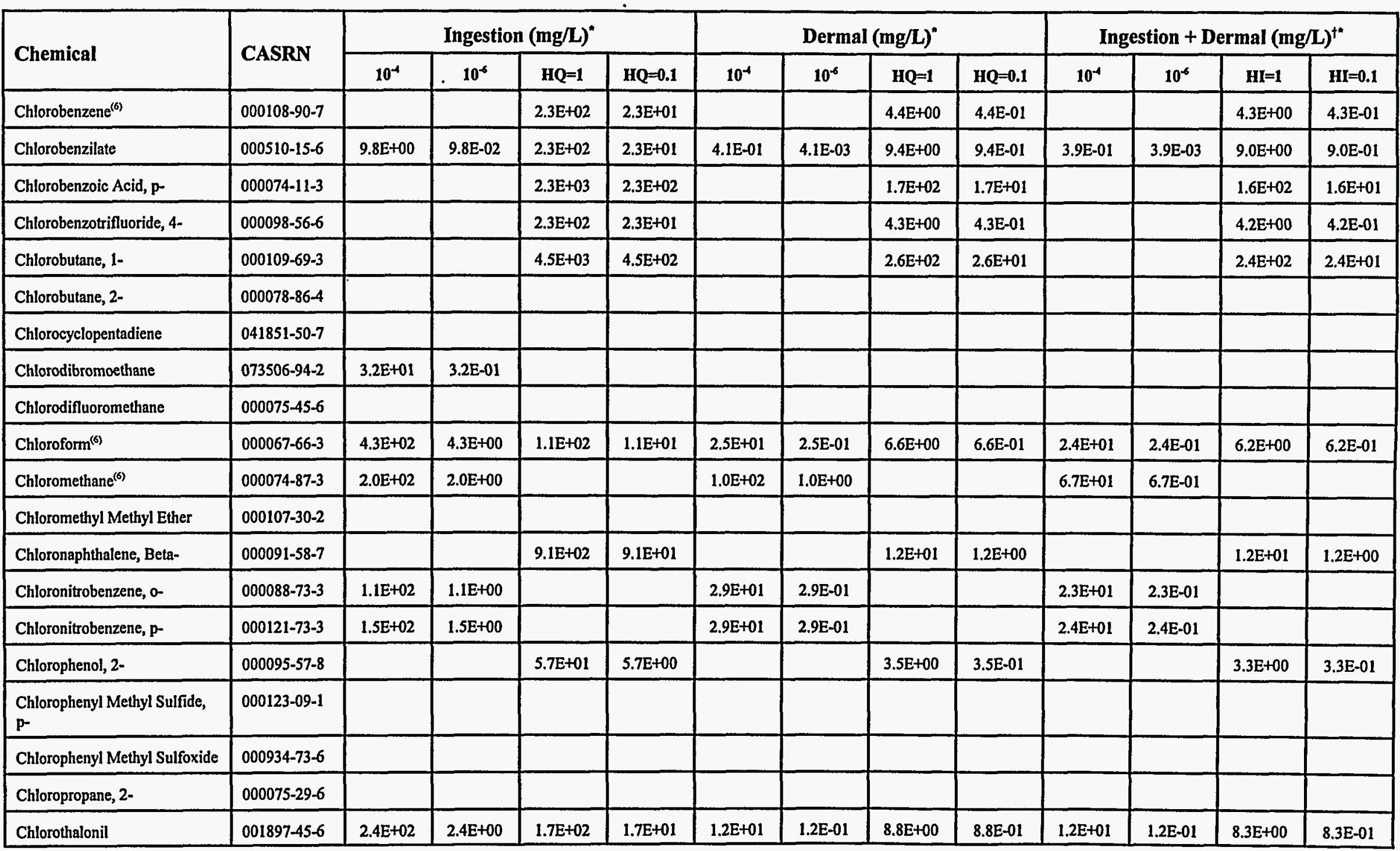


Table 3.4a. (continued)

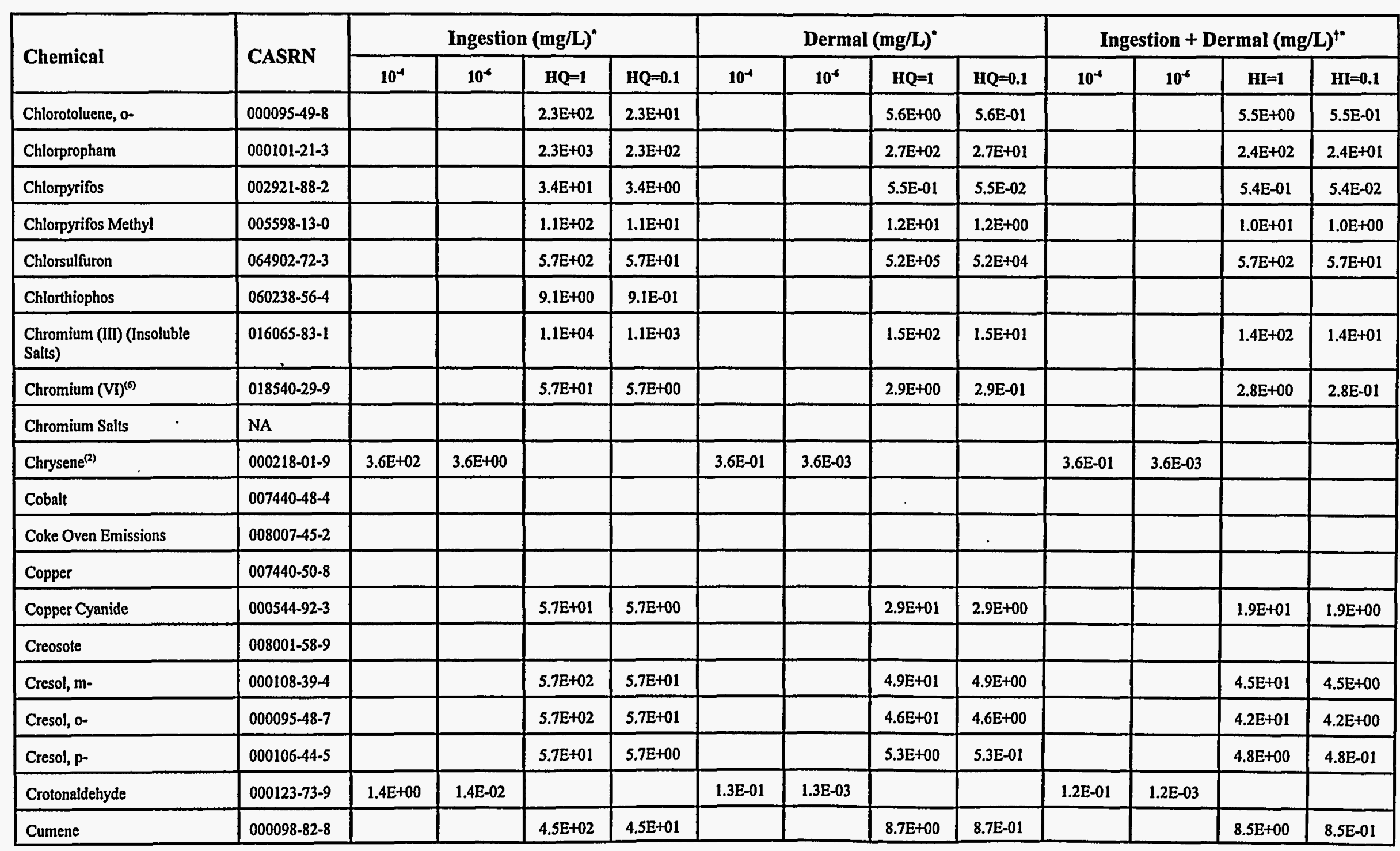

Nonradionuclides/groundwater/recreational

Page 9 of 36 


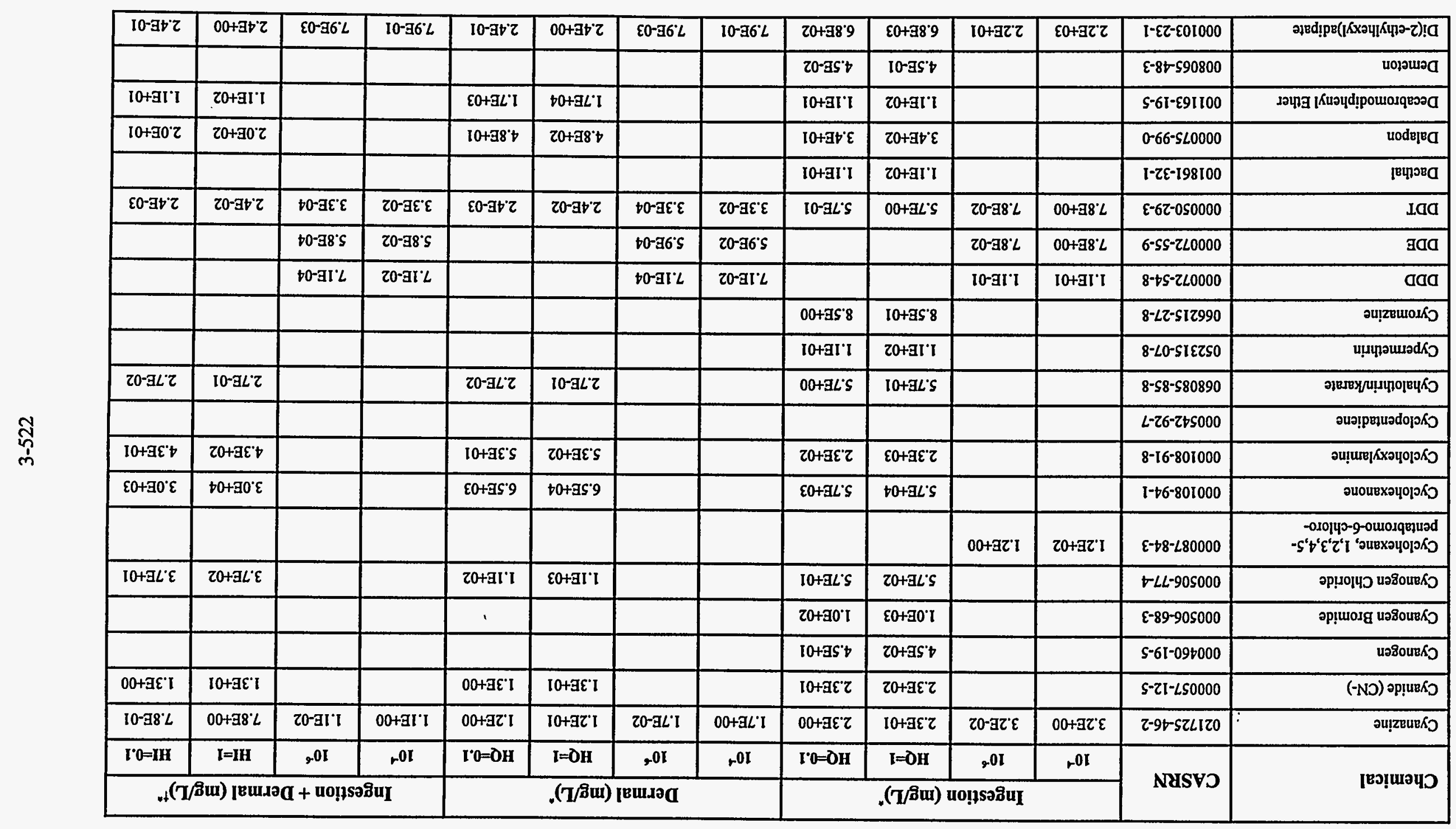

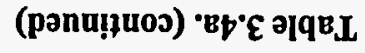


Table 3.4a. (continued)

\begin{tabular}{|c|c|c|c|c|c|c|c|c|c|c|c|c|c|}
\hline Chemical & CASRN & \multicolumn{4}{|c|}{ Ingestion (mg/L)" } & \multicolumn{4}{|c|}{ Dermal (mg/L)" } & \multicolumn{4}{|c|}{ Ingestion + Dermal $(\mathrm{mg} / \mathrm{L})^{\dagger^{\star}}$} \\
\hline Diallate & 002303-16-4 & $4.3 \mathrm{E}+01$ & $4.3 \mathrm{E}-01$ & & & $6.3 \mathrm{E}+02$ & $6.3 E+00$ & & & $4.1 \mathrm{E}+01$ & 4.1E-01 & & \\
\hline Dibenz[a,h]anthracene $e^{(2)}$ & $000053-70-3$ & $3.6 \mathrm{E}-01$ & $3.6 \mathrm{E}-03$ & & & $1.1 \mathrm{E}-04$ & $1.1 \mathrm{E}-06$ & & & $1.1 \mathrm{E}-04$ & $1.1 \mathrm{E}-06$ & & \\
\hline Dibromo-3-chloropropane, 1,2- & 000096-12-8 & $1.9 \mathrm{E}+00$ & $1.9 \mathrm{E}-02$ & & & $8.1 \mathrm{E}-01$ & 8.1E-03 & & & 5.7E-01 & $5.7 \mathrm{E}-03$ & & \\
\hline Dibromochloromethane & $000124-48-1$ & $3.2 \mathrm{E}+01$ & $3.2 \mathrm{E}-01$ & $2.3 \mathrm{E}+02$ & 2.3E+01 & $1.3 \mathrm{E}+01$ & $1.3 \mathrm{E}-01$ & $9.0 \mathrm{E}+01$ & $9.0 \mathrm{E}+00$ & $9.0 \mathrm{E}+00$ & $9.0 \mathrm{E}-02$ & $6.4 \mathrm{E}+01$ & $6.4 \mathrm{E}+00$ \\
\hline Dibromodichloromethane & 000594-18-3 & & & & & & & & & & & & \\
\hline Dibromodiphenyl Ether, p,p'- & $002050-47-7$ & & & & & & & & & & & & \\
\hline Dibromoethane, 1,2- & $000106-93-4$ & $3.1 \mathrm{E}-02$ & 3.1E-04 & & & $1.9 \mathrm{E}-02$ & $1.9 \mathrm{E}-04$ & & & $1.2 \mathrm{E}-02$ & $1.2 \mathrm{E}-04$ & & \\
\hline $\begin{array}{l}\text { Dibromomethane (Methylene } \\
\text { Bromide) }\end{array}$ & $000074-95-3$ & & & $1.1 \mathrm{E}+02$ & $1.1 \mathrm{E}+01$ & & & $2.0 \mathrm{E}+02$ & $2.0 \mathrm{E}+01$ & & & $7.2 \mathrm{E}+01$ & $7.2 \mathrm{E}+00$ \\
\hline \multicolumn{14}{|l|}{ Dichloroacetic Acid } \\
\hline Dichlorobenzene, $1,2^{-(3)}$ & $000095-50-1$ & & & $1.0 \mathrm{E}+03$ & $1.0 \mathrm{E}+02$ & & & $3.5 \mathrm{E}+01$ & $3.5 \mathrm{E}+00$ & & & $3.3 \mathrm{E}+01$ & $3.3 \mathrm{E}+00$ \\
\hline Dichlorobenzene, 1,3- & $000541-73-1$ & & & & & & & & & & & & \\
\hline Dichlorobenzene, 1,4- & $000106-46-7$ & $1.1 \mathrm{E}+02$ & $1.1 \mathrm{E}+00$ & & & $4.1 \mathrm{E}+00$ & 4.1E-02 & & & $4.0 \mathrm{E}+00$ & $4.0 \mathrm{E}-02$ & & \\
\hline Dichlorobenzidine, 3,3'- & $000091-94-1$ & $5.9 \mathrm{E}+00$ & $5.9 \mathrm{E}-02$ & & & 4.5E-01 & 4.5E-03 & & & $4.1 \mathrm{E}-01$ & 4.1E-03 & & \\
\hline Dichlorodifluoromethane & $000075-71-8$ & & & $2.3 \mathrm{E}+03$ & $2.3 \mathrm{E}+02$ & & & $1.1 \mathrm{E}+02$ & $1.1 \mathrm{E}+01$ & & & $1.1 \mathrm{E}+02$ & $1.1 E+01$ \\
\hline Dichloroethane, 1,1- & $000075-34-3$ & & & $1.1 E+03$ & $1.1 \mathrm{E}+02$ & & & $3.3 \mathrm{E}+02$ & $3.3 \mathrm{E}+01$ & & & $2.5 \mathrm{E}+02$ & $2.5 \mathrm{E}+01$ \\
\hline
\end{tabular}


Table 3.4a. (continued)

\begin{tabular}{|c|c|c|c|c|c|c|c|c|c|c|c|c|c|}
\hline Chemical & CASRN & \multicolumn{4}{|c|}{ Ingestion (mg/L)" } & \multicolumn{4}{|c|}{ Dermal (mg/L)" } & \multicolumn{4}{|c|}{ Ingestion + Dermal (mg/L) ${ }^{\dagger}$} \\
\hline Dichloroethane, 1,2- & $000107-06-2$ & $2.9 \mathrm{E}+01$ & $2.9 \mathrm{E}-01$ & & & $1.4 \mathrm{E}+01$ & $1.4 \mathrm{E}-01$ & & & $9.5 \mathrm{E}+00$ & $9.5 \mathrm{E}-02$ & & \\
\hline $\begin{array}{l}\text { Dichloroethylene, 1,2-(Mixed } \\
\text { Isomers) }\end{array}$ & $000540-59-0$ & & & $1.0 \mathrm{E}+02$ & $1.0 \mathrm{E}+01$ & & & $2.0 \mathrm{E}+02$ & $2.0 \mathrm{E}+01$ & & & $6.7 \mathrm{E}+01$ & $6.7 \mathrm{E}+00$ \\
\hline Dichlorophenol, 2,4- & 000120-83-2 & & & $3.4 \mathrm{E}+01$ & $3.4 \mathrm{E}+00$ & & & $1.2 \mathrm{E}+00$ & $1.2 \mathrm{E}-01$ & & & $1.2 \mathrm{E}+00$ & $1.2 \mathrm{E}-01$ \\
\hline $\begin{array}{l}\text { Dichlorophenoxy Acetic Acid, } \\
\text { 2,4- }\end{array}$ & 000094-75-7 & & & $1.1 \mathrm{E}+02$ & $1.1 \mathrm{E}+01$ & & & $4.6 \mathrm{E}+01$ & $4.6 \mathrm{E}+00$ & & & $3.3 \mathrm{E}+01$ & $3.3 \mathrm{E}+00$ \\
\hline $\begin{array}{l}\text { Dichlorophenoxy)butyric Acid, } \\
\text { 4-(2,4- }\end{array}$ & $000094-82-6$ & & & $9.1 E+01$ & $9.1 \mathrm{E}+00$ & & & $2.6 \mathrm{E}+01$ & $2.6 \mathrm{E}+00$ & & & $2.0 \mathrm{E}+01$ & $2.0 \mathrm{E}+00$ \\
\hline Dichlorvos & 000062-73-7 & $9.1 E+00$ & $9.1 \mathrm{E}-02$ & $5.7 \mathrm{E}+00$ & 5.7E-01 & $1.2 \mathrm{E}+01$ & $1.2 \mathrm{E}-01$ & $7.7 \mathrm{E}+00$ & 7.7E-01 & $5.3 \mathrm{E}+00$ & $5.3 \mathrm{E}-02$ & $3.3 \mathrm{E}+00$ & $3.3 \mathrm{E}-01$ \\
\hline Dicofol & $000115-32-2$ & & & & & & & & & & & & \\
\hline Dicyclopentadiene & $000077-73-6$ & & & $3.4 \mathrm{E}+02$ & $3.4 \mathrm{E}+01$ & & & 2.1E+01 & 2.1E+00 & & & $2.0 \mathrm{E}+01$ & $2.0 \mathrm{E}+00$ \\
\hline Dieldrin & $000060-57-1$ & $1.7 \mathrm{E}-01$ & $1.7 \mathrm{E}-03$ & $5.7 \mathrm{E}-01$ & $5.7 \mathrm{E}-02$ & $1.3 \mathrm{E}-02$ & 1.3E-04 & $4.6 \mathrm{E}-02$ & $4.6 \mathrm{E}-03$ & $1.2 \mathrm{E}-02$ & $1.2 \mathrm{E}-04$ & $4.2 \mathrm{E}-02$ & $4.2 \mathrm{E}-03$ \\
\hline Diethyl Phthalate & $000084-66-2$ & & & $9.1 \mathrm{E}+03$ & $9.1 \mathrm{E}+02$ & & & $4.4 \mathrm{E}+03$ & $4.4 \mathrm{E}+02$ & & & $3.0 \mathrm{E}+03$ & $3.0 E+02$ \\
\hline $\begin{array}{l}\text { Diethyl-p- } \\
\text { nitrophenylphosphate }\end{array}$ & $000311-45-5$ & & & & & & & & & & & & \\
\hline $\begin{array}{l}\text { Diethylene Glycol Dinitrate } \\
\text { (DEGDN) }\end{array}$ & $000693-21-0$ & & & & & & & & & & & & \\
\hline
\end{tabular}




\begin{tabular}{|c|c|c|c|c|c|c|c|c|c|c|c|c|c|}
\hline & & & & & & & & & & $20-301$ & $00+90^{\circ} \mathrm{I}$ & $L-t I-L S 0000$ & 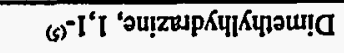 \\
\hline $20+30^{\circ} 1$ & $\mathfrak{E} 0+30^{\circ} \mathfrak{I}$ & & & $\varepsilon 0+\exists I^{\prime I}$ & $60+9 I^{\prime} \cdot 1$ & & & $20+9 I^{\prime} I$ & $\varepsilon 0+\mathrm{gl}\lceil\mathfrak{I}$ & & & $z-21-890000$ & 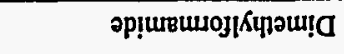 \\
\hline & & & & & & & & & & & & $L-0 \nleftarrow+8 S L O I$ & 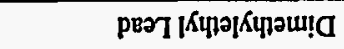 \\
\hline & & to-as'9 & $20-a s^{\prime} 9$ & & & t0- $-\mathrm{at} \cdot 8$ & $20-3 t \cdot 8$ & & & $\varepsilon 0-96 \%$ & $10-96 \%$ & $L-\varepsilon 6-611000$ & 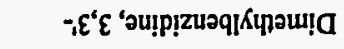 \\
\hline $10-\Xi I^{\prime} I$ & $00+3 I^{\prime} I$ & & & $10-3 Z 1$ & $00+3 z^{\prime} I$ & & & $00+A \varepsilon Z Z$ & $10+\exists \varepsilon \varepsilon$ & & & $L-69-121000$ & 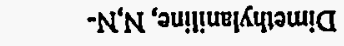 \\
\hline & & & & & & & & & & $20-a S^{\prime} \varepsilon$ & $00+3 S^{\prime} \varepsilon$ & {$[-89-560000$} & 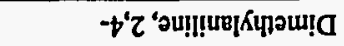 \\
\hline & & & & & & & & & & $20-396$ & $00+39 \cdot 6$ & $t-96-9 \varepsilon t i z 0$ & - ${ }^{6} \mathrm{C}$ ' \\
\hline & & & & & & & & & & & & $I-8 L-L L 0000$ & 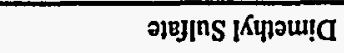 \\
\hline & & $00+3 I^{\prime} I$ & $20+911$ & & & $00+362$ & $20+3+2$ & & & $00+96^{\circ} \mathrm{I}$ & $20+96^{\circ} \mathrm{I}$ & $t-06-611000$ & 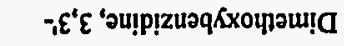 \\
\hline $20-96^{\prime} \varepsilon$ & $10-96 \cdot \varepsilon$ & & & $20-3 L \cdot b$ & $10-A L t$ & & & 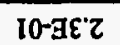 & $00+3 \varepsilon \tau$ & & & S-IS-090000 & әјеоцрәи! \\
\hline & & & & & & & & $10+3 E z$ & $20+\exists \varepsilon z$ & & & L-t9-06ZSSO & u!d!̣ழฺว \\
\hline $10+a 9 \cdot s$ & $20+39 \cdot s$ & & & $20+3 t^{1} 1$ & $\left.\varepsilon 0+3 b^{\prime}\right\rfloor$ & & & $10+9]^{\prime} 6$ & $20+91 \cdot 6$ & & & 9-SL-Sp+100 & 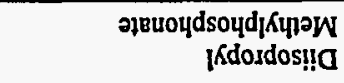 \\
\hline & & & & & & & & & & & & $9-\angle E=S \angle 0000$ & 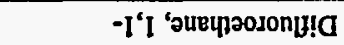 \\
\hline & & & & & & & & $10+a \varepsilon z$ & $20+9 \varepsilon z$ & & & $S-8 \varepsilon-\angle 9 \varepsilon S \varepsilon 0$ & นounzuəqnit!d \\
\hline & & & & & & & & $\cdot 10+91 \% 6$ & $20+91 \cdot 6$ & & & $9-8 \vdash-z z z \varepsilon t 0$ & IEnbozuวy!d \\
\hline & & $80-30 \%$ & $90-90 \%$ & & & $80-\Xi 1 ' Z$ & 90-al'z & & & 90-39's & $60-99 \cdot s$ & $\mathbb{I}-\varepsilon S-9 S 0000$ & 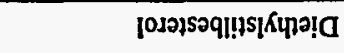 \\
\hline & & & & & & & & $10+3 z^{\prime} I$ & $20+\mathrm{az} \cdot 1$ & & & $s-68-\angle 19000$ & 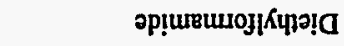 \\
\hline$\varepsilon 0+96 !$ & $50+96 . I$ & & & $20+3 Z^{\prime} I$ & $S 0+3 \tau^{\prime I}$ & & & $\varepsilon 0+g \varepsilon \tau$ & $+0+\exists \varepsilon z$ & & & 0-06-III000 & 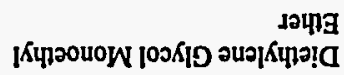 \\
\hline & & & & & & & & & & & & s-tદ-ZII000 & 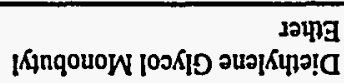 \\
\hline$I^{\prime} 0=I H$ & $\mathbf{I}=\mathbf{I H}$ & $.0 \mathrm{I}$ & .OI & $I^{\prime} 0=\partial H$ & $I=\partial \mathbf{H}$ & ,0I & rOI & $I^{\circ} 0=\mathrm{OH}$ & $\mathrm{I}=\mathrm{OH}$ & ,0I & , OI & \multirow{2}{*}{ N\&SVD } & \multirow{2}{*}{ ןвวุแนวนว } \\
\hline \multicolumn{4}{|c|}{ 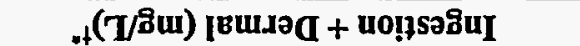 } & \multicolumn{4}{|c|}{ 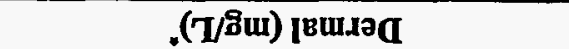 } & \multicolumn{4}{|c|}{ 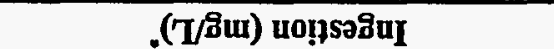 } & & \\
\hline
\end{tabular}


Table 3.4a. (continued)

\begin{tabular}{|c|c|c|c|c|c|c|c|c|c|c|c|c|c|}
\hline Chemical & CASRN & \multicolumn{4}{|c|}{ Ingestion (mg/L) } & \multicolumn{4}{|c|}{ Dermal (mg/L)" } & \multicolumn{4}{|c|}{ Ingestion + Dermal (mg/L) $)^{\dagger^{\bullet}}$} \\
\hline Dimethylhydrazine, 1,2- & $000540-73-8$ & & & & & & & & & & & & \\
\hline Dimethylphenol, 2,6- & $000576-26-1$ & & & $6.8 \mathrm{E}+00$ & $6.8 \mathrm{E}-01$ & & & $5.4 \mathrm{E}-01$ & $5.4 \mathrm{E}-02$ & & & $5.0 \mathrm{E}-01$ & $5.0 \mathrm{E}-02$ \\
\hline Dimethylphenol, 3,4- & $000095-65-8$ & & $:$ & $1.1 \mathrm{E}+01$ & $1.1 \mathrm{E}+00$ & & & 3.7E-01 & 3.7E-02 & & & $3.5 \mathrm{E}-01$ & $3.5 \mathrm{E}-02$ \\
\hline Dimethylterephthalate & $000120-61-6$ & & & $1.1 \mathrm{E}+03$ & $1.1 \mathrm{E}+02$ & & & & & & & & \\
\hline Dinitro-o-cresol, 4,6- & $000534-52-1$ & & & & & & & & & & & & \\
\hline $\begin{array}{l}\text { Dinitro-a-cyclohexyl Phenol, } \\
\text { 4,6- }\end{array}$ & $000131-89-5$ & & & $2.3 \mathrm{E}+01$ & 2.3E+00 & & & $5.3 \mathrm{E}+04$ & $5.3 \mathrm{E}+03$ & & & $2.3 \mathrm{E}+01$ & $2.3 \mathrm{E}+00$ \\
\hline Dinitrobenzene, 1,2- & $000528-29-0$ & & & $4.5 \mathrm{E}+00$ & $4.5 \mathrm{E}-01$ & & & $4.4 \mathrm{E}+00$ & $4.4 \mathrm{E}-01$ & & & $2.2 \mathrm{E}+00$ & 2.2E-01 \\
\hline Dinitrotoluene, 2,4- & $000121-14-2$ & $3.9 \mathrm{E}+00$ & $3.9 \mathrm{E}-02$ & 2.3E+01 & $2.3 \mathrm{E}+00$ & $2.2 \mathrm{E}+00$ & $2.2 \mathrm{E}-02$ & $1.3 \mathrm{E}+01$ & $1.3 \mathrm{E}+00$ & $1.4 \mathrm{E}+00$ & $1.4 \mathrm{E}-02$ & $8.3 \mathrm{E}+00$ & 8.3E-01 \\
\hline Dinitrotoluene, 2,6- & $000606-20-2$ & $3.9 \mathrm{E}+00$ & $3.9 \mathrm{E}-02$ & $1.1 \mathrm{E}+01$ & $1.1 \mathrm{E}+00$ & $3.4 \mathrm{E}+00$ & $3.4 \mathrm{E}-02$ & $1.0 \mathrm{E}+01$ & $1.0 \mathrm{E}+00$ & $1.8 \mathrm{E}+00$ & $1.8 \mathrm{E}-02$ & $5.3 \mathrm{E}+00$ & $5.3 \mathrm{E}-01$ \\
\hline Dinitrotoluene, 2-Amino-4,6- & $035572-78-2$ & & & & & & & & & & & & \\
\hline Dinoseb & $000088-85-7$ & & & $1.1 \mathrm{E}+01$ & $1.1 \mathrm{E}+00$ & & & $5.3 \mathrm{E}-01$ & $5.3 \mathrm{E}-02$ & & & $5.1 \mathrm{E}-01$ & $5.1 \mathrm{E}-02$ \\
\hline Dioxane, 1,4- & $000123-91-1$ & $2.4 \mathrm{E}+02$ & $2.4 \mathrm{E}+00$ & & & $1.2 E+03$ & $1.2 \mathrm{E}+01$ & & & $2.0 \mathrm{E}+02$ & $2.0 \mathrm{E}+00$ & & \\
\hline Diphenamid & $000957-51-7$ & & & $3.4 E+02$ & $3.4 \mathrm{E}+01$ & & & $1.9 \mathrm{E}+02$ & $1.9 \mathrm{E}+01$ & & & $1.2 \mathrm{E}+02$ & $1.2 \mathrm{E}+01$ \\
\hline Diphenylamine & $000122-39-4$ & & & $2.8 \mathrm{E}+02$ & $2.8 \mathrm{E}+01$ & & & $6.7 \mathrm{E}+00$ & $6.7 \mathrm{E}-01$ & & & $6.6 \mathrm{E}+00$ & $6.6 \mathrm{E}-01$ \\
\hline Diphenylhydrazine, $1,2-$ & $000122-66-7$ & $3.3 \mathrm{E}+00$ & $3.3 \mathrm{E}-02$ & & & $2.4 \mathrm{E}-01$ & $2.4 \mathrm{E}-03$ & & & $2.2 \mathrm{E}-01$ & $2.2 \mathrm{E}-03$ & & \\
\hline
\end{tabular}




\begin{tabular}{|c|c|c|c|c|c|c|c|c|c|c|c|c|c|}
\hline & & IO-aE:I & $10+9 E^{2} I$ & & & $10-98^{\prime} 1$ & $10+38^{\prime} I$ & & & $10-a s ' s$ & $10+35 . s$ & $s-88-0+1000$ & 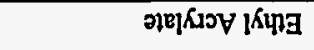 \\
\hline $20+31 \% s$ & E0+ar's & & & $\varepsilon 0+30^{\circ} \mathrm{I}$ & $50+90.1$ & & & $\mathfrak{E} 0+901$ & $60+30^{\circ} \mathrm{I}$ & & & 9-8L-It1000 & 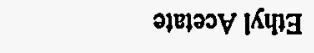 \\
\hline $20+3 L^{\circ} \varepsilon$ & $\varepsilon 0+\exists L \cdot \mathcal{E}$ & & & $\mathfrak{E O}+\mathfrak{A O}^{\prime} \mathcal{Z}$ & $50+90 \%$ & & & $20+3 s^{\prime} t$ & $\varepsilon 0+9 s^{\prime} \downarrow$ & & & $s-08-011000$ & 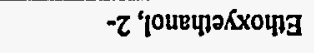 \\
\hline $20+90 z$ & $\varepsilon 0+\$ 10 z$ & & & $20+9 i ' s$ & $\mathfrak{E} 0+\mathfrak{I I}$ 'S & & & $20+a b \cdot \varepsilon$ & $\mathfrak{E} 0+\mathrm{g} \bullet \mathfrak{E}$ & & & 6-5I-III000 & 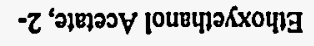 \\
\hline $20-\mathrm{aI} z$ & I0-aI'z & & & $20-3 I^{\prime} z$ & 10 -aI'z & & & $10-a L \cdot S$ & $00+3 L \cdot S$ & & & Z-ZI-£9ऽ000 & น0!เा日 \\
\hline & & & & & & & & $00+3 L \cdot S$ & $10+3 L \cdot S$ & & & $0-\angle 8-Z \angle 9910$ & uolydąıg \\
\hline & & & & & & & & & & & & $<-88-901000$ & 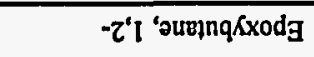 \\
\hline $00+36 \cdot I$ & $10+96 . I$ & $00+9 \varepsilon z$ & $20+\exists \varepsilon z$ & $10+3 \varepsilon \cdot 1$ & $20+3 \varepsilon^{\prime} I$ & $10+351$ & $\varepsilon 0+B S^{\prime} I$ & $00+\exists \varepsilon \tau$ & $10+9 \varepsilon z$ & $00+3 L^{\prime} z$ & $20+3 L^{\prime} \cdot \tau$ & 8-68-901000 & 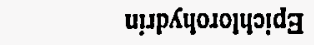 \\
\hline E0-aI’I & 20-ar'I & & & EO-aI’ & $20-a I^{\prime} I$ & & & $10-a b \cdot \varepsilon$ & $00+3 t \varepsilon \varepsilon$ & & & $8-0 z-z<0000$ & u!ıpug \\
\hline & & & & & & & & $10+3 \varepsilon z$ & $z 0+a \varepsilon z$ & & & $\mathcal{E}-\varepsilon L-S b 1000$ & \#вирория \\
\hline & & & & & & & & $00+88.9$ & $10+38^{\prime} 9$ & & & $L-6 Z-s 11000$ & นеуןกsopug \\
\hline & & & & & & & & $10+382$ & $z 0+987 z$ & & & $t+6-65<000$ & OLdG \\
\hline & & & & & & & & $00+3 s " t$ & $10+35 . t$ & & & $\varepsilon-0 I-6 \varepsilon+Z 00$ & әщ!pod \\
\hline $10-30 . t$ & $00+30 \%$ & & & $10-96 \%$ & $00+96 t$ & & & $00+\exists \varepsilon \tau$ & $10+\mathrm{A \varepsilon} z$ & & & $I-\$ S-0 \varepsilon \varepsilon 000$ & uodn! \\
\hline$\varepsilon 0-\Xi 0 \tau$ & $20-90 \% z$ & & & $\varepsilon 0-a ! \cdot z$ & $20-a i z$ & & & $20-a 5 \%$ & $10-a s^{\prime} b$ & & & $\leftarrow+0-862000$ & uopojyns!a \\
\hline & & & & & & & & & & & & $1-50-019200$ & 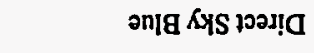 \\
\hline & & & & & & & & & & $\varepsilon 0-98 ' \tau$ & $10-38 \%$ & 9-98-IL0910 & S6 unosg poอม! \\
\hline & & & & & & & & & & $\mathfrak{E O - \Xi} \mathfrak{E}^{\mathfrak{E}} \mathcal{E}$ & $10-\exists \varepsilon \cdot \mathcal{E}$ & $z-9 t-209200$ & 9 әn|g วәכנ! \\
\hline & & & & & & & & & & 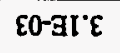 & I0-al' $\varepsilon$ & $L-L E-L \varepsilon 6100$ & 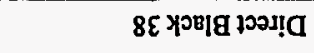 \\
\hline $00+95 \% 2$ & $10+\exists s z$ & & & $60+9 I^{\prime} \mathcal{E}$ & $S 0+\exists I^{\prime} \mathcal{E}$ & & & $00+3 s z$ & $10+9 S^{\prime} z$ & & & $L-00-580000$ & zenb!a \\
\hline$I^{\prime} 0=I H$ & $I=\mathbf{I H}$ & $90 \mathrm{I}$ & rOI & $I^{10}=0 H$ & $l=\partial H$ & $9.0 \mathrm{I}$ & rOI & $r^{\circ} 0=\partial H$ & $I=D H$ & $90 \mathrm{I}$ & rOI & מרזר & 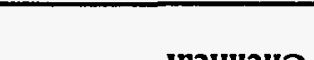 \\
\hline \multicolumn{4}{|c|}{ 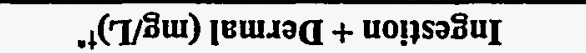 } & \multicolumn{4}{|c|}{. (T/8ిน) ןвшнә } & \multicolumn{4}{|c|}{ 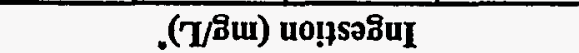 } & NAStr. & 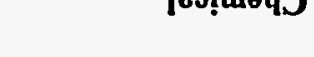 \\
\hline
\end{tabular}

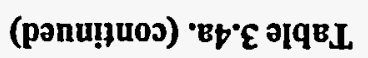


Table 3.4a. (continued)

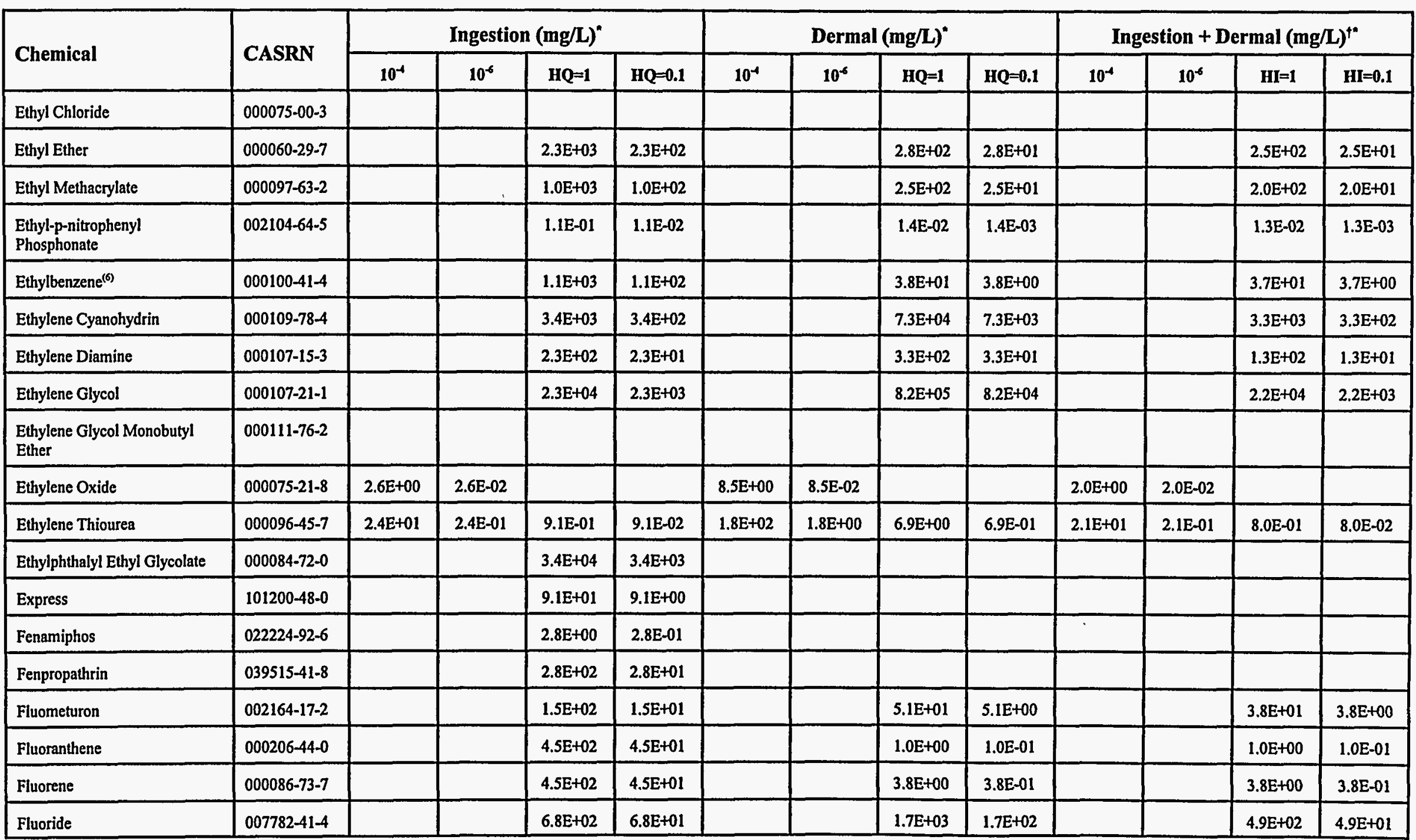




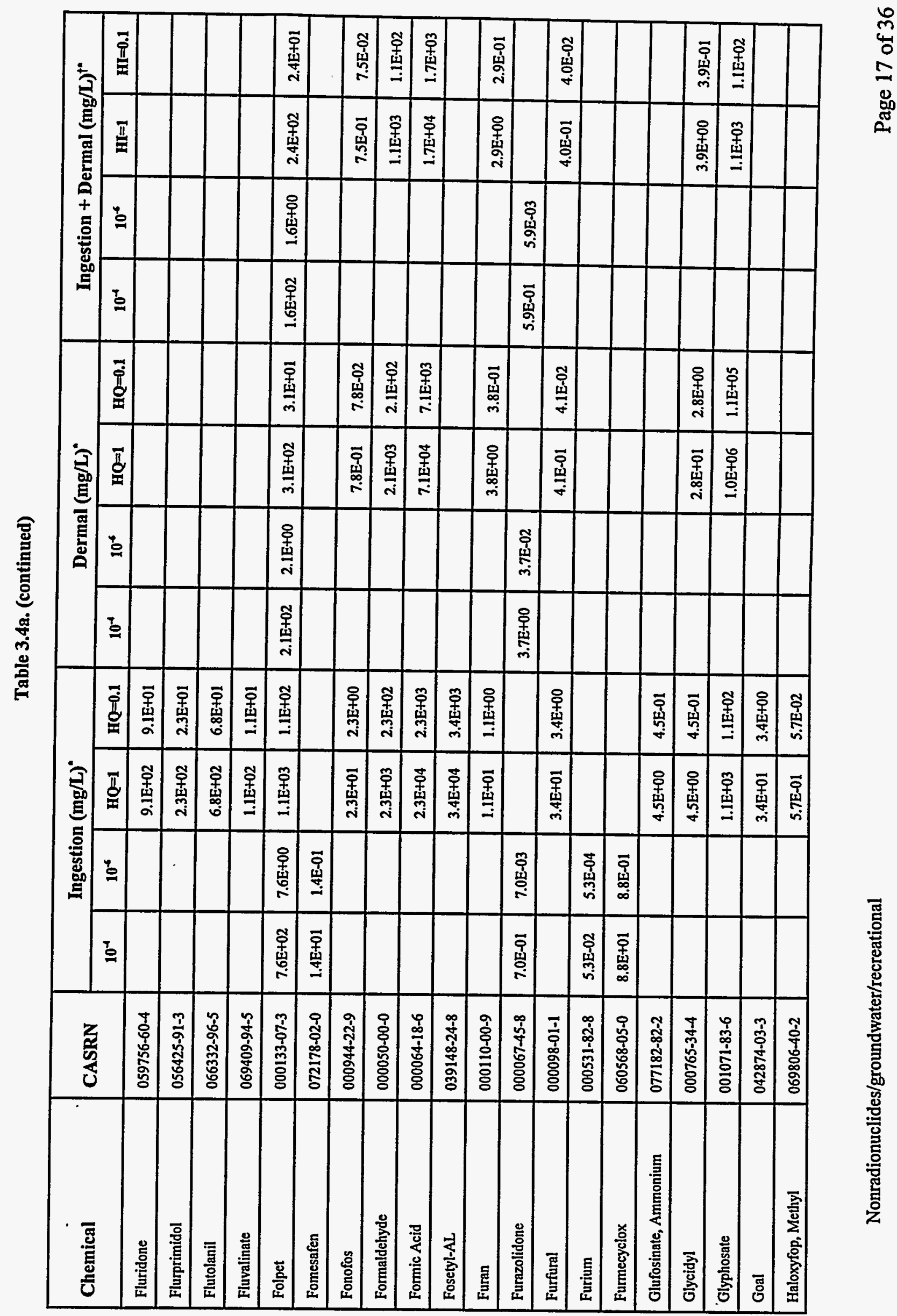


Table 3.4a. (continued)

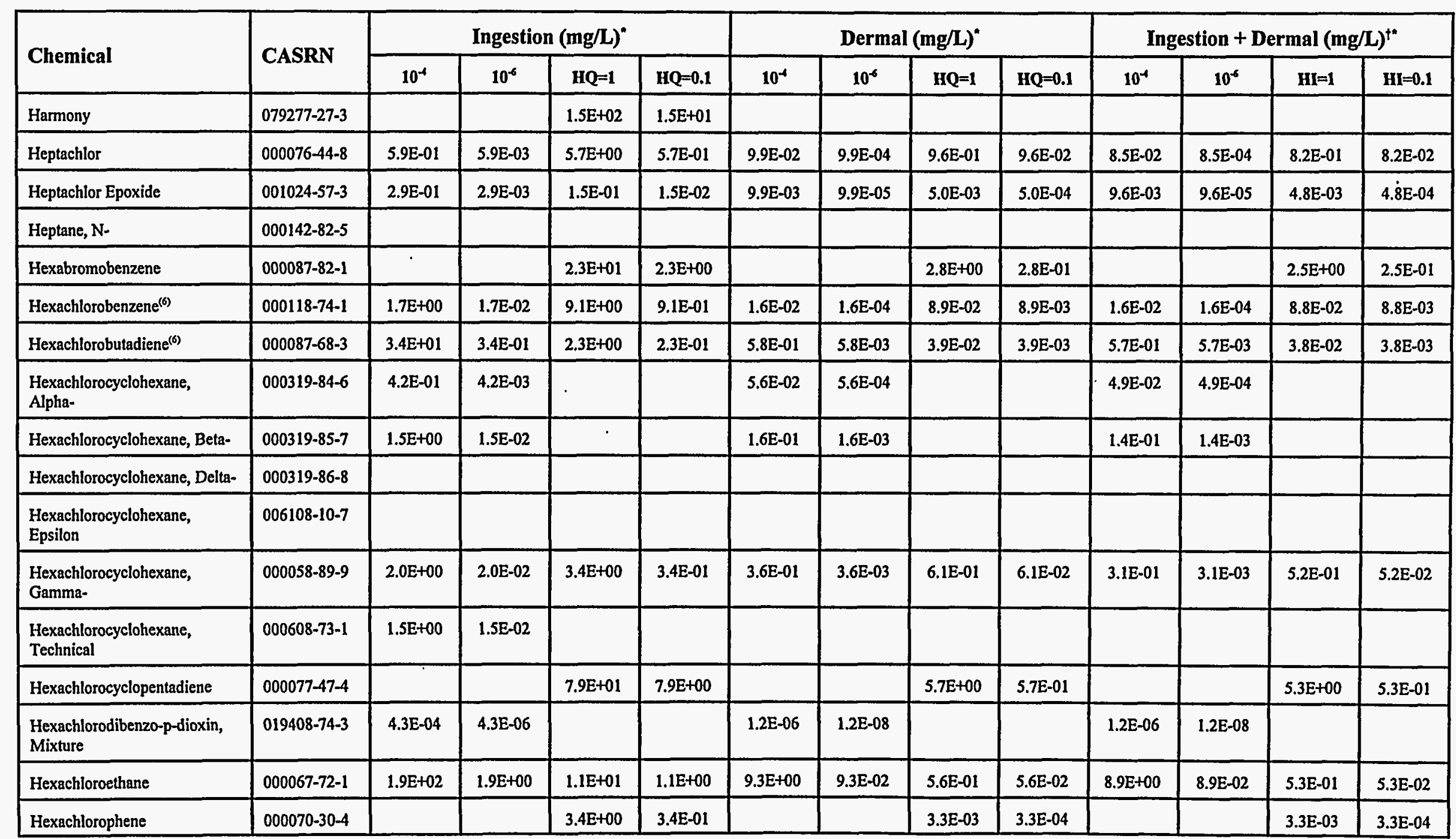


Table 3.4a. (continued)

\begin{tabular}{|c|c|c|c|c|c|c|c|c|c|c|c|c|c|}
\hline Chemical & CASRN & \multicolumn{4}{|c|}{ Ingestion (mg/L)" } & \multicolumn{4}{|c|}{ Dermal (mg/L)" } & \multicolumn{4}{|c|}{ Ingestion + Dermal (mg/L) ${ }^{\dagger^{*}}$} \\
\hline $\begin{array}{l}\text { Hexahydro-1,3,5-trinitro-1,3,5- } \\
\text { triazine (RDX) }\end{array}$ & $000121-82-4$ & $2.4 \mathrm{E}+01$ & $2.4 \mathrm{E}-01$ & $3.4 \mathrm{E}+01$ & $3.4 \mathrm{E}+00$ & $3.3 \mathrm{E}+00$ & $3.3 \mathrm{E}-02$ & 4.7E+00 & 4.7E-01 & $2.9 \mathrm{E}+00$ & $2.9 \mathrm{E}-02$ & $4.2 \mathrm{E}+00$ & $4.2 \mathrm{E}-01$ \\
\hline $\begin{array}{l}\text { Hexamethylene Diisocyanate, } \\
1,6-\end{array}$ & $000822-06-0$ & & & & & & & & & & & & \\
\hline Hexane, $N$ - & $000110-54-3$ & & $\cdot$ & $6.8 \mathrm{E}+02$ & $6.8 \mathrm{E}+01$ & & & $4.2 \mathrm{E}+00$ & 4.2E-01 & & & $4.2 \mathrm{E}+00$ & $4.2 \mathrm{E}-01$ \\
\hline Hexazinone & 051235-04-2 & & & 3.7E+02 & $3.7 E+01$ & & & $1.0 \mathrm{E}+06$ & $1.0 \mathrm{E}+06$ & & & $3.7 \mathrm{E}+02$ & $3.7 \mathrm{E}+01$ \\
\hline $\mathrm{HpCDD}, 2,3,7,8^{(4)}$ & $037871-00-4$ & $1.8 \mathrm{E}-03$ & $1.8 \mathrm{E}-05$ & & & & & & & & & & \\
\hline $\mathrm{HpCDF}, 2,3,7,8=(1)$ & $038998-75-3$ & $1.8 \mathrm{E}-03$ & $1.8 \mathrm{E}-05$ & & & & & & & & & & \\
\hline $\mathrm{HxCDD}, 2,3,7,8^{(4)}$ & $034465-46-8$ & $1.8 \mathrm{E}-04$ & $1.8 \mathrm{E}-06$ & & & & & & & & & & \\
\hline $\mathrm{HxCDF}, 2,3,7,8=(4)$ & $055684-94-1$ & $1.8 \mathrm{E}-04$ & $1.8 \mathrm{E}-06$ & & & & & & & & & & \\
\hline Hydrogen Cyanide & $000074-90-8$ & & & $2.3 \mathrm{E}+02$ & $2.3 \mathrm{E}+01$ & & & 4.1E+01 & 4.1E+00 & & & $3.5 \mathrm{E}+01$ & $3.5 \mathrm{E}+00$ \\
\hline Hydrogen Sulfide & $007783-06-4$ & & & $3.4 \mathrm{E}+01$ & $3.4 \mathrm{E}+00$ & & & $3.1 \mathrm{E}+00$ & 3.1E-01 & & & $2.9 \mathrm{E}+00$ & $2.9 \mathrm{E}-01$ \\
\hline Hydroquinone & $000123-31-9$ & & & $4.5 \mathrm{E}+02$ & $4.5 \mathrm{E}+01$ & & & $5.9 \mathrm{E}+02$ & $5.9 \mathrm{E}+01$ & & & $2.6 \mathrm{E}+02$ & $2.6 E+01$ \\
\hline Imazalit & $035554-44-0$ & & & $1.5 \mathrm{E}+02$ & $1.5 \mathrm{E}+01$ & & & & & & & & \\
\hline Imazaquin & $081335-37-7$ & & & $2.8 \mathrm{E}+03$ & $2.8 \mathrm{E}+02$ & & & & & & & & \\
\hline Indeno[1,2,3-cd]pyrene $e^{(2)}$ & $000193-39-5$ & $3.6 \mathrm{E}+00$ & $3.6 \mathrm{E}-02$ & & & $1.5 \mathrm{E}-03$ & $1.5 \mathrm{E}-05$ & & & $1.5 \mathrm{E}-03$ & $1.5 \mathrm{E}-05$ & & \\
\hline Iprodione & $036734-19-7$ & & & $4.5 \mathrm{E}+02$ & 4.5E+01 & & & & & & & & \\
\hline
\end{tabular}


Table 3.4a. (continued)

\begin{tabular}{|c|c|c|c|c|c|c|c|c|c|c|c|c|c|}
\hline Chemical & CASRN & \multicolumn{4}{|c|}{ Ingestion (mg/L) } & \multicolumn{4}{|c|}{ Dermal (mg/L)" } & \multicolumn{4}{|c|}{ Ingestion + Dermal (mg/L) $)^{{ }^{*}}$} \\
\hline Iron & $007439-89-6$ & & & & & & & & & & & & \\
\hline Isophorone & $000078-59-1$ & $2.8 \mathrm{E}+03$ & $2.8 \mathrm{E}+01$ & 2.3E+03 & $2.3 \mathrm{E}+02$ & $8.2 \mathrm{E}+02$ & $8.2 \mathrm{E}+00$ & $6.7 \mathrm{E}+02$ & $6.7 \mathrm{E}+01$ & $6.3 \mathrm{E}+02$ & $6.3 \mathrm{E}+00$ & $5.1 \mathrm{E}+02$ & $5.1 \mathrm{E}+01$ \\
\hline Isopropalin & $033820-53-0$ & & & $1.7 E+02$ & $1.7 \mathrm{E}+01$ & & & . & & & & & \\
\hline $\begin{array}{l}\text { Isopropyl Methyl Phosphonic } \\
\text { Acid }\end{array}$ & $001832-54-8$ & & & $1.1 \mathrm{E}+03$ & $1.1 \mathrm{E}+02$ & & & & & & & & \\
\hline Isoxaben & $082558-50-7$ & & & $5.7 \mathrm{E}+02$ & $5.7 \mathrm{E}+01$ & & & & & & & & \\
\hline Karate & $091465-08-6$ & & & 5.7E+01 & $5.7 E+00$ & & & & & & & & \\
\hline Kerb & $023950-58-5$ & & & $8.5 \mathrm{E}+02$ & $8.5 \mathrm{E}+01$ & & & & & & & & \\
\hline Linuron & $000330-55-2$ & & & $2.3 \mathrm{E}+01$ & $2.3 \mathrm{E}+00$ & & & & & & & & \\
\hline Lithium & $007439-93-2$ & & & & & & & & & & & & \\
\hline Londax & 083055-99-6 & & & $2.3 \mathrm{E}+03$ & $2.3 \mathrm{E}+02$ & & & & & & & & \\
\hline MCPA & $000094-74-6$ & & & $5.7 \mathrm{E}+00$ & 5.7E-01 & & & $1.5 \mathrm{E}+00$ & $1.5 \mathrm{E}-01$ & & & $1.2 \mathrm{E}+00$ & $1.2 \mathrm{E}-01$ \\
\hline MCPB & $000094-81-5$ & & & $1.1 \mathrm{E}+02$ & $1.1 \mathrm{E}+01$ & & & & & & & & \\
\hline MCPP & 000093-65-2 & & & $1.1 \mathrm{E}+01$ & $1.1 \mathrm{E}+00$ & & & $2.2 \mathrm{E}+00$ & 2.2E-01 & & & $1.9 \mathrm{E}+00$ & $1.9 \mathrm{E}-01$ \\
\hline Magnesium & $007439-95-4$ & & & & & & & & & & & & \\
\hline Malathion & $000121-75-5$ & & & $2.3 \mathrm{E}+02$ & $2.3 \mathrm{E}+01$ & & & $1.4 \mathrm{E}+02$ & $1.4 \mathrm{E}+01$ & & & $8.6 \mathrm{E}+01$ & $8.6 \mathrm{E}+00$ \\
\hline
\end{tabular}


Table 3.4a. (continued)

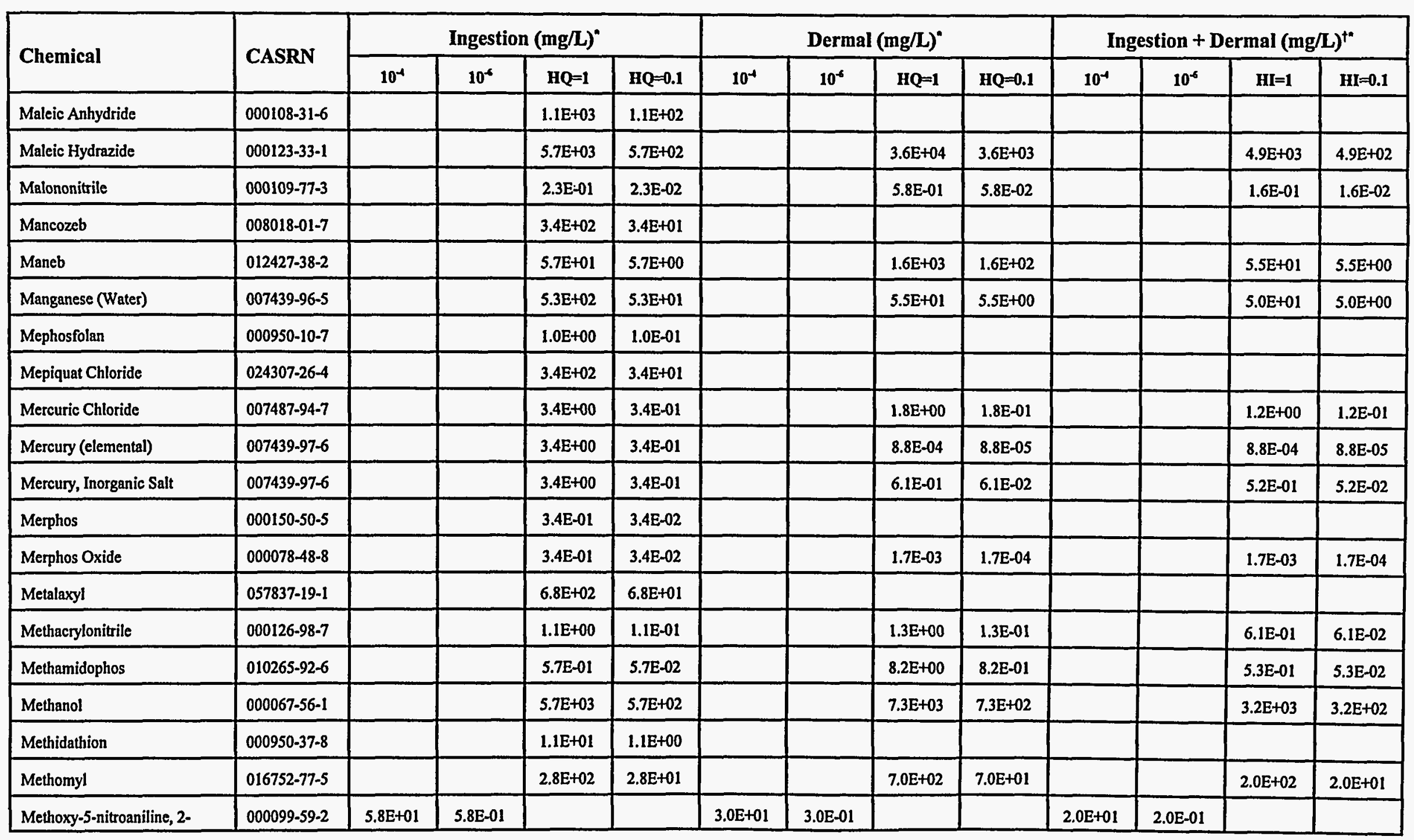


Table 3.4a. (continued)

\begin{tabular}{|c|c|c|c|c|c|c|c|c|c|c|c|c|c|}
\hline Chemical & CASRN & \multicolumn{4}{|c|}{ Ingestion (mg/L)" } & \multicolumn{4}{|c|}{ Dermal (mg/L) ${ }^{n}$} & \multicolumn{4}{|c|}{ Ingestion + Dermal $(\mathrm{mg} / \mathrm{L})^{\dagger^{*}}$} \\
\hline Methoxychlor & $000072-43-5$ & & & 5.7E+01 & $5.7 \mathrm{E}+00$ & & & $3.7 \mathrm{E}+00$ & $3.7 \mathrm{E}-01$ & & & $3.5 \mathrm{E}+00$ & $3.5 \mathrm{E}-01$ \\
\hline Methoxyethanol, 2- & $000109-86-4$ & & & $1.1 \mathrm{E}+01$ & $1.1 \mathrm{E}+00$ & & & 7.7E+01 & $7.7 \mathrm{E}+00$ & & & $9.9 \mathrm{E}+00$ & $9.9 \mathrm{E}-01$ \\
\hline Methyl Acetate & 000079-20-9 & & & $1.1 \mathrm{E}+04$ & $1.1 \mathrm{E}+03$ & & & $1.6 \mathrm{E}+04$ & $1.6 \mathrm{E}+03$ & & & $6.7 E+03$ & $6.7 \mathrm{E}+02$ \\
\hline Methyl Ethyl Ketone & 000078-93-3 & & & $6.8 \mathrm{E}+03$ & $6.8 \mathrm{E}+02$ & & & $1.3 \mathrm{E}+04$ & $1.3 \mathrm{E}+03$ & & & $4.5 \mathrm{E}+03$ & $4.5 \mathrm{E}+02$ \\
\hline Methyl Isobutyl Ketone & 000108-10-1 & & & $9.1 \mathrm{E}+02$ & $9.1 \mathrm{E}+01$ & & & $5.7 \mathrm{E}+02$ & $5.7 E+01$ & & & $3.5 \mathrm{E}+02$ & $3.5 \mathrm{E}+01$ \\
\hline Methyl Mercury & $022967-92-6$ & & & $1.1 \mathrm{E}+00$ & $1.1 \mathrm{E}-01$ & & & & & & & & \\
\hline Methyl Methacrylate & $000080-62-6$ & & & $9.1 \mathrm{E}+02$ & $9.1 \mathrm{E}+01$ & & & $4.8 \mathrm{E}+02$ & $4.8 \mathrm{E}+01$ & & & $3.1 \mathrm{E}+02$ & $3.1 E+01$ \\
\hline Methyl Parathion & $000298-00-0$ & & & $2.8 \mathrm{E}+00$ & $2.8 \mathrm{E}-01$ & & & $3.5 \mathrm{E}+00$ & $3.5 \mathrm{E}-01$ & & & $1.6 \mathrm{E}+00$ & $1.6 \mathrm{E}-01$ \\
\hline $\begin{array}{l}\text { Methyl tert-Butyl Ether } \\
\text { (MTBE) }\end{array}$ & $001634-04-4$ & & & & & & & & & & & & \\
\hline Methyl-5-Nitroaniline, 2- & 000099-55-8 & $8.0 \mathrm{E}+01$ & $8.0 \mathrm{E}-01$ & & & $1.7 E+01$ & $1.7 \mathrm{E}-01$ & & & $1.4 \mathrm{E}+01$ & $1.4 \mathrm{E}-01$ & & \\
\hline $\begin{array}{l}\text { Methylaniline Hydrochloride, } \\
2 .\end{array}$ & $000636-21-5$ & $1.5 \mathrm{E}+01$ & $1.5 \mathrm{E}-01$ & & & $9.0 \mathrm{E}+00$ & $9.0 \mathrm{E}-02$ & & & $5.6 \mathrm{E}+00$ & $5.6 \mathrm{E}-02$ & & \\
\hline Methylcyclohexane & $000108-87-2$ & & & & & & & & & & & & \\
\hline Methylene Chloride & $000075-09-2$ & $3.5 \mathrm{E}+02$ & $3.5 \mathrm{E}+00$ & $6.8 \mathrm{E}+02$ & $6.8 \mathrm{E}+01$ & $1.9 \mathrm{E}+02$ & $1.9 \mathrm{E}+00$ & 3.7E+02 & $3.7 \mathrm{E}+01$ & $1.2 \mathrm{E}+02$ & $1.2 \mathrm{E}+00$ & $2.4 \mathrm{E}+02$ & $2.4 E+01$ \\
\hline $\begin{array}{l}\text { Methylene-bis(2- } \\
\text { chloroaniline), 4,4- }\end{array}$ & $000101-14-4$ & $\begin{array}{c}2.0 \mathrm{E}+01 \\
.\end{array}$ & $2.0 \mathrm{E}-01$ & $7.9 \mathrm{E}+00$ & $7.9 \mathrm{E}-01$ & $9.4 \mathrm{E}-01$ & $9.4 \mathrm{E}-03$ & $3.7 \mathrm{E}-01$ & $3.7 \mathrm{E}-02$ & $9.0 \mathrm{E}-01$ & $9.0 \mathrm{E}-03$ & $3.5 \mathrm{E}-01$ & $3.5 \mathrm{E}-02$ \\
\hline
\end{tabular}




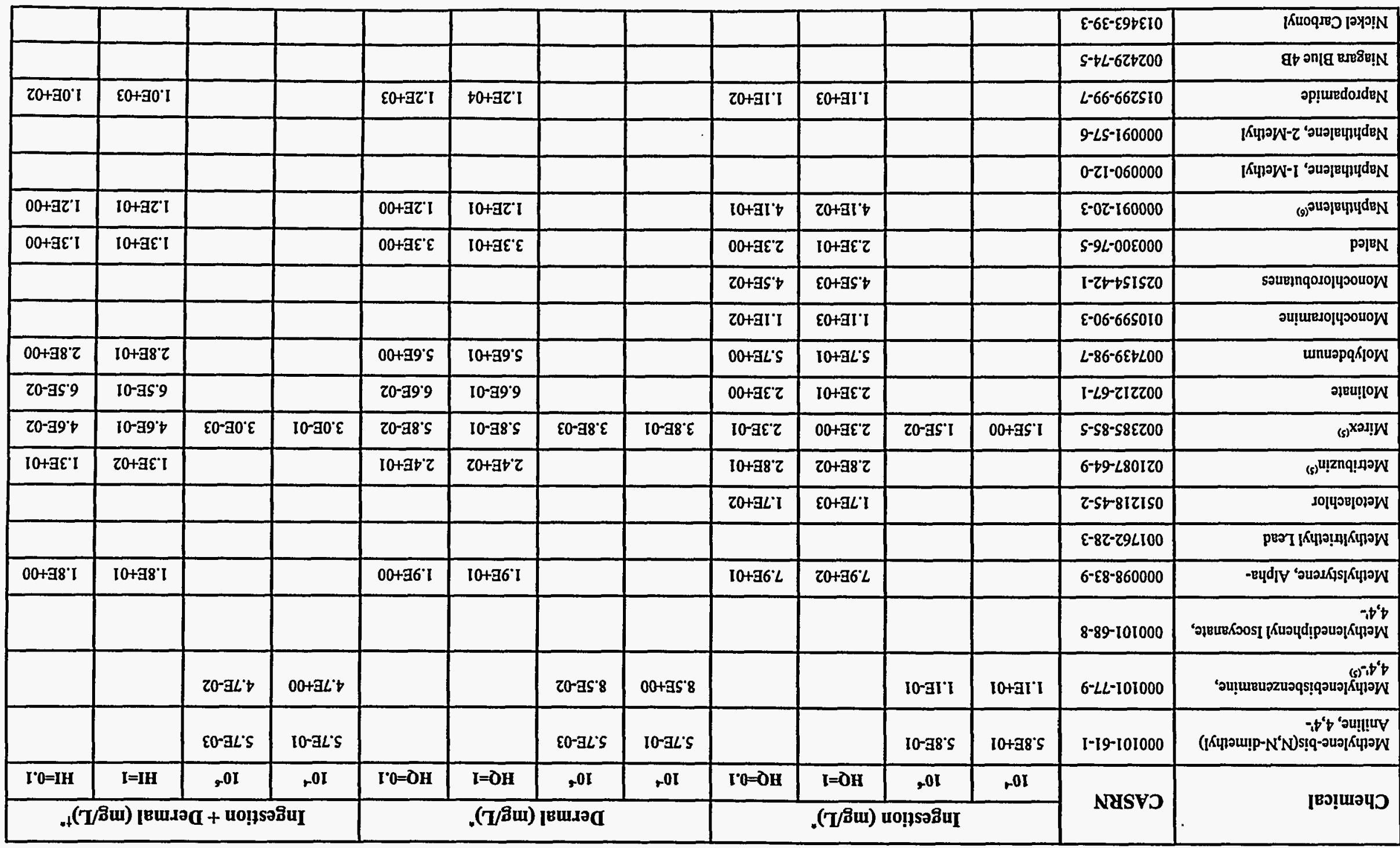

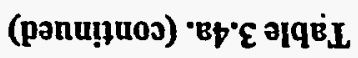


Table 3.4a. (continued)

\begin{tabular}{|c|c|c|c|c|c|c|c|c|c|c|c|c|c|}
\hline \multirow{2}{*}{ Chemical } & \multirow{2}{*}{ CASRN } & \multicolumn{4}{|c|}{ Ingestion $(\mathrm{mg} / \mathrm{L})^{*}$} & \multicolumn{4}{|c|}{ Dermal (mg/L)" } & \multicolumn{4}{|c|}{ Ingestion + Dermal $(\mathrm{mg} / \mathrm{L})^{\text {t*}^{*}}$} \\
\hline & & $10^{-1}$ & $10^{-6}$ & HQ $=1$ & $\mathrm{HQ}=0.1$ & $10^{-1}$ & $10^{-5}$ & $H Q=1$ & $H \mathrm{O}=0.1$ & $10^{-4}$ & $10^{-6}$ & $H I=1$ & $\mathrm{HI}=0.1$ \\
\hline Nickel Refinery Dust & $\mathrm{NA}$ & & & - & & & & & & & & & \\
\hline Nickel Soluble Salts & $007440-02-0$ & & & $2.3 \mathrm{E}+02$ & $2.3 \mathrm{E}+01$ & & & $1.6 \mathrm{E}+02$ & $1.6 \mathrm{E}+01$ & & & $9.3 \mathrm{E}+01$ & $9.3 \mathrm{E}+00$ \\
\hline Nickel Subsuifide & $012035-72-2$ & & & & & & & & & & & & \\
\hline Nitrapyrin $^{(3)}$ & $001929-82-4$ & & & $1.7 \mathrm{E}+01$ & $1.7 \mathrm{E}+00$ & & & $2.2 \mathrm{E}+00$ & $2.2 \mathrm{E}-01$ & & & $1.9 \mathrm{E}+00$ & $1.9 \mathrm{E}-01$ \\
\hline Nitrate & $014797-55-8$ & & & $1.8 \mathrm{E}+04$ & $1.8 \mathrm{E}+03$ & & & 2.3E+04 & $2.3 \mathrm{E}+03$ & & & $1.0 \mathrm{E}+04$ & $1.0 \mathrm{E}+03$ \\
\hline Nitric Oxide (s) & $010102-43-9$ & & & $1.1 \mathrm{E}+03$ & $1.1 \mathrm{E}+02$ & & & $5.9 \mathrm{E}+02$ & $5.9 \mathrm{E}+01$ & & & $3.9 \mathrm{E}+02$ & $3.9 \mathrm{E}+01$ \\
\hline Nitrite & $014797-65-0$ & & & $1.1 \mathrm{E}+03$ & $1.1 \mathrm{E}+02$ & & & $1.5 \mathrm{E}+03$ & $1.5 \mathrm{E}+02$ & & & $6.4 \mathrm{E}+02$ & $6.4 \mathrm{E}+01$ \\
\hline Nitroaniline, 2- & $000088-74-4$ & & & & & & & & & & & & \\
\hline Nitrobenzene & 000098-95-3 & & & $5.7 \mathrm{E}+00$ & $5.7 \mathrm{E}-01$ & & & $2.1 \mathrm{E}+00$ & $2.1 \mathrm{E}-01$ & & & $1.5 \mathrm{E}+00$ & $1.5 \mathrm{E}-01$ \\
\hline Nitrofurantoin & $000067-20-9$ & & & $7.9 \mathrm{E}+02$ & $7.9 \mathrm{E}+01$ & & & & & & & & \\
\hline Nitrofurazone & $000059-87-0$ & $1.8 \mathrm{E}+00$ & $1.8 \mathrm{E}-02$ & & & & & & & & & & \\
\hline Nitrogen Dioxide & $010102-44-0$ & & & $1.1 \mathrm{E}+04$ & $1.1 \mathrm{E}+03$ & & & 2.3E+04 & $2.3 \mathrm{E}+03$ & & & $7.6 \mathrm{E}+03$ & $7.6 \mathrm{E}+02$ \\
\hline Nitroguanidine & $000556-88-7$ & & & $1.1 \mathrm{E}+03$ & $1.1 \mathrm{E}+02$ & & & & & & & & \\
\hline Nitrophenol, 4- & $000100-02-7$ & & & & & & & & & & & & \\
\hline Nitropropane, $2^{(3)}$ & $000079-46-9$ & $2.8 \mathrm{E}-01$ & $2.8 \mathrm{E}-03$ & & & $5.8 \mathrm{E}-01$ & $5.8 \mathrm{E}-03$ & & & $1.9 \mathrm{E}-01$ & $1.9 \mathrm{E}-03$ & & \\
\hline Nitroso-N-ethylurea, $\mathrm{N}$ - & 000759-73-9 & $1.9 \mathrm{E}-02$ & $1.9 \mathrm{E}-04$ & & & 4.5E-02 & 4.5E-04 & & & $1.3 \mathrm{E}-02$ & $1.3 \mathrm{E}-04$ & & \\
\hline Nitroso-N-methylurea, N- & 000684-93-5 & & & & & & & & & & & & \\
\hline Nitroso-di-N-butylamine, N- & $000924-16-3$ & $4.9 \mathrm{E}-01$ & $4.9 \mathrm{E}-03$ & & & $2.1 \mathrm{E}-01$ & 2.1E-03 & & & $1.5 \mathrm{E}-01$ & $1.5 \mathrm{E}-03$ & & \\
\hline Nitroso-di-N-propylamine, $\mathrm{N}$ - & $000621-64-7$ & $3.8 \mathrm{E}-01$ & $3.8 \mathrm{E}-03$ & & & $8.7 \mathrm{E}-02$ & $8.7 \mathrm{E}-04$ & & & 7.1E-02 & 7.1E-04 & & \\
\hline Nitrosodiethanolamine, $\mathrm{N}$ - & $001116-54-7$ & $9.5 \mathrm{E}-01$ & $9.5 \mathrm{E}-03$ & & & $2.4 \mathrm{E}+02$ & $2.4 \mathrm{E}+00$ & & & $9.4 \mathrm{E}-01$ & $9.4 \mathrm{E}-03$ & & \\
\hline
\end{tabular}


Table 3.4a. (continued)

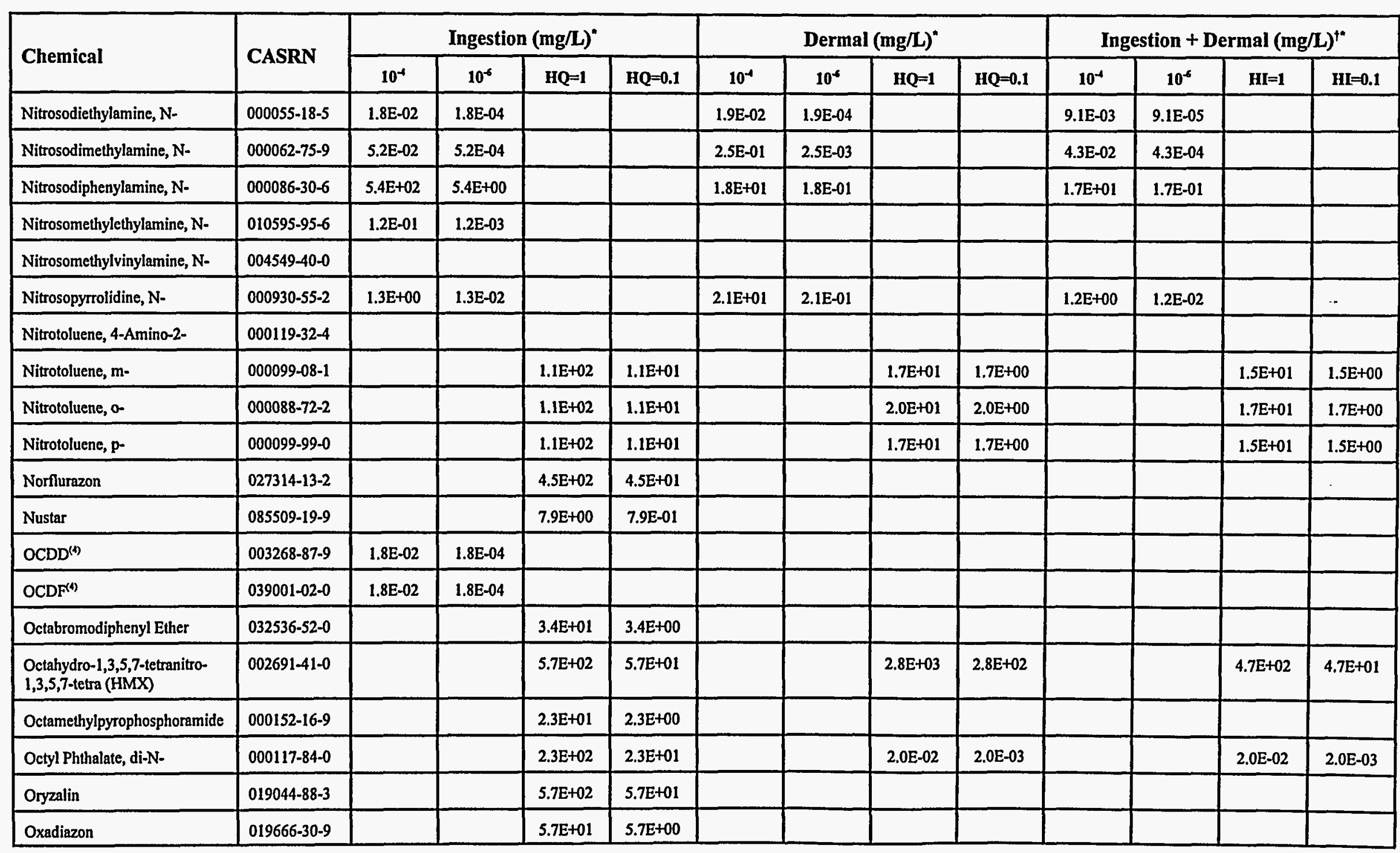

Nonradionuclides/groundwater/recreational

Page 25 of 36 
Table 3.4a. (continued)

\begin{tabular}{|c|c|c|c|c|c|c|c|c|c|c|c|c|c|}
\hline \multirow{2}{*}{ Chemical } & \multirow{2}{*}{ CASRN } & \multicolumn{4}{|c|}{ Ingestion (mg/L)" } & \multicolumn{4}{|c|}{ Dermal (mg/L)" } & \multicolumn{4}{|c|}{ Ingestion + Dermal (mg/L) ${ }^{\text {t*}^{*}}$} \\
\hline & & $10^{4}$ & $10^{-6}$ & $H Q=1$ & $\mathrm{HQ}=0.1$ & $10^{4}$ & $10^{-6}$ & $H Q=1$ & $H Q=0.1$ & $10^{-4}$ & $10^{-6}$ & $\mathbf{H I}=\mathbf{1}$ & $\mathrm{HI}=0.1$ \\
\hline Oxamyl & $023135-22-0$ & & & $2.8 \mathrm{E}+02$ & $2.8 \mathrm{E}+01$ & & & & & & & & \\
\hline Paclobutrazol & $076738-62-0$ & & & $1.5 \mathrm{E}+02$ & $1.5 \mathrm{E}+01$ & & & & & & & & \\
\hline Paraquat & $001910-42-5$ & & & $5.1 \mathrm{E}+01$ & $5.1 \mathrm{E}+00$ & & & 4.7E+05 & 4.7E+04 & & & $5.1 \mathrm{E}+01$ & $5.1 \mathrm{E}+00$ \\
\hline Parathion & $000056-38-2$ & & & $6.8 \mathrm{E}+01$ & $6.8 \mathrm{E}+00$ & & & $5.2 \mathrm{E}+00$ & $5.2 \mathrm{E}-01$ & & & $4.8 \mathrm{E}+00$ & $4.8 \mathrm{E}-01$ \\
\hline PeCDD, 2,3,7,8-(4) & 036088-22-9 & $3.5 \mathrm{E}-05$ & $3.5 \mathrm{E}-07$ & & & & & & & & & & \\
\hline PeCDF, $1,2,3,7,8-(4)$ & $057117-41-6$ & $3.5 \mathrm{E}-05$ & $3.5 \mathrm{E}-07$ & & & & & & & & & & \\
\hline PeCDF, 2,3,4,7,8-(4) & $057117-31-4$ & 3.5E-04 & 3.5E-06 & & & & & & & & & & \\
\hline Pebulate & 001114-71-2 & & & $5.7 \mathrm{E}+02$ & $5.7 \mathrm{E}+01$ & & & & & & . & & \\
\hline Pendimethalin & $040487-42-1$ & & & $4.5 \mathrm{E}+02$ & $4.5 \mathrm{E}+01$ & & & & & & & & \\
\hline Pentabromodiphenyl Ether & $032534-81-9$ & & & $2.3 \mathrm{E}+01$ & $2.3 E+00$ & & & & & & & & \\
\hline Pentachlorobenzene & 000608-93-5 & & & $9.1 \mathrm{E}+00$ & 9.1E-01 & & & $6.7 \mathrm{E}-02$ & $6.7 \mathrm{E}-03$ & & & $6.7 \mathrm{E}-02$ & $6.7 \mathrm{E}-03$ \\
\hline Pentachlorocyclopentadiene & $025329-35-5$ & & & & & & & & & & & & \\
\hline Pentachloronitrobenzene & $000082-68-8$ & $1.0 \mathrm{E}+01$ & $1.0 \mathrm{E}-01$ & $3.4 \mathrm{E}+01$ & $3.4 \mathrm{E}+00$ & $3.6 \mathrm{E}-01$ & $3.6 \mathrm{E}-03$ & $1.2 \mathrm{E}+00$ & $1.2 \mathrm{E}-01$ & $3.4 \mathrm{E}-01$ & 3.4E-03 & $1.2 \mathrm{E}+00$ & $1.2 \mathrm{E}-01$ \\
\hline Pentachlorophenol & $000087-86-5$ & $2.2 \mathrm{E}+01$ & $2.2 \mathrm{E}-01$ & $3.4 \mathrm{E}+02$ & $3.4 \mathrm{E}+01$ & 8.8E-02 & $8.8 \mathrm{E}-04$ & $1.4 \mathrm{E}+00$ & $1.4 \mathrm{E}-01$ & $8.7 \mathrm{E}-02$ & 8.7E-04 & $1.3 \mathrm{E}+00$ & $1.3 \mathrm{E}-01$ \\
\hline Pentyl Alcohol, N- & $000071-41-0$ & & & & & & & & & & & & \\
\hline Permethrin & $052645-53-1$ & & & $5.7 \mathrm{E}+02$ & $5.7 \mathrm{E}+01$ & & & $3.1 \mathrm{E}+02$ & $3.1 \mathrm{E}+01$ & & & $2.0 \mathrm{E}+02$ & $2.0 \mathrm{E}+01$ \\
\hline Phenanthrene & $000085-01-8$ & & & & & & & & & & & & \\
\hline Phenmedipham & $013684-63-4$ & & & $2.8 E+03$ & $2.8 \mathrm{E}+02$ & & & $1.0 \mathrm{E}+06$ & $1.1 \mathrm{E}+05$ & & & $2.8 \mathrm{E}+03$ & $2.8 \mathrm{E}+02$ \\
\hline Phenol & $000108-95-2$ & & & $6.8 E+03$ & $6.8 \mathrm{E}+02$ & & & $2.9 E+03$ & $2.9 \mathrm{E}+02$ & & & $2.0 \mathrm{E}+03$ & $2.0 \mathrm{E}+02$ \\
\hline Phenylenediamine, $\mathrm{m}$ - & $000108-45-2$ & & & $6.8 E+01$ & $6.8 \mathrm{E}+00$ & & & $7.2 \mathrm{E}+02$ & $7.2 \mathrm{E}+01$ & - & & $6.2 \mathrm{E}+01$ & $6.2 E+00$ \\
\hline
\end{tabular}


Table 3.4a. (continued)

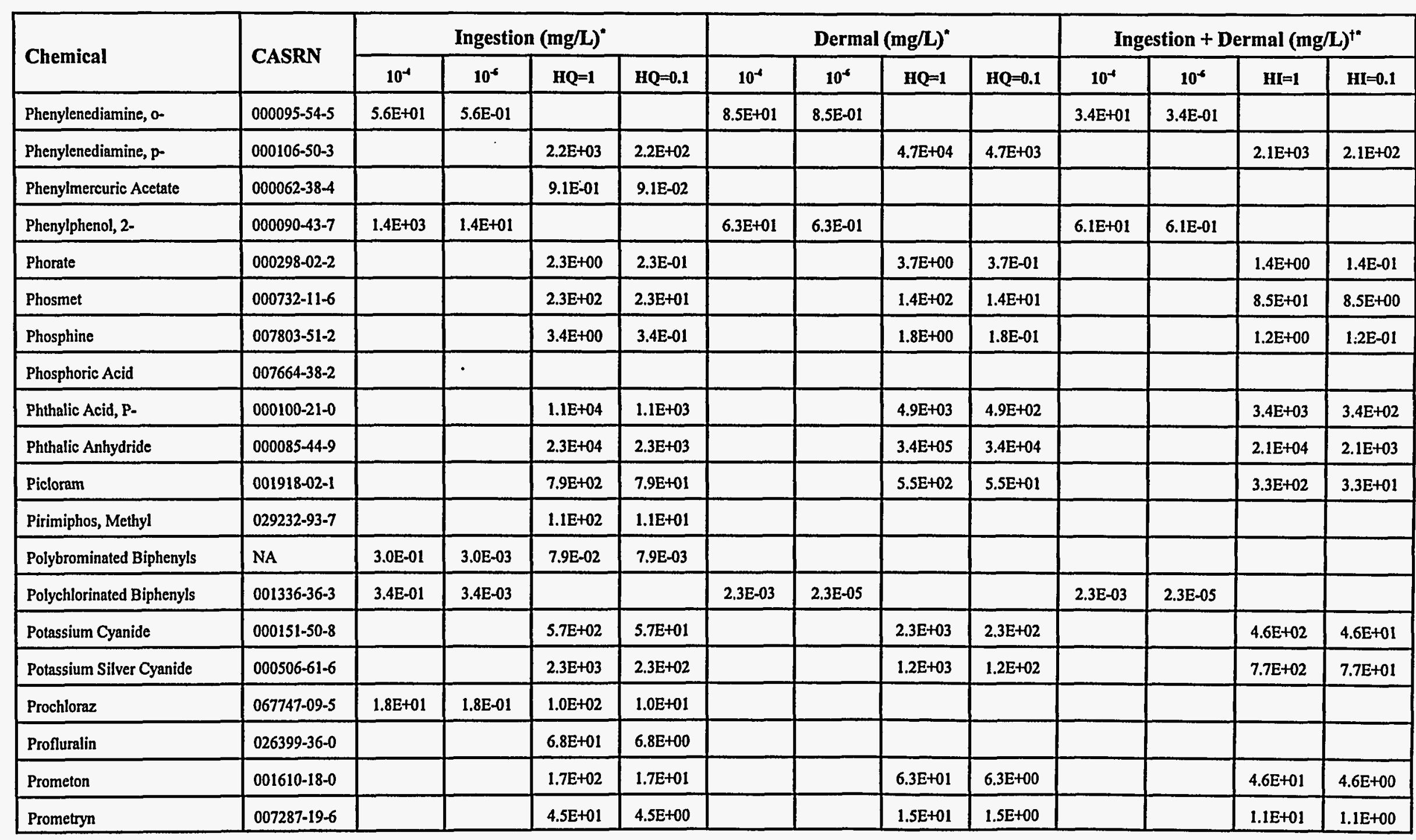


Table 3.4a. (continued)

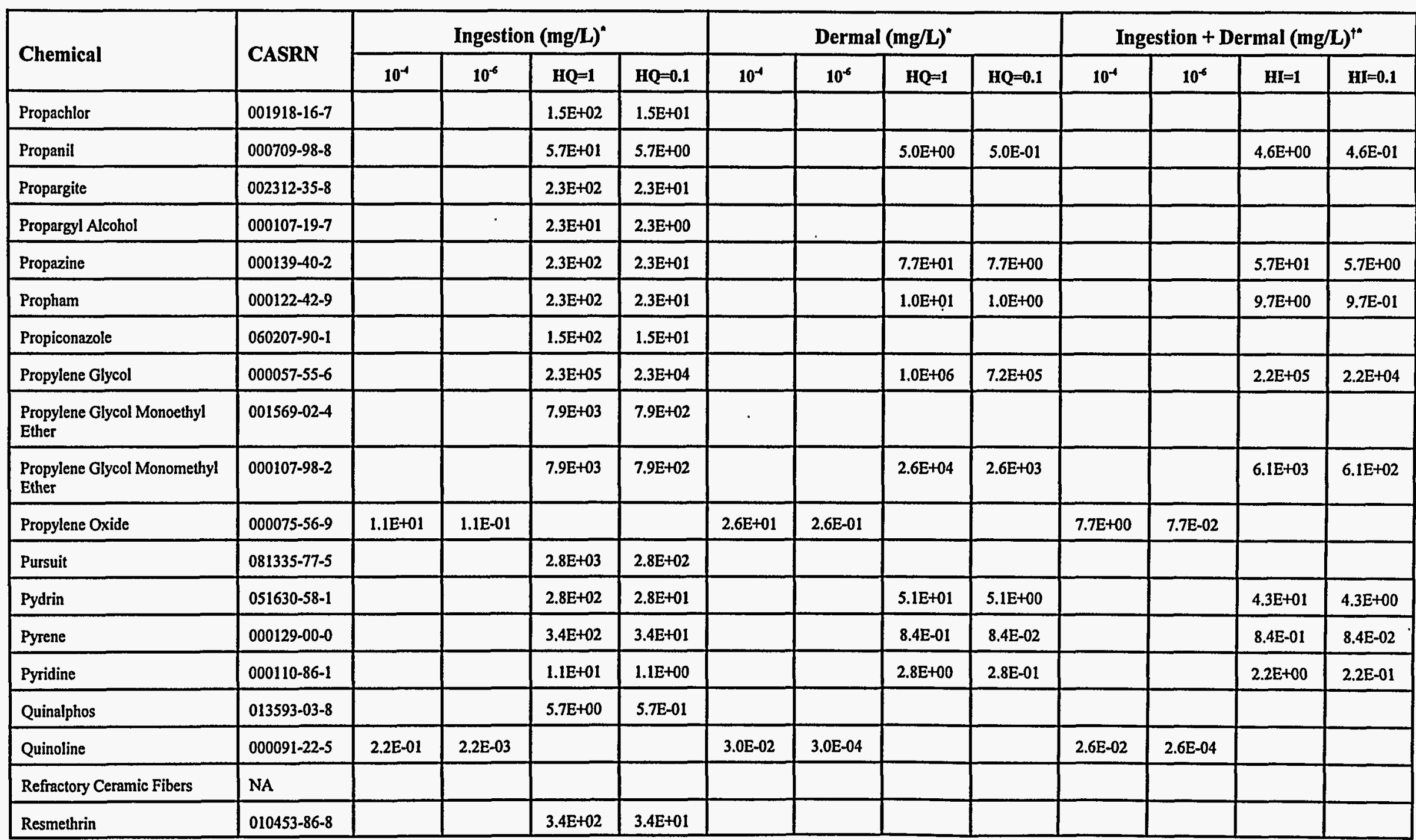


Table 3.4a. (continued)

\begin{tabular}{|c|c|c|c|c|c|c|c|c|c|c|c|c|c|}
\hline Chemical & CASRN & \multicolumn{4}{|c|}{ Ingestion (mg/L)" } & \multicolumn{4}{|c|}{ Dermal (mg/L)* } & \multicolumn{4}{|c|}{ Ingestion + Dermal $(\mathbf{m g} / \mathbf{L})^{\dagger^{*}}$} \\
\hline Ronnel & $000299-84-3$ & & & $5.7 \mathrm{E}+02$ & $5.7 \mathrm{E}+01$ & & & $8.4 \mathrm{E}+00$ & $8.4 \mathrm{E}-01$ & & & $8.3 E+00$ & $8.3 \mathrm{E}-01$ \\
\hline Savey & $078587-05-0$ & & & $2.8 \mathrm{E}+02$ & $2.8 \mathrm{E}+01$ & & & & . & & & & \\
\hline Selenious Acid & $007783-00-8$ & & & $5.7 \mathrm{E}+01$ & $5.7 \mathrm{E}+00$ & & & & & & & & \\
\hline Selenium & $007782-49-2$ & & & $5.7 \mathrm{E}+01$ & $5.7 \mathrm{E}+00$ & & & $6.4 E+01$ & $6.4 \mathrm{E}+00$ & & & $3.0 \mathrm{E}+01$ & $3: 0 \mathrm{E}+00$ \\
\hline Selenium Sulfide & $007446-34-6$ & & & & & & & & & & & & \\
\hline Selenourea & $000630-10-4$ & & & $5.7 \mathrm{E}+01$ & $5.7 \mathrm{E}+00$ & & & & & & & & \\
\hline Sethoxydim & 074051-80-2 & & & $1.0 \mathrm{E}+03$ & $1.0 \mathrm{E}+02$ & & & & & & & & \\
\hline Silver & $007440-22-4$ & & & $5.7 \mathrm{E}+01$ & $5.7 \mathrm{E}+00$ & & & $2.6 \mathrm{E}+01$ & $2.6 \mathrm{E}+00$ & & & $1.8 \mathrm{E}+01$ & $1.8 \mathrm{E}+00$ \\
\hline Sodium Acifluorfen & 062476-59-9 & & & $1.5 \mathrm{E}+02$ & $1.5 \mathrm{E}+01$ & & & & & & & & \\
\hline Sodium Azide & $026628-22-8$ & & & 4.5E+01 & $4.5 \mathrm{E}+00$ & & & 2.3E+01 & $2.3 \mathrm{E}+00$ & & & $1.5 \mathrm{E}+01$ & $1.5 \mathrm{E}+00$ \\
\hline Sodium Cyanide & $000143-33-9$ & & & $4.5 \mathrm{E}+02$ & $4.5 \mathrm{E}+01$ & & & $3.7 \mathrm{E}+03$ & $3.7 E+02$ & & & $4.1 \mathrm{E}+02$ & $4.1 \mathrm{E}+01$ \\
\hline $\begin{array}{l}\text { Sodium } \\
\text { Diethyldithiocarbamate }\end{array}$ & $000148-18-5$ & $9.8 \mathrm{E}+00$ & $9.8 \mathrm{E}-02$ & $3.4 \mathrm{E}+02$ & $3.4 \mathrm{E}+01$ & & & & & & & & \\
\hline Sodium Fluoroacetate & $000062-74-8$ & & & $2.3 \mathrm{E}-01$ & $2.3 \mathrm{E}-02$ & & & & & & & & \\
\hline Sodium Metavanadate & $013718-26-8$ & & & $1.1 \mathrm{E}+01$ & $1.1 \mathrm{E}+00$ & & & $5.9 \mathrm{E}+00$ & $5.9 \mathrm{E}-01$ & & & $3.9 \mathrm{E}+00$ & $3.9 \mathrm{E}-01$ \\
\hline Stirofos (Tetrachlorovinphos) & $000961-11-5$ & $1.1 \mathrm{E}+02$ & $1.1 \mathrm{E}+00$ & $3.4 \mathrm{E}+02$ & $3.4 \mathrm{E}+01$ & & & & & & & & \\
\hline
\end{tabular}

Nonradionuclides/groundwater/recreational

Page 29 of 36 
Table 3.4a. (continued)

\begin{tabular}{|c|c|c|c|c|c|c|c|c|c|c|c|c|c|}
\hline \multirow{2}{*}{ Chemical } & \multirow{2}{*}{ CASRN } & \multicolumn{4}{|c|}{ Ingestion $(\mathrm{mg} / \mathrm{L})^{*}$} & \multicolumn{4}{|c|}{ Dermal (mg/L)" } & \multicolumn{4}{|c|}{ Ingestion + Dermal $(\mathbf{m g} / \mathbf{L})^{\dagger^{\star}}$} \\
\hline & & $10^{-4}$ & $10^{-6}$ & $\mathrm{HQ}=1$ & $\mathrm{HQ}=0.1$ & $10^{-4}$ & $10^{-6}$ & $\mathrm{HQ}=1$ & $\mathrm{HQ}=0.1$ & $10^{-4}$ & $10^{-5}$ & $\mathbf{H} \mathbf{l}=\mathbf{1}$ & $\mathrm{HI}=0.1$ \\
\hline Strontium, Stable & $007440-24-6$ & & & $6.8 \mathrm{E}+03$ & $6.8 \mathrm{E}+02$ & & & $3.5 \mathrm{E}+03$ & $3.5 \mathrm{E}+02$ & & & $2.3 \mathrm{E}+03$ & $2.3 \mathrm{E}+02$ \\
\hline Strychnine & $000057-24-9$ & & & $3.4 \mathrm{E}+00$ & $3.4 \mathrm{E}-01$ & & & $1.1 \mathrm{E}+01$ & $1.1 \mathrm{E}+00$ & & & $2.6 \mathrm{E}+00$ & $2.6 \mathrm{E}-01$ \\
\hline Styrene $^{(6)}$ & $000100-42-5$ & & & $2.3 \mathrm{E}+03$ & $2.3 \mathrm{E}+02$ & & & $7.0 \mathrm{E}+00$ & 7.0E-01 & & & $7.0 \mathrm{E}+00$ & $7.0 \mathrm{E}-01$ \\
\hline Sulfate & $014808-79-8$ & & & & & & & & & & & & \\
\hline Systhane & 088671-89-0 & & & $2.8 \mathrm{E}+02$ & $2.8 \mathrm{E}+01$ & & & & & & & & \\
\hline TCDD, 2,3,7,8-(4) & 001746-01-6 & $1.8 \mathrm{E}-05$ & $1.8 \mathrm{E}-07$ & & & $1.6 \mathrm{E}-08$ & $1.6 \mathrm{E}-10$ & & & $1.6 \mathrm{E}-08$ & $1.6 \mathrm{E}-10$ & & \\
\hline TCDF, 2,3,7,8-(4) & $051207-31-9$ & $1.8 \mathrm{E}-04$ & $1.8 \mathrm{E}-06$ & & & & & & & & & & \\
\hline TCMTB & $021564-17-0$ & & & $3.4 E+02$ & $3.4 \mathrm{E}+01$ & & & & & & & & \\
\hline Tebuthiuron & 034014-18-1 & & & $7.9 E+02$ & $7.9 \mathrm{E}+01$ & & & $1.0 \mathrm{E}+04$ & $1.0 \mathrm{E}+03$ & & & $7.4 \mathrm{E}+02$ & $7.4 \mathrm{E}+01$ \\
\hline Temephos & $003383-96-8$ & & & $2.3 \mathrm{E}+02$ & $2.3 \mathrm{E}+01$ & & & & & & & & \\
\hline Terbacil & $005902-51-2$ & & & $1.5 \mathrm{E}+02$ & $1.5 \mathrm{E}+01$ & & & & & & & & \\
\hline Terbufos & 013071-79-9 & & & $2.8 \mathrm{E}-01$ & $2.8 \mathrm{E}-02$ & & & $2.7 \mathrm{E}-02$ & 2.7E-03 & & & $2.5 \mathrm{E}-02$ & $2.5 \mathrm{E}-03$ \\
\hline Terbutryn & $000886-50-0$ & & & $1.1 \mathrm{E}+01$ & $1.1 \mathrm{E}+00$ & & & $5.0 \mathrm{E}-01$ & $5.0 \mathrm{E}-02$ & & & $4.8 \mathrm{E}-01$ & $4.8 \mathrm{E}-02$ \\
\hline Tetrabutyl Lead & $001920-90-7$ & & & & & & & & & & & & \\
\hline Tetrachlorobenzene, 1,2,4,5- & $000095-94-3$ & & & $3.4 \mathrm{E}+00$ & 3.4E-01 & & & $4.1 \mathrm{E}-02$ & 4.1E-03 & & & $4.1 \mathrm{E}-02$ & $4.1 \mathrm{E}-03$ \\
\hline Tetrachloroethane, 1,1,1,2- & $000630-20-6$ & $1.0 \mathrm{E}+02$ & $1.0 E+00$ & $3.4 \mathrm{E}+02$ & $3.4 \mathrm{E}+01$ & $8.7 E+00$ & 8.7E-02 & $2.9 \mathrm{E}+01$ & $2.9 \mathrm{E}+00$ & $8.0 \mathrm{E}+00$ & $8.0 \mathrm{E}-02$ & $2.7 E+01$ & $2.7 E+00$ \\
\hline Tetrachloroethane, 1,1,2,2- & 000079-34-5 & $1.3 \mathrm{E}+01$ & $1.3 \mathrm{E}-01$ & & & $2.7 \mathrm{E}+00$ & $2.7 \mathrm{E}-02$ & & & $2.2 \mathrm{E}+00$ & $2.2 \mathrm{E}-02$ & & \\
\hline Tetrachloroethylene ${ }^{(6)}$ & $000127-18-4$ & $5.1 \mathrm{E}+01$ & $5.1 \mathrm{E}-01$ & $1.1 \mathrm{E}+02$ & $1.1 E+01$ & $3.5 \mathrm{E}-01$ & $3.5 \mathrm{E}-03$ & $7.9 \mathrm{E}-01$ & $7.9 \mathrm{E}-02$ & $3.5 \mathrm{E}-01$ & $3.5 \mathrm{E}-03$ & $7.9 \mathrm{E}-01$ & $7.9 \mathrm{E}-02$ \\
\hline Tetrachlorophenol, 2,3,4,6- & $000058-90-2$ & & & $3.4 \mathrm{E}+02$ & $3.4 \mathrm{E}+01$ & & & $7.3 \mathrm{E}+00$ & 7.3E-01 & & & $7.2 \mathrm{E}+00$ & $7.2 \mathrm{E}-01$ \\
\hline $\begin{array}{l}\text { Tetrachlorotoluene, p-alpha, } \\
\text { alpha, alpha- }\end{array}$ & $005216-25-1$ & $1.3 \mathrm{E}-01$ & $1.3 \mathrm{E}-03$ & & & & & & & & & & \\
\hline
\end{tabular}




\begin{tabular}{|c|c|c|c|c|c|c|c|c|c|c|c|c|c|}
\hline & & & & & & & & & & & & $9-\tau \varepsilon-0 b+\angle 00$ & 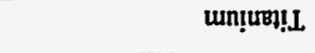 \\
\hline $20+96 \cdot 1$ & $\varepsilon 0+\not b^{\circ} I$ & & & $20+98^{\circ} \mathrm{I}$ & $\varepsilon 0+38^{\prime} \mathfrak{I}$ & & & $20+38^{\prime} 9$ & $\varepsilon 0+38^{\circ} 9$ & & & $s-1 \varepsilon-0 b+<00$ & u!L \\
\hline & & & & & & & & & & & & $1-6 z-0 t+\angle 00$ & un!̣oนL \\
\hline & & & & & & & & $00+a<\cdot s$ & $10+3 L ' S$ & & & 8-9Z-LEI000 & แณ!!̣L \\
\hline & & & & & & & & $10+9 I^{\prime} 6$ & $20+91 \% 6$ & & & $8-50-t 95 \varepsilon z 0$ & 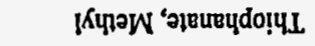 \\
\hline $10-9 \mathbf{b}^{\prime} \varepsilon$ & $00+3 b^{\circ} \varepsilon$ & & & $10+39 . L$ & $20+39{ }^{\circ} \mathrm{L}$ & & & $10-a b \varepsilon$ & $00+3 b \varepsilon$ & & & $\vdash 81-9616\{0$ & xourjo! $4 \mathrm{~L}$ \\
\hline & & & & & & & & $10+3 I I$ & $20+3 I^{\prime} I$ & & & $9-\angle L-6 b 28 Z 0$ & 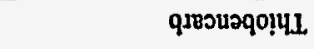 \\
\hline Z0-בI'E & $10-3 I^{\circ} \varepsilon$ & & & $20-3 c^{\circ} t$ & $10-3 L^{\circ} b$ & & & $20-\mathrm{al} 6$ & $10-a r^{\circ} 6$ & & & $9-81-9 t+b 00$ & गีEJ|nS un!||हपL \\
\hline zo-as' $\mathcal{E}$ & $10-9 S^{*} \mathcal{E}$ & & & $Z 0-\Xi E ' S$ & $10-a E^{\prime} S$ & & & $\mathrm{I0}-\mathrm{-3} 0^{\circ} \mathrm{I}$ & $00+30^{\circ} I$ & & & $0-25-6 \varepsilon 0210$ & 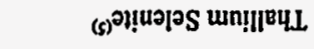 \\
\hline Z0-تI'E & I0-aI'E & & & $20-a L^{\prime} t$ & $10-a L^{\prime} t$ & & & $20-91 \% 6$ & I0-AI'6 & & & $0-2 I-I 6 L L 00$ & әр!บำนว แn!!|вчL \\
\hline & & & & & & & & $20-31 \% 6$ & $10-316$ & & & $6-\varepsilon L-\varepsilon \varepsilon S 900$ & 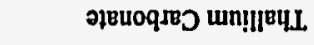 \\
\hline & & & & & & & & $10-30^{\circ} 1$ & $00+30^{\circ} 1$ & & & 8-89-£95000 & 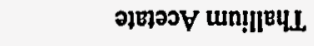 \\
\hline & & & & & & & & & & & & $0-8 z-0 t+\angle 00$ & 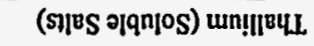 \\
\hline$z 0-A S \cdot \mathcal{E}$ & $10-\Xi S^{*} \mathcal{E}$ & & & $20-a \varepsilon ' s$ & I0-ą"S & & & $10-90^{\circ} \mathrm{I}$ & $00+30^{\circ} \mathrm{I}$ & & & t-st-201010 & 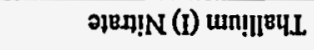 \\
\hline & & & & & & & & $20-36 \cdot \mathrm{L}$ & $10-96 . L$ & & & s-ZE-†IEI00 & 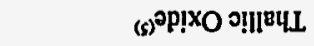 \\
\hline & & & & & & & & & & & & $\varepsilon-s L-0 t \downarrow \varepsilon 00$ & PBET |XdoudeñI \\
\hline & & & & & & & & & & & & $I-t L-s<0000$ & 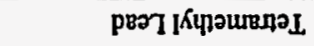 \\
\hline & & & & & & & & - & & & & $Z-L 6-I 18000$ & 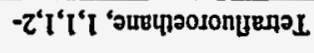 \\
\hline & & & & & & & & & & & & $\tau-00-8<0000$ & 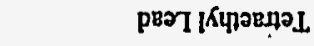 \\
\hline $10-98 \cdot I$ & $00+38 \cdot I$ & & & $10-392$ & $00+39 \cdot z$ & & & $10-a L S S$ & $00+3 L ' s$ & & & $s-\downarrow z-689 \varepsilon 00$ & 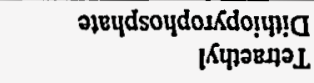 \\
\hline$I^{\prime} 0=\mathbf{I H}$ & $\mathfrak{l}=\mathbf{I H}$ & ,0I & roI & $I^{\circ} 0=0 H$ & $\mathfrak{l}=\mathbf{\partial H}$ & ,0I & roI & $I^{\circ} 0=\partial H$ & $I=\partial H$ & 9.0I & .0I & \multirow{2}{*}{ NXSYD } & \multirow{2}{*}{ [вว!ุนวัว } \\
\hline \multicolumn{4}{|c|}{ 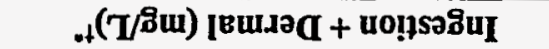 } & \multicolumn{4}{|c|}{.(T/8ిய) ןвщ.ә $\alpha$} & \multicolumn{4}{|c|}{.(T/8ిu) uo!̣sวรిนI } & & \\
\hline
\end{tabular}

(pənu!̣uos) 'вp' $\varepsilon$ ग1qв L 
Table 3.4a. (continued)

\begin{tabular}{|c|c|c|c|c|c|c|c|c|c|c|c|c|c|}
\hline \multirow{2}{*}{ Chemical } & \multirow{2}{*}{ CASRN } & \multicolumn{4}{|c|}{ Ingestion (mg/L)" } & \multicolumn{4}{|c|}{ Dermal (mg/L)" } & \multicolumn{4}{|c|}{ Ingestion + Dermal (mg/L) ${ }^{\dagger^{*}}$} \\
\hline & & $10^{-4}$ & $10^{-6}$ & $\mathrm{HQ}=1$ & $\mathrm{HQ}=0.1$ & $10^{-4}$ & $10^{-6}$ & $\mathrm{HQ}=1$ & $\mathrm{HQ}=0.1$ & $10^{-4}$ & $10^{-6}$ & $\mathrm{HI}=\mathbf{1}$ & $\mathrm{HI}=0.1$ \\
\hline Toluene $e^{(6)}$ & 000108-88-3 & & & $2.3 E+03$ & $2.3 \mathrm{E}+02$ & & & $1.0 \mathrm{E}+02$ & $1.0 \mathrm{E}+01$ & & & $1.0 \mathrm{E}+02$ & $1.0 \mathrm{E}+01$ \\
\hline $\begin{array}{l}\text { Toluene diisocyanate mixture } \\
\text { (TDI) }\end{array}$ & $026471-62-5$ & & & & & & & & & & & & \\
\hline Toluene-2,4-diamine & $000095-80-7$ & $8.3 \mathrm{E}-01$ & 8.3E-03 & & & $1.8 \mathrm{E}+00$ & $1.8 \mathrm{E}-02$ & & & $5.6 \mathrm{E}-01$ & $5.6 \mathrm{E}-03$ & & \\
\hline Toluene-2,5-diamine & $000095-70-5$ & & & $6.8 \mathrm{E}+03$ & $6.8 \mathrm{E}+02$ & & & & & & & & \\
\hline Toluene-2,6-diamine & $000823-40-5$ & & & $2.3 \mathrm{E}+03$ & $2.3 \mathrm{E}+02$ & & & & & & & & \\
\hline $\begin{array}{l}\text { Toluidine, 0- (Methylaniline, 2- } \\
\text { ) }\end{array}$ & $000095-53-4$ & $1.1 \mathrm{E}+01$ & $1.1 \mathrm{E}-01$ & & & $3.8 \mathrm{E}+00$ & $3.8 \mathrm{E}-02$ & & & $2.9 \mathrm{E}+00$ & $2.9 \mathrm{E}-02$ & & \\
\hline Toluidine, $p$ - & $000106-49-0$ & $1.4 \mathrm{E}+01$ & $1.4 \mathrm{E}-01$ & & & $1.6 \mathrm{E}+00$ & $1.6 \mathrm{E}-02$ & & & $1.4 \mathrm{E}+00$ & $1.4 \mathrm{E}-02$ & & \\
\hline Toxaphene & $008001-35-2$ & $2.4 \mathrm{E}+00$ & $2.4 \mathrm{E}-02$ & & & 2.1E-01 & 2.1E-03 & & & $1.9 \mathrm{E}-01$ & $1.9 \mathrm{E}-03$ & & \\
\hline Tralomethrin & $066841-25-6$ & & & $8.5 \mathrm{E}+01$ & $8.5 \mathrm{E}+00$ & & & & & & & & \\
\hline Triallate & $002303-17-5$ & & & $1.5 E+02$ & $1.5 \mathrm{E}+01$ & & & $1.1 \mathrm{E}+01$ & $1.1 \mathrm{E}+00$ & & & $1.0 \mathrm{E}+01$ & $1.0 \mathrm{E}+00$ \\
\hline Triasulfuron & 082097-50-5 & & & $1.1 \mathrm{E}+02$ & $1.1 \mathrm{E}+01$ & & & & & & & & \\
\hline Tribromobenzene, 1,2,4- & $000615-54-3$ & & & 5.7E+01 & $5.7 \mathrm{E}+00$ & & & & & & & & \\
\hline Tribromochloromethane & $000594-15-0$ & & & & & & & & & & & & \\
\hline Tribromodiphenyl Ether & 049690-94-0 & & & & & & & & & & & & \\
\hline Tributyltin Oxide & $000056-35-9$ & & & $3.4 \mathrm{E}-01$ & $3.4 \mathrm{E}-02$ & & & & & & & & \\
\hline $\begin{array}{l}\text { Trichloro-1,2,2-trifluoroethane, } \\
\text { 1,1,2- }\end{array}$ & $000076-13-1$ & & & $3.4 E+05$ & $3.4 E+04$ & & & 7.3E+04 & 7.3E+03 & & & $6.0 \mathrm{E}+04$ & $6.0 \mathrm{E}+03$ \\
\hline $\begin{array}{l}\text { Trichloro-2'- } \\
\text { hydroxydiphenylether }\end{array}$ & $003380-34-5$ & & & & & & & & & & & & \\
\hline Trichloroacetic Acid & & & & & & & & & & & & & \\
\hline
\end{tabular}

Nonradionuclides/groundwater/recreational

Page 32 of 36 
Table 3.4a. (continued)

\begin{tabular}{|c|c|c|c|c|c|c|c|c|c|c|c|c|c|}
\hline \multirow{2}{*}{ Chemical } & \multirow{2}{*}{ CASRN } & \multicolumn{4}{|c|}{ Ingestion (mg/L)" } & \multicolumn{4}{|c|}{ Dermal (mg/L)" } & \multicolumn{4}{|c|}{ Ingestion + Dermal $(\mathbf{m g} / \mathrm{L})^{\dagger *}$} \\
\hline & & $10^{-4}$ & $10^{6}$ & $H Q=1$ & $\mathrm{HQ}=0.1$ & $10^{-4}$ & $10^{6}$ & $\mathbf{H Q}=\mathbf{1}$ & $\mathrm{HQ}=0.1$ & $10^{-4}$ & $10^{6}$ & $H I=1$ & $\mathrm{HI}=0.1$ \\
\hline Trichloroaniline $\mathrm{HCl}, 2,4,6-$ & $033663-50-2$ & $9.1 \mathrm{E}+01$ & $9.1 \mathrm{E}-01$ & & & & & & & & & & \\
\hline Trichloroaniline, 2,4,6- & 000634-93-5 & $7.8 \mathrm{E}+01$ & $7.8 \mathrm{E}-01$ & & & & & & & & & & \\
\hline Trichlorobenzene, 1,2,4- & $000120-82-1$ & & & $1.1 \mathrm{E}+02$ & $1.1 \mathrm{E}+01$ & & & $2.8 \mathrm{E}+00$ & $2.8 \mathrm{E}-01$ & & & $2.8 \mathrm{E}+00$ & $2.8 \mathrm{E}-01$ \\
\hline Trichloroethane, 1,1,1-(5) & $000071-55-6$ & & & $1.0 \mathrm{E}+03$ & $1.0 \mathrm{E}+02$ & & & $1.4 \mathrm{E}+02$ & $1.4 \mathrm{E}+01$ & & & $1.2 E+02$ & $1.2 \mathrm{E}+01$ \\
\hline Trichloroethane, 1,1,2. & $000079-00-5$ & $4.6 E+01$ & $4.6 \mathrm{E}-01$ & $4.5 E+01$ & $4.5 \mathrm{E}+00$ & $1.2 \mathrm{E}+01$ & $1.2 \mathrm{E}-01$ & $1.1 \mathrm{E}+01$ & $1.1 \mathrm{E}+00$ & $9.2 E+00$ & $9.2 \mathrm{E}-02$ & $9.0 \mathrm{E}+00$ & $9.0 \mathrm{E}-01$ \\
\hline Trichloroethylene $^{(3)}$ & 000079-01-6 & $2.4 \mathrm{E}+02$ & $2.4 E+00$ & & & $5.8 \mathrm{E}+00$ & $5.8 \mathrm{E}-02$ & & & $5.7 \mathrm{E}+00$ & 5.7E-02 & & \\
\hline Trichlorofluoromethane & $000075-69-4$ & & & $3.4 \mathrm{E}+03$ & $3.4 \mathrm{E}+02$ & & & $1.2 \mathrm{E}+02$ & $1.2 \mathrm{E}+01$ & & & $1.1 \mathrm{E}+02$ & 1.1E+01 \\
\hline Trichlorophenol, 2,4,5- & $000095-95-4$ & & & $1.1 E+03$ & $1.1 \mathrm{E}+02$ & & & $3.9 \mathrm{E}+01$ & $3.9 \mathrm{E}+00$ & & & $3.8 \mathrm{E}+01$ & $3.8 \mathrm{E}+00$ \\
\hline Trichlorophenol, 2,4,6- & $000088-06-2$ & $2.4 \mathrm{E}+02$ & $2.4 E+00$ & & & $5.3 \mathrm{E}+00$ & $5.3 \mathrm{E}-02$ & & & $5.1 \mathrm{E}+00$ & 5.1E-02 & & \\
\hline $\begin{array}{l}\text { Trichlorophenoxy) Propionic } \\
\text { Acid, 2(2,4,5- }\end{array}$ & $000093-72-1$ & & & $9.1 \mathrm{E}+01$ & $9.1 \mathrm{E}+00$ & & & $4.6 \mathrm{E}+00$ & $4.6 \mathrm{E}-01$ & & & $4.4 \mathrm{E}+00$ & $4.4 \mathrm{E}-01$ \\
\hline $\begin{array}{l}\text { Trichlorophenoxyacetic Acid, } \\
\text { 2,4,5- }\end{array}$ & $000093-76-5$ & & & $1.1 \mathrm{E}+02$ & $1.1 \mathrm{E}+01$ & & & $4.0 \mathrm{E}+00$ & $4.0 \mathrm{E}-01$ & & & $3.9 \mathrm{E}+00$ & $3.9 \mathrm{E}-01$ \\
\hline Trichloropropane, 1,1,2- & $000598-77-6$ & & & $5.7 E+01$ & $5.7 \mathrm{E}+00$ & & & & & & & & \\
\hline Trichloropropane, 1,2,3- & $000096-18-4$ & $3.8 \mathrm{E}-01$ & $3.8 \mathrm{E}-03$ & $6.8 E+01$ & $6.8 \mathrm{E}+00$ & $1.8 \mathrm{E}-03$ & $1.8 \mathrm{E}-05$ & $3.2 \mathrm{E}-01$ & $3.2 \mathrm{E}-02$ & $1.8 \mathrm{E}-03$ & $1.8 \mathrm{E}-05$ & $3.2 \mathrm{E}-01$ & $3.2 \mathrm{E}-02$ \\
\hline Trichloropropene, 1,2,3- & $000096-19-5$ & & & $5.7 \mathrm{E}+01$ & $5.7 \mathrm{E}+00$ & & & & & & & & \\
\hline Trichlorotoluene, 2,3,6- & $002077-46-5$ & & & & & & & & & & & & \\
\hline Trichlorotoluene, alpha 2,6- & $002014-83-7$ & & & & & & & & & & & & \\
\hline Tridiphane & 058138-08-2 & & & $3.4 \mathrm{E}+01$ & $3.4 \mathrm{E}+00$ & & & & & & & & \\
\hline Triethyl Lead & $005224-23-7$ & & & & & & & & & & & & \\
\hline Triethylamine & $000121-44-8$ & & & & & & & & & & & & \\
\hline
\end{tabular}

Nonradionuclides/groundwater/recreational

Page 33 of 36 
Table 3.4a. (continued)

\begin{tabular}{|c|c|c|c|c|c|c|c|c|c|c|c|c|c|}
\hline \multirow{2}{*}{ Chemical } & \multirow{2}{*}{ CASRN } & \multicolumn{4}{|c|}{ Ingestion (mg/L)" } & \multicolumn{4}{|c|}{ Dermal (mg/L)" } & \multicolumn{4}{|c|}{ Ingestion + Dermal (mg/L) ${ }^{\dagger^{*}}$} \\
\hline & & $10^{-1}$ & $10^{-6}$ & $\mathrm{HQ}=1$ & $\mathrm{HQ}=0.1$ & $10^{-4}$ & $10^{-6}$ & $\mathrm{HQ}=1$ & $\mathrm{HQ}=0.1$ & $10^{-4}$ & $10^{-6}$ & $\mathrm{HI}=\mathbf{1}$ & $\mathrm{HI}=0.1$ \\
\hline Trifluralin & $001582-09-8$ & $3.4 \mathrm{E}+02$ & $3.4 \mathrm{E}+00$ & $8.5 \mathrm{E}+01$ & $8.5 \mathrm{E}+00$ & $4.5 \mathrm{E}+00$ & $4.5 \mathrm{E}-02$ & $1.1 \mathrm{E}+00$ & $1.1 \mathrm{E}-01$ & $4.4 \mathrm{E}+00$ & $4.4 \mathrm{E}-02$ & $1.1 \mathrm{E}+00$ & $1.1 \mathrm{E}-01$ \\
\hline Trimethyl Lead & $007442-13-9$ & & & & & & & & & & & & \\
\hline Trimethyl Phosphate & $000512-56-1$ & $7.2 \mathrm{E}+01$ & 7.2E-01 & & & & & & & & & & \\
\hline Trimethylethyl Lead & $001762-26-1$ & & & & & & & & & & & & \\
\hline Trinitrobenzene, 1,3,5- & 000099-35-4 & & & $5.7 \mathrm{E}-01$ & 5.7E-02 & & & $1.4 \mathrm{E}+00$ & $1.4 \mathrm{E}-01$ & & & 4.0E-01 & $4.0 \mathrm{E}-02$ \\
\hline Trinitrophenylmethylnitramine & $000479-45-8$ & & & $1.1 \mathrm{E}+02$ & $1.1 \mathrm{E}+01$ & & & $1.6 \mathrm{E}+02$ & $1.6 \mathrm{E}+01$ & & & $6.7 \mathrm{E}+01$ & $6.7 \mathrm{E}+00$ \\
\hline Trinitrotoluene, 2,4,6- & $000118-96-7$ & $8.8 \mathrm{E}+01$ & $8.8 \mathrm{E}-01$ & $5.7 \mathrm{E}+00$ & $5.7 \mathrm{E}-01$ & $4.1 \mathrm{E}+01$ & 4.1E-01 & $2.6 \mathrm{E}+00$ & $2.6 \mathrm{E}-01$ & $2.8 \mathrm{E}+01$ & $2.8 \mathrm{E}-01$ & $1.8 \mathrm{E}+00$ & $1.8 \mathrm{E}-01$ \\
\hline Tripropyl Lead & 006618-03-7 & & & & & & & & & & & & \\
\hline Uranium (Soluble Salts) & NA & & & $3.4 \mathrm{E}+01$ & $3.4 \mathrm{E}+00$ & & & 7.5E+01 & $7.5 \mathrm{E}+00$ & & & $2.3 \mathrm{E}+01$ & $2.3 \mathrm{E}+00$ \\
\hline Vanadium Pentoxide & $001314-62-1$ & & & $1.0 \mathrm{E}+02$ & $1.0 \mathrm{E}+01$ & & & $5.3 \mathrm{E}+01$ & $5.3 \mathrm{E}+00$ & & & $3.5 \mathrm{E}+01$ & $3.5 \mathrm{E}+00$ \\
\hline Vanadium Sulfate & $036907-42-3$ & & & 2.3E+02 & 2.3E+01 & & & $1.2 \mathrm{E}+02$ & $1.2 \mathrm{E}+01$ & & & $7.7 \mathrm{E}+01$ & $7.7 \mathrm{E}+00$ \\
\hline Vanadium, Metallic & $007440-62-2$ & & & $7.9 \mathrm{E}+01$ & $7.9 \mathrm{E}+00$ & & & $2.0 \mathrm{E}+00$ & $2.0 \mathrm{E}-01$ & & & $2.0 \mathrm{E}+00$ & $2.0 \mathrm{E}-01$ \\
\hline Vanadyl Sulfate & $027774-13-6$ & & & $2.3 \mathrm{E}+02$ & $2.3 \mathrm{E}+01$ & & & $1.2 \mathrm{E}+02$ & $1.2 E+01$ & & & 7.7E+01 & $7.7 \mathrm{E}+00$ \\
\hline Vernolate & $001929-77-7$ & & & $1.1 \mathrm{E}+01$ & $1.1 E+00$ & & & & & & & & \\
\hline Vinclozolin & $050471-44-8$ & & & $2.8 \mathrm{E}+02$ & $2.8 \mathrm{E}+01$ & & & & & & & & \\
\hline Vinyl Acetate & $000108-05-4$ & & & $1.1 \mathrm{E}+04$ & $1.1 \mathrm{E}+03$ & & & $1.6 \mathrm{E}+03$ & $1.6 \mathrm{E}+02$ & & & $1.4 \mathrm{E}+03$ & $1.4 E+02$ \\
\hline Vinyl Bromide & $000593-60-2$ & & & & & & & & & & & & \\
\hline Vinyl Chloride ${ }^{(6)}$ & $000075-01-4$ & $1.4 \mathrm{E}+00$ & $1.4 \mathrm{E}-02$ & & & $4.9 \mathrm{E}-01$ & $4.9 \mathrm{E}-03$ & & & $3.6 \mathrm{E}-01$ & $3.6 \mathrm{E}-03$ & & \\
\hline Warfarin & $000081-81-2$ & & & $3.4 \mathrm{E}+00$ & $3.4 \mathrm{E}-01$ & & & 9.4E-01 & $9.4 \mathrm{E}-02$ & & & 7.3E-01 & 7.3E-02 \\
\hline White Phosphorus & 007723-14-0 & & & $2.3 \mathrm{E}-01$ & $2.3 \mathrm{E}-02$ & & & $1.2 \mathrm{E}-01$ & $1.2 \mathrm{E}-02$ & & & $7.7 \mathrm{E}-02$ & $7.7 \mathrm{E}-03$ \\
\hline
\end{tabular}




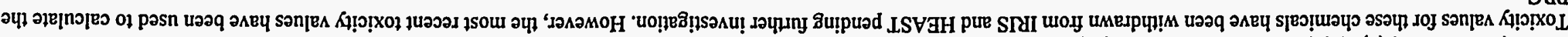

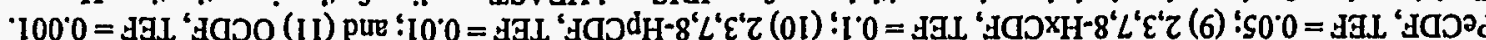

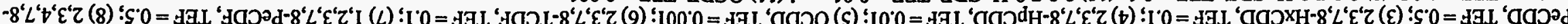

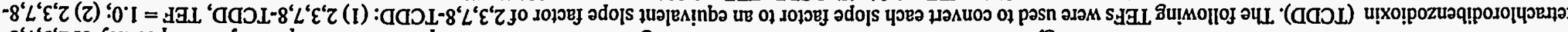

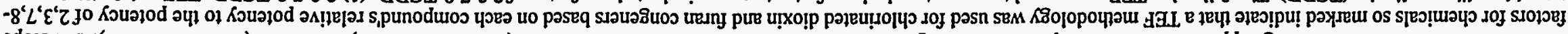

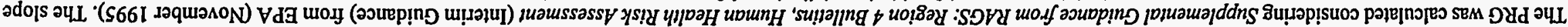

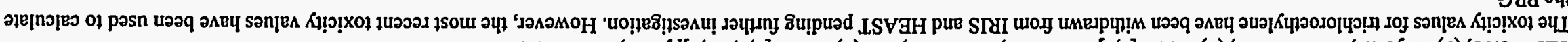

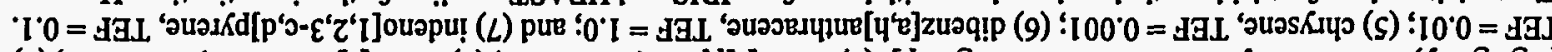

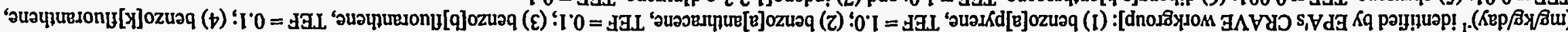

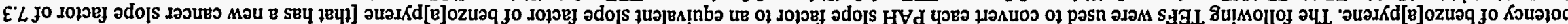

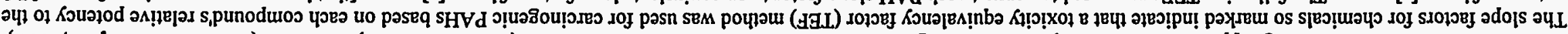

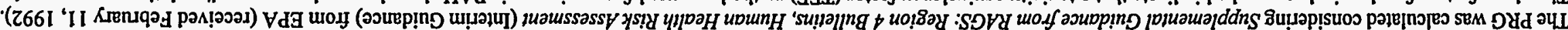

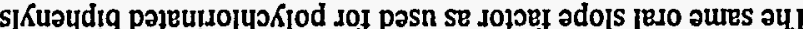

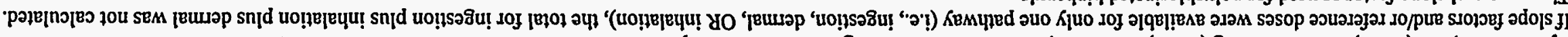

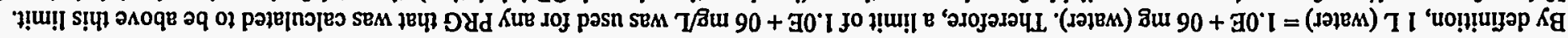

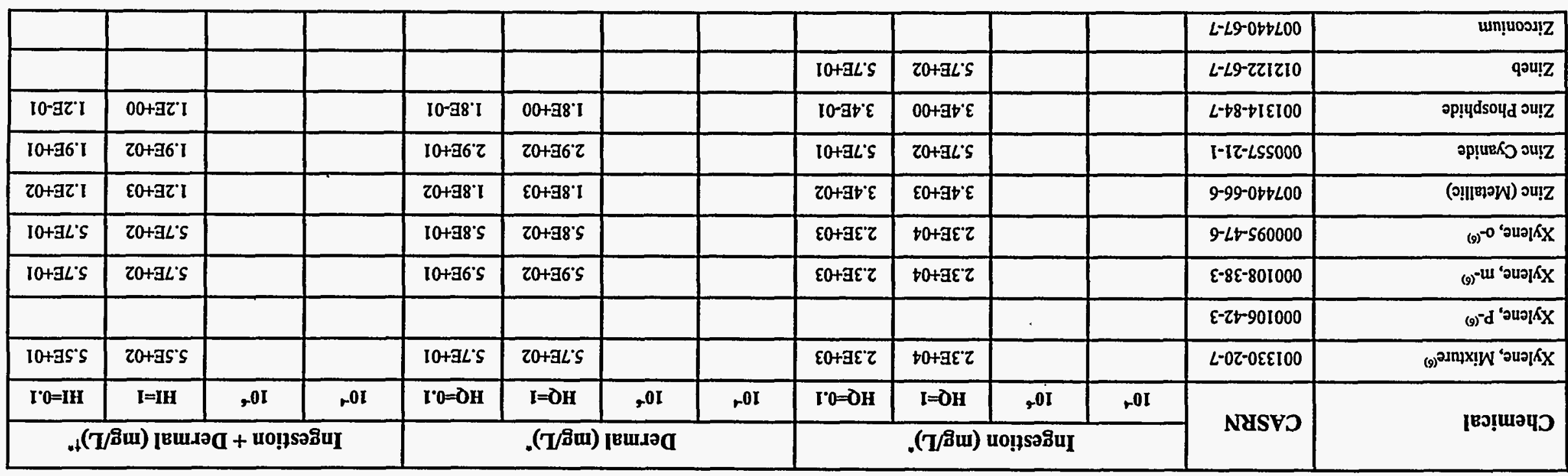

(ponu!ju00) 'вь'є गवвL 

Table 3.4b. Risk-based Preliminary Remediation Goals for radionuclides in groundwater (recreational scenario)

\begin{tabular}{|l|l|c|c|}
\hline \multirow{2}{*}{ Chemical } & \multirow{2}{*}{ CASRN } & \multicolumn{2}{|c|}{ Ingestion $(\mathrm{pCi} / \mathrm{L})$} \\
\cline { 3 - 4 } & & $10^{-4}$ & $10^{-6}$ \\
\hline Ac-225 & $014265-85-1$ & $1.0 \mathrm{E}+04$ & $1.0 \mathrm{E}+02$ \\
\hline Ac-227 & $014952-40-0$ & $4.2 \mathrm{E}+03$ & $4.2 \mathrm{E}+01$ \\
\hline Ac-227+D & $014952-40-0$ & $2.4 \mathrm{E}+03$ & $2.4 \mathrm{E}+01$ \\
\hline Ac-228 & $014331-83-0$ & $9.1 \mathrm{E}+05$ & $9.1 \mathrm{E}+03$ \\
\hline Ag-105 & $014928-14-4$ & $9.1 \mathrm{E}+05$ & $9.1 \mathrm{E}+03$ \\
\hline Ag-108 & $014391-65-2$ & $2.1 \mathrm{E}+08$ & $2.1 \mathrm{E}+06$ \\
\hline Ag-108m & $014391-65-2$ & $2.4 \mathrm{E}+05$ & $2.4 \mathrm{E}+03$ \\
\hline Ag-108m+D & $014391-65-2$ & $2.4 \mathrm{E}+05$ & $2.4 \mathrm{E}+03$ \\
\hline Ag-109m & $014378-38-2$ & $5.5 \mathrm{E}+09$ & $5.5 \mathrm{E}+07$ \\
\hline Ag-110 & $014391-76-5$ & $6.1 \mathrm{E}+08$ & $6.1 \mathrm{E}+06$ \\
\hline Ag-110m & $014391-76-5$ & $1.8 \mathrm{E}+05$ & $1.8 \mathrm{E}+03$ \\
\hline Ag-111 & $015760-04-0$ & $2.2 \mathrm{E}+05$ & $2.2 \mathrm{E}+03$ \\
\hline Am-241 & $014596-10-2$ & $4.5 \mathrm{E}+03$ & $4.5 \mathrm{E}+01$ \\
\hline Am-242 & $013981-54-9$ & $1.0 \mathrm{E}+06$ & $1.0 \mathrm{E}+04$ \\
\hline Am-242m & $013981-54-9$ & $5.1 \mathrm{E}+03$ & $5.1 \mathrm{E}+01$ \\
\hline Am-243 & $014993-75-0$ & $4.5 \mathrm{E}+03$ & $4.5 \mathrm{E}+01$ \\
\hline Am-243+D & $014993-75-0$ & $4.5 \mathrm{E}+03$ & $4.5 \mathrm{E}+01$ \\
\hline
\end{tabular}


Table 3.4b. (continued)

\begin{tabular}{|l|l|c|c|}
\hline \multirow{2}{*}{ Chemical $^{\dagger}$} & \multirow{2}{*}{ CASRN } & \multicolumn{2}{|c|}{ Ingestion (pCi/L) } \\
\cline { 3 - 4 } & & $10^{-4}$ & $10^{-6}$ \\
\hline Ar-41 & $014163-25-8$ & & \\
\hline At-217 & $017239-90-6$ & $1.6 \mathrm{E}+11$ & $1.6 \mathrm{E}+09$ \\
\hline Au-196 & $014914-16-0$ & $1.1 \mathrm{E}+06$ & $1.1 \mathrm{E}+04$ \\
\hline Au-198 & $010043-49-0$ & $2.8 \mathrm{E}+05$ & $2.8 \mathrm{E}+03$ \\
\hline Ba-131 & $014914-75-1$ & $8.7 \mathrm{E}+05$ & $8.7 \mathrm{E}+03$ \\
\hline Ba-133 & $013981-41-4$ & $5.5 \mathrm{E}+05$ & $5.5 \mathrm{E}+03$ \\
\hline Ba-133m & $013981-41-4$ & $5.4 \mathrm{E}+05$ & $5.4 \mathrm{E}+03$ \\
\hline Ba-137m & $013981-97-0$ & $6.1 \mathrm{E}+08$ & $6.1 \mathrm{E}+06$ \\
\hline Ba-139 & $014378-25-7$ & $4.9 \mathrm{E}+06$ & $4.9 \mathrm{E}+04$ \\
\hline Ba-140 & $014798-08-4$ & $1.3 \mathrm{E}+05$ & $1.3 \mathrm{E}+03$ \\
\hline Be-7 & $013966-02-4$ & $1.7 \mathrm{E}+07$ & $1.7 \mathrm{E}+05$ \\
\hline Bi-206 & $015776-19-9$ & $2.1 \mathrm{E}+05$ & $2.1 \mathrm{E}+03$ \\
\hline Bi-207 & $013982-38-2$ & $2.9 \mathrm{E}+05$ & $2.9 \mathrm{E}+03$ \\
\hline Bi-210 & $014331-79-4$ & $2.0 \mathrm{E}+05$ & $2.0 \mathrm{E}+03$ \\
\hline Bi-211 & $015229-37-5$ & $8.1 \mathrm{E}+07$ & $8.1 \mathrm{E}+05$ \\
\hline Bi-212 & $014913-49-6$ & $2.4 \mathrm{E}+06$ & $2.4 \mathrm{E}+04$ \\
\hline Bi-213 & $015776-20-2$ & $3.4 \mathrm{E}+06$ & $3.4 \mathrm{E}+04$ \\
\hline Bi-214 & $014733-03-0$ & $7.6 \mathrm{E}+06$ & $7.6 \mathrm{E}+04$ \\
\hline
\end{tabular}


Table 3.4b. (continued)

\begin{tabular}{|c|c|c|c|}
\hline \multirow{2}{*}{ Chemical $^{\dagger}$} & \multirow{2}{*}{ CASRN } & \multicolumn{2}{|c|}{ Ingestion (pCi/L) } \\
\hline & & $10^{-4}$ & $10^{-6}$ \\
\hline $\mathrm{Br}-82$ & $014686-69-2$ & $1.0 \mathrm{E}+06$ & $1.0 \mathrm{E}+04$ \\
\hline C-11 & $014333-33-6$ & $3.3 E+07$ & $3.3 E+05$ \\
\hline C-14 & $014762-75-5$ & $1.4 \mathrm{E}+06$ & $1.4 \mathrm{E}+04$ \\
\hline C-15 & $015929-23-4$ & $2.2 \mathrm{E}+09$ & $2.2 \mathrm{E}+07$ \\
\hline $\mathrm{Ca}-45$ & $013966-05-7$ & $7.3 \mathrm{E}+05$ & $7.3 E+03$ \\
\hline $\mathrm{Ca}-47$ & 001439-99-2 & $2.2 E+05$ & $2.2 \mathrm{E}+03$ \\
\hline Cd-109 & $014109-32-1$ & $1.8 \mathrm{E}+05$ & $1.8 \mathrm{E}+03$ \\
\hline Cd-115 & $014336-68-6$ & $2.0 \mathrm{E}+05$ & $2.0 \mathrm{E}+03$ \\
\hline $\mathrm{Cd}-115 \mathrm{~m}$ & $014336-68-6$ & $1.0 \mathrm{E}+05$ & $1.0 \mathrm{E}+03$ \\
\hline Ce-141 & $013967-74-3$ & $3.8 \mathrm{E}+05$ & $3.8 \mathrm{E}+03$ \\
\hline Ce-143 & $014119-19-8$ & $2.5 E+05$ & $2.5 \mathrm{E}+03$ \\
\hline Ce-144 & $014762-78-8$ & $5.0 \mathrm{E}+04$ & $5.0 \mathrm{E}+02$ \\
\hline Ce-144+D & $014762-78-8$ & $5.0 \mathrm{E}+04$ & $5.0 \mathrm{E}+02$ \\
\hline $\mathrm{Cl}-36$ & $013981-43-6$ & $6.6 \mathrm{E}+05$ & $6.6 \mathrm{E}+03$ \\
\hline $\mathrm{Cl}-38$ & $014158-34-0$ & $7.2 \mathrm{E}+06$ & 7.2E+04 \\
\hline $\mathrm{Cm}-242$ & $015510-73-3$ & $3.9 \mathrm{E}+04$ & $3.9 \mathrm{E}+02$ \\
\hline $\mathrm{Cm}-243$ & $015757-87-6$ & $5.9 \mathrm{E}+03$ & $5.9 \mathrm{E}+01$ \\
\hline $\mathrm{Cm}-243+\mathrm{D}$ & $015757-87-6$ & $5.9 \mathrm{E}+03$ & $5.9 \mathrm{E}+01$ \\
\hline
\end{tabular}


Table 3.4b. (continued)

\begin{tabular}{|l|l|c|c|}
\hline \multirow{2}{*}{ Chemical } & \multirow{2}{*}{ CASRN } & \multicolumn{2}{|c|}{ Ingestion (pCi/L) } \\
\cline { 3 - 4 } & & $10^{-4}$ & $10^{-6}$ \\
\hline Cm-244 & $013981-15-2$ & $7.0 \mathrm{E}+03$ & $7.0 \mathrm{E}+01$ \\
\hline Cm-245 & $015621-76-8$ & $4.4 \mathrm{E}+03$ & $4.4 \mathrm{E}+01$ \\
\hline Cm-246 & $015757-90-1$ & $4.5 \mathrm{E}+03$ & $4.5 \mathrm{E}+01$ \\
\hline Cm-247 & $015758-32-4$ & $4.8 \mathrm{E}+03$ & $4.8 \mathrm{E}+01$ \\
\hline Cm-248 & $015758-33-5$ & $1.1 \mathrm{E}+03$ & $1.1 \mathrm{E}+01$ \\
\hline Co-57 & $013981-50-5$ & $1.5 \mathrm{E}+06$ & $1.5 \mathrm{E}+04$ \\
\hline Co-58 & $013981-38-9$ & $5.3 \mathrm{E}+05$ & $5.3 \mathrm{E}+03$ \\
\hline Co-58m & $013981-38-9$ & $1.6 \mathrm{E}+07$ & $1.6 \mathrm{E}+05$ \\
\hline Co-60 & $010198-40-0$ & $7.8 \mathrm{E}+04$ & $7.8 \mathrm{E}+02$ \\
\hline Cr-51 & $014392-02-0$ & $1.1 \mathrm{E}+07$ & $1.1 \mathrm{E}+05$ \\
\hline Cs-131 & $014914-76-2$ & $8.2 \mathrm{E}+06$ & $8.2 \mathrm{E}+04$ \\
\hline Cs-134 & $013967-70-9$ & $3.1 \mathrm{E}+04$ & $3.1 \mathrm{E}+02$ \\
\hline Cs-134m & $013967-70-9$ & $3.3 \mathrm{E}+07$ & $3.3 \mathrm{E}+05$ \\
\hline Cs-135 & $015726-30-4$ & $3.3 \mathrm{E}+05$ & $3.3 \mathrm{E}+03$ \\
\hline Cs-136 & $014234-29-8$ & $1.9 \mathrm{E}+05$ & $1.9 \mathrm{E}+03$ \\
\hline Cs-137 & $010045-97-3$ & $4.7 \mathrm{E}+04$ & $4.7 \mathrm{E}+02$ \\
\hline Cs-137+D & $010045-97-3$ & $4.7 \mathrm{E}+04$ & $4.7 \mathrm{E}+02$ \\
\hline Cs-138 & $015758-29-9$ & $8.4 \mathrm{E}+06$ & $8.4 \mathrm{E}+04$ \\
\hline
\end{tabular}


Table 3.4b. (continued)

\begin{tabular}{|l|l|c|c|}
\hline \multirow{2}{*}{ Chemical } & \multirow{2}{*}{ CASRN } & \multicolumn{2}{|c|}{ Ingestion (pCi/L) } \\
\cline { 3 - 4 } & & $10^{-4}$ & $10^{-6}$ \\
\hline Cu-64 & $013981-25-4$ & $2.8 \mathrm{E}+06$ & $2.8 \mathrm{E}+04$ \\
\hline Dy-165 & $013967-64-1$ & $4.5 \mathrm{E}+06$ & $4.5 \mathrm{E}+04$ \\
\hline Dy-166 & $015840-01-4$ & $1.6 \mathrm{E}+05$ & $1.6 \mathrm{E}+03$ \\
\hline Er-169 & $015840-13-8$ & $7.0 \mathrm{E}+05$ & $7.0 \mathrm{E}+03$ \\
\hline Er-171 & $014391-45-8$ & $9.1 \mathrm{E}+05$ & $9.1 \mathrm{E}+03$ \\
\hline Eu-152 & $014683-23-9$ & $2.6 \mathrm{E}+05$ & $2.6 \mathrm{E}+03$ \\
\hline Eu-154 & $015585-10-1$ & $1.6 \mathrm{E}+05$ & $1.6 \mathrm{E}+03$ \\
\hline Eu-155 & $014391-16-3$ & $9.0 \mathrm{E}+05$ & $9.0 \mathrm{E}+03$ \\
\hline Eu-156 & $014280-35-4$ & $1.4 \mathrm{E}+05$ & $1.4 \mathrm{E}+03$ \\
\hline F-18 & $013981-56-1$ & $1.4 \mathrm{E}+07$ & $1.4 \mathrm{E}+05$ \\
\hline Fe-55 & $014681-59-5$ & $4.2 \mathrm{E}+06$ & $4.2 \mathrm{E}+04$ \\
\hline Fe-59 & $014596-12-4$ & $2.5 \mathrm{E}+05$ & $2.5 \mathrm{E}+03$ \\
\hline Fr-221 & $015756-41-9$ & $1.0 \mathrm{E}+07$ & $1.0 \mathrm{E}+05$ \\
\hline Fr-223 & $015756-98-6$ & $3.3 \mathrm{E}+06$ & $3.3 \mathrm{E}+04$ \\
\hline Ga-67 & $014119-09-6$ & $1.8 \mathrm{E}+06$ & $1.8 \mathrm{E}+04$ \\
\hline Ga-72 & $013982-22-4$ & $3.1 \mathrm{E}+05$ & $3.1 \mathrm{E}+03$ \\
\hline Gd-153 & $014276-65-4$ & $1.1 \mathrm{E}+06$ & $1.1 \mathrm{E}+04$ \\
\hline Gd-159 & $014041-42-0$ & $5.7 \mathrm{E}+05$ & $5.7 \mathrm{E}+03$ \\
\hline
\end{tabular}


Table 3.4b. (continued)

\begin{tabular}{|l|l|c|c|}
\hline \multirow{2}{*}{ Chemical } & \multirow{2}{*}{ CASRN } & \multicolumn{2}{|c|}{ Ingestion (pCi/L) } \\
\cline { 3 - 4 } & & $10^{-}$ & $10^{-6}$ \\
\hline Ge-71 & $014374-81-3$ & $1.3 \mathrm{E}+08$ & $1.3 \mathrm{E}+06$ \\
\hline H-3 & $010028-17-8$ & $2.1 \mathrm{E}+07$ & $2.1 \mathrm{E}+05$ \\
\hline Hg-197 & $013981-51-6$ & $1.3 \mathrm{E}+06$ & $1.3 \mathrm{E}+04$ \\
\hline Hg-203 & $013982-78-0$ & $5.6 \mathrm{E}+05$ & $5.6 \mathrm{E}+03$ \\
\hline Ho-166 & $013967-65-2$ & $2.0 \mathrm{E}+05$ & $2.0 \mathrm{E}+03$ \\
\hline I-122 & $018287-75-7$ & $6.9 \mathrm{E}+07$ & $6.9 \mathrm{E}+05$ \\
\hline I-123 & $015715-08-9$ & $2.7 \mathrm{E}+06$ & $2.7 \mathrm{E}+04$ \\
\hline I-125 & $014158-31-7$ & $5.7 \mathrm{E}+04$ & $5.7 \mathrm{E}+02$ \\
\hline I-126 & $014158-32-8$ & $3.1 \mathrm{E}+04$ & $3.1 \mathrm{E}+02$ \\
\hline I-129 & $015046-84-1$ & $8.1 \mathrm{E}+03$ & $8.1 \mathrm{E}+01$ \\
\hline I-130 & $014914-02-4$ & $3.1 \mathrm{E}+05$ & $3.1 \mathrm{E}+03$ \\
\hline I-131 & $010043-66-0$ & $4.1 \mathrm{E}+04$ & $4.1 \mathrm{E}+02$ \\
\hline I-132 & $014683-16-0$ & $2.2 \mathrm{E}+06$ & $2.2 \mathrm{E}+04$ \\
\hline I-133 & $014834-67-4$ & $1.4 \mathrm{E}+05$ & $1.4 \mathrm{E}+03$ \\
\hline I-134 & $014914-27-3$ & $6.4 \mathrm{E}+06$ & $6.4 \mathrm{E}+04$ \\
\hline I-135 & $014834-68-5$ & $6.5 \mathrm{E}+05$ & $6.5 \mathrm{E}+03$ \\
\hline In-113m & $014885-78-0$ & $1.8 \mathrm{E}+07$ & $1.8 \mathrm{E}+05$ \\
\hline In-114 & $013981-55-0$ & $3.3 \mathrm{E}+08$ & $3.3 \mathrm{E}+06$ \\
\hline
\end{tabular}


Table 3.4b. (continued)

\begin{tabular}{|l|l|c|c|}
\hline \multirow{2}{*}{ Chemical $^{\dagger}$} & \multirow{2}{*}{ CASRN } & \multicolumn{2}{|c|}{ Ingestion (pCi/L) } \\
\cline { 3 - 4 } & & $10^{-4}$ & $10^{-6}$ \\
\hline In-114m & $013981-55-0$ & $7.2 \mathrm{E}+04$ & $7.2 \mathrm{E}+02$ \\
\hline In-115 & $014191-71-0$ & $4.2 \mathrm{E}+04$ & $4.2 \mathrm{E}+02$ \\
\hline In-115m & $014191-71-0$ & $4.3 \mathrm{E}+06$ & $4.3 \mathrm{E}+04$ \\
\hline Ir-190 & $014981-91-0$ & $3.0 \mathrm{E}+05$ & $3.0 \mathrm{E}+03$ \\
\hline Ir-194 & $014158-35-1$ & $2.1 \mathrm{E}+05$ & $2.1 \mathrm{E}+03$ \\
\hline $\mathrm{K}-40$ & $013966-00-2$ & $1.2 \mathrm{E}+05$ & $1.2 \mathrm{E}+03$ \\
\hline $\mathrm{K}-42$ & $014378-21-3$ & $1.1 \mathrm{E}+06$ & $1.1 \mathrm{E}+04$ \\
\hline $\mathrm{Kr}-83 \mathrm{~m}$ & $013965-98-5$ & & \\
\hline $\mathrm{Kr}-85$ & $013983-27-2$ & & \\
\hline $\mathrm{Kr}-85 \mathrm{~m}$ & $013983-27-2$ & & \\
\hline $\mathrm{Kr}-87$ & $014809-68-8$ & & \\
\hline $\mathrm{Kr}-88$ & $014995-61-0$ & & \\
\hline $\mathrm{Kr}-89$ & $016316-03-3$ & & \\
\hline $\mathrm{Kr}-90$ & $015741-13-6$ & & $1.6 \mathrm{E}+03$ \\
\hline $\mathrm{La}-140$ & $013981-28-7$ & $1.6 \mathrm{E}+05$ & $5.0 \mathrm{E}+03$ \\
\hline $\mathrm{Lu}-177$ & $014265-75-9$ & $5.0 \mathrm{E}+05$ & \\
\hline $\mathrm{Mn}-52$ & $014092-99-0$ & $2.5 \mathrm{E}+05$ & \\
\hline $\mathrm{Mn}-54$ & $013966-31-9$ & $7.6 \mathrm{E}+05$ & \\
\hline
\end{tabular}


Table 3.4b. (continued)

\begin{tabular}{|l|l|c|c|}
\hline \multirow{2}{*}{ Chemical $^{\dagger}$} & \multirow{2}{*}{ CASRN } & \multicolumn{2}{|c|}{ Ingestion (pCi/L) } \\
\cline { 3 - 4 } & & $10^{-4}$ & $10^{-5}$ \\
\hline Mn-56 & $014681-52-8$ & $1.7 \mathrm{E}+06$ & $1.7 \mathrm{E}+04$ \\
\hline Mo-99 & $014119-15-4$ & $6.5 \mathrm{E}+05$ & $6.5 \mathrm{E}+03$ \\
\hline Na-22 & $013966-32-0$ & $1.8 \mathrm{E}+05$ & $1.8 \mathrm{E}+03$ \\
\hline Na-24 & $013982-04-2$ & $1.1 \mathrm{E}+06$ & $1.1 \mathrm{E}+04$ \\
\hline Nb-93m & $007440-03-1$ & $2.2 \mathrm{E}+06$ & $2.2 \mathrm{E}+04$ \\
\hline Nb-94 & $014681-63-1$ & $2.1 \mathrm{E}+05$ & $2.1 \mathrm{E}+03$ \\
\hline Nb-95 & $013967-76-5$ & $6.6 \mathrm{E}+05$ & $6.6 \mathrm{E}+03$ \\
\hline Nb-95m & $013967-76-5$ & $4.8 \mathrm{E}+05$ & $4.8 \mathrm{E}+03$ \\
\hline Nb-97 & $018496-04-3$ & $8.5 \mathrm{E}+06$ & $8.5 \mathrm{E}+04$ \\
\hline Nb-97m & $018496-04-3$ & $4.5 \mathrm{E}+08$ & $4.5 \mathrm{E}+06$ \\
\hline Nd-147 & $014269-74-0$ & $2.5 \mathrm{E}+05$ & $2.5 \mathrm{E}+03$ \\
\hline Nd-149 & $015749-81-2$ & $3.3 \mathrm{E}+06$ & $3.3 \mathrm{E}+04$ \\
\hline $\mathrm{Ni}-59$ & $014336-70-0$ & $8.0 \mathrm{E}+06$ & $8.0 \mathrm{E}+04$ \\
\hline Ni-63 & $013981-37-8$ & $2.7 \mathrm{E}+06$ & $2.7 \mathrm{E}+04$ \\
\hline Ni-65 & $014833-49-9$ & $2.6 \mathrm{E}+06$ & $2.6 \mathrm{E}+04$ \\
\hline Np-236 & $015700-36-4$ & $1.6 \mathrm{E}+06$ & $1.6 \mathrm{E}+04$ \\
\hline Np-237 & $013994-20-2$ & $5.0 \mathrm{E}+03$ & $5.0 \mathrm{E}+01$ \\
\hline Np-237+D & $013994-20-2$ & $4.9 \mathrm{E}+03$ & $4.9 \mathrm{E}+01$ \\
\hline
\end{tabular}


Table 3.4b. (continued)

\begin{tabular}{|l|l|c|c|}
\hline \multirow{2}{*}{ Chemical } & \multirow{2}{*}{ CASRN } & \multicolumn{2}{|c|}{ Ingestion (pCi/L) } \\
\cline { 3 - 4 } & & $10^{-4}$ & $10^{-6}$ \\
\hline Np-238 & $015766-25-3$ & $3.2 \mathrm{E}+05$ & $3.2 \mathrm{E}+03$ \\
\hline Np-239 & $013968-59-7$ & $3.5 \mathrm{E}+05$ & $3.5 \mathrm{E}+03$ \\
\hline Np-240 & $015690-84-3$ & $8.4 \mathrm{E}+06$ & $8.4 \mathrm{E}+04$ \\
\hline Np-240m & $015690-84-3$ & $6.1 \mathrm{E}+07$ & $6.1 \mathrm{E}+05$ \\
\hline Os-185 & $015766-50-4$ & $8.2 \mathrm{E}+05$ & $8.2 \mathrm{E}+03$ \\
\hline Os-191 & $014119-24-5$ & $4.9 \mathrm{E}+05$ & $4.9 \mathrm{E}+03$ \\
\hline Os-191m & $014119-24-5$ & $3.0 \mathrm{E}+06$ & $3.0 \mathrm{E}+04$ \\
\hline Os-193 & $016057-77-5$ & $3.4 \mathrm{E}+05$ & $3.4 \mathrm{E}+03$ \\
\hline $\mathrm{P}-32$ & $014596-37-3$ & $2.4 \mathrm{E}+05$ & $2.4 \mathrm{E}+03$ \\
\hline $\mathrm{P}-33$ & $015749-66-3$ & $1.9 \mathrm{E}+06$ & $1.9 \mathrm{E}+04$ \\
\hline Pa-231 & $014331-85-2$ & $9.9 \mathrm{E}+03$ & $9.9 \mathrm{E}+01$ \\
\hline Pa-233 & $013981-14-1$ & $3.2 \mathrm{E}+05$ & $3.2 \mathrm{E}+03$ \\
\hline Pa-234 & $015100-28-4$ & $7.0 \mathrm{E}+05$ & $7.0 \mathrm{E}+03$ \\
\hline Pa-234m & $015100-28-4$ & $3.1 \mathrm{E}+08$ & $3.1 \mathrm{E}+06$ \\
\hline Pb-203 & $014687-25-3$ & $1.4 \mathrm{E}+06$ & $1.4 \mathrm{E}+04$ \\
\hline Pb-209 & $014119-30-3$ & $7.1 \mathrm{E}+06$ & $7.1 \mathrm{E}+04$ \\
\hline Pb-210 & $014255-04-0$ & $2.2 \mathrm{E}+03$ & $2.2 \mathrm{E}+01$ \\
\hline Pb-210+D & $014255-04-0$ & $1.5 \mathrm{E}+03$ & $1.5 \mathrm{E}+01$ \\
\hline
\end{tabular}


Table 3.4b. (continued)

\begin{tabular}{|l|l|c|c|}
\hline \multirow{2}{*}{ Chemical $^{\dagger}$} & \multirow{2}{*}{ CASRN } & \multicolumn{2}{|c|}{ Ingestion (PCi/L) } \\
\cline { 3 - 4 } & & $10^{-4}$ & $10^{-6}$ \\
\hline Pb-211 & $015816-77-0$ & $4.4 \mathrm{E}+06$ & $4.4 \mathrm{E}+04$ \\
\hline Pb-212 & $015092-94-1$ & $8.2 \mathrm{E}+04$ & $8.2 \mathrm{E}+02$ \\
\hline Pb-214 & $015067-28-4$ & $5.0 \mathrm{E}+06$ & $5.0 \mathrm{E}+04$ \\
\hline Pd-100 & $015690-69-4$ & $4.0 \mathrm{E}+05$ & $4.0 \mathrm{E}+03$ \\
\hline Pd-101 & $015749-54-9$ & $4.0 \mathrm{E}+06$ & $4.0 \mathrm{E}+04$ \\
\hline Pd-103 & $014967-68-1$ & $1.4 \mathrm{E}+06$ & $1.4 \mathrm{E}+04$ \\
\hline Pd-107 & $017637-99-9$ & $7.1 \mathrm{E}+06$ & $7.1 \mathrm{E}+04$ \\
\hline Pd-109 & $014981-64-7$ & $4.4 \mathrm{E}+05$ & $4.4 \mathrm{E}+03$ \\
\hline Pm-147 & $014380-75-7$ & $1.1 \mathrm{E}+06$ & $1.1 \mathrm{E}+04$ \\
\hline Pm-148 & $014683-19-3$ & $1.0 \mathrm{E}+05$ & $1.0 \mathrm{E}+03$ \\
\hline Pm-148m & $014683-19-3$ & $1.5 \mathrm{E}+05$ & $1.5 \mathrm{E}+03$ \\
\hline Pm-149 & $015765-31-8$ & $2.7 \mathrm{E}+05$ & $2.7 \mathrm{E}+03$ \\
\hline Po-210 & $013981-52-7$ & $4.5 \mathrm{E}+03$ & $4.5 \mathrm{E}+01$ \\
\hline Po-212 & $015389-34-1$ & $3.3 \mathrm{E}+16$ & $3.3 \mathrm{E}+14$ \\
\hline Po-213 & $015756-57-7$ & $2.2 \mathrm{E}+15$ & $2.2 \mathrm{E}+13$ \\
\hline Po-214 & $015735-67-8$ & $7.0 \mathrm{E}+13$ & $7.0 \mathrm{E}+11$ \\
\hline Po-215 & $015706-52-2$ & $3.0 \mathrm{E}+12$ & $3.0 \mathrm{E}+10$ \\
\hline Po-216 & $015756-58-8$ & $1.7 \mathrm{E}+10$ & $1.7 \mathrm{E}+08$ \\
\hline
\end{tabular}


Table 3.4b. (continued)

\begin{tabular}{|l|l|c|c|}
\hline \multirow{2}{*}{ Chemical $^{\dagger}$} & \multirow{2}{*}{ CASRN } & \multicolumn{2}{|c|}{ Ingestion (pCi/L) } \\
\cline { 3 - 4 } & & $10^{-4}$ & $10^{-6}$ \\
\hline Po-218 & $015422-24-9$ & $2.9 \mathrm{E}+07$ & $2.9 \mathrm{E}+05$ \\
\hline Pr-142 & $014191-64-1$ & $2.1 \mathrm{E}+05$ & $2.1 \mathrm{E}+03$ \\
\hline Pr-143 & $014981-79-4$ & $2.2 \mathrm{E}+05$ & $2.2 \mathrm{E}+03$ \\
\hline Pr-144 & $014119-05-2$ & $1.8 \mathrm{E}+07$ & $1.8 \mathrm{E}+05$ \\
\hline Pr-144m & $014119-05-2$ & $4.6 \mathrm{E}+07$ & $4.6 \mathrm{E}+05$ \\
\hline Pt-191 & $015706-36-2$ & $9.9 \mathrm{E}+05$ & $9.9 \mathrm{E}+03$ \\
\hline Pt-193 & $015735-70-3$ & $9.1 \mathrm{E}+06$ & $9.1 \mathrm{E}+04$ \\
\hline Pt-193m & $015735-70-3$ & $5.9 \mathrm{E}+05$ & $5.9 \mathrm{E}+03$ \\
\hline Pt-197 & $015735-74-7$ & $7.0 \mathrm{E}+05$ & $7.0 \mathrm{E}+03$ \\
\hline Pt-197m & $015735-74-7$ & $4.6 \mathrm{E}+06$ & $4.6 \mathrm{E}+04$ \\
\hline Pu-236 & $015411-92-4$ & $1.9 \mathrm{E}+04$ & $1.9 \mathrm{E}+02$ \\
\hline Pu-238 & $013981-16-3$ & $5.0 \mathrm{E}+03$ & $5.0 \mathrm{E}+01$ \\
\hline Pu-239 & $015117-48-3$ & $4.7 \mathrm{E}+03$ & $4.7 \mathrm{E}+01$ \\
\hline Pu-240 & $014119-33-6$ & $4.7 \mathrm{E}+03$ & $4.7 \mathrm{E}+01$ \\
\hline Pu-241 & $014119-32-5$ & $2.8 \mathrm{E}+05$ & $2.8 \mathrm{E}+03$ \\
\hline Pu-241+D & $014119-32-5$ & $4.4 \mathrm{E}+03$ & $4.4 \mathrm{E}+01$ \\
\hline Pu-242 & $013982-10-0$ & $4.9 \mathrm{E}+03$ & $4.9 \mathrm{E}+01$ \\
\hline Pu-243 & $015706-37-3$ & $4.0 \mathrm{E}+06$ & $4.0 \mathrm{E}+04$ \\
\hline
\end{tabular}


0
4
0
ㄴ.
0
0
0
0

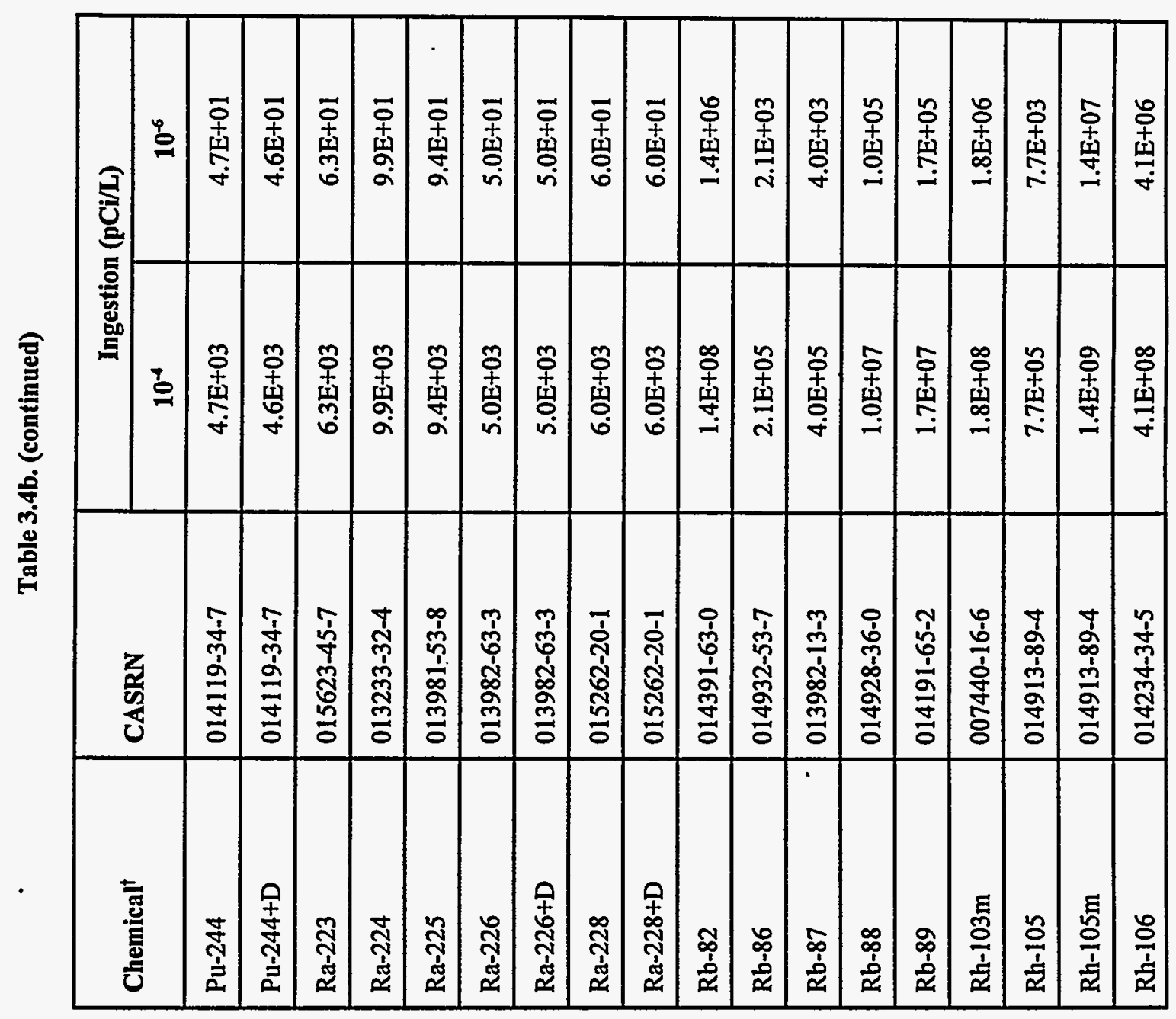

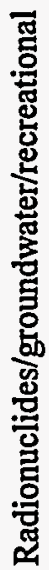


Table 3.4b. (continued)

\begin{tabular}{|l|l|c|c|}
\hline \multirow{2}{*}{ Chemical $^{\dagger}$} & \multirow{2}{*}{ CASRN } & \multicolumn{2}{|c|}{ Ingestion (pCi/L) } \\
\cline { 3 - 4 } & & $10^{-4}$ & $10^{-6}$ \\
\hline Rn-219 & $014835-02-0$ & & \\
\hline Rn-220 & $022481-48-7$ & & \\
\hline Rn-222+D & $014859-67-7$ & & \\
\hline Ru-103 & $013968-53-1$ & $4.5 \mathrm{E}+05$ & $4.5 \mathrm{E}+03$ \\
\hline Ru-105 & $014331-95-4$ & $1.3 \mathrm{E}+06$ & $1.3 \mathrm{E}+04$ \\
\hline Ru-106 & $013967-48-1$ & $4.3 \mathrm{E}+04$ & $4.3 \mathrm{E}+02$ \\
\hline $\mathrm{Ru}-106+\mathrm{D}$ & $013967-48-1$ & $4.3 \mathrm{E}+04$ & $4.3 \mathrm{E}+02$ \\
\hline $\mathrm{Ru}-97$ & $015758-35-7$ & $2.5 \mathrm{E}+06$ & $2.5 \mathrm{E}+04$ \\
\hline $\mathrm{S}-35$ & $015117-53-0$ & $3.6 \mathrm{E}+06$ & $3.6 \mathrm{E}+04$ \\
\hline $\mathrm{Sb}-122$ & $014374-79-9$ & $1.7 \mathrm{E}+05$ & $1.7 \mathrm{E}+03$ \\
\hline $\mathrm{Sb}-124$ & $014683-10-4$ & $1.4 \mathrm{E}+05$ & $1.4 \mathrm{E}+03$ \\
\hline $\mathrm{Sb}-125$ & $014234-35-6$ & $5.0 \mathrm{E}+05$ & $5.0 \mathrm{E}+03$ \\
\hline $\mathrm{Sb}-125+\mathrm{D}$ & $014234-35-6$ & $4.2 \mathrm{E}+05$ & $4.2 \mathrm{E}+03$ \\
\hline $\mathrm{Sb}-126$ & $015756-32-8$ & $1.5 \mathrm{E}+05$ & $1.5 \mathrm{E}+03$ \\
\hline $\mathrm{Sb}-126 \mathrm{~m}$ & $015756-32-8$ & $2.0 \mathrm{E}+07$ & $2.0 \mathrm{E}+05$ \\
\hline $\mathrm{Sb}-127$ & $013968-50-8$ & $1.7 \mathrm{E}+05$ & $1.7 \mathrm{E}+03$ \\
\hline $\mathrm{Sb}-129$ & $014331-88-5$ & $8.0 \mathrm{E}+05$ & $8.0 \mathrm{E}+03$ \\
\hline $\mathrm{Sc}-46$ & $013967-63-0$ & $2.6 \mathrm{E}+05$ & $2.6 \mathrm{E}+03$ \\
\hline
\end{tabular}


Table 3.4b. (continued)

\begin{tabular}{|l|l|c|c|}
\hline \multirow{2}{*}{ Chemical } & \multirow{2}{*}{ CASRN } & \multicolumn{2}{|c|}{ Ingestion (pCi/L) } \\
\cline { 3 - 4 } & & $10^{-4}$ & $10^{-6}$ \\
\hline Sc-47 & $014391-96-9$ & $5.0 \mathrm{E}+05$ & $5.0 \mathrm{E}+03$ \\
\hline Sc-48 & $014391-86-7$ & $2.2 \mathrm{E}+05$ & $2.2 \mathrm{E}+03$ \\
\hline Se-75 & $014265-71-5$ & $2.3 \mathrm{E}+05$ & $2.3 \mathrm{E}+03$ \\
\hline Si-31 & $014276-49-4$ & $2.9 \mathrm{E}+06$ & $2.9 \mathrm{E}+04$ \\
\hline Sm-147 & $014392-33-7$ & $5.9 \mathrm{E}+04$ & $5.9 \mathrm{E}+02$ \\
\hline Sm-151 & $015715-94-3$ & $3.2 \mathrm{E}+06$ & $3.2 \mathrm{E}+04$ \\
\hline Sm-153 & $015766-00-4$ & $3.7 \mathrm{E}+05$ & $3.7 \mathrm{E}+03$ \\
\hline Sn-113 & $013966-06-8$ & $4.0 \mathrm{E}+05$ & $4.0 \mathrm{E}+03$ \\
\hline Sn-121 & $014683-06-8$ & $1.2 \mathrm{E}+06$ & $1.2 \mathrm{E}+04$ \\
\hline Sn-121m & $014683-06-8$ & $7.4 \mathrm{E}+05$ & $7.4 \mathrm{E}+03$ \\
\hline Sn-125 & $014683-08-0$ & $8.8 \mathrm{E}+04$ & $8.8 \mathrm{E}+02$ \\
\hline Sn-126 & $015832-50-5$ & $7.0 \mathrm{E}+04$ & $7.0 \mathrm{E}+02$ \\
\hline Sr-82 & $014809-50-8$ & $5.7 \mathrm{E}+04$ & $5.7 \mathrm{E}+02$ \\
\hline Sr-85 & $013967-73-2$ & $1.1 \mathrm{E}+06$ & $1.1 \mathrm{E}+04$ \\
\hline Sr-85m & $013967-73-2$ & $8.2 \mathrm{E}+07$ & $8.2 \mathrm{E}+05$ \\
\hline Sr-89 & $014158-27-1$ & $1.4 \mathrm{E}+05$ & $1.4 \mathrm{E}+03$ \\
\hline Sr-90 & $010098-97-2$ & $3.6 \mathrm{E}+04$ & $3.6 \mathrm{E}+02$ \\
\hline Sr-90+D & $010098-97-2$ & $2.7 \mathrm{E}+04$ & $2.7 \mathrm{E}+02$ \\
\hline
\end{tabular}


Table 3.4b. (continued)

\begin{tabular}{|l|l|c|c|}
\hline \multirow{2}{*}{ Chemical $^{\dagger}$} & \multirow{2}{*}{ CASRN } & \multicolumn{2}{|c|}{ Ingestion (pCi/L) } \\
\cline { 3 - 4 } & & $10^{-4}$ & $10^{-6}$ \\
\hline Sr-91 & $014331-91-0$ & $5.3 \mathrm{E}+05$ & $5.3 \mathrm{E}+03$ \\
\hline Sr-92 & $014928-29-1$ & $7.3 \mathrm{E}+05$ & $7.3 \mathrm{E}+03$ \\
\hline Ta-182 & $013982-00-8$ & $2.1 \mathrm{E}+05$ & $2.1 \mathrm{E}+03$ \\
\hline Tb-158 & $015759-55-4$ & $3.5 \mathrm{E}+05$ & $3.5 \mathrm{E}+03$ \\
\hline Tb-160 & $013981-29-8$ & $1.9 \mathrm{E}+05$ & $1.9 \mathrm{E}+03$ \\
\hline Tc-95 & $014809-56-4$ & $2.2 \mathrm{E}+07$ & $2.2 \mathrm{E}+05$ \\
\hline Tc-95m & $014809-56-4$ & $1.2 \mathrm{E}+06$ & $1.2 \mathrm{E}+04$ \\
\hline Tc-96 & $014808-44-7$ & $6.5 \mathrm{E}+05$ & $6.5 \mathrm{E}+03$ \\
\hline Tc-96m & $014808-44-7$ & $5.7 \mathrm{E}+07$ & $5.7 \mathrm{E}+05$ \\
\hline Tc-97 & $015759-35-0$ & $9.4 \mathrm{E}+06$ & $9.4 \mathrm{E}+04$ \\
\hline Tc-97m & $015759-35-0$ & $1.2 \mathrm{E}+06$ & $1.2 \mathrm{E}+04$ \\
\hline Tc-99 & $014133-76-7$ & $1.1 \mathrm{E}+06$ & $1.1 \mathrm{E}+04$ \\
\hline Tc-99m & $014133-76-7$ & $2.7 \mathrm{E}+07$ & $2.7 \mathrm{E}+05$ \\
\hline Te-125m & $014390-73-9$ & $5.9 \mathrm{E}+05$ & $5.9 \mathrm{E}+03$ \\
\hline Te-127 & $013981-49-2$ & $1.7 \mathrm{E}+06$ & $1.7 \mathrm{E}+04$ \\
\hline Te-127m & $013981-49-2$ & $2.5 \mathrm{E}+05$ & $2.5 \mathrm{E}+03$ \\
\hline Te-129 & $014269-71-7$ & $1.0 \mathrm{E}+07$ & $1.0 \mathrm{E}+05$ \\
\hline Te-129m & $014269-71-7$ & $1.3 \mathrm{E}+05$ & $1.3 \mathrm{E}+03$ \\
\hline
\end{tabular}


Table 3.4b. (continued)

\begin{tabular}{|l|l|c|c|}
\hline \multirow{2}{*}{ Chemical } & \multirow{2}{*}{ CASRN } & \multicolumn{2}{|c|}{ Ingestion (pCi/L) } \\
\cline { 3 - 4 } & & $10^{-1}$ & $10^{-6}$ \\
\hline Te-131 & $014683-12-6$ & $3.8 \mathrm{E}+06$ & $3.8 \mathrm{E}+04$ \\
\hline Te-131m & $014683-12-6$ & $1.7 \mathrm{E}+05$ & $1.7 \mathrm{E}+03$ \\
\hline Te-132 & $014234-28-7$ & $1.2 \mathrm{E}+05$ & $1.2 \mathrm{E}+03$ \\
\hline Th-227 & $015623-47-9$ & $3.7 \mathrm{E}+04$ & $3.7 \mathrm{E}+02$ \\
\hline Th-228 & $014274-82-9$ & $2.4 \mathrm{E}+04$ & $2.4 \mathrm{E}+02$ \\
\hline Th-228+D & $014274-82-9$ & $6.4 \mathrm{E}+03$ & $6.4 \mathrm{E}+01$ \\
\hline Th-229 & $015594-54-4$ & $2.6 \mathrm{E}+04$ & $2.6 \mathrm{E}+02$ \\
\hline Th-229+D & $015594-54-4$ & $4.2 \mathrm{E}+03$ & $4.2 \mathrm{E}+01$ \\
\hline Th-230 & $014269-63-7$ & $4.0 \mathrm{E}+04$ & $4.0 \mathrm{E}+02$ \\
\hline Th-231 & $014932-40-2$ & $8.3 \mathrm{E}+05$ & $8.3 \mathrm{E}+03$ \\
\hline Th-232 & $007440-29-1$ & $4.5 \mathrm{E}+04$ & $4.5 \mathrm{E}+02$ \\
\hline Th-234 & $015065-10-8$ & $7.7 \mathrm{E}+04$ & $7.7 \mathrm{E}+02$ \\
\hline Tl-202 & $015720-57-7$ & $1.5 \mathrm{E}+06$ & $1.5 \mathrm{E}+04$ \\
\hline Tl-204 & $013968-51-9$ & $7.5 \mathrm{E}+05$ & $7.5 \mathrm{E}+03$ \\
\hline Tl-208 & $014913-50-9$ & $8.5 \mathrm{E}+07$ & $8.5 \mathrm{E}+05$ \\
\hline Tl-209 & $015690-73-0$ & $1.1 \mathrm{E}+08$ & $1.1 \mathrm{E}+06$ \\
\hline Tm-170 & $013981-30-1$ & $2.0 \mathrm{E}+05$ & $2.0 \mathrm{E}+03$ \\
\hline Tm-171 & $014333-45-0$ & $2.5 \mathrm{E}+06$ & $2.5 \mathrm{E}+04$ \\
\hline
\end{tabular}


Table 3.4b. (continued)

\begin{tabular}{|l|l|c|c|}
\hline \multirow{2}{*}{ Chemical } & \multirow{2}{*}{ CASRN } & \multicolumn{2}{|c|}{ Ingestion (pCi/L) } \\
\cline { 3 - 4 } & & $10^{-4}$ & $10^{-6}$ \\
\hline U-232 & $014158-29-3$ & $1.8 \mathrm{E}+04$ & $1.8 \mathrm{E}+02$ \\
\hline U-233 & $013968-55-3$ & $3.3 \mathrm{E}+04$ & $3.3 \mathrm{E}+02$ \\
\hline U-234 & $013966-29-5$ & $3.3 \mathrm{E}+04$ & $3.3 \mathrm{E}+02$ \\
\hline U-235 & $015117-96-1$ & $3.3 \mathrm{E}+04$ & $3.3 \mathrm{E}+02$ \\
\hline $\mathrm{U}-235+\mathrm{D}$ & $015117-96-1$ & $3.2 \mathrm{E}+04$ & $3.2 \mathrm{E}+02$ \\
\hline $\mathrm{U}-236$ & $013982-70-2$ & $3.5 \mathrm{E}+04$ & $3.5 \mathrm{E}+02$ \\
\hline $\mathrm{U}-237$ & $014269-75-1$ & $3.7 \mathrm{E}+05$ & $3.7 \mathrm{E}+03$ \\
\hline $\mathrm{U}-238$ & $007440-61-1$ & $3.5 \mathrm{E}+04$ & $3.5 \mathrm{E}+02$ \\
\hline $\mathrm{U}-238+\mathrm{D}$ & $007440-61-1$ & $2.4 \mathrm{E}+04$ & $2.4 \mathrm{E}+02$ \\
\hline $\mathrm{U}-240$ & $015687-53-3$ & $2.7 \mathrm{E}+05$ & $2.7 \mathrm{E}+03$ \\
\hline V-48 & $014331-97-6$ & $2.0 \mathrm{E}+05$ & $2.0 \mathrm{E}+03$ \\
\hline W-181 & $015749-46-9$ & $5.4 \mathrm{E}+06$ & $5.4 \mathrm{E}+04$ \\
\hline W-185 & $014932-41-3$ & $7.3 \mathrm{E}+05$ & $7.3 \mathrm{E}+03$ \\
\hline W-187 & $014983-48-3$ & $6.0 \mathrm{E}+05$ & $6.0 \mathrm{E}+03$ \\
\hline Xe-122 & $015151-09-4$ & & \\
\hline Xe-123 & $015700-10-4$ & & \\
\hline Xe-125 & $013994-18-8$ & & \\
\hline Xe-127 & $013994-19-9$ & & \\
\hline
\end{tabular}


Table 3.4b. (continued)

\begin{tabular}{|c|c|c|c|}
\hline \multirow{2}{*}{ Chemical $^{\dagger}$} & \multirow{2}{*}{ CASRN } & \multicolumn{2}{|c|}{ Ingestion ( $\mathrm{pCi} / \mathrm{L}$ ) } \\
\hline & & $10^{-4}$ & $10^{-6}$ \\
\hline $\mathrm{Xe}-129 \mathrm{~m}$ & 013965-99-6 & & \\
\hline $\mathrm{Xe}-131 \mathrm{~m}$ & 014683-11-5 & & \\
\hline Xe-133 & $014932-42-4$ & & \\
\hline $\mathrm{Xe}-133 \mathrm{~m}$ & $014932-42-4$ & & \\
\hline $\mathrm{Xe}-135$ & 014995-62-1 & & \\
\hline $\mathrm{Xe}-135 \mathrm{~m}$ & 014995-62-1 & & . \\
\hline $\mathrm{Xe}-137$ & 014835-21-3 & 9 & \\
\hline Xe-138 & 015751-81-2 & & \\
\hline Y-90 & 010098-91-6 & $9.9 \mathrm{E}+04$ & $9.9 \mathrm{E}+02$ \\
\hline Y-91 & 014234-24-3 & $1.1 \mathrm{E}+05$ & $1.1 \mathrm{E}+03$ \\
\hline $\mathrm{Y}-91 \mathrm{~m}$ & 014234-24-3 & $4.0 \mathrm{E}+07$ & $4.0 \mathrm{E}+05$ \\
\hline Y-92 & $015751-59-4$ & $7.6 \mathrm{E}+05$ & $7.6 \mathrm{E}+03$ \\
\hline Y-93 & $014981-70-5$ & $2.6 \mathrm{E}+05$ & $2.6 \mathrm{E}+03$ \\
\hline $\mathrm{Zn}-65$ & 013982-39-3 & $1.5 \mathrm{E}+05$ & $1.5 \mathrm{E}+03$ \\
\hline $\mathrm{Zn}-69$ & 013982-23-5 & $2.4 \mathrm{E}+07$ & $2.4 \mathrm{E}+05$ \\
\hline $\mathrm{Zn}-69 \mathrm{~m}$ & 013982-23-5 & $9.7 \mathrm{E}+05$ & $9.7 \mathrm{E}+03$ \\
\hline Zr-93 & $015751-77-6$ & $2.8 \mathrm{E}+06$ & $2.8 \mathrm{E}+04$ \\
\hline $\mathrm{Zr}-95$ & 013967-71-0 & $3.8 \mathrm{E}+05$ & $3.8 \mathrm{E}+03$ \\
\hline
\end{tabular}


Table 3.4b. (continued)

\begin{tabular}{|l|l|c|c|}
\hline \multirow{2}{*}{ Chemical $^{\dagger}$} & \multirow{2}{*}{ CASRN } & \multicolumn{2}{|c|}{ Ingestion (pCi/L) } \\
\cline { 3 - 4 } & & $10^{-4}$ & $10^{-6}$ \\
\hline Zr-97 & $014928-30-4$ & $1.4 \mathrm{E}+05$ & $1.4 \mathrm{E}+03$ \\
\hline
\end{tabular}

Note:

$\dagger$ Isotopes designated with an " $m$ " indicate that the isotope is metastable; isotopes designated with $a$ " $+D$ " indicate that the radionuclide slope factors include contributions from daughter products. 
3-568

, 
Table 3.4c. Risk-based Preliminary Remediation Goals for nonradionuclides in soil (recreational scenario)

\begin{tabular}{|c|c|c|c|c|c|c|c|c|c|c|c|c|c|c|c|c|c|c|c|}
\hline \multirow{2}{*}{ Chemical } & \multirow{2}{*}{ CASRN } & \multicolumn{6}{|c|}{ Ingestion (mg/kg) } & \multicolumn{4}{|c|}{ Dermal (mgkg) } & \multicolumn{4}{|c|}{ Inhalation $(\mathrm{mg} / \mathrm{kg})^{* *}$} & \multicolumn{4}{|c|}{$\begin{array}{c}\text { Ingestion + Dermal + Inhalation } \\
(\mathrm{mg} / \mathrm{kg})^{+* 1}\end{array}$} \\
\hline & & $10^{-4}$ & $10^{4}$ & $\begin{array}{l}\text { HQ }=1 \\
\text { Child }\end{array}$ & $\begin{array}{l}\text { HQ=1 } \\
\text { Adult }\end{array}$ & $\begin{array}{c}\mathrm{HQ}=0.1 \\
\text { Adult }\end{array}$ & $\begin{array}{l}\text { HQ }=0.1 \\
\text { Child }\end{array}$ & $10^{-4}$ & $10^{2}$ & $\mathrm{HQ}=\mathbf{1}$ & $\mathrm{HQ}=0.1$ & $10^{-4}$ & $10^{4}$ & $\mathrm{HQ}=1$ & $\mathrm{HQ}=0.1$ & $10^{-4}$ & $10^{-6}$ & $\mathrm{HI}=\mathbf{1}$ & $\mathrm{HI}=0.1$ \\
\hline ALAR & $001596-84-5$ & & & $1.0 \mathrm{E}+05$ & $1.0 \mathrm{E}+06$ & $1.0 \mathrm{E}+06$ & $1.3 E+05$ & & & $4.8 E+05$ & 4.8E+04 & & & & & - & & $4.6 \mathrm{E}+05$ & $4.6 \mathrm{E}+04$ \\
\hline Acenaphthene & $000083-32-9$ & & & $5.3 E+05$ & $1.0 \mathrm{E}+06$ & $4.9 E+05$ & $5.3 \mathrm{E}+04$ & & & $1.2 E+05$ & $1.2 E+04$ & & & & & & & $1.2 \mathrm{E}+05$ & $1.2 E+04$ \\
\hline Acenaphthylene & $000208-96-8$ & & & & & & & & & & & & & & & & & & \\
\hline Acephate & $030560-19.1$ & $8.2 \mathrm{E}+05$ & $8.2 E+03$ & $3.5 \mathrm{E}+04$ & $3.3 E+05$ & $3.3 \mathrm{E}+04$ & $3.5 E+03$ & $8.6 \mathrm{E}+04$ & $8.6 \mathrm{E}+02$ & $1.3 \mathrm{E}+04$ & $1.3 E+03$ & & & & & $8.3 E+04$ & $8.3 E+02$ & $1.2 \mathrm{E}+04$ & $1.2 E+03$ \\
\hline Acetaldehyde & $000075-07-0$ & & & & & & & & & & & $4.9 \mathrm{E}+04$ & $7.4 E+03$ & $4.9 E+04$ & $6.3 E+03$ & & & & \\
\hline Acetochior & $034256-82-1$ & & & $1.8 \mathrm{E}+0 \mathrm{~S}$ & $1,0 \mathrm{E}+06$ & $1.6 \mathrm{E}+05$ & $1.8 \mathrm{E}+04$ & & & $6.4 E+04$ & $6.4 E+03$ & & & & & & & $6.2 E+04$ & $6.2 \mathrm{E}+03$ \\
\hline Acetone & $000067-64-1$ & & & 8.8E+05 & $1.0 E+06$ & 8.2E+05 & $8.8 \mathrm{E}+04$ & & & $5.3 \mathrm{E}+05$ & $5.3 \mathrm{E}+04$ & & & & & & & $5.0 \mathrm{E}+05$ & $5.0 \mathrm{E}+04$ \\
\hline Acetone Cyanohydrin & 000075.86 .5 & & & $7.0 \mathrm{E}+03$ & $6.5 E+04$ & $6.5 E+03$ & $7.0 \mathrm{E}+02$ & & & $2.6 \mathrm{E}+03$ & $2.6 \mathrm{E}+02$ & & & $1.0 \mathrm{E}+06$ & $1.0 E+06$ & & & $2.5 \mathrm{E}+03$ & $2.5 \mathrm{E}+02$ \\
\hline Acetonitrile & 000075.05 .8 & & & $5.3 \mathrm{E}+04$ & $4.9 E+05$ & $4.9 E+04$ & $5.3 \mathrm{E}+03$ & & & $3.1 E+04$ & $3.1 \mathrm{E}+03$ & & & $1.0 \mathrm{E}+06$ & $1.0 E+06$ & & & $2.9 \mathrm{E}+04$ & $2.9 \mathrm{E}+03$ \\
\hline Acetophenone $e^{(3)}$ & $000098-86-2$ & & & $8.8 \mathrm{E}+05$ & $1.0 \mathrm{E}+06$ & $8.2 \mathrm{E}+05$ & $8.8 \mathrm{E}+04$ & & & $5.1 E+05$ & $5.1 E+04$ & & & $1.0 \mathrm{E}+06$ & $1.0 \mathrm{E}+06$ & & & $4.6 \mathrm{E}+05$ & $4.6 \mathrm{E}+04$ \\
\hline Acrolein'() & $000107-02.8$ & & & $1.8 \mathrm{E}+05$ & $1.0 E+06$ & $1.6 \mathrm{E}+05$ & $1.8 \mathrm{E}+04$ & & & $1.0 E+05$ & $1.0 \mathrm{E}+04$ & & & $3.1 E+01$ & $3.1 E+00$ & & & $2.5 E+01$ & $2.5 E+00$ \\
\hline Acrylamide & $000079-06-1$ & $1.6 \mathrm{E}+03$ & $1.6 \mathrm{E}+01$ & $1.8 \mathrm{E}+03$ & $1.6 \mathrm{E}+04$ & $1.6 \mathrm{E}+03$ & $1.8 \mathrm{E}+02$ & $1.7 \mathrm{E}+02$ & $1.7 E+\infty$ & $6.4 \mathrm{E}+02$ & $6.4 E+01$ & $1.0 \mathrm{E}+06$ & $9.1 E+05$ & & & $1.6 \mathrm{E}+02$ & $1.6 \mathrm{E}+00$ & $6.2 E+02$ & $6.2 \mathrm{E}+01$ \\
\hline Acrylic Acid & $000079-10.7$ & & & $1.0 \mathrm{E}+06$ & $1.0 E+06$ & $1.0 \mathrm{E}+06$ & 4. $4 \mathrm{E}+05$ & & & $1.0 \mathrm{E}+06$ & $1.6 \mathrm{E}+0 \mathrm{~S}$ & & & $1.0 \mathrm{E}+06$ & $1.0 \mathrm{E}+06$ & & & $1.0 \mathrm{E}+06$ & $1.5 E+05$ \\
\hline Aerylonitrile & $000107-13-1$ & $1.3 E+04$ & $1.3 \mathrm{E}+02$ & $8.8 E+03$ & $8.2 E+04$ & $8.2 E+03$ & $8.8 \mathrm{E}+02$ & $2.2 \mathrm{E}+03$ & $2.2 E+0 t$ & 5. $1 E+03$ & 5.1E+02 & $1.4 E+03$ & $1.4 E+01$ & $8.5 E+02$ & $8.5 \mathrm{E}+01$ & $8.5 \mathrm{E}+02$ & $8.5 E+00$ & $5.8 E+02$ & $5.8 \mathrm{E}+01$ \\
\hline Alactlor & $015972-60.8$ & $8.9 E+04$ & $8.9 E+02$ & $8.8 E+04$ & $8.2 \mathrm{E}+05$ & $8.2 \mathrm{E}+04$ & $8.8 \mathrm{E}+03$ & $9.4 \mathrm{E}+03$ & $9.4 \mathrm{E}+01$ & $3.2 \mathrm{E}+04$ & $3.2 E+03$ & & & & & $9.0 E+03$ & $9.0 \mathrm{E}+01$ & $3.1 \mathrm{E}+04$ & 3.IE+03 \\
\hline Aldicarb & $000116-06-3$ & & & $8.8 \mathrm{E}+03$ & $8.2 \mathrm{E}+04$ & $8.2 \mathrm{E}+03$ & $8.8 E+02$ & & & $3.2 \mathrm{E}+03$ & $3.2 \mathrm{E}+02$ & & & & & & & $3.1 \mathrm{E}+03$ & $3.1 E+02$ \\
\hline Addicarb Sulfone & $001646-88-4$ & & & $8.8 E+03$ & $8.2 \mathrm{E}+04$ & $8.2 E+03$ & $8.8 \mathrm{E}+02$ & & & $3.2 \mathrm{E}+03$ & $3.2 \mathrm{E}+02$ & & & & & & & $3.1 E+03$ & $3.1 E+02$ \\
\hline Aldrin & $000309 \cdot 00-2$ & 4.2E+02 & $4.2 \mathrm{E}+00$ & $2.6 \mathrm{E}+02$ & $2.5 \mathrm{E}+03$ & $2.5 \mathrm{E}+02$ & $2.6 \mathrm{E}+01$ & $4.4 \mathrm{E}+01$ & $4,4 \mathrm{E}-01$ & $9.6 \mathrm{E}+01$ & $9.6 \mathrm{E}+00$ & $1.0 \mathrm{E}+06$ & $2.4 \mathrm{E}+05$ & & & $4.2 \mathrm{E}+01$ & $4.2 \mathrm{E}-01$ & $9.3 E+01$ & $9.3 E+\infty 0$ \\
\hline Ally & 074223.64 .6 & & & $1.0 \mathrm{E}+06$ & $1.0 E+06$ & $1.0 \mathrm{E}+06$ & $2.2 E+05$ & & & $8.0 \mathrm{E}+0 \mathrm{~S}$ & $8.0 \mathrm{E}+04$ & & & & & & & $7.7 \mathrm{E}+05$ & $7.7 E+04$ \\
\hline Allyl Alcohol & 000107.18 .6 & & & 4.4E+04 & 4.1E+05 & 4.1E+04 & $4.4 E+03$ & & & $2.6 \mathrm{E}+04$ & $2.6 \mathrm{E}+03$ & & & & & & & $2.4 \mathrm{E}+04$ & $2.4 E+03$ \\
\hline Allyl Chloride & $000107-05-1$ & & & $4.4 \mathrm{E}+05$ & $1.0 \mathrm{E}+06$ & 4.1E+05 & 4.4E+04 & & & 2.6E+05 & $2.6 \mathrm{E}+04$ & & & $6.3 \mathrm{E}+02$ & 1.1E+02 & & & $6.3 \mathrm{E}+02$ & $9,2 \mathrm{E}+01$ \\
\hline Aluminum & $007429-90-5$ & & & & & & & & & & & & & & & & & & \\
\hline
\end{tabular}


Table 3.4c. (continued)

\begin{tabular}{|c|c|c|c|c|c|c|c|c|c|c|c|c|c|c|c|c|c|c|c|}
\hline \multirow{2}{*}{ Chemical } & \multirow{2}{*}{ CASRN } & \multicolumn{6}{|c|}{ Ingestion (mg/kg) } & \multicolumn{4}{|c|}{ Dermal (mgkg)* } & \multicolumn{4}{|c|}{ Inhalation (mg/kg) } & \multicolumn{4}{|c|}{$\begin{array}{c}\text { Ingestion + Dermal + Inhalation } \\
(\mathrm{mg} / \mathrm{kg})^{+* v}\end{array}$} \\
\hline & & $10^{-4}$ & $10^{4}$ & $\begin{array}{l}\mathrm{HQ}=1 \\
\text { Child }\end{array}$ & $\begin{array}{l}\text { HQ=1 } \\
\text { Adult }\end{array}$ & $\begin{array}{c}H Q=0.1 \\
\text { Adult }\end{array}$ & $\begin{array}{l}\text { HQ }=0.1 \\
\text { Child }\end{array}$ & $10^{-4}$ & $10^{-6}$ & $H Q=1$ & $\mathrm{HQ}=0.1$ & $10^{-4}$ & $10^{4}$ & $H Q=1$ & $\mathrm{HQ}=0.1$ & $10^{-4}$ & $10^{-6}$ & $H I=1$ & $\mathrm{HI}=0.1$ \\
\hline Aluminum Phosphide & $020859-73.8$ & & & $3.5 \mathrm{E}+03$ & $3.3 \mathrm{E}+04$ & $3.3 \mathrm{E}+03$ & $3.5 E+02$ & & & 5.tE +03 & $5.1 E+02$ & & & & & & & $4.4 E+03$ & $4.4 \mathrm{E}+02$ \\
\hline Amdro & $067485-29-4$ & & & $2.6 \mathrm{E}+03$ & 2.5E+04 & $2.5 \mathrm{E}+03$ & $2.6 \mathrm{E}+02$ & & & $9.6 \mathrm{E}+02$ & $9.6 \mathrm{E}+01$ & & & & & & & $9.3 \mathrm{E}+02$ & $9.3 E+01$ \\
\hline Ametryn & $000834-12-8$ & & & $7.9 \mathrm{E}+04$ & $7.4 \mathrm{E}+05$ & $7.4 \mathrm{E}+04$ & $7.9 \mathrm{E}+03$ & & & $2.9 \mathrm{E}+04$ & $2.9 E+03$ & & & & & & & $2.8 \mathrm{E}+04$ & $2.8 \mathrm{E}+03$ \\
\hline Aminophenol, m- & $000591-27.5$ & & & $6.1 E+05$ & $1.0 \mathrm{E}+06$ & 5.7E+05. & $6.1 E+04$ & & & $2.2 \mathrm{E}+05$ & $2.2 \mathrm{E}+04$ & & & & & & & $2.2 \mathrm{E}+0 \mathrm{~S}$ & $2.2 \mathrm{E}+04$ \\
\hline Aminopyridine, 4- & $000504-24-5$ & & & $1.8 \mathrm{E}+02$ & $1.6 \mathrm{E}+03$ & $1.6 \mathrm{E}+02$ & $1.8 \mathrm{E}+01$ & & & $6.4 E+01$ & $6.4 \mathrm{E}+00$ & & & & & & & $6.2 \mathrm{E}+01$ & $6.2 E+00$ \\
\hline Amitraz & $033089-61-1$ & & & $2.2 \mathrm{E}+04$ & $2.0 E+05$ & $2.0 \mathrm{E}+04$ & $2.2 \mathrm{E}+03$ & & & $8.0 \mathrm{E}+03$ & $8.0 \mathrm{E}+02$ & & & & & & & $7.7 \mathrm{E}+03$ & $7.7 \mathrm{E}+02$ \\
\hline Ammonia & $007664-41-7$ & & & & & & & & & & & & & $1.0 \mathrm{E}+06$ & $1.0 \mathrm{E}+06$ & & & & \\
\hline Ammonium Sulfamale & $007773-06-0$ & & & $1.0 \mathrm{E}+06$ & $1.0 \mathrm{E}+06$ & $1.0 \mathrm{E}+06$ & $1.8 \mathrm{E}+05$ & & & $1.0 \mathrm{E}+06$ & $2.6 \mathrm{E}+05$ & & & & & & & $1.0 \mathrm{E}+06$ & $2.2 \mathrm{E}+05$ \\
\hline Aniline & $000062-53-3$ & $1.0 \mathrm{E}+06$ & $1,3 \mathrm{E}+04$ & & & & & $1.3 \mathrm{E}+05$ & $1.3 \mathrm{E}+03$ & & & & & $1.0 \mathrm{E}+06$ & $1.0 \mathrm{E}+06$ & $1.3 \mathrm{E}+05$ & $1.3 \mathrm{E}+03$ & & \\
\hline Anthracene & $000120-12-7$ & & & $1.0 \mathrm{E}+06$ & $1.0 \mathrm{E}+06$ & $1.0 \mathrm{E}+06$ & $2.6 \mathrm{E}+05$ & & & $1.0 \mathrm{E}+06$ & $1.5 \mathrm{E}+05$ & & & & & & & $1.0 \mathrm{E}+06$ & $1.4 \mathrm{E}+05$ \\
\hline Antimony (metallic) & $007440-36.0$ & & & $3.5 E+03$ & $3,3 E+04$ & $3.3 \mathrm{E}+03$ & $3.5 \mathrm{E}+02$ & & & $5.1 \mathrm{E}+02$ & $5.1 \mathrm{E}+01$ & & & & & & & $5.1 E+02$ & $5.1 E+01$ \\
\hline Antimony Pentoxide & $001314.60-9$ & & & $4.4 E+03$ & 4.1E+04 & $4.1 \mathrm{E}+03$ & $4.4 E+02$ & & & $6.4 E+03$ & $6.4 \mathrm{E}+02$ & & & & & & & $5.6 \mathrm{E}+03$ & $5.6 \mathrm{E}+02$ \\
\hline $\begin{array}{l}\text { Antimony Potassium } \\
\text { Tartrate }\end{array}$ & $000304-61-0$ & & & $7.9 \mathrm{E}+03$ & $7.4 E+04$ & $7.4 E+03$ & $7.9 \mathrm{E}+02$ & & & $1.2 \mathrm{E}+04$ & $1.2 \mathrm{E}+03$ & & & & & & & $1.0 \mathrm{E}+04$ & $1.0 \mathrm{E}+03$ \\
\hline Antimony Tetroxide & $001332-81.6$ & & & $3.5 \mathrm{E}+03$ & $3,3 \mathrm{E}+04$ & $3.3 \mathrm{E}+03$ & $3.5 \mathrm{E}+02$ & & & $5.1 \mathrm{E}+03$ & $5.1 \mathrm{E}+02$ & & & & & & & $4.4 \mathrm{E}+03$ & $4.4 E+02$ \\
\hline Antimony Trioxide & $001309-64-4$ & & & $3.5 \mathrm{E}+03$ & $3.3 \mathrm{E}+04$ & $3.3 \mathrm{E}+03$ & $3.5 \mathrm{E}+02$ & & & $5.1 \mathrm{E}+03$ & $5.1 \mathrm{E}+02$ & & & $1.0 \mathrm{E}+06$ & $1.0 E+06$ & & & $4.4 E+03$ & $4.4 E+02$ \\
\hline Apollo & $074115-24-5$ & & & $1.1 \mathrm{E}+05$ & $1.0 \mathrm{E}+06$ & $1.1 \mathrm{E}+0 \mathrm{~s}$ & $1.1 \mathrm{E}+04$ & & & $4.2 E+04$ & $4.2 \mathrm{E}+03$ & & & & & & & $4.0 \mathrm{E}+04$ & $4.0 \mathrm{E}+03$ \\
\hline Aramite & 000140.57 .8 & $2.9 E+05$ & $2.9 E+03$ & $4.4 \mathrm{E}+05$ & $1.0 \mathrm{E}+06$ & 4.1E+05 & 4.4E+04 & $3.0 E+04$ & $3.0 \mathrm{E}+02$ & $1.6 \mathrm{E}+05$ & $1.6 \mathrm{E}+04$ & $1.0 \mathrm{E}+06$ & $1.0 \mathrm{E}+06$ & & & $2.9 E+04$ & $2.9 E+02$ & $1.5 E+05$ & $1.5 E+04$ \\
\hline Aroclor 1016 & $012674-11-2$ & & & $6.1 E+02$ & $5.7 \mathrm{E}+03$ & $5.7 E+02$ & $6.1 \mathrm{E}+01$ & & & $6.7 \mathrm{E}+01$ & $6.7 \mathrm{E}+00$ & & & & & & & $6.7 E+01$ & $6.7 \mathrm{E}+00$ \\
\hline Aroclor $122 f^{\prime \prime \prime}$ & $011104-28-2$ & $9.3 \mathrm{E}+02$ & $9.3 \mathrm{E}+00$ & & & & & $2.9 \mathrm{E}+01$ & $2.9 \mathrm{E}-01$ & & & & & & & $2.9 \mathrm{E}+01$ & $2.9 E-01$ & & \\
\hline Aroclor 1232(1) & $011141-16-5$ & $9.3 \mathrm{E}+02$ & $9.3 \mathrm{E}+00$ & & & & & $2.9 \mathrm{E}+01$ & $2.9 \mathrm{E}-01$ & & & & & & & $2.9 \mathrm{E}+01$ & $2.9 \mathrm{E}-01$ & & \\
\hline Aroclor 1242(1) & $053469-21-9$ & $9.3 \mathrm{E}+02$ & $9.3 \mathrm{E}+00$ & & & & & $2.9 \mathrm{E}+01$ & $2.9 \mathrm{E}-01$ & & & & & & & $2.9 \mathrm{E}+01$ & $2.9 \mathrm{E}-01$ & & \\
\hline Aroclor $1248^{(1)}$ & $012672-29-6$ & $9.3 \mathrm{E}+02$ & $9.3 \mathrm{E}+00$ & & & & & $2.9 E+01$ & $2.9 \mathrm{E}-01$ & & & & & & & $2.9 \mathrm{E}+01$ & $2.9 \mathrm{E}-01$ & & \\
\hline
\end{tabular}

Nonradionuclides/soil/recreational

Page 2 of 34 
Table 3.4c. (continued)

\begin{tabular}{|c|c|c|c|c|c|c|c|c|c|c|c|c|c|c|c|c|c|c|c|}
\hline \multirow{2}{*}{ Chemical } & \multirow{2}{*}{ CASRN } & \multicolumn{6}{|c|}{ Ingestion (mg/kg) } & \multicolumn{4}{|c|}{ Dermal (mgkg) } & \multicolumn{4}{|c|}{ Inbalation (mg/kg) } & \multicolumn{4}{|c|}{$\begin{array}{c}\text { Ingestion + Dermal + Inhalation } \\
(\mathrm{mg} / \mathrm{kg})^{+* \hat{*}}\end{array}$} \\
\hline & & $10^{-4}$ & $10^{6}$ & $\begin{array}{l}\text { HQ }=1 \\
\text { Child }\end{array}$ & $\begin{array}{l}\text { HQ=1 } \\
\text { Adult }\end{array}$ & $\begin{array}{c}\mathrm{HQ}=0.1 \\
\text { Adult }\end{array}$ & $\begin{array}{l}H Q=0.1 \\
\text { Child }\end{array}$ & $10^{-4}$ & $10^{4}$ & $H Q=1$ & $H Q=0.1$ & $10^{-4}$ & $10^{5}$ & $H Q=1$ & $\mathrm{HQ}=0.1$ & $10^{-4}$ & $10^{5}$ & HI=1 & $H I=0.1$ \\
\hline Aroclor 1254 & $011097-69-1$ & & & $1.8 \mathrm{E}+02$ & $1.6 \mathrm{E}+03$ & $1.6 \mathrm{E}+02$ & $1.8 \mathrm{E}+01$ & & & $1.9 \mathrm{E}+01$ & $1.9 \mathrm{E}+\infty 0$ & & & & & & & $1.9 E+01$ & $1.9 E+\infty 0$ \\
\hline Aroclor $1260^{111}$ & $011096-82.5$ & $9.3 \mathrm{E}+02$ & $9.3 \mathrm{E}+00$ & & & & & $2.9 \mathrm{E}+01$ & $2.9 \mathrm{E}-01$ & & & & & & & $2.9 E+01$ & $2.9 \mathrm{E}-01$ & & \\
\hline Arsenic Salts & $\mathrm{NA}$ & & & & & & & & & & & & & & & & & & \\
\hline Arsenic, Inorganic & $007440-38-2$ & $4.8 \mathrm{E}+03$ & $4.8 \mathrm{E}+01$ & $2.6 \mathrm{E}+03$ & 2.5E+04 & $2.5 \mathrm{E}+03$ & $2.6 \mathrm{E}+02$ & $4.1 E+03$ & $4.1 \mathrm{E}+01$ & $7.9 \mathrm{E}+03$ & $7.9 \mathrm{E}+02$ & $1.0 \mathrm{E}+06$ & $8.2 E+04$ & & & $3.1 \mathrm{E}+03$ & $3.1 \mathrm{E}+01$ & $6.0 \mathrm{E}+03$ & $60 E+02$ \\
\hline Arsine & $007784-42-1$ & & & & & & & & & & & & & $1.0 \mathrm{E}+06$ & $1.0 \mathrm{E}+06$ & & & & \\
\hline Asbestos & $001332-21-4$ & & & & & & & & & & & & & & & & & & \\
\hline Assure & $076578-14.8$ & & & $7.9 E+04$ & 7.4E+05 & $7.4 E+04$ & $7.9 \mathrm{E}+03$ & & & $2.9 E+04$ & $2.9 \mathrm{E}+03$ & & & & & & & $2.8 \mathrm{E}+04$ & $2.8 E+03$ \\
\hline Asulam & $003337-71-1$ & & & $4.4 \mathrm{E}+05$ & $1.0 \mathrm{E}+06$ & $4.1 \mathrm{E}+05$ & $4.4 \mathrm{E}+04$ & & & $1.6 \mathrm{E}+05$ & $1.6 \mathrm{E}+04$ & & & & & & & $1.5 E+05$ & $1.5 \mathrm{E}+04$ \\
\hline Alraxine & $001912-24-9$ & $3.2 E+04$ & $3.2 \mathrm{E}+02$ & $3.1 \mathrm{E}+05$ & $1.0 \mathrm{E}+06$ & $2.9 \mathrm{E}+0 \mathrm{~S}$ & $3.1 \mathrm{E}+04$ & $3.4 \mathrm{E}+03$ & $3.4 \mathrm{E}+01$ & $1.1 E+05$ & $1.1 E+04$ & & & & & $3.3 \mathrm{E}+03$ & $3.3 E+01$ & $1.1 \mathrm{E}+05$ & $1.1 E+04$ \\
\hline Avermectin Bl & $065195-55-3$ & & & $3.5 \mathrm{E}+03$ & $3.3 \mathrm{E}+04$ & $3.3 \mathrm{E}+03$ & $3.5 \mathrm{E}+02$ & & & $1.3 E+03$ & $1.3 \mathrm{E}+02$ & & & & & & & $1.2 E+03$ & $1.2 \mathrm{E}+02$ \\
\hline Azobenzene & $000103-33-3$ & $6.5 \mathrm{E}+0.4$ & $6.5 \mathrm{E}+02$ & & & & & $6.8 \mathrm{E}+03$ & $6.8 \mathrm{E}+01$ & & & $1.0 E+06$ & $1.0 E+06$ & & & $6.6 \mathrm{E}+03$ & $6.6 \mathrm{E}+01$ & & \\
\hline Barium & $007440-39-3$ & & & $6.18+05$ & $1.0 \mathrm{E}+06$ & $5.7 \mathrm{E}+05$ & $6.1 \mathrm{E}+04$ & & & $3.1 \mathrm{E}+05$ & $3.1 \mathrm{E}+04$ & & & $1.0 \mathrm{E}+06$ & $1.0 \mathrm{E}+06$ & & & $3.0 \mathrm{E}+05$ & $3.0 E+04$ \\
\hline Barium Cyanide & $000542-62-1$ & & & $8.8 \mathrm{E}+05$ & $1.0 \mathrm{E}+06$ & $8.2 E+0 S$ & $8.8 E+04$ & & & $1.0 \mathrm{E}+06$ & $1.3 E+05$ & & & & & & & $1.0 \mathrm{E}+06$ & 1.1E+OS \\
\hline Baygon & $000114-26-1$ & & & $3.5 E+04$ & 3.3E+0S & $3.3 \mathrm{E}+04$ & $3.5 \mathrm{E}+03$ & & & $1.3 \mathrm{E}+04$ & $1,3 E+03$ & & & & & & & $1.2 \mathrm{E}+04$ & $1.2 \mathrm{E}+03$ \\
\hline Bayleton & $043121-43.3$ & & & $2.6 \mathrm{E}+05$ & $1.0 \mathrm{E}+06$ & 2.5E+05 & $2.6 \mathrm{E}+04$ & & & $9.6 \mathrm{E}+04$ & $9.6 \mathrm{E}+03$ & & & & & & & $9.3 \mathrm{E}+04$ & $9.3 \mathrm{E}+03$ \\
\hline Baythroid & 068359.37 .5 & & & $2.2 \mathrm{E}+05$ & $1.0 \mathrm{E}+06$ & $2.0 \mathrm{E}+05$ & $2.2 E+04$ & & & $8.0 \mathrm{E}+04$ & $8.0 \mathrm{E}+03$ & & & & & & & $7.7 E+04$ & $7.7 E+03$ \\
\hline Benefin & $001861-40-1$ & & & $1.0 \mathrm{E}+06$ & $1.0 \mathrm{E}+06$ & $1.0 \mathrm{E}+06$ & $2.6 \mathrm{E}+05$ & & & $9.6 \mathrm{E}+05$ & $9.6 \mathrm{E}+04$ & & & & & & & $9.3 \mathrm{E}+05$ & $9.3 \mathrm{E}+04$ \\
\hline Benomyl & $017804-35-2$ & & & 4.4E+05 & $1,0 \mathrm{E}+06$ & $4.1 \mathrm{E}+05$ & $4.4 \mathrm{E}+04$ & & & $1.6 \mathrm{E}+05$ & $1.6 \mathrm{E}+04$ & & & & & & & $1.5 E+05$ & $1.5 \mathrm{E}+04$ \\
\hline Bentazon & $025057-89.0$ & & & $2.2 \mathrm{E}+04$ & $2.0 \mathrm{E}+05$ & $2.0 \mathrm{E}+04$ & $2.2 \mathrm{E}+03$ & & & $8.0 \mathrm{E}+03$ & $8.0 \mathrm{E}+02$ & & & & & . & & $7.7 E+03$ & $7.7 E+02$ \\
\hline Benz[a]anthracene ${ }^{m}$ & 000056.55 .3 & $9.8 \mathrm{E}+03$ & $9.8 \mathrm{E}+01$ & & & & & $6.4 \mathrm{E}+02$ & $6.4 \mathrm{E}+00$ & & & $1.0 \mathrm{E}+06$ & $1.0 \mathrm{E}+06$ & & & $6.2 \mathrm{E}+02$ & $6.2 \mathrm{E}+00$ & & \\
\hline Benzaldehyde & $000100-52-7$ & & & $8.8 \mathrm{E}+05$ & $1.0 E+06$ & $8.2 \mathrm{E}+05$ & $8.8 \mathrm{E}+04$ & & & $5.1 \mathrm{E}+05$ & $5.1 E+04$ & & & & & & & $4.8 E+05$ & $4.8 E+04$ \\
\hline Benzene & $000071-43-2$ & $2.5 \mathrm{E}+05$ & $2.5 \mathrm{E}+03$ & & & & & $5.0 E+04$ & $5.0 \mathrm{E}+02$ & & & $5.0 \mathrm{E}+02$ & $5.0 E+02$ & & & $5.0 E+02$ & $2.7 E+02$ & & \\
\hline
\end{tabular}

Nonradionuclides/soil/recreational

Page 3 of 34 
Table 3.4c. (continued)

\begin{tabular}{|c|c|c|c|c|c|c|c|c|c|c|c|c|c|c|c|c|c|c|c|}
\hline \multirow{2}{*}{ Chemical } & \multirow{2}{*}{ CASRN } & \multicolumn{6}{|c|}{ Ingestion (mg/kg) } & \multicolumn{4}{|c|}{ Dermal (mgkg)" } & \multicolumn{4}{|c|}{ Inhalation (mg/kg) } & \multicolumn{4}{|c|}{$\begin{array}{c}\text { Ingestion + Dermal + Inhalation } \\
(\mathrm{mg} / \mathrm{kg})^{t^{* v v}}\end{array}$} \\
\hline & & $10^{-4}$ & $10^{6}$ & $\begin{array}{l}\text { HQ=1 } \\
\text { Child }\end{array}$ & $\begin{array}{l}\text { HQ=1 } \\
\text { Adult }\end{array}$ & $\begin{array}{c}\mathrm{HQ}=0.1 \\
\text { Adult }\end{array}$ & $\begin{array}{c}\mathrm{HQ}=0.1 \\
\text { Child }\end{array}$ & $10^{-4}$ & $10^{6}$ & $H Q=1$ & $\mathrm{HQ}=0.1$ & $10^{-4}$ & $10^{4}$ & $H Q=1$ & $\mathrm{HQ}=0.1$ & $\mathrm{i}^{-4}$ & $10^{6}$ & $\mathrm{HI}=1$ & $\mathrm{HI}=0.1$ \\
\hline Benzene Hexachloride & NA & & & & & & & & & & & & & & & & & & \\
\hline Benzene, Ethyldimethyl & NA & & & & & & & & & & & & & & & & & & \\
\hline Benzene, Ethylmethyl & NA & & & & & & & & & & & & & & & & & & \\
\hline $\begin{array}{l}\text { Benzene, } \\
\text { Methylpropenyl }\end{array}$ & NA & & & & & & & & & & & & & & & & & & \\
\hline Benzene, Methylpropyl & $\mathrm{NA}$ & & & & & & & & & & & & & & & & & & \\
\hline Benzene, Trimethyl & $025551-13-7$ & & & & & & & & & & & & & & & & & & \\
\hline Benzenethiol & $000108-98-5$ & & & $8.8 \mathrm{E}+01$ & $8.2 \mathrm{E}+02$ & 8.2E+01 & $8.8 \mathrm{E}+00$ & & & $3,2 E+01$ & $3.2 E+\infty$ & & & & & & & $3.1 E+01$ & $3.1 \mathrm{E}+\infty$ \\
\hline Benzidine & $000092-87.5$ & $3.1 \mathrm{E}+01$ & $3.1 \mathrm{E}-01$ & $2.6 \mathrm{E}+04$ & $2.5 \mathrm{E}+05$ & 2.5E+04 & $2.6 \mathrm{E}+03$ & $5.2 \mathrm{E}+\infty 0$ & $5.2 \mathrm{E}-02$ & $1.5 E+04$ & $1.5 \mathrm{E}+03$ & $1.0 \mathrm{E}+06$ & $1.8 \mathrm{E}+04$ & & & $4.9 E+00$ & $4.9 \mathrm{E}-02$ & $1.5 \mathrm{E}+04$ & $1.5 \mathrm{E}+03$ \\
\hline Benzo[a]pyrene & $000050-32-8$ & $9.8 \mathrm{E}+02$ & $9.8 \mathrm{E}+00$ & & & & & $6.4 E+01$ & $6.4 \mathrm{E}-01$ & & & $1.0 \mathrm{E}+06$ & $1.0 E+06$ & & & $6.2 \mathrm{E}+01$ & $6.2 \mathrm{E}-01$ & & \\
\hline Benzo[b] fluoranthene $e^{(2)}$ & $000205-99-2$ & $9.8 \mathrm{E}+03$ & $9.8 \mathrm{E}+01$ & & & & & $6.4 \mathrm{E}+02$ & $6.4 E+00$ & & & $1.0 \mathrm{E}+06$ & $1.0 \mathrm{E}+06$ & & & $6.2 \mathrm{E}+02$ & $6.2 \mathrm{E}+00$ & & \\
\hline Benzo[B,h,i]perylene & $000191-24-2$ & & & & & & & & & & & & & & & & & & \\
\hline Benzo[k]nuoranthene $e^{(2)}$ & $000207-08.9$ & $9.8 \mathrm{E}+04$ & $9.8 \mathrm{E}+02$ & & & & & $6.4 \mathrm{E}+03$ & $6.4 E+01$ & & & $1.0 \mathrm{E}+06$ & $1.0 \mathrm{E}+06$ & & & $6.2 \mathrm{E}+03$ & $6.2 \mathrm{E}+01$ & & \\
\hline Benzoic Acid & $000065-85.0$ & & & $1.0 \mathrm{E}+06$ & $1.0 \mathrm{E}+06$ & $1.0 \mathrm{E}+06$ & $1.0 \mathrm{E}+06$ & & & $1.0 \mathrm{E}+06$ & $1.0 \mathrm{E}+06$ & & & & & & & $1.0 \mathrm{E}+06$ & $1.0 \mathrm{E}+06$ \\
\hline Benzotrichloride & $000098-07-7$ & $5.5 E+02$ & $5.5 \mathrm{E}+00$ & & & & & $5.8 \mathrm{E}+01$ & $5.8 \mathrm{E}-01$ & & & & & & & $5.6 E+01$ & $5.6 \mathrm{E}-01$ & & \\
\hline Benzyl Alcohol & $000100-51-6$ & & & $1.0 \mathrm{E}+06$ & $1.0 \mathrm{E}+06$ & $1.0 \mathrm{E}+06$ & $2.6 \mathrm{E}+05$ & & & $1.0 \mathrm{E}+06$ & $1.3 E+05$ & & & & & & & $1.0 \mathrm{E}+06$ & $1.2 \mathrm{E}+05$ \\
\hline Benzyl Chloride & $000100-44-7$ & $4.2 \mathrm{E}+04$ & $4.2 E+02$ & & & & & $7.1 \mathrm{E}+03$ & $7.1 \mathrm{E}+01$ & & & & & & & $6.6 \mathrm{E}+03$ & $6.6 \mathrm{E}+01$ & & \\
\hline Beryllium & $007440-41-7$ & $1.7 \mathrm{E}+03$ & $1.7 \mathrm{E}+01$ & $4.4 \mathrm{E}+04$ & 4. $1 E+0 S$ & 4.1E+04 & $4.4 E+03$ & $3.5 E+01$ & 3.5E-01 & $3.2 E+03$ & $3.2 \mathrm{E}+02$ & $1.0 \mathrm{E}+06$ & $4.9 E+05$ & & & $3.5 \mathrm{E}+01$ & 3.5E-01 & $3.2 \mathrm{E}+03$ & $3.2 E+02$ \\
\hline Bidrin & $000141-66-2$ & & & $8.8 \mathrm{E}+02$ & $8.2 E+03$ & $8.2 \mathrm{E}+02$ & $8.8 \mathrm{E}+01$ & & & $3.2 E+02$ & $3.2 \mathrm{E}+01$ & & & & & & & $3.1 \mathrm{E}+02$ & $3.1 E+0 I$ \\
\hline Biphenthrin & 082657.04 .3 & & & $1.3 \mathrm{E}+05$ & $1.0 E+06$ & $1.2 \mathrm{E}+05$ & $1.3 \mathrm{E}+04$ & & & $4.8 \mathrm{E}+04$ & $4.8 \mathrm{E}+03$ & & & & & & & 4.6E+04 & $4.6 \mathrm{E}+03$ \\
\hline Biphenyl, 1,1'- & $000092-52-4$ & & & $4.4 E+05$ & $1.0 \mathrm{E}+06$ & 4.1E+05 & 4.4E+04 & & & $1.6 \mathrm{E}+05$ & $1.6 \mathrm{E}+04$ & & & & & & & $1.5 E+05$ & $1.5 E+04$ \\
\hline $\begin{array}{l}\text { Bis(2-chloro-1- } \\
\text { methylethyl)ether } \\
\text { (Technical) }\end{array}$ & $000108-60-1$ & $1.0 \mathrm{E}+05$ & $1.0 \mathrm{E}+03$ & & & & & $1.7 E+04$ & $1.7 \mathrm{E}+02$ & & & $3.5 E+02$ & $3.5 \mathrm{E}+02$ & & & $3.5 E+02$ & $1.5 \mathrm{E}+02$ & & \\
\hline
\end{tabular}


Table 3.4c. (continued)

\begin{tabular}{|c|c|c|c|c|c|c|c|c|c|c|c|c|c|c|c|c|c|c|c|}
\hline \multirow{2}{*}{ Chemical } & \multirow{2}{*}{ CASRN } & \multicolumn{6}{|c|}{ Ingestion (mg/kg) } & \multicolumn{4}{|c|}{ Dermal (mgkg) } & \multicolumn{4}{|c|}{ Inhalation (mg/kg) } & \multicolumn{4}{|c|}{$\begin{array}{c}\text { Ingestion + Dermal + Inhalation } \\
(\mathrm{mg} / \mathrm{kg})^{+\cdot \mathrm{r}}\end{array}$} \\
\hline & & $10^{-4}$ & $10^{5}$ & $\begin{array}{l}\text { HQ=1 } \\
\text { Child }\end{array}$ & $\begin{array}{l}H Q=1 \\
\text { Adult }\end{array}$ & $\begin{array}{c}\mathrm{HQ}=0.1 \\
\text { Adult }\end{array}$ & $\begin{array}{l}\mathrm{HQ}=0.1 \\
\text { Child }\end{array}$ & $10^{-4}$ & $10^{4}$ & $H Q=1$ & $\mathrm{HQ}=0.1$ & $10^{-4}$ & $10^{5}$ & $H Q=1$ & $\mathrm{HQ}=0.1$ & $10^{-4}$ & $10^{6}$ & HII $=\mathbf{I}$ & $\mathrm{HI}=0.1$ \\
\hline $\begin{array}{l}\text { Bis(2- } \\
\text { chloroethoxy)methane }\end{array}$ & $000111-91-1$ & & & & & & & & & & & & & & & & & & \\
\hline Bis(2-chlioroethyl)ether & $000111-44-4$ & $6.5 E+03$ & $6.5 \mathrm{E}+01$ & & & & & $1.1 E+03$ & $1.1 \mathrm{E}+01$ & & & $6.4 E+02$ & $1.5 \mathrm{E}+02$ & & & $6.4 \mathrm{E}+02$ & $9.6 \mathrm{E}+00$ & & \\
\hline $\begin{array}{l}\text { Bis(2. } \\
\text { chloroisopropyl)ether }\end{array}$ & $039638-32-9$ & & & $3.5 \mathrm{E}+05$ & $1.0 \mathrm{E}+06$ & $3.3 E+05$ & $3.5 \mathrm{E}+04$ & & & $2.1 \mathrm{E}+05$ & 2.1E+04 & & & & & & & $1.9 \mathrm{E}+05$ & $1.9 \mathrm{E}+04$ \\
\hline $\begin{array}{l}\text { Bis(2. } \\
\text { ethylhexyl)phthalate }\end{array}$ & $\begin{array}{l}000117-81.7 \\
.\end{array}$ & $5.1 \mathrm{E}+05$ & $5.1 E+03$ & $1.8 \mathrm{E}+05$ & $1.0 \mathrm{E}+06$ & $1.6 \mathrm{E}+05$ & $1.8 \mathrm{E}+04$ & $2.0 \mathrm{E}+04$ & $2.0 \mathrm{E}+02$ & $2.4 \mathrm{E}+04$ & $2.4 E+03$ & & & & & $2.0 \mathrm{E}+04$ & $2.0 \mathrm{E}+02$ & $2.4 E+04$ & $2.4 \mathrm{E}+03$ \\
\hline Bis(chloromethyl)ether & $000542-88.1$ & $3.3 \mathrm{E}+01$ & $-3.3 \mathrm{E}-01$ & & & & & $5.5 \mathrm{E}+\infty$ & $5.5 \mathrm{E}-02$ & & & $4.9 \mathrm{E}+01$ & $4.9 \mathrm{E}-01$ & & & $4.6 \mathrm{E}+00$ & $4.6 \mathrm{E}-02$ & & \\
\hline Bisphenol A & $000080-05-7$ & & & $4.4 \mathrm{E}+05$ & $1.0 \mathrm{E}+06$ & $4.1 \mathrm{E}+05$ & 4.4E+04 & & & $1.6 \mathrm{E}+05$ & $1.6 \mathrm{E}+04$ & & & & & & & $1.5 \mathrm{E}+05$ & $1.5 \mathrm{E}+04$ \\
\hline $\begin{array}{l}\text { Boron And Borates } \\
\text { Only }\end{array}$ & $007440-42-8$ & & & $7.9 \mathrm{E}+05^{\circ}$ & $1.0 \mathrm{E}+06$ & $7.4 E+05$ & $7.9 E+04$ & & & $1.0 \mathrm{E}+06$ & $5.2 E+05$ & & & $1.0 \mathrm{E}+06$ & $1.0 \mathrm{E}+06$ & & & $1.0 \mathrm{E}+06$ & $3.0 \mathrm{E}+05$ \\
\hline Boron Trilluoride & $007637-07-2$ & & & & & & & & & & & & & $1.0 \mathrm{E}+06$ & $1.0 \mathrm{E}+06$ & & & & \\
\hline Bromochloromethane & $000074-97.5$ & & & & & & & & & & & & & & & & & & \\
\hline Bromodichloromethane & $000075.27-4$ & $1.2 \mathrm{E}+05$ & $1.2 \mathrm{E}+03$ & $1.8 \mathrm{E}+05$ & $1.0 \mathrm{E}+06$ & $1.6 \mathrm{E}+05$ & $1.8 \mathrm{E}+04$ & $2.4 E+04$ & $2.4 \mathrm{E}+02$ & $1.3 \mathrm{E}+05$ & $1.3 \mathrm{E}+04$ & & & & & $2.2 E+04$ & $2.2 \mathrm{E}+02$ & $1.2 \mathrm{E}+0 \mathrm{~S}$ & $1.2 \mathrm{E}+04$ \\
\hline $\begin{array}{l}\text { Bromodiphenyl Ether, } \\
\text { p- }\end{array}$ & $000101-55-3$ & & & & & & & & & & & & & & & & & & \\
\hline Bromoform & $000075-25-2$ & 9.1E+05 & $9.1 E+03$ & $1.8 \mathrm{E}+05$ & $1.0 \mathrm{E}+06$ & $1.6 \mathrm{E}+05$ & $1.8 \mathrm{E}+04$ & $1.1 \mathrm{E}+05$ & $1.1 \mathrm{E}+03$ & $7.7 E+04$ & $7.7 E+03$ & $1.0 \mathrm{E}+06$ & $1.0 E+06$ & & & $1.1 \mathrm{E}+05$ & $1.1 \mathrm{E}+03$ & $7.4 \mathrm{E}+04$ & $7.4 E+03$ \\
\hline Bromomethane $e^{(n)}$ & 000074.83 .9 & & & $1.2 E+04$ & I.IE+0S & $1.1 \mathrm{E}+04$ & $1.2 \mathrm{E}+03$ & & & $7.2 \mathrm{E}+03$ & $7.2 \mathrm{E}+02$ & & & $3.4 E+02$ & $1.9 \mathrm{E}+02$ & & & $3.4 \mathrm{E}+02$ & $1.2 \mathrm{E}+02$ \\
\hline Bromophos & $002104-96-3$ & & & 4.4E+04 & 4.1E+05 & $4.1 \mathrm{E}+04$ & $4.4 \mathrm{E}+03$ & & & $1.6 \mathrm{E}+04$ & $1.6 \mathrm{E}+03$ & & & & & & & $1.5 \mathrm{E}+04$ & $1.5 \mathrm{E}+03$ \\
\hline Bromotrichloromethane & $000075-62-7$ & & & & & & & & & & & & & & & & & & \\
\hline Bromoxynil & $001689-84.5$ & & & $1.8 \mathrm{E}+05$ & $1.0 \mathrm{E}+06$ & $1.6 \mathrm{E}+05$ & $1.8 E+04$ & & & $6.4 E+04$ & $6.4 E+03$ & & & & & & & $6.2 \mathrm{E}+04$ & $6.2 E+03$ \\
\hline Bromoxynil Octanoate & $001689-99-2$ & & & $1.8 \mathrm{E}+05$ & $1.0 E+06$ & $1.6 \mathrm{E}+0 \mathrm{~S}$ & $1.8 \mathrm{E}+04$ & & & $6.4 E+04$ & $6.4 E+03$ & & & & & & & $6.2 \mathrm{E}+04$ & $6.2 \mathrm{E}+03$ \\
\hline Butadiene, 1,3- & 000106.99 .0 & & & & & & & & & & & $3.2 \mathrm{E}+02$ & $3.2 \mathrm{E}+00$ & & & & & & \\
\hline Butanol, N- & $000071-36-3$ & & & $8.8 \mathrm{E}+05$ & $1.0 E+06$ & $8.2 \mathrm{E}+05$ & $8.8 E+04$ & & & $3.2 \mathrm{E}+05$ & $3.2 E+04$ & & & & & & & $3.1 E+05$ & $3.1 \mathrm{E}+04$ \\
\hline $\begin{array}{l}\text { Butanone-2, 4-chloro- } \\
\text { 4,4-difluoro }\end{array}$ & NA & & & & & & & & & & & & & & & & & & \\
\hline
\end{tabular}


Table 3.4c. (continued)

\begin{tabular}{|c|c|c|c|c|c|c|c|c|c|c|c|c|c|c|c|c|c|c|c|}
\hline \multirow{2}{*}{ Chemical } & \multirow{2}{*}{ CASRN } & \multicolumn{6}{|c|}{ Ingestion (mg/kg) } & \multicolumn{4}{|c|}{ Dermal (mgkg) } & \multicolumn{4}{|c|}{ Inhalation (mg/kg) } & \multicolumn{4}{|c|}{$\begin{array}{c}\text { Ingestion + Dermal + Inhalation } \\
(\mathrm{mg} / \mathrm{kg})^{\prime \cdot v}\end{array}$} \\
\hline & & $10^{-4}$ & $10^{6}$ & $\begin{array}{l}\text { HQ=1 } \\
\text { Child }\end{array}$ & $\begin{array}{l}\text { HQ=1 } \\
\text { Adult }\end{array}$ & $\begin{array}{c}\mathrm{HQ}=0.1 \\
\text { Adult }\end{array}$ & $\begin{array}{l}\text { HQ }=0.1 \\
\text { Child }\end{array}$ & $10^{-4}$ & $10^{4}$ & $\mathrm{HQ}=1$ & $\mathrm{HQ}=0.1$ & $10^{-4}$ & $10^{4}$ & $H Q=1$ & $\mathrm{HQ}=0.1$ & $10^{-4}$ & $10^{4}$ & $H I=1$ & $\mathrm{HI}=0.1$ \\
\hline Butyl Benzyl Phthlate & $000085-68-7$ & & & $1.0 \mathrm{E}+06$ & $1.0 \mathrm{E}+06$ & $1.0 \mathrm{E}+06$ & $1.8 \mathrm{E}+05$ & & & $7.8 \mathrm{E}+05$ & $7.8 \mathrm{E}+04$ & & & & & & & 7.5E+05 & $7.5 E+04$ \\
\hline Butylate & $002008-41-5$ & & & $4.4 \mathrm{E}+05$ & $1.0 \mathrm{E}+06$ & $4.1 \mathrm{E}+05$ & $4.4 \mathrm{E}+04$ & & & $1.6 \mathrm{E}+05$ & $1.6 \mathrm{E}+04$ & & & & & & & $1.5 \mathrm{E}+05$ & $1.5 \mathrm{E}+04$ \\
\hline Butylchioride, t- & $000507-20-0$ & & & & & & & & & & & & & & & & & & \\
\hline $\begin{array}{l}\text { Butylyhthaly! } \\
\text { Butylglycolate }\end{array}$ & $000085-70-1$ & & & $1.0 \mathrm{E}+06$ & $1.0 \mathrm{E}+06$ & $1.0 \mathrm{E}+06$ & $8.8 \mathrm{E}+05$ & & & $1.0 \mathrm{E}+06$ & $3.2 \mathrm{E}+0 \mathrm{~S}$ & & & & & & & $1.0 \mathrm{E}+06$ & 3.1E+05 \\
\hline Cacodylic Acid & $000075.60-5$ & & & $2.6 \mathrm{E}+04$ & $2.5 \mathrm{E}+05$ & $2.5 \mathrm{E}+04$ & $2.6 \mathrm{E}+03$ & & & $9.6 \mathrm{E}+03$ & $9.6 \mathrm{E}+02$ & & & & & & & $9.3 \mathrm{E}+03$ & $9.3 \mathrm{E}+02$ \\
\hline Cadmium (Diet) & $007440-43.9$ & & & $8.8 E+03$ & $8.2 \mathrm{E}+04$ & $8.2 \mathrm{E}+03$ & $8.8 \mathrm{E}+02$ & & & $6.4 \mathrm{E}+01$ & $6.4 \mathrm{E}+00$ & $1.0 \mathrm{E}+06$ & $6.7 E+05$ & & & & & $6.4 \mathrm{E}+01$ & $6.4 \mathrm{E}+00$ \\
\hline Calcium Cyanide & $000592.01-8$ & & & $3.5 \mathrm{E}+0 \dot{5}$ & $1.0 \mathrm{E}+06$ & $3.3 \mathrm{E}+05$ & 3.5E+04 & & & $5.1 \mathrm{E}+05$ & $5.1 \mathrm{E}+04$ & & & & & & & $4.4 \mathrm{E}+0 \mathrm{~S}$ & $4.4 \mathrm{E}+04$ \\
\hline Caprolactam & $000105-60-2$ & & & $1.0 \mathrm{E}+06$ & $1.0 \mathrm{E}+06$ & $1.0 \mathrm{E}+06$ & 4.4E+05 & & & $1.0 \mathrm{E}+06$ & $1.6 \mathrm{E}+05$ & & & & & & & $1.0 \mathrm{E}+06$ & $1.5 \mathrm{E}+05$ \\
\hline Captafol & $002425-06-1$ & $8.3 \mathrm{E}+05$ & $8.3 \mathrm{E}+03$ & $1.8 \mathrm{E}+04$ & $1.6 \mathrm{E}+05$ & $1.6 \mathrm{E}+04$ & $1.8 E+03$ & $8.7 E+04$ & $8.7 E+02$ & $6.4 \mathrm{E}+03$ & $6.4 \mathrm{E}+02$ & & & & & $8.4 E+04$ & $8.4 \mathrm{E}+02$ & $6.2 \mathrm{E}+03$ & $6.2 E+02$ \\
\hline Captan & $000133-06.2$ & $1.0 \mathrm{E}+06$ & $2.0 \mathrm{E}+04$ & $1.0 \mathrm{E}+06$ & $1.0 \mathrm{E}+06$ & $1.0 \mathrm{E}+06$ & $1.1 \mathrm{E}+05$ & 2.1E+05 & $2.1 \mathrm{E}+03$ & $4.2 \mathrm{E}+05$ & $4.2 \mathrm{E}+04$ & & & & & 2.1E+05 & $2.1 \mathrm{E}+03$ & $4.0 \mathrm{E}+05$ & 4.0E+04 \\
\hline Carbaryl & $000063-25-2$ & & & $8.8 \mathrm{E}+05$ & $1.0 \mathrm{E}+06$ & $8.2 \mathrm{E}+05$ & $8.8 \mathrm{E}+04$ & & & $3.2 \mathrm{E}+05$ & $3.2 \mathrm{E}+04$ & & & & & & & $3.1 E+05$ & $3.1 \mathrm{E}+04$ \\
\hline Carbazole & $000086-74-8$ & $3.6 \mathrm{E}+05$ & $3.6 \mathrm{E}+03$ & & & & & $5.2 \mathrm{E}+04$ & $5.2 \mathrm{E}+02$ & & & & & & & $5.0 \mathrm{E}+04$ & $5.0 \mathrm{E}+02$ & & \\
\hline Carbofuran & $001563.66-2$ & & & $4.4 \mathrm{E}+04$ & $4.1 \mathrm{E}+05$ & 4.1E+04 & $4.4 \mathrm{E}+03$ & & & $1.6 \mathrm{E}+04$ & $1.6 \mathrm{E}+03$ & & & & & & & $1.5 \mathrm{E}+04$ & $1.5 \mathrm{E}+03$ \\
\hline Carbon Disulfide & $000075-15-0$ & & & $8.8 E+05$ & $1.0 \mathrm{E}+06$ & $8.2 \mathrm{E}+05$ & $8.8 \mathrm{E}+04$ & & & $1.6 \mathrm{E}+04$ & $1.6 \mathrm{E}+03$ & & & $1.7 \mathrm{E}+03$ & $1.7 \mathrm{E}+03$ & & & $1.7 E+03$ & $1.3 \mathrm{E}+03$ \\
\hline Carbon Tetrachloride $e^{(1)}$ & $000056-23.5$ & 5.5E+04 & $5.5 \mathrm{E}+02$ & $6.1 \mathrm{E}+03$ & 5.7E+04 & $5.7 \mathrm{E}+03$ & $6.1 E+02$ & $7.5 E+03$ & $7.5 \mathrm{E}+01$ & $2.9 E+03$ & $2.9 \mathrm{E}+02$ & $1.0 \mathrm{E}+03$ & $3.4 E+02$ & & & $1.0 \mathrm{E}+03$ & $5.9 \mathrm{E}+01$ & $1.0 \mathrm{E}+03$ & $2.8 \mathrm{E}+02$ \\
\hline Carbosulfan & $055285-14.8$ & & & $8.8 \mathrm{E}+04$ & $8.2 \mathrm{E}+05$ & $8.2 \mathrm{E}+04$ & $8.8 \mathrm{E}+03$ & & & $3.2 E+04$ & $3.2 \mathrm{E}+03$ & & & & & & & $3.1 E+04$ & $3.1 \mathrm{E}+03$ \\
\hline Carboxin & $005234-68-4$ & & & $8.8 \mathrm{E}+05$ & $1.0 \mathrm{E}+06$ & $8.2 E+05$ & $8.8 \mathrm{E}+04$ & & & $3.2 E+05$ & $3.2 \mathrm{E}+04$ & & & & & & & $3.1 E+05$ & $3.1 E+04$ \\
\hline Chloral & $000075.87-6$ & & & $1.8 \mathrm{E}+04$ & $1.6 \mathrm{E}+05$ & $1.6 \mathrm{E}+04$ & $1.8 \mathrm{E}+03$ & & & $6.4 \mathrm{E}+03$ & $6.4 \mathrm{E}+02$ & & & & & & & $6.2 \mathrm{E}+03$ & $6.2 \mathrm{E}+02$ \\
\hline Chloramben & $000133-90-4$ & & & $1.3 \mathrm{E}+05$ & $1.0 \mathrm{E}+06$ & $1.2 \mathrm{E}+05$ & $1.3 \mathrm{E}+04$ & & & $4.8 \mathrm{E}+04$ & $4.8 \mathrm{E}+03$ & & & & & & & $4.6 \mathrm{E}+04$ & $4.6 \mathrm{E}+03$ \\
\hline Chloranil & $000118-75-2$ & $1.8 \mathrm{E}+04$ & $1.8 E+02$ & & & & & $1.9 E+03$ & $1.9 \mathrm{E}+01$ & & & & & & & $1.8 \mathrm{E}+03$ & $1.8 E+01$ & & \\
\hline Chlordane & 000057.74 .9 & S.5E+03 & $5.5 \mathrm{E}+01$ & $5.3 \mathrm{E}+02$ & $4.9 E+03$ & $4.9 E+02$ & $5.3 \mathrm{E}+01$ & $5.8 \mathrm{E}+02$ & $5.8 \mathrm{E}+\infty 0$ & $1.9 \mathrm{E}+02$ & $1.9 \mathrm{E}+0 \mathrm{I}$ & $1.0 \mathrm{E}+06$ & $1.0 \mathrm{E}+06$ & & & $5.6 \mathrm{E}+02$ & $5.6 \mathrm{E}+\infty$ & $1.9 \mathrm{E}+02$ & $1.9 \mathrm{E}+01$ \\
\hline Chloride & $016887.00-6$ & & & & & & & & & & & & & & & & & & \\
\hline
\end{tabular}


Table 3.4c. (continued)

\begin{tabular}{|c|c|c|c|c|c|c|c|c|c|c|c|c|c|c|c|c|c|c|c|}
\hline \multirow{2}{*}{ Chemical } & \multirow{2}{*}{ CASRN } & \multicolumn{6}{|c|}{ Ingestion (mg/kg) } & \multicolumn{4}{|c|}{ Dermal (mgkg)" } & \multicolumn{4}{|c|}{ Inhalation $(\mathrm{mg} / \mathrm{kg})^{\bullet *}$} & \multicolumn{4}{|c|}{$\begin{array}{c}\text { Ingestion + Dermal + In halation } \\
(\mathrm{mg} / \mathrm{kg})^{++8}\end{array}$} \\
\hline & & $10^{-4}$ & $10^{6}$ & $\begin{array}{l}\mathrm{HQ}=1 \\
\text { Child }\end{array}$ & $\begin{array}{l}\text { HQ=1 } \\
\text { Adult }\end{array}$ & $\begin{array}{c}\mathrm{HQ}=0.1 \\
\text { Adult }\end{array}$ & $\begin{array}{l}\mathrm{HQ}=0.1 \\
\text { Child }\end{array}$ & $10^{-4}$ & $10^{4}$ & $\mathrm{HQ}=1$ & $\mathrm{HQ}=0.1$ & $10^{-4}$ & $10^{4}$ & $H Q=1$ & $\mathrm{HQ}=0.1$ & $10^{-4}$ & $10^{-6}$ & $H I=1$ & $\mathrm{HI}=0.1$ \\
\hline Chlorimuron, Ethyl- & $090982-32-4$ & & & $1.8 E+05$ & $1.0 \mathrm{E}+05$ & $1.6 \mathrm{E}+05$ & $1.8 E+04$ & & & $6.4 \mathrm{E}+04$ & $6.4 E+03$ & & & & & & & $6.2 \mathrm{E}+04$ & $6.2 E+03$ \\
\hline Chlorine & $007782-50.5$ & & & $8.8 E+05$ & $1.0 \mathrm{E}+06$ & $8.2 E+05$ & $8.8 \mathrm{E}+04$ & & & $1.0 \mathrm{E}+06$ & $1.3 E+0 S$ & & & & & & & $1.0 \mathrm{E}+06$ & $1.1 \mathrm{E}+05$ \\
\hline Chlorine Dioxide & $010049-04-4$ & & & & & & & & & & & & & $1.0 \mathrm{E}+06$ & $1.0 \mathrm{E}+06$ & & & & \\
\hline Chlorite (Sodium Salt) & $007758-19-2$ & & & & & & & & & & & & & & & & & & \\
\hline $\begin{array}{l}\text { Chloro-1,1- } \\
\text { difluoroethane, 1- }\end{array}$ & $000075-68-3$ & & & & & & & & & & & & & $1.0 \mathrm{E}+06$ & $1.0 \mathrm{E}+06$ & & & & \\
\hline $\begin{array}{l}\text { Chloro-1,3-butadienc, } \\
2 \text { - }\end{array}$ & 000126.99 .8 & & & $1.8 \mathrm{E}+05$ & $1.0 \mathrm{E}+06$ & $1.6 \mathrm{E}+05$ & $1.8 \mathrm{E}+04$ & & & $1.0 \mathrm{E}+05$ & $1.0 E+04$ & & & $1.7 E+03$ & $1.7 \mathrm{E}+03$ & & & 1:7E+03 & $1.7 E+03$ \\
\hline $\begin{array}{l}\text { Chloro-2-methylaniline } \\
\mathrm{HCl}, 4 \text { - }\end{array}$ & $003165-93-3$ & $1.6 \mathrm{E}+04$ & $1.6 \mathrm{E}+02$ & & & & & $1.6 \mathrm{E}+03$ & $1.6 \mathrm{E}+01$ & & & & & & & $1.6 \mathrm{E}+03$ & $1.6 \mathrm{E}+01$ & & \\
\hline $\begin{array}{l}\text { Chloro-2-methylaniline, } \\
\text { 4. }\end{array}$ & $000095-69-2$ & $1.2 E+04$ & $1.2 \mathrm{E}+02$ & & & & & $1.3 \mathrm{E}+03$ & $1.3 \mathrm{E}+01$ & & & & & & & $1.2 \mathrm{E}+03$ & $1.2 \mathrm{E}+01$ & & \\
\hline Chloroacetic Acid & $000079-11.8$ & & & $1.8 \mathrm{E}+04$ & $1,6 \mathrm{E}+05$ & $1.6 \mathrm{E}+04$ & $1.8 \mathrm{E}+03$ & & & $6.4 \mathrm{E}+03$ & $6.4 \mathrm{E}+02$ & & & & & & & $6.2 \mathrm{E}+03$ & $6.2 \mathrm{E}+02$ \\
\hline $\begin{array}{l}\text { Chloroacetophenone, } \\
2 .\end{array}$ & $000532-27-4$ & & & & & & & & & & & & & $1.0 E+06$ & $1.0 \mathrm{E}+06$ & & & & \\
\hline Chloroaniline, p- & $000106-47.8$ & & & $3.5 \mathrm{E}+04$ & $3.3 E+05$ & $3.3 E+04$ & $3.5 \mathrm{E}+03$ & & & $1.3 E+04$ & $1.3 \mathrm{E}+03$ & & & & & & & $1.2 \mathrm{E}+04$ & $1.2 \mathrm{E}+03$ \\
\hline Chlorobenzene ${ }^{(1)}$ & $000108-90-7$ & & & $1.8 \mathrm{E}+05$ & $1.0 \mathrm{E}+06$ & $1.6 \mathrm{E}+05$ & $1.8 \mathrm{E}+04$ & & & $4.0 \mathrm{E}+04$ & $4.0 \mathrm{E}+03$ & & & $5.1 E+02$ & $5.1 \mathrm{E}+02$ & & & $5.1 E+02$ & $5.1 \mathrm{E}+02$ \\
\hline Chlorobenzilate & 000510.15 .6 & $2.6 \mathrm{E}+04$ & $2.6 \mathrm{E}+02$ & $1.8 \mathrm{E}+05$ & $1.0 \mathrm{E}+06$ & $1.6 \mathrm{E}+05$ & $1.8 \mathrm{E}+04$ & $2,8 E+03$ & $2.8 \mathrm{E}+01$ & $6.4 E+04$ & $6.4 E+03$ & $1.0 \mathrm{E}+06$ & $1.0 \mathrm{E}+06$ & & & $2.7 \mathrm{E}+03$ & $2.7 E+01$ & $6.2 E+04$ & $6.2 \mathrm{E}+03$ \\
\hline Chlorobenzoic Acid, p- & $000074-11-3$ & & & $1.0 E+06$ & $1.0 \mathrm{E}+06$ & $1.0 \mathrm{E}+06$ & $1.8 \mathrm{E}+05$ & & & $6.4 \mathrm{E}+05$ & $6.4 E+04$ & & & & & & & $6.2 E+05$ & $6.2 E+04$ \\
\hline $\begin{array}{l}\text { Chlorobenzotrifluoride, } \\
\text { 4- }\end{array}$ & 000098.56 .6 & & & $1.8 \mathrm{E}+05$ & $1.0 \mathrm{E}+06$ & $1.6 E+05$ & $1.8 \mathrm{E}+04$ & & & $6.4 E+04$ & $6.4 E+03$ & & & & & & & $6.2 \mathrm{E}+04$ & $6.2 \mathrm{E}+03$ \\
\hline Chlorobutene, 1- & 000109.69 .3 & & & $1.0 \mathrm{E}+06$ & $1.0 \mathrm{E}+06$ & $1.0 \mathrm{E}+06$ & $3.5 \mathrm{E}+05$ & & & $1.0 \mathrm{E}+06$ & 2.1E+05 & & & & & & & $1.0 \mathrm{E}+06$ & $1.9 \mathrm{E}+0 \mathrm{~S}$ \\
\hline Chlorobutane, 2. & $000078-86-4$ & & & & & & & & & & & & & & & & & & \\
\hline Chlorocyclopentadiene & 041851.50 .7 & & & & & & & & & & & & & & & & & & \\
\hline Chlorodibromoethane & $073506-94-2$ & $8.5 \mathrm{E}+04$ & $8.5 E+02$ & & & & & $1.4 \mathrm{E}+04$ & $1.4 E+02$ & & & & & & & $1.3 \mathrm{E}+04$ & $1.3 \mathrm{E}+02$ & & \\
\hline Chlorodifluoromethane & $000075-45.6$ & & & & & & & & & & & & & $1.0 E+06$ & $1.0 E+06$ & & & & \\
\hline
\end{tabular}


Table 3.4c. (continued)

\begin{tabular}{|c|c|c|c|c|c|c|c|c|c|c|c|c|c|c|c|c|c|c|c|}
\hline \multirow{2}{*}{ Chemical } & \multirow{2}{*}{ CASRN } & \multicolumn{6}{|c|}{ Ingestion (mg/kg) } & \multicolumn{4}{|c|}{ Dermal (mgkg) } & \multicolumn{4}{|c|}{ Inhalation $(\mathrm{mg} / \mathrm{kg})^{-\gamma}$} & \multicolumn{4}{|c|}{$\begin{array}{c}\text { Ingestion + Dermal + Inhalation } \\
(\mathrm{mg} / \mathrm{kg})^{+\vee v}\end{array}$} \\
\hline & & $10^{-4}$ & $10^{-6}$ & $\begin{array}{l}\text { HQ=1 } \\
\text { Child }\end{array}$ & $\begin{array}{l}H Q=1 \\
\text { Adult }\end{array}$ & $\begin{array}{c}\text { HQ }=0.1 \\
\text { Adult }\end{array}$ & $\begin{array}{c}\text { HQ }=0.1 \\
\text { Child }\end{array}$ & $10^{-4}$ & $10^{6}$ & $H Q=1$ & $\mathrm{HQ}=0.1$ & $10^{-4}$ & $10^{6}$ & $\mathrm{HQ}=1$ & $\mathrm{HQ}=0.1$ & $10^{-4}$ & $10^{4}$ & $H I=1$ & $\mathrm{HI}=0.1$ \\
\hline Chloroform ${ }^{(6)}$ & $000057-66-3$ & $1.0 \mathrm{E}+06$ & $1.2 \mathrm{E}+04$ & $8.8 E+04$ & $8.2 \mathrm{E}+05$ & $8.2 \mathrm{E}+04$ & $8.8 E+03$ & $4.9 \mathrm{E}+04$ & $4.9 E+02$ & $1.3 E+04$ & $1.3 \mathrm{E}+03$ & $1.5 \mathrm{E}+03$ & $2.1 \mathrm{E}+02$ & & & $1.5 \mathrm{E}+03$ & $1.5 \mathrm{E}+02$ & $1.5 \mathrm{E}+03$ & $1.3 \mathrm{E}+03$ \\
\hline Chloromethanee $^{(4)}$ & $000074-87.3$ & $5.5 \mathrm{E}+05$ & $5.5 \mathrm{E}+03$ & & & & & $9.2 E+04$ & $9.2 \mathrm{E}+02$ & & & $1.2 E+03$ & $4.9 E+02$ & & & $1.2 \mathrm{E}+03$ & $3.1 E+02$ & & \\
\hline $\begin{array}{l}\text { Chloromethyl Methyl } \\
\text { Ether }\end{array}$ & $000107-30-2$ & & & & & & & & & & & & & & & & & & \\
\hline $\begin{array}{l}\text { Chloronsphthalene, } \\
\text { Beta- }\end{array}$ & $000091-58-7$ & & & $7.0 \mathrm{E}+05$ & $1.0 \mathrm{E}+06$ & $6.5 \mathrm{E}+05$ & $7.0 E+04$ & & & 4.1E+05 & 4.1E+04 & & & & & & & $3.9 \mathrm{E}+05$ & $3.9 \mathrm{E}+04$ \\
\hline Chloronitrobenzene, o- & $000088-73-3$ & 2.9E+05 & $2,9 \mathrm{E}+03$ & & & & & $4.8 \mathrm{E}+04$ & $4.8 \mathrm{E}+02$ & & & & & & & 4.5E+04 & $4.5 \mathrm{E}+02$ & & \\
\hline Chloronitrobenzene, p- & $000121-73-3$ & $4.0 \mathrm{E}+05$ & $4.0 E+03$ & & & & & $6.7 \mathrm{E}+04$ & $6.7 \mathrm{E}+02$ & & & & & & & $6.3 E+04$ & $6.3 \mathrm{E}+02$ & & \\
\hline Chlorophenol, 2 . & $000095-57-8$ & & & $4.4 E+04$ & 4.1E+05 & 4.1E+04 & $4.4 E+03$ & & & $2.6 \mathrm{E}+04$ & $2.6 \mathrm{E}+03$ & & & & & & & $2.4 E+04$ & $2.4 \mathrm{E}+03$ \\
\hline $\begin{array}{l}\text { Chlorophenyl Methyl } \\
\text { Sulfide, p- }\end{array}$ & $000123-09-1$ & & & & & & & & & & & & & & & & & & \\
\hline $\begin{array}{l}\text { Chlorophenyl Methyl } \\
\text { Sulfoxide }\end{array}$ & $000934-73-6$ & & & & & & & & & . & & & & & & & & & \\
\hline Chloropropane, 2 - & $000075-29.6$ & & & & & & & & & & & & & $1.6 \mathrm{E}+03$ & $1.6 \mathrm{E}+03$ & & & & \\
\hline Chlorothalonil & $001897-45.6$ & $6.5 \mathrm{E}+05$ & $6.5 \mathrm{E}+03$ & $1.3 \mathrm{E}+05$ & $1.0 \mathrm{E}+06$ & $1.2 \mathrm{E}+05$ & $1.3 E+04$ & $6.8 \mathrm{E}+04$ & $6.8 \mathrm{E}+02$ & $4.8 \mathrm{E}+04$ & $4.8 \mathrm{E}+03$ & & & & & $6.6 \mathrm{E}+0.4$ & $6.6 \mathrm{E}+02$ & $4.6 \mathrm{E}+04$ & $4.6 \mathrm{E}+03$ \\
\hline Chlorotoluene, o- & $000095-49-8$ & & & $1.8 \mathrm{E}+05$ & $1.0 \mathrm{E}+06$ & $1.6 \mathrm{E}+05$ & $1.8 \mathrm{E}+04$ & & & $1.0 \mathrm{E}+05$ & $1.0 \mathrm{E}+04$ & & & & & & & $9.7 E+04$ & $9.7 E+03$ \\
\hline Chlorpropham & $000101-21-3$ & & & $1.0 \mathrm{E}+06$ & $1.0 \mathrm{E}+06$ & $1.0 \mathrm{E}+06$ & $1.8 \mathrm{E}+05$ & & & $6.4 \mathrm{E}+05$ & $6.4 E+04$ & & & & & & & $6.2 \mathrm{E}+0 \mathrm{~S}$ & $6.2 \mathrm{E}+04$ \\
\hline Chlorpyrifos & $002921.88-2$ & & & $2.6 \mathrm{E}+04$ & 2.SE+0S & $2.5 \mathrm{E}+04$ & $2.6 \mathrm{E}+03$ & & & $9.6 \mathrm{E}+03$ & $9.6 \mathrm{E}+02$ & & & & & & & $9.3 E+03$ & $9.3 \mathrm{E}+02$ \\
\hline Chlorpyrifos Methyl & $005398-13-0$ & & & $8.8 \mathrm{E}+04$ & $8.2 \mathrm{E}+05$ & $8.2 E+04$ & $8.8 \mathrm{E}+03$ & & & $3.2 E+04$ & $3.2 \mathrm{E}+03$ & & & & & & & $3.1 E+04$ & $3.1 E+03$ \\
\hline Chlorsulfuron & $064902-72-3$ & & & $4.4 E+05$ & $1.0 \mathrm{E}+06$ & 4.1E+0S & $4.4 E+04$ & & & $1.6 \mathrm{E}+05$ & $1.6 \mathrm{E}+04$ & & & & & & & $1.5 \mathrm{E}+05$ & $1.5 \mathrm{E}+04$ \\
\hline Chlorthiophos & $060238-56-4$ & & & $7.0 \mathrm{E}+03$ & $6.5 \mathrm{E}+04$ & $6.5 \mathrm{E}+03$ & $7.0 \mathrm{E}+02$ & & & $2.6 \mathrm{E}+03$ & $2.6 \mathrm{E}+02$ & & & & & & & $2.5 \mathrm{E}+03$ & $2.5 E+02$ \\
\hline $\begin{array}{l}\text { Chromium (III) } \\
\text { (Insoluble Salts) }\end{array}$ & $016065.83-1$ & & & $1.0 \mathrm{E}+06$ & $1.0 E+06$ & $1.0 \mathrm{E}+06$ & $8.8 \mathrm{E}+05$ & & & $3.2 \mathrm{E}+05$ & $3.2 E+04$ & & & & & & & $3.2 \mathrm{E}+05$ & $3.2 \mathrm{E}+04$ \\
\hline Chromium (VI) $)^{(6)}$ & $018540-29.9$ & & & 4.4E+04 & 4. $1 \mathrm{E}+05$ & 4.1E+04 & $4.4 E+03$ & & & $6.4 E+03$ & $6.4 E+02$ & $1.0 \mathrm{E}+06$ & $1.0 \mathrm{E}+05$ & & & & & $6.3 E+03$ & $6.3 \mathrm{E}+02$ \\
\hline Chromium Salts & $\mathrm{NA}$ & & & & & & & & & & & & & & & & & & \\
\hline
\end{tabular}


Table 3.4c. (continued)

\begin{tabular}{|c|c|c|c|c|c|c|c|c|c|c|c|c|c|c|c|c|c|c|c|}
\hline \multirow{2}{*}{ Chemical } & \multirow{2}{*}{ CASRN } & \multicolumn{6}{|c|}{ Ingestion (mg/kg) } & \multicolumn{4}{|c|}{ Dermal (mgkg) } & \multicolumn{4}{|c|}{ Inhalation (mg/kg) } & \multicolumn{4}{|c|}{$\begin{array}{c}\text { Ingestion + Dermal + Inhalation } \\
(\mathrm{mg} / \mathrm{kg})^{+*}\end{array}$} \\
\hline & & $10^{-4}$ & $10^{8}$ & $\begin{array}{l}\text { HQ=1 } \\
\text { Child }\end{array}$ & $\begin{array}{l}\mathrm{HQ}=1 \\
\text { Adult }\end{array}$ & $\begin{array}{l}\mathrm{HQ}=0.1 \\
\text { Adult }\end{array}$ & $\begin{array}{l}\text { HQ }=0.1 \\
\text { Child }\end{array}$ & $10^{-4}$ & $10^{4}$ & HQ=1 & $\mathrm{HQ}=0.1$ & $10^{-4}$ & $10^{4}$ & $H Q=1$ & $\mathrm{HQ}=0.1$ & $10^{-4}$ & $10^{-6}$ & $H I=1$ & $H \mathrm{I}=0.1$ \\
\hline Chrysene ${ }^{(2)}$ & $000218-01-9$ & $9.8 \mathrm{E}+05$ & $9.8 E+03$ & & & & & $6.4 E+04$ & $6.4 \mathrm{E}+02$ & & & $1.0 \mathrm{E}+06$ & $1.0 \mathrm{E}+06$ & & & $6.2 \mathrm{E}+04$ & $6.2 \mathrm{E}+02$ & & \\
\hline Cobalt & $007440-48-4$ & & & & & & & & & & & & & & & & & & \\
\hline Coke Oven Emissions & $008007-45-2$ & & & & & • & & & & & & $1.0 \mathrm{E}+06$ & $1.0 E+06$ & & & & & & \\
\hline Copper & $007440-50-8$ & & & & & & & & & & & & & & & & & & \\
\hline Copper Cyanide & $000544-92-3$ & & & $4,4 \mathrm{E}+04$ & $4.1 \mathrm{E}+05$ & $4.1 \mathrm{E}+04$ & $4.4 \mathrm{E}+03$ & & & $6.4 \mathrm{E}+04$ & $6.4 E+03$ & & & & & & & $5.6 \mathrm{E}+04$ & $5.6 \mathrm{E}+03$ \\
\hline Creosole & $008001-58-9$ & & & & & & & & & & & & & & & & & & \\
\hline Cresol, m- & $000108.39-4$ & & & $4.4 \mathrm{E}+05$ & $1.0 \mathrm{E}+06$ & $4.1 E+05$ & $4.4 \mathrm{E}+04$ & & & $1.6 \mathrm{E}+05$ & $1.6 \mathrm{E}+04$ & & & & & & & $1.5 E+05^{\circ}$ & 1.5E+04 \\
\hline Cresol, o- & $000095-48.7$ & & & $4,4 E+05$ & $1.0 E+06$ & $4.1 E+05$ & $4.4 \mathrm{E}+0.4$ & & & $1.6 \mathrm{E}+05$ & $1.6 \mathrm{E}+04$ & & & & & & & $1.5 E+05$ & $1.5 \mathrm{E}+04$ \\
\hline Cresol, p- & $000105-44-5$ & & & $4.4 \mathrm{E}+04$ & $4.1 \mathrm{E}+0 \mathrm{~S}$ & $4.1 E+04$ & $4.4 \mathrm{E}+03$ & & & $2.1 \mathrm{E}+04$ & $2.1 \mathrm{E}+03$ & & & & & & & $2.0 \mathrm{E}+04$ & $2.0 \mathrm{E}+03$ \\
\hline Crotonaldehyde & $000123-73-9$ & $3.8 \mathrm{E}+03$ & $3,8 \mathrm{E}+01$ & & & & & $3.9 \mathrm{E}+02$ & $3.9 \mathrm{E}+\infty 0$ & & & & & & & $3.8 \mathrm{E}+02$ & $3.8 \mathrm{E}+\infty 0$ & & \\
\hline Cumene & $000098-82-8$ & & & 3.5E+05 & $1.0 \mathrm{E}+06$ & 3.3E+05 & $3.5 \mathrm{E}+04$ & & & $2.1 \mathrm{E}+05$ & $2.1 E+04$ & & & $3.5 \mathrm{E}+02$ & $3.5 \mathrm{E}+02$ & & & $3.5 E+02$ & $3.5 \mathrm{E}+02$ \\
\hline Cyanazine & $021725-46-2$ & $8.5 \mathrm{E}+03$ & $8.5 E+01$ & $1.8 \mathrm{E}+04$ & $1.6 \mathrm{E}+05$ & $1.6 \mathrm{E}+04$ & $1.8 \mathrm{E}+03$ & $8.9 \mathrm{E}+02$ & $8.9 E+00$ & $6.4 \mathrm{E}+03$ & $6.4 \mathrm{E}+02$ & & & & & $8.6 \mathrm{E}+02$ & $8.6 \mathrm{E}+\infty$ & $6,2 \mathrm{E}+03$ & $6.2 \mathrm{E}+02$ \\
\hline Cyanide (CN-) & 000057.12 .5 & & & $1.8 \mathrm{E}+05$ & $1.0 \mathrm{E}+\infty 6$ & $1.6 \mathrm{E}+05$ & $1.8 \mathrm{E}+04$ & & & $2.2 \mathrm{E}+04$ & $2.2 \mathrm{E}+03$ & & & & & & & $2,2 \mathrm{E}+04$ & $2.2 E+03$ \\
\hline Cyanogen & $000460 \cdot 19.5$ & & & $3.5 \mathrm{E}+05$ & $1.0 \mathrm{E}+06$ & $3.3 E+05$ & 3.5E+04 & & & $2.1 \mathrm{E}+05$ & $2.1 \mathrm{E}+04$ & & & & & & & $1.9 E+05$ & $1.9 \mathrm{E}+04$ \\
\hline Cyanogen Bromide & $000506-68.3$ & & & $7.9 \mathrm{E}+05$ & $1.0 \mathrm{E}+06$ & 7.4E+05 & $7.9 E+04$ & & & $4.6 \mathrm{E}+05$ & $4.6 \mathrm{E}+0.4$ & & & & & & & $4.4 \mathrm{E}+05$ & $4.4 E+04$ \\
\hline Cyanogen Chloride & 000506.77 .4 & & & 4.4E+05 & $1.0 \mathrm{E}+06$ & $4,1 \mathrm{E}+05$ & $4.4 \mathrm{E}+04$ & & & $2.6 \mathrm{E}+05$ & $2.6 \mathrm{E}+04$ & & . & & & & & $2.4 E+05$ & $2.4 \mathrm{E}+04$ \\
\hline $\begin{array}{l}\text { Cyclohexane, 1,2,3,4,5- } \\
\text { pentabromo-6-chloro- }\end{array}$ & $000087-84-3$ & $3.1 \mathrm{E}+05$ & $3.1 \mathrm{E}+03$ & & & & & $3.3 \mathrm{E}+04$ & $3.3 \mathrm{E}+02$ & & & & & & & $3.1 \mathrm{E}+04$ & $3.1 \mathrm{E}+02$ & & \\
\hline Cyclohexanone & $000108-94-1$ & & & $1.0 \mathrm{E}+06$ & $1.0 \mathrm{E}+06$ & $1.0 \mathrm{E}+06$ & $1.0 E+06$ & & & $1.0 \mathrm{E}+06$ & $1.0 \mathrm{E}+06$ & & & & & & & $1.0 \mathrm{E}+06$ & $1.0 E+06$ \\
\hline Cyclohexylamine & $000108-91-8$ & & & $1.0 \mathrm{E}+06$ & $1,0 \mathrm{E}+06$ & $1.0 E+06$ & $1.8 \mathrm{E}+05$ & & & $6.4 \mathrm{E}+05$ & $6.4 E+04$ & & & & & & & $6.2 E+05$ & $6.2 E+04$ \\
\hline Cyclopentadiene & $000542-92.7$ & & & & & & & & & & & & & & & & & & \\
\hline Cyhalothrin/karate & 068085.85 .8 & & & 4.4E+04 & $4.1 \mathrm{E}+05$ & 4.IE+04 & $4.4 \mathrm{E}+03$ & & & $1.6 \mathrm{E}+04$ & $1.6 \mathrm{E}+03$ & & & & & & & $1.5 \mathrm{E}+04$ & $1.5 E+03$ \\
\hline Cypermethrin & $052315-07.8$ & & & $8.8 \mathrm{E}+04$ & $8.2 E+05$ & $8.2 E+04$ & $8.8 E+03$ & & & $3.2 \mathrm{E}+04$ & $3.2 E+03$ & & & & & & & $3.1 E+04$ & $3,1 E+03$ \\
\hline
\end{tabular}


Table 3.4c. (continued)

\begin{tabular}{|c|c|c|c|c|c|c|c|c|c|c|c|c|c|c|c|c|c|c|c|}
\hline \multirow{2}{*}{ Chemical } & \multirow{2}{*}{ CASRN } & \multicolumn{6}{|c|}{ Ingestion (mg/kg)" } & \multicolumn{4}{|c|}{ Dermal (mgkg) } & \multicolumn{4}{|c|}{ Inhalation (mg/kg) } & \multicolumn{4}{|c|}{$\begin{array}{c}\text { Ingestion + Dermal + Inhalation } \\
(\mathrm{mg} / \mathrm{kg})^{\mathrm{r} \cdot \mathrm{r}}\end{array}$} \\
\hline & & $10^{-4}$ & $10^{4}$ & $\begin{array}{l}\text { HQ=1 } \\
\text { Child }\end{array}$ & $\begin{array}{l}\text { HQ=1 } \\
\text { Adult }\end{array}$ & $\begin{array}{l}\text { HQ }=0.1 \\
\text { Adult }\end{array}$ & $\begin{array}{l}\text { HQ }=0.1 \\
\text { Child }\end{array}$ & $10^{-4}$ & $10^{4}$ & $H Q=1$ & $\mathrm{HQ}=0.1$ & $10^{4}$ & $10^{4}$ & $H Q=1$ & $\mathrm{HQ}=0.1$ & $10^{-4}$ & $10^{4}$ & HII $=\mathbf{I}$ & $\mathrm{HI}=0.1$ \\
\hline Cyromazine & 066215.27 .8 & & & $6.6 \mathrm{E}+04$ & $6.1 \mathrm{E}+05$ & $6.1 \mathrm{E}+04$ & $6.6 \mathrm{E}+03$ & & & 2.4E+04 & $2.4 \mathrm{E}+03$ & & & & & & & $2.3 E+04$ & $2.3 \mathrm{E}+03$ \\
\hline DDD & $000072-54-8$ & $3.0 \mathrm{E}+04$ & $3.0 \mathrm{E}+02$ & & & & & $4,4 \mathrm{E}+03$ & $4.4 \mathrm{E}+0 \mathrm{t}$ & & & & & & & $4.1 \mathrm{E}+03$ & $4.1 \mathrm{E}+01$ & & \\
\hline DDE & $000072-55.9$ & 2.1E+04 & $2.1 E+02$ & & & & & $3.1 E+03$ & $3.1 \mathrm{E}+01$ & & & & & & & $2.9 E+03$ & $2.9 E+01$ & & \\
\hline DDT & $000050-29-3$ & $2.1 E+04$ & 2.1E+02 & $4.4 \mathrm{E}+03$ & 4.1E+04 & $4.1 \mathrm{E}+03$ & $4.4 \mathrm{E}+02$ & $3.1 \mathrm{E}+03$ & 3.1E+01 & $2.2 \mathrm{E}+03$ & $2.2 \mathrm{E}+02$ & $1.0 \mathrm{E}+06$ & $1.0 \mathrm{E}+06$ & & & $2.9 \mathrm{E}+03$ & $2.9 \mathrm{E}+01$ & $2.1 \mathrm{E}+03$ & 2.1E+02 \\
\hline Dacthal & 001861.32 .1 & & & $8.8 \mathrm{E}+04$ & $8.2 \mathrm{E}+05$ & $8.2 E+04$ & $8.8 E+03$ & & & $3.2 \mathrm{E}+04$ & $3.2 \mathrm{E}+03$ & & & & & & & $3.1 \mathrm{E}+04$ & $3.1 E+03$ \\
\hline Dalapon & $000075-99-0$ & & & $2.6 \mathrm{E}+05$ & $1.0 \mathrm{E}+06$ & $2.5 \mathrm{E}+05$ & $2.6 \mathrm{E}+04$ & & & $9.6 \mathrm{E}+04$ & $9.6 \mathrm{E}+03$ & & & & & & & $9.3 \mathrm{E}+04$ & 9.3E+03 \\
\hline $\begin{array}{l}\text { Decabromodiphenyl } \\
\text { Ether }\end{array}$ & $001163-19-5$ & & . & $8.8 E+04$ & $8.2 E+05$ & $8.2 \mathrm{E}+04$ & $8.8 \mathrm{E}+03$ & & & $5.1 \mathrm{E}+04$ & $5.1 E+03$ & & & & & & & $4.8 \mathrm{E}+04$ & $4.8 \mathrm{E}+03$ \\
\hline Demeton & $008065-48-3$ & & & $3.5 \mathrm{E}+02$ & $3.3 \mathrm{E}+03$ & $3.3 \mathrm{E}+02$ & $3.5 \mathrm{E}+01$ & & & $1.3 E+02$ & $1.3 \mathrm{E}+01$ & & & & & & & $1.2 \mathrm{E}+02$ & $1.2 \mathrm{E}+01$ \\
\hline Di(2-ethylhexyl)adipate & $000103-23-1$ & $1.0 \mathrm{E}+06$ & $6.0 \mathrm{E}+04$ & $1.0 \mathrm{E}+06$ & $1.0 \mathrm{E}+06$ & $1.0 \mathrm{E}+06$ & $5.3 \mathrm{E}+05$ & $6.2 \mathrm{E}+0 \mathrm{~s}$ & $6.2 \mathrm{E}+03$ & $1.0 \mathrm{E}+06$ & $1.9 E+05$ & & & & & $6.0 \mathrm{E}+05$ & $6.0 \mathrm{E}+03$ & $1.0 \mathrm{E}+06$ & $1.9 \mathrm{E}+05$ \\
\hline Diallate & $002303-16-4$ & $1.2 E+05$ & $1.2 \mathrm{E}+03$ & & & & & $2.0 \mathrm{E}+04$ & $2.0 \mathrm{E}+02$ & & & & & & & $1.9 E+04$ & $1.9 \mathrm{E}+02$ & & \\
\hline Diaxinon & $000333-41-5$ & & & $7.9 \mathrm{E}+03$ & $7.4 \mathrm{E}+04$ & $7.4 E+03$ & $7.9 \mathrm{E}+02$ & & & $2.9 \mathrm{E}+03$ & $2.9 \mathrm{E}+02$ & & & & & & & $2.8 \mathrm{E}+03$ & $2.8 \mathrm{E}+02$ \\
\hline pibenz[a, h]anthracene ${ }^{n}$ & $000053.70-3$ & $9.8 \mathrm{E}+02$ & $9.8 \mathrm{E}+\infty 0$ & & & & & $6.4 \mathrm{E}+01$ & $6.4 \mathrm{E}-01$ & & & $1.0 \mathrm{E}+06$ & $1.0 \mathrm{E}+06$ & & & $6.2 \mathrm{E}+01$ & $6.2 \mathrm{E}-01$ & & \\
\hline $\begin{array}{l}\text { Dibromo-3- } \\
\text { chloropropane, 1,2- }\end{array}$ & $000096-12-8$ & $5.1 \mathrm{E}+03$ & $5.1 \mathrm{E}+01$ & & & & & $8.6 \mathrm{E}+02$ & $8.6 \mathrm{E}+\infty$ & & & $1.0 \mathrm{E}+06$ & $1.0 \mathrm{E}+06$ & $1.0 \mathrm{E}+06$ & $1.0 \mathrm{E}+06$ & $8.1 E+02$ & $8.1 E+\infty$ & & \\
\hline Dibromobenzene, 1,4- & $000106-37.6$ & & & $8.8 \mathrm{E}+04$ & $8.2 \mathrm{E}+05$ & $8.2 E+04$ & $8.8 \mathrm{E}+03$ & & & $5.1 \mathrm{E}+04$ & $5.1 \mathrm{E}+03$ & & & & & & & $4.8 \mathrm{E}+04$ & $4.8 \mathrm{E}+03$ \\
\hline Dibromoctioromethane & $000124-48-1$ & $8.5 \mathrm{E}+04$ & $8.5 E+02$ & $1.8 \mathrm{E}+05$ & $1.0 \mathrm{E}+06$ & $1.6 \mathrm{E}+05$ & $1.8 \mathrm{E}+04$ & $1.1 \mathrm{E}+04$ & $1.1 \mathrm{E}+02$ & 7.7E+04 & $7.7 \mathrm{E}+03$ & & & & & $1.0 \mathrm{E}+04$ & $1.0 \mathrm{E}+02$ & $7.4 \mathrm{E}+04$ & $7.4 E+03$ \\
\hline $\begin{array}{l}\text { Dibromodichlorometha } \\
\text { ne }\end{array}$ & $000594-18-3$ & & & & & & & & & & & & & & & & & & \\
\hline $\begin{array}{l}\text { Dibromodiphenyl } \\
\text { Ether, p.p'- }\end{array}$ & $002050-47.7$ & & & & & & & & & & & & & & & & & & \\
\hline Dibromoethane, 1,2. & $000106-93-4$ & $8.4 E+01$ & $8.4 \mathrm{E}-01$ & & & & & $1.4 E+01$ & $1.4 \mathrm{E}-01$ & & & $5.5 E+03$ & $1.7 \mathrm{E}+02$ & $3.1 \mathrm{E}+03$ & $3.1 E+02$ & $1.3 \mathrm{E}+01$ & $1.3 \mathrm{E}-01$ & & \\
\hline $\begin{array}{l}\text { Dibromomethane } \\
\text { (Methylene Bromide) }\end{array}$ & $000074-95-3$ & & & $8.8 \mathrm{E}+04$ & $8.2 \mathrm{E}+0 \mathrm{~S}$ & $8.2 E+04$ & $8.8 \mathrm{E}+03$ & & & $5.1 E+04$ & $5.1 \mathrm{E}+03$ & & & & & & & $4.8 \mathrm{E}+04$ & $4.8 \mathrm{E}+03$ \\
\hline Dibutyl Phthesate & $000084-74-2$ & & & $8.8 E+05$ & $1.0 \mathrm{E}+06$ & $8.2 E+05$ & $8.8 \mathrm{E}+04$ & & & $6.4 \mathrm{E}+05$ & $6.4 E+04$ & & & & & & & $6.0 \mathrm{E}+05$ & $6.0 \mathrm{E}+04$ \\
\hline
\end{tabular}


Table 3.4c. (continued)

\begin{tabular}{|c|c|c|c|c|c|c|c|c|c|c|c|c|c|c|c|c|c|c|c|}
\hline \multirow{2}{*}{ Chemical } & \multirow{2}{*}{ CASRN } & \multicolumn{6}{|c|}{ Ingestion (mg/kg) } & \multicolumn{4}{|c|}{ Dermal (mgkg) } & \multicolumn{4}{|c|}{ Inhalation (mg/kg) } & \multicolumn{4}{|c|}{$\begin{array}{c}\text { Ingestion + Dermal + Inhalation } \\
(\mathrm{mg} / \mathrm{kg})^{+\bullet i}\end{array}$} \\
\hline & & $10^{-1}$ & $10^{6}$ & $\begin{array}{l}\text { HQ=1 } \\
\text { Child }\end{array}$ & $\begin{array}{l}\text { HQ=1 } \\
\text { Adult }\end{array}$ & $\begin{array}{l}\mathrm{HQ}=0.1 \\
\text { Adult }\end{array}$ & $\begin{array}{l}\text { HQ }=0.1 \\
\text { Child }\end{array}$ & $10^{-4}$ & $10^{-4}$ & $\mathrm{HQ}=1$ & $\mathrm{HQ}=0.1$ & $10^{-4}$ & $10^{4}$ & $H Q=1$ & $\mathrm{HQ}=0.1$ & $10^{-1}$ & $10^{5}$ & $H I=1$ & $\mathrm{HI}=0.1$ \\
\hline Dicamba & $001918-00-9$ & & & $2.6 \mathrm{E}+05$ & $1.0 \mathrm{E}+06$ & 2.5E+05 & $2.6 \mathrm{E}+04$ & & & $9.6 \mathrm{E}+04$ & $9.6 \mathrm{E}+03$ & & & & & & & $9.3 \mathrm{E}+04$ & $9.3 \mathrm{E}+03$ \\
\hline Dichioro-2-butene, 1,4- & $000764-41-0$ & & & & & & & & & & & $1.4 E+03$ & $1.4 \mathrm{E}+01$ & & & & & & \\
\hline \multicolumn{20}{|l|}{ Dichloroucetic Acid } \\
\hline Dichlorobenzene, 1,2.(7) & $000095-50-1$ & & & $7.9 \mathrm{E}+0 \mathrm{~S}$ & $1.0 \mathrm{E}+06$ & 7.4E+0s & $7.9 \mathrm{E}+04$ & & & $4.6 \mathrm{E}+05$ & $4.6 \mathrm{E}+0.4$ & & & $5.1 E+02$ & $5.1 \mathrm{E}+02$ & & & $5.1 \mathrm{E}+02$ & $5.1 \mathrm{E}+02$ \\
\hline Dichlorobenzene, 1,3- & $000541-73-1$ & & & & & & & & & & & & & & & & & & \\
\hline Dichlorobenzene, 1,4- & $000106-46-7$ & $3.0 \mathrm{E}+05$ & $3.0 \mathrm{E}+03$ & & & & & $5.6 \mathrm{E}+04$ & $5.6 \mathrm{E}+02$ & & & & & $3.2 \mathrm{E}+01$ & $3.2 \mathrm{E}+01$ & $3,2 \mathrm{E}+01$ & $3.2 \mathrm{E}+01$ & & \\
\hline Dichlorobenridine, 3,3'- & $000091-94-1$ & $1.6 \mathrm{E}+04$ & $1.6 \mathrm{E}+02$ & & & & & $1.7 \mathrm{E}+03$ & $1.7 \mathrm{E}+01$ & & & & & & & $1.6 \mathrm{E}+03$ & $1.6 \mathrm{E}+01$ & & \\
\hline $\begin{array}{l}\text { Dichlorodifluorometha } \\
\text { ne }\end{array}$ & $000075-71-8$ & & & $1.0 \mathrm{E}+06$ & $1.0 \mathrm{E}+06$ & $1.0 \mathrm{E}+06$ & $1.8 \mathrm{E}+05$ & & & $3.0 \mathrm{E}+05$ & $3.0 \mathrm{E}+04$ & & & $1.0 \mathrm{E}+03$ & $1.0 \mathrm{E}+03$ & & & $1.0 \mathrm{E}+03$ & $1.0 \mathrm{E}+03$ \\
\hline Dichloroethane, 1,1- & $000075-34-3$ & & & 8.8E+05 & $1.0 \mathrm{E}+06$ & $8.2 \mathrm{E}+05$ & $8.8 E+04$ & & & $6.4 \mathrm{E}+05$ & $6.4 \mathrm{E}+04$ & & & $6.3 \mathrm{E}+02$ & $6.3 \mathrm{E}+01$ & & & $5.0 \mathrm{E}+02$ & $5.0 \mathrm{E}+01$ \\
\hline Dichloroethane, 1,2- & $000107-06-2$ & $7.9 \mathrm{E}+04$ & $7.9 \mathrm{E}+02$ & & & & & $1.6 \mathrm{E}+04$ & $1.6 \mathrm{E}+02$ & & & $6.7 \mathrm{E}+02$ & $2.2 \mathrm{E}+02$ & & & $6.7 \mathrm{E}+02$ & $9.1 E+01$ & & \\
\hline Dichloroethylene, 1,1- & $000075-35-4$ & $1.2 E+04$ & $1.2 \mathrm{E}+02$ & $7.9 \mathrm{E}+04$ & 7.4E+05 & 7.4E+04 & $7.9 E+03$ & $2.5 E+03$ & $2.5 \mathrm{E}+01$ & $5.8 \mathrm{E}+04$ & $5.8 \mathrm{E}+03$ & $5.0 \mathrm{E}+02$ & $5.0 \mathrm{E}+00$ & & & 4.1E+02 & 4. $1 \mathrm{E}+00$ & $1.2 \mathrm{E}+03$ & $1.2 \mathrm{E}+03$ \\
\hline $\begin{array}{l}\text { Diehloroethylene, 1,2- } \\
\text { (Mixed Isomers) }\end{array}$ & $000540.59-0$ & & & $7.9 \mathrm{E}+04$ & 7.4E+05 & 7.4E+04 & $7.9 \mathrm{E}+03$ & & & $4.6 \mathrm{E}+04$ & $4.6 \mathrm{E}+03$ & & & & & & & $4.4 \mathrm{E}+04$ & $4.4 E+03$ \\
\hline $\begin{array}{l}\text { Dichloroethylene, 1,2- } \\
\text { cis- }\end{array}$ & $000156-59-2$ & & & $8.8 \mathrm{E}+04$ & $8.2 E+05$ & $8.2 E+04$ & $8.8 \mathrm{E}+03$ & & & $6.4 \mathrm{E}+04$ & $6.4 \mathrm{E}+03$ & & & & & & & $6.0 \mathrm{E}+04$ & $6.0 \mathrm{E}+03$ \\
\hline $\begin{array}{l}\text { Dichioroethylene, 1,2- } \\
\text { trans- }\end{array}$ & 000156.60 .5 & & & $1.8 \mathrm{E}+05$ & $1.0 \mathrm{E}+06$ & $1.6 \mathrm{E}+05$ & $1.8 \mathrm{E}+04$ & & & $1.3 E+05$ & $1.3 E+04$ & & & & & & & $1.2 \mathrm{E}+05$ & $1.2 \mathrm{E}+04$ \\
\hline Dichjorophenol, 2,4- & 000120.83 .2 & & & $2.6 \mathrm{E}+04$ & $2.5 \mathrm{E}+05$ & $2.5 \mathrm{E}+04$ & $2.6 \mathrm{E}+03$ & & & $1.6 \mathrm{E}+04$ & $1.6 \mathrm{E}+03$ & & & & & & & $1.5 E+04$ & $1.5 \mathrm{E}+03$ \\
\hline $\begin{array}{l}\text { Dichlorophenoxy } \\
\text { Acetic Acid, 2,4. }\end{array}$ & $000094-75.7$ & & & 8.8E+04 & $8.2 \mathrm{E}+05$ & $8.2 \mathrm{E}+04$ & $8.8 \mathrm{E}+03$ & & & $5.1 \mathrm{E}+04$ & $5.1 E+03$ & & & & & & & $4.8 \mathrm{E}+04$ & $4.8 E+03$ \\
\hline $\begin{array}{l}\text { Dichjorophenoxy)butyi } \\
\text { cAcid, } 4 \cdot(2,4 \cdot\end{array}$ & $000094.82-6$ & & & $7.0 \mathrm{E}+04$ & $6.5 \mathrm{E}+05$ & $6.5 \mathrm{E}+04$ & $7,0 \mathrm{E}+03$ & & & $2.6 \mathrm{E}+04$ & $2.6 \mathrm{E}+03$ & & & & & & & $2.5 \mathrm{E}+04$ & $2.5 E+03$ \\
\hline Dichloropropane, 1,2. & $000078-87-5$ & $1.1 \mathrm{E}+05$ & $1.1 E+03$ & & & & & $1.6 \mathrm{E}+04$ & $1.6 \mathrm{E}+02$ & & & & & $4.8 E+02$ & $4.8 E+02$ & $4.8 \mathrm{E}+02$ & $1.5 \mathrm{E}+02$ & & \\
\hline Dichloropropanol, 2,3- & $000616-23-9$ & & & $2.6 \mathrm{E}+04$ & $2.5 E+05$ & $2.5 \mathrm{E}+04$ & $2.6 \mathrm{E}+03$ & & & $9.6 \mathrm{E}+03$ & $9.6 \mathrm{E}+02$ & & & & & & & $9.3 \mathrm{E}+03$ & $9.3 E+02$ \\
\hline Dictlotopropene, 1,3. & $000542-75-6$ & $4.0 E+04$ & $4.0 \mathrm{E}+02$ & $2.6 \mathrm{E}+03$ & $2.5 \mathrm{E}+04$ & $2.5 \mathrm{E}+03$ & $2.6 \mathrm{E}+02$ & $4.6 \mathrm{E}+03$ & $4.6 \mathrm{E}+01$ & $1.1 \mathrm{E}+03$ & $1.1 \mathrm{E}+02$ & $3.7 \mathrm{E}+03$ & $6.0 E+02$ & $3.7 E+03$ & $3.7 \mathrm{E}+03$ & 3.7E+03 & $4.1 E+01$ & $8.1 E+02$ & $8.1 E+01$ \\
\hline
\end{tabular}

Nonradionuclides/soil/recreational

Page 11 of 34 
Table 3.4c. (continued)

\begin{tabular}{|c|c|c|c|c|c|c|c|c|c|c|c|c|c|c|c|c|c|c|c|}
\hline \multirow{2}{*}{ Chemical } & \multirow{2}{*}{ CASRN } & \multicolumn{6}{|c|}{ Ingestion (mg/kg) } & \multicolumn{4}{|c|}{ Dermal (mgkg) } & \multicolumn{4}{|c|}{ Inhalation (mg/kg) } & \multicolumn{4}{|c|}{$\begin{array}{c}\text { Ingestion + Dermal + Inhalation } \\
(\mathrm{mg} / \mathrm{kg})^{+\bullet}\end{array}$} \\
\hline & & $10^{-4}$ & $10^{6}$ & $\begin{array}{l}\mathrm{HQ}=1 \\
\text { Child } \\
\end{array}$ & $\begin{array}{l}H Q=1 \\
\text { Adult } \\
\end{array}$ & $\begin{array}{c}\text { HQ=0.1 } \\
\text { Adult }\end{array}$ & $\begin{array}{c}\text { HQ=0.1 } \\
\text { Child } \\
\end{array}$ & $10^{-4}$ & $10^{-4}$ & $\mathrm{HQ}=1$ & $\mathrm{HQ}=0.1$ & $10^{-4}$ & $10^{6}$ & $\mathrm{HQ}=1$ & $\mathrm{HQ}=0.1$ & $10^{4}$ & $10^{5}$ & $\mathrm{HI}=\mathbf{1}$ & $\mathrm{HI}=0.1$ \\
\hline Dichlorvos & $000062.73-7$ & $2.5 \mathrm{E}+04$ & $2.5 \mathrm{E}+02$ & $4.4 E+03$ & $4.1 E+04$ & $4.1 \mathrm{E}+03$ & $4.4 \mathrm{E}+02$ & $2.6 \mathrm{E}+03$ & $2.6 \mathrm{E}+01$ & $1.6 \mathrm{E}+03$ & $1.6 \mathrm{E}+02$ & & & $1.0 \mathrm{E}+06$ & $1.0 E+06$ & $2.5 \mathrm{E}+03$ & $2.5 \mathrm{E}+01$ & $1.5 \mathrm{E}+03$ & $1.5 \mathrm{E}+02$ \\
\hline Dicofol & $000115-32-2$ & & & & & & & & & & & & & & & & & & \\
\hline Dieyclopentadiene & $000077-73 \cdot 6$ & & & 2.6E+05 & $1.0 \mathrm{E}+06$ & $2.5 \mathrm{E}+05$ & $2.6 \mathrm{E}+04$ & & & $1.5 \mathrm{E}+05$ & 1.5E+04 & & & $7.4 E+02$ & $3.4 \mathrm{E}+02$ & & & $7.4 \mathrm{E}+02$ & $2.6 \mathrm{E}+02$ \\
\hline Dieldrin & $000060-57.1$ & $4.5 \mathrm{E}+02$ & $4.5 \mathrm{E}+\infty 0$ & $4.4 \mathrm{E}+02$ & 4.1E+03 & $4.1 E+02$ & $4.4 E+01$ & 4. $\pi+01$ & 4.7E-01 & $1.6 \mathrm{E}+02$ & $1.6 \mathrm{E}+01$ & $1.0 \mathrm{E}+06$ & $2.6 \mathrm{E}+05$ & & & $4.5 \mathrm{E}+01$ & 4.5E-01 & $1.5 \mathrm{E}+02$ & $1.5 \mathrm{E}+01$ \\
\hline Diethyl Phthalate & $000084-66-2$ & & & $1.0 E+06$ & $1.0 E+06$ & $1.0 E+06$ & $7.0 E+05$ & & & $1.0 E+06$ & $4.6 \mathrm{E}+05$ & & & & & & & $1.0 E+06$ & $4.3 E+05$ \\
\hline $\begin{array}{l}\begin{array}{l}\text { Diethyl-p- } \\
\text { nitrophenylphosphate }\end{array} \\
\end{array}$ & $000311-45-5$ & & & & & 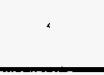 & & & & & & & & & & & & & \\
\hline $\begin{array}{l}\text { Diecthlene Glycol } \\
\text { Dinitrate (DECDN) }\end{array}$ & $000693-21-0$ & & & & & & & & & & & & & & & & & & \\
\hline $\begin{array}{l}\text { Diethylene Glycol } \\
\text { Monobutyl Ether }\end{array}$ & $000112-34-5$ & & & & & & & & & & & & & $1.0 \mathrm{E}+06$ & $1.0 E+06$ & & & & \\
\hline $\begin{array}{l}\text { Diethylene Glycol } \\
\text { Monoethyl Ether }\end{array}$ & $000111-90.0$ & & & $1.0 \mathrm{E}+06$ & $1.0 E+06$ & $1.0 E+06$ & $1.0 E+06$ & & & $1.0 \mathrm{E}+05$ & $6.4 \mathrm{E}+05$ & & & & & & & $1.0 E+06$ & $6.2 \mathrm{E}+05$ \\
\hline Diethylformamide & $000617-84-5$ & & & $9.6 \mathrm{E}+04$ & $9.0 \mathrm{E}+05$ & $9.0 E+04$ & $9.6 \mathrm{E}+03$ & & & $3.5 \mathrm{E}+04$ & $3.5 \mathrm{E}+03$ & & & & & & & $3.4 \mathrm{E}+04$ & $3.4 E+03$ \\
\hline Diethylstilbeserol & $000056-53-1$ & $1.5 \mathrm{E}+\infty 0$ & $1.5 \mathrm{E}-02$ & & & & & $1.6 \mathrm{E}-01$ & 1.6E-03 & & & $8.3 \mathrm{E}+05$ & $8.3 \mathrm{E}+03$ & & & $1.5 \mathrm{E}-01$ & $1.5 \mathrm{E}-03$ & & \\
\hline Diferzoquat & $043222-48-6$ & & & $7.0 \mathrm{E}+05$ & $1.0 \mathrm{E}+06$ & $6.5 \mathrm{E}+05$ & $7.0 E+04$ & & & $2.6 \mathrm{E}+05$ & $2.6 \mathrm{E}+04$ & & & & & & & $2.5 \mathrm{E}+05$ & $2.5 \mathrm{E}+04$ \\
\hline Diflubenzuron & $035367-38-5$ & & & $1.8 \mathrm{E}+05$ & $1.0 \mathrm{E}+06$ & $1.6 \mathrm{E}+05$ & $1.8 \mathrm{E}+04$ & & & $6.4 \mathrm{E}+04$ & $6.4 \mathrm{E}+03$ & & & & & & & $6.2 E+04^{\circ}$ & $6.2 E+03$ \\
\hline Dilluoroethene, 1,1 - & 000075.37 .6 & & & & & & & & & & & & & $1.0 \mathrm{E}+06$ & $1.0 E+06$ & & & & \\
\hline $\begin{array}{l}\text { Disopropyl } \\
\text { Methylphosphonate }\end{array}$ & $001445-75-6$ & & & $7.0 \mathrm{E}+05$ & $1.0 \mathrm{E}+06$ & $6.5 \mathrm{E}+05$ & $7.0 E+04$ & & & $2.6 \mathrm{E}+05$ & $2.6 \mathrm{E}+04$ & & & & & & & $2.5 \mathrm{E}+05$ & $2.5 \mathrm{E}+04$ \\
\hline Dimethipin & 055290.-64.7 & & & $1.8 \mathrm{E}+05$ & $1.0 \mathrm{E}+06$ & $1.6 \mathrm{E}+05$ & $1.8 E+04$ & & & $6.4 \mathrm{E}+04$ & $6.4 \mathrm{E}+03$ & & & & & & & $6.2 E+04$ & $6.2 E+03$ \\
\hline Dimethoate & $000060-51.5$ & & & $1.8 \mathrm{E}+03$ & $1.6 \mathrm{E}+04$ & $1.6 \mathrm{E}+03$ & $1.8 \mathrm{E}+02$ & & & $6.4 \mathrm{E}+02$ & $6.4 \mathrm{E}+01$ & & & & & & & $6.2 \mathrm{E}+02$ & $6.2 \mathrm{E}+01$ \\
\hline $\begin{array}{l}\text { Dimethoxbenzidines } \\
\text { 3,3'- }\end{array}$ & $000119.90-4$ & S.1E+os & $5.1 \mathrm{E}+03$ & & & & & $5.4 E+04$ & $5.4 E+02$ & & & & & & & $5.2 \mathrm{E}+04$ & $5.2 E+02$ & & \\
\hline Dimethyl Sulfate & $000077-78-1$ & & & & & & & & & & & & & & & & & & \\
\hline
\end{tabular}


Table 3.4c. (continued)

\begin{tabular}{|c|c|c|c|c|c|c|c|c|c|c|c|c|c|c|c|c|c|c|c|}
\hline \multirow{2}{*}{ Chemical } & \multirow{2}{*}{ CASRN } & \multicolumn{6}{|c|}{ Ingestion (mg/kg) } & \multicolumn{4}{|c|}{ Dermal (mgkg) } & \multicolumn{4}{|c|}{ Inhalation (mg/kg) } & \multicolumn{4}{|c|}{$\begin{array}{c}\text { Ingestion + Dermal + Inhalation } \\
(\mathbf{m g} / \mathrm{kg})^{+\cdot \mathrm{r}}\end{array}$} \\
\hline & & $10^{-4}$ & $10^{4}$ & $\begin{array}{l}\text { HQ=1 } \\
\text { Child }\end{array}$ & $\begin{array}{l}\text { HQ=1 } \\
\text { Adult }\end{array}$ & $\begin{array}{c}\text { HQ }=0.1 \\
\text { Adult }\end{array}$ & $\begin{array}{l}\text { HQ }=0.1 \\
\text { Child }\end{array}$ & $10^{-4}$ & $10^{-4}$ & $H Q=1$ & $H Q=0.1$ & $10^{4}$ & $10^{4}$ & $\mathbf{H Q}=\mathbf{I}$ & $\mathrm{HQ}=0.1$ & $10^{-1}$ & $10^{5}$ & $\cdot H I=1$ & $H I=0.1$ \\
\hline $\begin{array}{l}\text { Dimethylariline } \mathrm{HCl} \\
\text { 24. }\end{array}$ & $021436-96-4$ & $1.2 \mathrm{E}+04$ & $1.2 \mathrm{E}+02$ & & & & & $1.3 \mathrm{E}+03$ & $1.3 \mathrm{E}+01$ & & & & & & & $1.2 \mathrm{E}+03$ & $1.2 \mathrm{E}+01$ & & \\
\hline Dimethylaniline, 2,4- & $000095-68-1$ & $9.5 \mathrm{E}+03$ & $9.5 \mathrm{E}+01$ & & & & & $1.0 \mathrm{E}+03$ & $1.0 \mathrm{E}+01$ & & & & & & & $9.6 \mathrm{E}+02$ & $9.6 \mathrm{E}+\infty$ & & \\
\hline Dimethylariline, N,N- & $000121-69-7$ & & & $1.8 E+04$ & $1.6 \mathrm{E}+05$ & $1.6 \mathrm{E}+04$ & $1.8 \mathrm{E}+03$ & & & $6.4 \mathrm{E}+03$ & $6.4 \mathrm{E}+02$ & & & & & & & $6.2 E+03$ & $6.2 \mathrm{E}+02$ \\
\hline $\begin{array}{l}\text { Dimethylbenzidine, } \\
\text { 3,3*. }\end{array}$ & $000119-93-7$ & $7.8 \mathrm{E}+02$ & $7.8 \mathrm{E}+\infty 0$ & & & & & $8.2 \mathrm{E}+01$ & 8.2E-01 & & & & & & & $7.8 \mathrm{E}+01$ & $7.8 \mathrm{E}-0 \mathrm{t}$ & - & \\
\hline Dimethylethyl Lead & $107584-40-7$ & & & & & & & & & & & & & & & & & & \\
\hline Dimethylformamide & $000068-12-2$ & & & $8.8 E+05$ & $1.0 \mathrm{E}+06$ & $8.2 \mathrm{E}+05$ & $8.8 E+04$ & & & $3.2 \mathrm{E}+05$ & $3.2 \mathrm{E}+04$ & & & $1.0 \mathrm{E}+06$ & $1.0 E+06$ & & & $3.1 E+05$ & $3.1 E+04$ \\
\hline $\begin{array}{l}\text { Dimethylhydrazine, } \\
1,1^{(3)}\end{array}$ & $000057-14-7$ & $2.8 \mathrm{E}+03$ & 2.8E+01 & & & & & $2.9 \mathrm{E}+02$ & $2.9 \mathrm{E}+00$ & & & $1.0 \mathrm{E}+06$ & $1.0 \mathrm{E}+06$ & & & $2.8 \mathrm{E}+02$ & $2.8 \mathrm{E}+\infty 0$ & & \\
\hline $\begin{array}{l}\text { Dimethylhydraine, } \\
\text { 1,2- }\end{array}$ & $000540-73.8$ & & & & & & & & & & & & & & & & & & \\
\hline Dimethylphenol, 2,4- & $000105-67-9$ & & & $1.8 E+05$ & $1,0 \mathrm{E}+\infty 6$ & $1.6 \mathrm{E}+05$ & $1.8 \mathrm{E}+04$ & & & $1.0 \mathrm{E}+03$ & $1.0 E+04$ & & & & & & & $9.7 \mathrm{E}+04$ & $9.7 E+03$ \\
\hline Dimethylphenol, 2,6- & $000576-26-1$ & & & $5.3 \mathrm{E}+03$ & $4.9 E+04$ & $4.9 \mathrm{E}+03$ & $5.3 \mathrm{E}+02$ & & & $1.9 E+03$ & $1.9 E+02$ & & & & & & & $1.9 \mathrm{E}+03$ & $1.9 E+02$ \\
\hline Dimethylphenol, 3,4- & $000095-65.8$ & & & $8.8 \mathrm{E}+03$ & $8.2 E+04$ & $8.2 E+03$ & $8.8 E+02$ & & & $3.2 \mathrm{E}+03$ & $3.2 \mathrm{E}+02$ & & & & & & & $3.1 \mathrm{E}+03$ & 3.1E+02 \\
\hline Dimethylphthalate(s) & $000131-11.3$ & & & $1.0 \mathrm{E}+06$ & $1.08+06$ & $1.0 E+06$ & $1.0 \mathrm{E}+06$ & & & $1.0 \mathrm{E}+06$ & $1.0 \mathrm{E}+06$ & & & & & & & $1.0 \mathrm{E}+06$ & $1.0 \mathrm{E}+06$ \\
\hline Dimethylterephthalate & $000120-61-6$ & & & $8.8 E+05$ & $1.0 \mathrm{E}+06$ & $8.2 E+05$ & $8.8 \mathrm{E}+04$ & & & $3.2 \mathrm{E}+05$ & $3.2 E+04$ & & & & & & & $3.1 E+05$ & $3.1 E+04$ \\
\hline Dinitro-o-cresol, 4,6- & $000534-52-1$ & & & & & & & & & & & & & & & & & & \\
\hline $\begin{array}{l}\text { Dinitro-0-cyclohexyl } \\
\text { Phenol, 4,6* }\end{array}$ & $000131-89-5$ & & & $1,8 \mathrm{E}+04$ & $1.6 \mathrm{E}+05$ & $1.6 \mathrm{E}+04$ & $1.8 \mathrm{E}+03$ & & & $6.4 \mathrm{E}+03$ & $6,4 \mathrm{E}+02$ & & & & & & & $6.2 E+03$ & $6.2 E+02$ \\
\hline Dinitrobenzene, 1,2- & $000528-29.0$ & & & $3.5 \mathrm{E}+03$ & $3.3 \mathrm{E}+04$ & $3.3 \mathrm{E}+03$ & $3.5 E+02$ & & & $2.4 \mathrm{E}+03$ & $2.4 \mathrm{E}+02$ & & & & & & & $2.2 \mathrm{E}+03$ & $2.2 \mathrm{E}+02$ \\
\hline Dinitrobenzene, 1,3- & 000099.65 .0 & & & $8.8 \mathrm{E}+02$ & $8.2 E+03$ & $8.2 \mathrm{E}+02$ & $8.8 E+01$ & & & $4.2 \mathrm{E}+02$ & $4.2 E+01$ & & & & & & & $4.0 \mathrm{E}+02$ & $4.0 \mathrm{E}+01$ \\
\hline Dinitrobenzene, 1,4- & $000100-25-4$ & & & $3.5 \mathrm{E}+03$ & $3.3 \mathrm{E}+04$ & $3.3 \mathrm{E}+03$ & $3.5 E+02$ & & & $1.3 \mathrm{E}+03$ & $1.3 \mathrm{E}+02$ & & & & & & & $1.2 \mathrm{E}+03$ & $1.2 \mathrm{E}+02$ \\
\hline Dinitrophenol, 2,4. & $000051-28-5$ & & & $1.8 \mathrm{E}+04$ & $1.6 \mathrm{E}+0 \mathrm{~s}$ & $1.6 \mathrm{E}+04$ & $1.8 \mathrm{E}+03$ & & & $1.3 E+04$ & $1.3 E+03$ & & & & & & & $1.2 E+04$ & $1.2 \mathrm{E}+03$ \\
\hline Dinitrotoluene, 2,4- & $000121-14-2$ & $1.1 E+04$ & $1.1 \mathrm{E}+02$ & $1.8 \mathrm{E}+04$ & $1.6 \mathrm{E}+05$ & $1.6 \mathrm{E}+04$ & $1.8 E+03$ & $1.9 E+03$ & $1.9 E+01$ & $1.1 E+04$ & $1.1 E+03$ & & & & & $1.8 \mathrm{E}+03$ & $1.8 \mathrm{E}+0 \mathrm{t}$ & $1.0 E+04$ & $1.0 E+03$ \\
\hline
\end{tabular}


Table 3.4c. (continued)

\begin{tabular}{|c|c|c|c|c|c|c|c|c|c|c|c|c|c|c|c|c|c|c|c|}
\hline \multirow{2}{*}{ Chemical } & \multirow{2}{*}{ CASRN } & \multicolumn{6}{|c|}{ Ingestion (mg/kg) } & \multicolumn{4}{|c|}{ Dermal (mgkg)" } & \multicolumn{4}{|c|}{ Inhalation (mg/kg) } & \multicolumn{4}{|c|}{$\begin{array}{c}\text { Ingestion + Dermal + Inhalation } \\
(\mathrm{mg} / \mathrm{kg})^{+\bullet v}\end{array}$} \\
\hline & & $10^{-4}$ & $10^{6}$ & $\begin{array}{l}\text { HQ=1 } \\
\text { Child }\end{array}$ & $\begin{array}{l}\text { HQ=1 } \\
\text { Adult }\end{array}$ & $\begin{array}{c}\mathrm{HQ}=0.1 \\
\text { Adult }\end{array}$ & $\begin{array}{l}\text { HQ }=0.1 \\
\text { Child }\end{array}$ & $10^{-4}$ & $10^{-6}$ & $H Q=1$ & $\mathrm{HQ}=0.1$ & $10^{-4}$ & $10^{4}$ & $H Q=1$ & $\mathrm{HQ}=0.1$ & $10^{-1}$ & $10^{4}$ & $\mathrm{HII}=1$ & $\mathrm{HI}=0.1$ \\
\hline Dinitrotoluene, 2,6- & $000606-20-2$ & $1.1 E+04$ & $1.1 \mathrm{E}+02$ & $8.8 \mathrm{E}+03$ & $8.2 E+04$ & $8.2 E+03$ & $8.8 E+02$ & $1.9 E+03$ & $1.9 \mathrm{E}+01$ & $5.5 \mathrm{E}+03$ & $5.5 \mathrm{E}+02$ & & & & & $1.8 \mathrm{E}+03$ & $1.8 \mathrm{E}+01$ & $5.1 \mathrm{E}+03$ & $5.1 \mathrm{E}+02$ \\
\hline $\begin{array}{l}\text { Dinitrotoluene, } 2- \\
\text { Amino-4,6- }\end{array}$ & $035572-78-2$ & & & & & & & & & & & & & & & & & & \\
\hline Dinoseb & $000088-85.7$ & & & $8.8 \mathrm{E}+03$ & $8.2 \mathrm{E}+04$ & 8.2E+03 & $8.8 \mathrm{E}+02$ & & & $3.2 E+03$ & $3.2 E+02$ & & & & & & & $3.1 \mathrm{E}+03$ & $3.1 E+02$ \\
\hline Dioxane, 1,4- & $000123-91-1$ & $6.5 \mathrm{E}+05$ & $6.5 \mathrm{E}+03$ & & & & & $1.1 \mathrm{E}+0 \mathrm{~S}$ & $1.1 \mathrm{E}+03$ & & & & & & & $1.0 \mathrm{E}+05$ & $1.0 \mathrm{E}+03$ & & \\
\hline Diphenamid & $000957-51.7$ & & & $2.6 \mathrm{E}+05$ & $1.0 \mathrm{E}+06$ & $2.5 \mathrm{E}+05$ & $2.6 E+04$ & & & $9.6 \mathrm{E}+04$ & $9.6 \mathrm{E}+03$ & & & & & & & $9.3 \mathrm{E}+04$ & $9.3 \mathrm{E}+03$ \\
\hline Diphenylamine & $000122-39-4$ & & & $2.2 E+05$ & $1.0 E+06$ & $2.0 \mathrm{E}+05$ & $2.2 \mathrm{E}+04$ & & & $8.0 \mathrm{E}+04$ & $8.0 \mathrm{E}+03$ & & & & & & & $7.7 E+04$ & $7.7 \mathrm{E}+03$ \\
\hline Diphenylhydrazine, 1,2. & $000122-66-7$ & $8.9 \mathrm{E}+03$ & $8.9 E+01$ & & & & & $9.4 E+02$ & $9.4 \mathrm{E}+00$ & & & $1.0 \mathrm{E}+06$ & $1.0 \mathrm{E}+06$ & & & $9.0 \mathrm{E}+02$ & $9.0 \mathrm{E}+00$ & & \\
\hline Diquat & $000085-00-7$ & & & $1.9 E+04$ & $1.8 \mathrm{E}+05$ & $1.8 \mathrm{E}+04$ & $1.9 \mathrm{E}+03$ & & & $7.1 E+03$ & $7.1 \mathrm{E}+02$ & & & & & & & $6.8 \mathrm{E}+03$ & $6.8 \mathrm{E}+02$ \\
\hline Direct Black 38 & $001937-37-7$ & $8.3 \mathrm{E}+02$ & $8.3 \mathrm{E}+\infty$ & & & & & 8.7E+01 & 8.7E-01 & & & & & & & $8.4 E+01$ & $8.4 \mathrm{E}-01$ & & \\
\hline Direct Blue 6 & $002602-46-2$ & $8.8 \mathrm{E}+02$ & $8.8 \mathrm{E}+00$ & & & & & $9.3 \mathrm{E}+01$ & $9.3 \mathrm{E}-01$ & & & & & & & $8.9 E+01$ & $8.9 \mathrm{E}-01$ & & \\
\hline Direct Brown 95 & $016071-86-6$ & $7.7 \mathrm{E}+02$ & 7.7E+00 & & & & & $8.1 \mathrm{E}+01$ & $8.1 E-01$ & & & & & & & $7.8 \mathrm{E}+01$ & $7.8 \mathrm{E}-01$ & & \\
\hline Direct Sky Blue & $002610-05-1$ & & & & & & & & & - & & & & & & & & & \\
\hline Disulfoton & $000298-04-4$ & & & $3.5 E+02$ & $3.3 \mathrm{E}+03$ & 3.3E+02 & $3.5 \mathrm{E}+01$ & & & $1.3 E+02$ & $1.3 \mathrm{E}+01$ & & & & & & & $1.2 \mathrm{E}+02$ & $1.2 E+01$ \\
\hline Diuron & $000330-54-1$ & & & $1.8 \mathrm{E}+04$ & $1.6 \mathrm{E}+05$ & $1.6 \mathrm{E}+04$ & $1.8 \mathrm{E}+03$ & & & $6.4 E+03$ & $6.4 E+02$ & & & & & & & $6.2 \mathrm{E}+03$ & $6.2 \mathrm{E}+02$ \\
\hline Dodine & $002439-10-3$ & & & $3.5 E+04$ & $3.3 \mathrm{E}+05$ & $3.3 \mathrm{E}+04$ & $3.5 \mathrm{E}+03$ & & & $1.3 \mathrm{E}+04$ & $1.3 \mathrm{E}+03$ & & & & & & & $1.2 \mathrm{E}+04$ & $1.2 \mathrm{E}+03$ \\
\hline EPTC & $000759-94-4$ & & & $2.2 \mathrm{E}+0 \mathrm{~S}$ & $1.0 \mathrm{E}+06$ & $2.0 \mathrm{E}+05$ & $2.2 E+04$ & & & $8.0 E+04$ & $8.0 E+03$ & & & & & & & $7.7 E+04$ & $7.7 E+03$ \\
\hline Endosulfan & $000115-29.7$ & & & $5.3 \mathrm{E}+04$ & $4.9 \mathrm{E}+0 \mathrm{~s}$ & $4.9 \mathrm{E}+04$ & $5.3 \mathrm{E}+03$ & & & $1.9 \mathrm{E}+04$ & $1.9 E+03$ & & & & & & & $1.9 \mathrm{E}+04$ & $1.9 E+03$ \\
\hline Endothall & $000145-73-3$ & & & $1.8 \mathrm{E}+05$ & $1.0 \mathrm{E}+06$ & $1.6 \mathrm{E}+05$ & $1.8 \mathrm{E}+04$ & & & $6.4 E+04$ & $6.4 E+03$ & & & & & & & $6.2 \mathrm{E}+04$ & $6.2 \mathrm{E}+03$ \\
\hline Endrin & $000072-20-8$ & & & $2.6 \mathrm{E}+03$ & 2.5E+04 & $2.5 \mathrm{E}+03$ & $2.6 \mathrm{E}+02$ & & & $3.9 E+01$ & $3.9 E+00$ & & & & & & & $3.9 \mathrm{E}+01$ & $3.9 E+\infty 0$ \\
\hline Epictlorohydrin & $000106-89.8$ & $7.2 E+05$ & $7.2 E+03$ & $1.8 \mathrm{E}+04$ & $1.6 \mathrm{E}+05$ & $1.6 \mathrm{E}+04$ & $1.8 E+03$ & $1.2 \mathrm{E}+05$ & $1.2 E+03$ & $1.0 \mathrm{E}+04$ & $1.0 \mathrm{E}+03$ & $3.2 E+03$ & $3.2 E+03$ & $3.2 \mathrm{E}+03$ & $1.1 \mathrm{E}+03$ & $3.2 E+03$ & $1.1 E+03$ & $3.2 E+03$ & 4.1E+02 \\
\hline Epoxybutane, 1,2- & $000106-88-7$ & & & & & & & & & & & & & $1.0 \mathrm{E}+06$ & $1.0 \mathrm{E}+06$ & & & & \\
\hline Ethephon & $016672-87-0$ & & & 4. $4 E+04$ & 4.1E+0S & 4.1E+04 & $4.4 E+03$ & & & $1.6 \mathrm{E}+04$ & $1.6 \mathrm{E}+03$ & & & & & & & 1.5E+04 & 1.5E+03 \\
\hline
\end{tabular}


Table 3.4c. (continued)

\begin{tabular}{|c|c|c|c|c|c|c|c|c|c|c|c|c|c|c|c|c|c|c|c|}
\hline \multirow{2}{*}{ Chemical } & \multirow{2}{*}{ CASRN } & \multicolumn{6}{|c|}{ Ingestion (mg/kg) } & \multicolumn{4}{|c|}{ Dermal (mgkg) } & \multicolumn{4}{|c|}{ Inhalation (mg/kg) } & \multicolumn{4}{|c|}{$\begin{array}{c}\text { Ingestion }+ \text { Dermal }+ \text { Inhalation } \\
(\mathrm{mg} / \mathrm{kg})^{+\mathrm{s}}\end{array}$} \\
\hline & & $10^{-4}$ & $10^{5}$ & $\begin{array}{l}\text { HQ=1 } \\
\text { Child }\end{array}$ & $\begin{array}{l}\text { HQ=1 } \\
\text { Adult }\end{array}$ & $\begin{array}{l}\text { HQ }=0.1 \\
\text { Adult }\end{array}$ & $\begin{array}{c}\mathrm{HQ}=0.1 \\
\text { Child }\end{array}$ & $10^{4}$ & $10^{4}$ & $\mathrm{HQ}=1$ & $\mathrm{HQ}=0.1$ & $10^{-4}$ & $10^{6}$ & $H Q=1$ & $H Q=0.1$ & $10^{-4}$ & $10^{6}$ & $\mathrm{HI}=1$ & $\mathrm{HI}=0.1$ \\
\hline Ethion & $000563-12-2$ & & & $4.4 \mathrm{E}+03$ & 4.1E+04 & $4.1 \mathrm{E}+03$ & $4.4 \mathrm{E}+02$ & & & $1.6 \mathrm{E}+03$ & $1.6 \mathrm{E}+02$ & & & & & & & $1.5 E+03$ & $1.5 \mathrm{E}+02$ \\
\hline $\begin{array}{l}\text { Ethoxyethenol Acetate, } \\
\text { 2. }\end{array}$ & $000111-15-9$ & & & $1.0 \mathrm{E}+06$ & $1.0 \mathrm{E}+06$ & $1.0 \mathrm{E}+06$ & $2.6 \mathrm{E}+05$ & & & $9.6 \mathrm{E}+05$ & $9.6 \mathrm{E}+04$ & & & & & & & $9.3 \mathrm{E}+05$ & $9.3 E+04$ \\
\hline Ethoxyethanol, 2- & $000110-80.5$ & & & $1,0 \mathrm{E}+06$ & $1.0 \mathrm{E}+06$ & $1.0 \mathrm{E}+06$ & $3.5 \mathrm{E}+05$ & & & $1.0 \mathrm{E}+06$ & $1,3 E+05$ & & & $1.0 E+06$ & $1.0 \mathrm{E}+06$ & & & $1.0 \mathrm{E}+06$ & $1.2 \mathrm{E}+0 \mathrm{~S}$ \\
\hline Ethyl Acetale & $000141-78-6$ & & & $1.0 \mathrm{E}+06$ & $1.0 \mathrm{E}+06$ & $1.0 \mathrm{E}+06$ & $7.9 \mathrm{E}+05$ & & & $1.0 \mathrm{E}+06$ & $4.6 \mathrm{E}+05$ & & & & & & & $1.0 \mathrm{E}+06$ & $4.4 \mathrm{E}+0.5$ \\
\hline Ethyl Acrylate & $000140-88.5$ & $1.5 \mathrm{E}+05$ & $1.5 \mathrm{E}+03$ & & & & & $1.6 \mathrm{E}+04$ & $1.6 \mathrm{E}+02$ & & & & & & & $1.5 \mathrm{E}+04$ & $1.5 E+02$ & & \\
\hline Ethyl Chloride & $000075-00-3$ & & & & & & & & & & & & & $1.5 E+02$ & 1.SE+02 & & & & \\
\hline Ethyl Ether & $000060-29.7$ & & & $1.0 \mathrm{E}+06$ & $1.0 \mathrm{E}+06$ & $1.0 \mathrm{E}+06$ & $1.8 \mathrm{E}+05$ & & & $1.0 \mathrm{E}+06$ & $1.0 E+05$ & & & & & & & $9.7 E+05$ & $9.7 E+04$ \\
\hline Ethyl Methacrylate & 000097.63 .2 & & & $7.9 \mathrm{E}+05$ & $1.0 \mathrm{E}+06$ & $7.4 \mathrm{E}+05$ & $7.9 \mathrm{E}+04$ & & & $4.6 \mathrm{E}+05$ & $4.6 \mathrm{E}+04$ & & & & & & & $4.4 E+05$ & $4.4 E+04$ \\
\hline $\begin{array}{l}\text { Eethyl-p-nitrophenyl } \\
\text { Phosphonate }\end{array}$ & $002104-64-5$ & & & $8.8 \mathrm{E}+01$ & $8.2 \mathrm{E}+02$ & $8.2 \mathrm{E}+01$ & $8.8 E+\infty 0$ & & & $3.2 \mathrm{E}+01$ & $3.2 E+\infty$ & & & & & & & $3.1 E+01$ & 3.1E+00 \\
\hline Ethylbenzene" & $000100-41-4$ & & & $8.8 \mathrm{E}+05$ & $1.0 \mathrm{E}+06$ & $8.2 \mathrm{E}+0 \mathrm{~S}$ & $8.8 E+04$ & & & $6.2 E+05$ & $6.2 \mathrm{E}+04$ & & & $4.7 \mathrm{E}-01$ & $4.7 \mathrm{E}-01$ & & & 4.7E-01 & $4.7 \mathrm{E}-01$ \\
\hline Ethylene Cyznohydrin & 000109.78 .4 & & & $1.0 \mathrm{E}+06$ & $1.0 E+06$ & $1.0 \mathrm{E}+06$ & $2.6 \mathrm{E}+05$ & & & $9.6 \mathrm{E}+05$ & $9.6 \mathrm{E}+04$ & & & & & & & $9.3 \mathrm{E}+05$ & $9,3 \mathrm{E}+04$ \\
\hline Ethylene Diamine & $000107-15.3$ & & & $1.8 \mathrm{E}+05$ & $1.0 \mathrm{E}+06$ & $1.6 \mathrm{E}+05$ & $1.8 \mathrm{E}+04$ & & & $6.4 E+04$ & $6.4 E+03$ & & & & & & & $6.2 E+04$ & $6.2 E+03$ \\
\hline Ethylene Glycol & $000107.21-1$ & & & $1.0 \mathrm{E}+06$ & $1.0 \mathrm{E}+06$ & $1.0 \mathrm{E}+06$ & $1.0 \mathrm{E}+06$ & & & $1.0 \mathrm{E}+06$ & $6.4 E+05$ & & & & & & & $1.0 E+06$ & $6.2 E+05$ \\
\hline $\begin{array}{l}\text { Ethylene Glycol } \\
\text { Monobutyl Ether }\end{array}$ & $000111-76.2$ & & & & & & & & & & & & & $1,0 \mathrm{E}+06$ & $1.0 \mathrm{E}+06$ & & & & \\
\hline Ethylene Oxide & $000075-21-8$ & $7.0 \mathrm{E}+03$ & $7.0 \mathrm{E}+01$ & & & & & $1.2 E+03$ & $1.2 \mathrm{E}+0 \mathrm{I}$ & & & $5.4 \mathrm{E}+03$ & $5.4 E+01$ & & & $9.2 E+02$ & $9.2 E+\infty 0$ & & \\
\hline Ethylene Thiourea & $000096-45.7$ & 6.5E+04 & $6.5 \mathrm{E}+02$ & $7.0 \mathrm{E}+02$ & $6.5 \mathrm{E}+03$ & $6.5 E+02$ & $7.0 \mathrm{E}+01$ & $6.8 \mathrm{E}+03$ & $6.8 \mathrm{E}+0 \mathrm{t}$ & $2.6 \mathrm{E}+02$ & $2.6 \mathrm{E}+01$ & & & & & $6.6 \mathrm{E}+03$ & $6.6 \mathrm{E}+01$ & 2.5E+02 & 2.5E+01 \\
\hline $\begin{array}{l}\text { Ethylphthalyl Ethyl } \\
\text { Glycolate }\end{array}$ & $000084-72-0$ & & & $1.0 \mathrm{E}+06$ & $1.0 E+06$ & $1.0 E+\infty 6$ & $1.0 \mathrm{E}+06$ & & & $1.0 \mathrm{E}+06$ & $9.6 E+05$ & & & & & & & $1.0 \mathrm{E}+06$ & $9.3 E+0 S$ \\
\hline Express & $101200-48-0$ & & & $7.0 \mathrm{E}+04$ & $6.5 E+05$ & $6.5 E+04$ & $7.0 \mathrm{E}+03$ & & & $2.6 \mathrm{E}+04$ & $26 \mathrm{E}+03$ & & & & & & & 2.5E+04 & 2.5E+03 \\
\hline Fenamiphos & $022224.92-6$ & & & $2.2 \mathrm{E}+03$ & $2.0 E+04$ & $2.0 \mathrm{E}+03$ & $2.2 \mathrm{E}+02$ & & & $8.0 \mathrm{E}+02$ & $8.0 E+01$ & & & & & & & $7.7 E+02$ & $7.7 E+01$ \\
\hline Fenpropathrin & $039515-41-8$ & & & $2.2 \mathrm{E}+05$ & $1.0 \mathrm{E}+06$ & $2.0 \mathrm{E}+05$ & $2.2 \mathrm{E}+04$ & & & $8.0 \mathrm{E}+04$ & $8.0 E+03$ & & & & & & & $7.7 E+04$ & $7.7 \mathrm{E}+03$ \\
\hline Fluometuron & $002164-17.2$ & & & $1.1 \mathrm{E}+0 \mathrm{~S}$ & $1.0 E+06$ & $1.1 E+05$ & $1.1 E+04$ & & & $4.2 E+04$ & $4.2 \mathrm{E}+03$ & & & & & & & $4.0 \mathrm{E}+04^{\circ}$ & $4.0 E+03$ \\
\hline
\end{tabular}

Nonradionuclides/soil/recreational

Page 15 of 34 
Table 3.4c. (continued)

\begin{tabular}{|c|c|c|c|c|c|c|c|c|c|c|c|c|c|c|c|c|c|c|c|}
\hline \multirow{2}{*}{ Chemical } & \multirow{2}{*}{ CASRN } & \multicolumn{6}{|c|}{ Ingestion (mg/kg)" } & \multicolumn{4}{|c|}{ Dermal (mgkg) } & \multicolumn{4}{|c|}{ Inhalation (mg/kg) } & \multicolumn{4}{|c|}{$\begin{array}{c}\text { Ingestion + Dermal + Inhalation } \\
(\mathrm{mg} / \mathrm{kg})^{+\bullet r}\end{array}$} \\
\hline & & $10^{-4}$ & $10^{4}$ & $\begin{array}{l}\text { HQ=1 } \\
\text { Child }\end{array}$ & $\begin{array}{l}\text { HQ }=1 \\
\text { Adult }\end{array}$ & $\begin{array}{l}H Q=0.1 \\
\text { Adult }\end{array}$ & $\begin{array}{l}\mathrm{HQ}=0.1 \\
\text { Child }\end{array}$ & $10^{-1}$ & $10^{-6}$ & $\mathrm{HQ}=1$ & $H Q=0.1$ & $10^{-4}$ & $10^{4}$ & $\mathrm{HQ}=1$ & $\mathrm{HQ}=0.1$ & $10^{-4}$ & $10^{4}$ & $H I=1$ & $H I=0.1$ \\
\hline Fluoranthene & $000206-44-0$ & & & $3.5 \mathrm{E}+05$ & $1,0 \mathrm{E}+06$ & $3.3 E+05$ & $3.5 \mathrm{E}+04$ & & & $8.0 \mathrm{E}+04$ & $8.0 \mathrm{E}+03$ & & & & & & & $7.8 E+04$ & $7.8 E+03$ \\
\hline Fluorene & $000086-73-7$ & & & $3.5 E+05$ & $1.0 \mathrm{E}+06$ & $3.3 \mathrm{E}+05$ & $3.5 \mathrm{E}+04$ & & & $2.1 \mathrm{E}+05$ & $2.1 E+04$ & & & & & & & $1.9 \mathrm{E}+05$ & $1.9 E+04$ \\
\hline Fluoride & $\infty 07782-41-4$ & & & $5.3 \mathrm{E}+05$ & $1.0 \mathrm{E}+06$ & $4.9 E+05$ & $5.3 \mathrm{E}+04$ & & & $1.0 \mathrm{E}+06$ & 3.7E+05 & & & & & & & $1.0 E+06$ & 2.1E+05 \\
\hline Fluridone & $059756-60.4$ & & & $7.0 \mathrm{E}+05$ & $1.0 \mathrm{E}+06$ & $6.5 \mathrm{E}+05$ & $7.0 E+04$ & & & $2.6 \mathrm{E}+05$ & $2.6 \mathrm{E}+04$ & & & & & & & $2.5 \mathrm{E}+05$ & 2.5E+04 \\
\hline Flurprimidol & $056425.91-3$ & & & $1.8 \mathrm{E}+05$ & $1.0 \mathrm{~B}+06$ & $1.6 E+05$ & $1.8 \mathrm{E}+04$ & & & $6.4 \mathrm{E}+04$ & $6,4 E+03$ & & & & & & & $6.2 E+04$ & $6.2 E+03$ \\
\hline Flutolanil & $066332-96-5$ & & & $5.3 \mathrm{E}+05$ & $1.0 \mathrm{E}+06$ & $4.9 E+05$ & $5.3 \mathrm{E}+04$ & & & $1.9 E+05$ & $1.9 \mathrm{E}+04$ & & & & & & & $1.9 E+05$ & $1.9 \mathrm{E}+04$ \\
\hline Fluvalinate & $069409-94-5$ & & & $8.8 \mathrm{E}+04$ & $8.2 E+05$ & $8.2 E+04$ & $8.8 \mathrm{E}+03$ & & & $3.2 E+04$ & $3.2 E+03$ & & & & & & & $3.1 E+04$ & $3.1 \mathrm{E}+03$ \\
\hline Folpet & $000133-07-3$ & $1.0 E+06$ & $2.0 \mathrm{E}+04$ & $8.8 \mathrm{E}+05$ & $1.0 \mathrm{E}+06$ & B.2E+05 & $8.8 E+04$ & $2.1 \mathrm{E}+05$ & $2.1 \mathrm{E}+03$ & $3.2 \mathrm{E}+05$ & $3.2 \mathrm{E}+04$ & & & & & $2.1 \mathrm{E}+05$ & 2. $1 E+03$ & $3.1 \mathrm{E}+05$ & $3.1 E+04$ \\
\hline Fomesafen & $072178-02-0$ & $3.8 \mathrm{E}+04$ & $3.8 \mathrm{E}+02$ & & & & & $3.9 E+03$ & $3.9 \mathrm{E}+01$ & & & & & & & $3.8 \mathrm{E}+03$ & $3.8 \mathrm{E}+01$ & & \\
\hline Fonofos & $000944-22-9$ & & & $1.8 E+04$ & $1.6 \mathrm{E}+05$ & $1.6 \mathrm{E}+04$ & $1.8 \mathrm{E}+03$ & & & $6.4 E+03$ & $6.4 \mathrm{E}+02$ & & & & & & & $6.2 \mathrm{E}+03$ & $6.2 \mathrm{E}+02$ \\
\hline Formaldehyde & $000050-00-0$ & & & $1.0 \mathrm{E}+06$ & $1.0 \mathrm{E}+06$ & $1.0 \mathrm{E}+06$ & $1.8 \mathrm{E}+05$ & & & $1.0 \mathrm{E}+06$ & $1.0 \mathrm{E}+05$ & $1.0 \mathrm{E}+06$ & $1.0 \mathrm{E}+06$ & & & & & $9.7 \mathrm{E}+05$ & $9.7 \mathrm{E}+04$ \\
\hline Formic Acid & $000064-18-6$ & & & $1.0 \mathrm{E}+06$ & $1.0 \mathrm{E}+06$ & $1.0 \mathrm{E}+06$ & $1.0 \mathrm{E}+06$ & & & $1.0 \mathrm{E}+06$ & $6.4 \mathrm{E}+05$ & & & & & & & $1.0 \mathrm{E}+06$ & $6.2 E+0 S$ \\
\hline Fosetyl-AL & $039148-24-8$ & & & $1.0 E+06$ & $1.0 \mathrm{E}+06$ & $1.0 \mathrm{E}+06$ & $1.0 \mathrm{E}+06$ & & & $1.0 E+06$ & $9.6 \mathrm{E}+05$ & & & & & & & $1.0 \mathrm{E}+06$ & $9.3 \mathrm{E}+05$ \\
\hline Furan & $000110-00-9$ & & & $8.8 E+03$ & $8.2 \mathrm{E}+04$ & $8.2 E+03$ & $8.8 \mathrm{E}+02$ & & & $5.1 E+03$ & $5.1 E+02$ & & & & & & & $4.8 \mathrm{E}+03$ & 4.8E+02 \\
\hline Furazolidone & 000067.45 .8 & $1.9 E+03$ & $1.9 E+01$ & & & & & $2.0 E+02$ & $2.0 \mathrm{E}+00$ & & & & & & & $1.9 E+02$ & $1.9 E+\infty 0$ & & \\
\hline Furfural & $000098-01-1$ & & & $2.6 \mathrm{E}+04$ & 2.5E+05 & $2.5 E+04$ & $2.6 \mathrm{E}+03$ & & & $9.6 \mathrm{E}+03$ & $9.6 \mathrm{E}+02$ & & & $1.0 E+06$ & $1.0 E+06$ & & & $9.3 \mathrm{E}+03$ & $9.3 \mathrm{E}+02$ \\
\hline Furium & $000531-82-8$ & $1.4 \mathrm{E}+02$ & $1.4 \mathrm{E}+00$ & & & & & 1.5E+01 & $1.5 \mathrm{E}-01$ & & & & & & & $1.4 E+01$ & $1.4 E-01$ & & \\
\hline Furmecyclox & $060568-05.0$ & 2.4E+05 & $2.4 \mathrm{E}+03$ & & & & & $2.5 \mathrm{E}+04$ & $2.5 \mathrm{E}+02$ & & & & & & & $2.4 E+04$ & $2.4 \mathrm{E}+02$ & & \\
\hline $\begin{array}{l}\text { Glufosinate, } \\
\text { Ammoniumm }\end{array}$ & $077182-82-2$ & & & $3.5 \mathrm{E}+03$ & $3.3 E+04$ & $3.3 \mathrm{E}+03$ & $3.5 \mathrm{E}+02$ & & & $1.3 \mathrm{E}+03$ & $1.3 \mathrm{E}+02$ & & & & & & & $1.2 \mathrm{E}+03$ & $1.2 \mathrm{E}+02$ \\
\hline Glycidyl & $000765-34-4$ & & & $3.5 E+03$ & $3.3 E+04$ & 3.3E+03 & $3.5 \mathrm{E}+02$ & & & $1.3 \mathrm{E}+03$ & $1.3 \mathrm{E}+02$ & & & $1.0 \mathrm{E}+06$ & $1.0 \mathrm{E}+06$ & & & $1.2 E+03$ & $1.2 \mathrm{E}+02$ \\
\hline Glyphosate & $001071-83-6$ & & & $8.8 \mathrm{E}+05$ & $1.0 E+06$ & $8.2 E+05$ & $8.8 E+04$ & & & $3.2 E+05$ & $3.2 \mathrm{E}+04$ & & & & & & & 3.IE+05 & $3.1 \mathrm{E}+04$ \\
\hline Goal & $042874-03-3$ & & & $2.6 \mathrm{E}+04$ & $2.5 E+05$ & 2.5E+04 & $2.6 \mathrm{E}+03$ & & & $9.6 \mathrm{E}+03$ & $9.6 \mathrm{E}+02$ & & & & & & & $9.3 E+03$ & $9.3 \mathrm{E}+02$ \\
\hline
\end{tabular}

Nonradionuclides/soil/recreational

Page 16 of 34 
3-585

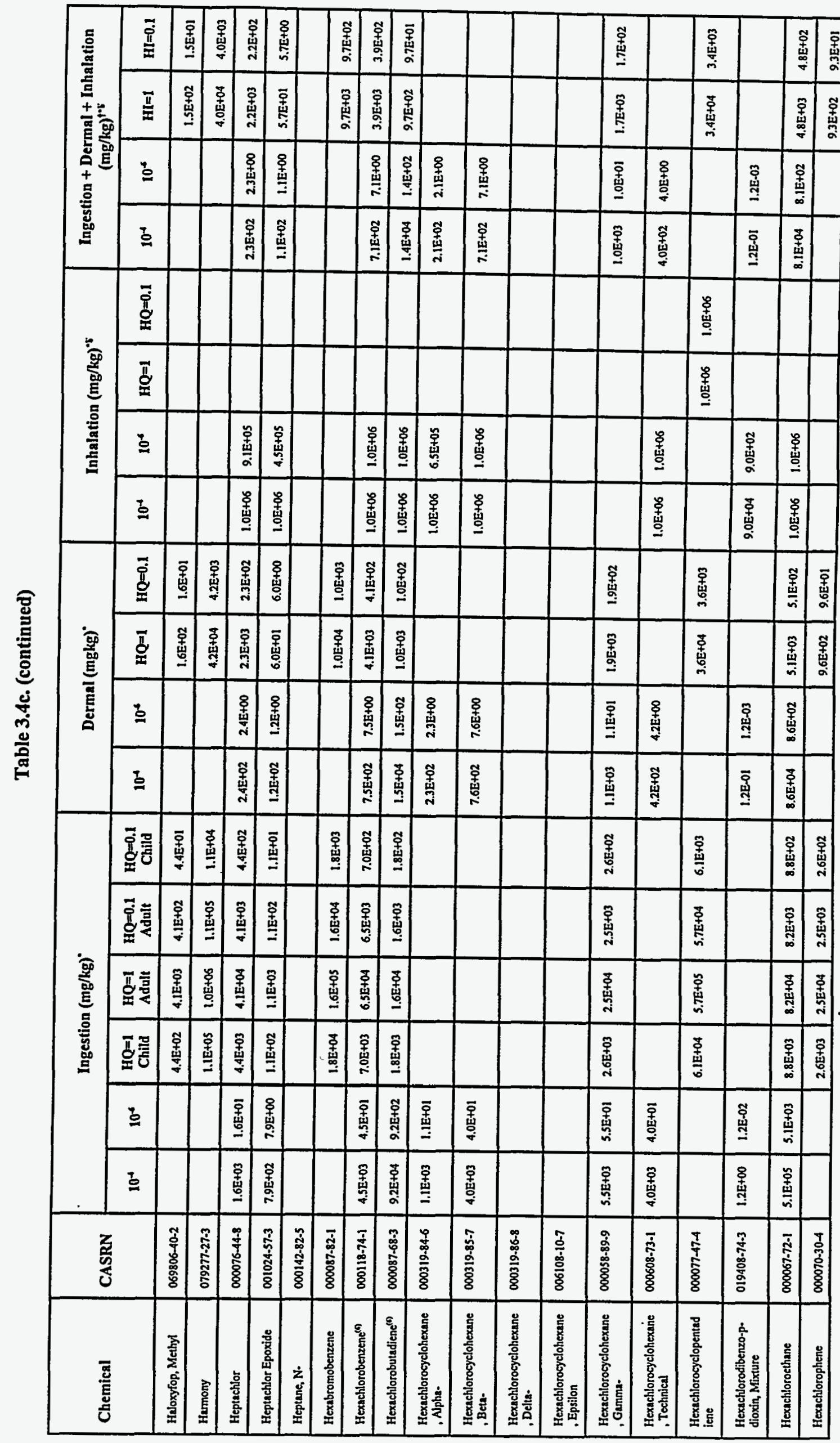


Table 3.4c. (continued)

\begin{tabular}{|c|c|c|c|c|c|c|c|c|c|c|c|c|c|c|c|c|c|c|c|}
\hline \multirow{2}{*}{ Chemical } & \multirow{2}{*}{ CASRN } & \multicolumn{6}{|c|}{ Ingestion $(\mathrm{mg} / \mathrm{kg})^{\bullet}$} & \multicolumn{4}{|c|}{ Dermal (mgkg) } & \multicolumn{4}{|c|}{ Inhalation (mg/kg) } & \multicolumn{4}{|c|}{$\begin{array}{c}\text { Ingestion + Dermal }+ \text { Inhalation } \\
(\mathrm{mg} / \mathrm{kg})^{++8}\end{array}$} \\
\hline & & $10^{4}$ & $10^{4}$ & $\begin{array}{l}\mathrm{HQ}=1 \\
\text { Child }\end{array}$ & $\begin{array}{l}H Q=1 \\
\text { Adult }\end{array}$ & $\begin{array}{c}\text { HQ }=0.1 \\
\text { Adult }\end{array}$ & $\begin{array}{l}\mathrm{HQ}=0.1 \\
\text { Child }\end{array}$ & $10^{-1}$ & $10^{4}$ & $H Q=1$ & $\mathrm{HQ}=0.1$ & $10^{-4}$ & $10^{4}$ & $H Q=1$ & $\mathrm{HQ}=0.1$ & $10^{-4}$ & $10^{4}$ & $\mathrm{HI}=1$ & $\mathrm{HI}=0.1$ \\
\hline $\begin{array}{l}\text { Hexahydro-1,3,5. } \\
\text { trinitro-1,3,5-triazine } \\
\text { (RDX) }\end{array}$ & 000121.82 .4 & $6.5 \mathrm{E}+04$ & $6.5 E+02$ & $2.6 \mathrm{E}+04$ & 2.5E+05 & $2.5 \mathrm{E}+04$ & $2.6 \mathrm{E}+03$ & $1.4 E+04$ & $1.4 \mathrm{E}+02$ & $1.9 \mathrm{E}+04$ & $1.9 \mathrm{E}+03$ & & & & & $1.3 \mathrm{E}+04$ & $1.3 \mathrm{E}+02$ & $1.8 \mathrm{E}+04$ & $1.8 \mathrm{E}+03$ \\
\hline $\begin{array}{l}\text { Hexamethylene } \\
\text { Disocyanate, 1,6- }\end{array}$ & $000822-06-0$ & & & & & & & & & & & & & $1.0 \mathrm{E}+06$ & $5.0 \mathrm{E}+05$ & & & & \\
\hline Hexane, N- & $000110-54-3$ & & & $5.3 E+05$ & $1.0 \mathrm{E}+06$ & $4.9 \mathrm{E}+05$ & $5.3 \mathrm{E}+04$ & & & $3.1 E+05$ & $3.1 \mathrm{E}+04$ & & & $9.1 \mathrm{E}+01$ & $9.1 \mathrm{E}+01$ & & & 9.1E+01 & $9.1 \mathrm{E}+0 \mathrm{t}$ \\
\hline Hexanone, 2- & $000591-78.6$ & & & & & & & & & & & & & & & & & & \\
\hline Hexazinone & $051235-04-2$ & & & $2.9 E+05$ & $1.0 \mathrm{E}+06$ & 2.7E+05 & $2.9 \mathrm{E}+04$ & & & $1.1 E+05$ & $1.1 E+04$ & & & & & & & $1.0 \mathrm{E}+05$ & $1.0 E+04$ \\
\hline $\mathrm{HpCDD}, 2,3,7,8.41)$ & $037871.00-4$ & $4.8 \mathrm{E}+00$ & $4.8 \mathrm{E}-02$ & & & & & $1.7 \mathrm{E}-01$ & 1.7E-03 & & & $2.7 \mathrm{E}+05$ & $2.7 \mathrm{E}+03$ & & & $1.6 \mathrm{E}-01$ & $1.6 \mathrm{E}-03$ & & \\
\hline $\mathrm{HpCDF}, 2,3,7,8^{(1)}$ & 038998.75 .3 & $4.8 \mathrm{E}+00$ & $4.8 \mathrm{E}-02$ & & & & & $5.0 \mathrm{E}-01$ & $5.0 \mathrm{E}-03$ & & & $2.7 \mathrm{E}+05$ & $2.7 \mathrm{E}+03$ & & & $4.8 \mathrm{E}-01$ & $4.8 \mathrm{E}-03$ & & \\
\hline HxCDD, 2,3,7,8.44) & $034465-46-8$ & $4.8 \mathrm{E}-01$ & $4.8 \mathrm{E}-03$ & & & & & $1.7 \mathrm{E}-02$ & 1.7E-04 & & & $2.7 \mathrm{E}+04$ & $2.7 E+02$ & & & $1.6 \mathrm{E}-02$ & $1.6 \mathrm{E}-04$ & & \\
\hline Hydrazine & 000302.01 .2 & $2.4 E+03$ & $2.4 E+01$ & & & & & 2.5E+02 & $2.5 \mathrm{E}+00$ & & & $1.0 E+06$ & $2.4 E+05$ & & & $2.4 E+02$ & $2.4 E+\infty$ & & \\
\hline Hydrazine Sulfate & $010034-93-2$ & $2.4 E+03$ & $2.4 E+01$ & & & & & $2.5 E+02$ & $2.5 \mathrm{E}+\infty$ & & & $1.0 \mathrm{E}+06$ & $2.4 E+05$ & & & $2,4 E+02$ & $2.4 \mathrm{E}+00$ & & \\
\hline Hydrogen Chloride & 007647.01 .0 & & & & & & & & & & & & & $1.0 \mathrm{E}+06$ & $1.0 \mathrm{E}+06$ & & & & \\
\hline Hydrogen Cyanide & $000074-90.8$ & & & $1.8 \mathrm{E}+05$ & $1.0 \mathrm{E}+06$ & $1.6 \mathrm{E}+05$ & $1.8 E+04$ & & & 2.2E+05 & $2.2 \mathrm{E}+04$ & & & $1.0 E+06$ & $1.0 \mathrm{E}+06$ & & & $1.9 \mathrm{E}+05$ & $1.9 \mathrm{E}+04$ \\
\hline Hydrogen Sulfide & $007783-06-4$ & & & $2.6 \mathrm{E}+04$ & $2.5 \mathrm{E}+05$ & 2.5E+04 & $2.6 \mathrm{E}+03$ & & & $3.9 \mathrm{E}+04$ & $3.9 E+03$ & & & $1.0 \mathrm{E}+06$ & $1.0 \mathrm{E}+06$ & & & $3.3 \mathrm{E}+04$ & $3.3 E+03$ \\
\hline Hydroquinone & 000123.31 .9 & & & $3.5 E+05$ & $1.0 \mathrm{E}+06$ & $3.3 \mathrm{E}+05$ & $3.5 \mathrm{E}+04$ & & & $1.3 \mathrm{E}+05$ & $1.3 E+04$ & & & & & & & $1.2 \mathrm{E}+05$ & $1.2 E+04$ \\
\hline Imazalit & $035554-44-0$ & & & $1.1 E+05$ & $1.0 \mathrm{E}+06$ & $1.1 E+05$ & $1.1 \mathrm{E}+04$ & & & $4.2 E+04$ & $4.2 \mathrm{E}+03$ & & & & & & & $4.0 \mathrm{E}+04$ & $4.0 E+03$ \\
\hline Imazaquin & 081335.37 .7 & & & $1.0 E+06$ & $1.0 \mathrm{E}+06$ & $1.0 \mathrm{E}+06$ & $2.2 \mathrm{E}+0 \mathrm{~S}$ & & & 8.0E+05 & $8.0 E+04$ & & & & & & & $7.7 \mathrm{E}+05$ & $7.7 \mathrm{E}+04$ \\
\hline $\begin{array}{l}\text { Indeno[1,2,3- } \\
\text { cd]pyrene } e^{(2)}\end{array}$ & $000193-39-5$ & $9.8 \mathrm{E}+03$ & $9.8 E+01$ & & & & & $6.4 \mathrm{E}+02$ & $6.4 E+\infty$ & & & $1.0 \mathrm{E}+06$ & $1.0 \mathrm{E}+06$ & & & $6.2 \mathrm{E}+02$ & $6.2 \mathrm{E}+\infty$ & & \\
\hline Iprodione & $036734-19-7$ & & & $3.5 E+05$ & $1.0 \mathrm{E}+06$ & $3.3 \mathrm{E}+05$ & $3.5 \mathrm{E}+04$ & & & $1.3 \mathrm{E}+05$ & $1.3 \mathrm{E}+04$ & & & & & & & $1.2 E+05$ & $1.2 \mathrm{E}+04$ \\
\hline Iron & $007439-89-6$ & & & & & & & & & & & & & & & & & & \\
\hline Isobuty! Alcohol & $000078-83-1$ & & & $1.0 E+06$ & $1.0 E+05$ & $1.0 \mathrm{E}+06$ & 2.6E+05 & & & $1.0 E+06$ & $1.5 E+05$ & & & & & & & $1.0 \mathrm{E}+06$ & $1.5 E+05$ \\
\hline
\end{tabular}


Table 3.4c. (continued)

\begin{tabular}{|c|c|c|c|c|c|c|c|c|c|c|c|c|c|c|c|c|c|c|c|}
\hline \multirow{2}{*}{ Chemical } & \multirow{2}{*}{ CASRN } & \multicolumn{6}{|c|}{ Ingestion (mg/kg) } & \multicolumn{4}{|c|}{ Dermal (mgkg) } & \multicolumn{4}{|c|}{ Inhalation $(\mathrm{mg} / \mathrm{kg})^{-x}$} & \multicolumn{4}{|c|}{$\begin{array}{c}\text { Ingestion + Dermal + Inhalation } \\
(\mathrm{mg} / \mathrm{kg})^{+* 1}\end{array}$} \\
\hline & & $10^{-4}$ & $10^{4}$ & $\begin{array}{l}\mathrm{HQ}=1 \\
\text { Child }\end{array}$ & $\begin{array}{l}H Q=1 \\
\text { Adult }\end{array}$ & $\begin{array}{c}\text { HQ }=0.1 \\
\text { Aduit }\end{array}$ & $\begin{array}{l}\text { HQ }=0.1 \\
\text { Child }\end{array}$ & $10^{-4}$ & $10^{-5}$ & $\mathrm{HQ}=1$ & $\mathrm{HQ}=0.1$ & $10^{-4}$ & $10^{4}$ & $H Q=1$ & $\mathrm{HQ}=0.1$ & $10^{-4}$ & $10^{4}$ & $H I=1$ & $H I=0.1$ \\
\hline Isophorone & $000078.59-1$ & $1.0 E+06$ & $7.5 E+04$ & $1.0 E+06$ & $1.0 \mathrm{E}+06$ & $1.0 \mathrm{E}+06$ & $1.8 \mathrm{E}+05$ & $7.9 E+05$ & $7.9 \mathrm{E}+03$ & $6.4 \mathrm{E}+05$ & $6.4 \mathrm{E}+04$ & & & & & $7.6 \mathrm{E}+05$ & $7.6 \mathrm{E}+03$ & $6.2 E+05$ & $6.2 E+04$ \\
\hline Isopropalin & $033820.53=0$ & & & $1.3 E+0 S$ & $1.0 \mathrm{E}+06$ & $1.2 \mathrm{E}+05$ & $1.3 \mathrm{E}+04$ & & & $4.8 \mathrm{E}+04$ & $4.8 \mathrm{E}+03$ & & & & & & & $4.6 E+04$ & $4.6 \mathrm{E}+03$ \\
\hline Isopropanol & $000067-63.0$ & & & & & & & & & & & & & & & & & & \\
\hline $\begin{array}{l}\text { Isopropyl Methyl } \\
\text { Phosphonic Acid }\end{array}$ & $001832-54.8$ & & & $8.8 E+05$ & $1.0 \mathrm{E}+06$ & $8.2 \mathrm{E}+05$ & $8.8 \mathrm{E}+04$ & & & $3.2 \mathrm{E}+05$ & $3.2 E+04$ & & & & & & & 3.1E+0S & $3.1 E+04$ \\
\hline Isoxaben & $082558.50-7$ & & & 4.4E+0s & $1.0 \mathrm{E}+06$ & $4.1 \mathrm{E}+05$ & 4.4E+04 & & & $1.6 \mathrm{E}+05$ & $1.6 \mathrm{E}+04$ & & & & & & & $1.5 E+05$ & $1.5 E+04$ \\
\hline Karate & $091465.08-6$ & & & $4.4 \mathrm{E}+04$ & 4.1E+05 & $4.1 E+04$ & $4.4 \mathrm{E}+03$ & & & $1.6 \mathrm{E}+04$ & $1.6 \mathrm{E}+03$ & & & & & & & $1.5 E+04$ & $1.5 \mathrm{E}+03$ \\
\hline Kerb & 023950.58 .5 & & & $6.6 \mathrm{E}+05$ & $1.0 \mathrm{E}+06$ & $6.1 \mathrm{E}+05$ & $6.6 \mathrm{E}+04$ & & & $2.4 E+05$ & $2.4 \mathrm{E}+04$ & & & & & & & $2.3 \mathrm{E}+05$ & $2.3 \mathrm{E}+04$ \\
\hline Lactofen & 077501.63 .4 & & & $1.8 \mathrm{E}+04$ & $1.6 \mathrm{E}+05$ & $1.6 \mathrm{E}+04$ & $1.8 \mathrm{E}+03$ & & & $6.4 E+03$ & $6.4 E+02$ & & & & & & & $6.2 E+03$ & $6.2 \mathrm{E}+02$ \\
\hline Lead Alkyls & NA & & & & & & & & & & & & & & & & & & \\
\hline Lead And Compounds & $007439.92-1$ & & & & & & & & & & & & & & & & & & \\
\hline Linuron & $000330.55-2$ & & & $1.8 \mathrm{E}+04$ & $1.6 \mathrm{E}+05$ & $1.6 \mathrm{E}+04$ & $1.8 \mathrm{E}+03$ & & & $6.4 E+03$ & $6.4 E+02$ & & & & & & & $6.2 \mathrm{E}+03$ & $6.2 \mathrm{E}+02$ \\
\hline Lithium & $007439-93-2$ & & & & & & & & & & & & & & & & & & \\
\hline Londax & 083055.99 .6 & & & $1.0 \mathrm{E}+06$ & $1.0 \mathrm{E}+06$ & $1.0 E+06$ & $1.8 \mathrm{E}+05$ & & & $6.4 E+05$ & $6.4 E+04$ & & & & & & & $6.2 \mathrm{E}+05$ & $6.2 \mathrm{E}+04$ \\
\hline MCPA & $000094-74-6$ & & & $4.4 E+03$ & 4. $1 \mathrm{E}+04$ & 4.1E+03 & 4.4E+02 & & & $1.6 \mathrm{E}+03$ & $1.6 \mathrm{E}+02$ & & & & & & & $1.5 \mathrm{E}+03$ & $1.5 \mathrm{E}+02$ \\
\hline MCPB & 000094.81 .5 & & & $8.8 E+04$ & $8.2 \mathrm{E}+05$ & 8.2E+04 & $8.8 E+03$ & & & $3.2 \mathrm{E}+04$ & $3.2 \mathrm{E}+03$ & & & & & & . & $3.1 E+04$ & $3.1 E+03$ \\
\hline MCPP & $000093-65-2$ & & & $8.8 \mathrm{E}+03$ & $8.2 E+04$ & $8.2 E+03$ & $8.8 \mathrm{E}+02$ & & & $3.2 \mathrm{E}+03$ & $3.2 E+02$ & & & & & & & 3. $1 \mathrm{E}+03$ & $3.1 E+02$ \\
\hline Magnesium & $007439.95-4$ & & & & & & & & & & & & & & & & & & \\
\hline Malathion & 000121.75 .5 & & & $1,8 \mathrm{E}+05$ & $1.0 E+06$ & $1.6 \mathrm{E}+05$ & $1.8 \mathrm{E}+04$ & & & $6.4 E+04$ & $6.4 E+03$ & & & & & & & $6.2 \mathrm{E}+04$ & $6.2 E+03$ \\
\hline Maleic Anhydride & $000108-31-6$ & & & $8.8 \mathrm{E}+05$ & $1.0 \mathrm{E}+06$ & $8.2 \mathrm{E}+05$ & $8.8 E+04$ & & & $3.2 \mathrm{E}+05$ & $3.2 \mathrm{E}+04$ & & & & & & & $3.1 E+05$ & 3.1E+04 \\
\hline Malecic Hydrazide & 000123.33 .1 & & & $1.0 \mathrm{E}+06$ & $1.0 \mathrm{E}+06$ & $1.0 \mathrm{E}+06$ & $4.4 E+05$ & & & $1.0 \mathrm{E}+06$ & $1.6 \mathrm{E}+0 \mathrm{~s}$ & & & & & & & $1.0 \mathrm{E}+06$ & 1.5E+05 \\
\hline Malononitrile & $000109-77 \cdot 3$ & & & $1.8 \mathrm{E}+02$ & $1.6 \mathrm{E}+03$ & $1.6 \mathrm{E}+02$ & $1.8 \mathrm{E}+01$ & & & $1.0 \mathrm{E}+02$ & $1.0 \mathrm{E}+01$ & & & & & & & $9.7 \mathrm{E}+01$ & $9.7 \mathrm{E}+00$ \\
\hline Mancozeb & $008018-01-7$ & & & $2.6 \mathrm{E}+05$ & $1.0 E+06$ & $2.5 \mathrm{E}+05$ & $2.6 \mathrm{E}+04$ & & & $9.6 E+04$ & $9.6 \mathrm{E}+03$ & & & & & & & $9.3 \mathrm{E}+04$ & $9.3 E+03$ \\
\hline
\end{tabular}


Table 3.4c. (continued)

\begin{tabular}{|c|c|c|c|c|c|c|c|c|c|c|c|c|c|c|c|c|c|c|c|}
\hline \multirow{2}{*}{ Chemical } & \multirow{2}{*}{ CASRN } & \multicolumn{6}{|c|}{ Ingestion (mg/kg) } & \multicolumn{4}{|c|}{ Dermal (mgkg) } & \multicolumn{4}{|c|}{ Inhalation (mg/kg) } & \multicolumn{4}{|c|}{ Ingestion $+\underset{(\mathrm{mg} / \mathrm{kg})^{+\vee v}}{\text { Dermal }+ \text { Inhalation }}$} \\
\hline & & $10^{-4}$ & $10^{4}$ & $\begin{array}{l}\text { HQ=1 } \\
\text { Child }\end{array}$ & $\begin{array}{l}\mathrm{HQ}=1 \\
\text { Adult }\end{array}$ & $\begin{array}{c}H Q=0.1 \\
\text { Adult }\end{array}$ & $\begin{array}{l}\text { HQ }=0.1 \\
\text { Child }\end{array}$ & $10^{-4}$ & $10^{4}$ & $\mathbf{H Q}=\mathbf{I}$ & $\mathrm{HQ}=0.1$ & $10^{-1}$ & $10^{-6}$ & $H Q=1$ & $\mathrm{HQ}=0.1$ & $10^{-1}$ & $10^{6}$ & HI $=1$ & $\mathrm{Hl}=0.1$ \\
\hline Maneb & $012427-38-2$ & & & $4.4 E+04$ & 4.1E+05 & 4.1E+04 & $4.4 E+03$ & & & $1.6 \mathrm{E}+04$ & $1.6 \mathrm{E}+03$ & & & & & & & $1.5 E+04$ & $1.5 E+03$ \\
\hline Manganese (Water) & 007439.96 .5 & & & 4.1E+05 & $1.0 \mathrm{E}+06$ & $3.8 E+05$ & 4.1E+04 & & & $1.2 \mathrm{E}+05$ & $1.2 \mathrm{E}+04$ & & & $1.0 \mathrm{E}+06$ & $1.0 \mathrm{E}+06$ & & & $1.2 \mathrm{E}+05$ & $1.2 \mathrm{E}+04$ \\
\hline Mephosfolan & $000950-10-7$ & & & $7.9 E+02$ & $7.4 E+03$ & $7.4 E+02$ & $7.9 \mathrm{E}+01$ & & & $2.9 E+02$ & $2.9 \mathrm{E}+01$ & & & & & & & $2.8 \mathrm{E}+02$ & $2.8 \mathrm{E}+01$ \\
\hline Mepiquat Chtoride & $024307-26-4$ & & & $2.6 \mathrm{E}+05$ & $1.0 \mathrm{E}+06$ & $2.5 \mathrm{E}+05$ & 2.6E+04 & & & $9.6 \mathrm{E}+04$ & $9.6 \mathrm{E}+03$ & & & & & & & $9.3 E+04$ & $9.3 E+03$ \\
\hline Mercuric Chloride & $007487-94-7$ & & & $2.6 \mathrm{E}+03$ & $2.5 \mathrm{E}+04$ & $2.5 \mathrm{E}+03$ & $2.6 \mathrm{E}+02$ & & & $3.9 E+03$ & $3.9 \mathrm{E}+02$ & & & & & & & $3.3 \mathrm{E}+03$ & $3.3 \mathrm{E}+02$ \\
\hline Mercury (elemental) & 007439.97 .6 & & & $2.6 \mathrm{E}+03$ & 2.5E+04 & $2.5 \mathrm{E}+03$ & $2.6 \mathrm{E}+02$ & & & $1.9 \mathrm{E}+00$ & $1.9 E-01$ & & & $1.0 \mathrm{E}+06$ & $1.0 \mathrm{E}+06$ & & & $1.9 \mathrm{E}+00$ & $1.9 \mathrm{E}-01$ \\
\hline Mercury, Inorganic Salt & $007439-97-6$ & & & $2.6 \mathrm{E}+03$ & $2.5 E+04$ & $2.5 \mathrm{E}+03$ & 2.6E+02 & $\cdot$ & & $1.3 \mathrm{E}+03$ & $1.3 E+02$ & & & $1.0 \mathrm{E}+06$ & $1.0 \mathrm{E}+06$ & & & $1.3 \mathrm{E}+03$ & $1.3 \mathrm{E}+02$ \\
\hline Merphos & $000150-50-5$ & & & $2.6 \mathrm{E}+02$ & $2.5 \mathrm{E}+03$ & $2.5 \mathrm{E}+02$ & $2.6 E+01$ & & . & $9.6 \mathrm{E}+01$ & $9.6 \mathrm{E}+00$ & & & & & & & $9.3 \mathrm{E}+01$ & $9.3 \mathrm{E}+00$ \\
\hline Merphos Oxide & $000078-48-8$ & & & $2.6 \mathrm{E}+02$ & $2.5 E+03$ & 2.5E+02 & 2.6E+01 & 冫 & & $9.6 \mathrm{E}+01$ & $9.6 \mathrm{E}+00$ & & & & & & & $9.3 E+01$ & $9.3 \mathrm{E}+\infty$ \\
\hline Metalaxyl & 057837-19-1 & & & $5.3 \mathrm{E}+05$ & $1.0 \mathrm{E}+06$ & $4.9 \mathrm{E}+05$ & $5.3 E+04$ & & & $1.9 \mathrm{E}+05$ & $1.9 \mathrm{E}+04$ & & & & & & & $1.9 \mathrm{E}+05$ & $1.9 \mathrm{E}+04$ \\
\hline Methacrylonitrile & $000126-98-7$ & & & $8.8 \mathrm{E}+02$ & $8.2 \mathrm{E}+03$ & $8.2 E+02$ & $8.8 E+01$ & & & S.1E+02 & $5.1 E+01$ & & & $9.3 E+03$ & $9.3 \mathrm{E}+02$ & & & $3.7 \mathrm{E}+02$ & $3.7 E+01$ \\
\hline Methamidophos & $010265.92-6$ & & & $4.4 E+02$ & $4.1 \mathrm{E}+03$ & $4.1 \mathrm{E}+02$ & $4.4 E+01$ & & & $1.6 \mathrm{E}+02$ & $1.6 \mathrm{E}+01$ & & & & & & & $1.5 E+02$ & $1.5 \mathrm{E}+01$ \\
\hline Methanol & $000067-56-1$ & & & $1.0 \mathrm{E}+06$ & $1.0 \mathrm{E}+06$ & $1.0 \mathrm{E}+06$ & $4.4 \mathrm{E}+05$ & & & $1.0 \mathrm{E}+06$ & $2.6 \mathrm{E}+0 \mathrm{~S}$ & & & & & & & $1.0 \mathrm{E}+06$ & $2.4 \mathrm{E}+05$ \\
\hline Methidathion & 000950.37 .8 & & & $8.8 E+03$ & $8.2 \mathrm{E}+04$ & $8.2 \mathrm{E}+03$ & $8.8 E+02$ & & & $3.2 \mathrm{E}+03$ & $3.2 \mathrm{E}+02$ & & & & & & & $3.1 \mathrm{E}+03$ & $3.1 \mathrm{E}+02$ \\
\hline Methomyl & $016752-77.5$ & & & 2.2E+0S & $1.0 E+06$ & $2.0 \mathrm{E}+05$ & $2.2 \mathrm{E}+04$ & & & $8.0 \mathrm{E}+04$ & $8.0 \mathrm{E}+03$ & & & & & & & $7.7 \mathrm{E}+04$ & $7.7 \mathrm{E}+03$ \\
\hline $\begin{array}{l}\text { Methoxy-S-nitroaniline, } \\
\text { 2- }\end{array}$ & 000099.59 .2 & $1.6 \mathrm{E}+05$ & $1.6 \mathrm{E}+03$ & & . & & & $1.6 \mathrm{E}+04$ & $1.6 \mathrm{E}+02$ & & & . & & & & $1.6 \mathrm{E}+04$ & $1.6 \mathrm{E}+02$ & & \\
\hline Methoxychlor & $000072-43-5$ & & & $4.4 \mathrm{E}+04$ & 4.1E+0s & $4.1 \mathrm{E}+04$ & $4.4 \mathrm{E}+03$ & & & $1.6 \mathrm{E}+04$ & $1.6 \mathrm{E}+03$ & & & & & & & $1.5 E+04$ & $1.5 E+03$ \\
\hline $\begin{array}{l}\text { Methoxyethanol } \\
\text { Actetate, 2- }\end{array}$ & $000110-49.6$ & & & $1.8 E+04$ & $1.6 \mathrm{E}+05$ & $1.6 \mathrm{E}+04$ & $1.8 \mathrm{E}+03$ & & & $6.4 E+03$ & $6.4 E+02$ & & & & & & & $6.2 \mathrm{E}+03$ & $6.2 \mathrm{E}+02$ \\
\hline Methoxyethanol, 2- & $000109.86-4$ & & & $8.8 \mathrm{E}+03$ & $8.2 \mathrm{E}+04$ & $8.2 E+03$ & $8.8 E+02$ & & & $3.2 \mathrm{E}+03$ & $3.2 \mathrm{E}+02$ & & & $1.0 \mathrm{E}+06$ & $1.0 \mathrm{E}+06$ & & & $3.1 E+03$ & $3.1 \mathrm{E}+02$ \\
\hline Methyl Acetate & 000079.20 .9 & & & $1.0 \mathrm{E}+06$ & $1.0 \mathrm{E}+06$ & $1.0 \mathrm{E}+06$ & $8.8 \mathrm{E}+05$ & & & $1.0 E+06$ & 3.2E+0S & & & & & & & $1.0 E+06$ & $3.1 \mathrm{E}+05$ \\
\hline Methyl Acrylate & $000096-33-3$ & & & $2.6 \mathrm{E}+05$ & $1.0 \mathrm{E}+06$ & 2.5E+05 & $2.6 \mathrm{E}+04$ & & & $9.6 \mathrm{E}+04$ & $9.6 \mathrm{E}+03$ & & & & & & & $9.3 \mathrm{E}+04$ & $9.3 E+03$ \\
\hline Methyl Ethyl Ketone & $000078-93-3$ & & & $1.0 E+06$ & $1.0 \mathrm{E}+06$ & $1.0 E+06$ & $5.3 \mathrm{E}+05$ & & & $1.0 \mathrm{E}+06$ & 3.1E+05 & & & $9.2 \mathrm{E}+03$ & $9.2 E+03$ & & & $9.2 E+03$ & $9.2 \mathrm{E}+03$ \\
\hline
\end{tabular}

Nonradionuclides/soil/recreational

Page 20 of 34 
Table 3.4c. (continued)

\begin{tabular}{|c|c|c|c|c|c|c|c|c|c|c|c|c|c|c|c|c|c|c|c|}
\hline \multirow{2}{*}{ Chemical } & \multirow{2}{*}{ CASRN } & \multicolumn{6}{|c|}{ Ingestion (mg/kg) } & \multicolumn{4}{|c|}{ Dermal (mgkg) } & \multicolumn{4}{|c|}{ Inhalation $(\mathrm{mg} / \mathrm{kg})^{-\mathrm{F}}$} & \multicolumn{4}{|c|}{$\begin{array}{c}\text { Ingestion + Dermal + Inhalation } \\
\left(\mathbf{m g} / \mathrm{kg}^{+* \mathrm{*}}\right.\end{array}$} \\
\hline & & $10^{-4}$ & $10^{4}$ & $\begin{array}{l}\text { HQ=1 } \\
\text { Child }\end{array}$ & $\begin{array}{l}\text { HQ=1 } \\
\text { Adult }\end{array}$ & $\begin{array}{c}\text { HQ }=0.1 \\
\text { Adult }\end{array}$ & $\begin{array}{c}\mathrm{HQ}=0.1 \\
\text { Child }\end{array}$ & $10^{-4}$ & $10^{5}$ & $\mathrm{HQ}=\mathbf{1}$ & $\mathrm{HQ}=0.1$ & $10^{-4}$ & $10^{5}$ & HQ=1 & $\mathrm{HQ}=0.1$ & $10^{-4}$ & $10^{6}$ & $H I=1$ & $\mathrm{HI}=0.1$ \\
\hline Methyl Isobutyl Ketone & $000108-10-1$ & & & $7.0 \mathrm{E}+05$ & $1.0 \mathrm{E}+06$ & $6.5 \mathrm{E}+05$ & $7.0 E+04$ & & & $4.1 E+05$ & $4.1 E+04$ & & & $7.0 \mathrm{E}+02$ & $7.0 E+02$ & & & $7.0 E+02$ & $7.0 E+02$ \\
\hline Methyl Mercury & 022967.92-6 & & & $8.8 E+02$ & $8.2 E+03$ & $8.2 E+02$ & $8.8 E+01$ & & & $5.8 \mathrm{E}+02$ & $5.8 \mathrm{E}+01$ & & & & & & & $5.4 E+02$ & $5.4 E+01$ \\
\hline Methyl Methaccylate & $000080-62-6$ & & & $7.0 E+05$ & $1.0 E+06$ & $6.5 \mathrm{E}+0.5$ & $7.0 E+04$ & & & $4.1 E+05$ & $4.1 E+04$ & & & & & & & $3.9 \mathrm{E}+05$ & $3.9 E+04$ \\
\hline Methyl Parathion & $000298-00-0$ & & & $2.2 E+03$ & $2.0 E+04$ & $2.0 E+03$ & $2.2 \mathrm{E}+02$ & & & $8.0 E+02$ & $8.0 E+01$ & & & & & & & $7.7 \mathrm{E}+02$ & $7.7 \mathrm{E}+01$ \\
\hline $\begin{array}{l}\text { Methy Styrene Mixed } \\
\text { Isomers) }\end{array}$ & $025013-15-4$ & & & $5.3 \mathrm{E}+04$ & $4.9 E+0.5$ & $4.9 E+04$ & $5.3 \mathrm{E}+03$ & & & $3.1 E+04$ & $3.1 E+03$ & & & $1.0 E+06$ & $1.0 E+06$ & & & $2.9 \mathrm{E}+04$ & $2.9 \mathrm{E}+03$ \\
\hline Methyl hydrazine(i) & $000000.34-4$ & $6.5 \mathrm{E}+03$ & $6.5 \mathrm{E}+01$ & & & & & $6.8 E+02$ & $6.8 \mathrm{E}+\infty 0$ & & & & & & & $6.6 \mathrm{E}+02$ & $6.6 \mathrm{E}+00$ & & \\
\hline $\begin{array}{l}\text { Methyl etr-Buty Ether } \\
\text { (MIBAE) }\end{array}$ & $001634-04-4$ & & & & & & & & & & & & & $1.0 E+06$ & $1.0 E+06$ & & & & \\
\hline $\begin{array}{l}\text { Mehyl-5-Nitroanilitine, } \\
2 .\end{array}$ & 000099.55 .8 & $2.2 \mathrm{E}+05$ & $2.2 E+03$ & & & & & $2.3 \mathrm{E}+04$ & $2.3 \mathrm{E}+02$ & & & & & & & $2.2 E+04$ & $2.2 E+02$ & & \\
\hline $\begin{array}{l}\text { Methylaniline } \\
\text { Hydrochloride, 2. }\end{array}$ & $000636-21.5$ & $4.0 E+04$ & $4.0 E+02$ & & & & & $4.2 E+03$ & $4.2 \mathrm{E}+01$ & & & & & & & $4.0 E+03$ & $4.0 E+01$ & & \\
\hline Methyleycloherane & $000108.87 \cdot 2$ & & & & & & & & & & & & & $2.4 E+02$ & $2.4 E+02$ & & & & \\
\hline Methylene Chloride & $000075-09-2$ & $9.5 \mathrm{E}+05$ & $9.5 \mathrm{E}+03$ & $5.3 E+05$ & $1.0 \mathrm{E}+06$ & $4.9 E+0.5$ & $5.3 E+04$ & $1.9 E+05$ & $1.9 E+03$ & $3.7 \mathrm{E}+05$ & $3.7 E+04$ & $1.0 E+03$ & $1.0 E+03$ & $1.0 E+03$ & $1.0 E+03$ & $1.0 \mathrm{E}+03$ & $1.0 E+03$ & $1.0 E+03$ & $1.0 E+03$ \\
\hline $\begin{array}{l}\text { Methylene-bis(2- } \\
\text { chloronaline), } 4,4-\end{array}$ & $000001-14-4$ & $5.5 \mathrm{E}+04$ & $5.5 E+02$ & $6.1 \mathrm{E}+03$ & $5.72+04$ & $5.7 E+03$ & $6.1 E+02$ & $5.8 \mathrm{E}+03$ & $5.8 \mathrm{E}+01$ & $2.2 \mathrm{E}+03$ & $2.2 \mathrm{E}+02$ & $1.0 \mathrm{E}+06$ & $1.0 \mathrm{E}+06$ & & & $5.6 \mathrm{E}+03$ & $5.6 \mathrm{E}+01$ & $2.2 \mathrm{E}+03$ & $2.2 \mathrm{E}+02$ \\
\hline $\begin{array}{l}\text { Methylene-bis (N,N- } \\
\text { dimethyl) Arufine, } 4,4-\end{array}$ & $000101 \cdot 61-1$ & $1.6 \mathrm{E}+05$ & $1.6 \mathrm{E}+03$ & & & & & $1.6 \mathrm{E}+04$ & $1.6 \mathrm{E}+02$ & & & & & & & $1.6 \mathrm{E}+04$ & $1.6 \mathrm{E}+02$ & & \\
\hline 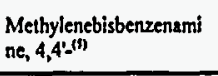 & $000101-77.9$ & $2.9 E+04$ & $2.9 E+02$ & . & & & & $3.0 E+03$ & $3.0 E+01$ & & & & & & & $2.9 \mathrm{E}+03$ & $2.9 \mathrm{E}+01$ & & \\
\hline $\begin{array}{l}\text { Methylenediphenyl } \\
\text { Isocyanate, 4,4:- }\end{array}$ & $000101-68 \cdot 8$ & & & & & & & & & & & & & $1.0 \mathrm{E}+06$ & $1.0 \mathrm{E}+06$ & & & & \\
\hline Methylsyrene, Apha. & 000098.83 .9 & & & $6.1 E+05$ & $1.0 \mathrm{E}+06$ & S.7E+os & $6.1 E+04$ & & & $3.6 \mathrm{E}+0 \mathrm{~S}$ & $3.6 \mathrm{E}+04$ & & & & & & & $3.4 E+05$ & $3.4 E+04$ \\
\hline Methyltriethyl Lead & $001762 \cdot 28-3$ & & & & & & & & & & & & & & & & & & \\
\hline Metoluchlor & $051218-45-2$ & & & $1.0 E+06$ & $1.0 E+06$ & $1.0 E+06$ & 1.3E+0. & & & $4.8 \mathrm{E}+05$ & $4.8 E+04$ & & & & & & & $4.6 \mathrm{E}+05$ & $4.6 \mathrm{E}+04$ \\
\hline Metribuzin" & $021087-64-9$ & & & $2.2 \mathrm{E}+05$ & $1.0 \mathrm{E}+06$ & $2.0 E+05$ & $2.2 \mathrm{E}+04$ & & & $8.0 E+04$ & $8.0 E+03$ & & & & & & & $7.7 E+04$ & $7.7 E+03$ \\
\hline
\end{tabular}


Table 3.4c. (continued)

\begin{tabular}{|c|c|c|c|c|c|c|c|c|c|c|c|c|c|c|c|c|c|c|c|}
\hline \multirow{2}{*}{ Chemical } & \multirow{2}{*}{ CASRN } & \multicolumn{6}{|c|}{ - $\quad$ Ingestion (mg/kg) } & \multicolumn{4}{|c|}{ Dermal (mgkg) } & \multicolumn{4}{|c|}{ Inhalation $(\mathrm{mg} / \mathrm{kg})^{\cdot \gamma}$} & \multicolumn{4}{|c|}{$\begin{array}{c}\text { Ingestion + Dermal + Inhalation } \\
(\mathrm{mg} / \mathrm{kg})^{+* v}\end{array}$} \\
\hline & & $10^{-4}$ & $10^{4}$ & $\begin{array}{l}\text { HQ=1 } \\
\text { Child }\end{array}$ & $\begin{array}{l}\text { HQ=1 } \\
\text { Adult }\end{array}$ & $\begin{array}{c}\mathrm{HQ}=0.1 \\
\text { Adult }\end{array}$ & $\begin{array}{c}\text { HQ }=0.1 \\
\text { Child }\end{array}$ & $10^{-4}$ & $10^{6}$ & $H Q=1$ & $\mathrm{HQ}=0.1$ & $10^{-4}$ & $10^{4}$ & $\mathrm{HQ}=1$ & $\mathrm{HQ}=0.1$ & $10^{-4}$ & $10^{-5}$ & $\mathrm{HI}=\mathbf{1}$ & $\mathrm{HI}=0.1$ \\
\hline Mirex") & $002385-85.5$ & $4.0 \mathrm{E}+03$ & $4.0 \mathrm{E}+01$ & $1.8 \mathrm{E}+03$ & $1.6 \mathrm{E}+04$ & $1.6 \mathrm{E}+03$ & $1.8 \mathrm{E}+02$ & $4.2 \mathrm{E}+02$ & $4.2 \mathrm{E}+00$ & $6.4 E+02$ & $6.4 \mathrm{E}+01$ & & & & & $4.0 \mathrm{E}+02$ & $4.0 E+00$ & $6.2 \mathrm{E}+02$ & $6.2 \mathrm{E}+01$ \\
\hline Molinate & $002212-67-1$ & & & $1.8 \mathrm{E}+04$ & $1.6 \mathrm{E}+05$ & $1.6 \mathrm{E}+04$ & $1.8 \mathrm{E}+03$ & & & $6.4 \mathrm{E}+03$ & $6.4 \mathrm{E}+02$ & & & & & & & $6.2 E+03$ & $6.2 E+02$ \\
\hline Molybdenum & $007439-98-7$ & & & 4.4E+04 & 4.1E+05 & $4.1 E+04$ & $4.4 E+03$ & & & $1.2 \mathrm{E}+05$ & $1.2 \mathrm{E}+04$ & & & & & & & $9.4 E+04$ & $9.4 E+03$ \\
\hline Monochloramine & 010599-90-3 & & & $8.8 \mathrm{E}+05$ & $1.0 \mathrm{E}+06$ & 8.2E+05 & $8.8 E+04$ & & & $3.2 \mathrm{E}+05$ & $3.2 \mathrm{E}+04$ & & & & & & & $3.1 \mathrm{E}+0 \mathrm{~S}$ & $3.1 \mathrm{E}+04$ \\
\hline Monochlorobutanes & $025154-42-1$ & & & $1.0 \mathrm{E}+06$ & $1.0 \mathrm{E}+06$ & $1.0 E+06$ & $3.5 E+05$ & & & $1.0 \mathrm{E}+06$ & $1.3 \mathrm{E}+05$ & & & & & & & $1.0 \mathrm{E}+06$ & $1.2 \mathrm{E}+05$ \\
\hline Naled & $000300-76-5$ & & & $1.8 \mathrm{E}+04$ & $1.6 \mathrm{E}+05$ & $1.6 \mathrm{E}+04$ & $1.8 \mathrm{E}+03$ & & & $6.4 E+03$ & $6.4 \mathrm{E}+02$ & & & & & & & $6.2 \mathrm{E}+03$ & $6.2 \mathrm{E}+02$ \\
\hline Naphthalene & $000091-20-3$ & & & $3.1 \mathrm{E}+05$ & $1.0 \mathrm{E}+06$ & $2.9 E+05$ & $3.1 E+04$ & & & $1.8 \mathrm{E}+05$ & $1.8 \mathrm{E}+04$ & & & & & & & $1.7 \mathrm{E}+05$ & $1.7 \mathrm{E}+04$ \\
\hline Naphthalene, 1-Methyl & $000090-12-0$ & & & & & & & & & & & & & & & & & & \\
\hline Naphthalene, 2-Methyl & $000091.57-6$ & & & & & & & & & & & & & & & & & & \\
\hline Napropamide & 015299.99 .7 & & & $8.8 \mathrm{E}+05$ & $1.0 \mathrm{E}+06$ & $8.2 \mathrm{E}+05$ & $8.8 \mathrm{E}+04$ & & & $3.2 \mathrm{E}+05$ & $3.2 \mathrm{E}+04$ & & & & & & & $3.1 \mathrm{E}+05$ & $3.1 E+04$ \\
\hline Niagara Blue 4B & $002429-74-5$ & & & & & & & & & & & & & & & & & & \\
\hline Nickel Carbonyl & $013463-39-3$ & & & & & & & & & & & & & & & & & & \\
\hline Nickel Refinery Dust & $\mathrm{NA}$ & & & & & & & & & & & $1.0 \mathrm{E}+06$ & $1.0 \mathrm{E}+06$ & & & & & & \\
\hline Nickel Soluble Salts & 007440.02 .0 & & & $1.8 \mathrm{E}+05$ & $1.0 \mathrm{E}+06$ & $1.6 \mathrm{E}+05$ & $1.8 \mathrm{E}+04$ & & & 3.5E+05 & $3.5 E+04$ & & & & & & & $2.9 \mathrm{E}+05$ & $2.9 \mathrm{E}+04$ \\
\hline Nickel Subsulfide & $012035-72-2$ & & & & & & & & & & & $1.0 \mathrm{E}+06$ & $1.0 E+06$ & & & & & & \\
\hline Nitrspyrin ${ }^{(3)}$ & $001929.82-4$ & & & $1.3 \mathrm{E}+04$ & $1.2 \mathrm{E}+05$ & $1.2 \mathrm{E}+04$ & $1.3 \mathrm{E}+03$ & & & $4.8 \mathrm{E}+03$ & $4.8 E+02$ & & & & & & & $4.6 \mathrm{E}+03$ & $4.6 \mathrm{E}+02$ \\
\hline Nitrate & $014797-55.8$ & & & $1.0 E+06$ & $1.0 E+06$ & $1.0 \mathrm{E}+06$ & $1.0 \mathrm{E}+06$ & & & $1.0 \mathrm{E}+06$ & $1.0 E+06$ & & & & & & & $1.0 \mathrm{E}+06$ & $1.0 \mathrm{E}+06$ \\
\hline Nitric Oxide ${ }^{(5)}$ & $010102-43-9$ & & & $8.8 \mathrm{E}+0 \mathrm{~S}$ & $1.0 \mathrm{E}+06$ & $8.2 \mathrm{E}+05$ & 8.8E+04 & & & $1.0 \mathrm{E}+06$ & $1.3 \mathrm{E}+05$ & & & & & & & $1.0 \mathrm{E}+06$ & $1.1 \mathrm{E}+05$ \\
\hline Nitrite & $014797-65-0$ & & & $8.8 \mathrm{E}+05$ & $1.0 \mathrm{E}+06$ & $8.2 E+05$ & $8.8 E+04$ & & & $1.0 \mathrm{E}+06$ & $3.2 \mathrm{E}+05$ & & & & & & & $1.0 \mathrm{E}+06$ & $2.3 \mathrm{E}+05$ \\
\hline Nitroaniline, 2 - & $000088-74-4$ & & & & & & & & & & & & & $9.6 \mathrm{E}+01$ & $9.6 \mathrm{E}+01$ & & & & \\
\hline Nitrobenzene & $000098-95.3$ & & & $4.4 E+03$ & 4.1E+04 & $4.1 \mathrm{E}+03$ & $4.4 E+02$ & & & $3.1 E+03$ & $3.1 \mathrm{E}+02$ & & & $3.2 \mathrm{E}+02$ & $9.6 \mathrm{E}+01$ & & & $3.2 \mathrm{E}+02$ & $5.8 \mathrm{E}+0 \mathrm{t}$ \\
\hline Nitrofurantoin & 000067.20 .9 & & & $6.1 \mathrm{E}+05$ & $1.0 E+06$ & $5.7 E+05$ & $6.1 E+04$ & & & $2.2 \mathrm{E}+05$ & $2.2 \mathrm{E}+04$ & & & & & & & $2.2 E+05$ & $2.2 \mathrm{E}+04$ \\
\hline
\end{tabular}

Nonradionuclides/soil/recreational

Page 22 of 34 
Table 3.4c. (continued)

\begin{tabular}{|c|c|c|c|c|c|c|c|c|c|c|c|c|c|c|c|c|c|c|c|}
\hline \multirow{2}{*}{ Chemical } & \multirow{2}{*}{ CASRN } & \multicolumn{6}{|c|}{ Ingestion (mg/kg) } & \multicolumn{4}{|c|}{ Dermal (mgkg) } & \multicolumn{4}{|c|}{ Inhalation (mg/kg)" } & \multicolumn{4}{|c|}{$\begin{array}{c}\text { Ingestion + Dermal + Inhalation } \\
(\mathrm{mg} / \mathrm{kg})^{+\mathrm{*}}\end{array}$} \\
\hline & & $10^{-4}$ & $10^{5}$ & $\begin{array}{l}\mathrm{HQ}=1 \\
\text { Child }\end{array}$ & $\begin{array}{l}\text { HQ=1 } \\
\text { Adult }\end{array}$ & $\begin{array}{c}\mathrm{HQ}=0.1 \\
\text { Adult }\end{array}$ & $\begin{array}{l}\mathrm{HQ}=0.1 \\
\text { Child }\end{array}$ & $10^{-4}$ & $10^{4}$ & $\mathrm{HQ}=1$ & $\mathrm{HQ}=0.1$ & $10^{-4}$ & $10^{4}$ & $H Q=1$ & $\mathrm{HQ}=0.1$ & $10^{-4}$ & $10^{6}$ & HI $=\mathbf{1}$ & $\mathrm{HI}=0.1$ \\
\hline Nitrofurzone & 000059.8770 & $4.8 E+03$ & $4.8 \mathrm{E}+01$ & & & & & $5.0 E+02$ & $5.0 E+\infty 0$ & & & & & & & $4.8 \mathrm{E}+02$ & $4.8 \mathrm{E}+00$ & & \\
\hline Nitrogen Dioxide(l) & $010102-44.0$ & & & $1.0 E+06$ & $1.0 E+06$ & $1.0 E+05$ & $8.8 E+05$ & & & $1.0 E+06$ & $1.0 \mathrm{E}+06$ & & & & & & & $1.0 E+06$ & $1.0 E+06$ \\
\hline Nitroguanidine & $000556-88-7$ & & & 8.8E+05 & $1.0 E+06$ & $8.2 E+05$ & $8.8 E+04$ & & & $3.2 E+05$ & $3.2 E+04$ & & & & & & & $3.1 E+05$ & $3.1 \mathrm{E}+04$ \\
\hline Nitrophenol, 4- & $000100-02.7$ & & & & & & & & & & & & & & & & & & \\
\hline Nitropropene, $2^{(1)}$ & $000079-46-9$ & 7.SE+02 & $7.5 E+\infty$ & & & & & $1.3 E+02$ & $1.3 \mathrm{E}+\infty$ & & & $9.4 \mathrm{E}+02$ & $9.4 E+00$ & 1.5E+04 & $1.5 \mathrm{E}+04$ & t. $. E+02$ & $1.1 E+\infty 0$ & & \\
\hline $\begin{array}{l}\text { Nitroso-N-ethylures, } \\
\text { N- }\end{array}$ & $000759 .-73-9$ & S.1E+01 & S.IE-01 & & & & & $5.4 E+\infty 0$ & $5.4 \mathrm{E}-02$ & & & & & & & $5.2 E+\infty 00$ & $5.2 \mathrm{E}-02$ & & \\
\hline $\begin{array}{l}\text { Nitroso-N-mehylurea, } \\
\text { N. }\end{array}$ & 000684.93 .5 & & & & & & & & & & & & & & & & & & \\
\hline $\begin{array}{l}\text { Nitroso-dit.N- } \\
\text { butylamine, N- }\end{array}$ & $000924-16 \cdot 3$ & $1.3 \mathrm{E}+03$ & $1.3 \mathrm{E}+01$ & & & & & $2.2 \mathrm{E}+02$ & $2.2 \mathrm{E}+\infty 0$ & & & $1.5 \mathrm{E}+03$ & $6.9 E+01$ & & & $2.0 \mathrm{E}+02$ & $2.0 \mathrm{E}+\infty 0$ & & \\
\hline $\begin{array}{l}\text { Nitroso-di.N. } \\
\text { propylamine, N- }\end{array}$ & $000621-64.7$ & $1.0 E+03$ & $1.0 \mathrm{E}+01$ & & & & & $5.4 \mathrm{E}+01$ & $5.4 E-01$ & & & & & & & $5.3 \mathrm{E}+01$ & $5.3 \mathrm{E}-01$ & & \\
\hline $\begin{array}{l}\text { Nitrosoditethanolamine, } \\
\text { N. }\end{array}$ & 001116.54 .7 & $2.6 \mathrm{E}+03$ & $2.6 \mathrm{E}+01$ & & & & & $2.7 E+02$ & $2.7 E+00$ & & & & & & & $2.6 \mathrm{E}+02$ & $2.6 \mathrm{E}+00$ & & \\
\hline $\begin{array}{l}\text { Nitrosodiethylamine, } \\
\text { N- }\end{array}$ & $000055.18-5$ & $4.8 \mathrm{E}+01$ & $4.8 \mathrm{E}-01$ & & & & & $5.0 E+00$ & $5.0 \mathrm{E}-02$ & & & $1.0 E+06$ & $2.7 \mathrm{E}+04$ & & & $4.8 \mathrm{E}+00$ & $4.8 \mathrm{E}-02$ & & \\
\hline $\begin{array}{l}\text { Nitrosodimethylamine, } \\
\text { N- }\end{array}$ & 000062.75 .9 & $1.4 E+02$ & $1.4 E+\infty 0$ & & & & & $1.5 \mathrm{E}+01$ & $1.5 E-01$ & & & $1.0 E+06$ & $8.0 E+04$ & & & $1.4 \mathrm{E}+01$ & $1.4 \mathrm{E}-01$ & & \\
\hline $\begin{array}{l}\text { Nitrosodiphenylamine, } \\
\text { N.- }\end{array}$ & $000086-30.6$ & $1.0 \mathrm{E}+06$ & I. $.5 \mathrm{E}+04$ & & & & & $7.7 \mathrm{E}+04$ & $7.7 E+02$ & & & & & & & 7.5E+04 & $7.5 E+02$ & & \\
\hline $\begin{array}{l}\text { Nitrosomethylehylemin } \\
\text { e, N- }\end{array}$ & $010595.95-6$ & $3.3 \mathrm{E}+02$ & $3.3 \mathrm{E}+\infty 0$ & & & & & $3.4 \mathrm{E}+01$ & $3.4 E-01$ & & & & & & & $3.3 \mathrm{E}+01$ & 3.3E-01 & & \\
\hline $\begin{array}{l}\text { Nitrosomethylinylamin } \\
e, N=\text {. N }\end{array}$ & $004549-40.0$ & & & & & & & & & & & & & & & & & & \\
\hline Nitrosopyrtolidine, N- & $000930.55-2$ & $3.4 E+03$ & $3.4 \mathrm{E}+01$ & & & & & $3.6 \mathrm{E}+02$ & $3.6 \mathrm{E}+00$ & & & $1.0 E+06$ & $1.0 E+06$ & & & $3.4 E+02$ & $3.4 \mathrm{E}+00$ & & \\
\hline $\begin{array}{l}\text { Nitrotoluene, 4-Amino- } \\
2-\text { - }\end{array}$ & $000119-32-4$ & & & & & & & & & & & & & & & & & & \\
\hline Nitrotoluene, $\mathrm{m}$ - & 000099-08-1 & & & $8.8 E+04$ & $8.2 \mathrm{E}+0 \mathrm{~S}$ & $8.2 E+04$ & $8.8 E+03$ & & & 5.IE+04 & $5.1 E+03$ & & & & & & & $4.8 \mathrm{E}+04$ & 4.8E+03 \\
\hline
\end{tabular}

Nonradionuclides/soil/recreational

Page 23 of 34 
Table 3.4c. (continued)

\begin{tabular}{|c|c|c|c|c|c|c|c|c|c|c|c|c|c|c|c|c|c|c|c|}
\hline \multirow{2}{*}{ Chemical } & \multirow{2}{*}{ CASRN } & \multicolumn{6}{|c|}{ Ingestion (mg/kg) } & \multicolumn{4}{|c|}{ Dermal (mgkg) } & \multicolumn{4}{|c|}{ Inhalation (mg/kg) ${ }^{\cdot v}$} & \multicolumn{4}{|c|}{$\begin{array}{c}\text { Ingestion + Dermal + Inhalation } \\
(\mathrm{mg} / \mathrm{kg})^{+\cdot v}\end{array}$} \\
\hline & & $10^{-4}$ & $10^{4}$ & $\begin{array}{l}\text { HQ=1 } \\
\text { Child }\end{array}$ & $\begin{array}{l}\text { HQ=1 } \\
\text { Adult }\end{array}$ & $\begin{array}{l}\mathrm{HQ}=0.1 \\
\text { Adult }\end{array}$ & $\begin{array}{l}\mathrm{HQ}=0.1 \\
\text { Child }\end{array}$ & $10^{-4}$ & $10^{6}$ & $H Q=1$ & $\mathrm{HQ}=0.1$ & $10^{-4}$ & $10^{6}$ & $\mathrm{HQ}=1$ & $\mathrm{HQ}=0.1$ & $10^{-4}$ & $10^{6}$ & $H I=1$ & $\mathrm{HI}=0.1$ \\
\hline Nitrotoluene, o. & $000088-72-2$ & & & $8.8 \mathrm{E}+04$ & $8.2 E+05$ & $8.2 \mathrm{E}+04$ & $8.8 E+03$ & & & $5.1 E+04$ & $5.1 \mathrm{E}+03$ & & & & & & & $4.8 \mathrm{E}+04$ & $4,8 \mathrm{E}+03$ \\
\hline Nitrotoluene, p- & $000099-99-0$ & & & $8.8 \mathrm{E}+04$ & $8.2 \mathrm{E}+05$ & $8.2 E+04$ & $8.8 \mathrm{E}+03$ & & & $5.1 E+04$ & $5.1 \mathrm{E}+03$ & & & & & & & $4.8 \mathrm{E}+04$ & $4.8 \mathrm{E}+03$ \\
\hline Norflurazon & $027314-13-2$ & & & $3.5 \mathrm{E}+05$ & $1.0 \mathrm{E}+06$ & 3.3E+05 & $3.5 \mathrm{E}+04$ & & & $1.3 E+05$. & $1.3 \mathrm{E}+04$ & & & & & & & $1.2 E+05$ & $1.2 \mathrm{E}+04$ \\
\hline Nustar & 085509-19-9 & & & $6.1 \mathrm{E}+03$ & $5.7 \mathrm{E}+0.4$ & $5.7 \mathrm{E}+03$ & $6.1 \mathrm{E}+02$ & & & $2.2 E+03$ & $2.2 E+02$ & & & & & & & $2.2 E+03$ & $2.2 E+02$ \\
\hline$O C D D^{(1)}$ & $003268-87-9$ & $4.8 \mathrm{E}+01$ & $4.8 \mathrm{E}-01$ & & & & & $1.7 E+\infty 0$ & $1.7 \mathrm{E}-02$ & & & $1.0 E+06$ & $2.7 \mathrm{E}+04$ & & & $1.6 \mathrm{E}+\infty 0$ & $1.6 \mathrm{E} \cdot 02$ & & \\
\hline $\operatorname{OCDF}^{(4)}$ & $039001-02-0$ & $4.8 \mathrm{E}+01$ & $4.8 \mathrm{E}-01$ & & & & & $5.0 \mathrm{E}+\infty 0$ & $5.0 \mathrm{E}-02$ & . & & $1.0 \mathrm{E}+06$ & $2.7 \mathrm{E}+04$ & & & $4.8 \mathrm{E}+00$ & $4,8 \mathrm{E}-02$ & & \\
\hline $\begin{array}{l}\text { Octabromodiphenyl } \\
\text { Ether }\end{array}$ & $032536-52.0$ & & & $2.6 \mathrm{E}+04$ & $2.5 \mathrm{E}+05$ & $2.5 \mathrm{E}+04$ & $2.6 \mathrm{E}+03$ & & & $9.6 \mathrm{E}+03$ & $9.6 \mathrm{E}+02$ & & & & & & & $9.3 \mathrm{E}+03$ & $9.3 \mathrm{E}+02$ \\
\hline $\begin{array}{l}\text { Oetehydro-1,3,5,7- } \\
\text { tetranitro-1,3,5,7-tetra } \\
(\mathrm{HM})\end{array}$ & $002691-41-0$ & & & $4.4 E+05$ & $1.0 \mathrm{E}+06$ & $4.1 \mathrm{E}+05$ & $4.4 \mathrm{E}+04$ & & & $4.8 \mathrm{E}+04$ & $4.8 E+03$ & & & & & & & $4.8 \mathrm{E}+04$ & $4.8 \mathrm{E}+03$ \\
\hline $\begin{array}{l}\text { Octamethylpyrophosph } \\
\text { oramide }\end{array}$ & $000152-16-9$ & & & $1.8 \mathrm{E}+04$ & $1.6 \mathrm{E}+05$ & $1.6 \mathrm{E}+04$ & $1.8 \mathrm{E}+03$ & & & $6.4 \mathrm{E}+03$ & $6.4 E+02$ & & & & & & & $6.2 \mathrm{E}+03$ & $6.2 E+02$ \\
\hline Octyl Phthalate, di-N- & $000117-84-0$ & & & $1.8 \mathrm{EE}+05$ & $1.0 \mathrm{E}+06$ & $1.6 \mathrm{E}+05$ & $1.8 \mathrm{E}+04$ & & & $1.2 \mathrm{E}+05$ & $1.2 \mathrm{E}+04$ & & & & & & & $1.1 \mathrm{E}+05$ & 1.1E+04 \\
\hline Oryzalin & $019044-88-3$ & & & $4.4 \mathrm{E}+05$ & $1.0 \mathrm{E}+06$ & $4.1 E+05$ & $4.4 \mathrm{E}+04$ & & & $1.6 \mathrm{E}+05$ & $1.6 \mathrm{E}+04$ & & & & & & & 1.5E+05 & $1.5 \mathrm{E}+04$ \\
\hline Oxadiazon & $019666-30-9$ & & & $4.4 \mathrm{E}+04$ & 4.1E+05 & $4.1 \mathrm{E}+04$ & $4.4 \mathrm{E}+03$ & & & $1.6 \mathrm{E}+04$ & $1.6 \mathrm{E}+03$ & & & & & & & $1.5 E+04$ & $1.5 \mathrm{E}+03$ \\
\hline Oxamyl & $023135-22-0$ & & & $2.2 \mathrm{E}+05$ & $1.0 \mathrm{E}+06$ & $2.0 \mathrm{E}+05$ & $2.2 \mathrm{E}+04$ & & & $8.0 \mathrm{E}+04$ & $8.0 E+03$ & & & & & & & $7.7 E+04$ & 7.7E+03 \\
\hline Paclobutrazol & $076738-62-0$ & & & $1.1 \mathrm{E}+05$ & $1.0 \mathrm{E}+06$ & $1.1 \mathrm{E}+05$ & $1.1 \mathrm{E}+04$ & & & $4.2 \mathrm{E}+04$ & $4.2 \mathrm{E}+03$ & & & & & & & $4.0 \mathrm{E}+04$ & $4.0 \mathrm{E}+03$ \\
\hline Paraquat & $001910.42-5$ & & & $3.9 E+04$ & 3.7E+05 & $3.7 E+04$ & $3.9 \mathrm{E}+03$ & & & $1.4 E+04$ & $1.4 E+03$ & & & & & & & $1.4 E+04$ & $1.4 \mathrm{E}+03$ \\
\hline Parathion & $000056-38-2$ & & & $5.3 \mathrm{E}+04$ & $4.9 \mathrm{E}+05$ & $4.9 \mathrm{E}+04$ & $5.3 \mathrm{E}+03$ & & & $1.9 E+04$ & $1.9 \mathrm{E}+03$ & & & & & & & $1.9 E+04$ & $1.9 E+03$ \\
\hline PeCDD, 2,3,7,8.(1) & $036088-22-9$ & $9.5 \mathrm{E}-02$ & $9.5 \mathrm{E}-04$ & & & & & $3.3 \mathrm{E}-03$ & 3.3E-05 & & & $5.4 \mathrm{E}+03$ & $5.4 \mathrm{E}+01$ & & & $3.3 \mathrm{E}-03$ & $3.3 \mathrm{E}-05$ & & \\
\hline PeCDF, 1,2,3,7,8.11) & $057117-41-6$ & $9.5 \mathrm{E}-02$ & $9.5 E-04$ & & & & & $1.0 \mathrm{E}-02$ & $1.0 \mathrm{E}-04$ & & & $5.4 \mathrm{E}+03$ & $5.4 \mathrm{E}+01$ & & & $9.6 \mathrm{E}-03$ & $9.6 \mathrm{E}-05$ & & \\
\hline PeCDF, 2,3,4,7,8=(1) & $057117-31-4$ & $9.5 \mathrm{E}-01$ & $9.5 \mathrm{E}-03$ & & & & & $1.0 \mathrm{E}-01$ & $1.0 \mathrm{E}-03$ & & & $5.4 \mathrm{E}+04$ & $5.4 \mathrm{E}+02$ & & & $9.6 \mathrm{E}-02$ & $9.6 \mathrm{E}-04$ & & \\
\hline Pebulate & $001114-71.2$ & & & $4.4 E+05$ & $1.0 \mathrm{E}+06$ & $4.1 \mathrm{E}+05$ & 4.4E+04 & & & $1.6 \mathrm{E}+05$ & $1.6 \mathrm{E}+04$ & & & & & & & $1.5 E+05$ & 1.5E+04 \\
\hline Pendimethalin & $040487-42-1$ & & & 3.5E+05 & $1.0 \mathrm{E}+06$ & $3.3 E+05$ & $3.5 \mathrm{E}+04$ & . & & $1.3 E+05$ & $1.3 \mathrm{E}+04$ & & & & & & & $1.2 E+05$ & $1.2 \mathrm{E}+04$ \\
\hline
\end{tabular}

Nonradionuclides/soil/recreational

Page 24 of 34 
Table 3.4c. (continued)

\begin{tabular}{|c|c|c|c|c|c|c|c|c|c|c|c|c|c|c|c|c|c|c|c|}
\hline \multirow{2}{*}{ Chemical } & \multirow{2}{*}{ CASRN } & \multicolumn{6}{|c|}{ Ingestion (mg/kg) } & \multicolumn{4}{|c|}{ Dermal (mgkg)" } & \multicolumn{4}{|c|}{ Inhalation (mg/kg) } & \multicolumn{4}{|c|}{$\begin{array}{c}\text { Ingestion + Dermal + Inhalation } \\
(\mathrm{mg} / \mathrm{kg})^{+\bullet v}\end{array}$} \\
\hline & & $10^{-4}$ & $10^{6}$ & $\begin{array}{l}\text { HQ=1 } \\
\text { Child }\end{array}$ & $\begin{array}{l}\text { HQ=1 } \\
\text { Adult }\end{array}$ & $\begin{array}{c}\mathrm{HQ}=0.1 \\
\text { Adult }\end{array}$ & $\begin{array}{c}\mathrm{HQ}=0.1 \\
\text { Child }\end{array}$ & $10^{-4}$ & $10^{4}$ & $\mathbf{H Q}=1$ & $\mathrm{HQ}=0.1$ & $10^{-4}$ & $10^{-5}$ & $H Q=1$ & $H Q=0.1$ & $10^{4}$ & $10^{5}$ & $\mathrm{HI}=\mathbf{1}$ & $\mathrm{HI}=0.1$ \\
\hline $\begin{array}{l}\text { Pentsbromodiphenyl } \\
\text { Ether }\end{array}$ & $032534-81-9$ & & & $1.8 E+04$ & $1.6 \mathrm{E}+05$ & $1.6 \mathrm{E}+04$ & $1.8 \mathrm{E}+03$ & & & $6.4 \mathrm{E}+03$ & $6.4 E+02$ & & & & & & & $6.2 E+03$ & $6.2 \mathrm{E}+02$ \\
\hline Pentachlorobenzene & $000608.93-5$ & & & $7.0 E+03$ & $6.5 E+04$ & $6.5 \mathrm{E}+03$ & $7.0 E+02$ & & & $4.1 \mathrm{E}+03$ & 4.1E+02 & & & & & & & $3.9 \mathrm{E}+03$ & $3.9 E+02$ \\
\hline $\begin{array}{l}\text { Pentachlorocyclopentad } \\
\text { iene }\end{array}$ & $025329-35.5$ & & & & & & & & & & & & & & & & & & \\
\hline $\begin{array}{l}\text { Pentachloronitrobenzen } \\
\text { e }\end{array}$ & $000082-68-8$ & $2.8 \mathrm{E}+04$ & $2.8 \mathrm{E}+02$ & $2.6 \mathrm{E}+04$ & $2.5 E+05$ & $2.5 \mathrm{E}+04$ & $2.6 E+03$ & $4.6 \mathrm{E}+03$ & $4.6 \mathrm{E}+01$ & 1.5E+04 & $1.5 E+03$ & & & & & $4.3 E+03$ & $4.3 E+01$ & $1.5 E+04$ & $1.5 \mathrm{E}+03$ \\
\hline Pentachlorophenol & $000087.86-5$ & $6.0 \mathrm{E}+04$ & $6.0 \mathrm{E}+02$ & $2.6 \mathrm{E}+05$ & $1.0 E+06$ & $2.5 E+05$ & $2.6 \mathrm{E}+04$ & $1.2 \mathrm{E}+04$ & $1.2 \mathrm{E}+02$ & $1.9 \mathrm{E}+0 \mathrm{~S}$ & $1.9 E+04$ & & & & & $1.2 E+04$ & $1.2 E+02$ & $1.8 \mathrm{E}+05$ & $1.8 \mathrm{E}+04$ \\
\hline Pentyl Alcohol, N. & $000071-41-0$ & & & & & & & & & & & & & & & & & & \\
\hline Permethrin & $052645-53-1$ & & & $4.4 \mathrm{E}+05$ & $1.0 \mathrm{E}+06$ & 4.1E+05 & 4.4E+04 & & & $1.6 E+05$ & $1.6 \mathrm{E}+04$ & & & & & & & $1.5 \mathrm{E}+05$ & $1.5 \mathrm{E}+04$ \\
\hline Phenanthrene & $000085-01-8$ & & & & & & & & & & & & & & & & & & \\
\hline Phenmedipham & 013684.63 .4 & & & $1.0 \mathrm{E}+06$ & $1.0 \mathrm{E}+06$ & $1.0 \mathrm{E}+06$ & $2.2 E+05$ & & & $8.0 E+05$ & $8.0 E+04$ & & & & & & & $7.7 \mathrm{E}+0 \mathrm{~S}$ & $7.7 E+04$ \\
\hline Phenol & $000108-95-2$ & & & $1.0 \mathrm{E}+06$ & $1.0 \mathrm{E}+06$ & $1.0 \mathrm{E}+06$ & $5.3 E+05$ & & & $1.0 \mathrm{E}+06$ & $3.5 E+05$ & & & & & & & $1.0 \mathrm{E}+06$ & $3.2 \mathrm{E}+05$ \\
\hline Phenylenediamine, m- & $000108-45-2$ & & & $5.3 \mathrm{E}+04$ & $4.9 E+05$ & 4.9E+04 & $5.3 \mathrm{E}+03$ & & & $1.9 \mathrm{E}+04$ & $1.9 E+03$ & & & & & & & $1.9 E+04$ & $1.9 \mathrm{E}+03$ \\
\hline Phenylenediamine, o- & 000095-54-5 & $1.5 E+05$ & $1.5 E+03$ & & & & & $1,6 \mathrm{E}+04$ & $1,6 \mathrm{E}+02$ & & & & & & & $1,5 \mathrm{E}+04$ & $1.5 E+02$ & & \\
\hline Phenylenediamine, p- & $000106-50-3$ & & & $1.0 \mathrm{E}+06$ & $1.0 \mathrm{E}+06$ & $1.0 E+06$ & t.7E+0s & & & $6.1 E+05$ & 6.IE+04 & & . & & & & & $5.9 E+05$ & $5.9 E+04$ \\
\hline Phenylmercuric Acctate & $000062.38-4$ & & & $7.0 \mathrm{E}+02$ & $6.5 E+03$ & $6.5 E+02$ & $7.0 \mathrm{E}+01$ & & & $2.6 \mathrm{E}+02$ & $2.6 \mathrm{E}+01$ & & & & & & & $2.5 E+02$ & $2.5 E+0 !$ \\
\hline Phenylphenol, 2. & $000090-43-7$ & $1.0 \mathrm{E}+06$ & 3.7E+04 & & & & & $3.9 \mathrm{E}+05^{\circ}$ & $3.9 \mathrm{E}+03$ & & & & & & & $3.7 E+05$ & $3.7 E+03$ & & \\
\hline Phorate & $000298-02-2$ & & & $1.8 \mathrm{E}+03$ & $1.6 \mathrm{E}+04$ & $1.6 \mathrm{E}+03$ & $1.8 \mathrm{E}+02$ & & & $6.4 E+02$ & $6.4 E+01$ & & & & & & & $6.2 \mathrm{E}+02$ & $6.2 \mathrm{E}+01$ \\
\hline Phosmet & $000732-11-6$ & & & $1.8 \mathrm{E}+05$ & $1.0 \mathrm{E}+06$ & $1.6 \mathrm{E}+05$ & $1.8 E+04$ & & & $6.4 \mathrm{E}+04$ & $6.4 E+03$ & & & & & & & $6.2 \mathrm{E}+04$ & $6.2 \mathrm{E}+03$ \\
\hline Phosphine & $007803.51-2$ & & & $26 \mathrm{E}+03$ & $2.5 \mathrm{E}+04$ & $2.5 E+03$ & $2.6 \mathrm{E}+02$ & & & $3.9 \mathrm{E}+03$ & $3.9 \mathrm{E}+02$ & & & $1.0 E+06$ & $1.0 \mathrm{E}+05$ & & & $3.3 \mathrm{E}+03$ & $3.3 \mathrm{E}+02$ \\
\hline Phosphoric Acid & $007664-38-2$ & & & & & & & & & & & $\cdot$ & & $1.0 \mathrm{E}+06$ & $1,0 \mathrm{E}+06$ & & & & \\
\hline Phthalic Acid, P. & $000100-21.0$ & & & $1.0 \mathrm{E}+06$ & $1.0 \mathrm{E}+06$ & $1.0 \mathrm{E}+06$ & $8.8 \mathrm{E}+05$ & & & $1.0 \mathrm{E}+06$ & $3.2 E+05$ & & & & & & & $1.0 \mathrm{E}+06$ & $3.1 \mathrm{E}+05$ \\
\hline Phathalic Arhydride & $000085-44.9$ & & & $1.0 E+06$ & $1.0 \mathrm{E}+06$ & $1.0 E+06$ & $1.0 E+06$ & & & $1.0 E+06$ & $6.4 E+0 S$ & & & $1.0 \mathrm{E}+06$ & $1.0 \mathrm{E}+06$ & & & $1.0 E+06$ & $6.2 \mathrm{E}+0 \mathrm{~s}$ \\
\hline
\end{tabular}


Table 3.4c. (continued)

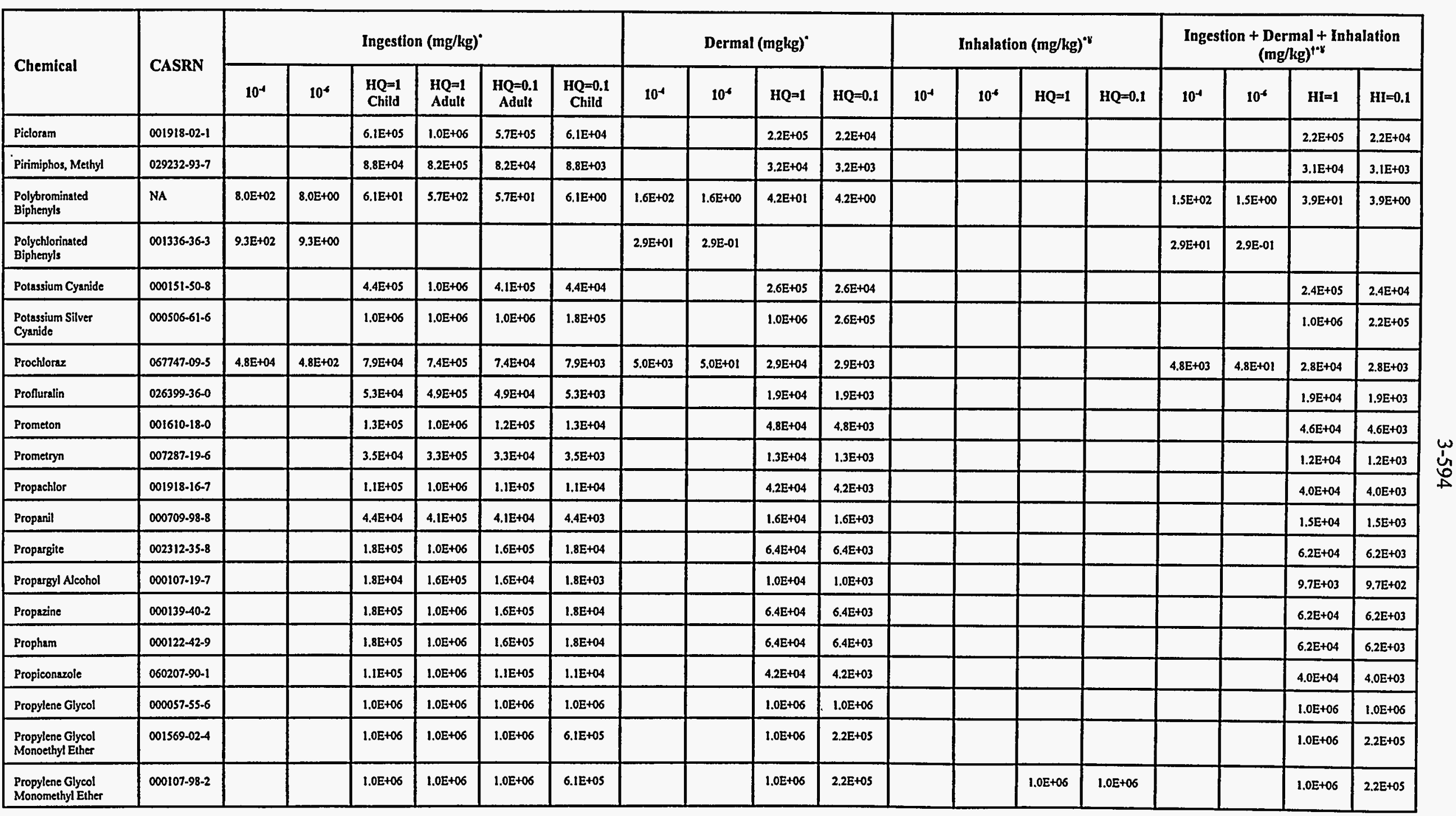

Nonradionuclides/soil/recreational

Page 26 of 34 
Table 3.4c. (continued)

\begin{tabular}{|c|c|c|c|c|c|c|c|c|c|c|c|c|c|c|c|c|c|c|c|}
\hline \multirow{2}{*}{ Chemical } & \multirow{2}{*}{ CASRN } & \multicolumn{6}{|c|}{ Ingestion (mg/kg) } & \multicolumn{4}{|c|}{ Dermal (mgkg) } & \multicolumn{4}{|c|}{ Inhalation (mg/kg) } & \multicolumn{4}{|c|}{$\begin{array}{c}\text { Ingestion + Dermal + Inhalation } \\
(\mathrm{mg} / \mathrm{kg})^{+* \varepsilon}\end{array}$} \\
\hline & & $10^{-4}$ & $10^{4}$ & $\begin{array}{l}\text { HQ=1 } \\
\text { Child }\end{array}$ & $\begin{array}{l}\text { HQ }=1 \\
\text { Adult }\end{array}$ & $\begin{array}{c}\mathrm{HQ}=0.1 \\
\text { Adult }\end{array}$ & $\begin{array}{l}\mathrm{HQ}=0.1 \\
\text { Child }\end{array}$ & $10^{-4}$ & $10^{5}$ & $H Q=1$ & $\mathrm{HQ}=0.1$ & $10^{-4}$ & $10^{4}$ & $H Q=1$ & $\mathrm{HQ}=0.1$ & $10^{-4}$ & $10^{6}$ & $\mathrm{HI}=1$ & $\mathrm{HI}=0.1$ \\
\hline Propylene Oxide & 000075.56 .9 & $3.0 \mathrm{E}+04$ & $3.0 \mathrm{E}+02$ & & & & & $5.0 E+03$ & $5.0 E+01$ & & & $2.8 E+04$ & 4.7E+03 & $2.8 \mathrm{E}+04$ & $2.2 E+04$ & 4.7E+03 & $4.7 E+01$ & & \\
\hline Pursuit & $081335-77.5$ & & & $1.0 \mathrm{E}+06$ & $1.0 \mathrm{E}+06$ & $1.0 \mathrm{E}+06$ & $2.2 \mathrm{E}+05$ & & & $8.0 \mathrm{E}+05$ & $8.0 \mathrm{E}+04$ & & & & & & & $7.7 E+05$ & $7.7 E+04$ \\
\hline Pydrin & $051630-58-1$ & & & $2.2 \mathrm{E}+05$ & $1.0 \mathrm{E}+\infty 6$ & $2.0 E+05$ & $2.2 \mathrm{E}+04$ & & & $8.0 \mathrm{E}+04$ & $8.0 \mathrm{E}+03$ & & & & & & & $7.7 E+04$ & 7.7E+03 \\
\hline Pyrene & $000129-00-0$ & & & 2.6E+05 & $1.0 \mathrm{E}+06$ & $2.5 \mathrm{E}+05$ & $2.6 \mathrm{E}+04$ & & & $6.0 E+04$ & $6.0 E+03$ & & & & & & & $5.8 E+04$ & $5.8 \mathrm{E}+03$ \\
\hline Pyridine & 000110.86 .1 & & & $8.8 E+03$ & $8.2 \mathrm{E}+04$ & $8.2 E+03$ & $8.8 \mathrm{E}+02$ & & & $3.2 E+03$ & $3.2 \mathrm{E}+02$ & & & & & & & $3.1 E+03$ & 3.1E+02 \\
\hline Quinalphos & $013593-03.8$ & & & $4.4 \mathrm{E}+03$ & $4.1 \mathrm{E}+04$ & $4.1 \mathrm{E}+03$ & $4.4 \mathrm{E}+02$ & & & $1.6 \mathrm{E}+03$ & $1.6 \mathrm{E}+02$ & & & & & & & $1.5 E+03$ & $1.5 \mathrm{E}+02$ \\
\hline Quinoline & $000091-22-5$ & $6.0 \mathrm{E}+02$ & $6.0 \mathrm{E}+\infty 0$ & & - & & & $6.2 \mathrm{E}+0 \mathrm{t}$ & $6.2 \mathrm{E}-01$ & & & & & & & $6.0 E+01$ & $6.0 \mathrm{E}-01$ & & \\
\hline $\begin{array}{l}\text { Refractory Ceramic } \\
\text { Fibers }\end{array}$ & $\mathrm{NA}$ & & & & & & & & & & & & & & & & & & \\
\hline Resmethrin & $010453-86-8$ & & & $2.6 \mathrm{E}+05$ & $1.0 \mathrm{E}+06$ & $2.5 \mathrm{E}+05$ & $2.6 \mathrm{E}+04$ & & & $9.6 \mathrm{E}+04$ & $9.6 \mathrm{E}+03$ & & & & & & & $9.3 \mathrm{E}+04$ & $9.3 E+03$ \\
\hline Ronnel & $000299-84-3$ & & & 4.4E+0S & $1.0 \mathrm{E}+\infty$ & 4.1E+05 & $4.4 E+04$ & & & $1.6 \mathrm{E}+05$ & $1.6 \mathrm{E}+04$ & & & & & & & $1.5 E+05$ & $1.5 \mathrm{E}+04$ \\
\hline Rotenone & $000083-79-4$ & & & 3.5E+04 & $3.3 \mathrm{E}+05$ & $3.3 \mathrm{E}+04$ & $3.5 \mathrm{E}+03$ & & & $1.3 \mathrm{E}+04$ & $1.3 \mathrm{E}+03$ & & & & & & & $1.2 E+04$ & $1.2 \mathrm{E}+03$ \\
\hline Sevey & $078587-05-0$ & & & $2.2 \mathrm{E}+05$ & $1.0 E+06$ & $2.0 \mathrm{E}+05$ & $2.2 \mathrm{E}+04$ & & & $8.0 E+04$ & $8.0 E+03$ & & & & & & & $7.7 E+04$ & $7.7 \mathrm{E}+03$ \\
\hline Selenious Acid & $007783 \cdot 00.8$ & & & $4.4 E+04$ & 4.1E+05 & $4.1 \mathrm{E}+04$ & $4.4 \mathrm{E}+03$ & & & $2.8 \mathrm{E}+04$ & $2.8 \mathrm{E}+03$ & & & & & & & $2.6 \mathrm{E}+04$ & $2.6 \mathrm{E}+03$ \\
\hline Selenite & $014124-67-5$ & & & & & & & & & & & & & & & & & & \\
\hline Selenium & $007782-49-2$ & & & $4.4 E+04$ & 4.IE+05 & $4.1 \mathrm{E}+04$ & $4.4 \mathrm{E}+03$ & & & $1.4 \mathrm{E}+05$ & $1.4 \mathrm{E}+04$ & & & & & & & $1, I \mathrm{E}+05$ & $1.1 \mathrm{E}+04$ \\
\hline Seienium Sulfide & $007446-34-6$ & & & & & & & & & & & & & & & & & & \\
\hline Selenourea & $000630-10-4$ & & & $4.4 E+04$ & 4. $1 \mathrm{E}+05$ & $4.1 \mathrm{E}+0.4$ & $4.4 E+03$ & & & $1.6 \mathrm{E}+04$ & $1.6 \mathrm{E}+03$ & & & & & & & $1.5 \mathrm{E}+04$ & $1.5 \mathrm{E}+03$ \\
\hline Sethoxydim & $074051-80-2$ & & & $7.9 E+05$ & $1.0 \mathrm{E}+06$ & 7.4E+05 & $7.9 E+04$ & & & $2.9 E+05$ & $2.9 E+04$ & & & & & & & $28 \mathrm{E}+0 \mathrm{~S}$ & $2.8 \mathrm{E}+04$ \\
\hline Silver & $007440-22-4$ & & & $4.4 E+04$ & 4.1E+05 & $4.1 \mathrm{E}+04$ & $4.4 \mathrm{E}+03$ & & & $5.8 \mathrm{E}+04$ & $5.8 \mathrm{E}+03$ & & & & & & & $5.1 E+04$ & $5.1 E+03$ \\
\hline Silver Cyanide & 000506.64 .9 & & & $8.8 E+05$ & $1.0 \mathrm{E}+06$ & $8.2 \mathrm{E}+0 \mathrm{~S}$ & $8.8 \mathrm{E}+04$ & & & $1.0 \mathrm{E}+06$ & $1.3 \mathrm{E}+05$ & & & & & & & $1.0 \mathrm{E}+06$ & $1.1 E+05$ \\
\hline Simarine & 000122.34 .9 & $6.0 \mathrm{E}+04$ & $6.0 \mathrm{E}+02$ & $4.4 E+04$ & 4.IE+0S & $4.1 E+04$ & $4.4 \mathrm{E}+03$ & $6.2 \mathrm{E}+03$ & $6.2 \mathrm{E}+01$ & $1.6 \mathrm{E}+04$ & $1.6 E+03$ & & & & & $6.0 \mathrm{E}+03$ & $6.0 \mathrm{E}+01$ & 1.5E+04 & $1.5 E+03$ \\
\hline Sodium & $007440-23-5$ & & & & & & & & & & & & & & & & & & \\
\hline
\end{tabular}

Nonradionuclides/soil/recreational

Page 27 of 34 
Table 3.4c. (continued)

\begin{tabular}{|c|c|c|c|c|c|c|c|c|c|c|c|c|c|c|c|c|c|c|c|}
\hline \multirow{2}{*}{ Chemical } & \multirow{2}{*}{ CASRN } & \multicolumn{6}{|c|}{ Ingestion (mg/kg) } & \multicolumn{4}{|c|}{ Dermal (mgkg) } & \multicolumn{4}{|c|}{ Inhalation (mg/kg) } & \multicolumn{4}{|c|}{$\begin{array}{c}\text { Ingestion + Dermal + Inhalation } \\
(\mathrm{mg} / \mathrm{kg})^{++v}\end{array}$} \\
\hline & & $10^{-4}$ & $10^{5}$ & $\begin{array}{l}H Q=1 \\
\text { Child }\end{array}$ & $\begin{array}{l}\text { HQ=I } \\
\text { Adult }\end{array}$ & $\begin{array}{c}H Q=0.1 \\
\text { Adult }\end{array}$ & $\begin{array}{c}H Q=0.1 \\
\text { Child }\end{array}$ & $10^{-4}$ & $10^{-4}$ & $\mathrm{HQ}=1$ & $\mathrm{HQ}=0.1$ & $10^{-4}$ & $10^{6}$ & $\mathbf{H Q}=1$ & $\mathrm{HQ}=0.1$ & $10^{-4}$ & $10^{4}$ & $\mathbf{H I}=1$ & $\mathrm{Hl}=0.1$ \\
\hline Sodium Acifluorfen & $062476-59.9$ & & & $1.1 \mathrm{E}+05$ & $1.0 \mathrm{E}+06$ & $1.1 \mathrm{E}+05$ & $1.1 \mathrm{E}+04$ & & & $4.2 E+04$ & $4.2 E+03$ & & & & & & & $4.0 \mathrm{E}+04$ & $4.0 E+03$ \\
\hline Sodium Azide & $026628-22-8$ & & & $3.5 \mathrm{E}+04$ & $3.3 E+05$ & $3.3 \mathrm{E}+04$ & $3.5 \mathrm{E}+03$ & & & $5.1 \mathrm{E}+04$ & $5.1 \mathrm{E}+03$ & & & & & & & $4.4 \mathrm{E}+04$ & $4.4 \mathrm{E}+03$ \\
\hline Sodium Cyanide & $000143-33.9$ & & & $3.5 \mathrm{E}+05$ & $1.0 \mathrm{E}+06$ & $3.3 \mathrm{E}+05$ & $3.5 \mathrm{E}+04$ & & & 2.1E+05 & 2.1E+04 & & & & & & & $1.9 E+05$ & $1.9 \mathrm{E}+04$ \\
\hline $\begin{array}{l}\text { Sodium } \\
\text { Diethyldithiocarbamate }\end{array}$ & $000148-18-5$ & $2.6 \mathrm{E}+04$ & $2.6 \mathrm{E}+02$ & $2.6 \mathrm{E}+05$ & $1.0 \mathrm{E}+06$ & $2.5 \mathrm{E}+05$ & $2.6 \mathrm{E}+04$ & $2.8 \mathrm{E}+03$ & $2.8 \mathrm{E}+01$ & $9.6 \mathrm{E}+04$ & $9.6 \mathrm{E}+03$ & & & & & $2.7 \mathrm{E}+03$ & $2.7 \mathrm{E}+01$ & $9.3 \mathrm{E}+04$ & $9.3 \mathrm{E}+03$ \\
\hline Sodium Fluoroacetate & $000062-74.8$ & & & $1.8 \mathrm{E}+02$ & $1.6 \mathrm{E}+03$ & $1.6 \mathrm{E}+02$ & $1.8 \mathrm{E}+01$ & & & $6.4 \mathrm{E}+01$ & $6.4 \mathrm{E}+00$ & & & & & & & $6.2 E+01$ & $6.2 \mathrm{E}+\infty$ \\
\hline Sodium Metavanadate & $013718-26-8$ & & & $8.8 \mathrm{E}+03$ & $8.2 \mathrm{E}+04$ & $8.2 \mathrm{E}+03$ & $8.8 \mathrm{E}+02$ & & & $1.3 E+04$ & $1.3 E+03$ & & & & & & & $1.1 \mathrm{E}+04$ & 1.1E+03 \\
\hline $\begin{array}{l}\text { Stirofos } \\
\text { (Tetrachlorovinphos) }\end{array}$ & $000961-11-5$ & $3.0 \mathrm{E}+05$ & $3.0 \mathrm{E}+03$ & $2.6 \mathrm{E}+05$ & $1.0 \mathrm{E}+06$ & $2.5 \mathrm{E}+05$ & $2.6 \mathrm{E}+04$ & $3.1 \mathrm{E}+04$ & $3.1 E+02$ & $9.6 \mathrm{E}+04$ & $9.6 \mathrm{E}+03$ & & & & & $3.0 \mathrm{E}+04$ & $3.0 \mathrm{E}+02$ & $9.3 E+04$ & $9.3 \mathrm{E}+03$ \\
\hline Strontium, Stable & $007440-24-6$ & & & $1.0 \mathrm{E}+06$ & $1.0 \mathrm{E}+06$ & $1.0 \mathrm{E}+06$ & $5,3 \mathrm{E}+05$ & & & $1.0 \mathrm{E}+06$ & $7.7 \mathrm{E}+05$ & & & & & & & $1.0 \mathrm{E}+06$ & $6.7 E+05$ \\
\hline Strychnine & $000057-24-9$ & & & $2.6 \mathrm{E}+03$ & $2.5 E+04$ & $2.5 \mathrm{E}+03$ & $2,6 \mathrm{E}+02$ & & & $9.6 \mathrm{E}+02$ & $9.6 \mathrm{E}+01$ & & & & & & & $9.3 \mathrm{E}+02$ & $9.3 E+01$ \\
\hline Styrene ${ }^{(1)}$ & $000100-42-5$ & & & $1.0 \mathrm{E}+06$ & $1.0 \mathrm{E}+06$ & $1.0 \mathrm{E}+06$ & $1.8 \mathrm{E}+05$ & & & $1.0 \mathrm{E}+06$ & $1.0 \mathrm{E}+05$ & & & $6.7 \mathrm{E}+02$ & $6.7 \mathrm{E}+02$ & & & $6.7 E+02$ & $6.7 \mathrm{E}+02$ \\
\hline Sulfate & $014808-79-8$ & & & & & & & & & & & & & & & & & & \\
\hline Systhane & $088671-89-0$ & & & $2.2 \mathrm{E}+05$ & $1.0 \mathrm{E}+06$ & $2.0 \mathrm{E}+05$ & $2.2 \mathrm{E}+04$ & & & $8.0 \mathrm{E}+04$ & $8.0 \mathrm{E}+03$ & & & & & & & $7.7 \mathrm{E}+04$ & 7.7E+03 \\
\hline TCDD, 2,3,7,8.(4) & $001746.01-6$ & $4.8 \mathrm{E}-02$ & 4.8E-04 & & & & & $1.7 \mathrm{E}-03$ & $1.7 \mathrm{E}-05$ & & & $2.7 \mathrm{E}+03$ & $2.7 \mathrm{E}+01$ & & & $1.6 \mathrm{E}-03$ & $1.6 \mathrm{E}-05$ & & \\
\hline TCDF, 2,3,7,8-(1) & $051207-31-9$ & 4.8E-01 & $4.8 \mathrm{E}-03$ & & & & & $5.0 \mathrm{E}-02$ & $5,0 \mathrm{E}-04$ & & & $2.7 E+04$ & $2.7 \mathrm{E}+02$ & & & $4.8 \mathrm{E}-02$ & $4.8 \mathrm{E}-04$ & & \\
\hline TCMTB & $021564-17.0$ & & & $2.6 \mathrm{E}+05$ & $1.0 \mathrm{E}+06$ & $2.5 E+05$ & $2.6 \mathrm{E}+04$ & & & $9.6 \mathrm{E}+04$ & $9.6 \mathrm{E}+03$ & & & & & & & $9.3 \mathrm{E}+04$ & $9.3 \mathrm{E}+03$ \\
\hline Tebuthiuron & $034014-18-1$ & & & $6.1 \mathrm{E}+05$ & $1.0 \mathrm{E}+06$ & $5.7 \mathrm{E}+05$ & $6.1 \mathrm{E}+04$ & & & $2.2 \mathrm{E}+05$ & $2.2 \mathrm{E}+04$ & & & & & & & $2.2 \mathrm{E}+05$ & $2.2 E+04$ \\
\hline Temephos & $003383.96-8$ & & & $1.8 \mathrm{E}+05$ & $1.0 \mathrm{E}+06$ & $1.6 \mathrm{E}+05$ & $1,8 E+04$ & & & $6.4 \mathrm{E}+04$ & $6.4 \mathrm{E}+03$ & & & & & & & $6.2 \mathrm{E}+04$ & $6.2 \mathrm{E}+03$ \\
\hline Terbacil & $005902-51 \cdot 2$ & & & $1.1 \mathrm{E}+05$ & $1.0 \mathrm{E}+06$ & $1.1 \mathrm{E}+05$ & $1.1 \mathrm{E}+04$ & & & $4.2 \mathrm{E}+04$ & $4.2 \mathrm{E}+03$ & & & & & & & $4.0 E+04$ & $4.0 \mathrm{E}+03$ \\
\hline Terbufos & $013071-79.9$ & & & $2.2 \mathrm{E}+02$ & $2.0 \mathrm{E}+03$ & $2.0 E+02$ & $2.2 E+01$ & & & $8.0 \mathrm{E}+01$ & $8.0 \mathrm{E}+00$ & & & & & & & $7.7 E+01$ & $7.7 \mathrm{E}+\infty$ \\
\hline Terbutryn & $000886-50-0$ & & & $8.8 \mathrm{E}+03$ & $8.2 \mathrm{E}+04$ & $8.2 E+03$ & $8.8 \mathrm{E}+02$ & & & $3.2 E+03$ & $3.2 \mathrm{E}+02$ & & & & & & & $3.1 E+03$ & 3.1E+02 \\
\hline Tetrabutyl Lead & $001920-90-7$ & & & & & & & & & & & & & & & & & & \\
\hline
\end{tabular}


Table 3.4c. (continued)

\begin{tabular}{|c|c|c|c|c|c|c|c|c|c|c|c|c|c|c|c|c|c|c|c|}
\hline \multirow{2}{*}{ Chemical } & \multirow{2}{*}{ CASRN } & \multicolumn{6}{|c|}{ Ingestion (mg/kg) } & \multicolumn{4}{|c|}{ Dermal (mgkg)" } & \multicolumn{4}{|c|}{ Inhalation (mg/kg) } & \multicolumn{4}{|c|}{$\begin{array}{c}\text { Ingestion + Dermal + Inhalation } \\
(\mathrm{mg} / \mathrm{kg})^{+\cdot \mathrm{r}}\end{array}$} \\
\hline & & $10^{-4}$ & $10^{6}$ & $\begin{array}{l}\text { HQ=1 } \\
\text { Child }\end{array}$ & $\begin{array}{l}\text { HQ=1 } \\
\text { Adult }\end{array}$ & $\begin{array}{c}H Q=0.1 \\
\text { Adult }\end{array}$ & $\begin{array}{l}\mathrm{HQ}=0.1 \\
\text { Child }\end{array}$ & $10^{-4}$ & $10^{6}$ & $H Q=1$ & $\mathrm{HQ}=0.1$ & $10^{4}$ & $10^{6}$ & $\mathrm{HQ}=1$ & $\mathrm{HQ}=0.1$ & $10^{-4}$ & $10^{4}$ & $\mathbf{H I}=\mathbf{l}$ & $\mathrm{HI}=0.1$ \\
\hline $\begin{array}{l}\text { Tetrachlorobenzene, } \\
1,2,4,5 \text {. }\end{array}$ & $000095-94-3$ & & & $2.6 \mathrm{E}+03$ & $2.5 E+04$ & $2.5 \mathrm{E}+03$ & $2.6 \mathrm{E}+02$ & & & $1.5 \mathrm{E}+03$ & $1.5 \mathrm{E}+02$ & & & & & & & $1.5 E+03$ & $1.5 E+02$ \\
\hline $\begin{array}{l}\text { Tetrachloroecthane, } \\
1,1,1,1,2-\end{array}$ & $000630-20-6$ & $2.8 \mathrm{E}+05$ & $2.8 \mathrm{E}+03$ & $2.6 \mathrm{E}+05$ & $1.0 \mathrm{E}+06$ & 2.5E+05 & $2.6 \mathrm{E}+04$ & $4.6 \mathrm{E}+04$ & $4.6 \mathrm{E}+02$ & $1.5 \mathrm{E}+05$ & $1.5 \mathrm{E}+04$ & 7.7E+02 & $7.7 \mathrm{E}+02$ & & & $7.7 E+02$ & $3.5 \mathrm{E}+02$ & $7.7 E+02$ & $7.7 \mathrm{E}+02$ \\
\hline $\begin{array}{l}\text { Tetrachloroethane, } \\
\text { 1.1,2,2. }\end{array}$ & $000079-34.5$ & $3.6 \mathrm{E}+04$ & $3.6 \mathrm{E}+02$ & & & & & $5.2 \mathrm{E}+03$ & $5.2 \mathrm{E}+01$ & & & $1.1 \mathrm{E}+03$ & $4.5 \mathrm{E}+02$ & & & $1.1 E+03$ & 4.5E+01 & & \\
\hline Tetrachloroethylene() & $000127-18-4$ & $1.4 \mathrm{E}+0 \mathrm{~S}$ & $1.4 \mathrm{E}+03$ & $8.8 E+04$ & $8.2 \mathrm{E}+05$ & $8.2 \mathrm{E}+04$ & $8.8 \mathrm{E}+03$ & $2.9 \mathrm{E}+04$ & $2.9 E+02$ & $6.4 \mathrm{E}+04$ & $6.4 E+03$ & $1.2 E+02$ & $1.2 \mathrm{E}+02$ & & & $1.2 \mathrm{E}+02$ & $1.2 \mathrm{E}+02$ & $1.2 \mathrm{E}+02$ & $1.2 \mathrm{E}+02$ \\
\hline $\begin{array}{l}\text { Tetrachlorophenol, } \\
2,3,4,6-\end{array}$ & $000058-90-2$ & & & $2.6 \mathrm{E}+05$ & $1.0 \mathrm{E}+06$ & 2.5E+05 & $2.6 \mathrm{E}+04$ & & & $9,6 E+04$ & $9.6 \mathrm{E}+03$ & & & & & & & $9.3 \mathrm{E}+04$ & $9.3 \mathrm{E}+03$ \\
\hline $\begin{array}{l}\text { Tetractlorotoluene, p- } \\
\text { alpha, alpha, alphas- }\end{array}$ & $005216-25-1$ & $3.6 \mathrm{E}+02$ & $3.6 \mathrm{E}+\infty 0$ & & & & & $6.0 \mathrm{E}+0 \mathrm{t}$ & $6.0 \mathrm{E}-01$ & & & & & & & $5.6 \mathrm{E}+01$ & $5,6 \mathrm{E}-01$ & & \\
\hline $\begin{array}{l}\text { Tetraethyl } \\
\text { Dithiopyrophosphate }\end{array}$ & $003689-24.5$ & & & $4.4 E+03$ & 4.1E+04 & 4.1E+03 & $4.4 \mathrm{E}+02$ & & & $1.6 \mathrm{E}+03$ & $1.6 \mathrm{E}+02$ & & & & & & & $1.5 \mathrm{E}+03$ & $1.5 E+02$ \\
\hline Tetrethyi Lead & $000078-00-2$ & & & & & & & & & & & & & & & & & & \\
\hline $\begin{array}{l}\text { Tetrafluoroethane, } \\
\text { 1,1,1,2. }\end{array}$ & $000811-97-2$ & & & & & & & & & & & & & $1.1 \mathrm{E}+02$ & $1.1 \mathrm{E}+02$ & & & & \\
\hline Tetramethyl Lead & $000075-74.1$ & & & & - & & & & & & & & & & & & & & \\
\hline Tetrapropyl Lead & $003440-75-3$ & & & & & & & & & & & & & & & & & & \\
\hline Thallic Oxide" & $001314-32-5$ & & & $6.1 \mathrm{E}+02$ & S.7E+03 & $5.7 \mathrm{E}+02$ & $6.1 \mathrm{E}+01$ & & & $2.2 \mathrm{E}+02$ & $2.2 \mathrm{E}+01$ & & & & & & & $2.2 E+02$ & $2.2 \mathrm{E}+01$ \\
\hline Thallium (l) Nitrate & $010102-45-1$ & & & $7.9 \mathrm{E}+02$ & $7.4 E+03$ & $7.4 \mathrm{E}+02$ & $7.9 E+01$ & & & $1.2 \mathrm{E}+03$ & $1.2 \mathrm{E}+02$ & & & & & & & $1.0 E+03$ & $1.0 \mathrm{E}+02$ \\
\hline $\begin{array}{l}\text { Thallium (Soluble } \\
\text { Salts) }\end{array}$ & $007440-28-0$ & & & & & & & & & & & & & & & & & & \\
\hline Thallium Acetate & 000563.68 .8 & & & $7.9 \mathrm{E}+02$ & 7.4E+03 & $7.4 \mathrm{E}+02$ & $7.9 E+01$ & & & $2.9 E+02$ & $2.9 E+01$ & & & & & & & $2.8 E+02$ & $2.8 \mathrm{E}+01$ \\
\hline Thallium Carbonste & 006533.73 .9 & & & $7.0 E+02$ & $6.5 \mathrm{E}+03$ & $6.5 \mathrm{E}+02$ & $7.0 \mathrm{E}+01$ & & & $2.6 \mathrm{E}+02$ & $2.6 \mathrm{E}+01$ & & & & & & & $2.5 \mathrm{E}+02$ & $2.5 \mathrm{E}+01$ \\
\hline Thallium Chloride & $007791-12-0$ & & & $7.0 E+02$ & $6.5 \mathrm{E}+03$ & $6.5 \mathrm{E}+02$ & $7.0 \mathrm{E}+01$ & & & $1.0 \mathrm{E}+03$ & $1.0 \mathrm{E}+02$ & & & & & & & $8.9 E+02$ & $8.9 E+01$ \\
\hline Thallium Selenite ${ }^{(3)}$ & $012039.52-0$ & & & $7.9 \mathrm{E}+02$ & $7.4 \mathrm{E}+03$ & $7.4 \mathrm{E}+02$ & $7.9 \mathrm{E}+01$ & & & $1.2 \mathrm{E}+03$ & $1.2 \mathrm{E}+02$ & & & & & & & $1.0 \mathrm{E}+03$ & $1.0 \mathrm{E}+02$ \\
\hline Thallium Sulfate & $007446-18-6$ & & & $7.0 \mathrm{E}+02$ & $6.5 \mathrm{E}+03$ & $6.5 \mathrm{E}+02$ & $7.0 E+01$ & & & $1.0 \mathrm{E}+03$ & $1.0 \mathrm{E}+02$ & & & & & & & $8.9 E+02$ & $8.9 E+0 I$ \\
\hline
\end{tabular}

Nonradionuclides/soil/recreational

Page 29 of 34 
Table 3.4c. (continued)

\begin{tabular}{|c|c|c|c|c|c|c|c|c|c|c|c|c|c|c|c|c|c|c|c|}
\hline \multirow{2}{*}{ Chemical } & \multirow{2}{*}{ CASRN } & \multicolumn{6}{|c|}{ Ingestion (mg/kg) } & \multicolumn{4}{|c|}{ Dermal (mgkg) } & \multicolumn{4}{|c|}{ Inhalation $(\mathrm{mg} / \mathrm{kg})^{\circ v}$} & \multicolumn{4}{|c|}{$\begin{array}{c}\text { Ingestion + Dermal + Inhalation } \\
(\mathrm{mg} / \mathrm{kg})^{+* v}\end{array}$} \\
\hline & & $10^{-4}$ & $10^{-6}$ & $\begin{array}{l}H Q=1 \\
\text { Child }\end{array}$ & $\begin{array}{l}H Q=1 \\
\text { Adult }\end{array}$ & $\begin{array}{c}\text { HQ }=0.1 \\
\text { Adult }\end{array}$ & $\begin{array}{l}\mathrm{HQ}=0.1 \\
\text { Child }\end{array}$ & $10^{4}$ & $10^{6}$ & $H Q=1$ & $\mathrm{HQ}=0.1$ & $10^{-4}$ & $10^{4}$ & $H Q=1$ & $\mathrm{HQ}=0.1$ & $10^{-4}$ & $10^{4}$ & $H I=1$ & $H \mathrm{I}=0.1$ \\
\hline Thiobencart & $028249.77-6$ & & & $8.8 \mathrm{E}+04$ & 8.2E+0S & 8.2E+04 & $8.8 E+03$ & & & $3.2 \mathrm{E}+04$ & $3.2 \mathrm{E}+03$ & & & & & & & $3.1 \mathrm{E}+04$ & $3.1 \mathrm{E}+03$ \\
\hline Thiofanox & $039196.18-4$ & & & 2.6E+03 & $2.5 \mathrm{E}+04$ & $2.5 \mathrm{E}+03$ & $2.6 \mathrm{E}+02$ & & & $9.6 \mathrm{E}+02$ & $9.6 \mathrm{E}+01$ & & & & & & & $9.3 \mathrm{E}+02$ & $9.3 \mathrm{E}+01$ \\
\hline Thiophanate, Methyl & $023564-05.8$ & & & $7.0 \mathrm{E}+05$ & $1.0 \mathrm{E}+06$ & $6.5 E+05$ & $7.0 \mathrm{E}+04$ & & & 2.6E+05 & $2.6 \mathrm{E}+04$ & & & & & & & $2.5 \mathrm{E}+05$ & $2,5 \mathrm{E}+04$ \\
\hline Thiram & $000137-26-8$ & & & 4.4E+04 & 4.1E+05 & 4.1E+04 & $4.4 E+03$ & & & $1.6 \mathrm{E}+04$ & $1.6 \mathrm{E}+03$ & & & & & & & $1.5 \mathrm{E}+04$ & $1.5 \mathrm{E}+03$ \\
\hline Thorium & $007440-29.1$ & & & & & & & & & & & & & & & & & & \\
\hline Tin & $007440-31-5$ & & & $1.0 \mathrm{E}+06$ & $1.0 \mathrm{E}+06$ & $1.0 \mathrm{E}+06$ & $5.3 \mathrm{E}+05$ & & & $1.0 E+06$ & $3.9 E+05$ & & & & & & & $1.0 \mathrm{E}+06$ & $3.6 \mathrm{E}+05$ \\
\hline Titarium & $007440-32-6$ & & & & & & & & & & & & & & & & & & \\
\hline Tolvend ${ }^{(0)}$ & $000108-88-3$ & & & $1.0 \mathrm{E}+06$ & $1.0 \mathrm{E}+06$ & $1.0 \mathrm{E}+06$ & $1.8 \mathrm{E}+05$ & & & $1.0 \mathrm{E}+06$ & $1.0 \mathrm{E}+05$ & & & $2.5 \mathrm{E}+02$ & $2.5 \mathrm{E}+02$ & & & $2.5 \mathrm{E}+02$ & $2.5 E+02$ \\
\hline $\begin{array}{l}\text { Toluene disocyanate } \\
\text { mixture (TDD) }\end{array}$ & $026471-62-5$ & & & & & & & & & & & & & $1.0 \mathrm{E}+06$ & $1.0 E+06$ & & & & \\
\hline Toluene-2,4-diamine & $000095-80.7$ & $2.2 \mathrm{E}+03$ & $2.2 \mathrm{E}+01$ & & & & & 2.3E+02 & $2.3 \mathrm{E}+\infty$ & & & & & & & $2.3 \mathrm{E}+02$ & $2.3 \mathrm{E}+00$ & & \\
\hline Toluene-2,5-diamine & $000095-70-5$ & & & $1.0 \mathrm{E}+06$ & $1.0 \mathrm{E}+06$ & $1.0 \mathrm{E}+06$ & $5.3 \mathrm{E}+05$ & & & $1.0 \mathrm{E}+06$ & $1.9 \mathrm{E}+05$ & & & & & & & $1.0 \mathrm{E}+06$ & $1.9 \mathrm{E}+05$ \\
\hline Toluene-2,6-diamine & $000823-40-5$ & & & $1.0 \mathrm{E}+06$ & $1.0 \mathrm{E}+06$ & $1.0 \mathrm{E}+06$ & $1.8 E+05$ & & & $6.4 E+05$ & $6.4 E+04$ & & & & & & & $6.2 \mathrm{E}+05$ & $6.2 E+04$ \\
\hline $\begin{array}{l}\text { Totuidine, o- } \\
\text { (Methylariline, 2-) }\end{array}$ & $000095-53-4$ & $3.0 E+04$ & $3.0 \mathrm{E}+02$ & & & & & $3.1 E+03$ & $3.1 E+01$ & & & & & & & $3.0 \mathrm{E}+03$ & $3.0 \mathrm{E}+01$ & & \\
\hline Toluidine, p- & $000106-49-0$ & $3.8 \mathrm{E}+04$ & $3.8 \mathrm{E}+02$ & & & & & $3.9 E+03$ & $3.9 E+01$ & & & & & & & $3.8 \mathrm{E}+03$ & $3.8 \mathrm{E}+01$ & & \\
\hline Toxaphene & $008001-35.2$ & $6.5 E+03$ & $6.5 \mathrm{E}+01$ & & & & & $6.8 \mathrm{E}+02$ & $6.8 \mathrm{E}+00$ & & & $1.0 \mathrm{E}+06$ & $1.0 \mathrm{E}+06$ & & & $6.6 \mathrm{E}+02$ & $6.6 \mathrm{E}+00$ & & \\
\hline Tralomethrin & $066841-25.6$ & & & $6.6 \mathrm{E}+04$ & $6.1 \mathrm{E}+05$ & $6.1 \mathrm{E}+04$ & $6.6 \mathrm{E}+03$ & & & $2.4 E+04$ & $2.4 E+03$ & & & & & & & $2.3 \mathrm{E}+04$ & 2.3E+03 \\
\hline Triallate & $002303-17.5$ & & & $1.1 E+05$ & $1.0 \mathrm{E}+06$ & $1.1 \mathrm{E}+05$ & $1.1 \mathrm{E}+04$ & & & $4.2 \mathrm{E}+04$ & $4.2 E+03$ & & & & & & & $4.0 \mathrm{E}+04$ & $4.0 E+03$ \\
\hline Triasulfuron & $082097-50.5$ & & & $8.8 \mathrm{E}+04$ & $8.2 \mathrm{E}+05$ & $8.2 \mathrm{E}+04$ & $8.8 E+03$ & & & $3.2 E+04$ & $3.2 \mathrm{E}+03$ & & & & & & & $3.1 E+04$ & $3.1 E+03$ \\
\hline $\begin{array}{l}\text { Tribromobenzene, } \\
\text { 1,2,4- }\end{array}$ & $000615-54-3$ & & & $4.4 E+04$ & 4.1E+05 & 4.1E+04 & $4.4 E+03$ & & & $2.6 E+04$ & $2.6 \mathrm{E}+03$ & & & & & & & $2.4 \mathrm{E}+04$ & $2.4 E+03$ \\
\hline $\begin{array}{l}\text { Tribromochloromethan } \\
\text { e }\end{array}$ & $000594-15.0$ & & & & & & & & & & & & & & & & & & \\
\hline
\end{tabular}




\begin{tabular}{|c|c|c|c|c|c|c|c|c|c|c|c|c|c|c|c|c|c|c|c|}
\hline & $\overline{\overline{0}}$ & & $\begin{array}{l}\stackrel{8}{4} \\
\text { 萦 }\end{array}$ & 窝 & & & & & 憲 & 薥 & 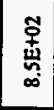 & & 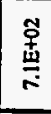 & 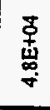 & & 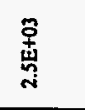 & 总 & 罍 & $\begin{array}{l}\text { 恿 } \\
\text { in }\end{array}$ \\
\hline & $\frac{\bar{u}}{\underline{z}}$ & & 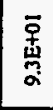 & 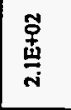 & & & & & 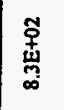 & 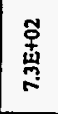 & 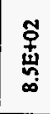 & & 总 & 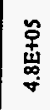 & & 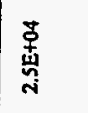 & 疍 & 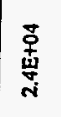 & 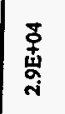 \\
\hline & 릉 & & & & & & 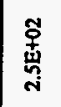 & 案 & & & 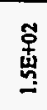 & 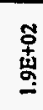 & & & $\begin{array}{l}\text { 总 } \\
\text { 总 } \\
0 \\
0\end{array}$ & & & & $\mid \begin{array}{l}8 \\
\text { 苾 } \\
-\end{array}$ \\
\hline & $\dot{\vec{b}}$ & & & & & & $\begin{array}{l}\text { 草 } \\
\text { 密 }\end{array}$ & 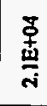 & & & 密 & 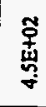 & & & $\begin{array}{l}\text { 蕚 } \\
\text { 总 }\end{array}$ & & & & 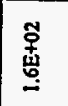 \\
\hline \multirow{4}{*}{ 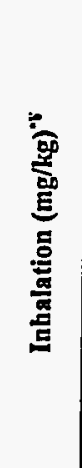 } & ర్d & & & 窋 & & & & & 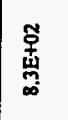 & 罩 & & & 草 & & & & & & \\
\hline & $\bar{g}$ & & & 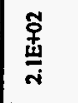 & & & & & 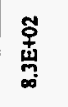 & 蜜 & & & 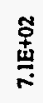 & & & & & & \\
\hline & 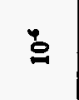 & & & & & & & & & & 㤐 & 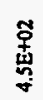 & & & 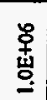 & & & & \\
\hline & $\stackrel{\square}{\varrho}$ & & & & & & & & & & 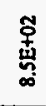 & 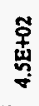 & & & 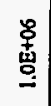 & & & & \\
\hline \multirow{4}{*}{ 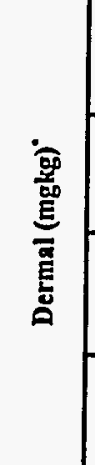 } & $\overrightarrow{0}$ & & 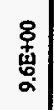 & 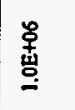 & & & & & 恿 & 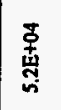 & 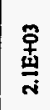 & & 予 & 虽 & & 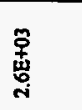 & 䍄 & 恿 & 䔰 \\
\hline & $\overline{0}$ & & 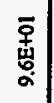 & 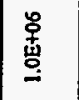 & . & & & & 䓌 & 亳 & 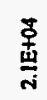 & & 总 & 鹤 & & 罍 & 总 & 䓌 & 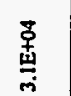 \\
\hline & $\dot{g}$ & & & & & & 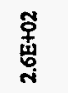 & 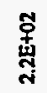 & & & 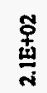 & 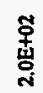 & & & 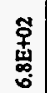 & & & & 密 \\
\hline & อ & & & & & & 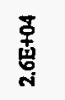 & $\begin{array}{l}\text { 预 } \\
\text { స్ }\end{array}$ & & & 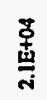 & 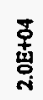 & & & 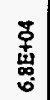 & & & & 熹 \\
\hline \multirow{6}{*}{ 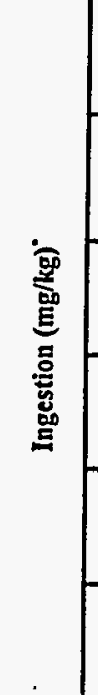 } & 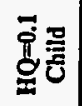 & & $\begin{array}{l}\underset{\mathbf{S}}{\mathbf{4}} \\
\underset{\sim}{*}\end{array}$ & 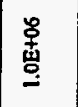 & & & & & 苾 & $\begin{array}{l}\text { 怘 } \\
\text { 蓼 }\end{array}$ & 恿 & & 总 & 怘 & & 兽 & 恿 & 罍 & 旁 \\
\hline & 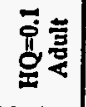 & & 窋 & $\begin{array}{l}\text { 早 } \\
\text { 产 } \\
-\end{array}$ & & & & & $\begin{array}{l}\text { 㤐 } \\
\text { 查 }\end{array}$ & 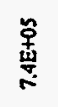 & 㽞 & & 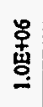 & 旁 & & 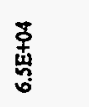 & 蒿 & 草 & 宧 \\
\hline & 栗害 & & 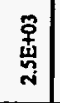 & 呆 & & & & & 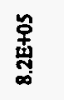 & 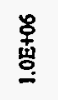 & 总 & & 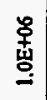 & $\begin{array}{l}\text { 草 } \\
\text { 㯖 }\end{array}$ & & 总 & 窑 & 总 & 嗙 \\
\hline & 氙总 & & 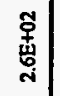 & 蔓 & & & & & 怘 & 亳 & 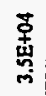 & & $\begin{array}{l}\text { 草 } \\
\text { 苟 }\end{array}$ & 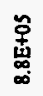 & & 喜 & 苦 & 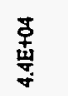 & 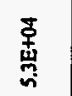 \\
\hline & $\dot{g}$ & & & & & & 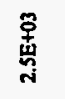 & 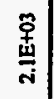 & & & 罍 & 总 & & & 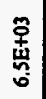 & & & & 恿 \\
\hline & 음 & & & & & & 总 & 总 & & & 嵒 & 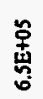 & & & 葛 & & & & $\begin{array}{l}\text { 窑 } \\
\text { 尊 }\end{array}$ \\
\hline \multicolumn{2}{|c|}{ 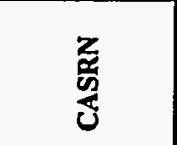 } & 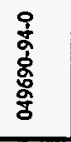 & 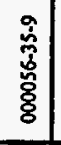 & 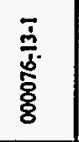 & 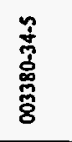 & & 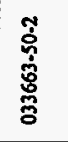 & 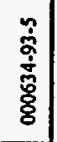 & 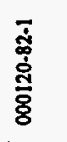 & 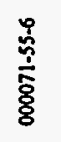 & 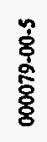 & 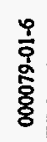 & 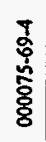 & 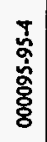 & 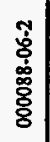 & 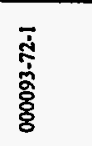 & 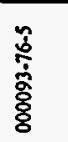 & 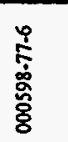 & 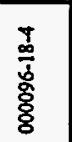 \\
\hline \multicolumn{2}{|c|}{ 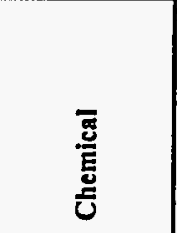 } & 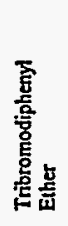 & 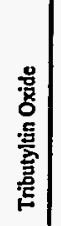 & 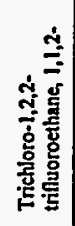 & 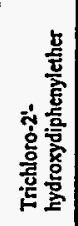 & 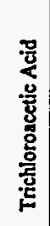 & 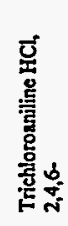 & 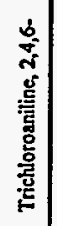 & 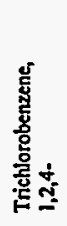 & 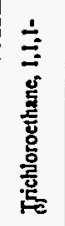 & 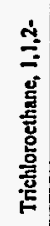 & 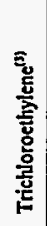 & 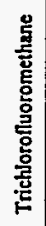 & 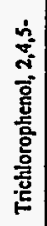 & 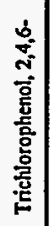 & 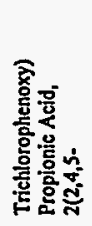 & 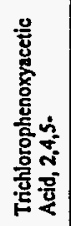 & 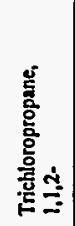 & 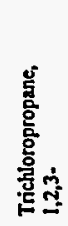 \\
\hline
\end{tabular}


Table 3.4c. (continued)

\begin{tabular}{|c|c|c|c|c|c|c|c|c|c|c|c|c|c|c|c|c|c|c|c|}
\hline \multirow{2}{*}{ Chemical } & \multirow{2}{*}{ CASRN } & \multicolumn{6}{|c|}{ Ingestion (mg/kg) } & \multicolumn{4}{|c|}{ Dermal (mgkg) } & \multicolumn{4}{|c|}{ Inhalation $(\mathrm{mg} / \mathrm{kg})^{-\gamma}$} & \multicolumn{4}{|c|}{$\begin{array}{c}\text { Ingestion + Dermal + Inhalation } \\
(\mathrm{mg} / \mathrm{kg})^{+\bullet \vartheta}\end{array}$} \\
\hline & & $10^{-4}$ & $10^{-5}$ & $\begin{array}{l}\text { HQ }=1 \\
\text { Child }\end{array}$ & $\begin{array}{l}\mathrm{HQ}=1 \\
\text { Adult }\end{array}$ & $\begin{array}{c}\text { HQ }=0.1 \\
\text { Adult }\end{array}$ & $\begin{array}{l}\text { HQ }=0.1 \\
\text { Child }\end{array}$ & $10^{-4}$ & $10^{4}$ & $H Q=1$ & $\mathrm{HQ}=0.1$ & $10^{-4}$ & $10^{-6}$ & $H Q=1$ & $\mathrm{HQ}=0.1$ & $10^{-4}$ & $10^{6}$ & $H I=1$ & $\mathrm{HI}=0.1$ \\
\hline $\begin{array}{l}\text { Trichloropropene, } \\
1,2,3-\end{array}$ & $000096-19-5$ & & & 4.4E+04 & 4.1E+05 & $4.1 \mathrm{E}+04$ & 4.4E+03 & & & $2.6 \mathrm{E}+04$ & $2.6 \mathrm{E}+03$ & & & & & & & $2.4 E+04$ & $2,4 E+03$ \\
\hline $\begin{array}{l}\text { Trichlorotoluene, } \\
2,3,6-\end{array}$ & $002077-46-5$ & & & & & & & & & & & & & & & & & & \\
\hline $\begin{array}{l}\text { Trichlorotoluene, alpha } \\
\text { 2,6. }\end{array}$ & $002014-83-7$ & & & & & & & & & & & & & & & & & & \\
\hline Tridiphane & $058138-08-2$ & & & $2.6 \mathrm{E}+04$ & $2.5 \mathrm{E}+05$ & $2.5 \mathrm{E}+04$ & $2.6 \mathrm{E}+03$ & & & $9.6 \mathrm{E}+03$ & $9.6 \mathrm{E}+02$ & & & & & & & $9.3 \mathrm{E}+03$ & $9.3 \mathrm{E}+02$ \\
\hline Triethyl Lead & $005224-23-7$ & & & & & & : & & & & & & & & & & & & \\
\hline Triethylamine & $000121-44.8$ & & & & & & & & & . & & & & $2.2 \mathrm{E}+04$ & $1.3 E+04$ & & & & \\
\hline Trifluralin & $001582.09-8$ & $9.3 \mathrm{E}+05$ & $9.3 \mathrm{E}+03$ & $6.6 \mathrm{E}+04$ & 6.1E+05 & $6.1 \mathrm{E}+04$ & $6.6 \mathrm{E}+03$ & $9.7 \mathrm{E}+04$ & $9.7 \mathrm{E}+02$ & $2.4 \mathrm{E}+04$ & $2.4 E+03$ & & & & & $9.4 E+04$ & $9.4 E+02$ & $2.3 \mathrm{E}+04$ & $2.3 \mathrm{E}+03$ \\
\hline Trimethyl Lead & $007442-13-9$ & & & & & & & & & & & & & & & & & & \\
\hline Trimethyl Phosphate & $000512-56-1$ & $1.9 \mathrm{E}+05$ & $1.9 \mathrm{E}+03$ & & & & & $2.0 \mathrm{E}+04$ & $2.0 \mathrm{E}+02$ & & & & & & & $2.0 \mathrm{E}+04$ & $2.0 \mathrm{E}+02$ & & \\
\hline Trimethylethyl Lead & $001762-26-1$ & & & & & & & & & & & & & & & & & & \\
\hline Trinitrobenzene, 1,3,5. & $000099-35-4$ & & & $4.4 E+02$ & $4.1 \mathrm{E}+03$ & $4.1 \mathrm{E}+02$ & 4.4E+01 & & & 2.1E+02 & $2.1 \mathrm{E}+01$ & & & & & & & $2.0 \mathrm{E}+02$ & $2.0 \mathrm{E}+01$ \\
\hline $\begin{array}{l}\text { Trinitrophenylmethylnit } \\
\text { ramine }\end{array}$ & $000479-45-8$ & & & $8.8 \mathrm{E}+04$ & 8.2E+05 & 8.2E+04 & $8.8 \mathrm{E}+03$ & & & $3.2 \mathrm{E}+04$ & $3.2 \mathrm{E}+03$ & & & & & & & $3.1 \mathrm{E}+04$ & $3.1 \mathrm{E}+03$ \\
\hline Trinitrotoluene, 2,4,6- & $000118-96-7$ & 2.4E+05 & $2.4 E+03$ & $4.4 \mathrm{E}+03$ & 4.1E+04 & $4.1 \mathrm{E}+03$ & $4.4 E+02$ & $3.0 \mathrm{E}+04$ & $3.0 \mathrm{E}+02$ & $1.9 E+03$ & $1.9 \mathrm{E}+02$ & & & & & $2.9 E+04$ & $2.9 E+02$ & $1.8 \mathrm{E}+03$ & $1.8 \mathrm{E}+02$ \\
\hline Tripropyl Lead & $006518-03-7$ & & & & & & & & & & & & & & & & & & \\
\hline $\begin{array}{l}\text { Uranium (Soluble } \\
\text { Salts) }\end{array}$ & NA & & & $2.6 \mathrm{E}+0.4$ & 2.5E+05 & $2.5 \mathrm{E}+04$ & $2.6 \mathrm{E}+03$ & & & $1.6 \mathrm{E}+05$ & $1.6 \mathrm{E}+04$ & & & & & & & $9.8 \mathrm{E}+04$ & $9.8 \mathrm{E}+03$ \\
\hline Vanadium Pentoxide & $001314-62-1$ & & & $7.9 \mathrm{E}+04$ & 7.4E+05 & 7.4E+04 & $7.9 E+03$ & & & $1.2 \mathrm{E}+05$ & $1.2 \mathrm{E}+04$ & & & & & & & $1.0 \mathrm{E}+05$ & $1.0 E+04$ \\
\hline Vanadium Sulfate & $036907-42-3$ & & & $1.8 E+05$ & $1.0 \mathrm{E}+06$ & $1.6 \mathrm{E}+05$ & $1.8 \mathrm{E}+04$ & & & $2.6 \mathrm{E}+05$ & $2.6 \mathrm{E}+04$ & & & & & & & $2.2 \mathrm{E}+05$ & $2.2 \mathrm{E}+04$ \\
\hline Vanadium, Metallic & $007440.62-2$ & & & $6.1 \mathrm{E}+04$ & $5.7 \mathrm{E}+05$ & $5.7 \mathrm{E}+04$ & $6.1 \mathrm{E}+03$ & & & $4.5 E+03$ & 4.SE+02 & & & & & & & $4.5 \mathrm{E}+03$ & $4.5 \mathrm{E}+02$ \\
\hline Vanadyl Sulfate & $027774-13-6$ & & & $1.8 \mathrm{E}+05$ & $1.0 E+06$ & $1.6 \mathrm{E}+0 \mathrm{~S}$ & $1.8 E+04$ & & & $2.6 \mathrm{E}+0 \mathrm{~S}$ & $2.6 E+04$ & & & & & & & $2.2 E+05$ & $2.2 \mathrm{E}+04$ \\
\hline Vernolate & $001929.77-7$ & & & $8.8 \mathrm{E}+03$ & $8.2 \mathrm{E}+04$ & $8.2 \mathrm{E}+03$ & $8.8 \mathrm{E}+02$ & & & $3.2 \mathrm{E}+03$ & $3.2 \mathrm{E}+02$ & & & & & & & $3.1 \mathrm{E}+03$ & $3.1 E+02$ \\
\hline
\end{tabular}


Table 3.4c. (continued)

\begin{tabular}{|c|c|c|c|c|c|c|c|c|c|c|c|c|c|c|c|c|c|c|c|}
\hline \multirow{2}{*}{ Chemical } & \multirow{2}{*}{ CASRN } & \multicolumn{6}{|c|}{ Ingestion (mg/kg) } & \multicolumn{4}{|c|}{ Dermal (mgkg) } & \multicolumn{4}{|c|}{ Inhalation (mg/kg)" } & \multicolumn{4}{|c|}{$\begin{array}{c}\text { Ingestion }+ \text { Dermal + Inhalation } \\
(\mathrm{mg} / \mathrm{kg})^{+\cdot \mathrm{b}}\end{array}$} \\
\hline & & $10^{-4}$ & $10^{-4}$ & $\begin{array}{l}\text { HQ }=1 \\
\text { Child }\end{array}$ & $\begin{array}{l}H Q=1 \\
\text { Adult }\end{array}$ & $\begin{array}{c}\mathrm{HQ}=0.1 \\
\text { Adult }\end{array}$ & $\begin{array}{c}\mathrm{HQ}=0.1 \\
\text { Child }\end{array}$ & $10^{-4}$ & $10^{4}$ & $H Q=1$ & $\mathrm{HQ}=0.1$ & $10^{-4}$ & $10^{5}$ & $H Q=1$ & $H Q=0.1$ & $10^{-4}$ & $10^{4}$ & $\mathrm{HI}=1$ & $\mathrm{HI}=\mathbf{0 . 1}$ \\
\hline Vinclozolin & $050471-44.8$ & & & $2.2 E+05$ & $1.0 \mathrm{E}+06$ & $2.0 \mathrm{E}+05$ & $2.2 E+04$ & & & 8.0E+04 & $8.0 \mathrm{E}+03$ & & & & & & & $7.7 E+04$ & $7.7 \mathrm{E}+03$ \\
\hline Vinyl Acetate & $000108-05-4$ & & & $1.0 \mathrm{E}+06$ & $1.0 \mathrm{E}+06$ & $1.0 E+06$ & $8.8 E+05$ & & & $1.0 E+06$ & $4.2 \mathrm{E}+05$ & & & $7.8 E+02$ & $7.8 E+02$ & & & $7.8 E+02$ & $7.8 \mathrm{E}+02$ \\
\hline Vinyl Bromide & $000593-60-2$ & & & & & & & & & & & $4.2 \mathrm{E}+03$ & $1.2 \mathrm{E}+02$ & $4.2 \mathrm{E}+03$ & $4.9 \mathrm{E}+02$ & & & & \\
\hline Vinyl Chloride ${ }^{(n)}$ & $000075-01-4$ & $3.8 \mathrm{E}+03$ & $3.8 \mathrm{E}+01$ & & & $\cdot$ & & $7.9 E+02$ & $7.9 \mathrm{E}+00$ & & & $2.7 E+\infty$ & 2.7E-02 & & & $2.7 E+\infty$ & $2.7 E-02$ & & \\
\hline Warfarin & 000081.81 .2 & & & $2.6 \mathrm{E}+03$ & $2.5 \mathrm{E}+0.4$ & $2.5 \mathrm{E}+03$ & $2.6 \mathrm{E}+02$ & & & $9.6 \mathrm{E}+02$ & $9.6 \mathrm{E}+01$ & & & & & & & $9.3 E+02$ & $9.3 E+01$ \\
\hline White Phosphonus & $007723-14-0$ & & & $1.8 \mathrm{E}+02$ & $1.6 \mathrm{E}+03$ & $1.6 \mathrm{E}+02$ & $1.8 \mathrm{E}+01$ & & & $2.6 \mathrm{E}+02$ & $2.6 \mathrm{E}+01$ & & & & & & & $2.2 \mathrm{E}+02$ & $2.2 E+01$ \\
\hline Xylene, Mixture & $001330-20-7$ & & & $1.0 \mathrm{E}+06$ & $1.0 \mathrm{E}+06$ & $1.0 \mathrm{E}+06$ & $1.0 \mathrm{E}+06$ & & & $1.0 \mathrm{E}+06$ & $1.0 \mathrm{E}+06$ & & & & & & & $1.0 \mathrm{E}+06$ & $1.0 E+06$ \\
\hline Xylene, $P_{-}^{(1)}$ & $000106-42-3$ & & & & & & & & & & & & & & & & & & \\
\hline Xylene, $m^{(6)}$ & $000108-38-3$ & & & $1.0 \mathrm{E}+06$ & $1.0 \mathrm{E}+06$ & $1.0 \mathrm{E}+06$ & $1.0 \mathrm{E}+06$ & & & $1.0 \mathrm{E}+06$ & $1.0 \mathrm{E}+06$ & & & & & & & $1.0 \mathrm{E}+06$ & $9.7 \mathrm{E}+05$ \\
\hline Xylene, o-(1) & $000095-47.6$ & & & $1.0 \mathrm{E}+06$ & $1.0 E+06$ & $1.0 \mathrm{E}+06$ & $1.0 \mathrm{E}+06$ & & & $1.0 \mathrm{E}+06$ & $1.0 \mathrm{E}+06$ & & & & & & & $1.0 \mathrm{E}+06$ & $9.7 E+05$ \\
\hline Zinc (Metallic) & $007440-66-6$ & & & $1.0 \mathrm{E}+06$ & $1.0 \mathrm{E}+06$ & $1.08+06$ & 2.6E+05 & & & $1.0 \mathrm{E}+06$ & $3.9 \mathrm{E}+05$ & & & & & & & $1.0 \mathrm{E}+06$ & $3.3 \mathrm{E}+0.5$ \\
\hline Zine Cyanide & $000557-21-1$ & & & $4.4 \mathrm{E}+05$ & $1.0 \mathrm{E}+06$ & $4.1 \mathrm{E}+05$ & $4.4 E+04$ & & & $6.4 E+05$ & $6.4 \mathrm{E}+04$ & & & & & & & $5.6 \mathrm{E}+05$ & $5.6 \mathrm{E}+04$ \\
\hline Zine Phosphide & $001314-84-7$ & & & $2.6 \mathrm{E}+03$ & $2.5 \mathrm{E}+0.4$ & $2.5 \mathrm{E}+03$ & $2.6 \mathrm{E}+02$ & & & $3.9 \mathrm{E}+03$ & $3.9 \mathrm{E}+02$ & & & & & & & $3.3 \mathrm{E}+03$ & $3.3 E+02$ \\
\hline Zineb & $012122-67-7$ & & & $4.4 E+05$ & $1.0 \mathrm{E}+06$ & 4.1E+05 & $4.4 E+04$ & & & $1.6 \mathrm{E}+05$ & $1.6 \mathrm{E}+04$ & & & & & & & $1.5 \mathrm{E}+05$ & $1.5 E+04$ \\
\hline Zirconium & $007440-67-7$ & & & & & & & & & & & & & & & & & & \\
\hline
\end{tabular}

Notes:

* By definition, $1 \mathrm{~kg}=1.0 \mathrm{E}+06 \mathrm{mg}$. Therefore, a limit of $1.0 \mathrm{E}+06 \mathrm{mg} / \mathrm{kg}$ was used for any PRG that was calculated to be above this limit.

$\dagger$ If slope factors and/or reference doses were available for only one pathway (i.e., ingestion, dermal, OR inhalation), the total for ingestion plus inhalation plus dermal was not calculated.

$¥$ The volatilization factor defines the relationship between the concentration of contaminants in soil and the volatilization of contaminants in air. The chemical concentration in soil (C) calculated using the volatilization factor must be compared with the soil saturation concentration $\left(C_{s u}\right)$. If $C$ is greater than $C_{s t y}$ then the $P R G$ is set equal to $C_{s a t}$.

(1) The same oral slope factor as used for polychlorinated biphenyls.

(2) The PRG was calculated considering Supplemental Guidance from RAGS: Region 4 Bulletins, Human Health Risk Assessment (Interim Guidance) from EPA (received February 11, 1992). The slope factors for chemicals so marked indicate that a toxicity equivalency factor (TEF) method was used for carcinogenic PAHs based on each compound's relative potency to the potency of benzo[a]pyrene. The following TEFs were used to convert each PAH slope factor to an equivalent slope factor of benzo[a]pyrene [that has a new cancer slope factor of 7.3 (mg/kg/day) ${ }^{-1}$ identified by EPA's CRAVE workgroup]: (1) benzo[a]pyrene, TEF $=1.0 ;(2)$ benzo[a]anthracene, TEF $=0.1 ;(3)$ benzo[b]fluoranthene, TEF $=0.1 ;(4)$ benzo[k]fluoranthene, TEF $=0.01$; 


\section{Table 3.4c. (continued)}

(5) chrysene, TEF $=0.001 ;(6)$ dibenz $[a, h]$ anthracene, $\operatorname{TEF}=1.0$; and $(7)$ indeno[ $[1,2,3-c, d]$ pyrene, $\operatorname{TEF}=0.1$

(3) The toxicity values for trichloroethylene have been withdrawn from IRIS and HEAST pending further investigation. However, the most recent toxicity values have been used to calculate the PRG.

(4) The PRG was calculated considering Supplemental Guidance from RAGS: Region 4 Bulletins, Human Health Risk Assessment (Interim Guidance) from EPA (November 1995). The slope factors for chemicals so marked indicate that a TEF methodology was used for chlorinated dioxin and furan congeners based on each compound's relative potency to the potency of 2,3,7,8tetrachlorodibenzodioxin (TCDD). The following TEFs were used to convert each slope factor to an equivalent slope factor of 2,3,7,8-TCDD: (1) 2,3,7,8-TCDD, TEF = 1.0; (2) 2,3,7,8PeCDD, TEF = 0.5; (3) 2,3,7,8-HxCDD, TEF =0.1; (4) 2,3,7,8-HpCDD, TEF =0.01; (5) OCDD, TEF =0.001; (6) 2,3,7,8-TCDF, TEF =0.1; (7) $1,2,3,7,8-\mathrm{PeCDF}, \mathrm{TEF}=0.5 ;(8) 2,3,4,7,8-$ $\mathrm{PeCDF}, \mathrm{TEF}=0.05 ;(9) 2,3,7,8-\mathrm{HxCDF}, \mathrm{TEF}=0.1 ;(10) 2,3,7,8-\mathrm{HpCDF}, \mathrm{TEF}=0.01 ;$ and $(11) \mathrm{OCDF}, \mathrm{TEF}=0.001$.

(5) Toxicity values for these chemicals have been withdrawn from IRIS and HEAST pending further investigation. However, the most recent toxicity values have been used to calculate the PRG.

(6) Toxicity values for these chemicals were obtained from the Superfund Health Risk Technical Support Center. 
Table 3.4d. Risk-based Preliminary Remediation Goals for radionuclides in soil (recreational scenario)

\begin{tabular}{|c|c|c|c|c|c|c|c|c|c|}
\hline \multirow[t]{2}{*}{ Chemical $^{\dagger}$} & \multirow[t]{2}{*}{ CASRN } & \multicolumn{2}{|c|}{$\begin{array}{l}\text { Ingestion } \\
\text { (pCi/g) }\end{array}$} & \multicolumn{2}{|c|}{$\begin{array}{l}\text { Inhalation } \\
\text { (pCi/g) }\end{array}$} & \multicolumn{2}{|c|}{$\begin{array}{l}\text { External Exposure } \\
(\mathrm{pCi} / \mathrm{g})^{+t}\end{array}$} & \multicolumn{2}{|c|}{$\begin{array}{c}\text { Ingestion + Inhalation } \\
\text { + External Exposure } \\
\text { (pCi/g) }^{\text {t+t }}\end{array}$} \\
\hline & & $10^{-4}$ & $10^{-6}$ & $10^{-4}$ & $10^{-6}$ & $10^{-4}$ & $10^{-6}$ & $10^{-4}$ & $10^{-6}$ \\
\hline Ac-225 & $014265-85-1$ & $6.3 E+04$ & $6.3 E+02$ & $2.3 \mathrm{E}+06$ & 2.3E+04 & $6.2 E+04$ & $6.2 \mathrm{E}+02$ & $3.4 \mathrm{E}+04$ & $3.4 \mathrm{E}+02$ \\
\hline Ac-227 & $014952-40-0$ & $2.5 \mathrm{E}+04$ & $2.5 \mathrm{E}+02$ & $1.3 \mathrm{E}+05$ & $1.3 \mathrm{E}+03$ & $2.1 \mathrm{E}+07$ & $2.1 \mathrm{E}+05$ & $3.0 \mathrm{E}+04$ & $3.0 \mathrm{E}+02$ \\
\hline Ac-227+D & $014952-40-0$ & $1.4 \mathrm{E}+04$ & $1.4 \mathrm{E}+02$ & $1.2 \mathrm{E}+05$ & $1.2 \mathrm{E}+03$ & 8.2E+02 & $8.2 \mathrm{E}+00$ & $7.8 \mathrm{E}+02$ & $7.8 \mathrm{E}+00$ \\
\hline Ac-228 & $014331-83-0$ & $5.5 \mathrm{E}+06$ & $5.5 \mathrm{E}+04$ & $2.9 \mathrm{E}+08$ & $2.9 E+06$ & $1.5 \mathrm{E}+02$ & $1.5 \mathrm{E}+00$ & $1.5 \mathrm{E}+02$ & $1.5 \mathrm{E}+00$ \\
\hline Ag-105 & $014928-14-4$ & $5.5 \mathrm{E}+06$ & $5.5 \mathrm{E}+04$ & $4.1 \mathrm{E}+09$ & $4.1 \mathrm{E}+07$ & & & $6.5 \mathrm{E}+06$ & $6.5 \mathrm{E}+04$ \\
\hline $\mathrm{Ag}-108$ & $014391-65-2$ & $1.3 \mathrm{E}+09$ & $1.3 \mathrm{E}+07$ & $1.0 \mathrm{E}+12$ & $1.0 \mathrm{E}+10$ & $8.4 \mathrm{E}+03$ & $8.4 \mathrm{E}+01$ & $8.4 \mathrm{E}+03$ & $8.4 \mathrm{E}+01$ \\
\hline $\mathrm{Ag}-108 \mathrm{~m}$ & $014391-65-2$ & $1.5 \mathrm{E}+06$ & $1.5 \mathrm{E}+04$ & $1.4 \mathrm{E}+08$ & $1.4 \mathrm{E}+06$ & $8.7 \mathrm{E}+01$ & 8.7E-01 & $8.7 E+01$ & 8.7E-01 \\
\hline$A g-108 m+D$ & 014391-65-2 & $1.5 E+06$ & $1.5 \mathrm{E}+04$ & $1.4 \mathrm{E}+08$ & $1.4 \mathrm{E}+06$ & $8.7 \mathrm{E}+01$ & $8.7 \mathrm{E}-01$ & $8.7 \mathrm{E}+01$ & 8.7E-01 \\
\hline $\mathrm{Ag}-109 \mathrm{~m}$ & $014378-38-2$ & $3.3 \mathrm{E}+10$ & $3.3 E+08$ & $2.8 \mathrm{E}+13$ & $2.8 \mathrm{E}+11$ & $4.2 E+05$ & $4.2 \mathrm{E}+03$ & $4.2 E+05$ & $4.2 E+03$ \\
\hline $\mathrm{Ag}-110$ & $014391-76-5$ & $3.6 \mathrm{E}+09$ & $3.6 \mathrm{E}+07$ & $3.0 \mathrm{E}+12$ & $3.0 \mathrm{E}+10$ & $4.3 \mathrm{E}+03$ & $4.3 E+01$ & $4.3 E+03$ & $4.3 E+01$ \\
\hline $\mathrm{Ag}-110 \mathrm{~m}$ & $014391-76-5$ & $1.1 \mathrm{E}+06$ & $1.1 \mathrm{E}+04$ & $3.0 \mathrm{E}+08$ & $3.0 \mathrm{E}+06$ & $4.6 \mathrm{E}+01$ & 4.6E-01 & $4.6 \mathrm{E}+01$ & $4.6 \mathrm{E}-01$ \\
\hline $\mathrm{Ag}-111$ & $015760-04-0$ & $1.3 E+06$ & $1.3 E+04$ & $1.8 \mathrm{E}+09$ & $1.8 \mathrm{E}+07$ & $5.7 \mathrm{E}+03$ & $5.7 \mathrm{E}+01$ & $5.7 \mathrm{E}+03$ & $5.7 \mathrm{E}+01$ \\
\hline Am-241 & $014596-10-2$ & $2.7 \mathrm{E}+04$ & $2.7 \mathrm{E}+02$ & $2.5 \mathrm{E}+05$ & $2.5 \mathrm{E}+03$ & $1.1 \mathrm{E}+05$ & $1.1 \mathrm{E}+03$ & $2.5 \mathrm{E}+04$ & $2.5 E+02$ \\
\hline Am-242 & $013981-54-9$ & $6.0 \mathrm{E}+06$ & $6.0 \mathrm{E}+04$ & $9.2 \mathrm{E}+08$ & $9.2 \mathrm{E}+06$ & $8.4 E+04$ & $8.4 \mathrm{E}+02$ & $8.4 \mathrm{E}+04$ & $8.4 \mathrm{E}+02$ \\
\hline $\mathrm{Am}-242 \mathrm{~m}$ & $013981-54-9$ & $3.0 \mathrm{E}+04$ & $3.0 \mathrm{E}+02$ & 2.7E+05 & $2.7 \mathrm{E}+03$ & $5.6 \mathrm{E}+06$ & $5.6 \mathrm{E}+04$ & $3.6 \mathrm{E}+04$ & $3.6 \mathrm{E}+02$ \\
\hline Am-243 & 014993-75-0 & $2.7 \mathrm{E}+04$ & $2.7 \mathrm{E}+02$ & $2.5 \mathrm{E}+05$ & $2.5 \mathrm{E}+03$ & $2.0 \mathrm{E}+04$ & $2.0 \mathrm{E}+02$ & $1.2 \mathrm{E}+04$ & $1.2 \mathrm{E}+02$ \\
\hline$A m-243+D$ & 014993-75-0 & $2.7 \mathrm{E}+04$ & $2.7 \mathrm{E}+02$ & $2.5 \mathrm{E}+05$ & $2.5 \mathrm{E}+03$ & $1.8 \mathrm{E}+03$ & $1.8 \mathrm{E}+01$ & $1.7 \mathrm{E}+03$ & $1.7 \mathrm{E}+01$ \\
\hline
\end{tabular}




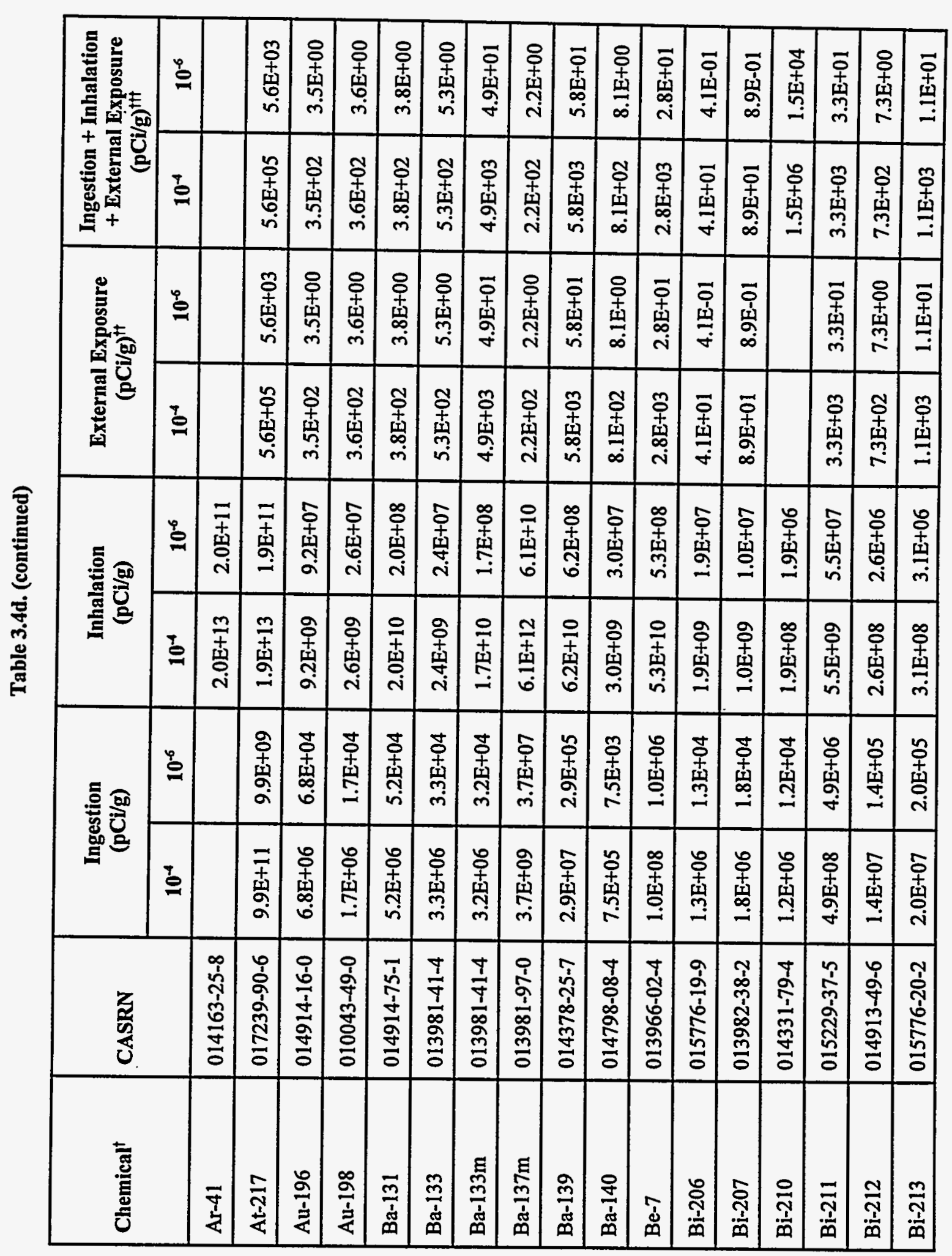

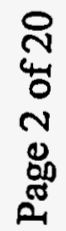


Table 3.4d. (continued)

\begin{tabular}{|c|c|c|c|c|c|c|c|c|c|}
\hline \multirow[t]{2}{*}{ Chemical $^{\dagger}$} & \multirow[t]{2}{*}{ CASRN } & \multicolumn{2}{|c|}{$\begin{array}{l}\text { Ingestion } \\
\text { (pCi/g) }\end{array}$} & \multicolumn{2}{|c|}{$\begin{array}{l}\text { Inhalation } \\
\text { (pCi/g) }\end{array}$} & \multicolumn{2}{|c|}{$\begin{array}{l}\text { External Exposure } \\
\qquad(\mathrm{pCi} / \mathrm{g})^{\dagger+}\end{array}$} & \multicolumn{2}{|c|}{$\begin{array}{c}\text { Ingestion + Inhalation } \\
+ \text { External Exposure } \\
(\text { pCi/g) }\end{array}$} \\
\hline & & $10^{-4}$ & $10^{-6}$ & $10^{-4}$ & $10^{-6}$ & $10^{-4}$ & $10^{-6}$ & $10^{-4}$ & $10^{-6}$ \\
\hline $\mathrm{Bi}-214$ & 014733-03-0 & $4.6 \mathrm{E}+07$ & $4.6 \mathrm{E}+05$ & $6.5 E+08$ & $6.5 \mathrm{E}+06$ & 8.1E+01 & $8.1 \mathrm{E}-01$ & $8.1 E+01$ & 8.1E-01 \\
\hline Br-82 & $014686-69-2$ & $6.3 \mathrm{E}+06$ & $6.3 E+04$ & $1.2 \mathrm{E}+10$ & $1.2 \mathrm{E}+08$ & $4.8 \mathrm{E}+01$ & $4.8 \mathrm{E}-01$ & $4.8 \mathrm{E}+01$ & $4.8 \mathrm{E}-01$ \\
\hline$C-11$ & $014333-33-6$ & $2.0 \mathrm{E}+08$ & $2.0 \mathrm{E}+06$ & $2.8 \mathrm{E}+11$ & $2.8 \mathrm{E}+09$ & $1.3 \mathrm{E}+02$ & $1.3 \mathrm{E}+00$ & $1.3 \mathrm{E}+02$ & $1.3 \mathrm{E}+00$ \\
\hline$C-14$ & $014762-75-5$ & $8.6 \mathrm{E}+06$ & $8.6 \mathrm{E}+04$ & $1.4 \mathrm{E}+12$ & $1.4 \mathrm{E}+10$ & & & $1.0 \mathrm{E}+07$ & $1.0 \mathrm{E}+05$ \\
\hline$C-15$ & $015929-23-4$ & $1.3 \mathrm{E}+10$ & $1.3 E+08$ & $1.2 \mathrm{E}+13$ & $1.2 \mathrm{E}+11$ & & & $1.6 \mathrm{E}+10$ & $1.6 \mathrm{E}+08$ \\
\hline $\mathrm{Ca}-45$ & 013966-05-7 & $4.4 \mathrm{E}+06$ & $4.4 \mathrm{E}+04$ & $3.8 \mathrm{E}+09$ & $3.8 \mathrm{E}+07$ & $1.3 \mathrm{E}+14$ & 1.3E+12 & $5.3 E+06$ & $5.3 E+04$ \\
\hline $\mathrm{Ca}-47$ & 001439-99-2 & $1.3 \mathrm{E}+06$ & $1.3 E+04$ & $1.8 \mathrm{E}+09$ & $1.8 \mathrm{E}+07$ & $1.2 \mathrm{E}+02$ & $1.2 \mathrm{E}+00$ & $1.2 \mathrm{E}+02$ & $1.2 \mathrm{E}+00$ \\
\hline Cd-109 & 014109-32-1 & $1.1 \mathrm{E}+06$ & $1.1 \mathrm{E}+04$ & $5.1 \mathrm{E}+08$ & $5.1 \mathrm{E}+06$ & $8.7 \mathrm{E}+05$ & 8.7E+03 & $5.2 \mathrm{E}+05$ & $5.2 \mathrm{E}+03$ \\
\hline Cd-115 & $014336-68-6$ & $1.2 \mathrm{E}+06$ & $1.2 \mathrm{E}+04$ & $1.9 \mathrm{E}+09$ & $1.9 \mathrm{E}+07$ & $6.9 \mathrm{E}+02$ & $6.9 \mathrm{E}+00$ & $6.9 \mathrm{E}+02$ & $6.9 \mathrm{E}+00$ \\
\hline Cd-115m & 014336-68-6 & $6.3 E+05$ & $6.3 \mathrm{E}+03$ & $5.6 \mathrm{E}+08$ & $5.6 \mathrm{E}+06$ & $5.7 \mathrm{E}+03$ & $5.7 \mathrm{E}+01$ & $5.6 \mathrm{E}+03$ & $5.6 \mathrm{E}+01$ \\
\hline $\mathrm{Ce}-141$ & $013967-74-3$ & $2.3 E+06$ & $2.3 E+04$ & $2.2 \mathrm{E}+09$ & $2.2 \mathrm{E}+07$ & $3.5 \mathrm{E}+03$ & $3.5 \mathrm{E}+01$ & $3.4 \mathrm{E}+03$ & $3.4 \mathrm{E}+01$ \\
\hline Ce-143 & $014119-19-8$ & $1.5 \mathrm{E}+06$ & $1.5 \mathrm{E}+04$ & $2.5 \mathrm{E}+09$ & $2.5 \mathrm{E}+07$ & $6.6 \mathrm{E}+02$ & $6.6 \mathrm{E}+00$ & $6.6 \mathrm{E}+02$ & $6.6 \mathrm{E}+00$ \\
\hline Ce-144 & 014762-78-8 & $3.0 \mathrm{E}+05$ & $3.0 \mathrm{E}+03$ & $8.8 \mathrm{E}+07$ & $8.8 \mathrm{E}+05$ & $1.9 \mathrm{E}+04$ & $1.9 \mathrm{E}+02$ & $1.8 \mathrm{E}+04$ & $1.8 \mathrm{E}+02$ \\
\hline Ce-144+D & $014762-78-8$ & $3.0 \mathrm{E}+05$ & $3.0 \mathrm{E}+03$ & $8.8 \mathrm{E}+07$ & $8.8 \mathrm{E}+05$ & $3.1 \mathrm{E}+03$ & $3.1 \mathrm{E}+01$ & $3.1 E+03$ & $3.1 \mathrm{E}+01$ \\
\hline $\mathrm{Cl}-36$ & 013981-43-6 & $4.0 \mathrm{E}+06$ & $4.0 \mathrm{E}+04$ & $7.3 E+09$ & $7.3 E+07$ & & & $4.8 \mathrm{E}+06$ & $4.8 \mathrm{E}+04$ \\
\hline Cl-38 & 014158-34-0 & $4.3 E+07$ & $4.3 E+05$ & $5.8 \mathrm{E}+10$ & $5.8 \mathrm{E}+08$ & $7.5 \mathrm{E}+01$ & $7.5 \mathrm{E}-01$ & $7.5 E+01$ & 7.5E-01 \\
\hline $\mathrm{Cm}-242$ & $015510-73-3$ & $2.3 E+05$ & $2.3 E+03$ & $3.0 \mathrm{E}+06$ & $3.0 \mathrm{E}+04$ & $2.1 \mathrm{E}+07$ & $2.1 \mathrm{E}+05$ & $2.7 \mathrm{E}+05$ & $2.7 E+03$ \\
\hline
\end{tabular}




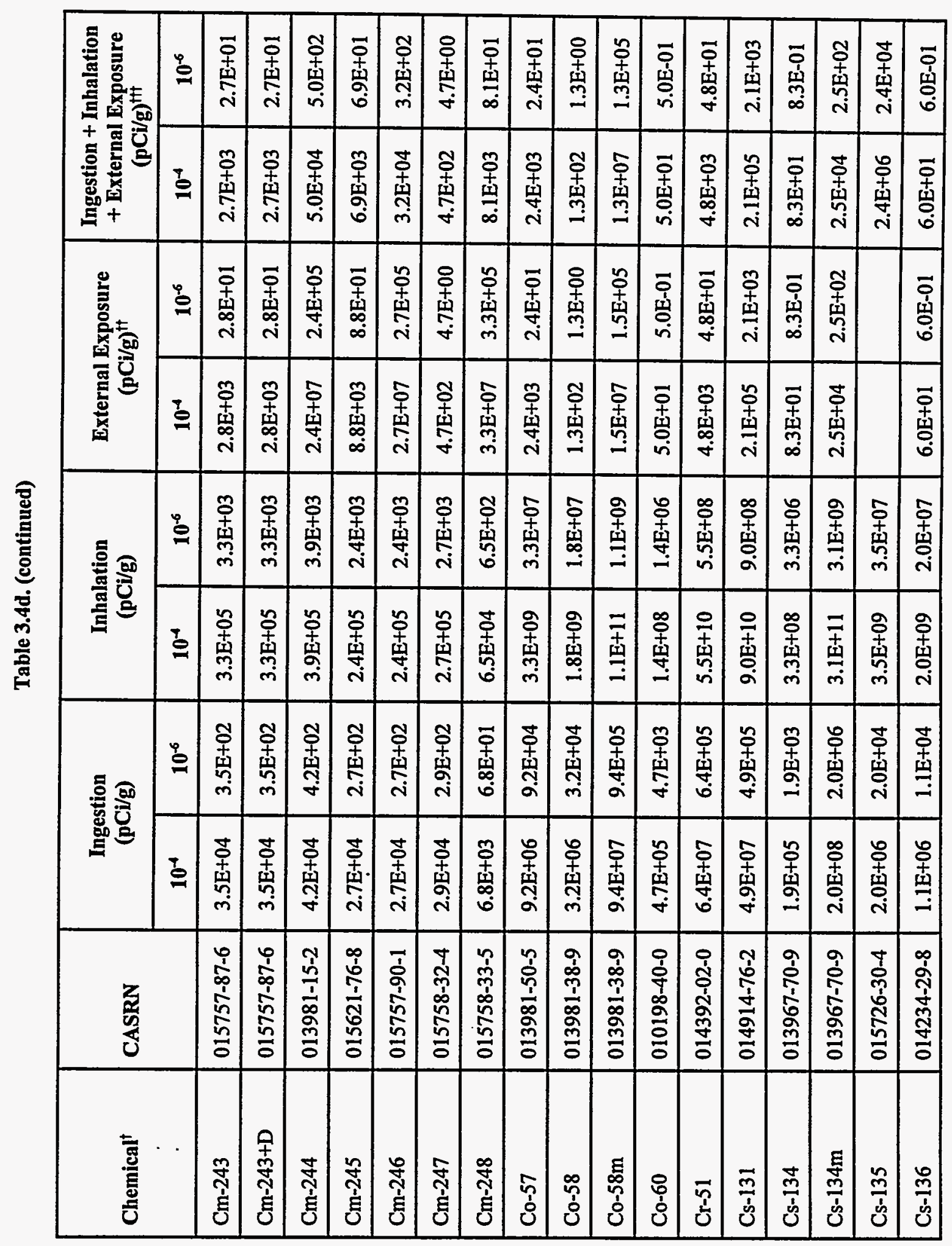

을
4
0
0
0
0
0
0 


\begin{tabular}{|c|c|c|c|c|c|c|c|c|c|c|c|c|c|c|c|c|c|c|}
\hline \multirow{2}{*}{ 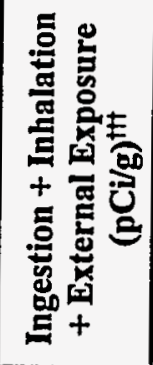 } & $\stackrel{9}{2}$ & $\begin{array}{c}m \\
\stackrel{9}{+} \\
\text { 孚 } \\
\text { m }\end{array}$ & $\begin{array}{l}8 \\
+ \\
\frac{1}{m} \\
\text { ஸे }\end{array}$ & 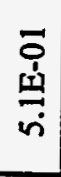 & 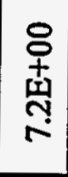 & 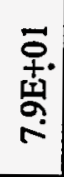 & 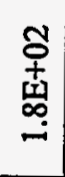 & 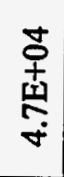 & 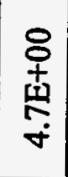 & 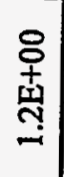 & $\begin{array}{l}8 \\
\text { 市 } \\
\text { 愛 } \\
\end{array}$ & $\begin{array}{l}\overline{0} \\
+ \\
\text { 吉 } \\
\infty\end{array}$ & 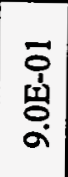 & 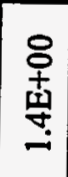 & $\begin{array}{l}n \\
\text { 웅 } \\
\text { 章 } \\
\text { m }\end{array}$ & $\begin{array}{c}8 \\
+ \\
\text { 至 } \\
\\
-\end{array}$ & 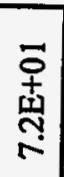 & 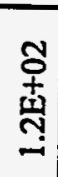 \\
\hline & $\underset{=}{\mathbf{S}}$ & 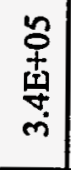 & 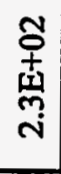 & 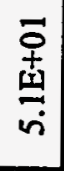 & 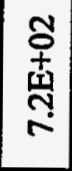 & $\begin{array}{c}m \\
\text { ò } \\
\text { à } \\
\text { à } \\
\end{array}$ & 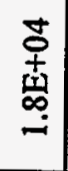 & 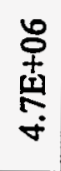 & 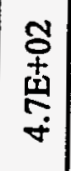 & 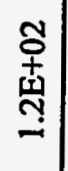 & 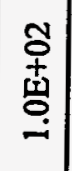 & $\begin{array}{c}m \\
⿱ \\
+9 \\
\text { I } \\
\infty \\
\infty\end{array}$ & $\begin{array}{l}\overrightarrow{0} \\
\text { 妾 } \\
a\end{array}$ & 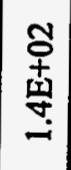 & $\begin{array}{l}5 \\
+ \\
\text { 帘 } \\
\text { m }\end{array}$ & 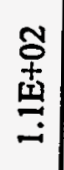 & 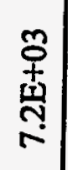 & $\begin{array}{c}\text { ષั } \\
+ \\
\text { 壳 } \\
\end{array}$ \\
\hline \multirow{2}{*}{ 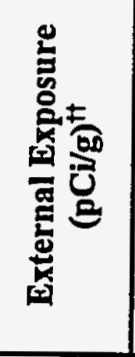 } & $\stackrel{\circ}{0}$ & & 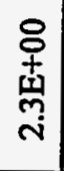 & 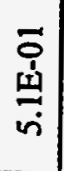 & 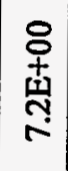 & $\begin{array}{l}\overline{0} \\
\text { + } \\
\text { ড⿳亠口了亍 } \\
\end{array}$ & 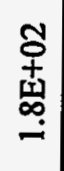 & 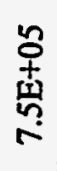 & $\begin{array}{l}8 \\
\stackrel{+}{+} \\
\stackrel{4}{9} \\
\dot{y}\end{array}$ & 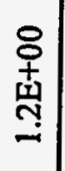 & $\begin{array}{l}8 \\
\text { +⿱ } \\
\text { 高 } \\
-\end{array}$ & $\begin{array}{l}\overline{0} \\
+ \\
0 \\
0 \\
\infty \\
\infty\end{array}$ & 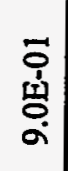 & 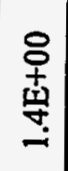 & & 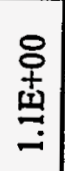 & 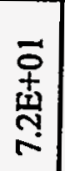 & 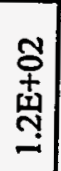 \\
\hline & 을 & & 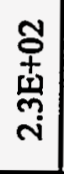 & 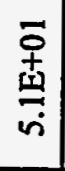 & 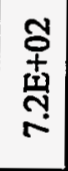 & 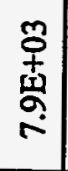 & 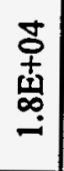 & $\begin{array}{c}5 \\
\vdots \\
5 \\
\\
5\end{array}$ & 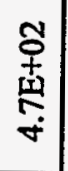 & $\begin{array}{c}\text { ㅇ. } \\
+ \\
\text { 离 } \\
\stackrel{-}{-}\end{array}$ & 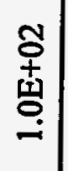 & 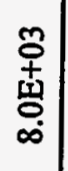 & $\begin{array}{l}\overrightarrow{0} \\
\text { 壱 } \\
\text { a }\end{array}$ & 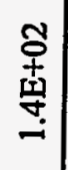 & & 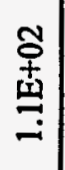 & $\begin{array}{l}\text { o } \\
+ \\
\text { 啇 } \\
\end{array}$ & 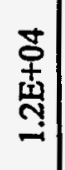 \\
\hline \multirow{2}{*}{ 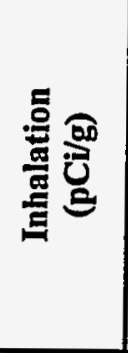 } & $\stackrel{\varphi}{\varrho}$ & 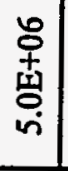 & 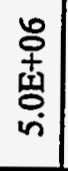 & 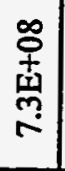 & 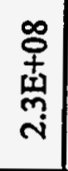 & 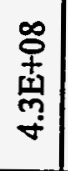 & 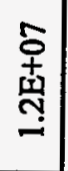 & 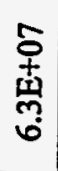 & $\begin{array}{c}\infty \\
\text { 古 } \\
\text { 孚 } \\
\text { m. }\end{array}$ & 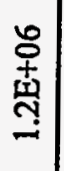 & 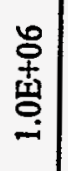 & $\begin{array}{l}\text { oे } \\
\text { 产 } \\
\text { å }\end{array}$ & $\begin{array}{c}5 \\
+1 \\
\text { 몽 } \\
-1\end{array}$ & 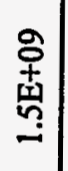 & 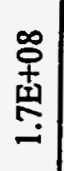 & 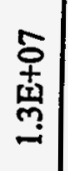 & 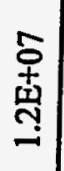 & 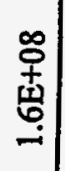 \\
\hline & $\stackrel{t}{\varrho}$ & 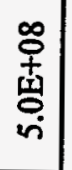 & 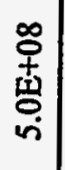 & 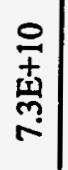 & 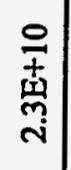 & $\begin{array}{l}\stackrel{9}{+} \\
\stackrel{4}{9} \\
\stackrel{\sim}{\forall}\end{array}$ & $\begin{array}{l}\text { oे } \\
\text { 索 } \\
\text { స్ }\end{array}$ & $\begin{array}{l}\text { g } \\
\text { + } \\
\text { 点 } \\
\text { b }\end{array}$ & $\begin{array}{l}\text { 음 } \\
\text { 盇 } \\
\end{array}$ & 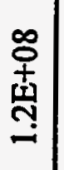 & 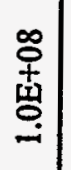 & $\begin{array}{l}\infty \\
\text { 市 } \\
\text { ज्ञ } \\
\text { à }\end{array}$ & 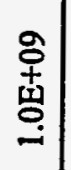 & $\begin{array}{l}\overrightarrow{7} \\
\text { 章 } \\
-\end{array}$ & 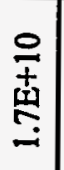 & 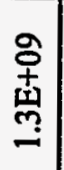 & $\begin{array}{c}\stackrel{8}{+} \\
\text { 壱 } \\
\text { స్} \\
-\end{array}$ & 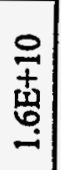 \\
\hline \multirow{2}{*}{ 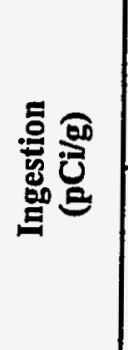 } & $\stackrel{\varphi}{\varrho}$ & 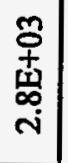 & 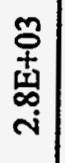 & 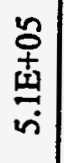 & 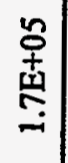 & 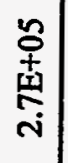 & 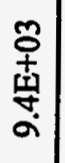 & 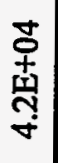 & 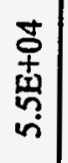 & 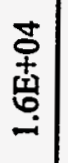 & 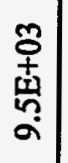 & $\begin{array}{l}\text { 夲 } \\
\text { 索 } \\
\text { ci }\end{array}$ & 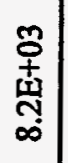 & 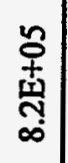 & 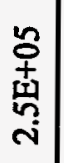 & 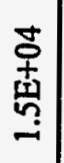 & 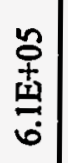 & 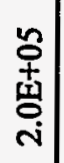 \\
\hline & $\stackrel{7}{g}$ & $\begin{array}{l}\text { n } \\
+ \\
+ \\
\infty \\
\infty \\
\text { i }\end{array}$ & $\begin{array}{l}n \\
o \\
+ \\
\text { 昰 } \\
\text { i }\end{array}$ & $\begin{array}{c}5 \\
\text { 畐 } \\
\text { ci }\end{array}$ & 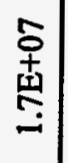 & $\begin{array}{c}5 \\
+ \\
+ \\
\text { ch } \\
\text { i }\end{array}$ & $\begin{array}{l}n \\
\text { † } \\
\text { w } \\
\text { ă }\end{array}$ & 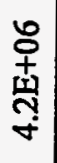 & 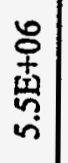 & 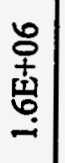 & 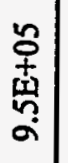 & 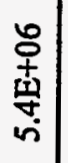 & $\begin{array}{l}n \\
0 \\
+ \\
\text { 壵 } \\
\infty\end{array}$ & $\begin{array}{l}5 \\
+ \\
\text { 吉 } \\
\infty\end{array}$ & $\begin{array}{l}\hat{0} \\
+ \\
\text { ân. } \\
\text { in }\end{array}$ & $\begin{array}{l}\text { o } \\
+ \\
\text { ț } \\
\end{array}$ & 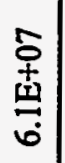 & $\begin{array}{l}\text { 우 } \\
\text { 㕝 } \\
\text { ㄱ }\end{array}$ \\
\hline 丞 & & 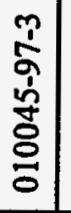 & 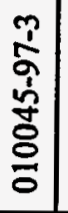 & 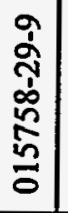 & 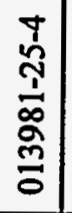 & 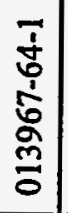 & 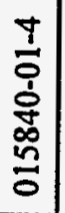 & 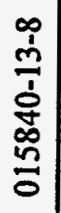 & 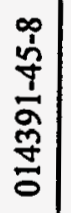 & 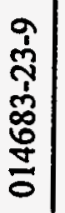 & 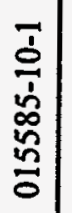 & $\begin{array}{l}\frac{n}{b} \\
\frac{1}{\dot{a}} \\
\frac{\tilde{g}}{\sigma} \\
\frac{3}{0}\end{array}$ & 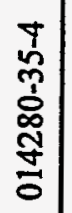 & 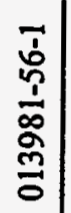 & 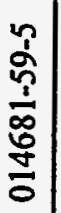 & 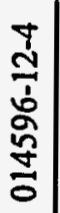 & 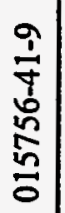 & 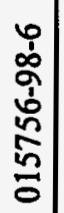 \\
\hline 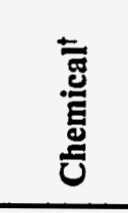 & & $\frac{\hat{m}}{\dot{v}}$ & $\frac{a}{\frac{1}{m}}$ & $\frac{\infty}{m}$ & $\begin{array}{l}\text { ষ্口 } \\
\stackrel{1}{3} \\
\end{array}$ & 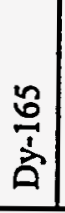 & $\begin{array}{l}8 \\
\frac{1}{2} \\
\frac{1}{0}\end{array}$ & $\frac{8}{\frac{1}{1}}$ & $\frac{\mathbb{1}}{\frac{1}{4}}$ & $\frac{\widetilde{N}}{\grave{1}}$ & 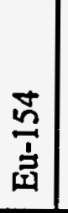 & $\frac{n}{2}$ & $\begin{array}{l}\stackrel{0}{2} \\
\text { 岂 }\end{array}$ & & & ฉి & సָ & 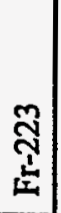 \\
\hline
\end{tabular}


Table 3.4d. (continued)

\begin{tabular}{|c|c|c|c|c|c|c|c|c|c|}
\hline \multirow[t]{2}{*}{ Chemical $^{\dagger}$} & \multirow[t]{2}{*}{ CASRN } & \multicolumn{2}{|c|}{$\begin{array}{l}\text { Ingestion } \\
\text { (pCi/g) }\end{array}$} & \multicolumn{2}{|c|}{$\begin{array}{l}\text { Inhalation } \\
\text { (pCi/g) }\end{array}$} & \multicolumn{2}{|c|}{$\begin{array}{l}\text { External Exposure } \\
\qquad(\mathrm{pCi} / \mathrm{g})^{++}\end{array}$} & \multicolumn{2}{|c|}{$\begin{array}{l}\text { Ingestion + Inhalation } \\
+ \text { External Exposure } \\
(\text { pCi/g) })^{++t}\end{array}$} \\
\hline & & $10^{-4}$ & $10^{-6}$ & $10^{-4}$ & $10^{-6}$ & $10^{-4}$ & $10^{-6}$ & $10^{-4}$ & $10^{-6}$ \\
\hline $\mathrm{Ga}-67$ & $014119-09-6$ & $1.1 \mathrm{E}+07$ & $1.1 \mathrm{E}+05$ & $1.9 \mathrm{E}+10$ & $1.9 E+08$ & $1.3 \mathrm{E}+03$ & $1.3 E+01$ & $1.3 \mathrm{E}+03$ & $1.3 \mathrm{E}+01$ \\
\hline Ga-72 & $013982-22-4$ & $1.9 \mathrm{E}+06$ & $1.9 \mathrm{E}+04$ & $4.4 \mathrm{E}+09$ & $4.4 \mathrm{E}+07$ & $4.3 \mathrm{E}+01$ & 4.3E- 01 & $4.3 \mathrm{E}+01$ & $4.3 \mathrm{E}-01$ \\
\hline Gd-153 & $014276-65-4$ & $6.7 \mathrm{E}+06$ & $6.7 E+04$ & $3.0 \mathrm{E}+09$ & $3.0 \mathrm{E}+07$ & $6.7 \mathrm{E}+03$ & $6.7 \mathrm{E}+01$ & $6.7 \mathrm{E}+03$ & $6.7 \mathrm{E}+01$ \\
\hline Gd-159 & $014041-42-0$ & $3.4 \mathrm{E}+06$ & $3.4 \mathrm{E}+04$ & $7.7 \mathrm{E}+09$ & $7.7 \mathrm{E}+07$ & $5.1 \mathrm{E}+03$ & $5.1 \mathrm{E}+01$ & $5.1 \mathrm{E}+03$ & $5.1 \mathrm{E}+01$ \\
\hline $\mathrm{Ge}-71$ & $014374-81-3$ & $7.5 \mathrm{E}+08$ & $7.5 \mathrm{E}+06$ & $1.6 \mathrm{E}+11$ & $1.6 \mathrm{E}+09$ & $3.1 \mathrm{E}+07$ & $3.1 E+05$ & $3.0 \mathrm{E}+07$ & $3.0 \mathrm{E}+05$ \\
\hline $\mathrm{H}-3$ & 010028-17-8 & $1.2 \mathrm{E}+08$ & $1.2 \mathrm{E}+06$ & $9.9 \mathrm{E}+10$ & $9.9 \mathrm{E}+08$ & & & $1.5 E+08$ & $1.5 \mathrm{E}+06$ \\
\hline $\mathrm{Hg}-197$ & 013981-51-6 & $7.5 \mathrm{E}+06$ & $7.5 \mathrm{E}+04$ & $1.4 \mathrm{E}+10$ & $1.4 \mathrm{E}+08$ & $8.9 \mathrm{E}+03$ & $8.9 \mathrm{E}+01$ & $8.9 \mathrm{E}+03$ & $8.9 E+01$ \\
\hline $\mathrm{Hg}-203$ & 013982-78-0 & $3.4 \mathrm{E}+06$ & $3.4 \mathrm{E}+04$ & $3.1 \mathrm{E}+09$ & $3.1 \mathrm{E}+07$ & $7.8 \mathrm{E}+02$ & $7.8 \mathrm{E}+00$ & $7.8 \mathrm{E}+02$ & $7.8 \mathrm{E}+00$ \\
\hline Ho-166 & $013967-65-2$ & $1.2 E+06$ & $1.2 \mathrm{E}+04$ & $2.3 \mathrm{E}+09$ & $2.3 \mathrm{E}+07$ & $7.0 \mathrm{E}+03$ & $7.0 \mathrm{E}+01$ & $7.0 \mathrm{E}+03$ & $7.0 \mathrm{E}+01$ \\
\hline I-122 & $018287-75-7$ & $4.1 \mathrm{E}+08$ & $4.1 \mathrm{E}+06$ & $4.3 E+11$ & $4.3 \mathrm{E}+09$ & $1.4 \mathrm{E}+02$ & $1.4 \mathrm{E}+00$ & $1.4 \mathrm{E}+02$ & $1.4 \mathrm{E}+00$ \\
\hline I-123 & 015715-08-9 & $1.6 \mathrm{E}+07$ & $1.6 \mathrm{E}+05$ & $3.2 \mathrm{E}+10$ & $3.2 \mathrm{E}+08$ & $1.9 E+03$ & $1.9 \mathrm{E}+01$ & $1.9 \mathrm{E}+03$ & $1.9 \mathrm{E}+01$ \\
\hline I-125 & $014158-31-7$ & $3.4 \mathrm{E}+05$ & $3.4 \mathrm{E}+03$ & $5.6 \mathrm{E}+08$ & $5.6 \mathrm{E}+06$ & $2.0 \mathrm{E}+05$ & $2.0 \mathrm{E}+03$ & $1.4 \mathrm{E}+05$ & $1.4 \mathrm{E}+03$ \\
\hline I-126 & $014158-32-8$ & $1.8 \mathrm{E}+05$ & $1.8 \mathrm{E}+03$ & $3.0 \mathrm{E}+08$ & $3.0 \mathrm{E}+06$ & $3.3 E+02$ & $3.3 E+00$ & $3.3 \mathrm{E}+02$ & $3.3 E+00$ \\
\hline I-129 & 015046-84-1 & $4.8 \mathrm{E}+04$ & $4.8 \mathrm{E}+02$ & $7.8 \mathrm{E}+07$ & $7.8 \mathrm{E}+05$ & $1.8 \mathrm{E}+05$ & $1.8 \mathrm{E}+03$ & $4.4 \mathrm{E}+04$ & $4.4 \mathrm{E}+02$ \\
\hline $\mathrm{I}-130$ & $014914-02-4$ & $1.8 \mathrm{E}+06$ & $1.8 \mathrm{E}+04$ & $3.6 \mathrm{E}+09$ & $3.6 \mathrm{E}+07$ & $6.1 \mathrm{E}+01$ & $6.1 \mathrm{E}-01$ & $6.1 \mathrm{E}+01$ & $6.1 \mathrm{E}-01$ \\
\hline $\mathrm{I}-131$ & $010043-66-0$ & $2.5 \mathrm{E}+05$ & $2.5 \mathrm{E}+03$ & $4.1 E+08$ & 4.1E+06 & $3.9 E+02$ & $3.9 \mathrm{E}+00$ & $3.9 \mathrm{E}+02$ & $3.9 \mathrm{E}+00$ \\
\hline $\mathrm{I}-132$ & 014683-16-0 & $1.3 \mathrm{E}+07$ & $1.3 E+05$ & $2.7 \mathrm{E}+10$ & $2.7 \mathrm{E}+08$ & $5.6 \mathrm{E}+01$ & $5.6 \mathrm{E}-01$ & $5.6 \mathrm{E}+01$ & $5.6 \mathrm{E}-01$ \\
\hline
\end{tabular}




\begin{tabular}{|c|c|c|c|c|c|c|c|c|c|c|c|c|c|c|c|c|c|c|}
\hline \multirow{2}{*}{ 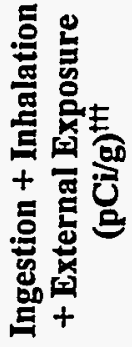 } & $\stackrel{\varphi}{\varrho}$ & 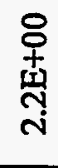 & $\begin{array}{l}\overline{0} \\
\dot{1} \\
\infty \\
\dot{0} \\
\dot{\forall}\end{array}$ & $\begin{array}{l}\overrightarrow{0} \\
0 \\
0 \\
0 \\
0\end{array}$ & 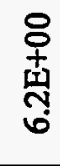 & 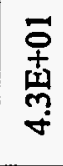 & $\begin{array}{l}\text { D } \\
+ \\
\text { i } \\
\text { i }\end{array}$ & m & $\begin{array}{l}\text { 울 } \\
\text { 恶 }\end{array}$ & $\begin{array}{l}\text { 용 } \\
\text { 索 } \\
\text { ․ }\end{array}$ & $\begin{array}{l}\overline{0} \\
+ \\
\text { 站 } \\
\stackrel{-}{-}\end{array}$ & $\begin{array}{l}8 \\
+ \\
+ \\
\text { 音 } \\
\infty\end{array}$ & $\begin{array}{l}8 \\
\text { +⿱ } \\
\text { 虫 } \\
\text { † }\end{array}$ & & & & & \\
\hline & $\stackrel{5}{=}$ & 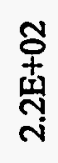 & $\begin{array}{l}\tilde{0} \\
+ \\
\text { 章 } \\
\dot{+}\end{array}$ & 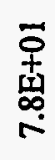 & 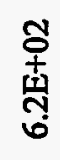 & $\begin{array}{l}\text { o } \\
+ \\
\text { 亲 } \\
m\end{array}$ & 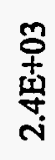 & 夯 & 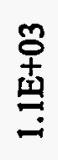 & \begin{tabular}{l}
\multirow{0}{0}{} \\
+ \\
옹
\end{tabular} & 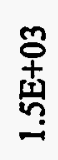 & \begin{tabular}{l}
\multirow{O}{0}{} \\
+ \\
몽 \\
$\infty$
\end{tabular} & 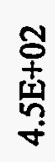 & & & & & \\
\hline \multirow{2}{*}{ 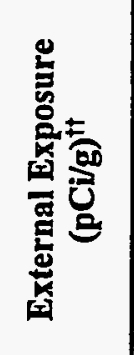 } & 웅 & 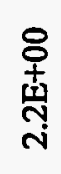 & $\begin{array}{l}\text { 官 } \\
\text { 离 } \\
\dot{+}\end{array}$ & 审 & $\begin{array}{l}8 \\
+ \\
+ \\
\text { ป్ } \\
6\end{array}$ & $\begin{array}{l}\overline{0} \\
+ \\
\text { 焉 }\end{array}$ & $\begin{array}{l}\overline{8} \\
+ \\
\text { 穼 } \\
\text { ণ }\end{array}$ & & $\begin{array}{l}\overrightarrow{0} \\
+1 \\
\text { 孚 } \\
-\end{array}$ & $\begin{array}{l}\text { 웅 } \\
\text { 点 } \\
\text { ․ }\end{array}$ & $\begin{array}{l}\text { 号 } \\
\text { 站 } \\
-\end{array}$ & $\begin{array}{l}8 \\
+ \\
1 \\
\text { 章 } \\
\infty\end{array}$ & 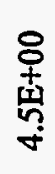 & & & & & \\
\hline & 흥 & 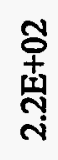 & $\begin{array}{l}\overrightarrow{0} \\
+ \\
\text { ज्ञ } \\
\infty \\
\dot{y}\end{array}$ & 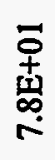 & 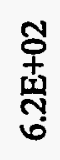 & 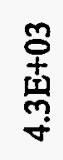 & $\begin{array}{c}m \\
+ \\
+1 \\
\text { 劣 } \\
\text { i }\end{array}$ & & 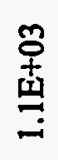 & 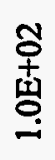 & 年 & 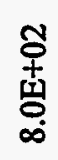 & 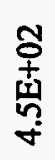 & & & & & \\
\hline \multirow{2}{*}{ 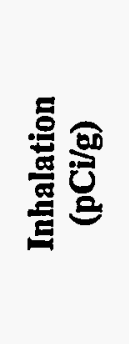 } & $\stackrel{9}{9}$ & 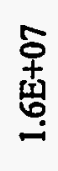 & 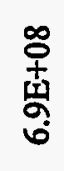 & 点 & 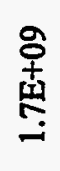 & $\frac{0}{\frac{1}{0}}$ & $\begin{array}{l}0 \\
⿱ 亠 \\
+1 \\
\infty \\
0 \\
\dot{0}\end{array}$ & $\begin{array}{l}\text { n } \\
+1 \\
\text { 帘 } \\
+\end{array}$ & $\begin{array}{l}\infty \\
0 \\
+ \\
\text { 孚 } \\
\text { ம் }\end{array}$ & $\frac{5}{\vdots}$ & 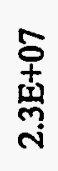 & $\begin{array}{c}5 \\
0 \\
\text { !n } \\
m\end{array}$ & 号 & $\underset{\text { in }}{\stackrel{+}{+}}$ & $\begin{array}{l}\not{F} \\
\dot{+1} \\
m \\
m\end{array}$ & 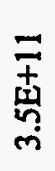 & $\frac{0}{\frac{1}{4}}$ & $\begin{array}{l}\stackrel{0}{7} \\
\text { 点 } \\
\text { \% }\end{array}$ \\
\hline & $\stackrel{\square}{\underline{\theta}}$ & 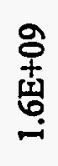 & $\begin{array}{l}\frac{0}{4} \\
\text { 章 } \\
\text { 6 }\end{array}$ & $\underset{\infty}{\stackrel{9}{+}}$ & 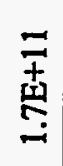 & 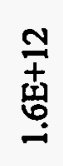 & $\begin{array}{l}\infty \\
0 \\
+ \\
\text { ty } \\
\infty \\
\text { r }\end{array}$ & $\begin{array}{l}5 \\
\text { 采 } \\
\text { 。 } \\
\dot{8}\end{array}$ & $\begin{array}{c}0 \\
+ \\
\dot{+} \\
\dot{q} \\
\dot{v}\end{array}$ & $\frac{g}{o}$ & $\begin{array}{l}\text { g } \\
\text { 章 } \\
\text { ñ } \\
\text { vis }\end{array}$ & $\begin{array}{l}g \\
+ \\
+ \\
\text { m } \\
\text { m }\end{array}$ & $\begin{array}{l}\frac{0}{ \pm} \\
\text { 章 }\end{array}$ & 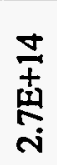 & $\begin{array}{l}m \\
\dot{m} \\
m \\
m \\
m\end{array}$ & $\begin{array}{l}\frac{m}{7} \\
\frac{1}{n} \\
n \\
n\end{array}$ & 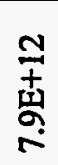 & 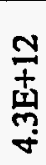 \\
\hline \multirow{2}{*}{ 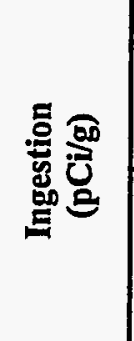 } & $\stackrel{9}{9}$ & 章 & $\begin{array}{l}n \\
\text { 呆 } \\
\text { nd } \\
\text { m }\end{array}$ & 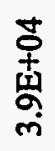 & 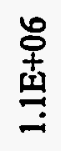 & $\begin{array}{l}5 \\
\text { ㅁ } \\
\text { 咅 } \\
\text { i }\end{array}$ & 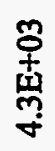 & 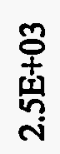 & $\begin{array}{l}\text { o } \\
+1 \\
\text { 吾 } \\
\text { n }\end{array}$ & 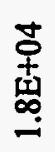 & 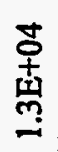 & $\underset{r}{\stackrel{n}{+}}$ & 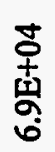 & & & & & \\
\hline & b & $\underset{\infty}{\infty}$ & $\begin{array}{l}5 \\
+ \\
+ \\
\infty \\
\infty \\
\text { m }\end{array}$ & $\begin{array}{l}0 \\
\text { †े } \\
\text { ⿷匚⿳丨コ丨 } \\
\text { ले }\end{array}$ & 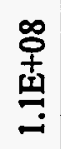 & $\begin{array}{c}9 \\
\stackrel{+}{+} \\
\text { 咅 } \\
\text { ci }\end{array}$ & 号 & 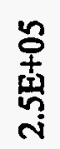 & $\begin{array}{l}\text { 웅 } \\
\text { in } \\
\text { i }\end{array}$ & 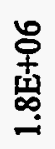 & 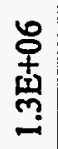 & 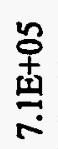 & $\begin{array}{l}\text { 옹 } \\
\text { 愛 } \\
\text { రీ }\end{array}$ & & & & & \\
\hline$\frac{3}{5}$ & & 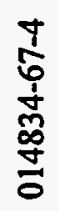 & $\frac{n}{\frac{n}{7}}$ & 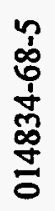 & $\begin{array}{l}0 \\
\infty \\
\infty \\
1 \\
1 \\
\infty \\
\infty \\
\pm \\
0\end{array}$ & 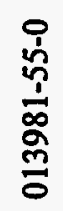 & 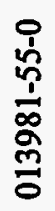 & $\frac{\frac{9}{1}}{\frac{1}{9}}$ & $\frac{\frac{0}{1}}{\frac{1}{\sigma}}$ & $\frac{\frac{0}{1}}{\frac{1}{0}}$ & $\begin{array}{l}\overrightarrow{\dot{s}} \\
\stackrel{\infty}{\infty} \\
\frac{\dot{m}}{\sigma}\end{array}$ & 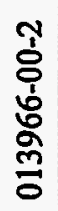 & 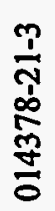 & 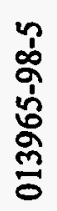 & 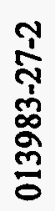 & 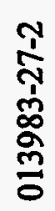 & 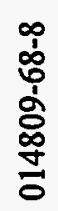 & $\begin{array}{l}\frac{0}{1} \\
\text { L } \\
\frac{1}{2} \\
\frac{\delta}{0}\end{array}$ \\
\hline 莺 & & $\stackrel{m}{m}$ & $\frac{⿱ 亠 凶}{ \pm}$ & $\stackrel{n}{\frac{n}{a}}$ & $\underset{⿱ 乛}{\stackrel{\Xi}{\Xi}}$ & $\stackrel{ \pm}{\Xi}$ & 息 & $\stackrel{\curvearrowleft}{\Xi}$ & 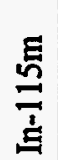 & $\frac{8}{\frac{8}{2}}$ & $\frac{8}{2}$ & $\frac{9}{4}$ & $\underset{y}{Y}$ & 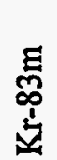 & 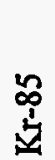 & 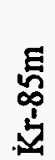 & \$) & 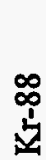 \\
\hline
\end{tabular}


Table 3.4d. (continued)

\begin{tabular}{|c|c|c|c|c|c|c|c|c|c|}
\hline \multirow[t]{2}{*}{ Chemical $^{\dagger}$} & \multirow[t]{2}{*}{ CASRN } & \multicolumn{2}{|c|}{$\begin{array}{c}\text { Ingestion } \\
\text { (pCi/g) }\end{array}$} & \multicolumn{2}{|c|}{$\begin{array}{l}\text { Inhalation } \\
\text { (pCi/g) }\end{array}$} & \multicolumn{2}{|c|}{$\begin{array}{l}\text { External Exposure } \\
(\mathrm{pCi} / \mathrm{g})^{+\dagger}\end{array}$} & \multicolumn{2}{|c|}{$\begin{array}{c}\text { Ingestion + Inhalation } \\
+ \text { External Exposure } \\
(\mathrm{pCi} / \mathrm{g})^{++1}\end{array}$} \\
\hline & & $10^{-4}$ & $10^{-6}$ & $10^{-4}$ & $10^{-6}$ & $10^{-4}$ & $10^{-6}$ & $10^{-4}$ & $10^{-6}$ \\
\hline Kr-89 & $016316-03-3$ & & & $5.9 \mathrm{E}+12$ & $5.9 \mathrm{E}+10$ & & & & \\
\hline $\mathrm{Kr}-90$ & 015741-13-6 & & & $6.0 \mathrm{E}+12$ & $6.0 \mathrm{E}+10$ & & & & \\
\hline La-140 & $013981-28-7$ & 9.4E+05 & $9.4 \mathrm{E}+03$ & $1.9 \mathrm{E}+09$ & $1.9 \mathrm{E}+07$ & $5.3 \mathrm{E}+01$ & $5.3 \mathrm{E}-01$ & $5.3 \mathrm{E}+01$ & $5.3 \mathrm{E}-01$ \\
\hline Lu-177 & $014265-75-9$ & $3.0 \mathrm{E}+06$ & $3.0 \mathrm{E}+04$ & $4.3 \mathrm{E}+09$ & $4.3 \mathrm{E}+07$ & $6.7 \mathrm{E}+03$ & $6.7 \mathrm{E}+01$ & $6.7 \mathrm{E}+03$ & $6.7 E+01$ \\
\hline $\mathrm{Mn}-52$ & 014092-99-0 & $1.5 \mathrm{E}+06$ & $1.5 E+04$ & $2.2 \mathrm{E}+09$ & $2.2 E+07$ & $3.6 \mathrm{E}+01$ & $3.6 \mathrm{E}-01$ & $3.6 \mathrm{E}+01$ & $3.6 \mathrm{E}-01$ \\
\hline $\mathrm{Mn}-54$ & 013966-31-9 & $4.5 E+06$ & $4.5 \mathrm{E}+04$ & $2.6 \mathrm{E}+09$ & $2.6 \mathrm{E}+07$ & $1.5 \mathrm{E}+02$ & $1.5 \mathrm{E}+00$ & $1.5 \mathrm{E}+02$ & $1.5 \mathrm{E}+00$ \\
\hline $\mathrm{Mn}-56$ & $014681-52-8$ & $1.0 \mathrm{E}+07$ & $1.0 \mathrm{E}+05$ & $1.8 \mathrm{E}+10$ & $1.8 \mathrm{E}+08$ & $7.0 \mathrm{E}+01$ & $7.0 \mathrm{E}-01$ & $7.0 \mathrm{E}+01$ & $7.0 \mathrm{E}-01$ \\
\hline Mo-99 & 014119-15-4 & $3.9 E+06$ & $3.9 E+04$ & $2.1 E+09$ & $2.1 \mathrm{E}+07$ & $8.9 \mathrm{E}+02$ & $8.9 \mathrm{E}+00$ & $8.9 E+02$ & $8.9 E+00$ \\
\hline $\mathrm{Na}-22$ & $013966-32-0$ & $1.1 E+06$ & $1.1 \mathrm{E}+04$ & $2.0 \mathrm{E}+09$ & $2.0 \mathrm{E}+07$ & $5.9 \mathrm{E}+01$ & $5.9 \mathrm{E}-01$ & $5.9 \mathrm{E}+01$ & $5.9 \mathrm{E}-01$ \\
\hline $\mathrm{Na}-24$ & 013982-04-2 & $6.4 E+06$ & $6.4 \mathrm{E}+04$ & $1.3 \mathrm{E}+10$ & $1.3 E+08$ & $2.7 \mathrm{E}+01$ & 2.7E-01 & $2.7 \mathrm{E}+01$ & 2.7E-01 \\
\hline Nb-93m & $007440-03-1$ & $1.3 \mathrm{E}+07$ & $1.3 E+05$ & $2.2 \mathrm{E}+09$ & $2.2 \mathrm{E}+07$ & $1.3 \mathrm{E}+07$ & $1.3 E+05$ & $7.3 E+06$ & $7.3 \mathrm{E}+04$ \\
\hline $\mathrm{Nb}-94$ & $014681-63-1$ & $1.3 \mathrm{E}+06$ & $1.3 E+04$ & $1.2 \mathrm{E}+08$ & $1.2 \mathrm{E}+06$ & $8.0 \mathrm{E}+01$ & $8.0 \mathrm{E}-01$ & $8.0 \mathrm{E}+01$ & $8.0 \mathrm{E}-01$ \\
\hline $\mathrm{Nb}-95$ & $013967-76-5$ & $4.0 \mathrm{E}+06$ & $4.0 \mathrm{E}+04$ & $3.1 E+09$ & $3.1 E+07$ & $1.7 \mathrm{E}+02$ & $1.7 \mathrm{E}+00$ & $1.7 \mathrm{E}+02$ & $1.7 \mathrm{E}+00$ \\
\hline $\mathrm{Nb}-95 \mathrm{~m}$ & $013967-76-5$ & $2.9 \mathrm{E}+06$ & $2.9 \mathrm{E}+04$ & $4.2 \mathrm{E}+09$ & $4.2 E+07$ & $5.6 \mathrm{E}+03$ & $5.6 \mathrm{E}+01$ & $5.6 \mathrm{E}+03$ & $5.6 \mathrm{E}+01$ \\
\hline $\mathrm{Nb}-97$ & 018496-04-3 & $5.1 \mathrm{E}+07$ & $5.1 \mathrm{E}+05$ & $4.5 E+10$ & $4.5 \mathrm{E}+08$ & $2.0 \mathrm{E}+02$ & $2.0 \mathrm{E}+00$ & $2.0 \mathrm{E}+02$ & $2.0 \mathrm{E}+00$ \\
\hline $\mathrm{Nb}-97 \mathrm{~m}$ & 018496-04-3 & $2.7 E+09$ & $2.7 \mathrm{E}+07$ & $2.9 E+12$ & $2.9 \mathrm{E}+10$ & $1.8 E+02$ & $1.8 \mathrm{E}+00$ & $1.8 \mathrm{E}+02$ & $1.8 \mathrm{E}+00$ \\
\hline Nd-147 & $014269-74-0$ & $1.5 \mathrm{E}+06$ & $1.5 \mathrm{E}+04$ & $2.0 \mathrm{E}+09$ & $2.0 \mathrm{E}+07$ & $1.5 \mathrm{E}+03$ & $1.5 \mathrm{E}+01$ & $1.5 \mathrm{E}+03$ & $1.5 \mathrm{E}+01$ \\
\hline
\end{tabular}




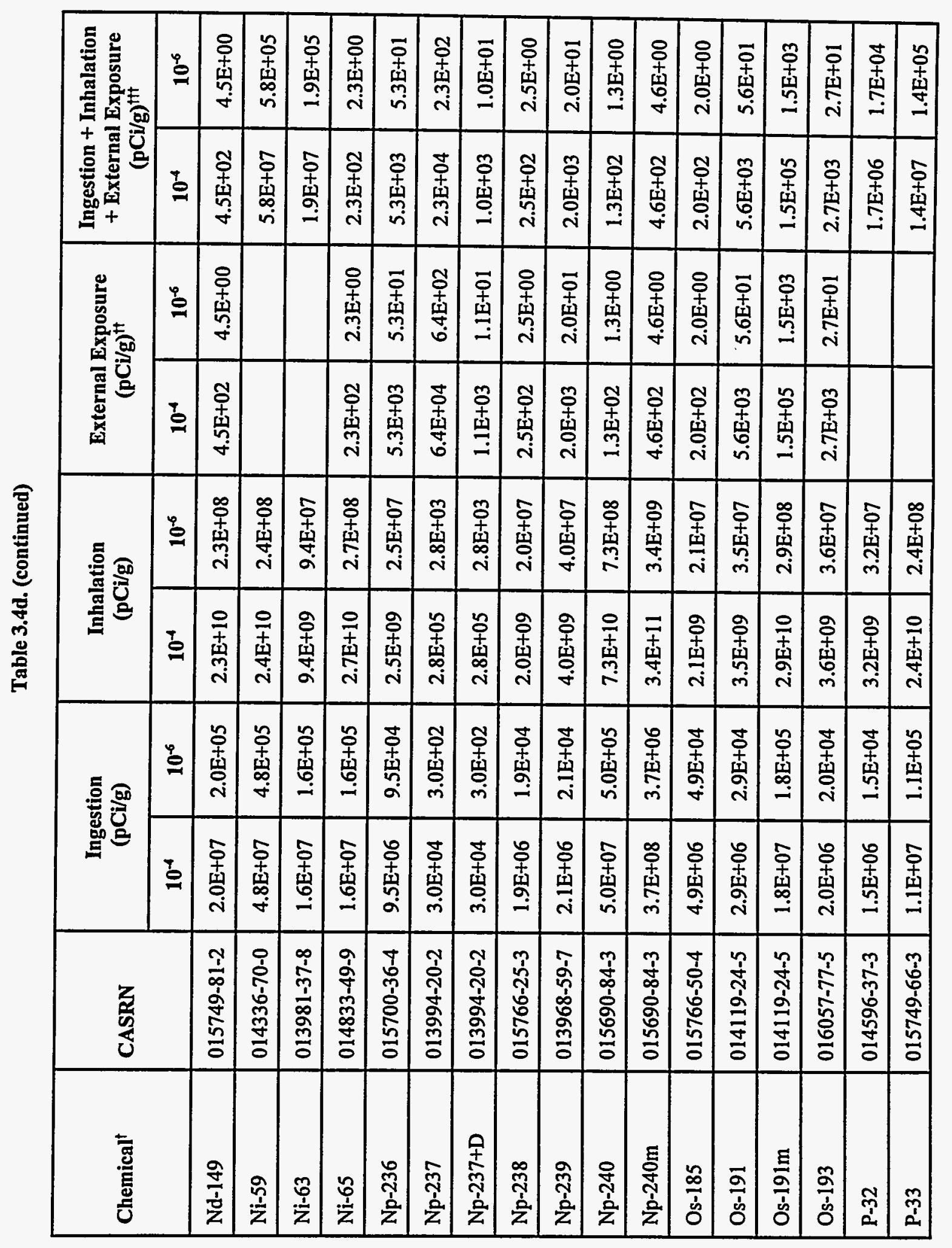


Table 3.4d. (continued)

\begin{tabular}{|c|c|c|c|c|c|c|c|c|c|}
\hline \multirow[t]{2}{*}{ Chemical $^{\dagger}$} & \multirow[t]{2}{*}{ CASRN } & \multicolumn{2}{|c|}{$\begin{array}{l}\text { Ingestion } \\
\text { (pCi/g) }\end{array}$} & \multicolumn{2}{|c|}{$\begin{array}{l}\text { Inhalation } \\
\text { (pCi/g) }\end{array}$} & \multicolumn{2}{|c|}{$\begin{array}{l}\text { External Exposure } \\
(\mathrm{pCi} / \mathrm{g})^{\dagger+}\end{array}$} & \multicolumn{2}{|c|}{$\begin{array}{c}\text { Ingestion + Inhalation } \\
+ \text { External Exposure } \\
(\mathrm{pCi} / \mathrm{g})^{++t}\end{array}$} \\
\hline & & $10^{-4}$ & $10^{-6}$ & $10^{-4}$ & $10^{-6}$ & $10^{-4}$ & $10^{-6}$ & $10^{-4}$ & $10^{-6}$ \\
\hline $\mathrm{Pa}-231$ & $014331-85-2$ & $6.0 \mathrm{E}+04$ & $6.0 \mathrm{E}+02$ & $3.9 \mathrm{E}+05$ & $3.9 \mathrm{E}+03$ & $1.8 \mathrm{E}+04$ & $1.8 \mathrm{E}+02$ & 1.4E+04 & $1.4 \mathrm{E}+02$ \\
\hline $\mathrm{Pa}-233$ & 013981-14-1 & $1.9 E+06$ & $1.9 \mathrm{E}+04$ & $1.9 \mathrm{E}+09$ & $1.9 \mathrm{E}+07$ & $1.1 \mathrm{E}+03$ & $1.1 \mathrm{E}+01$ & $1.1 E+03$ & $1.1 \mathrm{E}+01$ \\
\hline $\mathrm{Pa}-234$ & $015100-28-4$ & $4.2 \mathrm{E}+06$ & $4.2 \mathrm{E}+04$ & $7.3 \mathrm{E}+09$ & $7.3 \mathrm{E}+07$ & $7.4 \mathrm{E}+01$ & 7.4E-01 & $7.4 \mathrm{E}+01$ & $7.4 \mathrm{E}-01$ \\
\hline $\mathrm{Pa}-234 \mathrm{~m}$ & $015100-28=4$ & $1.9 \mathrm{E}+09$ & $1.9 \mathrm{E}+07$ & $1.5 \mathrm{E}+12$ & $1.5 \mathrm{E}+10$ & $1.2 \mathrm{E}+04$ & $1.2 \mathrm{E}+02$ & $1.2 \mathrm{E}+04$ & $1.2 \mathrm{E}+02$ \\
\hline $\mathrm{Pb}-203$ & $014687-25-3$ & $8.6 \mathrm{E}+06$ & $8.6 \mathrm{E}+04$ & $3.1 \mathrm{E}+10$ & $3.1 \mathrm{E}+08$ & $7.6 \mathrm{E}+02$ & $7.6 \mathrm{E}+00$ & $7.6 \mathrm{E}+02$ & $7.6 \mathrm{E}+00$ \\
\hline Pb-209 & 014119-30-3 & $4.3 \mathrm{E}+07$ & $4.3 E+05$ & $1.4 \mathrm{E}+11$ & $1.4 \mathrm{E}+09$ & & & $5.1 \mathrm{E}+07$ & $5.1 E+05$ \\
\hline $\mathrm{Pb}-210$ & 014255-04-0 & $1.3 E+04$ & $1.3 \mathrm{E}+02$ & $5.7 \mathrm{E}+06$ & $5.7 E+04$ & $4.3 E+06$ & $4.3 E+04$ & $1.6 \mathrm{E}+04$ & $1.6 \mathrm{E}+02$ \\
\hline $\mathrm{Pb}-210+\mathrm{D}$ & $014255-04-0$ & $8.8 \mathrm{E}+03$ & $8.8 \mathrm{E}+01$ & $2.5 \mathrm{E}+06$ & $2.5 E+04$ & $3.4 \mathrm{E}+06$ & $3.4 \mathrm{E}+04$ & $1.1 \mathrm{E}+04$ & $1.1 \mathrm{E}+02$ \\
\hline $\mathrm{Pb}-211$ & $015816-77-0$ & $2.6 \mathrm{E}+07$ & $2.6 \mathrm{E}+05$ & $9.2 \mathrm{E}+08$ & $9.2 \mathrm{E}+06$ & $2.6 \mathrm{E}+03$ & $2.6 \mathrm{E}+01$ & $2.6 \mathrm{E}+03$ & $2.6 \mathrm{E}+01$ \\
\hline $\mathrm{Pb}-212$ & 015092-94-1 & $4.9 \mathrm{E}+05$ & $4.9 \mathrm{E}+03$ & $2.5 \mathrm{E}+08$ & $2.5 \mathrm{E}+06$ & $1.6 \mathrm{E}+03$ & $1.6 \mathrm{E}+01$ & $1.6 \mathrm{E}+03$ & $1.6 \mathrm{E}+01$ \\
\hline $\mathrm{Pb}-214$ & $015067-28-4$ & $3.0 \mathrm{E}+07$ & $3.0 \mathrm{E}+05$ & $1.5 \mathrm{E}+09$ & $1.5 \mathrm{E}+07$ & $6.9 \mathrm{E}+02$ & $6.9 \mathrm{E}+00$ & $6.9 \mathrm{E}+02$ & $6.9 \mathrm{E}+00$ \\
\hline Pd-100 & $015690-69-4$ & $2.4 \mathrm{E}+06$ & $2.4 \mathrm{E}+04$ & $2.7 \mathrm{E}+09$ & $2.7 E+07$ & & & $2.9 \mathrm{E}+06$ & $2.9 \mathrm{E}+04$ \\
\hline Pd-101 & $015749-54-9$ & $2.4 \mathrm{E}+07$ & $2.4 E+05$ & $4.2 E+10$ & $4.2 \mathrm{E}+08$ & & & $2.9 \mathrm{E}+07$ & $2.9 \mathrm{E}+05$ \\
\hline Pd-103 & $014967-68-1$ & $8.5 \mathrm{E}+06$ & $8.5 E+04$ & $8.8 \mathrm{E}+09$ & $8.8 \mathrm{E}+07$ & $9.0 \mathrm{E}+05$ & $9.0 \mathrm{E}+03$ & $8.3 E+05$ & $8.3 \mathrm{E}+03$ \\
\hline Pd-107 & 017637-99-9 & $4.3 E+07$ & $4.3 E+05$ & $6.5 E+09$ & $6.5 \mathrm{E}+07$ & & & $5.1 \mathrm{E}+07$ & $5.1 \mathrm{E}+05$ \\
\hline Pd-109 & $014981-64-7$ & $2.7 E+06$ & $2.7 E+04$ & $4.8 E+09$ & $4.8 \mathrm{E}+07$ & $2.0 \mathrm{E}+05$ & $2.0 \mathrm{E}+03$ & $1.9 \mathrm{E}+05$ & $1.9 \mathrm{E}+03$ \\
\hline Pm-147 & $014380-75-7$ & $6.3 E+06$ & $6.3 E+04$ & $1.3 E+09$ & $1.3 E+07$ & $7.7 \mathrm{E}+07$ & $7.7 \mathrm{E}+05$ & $6.9 E+06$ & $6.9 E+04$ \\
\hline
\end{tabular}




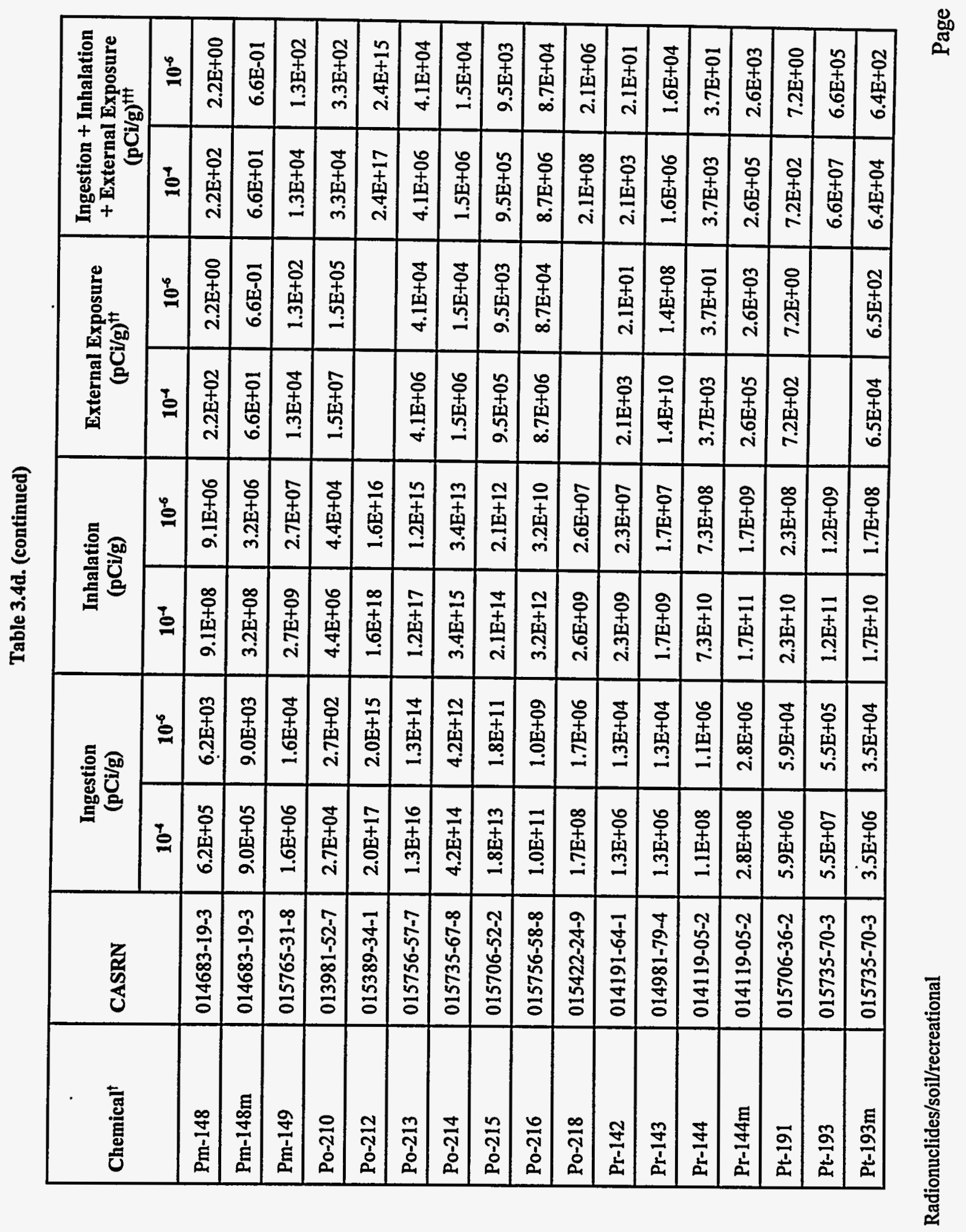


Table 3.4d. (continued)

\begin{tabular}{|c|c|c|c|c|c|c|c|c|c|}
\hline \multirow[t]{2}{*}{ Chemical $^{\dagger}$} & \multirow[t]{2}{*}{ CASRN } & \multicolumn{2}{|c|}{$\begin{array}{c}\text { Ingestion } \\
\text { (pCi/g) }\end{array}$} & \multicolumn{2}{|c|}{$\begin{array}{c}\text { Inhalation } \\
\text { (pCi/g) }\end{array}$} & \multicolumn{2}{|c|}{$\begin{array}{l}\text { External Exposure } \\
(\mathrm{pCi} / \mathrm{g})^{\dagger+}\end{array}$} & \multicolumn{2}{|c|}{$\begin{array}{c}\text { Ingestion + Inhalation } \\
+ \text { External Exposure } \\
(\text { pCi/g) }\end{array}$} \\
\hline & & $10^{-4}$ & $10^{-6}$ & $10^{-4}$ & $10^{-6}$ & $10^{-4}$ & $10^{-6}$ & $10^{-4}$ & $10^{-6}$ \\
\hline Pt-197 & 015735-74-7 & $4.2 \mathrm{E}+06$ & $4.2 E+04$ & $2.1 \mathrm{E}+10$ & $2.1 E+08$ & $1.5 \mathrm{E}+04$ & $1.5 \mathrm{E}+02$ & $1.5 \mathrm{E}+04$ & $1.5 \mathrm{E}+02$ \\
\hline Pt-197m & 015735-74-7 & $2.7 \mathrm{E}+07$ & $2.7 \mathrm{E}+05$ & $9.5 \mathrm{E}+10$ & $9.5 \mathrm{E}+08$ & $2.9 \mathrm{E}+03$ & $2.9 \mathrm{E}+01$ & $2.9 \mathrm{E}+03$ & $2.9 \mathrm{E}+01$ \\
\hline $\mathrm{Pu}-236$ & $015411-92-4$ & $1.2 \mathrm{E}+05$ & $1.2 \mathrm{E}+03$ & $7.1 E+05$ & $7.1 \mathrm{E}+03$ & 2.1E+07 & $2.1 E+05$ & $1.4 \mathrm{E}+05$ & $1.4 \mathrm{E}+03$ \\
\hline $\mathrm{Pu}-238$ & $013981-16-3$ & $3.0 \mathrm{E}+04$ & $3.0 \mathrm{E}+02$ & $3.5 \mathrm{E}+05$ & $3.5 \mathrm{E}+03$ & $2.5 \mathrm{E}+07$ & $2.5 E+05$ & $3.6 \mathrm{E}+04$ & $3.6 \mathrm{E}+02$ \\
\hline $\mathrm{Pu}-239$ & $015117-48-3$ & $2.8 \mathrm{E}+04$ & $2.8 \mathrm{E}+02$ & $3.4 \mathrm{E}+05$ & $3.4 \mathrm{E}+03$ & $3.9 \mathrm{E}+07$ & $3.9 \mathrm{E}+05$ & $3.4 \mathrm{E}+04$ & $3.4 \mathrm{E}+02$ \\
\hline Pu-240 & 014119-33-6 & $2.8 \mathrm{E}+04$ & $2.8 \mathrm{E}+02$ & $3.4 \mathrm{E}+05$ & $3.4 \mathrm{E}+03$ & $2.6 \mathrm{E}+07$ & $2.6 \mathrm{E}+05$ & $3.4 \mathrm{E}+04$ & $3.4 \mathrm{E}+02$ \\
\hline $\mathrm{Pu}-241$ & $014119-32-5$ & $1.7 \mathrm{E}+06$ & $1.7 \mathrm{E}+04$ & $3.4 \mathrm{E}+07$ & $3.4 \mathrm{E}+05$ & & & $2.0 \mathrm{E}+06$ & $2.0 \mathrm{E}+04$ \\
\hline $\mathrm{Pu}-241+\mathrm{D}$ & 014119-32-5 & $2.7 \mathrm{E}+04$ & $2.7 \mathrm{E}+02$ & $2.5 \mathrm{E}+05$ & $2.5 \mathrm{E}+03$ & $1.1 \mathrm{E}+05$ & $1.1 \mathrm{E}+03$ & $2.4 \mathrm{E}+04$ & $2.4 \mathrm{E}+02$ \\
\hline $\mathrm{Pu}-242$ & $013982-10-0$ & $3.0 \mathrm{E}+04$ & $3.0 \mathrm{E}+02$ & $3.6 \mathrm{E}+05$ & $3.6 \mathrm{E}+03$ & $3.1 \mathrm{E}+07$ & $3.1 \mathrm{E}+05$ & $3.5 E+04$ & $3.5 \mathrm{E}+02$ \\
\hline $\mathrm{Pu}-243$ & $015706-37-3$ & $2.4 E+07$ & $2.4 \mathrm{E}+05$ & $3.6 \mathrm{E}+10$ & $3.6 \mathrm{E}+08$ & $2.6 \mathrm{E}+04$ & $2.6 \mathrm{E}+02$ & $2.6 \mathrm{E}+04$ & $2.6 \mathrm{E}+02$ \\
\hline $\mathrm{Pu}-244$ & 014119-34-7 & $2.8 \mathrm{E}+04$ & $2.8 \mathrm{E}+02$ & $3.6 \mathrm{E}+05$ & $3.6 \mathrm{E}+03$ & $3.8 \mathrm{E}+07$ & $3.8 E+05$ & $3.4 E+04$ & $3.4 \mathrm{E}+02$ \\
\hline $\mathrm{Pu}-244+\mathrm{D}$ & 014119-34-7 & $2.8 \mathrm{E}+04$ & $2.8 \mathrm{E}+02$ & $3.6 E+05$ & $3.6 \mathrm{E}+03$ & $1.3 \mathrm{E}+02$ & $1.3 \mathrm{E}+00$ & $1.3 \mathrm{E}+02$ & $1.3 E+00$ \\
\hline $\mathrm{Ra}-223$ & $015623-45-7$ & $3.8 E+04$ & $3.8 \mathrm{E}+02$ & $2.6 \mathrm{E}+06$ & $2.6 \mathrm{E}+04$ & $2.0 \mathrm{E}+03$ & $2.0 \mathrm{E}+01$ & $1.9 E+03$ & $1.9 \mathrm{E}+01$ \\
\hline $\mathrm{Ra}-224$ & $013233-32-4$ & $6.0 \mathrm{E}+04$ & $6.0 \mathrm{E}+02$ & $4.2 E+06$ & $4.2 E+04$ & $2.0 \mathrm{E}+04$ & $2.0 \mathrm{E}+02$ & $1.5 \mathrm{E}+04$ & $1.5 \mathrm{E}+02$ \\
\hline $\mathrm{Ra}-224^{(1)}$ & $013233-32-4$ & $6.0 \mathrm{E}+04$ & $6.0 \mathrm{E}+02$ & $8.9 E+04$ & $8.9 E+02$ & $2.0 \mathrm{E}+04$ & $2.0 \mathrm{E}+02$ & $1.3 E+04$ & $1.3 \mathrm{E}+02$ \\
\hline $\mathrm{Ra}-225$ & $013981-53-8$ & $5.7 E+04$ & $5.7 \mathrm{E}+02$ & $4.0 \mathrm{E}+06$ & $4.0 \mathrm{E}+04$ & $2.8 \mathrm{E}+05$ & $2.8 \mathrm{E}+03$ & $5.5 E+04$ & $5.5 \mathrm{E}+02$ \\
\hline $\mathrm{Ra}-226$ & 013982-63-3 & $3.0 \mathrm{E}+04$ & $3.0 \mathrm{E}+02$ & $3.5 \mathrm{E}+06$ & $3.5 E+04$ & $3.7 \mathrm{E}+04$ & $3.7 \mathrm{E}+02$ & $1.8 \mathrm{E}+04$ & $1.8 \mathrm{E}+02$ \\
\hline
\end{tabular}




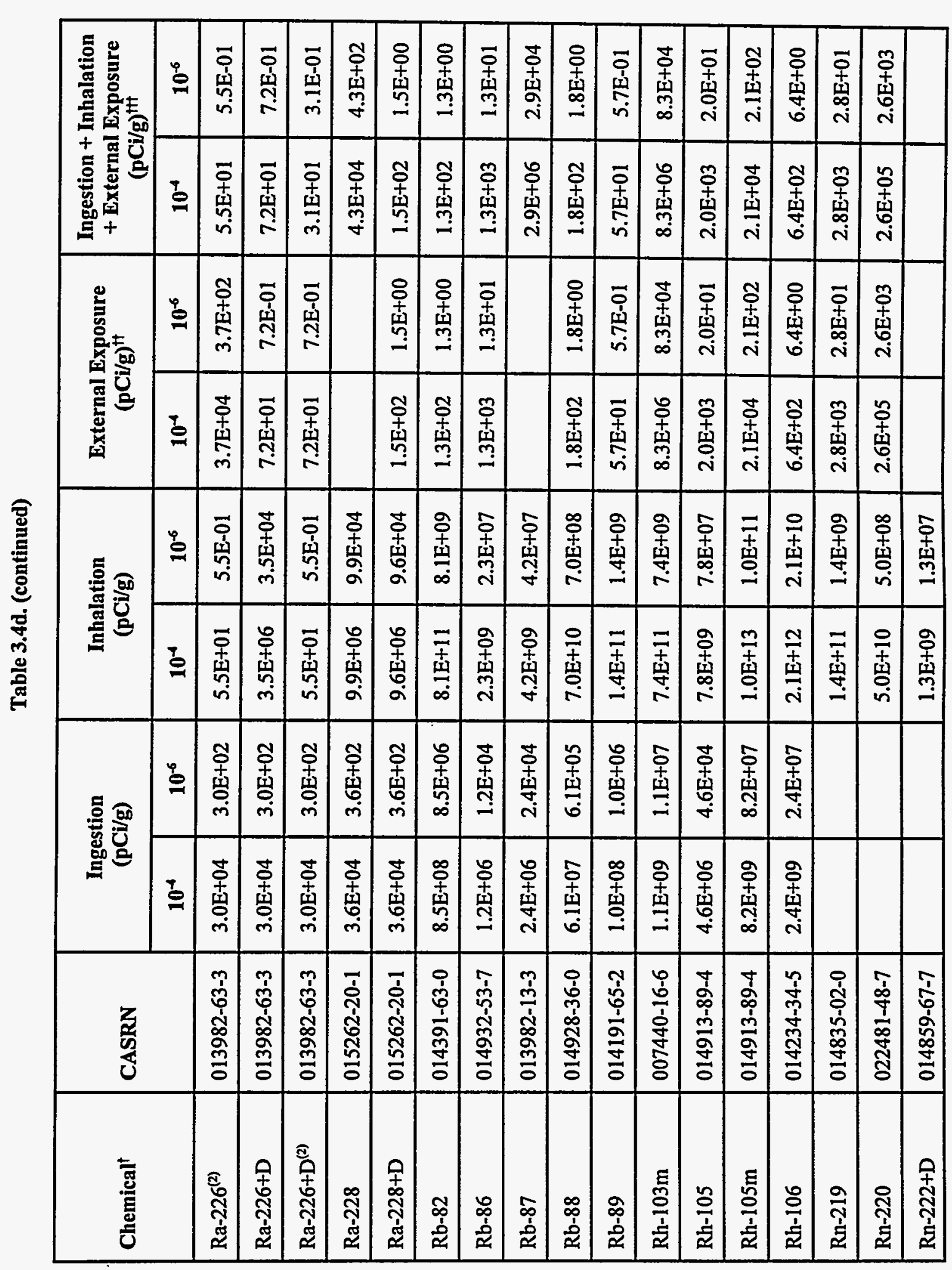


Table 3.4d. (continued)

\begin{tabular}{|c|c|c|c|c|c|c|c|c|c|}
\hline \multirow[t]{2}{*}{ Chemical $^{\dagger}$} & \multirow[t]{2}{*}{ CASRN } & \multicolumn{2}{|c|}{$\begin{array}{l}\text { Ingestion } \\
\text { (pCi/g) }\end{array}$} & \multicolumn{2}{|c|}{$\begin{array}{c}\text { Inhalation } \\
\text { (pCi/g) }\end{array}$} & \multicolumn{2}{|c|}{$\begin{array}{l}\text { External Exposure } \\
(\mathrm{pCi} / \mathrm{g})^{+}\end{array}$} & \multicolumn{2}{|c|}{$\begin{array}{c}\text { Ingestion + Inhalation } \\
\text { + External Exposure } \\
(\mathrm{pCi} / \mathrm{g})^{\mathrm{tt}}\end{array}$} \\
\hline & & $10^{-4}$ & $10^{-6}$ & $10^{-4}$ & $10^{-6}$ & $10^{-4}$ & $10^{-6}$ & $10^{-4}$ & $10^{-6}$ \\
\hline $\mathrm{Ru}-103$ & $013968-53-1$ & $2.7 \mathrm{E}+06$ & $2.7 E+04$ & $2.1 \mathrm{E}+09$ & $2.1 \mathrm{E}+07$ & $2.9 \mathrm{E}+02$ & $2.9 \mathrm{E}+00$ & $2.9 \mathrm{E}+02$ & $2.9 E+00$ \\
\hline $\mathrm{Ru}-105$ & $014331-95-4$ & $7.7 \mathrm{E}+06$ & $7.7 \mathrm{E}+04$ & $1.2 \mathrm{E}+10$ & $1.2 \mathrm{E}+08$ & $1.7 \mathrm{E}+02$ & $1.7 \mathrm{E}+00$ & $1.7 \mathrm{E}+02$ & $1.7 E+00$ \\
\hline $\mathrm{Ru}-106$ & $013967-48-1$ & $2.6 \mathrm{E}+05$ & $2.6 \mathrm{E}+03$ & $8.3 E+07$ & $8.3 \mathrm{E}+05$ & & & $3.1 E+05$ & $3.1 \mathrm{E}+03$ \\
\hline Ru-106+D & $013967-48-1$ & $2.6 \mathrm{E}+05$ & $2.6 \mathrm{E}+03$ & $8.3 E+07$ & $8.3 E+05$ & $6.4 \mathrm{E}+02$ & $6.4 \mathrm{E}+00$ & $6.4 \mathrm{E}+02$ & $6.4 \mathrm{E}+00$ \\
\hline Ru-97 & $015758-35-7$ & $1.5 \mathrm{E}+07$ & $1.5 \mathrm{E}+05$ & $2.3 E+10$ & $2.3 \mathrm{E}+08$ & $1.1 \mathrm{E}+03$ & $1.1 \mathrm{E}+01$ & $1.1 E+03$ & $1.1 \mathrm{E}+01$ \\
\hline S-35 & 015117-53-0 & $2.1 \mathrm{E}+07$ & $2.1 \mathrm{E}+05$ & $5.1 \mathrm{E}+10$ & $5.1 \mathrm{E}+08$ & & & $2.6 \mathrm{E}+07$ & $2.6 \mathrm{E}+05$ \\
\hline Sb-122 & $014374-79-9$ & $1.0 \mathrm{E}+06$ & $1.0 \mathrm{E}+04$ & $1.7 \mathrm{E}+09$ & $1.7 \mathrm{E}+07$ & $3.0 \mathrm{E}+02$ & $3.0 \mathrm{E}+00$ & $3.0 \mathrm{E}+02$ & $3.0 \mathrm{E}+00$ \\
\hline Sb-124 & $014683-10-4$ & $8.3 E+05$ & $8.3 \mathrm{E}+03$ & $7.2 E+08$ & $7.2 E+06$ & $6.6 \mathrm{E}+01$ & $6.6 \mathrm{E}-01$ & $6.6 \mathrm{E}+01$ & $6.6 \mathrm{E}-01$ \\
\hline Sb-125 & $014234-35-6$ & $3.0 \mathrm{E}+06$ & $3.0 \mathrm{E}+04$ & $1.8 \mathrm{E}+09$ & $1.8 \mathrm{E}+07$ & $3.6 \mathrm{E}+02$ & $3.6 \mathrm{E}+00$ & $3.6 \mathrm{E}+02$ & $3.6 \mathrm{E}+00$ \\
\hline Sb-125+D & 014234-35-6 & $2.5 \mathrm{E}+06$ & $2.5 \mathrm{E}+04$ & $1.6 \mathrm{E}+09$ & $1.6 \mathrm{E}+07$ & $3.6 \mathrm{E}+02$ & $3.6 \mathrm{E}+00$ & $3.6 \mathrm{E}+02$ & $3.6 \mathrm{E}+00$ \\
\hline Sb-126 & $015756-32-8$ & $9.1 E+05$ & $9.1 \mathrm{E}+03$ & $1.1 \mathrm{E}+09$ & $1.1 \mathrm{E}+07$ & $4.7 \mathrm{E}+01$ & 4.7E-01 & $4.7 \mathrm{E}+01$ & 4.7E-01 \\
\hline $\mathrm{Sb}-126 \mathrm{~m}$ & 015756-32-8 & $1.2 \mathrm{E}+08$ & $1.2 \mathrm{E}+06$ & $1.5 \mathrm{E}+11$ & $1.5 \mathrm{E}+09$ & $8.4 \mathrm{E}+01$ & 8.4E-01 & $8.4 \mathrm{E}+01$ & $8.4 \mathrm{E}-01$ \\
\hline Sb-127 & 013968-50-8 & $1.0 \mathrm{E}+06$ & $1.0 \mathrm{E}+04$ & $1.6 \mathrm{E}+09$ & $1.6 \mathrm{E}+07$ & $2.0 \mathrm{E}+02$ & $2.0 \mathrm{E}+00$ & $2.0 \mathrm{E}+02$ & $2.0 \mathrm{E}+00$ \\
\hline Sb-129 & 014331-88-5 & $4.8 E+06$ & $4.8 \mathrm{E}+04$ & $1.1 \mathrm{E}+10$ & $1.1 \mathrm{E}+08$ & $8.8 \mathrm{E}+01$ & $8.8 \mathrm{E}-01$ & $8.8 \mathrm{E}+01$ & $8.8 \mathrm{E}-01$ \\
\hline Sc-46 & 013967-63-0 & $1.6 \mathrm{E}+06$ & $1.6 \mathrm{E}+04$ & $7.3 \mathrm{E}+08$ & $7.3 E+06$ & $6.2 \mathrm{E}+01$ & $6.2 \mathrm{E}-01$ & $6.2 \mathrm{E}+01$ & $6.2 \mathrm{E}-01$ \\
\hline Sc-47 & 014391-96-9 & $3.0 \mathrm{E}+06$ & $3.0 \mathrm{E}+04$ & $4.7 \mathrm{E}+09$ & 4.7E+07 & $1.9 \mathrm{E}+03$ & $1.9 \mathrm{E}+01$ & $1.9 \mathrm{E}+03$ & $1.9 \mathrm{E}+01$ \\
\hline Sc-48 & $014391-86-7$ & $1.3 \mathrm{E}+06$ & $1.3 E+04$ & $2.3 E+09$ & $2.3 E+07$ & $3.7 \mathrm{E}+01$ & $3.7 \mathrm{E}-01$ & $3.7 \mathrm{E}+01$ & 3.7E-01 \\
\hline
\end{tabular}


Table 3.4d. (continued)

\begin{tabular}{|c|c|c|c|c|c|c|c|c|c|}
\hline \multirow[t]{2}{*}{ Chemical $^{\dagger}$} & \multirow[t]{2}{*}{ CASRN } & \multicolumn{2}{|c|}{$\begin{array}{l}\text { Ingestion } \\
\text { (pCi/g) }\end{array}$} & \multicolumn{2}{|c|}{$\begin{array}{l}\text { Inhalation } \\
\text { (pCi/g) }\end{array}$} & \multicolumn{2}{|c|}{$\begin{array}{l}\text { External Exposure } \\
(\mathrm{pCi} / \mathrm{g})^{++}\end{array}$} & \multicolumn{2}{|c|}{$\begin{array}{c}\text { Ingestion + Inhalation } \\
\text { + External Exposure } \\
(\text { (pCi/g) })^{+t+}\end{array}$} \\
\hline & & $10^{-4}$ & $10^{-6}$ & $10^{-4}$ & $10^{-6}$ & $10^{-4}$ & $10^{-6}$ & $10^{-4}$ & $10^{-6}$ \\
\hline Se-75 & $014265-71-5$ & $1.4 \mathrm{E}+06$ & $1.4 \mathrm{E}+04$ & $1.9 \mathrm{E}+09$ & $1.9 \mathrm{E}+07$ & $5.5 \mathrm{E}+02$ & $5.5 \mathrm{E}+00$ & $5.5 \mathrm{E}+02$ & $5.5 \mathrm{E}+00$ \\
\hline Si-31 & $014276-49-4$ & $1.8 \mathrm{E}+07$ & $1.8 \mathrm{E}+05$ & $2.9 E+10$ & $2.9 \mathrm{E}+08$ & $1.4 \mathrm{E}+05$ & $1.4 \mathrm{E}+03$ & $1.4 \mathrm{E}+05$ & $1.4 \mathrm{E}+03$ \\
\hline Sm-147 & 014392-33-7 & $3.5 \mathrm{E}+05$ & $3.5 \mathrm{E}+03$ & $1.4 \mathrm{E}+06$ & $1.4 \mathrm{E}+04$ & & ' & $4.2 E+05$ & $4.2 E+03$ \\
\hline Sm-151 & 015715-94-3 & $1.9 \mathrm{E}+07$ & $1.9 E+05$ & $2.1 \mathrm{E}+09$ & $2.1 \mathrm{E}+07$ & $1.7 E+09$ & $1.7 \mathrm{E}+07$ & $2.3 E+07$ & $2.3 \mathrm{E}+05$ \\
\hline Sm-153 & $015766-00-4$ & $2.2 \mathrm{E}+06$ & $2.2 \mathrm{E}+04$ & $4.4 \mathrm{E}+09$ & $4.4 \mathrm{E}+07$ & $1.0 \mathrm{E}+04$ & $1.0 \mathrm{E}+02$ & $1.0 \mathrm{E}+04$ & $1.0 \mathrm{E}+02$ \\
\hline Sn-113 & 013966-06-8 & $2.4 \mathrm{E}+06$ & $2.4 \mathrm{E}+04$ & $1.4 \mathrm{E}+09$ & $1.4 \mathrm{E}+07$ & $1.6 \mathrm{E}+05$ & $1.6 \mathrm{E}+03$ & $1.6 \mathrm{E}+05$ & $1.6 \mathrm{E}+03$ \\
\hline Sn-121 & 014683-06-8 & $7.3 E+06$ & 7.3E+04 & $1.6 \mathrm{E}+10$ & $1.6 \mathrm{E}+08$ & & & 8.7E+06 & $8.7 \mathrm{E}+04$ \\
\hline Sn-121m & 014683-06-8 & $4.4 E+06$ & 4.4E+04 & $1.3 \mathrm{E}+09$ & $1.3 E+07$ & & & $5.3 E+06$ & $5.3 E+04$ \\
\hline Sn-125 & 014683-08-0 & $5.3 \mathrm{E}+05$ & $5.3 E+03$ & $8.0 \mathrm{E}+08$ & $8.0 \mathrm{E}+06$ & $4.0 \mathrm{E}+02$ & $4.0 \mathrm{E}+00$ & $4.0 \mathrm{E}+02$ & $4.0 \mathrm{E}+00$ \\
\hline$S n-126$ & $015832-50-5$ & $4.2 \mathrm{E}+05$ & $4.2 \mathrm{E}+03$ & $2.2 \mathrm{E}+08$ & $2.2 \mathrm{E}+06$ & $1.5 \mathrm{E}+04$ & $1.5 \mathrm{E}+02$ & $1.4 \mathrm{E}+04$ & $1.4 \mathrm{E}+02$ \\
\hline Sr-82 & 014809-50-8 & $3.4 \mathrm{E}+05$ & $3.4 E+03$ & $1.1 \mathrm{E}+09$ & $1.1 \mathrm{E}+07$ & $5.4 \mathrm{E}+06$ & $5.4 \mathrm{E}+04$ & $3.8 E+05$ & $3.8 E+03$ \\
\hline Sr-85 & 013967-73-2 & $6.3 E+06$ & $6.3 E+04$ & $8.4 \mathrm{E}+09$ & $8.4 \mathrm{E}+07$ & $3.2 \mathrm{E}+02$ & $3.2 \mathrm{E}+00$ & $3.2 \mathrm{E}+02$ & $3.2 \mathrm{E}+00$ \\
\hline Sr-85m & $013967-73-2$ & $4.9 \mathrm{E}+08$ & $4.9 E+06$ & $1.3 \mathrm{E}+12$ & $1.3 \mathrm{E}+10$ & $9.3 \mathrm{E}+02$ & $9.3 \mathrm{E}+00$ & $9.3 \mathrm{E}+02$ & $9.3 E+00$ \\
\hline Sr-89 & 014158-27-1 & $8.6 \mathrm{E}+05$ & $8.6 \mathrm{E}+03$ & $2.6 \mathrm{E}+09$ & $2.6 \mathrm{E}+07$ & $9.0 \mathrm{E}+05$ & $9.0 \mathrm{E}+03$ & $4.8 \mathrm{E}+05$ & $4.8 E+03$ \\
\hline Sr-90 & $010098-97-2$ & $2.2 \mathrm{E}+05$ & $2.2 \mathrm{E}+03$ & $1.6 \mathrm{E}+08$ & $1.6 \mathrm{E}+06$ & & & $2.6 \mathrm{E}+05$ & $2.6 \mathrm{E}+03$ \\
\hline Sr-90+D & $010098-97-2$ & $1.6 \mathrm{E}+05$ & $1.6 \mathrm{E}+03$ & $1.4 \mathrm{E}+08$ & $1.4 \mathrm{E}+06$ & & & $1.9 \mathrm{E}+05$ & $1.9 \mathrm{E}+03$ \\
\hline Sr-91 & 014331-91-0 & $3.2 \mathrm{E}+06$ & $3.2 E+04$ & $1.2 \mathrm{E}+10$ & $1.2 \mathrm{E}+08$ & $1.8 \mathrm{E}+02$ & $1.8 \mathrm{E}+00$ & $1.8 \mathrm{E}+02$ & $1.8 \mathrm{E}+00$ \\
\hline
\end{tabular}


Table 3.4d. (continued)

\begin{tabular}{|c|c|c|c|c|c|c|c|c|c|}
\hline \multirow[t]{2}{*}{ Chemical $^{\dagger}$} & \multirow[t]{2}{*}{ CASRN } & \multicolumn{2}{|c|}{$\begin{array}{l}\text { Ingestion } \\
\text { (pCi/g) }\end{array}$} & \multicolumn{2}{|c|}{$\begin{array}{l}\text { Inhalation } \\
\text { (pCi/g) }\end{array}$} & \multicolumn{2}{|c|}{$\begin{array}{l}\text { External Exposure } \\
(\mathrm{pCi} / \mathrm{g})^{\dagger \dagger}\end{array}$} & \multicolumn{2}{|c|}{$\begin{array}{c}\text { Ingestion + Inhalation } \\
+ \text { External Exposure } \\
(\mathrm{pCi} / \mathrm{g})^{\dagger+t}\end{array}$} \\
\hline & & $10^{-4}$ & $10^{-6}$ & $10^{-4}$ & $10^{-6}$ & $10^{-4}$ & $10^{-6}$ & $10^{-4}$ & $10^{-6}$ \\
\hline Sr-92 & 014928-29-1 & $4.4 \mathrm{E}+06$ & $4.4 \mathrm{E}+04$ & $2.0 \mathrm{E}+10$ & $2.0 \mathrm{E}+08$ & $9.4 \mathrm{E}+01$ & $9.4 \mathrm{E}-01$ & $9.4 \mathrm{E}+01$ & $9.4 \mathrm{E}-01$ \\
\hline Ta-182 & $013982-00-8$ & $1.3 E+06$ & $1.3 \mathrm{E}+04$ & $5.8 \mathrm{E}+08$ & $5.8 \mathrm{E}+06$ & $1.0 \mathrm{E}+02$ & $1.0 \mathrm{E}+00$ & $1.0 \mathrm{E}+02$ & $1.0 \mathrm{E}+00$ \\
\hline Tb-158 & $015759-55-4$ & $2.1 E+06$ & $2.1 E+04$ & $1.4 \mathrm{E}+08$ & $1.4 \mathrm{E}+06$ & & & $2.5 \mathrm{E}+06$ & $2.5 \mathrm{E}+04$ \\
\hline Tb-160 & 013981-29-8 & $1.2 \mathrm{E}+06$ & $1.2 \mathrm{E}+04$ & $8.4 \mathrm{E}+08$ & $8.4 \mathrm{E}+06$ & $1.2 \mathrm{E}+02$ & $1.2 \mathrm{E}+00$ & $1.2 \mathrm{E}+02$ & $1.2 \mathrm{E}+00$ \\
\hline Tc-95 & $014809-56-4$ & $1.3 \mathrm{E}+08$ & $1.3 \mathrm{E}+06$ & $2.8 \mathrm{E}+11$ & $2.8 \mathrm{E}+09$ & $1.8 \mathrm{E}+02$ & $1.8 \mathrm{E}+00$ & $1.8 \mathrm{E}+02$ & $1.8 \mathrm{E}+00$ \\
\hline Tc-95m & $014809-56-4$ & $7.2 \mathrm{E}+06$ & $7.2 \mathrm{E}+04$ & $4.5 \mathrm{E}+09$ & $4.5 E+07$ & $2.3 \mathrm{E}+02$ & $2.3 E+00$ & $2.3 E+02$ & $2.3 E+00$ \\
\hline Tc-96 & $014808-44-7$ & $3.9 \mathrm{E}+06$ & $3.9 \mathrm{E}+04$ & $4.9 \mathrm{E}+09$ & $4.9 \mathrm{E}+07$ & $5.2 \mathrm{E}+01$ & $5.2 \mathrm{E}-01$ & $5.2 \mathrm{E}+01$ & $5.2 \mathrm{E}-01$ \\
\hline Tc-96m & $014808-44-7$ & $3.4 \mathrm{E}+08$ & $3.4 \mathrm{E}+06$ & $4.2 \mathrm{E}+11$ & $4.2 \mathrm{E}+09$ & $6.3 \mathrm{E}+03$ & $6.3 \mathrm{E}+01$ & $6.3 E+03$ & $6.3 \mathrm{E}+01$ \\
\hline Tc-97 & 015759-35-0 & $5.6 \mathrm{E}+07$ & $5.6 \mathrm{E}+05$ & $2.8 \mathrm{E}+10$ & $2.8 \mathrm{E}+08$ & $2.0 \mathrm{E}+06$ & $2.0 \mathrm{E}+04$ & $1.9 \mathrm{E}+06$ & $1.9 \mathrm{E}+04$ \\
\hline Tc-97m & 015759-35-0 & $7.4 \mathrm{E}+06$ & $7.4 \mathrm{E}+04$ & $4.9 E+09$ & $4.9 \mathrm{E}+07$ & $1.8 \mathrm{E}+06$ & $1.8 \mathrm{E}+04$ & $1.5 \mathrm{E}+06$ & $1.5 \mathrm{E}+04$ \\
\hline Tc-99 & 014133-76-7 & $6.3 \mathrm{E}+06$ & $6.3 E+04$ & $3.3 \mathrm{E}+09$ & $3.3 E+07$ & $7.9 \mathrm{E}+08$ & $7.9 E+06$ & $7.5 \mathrm{E}+06$ & $7.5 \mathrm{E}+04$ \\
\hline Tc-99m & 014133-76-7 & $1.6 \mathrm{E}+08$ & $1.6 \mathrm{E}+06$ & $2.7 \mathrm{E}+11$ & $2.7 E+09$ & $1.9 \mathrm{E}+03$ & $1.9 \mathrm{E}+01$ & $1.9 E+03$ & $1.9 \mathrm{E}+01$ \\
\hline Te-125m & 014390-73-9 & $3.5 \mathrm{E}+06$ & $3.5 \mathrm{E}+04$ & $3.3 \mathrm{E}+09$ & $3.3 E+07$ & $2.3 \mathrm{E}+05$ & $2.3 E+03$ & $2.1 E+05$ & $2.1 \mathrm{E}+03$ \\
\hline Te-127 & $013981-49-2$ & $1.0 \mathrm{E}+07$ & $1.0 \mathrm{E}+05$ & $2.2 \mathrm{E}+10$ & $2.2 \mathrm{E}+08$ & $3.0 \mathrm{E}+04$ & $3.0 \mathrm{E}+02$ & $3.0 \mathrm{E}+04$ & $3.0 \mathrm{E}+02$ \\
\hline $\mathrm{Te}-127 \mathrm{~m}$ & $013981-49-2$ & $1.5 \mathrm{E}+06$ & $1.5 \mathrm{E}+04$ & $7.3 E+08$ & $7.3 E+06$ & $6.9 \mathrm{E}+05$ & $6.9 \mathrm{E}+03$ & $4.9 E+05$ & $4.9 \mathrm{E}+03$ \\
\hline Te-129 & $014269-71-7$ & $6.0 \mathrm{E}+07$ & $6.0 \mathrm{E}+05$ & $6.5 \mathrm{E}+10$ & $6.5 \mathrm{E}+08$ & $3.3 \mathrm{E}+03$ & $3.3 \mathrm{E}+01$ & $3.3 E+03$ & $3.3 \mathrm{E}+01$ \\
\hline Te-129m & $014269-71-7$ & $7.6 \mathrm{E}+05$ & $7.6 \mathrm{E}+03$ & $7.2 \mathrm{E}+08$ & $7.2 \mathrm{E}+06$ & $7.0 \mathrm{E}+03$ & $7.0 \mathrm{E}+01$ & $7.0 \mathrm{E}+03$ & $7.0 \mathrm{E}+01$ \\
\hline
\end{tabular}




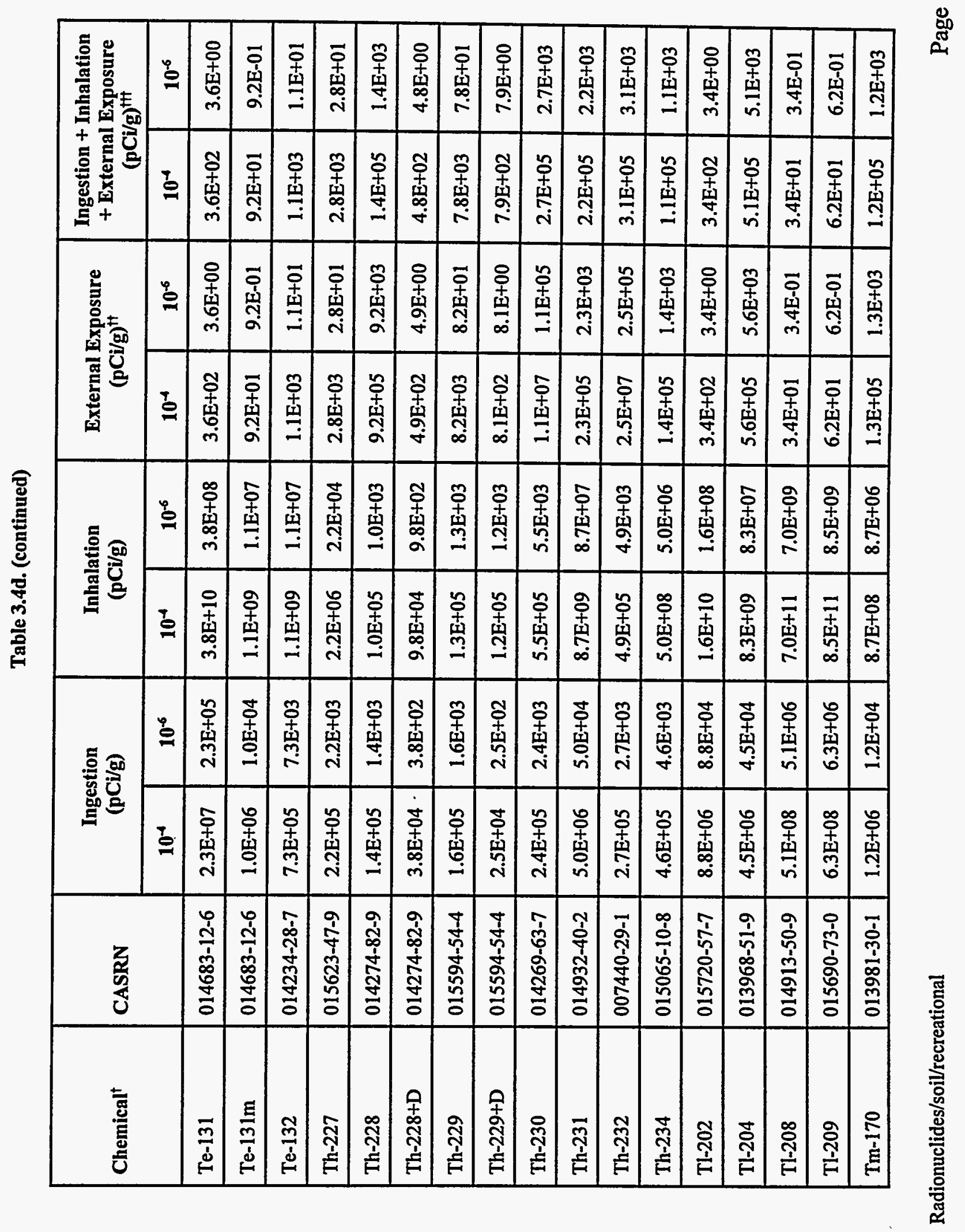


Table 3.4d. (continued)

\begin{tabular}{|c|c|c|c|c|c|c|c|c|c|}
\hline \multirow[t]{2}{*}{ Chemical $^{\dagger}$} & \multirow[t]{2}{*}{ CASRN } & \multicolumn{2}{|c|}{$\begin{array}{l}\text { Ingestion } \\
\text { (pCi/g) }\end{array}$} & \multicolumn{2}{|c|}{$\begin{array}{l}\text { Inhalation } \\
\text { (pCi/g) }\end{array}$} & \multicolumn{2}{|c|}{$\begin{array}{l}\text { External Exposure } \\
(\mathrm{pCi} / \mathrm{g})^{\dagger+}\end{array}$} & \multicolumn{2}{|c|}{$\begin{array}{c}\text { Ingestion + Inhalation } \\
+ \text { External Exposure } \\
(\mathrm{pCi} / \mathrm{g})^{\mathrm{t+t}}\end{array}$} \\
\hline & & $10^{-4}$ & $10^{-6}$ & $10^{-4}$ & $10^{-6}$ & $10^{-4}$ & $10^{-6}$ & $10^{-4}$ & $10^{-6}$ \\
\hline Tm-171 & $014333-45-0$ & $1.5 \mathrm{E}+07$ & $1.5 \mathrm{E}+05$ & $5.2 \mathrm{E}+09$ & $5.2 \mathrm{E}+07$ & $1.5 \mathrm{E}+06$ & $1.5 \mathrm{E}+04$ & $1.4 \mathrm{E}+06$ & $1.4 \mathrm{E}+04$ \\
\hline U-232 & 014158-29-3 & $1.1 \mathrm{E}+05$ & $1.1 \mathrm{E}+03$ & $1.8 \mathrm{E}+05$ & $1.8 \mathrm{E}+03$ & $1.4 \mathrm{E}+07$ & $1.4 \mathrm{E}+05$ & $1.3 \mathrm{E}+05$ & $1.3 E+03$ \\
\hline U-233 & 013968-55-3 & $2.0 \mathrm{E}+05$ & $2.0 \mathrm{E}+03$ & $6.8 \mathrm{E}+05$ & $6.8 \mathrm{E}+03$ & $1.4 \mathrm{E}+07$ & $1.4 \mathrm{E}+05$ & $2.3 \mathrm{E}+05$ & $2.3 \mathrm{E}+03$ \\
\hline U-234 & 013966-29-5 & $2.0 \mathrm{E}+05$ & $2.0 \mathrm{E}+03$ & $6.8 \mathrm{E}+05$ & $6.8 \mathrm{E}+03$ & $2.3 E+07$ & $2.3 E+05$ & $2.3 E+05$ & $2.3 E+03$ \\
\hline U-235 & $015117-96-1$ & $2.0 \mathrm{E}+05$ & $2.0 \mathrm{E}+03$ & $7.3 \mathrm{E}+05$ & $7.3 E+03$ & $1.9 \mathrm{E}+03$ & $1.9 \mathrm{E}+01$ & $1.8 \mathrm{E}+03$ & $1.8 \mathrm{E}+01$ \\
\hline $\mathrm{U}-235+\mathrm{D}$ & $015117-96-1$ & $1.9 \mathrm{E}+05$ & $1.9 \mathrm{E}+03$ & $7.3 E+05$ & $7.3 E+03$ & $1.8 \mathrm{E}+03$ & $1.8 \mathrm{E}+01$ & $1.8 \mathrm{E}+03$ & $1.8 \mathrm{E}+01$ \\
\hline U-236 & 013982-70-2 & 2.1E+05 & $2.1 \mathrm{E}+03$ & $7.2 \mathrm{E}+05$ & $7.2 \mathrm{E}+03$ & $2.8 \mathrm{E}+07$ & $2.8 \mathrm{E}+05$ & $2.5 \mathrm{E}+05$ & $2.5 \mathrm{E}+03$ \\
\hline U-237 & $014269-75-1$ & $2.2 E+06$ & $2.2 \mathrm{E}+04$ & $3.1 \mathrm{E}+09$ & $3.1 E+07$ & $3.6 \mathrm{E}+03$ & $3.6 \mathrm{E}+01$ & $3.5 \mathrm{E}+03$ & $3.5 \mathrm{E}+01$ \\
\hline U-238 & 007440-61-1 & $2.1 \mathrm{E}+05$ & $2.1 \mathrm{E}+03$ & $7.7 \mathrm{E}+05$ & $7.7 \mathrm{E}+03$ & $3.2 \mathrm{E}+07$ & $3.2 E+05$ & $2.4 \mathrm{E}+05$ & $2.4 \mathrm{E}+03$ \\
\hline $\mathrm{U}-238+\mathrm{D}$ & 007440-61-1 & $1.4 \mathrm{E}+05$ & $1.4 \mathrm{E}+03$ & $7.7 \mathrm{E}+05$ & $7.7 \mathrm{E}+03$ & $9.3 \mathrm{E}+03$ & $9.3 \mathrm{E}+01$ & $8.8 \mathrm{E}+03$ & $8.8 \mathrm{E}+01$ \\
\hline $\mathrm{U}-240$ & 015687-53-3 & $1.6 \mathrm{E}+06$ & $1.6 \mathrm{E}+04$ & $2.8 \mathrm{E}+09$ & $2.8 \mathrm{E}+07$ & $4.5 \mathrm{E}+06$ & $4.5 E+04$ & $1.4 \mathrm{E}+06$ & $1.4 \mathrm{E}+04$ \\
\hline$V-48$ & $014331-97-6$ & $1.2 E+06$ & $1.2 E+04$ & $1.4 \mathrm{E}+09$ & $1.4 \mathrm{E}+07$ & $4.3 E+01$ & $4.3 \mathrm{E}-01$ & $4.3 \mathrm{E}+01$ & 4.3E-01 \\
\hline W-181 & $015749-46-9$ & $3.3 \mathrm{E}+07$ & $3.3 E+05$ & $1.2 \mathrm{E}+11$ & $1.2 \mathrm{E}+09$ & $2.3 E+04$ & $2.3 E+02$ & $2.3 \mathrm{E}+04$ & $2.3 E+02$ \\
\hline W-185 & $014932-41-3$ & $4.4 E+06$ & $4.4 \mathrm{E}+04$ & $2.2 \mathrm{E}+10$ & $2.2 \mathrm{E}+08$ & $9.7 \mathrm{E}+06$ & $9.7 E+04$ & $3.4 \mathrm{E}+06$ & $3.4 \mathrm{E}+04$ \\
\hline W-187 & $014983-48-3$ & $3.6 \mathrm{E}+06$ & $3.6 \mathrm{E}+04$ & $1.8 \mathrm{E}+10$ & $1.8 \mathrm{E}+08$ & $3.0 \mathrm{E}+02$ & $3.0 \mathrm{E}+00$ & $3.0 \mathrm{E}+02$ & $3.0 \mathrm{E}+00$ \\
\hline $\mathrm{Xe}-122$ & 015151-09-4 & & & $3.1 E+12$ & $3.1 \mathrm{E}+10$ & & & & \\
\hline $\mathrm{Xe}-123$ & $015700-10-4$ & & & $1.1 E+13$ & $1.1 \mathrm{E}+11$ & & & & \\
\hline
\end{tabular}


Table 3.4d. (continued)

\begin{tabular}{|c|c|c|c|c|c|c|c|c|c|}
\hline \multirow[t]{2}{*}{ Chemical $^{\dagger}$} & \multirow[t]{2}{*}{ CASRN } & \multicolumn{2}{|c|}{$\begin{array}{l}\text { Ingestion } \\
\text { (pCi/g) }\end{array}$} & \multicolumn{2}{|c|}{$\begin{array}{l}\text { Inhalation } \\
\text { (pCi/g) }\end{array}$} & \multicolumn{2}{|c|}{$\begin{array}{l}\text { External Exposure } \\
(\mathrm{pCi} / \mathrm{g})^{t+}\end{array}$} & \multicolumn{2}{|c|}{$\begin{array}{c}\text { Ingestion + Inhalation } \\
+ \text { External Exposure } \\
(\text { pCi/g) }\end{array}$} \\
\hline & & $10^{-4}$ & $10^{-6}$ & $10^{-4}$ & $10^{-6}$ & $10^{-4}$ & $10^{-6}$ & $10^{-4}$ & $10^{-6}$ \\
\hline $\mathrm{Xe}-125$ & 013994-18-8 & & & $7.9 \mathrm{E}+12$ & $7.9 \mathrm{E}+10$ & & & & \\
\hline $\mathrm{Xe}-127$ & 013994-19-9 & & & $2.3 E+13$ & $2.3 E+11$ & & & & \\
\hline $\mathrm{Xe}-129 \mathrm{~m}$ & $013965-99-6$ & & & $1.7 E+13$ & $1.7 \mathrm{E}+11$ & & & & \\
\hline $\mathrm{Xe}-131 \mathrm{~m}$ & $014683-11-5$ & & & $2.3 E+13$ & $2.3 \mathrm{E}+11$ & & & & \\
\hline $\mathrm{Xe}-133$ & $014932-42-4$ & & & $2.3 E+13$ & $2.3 \mathrm{E}+11$ & & & & \\
\hline $\mathrm{Xe}-133 \mathrm{~m}$ & $014932-42-4$ & & & $1.9 \mathrm{E}+13$ & $1.9 \mathrm{E}+11$ & & & & \\
\hline Xe-135 & $014995-62-1$ & & & $1.3 \mathrm{E}+13$ & $1.3 \mathrm{E}+11$ & & & & \\
\hline $\mathrm{Xe}-135 \mathrm{~m}$ & 014995-62-1 & & & $5.1 \mathrm{E}+13$ & $5.1 \mathrm{E}+11$ & & & & \\
\hline $\mathrm{Xe}-137$ & $014835-21-3$ & & & $6.8 \mathrm{E}+12$ & $6.8 \mathrm{E}+10$ & & & & \\
\hline $\mathrm{Xe}-138$ & $015751-81-2$ & & & $4.6 \mathrm{E}+12$ & $4.6 \mathrm{E}+10$ & & & & \\
\hline Y-90 & $010098-91-6$ & $5.9 \mathrm{E}+05$ & $5.9 \mathrm{E}+03$ & $9.6 \mathrm{E}+08$ & $9.6 \mathrm{E}+06$ & & & $7.1 E+05$ & $7.1 \mathrm{E}+03$ \\
\hline$Y-91$ & $014234-24-3$ & $6.6 \mathrm{E}+05$ & $6.6 \mathrm{E}+03$ & $5.1 \mathrm{E}+08$ & $5.1 \mathrm{E}+06$ & $3.5 E+04$ & $3.5 \mathrm{E}+02$ & $3.3 E+04$ & $3.3 \mathrm{E}+02$ \\
\hline Y-91m & $014234-24-3$ & $2.4 \mathrm{E}+08$ & $2.4 \mathrm{E}+06$ & $3.2 \mathrm{E}+11$ & $3.2 \mathrm{E}+09$ & $2.6 \mathrm{E}+02$ & $2.6 \mathrm{E}+00$ & $2.6 \mathrm{E}+02$ & $2.6 \mathrm{E}+00$ \\
\hline Y-92 & $015751-59-4$ & $4.6 E+06$ & $4.6 \mathrm{E}+04$ & $5.9 \mathrm{E}+09$ & $5.9 \mathrm{E}+07$ & $5.0 \mathrm{E}+02$ & $5.0 \mathrm{E}+00$ & $5.0 \mathrm{E}+02$ & $5.0 \mathrm{E}+00$ \\
\hline Y-93 & $014981-70-5$ & $1.5 \mathrm{E}+06$ & $1.5 \mathrm{E}+04$ & $2.7 \mathrm{E}+09$ & $2.7 \mathrm{E}+07$ & $1.4 \mathrm{E}+03$ & $1.4 \mathrm{E}+01$ & $1.4 \mathrm{E}+03$ & $1.4 \mathrm{E}+01$ \\
\hline $\mathrm{Zn}-65$ & $013982-39-3$ & $9.0 \mathrm{E}+05$ & $9.0 \mathrm{E}+03$ & $9.5 \mathrm{E}+08$ & $9.5 \mathrm{E}+06$ & $2.1 E+02$ & $2.1 \mathrm{E}+00$ & $2.1 \mathrm{E}+02$ & $2.1 \mathrm{E}+00$ \\
\hline $\mathrm{Zn}-69$ & 013982-23-5 & $1.4 \mathrm{E}+08$ & $1.4 \mathrm{E}+06$ & $9.2 \mathrm{E}+10$ & $9.2 \mathrm{E}+08$ & $2.4 \mathrm{E}+07$ & $2.4 \mathrm{E}+05$ & $2.1 E+07$ & $2.1 \mathrm{E}+05$ \\
\hline
\end{tabular}


Table 3.4d. (continued)

\begin{tabular}{|c|c|c|c|c|c|c|c|c|c|}
\hline \multirow[t]{2}{*}{ Chemical $^{\dagger}$} & \multirow[t]{2}{*}{ CASRN } & \multicolumn{2}{|c|}{$\begin{array}{c}\text { Ingestion } \\
\text { (pCi/g) }\end{array}$} & \multicolumn{2}{|c|}{$\begin{array}{c}\text { Inhalation } \\
(\mathrm{pCi} / \mathrm{g})\end{array}$} & \multicolumn{2}{|c|}{$\begin{array}{c}\text { External Exposure } \\
(\text { (pCi/g) })^{t \dagger}\end{array}$} & \multicolumn{2}{|c|}{$\begin{array}{c}\text { Ingestion + Inhalation } \\
+ \text { External Exposure } \\
(\mathrm{pCi} / \mathrm{g})^{\mathrm{tt+}}\end{array}$} \\
\hline & & $10^{-4}$ & $10^{-6}$ & $10^{-4}$ & $10^{-6}$ & $10^{-4}$ & $10^{-6}$ & $10^{-4}$ & $10^{-6}$ \\
\hline $\mathrm{Zn}-69 \mathrm{~m}$ & $013982-23-5$ & $5.8 \mathrm{E}+06$ & $5.8 \mathrm{E}+04$ & $8.1 \mathrm{E}+09$ & $8.1 E+07$ & $3.4 \mathrm{E}+02$ & $3.4 \mathrm{E}+00$ & $3.4 \mathrm{E}+02$ & $3.4 \mathrm{E}+00$ \\
\hline Zr-93 & $015751-77-6$ & $1.7 \mathrm{E}+07$ & $1.7 \mathrm{E}+05$ & $1.8 \mathrm{E}+09$ & $1.8 \mathrm{E}+07$ & & & $2.0 \mathrm{E}+07$ & $2.0 \mathrm{E}+05$ \\
\hline $\mathrm{Zr}-95$ & $013967-71-0$ & $2.3 \mathrm{E}+06$ & $2.3 E+04$ & $1.5 \mathrm{E}+09$ & $1.5 \mathrm{E}+07$ & $1.7 \mathrm{E}+02$ & $1.7 \mathrm{E}+00$ & $1.7 \mathrm{E}+02$ & $1.7 \mathrm{E}+00$ \\
\hline Zr-97 & $014928-30-4$ & $8.5 \mathrm{E}+05$ & $8.5 E+03$ & $2.0 \mathrm{E}+09$ & $2.0 \mathrm{E}+07$ & $7.1 \mathrm{E}+02$ & $7.1 E+00$ & $7.1 \mathrm{E}+02$ & $7.1 \mathrm{E}+00$ \\
\hline
\end{tabular}

Notes:

$\dagger \quad$ Isotopes designated with an " $\mathrm{m}$ " indicate that the isotope is metastable; isotopes designated with a " $+\mathrm{D}$ " indicate that the radionuclide slope factors include contributions from daughter products.

i† If no PRG for external exposure was calculated, then no external exposure slope factors were available or the external exposure slope factor was equal to $0.00 \mathrm{E}+00$ (resulting in an undefined PRG for the external exposure pathway for soil under recreational land use).

ti† If slope factors and/or reference doses were available for only one pathway (i.e., ingestion, inhalation, OR external exposure), the total for ingestion plus inhalation plus external exposure was not calculated.

(1) The default volatilization factor of 200 for Ra-224 was calculated as the ratio of the average Ra-224 background concentration in soil to the average $\mathrm{Rn}-220$ background concentration in air. Inhalation slope factor values are for $\mathrm{Rn}-222$ plus decay products (i.e., $\mathrm{Rn}-222+\mathrm{D}$ ) formed from the radioactive decay of Ra-226 and for Rn-220+D from the decay of Ra-224. Slope factor values were taken from Table 4a of EPA's Health Effects Assessment Summary Tables (HEAST 1992).

(2) The default volatilization factor of 8 for Ra-226 was calculated as the ratio of the average natural background concentration of Ra-226 in soil to the corresponding average natural background concentration of Rn-222 in air. Inhalation slope factor values are for Rn-222 plus decay products (i.e., Rn$222+\mathrm{D}$ ) formed from the radioactive decay of $\mathrm{Ra}-226$ and for $\mathrm{Rn}-220+\mathrm{D}$ from the decay of Ra-224. Slope factor values were taken from Table $4 \mathrm{a}$ of EPA's Health Effects Assessment Summary Tables (HEAST 1992). 
Table 3.4e. Risk-based Preliminary Remediation Goals for nonradionuclides in fish (recreational scenario)

\begin{tabular}{|c|c|c|c|c|c|}
\hline \multirow{2}{*}{ Chemical } & \multirow{2}{*}{ CASRN } & \multicolumn{4}{|c|}{ Ingestion of fish (mg/kg) } \\
\hline & & $10^{-4}$ & $10^{-5}$ & $\mathbf{H Q}=\mathbf{1}$ & $H Q=0.1$ \\
\hline ALAR & $001596-84-5$ & & & $1.5 \mathrm{E}+03$ & $1.5 \mathrm{E}+02$ \\
\hline Acenaphthene & $000083-32-9$ & & & $5.9 \mathrm{E}+02$ & $5.9 \mathrm{E}+01$ \\
\hline Acenaphthylene & $000208-96-8$ & & & & \\
\hline Acephate & $030560-19-1$ & $2.6 \mathrm{E}+02$ & $2.6 \mathrm{E}+00$ & $3.9 \mathrm{E}+01$ & $3.9 E+00$ \\
\hline Acetaldehyde & $000075-07-0$ & & & & \\
\hline Acetochlor & $034256-82-1$ & & & $2.0 \mathrm{E}+02$ & $2.0 \mathrm{E}+01$ \\
\hline Acetone & $000067-64-1$ & & & $9.9 \mathrm{E}+02$ & $9.9 \mathrm{E}+01$ \\
\hline Acetone Cyanohydrin & $000075-86-5$ & & & $7.9 \mathrm{E}+00$ & $7.9 \mathrm{E}-01$ \\
\hline Acetonitrile & $000075-05-8$ & & & $5.9 \mathrm{E}+01$ & $5.9 \mathrm{E}+00$ \\
\hline Acetophenone $^{(s)}$ & $000098-86-2$ & & & $9.9 \mathrm{E}+02$ & $9.9 \mathrm{E}+01$ \\
\hline Acrolein $^{(6)}$ & $000107-02-8$ & & & $2.0 \mathrm{E}+02$ & $2.0 \mathrm{E}+01$ \\
\hline Acrylamide & $000079-06-1$ & 5.1E-01 & $5.1 \mathrm{E}-03$ & $2.0 \mathrm{E}+00$ & $2.0 \mathrm{E}-01$ \\
\hline Acrylic Acid & $000079-10-7$ & & & $4.9 E+03$ & $4.9 E+02$ \\
\hline Acrylonitrile & $000107-13-1$ & $4.3 \mathrm{E}+00$ & 4.3E-02 & $9.9 \mathrm{E}+00$ & $9.9 \mathrm{E}-01$ \\
\hline Alachlor & $015972-60-8$ & $2.9 \mathrm{E}+01$ & $2.9 \mathrm{E}-01$ & $9.9 \mathrm{E}+01$ & $9.9 \mathrm{E}+00$ \\
\hline Aldicarb & $000116-06-3$ & & & $9.9 \mathrm{E}+00$ & 9.9E-01 \\
\hline Aldicarb Sulfone & $001646-88-4$ & & & $9.9 \mathrm{E}+00$ & $.9 .9 \mathrm{E}-01$ \\
\hline Aldrin & $000309-00-2$ & $1.4 \mathrm{E}-01$ & $1.4 \mathrm{E}-03$ & $3.0 \mathrm{E}-01$ & $3.0 \mathrm{E}-02$ \\
\hline
\end{tabular}


Table 3.4e. (continued)

\begin{tabular}{|c|c|c|c|c|c|}
\hline \multirow{2}{*}{ Chemical } & \multirow{2}{*}{ CASRN } & \multicolumn{4}{|c|}{ Ingestion of fish (mg/kg) } \\
\hline & & $10^{-4}$ & $10^{-6}$ & $H Q=1$ & $\mathrm{HQ}=0.1$ \\
\hline Ally & $074223-64-6$ & & & $2.5 \mathrm{E}+03$ & $2.5 \mathrm{E}+02$ \\
\hline Allyl Alcohol & 000107-18-6 & & & $4.9 \mathrm{E}+01$ & $4.9 \mathrm{E}+00$ \\
\hline Allyl Chloride ${ }^{(5)}$ & 000107-05-1 & & & $4.9 \mathrm{E}+02$ & $4.9 \mathrm{E}+01$ \\
\hline Aluminnum & 007429-90-5 & & & & \\
\hline Aluminum Phosphide & 020859-73-8 & & & $3.9 \mathrm{E}+00$ & $3.9 \mathrm{E}-01$ \\
\hline Amdro & $067485-29-4$ & & & $3.0 \mathrm{E}+00$ & $3.0 \mathrm{E}-01$ \\
\hline Ametryn & $000834-12-8$ & & & $8.9 \mathrm{E}+01$ & $8.9 \mathrm{E}+00$ \\
\hline Aminophenol, m- & $000591-27-5$ & & & $6.9 \mathrm{E}+02$ & $6.9 \mathrm{E}+01$ \\
\hline Aminopyridine, 4- & 000504-24-5 & & & $2.0 \mathrm{E}-01$ & 2.0E-02 \\
\hline Amitraz & 033089-61-1 & & & $2.5 \mathrm{E}+01$ & $2.5 \mathrm{E}+00$ \\
\hline Ammonia & 007664-41-7 & & & & \\
\hline Ammonium Sulfamate & 007773-06-0 & & & $2.0 \mathrm{E}+03$ & $2.0 \mathrm{E}+02$ \\
\hline Aniline & 000062-53-3 & $4.0 \mathrm{E}+02$ & $4.0 \mathrm{E}+00$ & & \\
\hline Anthracene & 000120-12-7 & & & $3.0 \mathrm{E}+03$ & $3.0 \mathrm{E}+02$ \\
\hline Antimony (metallic) & 007440-36-0 & & & $3.9 \mathrm{E}+00$ & $3.9 \mathrm{E}-01$ \\
\hline Antimony Pentoxide & 001314-60-9 & & & $4.9 \mathrm{E}+00$ & $4.9 \mathrm{E}-01$ \\
\hline Antimony Potassium Tartrate & 000304-61-0 & & & $8.9 \mathrm{E}+00$ & $8.9 \mathrm{E}-01$ \\
\hline Antimony Tetroxide & 001332-81-6 & & & $3.9 \mathrm{E}+00$ & $3.9 \mathrm{E}-01$ \\
\hline
\end{tabular}


Table 3.4e. (continued)

\begin{tabular}{|c|c|c|c|c|c|}
\hline \multirow{2}{*}{ Chemical } & \multirow{2}{*}{ CASRN } & \multicolumn{4}{|c|}{ Ingestion of fish (mg/kg) } \\
\hline & & $10^{4}$ & $10^{-6}$ & $H Q=1$ & $\mathrm{HQ}=0.1$ \\
\hline Antimony Trioxide & 001309-64-4 & & & $3.9 \mathrm{E}+00$ & $3.9 \mathrm{E}-01$ \\
\hline Apollo & 074115-24-5 & & & $1.3 \mathrm{E}+02$ & $1.3 \mathrm{E}+01$ \\
\hline Aramite & $000140-57-8$ & $9.2 \mathrm{E}+01$ & $9.2 \mathrm{E}-01$ & $4.9 \mathrm{E}+02$ & $4.9 \mathrm{E}+01$ \\
\hline Aroclor 1016 & $012674-11-2$ & & & $6.9 \mathrm{E}-01$ & $6.9 \mathrm{E}-02$ \\
\hline Aroclor $1221^{(1)}$ & 011104-28-2 & $3.0 \mathrm{E}-01$ & $3.0 \mathrm{E}-03$ & & \\
\hline Aroclor $1232^{(1)}$ & $011141-16-5$ & $3.0 \mathrm{E}-01$ & $3.0 \mathrm{E}-03$ & & \\
\hline Aroclor $1242^{(1)}$ & 053469-21-9 & $3.0 \mathrm{E}-01$ & $3.0 \mathrm{E}-03$ & & \\
\hline Aroclor $1248^{(1)}$ & $012672-29-6$ & $3.0 \mathrm{E}-01$ & $3.0 \mathrm{E}-03$ & & \\
\hline Aroclor 1254 & $011097-69-1$ & & & 2.0E-01 & $2.0 \mathrm{E}-02$ \\
\hline Aroclor $1260^{(1)}$ & 011096-82-5 & $3.0 \mathrm{E}-01$ & $3.0 \mathrm{E}-03$ & & \\
\hline Arsenic Salts & NA & & & & \\
\hline Arsenic, Inorganic & 007440-38-2 & $1.5 \mathrm{E}+00$ & $1.5 \mathrm{E}-02$ & $3.0 \mathrm{E}+00$ & $3.0 \mathrm{E}-01$ \\
\hline Arsine & $007784-42-1$ & & & & \\
\hline Asbestos & $001332-21-4$ & & & & \\
\hline Assure & $076578-14-8$ & & & $8.9 E+01$ & $8.9 \mathrm{E}+00$ \\
\hline Asulam & $003337-71-1$ & & & $4.9 \mathrm{E}+02$ & $4.9 \mathrm{E}+01$ \\
\hline Atrazine & 001912-24-9 & $1.0 \mathrm{E}+01$ & $1.0 \mathrm{E}-01$ & $3.5 \mathrm{E}+02$ & $3.5 \mathrm{E}+01$ \\
\hline Avermectin B1 & 065195-55-3 & & & $3.9 \mathrm{E}+00$ & $3.9 \mathrm{E}-01$ \\
\hline
\end{tabular}


Table 3.4e. (continued)

\begin{tabular}{|l|l|l|l|l|l|}
\hline \multirow{2}{*}{ Chemical } & \multirow{2}{*}{ CASRN } & \multicolumn{4}{|c|}{ Ingestion of fish (mg/kg) } \\
\cline { 3 - 6 } & & $10^{-4}$ & $10^{-6}$ & HQ=1 & HQ=0.1 \\
\hline Azobenzene & $000103-33-3$ & $2.1 \mathrm{E}+01$ & $2.1 \mathrm{E}-01$ & & \\
\hline Barium & $007440-39-3$ & & & $6.9 \mathrm{E}+02$ & $6.9 \mathrm{E}+01$ \\
\hline Barium Cyanide & $000542-62-1$ & & & $9.9 \mathrm{E}+02$ & $9.9 \mathrm{E}+01$ \\
\hline Baygon & $000114-26-1$ & & & $3.9 \mathrm{E}+01$ & $3.9 \mathrm{E}+00$ \\
\hline Bayleton & $043121-43-3$ & & & $3.0 \mathrm{E}+02$ & $3.0 \mathrm{E}+01$ \\
\hline Baythroid & $068359-37-5$ & & & $2.5 \mathrm{E}+02$ & $2.5 \mathrm{E}+01$ \\
\hline Benefin & $001861-40-1$ & & & $3.0 \mathrm{E}+03$ & $3.0 \mathrm{E}+02$ \\
\hline Benomyl & $017804-35-2$ & & & $4.9 \mathrm{E}+02$ & $4.9 \mathrm{E}+01$ \\
\hline Bentazon & $025057-89-0$ & & & $2.5 \mathrm{E}+01$ & $2.5 \mathrm{E}+00$ \\
\hline Benz[a]anthracene ${ }^{(2)}$ & $000056-55-3$ & $3.2 \mathrm{E}+00$ & $3.2 \mathrm{E}-02$ & & \\
\hline Benzaldehyde & $000100-52-7$ & & & $9.9 \mathrm{E}+02$ & $9.9 \mathrm{E}+01$ \\
\hline Benzene & $000071-43-2$ & $7.9 \mathrm{E}+01$ & $7.9 \mathrm{E}-01$ & & \\
\hline Benzene Hexachloride & NA & & & & \\
\hline Benzene, Ethyldimethyl & NA & & & & \\
\hline Benzene, Ethylmethyl & NA & & & & \\
\hline Benzene, Methylpropenyl & NA & & & & \\
\hline Benzene, Methylpropyl & NA & & & & \\
\hline Benzene, Trimethyl & $025551-13-7$ & & & & \\
\hline
\end{tabular}


Table 3.4e. (continued)

\begin{tabular}{|c|c|c|c|c|c|}
\hline \multirow{2}{*}{ Chemical } & \multirow{2}{*}{ CASRN } & \multicolumn{4}{|c|}{ Ingestion of fish (mg/kg)" } \\
\hline & & $10^{-4}$ & $10^{-6}$ & $H Q=1$ & $\mathrm{HQ}=0.1$ \\
\hline Benzenethiol & $000108-98-5$ & & & $9.9 \mathrm{E}-02$ & $9.9 \mathrm{E}-03$ \\
\hline Benzidine & $000092-87-5$ & $1.0 \mathrm{E}-02$ & $1.0 \mathrm{E}-04$ & $3.0 \mathrm{E}+01$ & $3.0 \mathrm{E}+00$ \\
\hline Benzo[a]pyrene ${ }^{(2)}$ & $000050-32-8$ & 3.2E-01 & $3.2 \mathrm{E}-03$ & & \\
\hline Benzo[b]fluoranthene ${ }^{(2)}$ & $000205-99-2$ & $3.2 \mathrm{E}+00$ & $3.2 \mathrm{E}-02$ & & \\
\hline Benzo[g,h,i]perylene & 000191-24-2 & & & & \\
\hline Benzo[k]fluoranthene ${ }^{(2)}$ & 000207-08-9 & $3.2 \mathrm{E}+01$ & 3.2E-01 & & \\
\hline Benzoic Acid & $000065-85-0$ & & & $3.9 \mathrm{E}+04$ & $3.9 \mathrm{E}+03$ \\
\hline Benzotrichloride & 000098-07-7 & $1.8 \mathrm{E}-01$ & $1.8 \mathrm{E}-03$ & & \\
\hline Benzyl Alcohol & $000100-51-6$ & & & $3.0 \mathrm{E}+03$. & $3.0 \mathrm{E}+02$ \\
\hline Benzyl Chloride & $000100-44-7$ & $1.4 \mathrm{E}+01$ & $1.4 \mathrm{E}-01$ & & \\
\hline Beryllium & $007440-41-7$ & 5.3E-01 & $5.3 \mathrm{E}-03$ & $4.9 \mathrm{E}+01$ & $4.9 \mathrm{E}+00$ \\
\hline Bidrin & $000141-66-2$ & & & $9.9 \mathrm{E}-01$ & $9.9 \mathrm{E}-02$ \\
\hline Biphenthrin & 082657-04-3 & & & $1.5 E+02$ & $1.5 \mathrm{E}+01$ \\
\hline Biphenyl, 1,1'- & $000092-52-4$ & & & $4.9 \mathrm{E}+02$ & $4.9 \mathrm{E}+01$ \\
\hline $\begin{array}{l}\text { Bis(2-chloro-1-methylethyl)ether } \\
\text { (Technical) }\end{array}$ & 000108-60-1 & $3.3 \mathrm{E}+01$ & 3.3E-01 & & \\
\hline Bis(2-chloroethoxy)methane & 000111-91-1 & & & & \\
\hline Bis(2-chloroethyl)ether & $000111-44-4$ & $2.1 \mathrm{E}+00$ & 2.1E-02 & & \\
\hline
\end{tabular}




\begin{tabular}{|c|c|c|c|c|c|}
\hline & & & & $\mathbf{V N}$ & 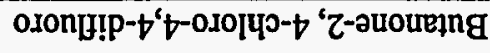 \\
\hline \multirow[t]{2}{*}{$10+96.6$} & $20+966$ & & & $\varepsilon-9 \varepsilon-I L 0000$ & $-N^{6}$ lourełng \\
\hline & & & & 0-66-90โ000 & $-\varepsilon^{c} I$ 'ouə!pelng \\
\hline $10+30 \% 2$ & $20+90^{\circ} Z$ & & & $\tau-66-689100$ & 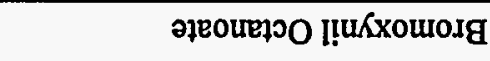 \\
\hline \multirow[t]{2}{*}{$10+30 \%$} & $20+B 0^{\circ} Z$ & & & s-t8-689100 & I!uKxowo.g \\
\hline & & & & $L-Z 9-S \angle 0000$ & әиечрәшодо|чр!пошодg \\
\hline $00+96^{\circ} t$ & $10+36^{\circ} t$ & & & $\varepsilon-96-t 0 I z 00$ & soydowosg \\
\hline $00+36 \div$ & $I 0+B b^{\circ} I$ & & & $6-\varepsilon 8-t<0000$ & (9) $)^{\text {atrqpowowosg }}$ \\
\hline \multirow[t]{2}{*}{$10+90 \%$} & $\mathrm{zO}+\mathrm{B0} \mathrm{Z}$ & $00+96 \tau$ & $20+36 \tau$ & $\tau-\varsigma Z-\varsigma \angle 0000$ & wrojourodg \\
\hline & & & & $\varepsilon-S S-101000$ & 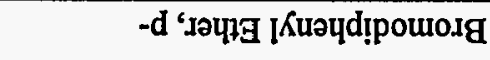 \\
\hline \multirow[t]{3}{*}{$10+307$} & $20+90^{\circ} Z$ & $10-\exists L^{\circ} \varepsilon$ & $10+\exists L^{\circ} \varepsilon$ & $t-L Z-S L 0000$ & әиечрәшологчрฺрошолg \\
\hline & & & & $s-\angle 6 ־ \downarrow \angle 0000$ & 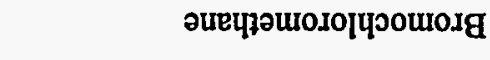 \\
\hline & & & & Z゙LO-LE9L0O & әр!̣onנJ!! L uodog \\
\hline $10+36^{\circ} 8$ & $20+36.8$ & & & $8-Z t-0 t b<00$ & 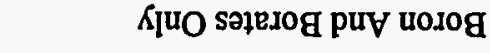 \\
\hline \multirow[t]{2}{*}{$10+966^{\circ}$} & $20+36$ & & & $L-\varsigma 0-080000$ & V jouəqds!g \\
\hline & & t0-90\% I & $20-30 \% I$ & $I-88-Z t S 000$ & 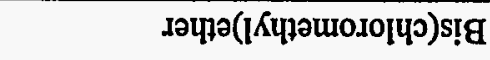 \\
\hline $10+90^{\circ} Z$ & $20+900^{\circ} \tau$ & $00+999^{\circ} I$ & $20+391$ & $L-I 8-\angle I I 000$ & 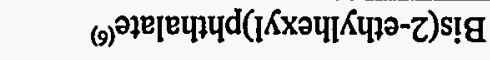 \\
\hline $10+96^{\circ} \varepsilon$ & $20+36^{\circ} \varepsilon$ & & & 6-乙E-8£96£0 & 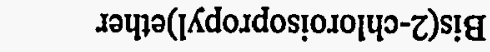 \\
\hline$I^{\prime} \mathbf{0}=\mathbf{\partial H}$ & $\mathbf{I}=\mathbf{O H}$ & $9-0 I$ & r.OI & \multirow{2}{*}{ NQSVD } & \multirow{2}{*}{ 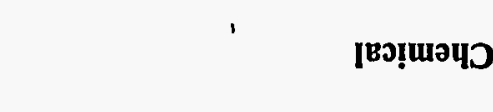 } \\
\hline & 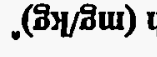 & Uọ̣seชి & & & \\
\hline
\end{tabular}


Table 3.4e. (continued)

\begin{tabular}{|l|l|l|c|c|c|}
\hline \multirow{2}{*}{ Chemical } & \multirow{2}{*}{ CASRN } & \multicolumn{3}{|c|}{ Ingestion of fish (mg/kg) } \\
\cline { 4 - 6 } & & $10^{-4}$ & $10^{-5}$ & HQ=1 & HQ=0.1 \\
\hline Butyl Benzyl Phthlate & $000085-68-7$ & & & $2.0 \mathrm{E}+03$ & $2.0 \mathrm{E}+02$ \\
\hline Butylate & $002008-41-5$ & & & $4.9 \mathrm{E}+02$ & $4.9 \mathrm{E}+01$ \\
\hline Butylchloride, t- & $000507-20-0$ & & & & \\
\hline Butylphthalyl Butylglycolate & $000085-70-1$ & & & $9.9 \mathrm{E}+03$ & $9.9 \mathrm{E}+02$ \\
\hline Cacodylic Acid & $000075-60-5$ & & & $3.0 \mathrm{E}+01$ & $3.0 \mathrm{E}+00$ \\
\hline Cadmium (Diet) & $007440-43-9$ & & & $9.9 \mathrm{E}+00$ & $9.9 \mathrm{E}-01$ \\
\hline Calcium Cyanide & $000592-01-8$ & & & $3.9 \mathrm{E}+02$ & $3.9 \mathrm{E}+01$ \\
\hline Caprolactam & $000105-60-2$ & & & $4.9 \mathrm{E}+03$ & $4.9 \mathrm{E}+02$ \\
\hline Captafol & $002425-06-1$ & $2.7 \mathrm{E}+02$ & $2.7 \mathrm{E}+00$ & $2.0 \mathrm{E}+01$ & $2.0 \mathrm{E}+00$ \\
\hline Captan & $000133-06-2$ & $6.6 \mathrm{E}+02$ & $6.6 \mathrm{E}+00$ & $1.3 \mathrm{E}+03$ & $1.3 \mathrm{E}+02$ \\
\hline Carbaryl & $000063-25-2$ & & & $9.9 \mathrm{E}+02$ & $9.9 \mathrm{E}+01$ \\
\hline Carbazole & $000086-74-8$ & $1.2 \mathrm{E}+02$ & $1.2 \mathrm{E}+00$ & & \\
\hline Carbofuran & $001563-66-2$ & & & $4.9 \mathrm{E}+01$ & $4.9 \mathrm{E}+00$ \\
\hline Carbon Disulfide & $000075-15-0$ & & & $9.9 \mathrm{E}+02$ & $9.9 \mathrm{E}+01$ \\
\hline Carbon Tetrachloride ${ }^{(6)}$ & $000056-23-5$ & $1.8 \mathrm{E}+01$ & $1.8 \mathrm{E}-01$ & $6.9 \mathrm{E}+00$ & $6.9 \mathrm{E}-01$ \\
\hline Carbosulfan & $055285-14-8$ & & & $9.9 \mathrm{E}+01$ & $9.9 \mathrm{E}+00$ \\
\hline Carboxin & $005234-68-4$ & & & $9.9 \mathrm{E}+02$ & $9.9 \mathrm{E}+01$ \\
\hline Chloral & $000075-87-6$ & & & $2.0 \mathrm{E}+01$ & $2.0 \mathrm{E}+00$ \\
\hline
\end{tabular}


Table 3.4e. (continued)

\begin{tabular}{|l|l|l|c|c|c|}
\hline \multirow{2}{*}{ Chemical } & \multirow{2}{*}{ CASRN } & \multicolumn{3}{|c|}{ Ingestion of fish (mg/kg) } \\
\cline { 4 - 6 } & & $10^{-4}$ & $10^{-6}$ & HQ=1 & HQ=0.1 \\
\hline Chloramben & $000133-90-4$ & & & $1.5 \mathrm{E}+02$ & $1.5 \mathrm{E}+01$ \\
\hline Chloranil & $000118-75-2$ & $5.7 \mathrm{E}+00$ & $5.7 \mathrm{E}-02$ & & \\
\hline Chlordane(6) & $000057-74-9$ & $1.8 \mathrm{E}+00$ & $1.8 \mathrm{E}-02$ & $5.9 \mathrm{E}-01$ & $5.9 \mathrm{E}-02$ \\
\hline Chloride & $016887-00-6$ & & & & \\
\hline Chlorimuron, Ethyl- & $090982-32-4$ & & & $2.0 \mathrm{E}+02$ & $2.0 \mathrm{E}+01$ \\
\hline Chlorine & $007782-50-5$ & & & $9.9 \mathrm{E}+02$ & $9.9 \mathrm{E}+01$ \\
\hline Chlorine Dioxide & $010049-04-4$ & & & & \\
\hline Chlorite (Sodium Salt) & $007758-19-2$ & & & & \\
\hline Chloro-1,1-difluoroethane, 1- & $000075-68-3$ & & & & \\
\hline Chloro-1,3-butadiene, 2- & $000126-99-8$ & & & $2.0 \mathrm{E}+02$ & $2.0 \mathrm{E}+01$ \\
\hline Chloro-2-methylaniline HCl, 4- & $003165-93-3$ & $5.0 \mathrm{E}+00$ & $5.0 \mathrm{E}-02$ & & \\
\hline Chloro-2-methylaniline, 4- & $000095-69-2$ & $4.0 \mathrm{E}+00$ & $4.0 \mathrm{E}-02$ & & \\
\hline Chloroacetic Acid & $000079-11-8$ & & & $2.0 \mathrm{E}+01$ & $2.0 \mathrm{E}+00$ \\
\hline Chloroacetophenone, 2- & $000532-27-4$ & & & & \\
\hline Chloroaniline, p- & $000106-47-8$ & & & $3.9 \mathrm{E}+01$ & $3.9 \mathrm{E}+00$ \\
\hline Chlorobenzene ${ }^{(6)}$ & $000108-90-7$ & & & $2.0 \mathrm{E}+02$ & $2.0 \mathrm{E}+01$ \\
\hline Chlorobenzilate & $000510-15-6$ & $8.5 \mathrm{E}+00$ & $8.5 \mathrm{E}-02$ & $2.0 \mathrm{E}+02$ & $2.0 \mathrm{E}+01$ \\
\hline Chlorobenzoic Acid, p- & $000074-11-3$ & & & $2.0 \mathrm{E}+03$ & $2.0 \mathrm{E}+02$ \\
\hline
\end{tabular}


Table 3.4e. (continued)

\begin{tabular}{|c|c|c|c|c|c|}
\hline \multirow{2}{*}{ Chemical } & \multirow{2}{*}{ CASRN } & \multicolumn{4}{|c|}{ Ingestion of fish (mg/kg)" } \\
\hline & & $10^{-4}$ & $10^{-6}$ & $\mathbf{H Q}=\mathbf{1}$ & $H Q=0.1$ \\
\hline Chlorobenzotrifluoride, 4- & $000098-56-6$ & & & $2.0 \mathrm{E}+02$ & $2.0 \mathrm{E}+01$ \\
\hline Chlorobutane, 1- & $000109-69-3$ & & & $3.9 \mathrm{E}+03$ & $3.9 \mathrm{E}+02$ \\
\hline Chlorobutane, 2- & $000078-86-4$ & & & & \\
\hline Chlorocyclopentadiene & 041851-50-7 & & & & \\
\hline Chlorodibromoethane & 073506-94-2 & $2.7 \mathrm{E}+01$ & 2.7E-01 & & \\
\hline Chlorodifluoromethane & $000075-45-6$ & & & & \\
\hline Chloroform $^{(6)}$ & $000067-66-3$ & $3.8 \mathrm{E}+02$ & $3.8 \mathrm{E}+00$ & $9.9 \mathrm{E}+01$ & $9.9 \mathrm{E}+00$ \\
\hline Chloromethane $^{(6)}$ & $000074-87-3$ & $1.8 \mathrm{E}+02$ & $1.8 \mathrm{E}+00$ & & \\
\hline Chloromethyl Methyl Ether & $000107-30-2$ & & & & \\
\hline Chloronaphthalene, Beta- & $000091-58-7$ & & & $7.9 E+02$ & $7.9 \mathrm{E}+01$ \\
\hline Chloronitrobenzene, o- & 000088-73-3 & $9.2 \mathrm{E}+01$ & $9.2 \mathrm{E}-01$ & & \\
\hline Chloronitrobenzene, $p$ - & $000121-73-3$ & $1.3 \mathrm{E}+02$ & $1.3 \mathrm{E}+00$ & & \\
\hline Chlorophenol, 2- & 000095-57-8 & & & $4.9 \mathrm{E}+01$ & $4.9 \mathrm{E}+00$ \\
\hline Chlorophenyl Methyl Sulfide, p- & $000123-09-1$ & & & & \\
\hline Chlorophenyl Methyl Sulfoxide & 000934-73-6 & & & & \\
\hline Chloropropane, 2- & 000075-29-6 & & & & \\
\hline Chlorothalonil & 001897-45-6 & $2.1 \mathrm{E}+02$ & $2.1 \mathrm{E}+00$ & $1.5 \mathrm{E}+02$ & $1.5 \mathrm{E}+01$ \\
\hline Chlorotoluene, o- & 000095-49-8 & & & $2.0 \mathrm{E}+02$ & $2.0 \mathrm{E}+01$ \\
\hline
\end{tabular}


Table 3.4e. (continued)

\begin{tabular}{|c|c|c|c|c|c|}
\hline \multirow{2}{*}{ Chemical } & \multirow{2}{*}{ CASRN } & \multicolumn{4}{|c|}{ Ingestion of fish (mg/kg) } \\
\hline & & $10^{-4}$ & $10^{-6}$ & $\mathbf{H Q}=\mathbf{1}$ & $H Q=0.1$ \\
\hline Chlorpropham & $000101-21-3$ & & & $2.0 \mathrm{E}+03$ & $2.0 \mathrm{E}+02$ \\
\hline Chlorpyrifos & $002921-88-2$ & & & $3.0 \mathrm{E}+01$ & $3.0 \mathrm{E}+00$ \\
\hline Chlorpyrifos Methyl & 005598-13-0 & & & $9.9 \mathrm{E}+01$ & $9.9 \mathrm{E}+00$ \\
\hline Chlorsulfuron & $064902-72-3$ & & & $4.9 \mathrm{E}+02$ & $4.9 \mathrm{E}+01$ \\
\hline Chlorthiophos & $060238-56-4$ & & & $7.9 \mathrm{E}+00$ & $7.9 \mathrm{E}-01$ \\
\hline Chromium (III) (Insoluble Salts) & $016065-83-1$ & & & $9.9 \mathrm{E}+03$ & $9.9 \mathrm{E}+02$ \\
\hline Chromium $(\mathrm{VI})^{(6)}$ & $018540-29-9$ & & & $4.9 \mathrm{E}+01$ & $4.9 \mathrm{E}+00$ \\
\hline Chromium Salts & NA & & & & \\
\hline Chrysene $^{(2)}$ & $000218-01-9$ & $3.2 \mathrm{E}+02$ & $3.2 \mathrm{E}+00$ & & \\
\hline Cobalt & $007440-48-4$ & & & & \\
\hline Coke Oven Emissions & $008007-45-2$ & & & & \\
\hline Copper & $007440-50-8$ & & & & \\
\hline Copper Cyanide & $000544-92-3$ & & & $4.9 \mathrm{E}+01$ & $4.9 \mathrm{E}+00$ \\
\hline Creosote & $008001-58-9$ & & & & \\
\hline Cresol, m- & $000108-39-4$ & & & $4.9 \mathrm{E}+02$ & $4.9 \mathrm{E}+01$ \\
\hline Cresol, o- & $000095-48-7$ & & & $4.9 \mathrm{E}+02$ & $4.9 \mathrm{E}+01$ \\
\hline Cresol, p- & $000106-44-5$ & & & $4.9 \mathrm{E}+01$ & $4.9 \mathrm{E}+00$ \\
\hline Crotonaldehyde & 000123-73-9 & $1.2 \mathrm{E}+00$ & $1.2 \mathrm{E}-02$ & & \\
\hline
\end{tabular}


Table 3.4e. (continued)

\begin{tabular}{|c|c|c|c|c|c|}
\hline \multirow{2}{*}{ Chemical } & \multirow{2}{*}{ CASRN } & \multicolumn{4}{|c|}{ Ingestion of fish (mg/kg) } \\
\hline & & $10^{-4}$ & $10^{-6}$ & $\mathbf{H Q}=\mathbf{1}$ & $\mathrm{HQ}=0.1$ \\
\hline Cumene & 000098-82-8 & & & $3.9 \mathrm{E}+02$ & $3.9 \mathrm{E}+01$ \\
\hline Cyanazine & $021725-46-2$ & $2.7 \mathrm{E}+00$ & 2.7E-02 & $2.0 \mathrm{E}+01$ & $2.0 \mathrm{E}+00$ \\
\hline Cyanide (CN-) & $000057-12-5$ & & & $2.0 \mathrm{E}+02$ & $2.0 \mathrm{E}+01$ \\
\hline Cyanogen & 000460-19-5 & & & $3.9 \mathrm{E}+02$ & $3.9 \mathrm{E}+01$ \\
\hline Cyanogen Bromide & 000506-68-3 & & & $8.9 \mathrm{E}+02$ & $8.9 E+01$ \\
\hline Cyanogen Chloride & 000506-77-4 & & & $4.9 \mathrm{E}+02$ & $4.9 \mathrm{E}+01$ \\
\hline $\begin{array}{l}\text { Cyclohexane, 1,2,3,4,5-pentabromo-6- } \\
\text { chloro- }\end{array}$ & 000087-84-3 & $1.0 \mathrm{E}+02$ & $1.0 \mathrm{E}+00$ & & \\
\hline Cyclohexanone & 000108-94-1 & & & $4.9 \mathrm{E}+04$ & $4.9 \mathrm{E}+03$ \\
\hline Cyclohexylamine & 000108-91-8 & & & $2.0 \mathrm{E}+03$ & $2.0 \mathrm{E}+02$ \\
\hline Cyclopentadiene & $000542-92-7$ & & & & \\
\hline Cyhalothrin/karate & 068085-85-8 & & & $4.9 E+01$ & $4.9 \mathrm{E}+00$ \\
\hline Cypermethrin & $052315-07-8$ & & & $9.9 \mathrm{E}+01$ & $9.9 \mathrm{E}+00$ \\
\hline Cyromazine & $066215-27-8$ & & & $7.4 \mathrm{E}+01$ & $7.4 \mathrm{E}+00$ \\
\hline DDD & $000072-54-8$ & $9.6 \mathrm{E}+00$ & $9.6 \mathrm{E}-02$ & & \\
\hline $\mathrm{DDE}$ & $000072-55-9$ & $6.8 \mathrm{E}+00$ & $6.8 \mathrm{E}-02$ & & \\
\hline DDT & 000050-29-3 & $6.8 \mathrm{E}+00$ & $6.8 \mathrm{E}-02$ & $4.9 \mathrm{E}+00$ & $4.9 \mathrm{E}-01$ \\
\hline Dacthal & 001861-32-1 & & & $9.9 \mathrm{E}+01$ & $9.9 \mathrm{E}+00$ \\
\hline
\end{tabular}


Table 3.4e. (continued)

\begin{tabular}{|l|l|l|c|c|c|}
\hline \multirow{2}{*}{ Chemical } & \multirow{2}{*}{ CASRN } & \multicolumn{3}{|c|}{ Ingestion of fish (mg/kg) } \\
\cline { 4 - 6 } & & $10^{-4}$ & $10^{-6}$ & HQ=1 & HQ=0.1 \\
\hline Dalapon & $000075-99-0$ & & & $3.0 \mathrm{E}+02$ & $3.0 \mathrm{E}+01$ \\
\hline Decabromodiphenyl Ether & $001163-19-5$ & & & $9.9 \mathrm{E}+01$ & $9.9 \mathrm{E}+00$ \\
\hline Demeton & $008065-48-3$ & & & $3.9 \mathrm{E}-01$ & $3.9 \mathrm{E}-02$ \\
\hline Di(2-ethylhexyl)adipate & $000103-23-1$ & $1.9 \mathrm{E}+03$ & $1.9 \mathrm{E}+01$ & $5.9 \mathrm{E}+03$ & $5.9 \mathrm{E}+02$ \\
\hline Diallate & $002303-16-4$ & $3.8 \mathrm{E}+01$ & $3.8 \mathrm{E}-01$ & & \\
\hline Diazinon & $000333-41-5$ & & & $8.9 \mathrm{E}+00$ & $8.9 \mathrm{E}-01$ \\
\hline Dibenz[a,h]anthracene ${ }^{(2)}$ & $000053-70-3$ & $3.2 \mathrm{E}-01$ & $3.2 \mathrm{E}-03$ & & \\
\hline Dibromo-3-chloropropane, 1,2- & $000096-12-8$ & $1.6 \mathrm{E}+00$ & $1.6 \mathrm{E}-02$ & & \\
\hline Dibromobenzene, 1,4- & $000106-37-6$ & & & $9.9 \mathrm{E}+01$ & $9.9 \mathrm{E}+00$ \\
\hline Dibromochloromethane & $000124-48-1$ & $2.7 \mathrm{E}+01$ & $2.7 \mathrm{E}-01$ & $2.0 \mathrm{E}+02$ & $2.0 \mathrm{E}+01$ \\
\hline Dibromodichloromethane & $000594-18-3$ & & & & \\
\hline Dibromodiphenyl Ether, p,p'- & $002050-47-7$ & & & & \\
\hline Dibromoethane, 1,2- & $000106-93-4$ & $2.7 \mathrm{E}-02$ & $2.7 \mathrm{E}-04$ &. & \\
\hline Dibromomethane (Methylene Bromide) & $000074-95-3$ & & & $9.9 \mathrm{E}+01$ & $9.9 \mathrm{E}+00$ \\
\hline Dibutyl Phthalate & $000084-74-2$ & & & $9.9 \mathrm{E}+02$ & $9.9 \mathrm{E}+01$ \\
\hline Dicamba & $001918-00-9$ & & & $3.0 \mathrm{E}+02$ & $3.0 \mathrm{E}+01$. \\
\hline Dichloro-2-butene, $1,4-$ & $000764-41-0$ & & & & \\
\hline Dichloroacetic Acid & & & & & \\
\hline
\end{tabular}


Table 3.4e. (continued)

\begin{tabular}{|l|l|l|c|c|c|}
\hline \multirow{2}{*}{ Chemical } & \multirow{2}{*}{ CASRN } & \multicolumn{3}{|c|}{ Ingestion of fish (mg/kg) } \\
\cline { 4 - 5 } & & $10^{-4}$ & $10^{-6}$ & HQ=1 & HQ=0.1 \\
\hline Dichlorobenzene, 1,2-(5) & $000095-50-1$ & & & $8.9 \mathrm{E}+02$ & $8.9 \mathrm{E}+01$ \\
\hline Dichlorobenzene, 1,3- & $000541-73-1$ & & & & \\
\hline Dichlorobenzene, 1,4- & $000106-46-7$ & $9.6 \mathrm{E}+01$ & $9.6 \mathrm{E}-01$ & & \\
\hline Dichlorobenzidine, 3,3- & $000091-94-1$ & $5.1 \mathrm{E}+00$ & $5.1 \mathrm{E}-02$ & & \\
\hline Dichlorodifluoromethane & $000075-71-8$ & & & $2.0 \mathrm{E}+03$ & $2.0 \mathrm{E}+02$ \\
\hline Dichloroethane, 1,1- & $000075-34-3$ & & & $9.9 \mathrm{E}+02$ & $9.9 \mathrm{E}+01$ \\
\hline Dichloroethane, 1,2- & $000107-06-2$ & $2.5 \mathrm{E}+01$ & $2.5 \mathrm{E}-01$ & & \\
\hline Dichloroethylene, 1,1- & $000075-35-4$ & $3.8 \mathrm{E}+00$ & $3.8 \mathrm{E}-02$ & $8.9 \mathrm{E}+01$ & $8.9 \mathrm{E}+00$ \\
\hline Dichloroethylene, 1,2-(Mixed Isomers) & $000540-59-0$ & & & $8.9 \mathrm{E}+01$ & $8.9 \mathrm{E}+00$ \\
\hline Dichloroethylene, 1,2-cis- & $000156-59-2$ & & & $9.9 \mathrm{E}+01$ & $9.9 \mathrm{E}+00$ \\
\hline Dichloroethylene, 1,2-trans- & $000156-60-5$ & & & $2.0 \mathrm{E}+02$ & $2.0 \mathrm{E}+01$ \\
\hline Dichlorophenol, 2,4- & $000120-83-2$ & & & $3.0 \mathrm{E}+01$ & $3.0 \mathrm{E}+00$ \\
\hline Dichlorophenoxy Acetic Acid, 2,4- & $000094-75-7$ & & & $9.9 \mathrm{E}+01$ & $9.9 \mathrm{E}+00$ \\
\hline Dichlorophenoxy)butyric Acid, 4-(2,4- & $000094-82-6$ & & & $7.9 \mathrm{E}+01$ & $7.9 \mathrm{E}+00$ \\
\hline Dichloropropane, 1,2- & $000078-87-5$ & $3.4 \mathrm{E}+01$ & $3.4 \mathrm{E}-01$ & & \\
\hline Dichloropropanol, 2,3- & $000616-23-9$ & & & $3.0 \mathrm{E}+01$ & $3.0 \mathrm{E}+00$ \\
\hline Dichloropropene, 1,3- & $000542-75-6$ & $1.3 \mathrm{E}+01$ & $1.3 \mathrm{E}-01$ & $3.0 \mathrm{E}+00$ & $3.0 \mathrm{E}-01$ \\
\hline Dichlorvos & $000062-73-7$ & $7.9 \mathrm{E}+00$ & $7.9 \mathrm{E}-02$ & $4.9 \mathrm{E}+00$ & $4.9 \mathrm{E}-01$ \\
\hline
\end{tabular}




\begin{tabular}{|c|c|c|c|c|c|}
\hline & & & & $I-8 L-L L 0000$ & 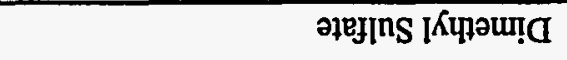 \\
\hline & & $00+899^{\circ} I$ & $20+39 \cdot 1$ & $t-06-6 I I 000$ & 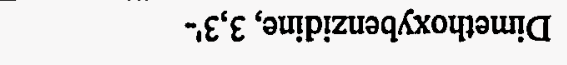 \\
\hline $10-30^{\circ} Z$ & $00+90 \%$ & & & S-IS-090000 & әยвочрәш! \\
\hline $10+30 \%$ & $20+30^{\circ} \tau$ & & & L-t9-06ZSSO & u!d!̣рәu! व \\
\hline \multirow[t]{2}{*}{$10+36 . \mathrm{L}$} & $20+96^{\circ} \mathrm{L}$ & & & $9-S L-S t D I 00$ & 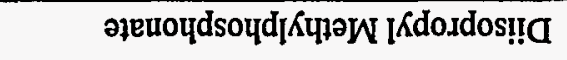 \\
\hline & & & & $9-\angle \varepsilon-S \angle 0000$ & 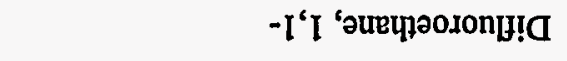 \\
\hline $10+902$ & $20+30 \cdot \tau$ & & & 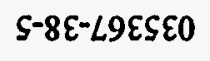 & uormzuaqny!d \\
\hline \multirow[t]{2}{*}{$10+36^{\circ} \mathrm{L}$} & $20+96^{\circ} \mathrm{L}$ & & & $9-8 t-z z Z \varepsilon \vdash 0$ & tenbozuəj! \\
\hline & & $90-96^{\circ} b$ & $t 0-966^{\circ} t$ & $I-\varepsilon \varsigma-9 \varsigma 0000$ & 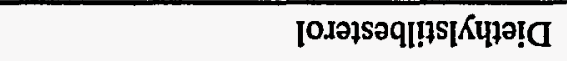 \\
\hline$I 0+g ! \cdot I$ & $20+B I \cdot I$ & & & $s-\nleftarrow 8-\angle[9000$ & 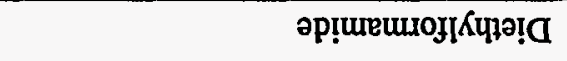 \\
\hline \multirow[t]{4}{*}{$\varepsilon 0+90^{\circ} Z$} & †0+G0 $Z$ & & & $0-06-111000$ & 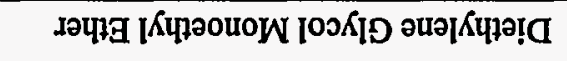 \\
\hline & & & & s-ヤ६-てI1000 & 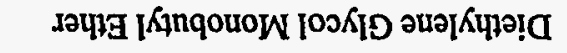 \\
\hline & & & & $0-\lfloor z-\varepsilon 69000$ & 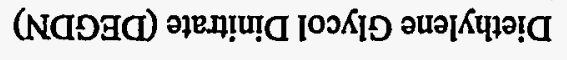 \\
\hline & & & & s-st-I I 1800 & 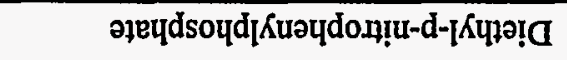 \\
\hline $20+96^{\circ} \mathrm{L}$ & $\varepsilon 0+96^{\circ} \mathrm{L}$ & & & $\tau-99-\$ 80000$ & 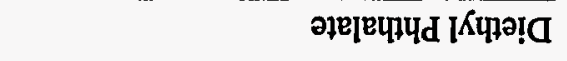 \\
\hline $20-366^{\circ}$ & $10-96 \bullet$ & $\varepsilon 0-\exists t^{\top} I$ & $\mathfrak{I 0}-a b \cdot I$ & $I-\angle S-090000$ & น!.ppəə! \\
\hline \multirow[t]{2}{*}{$10+90^{\circ} \varepsilon$} & $\tau 0+\not 0^{\circ} \varepsilon$ & & & $9-\varepsilon L-L L 0000$ & 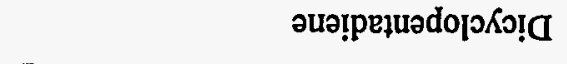 \\
\hline & & & & $\tau-Z \varepsilon-\varsigma I 1000$ & Iojos!a \\
\hline$I^{*} 0=\partial H$ & $\mathbf{I}=\mathbf{O H}$ & $9-0 I$ & r.OI & \multirow{2}{*}{ NצSYD } & \multirow{2}{*}{ ןвगุщวчว } \\
\hline & 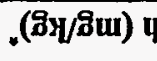 & 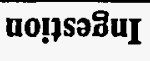 & & & \\
\hline
\end{tabular}


Table 3.4e. (continued)

\begin{tabular}{|l|l|l|l|l|l|}
\hline \multirow{2}{*}{ Chemical } & \multirow{2}{*}{ CASRN } & \multicolumn{3}{|c|}{ Ingestion of fish (mg/kg) } \\
\cline { 4 - 6 } & & $10^{-4}$ & $10^{-6}$ & HQ=1 & HQ=0.1 \\
\hline Dimethylaniline HCl, 2,4- & $021436-96-4$ & $4.0 \mathrm{E}+00$ & $4.0 \mathrm{E}-02$ & & \\
\hline Dimethylaniline, 2,4- & $000095-68-1$ & $3.1 \mathrm{E}+00$ & $3.1 \mathrm{E}-02$ & & \\
\hline Dimethylaniline, N,N- & $000121-69-7$ & & & $2.0 \mathrm{E}+01$ & $2.0 \mathrm{E}+00$ \\
\hline Dimethylbenzidine, 3,3- & $000119-93-7$ & $2.5 \mathrm{E}-01$ & $2.5 \mathrm{E}-03$ & & \\
\hline Dimethylethyl Lead & $107584-40-7$ & & & & \\
\hline Dimethylformamide & $000068-12-2$ & & & $9.9 \mathrm{E}+02$ & $9.9 \mathrm{E}+01$ \\
\hline Dimethylhydrazine, 1,1-(5) & $000057-14-7$ & $8.8 \mathrm{E}-01$ & $8.8 \mathrm{E}-03$ & & \\
\hline Dimethylhydrazine, 1,2- & $000540-73-8$ & & & & \\
\hline Dimethylphenol, 2,4- & $000105-67-9$ & & & $2.0 \mathrm{E}+02$ & $2.0 \mathrm{E}+01$ \\
\hline Dimethylphenol, 2,6- & $000576-26-1$ & & & $5.9 \mathrm{E}+00$ & $5.9 \mathrm{E}-01$ \\
\hline Dimethylphenol, 3,4- & $000095-65-8$ & & & $9.9 \mathrm{E}+00$ & $9.9 \mathrm{E}-01$ \\
\hline Dimethylphthalate ${ }^{(5)}$ & $000131-11-3$ & & & $9.9 \mathrm{E}+04$ & $9.9 \mathrm{E}+03$ \\
\hline Dimethylterephthalate & $000120-61-6$ & & & $9.9 \mathrm{E}+02$ & $9.9 \mathrm{E}+01$ \\
\hline Dinitro-0-cresol, 4,6- & $000534-52-1$ & & & & \\
\hline Dinitro-0-cyclohexyl Phenol, 4,6- & $000131-89-5$ & & & $2.0 \mathrm{E}+01$ & $2.0 \mathrm{E}+00$ \\
\hline Dinitrobenzene, 1,2- & $000528-29-0$ & & & $3.9 \mathrm{E}+00$ & $3.9 \mathrm{E}-01$ \\
\hline Dinitrobenzene, 1,3- & $000099-65-0$ & & & $9.9 \mathrm{E}-01$ & $9.9 \mathrm{E}-02$ \\
\hline Dinitrobenzene, 1,4- & $000100-25-4$ & & & $3.9 \mathrm{E}+00$ & $3.9 \mathrm{E}-01$ \\
\hline
\end{tabular}


Table 3.4e. (continued)

\begin{tabular}{|l|l|l|l|l|l|}
\hline \multirow{2}{*}{ Chemical } & \multirow{2}{*}{ CASRN } & \multicolumn{4}{|c|}{ Ingestion of fish (mg/kg)" } \\
\cline { 4 - 6 } & & $10^{-4}$ & $10^{-6}$ & HQ=1 & HQ=0.1 \\
\hline Dinitrophenol, 2,4- & $000051-28-5$ & & & $2.0 \mathrm{E}+01$ & $2.0 \mathrm{E}+00$ \\
\hline Dinitrotoluene, 2,4- & $000121-14-2$ & $3.4 \mathrm{E}+00$ & $3.4 \mathrm{E}-02$ & $2.0 \mathrm{E}+01$ & $2.0 \mathrm{E}+00$ \\
\hline Dinitrotoluene, 2,6- & $000606-20-2$ & $3.4 \mathrm{E}+00$ & $3.4 \mathrm{E}-02$ & $9.9 \mathrm{E}+00$ & $9.9 \mathrm{E}-01$ \\
\hline Dinitrotoluene, 2-Amino-4,6- & $035572-78-2$ & & & & \\
\hline Dinoseb & $000088-85-7$ & & & $9.9 \mathrm{E}+00$ & $9.9 \mathrm{E}-01$ \\
\hline Dioxane, 1,4- & $000123-91-1$ & $2.1 \mathrm{E}+02$ & $2.1 \mathrm{E}+00$ & & \\
\hline Diphenamid & $000957-51-7$ & & & $3.0 \mathrm{E}+02$ & $3.0 \mathrm{E}+01$ \\
\hline Diphenylamine & $000122-39-4$ & & & $2.5 \mathrm{E}+02$ & $2.5 \mathrm{E}+01$ \\
\hline Diphenylhydrazine, 1,2- & $000122-66-7$ & $2.9 \mathrm{E}+00$ & $2.9 \mathrm{E}-02$ & & \\
\hline Diquat & $000085-00-7$ & & & $2.2 \mathrm{E}+01$ & $2.2 \mathrm{E}+00$ \\
\hline Direct Black 38 & $001937-37-7$ & $2.7 \mathrm{E}-01$ & $2.7 \mathrm{E}-03$ & & \\
\hline Direct Blue 6 & $002602-46-2$ & $2.8 \mathrm{E}-01$ & $2.8 \mathrm{E}-03$ & & \\
\hline Direct Brown 95 & $016071-86-6$ & $2.5 \mathrm{E}-01$ & $2.5 \mathrm{E}-03$ & & \\
\hline Direct Sky Blue & $002610-05-1$ & & & & \\
\hline Disulfoton & $000298-04-4$ & & & $3.9 \mathrm{E}-01$ & $3.9 \mathrm{E}-02$ \\
\hline Diuron & $000330-54-1$ & & & $2.0 \mathrm{E}+01$ & $2.0 \mathrm{E}+00$ \\
\hline Dodine & $002439-10-3$ & & & $3.9 \mathrm{E}+01$ & $3.9 \mathrm{E}+00$ \\
\hline EPTC & $000759-94-4$ & & & $2.5 \mathrm{E}+02$ & $2.5 \mathrm{E}+01$ \\
\hline
\end{tabular}




\begin{tabular}{|c|c|c|c|c|c|}
\hline $10+90^{\circ} \mathrm{Z}$ & $20+90 \%$ & & & $\varepsilon-\varsigma I-\angle 0 I 000$ & 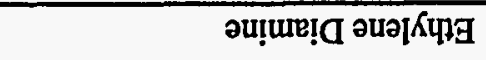 \\
\hline $20+\exists 0^{\circ} \varepsilon$ & $\varepsilon 0+\exists 0^{\circ} \varepsilon$ & & & $b-8 L-601000$ & 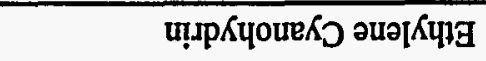 \\
\hline $10+36^{\circ} 6$ & $20+366$ & & & $b-1 b-001000$ & (9) \\
\hline$\varepsilon 0-86 \%$ & $20-96.6$ & & & s-t9-৮0IZ00 & 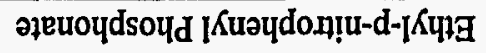 \\
\hline $10+36.8$ & $20+36 \cdot 8$ & & & $\tau-\varepsilon 9-\angle 60000$ & 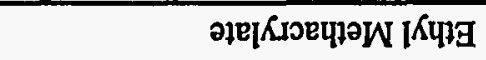 \\
\hline \multirow[t]{3}{*}{$20+30 \tau$} & $\mathcal{E} 0+\mathrm{H} 0^{\circ} \tau$ & & & $L-6 z-090000$ & 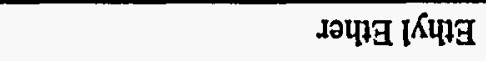 \\
\hline & & & & $\varepsilon-00-\varsigma \angle 0000$ & 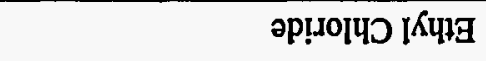 \\
\hline & & $10-98 \cdot b$ & $10+98^{\circ} b$ & $s-88-0+1000$ & 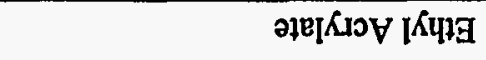 \\
\hline $20+96.8$ & $\varepsilon 0+96.8$ & & & $9-8 L-I t[000$ & 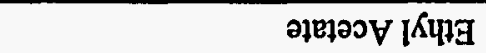 \\
\hline $20+96^{\circ} \varepsilon$ & $\mathcal{E} 0+\mathrm{g} 6^{\circ} \varepsilon$ & & & $s=08-0 I 1000$ & 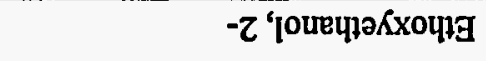 \\
\hline $20+200^{\circ} \varepsilon$ & $\varepsilon 0+\exists 0^{\circ} \varepsilon$ & & & 6-SI-III000 & 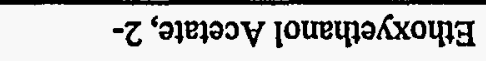 \\
\hline $10-96^{\circ} t$ & $00+36^{\circ} t$ & & & $\tau-z I-\varepsilon 9 S 000$ & นo!นเ日 \\
\hline \multirow[t]{2}{*}{$00+36^{\circ} b$} & $10+36^{\circ}$ & & & $0-\angle 8-Z L 99 I 0$ & นоपุdəપุี \\
\hline & & & & $L-88-90\lceil 000$ & 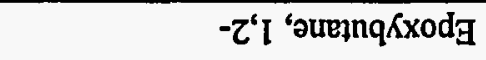 \\
\hline $00+30 \%$ & $10+30^{\circ} \tau$ & $00+g \varepsilon \cdot z$ & $\tau 0+\exists \varepsilon \varepsilon^{\prime} 乙$ & $8-68-901000$ & 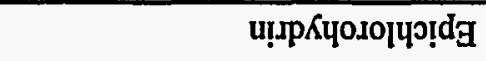 \\
\hline$\left[0-80^{\circ} \varepsilon\right.$ & $00+\exists 0^{\circ} \varepsilon$ & & & $8-0 z-z<0000$ & u!pug \\
\hline $10+30^{\circ} z$ & $20+300^{\circ}$ & & & $\varepsilon-\varepsilon L-S †[000$ & I[вч)орив \\
\hline $00+96 s$ & $10+36 \mathrm{~S}$ & & & $L-6 Z-S I I 000$ & ueyjnsopug \\
\hline$I^{\prime} 0=\partial H$ & $\mathbf{I}=\mathbf{O H}$ & $9.0 \mathrm{I}$ & ,OI & मNCyר & 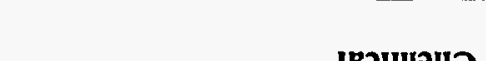 \\
\hline \multicolumn{4}{|c|}{ 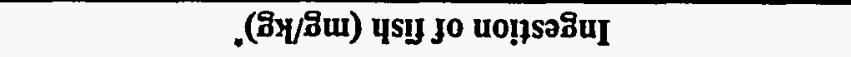 } & S & \\
\hline
\end{tabular}

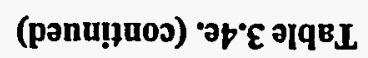


Table 3.4e. (continued)

\begin{tabular}{|c|c|c|c|c|c|}
\hline \multirow{2}{*}{ Chemical } & \multirow{2}{*}{ CASRN } & \multicolumn{4}{|c|}{ Ingestion of fish (mg/kg) } \\
\hline & & $10^{-4}$ & $10^{-6}$ & $H Q=1$ & $\mathbf{H Q}=0.1$ \\
\hline Ethylene Glycol & $000107-21-1$ & & & $2.0 \mathrm{E}+04$ & $2.0 \mathrm{E}+03$ \\
\hline Ethylene Glycol Monobutyl Ether & $000111-76-2$ & & & & \\
\hline Ethylene Oxide & $000075-21-8$ & $2.3 \mathrm{E}+00$ & 2.3E-02 & - & \\
\hline Ethylene Thiourea & $000096-45-7$ & $2.1 \mathrm{E}+01$ & $2.1 \mathrm{E}-01$ & $7.9 \mathrm{E}-01$ & 7.9E-02 \\
\hline Ethylphthalyl Ethyl Glycolate & $000084-72-0$ & & & $3.0 \mathrm{E}+04$ & $3.0 \mathrm{E}+03$ \\
\hline Express & $101200-48-0$ & & & $7.9 \mathrm{E}+01$ & $7.9 \mathrm{E}+00$ \\
\hline Fenamiphos & $022224-92-6$ & & & $2.5 \mathrm{E}+00$ & 2.5E-01 \\
\hline Fenpropathrin & $039515-41-8$ & & & $2.5 \mathrm{E}+02$ & $2.5 \mathrm{E}+01$ \\
\hline Fluometuron & $002164-17-2$ & & & $1.3 E+02$ & $1.3 \mathrm{E}+01$ \\
\hline Fluoranthene & $000206-44-0$ & & & $3.9 \mathrm{E}+02$ & $3.9 E+01$ \\
\hline Fluorene & $000086-73-7$ & & & $3.9 \mathrm{E}+02$ & $3.9 \mathrm{E}+01$ \\
\hline Fluoride & $007782-41-4$ & & & $5.9 E+02$ & $5.9 \mathrm{E}+01$ \\
\hline Fluridone & $059756-60-4$ & & & $7.9 \mathrm{E}+02$ & $7.9 \mathrm{E}+01$ \\
\hline Flurprimidol & $056425-91-3$ & & & $2.0 \mathrm{E}+02$ & $2.0 \mathrm{E}+01$ \\
\hline Flutolanil & $066332-96-5$ & & & $5.9 \mathrm{E}+02$ & $5.9 \mathrm{E}+01$ \\
\hline Fluvalinate & 069409-94-5 & & & $9.9 \mathrm{E}+01$ & $9.9 \mathrm{E}+00$ \\
\hline Folpet & $000133-07-3$ & $6.6 \mathrm{E}+02$ & $6.6 \mathrm{E}+00$ & $9.9 \mathrm{E}+02$ & $9.9 \mathrm{E}+01$ \\
\hline Fomesafen & $072178-02-0$ & $1.2 \mathrm{E}+01$ & $1.2 \mathrm{E}-01$ & & \\
\hline
\end{tabular}


Table 3.4e. (continued)

\begin{tabular}{|c|c|c|c|c|c|}
\hline \multirow{2}{*}{ Chemical } & \multirow{2}{*}{ CASRN } & \multicolumn{4}{|c|}{ Ingestion of fish (mg/kg) } \\
\hline & & $10^{-4}$ & $10^{-6}$ & $H Q=1$ & $\mathrm{HQ}=0.1$ \\
\hline Fonofos & 000944-22-9 & & & $2.0 \mathrm{E}+01$ & $2.0 \mathrm{E}+00$ \\
\hline Formaldehyde & $000050-00-0$ & & & $2.0 \mathrm{E}+03$ & $2.0 \mathrm{E}+02$ \\
\hline Formic Acid & 000064-18-6 & & & $2.0 \mathrm{E}+04$ & $2.0 \mathrm{E}+03$ \\
\hline Fosetyl-AL & $039148-24-8$ & & & $3.0 \mathrm{E}+04$ & $3.0 \mathrm{E}+03$ \\
\hline Furan & $000110-00-9$ & & & $9.9 \mathrm{E}+00$ & $9.9 \mathrm{E}-01$ \\
\hline Furazolidone & $000067-45-8$ & $6.1 \mathrm{E}-01$ & $6.1 \mathrm{E}-03$ & & \\
\hline Furfural & $000098-01-1$ & & & $3.0 \mathrm{E}+01$ & $3.0 \mathrm{E}+00$ \\
\hline Furium & $000531-82-8$ & $4.6 \mathrm{E}-02$ & $4.6 \mathrm{E}-04$ & & \\
\hline Furmecyclox & 060568-05-0 & $7.7 \mathrm{E}+01$ & 7.7E-01 & & \\
\hline Glufosinate, Ammonium & $077182-82-2$ & & & $3.9 \mathrm{E}+00$ & $3.9 \mathrm{E}-01$ \\
\hline Glycidyl & 000765-34-4 & & & $3.9 \mathrm{E}+00$ & $3.9 \mathrm{E}-01$ \\
\hline Glyphosate & 001071-83-6 & & & $9.9 \mathrm{E}+02$ & $9.9 \mathrm{E}+01$ \\
\hline Goal & 042874-03-3 & & & $3.0 \mathrm{E}+01$ & $3.0 \mathrm{E}+00$ \\
\hline Haloxyfop, Methyl & $069806-40-2$ & & & $4.9 \mathrm{E}-01$ & $4.9 \mathrm{E}-02$ \\
\hline Harmony & $079277-27-3$ & & & $1.3 \mathrm{E}+02$ & $1.3 \mathrm{E}+01$ \\
\hline Heptachlor & 000076-44-8 & $5.1 \mathrm{E}-01$ & $5.1 \mathrm{E}-03$ & $4.9 \mathrm{E}+00$ & $4.9 \mathrm{E}-01$ \\
\hline Heptachlor Epoxide & $001024-57-3$ & $2.5 \mathrm{E}-01$ & $2.5 \mathrm{E}-03$ & $1.3 \mathrm{E}-01$ & $1.3 \mathrm{E}-02$ \\
\hline Heptane, N- & $000142-82-5$ & & & & \\
\hline
\end{tabular}




\begin{tabular}{|c|c|c|c|c|c|}
\hline & & & & $9-8 L-I 6 S 000$ & $-\tau$ 'əuourXəH \\
\hline \multirow[t]{2}{*}{$10+36 . \mathrm{s}$} & $20+36.5$ & & & $\varepsilon-\downarrow s-0 \amalg 1000$ & $-\mathrm{N}$ 'əuеxəH \\
\hline & & & & $0-90-z 28000$ & 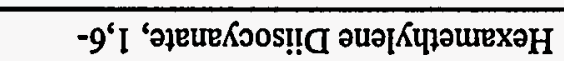 \\
\hline $00+\xi 0^{\circ} \varepsilon$ & {$\left[0+\exists 0^{\circ} \varepsilon\right.$} & I0-gI'Z & $I 0+B I Z$ & $b-Z 8-121000$ & 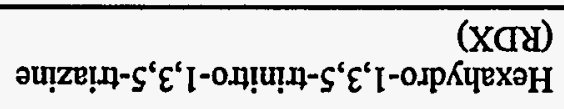 \\
\hline$\left[0^{-\Xi 0^{\circ} \varepsilon}\right.$ & $00+\exists 0^{\circ} \varepsilon$ & & & $\downarrow-0 \varepsilon-0<0000$ & әиәчdoxо|џәехәН \\
\hline \multirow[t]{2}{*}{ I0-966 6} & $00+96^{\circ} 6$ & $00+39^{\circ} I$ & $20+99^{\circ} I$ & $I-Z L-L 90000$ & әиецрәодо|ЧЈехәН \\
\hline & & $90-\exists L^{\circ} \varepsilon$ & to-g $\iota^{\circ} \varepsilon$ & $\varepsilon-t L-80 t 610$ & 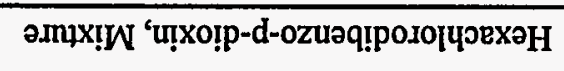 \\
\hline \multirow[t]{2}{*}{$00+36 \cdot 9$} & $10+96.9$ & & & $t-\angle t-\angle L 0000$ & 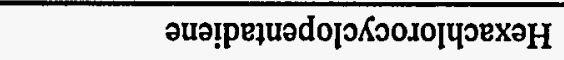 \\
\hline & & $Z 0-\exists \varepsilon^{*} I$ & $00+B \varepsilon \cdot I$ & {$[-\varepsilon L-809000$} & 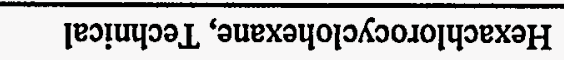 \\
\hline \multirow[t]{5}{*}{$10-90^{\circ} \varepsilon$} & $00+B 0^{\circ} \varepsilon$ & $20-98^{\circ} I$ & $00+98 \cdot I$ & $6-68-8 S 0000$ & 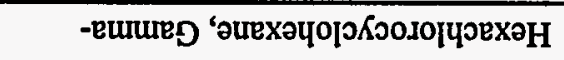 \\
\hline & & & & $L-0 I-80[900$ & 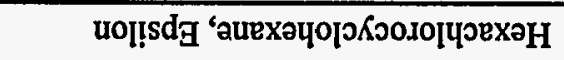 \\
\hline & & & & 8-98-6I $\varepsilon 000$ & 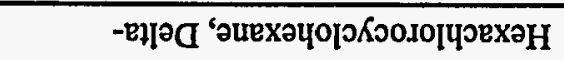 \\
\hline & & $20-\Xi \varepsilon^{*} I$ & $00+\exists \varepsilon^{\prime} L$ & L-\$8-6I $\varepsilon 000$ & 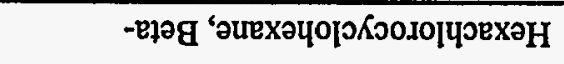 \\
\hline & & $\varepsilon 0^{-} \exists L^{\circ} \varepsilon$ & I0-GL'E & $9-t 8-6 I \varepsilon 000$ & 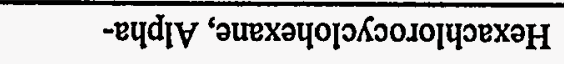 \\
\hline I0-30 2 & $00+\exists 0^{\circ} Z$ & $10-96^{\circ} 2$ & $10+36 z$ & $\varepsilon-89-\angle 80000$ & 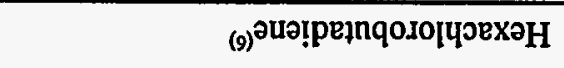 \\
\hline $10-96 \cdot L$ & $00+36 L$ & $20-9 \nabla^{\prime} I$ & $00+3 t^{\circ} I$ & $I-\nabla L-8 I 1000$ & 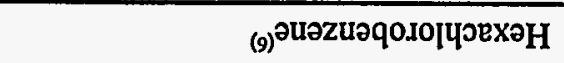 \\
\hline $00+\exists 0 \cdot \tau$ & $10+90 \%$ & & & {$[-Z 8-\angle 80000$} & әนәzuəqouодархәН \\
\hline$I^{\circ} \mathbf{0}=\partial_{H}$ & $\mathbf{I}=\mathbf{O} \mathbf{H}$ & $9.0 I$ & rOI & Drayn & 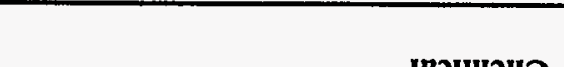 \\
\hline \multicolumn{4}{|c|}{ 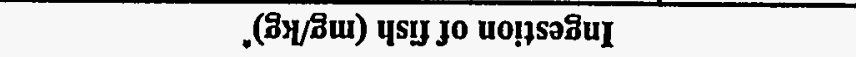 } & - & \\
\hline
\end{tabular}

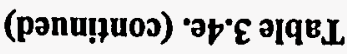


Table 3.4e. (continued)

\begin{tabular}{|c|c|c|c|c|c|}
\hline \multirow{2}{*}{ Chemical } & \multirow{2}{*}{ CASRN } & \multicolumn{4}{|c|}{ Ingestion of fish (mg/kg) } \\
\hline & & $10^{-4}$ & $10^{-6}$ & $\mathbf{H Q}=\mathbf{1}$ & $H Q=0.1$ \\
\hline Hexazinone & $051235-04-2$ & & & $3.3 E+02$ & $3.3 \mathrm{E}+01$ \\
\hline HpCDD, 2,3,7,8-(4) & $037871-00-4$ & $1.5 \mathrm{E}-03$ & $1.5 \mathrm{E}-05$ & & \\
\hline $\mathrm{HpCDF}, 2,3,7,8-^{(4)}$ & $038998-75-3$ & $1.5 \mathrm{E}-03$ & $1.5 \mathrm{E}-05$ & & \\
\hline $\mathrm{HxCDD}, 2,3,7,8{ }^{(4)}$ & $034465-46-8$ & $1.5 \mathrm{E}-04$ & $1.5 \mathrm{E}-06$ & & \\
\hline $\mathrm{HxCDF}, 2,3,7,8-(4)$ & $055684-94-1$ & $1.5 \mathrm{E}-04$ & $1.5 \mathrm{E}-06$ & & \\
\hline Hydrazine & $000302-01-2$ & 7.7E-01 & 7.7E-03 & & \\
\hline Hydrazine Sulfate & $010034-93-2$ & 7.7E-01 & 7.7E-03 & & \\
\hline Hydrogen Chloride & $007647-01-0$ & & & & \\
\hline Hydrogen Cyanide & $000074-90-8$ & & & $2.0 \mathrm{E}+02$ & $2.0 \mathrm{E}+01$ \\
\hline Hydrogen Sulfide & 007783-06-4 & & & $3.0 \mathrm{E}+01$ & $3.0 \mathrm{E}+00$ \\
\hline Hydroquinone & 000123-31-9 & & & $3.9 \mathrm{E}+02$ & $3.9 \mathrm{E}+01$ \\
\hline Imazalil & 035554-44-0 & & & $1.3 \mathrm{E}+02$ & $1.3 E+01$ \\
\hline Imazaquin & $081335-37-7$ & & & $2.5 \mathrm{E}+03$ & $2.5 \mathrm{E}+02$ \\
\hline Indeno $[1,2,3-c d]$ pyrene $^{(2)}$ & 000193-39-5 & $3.2 \mathrm{E}+00$ & $3.2 \mathrm{E}-02$ & & \\
\hline Iprọdione & $036734-19-7$ & & & $3.9 \mathrm{E}+02$ & $3.9 E+01$ \\
\hline Iron & $007439-89-6$ & & & & \\
\hline Isobutyl Alcohol & 000078-83-1 & & & $3.0 \mathrm{E}+03$ & $3.0 \mathrm{E}+02$ \\
\hline Isophorone & $000078-59-1$ & $2.4 \mathrm{E}+03$ & $2.4 \mathrm{E}+01$ & $2.0 \mathrm{E}+03$ & $2.0 \mathrm{E}+02$ \\
\hline
\end{tabular}


Table 3.4e. (continued)

\begin{tabular}{|c|c|c|c|c|c|}
\hline \multirow{2}{*}{ Chemical } & \multirow{2}{*}{ CASRN } & \multicolumn{4}{|c|}{ Ingestion of fish (mg/kg) } \\
\hline & & $10^{-4}$ & $10^{-6}$ & $\mathbf{H Q}=\mathbf{1}$ & $\mathrm{HQ}=0.1$ \\
\hline Isopropalin & 033820-53-0 & & & $1.5 \mathrm{E}+02$ & $1.5 \mathrm{E}+01$ \\
\hline Isopropanol & 000067-63-0 & & & & \\
\hline Isopropyl Methyl Phosphonic Acid & $001832-54-8$ & & & $9.9 \mathrm{E}+02$ & $9.9 \mathrm{E}+01$ \\
\hline Isoxaben & $082558-50-7$ & & & $4.9 E+02$ & $4.9 \mathrm{E}+01$ \\
\hline Karate & 091465-08-6 & & & $4.9 \mathrm{E}+01$ & $4.9 \mathrm{E}+00$ \\
\hline Kerb & 023950-58-5 & & & $7.4 \mathrm{E}+02$ & $7.4 \mathrm{E}+01$ \\
\hline Lactofen & $077501-63-4$ & & & $2.0 \mathrm{E}+01$ & $2.0 \mathrm{E}+00$ \\
\hline Lead Alkyls & NA & & & & \\
\hline Lead And Compounds & 007439-92-1 & & & & \\
\hline Linuron & $000330-55-2$ & & & $2.0 \mathrm{E}+01$ & $2.0 \mathrm{E}+00$ \\
\hline Lithium & 007439-93-2 & & & & \\
\hline Londax & 083055-99-6 & & & $2.0 \mathrm{E}+03$ & $2.0 \mathrm{E}+02$ \\
\hline MCPA & 000094-74-6 & & & $4.9 E+00$ & $4.9 \mathrm{E}-01$ \\
\hline MCPB & 000094-81-5 & & & $9.9 \mathrm{E}+01$ & $9.9 \mathrm{E}+00$ \\
\hline MCPP & 000093-65-2 & & & $9.9 \mathrm{E}+00$ & $9.9 \mathrm{E}-01$ \\
\hline Magnesium & $007439-95-4$ & & & & \\
\hline Malathion & 000121-75-5 & & & $2.0 \mathrm{E}+02$ & $2.0 \mathrm{E}+01$ \\
\hline Maleic Anhydride & 000108-31-6 & & & $9.9 \mathrm{E}+02$ & $9.9 \mathrm{E}+01$ \\
\hline
\end{tabular}


Table 3.4e. (continued)

\begin{tabular}{|c|c|c|c|c|c|}
\hline \multirow{2}{*}{ Chemical } & \multirow{2}{*}{ CASRN } & \multicolumn{4}{|c|}{ Ingestion of fish (mg/kg) } \\
\hline & & $10^{-4}$ & $10^{-6}$ & $H Q=1$ & $H Q=0.1$ \\
\hline Maleic Hydrazide & $000123-33-1$ & & & $4.9 \mathrm{E}+03$ & $4.9 \mathrm{E}+02$ \\
\hline Malononitrile & 000109-77-3 & & & $2.0 \mathrm{E}-01$ & $2.0 \mathrm{E}-02$ \\
\hline Mancozeb & 008018-01-7 & & & $3.0 \mathrm{E}+02$ & $3.0 \mathrm{E}+01$ \\
\hline Maneb & $012427-38-2$ & & & $4.9 \mathrm{E}+01$ & $4.9 \mathrm{E}+00$ \\
\hline Manganese (Diet) & 007439-96-5 & & & $1.4 E+03$ & $1.4 \mathrm{E}+02$ \\
\hline Mephosfolan & 000950-10-7 & & & $8.9 \mathrm{E}-01$ & $8.9 \mathrm{E}-02$ \\
\hline Mepiquat Chloride & $024307-26-4$ & & & $3.0 \mathrm{E}+02$ & $3.0 \mathrm{E}+01$ \\
\hline Mercuric Chloride & 007487-94-7 & & & $3.0 \mathrm{E}+00$ & $3.0 \mathrm{E}-01$ \\
\hline Mercury (elemental) & 007439-97-6 & & & $3.0 \mathrm{E}+00$ & $3.0 \mathrm{E}-01$ \\
\hline Mercury, Inorganic Salt & 007439-97-6 & & & $3.0 \mathrm{E}+00$ & $3.0 \mathrm{E}-01$ \\
\hline Merphos & $000150-50-5$ & & & $3.0 \mathrm{E}-01$ & $3.0 \mathrm{E}-02$ \\
\hline Merphos Oxide & 000078-48-8 & & & $3.0 \mathrm{E}-01$ & $3.0 \mathrm{E}-02$ \\
\hline Metalaxyl & $057837-19-1$ & & & $5.9 \mathrm{E}+02$ & $5.9 \mathrm{E}+01$ \\
\hline Methacrylonitrile & 000126-98-7 & & & $9.9 \mathrm{E}-01$ & $9.9 \mathrm{E}-02$ \\
\hline Methamidophos & $010265-92-6$ & & & $4.9 \mathrm{E}-01$ & $4.9 \mathrm{E}-02$ \\
\hline Methanol & 000067-56-1 & & & $4.9 \mathrm{E}+03$ & $4.9 \mathrm{E}+02$ \\
\hline Methidathion & 000950-37-8 & & & $9.9 E+00$ & $9.9 \mathrm{E}-01$ \\
\hline Methomyl & $016752-77-5$ & & & $2.5 \mathrm{E}+02$ & $2.5 \mathrm{E}+01$ \\
\hline
\end{tabular}




\begin{tabular}{|c|c|c|c|c|c|}
\hline $10+36.5$ & $20+86^{\circ} \mathrm{s}$ & $00+\operatorname{al} \varepsilon$ & $20+9 I^{\circ} \varepsilon$ & $\tau-60-\varsigma<0000$ & 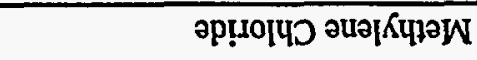 \\
\hline & & & & $\tau-\angle 8-80 I 000$ & 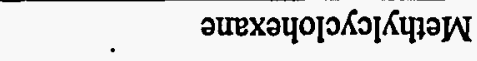 \\
\hline & & I0-ヨE'I & $I 0+A \varepsilon \cdot I$ & s-IZ-9\&9000 & 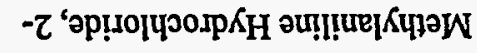 \\
\hline & & I0-90. L & $10+30^{\circ} \mathrm{C}$ & $8-\varsigma \varsigma-660000$ & 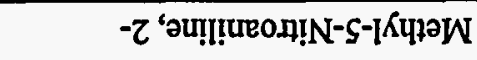 \\
\hline & & & & 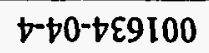 & 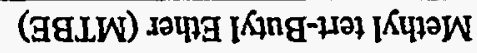 \\
\hline & & $z 0-\exists I \cdot z$ & $00+B I \cdot z$ & $\downarrow-\downarrow \varepsilon-090000$ & 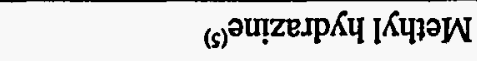 \\
\hline $00+36^{\circ} \varsigma$ & $10+36^{\circ} \mathrm{s}$ & & & $t-S I-\varepsilon I O S Z 0$ & 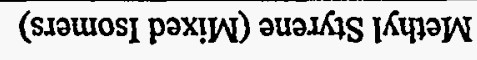 \\
\hline $10-3 s \cdot \tau$ & $00+9 s z$ & & & $0-00-86 Z 000$ & 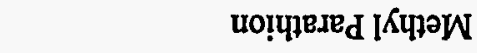 \\
\hline $10+36 . L$ & $20+96^{\circ} \mathrm{L}$ & & & $9-29-080000$ & 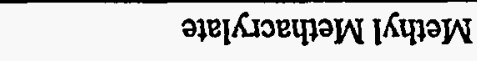 \\
\hline $20-966^{\circ} 6$ & $10-96.6$ & & & 9-Z6-८96ZZZ & 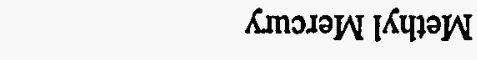 \\
\hline $10+96 \cdot \mathrm{L}$ & $20+86 \%$ & & & $I-0 I-80 I 000$ & 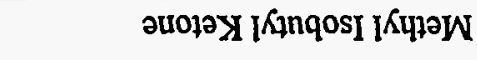 \\
\hline $20+96.5$ & $\varepsilon 0+\exists 6^{\circ} \mathrm{S}$ & & & $\varepsilon-\varepsilon 6-8 L 0000$ & 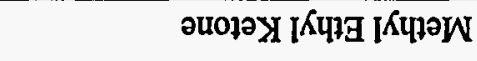 \\
\hline $10+90^{\circ} \varepsilon$ & $20+30^{\circ} \varepsilon$ & & & $\varepsilon-\varepsilon \varepsilon-960000$ & 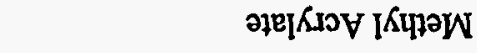 \\
\hline $20+366$ & $\varepsilon 0+966$ & & & $6-0 z-6<0000$ & 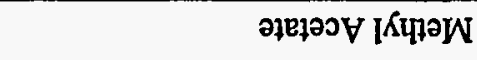 \\
\hline [0--966\% & $00+966$ & & & $t-98-601000$ & 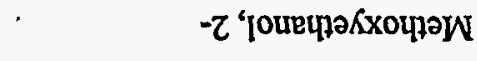 \\
\hline $00+30 \%$ & $10+90^{\circ} z$ & & & $9-6 t-011000$ & 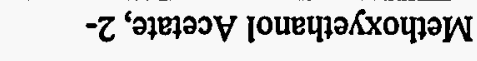 \\
\hline $00+36^{\circ} t$ & $10+86^{\circ} t$ & & & $s-\varepsilon t-Z L 0000$ & มоцчОКхочрәК \\
\hline & & $\mathrm{I} 0-\mathrm{g} 0^{\circ} \mathrm{S}$ & $10+30^{\circ} \mathrm{s}$ & $z-6 \varsigma-660000$ & 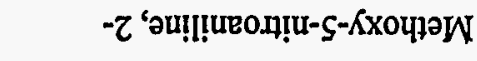 \\
\hline$I^{\prime} \mathbf{0}=\mathbf{\partial H}$ & $I=\mathbf{O H}$ & $9.0 I$ & rOI & \multirow{2}{*}{ N\&SYO } & \multirow{2}{*}{ [вวุщวนว } \\
\hline \multicolumn{4}{|c|}{ 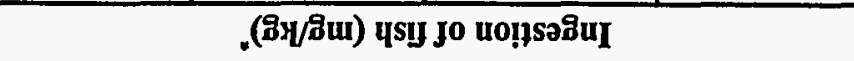 } & & \\
\hline
\end{tabular}

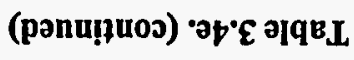


Table 3.4e. (continued)

\begin{tabular}{|l|l|l|c|c|c|}
\hline \multirow{2}{*}{ Chemical } & \multirow{2}{*}{ CASRN } & \multicolumn{3}{|c|}{ Ingestion of fish (mg/kg) } \\
\cline { 3 - 6 } & & $10^{-4}$ & $10^{-6}$ & HQ=1 & HQ=0.1 \\
\hline Methylene-bis(2-chloroaniline), 4,4'- & $000101-14-4$ & $1.8 \mathrm{E}+01$ & $1.8 \mathrm{E}-01$ & $6.9 \mathrm{E}+00$ & $6.9 \mathrm{E}-01$ \\
\hline $\begin{array}{l}\text { Methylene-bis(N,N-dimethyl) Aniline, } \\
\text { 4,4'- }\end{array}$ & $000101-61-1$ & $5.0 \mathrm{E}+01$ & $5.0 \mathrm{E}-01$ & & \\
\hline Methylenebisbenzenamine, 4,4'-(5) & $000101-77-9$ & $9.2 \mathrm{E}+00$ & $9.2 \mathrm{E}-02$ & & \\
\hline Methylenediphenyl Isocyanate, 4,4'- & $000101-68-8$ & & & & \\
\hline Methylstyrene, Alpha- & $000098-83-9$ & & & $6.9 \mathrm{E}+02$ & $6.9 \mathrm{E}+01$ \\
\hline Methyltriethyl Lead & $001762-28-3$ & & & & \\
\hline Metolachlor & $051218-45-2$ & & & $1.5 \mathrm{E}+03$ & $1.5 \mathrm{E}+02$ \\
\hline Metribuzin ${ }^{(5)}$ & $021087-64-9$ & & & $2.5 \mathrm{E}+02$ & $2.5 \mathrm{E}+01$ \\
\hline Mirex ${ }^{(3)}$ & $002385-85-5$ & $1.3 \mathrm{E}+00$ & $1.3 \mathrm{E}-02$ & $2.0 \mathrm{E}+00$ & $2.0 \mathrm{E}-01$ \\
\hline Molinate & $002212-67-1$ & & & $2.0 \mathrm{E}+01$ & $2.0 \mathrm{E}+00$ \\
\hline Molybdenum & $007439-98-7$ & & & $4.9 \mathrm{E}+01$ & $4.9 \mathrm{E}+00$ \\
\hline Monochloramine & $010599-90-3$ & & & $9.9 \mathrm{E}+02$ & $9.9 \mathrm{E}+01$ \\
\hline Monochlorobutanes & $025154-42-1$ & & & $3.9 \mathrm{E}+03$ & $3.9 \mathrm{E}+02$ \\
\hline Naled & $000300-76-5$ & & & $2.0 \mathrm{E}+01$ & $2.0 \mathrm{E}+00$ \\
\hline Naphthalene(6) & $000091-20-3$ & & & $3.5 \mathrm{E}+02$ & $3.5 \mathrm{E}+01$ \\
\hline Naphthalene, 1-Methyl & $000090-12-0$ & & & & \\
\hline Naphthalene, 2-Methyl & $000091-57-6$ & & & & \\
\hline
\end{tabular}


Table 3.4e. (continued)

\begin{tabular}{|c|c|c|c|c|c|}
\hline \multirow{2}{*}{ Chemical } & \multirow{2}{*}{ CASRN } & \multicolumn{4}{|c|}{ Ingestion of fish (mg/kg)" } \\
\hline & & $10^{-4}$ & $10^{-6}$ & $\mathbf{H Q}=\mathbf{1}$ & $H Q=0.1$ \\
\hline Napropamide & 015299-99-7 & & & $9.9 \mathrm{E}+02$ & $9.9 \mathrm{E}+01$ \\
\hline Niagara Blue 4B & $002429-74-5$ & & & & \\
\hline Nickel Carbonyl & 013463-39-3 & & & & \\
\hline Nickel Refinery Dust & NA & & & & \\
\hline Nickel Soluble Salts & $007440-02-0$ & & & $2.0 \mathrm{E}+02$ & $2.0 \mathrm{E}+01$ \\
\hline Nickel Subsulfide & $012035-72-2$ & & & & \\
\hline Nitrapyrin $^{(5)}$ & 001929-82-4 & & & $1.5 \mathrm{E}+01$ & $1.5 \mathrm{E}+00$ \\
\hline Nitrate & $014797-55-8$ & & & $1.6 \mathrm{E}+04$ & $1.6 \mathrm{E}+03$ \\
\hline Nitric Oxide ${ }^{(s)}$ & $010102-43-9$ & & & $9.9 \mathrm{E}+02$ & $9.9 \mathrm{E}+01$ \\
\hline Nitrite & $014797-65-0$ & & & $9.9 \mathrm{E}+02$ & $9.9 E+01$ \\
\hline Nitroaniline, 2- & $000088-74-4$ & & & & \\
\hline Nitrobenzene & 000098-95-3 & & & $4.9 \mathrm{E}+00$ & $4.9 \mathrm{E}-01$ \\
\hline Nitrofurantoin & 000067-20-9 & & & $6.9 \mathrm{E}+02$ & $6.9 \mathrm{E}+01$ \\
\hline Nitrofurazone & 000059-87-0 & $1.5 \mathrm{E}+00$ & $1.5 \mathrm{E}-02$ & & \\
\hline Nitrogen Dioxide ${ }^{(5)}$ & $010102-44-0$ & & & $9.9 \mathrm{E}+03$ & $9.9 \mathrm{E}+02$ \\
\hline Nitroguanidine & 000556-88-7 & & & $9.9 \mathrm{E}+02$ & $9.9 \mathrm{E}+01$ \\
\hline Nitrophenol, 4- & 000100-02-7 & & & & \\
\hline Nitropropane, 2-(5) & $000079-46-9$ & 2.4E-01 & $2.4 \mathrm{E}-03$ & & \\
\hline
\end{tabular}




\begin{tabular}{|c|c|c|c|c|c|}
\hline & & $b 0-\exists S^{*} I$ & $20-\mathrm{GS} \boldsymbol{S}^{\circ} \mathrm{I}$ & $6-\angle 8-89 Z E 00$ & (4) $व$ वगO \\
\hline $10-96^{\circ} 9$ & $00+369$ & & & $6-6 \mathrm{I}-60 S \$ 80$ & $\operatorname{IETSN} N$ \\
\hline $10+\exists 6^{\circ} \varepsilon$ & $20+36^{\circ} \varepsilon$ & & & 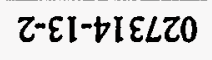 & uozemn」JION \\
\hline $00+366^{\circ}$ & $10+96^{\circ} 6$ & & & $0-66-660000$ & -d 'әuәnј010д!N \\
\hline $00+86^{\circ} 6$ & $10+36.6$ & & & $\tau-\tau L-880000$ & 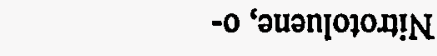 \\
\hline \multirow[t]{13}{*}{$00+36.6$} & $10+96.6$ & & & {$[-80-660000$} & -w 'อuən|010д!N \\
\hline & & & & t-ZE-6II000 & -Z-ou!urV-t 'әuәn|010म!!N \\
\hline & & $20-9 I \cdot I$ & $00+B I \cdot I$ & $z-\varsigma \varsigma-0 \varepsilon 6000$ & -N 'әu!p![0.uKdoson!N \\
\hline & & & & $0-0 t-6 b 5 t 00$ & 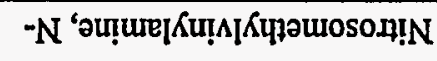 \\
\hline & & $\varepsilon 0^{-g} 0^{\circ} \mathrm{I}$ & I0-E0I & $9-\varsigma 6-\varsigma 6 \varsigma_{0 I 0}$ & 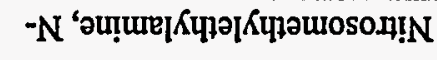 \\
\hline & & $00+3 L^{\circ} b$ & $20+B L \cdot b$ & $9-0 \varepsilon-980000$ & -N ‘ou!ure[Kuәчd!poso.д!N \\
\hline & & t0-GS't & $20-\not S S^{\prime} t$ & $6-S L-290000$ & 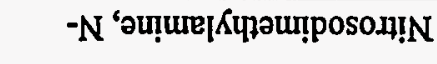 \\
\hline & & $t 0-\exists S^{\top} I$ & Z0-AS'I & $\varsigma-8 I-\varsigma \$ 0000$ & 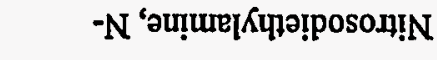 \\
\hline & & $\varepsilon 0-\exists z^{\prime} 8$ & I0-9Z'8 & L-tS-9III00 & -N 'әu!ur[oueqрә!роsoд!N \\
\hline & & $\varepsilon 0-\exists \varepsilon^{\prime} \varepsilon$ & 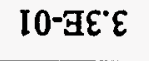 & L-t9-IZ9000 & -N 'əu!ure[Kdoud-N-!p-oson!N \\
\hline & & $\varepsilon 0-\exists \varepsilon^{\prime} \downarrow$ & $\mathfrak{1 0 - \Xi E " t ~}$ & $\varepsilon-9 I-t Z 6000$ & 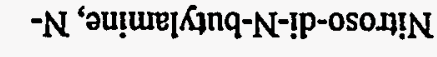 \\
\hline & & & & $s-\varepsilon 6-\$ 89000$ & -N 'еәл! \\
\hline & & t0-59*I & $20-89 \cdot 1$ & $6-\varepsilon L-6 S L 000$ & 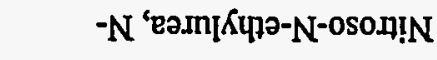 \\
\hline$I^{\prime} \mathbf{0}=\mathbf{O H}$ & $\mathbf{I}=\mathbf{O H}$ & $9-0 \mathrm{I}$ & rOI & NHCY? & 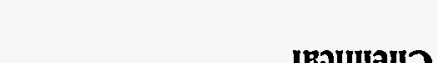 \\
\hline \multicolumn{4}{|c|}{ 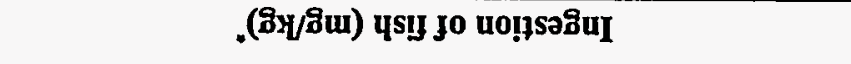 } & 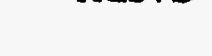 & \\
\hline
\end{tabular}


Table 3.4e. (continued)

\begin{tabular}{|c|c|c|c|c|c|}
\hline \multirow{2}{*}{ Chemical } & \multirow{2}{*}{ CASRN } & \multicolumn{4}{|c|}{ Ingestion of fish (mg/kg) } \\
\hline & & $10^{-4}$ & $10^{-6}$ & $\mathrm{HQ}=\mathbf{1}$ & $\mathrm{HQ}=0.1$ \\
\hline $\mathrm{OCDF}^{(4)}$ & $039001-02-0$ & $1.5 \mathrm{E}-02$ & $1.5 \mathrm{E}-04$ & & \\
\hline Octabromodiphenyl Ether & $032536-52-0$ & & & $3.0 \mathrm{E}+01$ & $3.0 \mathrm{E}+00$ \\
\hline $\begin{array}{l}\text { Octahydro-1,3,5,7-tetranitro-1,3,5,7- } \\
\text { tetra (HMX) }\end{array}$ & $002691-41-0$ & & & $4.9 E+02$ & $4.9 \mathrm{E}+01$ \\
\hline Octamethylpyrophosphoramide & $000152-16-9$ & & & $2.0 \mathrm{E}+01$ & $2.0 \mathrm{E}+00$ \\
\hline Octyl Phthalate, di-N- & 000117-84-0 & & & $2.0 \mathrm{E}+02$ & $2.0 \mathrm{E}+01$ \\
\hline Oryzalin & 019044-88-3 & & & $4.9 \mathrm{E}+02$ & $4.9 \mathrm{E}+01$ \\
\hline Oxadiazon & 019666-30-9 & & & $4.9 E+01$ & $4.9 \mathrm{E}+00$ \\
\hline Oxamyl & 023135-22-0 & & & $2.5 \mathrm{E}+02$ & $2.5 \mathrm{E}+01$ \\
\hline Paclobutrazol & $076738-62-0$ & & & $1.3 \mathrm{E}+02$ & $1.3 \mathrm{E}+01$ \\
\hline Paraquat & $001910-42-5$ & & & $4.4 \mathrm{E}+01$ & $4.4 \mathrm{E}+00$ \\
\hline Parathion & 000056-38-2 & & & $5.9 \mathrm{E}+01$ & $5.9 \mathrm{E}+00$ \\
\hline PeCDD, 2,3,7,8-(4) & 036088-22-9 & 3.1E-05 & 3.1E-07 & & \\
\hline PeCDF, $1,2,3,7,8-(4)$ & $057117-41-6$ & 3.1E-05 & $3.1 \mathrm{E}-07$ & & \\
\hline PeCDF, 2,3,4,7,8-(4) & $057117-31-4$ & $3.1 \mathrm{E}-04$ & $3.1 \mathrm{E}-06$ & & \\
\hline Pebulate & 001114-71-2 & & & $4.9 \mathrm{E}+02$ & $4.9 \mathrm{E}+01$ \\
\hline Pendimethalin & $040487-42-1$ & & & $3.9 \mathrm{E}+02$ & $3.9 \mathrm{E}+01$ \\
\hline Pentabromodiphenyl Ether & 032534-81-9 & & & $2.0 \mathrm{E}+01$ & $2.0 \mathrm{E}+00$ \\
\hline
\end{tabular}


Table 3.4e. (continued)

\begin{tabular}{|l|l|l|l|l|l|}
\hline \multirow{2}{*}{ Chemical } & \multirow{2}{*}{ CASRN } & \multicolumn{4}{|c|}{ Ingestion of fish (mg/kg) } \\
\cline { 5 - 6 } & & $10^{-1}$ & $10^{-6}$ & HQ=1 & HQ=0.1 \\
\hline Pentachlorobenzene & $000608-93-5$ & & & $7.9 \mathrm{E}+00$ & $7.9 \mathrm{E}-01$ \\
\hline Pentachlorocyclopentadiene & $025329-35-5$ & & & & \\
\hline Pentachloronitrobenzene & $000082-68-8$ & $8.8 \mathrm{E}+00$ & $8.8 \mathrm{E}-02$ & $3.0 \mathrm{E}+01$ & $3.0 \mathrm{E}+00$ \\
\hline Pentachlorophenol & $000087-86-5$ & $1.9 \mathrm{E}+01$ & $1.9 \mathrm{E}-01$ & $3.0 \mathrm{E}+02$ & $3.0 \mathrm{E}+01$ \\
\hline Pentyl Alcohol, N- & $000071-41-0$ & & & & \\
\hline Permethrin & $052645-53-1$ & & & $4.9 \mathrm{E}+02$ & $4.9 \mathrm{E}+01$ \\
\hline Phenanthrene & $000085-01-8$ & & & & \\
\hline Phenmedipham & $013684-63-4$ & & & $2.5 \mathrm{E}+03$ & $2.5 \mathrm{E}+02$ \\
\hline Phenol & $000108-95-2$ & & & $5.9 \mathrm{E}+03$ & $5.9 \mathrm{E}+02$ \\
\hline Phenylenediamine, $\mathrm{m}-$ & $000108-45-2$ & & & $5.9 \mathrm{E}+01$ & $5.9 \mathrm{E}+00$ \\
\hline Phenylenediamine, $0-$ & $000095-54-5$ & $4.9 \mathrm{E}+01$ & $4.9 \mathrm{E}-01$ & & \\
\hline Phenylenediamine, $\mathrm{p}-$ & $000106-50-3$ & & & $1.9 \mathrm{E}+03$ & $1.9 \mathrm{E}+02$ \\
\hline Phenylmercuric Acetate & $000062-38-4$ & & & $7.9 \mathrm{E}-01$ & $7.9 \mathrm{E}-02$ \\
\hline Phenylphenol, 2- & $000090-43-7$ & $1.2 \mathrm{E}+03$ & $1.2 \mathrm{E}+01$ & & \\
\hline Phorate & $000298-02-2$ & & & $2.0 \mathrm{E}+00$ & $2.0 \mathrm{E}-01$ \\
\hline Phosmet & $000732-11-6$ & & & $2.0 \mathrm{E}+02$ & $2.0 \mathrm{E}+01$ \\
\hline Phosphine & $007803-51-2$ & & & $3.0 \mathrm{E}+00$ & $3.0 \mathrm{E}-01$ \\
\hline Phosphoric Acid & $007664-38-2$ & & & \\
\hline
\end{tabular}


Table 3.4e. (continued)

\begin{tabular}{|l|l|l|c|c|c|}
\hline \multirow{2}{*}{ Chemical } & \multirow{2}{*}{ CASRN } & \multicolumn{3}{|c|}{ Ingestion of fish (mg/kg) $^{*}$} \\
\cline { 4 - 6 } & & $10^{-4}$ & $10^{-6}$ & HQ=1 & HQ=0.1 \\
\hline Phthalic Acid, P- & $000100-21-0$ & & & $9.9 \mathrm{E}+03$ & $9.9 \mathrm{E}+02$ \\
\hline Phthalic Anhydride & $000085-44-9$ & & & $2.0 \mathrm{E}+04$ & $2.0 \mathrm{E}+03$ \\
\hline Picloram & $001918-02-1$ & & & $6.9 \mathrm{E}+02$ & $6.9 \mathrm{E}+01$ \\
\hline Pirimiphos, Methyl & $029232-93-7$ & & & $9.9 \mathrm{E}+01$ & $9.9 \mathrm{E}+00$ \\
\hline Polybrominated Biphenyls & NA & $2.6 \mathrm{E}-01$ & $2.6 \mathrm{E}-03$ & $6.9 \mathrm{E}-02$ & $6.9 \mathrm{E}-03$ \\
\hline Polychlorinated Biphenyls & $001336-36-3$ & $3.0 \mathrm{E}-01$ & $3.0 \mathrm{E}-03$ & & \\
\hline Potassium Cyanide & $000151-50-8$ & & & $4.9 \mathrm{E}+02$ & $4.9 \mathrm{E}+01$ \\
\hline Potassium Silver Cyanide & $000506-61-6$ & & & $2.0 \mathrm{E}+03$ & $2.0 \mathrm{E}+02$ \\
\hline Prochloraz & $067747-09-5$ & $1.5 \mathrm{E}+01$ & $1.5 \mathrm{E}-01$ & $8.9 \mathrm{E}+01$ & $8.9 \mathrm{E}+00$ \\
\hline Profluralin & $026399-36-0$ & & & $5.9 \mathrm{E}+01$ & $5.9 \mathrm{E}+00$ \\
\hline Prometon & $001610-18-0$ & & & $1.5 \mathrm{E}+02$ & $1.5 \mathrm{E}+01$ \\
\hline Prometryn & $007287-19-6$ & & & $3.9 \mathrm{E}+01$ & $3.9 \mathrm{E}+00$ \\
\hline Propachlor & $001918-16-7$ & & & $1.3 \mathrm{E}+02$ & $1.3 \mathrm{E}+01$ \\
\hline Propanil & $000709-98-8$ & & & $4.9 \mathrm{E}+01$ & $4.9 \mathrm{E}+00$ \\
\hline Propargite & $002312-35-8$ & & & $2.0 \mathrm{E}+02$ & $2.0 \mathrm{E}+01$ \\
\hline Propargyl Alcohol & $000107-19-7$ & & & $2.0 \mathrm{E}+01$ & $2.0 \mathrm{E}+00$ \\
\hline Propazine & $000139-40-2$ & & & $2.0 \mathrm{E}+02$ & $2.0 \mathrm{E}+01$ \\
\hline Propham & $000122-42-9$ & & & $2.0 \mathrm{E}+02$ & $2.0 \mathrm{E}+01$ \\
\hline
\end{tabular}


Table 3.4e. (continued)

\begin{tabular}{|l|l|l|c|c|c|}
\hline \multirow{2}{*}{ Chemical } & \multirow{2}{*}{ CASRN } & \multicolumn{3}{|c|}{ Ingestion of fish (mg/kg) } \\
\cline { 4 - 6 } & & $10^{-4}$ & $10^{-6}$ & HQ=1 & HQ=0.1 \\
\hline Propiconazole & $060207-90-1$ & & & $1.3 \mathrm{E}+02$ & $1.3 \mathrm{E}+01$ \\
\hline Propylene Glycol & $000057-55-6$ & & & $2.0 \mathrm{E}+05$ & $2.0 \mathrm{E}+04$ \\
\hline Propylene Glycol Monoethyl Ether & $001569-02-4$ & & & $6.9 \mathrm{E}+03$ & $6.9 \mathrm{E}+02$ \\
\hline Propylene Glycol Monomethyl Ether & $000107-98-2$ & & & $6.9 \mathrm{E}+03$ & $6.9 \mathrm{E}+02$ \\
\hline Propylene Oxide & $000075-56-9$ & $9.6 \mathrm{E}+00$ & $9.6 \mathrm{E}-02$ & & \\
\hline Pursuit & $081335-77-5$ & & & $2.5 \mathrm{E}+03$ & $2.5 \mathrm{E}+02$ \\
\hline Pydrin & $051630-58-1$ & & & $2.5 \mathrm{E}+02$ & $2.5 \mathrm{E}+01$ \\
\hline Pyrene & $000129-00-0$ & & & $3.0 \mathrm{E}+02$ & $3.0 \mathrm{E}+01$ \\
\hline Pyridine & $000110-86-1$ & & & $9.9 \mathrm{E}+00$ & $9.9 \mathrm{E}-01$ \\
\hline Quinalphos & $013593-03-8$ & & & $4.9 \mathrm{E}+00$ & $4.9 \mathrm{E}-01$ \\
\hline Quinoline & $000091-22-5$ & $1.9 \mathrm{E}-01$ & $1.9 \mathrm{E}-03$ & & \\
\hline Refractory Ceramic Fibers & $\mathrm{NA}$ & & & & \\
\hline Resmethrin & $010453-86-8$ & & & $3.0 \mathrm{E}+02$ & $3.0 \mathrm{E}+01$ \\
\hline Ronnel & $000299-84-3$ & & & $4.9 \mathrm{E}+02$ & $4.9 \mathrm{E}+01$ \\
\hline Rotenone & $000083-79-4$ & & & $3.9 \mathrm{E}+01$ & $3.9 \mathrm{E}+00$ \\
\hline Savey & $078587-05-0$ & & & $2.5 \mathrm{E}+02$ & $2.5 \mathrm{E}+01$ \\
\hline Selenious Acid & $007783-00-8$ & & & $4.9 \mathrm{E}+01$ & $4.9 \mathrm{E}+00$ \\
\hline Selenite & $014124-67-5$ & & & \\
\hline
\end{tabular}


Table 3.4e. (continued)

\begin{tabular}{|l|l|l|c|c|c|}
\hline \multirow{2}{*}{ Chemical } & \multirow{2}{*}{ CASRN } & \multicolumn{3}{|c|}{ Ingestion of fish (mg/kg) } \\
\cline { 4 - 6 } & & $10^{-4}$ & $10^{-6}$ & HQ=1 & HQ=0.1 \\
\hline Selenium & $007782-49-2$ & & & $4.9 \mathrm{E}+01$ & $4.9 \mathrm{E}+00$ \\
\hline Selenium Sulfide & $007446-34-6$ & & & & \\
\hline Selenourea & $000630-10-4$ & & & $4.9 \mathrm{E}+01$ & $4.9 \mathrm{E}+00$ \\
\hline Sethoxydim & $074051-80-2$ & & & $8.9 \mathrm{E}+02$ & $8.9 \mathrm{E}+01$ \\
\hline Silver & $007440-22-4$ & & & $4.9 \mathrm{E}+01$ & $4.9 \mathrm{E}+00$ \\
\hline Silver Cyanide & $000506-64-9$ & & & $9.9 \mathrm{E}+02$ & $9.9 \mathrm{E}+01$ \\
\hline Simazine & $000122-34-9$ & $1.9 \mathrm{E}+01$ & $1.9 \mathrm{E}-01$ & $4.9 \mathrm{E}+01$ & $4.9 \mathrm{E}+00$ \\
\hline Sodium & $007440-23-5$ & & & & \\
\hline Sodium Acifluorfen & $062476-59-9$ & & & $1.3 \mathrm{E}+02$ & $1.3 \mathrm{E}+01$ \\
\hline Sodium Azide & $026628-22-8$ & & & $3.9 \mathrm{E}+01$ & $3.9 \mathrm{E}+00$ \\
\hline Sodium Cyanide & $000143-33-9$ & & & $3.9 \mathrm{E}+02$ & $3.9 \mathrm{E}+01$ \\
\hline Sodium Diethyldithiocarbamate & $000148-18-5$ & $8.5 \mathrm{E}+00$ & $8.5 \mathrm{E}-02$ & $3.0 \mathrm{E}+02$ & $3.0 \mathrm{E}+01$ \\
\hline Sodium Fluoroacetate & $000062-74-8$ & & & $2.0 \mathrm{E}-01$ & $2.0 \mathrm{E}-02$ \\
\hline Sodium Metavanadate & $013718-26-8$ & & & $9.9 \mathrm{E}+00$ & $9.9 \mathrm{E}-01$ \\
\hline Stirofos (Tetrachlorovinphos) & $000961-11-5$ & $9.6 \mathrm{E}+01$ & $9.6 \mathrm{E}-01$ & $3.0 \mathrm{E}+02$ & $3.0 \mathrm{E}+01$ \\
\hline Strontium, Stable & $007440-24-6$ & & & $5.9 \mathrm{E}+03$ & $5.9 \mathrm{E}+02$ \\
\hline Strychnine & $000057-24-9$ & & & $3.0 \mathrm{E}+00$ & $3.0 \mathrm{E}-01$ \\
\hline Styrene ${ }^{(6)}$ & $000100-42-5$ & & & $2.0 \mathrm{E}+03$ & $2.0 \mathrm{E}+02$ \\
\hline
\end{tabular}


Table 3.4e. (continued)

\begin{tabular}{|l|l|l|l|l|l|}
\hline \multirow{2}{*}{ Chemical } & \multirow{2}{*}{ CASRN } & \multicolumn{3}{|c|}{ Ingestion of fish (mg/kg) } \\
\cline { 5 - 6 } & & $10^{-4}$ & $10^{-6}$ & HQ=1 & HQ=0.1 \\
\hline Sulfate & $014808-79-8$ & & & & \\
\hline Systhane & $088671-89-0$ & & & $2.5 \mathrm{E}+02$ & $2.5 \mathrm{E}+01$ \\
\hline TCDD, 2,3,7,8-(4) & $001746-01-6$ & $1.5 \mathrm{E}-05$ & $1.5 \mathrm{E}-07$ & & \\
\hline TCDF, 2,3,7,8-(4) & $051207-31-9$ & $1.5 \mathrm{E}-04$ & $1.5 \mathrm{E}-06$ & & \\
\hline TCMTB & $021564-17-0$ & & & $3.0 \mathrm{E}+02$ & $3.0 \mathrm{E}+01$ \\
\hline Tebuthiuron & $034014-18-1$ & & & $6.9 \mathrm{E}+02$ & $6.9 \mathrm{E}+01$ \\
\hline Temephos & $003383-96-8$ & & & $2.0 \mathrm{E}+02$ & $2.0 \mathrm{E}+01$ \\
\hline Terbacil & $005902-51-2$ & & & $1.3 \mathrm{E}+02$ & $1.3 \mathrm{E}+01$ \\
\hline Terbufos & $013071-79-9$ & & & $2.5 \mathrm{E}-01$ & $2.5 \mathrm{E}-02$ \\
\hline Terbutryn & $000886-50-0$ & & & $9.9 \mathrm{E}+00$ & $9.9 \mathrm{E}-01$ \\
\hline Tetrabutyl Lead & $001920-90-7$ & & & & \\
\hline Tetrachlorobenzene, $1,2,4,5-$ & $000095-94-3$ & & & $3.0 \mathrm{E}+00$ & $3.0 \mathrm{E}-01$ \\
\hline Tetrachloroethane, $1,1,1,2-$ & $000630-20-6$ & $8.8 \mathrm{E}+01$ & $8.8 \mathrm{E}-01$ & $3.0 \mathrm{E}+02$ & $3.0 \mathrm{E}+01$ \\
\hline Tetrachloroethane, $1,1,2,2-$ & $000079-34-5$ & $1.2 \mathrm{E}+01$ & $1.2 \mathrm{E}-01$ & & \\
\hline Tetrachloroethylene ${ }^{(6)}$ & $000127-18-4$ & $4.4 \mathrm{E}+01$ & $4.4 \mathrm{E}-01$ & $9.9 \mathrm{E}+01$ & $9.9 \mathrm{E}+00$ \\
\hline Tetrachlorophenol, $2,3,4,6-$ & $000058-90-2$ & & & $3.0 \mathrm{E}+02$ & $3.0 \mathrm{E}+01$ \\
\hline $\begin{array}{l}\text { Tetrachlorotoluene, } \mathrm{p}-\mathrm{alpha} \\
\text { alpha- alpha, }\end{array}$ & $005216-25-1$ & $1.2 \mathrm{E}-01$ & $1.2 \mathrm{E}-03$ & & \\
\hline & & & & & \\
\hline
\end{tabular}


Table 3.4e. (continued)

\begin{tabular}{|l|l|l|c|c|c|}
\hline \multirow{2}{*}{ Chemical } & \multirow{2}{*}{ CASRN } & \multicolumn{4}{|c|}{ Ingestion of fish (mg/kg) } \\
\cline { 4 - 6 } & & $10^{-4}$ & $10^{-5}$ & HQ=1 & HQ=0.1 \\
\hline Tetraethyl Dithiopyrophosphate & $003689-24-5$ & & & $4.9 \mathrm{E}+00$ & $4.9 \mathrm{E}-01$ \\
\hline Tetraethyl Lead & $000078-00-2$ & & & & \\
\hline Tetrafluoroethane, 1,1,1,2- & $000811-97-2$ & & & & \\
\hline Tetramethyl Lead & $000075-74-1$ & & & & \\
\hline Tetrapropyl Lead & $003440-75-3$ & & & & \\
\hline Thallic Oxide ${ }^{(5)}$ & $001314-32-5$ & & & $6.9 \mathrm{E}-01$ & $6.9 \mathrm{E}-02$ \\
\hline Thallium (I) Nitrate & $010102-45-1$ & & & $8.9 \mathrm{E}-01$ & $8.9 \mathrm{E}-02$ \\
\hline Thallium (Soluble Salts) & $007440-28-0$ & & & & \\
\hline Thallium Acetate & $000563-68-8$ & & & $8.9 \mathrm{E}-01$ & $8.9 \mathrm{E}-02$ \\
\hline Thallium Carbonate & $006533-73-9$ & & & $7.9 \mathrm{E}-01$ & $7.9 \mathrm{E}-02$ \\
\hline Thallium Chloride & $007791-12-0$ & & & $7.9 \mathrm{E}-01$ & $7.9 \mathrm{E}-02$ \\
\hline Thallium Selenite ${ }^{(5)}$ & $012039-52-0$ & & & $8.9 \mathrm{E}-01$ & $8.9 \mathrm{E}-02$ \\
\hline Thallium Sulfate & $007446-18-6$ & & & $7.9 \mathrm{E}-01$ & $7.9 \mathrm{E}-02$ \\
\hline Thiobencarb & $028249-77-6$ & & & $9.9 \mathrm{E}+01$ & $9.9 \mathrm{E}+00$ \\
\hline Thiofanox & $039196-18-4$ & & & $3.0 \mathrm{E}+00$ & $3.0 \mathrm{E}-01$ \\
\hline Thiophanate, Methyl & $023564-05-8$ & & & $7.9 \mathrm{E}+02$ & $7.9 \mathrm{E}+01$ \\
\hline Thiram & $000137-26-8$ & & & $4.9 \mathrm{E}+01$ & $4.9 \mathrm{E}+00$ \\
\hline Thorium & $007440-29-1$ & & & & \\
\hline
\end{tabular}




\begin{tabular}{|c|c|c|c|c|c|}
\hline$† 0+30^{\circ} \varepsilon$ & $S 0+30^{\circ} \varepsilon$ & & & $I-\varepsilon I-9 L 0000$ & 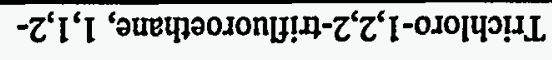 \\
\hline \multirow[t]{3}{*}{$z 0-\exists 0^{\circ} \varepsilon$} & $10-90^{\circ} \varepsilon$ & & & $6-\varsigma \varepsilon-9 \varsigma 0000$ & 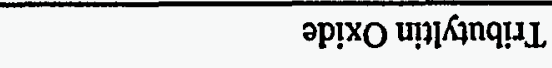 \\
\hline & & & & $0-t 6-0696 t 0$ & 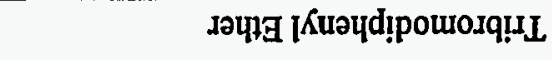 \\
\hline & & & & $0-S I-66 \$ 000$ & 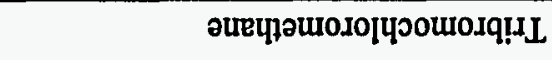 \\
\hline $00+96^{\circ} t$ & $10+36^{\circ} t$ & & & $\varepsilon-b \varsigma-\varsigma I 9000$ & $-b^{6} Z^{6} \mathrm{I}$ 'əuəzuəqourosq!II \\
\hline $00+36^{\circ} 6$ & $10+36 \cdot 6$ & & & $S-0 S-\angle 60 Z 80$ & uo.ny[nSe!̣I \\
\hline $10+g \varepsilon \varepsilon^{\circ} I$ & $20+B \varepsilon \varepsilon^{*} I$ & & & S-LI-EOEZOO & 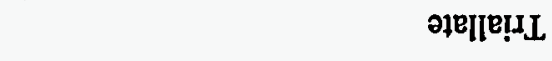 \\
\hline \multirow[t]{4}{*}{$00+3 t^{\circ} L$} & $10+3 b^{\circ} L$ & & & $9-\varsigma \tau-I \$ 8990$ & 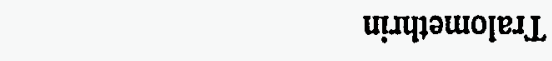 \\
\hline & & zo-gl'z & $00+\mathrm{gI} \tau$ & $Z-S E-I 00800$ & วuวydexol \\
\hline & & $I 0-a Z Z^{\prime} I$ & I0+GZ'I & $0-6 t-901000$ & -d 'วu!pinjol \\
\hline & & $20-39 \cdot 6$ & $00+39 \cdot 6$ & $t-\varepsilon \varsigma-\varsigma 60000$ & 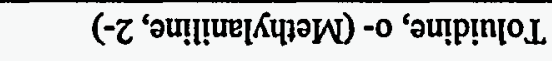 \\
\hline $20+90^{\circ} \mathrm{Z}$ & $\mathcal{E} 0+\exists 0^{\circ} \tau$ & & & $s-0 t-\varepsilon Z 8000$ & 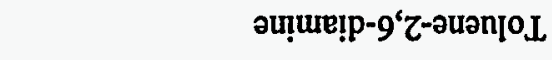 \\
\hline \multirow[t]{3}{*}{$20+966^{\circ} S$} & $\varepsilon 0+\exists 6^{\circ} \varsigma$ & & & $S-0 L-S 60000$ & 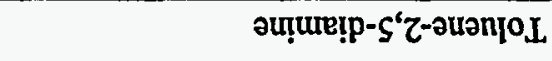 \\
\hline & & $\varepsilon 0-\exists \tau^{\circ} L$ & $10-a z^{\prime} L$ & $L-08-\$ 60000$ & 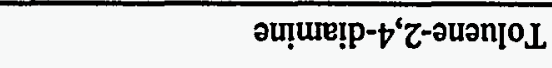 \\
\hline & & & & $s-Z 9-I<t 9 Z 0$ & 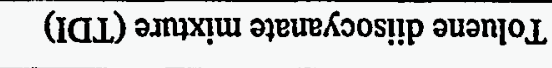 \\
\hline \multirow[t]{2}{*}{$20+30 \%$} & $\varepsilon 0+\exists 0^{\circ} \tau$ & & & $\varepsilon-88-801000$ & (9) $)^{\text {auanloL }}$ \\
\hline & & & & $9-Z \varepsilon-0 \not \triangleright L 00$ & um!̣uE!!I \\
\hline $20+96^{\circ} \mathrm{S}$ & $\mathcal{E} 0+86^{\circ} \mathrm{S}$ & & & $s-I \varepsilon=0 t t<00$ & u!. \\
\hline$I^{*} 0=\partial H$ & $\mathrm{I}=\partial \mathbf{H}$ & $\mathrm{s}-\mathrm{OI}$ & rOI & \multirow{2}{*}{ N\&SVD } & \multirow{2}{*}{ |вอฺ!นวนว } \\
\hline & .(ฮิy/శిน) 4 & นоำรวรిบI & & & \\
\hline
\end{tabular}

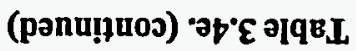


Table 3.4e. (continued)

\begin{tabular}{|c|c|c|c|c|c|}
\hline \multirow{2}{*}{ Chemical } & \multirow{2}{*}{ CASRN } & \multicolumn{4}{|c|}{ Ingestion of fish (mg/kg) } \\
\hline & & $10^{-4}$ & $10^{-6}$ & $\mathbf{H Q}=\mathbf{1}$ & $H Q=0.1$ \\
\hline Trichloro-2'-hydroxydiphenylether & $003380-34-5$ & & & & \\
\hline \multicolumn{6}{|l|}{ Trichloroacetic Acid } \\
\hline Trichloroaniline $\mathrm{HCl}, 2,4,6$ & 033663-50-2 & $7.9 \mathrm{E}+01$ & $7.9 \mathrm{E}-01$ & & \\
\hline Trichloroaniline, 2,4,6- & 000634-93-5 & $6.8 \mathrm{E}+01$ & $6.8 \mathrm{E}-01$ & & \\
\hline Trichlorobenzene, 1,2,4- & 000120-82-1 & & & $9.9 \mathrm{E}+01$ & $9.9 \mathrm{E}+00$ \\
\hline Trichloroethane, 1,1,1-(s) & $000071-55-6$ & & & $8.9 \mathrm{E}+02$ & $8.9 \mathrm{E}+01$ \\
\hline Trichloroethane, 1,1,2- & $000079-00-5$ & $4.0 \mathrm{E}+01$ & $4.0 \mathrm{E}-01$ & $3.9 \mathrm{E}+01$ & $3.9 \mathrm{E}+00$ \\
\hline Trichloroethylene $^{(3)}$ & 000079-01-6 & $2.1 \mathrm{E}+02$ & $2.1 \mathrm{E}+00$ & & \\
\hline Trichlorofluoromethane & $000075-69-4$ & & & $3.0 \mathrm{E}+03$ & $3.0 \mathrm{E}+02$ \\
\hline Trichlorophenol, 2,4,5- & $000095-95-4$ & & & $9.9 \mathrm{E}+02$ & $9.9 \mathrm{E}+01$ \\
\hline Trichlorophenol, 2,4,6- & 000088-06-2 & $2.1 \mathrm{E}+02$ & $2.1 \mathrm{E}+00$ & & \\
\hline $\begin{array}{l}\text { Trichlorophenoxy) Propionic Acid, } \\
2(2,4,5-\end{array}$ & 000093-72-1 & & & $7.9 \mathrm{E}+01$ & $7.9 \mathrm{E}+00$ \\
\hline Trichlorophenoxyacetic Acid, 2,4,5- & $000093-76-5$ & & & $9.9 \mathrm{E}+01$ & $9.9 \mathrm{E}+00$ \\
\hline Trichloropropane, 1,1,2- & $000598-77-6$ & & & $4.9 \mathrm{E}+01$ & $4.9 \mathrm{E}+00$ \\
\hline Trichloropropane, 1,2,3- & $000096-18-4$ & 3.3E-01 & $3.3 \mathrm{E}-03$ & $5.9 \mathrm{E}+01$ & $5.9 \mathrm{E}+00$ \\
\hline Trichloropropene, 1,2,3- & 000096-19-5 & & & $4.9 \mathrm{E}+01$ & $4.9 \mathrm{E}+00$ \\
\hline Trichlorotoluene, 2,3,6- & $002077-46-5$ & & & & \\
\hline
\end{tabular}


Table 3.4e. (continued)

\begin{tabular}{|l|l|l|l|l|l|}
\hline \multirow{2}{*}{ Chemical } & \multirow{2}{*}{ CASRN } & \multicolumn{3}{|c|}{ Ingestion of fish (mg/kg) } \\
\cline { 4 - 6 } & & $10^{-4}$ & $10^{-6}$ & HQ=1 & HQ=0.1 \\
\hline Trichlorotoluene, alpha 2,6- & $002014-83-7$ & & & & \\
\hline Tridiphane & $058138-08-2$ & & & $3.0 \mathrm{E}+01$ & $3.0 \mathrm{E}+00$ \\
\hline Triethyl Lead & $005224-23-7$ & & & & \\
\hline Triethylamine & $000121-44-8$ & & & & \\
\hline Trifluralin & $001582-09-8$ & $3.0 \mathrm{E}+02$ & $3.0 \mathrm{E}+00$ & $7.4 \mathrm{E}+01$ & $7.4 \mathrm{E}+00$ \\
\hline Trimethyl Lead & $007442-13-9$ & & & & \\
\hline Trimethyl Phosphate & $000512-56-1$ & $6.2 \mathrm{E}+01$ & $6.2 \mathrm{E}-01$ & & \\
\hline Trimethylethyl Lead & $001762-26-1$ & & & & \\
\hline Trinitrobenzene, 1,3,5- & $000099-35-4$ & & & $4.9 \mathrm{E}-01$ & $4.9 \mathrm{E}-02$ \\
\hline Trinitrophenylmethylnitramine & $000479-45-8$ & & & $9.9 \mathrm{E}+01$ & $9.9 \mathrm{E}+00$ \\
\hline Trinitrotoluene, 2,4,6- & $000118-96-7$ & $7.7 \mathrm{E}+01$ & $7.7 \mathrm{E}-01$ & $4.9 \mathrm{E}+00$ & $4.9 \mathrm{E}-01$ \\
\hline Tripropyl Lead & $006618-03-7$ & & & & \\
\hline Uranium (Soluble Salts) & NA & & & $3.0 \mathrm{E}+01$ & $3.0 \mathrm{E}+00$ \\
\hline Vanadium Pentoxide & $001314-62-1$ & & & $8.9 \mathrm{E}+01$ & $8.9 \mathrm{E}+00$ \\
\hline Vanadium Sulfate & $036907-42-3$ & & & $2.0 \mathrm{E}+02$ & $2.0 \mathrm{E}+01$ \\
\hline Vanadium, Metallic & $007440-62-2$ & & & $6.9 \mathrm{E}+01$ & $6.9 \mathrm{E}+00$ \\
\hline Vanadyl Sulfate & $027774-13-6$ & & & $2.0 \mathrm{E}+02$ & $2.0 \mathrm{E}+01$ \\
\hline Vernolate & $001929-77-7$ & & & $9.9 \mathrm{E}+00$ & $9.9 \mathrm{E}-01$ \\
\hline
\end{tabular}


Table 3.4e. (continued)

\begin{tabular}{|l|l|l|l|l|l|}
\hline \multirow{2}{*}{ Chemical } & \multirow{2}{*}{ CASRN } & \multicolumn{4}{|c|}{ Ingestion of fish (mg/kg) } \\
\cline { 4 - 6 } & & $10^{-4}$ & $10^{-6}$ & HQ=1 & HQ=0.1 \\
\hline Vinclozolin & $050471-44-8$ & & & $2.5 \mathrm{E}+02$ & $2.5 \mathrm{E}+01$ \\
\hline Vinyl Acetate & $000108-05-4$ & & & $9.9 \mathrm{E}+03$ & $9.9 \mathrm{E}+02$ \\
\hline Vinyl Bromide & $000593-60-2$ & & & & \\
\hline Vinyl Chloride ${ }^{(6)}$ & $000075-01-4$ & $1.2 \mathrm{E}+00$ & $1.2 \mathrm{E}-02$ & & \\
\hline Warfarin & $000081-81-2$ & & & $3.0 \mathrm{E}+00$ & $3.0 \mathrm{E}-01$ \\
\hline White Phosphorus & $007723-14-0$ & & & $2.0 \mathrm{E}-01$ & $2.0 \mathrm{E}-02$ \\
\hline Xylene, Mixture(6) & $001330-20-7$ & & & $2.0 \mathrm{E}+04$ & $2.0 \mathrm{E}+03$ \\
\hline Xylene, P-(6) & $000106-42-3$ & & & & \\
\hline Xylene, m- $^{(6)}$ & $000108-38-3$ & & & $2.0 \mathrm{E}+04$ & $2.0 \mathrm{E}+03$ \\
\hline Xylene, o-(6) $^{(6)}$ & $000095-47-6$ & & & $2.0 \mathrm{E}+04$ & $2.0 \mathrm{E}+03$ \\
\hline Zinc (Metallic) & $007440-66-6$ & & & $3.0 \mathrm{E}+03$ & $3.0 \mathrm{E}+02$ \\
\hline Zinc Cyanide & $000557-21-1$ & & & $4.9 \mathrm{E}+02$ & $4.9 \mathrm{E}+01$ \\
\hline Zinc Phosphide & $001314-84-7$ & & & $3.0 \mathrm{E}+00$ & $3.0 \mathrm{E}-01$ \\
\hline Zineb & $012122-67-7$ & & & $4.9 \mathrm{E}+02$ & $4.9 \mathrm{E}+01$ \\
\hline Zirconium & $007440-67-7$ & & & & \\
\hline
\end{tabular}


Notes:

* By definition, $1 \mathrm{~kg}=1.0 \mathrm{E}+06 \mathrm{mg}$. Therefore, a limit of $1.0 \mathrm{E}+06 \mathrm{mg} / \mathrm{kg}$ was used for any PRG that was calculated to be above this limit.

(1) The same oral slope factor as used for polychlorinated biphenyls.

(2) The PRG was calculated considering Supplemental Guidance from RAGS: Region 4 Bulletins, Human Health Risk Assessment (Interim Guidance) from EPA (received February 11, 1992). The slope factors for chemicals so marked indicate that a toxicity equivalency factor (TEF) method was used for carcinogenic PAHs based on each compound's relative potency to the potency of benzo[a]pyrene. The following TEFs were used to convert each PAH slope factor to an equivalent slope factor of benzo[a]pyrene [that has a new cancer slope factor of $7.3(\mathrm{mg} / \mathrm{kg} / \text { day) })^{-1}$ identified by EPA's CRAVE workgroup]: (1) benzo[a]pyrene, TEF $=1.0$; (2) benzo[a]anthracene, TEF $=0.1$; (3) benzo[b]fluoranthene, TEF $=0.1$; (4) benzo[k]fluoranthene, $\mathrm{TEF}=0.01 ;(5)$ chrysene, $\mathrm{TEF}=0.001$; (6) dibenz[a,h]anthracene, TEF $=1.0$; and (7) indeno[1,2,3-c,d]pyrene, TEF $=0.1$.

(3) The toxicity values for trichloroethylene have been withdrawn from IRIS and HEAST pending further investigation. However, the most recent toxicity values have been used to calculate the PRG.

(4) The PRG was calculated considering Supplemental Guidance from RAGS: Region 4 Bulletins, Human Health Risk Assessment (Interim Guidance) from EPA (November 1995). The slope factors for chemicals so marked indicate that a TEF methodology was used for chlorinated dioxin and furan congeners based on each compound's relative potency to the potency of 2,3,7,8-tetrachlorodibenzodioxin (TCDD). The following TEFs were used to convert each slope factor to an equivalent slope factor of 2,3,7,8-TCDD: (1) 2,3,7,8-TCDD, TEF = 1.0; (2) 2,3,7,8-PeCDD, TEF =0.5; (3) $2,3,7,8$-HxCDD, TEF $=0.1$; (4) 2,3,7,8-HpCDD, TEF $=0.01$; (5) OCDD, TEF $=0.001$; (6) 2,3,7,8-TCDF, TEF $=0.1$; (7) $1,2,3,7,8$-PeCDF, TEF $=0.5$; (8) 2,3,4,7,8-PeCDF, TEF $=0.05$; (9) 2,3,7,8-HxCDF, $\mathrm{TEF}=0.1 ;(10)$ $2,3,7,8-\mathrm{HpCDF}, \mathrm{TEF}=0.01 ;$ and $(11) \mathrm{OCDF}, \mathrm{TEF}=0.001$.

(5) Toxicity values for these chemicals have been withdrawn from IRIS and HEAST pending further investigation. However, the most recent toxicity values have been used to calculate the PRG.

(6) Toxicity values for these chemicals were obtained from the Superfund Health Risk Technical Support Center. 
Table 3.4f. Risk-based Preliminary Remediation Goals for radionuclides in fish (recreational scenario)

\begin{tabular}{|l|l|c|c|}
\hline \multirow{2}{*}{ Chemical } & \multirow{2}{*}{ CASRN } & \multicolumn{2}{|c|}{ Ingestion of fish (pCi/g) } \\
\cline { 3 - 4 } & & $10^{-4}$ & $10^{-6}$ \\
\hline Ac-225 & $014265-85-1$ & $9.1 \mathrm{E}+00$ & $9.1 \mathrm{E}-02$ \\
\hline Ac-227 & $014952-40-0$ & $3.7 \mathrm{E}+00$ & $3.7 \mathrm{E}-02$ \\
\hline Ac-227+D & $014952-40-0$ & $2.1 \mathrm{E}+00$ & $2.1 \mathrm{E}-02$ \\
\hline Ac-228 & $014331-83-0$ & $7.9 \mathrm{E}+02$ & $7.9 \mathrm{E}+00$ \\
\hline Ag-105 & $014928-14-4$ & $7.9 \mathrm{E}+02$ & $7.9 \mathrm{E}+00$ \\
\hline Ag-108 & $014391-65-2$ & $1.9 \mathrm{E}+05$ & $1.9 \mathrm{E}+03$ \\
\hline Ag-108m & $014391-65-2$ & $2.1 \mathrm{E}+02$ & $2.1 \mathrm{E}+00$ \\
\hline Ag-108m+D & $014391-65-2$ & $2.1 \mathrm{E}+02$ & $2.1 \mathrm{E}+00$ \\
\hline Ag-109m & $014378-38-2$ & $4.7 \mathrm{E}+06$ & $4.7 \mathrm{E}+04$ \\
\hline Ag-110 & $014391-76-5$ & $5.3 \mathrm{E}+05$ & $5.3 \mathrm{E}+03$ \\
\hline Ag-110m & $014391-76-5$ & $1.5 \mathrm{E}+02$ & $1.5 \mathrm{E}+00$ \\
\hline Ag-111 & $015760-04-0$ & $1.9 \mathrm{E}+02$ & $1.9 \mathrm{E}+00$ \\
\hline Am-241 & $014596-10-2$ & $3.9 \mathrm{E}+00$ & $3.9 \mathrm{E}-02$ \\
\hline Am-242 & $013981-54-9$ & $8.7 \mathrm{E}+02$ & $8.7 \mathrm{E}+00$ \\
\hline Am-242m & $013981-54-9$ & $4.4 \mathrm{E}+00$ & $4.4 \mathrm{E}-02$ \\
\hline Am-243 & $014993-75-0$ & $3.9 \mathrm{E}+00$ & $3.9 \mathrm{E}-02$ \\
\hline Am-243+D & $014993-75-0$ & $3.9 \mathrm{E}+00$ & $3.9 \mathrm{E}-02$ \\
\hline Ar-41 & $014163-25-8$ & & \\
\hline
\end{tabular}


Table 3.4f. (continued)

\begin{tabular}{|l|l|c|c|}
\hline \multirow{2}{*}{ Chemical $^{\dagger}$} & \multirow{2}{*}{ CASRN } & \multicolumn{2}{|c|}{ Ingestion of fish (pCi/g) } \\
\cline { 3 - 4 } & & $10^{-4}$ & $10^{-6}$ \\
\hline At-217 & $017239-90-6$ & $1.4 \mathrm{E}+08$ & $1.4 \mathrm{E}+06$ \\
\hline Au-196 & $014914-16-0$ & $9.9 \mathrm{E}+02$ & $9.9 \mathrm{E}+00$ \\
\hline Au-198 & $010043-49-0$ & $2.4 \mathrm{E}+02$ & $2.4 \mathrm{E}+00$ \\
\hline Ba-131 & $014914-75-1$ & $7.6 \mathrm{E}+02$ & $7.6 \mathrm{E}+00$ \\
\hline Ba-133 & $013981-41-4$ & $4.8 \mathrm{E}+02$ & $4.8 \mathrm{E}+00$ \\
\hline Ba-133m & $013981-41-4$ & $4.7 \mathrm{E}+02$ & $4.7 \mathrm{E}+00$ \\
\hline Ba-137m & $013981-97-0$ & $5.3 \mathrm{E}+05$ & $5.3 \mathrm{E}+03$ \\
\hline Ba-139 & $014378-25-7$ & $4.2 \mathrm{E}+03$ & $4.2 \mathrm{E}+01$ \\
\hline Ba-140 & $014798-08-4$ & $1.1 \mathrm{E}+02$ & $1.1 \mathrm{E}+00$ \\
\hline Be-7 & $013966-02-4$ & $1.5 \mathrm{E}+04$ & $1.5 \mathrm{E}+02$ \\
\hline Bi-206 & $015776-19-9$ & $1.8 \mathrm{E}+02$ & $1.8 \mathrm{E}+00$ \\
\hline Bi-207 & $013982-38-2$ & $2.5 \mathrm{E}+02$ & $2.5 \mathrm{E}+00$ \\
\hline Bi-210 & $014331-79-4$ & $1.8 \mathrm{E}+02$ & $1.8 \mathrm{E}+00$ \\
\hline Bi-211 & $015229-37-5$ & $7.1 \mathrm{E}+04$ & $7.1 \mathrm{E}+02$ \\
\hline Bi-212 & $014913-49-6$ & $2.1 \mathrm{E}+03$ & $2.1 \mathrm{E}+01$ \\
\hline Bi-213 & $015776-20-2$ & $2.9 \mathrm{E}+03$ & $2.9 \mathrm{E}+01$ \\
\hline Bi-214 & $014733-03-0$ & $6.6 \mathrm{E}+03$ & $6.6 \mathrm{E}+01$ \\
\hline Br-82 & $014686-69-2$ & $9.1 \mathrm{E}+02$ & $9.1 \mathrm{E}+00$ \\
\hline
\end{tabular}


Table 3.4f. (continued)

\begin{tabular}{|l|l|c|c|}
\hline \multirow{2}{*}{ Chemical } & \multirow{2}{*}{ CASRN } & \multicolumn{2}{|c|}{ Ingestion of fish (pCi/g) } \\
\cline { 3 - 4 } & & $10^{-4}$ & $10^{-6}$ \\
\hline C-11 & $014333-33-6$ & $2.9 \mathrm{E}+04$ & $2.9 \mathrm{E}+02$ \\
\hline C-14 & $014762-75-5$ & $1.2 \mathrm{E}+03$ & $1.2 \mathrm{E}+01$ \\
\hline C-15 & $015929-23-4$ & $1.9 \mathrm{E}+06$ & $1.9 \mathrm{E}+04$ \\
\hline Ca-45 & $013966-05-7$ & $6.4 \mathrm{E}+02$ & $6.4 \mathrm{E}+00$ \\
\hline Ca-47 & $001439-99-2$ & $1.9 \mathrm{E}+02$ & $1.9 \mathrm{E}+00$ \\
\hline Cd-109 & $014109-32-1$ & $1.6 \mathrm{E}+02$ & $1.6 \mathrm{E}+00$ \\
\hline Cd-115 & $014336-68-6$ & $1.8 \mathrm{E}+02$ & $1.8 \mathrm{E}+00$ \\
\hline Cd-115m & $014336-68-6$ & $9.1 \mathrm{E}+01$ & $9.1 \mathrm{E}-01$ \\
\hline Ce-141 & $013967-74-3$ & $3.3 \mathrm{E}+02$ & $3.3 \mathrm{E}+00$ \\
\hline Ce-143 & $014119-19-8$ & $2.2 \mathrm{E}+02$ & $2.2 \mathrm{E}+00$ \\
\hline Ce-144 & $014762-78-8$ & $4.3 \mathrm{E}+01$ & $4.3 \mathrm{E}-01$ \\
\hline Ce-144+D & $014762-78-8$ & $4.3 \mathrm{E}+01$ & $4.3 \mathrm{E}-01$ \\
\hline Cl-36 & $013981-43-6$ & $5.8 \mathrm{E}+02$ & $5.8 \mathrm{E}+00$ \\
\hline Cl-38 & $014158-34-0$ & $6.2 \mathrm{E}+03$ & $6.2 \mathrm{E}+01$ \\
\hline Cm-242 & $015510-73-3$ & $3.4 \mathrm{E}+01$ & $3.4 \mathrm{E}-01$ \\
\hline Cm-243 & $015757-87-6$ & $5.1 \mathrm{E}+00$ & $.5 .1 \mathrm{E}-02$ \\
\hline Cm-243+D & $015757-87-6$ & $5.1 \mathrm{E}+00$ & $5.1 \mathrm{E}-02$ \\
\hline Cm-244 & $013981-15-2$ & $6.1 \mathrm{E}+00$ & $6.1 \mathrm{E}-02$ \\
\hline
\end{tabular}


Table 3.4f. (continued)

\begin{tabular}{|l|l|c|c|}
\hline \multirow{2}{*}{ Chemical $^{\dagger}$} & \multirow{2}{*}{ CASRN } & \multicolumn{2}{|c|}{ Ingestion of fish (pCi/g) } \\
\cline { 3 - 4 } & & $10^{-4}$ & $10^{-6}$ \\
\hline Cm-245 & $015621-76-8$ & $3.8 \mathrm{E}+00$ & $3.8 \mathrm{E}-02$ \\
\hline Cm-246 & $015757-90-1$ & $3.9 \mathrm{E}+00$ & $3.9 \mathrm{E}-02$ \\
\hline Cm-247 & $015758-32-4$ & $4.2 \mathrm{E}+00$ & $4.2 \mathrm{E}-02$ \\
\hline Cm-248 & $015758-33-5$ & $9.8 \mathrm{E}-01$ & $9.8 \mathrm{E}-03$ \\
\hline Co-57 & $013981-50-5$ & $1.3 \mathrm{E}+03$ & $1.3 \mathrm{E}+01$ \\
\hline Co-58 & $013981-38-9$ & $4.6 \mathrm{E}+02$ & $4.6 \mathrm{E}+00$ \\
\hline Co-58m & $013981-38-9$ & $1.4 \mathrm{E}+04$ & $1.4 \mathrm{E}+02$ \\
\hline Co-60 & $010198-40-0$ & $6.8 \mathrm{E}+01$ & $6.8 \mathrm{E}-01$ \\
\hline Cr-51 & $014392-02-0$ & $9.3 \mathrm{E}+03$ & $9.3 \mathrm{E}+01$ \\
\hline Cs-131 & $014914-76-2$ & $7.1 \mathrm{E}+03$ & $7.1 \mathrm{E}+01$ \\
\hline Cs-134 & $013967-70-9$ & $2.7 \mathrm{E}+01$ & $2.7 \mathrm{E}-01$ \\
\hline Cs-134m & $013967-70-9$ & $2.8 \mathrm{E}+04$ & $2.8 \mathrm{E}+02$ \\
\hline Cs-135 & $015726-30-4$ & $2.8 \mathrm{E}+02$ & $2.8 \mathrm{E}+00$ \\
\hline Cs-136 & $014234-29-8$ & $1.7 \mathrm{E}+02$ & $1.7 \mathrm{E}+00$ \\
\hline Cs-137 & $010045-97-3$ & $4.1 \mathrm{E}+01$ & $4.1 \mathrm{E}-01$ \\
\hline Cs-137+D & $010045-97-3$ & $4.1 \mathrm{E}+01$ & $4.1 \mathrm{E}-01$ \\
\hline Cs-138 & $015758-29-9$ & $7.3 \mathrm{E}+03$ & $7.3 \mathrm{E}+01$ \\
\hline Cu-64 & $013981-25-4$ & $2.4 \mathrm{E}+03$ & $2.4 \mathrm{E}+01$ \\
\hline
\end{tabular}


Table 3.4f. (continued)

\begin{tabular}{|l|l|c|c|}
\hline \multirow{2}{*}{ Chemical $^{\dagger}$} & \multirow{2}{*}{ CASRN } & \multicolumn{2}{|c|}{ Ingestion of fish (pCi/g) } \\
\cline { 3 - 4 } & & $10^{-4}$ & $10^{-6}$ \\
\hline Dy-165 & $013967-64-1$ & $3.9 \mathrm{E}+03$ & $3.9 \mathrm{E}+01$ \\
\hline Dy-166 & $015840-01-4$ & $1.4 \mathrm{E}+02$ & $1.4 \mathrm{E}+00$ \\
\hline Er-169 & $015840-13-8$ & $6.1 \mathrm{E}+02$ & $6.1 \mathrm{E}+00$ \\
\hline Er-171 & $014391-45-8$ & $7.9 \mathrm{E}+02$ & $7.9 \mathrm{E}+00$ \\
\hline Eu-152 & $014683-23-9$ & $2.2 \mathrm{E}+02$ & $2.2 \mathrm{E}+00$ \\
\hline Eu-154 & $015585-10-1$ & $1.4 \mathrm{E}+02$ & $1.4 \mathrm{E}+00$ \\
\hline Eu-155 & $014391-16-3$ & $7.8 \mathrm{E}+02$ & $7.8 \mathrm{E}+00$ \\
\hline Eu-156 & $014280-35-4$ & $1.2 \mathrm{E}+02$ & $1.2 \mathrm{E}+00$ \\
\hline F-18 & $013981-56-1$ & $1.2 \mathrm{E}+04$ & $1.2 \mathrm{E}+02$ \\
\hline Fe-55 & $014681-59-5$ & $3.7 \mathrm{E}+03$ & $3.7 \mathrm{E}+01$ \\
\hline Fe-59 & $014596-12-4$ & $2.2 \mathrm{E}+02$ & $2.2 \mathrm{E}+00$ \\
\hline Fr-221 & $015756-41-9$ & $8.9 \mathrm{E}+03$ & $8.9 \mathrm{E}+01$ \\
\hline Fr-223 & $015756-98-6$ & $2.9 \mathrm{E}+03$ & $2.9 \mathrm{E}+01$ \\
\hline Ga-67 & $014119-09-6$ & $1.5 \mathrm{E}+03$ & $1.5 \mathrm{E}+01$ \\
\hline Ga-72 & $013982-22-4$ & $2.7 \mathrm{E}+02$ & $2.7 \mathrm{E}+00$ \\
\hline Gd-153 & $014276-65-4$ & $9.7 \mathrm{E}+02$ & $9.7 \mathrm{E}+00$ \\
\hline Gd-159 & $014041-42-0$ & $4.9 \mathrm{E}+02$ & $4.9 \mathrm{E}+00$ \\
\hline Ge-71 & $014374-81-3$ & $1.1 \mathrm{E}+05$ & $1.1 \mathrm{E}+03$ \\
\hline
\end{tabular}


Table 3.4f. (continued)

\begin{tabular}{|l|l|c|c|}
\hline \multirow{2}{*}{ Chemical } & \multirow{2}{*}{ CASRN } & \multicolumn{2}{|c|}{ Ingestion of fish (pCi/g) } \\
\cline { 2 - 4 } & & $10^{-4}$ & $10^{-6}$ \\
\hline H-3 & $010028-17-8$ & $1.8 \mathrm{E}+04$ & $1.8 \mathrm{E}+02$ \\
\hline Hg-197 & $013981-51-6$ & $1.1 \mathrm{E}+03$ & $1.1 \mathrm{E}+01$ \\
\hline Hg-203 & $013982-78-0$ & $4.9 \mathrm{E}+02$ & $4.9 \mathrm{E}+00$ \\
\hline Ho-166 & $013967-65-2$ & $1.7 \mathrm{E}+02$ & $1.7 \mathrm{E}+00$ \\
\hline I-122 & $018287-75-7$ & $6.0 \mathrm{E}+04$ & $6.0 \mathrm{E}+02$ \\
\hline I-123 & $015715-08-9$ & $2.4 \mathrm{E}+03$ & $2.4 \mathrm{E}+01$ \\
\hline I-125 & $014158-31-7$ & $5.0 \mathrm{E}+01$ & $5.0 \mathrm{E}-01$ \\
\hline I-126 & $014158-32-8$ & $2.7 \mathrm{E}+01$ & $2.7 \mathrm{E}-01$ \\
\hline I-129 & $015046-84-1$ & $7.0 \mathrm{E}+00$ & $7.0 \mathrm{E}-02$ \\
\hline I-130 & $014914-02-4$ & $2.7 \mathrm{E}+02$ & $2.7 \mathrm{E}+00$ \\
\hline I-131 & $010043-66-0$ & $3.6 \mathrm{E}+01$ & $3.6 \mathrm{E}-01$ \\
\hline I-132 & $014683-16-0$ & $1.9 \mathrm{E}+03$ & $1.9 \mathrm{E}+01$ \\
\hline I-133 & $014834-67-4$ & $1.2 \mathrm{E}+02$ & $1.2 \mathrm{E}+00$ \\
\hline I-134 & $014914-27-3$ & $5.6 \mathrm{E}+03$ & $5.6 \mathrm{E}+01$ \\
\hline I-135 & $014834-68-5$ & $5.7 \mathrm{E}+02$ & $5.7 \mathrm{E}+00$ \\
\hline In-113m & $014885-78-0$ & $1.5 \mathrm{E}+04$ & $1.5 \mathrm{E}+02$ \\
\hline In-114 & $013981-55-0$ & $2.8 \mathrm{E}+05$ & $2.8 \mathrm{E}+03$ \\
\hline In-114m & $013981-55-0$ & $6.2 \mathrm{E}+01$ & $6.2 \mathrm{E}-01$ \\
\hline
\end{tabular}


Table 3.4f. (continued)

\begin{tabular}{|l|l|c|c|}
\hline \multirow{2}{*}{ Chemical } & \multirow{2}{*}{ CASRN } & \multicolumn{2}{|c|}{ Ingestion of fish (pCi/g) } \\
\cline { 3 - 4 } & & $10^{-4}$ & $10^{-6}$ \\
\hline In-115 & $014191-71-0$ & $3.7 \mathrm{E}+01$ & $3.7 \mathrm{E}-01$ \\
\hline In-115m & $014191-71-0$ & $3.8 \mathrm{E}+03$ & $3.8 \mathrm{E}+01$ \\
\hline Ir-190 & $014981-91-0$ & $2.6 \mathrm{E}+02$ & $2.6 \mathrm{E}+00$ \\
\hline Ir-194 & $014158-35-1$ & $1.8 \mathrm{E}+02$ & $1.8 \mathrm{E}+00$ \\
\hline $\mathrm{K}-40$ & $013966-00-2$ & $1.0 \mathrm{E}+02$ & $1.0 \mathrm{E}+00$ \\
\hline $\mathrm{K}-42$ & $014378-21-3$ & $1.0 \mathrm{E}+03$ & $1.0 \mathrm{E}+01$ \\
\hline $\mathrm{Kr}-83 \mathrm{~m}$ & $013965-98-5$ & & \\
\hline $\mathrm{Kr}-85$ & $013983-27-2$ & & \\
\hline $\mathrm{Kr}-85 \mathrm{~m}$ & $013983-27-2$ & & \\
\hline $\mathrm{Kr}-87$ & $014809-68-8$ & & \\
\hline $\mathrm{Kr}-88$ & $014995-61-0$ & & \\
\hline $\mathrm{Kr}-89$ & $016316-03-3$ & & \\
\hline $\mathrm{Kr}-90$ & $015741-13-6$ & & $1.5 \mathrm{E}+01$ \\
\hline $\mathrm{La}-140$ & $013981-28-7$ & $1.4 \mathrm{E}+02$ & \\
\hline $\mathrm{Lu}-177$ & $014265-75-9$ & $4.4 \mathrm{E}+02$ & \\
\hline $\mathrm{Mn}-52$ & $014092-99-0$ & $2.1 \mathrm{E}+02$ & \\
\hline $\mathrm{Mn}-54$ & $013966-31-9$ & $6.6 \mathrm{E}+02$ & \\
\hline $\mathrm{Mn}-56$ & $014681-52-8$ & $1.5 \mathrm{E}+03$ & \\
\hline
\end{tabular}


Table 3.4f. (continued)

\begin{tabular}{|l|l|c|c|}
\hline \multirow{2}{*}{ Chemical } & \multirow{2}{*}{ CASRN } & \multicolumn{2}{|c|}{ Ingestion of fish (pCi/g) } \\
\cline { 2 - 4 } & & $10^{-4}$ & $10^{-6}$ \\
\hline Mo-99 & $014119-15-4$ & $5.7 \mathrm{E}+02$ & $5.7 \mathrm{E}+00$ \\
\hline $\mathrm{Na}-22$ & $013966-32-0$ & $1.6 \mathrm{E}+02$ & $1.6 \mathrm{E}+00$ \\
\hline Na-24 & $013982-04-2$ & $9.3 \mathrm{E}+02$ & $9.3 \mathrm{E}+00$ \\
\hline $\mathrm{Nb}-93 \mathrm{~m}$ & $007440-03-1$ & $1.9 \mathrm{E}+03$ & $1.9 \mathrm{E}+01$ \\
\hline $\mathrm{Nb}-94$ & $014681-63-1$ & $1.9 \mathrm{E}+02$ & $1.9 \mathrm{E}+00$ \\
\hline $\mathrm{Nb}-95$ & $013967-76-5$ & $5.7 \mathrm{E}+02$ & $5.7 \mathrm{E}+00$ \\
\hline $\mathrm{Nb}-95 \mathrm{~m}$ & $013967-76-5$ & $4.2 \mathrm{E}+02$ & $4.2 \mathrm{E}+00$ \\
\hline $\mathrm{Nb}-97$ & $018496-04-3$ & $7.3 \mathrm{E}+03$ & $7.3 \mathrm{E}+01$ \\
\hline $\mathrm{Nb}-97 \mathrm{~m}$ & $018496-04-3$ & $3.9 \mathrm{E}+05$ & $3.9 \mathrm{E}+03$ \\
\hline $\mathrm{Nd}-147$ & $014269-74-0$ & $2.2 \mathrm{E}+02$ & $2.2 \mathrm{E}+00$ \\
\hline $\mathrm{Nd}-149$ & $015749-81-2$ & $2.8 \mathrm{E}+03$ & $2.8 \mathrm{E}+01$ \\
\hline $\mathrm{Ni}-59$ & $014336-70-0$ & $7.0 \mathrm{E}+03$ & $7.0 \mathrm{E}+01$ \\
\hline $\mathrm{Ni}-63$ & $013981-37-8$ & $2.3 \mathrm{E}+03$ & $2.3 \mathrm{E}+01$ \\
\hline $\mathrm{Ni}-65$ & $014833-49-9$ & $2.3 \mathrm{E}+03$ & $2.3 \mathrm{E}+01$ \\
\hline $\mathrm{Np}-236$ & $015700-36-4$ & $1.4 \mathrm{E}+03$ & $1.4 \mathrm{E}+01$ \\
\hline $\mathrm{Np}-237$ & $013994-20-2$ & $4.4 \mathrm{E}+00$ & $4.4 \mathrm{E}-02$ \\
\hline $\mathrm{Np}-237+\mathrm{D}$ & $013994-20-2$ & $4.3 \mathrm{E}+00$ & $4.3 \mathrm{E}-02$ \\
\hline $\mathrm{Np}-238$ & $015766-25-3$ & $2.8 \mathrm{E}+02$ & $2.8 \mathrm{E}+00$ \\
\hline
\end{tabular}


Table 3.4f. (continued)

\begin{tabular}{|l|l|c|c|}
\hline \multirow{2}{*}{ Chemical $^{\dagger}$} & \multirow{2}{*}{ CASRN } & \multicolumn{2}{|c|}{ Ingestion of fish (pCi/g) } \\
\cline { 2 - 4 } & & $10^{-4}$ & $10^{-6}$ \\
\hline Np-239 & $013968-59-7$ & $3.0 \mathrm{E}+02$ & $3.0 \mathrm{E}+00$ \\
\hline Np-240 & $015690-84-3$ & $7.3 \mathrm{E}+03$ & $7.3 \mathrm{E}+01$ \\
\hline Np-240m & $015690-84-3$ & $5.3 \mathrm{E}+04$ & $5.3 \mathrm{E}+02$ \\
\hline Os-185 & $015766-50-4$ & $7.1 \mathrm{E}+02$ & $7.1 \mathrm{E}+00$ \\
\hline Os-191 & $014119-24-5$ & $4.2 \mathrm{E}+02$ & $4.2 \mathrm{E}+00$ \\
\hline Os-191m & $014119-24-5$ & $2.6 \mathrm{E}+03$ & $2.6 \mathrm{E}+01$ \\
\hline Os-193 & $016057-77-5$ & $2.9 \mathrm{E}+02$ & $2.9 \mathrm{E}+00$ \\
\hline $\mathrm{P}-32$ & $014596-37-3$ & $2.1 \mathrm{E}+02$ & $2.1 \mathrm{E}+00$ \\
\hline $\mathrm{P}-33$ & $015749-66-3$ & $1.6 \mathrm{E}+03$ & $1.6 \mathrm{E}+01$ \\
\hline $\mathrm{Pa}-231$ & $014331-85-2$ & $8.6 \mathrm{E}+00$ & $8.6 \mathrm{E}-02$ \\
\hline $\mathrm{Pa}-233$ & $013981-14-1$ & $2.7 \mathrm{E}+02$ & $2.7 \mathrm{E}+00$ \\
\hline $\mathrm{Pa}-234$ & $015100-28-4$ & $6.0 \mathrm{E}+02$ & $6.0 \mathrm{E}+00$ \\
\hline $\mathrm{Pa}-234 \mathrm{~m}$ & $015100-28-4$ & $2.7 \mathrm{E}+05$ & $2.7 \mathrm{E}+03$ \\
\hline $\mathrm{Pb}-203$ & $014687-25-3$ & $1.2 \mathrm{E}+03$ & $1.2 \mathrm{E}+01$ \\
\hline $\mathrm{Pb}-209$ & $014119-30-3$ & $6.2 \mathrm{E}+03$ & $6.2 \mathrm{E}+01$ \\
\hline $\mathrm{Pb}-210$ & $014255-04-0$ & $1.9 \mathrm{E}+00$ & $1.9 \mathrm{E}-02$ \\
\hline $\mathrm{Pb}-210+\mathrm{D}$ & $014255-04-0$ & $1.3 \mathrm{E}+00$ & $1.3 \mathrm{E}-02$ \\
\hline $\mathrm{Pb}-211$ & $015816-77-0$ & $3.8 \mathrm{E}+03$ & $3.8 \mathrm{E}+01$ \\
\hline & & & \\
\hline
\end{tabular}


Table 3.4f. (continued)

\begin{tabular}{|l|l|c|c|}
\hline \multirow{2}{*}{ Chemical $^{\dagger}$} & \multirow{2}{*}{ CASRN } & \multicolumn{2}{|c|}{ Ingestion of fish (pCi/g) } \\
\cline { 2 - 4 } & & $10^{-4}$ & $10^{-6}$ \\
\hline Pb-212 & $015092-94-1$ & $7.1 \mathrm{E}+01$ & $7.1 \mathrm{E}-01$ \\
\hline Pb-214 & $015067-28-4$ & $4.4 \mathrm{E}+03$ & $4.4 \mathrm{E}+01$ \\
\hline Pd-100 & $015690-69-4$ & $3.4 \mathrm{E}+02$ & $3.4 \mathrm{E}+00$ \\
\hline Pd-101 & $015749-54-9$ & $3.4 \mathrm{E}+03$ & $3.4 \mathrm{E}+01$ \\
\hline Pd-103 & $014967-68-1$ & $1.2 \mathrm{E}+03$ & $1.2 \mathrm{E}+01$ \\
\hline Pd-107 & $017637-99-9$ & $6.2 \mathrm{E}+03$ & $6.2 \mathrm{E}+01$ \\
\hline Pd-109 & $014981-64-7$ & $3.9 \mathrm{E}+02$ & $3.9 \mathrm{E}+00$ \\
\hline Pm-147 & $014380-75-7$ & $9.1 \mathrm{E}+02$ & $9.1 \mathrm{E}+00$ \\
\hline Pm-148 & $014683-19-3$ & $8.9 \mathrm{E}+01$ & $8.9 \mathrm{E}-01$ \\
\hline Pm-148m & $014683-19-3$ & $1.3 \mathrm{E}+02$ & $1.3 \mathrm{E}+00$ \\
\hline Pm-149 & $015765-31-8$ & $2.3 \mathrm{E}+02$ & $2.3 \mathrm{E}+00$ \\
\hline Po-210 & $013981-52-7$ & $3.9 \mathrm{E}+00$ & $3.9 \mathrm{E}-02$ \\
\hline Po-212 & $015389-34-1$ & $2.9 \mathrm{E}+13$ & $2.9 \mathrm{E}+11$ \\
\hline Po-213 & $015756-57-7$ & $1.9 \mathrm{E}+12$ & $1.9 \mathrm{E}+10$ \\
\hline Po-214 & $015735-67-8$ & $6.1 \mathrm{E}+10$ & $6.1 \mathrm{E}+08$ \\
\hline Po-215 & $015706-52-2$ & $2.6 \mathrm{E}+09$ & $2.6 \mathrm{E}+07$ \\
\hline Po-216 & $015756-58-8$ & $1.5 \mathrm{E}+07$ & $1.5 \mathrm{E}+05$ \\
\hline Po-218 & $015422-24-9$ & $2.5 \mathrm{E}+04$ & $2.5 \mathrm{E}+02$ \\
\hline
\end{tabular}


Table 3.4f. (continued)

\begin{tabular}{|l|l|c|c|}
\hline \multirow{2}{*}{ Chemical $^{\dagger}$} & \multirow{2}{*}{ CASRN } & \multicolumn{2}{|c|}{$i$ Ingestion of fish (pCi/g) } \\
\cline { 2 - 4 } & & $10^{-4}$ & $10^{-6}$ \\
\hline Pr-142 & $014191-64-1$ & $1.8 \mathrm{E}+02$ & $1.8 \mathrm{E}+00$ \\
\hline Pr-143 & $014981-79-4$ & $1.9 \mathrm{E}+02$ & $1.9 \mathrm{E}+00$ \\
\hline Pr-144 & $014119-05-2$ & $1.6 \mathrm{E}+04$ & $1.6 \mathrm{E}+02$ \\
\hline Pr-144m & $014119-05-2$ & $4.0 \mathrm{E}+04$ & $4.0 \mathrm{E}+02$ \\
\hline Pt-191 & $015706-36-2$ & $8.6 \mathrm{E}+02$ & $8.6 \mathrm{E}+00$ \\
\hline Pt-193 & $015735-70-3$ & $7.9 \mathrm{E}+03$ & $7.9 \mathrm{E}+01$ \\
\hline Pt-193m & $015735-70-3$ & $5.1 \mathrm{E}+02$ & $5.1 \mathrm{E}+00$ \\
\hline Pt-197 & $015735-74-7$ & $6.1 \mathrm{E}+02$ & $6.1 \mathrm{E}+00$ \\
\hline Pt-197m & $015735-74-7$ & $4.0 \mathrm{E}+03$ & $4.0 \mathrm{E}+01$ \\
\hline Pu-236 & $015411-92-4$ & $1.7 \mathrm{E}+01$ & $1.7 \mathrm{E}-01$ \\
\hline Pu-238 & $013981-16-3$ & $4.4 \mathrm{E}+00$ & $4.4 \mathrm{E}-02$ \\
\hline Pu-239 & $015117-48-3$ & $4.1 \mathrm{E}+00$ & $4.1 \mathrm{E}-02$ \\
\hline Pu-240 & $014119-33-6$ & $4.1 \mathrm{E}+00$ & $4.1 \mathrm{E}-02$ \\
\hline Pu-241 & $014119-32-5$ & $2.5 \mathrm{E}+02$ & $2.5 \mathrm{E}+00$ \\
\hline Pu-241+D & $014119-32-5$ & $3.9 \mathrm{E}+00$ & $3.9 \mathrm{E}-02$ \\
\hline Pu-242 & $013982-10-0$ & $4.3 \mathrm{E}+00$ & $4.3 \mathrm{E}-02$ \\
\hline Pu-243 & $015706-37-3$ & $3.5 \mathrm{E}+03$ & $3.5 \mathrm{E}+01$ \\
\hline Pu-244 & $014119-34-7$ & $4.1 \mathrm{E}+00$ & $4.1 \mathrm{E}-02$ \\
\hline & & & \\
\hline
\end{tabular}


Table 3.4f. (continued)

\begin{tabular}{|l|l|c|c|}
\hline \multirow{2}{*}{ Chemical $^{\dagger}$} & \multirow{2}{*}{ CASRN } & \multicolumn{2}{|c|}{ Ingestion of fish (pCi/g) } \\
\cline { 2 - 4 } & & $10^{-4}$ & $10^{-6}$ \\
\hline Pu-244+D & $014119-34-7$ & $4.0 \mathrm{E}+00$ & $4.0 \mathrm{E}-02$ \\
\hline Ra-223 & $015623-45-7$ & $5.5 \mathrm{E}+00$ & $5.5 \mathrm{E}-02$ \\
\hline Ra-224 & $013233-32-4$ & $8.6 \mathrm{E}+00$ & $8.6 \mathrm{E}-02$ \\
\hline Ra-225 & $013981-53-8$ & $8.2 \mathrm{E}+00$ & $8.2 \mathrm{E}-02$ \\
\hline Ra-226 & $013982-63-3$ & $4.4 \mathrm{E}+00$ & $4.4 \mathrm{E}-02$ \\
\hline Ra-226+D & $013982-63-3$ & $4.3 \mathrm{E}+00$ & $4.3 \mathrm{E}-02$ \\
\hline Ra-228 & $015262-20-1$ & $5.2 \mathrm{E}+00$ & $5.2 \mathrm{E}-02$ \\
\hline Ra-228+D & $015262-20-1$ & $5.2 \mathrm{E}+00$ & $5.2 \mathrm{E}-02$ \\
\hline Rb-82 & $014391-63-0$ & $1.2 \mathrm{E}+05$ & $1.2 \mathrm{E}+03$ \\
\hline Rb-86 & $014932-53-7$ & $1.8 \mathrm{E}+02$ & $1.8 \mathrm{E}+00$ \\
\hline Rb-87 & $013982-13-3$ & $3.5 \mathrm{E}+02$ & $3.5 \mathrm{E}+00$ \\
\hline Rb-88 & $014928-36-0$ & $8.8 \mathrm{E}+03$ & $8.8 \mathrm{E}+01$ \\
\hline Rb-89 & $014191-65-2$ & $1.5 \mathrm{E}+04$ & $1.5 \mathrm{E}+02$ \\
\hline Rh-103m & $007440-16-6$ & $1.6 \mathrm{E}+05$ & $1.6 \mathrm{E}+03$ \\
\hline Rh-105 & $014913-89-4$ & $6.7 \mathrm{E}+02$ & $6.7 \mathrm{E}+00$ \\
\hline Rh-105m & $014913-89-4$ & $1.2 \mathrm{E}+06$ & $1.2 \mathrm{E}+04$ \\
\hline Rh-106 & $014234-34-5$ & $3.5 \mathrm{E}+05$ & $3.5 \mathrm{E}+03$ \\
\hline Rn-219 & $014835-02-0$ & & \\
\hline
\end{tabular}


Table 3.4f. (continued)

\begin{tabular}{|l|l|l|l|}
\hline \multirow{2}{*}{ Chemical } & \multirow{2}{*}{ CASRN } & \multicolumn{2}{|c|}{ Ingestion of fish (pCi/g) } \\
\cline { 3 - 4 } & & $10^{-4}$ & $10^{-6}$ \\
\hline Rn-220 & $022481-48-7$ & & \\
\hline Rn-222+D & $014859-67-7$ & & $3.9 \mathrm{E}+00$ \\
\hline Ru-103 & $013968-53-1$ & $3.9 \mathrm{E}+02$ & $1.1 \mathrm{E}+01$ \\
\hline Ru-105 & $014331-95-4$ & $1.1 \mathrm{E}+03$ & $3.7 \mathrm{E}-01$ \\
\hline Ru-106 & $013967-48-1$ & $3.7 \mathrm{E}+01$ & $3.7 \mathrm{E}-01$ \\
\hline Ru-106+D & $013967-48-1$ & $3.7 \mathrm{E}+01$ & $2.2 \mathrm{E}+01$ \\
\hline Ru-97 & $015758-35-7$ & $2.2 \mathrm{E}+03$ & $3.1 \mathrm{E}+01$ \\
\hline $\mathrm{S}-35$ & $015117-53-0$ & $3.1 \mathrm{E}+03$ & $1.5 \mathrm{E}+00$ \\
\hline Sb-122 & $014374-79-9$ & $1.5 \mathrm{E}+02$ & $1.2 \mathrm{E}+00$ \\
\hline Sb-124 & $014683-10-4$ & $1.2 \mathrm{E}+02$ & $4.3 \mathrm{E}+00$ \\
\hline Sb-125 & $014234-35-6$ & $4.3 \mathrm{E}+02$ & $3.6 \mathrm{E}+00$ \\
\hline Sb-125+D & $014234-35-6$ & $3.6 \mathrm{E}+02$ & $1.3 \mathrm{E}+00$ \\
\hline Sb-126 & $015756-32-8$ & $1.3 \mathrm{E}+02$ & $1.8 \mathrm{E}+02$ \\
\hline Sb-126m & $015756-32-8$ & $1.8 \mathrm{E}+04$ & $1.5 \mathrm{E}+00$ \\
\hline Sb-127 & $013968-50-8$ & $1.5 \mathrm{E}+02$ & $6.9 \mathrm{E}+00$ \\
\hline Sb-129 & $014331-88-5$ & $6.9 \mathrm{E}+02$ & $2.2 \mathrm{E}+00$ \\
\hline Sc-46 & $013967-63-0$ & $2.2 \mathrm{E}+02$ & $4.4 \mathrm{E}+00$ \\
\hline Sc-47 & $014391-96-9$ & $4.4 \mathrm{E}+02$ & \\
\hline
\end{tabular}


Table 3.4f. (continued)

\begin{tabular}{|l|l|c|c|}
\hline \multirow{2}{*}{ Chemical $^{\dagger}$} & \multirow{2}{*}{ CASRN } & \multicolumn{2}{|c|}{ Ingestion of fish (pCi/g) } \\
\cline { 3 - 4 } & & $10^{-4}$ & $10^{-6}$ \\
\hline Sc-48 & $014391-86-7$ & $1.9 \mathrm{E}+02$ & $1.9 \mathrm{E}+00$ \\
\hline Se-75 & $014265-71-5$ & $2.0 \mathrm{E}+02$ & $2.0 \mathrm{E}+00$ \\
\hline Si-31 & $014276-49-4$ & $2.6 \mathrm{E}+03$ & $2.6 \mathrm{E}+01$ \\
\hline Sm-147 & $014392-33-7$ & $5.1 \mathrm{E}+01$ & $5.1 \mathrm{E}-01$ \\
\hline Sm-151 & $015715-94-3$ & $2.8 \mathrm{E}+03$ & $2.8 \mathrm{E}+01$ \\
\hline Sm-153 & $015766-00-4$ & $3.2 \mathrm{E}+02$ & $3.2 \mathrm{E}+00$ \\
\hline Sn-113 & $013966-06-8$ & $3.5 \mathrm{E}+02$ & $3.5 \mathrm{E}+00$ \\
\hline Sn-121 & $014683-06-8$ & $1.1 \mathrm{E}+03$ & $1.1 \mathrm{E}+01$ \\
\hline Sn-121m & $014683-06-8$ & $6.4 \mathrm{E}+02$ & $6.4 \mathrm{E}+00$ \\
\hline Sn-125 & $014683-08-0$ & $7.7 \mathrm{E}+01$ & $7.7 \mathrm{E}-01$ \\
\hline Sn-126 & $015832-50-5$ & $6.1 \mathrm{E}+01$ & $6.1 \mathrm{E}-01$ \\
\hline Sr-82 & $014809-50-8$ & $5.0 \mathrm{E}+01$ & $5.0 \mathrm{E}-01$ \\
\hline Sr-85 & $013967-73-2$ & $9.2 \mathrm{E}+02$ & $9.2 \mathrm{E}+00$ \\
\hline Sr-85m & $013967-73-2$ & $7.1 \mathrm{E}+04$ & $7.1 \mathrm{E}+02$ \\
\hline Sr-89 & $014158-27-1$ & $1.2 \mathrm{E}+02$ & $1.2 \mathrm{E}+00$ \\
\hline Sr-90 & $010098-97-2$ & $3.1 \mathrm{E}+01$ & $3.1 \mathrm{E}-01$ \\
\hline Sr-90+D & $010098-97-2$ & $2.3 \mathrm{E}+01$ & $2.3 \mathrm{E}-01$ \\
\hline Sr-91 & $014331-91-0$ & $4.6 \mathrm{E}+02$ & $4.6 \mathrm{E}+00$ \\
\hline
\end{tabular}


Table 3.4f. (continued)

\begin{tabular}{|l|l|c|c|}
\hline \multirow{2}{*}{ Chemical $^{\dagger}$} & \multirow{2}{*}{ CASRN } & \multicolumn{2}{|c|}{ Ingestion of fish (pCi/g) } \\
\cline { 3 - 4 } & & $10^{-4}$ & $10^{-6}$ \\
\hline Sr-92 & $014928-29-1$ & $6.3 \mathrm{E}+02$ & $6.3 \mathrm{E}+00$ \\
\hline Ta-182 & $013982-00-8$ & $1.8 \mathrm{E}+02$ & $1.8 \mathrm{E}+00$ \\
\hline Tb-158 & $015759-55-4$ & $3.1 \mathrm{E}+02$ & $3.1 \mathrm{E}+00$ \\
\hline Tb-160 & $013981-29-8$ & $1.7 \mathrm{E}+02$ & $1.7 \mathrm{E}+00$ \\
\hline Tc-95 & $014809-56-4$ & $1.9 \mathrm{E}+04$ & $1.9 \mathrm{E}+02$ \\
\hline Tc-95m & $014809-56-4$ & $1.0 \mathrm{E}+03$ & $1.0 \mathrm{E}+01$ \\
\hline Tc-96 & $014808-44-7$ & $5.6 \mathrm{E}+02$ & $5.6 \mathrm{E}+00$ \\
\hline Tc-96m & $014808-44-7$ & $4.9 \mathrm{E}+04$ & $4.9 \mathrm{E}+02$ \\
\hline Tc-97 & $015759-35-0$ & $8.1 \mathrm{E}+03$ & $8.1 \mathrm{E}+01$ \\
\hline Tc-97m & $015759-35-0$ & $1.1 \mathrm{E}+03$ & $1.1 \mathrm{E}+01$ \\
\hline Tc-99 & $014133-76-7$ & $9.2 \mathrm{E}+02$ & $9.2 \mathrm{E}+00$ \\
\hline Tc-99m & $014133-76-7$ & $2.3 \mathrm{E}+04$ & $2.3 \mathrm{E}+02$ \\
\hline Te-125m & $5.1 \mathrm{E}+02$ & $5.1 \mathrm{E}+00$ \\
\hline Te-127 & $014390-73-9$ & $1.5 \mathrm{E}+03$ & $1.5 \mathrm{E}+01$ \\
\hline Te-127m & $013981-49-2$ & $2.1 \mathrm{E}+02$ & $2.1 \mathrm{E}+00$ \\
\hline Te-129 & $013981-49-2$ & $8.7 \mathrm{E}+03$ & $8.7 \mathrm{E}+01$ \\
\hline Te-129m & $014269-71-7$ & $1.1 \mathrm{E}+02$ & $1.1 \mathrm{E}+00$ \\
\hline Te-131 & $014269-71-7$ & $3.3 \mathrm{E}+03$ & $3.3 \mathrm{E}+01$ \\
\hline & $014683-12-6$ & & \\
\hline
\end{tabular}


Table 3.4f. (continued)

\begin{tabular}{|l|l|c|c|}
\hline \multirow{2}{*}{ Chemical } & \multirow{2}{*}{ CASRN } & \multicolumn{2}{|c|}{ Ingestion of fish (pCi/g) } \\
\cline { 3 - 4 } & & $10^{-4}$ & $10^{-6}$ \\
\hline Te-131m & $014683-12-6$ & $1.5 \mathrm{E}+02$ & $1.5 \mathrm{E}+00$ \\
\hline Te-132 & $014234-28-7$ & $1.1 \mathrm{E}+02$ & $1.1 \mathrm{E}+00$ \\
\hline Th-227 & $015623-47-9$ & $3.2 \mathrm{E}+01$ & $3.2 \mathrm{E}-01$ \\
\hline Th-228 & $014274-82-9$ & $2.0 \mathrm{E}+01$ & $2.0 \mathrm{E}-01$ \\
\hline Th-228+D & $014274-82-9$ & $5.6 \mathrm{E}+00$ & $5.6 \mathrm{E}-02$ \\
\hline Th-229 & $015594-54-4$ & $2.3 \mathrm{E}+01$ & $2.3 \mathrm{E}-01$ \\
\hline Th-229+D & $015594-54-4$ & $3.6 \mathrm{E}+00$ & $3.6 \mathrm{E}-02$ \\
\hline Th-230 & $014269-63-7$ & $3.4 \mathrm{E}+01$ & $3.4 \mathrm{E}-01$ \\
\hline Th-231 & $014932-40-2$ & $7.2 \mathrm{E}+02$ & $7.2 \mathrm{E}+00$ \\
\hline Th-232 & $007440-29-1$ & $3.9 \mathrm{E}+01$ & $3.9 \mathrm{E}-01$ \\
\hline Th-234 & $015065-10-8$ & $6.7 \mathrm{E}+01$ & $6.7 \mathrm{E}-01$ \\
\hline Tl-202 & $015720-57-7$ & $1.3 \mathrm{E}+03$ & $1.3 \mathrm{E}+01$ \\
\hline Tl-204 & $013968-51-9$ & $6.5 \mathrm{E}+02$ & $6.5 \mathrm{E}+00$ \\
\hline Tl-208 & $014913-50-9$ & $7.3 \mathrm{E}+04$ & $7.3 \mathrm{E}+02$ \\
\hline Tl-209 & $015690-73-0$ & $9.2 \mathrm{E}+04$ & $9.2 \mathrm{E}+02$ \\
\hline Tm-170 & $013981-30-1$ & $1.7 \mathrm{E}+02$ & $1.7 \mathrm{E}+00$ \\
\hline Tm-171 & $014333-45-0$ & $2.2 \mathrm{E}+03$ & $2.2 \mathrm{E}+01$ \\
\hline U-232 & $014158-29-3$ & $1.6 \mathrm{E}+01$ & $1.6 \mathrm{E}-01$ \\
\hline
\end{tabular}


Table 3.4f. (continued)

\begin{tabular}{|l|l|c|c|}
\hline \multirow{2}{*}{ Chemical } & \multirow{2}{*}{ CASRN } & \multicolumn{2}{|c|}{ Ingestion of fish (pCi/g) } \\
\cline { 3 - 4 } & & $10^{-4}$ & $10^{-6}$ \\
\hline U-233 & $013968-55-3$ & $2.9 \mathrm{E}+01$ & $2.9 \mathrm{E}-01$ \\
\hline U-234 & $013966-29-5$ & $2.9 \mathrm{E}+01$ & $2.9 \mathrm{E}-01$ \\
\hline U-235 & $015117-96-1$ & $2.8 \mathrm{E}+01$ & $2.8 \mathrm{E}-01$ \\
\hline U-235+D & $015117-96-1$ & $2.7 \mathrm{E}+01$ & $2.7 \mathrm{E}-01$ \\
\hline U-236 & $013982-70-2$ & $3.1 \mathrm{E}+01$ & $3.1 \mathrm{E}-01$ \\
\hline U-237 & $014269-75-1$ & $3.2 \mathrm{E}+02$ & $3.2 \mathrm{E}+00$ \\
\hline U-238 & $007440-61-1$ & $3.0 \mathrm{E}+01$ & $3.0 \mathrm{E}-01$ \\
\hline U-238+D & $007440-61-1$ & $2.1 \mathrm{E}+01$ & $2.1 \mathrm{E}-01$ \\
\hline U-240 & $015687-53-3$ & $2.4 \mathrm{E}+02$ & $2.4 \mathrm{E}+00$ \\
\hline V-48 & $014331-97-6$ & $1.7 \mathrm{E}+02$ & $1.7 \mathrm{E}+00$ \\
\hline W-181 & $015749-46-9$ & $4.7 \mathrm{E}+03$ & $4.7 \mathrm{E}+01$ \\
\hline W-185 & $014932-41-3$ & $6.3 \mathrm{E}+02$ & $6.3 \mathrm{E}+00$ \\
\hline W-187 & $014983-48-3$ & $5.2 \mathrm{E}+02$ & $5.2 \mathrm{E}+00$ \\
\hline Xe-122 & $015151-09-4$ & & \\
\hline Xe-123 & $015700-10-4$ & & \\
\hline Xe-125 & $013994-18-8$ & & \\
\hline Xe-127 & $013994-19-9$ & & \\
\hline Xe-129m & $013965-99-6$ & & \\
\hline
\end{tabular}


Table 3.4f. (continued)

\begin{tabular}{|c|c|c|c|}
\hline \multirow{2}{*}{ Chemical $^{\dagger}$} & \multirow{2}{*}{ CASRN } & \multicolumn{2}{|c|}{ Ingestion of fish (pCi/g) } \\
\hline & & $10^{-4}$ & $10^{-6}$ \\
\hline $\mathrm{Xe}-131 \mathrm{~m}$ & 014683-11-5 & & \\
\hline Xe-133 & $014932-42-4$ & & \\
\hline $\mathrm{Xe}-133 \mathrm{~m}$ & $014932-42-4$ & & \\
\hline $\mathrm{Xe}-135$ & $014995-62-1$ & & \\
\hline $\mathrm{Xe}-135 \mathrm{~m}$ & 014995-62-1 & & \\
\hline $\mathrm{Xe}-137$ & 014835-21-3 & & \\
\hline $\mathrm{Xe}-138$ & $015751-81-2$ & & \\
\hline$Y-90$ & 010098-91-6 & $8.6 \mathrm{E}+01$ & $8.6 \mathrm{E}-01$ \\
\hline Y-91 & $014234-24-3$ & $9.5 \mathrm{E}+01$ & $9.5 \mathrm{E}-01$ \\
\hline $\mathrm{Y}-91 \mathrm{~m}$ & $014234-24-3$ & $3.5 \mathrm{E}+04$ & $3.5 \mathrm{E}+02$ \\
\hline Y-92 & $015751-59-4$ & $6.6 \mathrm{E}+02$ & $6.6 \mathrm{E}+00$ \\
\hline Y-93 & $014981-70-5$ & $2.2 \mathrm{E}+02$ & $2.2 \mathrm{E}+00$ \\
\hline $\mathrm{Zn}-65$ & $013982-39-3$ & $1.3 \mathrm{E}+02$ & $1.3 \mathrm{E}+00$ \\
\hline $\mathrm{Zn}-69$ & $013982-23-5$ & $2.1 \mathrm{E}+04$ & $2.1 \mathrm{E}+02$ \\
\hline $\mathrm{Zn}-69 \mathrm{~m}$ & 013982-23-5 & $8.5 \mathrm{E}+02$ & $8.5 \mathrm{E}+00$ \\
\hline $\mathrm{Zr}-93$ & $015751-77-6$ & $2.5 \mathrm{E}+03$ & $2.5 \mathrm{E}+01$ \\
\hline $\mathrm{Zr}-95$ & $013967-71-0$ & $3.3 \mathrm{E}+02$ & $3.3 \mathrm{E}+00$ \\
\hline $\mathrm{Zr}-97$ & 014928-30-4 & $1.2 \mathrm{E}+02$ & $1.2 \mathrm{E}+00$ \\
\hline
\end{tabular}




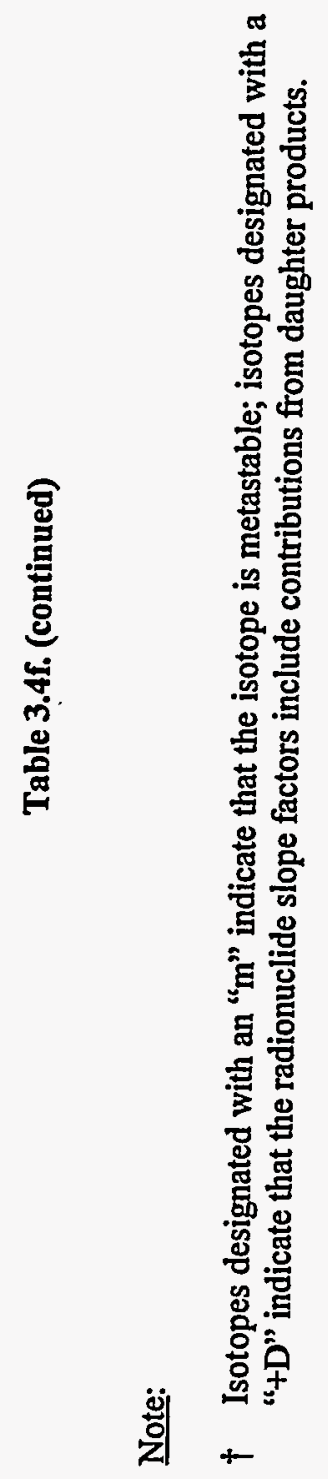

폼 
3-682 
Table 3.4g. Risk-based Preliminary Remediation Goals for water based on ingestion of fish for nonradionuclides (recreational scenario)

\begin{tabular}{|c|c|c|c|c|c|}
\hline \multirow{2}{*}{ Chemical } & \multirow{2}{*}{ CASRN } & \multicolumn{4}{|c|}{ Ingestion of Fish (concentration in water) (mg/L)" } \\
\hline & & $10^{-4}$ & $10^{-6}$ & $\mathbf{H Q}=\mathbf{1}$ & HQ $=0.1$ \\
\hline ALAR & 001596-84-5 & & & & \\
\hline Acenaphthene & $000083-32-9$ & & & $5.4 \mathrm{E}-01$ & $5.4 \mathrm{E}-02$ \\
\hline Acenaphthylene & 000208-96-8 & & & & \\
\hline Acephate & $030560-19-1$ & $2.0 \mathrm{E}+03$ & $2.0 \mathrm{E}+01$ & $3.0 \mathrm{E}+02$ & $3.0 \mathrm{E}+01$ \\
\hline Acetaldehyde & $000075-07-0$ & & & & \\
\hline Acetochlor & $034256-82-1$ & & & & \\
\hline Acetone & $000067-64-1$ & & & $2.5 \mathrm{E}+03$ & $2.5 \mathrm{E}+02$ \\
\hline Acetone Cyanohydrin & 000075-86-5 & & & & \\
\hline Acetonitrile & 000075-05-8 & & & $1.8 \mathrm{E}+02$ & $1.8 \mathrm{E}+01$ \\
\hline Acetophenone $^{(3)}$ & 000098-86-2 & & & $8.5 E+01$ & $8.5 \mathrm{E}+00$ \\
\hline Acrolein $^{(6)}$ & $000107-02-8$ & & & $4.0 \mathrm{E}+02$ & $4.0 \mathrm{E}+01$ \\
\hline Acrylamide & 000079-06-1 & $2.8 \mathrm{E}+00$ & $2.8 \mathrm{E}-02$ & $1.1 \mathrm{E}+01$ & $1.1 \mathrm{E}+00$ \\
\hline Acrylic Acid & 000079-10-7 & & & $6.3 E+03$ & $6.3 \mathrm{E}+02$ \\
\hline Acrylonitrile & $000107-13-1$ & $4.7 \mathrm{E}+00$ & 4.7E-02 & $1.1 \mathrm{E}+01$ & $1.1 \mathrm{E}+00$ \\
\hline Alachlor & $015972-60-8$ & $3.1 \mathrm{E}-01$ & $3.1 \mathrm{E}-03$ & $1.0 \mathrm{E}+00$ & $1.0 \mathrm{E}-01$ \\
\hline Aldicarb & 000116-06-3 & & & $2.3 E+00$ & 2.3E-01 \\
\hline Aldicarb Sulfone & $001646-88-4$ & & & & \\
\hline Aldrin & $000309-00-2$ & $1.2 \mathrm{E}-03$ & $1.2 \mathrm{E}-05$ & $2.6 \mathrm{E}-03$ & $2.6 \mathrm{E}-04$ \\
\hline
\end{tabular}


Table 3.4g. (continued)

\begin{tabular}{|c|c|c|c|c|c|}
\hline \multirow{2}{*}{ Chemical } & \multirow{2}{*}{ CASRN } & \multicolumn{4}{|c|}{ Ingestion of Fish (concentration in water) (mg/L)" } \\
\hline & & $10^{-4}$ & $10^{-5}$ & $\mathbf{H Q}=\mathbf{1}$ & $H Q=0.1$ \\
\hline Ally & 074223-64-6 & & & $4.1 \mathrm{E}+03$ & $4.1 \mathrm{E}+02$ \\
\hline Allyl Alcohol & 000107-18-6 & & & $1.2 \mathrm{E}+02$ & $1.2 \mathrm{E}+01$ \\
\hline Allyl Chloride ${ }^{(3)}$ & 000107-05-1 & & & $7.2 \mathrm{E}+01$ & $7.2 \mathrm{E}+00$ \\
\hline Aluminum & 007429-90-5 & & & & \\
\hline Aluminum Phosphide & 020859-73-8 & & & & \\
\hline Amdro & 067485-29-4 & & & & \\
\hline Ametryn & 000834-12-8 & & & $6.5 \mathrm{E}+00$ & $6.5 \mathrm{E}-01$ \\
\hline Aminophenol, m- & 000591-27-5 & & & $8.1 \mathrm{E}+02$ & 8.1E+01 \\
\hline Aminopyridine, 4- & 000504-24-5 & & & 2.1E-01 & 2.1E-02 \\
\hline Amitraz & 033089-61-1 & & & & \\
\hline Ammonia & 007664-41-7 & & & & \\
\hline Ammonium Sulfamate & 007773-06-0 & & & & \\
\hline Aniline & 000062-53-3 & $1.4 \mathrm{E}+02$ & $1.4 \mathrm{E}+00$ & & \\
\hline Anthracene & 000120-12-7 & & & $2.3 \mathrm{E}+00$ & 2.3E-01 \\
\hline Antimony (metallic) & 007440-36-0 & & & $3.9 \mathrm{E}-02$ & $3.9 \mathrm{E}-03$ \\
\hline Antimony Pentoxide & $001314-60-9$ & & & & \\
\hline Antimony Potassium Tartrate & $000304-61-0$ & & & & \\
\hline Antimony Tetroxide & $001332-81-6$ & & & & \\
\hline
\end{tabular}




\section{Table 3.4g. (continued)}

\begin{tabular}{|c|c|c|c|c|c|}
\hline \multirow{2}{*}{ Chemical } & \multirow{2}{*}{ CASRN } & \multicolumn{4}{|c|}{ Ingestion of Fish (concentration in water) (mg/L) } \\
\hline & & $10^{-4}$ & $10^{-6}$ & $\mathbf{H Q}=\mathbf{1}$ & $\mathrm{HQ}=\mathbf{0 . 1}$ \\
\hline Antimony Trioxide & $001309-64-4$ & & & & \\
\hline Apollo & $074115-24-5$ & & & & \\
\hline Aramite & $000140-57-8$ & & & & \\
\hline Aroclor 1016 & $012674-11-2$ & & & $3.8 \mathrm{E}-05$ & 3.8E-06 \\
\hline Aroclor $1221^{(1)}$ & 011104-28-2 & $3.9 \mathrm{E}-04$ & $3.9 \mathrm{E}-06$ & & \\
\hline Aroclor $1232^{(1)}$ & $011141-16-5$ & $1.9 \mathrm{E}-03$ & $1.9 \mathrm{E}-05$ & & \\
\hline Aroclor $1242^{(1)}$ & $053469-21-9$ & $3.9 \mathrm{E}-04$ & $3.9 \mathrm{E}-06$ & & \\
\hline Aroclor $1248^{(1)}$ & $012672-29-6$ & $2.0 \mathrm{E}-05$ & $2.0 \mathrm{E}-07$ & & \\
\hline Aroclor 1254 & $011097-69-1$ & & & $9.2 \mathrm{E}-06$ & 9.2E-07 \\
\hline Aroclor $1260^{(1)}$ & 011096-82-5 & $2.0 \mathrm{E}-06$ & $2.0 \mathrm{E}-08$ & & \\
\hline Arsenic Salts & NA & & & & \\
\hline Arsenic, Inorganic & $007440-38-2$ & & & & \\
\hline Arsine & $007784-42-1$ & & & & \\
\hline Asbestos & $001332-21-4$ & & & & \\
\hline Assure & 076578-14-8 & & & & \\
\hline Asulam & 003337-71-1 & & & & \\
\hline Atrazine & 001912-24-9 & $1.6 \mathrm{E}-01$ & $1.6 \mathrm{E}-03$ & $5.2 \mathrm{E}+00$ & $5.2 \mathrm{E}-01$ \\
\hline Avermectin B1 & 065195-55-3 & & & & \\
\hline
\end{tabular}


Table 3.4g. (continued)

\begin{tabular}{|c|c|c|c|c|c|}
\hline \multirow{2}{*}{ Chemical } & \multirow{2}{*}{ CASRN } & \multicolumn{4}{|c|}{ Ingestion of Fish (concentration in water) (mg/L)" } \\
\hline & & $10^{-4}$ & $10^{-6}$ & $\mathbf{H Q}=\mathbf{1}$ & $\mathrm{HQ}=\mathbf{0 . 1}$ \\
\hline Azobenzene & 000103-33-3 & 4.6E-02 & 4.6E-04 & & \\
\hline Barium & 007440-39-3 & & & $1.7 \mathrm{E}+02$ & $1.7 \mathrm{E}+01$ \\
\hline Barium Cyanide & $000542-62-1$ & & & & \\
\hline Baygon & 000114-26-1 & & & $4.1 \mathrm{E}+00$ & 4.1E-01 \\
\hline Bayleton & 043121-43-3 & & & $9.0 \mathrm{E}+00$ & $9.0 \mathrm{E}-01$ \\
\hline Baythroid & $068359-37-5$ & & & & \\
\hline Benefin & $001861-40-1$ & & & $1.8 \mathrm{E}+00$ & $1.8 \mathrm{E}-01$ \\
\hline Benomyl & 017804-35-2 & & & $1.5 \mathrm{E}+01$ & $1.5 \mathrm{E}+00$ \\
\hline Bentazon & $025057-89-0$ & & & $6.1 \mathrm{E}+00$ & 6.1E-01 \\
\hline Benz$[a]$ anthracene $e^{(2)}$ & 000056-55-3 & 2.5E-04 & $2.5 \mathrm{E}-06$ & & \\
\hline Benzaldehyde & $000100-52-7$ & & & $3.0 \mathrm{E}+01$ & $3.0 \mathrm{E}+00$ \\
\hline Benzene & $000071-43-2$ & $3.4 \mathrm{E}+00$ & $3.4 \mathrm{E}-02$ & & \\
\hline Benzene Hexachloride & NA & & & & \\
\hline Benzene, Ethyldimethyl & NA & & & & \\
\hline Benzene, Ethylmethyl & NA & & & & \\
\hline Benzene, Methylpropenyl & NA & & & & \\
\hline Benzene, Methylpropyl & NA & & & & \\
\hline Benzene, Trimethyl & 025551-13-7 & & & & \\
\hline
\end{tabular}


Table 3.4g. (continued)

\begin{tabular}{|c|c|c|c|c|c|}
\hline \multirow{2}{*}{ Chemical } & \multirow{2}{*}{ CASRN } & \multicolumn{4}{|c|}{ Ingestion of Fish (concentration in water) (mg/L)" } \\
\hline & & $10^{-4}$ & $10^{-6}$ & $\mathbf{H Q}=\mathbf{1}$ & $\mathrm{HQ}=\mathbf{0 . 1}$ \\
\hline Benzenethiol & 000108-98-5 & & & 2.1E-03 & 2.1E-04 \\
\hline Benzidine & 000092-87-5 & $1.7 \mathrm{E}-03$ & $1.7 \mathrm{E}-05$ & $5.2 \mathrm{E}+00$ & $5.2 \mathrm{E}-01$ \\
\hline Benzo[a]pyrene ${ }^{(2)}$ & 000050-32-8 & $1.2 \mathrm{E}-05$ & $1.2 \mathrm{E}-07$ & & \\
\hline Benzo[b]fluoranthene ${ }^{(2)}$ & $000205-99-2$ & $1.2 \mathrm{E}-04$ & $1.2 \mathrm{E}-06$ & & \\
\hline Benzo[g,h,i]perylene & 000191-24-2 & & & & \\
\hline Benzo[k]fluoranthene ${ }^{(2)}$ & 000207-08-9 & $3.6 \mathrm{E}-04$ & $3.6 \mathrm{E}-06$ & & \\
\hline Benzoic Acid & $000065-85-0$ & & & $2.4 \mathrm{E}+03$ & $2.4 \mathrm{E}+02$ \\
\hline Benzotrichloride & 000098-07-7 & $1.9 \mathrm{E}-03$ & $1.9 \mathrm{E}-05$ & & \\
\hline Benzyl Alcohol & 000100-51-6 & & & $7.3 \mathrm{E}+02$ & 7.3E+01 \\
\hline Benzyl Chloride & $000100-44-7$ & 4.1E-01 & $4.1 \mathrm{E}-03$ & & \\
\hline Beryllium & $007440-41-7$ & $5.3 \mathrm{E}-03$ & 5.3E-05 & $4.9 \mathrm{E}-01$ & $4.9 \mathrm{E}-02$ \\
\hline Bidrin & $000141-66-2$ & & & $5.5 \mathrm{E}+00$ & $5.5 \mathrm{E}-01$ \\
\hline Biphenthrin & 082657-04-3 & & & & \\
\hline Biphenyl, 1,1'- & $000092-52-4$ & & & $6.4 \mathrm{E}-01$ & $6.4 \mathrm{E}-02$ \\
\hline $\begin{array}{l}\text { Bis(2-chloro-1-methylethyl)ether } \\
\text { (Technical) }\end{array}$ & $000108-60-1$ & $6.1 \mathrm{E}-01$ & $6.1 \mathrm{E}-03$ & & \\
\hline Bis(2-chloroethoxy)methane & $000111-91-1$ & & & & \\
\hline Bis(2-chloroethyl)ether & $000111-44-4$ & 3.7E-01 & 3.7E-03 & & \\
\hline
\end{tabular}




\begin{tabular}{|c|c|c|c|c|c|}
\hline & & & & $\mathrm{VN}$ & 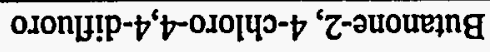 \\
\hline \multirow[t]{3}{*}{ I0+at's } & $20+3 t^{\circ} S$ & & & $\varepsilon-9 \varepsilon-I L 0000$ & $-\mathrm{N}$ ' louęng \\
\hline & & & & $0-66-901000$ & $-\varepsilon^{6} I$ 'әuә!pęng \\
\hline & & & & $z-66-689100$ & 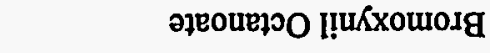 \\
\hline \multirow[t]{3}{*}{$10+3 b^{\circ} I$} & $20+3 t^{\circ} 1$ & & & $\varsigma-t 8-689100$ & I!UKxomosg \\
\hline & & & & $L-Z 9-\varsigma L 0000$ & 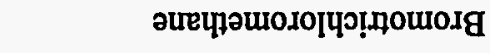 \\
\hline & & & & $\varepsilon-96-\downarrow 0 I Z 00$ & soydourosg \\
\hline $10-36^{\circ} Z$ & $00+362$ & & & $6-\varepsilon 8-\downarrow L 0000$ & (g) $)^{\text {areqpourourosg }}$ \\
\hline \multirow[t]{2}{*}{$10-90^{\circ} \mathrm{S}$} & $00+30^{\circ} \mathrm{S}$ & $20-9 b^{\circ} L$ & $00+B \nabla^{\circ} L$ & $\tau-\varsigma \tau-s L 0000$ & uwojourosg \\
\hline & & & & $\varepsilon-\varsigma \varsigma-101000$ & 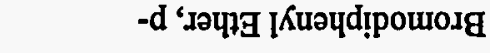 \\
\hline \multirow[t]{4}{*}{$10-3 S^{\prime} 8$} & $00+3 S^{\prime} 8$ & $20-89^{\circ} \mathrm{I}$ & $00+39$ I & $t-L Z-\varsigma L 0000$ & 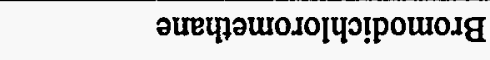 \\
\hline & & & & $s-\angle 6-t<0000$ & әигчрәшиолорчวошолg \\
\hline & & & & $Z-\angle 0-\angle E 9 L 00$ & әp!̣on|J!1L uodog \\
\hline & & & & $8-Z ち 0 t \downarrow L 00$ & Кuo səłexog pur uosog \\
\hline \multirow[t]{2}{*}{ I0-ES'I } & $00+a S \cdot I$ & & & $L-S 0-080000$ & V jouaqds!g \\
\hline & & S0:-7I\%6 & $\varepsilon 0-9]^{\circ} 6$ & $I-88-z b \varsigma 000$ & 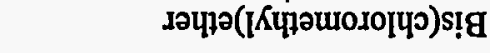 \\
\hline$\varepsilon 0-\underline{\varepsilon} \varepsilon^{*} 9$ & 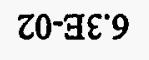 & $\nabla 0-\not \varepsilon \varepsilon$ & Z0-घE'S & $L-18-L I 1000$ & 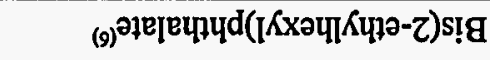 \\
\hline $00+\exists L^{\prime} I$ & $I 0+B L^{\prime} I$ & & & $6-\tau \varepsilon-8 \varepsilon 96 \varepsilon 0$ & 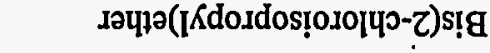 \\
\hline$I^{\prime} \mathbf{0}=\mathbf{D H}$ & $I=\mathbf{O H}$ & $9.0 \mathrm{I}$ & rOI & \multirow{2}{*}{ NYSVD } & \multirow{2}{*}{ 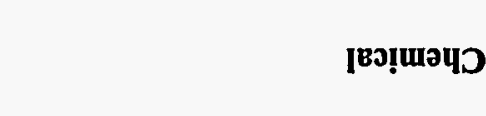 } \\
\hline.$\left(\mathrm{T} / \mathbf{5}^{2}\right)$ & M u! นo! & )) ys! & & & \\
\hline
\end{tabular}

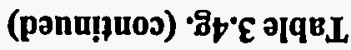


Table 3.4g. (continued)

\begin{tabular}{|c|c|c|c|c|c|}
\hline \multirow{2}{*}{ Chemical } & \multirow{2}{*}{ CASRN } & \multicolumn{4}{|c|}{ Ingestion of Fish (concentration in water) (mg/L)" } \\
\hline & & $10^{-4}$ & $10^{-6}$ & $\mathbf{H Q}=\mathbf{1}$ & HQ $=0.1$ \\
\hline Butyl Benzyl Phthlate & 000085-68-7 & & & $6.3 \mathrm{E}-01$ & $6.3 \mathrm{E}-02$ \\
\hline Butylate & 002008-41-5 & & & & \\
\hline Butylchloride, t- & $000507-20-0$ & & & & \\
\hline Butylphthalyl Butylglycolate & 000085-70-1 & & & & \\
\hline Cacodylic Acid & $000075-60-5$ & & & $5.0 \mathrm{E}+01$ & $5.0 \mathrm{E}+00$ \\
\hline Cadmium (Diet) & 007440-43-9 & & & $4.9 \mathrm{E}-02$ & $4.9 \mathrm{E}-03$ \\
\hline Calcium Cyanide & 000592-01-8 & & & & \\
\hline Caprolactam & 000105-60-2 & & & $1.2 \mathrm{E}+04$ & $1.2 \mathrm{E}+03$ \\
\hline Captafol & 002425-06-1 & $5.6 \mathrm{E}+00$ & $5.6 \mathrm{E}-02$ & 4.1E-01 & 4.1E-02 \\
\hline Captan & 000133-06-2 & $2.0 \mathrm{E}+01$ & $2.0 \mathrm{E}-01$ & $3.9 \mathrm{E}+01$ & $3.9 \mathrm{E}+00$ \\
\hline Carbaryl & 000063-25-2 & & & $3.0 \mathrm{E}+01$ & $3.0 \mathrm{E}+00$ \\
\hline Carbazole & 000086-74-8 & $2.5 \mathrm{E}-01$ & $2.5 \mathrm{E}-03$ & & \\
\hline Carbofuran & 001563-66-2 & & & $5.1 \mathrm{E}+00$ & $5.1 \mathrm{E}-01$ \\
\hline Carbon Disulfide & $000075-15-0$ & & & $3.6 \mathrm{E}+01$ & $3.6 \mathrm{E}+00$ \\
\hline Carbon Tetrachloride ${ }^{(6)}$ & 000056-23-5 & $2.2 \mathrm{E}-01$ & $2.2 \mathrm{E}-03$ & $8.7 \mathrm{E}-02$ & 8.7E-03 \\
\hline Carbosulfan & 055285-14-8 & & & & \\
\hline Carboxin & 005234-68-4 & & & $4.2 \mathrm{E}+01$ & $4.2 \mathrm{E}+00$ \\
\hline Chloral & 000075-87-6 & & & & \\
\hline
\end{tabular}


Table 3.4g. (continued)

\begin{tabular}{|c|c|c|c|c|c|}
\hline \multirow{2}{*}{ Chemical } & \multirow{2}{*}{ CASRN } & \multicolumn{4}{|c|}{ Ingestion of Fish (concentration in water) (mg/L)" } \\
\hline & & $10^{-4}$ & $10^{-6}$ & $\mathbf{H Q}=\mathbf{1}$ & HQ $=0.1$ \\
\hline Chloramben & 000133-90-4 & & & & \\
\hline Chloranil & 000118-75-2 & & & & \\
\hline Chlordane $^{(6)}$ & 000057-74-9 & $2.0 \mathrm{E}-04$ & $2.0 \mathrm{E}-06$ & $6.6 \mathrm{E}-05^{\circ}$ & $6.6 \mathrm{E}-06$ \\
\hline Chloride & 016887-00-6 & & & & \\
\hline Chlorimuron, Ethyl- & $.090982-32-4$ & & & & \\
\hline Chlorine & 007782-50-5 & & & & \\
\hline Chlorine Dioxide & 010049-04-4 & & & & \\
\hline Chlorite (Sodium Salt) & 007758-19-2 & & & & \\
\hline Chloro-1,1-difluoroethane, 1- & $000075-68-3$ & & & & \\
\hline Chloro-1,3-butadiene, 2- & 000126-99-8 & & & $1.4 \mathrm{E}+01$ & $1.4 \mathrm{E}+00$ \\
\hline Chloro-2-methylaniline $\mathrm{HCl}, 4-$ & 003165-93-3 & & & & \\
\hline Chloro-2-methylaniline, 4- & 000095-69-2 & & & & \\
\hline Chloroacetic Acid & $000079-11-8$ & & & $4.1 \mathrm{E}+00$ & 4.1E-01 \\
\hline Chloroacetophenone, 2- & 000532-27-4 & & & & \\
\hline Chloroaniline, $\mathrm{p}$ - & 000106-47-8 & & & $5.0 \mathrm{E}-01$ & $5.0 \mathrm{E}-02$ \\
\hline Chlorobenzene $^{(6)}$ & 000108-90-7 & & & $2.5 \mathrm{E}+00$ & $2.5 \mathrm{E}-01$ \\
\hline Chlorobenzilate & 000510-15-6 & $5.5 \mathrm{E}-03$ & $5.5 \mathrm{E}-05$ & 1.3E-01 & $1.3 \mathrm{E}-02$ \\
\hline Chlorobenzoic Acid, p- & 000074-11-3 & & & $3.0 \mathrm{E}+01$ & $3.0 \mathrm{E}+00$ \\
\hline
\end{tabular}


Table 3.4g. (continued)

\begin{tabular}{|c|c|c|c|c|c|}
\hline \multirow{2}{*}{ Chemical } & \multirow{2}{*}{ CASRN } & \multicolumn{4}{|c|}{ Ingestion of Fish (concentration in water) (mg/L) } \\
\hline & & $10^{-4}$ & $10^{-6}$ & $\mathbf{H Q}=\mathbf{1}$ & $\mathrm{HQ}=0.1$ \\
\hline Chlorobenzotrifluoride, 4- & $000098-56-6$ & & & 4.8E-01 & 4.8E-02 \\
\hline Chlorobutane, 1- & $000109-69-3$ & & & $7.1 \mathrm{E}+01$ & 7.1E+00 \\
\hline Chlorobutane, 2- & $000078-86-4$ & & & & \\
\hline Chlorocyclopentadiene & 041851-50-7 & & & & \\
\hline Chlorodibromoethane & $073506-94-2$ & & & & \\
\hline Chlorodifluoromethane & $000075-45-6$ & & & & \\
\hline Chloroform ${ }^{(6)}$ & $000067-66-3$ & $1.9 \mathrm{E}+01$ & $1.9 \mathrm{E}-01$ & $5.1 \mathrm{E}+00$ & $5.1 \mathrm{E}-01$ \\
\hline Chloromethane $^{(6)}$ & 000074-87-3 & $6.1 \mathrm{E}+01$ & $6.1 \mathrm{E}-01$ & & \\
\hline Chloromethyl Methyl Ether & $000107-30-2$ & & & & \\
\hline Chloronaphthalene, Beta- & $000091-58-7$ & & & $1.0 \mathrm{E}+00$ & $1.0 \mathrm{E}-01$ \\
\hline Chloronitrobenzene, o- & $000088-73-3$ & $3.3 \mathrm{E}+00$ & $3.3 \mathrm{E}-02$ & & \\
\hline Chloronitrobenzene, $\mathrm{p}$ - & $000121-73-3$ & $3.3 \mathrm{E}+00$ & $3.3 \mathrm{E}-02$ & & \\
\hline Chlorophenol, 2- & $000095-57-8$ & & & $1.8 \mathrm{E}+00$ & $1.8 \mathrm{E}-01$ \\
\hline Chlorophenyl Methyl Sulfide, p- & $000123-09-1$ & & & & \\
\hline Chlorophenyl Methyl Sulfoxide & $000934-73-6$ & & & & \\
\hline Chloropropane, 2- & $000075-29-6$ & & & & \\
\hline Chlorothalonil & $001897-45-6$ & $3.9 \mathrm{E}-01$ & $3.9 \mathrm{E}-03$ & 2.7E-01 & 2.7E-02 \\
\hline Chlorotoluene, o- & $000095-49-8$ & & & 8.7E-01 & $8.7 \mathrm{E}-02$ \\
\hline
\end{tabular}


Table 3.4g. (continued)

\begin{tabular}{|c|c|c|c|c|c|}
\hline \multirow{2}{*}{ Chemical } & \multirow{2}{*}{ CASRN } & \multicolumn{4}{|c|}{ Ingestion of Fish (concentration in water) (mg/L)" } \\
\hline & & $10^{-4}$ & $10^{-6}$ & $H Q=1$ & $H Q=0.1$ \\
\hline Chlorpropham & 000101-21-3 & & & $2.1 \mathrm{E}+01$ & $2.1 \mathrm{E}+00$ \\
\hline Chlorpyrifos & 002921-88-2 & & & 4.7E-03 & 4.7E-04 \\
\hline Chlorpyrifos Methyl & 005598-13-0 & & & $9.0 \mathrm{E}-02$ & $9.0 \mathrm{E}-03$ \\
\hline Chlorsulfuron & 064902-72-3 & & & 8.7E+03 & 8.7E+02 \\
\hline Chlorthiophos & 060238-56-4 & & & & \\
\hline Chromium (III) (Insoluble Salts) & 016065-83-1 & & & $4.9 \mathrm{E}+01$ & $4.9 \mathrm{E}+00$ \\
\hline Chromium $(\mathrm{VI})^{(6)}$ & 018540-29-9 & & & $2.5 \mathrm{E}-01$ & 2.5E-02 \\
\hline Chromium Salts & NA & & & & \\
\hline Chrysene $^{(2)}$ & 000218-01-9 & $2.5 \mathrm{E}-02$ & $2.5 \mathrm{E}-04$ & & \\
\hline Cobalt & $007440-48-4$ & & & & \\
\hline Coke Oven Emissions & $008007-45-2$ & & & & \\
\hline Copper & $007440-50-8$ & & & & \\
\hline Copper Cyanide & 000544-92-3 & & & & \\
\hline Creosote & 008001-58-9 & & & & \\
\hline Cresol, m- & 000108-39-4 & & & $2.5 \mathrm{E}+01$ & $2.5 \mathrm{E}+00$ \\
\hline Cresol, o- & 000095-48-7 & & & $3.0 \mathrm{E}+01$ & $3.0 \mathrm{E}+00$ \\
\hline Cresol, p- & $000106-44-5$ & & & $3.0 \mathrm{E}+00$ & 3.0E-01 \\
\hline Crotonaldehyde & 000123-73-9 & $8.8 \mathrm{E}-02$ & $8.8 \mathrm{E}-04$ & & \\
\hline
\end{tabular}


Table 3.4g. (continued)

\begin{tabular}{|c|c|c|c|c|c|}
\hline \multirow{2}{*}{ Chemical } & \multirow{2}{*}{ CASRN } & \multicolumn{4}{|c|}{ Ingestion of Fish (concentration in water) (mg/L)" } \\
\hline & & $10^{-4}$ & $10^{-6}$ & $\mathbf{H Q}=\mathbf{1}$ & $\mathrm{HQ}=0.1$ \\
\hline Cumene & 000098-82-8 & & & $1.5 \mathrm{E}+00$ & $1.5 \mathrm{E}-01$ \\
\hline Cyanazine & $021725-46-2$ & $9.6 \mathrm{E}-02$ & $9.6 \mathrm{E}-04$ & $6.9 \mathrm{E}-01$ & $6.9 \mathrm{E}-02$ \\
\hline Cyanide (CN-) & $000057-12-5$ & & & $4.9 \mathrm{E}+01$ & $4.9 \mathrm{E}+00$ \\
\hline Cyanogen & 000460-19-5 & & & & \\
\hline Cyanogen Bromide & $000506-68-3$ & & & & \\
\hline Cyanogen Chloride & $000506-77-4$ & & & $5.9 \mathrm{E}+02$ & $5.9 \mathrm{E}+01$ \\
\hline $\begin{array}{l}\text { Cyclohexane, 1,2,3,4,5-pentabromo-6- } \\
\text { chloro- }\end{array}$ & 000087-84-3 & & & & \\
\hline Cyclohexanone & $000108-94-1$ & & & $2.0 \mathrm{E}+04$ & $2.0 \mathrm{E}+03$ \\
\hline Cyclohexylamine & $000108-91-8$ & & & $2.4 \mathrm{E}+02$ & $2.4 \mathrm{E}+01$ \\
\hline Cyclopentadiene & $000542-92-7$ & & & & \\
\hline Cyhalothrin/karate & $068085-85-8$ & & & $4.8 \mathrm{E}-04$ & 4.8E-05 \\
\hline Cypermethrin & $052315-07-8$ & & & & \\
\hline Cyromazine & $066215-27-8$ & & & & \\
\hline $\mathrm{DDD}$ & $000072-54-8$ & $6.4 \mathrm{E}-04$ & $6.4 \mathrm{E}-06$ & & \\
\hline DDE & $000072-55-9$ & $5.3 \mathrm{E}-04$ & 5.3E-06 & & \\
\hline DDT & $000050-29-3$ & $1.7 \mathrm{E}-04$ & $1.7 \mathrm{E}-06$ & $1.2 \mathrm{E}-04$ & $1.2 \mathrm{E}-05$ \\
\hline Dacthal & $001861-32-1$ & & & & \\
\hline
\end{tabular}


Table 3.4g. (continued)

\begin{tabular}{|l|l|l|l|l|c|}
\hline \multirow{2}{*}{ Chemical } & \multirow{2}{*}{ CASRN } & \multicolumn{2}{|c|}{ Ingestion of Fish (concentration in water) (mg/L) } \\
\cline { 4 - 6 } & & $10^{-4}$ & $10^{-6}$ & HQ=1 & HQ=0.1 \\
\hline Dalapon & $000075-99-0$ & & & $1.3 \mathrm{E}+02$ & $1.3 \mathrm{E}+01$ \\
\hline Decabromodiphenyl Ether & $001163-19-5$ & & & $1.7 \mathrm{E}-02$ & $1.7 \mathrm{E}-03$ \\
\hline Demeton & $008065-48-3$ & & & \\
\hline Di(2-ethylhexyl)adipate & $000103-23-1$ & $3.8 \mathrm{E}-03$ & $3.8 \mathrm{E}-05$ & $1.2 \mathrm{E}-02$ & $1.2 \mathrm{E}-03$ \\
\hline Diallate & $002303-16-4$ & $1.8 \mathrm{E}+01$ & $1.8 \mathrm{E}-01$ & & \\
\hline Diazinon & $000333-41-5$ & & & $1.9 \mathrm{E}-02$ & $1.9 \mathrm{E}-03$ \\
\hline Dibenz[a,h]anthracene ${ }^{(2)}$ & $000053-70-3$ & $3.6 \mathrm{E}-06$ & $3.6 \mathrm{E}-08$ & & \\
\hline Dibromo-3-chloropropane, 1,2- & $000096-12-8$ & $2.9 \mathrm{E}-02$ & $2.9 \mathrm{E}-04$ & & \\
\hline Dibromobenzene, 1,4- & $000106-37-6$ & & & $2.2 \mathrm{E}-01$ & $2.2 \mathrm{E}-02$ \\
\hline Dibromochloromethane & $000124-48-1$ & $9.9 \mathrm{E}-01$ & $9.9 \mathrm{E}-03$ & $7.1 \mathrm{E}+00$ & $7.1 \mathrm{E}-01$ \\
\hline Dibromodichloromethane & $000594-18-3$ & & & & \\
\hline Dibromodiphenyl Ether, p,p'- & $002050-47-7$ & & & & \\
\hline Dibromoethane, 1,2- & $000106-93-4$ & $1.4 \mathrm{E}-03$ & $1.4 \mathrm{E}-05$ & & \\
\hline Dibromomethane (Methylene Bromide) & $000074-95-3$ & & & $2.1 \mathrm{E}+01$ & $2.1 \mathrm{E}+00$ \\
\hline Dibutyl Phthalate & $000084-74-2$ & & & $3.2 \mathrm{E}-01$ & $3.2 \mathrm{E}-02$ \\
\hline Dicamba & $001918-00-9$ & & & $2.6 \mathrm{E}+00$ & $2.6 \mathrm{E}-01$ \\
\hline Dichloro-2-butene, 1,4- & $000764-41-0$ & & & \\
\hline Dichloroacetic Acid & & & & & \\
\hline
\end{tabular}




\begin{tabular}{|c|c|c|c|c|c|}
\hline$z 0-\Xi t^{\prime} z$ & $10-a+z$ & $\varepsilon 0-96^{\circ} \varepsilon$ & {$\left[0-\pi 6^{\circ} \varepsilon\right.$} & L-EL-Z90000 & sonxolyग! \\
\hline \multirow[t]{3}{*}{ Z0-GI'E } & I0-gI' $\varepsilon$ & $20-\exists \varepsilon^{\prime} I$ & $00+G \varepsilon^{\prime} I$ & $9-S L-\tau+S 000$ & 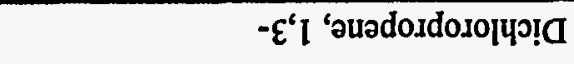 \\
\hline & & & & $6-\varepsilon z-9[9000$ & 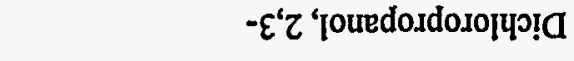 \\
\hline & & $20-\exists L \cdot I$ & $00+9 L \cdot I$ & $S=\angle 8-8 \angle 0000$ & 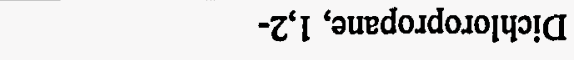 \\
\hline $20-98 \cdot \tau$ & $10-98^{\prime} Z$ & & & 9-Z8- & 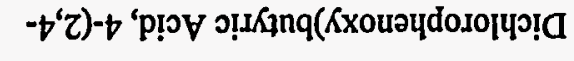 \\
\hline 10-gI'Z & $00+3 I^{\prime} \mathrm{Z}$ & & & $L-S L-\$ 60000$ & 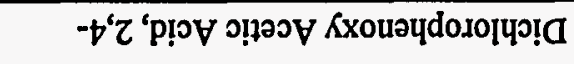 \\
\hline$Z 0-\mathrm{Al}{ }^{\prime} \varepsilon$ & $\left\lfloor 0-\exists\left[{ }^{\circ} \varepsilon\right.\right.$ & & & $\tau-\varepsilon 8-0 z 1000$ & 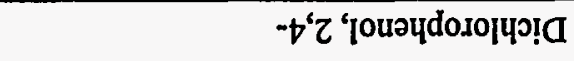 \\
\hline $10+3 t+I$ & $20+B 6 \cdot 1$ & & & ৎ-09-9S1000 & 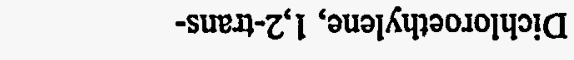 \\
\hline $10-30 * 9$ & $00+70^{\circ} 9$ & & & $\tau-6 \varsigma-9 S 1000$ & 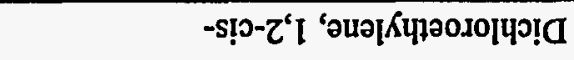 \\
\hline $00+B \varsigma^{*} 9$ & $10+3 S^{\prime} 9$ & & & $0-6 \varsigma-0 t \varsigma 000$ & 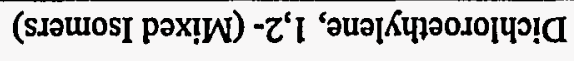 \\
\hline \multirow[t]{2}{*}{$0-3 S^{\circ} 9$} & $00+3 S^{\prime} 9$ & $\varepsilon 0-98 \cdot z$ & I0-98'z & $t-S E-\varsigma L 0000$ & 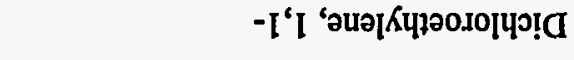 \\
\hline & & $20-g I \cdot \varepsilon$ & $00+a I^{\circ} \varepsilon$ & $z-90-\angle 01000$ & -Z'I 'әนвцрәодоцગ! \\
\hline $00+g Z L$ & $10+a Z^{\prime} L$ & & & $\varepsilon-\triangleright \varepsilon-\varsigma L 0000$ & 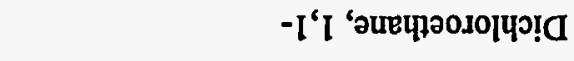 \\
\hline \multirow[t]{4}{*}{$00+B I L$} & $I 0+B I^{\circ} L$ & & & 8-IL-SL0000 & 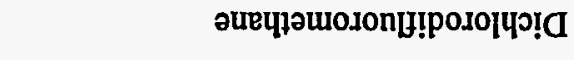 \\
\hline & & $t 0-36 . I$ & $20-96 \cdot I$ & 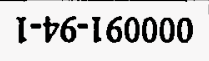 & 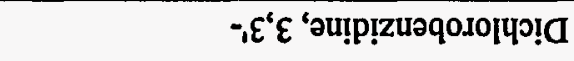 \\
\hline & & $\varepsilon 0-a z \succ b$ & $10-a Z^{\prime} b$ & $L-9 b-901000$ & 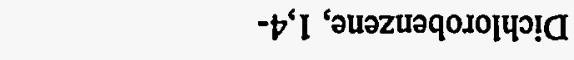 \\
\hline & & & & $\mathfrak{l}-\varepsilon L-I t S 000$ & 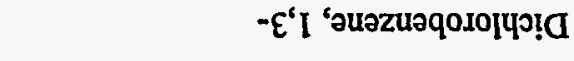 \\
\hline $10-\exists 6^{\circ} \varepsilon$ & $00+\exists 6^{\circ} \varepsilon$ & & & $\lfloor-0 \varsigma-\varsigma 60000$ & 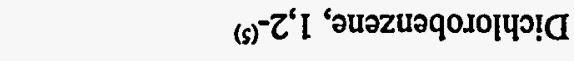 \\
\hline$I^{\prime} \mathbf{0}=\mathbf{\partial H}$ & $I=\partial H$ & $9-0 I$ & rOI & \multirow{2}{*}{ NASYD } & \multirow{2}{*}{ |вอ!ุแวบว } \\
\hline.$\left(T / s^{s}\right.$ & A u!̣ uo! & 03) чS!! & & & \\
\hline
\end{tabular}

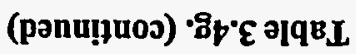




\begin{tabular}{|c|c|c|c|c|c|}
\hline & & & & $I-8 L-L L 0000$ & 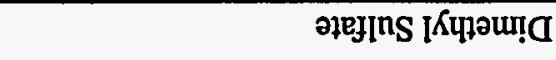 \\
\hline & & I0-GZ'I & $I 0+B z \cdot I$ & $t-06-611000$ & $-\varepsilon^{\prime} \varepsilon$ 'әи!р!zuәq кхочрәи! \\
\hline \multirow[t]{2}{*}{$\varepsilon 0^{-}-\underline{\mathbf{C}} 0^{\circ} \varepsilon$} & $20^{-}-80^{\circ} \varepsilon$ & & & S-IS-090000 & әџеочрәи! \\
\hline & & & & L-†9-06ZSSO & 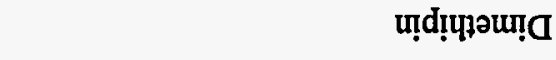 \\
\hline \multirow[t]{6}{*}{$10+9 \tau^{\prime} z$} & $z 0+3 z z$ & & & 9-SL-Stt100 & 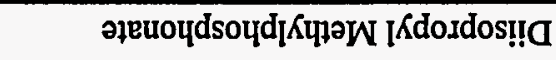 \\
\hline & & & & $9-\angle E-S L 0000$ & 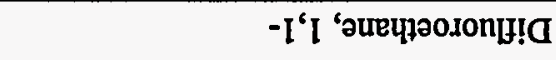 \\
\hline & & & & S-8E-L9ESE0 & uornzuəqnเป! \\
\hline & & & & 9-8t-ZZZ£Ь0 & zenbozuəy!d \\
\hline & & $0 I-\exists S S S$ & 80 -aS'S & {$[-\varepsilon \varsigma-9 \varsigma 0000$} & 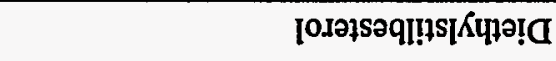 \\
\hline & & & & $S-b 8-\angle[9000$ & 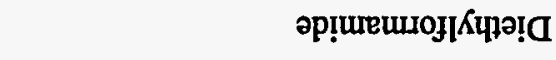 \\
\hline \multirow[t]{4}{*}{$\varepsilon 0+96^{\circ} \varepsilon$} & $t 0+36^{\circ} \varepsilon$ & & & 0-06-I I I 1000 & 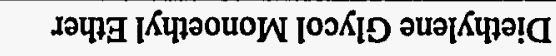 \\
\hline & & & & s-ヤย-乙II000 & 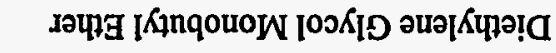 \\
\hline & & & & $0-I Z-\varepsilon 69000$ & 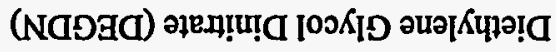 \\
\hline & & & & S-St-I I $\varepsilon 000$ & 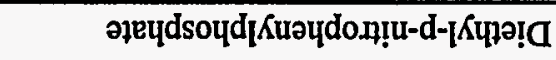 \\
\hline $\mathfrak{l 0}+3 L^{\prime} \mathfrak{I}$ & $20+a<\cdot I$ & & & $\tau-99-680000$ & 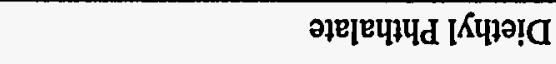 \\
\hline$S_{0}-\exists L \cdot \tau$ & $b 0-\exists L \cdot Z$ & $\mathrm{LO}-\mathrm{G8} 8^{\circ} \mathrm{L}$ & SO-G8. L & $I-\angle S-090000$ & นฺฺрाə!ด \\
\hline \multirow[t]{2}{*}{$10-\exists Z^{\circ} \varepsilon$} & $00+\theta Z^{\circ} \varepsilon$ & & & $9-\varepsilon L-L L 0000$ & 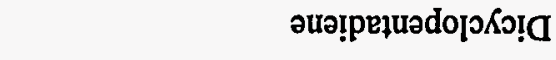 \\
\hline & & & & $\tau-\tau \varepsilon-\varsigma I 1000$ & [0]0ग!ब \\
\hline $\mathbf{I}^{*} \mathbf{0}=\partial \mathbf{H}$ & $\mathbf{I}=\mathbf{O H}$ & $9-0 \mathrm{I}$ & rOI & \multirow{2}{*}{ N\&SWD } & \multirow{2}{*}{ 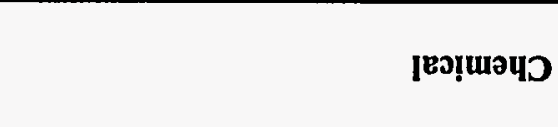 } \\
\hline .(T/8 & IBM U!̣ UO!! & 100) ys!t ] & & & \\
\hline
\end{tabular}

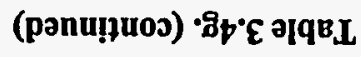


Table 3.4g. (continued)

\begin{tabular}{|c|c|c|c|c|c|}
\hline \multirow{2}{*}{ Chemical } & \multirow{2}{*}{ CASRN } & \multicolumn{4}{|c|}{ Ingestion of Fish (concentration in water) (mg/L)" } \\
\hline & & $10^{-4}$ & $10^{-6}$ & $\mathbf{H Q}=\mathbf{1}$ & $H Q=0.1$ \\
\hline Dimethylaniline $\mathrm{HCl}, 2,4-$ & 021436-96-4 & & & & \\
\hline Dimethylaniline, 2,4- & 000095-68-1 & & & & \\
\hline Dimethylaniline, N,N- & 000121-69-7 & & & $3.5 \mathrm{E}-01$ & 3.5E-02 \\
\hline Dimethylbenzidine, 3,3'- & 000119-93-7 & $7.6 \mathrm{E}-03$ & 7.6E-05 & & \\
\hline Dimethylethyl Lead & $107584-40-7$ & & & & \\
\hline Dimethylformamide & $000068-12-2$ & & & $9.6 \mathrm{E}+03$ & $9.6 \mathrm{E}+02$ \\
\hline Dimethylhydrazine, $1,1-^{(5)}$ & 000057-14-7 & & & & \\
\hline Dimethylhydrazine, 1,2- & 000540-73-8 & & & & \\
\hline Dimethylphenol, 2,4- & 000105-67-9 & & & $6.0 \mathrm{E}+00$ & $6.0 \mathrm{E}-01$ \\
\hline Dimethylphenol, 2,6- & 000576-26-1 & & & $1.6 \mathrm{E}-01$ & $1.6 \mathrm{E}-02$ \\
\hline Dimethylphenol, 3,4- & 000095-65-8 & & & $3.6 \mathrm{E}-01$ & $3.6 \mathrm{E}-02$ \\
\hline Dimethylphthalate ${ }^{(3)}$ & 000131-11-3 & & & $1.0 \mathrm{E}+04$ & $1.0 \mathrm{E}+03$ \\
\hline Dimethylterephthalate & 000120-61-6 & & & $8.8 \mathrm{E}+00$ & $8.8 \mathrm{E}-01$ \\
\hline Dinitro-o-cresol, 4,6- & $000534-52-1$ & & & & \\
\hline Dinitro-o-cyclohexyl Phenol, 4,6- & 000131-89-5 & & & $3.8 \mathrm{E}+03$ & $3.8 \mathrm{E}+02$ \\
\hline Dinitrobenzene, 1,2- & $000528-29-0$ & & & 4.1E-01 & 4.1E-02 \\
\hline Dinitrobenzene, 1,3- & 000099-65-0 & & & $1.0 \mathrm{E}-01$ & $1.0 \mathrm{E}-02$ \\
\hline Dinitrobenzene, 1,4- & 000100-25-4 & & & $4.9 \mathrm{E}-01$ & $4.9 \mathrm{E}-02$ \\
\hline
\end{tabular}




\begin{tabular}{|c|c|c|c|c|c|}
\hline & & & & $\downarrow-\downarrow 6-6 \varsigma<000$ & DIdヨ \\
\hline & & & & $\varepsilon-0 I-6 \varepsilon+Z 00$ & әuฺpod \\
\hline $20-\mathrm{A} 0^{\circ} \varepsilon$ & $10-900$ & & & 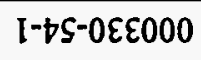 & uom!̣ \\
\hline \multirow[t]{5}{*}{ S0-Al'9 } & t0-3I'9 & & & $t-t 0-862000$ & uoloy[ns! \\
\hline & & & & I-S0-0I9Z00 & әnโg אYS ฆәп! \\
\hline & & & & 9-98-I L 0910 & \$6 uм01g pวد!ם \\
\hline & & & & $z-9 b-209 z 00$ & 9 әnןg 102ग!ם \\
\hline & & & & $L-L E-L E 6100$ & 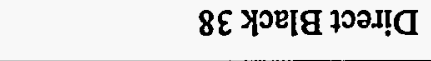 \\
\hline \multirow[t]{2}{*}{$Z 0+B L L$} & $\varepsilon 0+B L L$ & & & $L-00-\$ 80000$ & jenbịa \\
\hline & & $\triangleright 0-\exists I^{\circ} \varepsilon$ & $20-\exists\left[I^{\circ} \varepsilon\right.$ & L-99-ZZI000 & 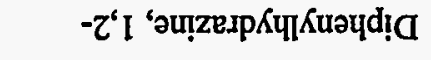 \\
\hline $20-a z^{\prime} 6$ & [0--9 $9 z^{*} 6$ & & & $t-6 \varepsilon-2 Z 1000$ & 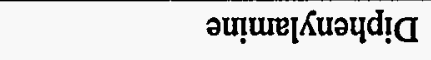 \\
\hline \multirow[t]{2}{*}{$00+3 I^{\bullet} I$} & $I 0+B I \cdot I$ & & & $L-I S-L S 6000$ & p!ureuəud!̣ \\
\hline & & $00+B L \cdot S$ & $20+B L S S$ & $I-I 6-\varepsilon Z I 000$ & $-t^{6}$ I 'ouexo!̣ \\
\hline \multirow[t]{2}{*}{$\varepsilon 0-899^{\circ} 乙$} & $20-899^{\circ} z$ & & & $L-\$ 8-880000$ & qəsou!a \\
\hline & & & & Z-8L-ZLSৎEO & 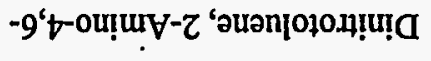 \\
\hline 20-تิS*8 & $10-9 S^{\prime} 8$ & $\varepsilon 0-\exists 6 \tau$ & $10-36^{\circ} \tau$ & $z-0 z-909000$ & 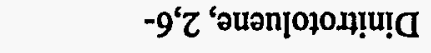 \\
\hline$I 0-80^{\circ} I$ & $00+30^{\circ} I$ & $\varepsilon 0-\exists L^{\prime} I$ & $I 0-\exists L^{\prime} I$ & $Z-b I-I Z I 000$ & - \\
\hline $10-a b^{\prime} Z$ & $00+\exists \nabla^{\circ} Z$ & & & S-8Z-IS0000 & 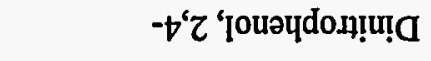 \\
\hline$I^{\prime} \mathbf{0}=\mathbf{D H}$ & $\mathbf{I}=\mathbf{O H}$ & $9.0 I$ & $r \mathrm{OI}$ & \multirow{2}{*}{ NUSYD } & \multirow{2}{*}{ |вว!นวนว } \\
\hline.$\left(\mathrm{T} / \mathrm{s}^{2}\right.$ & M u! uo! & (00) 4s! & & & \\
\hline
\end{tabular}

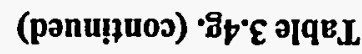


Table 3.4g. (continued)

\begin{tabular}{|c|c|c|c|c|c|}
\hline \multirow{2}{*}{ Chemical } & \multirow{2}{*}{ CASRN } & \multicolumn{4}{|c|}{ Ingestion of Fish (concentration in water) $(\mathrm{mg} / \mathrm{L})$} \\
\hline & & $10^{-4}$ & $10^{-6}$ & $\mathbf{H Q}=\mathbf{1}$ & $\mathrm{HQ}=0.1$ \\
\hline Endosulfan & 000115-29-7 & & & & \\
\hline Endothall & 000145-73-3 & & & & \\
\hline Endrin & 000072-20-8 & & & $1.6 \mathrm{E}-03$ & $1.6 \mathrm{E}-04$ \\
\hline Epichlorohydrin & 000106-89-8 & $5.7 \mathrm{E}+02$ & $5.7 \mathrm{E}+00$ & $4.8 \mathrm{E}+01$ & $4.8 \mathrm{E}+00$ \\
\hline Epoxybutane, 1,2- & 000106-88-7 & & & & \\
\hline Ethephon & $016672-87-0$ & & & & \\
\hline Ethion & $000563-12-2$ & & & $1.2 \mathrm{E}-03$ & $1.2 \mathrm{E}-04$ \\
\hline Ethoxyethanol Acetate, 2- & 000111-15-9 & & & $1.6 \mathrm{E}+03$ & $1.6 \mathrm{E}+02$ \\
\hline Ethoxyethanol, 2- & $000110-80-5$ & & & $8.0 \mathrm{E}+03$ & $8.0 \mathrm{E}+02$ \\
\hline Ethyl Acetate & $000141-78-6$ & & & $3.7 \mathrm{E}+03$ & $3.7 \mathrm{E}+02$ \\
\hline Ethyl Acrylate & 000140-88-5 & $8.4 \mathrm{E}+00$ & $8.4 \mathrm{E}-02$ & & \\
\hline Ethyl Chloride & $000075-00-3$ & & & & \\
\hline Ethyl Ether & $000060-29-7$ & & & $7.1 \mathrm{E}+02$ & $7.1 \mathrm{E}+01$ \\
\hline Ethyl Methacrylate & $000097-63-2$ & & & $5.4 \mathrm{E}+01$ & $5.4 \mathrm{E}+00$ \\
\hline Ethyl-p-nitrophenyl Phosphonate & $002104-64-5$ & & & $2.2 \mathrm{E}-04$ & $2.2 \mathrm{E}-05$ \\
\hline Ethylbenzene ${ }^{(6)}$ & $000100-41-4$ & & & $7.4 \mathrm{E}+00$ & 7.4E-01 \\
\hline Ethylene Cyanohydrin & $000109-78-4$ & & & $6.9 \mathrm{E}+04$ & $6.9 \mathrm{E}+03$ \\
\hline Ethylene Diamine & 000107-15-3 & & & $3.1 \mathrm{E}+02$ & $3.1 \mathrm{E}+01$ \\
\hline
\end{tabular}


Table 3.4g. (continued)

\begin{tabular}{|c|c|c|c|c|c|}
\hline \multirow{2}{*}{ Chemical } & \multirow{2}{*}{ CASRN } & \multicolumn{4}{|c|}{ Ingestion of Fish (concentration in water) (mg/L)* } \\
\hline & & $10^{-4}$ & $10^{-6}$ & $\mathbf{H Q}=\mathbf{1}$ & HQ $=0.1$ \\
\hline Ethylene Glycol & 000107-21-1 & & & $9.3 \mathrm{E}+05$ & $9.3 \mathrm{E}+04$ \\
\hline Ethylene Glycol Monobutyl Ether & 000111-76-2 & & & & \\
\hline Ethylene Oxide & 000075-21-8 & $6.5 \mathrm{E}+00$ & $6.5 \mathrm{E}-02$ & & \\
\hline Ethylene Thiourea & $000096-45-7$ & $1.1 \mathrm{E}+02$ & $1.1 \mathrm{E}+00$ & $4.3 \mathrm{E}+00$ & $4.3 \mathrm{E}-01$ \\
\hline Ethylphthalyl Ethyl Glycolate & $000084-72-0$ & & & & \\
\hline Express & $101200-48-0$ & & & & \\
\hline Fenamiphos & $022224-92-6$ & & & & \\
\hline Fenpropathrin & 039515-41-8 & & & & \\
\hline Fluometuron & $002164-17-2$ & & & $3.3 \mathrm{E}+00$ & 3.3E-01 \\
\hline Fluoranthene & 000206-44-0 & & & $1.3 \mathrm{E}-01$ & $1.3 \mathrm{E}-02$ \\
\hline Fluorene & 000086-73-7 & & & 3.0E-01 & 3.0E-02 \\
\hline Fluoride & $007782-41-4$ & & & & \\
\hline Fluridone & 059756-60-4 & & & & \\
\hline Flurprimidol & $056425-91-3$ & & & & \\
\hline Flutolanil & $066332-96-5$ & & & & \\
\hline Fluvalinate & 069409-94-5 & & & & \\
\hline Folpet & 000133-07-3 & $4.9 \mathrm{E}+00$ & $4.9 \mathrm{E}-02$ & $7.4 \mathrm{E}+00$ & 7.4E-01 \\
\hline Fomesafen & $072178-02-0$ & & & & \\
\hline
\end{tabular}


Table 3.4g. (continued)

\begin{tabular}{|c|c|c|c|c|c|}
\hline \multirow{2}{*}{ Chemical } & \multirow{2}{*}{ CASRN } & \multicolumn{4}{|c|}{ Ingestion of Fish (concentration in water) (mg/L)" } \\
\hline & & $10^{-4}$ & $10^{-6}$ & $\mathbf{H Q}=\mathbf{1}$ & $\mathrm{HQ}=0.1$ \\
\hline Fonofos & 000944-22-9 & & & $3.4 \mathrm{E}-02$ & 3.4E-03 \\
\hline Formaldehyde & 000050-00-0 & & & $3.3 \mathrm{E}+03$ & $3.3 \mathrm{E}+02$ \\
\hline Formic Acid & 000064-18-6 & & & $8.6 \mathrm{E}+04$ & $8.6 \mathrm{E}+03$ \\
\hline Fosetyl-AL & 039148-24-8 & & & & \\
\hline Furan & 000110-00-9 & & & $1.7 \mathrm{E}+00$ & $1.7 \mathrm{E}-01$ \\
\hline Furazolidone & 000067-45-8 & 3.1E-01 & 3.1E-03 & & \\
\hline Furfural & 000098-01-1 & & & $1.1 \mathrm{E}-01$ & 1.1E-02 \\
\hline Furium & 000531-82-8 & & & & \\
\hline Furmecyclox & 060568-05-0 & & & & \\
\hline Glufosinate, Ammonium & 077182-82-2 & & & & \\
\hline Glycidyl & $000765-34-4$ & & & $2.4 \mathrm{E}+01$ & $2.4 \mathrm{E}+00$ \\
\hline Glyphosate & 001071-83-6 & & & $3.2 E+05$ & $3.2 E+04$ \\
\hline Goal & $042874-03-3$ & & & & \\
\hline Haloxyfop, Methyl & $069806-40-2$ & & & & \\
\hline Harmony & $079277-27-3$ & & & & \\
\hline Heptachlor & $000076-44-8$ & 4.7E-04 & 4.7E-06 & $4.5 \mathrm{E}-03$ & 4.5E-04 \\
\hline Heptachlor Epoxide & $001024-57-3$ & $3.4 \mathrm{E}-05$ & $3.4 \mathrm{E}-07$ & $1.7 \mathrm{E}-05$ & $1.7 \mathrm{E}-06$ \\
\hline Heptane, N- & $000142-82-5$ & & & & \\
\hline
\end{tabular}




\begin{tabular}{|c|c|c|c|c|c|}
\hline & & & & $9-8 L-I 6 S 000$ & $-\tau^{\prime}$ 'วuouexəH \\
\hline \multirow[t]{2}{*}{ I0-GI'I } & $00+B I^{\circ} \mathrm{I}$ & & & $\varepsilon-\downarrow \varsigma-0 I L 000$ & -N 'әưxəH \\
\hline & & & & $0-90-z z 8000$ & 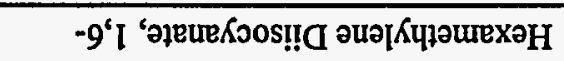 \\
\hline $20-99^{\circ} I$ & I0-39・ I & $\varepsilon 0-\exists I \cdot I$ & $I 0-A I \cdot I$ & $t-Z 8-I Z 1000$ & 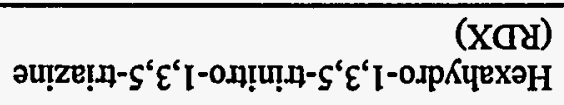 \\
\hline $90-80^{\circ} \mathrm{I}$ & 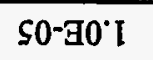 & & & $t-0 \varepsilon-0<0000$ & 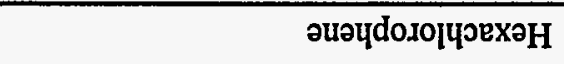 \\
\hline \multirow[t]{2}{*}{$\varepsilon 0-38 \cdot I$} & Z0-G8'I & $\mathcal{E} 0-\exists 0^{\circ} \varepsilon$ & $10-g 0^{\circ} \varepsilon$ & I-ZL-L90000 & әиецрәодоцџехәН \\
\hline & & II-GI'S & $60-\mathrm{gI} \mathrm{I}^{\circ} \mathrm{S}$ & $\varepsilon-\downarrow L-80 t 610$ & 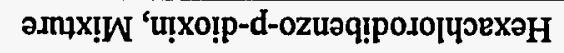 \\
\hline \multirow[t]{2}{*}{ 20-AI'I } & I0-GI’I & & & $t-\angle t-L L 0000$ & 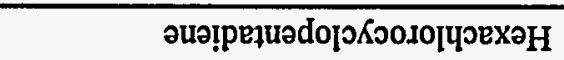 \\
\hline & & & & $I-\varepsilon L-809000$ & 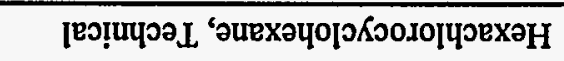 \\
\hline \multirow[t]{5}{*}{$+0-\exists L \cdot L$} & $\varepsilon 0-\exists L L^{\circ} L$ & so- $399^{\circ}$ & $\varepsilon 0-\exists 9^{\circ} t$ & $6-68-850000$ & 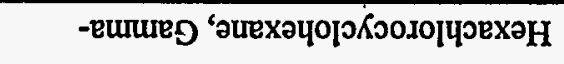 \\
\hline & & & & $L-0 I-80 I 900$ & 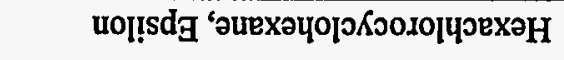 \\
\hline & & & & 8-98-6IE000 & 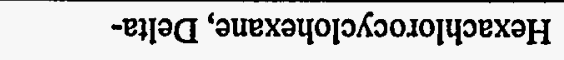 \\
\hline & & SO- $90^{\circ} Z$ & $\varepsilon 0^{-g} 0^{\circ} z$ & $L-\varsigma 8-61 \varepsilon 000$ & 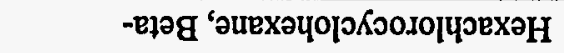 \\
\hline & & $90-\exists L^{\prime} 9$ & $+0-\exists\llcorner\cdot 9$ & $9-\downarrow 8-6 I \varepsilon 000$ & 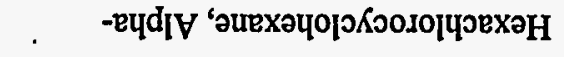 \\
\hline$S 0-A S^{\circ} L$ & $t 0-\exists S^{\circ} L$ & t0-GI'I & $20-\mathrm{GI} \cdot \mathrm{I}$ & $\varepsilon-89-\angle 80000$ & 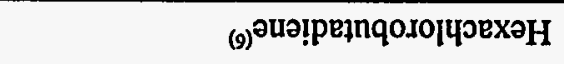 \\
\hline t0-GE'I & $\varepsilon 0-\exists \varepsilon^{\prime \prime} I$ & $90-\exists \varepsilon \varepsilon^{\prime} \tau$ & เ0-घE'z & {$[-t<-811000$} & 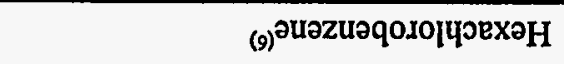 \\
\hline S0-घz'8 & $t 0-9 Z^{\prime} 8$ & & & {$[-Z 8-\angle 80000$} & әuәzuәqouо.дехәH \\
\hline$I^{\prime} 0=\partial H$ & $I=O H$ & $9-0 I$ & rOI & \multirow{2}{*}{ NQSWJ } & \multirow{2}{*}{ ןвอุயวนว } \\
\hline . & a u! uo & 00) qs!d & & & \\
\hline
\end{tabular}

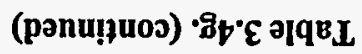




\begin{tabular}{|c|c|c|c|c|c|}
\hline$I 0+\exists L^{\cdot} I$ & $20+B<\cdot I$ & $00+9 I^{\prime} \mathrm{Z}$ & $\mathrm{ZO}+\mathrm{Al} \mathrm{l}^{2} \mathrm{Z}$ & $I-6 S-8<0000$ & 2uoroudosI \\
\hline \multirow[t]{6}{*}{$20+\exists Z^{\prime} I$} & $\varepsilon 0+\mathrm{g} \tau^{\prime} \mathrm{I}$ & & & I- $\varepsilon 8-8\llcorner 0000$ & 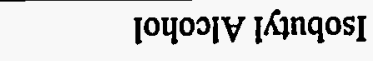 \\
\hline & & & & $9-68-6 \varepsilon t<00$ & todI \\
\hline & & & & $L-6 I-\downarrow \varepsilon L 9 \varepsilon 0$ & วuo!poudI \\
\hline & & $\angle 0-\exists Z Z^{\prime} S$ & So-az's & $s-6 \varepsilon-\varepsilon 61000$ & $(z)^{\partial u \partial I K d\left[p \rho-\varepsilon^{c} \tau^{6} I\right] 0 u \partial p u I}$ \\
\hline & & & & L-LE-SEEI 80 & u!̣nbezeurI \\
\hline & & & & $0-t b-t S S S S E 0$ & I!lezeuI \\
\hline $10+79^{\circ} \mathrm{Z}$ & $20+992$ & & & $6-I \varepsilon-\varepsilon Z 1000$ & auoựnbosp $\kappa_{H}$ \\
\hline I0-9SS'6 & $00+a S \% 6$ & & & $t-90-\varepsilon 8<L 00$ & 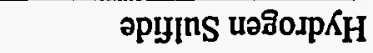 \\
\hline \multirow[t]{8}{*}{$I 0+g L \cdot I$} & $20+3 L^{\circ} I$ & & & $8-06 ־+\angle 0000$ & 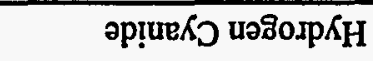 \\
\hline & & & & $0-10-\angle t 9 L 00$ & 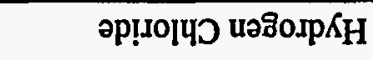 \\
\hline & & $10-a[\cdot S$ & $10+3[\cdot S$ & $\tau-\varepsilon 6-\downarrow \varepsilon 0010$ & 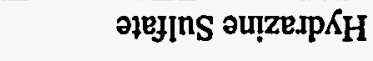 \\
\hline & & I0-GI'S & I0+gl's & $\tau-10-Z 0 \varepsilon 000$ & әu!ze.Jp $\kappa_{H}$ \\
\hline & & & & $I-t 6-\downarrow 89 S S 0$ & $\left.(n)^{-8} 8^{6} L^{6} \varepsilon^{6} Z^{6}\right] \alpha \supset \times H$ \\
\hline & & & & $8-9 t-\varsigma 9 t \succ \varepsilon 0$ & 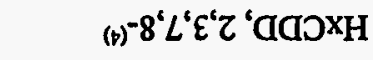 \\
\hline & & & & $\varepsilon=S L-8668 \varepsilon 0$ & ()$\left.^{-}\right)^{-8} L^{6} \varepsilon^{6} Z^{6} H \sigma^{6} \partial^{d} H$ \\
\hline & & & & $t=00-T \angle 8 \angle E 0^{\circ}$ & 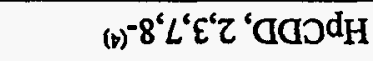 \\
\hline$S 0+g Z I$ & $90+B 0^{\circ} I$ & & & 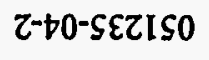 & วนоน!zexəH \\
\hline$I^{\prime} 0=\partial H$ & $\mathbf{I}=\mathbf{O H}$ & $g-0 I$ & rOI & \multirow{2}{*}{ NASVD } & \multirow{2}{*}{ |вכ!யข्पว } \\
\hline . (T/8 & BM u! uo! & oD) पs! J J & & & \\
\hline
\end{tabular}

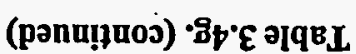


Table 3.4g. (continued)

\begin{tabular}{|c|c|c|c|c|c|}
\hline \multirow{2}{*}{ Chemical } & \multirow{2}{*}{ CASRN } & \multicolumn{4}{|c|}{ Ingestion of Fish (concentration in water) (mg/L)" } \\
\hline & & $10^{-4}$ & $10^{-6}$ & $\mathbf{H Q}=1$ & $\mathrm{HQ}=0.1$ \\
\hline Isopropalin & $033820-53-0$ & & & & \\
\hline Isopropanol & $000067-63-0$ & & & & \\
\hline Isopropyl Methyl Phosphonic Acid & 001832-54-8 & & & & \\
\hline Isoxaben & 082558-50-7 & & & & \\
\hline Karate & 091465-08-6 & & & & \\
\hline Kerb & 023950-58-5 & & & & \\
\hline Lactofen & $077501-63-4$ & & & & \\
\hline Lead Alkyls & NA & & & & \\
\hline Lead And Compounds & $007439-92-1$ & & & & \\
\hline Linuron & $000330-55-2$ & & & & \\
\hline Lithium & 007439-93-2 & & & & \\
\hline Londax & $083055-99-6$ & & & & \\
\hline MCPA & $000094-74-6$ & & & $1.5 \mathrm{E}-01$ & $1.5 \mathrm{E}-02$ \\
\hline MCPB & 000094-81-5 & & & & \\
\hline MCPP & $000093-65-2$ & & & $1.8 \mathrm{E}-01$ & $1.8 \mathrm{E}-02$ \\
\hline Magnesium & 007439-95-4 & & & & \\
\hline Malathion & $000121-75-5$ & & & $2.1 \mathrm{E}+00$ & $2.1 \mathrm{E}-01$ \\
\hline Maleic Anhydride & $000108-31-6$ & & & & \\
\hline
\end{tabular}


Table 3.4g. (continued)

\begin{tabular}{|c|c|c|c|c|c|}
\hline \multirow{2}{*}{ Chemical } & \multirow{2}{*}{ CASRN } & \multicolumn{4}{|c|}{ Ingestion of Fish (concentration in water) (mg/L)" } \\
\hline & & $10^{-4}$ & $10^{-6}$ & $\mathbf{H Q}=\mathbf{1}$ & $\mathrm{HQ}=\mathbf{0 . 1}$ \\
\hline Maleic Hydrazide & 000123-33-1 & & & $1.7 \mathrm{E}+04$ & $1.7 \mathrm{E}+03$ \\
\hline Malononitrile & $000109-77-3$ & & & $3.1 \mathrm{E}-01$ & 3.1E-02 \\
\hline Mancozeb & 008018-01-7 & & & & \\
\hline Maneb & $012427-38-2$ & & & $8.4 E+01$ & $8.4 \mathrm{E}+00$ \\
\hline Manganese (Diet) & 007439-96-5 & & & $3.5 \mathrm{E}+00$ & 3.5E-01 \\
\hline Mephosfolan & $000950-10-7$ & & & & \\
\hline Mepiquat Chloride & $024307-26-4$ & & & & \\
\hline Mercuric Chloride & 007487-94-7 & & & & \\
\hline Mercury (elemental) & 007439-97-6 & & & $3.0 \mathrm{E}-03$ & 3.0E-04 \\
\hline Mercury, Inorganic Salt & 007439-97-6 & & & $3.0 \mathrm{E}-03$ & $3.0 \mathrm{E}-04$ \\
\hline Merphos & $000150-50-5$ & & & & \\
\hline Merphos Oxide & $000078-48-8$ & & & 2.3E-05 & 2.3E-06 \\
\hline Metalaxyl & $057837-19-1$ & & & & \\
\hline Methacrylonitrile & $000126-98-7$ & & & $6.5 \mathrm{E}-01$ & $6.5 \mathrm{E}-02$ \\
\hline Methamidophos & $010265-92-6$ & & & $2.7 E+00$ & 2.7E-01 \\
\hline Methanol & $000067-56-1$ & & & $3.2 E+04$ & $3.2 \mathrm{E}+03$ \\
\hline Methidathion & $000950-37-8$ & & & & \\
\hline Methomyl & $016752-77-5$ & & & $1.5 \mathrm{E}+02$ & $1.5 \mathrm{E}+01$ \\
\hline
\end{tabular}


Table 3.4g. (continued)

\begin{tabular}{|l|l|l|l|l|l|}
\hline \multirow{2}{*}{ Chemical } & \multirow{2}{*}{ CASRN } & \multicolumn{3}{|c|}{ Ingestion of Fish (concentration in water) (mg/L)" } \\
\cline { 4 - 6 } & & $10^{-4}$ & $10^{-6}$ & HQ=1 & HQ=0.1 \\
\hline Methoxy-5-nitroaniline, 2- & $000099-59-2$ & $6.2 \mathrm{E}+00$ & $6.2 \mathrm{E}-02$ & & \\
\hline Methoxychlor & $000072-43-5$ & & & $3.8 \mathrm{E}-02$ & $3.8 \mathrm{E}-03$ \\
\hline Methoxyethanol Acetate, 2- & $000110-49-6$ & & & & \\
\hline Methoxyethanol, 2- & $000109-86-4$ & & & $6.4 \mathrm{E}+01$ & $6.4 \mathrm{E}+00$ \\
\hline Methyl Acetate & $000079-20-9$ & & & $1.2 \mathrm{E}+04$ & $1.2 \mathrm{E}+03$ \\
\hline Methyl Acrylate & $000096-33-3$ & & & $1.6 \mathrm{E}+02$ & $1.6 \mathrm{E}+01$ \\
\hline Methyl Ethyl Ketone & $000078-93-3$ & & & $6.3 \mathrm{E}+03$ & $6.3 \mathrm{E}+02$ \\
\hline Methyl Isobutyl Ketone & $000108-10-1$ & & & $1.6 \mathrm{E}+02$ & $1.6 \mathrm{E}+01$ \\
\hline Methyl Mercury & $022967-92-6$ & & & & \\
\hline Methyl Methacrylate & $000080-62-6$ & & & $1.4 \mathrm{E}+02$ & $1.4 \mathrm{E}+01$ \\
\hline Methyl Parathion & $000298-00-0$ & & & $1.5 \mathrm{E}-01$ & $1.5 \mathrm{E}-02$ \\
\hline Methyl Styrene (Mixed Isomers) & $025013-15-4$ & & & $2.9 \mathrm{E}-01$ & $2.9 \mathrm{E}-02$ \\
\hline Methyl hydrazine(s) & $000060-34-4$ & & & & \\
\hline Methyl tert-Butyl Ether (MTBE) & $001634-04-4$ & & & & \\
\hline Methyl-5-Nitroaniline, 2- & $000099-55-8$ & $3.6 \mathrm{E}+00$ & $3.6 \mathrm{E}-02$ & & \\
\hline Methylaniline Hydrochloride, 2- & $000636-21-5$ & $2.2 \mathrm{E}+01$ & $2.2 \mathrm{E}-01$ & & \\
\hline Methylcyclohexane & $000108-87-2$ & & & & \\
\hline Methylene Chloride & $000075-09-2$ & $5.4 \mathrm{E}+01$ & $5.4 \mathrm{E}-01$ & $1.0 \mathrm{E}+02$ & $1.0 \mathrm{E}+01$ \\
\hline
\end{tabular}


Table 3.4g. (continued)

\begin{tabular}{|c|c|c|c|c|c|}
\hline \multirow{2}{*}{ Chemical } & \multirow{2}{*}{ CASRN } & \multicolumn{4}{|c|}{ Ingestion of Fish (concentration in water) $(\mathrm{mg} / \mathrm{L})$} \\
\hline & & $10^{-4}$ & $10^{-6}$ & $\mathbf{H Q}=\mathbf{1}$ & $H Q=0.1$ \\
\hline Methylene-bis(2-chloroaniline), 4,4'- & 000101-14-4 & $3.3 \mathrm{E}-02$ & 3.3E-04 & $1.3 \mathrm{E}-02$ & $1.3 \mathrm{E}-03$ \\
\hline Methylene-bis(N,N-dimethyl) Aniline, 4,4'- & $000101-61-1$ & $1.9 \mathrm{E}-02$ & $1.9 \mathrm{E}-04$ & & \\
\hline Methylenebisbenzenamine, 4,4-(s) & $000101-77-9$ & $9.5 \mathrm{E}-01$ & $9.5 \mathrm{E}-03$ & & \\
\hline Methylenediphenyl Isocyanate, 4,4- & 000101-68-8 & & & & \\
\hline Methylstyrene, Alpha- & 000098-83-9 & & & $3.3 \mathrm{E}+00$ & 3.3E-01 \\
\hline Methyltriethyl Lead & 001762-28-3 & & & & \\
\hline Metolachlor & $051218-45-2$ & & & & \\
\hline Metribuzin $^{(s)}$ & 021087-64-9 & & & $2.1 E+01$ & 2.1E+00 \\
\hline Mirex $^{(5)}$ & 002385-85-5 & 2.1E-04 & $2.1 \mathrm{E}-06$ & 3.3E-04 & 3.3E-05 \\
\hline Molinate & $002212-67-1$ & & & $7.0 \mathrm{E}-02$ & $7.0 \mathrm{E}-03$ \\
\hline Molybdenum & 007439-98-7 & & & $4.9 \mathrm{E}+00$ & 4.9E-01 \\
\hline Monochloramine & 010599-90-3 & & & & \\
\hline Monochlorobutanes & $025154-42-1$ & & & & \\
\hline Naled & $000300-76-5$ & & & $2.5 \mathrm{E}-01$ & $2.5 \mathrm{E}-02$ \\
\hline Naphthalene $^{(6)}$ & 000091-20-3 & & & $1.9 E+00$ & $1.9 \mathrm{E}-01$ \\
\hline Naphthalene, 1-Methyl & $000090-12-0$ & & & & \\
\hline Naphthalene, 2-Methyl & $000091-57-6$ & & & & \\
\hline Napropamide & 015299-99-7 & & & $5.3 E+02$ & $5.3 E+01$ \\
\hline
\end{tabular}


Table 3.4g. (continued)

\begin{tabular}{|c|c|c|c|c|c|}
\hline \multirow{2}{*}{ Chemical } & \multirow{2}{*}{ CASRN } & \multicolumn{4}{|c|}{ Ingestion of Fish (concentration in water) (mg/L)" } \\
\hline & & $10^{-4}$ & $10^{-6}$ & $H Q=1$ & $\mathrm{HQ}=0.1$ \\
\hline Niagara Blue 4B & 002429-74-5 & & & & \\
\hline Nickel Carbonyl & 013463-39-3 & & & & \\
\hline Nickel Refinery Dust & NA & & & & \\
\hline Nickel Soluble Salts & $007440-02-0$ & & & $2.0 \mathrm{E}+00$ & 2.0E-01 \\
\hline Nickel Subsulfide & $012035-72-2$ & & & & \\
\hline Nitrapyrin $^{(5)}$ & 001929-82-4 & & & $1.3 \mathrm{E}-01$ & $1.3 \mathrm{E}-02$ \\
\hline Nitrate & 014797-55-8 & & & & \\
\hline Nitric Oxide ${ }^{(3)}$ & 010102-43-9 & & & & \\
\hline Nitrite & 014797-65-0 & & & & \\
\hline Nitroaniline, 2- & 000088-74-4 & & & & \\
\hline Nitrobenzene & 000098-95-3 & & & $3.6 \mathrm{E}-01$ & 3.6E-02 \\
\hline Nitrofurantoin & 000067-20-9 & & & & \\
\hline Nitrofurazone & 000059-87-0 & & & & \\
\hline Nitrogen Dioxide ${ }^{(5)}$ & $010102-44-0$ & & & & \\
\hline Nitroguanidine & 000556-88-7 & & & & \\
\hline Nitrophenol, 4- & 000100-02-7 & & & & \\
\hline Nitropropane, $2^{-(5)}$ & $000079-46-9$ & $1.6 \mathrm{E}-01$ & $1.6 \mathrm{E}-03$ & & \\
\hline Nitroso-N-ethylurea, N- & 000759-73-9 & $1.9 \mathrm{E}-02$ & $1.9 \mathrm{E}-04$ & & \\
\hline
\end{tabular}




\begin{tabular}{|c|c|c|c|c|c|}
\hline & & & & $0-Z 0-1006 \varepsilon 0$ & (b) $)$ ICDO \\
\hline & & $I I-\exists S \cdot S$ & 60-gS'S & $6-\angle 8-89 Z \varepsilon 00$ & $\left.{ }_{(h)}\right) \triangle ब \supset 0$ \\
\hline & & & & $6-6 I-60 S \varsigma 80$ & IetsnN \\
\hline & & & & $\tau-\varepsilon I-\downarrow I \varepsilon<Z 0$ & uozesnfJuN \\
\hline $10-3 S^{\prime} \tau$ & $00+B S Z$ & & & $0-66-660000$ & -d 'วuən|0зод!N \\
\hline $10-90^{\circ} \varepsilon$ & $00+90^{\circ} \varepsilon$ & & & $\tau-Z L-880000$ & -o ‘วuənןołon! $\mathrm{N}$ \\
\hline I0- $\exists S^{\prime} Z$ & $00+a s^{\prime} z$ & & & {$[-80-660000$} & 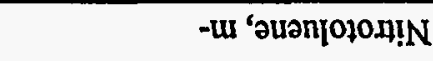 \\
\hline & & & & $b-\tau \varepsilon-611000$ & 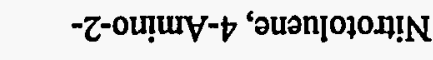 \\
\hline & & I0-GE & $I 0+\exists \varepsilon \cdot I$ & $z-\varsigma s-0 \varepsilon 6000$ & -N 'ou!p!no.uKdoson!N \\
\hline & & & & $0-0 t-6 t S t 00$ & 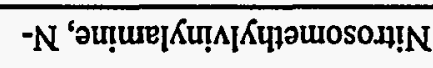 \\
\hline & & & & $9-\varsigma 6-\varsigma 6 S_{0} \mathrm{I0}$ & 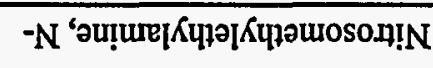 \\
\hline & & Zo-बS'E & $00+B S^{\circ} \varepsilon$ & $9-0 \varepsilon-980000$ & - $N$ 'әu!ure[Kuəud!poson!̣ \\
\hline & & EO-GI'Z & I0-gI' $\tau$ & $6-S L-Z 90000$ & -N 'әu!ure|Кцџәu!posoд! $N$ \\
\hline & & t0-GI'I & Z0-GI'I & $\varsigma-8 I-\varsigma \varsigma 0000$ & 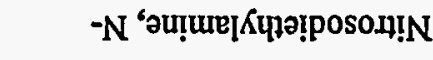 \\
\hline & & 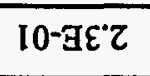 & $\mathrm{I} 0+\mathrm{B} \varepsilon \tau$ & $L-t S-9 I I I 00$ & 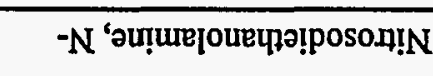 \\
\hline & & $t 0-388^{\circ} t$ & $20-38^{\circ} t$ & L-t9-IZ9000 & -N 'auture[Kdord- $N$-!p-oson! $N$ \\
\hline & & t0--39'乙 & $20-39^{\circ} \tau$ & $\varepsilon-9[-t Z 6000$ & 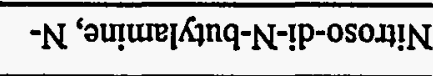 \\
\hline & & & & $s-\varepsilon 6-t 89000$ & 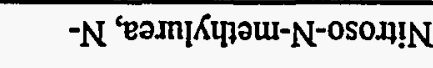 \\
\hline$I^{\prime} 0=\partial H$ & $\mathrm{I}=\mathbf{\partial H}$ & $9.0 \mathrm{I}$ & rOI & \multirow{2}{*}{ NYSYD } & \multirow{2}{*}{ โвวุแอนว } \\
\hline \multicolumn{4}{|c|}{ 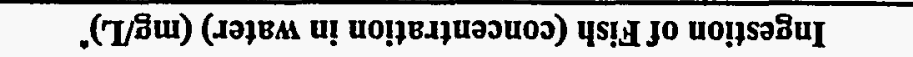 } & & \\
\hline
\end{tabular}

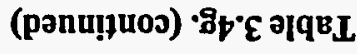


Table 3.4g. (continued)

\begin{tabular}{|c|c|c|c|c|c|}
\hline \multirow{2}{*}{ Chemical } & \multirow{2}{*}{ CASRN } & \multicolumn{4}{|c|}{ Ingestion of Fish (concentration in water) $(\mathrm{mg} / \mathrm{L})^{*}$} \\
\hline & & $10^{-4}$ & $10^{-6}$ & $\mathbf{H Q}=1$ & $\mathrm{HQ}=\mathbf{0 . 1}$ \\
\hline Octabromodiphenyl Ether & $032536-52-0$ & & & & \\
\hline $\begin{array}{l}\text { Octahydro-1,3,5,7-tetranitro-1,3,5,7-tetra } \\
\text { (HMX) }\end{array}$ & 002691-41-0 & & & $3.0 \mathrm{E}+02$ & $3.0 \mathrm{E}+01$ \\
\hline Octamethylpyrophosphoramide & 000152-16-9 & & & & \\
\hline Octyl Phthalate, di-N- & 000117-84-0 & & & 3.4E-05 & 3.4E-06 \\
\hline Oryzalin & 019044-88-3 & & & & \\
\hline Oxadiazon & 019666-30-9 & & & & \\
\hline Oxamyl & 023135-22-0 & & & & \\
\hline Paclobutrazol & $076738-62-0$ & & & & \\
\hline Paraquat & $001910-42-5$ & & & $1.2 \mathrm{E}+05$ & $1.2 \mathrm{E}+04$ \\
\hline Parathion & 000056-38-2 & & & $1.3 \mathrm{E}-01$ & $1.3 \mathrm{E}-02$ \\
\hline PeCDD, 2,3,7,8-(4) & 036088-22-9 & $6.0 \mathrm{E}-10$ & $6.0 \mathrm{E}-12$ & & \\
\hline PeCDF, $1,2,3,7,8_{-(4)}^{(4)}$ & $057117-41-6$ & & & & \\
\hline PeCDF, $2,3,4,7,8-^{(4)}$ & $057117-31-4$ & & & & \\
\hline Pebulate & 001114-71-2 & & & & \\
\hline Pendimethalin & $040487-42-1$ & & & & \\
\hline Pentabromodiphenyl Ether & 032534-81-9 & & & & \\
\hline Pentachlorobenzene & $000608-93-5$ & & & $1.5 \mathrm{E}-03$ & $1.5 \mathrm{E}-04$ \\
\hline
\end{tabular}


Table 3.4g. (continued)

\begin{tabular}{|l|l|l|c|c|c|}
\hline \multirow{2}{*}{ Chemical } & \multirow{2}{*}{ CASRN } & \multicolumn{3}{|c|}{ Ingestion of Fish (concentration in water) (mg/L) } \\
\cline { 4 - 6 } & & $10^{-4}$ & $10^{-6}$ & HQ=1 & HQ=0.1 \\
\hline Pentachlorocyclopentadiene & $025329-35-5$ & & & & \\
\hline Pentachloronitrobenzene & $000082-68-8$ & $4.8 \mathrm{E}-03$ & $4.8 \mathrm{E}-05$ & $1.6 \mathrm{E}-02$ & $1.6 \mathrm{E}-03$ \\
\hline Pentachlorophenol & $000087-86-5$ & $1.1 \mathrm{E}-03$ & $1.1 \mathrm{E}-05$ & $1.6 \mathrm{E}-02$ & $1.6 \mathrm{E}-03$ \\
\hline Pentyl Alcohol, N- & $000071-41-0$ & & & & \\
\hline Permethrin & $052645-53-1$ & & & $1.8 \mathrm{E}+00$ & $1.8 \mathrm{E}-01$ \\
\hline Phenanthrene & $000085-01-8$ & & & & \\
\hline Phenmedipham & $013684-63-4$ & & & $4.1 \mathrm{E}+04$ & $4.1 \mathrm{E}+03$ \\
\hline Phenol & $000108-95-2$ & & & $7.3 \mathrm{E}+02$ & $7.3 \mathrm{E}+01$ \\
\hline Phenylenediamine, m- & $000108-45-2$ & & & $3.7 \mathrm{E}+02$ & $3.7 \mathrm{E}+01$ \\
\hline Phenylenediamine, $0-$ & $000095-54-5$ & $3.8 \mathrm{E}+01$ & $3.8 \mathrm{E}-01$ & & \\
\hline Phenylenediamine, p- & $000106-50-3$ & & & $2.6 \mathrm{E}+04$ & $2.6 \mathrm{E}+03$ \\
\hline Phenylmercuric Acetate & $000062-38-4$ & & & & \\
\hline Phenylphenol, 2- & $000090-43-7$ & $8.9 \mathrm{E}+00$ & $8.9 \mathrm{E}-02$ & & \\
\hline Phorate & $000298-02-2$ & & & $1.7 \mathrm{E}-01$ & $1.7 \mathrm{E}-02$ \\
\hline Phosmet & $000732-11-6$ & & & $2.5 \mathrm{E}+00$ & $2.5 \mathrm{E}-01$ \\
\hline Phosphine & $007803-51-2$ & & & & \\
\hline Phosphoric Acid & $007664-38-2$ & & & & \\
\hline Phthalic Acid, P- & $000100-21-0$ & & & & \\
\hline
\end{tabular}




\begin{tabular}{|c|c|c|c|c|c|}
\hline & & & & [-06-LOZ090 & P[ozeuoग!do]d \\
\hline I0-gZ' I & $00+\mathrm{AZ} \cdot \mathrm{I}$ & & & $6-Z t-2 Z 1000$ & urequdold \\
\hline \multirow[t]{3}{*}{$10-90 \%$} & $00+30^{\circ} \mathrm{S}$ & & & $z-0 t-6 \varepsilon l 000$ & aų̣zedold $_{\mathbf{d}}$ \\
\hline & & & & $\angle-6 I-\angle 01000$ & 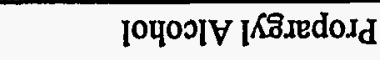 \\
\hline & & & & 8-SE-ZI EZ00 & 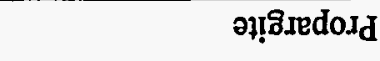 \\
\hline \multirow[t]{2}{*}{$20-99^{\circ} \varepsilon$} & 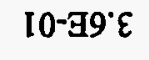 & & & $8-86-60<000$ & l!uredold \\
\hline & & & & $L-9 I-8 I 6 I 00$ & IOIYJEdord \\
\hline $20-a b \cdot 8$ & $10-96 t^{\circ} 8$ & & & $9-6 I-\angle 8 Z \angle 00$ & 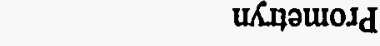 \\
\hline \multirow[t]{4}{*}{$10-9 S^{\prime} t$} & $00+a s^{\circ} t$ & & & $0-8[-019100$ & uolourold \\
\hline & & & & $0-9 \varepsilon-66 \varepsilon 9 Z 0$ & u!̣emfjo d \\
\hline & & & & $s-60-\angle t<L 90$ & zexo|५ว0.1d \\
\hline & & & & $9-19-905000$ & 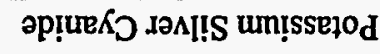 \\
\hline \multirow[t]{4}{*}{$20+Z \varepsilon^{\prime} I$} & $\varepsilon 0+\mathrm{G} \varepsilon^{\prime} I$ & & & 8-0S-ISI000 & әp!UR \\
\hline & & $L 0-B b^{\prime} I$ & SO-Bt:I & $\varepsilon-9 \varepsilon-9 \varepsilon \varepsilon 100$ & 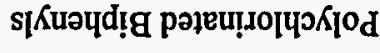 \\
\hline & & & & $\forall N$ & 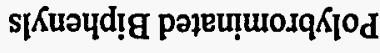 \\
\hline & & & & $L-\varepsilon 6-Z \varepsilon Z 6 Z 0$ & IКцрәW 'soud!̣u!̣!d \\
\hline $00+88 . I$ & $10+98^{\circ} I$ & & & [-20-8I6]00 & urexolo!d \\
\hline$\varepsilon 0+36^{\circ} 6$ & $t 0+96^{\circ} 6$ & & & $6-b t-\varsigma 80000$ & 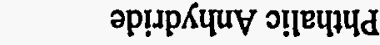 \\
\hline$I^{\prime} 0=\partial H$ & $\mathbf{I}=\mathbf{O H}$ & $9-0 I$ & rOI & \multirow{2}{*}{ N\&SVD } & \multirow{2}{*}{ [вอุแนขว } \\
\hline.$\left(T / \mathbf{s}^{5}\right.$ & BM U!̣ Uo! & כ0) чs!! & & & \\
\hline
\end{tabular}


Table 3.4g. (continued)

\begin{tabular}{|c|c|c|c|c|c|}
\hline \multirow{2}{*}{ Chemical } & \multirow{2}{*}{ CASRN } & \multicolumn{4}{|c|}{ Ingestion of Fish (concentration in water) (mg/L)" } \\
\hline & & $10^{-4}$ & $10^{-6}$ & HQ $=1$ & $\mathrm{HQ}=0.1$ \\
\hline Propylene Glycol & $000057-55-6$ & & & $1.0 \mathrm{E}+06$ & $6.6 \mathrm{E}+05$ \\
\hline Propylene Glycol Monoethyl Ether & $001569-02-4$ & & & & \\
\hline Propylene Glycol Monomethyl Ether & $000107-98-2$ & & & $1.6 \mathrm{E}+04$ & $1.6 \mathrm{E}+03$ \\
\hline Propylene Oxide & $000075-56-9$ & $1.5 \mathrm{E}+01$ & $1.5 \mathrm{E}-01$ & . & \\
\hline Pursuit & $081335-77-5$ & & & & \\
\hline Pydrin & $051630-58-1$ & & & $1.8 \mathrm{E}-01$ & $1.8 \mathrm{E}-02$ \\
\hline Pyrene & $000129-00-0$ & & & 9.5E-02 & 9.5E-03 \\
\hline Pyridine & 000110-86-1 & & & $1.7 \mathrm{E}+00$ & 1.7E-01 \\
\hline Quinalphos & 013593-03-8 & & & & \\
\hline Quinoline & $000091-22-5$ & $8.3 \mathrm{E}-03$ & 8.3E-05 & & \\
\hline Refractory Ceramic Fibers & NA & & & & \\
\hline Resmethrin & $010453-86-8$ & & & & \\
\hline Ronnel & 000299-84-3 & & & $1.1 \mathrm{E}-01$ & $1.1 \mathrm{E}-02$ \\
\hline Rotenone & $000083-79-4$ & & & $5.1 \mathrm{E}-02$ & 5.1E-03 \\
\hline Savey & 078587-05-0 & & & & \\
\hline Selenious Acid & $007783-00-8$ & & & & \\
\hline Selenite & $014124-67-5$ & & & & \\
\hline Selenium & $007782-49-2$ & & & & \\
\hline
\end{tabular}


Table 3.4g. (continued)

\begin{tabular}{|c|c|c|c|c|c|}
\hline \multirow{2}{*}{ Chemical } & \multirow{2}{*}{ CASRN } & \multicolumn{4}{|c|}{ Ingestion of Fish (concentration in water) (mg/L)" } \\
\hline & & $10^{-4}$ & $10^{-6}$ & $H Q=1$ & $\mathrm{HQ}=0.1$ \\
\hline Selenium Sulfide & $007446-34-6$ & & & & \\
\hline Selenourea & $000630-10-4$ & & & & \\
\hline Sethoxydim & $074051-80-2$ & & & & \\
\hline Silver & $007440-22-4$ & & & $9.9 \mathrm{E}+00$ & $9.9 \mathrm{E}-01$ \\
\hline Silver Cyanide & 000506-64-9 & & & & \\
\hline Simazine & 000122-34-9 & $6.9 \mathrm{E}-01$ & $6.9 \mathrm{E}-03$ & $1.8 \mathrm{E}+00$ & $1.8 \mathrm{E}-01$ \\
\hline Sodium & $007440-23-5$ & & & & \\
\hline Sodium Acifluorfen & 062476-59-9 & & & & \\
\hline Sodium Azide & $026628-22-8$ & & & & \\
\hline Sodium Cyanide & 000143-33-9 & & & $2.8 \mathrm{E}+03$ & $2.8 \mathrm{E}+02$ \\
\hline Sodium Diethyldithiocarbamate & 000148-18-5 & & & & \\
\hline Sodium Fluoroacetate & 000062-74-8 & & & & \\
\hline Sodium Metavanadate & 013718-26-8 & & & & \\
\hline Stirofos (Tetrachlorovinphos) & 000961-11-5 & & & & \\
\hline Strontium, Stable & $007440-24-6$ & & & $9.9 \mathrm{E}+01$ & $9.9 \mathrm{E}+00$ \\
\hline Strychnine & 000057-24-9 & & & $1.8 \mathrm{E}-01$ & $1.8 \mathrm{E}-02$ \\
\hline Styrene ${ }^{(6)}$ & $000100-42-5$ & & & $2.1 \mathrm{E}+01$ & $2.1 \mathrm{E}+00$ \\
\hline Sulfate & 014808-79-8 & & & & \\
\hline
\end{tabular}


Table 3.4g. (continued)

\begin{tabular}{|c|c|c|c|c|c|}
\hline \multirow{2}{*}{ Chemical } & \multirow{2}{*}{ CASRN } & \multicolumn{4}{|c|}{ Ingestion of Fish (concentration in water) (mg/L)* } \\
\hline & & $10^{-4}$ & $10^{-6}$ & $\mathbf{H Q}=\mathbf{1}$ & $\mathrm{HQ}=0.1$ \\
\hline Systhane & 088671-89-0 & & & & \\
\hline TCDD, 2,3,7,8-(4) & $001746-01-6$ & $1.8 \mathrm{E}-10$ & $1.8 \mathrm{E}-12$ & & \\
\hline TCDF, $2,3,7,8-(4)$ & $051207-31-9$ & $9.8 \mathrm{E}-09$ & $9.8 \mathrm{E}-11$ & & \\
\hline TCMTB & $021564-17-0$ & & & & \\
\hline Tebuthiuron & 034014-18-1 & & & $9.0 \mathrm{E}+02$ & $9.0 \mathrm{E}+01$ \\
\hline Temephos & 003383-96-8 & & & & \\
\hline Terbacil & $005902-51-2$ & & & & \\
\hline Terbufos & 013071-79-9 & & & $6.7 \mathrm{E}-04$ & $6.7 \mathrm{E}-05$ \\
\hline Terbutryn & 000886-50-0 & & & 2.4E-02 & $2.4 \mathrm{E}-03$ \\
\hline Tetrabutyl Lead & 001920-90-7 & & & & \\
\hline Tetrachlorobenzene, 1,2,4,5- & 000095-94-3 & & & $1.6 \mathrm{E}-03$ & $1.6 \mathrm{E}-04$ \\
\hline Tetrachloroethane, 1,1,1,2- & 000630-20-6 & $7.9 \mathrm{E}-01$ & $7.9 \mathrm{E}-03$ & $2.6 \mathrm{E}+00$ & $2.6 \mathrm{E}-01$ \\
\hline Tetrachloroethane, 1,1,2,2- & 000079-34-5 & $2.9 \mathrm{E}-01$ & $2.9 \mathrm{E}-03$ & & \\
\hline Tetrachloroethylene ${ }^{(6)}$ & $000127-18-4$ & $7.9 \mathrm{E}-01$ & $7.9 \mathrm{E}-03$ & $1.8 \mathrm{E}+00$ & $1.8 \mathrm{E}-01$ \\
\hline Tetrachlorophenol, 2,3,4,6- & $000058-90-2$ & & & $3.8 \mathrm{E}-01$ & $3.8 \mathrm{E}-02$ \\
\hline Tetrachlorotoluene, p- alpha, alpha, alpha- & 005216-25-1 & & & & \\
\hline Tetraethyl Dithiopyrophosphate & 003689-24-5 & & & $4.4 \mathrm{E}-02$ & 4.4E-03 \\
\hline Tetraethyl Lead & $000078-00-2$ & & & & \\
\hline
\end{tabular}


Table 3.4g. (continued)

\begin{tabular}{|c|c|c|c|c|c|}
\hline \multirow{2}{*}{ Chemical } & \multirow{2}{*}{ CASRN } & \multicolumn{4}{|c|}{ Ingestion of Fish (concentration in water) (mg/L)" } \\
\hline & & $10^{-4}$ & $10^{-6}$ & $\mathbf{H Q}=\mathbf{1}$ & $\mathrm{HQ}=\mathbf{0 . 1}$ \\
\hline Tetrafluoroethane, $1,1,1,2$ - & $000811-97-2$ & & & & \\
\hline Tetramethyl Lead & 000075-74-1 & & & & \\
\hline Tetrapropyl Lead & 003440-75-3 & & & & \\
\hline Thallic Oxide ${ }^{(5)}$ & 001314-32-5 & & & & \\
\hline Thallium (I) Nitrate & $010102-45-1$ & & & & \\
\hline Thallium (Soluble Salts) & 007440-28-0 & & & & \\
\hline Thallium Acetate & 000563-68-8 & & & & \\
\hline Thallium Carbonate & 006533-73-9 & & & & \\
\hline Thallium Chloride & 007791-12-0 & & & & \\
\hline Thallium Selenite ${ }^{(3)}$ & $012039-52-0$ & & & & \\
\hline Thallium Sulfate & 007446-18-6 & & & & \\
\hline Thiobencarb & $028249-77-6$ & & & & \\
\hline Thiofanox & 039196-18-4 & & & $9.3 \mathrm{E}+01$ & $9.3 \mathrm{E}+00$ \\
\hline Thiophanate, Methyl & 023564-05-8 & & & & \\
\hline Thiram & 000137-26-8 & & & & \\
\hline Thorium & 007440-29-1 & & & & \\
\hline Tin & 007440-31-5 & & & $2.0 \mathrm{E}+00$ & $2.0 \mathrm{E}-01$ \\
\hline Titanium & $007440-32-6$ & & & & \\
\hline
\end{tabular}




\begin{tabular}{|c|c|c|c|c|c|}
\hline & & & & & 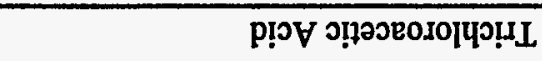 \\
\hline & & & & 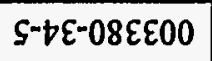 & 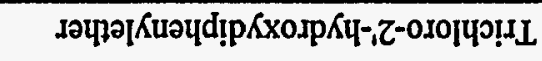 \\
\hline \multirow[t]{6}{*}{$20+\exists \varepsilon \subseteq$} & $\varepsilon 0+B \varepsilon \cdot \mathcal{S}$ & & & {$[-\varepsilon[-9<0000$} & 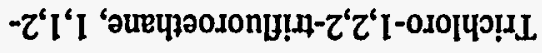 \\
\hline & & & & $6-\varsigma \varepsilon-9 \varsigma 0000$ & 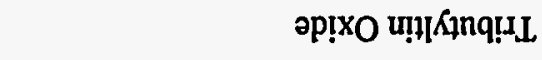 \\
\hline & & & & $0-t 6-0696+0$ & 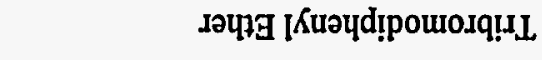 \\
\hline & & & & $0-\varsigma[-66 \varsigma 000$ & 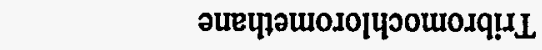 \\
\hline & & & & $\varepsilon-\triangleright S-S I 9000$ & 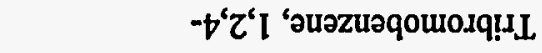 \\
\hline & & & & $S-0 S-\angle 60280$ & uodny|nse!IL \\
\hline \multirow[t]{9}{*}{ zo-al'z } & I0-g]'Z & & & S-LI-EOEZ00 & 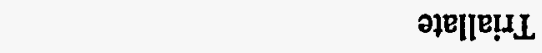 \\
\hline & & & & $9-\varsigma Z-I \$ 8990$ & 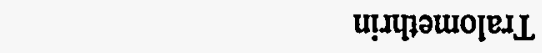 \\
\hline & & $90-90 \% 8$ & $50-30.8$ & $\tau-\varsigma \varepsilon-100800$ & aurudexol \\
\hline & & $\varepsilon 0-\Xi z^{\prime} 9$ & $10-3 z^{\prime} 9$ & $0-6 b-901000$ & -d 'әu!p!nı๐ \\
\hline & & $20-\exists L \cdot I$ & $00+B \angle L^{\prime} I$ & $b-\varepsilon \varsigma-\varsigma 60000$ & 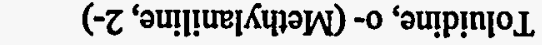 \\
\hline & & & & s-0t-£Z8000 & 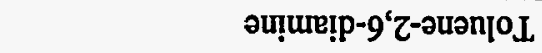 \\
\hline & & & & $S-0<-\$ 60000$ & 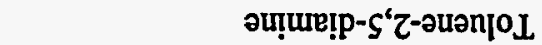 \\
\hline & & $\varepsilon 0-\exists L \cdot 9$ & $10-3<\cdot 9$ & $L-08-\$ 60000$ & 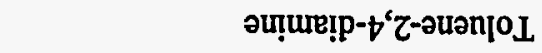 \\
\hline & & & & $s-Z 9-\llbracket<t 9 Z 0$ & 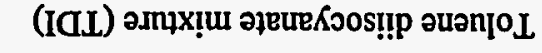 \\
\hline $00+90^{\circ} \varepsilon$ & $10+90^{\circ} \varepsilon$ & & & $\varepsilon-88-801000$ & 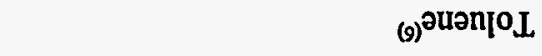 \\
\hline$I^{*} \mathbf{0}=\mathbf{\partial H}$ & $\mathbf{I}=\mathbf{\partial H}$ & $9-0 \mathrm{I}$ & ,OI & & \\
\hline \multicolumn{4}{|c|}{ 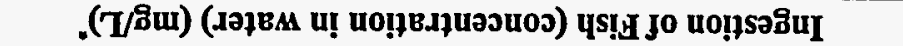 } & & \\
\hline
\end{tabular}

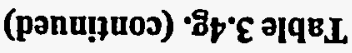


Table 3.4g. (continued)

\begin{tabular}{|l|l|l|l|l|l|}
\hline \multirow{2}{*}{ Chemical } & \multirow{2}{*}{ CASRN } & \multicolumn{2}{|c|}{ Ingestion of Fish (concentration in water) (mg/L) } \\
\cline { 5 - 6 } & & $10^{-4}$ & $10^{-6}$ & HQ=1 & HQ=0.1 \\
\hline Trichloroaniline HCl, 2,4,6- & $033663-50-2$ & & & & \\
\hline Trichloroaniline, 2,4,6- & $000634-93-5$ & & & & \\
\hline Trichlorobenzene, 1,2,4- & $000120-82-1$ & & & $1.5 \mathrm{E}-01$ & $1.5 \mathrm{E}-02$ \\
\hline Trichloroethane, 1,1,1-(5) & $000071-55-6$ & & & $1.9 \mathrm{E}+01$ & $1.9 \mathrm{E}+00$ \\
\hline Trichloroethane, 1,1,2- & $000079-00-5$ & $2.1 \mathrm{E}+00$ & $2.1 \mathrm{E}-02$ & $2.0 \mathrm{E}+00$ & $2.0 \mathrm{E}-01$ \\
\hline Trichloroethylene ${ }^{(3)}$ & $000079-01-6$ & $5.3 \mathrm{E}+00$ & $5.3 \mathrm{E}-02$ & & \\
\hline Trichlorofluoromethane & $000075-69-4$ & & & $6.3 \mathrm{E}+01$ & $6.3 \mathrm{E}+00$ \\
\hline Trichlorophenol, 2,4,5- & $000095-95-4$ & & & $2.2 \mathrm{E}+00$ & $2.2 \mathrm{E}-01$ \\
\hline Trichlorophenol, 2,4,6- & $000088-06-2$ & $5.5 \mathrm{E}-01$ & $5.5 \mathrm{E}-03$ & & \\
\hline Trichlorophenoxy) Propionic Acid, 2(2,4,5- & $000093-72-1$ & & & $1.5 \mathrm{E}-01$ & $1.5 \mathrm{E}-02$ \\
\hline Trichlorophenoxyacetic Acid, 2,4,5- & $000093-76-5$ & & & $1.5 \mathrm{E}-01$ & $1.5 \mathrm{E}-02$ \\
\hline Trichloropropane, 1,1,2- & $000598-77-6$ & & & \\
\hline Trichloropropane, 1,2,3- & $000096-18-4$ & $1.8 \mathrm{E}-04$ & $1.8 \mathrm{E}-06$ & $3.2 \mathrm{E}-02$ & $3.2 \mathrm{E}-03$ \\
\hline Trichloropropene, 1,2,3- & $000096-19-5$ & & & & \\
\hline Trichlorotoluene, 2,3,6- & $002077-46-5$ & & & & \\
\hline Trichlorotoluene, alpha 2,6- & $002014-83-7$ & & & & \\
\hline Tridiphane & $058138-08-2$ & & & & \\
\hline Triethyl Lead & $005224-23-7$ & & & & \\
\hline
\end{tabular}


Table 3.4g. (continued)

\begin{tabular}{|c|c|c|c|c|c|}
\hline \multirow{2}{*}{ Chemical } & \multirow{2}{*}{ CASRN } & \multicolumn{4}{|c|}{ Ingestion of Fish (concentration in water) (mg/L) } \\
\hline & & $10^{-4}$ & $10^{-6}$ & $\mathbf{H Q}=\mathbf{1}$ & $H Q=0.1$ \\
\hline Triethylamine & $000121-44-8$ & & & & \\
\hline Trifluralin & $001582-09-8$ & 4.8E-02 & $4.8 \mathrm{E}-04$ & $1.2 \mathrm{E}-02$ & $1.2 \mathrm{E}-03$ \\
\hline Trimethyl Lead & 007442-13-9 & & & & \\
\hline Trimethyl Phosphate & $000512-56-1$ & & & & \\
\hline Trimethylethyl Lead & $001762-26-1$ & & & & \\
\hline Trinitrobenzene, 1,3,5- & 000099-35-4 & & & $1.0 \mathrm{E}-01$ & $1.0 \mathrm{E}-02$ \\
\hline Trinitrophenylmethylnitramine & $000479-45-8$ & & & $5.1 E+00$ & 5.1E-01 \\
\hline Trinitrotoluene, 2,4,6- & 000118-96-7 & $2.3 \mathrm{E}+00$ & 2.3E-02 & $1.5 \mathrm{E}-01$ & $1.5 \mathrm{E}-02$ \\
\hline Tripropyl Lead & 006618-03-7 & & & & \\
\hline Uranium (Soluble Salts) & NA & & & $3.0 \mathrm{E}+00$ & $3.0 \mathrm{E}-01$ \\
\hline Vanadium Pentoxide & $001314-62-1$ & & & & \\
\hline Vanadium Sulfate & $036907-42-3$ & & & & \\
\hline Vanadium, Metallic & $007440-62-2$ & & & $6.9 \mathrm{E}+00$ & $6.9 \mathrm{E}-01$ \\
\hline Vanadyl Sulfate & $027774-13-6$ & & & & \\
\hline Vernolate & $001929-77-7$ & & & & \\
\hline Vinclozolin & $050471-44-8$ & & & & \\
\hline Vinyl Acetate & $000108-05-4$ & & & $4.7 \mathrm{E}+03$ & 4.7E+02 \\
\hline Vinyl Bromide & $000593-60-2$ & & & & \\
\hline
\end{tabular}


Table 3.4g. (continued)

\begin{tabular}{|c|c|c|c|c|c|}
\hline \multirow{2}{*}{ Chemical } & \multirow{2}{*}{ CASRN } & \multicolumn{4}{|c|}{ Ingestion of Fish (concentration in water) $(\mathrm{mg} / \mathrm{L})^{\bullet}$} \\
\hline & & $10^{-4}$ & $10^{-6}$ & $H Q=1$ & $\mathrm{HQ}=0.1$ \\
\hline Vinyl Chloride $^{(6)}$ & $000075-01-4$ & $1.8 \mathrm{E}-01$ & $1.8 \mathrm{E}-03$ & & \\
\hline Warfarin & $000081-81-2$ & & & $1.9 \mathrm{E}-02$ & $1.9 \mathrm{E}-03$ \\
\hline White Phosphorus & $007723-14-0$ & & & & \\
\hline Xylene, Mixture ${ }^{(6)}$ & 001330-20-7 & & & $1.0 \mathrm{E}+02$ & $1.0 \mathrm{E}+01$ \\
\hline Xylene, P-(6) & $000106-42-3$ & & & & \\
\hline Xylene, $\mathrm{m}^{(6)}$ & 000108-38-3 & & & $1.2 \mathrm{E}+02$ & $1.2 \mathrm{E}+01$ \\
\hline Xylene, $0^{(6)}$ & $000095-47-6$ & & & $1.2 \mathrm{E}+02$ & $1.2 \mathrm{E}+01$ \\
\hline Zinc (Metallic) & $007440-66-6$ & & & $3.0 \mathrm{E}+00$ & 3.0E-01 \\
\hline Zinc Cyanide & $000557-21-1$ & & & & \\
\hline Zinc Phosphide & 001314-84-7 & & & & \\
\hline Zineb & $012122-67-7$ & & & & \\
\hline Zirconium & $007440-67-7$ & & & & \\
\hline
\end{tabular}

Notes:

* By definition, $1 \mathrm{~L}$ (water) $=1.0 \mathrm{E}+06 \mathrm{mg}$ (water). Therefore, a limit of $1.0 \mathrm{E}+06 \mathrm{mg} / \mathrm{L}$ was used for any PRG that was calculated to be above this limit.

(1) The same oral slope factor as used for polychlorinated biphenyls.

(2) The PRG was calculated considering Supplemental Guidance from RAGS: Region 4 Bulletins, Human Health Risk Assessment (Interim Guidance) from EPA (received February 11, 1992). The slope factors for chemicals so marked indicate that a toxicity equivalency factor (TEF) method was used for carcinogenic PAHs based on each compound's relative potency to the potency of benzo[a]pyrene. The following TEFs were used to convert each PAH slope factor to an equivalent slope factor of benzo[a]pyrene [that has a new cancer slope factor of $7.3(\mathrm{mg} / \mathrm{kg} / \text { day) })^{-1}$ identified by EPA's CRAVE workgroup]: (1) benzo[a]pyrene, TEF $=1.0 ;(2)$ 


\section{Table 3.4g. (continued)}

benzo[a]anthracene, $\mathrm{TEF}=0.1$; (3) benzo[b]fluoranthene, TEF $=0.1$; (4) benzo[k]fluoranthene, $\mathrm{TEF}=0.01$; (5) chrysene, $\mathrm{TEF}=$ $0.001 ;(6)$ dibenz $[\mathrm{a}, \mathrm{h}]$ anthracene, TEF $=1.0$; and $(7)$ indeno[1,2,3-c,d]pyrene, $\mathrm{TEF}=0.1$.

(3) The toxicity values for trichloroethylene have been withdrawn from IRIS and HEAST pending further investigation. However, the most recent toxicity values have been used to calculate the PRG.

(4) The PRG was calculated considering Supplemental Guidance from RAGS: Region 4 Bulletins, Human Health Risk Assessment (Interim Guidance) from EPA (November 1995). The slope factors for chemicals so marked indicate that a TEF methodology was used for chlorinated dioxin and furan congeners based on each compound's relative potency to the potency of 2,3,7,8tetrachlorodibenzodioxin (TCDD). The following TEFs were used to convert each slope factor to an equivalent slope factor of 2,3,7,8-TCDD: (1) 2,3,7,8-TCDD, TEF = 1.0; (2) 2,3,7,8-PeCDD, TEF =0.5; (3) 2,3,7,8-HxCDD, TEF = 0.1; (4) 2,3,7,8-HpCDD, $\mathrm{TEF}=0.01 ;(5) \mathrm{OCDD}, \mathrm{TEF}=0.001 ;(6) 2,3,7,8-\mathrm{TCDF}, \mathrm{TEF}=0.1 ;(7) 1,2,3,7,8-\mathrm{PeCDF}, \mathrm{TEF}=0.5 ;(8) 2,3,4,7,8-\mathrm{PeCDF}, \mathrm{TEF}=$ $0.05 ;(9) 2,3,7,8-\mathrm{HxCDF}, \mathrm{TEF}=0.1 ;(10) 2,3,7,8-\mathrm{HpCDF}, \mathrm{TEF}=0.01$; and (11) OCDF, TEF $=0.001$.

(5) Toxicity values for these chemicals have been withdrawn from IRIS and HEAST pending further investigation. However, the most recent toxicity values have been used to calculate the PRG.

(6) Toxicity values for these chemicals were obtained from the Superfund Health Risk Technical Support Center. 
Table 3.4h. Risk-based Preliminary Remediation Goals for water based on ingestion of fish for radionuclides (recreational scenario)

\begin{tabular}{|l|l|c|c|}
\hline \multirow{2}{*}{ Chemical } & \multirow{2}{*}{ CASRN } & \multicolumn{2}{|c|}{$\begin{array}{c}\text { Ingestion of Fish (concentration in water) } \\
\text { (pCi/L) }\end{array}$} \\
\cline { 3 - 4 } & & $10^{-4}$ & $10^{-6}$ \\
\hline Ac-225 & $014265-85-1$ & $3.6 \mathrm{E}+02$ & $3.6 \mathrm{E}+00$ \\
\hline Ac-227 & $014952-40-0$ & $1.5 \mathrm{E}+02$ & $1.5 \mathrm{E}+00$ \\
\hline Ac-227+D & $014952-40-0$ & $4.5 \mathrm{E}+00$ & $4.5 \mathrm{E}-02$ \\
\hline Ac-228 & $014331-83-0$ & $3.2 \mathrm{E}+04$ & $3.2 \mathrm{E}+02$ \\
\hline Ag-105 & $014928-14-4$ & $1.6 \mathrm{E}+05$ & $1.6 \mathrm{E}+03$ \\
\hline Ag-108 & $014391-65-2$ & $3.7 \mathrm{E}+07$ & $3.7 \mathrm{E}+05$ \\
\hline Ag-108m & $014391-65-2$ & $4.3 \mathrm{E}+04$ & $4.3 \mathrm{E}+02$ \\
\hline Ag-108m+D & $014391-65-2$ & $4.3 \mathrm{E}+04$ & $4.3 \mathrm{E}+02$ \\
\hline Ag-109m & $014378-38-2$ & $9.5 \mathrm{E}+08$ & $9.5 \mathrm{E}+06$ \\
\hline Ag-110 & $014391-76-5$ & $1.1 \mathrm{E}+08$ & $1.1 \mathrm{E}+06$ \\
\hline Ag-110m & $014391-76-5$ & $3.1 \mathrm{E}+04$ & $3.1 \mathrm{E}+02$ \\
\hline Ag-111 & $015760-04-0$ & $3.8 \mathrm{E}+04$ & $3.8 \mathrm{E}+02$ \\
\hline Am-241 & $014596-10-2$ & $1.3 \mathrm{E}+02$ & $1.3 \mathrm{E}+00$ \\
\hline Am-242 & $013981-54-9$ & $2.9 \mathrm{E}+04$ & $2.9 \mathrm{E}+02$ \\
\hline Am-242m & $013981-54-9$ & $1.5 \mathrm{E}+02$ & $1.5 \mathrm{E}+00$ \\
\hline Am-243 & $014993-75-0$ & $1.3 \mathrm{E}+02$ & $1.3 \mathrm{E}+00$ \\
\hline Am-243+D & $014993-75-0$ & $6.5 \mathrm{E}+01$ & $6.5 \mathrm{E}-01$ \\
\hline
\end{tabular}


Table 3.4h. (continued)

\begin{tabular}{|l|l|c|c|}
\hline \multirow{2}{*}{ Chemical $^{\dagger}$} & \multirow{2}{*}{ CASRN } & \multicolumn{2}{|c|}{$\begin{array}{c}\text { Ingestion of Fish (concentration in water) } \\
\text { (pCi/L) }\end{array}$} \\
\cline { 3 - 4 } & & $10^{-4}$ & $10^{-6}$ \\
\hline Ar-41 & $014163-25-8$ & & \\
\hline At-217 & $017239-90-6$ & & \\
\hline Au-196 & $014914-16-0$ & & \\
\hline Au-198 & $010043-49-0$ & & $1.9 \mathrm{E}+03$ \\
\hline Ba-131 & $014914-75-1$ & $1.9 \mathrm{E}+05$ & $1.2 \mathrm{E}+03$ \\
\hline Ba-133 & $013981-41-4$ & $1.2 \mathrm{E}+05$ & $1.2 \mathrm{E}+03$ \\
\hline Ba-133m & $013981-41-4$ & $1.2 \mathrm{E}+05$ & $1.3 \mathrm{E}+06$ \\
\hline Ba-137m & $013981-97-0$ & $1.3 \mathrm{E}+08$ & $1.1 \mathrm{E}+04$ \\
\hline Ba-139 & $014378-25-7$ & $1.1 \mathrm{E}+06$ & $2.7 \mathrm{E}+02$ \\
\hline Ba-140 & $014798-08-4$ & $2.7 \mathrm{E}+04$ & $1.5 \mathrm{E}+03$ \\
\hline Be-7 & $013966-02-4$ & $1.5 \mathrm{E}+05$ & $1.8 \mathrm{E}+02$ \\
\hline Bi-206 & $015776-19-9$ & $1.8 \mathrm{E}+04$ & $2.5 \mathrm{E}+02$ \\
\hline Bi-207 & $013982-38-2$ & $2.5 \mathrm{E}+04$ & $1.8 \mathrm{E}+02$ \\
\hline Bi-210 & $014331-79-4$ & $1.8 \mathrm{E}+04$ & $7.1 \mathrm{E}+04$ \\
\hline Bi-211 & $015229-37-5$ & $7.1 \mathrm{E}+06$ & $2.1 \mathrm{E}+03$ \\
\hline Bi-212 & $014913-49-6$ & $2.1 \mathrm{E}+05$ & $2.9 \mathrm{E}+03$ \\
\hline Bi-213 & $015776-20-2$ & $2.9 \mathrm{E}+05$ & \\
\hline
\end{tabular}


Table 3.4h. (continued)

\begin{tabular}{|l|l|c|c|}
\hline \multirow{2}{*}{ Chemical $^{\dagger}$} & \multirow{2}{*}{ CASRN } & \multicolumn{2}{|c|}{$\begin{array}{c}\text { Ingestion of Fish (concentration in water) } \\
\text { (pCi/L) }\end{array}$} \\
\cline { 3 - 4 } & & $10^{-4}$ & $10^{-6}$ \\
\hline Bi-214 & $014733-03-0$ & $6.6 \mathrm{E}+05$ & $6.6 \mathrm{E}+03$ \\
\hline Br-82 & $014686-69-2$ & $2.3 \mathrm{E}+03$ & $2.3 \mathrm{E}+01$ \\
\hline $\mathrm{C}-11$ & $014333-33-6$ & & \\
\hline $\mathrm{C}-14$ & $014762-75-5$ & & \\
\hline $\mathrm{C}-15$ & $015929-23-4$ & & \\
\hline $\mathrm{Ca}-45$ & $013966-05-7$ & & $8.0 \mathrm{E}+00$ \\
\hline $\mathrm{Ca}-47$ & $001439-99-2$ & & $8.8 \mathrm{E}+00$ \\
\hline $\mathrm{Cd}-109$ & $014109-32-1$ & $8.0 \mathrm{E}+02$ & $4.5 \mathrm{E}+00$ \\
\hline $\mathrm{Cd}-115$ & $014336-68-6$ & $8.8 \mathrm{E}+02$ & $1.1 \mathrm{E}+02$ \\
\hline $\mathrm{Cd}-115 \mathrm{~m}$ & $014336-68-6$ & $4.5 \mathrm{E}+02$ & $7.3 \mathrm{E}+01$ \\
\hline $\mathrm{Ce}-141$ & $013967-74-3$ & $1.1 \mathrm{E}+04$ & $1.4 \mathrm{E}+01$ \\
\hline $\mathrm{Ce}-143$ & $014119-19-8$ & $7.3 \mathrm{E}+03$ & $1.9 \mathrm{E}+00$ \\
\hline $\mathrm{Ce}-144$ & $014762-78-8$ & $1.4 \mathrm{E}+03$ & \\
\hline $\mathrm{Ce}-144+\mathrm{D}$ & $014762-78-8$ & $1.9 \mathrm{E}+02$ & \\
\hline $\mathrm{Cl}-36$ & $013981-43-6$ & & \\
\hline $\mathrm{Cl}-38$ & $014158-34-0$ & & \\
\hline $\mathrm{Cm}-242$ & $015510-73-3$ & $1.1 \mathrm{E}+03$ & \\
\hline & & & \\
\hline
\end{tabular}


Table 3.4h. (continued)

\begin{tabular}{|l|l|c|c|}
\hline \multirow{2}{*}{ Chemical } & \multirow{2}{*}{ CASRN } & \multicolumn{2}{|c|}{ Ingestion of Fish (concentration in water) } \\
\cline { 3 - 4 } & & $10^{-4}$ & $10^{-6}$ \\
\hline Cm-243 & $015757-87-6$ & $1.7 \mathrm{E}+02$ & $1.7 \mathrm{E}+00$ \\
\hline Cm-243+D & $015757-87-6$ & $5.2 \mathrm{E}+00$ & $5.2 \mathrm{E}-02$ \\
\hline Cm-244 & $013981-15-2$ & $2.0 \mathrm{E}+02$ & $2.0 \mathrm{E}+00$ \\
\hline Cm-245 & $015621-76-8$ & $1.3 \mathrm{E}+02$ & $1.3 \mathrm{E}+00$ \\
\hline Cm-246 & $015757-90-1$ & $1.3 \mathrm{E}+02$ & $1.3 \mathrm{E}+00$ \\
\hline Cm-247 & $015758-32-4$ & $1.4 \mathrm{E}+02$ & $1.4 \mathrm{E}+00$ \\
\hline Cm-248 & $015758-33-5$ & $3.3 \mathrm{E}+01$ & $3.3 \mathrm{E}-01$ \\
\hline Co-57 & $013981-50-5$ & $4.4 \mathrm{E}+03$ & $4.4 \mathrm{E}+01$ \\
\hline Co-58 & $013981-38-9$ & $1.5 \mathrm{E}+03$ & $1.5 \mathrm{E}+01$ \\
\hline Co-58m & $013981-38-9$ & $4.5 \mathrm{E}+04$ & $4.5 \mathrm{E}+02$ \\
\hline Co-60 & $010198-40-0$ & $2.3 \mathrm{E}+02$ & $2.3 \mathrm{E}+00$ \\
\hline Cr-51 & $014392-02-0$ & $4.7 \mathrm{E}+04$ & $4.7 \mathrm{E}+02$ \\
\hline Cs-131 & $014914-76-2$ & $3.6 \mathrm{E}+03$ & $3.6 \mathrm{E}+01$ \\
\hline Cs-134 & $013967-70-9$ & $1.4 \mathrm{E}+01$ & $1.4 \mathrm{E}-01$ \\
\hline Cs-134m & $013967-70-9$ & $1.4 \mathrm{E}+04$ & $1.4 \mathrm{E}+02$ \\
\hline Cs-135 & $015726-30-4$ & $1.4 \mathrm{E}+02$ & $1.4 \mathrm{E}+00$ \\
\hline Cs-136 & $014234-29-8$ & $8.3 \mathrm{E}+01$ & $8.3 \mathrm{E}-01$ \\
\hline & & & \\
\hline
\end{tabular}


Table 3.4h. (continued)

\begin{tabular}{|l|l|c|c|}
\hline \multirow{2}{*}{ Chemical $^{\dagger}$} & \multirow{2}{*}{ CASRN } & \multicolumn{2}{|c|}{$\begin{array}{c}\text { Ingestion of Fish (concentration in water) } \\
\text { (pCi/L) }\end{array}$} \\
\cline { 3 - 4 } & & $10^{-4}$ & $10^{-6}$ \\
\hline Cs-137 & $010045-97-3$ & $2.0 \mathrm{E}+01$ & $2.0 \mathrm{E}-01$ \\
\hline Cs-137+D & $010045-97-3$ & $2.0 \mathrm{E}+01$ & $2.0 \mathrm{E}-01$ \\
\hline Cs-138 & $015758-29-9$ & $3.7 \mathrm{E}+03$ & $3.7 \mathrm{E}+01$ \\
\hline Cu-64 & $013981-25-4$ & $1.2 \mathrm{E}+04$ & $1.2 \mathrm{E}+02$ \\
\hline Dy-165 & $013967-64-1$ & & \\
\hline Dy-166 & $015840-01-4$ & & \\
\hline Er-169 & $015840-13-8$ & & \\
\hline Er-171 & $014391-45-8$ & & \\
\hline Eu-152 & $014683-23-9$ & $4.5 \mathrm{E}+03$ & \\
\hline Eu-154 & $015585-10-1$ & $2.7 \mathrm{E}+03$ & \\
\hline Eu-155 & $014391-16-3$ & $1.6 \mathrm{E}+04$ & $1.6 \mathrm{E}+02$ \\
\hline Eu-156 & $014280-35-4$ & $2.4 \mathrm{E}+03$ & $2.4 \mathrm{E}+01$ \\
\hline F-18 & $013981-56-1$ & & \\
\hline Fe-55 & $014681-59-5$ & $1.8 \mathrm{E}+04$ & $1.8 \mathrm{E}+02$ \\
\hline Fe-59 & $014596-12-4$ & $1.1 \mathrm{E}+03$ & \\
\hline Fr-221 & $015756-41-9$ & & \\
\hline Fr-223 & $015756-98-6$ & & \\
\hline
\end{tabular}


Table 3.4h. (continued)

\begin{tabular}{|l|l|c|c|}
\hline \multirow{2}{*}{ Chemical } & \multirow{2}{*}{ CASRN } & \multicolumn{2}{|c|}{$\begin{array}{c}\text { Ingestion of Fish (concentration in water) } \\
\text { (pCi/L) }\end{array}$} \\
\cline { 3 - 4 } & & $10^{-4}$ & $10^{-6}$ \\
\hline Ga-67 & $014119-09-6$ & & \\
\hline Ga-72 & $013982-22-4$ & & \\
\hline Gd-153 & $014276-65-4$ & & \\
\hline Gd-159 & $014041-42-0$ & & $1.8 \mathrm{E}+05$ \\
\hline Ge-71 & $014374-81-3$ & & $1.1 \mathrm{E}+01$ \\
\hline H-3 & $010028-17-8$ & $1.8 \mathrm{E}+07$ & $4.9 \mathrm{E}+00$ \\
\hline Hg-197 & $013981-51-6$ & $1.1 \mathrm{E}+03$ & \\
\hline Hg-203 & $013982-78-0$ & $4.9 \mathrm{E}+02$ & $1.5 \mathrm{E}+04$ \\
\hline Ho-166 & $013967-65-2$ & & $5.9 \mathrm{E}+02$ \\
\hline I-122 & $018287-75-7$ & $1.5 \mathrm{E}+06$ & $1.2 \mathrm{E}+01$ \\
\hline I-123 & $015715-08-9$ & $5.9 \mathrm{E}+04$ & $6.7 \mathrm{E}+00$ \\
\hline I-125 & $014158-31-7$ & $1.2 \mathrm{E}+03$ & $1.7 \mathrm{E}+00$ \\
\hline I-126 & $014158-32-8$ & $6.7 \mathrm{E}+02$ & $6.6 \mathrm{E}+01$ \\
\hline I-129 & $015046-84-1$ & $1.7 \mathrm{E}+02$ & $8.9 \mathrm{E}+00$ \\
\hline I-130 & $014914-02-4$ & $6.6 \mathrm{E}+03$ & \\
\hline I-131 & $010043-66-0$ & $8.9 \mathrm{E}+02$ & \\
\hline I-132 & $014683-16-0$ & $4.9 \mathrm{E}+04$ & \\
\hline & & & \\
\hline
\end{tabular}


Table 3.4h. (continued)

\begin{tabular}{|l|l|c|c|}
\hline \multirow{2}{*}{ Chemical } & \multirow{2}{*}{ CASRN } & \multicolumn{2}{|c|}{$\begin{array}{c}\text { Ingestion of Fish (concentration in water) } \\
\text { (pCiL) }\end{array}$} \\
\cline { 3 - 4 } & & $10^{-4}$ & $10^{-6}$ \\
\hline I-133 & $014834-67-4$ & $3.0 \mathrm{E}+03$ & $3.0 \mathrm{E}+01$ \\
\hline I-134 & $014914-27-3$ & $1.4 \mathrm{E}+05$ & $1.4 \mathrm{E}+03$ \\
\hline I-135 & $014834-68-5$ & $1.4 \mathrm{E}+04$ & $1.4 \mathrm{E}+02$ \\
\hline In-113m & $014885-78-0$ & & \\
\hline In-114 & $013981-55-0$ & & \\
\hline In-114m & $013981-55-0$ & & \\
\hline In-115 & $014191-71-0$ & & \\
\hline In-115m & $014191-71-0$ & & \\
\hline Ir-190 & $014981-91-0$ & & \\
\hline Ir-194 & $014158-35-1$ & & \\
\hline K-40 & $013966-00-2$ & & \\
\hline K-42 & $014378-21-3$ & & \\
\hline Kr-83m & $013965-98-5$ & & \\
\hline Kr-85 & $013983-27-2$ & & \\
\hline Kr-85m & $013983-27-2$ & & \\
\hline Kr-87 & $014809-68-8$ & & \\
\hline Kr-88 & $014995-61-0$ & & \\
\hline
\end{tabular}


Table 3.4h. (continued)

\begin{tabular}{|l|l|c|c|}
\hline \multirow{2}{*}{ Chemical $^{\dagger}$} & \multirow{2}{*}{ CASRN } & \multicolumn{2}{|c|}{$\begin{array}{c}\text { Ingestion of Fish (concentration in water) } \\
\text { (pCi/L) }\end{array}$} \\
\cline { 3 - 4 } & & $10^{-4}$ & $10^{-6}$ \\
\hline Kr-89 & $016316-03-3$ & & \\
\hline Kr-90 & $015741-13-6$ & & $4.5 \mathrm{E}+01$ \\
\hline Lu-140 & $013981-28-7$ & $4.5 \mathrm{E}+03$ & \\
\hline $\mathrm{Mn}-52$ & $014265-75-9$ & & $5.3 \mathrm{E}+00$ \\
\hline $\mathrm{Mn}-54$ & $014092-99-0$ & $5.3 \mathrm{E}+02$ & $1.6 \mathrm{E}+01$ \\
\hline $\mathrm{Mn}-56$ & $013966-31-9$ & $1.6 \mathrm{E}+03$ & $3.8 \mathrm{E}+01$ \\
\hline $\mathrm{Mo}-99$ & $014681-52-8$ & $3.8 \mathrm{E}+03$ & $5.7 \mathrm{E}+02$ \\
\hline $\mathrm{Na}-22$ & $014119-15-4$ & $5.7 \mathrm{E}+04$ & $8.0 \mathrm{E}+01$ \\
\hline $\mathrm{Na}-24$ & $013966-32-0$ & $8.0 \mathrm{E}+03$ & $4.7 \mathrm{E}+02$ \\
\hline $\mathrm{Nb}-93 \mathrm{~m}$ & $013982-04-2$ & $4.7 \mathrm{E}+04$ & $6.5 \mathrm{E}+01$ \\
\hline $\mathrm{Nb}-94$ & $007440-03-1$ & $6.5 \mathrm{E}+03$ & $6.2 \mathrm{E}+00$ \\
\hline $\mathrm{Nb}-95$ & $014681-63-1$ & $6.2 \mathrm{E}+02$ & $1.9 \mathrm{E}+01$ \\
\hline $\mathrm{Nb}-95 \mathrm{~m}$ & $013967-76-5$ & $1.9 \mathrm{E}+03$ & $1.4 \mathrm{E}+01$ \\
\hline $\mathrm{Nb}-97$ & $013967-76-5$ & $1.4 \mathrm{E}+03$ & $2.4 \mathrm{E}+02$ \\
\hline $\mathrm{Nb}-97 \mathrm{~m}$ & $018496-04-3$ & $2.4 \mathrm{E}+04$ & $1.3 \mathrm{E}+04$ \\
\hline $\mathrm{Nd}-147$ & $018496-04-3$ & $1.3 \mathrm{E}+06$ & $2.2 \mathrm{E}+01$ \\
\hline & $014269-74-0$ & $2.2 \mathrm{E}+03$ & \\
\hline
\end{tabular}


Table 3.4h. (continued)

\begin{tabular}{|l|l|c|c|}
\hline \multirow{2}{*}{ Chemical $^{\dagger}$} & \multirow{2}{*}{ CASRN } & \multicolumn{2}{|c|}{$\begin{array}{c}\text { Ingestion of Fish (concentration in water) } \\
\text { (pCi/L) }\end{array}$} \\
\cline { 3 - 4 } & & $10^{-4}$ & $10^{-6}$ \\
\hline Nd-149 & $015749-81-2$ & $2.8 \mathrm{E}+04$ & $2.8 \mathrm{E}+02$ \\
\hline Ni-59 & $014336-70-0$ & $7.0 \mathrm{E}+04$ & $7.0 \mathrm{E}+02$ \\
\hline Ni-63 & $013981-37-8$ & $2.3 \mathrm{E}+04$ & $2.3 \mathrm{E}+02$ \\
\hline Ni-65 & $014833-49-9$ & $2.3 \mathrm{E}+04$ & $2.3 \mathrm{E}+02$ \\
\hline Np-236 & $015700-36-4$ & $4.6 \mathrm{E}+04$ & $4.6 \mathrm{E}+02$ \\
\hline Np-237 & $013994-20-2$ & $1.5 \mathrm{E}+02$ & $1.5 \mathrm{E}+00$ \\
\hline Np-237+D & $013994-20-2$ & $1.1 \mathrm{E}+02$ & $1.1 \mathrm{E}+00$ \\
\hline Np-238 & $015766-25-3$ & $9.4 \mathrm{E}+03$ & $9.4 \mathrm{E}+01$ \\
\hline Np-239 & $013968-59-7$ & $1.0 \mathrm{E}+04$ & $1.0 \mathrm{E}+02$ \\
\hline Np-240 & $015690-84-3$ & $2.4 \mathrm{E}+05$ & $2.4 \mathrm{E}+03$ \\
\hline Np-240m & $015690-84-3$ & $1.8 \mathrm{E}+06$ & $1.8 \mathrm{E}+04$ \\
\hline Os-185 & $015766-50-4$ & & \\
\hline Os-191 & $014119-24-5$ & & $3.2 \mathrm{E}-02$ \\
\hline Os-191m & $014119-24-5$ & & \\
\hline Os-193 & $016057-77-5$ & & \\
\hline P-32 & $014596-37-3$ & $4.2 \mathrm{E}+00$ & \\
\hline P-33 & $015749-66-3$ & $3.3 \mathrm{E}+01$ & \\
\hline
\end{tabular}


Table 3.4h. (continued)

\begin{tabular}{|l|l|c|c|}
\hline \multirow{2}{*}{ Chemical } & \multirow{2}{*}{ CASRN } & \multicolumn{2}{|c|}{ Ingestion of Fish (concentration in water) } \\
\cline { 3 - 4 } & & $10^{-4}$ & $10^{-6}$ \\
\hline $\mathrm{Pa}-231$ & $014331-85-2$ & $8.6 \mathrm{E}+02$ & $8.6 \mathrm{E}+00$ \\
\hline $\mathrm{Pa}-233$ & $013981-14-1$ & $2.7 \mathrm{E}+04$ & $2.7 \mathrm{E}+02$ \\
\hline $\mathrm{Pa}-234$ & $015100-28-4$ & $6.0 \mathrm{E}+04$ & $6.0 \mathrm{E}+02$ \\
\hline $\mathrm{Pa}-234 \mathrm{~m}$ & $015100-28-4$ & $2.7 \mathrm{E}+07$ & $2.7 \mathrm{E}+05$ \\
\hline $\mathrm{Pb}-203$ & $014687-25-3$ & $4.2 \mathrm{E}+03$ & $4.2 \mathrm{E}+01$ \\
\hline $\mathrm{Pb}-209$ & $014119-30-3$ & $2.1 \mathrm{E}+04$ & $2.1 \mathrm{E}+02$ \\
\hline $\mathrm{Pb}-210$ & $014255-04-0$ & $6.4 \mathrm{E}+00$ & $6.4 \mathrm{E}-02$ \\
\hline $\mathrm{Pb}-210+\mathrm{D}$ & $014255-04-0$ & $3.5 \mathrm{E}+00$ & $3.5 \mathrm{E}-02$ \\
\hline $\mathrm{Pb}-211$ & $015816-77-0$ & $1.3 \mathrm{E}+04$ & $1.3 \mathrm{E}+02$ \\
\hline $\mathrm{Pb}-212$ & $015092-94-1$ & $2.4 \mathrm{E}+02$ & $2.4 \mathrm{E}+00$ \\
\hline $\mathrm{Pb}-214$ & $015067-28-4$ & $1.5 \mathrm{E}+04$ & $1.5 \mathrm{E}+02$ \\
\hline $\mathrm{Pd}-100$ & & & \\
\hline $\mathrm{Pd}-101$ & $015690-69-4$ & & $3.0 \mathrm{E}+02$ \\
\hline $\mathrm{Pd}-103$ & $015749-54-9$ & & \\
\hline $\mathrm{Pd}-107$ & $014967-68-1$ & & \\
\hline $\mathrm{Pd}-109$ & $017637-99-9$ & & \\
\hline $\mathrm{Pm}-147$ & $014981-64-7$ & & \\
\hline
\end{tabular}


Table 3.4h.(continued)

\begin{tabular}{|l|l|c|c|}
\hline \multirow{2}{*}{ Chemical $^{\dagger}$} & \multirow{2}{*}{ CASRN } & \multicolumn{2}{|c|}{$\begin{array}{c}\text { Ingestion of Fish (concentration in water) } \\
\text { (pCi/L) }\end{array}$} \\
\cline { 3 - 4 } & & $10^{-4}$ & $10^{-6}$ \\
\hline Pm-148 & $014683-19-3$ & $3.0 \mathrm{E}+03$ & $3.0 \mathrm{E}+01$ \\
\hline Pm-148m & $014683-19-3$ & $4.3 \mathrm{E}+03$ & $4.3 \mathrm{E}+01$ \\
\hline Pm-149 & $015765-31-8$ & $7.8 \mathrm{E}+03$ & $7.8 \mathrm{E}+01$ \\
\hline Po-210 & $013981-52-7$ & $7.9 \mathrm{E}+01$ & $7.9 \mathrm{E}-01$ \\
\hline Po-212 & $015389-34-1$ & $5.7 \mathrm{E}+14$ & $5.7 \mathrm{E}+12$ \\
\hline Po-213 & $015756-57-7$ & $3.8 \mathrm{E}+13$ & $3.8 \mathrm{E}+11$ \\
\hline Po-214 & $015735-67-8$ & $1.2 \mathrm{E}+12$ & $1.2 \mathrm{E}+10$ \\
\hline Po-215 & $015706-52-2$ & $5.2 \mathrm{E}+10$ & $5.2 \mathrm{E}+08$ \\
\hline Po-216 & $015756-58-8$ & $2.9 \mathrm{E}+08$ & $2.9 \mathrm{E}+06$ \\
\hline Po-218 & $015422-24-9$ & $5.1 \mathrm{E}+05$ & $5.1 \mathrm{E}+03$ \\
\hline Pr-142 & $014191-64-1$ & $1.8 \mathrm{E}+03$ & $1.8 \mathrm{E}+01$ \\
\hline Pr-143 & $014981-79-4$ & $1.9 \mathrm{E}+03$ & $1.9 \mathrm{E}+01$ \\
\hline Pr-144 & $014119-05-2$ & $1.6 \mathrm{E}+05$ & $1.6 \mathrm{E}+03$ \\
\hline Pr-144m & $014119-05-2$ & $4.0 \mathrm{E}+05$ & $4.0 \mathrm{E}+03$ \\
\hline Pt-191 & $015706-36-2$ & & \\
\hline Pt-193 & $015735-70-3$ & & \\
\hline Pt-193m & $015735-70-3$ & & \\
\hline
\end{tabular}


Table 3.4h. (continued)

\begin{tabular}{|l|l|l|l|}
\hline \multirow{2}{*}{ Chemical $^{\dagger}$} & \multirow{2}{*}{ CASRN } & \multicolumn{2}{|c|}{$\begin{array}{c}\text { Ingestion of Fish (concentration in water) } \\
\text { (pCi/) }\end{array}$} \\
\cline { 3 - 4 } & & $10^{-4}$ & $10^{-6}$ \\
\hline Pt-197 & $015735-74-7$ & & \\
\hline Pt-197m & $015735-74-7$ & & $5.6 \mathrm{E}+00$ \\
\hline Pu-236 & $015411-92-4$ & $5.6 \mathrm{E}+02$ & $1.5 \mathrm{E}+00$ \\
\hline Pu-238 & $013981-16-3$ & $1.5 \mathrm{E}+02$ & $1.4 \mathrm{E}+00$ \\
\hline Pu-239 & $015117-48-3$ & $1.4 \mathrm{E}+02$ & $1.4 \mathrm{E}+00$ \\
\hline Pu-240 & $014119-33-6$ & $1.4 \mathrm{E}+02$ & $8.2 \mathrm{E}+01$ \\
\hline Pu-241 & $014119-32-5$ & $8.2 \mathrm{E}+03$ & $5.5 \mathrm{E}-01$ \\
\hline Pu-241+D & $014119-32-5$ & $5.5 \mathrm{E}+01$ & $1.4 \mathrm{E}+00$ \\
\hline Pu-242 & $013982-10-0$ & $1.4 \mathrm{E}+02$ & $1.2 \mathrm{E}+03$ \\
\hline Pu-243 & $015706-37-3$ & $1.2 \mathrm{E}+05$ & $1.4 \mathrm{E}+00$ \\
\hline Pu-244 & $014119-34-7$ & $1.4 \mathrm{E}+02$ & $5.8 \mathrm{E}-01$ \\
\hline Pu-244+D & $014119-34-7$ & $5.8 \mathrm{E}+01$ & $1.1 \mathrm{E}+00$ \\
\hline Ra-223 & $015623-45-7$ & $1.1 \mathrm{E}+02$ & $1.7 \mathrm{E}+00$ \\
\hline Ra-224 & $013233-32-4$ & $1.7 \mathrm{E}+02$ & $1.6 \mathrm{E}+00$ \\
\hline Ra-225 & $013981-53-8$ & $1.6 \mathrm{E}+02$ & $8.7 \mathrm{E}-01$ \\
\hline Ra-226 & $013982-63-3$ & $8.7 \mathrm{E}+01$ & $9.4 \mathrm{E}-02$ \\
\hline Ra-226+D & $013982-63-3$ & $9.4 \mathrm{E}+00$ & \\
\hline
\end{tabular}


Table 3.4h. (continued)

\begin{tabular}{|c|c|c|c|}
\hline \multirow[t]{2}{*}{ Chemical ${ }^{\dagger}$} & \multirow[t]{2}{*}{ CASRN } & \multicolumn{2}{|c|}{$\begin{array}{l}\text { Ingestion of Fish (concentration in water) } \\
(\mathrm{pCi} / \mathrm{L})\end{array}$} \\
\hline & & $10^{-4}$ & $10^{-6}$ \\
\hline $\mathrm{Ra}-228$ & $015262-20-1$ & $1.0 \mathrm{E}+02$ & $1.0 \mathrm{E}+00$ \\
\hline $\mathrm{Ra}-228+\mathrm{D}$ & $015262-20-1$ & $1.0 \mathrm{E}+02$ & $1.0 \mathrm{E}+00$ \\
\hline $\mathrm{Rb}-82$ & 014391-63-0 & $6.1 E+04$ & $6.1 E+02$ \\
\hline $\mathrm{Rb}-86$ & $014932-53-7$ & $9.0 \mathrm{E}+01$ & $9.0 \mathrm{E}-01$ \\
\hline Rb-87 & $013982-13-3$ & $1.7 \mathrm{E}+02$ & $1.7 \mathrm{E}+00$ \\
\hline Rb-88 & 014928-36-0 & $4.4 \mathrm{E}+03$ & $4.4 \mathrm{E}+01$ \\
\hline $\mathrm{Rb}-89$ & 014191-65-2 & $7.4 \mathrm{E}+03$ & $7.4 \mathrm{E}+01$ \\
\hline $\mathrm{Rh}-103 \mathrm{~m}$ & 007440-16-6 & $1.6 \mathrm{E}+07$ & $1.6 \mathrm{E}+05$ \\
\hline $\mathrm{Rh}-105$ & 014913-89-4 & $6.7 E+04$ & $6.7 \mathrm{E}+02$ \\
\hline $\mathrm{Rh}-105 \mathrm{~m}$ & $014913-89-4$ & $1.2 \mathrm{E}+08$ & $1.2 \mathrm{E}+06$ \\
\hline Rh-106 & 014234-34-5 & $3.5 \mathrm{E}+07$ & $3.5 \mathrm{E}+05$ \\
\hline $\mathrm{Rn}-219$ & $014835-02-0$ & & \\
\hline $\mathrm{Rn}-220$ & $022481-48-7$ & & \\
\hline$R n-222+D$ & $014859-67-7$ & & \\
\hline $\mathrm{Ru}-103$ & $013968-53-1$ & $3.9 \mathrm{E}+04$ & $3.9 E+02$ \\
\hline Ru-105 & $014331-95-4$ & $1.1 \mathrm{E}+05$ & $1.1 \mathrm{E}+03$ \\
\hline Ru-106 & $013967-48-1$ & $3.7 \mathrm{E}+03$ & $3.7 \mathrm{E}+01$ \\
\hline
\end{tabular}


Table 3.4h. (continued)

\begin{tabular}{|l|l|c|c|}
\hline \multirow{2}{*}{ Chemical } & \multirow{2}{*}{ CASRN } & \multicolumn{2}{|c|}{$\begin{array}{c}\text { Ingestion of Fish (concentration in water) } \\
\text { (pCi/L) }\end{array}$} \\
\cline { 3 - 4 } & & $10^{-4}$ & $10^{-6}$ \\
\hline Ru-106+D & $013967-48-1$ & $1.9 \mathrm{E}+03$ & $1.9 \mathrm{E}+01$ \\
\hline Ru-97 & $015758-35-7$ & $2.2 \mathrm{E}+05$ & $2.2 \mathrm{E}+03$ \\
\hline S-35 & $015117-53-0$ & $3.9 \mathrm{E}+03$ & $3.9 \mathrm{E}+01$ \\
\hline Sb-122 & $014374-79-9$ & $1.5 \mathrm{E}+03$ & $1.5 \mathrm{E}+01$ \\
\hline Sb-124 & $014683-10-4$ & $1.2 \mathrm{E}+03$ & $1.2 \mathrm{E}+01$ \\
\hline Sb-125 & $014234-35-6$ & $4.3 \mathrm{E}+03$ & $4.3 \mathrm{E}+01$ \\
\hline Sb-125+D & $014234-35-6$ & $7.3 \mathrm{E}+02$ & $7.3 \mathrm{E}+00$ \\
\hline Sb-126 & $015756-32-8$ & $1.3 \mathrm{E}+03$ & $1.3 \mathrm{E}+01$ \\
\hline Sb-126m & $015756-32-8$ & $1.8 \mathrm{E}+05$ & $1.8 \mathrm{E}+03$ \\
\hline Sb-127 & $013968-50-8$ & $1.5 \mathrm{E}+03$ & $1.5 \mathrm{E}+01$ \\
\hline Sb-129 & $014331-88-5$ & $6.9 \mathrm{E}+03$ & $6.9 \mathrm{E}+01$ \\
\hline Sc-46 & $013967-63-0$ & $2.2 \mathrm{E}+03$ & $2.2 \mathrm{E}+01$ \\
\hline Sc-47 & $014391-96-9$ & $4.4 \mathrm{E}+03$ & $4.4 \mathrm{E}+01$ \\
\hline Sc-48 & $014391-86-7$ & $1.9 \mathrm{E}+03$ & $1.9 \mathrm{E}+01$ \\
\hline Se-75 & $014265-71-5$ & & \\
\hline Si-31 & $014276-49-4$ & & \\
\hline Sm-147 & $014392-33-7$ & & \\
\hline & & & \\
\hline
\end{tabular}


Table 3.4h. (continued)

\begin{tabular}{|l|l|c|c|}
\hline \multirow{2}{*}{ Chemical $^{\dagger}$} & \multirow{2}{*}{ CASRN } & \multicolumn{2}{|c|}{$\begin{array}{c}\text { Ingestion of Fish (concentration in water) } \\
\text { (pCi/L) }\end{array}$} \\
\cline { 3 - 4 } & & $10^{-4}$ & $10^{-6}$ \\
\hline Sm-151 & $015715-94-3$ & & \\
\hline Sm-153 & $015766-00-4$ & & $1.2 \mathrm{E}+00$ \\
\hline Sn-113 & $013966-06-8$ & $1.2 \mathrm{E}+02$ & $3.5 \mathrm{E}+00$ \\
\hline Sn-121 & $014683-06-8$ & $3.5 \mathrm{E}+02$ & $2.1 \mathrm{E}+00$ \\
\hline Sn-121m & $014683-06-8$ & $2.1 \mathrm{E}+02$ & $2.6 \mathrm{E}-01$ \\
\hline Sn-125 & $014683-08-0$ & $2.6 \mathrm{E}+01$ & $2.0 \mathrm{E}-01$ \\
\hline Sn-126 & $015832-50-5$ & $2.0 \mathrm{E}+01$ & $8.3 \mathrm{E}+00$ \\
\hline Sr-82 & $014809-50-8$ & $8.3 \mathrm{E}+02$ & $1.5 \mathrm{E}+02$ \\
\hline Sr-85 & $013967-73-2$ & $1.5 \mathrm{E}+04$ & $1.2 \mathrm{E}+04$ \\
\hline Sr-85m & $013967-73-2$ & $1.2 \mathrm{E}+06$ & $2.1 \mathrm{E}+01$ \\
\hline Sr-89 & $014158-27-1$ & $2.1 \mathrm{E}+03$ & $5.2 \mathrm{E}+00$ \\
\hline Sr-90 & $010098-97-2$ & $5.2 \mathrm{E}+02$ & $2.6 \mathrm{E}+00$ \\
\hline Sr-90+D & $010098-97-2$ & $2.6 \mathrm{E}+02$ & $7.6 \mathrm{E}+01$ \\
\hline Sr-91 & $014331-91-0$ & $7.6 \mathrm{E}+03$ & $1.1 \mathrm{E}+02$ \\
\hline Sr-92 & $014928-29-1$ & $1.1 \mathrm{E}+04$ & $1.8 \mathrm{E}+01$ \\
\hline Ta-182 & $013982-00-8$ & $1.8 \mathrm{E}+03$ & \\
\hline Tb-158 & $015759-55-4$ & & \\
\hline
\end{tabular}


Table 3.4h. (continued)

\begin{tabular}{|l|l|c|c|}
\hline \multirow{2}{*}{ Chemical } & \multirow{2}{*}{ CASRN } & \multicolumn{2}{|c|}{$\begin{array}{c}\text { Ingestion of Fish (concentration in water) } \\
\text { (pCi/L) }\end{array}$} \\
\cline { 3 - 4 } & & $10^{-4}$ & $10^{-6}$ \\
\hline Tb-160 & $013981-29-8$ & & \\
\hline Tc-95 & $014809-56-4$ & $9.4 \mathrm{E}+05$ & $9.4 \mathrm{E}+03$ \\
\hline Tc-95m & $014809-56-4$ & $5.2 \mathrm{E}+04$ & $5.2 \mathrm{E}+02$ \\
\hline Tc-96 & $014808-44-7$ & $2.8 \mathrm{E}+04$ & $2.8 \mathrm{E}+02$ \\
\hline Tc-96m & $014808-44-7$ & $2.5 \mathrm{E}+06$ & $2.5 \mathrm{E}+04$ \\
\hline Tc-97 & $015759-35-0$ & $4.1 \mathrm{E}+05$ & $4.1 \mathrm{E}+03$ \\
\hline Tc-97m & $015759-35-0$ & $5.4 \mathrm{E}+04$ & $5.4 \mathrm{E}+02$ \\
\hline Tc-99 & $014133-76-7$ & $4.6 \mathrm{E}+04$ & $4.6 \mathrm{E}+02$ \\
\hline Tc-99m & $014133-76-7$ & $1.2 \mathrm{E}+06$ & $1.2 \mathrm{E}+04$ \\
\hline Te-125m & $014390-73-9$ & $1.3 \mathrm{E}+03$ & $1.3 \mathrm{E}+01$ \\
\hline Te-127 & $013981-49-2$ & $3.8 \mathrm{E}+03$ & $3.8 \mathrm{E}+01$ \\
\hline Te-127m & $013981-49-2$ & $5.3 \mathrm{E}+02$ & $5.3 \mathrm{E}+00$ \\
\hline Te-129 & $014269-71-7$ & $2.2 \mathrm{E}+04$ & $2.2 \mathrm{E}+02$ \\
\hline Te-129m & $014269-71-7$ & $2.7 \mathrm{E}+02$ & $2.7 \mathrm{E}+00$ \\
\hline Te-131 & $014683-12-6$ & $8.2 \mathrm{E}+03$ & $8.2 \mathrm{E}+01$ \\
\hline Te-131m & $014683-12-6$ & $3.6 \mathrm{E}+02$ & $3.6 \mathrm{E}+00$ \\
\hline Te-132 & $014234-28-7$ & $2.6 \mathrm{E}+02$ & $2.6 \mathrm{E}+00$ \\
\hline
\end{tabular}


Table 3.4h. (continued)

\begin{tabular}{|l|l|c|c|}
\hline \multirow{2}{*}{ Chemical } & \multirow{2}{*}{ CASRN } & \multicolumn{2}{|c|}{$\begin{array}{c}\text { Ingestion of Fish (concentration in water) } \\
\text { (pCi/L) }\end{array}$} \\
\cline { 3 - 4 } & & $10^{-4}$ & $10^{-6}$ \\
\hline Th-227 & $015623-47-9$ & $3.2 \mathrm{E}+02$ & $3.2 \mathrm{E}+00$ \\
\hline Th-228 & $014274-82-9$ & $2.0 \mathrm{E}+02$ & $2.0 \mathrm{E}+00$ \\
\hline Th-228+D & $014274-82-9$ & $9.9 \mathrm{E}+00$ & $9.9 \mathrm{E}-02$ \\
\hline Th-229 & $015594-54-4$ & $2.3 \mathrm{E}+02$ & $2.3 \mathrm{E}+00$ \\
\hline Th-229+D & $015594-54-4$ & $6.9 \mathrm{E}+00$ & $6.9 \mathrm{E}-02$ \\
\hline Th-230 & $014269-63-7$ & $3.4 \mathrm{E}+02$ & $3.4 \mathrm{E}+00$ \\
\hline Th-231 & $014932-40-2$ & $7.2 \mathrm{E}+03$ & $7.2 \mathrm{E}+01$ \\
\hline Th-232 & $007440-29-1$ & $3.9 \mathrm{E}+02$ & $3.9 \mathrm{E}+00$ \\
\hline Th-234 & $015065-10-8$ & $6.7 \mathrm{E}+02$ & $6.7 \mathrm{E}+00$ \\
\hline Tl-202 & $015720-57-7$ & & \\
\hline Tl-204 & $013968-51-9$ & & \\
\hline Tl-208 & $014913-50-9$ & & \\
\hline Tl-209 & $015690-73-0$ & & \\
\hline Tm-170 & $013981-30-1$ & & \\
\hline Tm-171 & $014333-45-0$ & & \\
\hline U-232 & $014158-29-3$ & & \\
\hline U-233 & $013968-55-3$ & & \\
\hline
\end{tabular}


Table 3.4h. (continued)

\begin{tabular}{|l|l|c|c|}
\hline \multirow{2}{*}{ Chemical } & \multirow{2}{*}{ CASRN } & \multicolumn{2}{|c|}{$\begin{array}{c}\text { Ingestion of Fish (concentration in water) } \\
\text { (pCi/L) }\end{array}$} \\
\cline { 3 - 4 } & & $10^{-4}$ & $10^{-6}$ \\
\hline U-234 & $013966-29-5$ & $2.9 \mathrm{E}+03$ & $2.9 \mathrm{E}+01$ \\
\hline $\mathrm{U}-235$ & $015117-96-1$ & $2.8 \mathrm{E}+03$ & $2.8 \mathrm{E}+01$ \\
\hline $\mathrm{U}-235+\mathrm{D}-236$ & $015117-96-1$ & $2.5 \mathrm{E}+02$ & $2.5 \mathrm{E}+00$ \\
\hline $\mathrm{U}-237$ & $013982-70-2$ & $3.1 \mathrm{E}+03$ & $3.1 \mathrm{E}+01$ \\
\hline $\mathrm{U}-238$ & $014269-75-1$ & $3.2 \mathrm{E}+04$ & $3.2 \mathrm{E}+02$ \\
\hline $\mathrm{U}-238+\mathrm{D}$ & $007440-61-1$ & $3.0 \mathrm{E}+03$ & $3.0 \mathrm{E}+01$ \\
\hline $\mathrm{U}-240$ & $007440-61-1$ & $1.6 \mathrm{E}+02$ & $1.6 \mathrm{E}+00$ \\
\hline $\mathrm{V}-48$ & $015687-53-3$ & $2.4 \mathrm{E}+04$ & $2.4 \mathrm{E}+02$ \\
\hline W-181 & $014331-97-6$ & $1.7 \mathrm{E}+04$ & $1.7 \mathrm{E}+02$ \\
\hline $\mathrm{W}-185$ & $015749-46-9$ & $4.7 \mathrm{E}+05$ & $4.7 \mathrm{E}+03$ \\
\hline W-187 & $014932-41-3$ & $6.3 \mathrm{E}+04$ & $6.3 \mathrm{E}+02$ \\
\hline Xe-122 & $014983-48-3$ & $5.2 \mathrm{E}+04$ & $5.2 \mathrm{E}+02$ \\
\hline Xe-123 & $015151-09-4$ & & \\
\hline Xe-125 & $015700-10-4$ & & \\
\hline Xe-127 & $013994-18-8$ & & \\
\hline Xe-129m & $013994-19-9$ & & \\
\hline & $013965-99-6$ & & \\
\hline
\end{tabular}


Table 3.4h. (continued)

\begin{tabular}{|l|l|c|c|}
\hline \multirow{2}{*}{ Chemical $^{\dagger}$} & \multirow{2}{*}{ CASRN } & \multicolumn{2}{|c|}{$\begin{array}{c}\text { Ingestion of Fish (concentration in water) } \\
\text { (pCi/L) }\end{array}$} \\
\cline { 3 - 4 } & & $10^{-4}$ & $10^{-6}$ \\
\hline Xe-131m & $014683-11-5$ & & \\
\hline Xe-133 & $014932-42-4$ & & \\
\hline Xe-133m & $014932-42-4$ & & \\
\hline Xe-135 & $014995-62-1$ & & \\
\hline Xe-135m & $014995-62-1$ & & \\
\hline Xe-137 & $014835-21-3$ & & \\
\hline Xe-138 & $015751-81-2$ & & $2.9 \mathrm{E}+01$ \\
\hline $\mathrm{Y}-90$ & $010098-91-6$ & $2.9 \mathrm{E}+03$ & $3.2 \mathrm{E}+01$ \\
\hline $\mathrm{Y}-91$ & $014234-24-3$ & $3.2 \mathrm{E}+03$ & $1.2 \mathrm{E}+04$ \\
\hline $\mathrm{Y}-91 \mathrm{~m}$ & $014234-24-3$ & $1.2 \mathrm{E}+06$ & $2.2 \mathrm{E}+02$ \\
\hline $\mathrm{Y}-92$ & $015751-59-4$ & $2.2 \mathrm{E}+04$ & $7.5 \mathrm{E}+01$ \\
\hline $\mathrm{Y}-93$ & $014981-70-5$ & $7.5 \mathrm{E}+03$ & $1.3 \mathrm{E}+00$ \\
\hline $\mathrm{Zn}-65$ & $013982-39-3$ & $1.3 \mathrm{E}+02$ & $2.1 \mathrm{E}+02$ \\
\hline $\mathrm{Zn}-69$ & $013982-23-5$ & $2.1 \mathrm{E}+04$ & $8.5 \mathrm{E}+00$ \\
\hline $\mathrm{Zn}-69 \mathrm{~m}$ & $013982-23-5$ & $8.5 \mathrm{E}+02$ & \\
\hline $\mathrm{Zr}-93$ & $015751-77-6$ & $8.2 \mathrm{E}+03$ & $1.1 \mathrm{E}+01$ \\
\hline $\mathrm{Zr}-95$ & $013967-71-0$ & & \\
\hline & & & \\
\hline
\end{tabular}




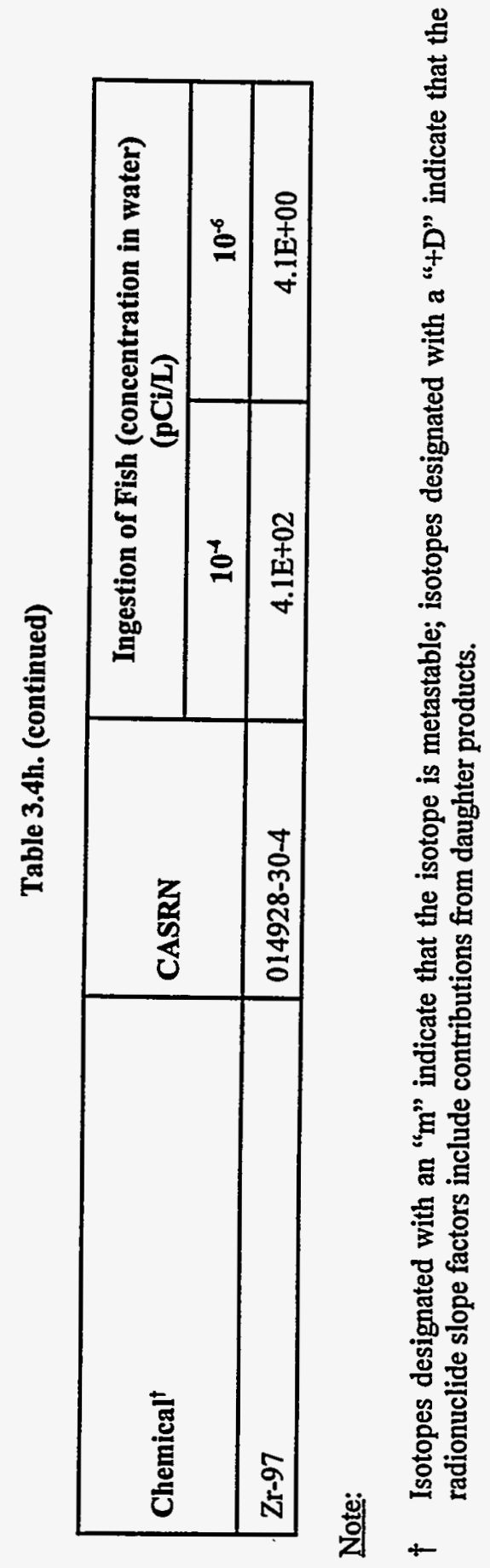

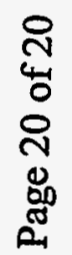


Table 3.5a. Risk-based Preliminary Remediation Goals for nonradionuclides in soil (excavation scenario)

\begin{tabular}{|c|c|c|c|c|c|c|c|c|c|c|c|c|c|c|c|c|c|}
\hline \multirow[t]{2}{*}{ Chemical } & \multirow[t]{2}{*}{ CASRN } & \multicolumn{4}{|c|}{ Ingestion (mg/kg) } & \multicolumn{4}{|c|}{ Inhalation (mg/kg) } & \multicolumn{4}{|c|}{ Dermal (mg/kg) } & \multicolumn{4}{|c|}{$\begin{array}{l}\text { Ingestion + Inhalation + } \\
\text { Dermal (mg/kg) })^{+* k}\end{array}$} \\
\hline & & $10^{-4}$ & $10^{4}$ & $\mathrm{HQ}=1$ & $\mathrm{HQ}=0.1$ & $10^{-4}$ & $10^{4}$ & $H Q=1$ & $\mathrm{HQ}=0.1$ & $10^{-4}$ & $10^{5}$ & $H Q=1$ & $\mathrm{HQ}=0.1$ & $10^{-4}$ & $10^{4}$ & $H \mathrm{II}=1$ & $\mathrm{HI}=0.1$ \\
\hline ALAR & $001596-84.5$ & & & $4.0 \mathrm{E}+05$ & $4.0 E+04$ & & & & & & & $1.0 \mathrm{E}+06$ & $3.0 \mathrm{E}+05$ & & & 3.5E+0S & $3.5 E+04$ \\
\hline Acenaphthene & $000083-32-9$ & & & $1.0 \mathrm{E}+06$ & $1.6 \mathrm{E}+05$ & & & & & & & $1.0 \mathrm{E}+06$ & $7.5 E+05$ & & & $1.0 \mathrm{E}+06$ & $1.3 \mathrm{E}+05$ \\
\hline Acenaphthylene & $000208-96.8$ & & & & & & & & & & & & & & & & \\
\hline Acephate & $030560-19-1$ & $1.0 \mathrm{E}+06$ & $2.1 \mathrm{E}+04$ & $1.1 \mathrm{E}+04$ & $1.1 \mathrm{E}+03$ & & & & & $1.0 \mathrm{E}+06$ & $1.6 \mathrm{E}+05$ & $8.1 \mathrm{E}+04$ & $8.1 E+03$ & $1.0 \mathrm{E}+06$ & $1.9 \mathrm{E}+04$ & $9.4 E+03$ & $9.4 \mathrm{E}+02$ \\
\hline Acetaldehyde & $000075 \cdot 07-0$ & & & & & $4.9 \mathrm{E}+04$ & $3.5 \mathrm{E}+04$ & $9.8 \mathrm{E}+03$ & $9.8 \mathrm{E}+02$ & & & & & & & & \\
\hline Acetochlor & $034256-82-1$ & & & $3.3 E+04$ & $5.3 \mathrm{E}+03$ & & & & & & & $4.0 \mathrm{E}+05$ & $4.0 \mathrm{E}+04$ & & & 4.7E+04 & $4.7 E+03$ \\
\hline Acetone & 000067.64 .1 & & & $1.0 \mathrm{E}+06$ & $2.7 \mathrm{E}+05$ & & & & & & & $1.0 \mathrm{E}+08$ & $1.0 \mathrm{E}+06$ & & & $1.0 \mathrm{E}+06$ & $2.5 \mathrm{E}+05$ \\
\hline Acetone Cyanohydrin & $000075-86.5$ & & & 2.1E+04 & $2.1 \mathrm{E}+03$ & & & $1.0 \mathrm{E}+05$ & $1.0 \mathrm{E}+06$ & & & $1.6 \mathrm{E}+05$ & $1.6 \mathrm{E}+04$ & & & $1.9 \mathrm{E}+04$ & $1.9 \mathrm{E}+03$ \\
\hline Acetonitrile & $000075-05-8$ & & & $1.6 \mathrm{E}+05$ & $1.6 \mathrm{E}+04$ & & & $1.0 \mathrm{E}+06$ & $1.0 \mathrm{E}+06$ & & & $1.0 \mathrm{E}+06$ & $1.9 E+0 S$ & & & $1.5 \mathrm{E}+05$ & $1.5 \mathrm{E}+04$ \\
\hline Acetophenone ${ }^{(3)}$ & $000098-86-2$ & & & $1.0 \mathrm{E}+06$ & $2.7 \mathrm{E}+05$ & & & $1.0 \mathrm{E}+06$ & $1.6 \mathrm{E}+05$ & & & $1.0 \mathrm{E}+06$ & $1.0 \mathrm{E}+06$ & & & $9.4 E+05$ & $9.4 E+04$ \\
\hline Acrolein ${ }^{(0)}$ & $000107-02-8$ & & & $5.3 \mathrm{E}+04$ & $5.3 \mathrm{E}+03$ & & & $4.9 \mathrm{E}+00$ & $4.9 \mathrm{E}-01$ & & & $6.5 \mathrm{E}+05$ & $6.5 \mathrm{E}+04$ & & & $4.9 E+00$ & $4.9 \mathrm{E}-01$ \\
\hline Acrylamide & $000079-06-1$ & 4.1E+03 & 4.1E+01 & $5.3 \mathrm{E}+03$ & $5,3 \mathrm{E}+02$ & $1.0 \mathrm{E}+06$ & $1.0 \mathrm{E}+06$ & & & $3.1 E+04$ & $3.1 E+02$ & $4.0 \mathrm{E}+04$ & $4.0 \mathrm{E}+03$ & $3.7 E+03$ & $3.7 \mathrm{E}+01$ & 4.7E+03 & 4.7E+02 \\
\hline Acrylic Acid & $000079-10-7$ & & & $1.0 \mathrm{E}+06$ & $1.3 \mathrm{E}+05$ & & & $1.0 \mathrm{E}+06$ & $1.0 \mathrm{E}+06$ & & & $1.0 \mathrm{E}+06$ & $1.0 \mathrm{E}+06$ & & & $1.0 \mathrm{E}+06$ & $1.2 \mathrm{E}+05$ \\
\hline Acrylonitrile & $000107-13-1$ & 3.5E+04 & $3.5 \mathrm{E}+02$ & 2.7E+04 & $2.7 E+03$ & $1.8 \mathrm{E}+03$ & $6.8 \mathrm{E}+01$ & $1.3 \mathrm{E}+02$ & $1.3 \mathrm{E}+01$ & 4.2E+05 & $4.2 \mathrm{E}+03$ & $3.2 \mathrm{E}+05$ & $3.2 \mathrm{E}+04$ & $1.8 \mathrm{E}+03$ & $5.6 \mathrm{E}+01$ & $1.3 \mathrm{E}+02$ & $1.3 \mathrm{E}+01$ \\
\hline Nachlor & 015972.60 .8 & $2.3 \mathrm{E}+05$ & $2.3 E+03$ & 2.7E+04 & $2.7 \mathrm{E}+03$ & & & & & $1.0 \mathrm{E}+06$ & $1.8 \mathrm{E}+04$ & $2.0 E+05$ & $2.0 \mathrm{E}+04$ & 2.1E+05 & $2.1 E+03$ & $2.4 E+04$ & $2.4 E+03$ \\
\hline Aldicarb & $000116-06-3$ & & & $2.7 \mathrm{E}+03$ & $2.7 \mathrm{E}+02$ & & & & & & & $2.0 \mathrm{E}+04$ & $2.0 \mathrm{E}+03$ & & & $2.4 E+03$ & $2.4 E+02$ \\
\hline Aldicarb Sulfone & $001646.88-4$ & & & $2.7 \mathrm{E}+03$ & $2.7 \mathrm{E}+02$ & & & & & & & $2.0 \mathrm{E}+04$ & $2.0 \mathrm{E}+03$ & & & $2.4 \mathrm{E}+03$ & $2.4 \mathrm{E}+02$ \\
\hline Addrin & $000309-00-2$ & $1.1 \mathrm{E}+03$ & $1.1 E+01$ & $8.0 \mathrm{E}+01$ & $8.0 \mathrm{E}+00$ & $1.0 \mathrm{E}+06$ & $1.0 E+06$ & & & $8.3 E+03$ & $8.3 \mathrm{E}+01$ & $6.1 \mathrm{E}+02$ & $6.1 E+01$ & $9.7 E+02$ & $9.7 \mathrm{E}+00$ & $7.1 E+01$ & 7. $1 E+\infty$ \\
\hline Ally & $074223-64-6$ & & & $6.7 \mathrm{E}+0 \mathrm{~S}$ & $6.7 \mathrm{E}+04$ & & & & & & & $1.0 \mathrm{E}+06$ & 5.1E+05 & & & $5.9 E+05$ & $5.9 E+04$ \\
\hline Allyt Alcohol & $000107-18-6$ & & & $1.3 E+05$ & $1.3 \mathrm{E}+04$ & & & & & & & $1.0 \mathrm{E}+06$ & $1,6 \mathrm{E}+05$ & & & $1.2 \mathrm{E}+05$ & $1.2 \mathrm{E}+04$ \\
\hline Alyyl Chloride ${ }^{(3)}$ & $000107-05-1$ & & & $1.3 E+0 S$ & $1.3 \mathrm{E}+04$ & & & $6.3 \mathrm{E}+02$ & $1.8 \mathrm{E}+02$ & & & $1.0 \mathrm{E}+06$ & $1.6 \mathrm{E}+0 \mathrm{~S}$ & & & $6.3 E+02$ & $1.8 \mathrm{E}+02$ \\
\hline Aluminum & $007429-90-5$ & & & & & & & & & & & & & & & & \\
\hline Aluminum Phosphide & 020859.73 .8 & & & $1.1 \mathrm{E}+03$ & $1.1 \mathrm{E}+02$ & & & & & & & $3.2 \mathrm{E}+04$ & $3.2 \mathrm{E}+03$ & & & $1.0 \mathrm{E}+03$ & $1.0 E+02$ \\
\hline
\end{tabular}

Nonradionuclides/soil/excavation

Page 1 of 32 
Table 3.5a. (continued)

\begin{tabular}{|c|c|c|c|c|c|c|c|c|c|c|c|c|c|c|c|c|c|}
\hline \multirow[t]{2}{*}{ Chemical } & \multirow[t]{2}{*}{ CASRN } & \multicolumn{4}{|c|}{ Ingestion $(\mathrm{mg} / \mathrm{kg})^{\circ}$} & \multicolumn{4}{|c|}{ Inhalation $(\mathrm{mg} / \mathrm{kg})^{\circ y}$} & \multicolumn{4}{|c|}{ Dermal (mg/kg) } & \multicolumn{4}{|c|}{$\begin{array}{c}\text { Ingestion + Inhalation + } \\
\text { Dermal (mg/kg) })^{+*}\end{array}$} \\
\hline & & $10^{-4}$ & $10^{6}$ & $H Q=1$ & $\mathrm{HQ}=0.1$ & $10^{-4}$ & $10^{4}$ & $H Q=1$ & $H Q=0.1$ & $10^{4}$ & $10^{4}$ & $\mathbf{H Q}=1$ & $H Q=0.1$ & $10^{-4}$ & $10^{6}$ & $H I=1$ & $\mathrm{HI}=0.1$ \\
\hline Amdro & $067485-29-4$ & & & $8,0 E+02$ & $8.0 \mathrm{E}+01$ & & & & & & & $6.1 E+03$ & $6,1 \mathrm{E}+02$ & & & $7.1 E+02$ & $7.1 \mathrm{E}+01$ \\
\hline Ametryn & $000834-12-8$ & & & $2.4 \mathrm{E}+05$ & $2.4 \mathrm{E}+04$ & & & & & & & $1.0 \mathrm{E}+06$ & $1.8 \mathrm{E}+05$ & & & $2.1 \mathrm{E}+05$ & 2.1E+04 \\
\hline Aminophenol, m- & $000591 \cdot 27.5$ & & & $1.0 \mathrm{E}+06$ & $1.9 \mathrm{E}+05$ & & & & & & & $1.0 \mathrm{E}+06$ & $1.0 \mathrm{E}+06$ & & & $1.0 \mathrm{E}+06$ & $1.6 \mathrm{E}+05$ \\
\hline Aminopyridine, 4- & $000504-24.5$ & & & $5.3 E+02$ & $5.3 E+01$ & & & & & & & $4.0 E+03$ & $4.0 \mathrm{E}+02$ & & & $4.7 E+02$ & $4.7 \mathrm{E}+01$ \\
\hline Amitraz & $033089.61-1$ & & & $6.7 E+03$ & $6.7 \mathrm{E}+02$ & & & & & & & $5.1 \mathrm{E}+04$ & $5.1 \mathrm{E}+03$ & & & $5.9 \mathrm{E}+03$ & $5.9 \mathrm{E}+02$ \\
\hline Ammonia & $007664-41 \cdot 7$ & & & & & & & $1.0 \mathrm{E}+06$ & $1.0 \mathrm{E}+06$ & & & & & & & & \\
\hline Ammonium Sulfamate & $007773-06-0$ & & & $5.3 \mathrm{E}+05$ & $5.3 \mathrm{E}+04$ & & & & & & & $1.0 \mathrm{E}+06$ & $1.0 \mathrm{E}+06$ & & & $5.2 \mathrm{E}+05$ & $5.2 \mathrm{E}+04$ \\
\hline Aniline & $000052-53-3$ & $1.0 \mathrm{E}+06$ & $3.3 \mathrm{E}+04$ & & & & & $1.0 \mathrm{E}+06$ & $1.0 \mathrm{E}+06$ & $1.0 \mathrm{E}+06$ & $2.5 \mathrm{E}+05$ & & & $1.0 \mathrm{E}+06$ & $2.9 \mathrm{E}+04$ & & \\
\hline Anthracene & $000120-12-7$ & & & $1.0 \mathrm{E}+06$ & 8.0E+05 & & & & & & & $1.0 \mathrm{E}+06$ & $1.0 \mathrm{E}+06$ & & & $1.0 \mathrm{E}+06$ & 7.3E+05 \\
\hline Antimony (metallic) & 007440.36 .0 & & & $1.1 E+03$ & $1.1 \mathrm{E}+02$ & & & & & & & $3.2 E+03$ & $3.2 \mathrm{E}+02$ & & & $8.0 \mathrm{E}+02$ & $8.0 \mathrm{E}+01$ \\
\hline Antimony Pentoxide & $001314.60-9$ & & & $1.3 \mathrm{E}+03$ & 1.3E+02 & & & & & & & $4.0 \mathrm{E}+04$ & $4.0 \mathrm{E}+03$ & & & $1.3 \mathrm{E}+03$ & $1.3 E+02$ \\
\hline $\begin{array}{l}\text { Antimony Potassium } \\
\text { Tastrate }\end{array}$ & $000304.61-0$ & & & $2.4 \mathrm{E}+03$ & $2.4 E+02$ & & & & & & & $7.3 \mathrm{E}+04$ & $7.3 \mathrm{E}+03$ & & & $2.3 E+03$ & $2.3 E+02$ \\
\hline Antimony Tetroxide & $001332.81-6$ & & & $1.1 \mathrm{E}+03$ & $1.1 E+02$ & & & & & & & $3.2 \mathrm{E}+04$ & $3.2 E+03$ & & & $1.0 \mathrm{E}+03$ & $1.0 \mathrm{E}+02$ \\
\hline Antimony Trioxide & $001309.64-4$ & & & $1.1 \mathrm{E}+03$ & 1.1E+02 & & & $1.0 \mathrm{E}+06$ & $1.0 \mathrm{E}+06$ & & & $3.2 \mathrm{E}+04$ & $3.2 \mathrm{E}+03$ & & & $1.0 \mathrm{E}+03$ & $1.0 E+02$ \\
\hline Apollo & $074115-24-5$ & & & $3.5 \mathrm{E}+04$ & $3.5 \mathrm{E}+03$ & & & & & & & $2.6 \mathrm{E}+05$ & $2.6 \mathrm{E}+04$ & & & $3.1 E+04$ & $3.1 E+03$ \\
\hline Aramite & $000140-57-8$ & $7.5 E+05$ & 7.5E+03 & 2.7E+0s & $2.7 E+04$ & $1.0 \mathrm{E}+06$ & $1.0 \mathrm{E}+06$ & & & $1.0 \mathrm{E}+06$ & $5.7 \mathrm{E}+04$ & $1.0 \mathrm{E}+06$ & $2.0 \mathrm{E}+05$ & $6.6 \mathrm{E}+05$ & $6.6 \mathrm{E}+03$ & $2.4 E+05$ & $2.4 E+04$ \\
\hline Aroclor 1016 & $012674-11-2$ & & & $1.9 \mathrm{E}+02$ & $1.9 \mathrm{E}+01$ & & & & & & & $4.2 \mathrm{E}+02$ & 4.2E+01 & & & 1.3E+02 & $1.3 \mathrm{E}+0 \mathrm{t}$ \\
\hline Aroclor $1221^{(1)}$ & $011104-28-2$ & $2.4 E+03$ & $2.4 E+01$ & & & & & & & $5.5 \mathrm{E}+03$ & 5.5E+01 & & & $1.7 E+03$ & $1.7 \mathrm{E}+01$ & & \\
\hline Aroclor $1232^{(1)}$ & $011141-16-5$ & $2.4 E+03$ & $2.4 E+01$ & & & & & & & $5.5 \mathrm{E}+03$ & $5.5 \mathrm{E}+01$ & & & $1.7 E+03$ & $1.7 \mathrm{E}+01$ & & \\
\hline Aroclor $1242^{(1)}$ & $053469-21.9$ & $2.4 E+03$ & $2.4 E+01$ & & & & & & & $5.5 \mathrm{E}+03$ & $5.5 \mathrm{E}+01$ & & & $1.7 E+03$ & $1.7 \mathrm{E}+01$ & & \\
\hline Aroclor $1248^{(1)}$ & $012672-29.6$ & $2.4 \mathrm{E}+03$ & $2.4 E+01$ & & & & & & & $5.5 E+03$ & $5.5 \mathrm{E}+01$ & & & $1.7 E+03$ & $1.7 \mathrm{E}+01$ & & \\
\hline Aroclor 1254 & $011097-69-1$ & & & $1.3 \mathrm{E}+02$ & $1.3 \mathrm{E}+01$ & & & & & & & $3.0 E+02$ & $3.0 E+01$ & & & $9.2 E+01$ & $9.2 E+00$ \\
\hline
\end{tabular}


Table 3.5a. (continued)

\begin{tabular}{|c|c|c|c|c|c|c|c|c|c|c|c|c|c|c|c|c|c|}
\hline \multirow{2}{*}{ Chemical } & \multirow[t]{2}{*}{ CASRN } & \multicolumn{4}{|c|}{ Ingestion (mg/kg) } & \multicolumn{4}{|c|}{ Inhalation (mg/kg) } & \multicolumn{4}{|c|}{ Dermal (mg/kg) } & \multicolumn{4}{|c|}{$\begin{array}{l}\text { Ingestion + Inhalation + } \\
\text { Dermal }\left(\mathrm{mg} / \mathrm{kg}^{+{ }^{+* \gamma}}\right.\end{array}$} \\
\hline & & $10^{-4}$ & $10^{5}$ & $\mathrm{HQ}=1$ & $\mathrm{HQ}=0.1$ & $10^{-4}$ & $10^{6}$ & $H Q=1$ & $\mathrm{HQ}=0.1$ & $10^{-4}$ & $10^{4}$ & $H Q=1$ & $\mathrm{HQ}=0.1$ & $10^{4}$ & $10^{4}$ & HII $=1$ & $\mathrm{HI}=0.1$ \\
\hline Aroclor $1260^{(1)}$ & $011096-82.5$ & $2.4 E+03$ & $2,4 E+01$ & & & & & & & $5.5 \mathrm{E}+03$ & $5.5 \mathrm{E}+01$ & & & $1.7 \mathrm{E}+03$ & $1.7 \varepsilon+01$ & & \\
\hline Arserric Salts & NA & & & & & & & & & & & & & & & & \\
\hline Arsenic, Inorgenic & $007440-38-2$ & $1.2 E+04$ & $1.2 \mathrm{E}+02$ & $8.0 \mathrm{E}+02$ & $8.0 \mathrm{E}+01$ & $1.0 \mathrm{E}+06$ & $3.8 \mathrm{E}+05$ & & & 7.7E+05 & 7.7E+03 & $5.0 \mathrm{E}+04$ & $5.0 \mathrm{E}+03$ & $1.2 E+04$ & $1.2 \mathrm{E}+02$ & $7.9 E+02$ & $7.9 E+01$ \\
\hline Arsine & $007784-42-1$ & & & & & & & $1.0 \mathrm{E}+06$ & $3,9 E+05$ & & & & & & & & \\
\hline Asbestos & $001332-21-4$ & & & & & & & & & & & & & & & & \\
\hline Assure & $076578-14-8$ & & & $2.4 \mathrm{E}+04$ & $2.4 E+03$ & & & & & & & $1.8 \mathrm{E}+05$ & $1.8 \mathrm{E}+04$ & & & $2.1 \mathrm{E}+04$ & $2.1 E+03$ \\
\hline Asulam & $003337-71-1$ & & & 1.JE+0S & $1.3 E+04$ & & & & & & & $1.0 \mathrm{E}+06$ & $1.0 \mathrm{E}+0 \mathrm{~s}$ & & & $1.2 \mathrm{E}+05$ & $1.2 \mathrm{E}+04$ \\
\hline Atrazine & $001912-24-9$ & $8.4 E+04$ & $8.4 E+02$ & $9.3 E+04$ & $9.3 \mathrm{E}+03$ & & & & & $6.4 E+05$ & $6.4 \mathrm{E}+03$ & 7.1E+05 & $7.1 \mathrm{E}+04$ & $7.4 \mathrm{E}+04$ & $7.4 \mathrm{E}+02$ & $8.2 \mathrm{E}+04$ & 8. $2 E+03$ \\
\hline Avermectin Bl & $065195.55-3$ & & & $1.1 \mathrm{E}+03$ & $1.1 E+02$ & & & & & & & 8. $1 E+03$ & $8.1 E+02$ & & & $9.4 \mathrm{E}+02$ & $9.4 E+0 i$ \\
\hline Azoberzene & $000103-33 \cdot 3$ & $1.7 E+05$ & $1.7 E+03$ & & & $1.0 \mathrm{E}+06$ & $1.0 \mathrm{E}+06$ & & & $1.0 E+06$ & $1.3 \mathrm{E}+04$ & & & 1.5E+05 & $1.5 E+03$ & & \\
\hline Barium & $007440-39-3$ & & & $1.9 E+05$ & $1.9 E+04$ & & & $1.0 \mathrm{E}+06$ & $1.0 \mathrm{E}+06$ & & & $1.0 E+06$ & $2.0 \mathrm{E}+05$ & & & 1.7E+OS & $1.7 \mathrm{E}+04$ \\
\hline Burium Cyanide & $000542-62-1$ & & & $2.7 \mathrm{E}+05$ & 2.7E+04 & & & & & & & $1.0 \mathrm{E}+06$ & 8.1E+05 & & & $2.6 \mathrm{E}+05$ & $2.6 \mathrm{E}+04$ \\
\hline Baygon & $000114-26-1$ & & & 1. $1 E+04$ & 1. $I E+03$ & & & & & & & 8.1E+04 & 8. $1 E+03$ & & & $9.4 \mathrm{E}+03$ & $9.4 E+02$ \\
\hline Bayleton & $043121-43-3$ & & & $8.0 E+04$ & $8.0 E+03$ & & & & & & & $6.1 E+05$ & $6.1 E+04$ & & & $7.1 \mathrm{E}+04$ & $7.1 \mathrm{E}+03$ \\
\hline Baythroid & 068359.37 .5 & & & $6.7 \mathrm{E}+04$ & $6.7 \mathrm{E}+03$ & & & & & & & $5.1 E+05$ & $5.1 \mathrm{E}+04$ & & & $5.9 E+04$ & $5.9 E+03$ \\
\hline Benefin & $001861-40-1$ & & & $8.0 \mathrm{E}+05$ & $8.0 E+04$ & & & & & & & $1.0 \mathrm{E}+06$ & $6.1 E+05$ & & & 7.1E+05 & 7.1E+04 \\
\hline Benomil & $017804-35-2$ & & & $1,3 \mathrm{E}+05$ & $1.3 E+04$ & & & & & & & $1.0 \mathrm{E}+06$ & $1.0 \mathrm{E}+05$ & & & $1.2 \mathrm{E}+05$ & $1.2 \mathrm{E}+04$ \\
\hline Bentazon & $025057-89-0$ & & & $6.7 \mathrm{E}+03$ & $6.7 E+02$ & & & & & & & $5.1 E+04$ & $5.1 \mathrm{E}+03$ & & & $5.9 E+03$ & $5.9 E+02$ \\
\hline Benz[a]enthracente & $000056-55-3$ & 2.6E+04 & 2.6E+02 & & & $1.0 \mathrm{E}+06$ & $1.0 \mathrm{E}+06$ & & & $1.2 \mathrm{E}+05$ & $1.2 \mathrm{E}+03$ & & & $2.1 \mathrm{E}+04$ & $2.1 E+02$ & & \\
\hline Benzaldehyde & $000100-52-7$ & & & $1.0 E+06$ & $2.7 E+05$ & & & & & & & $1.0 \mathrm{E}+06$ & $1.0 \mathrm{E}+06$ & & & $1.0 \mathrm{E}+06$ & $2.4 \mathrm{E}+05$ \\
\hline Benzene & 000071.43 .2 & 6.4E+0S & $6.4 E+03$ & & & $5.0 \mathrm{E}+02$ & $5.0 \mathrm{E}+02$ & & & $1.0 \mathrm{E}+06$ & 9.5E+04 & & & $5.0 \mathrm{E}+02$ & $5.0 E+02$ & & \\
\hline Benzene Hexachloride & $\mathrm{NA}$ & & & & & & & & & & & & & & & & \\
\hline Benzene, Ethyldimethyl & NA & & & & & & & & & & & & & & & & \\
\hline
\end{tabular}

Nonradionuclides/soil/excavation

Page 3 of 32 
Table 3.5a. (continued)

\begin{tabular}{|c|c|c|c|c|c|c|c|c|c|c|c|c|c|c|c|c|c|}
\hline \multirow[t]{2}{*}{ Chemical } & \multirow[t]{2}{*}{ CASRN } & \multicolumn{4}{|c|}{ Ingestion (mg/kg) } & \multicolumn{4}{|c|}{ Inhalation (mg/kg) } & \multicolumn{4}{|c|}{ Dermal (mg/kg) } & \multicolumn{4}{|c|}{$\begin{array}{c}\text { Ingestion + Inhalation }+ \\
\text { Dermal }(\mathrm{mg} / \mathrm{kg})^{+\bullet *}\end{array}$} \\
\hline & & $10^{-4}$ & $10^{4}$ & $H Q=1$ & $H Q=0.1$ & $10^{-4}$ & $10^{6}$ & $\mathrm{HQ}=1$ & $\mathrm{HQ}=0.1$ & $10^{-1}$ & $10^{4}$ & $H Q=1$ & $\mathrm{HQ}=0.1$ & $10^{4}$ & $10^{4}$ & $H I=1$ & $H I=0.1$ \\
\hline Benzene, Ethylmethyl & NA & & & & & & & & & & & & & & & & \\
\hline Benzene, Methylpropenyl & NA & & & & & & & & & & & & & & & & \\
\hline Benzene, Methylpropyl & NA & & & & & & & & & & & & & & & & \\
\hline Benzene, Trimethyl & $025551-13-7$ & & & & & & & & & & & & & & & & \\
\hline Benzenethiol & $000108.98-5$ & & & $2.7 \mathrm{E}+02$ & $2.7 E+01$ & & & & & & & $2.0 E+03$ & $2.0 \mathrm{E}+02$ & & & $2.4 E+02$ & $2.4 \mathrm{E}+01$ \\
\hline Benzidine & $000092-87.5$ & $8.1 E+01$ & 8.1E-01 & $8.0 E+03$ & $8.0 \mathrm{E}+02$ & $1.0 \mathrm{E}+06$ & $8.3 E+04$ & & & $9.8 \mathrm{E}+02$ & $9.8 \mathrm{E}+\infty$ & $9.7 E+04$ & $9.7 \mathrm{E}+03$ & $7.5 \mathrm{E}+01$ & $7.5 \mathrm{E}-01$ & $7.4 E+03$ & $7.4 \mathrm{E}+02$ \\
\hline Benzo[a]pyrene $e^{(x)}$ & $000050-32-8$ & $2.6 \mathrm{E}+03$ & $2.6 \mathrm{E}+01$ & & & $1.0 \mathrm{E}+06$ & $1.0 \mathrm{E}+06$ & & & $1.2 \mathrm{E}+04$ & $1.2 E+02$ & & & $2.1 \mathrm{E}+03$ & $2.1 E+01$ & & \\
\hline Benzo[b] lluoranthene $e^{(x)}$ & $000205-99-2$ & $2.6 \mathrm{E}+04$ & $2.6 \mathrm{E}+02$ & & & $1.0 \mathrm{E}+06$ & $1.0 \mathrm{E}+06$ & & & $1.2 \mathrm{E}+05$ & $1.2 \mathrm{E}+03$ & & & $2.1 \mathrm{E}+04$ & $2.1 E+02$ & & \\
\hline Benzo[g,h,i]perylene & $000191-24-2$ & & & & & & & & & & & & & & & & \\
\hline Benzo[k] liuoranthene $e^{(x)}$ & $000207.08-9$ & $2.6 \mathrm{E}+05$ & $2.6 \mathrm{E}+03$ & & & $1.0 \mathrm{E}+06$ & $1.0 \mathrm{E}+06$ & & & $1.0 \mathrm{E}+06$ & 1.2E+04 & & & $2.1 \mathrm{E}+05$ & $2.1 \mathrm{E}+03$ & & \\
\hline Benzoic Acid & 000065.85 .0 & & & $1.0 \mathrm{E}+06$ & $1.0 \mathrm{E}+06$ & & & & & & & $1.0 \mathrm{E}+06$ & $1.0 \mathrm{E}+06$ & & & $1.0 \mathrm{E}+06$ & $1.0 \mathrm{E}+06$ \\
\hline Benzotrichloride & $000098-07-7$ & $1.4 E+03$ & $1.4 E+01$ & & & & & & & $1.1 \mathrm{E}+04$ & $1.1 \mathrm{E}+02$ & & & $1.3 \mathrm{E}+03$ & $1.3 \mathrm{E}+01$ & & \\
\hline Benzyl Alcohol & $000100-51-6$ & & & $1.0 \mathrm{E}+06$ & 2.7E+0S & & & & & & & $1.0 \mathrm{E}+06$ & $1.0 E+06$ & & & $1.0 E+06$ & $2.4 E+05$ \\
\hline Benzyl Chloride & $000100-44-7$ & $1.1 \mathrm{E}+05$ & 1.1E+03 & & & & & & & $1.0 \mathrm{E}+06$ & $1.3 \mathrm{E}+04$ & & & $1.0 \mathrm{E}+05$ & $1.0 \mathrm{E}+03$ & & \\
\hline Beryllium & $007440-41-7$ & $4.3 E+03$ & 4.3E+01 & $1.3 \mathrm{E}+04$ & $1.3 \mathrm{E}+03$ & $1.0 \mathrm{E}+06$ & $1.0 \mathrm{E}+06$ & & & $6.6 \mathrm{E}+03$ & $6.6 \mathrm{E}+01$ & $2.0 \mathrm{E}+04$ & $2.0 \mathrm{E}+03$ & $2,6 \mathrm{E}+03$ & $2.6 \mathrm{E}+01$ & $8.0 E+03$ & $8.0 \mathrm{E}+02$ \\
\hline Bidrin & $000141-66-2$ & & & $2.7 \mathrm{E}+02$ & 2.7E+0I & & & & & & & $2.0 \mathrm{E}+03$ & $2.0 \mathrm{E}+02$ & & & $2.4 \mathrm{E}+02$ & $2.4 E+01$ \\
\hline Biphenthrin & $082657.04 \cdot 3$ & & & $4.0 \mathrm{E}+04$ & $4.0 \mathrm{E}+03$ & & & & & & & $3.0 \mathrm{E}+05$ & $3.0 E+04$ & & & $3.5 \mathrm{E}+04$ & $3.5 E+03$ \\
\hline Biphenyl, 1,1:- & $000092-52-4$ & & & $1.3 \mathrm{E}+05$ & $1.3 \mathrm{E}+04$ & & & & & & & $1.0 \mathrm{E}+06$ & $1.0 \mathrm{E}+05$ & & & $1.2 E+05$ & $1.2 \mathrm{E}+04$ \\
\hline $\begin{array}{l}\text { Bis(2-chloro-1- } \\
\text { methylethy)ether } \\
\text { (Technical) }\end{array}$ & $000108-60-1$ & $2.7 \mathrm{E}+05$ & $2.7 \mathrm{E}+03$ & & & $3.5 \mathrm{E}+02$ & $3.5 \mathrm{E}+02$ & & & $1.0 \mathrm{E}+06$ & $3.2 \mathrm{E}+04$ & & & 3.SE+02 & $3.5 \mathrm{E}+02$ & & \\
\hline $\begin{array}{l}\text { Bis(2- } \\
\text { clloroethoxy)methane }\end{array}$ & $000111-91-1$ & & & & & & & & & & & & & & & & \\
\hline Bis(2-chloroethyl)ether & $000111-44-4$ & $1.7 E+04$ & $1.7 \mathrm{E}+02$ & & & $6.4 E+02$ & $6.4 \mathrm{E}+02$ & & & 2.1E+05 & $2.1 E+03$ & & & $6.4 E+02$ & $1.3 \mathrm{E}+02$ & & \\
\hline
\end{tabular}


Table 3.5a. (continued)

\begin{tabular}{|c|c|c|c|c|c|c|c|c|c|c|c|c|c|c|c|c|c|}
\hline \multirow[t]{2}{*}{ Chemical } & \multirow[t]{2}{*}{ CASRN } & \multicolumn{4}{|c|}{ Ingestion (mg/kg) } & \multicolumn{4}{|c|}{ Inhalation (mg/kg) } & \multicolumn{4}{|c|}{ Dermal (mg/kg) } & \multicolumn{4}{|c|}{$\begin{array}{c}\text { Ingestion + Inhalation + } \\
\text { Dermal (mg/kg) })^{+\bullet ళ}\end{array}$} \\
\hline & & $10^{-4}$ & $10^{4}$ & $H Q=1$ & $\mathrm{HQ}=0.1$ & $10^{-4}$ & $10^{4}$ & $H Q=1$ & $\mathrm{HQ}=0.1$ & $10^{-4}$ & $10^{5}$ & $\mathrm{HQ}=1$ & $\mathrm{HQ}=0.1$ & $10^{-4}$ & $10^{5}$ & $H=1$ & $\mathrm{HI}=0.1$ \\
\hline $\begin{array}{l}\text { Bis(2- } \\
\text { chloroisopropyl)ether }\end{array}$ & $039638-32.9$ & & & $1.1 \mathrm{E}+05$ & $1.1 \mathrm{E}+04$ & . & & & & & & $8.1 E+05$ & 8.1E+04 & & & $9.4 E+04$ & $9.4 \mathrm{E}+03$ \\
\hline $\begin{array}{l}\text { Bis(2. } \\
\text { ethylhexyl)phthalate(e) }\end{array}$ & $000117-81-7$ & $1.0 \mathrm{E}+06$ & $1.3 \mathrm{E}+04$ & $5.3 \mathrm{E}+04$ & $5.3 \mathrm{E}+03$ & & & & & $1.0 \mathrm{E}+06$ & $3.8 \mathrm{E}+04$ & $1.5 E+05$ & $1.5 \mathrm{E}+04$ & $9.9 \mathrm{E}+05$ & $9.9 E+03$ & $4.0 E+04$ & $4.0 E+03$ \\
\hline Bis(chloromethyl)ether & $000542-88-1$ & $8.5 E+01$ & $8.5 \mathrm{E}-01$ & & & $2,3 \mathrm{E}+02$ & $2.3 E+\infty 0$ & & & $1.0 E+03$ & $1.0 \mathrm{E}+01$ & & & $5.8 E+01$ & $5.8 \mathrm{E}-01$ & & \\
\hline Bisphenol A & $000080.05-7$ & & & $1.0 \mathrm{E}+06$ & $1.6 \mathrm{E}+05$ & & & & & & & $1.0 E+06$ & $1.0 E+06$ & & & $1,0 E+06$ & $1.4 E+05$ \\
\hline Boron And Borntes Only & $007440-42-8$ & & & $2.4 E+05$ & $2.4 E+04$ & & & $1.0 \mathrm{E}+06$ & $1.0 \mathrm{E}+06$ & & & $1.0 \mathrm{E}+06$ & $1.0 E+06$ & & & $2.4 E+05$ & $2.4 \mathrm{E}+04$ \\
\hline Boron Trinuoride & $007637.07-2$ & & & & & & & $1.0 \mathrm{E}+06$ & $1.0 \mathrm{E}+06$ & & & & & & & & \\
\hline Bromochloromethane & $000074-97-5$ & & & & & & & & & & & & & & & & \\
\hline Bromodichloromethane & $000075-27-4$ & $3.0 \mathrm{E}+05$ & $3.0 E+03$ & $5.3 \mathrm{E}+04$ & $5.3 E+03$ & & & & & $1.0 \mathrm{E}+06$ & $4.5 \mathrm{E}+04$ & $7.9 E+05$ & $7.9 \mathrm{E}+04$ & $2.8 \mathrm{E}+05$ & $2.8 E+03$ & $5.0 \mathrm{E}+04$ & $5.0 \mathrm{E}+03$ \\
\hline Bromodiphenyl Ether, p- & $000101-55.3$ & & & & & & & & & & & & & & & & \\
\hline Bromoform & $000075-25-2$ & $1.0 E+06$ & $2.4 \mathrm{E}+04$ & $5.3 E+05$ & $5.3 E+04$ & $1.0 E+06$ & $1.0 \mathrm{E}+06$ & & & $1.0 \mathrm{E}+06$ & $2.1 E+05$ & $1.0 \mathrm{E}+06$ & $4.9 E+05$ & $1,0 \mathrm{E}+06$ & 2.1E+04 & $4.8 \mathrm{E}+05$ & $4.8 \mathrm{E}+04$ \\
\hline Bromomethane $\mathrm{e}^{\text {(n) }}$ & $000074-83-9$ & & & $1.2 \mathrm{E}+04$ & $1.2 \mathrm{E}+03$ & & & $3.4 \mathrm{E}+02$ & $3.4 E+02$ & & & $4.5 E+05$ & $4.5 E+04$ & & & $3.4 E+02$ & $3.4 E+02$ \\
\hline Bromophos & $002104-96-3$ & & & $1.3 \mathrm{E}+0 \mathrm{~S}$ & $1.3 \mathrm{E}+04$ & & & & & & & $1.0 \mathrm{E}+06$ & $1.0 E+05$ & & & $1.2 \mathrm{E}+05$ & $1.2 E+04$ \\
\hline Bromotrichloromethane & $000075-62-7$ & & & & & & & & & & & & & & & & \\
\hline Bromoxynil & $001689.84-5$ & & & $5.3 E+04$ & $5.3 \mathrm{E}+03$ & & & & & & & $4.0 E+05$ & $4.0 E+04$ & & & $4.7 E+04$ & $4.7 E+03$ \\
\hline Bromoxynil Octanoate & 001689.99 .2 & & & $5.3 E+04$ & $5.3 \mathrm{E}+03$ & & & & & & & $4.0 E+05$ & $4.0 E+04$ & & & 4.7E+04 & $4.7 \mathrm{E}+03$ \\
\hline Butadiene, 1,3- & $000106-99-0$ & & & & & $3.2 \mathrm{E}+02$ & $1.5 \mathrm{E}+01$ & & & & & & & & & & \\
\hline Butanol, N. & $000071-36-3$ & & & $1.0 E+06$ & $2.7 \mathrm{E}+05$ & & & & & & & $1.0 \mathrm{E}+06$ & $1.0 E+06$ & & & $1.0 E+06$ & $2.4 \mathrm{E}+0 \mathrm{~S}$ \\
\hline $\begin{array}{l}\text { Butanone-2, 4-chloro-4,4- } \\
\text { diffuoro }\end{array}$ & NA & & & & & & & & & & & & & & & & \\
\hline Buty/ Berxyl Phthlate & $000085-68.7$ & & & $1.0 \mathrm{E}+06$ & $5.3 \mathrm{E}+05$ & & & & & & & $1.0 \mathrm{E}+06$ & $1.0 \mathrm{E}+06$ & & & $1.0 \mathrm{E}+06$ & $4.8 \mathrm{E}+05$ \\
\hline Butylate & $002008-41-5$ & & & $1.3 \mathrm{E}+05$ & $1.3 \mathrm{E}+04$ & & & & & & & $1.0 \mathrm{E}+06$ & $1.0 E+05$ & & & $1.2 E+05$ & $1.2 \mathrm{E}+04$ \\
\hline Butylchloride, t. & $000507-20-0$ & & & & & & & & & & & & & & & & \\
\hline
\end{tabular}


Table 3.5a. (continued)

\begin{tabular}{|c|c|c|c|c|c|c|c|c|c|c|c|c|c|c|c|c|c|}
\hline \multirow[t]{2}{*}{ Chemical } & \multirow[t]{2}{*}{ CASRN } & \multicolumn{4}{|c|}{ Ingestion (mg/kg) } & \multicolumn{4}{|c|}{ Inhalation (mg/kg) } & \multicolumn{4}{|c|}{ Dermal (mg/kg) } & \multicolumn{4}{|c|}{$\begin{array}{c}\text { Ingestion + Inhalation }+ \\
\text { Dermal }(\mathrm{mg} / \mathrm{kg})^{+* *}\end{array}$} \\
\hline & & $10^{4}$ & $10^{4}$ & $\mathbf{H Q}=\mathbf{1}$ & $\mathrm{HQ}=0.1$ & $10^{-4}$ & $10^{4}$ & $H Q=1$ & $\mathrm{HQ}=0.1$ & $10^{-4}$ & $10^{4}$ & $H Q=1$ & $\mathrm{HQ}=0.1$ & $10^{-4}$ & $10^{6}$ & $H I=1$ & $\mathrm{HI}=0.1$ \\
\hline $\begin{array}{l}\text { Butylyphthalyl } \\
\text { Butylglycolate }\end{array}$ & $000085-70-1$ & & & $1.0 \mathrm{E}+06$ & $2.7 \mathrm{E}+0 \mathrm{~S}$ & & & & & & & $1.0 E+06$ & $1.0 E+06$ & & & $1.0 \mathrm{E}+06$ & $2.4 \mathrm{E}+05$ \\
\hline Cacodylic Acid & $000075-60-5$ & & & $8.0 \mathrm{E}+04$ & $8.0 \mathrm{E}+03$ & & & & & & & $6.1 E+05$ & $6.1 \mathrm{E}+04$ & & & $7.1 E+04$ & $7.1 \mathrm{E}+03$ \\
\hline Cadmium (Diet) & $007440-43-9$ & & & $2.7 \mathrm{E}+03$ & $2.7 \mathrm{E}+02$ & $1.0 \mathrm{E}+06$ & $1.0 E+06$ & & & & & $4.0 E+02$ & 4.0E+01 & & & $3.5 \mathrm{E}+02$ & $3.5 \mathrm{E}+01$ \\
\hline Calcium Cyanide & $000592-01-8$ & & & $1.1 E+05$ & $1.1 \mathrm{E}+04$ & & & & & & & $1.0 \mathrm{E}+06$ & $3.2 \mathrm{E}+05$ & & & $1.0 \mathrm{E}+05$ & $1.0 \mathrm{E}+04$ \\
\hline Caproiactam & $000105-60-2$ & & & $1.0 \mathrm{E}+06$ & $1.3 \mathrm{E}+05$ & & & & & & & $1.0 \mathrm{E}+06$ & $1.0 \mathrm{E}+06$ & & & $1.0 \mathrm{E}+06$ & $1.2 \mathrm{E}+05$ \\
\hline Captafo! & $002425-06-1$ & $1.0 E+06$ & $2.2 E+04$ & $5,3 \mathrm{E}+03$ & $5.3 \mathrm{E}+02$ & & & & & $1.0 \mathrm{E}+06$ & $1.6 \mathrm{E}+05$ & $4.0 E+04$ & $4.0 E+03$ & $1.0 \mathrm{E}+06$ & $1.9 \mathrm{E}+04$ & 4.7E+03 & $4.7 E+02$ \\
\hline Cuptan & $000133.06-2$ & $1.0 \mathrm{E}+06$ & $5.3 \mathrm{E}+04$ & 3.3E+05 & $3.5 \mathrm{E}+04$ & & & & & $1.0 \mathrm{E}+06$ & $4.0 E+05$ & $1.0 \mathrm{E}+06$ & $2.6 \mathrm{E}+05$ & $1.0 E+06$ & 4.7E+04 & $3.1 E+05$ & 3.1E+04 \\
\hline Carbaryl & $000063-25-2$ & & & $2.7 \mathrm{E}+05$ & $2.7 \mathrm{E}+04$ & & & & & & & $1.0 \mathrm{E}+06$ & $2.0 \mathrm{E}+05$ & & & $2.4 \mathrm{E}+05$ & $2.4 E+04$ \\
\hline Carbazole & 000086.74 .8 & $9.3 \mathrm{E}+05$ & $9,3 E+03$ & & & & & & & $1.0 \mathrm{E}+06$ & $9.9 E+04$ & & & $8.5 \mathrm{E}+05$ & $8.5 E+03$ & & \\
\hline Carbofuran & $001563-66-2$ & & & $1.3 \mathrm{E}+04$ & $1.3 E+03$ & & & & & & & $1.0 \mathrm{E}+05$ & $1.0 \mathrm{E}+04$ & & & $1.2 \mathrm{E}+04$ & 1.2E+03 \\
\hline Carbon Disulfide & $000075-15-0$ & & & $2.7 E+05$ & 2.7E+04 & & & $1.7 \mathrm{E}+03$ & $3.6 \mathrm{E}+02$ & & & $1.0 \mathrm{E}+05$ & $1.0 \mathrm{E}+04$ & & & $1.7 E+03$ & $3.4 \mathrm{E}+02$ \\
\hline Carbon Tetrachloride' & $000056-23-5$ & $1.4 \mathrm{E}+0 \mathrm{~S}$ & $1.4 \mathrm{E}+03$ & $6.3 \mathrm{E}+03$ & $6.3 \mathrm{E}+02$ & $1.0 \mathrm{E}+03$ & $1.0 E+03$ & & & $1.0 \mathrm{E}+06$ & $1.4 E+04$ & $1.8 \mathrm{E}+05$ & $1.8 \mathrm{E}+04$ & $1.0 \mathrm{E}+03$ & $7.2 \mathrm{E}+02$ & $1.0 E+03$ & $6.1 \mathrm{E}+02$ \\
\hline Cerbosulfin & $055285-14-8$ & & & 2.7E+04 & $2.7 E+03$ & & & & & & & $2.0 \mathrm{E}+05$ & $2.0 \mathrm{E}+04$ & & & $2.4 E+04$ & $2.4 \mathrm{E}+03$ \\
\hline Carboxin & $005234.68-4$ & & & 2.7E+05 & $2.7 E+04$ & & & & & & & $1.0 \mathrm{E}+06$ & $2.0 \mathrm{E}+05$ & & & $2.4 E+05$ & $2.4 \mathrm{E}+04$ \\
\hline Chloral & $000075-87-6$ & & & $5.3 \mathrm{E}+04$ & $5.3 \mathrm{E}+03$ & & & & & & & $4.0 \mathrm{E}+05$ & $4.0 E+04$ & & & $4.7 \mathrm{E}+04$ & $4.7 E+03$ \\
\hline Chloramben & $000133-90-4$ & & & $4.0 \mathrm{E}+04$ & $4.0 \mathrm{E}+03$ & & & & & & & $3.0 \mathrm{E}+05$ & $3.0 E+04$ & & & $3.5 \mathrm{E}+04$ & $3.5 \mathrm{E}+03$ \\
\hline Chloranil & $000118-75-2$ & $4.6 \mathrm{E}+04$ & $4.6 \mathrm{E}+02$ & & & & & & & $3.5 \mathrm{E}+05$ & $3.5 \mathrm{E}+03$ & & & $4.1 \mathrm{E}+04$ & $4.1 E+02$ & & \\
\hline Chlordane ${ }^{19}$ & $000057-74.9$ & $1.4 \mathrm{E}+04$ & $1.4 \mathrm{E}+02$ & $1.6 \mathrm{E}+02$ & $1.6 \mathrm{E}+01$ & $1.0 \mathrm{E}+06$ & $1.0 \mathrm{E}+06$ & & & 1.1E+05 & $1.1 E+03$ & $1.2 \mathrm{E}+03$ & $1.2 \mathrm{E}+02$ & $1.3 \mathrm{E}+04$ & $1.3 \mathrm{E}+02$ & $1.4 E+02$ & $1.4 E+01$ \\
\hline Chloride & $016887-00-6$ & & & & & & & & & & & & & & & & \\
\hline Chlorimuron, Ethyl- & $090982-32-4$ & & & $5.3 \mathrm{E}+04$ & $5.3 \mathrm{E}+03$ & & & & & & & $4.0 \mathrm{E}+05$ & $4.0 \mathrm{E}+04$ & & & 4. $7 \mathrm{E}+04$ & $4.7 \mathrm{E}+03$ \\
\hline Chlorine & $007782-50.5$ & & & 2.7E+05 & 2.7E+04 & & & & & & & $1.0 \mathrm{E}+06$ & $8.1 E+05$ & & & 2.6E+05 & $2.6 \mathrm{E}+04$ \\
\hline Chlorine Dioxide & $010049-04-4$ & & & & & & & $1.0 \mathrm{E}+06$ & $1.0 \mathrm{E}+06$ & & & & & & & & \\
\hline
\end{tabular}


Table 3.5a. (continued)

\begin{tabular}{|c|c|c|c|c|c|c|c|c|c|c|c|c|c|c|c|c|c|}
\hline \multirow[t]{2}{*}{ Chemical } & \multirow[t]{2}{*}{ CASRN } & \multicolumn{4}{|c|}{ Ingestion (mg/kg) } & \multicolumn{4}{|c|}{ Inbalation (mg/kg) } & \multicolumn{4}{|c|}{ Dermal (mg/kg) } & \multicolumn{4}{|c|}{$\begin{array}{c}\text { Ingestion + Inhalation + } \\
\text { Dermal (mg/kg) })^{+v}\end{array}$} \\
\hline & & $10^{-4}$ & $10^{4}$ & $H Q=1$ & $\mathrm{HQ}=0.1$ & $10^{-4}$ & $10^{6}$ & $\mathrm{HQ}=\mathbf{I}$ & $\mathrm{HQ}=0.1$ & $10^{-4}$ & $10^{6}$ & $H Q=1$ & $\mathrm{HQ}=0.1$ & $10^{-4}$ & $10^{6}$ & $\mathrm{HI}=1$ & $\mathrm{HI}=0.1$ \\
\hline Chlorite (Sodium Salt) & $007758-19-2$ & & & & & & & & & & & & & & & & \\
\hline $\begin{array}{l}\text { Chloro-1,1- } \\
\text { difluoroethane, 1. }\end{array}$ & $000075-68-3$ & & & & & & & $1.0 \mathrm{E}+06$ & $1.0 \mathrm{E}+06$ & & & & & & & & \\
\hline Chloro-1,3-butadiene, 2- & $000126-99-8$ & & & $5.3 \mathrm{E}+04$ & $5.3 \mathrm{E}+03$ & & & $1.7 E+03$ & $1.7 \mathrm{E}+03$ & & & $4.0 \mathrm{E}+05$ & $4.0 \mathrm{E}+04$ & & & $1.7 E+03$ & $1.7 E+03$ \\
\hline $\begin{array}{l}\text { Chloro-2-methylaniline } \\
\text { BCl, } 4 \text { - }\end{array}$ & $003165-93-3$ & 4.1E+04 & 4.1E+02 & & & & & & & $3.1 \mathrm{E}+05$ & $3.1 E+03$ & & & $3.6 \mathrm{E}+04$ & $3.6 \mathrm{E}+02$ & & \\
\hline CHoro-2-methyleriline, 4 & $000095-69-2$ & $3.2 E+04$ & $3.2 \mathrm{E}+02$ & & & & & & & $2.4 \mathrm{E}+05$ & $2.4 \mathrm{E}+03$ & & & $2.8 \mathrm{E}+04$ & $2.8 \mathrm{E}+02$ & & \\
\hline Chloroacetic Acid & 000079.11 .8 & & & $5.3 \mathrm{E}+04$ & $5.3 \mathrm{E}+03$ & & & & & & & $4.0 E+05$ & $4.0 \mathrm{E}+04$ & & & $4.7 E+04$ & 4.7E+03 \\
\hline Chloroacetophenone, 2- & $000532-27-4$ & & & & & & & $1.0 \mathrm{E}+06$ & $2.3 \mathrm{E}+05$ & & & & & & & & \\
\hline ChLoroaniline, p- & $000106-47.8$ & & & $1.1 \mathrm{E}+04$ & $1.1 \mathrm{E}+03$ & & & & & & & $8.1 E+04$ & $8.1 E+03$ & & & $9.4 E+03$ & $9.4 E+02$ \\
\hline Chlorobenzene $e^{(1)}$ & $000108-90-7$ & & & $5.3 \mathrm{E}+05$ & $5.3 \mathrm{E}+04$ & & & $5.1 \mathrm{E}+02$ & $5.1 \mathrm{E}+02$ & & & $1.0 \mathrm{E}+06$ & $2.5 \mathrm{E}+05$ & & & $5.1 E+02$ & $5.1 \mathrm{E}+02$ \\
\hline Chlorobenzilate & $000510-15-6$ & $6.9 \mathrm{E}+04$ & $6.9 E+02$ & $5.3 E+04$ & $3.3 \mathrm{E}+03$ & $1.0 E+06$ & $1.0 \mathrm{E}+06$ & & & $5.2 E+05$ & $5.2 \mathrm{E}+03$ & $4.0 \mathrm{E}+0 \mathrm{~S}$ & $4.0 \mathrm{E}+04$ & $6.1 \mathrm{E}+04$ & $6.1 E+02$ & 4.7E+04 & $4.7 E+03$ \\
\hline Chlorobenzoic Acid, p- & $000074-11-3$ & & & $1.0 \mathrm{E}+06$ & $5.3 \mathrm{E}+05$ & & & & & & & $1.0 \mathrm{E}+06$ & $1.0 \mathrm{E}+06$ & & & $1.0 \mathrm{E}+06$ & 4.7E+05 \\
\hline Chlorobenzotrilluoride, 4 - & $000098-56-6$ & & & $5.3 E+05$ & $5.3 \mathrm{E}+04$ & & & & & & & $1.0 \mathrm{E}+06$ & $4.0 \mathrm{E}+05$ & & & $4.7 \mathrm{E}+05$ & 4.7E+04 \\
\hline Chlorobutane, 1- & 000109.69 .3 & & & $1.0 \mathrm{E}+06$ & $2.4 \mathrm{E}+05$ & & & & & & & $1.0 \mathrm{E}+06$ & $1.0 \mathrm{E}+06$ & & & $1.0 \mathrm{E}+06$ & $2.1 E+05$ \\
\hline Chlorobutane, 2- & $000078-86-4$ & & . & & & & & & & & & & & & & & \\
\hline Chlorocyclopentadiene & 041851.50 .7 & & & & & & & & & & & & & & & & \\
\hline Chlorodibromoethare & $073506-94-2$ & $2.2 E+0 S$ & $2.2 E+03$ & & & & & & & $1.0 \mathrm{E}+06$ & 2.7E+04 & & & $2.0 \mathrm{E}+05$ & $2.0 E+03$ & & \\
\hline Chlorodifluoromethane & $000075-45-6$ & & & & & & & $1.0 \mathrm{E}+06$ & $1.0 \mathrm{E}+06$ & & & & & & & & \\
\hline Chloroform & 000067.66 .3 & $1.0 \mathrm{E}+06$ & $3.1 E+04$ & $2.7 E+04$ & $2.7 E+03$ & $1.5 E+03$ & $9.8 \mathrm{E}+02$ & & & $1.0 \mathrm{E}+06$ & $9.3 E+04$ & 8.1E+04 & $8.1 \mathrm{E}+03$ & $1.5 E+03$ & $9.4 \mathrm{E}+02$ & $1.5 \mathrm{E}+03$ & $1.5 \mathrm{E}+03$ \\
\hline Chloromethane $e^{\text {(n) }}$ & 000074.87 .3 & $1.0 \mathrm{E}+06$ & $1.4 \mathrm{E}+04$ & & & $1.2 \mathrm{E}+03$ & $1.2 \mathrm{E}+03$ & $1.2 \mathrm{E}+03$ & $1.2 \mathrm{E}+03$ & $1.0 \mathrm{E}+06$ & $1.7 E+05$ & & & $1.2 E+03$ & $1.2 \mathrm{E}+03$ & & \\
\hline $\begin{array}{l}\text { Chioromethyl Methyl } \\
\text { Ether }\end{array}$ & $000107-30-2$ & & & & & & & & & & & & & & & & \\
\hline Chioronsphthisene, Beta- & $000091-58-7$ & & & $2.1 \mathrm{E}+05$ & 2.1E+04 & & & & & & & $1.0 \mathrm{E}+06$ & $2.6 \mathrm{E}+05$ & & & $2.0 \mathrm{E}+05$ & $2.0 \mathrm{E}+04$ \\
\hline ChHoronitrobenzene, o- & $000088-73-3$ & $7.5 E+05$ & $7.5 E+03$ & & & & & & & $1.0 \mathrm{E}+06$ & $9.1 E+04$ & & & $6.9 \mathrm{E}+0 \mathrm{~s}$ & $6.9 \mathrm{E}+03$ & & \\
\hline
\end{tabular}


Table 3.5a. (continued)

\begin{tabular}{|c|c|c|c|c|c|c|c|c|c|c|c|c|c|c|c|c|c|}
\hline \multirow[t]{2}{*}{ Chemical } & \multirow[t]{2}{*}{ CASRN } & \multicolumn{4}{|c|}{ Ingestion (mg/kg) } & \multicolumn{4}{|c|}{ Inhalation (mg/kg) } & \multicolumn{4}{|c|}{ Dermal (mg/kg) } & \multicolumn{4}{|c|}{$\begin{array}{l}\text { Ingestion + Inbalation + } \\
\text { Dermal (mg/kg) }{ }^{+*}\end{array}$} \\
\hline & & $10^{-4}$ & $10^{4}$ & $H Q=1$ & $H Q=0.1$ & $10^{-4}$ & $10^{4}$ & $\mathrm{HQ}=1$ & $H Q=0.1$ & $10^{-4}$ & $10^{5}$ & $H Q=1$ & $\mathrm{HQ}=0.1$ & $10^{-4}$ & $10^{6}$ & $H I=1$ & $\mathrm{HI}=0.1$ \\
\hline Chloronitrobenzene, p- & $000121-73-3$ & $1.0 \mathrm{E}+06$ & $1.0 \mathrm{E}+04$ & & & & & & & $1.0 \mathrm{E}+06$ & $1.3 E+05$ & & & $9.6 \mathrm{E}+05$ & $9.6 \mathrm{E}+03$ & & \\
\hline Chlorophenol, 2- & $000095-57-8$ & & & $1.3 \mathrm{E}+05$ & $1.3 E+04$ & & & & & & & $1.0 \mathrm{E}+06$ & $1.0 \mathrm{E}+05$ & & & $1.2 \mathrm{E}+05$ & $1.2 \mathrm{E}+04$ \\
\hline $\begin{array}{l}\text { Chlorophenyl Methyl } \\
\text { Sulfide, p- }\end{array}$ & $000123.09-1$ & & & & & & & & & & & & & & & & \\
\hline $\begin{array}{l}\text { Chlorophenyl Methyl } \\
\text { Sulfoxide }\end{array}$ & $000934-73-6$ & & & & & & & & & & & & & & & & \\
\hline Chloropropene, 2 . & $000075-29.6$ & & & & & & & $1.6 \mathrm{E}+03$ & $1.6 \mathrm{E}+03$ & & & & & & & & \\
\hline Chlorothalonil & $001897-45-6$ & $1.0 \mathrm{E}+06$ & $1.7 \mathrm{E}+04$ & $4.0 E+04$ & $4.0 \mathrm{E}+03$ & & & & & $1.08+06$ & $1.3 E+05$ & $3.0 \mathrm{E}+05$ & $3.0 E+04$ & $1.0 \mathrm{E}+06$ & $1.5 \mathrm{E}+04$ & 3.5E+04 & 3.5E+03 \\
\hline Chlorotoluene, o- & $000095-49-8$ & & & $5.3 E+05$ & $5.3 \mathrm{E}+04$ & & & & & & & $1.0 \mathrm{E}+06$ & $6.5 \mathrm{E}+05$ & & & $4.9 \mathrm{E}+05$ & $4.9 E+04$ \\
\hline Chlorpropham & $000101-21-3$ & & & $5.3 \mathrm{E}+05$ & $5.3 \mathrm{E}+04$ & & & & & & & $1.0 \mathrm{E}+06$ & $4.0 \mathrm{E}+05$ & & & $4.7 E+05$ & $4.7 E+04$ \\
\hline Chlorpyrifos & $002921-88-2$ & & & $8.0 \mathrm{E}+03$ & $8.0 \mathrm{E}+02$ & & & & & & & $6.1 \mathrm{IE}+04$ & $6.1 \mathrm{E}+03$ & & & $7.1 E+03$ & 7.1E+02 \\
\hline Chlorpyrifos Methyl & $005598-13 \cdot 0$ & & & $2.7 \mathrm{E}+04$ & $2.7 \mathrm{E}+03$ & & & & & & & $2.0 E+05$ & $2.0 E+04$ & & & 2.4E+04 & $2.4 E+03$ \\
\hline Chlorsulfuron & $064902-72-3$ & & & $1.3 \mathrm{E}+05$ & $1.3 \mathrm{E}+04$ & & & & & & & $1.0 \mathrm{E}+06$ & $1.0 \mathrm{E}+05$ & & & $1.2 E+05$ & $1.2 E+04$ \\
\hline Chlorthiophos & $060238-56-4$ & & & $2.1 \mathrm{E}+03$ & $2.1 \mathrm{E}+02$ & & & & & & & $1.6 \mathrm{E}+04$ & $1.6 \mathrm{E}+03$ & & & $1.9 E+03$ & $1.9 E+02$ \\
\hline $\begin{array}{l}\text { Chromium (III) (Insoluble } \\
\text { Salts) }\end{array}$ & $016085-83-1$ & & & $1.0 \mathrm{E}+06$ & $2.7 \mathrm{E}+0 \mathrm{~S}$ & & & & & & & $1.0 E+06$ & $2.0 \mathrm{E}+05$ & & & $1.0 \mathrm{E}+06$ & $1.1 \mathrm{E}+05$ \\
\hline Chromium (VI) ${ }^{(1)}$ & $018540-29-9$ & & & $5.3 E+04$ & $5.3 \mathrm{E}+03$ & $1.0 \mathrm{E}+06$ & $4.7 \mathrm{E}+0 \mathrm{~S}$ & & & & & $1.6 \mathrm{E}+05$ & $1.6 \mathrm{E}+04$ & & & $4.0 \mathrm{E}+04$ & $4.0 \mathrm{E}+03$ \\
\hline Chromium Saits & $\mathrm{NA}$ & & & & & & & & & & & & & & & & \\
\hline Chrysene ${ }^{(n)}$ & 000218.01 .9 & $1.0 \mathrm{E}+06$ & $2.6 \mathrm{E}+04$ & & & $1.0 \mathrm{E}+06$ & $1.0 \mathrm{E}+06$ & & & $1.0 \mathrm{E}+06$ & $1.2 \mathrm{E}+05$ & & & $1.0 \mathrm{E}+06$ & $2.1 \mathrm{E}+04$ & & \\
\hline Cobalt & $007440-48-4$ & & & & & & & & & & & & & & & & \\
\hline Coke Oven Emissions & $008007-45-2$ & & & & & $1.0 \mathrm{E}+06$ & $1.0 \mathrm{E}+06$ & & & & & & & & & & \\
\hline Copper & $007440-50.8$ & & & & & & & & & & & & & $\cdot$ & & & \\
\hline Copper Cyaride & $000544-92-3$ & & & $1.3 E+05$ & $1.3 \mathrm{E}+04$ & & & & & & & $1.0 \mathrm{E}+06$ & $4.0 \mathrm{E}+05$ & & & $1.3 \mathrm{E}+05$ & $1.3 \mathrm{E}+04$ \\
\hline Creosote & $008001-58.9$ & & & & & & & & & & & & & & & & \\
\hline Cresol, m- & $000108-39-4$ & & & $1.0 E+06$ & $1.3 \mathrm{E}+05$ & & & & & & & $1.0 E+06$ & $1.0 \mathrm{E}+06$ & & & $1.0 \mathrm{E}+06$ & $1.2 E+05$ \\
\hline
\end{tabular}

Nonradionuclides/soil/excavation

Page 8 of 32 
Table 3.5a. (continued)

\begin{tabular}{|c|c|c|c|c|c|c|c|c|c|c|c|c|c|c|c|c|c|}
\hline \multirow{2}{*}{ Chemical } & \multirow[t]{2}{*}{ CASRN } & \multicolumn{4}{|c|}{ Ingestion (mg/kg) } & \multicolumn{4}{|c|}{ Inhalation (mg/kg) ${ }^{\cdot \gamma}$} & \multicolumn{4}{|c|}{ Dermal (mg/kg) } & \multicolumn{4}{|c|}{$\begin{array}{c}\text { Ingestion + Inhalation + } \\
\text { Dermal (mg/kg) }\end{array}$} \\
\hline & & $10^{-4}$ & $10^{4}$ & $H Q=1$ & $\mathrm{HQ}=0.1$ & $10^{-4}$ & $10^{4}$ & $\mathbf{H Q}=\mathbf{1}$ & $\mathrm{HQ}=0.1$ & $10^{-4}$ & $10^{5}$ & $\mathrm{HQ}=1$ & HQ $=0.1$ & $10^{-4}$ & $10^{4}$ & $\mathrm{HI}=\mathbf{1}$ & $\mathrm{HI}=0.1$ \\
\hline Cresol, o- & $000095-48-7$ & & & $1.0 \mathrm{E}+06$ & $1.3 \mathrm{E}+05$ & & & & & & & $1.0 \mathrm{E}+06$ & $1.0 E+06$ & & & $1.0 E+06$ & $1.2 \mathrm{E}+05$ \\
\hline Cresol, p- & $000106-44-5$ & & & $1.3 \mathrm{E}+04$ & $1.3 \mathrm{E}+03$ & & & & & & & $1.3 \mathrm{E}+05$ & $1.3 \mathrm{E}+04$ & & & $1.2 E+04$ & $1.2 \mathrm{E}+03$ \\
\hline Crotonaldehyde & $000123-73-9$ & $9.8 \mathrm{E}+03$ & $9.8 \mathrm{E}+01$ & & & - & & & & $7.4 E+04$ & $7.4 E+02$ & & & 8.7E+03 & $8.7 \mathrm{E}+01$ & & \\
\hline Cumene & 000098.82 .8 & & & $1.0 \mathrm{E}+06$ & $1.1 \mathrm{E}+05$ & & & $3.5 E+02$ & $3.5 \mathrm{E}+02$ & & & $1.0 \mathrm{E}+06$ & $8.1 E+05$ & & & $3.5 E+02$ & $3.5 \mathrm{E}+02$ \\
\hline Cyanazine & $021725-46-2$ & $2.2 E+04$ & $2.2 \mathrm{E}+02$ & $5.3 \mathrm{E}+03$ & $5.3 \mathrm{E}+02$ & & & & & $1.7 E+05$ & $1.7 \mathrm{E}+03$ & $4.0 \mathrm{E}+04$ & $4.0 E+03$ & $2.0 E+04$ & $2.0 \mathrm{E}+02$ & 4.7E+03 & $4.7 \mathrm{E}+02$ \\
\hline Cyenide (CN-) & $000057-12-5$ & & & $5.3 E+04$ & $5.3 \mathrm{E}+03$ & & & & & & & $1.4 E+05$ & $1.4 E+04$ & & & $3.8 \mathrm{E}+04$ & $3.8 \mathrm{E}+03$ \\
\hline Cyanogen & $000460-19-5$ & & & $1.1 \mathrm{E}+05$ & $1.1 E+04$ & & & & & & & $1.0 \mathrm{E}+06$ & $1.3 E+05$ & & & $9.8 \mathrm{E}+04$ & $9.8 \mathrm{E}+03$ \\
\hline Cyanogen Bromide & $000506-68 \cdot 3$ & & & $2.4 E+05$ & $2.4 E+04$ & & & & & & & $1.0 \mathrm{E}+06$ & $2.9 \mathrm{E}+05$ & & & $2.2 E+05$ & $2.2 \mathrm{E}+04$ \\
\hline Cyanogen Chloride & $000506-77-4$ & & & $1.3 \mathrm{E}+05$ & $1.3 \mathrm{E}+04$ & & & & & & & $1.0 E+06$ & $1.6 \mathrm{E}+05$ & & & $1.2 E+05$ & $1.2 E+04$ \\
\hline $\begin{array}{l}\text { Cyclohexane, 1,2,3,4,5- } \\
\text { pentabromo-6-chlooro- }\end{array}$ & $000087-84-3$ & $8.1 E+05$ & 8.1E+03 & & & & & & & $1.0 \mathrm{E}+06$ & $6.2 \mathrm{E}+04$ & & & $7.2 E+05$ & $7.2 E+03$ & & \\
\hline Cyclohexanone & $000108-94 \cdot 1$ & & & $1.0 \mathrm{E}+06$ & $1,0 \mathrm{E}+06$ & & & & & & & $1.0 \mathrm{E}+06$ & $1.0 E+06$ & & & $1.0 \mathrm{E}+06$ & $1.0 \mathrm{E}+06$ \\
\hline Cyclohexylamine & $000108.91-8$ & & & $8.0 \mathrm{E}+05$ & $8.0 \mathrm{E}+04$ & & & & & & & $1.0 \mathrm{E}+06$ & $6.1 \mathrm{E}+05$ & & & $7.1 E+05$ & 7.1E+04 \\
\hline Cyclopentadiene & $000542-92-7$ & & & & & & & $1.0 \mathrm{E}+06$ & $1,0 \mathrm{E}+06$ & & & & & & & & \\
\hline Cyhalothrin/karte & $068085-85.8$ & & & $1.3 E+04$ & $1.3 \mathrm{E}+03$ & & & & & & & $1.0 \mathrm{E}+0 \mathrm{~S}$ & $1.0 \mathrm{E}+04$ & & & $1.2 E+04$ & $1.2 E+03$ \\
\hline Cypermethrin & 052315.07 .8 & & & $2.7 \mathrm{E}+04$ & $2.7 E+03$ & & & & & & & $2.0 \mathrm{E}+05$ & $2.0 E+04$ & & & $2.4 E+04$ & $2.4 E+03$ \\
\hline Cyromaxine & $066215-27-8$ & & & $2.0 \mathrm{E}+04$ & $2.0 E+03$ & & & & & & & $1.5 \mathrm{E}+05$ & $1.5 \mathrm{E}+04$ & & & $1.8 \mathrm{E}+04$ & $1.8 E+03$ \\
\hline DDD & $000072-54-8$ & $7.8 \mathrm{E}+04$ & $7.8 \mathrm{E}+02$ & & & & & & & $8.3 \mathrm{E}+05$ & $8.3 E+03$ & & & 7.1E+04 & $7.1 \mathrm{E}+02$ & & \\
\hline DDE & $000072.55-9$ & $3.5 \mathrm{E}+04$ & $5.5 E+02$ & & & & & & & $5.8 \mathrm{E}+05$ & $5.8 \mathrm{E}+03$ & & & $5.0 \mathrm{E}+04$ & $5.0 E+02$ & & \\
\hline DDT & $000050-29-3$ & S.5E+04 & $5.5 \mathrm{E}+02$ & $1.3 \mathrm{E}+03$ & $1.3 \mathrm{E}+02$ & $1.0 E+06$ & $1.0 \mathrm{E}+06$ & & & $5.8 E+05$ & $5.8 \mathrm{E}+03$ & $1.4 E+04$ & $1.4 E+03$ & $5.0 E+04$ & $5.0 \mathrm{E}+02$ & $1.2 E+03$ & $1.2 \mathrm{E}+02$ \\
\hline Deethal & $001861-32-1$ & & & $2.7 \mathrm{E}+04$ & $2.7 \mathrm{E}+03$ & & & & & & & $2.0 \mathrm{E}+05$ & $2.0 \mathrm{E}+04$ & & & $2.4 E+04$ & $2.4 E+03$ \\
\hline Dalapon & 000075.99 .0 & & & $8.0 \mathrm{E}+04$ & $8.0 \mathrm{E}+03$ & & & & & & & $6.1 E+05$ & $6.1 \mathrm{E}+04$ & & & $7.1 E+04$ & $7.1 E+03$ \\
\hline Decabromodiphenyl Ether & $001163-19-5$ & & & $2.7 \mathrm{E}+04$ & $2.7 E+03$ & & & & & & & $2.0 \mathrm{E}+05$ & $2.0 E+04$ & & & $2.4 E+04$ & $2.4 \mathrm{E}+03$ \\
\hline
\end{tabular}




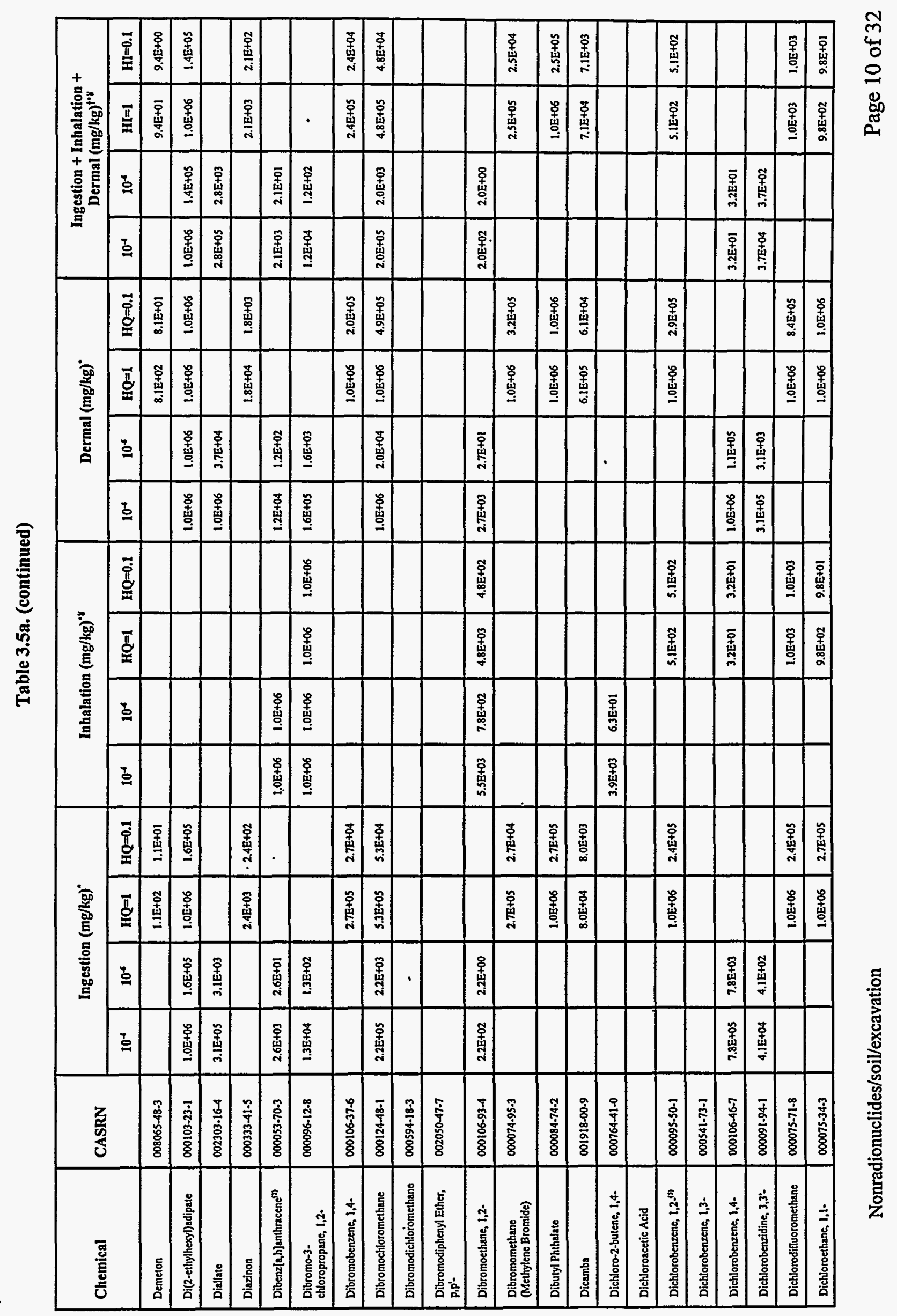


Table 3.5a. (continued)

\begin{tabular}{|c|c|c|c|c|c|c|c|c|c|c|c|c|c|c|c|c|c|}
\hline \multirow[t]{2}{*}{ Chemical } & \multirow[t]{2}{*}{ CASRN } & \multicolumn{4}{|c|}{ Ingestion (mg/kg) } & \multicolumn{4}{|c|}{ Inhalation (mg/kg) } & \multicolumn{4}{|c|}{ Dermal (mg/kg) } & \multicolumn{4}{|c|}{$\begin{array}{c}\text { Ingestion + Inhalation + } \\
\text { Dermal (mg/kg) }\end{array}$} \\
\hline & & $10^{-4}$ & $10^{6}$ & $H Q=1$ & $\mathrm{HQ}=0.1$ & $10^{-4}$ & $10^{6}$ & $\mathrm{HQ}=1$ & $H Q=0.1$ & $10^{-4}$ & $10^{6}$ & $\mathrm{HQ}=\mathbf{I}$ & $\mathrm{HQ}=0.1$ & $10^{-4}$ & $10^{4}$ & $H I=1$ & $\mathrm{HI}=0.1$ \\
\hline Dichloroethane, 1,2- & $000107-06.2$ & $2.0 \mathrm{E}+05$ & $2.0 E+03$ & & & $6.7 \mathrm{E}+02$ & $6.7 \mathrm{E}+02$ & & & $1.0 \mathrm{E}+06$ & $3.1 \mathrm{E}+04$ & & & $6.7 E+02$ & $6.7 \mathrm{E}+02$ & & \\
\hline Dichloroethylene, 1,1- & $000075-35-4$ & $3.1 \mathrm{E}+04$ & $3.1 E+02$ & 2.4E+04 & $2.4 E+03$ & $1.2 \mathrm{E}+03$ & $2.4 E+01$ & & & 4. $7 \mathrm{E}+05$ & 4.7E+03 & $3.6 \mathrm{E}+05$ & $3.6 \mathrm{E}+04$ & $1.2 E+03$ & $2.2 E+01$ & $1.2 \mathrm{E}+03$ & $1.2 \mathrm{E}+03$ \\
\hline $\begin{array}{l}\text { Dichlorocthylene, 1,2- } \\
\text { (Mixed Isomera) }\end{array}$ & $000540-59-0$ & & & $2.4 E+04$ & $2.4 E+03$ & & & & & & & $2.9 \mathrm{E}+05$ & $2.9 E+04$ & & & $2.2 E+04$ & $2.2 \mathrm{E}+03$ \\
\hline Dichloroethylene, 1,2-cis- & $000156-59-2$ & & & $2.7 \mathrm{E}+05$ & $2.7 E+04$ & & & & & & & $1.0 E+06$ & $4.0 \mathrm{E}+05$ & & & 2.5E+05 & $2.5 E+04$ \\
\hline $\begin{array}{l}\text { Dichloroethylene, 1,2- } \\
\text { trans- }\end{array}$ & $000156-60-5$ & & & $5.3 \mathrm{E}+05$ & $5.3 E+04$ & & & & & & & $1.0 \mathrm{E}+06$ & $8.1 E+05$ & & & $5.0 \mathrm{E}+05$ & $5.0 \mathrm{E}+04$ \\
\hline Dichlorophenol, 2,4• & $000120-83-2$ & & & $8.0 \mathrm{E}+03$ & $8.0 E+02$ & & & & & & & $9,9 E+04$ & $9.9 E+03$ & & & $7.4 E+03$ & $7.4 \mathrm{E}+02$ \\
\hline $\begin{array}{l}\text { Dichtorophenoxy Acetic } \\
\text { Acid, } 2,4 \text { - }\end{array}$ & $000094-75.7$ & & & 2.7E+04 & $2.7 E+03$ & . & & & & & & $2.0 \mathrm{E}+05$ & $2.0 \mathrm{E}+04$ & & & $2.4 \mathrm{E}+04$ & $2.4 \mathrm{E}+03$ \\
\hline $\begin{array}{l}\text { Dichorophenoxy)butyric } \\
\text { Acid, } 4-(2,4-\end{array}$ & $000094-82-6$ & & & $2.1 E+05$ & 2.1E+04 & & & & & & & $1.0 \mathrm{E}+06$ & $1.6 \mathrm{E}+05$ & & & $1.9 \mathrm{E}+05$ & $1.9 E+04$ \\
\hline Dichloropropane, 1,2- & $000078-87.5$ & 2.7E+0S & $2.7 \mathrm{E}+03$ & & & & & $4.8 \mathrm{E}+02$ & $4.8 \mathrm{E}+02$ & $1.0 \mathrm{E}+06$ & $3.1 E+04$ & & & $4.8 E+02$ & $4.8 E+02$ & & \\
\hline Dichloropropanol, 2,3- & 000616.23 .9 & & & $8.0 E+03$ & $8.0 \mathrm{E}+02$ & & & & & & & $6.1 \mathrm{E}+04$ & $6.1 E+03$ & & & 7.1E+03 & $7.1 \mathrm{E}+02$ \\
\hline Dichloropropene, 1,3- & $000542-75-6$ & $1.0 \mathrm{E}+05$ & $1.0 E+03$ & $8.0 \mathrm{E}+03$ & $8.0 \mathrm{E}+02$ & $3.7 \mathrm{E}+03$ & $2.8 E+03$ & $3.7 \mathrm{E}+03$ & $3.0 \mathrm{E}+03$ & $8.7 E+0 S$ & $8.7 \mathrm{E}+03$ & $6.7 E+04$ & $6.7 E+03$ & $3.7 E+03$ & $7.0 \mathrm{E}+02$ & $3.7 \mathrm{E}+03$ & $5.8 \mathrm{E}+02$ \\
\hline Dichlorvos & $000062-73.7$ & $6.4 E+04$ & $6.4 \mathrm{E}+02$ & $1.3 \mathrm{E}+03$ & $1.3 \mathrm{E}+02$ & & & $1.0 \mathrm{E}+06$ & $1.0 \mathrm{E}+06$ & $4.9 E+05$ & $4.9 \mathrm{E}+03$ & $1.0 \mathrm{E}+04$ & $1.0 \mathrm{E}+03$ & $5.7 E+04$ & $5.7 E+02$ & $1.2 E+03$ & $1.2 \mathrm{E}+02$ \\
\hline Dicofol & $000115-32-2$ & & & & & & & & & & & & & & & & \\
\hline Dicyclopentadiene & 000077.73 .6 & & & $8.0 \mathrm{E}+05$ & $8.0 \mathrm{E}+04$ & & & 7.4E+02 & $5.3 \mathrm{E}+02$ & & & $1.0 \mathrm{E}+06$ & $6.1 \mathrm{E}+0 \mathrm{~S}$ & & & $7,4 \mathrm{E}+02$ & $5,2 \mathrm{E}+02$ \\
\hline Dieldrin & 000060.57 .1 & $1.2 \mathrm{E}+03$ & $1.2 \mathrm{E}+01$ & $1.3 \mathrm{E}+02$ & $1.3 \mathrm{E}+01$ & $1.0 \mathrm{E}+06$ & $1.0 \mathrm{E}+06$ & & & $8.8 \mathrm{E}+03$ & $8.8 \mathrm{E}+01$ & $1.0 \mathrm{E}+03$ & $1.0 E+02$ & $1.0 \mathrm{E}+03$ & $1.0 \mathrm{E}+01$ & $1.2 \mathrm{E}+02$ & $1,2 \mathrm{E}+01$ \\
\hline Diethyl Phthalate & $000084-66-2$ & & & $1.0 \mathrm{E}+06$ & $1.0 \mathrm{E}+06$ & & & & & & & $1.0 \mathrm{E}+06$ & $1.0 \mathrm{E}+06$ & & & $1.0 \mathrm{E}+06$ & $1.0 \mathrm{E}+06$ \\
\hline $\begin{array}{l}\text { Diethyl-p. } \\
\text { nitrophenylphosphate }\end{array}$ & $000311-45.5$ & & & & & & & & & & & & & & & & \\
\hline $\begin{array}{l}\text { Diethylene Glycol } \\
\text { Dinitrate (DEGDN) }\end{array}$ & $000693-21-0$ & & & & & & & & & & & & & & & & \\
\hline $\begin{array}{l}\text { Diethylene Glycol } \\
\text { Monobutyl Ether }\end{array}$ & 000112.34 .5 & & & & & & & $1.0 \mathrm{E}+06$ & $1.0 \mathrm{E}+06$ & & & & & & & & \\
\hline
\end{tabular}


Table 3.5a. (continued)

\begin{tabular}{|c|c|c|c|c|c|c|c|c|c|c|c|c|c|c|c|c|c|}
\hline \multirow[t]{2}{*}{ Chemical } & \multirow[t]{2}{*}{ CASRN } & \multicolumn{4}{|c|}{ Ingestion (mg/kg) } & \multicolumn{4}{|c|}{ Inhalation (mg/kg) } & \multicolumn{4}{|c|}{ Dermal (mg/kg) } & \multicolumn{4}{|c|}{$\begin{array}{l}\text { Ingestion + Inhalation + } \\
\text { Dermal }(\mathrm{mg} / \mathrm{kg})^{+*}\end{array}$} \\
\hline & & $10^{-4}$ & $10^{6}$ & $H Q=1$ & $H Q=0.1$ & $10^{4}$ & $10^{6}$ & $\mathbf{H Q}=1$ & $\mathrm{HQ}=0.1$ & $10^{-4}$ & $10^{6}$ & $\mathrm{HQ}=1$ & $\mathrm{HQ}=0.1$ & $10^{-4}$ & $10^{4}$ & $\mathrm{HI}=1$ & $\mathrm{HI}=0.1$ \\
\hline $\begin{array}{l}\text { Diethylene Glycol } \\
\text { Monoethyl Ether }\end{array}$ & $000111-90.0$ & & & $1.0 E+06$ & $1.0 \mathrm{E}+06$ & & & & & & & $1.0 \mathrm{E}+06$ & $1.0 \mathrm{E}+06$ & & & $1.0 \mathrm{E}+06$ & $1.0 \mathrm{E}+06$ \\
\hline Diethylformamide & $000617-84-5$ & & & $2.9 \mathrm{E}+04$ & $2.9 \mathrm{E}+03$ & & & & & & & $2.2 \mathrm{E}+05$ & $2.2 E+04$ & & & 2.6E+04 & $2.6 \mathrm{E}+03$ \\
\hline Diethylstilibesterol & $000056-53-1$ & $4.0 \mathrm{E}+\infty 0$ & $4.0 \mathrm{E}-02$ & & & $1.0 \mathrm{E}+06$ & $3.9 E+04$ & & & $3.0 \mathrm{E}+01$ & $3.0 \mathrm{E}-01$ & & & $3.5 \mathrm{E}+\infty$ & $3.5 \mathrm{E}-02$ & & \\
\hline Difenzoquat & $043222-48.6$ & & & $2.1 \mathrm{E}+05$ & $2.1 E+04$ & & & & & & & $1.0 \mathrm{E}+06$ & $1.6 \mathrm{E}+05^{\circ}$ & & & $1.9 \mathrm{E}+05$ & $1.9 E+04$ \\
\hline Diflubenzuron & $035367-38-5$ & & & $5.3 \mathrm{E}+04$ & $5.3 \mathrm{E}+03$ & & & & & & & $4.0 E+05$ & $4.0 \mathrm{E}+04$ & & & $4.7 E+04$ & $4.7 E+03$ \\
\hline Difluoroethane, 1,1- & $000075-37-6$ & & & & & & & $1.0 \mathrm{E}+06$ & $1,0 \mathrm{E}+06$ & & & & & & & & \\
\hline $\begin{array}{l}\text { Disisopropyl } \\
\text { Methylphosphonate }\end{array}$ & 001445.75 .6 & & & $2.1 \mathrm{E}+05$ & $2.1 \mathrm{E}+04$ & & & & & & & $1.0 \mathrm{E}+06$ & $1.6 \mathrm{E}+05$ & & & $1.9 \mathrm{E}+05$ & $1.9 \mathrm{E}+04$ \\
\hline Dimethipin & $055290-64-7$ & & & $5.3 \mathrm{E}+04$ & $5.3 \mathrm{E}+03$ & & & & & & & $4.0 \mathrm{E}+0 \mathrm{~S}$ & $4.0 \mathrm{E}+04$ & & & $4.7 E+04$ & $4.7 \mathrm{E}+03$ \\
\hline Dimethoate & $000060-51-5$ & & & $5.3 \mathrm{E}+02$ & $5.3 \mathrm{E}+01$ & & & & & & & $4.0 \mathrm{E}+03$ & $4.0 \mathrm{E}+02$ & & & 4.7E+02 & $4.7 \mathrm{E}+01$ \\
\hline Dimethoxybenzidine, 3,3- & $000119.90-4$ & $1.0 \mathrm{E}+06$ & $1.3 \mathrm{E}+04$ & & & & & & & $1.0 \mathrm{E}+06$ & $1.0 \mathrm{E}+05$ & & & $1.0 \mathrm{E}+06$ & $1.2 E+04$ & & \\
\hline Dimethyl Sulfate & $000077-78-1$ & & & & & & & & & & & & & & & & \\
\hline Dimethylianiline $\mathrm{HCl}, 2,4-$ & $021436-96-4$ & $3.2 \mathrm{E}+04$ & $3.2 E+02$ & & & & & & & 2.4E+05 & $2.4 \mathrm{E}+03$ & & & $2,8 \mathrm{E}+04$ & $2.8 \mathrm{E}+02$ & & \\
\hline Dimethylaniline, 2,4- & $000095.68-1$ & 2.5E+04 & $2.5 \mathrm{E}+02$ & & & & & & & $1.9 \mathrm{E}+05$ & $1.9 \mathrm{E}+03$ & & & $2.2 E+04$ & $2.2 E+02$ & & \\
\hline Dimethylaniline, N,N- & $000121-69-7$ & & & $5.3 \mathrm{E}+04$ & $5.3 \mathrm{E}+03$ & & & & & & & $4.0 \mathrm{E}+05$ & $4.0 \mathrm{E}+0.4$ & & & $4.7 \mathrm{E}+04$ & $4.7 \mathrm{E}+03$ \\
\hline Dimethylbenzidine, 3,3'- & $000119-93-7$ & $2.0 \mathrm{E}+03$ & $2.0 \mathrm{E}+01$ & & & & & & & $1.5 \mathrm{E}+04$ & $1.5 \mathrm{E}+02$ & & & $1.8 \mathrm{E}+03$ & $1.8 \mathrm{E}+01$ & & \\
\hline Dimethylethyl Lead & $107584-40-7$ & & & & & & & & & & & & & & & & \\
\hline Dimethylformamide & $000068-12-2$ & & & $1.0 \mathrm{E}+06$ & $2.7 \mathrm{E}+05$ & & & $1.0 \mathrm{E}+06$ & $1.0 \mathrm{E}+06$ & & & $1.0 \mathrm{E}+06$ & $1.0 \mathrm{E}+06$ & & & $1.0 E+06$ & $2.3 \mathrm{E}+05$ \\
\hline Dimethylhydrazine, $1,11^{(3)}$ & $000057-14-7$ & $7.2 E+03$ & $7.2 \mathrm{E}+01$ & & & $1.0 \mathrm{E}+06$ & $1.0 \mathrm{E}+06$ & & & $5.4 \mathrm{E}+04$ & $5.4 \mathrm{E}+02$ & & & $6.3 \mathrm{E}+03$ & $6.3 \mathrm{E}+01$ & & \\
\hline Dimethylhydrazine, 1,2- & 000540.73 .8 & & & & & & & & & & & & & & & & \\
\hline Dimethylphenol, 2,4- & $000105-67-9$ & & & $5.3 \mathrm{E}+05$ & $5.3 \mathrm{E}+04$ & & & & & & & $1.0 \mathrm{E}+06$ & $4.0 \mathrm{E}+05$ & & & $4.7 E+05$ & 4.7E+04 \\
\hline Dimethylphenol, 2,6. & $000576-26-1$ & & & $1.6 \mathrm{E}+04$ & $1.6 \mathrm{E}+03$ & & & & & & & $1.2 \mathrm{E}+05$ & $1.2 \mathrm{E}+04$ & & & $1.4 E+04$ & $1.4 \mathrm{E}+03$ \\
\hline Dimethylphenol, 3,4- & $000095-65.8$ & & & $2.7 \mathrm{E}+04$ & $2.7 \mathrm{E}+03$ & & & & & & & $2.0 \mathrm{E}+0 \mathrm{~S}$ & $2.0 E+04$ & & & $2.4 E+04$ & $2.4 \mathrm{E}+03$ \\
\hline
\end{tabular}

Nonradionuclides/soil/excavation

Page 12 of 32 
Table 3.5a. (continued)

\begin{tabular}{|c|c|c|c|c|c|c|c|c|c|c|c|c|c|c|c|c|c|}
\hline \multirow[t]{2}{*}{ Chemical } & \multirow[t]{2}{*}{ CASRN } & \multicolumn{4}{|c|}{ Ingestion (mg/kg) } & \multicolumn{4}{|c|}{ Inhalation $(\mathrm{mg} / \mathrm{kg})^{\cdot \mathbf{y}}$} & \multicolumn{4}{|c|}{ Dermal (mg/kg) } & \multicolumn{4}{|c|}{$\begin{array}{c}\text { Ingestion + Inhalation + } \\
\text { Dermal (mg/kg) }\end{array}$} \\
\hline & & $10^{-4}$ & $10^{6}$ & $H Q=1$ & $\mathrm{HQ}=0.1$ & $10^{-4}$ & $10^{4}$ & $\mathrm{HQ}=1$ & $\mathrm{HQ}=0.1$ & $10^{-4}$ & $10^{5}$ & $\mathrm{HQ}=1$ & $\mathrm{HQ}=0.1$ & $10^{-4}$ & $10^{4}$ & $H I=1$ & $\mathrm{Hl}=0.1$ \\
\hline Dimethylphthalate & $000131-11-3$ & & & $1.0 \mathrm{E}+06$ & $1.0 \mathrm{E}+06$ & & & & & & & $1.0 \mathrm{E}+06$ & $1.0 \mathrm{E}+06$ & & & $1.0 E+06$ & $1.0 \mathrm{E}+06$ \\
\hline Dimethylterephthalate & $000120.61-6$ & & & 2.7E+05 & 2. $\pi E+04$ & & & & & & & $1.0 \mathrm{E}+06$ & $2.0 E+05$ & & & $2.4 E+05$ & $2.4 E+04$ \\
\hline Dinitro-a-cresol, 4,6- & $000534-52-1$ & & & & & & & & & & & & & & & & \\
\hline $\begin{array}{l}\text { Dinitro-a-ycyclohexyl } \\
\text { Phenol, 4,6. }\end{array}$ & $000131-89-5$ & & & $5.3 \mathrm{E}+03$ & $5.3 E+02$ & & & & & & & $4.0 E+04$ & $4.0 E+03$ & & & $4.7 E+03$ & $4.7 E+02$ \\
\hline Dinitrobenzene, 1,2- & $000528-29-0$ & & & $1.1 \mathrm{E}+04$ & $1.1 E+03$ & & & & & & & $1.5 E+05$ & $1.5 \mathrm{E}+04$ & & & $9.9 \mathrm{E}+03$ & $9.9 \mathrm{E}+02$ \\
\hline Dinitrobenzene, 1,3- & $000099-65-0$ & & & $2.7 \mathrm{E}+03$ & $2 . \pi E+02$ & & & & & & & $2.6 \mathrm{E}+04$ & $2.6 E+03$ & & & $2.4 E+03$ & $2.4 E+02$ \\
\hline Dinitrobenzene, 1,4- & $000100-25-4$ & & & 1. $1 E+04$ & $1.1 E+03$ & & & & & & & $8.1 \mathrm{E}+04$ & $8.1 E+03$ & & & $9.4 \mathrm{E}+03$ & $9.4 \mathrm{E}+02$ \\
\hline Dinitrophenol, 2,4. & 000051.28 .5 & & & $5.3 \mathrm{E}+03$ & $5.3 E+02$ & & & & & & & $8.1 E+04$ & $8.1 E+03$ & & & $5.0 \mathrm{E}+03$ & $5.0 \mathrm{E}+02$ \\
\hline Dinitrotoluene, 2,4- & $000121-14-2$ & $2.7 \varepsilon+04$ & $2.7 E+02$ & $5.3 \mathrm{E}+03$ & $5.3 E+02$ & & & & & $3.5 \mathrm{E}+05$ & $3.5 E+03$ & $6.9 \mathrm{E}+04$ & $6.9 E+03$ & $2.5 E+04$ & $2.5 \mathrm{E}+02$ & $4.9 \mathrm{E}+03$ & $4.9 E+02$ \\
\hline Dinitrotoluene, 2,6- & $000606-20-2$ & 2.7E+04 & $2.7 \mathrm{E}+02$ & $2.7 E+04$ & $2.7 \mathrm{E}+03$ & & & & & $3.5 \mathrm{E}+05$ & $3.5 \mathrm{E}+03$ & $3.4 E+05$ & $3.4 \mathrm{E}+04$ & $2.5 E+04$ & $2.5 \mathrm{E}+02$ & $2.5 \mathrm{E}+04$ & $2.5 \mathrm{E}+03$ \\
\hline $\begin{array}{l}\text { Dinitrotoluene, 2-Amino- } \\
\text { 4,6- }\end{array}$ & $035572-78-2$ & & & & & & & & & & & & & & & & \\
\hline Dinoseb & $000088.85-7$ & & & $2.7 \mathrm{E}+03$ & $2.7 \mathrm{E}+02$ & & & & & & & $2.0 \mathrm{E}+04$ & $2.0 \mathrm{E}+03$ & & & $2.4 \mathrm{E}+03$ & $2.4 E+02$ \\
\hline Dioxsлe, 1,4- & $000123.91-1$ & $1.0 \mathrm{E}+06$ & $1.7 \mathrm{E}+04$ & & & & & & & $1.0 E+06$ & $2.1 \mathrm{E}+05$ & & & $1.0 \mathrm{E}+06$ & $1.6 \mathrm{E}+04$ & & \\
\hline Diphenamid & $000957.51-7$ & & & $8.0 \mathrm{E}+04$ & $8.0 \mathrm{E}+03$ & & & & & & & $6.1 E+05$ & $6.1 E+04$ & & & $7.1 \mathrm{E}+04$ & $7.1 \mathrm{E}+03$ \\
\hline Diphenylamine & $000122-39-4$ & & & $6.7 E+04$ & $6.7 E+03$ & & & & & & & $5.1 E+05$ & $5.1 \mathrm{E}+04$ & & & $5.9 E+04$ & $5.9 E+03$ \\
\hline Diphenylhydrazine, 1,2- & $000122-66-7$ & $2.3 \mathrm{E}+04$ & $2.3 \mathrm{E}+02$ & & & $1.0 \mathrm{E}+06$ & $1.0 \mathrm{E}+06$ & & & $1.8 E+05$ & $1.8 E+03$ & & & $2.1 E+04$ & $2.1 E+02$ & & \\
\hline Diquat & $000085-00-7$ & & & $5.9 \mathrm{E}+03$ & $5.9 \mathrm{E}+02$ & & & & & & & 4.4E+04 & $4.4 E+03$ & & & $5.2 \mathrm{E}+03$ & $5.2 \mathrm{E}+02$ \\
\hline Direct Black 38 & $001937-37-7$ & $2.2 E+03$ & $2.2 \mathrm{E}+01$ & & & & & & & $1.6 \mathrm{E}+04$ & $1.6 \mathrm{E}+02$ & & & $1.9 E+03$ & $1.9 \mathrm{E}+01$ & & \\
\hline Direct Blue 6 & $002602-46-2$ & $2.3 E+03$ & $2.3 E+01$ & & & & & & & $1.7 \mathrm{E}+04$ & $1.7 E+02$ & & & $2.0 E+03$ & $2.0 E+01$ & & \\
\hline Direct Brown 95 & $016071-86-6$ & $2.0 \mathrm{E}+03$ & $2.0 E+01$ & & & & & & & 1.5E+04 & $1.5 E+02$ & & & $1.8 \mathrm{E}+03$ & $1.8 \mathrm{E}+01$ & & \\
\hline Direet Sky Blue & $002610-05-1$ & & & & & & & & & & & & & & & & \\
\hline Disulfoton & $000298-04-4$ & & & 1.1. E+02 & 1.1E+01 & & & & & & & $8.1 E+02$ & $8.1 E+01$ & & & $9.4 E+01$ & $9.4 E+00$ \\
\hline
\end{tabular}

Nonradionuclides/soil/excavation

Page 13 of 32 
Table 3.5a. (continued)

\begin{tabular}{|c|c|c|c|c|c|c|c|c|c|c|c|c|c|c|c|c|c|}
\hline \multirow[t]{2}{*}{ Chemical } & \multirow[t]{2}{*}{ CASRN } & \multicolumn{4}{|c|}{ Ingestion (mg/kg) } & \multicolumn{4}{|c|}{ Inhalation (mg/kg) } & \multicolumn{4}{|c|}{ Dermal (mg/kg) } & \multicolumn{4}{|c|}{$\begin{array}{l}\text { Ingestion + Inhalation + } \\
\text { Dermal (mg/kg) })^{+\vee v}\end{array}$} \\
\hline & & $10^{-4}$ & $10^{4}$ & $\mathrm{HQ}=1$ & $\mathrm{HQ}=0.1$ & $10^{-4}$ & $10^{6}$ & $H Q=1$ & $\mathrm{HQ}=0.1$ & $10^{-4}$ & $10^{4}$ & $H Q=1$ & $\mathrm{HQ}=0.1$ & $10^{-4}$ & $10^{4}$ & $\mathrm{HI}=1$ & $\mathrm{HI}=0.1$ \\
\hline Diuron & $000330-54-1$ & & & $5.3 \mathrm{E}+03$ & $5.3 E+02$ & & & & & & & $4.0 \mathrm{E}+04$ & $4.0 \mathrm{E}+03$ & & & $4.7 E+03$ & $4.7 \mathrm{E}+02$ \\
\hline Dodine & $002439-10-3$ & & & $1.1 E+04$ & $1.1 \mathrm{E}+03$ & & & & & & & $8.1 E+04$ & $8.1 E+03$ & & & $9.4 \mathrm{E}+03$ & $9.4 E+02$ \\
\hline EPTC & $000759.94-4$ & & & $6.7 \mathrm{E}+04$ & $6.7 \mathrm{E}+03$ & & & & & & & $5.1 \mathrm{E}+05$ & 5.1E+04 & & & $5.9 \mathrm{E}+04$ & $5.9 E+03$ \\
\hline Endosulfan & $000115-29-7$ & & & $1.6 \mathrm{E}+04$ & $1.6 \mathrm{E}+03$ & & & & & & & $1.2 \mathrm{E}+05$ & $1.2 E+04$ & & & $1.4 \mathrm{E}+04$ & $1.4 \mathrm{E}+03$ \\
\hline Endothall & $000145-73-3$ & & & $5.3 \mathrm{E}+04$ & $5.3 \mathrm{E}+03$ & & & & & & & $4.0 \mathrm{E}+05$ & $4.0 \mathrm{E}+04$ & & & 4. $7 E+04$ & $4.7 E+03$ \\
\hline Endrin & $000072-20-8$ & & & $8.0 \mathrm{E}+02$ & $8.0 E+01$ & & & & & & & $2.4 \mathrm{E}+02$ & $2.4 E+01$ & & & $1.9 \mathrm{E}+02$ & $1.9 \mathrm{E}+01$ \\
\hline Epiehtorohydrin & $000106-89.8$ & $1.0 \mathrm{E}+06$ & $1.9 \mathrm{E}+04$ & $5.3 \mathrm{E}+03$ & $5.3 \mathrm{E}+02$ & $3.2 E+03$ & $3.2 E+03$ & $3.2 E+03$ & $1.7 \mathrm{E}+03$ & $1.0 \mathrm{E}+06$ & $2.3 \mathrm{E}+05$ & $6.5 \mathrm{E}+04$ & $6.5 \mathrm{E}+03$ & $3.2 \mathrm{E}+03$ & $3.2 \mathrm{E}+03$ & $3.2 \mathrm{E}+03$ & $3.8 \mathrm{E}+02$ \\
\hline Epoxybutane, 1,2- & $000106-88-7$ & & & & & & & $1.0 \mathrm{E}+06$ & $1.0 \mathrm{E}+06$ & & & & & & & & \\
\hline Ethephon & $016672-87-0$ & & & $1.3 \mathrm{E}+04$ & $1.3 \mathrm{E}+03$ & & & & & & & $1.0 E+05$ & $1.0 E+04$ & & & $1.2 \mathrm{E}+04$ & $1.2 \mathrm{E}+03$ \\
\hline Ethion & $000563-12-2$ & & & $1.3 \mathrm{E}+03$ & $1.3 \mathrm{E}+02$ & & & & & & & $1.0 \mathrm{E}+04$ & $1.0 \mathrm{E}+03$ & & & $1.2 \mathrm{E}+03$ & $1.2 E+02$ \\
\hline Ethoxyethanol Acetate, 2- & $000111-15-9$ & & & $8.0 E+05$ & $8.0 \mathrm{E}+04$ & & & & & & & $1.0 \mathrm{E}+06$ & 6.1E+05 & & & $7.1 \mathrm{E}+05$ & $7.1 E+04$ \\
\hline Ethoxyethanol, 2 - & $000110-80-5$ & & & $1.0 \mathrm{E}+06$ & $1.3 E+05$ & & & $1.0 E+06$ & $1.0 \mathrm{E}+06$ & & & $1.0 \mathrm{E}+06$ & $1.0 \mathrm{E}+06$ & & & $1.0 \mathrm{E}+06$ & $1.2 \mathrm{E}+05$ \\
\hline Ethyl Acetate & $000141-78-6$ & & & $1.0 \mathrm{E}+06$ & $1.0 E+06$ & & & & & & & $1.0 E+06$ & $1.0 \mathrm{E}+06$ & & & $1.0 \mathrm{E}+06$ & $1.0 \mathrm{E}+06$ \\
\hline Ethyl Acryiatc & $000140-88-5$ & $3.9 E+05$ & $3.9 \mathrm{E}+03$ & & & & & & & $1.0 \mathrm{E}+06$ & $2.9 \mathrm{E}+04$ & & & $3.4 E+05$ & $3.4 \mathrm{E}+03$ & & \\
\hline Ethyl Chloride & $000075-00-3$ & & & & & & & $1.5 \mathrm{E}+02$ & $1.5 \mathrm{E}+02$ & & & & & & & & \\
\hline Ethyl Ether & $000060-29-7$ & & & $1.0 \mathrm{E}+06$ & $5.3 \mathrm{E}+05$ & & & & & & & $1.0 E+06$ & $1.0 \mathrm{E}+06$ & & & $1.0 \mathrm{E}+06$ & $4.9 \mathrm{E}+05$ \\
\hline Ethyl Methactylate & $000097-63-2$ & & & $2.4 E+05$ & $2.4 E+04$ & & & & & & & $1.0 E+06$ & $2.9 \mathrm{E}+05$ & & & 2.2E+05 & $2.2 \mathrm{E}+04$ \\
\hline $\begin{array}{l}\text { Ethyl-p.pitrophenyl } \\
\text { Phosphionate }\end{array}$ & $002104-64-5$ & & & $2.7 E+01$ & $2.7 E+00$ & & & & & & & $2.0 E+02$ & $2.0 \mathrm{E}+01$ & & & $2.4 E+01$ & $2.4 E+\infty$ \\
\hline Ethylbenzene ${ }^{19}$ & $000100-41-4$ & & & $2.6 \mathrm{E}+05$ & $2.6 \mathrm{E}+04$ & & & 4.TE-01 & 4.7E-01 & & & $1.0 \mathrm{E}+06$ & $3.8 \mathrm{E}+05$ & & & 4.7E-01 & 4.7E-01 \\
\hline Ethylene Cyanohydrin & $000109-78-4$ & & & $8.0 E+05$ & $8.0 E+04$ & & & & & & & $1.0 \mathrm{E}+06$ & $6.1 \mathrm{E}+05$ & & & $7.1 \mathrm{E}+05$ & $7.1 E+04$ \\
\hline Ethylene Diamine & $000107-15-3$ & & & $5.3 \mathrm{E}+0 \mathrm{~S}$ & $5.3 \mathrm{E}+04$ & & & & & & & $1.0 \mathrm{E}+06$ & $4.0 E+05$ & & & 4.7E+05 & $4.7 E+04$ \\
\hline Ethylene Glycol & $000107-21-1$ & & & $1.0 \mathrm{E}+06$ & 5.3E+05 & & & & & & & $1.0 \mathrm{E}+06$ & $1.0 \mathrm{E}+06$ & & & $1.0 E+06$ & 4.7E+0S \\
\hline
\end{tabular}


Table 3.5a. (continued)

\begin{tabular}{|c|c|c|c|c|c|c|c|c|c|c|c|c|c|c|c|c|c|}
\hline \multirow[t]{2}{*}{ Chemical } & \multirow[t]{2}{*}{ CASRN } & \multicolumn{4}{|c|}{ Ingestion (mg/kg) } & \multicolumn{4}{|c|}{ Inhalation (mg/kg) ${ }^{-\gamma}$} & \multicolumn{4}{|c|}{ Dermal (mg/kg)" } & \multicolumn{4}{|c|}{$\begin{array}{c}\text { Ingestion + Inhalation + } \\
\text { Dermal (mg/kg) }\end{array}$} \\
\hline & & $10^{-4}$ & $10^{4}$ & $H Q=1$ & $\mathrm{HQ}=0.1$ & $10^{-4}$ & $10^{4}$ & $H Q=1$ & $\mathrm{HQ}=0.1$ & $10^{-4}$ & $10^{4}$ & $H Q=1$ & $\mathrm{HQ}=0.1$ & $10^{-4}$ & $10^{5}$ & $\mathrm{HI}=1$ & $\mathrm{HI}=0.1$ \\
\hline $\begin{array}{l}\text { Ethyiene Glycol } \\
\text { Monobuty Ether }\end{array}$ & $000111-76-2$ & & & & & & & $1.0 \mathrm{E}+06$ & $1.0 \mathrm{E}+06$ & & & & & & & & \\
\hline Ethylene Oxide & $000075-21-8$ & $1.8 \mathrm{E}+04$ & $1.8 \mathrm{E}+02$ & & & $2.5 \mathrm{E}+04$ & $2.5 \mathrm{E}+02$ & & & $2.2 \mathrm{E}+05$ & $2.2 E+03$ & & & $1.0 \mathrm{E}+04$ & $1.0 \mathrm{E}+02$ & & \\
\hline Ethylene Thiourca & 000096.45 .7 & $1.7 E+05$ & $1.7 E+03$ & $2.1 E+02$ & 2. $I E+01$ & & & & & $1.0 \mathrm{E}+06$ & $1.3 \mathrm{E}+04$ & $1.6 \mathrm{E}+03$ & $1.6 \mathrm{E}+02$ & $1.5 \mathrm{E}+05$ & $1.5 E+03$ & $1.9 \mathrm{E}+02$ & $1.9 \mathrm{E}+01$ \\
\hline $\begin{array}{l}\text { Ethylphthaly' Ethyl } \\
\text { Glycolute }\end{array}$ & $000084-72-0$ & & & $1.0 \mathrm{E}+06$ & $8.0 E+05$ & & & & & & & $1.0 \mathrm{E}+06$ & $1.0 \mathrm{E}+06$ & & & $1.0 \mathrm{E}+06$ & $7.1 E+05$ \\
\hline Express & $101200-48-0$ & & & $2.1 E+04$ & 2.1E+03 & & & & & & & $1.6 \mathrm{E}+05$ & $1.6 \mathrm{E}+04$ & & & $1.9 \mathrm{E}+04$ & $1.9 E+03$ \\
\hline Fenamiphos & $022224-92-6$ & & & $6.7 E+02$ & $6.7 \mathrm{E}+01$ & & & & & & & S.1E+03 & $5.1 \mathrm{E}+02$ & & & $5.9 \mathrm{E}+02$ & $5.9 E+01$ \\
\hline Fenpropathrin & $039515-41-8$ & & & $6.7 \mathrm{E}+04$ & $6.7 \mathrm{E}+03$ & & & & & & & $5.1 E+0 S$ & $5.1 \mathrm{E}+04$ & & & $5.9 \mathrm{E}+04$ & $5.9 \mathrm{E}+03$ \\
\hline Fluometuron & $002164-17-2$ & & & $3.5 \mathrm{E}+04$ & $3.5 \mathrm{E}+03$ & & & & & & & $2.6 \mathrm{E}+05$ & $2.6 \mathrm{E}+04$ & & & $3.1 E+04$ & $3.1 \mathrm{E}+03$ \\
\hline Fluoranthene & $000206-44-0$ & & & $1.0 \mathrm{E}+06$ & 1. $1 E+05$ & & & & & & & $1.0 \mathrm{E}+06$ & $5.0 \mathrm{E}+05$ & & & $8,8 \mathrm{E}+05$ & $8.8 E+04$ \\
\hline Fluorene & $000086-73.7$ & & & $1.0 \mathrm{E}+06$ & $1.1 \mathrm{E}+05$ & & & & & & & $1.0 E+06$ & $8.1 E+05$ & & & $9.4 \mathrm{E}+05$ & $9.4 E+04$ \\
\hline Fluoride & $007782-41-4$ & & & $1.6 \mathrm{E}+05$ & $1.6 E+04$ & & & & & & & $1.0 \mathrm{E}+06$ & $1.0 \mathrm{E}+06$ & & & $1.6 \mathrm{E}+05$ & $1.6 \mathrm{E}+04$ \\
\hline Fluridone & $059756-60-4$ & & & 2.1E+05 & 2.1E+04 & & & & & & & $1.0 \mathrm{E}+06$ & $1.6 \mathrm{E}+05$ & & & $1.9 E+05$ & $1.9 \mathrm{E}+04$ \\
\hline Flurprimidol & $056425-91-3$ & & & $5.3 E+04$ & $5.3 \mathrm{E}+03$ & & & & & & & $4.0 E+05$ & $4.0 \mathrm{E}+04$ & & & 4.7E+04 & 4. $7 \mathrm{E}+03$ \\
\hline Flutolunil & $066332-96-5$ & & & $1.6 \mathrm{E}+05$ & $1.6 \mathrm{E}+04$ & & & & & & & $1.0 \mathrm{E}+06$ & $1.2 \mathrm{E}+05$ & & & $1.4 E+05$ & $1.4 \mathrm{E}+04$ \\
\hline Fluvalinate & $069409.94-5$ & & & $2.7 \mathrm{E}+04$ & $2.7 E+03$ & & & & & & & $2.0 \mathrm{E}+0 \mathrm{~S}$ & $2.0 E+04$ & & & $2.4 \mathrm{E}+04$ & $2.4 \mathrm{E}+03$ \\
\hline Foipet & $000133.07-3$ & $1.0 \mathrm{E}+06$ & $5.3 E+04$ & $2.7 \mathrm{E}+0 \mathrm{~S}$ & 2.7E+04 & & & & & $1.0 \mathrm{E}+06$ & 4.0E+05 & $1.0 \mathrm{E}+06$ & $2.0 \mathrm{E}+0 \mathrm{~S}$ & $1.0 \mathrm{E}+06$ & $4.7 E+04$ & $2.4 \mathrm{E}+05$ & $2.4 \mathrm{E}+04$ \\
\hline Fomessafen & $072178-02-0$ & $9.8 \mathrm{E}+04$ & $9.8 E+02$ & & & & & & & $7.4 \mathrm{E}+05$ & $7.4 E+03$ & & & $8.7 E+04$ & $8.7 \mathrm{E}+02$ & & \\
\hline Fonofos & $000944-22.9$ & & & $5.3 \mathrm{E}+03$ & $5.3 \mathrm{E}+02$ & & & & & & & $4.0 \mathrm{E}+04$ & $4.0 E+03$ & & & 4.7E+03 & 4.7E+02 \\
\hline Formaldehyde & $000050-00-0$ & & & $5.3 \mathrm{E}+05$ & $5.3 \mathrm{E}+04$ & $1.0 \mathrm{E}+06$ & $1,0 \mathrm{E}+06$ & & & & & $1.0 \mathrm{E}+06$ & $6.5 E+05$ & & & $4.9 E+05$ & $4.9 E+04$ \\
\hline Formic Acid & $000064 \cdot 18-6$ & & & $1.0 \mathrm{E}+06$ & $5.3 \mathrm{E}+05$ & & & & & & & $1.0 \mathrm{E}+06$ & $1.0 \mathrm{E}+06$ & & & $1.0 \mathrm{E}+06$ & 4.7E+05 \\
\hline Fosetyl-AL & $039148-24-8$ & & & $1.0 E+06$ & $8.0 E+05$ & & & & & & & $1.0 \mathrm{E}+06$ & $1.0 \mathrm{E}+06$ & & & $1.0 E+06$ & $7.1 E+05$ \\
\hline Furan & $000110-00-9$ & & & $2.7 E+04$ & $2.7 E+03$ & & & & & & & $2.0 E+05$ & $2.0 E+04$ & & & $2.4 E+04$ & $2.4 E+03$ \\
\hline
\end{tabular}

Nonradionuclides/soil/excavation

Page 15 of 32 
Table 3.5a. (continued)

\begin{tabular}{|c|c|c|c|c|c|c|c|c|c|c|c|c|c|c|c|c|c|}
\hline \multirow[t]{2}{*}{ Chemical } & \multirow{2}{*}{$\begin{array}{c}\text { CASRN } \\
\cdot\end{array}$} & \multicolumn{4}{|c|}{ Ingestion (mg/kg) } & \multicolumn{4}{|c|}{ Inbalation (mg/kg) } & \multicolumn{4}{|c|}{ Dermal (mg/kg) } & \multicolumn{4}{|c|}{$\begin{array}{c}\text { Ingestion }+ \text { Inhalation + } \\
\text { Dermal (mg/kg) })^{+\vee}\end{array}$} \\
\hline & & $10^{-1}$ & $10^{4}$ & $H Q=1$ & $\mathrm{HQ}=0.1$ & $10^{-4}$ & $10^{4}$ & $H Q=1$ & $\mathrm{HQ}=0.1$ & $10^{-4}$ & $10^{6}$ & $H Q=1$ & $\mathrm{HQ}=0.1$ & $10^{4}$ & $10^{4}$ & $H I=1$ & $H I=0.1$ \\
\hline Furazolidone & $000067-45-8$ & $4.9 \mathrm{E}+03$ & $4.9 \mathrm{E}+01$ & & & & & & & 3.TE+04 & 3.7E+02 & & & $4.3 \mathrm{E}+03$ & $4.3 \mathrm{E}+01$ & & \\
\hline Furfural & $000098.01-1$ & & & $8.0 \mathrm{E}+04$ & $8.0 \mathrm{E}+03$ & & & $1.0 E+06$ & $1.0 E+06$ & & & $6.1 E+05$ & 6. $1 E+04$ & & & $7.1 E+04$ & $7.1 E+03$ \\
\hline Furium & $000531-82-8$ & $3.7 \mathrm{E}+02$ & $3.7 \mathrm{E}+00$ & & & & & & & $2.8 E+03$ & $2.8 E+01$ & & & $3.3 \mathrm{E}+02$ & $3.3 \mathrm{E}+00$ & & \\
\hline Furmecyclox & $060568-05-0$ & $6.2 \mathrm{E}+05$ & $6.2 E+03$ & & & & & & & $1.0 \mathrm{E}+06$ & $4.7 \mathrm{E}+04$ & & & $5.5 \mathrm{E}+05$ & $5.5 \mathrm{E}+03$ & & \\
\hline Glufosinate, Ammonium & $077182-82-2$ & & & $1.1 \mathrm{E}+03$ & $1.1 \mathrm{E}+02$ & & & & & & & $8.1 E+03$ & 8.1E+02 & & & $9.4 E+02$ & $9.4 \mathrm{E}+01$ \\
\hline Glycidyl & $000765-34-4$ & & & $1.1 \mathrm{E}+04$ & $1.1 E+03$ & & & $1.0 \mathrm{E}+06$ & $1.0 \mathrm{E}+06$ & & & $8.1 \mathrm{E}+04$ & $8.1 E+03$ & & & $9.4 E+03$ & $9.4 E+02$ \\
\hline Glyphosate & $001071-83-6$ & & & $2.7 \mathrm{E}+05$ & $2.7 \mathrm{E}+04$ & & & & & & & $1.0 \mathrm{E}+06$ & $2.0 E+05$ & & & $2.4 E+05$ & $2.4 \mathrm{E}+04$ \\
\hline God & 042874-03-3 & & & $8.0 \mathrm{E}+03$ & $8.0 \mathrm{E}+02$ & & & & & & & $6.1 E+04$ & $6.1 E+03$ & & & 7.1E+03 & 7.1E+02 \\
\hline Haloxyfop, Methyl & $069806-40-2$ & & & $1.3 \mathrm{E}+02$ & $1.3 \mathrm{E}+01$ & & & & & & & $1.0 \mathrm{E}+03$ & $1.0 E+02$ & & & $1.2 \mathrm{E}+02$ & $1.2 \mathrm{E}+01$ \\
\hline Harmony & $079277-27-3$ & & & $3.5 E+04$ & $3.5 \mathrm{E}+03$ & & & & & & & $2.6 \mathrm{E}+05$ & $2.6 \mathrm{E}+04$ & & & 3.1E+04 & $3.1 \mathrm{E}+03$ \\
\hline Heptachlor & $000076-44-8$ & $4.1 \mathrm{E}+03$ & 4.1E+01 & $1.3 \mathrm{E}+03$ & $1.3 \mathrm{E}+02$ & $1.0 \mathrm{E}+06$ & $1.0 \mathrm{E}+06$ & & & $4.5 \mathrm{E}+04$ & $4.5 \mathrm{E}+02$ & $1.5 \mathrm{E}+04$ & $1.5 \mathrm{E}+03$ & $3.8 \mathrm{E}+03$ & $3.8 \mathrm{E}+01$ & $1.2 \mathrm{E}+03$ & $1.2 \mathrm{E}+02$ \\
\hline Heptachlor Epoxide & $001024-57-3$ & $2.0 \mathrm{E}+03$ & $2.0 E+01$ & $3.5 \mathrm{E}+01$ & $3.5 \mathrm{E}+00$ & $1.0 \mathrm{E}+06$ & $1.0 \mathrm{E}+06$ & & & $2.2 \mathrm{E}+04$ & $2.2 E+02$ & $3.8 \mathrm{E}+02$ & $3.8 \mathrm{E}+01$ & $1.9 E+03$ & $1.9 \mathrm{E}+01$ & $3.2 \mathrm{E}+01$ & $3.2 \mathrm{E}+00$ \\
\hline Heptane, N. & $000142-82-5$ & & & & & & & & & & & & & & & & \\
\hline Hexabromobenzene & $000087-82-1$ & & & $5.3 \mathrm{E}+04$ & $5.3 \mathrm{E}+03$ & & & & & & & $4.0 \mathrm{E}+05$ & $4.0 E+04$ & & & 4.7E+04 & 4. TE+03 \\
\hline Hexachlorobenzene ${ }^{(9)}$ & $000118-74-1$ & $1.2 \mathrm{E}+04$ & $1.2 \mathrm{E}+02$ & $2.1 \mathrm{E}+03$ & $2.1 \mathrm{E}+02$ & $1.0 \mathrm{E}+06$ & $1.0 \mathrm{E}+06$ & & & $1.4 E+05$ & $1.4 \mathrm{E}+03$ & $2.6 \mathrm{E}+04$ & $2.6 \mathrm{E}+03$ & $1.1 \mathrm{E}+04$ & $1.1 \mathrm{E}+02$ & $2.0 \mathrm{E}+03$ & $2.0 E+02$ \\
\hline Hexachlorobutadiene ${ }^{(\theta)}$ & $000087-68-3$ & $2.4 E+05$ & $2.4 \mathrm{E}+03$ & $1.8 \mathrm{E}+03$ & $1.8 \mathrm{E}+02$ & $1.0 \mathrm{E}+06$ & $1.0 \mathrm{E}+06$ & & & $1.0 \mathrm{E}+06$ & $2.9 \mathrm{E}+04$ & $1.4 E+04$ & $1.4 E+03$ & $2.2 \mathrm{E}+05$ & $2.2 \mathrm{E}+03$ & $1.6 \mathrm{E}+03$ & $1.6 \mathrm{E}+02$ \\
\hline $\begin{array}{l}\text { Hexachlorocyciohexane, } \\
\text { Nphas- }\end{array}$ & $000319-84-6$ & $3.0 \mathrm{E}+03$ & $3.0 \mathrm{E}+01$ & & & $1.0 \mathrm{E}+06$ & $1.0 \mathrm{E}+06$ & & & $4.4 \mathrm{E}+04$ & $4.4 \mathrm{E}+02$ & & & $2.8 \mathrm{E}+03$ & $2.8 \mathrm{E}+01$ & & \\
\hline $\begin{array}{l}\text { Hexachlorocyclohexane, } \\
\text { Beta- }\end{array}$ & 000319.85 .7 & $1.0 \mathrm{E}+04$ & $1.0 \mathrm{E}+02$ & & & $1.0 \mathrm{E}+06$ & $1.0 \mathrm{E}+06$ & & & $1.4 \mathrm{E}+05$ & $1.4 \mathrm{E}+03$ & & & $9.7 \mathrm{E}+03$ & $9.7 E+01$ & & \\
\hline $\begin{array}{l}\text { Hexachtorocyclohexane, } \\
\text { Delia- }\end{array}$ & 000319.86 .8 & & & & & & & & & & & & & & & & \\
\hline $\begin{array}{l}\text { Hexacthorocyclohexane, } \\
\text { Epsilon }\end{array}$ & $006108-10.7$ & & & & & & & & & & & & & & & & \\
\hline $\begin{array}{l}\text { Hexachlorocyclohexane, } \\
\text { Gamma- }\end{array}$ & $000058-89-9$ & $1.4 E+04$ & $1.4 E+02$ & $8.0 E+03$ & $8.0 \mathrm{E}+02$ & & & & & $2.1 E+05$ & $2.1 E+03$ & $1.2 \mathrm{E}+05$ & $1.2 \mathrm{E}+04$ & $1.3 \mathrm{E}+04$ & $1.3 E+02$ & $7.5 E+03$ & $7.5 E+02$ \\
\hline
\end{tabular}

Nonradionuclides/soil/excavation

Page 16 of 32 
Table 3.5a. (continued)

\begin{tabular}{|c|c|c|c|c|c|c|c|c|c|c|c|c|c|c|c|c|c|}
\hline \multirow[t]{2}{*}{ Chemical } & \multirow[t]{2}{*}{ CASRN } & \multicolumn{4}{|c|}{ Ingestion (mg/kg) } & \multicolumn{4}{|c|}{ Inhalation (mg/kg) } & \multicolumn{4}{|c|}{ Dermal (mg/kg)" } & \multicolumn{4}{|c|}{$\begin{array}{c}\text { Ingestion + Inhalation + } \\
\text { Dermal (mg/kg) }\end{array}$} \\
\hline & & $10^{-4}$ & $10^{4}$ & $H Q=1$ & $\mathrm{HQ}=0.1$ & $10^{-4}$ & $10^{6}$ & $\mathbf{H Q}=\mathbf{I}$ & $\mathrm{HQ}=0.1$ & $10^{-4}$ & $10^{4}$ & $H Q=1$ & $\mathrm{HQ}=0.1$ & $10^{-4}$ & $10^{4}$ & $H I=1$ & $\mathrm{HI}=0.1$ \\
\hline $\begin{array}{l}\text { Hexachlorocyclohexane, } \\
\text { Technical }\end{array}$ & $000608-73-1$ & $1.0 E+04$ & $1.0 \mathrm{E}+02$ & & & $1.0 \mathrm{E}+06$ & $1.0 E+06$ & & & $7.9 \mathrm{E}+04$ & $7.9 \mathrm{E}+02$ & & & $9.1 E+03$ & $9.1 E+01$ & & \\
\hline $\begin{array}{l}\text { Hexachlorocyclopentudien } \\
\text { e }\end{array}$ & $000077-47-4$ & & & $1.9 E+05$ & $1.9 E+04$ & & & $1.0 \mathrm{E}+06$ & $1.0 \mathrm{E}+06$ & & & $1.0 \mathrm{E}+06$ & $1.4 \mathrm{E}+05$ & & & $1.6 \mathrm{E}+05$ & $1.6 \mathrm{E}+04$ \\
\hline $\begin{array}{l}\text { Hexachlorodibenzo-p- } \\
\text { dioxin, Mixture }\end{array}$ & $019408-74-3$ & $3.0 \mathrm{E}+00$ & $3.0 \mathrm{E}-02$ & & & $4.2 \mathrm{E}+05$ & $4.2 \mathrm{E}+03$ & & & 2.3E+01 & $2.3 E-01$ & & & $2.7 \mathrm{E}+00$ & 2.7E-02 & & \\
\hline Hexachloroethane & $000067-72-1$ & $1.0 E+06$ & $1.3 \mathrm{E}+04$ & $2.7 E+04$ & $2.7 \pm+03$ & $1.0 \mathrm{E}+06$ & $1.0 \mathrm{E}+06$ & & & $1.0 \mathrm{E}+06$ & $1.6 \mathrm{E}+05$ & $2.0 E+05$ & $2.0 \mathrm{E}+04$ & $1.0 \mathrm{E}+06$ & $1.2 E+04$ & $2.4 E+04$ & $2.4 E+03$ \\
\hline Hexachlorophene & $000070-30-4$ & & & $8.0 \mathrm{E}+03$ & $8.0 \mathrm{E}+02$ & & & & & & & $6.1 E+04$ & $6.1 E+03$ & & & $7,1 \mathrm{E}+03$ & $7.1 \mathrm{E}+02$ \\
\hline $\begin{array}{l}\text { Hexahydro-1,3,5-trinitro- } \\
\text { 1,3,5-5rikine (RDX) }\end{array}$ & $000121-82-4$ & $1.7 E+05$ & 1.7E+03 & $8.0 E+03$ & $8.0 \mathrm{E}+02$ & & & & & $1.0 \mathrm{E}+06$ & $2.6 \mathrm{E}+04$ & $1.2 \mathrm{E}+05$ & $1.2 \mathrm{E}+04$ & $1.6 \mathrm{E}+05$ & $1.6 \mathrm{E}+03$ & 7.5E+03 & 7.5E+02 \\
\hline $\begin{array}{l}\text { Hexamethylene } \\
\text { Ditsocyanate, 1,6- }\end{array}$ & $000822-06-0$ & & & & & & & $7.8 \mathrm{E}+05$ & $7.8 \mathrm{E}+04$ & & & & & & & & \\
\hline Hexane, N. & $000110-54-3$ & & & $1.0 \mathrm{E}+06$ & $1.6 \mathrm{E}+05$ & & & $9.1 E+01$ & $9.1 \mathrm{E}+01$ & & & $1.0 \mathrm{E}+06$ & $1.0 \mathrm{E}+06$ & & & $9.1 E+01$ & $9.1 E+01$ \\
\hline Hexanone, 2 - & $000591-78-6$ & & & & & & & & & & & & & & & & \\
\hline Hexarinone & $051235-04-2$ & & & $8.8 E+04$ & $8.8 \mathrm{E}+03$ & & & & & & & $6.7 \mathrm{E}+05$ & $6.7 \mathrm{E}+04$ & & & $7.8 \mathrm{E}+04$ & $7.8 \mathrm{E}+03$ \\
\hline $\mathrm{HpCDD}, 2,3,7,8$-(1) & $037871-00-4$ & $1.2 \mathrm{E}+01$ & $1,2 \mathrm{E}-01$ & & & $1.0 \mathrm{E}+06$ & $1.3 \mathrm{E}+04$ & & & $3.1 \mathrm{E}+01$ & $3.1 \mathrm{E}-01$ & & & $8.9 \mathrm{E}+00$ & 8.9E-02. & & \\
\hline $\mathrm{HpCDF}, 2,3,7,8(1)$ & 038998-75-3 & $1.2 \mathrm{E}+01$ & $1.2 \mathrm{E}-01$ & & & $1,0 \mathrm{E}+06$ & $1.3 \mathrm{E}+04$ & & & 9.4E+0! & $9.4 \mathrm{E}-01$ & & & $1.1 \mathrm{E}+01$ & t.1E-01 & & \\
\hline $\mathrm{HxCDD}, 2,3,7,8^{(1)}$ & $034465-46-8$ & $1.2 \mathrm{E}+00$ & $1.2 \mathrm{E}-02$ & & & $1.3 E+05$ & $1.3 \mathrm{E}+03$ & & & $3.1 E+\infty 0$ & $3.1 \mathrm{E}-02$ & & & 8.9E-01 & $8.9 \mathrm{E}-03$ & & \\
\hline$H x C D F, 2,3,7,8^{(1)}$ & $055684-94-1$ & $1.2 E+\infty 0$ & $1.2 \mathrm{E}-02$ & & & $1.3 \mathrm{E}+05$ & $1,3 \mathrm{E}+03$ & & & $9.4 \mathrm{E}+00$ & $9.4 \mathrm{E}-02$ & & & $1.1 \mathrm{E}+\infty$ & $1.1 \mathrm{E}-02$ & & \\
\hline Hydraxine & $000302-01-2$ & $6.2 E+03$ & $6.2 \mathrm{E}+01$ & & & $1.0 \mathrm{E}+06$ & $1.0 E+06$ & & & $4.7 \mathrm{E}+04$ & 4.7E+02 & & & $5.5 E+03$ & $5.5 \mathrm{E}+01$ & & \\
\hline Hydraxine Sulfate & $010034.93-2$ & $6.2 \mathrm{E}+03$ & $6.2 \mathrm{E}+01$ & & & $1.0 \mathrm{E}+06$ & $1.0 \mathrm{E}+06$ & & & 4.7E+04 & $4.7 E+02$ & & & $5.5 E+03$ & $5.5 \mathrm{E}+01$ & & \\
\hline Hydrogen Chloride & $007647-01.0$ & & & & & & & $1.0 \mathrm{E}+06$ & $1.0 \mathrm{E}+06$ & & & & & & & & \\
\hline Hydrogen Cyanide & $000074-90-8$ & & & $5.3 E+04$ & $5.3 \mathrm{E}+03$ & & & $1.0 \mathrm{E}+06$ & $1.0 \mathrm{E}+06$ & & & $1.0 \mathrm{E}+06$ & $1.4 \mathrm{E}+05$ & & & $5,1 E+04$ & $5.1 \mathrm{E}+03$ \\
\hline Hydrogen Sulfide & $007783-06-4$ & & & $8.0 E+04$ & $8.0 \mathrm{E}+03$ & & & $1.0 \mathrm{E}+06$ & $1.0 \mathrm{E}+06$ & & & $1.0 \mathrm{E}+06$ & $2.4 \mathrm{E}+05$ & & & $7.7 E+04$ & $7.7 \mathrm{E}+03$ \\
\hline Hydroquinone & $000123-31.9$ & & & $1.0 \mathrm{E}+06$ & $1.1 E+05$ & & & & & & & $1.0 \mathrm{E}+06$ & $8.1 \mathrm{E}+05$ & & & $9.4 \mathrm{E}+05$ & $9.4 \mathrm{E}+04$ \\
\hline Imazalil & $035554-44-0$ & & & $3.5 \mathrm{E}+04$ & $3.5 \mathrm{E}+03$ & & & & & & & $2.6 \mathrm{E}+05$ & $2.6 \mathrm{E}+04$ & & & 3.1E+04 & $3.1 \mathrm{E}+03$ \\
\hline
\end{tabular}

Nonradionuclides/soil/excavation

Page 17 of 32 
Table 3.5a. (continued)

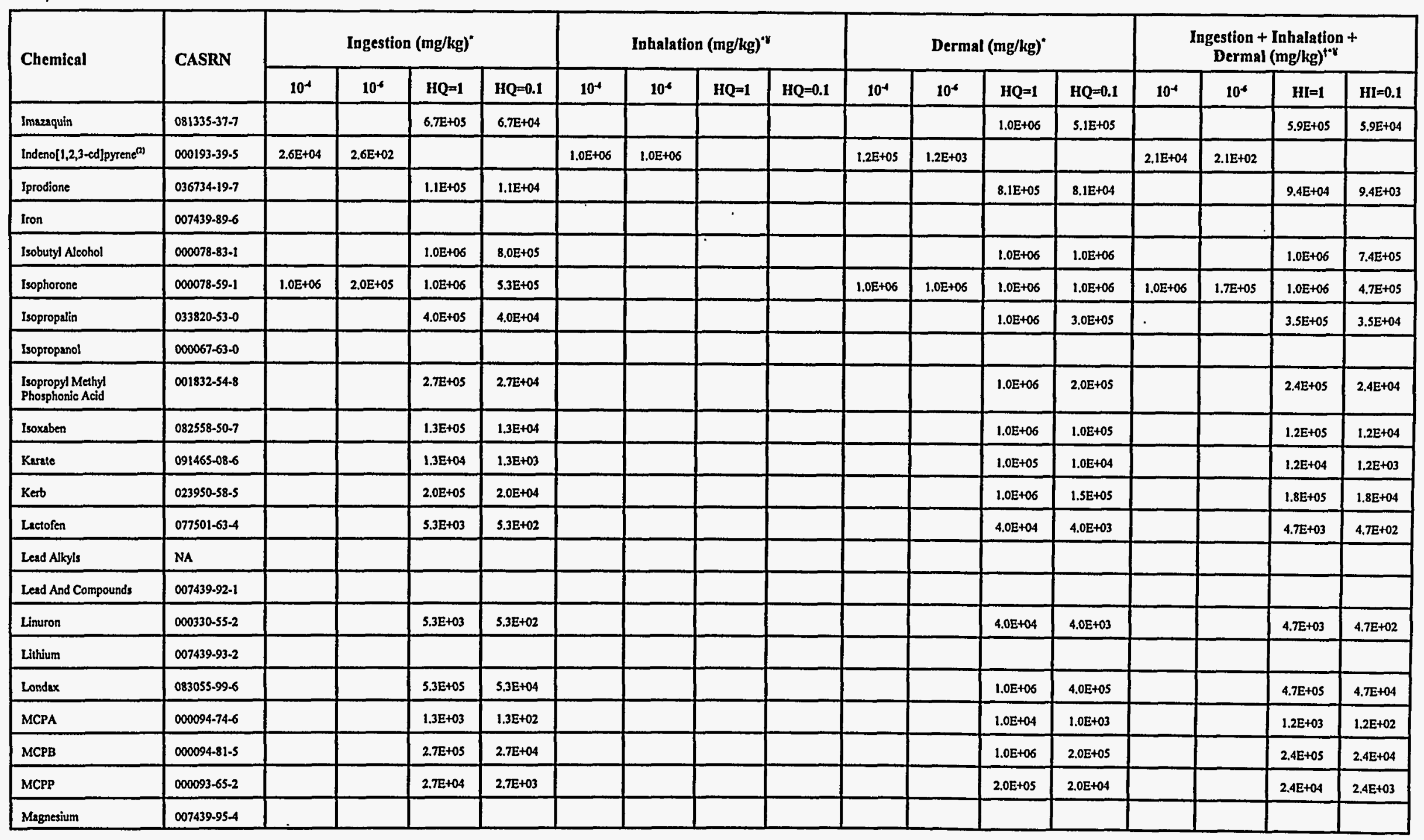


Table 3.5a. (continued)

\begin{tabular}{|c|c|c|c|c|c|c|c|c|c|c|c|c|c|c|c|c|c|}
\hline \multirow[t]{2}{*}{ Chemical } & \multirow[t]{2}{*}{ CASRN } & \multicolumn{4}{|c|}{ Ingestion (mg/kg) } & \multicolumn{4}{|c|}{ Inhalation (mg/kg) } & \multicolumn{4}{|c|}{ Dermal (mg/kg) } & \multicolumn{4}{|c|}{$\begin{array}{l}\text { Ingestion + Inhalation + } \\
\text { Dermal (mg/kg) })^{* *}\end{array}$} \\
\hline & & $10^{-4}$ & $10^{-4}$ & $\mathrm{HQ}=1$ & $\mathrm{HQ}=0.1$ & $10^{4}$ & $10^{4}$ & $\mathrm{HQ}=1$ & $\mathrm{HQ}=0.1$ & $10^{-4}$ & $10^{5}$ & $H Q=1$ & $\mathrm{HQ}=0.1$ & $10^{-4}$ & $10^{6}$ & $\mathrm{HI}=1$ & $\mathrm{HI}=0.1$ \\
\hline Malathion & $000121-75-5$ & & & $5.3 E+04$ & $5.3 E+03$ & & & & & & & $4.0 \mathrm{E}+05$ & $4.0 \mathrm{E}+04$ & & & 4.7E+04 & $4.7 \mathrm{E}+03$ \\
\hline Maleic Anhydride & $000108-31-6$ & & & $2.7 \mathrm{E}+05$ & $2.7 E+04$ & & & & & & & $1.0 \mathrm{E}+06$ & $2.0 \mathrm{E}+05$ & & & $2.4 E+05$ & 2.4E+04 \\
\hline Maleic Hydrazide & $000123-33-1$ & & & $1.0 E+06$ & $1.3 E+05$ & & & & & & & $1.0 \mathrm{E}+06$ & $1.0 \mathrm{E}+06$ & & & $1.0 \mathrm{E}+06$ & $1.2 \mathrm{E}+05$ \\
\hline Malononitrile & $000109-77.3$ & & & $5.3 E+02$ & $5.3 E+01$ & & & & & & & $6.5 E+03$ & $6.5 \mathrm{E}+02$ & & & $4.9 \mathrm{E}+02$ & $4.9 \mathrm{E}+01$ \\
\hline Mancozeb & $008018-01-7$ & & & $8.0 E+04$ & $8.0 E+03$ & & & & & & & $6.1 \mathrm{E}+05$ & $6.1 \mathrm{E}+04$ & & & $7.1 E+04$ & $7.1 E+03$ \\
\hline Maneb & $012427-38-2$ & & & $1.3 E+05$ & $1.3 E+04$ & & & & & & & $1.0 \mathrm{E}+06$ & $1.0 \mathrm{E}+05$ & & & $1.2 \mathrm{E}+05$ & $1.2 \mathrm{E}+04$ \\
\hline Manganese (Water) & $007439-96-5$ & & & $1.3 E+04$ & $1.3 E+03$ & & & $1.0 \mathrm{E}+06$ & $3.9 E+05$ & & & $8,1 E+04$ & $8.1 E+03$ & & & $1.1 \mathrm{E}+04$ & $1.1 \mathrm{E}+03$ \\
\hline Mephosfolan & $000950-10-7$ & & & $2.4 E+03$ & $2.4 E+02$ & & & & & & & $1.8 \mathrm{E}+04$ & $1.8 \mathrm{E}+03$ & & & $2.1 E+03$ & $2.1 \mathrm{E}+02$ \\
\hline Mepiquat Chloride & $024307-26-4$ & & & $8.0 E+04$ & $8.0 E+03$ & & & & & & & $6.1 \mathrm{E}+05$ & $6.1 E+04$ & & & $7.1 E+04$ & $7.1 E+03$ \\
\hline Mercuric Chloride & $007487-94-7$ & & & $8.0 E+02$ & $8.0 E+01$ & & & & & & & $2.4 \mathrm{E}+04$ & $2.4 \mathrm{E}+03$ & & & $7.7 E+02$ & 7.7E+01 \\
\hline Mercury (elemental) & $007439-97-6$ & & & $8.0 \mathrm{E}+02$ & $8.0 E+01$ & & & $1,0 \mathrm{E}+06$ & $1.0 \mathrm{E}+06$ & & & $1.2 \mathrm{E}+01$ & $1.2 E+\infty$ & & & $1.2 \mathrm{E}+01$ & $1.2 \mathrm{E}+00$ \\
\hline Mercury, Inorganie Salt & $007439-97.6$ & & & $8.0 \mathrm{E}+02$ & $8.0 E+01$ & & & $1.0 \mathrm{E}+06$ & $1.0 E+06$ & & & $8.5 \mathrm{E}+03$ & $8.5 E+02$ & & & $7.3 \mathrm{E}+02$ & $7.3 E+01$ \\
\hline Merphos & $000150.50-5$ & & & $8.0 \mathrm{E}+02$ & $8.0 E+01$ & & & & & & & $6.1 \mathrm{E}+03$ & $6.1 E+02$ & & & $7.1 \mathrm{E}+02$ & $7,1 E+01$ \\
\hline Merphos Oxide & $000078-48-8$ & & & $8.0 \mathrm{E}+02$ & $8.0 \mathrm{E}+01$ & & & & & & & $6.1 \mathrm{E}+03$ & $6.1 E+02$ & & & $7.1 \mathrm{E}+02$ & $7.1 E+01$ \\
\hline Metaluxyl & $057837-19-1$ & & & $1.6 \mathrm{E}+05$ & $1.6 \mathrm{E}+04$ & & & & & & & $1.0 \mathrm{E}+06$ & $1.2 E+05$ & & & $1,4 E+05$ & $1.4 E+04$ \\
\hline Methacrylonitrile & $000126-98-7$ & & & $2.7 E+03$ & $2.7 \mathrm{E}+02$ & & & $1,2 E+04$ & 1.5E+03 & & & $3.2 \mathrm{E}+04$ & $3.2 \mathrm{E}+03$ & & & $2.1 E+03$ & 2.1E+02 \\
\hline Methamidophos & $010265-92-6$ & & & $1.3 \mathrm{E}+02$ & $1.3 \mathrm{E}+01$ & & & & & & & $1.0 E+03$ & $1.0 \mathrm{E}+02$ & & & $1.2 \mathrm{E}+02$ & $1.2 E+01$ \\
\hline Methanol & $000067-56-1$ & & & $1.0 E+06$ & $1.0 \mathrm{E}+06$ & & & & & & & $1.0 \mathrm{E}+06$ & $1.0 \mathrm{E}+06$ & & & $1.0 E+06$ & $1.0 \mathrm{E}+06$ \\
\hline Methidathion & $000950-37.8$ & & & $2.7 \mathrm{E}+03$ & $2.7 \mathrm{E}+02$ & & & & & & & $2.0 E+04$ & $2.0 \mathrm{E}+03$ & & & $2.4 E+03$ & $2.4 \mathrm{E}+02$ \\
\hline Methomyl & 016752.77 .5 & & & $6.7 E+04$ & $6.7 \mathrm{E}+03$ & & & & & & & $5.1 \mathrm{E}+05$ & $5.1 E+04$ & & & $5.9 \mathrm{E}+04$ & $5.9 E+03$ \\
\hline Methoxy-5-nitroaniline, 2 - & $000099.59-2$ & $4.1 E+05$ & 4.1E+03 & & & & & & & $1.0 \mathrm{E}+06$ & $3.1 E+04$ & & & $3.6 \mathrm{E}+05$ & $3.6 \mathrm{E}+03$ & & \\
\hline Methoxychlor & $000072-43-5$ & & & $1.3 \mathrm{E}+04$ & $1.3 \mathrm{E}+03$ & & & & & & & $1.0 E+05$ & $1.0 E+04$ & & & $1.2 \mathrm{E}+04$ & $1.2 E+03$ \\
\hline
\end{tabular}




\begin{tabular}{|c|c|c|c|c|c|c|c|c|c|c|c|c|c|c|c|c|c|}
\hline & & $20+399$ & $50+39 \%$ & & & $\varepsilon 0+a l \cdot S$ & $50+31 \cdot S$ & & & & & & & $20+356$ & botas L & 6-LL-101000 & 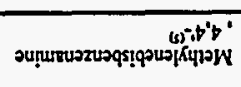 \\
\hline & & $\varepsilon 0+\exists 9^{\prime} \varepsilon$ & $50+399^{\circ} \varepsilon$ & & & botal' & $90+30 \% 1$ & & & & & & & $\varepsilon 0+91 \%$ & so+al't & $1-19-101000$ & 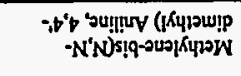 \\
\hline $20+391$ & $\varepsilon 0+39 \%$ & $\varepsilon 0+\exists \varepsilon 1$ & $50+3 E t$ & $\{0+\exists \mapsto 1$ & $50+36 \cdot i$ & totall & $90+30 \% t$ & & & $90+30 \% t$ & $90+30 \% 1$ & $20+36 t$ & $\varepsilon 0+36 \mathrm{I}$ & $\varepsilon 0+I b t$ & so+abli 1 & $t+1-101000$ & 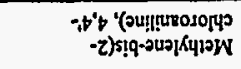 \\
\hline $20+301$ & $\varepsilon 0+30^{\circ} 1$ & $\varepsilon 0+301$ & $\varepsilon 0+30 \%$ & $50+a k z$ & $90+301$ & $50+39 \cdot \varepsilon$ & $90+30^{\circ} \mathrm{t}$ & $80+30^{\circ} \mathrm{I}$ & $50+301$ & $\varepsilon 0+30^{\circ} t$ & $60+200^{\circ} 1$ & $60+391$ & $50+39 \cdot 1$ & $50+3 s z$ & $90+30 \div 1$ & $2-60-510000$ & 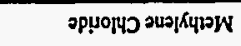 \\
\hline & & & & & & & & $20+362$ & $20+361 \tau$ & & & & & & & $z<\angle 8-801000$ & 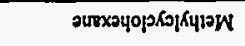 \\
\hline & & $20+91 \%$ & $50+91 \cdot 6$ & & & $\varepsilon 0+36 L$ & $50+36^{\circ} \mathrm{L}$ & & & & & & & $80+301$ & $50+30.1$ & $s-1 z-969000$ & 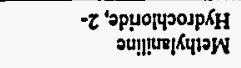 \\
\hline & & $\varepsilon 0+30 \%$ & $50+30 \% 5$ & & & $50+a \varepsilon$ & $90+301$ & & & & & & & $\varepsilon 0+39 \cdot s$ & $50+39 \% s$ & $8-55 \cdot 6600000$ & 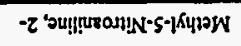 \\
\hline & & & & & & & & $90+30.1$ & $90+3011$ & & & & & & & $F+\$ 0-1 E 9100$ & 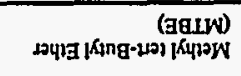 \\
\hline & & $20+351$ & $50+951$ & & & $\varepsilon 0+3 \varepsilon^{\prime} \mid$ & $50+3 \mathrm{at}$ & & & & & & & $20+a b i$ & $50+32<1$ & $t+D E-090000$ & 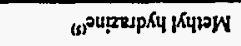 \\
\hline$\varepsilon 0+9 s \div 1$ & $50+35: 1$ & & & $50+36 t$ & $50+36.1$ & & & $90+30 \mathrm{t}$ & $90+3011$ & & & $\varepsilon 0+39 \%$ & $60+391$ & & & Fsl-Eloszo & 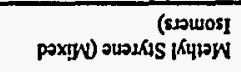 \\
\hline $20+326$ & $60+32 t$ & & & $\varepsilon 0+90^{\circ} \mathrm{b}$ & $60+301$ & & & & & & & $20+a k s$ & $\varepsilon 0+a \varepsilon s$ & & & $0-00-86 z 000$ & Wo:qh) \\
\hline $50+30 z$ & $50+30 z$ & & & $50+79$ & $90+30 \% 1$ & & & & & & & po+aiz & $50+a 12$ & & & $9-295-080000$ & 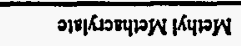 \\
\hline $10+95 z$ & $z 0+\exists s z$ & & & $20+99^{\prime} \varepsilon$ & $\varepsilon 0+399^{\prime} \varepsilon$ & & & & & & & $10+3 L 2$ & $20+3 L Z$ & & & $9-26 \sigma-\angle 96 z z 0$ & 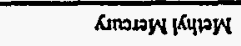 \\
\hline $20+30 \% 6$ & $20+90 \% \mathrm{~L}$ & & & $90+30 \%$ & $90+3011$ & & & $20+30 \%$ & $20+30.2$ & & & sotalz & $90+3011$ & & & $1-01-801000$ & 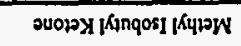 \\
\hline$\varepsilon 0+\exists z^{\prime} 6$ & $\varepsilon 0+a z$ & & & $90+30 \%$ & $90+301$ & & & $\varepsilon 0+3 z^{\prime} 6$ & $50+3 z^{\prime} 6$ & & & $s 0+\nexists \varepsilon \dot{s}$ & $90+301$ & & & $\varepsilon-86-8<0000$ & 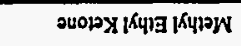 \\
\hline $60+3 I^{\prime} \cdot L$ & $50+3 I^{\prime} \mathrm{L}$ & & & $50+31 \cdot 9$ & sotar's & & & & & & & $\varepsilon 0+30^{\prime} 8$ & $60+30.8$ & & & $\varepsilon-\varepsilon \varepsilon-960000$ & 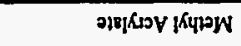 \\
\hline $90+50 \div 1$ & $90+20 \% 1$ & & & $90+20^{\circ} 1$ & $90+30^{\prime} \mathrm{t}$ & & & & & & & $90+30 \mathrm{~T}$ & $90+30.1$ & & & $6-02-600000$ & 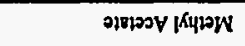 \\
\hline$\varepsilon 0+a b z$ & $50+26 z$ & & & $60+302 z$ & $50+30 z$ & & & $90+301$ & $90+301$ & & & $\varepsilon 0+z l z$ & $50+21 / 2$ & & & $598-601000$ & 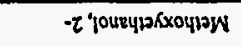 \\
\hline$\varepsilon 0+3 L$ & $50+31 \%$ & & & $50+30 \%$ & $50+30.6$ & & & & & & & Eo+aEs & botacis & & & $9-65011000$ & 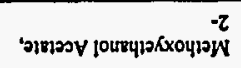 \\
\hline$I 0=1 H$ & $I=I H$ & , ol & .01 & $I^{\prime} 0=\mathrm{OH}$ & $I=\partial H$ & , $0 \mathrm{I}$ & rOI & $r^{\prime} O=D H$ & $l=\partial H$ & .01 & rOI & $\Gamma^{\prime} 0=0 \mathrm{H}$ & $I=\partial H$ & ,ol & ros & \multirow[b]{2}{*}{ N\&SYO } & \multirow[b]{2}{*}{4043} \\
\hline \multicolumn{4}{|c|}{ 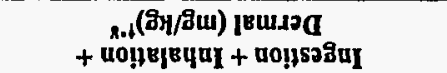 } & \multicolumn{4}{|c|}{ 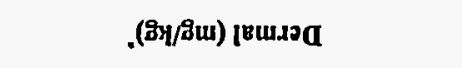 } & \multicolumn{4}{|c|}{ 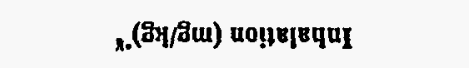 } & \multicolumn{4}{|c|}{ 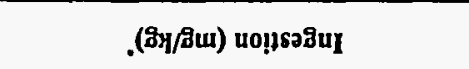 } & & \\
\hline
\end{tabular}

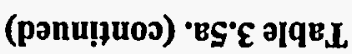


Table 3.5a. (continued)

\begin{tabular}{|c|c|c|c|c|c|c|c|c|c|c|c|c|c|c|c|c|c|}
\hline \multirow{2}{*}{ Chemical } & \multirow{2}{*}{ CASRN } & \multicolumn{4}{|c|}{ Ingestion (mg/kg) } & \multicolumn{4}{|c|}{ Inhalation (mg/kg) } & \multicolumn{4}{|c|}{ Dermal (mg/kg)" } & \multicolumn{4}{|c|}{$\begin{array}{c}\text { Ingestion + Inhalation + } \\
\text { Dermal (mg/kg) })^{+\vee \gamma}\end{array}$} \\
\hline & & $10^{-4}$ & $10^{5}$ & $H Q=1$ & $\mathrm{HQ}=0.1$ & $10^{-4}$ & $10^{4}$ & $H Q=1$ & $\mathrm{HQ}=0.1$ & $10^{-4}$ & $10^{6}$ & $H Q=1$ & $\mathrm{HQ}=0.1$ & $10^{-4}$ & $10^{5}$ & $\mathrm{HI}=1$ & $\mathrm{HI}=0.1$ \\
\hline $\begin{array}{l}\text { Methylenodiphenyl } \\
\text { Isocyanute, 4,4-: }\end{array}$ & $000101-68-8$ & & & & & & & $1.0 E+06$ & $1.6 \mathrm{E}+05$ & & & & & & & & \\
\hline Methylstyrene, Alphs. & $000098-83.9$ & & & $1.0 \mathrm{E}+06$ & $1.9 \mathrm{E}+05$ & & & & & & & $1.0 E+06$ & $1.0 \mathrm{E}+06$ & & & $1.0 \mathrm{E}+06$ & $1.7 \mathrm{E}+05$ \\
\hline Methyltriethyl Lead & $001762-28-3$ & & & & & & & & & & & & & & & & \\
\hline Metolachlor & $051218-45-2$ & & & $4.0 \mathrm{E}+05$ & $4.0 \mathrm{E}+04$ & & & & & & & $1.0 E+06$ & $3.0 E+05$ & & & 3.5E+05 & $3.5 \mathrm{E}+04$ \\
\hline Metribuzin'(s) & $021087-64-9$ & & & $6.7 \mathrm{E}+04$ & $6.7 E+03$ & & & & & & & S.IE+05 & $5.1 E+04$ & & & $5.9 E+04$ & $5.9 \mathrm{E}+03$ \\
\hline Mirex" & $002385-85-5$ & $1.0 \mathrm{E}+04$ & $1.0 \mathrm{E}+02$ & $5.3 E+02$ & $5.3 \mathrm{E}+01$ & & & & & $7.9 E+04$ & $7.9 E+02$ & $4.0 E+03$ & $4.0 \mathrm{E}+02$ & $9.1 E+03$ & $9.1 E+01$ & $4.7 E+02$ & 4.7E+01 \\
\hline Molinate & $002212-67.1$ & & & $5.3 E+03$ & $5.3 \mathrm{E}+02$ & & & & & & & $4.0 E+04$ & $4.0 \mathrm{E}+03$ & & & $4.7 E+03$ & $4.7 E+02$ \\
\hline Molybdenum & $007439-98-7$ & & & $1.3 E+04$ & $1.3 E+03$ & & & & & & & 7.7E+05 & $7.7 E+04$ & & & $1.3 E+04$ & $1.3 E+03$ \\
\hline Monoctloramine & $010599-90-3$ & & & 2.TE+0s & $2.7 \mathrm{E}+04$ & & & & & & & $1.0 \mathrm{E}+06$ & $2.0 \mathrm{E}+05$ & & & $2.4 E+05$ & $2.4 E+04$ \\
\hline Monochlorobutanes & $025154-42-1$ & & & $1.0 E+06$ & $1.1 \mathrm{E}+05$ & & & & & & & $1.0 \mathrm{E}+06$ & 8.1E+05 & & & $9.4 \mathrm{E}+0.5$ & $9.4 E+04$ \\
\hline Naled & $000300-76-5$ & & & $5.3 \mathrm{E}+03$ & $5.3 \mathrm{E}+02$ & & & & & & & $4.0 \mathrm{E}+04$ & $4.0 \mathrm{E}+03$ & & & $4.7 \mathrm{E}+03$ & $4.7 \mathrm{E}+02$ \\
\hline Naphthalene $e^{(1)}$ & $000091-20-3$ & & & $9.5 \mathrm{E}+04$ & $9.5 \mathrm{E}+03$ & & & & & & & $1.0 \mathrm{E}+06$ & $1.2 \mathrm{E}+0 \mathrm{~S}$ & & & $8.8 \mathrm{E}+04$ & $8.8 E+03$ \\
\hline Naphthalene, 1-Methyl & $000090-12-0$ & & & & & & & & & & & & & & & & \\
\hline Naphthalene, 2-Methyl & $000091-57-6$ & & & & & & & & & & & & & & & & \\
\hline Napropamide & $015299.99-7$ & & & $2.7 \mathrm{E}+05$ & $2.7 E+04$ & & & & & & & $1.0 \mathrm{E}+06$ & $2.0 \mathrm{E}+05$ & & & $2.4 E+05$ & $2.4 E+04$ \\
\hline Nizgara Blue 4B & $002429.74-5$ & & & & & & & & & & & & & & & & \\
\hline Nickel Carbonyl & $013463-39-3$ & & & & & & & & & & & & & & & & \\
\hline Nickel Refinery Dust & $\mathrm{NA}$ & & & & & $1.0 \mathrm{E}+06$ & $1.0 \mathrm{E}+06$ & & & & & & & & & & \\
\hline Nickel Soluble Salts & $007440-02-0$ & & & $5,3 E+04$ & $5.3 \mathrm{E}+03$ & & & & & & & $1.0 \mathrm{E}+06$ & $2.2 \mathrm{E}+05$ & & & $5.2 E+04$ & $5.2 \mathrm{E}+03$ \\
\hline Nickel Subsulfide & $012035-72-2$ & & & & & $1.0 \mathrm{E}+06$ & $1.0 E+06$ & & & & & & & & & & \\
\hline Nitrapyrin' & $001929-82-4$ & & & $4.0 \mathrm{E}+03$ & $4.0 E+02$ & & & & & & & $3.0 \mathrm{E}+04$ & $3.0 E+03$ & & & $3.5 \mathrm{E}+03$ & $3.5 E+02$ \\
\hline Nitrate & 014797.55 .8 & & & $1.0 \mathrm{E}+06$ & $4.3 \mathrm{E}+05$ & & & & & & & $1.0 E+06$ & $1.0 \mathrm{E}+06$ & & & $1.0 E+06$ & $4.2 E+05$ \\
\hline
\end{tabular}

Nonradionuclides/soil/excavation

Page 21 of 32 
Table 3.5a. (continued)

\begin{tabular}{|c|c|c|c|c|c|c|c|c|c|c|c|c|c|c|c|c|c|}
\hline \multirow[t]{2}{*}{ Chemical } & \multirow[t]{2}{*}{ CASRN } & \multicolumn{4}{|c|}{ Ingestion (mg/kg) } & \multicolumn{4}{|c|}{ Inhalation $(\mathrm{mg} / \mathrm{kg})^{\cdot x}$} & \multicolumn{4}{|c|}{ Dermal (mg/kg) } & \multicolumn{4}{|c|}{$\begin{array}{l}\text { Ingestion }+ \text { Inhalation }+ \\
\text { Dermal }(\mathrm{mg} / \mathrm{kg})^{+* y}\end{array}$} \\
\hline & & $10^{-4}$ & $10^{4}$ & $H Q=1$ & $H Q=0.1$ & $10^{-4}$ & $10^{4}$ & $H Q=1$ & $H Q=0.1$ & $10^{-1}$ & $10^{-6}$ & $\mathrm{HQ}=1$ & $H Q=0.1$ & $10^{-4}$ & $10^{4}$ & $H I=1$ & $\mathrm{HI}=0.1$ \\
\hline Nitric Oxide & $010102-43-9$ & & & $2.7 \mathrm{E}+05$ & 2.7E+04 & & & & & & & $1.0 \mathrm{E}+06$ & $8.1 \mathrm{E}+05$ & & & $2.6 E+05$ & $2.6 \mathrm{E}+04$ \\
\hline Nitrite & $014797.65-0$ & & & 2.7E+05 & $2.7 \mathrm{E}+04$ & & & & & & & $1.0 E+06$ & $1.0 \mathrm{E}+06$ & & & $2.6 E+05$ & $2.6 E+04$ \\
\hline Nitroaniline, 2- & $000088-74-4$ & & & & & & & $9.6 \mathrm{E}+01$ & $9.6 \mathrm{E}+01$ & & & & & & & & \\
\hline Nitrobenzene & 000098.95 .3 & & & $1.3 \mathrm{E}+04$ & $1.3 E+03$ & & & $3.2 E+02$ & $1.5 E+02$ & & & $2.0 E+05$ & $2.0 \mathrm{E}+04$ & & & $3.2 \mathrm{E}+02$ & $1.3 \mathrm{E}+02$ \\
\hline Nitrofurantoin & 000067.20 .9 & & & $1.0 \mathrm{E}+06$ & $1.9 \mathrm{E}+05$ & & & & & & & $1.0 \mathrm{E}+06$ & $1.0 \mathrm{E}+06$ & & & $1.0 \mathrm{E}+06$ & $1.6 \mathrm{E}+05$ \\
\hline Nitrofurazone & $000059-87.0$ & $1.2 \mathrm{E}+04$ & $1.2 \mathrm{E}+02$ & & & & & & & $9.4 E+04$ & $9.4 E+02$ & & & $1.1 E+04$ & 1. $1 \mathrm{E}+02$ & & \\
\hline Nitrogen Dioxide $e^{(7)}$ & $010102-44-0$ & & & $1.0 \mathrm{E}+06$ & $2.7 \mathrm{E}+05$ & & & & & & & $1.0 \mathrm{E}+06$ & $1.0 \mathrm{E}+06$ & & & $1.0 \mathrm{E}+06$ & $2.6 \mathrm{E}+05$ \\
\hline Nitroguanidine & $000556-88-7$ & & & $2.7 \mathrm{E}+05$ & $2.7 E+04$ & & & & & & & $1.0 E+06$ & $2.0 \mathrm{E}+05$ & & & $2.4 E+05$ & $2.4 E+04$ \\
\hline Nitrophenol, 4- & $000100-02-7$ & & & & & & & & & & & & & & & & \\
\hline Nitropropane, 2.17) & $000079-46.9$ & $2.0 E+03$ & $2.0 \mathrm{E}+01$ & & & $4.4 \mathrm{E}+03$ & $4.4 \mathrm{E}+01$ & $1.5 \mathrm{E}+04$ & $3.4 E+03$ & $2.4 \mathrm{E}+04$ & $2.4 E+02$ & & & $1.3 \mathrm{E}+03$ & $1.3 \mathrm{E}+01$ & & \\
\hline Nitroso-N-ethylurea, $\mathrm{N}$ - & 000759.73 .9 & $1.3 \mathrm{E}+02$ & $1.3 \mathrm{E}+00$ & & & & & & & $1.0 \mathrm{E}+03$ & $1.0 \mathrm{E}+01$ & & & $1.2 \mathrm{E}+02$ & $1.2 \mathrm{E}+00$ & & \\
\hline Nitroso-N-methylurea, $\mathrm{N}$ - & $000684-93-5$ & & & & & & & & & & & & & & & & \\
\hline $\begin{array}{l}\text { Nitroso-di-N-butylamine, } \\
\text { N. }\end{array}$ & $000924-16-3$ & $3.5 E+03$ & $3.5 \mathrm{E}+01$ & & & $1.5 \mathrm{E}+03$ & $3.2 \mathrm{E}+02$ & & & $4.2 E+04$ & $4.2 \mathrm{E}+02$ & & & $1.5 E+03$ & $2.9 E+01$ & & \\
\hline $\begin{array}{l}\text { Nitroso-dijN- } \\
\text { propylamine, } \mathrm{N} \text {. }\end{array}$ & $000621-64-7$ & $2.7 \mathrm{E}+03$ & 2.7E+01 & & & & & & & $1.0 \mathrm{E}+04$ & $1.0 \mathrm{E}+02$ & & & $2.1 \mathrm{E}+03$ & $2.1 E+01$ & & \\
\hline Nitrosodiethanolamine, $\mathrm{N}$ - & $001116.54-7$ & $6.7 E+03$ & $6.7 E+01$ & & & & & & & S.IE+04 & $5.1 \mathrm{E}+02$ & & & $5.9 \mathrm{E}+03$ & $5.9 E+01$ & & \\
\hline Nitrosodiethylamine, N- & $000055-18.5$ & $1.2 \mathrm{E}+02$ & $1.2 \mathrm{E}+\infty$ & & & $1.0 \mathrm{E}+06$ & $1.3 \mathrm{E}+05$ & & & $9.4 \mathrm{E}+02$ & $9.4 E+00$ & & & $1.1 \mathrm{E}+02$ & $1.1 \mathrm{E}+00$ & & \\
\hline Nitrosodimethylamine, $N$ - & $000062-75-9$ & $3.7 E+02$ & $3.7 \mathrm{E}+00$ & & & $1.0 \mathrm{E}+06$ & $3.8 \mathrm{E}+05$ & & & $2.8 \mathrm{E}+03$ & $2.8 \mathrm{E}+01$ & & & $3.2 \mathrm{E}+02$ & $3.2 \mathrm{E}+00$ & & \\
\hline Nitrosodiphenylamine, N- & $000086-30-6$ & $1.0 \mathrm{E}+06$ & $3.8 \mathrm{E}+04$ & & & & & & & $1.0 \mathrm{E}+06$ & $1.4 \mathrm{E}+05$ & & & $1.0 \mathrm{E}+06$ & $3.0 \mathrm{E}+04$ & & \\
\hline $\begin{array}{l}\text { Nitrosomethylethylamine, } \\
\text { N- }\end{array}$ & $010595-95-6$ & $8.5 E+02$ & $8.5 E+\infty$ & & & & & & & $6.4 E+03$ & $6.4 \mathrm{E}+01$ & & & $7.5 \mathrm{E}+02$ & $7.5 E+\infty$ & & \\
\hline $\begin{array}{l}\text { Nitrosomethylvinylaming, } \\
\text { N- }\end{array}$ & $004549-40.0$ & & & & & & & & & & & & & & & & \\
\hline Nitrosopyrrolidine, N- & $000930-55-2$ & $8.9 E+03$ & $8.9 E+01$ & & & $1.0 E+06$ & $1.0 \mathrm{E}+06$ & & & $6.7 \mathrm{E}+04$ & $6.7 E+02$ & & & $7.8 E+03$ & $7.8 E+01$ & & \\
\hline
\end{tabular}


Table 3.5a. (continued)

\begin{tabular}{|c|c|c|c|c|c|c|c|c|c|c|c|c|c|c|c|c|c|}
\hline \multirow[t]{2}{*}{ Chemical } & \multirow[t]{2}{*}{ CASRN } & \multicolumn{4}{|c|}{ Ingestion (mg/kg)' } & \multicolumn{4}{|c|}{ Inhalation (mg/kg) } & \multicolumn{4}{|c|}{ Dermal (mg/kg)" } & \multicolumn{4}{|c|}{$\begin{array}{c}\text { Ingestion + Inhalation + } \\
\text { Dermal (mg/kg })^{+* k}\end{array}$} \\
\hline & & $10^{-4}$ & $10^{5}$ & $H Q=1$ & $\mathrm{HQ}=0.1$ & $10^{-4}$ & $10^{-6}$ & $H Q=1$ & $\mathrm{HQ}=0.1$ & $10^{-4}$ & $10^{4}$ & $H Q=1$ & $\mathrm{HQ}=0.1$ & $10^{-4}$ & $10^{-4}$ & $\mathrm{HI}=1$ & $\mathrm{HI}=0.1$ \\
\hline Nitrotoluene, 4-Amino-2- & $000119-32-4$ & & & & & & & & & & & & & & & & \\
\hline Nitrotoluene, $m$ - & $000099-08-1$ & & & 2.7E+05 & $2.7 E+04$ & & & & & & & $1.0 \mathrm{E}+06$ & $2.0 E+05$ & & & $2.4 E+05$ & $2.4 E+04$ \\
\hline Nitrotoluene, $\alpha$. & $000088.72-2$ & & & $2.7 E+05$ & $2.7 E+04$ & & & & & & & $1.0 \mathrm{E}+0.5$ & $2.0 \mathrm{E}+05$ & & & $2.4 E+0 S$ & 2.4E+04 \\
\hline Nitrotoluene, p- & $000099-99-0$ & & & $2.7 \mathrm{E}+05$ & $2.7 E+04$ & & & & & & & $1.0 \mathrm{E}+06$ & $2.0 E+05$ & & & 2.4E+0s & 2.4E+04 \\
\hline Norflurazon & $027314-13-2$ & & & $1.1 E+05$ & $1.1 \mathrm{E}+04$ & & & & & & & $8.1 \mathrm{E}+05$ & 8.1E+04 & & & $9.4 E+04$ & $9.4 E+03$ \\
\hline Nustar & 085509-19-9 & & & $1.9 E+03$ & $1.9 \mathrm{E}+02$ & & & & & & & $1.4 E+04$ & $1.4 \mathrm{E}+03$ & & & $1.6 \mathrm{E}+03$ & $1.6 \mathrm{E}+02$ \\
\hline$O C D^{(i)}$ & $003268-87-9$ & $1.2 \mathrm{E}+02$ & $1.2 \mathrm{E}+\infty$ & & & $1.0 \mathrm{E}+06$ & $1.3 \mathrm{E}+05$ & & & $3.1 E+02$ & $3.1 E+\infty$ & & & $8.9 \mathrm{E}+01$ & $8.9 E-01$ & & \\
\hline OCDF' & $039001-02-0$ & $1.2 E+02$ & $1.2 \mathrm{E}+00$ & & & $1.0 E+06$ & $1.3 E+05$ & & & $9.4 \mathrm{E}+02$ & $9.4 \mathrm{E}+\infty 0$ & & & $1.1 E+02$ & $1.1 E+\infty 0$ & & \\
\hline Octabromodiphenyl Ether & $032536-52-0$ & & & $8.0 E+04$ & $8.0 \mathrm{E}+03$ & & & & & & & $6.1 E+03$ & $6.1 \mathrm{E}+04$ & & & $7.1 \mathrm{E}+04$ & $7.1 E+03$ \\
\hline $\begin{array}{l}\text { Octahydro-1,3,5,7. } \\
\text { tetranitro-1,3,5,7-tetra } \\
\text { (HMX) }\end{array}$ & $002691-41-0$ & & & $1.3 E+05$ & $1.3 \mathrm{E}+04$ & & & & & & & $3.0 \mathrm{E}+05$ & $3.0 \mathrm{E}+04$ & & & $9.2 \mathrm{E}+04$ & $9.2 E+03$ \\
\hline $\begin{array}{l}\text { Octamelhylpyrophosphors } \\
\text { mide }\end{array}$ & $000152-16-9$ & & & $5.3 \mathrm{E}+03$ & $5.3 E+02$ & & & & & & & $4.0 \mathrm{E}+04$ & $4.0 \mathrm{E}+03$ & & & 4.7E+03 & $4.7 E+02$ \\
\hline Octyl Phthalate, di-N. & $000117-84.0$ & & & $5.3 E+04$ & $5.3 \mathrm{E}+03$ & & & & & & & 7.3E+05 & $7.3 \mathrm{E}+04$ & & & $5.0 \mathrm{E}+04$ & $5.0 E+03$ \\
\hline Oryzalin & 019044-88-3 & & & $1.3 E+05$ & $1.3 \mathrm{E}+04$ & & & & & & & $1.0 E+06$ & $1.0 \mathrm{E}+0 \mathrm{~S}$ & & & $1.2 E+05$ & $1.2 \mathrm{E}+04$ \\
\hline Oxadiazon & $019666-30-9$ & & & $1.3 \mathrm{E}+04$ & $1.3 E+03$ & & & & & & & $1.0 \mathrm{E}+05$ & $1.0 \mathrm{E}+04$ & & & $1.2 E+04$ & $1.2 \mathrm{E}+03$ \\
\hline Oxamyl & $023135-22.0$ & & & $6.7 E+04$ & $6.7 \mathrm{E}+03$ & & & & & & & $5.1 \mathrm{E}+05$ & $5.1 \mathrm{E}+0.4$ & & & $5.9 \mathrm{E}+04$ & $5.9 \mathrm{E}+03$ \\
\hline Paclobutrazol & 076738.62 .0 & & & $3.5 \mathrm{E}+04$ & $3.5 \mathrm{E}+03$ & & & & & & & $2.6 E+0 S$ & $2.6 \mathrm{E}+04$ & & & $3.1 \mathrm{E}+04$ & $3.1 E+03$ \\
\hline Paraquat & $001910-42-5$ & & & $1.2 E+04$ & $1.2 \mathrm{E}+03$ & & & & & & & $9.1 E+04$ & 9.1E+03 & & & $1.1 \mathrm{E}+04$ & $1.1 E+03$ \\
\hline Parathion & $000056-38-2$ & & & $1.6 \mathrm{E}+04$ & $1.6 \mathrm{E}+03$ & & & & & & & $1.2 \mathrm{E}+0 \mathrm{~s}$ & $1.2 E+04$ & & & $1.4 \mathrm{E}+04$ & $1.4 E+03$ \\
\hline PeCDD, 2,3,7,8.(1) & $036088.22-9$ & 2.5E-01 & 2.5E-03 & & & $2.6 \mathrm{E}+04$ & $2.6 \mathrm{E}+02$ & & & $6.3 \mathrm{E}-01$ & $6.3 \mathrm{E}-03$ & & & $1.8 \mathrm{E}-01$ & $1.8 \mathrm{E}-03$ & & \\
\hline PeCDF, 1,2,3,7,8=(1) & $057117-41-6$ & 2.5E-01 & 2.5E-03 & & & $2.6 \mathrm{E}+04$ & 2.6E+02 & & & $1.9 \mathrm{E}+00$ & $1.9 \mathrm{E}-02$ & & & 2.2E-01 & $2.2 \mathrm{E}-03$ & & \\
\hline PeCDF, 2,3,4,7,8-(1) & $057117-31-4$ & $2.5 \mathrm{E}+\infty 0$ & $2.5 E-02$ & & & $2.6 \mathrm{E}+05$ & $2.6 \mathrm{E}+03$ & & & $1.9 E+01$ & $1.9 \mathrm{E}-01$ & & & $2.2 \mathrm{E}+00$ & $2.2 \mathrm{E}-02$ & & \\
\hline Pebulate & $001114-71-2$ & & & $1.3 \mathrm{E}+05$ & $1.3 E+04$ & & & & & & & $1.0 \mathrm{E}+06$ & $1.0 \mathrm{E}+0 \mathrm{~S}$ & & & $1.2 E+05$ & $1.2 \mathrm{E}+04$ \\
\hline
\end{tabular}

Nonradionuclides/soil/excavation

Page 23 of 32 
Table 3.5a. (continued)

\begin{tabular}{|c|c|c|c|c|c|c|c|c|c|c|c|c|c|c|c|c|c|}
\hline \multirow[t]{2}{*}{ Chemical } & \multirow[t]{2}{*}{ CASRN } & \multicolumn{4}{|c|}{ Ingestion (mg/kg) } & \multicolumn{4}{|c|}{ Inhalation $(\mathrm{mg} / \mathrm{kg})^{-y}$} & \multicolumn{4}{|c|}{ Dermal (mg/kg) } & \multicolumn{4}{|c|}{$\begin{array}{c}\text { Ingestion + Inhalation + } \\
\text { Dermal (mg/kg) })^{+\vee v}\end{array}$} \\
\hline & & $10^{4}$ & $10^{4}$ & $H Q=1$ & $\mathrm{HQ}=0.1$ & $10^{-4}$ & $10^{6}$ & $\mathrm{HQ}=1$ & $H Q=0.1$ & $10^{-4}$ & $10^{6}$ & $\mathbf{H Q}=1$ & $\mathrm{HQ}=0.1$ & $10^{-1}$ & $10^{-6}$ & $H I=1$ & $\mathrm{HI}=0.1$ \\
\hline Pendimethalin & $040487-42-1$ & & & $1.1 \mathrm{E}+05$ & $1.1 E+04$ & & & & & & & 8.1E+05 & 8.1E+04 & & & $9.4 E+04$ & $9.4 E+03$ \\
\hline Pentabromodiphenyl Ether & 032534.81 .9 & & & $5.3 \mathrm{E}+04$ & $5.3 \mathrm{E}+03$ & & & & & & & $4.0 E+05$ & $4.0 \mathrm{E}+04$ & & & 4.7E+04 & $4.7 E+03$ \\
\hline Pentachlorobenzene & $000608-93-5$ & & & $2.1 E+04$ & $2.1 E+03$ & & & & & & & $1.6 \mathrm{E}+05$ & $1.6 \mathrm{E}+04$ & & & $1.9 \mathrm{E}+04$ & $1.9 E+03$ \\
\hline $\begin{array}{l}\text { Pentachlorocyclopentadie } \\
\text { ne }\end{array}$ & $025329-35-5$ & & & & & & & & & & & & & & & & \\
\hline Pentachloronitrobenzene & $000082-68-8$ & $7.2 \mathrm{E}+04$ & $7.2 \mathrm{E}+02$ & $8,0 \mathrm{E}+03$ & $8.0 \mathrm{E}+02$ & & & & & $8.7 \mathrm{E}+0 \mathrm{~S}$ & $8.7 E+03$ & $6.1 E+04$ & $6.1 \mathrm{E}+03$ & $6.6 \mathrm{E}+04$ & $6.6 \mathrm{E}+02$ & $7.1 \mathrm{E}+03$ & 7.1E+02 \\
\hline Pentachlorophenol & $000087-86-5$ & $1.6 \mathrm{E}+05$ & $1.6 \mathrm{E}+03$ & $8.0 \mathrm{E}+04$ & $8.0 \mathrm{E}+03$ & & & & & $1.0 \mathrm{E}+06$ & $2.4 \mathrm{E}+04$ & $1.0 \mathrm{E}+06$ & $1.2 \mathrm{E}+05$ & $1.5 \mathrm{E}+05$ & $1.5 E+03$ & $7.5 E+04$ & $7.5 E+03$ \\
\hline Pentyl Alcohol, N- & $000071-41 \cdot 0$ & & & & & & & & & & & & & & & & \\
\hline Permethrin & $052645-53-1$ & & & $1.3 \mathrm{E}+05$ & $1.3 \mathrm{E}+04$ & & & & & & & $1.0 \mathrm{E}+06$ & $1.0 \mathrm{E}+05$ & & & $1.2 \mathrm{E}+05$ & $1.2 \mathrm{E}+04$ \\
\hline Phenanthrene & $000085-01-8$ & & & & & & & & & & & & & & & & \\
\hline Phenmedipharn & 013684.63 .4 & & & $6.7 E+05$ & $6.7 \mathrm{E}+04$ & & & & & & & $1.0 \mathrm{E}+06$ & $5.1 E+05$ & & & $5.9 \mathrm{E}+05$ & $5.9 E+04$ \\
\hline Phenol & $000108-95-2$ & & & $1.0 \mathrm{E}+06$ & $1.6 \mathrm{E}+05$ & & & & & & & $1.0 \mathrm{E}+06$ & $1.0 \mathrm{E}+06$ & & & $1.0 \mathrm{E}+06$ & 1.5E+05 \\
\hline Phenylenediamine, $\mathrm{m}$ - & $000108-45-2$ & & & $1.6 \mathrm{E}+05$ & $1.6 \mathrm{E}+04$ & & & & & & & $1.0 \mathrm{E}+06$ & $1.2 \mathrm{E}+05$ & & & $1.4 E+05$ & $1.4 E+04$ \\
\hline Phenylenediamine, o- & $000095-54-5$ & $4.0 \mathrm{E}+05$ & $4.0 \mathrm{E}+03$ & & & & & & & $1.0 \mathrm{E}+06$ & $3.0 \mathrm{E}+04$ & & & $3.5 E+05$ & $3.5 \mathrm{E}+03$ & & \\
\hline Phenylenediamine, p- & $000106-50-3$ & & & S.IE+0S & $5.1 \mathrm{E}+04$ & & & & & & & $1.0 \mathrm{E}+06$ & $3.8 \mathrm{E}+05$ & & & 4.5E+OS & $4.5 \mathrm{E}+04$ \\
\hline Phenylmercuric Acetate & $000062-38-4$ & & & $2.1 E+02$ & $2.1 \mathrm{E}+01$ & & & & & & & $1.6 \mathrm{E}+03$ & $1.6 \mathrm{E}+02$ & & & $1.9 \mathrm{E}+02$ & $1.9 \mathrm{E}+01$ \\
\hline Phenylphenol, 2. & $000090-43-7$ & $1.0 \mathrm{E}+06$ & $9.6 \mathrm{E}+04$ & & & & & & & $1.0 \mathrm{E}+06$ & 7.3E+05 & & & $1.0 \mathrm{E}+06$ & 8.5E+04 & & \\
\hline Phorate & $000298-02-2$ & & & $5.3 \mathrm{E}+02$ & $5.3 \mathrm{E}+01$ & & & & & & & $4.0 \mathrm{E}+03$ & $4.0 \mathrm{E}+02$ & & & 4.7E+02 & 4.7E+01 \\
\hline Phosmet & $000732-11-6$ & & & $5.3 \mathrm{E}+04$ & $5.3 \mathrm{E}+03$ & & & & & & & $4.0 E+05$ & $4.0 E+04$ & & & $4.7 \mathrm{E}+04$ & 4.7E+03 \\
\hline Phosphine & $007803-51-2$ & & & $8.0 E+02$ & $8.0 \mathrm{E}+01$ & & & $1.0 \mathrm{E}+06$ & $1.0 \mathrm{E}+06$ & & & $2.4 \mathrm{E}+04$ & $2.4 E+03$ & & & $7.7 E+02$ & $7.7 E+01$ \\
\hline Phosphoric Acid & $007664-38-2$ & & & & & & & $1.0 \mathrm{E}+06$ & $1.0 \mathrm{E}+06$ & & & & & & & & \\
\hline Phthalic Acid, P. & $000100-21 \cdot 0$ & & & $1.0 E+06$ & 2.7E+05 & & & & & & & $1.0 \mathrm{E}+06$ & $1.0 E+06$ & & & $1.0 \mathrm{E}+06$ & $2.4 E+05$ \\
\hline Phthalic Arhydride & $000085-44-9$ & & & $1.0 \mathrm{E}+06$ & $5.3 \mathrm{E}+05$ & & & $1.08+06$ & $1.0 E+06$ & & & $1.0 E+06$ & $1.0 E+06$ & & & $1.0 \mathrm{E}+06$ & $4.7 \mathrm{E}+05$ \\
\hline
\end{tabular}




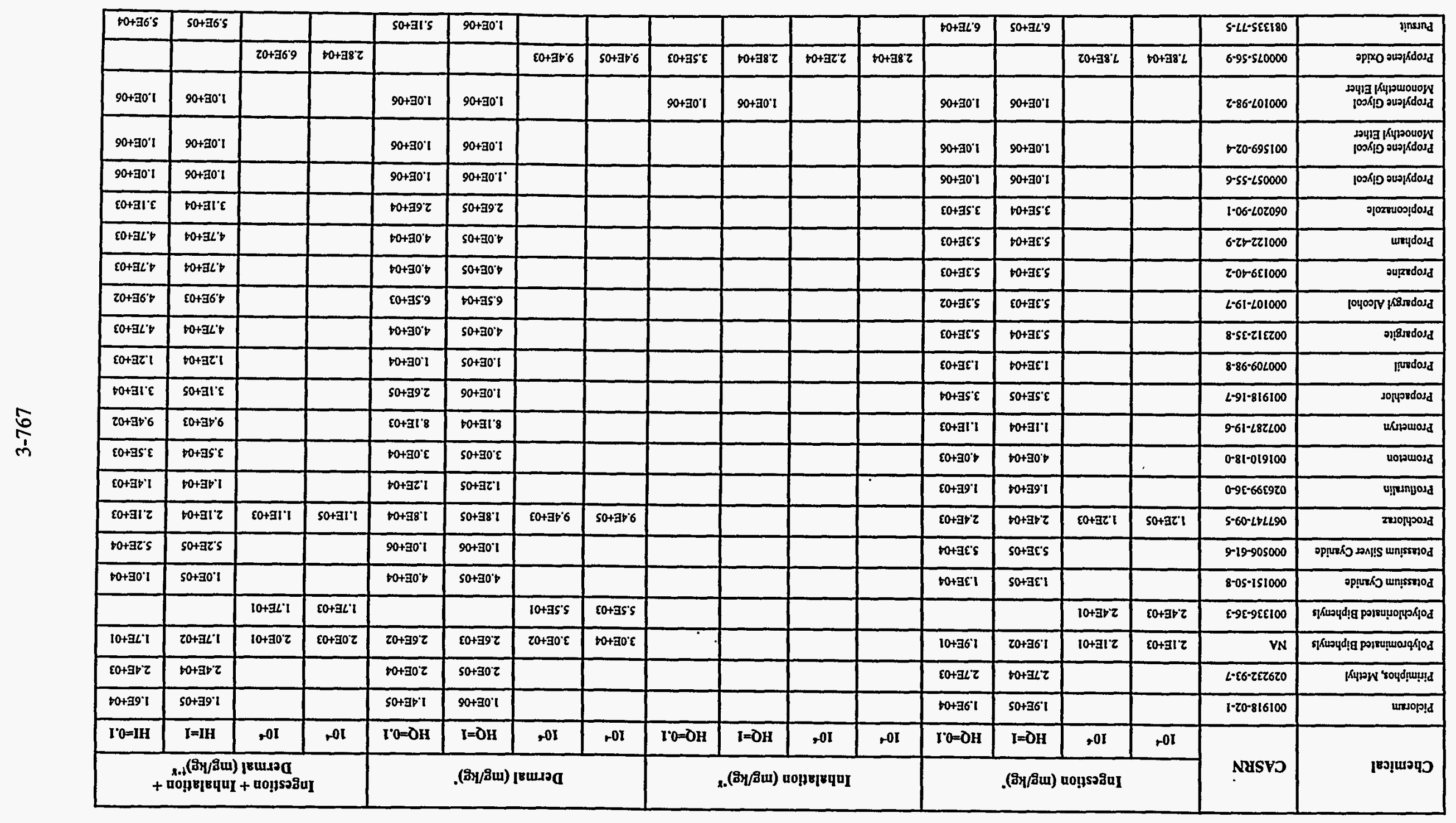

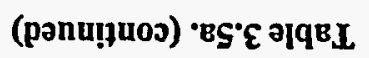


Table 3.5a. (continued)

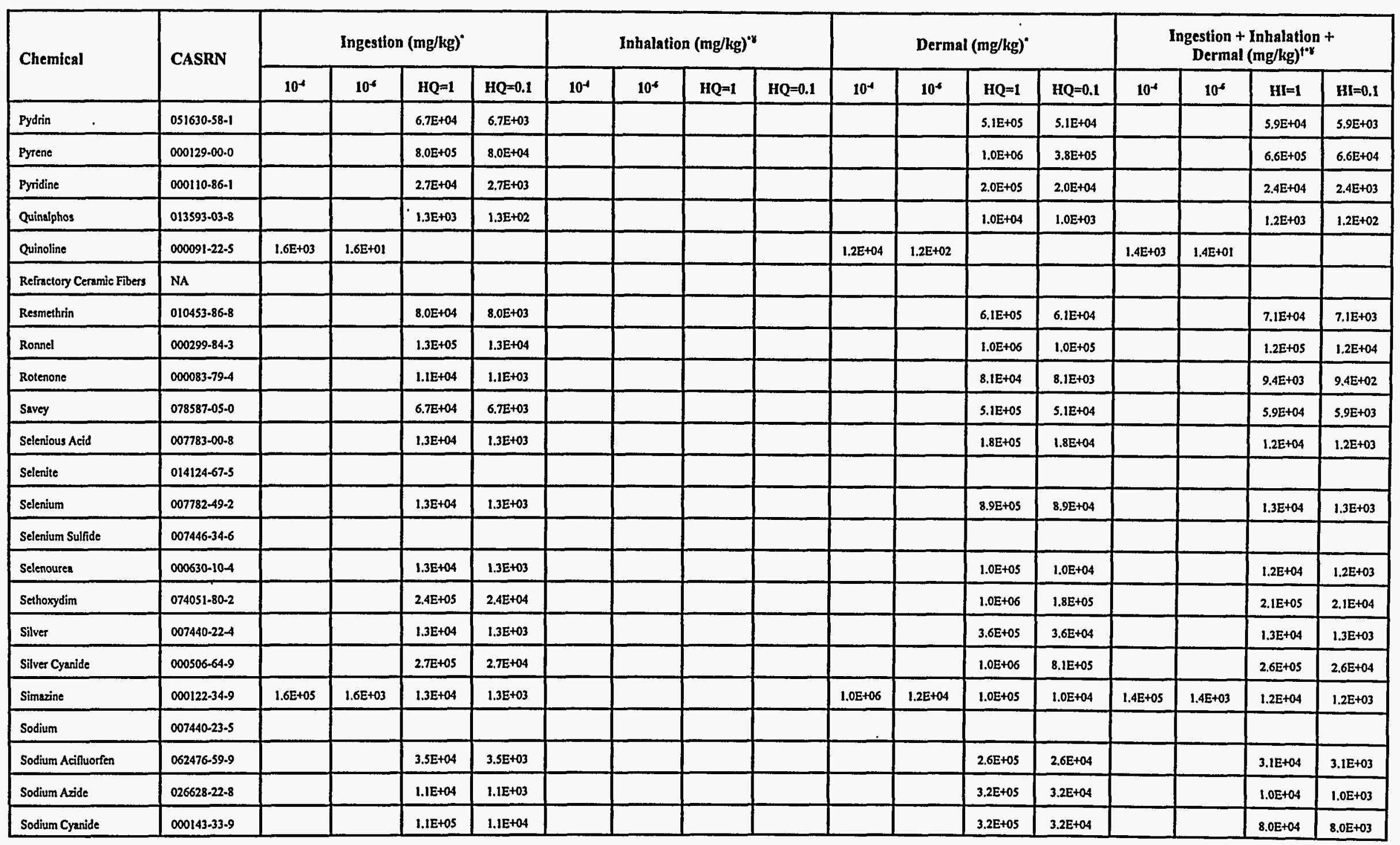


Table 3.5a. (continued)

\begin{tabular}{|c|c|c|c|c|c|c|c|c|c|c|c|c|c|c|c|c|c|}
\hline \multirow[t]{2}{*}{ Chemical } & \multirow[t]{2}{*}{ CASRN } & \multicolumn{4}{|c|}{ Ingestion (mg/kg) } & \multicolumn{4}{|c|}{ Inhalation (mg/kg) } & \multicolumn{4}{|c|}{ Dermal (mg/kg) } & \multicolumn{4}{|c|}{$\begin{array}{c}\text { Ingestion + Inhalation + } \\
\text { Dermal (mg/kg) }\end{array}$} \\
\hline & & $10^{-4}$ & $10^{4}$ & $H Q=1$ & $\mathrm{HQ}=0.1$ & $10^{-4}$ & $10^{6}$ & $\mathrm{HQ}=\mathbf{I}$ & $\mathrm{HQ}=0.1$ & $10^{-4}$ & $10^{4}$ & $H Q=1$ & $\mathrm{HQ}=0.1$ & $10^{4}$ & $10^{4}$ & $\mathrm{HI}=\mathrm{I}$ & $\mathrm{HI}=0.1$ \\
\hline $\begin{array}{l}\text { Sodium } \\
\text { Diethyldithiocarbamste }\end{array}$ & $000148-18-5$ & $6.9 E+04$ & $6.9 E+02$ & $8.0 \mathrm{E}+05$ & $8.0 E+04$ & & & & & $5.2 \mathrm{E}+05$ & $5.2 \mathrm{E}+03$ & $1.0 E+06$ & $6.1 \mathrm{E}+05$ & $6.1 E+04$ & $6.1 \mathrm{E}+02$ & 7.1E+05 & $7.1 E+04$ \\
\hline Sodium Fluoroacetate & $000062-74-8$ & & & $5.3 \mathrm{E}+01$ & $5.3 E+\infty$ & & & & & & & $4.0 \mathrm{E}+02$ & $4.0 E+01$ & & & $4.7 E+01$ & 4.7E+00 \\
\hline Sodium Metrvanadate & $013718-26-8$ & & & $2.7 \mathrm{E}+04$ & $2.7 E+03$ & & & & & & & $8.1 E+05$ & $8.1 E+04$ & & & $2.6 \mathrm{E}+04$ & $2.6 \mathrm{E}+03$ \\
\hline $\begin{array}{l}\text { Stirofos } \\
\text { (Tetrachiorovinphos) }\end{array}$ & $000961-11-5$ & $7,8 \mathrm{E}+05$ & $7.8 \mathrm{E}+03$ & $8.0 \mathrm{E}+04$ & $8.0 E+03$ & & & & & $1.0 \mathrm{E}+06$ & $5.9 E+04$ & $6.1 E+05$ & $6.1 \mathrm{E}+04$ & $6.9 \mathrm{E}+05$ & $6.9 E+03$ & $7.1 \mathrm{E}+04$ & $7.1 E+03$ \\
\hline Strontium, Suble & $007440-24-6$ & & & $1.0 \mathrm{E}+06$ & $1.6 \mathrm{E}+05$ & & & & & & & $1.0 \mathrm{E}+06$ & $1.0 \mathrm{E}+06$ & & & $1.0 \mathrm{E}+06$ & 1.5E+05 \\
\hline Stryehrine & $000057-24-9$ & & & $8.0 E+03$ & $8.0 \mathrm{E}+02$ & & & & & & & $6.1 \mathrm{E}+04$ & $6.1 \mathrm{E}+03$ & & & $7.1 \mathrm{E}+03$ & 7.1E+02 \\
\hline Styrene ${ }^{(9)}$ & $000100-42-5$ & & & $5.3 \mathrm{E}+0 \mathrm{~S}$ & $5.3 E+04$ & & & $6.7 E+02$ & $6.7 \mathrm{E}+02$ & & & $1.0 \mathrm{E}+06$ & $6.5 E+05$ & & & $6.7 \mathrm{E}+02$ & $6.7 \mathrm{E}+02$ \\
\hline Sulfate & $014808-79-8$ & & & & & & & & & & & & & & & & \\
\hline Systhane & $088671-89-0$ & & & $6.7 E+04$ & $6.7 \mathrm{E}+03$ & & & & & & & $5.1 \mathrm{E}+05$ & $5.1 \mathrm{E}+04$ & & & $5.9 \mathrm{E}+04$ & $5.9 \mathrm{E}+03$ \\
\hline TCDD, 2,3,7,8.3' & $001746-01-6$ & $1.2 \mathrm{E}-01$ & $1.2 \mathrm{E}-03$ & & & $1.3 \mathrm{E}+04$ & $1.3 E+02$ & & & $3.1 \mathrm{E}-01$ & $3.1 \mathrm{E}-03$ & & & $8.9 \mathrm{E}-02$ & $8.9 \mathrm{E}-04$ & & \\
\hline TCDF, 2,3,7,8.(1) & $051207-31-9$ & $1.2 \mathrm{E}+00$ & $1.2 \mathrm{E}-02$ & & & $1.3 E+05$ & $1.3 \mathrm{E}+03$ & & & $9.4 \mathrm{E}+00$ & 9.4E-02 & & & $1.1 \mathrm{E}+\infty$ & $1.1 \mathrm{E}-02$ & & \\
\hline TCMTB & $021564-17.0$ & & & $8.0 \mathrm{E}+05$ & $8.0 \mathrm{E}+04$ & & & & & & & $1.0 \mathrm{E}+06$ & $6.1 E+05$ & & & $7.1 E+05$ & $7.1 E+04$ \\
\hline Tebuthiuron & $034014-18-1$ & & & $1.9 \mathrm{E}+05$ & $1.9 \mathrm{E}+04$ & & & & & & & $1.0 \mathrm{E}+06$ & $1.4 E+05$ & & & $1.6 \mathrm{E}+05$ & $1.6 \mathrm{E}+04$ \\
\hline Temephos & $003383-96.8$ & & & $5.3 E+05$ & $5.3 \mathrm{E}+04$ & & & & & & & $1.0 \mathrm{E}+06$ & $4.0 \mathrm{E}+05$ & & & $4.7 \mathrm{E}+05$ & $4.7 E+04$ \\
\hline Terbacil & $005902-51-2$ & & & $3.5 \mathrm{E}+04$ & $3.5 \mathrm{E}+03$ & & & & & & & $2.6 \mathrm{E}+05$ & $2.6 \mathrm{E}+04$ & & & $3.1 \mathrm{E}+04$ & $3.1 \mathrm{E}+03$ \\
\hline Terbufos & $013071-79-9$ & & & $6.7 \mathrm{E}+01$ & $6.7 E+\infty 0$ & & & & & & & $5.1 E+02$ & $5.1 \mathrm{E}+01$ & & & $5.9 E+01$ & $5.9 \mathrm{E}+00$ \\
\hline Tetbutryn & $000886-50-0$ & & & $2.7 \mathrm{E}+03$ & $2.7 E+02$ & & & & & & & $2.0 \mathrm{E}+04$ & $2.0 \mathrm{E}+03$ & & & $2.4 E+03$ & $2.4 E+02$ \\
\hline Tetrabutyl Lead & $001920-90-7$ & & & & & & & & & & & & & & & & \\
\hline $\begin{array}{l}\text { Tetrachlorobenzene, } \\
\text { 1,2,4,5. }\end{array}$ & $000095-94.3$ & & & $8.0 \mathrm{E}+03$ & $8.0 E+02$ & & & & & & & $6.1 E+04$ & $6.1 E+03$ & & & $7.1 E+03$ & 7.1E+02 \\
\hline $\begin{array}{l}\text { Tetrachloroethane, } \\
\text { 1,1,1,2- }\end{array}$ & $000630-20-6$ & $7.2 E+05$ & $7.2 \mathrm{E}+03$ & $8.0 \mathrm{E}+04$ & $8.0 \mathrm{E}+03$ & $7.7 E+02$ & $7.7 E+02$ & & & $1.0 \mathrm{E}+06$ & $8.7 \mathrm{E}+04$ & $9.7 E+05$ & $9.7 E+04$ & 7.7E+02 & 7.7E+02 & $7.7 \mathrm{E}+02$ & $7.7 \mathrm{E}+02$ \\
\hline $\begin{array}{l}\text { Tetrachloroethane, } \\
1,1,2,2-\end{array}$ & $000079-34-5$ & $9.3 \mathrm{E}+04$ & $9.3 \mathrm{E}+02$ & & & $1.1 \mathrm{E}+03$ & $1.1 E+03$ & & & $9.9 E+05$ & $9.9 E+03$ & & & $1.1 E+03$ & $6.1 E+02$ & & \\
\hline
\end{tabular}

Nonradionuclides/soil/excavation

Page 27 of 32 


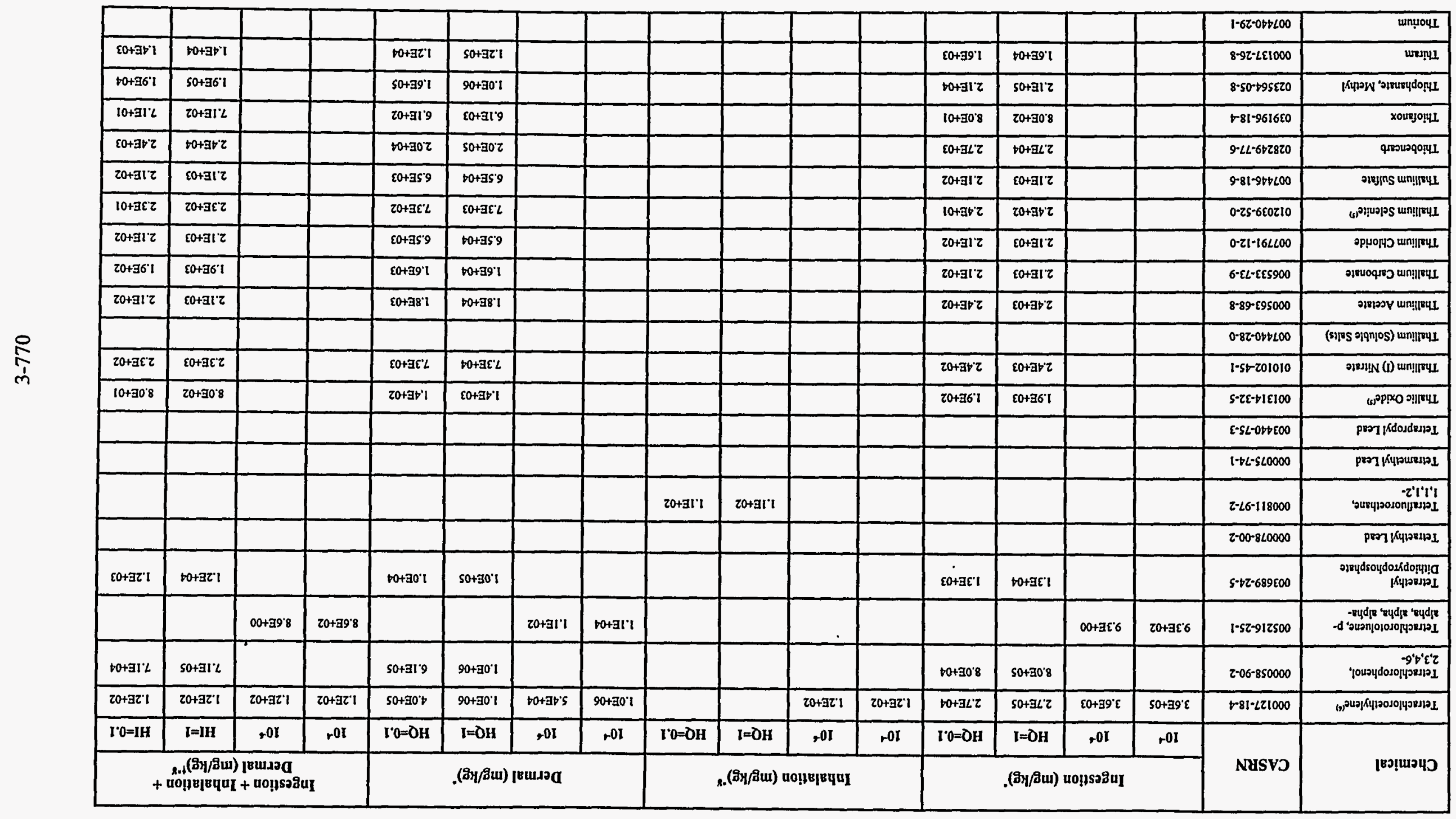


Table 3.5a. (continued)

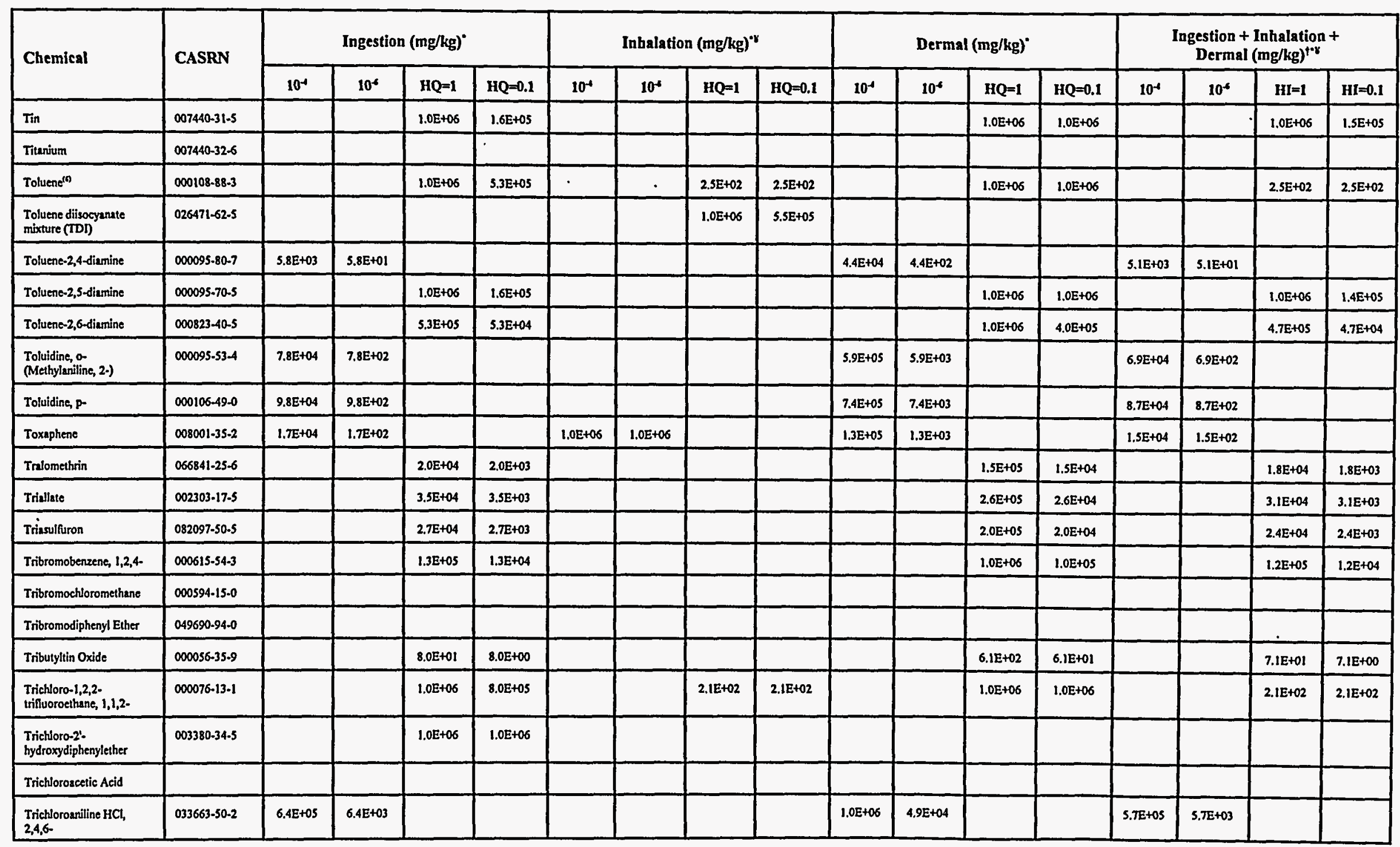

Nonradionuclides/soil/excavation

Page 29 of 32 
Table 3.5a. (continued)

\begin{tabular}{|c|c|c|c|c|c|c|c|c|c|c|c|c|c|c|c|c|c|}
\hline \multirow[t]{2}{*}{ Chemical } & \multirow[t]{2}{*}{ CASRN } & \multicolumn{4}{|c|}{ Ingestion (mg/kg) } & \multicolumn{4}{|c|}{ Inhalation $(\mathrm{mg} / \mathrm{kg})^{-8}$} & \multicolumn{4}{|c|}{ Dermal (mg/kg)" } & \multicolumn{4}{|c|}{$\begin{array}{c}\text { Ingestion + Inhalation + } \\
\text { Dermal }(\mathrm{mg} / \mathrm{kg})^{* * *}\end{array}$} \\
\hline & & $10^{-4}$ & $10^{5}$ & $\mathrm{HQ}=1$ & $\mathrm{HQ}=0.1$ & $10^{-4}$ & $10^{4}$ & $\mathrm{HQ}=1$ & $\mathrm{HQ}=0.1$ & $10^{4}$ & $10^{4}$ & $\mathrm{HQ}=1$ & $\mathrm{HQ}=0.1$ & $10^{-4}$ & $10^{6}$ & $H I=1$ & $\mathrm{HI}=0.1$ \\
\hline Trichloroaniline, 2,4,6- & $000634-93.5$ & $5.5 \mathrm{E}+05$ & $5.5 \mathrm{E}+03$ & & & & & & & $1.0 \mathrm{E}+06$ & $4.2 \mathrm{E}+04$ & & & $4.8 \mathrm{E}+05$ & $4.8 \mathrm{E}+03$ & & \\
\hline Trichlorobenzene, 1,2,4- & $000120-82-1$ & & & $2.7 \mathrm{E}+04$ & $2.7 \mathrm{E}+03$ & & & 8.3E+02 & $8.3 \mathrm{E}+02$ & & & $3.9 \mathrm{E}+05$ & $3.9 \mathrm{E}+04$ & & & $8.3 \mathrm{E}+02$ & $8.3 \mathrm{E}+02$ \\
\hline Trichloroecthane, 1,1,1-1) & $000071-55.6$ & & & 2.4E+05 & $2.4 \mathrm{E}+04$ & & & 7.3E+02 & 7.3E+02 & & & $1.0 \mathrm{E}+06$ & $3.3 \mathrm{E}+05$ & & & 7.3E+02 & $7.3 \mathrm{E}+02$ \\
\hline Trichloroethane, 1,1,2- & $000079-00-5$ & $3.3 \mathrm{E}+05$ & $3.3 \mathrm{E}+03$ & $1.1 \mathrm{E}+05$ & $1.1 \mathrm{E}+04$ & $8.5 E+02$ & $8.5 \mathrm{E}+02$ & & & $1.0 \mathrm{E}+06$ & $4.0 \mathrm{E}+04$ & $1.0 \mathrm{E}+06$ & $1.3 \mathrm{E}+05$ & $8.5 \mathrm{E}+02$ & $8.5 \mathrm{E}+02$ & $8.5 \mathrm{E}+02$ & $8.5 \mathrm{E}+02$ \\
\hline Trichloroethylene & $000079.01-6$ & $1.0 \mathrm{E}+06$ & $1.7 \mathrm{E}+04$ & & & 4.5E+02 & $4.5 \mathrm{E}+02$ & & & $1.0 \mathrm{E}+06$ & $3.9 E+04$ & & & 4.5E+02 & $4.5 \mathrm{E}+02$ & & \\
\hline Trichlorofluoromethane & $000075-69-4$ & & & $1.0 \mathrm{E}+06$ & $1.9 \mathrm{E}+05$ & & & $7.1 \mathrm{E}+02$ & 7.1E+02 & & & $1.0 \mathrm{E}+06$ & $6.5 \mathrm{E}+05$ & & & $7.1 \mathrm{E}+02$ & $7.1 \mathrm{E}+02$ \\
\hline Trichlorophenol, 2,4,5- & $000095-95-4$ & & & $1.0 \mathrm{E}+06$ & 2.7E+0S & & & & & & & $1.0 \mathrm{E}+06$ & $1.0 \mathrm{E}+06$ & & & $1.0 \mathrm{E}+06$ & $2.4 \mathrm{E}+05$ \\
\hline Trichlorophenol, 2,4,6- & $000088-06-2$ & $1.0 \mathrm{E}+06$ & $1.7 \mathrm{E}+04$ & & & $1.0 \mathrm{E}+06$ & $1.0 \mathrm{E}+06$ & & & $1.0 \mathrm{E}+06$ & $1.3 \mathrm{E}+05$ & & & $1.0 \mathrm{E}+06$ & $1.5 \mathrm{E}+04$ & & \\
\hline $\begin{array}{l}\text { Trichlorophenoxy) } \\
\text { Propionic Acid, 2(2,4,5- }\end{array}$ & $000093-72-1$ & & & $2.1 \mathrm{E}+04$ & 2. $1 \mathrm{E}+03$ & & & & & & & $1.6 \mathrm{E}+05$ & $1.6 \mathrm{E}+04$ & & & $1.9 E+04$ & $1.9 E+03$ \\
\hline $\begin{array}{l}\text { Trichlorophenoxyactic } \\
\text { Acid, 2,4,5. }\end{array}$ & $000093.76-5$ & & & $2.7 E+05$ & 2.7E+04 & & & & & & & $1.0 \mathrm{E}+06$ & $2.0 \mathrm{E}+05$ & & & $2.4 \mathrm{E}+05$ & $2.4 E+04$ \\
\hline Trichloropropane, 1,1,2- & $000598-77-6$ & & & $1.3 \mathrm{E}+0 \mathrm{~S}$ & $1.3 \mathrm{E}+04$ & & & & & & & $1.0 \mathrm{E}+06$ & $1.0 E+05$ & & & $1.2 \mathrm{E}+05$ & $1.2 \mathrm{E}+04$ \\
\hline Trichloropropane, 1,2,3- & $000096-18-4$ & $2.7 \mathrm{E}+03$ & $2.7 \mathrm{E}+01$ & $1.6 \mathrm{E}+05$ & $1.6 \mathrm{E}+04$ & & & & & $3.2 \mathrm{E}+04$ & $3.2 \mathrm{E}+02$ & $1.0 \mathrm{E}+06$ & $1.9 \mathrm{E}+05$ & $2.5 E+03$ & $2.5 \mathrm{E}+01$ & $1.5 \mathrm{E}+05$ & $1.5 E+04$ \\
\hline Trichloropropene, 1,2,3. & $000096-19-5$ & & & $1.3 \mathrm{E}+04$ & $1.3 \mathrm{E}+03$ & & & & & & & $1.0 \mathrm{E}+05$ & $1.0 \mathrm{E}+04$ & & & $1.2 \mathrm{E}+04$ & $1.2 \mathrm{E}+03$ \\
\hline Trichlorotoluene, 2,3,6- & $002077-46-5$ & & & $1.3 \mathrm{E}+02$ & $1.3 \mathrm{E}+01$ & & & & & & & & & & & & \\
\hline $\begin{array}{l}\text { Trichloratoluene, alpha } \\
2,6-\end{array}$ & $002014-83-7$ & & & $1.3 \mathrm{E}+02$ & $1.3 \mathrm{E}+01$ & & & & & & & & & & & & \\
\hline Tridiphane & $058138-08-2$ & & & $8.0 E+03$ & $8.0 \mathrm{E}+02$ & & & & & & & $6.1 \mathrm{E}+04$ & $6.1 \mathrm{E}+03$ & & & $7.1 E+03$ & $7.1 E+02$ \\
\hline Triethyl Lead & $005224-23-7$ & & & & & & & & & & & & & & & & \\
\hline Triethylamine & $000121-44-8$ & & & & & & & $2.1 E+04$ & $2.1 E+03$ & & & & & & & & \\
\hline Trifluralin & $001582.09-8$ & $1.0 \mathrm{E}+06$ & $2.4 E+04$ & $2.0 \mathrm{E}+04$ & $2.0 \mathrm{E}+03$ & & & & & $1.0 \mathrm{E}+06$ & $1.8 \mathrm{E}+05$ & $1.5 \mathrm{E}+0 \mathrm{~S}$ & $1.5 \mathrm{E}+04$ & $1.0 \mathrm{E}+06$ & $2.1 \mathrm{E}+04$ & $1.8 \mathrm{E}+04$ & $1.8 E+03$ \\
\hline Trimethyl Lead & $007442-13-9$ & & & & & & & & & & & & & & & & \\
\hline Trimethyl Phosphate & 000512.56 .1 & $5.0 E+05$ & $5.0 \mathrm{E}+03$ & & & & & & & $1.0 \mathrm{E}+06$ & $3.8 \mathrm{E}+04$ & & & $4.4 \mathrm{E}+05$ & $4.4 E+03$ & & \\
\hline Trimethylethyl Lesd & $001762-26-1$ & & & & & & & & & & & & & & & & \\
\hline
\end{tabular}


Table 3.5a. (continued)

\begin{tabular}{|c|c|c|c|c|c|c|c|c|c|c|c|c|c|c|c|c|c|}
\hline \multirow[t]{2}{*}{ Chemical } & \multirow[t]{2}{*}{ CASRN } & \multicolumn{4}{|c|}{ Ingestion (mg/kg) } & \multicolumn{4}{|c|}{ Inhalation (mg/kg) } & \multicolumn{4}{|c|}{ Dermal (mg/kg) } & \multicolumn{4}{|c|}{$\begin{array}{c}\text { Ingestion + Inhalation + } \\
\text { Dermal (mg/kg) }{ }^{1 * v}\end{array}$} \\
\hline & & $10^{-4}$ & $10^{6}$ & $H Q=1$ & $\mathrm{HQ}=0.1$ & $10^{-4}$ & $10^{*}$ & $H Q=1$ & $\mathrm{HQ}=0.1$ & $10^{-4}$ & $10^{4}$ & $H Q=1$ & $\mathrm{HQ}=0.1$ & $10^{-4}$ & $10^{4}$ & $H I=1$ & $\mathrm{Hl}=0.1$ \\
\hline Trinitrobenzene, 1,3,5- & $000099-35-4$ & & & $1.3 \mathrm{E}+03$ & $1.3 E+02$ & & & & & & & $1.3 E+04$ & $1.3 E+03$ & & & $1.2 \mathrm{E}+03$ & $1.2 \mathrm{E}+02$ \\
\hline $\begin{array}{l}\text { Trinitrophenylmethylnitra } \\
\text { mine }\end{array}$ & $000479-45.8$ & & & 2.7E+05 & 2.7E+04 & & & & & & & $1.0 \mathrm{E}+06$ & $2.0 \mathrm{E}+0 \mathrm{~S}$ & & & $2.4 \mathrm{E}+05$ & $2.4 E+04$ \\
\hline Trinitrotoluene, 2,4,6- & $000118-96-7$ & $6.2 E+05$ & $6.2 \mathrm{E}+03$ & $1.3 E+03$ & $1.3 E+02$ & & & & & $1.0 \mathrm{E}+06$ & $5.7 E+04$ & $1.2 \mathrm{E}+04$ & $1.2 E+03$ & $5.6 \mathrm{E}+05$ & $5.6 \mathrm{E}+03$ & $1.2 \mathrm{E}+03$ & $1.2 E+02$ \\
\hline Tripropyl Lead & $006618-03-7$ & & & & & & & & & & & & & & & & \\
\hline Uranium (Soluble Salts) & NA & & & $8.0 \mathrm{E}+03$ & $8.0 E+02$ & & & & & & & $1.0 \mathrm{E}+06$ & $1.0 E+05$ & & & $7.9 E+03$ & $7.9 \mathrm{E}+02$ \\
\hline Vanadium Pentoxide & $001314-62-1$ & & & $2.4 E+04$ & $2.4 E+03$ & & & & & & & 7.3E+05 & $7.3 E+04$ & & & $2.3 E+04$ & 2.3E+03 \\
\hline Vanadium Sulfate & $036907-42-3$ & & & $5.3 \mathrm{E}+04$ & $5.3 E+03$ & & & & & & & $1.0 E+06$ & $1.6 \mathrm{E}+05$ & & & $5.2 \mathrm{E}+04$ & $5.2 \mathrm{E}+03$ \\
\hline Venadium, Metallic & $007440-62-2$ & & & $1.9 E+04$ & $1.9 E+03$ & & & & & & & $2.8 \mathrm{E}+04$ & $2.8 \mathrm{E}+03$ & & & $1.1 \mathrm{E}+04$ & 1.IE+03 \\
\hline Vanadyl Sulfate & $027774-13-6$ & & & $5.3 \mathrm{E}+04$ & $5.3 \mathrm{E}+03$ & & & & & & & $1.0 \mathrm{E}+06$ & $1.6 \mathrm{E}+05$ & & & $5.2 \mathrm{E}+04$ & $5.2 \mathrm{E}+03$ \\
\hline Vernolate & $001929-77-7$ & & & $2.7 E+04$ & $2.7 E+03$ & • & & & & & & $2.0 \mathrm{E}+05$ & $2.0 \mathrm{E}+04$ & & & $2.4 E+04$ & $2.4 E+03$ \\
\hline Vinclozolin & $050471-44-8$ & & & $6.7 E+04$ & $6.7 \mathrm{E}+03$ & & & & & & & $5.1 E+05$ & $5.1 \mathrm{E}+04$ & & & $5.9 E+04$ & $5.9 E+03$ \\
\hline Vinyl Acetate & $000108.05-4$ & & • & $1.0 E+06$ & 2.7E+0S & & & $7.8 \mathrm{E}+02$ & $7.8 \mathrm{E}+02$ & & & $1.0 E+06$ & $1.0 E+06$ & & & $7.8 E+02$ & $7.8 \mathrm{E}+02$ \\
\hline Vinyl Bromide & 000593.60 .2 & & & & & $4.2 \mathrm{E}+03$ & $3.7 \mathrm{E}+02$ & 7.7E+02 & $7.7 \mathrm{E}+0 \mathrm{I}$ & & & & & & & & \\
\hline Vinyl Chloride $e^{10}$ & $000075.01-4$ & $9.8 E+03$ & $9.8 \mathrm{E}+01$ & & & $1.3 E+01$ & $1.3 \mathrm{E}-01$ & & & $1.5 \mathrm{E}+05$ & $1.5 E+03$ & & & t.3E+0I & 1.3E-01 & & \\
\hline Werfarin & 000081.81 .2 & . & & $8.0 \mathrm{E}+02$ & $8.0 E+01$ & & & & & & & $6.1 E+03$ & $6.1 E+02$ & & & $7.1 E+02$ & $7.1 E+01$ \\
\hline White Phosphorus & $007723-14-0$ & & & $5.3 \mathrm{E}+01$ & $5.3 \mathrm{E}+\infty$ & & & & & & & $1.6 \mathrm{E}+03$ & $1.6 \mathrm{E}+02$ & & & $5.2 E+01$ & $5.2 \mathrm{E}+\infty$ \\
\hline Xylene, Mixture & $001330-20.7$ & & & $9.5 \mathrm{E}+05$ & $9.5 \mathrm{E}+04$ & & & & & & & $1.0 \mathrm{E}+06$ & $1.0 \mathrm{E}+06$ & & & $8.9 E+05$ & $8.9 \mathrm{E}+04$ \\
\hline Xylene, P.(6) & $000106-42.3$ & & & $7.1 \mathrm{E}+05$ & $7.1 \mathrm{E}+04$ & & & & & & & & & & & & \\
\hline Xylene, $m \cdot{ }^{n}$ & $000108-38-3$ & & & $1.8 \mathrm{E}+05$ & $1.8 \mathrm{E}+04$ & & & & & & & $1.0 \mathrm{E}+06$ & 2.2E+05 & & & $1.6 \mathrm{E}+05$ & $1.6 \mathrm{E}+04$ \\
\hline Xylene, o. (1) & $000095-47.6$ & & & $1.0 \mathrm{E}+06$ & $5.3 E+05$ & & & & & & & $1.0 \mathrm{E}+06$ & $1.0 \mathrm{E}+06$ & & & $1.0 \mathrm{E}+06$ & $4.9 \mathrm{E}+05$ \\
\hline Zine (Metallic) & $007440-66-6$ & & & $8.0 \mathrm{E}+05$ & $8.0 E+04$ & & & & & & & $1.0 \mathrm{E}+06$ & $1.0 \mathrm{E}+06$ & & & $7.7 \mathrm{E}+05$ & $7.7 \mathrm{E}+04$ \\
\hline Zinc Cyanide & $000557-21-1$ & & & $1.3 \mathrm{E}+05$ & $1.3 E+04$ & & & & & & & $1.0 \mathrm{E}+06$ & 4.0E+05 & & & $1.3 E+05$ & 1.3E+04 \\
\hline
\end{tabular}


Table 3.5a. (continued)

\begin{tabular}{|c|c|c|c|c|c|c|c|c|c|c|c|c|c|c|c|c|c|}
\hline \multirow[t]{2}{*}{ Chemical } & \multirow[t]{2}{*}{ CASRN } & \multicolumn{4}{|c|}{ Ingestion (mg/kg) } & \multicolumn{4}{|c|}{ Inhalation (mg/kg) } & \multicolumn{4}{|c|}{ Dermal (mg/kg) } & \multicolumn{4}{|c|}{$\begin{array}{c}\text { Ingestion + Inhalation + } \\
\text { Dermal }(\mathrm{mg} / \mathrm{kg})^{+\bullet}\end{array}$} \\
\hline & & $10^{-4}$ & $10^{6}$ & $\mathrm{HQ}=1$ & $\mathrm{HQ}=0.1$ & $10^{-4}$ & $10^{4}$ & $\mathrm{HQ}=\mathbf{1}$ & $\mathrm{HQ}=0.1$ & $10^{-1}$ & $10^{5}$ & $\mathrm{HQ}=1$ & $\mathrm{HQ}=0.1$ & $10^{-4}$ & $10^{4}$ & $\mathrm{HI}=1$ & $\mathrm{HI}=0.1$ \\
\hline Zinc Phosphide & 001314.84 .7 & & & $8.0 E+03$ & $8.0 E+02$ & & & & & & & $2.4 \mathrm{E}+05$ & $2.4 \mathrm{E}+04$ & & & $7.7 \mathrm{E}+03$ & $7.7 E+02$ \\
\hline Zineb & $012122-67.7$ & & & $1.3 E+05$ & $1.3 \mathrm{E}+04$ & & & & & & & $1.0 E+06$ & $1.0 E+05$ & & & $1.2 \mathrm{E}+05$ & $1.2 \mathrm{E}+04$ \\
\hline Zirconium & $007440-67-7$ & & & & & & & & & & & & & & & & \\
\hline
\end{tabular}

Notes:

* By definition, $1 \mathrm{~kg}=1.0 \mathrm{E}+06 \mathrm{mg}$. Therefore, a limit of $1.0 \mathrm{E}+06 \mathrm{mg} / \mathrm{kg}$ was used for any PRG that was calculated to be above this limit.

$\dagger$ If slope factors and/or reference doses were available for only one pathway (i.e., ingestion, dermal, OR inhalation), the total for ingestion plus inhalation plus dermal was not calculated.

\# The volatilization factor defines the relationship between the concentration of contaminants in soil and the volatilization of contaminants in air. The chemical concentration in soil (C) calculated using the volatilization factor must be compared with the soil saturation concentration $\left(\mathrm{C}_{\mathrm{sat}}\right)$. If $\mathrm{C}$ is greater than $\mathrm{C}_{\text {sat }}$, then the $P R G$ is set equal to $\mathrm{C}_{\text {sat* }}$

(1) The same oral slope factor as used for polychlorinated biphenyls.

(2) The PRG was calculated considering Supplemental Guidance from RAGS: Region 4 Bulletins, Human Health Risk Assessment (Interim Guidance) from EPA (received February 11, 1992). The slope factors for chemicals so marked indicate that a toxicity equivalency factor (TEF) method was used for carcinogenic PAHs based on each compound's relative potency to the potency of benzo[a]pyrene. The following TEFs were used to convert each PAH slope factor to an equivalent slope factor of benzo[a]pyrene [that has a new cancer slope factor of $7.3(\mathrm{mg} / \mathrm{kg} / \mathrm{day})^{-1}$ identified by EPA's CRAVE workgroup]: (1) benzo[a]pyrene, TEF $=1.0 ;(2)$ benzo[a]anthracene, $\mathrm{TEF}=0.1 ;(3)$ benzo[b]fluoranthene, TEF $=0.1 ;$ (4) benzo[k]fluoranthene, TEF $=0.01 ;(5)$ chrysene, TEF $=0.001 ;(6)$ dibenz $[\mathrm{a}$,h]anthracene, TEF $=1.0$; and $(7)$ indeno[ $[1,2,3-c, d]$ pyrene, $\mathrm{TEF}=0.1$.

(3) The toxicity values for trichloroethylene have been withdrawn from IRIS and HEAST pending further investigation. However, the most recent toxicity values have been used to calculate the PRG.

(4) The PRG was calculated considering Supplemental Guidance from RAGS: Region 4 Bulletins, Human Health Risk Assessment (Interim Guidance) from EPA (November 1995). The slope factors for chemicals so marked indicate that a TEF methodology was used for chlorinated dioxin and furan congeners based on each compound's relative potency to the potency of 2,3,7,8-tetrachlorodibenzodioxin (TCDD). The following TEFs were used to convert each slope factor to an equivalent slope factor of 2,3,7,8TCDD: (1) 2,3,7,8-TCDD, TEF = 1.0; (2) 2,3,7,8-PeCDD, TEF = 0.5; (3) 2,3,7,8-HxCDD, TEF =0.1; (4) 2,3,7,8-HpCDD, TEF =0.01; (5) OCDD, TEF =0.001; (6) $2,3,7,8-\mathrm{TCDF}, \mathrm{TEF}=0.1 ;(7) 1,2,3,7,8-\mathrm{PeCDF}, \mathrm{TEF}=0.5 ;(8) 2,3,4,7,8-\mathrm{PeCDF}, \mathrm{TEF}=0.05 ;(9) 2,3,7,8-\mathrm{HxCDF}, \mathrm{TEF}=0.1 ;(10) 2,3,7,8-\mathrm{HpCDF}, \mathrm{TEF}=0.01 ;$ and (11) OCDF, TEF $=0.001$.

(5) Toxicity values for these chemicals have been withdrawn from IRIS and HEAST pending further investigation. However, the most recent toxicity values have been used to calculate the PRG.

(6) Toxicity values for these chemicals were obtained from the Superfund Health Risk Technical Support Center. 
Table 3.5b. Risk-based Preliminary Remediation Goals for radionuclides in soil (excavation scenario)

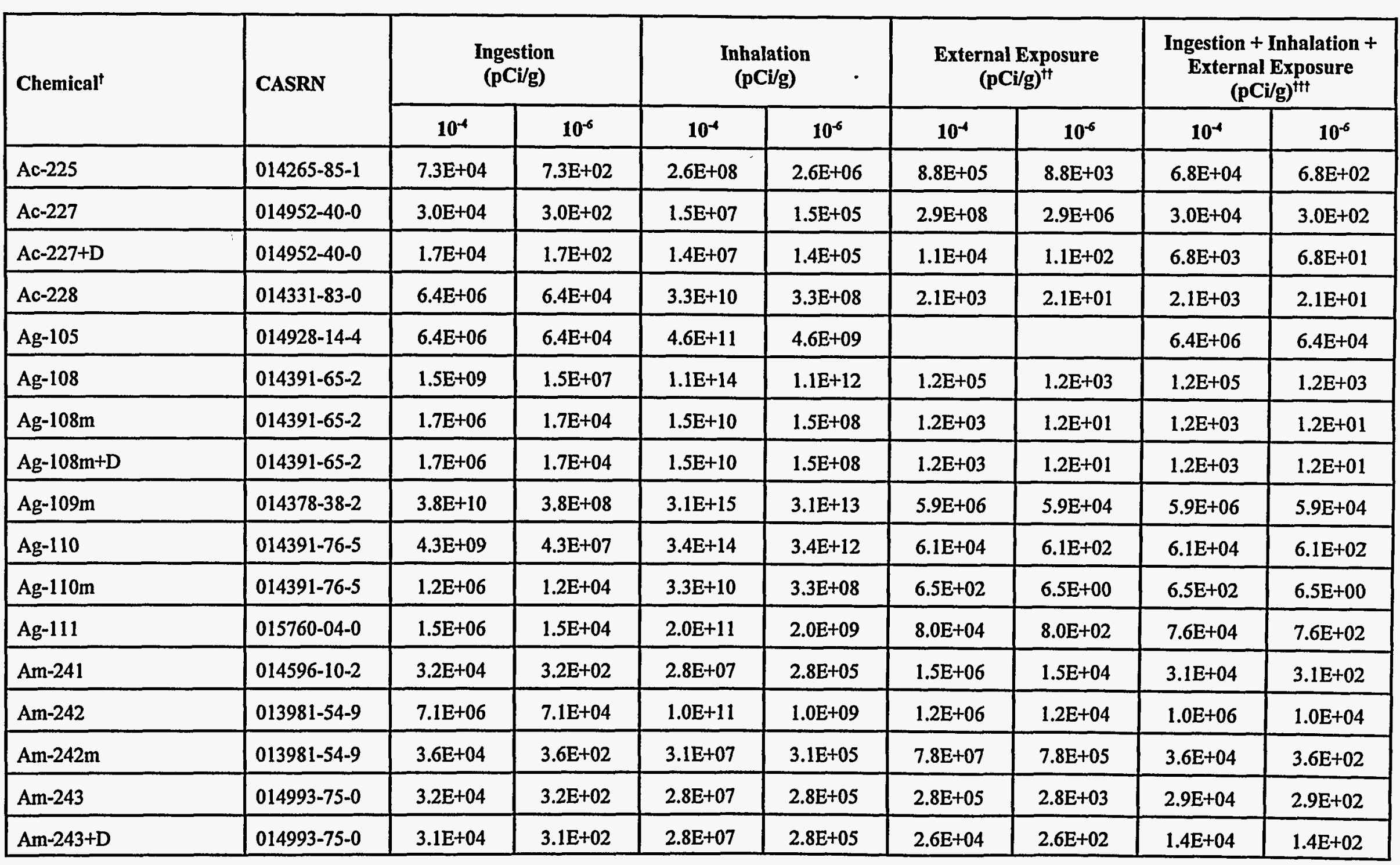


Table 3.5b. (continued)

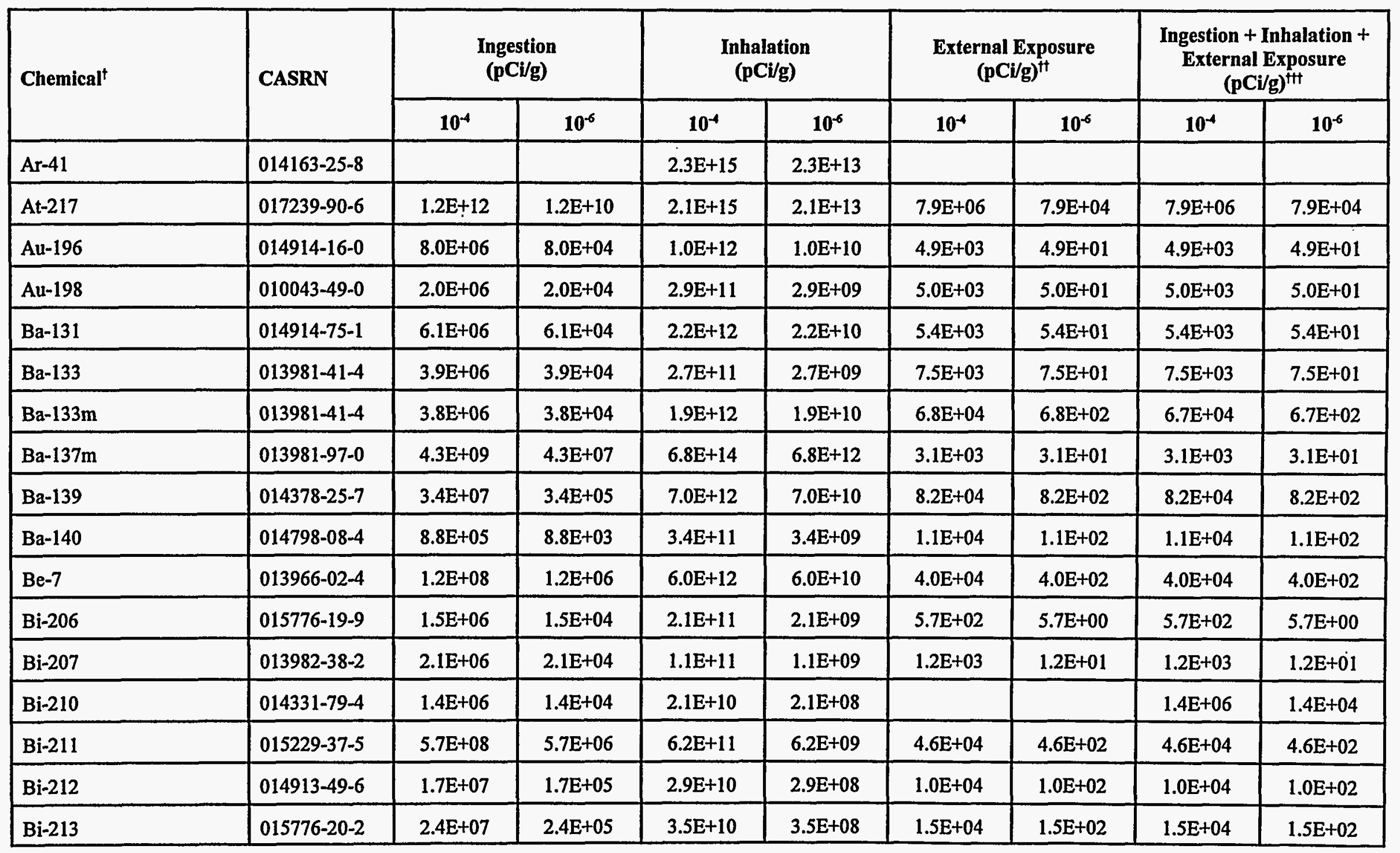


Table 3.5b. (continued)

\begin{tabular}{|c|c|c|c|c|c|c|c|c|c|}
\hline \multirow[t]{2}{*}{ Chemical $^{\dagger}$} & \multirow[t]{2}{*}{ CASRN } & \multicolumn{2}{|c|}{$\begin{array}{l}\text { Ingestion } \\
\text { (pCi/g) }\end{array}$} & \multicolumn{2}{|c|}{$\begin{array}{l}\text { Inhalation } \\
\text { (pCi/g) }\end{array}$} & \multicolumn{2}{|c|}{$\begin{array}{l}\text { External Exposure } \\
(\mathrm{pCi} / \mathrm{g})^{\mathrm{tt}}\end{array}$} & \multicolumn{2}{|c|}{$\begin{array}{c}\text { Ingestion + Inhalation + } \\
\text { External Exposure } \\
(\mathrm{pCi} / \mathrm{g})^{+1+}\end{array}$} \\
\hline & & $10^{-4}$ & $10^{-6}$ & $10^{-4}$ & $10^{-6}$ & $10^{-4}$ & $10^{-6}$ & $10^{-4}$ & $10^{-5}$ \\
\hline $\mathrm{Bi}-214$ & 014733-03-0 & $5.3 E+07$ & $5.3 E+05$ & $7.3 E+10$ & $7.3 \mathrm{E}+08$ & $1.1 E+03$ & $1.1 \mathrm{E}+01$ & $1.1 \mathrm{E}+03$ & $1.1 \mathrm{E}+01$ \\
\hline $\mathrm{Br}-82$ & $014686-69-2$ & $7.3 E+06$ & $7.3 E+04$ & $1.4 \mathrm{E}+12$ & $1.4 \mathrm{E}+10$ & $6.8 \mathrm{E}+02$ & $6.8 \mathrm{E}+00$ & $6.8 \mathrm{E}+02$ & $6.8 \mathrm{E}+00$ \\
\hline C-14 & $014762-75-5$ & $1.0 \mathrm{E}+07$ & $1.0 \mathrm{E}+05$ & $1.5 \mathrm{E}+14$ & $1.5 \mathrm{E}+12$ & & & $1.0 \mathrm{E}+07$ & $1.0 \mathrm{E}+05$ \\
\hline$C-15$ & 015929-23-4 & $1.6 \mathrm{E}+10$ & $1.6 \mathrm{E}+08$ & $1.3 \mathrm{E}+15$ & $1.3 \dot{E}+13$ & & & $1.6 \mathrm{E}+10$ & $1.6 \mathrm{E}+08$ \\
\hline $\mathrm{Ca}-45$ & 013966-05-7 & $5.2 \mathrm{E}+06$ & $5.2 \mathrm{E}+04$ & $4.3 \mathrm{E}+11$ & $4.3 E+09$ & $1.8 \mathrm{E}+15$ & $1.8 \mathrm{E}+13$ & $5.2 E+06$ & $5.2 \mathrm{E}+04$ \\
\hline $\mathrm{Ca}-47$ & 001439-99-2 & $1.6 \mathrm{E}+06$ & $1.6 \mathrm{E}+04$ & $2.1 E+11$ & $2.1 E+09$ & $1.7 \mathrm{E}+03$ & $1.7 \mathrm{E}+01$ & $1.7 \mathrm{E}+03$ & $1.7 \mathrm{E}+01$ \\
\hline $\mathrm{Ce}-141$ & 013967-74-3 & $2.7 \mathrm{E}+06$ & $2.7 E+04$ & $2.5 \mathrm{E}+11$ & $2.5 \mathrm{E}+09$ & $4.9 \mathrm{E}+04$ & $4.9 \mathrm{E}+02$ & $4.8 \mathrm{E}+04$ & $4.8 \mathrm{E}+02$ \\
\hline $\mathrm{Ce}-143$ & 014119-19-8 & $1.8 \mathrm{E}+06$ & $1.8 \mathrm{E}+04$ & $2.8 \mathrm{E}+11$ & $2.8 \mathrm{E}+09$ & $9.3 E+03$ & $9.3 E+01$ & $9.3 E+03$ & $9.3 \mathrm{E}+01$ \\
\hline $\mathrm{Ce}-144$ & 014762-78-8 & $3.5 \mathrm{E}+05$ & $3.5 E+03$ & $9.9 E+09$ & $9.9 E+07$ & 2.7E+05 & $2.7 \mathrm{E}+03$ & $1.5 \mathrm{E}+05$ & $1.5 \mathrm{E}+03$ \\
\hline Ce-144+D & 014762-78-8 & $3.5 \mathrm{E}+05$ & $3.5 \mathrm{E}+03$ & $9.9 \mathrm{E}+09$ & $9.9 E+07$ & 4.4E+04 & $4.4 \mathrm{E}+02$ & $3.9 E+04$ & $3.9 E+02$ \\
\hline $\mathrm{Cl}-36$ & 013981-43-6 & $4.7 E+06$ & $4.7 \mathrm{E}+04$ & $8.2 E+11$ & $8.2 E+09$ & & & $4.7 \mathrm{E}+06$ & 4.7E+04 \\
\hline Cl-38 & 014158-34-0 & $5.0 \mathrm{E}+07$ & $5.0 \mathrm{E}+05$ & $6.6 \mathrm{E}+12$ & $6.6 \mathrm{E}+10$ & $1.1 \mathrm{E}+03$ & $1.1 \mathrm{E}+01$ & $1.1 \mathrm{E}+03$ & $1.1 \mathrm{E}+01$ \\
\hline $\mathrm{Cm}-242$ & 015510-73-3 & $2.7 \mathrm{E}+05$ & $2.7 \mathrm{E}+03$ & $3.4 \mathrm{E}+08$ & $3.4 \mathrm{E}+06$ & $2.9 E+08$ & $2.9 \mathrm{E}+06$ & $2.7 E+05$ & $2.7 \mathrm{E}+03$ \\
\hline
\end{tabular}


Table 3.5b. (continued)

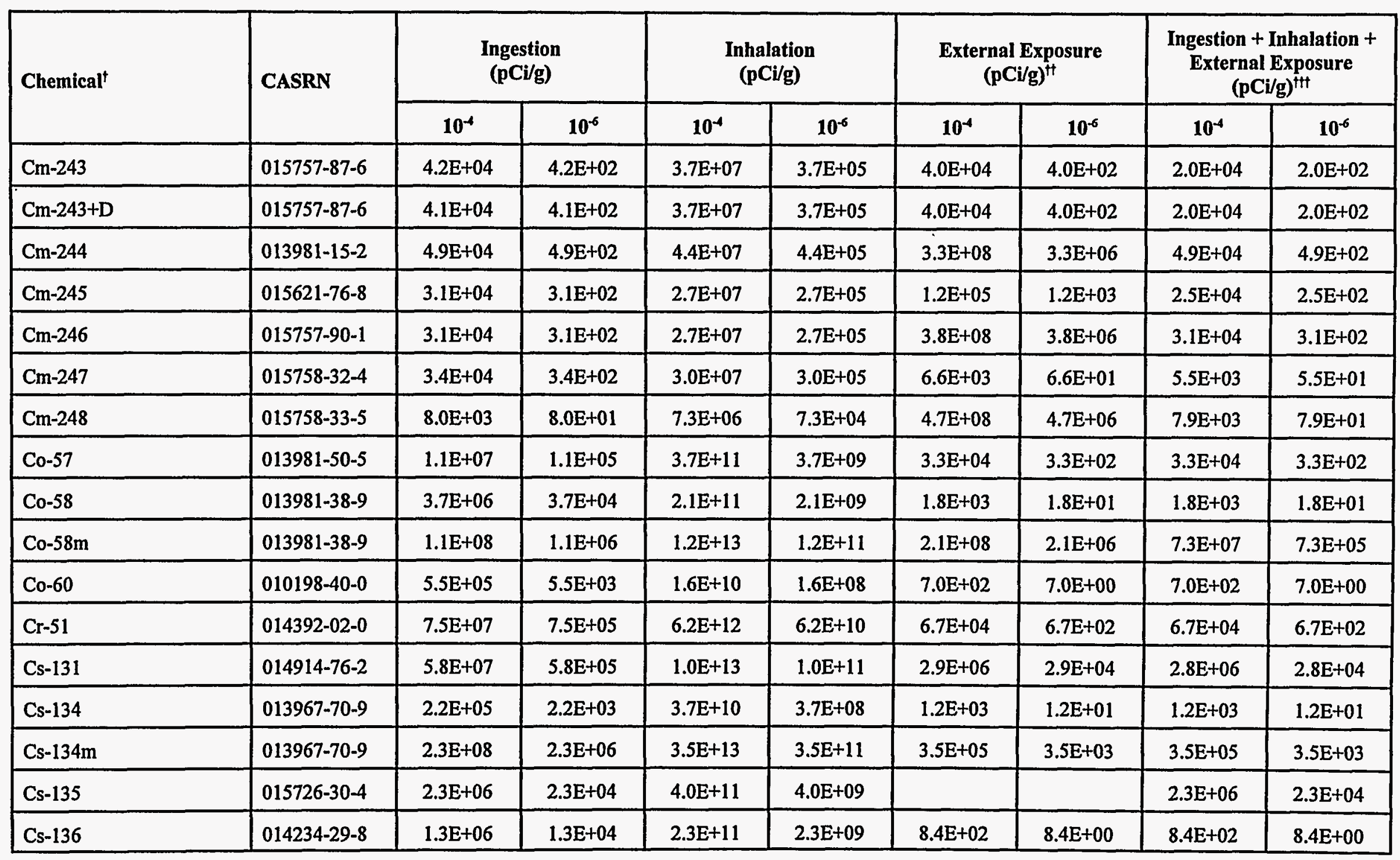


Table 3.5b. (continued)

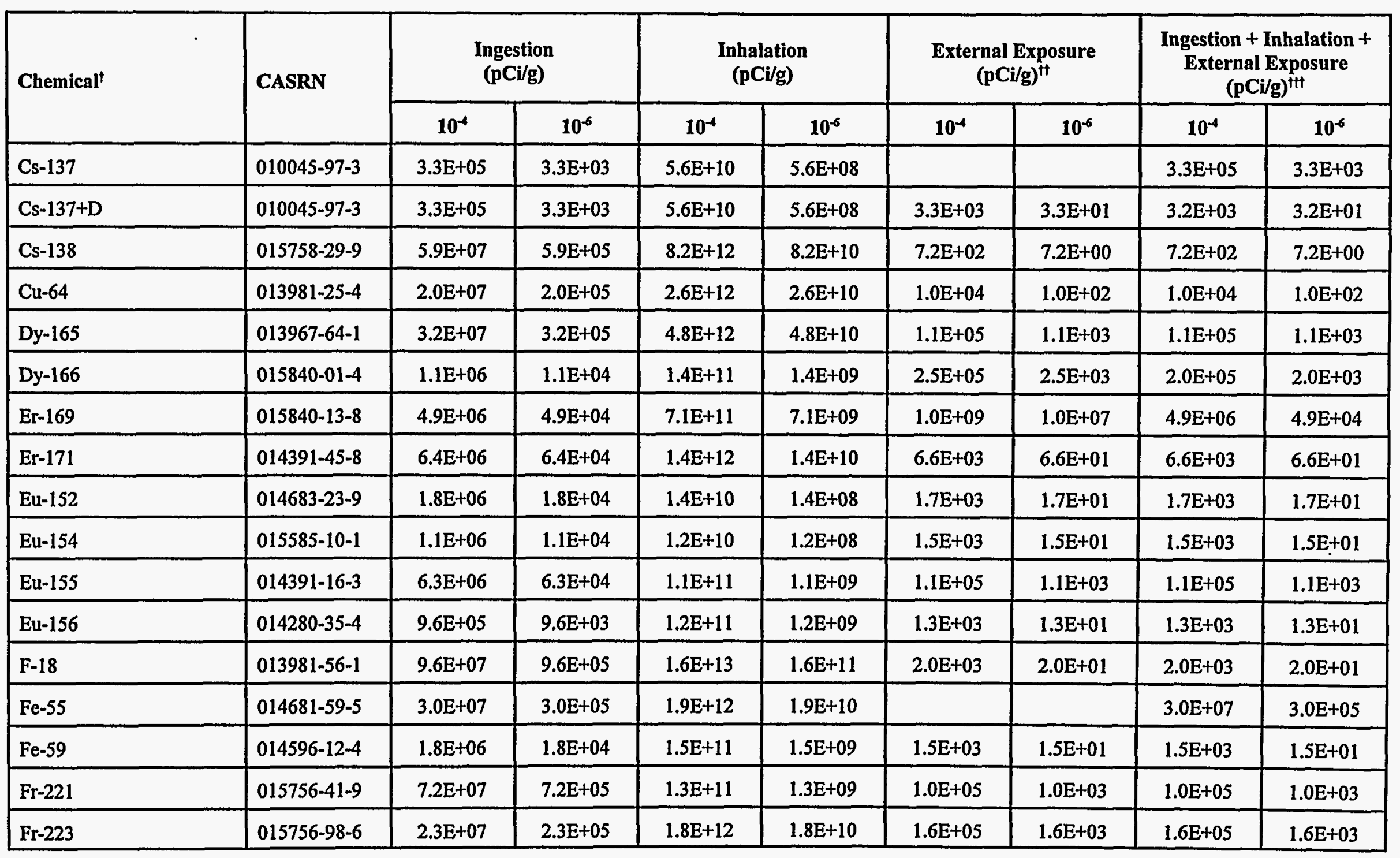


Table 3.5b. (continued)

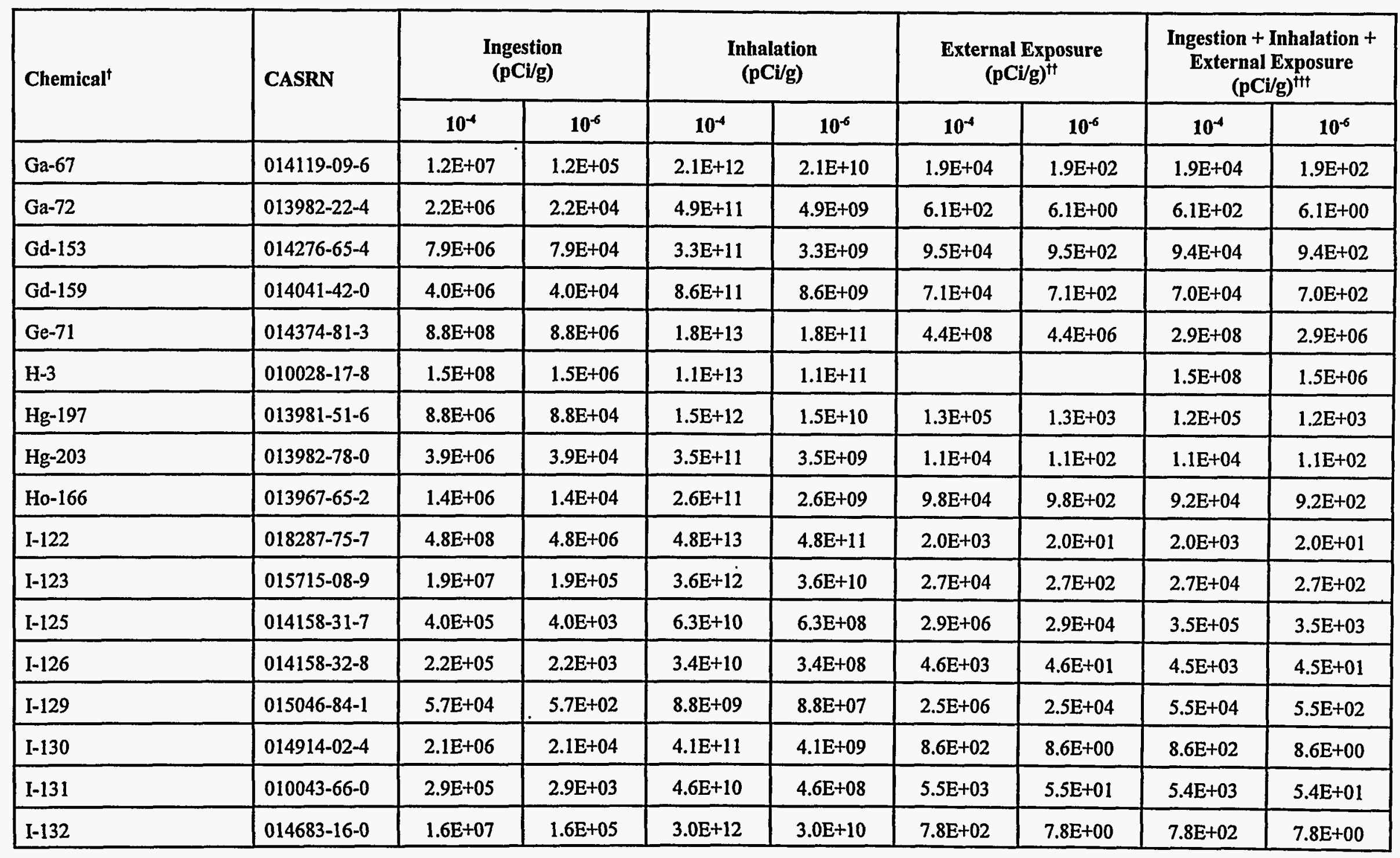


Table 3.5b. (continued)

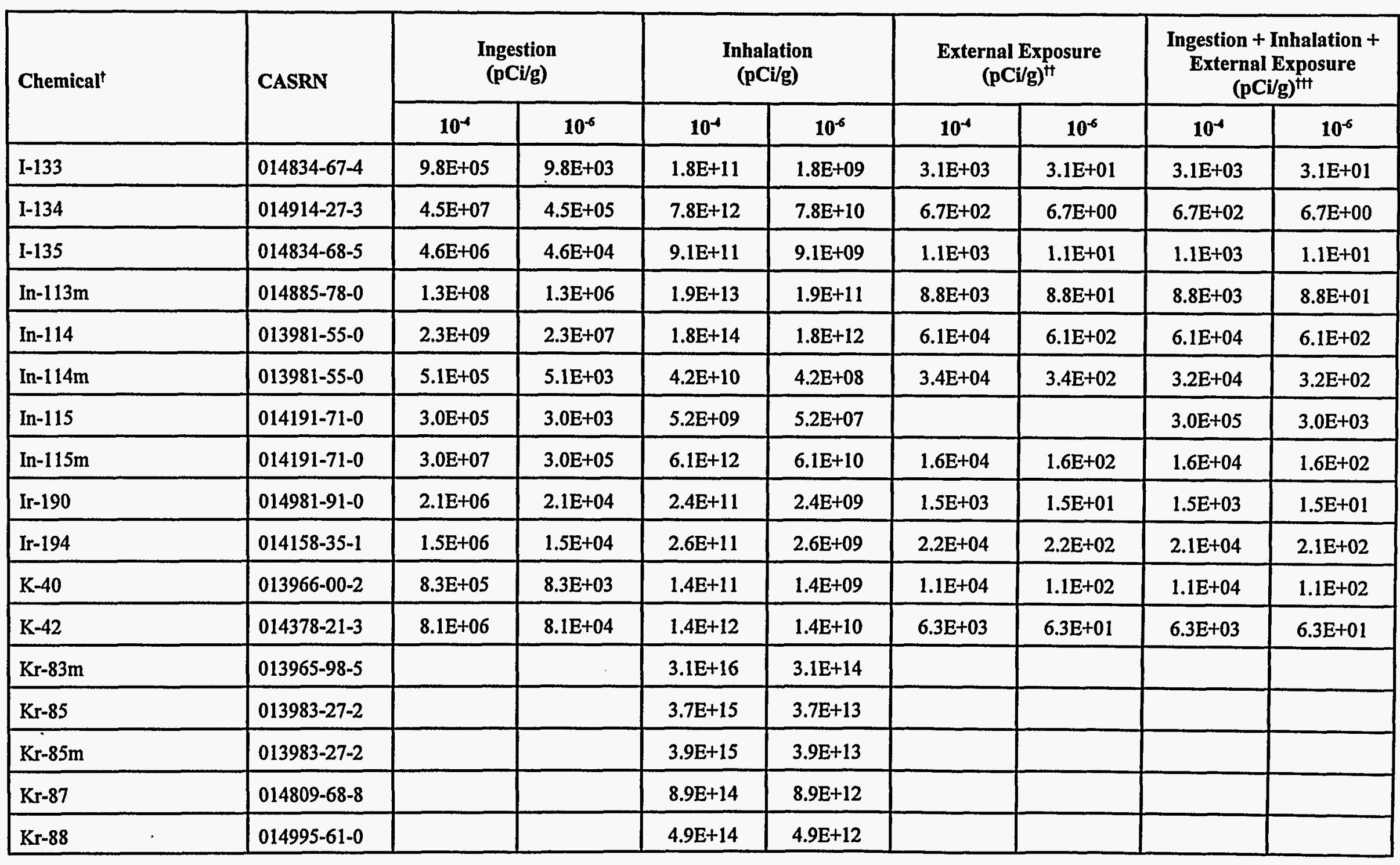


Table 3.5b. (continued)

\begin{tabular}{|c|c|c|c|c|c|c|c|c|c|}
\hline \multirow[t]{2}{*}{ Chemical $^{\dagger}$} & \multirow[t]{2}{*}{ CASRN } & \multicolumn{2}{|c|}{$\begin{array}{l}\text { Ingestion } \\
\text { (pCi/g) }\end{array}$} & \multicolumn{2}{|c|}{$\begin{array}{l}\text { Inhalation } \\
\text { (pCi/g) }\end{array}$} & \multicolumn{2}{|c|}{$\begin{array}{l}\text { External Exposure } \\
(\mathrm{pCi} / \mathrm{g})^{++}\end{array}$} & \multicolumn{2}{|c|}{$\begin{array}{c}\text { Ingestion + Inhalation + } \\
\text { External Exposure } \\
(\mathrm{pCi} / \mathrm{g})^{+t+}\end{array}$} \\
\hline & & $10^{-4}$ & $10^{-6}$ & $10^{-4}$ & $10^{-6}$ & $10^{-4}$ & $10^{-6}$ & $10^{-4}$ & $10^{-6}$ \\
\hline $\mathrm{Kr}-89$ & 016316-03-3 & & & $6.7 E+14$ & $6.7 \mathrm{E}+12$ & & & & \\
\hline $\mathrm{Kr}-90$ & 015741-13-6 & & & $6.7 \mathrm{E}+14$ & $6.7 \mathrm{E}+12$ & & & & . \\
\hline La-140 & 013981-28-7 & $1.1 \mathrm{E}+06$ & $1.1 \mathrm{E}+04$ & $2.1 \mathrm{E}+11$ & $2.1 E+09$ & $7.5 \mathrm{E}+02$ & $7.5 \mathrm{E}+00$ & $7.5 E+02$ & $7.5 \mathrm{E}+00$ \\
\hline Lu-177 & 014265-75-9 & $3.5 \mathrm{E}+06$ & $3.5 \mathrm{E}+04$ & $4.9 E+11$ & $4.9 E+09$ & $9.5 \mathrm{E}+04$ & $9.5 \mathrm{E}+02$ & $9.2 \mathrm{E}+04$ & $9.2 \mathrm{E}+02$ \\
\hline $\mathrm{Mn}-52$ & 014092-99-0 & $1.7 \mathrm{E}+06$ & $1.7 E+04$ & $2.4 \mathrm{E}+11$ & $2.4 \mathrm{E}+09$ & $5.1 E+02$ & $5.1 \mathrm{E}+00$ & $5.1 \mathrm{E}+02$ & $5.1 \mathrm{E}+00$ \\
\hline $\mathrm{Mn}-54$ & 013966-31-9 & $5.3 \mathrm{E}+06$ & $5.3 \mathrm{E}+04$ & $2.9 \mathrm{E}+11$ & $2.9 \mathrm{E}+09$ & $2.1 \mathrm{E}+03$ & $2.1 \mathrm{E}+01$ & $2.1 \mathrm{E}+03$ & $2.1 \mathrm{E}+01$ \\
\hline $\mathrm{Mn}-56$ & $014681-52-8$ & $1.2 E+07$ & $1.2 \mathrm{E}+05$ & $2.1 \mathrm{E}+12$ & $2.1 \mathrm{E}+10$ & $9.8 \mathrm{E}+02$ & $9.8 \mathrm{E}+00$ & $9.8 \mathrm{E}+02$ & $9.8 \mathrm{E}+00$ \\
\hline Mo-99 & $014119-15-4$ & $4.6 \mathrm{E}+06$ & $4.6 \mathrm{E}+04$ & $2.4 \mathrm{E}+11$ & $2.4 \mathrm{E}+09$ & $1.3 \mathrm{E}+04$ & $1.3 E+02$ & $1.3 \mathrm{E}+04$ & $1.3 \mathrm{E}+02$ \\
\hline $\mathrm{Na}-22$ & $013966-32-0$ & $1.3 \mathrm{E}+06$ & $1.3 \mathrm{E}+04$ & $2.2 \mathrm{E}+11$ & $2.2 E+09$ & $8.4 E+02$ & $8.4 \mathrm{E}+00$ & $8.4 \mathrm{E}+02$ & $8.4 \mathrm{E}+00$ \\
\hline $\mathrm{Na}-24$ & $013982-04-2$ & $7.5 \mathrm{E}+06$ & $7.5 \mathrm{E}+04$ & $1.4 \mathrm{E}+12$ & $1.4 \mathrm{E}+10$ & $3.9 \mathrm{E}+02$ & $3.9 \mathrm{E}+00$ & $3.9 \mathrm{E}+02$ & $3.9 \mathrm{E}+00$ \\
\hline $\mathrm{Nb}-93 \mathrm{~m}$ & 007440-03-1 & $1.6 \mathrm{E}+07$ & $1.6 \mathrm{E}+05$ & $2.5 E+11$ & $2.5 \mathrm{E}+09$ & $1.9 \mathrm{E}+08$ & $1.9 \mathrm{E}+06$ & $1.4 \mathrm{E}+07$ & $1.4 \mathrm{E}+05$ \\
\hline $\mathrm{Nb}-94$ & $014681-63-1$ & $1.5 \mathrm{E}+06$ & $1.5 \mathrm{E}+04$ & $1.3 \mathrm{E}+10$ & $1.3 \mathrm{E}+08$ & $1.1 \mathrm{E}+03$ & $1.1 \mathrm{E}+01$ & $1.1 \mathrm{E}+03$ & $1.1 \mathrm{E}+01$ \\
\hline $\mathrm{Nb}-95$ & $013967-76-5$ & $4.6 \mathrm{E}+06$ & $4.6 \mathrm{E}+04$ & $3.4 \mathrm{E}+11$ & $3.4 \mathrm{E}+09$ & $2.3 \mathrm{E}+03$ & $2.3 \mathrm{E}+01$ & $2.3 E+03$ & $2.3 \mathrm{E}+01$ \\
\hline $\mathrm{Nb}-95 \mathrm{~m}$ & $013967-76-5$ & $3.4 \mathrm{E}+06$ & $3.4 \mathrm{E}+04$ & $4.8 \mathrm{E}+11$ & $4.8 \mathrm{E}+09$ & $7.9 E+04$ & $7.9 \mathrm{E}+02$ & $7.7 \mathrm{E}+04$ & $7.7 \mathrm{E}+02$ \\
\hline $\mathrm{Nb}-97$ & $018496-04-3$ & $6.0 \mathrm{E}+07$ & $6.0 \mathrm{E}+05$ & $5.0 \mathrm{E}+12$ & $5.0 \mathrm{E}+10$ & $2.7 \mathrm{E}+03$ & $2.7 \mathrm{E}+01$ & $2.7 \mathrm{E}+03$ & $2.7 \mathrm{E}+01$ \\
\hline $\mathrm{Nb}-97 \mathrm{~m}$ & 018496-04-3 & $3.2 \mathrm{E}+09$ & $3.2 \mathrm{E}+07$ & $3.2 \mathrm{E}+14$ & $3.2 E+12$ & $2.5 \mathrm{E}+03$ & $2.5 \mathrm{E}+01$ & $2.5 \mathrm{E}+03$ & $2.5 \mathrm{E}+01$ \\
\hline Nd-147 & $014269-74-0$ & $1.8 \mathrm{E}+06$ & $1.8 \mathrm{E}+04$ & $2.2 \mathrm{E}+11$ & $2.2 E+09$ & $2.1 E+04$ & $2.1 \mathrm{E}+02$ & $2.1 \mathrm{E}+04$ & $2.1 E+02$ \\
\hline
\end{tabular}


Table 3.5b. (continued)

\begin{tabular}{|c|c|c|c|c|c|c|c|c|c|}
\hline \multirow[t]{2}{*}{ Chemical $^{\dagger}$} & \multirow[t]{2}{*}{ CASRN } & \multicolumn{2}{|c|}{$\begin{array}{c}\text { Ingestion } \\
\text { (pCi/g) }\end{array}$} & \multicolumn{2}{|c|}{$\begin{array}{c}\text { Inhalation } \\
\text { (pCi/g) }\end{array}$} & \multicolumn{2}{|c|}{$\begin{array}{l}\text { External Exposure } \\
\qquad(\mathrm{pCi} / \mathrm{g})^{+t}\end{array}$} & \multicolumn{2}{|c|}{$\begin{array}{c}\text { Ingestion + Inhalation + } \\
\text { External Exposure } \\
(\mathrm{pCi} / \mathrm{g})^{\mathrm{Ht}}\end{array}$} \\
\hline & & $10^{-4}$ & $10^{-6}$ & $10^{-4}$ & $10^{-6}$ & $10^{-4}$ & $10^{-6}$ & $10^{-4}$ & $10^{-6}$ \\
\hline Nd-149 & $015749-81-2$ & $2.3 E+07$ & $2.3 \mathrm{E}+05$ & $2.5 \mathrm{E}+12$ & $2.5 \mathrm{E}+10$ & $6.3 E+03$ & $6.3 \mathrm{E}+01$ & $6.3 \mathrm{E}+03$ & $6.3 \mathrm{E}+01$ \\
\hline Ni-59 & $014336-70-0$ & $5.6 \mathrm{E}+07$ & $5.6 \mathrm{E}+05$ & $2.7 \mathrm{E}+12$ & $2.7 \mathrm{E}+10$ & & & $5.6 \mathrm{E}+07$ & $5.6 \mathrm{E}+05$ \\
\hline $\mathrm{Ni}-63$ & 013981-37-8 & $1.9 E+07$ & $1.9 \mathrm{E}+05$ & $1.1 \mathrm{E}+12$ & $1.1 \mathrm{E}+10$ & & & $1.9 E+07$ & $1.9 E+05$ \\
\hline $\mathrm{Ni}-65$ & $014833-49-9$ & $1.9 \mathrm{E}+07$ & $1.9 \mathrm{E}+05$ & $3.0 \mathrm{E}+12$ & $3.0 \mathrm{E}+10$ & $3.2 \mathrm{E}+03$ & $3.2 \mathrm{E}+01$ & $3.2 \mathrm{E}+03$ & $3.2 \mathrm{E}+01$ \\
\hline Np-236 & $015700-36-4$ & $1.1 \mathrm{E}+07$ & $1.1 E+05$ & $2.8 \mathrm{E}+11$ & $2.8 \mathrm{E}+09$ & $7.4 \mathrm{E}+04$ & $7.4 \mathrm{E}+02$ & $7.4 \mathrm{E}+04$ & $7.4 \mathrm{E}+02$ \\
\hline Np-237 & $013994-20-2$ & $3.5 E+04$ & $3.5 \mathrm{E}+02$ & $3.1 E+07$ & $3.1 E+05$ & $9.1 \mathrm{E}+05$ & $9.1 \mathrm{E}+03$ & $3.4 \mathrm{E}+04$ & $3.4 \mathrm{E}+02$ \\
\hline $\mathrm{Np}-237+\mathrm{D}$ & 013994-20-2 & $3.5 \mathrm{E}+04$ & $3.5 \mathrm{E}+02$ & $3.1 E+07$ & $3.1 E+05$ & $1.5 \mathrm{E}+04$ & $1.5 E+02$ & $1.0 \mathrm{E}+04$ & $1.0 \mathrm{E}+02$ \\
\hline Np-238 & $015766-25-3$ & $2.3 \mathrm{E}+06$ & $2.3 E+04$ & $2.3 E+11$ & $2.3 E+09$ & $3.5 \mathrm{E}+03$ & $3.5 \mathrm{E}+01$ & $3.5 \mathrm{E}+03$ & $3.5 \mathrm{E}+01$ \\
\hline Np-239 & 013968-59-7 & $2.4 \mathrm{E}+06$ & $2.4 \mathrm{E}+04$ & $4.4 \mathrm{E}+11$ & $4.4 \mathrm{E}+09$ & $2.8 \mathrm{E}+04$ & $2.8 \mathrm{E}+02$ & $2.8 \mathrm{E}+04$ & $2.8 \mathrm{E}+02$ \\
\hline $\mathrm{Np}-240$ & $015690-84-3$ & $5.9 \mathrm{E}+07$ & $5.9 \mathrm{E}+05$ & $8.2 \mathrm{E}+12$ & $8.2 \mathrm{E}+10$ & $1.9 E+03$ & $1.9 \mathrm{E}+01$ & $1.9 \mathrm{E}+03$ & $1.9 \mathrm{E}+01$ \\
\hline $\mathrm{Np}-240 \mathrm{~m}$ & $015690-84-3$ & $4.3 \mathrm{E}+08$ & $4.3 E+06$ & $3.8 E+13$ & $3.8 \mathrm{E}+11$ & $6.5 \mathrm{E}+03$ & $6.5 \mathrm{E}+01$ & $6.5 \mathrm{E}+03$ & $6.5 \mathrm{E}+01$ \\
\hline Os-185 & $015766-50-4$ & $5.8 \mathrm{E}+06$ & $5.8 \mathrm{E}+04$ & $2.3 \mathrm{E}+11$ & $2.3 \mathrm{E}+09$ & $2.8 \mathrm{E}+03$ & $2.8 \mathrm{E}+01$ & $2.8 \mathrm{E}+03$ & $2.8 \mathrm{E}+01$ \\
\hline Os-191 . & 014119-24-5 & $3.4 \mathrm{E}+06$ & $3.4 \mathrm{E}+04$ & $4.0 \mathrm{E}+11$ & $4.0 \mathrm{E}+09$ & $7.8 \mathrm{E}+04$ & $7.8 \mathrm{E}+02$ & $7.7 E+04$ & $7.7 E+02$ \\
\hline Os-191m & 014119-24-5 & $2.1 \mathrm{E}+07$ & $2.1 \mathrm{E}+05$ & $3.2 \mathrm{E}+12$ & $3.2 \mathrm{E}+10$ & $2.1 \mathrm{E}+06$ & $2.1 E+04$ & $1.9 \mathrm{E}+06$ & $1.9 \mathrm{E}+04$ \\
\hline Os-193 & $016057-77-5$ & $2.4 \mathrm{E}+06$ & $2.4 E+04$ & $4.0 \mathrm{E}+11$ & $4.0 \mathrm{E}+09$ & $3.8 \mathrm{E}+04$ & $3.8 \mathrm{E}+02$ & $3.7 \mathrm{E}+04$ & $3.7 \mathrm{E}+02$ \\
\hline P-32 & 014596-37-3 & $1.7 \mathrm{E}+06$ & $1.7 E+04$ & $3.7 \mathrm{E}+11$ & $3.7 \mathrm{E}+09$ & & & $1.7 \mathrm{E}+06$ & $1.7 \mathrm{E}+04$ \\
\hline P-33 & $015749-66-3$ & $1.3 E+07$ & $1.3 \mathrm{E}+05$ & $2.7 \mathrm{E}+12$ & $2.7 \mathrm{E}+10$ & & & $1.3 \mathrm{E}+07$ & $1.3 E+05$ \\
\hline
\end{tabular}

Radionuclides/soil/excavation 
Table 3.5b. (continued)

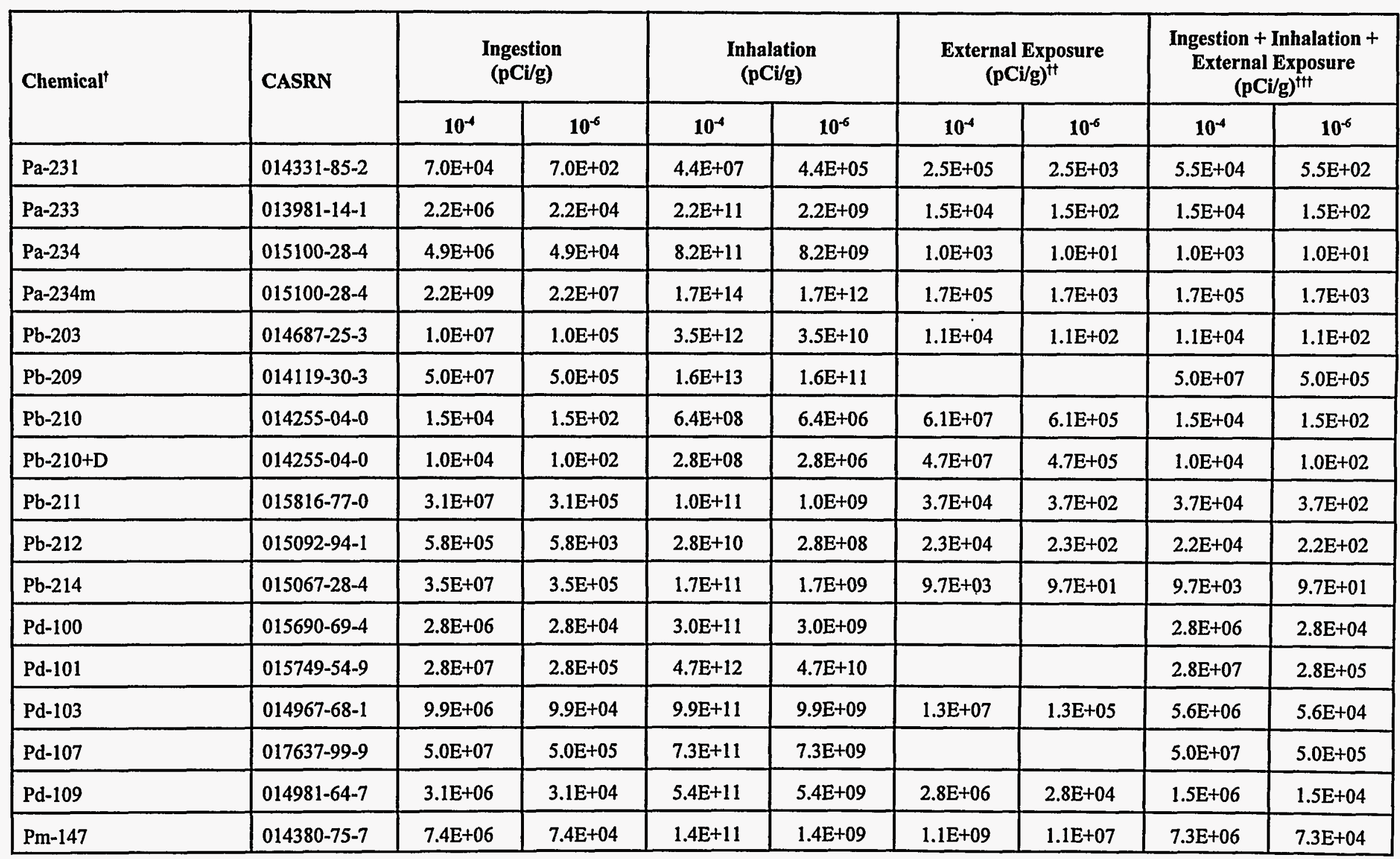


Table 3.5b. (continued)

\begin{tabular}{|c|c|c|c|c|c|c|c|c|c|}
\hline \multirow[t]{2}{*}{ Chemical $^{\dagger}$} & \multirow[t]{2}{*}{ CASRN } & \multicolumn{2}{|c|}{$\begin{array}{c}\text { Ingestion } \\
\text { (pCi/g) }\end{array}$} & \multicolumn{2}{|c|}{$\begin{array}{c}\text { Inhalation } \\
\text { (pCi/g) }\end{array}$} & \multicolumn{2}{|c|}{$\begin{array}{c}\text { External Exposure } \\
(\mathrm{pCi} / \mathrm{g})^{\dagger+}\end{array}$} & \multicolumn{2}{|c|}{$\begin{array}{c}\text { Ingestion + Inhalation + } \\
\text { External Exposure } \\
(\mathrm{pCi} / \mathrm{g})^{\dagger+t}\end{array}$} \\
\hline & & $10^{-4}$ & $10^{-6}$ & $10^{-4}$ & $10^{-6}$ & $10^{-4}$ & $10^{-6}$ & $10^{-4}$ & $10^{-6}$ \\
\hline Pm-148 & 014683-19-3 & $7.2 E+05$ & $7.2 \mathrm{E}+03$ & $1.0 \mathrm{E}+11$ & $1.0 \mathrm{E}+09$ & $3.1 \mathrm{E}+03$ & $3.1 \mathrm{E}+01$ & $3.1 \mathrm{E}+03$ & $3.1 \mathrm{E}+01$ \\
\hline Pm-148m & 014683-19-3 & $1.0 \mathrm{E}+06$ & $1.0 \mathrm{E}+04$ & $3.6 \mathrm{E}+10$ & $3.6 \mathrm{E}+08$ & $9.3 E+02$ & $9.3 E+00$ & $9.3 E+02$ & $9.3 \mathrm{E}+00$ \\
\hline Pm-149 & 015765-31-8 & $1.9 \mathrm{E}+06$ & $1.9 \mathrm{E}+04$ & $3.0 \mathrm{E}+11$ & $3.0 \mathrm{E}+09$ & $1.9 \mathrm{E}+05$ & $1.9 \mathrm{E}+03$ & $1.7 \mathrm{E}+05$ & $1.7 \mathrm{E}+03$ \\
\hline Po-210 & $013981-52-7$ & $3.2 E+04$ & $3.2 \mathrm{E}+02$ & $5.0 \mathrm{E}+08$ & $5.0 \mathrm{E}+06$ & $2.1 E+08$ & $2.1 \mathrm{E}+06$ & $3.2 \mathrm{E}+04$ & $3.2 \mathrm{E}+02$ \\
\hline Po-212 & 015389-34-1 & $2.3 E+17$ & $2.3 \mathrm{E}+15$ & $1.8 \mathrm{E}+20$ & $1.8 \mathrm{E}+18$ & & & $2.3 \mathrm{E}+17$ & $2.3 \mathrm{E}+15$ \\
\hline Po-213 & $015756-57-7$ & $1.6 \mathrm{E}+16$ & $1.6 \mathrm{E}+14$ & $1.4 \mathrm{E}+19$ & $1.4 \mathrm{E}+17$ & $5.8 \mathrm{E}+07$ & $5.8 \mathrm{E}+05$ & $5.8 \mathrm{E}+07$ & $5.8 \mathrm{E}+05$ \\
\hline Po-214 & 015735-67-8 & $4.9 \mathrm{E}+14$ & $4.9 \mathrm{E}+12$ & $3.9 E+17$ & $3.9 \mathrm{E}+15$ & $2.1 \mathrm{E}+07$ & $2.1 E+05$ & $2.1 E+07$ & $2.1 E+05$ \\
\hline Po-215 & $015706-52-2$ & $2.1 \mathrm{E}+13$ & $2.1 \mathrm{E}+11$ & $2.4 \mathrm{E}+16$ & $2.4 \mathrm{E}+14$ & $1.3 \mathrm{E}+07$ & $1.3 E+05$ & $1.3 \mathrm{E}+07$ & $1.3 E+05$ \\
\hline Po-216 & 015756-58-8 & $1.2 \mathrm{E}+11$ & $1.2 \mathrm{E}+09$ & $3.6 \mathrm{E}+14$ & $3.6 \mathrm{E}+12$ & $1.2 \mathrm{E}+08$ & $1.2 \mathrm{E}+06$ & $1.2 E+08$ & $1.2 \mathrm{E}+06$ \\
\hline Po-218 & $015422-24-9$ & $2.1 \mathrm{E}+08$ & $2.1 \mathrm{E}+06$ & $2.9 \mathrm{E}+11$ & $2.9 \mathrm{E}+09$ & & & $2.0 \mathrm{E}+08$ & $2.0 \mathrm{E}+06$ \\
\hline $\operatorname{Pr}-142$ & $014191-64-1$ & $1.5 \mathrm{E}+06$ & $1.5 \mathrm{E}+04$ & $2.6 \mathrm{E}+11$ & $2.6 \mathrm{E}+09$ & $2.9 \mathrm{E}+04$ & $2.9 \mathrm{E}+02$ & $2.9 \mathrm{E}+04$ & $2.9 \mathrm{E}+02$ \\
\hline $\operatorname{Pr}-143$ & $014981-79-4$ & $1.6 \mathrm{E}+06$ & $1.6 \mathrm{E}+04$ & $1.9 \mathrm{E}+11$ & $1.9 \mathrm{E}+09$ & $2.0 \mathrm{E}+11$ & $2.0 \mathrm{E}+09$ & $1.6 \mathrm{E}+06$ & $1.6 \mathrm{E}+04$ \\
\hline Pr-144 & $014119-05-2$ & $1.3 \mathrm{E}+08$ & $1.3 \mathrm{E}+06$ & $8.2 \mathrm{E}+12$ & $8.2 \mathrm{E}+10$ & $5.1 E+04$ & $5.1 \mathrm{E}+02$ & $5.1 \mathrm{E}+04$ & $5.1 \mathrm{E}+02$ \\
\hline Pr-144m & $014119-05-2$ & $3.2 \mathrm{E}+08$ & $3.2 E+06$ & $1.9 \mathrm{E}+13$ & $1.9 \mathrm{E}+11$ & $3.7 \mathrm{E}+06$ & 3.7E+04 & $3.7 \mathrm{E}+06$ & $3.7 \mathrm{E}+04$ \\
\hline Pt-191 & $015706-36-2$ & $6.9 \mathrm{E}+06$ & $6.9 \mathrm{E}+04$ & $2.6 \mathrm{E}+12$ & $2.6 \mathrm{E}+10$ & $1.0 \mathrm{E}+04$ & $1.0 \mathrm{E}+02$ & $1.0 \mathrm{E}+04$ & $1.0 \mathrm{E}+02$ \\
\hline Pt-193 & $015735-70-3$ & $6.4 E+07$ & $6.4 \mathrm{E}+05$ & $1.4 \mathrm{E}+13$ & $1.4 \mathrm{E}+11$ & & & $6.4 \mathrm{E}+07$ & $6.4 \mathrm{E}+05$ \\
\hline Pt-193m & $015735-70-3$ & $4.2 E+06$ & $4.2 E+04$ & $1.9 \mathrm{E}+12$ & $1.9 \mathrm{E}+10$ & $9.2 \mathrm{E}+05$ & $9.2 E+03$ & $7.5 \mathrm{E}+05$ & $7.5 \mathrm{E}+03$ \\
\hline
\end{tabular}


Table 3.5b. (continued)

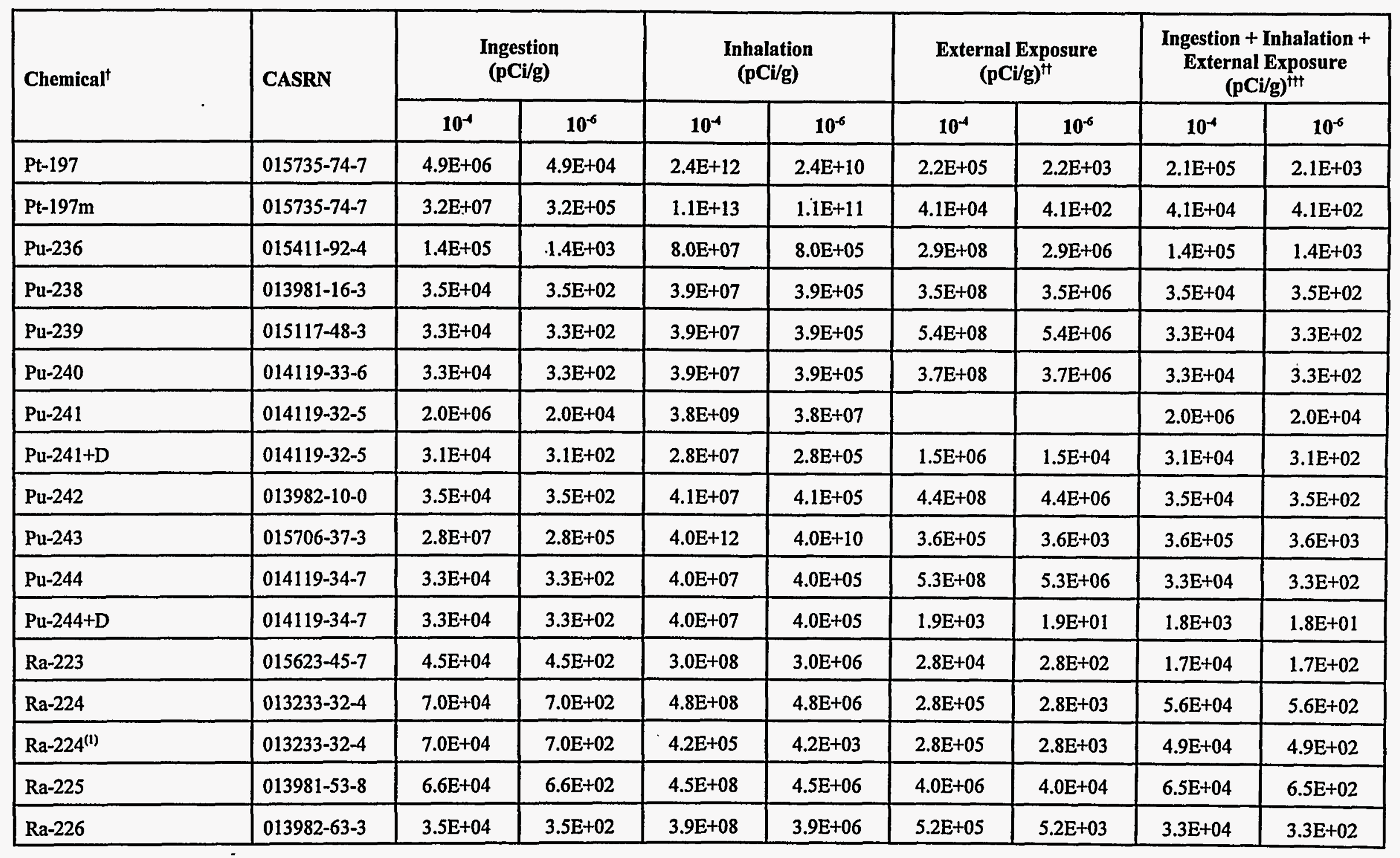


Table 3.5b. (continued)

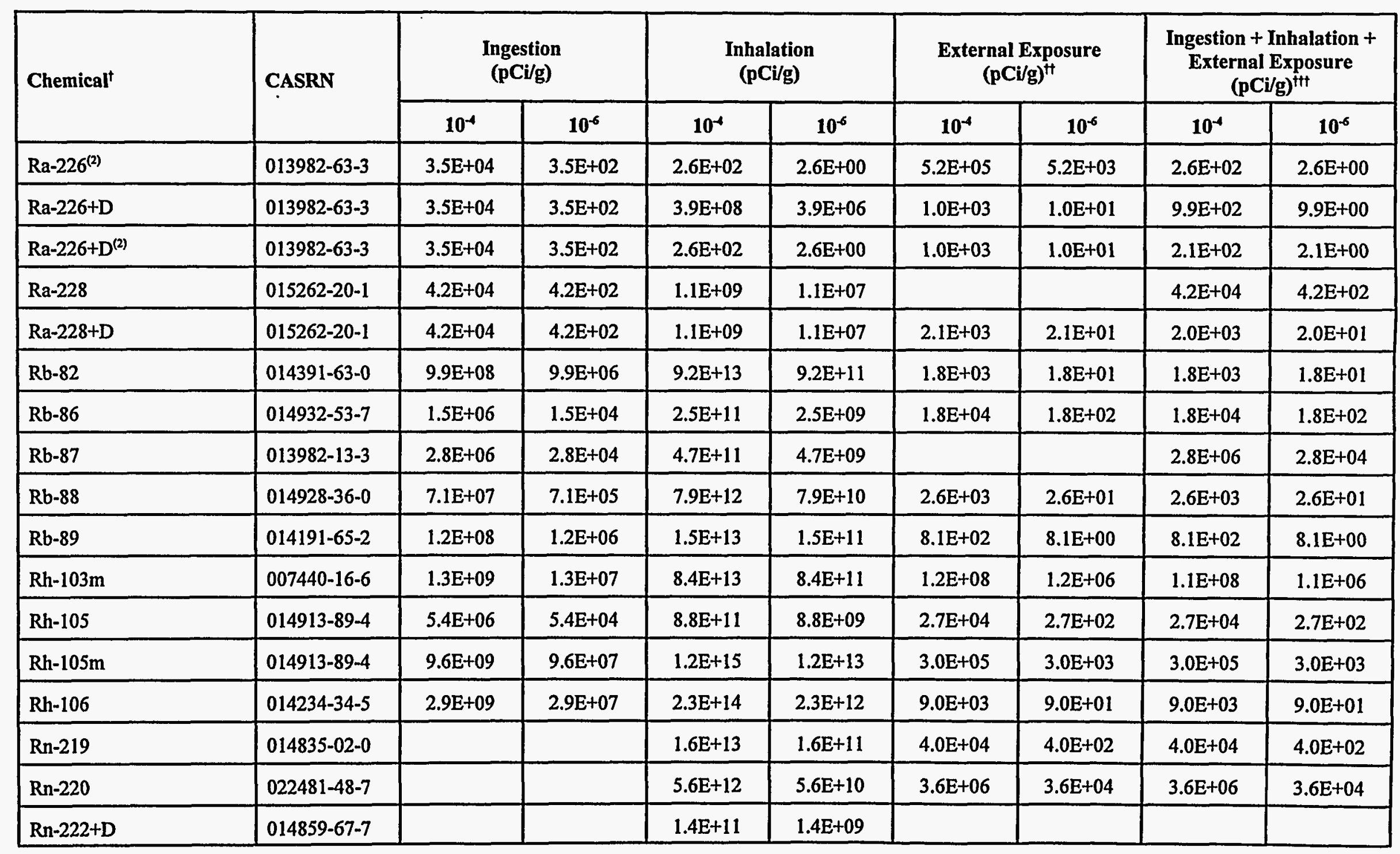


Table 3.5b. (continued)

\begin{tabular}{|c|c|c|c|c|c|c|c|c|c|}
\hline \multirow[t]{2}{*}{ Chemical $^{\dagger}$} & \multirow[t]{2}{*}{ CASRN } & \multicolumn{2}{|c|}{$\begin{array}{l}\text { Ingestion } \\
\text { (pCi/g) }\end{array}$} & \multicolumn{2}{|c|}{$\begin{array}{l}\text { Inhalation } \\
\text { (pCi/g) }\end{array}$} & \multicolumn{2}{|c|}{$\begin{array}{l}\text { External Exposure } \\
(\text { (pCi/g) })^{\dagger t}\end{array}$} & \multicolumn{2}{|c|}{$\begin{array}{c}\text { Ingestion + Inhalation + } \\
\text { External Exposure } \\
(\mathrm{pCi} / \mathrm{g})^{+++}\end{array}$} \\
\hline & & $10^{-4}$ & $10^{-6}$ & $10^{-4}$ & $10^{-6}$ & $10^{-4}$ & $10^{-6}$ & $10^{-4}$ & $10^{-6}$ \\
\hline Ru-103 & 013968-53-1 & $3.1 E+06$ & $3.1 \mathrm{E}+04$ & $2.3 E+11$ & $2.3 \mathrm{E}+09$ & $4.0 \mathrm{E}+03$ & $4.0 \mathrm{E}+01$ & $4.0 \mathrm{E}+03$ & $4.0 \mathrm{E}+01$ \\
\hline Ru-105 & $014331-95-4$ & $9.1 \mathrm{E}+06$ & $9.1 \mathrm{E}+04$ & $1.3 \mathrm{E}+12$ & $1.3 \mathrm{E}+10$ & $2.4 \mathrm{E}+03$ & $2.4 \mathrm{E}+01$ & $2.4 \mathrm{E}+03$ & $2.4 \mathrm{E}+01$ \\
\hline Ru-97 & 015758-35-7 & $1.8 \mathrm{E}+07$ & $1.8 \mathrm{E}+05$ & $2.6 \mathrm{E}+12$ & $2.6 \mathrm{E}+10$ & $1.5 \mathrm{E}+04$ & $1.5 \mathrm{E}+02$ & $1.5 \mathrm{E}+04$ & $1.5 \mathrm{E}+02$ \\
\hline S-35 & 015117-53-0 & $2.5 \mathrm{E}+07$ & $2.5 \mathrm{E}+05$ & $5.8 \mathrm{E}+12$ & $5.8 \mathrm{E}+10$ & & & $2.5 \mathrm{E}+07$ & $2.5 \mathrm{E}+05$ \\
\hline $\mathrm{Sb}-122$ & 014374-79-9 & $1.2 \mathrm{E}+06$ & $1.2 \mathrm{E}+04$ & $2.0 \mathrm{E}+11$ & $2.0 \mathrm{E}+09$ & $4.3 E+03$ & $4.3 \mathrm{E}+01$ & $4.2 \mathrm{E}+03$ & $4.2 \mathrm{E}+01$ \\
\hline Sb-124 & $014683-10-4$ & $9.7 E+05$ & $9.7 \mathrm{E}+03$ & $8.1 E+10$ & $8.1 \mathrm{E}+08$ & $9.3 \mathrm{E}+02$ & $9.3 \mathrm{E}+00$ & $9.3 E+02$ & $9.3 \mathrm{E}+00$ \\
\hline Sb-126 & 015756-32-8 & $1.1 \mathrm{E}+06$ & $1.1 \mathrm{E}+04$ & $1.3 \mathrm{E}+11$ & $1.3 \mathrm{E}+09$ & $6.6 \mathrm{E}+02$ & $6.6 \mathrm{E}+00$ & $6.6 \mathrm{E}+02$ & $6.6 \mathrm{E}+00$ \\
\hline $\mathrm{Sb}-126 \mathrm{~m}$ & $015756-32-8$ & $1.4 \mathrm{E}+08$ & $1.4 \mathrm{E}+06$ & $1.7 \mathrm{E}+13$ & $1.7 \mathrm{E}+11$ & $1.2 \mathrm{E}+03$ & $1.2 \mathrm{E}+01$ & $1.2 \mathrm{E}+03$ & $1.2 \mathrm{E}+01$ \\
\hline Sb-127 & 013968-50-8 & $1.2 \mathrm{E}+06$ & $1.2 \mathrm{E}+04$ & $1.8 \mathrm{E}+11$ & $1.8 \mathrm{E}+09$ & $2.9 \mathrm{E}+03$ & $2.9 \mathrm{E}+01$ & $2.8 \mathrm{E}+03$ & $2.8 \mathrm{E}+01$ \\
\hline Sb-129 & $014331-88-5$ & $5.6 \mathrm{E}+06$ & $5.6 E+04$ & $1.2 \mathrm{E}+12$ & $1.2 \mathrm{E}+10$ & $1.2 \mathrm{E}+03$ & $1.2 \mathrm{E}+01$ & $1.2 \mathrm{E}+03$ & $1.2 \mathrm{E}+01$ \\
\hline Sc-46 & 013967-63-0 & $1.8 \mathrm{E}+06$ & $1.8 \mathrm{E}+04$ & $8.2 \mathrm{E}+10$ & $8.2 E+08$ & $8.7 \mathrm{E}+02$ & $8.7 E+00$ & $8.7 \mathrm{E}+02$ & $8.7 \mathrm{E}+00$ \\
\hline Sc-47 & 014391-96-9 & $3.5 \mathrm{E}+06$ & $3.5 \mathrm{E}+04$ & $5.3 \mathrm{E}+11$ & $5.3 \mathrm{E}+09$ & $2.7 \mathrm{E}+04$ & $2.7 \mathrm{E}+02$ & $2.7 E+04$ & $2.7 E+02$ \\
\hline Sc-48 & $014391-86-7$ & $1.6 \mathrm{E}+06$ & $1.6 \mathrm{E}+04$ & $2.6 \mathrm{E}+11$ & $2.6 \mathrm{E}+09$ & $5.2 \mathrm{E}+02$ & $5.2 \mathrm{E}+00$ & $5.2 E+02$ & $5.2 \mathrm{E}+00$ \\
\hline
\end{tabular}


Table 3.5b. (continued)

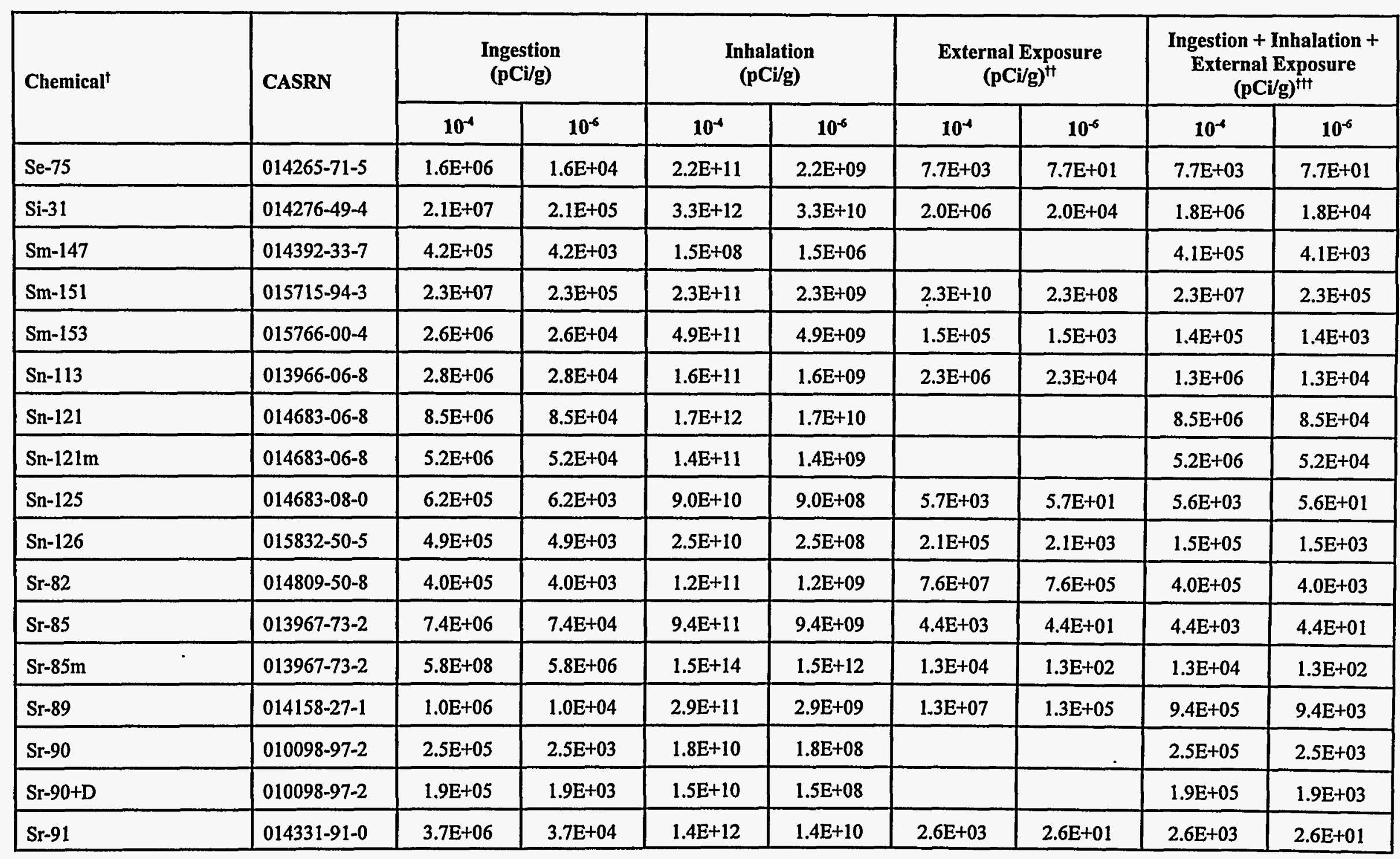


Table 3.5b. (continued)

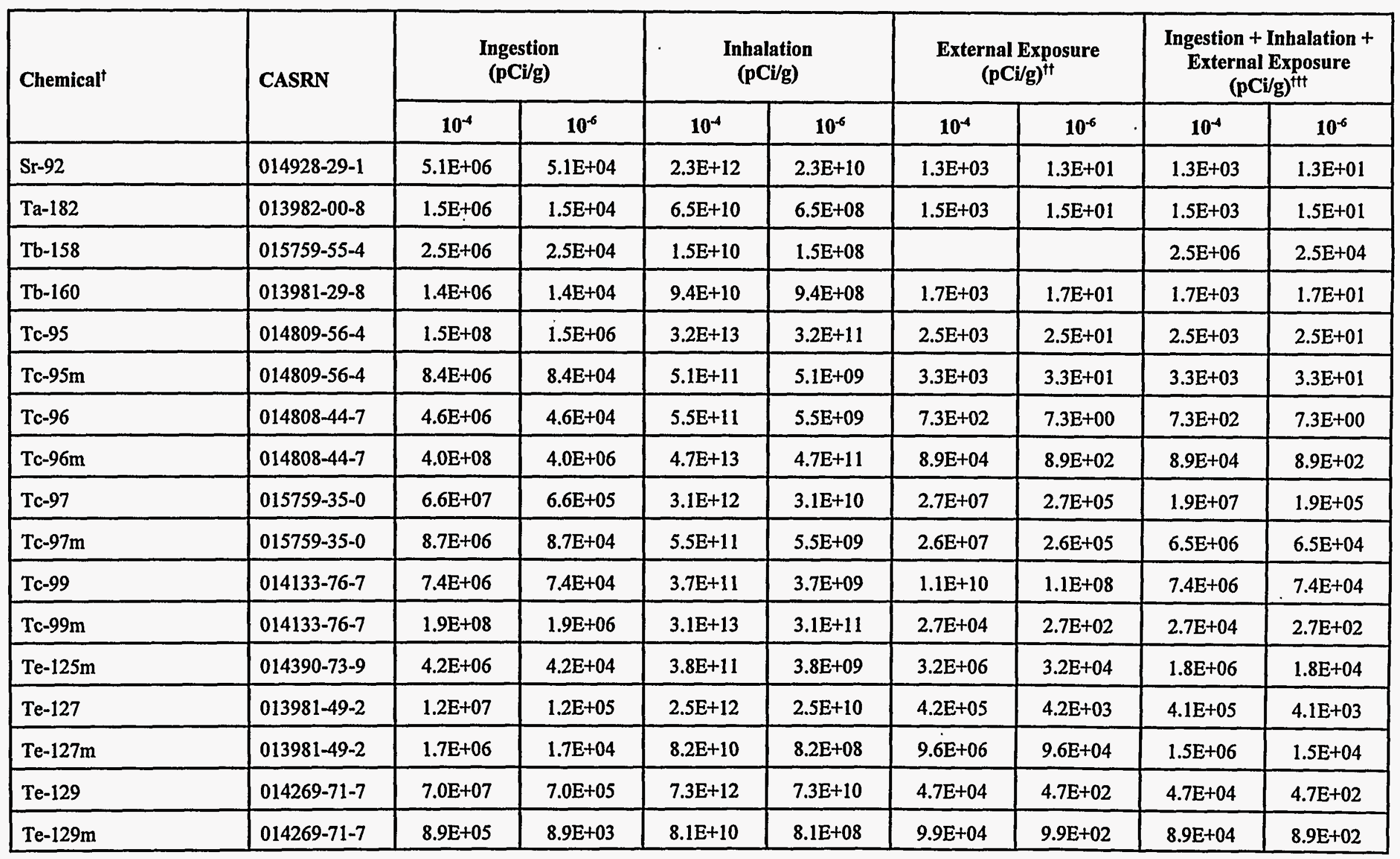


Table 3.5b. (continued)

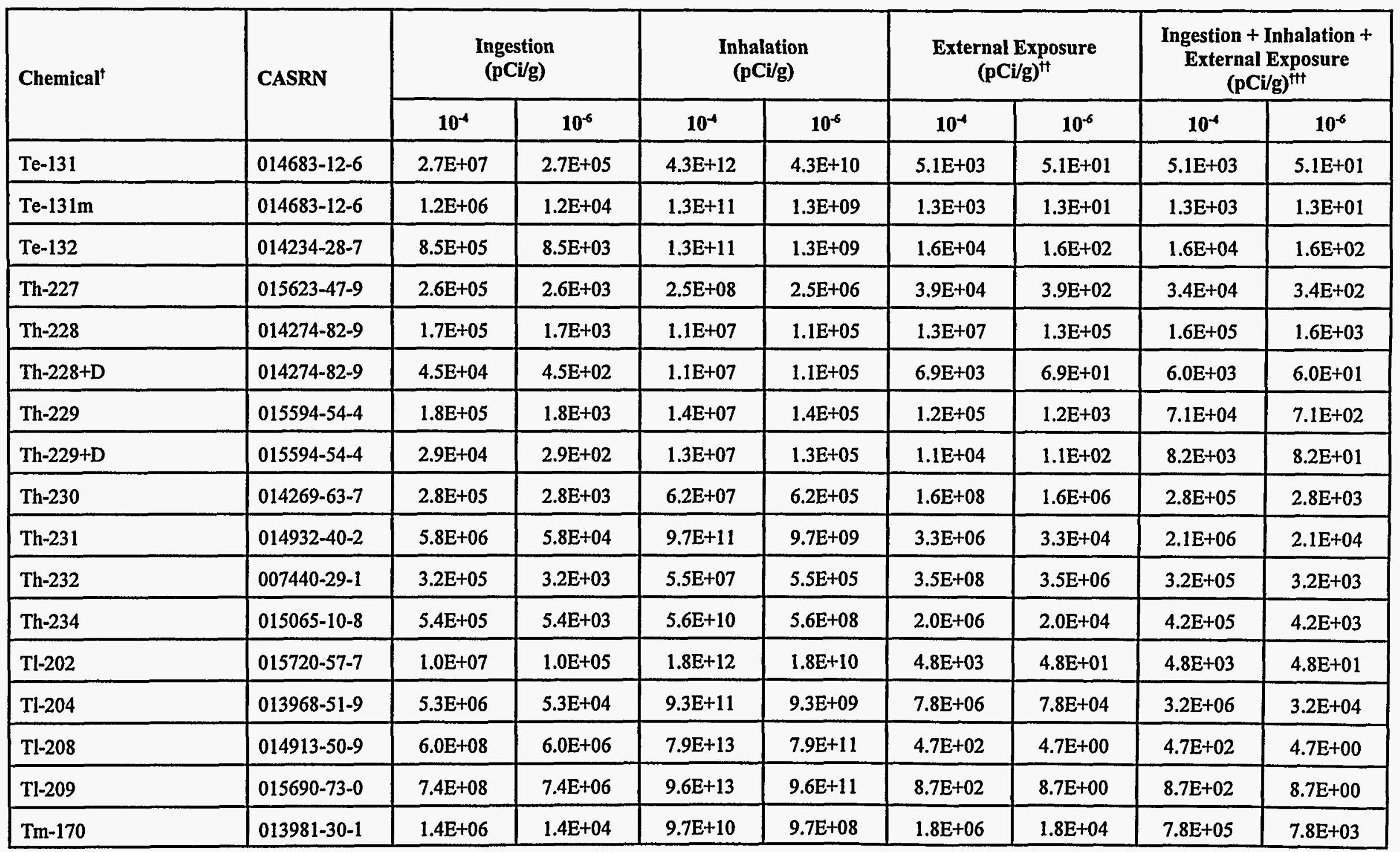


Table 3.5b. (continued)

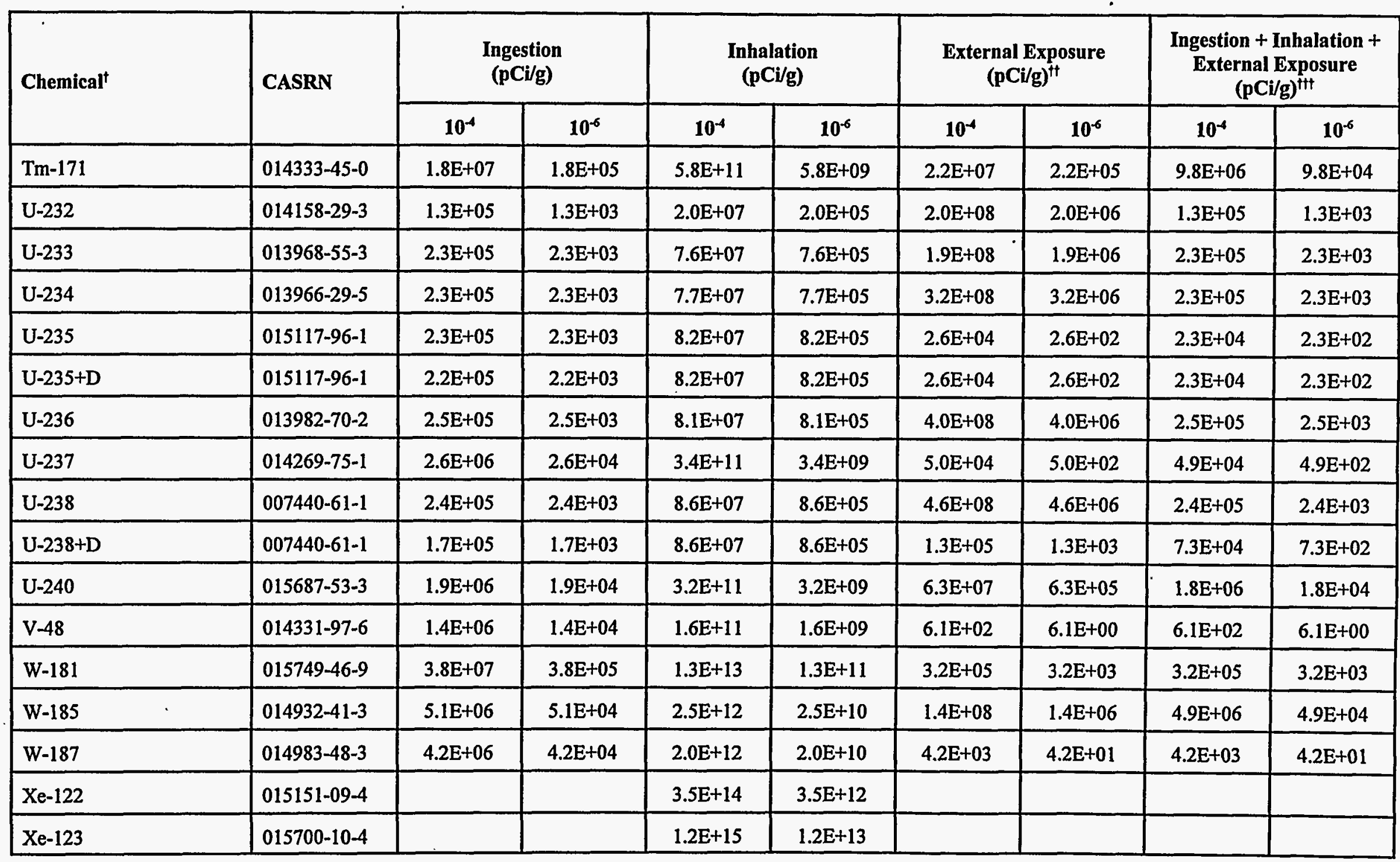


Table 3.5b. (continued)

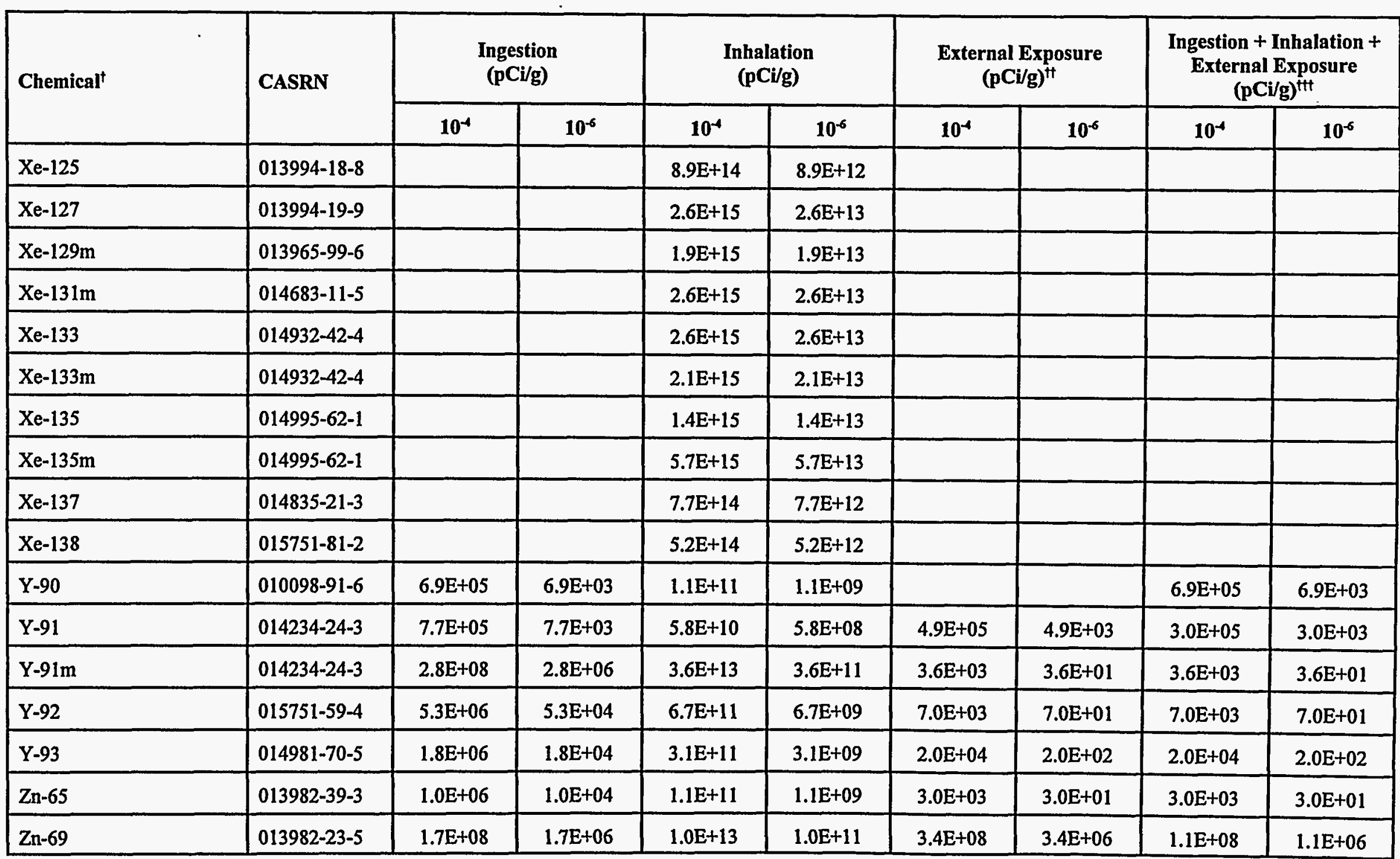


Table 3.5b. (continued)

\begin{tabular}{|l|l|c|c|c|c|c|c|c|c|}
\hline \multirow{2}{*}{ Chemical $^{\dagger}$} & \multirow{2}{*}{ CASRN } & \multicolumn{2}{|c|}{$\begin{array}{c}\text { Ingestion } \\
\text { (pCi/g) }\end{array}$} & \multicolumn{2}{|c|}{$\begin{array}{c}\text { Inhalation } \\
\text { (pCi/g) }\end{array}$} & \multicolumn{3}{|c|}{$\begin{array}{c}\text { External Exposure } \\
(\mathrm{pCi} / \mathrm{g})^{\mathrm{H}}\end{array}$} & $\begin{array}{c}\text { Ingestion + Inhalation + } \\
\text { External Exposure } \\
(\mathrm{pCi} / \mathrm{g})^{\mathrm{Ht}}\end{array}$ \\
\cline { 3 - 10 } & & $10^{-4}$ & $10^{-6}$ & $10^{-4}$ & $10^{-6}$ & $10^{-4}$ & $10^{-6}$ & $10^{-4}$ & $10^{-6}$ \\
\hline $\mathrm{Zn}-69 \mathrm{~m}$ & $013982-23-5$ & $6.9 \mathrm{E}+06$ & $6.9 \mathrm{E}+04$ & $9.2 \mathrm{E}+11$ & $9.2 \mathrm{E}+09$ & $4.8 \mathrm{E}+03$ & $4.8 \mathrm{E}+01$ & $4.8 \mathrm{E}+03$ & $4.8 \mathrm{E}+01$ \\
\hline $\mathrm{Zr}-93$ & $015751-77-6$ & $2.0 \mathrm{E}+07$ & $2.0 \mathrm{E}+05$ & $2.0 \mathrm{E}+11$ & $2.0 \mathrm{E}+09$ & & & $2.0 \mathrm{E}+07$ & $2.0 \mathrm{E}+05$ \\
\hline $\mathrm{Zr}-95$ & $013967-71-0$ & $2.7 \mathrm{E}+06$ & $2.7 \mathrm{E}+04$ & $1.7 \mathrm{E}+11$ & $1.7 \mathrm{E}+09$ & $2.4 \mathrm{E}+03$ & $2.4 \mathrm{E}+01$ & $2.4 \mathrm{E}+03$ & $2.4 \mathrm{E}+01$ \\
\hline $\mathrm{Zr}-97$ & $014928-30-4$ & $1.0 \mathrm{E}+06$ & $1.0 \mathrm{E}+04$ & $2.3 \mathrm{E}+11$ & $2.3 \mathrm{E}+09$ & $1.0 \mathrm{E}+04$ & $1.0 \mathrm{E}+02$ & $9.9 \mathrm{E}+03$ & $9.9 \mathrm{E}+01$ \\
\hline
\end{tabular}

\section{Notes:}

$\dagger \quad$ Isotopes designated with an " $\mathrm{m}$ " indicate that the isotope is metastable; isotopes designated with a " $+\mathrm{D}$ " indicate that the radionuclide slope factors include contributions from daughter products.

i† If no PRG for external exposure was calculated, then no external exposure slope factors were available or the external exposure slope factor was equal to $0.00 \mathrm{E}+00$ (resulting in an undefined PRG for the external exposure pathway for soil under excavation land use).

†† If slope factors and/or reference doses were available for only one pathway (i.e., ingestion, inhalation, OR external exposure), the total for ingestion plus inhalation plus external exposure was not calculated.

(1) The default volatilization factor of 200 for Ra-224 was calculated as the ratio of the average Ra-224 background concentration in soil to the average Rn-220 background concentration in air. Inhalation slope factor values are for Rn-222 plus decay products (i.e., Rn-222+D) formed from the radioactive decay of Ra-226 and for Rn-220+D from the decay of Ra-224. Slope factor values were taken from Table 4a of EPA's Health Effects Assessment Summary Tables (HEAST 1992).

(2) The default volatilization factor of 8 for Ra-226 was calculated as the ratio of the average natural background concentration of Ra-226 in soil to the corresponding average natural background concentration of Rn-222 in air. Inhalation slope factor values are for Rn-222 plus decay products (i.e., Rn-222+D) formed from the radioactive decay of Ra-226 and for Rn-220+D from the decay of Ra-224. Slope factor values were taken from Table 4a of EPA's Health Effects Assessment Summary Tables (HEAST 1992). 
Table 3.6a. Toxicity values used in calculating risk-based Preliminary Remediation Goals (nonradionuclides)

\begin{tabular}{|c|c|c|c|c|c|c|c|c|c|c|c|c|c|}
\hline Chemical & CASRN & $\begin{array}{c}\text { Oral } \\
\text { Chronic } \\
\text { RID } \\
\text { (mg/kg- } \\
\text { day) }\end{array}$ & $\begin{array}{c}\text { Dermal } \\
\text { Chronic } \\
\text { Rm } \\
\text { (mg/kg- } \\
\text { day) }\end{array}$ & $\begin{array}{c}\text { Oral Sub- } \\
\text { chronic } \\
\text { RfD } \\
\text { (mg/kg- } \\
\text { day) }\end{array}$ & $\begin{array}{c}\text { Dermal } \\
\text { Sub- } \\
\text { chronic } \\
\text { Rm } \\
\text { (mg/kg- } \\
\text { day) }\end{array}$ & $\begin{array}{c}\text { Inhalation } \\
\text { Chronic } \\
\text { RID } \\
\text { (mg/kg- } \\
\text { day) }\end{array}$ & $\begin{array}{l}\text { Inhalation } \\
\text { Sub- } \\
\text { chronic } \\
\text { RfD } \\
\text { (mg/kg- } \\
\text { day) }^{\dagger}\end{array}$ & $\begin{array}{c}\text { Oral } \\
\text { Slope } \\
\text { Factor } \\
\text { (mg/kg- } \\
\text { day) }\end{array}$ & $\begin{array}{l}\text { Inhalation } \\
\text { Slope } \\
\text { Factor } \\
\text { (mg/kg- } \\
\text { day) }\end{array}$ & $\begin{array}{l}\text { Dermal } \\
\text { Slope } \\
\text { Factor } \\
\text { (mg/kg- } \\
\text { day) }\end{array}$ & $\begin{array}{c}\text { GI } \\
\text { Absorp- } \\
\text { tion } \\
\text { Factor }\end{array}$ & $\begin{array}{l}\text { Dermal } \\
\text { Absorp- } \\
\text { tion } \\
\text { Factor }\end{array}$ & $\begin{array}{c}\text { Date Toxicity } \\
\text { Value } \\
\text { Withdrawn } \\
\text { from } \\
\text { IRIS/HEAST }\end{array}$ \\
\hline ALAR & $001596-84-5$ & $1.5 \mathrm{E}-01^{2}$ & 7.5E-02 & & & & & & & & 0.50 & 0.010 & \\
\hline Acenaphthene & $000083-32-9$ & $6.0 \mathrm{E}-02^{2}$ & $1.9 \mathrm{E}-02$ & $6.0 \mathrm{E}-01^{b}$ & $1.9 \mathrm{E}-01$ & & & & & & 0.31 & 0.010 & \\
\hline Acenaphthylene & $000208-96-8$ & & & & & & & & & & 0.31 & 0.010 & \\
\hline Acephate & $030560-19-1$ & $4.0 \mathrm{E}-03^{\circ}$ & $2.0 \mathrm{E}-03$ & $4.0 \mathrm{E}-03^{\mathrm{b}}$ & $2.0 \mathrm{E}-03$ & & & 8.7E-03' & & $1.7 \mathrm{E}-02$ & 0.50 & 0.010 & \\
\hline Acetaldehyde & $000075-07-0$ & & & & & $2.6 \mathrm{E}-03^{\circ}$ & & & $7.7 \mathrm{E}-03^{\mathrm{u}}$ & & 0.80 & 0.010 & \\
\hline Acetochlor & $034256-82-1$ & $2.0 \mathrm{E}-02^{\circ}$ & $1.0 \mathrm{E}-02$ & & & & & & & & 0.50 & 0.010 & \\
\hline Acetone & $000067-64-1$ & $1.0 \mathrm{E}-01^{2}$ & 8.3E-02 & $1.0 \mathrm{E}+00^{b}$ & 8.3E-01 & & & & & & 0.83 & 0.010 & \\
\hline Acetone Cyanohydrin & $000075-86-5$ & $8.0 \mathrm{E}-04^{\mathrm{b}}$ & $4.0 \mathrm{E}-04$ & $8.0 \mathrm{E}-03^{\mathrm{b}}$ & $4.0 \mathrm{E}-03$ & $2.9 \mathrm{E}-03^{\mathrm{bc}}$ & $2.9 \mathrm{E}-02^{\mathrm{b}, \mathrm{c}}$ & & & & 0.50 & 0.010 & \\
\hline Acetonitrile & $000075-05-8$ & $6.0 \mathrm{E}-03^{2}$ & $4.8 \mathrm{E}-03$ & $6.0 \mathrm{E}-02^{\mathrm{b}}$ & 4.8E-02 & $1.4 \mathrm{E}-02^{\mathrm{b}, \mathrm{e}}$ & $1.4 \mathrm{E}-01^{\mathrm{b}, \mathrm{c}}$ & & & & 0.80 & 0.010 & \\
\hline Acetophenone ${ }^{(3)}$ & $000098-86-2$ & $1.0 \mathrm{E}-01^{2}$ & $8.0 \mathrm{E}-02$ & $1.0 \mathrm{E}+00^{b}$ & $5.0 \mathrm{E}-01$ & $5.7 \mathrm{E}-06^{\mathrm{w}}$ & & & & & 0.80 & 0.010 & 9/93 \\
\hline Acrolein $^{(6)}$ & $000107-02-8$ & $2.0 \mathrm{E}-02^{\mathrm{b}}$ & $1.6 \mathrm{E}-02$ & & & 5.7E-06 & & & & & 0.80 & 0.010 & \\
\hline Acrylamide & $000079-06-1$ & $2.0 \mathrm{E}-04^{\circ}$ & $1.0 \mathrm{E}-04$ & $2.0 \mathrm{E}-03^{\mathrm{b}}$ & $1.0 \mathrm{E}-03$ & & & $4.5 \mathrm{E}+00^{\circ}$ & $4.5 E+00^{b}$ & $9.0 \mathrm{E}+00$ & 0.50 & 0.010 & \\
\hline Acrylic Acid & $000079-10-7$ & $5.0 \mathrm{E}-01^{2}$ & $2.5 \mathrm{E}-01$ & $5.0 \mathrm{E}-01^{\mathrm{b}}$ & 2.5E-01 & $2.9 \mathrm{E}-04^{2}$ & $8.6 \mathrm{E}-04^{b}$ & & & & 0.50 & 0.010 & \\
\hline Acrylonitrile & $000107-13-1$ & $1.0 \mathrm{E}-03^{\mathrm{b}}$ & $8.0 \mathrm{E}-04$ & $1.0 \mathrm{E}-02^{\mathrm{b}}$ & $8.0 \mathrm{E}-03$ & $5.7 \mathrm{E}-04^{4}$ & & $5.4 \mathrm{E}-0 \mathrm{I}^{*}$ & $2.4 \mathrm{E}-01^{b}$ & $6.8 \mathrm{E}-01$ & 0.80 & 0.010 & \\
\hline Alachlor & $015972-60-8$ & $1.0 \mathrm{E}-02^{2}$ & $5.0 \mathrm{E}-03$ & $1.0 \mathrm{E}-02^{\mathrm{b}}$ & $5.0 \mathrm{E}-03$ & & & $8.0 \mathrm{E}-02^{\mathrm{b}}$ & & $1.6 \mathrm{E}-01$ & 0.50 & 0.010 & \\
\hline Aldicarb & $000116-06-3$ & $1.0 \mathrm{E}-03^{2}$ & $5.0 \mathrm{E}-04$ & $1.0 \mathrm{E}-03^{\mathrm{b}}$ & $5.0 \mathrm{E}-04$ & & & & & & 0.50 & 0.010 & \\
\hline Aldicarb Sulfone & $001646-88-4$ & $1.0 \mathrm{E}-03^{\circ}$ & $5.0 \mathrm{E}-04$ & & & & & & & & 0.50 & 0.010 & \\
\hline Aldrin & $000309-00-2$ & $3.0 \mathrm{E}-05^{\circ}$ & $1.5 \mathrm{E}-05$ & $3.0 \mathrm{E}-05^{\mathrm{b}}$ & $1.5 \mathrm{E}-05$ & & & $1.7 \mathrm{E}+01^{*}$ & $1.7 \mathrm{E}+01^{b}$ & $3.4 \mathrm{E}+01$ & 0.50 & 0.010 & \\
\hline Ally & $074223-64-6$ & 2.5E-01" & $1.3 \mathrm{E}-01$ & & & & & & & & 0.50 & 0.010 & \\
\hline
\end{tabular}

Toxicity values used in PRGs calculations/nonradionuclides

Page 1 of 39 
Table 3.6a. (continued)

\begin{tabular}{|c|c|c|c|c|c|c|c|c|c|c|c|c|c|}
\hline Chemical & CASRN & $\begin{array}{c}\text { Oral } \\
\text { Chronic } \\
\text { RID } \\
\text { (mg/kg- } \\
\text { day) }\end{array}$ & $\begin{array}{c}\text { Dermal } \\
\text { Chronic } \\
\text { RID } \\
\text { (mg/kg- } \\
\text { day) }\end{array}$ & $\begin{array}{c}\text { Oral Sub- } \\
\text { chronic } \\
\text { RmD } \\
\text { (mg/kg- } \\
\text { day) }\end{array}$ & $\begin{array}{c}\text { Dermal } \\
\text { Sub- } \\
\text { chronic } \\
\text { RmD } \\
\text { (mg/kg- } \\
\text { day) }\end{array}$ & $\begin{array}{c}\text { Inhalation } \\
\text { Chronic } \\
\text { Rm } \\
\text { (mg/kg- } \\
\text { day) }^{\dagger}\end{array}$ & $\begin{array}{c}\text { Inhaiation } \\
\text { Sub- } \\
\text { chronic } \\
\text { RID } \\
\text { (mg/kg- } \\
\text { day) }\end{array}$ & $\begin{array}{c}\text { Oral } \\
\text { Slope } \\
\text { Factor } \\
\text { (mg/kg- }^{\text {day) }}{ }^{-1}\end{array}$ & $\begin{array}{c}\text { Inhalation } \\
\text { Slope } \\
\text { Factor } \\
\text { (mg/kg- } \\
\text { day) }\end{array}$ & $\begin{array}{c}\text { Dermal } \\
\text { Slope } \\
\text { Factor } \\
\text { (mg/kg- } \\
\text { day) }\end{array}$ & $\begin{array}{c}\text { GI } \\
\text { Absorp- } \\
\text { tion } \\
\text { Factor }\end{array}$ & $\begin{array}{c}\text { Dermal } \\
\text { Absorp- } \\
\text { tion } \\
\text { Factor }\end{array}$ & $\begin{array}{l}\text { Date Toxicity } \\
\text { Value } \\
\text { Withdrawn } \\
\text { from } \\
\text { IRIS/HEAST }\end{array}$ \\
\hline Allyl Alcohol & $000107-18-6$ & $5.0 \mathrm{E}-03^{\prime \prime}$ & $4.0 \mathrm{E}-03$ & $5.0 \mathrm{E}-02^{b}$ & $4.0 \mathrm{E}-02$ & & & & & & 0.80 & 0.010 & \\
\hline Allyl Chloride ${ }^{(3)}$ & $000107-05-1$ & $5.0 \mathrm{E}-02^{\mathrm{w}}$ & $4.0 \mathrm{E}-02$ & & & $2.9 \mathrm{E}-04^{*}$ & $2.9 \mathrm{E}-03^{b}$ & & & & 0.80 & 0.010 & $9 / 93$ \\
\hline Aluminum & $007429-90-5$ & & & & & & & & & & 0.10 & 0.001 & \\
\hline Aluminum Phosphide & $020859-73-8$ & $4.0 \mathrm{E}-04^{2}$ & $8.0 \mathrm{E}-05$ & $4.0 \mathrm{E}-04^{\mathrm{b}}$ & $8.0 \mathrm{E}-05$ & & & & & & 0.20 & 0.001 & \\
\hline Amdro & $067485-29-4$ & $3.0 \mathrm{E}-04^{n}$ & $1.5 \mathrm{E}-04$ & & & & & & & & 0.50 & 0.010 & \\
\hline Ametryn & $000834-12-8$ & $9.0 \mathrm{E}-03^{*}$ & $4.5 \mathrm{E}-03$ & $9.0 \mathrm{E}-02^{\mathrm{b}}$ & 4.5E-02 & & & & & & 0.50 & 0.010 & \\
\hline Aminophenol, $\mathrm{m}$ - & $000591-27-5$ & $7.0 \mathrm{E}-02^{\mathrm{b}}$ & $3.5 \mathrm{E}-02$ & $7.0 \mathrm{E}-01^{\mathrm{b}}$ & $3.5 \mathrm{E}-01$ & & & & & & 0.50 & 0.010 & \\
\hline Aminopyridine, 4- & $000504-24-5$ & $2.0 \mathrm{E}-05^{\mathrm{b}}$ & $1.0 \mathrm{E}-05$ & $2.0 \mathrm{E}-04^{\mathrm{b}}$ & $1.0 \mathrm{E}-04$ & & & & & & 0.50 & 0.010 & \\
\hline Amitraz & 033089-61-1 & $2.5 \mathrm{E}-03^{4}$ & $1.3 \mathrm{E}-03$ & & & & & & & & 0.50 & 0.010 & \\
\hline Ammonia & $007664-41-7$ & & & & & $2.9 \mathrm{E}-02^{n}$ & $2.9 \mathrm{E}-02^{b}$ & & & & 0.20 & 0.001 & \\
\hline Ammonium Sulfamate & $007773-06-0$ & $2.0 \mathrm{E}-01^{4}$ & 4.0E-02 & & & & & & & & 0.20 & 0.001 & \\
\hline Aniline & $000062-53-3$ & & & & & $2.9 \mathrm{E}-04^{\circ}$ & $2.9 \mathrm{E}-03^{b}$ & $5.7 \mathrm{E}-03^{\prime}$ & & $1.1 \mathrm{E}-02$ & 0.50 & 0.010 & \\
\hline Anthracene & $000120-12-7$ & $3.0 \mathrm{E}-01^{*}$ & 2.3E-01 & $3.0 \mathrm{E}+00^{\mathrm{b}}$ & $2.3 \mathrm{E}+00$ & & & & & & 0.76 & 0.010 & \\
\hline Antimony (metallic) & $007440-36-0$ & $4.0 \mathrm{E}-04^{4}$ & 8.0E-06 & $4.0 \mathrm{E}-04^{\mathrm{b}}$ & $8.0 \mathrm{E}-06$ & & & & & & 0.02 & 0.001 & \\
\hline Antimony Pentoxide & $001314-60-9$ & $5.0 \mathrm{E}-04^{b}$ & $1.0 \mathrm{E}-04$ & $5.0 \mathrm{E}-04^{2}$ & $1.0 \mathrm{E}-04$ & & & & & & 0.20 & 0.001 & \\
\hline Antimony Potassium Tartrate & $000304-61-0$ & $9.0 \mathrm{E}-04^{b}$ & $1.8 \mathrm{E}-04$ & $9.0 \mathrm{E}-04^{\mathrm{b}}$ & $1.8 \mathrm{E}-04$ & & & & & & 0.20 & 0.001 & \\
\hline Antimony Tetroxide & $001332-81-6$ & $4.0 \mathrm{E}-04^{\circ}$ & $8.0 \mathrm{E}-05$ & $4.0 \mathrm{E}-04^{\mathrm{b}}$ & $8.0 \mathrm{E}-05$ & & & & . & & 0.20 & 0.001 & \\
\hline Antimony Trioxide & $001309-64-4$ & $4.0 \mathrm{E}-04^{\mathrm{b}}$ & 8.0E-05 & $4.0 \mathrm{E}-04^{\mathrm{b}}$ & $8.0 \mathrm{E}-05$ & $5.7 \mathrm{E}-05^{4}$ & & & & & 0.20 & 0.001 & \\
\hline Apollo & $074115-24-5$ & $1.3 \mathrm{E}-02^{\prime}$ & $6.5 \mathrm{E}-03$ & & & & & & & & 0.50 & 0.010 & \\
\hline
\end{tabular}


Table 3.6a. (continued)

\begin{tabular}{|c|c|c|c|c|c|c|c|c|c|c|c|c|c|}
\hline Chemical & CASRN & $\begin{array}{c}\text { Oral } \\
\text { Chronic } \\
\text { RID } \\
\text { (mg/kg- } \\
\text { day) }\end{array}$ & $\begin{array}{c}\text { Dermal } \\
\text { Chronic } \\
\text { Rm } \\
\text { (mg/kg- } \\
\text { day) }\end{array}$ & $\begin{array}{l}\text { Oral Sub- } \\
\text { chronic } \\
\text { RfD } \\
\text { (mg/kg- } \\
\text { day) }\end{array}$ & $\begin{array}{c}\text { Dermal } \\
\text { Sub- } \\
\text { chronic } \\
\text { RfD } \\
\text { (mg/kg- } \\
\text { day) }\end{array}$ & $\begin{array}{c}\text { Inhalation } \\
\text { Chronic } \\
\text { RfD } \\
\text { (mg/kg- } \\
\text { day) }\end{array}$ & $\begin{array}{c}\text { Inbalation } \\
\text { Sub- } \\
\text { chronic } \\
\text { RfD } \\
\text { (mg/kg- } \\
\text { day) }\end{array}$ & $\begin{array}{l}\text { Oral } \\
\text { Slope } \\
\text { Factor } \\
\text { (mg/kg- } \\
\text { day) }\end{array}$ & $\begin{array}{l}\text { Inhalation } \\
\text { Slope } \\
\text { Factor } \\
\text { (mg/kg- } \\
\text { day) }\end{array}$ & $\begin{array}{l}\text { Dermal } \\
\text { Slope } \\
\text { Factor } \\
\text { (mg/kg- } \\
\text { day) }\end{array}$ & $\begin{array}{c}\text { GI } \\
\text { Absorp- } \\
\text { tion } \\
\text { Factor }\end{array}$ & $\begin{array}{c}\text { Dermal } \\
\text { Absorp- } \\
\text { tion } \\
\text { Factor }\end{array}$ & $\begin{array}{c}\text { Date Toxicity } \\
\text { Value } \\
\text { Withdrawn } \\
\text { from } \\
\text { IRIS/REAST }\end{array}$ \\
\hline Aramite & $000140-57-8$ & $5.0 \mathrm{E}-02 \mathrm{~b}$ & $2.5 \mathrm{E}-02$ & $1.0 \mathrm{E}-01^{b}$ & $5.0 \mathrm{E}-02$ & & & $2.5 \mathrm{E}-02^{\prime}$ & $2.5 \mathrm{E}-02^{b}$ & $5.0 \mathrm{E}-02$ & 0.50 & 0.010 & \\
\hline Aroclor 1016 & $012674-11-2$ & $7.0 \mathrm{E}-05^{\prime}$ & $6.3 \mathrm{E}-05$ & & & & & & & & 0.90 & 0.060 & \\
\hline Aroclor $1221^{(1)}$ & $011104-28-2$ & & & & & & & $7.7 \mathrm{E}+00^{(2)}$ & & $8.6 E+00$ & 0.90 & 0.060 & \\
\hline Aroclor $1232^{(1)}$ & $011141-16-5$ & & & & & & & $7.7 \mathrm{E}+00^{(2)}$ & & $8.6 \mathrm{E}+00$ & 0.90 & 0.060 & \\
\hline Aroclor $1242^{(1)}$ & $053469-21-9$ & & & & & & & $7.7 \mathrm{E}+00^{(2)}$ & & $8.6 E+00$ & 0.90 & 0.060 & \\
\hline Aroclor $1248^{(1)}$ & $012672-29-6$ & & & & & & & $7.7 \mathrm{E}+00^{(2)}$ & & $8.6 \mathrm{E}+00$ & 0.90 & 0.060 & \\
\hline Aroclor 1254 & $011097-69-1$ & $2.0 \mathrm{E}-05^{2}$ & $1.8 \mathrm{E}-05$ & $5.0 \mathrm{E}-05^{\mathrm{b}}$ & 4.5E-05 & & & & & & 0.90 & 0.060 & \\
\hline Aroclor $1260^{(1)}$ & $011096-82-5$ & & & & & & & 7.7E+00 & & $8.6 \mathrm{E}+00$ & 0.90 & 0.060 & \\
\hline Arsenic Salts & NA & & & & & & & & & & 0.80 & 0.001 & \\
\hline Arsenic, Inorganic & $007440-38-2$ & $3.0 \mathrm{E}-04^{\prime \prime}$ & $1.2 \mathrm{E}-04$ & $3.0 \mathrm{E}-04^{b}$ & $1.2 \mathrm{E}-04$ & & & $1.5 \mathrm{E}+00^{\circ}$ & $5.0 \mathrm{E}+01^{b}$ & $3.7 \mathrm{E}+00$ & 0.41 & 0.001 & \\
\hline Arsine & $007784-42-1$ & & & & & $1.4 \mathrm{E}-05^{n}$ & & & & & 0.20 & 0.001 & \\
\hline Asbestos & $001332-21-4$ & & & & & & & & & & 0.20 & 0.001 & \\
\hline Assure & $076578-14-8$ & $9.0 \mathrm{E}-03^{\circ}$ & $4.5 \mathrm{E}-03$ & & & & & & & & 0.50 & 0.010 & \\
\hline Asulam & $003337-71-1$ & $5.0 \mathrm{E}-02^{\prime \prime}$ & $2.5 \mathrm{E}-02$ & & . & & & & & & 0.50 & 0.010 & \\
\hline Atrazine & $001912-24-9$ & $3.5 \mathrm{E}-02^{2}$ & $1.8 \mathrm{E}-02$ & $3.5 \mathrm{E}-02^{b}$ & $1.8 \mathrm{E}-02$ & & & $2.2 \mathrm{E}-01^{\mathrm{b}}$ & & $4.4 \mathrm{E}-01$ & 0.50 & 0.010 & \\
\hline Avermectin B1 & $065195-55-3$ & $4.0 \mathrm{E}-04^{4}$ & 2.0E-04 & & & & & & & & 0.50 & 0.010 & \\
\hline Azobenzene & $000103-33-3$ & & & & & & & $1.1 \mathrm{E}-01^{\circ}$ & $1.1 \mathrm{E}-01^{b}$ & $2.2 \mathrm{E}-01$ & 0.50 & 0.010 & \\
\hline Barium & $007440-39-3$ & $7.0 \mathrm{E}-02^{2}$ & $4.9 \mathrm{E}-03$ & $7.0 \mathrm{E}-02^{b}$ & $4.9 \mathrm{E}-03$ & $1.4 \mathrm{E}-04^{\mathrm{b}, \mathrm{c}}$ & $1.4 \mathrm{E}-03^{b, \varepsilon}$ & & & & 0.07 & 0.001 & \\
\hline Barium Cyanide & $000542-62-1$ & $1.0 \mathrm{E}-01^{b}$ & $2.0 \mathrm{E}-02$ & $1.0 \mathrm{E}-01^{6}$ & $2.0 \mathrm{E}-02$ & & & & & & 0.20 & 0.001 & \\
\hline
\end{tabular}

Toxicity values used in PRGs calculations/nonradionuclides

Page 3 of 39 


\begin{tabular}{|c|c|c|c|c|c|c|c|c|c|c|c|c|c|}
\hline & $010^{\circ} 0$ & IE'0 & $00+3 t z$ & (2) $10-9 I^{\circ} \varepsilon$ & $.70-9 E^{\circ} L$ & & & & & & & $z-66-502000$ & (z) $)^{\text {พuวนp }}$ \\
\hline & $010^{\circ} 0$ & IE'0 & $10+367$ & $\left(200+9 r^{\circ} \varepsilon\right.$ & $.00+B E L$ & & & & & & & 8-ZE-0S0000 & 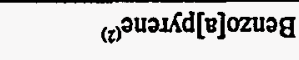 \\
\hline & $010^{\circ} 0$ & $08^{\circ} 0$ & $20+362 \tau$ & ${ }_{9} 20+9 \varepsilon z$ & $.20+9 \varepsilon z$ & & & $\varepsilon 0-\Xi \bullet \tau$ & ${ }_{q} \varepsilon 0-\exists 0^{\circ} \varepsilon$ & $\varepsilon 0-a+z$ & . $\varepsilon 0-\exists 0^{\circ} \varepsilon$ & $s-\angle 8-260000$ & әu!p!zuวg \\
\hline & $010^{\circ} 0$ & $05^{\circ} 0$ & & & & & & SO-so's & $\$ 70-901$ & $90-30^{\circ} \mathrm{s}$ & $\$ \$ 0-30 \% 1$ & $5-86-801000$ & 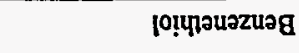 \\
\hline & $010^{\circ} 0$ & $\angle 60$ & & & & & & & & & & L-EI-ISSऽZZO & 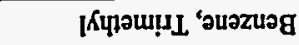 \\
\hline & $010^{\circ} 0$ & $\angle 6^{\circ} 0$ & & & & & & & & & & $\mathrm{VN}$ & 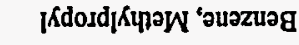 \\
\hline & $010^{\circ} 0$ & $\angle 60$ & & & & & & & & & & $\mathrm{VN}$ & 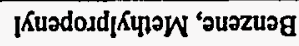 \\
\hline & $010^{\circ} 0$ & $\angle 60$ & & & & & & & & & & $\mathbf{V N}$ & 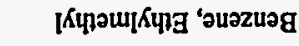 \\
\hline . & $010^{\circ} 0$ & $\angle 60^{\circ}$ & & & & & & & & & & VN & 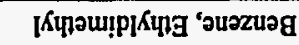 \\
\hline & $010^{\circ} 0$ & $\angle 60$ & & & & & & & & & & VN & әр!ฺо|นวвхәН әиәzนวg \\
\hline & $010^{\circ} 0$ & $\angle 6^{\circ} 0$ & $20-90^{\circ} \varepsilon$ & $820-36 \tau$ & $.20-36^{\prime} \tau$ & & & & & & & $\tau-\varepsilon t-l<0000$ & วนวzuวg \\
\hline & 0100 & $08^{\circ} 0$ & & & & & & $10-30 \% \mathrm{~S}$ & ${ }_{9} 00+90^{\circ} I$ & $20-50.8$ & $.10-90^{\circ} 1$ & L-ZS-001000 & әрКчәрןвzиая \\
\hline & 0100 & IE'0 & $00+3+2$ & (2) $10-\exists I^{\prime} \mathcal{E}$ & $.10-a E * L$ & & & & & & & $\varepsilon-\varsigma \varsigma-9 S 0000$ & 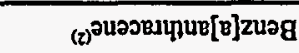 \\
\hline & $010^{\circ} 0$ & OS'0 & & & & & & & & $\mathfrak{E O}-\mathfrak{A E} \mathfrak{E}^{\prime} \mathbf{I}$ & $. \varepsilon 0-\exists S \tau$ & $0-68-\angle S O S Z 0$ & uoztyueg \\
\hline & $010^{\circ} 0$ & OS'O & & & & & & & & zo-as'z & $.20-30 \% \mathrm{~S}$ & $Z-S E-t 08 L I 0$ & I Kuourg \\
\hline & $010^{\circ} 0$ & $0 S^{\circ} 0$ & & & & & & I0-AS I I & จ $10-30^{\circ} \varepsilon$ & I0-as'I & $.10-30^{\circ} \varepsilon$ & I-0t-198100 & ugวurg \\
\hline & $010^{\circ} 0$ & $0 S^{\prime} 0$ & & & & & & & & ZO-AE'I & $.20-9 s 2$ & $\mathcal{S}-\angle E^{-} 6 S\{890$ & ptodqpरвg \\
\hline & $010^{\circ} 0$ & $0 S^{\prime} 0$ & & & & & & & & Z0-as.I & $.20-30^{\circ} \varepsilon$ & $\varepsilon=\varepsilon t-I Z I \varepsilon t 0$ & uopગאrg \\
\hline & $010^{\circ} 0$ & $0 S^{\circ} 0$ & & & & & & & & $\varepsilon 0-\mathrm{E} 0 \%$ & $. \varepsilon 0-a 0^{\circ} t$ & {$[-9 z-t\} 1000$} & น08אВg \\
\hline 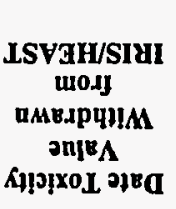 & 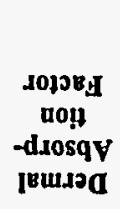 & 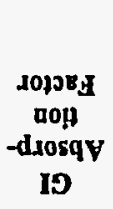 & 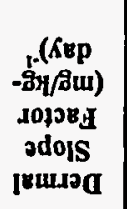 & 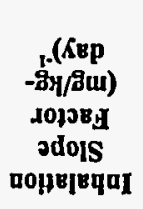 & 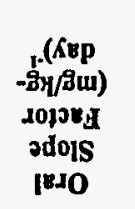 & 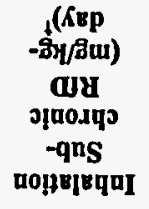 & 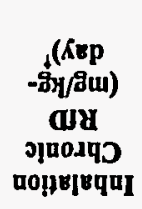 & 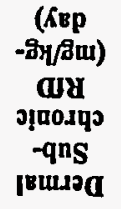 & 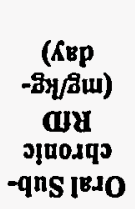 & 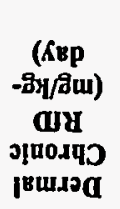 & 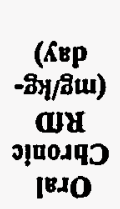 & NUSWD & |вэฺ \\
\hline
\end{tabular}

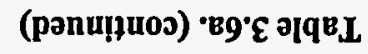


Table 3.6a. (continued)

\begin{tabular}{|c|c|c|c|c|c|c|c|c|c|c|c|c|c|}
\hline Chemical & CASRN & $\begin{array}{c}\text { Oral } \\
\text { Chronic } \\
\text { RTD } \\
\text { (mg/kg- } \\
\text { day) }\end{array}$ & $\begin{array}{c}\text { Dermal } \\
\text { Chronic } \\
\text { RRD } \\
\text { (mg/kg- } \\
\text { day) }\end{array}$ & $\begin{array}{c}\text { Oral Sub- } \\
\text { chronic } \\
\text { RfD } \\
\text { (mg/kg- } \\
\text { day) }\end{array}$ & $\begin{array}{c}\text { Dermal } \\
\text { Sub- } \\
\text { chronic } \\
\text { RDD } \\
\text { (mg/kg- } \\
\text { day) }\end{array}$ & $\begin{array}{c}\text { Inhalation } \\
\text { Chronic } \\
\text { RID } \\
\text { (mg/kg- } \\
\text { day) }^{\dagger}\end{array}$ & $\begin{array}{c}\text { Inhalation } \\
\text { Sub- } \\
\text { chronic } \\
\text { RmD } \\
\text { (mg/kg- } \\
\text { day) }\end{array}$ & $\begin{array}{c}\text { Oral } \\
\text { Slope } \\
\text { Factor } \\
\text { (mg/kg- } \\
\text { day) }\end{array}$ & $\begin{array}{l}\text { Inhalation } \\
\text { Slope } \\
\text { Factor } \\
\text { (mg/kg- } \\
\text { day) }^{-1}\end{array}$ & $\begin{array}{l}\text { Dermal } \\
\text { Slope } \\
\text { Factor } \\
\text { (mg/kg- } \\
\text { day) }^{-1}\end{array}$ & $\begin{array}{c}\text { Gl } \\
\text { Absorp- } \\
\text { tion } \\
\text { Factor }\end{array}$ & $\begin{array}{c}\text { Dermal } \\
\text { Absorp- } \\
\text { tion } \\
\text { Factor }\end{array}$ & $\begin{array}{c}\text { Date Toxicity } \\
\text { Value } \\
\text { Withdrawn } \\
\text { from } \\
\text { IRIS/HEAST }\end{array}$ \\
\hline Benzo[g,h,i]perylene & $000191-24-2$ & & & & & & & & & & 0.31 & 0.010 & \\
\hline Benzo[k]fluoranthene $e^{(2)}$ & 000207-08-9 & & & & & & & 7.3E-02 & $3.1 \mathrm{E}-02^{(2)}$ & $2.4 \mathrm{E}-01$ & 0.31 & 0.010 & \\
\hline Benzoic Acid & $000065-85-0$ & $4.0 \mathrm{E}+00^{\circ}$ & $4.0 \mathrm{E}+00$ & $4.0 \mathrm{E}+00^{b}$ & $4.0 \mathrm{E}+00$ & & & & & & 1.00 & 0.010 & \\
\hline Benzotrichloride & $000098-07-7$ & & & & & & & $1.3 \mathrm{E}+01^{2}$ & & $2.6 \mathrm{E}+01$ & 0.50 & 0.010 & \\
\hline Benzyl Alcohol & $000100-51-6$ & $3.0 \mathrm{E}-01^{\mathrm{b}}$ & $2.0 \mathrm{E}-01$ & $1.0 \mathrm{E}+00^{\mathrm{b}}$ & $6.6 \mathrm{E}-01$ & & & & & & 0.66 & 0.010 & \\
\hline Benzyl Chloride & $000100-44-7$ & & & & & & & $1.7 \mathrm{E}-01^{*}$ & & 2.1E-01 & 0.80 & 0.010 & \\
\hline Beryllium & $007440-41-7$ & $5.0 \mathrm{E}-03^{\circ}$ & $5.0 \mathrm{E}-05$ & $5.0 \mathrm{E}-03^{\mathrm{b}}$ & $5.0 \mathrm{E}-05$ & & & $4.3 \mathrm{E}+00^{2}$ & $8.4 \mathrm{E}+00^{\mathrm{b}}$ & $4.3 E+02$ & 0.01 & 0.001 & \\
\hline Bidrin & $000141-66-2$ & $1.0 \mathrm{E}-04^{\circ}$ & $5.0 \mathrm{E}-05$ & & & & & & & & 0.50 & 0.010 & \\
\hline Biphenthrin & 082657-04-3 & $1.5 \mathrm{E}-02^{2}$ & 7.5E-03 & & & & & & & & 0.50 & 0.010 & \\
\hline Biphenyl, 1,1'- & $000092-52-4$ & $5.0 \mathrm{E}-02^{\mathrm{a}}$ & $2.5 \mathrm{E}-02$ & $5.0 \mathrm{E}-02^{b}$ & $2.5 \mathrm{E}-02$ & & & & & & 0.50 & 0.010 & \\
\hline $\begin{array}{l}\text { Bis(2-chloro-1- } \\
\text { methylethyl)ether (Technical) }\end{array}$ & $000108-60-1$ & & & & & & & $7.0 \mathrm{E}-02^{\mathrm{b}}$ & $3.5 \mathrm{E}-02^{\mathrm{b}}$ & $8.8 \mathrm{E}-02$ & 0.80 & 0.010 & \\
\hline Bis(2-chloroethoxy)methane & $000111-91-1$ & & & & & & & & & & 0.50 & 0.010 & \\
\hline Bis(2-chloroethyl)ether & $000111-44-4$ & & & & & & & $1.1 \mathrm{E}+00^{2}$ & $1.1 \mathrm{E}+00^{\circ}$ & $1.4 \mathrm{E}+00$ & 0.80 & 0.010 & \\
\hline Bis(2-chloroisopropyl)ether & 039638-32-9 & $4.0 \mathrm{E}-02^{*}$ & $3.2 \mathrm{E}-02$ & $4.0 \mathrm{E}-02^{\mathrm{b}}$ & $2.0 \mathrm{E}-02$ & & & & & & 0.80 & 0.010 & \\
\hline Bis(2-ethylhexyl)phthalate ${ }^{(6)}$ & $000117-81-7$ & $2.0 \mathrm{E}-02^{2}$ & $3.8 \mathrm{E}-03$ & $2.0 \mathrm{E}-02^{b, 8}$ & $3.8 \mathrm{E}-03$ & & & $1.4 \mathrm{E}-02^{*}$ & & 7.4E-02 & 0.19 & 0.010 & \\
\hline Bis(chloromethyl)ether & $000542-88-1$ & & & & & & & $2.2 \mathrm{E}+02^{8}$ & $2.2 \mathrm{E}+02^{\mathrm{b}}$ & $2.8 \mathrm{E}+02$ & 0.80 & 0.010 & \\
\hline Bisphenol A & $000080-05-7$ & $5.0 \mathrm{E}-02^{2}$ & 2.5E-02 & $6.0 \mathrm{E}-01^{\mathrm{b}}$ & $3.0 \mathrm{E}-01$ & & & & & & 0.50 & 0.010 & \\
\hline Boron And Borates Only & $007440-42-8$ & $9.0 \mathrm{E}-02^{\circ}$ & 8.1E-02 & $9.0 \mathrm{E}-02^{\mathrm{b}}$ & 8.1E-02 & $5.7 \mathrm{E}-03^{\mathrm{b}}$ & 5.7E- $03^{b}$ & & & & 0.90 & 0.001 & \\
\hline
\end{tabular}

Toxicity values used in PRGs calculations/nonradionuclides

Page 5 of 39 
Table 3.6a. (continued)

\begin{tabular}{|c|c|c|c|c|c|c|c|c|c|c|c|c|c|}
\hline Chemical & CASRN & $\begin{array}{c}\text { Oral } \\
\text { Chronic } \\
\text { RfD } \\
\text { (mg/kg- } \\
\text { day) }\end{array}$ & $\begin{array}{c}\text { Dermal } \\
\text { Chronic } \\
\text { RfD } \\
\text { (mg/kg- } \\
\text { day) }\end{array}$ & $\begin{array}{c}\text { Oral Sub- } \\
\text { chronic } \\
\text { Rm } \\
\text { (mg/kg- } \\
\text { day) }\end{array}$ & $\begin{array}{c}\text { Dermal } \\
\text { Sub- } \\
\text { chronic } \\
\text { RfD } \\
\text { (mg/kg- } \\
\text { day) }\end{array}$ & $\begin{array}{c}\text { Inhalation } \\
\text { Chronic } \\
\text { RmD } \\
\text { (mg/kg- } \\
\text { day) })^{\dagger}\end{array}$ & $\begin{array}{c}\text { Inhalation } \\
\text { Sub- } \\
\text { chronic } \\
\text { RID } \\
\text { (mg/kg- } \\
\text { day) }{ }^{\dagger}\end{array}$ & $\begin{array}{c}\text { Oral } \\
\text { Slope } \\
\text { Factor } \\
\text { (mg/kg- } \\
\text { day) }\end{array}$ & $\begin{array}{c}\text { Inhalation } \\
\text { Slope } \\
\text { Factor } \\
\text { (mg/kg- } \\
\text { day) })^{-1}\end{array}$ & $\begin{array}{c}\text { Dermal } \\
\text { Slope } \\
\text { Factor } \\
\text { (mg/kg- } \\
\text { day) }\end{array}$ & $\begin{array}{c}\text { GI } \\
\text { Absorp- } \\
\text { tion } \\
\text { Factor }\end{array}$ & $\begin{array}{c}\text { Dermal } \\
\text { Absorp- } \\
\text { tion } \\
\text { Factor }\end{array}$ & $\begin{array}{l}\text { Date Toxicity } \\
\text { Value } \\
\text { Withdrawn } \\
\text { from } \\
\text { IRIS/HEAST }\end{array}$ \\
\hline Boron Trifluoride & $007637-07-2$ & & & & & $2.0 \mathrm{E}-04^{\mathrm{b}}$ & $2.0 \mathrm{E}-03^{b}$ & & & & 0.20 & 0.001 & \\
\hline Bromochloromethane & $000074-97-5$ & & & & & & & & & & 0.80 & 0.010 & \\
\hline Bromodichloromethane & $000075-27-4$ & 2.0E-02 & $2.0 \mathrm{E}-02$ & $2.0 \mathrm{E}-02^{\mathrm{b}}$ & $2.0 \mathrm{E}-02$ & & & $6.2 \mathrm{E}-02^{n}$ & & $6.3 \mathrm{E}-02$ & 0.98 & 0.010 & \\
\hline Bromodiphenyl Ether, $\mathrm{p}$ - & $000101-55-3$ & & & & & & & & & & 0.50 & 0.010 & \\
\hline Bromoform & $000075-25-2$ & $2.0 \mathrm{E}-02^{2}$ & $1.2 \mathrm{E}-02$ & $2.0 \mathrm{E}-01^{\mathrm{b}}$ & $1.2 \mathrm{E}-01$ & & & $7.9 E-03^{\circ}$ & $3.9 \mathrm{E}-03^{\mathrm{b}}$ & $1.3 \mathrm{E}-02$ & 0.60 & 0.010 & \\
\hline Bromomethane ${ }^{(6)}$ & $000074-83-9$ & $1.4 \mathrm{E}-03^{2}$ & 1.1E-03 & $4.7 \mathrm{E}-03^{\mathrm{b}, \mathrm{e}}$ & $1.1 \mathrm{E}-02$ & $1.4 \mathrm{E}-03^{\circ}$ & $4.8 \mathrm{E}-02^{\mathrm{b}, 0}$ & & & & 0.80 & 0.010 & \\
\hline Bromophos & $002104-96-3$ & $5.0 \mathrm{E}-03^{6}$ & $2.5 \mathrm{E}-03$ & $5.0 \mathrm{E}-02^{6}$ & $2.5 \mathrm{E}-02$ & & & & & & 0.50 & 0.010 & \\
\hline Bromotrichloromethane & $000075-62-7$ & & & & & & & & & & 0.80 & 0.010 & \\
\hline Bromoxynil & $001689-84-5$ & $2.0 \mathrm{E}-02^{2}$ & $1.0 \mathrm{E}-02$ & $2.0 \mathrm{E}-02^{6}$ & $1.0 \mathrm{E}-02$ & & & & & & 0.50 & 0.010 & \\
\hline Bromoxynil Octanoate & $001689-99-2$ & $2.0 \mathrm{E}-02^{2}$ & $1.0 \mathrm{E}-02$ & $2.0 \mathrm{E}-02^{\mathrm{b}}$ & $1.0 \mathrm{E}-02$ & & & & & & 0.50 & 0.010 & \\
\hline Butadiene, 1,3- & 000106-99-0 & & & & & & & & $1.8 \mathrm{E}+00^{\mathrm{b}}$ & & 0.80 & 0.010 & \\
\hline Butanol, N- & $000071-36-3$ & $1.0 \mathrm{E}-01^{4}$ & $5.0 \mathrm{E}-02$ & $1.0 \mathrm{E}+00^{\mathrm{b}}$ & $5.0 \mathrm{E}-01$ & & & & & & 0.50 & 0.010 & \\
\hline $\begin{array}{l}\text { Butanone-2, 4-chloro-4,4- } \\
\text { difluoro }\end{array}$ & NA & & & & & & & & & & 0.80 & 0.010 & \\
\hline Butyl Benzyl Phthlate & $000085-68-7$ & $2.0 \mathrm{E}-01^{\star}$ & $1.2 \mathrm{E}-01$ & $2.0 \mathrm{E}+00^{6}$ & $1.2 \mathrm{E}+00$ & & & & & & 0.61 & 0.010 & \\
\hline Butylate & $002008-41-5$ & $5.0 \mathrm{E}-02^{*}$ & 2.5E-02 & $5.0 \mathrm{E}-02^{6}$ & 2.5E-02 & & & & & & 0.50 & 0.010 & \\
\hline Butylchloride, t- & $000507-20-0$ & & & & & & & & & & 0.50 & 0.010 & \\
\hline Butylphthalyl Butylglycolate & $000085-70-1$ & $1.0 \mathrm{E}+00^{\circ}$ & $5.0 \mathrm{E}-01$ & & & & . & & & & 0.50 & 0.010 & \\
\hline Cacodylic Acid & $000075-60-5$ & $3.0 \mathrm{E}-03^{\mathrm{b}}$ & $1.5 \mathrm{E}-03$ & $3.0 \mathrm{E}-02^{\mathrm{b}}$ & $1.5 \mathrm{E}-02$ & & & & & & 0.50 & 0.010 & \\
\hline
\end{tabular}


Table 3.6a. (continued)

\begin{tabular}{|c|c|c|c|c|c|c|c|c|c|c|c|c|c|}
\hline Chemical & CASRN & $\begin{array}{c}\text { Oral } \\
\text { Chronic } \\
\text { RfD } \\
\text { (mg/kg- } \\
\text { day) }\end{array}$ & $\begin{array}{c}\text { Dermal } \\
\text { Chronic } \\
\text { Rm } \\
\text { (mg/kg- } \\
\text { day) }\end{array}$ & $\begin{array}{c}\text { Oral Sub- } \\
\text { chronic } \\
\text { RDD } \\
\text { (mg/kg- } \\
\text { day) }\end{array}$ & $\begin{array}{l}\text { Dermal } \\
\text { Sub- } \\
\text { chronic } \\
\text { RfD } \\
\text { (mg/kg- } \\
\text { day) }\end{array}$ & $\begin{array}{c}\text { Inhalation } \\
\text { Chronic } \\
\text { RfD } \\
\text { (mg/kg- } \\
\text { day) }\end{array}$ & $\begin{array}{l}\text { Inhalation } \\
\text { Sub- } \\
\text { chronic } \\
\text { RfD } \\
\text { (mg/kg- } \\
\text { day) }\end{array}$ & $\begin{array}{c}\text { Oral } \\
\text { Slope } \\
\text { Factor } \\
\text { (mg/kg- } \\
\text { day) }\end{array}$ & $\begin{array}{l}\text { Inhalation } \\
\text { Slope } \\
\text { Factor } \\
\text { (mg/kg- } \\
\text { day) }\end{array}$ & $\begin{array}{l}\text { Dermal } \\
\text { Slope } \\
\text { Factor } \\
\text { (mg/kg- } \\
\text { day) }\end{array}$ & $\begin{array}{c}\text { GI } \\
\text { Absorp- } \\
\text { tion } \\
\text { Factor }\end{array}$ & $\begin{array}{c}\text { Dermal } \\
\text { Absorp- } \\
\text { tion } \\
\text { Factor }\end{array}$ & $\begin{array}{c}\text { Date Toxicity } \\
\text { Value } \\
\text { Withdrawn } \\
\text { from } \\
\text { IRIS/HEAST }\end{array}$ \\
\hline Cadmium (Diet) & $007440-43-9$ & $1.0 \mathrm{E}-03^{2}$ & $1.0 \mathrm{E}-05$ & & & & & & $6.1 \mathrm{E}+00^{b}$ & & 0.01 & 0.010 & \\
\hline Cadmium (Water) & $007440-43-9$ & $5.0 \mathrm{E}-04^{\circ}$ & $5.0 \mathrm{E}-06$ & & & & & & $6.1 \mathrm{E}+00^{\mathrm{b}}$ & & 0.01 & 0.010 & \\
\hline Calcium Cyanide & $000592-01-8$ & $4.0 \mathrm{E}-02^{2}$ & 8.0E-03 & $4.0 \mathrm{E}-02^{\mathrm{b}}$ & 8.0E-03 & & & & & & 0.20 & 0.001 & \\
\hline Caprolactam & $000105-60-2$ & $5.0 \mathrm{E}-01^{*}$ & $2.5 \mathrm{E}-01$ & $5.0 \mathrm{E}-01^{\mathrm{b}}$ & 2.5E-01 & & & & & & 0.50 & 0.010 & \\
\hline Captafol & $002425-06-1$ & $2.0 \mathrm{E}-03^{n}$ & $1.0 \mathrm{E}-03$ & $2.0 \mathrm{E}-03^{\mathrm{b}}$ & $1.0 \mathrm{E}-03$ & & & $8.6 \mathrm{E}-03^{b}$ & & $1.7 \mathrm{E}-02$ & 0.50 & 0.010 & \\
\hline Captan & $000133-06-2$ & $1.3 \mathrm{E}-01^{\circ}$ & $6.5 \mathrm{E}-02$ & $1.3 \mathrm{E}-0 \mathrm{I}^{\mathrm{b}}$ & $6.5 \mathrm{E}-02$ & & & $3.5 \mathrm{E}-03^{\mathrm{b}}$ & & $7.0 \mathrm{E}-03$ & 0.50 & 0.010 & \\
\hline Carbaryl & $000063-25-2$ & $1.0 \mathrm{E}-01^{2}$ & $5.0 \mathrm{E}-02$ & $1.0 \mathrm{E}-01^{b}$ & $5.0 \mathrm{E}-02$ & & & & & & 0.50 & 0.010 & \\
\hline Carbazole & $000086-74-8$ & & & & & & & $2.0 \mathrm{E}-02^{\mathrm{b}}$ & & $2.9 \mathrm{E}-02$ & 0.70 & 0.010 & \\
\hline Carbofuran & $001563-66-2$ & $5.0 \mathrm{E}-03^{2}$ & $2.5 \mathrm{E}-03$ & $5.0 \mathrm{E}-03^{b}$ & $2.5 \mathrm{E}-03$ & & & & & & 0.50 & 0.010 & \\
\hline Carbon Disulfide & $000075-15-0$ & $1.0 \mathrm{E}-01 \mathrm{a}$ & $6.3 \mathrm{E}-02$ & $1.0 \mathrm{E}-01^{b}$ & $6.3 \mathrm{E}-02$ & $2.0 \mathrm{E}-0 \mathrm{I}^{*}$ & $2.9 \mathrm{E}-03^{b}$ & & & & 0.63 & 0.250 & \\
\hline Carbon Tetrachloride $e^{(\theta)}$ & $000056-23-5$ & 7.0E-04 & 4.6E-04 & $2.4 \mathrm{E}-03^{\mathrm{b},}$ & 4.6E-03 & & & $1.3 \mathrm{E}-01^{2}$ & $5.3 \mathrm{E}-02^{\mathrm{b}}$ & $2.0 \mathrm{E}-01$ & 0.65 & 0.010 & \\
\hline Carbosulfan & $055285-14-8$ & $1.0 \mathrm{E}-02^{2}$ & $5.0 \mathrm{E}-03$ & & & & & & & & 0.50 & 0.010 & \\
\hline Carboxin & $005234-68-4$ & $1.0 \mathrm{E}-01^{\bullet}$ & $5.0 \mathrm{E}-02$ & & & & & & & & 0.50 & 0.010 & \\
\hline Chloral & $000075-87-6$ & $2.0 \mathrm{E}-03^{2}$ & $1.0 \mathrm{E}-03$ & $2.0 \mathrm{E}-02^{b}$ & $1.0 \mathrm{E}-02$ & & & & & & 0.50 & 0.010 & \\
\hline Chloramben & $000133-90-4$ & $1.5 \mathrm{E}-02^{2}$ & 7.5E-03 & & & & & & & & 0.50 & 0.010 & \\
\hline Chloranil & $000118-75-2$ & & & & & & & $4.0 \mathrm{E}-01^{b}$ & & 8.1E-01 & 0.50 & 0.010 & \\
\hline Chlordane ${ }^{(6)}$ & $000057-74-9$ & $6.0 \mathrm{E}-05^{2}$ & $3.0 \mathrm{E}-05$ & $6.0 \mathrm{E}-05^{b}$ & $3.0 \mathrm{E}-05$ & & & $1.3 \mathrm{E}+00^{\circ}$ & 1.3E $+00^{6}$ & $2.6 \mathrm{E}+00$ & 0.50 & 0.010 & \\
\hline Chloride & $016887-00-6$ & & & & & & & . & & & 1.00 & 0.001 & \\
\hline Chlorimuron, Ethyl. & $090982-32-4$ & $2.0 \mathrm{E}-02$ & $1.0 \mathrm{E}-02$ & & & & & & & & 0.50 & 0.010 & \\
\hline
\end{tabular}

Toxicity values used in PRGs calculations/nonradionuclides

Page 7 of 39 
Table 3.6a. (continued)

\begin{tabular}{|c|c|c|c|c|c|c|c|c|c|c|c|c|c|}
\hline Chemical & CASRN & $\begin{array}{c}\text { Oral } \\
\text { Chronic } \\
\text { RfD } \\
\text { (mg/kg- } \\
\text { day) }\end{array}$ & $\begin{array}{c}\text { Dermal } \\
\text { Chronic } \\
\text { RfD } \\
\text { (mg/kg- } \\
\text { day) }\end{array}$ & $\begin{array}{c}\text { Oral Sub- } \\
\text { chronic } \\
\text { Rm } \\
\text { (mg/kg- } \\
\text { day) }\end{array}$ & $\begin{array}{c}\text { Dermal } \\
\text { Sub- } \\
\text { chronic } \\
\text { RID } \\
\text { (mg/kg- } \\
\text { day) }\end{array}$ & $\begin{array}{c}\text { Inhalation } \\
\text { Chronic } \\
\text { RfD } \\
\text { (mg/kg- } \\
\text { day) }\end{array}$ & $\begin{array}{c}\text { Inhalation } \\
\text { Sub- } \\
\text { chronic } \\
\text { RmD } \\
\text { (mg/kg- } \\
\text { day) }^{\dagger}\end{array}$ & $\begin{array}{c}\text { Oral } \\
\text { Slope } \\
\text { Factor } \\
\text { (mg/kg- } \\
\text { day) }\end{array}$ & $\begin{array}{l}\text { Inhalation } \\
\text { Slope } \\
\text { Factor } \\
\text { (mg/kg- } \\
\text { day) }\end{array}$ & $\begin{array}{l}\text { Dermal } \\
\text { Slope } \\
\text { Factor } \\
\text { (mg/kg- } \\
\text { day) }\end{array}$ & $\begin{array}{c}\text { GI } \\
\text { Absorp- } \\
\text { tion } \\
\text { Factor }\end{array}$ & $\begin{array}{c}\text { Dermal } \\
\text { Absorp- } \\
\text { tion } \\
\text { Factor }\end{array}$ & $\begin{array}{l}\text { Date Toxicity } \\
\text { Value } \\
\text { Withdrawn } \\
\text { from } \\
\text { IRIS/HEAST }\end{array}$ \\
\hline Chlorine & $007782-50-5$ & $1.0 \mathrm{E}-01^{2}$ & $2.0 \mathrm{E}-02$ & & & & & & & & 0.20 & 0.001 & \\
\hline Chlorine Dioxide & $010049-04-4$ & & & & & $5.7 \mathrm{E}-05^{*}$ & & & & & 0.50 & 0.010 & \\
\hline Chloro-1,1-difluoroethane, 1- & $000075-68-3$ & & & & & $1.4 \mathrm{E}+01^{2}$ & & & & & 0.80 & 0.010 & \\
\hline Chloro-1,3-butadiene, 2- & $000126-99-8$ & $2.0 \mathrm{E}-02^{\mathrm{b}, \mathrm{c}}$ & $1.6 \mathrm{E}-02$ & $2.0 \mathrm{E}-02^{\mathrm{b}, \mathrm{c}}$ & $1.0 \mathrm{E}-02$ & $2.0 \mathrm{E}-03^{b}$ & $2.0 \mathrm{E}-02^{\mathrm{b}}$ & & & & 0.80 & 0.010 & \\
\hline Chloro-2-methylaniline $\mathrm{HCl}, 4$ - & 003165-93-3 & & & & & & & $4.6 \mathrm{E}-01^{\mathrm{b}}$ & & 9.2E-01 & 0.50 & 0.010 & \\
\hline Chloro-2-methylaniline, 4- & $000095-69-2$ & & & & & & & $5.8 \mathrm{E}-01^{\mathrm{b}}$ & & $1.2 \mathrm{E}+00$ & 0.50 & 0.010 & \\
\hline Chloroacetic Acid & $000079-11-8$ & $2.0 \mathrm{E}-03^{b}$ & $1.0 \mathrm{E}-03$ & $2.0 \mathrm{E}-02^{\mathrm{b}}$ & $1.0 \mathrm{E}-02$ & & & & & & 0.50 & 0.010 & \\
\hline Chloroaniline, p- & $000106-47-8$ & $4.0 \mathrm{E}-03^{\mathrm{e}}$ & $2.0 \mathrm{E}-03$ & $4.0 \mathrm{E}-03^{b}$ & $2.0 \mathrm{E}-03$ & & & & & & 0.50 & 0.010 & \\
\hline Chlorobenzene ${ }^{(6)}$ & 000108-90-7 & $2.0 \mathrm{E}-02^{2}$ & $6.2 \mathrm{E}-03$ & $2.0 \mathrm{E}-01^{\mathrm{b}, \mathrm{e}}$ & $6.2 \mathrm{E}-02$ & $5.7 \mathrm{E}-03^{\mathrm{b}, \mathrm{c}}$ & & & & & 0.31 & 0.010 & \\
\hline Chlorobenzilate & $000510-15-6$ & $2.0 \mathrm{E}-02^{2}$ & $1.0 \mathrm{E}-02$ & $2.0 \mathrm{E}-02^{\mathrm{b}}$ & $1.0 \mathrm{E}-02$ & & & $2.7 \mathrm{E}-01^{\mathrm{b}}$ & $2.7 \mathrm{E}-01^{\mathrm{b}}$ & $5.4 \mathrm{E}-01$ & 0.50 & 0.010 & \\
\hline Chlorobenzoic Acid, p- & $000074-11-3$ & $2.0 \mathrm{E}-01^{b}$ & $1.0 \mathrm{E}-01$ & $2.0 \mathrm{E}+00^{b}$ & $1.0 \mathrm{E}+00$ & & & & & & 0.50 & 0.010 & \\
\hline Chlorobenzotrifluoride, 4- & $000098-56-6$ & $2.0 \mathrm{E}-02^{\mathrm{b}}$ & $1.0 \mathrm{E}-02$ & $2.0 \mathrm{E}-01^{\mathrm{b}}$ & $1.0 \mathrm{E}-01$ & & & & & & 0.50 & 0.010 & \\
\hline Chlorobutane, 1- & 000109-69-3 & $4.0 \mathrm{E}-01^{\mathrm{b}}$ & 3.2E-01 & $9.0 \mathrm{E}-01^{\mathrm{b}}$ & 4.5E-01 & & & & & & 0.80 & 0.010 & \\
\hline Chlorobutane, 2- & $000078-86-4$ & & & & & & & & & & 0.50 & 0.010 & \\
\hline Chlorocyclopentadiene & $041851-50-7$ & & & & & & & & & & 0.50 & 0.010 & \\
\hline Chlorodibromoethane & $073506-94-2$ & & & & & & & $8.4 \mathrm{E}-02^{b}$ & & $1.1 \mathrm{E}-01$ & 0.80 & 0.010 & \\
\hline Chlorodifluoromethane & $000075-45-6$ & & & & & $1.4 \mathrm{E}+01^{*}$ & & & & & 0.80 & 0.010 & \\
\hline
\end{tabular}


Table 3.6a. (continued)

\begin{tabular}{|c|c|c|c|c|c|c|c|c|c|c|c|c|c|}
\hline Chemical & CASRN & $\begin{array}{c}\text { Oral } \\
\text { Chronic } \\
\text { RfD } \\
\text { (mg/kg- } \\
\text { day) }\end{array}$ & $\begin{array}{c}\text { Dermal } \\
\text { Chronic } \\
\text { Rm } \\
\text { (mg/kg- } \\
\text { day) }\end{array}$ & $\begin{array}{l}\text { Oral Sub- } \\
\text { chronic } \\
\text { RD } \\
\text { (mg/kg- } \\
\text { day) }\end{array}$ & $\begin{array}{l}\text { Dermal } \\
\text { Sub- } \\
\text { chronic } \\
\text { Rid } \\
\text { (mg/kg- } \\
\text { day) }\end{array}$ & $\begin{array}{c}\text { Inhalation } \\
\text { Chronic } \\
\text { RfD } \\
\text { (mg/kg- } \\
\text { day) }\end{array}$ & $\begin{array}{c}\text { Inhalation } \\
\text { Sub- } \\
\text { chronic } \\
\text { RfD } \\
\text { (mg/kg- } \\
\text { day) }\end{array}$ & $\begin{array}{c}\text { Oral } \\
\text { Slope } \\
\text { Factor } \\
\text { (mg/kg- } \\
\text { day) }^{-1}\end{array}$ & $\begin{array}{l}\text { Inhalation } \\
\text { Slope } \\
\text { Factor } \\
\text { (mg/kg- } \\
\text { day) }\end{array}$ & $\begin{array}{c}\text { Dermal } \\
\text { Slope } \\
\text { Factor } \\
\text { (mg/kg- } \\
\text { day) }\end{array}$ & $\begin{array}{c}\text { GI } \\
\text { Absorp- } \\
\text { tion } \\
\text { Factor }\end{array}$ & $\begin{array}{c}\text { Dermal } \\
\text { Absorp- } \\
\text { tion } \\
\text { Factor }\end{array}$ & $\begin{array}{l}\text { Date Toxicity } \\
\text { Value } \\
\text { Withdrawn } \\
\text { from } \\
\text { IRIS/HEAST }\end{array}$ \\
\hline Chloroform ${ }^{(6)}$ & $000067-66-3$ & $1.0 \mathrm{E}-02^{2}$ & $2.0 \mathrm{E}-03$ & $1.0 \mathrm{E}-02^{\mathrm{b}, 0}$ & $2.0 \mathrm{E}-03$ & & & $6.1 \mathrm{E}-03^{*}$ & $8.1 E-02^{b}$ & $3.1 \mathrm{E}-02$ & 0.20 & 0.010 & \\
\hline Chloromethane $e^{(6)}$ & $000074-87-3$ & & & & & & $2.6 \mathrm{E}+00^{\circ}$ & $1.3 \mathrm{E}-02^{\mathrm{b}}$ & $6.3 \mathrm{E}-03^{\mathrm{b}}$ & $1.6 \mathrm{E}-02$ & 0.80 & 0.010 & \\
\hline Chloromethyl Methyl Ether & $000107-30-2$ & & & & & & & & & & 0.50 & 0.010 & \\
\hline Chloronaphthalene, Beta- & $000091-58-7$ & $8.0 \mathrm{E}-02^{4}$ & $6.4 \mathrm{E}-02$ & & & & & & & & 0.80 & 0.010 & \\
\hline Chloronitrobenzene, o- & $000088-73-3$ & & & & & & & $2.5 \mathrm{E}-02^{b}$ & & 3.1E-02. & 0.80 & 0.010 & \\
\hline Chloronitrobenzene, p- & $000121-73-3$ & & & & & & & $1.8 \mathrm{E}-02^{b}$ & & 2.3E-02 & 0.80 & 0.010 & \\
\hline Chlorophenol, 2- & $000095-57-8$ & $5.0 \mathrm{E}-03^{a}$ & 4.0E-03 & $5.0 \mathrm{E}-02^{b}$ & 2.5E-02 & & & & & & 0.80 & 0.010 & \\
\hline $\begin{array}{l}\text { Chlorophenyl Methyl Sulfide, } \\
\text { p- }\end{array}$ & $000123-09-1$ & & & & & & & & & & 0.50 & 0.010 & \\
\hline $\begin{array}{l}\text { Chlorophenyl Methyl } \\
\text { Sulfoxide }\end{array}$ & $000934-73-6$ & & & & & & & & & & 0.50 & 0.010 & \\
\hline Chloropropane, 2- & $000075-29-6$ & & & & & $2.9 \mathrm{E}-02^{\mathrm{b}}$ & $2.9 \mathrm{E}-01^{\mathrm{b}}$ & & & & 0.80 & 0.010 & \\
\hline Chlorothalonil & $001897-45-6$ & $1.5 \mathrm{E}-02^{2}$ & 7.5E-03 & $1.5 \mathrm{E}-02^{\mathrm{b}}$ & 7.5E-03 & & & $1.1 \mathrm{E}-02^{\mathrm{b}}$ & & 2.2E-02 & 0.50 & 0.010 & \\
\hline Chlorotoluene, o- & $000095-49-8$ & $2.0 \mathrm{E}-02^{\mathrm{a}}$ & $1.6 \mathrm{E}-02$ & $2.0 \mathrm{E}-01^{\mathrm{b}}$ & $1.6 \mathrm{E}-01$ & & & & & & 0.80 & 0.010 & \\
\hline Chlorpropham & $000101-21-3$ & $2.0 \mathrm{E}-01^{*}$ & $1.0 \mathrm{E}-01$ & & & & & & . & & 0.50 & 0.010 & \\
\hline Chlorpyrifos & $002921-88-2$ & $3.0 \mathrm{E}-03^{n}$ & $1.5 \mathrm{E}-03$ & $3.0 \mathrm{E}-03^{\mathrm{b}}$ & $1.5 \mathrm{E}-03$ & & & & & & 0.50 & 0.010 & \\
\hline Chlorpyrifos Methyl & $005598-13-0$ & $1.0 \mathrm{E}-02^{\mathrm{b}}$ & $5.0 \mathrm{E}-03$ & $1.0 \mathrm{E}-02^{\mathrm{b}}$ & $5.0 \mathrm{E}-03$ & & & & & & 0.50 & 0.010 & \\
\hline Chlorsulfuron & $064902-72-3$ & $5.0 \mathrm{E}-02^{2}$ & $2.5 \mathrm{E}-02$ & & & & & & & & 0.50 & 0.010 & \\
\hline Chlorthiophos & $060238-56-4$ & $8.0 \mathrm{E}-04^{\mathrm{b}}$ & 4.0E-04 & $8.0 \mathrm{E}-04^{b}$ & 4.0E-04 & & & & & & 0.50 & 0.010 & \\
\hline
\end{tabular}


Table 3.6a. (continued)

\begin{tabular}{|c|c|c|c|c|c|c|c|c|c|c|c|c|c|}
\hline Chemical & CASRN & $\begin{array}{c}\text { Oral } \\
\text { Chronic } \\
\text { RmD } \\
\text { (mg/kg- } \\
\text { day) }\end{array}$ & $\begin{array}{c}\text { Dermal } \\
\text { Chronic } \\
\text { RmD } \\
\text { (mg/kg- } \\
\text { day) }\end{array}$ & $\begin{array}{c}\text { Oral Sub- } \\
\text { chronic } \\
\text { Rm } \\
\text { (mg/kg- } \\
\text { day) }\end{array}$ & $\begin{array}{c}\text { Dermal } \\
\text { Sub- } \\
\text { chronic } \\
\text { RfD } \\
\text { (mg/kg- } \\
\text { day) }\end{array}$ & $\begin{array}{c}\text { Inhalation } \\
\text { Chronic } \\
\text { RfD } \\
\text { (mg/kg- }^{\text {day) }}\end{array}$ & $\begin{array}{c}\text { Inhalation } \\
\text { Sub- } \\
\text { chronic } \\
\text { RfD } \\
\text { (mg/kg- } \\
\text { day) })^{\dagger}\end{array}$ & $\begin{array}{c}\text { Oral } \\
\text { Slope } \\
\text { Factor } \\
\text { (mg/kg- } \\
\text { day) }\end{array}$ & $\begin{array}{l}\text { Inhalation } \\
\text { Slope } \\
\text { Factor } \\
\text { (mg/kg- }^{\text {day) }}{ }^{-1}\end{array}$ & $\begin{array}{c}\text { Dermal } \\
\text { Slope } \\
\text { Factor } \\
\text { (mg/kg- } \\
\text { day) }\end{array}$ & $\begin{array}{c}\text { GI } \\
\text { Absorp- } \\
\text { tion } \\
\text { Factor }\end{array}$ & $\begin{array}{c}\text { Dermal } \\
\text { Absorp- } \\
\text { tion } \\
\text { Factor }\end{array}$ & $\begin{array}{l}\text { Date Toxicity } \\
\text { Value } \\
\text { Withdrawn } \\
\text { from } \\
\text { IRIS/HEAST }\end{array}$ \\
\hline $\begin{array}{l}\text { Chromium (III) (Insoluble } \\
\text { Salts) }\end{array}$ & $016065-83-1$ & $1.0 \mathrm{E}+00^{4}$ & $5.0 \mathrm{E}-03$ & $1.0 \mathrm{E}+00^{b}$ & $5.0 \mathrm{E}-03$ & - & & & & & 0.01 & 0.001 & \\
\hline Chromium (VI) ${ }^{(6)}$ & $018540-29-9$ & $5.0 \mathrm{E}-03^{4}$ & $1.0 \mathrm{E}-04$ & $2.0 \mathrm{E}-02^{\mathrm{b}, \mathrm{e}}$ & $4.0 \mathrm{E}-04$ & & & & $4.1 \mathrm{E}+01^{\mathrm{b}}$ & & 0.02 & 0.001 & \\
\hline Chromium Salts & NA & & & 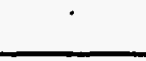 & & & & & & & 0.02 & 0.001 & \\
\hline Chrysene $^{(2)}$ & $000218-01-9$ & & & & & & & 7.3E-03' & $3.1 \mathrm{E}-03^{(2)}$ & $2.4 \mathrm{E}-02$ & 0.31 & 0.010 & \\
\hline Cobalt & $007440-48-4$ & & & & & & & & & & 0.80 & 0.001 & \\
\hline Coke Oven Emissions & $008007-45-2$ & & & & & & & & $2.2 \mathrm{E}+00^{b}$ & & 0.50 & 0.010 & \\
\hline Copper & $007440-50-8$ & & & & & & & & & & 0.30 & 0.001 & \\
\hline Copper Cyanide & $000544-92-3$ & $5.0 \mathrm{E}-03^{*}$ & $1.0 \mathrm{E}-03$ & $5.0 \mathrm{E}-02^{\mathrm{b}}$ & $1.0 \mathrm{E}-02$ & & & & & & 0.20 & 0.001 & \\
\hline Creosote & $008001-58-9$ & & & & & & & & & & 0.50 & 0.010 & \\
\hline Cresol, m- & $000108-39-4$ & $5.0 \mathrm{E}-02^{2}$ & $2.5 \mathrm{E}-02$ & $5.0 \mathrm{E}-01^{b}$ & $2.5 \mathrm{E}-01$ & & & & & & 0.50 & 0.010 & \\
\hline Cresol, o & $000095-48-7$ & $5.0 \mathrm{E}-02^{2}$ & $2.5 \mathrm{E}-02$ & $5.0 \mathrm{E}-01^{\mathrm{b}}$ & $2.5 \mathrm{E}-01$ & & & & & & 0.50 & 0.010 & \\
\hline Cresol, p- & $000106-44-5$ & $5.0 \mathrm{E}-03^{\mathrm{b}}$ & $3.3 \mathrm{E}-03$ & $5.0 \mathrm{E}-03 \mathrm{~b}$ & $3.3 \mathrm{E}-03$ & & & & & & 0.65 & 0.010 & \\
\hline Crotonaldehyde & $000123-73-9$ & & & & & & & $1.9 \mathrm{E}+00^{b}$ & & $3.8 \mathrm{E}+00$ & 0.50 & 0.010 & \\
\hline Cumene & $000098-82-8$ & $4.0 \mathrm{E}-02^{n}$ & $3.2 \mathrm{E}-02$ & $4.0 \mathrm{E}-01^{\mathrm{b}}$ & $2.0 \mathrm{E}-01$ & $2.6 \mathrm{E}-03^{\mathrm{b}}$ & $2.6 \mathrm{E}-02^{b}$ & & & & 0.80 & 0.010 & \\
\hline Cyanazine & $021725-46-2$ & $2.0 \mathrm{E}-03^{b}$ & $1.0 \mathrm{E}-03$ & $2.0 \mathrm{E}-03^{\mathrm{b}}$ & $1.0 \mathrm{E}-03$ & & & $8.4 \mathrm{E}-01^{\mathrm{b}}$ & & $1.7 \mathrm{E}+00$ & 0.50 & 0.010 & \\
\hline Cyanide (CN-) & $000057-12-5$ & $2.0 \mathrm{E}-02^{2}$ & $3.4 \mathrm{E}-03$ & $2.0 \mathrm{E}-02^{\mathrm{b}}$ & $3.4 \mathrm{E}-03$ & & & & & & 0.17 & 0.010 & \\
\hline Cyanogen & 000460-19-5 & $4.0 \mathrm{E}-02^{2}$ & $3.2 \mathrm{E}-02$ & $4.0 \mathrm{E}-02^{\mathrm{b}}$ & $3.2 \mathrm{E}-02$ & & & & & & 0.80 & 0.010 & \\
\hline Cyanogen Bromide & $000506-68-3$ & $9.0 \mathrm{E}-02^{\circ}$ & $7.2 \mathrm{E}-02$ & $9.0 \mathrm{E}-02^{\mathrm{b}}$ & $7.2 \mathrm{E}-02$ & & & & & & 0.80 & 0.010 & \\
\hline
\end{tabular}


Table 3.6a. (continued)

\begin{tabular}{|c|c|c|c|c|c|c|c|c|c|c|c|c|c|}
\hline Chemical & CASRN & $\begin{array}{c}\text { Oral } \\
\text { Chronic } \\
\text { RiD } \\
\text { (mg/kg- } \\
\text { day) }\end{array}$ & $\begin{array}{c}\text { Dermal } \\
\text { Chronic } \\
\text { RID } \\
\text { (mg/kg- } \\
\text { day) }\end{array}$ & $\begin{array}{l}\text { Oral Sub- } \\
\text { chronic } \\
\text { RD } \\
\text { (mg/kg- } \\
\text { day) }\end{array}$ & $\begin{array}{c}\text { Dermal } \\
\text { Sub- } \\
\text { chronic } \\
\text { RD } \\
\text { (mg/kg- } \\
\text { day) } \\
\end{array}$ & $\begin{array}{c}\text { Inhalation } \\
\text { Chronic } \\
\text { RD } \\
\text { (mg/kg- } \\
\text { day) }\end{array}$ & $\begin{array}{c}\text { Inhalation } \\
\text { Sub- } \\
\text { chronic } \\
\text { RID } \\
\text { (mglkg- } \\
\text { day) }\end{array}$ & $\begin{array}{c}\text { Oral } \\
\text { Slope } \\
\text { Factor } \\
\text { (mg/kg- } \\
\text { day) }\end{array}$ & $\begin{array}{l}\text { Inbalation } \\
\text { Slope } \\
\text { Factor } \\
\text { (mg/kg- } \\
\text { day) }^{-1}\end{array}$ & $\begin{array}{l}\text { Dermal } \\
\text { Slope } \\
\text { Factor } \\
\text { (mg/kg- }^{\text {(day) }} \\
\text { day }^{-1}\end{array}$ & $\begin{array}{c}\text { GI } \\
\text { Absorp- } \\
\text { tion } \\
\text { Factor }\end{array}$ & $\begin{array}{c}\text { Dermal } \\
\text { Absorp- } \\
\text { tion } \\
\text { Factor }\end{array}$ & $\begin{array}{l}\text { Date Toxicity } \\
\text { Value } \\
\text { Withdrawn } \\
\text { from } \\
\text { IRIS/HEAST }\end{array}$ \\
\hline Cyanogen Chloride & $000506-77-4$ & $5.0 \mathrm{E}-02^{\circ}$ & $4.0 \mathrm{E}-02$ & & & & & & & & 0.80 & 0.010 & \\
\hline $\begin{array}{l}\text { Cyclohexane, 1,2,3,4,5- } \\
\text { pentabromo-6-chloro- }\end{array}$ & $000087-84-3$ & & & & & & & $2.3 \mathrm{E}-02^{b}$ & & $4.6 \mathrm{E}-02$ & 0.50 & 0.010 & \\
\hline Cyclohexanone & $000108-94-1$ & $5.0 \mathrm{E}+00^{2}$ & $4.0 \mathrm{E}+00$ & & & & & & & & 0.80 & 0.010 & \\
\hline Cyclohexylamine & $000108-91: 8$ & $2.0 \mathrm{E}-01^{*}$ & $1.0 \mathrm{E}-01$ & $3.0 \mathrm{E}-01^{\mathrm{b}}$ & $1.5 \mathrm{E}-01$ & & & & & & 0.50 & 0.010 & \\
\hline Cyclopentadiene & $000542-92-7$ & & & & & & $8.6 \mathrm{E}-01^{\mathrm{b}, \mathrm{C}}$ & & & & 0.50 & 0.010 & \\
\hline Cyhalothrin/karate & $068085-85-8$ & $5.0 \mathrm{E}-03^{\circ}$ & $2.5 \mathrm{E}-03$ & & & & & & & & 0.50 & 0.010 & \\
\hline Cypermethrin & $052315-07-8$ & $1.0 \mathrm{E}-02^{\circ}$ & $5.0 \mathrm{E}-03$ & & & & & & & & 0.50 & 0.010 & \\
\hline Cyromazine & $066215-27-8$ & 7.5E-03" & $3.8 \mathrm{E}-03$ & & & & & & & & 0.50 & 0.010 & \\
\hline DDD & $000072-54-8$ & & & & & & & $2.4 \mathrm{E}-01^{\circ}$ & & $3.4 \mathrm{E}-01$ & 0.70 & 0.010 & \\
\hline DDE & $000072-55-9$ & & & & & & & $3.4 \mathrm{E}-01^{*}$ & & $4.9 \mathrm{E}-01$ & 0.70 & 0.010 & \\
\hline DDT & $000050-29-3$ & $5.0 \mathrm{E}-04^{\circ}$ & $3.5 \mathrm{E}-04$ & $5.0 \mathrm{E}-04^{b}$ & 3.5E-04 & & & $3.4 \mathrm{E}-01^{*}$ & $3.4 \mathrm{E}-01^{\mathrm{b}}$ & $4.9 \mathrm{E}-01$ & 0.70 & 0.010 & \\
\hline Dacthal & $001861-32-1$ & $1.0 \mathrm{E}-02^{2}$ & $5.0 \mathrm{E}-03$ & $1.0 \mathrm{E}-02^{\mathrm{b}}$ & $5.0 \mathrm{E}-03$ & & & & & & 0.50 & 0.010 & \\
\hline Dalapon & $000075-99-0$ & $3.0 \mathrm{E}-02^{2}$ & $1.5 \mathrm{E}-02$ & $3.0 \mathrm{E}-02^{\mathrm{b}}$ & $1.5 \mathrm{E}-02$ & & & & & & 0.50 & 0.010 & \\
\hline Decabromodiphenyl Ether & 001163-19-5 & 1.0E-02" & $8.0 \mathrm{E}-03$ & $1.0 \mathrm{E}-02^{\mathrm{b}}$ & $5.0 \mathrm{E}-03$ & & & & & & 0.80 & 0.010 & \\
\hline Demeton & $008065-48-3$ & $4.0 \mathrm{E}-05^{\circ}$ & $2.0 \mathrm{E}-05$ & & & & & & & & 0.50 & 0.010 & \\
\hline Di(2-ethylhexyl)adipate & $000103-23-1$ & $6.0 \mathrm{E}-01^{\circ}$ & $3.0 \mathrm{E}-01$ & & & & & $1.2 \mathrm{E}-03^{\circ}$ & & $2.4 \mathrm{E}-03$ & 0.50 & 0.010 & \\
\hline Diallate & $002303-16-4$ & & & & & & & $6.1 \mathrm{E}-02^{\mathrm{b}}$ & & $7.6 \mathrm{E}-02$ & 0.80 & 0.010 & \\
\hline Diazinon & $000333-41-5$ & $9.0 \mathrm{E}-04^{b}$ & 4.5E-04 & $9.0 \mathrm{E}-04^{\mathrm{b}}$ & 4.5E-04 & & & & & & 0.50 & 0.010 & \\
\hline
\end{tabular}

Toxicity values used in PRGs calculations/nonradionuclides

Page 11 of 39 
3-806

\begin{tabular}{|c|c|c|c|c|c|c|c|c|c|c|c|c|c|c|c|c|c|c|}
\hline 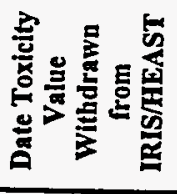 & & & & & & & & & & & & & ๙ু & & & & & \\
\hline 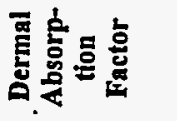 & $\begin{array}{l}0 \\
0 \\
0\end{array}$ & 응 & 응 & 응 & 융 & 응 & 응 & : & 웅 & 응 & 영 & $\stackrel{\circ}{\circ}$ & $\stackrel{\circ}{\circ}$ & O̊. & 웅 & $\stackrel{\circ}{\circ}$ & 융 & 영 \\
\hline
\end{tabular}

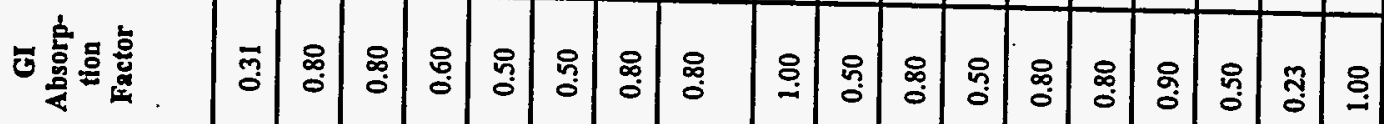

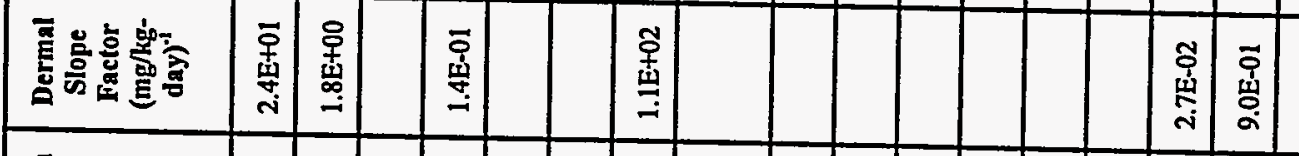

\begin{tabular}{|c|c|c|c|c|c|c|c|c|c|c|c|c|c|c|c|c|c|c|}
\hline 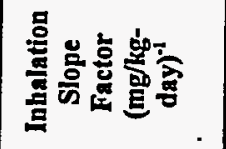 & 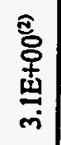 & 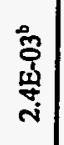 & & & & & 蒗 & & & & 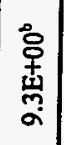 & & & & & & & \\
\hline 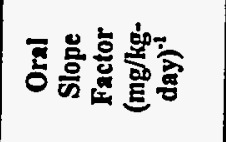 & 㝘 & 辛 & & 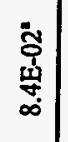 & & & $\begin{array}{l}\dot{\bar{\phi}} \\
\text { 索 } \\
\infty\end{array}$ & & & & & & & & 芯 & 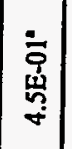 & & \\
\hline 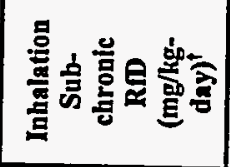 & & & & & & & $\begin{array}{l}\stackrel{0}{5} \\
\stackrel{4}{w} \\
\dot{w}\end{array}$ & & & & & & 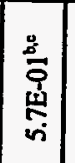 & & 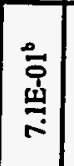 & & 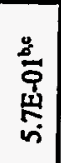 & 总 \\
\hline 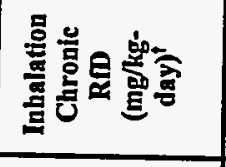 & & 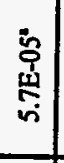 & & & & & 草 & & & & & & 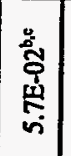 & & 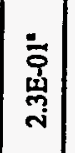 & & 苔 & 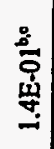 \\
\hline 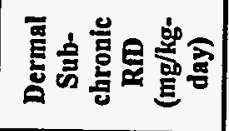 & & & 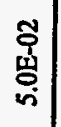 & 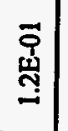 & & & & 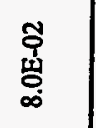 & 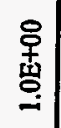 & 苍 & & & & & & & 葛 & 冨 \\
\hline 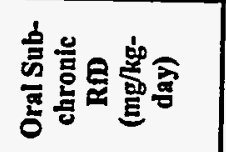 & & & 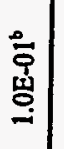 & 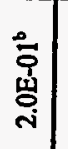 & & & & 悹 & 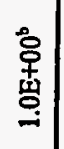 & 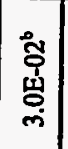 & & & 产 & & & & 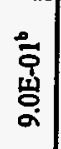 & 总 \\
\hline 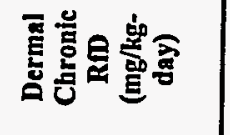 & & & 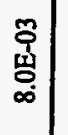 & 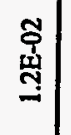 & & & & 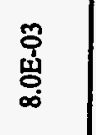 & 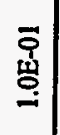 & 寅 & & & 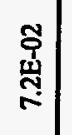 & & & & 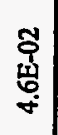 & 宮 \\
\hline 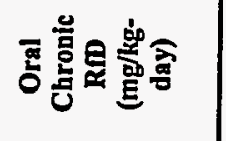 & & & 预 & 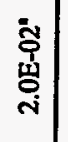 & & & & 产 & 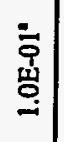 & 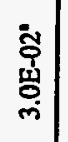 & & & 葶 & & & & 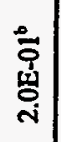 & 高 \\
\hline 宽 & 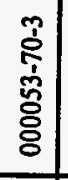 & 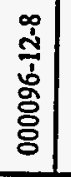 & 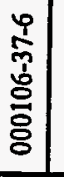 & 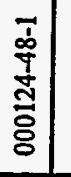 & 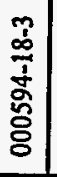 & 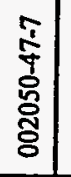 & 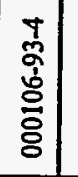 & 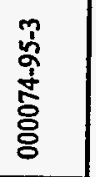 & 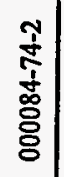 & $\begin{array}{l}a \\
\dot{\delta} \\
\dot{0} \\
\frac{0}{2} \\
\overline{8}\end{array}$ & $\begin{array}{l}\frac{9}{7} \\
\frac{7}{0} \\
\frac{0}{0}\end{array}$ & & 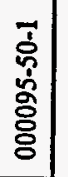 & 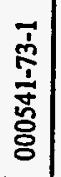 & 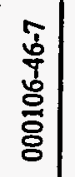 & $\begin{array}{l}\frac{7}{5} \\
\frac{1}{8} \\
\text { ठे }\end{array}$ & 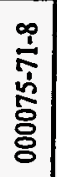 & 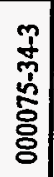 \\
\hline 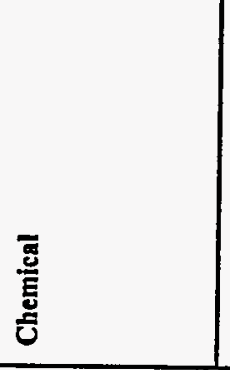 & 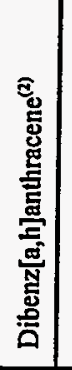 & 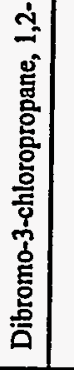 & 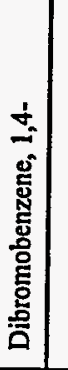 & 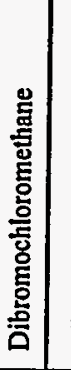 & 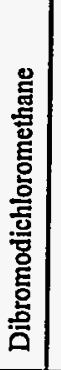 & 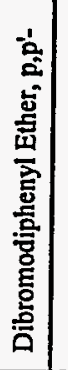 & 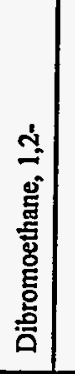 & 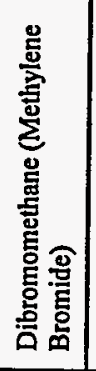 & 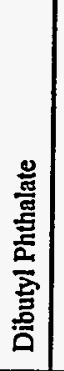 & हू. & 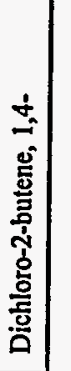 & 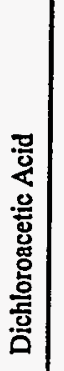 & 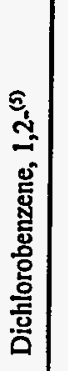 & 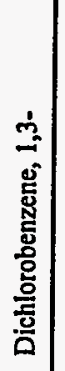 & 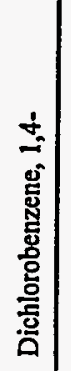 & 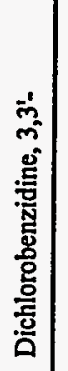 & 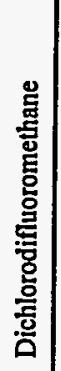 & 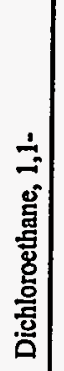 \\
\hline
\end{tabular}


Table 3.6a. (continued)

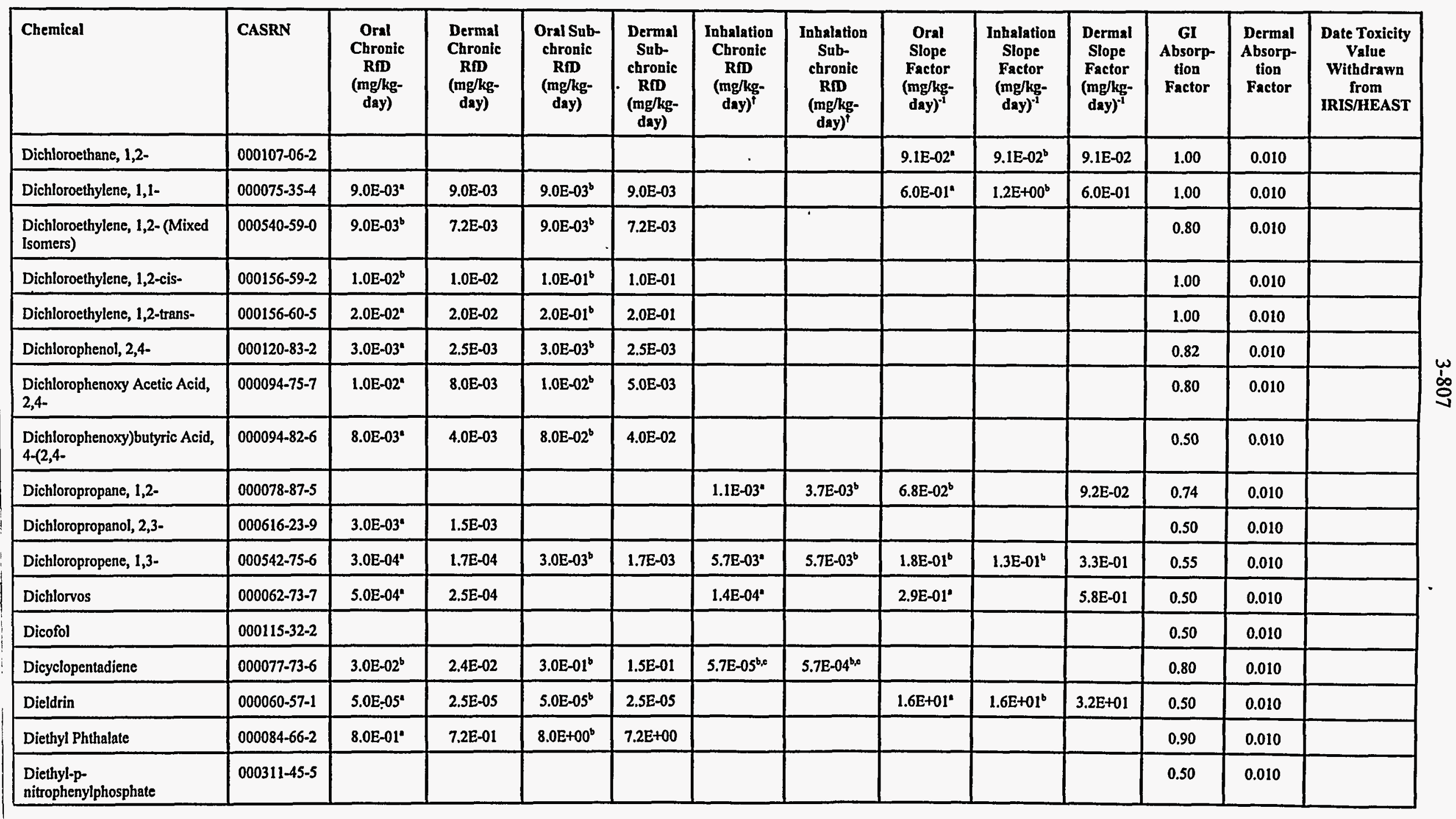

Toxicity values used in PRGs calculations/nonradionuclides

Page 13 of 39 
Table 3.6a. (continued)

\begin{tabular}{|c|c|c|c|c|c|c|c|c|c|c|c|c|c|}
\hline Chemical & CASRN & $\begin{array}{c}\text { Oral } \\
\text { Chronic } \\
\text { RDD } \\
\text { (mg/kg- } \\
\text { day) }\end{array}$ & $\begin{array}{c}\text { Dermal } \\
\text { Chronic } \\
\text { RfD } \\
\text { (mg/kg- } \\
\text { day) }\end{array}$ & $\begin{array}{c}\text { Oral Sub- } \\
\text { chronic } \\
\text { RfD } \\
\text { (mg/kg- } \\
\text { day) }\end{array}$ & $\begin{array}{c}\text { Dermal } \\
\text { Sub- } \\
\text { chronic } \\
\text { RmD } \\
\text { (mg/kg- } \\
\text { day) }\end{array}$ & $\begin{array}{c}\text { Inhalation } \\
\text { Chronic } \\
\text { RID } \\
\text { (mg/kg- } \\
\text { day })^{\dagger}\end{array}$ & $\begin{array}{c}\text { Inhalation } \\
\text { Sub- } \\
\text { chronic } \\
\text { RID } \\
\text { (mg/kg- } \\
\text { day) }\end{array}$ & $\begin{array}{c}\text { Oral } \\
\text { Slope } \\
\text { Factor } \\
\text { (mg/kg- } \\
\text { day) }{ }^{-1}\end{array}$ & $\begin{array}{l}\text { Inhalation } \\
\text { Slope } \\
\text { Factor } \\
\text { (mg/kg- } \\
\text { day) }\end{array}$ & $\begin{array}{l}\text { Dermal } \\
\text { Slope } \\
\text { Factor } \\
\text { (mg/kg- } \\
\text { day) }\end{array}$ & $\begin{array}{c}\text { GI } \\
\text { Absorp- } \\
\text { tion } \\
\text { Factor }\end{array}$ & $\begin{array}{c}\text { Dermal } \\
\text { Absorp- } \\
\text { tion } \\
\text { Factor }\end{array}$ & $\begin{array}{c}\text { Date Toxicity } \\
\text { Value } \\
\text { Withdrawn } \\
\text { from } \\
\text { IRIS/HEAST }\end{array}$ \\
\hline $\begin{array}{l}\text { Diethylene Glycol Dinitrate } \\
\text { (DEGDN) }\end{array}$ & 000693-21-0 & & & & & & & & & & 0.50 & 0.010 & \\
\hline $\begin{array}{l}\text { Diethylene Glycol Monobutyl } \\
\text { Ether }\end{array}$ & $000112-34-5$ & & & & & $5.7 \mathrm{E}-03^{\mathrm{b}}$ & $5.7 \mathrm{E}-02^{\mathrm{b}}$ & & & & 0.50 & 0.010 & \\
\hline $\begin{array}{l}\text { Diethylene Glycol Monoethyl } \\
\text { Ether }\end{array}$ & $000111-90-0$ & $2.0 \mathrm{E}+00^{\mathrm{b}}$ & $1.0 \mathrm{E}+00$ & $5.0 \mathrm{E}+00^{6}$ & $2.5 \mathrm{E}+00$ & & . & & & & 0.50 & 0.010 & \\
\hline Diethylformamide & $000617-84-5$ & $1.1 \mathrm{E}-02^{\mathrm{b}}$ & $5.5 \mathrm{E}-03$ & $1.1 \mathrm{E}-02^{\mathrm{b}}$ & $5.5 \mathrm{E}-03$ & & & & & & 0.50 & 0.010 & \\
\hline Diethylstilbesterol & $000056-53-1$ & & & & & & & $4.7 \mathrm{E}+03^{b}$ & $4.9 \mathrm{E}+02^{b}$ & $9.4 \mathrm{E}+03$ & 0.50 & 0.010 & \\
\hline Difenzoquat & $043222-48-6$ & $8.0 \mathrm{E}-02^{\circ}$ & $4.0 \mathrm{E}-02$ & & & & & & & & 0.50 & 0.010 & \\
\hline Diflubenzuron & $035367-38-5$ & $2.0 \mathrm{E}-02^{2}$ & $1.0 \mathrm{E}-02$ & & & & & & & & 0.50 & 0.010 & \\
\hline Difluoroethane, 1,1- & $000075-37-6$ & & & & & $1.1 \mathrm{E}+01^{\circ}$ & & & & & 0.80 & 0.010 & \\
\hline $\begin{array}{l}\text { Diisopropyl } \\
\text { Methylphosphonate }\end{array}$ & $001445-75-6$ & $8.0 \mathrm{E}-02^{n}$ & $4.0 \mathrm{E}-02$ & & & & & & & & 0.50 & 0.010 & \\
\hline Dimethipin & $055290-64-7$ & $2.0 \mathrm{E}-02^{2}$ & $1.0 \mathrm{E}-02$ & & & & & & & & 0.50 & 0.010 & \\
\hline Dimethoate & $000060-51-5$ & $2.0 \mathrm{E}-04^{\prime}$ & $1.0 \mathrm{E}-04$ & $2.0 \mathrm{E}-04^{\mathrm{b}}$ & $1.0 \mathrm{E}-04$ & & & & & & 0.50 & 0.010 & \\
\hline Dimethoxybenzidine, 3,3'- & $000119-90-4$ & & & & & & & $1.4 \mathrm{E}-02^{\mathrm{b}}$ & & $2.8 \mathrm{E}-02$ & 0.50 & 0.010 & \\
\hline Dimethyl Sulfate & $000077-78-1$ & & & & & & & & & & 0.50 & 0.010 & \\
\hline Dimethylaniline $\mathrm{HCl}, 2,4-$ & $021436-96-4$ & & & & & & & $5.8 \mathrm{E}-01^{b}$ & & $1.2 \mathrm{E}+00$ & 0.50 & 0.010 & \\
\hline Dimethylaniline, 2,4- & $000095-68-1$ & & & & & & & $7.5 \mathrm{E}-01^{\mathrm{b}}$ & • & $1.5 \mathrm{E}+00$ & 0.50 & 0.010 & \\
\hline Dimethylaniline, $\mathrm{N}, \mathrm{N}$ - & $000121-69-7$ & $2.0 \mathrm{E}-03^{\circ}$ & $1.0 \mathrm{E}-03$ & $2.0 \mathrm{E}-02^{\mathrm{b}}$ & $1.0 \mathrm{E}-02$ & & & & & & 0.50 & 0.010 & \\
\hline Dimethylbenzidine, 3,3'- & $000119-93-7$ & & & & & & & $9.2 \mathrm{E}+00^{6}$ & & $1.8 \mathrm{E}+01$ & 0.50 & 0.010 & \\
\hline
\end{tabular}

Toxicity values used in PRGs calculations/nonradionuclides

Page 14 of 39 
Table 3.6a. (continued)

\begin{tabular}{|c|c|c|c|c|c|c|c|c|c|c|c|c|c|}
\hline Chemical & CASRN & $\begin{array}{c}\text { Oral } \\
\text { Chronic } \\
\text { Rm } \\
\text { (mg/kg- } \\
\text { day) }\end{array}$ & $\begin{array}{c}\text { Dermal } \\
\text { Chronic } \\
\text { RD } \\
\text { (mg/kg- } \\
\text { day) }\end{array}$ & $\begin{array}{c}\text { Oral Sub- } \\
\text { chronic } \\
\text { RD } \\
\text { (mg/kg- } \\
\text { day) }\end{array}$ & $\begin{array}{c}\text { Dermal } \\
\text { Sub- } \\
\text { chronic } \\
\text { RD } \\
\text { (mg/kg- } \\
\text { day) } \\
\end{array}$ & $\begin{array}{c}\text { Inhalation } \\
\text { Chronic } \\
\text { RID } \\
\text { (mg//kg- } \\
\text { day) }\end{array}$ & $\begin{array}{c}\text { Inhalation } \\
\text { Sub- } \\
\text { chronic } \\
\text { RDD } \\
\text { (mg/kg- } \\
\text { day) } \\
\end{array}$ & $\begin{array}{c}\text { Oral } \\
\text { Slope } \\
\text { Factor } \\
(\mathrm{mg} / \mathrm{kg}- \\
\text { day })^{-1}\end{array}$ & $\begin{array}{l}\text { Inhalation } \\
\text { Slope } \\
\text { Factor } \\
\text { (mg/kg- } \\
\text { day) }\end{array}$ & $\begin{array}{l}\text { Dermal } \\
\text { Slope } \\
\text { Factor } \\
\text { (mg/kg- } \\
\text { day) })^{-1}\end{array}$ & $\begin{array}{c}\text { GI } \\
\text { Absorp- } \\
\text { tion } \\
\text { Factor }\end{array}$ & $\begin{array}{c}\text { Dermal } \\
\text { Absorp- } \\
\text { tion } \\
\text { Factor }\end{array}$ & $\begin{array}{c}\text { Date Toxicity } \\
\text { Value } \\
\text { Withdrawn } \\
\text { from } \\
\text { IRIS/HEAST }\end{array}$ \\
\hline Dimethylethyl Lead & $107584-40-7$ & & & & & & & & & & 0.50 & 0.010 & \\
\hline Dimethylformamide & $000068-12-2$ & $1.0 \mathrm{E}-01^{b}$ & $5.0 \mathrm{E}-02$ & $1.0 E+00^{b}$ & $5.0 \mathrm{E}-01$ & 8.6E-03" & $8.6 \mathrm{E}-03^{\mathrm{b}}$ & & & & 0.50 & 0.010 & \\
\hline Dimethylhydrazine, $1,11^{(5)}$ & $000057-14-7$ & & & & & & & $2.6 \mathrm{E}+00^{w}$ & $3.5 \mathrm{E}+00^{\mathrm{w}}$ & $5.2 \mathrm{E}+00$ & 0.50 & 0.010 & 7794 \\
\hline Dimethylhydrazine, 1,2- & $000540-73-8$ & & & & & & & & & & 0.50 & 0.010 & \\
\hline Dimethylphenol, 2,4- & $000105-67-9$ & $2.0 \mathrm{E}-02^{*}$ & $1.6 \mathrm{E}-02$ & $2.0 \mathrm{E}-01^{\mathrm{b}}$ & $1.0 \mathrm{E}-01$ & & & & & & 0.80 & 0.010 & \\
\hline Dimethylphenol, 2,6- & $000576-26-1$ & $6.0 \mathrm{E}-04^{*}$ & 3.0E-04 & $6.0 \mathrm{E}-03^{\mathrm{b}}$ & $3.0 \mathrm{E}-03$ & & & & & & 0.50 & 0.010 & \\
\hline Dimethylphenol, 3,4- & $000095-65-8$ & $1.0 \mathrm{E}-03^{n}$ & $5.0 \mathrm{E}-04$ & $1.0 \mathrm{E}-02^{\mathrm{b}}$ & $5.0 \mathrm{E}-03$ & & & & & & 0.50 & 0.010 & \\
\hline Dimethylphthalate ${ }^{(3)}$ & $000131-11-3$ & $1.0 \mathrm{E}+01^{w}$ & $9.0 \mathrm{E}+00$ & & & & & & & & 0.90 & 0.010 & $11 / 94$ \\
\hline Dimethylterephthalate & $000120-61-6$ & $1.0 \mathrm{E}-01^{*}$ & $5.0 \mathrm{E}-02$ & $1.0 \mathrm{E}-01^{\mathrm{b}}$ & $5.0 \mathrm{E}-02$ & & & & & & 0.50 & 0.010 & \\
\hline Dinitro-o-cresol, 4,6- & $000534-52-1$ & & & & & & & & & & 1.00 & 0.010 & \\
\hline $\begin{array}{l}\text { Dinitro-0-cyclohexyl Phenol, } \\
\text { 4,6- }\end{array}$ & 000131-89-5 & $2.0 \mathrm{E}-03^{\circ}$ & $1.0 \mathrm{E}-03$ & & & & & & & & 0.50 & 0.010 & \\
\hline Dinitrobenzene, 1,2- & $000528-29-0$ & $4.0 \mathrm{E}-04^{b}$ & 3.7E-04 & $4.0 \mathrm{E}-03^{\mathrm{b}}$ & 3.7E-03 & & & & & & 0.93 & 0.010 & \\
\hline Dinitrobenzene, 1,3- & $000099-65-0$ & $1.0 \mathrm{E}-04^{\circ}$ & $6.5 \mathrm{E}-05$ & $1.0 \mathrm{E}-03^{\mathrm{b}}$ & $6.5 \mathrm{E}-04$ & & & & & & 0.65 & 0.010 & \\
\hline Dinitrobenzene, 1,4- & $000100-25-4$ & $4.0 \mathrm{E}-04^{b}$ & $2.0 \mathrm{E}-04$ & $4.0 \mathrm{E}-03^{b}$ & $2.0 \mathrm{E}-03$ & & & & & & 0.50 & 0.010 & \\
\hline Dinitrophenol, 2,4- & $000051-28-5$ & 2.0E-03' & $2.0 \mathrm{E}-03$ & $2.0 \mathrm{E}-03^{b}$ & $2.0 \mathrm{E}-03$ & & & & & & 1.00 & 0.010 & \\
\hline Dinitrotoluene, 2,4- & $000121-14-2$ & $2.0 \mathrm{E}-03^{2}$ & $1.7 \mathrm{E}-03$ & $2.0 \mathrm{E}-03^{\circ}$ & $1.7 \mathrm{E}-03$ & & & $6.8 \mathrm{E}-01^{48}$ & & $8.0 \mathrm{E}-01$ & 0.85 & 0.010 & \\
\hline Dinitrotoluene, 2,6- & $000606-20-2$ & $1.0 \mathrm{E}-03^{b}$ & $8.5 \mathrm{E}-04$ & $1.0 \mathrm{E}-02^{\mathrm{b}}$ & $8.5 \mathrm{E}-03$ & & & $6.8 \mathrm{E}-01^{45}$ & & 8.0E-01 & 0.85 & 0.010 & \\
\hline Dinitrotoluene, 2-Amino-4,6- & $035572-78-2$ & & & & & & & & & & 0.50 & 0.010 & \\
\hline
\end{tabular}


Table 3.6a. (continued)

\begin{tabular}{|c|c|c|c|c|c|c|c|c|c|c|c|c|c|}
\hline Chemical & CASRN & $\begin{array}{c}\text { Oral } \\
\text { Chronic } \\
\text { RID } \\
\text { (mg/kg- } \\
\text { day) }\end{array}$ & $\begin{array}{c}\text { Dermal } \\
\text { Chronic } \\
\text { Rm } \\
\text { (mg/kg- } \\
\text { day) }\end{array}$ & $\begin{array}{c}\text { Oral Sub- } \\
\text { chronlc } \\
\text { RfD } \\
\text { (mg/kg- } \\
\text { day) }\end{array}$ & $\begin{array}{c}\text { Dermal } \\
\text { Sub- } \\
\text { chronic } \\
\text { RmD } \\
\text { (mg/kg- } \\
\text { day) }\end{array}$ & $\begin{array}{c}\text { Inhalation } \\
\text { Chronic } \\
\text { RfD } \\
\text { (mg/kg- } \\
\text { day) }\end{array}$ & $\begin{array}{c}\text { Inhalation } \\
\text { Sub- } \\
\text { chronic } \\
\text { RmD } \\
\text { (mg/kg- } \\
\text { day) }^{\dagger}\end{array}$ & $\begin{array}{c}\text { Oral } \\
\text { Slope } \\
\text { Factor } \\
\text { (mg/kg- } \\
\text { day) }\end{array}$ & $\begin{array}{l}\text { Inhalation } \\
\text { Slope } \\
\text { Factor } \\
\text { (mg/kg- } \\
\text { day) }^{-1}\end{array}$ & $\begin{array}{l}\text { Dermal } \\
\text { Slope } \\
\text { Factor } \\
\text { (mg/kg- } \\
\text { day) }^{-1}\end{array}$ & $\begin{array}{c}\text { GI } \\
\text { Absorp- } \\
\text { tion } \\
\text { Factor }\end{array}$ & $\begin{array}{c}\text { Dermal } \\
\text { Absorp- } \\
\text { tion } \\
\text { Factor }\end{array}$ & $\begin{array}{l}\text { Date Toxicity } \\
\text { Value } \\
\text { Withdrawn } \\
\text { from } \\
\text { IRIS/HEAST }\end{array}$ \\
\hline Dinoseb & $000088-85-7$ & $1.0 \mathrm{E}-03^{2}$ & $5.0 \mathrm{E}-04$ & $1.0 \mathrm{E}-03^{6}$ & $5.0 \mathrm{E}-04$ & & & & & & 0.50 & 0.010 & \\
\hline Dioxane, 1,4- & $000123-91-1$ & & & & & & & $1.1 \mathrm{E}-02^{\prime}$ & & $1.4 \mathrm{E}-02$ & 0.80 & 0.010 & \\
\hline Diphenamid & $000957-51-7$ & $3.0 \mathrm{E}-02^{*}$ & $1.5 \mathrm{E}-02$ & & & & & & & & 0.50 & 0.010 & \\
\hline Diphenylamine & $000122-39-4$ & $2.5 \mathrm{E}-02^{\prime}$ & $1.3 \mathrm{E}-02$ & $2.5 \mathrm{E}-02^{b}$ & $1.3 \mathrm{E}-02$ & & & & & & 0.50 & 0.010 & \\
\hline Diphenylhydrazine, 1,2- & $000122-66-7$ & & & & & & & $8.0 \mathrm{E}-01^{\prime \prime}$ & $8.0 \mathrm{E}-01^{b}$ & $1.6 \mathrm{E}+00$ & 0.50 & 0.010 & \\
\hline Diquat & $000085-00-7$ & $2.2 \mathrm{E}-03^{\prime \prime}$ & $1.1 \mathrm{E}-03$ & & & & & & & & 0.50 & 0.010 & \\
\hline Direct Black 38 & $001937-37-7$ & & & & & & & $8.6 \mathrm{E}+00^{b}$ & & $1.7 \mathrm{E}+01$ & 0.50 & 0.010 & \\
\hline Direct Blue 6 & $002602-46-2$ & & & & & & & $8.1 E+00^{b}$ & & $1.6 \mathrm{E}+01$ & 0.50 & 0.010 & \\
\hline Direct Brown 95 & $016071-86-6$ & & & & & & & $9.3 \mathrm{E}+00^{\circ}$ & & $1.9 E+01$ & 0.50 & 0.010 & \\
\hline Direct Sky Blue & $002610-05-1$ & & & & & & & & & & 0.50 & 0.010 & \\
\hline Disulfoton & $000298-04-4$ & 4.0E- $05^{n}$ & $2.0 \mathrm{E}-05$ & $4.0 \mathrm{E}-05^{\mathrm{b}}$ & $2.0 \mathrm{E}-05$ & & & & & & 0.50 & 0.010 & \\
\hline Diuron & $000330-54-1$ & $2.0 \mathrm{E}-03^{\prime \prime}$ & $1.0 \mathrm{E}-03$ & & & & & & & & 0.50 & 0.010 & \\
\hline Dodine & $002439-10-3$ & 4.0E-03" & $2.0 \mathrm{E}-03$ & & & & & & & & 0.50 & 0.010 & \\
\hline EPTC & $000759-94-4$ & $2.5 \mathrm{E}-02^{2}$ & $1.3 \mathrm{E}-02$ & $2.5 \mathrm{E}-02^{\mathrm{b}}$ & $1.3 \mathrm{E}-02$ & & & & & & 0.50 & 0.010 & \\
\hline Endosulfan & $000115-29-7$ & $6.0 \mathrm{E}-03^{3}$ & $3.0 \mathrm{E}-03$ & $6.0 \mathrm{E}-03^{\mathrm{b}}$ & $3.0 \mathrm{E}-03$ & & & & & & 0.50 & 0.010 & \\
\hline Endothall & $000145-73-3$ & $2.0 \mathrm{E}-02^{\prime}$ & $1.0 \mathrm{E}-02$ & $2.0 \mathrm{E}-02^{\mathrm{b}}$ & $1.0 \mathrm{E}-02$ & & & & & & 0.50 & 0.010 & \\
\hline Endrin & $000072-20-8$ & $3.0 \mathrm{E}-04^{2}$ & $6.0 \mathrm{E}-06$ & $3.0 \mathrm{E}-04^{b}$ & $6.0 \mathrm{E}-06$ & & . & & & & 0.02 & 0.010 & \\
\hline Epichlorohydrin & $000106-89-8$ & $2.0 \mathrm{E}-03^{b}$ & $1.6 \mathrm{E}-03$ & $2.0 \mathrm{E}-03^{b}$ & $1.6 \mathrm{E}-03$ & $2.9 \mathrm{E}-04^{\prime}$ & $2.9 \mathrm{E}-03^{b}$ & $9.9 \mathrm{E}-03^{\circ}$ & $4.2 \mathrm{E}-03^{\mathrm{b}}$ & $1.2 \mathrm{E}-02$ & 0.80 & 0.010 & \\
\hline Epoxybutane, 1,2- & $000106-88-7$ & & & & & $5.7 \mathrm{E}-03^{\circ}$ & & & & & 0.50 & 0.010 & \\
\hline
\end{tabular}

Toxicity values used in PRGs calculations/nonradionuclides

Page 16 of 39 
Table 3.6a. (continued)

\begin{tabular}{|c|c|c|c|c|c|c|c|c|c|c|c|c|c|}
\hline Chemical & CASRN & $\begin{array}{c}\text { Oral } \\
\text { Chronic } \\
\text { RfD } \\
\text { (mg/kg- } \\
\text { day) }\end{array}$ & $\begin{array}{c}\text { Dermal } \\
\text { Chronic } \\
\text { Rm } \\
\text { (mg/kg- } \\
\text { day) }\end{array}$ & $\begin{array}{l}\text { Oral Sub- } \\
\text { chronic } \\
\text { RID } \\
\text { (mg/kg- } \\
\text { day) }\end{array}$ & $\begin{array}{c}\text { Dermal } \\
\text { Sub- } \\
\text { chronic } \\
\text { RfD } \\
\text { (mg/kg- } \\
\text { day) }\end{array}$ & $\begin{array}{c}\text { Inhalation } \\
\text { Chronic } \\
\text { RfD } \\
\text { (mg/kg- } \\
\text { day) }\end{array}$ & $\begin{array}{c}\text { Inhalation } \\
\text { Sub- } \\
\text { chronic } \\
\text { Rm } \\
\text { (mg/kg- } \\
\text { day) }\end{array}$ & $\begin{array}{l}\text { Oral } \\
\text { Slope } \\
\text { Factor } \\
\text { (mg/kg- } \\
\text { day) }\end{array}$ & $\begin{array}{l}\text { Inhalation } \\
\text { Slope } \\
\text { Factor } \\
\text { (mg/kg- } \\
\text { day) }\end{array}$ & $\begin{array}{l}\text { Dermal } \\
\text { Slope } \\
\text { Factor } \\
\text { (mg/kg- } \\
\text { day) }\end{array}$ & $\begin{array}{c}\text { GI } \\
\text { Absorp- } \\
\text { tion } \\
\text { Factor }\end{array}$ & $\begin{array}{c}\text { Dermal } \\
\text { Absorp- } \\
\text { tion } \\
\text { Factor }\end{array}$ & $\begin{array}{c}\text { Date Toxicity } \\
\text { Value } \\
\text { Withdrawn } \\
\text { from } \\
\text { IRIS/HEAST }\end{array}$ \\
\hline Ethephon & $016672-87-0$ & $5.0 \mathrm{E}-03^{2}$ & $2.5 \mathrm{E}-03$ & & & & & & & & 0.50 & 0.010 & \\
\hline Ethion & $000563-12-2$ & $5.0 \mathrm{E}-04^{\prime}$ & $2.5 \mathrm{E}-04$ & & & & & & & & 0.50 & 0.010 & \\
\hline Ethoxyethanol Acetate, 2- & $000111-15-9$ & $3.0 \mathrm{E}-01^{\mathrm{bcc}}$ & $1.5 \mathrm{E}-01$ & $3.0 \mathrm{E}-01^{\mathrm{bec}}$ & $1.5 \mathrm{E}-01$ & & & & & & 0.50 & 0.010 & \\
\hline Ethoxyethanol, 2- & $000110-80-5$ & $4.0 \mathrm{E}-01^{b}$ & 2.0E-01 & $5.0 \mathrm{E}-01^{\mathrm{b}}$ & $2.5 \mathrm{E}-01$ & $5.7 \mathrm{E}-02^{\prime}$ & $5.7 \mathrm{E}-01^{\mathrm{b}}$ & & & & 0.50 & 0.010 & \\
\hline Ethyl Acetate & $000141-78-6$ & $9.0 \mathrm{E}-01^{\prime}$ & $7.2 \mathrm{E}-01$ & $9.0 \mathrm{E}+00^{\circ}$ & $7.2 E+00$ & & & & & & 0.80 & 0.010 & \\
\hline Ethyl Acrylate & $000140-88-5$ & & & & & & & $4.8 \mathrm{E}-02^{\mathrm{b}}$ & & $9.6 \mathrm{E}-02$ & 0.50 & 0.010 & \\
\hline Ethyl Chloride & $000075-00-3$ & & & & & $2.9 \mathrm{E}+00^{2}$ & $2.9 \mathrm{E}+00^{b}$ & & & & 0.80 & 0.010 & \\
\hline Ethyl Ether & 000060-29-7 & $2.0 \mathrm{E}-01^{\prime}$ & $1.6 \mathrm{E}-01$ & $2.0 \mathrm{E}+00^{b}$ & $1.6 \mathrm{E}+00$ & & & & & & 0.80 & 0.010 & \\
\hline Ethyl Methacrylate & $000097-63-2$ & $9.0 \mathrm{E}-02^{\mathrm{b}}$ & $7.2 \mathrm{E}-02$ & $9.0 \mathrm{E}-02^{\mathrm{b}}$ & $7.2 \mathrm{E}-02$ & & & & & , & 0.80 & 0.010 & \\
\hline $\begin{array}{l}\text { Ethyl-p-nitrophenyl } \\
\text { Phosphonate }\end{array}$ & 002104-64-5 & $1.0 \mathrm{E}-05^{*}$ & $5.0 \mathrm{E}-06$ & & & & & & & & 0.50 & 0.010 & \\
\hline Ethylbenzene $e^{(6)}$ & $000100-41-4$ & $1.0 \mathrm{E}-01^{\circ}$ & 9.7E-02 & 9.7E-02" & $9.4 \mathrm{E}-02$ & $2.9 \mathrm{E}-01^{\bullet}$ & & & & & 0.97 & 0.010 & \\
\hline Ethylene Cyanohydrin & $000109-78-4$ & $3.0 \mathrm{E}-01^{\mathrm{b}}$ & $1.5 \mathrm{E}-01$ & $3.0 \mathrm{E}-01^{\mathrm{b}}$ & $1.5 \mathrm{E}-01$ & & & & & & 0.50 & 0.010 & \\
\hline Ethylene Diamine & $000107-15-3$ & $2.0 \mathrm{E}-02^{\mathrm{b}}$ & $1.0 \mathrm{E}-02$ & $2.0 \mathrm{E}-01^{b}$ & $1.0 \mathrm{E}-01$ & & & & & & 0.50 & 0.010 & \\
\hline Ethylene Glycol & $000107-21-1$ & $2.0 \mathrm{E}+00^{4}$ & $1.0 \mathrm{E}+00$ & $2.0 \mathrm{E}+00^{6}$ & $1.0 \mathrm{E}+00$ & & & & & & 0.50 & 0.010 & \\
\hline $\begin{array}{l}\text { Ethylene Glycol Monobutyl } \\
\text { Ether }\end{array}$ & $000111-76-2$ & & & & & $5.7 \mathrm{E}-03^{b}$ & $5.7 \mathrm{E}-02^{\mathrm{b}}$ & & & & 0.50 & 0.010 & \\
\hline Ethylene Oxide & $000075-21-8$ & & & & & & & $1.0 \mathrm{E}+00^{6}$ & $3.5 \mathrm{E}-01^{b}$ & $1.3 \mathrm{E}+00$ & 0.80 & 0.010 & \\
\hline Ethylene Thiourea & $000096-45-7$ & $8.0 \mathrm{E}-05^{\circ}$ & 4.0E-05 & $8.0 \mathrm{E}-05^{b}$ & 4.0E-05 & & & $1.1 \mathrm{E}-01^{b}$ & & 2.2E-01 & 0.50 & 0.010 & \\
\hline Ethylphthalyl Ethyl Glycolate & $000084-72-0$ & $3.0 \mathrm{E}+00^{2}$ & $1.5 \mathrm{E}+00$ & & & & & & & & 0.50 & 0.010 & \\
\hline
\end{tabular}

Toxicity values used in PRGs calculations/nonradionuclides

Page 17 of 39 
Table 3.6a. (continued)

\begin{tabular}{|c|c|c|c|c|c|c|c|c|c|c|c|c|c|}
\hline Chemical & CASRN & $\begin{array}{c}\text { Oral } \\
\text { Chronic } \\
\text { RDD } \\
\text { (mg/kg- } \\
\text { day) }\end{array}$ & $\begin{array}{c}\text { Dermal } \\
\text { Chronic } \\
\text { RID } \\
\text { (mg/kg- } \\
\text { day) }\end{array}$ & $\begin{array}{l}\text { Oral Sub- } \\
\text { chronic } \\
\text { Rm } \\
\text { (mg/kg- } \\
\text { day) }\end{array}$ & $\begin{array}{c}\text { Dermal } \\
\text { Sub- } \\
\text { chronic } \\
\text { RmD } \\
\text { (mg/kg- } \\
\text { day) }\end{array}$ & $\begin{array}{c}\text { Inhalation } \\
\text { Chronic } \\
\text { RDD } \\
\text { (mg/kg- } \\
\text { day) }^{\dagger}\end{array}$ & $\begin{array}{l}\text { Inhalation } \\
\text { Sub- } \\
\text { chronic } \\
\text { RfD } \\
\text { (mg/kg- } \\
\text { day) }^{\dagger}\end{array}$ & $\begin{array}{c}\text { Oral } \\
\text { Slope } \\
\text { Factor } \\
\text { (mg/kg- } \\
\text { day) }\end{array}$ & $\begin{array}{l}\text { Inhalation } \\
\text { Slope } \\
\text { Factor } \\
\text { (mg/kg- } \\
\text { day) }\end{array}$ & $\begin{array}{l}\text { Dermal } \\
\text { Slope } \\
\text { Factor } \\
\text { (mg/kg- } \\
\text { day) }\end{array}$ & $\begin{array}{c}\text { GI } \\
\text { Absorp- } \\
\text { tion } \\
\text { Factor }\end{array}$ & $\begin{array}{c}\text { Dermal } \\
\text { Absorp- } \\
\text { tion } \\
\text { Factor }\end{array}$ & $\begin{array}{l}\text { Date Toxicity } \\
\text { Value } \\
\text { Withdrawn } \\
\text { from } \\
\text { IRIS/HEAST }\end{array}$ \\
\hline Express & $101200-48-0$ & $8.0 \mathrm{E}-03^{\prime \prime}$ & $4.0 \mathrm{E}-03$ & & & & & & & & 0.50 & 0.010 & \\
\hline Fenamiphos & $022224-92-6$ & $2.5 \mathrm{E}-04^{\circ}$ & $1.3 \mathrm{E}-04$ & & & & & & & & 0.50 & 0.010 & \\
\hline Fenpropathrin & $039515-41-8$ & 2.5E-02" & $1.3 \mathrm{E}-02$ & & & & & & & & 0.50 & 0.010 & \\
\hline Fluometuron & $002164-17-2$ & $1.3 \mathrm{E}-02^{\prime \prime}$ & $6.5 \mathrm{E}-03$ & & & & & & & & 0.50 & 0.010 & \\
\hline Fluoranthene & $000206-44-0$ & $4.0 \mathrm{E}-02^{*}$ & $1.2 \mathrm{E}-02$ & $4.0 \mathrm{E}-01^{\mathrm{b}}$ & $1.2 \mathrm{E}-01$ & & & & & & 0.31 & 0.010 & \\
\hline Fluorene & $000086-73-7$ & $4.0 \mathrm{E}-02^{\mathrm{a}}$ & $3.2 \mathrm{E}-02$ & $4.0 \mathrm{E}-01^{\mathrm{b}}$ & $2.0 \mathrm{E}-01$ & & & & & & 0.80 & 0.010 & \\
\hline Fluoride & $007782-41-4$ & $6.0 \mathrm{E}-02^{2}$ & $5.8 \mathrm{E}-02$ & $6.0 \mathrm{E}-02^{\mathrm{b}}$ & $5.8 \mathrm{E}-02$ & & & & & & 0.97 & 0.001 & \\
\hline Fluridone & $059756-60-4$ & 8.0E-02 & $4.0 \mathrm{E}-02$ & $8.0 \mathrm{E}-02^{\mathrm{b}}$ & $4.0 \mathrm{E}-02$ & & & & & & 0.50 & 0.010 & \\
\hline Flurprimidol & $056425-91-3$ & $2.0 \mathrm{E}-02^{2}$ & $1.0 \mathrm{E}-02$ & & & & & & & & 0.50 & 0.010 & \\
\hline Flutolanil & $066332-96-5$ & $6.0 \mathrm{E}-02^{2}$ & $3.0 \mathrm{E}-02$ & & & & & & & & 0.50 & 0.010 & \\
\hline Fluvalinate & 069409-94-5 & $1.0 \mathrm{E}-02^{2}$ & $5.0 \mathrm{E}-03$ & & & & & & & & 0.50 & 0.010 & \\
\hline Folpet & $000133-07-3$ & $1.0 \mathrm{E}-01^{2}$ & $5.0 \mathrm{E}-02$ & $1.0 \mathrm{E}-01^{6}$ & $5.0 \mathrm{E}-02$ & & & 3.5E-03" & & $7.0 \mathrm{E}-03$ & 0.50 & 0.010 & \\
\hline Fomesafen & $072178-02-0$ & & & & & & & $1.9 \mathrm{E}-01^{\circ}$ & & $3.8 \mathrm{E}-01$ & 0.50 & 0.010 & \\
\hline Fonofos & $000944-22-9$ & $2.0 \mathrm{E}-03^{2}$ & $1.0 \mathrm{E}-03$ & & & & & & & & 0.50 & 0.010 & \\
\hline Formaldehyde & $000050-00-0$ & $2.0 \mathrm{E}-01^{*}$ & $1.6 \mathrm{E}-01$ & $2.0 \mathrm{E}-01^{b}$ & $1.6 \mathrm{E}-01$ & & & & $4.5 \mathrm{E}-02^{b}$ & & 0.80 & 0.010 & \\
\hline Formic Acid & $000064-18-6$ & $2.0 \mathrm{E}+00^{\circ}$ & $1.0 E+00$ & $2.0 \mathrm{E}+00^{b}$ & $1.0 \mathrm{E}+00$ & & & & & & 0.50 & 0.010 & \\
\hline Fosetyl-AL & $039148-24-8$ & $3.0 \mathrm{E}+00^{\prime}$ & $1.5 E+00$ & & & & & & & & 0.50 & 0.010 & \\
\hline Furan & $000110-00-9$ & $1.0 \mathrm{E}-03^{\circ}$ & 8.0E-04 & $1.0 \mathrm{E}-02^{\mathrm{b}}$ & $5.0 \mathrm{E}-03$ & & & & & & 0.80 & 0.010 & \\
\hline Furazolidone & $000067-45-8$ & & & & & & & $3.8 \mathrm{E}+00^{5}$ & & $7.6 \mathrm{E}+00$ & 0.50 & 0.010 & \\
\hline
\end{tabular}


Table 3.6a. (continued)

\begin{tabular}{|c|c|c|c|c|c|c|c|c|c|c|c|c|c|}
\hline Chemical & CASRN & $\begin{array}{c}\text { Oral } \\
\text { Chronic } \\
\text { RID } \\
\text { (mg/kg- } \\
\text { day) }\end{array}$ & $\begin{array}{c}\text { Dermal } \\
\text { Chronic } \\
\text { RmD } \\
\text { (mg/kg- } \\
\text { day) }\end{array}$ & $\begin{array}{l}\text { Oral Sub- } \\
\text { chronic } \\
\text { RfD } \\
\text { (mg/kg- } \\
\text { day) }\end{array}$ & $\begin{array}{c}\text { Dermal } \\
\text { Sub- } \\
\text { chronic } \\
\text { Rm } \\
\text { (mg/kg- } \\
\text { day) }\end{array}$ & $\begin{array}{c}\text { Inhalation } \\
\text { Chronic } \\
\text { RmD } \\
\text { (mg/kg- } \\
\text { day) }^{\dagger}\end{array}$ & $\begin{array}{c}\text { Inhalation } \\
\text { Sub- } \\
\text { chronic } \\
\text { Rm } \\
\text { (mg/kg- } \\
\text { day) }\end{array}$ & $\begin{array}{c}\text { Oral } \\
\text { Slope } \\
\text { Factor } \\
\text { (mg/kg- } \\
\text { day) }\end{array}$ & $\begin{array}{l}\text { Inhalation } \\
\text { Slope } \\
\text { Factor } \\
\text { (mg/kg- } \\
\text { day) }\end{array}$ & $\begin{array}{l}\text { Dermal } \\
\text { Slope } \\
\text { Factor } \\
\text { (mg/kg- } \\
\text { day) }\end{array}$ & $\begin{array}{c}\text { GI } \\
\text { Absorp- } \\
\text { tion } \\
\text { Factor }\end{array}$ & $\begin{array}{c}\text { Dermal } \\
\text { Absorp- } \\
\text { tion } \\
\text { Factor }\end{array}$ & $\begin{array}{c}\text { Date Toxicity } \\
\text { Value } \\
\text { Withdrawn } \\
\text { from } \\
\text { IRIS/HEAST }\end{array}$ \\
\hline Furfural & $000098-01-1$ & $3.0 \mathrm{E}-03^{\circ}$ & $1.5 \mathrm{E}-03$ & $3.0 \mathrm{E}-02^{b}$ & $1.5 \mathrm{E}-02$ & $1.4 \mathrm{E}-02^{\mathrm{b}, \mathrm{e}}$ & $1.4 \mathrm{E}-01^{\mathrm{b}, \mathrm{c}}$ & & & & 0.50 & 0.010 & \\
\hline Furium & $000531-82-8$ & & & & & & & $5.0 \mathrm{E}+01^{\mathrm{b}}$ & & $1.0 E+02$ & 0.50 & 0.010 & \\
\hline Furmecyclox & $060568-05-0$ & & & & & & & $3.0 \mathrm{E}-02^{\mathrm{x}}$ & & $6.0 \mathrm{E}-02$ & 0.50 & 0.010 & \\
\hline Glufosinate, Ammonium & $077182-82-2$ & $4.0 \mathrm{E}-04^{\prime}$ & $2.0 \mathrm{E}-04$ & & & & & & & & 0.50 & 0.010 & \\
\hline Glycidyl & $000765-34-4$ & 4.0E-04" & $2.0 \mathrm{E}-04$ & $4.0 \mathrm{E}-03^{\mathrm{b}}$ & $2.0 \mathrm{E}-03$ & $2.9 \mathrm{E}-04^{b}$ & $2.9 \mathrm{E}-03^{b}$ & & & & 0.50 & 0.010 & \\
\hline Glyphosate & $001071-83-6$ & $1.0 \mathrm{E}-01^{\circ}$ & $5.0 \mathrm{E}-02$ & & & & & & & & 0.50 & 0.010 & \\
\hline Goal & $042874-03-3$ & $3.0 \mathrm{E}-03^{\prime}$ & $1.5 \mathrm{E}-03$ & & & & & & & & 0.50 & 0.010 & \\
\hline Haloxyfop, Methyl & $069806-40-2$ & $5.0 \mathrm{E}-05^{\circ}$ & 2.5E-05 & & & & & & & & 0.50 & 0.010 & \\
\hline Heptachlor & $000076-44-8$ & $5.0 \mathrm{E}-04^{\prime}$ & $3.6 \mathrm{E}-04$ & $5.0 \mathrm{E}-04^{\mathrm{b}}$ & $3.6 \mathrm{E}-04$ & & & $4.5 \mathrm{E}+00^{\circ}$ & $4.5 \mathrm{E}+00^{\circ}$ & $6.3 E+00$ & 0.72 & 0.010 & \\
\hline Heptachlor Epoxide & $001024-57-3$ & $1.3 \mathrm{E}-05^{2}$ & $9.4 \mathrm{E}-06$ & $1.3 \mathrm{E}-05^{b}$ & 9.4E-06 & & & $9.1 E+00^{\prime \prime}$ & $9.1 \mathrm{E}+00^{\mathrm{b}}$ & $1.3 \mathrm{E}+01$ & 0.72 & 0.010 & \\
\hline Heptane, $\mathrm{N}$ - & $000142-82-5$ & & & & & & & & & & 0.80 & 0.010 & \\
\hline Hexabromobenzene & $000087-82-1$ & $2.0 \mathrm{E}-03^{\prime}$ & $1.6 \mathrm{E}-03$ & $2.0 \mathrm{E}-02^{\mathrm{b}}$ & $1.0 \mathrm{E}-02$ & & & & & & 0.80 & 0.010 & \\
\hline Hexachlorobenzene ${ }^{(6)}$ & $000118-74-1$ & 8.0E-04" & $6.4 \mathrm{E}-04$ & & & & & $1.6 \mathrm{E}+00^{2}$ & $1.6 \mathrm{E}+00^{\mathrm{b}}$ & $2.0 \mathrm{E}+00$ & 0.80 & 0.010 & \\
\hline Hexachlorobutadiene $\mathrm{e}^{(\mathrm{)})}$ & $000087-68-3$ & $2.0 \mathrm{E}-04^{\mathrm{b}}$ & $1.6 \mathrm{E}-04$ & $6.7 \mathrm{E}-04^{\circ}$ & 3.3E-04 & & & $7.8 \mathrm{E}-02^{2}$ & $7.8 \mathrm{E}-02^{b}$ & $9.8 \mathrm{E}-02$ & 0.80 & 0.010 & \\
\hline $\begin{array}{l}\text { Hexachlorocyclohexane, } \\
\text { Alpha- }\end{array}$ & $000319-84-6$ & & & & & & & $6.3 \mathrm{E}+00^{4}$ & $6.3 \mathrm{E}+00^{6}$ & $6.5 \mathrm{E}+00$ & 0.97 & 0.010 & \\
\hline Hexachlorocyclohexane, Beta- & $000319-85-7$ & & & & & & & $1.8 \mathrm{E}+00^{\prime}$ & $1.8 \mathrm{E}+00^{b}$ & $2.0 E+00$ & 0.91 & 0.010 & \\
\hline Hexachlorocyclohexane, Delta- & $000319-86-8$ & & & & & & & & & & 0.50 & 0.010 & \\
\hline
\end{tabular}

Toxicity values used in PRGs calculations/nonradionuclides

Page 19 of 39 
Table 3.6a. (continued)

\begin{tabular}{|c|c|c|c|c|c|c|c|c|c|c|c|c|c|}
\hline Chemical & CASRN & $\begin{array}{c}\text { Oral } \\
\text { Chronic } \\
\text { RID } \\
\text { (mg/kg- } \\
\text { day) }\end{array}$ & $\begin{array}{c}\text { Dermal } \\
\text { Chronic } \\
\text { RfD } \\
\text { (mg/kg- } \\
\text { day) }\end{array}$ & $\begin{array}{c}\text { Oral Sub- } \\
\text { chronic } \\
\text { RfD } \\
\text { (mg/kg- } \\
\text { day) }\end{array}$ & $\begin{array}{c}\text { Dermal } \\
\text { Sub- } \\
\text { chronic } \\
\text { Rm } \\
\text { (mg/kg- } \\
\text { day) }\end{array}$ & $\begin{array}{c}\text { Inhalation } \\
\text { Chronic } \\
\text { RID } \\
\text { (mg/kg- } \\
\text { day) }\end{array}$ & $\begin{array}{c}\text { Inhalation } \\
\text { Sub- } \\
\text { chronic } \\
\text { RID } \\
\text { (mg/kg- } \\
\text { day) }\end{array}$ & $\begin{array}{c}\text { Oral } \\
\text { Slope } \\
\text { Factor } \\
\text { (mg/kg- } \\
\text { day) }^{-1}\end{array}$ & $\begin{array}{l}\text { Inbalation } \\
\text { Slope } \\
\text { Factor } \\
\text { (mg/kg- } \\
\text { day) })^{-1}\end{array}$ & $\begin{array}{c}\text { Dermal } \\
\text { Slope } \\
\text { Factor } \\
\text { (mg/kg. } \\
\text { day) }\end{array}$ & $\begin{array}{c}\text { GI } \\
\text { Absorp- } \\
\text { tion } \\
\text { Factor }\end{array}$ & $\begin{array}{c}\text { Dermal } \\
\text { Absorp- } \\
\text { tion } \\
\text { Factor }\end{array}$ & $\begin{array}{c}\text { Date Toxicity } \\
\text { Value } \\
\text { Withdrawn } \\
\text { from } \\
\text { IRIS/HEAST }\end{array}$ \\
\hline $\begin{array}{l}\text { Hexachlorocyclohexane, } \\
\text { Epsilon }\end{array}$ & $006108-10-7$ & & & & & & & & & & 0.50 & 0.010 & \\
\hline $\begin{array}{l}\text { Hexachlorocyclohexane, } \\
\text { Gamma- }\end{array}$ & $000058-89-9$ & $3.0 \mathrm{E}-04^{*}$ & $2.9 \mathrm{E}-04$ & $3.0 \mathrm{E}-03^{b}$ & $2.9 \mathrm{E}-03$ & & & $1.3 \mathrm{E}+00^{b}$ & & $1.3 \mathrm{E}+00$ & 0.97 & 0.010 & \\
\hline $\begin{array}{l}\text { Hexachlorocyclohexane, } \\
\text { Technical }\end{array}$ & $000608-73-1$ & & & & & & & $1.8 \mathrm{E}+00^{\circ}$ & $1.8 \mathrm{E}+00^{b}$ & $3.6 \mathrm{E}+00$ & 0.50 & 0.010 & \\
\hline Hexachlorocyclopentadiene & $000077-47-4$ & 7.0E-03" & $5.6 \mathrm{E}-03$ & $7.0 \mathrm{E}-02^{b}$ & $3.5 \mathrm{E}-02$ & $2.0 \mathrm{E}-05^{b}$ & $2.0 \mathrm{E}-04^{b}$ & & & & 0.80 & 0.010 & \\
\hline $\begin{array}{l}\text { Hexachlorodibenzo-p-dioxin, } \\
\text { Mixture }\end{array}$ & $019408-74-3$ & & & & & & & $6.2 \mathrm{E}+03^{\circ}$ & $4.6 \mathrm{E}+03^{u}$ & $1.2 \mathrm{E}+04$ & 0.50 & 0.010 & \\
\hline Hexachloroethane & $000067-72-1$ & $1.0 \mathrm{E}-03^{\prime}$ & $8.0 \mathrm{E}-04$ & $1.0 \mathrm{E}-02^{b}$ & $5.0 \mathrm{E}-03$ & & & $1.4 \mathrm{E}-02^{2}$ & $1.4 \mathrm{E}-02^{b}$ & $1.8 \mathrm{E}-02$ & 0.80 & 0.010 & \\
\hline Hexachlorophene & $000070-30-4$ & $3.0 \mathrm{E}-04^{\prime}$ & $1.5 \mathrm{E}-04$ & $3.0 \mathrm{E}-03^{\mathrm{b}}$ & $1.5 \mathrm{E}-03$ & & & & & & 0.50 & 0.010 & \\
\hline $\begin{array}{l}\text { Hexahydro-1,3,5-trinitro-1,3,5- } \\
\text { triazine (RDX) }\end{array}$ & $000121-82-4$ & $3.0 \mathrm{E}-03^{2}$ & $3.0 \mathrm{E}-03$ & $3.0 \mathrm{E}-03^{\mathrm{b}}$ & $3.0 \mathrm{E}-03$ & & & $1.1 \mathrm{E}-01^{\circ}$ & & $1.1 \mathrm{E}-01$ & 1.00 & 0.010 & \\
\hline $\begin{array}{l}\text { Hexamethylene Diisocyanate, } \\
1,6-\end{array}$ & $000822-06-0$ & & & & & $2.9 \mathrm{E}-06^{n}$ & & & & & 0.50 & 0.010 & \\
\hline Hexane, $\mathrm{N}$ - & $000110-54-3$ & $6.0 \mathrm{E}-02^{b}$ & $4.8 \mathrm{E}-02$ & $6.0 \mathrm{E}-01^{b}$ & $4.8 \mathrm{E}-01$ & $5.7 \mathrm{E}-02^{*}$ & $5.7 \mathrm{E}-02^{b}$ & & & & 0.80 & 0.010 & \\
\hline Hexanone, 2- & $000591-78-6$ & & & & & & & & & & 0.66 & 0.010 & \\
\hline Hexazinone & $051235-04-2$ & $3.3 \mathrm{E}-02^{2}$ & $1.7 \mathrm{E}-02$ & & & & & & & & 0.50 & 0.010 & \\
\hline $\mathrm{HpCDD}, 2,3,7,8^{-(4)}$ & $037871-00-4$ & & & & & & & $1.5 \mathrm{E}+03^{(2)}$ & $1.5 \mathrm{E}+03^{(2)}$ & $3.0 \mathrm{E}+03$ & 0.50 & 0.030 & \\
\hline $\mathrm{HpCDF}, 2,3,7,8^{(4)}$ & 038998-75-3 & & & & & & & $1.5 \mathrm{E}+03^{(2)}$ & $1.5 \mathrm{E}+03^{(2)}$ & $3.0 \mathrm{E}+03$ & 0.50 & 0.010 & \\
\hline $\mathrm{HxCDD}, 2,3,7,8^{-(1)}$ & $034465-46-8$ & & & & & & & $1.5 \mathrm{E}+04^{(2)}$ & $1.5 \mathrm{E}+04^{(2)}$ & $3.0 \mathrm{E}+04$ & 0.50 & 0.030 & \\
\hline $\mathrm{HxCDF}, 2,3,7,8-_{(d)}^{(d)}$ & $0556884-94-1$ & & & & & & & $1.5 \mathrm{E}+04^{(2)}$ & $1.5 \mathrm{E}+04^{(2)}$ & $3.0 \mathrm{E}+04$ & 0.50 & 0.010 & \\
\hline
\end{tabular}

Toxicity values used in PRGs calculations/nonradionuclides

Page 20 of 39 
Table 3.6a. (continued)

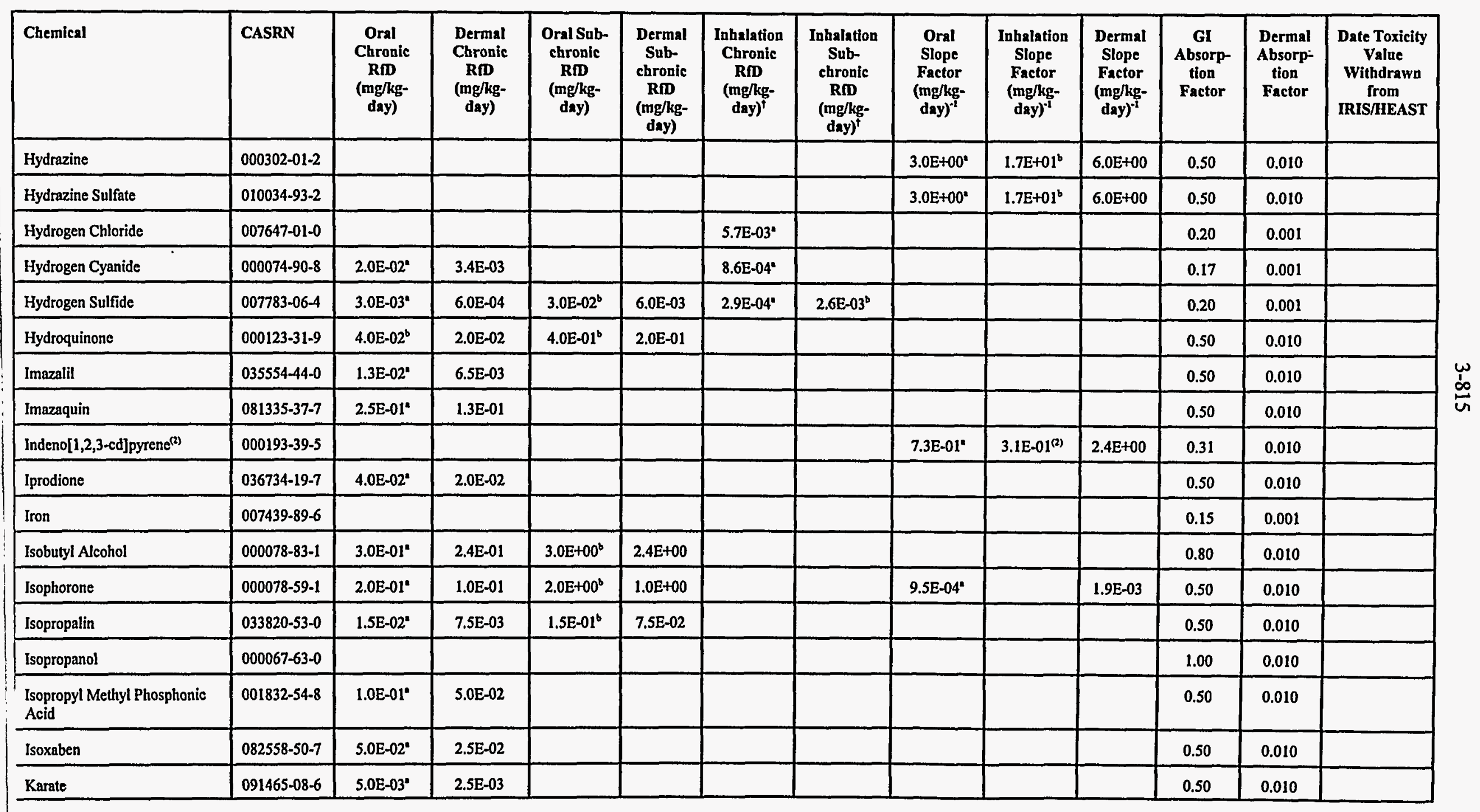

Toxicity values used in PRGs calculations/nonradionuclides

Page 21 of 39 
Table 3.6a. (continued)

\begin{tabular}{|c|c|c|c|c|c|c|c|c|c|c|c|c|c|}
\hline Chemical & CASRN & $\begin{array}{c}\text { Oral } \\
\text { Chronic } \\
\text { RDD } \\
\text { (mg/kg- } \\
\text { day) }\end{array}$ & $\begin{array}{c}\text { Dermal } \\
\text { Chronic } \\
\text { RfD } \\
\text { (mg/kg- } \\
\text { day) }\end{array}$ & $\begin{array}{c}\text { Oral Sub- } \\
\text { chronic } \\
\text { RRD } \\
\text { (mg/kg- } \\
\text { day) }\end{array}$ & $\begin{array}{c}\text { Dermal } \\
\text { Sub- } \\
\text { chronic } \\
\text { RD } \\
\text { (mg/kg- } \\
\text { day) }\end{array}$ & $\begin{array}{c}\text { Inhalation } \\
\text { Chronic } \\
\text { RDD } \\
(\mathbf{m g} / \mathrm{kg} \\
\text { day) }\end{array}$ & $\begin{array}{c}\text { Inhalation } \\
\text { Sub- } \\
\text { chronic } \\
\text { RD } \\
\text { (mg/kg- } \\
\text { day) }\end{array}$ & $\begin{array}{c}\text { Oral } \\
\text { Slope } \\
\text { Factor } \\
\text { (mg/kg- } \\
\text { day) }\end{array}$ & $\begin{array}{l}\text { Inhalation } \\
\text { Stope } \\
\text { Factor } \\
\text { (mg/kg- } \\
\text { day) }\end{array}$ & $\begin{array}{l}\text { Dermal } \\
\text { Slope } \\
\text { Factor } \\
\text { (mg/kg- } \\
\text { day) }\end{array}$ & $\begin{array}{c}\text { GI } \\
\text { Absorp- } \\
\text { tion } \\
\text { Factor }\end{array}$ & $\begin{array}{c}\text { Dermal } \\
\text { Absorp- } \\
\text { tion } \\
\text { Factor }\end{array}$ & $\begin{array}{c}\text { Date Toxicity } \\
\text { Value } \\
\text { Withdrawn } \\
\text { from } \\
\text { IRIS/HEAST }\end{array}$ \\
\hline Kerb & $023950-58-5$ & 7.5E-02 & $3.8 \mathrm{E}-02$ & $7.5 \mathrm{E}-02^{\mathrm{b}}$ & $3.8 \mathrm{E}-02$ & & & & & & 0.50 & 0.010 & \\
\hline Lactofen & $077501-63-4$ & $2.0 \mathrm{E}-03^{a}$ & $1.0 \mathrm{E}-03$ & & & & & & & & 0.50 & 0.010 & \\
\hline Lead Alkyls & NA & & & & & & & & & & 0.50 & 0.010 & \\
\hline Lead And Compounds & $007439-92-1$ & & & & & & & & & & 0.15 & 0.001 & \\
\hline Linuron & $000330-55-2$ & $2.0 \mathrm{E}-03^{a}$ & $1.0 \mathrm{E}-03$ & $2.0 \mathrm{E}-03^{\mathrm{b}}$ & $1.0 \mathrm{E}-03$ & & & & & & 0.50 & 0.010 & \\
\hline Lithium & 007439-93-2 & & & & & & & & & & 0.80 & 0.001 & \\
\hline Londax & $083055-99-6$ & $2.0 \mathrm{E}-01^{\bullet}$ & $1.0 \mathrm{E}-01$ & & & & & & & & 0.50 & 0.010 & \\
\hline MCPA & $000094-74-6$ & $5.0 \mathrm{E}-04^{n}$ & 2.5E-04 & $5.0 \mathrm{E}-04^{b}$ & 2.5E-04 & & & & & & 0.50 & 0.010 & \\
\hline MCPB & $000094-81-5$ & $1.0 \mathrm{E}-02$ & $5.0 \mathrm{E}-03$ & $1.0 \mathrm{E}-01^{\mathrm{b}}$ & $5.0 \mathrm{E}-02$ & & & & & & 0.50 & 0.010 & \\
\hline MCPP & $000093-65-2$ & $1.0 \mathrm{E}-03^{\circ}$ & $5.0 \mathrm{E}-04$ & $1.0 \mathrm{E}-02^{\mathrm{b}}$ & $5.0 \mathrm{E}-03$ & & & & & & 0.50 & 0.010 & \\
\hline Magnesium & 007439-95-4 & & & & & & & & & & 0.20 & 0.001 & \\
\hline Malathion & $000121-75-5$ & $2.0 \mathrm{E}-02^{\prime \prime}$ & $1.0 \mathrm{E}-02$ & $2.0 \mathrm{E}-02^{\mathrm{b}}$ & $1.0 \mathrm{E}-02$ & & & & & , & 0.50 & 0.010 & \\
\hline Maleic Anhydride & $000108-31-6$ & $1.0 \mathrm{E}-01^{2}$ & $5.0 \mathrm{E}-02$ & $1.0 \mathrm{E}-01^{\mathrm{b}}$ & $5.0 \mathrm{E}-02$ & & & & & & 0.50 & 0.010 & \\
\hline Maleic Hydrazide & $000123-33-1$ & $5.0 \mathrm{E}-01^{*}$ & $2.5 \mathrm{E}-01$ & $5.0 \mathrm{E}-01^{\mathrm{b}}$ & $2.5 \mathrm{E}-01$ & & & & & & 0.50 & 0.010 & \\
\hline Malononitrile & $000109-77-3$ & $2.0 \mathrm{E}-05^{b}$ & $1.6 \mathrm{E}-05$ & $2.0 \mathrm{E}-04^{\mathrm{b}}$ & $1.6 \mathrm{E}-04$ & & & & & & 0.80 & 0.010 & \\
\hline Mancozeb & $008018-01-7$ & $3.0 \mathrm{E}-02^{\mathrm{b}}$ & $1.5 \mathrm{E}-02$ & $3.0 \mathrm{E}-02^{\mathrm{b}}$ & $1.5 \mathrm{E}-02$ & & & & & & 0.50 & 0.010 & \\
\hline Maneb & $012427-38-2$ & $5.0 \mathrm{E}-03^{\circ}$ & 2.5E-03 & $5.0 \mathrm{E}-02^{\mathrm{b}}$ & $2.5 \mathrm{E}-02$ & & & & & & 0.50 & 0.010 & \\
\hline Manganese (Diet) & $007439-96-5$ & $1.4 \mathrm{E}-01^{2 \mathrm{~m}}$ & $5.6 \mathrm{E}-03$ & $1.4 \mathrm{E}-01^{\mathrm{b}}$ & $5.6 \mathrm{E}-03$ & $1.4 \mathrm{E}-05^{4}$ & & & & & 0.04 & 0.001 & \\
\hline Manganese (Water) & 007439-96-5 & 4.7E- $-02^{2 \mathrm{~mm}}$ & $1.9 \mathrm{E}-03$ & $5.0 \mathrm{E}-03^{b}$ & $2.0 \mathrm{E}-04$ & $1.4 \mathrm{E}-05^{\circ}$ & & & & & 0.04 & 0.001 & \\
\hline
\end{tabular}


Table 3.6a. (continued)

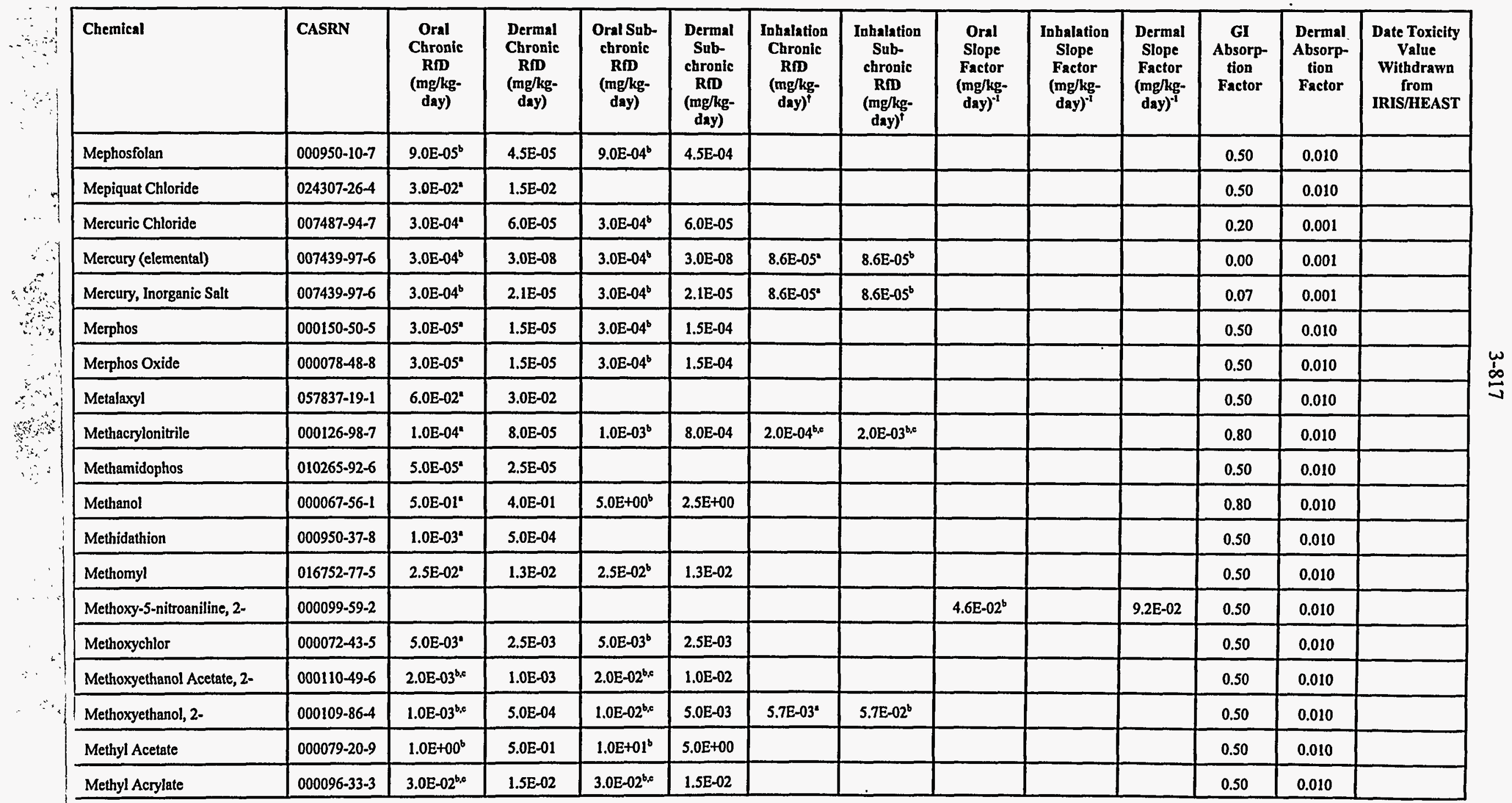


Table 3.6a. (continued)

\begin{tabular}{|c|c|c|c|c|c|c|c|c|c|c|c|c|c|}
\hline Chemical & CASRN & $\begin{array}{c}\text { Oral } \\
\text { Chronic } \\
\text { RiD } \\
\text { (mg/kg- } \\
\text { day) }\end{array}$ & $\begin{array}{c}\text { Dermal } \\
\text { Chronic } \\
\text { RfD } \\
\text { (mg/kg- } \\
\text { day) }\end{array}$ & $\begin{array}{l}\text { Oral Sub- } \\
\text { chronic } \\
\text { RiD } \\
\text { (mg/kg- } \\
\text { day) }\end{array}$ & $\begin{array}{c}\text { Dermal } \\
\text { Sub- } \\
\text { chronic } \\
\text { RfD } \\
\text { (mg/kg- } \\
\text { day) }\end{array}$ & $\begin{array}{c}\text { Inhalation } \\
\text { Chronic } \\
\text { RDD } \\
\text { (mg/kg- } \\
\text { day) }\end{array}$ & $\begin{array}{c}\text { Inhalation } \\
\text { Sub- } \\
\text { chronic } \\
\text { RD } \\
\text { (mg/kg- } \\
\text { day) }\end{array}$ & $\begin{array}{c}\text { Oral } \\
\text { Slope } \\
\text { Factor } \\
\text { (mg/kg- } \\
\text { day) }\end{array}$ & $\begin{array}{l}\text { Inhalation } \\
\text { Slope } \\
\text { Factor } \\
\text { (mg/kg- } \\
\text { day) }^{-1}\end{array}$ & $\begin{array}{l}\text { Dermal } \\
\text { Slope } \\
\text { Factor } \\
\text { (mg/kg- } \\
\text { day }^{-1}\end{array}$ & $\begin{array}{c}\text { Gl } \\
\text { Absorp- } \\
\text { tion } \\
\text { Factor }\end{array}$ & $\begin{array}{c}\text { Dermal } \\
\text { Absorp- } \\
\text { tion } \\
\text { Factor }\end{array}$ & $\begin{array}{c}\text { Date Toxicity } \\
\text { Value } \\
\text { Withdrawn } \\
\text { from } \\
\text { IRIS/HEAST }\end{array}$ \\
\hline Methyl Ethyl Ketone & 000078-93-3 & $6.0 \mathrm{E}-01^{*}$ & $4.8 \mathrm{E}-01$ & $2.0 E+00^{b}$ & $1.6 \mathrm{E}+00$ & $2.9 \mathrm{E}-01^{1}$ & $2.9 \mathrm{E}-01^{\mathrm{b}}$ & & & & 0.80 & 0.010 & \\
\hline Methyl Isobutyl Ketone & $000108-10-1$ & $8.0 \mathrm{E}-02^{\mathrm{b}}$ & $6.4 \mathrm{E}-02$ & $8.0 \mathrm{E}-01^{\mathrm{b}}$ & $6.4 \mathrm{E}-01$ & $2.3 \mathrm{E}-02^{\mathrm{b}, \mathrm{c}}$ & 2.3E- $-01^{b, c}$ & & & & 0.80 & 0.010 & \\
\hline Methyl Mercury & $022967-92-6$ & $1.0 \mathrm{E}-04^{\prime}$ & $9.0 \mathrm{E}-05$ & $1.0 \mathrm{E}-04^{\mathrm{b}}$ & $9.0 \mathrm{E}-05$ & & & & & & 0.90 & 0.010 & \\
\hline Methyl Methacrylate & $000080-62-6$ & $8.0 \mathrm{E}-02^{\mathrm{b}}$ & $6.4 \mathrm{E}-02$ & $8.0 \mathrm{E}-02^{\mathrm{b}}$ & $6.4 \mathrm{E}-02$ & & & & & & 0.80 & 0.010 & \\
\hline Methyl Parathion & $000298-00-0$ & $2.5 \mathrm{E}-04^{\circ}$ & $1.3 \mathrm{E}-04$ & $2.0 \mathrm{E}-03^{\mathrm{b}}$ & $1.0 \mathrm{E}-03$ & & & & & & 0.50 & 0.010 & \\
\hline $\begin{array}{l}\text { Methyl Styrene (Mixed } \\
\text { Isomers) }\end{array}$ & $025013-15-4$ & $6.0 \mathrm{E}-03^{\mathrm{bc}}$ & $4.8 \mathrm{E}-03$ & $6.0 \mathrm{E}-03^{\mathrm{b}, \mathrm{c}}$ & $4.8 \mathrm{E}-03$ & $1.1 \mathrm{E}-02^{\mathrm{b}, \mathrm{s}}$ & $1.1 \mathrm{E}-02^{\mathrm{b}, \mathrm{e}}$ & & & & 0.80 & 0.010 & \\
\hline Methyl hydrazine( ${ }^{(3)}$ & $000060-34-4$ & & & & & & & $1.1 \mathrm{E}+00^{\mathrm{w}}$ & & $2.2 \mathrm{E}+00$ & 0.50 & 0.010 & $7 / 94$ \\
\hline $\begin{array}{l}\text { Methyl tert-Butyl Ether } \\
\text { (MTBE) }\end{array}$ & $001634-04-4$ & & & & & $8.6 \mathrm{E}-01^{*}$ & & & & & 0.80 & 0.010 & \\
\hline Methyl-5-Nitroaniline, 2- & $000099-55-8$ & & & & & & & $3.3 \mathrm{E}-02^{\mathrm{b}}$ & & $6.6 \mathrm{E}-02$ & 0.50 & 0.010 & \\
\hline $\begin{array}{l}\text { Methylaniline Hydrochloride, } \\
\text { 2. }\end{array}$ & $000636-21-5$ & & & & & & & $1.8 \mathrm{E}-01^{\mathrm{b}}$ & & $3.6 \mathrm{E}-01$ & 0.50 & 0.010 & \\
\hline Methylcyclohexane & $000108-87-2$ & & & & & $8.6 \mathrm{E}-01^{\mathrm{b}}$ & $8.6 \mathrm{E}-01^{b}$ & & & & 0.80 & 0.010 & \\
\hline Methylene Chloride & $000075-09-2$ & $6.0 \mathrm{E}-02$ & $5.7 \mathrm{E}-02$ & $6.0 \mathrm{E}-02^{b}$ & $5.7 \mathrm{E}-02$ & $8.6 \mathrm{E}-01^{6}$ & $8.6 \mathrm{E}-01^{\mathrm{b}}$ & 7.5E-03' & $1.6 \mathrm{E}-03^{4}$ & $7.9 \mathrm{E}-03$ & 0.95 & 0.010 & \\
\hline $\begin{array}{l}\text { Methylene-bis(2- } \\
\text { chloroaniline), 4,4- }\end{array}$ & $000101-14-4$ & $7.0 \mathrm{E}-04^{\mathrm{b}}$ & $3.5 \mathrm{E}-04$ & $7.0 \mathrm{E}-04^{b}$ & 3.5E-04 & & & $1.3 \mathrm{E}-01^{\mathrm{b}}$ & $1.3 \mathrm{E}-01^{b}$ & $2.6 \mathrm{E}-01$ & 0.50 & 0.010 & \\
\hline $\begin{array}{l}\text { Methylene-bis(N,N-dimethyl) } \\
\text { Aniline, 4,4- }\end{array}$ & $000101-61-1$ & & & & & & & 4.6E-02: & & $9.2 \mathrm{E}-02$ & 0.50 & 0.010 & \\
\hline $\begin{array}{l}\text { Methylenebisbenzenamine, } \\
4,4^{-(5)}\end{array}$ & $000101-77-9$ & & & & & & & $2.5 \mathrm{E}-01^{\mathrm{w}}$ & & $5.0 \mathrm{E}-01$ & 0.50 & 0.010 & 2/94 \\
\hline
\end{tabular}


Table 3.6a. (continued)

\begin{tabular}{|c|c|c|c|c|c|c|c|c|c|c|c|c|c|}
\hline Chemical & CASRN & $\begin{array}{c}\text { Oral } \\
\text { Chronic } \\
\text { RfD } \\
\text { (mg/kg- } \\
\text { day) }\end{array}$ & $\begin{array}{c}\text { Dermal } \\
\text { Chronic } \\
\text { RID } \\
\text { (mg/kg- } \\
\text { day) }\end{array}$ & $\begin{array}{c}\text { Oral Sub- } \\
\text { chronic } \\
\text { Rm } \\
\text { (mg/kg- } \\
\text { day) }\end{array}$ & $\begin{array}{c}\text { Dermal } \\
\text { Sub- } \\
\text { chronic } \\
\text { RID } \\
\text { (mg/kg- } \\
\text { day) } \\
\end{array}$ & $\begin{array}{c}\text { Inhalation } \\
\text { Chronic } \\
\text { RfD } \\
\text { (mg/kg- } \\
\text { day) }\end{array}$ & $\begin{array}{c}\text { Inhalation } \\
\text { Sub- } \\
\text { chronic } \\
\text { RmD } \\
\text { (mg/kg- } \\
\text { day) }\end{array}$ & $\begin{array}{c}\text { Oral } \\
\text { Slope } \\
\text { Factor } \\
\text { (mg/kg- } \\
\text { day) })^{-1}\end{array}$ & $\begin{array}{l}\text { Inhalation } \\
\text { Slope } \\
\text { Factor } \\
\text { (mg/kg- } \\
\text { day) }{ }^{-1}\end{array}$ & $\begin{array}{l}\text { Dermal } \\
\text { Slope } \\
\text { Factor } \\
\text { (mg/kg- } \\
\text { day) }{ }^{-1}\end{array}$ & $\begin{array}{c}\text { Gl } \\
\text { Absorp- } \\
\text { tion } \\
\text { Factor }\end{array}$ & $\begin{array}{c}\text { Dermal } \\
\text { Absorp- } \\
\text { tion } \\
\text { Factor }\end{array}$ & $\begin{array}{l}\text { Date Toxicity } \\
\text { Value } \\
\text { Withdrawn } \\
\text { from } \\
\text { IRIS/HEAST }\end{array}$ \\
\hline $\begin{array}{l}\text { Methylenediphenyl Isocyanate, } \\
4,4^{\prime}-\end{array}$ & $000101-68-8$ & & & & & $5.7 \mathrm{E}-06^{\circ}$ & $5.7 \mathrm{E}-06^{b}$ & & & & 0.80 & 0.010 & \\
\hline Methylstyrene, Alpha- & 000098-83-9 & $7.0 \mathrm{E}-02^{\mathrm{b}, \mathrm{c}}$ & $5.6 \mathrm{E}-02$ & $7.0 \mathrm{E}-01^{\mathrm{ho}}$ & $5.6 \mathrm{E}-01$ & & & & & & 0.80 & 0.010 & \\
\hline Methyltriethyl Lead & $001762-28-3$ & & & & & & & & & & 0.50 & 0.010 & \\
\hline Metolachlor & $051218-45-2$ & $1.5 \mathrm{E}-01^{\bullet}$ & $7.5 \mathrm{E}-02$ & $1.5 \mathrm{E}-01^{\mathrm{b}}$ & $7.5 \mathrm{E}-02$ & & & & & & 0.50 & 0.010 & \\
\hline Metribuzin $^{(3)}$ & $021087-64-9$ & $2.5 \mathrm{E}-02^{2}$ & $1.3 E-02$ & $2.5 \mathrm{E}-02^{\mathrm{w}}$ & & & & & & & 0.50 & 0.010 & 9/95 \\
\hline Mirex $^{(5)}$ & $002385-85-5$ & $2.0 \mathrm{E}-04^{\circ}$ & $1.0 \mathrm{E}-04$ & $2.0 \mathrm{E}-04^{\mathrm{b}}$ & $1.0 \mathrm{E}-04$ & & & $1.8 \mathrm{E}+00^{\mathrm{w}}$ & & $3.6 \mathrm{E}+00$ & 0.50 & 0.010 & $2 / 94$ \\
\hline Molinate & $002212-67-1$ & $2.0 \mathrm{E}-03^{*}$ & $1.0 \mathrm{E}-03$ & $2.0 \mathrm{E}-03^{\mathrm{b}}$ & $1.0 \mathrm{E}-03$ & & & & & & 0.50 & 0.010 & \\
\hline Molybdenum & $007439-98-7$ & $5.0 \mathrm{E}-03^{\circ}$ & $1.9 \mathrm{E}-03$ & $5.0 \mathrm{E}-03^{\mathrm{b}}$ & $1.9 \mathrm{E}-03$ & & & & & & 0.38 & 0.001 & \\
\hline Monochloramine & $010599-90-3$ & $1.0 \mathrm{E}-01^{*}$ & $5.0 \mathrm{E}-02$ & $1.0 \mathrm{E}-01^{\mathrm{b}}$ & $5.0 \mathrm{E}-02$ & & & & & & 0.50 & 0.010 & \\
\hline Monochlorobutanes & $025154-42-1$ & $4.0 \mathrm{E}-01^{\mathrm{b}}$ & $2.0 \mathrm{E}-01$ & & & & & & & & 0.50 & 0.010 & \\
\hline Naled & $000300-76-5$ & $2.0 \mathrm{E}-03^{4}$ & $1.0 \mathrm{E}-03$ & & & & . & & & & 0.50 & 0.010 & \\
\hline Naphthalene $e^{(6)}$ & $000091-20-3$ & $3.6 \mathrm{E}-02^{\circ}$ & $2.9 \mathrm{E}-02$ & & & & & & & & 0.80 & 0.010 & \\
\hline Naphthalene, 1-Methyl & $000090-12-0$ & & & & & & & & & & 0.80 & 0.010 & \\
\hline Naphthalene, 2-Methyl & $000091-57-6$ & & & & & & & & & & 0.80 & 0.010 & \\
\hline Napropamide & $015299-99-7$ & $1.0 \mathrm{E}-01^{\bullet}$ & $5.0 \mathrm{E}-02$ & & & & & & & & 0.50 & 0.010 & \\
\hline Niagara Blue 4B & $002429-74-5$ & & & & & & & & & & 0.50 & 0.010 & \\
\hline Nickel Carbonyl & $013463-39-3$ & & & & & & & & & & 0.50 & 0.010 & \\
\hline Nickel Refinery Dust & NA & & & & & & & & $8.4 \mathrm{E}-01 \mathrm{~b}$ & & 0.20 & 0.001 & \\
\hline
\end{tabular}


Table 3.6a. (continued)

\begin{tabular}{|c|c|c|c|c|c|c|c|c|c|c|c|c|c|}
\hline Chemical & CASRN & $\begin{array}{c}\text { Oral } \\
\text { Chronic } \\
\text { RID } \\
\text { (mg/kg- } \\
\text { day) }\end{array}$ & $\begin{array}{c}\text { Dermal } \\
\text { Chronic } \\
\text { RfD } \\
\text { (mg/kg- } \\
\text { day) }\end{array}$ & $\begin{array}{l}\text { Oral Sub- } \\
\text { chronic } \\
\text { RiD } \\
\text { (mg/kg- } \\
\text { day) }\end{array}$ & $\begin{array}{l}\text { Dermal } \\
\text { Sub- } \\
\text { chronic } \\
\mathbf{R D} \\
\text { (mg/kg- } \\
\text { day) }\end{array}$ & $\begin{array}{c}\text { Inhalation } \\
\text { Chronic } \\
\text { RRD } \\
\text { (mg/kg- } \\
\text { day) }\end{array}$ & $\begin{array}{c}\text { Inhalation } \\
\text { Sub- } \\
\text { chronic } \\
\mathbf{R D D} \\
(\mathbf{m g} / \mathrm{kg} \text { - } \\
\text { day) }\end{array}$ & $\begin{array}{c}\text { Oral } \\
\text { Slope } \\
\text { Factor } \\
\text { (mg/kg- } \\
\text { day) }\end{array}$ & $\begin{array}{c}\text { Inhalation } \\
\text { Slope } \\
\text { Factor } \\
\text { (mg/kg- } \\
\text { day) }\end{array}$ & $\begin{array}{l}\text { Dermal } \\
\text { Slope } \\
\text { Factor } \\
\text { (mg/kg- } \\
\text { day) }\end{array}$ & $\begin{array}{c}\text { GI } \\
\text { Absorp- } \\
\text { tion } \\
\text { Factor }\end{array}$ & $\begin{array}{c}\text { Dermal } \\
\text { Absorp- } \\
\text { tion } \\
\text { Factor }\end{array}$ & $\begin{array}{l}\text { Date Toxicity } \\
\text { Value } \\
\text { Withdrawn } \\
\text { from } \\
\text { IRIS/HEAST }\end{array}$ \\
\hline Nickel Soluble Salts & $007440-02-0$ & $2.0 \mathrm{E}-02^{*}$ & $5.4 \mathrm{E}-03$ & $2.0 \mathrm{E}-02^{\mathrm{b}}$ & $5.4 \mathrm{E}-03$ & & & & & & 0.27 & 0.001 & \\
\hline Nickel Subsulfide & $012035-72-2$ & & & & & & & & $1.7 \mathrm{E}+00^{b}$ & & 0.20 & 0.001 & \\
\hline Nitrapyrin ${ }^{(3)}$ & $001929-82-4$ & $1.5 \mathrm{E}-03^{w}$ & $7.5 \mathrm{E}-04$ & & & & & & & & 0.50 & 0.010 & 9/93 \\
\hline Nitrate & $014797-55-8$ & $1.6 \mathrm{E}+00^{\circ}$ & $8.0 \mathrm{E}-01$ & & & & & & & & 0.50 & 0.001 & \\
\hline Nitric Oxide ${ }^{(3)}$ & $010102-43-9$ & $1.0 \mathrm{E}-01^{\mathrm{w}}$ & $2.0 \mathrm{E}-02$ & & & & & & & & 0.20 & 0.001 & $11 / 94$ \\
\hline Nitrite & $014797-65-0$ & $1.0 \mathrm{E}-01^{\circ}$ & $5.0 \mathrm{E}-02$ & $1.0 \mathrm{E}-01^{6}$ & $5.0 \mathrm{E}-02$ & & & & & & 0.50 & 0.001 & \\
\hline Nitroaniline, 2- & $000088-74-4$ & & & & & $5.7 \mathrm{E}-05^{b}$ & $5.7 \mathrm{E}-04^{b}$ & & & & 0.80 & 0.010 & \\
\hline Nitrobenzene & 000098-95-3 & $5.0 \mathrm{E}-04^{\circ}$ & $4.9 \mathrm{E}-04$ & $5.0 \mathrm{E}-03^{\mathrm{b}}$ & $4.9 \mathrm{E}-03$ & $5.7 \mathrm{E}-04^{\mathrm{b}, \mathrm{C}}$ & $5.7 \mathrm{E}-03^{\mathrm{b}, \mathrm{e}}$ & & & & 0.97 & 0.010 & \\
\hline Nitrofurantoin & $000067-20-9$ & $7.0 \mathrm{E}-02^{\mathrm{b}}$ & $3.5 \mathrm{E}-02$ & 7.0E-01 & 3.5E-01 & & & & & & 0.50 & 0.010 & \\
\hline Nitrofurazone & $000059-87-0$ & & & & & & & $1.5 \mathrm{E}+00^{\mathrm{b}}$ & & $3.0 \mathrm{E}+00$ & 0.50 & 0.010 & \\
\hline Nitrogen Dioxide & $010102-44-0$ & $1.0 \mathrm{E}+00^{\mathrm{wl}}$ & $8.0 \mathrm{E}-01$ & $1.0 \mathrm{E}+00^{\mathrm{m} 2}$ & & & & & & & 0.20 & 0.001 & $11 / 94,11 / 95$ \\
\hline Nitroguanidine & $000556-88-7$ & $1.0 \mathrm{E}-01^{\circ}$ & $5.0 \mathrm{E}-02$ & & & & & & & & 0.50 & 0.010 & \\
\hline Nitrophenol, 4- & $000100-02-7$ & & & & & & & & & & 1.00 & 0.010 & \\
\hline Nitropropane, $2-(5)$ & $000079-46-9$ & & & & & $5.7 \mathrm{E}-03^{\circ}$ & $5.7 \mathrm{E}-03^{\mathrm{b}}$ & $9.5 \mathrm{E}+00^{w}$ & $9.4 \mathrm{E}+00^{6}$ & $1.2 \mathrm{E}+01$ & 0.80 & 0.010 & $5 / 95$ \\
\hline Nitroso-N-ethylurea, $\mathrm{N}$ - & 000759-73-9 & & & & & & & $1.4 \mathrm{E}+02^{\mathrm{b}}$ & & $2.8 \mathrm{E}+02$ & 0.50 & 0.010 & \\
\hline Nitroso-N-methylurea, N- & $000684-93-5$ & & & & & & & & & & 0.50 & $0.010^{\circ}$ & \\
\hline Nitroso-di-N-butylamine, $\mathrm{N}$ - & $000924-16-3$ & & & & & & & $5.4 \mathrm{E}+00^{2}$ & $5.4 \mathrm{E}+00^{\circ}$ & $6.8 \mathrm{E}+00$ & 0.80 & 0.010 & \\
\hline Nitroso-di-N-propylamine, N- & $000621-64-7$ & & & & & & & $7.0 \mathrm{E}+00^{\circ}$ & & $2.8 \mathrm{E}+01$ & 0.25 & 0.010 & \\
\hline Nitrosodiethanolamine, N- & $001116-54-7$ & & & & & & & $2.8 \mathrm{E}+00^{\circ}$ & & $5.6 \mathrm{E}+00$ & 0.50 & 0.010 & \\
\hline
\end{tabular}


Table 3.6a. (continued)

\begin{tabular}{|c|c|c|c|c|c|c|c|c|c|c|c|c|c|}
\hline Chemical & CASRN & $\begin{array}{c}\text { Oral } \\
\text { Chronic } \\
\text { Rm } \\
\text { (mg/kg- } \\
\text { day) }\end{array}$ & $\begin{array}{c}\text { Dermal } \\
\text { Chronic } \\
\text { Rm } \\
\text { (mg/kg- } \\
\text { day) }\end{array}$ & $\begin{array}{c}\text { Oral Sub- } \\
\text { chronic } \\
\text { RID } \\
\text { (mg/kg- } \\
\text { day) }\end{array}$ & $\begin{array}{l}\text { Dermal } \\
\text { Sub- } \\
\text { chronic } \\
\mathbf{R m} \\
(\mathrm{mg} / \mathrm{kg}- \\
\text { day) }\end{array}$ & $\begin{array}{c}\text { Inhalation } \\
\text { Chronic } \\
\text { RID } \\
\text { (mg/kg- } \\
\text { day) })^{\dagger}\end{array}$ & $\begin{array}{l}\text { Inhalation } \\
\text { Sub- } \\
\text { chronic } \\
\text { Rm } \\
\text { (mg/kg- } \\
\text { day) }\end{array}$ & $\begin{array}{l}\text { Oral } \\
\text { Slope } \\
\text { Factor } \\
\text { (mg/kg- } \\
\text { day) }\end{array}$ & $\begin{array}{l}\text { Inbalation } \\
\text { Slope } \\
\text { Factor } \\
\text { (mg/kg- } \\
\text { day) }{ }^{-1}\end{array}$ & $\begin{array}{l}\text { Dermal } \\
\text { Slope } \\
\text { Factor } \\
\text { (mg/kg- } \\
\text { day) }\end{array}$ & $\begin{array}{c}\text { GI } \\
\text { Absorp- } \\
\text { tion } \\
\text { Factor }\end{array}$ & $\begin{array}{c}\text { Dermal } \\
\text { Absorp- } \\
\text { tion } \\
\text { Factor }\end{array}$ & $\begin{array}{c}\text { Date Toxicity } \\
\text { Value } \\
\text { Withdrawn } \\
\text { from } \\
\text { IRIS/HEAST }\end{array}$ \\
\hline Nitrosodiethylamine, N- & $000055-18-5$ & & & & & & & $1.5 \mathrm{E}+02^{4}$ & $1.5 E+02^{b}$ & $3.0 E+02$ & 0.50 & 0.010 & \\
\hline Nitrosodimethylamine, $\mathrm{N}$ - & $000062-75-9$ & & & & & & & $5.1 E+01^{\circ}$ & $5.1 \mathrm{E}+01^{\mathrm{b}}$ & $1.0 \mathrm{E}+02$ & 0.50 & 0.010 & \\
\hline Nitrosodiphenylamine, $\mathrm{N}$ - & $000086-30-6$ & & & & & & & 4.9E- $03^{2}$ & & $2.0 \mathrm{E}-02$ & 0.25 & 0.010 & \\
\hline Nitrosomethylethylamine, N- & $010595-95-6$ & & & & & & & $2.2 \mathrm{E}+01^{*}$ & & $4.4 \mathrm{E}+01$ & 0.50 & 0.010 & \\
\hline Nitrosomethylvinylamine, $\mathrm{N}$ - & $.004549-40-0$ & & & & & & & & & & 0.50 & 0.010 & \\
\hline Nitrosopyrrolidine, N- & $000930-55-2$ & & & & & & & $2.1 \mathrm{E}+00^{\circ}$ & $2.1 E+00^{b}$ & $4.2 \mathrm{E}+00$ & 0.50 & 0.010 & \\
\hline Nitrotoluene, 4-Amino-2- & $000119-32-4$ & & & & & & & & & & 0.50 & 0.010 & \\
\hline Nitrotoluene, m- & $000099-08-1$ & $1.0 \mathrm{E}-02^{\mathrm{b}}$ & $8.0 \mathrm{E}-03$ & $1.0 \mathrm{E}-01^{\mathrm{b}}$ & $5.0 \mathrm{E}-02$ & & & & & & 0.80 & 0.010 & \\
\hline Nitrotoluene, o- & $000088-72-2$ & $1.0 \mathrm{E}-02^{b}$ & $8.0 \mathrm{E}-03$ & $1.0 \mathrm{E}-01^{\mathrm{b}}$ & $5.0 \mathrm{E}-02$ & & & & & & 0.80 & 0.010 & \\
\hline Nitrotoluene, $\mathrm{p}$ - & $000099-99-0$ & $1.0 \mathrm{E}-02^{b}$ & $8.0 \mathrm{E}-03$ & $1.0 \mathrm{E}-01^{\mathrm{b}}$ & $5.0 \mathrm{E}-02$ & & & & & & 0.80 & 0.010 & \\
\hline Norflurazon & $027314-13-2$ & $4.0 \mathrm{E}-02^{\circ}$ & $2.0 \mathrm{E}-02$ & & & & & & & & 0.50 & 0.010 & \\
\hline Nustar & $085509-19-9$ & $7.0 \mathrm{E}-04^{\circ}$ & $3.5 \mathrm{E}-04$ & & & & & & & & 0.50 & 0.010 & \\
\hline $\mathrm{OCDD}^{(1)}$ & $003268-87-9$ & & & & & & & $1.5 \mathrm{E}+02^{(2)}$ & $1.5 \mathrm{E}+02^{(2)}$ & $3.0 \mathrm{E}+02$ & 0.50 & 0.030 & \\
\hline$O C D F^{(4)}$ & $039001-02-0$ & & & & & & & $1.5 \mathrm{E}+02^{(2)}$ & $1.5 \mathrm{E}+02^{(2)}$ & $3.0 \mathrm{E}+02$ & 0.50 & 0.010 & \\
\hline Octabromodiphenyl Ether & $032536-52-0$ & 3.0E- $-03^{2}$ & $1.5 \mathrm{E}-03$ & $3.0 \mathrm{E}-02^{\mathrm{b}}$ & $1.5 \mathrm{E}-02$ & & & & & & 0.50 & 0.010 & \\
\hline $\begin{array}{l}\text { Octahydro-1,3,5,7-tetranitro- } \\
\text { 1,3,5,7-tetra (HMX) }\end{array}$ & $002691-41-0$ & $5.0 \mathrm{E}-02 \mathrm{a}$ & $7.5 \mathrm{E}-03$ & & & & & & & & 0.15 & 0.010 & \\
\hline $\begin{array}{l}\text { Octamethyipyrophosphoramid } \\
\text { e }\end{array}$ & $000152-16-9$ & $2.0 \mathrm{E}-03^{b}$ & $1.0 \mathrm{E}-03$ & $2.0 \mathrm{E}-03^{b}$ & $1.0 \mathrm{E}-03$ & & & & & & 0.50 & 0.010 & \\
\hline Octyl Phthalate, di-N- & $000117-84-0$ & $2.0 \mathrm{E}-02^{b}$ & $1.8 \mathrm{E}-02$ & $2.0 \mathrm{E}-02^{b}$ & $1.8 \mathrm{E}-02$ & & & & & & 0.90 & 0.010 & \\
\hline
\end{tabular}

Toxicity values used in PRGs calculations/nonradionuclides

Page 27 of 39 
Table 3.6a. (continued)

\begin{tabular}{|c|c|c|c|c|c|c|c|c|c|c|c|c|c|}
\hline Chemical & CASRN & $\begin{array}{c}\text { Oral } \\
\text { Chronic } \\
\text { RfD } \\
\text { (mg/kg- } \\
\text { day) }\end{array}$ & $\begin{array}{c}\text { Dermal } \\
\text { Chronic } \\
\text { RmD } \\
\text { (mg/kg- } \\
\text { day) }\end{array}$ & $\begin{array}{c}\text { Oral Sub- } \\
\text { chronic } \\
\text { Rm } \\
\text { (mg/kg- } \\
\text { day) }\end{array}$ & $\begin{array}{c}\text { Dermal } \\
\text { Sub- } \\
\text { chronic } \\
\text { RfD } \\
\text { (mg/kg- } \\
\text { day) }\end{array}$ & $\begin{array}{c}\text { Inhalation } \\
\text { Chronic } \\
\text { RfD } \\
\text { (mg/kg- } \\
\text { day) }^{\dagger}\end{array}$ & $\begin{array}{c}\text { Inhalation } \\
\text { Sub- } \\
\text { chronic } \\
\text { RfD } \\
\text { (mg/kg- } \\
\text { day) })^{\dagger}\end{array}$ & $\begin{array}{c}\text { Oral } \\
\text { Slope } \\
\text { Factor } \\
\text { (mg/kg- } \\
\text { day) }\end{array}$ & $\begin{array}{l}\text { Inhalation } \\
\text { Slope } \\
\text { Factor } \\
\text { (mg/kg- } \\
\text { day) }\end{array}$ & $\begin{array}{c}\text { Dermal } \\
\text { Slope } \\
\text { Factor } \\
\text { (mg/kg- } \\
\text { day) }^{-1}\end{array}$ & $\begin{array}{c}\text { GI } \\
\text { Absorp- } \\
\text { tion } \\
\text { Factor }\end{array}$ & $\begin{array}{c}\text { Dermal } \\
\text { Absorp- } \\
\text { tion } \\
\text { Factor }\end{array}$ & $\begin{array}{l}\text { Date Toxicity } \\
\text { Value } \\
\text { Withdrawn } \\
\text { from } \\
\text { IRIS/HEAST }\end{array}$ \\
\hline Oryzalin & $019044-88-3$ & $5.0 \mathrm{E}-02^{\circ}$ & $2.5 \mathrm{E}-02$ & & & & & & & & 0.50 & 0.010 & \\
\hline Oxadiazon & 019666-30-9 & $5.0 \mathrm{E}-03^{2}$ & 2.5E-03 & & & & & & & & 0.50 & 0.010 & \\
\hline Oxamyl & $023135-22-0$ & $2.5 \mathrm{E}-02^{2}$ & $1.3 \mathrm{E}-02$ & & & & & & & & 0.50 & 0.010 & \\
\hline Paclobutrazol & $076738-62-0$ & $1.3 \mathrm{E}-02^{n}$ & $6.5 \mathrm{E}-03$ & & & & & & & & 0.50 & 0.010 & \\
\hline Paraquat & $001910-42-5$ & $4.5 \mathrm{E}-03^{n}$ & $2.3 \mathrm{E}-03$ & & & & & & & & 0.50 & 0.010 & \\
\hline Parathion & $000056-38-2$ & $6.0 \mathrm{E}-03^{\mathrm{b}}$ & $3.0 \mathrm{E}-03$ & $6.0 \mathrm{E}-03 \mathrm{~b}$ & $3.0 \mathrm{E}-03$ & & & & & & 0.50 & 0.010 & \\
\hline PeCDD, 2,3,7,8-(4) & $036088-22-9$ & & & & & & & $7.5 \mathrm{E}+04^{(2)}$ & $7.5 \mathrm{E}+04^{(2)}$ & $1.5 \mathrm{E}+05$ & 0.50 & 0.030 & \\
\hline PeCDF, $1,2,3,7,8-^{(4)}$ & $057117-41-6$ & & & & & & & $7.5 \mathrm{E}+04^{(2)}$ & $7.5 \mathrm{E}+04^{(2)}$ & $1.5 \mathrm{E}+05$ & 0.50 & 0.010 & \\
\hline $\mathrm{PeCDF}, 2,3,4,7,8-(4)$ & $057117-31-4$ & & & & & & & $7.5 \mathrm{E}+03^{(2)}$ & $7.5 \mathrm{E}+03^{(2)}$ & $1.5 \mathrm{E}+04$ & 0.50 & 0.010 & \\
\hline Pebulate & 001114-71-2 & $5.0 \mathrm{E}-02^{\mathrm{b}}$ & $2.5 \mathrm{E}-02$ & $5.0 \mathrm{E}-02^{\mathrm{b}}$ & $2.5 \mathrm{E}-02$ & & & & & & 0.50 & 0.010 & \\
\hline Pendimethalin & $040487-42-1$ & $4.0 \mathrm{E}-02^{\circ}$ & $2,0 \mathrm{E}-02$ & $4.0 \mathrm{E}-02^{\mathrm{b}}$ & $2.0 \mathrm{E}-02$ & & & & & & 0.50 & 0.010 & \\
\hline Pentabromodiphenyl Ether & $032534-81-9$ & $2.0 \mathrm{E}-03^{\prime}$ & $1.0 \mathrm{E}-03$ & $2.0 \mathrm{E}-02^{\mathrm{b}}$ & $1.0 \mathrm{E}-02$ & & & & & & 0.50 & 0.010 & \\
\hline Pentachlorobenzene & 000608-93-5 & $8.0 \mathrm{E}-04^{\circ}$ & $6.4 \mathrm{E}-04$ & $8.0 \mathrm{E}-03^{b}$ & $4.0 \mathrm{E}-03$ & & & & & & 0.80 & 0.010 & \\
\hline Pentachlorocyclopentadiene & $025329-35-5$ & & & & & & & & & & 0.50 & 0.010 & \\
\hline Pentachloronitrobenzene & $000082-68-8$ & $3.0 \mathrm{E}-03^{2}$ & $2.4 \mathrm{E}-03$ & $3.0 \mathrm{E}-03^{6}$ & $1.5 \mathrm{E}-03$ & & & $2.6 \mathrm{E}-01^{b}$ & & 3.3E-01 & 0.80 & 0.010 & \\
\hline Pentachlorophenol & $000087-86-5$ & $3.0 \mathrm{E}-02^{\mathrm{n}}$ & $3.0 \mathrm{E}-02$ & $3.0 \mathrm{E}-02^{b}$ & $3.0 \mathrm{E}-02$ & & & $1.2 \mathrm{E}-01^{\prime \prime}$ & & $1.2 \mathrm{E}-01$ & 1.00 & 0.010 & \\
\hline Pentyl Alcohol, N- & $000071-41-0$ & & & & & & & & & & 0.50 & 0.010 & \\
\hline Permethrin & $052645-53-1$ & $5.0 \mathrm{E}-02^{2}$ & 2.5E-02 & & & & & & & & 0.50 & 0.010 & \\
\hline Phenanthrene & $000085-01-8$ & & & & & & & & & & 0.73 & 0.010 & \\
\hline
\end{tabular}


Table 3.6a. (continued)

\begin{tabular}{|c|c|c|c|c|c|c|c|c|c|c|c|c|c|}
\hline Chemical & CASRN & $\begin{array}{c}\text { Oral } \\
\text { Chronic } \\
\text { RfD } \\
\text { (mg/kg- } \\
\text { day) }\end{array}$ & $\begin{array}{c}\text { Dermal } \\
\text { Chronic } \\
\text { RID } \\
\text { (mg/kg- } \\
\text { day) }\end{array}$ & $\begin{array}{l}\text { Oral Sub- } \\
\text { chronic } \\
\text { RmD } \\
\text { (mg/kg- } \\
\text { day) }\end{array}$ & $\begin{array}{c}\text { Dermal } \\
\text { Sub- } \\
\text { chronic } \\
\text { Rm } \\
\text { (mg/kg- } \\
\text { day) }\end{array}$ & $\begin{array}{c}\text { Inhalation } \\
\text { Chronic } \\
\text { RD } \\
\text { (mg/kg- } \\
\text { day) }^{\dagger}\end{array}$ & $\begin{array}{c}\text { Inhalation } \\
\text { Sub- } \\
\text { chronic } \\
\text { RfD } \\
\text { (mg/kg- } \\
\text { day) }\end{array}$ & $\begin{array}{l}\text { Oral } \\
\text { Slope } \\
\text { Factor } \\
\text { (mg/kg- } \\
\text { day) }\end{array}$ & $\begin{array}{l}\text { Inhalation } \\
\text { Slope } \\
\text { Factor } \\
\text { (mg/kg- } \\
\text { day) }\end{array}$ & $\begin{array}{l}\text { Dermal } \\
\text { Slope } \\
\text { Factor } \\
\text { (mg/kg- } \\
\text { day) }\end{array}$ & $\begin{array}{c}\text { GI } \\
\text { Absorp- } \\
\text { tion } \\
\text { Factor }\end{array}$ & $\begin{array}{c}\text { Dermal } \\
\text { Absorp- } \\
\text { tion } \\
\text { Factor }\end{array}$ & $\begin{array}{c}\text { Date Toxicity } \\
\text { Value } \\
\text { Withdrawn } \\
\text { from } \\
\text { IRIS/HEAST }\end{array}$ \\
\hline Phenmedipham & $013684-63-4$ & $2.5 \mathrm{E}-01^{*}$ & $1.3 \mathrm{E}-01$ & & & & & & & & 0.50 & 0.010 & \\
\hline Phenol & $000108-95-2$ & $6.0 \mathrm{E}-01^{*}$ & $5.4 \mathrm{E}-01$ & $6.0 \mathrm{E}-01^{\mathrm{t}}$ & $5.4 \mathrm{E}-01$ & & & . & & & 0.90 & 0.010 & \\
\hline Phenylenediamine, m- & $000108-45-2$ & $6.0 \mathrm{E}-03^{8}$ & $3.0 \mathrm{E}-03$ & $6.0 \mathrm{E}-02^{\circ}$ & $3.0 \mathrm{E}-02$ & & & & & & 0.50 & 0.010 & \\
\hline Phenylenediamine, 0 - & $000095-54-5$ & & & & & & & 4.7E- $02^{b}$ & & $9.4 \mathrm{E}-02$ & 0.50 & 0.010 & \\
\hline Phenylenediamine, p- & $000106-50-3$ & $1.9 \mathrm{E}-01^{b}$ & $9.5 \mathrm{E}-02$ & & & & & & & & 0.50 & 0.010 & \\
\hline Phenylmercuric Acetate & $000062-38-4$ & $8.0 \mathrm{E}-05^{\mathrm{e}}$ & $4.0 \mathrm{E}-05$ & $8.0 \mathrm{E}-05^{b}$ & $4.0 \mathrm{E}-05$ & & & & & & 0.50 & 0.010 & \\
\hline Phenylphenol, 2- & $000090-43-7$ & & & & & & & $1.9 \mathrm{E}-03^{b}$ & & $3.9 \mathrm{E}-03$ & 0.50 & 0.010 & \\
\hline Phorate & $000298-02-2$ & $2.0 \mathrm{E}-04^{b}$ & $1.0 \mathrm{E}-04$ & $2.0 \mathrm{E}-04^{\mathrm{b}}$ & $1.0 \mathrm{E}-04$ & & & & & & 0.50 & 0.010 & \\
\hline Phosmet & $000732-11-6$ & $2.0 \mathrm{E}-02^{*}$ & $1.0 \mathrm{E}-02$ & & & & & & & & 0.50 & 0.010 & \\
\hline Phosphine & $007803-51-2$ & $3.0 \mathrm{E}-04^{n}$ & $6.0 \mathrm{E}-05$ & $3.0 \mathrm{E}-04^{b}$ & $6.0 \mathrm{E}-05$ & $8.6 \mathrm{E}-05^{a}$ & $8.6 \mathrm{E}-05^{b}$ & & & & 0.20 & 0.001 & \\
\hline Phosphoric Acid & $007664-38-2$ & & & & & $2.9 \mathrm{E}-03^{\prime}$ & & & & & 0.20 & 0.001 & \\
\hline Phthalic Acid, P. & $000100-21-0$ & $1.0 \mathrm{E}+00^{\mathrm{b}}$ & $5.0 \mathrm{E}-01$ & $1.0 \mathrm{E}+00^{\circ}$ & $5.0 \mathrm{E}-01$ & & & & & & 0.50 & 0.010 & \\
\hline Phthalic Anhydride & $000085-44-9$ & $2.0 \mathrm{E}+00^{\circ}$ & $1.0 \mathrm{E}+00$ & $2.0 \mathrm{E}+00^{b}$ & $1.0 \mathrm{E}+00$ & $3.4 \mathrm{E}-02^{\mathrm{b}}$ & $3.4 \mathrm{E}-02^{\mathrm{b}}$ & & & & 0.50 & 0.010 & \\
\hline Picloram & $001918-02-1$ & $7.0 \mathrm{E}-02^{\prime \prime}$ & 3.5E-02 & & & & & & & & 0.50 & 0.010 & \\
\hline Pirimiphos, Methyl & $029232-93-7$ & $1.0 \mathrm{E}-02^{2}$ & $5.0 \mathrm{E}-03$ & & & & & & & & 0.50 & 0.010 & \\
\hline Polybrominated Biphenyls & NA & $7.0 \mathrm{E}-06^{\mathrm{b}}$ & $6.5 \mathrm{E}-06$ & $7.0 \mathrm{E}-05^{b}$ & $6.5 \mathrm{E}-05$ & & & $8.9 \mathrm{E}+00^{b}$ & & $9.6 \mathrm{E}+00$ & 0.93 & 0.010 & \\
\hline Polychlorinated Biphenyls & $001336-36-3$ & & & & & & & $7.7 \mathrm{E}+00^{\circ}$ & & $8.6 \mathrm{E}+00$ & 0.90 & 0.060 & \\
\hline Potassium Cyanide & $000151-50-8$ & $5.0 \mathrm{E}-02^{2}$ & 4.0E-02 & $5.0 \mathrm{E}-02^{\mathrm{b}}$ & $1.0 \mathrm{E}-02$ & & & & & & 0.80 & 0.010 & \\
\hline Potassium Silver Cyanide & $000506-61-6$ & $2.0 \mathrm{E}-01^{\bullet}$ & $4.0 \mathrm{E}-02$ & $2.0 \mathrm{E}-01^{b}$ & $4.0 \mathrm{E}-02$ & & & & & & 0.20 & 0.001 & \\
\hline
\end{tabular}

Toxicity values used in PRGs calculations/nonradionuclides

Page 29 of 39 
Table 3.6a. (continued)

\begin{tabular}{|c|c|c|c|c|c|c|c|c|c|c|c|c|c|}
\hline Chemical & CASRN & $\begin{array}{c}\text { Oral } \\
\text { Chronic } \\
\text { RfD } \\
\text { (mg/kg- } \\
\text { day) }\end{array}$ & $\begin{array}{c}\text { Dermal } \\
\text { Chronic } \\
\text { Rm } \\
\text { (mg/kg- } \\
\text { day) }\end{array}$ & $\begin{array}{c}\text { Oral Sub- } \\
\text { chronic } \\
\text { RID } \\
\text { (mg/kg- } \\
\text { day) }\end{array}$ & $\begin{array}{c}\text { Dermal } \\
\text { Sub- } \\
\text { chronic } \\
\text { Rm } \\
\text { (mg/kg- } \\
\text { day) }\end{array}$ & $\begin{array}{c}\text { Inhalation } \\
\text { Chronic } \\
\text { RID } \\
\text { (mg/kg- } \\
\text { day) }\end{array}$ & $\begin{array}{c}\text { Inhalation } \\
\text { Sub- } \\
\text { chronic } \\
\text { RmD } \\
\text { (mg/kg- } \\
\text { day) }\end{array}$ & $\begin{array}{c}\text { Oral } \\
\text { Slope } \\
\text { Factor } \\
\text { (mg/kg- } \\
\text { day) }\end{array}$ & $\begin{array}{l}\text { Inhalation } \\
\text { Slope } \\
\text { Factor } \\
\text { (mg/kg- } \\
\text { day) }\end{array}$ & $\begin{array}{l}\text { Dermal } \\
\text { Slope } \\
\text { Factor } \\
\text { (mg/kg- } \\
\text { day) }\end{array}$ & $\begin{array}{c}\text { GI } \\
\text { Absorp- } \\
\text { tion } \\
\text { Factor }\end{array}$ & $\begin{array}{c}\text { Dermal } \\
\text { Absorp- } \\
\text { tion } \\
\text { Factor }\end{array}$ & $\begin{array}{c}\text { Date Toxicity } \\
\text { Value } \\
\text { Withdrawn } \\
\text { from } \\
\text { IRIS/HEAST }\end{array}$ \\
\hline Prochloraz & $067747-09-5$ & $9.0 \mathrm{E}-03^{2}$ & $4.5 \mathrm{E}-03$ & & & & & $1.5 \mathrm{E}-01^{\circ}$ & & $3.0 \mathrm{E}-01$ & 0.50 & 0.010 & \\
\hline Profluralin & $026399-36-0$ & $6.0 \mathrm{E}-03^{\mathrm{b}}$ & $3.0 \mathrm{E}-03$ & $6.0 \mathrm{E}-03^{b}$ & $3.0 \mathrm{E}-03$ & & & & & & 0.50 & 0.010 & \\
\hline Prometon & $001610-18-0$ & 1.5E-02' & 7.5E-03 & & & & & & & & 0.50 & 0.010 & \\
\hline Prometryn & $007287-19-6$ & $4.0 \mathrm{E}-03^{2}$ & $2.0 \mathrm{E}-03$ & & & & & & & & 0.50 & 0.010 & \\
\hline Propachlor & $001918-16-7$ & $1.3 \mathrm{E}-02^{2}$ & $6.5 \mathrm{E}-03$ & $1.3 \mathrm{E}-01^{b}$ & $6.5 \mathrm{E}-02$ & & & & & & 0.50 & 0.010 & \\
\hline Propanil & $000709-98-8$ & $5.0 \mathrm{E}-03^{n}$ & $2.5 \mathrm{E}-03$ & & & & & & & & 0.50 & 0.010 & \\
\hline Propargite & $002312-35-8$ & 2.0E-02' & $1.0 \mathrm{E}-02$ & & & & & & & & 0.50 & 0.010 & \\
\hline Propargyl Alcohol & $000107-19-7$ & $2.0 \mathrm{E}-03^{\prime}$ & $1.6 \mathrm{E}-03$ & & & & & & & & 0.80 & 0.010 & \\
\hline Propazine & $000139-40-2$ & $2.0 \mathrm{E}-02^{\prime \prime}$ & $1.0 \mathrm{E}-02$ & $2.0 \mathrm{E}-02^{\mathrm{b}}$ & $1.0 \mathrm{E}-02$ & & & & & & 0.50 & 0.010 & \\
\hline Propham & $000122-42-9$ & $2.0 \mathrm{E}-02^{\prime}$ & $1.0 \mathrm{E}-02$ & & & & & & & & 0.50 & 0.010 & \\
\hline Propiconazole & $060207-90-1$ & 1.3E-02: & $6.5 \mathrm{E}-03$ & & & & & & & & 0.50 & 0.010 & \\
\hline Propylene Glycol & $000057-55-6$ & $2.0 \mathrm{E}+01^{\mathrm{b}}$ & $1.0 \mathrm{E}+01$ & $3.0 \mathrm{E}+01^{\mathrm{b}}$ & $1.5 \mathrm{E}+01$ & & & & & & 0.50 & 0.010 & \\
\hline $\begin{array}{l}\text { Propylene Glycol Monoethyl } \\
\text { Ether }\end{array}$ & $001569-02-4$ & $7.0 \mathrm{E}-01^{b}$ & $3.5 \mathrm{E}-01$ & $7.0 \mathrm{E}+00^{6}$ & $3.5 \mathrm{E}+00$ & & & & & & 0.50 & 0.010 & \\
\hline $\begin{array}{l}\text { Propylene Glycol Monomethyl } \\
\text { Ether }\end{array}$ & $000107-98-2$ & $7.0 \mathrm{E}-01^{b}$ & $3.5 \mathrm{E}-01$ & $7.0 \mathrm{E}+00^{\mathrm{b}}$ & $3.5 \mathrm{E}+00$ & 5.7E-01" & $5.7 \mathrm{E}+00^{b}$ & & & & 0.50 & 0.010 & \\
\hline Propylene Oxide & $000075-56-9$ & & & & & $8.6 \mathrm{E}-03^{2}$ & $8.6 \mathrm{E}-03^{b}$ & $2.4 \mathrm{E}-01^{*}$ & $1.3 \mathrm{E}-02^{\mathrm{b}}$ & $3.0 \mathrm{E}-01$ & 0.80 & 0.010 & \\
\hline Pursuit & $081335-77-5$ & $2.5 \mathrm{E}-01^{\circ}$ & $1.3 \mathrm{E}-01$ & & & & & & & & 0.50 & 0.010 & \\
\hline Pydrin & $051630-58-1$ & 2.5E-02" & $1.3 \mathrm{E}-02$ & & & & & & & & 0.50 & 0.010 & \\
\hline Pyrene & $000129-00-0$ & $3.0 \mathrm{E}-02^{\circ}$ & $9.3 \mathrm{E}-03$ & $3.0 \mathrm{E}-01^{b}$ & $9.3 \mathrm{E}-02$ & & & & & & 0.31 & 0.010 & \\
\hline
\end{tabular}

Toxicity values used in PRGs calculations/nonradionuclides

Page 30 of 39 
Table 3.6a. (continued)

\begin{tabular}{|c|c|c|c|c|c|c|c|c|c|c|c|c|c|}
\hline Chemical & CASRN & $\begin{array}{c}\text { Oral } \\
\text { Chronic } \\
\text { RD } \\
\text { (mg/kg- } \\
\text { day) }\end{array}$ & $\begin{array}{c}\text { Dermal } \\
\text { Chronic } \\
\text { RfD } \\
\text { (mg/kg- } \\
\text { day) }\end{array}$ & $\begin{array}{l}\text { Oral Sub- } \\
\text { chronic } \\
\text { RID } \\
\text { (mg/kg- } \\
\text { day) }\end{array}$ & $\begin{array}{c}\text { Dermal } \\
\text { Sub- } \\
\text { chronic } \\
\text { RRD } \\
\text { (mg/kg- } \\
\text { day) }\end{array}$ & $\begin{array}{c}\text { Inhalation } \\
\text { Chronic } \\
\text { RfD } \\
\text { (mg/kg- } \\
\text { day) }\end{array}$ & $\begin{array}{c}\text { Inhalation } \\
\text { Sub- } \\
\text { chronic } \\
\text { RRD } \\
\text { (mg/kg- } \\
\text { day) } \\
\end{array}$ & $\begin{array}{c}\text { Oral } \\
\text { Slope } \\
\text { Factor } \\
\text { (mg/kg- } \\
\text { day) })^{-1}\end{array}$ & $\begin{array}{c}\text { Inhalation } \\
\text { Slope } \\
\text { Factor } \\
\text { (mg/kg- } \\
\text { day) }\end{array}$ & $\begin{array}{l}\text { Dermal } \\
\text { Slope } \\
\text { Factor } \\
\text { (mg/kg- } \\
\text { day) })^{-1}\end{array}$ & $\begin{array}{c}\text { GI } \\
\text { Absorp- } \\
\text { tion } \\
\text { Factor }\end{array}$ & $\begin{array}{c}\text { Dermal } \\
\text { Absorp- } \\
\text { tion } \\
\text { Factor }\end{array}$ & $\begin{array}{l}\text { Date Toxicity } \\
\text { Value } \\
\text { Withdrawn } \\
\text { from } \\
\text { IRIS/HEAST }\end{array}$ \\
\hline Pyridine & $000110-86-1$ & $1.0 \mathrm{E}-03^{\circ}$ & $5.0 \mathrm{E}-04$ & $1.0 \mathrm{E}-02^{\mathrm{b}}$ & $5.0 \mathrm{E}-03$ & & & & & & 0.50 & 0.010 & \\
\hline Quinalphos & $013593-03-8$ & $5.0 \mathrm{E}-04^{*}$ & 2.5E-04 & & & & & & & & 0.50 & 0.010 & \\
\hline Quinoline & $000091-22-5$ & & & & & & & $1.2 \mathrm{E}+01^{\mathrm{b}}$ & & $2.4 \mathrm{E}+01$ & 0.50 & 0.010 & \\
\hline Refractory Ceramic Fibers & $\mathrm{NA}$ & & & & & & & & & & 0.50 & 0.010 & \\
\hline Resmethrin & $010453-86-8$ & 3.0E-02" & $1.5 \mathrm{E}-02$ & & & & & & & & 0.50 & 0.010 & \\
\hline Ronnel & $000299-84-3$ & $5.0 \mathrm{E}-02^{\mathrm{b}}$ & $2.5 \mathrm{E}-02$ & $5.0 \mathrm{E}-02^{\mathrm{b}}$ & $2.5 \mathrm{E}-02$ & & & & & & 0.50 & 0.010 & \\
\hline Rotenone & $000083-79-4$ & $4.0 \mathrm{E}-03^{\prime}$ & $2.0 \mathrm{E}-03$ & & & & & & & & 0.50 & 0.010 & \\
\hline Savey & $078587-05-0$ & $2.5 \mathrm{E}-02^{\circ}$ & $1.3 \mathrm{E}-02$ & & & & & & & & 0.50 & 0.010 & \\
\hline Selenious Acid & $007783-00-8$ & $5.0 \mathrm{E}-03^{2}$ & $4.4 \mathrm{E}-03$ & $5.0 \mathrm{E}-03^{\mathrm{b}}$ & 4.4E-03 & & & & & & 0.87 & 0.010 & \\
\hline Selenite & $014124-67-5$ & & & & & & & & & & 0.70 & 0.010 & \\
\hline Selenium & $007782-49-2$ & 5.0E-0 $03^{2}$ & $2.2 \mathrm{E}-03$ & $5.0 \mathrm{E}-03^{b}$ & $2.2 \mathrm{E}-03$ & & & & & & 0.44 & 0.001 & \\
\hline Selenium Sulfide & $007446-34-6$ & & & & & & & & & & 0.20 & 0.001 & \\
\hline Selenourea & $000630-10-4$ & $5.0 \mathrm{E}-03^{\mathrm{b}}$ & $2.5 \mathrm{E}-03$ & $5.0 \mathrm{E}-03^{\mathrm{b}}$ & $2.5 \mathrm{E}-03$ & & & & & & 0.50 & 0.010 & \\
\hline Sethoxydim & $074051-80-2$ & 9.0E-02" & $4.5 \mathrm{E}-02$ & & & & & & & & 0.50 & 0.010 & \\
\hline Silver & $007440-22-4$ & $5.0 \mathrm{E}-03^{2}$ & $9.0 \mathrm{E}-04$ & $5.0 \mathrm{E}-03^{\mathrm{b}}$ & $9.0 \mathrm{E}-04$ & & & & & & 0.18 & 0.001 & \\
\hline Silver Cyanide & $000506-64-9$ & $1.0 \mathrm{E}-01^{*}$ & $2.0 \mathrm{E}-02$ & $1.0 \mathrm{E}-01^{\mathrm{b}}$ & $2.0 \mathrm{E}-02$ & & & & & & 0.20 & 0.001 & \\
\hline Simazine & $000122-34-9$ & $5.0 \mathrm{E}-03^{\circ}$ & $2.5 \mathrm{E}-03$ & $5.0 \mathrm{E}-03^{\mathrm{b}}$ & $2.5 \mathrm{E}-03$ & & & $1.2 \mathrm{E}-01^{\mathrm{b}}$ & & $2.4 \mathrm{E}-01$ & 0.50 & 0.010 & \\
\hline Sodium & $007440-23-5$ & & & & & & & & & & 1.00 & 0.001 & \\
\hline Sodium Acifluorfen & $062476-59-9$ & $1.3 \mathrm{E}-02^{2}$ & $6.5 \mathrm{E}-03$ & & & & & & & & 0.50 & 0.010 & \\
\hline
\end{tabular}


Table 3.6a. (continued)

\begin{tabular}{|c|c|c|c|c|c|c|c|c|c|c|c|c|c|}
\hline Chemical & CASRN & $\begin{array}{l}\text { Oral } \\
\text { Chronic } \\
\text { Rm } \\
\text { (mg/kg- } \\
\text { day) }\end{array}$ & $\begin{array}{c}\text { Dermal } \\
\text { Chronic } \\
\text { RID } \\
\text { (mg/kg- } \\
\text { day) }\end{array}$ & $\begin{array}{l}\text { Oral Sub- } \\
\text { chronic } \\
\text { RfD } \\
\text { (mg/kg } \\
\text { day) }\end{array}$ & $\begin{array}{c}\text { Dermal } \\
\text { Sub- } \\
\text { chronic } \\
\text { RfD } \\
\text { (mg/kg- } \\
\text { day) }\end{array}$ & $\begin{array}{c}\text { Inbalation } \\
\text { Chronic } \\
\text { RfD } \\
\text { (mg/kg- } \\
\text { day) }\end{array}$ & $\begin{array}{c}\text { Inhalation } \\
\text { Sub- } \\
\text { chronic } \\
\text { RfD } \\
\text { (mg/kg- } \\
\text { day) }^{\dagger}\end{array}$ & $\begin{array}{c}\text { Oral } \\
\text { Slope } \\
\text { Factor } \\
\text { (mg/kg- } \\
\text { day) }\end{array}$ & $\begin{array}{c}\text { Inhalation } \\
\text { Slope } \\
\text { Factor } \\
\text { (mg/kg- } \\
\text { day) }\end{array}$ & $\begin{array}{c}\text { Dermal } \\
\text { Slope } \\
\text { Factor } \\
\text { (mg/kg- } \\
\text { day) }^{-1}\end{array}$ & $\begin{array}{c}\text { GI } \\
\text { Absorp- } \\
\text { tion } \\
\text { Factor }\end{array}$ & $\begin{array}{c}\text { Dermal } \\
\text { Absorp- } \\
\text { tion } \\
\text { Factor }\end{array}$ & $\begin{array}{l}\text { Date Toxicity } \\
\text { Value } \\
\text { Withdrawn } \\
\text { from } \\
\text { IRIS/HEAST }\end{array}$ \\
\hline Sodium Azide & $026628-22-8$ & $4.0 \mathrm{E}-03^{\circ}$ & $8.0 \mathrm{E}-04$ & & & & & & & & 0.20 & 0.001 & \\
\hline Sodium Cyanide & 000143-33-9 & $4.0 \mathrm{E}-02^{2}$ & 3.2E-02 & $4.0 \mathrm{E}-02^{\mathrm{b}}$ & $8.0 \mathrm{E}-03$ & & & & & & 0.80 & 0.010 & \\
\hline $\begin{array}{l}\text { Sodium } \\
\text { Diethyldithiocarbamate }\end{array}$ & 000148-18-5 & $3.0 \mathrm{E}-02^{\prime}$ & $1.5 \mathrm{E}-02$ & $3.0 \mathrm{E}-01^{b}$ & $1.5 \mathrm{E}-01$ & & & $2.7 \mathrm{E}-01^{\mathrm{b}}$ & & 5.4E-01 & 0.50 & 0.010 & \\
\hline Sodium Fluoroacetate & $000062-74-8$ & $2.0 \mathrm{E}-05^{4}$ & $1.0 \mathrm{E}-05$ & & & & & & & & 0.50 & 0.010 & \\
\hline Sodium Metavanadate & $013718-26-8$ & $1.0 \mathrm{E}-03^{b}$ & $2.0 \mathrm{E}-04$ & $1.0 \mathrm{E}-02^{\mathrm{b}}$ & $2.0 \mathrm{E}-03$ & & & & & & 0.20 & 0.001 & \\
\hline Stirofos (Tetrachlorovinphos) & $000961-11-5$ & $3.0 \mathrm{E}-02^{4}$ & $1.5 \mathrm{E}-02$ & $3.0 \mathrm{E}-02^{b}$ & $1.5 \mathrm{E}-02$ & & & $2.4 \mathrm{E}-02^{b}$ & & $4.8 \mathrm{E}-02$ & 0.50 & 0.010 & \\
\hline Strontium, Stable & $007440-24-6$ & $6.0 \mathrm{E}-01^{*}$ & $1.2 \mathrm{E}-01$ & $6.0 \mathrm{E}-01^{\mathrm{b}}$ & $1.2 \mathrm{E}-01$ & & & & & & 0.20 & 0.001 & \\
\hline Strychnine & $000057-24-9$ & $3.0 \mathrm{E}-04^{\prime \prime}$ & $1.5 \mathrm{E}-04$ & $3.0 \mathrm{E}-03^{\mathrm{b}}$ & $1.5 \mathrm{E}-03$ & & & & & & 0.50 & 0.010 & \\
\hline Styrene $e^{(6)}$ & $000100-42-5$ & $2.0 \mathrm{E}-01^{\circ}$ & $1.6 \mathrm{E}-01$ & & & $2.9 \mathrm{E}-01^{\bullet}$ & $8.6 \mathrm{E}-01^{\mathrm{b}}$ & & & & 0.80 & 0.010 & \\
\hline Sulfate & 014808-79-8 & & & & & & & & & & 0.20 & 0.001 & \\
\hline Systhane & $088671-89-0$ & $2.5 \mathrm{E}-02^{2}$ & $1.3 \mathrm{E}-02$ & & & & & & & & 0.50 & 0.010 & \\
\hline TCDD, 2,3,7,8-(4) & $001746-01-6$ & & & & & & & $1.5 \mathrm{E}+05^{\mathrm{b}}$ & $1.5 \mathrm{E}+05^{\mathrm{b}}$ & $3.0 \mathrm{E}+05$ & 0.50 & 0.030 & \\
\hline TCDF, 2,3,7,8-(4) & $051207-31-9$ & & & & & & & $1.5 \mathrm{E}+04^{(2)}$ & $1.5 \mathrm{E}+04^{(2)}$ & $3.0 \mathrm{E}+04$ & 0.50 & 0.010 & \\
\hline TCMTB & $021564-17-0$ & $3.0 \mathrm{E}-02^{\mathrm{b}}$ & $1.5 \mathrm{E}-02$ & $3.0 \mathrm{E}-01^{\mathrm{b}}$ & $1.5 \mathrm{E}-01$ & & & & & & 0.50 & 0.010 & \\
\hline Tebuthiuron & 034014-18-1 & $7.0 \mathrm{E}-02^{2}$ & $3.5 \mathrm{E}-02$ & & & & & & & & 0.50 & 0.010 & \\
\hline Temephos & $003383-96-8$ & $2.0 \mathrm{E}-02^{\mathrm{b}}$ & $1.0 \mathrm{E}-02$ & $2.0 \mathrm{E}-01^{\mathrm{b}}$ & $1.0 \mathrm{E}-01$ & & & & & & 0.50 & 0.010 & \\
\hline Terbacil & $005902-51-2$ & $1.3 \mathrm{E}-02^{\circ}$ & $6.5 \mathrm{E}-03$ & & & & & & & & 0.50 & 0.010 & \\
\hline Terbufos & $013071-79-9$ & $2.5 \mathrm{E}-05^{\mathrm{b}}$ & $1.3 \mathrm{E}-05$ & $2.5 \mathrm{E}-05^{b}$ & $1.3 \mathrm{E}-05$ & & & & & & 0.50 & 0.010 & \\
\hline
\end{tabular}

Toxicity values used in PRGs calculations/nonradionuclides

Page 32 of 39 
Table 3.6a. (continued)

\begin{tabular}{|c|c|c|c|c|c|c|c|c|c|c|c|c|c|}
\hline Chemical & CASRN & $\begin{array}{c}\text { Oral } \\
\text { Chronic } \\
\text { RmD } \\
\text { (mg/kg- } \\
\text { day) }\end{array}$ & $\begin{array}{c}\text { Dermal } \\
\text { Chronic } \\
\text { RfD } \\
\text { (mg/kg- } \\
\text { day) }\end{array}$ & $\begin{array}{l}\text { Oral Sub- } \\
\text { chronic } \\
\text { Rm } \\
\text { (mg/kg- } \\
\text { day) }\end{array}$ & $\begin{array}{c}\text { Dermal } \\
\text { Sub- } \\
\text { chronic } \\
\text { RID } \\
\text { (mg/kg- } \\
\text { day) }\end{array}$ & $\begin{array}{c}\text { Inhalation } \\
\text { Chronic } \\
\text { RD } \\
\text { (mg/kg- } \\
\text { day) }\end{array}$ & $\begin{array}{c}\text { Inhalation } \\
\text { Sub- } \\
\text { chronic } \\
\text { RD } \\
\text { (mg/kg- } \\
\text { day) }\end{array}$ & $\begin{array}{c}\text { Oral } \\
\text { Slope } \\
\text { Factor } \\
\text { (mg/kg- } \\
\text { day) })^{-1}\end{array}$ & $\begin{array}{c}\text { Inhalation } \\
\text { Slope } \\
\text { Factor } \\
\text { (mg/kg- } \\
\text { day) }\end{array}$ & $\begin{array}{l}\text { Dermal } \\
\text { Slope } \\
\text { Factor } \\
\text { (mg/kg- } \\
\text { day) }\end{array}$ & $\begin{array}{c}\text { GI } \\
\text { Absorp- } \\
\text { tion } \\
\text { Factor }\end{array}$ & $\begin{array}{c}\text { Dermal } \\
\text { Absorp- } \\
\text { tion } \\
\text { Factor }\end{array}$ & $\begin{array}{c}\text { Date Toxicity } \\
\text { Value } \\
\text { Withdrawn } \\
\text { from } \\
\text { IRIS/HEAST }\end{array}$ \\
\hline Terbutryn & $000886-50-0$ & $1.0 \mathrm{E}-03^{2}$ & $5.0 \mathrm{E}-04$ & & & & & & & & 0.50 & 0.010 & \\
\hline Tetrabutyl Lead & $001920-90-7$ & & & & & & & & & & 0.50 & 0.010 & \\
\hline Tetrachloroethane, 1,1,1,2- & $000630-20-6$ & $3.0 \mathrm{E}-02^{\mathrm{E}}$ & 2.4E-02 & $3.0 \mathrm{E}-02^{\mathrm{b}}$ & $2.4 \mathrm{E}-02$ & & & $2.6 \mathrm{E}-02^{2}$ & $2.6 \mathrm{E}-02^{\mathrm{b}}$ & 3.3E-02 & 0.80 & 0.010 & \\
\hline Tetrachloroethane, 1,1,2,2- & $000079-34-5$ & & & & & & & $2.0 \mathrm{E}-01^{\circ}$ & $2.0 \mathrm{E}-01^{\mathrm{b}}$ & $2.9 \mathrm{E}-01$ & 0.70 & 0.010 & \\
\hline Tetrachloroethylene ${ }^{(6)}$ & $000127-18-4$ & $1.0 \mathrm{E}-02^{\mathrm{z}}$ & $1.0 \mathrm{E}-02$ & $1.0 \mathrm{E}-01^{\mathrm{b}}$ & $1.0 \mathrm{E}-01$ & & & $5.2 \mathrm{E}-02^{\circ}$ & $2.0 \mathrm{E}-03^{\circ}$ & $5.2 \mathrm{E}-02$ & 1.00 & 0.010 & \\
\hline Tetrachlorophenol, 2,3,4,6- & $000058-90-2$ & $3.0 \mathrm{E}-02^{\mathrm{Z}}$ & $1.5 \mathrm{E}-02$ & $3.0 \mathrm{E}-01^{\mathrm{b}}$ & $1.5 \mathrm{E}-01$ & & & & & & 0.50 & 0.010 & \\
\hline $\begin{array}{l}\text { Tetrachlorotoluene, p-alpha, } \\
\text { alpha, alpha- }\end{array}$ & $005216-25-1$ & & & & & & & $2.0 \mathrm{E}+01^{b}$ & & $2.5 \mathrm{E}+01$ & 0.80 & 0.010 & \\
\hline $\begin{array}{l}\text { Tetraethyl } \\
\text { Dithiopyrophosphate }\end{array}$ & $003689-24-5$ & $5.0 \mathrm{E}-04^{4}$ & 2.5E-04 & $5.0 \mathrm{E}-03^{b}$ & 2.5E-03 & & & & & & 0.50 & 0.010 & \\
\hline Tetraethyl Lead & $000078-00-2$ & & & & & & & & & & 0.50 & 0.010 & \\
\hline Tetrafluoroethane, 1,1,1,2- & $000811-97-2$ & & & & & $2.3 \mathrm{E}+01^{\prime \prime}$ & & & & & 0.80 & 0.010 & \\
\hline Tetramethyl Lead & $000075-74-1$ & & & & & & & & & & 0.50 & 0.010 & \\
\hline Tetrapropyl Lead & 003440-75-3 & & & & & & & & & & 0.50 & 0.010 & \\
\hline Thallic Oxide $e^{(3)}$ & $001314-32-5$ & $7.0 \mathrm{E}-05^{\mathrm{w}}$ & 3.5E-05 & $7.0 \mathrm{E}-04^{\mathrm{w}}$ & & & & & & & 0.50 & 0.010 & $5 / 95$ \\
\hline Thallium (I) Nitrate & $010102-45-1$ & $9.0 \mathrm{E}-05^{\circ}$ & $1.8 \mathrm{E}-05$ & $9.0 \mathrm{E}-04^{b}$ & $1.8 \mathrm{E}-04$ & & & & & & 0.20 & 0.001 & \\
\hline Thallium (Soluble Salts) & $007440-28-0$ & & & & & & & & & & 0.15 & 0.001 & \\
\hline Thallium Acetate & $000563-68-8$ & $9.0 \mathrm{E}-05^{n}$ & $4.5 \mathrm{E}-05$ & $9.0 \mathrm{E}-04^{\mathrm{b}}$ & 4.5E-04 & & & & & & 0.50 & 0.010 & \\
\hline Thallium Carbonate & 006533-73-9 & $8.0 \mathrm{E}-05^{*}$ & 4.0E-05 & $8.0 \mathrm{E}-04^{b}$ & 4.0E-04 & & & & & & 0.50 & 0.010 & \\
\hline
\end{tabular}

Toxicity values used in PRGs calculations/nonradionuclides

Page 33 of 39 
Table 3.6a. (continued)

\begin{tabular}{|c|c|c|c|c|c|c|c|c|c|c|c|c|c|}
\hline Chemical & CASRN & $\begin{array}{c}\text { Oral } \\
\text { Chronic } \\
\text { RID } \\
\text { (mg/kg- } \\
\text { day) }\end{array}$ & $\begin{array}{c}\text { Dermal } \\
\text { Chronic } \\
\text { RID } \\
\text { (mg/kg- } \\
\text { day) }\end{array}$ & $\begin{array}{c}\text { Oral Sub- } \\
\text { chronic } \\
\text { RD } \\
\text { (mg/kg- } \\
\text { day) }\end{array}$ & $\begin{array}{c}\text { Dermal } \\
\text { Sub- } \\
\text { chronic } \\
\text { RfD } \\
\text { (mg/kg- } \\
\text { day) }\end{array}$ & $\begin{array}{c}\text { Inhalation } \\
\text { Chronic } \\
\text { RID } \\
\text { (mg/kg- } \\
\text { day) }^{\dagger}\end{array}$ & $\begin{array}{c}\text { Inhalation } \\
\text { Sub- } \\
\text { chronic } \\
\text { RmD } \\
\text { (mg/kg- } \\
\text { day) }\end{array}$ & $\begin{array}{c}\text { Oral } \\
\text { Slope } \\
\text { Factor } \\
\text { (mg/kg- } \\
\text { day) }\end{array}$ & $\begin{array}{l}\text { Inhalation } \\
\text { Slope } \\
\text { Factor } \\
\text { (mg/kg- } \\
\text { day) }^{-1}\end{array}$ & $\begin{array}{l}\text { Dermal } \\
\text { Slope } \\
\text { Factor } \\
\text { (mg/kg- } \\
\text { day) }\end{array}$ & $\begin{array}{c}\text { GI } \\
\text { Absorp- } \\
\text { tion } \\
\text { Factor }\end{array}$ & $\begin{array}{c}\text { Dermal } \\
\text { Absorp- } \\
\text { tion } \\
\text { Factor }\end{array}$ & $\begin{array}{l}\text { Date Toxicity } \\
\text { Value } \\
\text { Withdrawn } \\
\text { from } \\
\text { IRIS/HEAST }\end{array}$ \\
\hline Thallium Chloride & $007791-12-0$ & $8.0 \mathrm{E}-05^{\circ}$ & $1.6 \mathrm{E}-05$ & $8.0 \mathrm{E}-04^{b}$ & $1.6 \mathrm{E}-04$ & & & & & & 0.20 & 0.001 & \\
\hline Thallium Selenite $^{(3)}$ & $012039-52-0$ & $9.0 \mathrm{E}-05^{\mathrm{w}}$ & $1.8 \mathrm{E}-05$ & & & & & & & & 0.20 & 0.001 & $9 / 93$ \\
\hline Thallium Sulfate & $007446-18-6$ & $8.0 \mathrm{E}-05^{2}$ & $1.6 \mathrm{E}-05$ & $8.0 \mathrm{E}-04^{b}$ & $1.6 \mathrm{E}-04$ & & & & & & 0.20 & 0.001 & \\
\hline Thiobencarb & $028249-77-6$ & $1.0 \mathrm{E}-02^{\prime}$ & $5.0 \mathrm{E}-03$ & & & & & & & & 0.50 & 0.010 & \\
\hline Thiofanox & $039196-18-4$ & $3.0 \mathrm{E}-04^{\mathrm{b}}$ & $1.5 \mathrm{E}-04$ & $3.0 \mathrm{E}-04^{\mathrm{b}}$ & $1.5 \mathrm{E}-04$ & & & & & & 0.50 & 0.010 & \\
\hline Thiophanate, Methyl & $023564-05-8$ & $8.0 \mathrm{E}-02^{2}$ & $4.0 \mathrm{E}-02$ & & & & & & & & 0.50 & 0.010 & \\
\hline Thiram & $000137-26-8$ & $5.0 \mathrm{E}-03^{\circ}$ & $2.5 \mathrm{E}-03$ & $6.0 \mathrm{E}-03^{\mathrm{b}}$ & $3.0 \mathrm{E}-03$ & & & & & & 0.50 & 0.010 & \\
\hline Thorium & $007440-29-1$ & & & & & & & & & & 0.01 & 0.001 & \\
\hline Tin & $007440-31-5$ & $6.0 \mathrm{E}-01^{b}$ & $6.0 \mathrm{E}-02$ & $6.0 \mathrm{E}-01^{b}$ & $6.0 \mathrm{E}-02$ & & & & & & 0.10 & 0.001 & \\
\hline Titanium & $007440-32-6$ & & & & & & & & & & 0.03 & 0.001 & \\
\hline Toluene $e^{(6)}$ & $000108-88-3$ & $2.0 \mathrm{E}-01^{\circ}$ & $1.6 \mathrm{E}-01$ & $2.0 \mathrm{E}+00^{b}$ & $1.6 \mathrm{E}+00$ & 1.1E-01" & $3.2 \mathrm{E}-01^{\mathrm{b}, \mathrm{e}}$ & & & & 0.80 & 0.010 & \\
\hline $\begin{array}{l}\text { Toluene diisocyanate mixture } \\
\text { (TDI) }\end{array}$ & $026471-62-5$ & & & & & $2.0 \mathrm{E}-05^{\prime}$ & & & & & 0.80 & 0.010 & \\
\hline Toluene-2,4-diamine & $000095-80-7$ & & & & & & & $3.2 \mathrm{E}+00^{6}$ & & $6.4 \mathrm{E}+00$ & 0.50 & 0.010 & \\
\hline Toluene-2,5-diamine & $000095-70-5$ & $6.0 \mathrm{E}-01^{\mathrm{b}}$ & $3.0 \mathrm{E}-01$ & $6.0 \mathrm{E}-01^{\mathrm{b}}$ & $3.0 \mathrm{E}-01$ & & & & & & 0.50 & 0.010 & \\
\hline Toluene-2,6-diamine & $000823-40-5$ & $2.0 \mathrm{E}-01^{\mathrm{b}}$ & $1.0 \mathrm{E}-01$ & $2.0 \mathrm{E}-01^{b}$ & $1.0 \mathrm{E}-01$ & & & & & & 0.50 & 0.010 & \\
\hline $\begin{array}{l}\text { Toluidine, o- (Methylaniline, } \\
\text { 2-) }\end{array}$ & $000095-53-4$ & & & & & & & $2.4 \mathrm{E}-01^{b}$ & & $4.8 \mathrm{E}-01$ & 0.50 & 0.010 & \\
\hline Toluidine, p- & $000106-49-0$ & & & & & & & $1.9 \mathrm{E}-01^{b}$ & & $3.8 \mathrm{E}-01$ & 0.50 & 0.010 & \\
\hline Toxaphene & $008001-35-2$ & & & & & & & $1.1 \mathrm{E}+00^{\circ}$ & $1.1 \mathrm{E}+00^{b}$ & $2.2 \mathrm{E}+00$ & 0.50 & 0.010 & \\
\hline
\end{tabular}


Table 3.6a. (continued)

\begin{tabular}{|c|c|c|c|c|c|c|c|c|c|c|c|c|c|}
\hline Chemical & CASRN & $\begin{array}{c}\text { Oral } \\
\text { Chronic } \\
\text { Rm } \\
\text { (mg/kg- } \\
\text { day) }\end{array}$ & $\begin{array}{c}\text { Dermal } \\
\text { Chronic } \\
\text { RmD } \\
\text { (mg/kg- } \\
\text { day) }\end{array}$ & $\begin{array}{c}\text { Oral Sub- } \\
\text { chronic } \\
\text { RmD } \\
\text { (mg/kg- } \\
\text { day) }\end{array}$ & $\begin{array}{l}\text { Dermal } \\
\text { Sub- } \\
\text { chronic } \\
\text { RID } \\
\text { (mg/kg- } \\
\text { day) }\end{array}$ & $\begin{array}{c}\text { Inhalation } \\
\text { Chronic } \\
\text { RID } \\
\text { (mg/kg- } \\
\text { day) }\end{array}$ & $\begin{array}{c}\text { Inhalation } \\
\text { Sub- } \\
\text { chronic } \\
\text { RfD } \\
\text { (mg/kg- } \\
\text { day) }\end{array}$ & $\begin{array}{c}\text { Oral } \\
\text { Slope } \\
\text { Factor } \\
\text { (mg/kg- } \\
\text { day) }\end{array}$ & $\begin{array}{l}\text { Inhalation } \\
\text { Slope } \\
\text { Factor } \\
\text { (mg/kg- } \\
\text { day) }\end{array}$ & $\begin{array}{l}\text { Dermal } \\
\text { Slope } \\
\text { Factor } \\
\text { (mg/kg- } \\
\text { day) }\end{array}$ & $\begin{array}{c}\text { GI } \\
\text { Absorp- } \\
\text { tion } \\
\text { Factor }\end{array}$ & $\begin{array}{c}\text { Dermal } \\
\text { Absorp- } \\
\text { tion } \\
\text { Factor }\end{array}$ & $\begin{array}{l}\text { Date Toxicity } \\
\text { Value } \\
\text { Withdrawn } \\
\text { from } \\
\text { IRIS/HEAST }\end{array}$ \\
\hline Tralomethrin & $066841-25-6$ & 7.5E-03" & $3.8 \mathrm{E}-03$ & & & & & & & & 0.50 & 0.010 & \\
\hline Triallate & $002303-17-5$ & $1.3 \mathrm{E}-02^{a}$ & $6.5 \mathrm{E}-03$ & $1.3 \mathrm{E}-02^{\mathrm{b}}$ & $6.5 \mathrm{E}-03$ & & & & & & 0.50 & 0.010 & \\
\hline Triasulfuron & $082097-50-5$ & $1.0 \mathrm{E}-02^{\prime}$ & $5.0 \mathrm{E}-03$ & & & & & & & & 0.50 & 0.010 & \\
\hline Tribromobenzene, 1,2,4- & $000615-54-3$ & $5.0 \mathrm{E}-03^{\prime}$ & $4.0 \mathrm{E}-03$ & $5.0 \mathrm{E}-02^{\mathrm{b}}$ & $2.5 \mathrm{E}-02$ & & & & & & 0.80 & 0.010 & \\
\hline Tribromochloromethane & $000594-15-0$ & & & & & & & & & & 0.50 & 0.010 & \\
\hline Tribromodiphenyl Ether & $049690-94-0$ & & & & & & & & & & 0.50 & 0.010 & \\
\hline Tributyltin Oxide & $000056-35-9$ & $3.0 \mathrm{E}-05^{\prime}$ & $1.5 \mathrm{E}-05$ & & & & & & & & 0.50 & 0.010 & \\
\hline $\begin{array}{l}\text { Trichloro-1,2,2-trifluoroethane, } \\
\text { 1,1,2- }\end{array}$ & $000076-13-1$ & $3.0 \mathrm{E}+01^{\circ}$ & $2.4 \mathrm{E}+01$ & $3.0 \mathrm{E}+00^{6}$ & $2.4 \mathrm{E}+00$ & $8.6 \mathrm{E}+00^{b}$ & $8.6 \mathrm{E}+00^{\circ}$ & & & & 0.80 & 0.010 & \\
\hline $\begin{array}{l}\text { Trichloro-2'- } \\
\text { hydroxydiphenylether }\end{array}$ & $003380-34-5$ & & & $4.0 \mathrm{E}+00^{6}$ & & & & & & & 0.50 & 0.010 & \\
\hline Trichloroacetic Acid & & & & & & & & & & & 0.50 & 0.010 & \\
\hline Trichloroaniline $\mathrm{HCl}, 2,4,6-$ & $033663-50-2$ & & & & & & & $2.9 \mathrm{E}-02^{\mathrm{b}}$ & & $5.8 \mathrm{E}-02$ & 0.50 & 0.010 & \\
\hline Trichloroaniline, 2,4,6- & $000634-93-5$ & & & & & & & $3.4 \mathrm{E}-02^{\mathrm{b}}$ & & $6.8 \mathrm{E}-02$ & 0.50 & 0.010 & \\
\hline Trichlorobenzene, 1,2,4- & $000120-82-1$ & $1.0 \mathrm{E}-02^{\prime}$ & $9.7 \mathrm{E}-03$ & $1.0 \mathrm{E}-02^{\mathrm{b}}$ & $9.7 \mathrm{E}-03$ & $5.7 \mathrm{E}-02^{\mathrm{b}}$ & $5.7 \mathrm{E}-01^{b}$ & & & & 0.97 & 0.010 & \\
\hline Trichloroethane, 1,1,1-(3) & $000071-55-6$ & $9.0 \mathrm{E}-02^{\mathrm{e}, \mathrm{w}}$ & $8.1 \mathrm{E}-02$ & & & $2.9 \mathrm{E}-01^{\circ}$ & & & & & 0.90 & 0.010 & 9/93 \\
\hline Trichloroethane, 1,1,2- & $000079-00-5$ & 4.0E-03' & $3.2 \mathrm{E}-03$ & $4.0 \mathrm{E}-02^{\mathrm{b}}$ & $3.2 \mathrm{E}-02$ & & & 5.7E-02' & $5.7 \mathrm{E}-02^{b}$ & $7.0 \mathrm{E}-02$ & 0.81 & 0.010 & \\
\hline Trichloroethylene ${ }^{(3)}$ & $000079-01-6$ & & & & & & & $1.1 \mathrm{E}-02^{\mathrm{e}}$ & $6.0 \mathrm{E}-03^{\circ}$ & 7.3E-02 & 0.15 & 0.010 & \\
\hline Trichlorofluoromethane & $000075-69-4$ & $3.0 \mathrm{E}-01^{\prime}$ & $6.9 \mathrm{E}-02$ & $7.0 \mathrm{E}-01^{b}$ & $1.6 \mathrm{E}-01$ & $2.0 \mathrm{E}-01^{\mathrm{b}, \mathrm{c}}$ & $2.0 \mathrm{E}+00^{\mathrm{b}, \mathrm{c}}$ & & & & 0.23 & 0.010 & \\
\hline Trichlorophenol, 2,4,5- & $000095-95-4$ & $1.0 \mathrm{E}-01^{*}$ & 8.0E-02 & $1.0 \mathrm{E}+00^{6}$ & $5.0 \mathrm{E}-01$ & & & & & & 0.80 & 0.010 & \\
\hline
\end{tabular}

Toxicity values used in PRGs calculations/nonradionuclides

Page 35 of 39 
Table 3.6a. (continued)

\begin{tabular}{|c|c|c|c|c|c|c|c|c|c|c|c|c|c|}
\hline Chemical & CASRN & $\begin{array}{c}\text { Oral } \\
\text { Chronic } \\
\text { RDD } \\
\text { (mg/kg. } \\
\text { day) }\end{array}$ & $\begin{array}{c}\text { Dermal } \\
\text { Chronic } \\
\text { RTD } \\
\text { (mg/kg- } \\
\text { day) }\end{array}$ & $\begin{array}{l}\text { Oral Sub- } \\
\text { chronic } \\
\text { RDD } \\
\text { (mg/kg- } \\
\text { day) }\end{array}$ & $\begin{array}{c}\text { Dermal } \\
\text { Sub- } \\
\text { chronic } \\
\text { RRD } \\
\text { (mg/kg- } \\
\text { day) } \\
\end{array}$ & $\begin{array}{c}\text { Inhalation } \\
\text { Chronic } \\
\text { RID } \\
\text { (mg/kg- } \\
\text { day) }\end{array}$ & $\begin{array}{c}\text { Inhalation } \\
\text { Sub- } \\
\text { chronic } \\
\text { RR } \\
\text { (mg/kg- } \\
\text { day) }\end{array}$ & $\begin{array}{c}\text { Oral } \\
\text { Slope } \\
\text { Factor } \\
(\mathrm{mg} / \mathrm{kg}- \\
\text { day) }\end{array}$ & $\begin{array}{c}\text { Inhalation } \\
\text { Slope } \\
\text { Factor } \\
\text { (mg/kg- } \\
\text { day) }^{-1}\end{array}$ & $\begin{array}{l}\text { Dermal } \\
\text { Slope } \\
\text { Factor } \\
\text { (mg/kg- } \\
\text { day) }\end{array}$ & $\begin{array}{c}\text { GI } \\
\text { Absorp- } \\
\text { tion } \\
\text { Factor }\end{array}$ & $\begin{array}{c}\text { Dermal } \\
\text { Absorp- } \\
\text { tion } \\
\text { Factor }\end{array}$ & $\begin{array}{c}\text { Date Toxicity } \\
\text { Value } \\
\text { Withdrawn } \\
\text { from } \\
\text { IRIS/HEAST }\end{array}$ \\
\hline Trichlorophenol, 2,4,6- & $000088-06-2$ & & & & & & & $1.1 \mathrm{E}-02^{\circ}$ & $1.0 \mathrm{E}-02^{\mathrm{b}}$ & $2.2 \mathrm{E}-02$ & 0.50 & 0.010 & \\
\hline $\begin{array}{l}\text { Trichlorophenoxy) Propionic } \\
\text { Acid, 2(2,4,5- }\end{array}$ & $000093-72-1$ & 8.0E-03: & $4.0 \mathrm{E}-03$ & $8.0 \mathrm{E}-03^{\mathrm{b}}$ & $4.0 \mathrm{E}-03$ & & & & & & 0.50 & 0.010 & \\
\hline $\begin{array}{l}\text { Trichlorophenoxyacetic Acid, } \\
\text { 2,4,5- }\end{array}$ & $000093-76-5$ & $1.0 \mathrm{E}-02^{2}$ & $5.0 \mathrm{E}-03$ & $1.0 \mathrm{E}-01^{\mathrm{b}}$ & $5.0 \mathrm{E}-02$ & & & & & & 0.50 & 0.010 & \\
\hline Trichloropropane, 1,1,2- & $000598-77-6$ & $5.0 \mathrm{E}-03^{n}$ & $4.0 \mathrm{E}-03$ & $5.0 \mathrm{E}-02^{\mathrm{b}}$ & $2.5 \mathrm{E}-02$ & & & & & & 0.80 & 0.010 & \\
\hline Trichloropropane, 1,2,3- & $000096-18-4$ & $6.0 \mathrm{E}-03^{*}$ & $4.8 \mathrm{E}-03$ & $6.0 \mathrm{E}-02^{\mathrm{b}}$ & $4.8 \mathrm{E}-02$ & & & 7.0E+00 & & $8.8 \mathrm{E}+00$ & 0.80 & 0.010 & \\
\hline Trichloropropene, 1,2,3- & $000096-19-5$ & $5.0 \mathrm{E}-03^{\mathrm{b}}$ & $4.0 \mathrm{E}-03$ & $5.0 \mathrm{E}-03^{b}$ & $2.5 \mathrm{E}-03$ & & & & & & 0.80 & 0.010 & \\
\hline Trichlorotoluene, 2,3,6- & $002077-46-5$ & & & $5.0 \mathrm{E}-05^{b}$ & & & & & & & 0.50 & 0.010 & \\
\hline Trichlorotoluene, alpha 2,6- & $002014-83-7$ & & & $5.0 \mathrm{E}-05^{b}$ & & & & & & & 0.50 & 0.010 & \\
\hline Tridiphane & 058138-08-2 & 3.0E-03' & $1.5 \mathrm{E}-03$ & & & & & & & & 0.50 & 0.010 & \\
\hline Triethyl Lead & $005224-23-7$ & & & & & & & & & & 0.50 & 0.010 & \\
\hline Triethylamine & $000121-44-8$ & & & & & $2.0 \mathrm{E}-03^{*}$ & & & & & 0.80 & 0.010 & \\
\hline Trifluralin & 001582-09-8 & $7.5 \mathrm{E}-03^{\circ}$ & $3.8 \mathrm{E}-03$ & $7.5 \mathrm{E}-03^{\mathrm{b}}$ & $3.8 \mathrm{E}-03$ & & & $7.7 \mathrm{E}-03^{\circ}$ & & $1.5 \mathrm{E}-02$ & 0.50 & 0.010 & \\
\hline Trimethyl Lead & 007442-13-9 & & & & & & & & & & 0.50 & 0.010 & \\
\hline Trimethyl Phosphate & $000512-56-1$ & & & & & & & $3.7 \mathrm{E}-02^{\mathrm{b}}$ & & $7.4 \mathrm{E}-02$ & 0.50 & 0.010 & \\
\hline Trimethylethyl Lead & $001762-26-1$ & & & & & & & & & & 0.50 & 0.010 & \\
\hline Trinitrobenzene, 1,3,5- & $000099-35-4$ & $5.0 \mathrm{E}-05^{n}$ & $3.3 \mathrm{E}-05$ & $5.0 \mathrm{E}-04^{\mathrm{b}}$ & $3.3 \mathrm{E}-04$ & & & & & & 0.65 & 0.010 & \\
\hline Trinitrophenylmethylnitramine & $000479-45-8$ & $1.0 \mathrm{E}-02^{\mathrm{b}}$ & $5.0 \mathrm{E}-03$ & $1.0 \mathrm{E}-01^{\mathrm{b}}$ & $5.0 \mathrm{E}-02$ & & & & & & 0.50 & 0.010 & \\
\hline Trinitrotoluene, 2,4,6- & $000118-96-7$ & $5.0 \mathrm{E}-04^{2}$ & $3.0 \mathrm{E}-04$ & $5.0 \mathrm{E}-04^{\mathrm{b}}$ & $3.0 \mathrm{E}-04$ & & & $3.0 \mathrm{E}-02^{2}$ & & $5.0 \mathrm{E}-02$ & 0.60 & 0.010 & \\
\hline
\end{tabular}

Toxicity values used in PRGs calculations/nonradionuclides

Page 36 of 39 
Table 3.6a. (continued)

\begin{tabular}{|c|c|c|c|c|c|c|c|c|c|c|c|c|c|}
\hline Chemical & CASRN & $\begin{array}{c}\text { Oral } \\
\text { Chronic } \\
\text { RiD } \\
\text { (mg/kg- } \\
\text { day) }\end{array}$ & $\begin{array}{c}\text { Dermal } \\
\text { Chronic } \\
\text { RID } \\
\text { (mg/kg- } \\
\text { day) }\end{array}$ & $\begin{array}{c}\text { Oral Sub- } \\
\text { chronic } \\
\text { RfD } \\
\text { (mg/kg- } \\
\text { day) }\end{array}$ & $\begin{array}{l}\text { Dermal } \\
\text { Sub- } \\
\text { chronic } \\
\text { Rm } \\
\text { (mg/kg- } \\
\text { day) }\end{array}$ & $\begin{array}{l}\text { Inhalation } \\
\text { Chronic } \\
\text { Rm } \\
\text { (mg/kg- } \\
\text { day) }^{f}\end{array}$ & $\begin{array}{c}\text { Inhalation } \\
\text { Sub- } \\
\text { chronic } \\
\text { RmD } \\
\text { (mg/kg- } \\
\text { day) }\end{array}$ & $\begin{array}{c}\text { Oral } \\
\text { Slope } \\
\text { Factor } \\
\text { (mg/kg- } \\
\text { day) }\end{array}$ & $\begin{array}{l}\text { Inhalation } \\
\text { Slope } \\
\text { Factor } \\
\text { (mg/kg- } \\
\text { day) }{ }^{-1}\end{array}$ & $\begin{array}{l}\text { Dermal } \\
\text { Slope } \\
\text { Factor } \\
\text { (mg/kg- } \\
\text { day) }\end{array}$ & $\begin{array}{c}\text { GI } \\
\text { Absorp- } \\
\text { tion } \\
\text { Factor }\end{array}$ & $\begin{array}{l}\text { Dermal } \\
\text { Absorp- } \\
\text { tion } \\
\text { Factor }\end{array}$ & $\begin{array}{c}\text { Date Toxicity } \\
\text { Value } \\
\text { Withdrawn } \\
\text { from } \\
\text { IRIS/HEAST }\end{array}$ \\
\hline Tripropyl Lead & $006618-03-7$ & & & & & & & & & & 0.50 & 0.010 & \\
\hline Uranium (Soluble Salts) & NA & $3.0 \mathrm{E}-03^{\circ}$ & $2.6 \mathrm{E}-03$ & & & & & & & & 0.85 & 0.001 & \\
\hline Vanadium Pentoxide & $001314-62-1$ & $9.0 \mathrm{E}-03^{*}$ & $1.8 \mathrm{E}-03$ & $9.0 \mathrm{E}-03^{b}$ & $1.8 \mathrm{E}-03$ & & & & & & 0.20 & 0.001 & \\
\hline Vanadium Sulfate & $036907-42-3$ & $2.0 \mathrm{E}-02^{\mathrm{b}}$ & $4.0 \mathrm{E}-03$ & $2.0 \mathrm{E}-02^{b}$ & $4.0 \mathrm{E}-03$ & & & & & & 0.20 & 0.001 & \\
\hline Vanadium, Metallic & $007440-62-2$ & $7.0 \mathrm{E}-03^{\mathrm{b}}$ & $7.0 \mathrm{E}-05$ & $7.0 \mathrm{E}-03^{\mathrm{b}}$ & $7.0 \mathrm{E}-05$ & & & & & & 0.01 & 0.001 & \\
\hline Vanadyl Sulfate & $027774-13-6$ & $2.0 \mathrm{E}-02^{\mathrm{b}}$ & $4.0 \mathrm{E}-03$ & & & & & & & & 0.20 & 0.001 & \\
\hline Vernolate & $001929-77-7$ & $1.0 \mathrm{E}-03^{\circ}$ & $5.0 \mathrm{E}-04$ & $1.0 \mathrm{E}-02^{\mathrm{b}}$ & $5.0 \mathrm{E}-03$ & & & & & & 0.50 & 0.010 & \\
\hline Vinclozolin & $050471-44-8$ & $2.5 \mathrm{E}-02^{4}$ & $1.3 \mathrm{E}-02$ & & & & & . & & & 0.50 & 0.010 & \\
\hline Vinyl Acetate & $000108-05-4$ & $1.0 \mathrm{E}+00^{b}$ & $6.5 \mathrm{E}-01$ & $1.0 \mathrm{E}+00^{6}$ & $6.5 \mathrm{E}-01$ & $5.7 \mathrm{E}-02^{2}$ & $5.7 \mathrm{E}-02^{b}$ & & & & 0.65 & 0.010 & \\
\hline Vinyl Bromide & $000593-60-2$ & & & & & $8.6 \mathrm{E}-04^{4}$ & $8.6 \mathrm{E}-04^{\mathrm{b}}$ & & $1.1 \mathrm{E}-01^{\mathrm{b}}$ & & 0.80 & 0.010 & \\
\hline Vinyl Chloride ${ }^{(6)}$ & $000075-01-4$ & & & & & & & $1.9 \mathrm{E}+00^{\circ}$ & $3.0 \mathrm{E}-01^{\mathrm{b}}$ & $1.9 \mathrm{E}+00$ & 1.00 & 0.010 & \\
\hline Warfarin & $000081-81-2$ & $3.0 \mathrm{E}-04^{\circ}$ & $1.5 \mathrm{E}-04$ & $3.0 \mathrm{E}-04^{\mathrm{b}}$ & $1.5 \mathrm{E}-04$ & & & & & & 0.50 & 0.010 & \\
\hline White Phosphorus & $007723-14-0$ & 2.0E-05 & $4.0 \mathrm{E}-06$ & & & & & & & & 0.20 & 0.001 & \\
\hline Xylene, Mixture ${ }^{(6)}$ & $001330-20-7$ & $2.0 \mathrm{E}+00^{\circ}$ & $1.8 \mathrm{E}+00$ & $3.6 \mathrm{E}-01^{\circ}$ & 3.3E-01 & & & & & & 0.92 & 0.010 & \\
\hline Xylene, $P_{-}(6)$ & $000106-42-3$ & & & $2.7 \mathrm{E}-01^{\circ}$ & & & & & & & 0.80 & 0.010 & \\
\hline Xylene, $m^{(6)}$ & $000108-38-3$ & $2.0 \mathrm{E}+00^{\mathrm{b}}$ & $1.6 \mathrm{E}+00$ & $6.7 \mathrm{E}-02^{\circ}$ & $5.3 \mathrm{E}-02$ & & & & & & 0.80 & 0.010 & \\
\hline Xylene, $0 \cdot\left({ }^{(6)}\right.$ & $000095-47-6$ & $2.0 \mathrm{E}+00^{6}$ & $1.6 \mathrm{E}+00$ & & & & & & & & 0.80 & 0.010 & \\
\hline Zinc (Metallic) & $007440-66-6$ & $3.0 \mathrm{E}-0 \mathrm{I}^{\circ}$ & $6.0 \mathrm{E}-02$ & $3.0 \mathrm{E}-01^{\mathrm{b}}$ & $6.0 \mathrm{E}-02$ & & & & & & 0.20 & 0.001 & \\
\hline Zinc Cyanide & $000557-21-1$ & $5.0 \mathrm{E}-02^{\circ}$ & $1.0 \mathrm{E}-02$ & $5.0 \mathrm{E}-02^{b}$ & $1.0 \mathrm{E}-02$ & & & & & & 0.20 & 0.001 & \\
\hline
\end{tabular}


Table 3.6a. (continued)

\begin{tabular}{|c|c|c|c|c|c|c|c|c|c|c|c|c|c|}
\hline Chemical & CASRN & $\begin{array}{c}\text { Oral } \\
\text { Chronic } \\
\text { RfD } \\
\text { (mg/kg- } \\
\text { day) }\end{array}$ & $\begin{array}{c}\text { Dermal } \\
\text { Chronic } \\
\text { RfD } \\
\text { (mg/kg- } \\
\text { day) }\end{array}$ & $\begin{array}{c}\text { Oral Sub- } \\
\text { chronic } \\
\text { RID } \\
\text { (mg/kg- } \\
\text { day) }\end{array}$ & $\begin{array}{c}\text { Dermal } \\
\text { Sub- } \\
\text { chronic } \\
\text { Rm } \\
\text { (mg/kg- } \\
\text { day) }\end{array}$ & $\begin{array}{c}\text { Inhalation } \\
\text { Chronic } \\
\text { RmD } \\
\text { (mg/kg- } \\
\text { day) }\end{array}$ & $\begin{array}{l}\text { Inhalation } \\
\text { Sub- } \\
\text { chronic } \\
\text { RfD } \\
\text { (mg/kg- } \\
\text { day) }\end{array}$ & $\begin{array}{c}\text { Oral } \\
\text { Slope } \\
\text { Factor } \\
\text { (mg/kg- } \\
\text { day) }\end{array}$ & $\begin{array}{c}\text { Inhalation } \\
\text { Slope } \\
\text { Factor } \\
\text { (mg/kg- } \\
\text { day) }\end{array}$ & $\begin{array}{l}\text { Dermal } \\
\text { Slope } \\
\text { Factor } \\
\text { (mg/kg- } \\
\text { day) })^{-1}\end{array}$ & $\begin{array}{c}\text { GI } \\
\text { Absorp- } \\
\text { tion } \\
\text { Factor }\end{array}$ & $\begin{array}{l}\text { Dermal } \\
\text { Absorp- } \\
\text { tion } \\
\text { Factor }\end{array}$ & $\begin{array}{c}\text { Date Toxicity } \\
\text { Value } \\
\text { Withdrawn } \\
\text { from } \\
\text { IRIS/HEAST }\end{array}$ \\
\hline Zinc Phosphide & $001314-84-7$ & $3.0 \mathrm{E}-04^{\prime}$ & $6.0 \mathrm{E}-05$ & $3.0 \mathrm{E}-03^{b}$ & $6.0 \mathrm{E}-04$ & & & & & & 0.20 & 0.001 & \\
\hline Zineb & $012122-67-7$ & $5.0 \mathrm{E}-02^{2}$ & $2.5 \mathrm{E}-02$ & $5.0 \mathrm{E}-02^{\mathrm{b}}$ & 2.5E-02 & & & & & & 0.50 & 0.010 & \\
\hline Zirconium & $007440-67-7$ & & & & & & & & & & 0.80 & 0.001 & \\
\hline
\end{tabular}

Notes:

$t \quad$ The $\mathrm{RDD}$ value for inhalation exposure is generally reported as a concentration in air (RfC) in $\mathrm{mg} / \mathrm{m}^{3}$ for continuous, 24-hour/day exposure. A human body weight of $70 \mathrm{~kg}$ and an inhalation rate of $20 \mathrm{~m} / \mathrm{day}$ are used to convert to an inhaled intake expressed in units of mg/kg $\times$ day (Risk Assessment Guidance for Superfimd, Volume 1: Human Health Evaluation Mamual (Part A), Interim Final, EPA/540/1-89/002, December 1989).
19ed to convert to an inhaled intake expressed in units

a Source: Integrated Risk Information System (IRIS)

b Source: Health and Environmental Affects Summary Table (HEAST) 1995

c This value was derived from methodology that is not current with the interim inhalation methodology used by the RfD/RfC Work Group (see HEAST Table 2 for details).

d Lead alkyls-See IRIS cover sheet for further explanation.

c Contact the Superfund Health Risk Technical Support Center: (513) 569-7300.

f HEAST concluded that toxicity data were inadequate for calculation of oral RfDs for copper and substituted the current drinking water standard (MCLG) of $1.3 \mathrm{mg} / \mathrm{L}$

g Listed as "Dinitrotoluene mixture, 2,4-/2,6-" in IRIS. The value is based on a study using technical grade DNT.

m IRIS no longer separates manganese values for chronic oral RfDs into water and diet RDs. The chronic oral RID is now for the total oral intake of manganese. HEAST manganese values remain separated into subchronic oral RfD (water) and subchronic oral RfD (diet). Since it was necessary to keep the RfD categories for both diet and water on the table to list HEAST values, the IRIS chronic oral RfD for water was changed from 5.00E-03 to 1.40E-01 (the new manganese value for total oral intake) and footnoted " $m$."

r The PRG was calculated considering Supplemental Gufdance from RAGS: Region 4 Bulletins, Human Health Risk Assessment (Interim Guidance) [received February 11 , 1992, (benzo[a]pyrene) and November 1995(dioxins and furans)]. The slope factors for chemicals so marked indicate that a toxicity equivalency factor (TEF) method was used for carcinogenic PAHs based on each compound's relative potency to the potency of benzo[a]pyrene. The following TEFs were used to convert each PAH slope factor to an equivalent slope factor of benzo[a]pyrene [that has a new cancer slope factor of 7.3 (mg/kg/day) ${ }^{-1}$ identified by EPA's CRAVE workgroup]: (1) benzo[a]pyrene, TEF $=1.0 ;$ (2) benzo[a]anthracene, TEF $=0.1 ;(3)$ benzo[b]fluoranthene, TEF $=0.1 ;(4)$ benzo[k]fluoranthene, TEF $=0.01 ;(5)$ chrysene, TEF $=0.001 ;(6)$ dibenz $[a, h]$ anthracene, $T E F=1.0$; and $(7)$ indeno[1,2,3-c,d]pyrene, $T E F=0.1$. In addition, the slope factors for chemicals so marked indicate that a TEF methodology was used for chlorinated dioxin and furan congeners based on each compound's relative potency to the potency of 2,3,7,8-tetrachlorodibenzodioxin (TCDD). The following TEFs were used to convert each slope factor to an equivalent slope factor of $2,3,7,8-$ TCDD: (1) 2,3,7,8-TCDD, TEF = 1.0; (2) 2,3,7,8-PeCDD, TEF =0.5; (3) 2,3,7,8-HxCDD, TEF =0.1; (4) 2,3,7,8-HpCDD, TEF $=0.01 ;(5)$ OCDD, TEF $=0.001 ;(6) 2,3,7,8-\mathrm{TCDF}, \mathrm{TEF}=0.1 ;(7) 1,2,3,7,8-\mathrm{PeCDF}$, $\mathrm{TEF}=0.5 ;(8) 2,3,4,7,8-\mathrm{PeCDF}, \mathrm{TEF}=0.05 ;(9) 2,3,7,8-\mathrm{HxCDF}, \mathrm{TEF}=0.1 ;(10) 2,3,7,8-\mathrm{HpCDF}, \mathrm{TEF}=0.01 ;$ and $(11) \mathrm{OCDF}, \mathrm{TEF}=0.001$.

u The SFI was calculated from inhalation unit risk as described in Supplemental Guidance from RAGS: Region 4 Bulletins, Human Health Risk Assessment (Interim Guidance) (November 1995).

w The toxicity value was withdrawn.

(1) The same oral slope factor as used for polychlorinated biphenyls.

(2) The PRG was calculated considering Supplemental Guidance from RAGS: Region 4 Bulletins, Human Health Risk Assessment (Interim Guidance) [received February 11, 1992, (benzo[a]pyrene) and November 1995(dioxins and furans)]. The slope factors for chemicals so marked indicate that a toxicity equivalency factor (TEF) method was used for carcinogenic PAHs based on each compound's relative potency to the potency of benzo[a]pyrene. The following TEFs were used to convert each PAH slope factor to an equivalent slope factor of benzo[a]pyrene [that has a new cancer slope factor of 7.3 (mg/kg/day) $)^{-1}$ identified by EPA's CRAVE workgroup]: (1) benzo[a]pyrene, TEF $=1.0$; (2) benzo[a] anthracene, TEF $=0.1 ;$ (3) benzo[b]fluoranthene, TEF $=0.1 ;(4)$ benzo[k]fluoranthene, TEF $=0.01 ;(5)$ chrysene, TEF $=0.001 ;(6)$ dibenz [a, h]anthracene, $\mathrm{TEF}=1.0$; and $(7)$ indeno[1,2,3-c,d]pyrene, $\mathrm{TEF}=0.1$. In addition, the slope factors for chemicals so marked indicate that a TEF methodology was used for chlorinated dioxin and furan 


\section{Table 3.6a. (continued)}

congeners based on each compound's relative potency to the potency of 2,3,7,8-tetrachlorodibenzodioxin (TCDD). The following TEFs were used to convert each slope factor to an equivalent slope factor of 2,3,7,8-

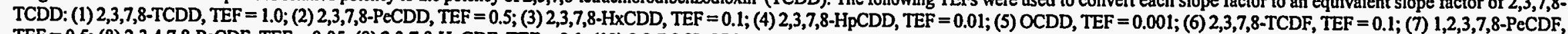
$\mathrm{TEF}=0.5 ;(8) 2,3,4,7,8-\mathrm{PeCDF}, \mathrm{TEF}=0.05 ;(9) 2,3,7,8-\mathrm{HxCDF}, \mathrm{TEF}=0.1 ;(10) 2,3,7,8-\mathrm{HPCDF}, \mathrm{TEF}=0.01$; and (11) OCDF, TEF $=0.001$

(3) The toxicity values for trichloroethylene have been withdrawn from IRIS and HEAST pending further investigation. However, the most recent toxicity values have been used to calculate the PRG.

(4) The PRG was calculated considering Supplemental Guidance from RAGS: Region 4 Bulletins, Human Heallh Risk Assessment (Interim Guidance) from EPA (November 1995). The slope factors for chemicals so marked indicate that a TEF methodology was used for chlorinated dioxin and furan congeners based on each compound's relative potency to the potency of 237, (3) TEFs were used to convert each slope factor to an equivalent slope factor 23,78 -TCDD: (1) 23,7,-TCDD TEF $=10 ;$ (2) 23,78-PeCDD $=0.01 ;(5)$ OCDD, TEF $=0.001 ;(6) 2,3,7,8-\mathrm{TCDF}, \operatorname{TEF}=0.1 ;$ (7) 1,2,3,7,8-PeCDF, TEF $=0.5 ;(8) 2,3,4,7,8-\mathrm{PeCDF}, \mathrm{TEF}=0.05 ;(9) 2,3,7,8-\mathrm{HxCDF}, \mathrm{TEF}=0.1 ;(10) 2,3,7,8-\mathrm{HpCDF}, \mathrm{TEF}=0.01 ;$ and $(11)$ OCDF, TEF $=0.001$.

(5) Toxicity values for these chemicals have been withdrawn from IRIS and HEAST pending further investigation. However, the most recent toxicity values have been used to calculate the PRG.

(6) Toxicity values for these chemicals were obtained from the Superfund Health Risk Technical Support Center. 
$+$ 
Table 3.6b. Chemical-specific parameters used in calculating risk-based Preliminary Remediation Goals (nonradionuclides)

\begin{tabular}{|c|c|c|c|c|c|c|c|c|c|}
\hline Chemical & CASRN & $\begin{array}{l}\text { Perme- } \\
\text { ability } \\
\text { Constant } \\
\text { (cm/hr) } \\
\text { [Kpl }\end{array}$ & $\begin{array}{c}\text { Volatil- } \\
\text { ization } \\
\text { Factor } \\
\left(\mathrm{m}^{3} / \mathrm{kg}\right) \\
{[\mathrm{VF}]}\end{array}$ & $\begin{array}{c}\text { Soil } \\
\text { Satura- } \\
\text { tion Conc. } \\
\text { (mg/kg) } \\
\text { [CSAT] }\end{array}$ & $\begin{array}{c}\text { Soil-to- } \\
\text { Plant } \\
\text { Uptake } \\
\left(\mathbf{B}_{\mathbf{v}}\right)\end{array}$ & $\begin{array}{c}\text { Soil-to- } \\
\text { Wet Plant } \\
\text { Uptake } \\
\left(B_{\mathrm{rm}}\right)\end{array}$ & $\begin{array}{c}\text { Bioaccum- } \\
\text { ulation } \\
\text { Factor for } \\
\text { Fish } \\
\text { (L/kg) } \\
\text { [BF] }\end{array}$ & $\begin{array}{c}\text { Biotransfer } \\
\text { Factor for } \\
\text { Beef } \\
\text { (day/kg) } \\
{\left[F_{\mathbf{B}}\right]}\end{array}$ & $\begin{array}{c}\text { Biotransfer } \\
\text { Factor for } \\
\text { Milk } \\
\text { (day/kg) } \\
{\left[\mathrm{F}_{\mathrm{w}}\right]}\end{array}$ \\
\hline ALAR & 001596-84-5 & & & & & & & & \\
\hline Acenaphthene & $000083-32-9$ & $2.5 \mathrm{E}-01$ & & & $1.2 \mathrm{E}-01$ & $2.5 \mathrm{E}-02$ & $1.1 \mathrm{E}+03$ & $5.0 \mathrm{E}-04$ & $1.6 \mathrm{E}-04$ \\
\hline Acenaphthylene & 000208-96-8 & $9.5 \mathrm{E}-02$ & & . & $2.7 \mathrm{E}-01$ & $5.5 \mathrm{E}-02$ & $3.8 \mathrm{E}+02$ & $1.3 \mathrm{E}-04$ & 4.0E-05 \\
\hline Acephate & $030560-19-1$ & $3.6 \mathrm{E}-05$ & & & $1.2 \mathrm{E}+02$ & $2.4 \mathrm{E}+01$ & $1.3 \mathrm{E}-01$ & $3.5 \mathrm{E}-09$ & $1.1 \mathrm{E}-09$ \\
\hline Acetaldehyde & 000075-07-0 & $7.2 \mathrm{E}-04$ & $6.0 \mathrm{E}+04$ & $4.9 \mathrm{E}+04$ & $5.1 \mathrm{E}+01$ & $1.0 \mathrm{E}+01$ & 4.0E-01 & $1.5 \mathrm{E}-08$ & 4.8E-09 \\
\hline Acetochlor & 034256-82-1 & & & & & & & & \\
\hline Acetone & 000067-64-1 & $5.7 \mathrm{E}-04$ & & & $5.2 \mathrm{E}+01$ & $1.1 \mathrm{E}+01$ & $3.9 \mathrm{E}-01$ & $1.4 \mathrm{E}-08$ & $4.5 \mathrm{E}-09$ \\
\hline Acetone Cyanohydrin & $000075-86-5$ & & & & & & & & \\
\hline Acetonitrile & 000075-05-8 & $6.1 \mathrm{E}-04$ & & & $6.0 \mathrm{E}+01$ & $1.2 \mathrm{E}+01$ & $3.2 \mathrm{E}-01$ & $1.1 \mathrm{E}-08$ & $3.6 \mathrm{E}-09$ \\
\hline Acetophenone & 000098-86-2 & $5.7 \mathrm{E}-03$ & & & $3.9 E+00$ & $8.0 \mathrm{E}-01$ & $1.2 \mathrm{E}+01$ & $1.3 \mathrm{E}-06$ & $4.0 \mathrm{E}-07$ \\
\hline Acrolein & $000107-02-8$ & $7.4 \mathrm{E}-04$ & $1.3 \mathrm{E}+04$ & $4.8 \mathrm{E}+03$ & $4.3 \mathrm{E}+01$ & $8.8 \mathrm{E}+00$ & $4.9 \mathrm{E}-01$ & $2.0 \mathrm{E}-08$ & $6.3 \mathrm{E}-09$ \\
\hline Acrylamide & $000079-06-1$ & $2.4 \mathrm{E}-04$ & & & $9.3 \mathrm{E}+01$ & $1.9 \mathrm{E}+01$ & $1.8 \mathrm{E}-01$ & $5.3 \mathrm{E}-09$ & $1.7 \mathrm{E}-09$ \\
\hline Acrylic Acid & $000079-10-7$ & $9.0 \mathrm{E}-04$ & & & $3.1 \mathrm{E}+01$ & $6.2 \mathrm{E}+00$ & $7.8 \mathrm{E}-01$ & $3.6 \mathrm{E}-08$ & $1.1 \mathrm{E}-08$ \\
\hline Acrylonitrile & $000107-13-1$ & $1.4 \mathrm{E}-03$ & $3.6 \mathrm{E}+03$ & $1.8 \mathrm{E}+03$ & $2.7 \mathrm{E}+01$ & $5.5 \mathrm{E}+00$ & $9.1 \mathrm{E}-01$ & $4.4 \mathrm{E}-08$ & $1.4 \mathrm{E}-08$ \\
\hline Alachlor & $015972-60-8$ & $4.9 \mathrm{E}-03$ & & & $7.9 \mathrm{E}-01$ & $1.6 \mathrm{E}-01$ & $9.4 \mathrm{E}+01$ & $2.0 \mathrm{E}-05$ & $6.3 \mathrm{E}-06$ \\
\hline Aldicarb & $000116-06-3$ & 8.4E-04 & & & $8.4 \mathrm{E}+00$ & $1.7 \mathrm{E}+00$ & $4.3 \mathrm{E}+00$ & $3.4 \mathrm{E}-07$ & $1.1 \mathrm{E}-07$ \\
\hline Aldicarb Sulfone & 001646-88-4 & & & & & & & & \\
\hline
\end{tabular}

Chemical-specific parameters used to calculate PRGs/nonradionuclides

Page 1 of 41 
Table 3.6b. (continued)

\begin{tabular}{|c|c|c|c|c|c|c|c|c|c|}
\hline Chemical & CASRN & $\begin{array}{c}\text { Perme- } \\
\text { ability } \\
\text { Constant } \\
\text { (cm/hr) } \\
{[\mathrm{Kp}]}\end{array}$ & $\begin{array}{l}\text { Volatil- } \\
\text { ization } \\
\text { Factor } \\
\left(\mathrm{m}^{3} / \mathrm{kg}\right) \\
{[\mathrm{VF}]}\end{array}$ & $\begin{array}{c}\text { Soil } \\
\text { Satura- } \\
\text { tion Conc. } \\
\text { (mg/kg) } \\
\text { [CSAT] }\end{array}$ & $\begin{array}{c}\text { Soil-to- } \\
\text { Plant } \\
\text { Uptake } \\
\left(B_{v}\right)\end{array}$ & $\begin{array}{c}\text { Soil-to- } \\
\text { Wet Plant } \\
\text { Uptake } \\
\left(B_{m}\right)\end{array}$ & $\begin{array}{c}\text { Bioaccum- } \\
\text { ulation } \\
\text { Factor for } \\
\text { Fish } \\
\text { (L/kg) } \\
\text { [BF] } \\
\end{array}$ & $\begin{array}{c}\text { Biotransfer } \\
\text { Factor for } \\
\text { Beef } \\
\text { (day/kg) } \\
{\left[F_{\mathrm{b}}\right]}\end{array}$ & $\begin{array}{c}\text { Biotransfer } \\
\text { Factor for } \\
\text { Milk } \\
\text { (day/kg) } \\
{\left[\mathrm{F}_{\mathrm{m}}\right]}\end{array}$ \\
\hline Aldrin & 000309-00-2 & $1.6 \mathrm{E}-03$ & & & $6.9 \mathrm{E}-01$ & $1.4 \mathrm{E}-01$ & $1.1 \mathrm{E}+02$ & $2.5 \mathrm{E}-05$ & $7.9 \mathrm{E}-06$ \\
\hline Ally & $074223-64-6$ & $9.2 \mathrm{E}-06$ & & & $3.7 \mathrm{E}+01$ & $7.6 \mathrm{E}+00$ & $6.0 \mathrm{E}-01$ & $2.6 \mathrm{E}-08$ & $8.2 \mathrm{E}-09$ \\
\hline Allyl Alcohol & $000107-18-6$ & $5.9 \mathrm{E}-04$ & & & $5.1 \mathrm{E}+01$ & $1.0 \mathrm{E}+01$ & $4.0 \mathrm{E}-01$ & $1.5 \mathrm{E}-08$ & 4.8E-09 \\
\hline Allyl Chloride & 000107-05-1 & $7.0 \mathrm{E}-03$ & $9.8 \mathrm{E}+03$ & $6.3 \mathrm{E}+02$ & $5.9 \mathrm{E}+00$ & $1.2 \mathrm{E}+00$ & $6.8 \mathrm{E}+00$ & $6.3 \mathrm{E}-07$ & $2.0 \mathrm{E}-07$ \\
\hline Aluminum & 007429-90-5 & $1.0 \mathrm{E}-03$ & & & $4.0 \mathrm{E}-03$ & $1.0 \mathrm{E}-03$ & & $1.5 \mathrm{E}-03$ & $2.0 \mathrm{E}-04$ \\
\hline Aluminum Phosphide & 020859-73-8 & $1.0 \mathrm{E}-03$ & & & & & & & \\
\hline Amdro & $067485-29-4$ & & & & & & & & \\
\hline Ametryn & $000834-12-8$ & 7.3E-04 & & & $3.4 \mathrm{E}+00$ & $7.0 \mathrm{E}-01$ & $1.4 \mathrm{E}+01$ & $1.6 \mathrm{E}-06$ & $5.0 \mathrm{E}-07$ \\
\hline Aminophenol, m- & $000591-27-5$ & $5.8 \mathrm{E}-04$ & & & $2.9 \mathrm{E}+01$ & $5.8 \mathrm{E}+00$ & $8.5 \mathrm{E}-01$ & 4.1E-08 & $1.3 \mathrm{E}-08$ \\
\hline Aminopyridine, 4- & 000504-24-5 & 8.0E-04 & & & $2.6 \mathrm{E}+01$ & $5.3 \mathrm{E}+00$ & $9.6 \mathrm{E}-01$ & $4.8 \mathrm{E}-08$ & $1.5 \mathrm{E}-08$ \\
\hline Amitraz & 033089-61-1 & & & & & & & & \\
\hline Ammonia & $007664-41-7$ & $1.5 \mathrm{E}-03$ & & & $3.8 \mathrm{E}+01$ & $7.7 \mathrm{E}+00$ & $5.9 \mathrm{E}-01$ & $2.5 \mathrm{E}-08$ & $7.9 \mathrm{E}-09$ \\
\hline Ammonium Sulfamate & 007773-06-0 & $1.0 \mathrm{E}-03$ & & & & & & & \\
\hline Aniline & $000062-53-3$ & 4.1E-02 & & & $1.1 \mathrm{E}+01$ & $2.3 \mathrm{E}+00$ & $2.8 \mathrm{E}+00$ & $2.0 \mathrm{E}-07$ & $6.3 \mathrm{E}-08$ \\
\hline Anthracene & $000120-12-7$ & $2.2 \mathrm{E}-01$ & & & $1.1 \mathrm{E}-01$ & $2.2 \mathrm{E}-02$ & $1.3 \mathrm{E}+03$ & $6.3 \mathrm{E}-04$ & $2.0 \mathrm{E}-04$ \\
\hline Antimony (metallic) & $007440-36-0$ & $1.0 \mathrm{E}-03$ & & & $5.0 \mathrm{E}-02$ & $1.0 \mathrm{E}-02$ & $1.0 \mathrm{E}+02$ & $4.0 \mathrm{E}-05$ & $2.5 \mathrm{E}-05$ \\
\hline Antimony Pentoxide & 001314-60-9 & $1.0 \mathrm{E}-03$ & & & & & & & \\
\hline
\end{tabular}




\begin{tabular}{|c|c|c|c|c|c|c|c|c|c|}
\hline & & & & & & & & $8-t I-8 L S 9 L 0$ & anssy \\
\hline & & & & & & & $\varepsilon 0^{-g} 0^{\circ} \mathrm{I}$ & t-IZ-ZEEIOO & solsoqsy \\
\hline & & & & & & & $\mathcal{E} 0-\mathrm{G} 0^{\circ} \mathrm{I}$ & {$[-2 \downarrow * \downarrow 8<<00$} & әU!sst \\
\hline \multirow[t]{2}{*}{ S0-30'9 } & $\varepsilon 0^{-} \Xi 0^{\circ} \tau$ & & $20-30^{\circ} I$ & $20-30 \%$ & & & $\varepsilon 0^{-30} 0^{\circ} \mathrm{I}$ & $\tau-8 \varepsilon-0 t+\angle 00$ & 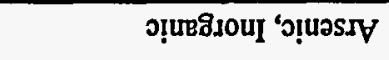 \\
\hline & & & & & & & $\varepsilon 0-30^{\circ} \mathrm{I}$ & $\forall N$ & SIIES ग!UวS.V \\
\hline $20-96.6$ & I0-ヨI' $\varepsilon$ & $S 0+Z S^{\prime} 1$ & $\$ 0-96 . \mathrm{s}$ & $\varepsilon 0-96 \cdot z$ & & & $00+a I \cdot I$ & $s=28-960110$ & (1)09ZI 100001V \\
\hline$\varepsilon 0-36 . \mathrm{L}$ & $20-\exists \varsigma^{\prime} Z$ & $t 0+81 \cdot z$ & $\varepsilon 0-\exists S^{\prime} \tau$ & Z0-aE'I & & & $10-\exists \varsigma^{\circ} \mathcal{E}$ & I-69-L60โ I0 & tSZI I0100.V \\
\hline $\mathfrak{E} 0-\mathrm{g} 00^{\circ} \mathrm{S}$ & $20-39 \cdot 1$ & $50+3 S^{\prime} l$ & $\varepsilon 0-\exists \varepsilon \varepsilon^{\prime} \varepsilon$ & $20-99 \cdot 1$ & & & $10-\exists L^{\circ} \varepsilon$ & $9-6 Z-Z \angle 9 Z 10$ & (1)8मZI sopoory \\
\hline $50-96 \cdot 6$ & $\not 0-\exists\left[I^{\circ} \varepsilon\right.$ & $20+7 L L$ & $20-\bar{a} \tau^{\circ} \varepsilon$ & $10-39 \cdot I$ & & & $z 0-\exists L^{\circ} \mathcal{E}$ & $6-1 Z-69+\varepsilon S 0$ & 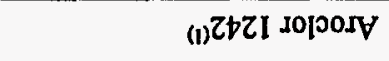 \\
\hline SO-ÄE'I & $50-30^{\circ} \mathrm{t}$ & $20+39^{\circ} \mathrm{I}$ & I0-ZII'I & $10-\exists E^{\prime} S$ & & & $20-a+1$ & $s-9 I-I t+1 L 0$ & (1) ZEZI دорољУ \\
\hline $50-96.6$ & $+0-\overline{G l} \mathcal{E}$ & $20+3 L \cdot L$ & $20-a \tau \cdot \varepsilon$ & $10-39 \cdot 1$ & & & $20-9 z^{\circ} 6$ & $z=8 z=601110$ & (1) IZZI Jo|josY \\
\hline \multirow[t]{6}{*}{$\varepsilon 0-\exists \varepsilon^{*} 9$} & $20-30^{\circ} \mathrm{Z}$ & $t 0+38^{\prime} I$ & $\varepsilon 0-\mathrm{g} 6 \cdot \tau$ & $20-a b 1$ & & & $10-96 . \mathrm{L}$ & $Z-I I-t<9 Z I 0$ & 9101 dopory \\
\hline & & & & & & & & $8-L S-0+1000$ & วฺ̣uerV \\
\hline & & & & & & & & $s-t Z$ sIItLO & ollody \\
\hline & & & & & & & $\varepsilon 0^{-}-90^{\circ} I$ & $t-t 9-60 \varepsilon 100$ & כp!Xo!IL Kuou!̣u \\
\hline & & & & & & & $\varepsilon 0-300^{\circ}$ & 9-โ8-ZEEI00 & 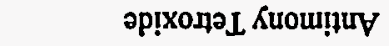 \\
\hline & & & & & & & $\varepsilon 0-\mathbf{g} 0$ I & $0-19-$ เ0E000 & 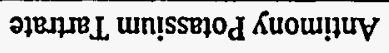 \\
\hline 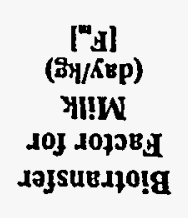 & 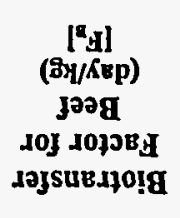 & 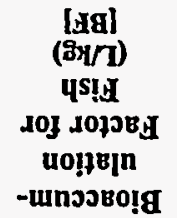 & 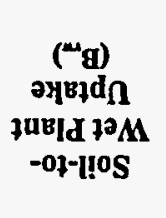 & 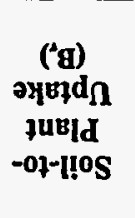 & 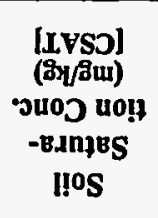 & 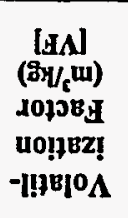 & 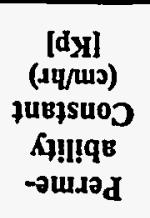 & N\&SVD & ןвอ!นวนว \\
\hline
\end{tabular}

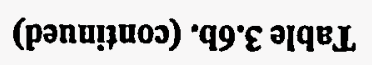


Table 3.6b. (continued)

\begin{tabular}{|c|c|c|c|c|c|c|c|c|c|}
\hline Chemical & CASRN & $\begin{array}{l}\text { Perme- } \\
\text { ability } \\
\text { Constant } \\
\text { (cm/hr) } \\
\text { [Kp] }\end{array}$ & $\begin{array}{l}\text { Volatil- } \\
\text { ization } \\
\text { Factor } \\
\left(\mathrm{m}^{3} / \mathrm{kg}\right) \\
\text { [VF] }\end{array}$ & $\begin{array}{c}\text { Soil } \\
\text { Satura- } \\
\text { tion Conc. } \\
\text { (mg/kg) } \\
\text { [CSAT] }\end{array}$ & $\begin{array}{c}\text { Soil-to- } \\
\text { Plant } \\
\text { Uptake } \\
\text { (Bs) }\end{array}$ & $\begin{array}{c}\text { Soil-to- } \\
\text { Wet Plant } \\
\text { Uptake } \\
\left(B_{m}\right)\end{array}$ & $\begin{array}{l}\text { Bioaccum- } \\
\text { ulation } \\
\text { Factor for } \\
\text { Fish } \\
\text { (L/kg) } \\
\text { [BF] }\end{array}$ & $\begin{array}{c}\text { Biotransfer } \\
\text { Factor for } \\
\text { Beef } \\
\text { (day/kg) } \\
{\left[F_{\mathrm{g}}\right]}\end{array}$ & $\begin{array}{c}\text { Biotransfer } \\
\text { Factor for } \\
\text { Milk } \\
\text { (day/kg) } \\
{\left[\mathrm{F}_{\mathrm{m}}\right]}\end{array}$ \\
\hline Asulam & $003337-71-1$ & & & & & & & & \\
\hline Atrazine & $001912-24-9$ & $7.6 \mathrm{E}-03$ & & & $1.0 \mathrm{E}+00$ & 2.1E-01 & $6.6 \mathrm{E}+01$ & $1.3 \mathrm{E}-05$ & $4.0 \mathrm{E}-06$ \\
\hline Avermectin B1 & $065195-55-3$ & & & & & & & & \\
\hline Azobenzene & 000103-33-3 & $7.4 \mathrm{E}-02$ & & & $2.4 \mathrm{E}-01$ & $4.8 \mathrm{E}-02$ & $4.5 \mathrm{E}+02$ & $1.6 \mathrm{E}-04$ & $5.0 \mathrm{E}-05$ \\
\hline Barium & $007440-39-3$ & $1.0 \mathrm{E}-03$ & & & $1.0 \mathrm{E}-01$ & $3.0 \mathrm{E}-03$ & $4.0 \mathrm{E}+00$ & $2.0 \mathrm{E}-04$ & 4.8E-04 \\
\hline Barium Cyanide & $000542-62-1$ & $1.0 \mathrm{E}-03$ & & & & & & & \\
\hline Baygon & $000114-26-1$ & $1.4 \mathrm{E}-03$ & & & $4.5 \mathrm{E}+00$ & $9.1 \mathrm{E}-01$ & $9.7 \mathrm{E}+00$ & $1.0 \mathrm{E}-06$ & 3.1E-07 \\
\hline Bayleton & $043121-43-3$ & $1.3 \mathrm{E}-03$ & & & $1.8 \mathrm{E}+00$ & $3.6 \mathrm{E}-01$ & $3.3 \mathrm{E}+01$ & $5.0 \mathrm{E}-06$ & $1.6 \mathrm{E}-06$ \\
\hline Baythroid & $068359-37-5$ & & & & & & & & \\
\hline Benefin & $001861-40-1$ & $2.9 \mathrm{E}-02$ & & & $8.8 \mathrm{E}-02$ & $1.8 \mathrm{E}-02$ & $1.7 E+03$ & 8.7E-04 & 2.7E-04 \\
\hline Benomyl & $017804-35-2$ & $1.4 \mathrm{E}-03$ & & & $1.8 \mathrm{E}+00$ & $3.6 \mathrm{E}-01$ & $3.3 E+01$ & $5.0 \mathrm{E}-06$ & $1.6 \mathrm{E}-06$ \\
\hline Bentazon & $025057-89-0$ & $3.9 \mathrm{E}-04$ & & & $8.7 \mathrm{E}+00$ & $1.8 \mathrm{E}+00$ & $4.0 \mathrm{E}+00$ & 3.1E-07 & $9.9 \mathrm{E}-08$ \\
\hline Benz[a]anthracene $e^{(2)}$ & $000056-55-3$ & 8.1E-01 & & & $1.9 \mathrm{E}-02$ & $3.8 \mathrm{E}-03$ & $1.3 E+04$ & $1.3 \mathrm{E}-02$ & $4.0 \mathrm{E}-03$ \\
\hline Benzaldehyde & $000100-52-7$ & $1.8 \mathrm{E}-02$ & & & $1.8 \mathrm{E}+00$ & $3.6 \mathrm{E}-01$ & $3.3 \mathrm{E}+01$ & 5.0E-06 & $1.6 \mathrm{E}-06$ \\
\hline Benzene & $000071-43-2$ & 2.1E-02 & $1.9 \mathrm{E}+04$ & $5.0 \mathrm{E}+02$ & $2.3 E+00$ & 4.7E-01 & $2.3 \mathrm{E}+01$ & 3.1E-06 & $9.9 \mathrm{E}-07$ \\
\hline Benzene Hexachloride & NA & & & . & & & & - & \\
\hline Benzene, Ethyldimethyl & NA & & & & & & & & \\
\hline
\end{tabular}


Table 3.6b. (continued)

\begin{tabular}{|c|c|c|c|c|c|c|c|c|c|}
\hline Chemical & CASRN & $\begin{array}{c}\text { Perme- } \\
\text { ability } \\
\text { Constant } \\
\text { (cm/hr) } \\
\text { [Kp] }\end{array}$ & $\begin{array}{l}\text { Volatil- } \\
\text { ization } \\
\text { Factor } \\
\left(\mathbf{m}^{3} / \mathbf{k g}\right) \\
{[\mathrm{VF}]}\end{array}$ & $\begin{array}{c}\text { Soil } \\
\text { Satura- } \\
\text { tion Conc. } \\
\text { (mg/kg) } \\
\text { [CSAT] }\end{array}$ & $\begin{array}{c}\text { Soil-to- } \\
\text { Plant } \\
\text { Uptake } \\
\text { (B, }\end{array}$ & $\begin{array}{c}\text { Soil-to- } \\
\text { Wet Plant } \\
\text { Uptake } \\
\text { (Bm) }\end{array}$ & $\begin{array}{l}\text { Bioaccum- } \\
\text { ulation } \\
\text { Factor for } \\
\text { Fish } \\
\text { (L/kg) } \\
\text { [BF] }\end{array}$ & $\begin{array}{c}\text { Biotransfer } \\
\text { Factor for } \\
\text { Beef } \\
\text { (day/kg) } \\
{\left[F_{\mathrm{g}}\right]}\end{array}$ & $\begin{array}{c}\text { Biotransfer } \\
\text { Factor for } \\
\text { Milk } \\
\text { (day/kg) } \\
\text { [F. }\end{array}$ \\
\hline Benzene, Ethylmethyl & NA & & & & & & & & \\
\hline Benzene, Methylpropenyl & NA & & & & & & & & \\
\hline Benzene, Methylpropyl & NA & & & & & & & & \\
\hline Benzene, Trimethyl & $025551-13-7$ & $9.1 \mathrm{E}-02$ & & & 4.1E-01 & $8.2 \mathrm{E}-02$ & $2.3 \mathrm{E}+02$ & $6.3 \mathrm{E}-05$ & $2.0 \mathrm{E}-05$ \\
\hline Benzenethiol & $000108-98-5$ & $2.4 \mathrm{E}-02$ & & & $1.3 \mathrm{E}+00$ & 2.7E-01 & $4.7 \mathrm{E}+01$ & 7.9E-06 & $2.5 \mathrm{E}-06$ \\
\hline Benzidine & $000092-87-5$ & $1.3 \mathrm{E}-03$ & & & $6.7 \mathrm{E}+00$ & $1.4 \mathrm{E}+00$ & $5.7 E+00$ & $5.0 \mathrm{E}-07$ & $1.6 \mathrm{E}-07$ \\
\hline Benzo[a]pyrene $e^{(2)}$ & $000050-32-8$ & $1.2 \mathrm{E}+00$ & & & $1.1 \mathrm{E}-02$ & $2.2 \mathrm{E}-03$ & $2.5 \mathrm{E}+04$ & 3.1E-02 & $9.9 \mathrm{E}-03$ \\
\hline Benzo[b]fluoranthene $e^{(2)}$ & $000205-99-2$ & $1.2 \mathrm{E}+00$ & & & $1.1 \mathrm{E}-02$ & $2.2 \mathrm{E}-03$ & $2.5 \mathrm{E}+04$ & $3.1 \mathrm{E}-02$ & $9.9 \mathrm{E}-03$ \\
\hline Benzo $[\mathrm{g}, \mathrm{h}, \mathrm{i}]$ perylene & $000191-24-2$ & $1.8 \mathrm{E}+00$ & & & $5.6 \mathrm{E}-03$ & $1.1 \mathrm{E}-03$ & $6.1 \mathrm{E}+04$ & $1.0 \mathrm{E}-01$ & 3.1E-02 \\
\hline Benzo[k]fluoranthene $\mathrm{e}^{(2)}$ & $000207-08-9$ & $6.0 \mathrm{E}-01$ & & & $4.3 \mathrm{E}-03$ & 8.8E-04 & $8.7 E+04$ & $1.6 \mathrm{E}-01$ & $5.0 \mathrm{E}-02$ \\
\hline Benzoic Acid & $000065-85-0$ & 7.3E-03 & & & $3.0 \mathrm{E}+00$ & $6.1 \mathrm{E}-01$ & $1.6 \mathrm{E}+01$ & $2.0 \mathrm{E}-06$ & $6.3 \mathrm{E}-07$ \\
\hline Benzotrichloride & $000098-07-7$ & $1.5 \mathrm{E}-02$ & & & $7.9 \mathrm{E}-01$ & $1.6 \mathrm{E}-01$ & $9.4 \mathrm{E}+01$ & $2.0 \mathrm{E}-05$ & $6.3 \mathrm{E}-06$ \\
\hline Benzyl Alcohol & $000100-51-6$ & $6.0 \mathrm{E}-03$ & & & $8.7 E+00$ & $1.8 E+00$ & $4.0 \mathrm{E}+00$ & $3.1 \mathrm{E}-07$ & $9.9 \mathrm{E}-08$ \\
\hline Benzyl Chloride & $000100-44-7$ & $1.4 \mathrm{E}-02$ & & & $1.8 \mathrm{E}+00$ & $3.6 \mathrm{E}-01$ & $3.3 \mathrm{E}+01$ & $5.0 \mathrm{E}-06$ & $1.6 \mathrm{E}-06$ \\
\hline Beryllium & $007440-41-7$ & $1.0 \mathrm{E}-03$ & & & $1.0 \mathrm{E}-02$ & $2.5 \mathrm{E}-03$ & $1.0 \mathrm{E}+02$ & $1.0 \mathrm{E}-03$ & $9.0 \mathrm{E}-07$ \\
\hline Bidrin & $000141-66-2$ & $2.2 \mathrm{E}-05$ & & & $9.4 \mathrm{E}+01$ & $1.9 \mathrm{E}+01$ & $1.8 \mathrm{E}-01$ & $5.2 \mathrm{E}-09$ & $1.7 \mathrm{E}-09$ \\
\hline Biphenthrin & $082657-04-3$ & & & & & & & & \\
\hline
\end{tabular}




\begin{tabular}{|c|c|c|c|c|c|c|c|c|c|}
\hline & & & & & & & & $\angle-Z 9-S \angle 0000$ & 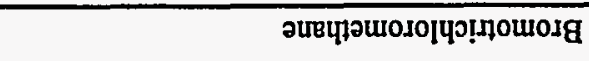 \\
\hline & & & & & & & & $\varepsilon-96-\downarrow 01200$ & soųdourosg \\
\hline$\angle 0-G E^{\prime} I$ & $\angle 0-90^{\circ}{ }^{\circ}$ & $00+38$ & $00+39 \cdot I$ & $00+3 L i$ & $20+97 \cdot \varepsilon$ & $\varepsilon 0+\exists \varepsilon^{\cdot} \varepsilon$ & $\varepsilon 0-\exists S^{\circ} \varepsilon$ & $6-\varepsilon 8-\downarrow<0000$ & 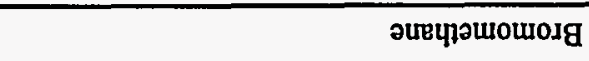 \\
\hline $90-80^{\circ} z$ & $90-\exists \varepsilon^{\prime} 9$ & $10+96 \varepsilon$ & $I 0-\mathrm{Gl} \varepsilon$ & $00+95 \cdot 1$ & & & $\varepsilon 0-a 9 \cdot z$ & $z-s z-s \angle 0000$ & wrojowosg \\
\hline $50-36.6$ & $\varepsilon 0-\exists I I^{*} \varepsilon$ & $\varepsilon 0+3 t b$ & $\varepsilon 0-9 \varsigma^{\prime} 8$ & $z 0-a z t$ & & & I0-ヨ๖て & $\varepsilon-\varsigma s-101000$ & 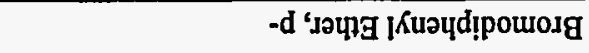 \\
\hline$\angle 0-36.6$ & $90-\mathrm{aI} \mathcal{} \varepsilon$ & $10+\exists E z$ & $10-3 L^{\prime} t$ & $00+9 \varepsilon z$ & & & $\mathcal{E} 0-98$ ' $\mathcal{S}$ & $t-\angle Z-\varsigma \angle 0000$ & 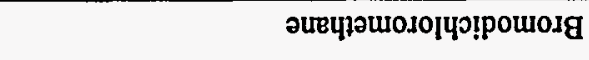 \\
\hline$\angle 0-90^{\circ} Z$ & $\angle 0-\exists \varepsilon^{*} 9$ & $00+98^{\prime} 9$ & $00+9 Z^{\prime} I$ & $00+36 \mathrm{~s}$ & & & $\varepsilon 0-\exists\left[{ }^{`} \mathcal{E}\right.$ & $s=\angle 6^{-} ヤ \angle 0000$ & 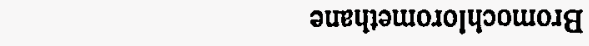 \\
\hline & & & & & & & $\varepsilon 0^{-} \mathrm{G} 0^{\circ} \mathrm{I}$ & $\tau-L 0-\angle E 9 L 00$ & 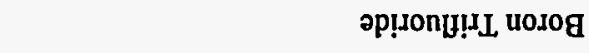 \\
\hline E0-As'I I & t0-30'8 & & $00+90.1$ & $00+90^{\circ} t$ & & & $\varepsilon 0-90 \%$ & $8-z t-0 t t<00$ & 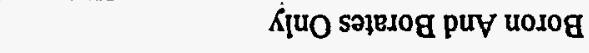 \\
\hline S0-aI' $\varepsilon$ & $\forall 0^{-}-\mathrm{G} 0^{\circ} \mathrm{I}$ & $z 0+A Z^{\prime} \mathcal{E}$ & $z 0-\exists \varepsilon^{*} 9$ & I0-gI' $\varepsilon$ & & & $z 0-98 \cdot \tau$ & L-S0-080000 & $\forall$ louryds!g \\
\hline $80-96 ! I$ & $80-80.9$ & $00+9 I \cdot I$ & $00+39 \%$ & $10+9 \varepsilon \tau$ & $\varepsilon 0+a t * 6$ & $\mathrm{SO}+\mathrm{GI} \cdot \mathrm{I}$ & $50-3 I^{\prime} L$ & $I-88-Z \pitchfork \varsigma 000$ & 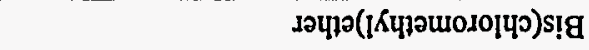 \\
\hline เ0-ฮદ'9 & $\varepsilon 0-\exists 0^{\circ} \tau$ & $\varepsilon 0+\exists I \cdot \varepsilon$ & $20-9 I \cdot I$ & Z0-gs's & & & $z 0-\exists \varepsilon z$ & $L-18-L I 1000$ & 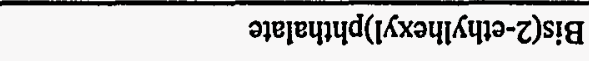 \\
\hline$\angle 0-966$ & $90-\mathrm{gI} \cdot \mathcal{E}$ & $10+9 \varepsilon z$ & $10-\mathrm{a} L^{\prime} \boldsymbol{t}$ & $00+a \varepsilon Z$ & & & $\varepsilon 0-\exists \varepsilon \subset \mathcal{S}$ & 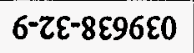 & 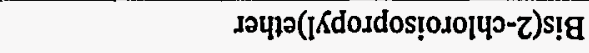 \\
\hline L0-G9'I & $\angle 0^{-30} \mathrm{~S}$ & $00+g L \cdot S$ & $00+9 b^{\circ} I$ & $00+3<\cdot 9$ & $20+36 \div 9$ & $S 0+38^{\circ} I$ & $\varepsilon 0-$ Gl'z & $t-t 6-111000$ & 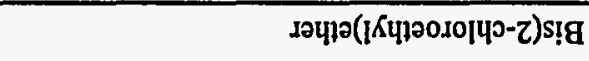 \\
\hline$\angle 0-399^{\circ} \mathrm{I}$ & $\angle 0^{-} \mathrm{-9} 0^{\circ} \mathrm{S}$ & $00+9 L ' S$ & $00+3+1$ & $00+3 L^{\prime} 9$ & & & $\varepsilon 0-\exists t^{\prime} I$ & I-I6-I I I000 & 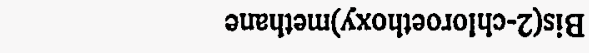 \\
\hline $90-30^{\circ} \varepsilon$ & $90-95 \% 6$ & $10+9 t s$ & I0-as'z & $00+\mathrm{g} \tau^{\prime} \mathrm{I}$ & $20+9 S^{\prime} \varepsilon$ & $S 0+3 b \cdot I$ & $20-3 Z^{\prime} \mathrm{I}$ & {$[-09-801000$} & 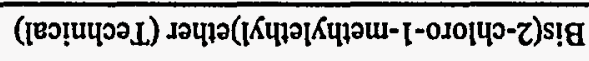 \\
\hline S0-96.6 & t0-al' $\mathcal{E}$ & $20+3 L \cdot L$ & $z 0-\exists \tau^{\prime} \mathcal{E}$ & $10-39 \cdot I$ & & & $I 0-98 \cdot I$ & $t-2 s-260000$ & 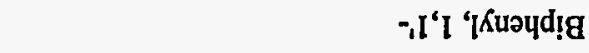 \\
\hline 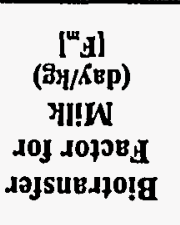 & 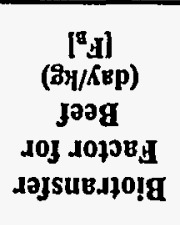 & 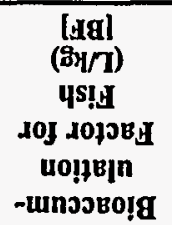 & 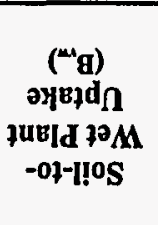 & $\begin{array}{c}\text { (Ag) } \\
\text { oytud } \\
\text { fut]d } \\
-0\}-1 ! 0 S\end{array}$ & 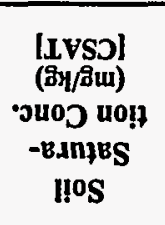 & 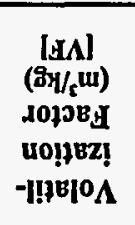 & $\begin{array}{c}\text { [dy] } \\
\text { (dq/uo) } \\
\text { fukjsuoJ } \\
\text { Sł!!!q8 } \\
\text {-əurdod }\end{array}$ & N\&SYO & ןвว!นщวчว \\
\hline
\end{tabular}

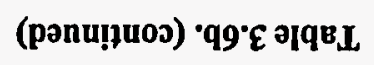


Table 3.6b. (continued)

\begin{tabular}{|c|c|c|c|c|c|c|c|c|c|}
\hline Chemical & CASRN & $\begin{array}{l}\text { Perme- } \\
\text { ability } \\
\text { Constant } \\
\text { (cm/hr) } \\
{[\mathrm{Kp}]}\end{array}$ & $\begin{array}{c}\text { Volatil- } \\
\text { ization } \\
\text { Factor } \\
\left(\mathrm{m}^{3} / \mathrm{kg}\right) \\
{[\text { [VF] }}\end{array}$ & $\begin{array}{c}\text { Soil } \\
\text { Satura- } \\
\text { tion Conc. } \\
\text { (mg/kg) } \\
{[\text { [CSAT] }}\end{array}$ & $\begin{array}{c}\text { Soil-to- } \\
\text { Plant } \\
\text { Uptake } \\
\left(B_{\text {, }}\right)\end{array}$ & $\begin{array}{c}\text { Soil-to- } \\
\text { Wet Plant } \\
\text { Uptake } \\
\left(B_{n m}\right)\end{array}$ & $\begin{array}{l}\text { Bioaccum- } \\
\text { ulation } \\
\text { Factor for } \\
\text { Fish } \\
\text { (L/kg) } \\
\text { [BF] }\end{array}$ & $\begin{array}{c}\text { Biotransfer } \\
\text { Factor for } \\
\text { Beef } \\
\text { (day/kg) } \\
{\left[\mathrm{F}_{\mathbf{g}}\right]}\end{array}$ & $\begin{array}{c}\text { Biotransfer } \\
\text { Factor for } \\
\text { Milk } \\
\text { (day/kg) } \\
\text { [F }\end{array}$ \\
\hline Bromoxynil & $001689-84-5$ & 8.7E-05 & & & $2.0 \mathrm{E}+01$ & $4.0 \mathrm{E}+00$ & $1.4 \mathrm{E}+00$ & 7.7E-08 & $2.4 \mathrm{E}-08$ \\
\hline Bromoxynil Octanoate & $001689-99-2$ & & & & & & & & \\
\hline Butadiene, 1,3- & $000106-99-0$ & 2.3E-02 & $6.1 \mathrm{E}+03$ & $3.2 \mathrm{E}+02$ & $2.6 \mathrm{E}+00$ & $5.3 \mathrm{E}-01$ & $1.9 \mathrm{E}+01$ & $2.5 \mathrm{E}-06$ & $7.9 \mathrm{E}-07$ \\
\hline Butanol, N- & $000071-36-3$ & $2.5 \mathrm{E}-03$ & & & $1.6 \mathrm{E}+01$ & $3.2 \mathrm{E}+00$ & $1.8 \mathrm{E}+00$ & 1.1E-07 & $3.5 \mathrm{E}-08$ \\
\hline Butanone-2, 4-chloro-4,4-difluoro & NA & & & & & & & & \\
\hline Butyl Benzyl Phthlate & $000085-68-7$ & $7.1 \mathrm{E}-02$ & & & $5.5 \mathrm{E}-02$ & $1.1 \mathrm{E}-02$ & $3.1 \mathrm{E}+03$ & 2.0E-03 & $6.3 \mathrm{E}-04$ \\
\hline Butylate & $002008-41-5$ & & & & & & & & \\
\hline Butylchloride, t- & $000507-20-0$ & & & & & & & & \\
\hline Butylphthalyl Butylglycolate & 000085-70-1 & & & & & & & & \\
\hline Cacodylic Acid & $000075-60-5$ & 2.7E-04 & & & $3.8 \mathrm{E}+01$ & $7.7 \mathrm{E}+00$ & $5.9 \mathrm{E}-01$ & $2.5 \mathrm{E}-08$ & $7.9 \mathrm{E}-09$ \\
\hline Cadmium (Diet) & 007440-43-9 & $1.0 \mathrm{E}-03$ & & & $5.5 \mathrm{E}-01$ & $1.4 \mathrm{E}-01$ & $2.0 \mathrm{E}+02$ & $4.0 \mathrm{E}-04$ & $1.0 \mathrm{E}-03$ \\
\hline Cadmium (Water) & $007440-43-9$ & $1.0 \mathrm{E}-03$ & & & $5.5 \mathrm{E}-01$ & $1.4 \mathrm{E}-01$ & $2.0 \mathrm{E}+02$ & $4.0 \mathrm{E}-04$ & $1.0 \mathrm{E}-03$ \\
\hline Calcium Cyanide & $000592-01-8$ & $1.0 \mathrm{E}-03$ & & & & & & & \\
\hline Caprolactam & $000105-60-2$ & $2.8 \mathrm{E}-04$ & & & $4.9 \mathrm{E}+01$ & $9.9 \mathrm{E}+00$ & $4.2 \mathrm{E}-01$ & $1.6 \mathrm{E}-08$ & 5.1E-09 \\
\hline Captafol & $002425-06-1$ & $8.6 \mathrm{E}-04$ & & & $1.3 \mathrm{E}+00$ & 2.7E-01 & $4.8 \mathrm{E}+01$ & $8.1 \mathrm{E}-06$ & 2.6E-06 \\
\hline Captan & $000133-06-2$ & $1.3 \mathrm{E}-03$ & & & $1.8 \mathrm{E}+00$ & $3.6 \mathrm{E}-01$ & $3.3 E+01$ & $5.0 \mathrm{E}-06$ & $1.6 \mathrm{E}-06$ \\
\hline Carbaryl & $000063-25-2$ & 4.8E-03 & & & $1.8 \mathrm{E}+00$ & $3.6 \mathrm{E}-01$ & $3.3 \mathrm{E}+01$ & $5.0 \mathrm{E}-06$ & $1.6 \mathrm{E}-06$ \\
\hline
\end{tabular}




\begin{tabular}{|c|c|c|c|c|c|c|c|c|c|}
\hline$\angle O^{-9} 0^{\circ} \mathrm{S}$ & $90-39^{\circ} \mathrm{I}$ & $10+37.1$ & $10-90^{\circ} \mathrm{L}$ & $00+9 b^{\prime} \varepsilon$ & $\varepsilon 0+g<\cdot I$ & $t 0+a \varepsilon$ & $20-90^{\circ} I$ & 8-66-9z1000 & 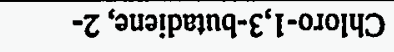 \\
\hline \multirow[t]{3}{*}{$\angle 0-\exists I{ }^{`} \varepsilon$} & $90-30 . I$ & $00+9 L^{\prime} 6$ & I0-9I'6 & $00+3 S$ & & & & $\varepsilon-89-\varsigma<0000$ & 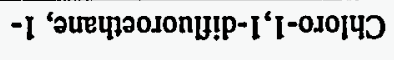 \\
\hline & & & & & & & $\mathcal{E} 0-90^{\circ} \mathrm{I}$ & $\tau-6 I-8 S L L 00$ & 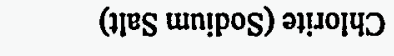 \\
\hline & & & & & & & & $t-t 0-6+0010$ & әp!xo!̣ әu!ฺ이 \\
\hline \multirow[t]{3}{*}{$20-3 L \cdot I$} & $20-30^{\circ} \tau$ & & $10+98 . I$ & $10+90^{\circ} \mathrm{L}$ & & & 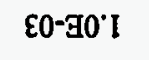 & $\mathcal{S}-0 S-Z 8 \angle L 00$ & วய!1이น \\
\hline & & & & & & & & $t-Z \varepsilon-286060$ & 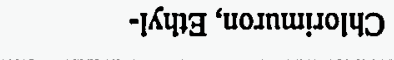 \\
\hline & & & & & & & $\varepsilon 0-90^{\circ} \mathrm{I}$ & $9-00-\angle 88910$ & әр!1익 \\
\hline \multirow[t]{4}{*}{$\varepsilon 0-\exists \varsigma^{\prime} \tau$} & $\varepsilon 0-96 . L$ & $\varepsilon 0+368$ & $\varepsilon 0^{-\mathrm{g} 0^{\circ} \mathrm{S}}$ & $20-9 s^{\prime} z$ & & & $z 0-g z \cdot s$ & $6-\nleftarrow L-L S 0000$ & әитрхорчว \\
\hline & & - & & & & & & $Z=S L=8 I 1000$ & โ!นसม이 \\
\hline & & & & & & & & $t-06-\varepsilon \varepsilon 1000$ & แəqurexo|นว \\
\hline & & & & & & & & $9-\angle 8-S \angle 0000$ & ๘ЕมОГนว \\
\hline \multirow[t]{2}{*}{$\angle 0-966$} & $90-\exists I I^{`} \varepsilon$ & $10+9 \varepsilon z$ & $10-3 L^{\prime} t$ & $00+\mathrm{A} \varepsilon z$ & & & $\varepsilon 0-\Xi \tau^{\prime} Z$ & $\downarrow-89-\downarrow \varepsilon Z S 00$ & u!xoqreכ \\
\hline & & & & & & & & $8-t I-s 8 Z S \varsigma 0$ & UE్f!nsoqIBD \\
\hline $90-30 . \mathrm{S}$ & S0-79'I & $10+96 . L$ & $I 0-98 \cdot I$ & $10-90 \% 6$ & $\varepsilon 0+30^{\circ} I$ & $70+361$ & $z 0-\overline{a z} z$ & $s-\varepsilon z-9 \$ 0000$ & 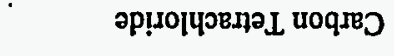 \\
\hline 90-घE'l & $90-90 \%$ & $10+9872$ & $10-3 I^{\circ}$ & $00+30^{\circ} z$ & $\varepsilon 0+9 L^{\prime} \mathfrak{I}$ & $10+902$ & $z 0-a t z$ & $0-S I-S \angle 0000$ & әрIyns! \\
\hline$L 0-\mathrm{GI} \varepsilon$ & $90-\mathrm{G0} \mathrm{I}$ & $00+3 L^{\prime} 6$ & I0-9I'6 & $00+9 S^{\prime} t$ & & & $\varepsilon 0-g Z$ I & Z-99-६9SI00 & ursnjoqges \\
\hline$s 0-90$ 's & to-39*I & $20+9 S^{\prime} t$ & $20-98^{\circ} t$ & $10-367 z$ & & & $20-\mathrm{al} 6$ & $8-t L-980000$ & ग0zвqIEว \\
\hline 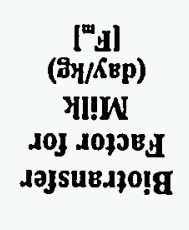 & 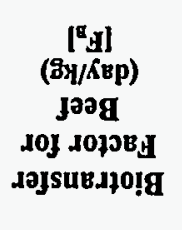 & 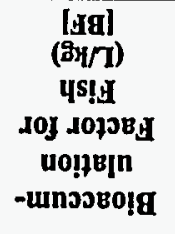 & 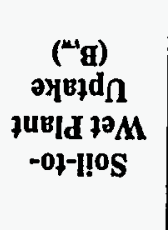 & 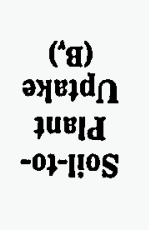 & 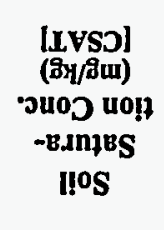 & 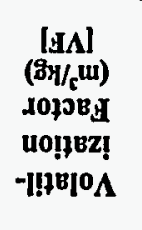 & 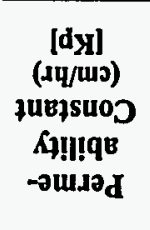 & NASWD & |вว!шวчว \\
\hline
\end{tabular}

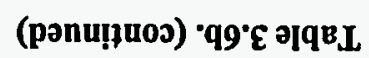


Table 3.6b. (continued)

\begin{tabular}{|c|c|c|c|c|c|c|c|c|c|}
\hline Chemical & CASRN & $\begin{array}{c}\text { Perme- } \\
\text { ability } \\
\text { Constant } \\
\text { (cm/hr) } \\
\text { [Kp] }\end{array}$ & $\begin{array}{l}\text { Volatil- } \\
\text { ization } \\
\text { Factor } \\
\left(\mathbf{m}^{3} / \mathbf{k g}\right) \\
{[\mathrm{VF}]}\end{array}$ & $\begin{array}{c}\text { Soil } \\
\text { Satura- } \\
\text { tion Conc. } \\
\text { (mg/kg) } \\
\text { [CSAT] }\end{array}$ & $\begin{array}{c}\text { Soil-to- } \\
\text { Plant } \\
\text { Uptake } \\
\text { (B) }\end{array}$ & $\begin{array}{c}\text { Soil-to- } \\
\text { Wet Plant } \\
\text { Uptake } \\
\text { (Bm) }\end{array}$ & $\begin{array}{l}\text { Bioaccum- } \\
\text { ulation } \\
\text { Factor for } \\
\text { Fish } \\
\text { (L/kg) } \\
\text { [BF] }\end{array}$ & $\begin{array}{c}\text { Biotransfer } \\
\text { Factor for } \\
\text { Beef } \\
\text { (day/kg) } \\
{\left[F_{\mathrm{a}}\right]}\end{array}$ & $\begin{array}{c}\text { Biotransfer } \\
\text { Factor for } \\
\text { Milk } \\
\text { (day/kg) } \\
{\left[\mathrm{F}_{\mathrm{w}}\right]}\end{array}$ \\
\hline Chloro-2-methylaniline $\mathrm{HCl}, 4-$ & $003165-93-3$ & & & & & & & & \\
\hline Chloro-2-methylaniline, 4- & $000095-69-2$ & & & & & & & & \\
\hline Chloroacetic Acid & $000079-11-8$ & $3.6 \mathrm{E}-03$ & & & $7.7 \mathrm{E}+00$ & $1.6 \mathrm{E}+00$ & $4.8 \mathrm{E}+00$ & 4.0E-07 & $1.3 \mathrm{E}-07$ \\
\hline Chloroacetophenone, 2- & $000532-27-4$ & $6.6 \mathrm{E}-03$ & & & $2.3 \mathrm{E}+00$ & 4.7E-01 & $2.3 \mathrm{E}+01$ & 3.1E-06 & $9.7 \mathrm{E}-07$ \\
\hline Chloroaniline, $\mathrm{p}$ - & $000106-47-8$ & $3.1 \mathrm{E}-02$ & & & $9.0 \mathrm{E}-01$ & $1.8 \mathrm{E}-01$ & $7.9 \mathrm{E}+01$ & $1.6 \mathrm{E}-05$ & $5.0 \mathrm{E}-06$ \\
\hline Chlorobenzene & $000108-90-7$ & 4.1E-02 & $4.9 \mathrm{E}+04$ & $5.1 \mathrm{E}+02$ & $9.0 \mathrm{E}-01$ & $1.8 \mathrm{E}-01$ & $7.9 \mathrm{E}+01$ & $1.6 \mathrm{E}-05$ & $5.0 \mathrm{E}-06$ \\
\hline Chlorobenzilate & $000510-15-6$ & 3.1E-02 & & & $9.3 \mathrm{E}-02$ & $1.9 \mathrm{E}-02$ & $1.5 \mathrm{E}+03$ & $7.9 \mathrm{E}-04$ & $2.5 \mathrm{E}-04$ \\
\hline Chlorobenzoic Acid, p- & $000074-11-3$ & $1.7 \mathrm{E}-02$ & & & $1.0 \mathrm{E}+00$ & 2.1E-01 & $6.6 \mathrm{E}+01$ & $1.3 \mathrm{E}-05$ & $4.0 \mathrm{E}-06$ \\
\hline Chlorobenzotrifluoride, 4- & $000098-56-6$ & $6.8 \mathrm{E}-02$ & & & $2.6 \mathrm{E}-01$ & $5.2 \mathrm{E}-02$ & 4.1E+02 & $1.4 \mathrm{E}-04$ & 4.3E-05 \\
\hline Chlorobutane, 1- & $000109-69-3$ & $3.6 \mathrm{E}-02$ & & & $1.2 \mathrm{E}+00$ & $2.4 \mathrm{E}-01$ & $5.6 \mathrm{E}+01$ & $1.0 \mathrm{E}-05$ & $3.1 \mathrm{E}-06$ \\
\hline Chlorobutane, 2- & $000078-86-4$ & & & & & & & & \\
\hline Chlorocyclopentadiene & $041851-50-7$ & & & & & & & & \\
\hline Chlorodibromoethane & $073506-94-2$ & & & & & & & & \\
\hline Chlorodifluoromethane & $000075-45-6$ & 3.3E-03 & & & $9.0 \mathrm{E}+00$ & $1.8 \mathrm{E}+00$ & $3.9 \mathrm{E}+00$ & 3.0E-07 & $9.5 \mathrm{E}-08$ \\
\hline Chloroform & $000067-66-3$ & $8.9 \mathrm{E}-03$ & $1.8 \mathrm{E}+04$ & $1.5 \mathrm{E}+03$ & $2.6 \mathrm{E}+00$ & 5.3E-01 & $1.9 \mathrm{E}+01$ & $2.5 \mathrm{E}-06$ & 7.9E-07 \\
\hline Chloromethane & $000074-87-3$ & $4.2 \mathrm{E}-03$ & $3.3 \mathrm{E}+03$ & $1.2 \mathrm{E}+03$ & $1.1 \mathrm{E}+01$ & $2.3 E+00$ & $2.9 \mathrm{E}+00$ & $2.0 \mathrm{E}-07$ & $6.4 \mathrm{E}-08$ \\
\hline Chloromethyl Methyl Ether & $000107-30-2$ & & & & & & & & \\
\hline
\end{tabular}


Table 3.6b. (continued)

\begin{tabular}{|c|c|c|c|c|c|c|c|c|c|}
\hline Chemical & CASRN & $\begin{array}{c}\text { Perme- } \\
\text { ability } \\
\text { Constant } \\
\text { (cm/hr) } \\
\text { [Kp] }\end{array}$ & $\begin{array}{c}\text { Volatil- } \\
\text { ization } \\
\text { Factor } \\
\left(\mathrm{m}^{3} / \mathrm{kg}\right) \\
{[\mathrm{VF}]}\end{array}$ & $\begin{array}{c}\text { Soil } \\
\text { Satura- } \\
\text { tion Conc. } \\
\text { (mg/kg) } \\
\text { [CSAT] }\end{array}$ & $\begin{array}{c}\text { Soil-to- } \\
\text { Plant } \\
\text { Uptake } \\
\left(B_{v}\right)\end{array}$ & $\begin{array}{c}\text { Soil-to- } \\
\text { Wet Plant } \\
\text { Uptake } \\
\left(B_{\mathrm{rm}}\right)\end{array}$ & $\begin{array}{l}\text { Bioaccum- } \\
\text { ulation } \\
\text { Factor for } \\
\text { Fish } \\
(\mathrm{L} / \mathrm{kg}) \\
{[\mathrm{BF}]}\end{array}$ & $\begin{array}{c}\text { Biotransfer } \\
\text { Factor for } \\
\text { Beef } \\
\text { (day/kg) } \\
{\left[F_{\mathbb{b}}\right]}\end{array}$ & $\begin{array}{c}\text { Biotransfer } \\
\text { Factor for } \\
\text { Milk } \\
\text { (day/kg) } \\
{\left[F_{\mathrm{m}}\right]}\end{array}$ \\
\hline Chloronaphthalene, Beta- & $000091-58-7$ & $1.6 \mathrm{E}-01$ & & & $1.6 \mathrm{E}-01$ & $3.2 \mathrm{E}-02$ & $7.7 \mathrm{E}+02$ & $3.1 \mathrm{E}-04$ & $9.9 \mathrm{E}-05$ \\
\hline Chloronitrobenzene, o- & $000088-73-3$ & $7.6 \mathrm{E}-03$ & & & $2.0 \mathrm{E}+00$ & $4.1 \mathrm{E}-01$ & $2.8 \mathrm{E}+01$ & $4.0 \mathrm{E}-06$ & $1.3 \mathrm{E}-06$ \\
\hline Chloronitrobenzene, $\mathrm{p}$ - & $000121-73-3$ & $1.1 \mathrm{E}-02$ & & & $1.5 \mathrm{E}+00$ & $3.1 \mathrm{E}-01$ & $3.9 \mathrm{E}+01$ & $6.3 \mathrm{E}-06$ & $2.0 \mathrm{E}-06$ \\
\hline Chlorophenol, 2- & $000095-57-8$ & 3.3E-02 & & & $2.0 \mathrm{E}+00$ & 4.1E-01 & $2.8 \mathrm{E}+01$ & $4.0 \mathrm{E}-06$ & $1.3 \mathrm{E}-06$ \\
\hline Chlorophenyl Methyl Sulfide, p- & $000123-09-1$ & & & & & & & & \\
\hline Chlorophenyl Methyl Sulfoxide & $000934-73-6$ & & & & & & & & \\
\hline Chloropropane, 2- & $000075-29-6$ & & $1.5 \mathrm{E}+04$ & $1.6 \mathrm{E}+03$ & & & & & \\
\hline Chlorothalonil & $001897-45-6$ & $2.5 \mathrm{E}-02$ & & & 2.1E-01 & $4.2 \mathrm{E}-02$ & $5.4 \mathrm{E}+02$ & $2.0 \mathrm{E}-04$ & $6.3 \mathrm{E}-05$ \\
\hline Chlorotoluene, o- & $000095-49-8$ & $8.4 \mathrm{E}-02$ & & & $4.1 \mathrm{E}-01$ & $8.2 \mathrm{E}-02$ & $2.3 \mathrm{E}+02$ & $6.3 \mathrm{E}-05$ & $2.0 \mathrm{E}-05$ \\
\hline Chlorpropham & $000101-21-3$ & $1.1 \mathrm{E}-02$ & & & $7.9 \mathrm{E}-01$ & $1.6 \mathrm{E}-01$ & $9.4 \mathrm{E}+\dot{0} 1$ & $2.0 \mathrm{E}-05$ & $6.3 \mathrm{E}-06$ \\
\hline Chlorpyrifos & $002921-88-2$ & $8.0 \mathrm{E}-02$ & & & $3.2 \mathrm{E}-02$ & $6.5 \mathrm{E}-03$ & $6.3 \mathrm{E}+03$ & $5.0 \mathrm{E}-03$ & $1.6 \mathrm{E}-03$ \\
\hline Chlorpyrifos Methyl & $005598-13-0$ & $1.3 \mathrm{E}-02$ & & & $1.2 \mathrm{E}-01$ & $2.5 \mathrm{E}-02$ & $1.1 \mathrm{E}+03$ & $5.0 \mathrm{E}-04$ & $1.6 \mathrm{E}-04$ \\
\hline Chlorsulfuron & $064902-72-3$ & $1.4 \mathrm{E}-06$ & & & $2.3 \mathrm{E}+02$ & $4.6 \mathrm{E}+01$ & $5.6 \mathrm{E}-02$ & $1.1 \mathrm{E}-09$ & $3.6 \mathrm{E}-10$ \\
\hline Chlorthiophos & $060238-56-4$ & & & & & & & & \\
\hline Chromium (III) (Insoluble Salts) & $016065-83-1$ & $1.0 \mathrm{E}-03$ & & & $4.0 \mathrm{E}-02$ & $1.0 \mathrm{E}-04$ & $2.0 \mathrm{E}+02$ & $9.0 \mathrm{E}-03$ & $1.0 \mathrm{E}-05$ \\
\hline Chromium (VI) & $018540-29-9$ & $1.0 \mathrm{E}-03$ & & & $4.0 \mathrm{E}-02$ & $1.0 \mathrm{E}-04$ & $2.0 \mathrm{E}+02$ & $9.0 \mathrm{E}-03$ & $1.0 \mathrm{E}-05$ \\
\hline Chromium Salts & NA & $1.0 \mathrm{E}-03$ & & & & & $2.0 \mathrm{E}+02$ & $9.0 \mathrm{E}-03$ & $1.0 \mathrm{E}-05$ \\
\hline
\end{tabular}


Table 3.6b. (continued)

\begin{tabular}{|c|c|c|c|c|c|c|c|c|c|}
\hline Chemical & CASRN & $\begin{array}{c}\text { Perme- } \\
\text { ability } \\
\text { Constant } \\
\text { (cm/hr) } \\
\text { [Kp] }\end{array}$ & $\begin{array}{c}\text { Volatil- } \\
\text { ization } \\
\text { Factor } \\
\left(\mathrm{m}^{3} / \mathrm{kg}\right) \\
{[\mathrm{VF}]}\end{array}$ & $\begin{array}{c}\text { Soil } \\
\text { Satura- } \\
\text { tion Conc. } \\
\text { (mg/kg) } \\
\text { [CSAT] }\end{array}$ & $\begin{array}{c}\text { Soil-to- } \\
\text { Plant } \\
\text { Uptake } \\
\left(B_{n}\right)\end{array}$ & $\begin{array}{c}\text { Soil-to- } \\
\text { Wet Plant } \\
\text { Uptake } \\
\left(B_{\text {na }}\right)\end{array}$ & $\begin{array}{c}\text { Bioaccum- } \\
\text { ulation } \\
\text { Factor for } \\
\text { Fish } \\
\text { (L/kg) } \\
\text { [BF] }\end{array}$ & $\begin{array}{c}\text { Biotransfer } \\
\text { Factor for } \\
\text { Beef } \\
\text { (day/kg) } \\
\left.\text { [F } F_{\mathrm{g}}\right]\end{array}$ & $\begin{array}{c}\text { Biotransfer } \\
\text { Factor for } \\
\text { Milk } \\
\text { (day/kg) } \\
\text { [F, }\end{array}$ \\
\hline Chrysene $^{(2)}$ & $000218-01-9$ & $8.1 \mathrm{E}-01$ & & & $1.9 \mathrm{E}-02$ & $3.8 \mathrm{E}-03$ & $1.3 \mathrm{E}+04$ & $1.3 \mathrm{E}-02$ & $4.0 \mathrm{E}-03$ \\
\hline Cobalt & $007440-48-4$ & $1.0 \mathrm{E}-03$ & & & $5.4 \mathrm{E}-02$ & 2.3E-02 & $3.0 \mathrm{E}+02$ & $1.0 \mathrm{E}-04$ & 7.0E-05 \\
\hline Coke Oven Emissions & $008007-45-2$ & $1.6 \mathrm{E}+00$ & & & $6.5 \mathrm{E}-03$ & $1.3 \mathrm{E}-03$ & $5.1 \mathrm{E}+04$ & $7.9 \mathrm{E}-02$ & $2.5 \mathrm{E}-02$ \\
\hline Copper & $007440-50-8$ & $1.0 \mathrm{E}-03$ & & & $8.0 \mathrm{E}-01$ & $8.0 \mathrm{E}-02$ & $2.0 \mathrm{E}+02$ & $9.0 \mathrm{E}-03$ & $1.5 \mathrm{E}-03$ \\
\hline Copper Cyanide & $000544-92-3$ & $1.0 \mathrm{E}-03$ & & , & & & & & \\
\hline Creosote & $008001-58-9$ & & & & & & & & \\
\hline Cresol, m- & $000108-39-4$ & $1.5 \mathrm{E}-02$ & & & $2.6 \mathrm{E}+00$ & $5.3 \mathrm{E}-01$ & $1.9 \mathrm{E}+01$ & $2.5 \mathrm{E}-06$ & $7.9 \mathrm{E}-07$ \\
\hline Cresol, 0- & $000095-48-7$ & $1.6 \mathrm{E}-02$ & & & $3.0 \mathrm{E}+00$ & $6.1 \mathrm{E}-01$ & $1.6 \mathrm{E}+01$ & $2.0 \mathrm{E}-06$ & $6.3 \mathrm{E}-07$ \\
\hline Cresol, p- & $000106-44-5$ & $1.8 \mathrm{E}-02$ & & & $3.0 \mathrm{E}+00$ & $6.1 \mathrm{E}-01$ & $1.6 \mathrm{E}+01$ & $2.0 \mathrm{E}-06$ & $6.3 \mathrm{E}-07$ \\
\hline Crotonaldehyde & $000123-73-9$ & $1.4 \mathrm{E}-02$ & & & $3.4 \mathrm{E}+00$ & $7.0 \mathrm{E}-01$ & $1.4 \mathrm{E}+01$ & $1.6 \mathrm{E}-06$ & $5.0 \mathrm{E}-07$ \\
\hline Cumene & $000098-82-8$ & $1.1 \mathrm{E}-01$ & $7.0 \mathrm{E}+04$ & $3.5 \mathrm{E}+02$ & $3.5 \mathrm{E}-01$ & $7.2 \mathrm{E}-02$ & $2.7 \mathrm{E}+02$ & 7.9E-05 & $2.5 \mathrm{E}-05$ \\
\hline Cyanazine & $021725-46-2$ & $2.4 \mathrm{E}-03$ & & & $2.0 \mathrm{E}+00$ & $4.0 \mathrm{E}-01$ & $2.9 \mathrm{E}+01$ & 4.1E-06 & $1.3 \mathrm{E}-06$ \\
\hline Cyanide (CN-) & $000057-12-5$ & $7.5 \mathrm{E}-03$ & & & $8.7 \mathrm{E}+00$ & $1.8 \mathrm{E}+00$ & $4.0 \mathrm{E}+00$ & 3.1E-07 & $9.9 \mathrm{E}-08$ \\
\hline Cyanogen & $000460-19-5$ & & & & & & & & \\
\hline Cyanogen Bromide & $000506-68-3$ & & & & & & & & \\
\hline Cyanogen Chloride & $000506-77-4$ & $1.1 \mathrm{E}-03$ & & & $2.9 \mathrm{E}+01$ & $5.9 \mathrm{E}+00$ & $8.4 \mathrm{E}-01$ & $4.0 \mathrm{E}-08$ & $1.3 \mathrm{E}-08$ \\
\hline Cyclohexane, 1,2,3,4,5-pentabromo-6-chloro- & $000087-84-3$ & & & & & & & & \\
\hline
\end{tabular}


It ร0 そI วภือd

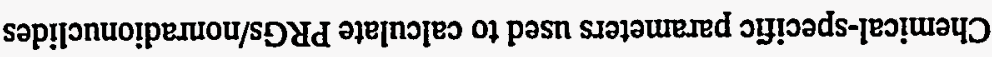

\begin{tabular}{|c|c|c|c|c|c|c|c|c|c|}
\hline $20-\mathrm{g} 0^{\circ} \mathrm{S}$ & I0-99. I & $10+3 L^{\prime} 8$ & t0-38.8 & $\varepsilon 0-\exists \varepsilon^{\prime} \downarrow$ & & & $00+3 L^{\prime} \tau$ & $\varepsilon-0 L-\varepsilon S 0000$ & 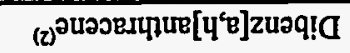 \\
\hline S0-G0's & $t 0-39 \cdot I$ & $20+9 s+t$ & $20-38.7$ & $10-3+2$ & & & Z0-ヨЕ'I & 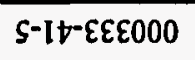 & uou!̣ze!d \\
\hline $80-3 z^{\circ} t$ & $\angle 0-\exists E^{\prime} I$ & $00+31 z$ & $00+36 z$ & $I 0+a t^{\circ} I$ & & & $t 0-\exists t \cdot I$ & $t-9 I-\varepsilon 0 \varepsilon Z 00$ & ગષ્|ા! \\
\hline \multirow[t]{2}{*}{$\mathrm{I} 0-30^{\circ} \mathrm{S}$} & $00+39 \cdot I$ & $\mathrm{~S} 0+\mathrm{g} 00^{\circ} \mathrm{S}$ & to-aE'z & E0-gI’I & & & $00+39^{\circ} \mathcal{E}$ & 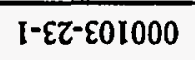 & 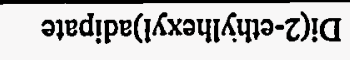 \\
\hline & & & & & & & & $\varepsilon-8 t-\varsigma 90800$ & นорәшад \\
\hline$\varepsilon 0-a b \cdot I$ & $\varepsilon 0-\exists \varepsilon^{\prime} \downarrow$ & $\varepsilon 0+\exists L ' s$ & $\varepsilon 0^{-}-\mathrm{G} 0^{\circ} \mathrm{L}$ & $z 0-\Xi \varsigma^{\prime} \varepsilon$ & & & SO-Gt'I & S-6I-E9I L00 & 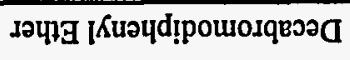 \\
\hline \multirow[t]{2}{*}{$80-\exists L^{\prime} b$} & 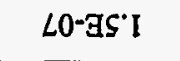 & $00+\exists \varepsilon z$ & $00+B L^{\prime} Z$ & $I 0+\exists \varepsilon \cdot I$ & & & to-al'6 & $0-66-\varsigma \angle 0000$ & uodeped \\
\hline & & & & & & & & $\mathrm{I}-Z \varepsilon-198 \mathrm{I} 00$ & гечртеव \\
\hline $20-98 \cdot I$ & $20-3 L \cdot S$ & $t 0+90 . t$ & $\varepsilon 0-$-g9`I & $\mathcal{E} 0-98^{\circ} \mathrm{L}$ & & & $10-9 E^{\prime \prime} t$ & $\varepsilon-6 Z-0 S 0000$ & Lad \\
\hline$\varepsilon 0-90^{\circ} \mathrm{t}$ & Z0-GE'I & tO+ $\mathrm{AE} 1$ & $\mathcal{E} 0-\bar{a} 8^{\circ} \varepsilon$ & $20-36 \cdot 1$ & & & $10-3 t^{2} z$ & $6-\varsigma S-Z L 0000$ & gad \\
\hline \multirow[t]{3}{*}{$\varepsilon 0^{-g} 0^{\circ} \mathrm{S}$} & $20-39 \cdot 1$ & $t 0+9 S I$ & $\mathcal{E} 0-\exists \varepsilon^{*} \varepsilon$ & $20-99 \cdot 1$ & & & $10-98 ' Z$ & $8-t s-Z<0000$ & $a \sigma a$ \\
\hline & & & & & & & & $8-L Z-S I Z 990$ & au!zeuodß刀 \\
\hline & & & & & & & & 8-LO-SIEZSO & 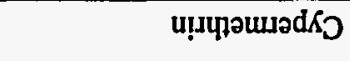 \\
\hline $20-\exists \varepsilon \cdot 9$ & $10-\exists 0^{\prime} z$ & $S 0+30 \% I$ & $+0-\mathrm{G} L L^{\circ} L$ & $\mathcal{E} 0-98^{\circ} \mathcal{E}$ & & & $10-\Xi L \cdot z$ & 8-\$8-\$80890 & गұегех/и!чиоречКЈ \\
\hline $90-39 \cdot 1$ & $90^{-}-30^{\circ} \mathrm{S}$ & $10+\exists \varepsilon^{\prime} \varepsilon$ & $10-39^{\circ} \varepsilon$ & $00+98^{\prime} I$ & & & $20-g z^{\prime} \varepsilon$ & $L-Z 6-Z \not S 000$ & 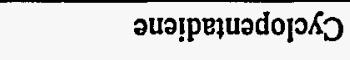 \\
\hline$\angle 0-a S^{\prime} Z$ & $\angle 0-96 \angle$ & $00+\operatorname{al} \cdot 8$ & $00+30.1$ & $00+a I^{\prime} s$ & & & $\varepsilon 0-\mathrm{gS}$ 'S & $8-16-801000$ & 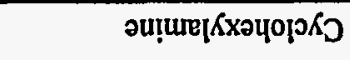 \\
\hline $80-\mathrm{sI}{ }^{\prime} \mathrm{S}$ & $\angle 0-399^{\circ} I$ & $00+3 t z$ & $00+39 \cdot 2$ & $10+\exists E \cdot I$ & & & $\varepsilon 0-388^{\prime I}$ & $\mathfrak{l}-\$ 6-801000$ & 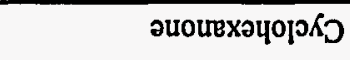 \\
\hline 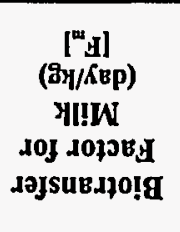 & 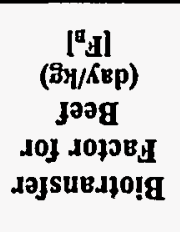 & 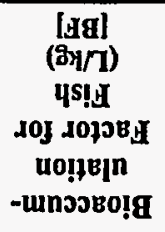 & 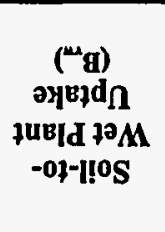 & $\begin{array}{c}\text { ('g) } \\
\text { oykzd } \\
\text { JuE|d } \\
-01-1 ! 0 S\end{array}$ & 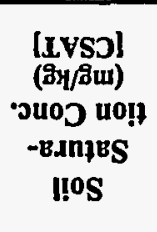 & 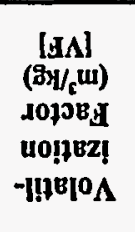 & 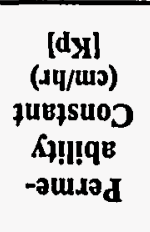 & N\&SVO & 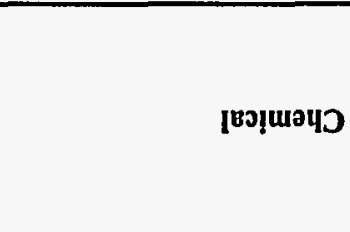 \\
\hline
\end{tabular}

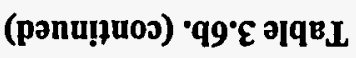


Table 3.6b. (continued)

\begin{tabular}{|c|c|c|c|c|c|c|c|c|c|}
\hline Chemical & CASRN & $\begin{array}{c}\text { Perme- } \\
\text { ability } \\
\text { Constant } \\
\text { (cm/hr) } \\
\text { [Kp] }\end{array}$ & $\begin{array}{l}\text { Volatil- } \\
\text { ization } \\
\text { Factor } \\
\left(\mathrm{m}^{3} / \mathrm{kg}\right) \\
{[\mathrm{VF}]}\end{array}$ & $\begin{array}{c}\text { Soil } \\
\text { Satura- } \\
\text { tion Conc. } \\
\text { (mg/kg) } \\
\text { [CSAT] }\end{array}$ & $\begin{array}{c}\text { Soil-to- } \\
\text { Plant } \\
\text { Uptake } \\
\left(B_{1}\right)\end{array}$ & $\begin{array}{c}\text { Soil-to- } \\
\text { Wet Plant } \\
\text { Uptake } \\
\left(B_{\text {rm }}\right)\end{array}$ & $\begin{array}{c}\text { Bioaccum- } \\
\text { ulation } \\
\text { Factor for } \\
\text { Fish } \\
\text { (L/kg) } \\
\text { [BF] }\end{array}$ & $\begin{array}{c}\text { Biotransfer } \\
\text { Factor for } \\
\text { Beef } \\
\text { (day/kg) } \\
{\left[\mathbf{F}_{\mathbf{y}}\right]}\end{array}$ & $\begin{array}{c}\text { Biotransfer } \\
\text { Factor for } \\
\text { Milk } \\
\text { (day/kg) } \\
\text { [F.] }\end{array}$ \\
\hline Dibromo-3-chloropropane, 1,2- & $000096-12-8$ & $4.8 \mathrm{E}-03$ & & & $1.2 \mathrm{E}+00$ & $2.4 \mathrm{E}-01$ & $5.6 \mathrm{E}+01$ & $1.0 \mathrm{E}-05$ & $3.1 \mathrm{E}-06$ \\
\hline Dibromobenzene, 1,4- & $000106-37-6$ & $3.5 \mathrm{E}-02$ & & & $2.4 \mathrm{E}-01$ & $4.8 \mathrm{E}-02$ & $4.5 \mathrm{E}+02$ & $1.6 \mathrm{E}-04$ & $5.0 \mathrm{E}-05$ \\
\hline Dibromochloromethane & $000124-48-1$ & $3.9 \mathrm{E}-03$ & & & $2.0 \mathrm{E}+00$ & 4.1E-01 & $2.8 \mathrm{E}+01$ & 4.0E-06 & $1.3 \mathrm{E}-06$ \\
\hline Dibromodichloromethane & $000594-18-3$ & & & & & & & & \\
\hline Dibromodiphenyl Ether, p,p'- & $002050-47-7$ & & & & & & & & \\
\hline Dibromoethane, 1,2- & $000106-93-4$ & 3.3E-03 & $1.3 \mathrm{E}+05$ & $5.5 \mathrm{E}+03$ & $2.6 \mathrm{E}+00$ & $5.3 \mathrm{E}-01$ & $1.9 \mathrm{E}+01$ & $2.5 \mathrm{E}-06$ & $7.9 \mathrm{E}-07$ \\
\hline Dibromomethane (Methylene Bromide) & $000074-95-3$ & $1.2 \mathrm{E}-03$ & & & $7.7 \mathrm{E}+00$ & $1.6 \mathrm{E}+00$ & $4.8 \mathrm{E}+00$ & 4.0E-07 & $1.3 \mathrm{E}-07$ \\
\hline Dibutyl Phthalate & $000084-74-2$ & $1.2 \mathrm{E}-01$ & & & $5.5 \mathrm{E}-02$ & $1.1 \mathrm{E}-02$ & $3.1 \mathrm{E}+03$ & $2.0 \mathrm{E}-03$ & $6.3 \mathrm{E}-04$ \\
\hline Dicamba & 001918-00-9 & $1.2 \mathrm{E}-02$ & & & $6.9 \mathrm{E}-01$ & $1.4 \mathrm{E}-01$ & $1.1 \mathrm{E}+02$ & $2.5 \mathrm{E}-05$ & $7.9 \mathrm{E}-06$ \\
\hline Dichloro-2-butene, 1,4- & $000764-41-0$ & & $1.3 \mathrm{E}+05$ & $3.9 \mathrm{E}+03$ & & & & & \\
\hline \multicolumn{10}{|l|}{ Dichloroacetic Acid } \\
\hline Dichlorobenzene, 1,2- & $000095-50-1$ & $6.1 \mathrm{E}-02$ & $1.6 \mathrm{E}+05$ & $5.1 \mathrm{E}+02$ & 4.1E-01 & $8.2 \mathrm{E}-02$ & $2.3 \mathrm{E}+02$ & $6.3 \mathrm{E}-05$ & $2.0 \mathrm{E}-05$ \\
\hline Dichlorobenzene, 1,3- & 000541-73-1 & $8.7 \mathrm{E}-02$ & & & 3.1E-01 & $6.3 \mathrm{E}-02$ & $3.2 \mathrm{E}+02$ & $1.0 \mathrm{E}-04$ & 3.1E-05 \\
\hline Dichlorobenzene, 1,4- & $000106-46-7$ & $6.2 \mathrm{E}-02$ & $4.0 \mathrm{E}+04$ & $3.2 \mathrm{E}+01$ & 4.1E-01 & $8.2 \mathrm{E}-02$ & $2.3 \mathrm{E}+02$ & $6.3 \mathrm{E}-05$ & $2.0 \mathrm{E}-05$ \\
\hline Dichlorobenzidine, 3,3'- & 000091-94-1 & $1.7 \mathrm{E}-02$ & & & 3.5E-01 & $7.2 \mathrm{E}-02$ & $2.7 \mathrm{E}+02$ & $7.9 \mathrm{E}-05$ & $2.5 \mathrm{E}-05$ \\
\hline Dichlorodifluoromethane & $000075-71-8$ & $1.2 \mathrm{E}-02$ & $1.1 \mathrm{E}+03$ & $1.0 \mathrm{E}+03$ & $2.0 \mathrm{E}+00$ & $4.1 \mathrm{E}-01$ & $2.8 \mathrm{E}+01$ & 4.0E-06 & $1.3 \mathrm{E}-06$ \\
\hline Dichloroethane, 1,1- & $000075-34-3$ & $8.9 \mathrm{E}-03$ & $1.1 \mathrm{E}+01$ & $1.4 \mathrm{E}+05$ & $3.4 \mathrm{E}+00$ & $7.0 \mathrm{E}-01$ & $1.4 \mathrm{E}+01$ & $1.6 \mathrm{E}-06$ & $5.0 \mathrm{E}-07$ \\
\hline
\end{tabular}


Table 3.6b. (continued)

\begin{tabular}{|c|c|c|c|c|c|c|c|c|c|}
\hline Chemical & CASRN & $\begin{array}{c}\text { Perme- } \\
\text { ability } \\
\text { Constant } \\
\text { (cm/hr) } \\
\text { [Kp] }\end{array}$ & $\begin{array}{l}\text { Volatil- } \\
\text { ization } \\
\text { Factor } \\
\left(\mathrm{m}^{3} / \mathrm{kg}\right) \\
{[\mathrm{VF}]}\end{array}$ & $\begin{array}{c}\text { Soil } \\
\text { Satura- } \\
\text { tion Conc. } \\
\text { (mg/kg) } \\
\text { [CSAT] }\end{array}$ & $\begin{array}{c}\text { Soil-to- } \\
\text { Plant } \\
\text { Uptake } \\
\text { (B) }\end{array}$ & $\begin{array}{c}\text { Soil-to- } \\
\text { Wet Plant } \\
\text { Uptake } \\
\left(B_{m}\right)\end{array}$ & $\begin{array}{l}\text { Bioaccum- } \\
\text { ulation } \\
\text { Factor for } \\
\text { Fish } \\
\text { (L/kg) } \\
\text { [BF] }\end{array}$ & $\begin{array}{c}\text { Biotransfer } \\
\text { Factor for } \\
\text { Beef } \\
\text { (day/kg) } \\
{\left[F_{\mathrm{v}}\right]}\end{array}$ & $\begin{array}{c}\text { Biotransfer } \\
\text { Factor for } \\
\text { Milk } \\
\text { (day/kg) } \\
{\left[\mathrm{F}_{\mathrm{m}}\right]}\end{array}$ \\
\hline Dichloroethane, 1,2- & $000107-06-2$ & $5.3 \mathrm{E}-03$ & $2.1 \mathrm{E}+04$ & $6.7 \mathrm{E}+02$ & $5.1 \mathrm{E}+00$ & $1.0 \mathrm{E}+00$ & $8.1 E+00$ & 7.9E-07 & 2.5E-07 \\
\hline Dichloroethylene, 1,1- & $000075-35-4$ & $8.9 \mathrm{E}-03$ & $6.3 E+03$ & $1.2 \mathrm{E}+03$ & $3.4 \mathrm{E}+00$ & $7.0 \mathrm{E}-01$ & $1.4 \mathrm{E}+01$ & $1.6 \mathrm{E}-06$ & $5.0 \mathrm{E}-07$ \\
\hline Dichloroethylene, 1,2-(Mixed Isomers) & 000540-59-0 & $1.1 \mathrm{E}-03$ & & & $2.0 \mathrm{E}+01$ & 4.1E+00 & $1.4 \mathrm{E}+00$ & 7.5E-08 & $2.4 \mathrm{E}-08$ \\
\hline Dichloroethylene, 1,2-cis- & $000156-59-2$ & $1.0 \mathrm{E}-02$ & & & $3.0 \mathrm{E}+00$ & $6.1 \mathrm{E}-01$ & $1.6 \mathrm{E}+01$ & $2.0 \mathrm{E}-06$ & $6.3 \mathrm{E}-07$ \\
\hline Dichloroethylene, 1,2-trans- & $000156-60-5$ & $1.1 \mathrm{E}-03$ & & & $2.0 \mathrm{E}+01$ & $4.1 E+00$ & $1.4 \mathrm{E}+00$ & 7.5E-08 & $2.4 \mathrm{E}-08$ \\
\hline Dichlorophenol, 2,4- & $000120-83-2$ & $6.0 \mathrm{E}-02$ & & & $7.9 \mathrm{E}-01$ & $1.6 \mathrm{E}-01$ & $9.4 \mathrm{E}+01$ & 2.0E-05 & $6.3 \mathrm{E}-06$ \\
\hline Dichlorophenoxy Acetic Acid, 2,4- & $000094-75-7$ & 5.1E-03 & & & $1.3 \mathrm{E}+00$ & 2.7E-01 & $4.7 \mathrm{E}+01$ & $7.9 \mathrm{E}-06$ & 2.5E-06 \\
\hline Dichlorophenoxy)butyric Acid, 4-(2,4- & $000094-82-6$ & $4.5 \mathrm{E}-03$ & & & $3.4 \mathrm{E}-01$ & $6.9 \mathrm{E}-02$ & $2.8 \mathrm{E}+02$ & $8.5 \mathrm{E}-05$ & 2.7E-05 \\
\hline Dichloropropane, 1,2- & $000078-87-5$ & $1.0 \mathrm{E}-02$ & $2.4 \mathrm{E}+04$ & $4.8 \mathrm{E}+02$ & $2.6 \mathrm{E}+00$ & 5.3E-01 & $1.9 \mathrm{E}+01$ & 2.5E-06 & 7.9E-07 \\
\hline Dichloropropanol, 2,3- & $000616-23-9$ & & & & & & & & \\
\hline Dichloropropene, 1,3- & $000542-75-6$ & $5.5 \mathrm{E}-03$ & $8.2 \mathrm{E}+04$ & $3.7 \mathrm{E}+03$ & $4.5 \mathrm{E}+00$ & 9.1E-01 & $9.7 \mathrm{E}+00$ & $1.0 \mathrm{E}-06$ & 3.1E-07 \\
\hline Dichlorvos & $000062-73-7$ & 9.5E-04 & & & $2.5 \mathrm{E}+00$ & $5.1 \mathrm{E}-01$ & $2.1 \mathrm{E}+01$ & 2.7E-06 & 8.5E-07 \\
\hline Dicofol & $000115-32-2$ & 2.3E-01 & & & $1.1 \mathrm{E}-02$ & $2.2 \mathrm{E}-03$ & $2.5 \mathrm{E}+04$ & $3.1 \mathrm{E}-02$ & $9.9 \mathrm{E}-03$ \\
\hline Dicyclopentadiene & $000077-73-6$ & $3.4 \mathrm{E}-02$ & $1.4 \mathrm{E}+05$ & $7.4 \mathrm{E}+02$ & $8.0 \mathrm{E}-01$ & $1.6 \mathrm{E}-01$ & $9.3 \mathrm{E}+01$ & $1.9 \mathrm{E}-05$ & $6.1 \mathrm{E}-06$ \\
\hline Dieldrin & $000060-57-1$ & $1.6 \mathrm{E}-02$ & & & $8.2 \mathrm{E}-02$ & $1.7 \mathrm{E}-02$ & $1.8 \mathrm{E}+03$ & $1.0 \mathrm{E}-03$ & 3.1E-04 \\
\hline Diethyl Phthalate & $000084-66-2$ & $4.8 \mathrm{E}-03$ & & & $1.3 \mathrm{E}+00$ & $2.7 \mathrm{E}-01$ & $4.7 E+01$ & $7.9 \mathrm{E}-06$ & 2.5E-06 \\
\hline Diethyl-p-nitrophenylphosphate & $000311-45-5$ & & & & & & & & \\
\hline
\end{tabular}


Table 3.6b. (continued)

\begin{tabular}{|c|c|c|c|c|c|c|c|c|c|}
\hline Chemical & CASRN & $\begin{array}{c}\text { Perme- } \\
\text { ability } \\
\text { Constant } \\
\text { (cm/hr) } \\
\text { [Kp] }\end{array}$ & $\begin{array}{c}\text { Volatil- } \\
\text { ization } \\
\text { Factor } \\
\left.\text { ( } \mathrm{m}^{3} / \mathrm{kg}\right) \\
{[\mathrm{VF}]}\end{array}$ & $\begin{array}{c}\text { Soil } \\
\text { Satura- } \\
\text { tion Conc. } \\
\text { (mg/kg) } \\
\text { [CSAT] }\end{array}$ & $\begin{array}{c}\text { Soil-to- } \\
\text { Plant } \\
\text { Uptake } \\
\left(B_{r}\right)\end{array}$ & $\begin{array}{c}\text { Soil-to- } \\
\text { Wet Plant } \\
\text { Uptake } \\
\left(B_{m m}\right)\end{array}$ & $\begin{array}{c}\text { Bioaccum- } \\
\text { ulation } \\
\text { Factor for } \\
\text { Fish } \\
\text { (L/kg) } \\
\text { [BF] } \\
\end{array}$ & $\begin{array}{c}\text { Biotransfer } \\
\text { Factor for } \\
\text { Beef } \\
\text { (day/kg) } \\
{\left[F_{3}\right]}\end{array}$ & $\begin{array}{c}\text { Biotransfer } \\
\text { Factor for } \\
\text { Milk } \\
\text { (day/kg) } \\
\text { [F }\end{array}$ \\
\hline Diethylene Glycol Dinitrate (DEGDN) & 000693-21-0 & & & & & & & & \\
\hline Diethylene Glycol Monobutyl Ether & $000112-34-5$ & 4.4E-05 & & & $1.3 \mathrm{E}+02$ & $2.6 \mathrm{E}+01$ & $1.2 \mathrm{E}-01$ & $3.0 \mathrm{E}-09$ & $9.5 \mathrm{E}-10$ \\
\hline Diethylene Glycol Monoethyl Ether & $000111-90-0$ & 2.5E-04 & & & $4.2 \mathrm{E}+01$ & $8.6 \mathrm{E}+00$ & $5.1 \mathrm{E}-01$ & 2.1E-08 & $6.6 \mathrm{E}-09$ \\
\hline Diethylformamide & $000617-84-5$ & & & & & & & & \\
\hline Diethylstilbesterol & $000056-53-1$ & 3.5E-01 & & & $2.5 \mathrm{E}-02$ & $5.0 \mathrm{E}-03$ & $8.9 \mathrm{E}+03$ & $7.9 \mathrm{E}-03$ & $2.5 \mathrm{E}-03$ \\
\hline Difenzoquat & $043222-48-6$ & & & & & & & & \\
\hline Diflubenzuron & $035367-38-5$ & & & & & & & & \\
\hline Difluoroethane, 1,1- & $000075-37-6$ & $2.6 \mathrm{E}-03$ & & & $1.4 \mathrm{E}+01$ & $2.8 \mathrm{E}+00$ & $2.2 \mathrm{E}+00$ & $1.4 \mathrm{E}-07$ & $4.4 \mathrm{E}-08$ \\
\hline Diisopropyl Methylphosphonate & 001445-75-6 & 8.2E-04 & & & $9.6 \mathrm{E}+00$ & $1.9 \mathrm{E}+00$ & $3.6 \mathrm{E}+00$ & 2.7E-07 & $8.5 \mathrm{E}-08$ \\
\hline Dimethipin & $055290-64-7$ & & & & & & & & \\
\hline Dimethoate & $000060-51-5$ & $6.3 \mathrm{E}-03$ & & & $1.0 \mathrm{E}+00$ & $2.1 \mathrm{E}-01$ & $6.6 \mathrm{E}+01$ & $1.3 \mathrm{E}-05$ & $4.0 \mathrm{E}-06$ \\
\hline Dimethoxybenzidine, 3,3'- & 000119-90-4 & $1.0 \mathrm{E}-03$ & & & $3.4 \mathrm{E}+00$ & $7.0 \mathrm{E}-01$ & $1.4 \mathrm{E}+01$ & $1.6 \mathrm{E}-06$ & $5.0 \mathrm{E}-07$ \\
\hline Dimethyl Sulfate & $000077-78-1$ & $2.2 \mathrm{E}-03$ & & & 7.7E+00 & $1.6 \mathrm{E}+00$ & $4.8 \mathrm{E}+00$ & $4.0 \mathrm{E}-07$ & $1.3 \mathrm{E}-07$ \\
\hline Dimethylaniline $\mathrm{HCl}, 2,4-$ & $021436-96-4$ & & & & & & & & \\
\hline Dimethylaniline, 2,4- & $000095-68-1$ & & & & & & & & \\
\hline Dimethylaniline, N,N- & $000121-69-7$ & $2.4 \mathrm{E}-02$ & & & $1.2 \mathrm{E}+00$ & $2.4 \mathrm{E}-01$ & $5.6 \mathrm{E}+01$ & $1.0 \mathrm{E}-05$ & $3.1 \mathrm{E}-06$ \\
\hline Dimethylbenzidine, 3,3'- & 000119-93-7 & 4.4E-03 & & & $1.8 \mathrm{E}+00$ & $3.6 \mathrm{E}-01$ & $3.3 \mathrm{E}+01$ & $5.0 \mathrm{E}-06$ & $1.6 \mathrm{E}-06$ \\
\hline
\end{tabular}




\begin{tabular}{|c|c|c|c|c|c|c|c|c|c|}
\hline$\angle 0-300^{\circ} \mathrm{B}$ & $90-\exists \varepsilon^{*} I$ & $\mathrm{I} 0+\mathrm{g} z \cdot \mathrm{I}$ & $10-90.8$ & $00+36 \varepsilon$ & & & $\varepsilon 0-3 \zeta Z$ & $\tau-0 z-909000$ & $-9^{\circ}$ 乙 'วนวก \\
\hline$L 0-96 . \mathrm{L}$ & $90-\mathrm{as} z$ & $10+961$ & I0-GE'S & $00+39 z$ & & & $\varepsilon 0-\bar{A} 8^{\circ} \varepsilon$ & $Z-カ I-I Z 1000$ & 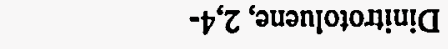 \\
\hline$\angle 0-A S Z \tau$ & $\angle 0-36^{\circ} \mathrm{L}$ & $00+91 \cdot 8$ & $00+301$ & $00+3 I \cdot S$ & & & $\varepsilon 0-\exists Z \varepsilon$ & $s-8 z-I S 0000$ & 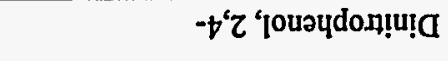 \\
\hline$\angle 0-\exists S Z$ & $\angle 0-36 \% \mathrm{~L}$ & $00+31 \cdot 8$ & $00+30 \cdot 1$ & $00+3 i \cdot s$ & & & $\varepsilon 0-\exists I ' Z$ & $t-s Z-001000$ & -†'I 'วนวzนวgод!u! \\
\hline$\angle 0-\exists I \cdot \varepsilon$ & $90-30.1$ & $00+3<\cdot 6$ & I0-9l'6 & $00+\mathrm{As} " \mathrm{t}$ & & & $\varepsilon 0-a+z$ & $0-\$ 9-660000$ & 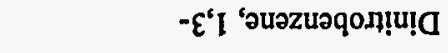 \\
\hline$\angle 0^{-} \exists I^{\circ} \mathcal{E}$ & $90-90 \div I$ & $00+3 L^{\prime} 6$ & $10-916$ & $00+9 S^{\prime} t$ & & & $\varepsilon 0-\exists S^{\prime} z$ & $0-6 z-8 z 5000$ & $-\tau^{\prime}$ เ 'วuวzuวqод!u! \\
\hline$I I-39 \cdot I$ & II- 100 S & $\varepsilon 0-\exists z ' \varsigma$ & $20+98 \cdot z$ & $\varepsilon 0+3 t \cdot I$ & & & $\angle 0-\mathrm{a} S \mathrm{~S}$ & S-68-IEI000 & 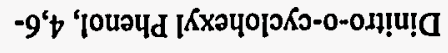 \\
\hline $90-30^{\circ} t$ & SO-GE'I & $10+399^{9}$ & $10-g 1 ' z$ & $00+90^{\circ} I$ & & & $\varepsilon 0-78^{\circ} 6$ & $I-Z S-\downarrow \varepsilon S 000$ & 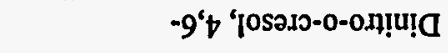 \\
\hline $90-96^{\circ} \mathrm{L}$ & SO-AS'Z & $20+3 I \cdot I$ & $10-a t+1$ & $10-369$ & & & & 9-19-0Z1000 & 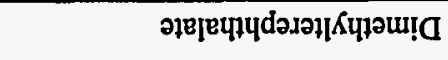 \\
\hline$L 0-G I^{\prime} \varepsilon$ & $90-90^{\circ} I$ & $00+3<\cdot 6$ & $10-91 \cdot 6$ & $00+9 s^{\prime} t$ & & & $\varepsilon 0-39 \cdot I$ & $\varepsilon=I I-I E I 000$ & 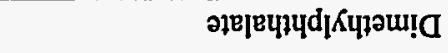 \\
\hline $90-\exists \varepsilon^{*} I$ & $90-30^{\circ} \mathrm{t}$ & $10+982$ & $10-\mathrm{al} b$ & $00+30 r$ & & & $20-30.6$ & $8-\varsigma 9-\$ 60000$ & 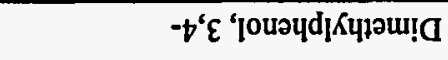 \\
\hline $90-78 \cdot I$ & $90-\exists L \cdot S$ & $10+\exists L^{\circ} \varepsilon$ & $10-\exists \varepsilon \_\varepsilon$ & $00+39 \cdot I$ & & & 20-89. I & $\mathfrak{I}-9 Z-9<5000$ & 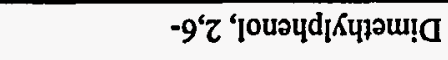 \\
\hline \multirow[t]{3}{*}{$90-39 \cdot 1$} & $90-30^{\circ} \mathrm{S}$ & $10+9 \varepsilon^{\circ} \varepsilon$ & I0- $99^{\circ} \varepsilon$ & $00+98 \cdot 1$ & & & I0-aI’I & 6-L9-501000 & 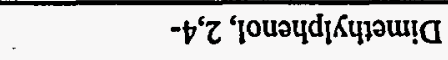 \\
\hline & & & & & & & & $8-\varepsilon L-0 b S 000$ & 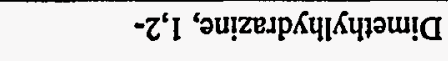 \\
\hline & & & & . & & & & $L-t I-L S 0000$ & 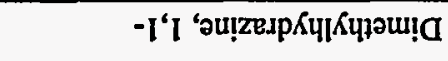 \\
\hline \multirow[t]{2}{*}{$0 I-366^{\circ} L$} & $60-9 S^{\prime} Z$ & I0-G0*I & $10+36 z$ & $20+a b^{\prime} I$ & & & $t 0-\exists \varepsilon \varepsilon^{-I}$ & $z-2 l-890000$ & 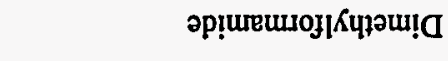 \\
\hline & & & & & & & & $\angle-0 t-t 8 S \angle 0 I$ & 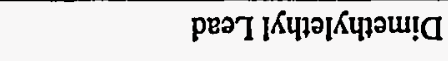 \\
\hline 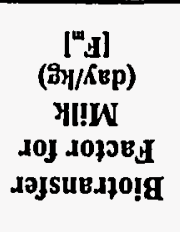 & 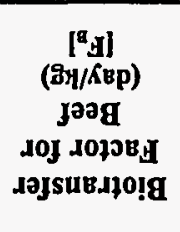 & 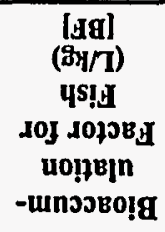 & 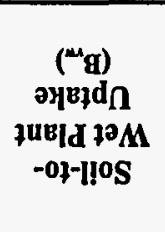 & 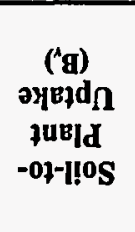 & 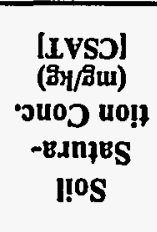 & 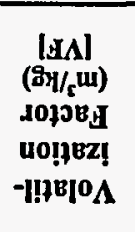 & 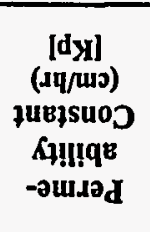 & N\&SWD & 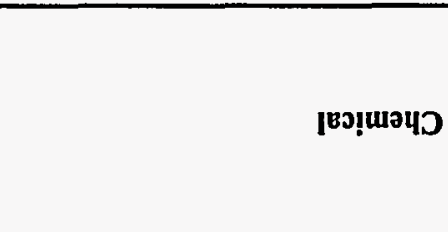 \\
\hline
\end{tabular}

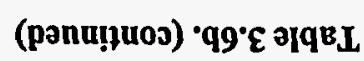




\begin{tabular}{|c|c|c|c|c|c|c|c|c|c|}
\hline & & & & & & & & $\varepsilon-\varepsilon L-s \neq 1000$ & ाечрория \\
\hline & & . & & & & & & $L-6 Z-\varsigma 11000$ & uej|nsopug \\
\hline & & & & & & & & $t-t 6-6 S L 000$ & OId \\
\hline & & & & & & & & $\varepsilon-0 l-6 \varepsilon+Z 00$ & әu!pod \\
\hline $90-30 \%$ & SO-GE'I & $10+39 \cdot 9$ & I0-घI'Z & $00+90^{\circ} \mathrm{I}$ & & & $\varepsilon 0-30 \cdot 9$ & $I-t s-0 \varepsilon \varepsilon 000$ & uodn!a \\
\hline \multirow[t]{5}{*}{$S 0-36^{\circ} \mathrm{L}$} & $+0-a s \cdot z$ & $20+a s=9$ & $Z 0^{-\Xi} \Omega L^{\prime} \mathcal{E}$ & $10-981$ & & & $20-38^{\prime} z$ & $t-50-862000$ & uoloj|ns!d \\
\hline & & & & & & & . & I-\$0-019Z00 & 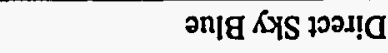 \\
\hline & & & & & & & & 9-98-I I 09I0 & S6 uм0دg pod!ם \\
\hline & & & & & & & & $z-9 t-209200$ & 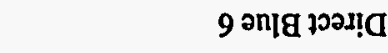 \\
\hline & & & & & & & & L-LE-LE6100 & 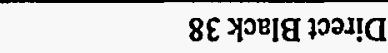 \\
\hline$Z I-G 0^{\circ} \mathrm{L}$ & $\mathrm{II}-\mathrm{az} z$ & $\varepsilon 0-388^{\prime} z$ & $20+3 S^{\prime \prime} t$ & $\varepsilon 0+\mathrm{g} z \bar{z}$ & & & $\angle 0^{-3} 0^{\circ} I$ & $L-00-\$ 80000$ & zenb!̣a \\
\hline $90-3 \varepsilon^{\prime} 9$ & S0-90'Z & $10+3+6$ & $10-39^{\circ} \mathrm{I}$ & $10-96 . L$ & & & $20-38 . I$ & L-99-ZZ1000 & 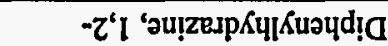 \\
\hline SO-GS'Z & S0-36 L & $20+a<\cdot z$ & $z 0-\exists z^{\prime} L$ & 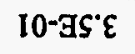 & & & $20-30 \cdot s$ & t-6£-ZZ1000 & oụure[Ruą̧d!̣ \\
\hline $90-\exists Z^{\prime} I$ & $90-\exists L^{\circ} \varepsilon$ & $10+399^{\prime} 2$ & $I 0-\exists z^{\prime} \downarrow$ & $00+g 1 \cdot z$ & & & $\varepsilon 0-\overline{\varepsilon \varepsilon} \tau$ & $L-I S-L S 6000$ & p!wreuəud!a \\
\hline $60-3 z z^{2}$ & $80-\exists \varepsilon^{\prime} I$ & $I 0-\mathrm{a} L^{\prime} \mathcal{E}$ & $10+3 I^{\prime} I$ & $10+3 t s$ & & & $50-30 \%$ & $\mathfrak{I}-\mathbb{I} 6-\mathcal{E Z I 0 0 0}$ & - t' I 'auexo!d \\
\hline \multirow[t]{2}{*}{$50-30^{\circ} \mathrm{t}$} & $\forall 0-\exists \varepsilon=I$ & $20+38^{\circ} \varepsilon$ & $z 0-\mathrm{GS} S$ & $10-\exists L \cdot 2$ & & & zo-38'z & L-\$8-880000 & qəsou!d \\
\hline & & & & & & & & $Z-8 L-Z L S S E O$ & -9'b-ou!ury-z 'auənjojon!u!a \\
\hline 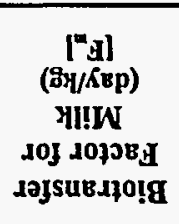 & 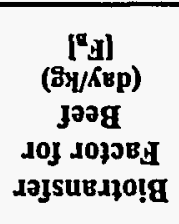 & 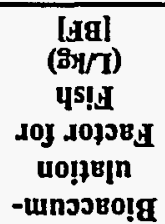 & 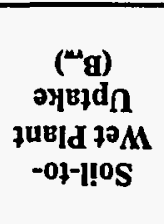 & 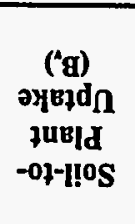 & 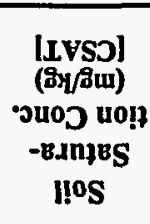 & 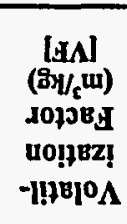 & 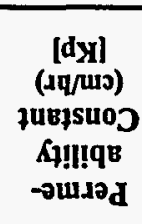 & NQSWD & 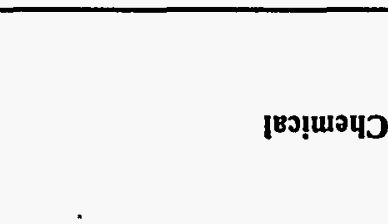 \\
\hline
\end{tabular}

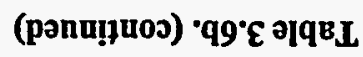


Table 3.6b. (continued)

\begin{tabular}{|c|c|c|c|c|c|c|c|c|c|}
\hline Chemical & CASRN & $\begin{array}{c}\text { Perme- } \\
\text { ability } \\
\text { Constant } \\
\text { (cm/hr) } \\
{[\mathrm{Kpl}]}\end{array}$ & $\begin{array}{l}\text { Volatil- } \\
\text { ization } \\
\text { Factor } \\
\left(\mathrm{m}^{3} / \mathrm{kg}\right) \\
{[\mathrm{VF}]}\end{array}$ & $\begin{array}{c}\text { Soil } \\
\text { Satura- } \\
\text { tion Conc. } \\
\text { (mg/kg) } \\
\text { [CSAT] }\end{array}$ & $\begin{array}{c}\text { Soil-to- } \\
\text { Plant } \\
\text { Uptake } \\
\left(B_{v}\right)\end{array}$ & $\begin{array}{c}\text { Soil-to- } \\
\text { Wet Plant } \\
\text { Uptake } \\
\text { (B B })\end{array}$ & $\begin{array}{c}\text { Bioaccum- } \\
\text { ulation } \\
\text { Factor for } \\
\text { Fish } \\
\text { (L/kg) } \\
\text { [BF] }\end{array}$ & $\begin{array}{c}\text { Biotransfer } \\
\text { Factor for } \\
\text { Beef } \\
\text { (day/kg) } \\
{\left[F_{\mathrm{g}}\right]}\end{array}$ & $\begin{array}{c}\text { Biotransfer } \\
\text { Factor for } \\
\text { Milk } \\
\text { (day/kg) } \\
{\left[\mathrm{F}_{m}\right]}\end{array}$ \\
\hline Endrin & $000072-20-8$ & $1.6 \mathrm{E}-02$ & & & $8.2 \mathrm{E}-02$ & $1.7 \mathrm{E}-02$ & $1.8 \mathrm{E}+03$ & $1.0 \mathrm{E}-03$ & $3.1 \mathrm{E}-04$ \\
\hline Epichlorohydrin & 000106-89-8 & 3.7E-04 & $9.5 \mathrm{E}+04$ & $3.2 \mathrm{E}+03$ & $5.0 \mathrm{E}+01$ & $1.0 \mathrm{E}+01$ & 4.1E-01 & $1.5 \mathrm{E}-08$ & $4.9 \mathrm{E}-09$ \\
\hline Epoxybutane, 1,2- & $000106-88-7$ & & & & & & & & \\
\hline Ethephon & $016672-87-0$ & & & & & & & & \\
\hline Ethion & $000563-12-2$ & $3.4 \mathrm{E}-02$ & & & $4.3 \mathrm{E}-02$ & $8.8 \mathrm{E}-03$ & $4.2 \mathrm{E}+03$ & $3.0 \mathrm{E}-03$ & 9.3E-04 \\
\hline Ethoxyethanol Acetate, 2- & 000111-15-9 & $8.6 \mathrm{E}-04$ & & & $1.6 \mathrm{E}+01$ & $3.2 \mathrm{E}+00$ & $1.8 \mathrm{E}+00$ & $1.1 \mathrm{E}-07$ & $3.5 \mathrm{E}-08$ \\
\hline Ethoxyethanol, 2- & 000110-80-5 & $3.0 \mathrm{E}-04$ & & & $4.3 \mathrm{E}+01$ & $8.8 \mathrm{E}+00$ & $4.9 \mathrm{E}-01$ & $2.0 \mathrm{E}-08$ & $6.3 \mathrm{E}-09$ \\
\hline Ethyl Acetate & $000141-78-6$ & $2.1 \mathrm{E}-03$ & & & $1.3 \mathrm{E}+01$ & $2.6 \mathrm{E}+00$ & $2.4 \mathrm{E}+00$ & $1.6 \mathrm{E}-07$ & $5.1 \mathrm{E}-08$ \\
\hline Ethyl Acrylate & 000140-88-5 & $4.0 \mathrm{E}-03$ & & & $6.7 \mathrm{E}+00$ & $1.4 \mathrm{E}+00$ & $5.7 \mathrm{E}+00$ & $5.0 \mathrm{E}-07$ & $1.6 \mathrm{E}-07$ \\
\hline Ethyl Chloride & $000075-00-3$ & $8.0 \mathrm{E}-03$ & $1.9 \mathrm{E}+03$ & $1.5 \mathrm{E}+02$ & $5.9 \mathrm{E}+00$ & $1.2 \mathrm{E}+00$ & $6.8 \mathrm{E}+00$ & $6.3 \mathrm{E}-07$ & $2.0 \mathrm{E}-07$ \\
\hline Ethyl Ether & 000060-29-7 & $1.7 \mathrm{E}-02$ & & & $1.2 \mathrm{E}+01$ & $2.3 \mathrm{E}+00$ & $2.8 \mathrm{E}+00$ & $1.9 \mathrm{E}-07$ & $6.1 \mathrm{E}-08$ \\
\hline Ethyl Methacrylate & $000097-63-2$ & $8.6 \mathrm{E}-03$ & & & $3.0 \mathrm{E}+00$ & $6.1 \mathrm{E}-01$ & $1.6 \mathrm{E}+01$ & $2.0 \mathrm{E}-06$ & $6.3 \mathrm{E}-07$ \\
\hline Ethyl-p-nitrophenyl Phosphonate & $002104-64-5$ & $1.0 \mathrm{E}-02$ & & & $2.4 \mathrm{E}-01$ & $4.8 \mathrm{E}-02$ & $4.5 \mathrm{E}+02$ & $1.6 \mathrm{E}-04$ & $5.0 \mathrm{E}-05$ \\
\hline Ethylbenzene & $000100-41-4$ & 7.4E-02 & $2.1 E+04$ & $4.7 \mathrm{E}-01$ & $6.1 \mathrm{E}-01$ & $1.2 \mathrm{E}-01$ & $1.3 \mathrm{E}+02$ & 3.1E-05 & $9.9 \mathrm{E}-06$ \\
\hline Ethylene Cyanohydrin & 000109-78-4 & $6.0 \mathrm{E}-05$ & & & $2.8 \mathrm{E}+02$ & $5.7 \mathrm{E}+01$ & 4.3E-02 & $7.9 \mathrm{E}-10$ & $2.5 \mathrm{E}-10$ \\
\hline Ethylene Diamine & $000107-15-3$ & $8.9 \mathrm{E}-04$ & & & $3.6 \mathrm{E}+01$ & $7.2 \mathrm{E}+00$ & $6.4 \mathrm{E}-01$ & $2.8 \mathrm{E}-08$ & $8.9 \mathrm{E}-09$ \\
\hline Ethylene Glycol & $000107-21-1$ & $3.6 \mathrm{E}-05$ & & & $4.8 \mathrm{E}+02$ & $9.7 \mathrm{E}+01$ & 2.1E-02 & $3.1 \mathrm{E}-10$ & $9.9 \mathrm{E}-11$ \\
\hline
\end{tabular}


Table 3.6b. (continued)

\begin{tabular}{|c|c|c|c|c|c|c|c|c|c|}
\hline Chemical & CASRN & $\begin{array}{c}\text { Perme- } \\
\text { ability } \\
\text { Constant } \\
\text { (cm/hr) } \\
\text { [Kp] }\end{array}$ & $\begin{array}{l}\text { Volatil- } \\
\text { ization } \\
\text { Factor } \\
\left(\mathrm{m}^{3} / \mathrm{kg}\right) \\
{[\mathrm{VF}]}\end{array}$ & $\begin{array}{c}\text { Soil } \\
\text { Satura- } \\
\text { tion Conc. } \\
\text { (mg/kg) } \\
\text { [CSAT] }\end{array}$ & $\begin{array}{c}\text { Soil-to- } \\
\text { Plant } \\
\text { Uptake } \\
\text { (B) }\end{array}$ & $\begin{array}{c}\text { Soil-to- } \\
\text { Wet Plant } \\
\text { Uptake } \\
\left(B_{\mathrm{rm}}\right)\end{array}$ & $\begin{array}{c}\text { Bioaccum- } \\
\text { ulation } \\
\text { Factor for } \\
\text { Fish } \\
\text { (L/kg) } \\
\text { [BF] } \\
\end{array}$ & $\begin{array}{c}\text { Biotransfer } \\
\text { Factor for } \\
\text { Beef } \\
\text { (day/kg) } \\
{\left[F_{\mathrm{B}}\right]}\end{array}$ & $\begin{array}{c}\text { Biotransfer } \\
\text { Factor for } \\
\text { Milk } \\
\text { (day/kg) } \\
\text { [F.] }\end{array}$ \\
\hline Ethylene Glycol Monobutyl Ether & 000111-76-2 & $1.2 \mathrm{E}-02$ & & & $1.3 \mathrm{E}+01$ & $2.5 \mathrm{E}+00$ & $2.5 \mathrm{E}+00$ & $1.7 \mathrm{E}-07$ & $5.3 \mathrm{E}-08$ \\
\hline Ethylene Oxide & $000075-21-8$ & $6.3 \mathrm{E}-04$ & $2.0 \mathrm{E}+04$ & $2.9 \mathrm{E}+04$ & $5.7 \mathrm{E}+01$ & $1.1 \mathrm{E}+01$ & 3.5E-01 & $1.3 \mathrm{E}-08$ & 4.0E-09 \\
\hline Ethylene Thiourea & 000096-45-7 & $1.7 \mathrm{E}-04$ & & & $9.2 \mathrm{E}+01$ & $1.9 \mathrm{E}+01$ & $1.9 \mathrm{E}-01$ & $5.5 \mathrm{E}-09$ & $1.7 \mathrm{E}-09$ \\
\hline Ethylphthalyl Ethyl Glycolate & $000084-72-0$ & & & & & & & & \\
\hline Express & $101200-48-0$ & & & & & & & & \\
\hline Fenamiphos & $022224-92-6$ & & & & & & & & \\
\hline Fenpropathrin & 039515-41-8 & & & & & & & & \\
\hline Fluometuron & $002164-17-2$ & 3.7E-03 & & & $1.5 \mathrm{E}+00$ & $3.1 \mathrm{E}-01$ & $3.9 \mathrm{E}+01$ & $6.3 \mathrm{E}-06$ & $2.0 \mathrm{E}-06$ \\
\hline Fluoranthene & $000206-44-0$ & $3.6 \mathrm{E}-01$ & & & $5.5 \mathrm{E}-02$ & $1.1 \mathrm{E}-02$ & $3.1 \mathrm{E}+03$ & $2.0 \mathrm{E}-03$ & $6.3 \mathrm{E}-04$ \\
\hline Fluorene & 000086-73-7 & $2.5 \mathrm{E}-01$ & & & $1.1 \mathrm{E}-01$ & 2.2E-02 & $1.3 E+03$ & $6.3 \mathrm{E}-04$ & $2.0 \mathrm{E}-04$ \\
\hline Fluoride & $007782-41-4$ & $1.0 \mathrm{E}-03$ & & & & & & & \\
\hline Fluridone & $059756-60-4$ & & & & & & & & \\
\hline Flurprimidol & $056425-91-3$ & & & & & & & & \\
\hline Flutolanil & 066332-96-5 & & & & & & & & \\
\hline Fluvalinate & $069409-94-5$ & & & & & & & & \\
\hline Folpet & $000133-07-3$ & $4.7 \mathrm{E}-03$ & & & $6.1 \mathrm{E}-01$ & $1.2 \mathrm{E}-01$ & $1.3 \mathrm{E}+02$ & $3.1 \mathrm{E}-05$ & $9.9 \mathrm{E}-06$ \\
\hline Fomesafen & $072178-02-0$ & & & & & & & & \\
\hline
\end{tabular}


Table 3.6b. (continued)

\begin{tabular}{|c|c|c|c|c|c|c|c|c|c|}
\hline Chemical & CASRN & $\begin{array}{c}\text { Perme- } \\
\text { ability } \\
\text { Constant } \\
\text { (cm/hr) } \\
\text { [Kp] }\end{array}$ & $\begin{array}{l}\text { Volatil- } \\
\text { ization } \\
\text { Factor } \\
\left(\mathrm{m}^{3} / \mathrm{kg}\right) \\
{[\mathrm{VF}]}\end{array}$ & $\begin{array}{c}\text { Soil } \\
\text { Satura- } \\
\text { tion Conc. } \\
\text { (mg/kg) } \\
\text { [CSAT] }\end{array}$ & $\begin{array}{c}\text { Soil-to- } \\
\text { Plant } \\
\text { Uptake } \\
\left(\mathbf{B}_{\mathrm{r}}\right)\end{array}$ & $\begin{array}{c}\text { Soil-to- } \\
\text { Wet Plant } \\
\text { Uptake } \\
\left.\text { (B } \text { B }_{v *}\right)\end{array}$ & $\begin{array}{l}\text { Bioaccum- } \\
\text { ulation } \\
\text { Factor for } \\
\text { Fish } \\
\text { (L/kg) } \\
\text { [BF] }\end{array}$ & $\begin{array}{c}\text { Biotransfer } \\
\text { Factor for } \\
\text { Beef } \\
\text { (day/kg) } \\
{\left[F_{\mathrm{B}}\right]}\end{array}$ & $\begin{array}{l}\text { Biotransfer } \\
\text { Factor for } \\
\text { Milk } \\
\text { (day/kg) } \\
\text { [F }\end{array}$ \\
\hline Fonofos & 000944-22-9 & $3.8 \mathrm{E}-02$ & & & $2.0 \mathrm{E}-01$ & $4.0 \mathrm{E}-02$ & $5.8 \mathrm{E}+02$ & 2.2E-04 & $6.9 \mathrm{E}-05$ \\
\hline Formaldehyde & 000050-00-0 & $2.2 \mathrm{E}-03$ & & & $3.8 \mathrm{E}+01$ & $7.7 \mathrm{E}+00$ & $5.9 \mathrm{E}-01$ & $2.5 \mathrm{E}-08$ & $7.9 \mathrm{E}-09$ \\
\hline Formic Acid & 000064-18-6 & 4.1E-04 & & & $7.8 \mathrm{E}+01$ & $1.6 \mathrm{E}+01$ & 2.3E-01 & $7.2 \mathrm{E}-09$ & 2.3E-09 \\
\hline Fosetyl-AL & $039148-24-8$ & & & & & & & & \\
\hline Furan & $000110-00-9$ & $6.1 \mathrm{E}-03$ & & & $6.7 \mathrm{E}+00$ & $1.4 \mathrm{E}+00$ & $5.7 \mathrm{E}+00$ & $5.0 \mathrm{E}-07$ & $1.6 \mathrm{E}-07$ \\
\hline Furazolidone & $000067-45-8$ & $2.5 \mathrm{E}-04$ & & & $1.5 \mathrm{E}+01$ & $3.1 \mathrm{E}+00$ & $1.9 \mathrm{E}+00$ & $1.2 \mathrm{E}-07$ & $3.8 \mathrm{E}-08$ \\
\hline Furfural & 000098-01-1 & $1.1 \mathrm{E}-01$ & & & $3.5 \mathrm{E}-01$ & $7.2 \mathrm{E}-02$ & $2.7 \mathrm{E}+02$ & $7.9 \mathrm{E}-05$ & 2.5E-05 \\
\hline Furium & $000531-82-8$ & & & & & & & & \\
\hline Furmecyclox & $060568-05-0$ & & & & & & & & \\
\hline Glufosinate, Ammonium & $077182-82-2$ & & & & & & & & \\
\hline Glycidyl & $000765-34-4$ & $2.1 \mathrm{E}-04$ & & & $1.0 \mathrm{E}+02$ & $2.0 \mathrm{E}+01$ & $1.6 \mathrm{E}-01$ & 4.7E-09 & $1.5 \mathrm{E}-09$ \\
\hline Glyphosate & $001071-83-6$ & $1.3 \mathrm{E}-06$ & & & $2.1 \mathrm{E}+03$ & $4.2 \mathrm{E}+02$ & $3.1 \mathrm{E}-03$ & 2.5E-11 & $7.9 \mathrm{E}-12$ \\
\hline Goal & $042874-03-3$ & & & & & & & & \\
\hline Haloxyfop, Methyl & $069806-40-2$ & & & & & & & & \\
\hline Harmony & $079277-27-3$ & & & & & & & & \\
\hline Heptachlor & $000076-44-8$ & $1.1 \mathrm{E}-02$ & & & $1.2 \mathrm{E}-01$ & $2.5 \mathrm{E}-02$ & $1.1 \mathrm{E}+03$ & $5.0 \mathrm{E}-04$ & $1.6 \mathrm{E}-04$ \\
\hline Heptachlor Epoxide & $001024-57-3$ & $5.5 \mathrm{E}-02$ & & & 2.8E-02 & $5.7 \mathrm{E}-03$ & $7.5 \mathrm{E}+03$ & $6.3 \mathrm{E}-03$ & $2.0 \mathrm{E}-03$ \\
\hline
\end{tabular}


Table 3.6b. (continued)

\begin{tabular}{|c|c|c|c|c|c|c|c|c|c|}
\hline Chemical & CASRN & $\begin{array}{c}\text { Perme- } \\
\text { ability } \\
\text { Constant } \\
\text { (cm/hr) } \\
\text { [Kp] }\end{array}$ & $\begin{array}{l}\text { Volatil- } \\
\text { ization } \\
\text { Factor } \\
\left(\mathrm{m}^{3} / \mathrm{kg}\right) \\
{[\mathrm{VF}]}\end{array}$ & $\begin{array}{c}\text { Soil } \\
\text { Satura- } \\
\text { tion Conc. } \\
\text { (mg/kg) } \\
\text { [CSAT] }\end{array}$ & $\begin{array}{c}\text { Soil-to- } \\
\text { Plant } \\
\text { Uptake } \\
\left(B_{\text {B }}\right)\end{array}$ & $\begin{array}{c}\text { Soil-to- } \\
\text { Wet Plant } \\
\text { Uptake } \\
\left(B_{m}\right)\end{array}$ & $\begin{array}{l}\text { Bioaccum- } \\
\text { ulation } \\
\text { Factor for } \\
\text { Fish } \\
\text { (L/kg) } \\
\text { [BF] }\end{array}$ & $\begin{array}{c}\text { Biotransfer } \\
\text { Factor for } \\
\text { Beef } \\
(d a y / k g) \\
{\left[F_{s}\right]}\end{array}$ & $\begin{array}{l}\text { Biotransfer } \\
\text { Factor for } \\
\text { Milk } \\
\text { (day/kg) } \\
\text { [F }]\end{array}$ \\
\hline Heptane, N- & $000142-82-5$ & $1.0 \mathrm{E}+00$ & & & $7.1 \mathrm{E}-02$ & $1.4 \mathrm{E}-02$ & $2.2 E+03$ & $1.3 \mathrm{E}-03$ & $4.0 \mathrm{E}-04$ \\
\hline Hexabromobenzene & $000087-82-1$ & $1.7 \mathrm{E}-02$ & & & $1.1 \mathrm{E}-02$ & 2.3E-03 & $2.4 \mathrm{E}+04$ & $2.9 \mathrm{E}-02$ & 9.3E-03 \\
\hline Hexachlorobenzene & $000118-74-1$ & $2.1 \mathrm{E}-01$ & & & $3.2 \mathrm{E}-02$ & $6.5 \mathrm{E}-03$ & $6.3 \mathrm{E}+03$ & $5.0 \mathrm{E}-03$ & $1.6 \mathrm{E}-03$ \\
\hline Hexachlorobutadiene & $000087-68-3$ & $1.2 \mathrm{E}-01$ & & & $6.2 \mathrm{E}-02$ & $1.3 \mathrm{E}-02$ & $2.6 \mathrm{E}+03$ & $1.6 \mathrm{E}-03$ & $5.0 \mathrm{E}-04$ \\
\hline Hexachlorocyclohexane, Alpha- & $000319-84-6$ & $1.9 \mathrm{E}-02$ & & & 2.1E-01 & 4.2E-02 & $5.4 \mathrm{E}+02$ & 2.0E-04 & $6.3 \mathrm{E}-05$ \\
\hline Hexachlorocyclohexane, Beta- & 000319-85-7 & $2.2 \mathrm{E}-02$ & & & $1.8 \mathrm{E}-01$ & $3.7 \mathrm{E}-02$ & $6.5 \mathrm{E}+02$ & $2.5 \mathrm{E}-04$ & $7.9 \mathrm{E}-05$ \\
\hline Hexachlorocyclohexane, Delta- & $000319-86-8$ & 3.1E-03 & & & $9.0 \mathrm{E}-01$ & $1.8 \mathrm{E}-01$ & $7.9 \mathrm{E}+01$ & $1.6 \mathrm{E}-05$ & $5.0 \mathrm{E}-06$ \\
\hline Hexachlorocyclohexane, Epsilon & 006108-10-7 & & & & & & & & \\
\hline Hexachlorocyclohexane, Gamma- & 000058-89-9 & $1.4 \mathrm{E}-02$ & & & 2.7E-01 & $5.5 \mathrm{E}-02$ & $3.8 \mathrm{E}+02$ & $1.3 \mathrm{E}-04$ & $4.0 \mathrm{E}-05$ \\
\hline Hexachlorocyclohexane, Technical & 000608-73-1 & & & & & & & & \\
\hline Hexachlorocyclopentadiene & $000077-47-4$ & $2.9 \mathrm{E}-02$ & & & $1.8 \mathrm{E}-01$ & 3.7E-02 & $6.5 \mathrm{E}+02$ & 2.5E-04 & $7.9 \mathrm{E}-05$ \\
\hline Hexachlorodibenzo-p-dioxin, Mixture & 019408-74-3 & 4.5E-01 & & & 4.9E-03 & $1.0 \mathrm{E}-03$ & $7.3 \mathrm{E}+04$ & $1.3 \mathrm{E}-01$ & $4.0 \mathrm{E}-02$ \\
\hline Hexachloroethane & 000067-72-1 & 4.2E-02 & & & $2.1 \mathrm{E}-01$ & $4.2 \mathrm{E}-02$ & $5.4 \mathrm{E}+02$ & $2.0 \mathrm{E}-04$ & $6.3 \mathrm{E}-05$ \\
\hline Hexachlorophene & $000070-30-4$ & $1.3 \mathrm{E}+00$ & & & $1.7 \mathrm{E}-03$ & 3.4E-04 & $3.0 \mathrm{E}+05$ & $7.9 \mathrm{E}-01$ & $2.5 \mathrm{E}-01$ \\
\hline Hexahydro-1,3,5-trinitro-1,3,5-triazine (RDX) & $000121-82-4$ & $1.9 \mathrm{E}-02$ & & & 4.6E-01 & $9.4 \mathrm{E}-02$ & $1.9 \mathrm{E}+02$ & $5.0 \mathrm{E}-05$ & $1.6 \mathrm{E}-05$ \\
\hline Hexamethylene Diisocyanate, 1,6- & 000822-06-0 & . & & & & & & & \\
\hline Hexane, N- & $000110-54-3$ & 3.3E-01 & $6.1 \mathrm{E}+03$ & $9.1 \mathrm{E}+01$ & 2.1E-01 & $4.2 \mathrm{E}-02$ & $5.4 \mathrm{E}+02$ & 2.0E-04 & $6.3 \mathrm{E}-05$ \\
\hline
\end{tabular}


Table 3.6b. (continued)

\begin{tabular}{|c|c|c|c|c|c|c|c|c|c|}
\hline Chemical & CASRN & $\begin{array}{l}\text { Perme- } \\
\text { ability } \\
\text { Constant } \\
(\mathrm{cm} / \mathrm{hr}) \\
{[\mathrm{Kp}]}\end{array}$ & $\begin{array}{l}\text { Volatil- } \\
\text { ization } \\
\text { Factor } \\
\left(m^{3} / \mathrm{kg}\right) \\
{[\mathrm{VF})}\end{array}$ & $\begin{array}{c}\text { Soil } \\
\text { Satura- } \\
\text { tion Conc. } \\
\text { (mg/kg) } \\
\text { [CSAT] }\end{array}$ & $\begin{array}{c}\text { Soil-to- } \\
\text { Plant } \\
\text { Uptake } \\
\left(B_{\text {B }}\right)\end{array}$ & $\begin{array}{c}\text { Soil-to- } \\
\text { Wet Plant } \\
\text { Uptake } \\
\left(B_{\mathrm{m}}\right)\end{array}$ & $\begin{array}{c}\text { Bioaccum- } \\
\text { ulation } \\
\text { Factor for } \\
\text { Fish } \\
\text { (U/kg) } \\
\text { [BF] }\end{array}$ & $\begin{array}{c}\text { Biotransfer } \\
\text { Factor for } \\
\text { Beef } \\
\text { (day/kg) } \\
{\left[F_{\mathrm{B}}\right]}\end{array}$ & $\begin{array}{c}\text { Biotransfer } \\
\text { Factor for } \\
\text { Milk } \\
\text { (day/kg) } \\
{\left[F_{m} \mid\right.}\end{array}$ \\
\hline Hexanone, 2- & 000591-78-6 & $4.6 \mathrm{E}-03$ & & & $5.9 \mathrm{E}+00$ & $1.2 \mathrm{E}+00$ & $6.8 \mathrm{E}+00$ & $6.3 \mathrm{E}-07$ & $2.0 \mathrm{E}-07$ \\
\hline Hexazinone & $051235-04-2$ & 4.1E-08 & & & $1.4 \mathrm{E}+04$ & $2.7 \mathrm{E}+03$ & $2.7 \mathrm{E}-04$ & $1.0 \mathrm{E}-12$ & $3.1 \mathrm{E}-13$ \\
\hline $\mathrm{HpCDD}, 2,3,7,8^{(3)}$ & $037871-00-4$ & & & & & & & & \\
\hline $\mathrm{HpCDF}, 2,3,7,8-{ }^{(3)}$ & 038998-75-3 & & & & & & & & \\
\hline $\mathrm{HxCDD}, 2,3,7,8-^{(3)}$ & $034465-46-8$ & & & & & & & & \\
\hline $\mathrm{HxCDF}, 2,3,7,8^{(3)}$ & 055684-94-1 & & & & & & & & \\
\hline Hydrazine & $000302-01-2$ & 4.1E-05 & & & $6.3 \mathrm{E}+02$ & $1.3 E+02$ & $1.5 \mathrm{E}-02$ & $2.0 \mathrm{E}-10$ & $6.3 \mathrm{E}-11$ \\
\hline Hydrazine Sulfate & 010034-93-2 & 4.1E-05 & & & $6.3 \mathrm{E}+02$ & $1.3 E+02$ & $1.5 \mathrm{E}-02$ & 2.0E-10 & $6.3 \mathrm{E}-11$ \\
\hline Hydrogen Chloride & $007647-01-0$ & $1.0 \mathrm{E}-03$ & & & & & & & \\
\hline Hydrogen Cyanide & 000074-90-8 & $2.4 \mathrm{E}-03$ & & & $2.3 \mathrm{E}+01$ & $4.6 \mathrm{E}+00$ & $1.1 \mathrm{E}+00$ & $6.0 \mathrm{E}-08$ & $1.9 \mathrm{E}-08$ \\
\hline Hydrogen Sulfide & $007783-06-4$ & $5.6 \mathrm{E}-03$ & & & $1.1 \mathrm{E}+01$ & $2.2 \mathrm{E}+00$ & $3.1 \mathrm{E}+00$ & 2.2E-07 & 7.0E-08 \\
\hline Hydroquinone & 000123-31-9 & $1.0 \mathrm{E}-03$ & & & $1.8 \mathrm{E}+01$ & $3.7 \mathrm{E}+00$ & $1.5 \mathrm{E}+00$ & $8.9 \mathrm{E}-08$ & $2.8 \mathrm{E}-08$ \\
\hline Imazalil & $035554-44-0$ & & & & & & & & \\
\hline Imazaquin & $081335-37-7$ & & & & & & & & \\
\hline Indeno[1,2,3-cd]pyrene ${ }^{(2)}$ & 000193-39-5 & $1.9 \mathrm{E}+00$ & & & $5.6 \mathrm{E}-03$ & $1.1 \mathrm{E}-03$ & $6.1 \mathrm{E}+04$ & $1.0 \mathrm{E}-01$ & $3.1 \mathrm{E}-02$ \\
\hline Iprodione & 036734-19-7 & & & & & & & & \\
\hline Iron & 007439-89-6 & $1.0 \mathrm{E}-03$ & & & $1.0 \mathrm{E}-02$ & $4.0 \mathrm{E}-04$ & $2.0 \mathrm{E}+02$ & $2.0 \mathrm{E}-02$ & $3.0 \mathrm{E}-05$ \\
\hline
\end{tabular}


Table 3.6b. (continued)

\begin{tabular}{|c|c|c|c|c|c|c|c|c|c|}
\hline Chemical & CASRN & $\begin{array}{c}\text { Perme- } \\
\text { ability } \\
\text { Constant } \\
\text { (cm/hr) } \\
{[\mathrm{Kp}]}\end{array}$ & $\begin{array}{c}\text { Volatil- } \\
\text { ization } \\
\text { Factor } \\
\left(\mathrm{m}^{3} / \mathrm{kg}\right) \\
{[\mathrm{VF}]}\end{array}$ & $\begin{array}{c}\text { Soil } \\
\text { Satura- } \\
\text { tion Conc. } \\
\text { (mg/kg) } \\
\text { [CSAT] }\end{array}$ & $\begin{array}{l}\text { Soil-to- } \\
\text { Plant } \\
\text { Uptake } \\
\left(B_{\text {f }}\right)\end{array}$ & $\begin{array}{c}\text { Soil-to- } \\
\text { Wet Plant } \\
\text { Uptake } \\
\text { (B }\end{array}$ & $\begin{array}{c}\text { Bioaccum- } \\
\text { ulation } \\
\text { Factor for } \\
\text { Fish } \\
\text { (L/kg) } \\
\text { [BF] }\end{array}$ & $\begin{array}{c}\text { Biotransfer } \\
\text { Factor for } \\
\text { Beef } \\
\text { (day/kg) } \\
{\left[F_{2}\right]}\end{array}$ & $\begin{array}{c}\text { Biotransfer } \\
\text { Factor for } \\
\text { Milk } \\
\text { (day/kg) } \\
{\left[F_{\mathrm{w}}\right]}\end{array}$ \\
\hline Isobutyl Alcohol & $000078-83-1$ & $2.6 \mathrm{E}-03$ & & & $1.3 \mathrm{E}+01$ & $2.5 \mathrm{E}+00$ & $2.5 \mathrm{E}+00$ & $1.7 \mathrm{E}-07$ & $5.3 \mathrm{E}-08$ \\
\hline Isophorone & $000078-59-1$ & $4.4 \mathrm{E}-03$ & & & $3.9 \mathrm{E}+00$ & $8.0 \mathrm{E}-01$ & $1.2 \mathrm{E}+01$ & $1.3 \mathrm{E}-06$ & $4.0 \mathrm{E}-07$ \\
\hline Isopropalin & $033820-53-0$ & & & & & & & & \\
\hline Isopropanol & $000067-63-0$ & $8.9 \mathrm{E}-04$ & & & $3.6 \mathrm{E}+01$ & $7.2 \mathrm{E}+00$ & $6.4 \mathrm{E}-01$ & $2.8 \mathrm{E}-08$ & $8.8 \mathrm{E}-09$ \\
\hline Isopropyl Methyl Phosphonic Acid & $001832-54-8$ & & & & & & & & \\
\hline Isoxaben & $082558-50-7$ & & & & & & & & \\
\hline Karate & 091465-08-6 & & & & & & & & \\
\hline Kerb & $023950-58-5$ & & & & & & & & \\
\hline Lactofen & $077501-63-4$ & & & & & & & & \\
\hline Lead Alkyls & NA & & & & & & & & \\
\hline Lead And Compounds & $007439-92-1$ & $1.0 \mathrm{E}-03$ & & & $9.0 \mathrm{E}-02$ & $7.6 \mathrm{E}-04$ & $3.0 \mathrm{E}+02$ & $4.0 \mathrm{E}-04$ & 3.0E-04 \\
\hline Linuron & $000330-55-2$ & & & & & & & & \\
\hline Lithium & 007439-93-2 & $1.0 \mathrm{E}-03$ & & & 2.5E-02 & $6.3 \mathrm{E}-03$ & & $1.0 \mathrm{E}-02$ & $2.0 \mathrm{E}-02$ \\
\hline Londax & $083055-99-6$ & & & & & & & & \\
\hline MCPA & $000094-74-6$ & $4.9 \mathrm{E}-03$ & & & $1.8 \mathrm{E}+00$ & $3.6 \mathrm{E}-01$ & $3.3 \mathrm{E}+01$ & $5.0 \mathrm{E}-06$ & $1.6 \mathrm{E}-06$ \\
\hline MCPB & $000094-81-5$ & & & & & & & & \\
\hline MCPP & $000093-65-2$ & $6.6 \mathrm{E}-03$ & & & $1.2 \mathrm{E}+00$ & $2.4 \mathrm{E}-01$ & $5.6 \mathrm{E}+01$ & $1.0 \mathrm{E}-05$ & $3.1 \mathrm{E}-06$ \\
\hline
\end{tabular}


Table 3.6b. (continued)

\begin{tabular}{|c|c|c|c|c|c|c|c|c|c|}
\hline Chemical & CASRN & $\begin{array}{c}\text { Perme- } \\
\text { ability } \\
\text { Constant } \\
\text { (cm/hr) } \\
{[\mathrm{Kp}]}\end{array}$ & $\begin{array}{l}\text { Volatil- } \\
\text { ization } \\
\text { Factor } \\
\left(\mathrm{m}^{3} / \mathrm{kg}\right) \\
{[\mathrm{VF}]}\end{array}$ & $\begin{array}{c}\text { Soil } \\
\text { Satura- } \\
\text { tion Conc. } \\
\text { (mg/kg) } \\
\text { [CSAT] }\end{array}$ & $\begin{array}{c}\text { Soil-to- } \\
\text { Plant } \\
\text { Uptake } \\
\left(\mathbf{B}_{\mathbf{r}}\right)\end{array}$ & $\begin{array}{c}\text { Soil-to- } \\
\text { Wet Plant } \\
\text { Uptake } \\
\left(B_{m}\right)\end{array}$ & $\begin{array}{l}\text { Bioaccum- } \\
\text { ulation } \\
\text { Factor for } \\
\text { Fish } \\
\text { (L/kg) } \\
\text { [BF] }\end{array}$ & $\begin{array}{c}\text { Biotransfer } \\
\text { Factor for } \\
\text { Beef } \\
\text { (day/kg) } \\
{\left[\mathrm{F}_{\mathrm{B}}\right]}\end{array}$ & $\begin{array}{c}\text { Biotransfer } \\
\text { Factor for } \\
\text { Milk } \\
\text { (day/kg) } \\
{\left[F_{\mathrm{m}}\right]}\end{array}$ \\
\hline Magnesium & $007439-95-4$ & $1.0 \mathrm{E}-03$ & & & $1.0 \mathrm{E}+00$ & $2.5 \mathrm{E}-01$ & & $5.0 \mathrm{E}-03$ & $4.0 \mathrm{E}-03$ \\
\hline Malathion & $000121-75-5$ & 2.1E-03 & & & $7.9 \mathrm{E}-01$ & $1.6 \mathrm{E}-01$ & $9.4 \mathrm{E}+01$ & $2.0 \mathrm{E}-05$ & $6.3 \mathrm{E}-06$ \\
\hline Maleic Anhydride & 000108-31-6 & & & & & & & & \\
\hline Maleic Hydrazide & 000123-33-1 & 2.1E-04 & & & $6.5 \mathrm{E}+01$ & $1.3 \mathrm{E}+01$ & $2.9 \mathrm{E}-01$ & $1.0 \mathrm{E}-08$ & $3.1 \mathrm{E}-09$ \\
\hline Malononitrile & 000109-77-3 & $8.0 \mathrm{E}-04$ & & & $3.6 \mathrm{E}+01$ & $7.3 \mathrm{E}+00$ & $6.3 \mathrm{E}-01$ & $2.7 \mathrm{E}-08$ & 8.7E-09 \\
\hline Mancozeb & 008018-01-7 & & & & & & & & \\
\hline Maneb & $012427-38-2$ & $4.6 \mathrm{E}-05$ & & & $3.8 \mathrm{E}+01$ & $7.7 \mathrm{E}+00$ & $5.9 \mathrm{E}-01$ & $2.5 \mathrm{E}-08$ & $7.9 \mathrm{E}-09$ \\
\hline Manganese (Diet) & 007439-96-5 & $1.0 \mathrm{E}-03$ & & & $6.8 \mathrm{E}-01$ & $6.9 \mathrm{E}-02$ & $4.0 \mathrm{E}+02$ & $5.0 \mathrm{E}-04$ & $3.0 \mathrm{E}-05$ \\
\hline Manganese (Water) & 007439-96-5 & $1.0 \mathrm{E}-03$ & & & $6.8 \mathrm{E}-01$ & $6.9 \mathrm{E}-02$ & $4.0 \mathrm{E}+02$ & $5.0 \mathrm{E}-04$ & $3.0 \mathrm{E}-05$ \\
\hline Mephosfolan & 000950-10-7 & & & & & & & & \\
\hline Mepiquat Chloride & $024307-26-4$ & & & & & & & & \\
\hline Mercuric Chloride & $007487-94-7$ & $1.0 \mathrm{E}-03$ & & & & & & & \\
\hline Mercury (elemental) & 007439-97-6 & $1.0 \mathrm{E}-03$ & & & $1.0 \mathrm{E}+00$ & $3.0 \mathrm{E}-01$ & $1.0 \mathrm{E}+03$ & $1.0 \mathrm{E}-02$ & 4.7E-04 \\
\hline Mercury, Inorganic Salt & 007439-97-6 & $1.0 \mathrm{E}-03$ & & & $1.0 \mathrm{E}+00$ & $3.0 \mathrm{E}-01$ & $1.0 \mathrm{E}+03$ & $1.0 \mathrm{E}-02$ & 4.7E-04 \\
\hline Merphos & $000150-50-5$ & & & & & & & & \\
\hline Merphos Oxide & $000078-48-8$ & $2.6 \mathrm{E}-01$ & & & $1.9 \mathrm{E}-02$ & $3.8 \mathrm{E}-03$ & $1.3 \mathrm{E}+04$ & $1.3 \mathrm{E}-02$ & $4.0 \mathrm{E}-03$ \\
\hline Metalaxyl & 057837-19-1 & & & & & & & & \\
\hline
\end{tabular}


Table 3.6b. (continued)

\begin{tabular}{|c|c|c|c|c|c|c|c|c|c|}
\hline Chemical & CASRN & $\begin{array}{c}\text { Perme- } \\
\text { ability } \\
\text { Constant } \\
\text { (cm/hr) } \\
\text { [Kp] }\end{array}$ & $\begin{array}{l}\text { Volatil- } \\
\text { ization } \\
\text { Factor } \\
\left(\mathrm{m}^{3} / \mathbf{k g}\right) \\
{[\mathrm{VF}]}\end{array}$ & $\begin{array}{c}\text { Soil } \\
\text { Satura- } \\
\text { tion Conc. } \\
(\mathrm{mg} / \mathrm{kg}) \\
{[\mathrm{CSAT}]}\end{array}$ & $\begin{array}{c}\text { Soil-to- } \\
\text { Plant } \\
\text { Uptake } \\
\text { (B) }\end{array}$ & $\begin{array}{c}\text { Soil-to- } \\
\text { Wet Plant } \\
\text { Uptake } \\
\text { (Bm) }\end{array}$ & $\begin{array}{l}\text { Bioaccum- } \\
\text { ulation } \\
\text { Factor for } \\
\text { Fish } \\
\text { (L/kg) } \\
\text { [BF] }\end{array}$ & $\begin{array}{c}\text { Biotransfer } \\
\text { Factor for } \\
\text { Beef } \\
\text { (day/kg) } \\
{\left[F_{8}\right]}\end{array}$ & $\begin{array}{c}\text { Biotransfer } \\
\text { Factor for } \\
\text { Milk } \\
\text { (day/kg) } \\
{\left[\mathbf{F}_{m}\right]}\end{array}$ \\
\hline Methacrylonitrile & $000126-98-7$ & $1.8 \mathrm{E}-03$ & $1.1 \mathrm{E}+05$ & $1.2 \mathrm{E}+04$ & $1.8 \mathrm{E}+01$ & $3.7 \mathrm{E}+00$ & $1.5 \mathrm{E}+00$ & 8.7E-08 & 2.7E-08 \\
\hline Methamidophos & $010265-92-6$ & $8.9 \mathrm{E}-05$ & & & $9.2 \mathrm{E}+01$ & $1.9 \mathrm{E}+01$ & $1.9 \mathrm{E}-01$ & $5.5 \mathrm{E}-09$ & $1.7 \mathrm{E}-09$ \\
\hline Methanol & $000067-56-1$ & $1.6 \mathrm{E}-03$ & & & $1.1 \mathrm{E}+02$ & $2.2 \mathrm{E}+01$ & $1.5 \mathrm{E}-01$ & $4.2 \mathrm{E}-09$ & $1.3 \mathrm{E}-09$ \\
\hline Methidathion & $000950-37-8$ & & & & & & & & \\
\hline Methomyl & $016752-77-5$ & $5.2 \mathrm{E}-04$ & & & $1.7 \mathrm{E}+01$ & $3.5 \mathrm{E}+00$ & $1.7 \mathrm{E}+00$ & $1.0 \mathrm{E}-07$ & 3.1E-08 \\
\hline Methoxy-5-nitroaniline, 2- & $000099-59-2$ & $2.5 \mathrm{E}-03$ & & & $5.1 \mathrm{E}+00$ & $1.0 \mathrm{E}+00$ & $8.1 E+00$ & $7.9 \mathrm{E}-07$ & 2.5E-07 \\
\hline Methoxychlor & $000072-43-5$ & $2.0 \mathrm{E}-02$ & & & $1.1 \mathrm{E}-01$ & $2.2 \mathrm{E}-02$ & $1.3 \mathrm{E}+03$ & $6.3 \mathrm{E}-04$ & $2.0 \mathrm{E}-04$ \\
\hline Methoxyethanol Acetate, 2- & $000110-49-6$ & & & & & & & & \\
\hline Methoxyethanol, 2- & $000109-86-4$ & $1.9 \mathrm{E}-04$ & & & $1.1 E+02$ & $2.2 \mathrm{E}+01$ & $1.5 \mathrm{E}-01$ & 4.2E-09 & $1.3 \mathrm{E}-09$ \\
\hline Methyl Acetate & $000079-20-9$ & $9.0 \mathrm{E}-04$ & & & $3.0 \mathrm{E}+01$ & $6.1 \mathrm{E}+00$ & 8.1E-01 & $3.8 \mathrm{E}-08$ & $1.2 \mathrm{E}-08$ \\
\hline Methyl Acrylate & $000096-33-3$ & 1.7E-03 & & & $1.6 \mathrm{E}+01$ & $3.1 \mathrm{E}+00$ & $1.9 \mathrm{E}+00$ & $1.2 \mathrm{E}-07$ & 3.7E-08 \\
\hline Methyl Ethyl Ketone & $000078-93-3$ & 1.1E-03 & $2.5 \mathrm{E}+04$ & $9.2 \mathrm{E}+03$ & $2.6 \mathrm{E}+01$ & $5.4 \mathrm{E}+00$ & 9.4E-01 & 4.7E-08 & $1.5 \mathrm{E}-08$ \\
\hline Methyl Isobutyl Ketone & $000108-10-1$ & 3.3E-03 & $6.5 \mathrm{E}+04$ & $7.0 \mathrm{E}+02$ & $7.7 \mathrm{E}+00$ & $1.6 \mathrm{E}+00$ & $4.8 \mathrm{E}+00$ & 4.0E-07 & $1.3 \mathrm{E}-07$ \\
\hline Methyl Mercury & $022967-92-6$ & & & & & & & & \\
\hline Methyl Methacrylate & $000080-62-6$ & $3.9 \mathrm{E}-03$ & & & $6.7 \mathrm{E}+00$ & $1.4 \mathrm{E}+00$ & $5.7 \mathrm{E}+00$ & $5.0 \mathrm{E}-07$ & $1.6 \mathrm{E}-07$ \\
\hline Methyl Parathion & $000298-00-0$ & $1.1 \mathrm{E}-03$ & & & $3.0 \mathrm{E}+00$ & $6.1 \mathrm{E}-01$ & $1.6 \mathrm{E}+01$ & $2.0 \mathrm{E}-06$ & $6.3 \mathrm{E}-07$ \\
\hline Methyl Styrene (Mixed Isomers) & $025013-15-4$ & 8.7E-02 & & & 4.3E-01 & 8.8E-02 & $2.1 E+02$ & $5.6 \mathrm{E}-05$ & $1.8 \mathrm{E}-05$ \\
\hline
\end{tabular}

Chemical-specific parameters used to calculate PRGs/nonradionuclides

Page 25 of 41 
Table 3.6b. (continued)

\begin{tabular}{|c|c|c|c|c|c|c|c|c|c|}
\hline Chemical & CASRN & $\begin{array}{c}\text { Perme- } \\
\text { ability } \\
\text { Constant } \\
\text { (cm/hr) } \\
\text { [Kp] }\end{array}$ & $\begin{array}{c}\text { Volatil- } \\
\text { ization } \\
\text { Factor } \\
\left(\mathrm{m}^{3} / \mathrm{kg}\right) \\
{[\mathrm{VF}]}\end{array}$ & $\begin{array}{c}\text { Soil } \\
\text { Satura- } \\
\text { tion Conc. } \\
\text { (mg/kg) } \\
\text { [CSAT] }\end{array}$ & $\begin{array}{c}\text { Soil-to- } \\
\text { Plant } \\
\text { Uptake } \\
\left(B_{7}\right)\end{array}$ & $\begin{array}{c}\text { Soil-to- } \\
\text { Wet Plant } \\
\text { Uptake } \\
\left(B_{\mathrm{rm}}\right)\end{array}$ & $\begin{array}{l}\text { Bioaccum- } \\
\text { ulation } \\
\text { Factor for } \\
\text { Fish } \\
\text { (L/kg) } \\
\text { [BF] }\end{array}$ & $\begin{array}{c}\text { Biotransfer } \\
\text { Factor for } \\
\text { Beef } \\
\text { (day/kg) } \\
{\left[\mathrm{F}_{\mathrm{B}}\right]}\end{array}$ & 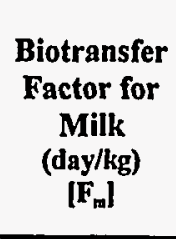 \\
\hline Methyl hydrazine & $000060-34-4$ & & & & & & & & \\
\hline Methyl tert-Butyl Ether (MTBE) & $001634-04-4$ & & & & & & & & \\
\hline Methyl-5-Nitroaniline, 2- & 000099-55-8 & $5.9 \mathrm{E}-03$ & & & $2.6 \mathrm{E}+00$ & 5.3E-01 & $1.9 \mathrm{E}+01$ & 2.5E-06 & $7.9 \mathrm{E}-07$ \\
\hline Methylaniline Hydrochloride, 2- & $000636-21-5$ & 2.1E-03 & & & $3.8 \mathrm{E}+01$ & $7.7 \mathrm{E}+00$ & $5.9 \mathrm{E}-01$ & $2.5 \mathrm{E}-08$ & $7.9 \mathrm{E}-09$ \\
\hline Methylcyclohexane & $000108-87-2$ & $5.1 \mathrm{E}-02$ & $1.8 \mathrm{E}+04$ & $2.4 \mathrm{E}+02$ & $8.3 \mathrm{E}-01$ & $1.7 \mathrm{E}-01$ & $8.8 \mathrm{E}+01$ & $1.8 \mathrm{E}-05$ & $5.7 \mathrm{E}-06$ \\
\hline Methylene Chloride & 000075-09-2 & $4.5 \mathrm{E}-03$ & $9.3 \mathrm{E}+03$ & $1.0 \mathrm{E}+03$ & $6.7 \mathrm{E}+00$ & $1.4 \mathrm{E}+00$ & $5.7 \mathrm{E}+00$ & $5.0 \mathrm{E}-07$ & $1.6 \mathrm{E}-07$ \\
\hline Methylene-bis(2-chloroaniline), 4,4'- & $000101-14-4$ & $2.8 \mathrm{E}-02$ & & & $2.1 \mathrm{E}-01$ & $4.2 \mathrm{E}-02$ & $5.4 \mathrm{E}+02$ & $2.0 \mathrm{E}-04$ & $6.3 \mathrm{E}-05$ \\
\hline Methylene-bis(N,N-dimethyl) Aniline, 4,4'- & $000101-61-1$ & $1.3 \mathrm{E}-01$ & & & $6.2 \mathrm{E}-02$ & $1.3 \mathrm{E}-02$ & $2.6 \mathrm{E}+03$ & $1.6 \mathrm{E}-03$ & $5.0 \mathrm{E}-04$ \\
\hline Methylenebisbenzenamine, 4,4'- & $000101-77-9$ & $1.6 \mathrm{E}-03$ & & & $4.5 \mathrm{E}+00$ & $9.1 \mathrm{E}-01$ & $9.7 \mathrm{E}+00$ & $1.0 \mathrm{E}-06$ & $3.1 \mathrm{E}-07$ \\
\hline Methylenediphenyl Isocyanate, 4,4'- & 000101-68-8 & & & & & & & & \\
\hline Methylstyrene, Alpha- & 000098-83-9 & 8.7E-02 & & & 4.3E-01 & $8.8 \mathrm{E}-02$ & $2.1 \mathrm{E}+02$ & $5.6 \mathrm{E}-05$ & $1.8 \mathrm{E}-05$ \\
\hline Methyltriethyl Lead & $001762-28-3$ & & & & & & & & \\
\hline Metolachlor & $051218-45-2$ & & & & & & & & \\
\hline Metribuzin & $021087-64-9$ & $1.5 \mathrm{E}-03$ & & & $3.9 \mathrm{E}+00$ & $8.0 \mathrm{E}-01$ & $1.2 \mathrm{E}+01$ & $1.3 \mathrm{E}-06$ & $4.0 \mathrm{E}-07$ \\
\hline Mirex & $002385-85-5$ & $5.0 \mathrm{E}-03$ & & & $3.3 E-02$ & $6.7 \mathrm{E}-03$ & $6.1 \mathrm{E}+03$ & $4.8 \mathrm{E}-03$ & $1.5 \mathrm{E}-03$ \\
\hline Molinate & $002212-67-1$ & $4.4 \mathrm{E}-02$ & & & 3.4E-01 & $6.9 \mathrm{E}-02$ & $2.8 \mathrm{E}+02$ & 8.5E-05 & 2.7E-05 \\
\hline Molybdenum & 007439-98-7 & $1.0 \mathrm{E}-03$ & & & 4.0E-01 & $8.0 \mathrm{E}-02$ & $1.0 \mathrm{E}+01$ & $1.0 \mathrm{E}-03$ & $1.7 \mathrm{E}-03$ \\
\hline
\end{tabular}


Table 3.6b. (continued)

\begin{tabular}{|c|c|c|c|c|c|c|c|c|c|}
\hline Chemical & CASRN & $\begin{array}{c}\text { Perme- } \\
\text { ability } \\
\text { Constant } \\
\text { (cm/hr) } \\
{[\mathrm{Kp}]}\end{array}$ & $\begin{array}{l}\text { Volatil- } \\
\text { ization } \\
\text { Factor } \\
\left(\mathrm{m}^{3} / \mathrm{kg}\right) \\
{[\mathrm{VF}]}\end{array}$ & $\begin{array}{c}\text { Soil } \\
\text { Satura- } \\
\text { tion Conc. } \\
\text { (mg/kg) } \\
{[\text { CSAT] }}\end{array}$ & $\begin{array}{c}\text { Soil-to- } \\
\text { Plant } \\
\text { Uptake } \\
\left(B_{v}\right)\end{array}$ & $\begin{array}{c}\text { Soil-to- } \\
\text { Wet Plant } \\
\text { Uptake } \\
\left(B_{\mathrm{ma}}\right)\end{array}$ & $\begin{array}{l}\text { Bioaccum- } \\
\text { ulation } \\
\text { Factor for } \\
\text { Fish } \\
\text { (L/kg) } \\
\text { [BF] }\end{array}$ & $\begin{array}{c}\text { Biotransfer } \\
\text { Factor for } \\
\text { Beef } \\
\text { (day/kg) } \\
{\left[\mathrm{F}_{\mathrm{z}}\right]}\end{array}$ & $\begin{array}{c}\text { Biotransfer } \\
\text { Factor for } \\
\text { Milk } \\
\text { (day/kg) } \\
\text { [F.] }\end{array}$ \\
\hline Monochloramine & 010599-90-3 & & & & & & & & \\
\hline Monochlorobutanes & $025154-42-1$ & & & & & & & & \\
\hline Naled & $000300-76-5$ & $8.8 \mathrm{E}-04$ & & & $9.0 \mathrm{E}-01$ & $1.8 \mathrm{E}-01$ & $7.9 \mathrm{E}+01$ & $1.6 \mathrm{E}-05$ & $5.0 \mathrm{E}-06$ \\
\hline Naphthalene & $000091-20-3$ & $6.9 \mathrm{E}-02$ & & & $4.6 \mathrm{E}-01$ & $9.4 \mathrm{E}-02$ & $1.9 \mathrm{E}+02$ & $5.0 \mathrm{E}-05$ & $1.6 \mathrm{E}-05$ \\
\hline Naphthalene, 1-Methyl & $000090-12-0$ & & & & & & & & \\
\hline Naphthalene, 2-Methyl & $000091-57-6$ & $1.5 \mathrm{E}-01$ & & & $2.1 \mathrm{E}-01$ & 4.2E-02 & $5.4 \mathrm{E}+02$ & 2.0E-04 & $6.3 \mathrm{E}-05$ \\
\hline Napropamide & $015299-99-7$ & $1.2 \mathrm{E}-04$ & & & $1.6 \mathrm{E}+01$ & $3.2 \mathrm{E}+00$ & $1.9 \mathrm{E}+00$ & $1.1 \mathrm{E}-07$ & $3.6 \mathrm{E}-08$ \\
\hline Niagara Blue 4B & $002429-74-5$ & & & & & & & & \\
\hline Nickel Carbonyl & 013463-39-3 & & & & & & & & \\
\hline Nickel Refinery Dust & NA & $1.0 \mathrm{E}-03$ & & & & & & & \\
\hline Nickel Soluble Salts & 007440-02-0 & $1.0 \mathrm{E}-03$ & & & $1.8 \mathrm{E}-01$ & $5.0 \mathrm{E}-02$ & $1.0 \mathrm{E}+02$ & $5.0 \mathrm{E}-03$ & $1.6 \mathrm{E}-02$ \\
\hline Nickel Subsulfide & $012035-72-2$ & $1.0 \mathrm{E}-03$ & & & & & & & \\
\hline Nitrapyrin & 001929-82-4 & $1.0 \mathrm{E}-02$ & & & $6.9 \mathrm{E}-01$ & $1.4 \mathrm{E}-01$ & $1.1 \mathrm{E}+02$ & $2.5 \mathrm{E}-05$ & 7.9E-06 \\
\hline Nitrate & $014797-55-8$ & $1.0 \mathrm{E}-03$ & & & & & & & \\
\hline Nitric Oxide & 010102-43-9 & $1.0 \mathrm{E}-03$ & & & & & & & \\
\hline Nitrite & $014797-65-0$ & $1.0 \mathrm{E}-03$ & & & & & & & \\
\hline Nitroaniline, 2- & $000088-74-4$ & $5.2 \mathrm{E}-03$ & $7.0 \mathrm{E}+04$ & $9.6 \mathrm{E}+01$ & $3.4 \mathrm{E}+00$ & 7.0E-01 & $1.4 \mathrm{E}+01$ & $1.6 \mathrm{E}-06$ & $5.0 \mathrm{E}-07$ \\
\hline
\end{tabular}


Table 3.6b. (continued)

\begin{tabular}{|c|c|c|c|c|c|c|c|c|c|}
\hline Chemical & CASRN & $\begin{array}{l}\text { Perme- } \\
\text { ability } \\
\text { Constant } \\
\text { (cm/hr) } \\
\text { [Kp] }\end{array}$ & $\begin{array}{l}\text { Volatil- } \\
\text { ization } \\
\text { Factor } \\
\left(\mathrm{m}^{3} / \mathrm{kg}\right) \\
{[\mathrm{VF}]}\end{array}$ & $\begin{array}{c}\text { Soil } \\
\text { Satura- } \\
\text { tion Conc. } \\
\text { (mg/kg) } \\
\text { [CSAT] }\end{array}$ & $\begin{array}{l}\text { Soil-to- } \\
\text { Plant } \\
\text { Uptake } \\
\left(B_{v}\right)\end{array}$ & $\begin{array}{l}\text { Soil-to- } \\
\text { Wet Plant } \\
\text { Uptake } \\
\left(B_{n m}\right)\end{array}$ & $\begin{array}{l}\text { Bioaccum- } \\
\text { ulation } \\
\text { Factor for } \\
\text { Fish } \\
\text { (L/kg) } \\
\text { [BF] }\end{array}$ & $\begin{array}{c}\text { Biotransfer } \\
\text { Factor for } \\
\text { Beef } \\
\text { (day/kg) } \\
{\left[\mathrm{F}_{\mathrm{B}}\right]}\end{array}$ & $\begin{array}{c}\text { Biotransfer } \\
\text { Factor for } \\
\text { Milk } \\
\text { (day/kg) } \\
{\left[F_{\mathrm{m}}\right]}\end{array}$ \\
\hline Nitrobenzene & $000098-95-3$ & $6.9 \mathrm{E}-03$ & $4.1 \mathrm{E}+03$ & $3.2 \mathrm{E}+02$ & $3.4 \mathrm{E}+00$ & $7.0 \mathrm{E}-01$ & $1.4 \mathrm{E}+01$ & $1.6 \mathrm{E}-06$ & $5.0 \mathrm{E}-07$ \\
\hline Nitrofurantoin & $000067-20-9$ & & & & & & & & \\
\hline Nitrofurazone & $000059-87-0$ & & & & & & & & \\
\hline Nitrogen Dioxide & 010102-44-0 & $1.0 \mathrm{E}-03$ & & & & & & & \\
\hline Nitroguanidine & 000556-88-7 & & & & & & & & \\
\hline Nitrophenol, 4- & $000100-02-7$ & $6.0 \mathrm{E}-03$ & & & $3.0 \mathrm{E}+00$ & $6.1 \mathrm{E}-01$ & $1.6 \mathrm{E}+01$ & $2.0 \mathrm{E}-06$ & $6.3 \mathrm{E}-07$ \\
\hline Nitropropane, 2- & 000079-46-9 & $1.0 \mathrm{E}-03$ & $9.2 \mathrm{E}+04$ & $1.5 \mathrm{E}+04$ & $1.8 \mathrm{E}+01$ & $3.7 \mathrm{E}+00$ & $1.5 \mathrm{E}+00$ & $8.9 \mathrm{E}-08$ & $2.8 \mathrm{E}-08$ \\
\hline Nitroso-N-ethylurea, N- & 000759-73-9 & $5.4 \mathrm{E}-04$ & & & $2.8 \mathrm{E}+01$ & $5.7 \mathrm{E}+00$ & $8.8 \mathrm{E}-01$ & $4.2 \mathrm{E}-08$ & $1.3 \mathrm{E}-08$ \\
\hline Nitroso-N-methylurea, N- & 000684-93-5 & 4.3E-04 & & & $4.0 \mathrm{E}+01$ & $8.0 \mathrm{E}+00$ & $5.6 \mathrm{E}-01$ & 2.3E-08 & 7.4E-09 \\
\hline Nitroso-di-N-butylamine, $\mathrm{N}$ - & 000924-16-3 & $4.8 \mathrm{E}-03$ & $3.9 \mathrm{E}+05$ & $1.5 \mathrm{E}+03$ & $3.0 \mathrm{E}+00$ & $6.1 \mathrm{E}-01$ & $1.6 \mathrm{E}+01$ & $2.0 \mathrm{E}-06$ & $6.3 \mathrm{E}-07$ \\
\hline Nitroso-di-N-propylamine, N- & $000621-64-7$ & $2.8 \mathrm{E}-03$ & & & $5.9 \mathrm{E}+00$ & $1.2 \mathrm{E}+00$ & $6.8 \mathrm{E}+00$ & $6.3 \mathrm{E}-07$ & 2.0E-07 \\
\hline Nitrosodiethanolamine, N- & 001116-54-7 & $5.0 \mathrm{E}-06$ & & & $3.2 \mathrm{E}+02$ & $6.5 \mathrm{E}+01$ & $3.6 \mathrm{E}-02$ & $6.3 \mathrm{E}-10$ & $2.0 \mathrm{E}-10$ \\
\hline Nitrosodiethylamine, N- & $000055-18-5$ & $1.2 \mathrm{E}-03$ & & & $2.0 \mathrm{E}+01$ & $4.1 \mathrm{E}+00$ & $1.4 \mathrm{E}+00$ & 7.5E-08 & $2.4 \mathrm{E}-08$ \\
\hline Nitrosodimethylamine, N- & $000062-75-9$ & 2.7E-04 & & & $8.1 E+01$ & $1.6 \mathrm{E}+01$ & 2.2E-01 & $6.7 \mathrm{E}-09$ & 2.1E-09 \\
\hline Nitrosodiphenylamine, N- & $000086-30-6$ & $2.0 \mathrm{E}-02$ & & & $6.1 \mathrm{E}-01$ & $1.2 \mathrm{E}-01$ & $1.3 \mathrm{E}+02$ & $3.1 \mathrm{E}-05$ & $9.9 \mathrm{E}-06$ \\
\hline Nitrosomethylethylamine, N- & 010595-95-6 & & & & & & & & \\
\hline Nitrosomethylvinylamine, N- & 004549-40-0 & 5.7E-04 & & & $3.8 \mathrm{E}+01$ & $7.7 \mathrm{E}+00$ & $5.9 \mathrm{E}-01$ & 2.5E-08 & 7.9E-09 \\
\hline
\end{tabular}


Table 3.6b. (continued)

\begin{tabular}{|c|c|c|c|c|c|c|c|c|c|}
\hline Chemical & CASRN & $\begin{array}{c}\text { Perme- } \\
\text { ability } \\
\text { Constant } \\
\text { (cm/hr) } \\
\text { [Kp] }\end{array}$ & $\begin{array}{l}\text { Volatil- } \\
\text { ization } \\
\text { Factor } \\
\left(\mathrm{m}^{3} / \mathrm{kg}\right) \\
{[\mathrm{VF}]}\end{array}$ & $\begin{array}{c}\text { Soil } \\
\text { Satura- } \\
\text { tion Conc. } \\
\text { (mg/kg) } \\
\text { [CSAT] }\end{array}$ & $\begin{array}{c}\text { Soil-to- } \\
\text { Plant } \\
\text { Uptake } \\
\left(B_{r}\right)\end{array}$ & $\begin{array}{c}\text { Soil-to- } \\
\text { Wet Plant } \\
\text { Uptake } \\
\left(B_{n m}\right)\end{array}$ & $\begin{array}{l}\text { Bioaccum- } \\
\text { ulation } \\
\text { Factor for } \\
\text { Fish } \\
\text { (L/kg) } \\
\text { [BF] }\end{array}$ & $\begin{array}{c}\text { Biotransfer } \\
\text { Factor for } \\
\text { Beef } \\
\text { (day/kg) } \\
{\left[F_{\mathbf{B}}\right]}\end{array}$ & $\begin{array}{c}\text { Biotransfer } \\
\text { Factor for } \\
\text { Milk } \\
\text { (day/kg) } \\
\text { [F.] }\end{array}$ \\
\hline Nitrosopyrrolidine, N- & 000930-55-2 & 7.7E-05 & & & $1.7 \mathrm{E}+02$ & $3.3 \mathrm{E}+01$ & $8.6 \mathrm{E}-02$ & $2.0 \mathrm{E}-09$ & $6.3 \mathrm{E}-10$ \\
\hline Nitrotoluene, 4-Amino-2- & 000119-32-4 & & & & & & & & \\
\hline Nitrotoluene, m- & 000099-08-1 & $1.4 \mathrm{E}-02$ & & & $1.5 \mathrm{E}+00$ & $3.1 \mathrm{E}-01$ & $3.9 \mathrm{E}+01$ & $6.3 \mathrm{E}-06$ & $2.0 \mathrm{E}-06$ \\
\hline Nitrotoluene, 0 - & 000088-72-2 & $1.2 \mathrm{E}-02$ & & & $1.8 \mathrm{E}+00$ & $3.6 \mathrm{E}-01$ & $3.3 \mathrm{E}+01$ & $5.0 \mathrm{E}-06$ & $1.6 \mathrm{E}-06$ \\
\hline Nitrotoluene, $\mathrm{p}$ - & 000099-99-0 & $1.4 \mathrm{E}-02$ & & & $1.5 \mathrm{E}+00$ & 3.1E-01 & $3.9 \mathrm{E}+01$ & $6.3 \mathrm{E}-06$ & $2.0 \mathrm{E}-06$ \\
\hline Norflurazon & $027314-13-2$ & & & & & & & & \\
\hline Nustar & 085509-19-9 & & & & & & & & \\
\hline $\mathrm{OCDD}^{(3)}$ & 003268-87-9 & & & & $3.1 \mathrm{E}-04$ & $6.2 \mathrm{E}-05$ & $2.8 \mathrm{E}+06$ & $1.5 \mathrm{E}+01$ & $4.8 \mathrm{E}+00$ \\
\hline $\mathrm{OCDF}^{(3)}$ & $039001-02-0$ & & & & & & & & \\
\hline Octabromodiphenyl Ether & $032536-52-0$ & & & & & & & & \\
\hline $\begin{array}{l}\text { Octahydro-1,3,5,7-tetranitro-1,3,5,7-tetra } \\
\text { (HMX) }\end{array}$ & $002691-41-0$ & $7.8 \mathrm{E}-05$ & & & $1.7 \mathrm{E}+01$ & $3.5 \mathrm{E}+00$ & $1.7 \mathrm{E}+00$ & $9.7 \mathrm{E}-08$ & $3.1 \mathrm{E}-08$ \\
\hline Octamethylpyrophosphoramide & $000152-16-9$ & & & & & & & & \\
\hline Octyl Phthalate, di-N- & $000117-84-0$ & $2.7 \mathrm{E}+01$ & & & $1.8 \mathrm{E}-04$ & $3.6 \mathrm{E}-05$ & $5.8 \mathrm{E}+06$ & $4.0 \mathrm{E}+01$ & $1.3 \mathrm{E}+01$ \\
\hline Oryzalin & $019044-88-3$ & & & & & & & & \\
\hline Oxadiazon & 019666-30-9 & & & & & & & & \\
\hline Oxamyl & 023135-22-0 & & & & & & & & \\
\hline Paclobutrazol & $076738-62-0$ & & & & & & & & \\
\hline
\end{tabular}


Table 3.6b. (continued)

\begin{tabular}{|c|c|c|c|c|c|c|c|c|c|}
\hline Chemical & CASRN & $\begin{array}{c}\text { Perme- } \\
\text { ability } \\
\text { Constant } \\
\text { (cm/hr) } \\
\text { [Kp] }\end{array}$ & $\begin{array}{l}\text { Volatil- } \\
\text { ization } \\
\text { Factor } \\
\left(\mathrm{m}^{3} / \mathrm{kg}\right) \\
\text { [VF] }\end{array}$ & $\begin{array}{l}\text { Soil } \\
\text { Satura- } \\
\text { tion Conc. } \\
\text { (mg/kg) } \\
\text { [CSAT] }\end{array}$ & $\begin{array}{c}\text { Soil-to- } \\
\text { Plant } \\
\text { Uptake } \\
\text { (B) }\end{array}$ & $\begin{array}{c}\text { Soil-to- } \\
\text { Wet Plant } \\
\text { Uptake } \\
\text { (B, })\end{array}$ & $\begin{array}{l}\text { Bioaccum- } \\
\text { ulation } \\
\text { Factor for } \\
\text { Fish } \\
\text { (L/kg) } \\
{[\mathrm{BF}]}\end{array}$ & $\begin{array}{c}\text { Biotransfer } \\
\text { Factor for } \\
\text { Beef } \\
\text { (day/kg) } \\
{\left[F_{\mathrm{B}}\right]}\end{array}$ & 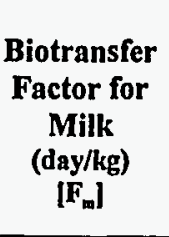 \\
\hline Paraquat & $001910-42-5$ & $1.4 \mathrm{E}-07$ & & & $1.1 \mathrm{E}+04$ & $2.2 \mathrm{E}+03$ & 3.7E-04 & $1.5 \mathrm{E}-12$ & $4.8 \mathrm{E}-13$ \\
\hline Parathion & $000056-38-2$ & $1.7 \mathrm{E}-02$ & & & $2.4 \mathrm{E}-01$ & $4.8 \mathrm{E}-02$ & $4.5 \mathrm{E}+02$ & $1.6 \mathrm{E}-04$ & $5.0 \mathrm{E}-05$ \\
\hline $\operatorname{PeCDD}, 2,3,7,8_{-}^{(3)}$ & $036088-22-9$ & & & & $6.5 \mathrm{E}-03$ & $1.3 \mathrm{E}-03$ & $5.1 E+04$ & 7.9E-02 & $2.5 \mathrm{E}-02$ \\
\hline PeCDF, $1,2,3,7,8-^{(3)}$ & $057117-41-6$ & & & & & & & & \\
\hline PeCDF, $2,3,4,7,8-^{(3)}$ & $057117-31-4$ & & & & & & & & \\
\hline Pebulate & $001114-71-2$ & & & & & & & & . \\
\hline Pendimethalin & $040487-42-1$ & & & & & & & & \\
\hline Pentachlorobenzene & $000608-93-5$ & $2.8 \mathrm{E}-01$ & & & 3.7E-02 & $7.4 \mathrm{E}-03$ & $5.3 \mathrm{E}+03$ & $4.0 \mathrm{E}-03$ & $1.3 \mathrm{E}-03$ \\
\hline Pentachlorocyclopentadiene & 025329-35-5 & & & & & & & & \\
\hline Pentachloronitrobenzene & $000082-68-8$ & $5.9 \mathrm{E}-02$ & & & $8.2 \mathrm{E}-02$ & $1.7 \mathrm{E}-02$ & $1.8 \mathrm{E}+03$ & $1.0 \mathrm{E}-03$ & $3.1 E-04$ \\
\hline Pentachlorophenol & $000087-86-5$ & $6.5 \mathrm{E}-01$ & & & $1.4 \mathrm{E}-02$ & $2.9 \mathrm{E}-03$ & $1.8 \mathrm{E}+04$ & $2.0 \mathrm{E}-02$ & $6.3 \mathrm{E}-03$ \\
\hline Pentyl Alcohol, N- & $000071-41-0$ & & & & & & & & \\
\hline Permethrin & $052645-53-1$ & $2.4 \mathrm{E}-03$ & & & $3.5 \mathrm{E}-01$ & $7.2 \mathrm{E}-02$ & $2.7 \mathrm{E}+02$ & $7.9 \mathrm{E}-05$ & $2.5 \mathrm{E}-05$ \\
\hline Phenanthrene & $000085-01-8$ & 2.7E-01 & & & 8.2E-02 & $1.7 \mathrm{E}-02$ & $1.8 \mathrm{E}+03$ & $1.0 \mathrm{E}-03$ & $3.1 \mathrm{E}-04$ \\
\hline Phenmedipham & $013684-63-4$ & $3.4 \mathrm{E}-06$ & & & $2.2 \mathrm{E}+02$ & $4.4 \mathrm{E}+01$ & $6.1 \mathrm{E}-02$ & $1.3 \mathrm{E}-09$ & $4.0 \mathrm{E}-10$ \\
\hline Phenol & $000108-95-2$ & $5.5 \mathrm{E}-03$ & & & $5.1 E+00$ & $1.0 \mathrm{E}+00$ & $8.1 \mathrm{E}+00$ & 7.9E-07 & $2.5 \mathrm{E}-07$ \\
\hline
\end{tabular}


Table 3.6b. (continued)

\begin{tabular}{|c|c|c|c|c|c|c|c|c|c|}
\hline Chemical & CASRN & $\begin{array}{c}\text { Perme- } \\
\text { ability } \\
\text { Constant } \\
\text { (cm/hr) } \\
\text { [Kp] }\end{array}$ & $\begin{array}{l}\text { Volatil- } \\
\text { ization } \\
\text { Factor } \\
\left(\mathrm{m}^{3} / \mathrm{kg}\right) \\
{[\mathrm{VF}]}\end{array}$ & $\begin{array}{c}\text { Soil } \\
\text { Satura- } \\
\text { tion Conc. } \\
\text { (mg/kg) } \\
\text { [CSAT] }\end{array}$ & $\begin{array}{c}\text { Soil-to- } \\
\text { Plant } \\
\text { Uptake } \\
\left(\mathbf{B}_{v}\right)\end{array}$ & $\begin{array}{c}\text { Soil-to- } \\
\text { Wet Plant } \\
\text { Uptake } \\
\text { (B B })\end{array}$ & $\begin{array}{l}\text { Bioaccum- } \\
\text { ulation } \\
\text { Factor for } \\
\text { Fish } \\
\text { (L/kg) } \\
\text { [BF] }\end{array}$ & $\begin{array}{c}\text { Biotransfer } \\
\text { Factor for } \\
\text { Beef } \\
\text { (day/kg) } \\
{\left[\mathrm{F}_{\mathrm{B}}\right]}\end{array}$ & $\begin{array}{c}\text { Biotransfer } \\
\text { Factor for } \\
\text { Milk } \\
\text { (day/kg) } \\
\text { [F }\end{array}$ \\
\hline Phenylenediamine, $m$ - & $000108-45-2$ & $1.2 \mathrm{E}-04$ & & & $1.0 \mathrm{E}+02$ & $2.1 \mathrm{E}+01$ & $1.6 \mathrm{E}-01$ & 4.4E-09 & $1.4 \mathrm{E}-09$ \\
\hline Phenylenediamine, o- & 000095-54-5 & 8.6E-04 & & & $2.1 \mathrm{E}+01$ & $4.3 \mathrm{E}+00$ & $1.3 E+00$ & $6.9 \mathrm{E}-08$ & $2.2 \mathrm{E}-08$ \\
\hline Phenylenediamine, $p$ - & 000106-50-3 & $5.9 \mathrm{E}-05$ & & & $1.9 \mathrm{E}+02$ & $3.8 \mathrm{E}+01$ & $7.2 \mathrm{E}-02$ & $1.6 \mathrm{E}-09$ & $5.0 \mathrm{E}-10$ \\
\hline Phenylmercuric Acetate & $000062-38-4$ & & & & & & & & \\
\hline Phenylphenol, 2- & 000090-43-7 & $2.8 \mathrm{E}-02$ & & & $6.1 \mathrm{E}-01$ & $1.2 \mathrm{E}-01$ & $1.3 \mathrm{E}+02$ & $3.1 \mathrm{E}-05$ & $9.9 \mathrm{E}-06$ \\
\hline Phorate & 000298-02-2 & $7.9 \mathrm{E}-04$ & & & $3.9 \mathrm{E}+00$ & $8.0 \mathrm{E}-01$ & $1.2 \mathrm{E}+01$ & $1.3 \mathrm{E}-06$ & 4.0E-07 \\
\hline Phosmet & $000732-11-6$ & 2.1E-03 & & & $9.0 \mathrm{E}-01$ & $1.8 \mathrm{E}-01$ & $7.9 \mathrm{E}+01$ & $1.6 \mathrm{E}-05$ & $5.0 \mathrm{E}-06$ \\
\hline Phosphine & $007803-51-2$ & $1.0 \mathrm{E}-03$ & & & & & & & \\
\hline Phosphoric Acid & $007664-38-2$ & $1.0 \mathrm{E}-03$ & & & & & & & \\
\hline Phthalic Acid, P. & $000100-21-0$ & $3.0 \mathrm{E}-03$ & & & $3.9 \mathrm{E}+00$ & $8.0 \mathrm{E}-01$ & $1.2 \mathrm{E}+01$ & $1.3 \mathrm{E}-06$ & $4.0 \mathrm{E}-07$ \\
\hline Phthalic Anhydride & $000085-44-9$ & $8.6 \mathrm{E}-05$ & & & $8.7 E+01$ & $1.8 \mathrm{E}+01$ & $2.0 \mathrm{E}-01$ & $6.0 \mathrm{E}-09$ & $1.9 \mathrm{E}-09$ \\
\hline Picloram & 001918-02-1 & $1.9 \mathrm{E}-03$ & & & $1.5 \mathrm{E}+00$ & 3.1E-01 & $3.9 \mathrm{E}+01$ & $6.3 \mathrm{E}-06$ & $2.0 \mathrm{E}-06$ \\
\hline Pirimiphos, Methyl & $029232-93-7$ & & & & & & & & \\
\hline Polybrominated Biphenyls & $\mathrm{NA}$ & & & & & & & & \\
\hline Polychlorinated Biphenyls & $001336-36-3$ & $3.5 \mathrm{E}-01$ & & & $1.3 \mathrm{E}-02$ & $2.5 \mathrm{E}-03$ & $2.1 \mathrm{E}+04$ & 2.5E-02 & $7.9 \mathrm{E}-03$ \\
\hline Potassium Cyanide & $000151-50.8$ & 5.1E-04 & & & $5.3 \mathrm{E}+01$ & $1.1 E+01$ & $3.8 \mathrm{E}-01$ & $1.4 \mathrm{E}-08$ & $4.4 \mathrm{E}-09$ \\
\hline Potassium Silver Cyanide & $000506-61-6$ & $1.0 \mathrm{E}-03$ & & & & & & & \\
\hline
\end{tabular}


Table 3.6b. (continued)

\begin{tabular}{|c|c|c|c|c|c|c|c|c|c|}
\hline Chemical & CASRN & $\begin{array}{c}\text { Perme- } \\
\text { ability } \\
\text { Constant } \\
\text { (cm/hr) } \\
{[\mathrm{Kp}]}\end{array}$ & $\begin{array}{c}\text { Volatil- } \\
\text { ization } \\
\text { Factor } \\
\left(\mathbf{m}^{3} / \mathrm{kg}\right) \\
{[\mathrm{VF}]}\end{array}$ & $\begin{array}{c}\text { Soil } \\
\text { Satura- } \\
\text { tion Conc. } \\
\text { (mg/kg) } \\
\text { [CSAT] }\end{array}$ & $\begin{array}{c}\text { Soil-to- } \\
\text { Plant } \\
\text { Uptake } \\
\left(B_{v}\right)\end{array}$ & $\begin{array}{c}\text { Soil-to- } \\
\text { Wet Plant } \\
\text { Uptake } \\
\left(B_{(m)}\right)\end{array}$ & $\begin{array}{c}\text { Bioaccum- } \\
\text { ulation } \\
\text { Factor for } \\
\text { Fish } \\
\text { (L/kg) } \\
\text { [BF] }\end{array}$ & $\begin{array}{c}\text { Biotransfer } \\
\text { Factor for } \\
\text { Beef } \\
\text { (day/kg) } \\
{\left[F_{\mathrm{B}}\right]}\end{array}$ & $\begin{array}{c}\text { Biotransfer } \\
\text { Factor for } \\
\text { Milk } \\
\text { (day/kg) } \\
\left.\text { [F } F_{m}\right]\end{array}$ \\
\hline Prochloraz & $067747-09-5$ & & & & & & & & \\
\hline Profluralin & $026399-36-0$ & & & & & & & & \\
\hline Prometon & $001610-18-0$ & 3.5E-03 & & & $1.8 \mathrm{E}+00$ & $3.6 \mathrm{E}-01$ & $3.3 \mathrm{E}+01$ & $5.0 \mathrm{E}-06$ & $1.6 \mathrm{E}-06$ \\
\hline Prometryn & 007287-19-6 & $3.8 \mathrm{E}-03$ & & & $1.3 \mathrm{E}+00$ & $2.7 \mathrm{E}-01$ & $4.7 \mathrm{E}+01$ & 7.9E-06 & $2.5 \mathrm{E}-06$ \\
\hline Propachlor & 001918-16-7 & & & & & & & & \\
\hline Propanil & 000709-98-8 & $1.5 \mathrm{E}-02$ & & & $5.9 \mathrm{E}-01$ & $1.2 \mathrm{E}-01$ & $1.4 \mathrm{E}+02$ & 3.3E-05 & $1.0 \mathrm{E}-05$ \\
\hline Propargite & $002312-35-8$ & & & & & & & & \\
\hline Propargyl Alcohol & $000107-19-7$ & & & & & & & & \\
\hline Propazine & $000139-40-2$ & $3.8 \mathrm{E}-03$ & & & $1.5 \mathrm{E}+00$ & $3.1 \mathrm{E}-01$ & $3.9 \mathrm{E}+01$ & $6.3 \mathrm{E}-06$ & $2.0 \mathrm{E}-06$ \\
\hline Propham & $000122-42-9$ & $2.9 \mathrm{E}-02$ & & & $5.3 \mathrm{E}-01$ & $1.1 \mathrm{E}-01$ & $1.6 \mathrm{E}+02$ & 4.0E-05 & $1.3 \mathrm{E}-05$ \\
\hline Propiconazole & 060207-90-1 & & & & & & & & \\
\hline Propylene Glycol & $000057-55-6$ & 4.1E-05 & & & $3.7 E+02$ & $7.5 \mathrm{E}+01$ & $3.0 \mathrm{E}-02$ & $5.0 \mathrm{E}-10$ & $1.6 \mathrm{E}-10$ \\
\hline Propylene Glycol Monoethyl Ether & $001569-02-4$ & & & & & & & & \\
\hline Propylene Glycol Monomethyl Ether & $000107-98-2$ & $4.0 \mathrm{E}-04$ & & & $4.8 \mathrm{E}+01$ & $9.8 \mathrm{E}+00$ & 4.3E-01 & $1.7 \mathrm{E}-08$ & $5.2 \mathrm{E}-09$ \\
\hline Propylene Oxide & $000075-56-9$ & $8.9 \mathrm{E}-04$ & $6.4 \mathrm{E}+04$ & $2.8 \mathrm{E}+04$ & $3.7 \mathrm{E}+01$ & $7.4 \mathrm{E}+00$ & $6.2 \mathrm{E}-01$ & 2.7E-08 & $8.5 \mathrm{E}-09$ \\
\hline Pursuit & $081335-77-5$ & & & & & . & & & \\
\hline Pydrin & $051630-58-1$ & $7.2 \mathrm{E}-03$ & & & $1.0 \mathrm{E}-01$ & 2.1E-02 & $1.3 \mathrm{E}+03$ & $6.6 \mathrm{E}-04$ & 2.1E-04 \\
\hline
\end{tabular}


Table 3.6b. (continued)

\begin{tabular}{|c|c|c|c|c|c|c|c|c|c|}
\hline Chemical & CASRN & $\begin{array}{c}\text { Perme- } \\
\text { ability } \\
\text { Constant } \\
\text { (cm/hr) } \\
\text { [Kp] }\end{array}$ & $\begin{array}{c}\text { Volatil- } \\
\text { ization } \\
\text { Factor } \\
\left(\mathbf{m}^{3} / \mathbf{k g}\right) \\
{[\mathrm{VF}]}\end{array}$ & $\begin{array}{c}\text { Soil } \\
\text { Satura- } \\
\text { tion Conc. } \\
\text { (mg/kg) } \\
\text { [CSAT] }\end{array}$ & $\begin{array}{c}\text { Soil-to- } \\
\text { Plant } \\
\text { Uptake } \\
\text { (B) }\end{array}$ & $\begin{array}{c}\text { Soil-to- } \\
\text { Wet Plant } \\
\text { Uptake } \\
\text { (Bn) }\end{array}$ & $\begin{array}{l}\text { Bioaccum- } \\
\text { ulation } \\
\text { Factor for } \\
\text { Fish } \\
\text { (L/kg) } \\
{[\mathrm{BF}]}\end{array}$ & $\begin{array}{c}\text { Biotransfer } \\
\text { Factor for } \\
\text { Beef } \\
\text { (day/kg) } \\
{\left[F_{\mathrm{g}}\right]}\end{array}$ & $\begin{array}{c}\text { Biotransfer } \\
\text { Factor for } \\
\text { Milk } \\
\text { (day/kg) } \\
{\left[\mathrm{F}_{\mathrm{m}}\right]}\end{array}$ \\
\hline Pyrene & $000129-00-0$ & $3.2 \mathrm{E}-01$ & & & $5.5 \mathrm{E}-02$ & $1.1 \mathrm{E}-02$ & $3.1 E+03$ & $2.0 \mathrm{E}-03$ & $6.3 \mathrm{E}-04$ \\
\hline Pyridine & $000110-86-1$ & $5.3 \mathrm{E}-03$ & & & $6.7 \mathrm{E}+00$ & $1.4 \mathrm{E}+00$ & $5.7 \mathrm{E}+00$ & $5.0 \mathrm{E}-07$ & $1.6 \mathrm{E}-07$ \\
\hline Quinalphos & $013593-03-8$ & & & & & & & & \\
\hline Quinoline & $000091-22-5$ & $9.6 \mathrm{E}-03$ & & & $2.3 \mathrm{E}+00$ & 4.7E-01 & $2.3 \mathrm{E}+01$ & $3.1 \mathrm{E}-06$ & 9.9E-07 \\
\hline Refractory Ceramic Fibers & NA & & & & & & & & \\
\hline Resmethrin & 010453-86-8 & & & & & & & & \\
\hline Ronnel & $000299-84-3$ & 8.7E-02 & & & 4.2E-02 & $8.5 \mathrm{E}-03$ & $4.4 \mathrm{E}+03$ & $3.1 \mathrm{E}-03$ & $9.9 \mathrm{E}-04$ \\
\hline Rotenone & $000083-79-4$ & $6.1 \mathrm{E}-03$ & & & $1.6 \mathrm{E}-01$ & $3.2 \mathrm{E}-02$ & $7.7 E+02$ & 3.1E-04 & $9.9 \mathrm{E}-05$ \\
\hline Savey & $078587-05-0$ & & & & & & & & \\
\hline Selenious Acid & $007783-00-8$ & & & & & & & & \\
\hline Selenite & $014124-67-5$ & & & & & & & & \\
\hline Selenium & $007782-49-2$ & $1.0 \mathrm{E}-03$ & & & $5.0 \mathrm{E}-01$ & $1.0 \mathrm{E}-01$ & & $1.0 \mathrm{E}-01$ & $1.0 \mathrm{E}-02$ \\
\hline Selenium Sulfide & $007446-34-6$ & $1.0 \mathrm{E}-03$ & & & & & & & \\
\hline Selenourea & $000630-10-4$ & & & & & & & & \\
\hline Sethoxydim & $074051-80-2$ & & & & & & & & \\
\hline Silver & $007440-22-4$ & $1.0 \mathrm{E}-03$ & & & $1.0 \mathrm{E}+00$ & $2.2 \mathrm{E}-05$ & $5.0 \mathrm{E}+00$ & $3.0 \mathrm{E}-03$ & $5.0 \mathrm{E}-05$ \\
\hline Silver Cyanide & $000506-64-9$ & $1.0 \mathrm{E}-03$ & & & & & & & \\
\hline
\end{tabular}


Table 3.6b. (continued)

\begin{tabular}{|c|c|c|c|c|c|c|c|c|c|}
\hline Chemical & CASRN & $\begin{array}{c}\text { Perme- } \\
\text { ability } \\
\text { Constant } \\
\text { (cm/hr) } \\
{[\mathrm{Kpl}}\end{array}$ & $\begin{array}{c}\text { Volatil- } \\
\text { ization } \\
\text { Factor } \\
\left(\mathrm{m}^{3} / \mathrm{kg}\right) \\
\text { [VF] }\end{array}$ & $\begin{array}{c}\text { Soil } \\
\text { Satura- } \\
\text { tion Conc. } \\
\text { (mg/kg) } \\
\text { [CSAT] }\end{array}$ & $\begin{array}{c}\text { Soil-to- } \\
\text { Plant } \\
\text { Uptake } \\
\left(B_{v}\right)\end{array}$ & $\begin{array}{c}\text { Soil-to- } \\
\text { Wet Plant } \\
\text { Uptake } \\
\left(B_{m}\right)\end{array}$ & $\begin{array}{l}\text { Bioaccum- } \\
\text { ulation } \\
\text { Factor for } \\
\text { Fish } \\
\text { (L/kg) } \\
\text { [BF] }\end{array}$ & $\begin{array}{c}\text { Biotransfer } \\
\text { Factor for } \\
\text { Beef } \\
\text { (day/kg) } \\
{\left[F_{B}\right]}\end{array}$ & $\begin{array}{c}\text { Biotransfer } \\
\text { Factor for } \\
\text { Milk } \\
\text { (day/kg) } \\
{\left[F_{m}\right]}\end{array}$ \\
\hline Simazine & $000122-34-9$ & 4.1E-03 & & & $2.0 \mathrm{E}+00$ & 4.1E-01 & $2.8 \mathrm{E}+01$ & $4.0 \mathrm{E}-06$ & $1.3 \mathrm{E}-06$ \\
\hline Sodium & 007440-23-5 & $1.0 \mathrm{E}-03$ & & & $2.0 \mathrm{E}-01$ & $3.0 \mathrm{E}-02$ & $2.0 \mathrm{E}+01$ & $8.0 \mathrm{E}-02$ & $1.6 \mathrm{E}-02$ \\
\hline Sodium Acifluorfen & 062476-59-9 & & & & & & & & \\
\hline Sodium Azide & $026628-22-8$ & $1.0 \mathrm{E}-03$ & & & & & & & \\
\hline Sodium Cyanide & 000143-33-9 & $2.5 \mathrm{E}-04$ & & & $1.1 \mathrm{E}+02$ & $2.3 \mathrm{E}+01$ & $1.4 \mathrm{E}-01$ & $3.8 \mathrm{E}-09$ & $1.2 \mathrm{E}-09$ \\
\hline Sodium Diethyldithiocarbamate & 000148-18-5 & & & & & & & & \\
\hline Sodium Fluoroacetate & $000062-74-8$ & & & & & & & & \\
\hline Sodium Metavanadate & $013718-26-8$ & $1.0 \mathrm{E}-03$ & & & & & & & \\
\hline Stirofos (Tetrachlorovinphos) & 000961-11-5 & & & & & & & & \\
\hline Strontium, Stable & $007440-24-6$ & $1.0 \mathrm{E}-03$ & & & $1.1 \mathrm{E}+00$ & $2.1 \mathrm{E}-01$ & $6.0 \mathrm{E}+01$ & $8.0 \mathrm{E}-03$ & $2.8 \mathrm{E}-03$ \\
\hline Strychnine & $000057-24-9$ & $3.9 \mathrm{E}-04$ & & & $3.0 \mathrm{E}+00$ & $6.1 \mathrm{E}-01$ & $1.6 \mathrm{E}+01$ & $2.0 \mathrm{E}-06$ & $6.3 \mathrm{E}-07$ \\
\hline Styrene & $000100-42-5$ & $6.7 \mathrm{E}-01$ & $6.8 \mathrm{E}+04$ & $6.7 \mathrm{E}+02$ & $7.9 \mathrm{E}-01$ & $1.6 \mathrm{E}-01$ & $9.4 \mathrm{E}+01$ & $2.0 \mathrm{E}-05$ & $6.3 \mathrm{E}-06$ \\
\hline Sulfate & $014808-79-8$ & $1.0 \mathrm{E}-03$ & & & & & & & \\
\hline Systhane & 088671-89-0 & & & & & & & & \\
\hline TCDD, 2,3,7,8-(3) & 001746-01-6 & $1.4 \mathrm{E}+00$ & & & $4.3 \mathrm{E}-03$ & $8.8 \mathrm{E}-04$ & $8.7 \mathrm{E}+04$ & $1.6 \mathrm{E}-01$ & $5.0 \mathrm{E}-02$ \\
\hline $\mathrm{TCDF}, 2,3,7,8-^{(3)}$ & $051207-31-9$ & & & & $1.6 \mathrm{E}-02$ & $3.2 \mathrm{E}-03$ & $1.6 \mathrm{E}+04$ & $1.7 \mathrm{E}-02$ & $5.2 \mathrm{E}-03$ \\
\hline TCMTB & $021564-17-0$ & & & & & & & & \\
\hline
\end{tabular}


Table 3.6b. (continued)

\begin{tabular}{|c|c|c|c|c|c|c|c|c|c|}
\hline Chemical & CASRN & $\begin{array}{c}\text { Perme- } \\
\text { ability } \\
\text { Constant } \\
\text { (cm/hr) } \\
\text { [Kp] }\end{array}$ & $\begin{array}{l}\text { Volatil- } \\
\text { ization } \\
\text { Factor } \\
\left(\mathrm{m}^{3} / \mathrm{kg}\right) \\
{[\mathrm{VF}]}\end{array}$ & $\begin{array}{c}\text { Soil } \\
\text { Satura- } \\
\text { tion Conc. } \\
\text { (mg/kg) } \\
\text { [CSAT] }\end{array}$ & $\begin{array}{c}\text { Soil-to- } \\
\text { Plant } \\
\text { Uptake } \\
\left(\mathbf{B}_{v}\right)\end{array}$ & $\begin{array}{c}\text { Soil-to- } \\
\text { Wet Plant } \\
\text { Uptake } \\
\text { (B, }\end{array}$ & $\begin{array}{l}\text { Bioaccum- } \\
\text { ulation } \\
\text { Factor for } \\
\text { Fish } \\
\text { (L/kg) } \\
\text { [BF] }\end{array}$ & $\begin{array}{c}\text { Biotransfer } \\
\text { Factor for } \\
\text { Beef } \\
\text { (day/kg) } \\
{\left[\mathrm{F}_{\mathbf{B}}\right]}\end{array}$ & $\begin{array}{c}\text { Biotransfer } \\
\text { Factor for } \\
\text { Milk } \\
\text { (day/kg) } \\
\text { [F. }\end{array}$ \\
\hline Tebuthiuron & 034014-18-1 & $9.9 \mathrm{E}-05$ & & & $3.1 \mathrm{E}+01$ & $6.3 \mathrm{E}+00$ & $7.7 \mathrm{E}-01$ & 3.5E-08 & $1.1 \mathrm{E}-08$ \\
\hline Temephos & $003383-96-8$ & & & & & & & & \\
\hline Terbacil & $005902-51-2$ & & & & & & & & \\
\hline Terbufos & $013071-79-9$ & $1.4 \mathrm{E}-02$ & & & $2.8 \mathrm{E}-01$ & 5.7E-02 & $3.7 \mathrm{E}+02$ & $1.2 \mathrm{E}-04$ & $3.8 \mathrm{E}-05$ \\
\hline Terbutryn & 000886-50-0 & $2.9 \mathrm{E}-02$ & & & $2.6 \mathrm{E}-01$ & $5.2 \mathrm{E}-02$ & $4.1 \mathrm{E}+02$ & $1.4 \mathrm{E}-04$ & 4.3E-05 \\
\hline Tetrabutyl Lead & $001920-90-7$ & & & & & & & & \\
\hline Tetrachlorobenzene, 1,2,4,5- & $000095-94-3$ & $1.7 \mathrm{E}-01$ & & & $8.2 \mathrm{E}-02$ & $1.7 \mathrm{E}-02$ & $1.8 \mathrm{E}+03$ & $1.0 \mathrm{E}-03$ & 3.1E-04 \\
\hline Tetrachloroethane, 1,1,1,2- & $000630-20-6$ & $2.4 \mathrm{E}-02$ & $5.3 \mathrm{E}+04$ & $7.7 \mathrm{E}+02$ & $6.9 \mathrm{E}-01$ & $1.4 \mathrm{E}-01$ & $1.1 \mathrm{E}+02$ & 2.5E-05 & $7.9 \mathrm{E}-06$ \\
\hline Tetrachloroethane, 1,1,2,2- & $000079-34-5$ & $9.0 \mathrm{E}-03$ & $9.4 \mathrm{E}+04$ & $1.1 \mathrm{E}+03$ & $1.5 \mathrm{E}+00$ & $3.1 \mathrm{E}-01$ & $3.9 \mathrm{E}+01$ & $6.3 \mathrm{E}-06$ & $2.0 \mathrm{E}-06$ \\
\hline Tetrachloroethylene & $000127-18-4$ & 3.7E-01 & $2.2 \mathrm{E}+04$ & $1.2 \mathrm{E}+02$ & $1.2 \mathrm{E}+00$ & $2.4 \mathrm{E}-01$ & $5.6 \mathrm{E}+01$ & $1.0 \mathrm{E}-05$ & $3.1 \mathrm{E}-06$ \\
\hline Tetrachlorophenol, 2,3,4,6. & $000058-90-2$ & $6.0 \mathrm{E}-02$ & & & $1.6 \mathrm{E}-01$ & $3.2 \mathrm{E}-02$ & $7.7 \mathrm{E}+02$ & 3.1E-04 & $9.9 \mathrm{E}-05$ \\
\hline Tetrachlorotoluene, $\mathrm{p}$ - alpha, alpha, alpha- & $005216-25-1$ & & & & & & & & \\
\hline Tetraethyl Dithiopyrophosphate & 003689-24-5 & $2.8 \mathrm{E}-03$ & & & $6.9 \mathrm{E}-01$ & $1.4 \mathrm{E}-01$ & $1.1 \mathrm{E}+02$ & $2.5 \mathrm{E}-05$ & $7.9 \mathrm{E}-06$ \\
\hline Tetraethyl Lead & $000078-00-2$ & & & & & & & & \\
\hline Tetrafluoroethane, 1,1,1,2- & $000811-97-2$ & $3.6 \mathrm{E}-03$ & $7.9 \mathrm{E}+02$ & $1.1 \mathrm{E}+02$ & $6.9 \mathrm{E}+00$ & $1.4 \mathrm{E}+00$ & $5.5 \mathrm{E}+00$ & 4.7E-07 & $1.5 \mathrm{E}-07$ \\
\hline Tetramethyl Lead & $000075-74-1$ & & & & & & & & \\
\hline Tetrapropyl Lead & $003440-75-3$ & & & & & & & & \\
\hline
\end{tabular}

Chemical-specific parameters used to calculate PRGs/nonradionuclides

Page 35 of 41 
Table 3.6b. (continued)

\begin{tabular}{|c|c|c|c|c|c|c|c|c|c|}
\hline Chemical & CASRN & $\begin{array}{c}\text { Perme- } \\
\text { ability } \\
\text { Constant } \\
\text { (cm/hr) } \\
\text { [Kp] }\end{array}$ & $\begin{array}{l}\text { Volatil- } \\
\text { ization } \\
\text { Factor } \\
\left(\mathrm{m}^{3} / \mathrm{kg}\right) \\
{[\mathrm{VF}]}\end{array}$ & $\begin{array}{c}\text { Soil } \\
\text { Satura- } \\
\text { tion Conc. } \\
\text { (mg/kg) } \\
\text { [CSAT] }\end{array}$ & $\begin{array}{c}\text { Soil-to- } \\
\text { Plant } \\
\text { Uptake } \\
\text { (B) }\end{array}$ & $\begin{array}{c}\text { Soil-to- } \\
\text { Wet Plant } \\
\text { Uptake } \\
\text { (B B })\end{array}$ & $\begin{array}{l}\text { Bioaccum- } \\
\text { ulation } \\
\text { Factor for } \\
\text { Fish } \\
\text { (L/kg) } \\
\text { [BF] }\end{array}$ & $\begin{array}{c}\text { Biotransfer } \\
\text { Factor for } \\
\text { Beef } \\
\text { (day/kg) } \\
{\left[F_{B}\right]}\end{array}$ & $\begin{array}{c}\text { Biotransfer } \\
\text { Factor for } \\
\text { Milk } \\
\text { (day/kg) } \\
{\left[F_{\mathrm{m}}\right]}\end{array}$ \\
\hline Thallic Oxide & $001314-32-5$ & & & & & & & & \\
\hline Thallium (I) Nitrate & $010102-45-1$ & $1.0 \mathrm{E}-03$ & & & & & & & \\
\hline Thallium (Soluble Salts) & $007440-28-0$ & $1.0 \mathrm{E}-03$ & & & $4.0 \mathrm{E}-03$ & $1.0 \mathrm{E}-03$ & & $4.0 \mathrm{E}-02$ & $2.0 \mathrm{E}-03$ \\
\hline Thallium Acetate & $000563-68-8$ & & & & & & & & \\
\hline Thallium Carbonate & $006533-73-9$ & & & & & & & & \\
\hline Thallium Chloride & $007791-12-0$ & $1.0 \mathrm{E}-03$ & & & & & & & \\
\hline Thallium Selenite & $012039-52-0$ & $1.0 \mathrm{E}-03$ & & & & & & & \\
\hline Thallium Sulfate & $007446-18-6$ & $1.0 \mathrm{E}-03$ & & & & & & & \\
\hline Thiobencarb & $028249-77-6$ & & & & & & & & \\
\hline Thiofanox & 039196-18-4 & $5.8 \mathrm{E}-06$ & & & $3.5 \mathrm{E}+02$ & $7.2 \mathrm{E}+01$ & $3.2 \mathrm{E}-02$ & $5.3 \mathrm{E}-10$ & $1.7 \mathrm{E}-10$ \\
\hline Thiophanate, Methyl & $023564-05-8$ & & & & & & & & \\
\hline Thiram & $000137-26-8$ & & & & & & & - & \\
\hline Thorium & $007440-29-1$ & $1.0 \mathrm{E}-03$ & & & $1.1 \mathrm{E}-02$ & $1.4 \mathrm{E}-04$ & $1.0 \mathrm{E}+02$ & $1.0 \mathrm{E}-04$ & $5.0 \mathrm{E}-06$ \\
\hline Tin & $007440-31-5$ & $1.0 \mathrm{E}-03$ & & & $1.0 \mathrm{E}+00$ & $3.0 \mathrm{E}-01$ & $3.0 \mathrm{E}+03$ & $1.0 \mathrm{E}-02$ & $1.0 \mathrm{E}-03$ \\
\hline Titanium & $007440-32-6$ & $1.0 \mathrm{E}-03$ & & & $5.5 \mathrm{E}-03$ & $1.4 \mathrm{E}-03$ & & $3.0 \mathrm{E}-02$ & $1.0 \mathrm{E}-02$ \\
\hline Toluene & $000108-88-3$ & $4.5 \mathrm{E}-02$ & $2.5 \mathrm{E}+04$ & $2.5 \mathrm{E}+02$ & $1.0 \mathrm{E}+00$ & 2.1E-01 & $6.6 \mathrm{E}+01$ & $1.3 \mathrm{E}-05$ & $4.0 \mathrm{E}-06$ \\
\hline Toluene diisocyanate mixture (TDI) & $026471-62-5$ & 6.1E-02 & & & 3.0E-01 & $6.1 \mathrm{E}-02$ & $3.3 \mathrm{E}+02$ & $1.0 \mathrm{E}-04$ & 3.3E-05 \\
\hline
\end{tabular}


Table 3.6b. (continued)

\begin{tabular}{|c|c|c|c|c|c|c|c|c|c|}
\hline Chemical & CASRN & $\begin{array}{c}\text { Perme- } \\
\text { ability } \\
\text { Constant } \\
\text { (cm/hr) } \\
\text { [Kp] }\end{array}$ & $\begin{array}{c}\text { Volatil- } \\
\text { ization } \\
\text { Factor } \\
\left(\mathrm{m}^{3} / \mathrm{kg}\right) \\
{[\mathrm{VF}]}\end{array}$ & $\begin{array}{c}\text { Soil } \\
\text { Satura- } \\
\text { tion Conc. } \\
\text { (mg/kg) } \\
\text { [CSAT] }\end{array}$ & $\begin{array}{c}\text { Soil-to- } \\
\text { Plant } \\
\text { Uptake } \\
\left(B_{r}\right)\end{array}$ & $\begin{array}{c}\text { Soil-to- } \\
\text { Wet Plant } \\
\text { Uptake } \\
\left(B_{m}\right)\end{array}$ & $\begin{array}{l}\text { Bioaccum- } \\
\text { ulation } \\
\text { Factor for } \\
\text { Fish } \\
\text { (L/kg) } \\
\text { [BF] }\end{array}$ & $\begin{array}{c}\text { Biotransfer } \\
\text { Factor for } \\
\text { Beef } \\
\text { (day/kg) } \\
{\left[F_{\mathrm{g}}\right]}\end{array}$ & $\begin{array}{c}\text { Biotransfer } \\
\text { Factor for } \\
\text { Milk } \\
\text { (day/kg) } \\
\text { [F.] }\end{array}$ \\
\hline Toluene-2,4-diamine & $000095-80-7$ & $6.0 \mathrm{E}-04$ & & & $2.4 \mathrm{E}+01$ & $4.9 \mathrm{E}+00$ & $1.1 \mathrm{E}+00$ & $5.5 \mathrm{E}-08$ & $1.7 \mathrm{E}-08$ \\
\hline Toluene-2,5-diamine & $000095-70-5$ & & & & & & & & \\
\hline Toluene-2,6-diamine & $000823-40-5$ & & & & & & & & \\
\hline Toluidine, 0- (Methylaniline, 2-) & 000095-53-4 & $3.7 \mathrm{E}-03$ & & & $6.7 E+00$ & $1.4 \mathrm{E}+00$ & $5.7 \mathrm{E}+00$ & $5.0 \mathrm{E}-07$ & $1.6 \mathrm{E}-07$ \\
\hline Toluidine, p- & 000106-49-0 & $1.1 \mathrm{E}-02$ & & & $2.6 \mathrm{E}+00$ & $5.3 \mathrm{E}-01$ & $1.9 \mathrm{E}+01$ & $2.5 \mathrm{E}-06$ & $7.9 \mathrm{E}-07$ \\
\hline Toxaphene & $008001-35-2$ & $1.5 \mathrm{E}-02$ & & & $6.2 \mathrm{E}-02$ & $1.3 \mathrm{E}-02$ & $2.6 \mathrm{E}+03$ & $1.6 \mathrm{E}-03$ & $5.0 \mathrm{E}-04$ \\
\hline Tralomethrin & $066841-25-6$ & & & & & & & & \\
\hline Triallate & $002303-17-5$ & $1.8 \mathrm{E}-02$ & & & $1.9 \mathrm{E}-01$ & $3.8 \mathrm{E}-02$ & $6.2 \mathrm{E}+02$ & 2.4E-04 & 7.5E-05 \\
\hline Triasulfuron & 082097-50-5 & & & & & & & & \\
\hline Tribromobenzene, 1,2,4- & 000615-54-3 & & & & & & & & \\
\hline Tribromochloromethane & 000594-15-0 & & & & & & & & \\
\hline Tribromodiphenyl Ether & 049690-94-0 & & & & & & & & \\
\hline Tributyltin Oxide & 000056-35-9 & & & & & & & & \\
\hline Trichloro-1,2,2-trifluoroethane, 1,1,2- & $000076-13-1$ & $9.6 \mathrm{E}-03$ & $5.8 \mathrm{E}+03$ & $2.1 \mathrm{E}+02$ & $1.2 \mathrm{E}+00$ & $2.4 \mathrm{E}-01$ & $5.6 \mathrm{E}+01$ & $1.0 \mathrm{E}-05$ & 3.1E-06 \\
\hline Trichloro-2'-hydroxydiphenylether & $003380-34-5$ & & & & & & & & \\
\hline \multicolumn{10}{|l|}{ Trichloroacetic Acid } \\
\hline Trichloroaniline $\mathrm{HCl}, 2,4,6-$ & $033663-50-2$ & & & & & & & & \\
\hline
\end{tabular}


Table 3.6b. (continued)

\begin{tabular}{|c|c|c|c|c|c|c|c|c|c|}
\hline Chemical & CASRN & $\begin{array}{c}\text { Perme- } \\
\text { ability } \\
\text { Constant } \\
\text { (cm/hr) } \\
{[\mathrm{Kp}]}\end{array}$ & $\begin{array}{c}\text { Volatil- } \\
\text { ization } \\
\text { Factor } \\
\left(\mathrm{m}^{3} / \mathrm{kg}\right) \\
{[\mathrm{VF}]}\end{array}$ & $\begin{array}{c}\text { Soil } \\
\text { Satura- } \\
\text { tion Conc. } \\
\text { (mg/kg) } \\
\text { [CSAT] }\end{array}$ & $\begin{array}{c}\text { Soil-to- } \\
\text { Plant } \\
\text { Uptake } \\
\left(B_{v}\right)\end{array}$ & $\begin{array}{c}\text { Soil-to- } \\
\text { Wet Plant } \\
\text { Uptake } \\
\left(B_{m m}\right)\end{array}$ & $\begin{array}{c}\text { Bioaccum- } \\
\text { ulation } \\
\text { Factor for } \\
\text { Fish } \\
\text { (L/kg) } \\
\text { [BF] }\end{array}$ & $\begin{array}{c}\text { Biotransfer } \\
\text { Factor for } \\
\text { Beef } \\
\text { (day/kg) } \\
{\left[\mathbf{F}_{\mathbf{B}}\right]}\end{array}$ & $\begin{array}{c}\text { Biotransfer } \\
\text { Factor for } \\
\text { Milk } \\
\text { (day/kg) } \\
{\left[F_{\mathrm{w}}\right]}\end{array}$ \\
\hline Trichloroaniline, 2,4,6- & 000634-93-5 & & & & & & & & \\
\hline Trichlorobenzene, 1,2,4- & $000120-82-1$ & $1.0 \mathrm{E}-01$ & $3.6 \mathrm{E}+05$ & $8.3 \mathrm{E}+02$ & $1.8 \mathrm{E}-01$ & $3.7 \mathrm{E}-02$ & $6.5 \mathrm{E}+02$ & $2.5 \mathrm{E}-04$ & $7.9 \mathrm{E}-05$ \\
\hline Trichloroethane, 1,1,1- & $000071-55-6$ & $1.7 \mathrm{E}-02$ & $1.7 \mathrm{E}+04$ & $7.3 \mathrm{E}+02$ & $1.3 \mathrm{E}+00$ & $2.7 \mathrm{E}-01$ & $4.7 \mathrm{E}+01$ & $7.9 \mathrm{E}-06$ & $2.5 \mathrm{E}-06$ \\
\hline Trichloroethane, 1,1,2- & $000079-00-5$ & 8.4E-03 & $3.5 \mathrm{E}+04$ & $8.5 \mathrm{E}+02$ & $2.6 \mathrm{E}+00$ & $5.3 \mathrm{E}-01$ & $1.9 \mathrm{E}+01$ & $2.5 \mathrm{E}-06$ & $7.9 \mathrm{E}-07$ \\
\hline Trichloroethylene & 000079-01-6 & $1.6 \mathrm{E}-02$ & $1.9 \mathrm{E}+04$ & $4.5 \mathrm{E}+02$ & $1.5 \mathrm{E}+00$ & $3.1 \mathrm{E}-01$ & $3.9 \mathrm{E}+01$ & $6.3 \mathrm{E}-06$ & $2.0 \mathrm{E}-06$ \\
\hline Trichlorofluoromethane & $000075-69-4$ & $1.7 \mathrm{E}-02$ & $5.8 \mathrm{E}+03$ & $7.1 \mathrm{E}+02$ & $1.3 \mathrm{E}+00$ & 2.7E-01 & $4.7 \mathrm{E}+01$ & 7.9E-06 & $2.5 \mathrm{E}-06$ \\
\hline Trichlorophenol, 2,4,5- & 000095-95-4 & $5.9 \mathrm{E}-02$ & & & $2.4 \mathrm{E}-01$ & $4.8 \mathrm{E}-02$ & $4.5 \mathrm{E}+02$ & $1.6 \mathrm{E}-04$ & $5.0 \mathrm{E}-05$ \\
\hline Trichlorophenol, 2,4,6- & 000088-06-2 & $5.9 \mathrm{E}-02$ & & & 2.7E-01 & $5.5 \mathrm{E}-02$ & $3.8 \mathrm{E}+02$ & $1.3 \mathrm{E}-04$ & 4.0E-05 \\
\hline Trichlorophenoxy) Propionic Acid, 2(2,4,5- & $000093-72-1$ & $2.5 \mathrm{E}-02$ & & & $2.1 \mathrm{E}-01$ & $4.2 \mathrm{E}-02$ & $5.4 \mathrm{E}+02$ & $2.0 \mathrm{E}-04$ & $6.3 \mathrm{E}-05$ \\
\hline Trichlorophenoxyacetic Acid, 2,4,5- & $000093-76-5$ & 3.6É-02 & & & $1.8 \mathrm{E}-01$ & $3.7 \mathrm{E}-02$ & $6.5 \mathrm{E}+02$ & $2.5 \mathrm{E}-04$ & $7.9 \mathrm{E}-05$ \\
\hline Trichloropropane, 1,1,2- & $000598-77-6$ & & & & & & & & \\
\hline Trichloropropane, 1,2,3- & 000096-18-4 & 4.4E-01 & & & $8.2 \mathrm{E}-02$ & $1.7 \mathrm{E}-02$ & $1.8 \mathrm{E}+03$ & $1.0 \mathrm{E}-03$ & $3.1 \mathrm{E}-04$ \\
\hline Trichloropropene, 1,2,3- & $000096-19-5$ & & & & & & & & \\
\hline Trichlorotoluene, 2,3,6- & $002077-46-5$ & & & & & & & & \\
\hline Trichlorotoluene, alpha 2,6- & 002014-83-7 & & & & & & & & \\
\hline Tridiphane & 058138-08-2 & & & & & & & & \\
\hline Triethyl Lead & $005224-23-7$ & & & & & & & & \\
\hline
\end{tabular}


Table 3.6b. (continued)

\begin{tabular}{|c|c|c|c|c|c|c|c|c|c|}
\hline Chemical & CASRN & $\begin{array}{l}\text { Perme- } \\
\text { ability } \\
\text { Constant } \\
\text { (cm/hr) } \\
\text { [Kp] }\end{array}$ & $\begin{array}{c}\text { Volatil- } \\
\text { ization } \\
\text { Factor } \\
\left(\mathbf{m}^{3} / \mathbf{k g}\right) \\
\text { [VF] }\end{array}$ & $\begin{array}{c}\text { Soil } \\
\text { Satura- } \\
\text { tion Conc. } \\
\text { (mg/kg) } \\
\text { [CSAT] }\end{array}$ & $\begin{array}{c}\text { Soil-to- } \\
\text { Plant } \\
\text { Uptake } \\
\text { (B) }\end{array}$ & $\begin{array}{c}\text { Soil-to- } \\
\text { Wet Plant } \\
\text { Uptake } \\
\left(B_{m}\right)\end{array}$ & $\begin{array}{l}\text { Bioaccum- } \\
\text { ulation } \\
\text { Factor for } \\
\text { Fish } \\
\text { (L/kg) } \\
\text { [BF] }\end{array}$ & $\begin{array}{c}\text { Biotransfer } \\
\text { Factor for } \\
\text { Beef } \\
\text { (day/kg) } \\
{\left[F_{\mathrm{B}}\right]}\end{array}$ & $\begin{array}{c}\text { Biotransfer } \\
\text { Factor for } \\
\text { Milk } \\
\text { (day/kg) } \\
\text { [F }]\end{array}$ \\
\hline Triethylamine & $000121-44-8$ & 4.5E-03 & $1.7 \mathrm{E}+05$ & $2.2 \mathrm{E}+04$ & $5.9 \mathrm{E}+00$ & $1.2 \mathrm{E}+00$ & $6.8 \mathrm{E}+00$ & $6.3 \mathrm{E}-07$ & $2.0 \mathrm{E}-07$ \\
\hline Trifluralin & $001582-09-8$ & $9.9 \mathrm{E}-02$ & & & $3.2 \mathrm{E}-02$ & $6.5 \mathrm{E}-03$ & $6.3 \mathrm{E}+03$ & $5.0 \mathrm{E}-03$ & $1.6 \mathrm{E}-03$ \\
\hline Trimethyl Lead & $007442-13-9$ & & & & & & & & \\
\hline Trimethyl Phosphate & $000512-56-1$ & & & & & & & & \\
\hline Trimethylethyl Lead & $001762-26-1$ & & & & & & & & \\
\hline Trinitrobenzene, 1,3,5- & $000099-35-4$ & $6.8 \mathrm{E}-04$ & & & 7.7E+00 & $1.6 \mathrm{E}+00$ & $4.8 \mathrm{E}+00$ & 4.0E-07 & $1.3 \mathrm{E}-07$ \\
\hline Trinitrophenylmethylnitramine & $000479-45-8$ & $8.9 \mathrm{E}-04$ & & & $2.6 \mathrm{E}+00$ & $5.3 \mathrm{E}-01$ & $1.9 \mathrm{E}+01$ & 2.5E-06 & $7.9 \mathrm{E}-07$ \\
\hline Trinitrotoluene, 2,4,6- & $000118-96-7$ & $3.4 \mathrm{E}-03$ & & & $1.8 \mathrm{E}+00$ & $3.6 \mathrm{E}-01$ & $3.3 \mathrm{E}+01$ & $5.0 \mathrm{E}-06$ & $1.6 \mathrm{E}-06$ \\
\hline Tripropyl Lead & $006618-03-7$ & & & & & & & & \\
\hline Uranium (Soluble Salts) & NA & $1.0 \mathrm{E}-03$ & & & 2.3E-02 & $6.3 \mathrm{E}-04$ & $1.0 \mathrm{E}+01$ & $3.0 \mathrm{E}-04$ & 4.0E-04 \\
\hline Vanadium Pentoxide & $001314-62-1$ & $1.0 \mathrm{E}-03$ & & & & & & & \\
\hline Vanadium Sulfate & $036907-42-3$ & $1.0 \mathrm{E}-03$ & & & & & & & \\
\hline Vanadium, Metallic & $007440-62-2$ & $1.0 \mathrm{E}-03$ & & & $5.5 \mathrm{E}-03$ & $1.4 \mathrm{E}-03$ & $1.0 \mathrm{E}+01$ & $2.5 \mathrm{E}-03$ & $2.0 \mathrm{E}-05$ \\
\hline Vanadyl Sulfate & $027774-13-6$ & $1.0 \mathrm{E}-03$ & & & & & & & \\
\hline Vernolate & $001929-77-7$ & & & & & & & & \\
\hline Vinclozolin & $050471-44-8$ & & & & & & & & \\
\hline Vinyl Acetate & $000108-05-4$ & $1.2 \mathrm{E}-02$ & $1.3 E+04$ & $7.8 \mathrm{E}+02$ & $1.4 \mathrm{E}+01$ & $2.9 \mathrm{E}+00$ & $2.1 \mathrm{E}+00$ & 1.3E-07 & $4.2 \mathrm{E}-08$ \\
\hline
\end{tabular}

Chemical-specific parameters used to calculate PRGs/nonradionuclides

Page 39 of 41 
Table 3.6b. (continued)

\begin{tabular}{|c|c|c|c|c|c|c|c|c|c|}
\hline Chemical & CASRN & $\begin{array}{c}\text { Perme- } \\
\text { ability } \\
\text { Constant } \\
\text { (cm/hr) } \\
\text { [Kp] }\end{array}$ & $\begin{array}{c}\text { Volatil- } \\
\text { ization } \\
\text { Factor } \\
\left(\mathbf{m}^{3} / \mathbf{k g}\right) \\
{[\mathrm{VF}]}\end{array}$ & $\begin{array}{c}\text { Soil } \\
\text { Satura- } \\
\text { tion Conc. } \\
\text { (mg/kg) } \\
\text { [CSAT] }\end{array}$ & $\begin{array}{c}\text { Soil-to- } \\
\text { Plant } \\
\text { Uptake } \\
\left(B_{v}\right)\end{array}$ & $\begin{array}{c}\text { Soil-to- } \\
\text { Wet Plant } \\
\text { Uptake } \\
\left(B_{m}\right)\end{array}$ & $\begin{array}{l}\text { Bioaccum- } \\
\text { ulation } \\
\text { Factor for } \\
\text { Fish } \\
\text { (L/kg) } \\
\text { [BF] }\end{array}$ & $\begin{array}{c}\text { Biotransfer } \\
\text { Factor for } \\
\text { Beef } \\
\text { (day/kg) } \\
{\left[F_{\mathrm{B}}\right]}\end{array}$ & $\begin{array}{c}\text { Biotransfer } \\
\text { Factor for } \\
\text { Milk } \\
\text { (day/kg) } \\
{\left[\mathrm{F}_{\mathrm{m}}\right]}\end{array}$ \\
\hline Vinyl Bromide & $000593-60-2$ & $5.5 \mathrm{E}-03$ & $1.4 \mathrm{E}+04$ & $4.2 \mathrm{E}+03$ & $4.5 \mathrm{E}+00$ & 9.1E-01 & $9.7 \mathrm{E}+00$ & $1.0 \mathrm{E}-06$ & 3.1E-07 \\
\hline Vinyl Chloride & $000075-01-4$ & 7.3E-03 & $8.6 \mathrm{E}+00$ & $2.7 \mathrm{E}+03$ & $5.9 \mathrm{E}+00$ & $1.2 \mathrm{E}+00$ & $6.8 \mathrm{E}+00$ & $6.3 \mathrm{E}-07$ & $2.0 \mathrm{E}-07$ \\
\hline Warfarin & $000081-81-2$ & 4.7E-03 & & & $5.3 \mathrm{E}-01$ & $1.1 E-01$ & $1.6 \mathrm{E}+02$ & 4.0E-05 & $1.3 \mathrm{E}-05$ \\
\hline White Phosphorus & $007723-14-0$ & $1.0 \mathrm{E}-03$ & & & & & & & \\
\hline Xylene, Mixture & $001330-20-7$ & $9.5 \mathrm{E}-02$ & & & $4.6 \mathrm{E}-01$ & $9.4 \mathrm{E}-02$ & $1.9 \mathrm{E}+02$ & $5.0 \mathrm{E}-05$ & $1.6 \mathrm{E}-05$ \\
\hline Xylene, $\mathrm{P}$ - & $000106-42-3$ & 8.0E-02 & & & $5.3 \mathrm{E}-01$ & $1.1 \mathrm{E}-01$ & $1.6 \mathrm{E}+02$ & $4.0 \mathrm{E}-05$ & $1.3 \mathrm{E}-05$ \\
\hline Xylene, m- & $000108-38-3$ & 8.0E-02 & & & $5.3 \mathrm{E}-01$ & $1.1 \mathrm{E}-01$ & $1.6 \mathrm{E}+02$ & $4.0 \mathrm{E}-05$ & $1.3 \mathrm{E}-05$ \\
\hline Xylene, 0- & $000095-47-6$ & $8.0 \mathrm{E}-02$ & & & $5.3 \mathrm{E}-01$ & $1.1 \mathrm{E}-01$ & $1.6 \mathrm{E}+02$ & $4.0 \mathrm{E}-05$ & $1.3 \mathrm{E}-05$ \\
\hline Zinc (Metallic) & $007440-66-6$ & $1.0 \mathrm{E}-03$ & & & $9.9 \mathrm{E}-01$ & $2.6 \mathrm{E}-01$ & $1.0 \mathrm{E}+03$ & $1.0 \mathrm{E}-01$ & $1.0 \mathrm{E}-02$ \\
\hline Zinc Cyanide & $000557-21-1$ & $1.0 \mathrm{E}-03$ & & & & & & & \\
\hline Zinc Phosphide & $001314-84-7$ & $1.0 \mathrm{E}-03$ & & & & & & & \\
\hline Zineb & $012122-67-7$ & & & & & & & & \\
\hline Zirconium & $007440-67-7$ & $1.0 \mathrm{E}-03$ & & & $1.0 \mathrm{E}-03$ & $1.0 \mathrm{E}-04$ & $3.0 \mathrm{E}+02$ & $1.0 \mathrm{E}-06$ & $5.5 \mathrm{E}-07$ \\
\hline
\end{tabular}

Notes:

(1) The same oral slope factor as used for polychlorinated biphenyls.

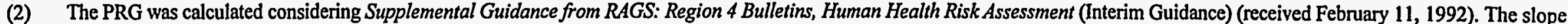
factors for chemicals so marked indicate that a toxicity equivalency factor (TEF) method was used for carcinogenic PAHs based on each compound's relative potency to the potency of benzo[a]pyrene. The following TEFs were used to convert each PAH slope factor to an equivalent slope factor of benzo[a]pyrene [that has a new cancer slope factor of 7.3 (mg/kg/day) ${ }^{-1}$ identified by EPA's CRAVE workgroup]: (1) benzo[a]pyrene, TEF $=1.0 ;(2)$ benzo[a]anthracene, TEF $=0.1 ;(3)$ benzo[b]fluoranthene, TEF $=0.1 ;(4)$ benzo[k]fluoranthene, TEF $=0.01$; (5) chrysene, TEF $=0.001 ;(6)$ dibenz $[\mathrm{a}, \mathrm{h}]$ anthracene, $\mathrm{TEF}=1.0$; and (7) indeno $[1,2,3-\mathrm{c}, \mathrm{d}]$ pyrene, $\mathrm{TEF}=0.1$. 


\section{Table 3.6b. (continued)}

(3) The PRG was calculated considering Supplemental Guidance from RAGS: Region 4 Bulletins, Human Health Risk Assessment (Interim Guidance) from EPA (November 1995). The slope factors for chemicals so marked indicate that a TEF methodology was used for chlorinated dioxin and furan congeners based on each compound's relative potency to the potency of 2,3,7,8tetrachlorodibenzodioxin (TCDD). The following TEFs were used to convert each slope factor to an equivalent slope factor of 2,3,7,8-TCDD: (1) 2,3,7,8-TCDD, TEF =1.0; (2) 2,3,7,8PeCDD, TEF $=0.5$; (3) 2,3,7,8-HxCDD, TEF $=0.1$; (4) $2,3,7,8-\mathrm{HpCDD}, \mathrm{TEF}=0.01$; (5) OCDD, TEF $=0.001 ;(6) 2,3,7,8-\mathrm{TCDF}, \mathrm{TEF}=0.1 ;$ (7) $1,2,3,7,8-\mathrm{PeCDF}$, TEF $=0.5 ;(8)$ $2,3,4,7,8-\mathrm{PeCDF}, \mathrm{TEF}=0.05 ;(9) 2,3,7,8-\mathrm{HxCDF}, \mathrm{TEF}=0.1 ;(10) 2,3,7,8-\mathrm{HpCDF}, \mathrm{TEF}=0.01$; and $(11) \mathrm{OCDF}, \mathrm{TEF}=0.001$. 
3-876

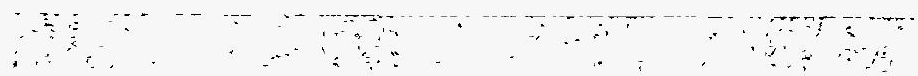


Table 3.6c. Chemical-specific parameters used in calculating risk-based Preliminary Remediation Goals (radionuclides)

\begin{tabular}{|c|c|c|c|c|c|c|c|c|c|c|}
\hline Chemical $^{\dagger}$ & CASRN & $\begin{array}{c}\text { Ingestion } \\
\text { SF } \\
\left(\mathrm{pCi}^{-1}\right)^{-1}\end{array}$ & $\begin{array}{c}\text { Inhalation } \\
\text { SF } \\
(\mathrm{PC} i)^{-2}\end{array}$ & $\begin{array}{c}\text { External } \\
\text { Exposure } \\
\text { SF } \\
\frac{\mathrm{g}}{\mathrm{pCl}-\mathrm{yr}}\end{array}$ & $\begin{array}{c}\text { Half-life } \\
\text { (days) } \\
{\left[\mathbf{T}_{k}\right]}\end{array}$ & $\begin{array}{c}\text { Soil-to- } \\
\text { Plant } \\
\text { Uptake } \\
\left(B_{r}\right)\end{array}$ & $\begin{array}{c}\text { Soil-to- } \\
\text { Wet Plant } \\
\text { Uptake } \\
\left(\mathbf{B}_{\mathbf{v w}}\right)\end{array}$ & $\begin{array}{l}\text { Bioaccum- } \\
\text { ulation } \\
\text { Factor for } \\
\text { Fish } \\
\text { (I/kg) } \\
\text { [BF] }\end{array}$ & 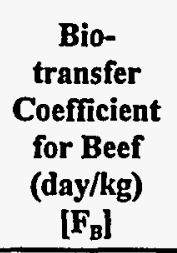 & $\begin{array}{c}\text { Bio- } \\
\text { transfer } \\
\text { Coefficient } \\
\text { for Milk } \\
\text { (day/kg) } \\
{\left[\mathrm{F}_{\mathrm{m}}\right]}\end{array}$ \\
\hline Ac-225 & $014265-85-1$ & $1.42 \mathrm{E}-10$ & $4.16 \mathrm{E}-09$ & $7.81 \mathrm{E}-09$ & $1.00 \mathrm{E}+01$ & $3.5 \mathrm{E}-03$ & $8.8 \mathrm{E}-04$ & & $2.5 \mathrm{E}-05$ & $2.0 \mathrm{E}-05$ \\
\hline Ac-227 & $014952-40-0$ & $3.52 \mathrm{E}-10$ & $7.08 \mathrm{E}-08$ & $2.35 \mathrm{E}-11$ & $7.96 \mathrm{E}+03$ & $3.5 \mathrm{E}-03$ & $8.8 \mathrm{E}-04$ & & $2.5 \mathrm{E}-05$ & $2.0 \mathrm{E}-05$ \\
\hline$A c-227+D$ & $014952-40-0$ & $6.26 \mathrm{E}-10$ & $7.87 \mathrm{E}-08$ & 5.97E-07 & $7.96 \mathrm{E}+03$ & $2.6 \mathrm{E}-01$ & $1.9 \mathrm{E}-02$ & $4.6 \mathrm{E}+02$ & $4.8 \mathrm{E}-02$ & 2.3E-02 \\
\hline Ac-228 & $014331-83-0$ & $1.62 \mathrm{E}-12$ & 3.27E-11 & $3.28 \mathrm{E}-06$ & 2.55E-01 & $3.5 \mathrm{E}-03$ & $8.8 \mathrm{E}-04$ & & $2.5 \mathrm{E}-05$ & $2.0 \mathrm{E}-05$ \\
\hline $\mathrm{Ag}-105$ & $014928-14-4$ & $1.63 \mathrm{E}-12$ & 2.33E-12 & & $4.13 E+01$ & $1.0 \mathrm{E}+00$ & 2.2E-05 & $5.0 \mathrm{E}+00$ & $3.0 \mathrm{E}-03$ & $5.0 \mathrm{E}-05$ \\
\hline Ag-108 & $014391-65-2$ & $6.94 \mathrm{E}-15$ & $9.43 \mathrm{E}-15$ & $5.78 \mathrm{E}-08$ & $1.65 \mathrm{E}-03$ & $1.0 \mathrm{E}+00$ & 2.2E-05 & $5.0 \mathrm{E}+00$ & $3.0 \mathrm{E}-03$ & $5.0 \mathrm{E}-05$ \\
\hline $\mathrm{Ag}-108 \mathrm{~m}$ & $014391-65-2$ & $6.05 \mathrm{E}-12$ & $7.02 \mathrm{E}-11$ & 5.61E-06 & $4.64 \mathrm{E}+04$ & $1.0 \mathrm{E}+00$ & 2.2E-05 & $5.0 \mathrm{E}+00$ & $3.0 \mathrm{E}-03$ & $5.0 \mathrm{E}-05$ \\
\hline$A g-108 m+D$ & $014391-65-2$ & $6.05 \mathrm{E}-12$ & $7.02 \mathrm{E}-11$ & 5.61E-06 & $4.64 \mathrm{E}+04$ & $1.0 \mathrm{E}+00$ & 2.2E-05 & $5.0 \mathrm{E}+00$ & $3.0 \mathrm{E}-03$ & $5.0 \mathrm{E}-05$ \\
\hline $\mathrm{Ag}-109 \mathrm{~m}$ & $014378-38-2$ & $2.71 \mathrm{E}-16$ & $3.46 \mathrm{E}-16$ & $1.16 \mathrm{E}-09$ & $4.58 \mathrm{E}-04$ & $1.0 \mathrm{E}+00$ & 2.2E-05 & $5.0 \mathrm{E}+00$ & $3.0 \mathrm{E}-03$ & $5.0 \mathrm{E}-05$ \\
\hline Ag- 110 & $014391-76-5$ & $2.44 \mathrm{E}-15$ & $3.16 \mathrm{E}-15$ & 1.13E-07 & $2.85 \mathrm{E}-04$ & $1.0 \mathrm{E}+00$ & $2.2 \mathrm{E}-05$ & $5.0 \mathrm{E}+00$ & $3.0 \mathrm{E}-03$ & $5.0 \mathrm{E}-05$ \\
\hline $\mathrm{Ag}-110 \mathrm{~m}$ & $014391-76-5$ & $8.43 \mathrm{E}-12$ & 3.21E-11 & $1.05 \mathrm{E}-05$ & $2.50 \mathrm{E}+02$ & $1.0 \mathrm{E}+00$ & 2.2E-05 & $5.0 \mathrm{E}+00$ & $3.0 \mathrm{E}-03$ & $5.0 \mathrm{E}-05$ \\
\hline $\mathrm{Ag}-111$ & $015760-04-0$ & $6.83 \mathrm{E}-12$ & $5.24 \mathrm{E}-12$ & 8.51E-08 & $7.46 \mathrm{E}+00$ & $1.0 \mathrm{E}+00$ & $2.2 \mathrm{E}-05$ & $5.0 \mathrm{E}+00$ & $3.0 \mathrm{E}-03$ & $5.0 \mathrm{E}-05$ \\
\hline Am-241 & $014596-10-2$ & $3.28 \mathrm{E}-10$ & 3.85E-08 & $4.59 \mathrm{E}-09$ & $1.58 \mathrm{E}+05$ & $1.2 \mathrm{E}-03$ & $2.4 \mathrm{E}-05$ & $3.0 \mathrm{E}+01$ & $4.0 \mathrm{E}-05$ & $1.5 \mathrm{E}-06$ \\
\hline Am-242 & $013981-54-9$ & $1.47 \mathrm{E}-12$ & $1.04 \mathrm{E}-11$ & $5.76 \mathrm{E}-09$ & $6.67 \mathrm{E}-01$ & $1.2 \mathrm{E}-03$ & 2.4E-05 & $3.0 \mathrm{E}+01$ & 4.0E-05 & $1.5 \mathrm{E}-06$ \\
\hline Am-242m & 013981-54-9 & $2.92 \mathrm{E}-10$ & $3.49 \mathrm{E}-08$ & $8.76 \mathrm{E}-11$ & $5.55 \mathrm{E}+04$ & $1.2 \mathrm{E}-03$ & $2.4 \mathrm{E}-05$ & $3.0 \mathrm{E}+01$ & $4.0 \mathrm{E}-05$ & $1.5 \mathrm{E}-06$ \\
\hline Am-243 & 014993-75-0 & $3.27 \mathrm{E}-10$ & $3.82 \mathrm{E}-08$ & $2.43 \mathrm{E}-08$ & $2.69 \mathrm{E}+06$ & $1.2 \mathrm{E}-03$ & $2.4 \mathrm{E}-05$ & $3.0 \mathrm{E}+01$ & $4.0 \mathrm{E}-05$ & $1.5 \mathrm{E}-06$ \\
\hline$A m-243+D$ & 014993-75-0 & $3.31 \mathrm{E}-10$ & $3.82 \mathrm{E}-08$ & $2.66 \mathrm{E}-07$ & $2.69 E+06$ & 7.0E-02 & $2.9 \mathrm{E}-03$ & $6.0 \mathrm{E}+01$ & $1.0 \mathrm{E}-03$ & $6.5 \mathrm{E}-06$ \\
\hline $\mathrm{Ar}-41$ & $014163-25-8$ & & $4.71 \mathrm{E}-16$ & & 7.63E-02 & $0.0 \mathrm{E}+00$ & $0.0 \mathrm{E}+00$ & & $0.0 \mathrm{E}+00$ & $0.0 \mathrm{E}+00$ \\
\hline At-217 & $017239-90-6$ & $8.99 \mathrm{E}-18$ & $5.14 \mathrm{E}-16$ & $8.71 \mathrm{E}-10$ & $3.74 \mathrm{E}-07$ & $1.0 \mathrm{E}+00$ & $2.5 \mathrm{E}-01$ & & $1.0 \mathrm{E}-02$ & $1.0 \mathrm{E}-02$ \\
\hline
\end{tabular}


Table 3.6c. (continued)

\begin{tabular}{|c|c|c|c|c|c|c|c|c|c|c|}
\hline Chemical $^{\dagger}$ & CASRN & $\begin{array}{c}\text { Ingestion } \\
\text { SF } \\
(\mathrm{pCi})^{-1}\end{array}$ & $\begin{array}{c}\text { Inhalation } \\
\text { SF } \\
(\text { (pCi) })^{-1}\end{array}$ & $\begin{array}{c}\text { External } \\
\text { Exposure } \\
\text { SF } \\
\frac{\mathrm{g}}{\mathrm{pCi}-\mathrm{yr}}\end{array}$ & $\begin{array}{c}\text { Half-life } \\
\text { (days) } \\
{\left[T_{R}\right]}\end{array}$ & $\begin{array}{c}\text { Soil-to- } \\
\text { Plant } \\
\text { Uptake } \\
\left(\mathbf{B}_{\mathbf{v}}\right)\end{array}$ & $\begin{array}{c}\text { Soil-to- } \\
\text { Wet Plant } \\
\text { Uptake } \\
\left(B_{\mathrm{vw}}\right)\end{array}$ & $\begin{array}{c}\text { Bioaccum- } \\
\text { ulation } \\
\text { Factor for } \\
\text { Fish } \\
(\mathrm{L} / \mathrm{kg}) \\
{[\mathrm{BF}]} \\
\end{array}$ & $\begin{array}{c}\text { Bio- } \\
\text { transfer } \\
\text { Coefficient } \\
\text { for Beef } \\
\text { (day/kg) } \\
{\left[\mathrm{F}_{\mathrm{B}}\right]} \\
\end{array}$ & $\begin{array}{c}\text { Bio- } \\
\text { transfer } \\
\text { Coefficient } \\
\text { for Milk } \\
\text { (day/kg) } \\
{\left[\mathrm{F}_{\mathrm{m}}\right]}\end{array}$ \\
\hline Au-196 & $014914-16-0$ & $1.30 \mathrm{E}-12$ & $1.04 \mathrm{E}-12$ & $1.41 \mathrm{E}-06$ & $6.18 \mathrm{E}+00$ & $4.0 \mathrm{E}-01$ & $1.0 \mathrm{E}-01$ & & $5.0 \mathrm{E}-03$ & $1.0 \mathrm{E}-05$ \\
\hline Au-198 & $010043-49-0$ & $5.28 \mathrm{E}-12$ & $3.64 \mathrm{E}-12$ & 1.37E-06 & $2.70 \mathrm{E}+00$ & $4.0 \mathrm{E}-01$ & $1.0 \mathrm{E}-01$ & & $5.0 \mathrm{E}-03$ & $1.0 \mathrm{E}-05$ \\
\hline $\mathrm{Ba}-131$ & $014914-75-1$ & $1.70 \mathrm{E}-12$ & $4.79 \mathrm{E}-13$ & $1.27 \mathrm{E}-06$ & $1.18 \mathrm{E}+01$ & $1.0 \mathrm{E}-01$ & $3.0 \mathrm{E}-03$ & $4.0 \mathrm{E}+00$ & $2.0 \mathrm{E}-04$ & $4.8 \mathrm{E}-04$ \\
\hline Ba-133 & $013981-41-4$ & $2.70 \mathrm{E}-12$ & 4.03E-12 & 9.15E-07 & $3.83 E+03$ & $1.0 \mathrm{E}-01$ & $3.0 \mathrm{E}-03$ & $4.0 \mathrm{E}+00$ & $2.0 \mathrm{E}-04$ & $4.8 \mathrm{E}-04$ \\
\hline Ba-133m & $013981-41-4$ & $2.76 \mathrm{E}-12$ & $5.60 \mathrm{E}-13$ & $1.00 \mathrm{E}-07$ & $1.62 \mathrm{E}+00$ & $1.0 \mathrm{E}-01$ & $3.0 \mathrm{E}-03$ & $4.0 \mathrm{E}+00$ & $2.0 \mathrm{E}-04$ & 4.8E-04 \\
\hline Ba-137m & 013981-97-0 & $2.43 \mathrm{E}-15$ & $1.57 \mathrm{E}-15$ & 2.21E-06 & $1.77 \mathrm{E}-03$ & $1.0 \mathrm{E}-01$ & $3.0 \mathrm{E}-03$ & $4.0 \mathrm{E}+00$ & $2.0 \mathrm{E}-04$ & $4.8 \mathrm{E}-04$ \\
\hline Ba-139 & $014378-25-7$ & $3.04 \mathrm{E}-13$ & $1.53 \mathrm{E}-13$ & $8.35 \mathrm{E}-08$ & $5.77 \mathrm{E}-02$ & $1.0 \mathrm{E}-01$ & $3.0 \mathrm{E}-03$ & $4.0 \mathrm{E}+00$ & $2.0 \mathrm{E}-04$ & $4.8 \mathrm{E}-04$ \\
\hline $\mathrm{Ba}-140$ & 014798-08-4 & $1.18 \mathrm{E}-11$ & $3.17 \mathrm{E}-12$ & $6.00 \mathrm{E}-07$ & $1.28 \mathrm{E}+01$ & $1.0 \mathrm{E}-01$ & $3.0 \mathrm{E}-03$ & $4.0 \mathrm{E}+00$ & 2.0E-04 & $4.8 \mathrm{E}-04$ \\
\hline $\mathrm{Be}-7$ & $013966-02-4$ & $8.64 \mathrm{E}-14$ & $1.78 \mathrm{E}-13$ & $1.73 \mathrm{E}-07$ & $5.34 \mathrm{E}+01$ & $1.0 \mathrm{E}-02$ & $2.5 \mathrm{E}-03$ & $1.0 \mathrm{E}+02$ & $1.0 \mathrm{E}-03$ & $9.0 \mathrm{E}-07$ \\
\hline Bi-206 & 015776-19-9 & $7.11 \mathrm{E}-12$ & $5.07 \mathrm{E}-12$ & $1.20 \mathrm{E}-05$ & $6.24 \mathrm{E}+00$ & $3.5 \mathrm{E}-02$ & $8.8 \mathrm{E}-03$ & $1.0 \mathrm{E}+01$ & 4.0E-04 & $5.0 \mathrm{E}-04$ \\
\hline Bi-207 & $013982-38-2$ & 5.05E-12 & $9.42 \mathrm{E}-12$ & $5.49 \mathrm{E}-06$ & $1.22 \mathrm{E}+04$ & $3.5 \mathrm{E}-02$ & $8.8 \mathrm{E}-03$ & $1.0 \mathrm{E}+01$ & $4.0 \mathrm{E}-04$ & $5.0 \mathrm{E}-04$ \\
\hline Bi-210 & $014331-79-4$ & $7.29 \mathrm{E}-12$ & $5.12 \mathrm{E}-11$ & $0.00 \mathrm{E}+00$ & $5.01 \mathrm{E}+00$ & $3.5 \mathrm{E}-02$ & $8.8 \mathrm{E}-03$ & $1.0 \mathrm{E}+01$ & $4.0 \mathrm{E}-04$ & $5.0 \mathrm{E}-04$ \\
\hline Bi-211 & $015229-37-5$ & $1.82 \mathrm{E}-14$ & $1.74 \mathrm{E}-12$ & $1.48 \mathrm{E}-07$ & $1.48 \mathrm{E}-03$ & $3.5 \mathrm{E}-02$ & $8.8 \mathrm{E}-03$ & $1.0 \mathrm{E}+01$ & $4.0 \mathrm{E}-04$ & $5.0 \mathrm{E}-04$ \\
\hline $\mathrm{Bi}-212$ & 014913-49-6 & $6.20 \mathrm{E}-13$ & $3.65 \mathrm{E}-11$ & $6.67 \mathrm{E}-07$ & 4.21E-02 & $3.5 \mathrm{E}-02$ & $8.8 \mathrm{E}-03$ & $1.0 \mathrm{E}+01$ & $4.0 \mathrm{E}-04$ & $5.0 \mathrm{E}-04$ \\
\hline $\mathrm{Bi}-213$ & $015776-20-2$ & $4.40 \mathrm{E}-13$ & $3.09 \mathrm{E}-11$ & 4.62E-07 & 3.17E-02 & $3.5 \mathrm{E}-02$ & $8.8 \mathrm{E}-03$ & $1.0 \mathrm{E}+01$ & $4.0 \mathrm{E}-04$ & $5.0 \mathrm{E}-04$ \\
\hline $\mathrm{Bi}-214$ & 014733-03-0 & $1.95 \mathrm{E}-13$ & $1.46 \mathrm{E}-11$ & $6.02 \mathrm{E}-06$ & $1.38 \mathrm{E}-02$ & $3.5 \mathrm{E}-02$ & $8.8 \mathrm{E}-03$ & $1.0 \mathrm{E}+01$ & $4.0 \mathrm{E}-04$ & $5.0 \mathrm{E}-04$ \\
\hline $\mathrm{Br}-82$ & $014686-69-2$ & $1.42 \mathrm{E}-12$ & $7.86 \mathrm{E}-13$ & $1.01 \mathrm{E}-05$ & $1.47 \mathrm{E}+00$ & $1.5 \mathrm{E}+00$ & $3.8 \mathrm{E}-01$ & $4.0 \mathrm{E}+02$ & $2.5 \mathrm{E}-02$ & $2.0 \mathrm{E}-02$ \\
\hline C-11 & 014333-33-6 & 4.49E-14 & $3.38 \mathrm{E}-14$ & $3.61 \mathrm{E}-06$ & $1.42 \mathrm{E}-02$ & & & & & \\
\hline $\mathrm{C}-14$ & $014762-75-5$ & $1.03 \mathrm{E}-12$ & $6.99 \mathrm{E}-15$ & $0.00 \mathrm{E}+00$ & $2.09 \mathrm{E}+06$ & & & & & \\
\hline
\end{tabular}


Table 3.6c. (continued)

\begin{tabular}{|c|c|c|c|c|c|c|c|c|c|c|}
\hline Chemical $^{\dagger}$ & CASRN & $\begin{array}{c}\text { Ingestion } \\
\text { SF } \\
(\mathrm{pCi})^{-4}\end{array}$ & $\begin{array}{c}\text { Inhalation } \\
\text { SF } \\
(\mathrm{pCi})^{-2}\end{array}$ & $\begin{array}{c}\text { External } \\
\text { Exposure } \\
\text { SF } \\
\frac{\mathbf{g}}{\mathbf{p C i}-\mathbf{y r}}\end{array}$ & $\begin{array}{c}\text { Half-life } \\
\text { (days) } \\
{\left[\mathrm{T}_{\mathrm{R}}\right]}\end{array}$ & $\begin{array}{c}\text { Soil-to- } \\
\text { Plant } \\
\text { Uptake } \\
\text { (B, }\end{array}$ & $\begin{array}{c}\text { Soil-to- } \\
\text { Wet Plant } \\
\text { Uptake } \\
\left(B_{v_{w}}\right)\end{array}$ & $\begin{array}{l}\text { Bioaccum- } \\
\text { ulation } \\
\text { Factor for } \\
\text { Fish } \\
\text { (L/kg) } \\
\text { [BF] }\end{array}$ & $\begin{array}{c}\text { Bio- } \\
\text { transfer } \\
\text { Coeffrcient } \\
\text { for Beef } \\
\text { (day/kg) } \\
{\left[F_{B}\right]}\end{array}$ & $\begin{array}{c}\text { Bio- } \\
\text { transfer } \\
\text { Coefficient } \\
\text { for Milk } \\
\text { (day/kg) } \\
{\left[\mathrm{F}_{\mathrm{m}}\right]}\end{array}$ \\
\hline$C-15$ & $015929-23-4$ & $6.62 \mathrm{E}-16$ & $8.06 \mathrm{E}-16$ & & $2.84 \mathrm{E}-05$ & & & & & \\
\hline $\mathrm{Ca}-45$ & $013966-05-7$ & $2.02 \mathrm{E}-12$ & $2.51 \mathrm{E}-12$ & $3.88 \mathrm{E}-18$ & $1.63 \mathrm{E}+02$ & $3.5 \mathrm{E}+00$ & $8.8 \mathrm{E}-01$ & & $2.0 \mathrm{E}-03$ & 3.0E-03 \\
\hline $\mathrm{Ca}-47$ & 001439-99-2 & $6.66 \mathrm{E}-12$ & $5.22 \mathrm{E}-12$ & 4.12E-06 & $4.54 \mathrm{E}+00$ & $3.5 \mathrm{E}+00$ & $8.8 \mathrm{E}-01$ & & $2.0 \mathrm{E}-03$ & $3.0 \mathrm{E}-03$ \\
\hline Cd-109 & $014109-32-1$ & $8.01 E-12$ & $1.85 \mathrm{E}-11$ & $5.62 \mathrm{E}-10$ & $4.64 \mathrm{E}+02$ & $5.5 \mathrm{E}-01$ & $1.4 \mathrm{E}-01$ & $2.0 \mathrm{E}+02$ & $4.0 \mathrm{E}-04$ & $1.0 \mathrm{E}-03$ \\
\hline Cd-115 & $014336-68-6$ & $7.29 \mathrm{E}-12$ & $4.93 \mathrm{E}-12$ & 7.02E-07 & $2.23 E+00$ & $5.5 \mathrm{E}-01$ & $1.4 \mathrm{E}-01$ & $2.0 \mathrm{E}+02$ & $4.0 \mathrm{E}-04$ & $1.0 \mathrm{E}-03$ \\
\hline Cd-115m & $014336-68-6$ & $1.42 \mathrm{E}-11$ & $1.70 \mathrm{E}-11$ & 8.55E-08 & $4.46 \mathrm{E}+01$ & $5.5 \mathrm{E}-01$ & $1.4 \mathrm{E}-01$ & $2.0 \mathrm{E}+02$ & $4.0 \mathrm{E}-04$ & $1.0 \mathrm{E}-03$ \\
\hline Ce-141 & $013967-74-3$ & $3.91 \mathrm{E}-12$ & $4.32 \mathrm{E}-12$ & 1.41E-07 & $3.25 \mathrm{E}+01$ & $5.0 \mathrm{E}-02$ & $3.0 \mathrm{E}-03$ & $3.0 \mathrm{E}+01$ & 2.0E-05 & $3.0 \mathrm{E}-05$ \\
\hline Ce-143 & $014119-19-8$ & $5.91 \mathrm{E}-12$ & $3.84 \mathrm{E}-12$ & 7.32E-07 & $1.38 \mathrm{E}+00$ & $5.0 \mathrm{E}-02$ & $3.0 \mathrm{E}-03$ & $3.0 \mathrm{E}+01$ & $2.0 \mathrm{E}-05$ & $3.0 \mathrm{E}-05$ \\
\hline Ce-144 & $014762-78-8$ & $2.96 \mathrm{E}-11$ & $1.08 \mathrm{E}-10$ & $2.58 \mathrm{E}-08$ & $2.84 \mathrm{E}+02$ & $5.0 \mathrm{E}-02$ & $3.0 \mathrm{E}-03$ & $3.0 \mathrm{E}+01$ & $2.0 \mathrm{E}-05$ & $3.0 \mathrm{E}-05$ \\
\hline Ce-144+D & $014762-78-8$ & $2.97 \mathrm{E}-11$ & $1.08 \mathrm{E}-10$ & $1.56 \mathrm{E}-07$ & $2.84 \mathrm{E}+02$ & $9.0 \mathrm{E}-02$ & $7.0 \mathrm{E}-03$ & $2.3 E+02$ & $6.2 \mathrm{E}-04$ & $7.0 \mathrm{E}-05$ \\
\hline $\mathrm{Cl}-36$ & $013981-43-6$ & $2.23 \mathrm{E}-12$ & $1.30 \mathrm{E}-12$ & $0.00 \mathrm{E}+00$ & $1.10 \mathrm{E}+08$ & $7.0 \mathrm{E}+01$ & $1.8 \mathrm{E}+01$ & & $2.0 \mathrm{E}-02$ & $1.7 \mathrm{E}-02$ \\
\hline $\mathrm{Cl}-38$ & $014158-34-0$ & $2.07 \mathrm{E}-13$ & $1.63 \mathrm{E}-13$ & $6.47 \mathrm{E}-06$ & $2.58 \mathrm{E}-02$ & $7.0 \mathrm{E}+01$ & $1.8 \mathrm{E}+0 \mathrm{I}$ & & $2.0 \mathrm{E}-02$ & $1.7 \mathrm{E}-02$ \\
\hline $\mathrm{Cm}-242$ & $015510-73-3$ & 3.83E-11 & $3.16 \mathrm{E}-09$ & $2.34 \mathrm{E}-11$ & $1.63 E+02$ & $1.1 \mathrm{E}-03$ & $5.9 \mathrm{E}-05$ & $3.0 \mathrm{E}+01$ & $2.0 \mathrm{E}-05$ & $4.0 \mathrm{E}-07$ \\
\hline $\mathrm{Cm}-243$ & $015757-87-6$ & $2.51 \mathrm{E}-10$ & $2.89 \mathrm{E}-08$ & 1.71E-07 & $1.04 \mathrm{E}+04$ & $1.1 \mathrm{E}-03$ & $5.9 \mathrm{E}-05$ & $3.0 \mathrm{E}+01$ & $2.0 \mathrm{E}-05$ & $4.0 \mathrm{E}-07$ \\
\hline $\mathrm{Cm}-243+\mathrm{D}$ & $015757-87-6$ & 2.52E-10 & $2.90 \mathrm{E}-08$ & $1.72 \mathrm{E}-07$ & $1.04 \mathrm{E}+04$ & $4.4 \mathrm{E}-01$ & $2.3 \mathrm{E}-02$ & $9.9 \mathrm{E}+02$ & $4.8 \mathrm{E}-02$ & $5.2 \mathrm{E}-03$ \\
\hline $\mathrm{Cm}-244$ & $013981-15-2$ & $2.11 \mathrm{E}-10$ & $2.43 \mathrm{E}-08$ & $2.07 \mathrm{E}-11$ & $6.61 E+03$ & 1.1E-03 & $5.9 \mathrm{E}-05$ & $3.0 \mathrm{E}+01$ & $2.0 \mathrm{E}-05$ & $4.0 \mathrm{E}-07$ \\
\hline $\mathrm{Cm}-245$ & $015621-76-8$ & 3.35E-10 & $3.92 \mathrm{E}-08$ & $5.51 \mathrm{E}-08$ & $3.10 \mathrm{E}+06$ & $1.1 \mathrm{E}-03$ & $5.9 \mathrm{E}-05$ & $3.0 \mathrm{E}+01$ & $2.0 \mathrm{E}-05$ & $4.0 \mathrm{E}-07$ \\
\hline $\mathrm{Cm}-246$ & $015757-90-1$ & 3.32E-10 & $3.90 \mathrm{E}-08$ & $1.81 \mathrm{E}-11$ & $1.73 E+06$ & $1.1 \mathrm{E}-03$ & $5.9 \mathrm{E}-05$ & $3.0 \mathrm{E}+01$ & $2.0 \mathrm{E}-05$ & $4.0 \mathrm{E}-07$ \\
\hline $\mathrm{Cm}-247$ & $015758-32-4$ & $3.09 \mathrm{E}-10$ & $3.58 \mathrm{E}-08$ & $1.03 \mathrm{E}-06$ & $5.69 E+09$ & $1.1 \mathrm{E}-03$ & $5.9 \mathrm{E}-05$ & $3.0 \mathrm{E}+01$ & $2.0 \mathrm{E}-05$ & $4.0 \mathrm{E}-07$ \\
\hline
\end{tabular}

Chemical-specific parameters used in calculating PRGs/radionuclides

Page 3 of 18 
Table 3.6c. (continued)

\begin{tabular}{|c|c|c|c|c|c|c|c|c|c|c|}
\hline Chemical $^{\dagger}$ & CASRN & $\begin{array}{c}\text { Ingestion } \\
\text { SF } \\
(\mathrm{pCI})^{-1}\end{array}$ & $\begin{array}{c}\text { Inhalation } \\
\text { SF } \\
(\mathrm{pCi})^{-1}\end{array}$ & $\begin{array}{c}\text { External } \\
\text { Exposure } \\
\text { SF } \\
\frac{\mathbf{g}}{\mathbf{p C l}-\mathbf{y r}}\end{array}$ & $\begin{array}{c}\text { Half-life } \\
\text { (days) } \\
{\left[\mathbf{T}_{\mathbf{n}}\right]}\end{array}$ & $\begin{array}{c}\text { Soil-to- } \\
\text { Plant } \\
\text { Uptake } \\
\left(\mathbf{B}_{v}\right)\end{array}$ & $\begin{array}{c}\text { Soil-to- } \\
\text { Wet Plant } \\
\text { Uptake } \\
\left(\mathbf{B}_{\mathrm{vw}}\right)\end{array}$ & $\begin{array}{l}\text { Bioaccum- } \\
\text { ulation } \\
\text { Factor for } \\
\text { Fish } \\
\text { (L/kg) } \\
\text { [BF] }\end{array}$ & $\begin{array}{c}\text { Bio- } \\
\text { transfer } \\
\text { Coefficient } \\
\text { for Beef } \\
\text { (day/kg) } \\
{\left[\mathrm{F}_{\mathrm{B}}\right]}\end{array}$ & $\begin{array}{c}\text { Bio- } \\
\text { transfer } \\
\text { Coefficient } \\
\text { for Milk } \\
\text { (day/kg) } \\
{\left[\mathrm{F}_{\mathrm{m}}\right]}\end{array}$ \\
\hline $\mathrm{Cm}-248$ & 015758-33-5 & $1.31 \mathrm{E}-09$ & $1.46 \mathrm{E}-07$ & $1.47 \mathrm{E}-11$ & $1.24 \mathrm{E}+08$ & $1.1 \mathrm{E}-03$ & $5.9 \mathrm{E}-05$ & $3.0 \mathrm{E}+01$ & $2.0 \mathrm{E}-05$ & 4.0E-07 \\
\hline Co-57 & 013981-50-5 & $9.71 \mathrm{E}-13$ & $2.88 \mathrm{E}-12$ & 2.07E-07 & $2.71 \mathrm{E}+02$ & $5.4 \mathrm{E}-02$ & $2.3 \mathrm{E}-02$ & $3.0 \mathrm{E}+02$ & $1.0 \mathrm{E}-04$ & 7.0E-05 \\
\hline Co-58 & 013981-38-9 & $2.82 \mathrm{E}-12$ & $5.17 \mathrm{E}-12$ & 3.73E-06 & $7.08 \mathrm{E}+01$ & $5.4 \mathrm{E}-02$ & 2.3E-02 & $3.0 \mathrm{E}+02$ & $1.0 \mathrm{E}-04$ & 7.0E-05 \\
\hline $\mathrm{Co}-58 \mathrm{~m}$ & 013981-38-9 & $9.46 \mathrm{E}-14$ & $8.90 \mathrm{E}-14$ & $3.21 \mathrm{E}-11$ & 3.81E-01 & $5.4 \mathrm{E}-02$ & $2.3 \mathrm{E}-02$ & $3.0 \mathrm{E}+02$ & $1.0 \mathrm{E}-04$ & 7.0E-05 \\
\hline Co-60 & $010198-40-0$ & $1.89 \mathrm{E}-11$ & $6.88 \mathrm{E}-11$ & $9.76 \mathrm{E}-06$ & $1.92 \mathrm{E}+03$ & $5.4 \mathrm{E}-02$ & 2.3E-02 & $3.0 \mathrm{E}+02$ & $1.0 \mathrm{E}-04$ & 7.0E-05 \\
\hline Cr:51 & $014392-02-0$ & $1.38 \mathrm{E}-13$ & $1.74 \mathrm{E}-13$ & $1.02 \mathrm{E}-07$ & 2.77E+01 & $4.0 \mathrm{E}-02$ & $1.0 \mathrm{E}-04$ & $2.0 \mathrm{E}+02$ & $9.0 \mathrm{E}-03$ & $1.0 \mathrm{E}-05$ \\
\hline Cs-131 & $014914-76-2$ & $1.80 \mathrm{E}-13$ & $1.06 \mathrm{E}-13$ & 2.34E-09 & $9.69 \mathrm{E}+00$ & $1.1 \mathrm{E}-01$ & $1.4 \mathrm{E}-02$ & $2.0 \mathrm{E}+03$ & $5.0 \mathrm{E}-02$ & $7.9 \mathrm{E}-03$ \\
\hline Cs-134 & $013967-70-9$ & 4.73E-11 & $2.89 \mathrm{E}-11$ & $5.88 \mathrm{E}-06$ & $7.52 \mathrm{E}+02$ & $1.1 \mathrm{E}-01$ & $1.4 \mathrm{E}-02$ & $2.0 \mathrm{E}+03$ & $5.0 \mathrm{E}-02$ & $7.9 \mathrm{E}-03$ \\
\hline Cs-134m & $013967-70-9$ & $4.54 \mathrm{E}-14$ & $3.10 \mathrm{E}-14$ & $1.96 \mathrm{E}-08$ & $1.21 \mathrm{E}-01$ & $1.1 \mathrm{E}-01$ & $1.4 \mathrm{E}-02$ & $2.0 \mathrm{E}+03$ & $5.0 \mathrm{E}-02$ & $7.9 \mathrm{E}-03$ \\
\hline Cs-135 & $015726-30-4$ & $4.53 \mathrm{E}-12$ & 2.71E-12 & $0.00 \mathrm{E}+00$ & $8.40 \mathrm{E}+08$ & $1.1 \mathrm{E}-01$ & $1.4 \mathrm{E}-02$ & $2.0 \mathrm{E}+03$ & $5.0 \mathrm{E}-02$ & $7.9 \mathrm{E}-03$ \\
\hline Cs-136 & $014234-29-8$ & $7.74 \mathrm{E}-12$ & $4.65 \mathrm{E}-12$ & $8.13 \mathrm{E}-06$ & $1.32 \mathrm{E}+01$ & $1.1 \mathrm{E}-01$ & $1.4 \mathrm{E}-02$ & $2.0 E+03$ & $5.0 \mathrm{E}-02$ & $7.9 \mathrm{E}-03$ \\
\hline Cs-137 & $010045-97-3$ & $3.16 \mathrm{E}-11$ & $1.91 \mathrm{E}-11$ & $0.00 \mathrm{E}+00$ & $1.10 \mathrm{E}+04$ & $1.1 \mathrm{E}-01$ & $1.4 \mathrm{E}-02$ & $2.0 \mathrm{E}+03$ & $5.0 \mathrm{E}-02$ & $7.9 \mathrm{E}-03$ \\
\hline Cs- $137+D$ & $010045-97-3$ & $3.16 \mathrm{E}-11$ & $1.91 \mathrm{E}-11$ & $2.09 \mathrm{E}-06$ & $1.10 \mathrm{E}+04$ & $2.1 \mathrm{E}-01$ & $1.7 \mathrm{E}-02$ & $2.0 \mathrm{E}+03$ & $5.0 \mathrm{E}-02$ & $8.4 \mathrm{E}-03$ \\
\hline Cs-138 & 015758-29-9 & $1.76 \mathrm{E}-13$ & $1.30 \mathrm{E}-13$ & $9.45 \mathrm{E}-06$ & $2.24 \mathrm{E}-02$ & 1.1E-01 & $1.4 \mathrm{E}-02$ & $2.0 \mathrm{E}+03$ & $5.0 \mathrm{E}-02$ & $7.9 \mathrm{E}-03$ \\
\hline Cu-64 & $013981-25-4$ & $5.25 \mathrm{E}-13$ & $4.18 \mathrm{E}-13$ & $6.72 \mathrm{E}-07$ & $5.29 \mathrm{E}-01$ & $8.0 \mathrm{E}-01$ & 8.0E-02 & $2.0 \mathrm{E}+02$ & $9.0 \mathrm{E}-03$ & $1.5 \mathrm{E}-03$ \\
\hline Dy-165 & $013967-64-1$ & $3.26 \mathrm{E}-13$ & $2.24 \mathrm{E}-13$ & $6.18 \mathrm{E}-08$ & $9.71 \mathrm{E}-02$ & $1.0 \mathrm{E}-02$ & $2.5 \mathrm{E}-03$ & & $5.5 \mathrm{E}-03$ & $2.0 \mathrm{E}-05$ \\
\hline Dy-166 & $015840-01-4$ & $9.42 \mathrm{E}-12$ & $7.82 \mathrm{E}-12$ & 2.72E-08 & $3.40 \mathrm{E}+00$ & $1.0 \mathrm{E}-02$ & 2.5E-03 & & $5.5 \mathrm{E}-03$ & 2.0E-05 \\
\hline Er-169 & 015840-13-8 & $2.12 \mathrm{E}-12$ & $1.51 \mathrm{E}-12$ & $6.52 \mathrm{E}-12$ & $9.40 \mathrm{E}+00$ & $1.0 \mathrm{E}-02$ & $2.5 \mathrm{E}-03$ & & $4.0 \mathrm{E}-03$ & $2.0 \mathrm{E}-05$ \\
\hline Er-171 & 014391-45-8 & $1.63 \mathrm{E}-12$ & $7.50 \mathrm{E}-13$ & $1.04 \mathrm{E}-06$ & 3.13E-01 & $1.0 \mathrm{E}-02$ & 2.5E-03 & & $4.0 \mathrm{E}-03$ & 2.0E-05 \\
\hline
\end{tabular}

Chemical-specific varameters used in calculatino PRGs/radinnurlidec . 


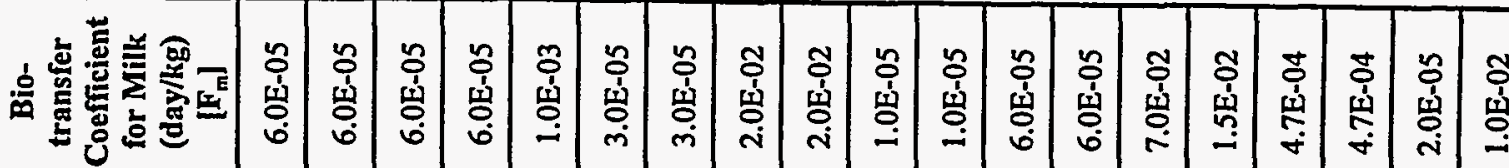

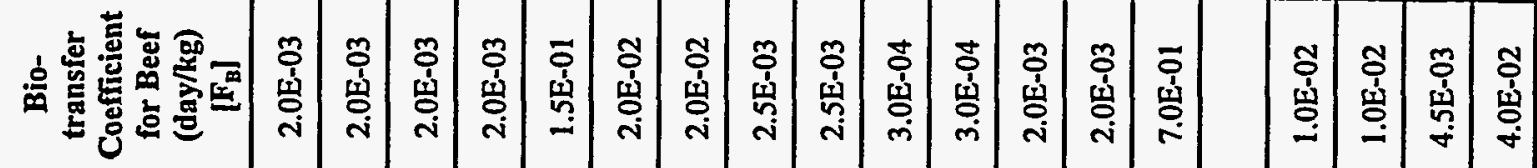

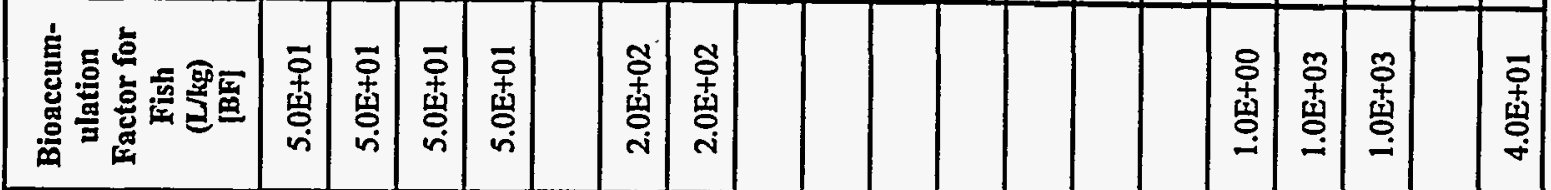

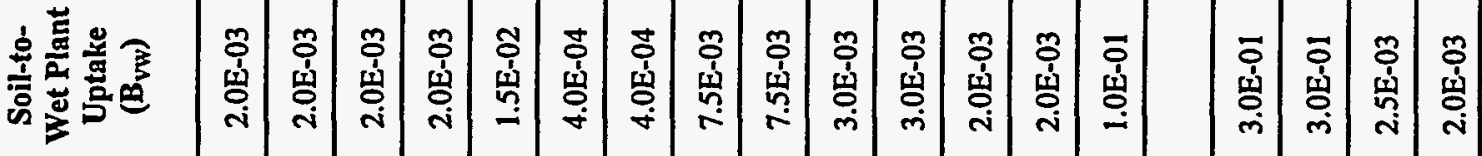

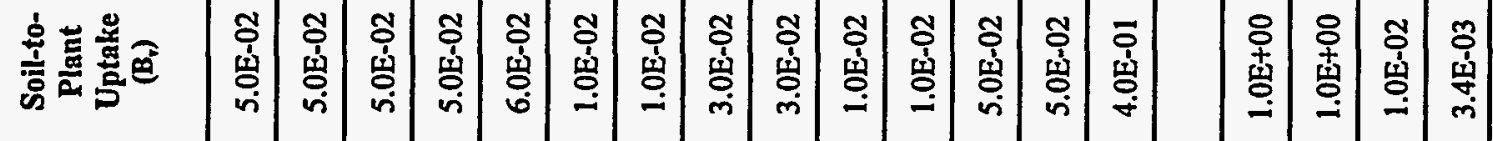

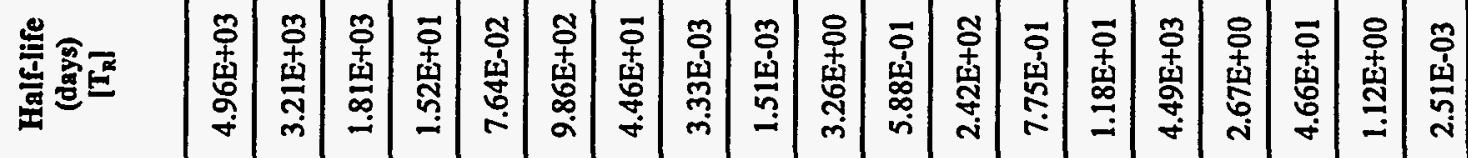

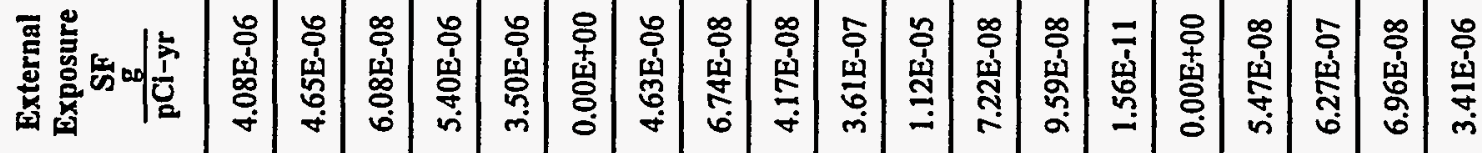

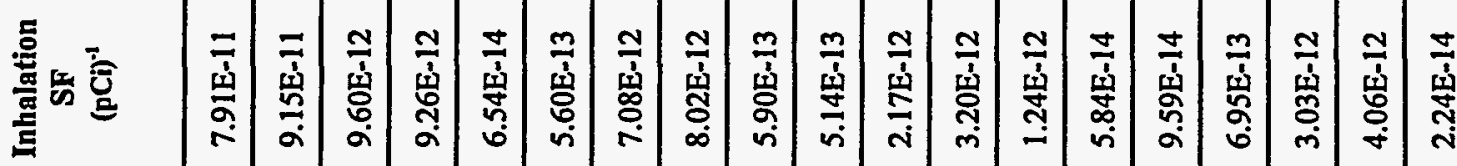

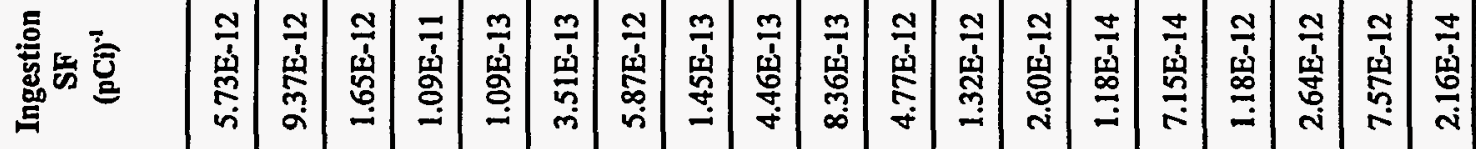

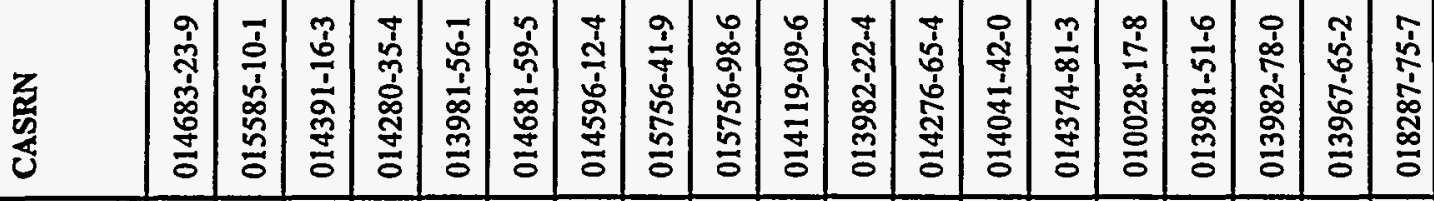

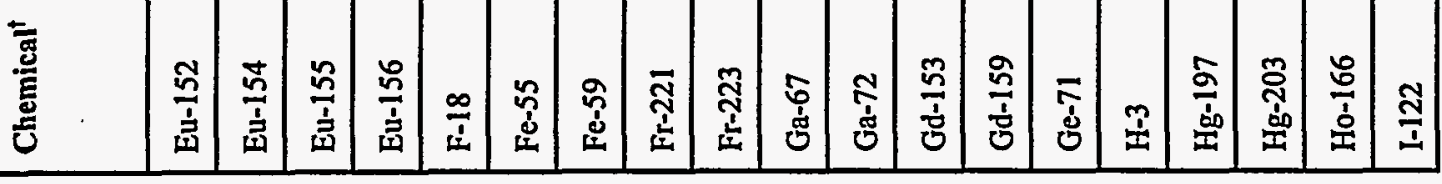


Table 3.6c. (continued)

\begin{tabular}{|c|c|c|c|c|c|c|c|c|c|c|}
\hline Chemical $^{\dagger}$ & CASRN & $\begin{array}{c}\text { Ingestion } \\
\text { SF } \\
(\mathrm{pCi})^{-1}\end{array}$ & $\begin{array}{c}\text { Inhalation } \\
\text { SF } \\
(\mathbf{p C i})^{-1}\end{array}$ & $\begin{array}{c}\text { External } \\
\text { Exposure } \\
\text { SF } \\
\frac{\mathbf{g}}{\mathbf{p C i}-\mathbf{y r}}\end{array}$ & $\begin{array}{l}\text { Half-life } \\
\text { (days) } \\
{\left[\mathbf{T}_{\mathbf{R}} \mid\right.}\end{array}$ & $\begin{array}{c}\text { Soil-to- } \\
\text { Plant } \\
\text { Uptake } \\
\left(B_{r}\right)\end{array}$ & $\begin{array}{c}\text { Soil-to- } \\
\text { Wet Plant } \\
\text { Uptake } \\
\left(\mathbf{B}_{\mathrm{vw}}\right)\end{array}$ & $\begin{array}{l}\text { Bioaccum- } \\
\text { ulation } \\
\text { Factor for } \\
\text { Fish } \\
\text { (L/kg) } \\
\text { [BF] }\end{array}$ & $\begin{array}{c}\text { Bio- } \\
\text { transfer } \\
\text { Coefficient } \\
\text { for Beef } \\
(\text { day/kg) } \\
{\left[F_{B}\right]}\end{array}$ & $\begin{array}{c}\text { Bio- } \\
\text { transfer } \\
\text { Coefficient } \\
\text { for Milk } \\
\text { (day/kg) } \\
{\left[F_{m}\right]}\end{array}$ \\
\hline I-123 & 015715-08-9 & $5.42 \mathrm{E}-13$ & $2.94 \mathrm{E}-13$ & $2.52 \mathrm{E}-07$ & $5.46 \mathrm{E}-01$ & $3.4 \mathrm{E}-03$ & $2.0 \mathrm{E}-03$ & $4.0 \mathrm{E}+01$ & $4.0 \mathrm{E}-02$ & $1.0 \mathrm{E}-02$ \\
\hline I-125 & 014158-31-7 & $2.58 \mathrm{E}-11$ & $1.71 \mathrm{E}-11$ & $2.39 \mathrm{E}-09$ & $6.01 E+01$ & $3.4 \mathrm{E}-03$ & 2.0E-03 & $4.0 \mathrm{E}+01$ & $4.0 \mathrm{E}-02$ & $1.0 \mathrm{E}-02$ \\
\hline I-126 & $014158-32-8$ & $4.82 \mathrm{E}-11$ & $3.15 \mathrm{E}-11$ & $1.49 \mathrm{E}-06$ & $1.29 \mathrm{E}+01$ & $3.4 \mathrm{E}-03$ & $2.0 \mathrm{E}-03$ & $4.0 \mathrm{E}+01$ & $4.0 \mathrm{E}-02$ & $1.0 \mathrm{E}-02$ \\
\hline I-129 & 015046-84-1 & $1.84 \mathrm{E}-10$ & $1.22 \mathrm{E}-10$ & 2.69E-09 & $5.73 E+09$ & $3.4 \mathrm{E}-03$ & $2.0 \mathrm{E}-03$ & $4.0 \mathrm{E}+01$ & $4.0 \mathrm{E}-02$ & $1.0 \mathrm{E}-02$ \\
\hline I-130 & $014914-02-4$ & $4.85 \mathrm{E}-12$ & $2.61 \mathrm{E}-12$ & 7.93E-06 & $5.17 \mathrm{E}-01$ & $3.4 \mathrm{E}-03$ & $2.0 \mathrm{E}-03$ & $4.0 \mathrm{E}+01$ & $4.0 \mathrm{E}-02$ & $1.0 \mathrm{E}-02$ \\
\hline I-13I & $010043-66-0$ & 3.62E-11 & $2.33 \mathrm{E}-11$ & $1.25 \mathrm{E}-06$ & $8.04 E+00$ & $3.4 \mathrm{E}-03$ & 2.0E-03 & $4.0 \mathrm{E}+01$ & $4.0 \mathrm{E}-02$ & $1.0 \mathrm{E}-02$ \\
\hline I-132 & $014683-16-0$ & $6.62 \mathrm{E}-13$ & $3.52 \mathrm{E}-13$ & 8.75E-06 & $9.58 \mathrm{E}-02$ & $3.4 \mathrm{E}-03$ & $2.0 \mathrm{E}-03$ & $4.0 \mathrm{E}+01$ & $4.0 \mathrm{E}-02$ & $1.0 \mathrm{E}-02$ \\
\hline I-133 & $014834-67-4$ & $1.06 \mathrm{E}-11$ & $6.02 \mathrm{E}-12$ & $2.20 \mathrm{E}-06$ & 8.67E-01 & $3.4 \mathrm{E}-03$ & $2.0 \mathrm{E}-03$ & $4.0 \mathrm{E}+01$ & 4.0E-02 & $1.0 \mathrm{E}-02$ \\
\hline I-134 & $014914-27-3$ & $2.31 \mathrm{E}-13$ & $1.38 \mathrm{E}-13$ & $1.02 \mathrm{E}-05$ & $3.65 \mathrm{E}-02$ & $3.4 \mathrm{E}-03$ & $2.0 \mathrm{E}-03$ & $4.0 \mathrm{E}+01$ & $4.0 \mathrm{E}-02$ & $1.0 \mathrm{E}-02$ \\
\hline I-135 & $014834-68-5$ & $2.27 \mathrm{E}-12$ & $1.18 \mathrm{E}-12$ & $6.23 \mathrm{E}-06$ & 2.75E-01 & $3.4 \mathrm{E}-03$ & $2.0 \mathrm{E}-03$ & $4.0 \mathrm{E}+01$ & $4.0 \mathrm{E}-02$ & $1.0 \mathrm{E}-02$ \\
\hline In-113m & $014885-78-0$ & $8.30 \mathrm{E}-14$ & $5.77 \mathrm{E}-14$ & 7.82E-07 & $6.92 \mathrm{E}-02$ & $1.0 \mathrm{E}-02$ & $3.0 \mathrm{E}-03$ & & $4.0 \mathrm{E}-03$ & $2.0 \mathrm{E}-04$ \\
\hline In-114 & 013981-55-0 & 4.53E-15 & $5.81 \mathrm{E}-15$ & 1.13E-07 & 8.32E-04 & $1.0 \mathrm{E}-02$ & 3.0E-03 & & $4.0 \mathrm{E}-03$ & $2.0 \mathrm{E}-04$ \\
\hline In-114m & $013981-55-0$ & $2.06 \mathrm{E}-11$ & $2.53 \mathrm{E}-11$ & $2.00 \mathrm{E}-07$ & $4.95 \mathrm{E}+01$ & $1.0 \mathrm{E}-02$ & $3.0 \mathrm{E}-03$ & & $4.0 \mathrm{E}-03$ & $2.0 \mathrm{E}-04$ \\
\hline In-115 & $014191-71-0$ & $3.49 \mathrm{E}-11$ & $2.07 \mathrm{E}-10$ & $0.00 \mathrm{E}+00$ & $1.68 \mathrm{E}+18$ & $1.0 \mathrm{E}-02$ & $3.0 \mathrm{E}-03$ & & $4.0 \mathrm{E}-03$ & $2.0 \mathrm{E}-04$ \\
\hline In-115m & $014191-71-0$ & $3.42 \mathrm{E}-13$ & $1.75 \mathrm{E}-13$ & $4.29 \mathrm{E}-07$ & $1.82 \mathrm{E}-01$ & $1.0 \mathrm{E}-02$ & $3.0 \mathrm{E}-03$ & & $4.0 \mathrm{E}-03$ & $2.0 \mathrm{E}-04$ \\
\hline Ir-190 & $014981-91-0$ & $4.95 \mathrm{E}-12$ & $4.49 \mathrm{E}-12$ & 4.65E-06 & $1.18 \mathrm{E}+01$ & 2.0E-01 & $3.0 \mathrm{E}-02$ & & $2.0 \mathrm{E}-03$ & 2.0E-06 \\
\hline Ir-194 & 014158-35-1 & $7.00 \mathrm{E}-12$ & $4.18 \mathrm{E}-12$ & $3.17 \mathrm{E}-07$ & $8.00 \mathrm{E}-01$ & $2.0 \mathrm{E}-01$ & $3.0 \mathrm{E}-02$ & & $2.0 \mathrm{E}-03$ & 2.0E-06 \\
\hline $\mathrm{K}-40$ & $013966-00-2$ & $1.25 \mathrm{E}-11$ & $7.46 \mathrm{E}-12$ & $6.11 \mathrm{E}-07$ & $4.67 \mathrm{E}+11$ & $3.0 \mathrm{E}+00$ & 3.0E-01 & & $2.0 \mathrm{E}-02$ & $7.2 \mathrm{E}-03$ \\
\hline $\mathrm{K}-42$ & $014378-21-3$ & $1.29 \mathrm{E}-12$ & $7.56 \mathrm{E}-13$ & $1.09 \mathrm{E}-06$ & $5.17 \mathrm{E}-01$ & $3.0 \mathrm{E}+00$ & $3.0 \mathrm{E}-01$ & & $2.0 \mathrm{E}-02$ & 7.2E-03 \\
\hline
\end{tabular}


Table 3.6c. (continued)

\begin{tabular}{|c|c|c|c|c|c|c|c|c|c|c|}
\hline Chemical $^{\dagger}$ & CASRN & $\begin{array}{c}\text { Ingestion } \\
\text { SF } \\
(\mathrm{pCi})^{-1}\end{array}$ & $\begin{array}{c}\text { Inhalation } \\
\text { SF } \\
(\mathrm{pCi})^{-1}\end{array}$ & $\begin{array}{c}\text { External } \\
\text { Exposure } \\
\text { SF } \\
\frac{\mathrm{g}}{\mathrm{pCl}-\mathrm{yr}}\end{array}$ & $\begin{array}{c}\text { Half-life } \\
\text { (days) } \\
{\left[T_{R}\right]}\end{array}$ & $\begin{array}{c}\text { Soil-to- } \\
\text { Plant } \\
\text { Uptake } \\
\text { (B) }\end{array}$ & $\begin{array}{c}\text { Soil-to- } \\
\text { Wet Plant } \\
\text { Uptake } \\
\left(B_{\mathrm{ww}}\right)\end{array}$ & $\begin{array}{c}\text { Bioaccum- } \\
\text { ulation } \\
\text { Factor for } \\
\text { Fish } \\
\text { (L/kg) } \\
\text { [BF] }\end{array}$ & $\begin{array}{c}\text { Bio- } \\
\text { transfer } \\
\text { Coefficient } \\
\text { for Beef } \\
\text { (day/kg) } \\
{\left[F_{\mathrm{B}}\right]}\end{array}$ & $\begin{array}{c}\text { Bio- } \\
\text { transfer } \\
\text { Coefficient } \\
\text { for Milk } \\
\text { (day/kg) } \\
{\left[\mathrm{F}_{\mathrm{m}}\right]}\end{array}$ \\
\hline $\mathrm{Kr}-83 \mathrm{~m}$ & $013965-98-5$ & & $3,48 \mathrm{E}-17$ & & $7.63 \mathrm{E}-02$ & $0.0 \mathrm{E}+00$ & $0.0 \mathrm{E}+00$ & & $0.0 \mathrm{E}+00$ & $0.0 \mathrm{E}+00$ \\
\hline $\mathrm{Kr}-85$ & $013983-27-2$ & & $2.87 \mathrm{E}-16$ & & $3.91 \mathrm{E}+03$ & $0.0 \mathrm{E}+00$ & $0.0 E+00$ & & $0.0 \mathrm{E}+00$ & $0.0 \mathrm{E}+00$ \\
\hline $\mathrm{Kr}-85 \mathrm{~m}$ & $013983-27-2$ & & 2.75E-16 & & $1.87 \mathrm{E}-01$ & $0.0 \mathrm{E}+00$ & $0.0 \mathrm{E}+00$ & & $0.0 \mathrm{E}+00$ & $0.0 \mathrm{E}+00$ \\
\hline $\mathrm{Kr}-87$ & $014809-68-8$ & & $1.20 \mathrm{E}-15$ & & $5.30 \mathrm{E}-02$ & $0.0 \mathrm{E}+00$ & $0.0 \mathrm{E}+00$ & & $0.0 \mathrm{E}+00$ & $0.0 \mathrm{E}+00$ \\
\hline $\mathrm{Kr}-88$ & $014995-61-0$ & & $2.20 \mathrm{E}-15$ & & $1.18 \mathrm{E}-01$ & $0.0 \mathrm{E}+00$ & $0.0 \mathrm{E}+00$ & & $0,0 \mathrm{E}+00$ & $0.0 \mathrm{E}+00$ \\
\hline $\mathrm{Kr}-89$ & $016316-03-3$ & & $1.61 \mathrm{E}-15$ & & $2.19 \mathrm{E}-03$ & $0.0 \mathrm{E}+00$ & $0.0 \mathrm{E}+00$ & & $0.0 \mathrm{E}+00$ & $0.0 \mathrm{E}+00$ \\
\hline $\mathrm{Kr}-90$ & $015741-13-6$ & & $1.60 \mathrm{E}-15$ & & $3.74 \mathrm{E}-04$ & $0.0 \mathrm{E}+00$ & $0.0 \mathrm{E}+00$ & & $0.0 \mathrm{E}+00$ & $0.0 \mathrm{E}+00$ \\
\hline La-140 & $013981-28-7$ & $9.46 \mathrm{E}-12$ & $5.10 \mathrm{E}-12$ & $9.11 \mathrm{E}-06$ & $1.68 \mathrm{E}+00$ & $5.2 \mathrm{E}-03$ & $4.0 \mathrm{E}-04$ & $3.0 \mathrm{E}+01$ & 3.0E-04 & $2.0 \mathrm{E}-05$ \\
\hline Lu-177 & $014265-75-9$ & $2.95 \mathrm{E}-12$ & $2.20 \mathrm{E}-12$ & $7.22 \mathrm{E}-08$ & $6.71 E+00$ & $1.0 \mathrm{E}-02$ & $2.5 \mathrm{E}-03$ & & $4.5 \mathrm{E}-03$ & $2.0 \mathrm{E}-05$ \\
\hline $\mathrm{Mn}-52$ & 014092-99-0 & $6.01 \mathrm{E}-12$ & $4.40 \mathrm{E}-12$ & $1.34 \mathrm{E}-05$ & $5.59 \mathrm{E}+00$ & $6.8 \mathrm{E}-01$ & $6.9 \mathrm{E}-02$ & $4.0 \mathrm{E}+02$ & $5.0 \mathrm{E}-04$ & $3.0 \mathrm{E}-05$ \\
\hline $\mathrm{Mn}-54$ & $013966-31-9$ & $1.96 \mathrm{E}-12$ & $3.69 \mathrm{E}-12$ & $3.26 \mathrm{E}-06$ & $3.13 \mathrm{E}+02$ & $6.8 \mathrm{E}-01$ & $6.9 \mathrm{E}-02$ & $4.0 \mathrm{E}+02$ & $5.0 \mathrm{E}-04$ & $3.0 \mathrm{E}-05$ \\
\hline Mn-56 & $014681-52-8$ & 8.57E-13 & $5.21 \mathrm{E}-13$ & $6.95 \mathrm{E}-06$ & $1.08 \mathrm{E}-01$ & $6.8 \mathrm{E}-01$ & $6.9 \mathrm{E}-02$ & $4.0 \mathrm{E}+02$ & $5.0 \mathrm{E}-04$ & $3.0 \mathrm{E}-05$ \\
\hline Mo-99 & $014119-15-4$ & $2.27 \mathrm{E}-12$ & $4.48 \mathrm{E}-12$ & $5.46 \mathrm{E}-07$ & $2.75 \mathrm{E}+00$ & $4.0 \mathrm{E}-01$ & $8.0 \mathrm{E}-02$ & $1.0 \mathrm{E}+01$ & $1.0 \mathrm{E}-03$ & $1.7 \mathrm{E}-03$ \\
\hline $\mathrm{Na}-22$ & $013966-32-0$ & $8.02 \mathrm{E}-12$ & $4.88 \mathrm{E}-12$ & $8.18 \mathrm{E}-06$ & $9.49 E+02$ & $2.0 \mathrm{E}-01$ & $3.0 \mathrm{E}-02$ & $2.0 \mathrm{E}+01$ & $8.0 \mathrm{E}-02$ & $1.6 \mathrm{E}-02$ \\
\hline $\mathrm{Na}-24$ & $013982-04-2$ & $1.38 \mathrm{E}-12$ & $7.51 \mathrm{E}-13$ & 1.77E-05 & $6.25 \mathrm{E}-01$ & $2.0 \mathrm{E}-01$ & $3.0 \mathrm{E}-02$ & $2.0 \mathrm{E}+01$ & $8.0 \mathrm{E}-02$ & $1.6 \mathrm{E}-02$ \\
\hline $\mathrm{Nb}-93 \mathrm{~m}$ & $007440-03-1$ & $6.64 \mathrm{E}-13$ & 4.33E-12 & $3.64 \mathrm{E}-11$ & $5.33 E+03$ & $1.0 \mathrm{E}-01$ & 4.3E-03 & $3.0 \mathrm{E}+02$ & $3.0 \mathrm{E}-07$ & $4.1 \mathrm{E}-07$ \\
\hline $\mathrm{Nb}-94$ & $014681-63-1$ & $6.91 \mathrm{E}-12$ & $8.20 \mathrm{E}-11$ & $6.08 \mathrm{E}-06$ & $7.41 \mathrm{E}+06$ & $1.0 \mathrm{E}-01$ & $4.3 \mathrm{E}-03$ & $3.0 \mathrm{E}+02$ & $3.0 \mathrm{E}-07$ & 4.1E-07 \\
\hline $\mathrm{Nb}-95$ & $013967-76-5$ & $2.25 \mathrm{E}-12$ & $3.11 \mathrm{E}-12$ & $2.94 \mathrm{E}-06$ & $3.51 \mathrm{E}+01$ & $1.0 \mathrm{E}-01$ & $4.3 \mathrm{E}-03$ & $3.0 \mathrm{E}+02$ & $3.0 \mathrm{E}-07$ & 4.1E-07 \\
\hline $\mathrm{Nb}-95 \mathrm{~m}$ & $013967-76-5$ & $3.06 \mathrm{E}-12$ & $2.25 \mathrm{E}-12$ & 8.71E-08 & $3.61 \mathrm{E}+00$ & $1.0 \mathrm{E}-01$ & $4.3 \mathrm{E}-03$ & $3.0 \mathrm{E}+02$ & 3.0E-07 & 4.1E-07 \\
\hline
\end{tabular}


Table 3.6c. (continued)

\begin{tabular}{|c|c|c|c|c|c|c|c|c|c|c|}
\hline Chemical $^{\dagger}$ & CASRN & $\begin{array}{l}\text { Ingestion } \\
\text { SF } \\
(\mathrm{pCi})^{-1}\end{array}$ & $\begin{array}{c}\text { Inhalation } \\
\text { SF } \\
(\mathrm{pCi})^{-1}\end{array}$ & $\begin{array}{c}\text { External } \\
\text { Exposure } \\
\text { SF } \\
\frac{\mathbf{g}}{\mathbf{p C l}-\mathbf{y r}}\end{array}$ & $\begin{array}{c}\text { Half-life } \\
\text { (days) } \\
{\left[T_{R}\right]}\end{array}$ & $\begin{array}{c}\text { Soil-to- } \\
\text { Plant } \\
\text { Uptake } \\
\text { (B) }\end{array}$ & $\begin{array}{c}\text { Soil-to- } \\
\text { Wet Plant } \\
\text { Uptake } \\
\left(\mathbf{B}_{\mathrm{vw}}\right)\end{array}$ & $\begin{array}{l}\text { Bioaccum- } \\
\text { ulation } \\
\text { Factor for } \\
\text { Fish } \\
\text { (L/kg) } \\
\text { [BF] }\end{array}$ & $\begin{array}{c}\text { Bio- } \\
\text { transfer } \\
\text { Coefficient } \\
\text { for Beef } \\
(\text { day/kg) } \\
{\left[F_{B}\right]}\end{array}$ & $\begin{array}{c}\text { Bio- } \\
\text { transfer } \\
\text { Coefficient } \\
\text { for Milk } \\
\text { (day/kg) } \\
{\left[\mathrm{F}_{\mathrm{m}}\right]} \\
\end{array}$ \\
\hline $\mathrm{Nb}-97$ & 018496-04-3 & $1.75 \mathrm{E}-13$ & $2.13 \mathrm{E}-13$ & $2.49 \mathrm{E}-06$ & 5.01E-02 & $1.0 \mathrm{E}-01$ & $4.3 \mathrm{E}-03$ & $3.0 \mathrm{E}+02$ & $3.0 \mathrm{E}-07$ & 4.1E-07 \\
\hline $\mathrm{Nb}-97 \mathrm{~m}$ & 018496-04-3 & $3.27 \mathrm{E}-15$ & $3.34 \mathrm{E}-15$ & $2.78 \mathrm{E}-06$ & $6.94 \mathrm{E}-04$ & $1.0 \mathrm{E}-01$ & $4.3 \mathrm{E}-03$ & $3.0 \mathrm{E}+02$ & $3.0 \mathrm{E}-07$ & 4.1E-07 \\
\hline Nd-147 & $014269-74-0$ & $5.88 \mathrm{E}-12$ & $4.84 \mathrm{E}-12$ & $3.22 \mathrm{E}-07$ & $1.10 E+01$ & $2.0 \mathrm{E}-02$ & $2.0 \mathrm{E}-03$ & $1.0 \mathrm{E}+02$ & $3.0 \mathrm{E}-04$ & $2.0 \mathrm{E}-05$ \\
\hline Nd-149 & $015749-81-2$ & $4.55 \mathrm{E}-13$ & $4.22 \mathrm{E}-13$ & $1.08 \mathrm{E}-06$ & $7.21 E-02$ & $2.0 \mathrm{E}-02$ & $2.0 \mathrm{E}-03$ & $1.0 \mathrm{E}+02$ & $3.0 \mathrm{E}-04$ & $2.0 \mathrm{E}-05$ \\
\hline Ni-59 & $014336-70-0$ & $1.85 \mathrm{E}-13$ & $4.01 \mathrm{E}-13$ & $0.00 \mathrm{E}+00$ & $2.74 \mathrm{E}+07$ & $1.8 \mathrm{E}-01$ & $5.0 \mathrm{E}-02$ & $1.0 \mathrm{E}+02$ & $5.0 \mathrm{E}-03$ & $1.6 \mathrm{E}-02$ \\
\hline $\mathrm{Ni}-63$ & $013981-37-8$ & $5.50 \mathrm{E}-13$ & $1.01 \mathrm{E}-12$ & $0.00 \mathrm{E}+00$ & $3.65 \mathrm{E}+04$ & $1.8 \mathrm{E}-01$ & $5.0 \mathrm{E}-02$ & $1.0 \mathrm{E}+02$ & $5.0 \mathrm{E}-03$ & $1.6 \mathrm{E}-02$ \\
\hline $\mathrm{Ni}-65$ & $014833-49-9$ & $5.62 \mathrm{E}-13$ & $3.59 \mathrm{E}-13$ & $2.14 \mathrm{E}-06$ & $1.05 \mathrm{E}-01$ & $1.8 \mathrm{E}-01$ & $5.0 \mathrm{E}-02$ & $1.0 \mathrm{E}+02$ & $5.0 \mathrm{E}-03$ & $1.6 \mathrm{E}-02$ \\
\hline $\mathrm{Np}-236$ & $015700-36-4$ & $9.31 \mathrm{E}-13$ & $3.87 \mathrm{E}-12$ & $9.22 \mathrm{E}-08$ & $4.20 \mathrm{E}+07$ & $6.9 \mathrm{E}-02$ & $2.9 \mathrm{E}-03$ & $3.0 \mathrm{E}+01$ & $1.0 \mathrm{E}-03$ & $5.0 \mathrm{E}-06$ \\
\hline $\mathrm{Np}-237$ & $013994-20-2$ & 2.95E-10 & $3.45 \mathrm{E}-08$ & $7.56 \mathrm{E}-09$ & $7.81 E+08$ & $6.9 \mathrm{E}-02$ & $2.9 \mathrm{E}-03$ & $3.0 \mathrm{E}+01$ & $1.0 \mathrm{E}-03$ & $5.0 \mathrm{E}-06$ \\
\hline $\mathrm{Np}-237+\mathrm{D}$ & $013994-20-2$ & $3.00 \mathrm{E}-10$ & $3.45 \mathrm{E}-08$ & $4.62 \mathrm{E}-07$ & $7.81 E+08$ & $7.2 \mathrm{E}-02$ & $3.5 \mathrm{E}-03$ & $4.0 \mathrm{E}+01$ & $1.0 \mathrm{E}-03$ & $1.0 \mathrm{E}-05$ \\
\hline $\mathrm{Np}-238$ & $015766-25-3$ & $4.56 \mathrm{E}-12$ & $4.68 \mathrm{E}-12$ & $1.95 \mathrm{E}-06$ & $2.12 \mathrm{E}+00$ & $6.9 \mathrm{E}-02$ & $2.9 \mathrm{E}-03$ & $3.0 \mathrm{E}+01$ & $1.0 \mathrm{E}-03$ & $5.0 \mathrm{E}-06$ \\
\hline $\mathrm{Np}-239$ & $013968-59-7$ & $4.27 \mathrm{E}-12$ & $2.41 \mathrm{E}-12$ & $2.42 \mathrm{E}-07$ & $2.36 \mathrm{E}+00$ & $6.9 \mathrm{E}-02$ & $2.9 \mathrm{E}-03$ & $3.0 \mathrm{E}+01$ & $1.0 \mathrm{E}-03$ & $5.0 \mathrm{E}-06$ \\
\hline $\mathrm{Np}-240$ & $015690-84-3$ & 1.77E-13 & $1.31 \mathrm{E}-13$ & 3.65E-06 & 4.51E-02 & $6.9 \mathrm{E}-02$ & $2.9 \mathrm{E}-03$ & $3.0 \mathrm{E}+01$ & $1.0 \mathrm{E}-03$ & $5.0 \mathrm{E}-06$ \\
\hline $\mathrm{Np}-240 \mathrm{~m}$ & $015690-84-3$ & $2.42 \mathrm{E}-14$ & $2.83 E-14$ & $1.05 \mathrm{E}-06$ & $5.14 \mathrm{E}-03$ & $6.9 \mathrm{E}-02$ & $2.9 \mathrm{E}-03$ & $3.0 \mathrm{E}+01$ & $1.0 \mathrm{E}-03$ & $5.0 \mathrm{E}-06$ \\
\hline Os-185 & $015766-50-4$ & $1.80 \mathrm{E}-12$ & $4.62 E-12$ & $2.45 \mathrm{E}-06$ & $9.36 \mathrm{E}+01$ & $1.5 \mathrm{E}-02$ & $3.8 \mathrm{E}-03$ & & $4.0 \mathrm{E}-01$ & $5.0 \mathrm{E}-03$ \\
\hline Os-191 & $014119-24-5$ & $3.04 \mathrm{E}-12$ & $2.70 \mathrm{E}-12$ & $8.74 \mathrm{E}-08$ & $1.54 \mathrm{E}+01$ & $1.5 \mathrm{E}-02$ & $3.8 \mathrm{E}-03$ & & $4.0 \mathrm{E}-01$ & $5.0 \mathrm{E}-03$ \\
\hline Os-191m & $014119-24-5$ & $4.95 \mathrm{E}-13$ & $3.32 \mathrm{E}-13$ & 3.22E-09 & $5.42 \mathrm{E}-01$ & $1.5 \mathrm{E}-02$ & $3.8 \mathrm{E}-03$ & & $4.0 \mathrm{E}-01$ & $5.0 \mathrm{E}-03$ \\
\hline Os-193 & $016057-77-5$ & $4.36 \mathrm{E}-12$ & $2.68 \mathrm{E}-12$ & $1.82 \mathrm{E}-07$ & $1.25 \mathrm{E}+00$ & $1.5 \mathrm{E}-02$ & $3.8 \mathrm{E}-03$ & & $4.0 \mathrm{E}-01$ & $5.0 \mathrm{E}-03$ \\
\hline P-32 & $014596-37-3$ & $6.11 E-12$ & $2.93 \mathrm{E}-12$ & $0.00 \mathrm{E}+00$ & $1.43 \mathrm{E}+01$ & $3.0 \mathrm{E}+00$ & $1.0 \mathrm{E}+00$ & $5.0 \mathrm{E}+04$ & $5.0 \mathrm{E}-02$ & $1.6 \mathrm{E}-02$ \\
\hline
\end{tabular}


Table 3.6c. (continued)

\begin{tabular}{|c|c|c|c|c|c|c|c|c|c|c|}
\hline Chemical $^{\dagger}$ & CASRN & $\begin{array}{c}\text { Ingestion } \\
\text { SF } \\
(\mathrm{pCi})^{-1}\end{array}$ & $\begin{array}{c}\text { Inhalation } \\
\text { SF } \\
(\mathrm{pCi})^{-1}\end{array}$ & $\begin{array}{c}\text { External } \\
\text { Exposure } \\
\text { SF } \\
\frac{\mathbf{g}}{\mathbf{p C i}-\mathbf{y r}}\end{array}$ & $\begin{array}{c}\text { Half-life } \\
\text { (days) } \\
{\left[\mathbf{T}_{\mathrm{R}}\right]}\end{array}$ & $\begin{array}{l}\text { Soil-to- } \\
\text { Plant } \\
\text { Uptake } \\
\left(B_{v}\right)\end{array}$ & $\begin{array}{c}\text { Soil-to- } \\
\text { Wet Plant } \\
\text { Uptake } \\
\left(B_{\mathrm{vw}}\right)\end{array}$ & $\begin{array}{l}\text { Bioaccum- } \\
\text { ulation } \\
\text { Factor for } \\
\text { Fish } \\
\text { (L/kg) } \\
\text { [BF] }\end{array}$ & $\begin{array}{c}\text { Bio- } \\
\text { transfer } \\
\text { Coefficient } \\
\text { for Beef } \\
\text { (day/kg) } \\
{\left[F_{B}\right]} \\
\end{array}$ & $\begin{array}{c}\text { Bio- } \\
\text { transfer } \\
\text { Coefficient } \\
\text { for Milk } \\
\text { (day/kg) } \\
{\left[F_{\mathrm{m}}\right]}\end{array}$ \\
\hline P-33 & $015749-66-3$ & $7.81 \mathrm{E}-13$ & $3.96 \mathrm{E}-13$ & $0.00 \mathrm{E}+00$ & $2.54 \mathrm{E}+01$ & $3.0 \mathrm{E}+00$ & $1.0 \mathrm{E}+00$ & $5.0 \mathrm{E}+04$ & $5.0 \mathrm{E}-02$ & $1.6 \mathrm{E}-02$ \\
\hline $\mathrm{Pa}-231$ & $014331-85-2$ & $1.49 \mathrm{E}-10$ & $2.42 \mathrm{E}-08$ & 2.71E-08 & $1.36 \mathrm{E}+07$ & $2.5 \mathrm{E}-03$ & 6.3E-04 & $1.0 \mathrm{E}+01$ & $1.0 \mathrm{E}-05$ & $5.0 \mathrm{E}-06$ \\
\hline $\mathrm{Pa}-233$ & $013981-14-1$ & $4.69 \mathrm{E}-12$ & $4.92 \mathrm{E}-12$ & $4.54 \mathrm{E}-07$ & $2.70 \mathrm{E}+01$ & $2.5 \mathrm{E}-03$ & $6.3 \mathrm{E}-04$ & $1.0 \mathrm{E}+01$ & $1.0 \mathrm{E}-05$ & $5.0 \mathrm{E}-06$ \\
\hline $\mathrm{Pa}-234$ & $015100-28-4$ & 2.13E-12 & $1.30 \mathrm{E}-12$ & $6.60 \mathrm{E}-06$ & $2.79 \mathrm{E}-01$ & $2.5 \mathrm{E}-03$ & $6.3 \mathrm{E}-04$ & $1.0 \mathrm{E}+01$ & $1.0 \mathrm{E}-05$ & $5.0 \mathrm{E}-06$ \\
\hline $\mathrm{Pa}-234 \mathrm{~m}$ & $015100-28-4$ & $4.77 \mathrm{E}-15$ & $6.27 \mathrm{E}-15$ & 4.05E-08 & 8.13E-04 & $2.5 \mathrm{E}-03$ & $6.3 \mathrm{E}-04$ & $1.0 \mathrm{E}+01$ & $1.0 \mathrm{E}-05$ & $5.0 \mathrm{E}-06$ \\
\hline $\mathrm{Pb}-203$ & $014687-25-3$ & $1.03 E-12$ & $3.10 \mathrm{E}-13$ & $6.40 \mathrm{E}-07$ & $2.17 \mathrm{E}+00$ & $9.0 \mathrm{E}-02$ & $7.6 \mathrm{E}-04$ & $3.0 \mathrm{E}+02$ & $4.0 \mathrm{E}-04$ & $3.0 \mathrm{E}-04$ \\
\hline $\mathrm{Pb}-209$ & $014119-30-3$ & $2.09 \mathrm{E}-13$ & $6.85 \mathrm{E}-14$ & $0.00 \mathrm{E}+00$ & $1.35 \mathrm{E}-01$ & $9.0 \mathrm{E}-02$ & $7.6 \mathrm{E}-04$ & $3.0 \mathrm{E}+02$ & $4.0 \mathrm{E}-04$ & $3.0 \mathrm{E}-04$ \\
\hline $\mathrm{Pb}-210$ & $014255-04-0$ & $6.75 \mathrm{E}-10$ & $1.67 \mathrm{E}-09$ & $1.12 \mathrm{E}-10$ & $8.14 E+03$ & $9.0 \mathrm{E}-02$ & $7.6 \mathrm{E}-04$ & $3.0 \mathrm{E}+02$ & $4.0 \mathrm{E}-04$ & $3.0 \mathrm{E}-04$ \\
\hline $\mathrm{Pb}-210+\mathrm{D}$ & $014255-04-0$ & $1.01 \mathrm{E}-09$ & $3.86 \mathrm{E}-09$ & $1.45 \mathrm{E}-10$ & $8.14 \mathrm{E}+03$ & $2.2 \mathrm{E}-01$ & $9.6 \mathrm{E}-03$ & $3.6 \mathrm{E}+02$ & $5.8 \mathrm{E}-03$ & $1.1 \mathrm{E}-03$ \\
\hline $\mathrm{Pb}-211$ & $015816-77-0$ & $3.38 \mathrm{E}-13$ & $1.03 \mathrm{E}-11$ & $1.85 \mathrm{E}-07$ & $2.51 \mathrm{E}-02$ & $9.0 \mathrm{E}-02$ & $7.6 \mathrm{E}-04$ & $3.0 \mathrm{E}+02$ & $4.0 \mathrm{E}-04$ & $3.0 \mathrm{E}-04$ \\
\hline $\mathrm{Pb}-212$ & $015092-94-1$ & $1.80 \mathrm{E}-11$ & $3.85 \mathrm{E}-11$ & $3.00 \mathrm{E}-07$ & $4.42 \mathrm{E}-01$ & $9.0 \mathrm{E}-02$ & $7.6 \mathrm{E}-04$ & $3.0 \mathrm{E}+02$ & $4.0 \mathrm{E}-04$ & $3.0 \mathrm{E}-04$ \\
\hline $\mathrm{Pb}-214$ & $015067-28-4$ & $2.94 \mathrm{E}-13$ & $6.23 \mathrm{E}-12$ & $7.09 \mathrm{E}-07$ & $1.86 \mathrm{E}-02$ & $9.0 \mathrm{E}-02$ & $7.6 \mathrm{E}-04$ & $3.0 \mathrm{E}+02$ & $4.0 \mathrm{E}-04$ & $3.0 \mathrm{E}-04$ \\
\hline $\mathrm{Pd}-100$ & $015690-69-4$ & $3.74 \mathrm{E}-12$ & $3.55 \mathrm{E}-12$ & & $3.64 \mathrm{E}+00$ & $1.5 \mathrm{E}-01$ & $3.8 \mathrm{E}-02$ & & $4.0 \mathrm{E}-03$ & $1.0 \mathrm{E}-02$ \\
\hline Pd-101 & $015749-54-9$ & $3.74 \mathrm{E}-13$ & $2.29 \mathrm{E}-13$ & & $3.53 \mathrm{E}-01$ & $1.5 \mathrm{E}-01$ & $3.8 \mathrm{E}-02$ & & $4.0 \mathrm{E}-03$ & $1.0 \mathrm{E}-02$ \\
\hline Pd-103 & $014967-68-1$ & $1.05 \mathrm{E}-12$ & $1.08 \mathrm{E}-12$ & $5.38 \mathrm{E}-10$ & $1.70 \mathrm{E}+01$ & $1.5 \mathrm{E}-01$ & $3.8 \mathrm{E}-02$ & & $4.0 \mathrm{E}-03$ & $1.0 \mathrm{E}-02$ \\
\hline Pd-107 & $017637-99-9$ & $2.09 \mathrm{E}-13$ & $1.46 \mathrm{E}-12$ & $0.00 \mathrm{E}+00$ & $2.37 E+09$ & $1.5 \mathrm{E}-01$ & $3.8 \mathrm{E}-02$ & & $4.0 \mathrm{E}-03$ & $1.0 \mathrm{E}-02$ \\
\hline Pd-109 & $01498 \mathrm{I}-64-7$ & 3.33E-12 & $1.99 \mathrm{E}-12$ & 2.43E-09 & $5.63 \mathrm{E}-01$ & $1.5 \mathrm{E}-01$ & $3.8 \mathrm{E}-02$ & & $4.0 \mathrm{E}-03$ & $1.0 \mathrm{E}-02$ \\
\hline $\mathrm{Pm}-147$ & $014380-75-7$ & $1.41 \mathrm{E}-12$ & $7.49 \mathrm{E}-12$ & $6.35 \mathrm{E}-12$ & $9.56 \mathrm{E}+02$ & $1.0 \mathrm{E}-02$ & $2.5 \mathrm{E}-03$ & $3.0 E+01$ & $5.0 \mathrm{E}-03$ & 2.0E-05 \\
\hline$P m-148$ & $014683-19-3$ & $1.44 \mathrm{E}-11$ & $1.05 \mathrm{E}-11$ & 2.21E-06 & $5.37 E+00$ & $1.0 \mathrm{E}-02$ & 2.5E-03 & $3.0 \mathrm{E}+01$ & $5.0 \mathrm{E}-03$ & $2.0 \mathrm{E}-05$ \\
\hline
\end{tabular}

Chemical-specific parameters used in calculating PRGs/radionuclides

Page 9 of 18 
Table 3.6c. (continued)

\begin{tabular}{|c|c|c|c|c|c|c|c|c|c|c|}
\hline Chemical $^{\dagger}$ & CASRN & $\begin{array}{c}\text { Ingestion } \\
\text { SF } \\
(\mathrm{pCC})^{-1}\end{array}$ & $\begin{array}{c}\text { Inhalation } \\
\text { SF } \\
(\mathrm{pCi})^{-1}\end{array}$ & $\begin{array}{c}\text { External } \\
\text { Exposure } \\
\text { SF } \\
\frac{\mathbf{g}}{\mathbf{p C i}-\mathrm{yr}}\end{array}$ & $\begin{array}{c}\text { Half-life } \\
\text { (days) } \\
{\left[\mathbf{T}_{\mathrm{R}}\right]}\end{array}$ & $\begin{array}{c}\text { Soil-to- } \\
\text { Plant } \\
\text { Uptake } \\
\left(B_{y}\right)\end{array}$ & $\begin{array}{c}\text { Soil-to- } \\
\text { Wet Plant } \\
\text { Uptake } \\
\left(\mathbf{B}_{\mathrm{vw}}\right)\end{array}$ & $\begin{array}{l}\text { Bioaccum- } \\
\text { ulation } \\
\text { Factor for } \\
\text { Fish } \\
\text { (L/kg) } \\
\text { [BF] }\end{array}$ & $\begin{array}{c}\text { Bio- } \\
\text { transfer } \\
\text { Coefficient } \\
\text { for Beef } \\
(\text { day } / \mathrm{kg}) \\
{\left[\mathrm{F}_{\mathrm{B}}\right]}\end{array}$ & $\begin{array}{c}\text { Bio- } \\
\text { transfer } \\
\text { Coefficient } \\
\text { for Milk } \\
\text { (day/kg) } \\
{\left[F_{m}\right]}\end{array}$ \\
\hline $\mathrm{Pm}-148 \mathrm{~m}$ & $014683-19-3$ & 9.93E-12 & $2.95 \mathrm{E}-11$ & 7.32E-06 & $4.13 E+01$ & $1.0 \mathrm{E}-02$ & $2.5 \mathrm{E}-03$ & $3.0 \mathrm{E}+01$ & $5.0 \mathrm{E}-03$ & $2.0 \mathrm{E}-05$ \\
\hline Pm-149 & $015765-31-8$ & $5.52 \mathrm{E}-12$ & $3.57 \mathrm{E}-12$ & $3.65 \mathrm{E}-08$ & $2.21 E+00$ & $1.0 \mathrm{E}-02$ & $2.5 \mathrm{E}-03$ & $3.0 \mathrm{E}+01$ & $5.0 \mathrm{E}-03$ & $2.0 \mathrm{E}-05$ \\
\hline Po-210 & $013981-52-7$ & $3.26 \mathrm{E}-10$ & 2.14E-09 & $3.30 \mathrm{E}-11$ & $1.38 \mathrm{E}+02$ & $9.0 \mathrm{E}-02$ & $9.1 \mathrm{E}-05$ & $5.0 \mathrm{E}+01$ & $5.0 \mathrm{E}-03$ & $3.4 \mathrm{E}-04$ \\
\hline Po-212 & 015389-34-1 & $4.51 \mathrm{E}-23$ & $5.93 \mathrm{E}-21$ & $0.00 \mathrm{E}+00$ & $3.45 \mathrm{E}-12$ & $9.0 \mathrm{E}-02$ & $9.1 \mathrm{E}-05$ & $5.0 \mathrm{E}+01$ & $5.0 \mathrm{E}-03$ & $3.4 \mathrm{E}-04$ \\
\hline Po-213 & $015756-57-7$ & $6.70 \mathrm{E}-22$ & $7.80 \mathrm{E}-20$ & $1.18 \mathrm{E}-10$ & $4.86 \mathrm{E}-11$ & $9.0 \mathrm{E}-02$ & $9.1 \mathrm{E}-05$ & $5.0 \mathrm{E}+01$ & $5.0 \mathrm{E}-03$ & 3.4E-04 \\
\hline Po-214 & $015735-67-8$ & 2.12E-20 & 2.77E-18 & $3.23 \mathrm{E}-10$ & $1.90 \mathrm{E}-09$ & $9.0 \mathrm{E}-02$ & 9.1E-05 & $5.0 \mathrm{E}+01$ & $5.0 \mathrm{E}-03$ & $3.4 \mathrm{E}-04$ \\
\hline Po-215 & $015706-52-2$ & $4.99 \mathrm{E}-19$ & $4.48 \mathrm{E}-17$ & $5.11 \mathrm{E}-10$ & $2.06 \mathrm{E}-08$ & $9.0 \mathrm{E}-02$ & $9.1 \mathrm{E}-05$ & $5.0 \mathrm{E}+01$ & $5.0 \mathrm{E}-03$ & $3.4 \mathrm{E}-04$ \\
\hline Po-216 & $015756-58-8$ & $8.79 \mathrm{E}-17$ & $2.95 \mathrm{E}-15$ & $5.62 \mathrm{E}-11$ & $1.69 \mathrm{E}-06$ & $9.0 \mathrm{E}-02$ & 9.1E-05 & $5.0 \mathrm{E}+01$ & $5.0 \mathrm{E}-03$ & 3.4E-04 \\
\hline Po-218 & $015422-24-9$ & $5.08 \mathrm{E}-14$ & $\cdot 3.69 \mathrm{E}-12$ & $0.00 \mathrm{E}+00$ & 2.12E-03 & $9.0 \mathrm{E}-02$ & $9.1 \mathrm{E}-05$ & $5.0 \mathrm{E}+01$ & $5.0 \mathrm{E}-03$ & $3.4 \mathrm{E}-04$ \\
\hline Pr-142 & $014191-64-1$ & $6.98 \mathrm{E}-12$ & $4.16 \mathrm{E}-12$ & 2.34E-07 & $7.96 \mathrm{E}-01$ & $2.0 \mathrm{E}-02$ & $2.0 \mathrm{E}-03$ & $1.0 \mathrm{E}+02$ & $3.0 \mathrm{E}-04$ & $2.0 \mathrm{E}-05$ \\
\hline Pr-143 & $014981-79-4$ & $6.60 \mathrm{E}-12$ & $5.60 \mathrm{E}-12$ & $3.41 \mathrm{E}-14$ & $1.36 \mathrm{E}+01$ & $2.0 \mathrm{E}-02$ & $2.0 \mathrm{E}-03$ & $1.0 \mathrm{E}+02$ & $3.0 \mathrm{E}-04$ & $2.0 \mathrm{E}-05$ \\
\hline $\mathrm{Pr}-144$ & $014119-05-2$ & $8.08 \mathrm{E}-14$ & $1.31 \mathrm{E}-13$ & 1.33E-07 & $1.20 \mathrm{E}-02$ & $2.0 \mathrm{E}-02$ & $2.0 \mathrm{E}-03$ & $1.0 \mathrm{E}+02$ & $3.0 \mathrm{E}-04$ & $2.0 \mathrm{E}-05$ \\
\hline Pr-144m & $014119-05-2$ & 3.23E-14 & $5.61 \mathrm{E}-14$ & $1.85 \mathrm{E}-09$ & $5.00 \mathrm{E}-03$ & $2.0 \mathrm{E}-02$ & $2.0 \mathrm{E}-03$ & $1.0 \mathrm{E}+02$ & $3.0 \mathrm{E}-04$ & $2.0 \mathrm{E}-05$ \\
\hline Pt-191 & $015706-36-2$ & $1.50 \mathrm{E}-12$ & $4.13 \mathrm{E}-13$ & $6.74 \mathrm{E}-07$ & $2.71 \mathrm{E}+00$ & $9.5 \mathrm{E}-02$ & 2.4E-02 & & $4.0 \mathrm{E}-03$ & $5.0 \mathrm{E}-03$ \\
\hline Pt-193 & $015735-70-3$ & $1.62 \mathrm{E}-13$ & $7.89 \mathrm{E}-14$ & $0.00 \mathrm{E}+00$ & $1.83 E+04$ & $9.5 \mathrm{E}-02$ & $2.4 \mathrm{E}-02$ & & $4.0 \mathrm{E}-03$ & $5.0 \mathrm{E}-03$ \\
\hline Pt-193m & $015735-70-3$ & $2.51 \mathrm{E}-12$ & $5.76 \mathrm{E}-13$ & 7.44E-09 & $4.33 \mathrm{E}+00$ & $9.5 \mathrm{E}-02$ & $2.4 \mathrm{E}-02$ & & $4.0 \mathrm{E}-03$ & $5.0 \mathrm{E}-03$ \\
\hline Pt-197 & $015735-74-7$ & $2.12 \mathrm{E}-12$ & $4.54 \mathrm{E}-13$ & $3.15 \mathrm{E}-08$ & 7.63E-01 & $9.5 \mathrm{E}-02$ & $2.4 \mathrm{E}-02$ & & $4.0 \mathrm{E}-03$ & $5.0 \mathrm{E}-03$ \\
\hline Pt-197m & $015735-74-7$ & $3.25 \mathrm{E}-13$ & $1.00 \mathrm{E}-13$ & $1.65 \mathrm{E}-07$ & $6.56 \mathrm{E}-02$ & $9.5 \mathrm{E}-02$ & 2.4E-02 & & $4.0 \mathrm{E}-03$ & $5.0 \mathrm{E}-03$ \\
\hline Pu-236 & $015411-92-4$ & $7.68 \mathrm{E}-11$ & $1.34 \mathrm{E}-08$ & 2.32E-11 & $1.04 \mathrm{E}+03$ & $3.4 \mathrm{E}-04$ & $4.9 \mathrm{E}-06$ & $3.0 \mathrm{E}+01$ & $1.0 \mathrm{E}-05$ & $1.1 \mathrm{E}-06$ \\
\hline
\end{tabular}


Table 3.6c. (continued)

\begin{tabular}{|c|c|c|c|c|c|c|c|c|c|c|}
\hline Chemical $^{\dagger}$ & CASRN & $\begin{array}{c}\text { Ingestion } \\
\text { SF } \\
(\mathrm{pCi})^{-1}\end{array}$ & $\begin{array}{c}\text { Inhalation } \\
\text { SF } \\
(\mathrm{pCi})^{-1}\end{array}$ & $\begin{array}{c}\text { External } \\
\text { Exposure } \\
\frac{\text { SF }}{\mathbf{g}} \\
\mathbf{p C i}-\mathbf{y r}\end{array}$ & $\begin{array}{c}\text { Half-life } \\
\text { (days) } \\
{\left[T_{R}\right]}\end{array}$ & $\begin{array}{c}\text { Soil-to- } \\
\text { Plant } \\
\text { Uptake } \\
\left(\mathrm{B}_{\mathrm{r}}\right)\end{array}$ & $\begin{array}{c}\text { Soil-to- } \\
\text { Wet Plant } \\
\text { Uptake } \\
\left(\mathbf{B}_{\mathbf{v w}}\right)\end{array}$ & $\begin{array}{l}\text { Bioaccum- } \\
\text { ulation } \\
\text { Factor for } \\
\text { Fish } \\
\text { (L/kg) } \\
\text { [BF] }\end{array}$ & $\begin{array}{c}\text { Bio- } \\
\text { transfer } \\
\text { Coefficient } \\
\text { for Beef } \\
\text { (day/kg) } \\
{\left[\mathrm{F}_{\mathrm{B}}\right]} \\
\end{array}$ & $\begin{array}{c}\text { Bio- } \\
\text { transfer } \\
\text { Coefficient } \\
\text { for Milk } \\
\text { (day/kg) } \\
{\left[F_{\mathrm{m}}\right]}\end{array}$ \\
\hline $\mathrm{Pu}-238$ & $013981-16-3$ & $2.95 \mathrm{E}-10$ & $2.74 \mathrm{E}-08$ & $1.94 \mathrm{E}-11$ & $3.20 \mathrm{E}+04$ & $3.4 \mathrm{E}-04$ & $4.9 \mathrm{E}-06$ & $3.0 \mathrm{E}+01$ & $1.0 \mathrm{E}-05$ & $1.1 \mathrm{E}-06$ \\
\hline $\mathrm{Pu}-239$ & $015117-48-3$ & $3.16 \mathrm{E}-10$ & 2.78E-08 & $1.26 \mathrm{E}-11$ & $8.80 \mathrm{E}+06$ & $3.4 \mathrm{E}-04$ & $4.9 \mathrm{E}-06$ & $3.0 \mathrm{E}+01$ & $1.0 \mathrm{E}-05$ & $1.1 \mathrm{E}-06$ \\
\hline Pu-240 & 014119-33-6 & 3.15E-10 & $2.78 \mathrm{E}-08$ & $1.87 \mathrm{E}-11$ & $2.40 \mathrm{E}+06$ & $3.4 \mathrm{E}-04$ & $4.9 \mathrm{E}-06$ & $3.0 \mathrm{E}+01$ & $1.0 \mathrm{E}-05$ & $1.1 \mathrm{E}-06$ \\
\hline $\mathrm{Pu}-241$ & $014119-32-5$ & $5.20 \mathrm{E}-12$ & $2.81 \mathrm{E}-10$ & $0.00 \mathrm{E}+00$ & $5.26 \mathrm{E}+03$ & $3.4 \mathrm{E}-04$ & $4.9 \mathrm{E}-06$ & $3.0 \mathrm{E}+01$ & $1.0 \mathrm{E}-05$ & $1.1 \mathrm{E}-06$ \\
\hline Pu-241+D & $014119-32-5$ & 3.33E-10 & $3.88 \mathrm{E}-08$ & 4.59E-09 & $5.26 \mathrm{E}+03$ & $2.5 \mathrm{E}-02$ & $6.6 \mathrm{E}-04$ & $7.0 \mathrm{E}+01$ & 3.5E-04 & $4.0 \mathrm{E}-04$ \\
\hline Pu-242 & $013982-10-0$ & $3.00 \mathrm{E}-10$ & $2.64 \mathrm{E}-08$ & $1.55 \mathrm{E}-11$ & $1.37 \mathrm{E}+08$ & $3.4 \mathrm{E}-04$ & $4.9 \mathrm{E}-06$ & $3.0 \mathrm{E}+01$ & $1.0 \mathrm{E}-05$ & $1.1 \mathrm{E}-06$ \\
\hline Pu-243 & $015706-37-3$ & $3.69 \mathrm{E}-13$ & $2.67 \mathrm{E}-13$ & $1.89 \mathrm{E}-08$ & 2.07E-01 & $3.4 \mathrm{E}-04$ & $4.9 \mathrm{E}-06$ & $3.0 \mathrm{E}+01$ & $1.0 \mathrm{E}-05$ & $1.1 \mathrm{E}-06$ \\
\hline Pu-244 & $014119-34-7$ & $3.13 \mathrm{E}-10$ & 2.67E-08 & $1.29 \mathrm{E}-11$ & $3.01 \mathrm{E}+10$ & $3.4 \mathrm{E}-04$ & $4.9 \mathrm{E}-06$ & $3.0 \mathrm{E}+01$ & $1.0 \mathrm{E}-05$ & 1.1E-06 \\
\hline $\mathrm{Pu}-244+\mathrm{D}$ & $014119-34-7$ & $3.19 \mathrm{E}-10$ & 2.67E-08 & 3.65E-06 & $3.01 E+10$ & $9.2 \mathrm{E}-02$ & $3.5 \mathrm{E}-03$ & $7.0 \mathrm{E}+01$ & $1.3 \mathrm{E}-03$ & 4.1E-04 \\
\hline $\mathrm{Ra}-223$ & 015623-45-7 & $2.34 \mathrm{E}-10$ & $3.60 \mathrm{E}-09$ & 2.44E-07 & $1.14 \mathrm{E}+01$ & 8.0E-02 & $9.3 \mathrm{E}-03$ & $5.0 \mathrm{E}+01$ & $9.0 \mathrm{E}-04$ & $1.3 \mathrm{E}-03$ \\
\hline $\mathrm{Ra}-224$ & $013233-32-4$ & 1.49E-10 & 2.25E-09 & $2.48 \mathrm{E}-08$ & $3.62 \mathrm{E}+00$ & 8.0E-02 & 9.3E-03 & $5.0 \mathrm{E}+01$ & $9.0 \mathrm{E}-04$ & $1.3 \mathrm{E}-03$ \\
\hline $\mathrm{Ra}-225$ & $013981-53-8$ & $1.57 \mathrm{E}-10$ & 2.38E-09 & 1.71E-09 & $1.48 \mathrm{E}+01$ & $8.0 \mathrm{E}-02$ & 9.3E-03 & $5.0 \mathrm{E}+01$ & $9.0 \mathrm{E}-04$ & $1.3 \mathrm{E}-03$ \\
\hline $\mathrm{Ra}-226$ & $013982-63-3$ & 2.95E-10 & 2.72E-09 & 1.31E-08 & $5.84 \mathrm{E}+05$ & $8.0 \mathrm{E}-02$ & 9.3E-03 & $5.0 \mathrm{E}+01$ & $9.0 \mathrm{E}-04$ & $1.3 \mathrm{E}-03$ \\
\hline $\mathrm{Ra}-226+\mathrm{D}$ & $013982-63-3$ & $2.96 \mathrm{E}-10$ & 2.75E-09 & $6.74 \mathrm{E}-06$ & $5.84 \mathrm{E}+05$ & $3.9 \mathrm{E}-01$ & $1.9 \mathrm{E}-02$ & $4.6 \mathrm{E}+02$ & $1.2 \mathrm{E}-02$ & $2.8 \mathrm{E}-03$ \\
\hline $\mathrm{Ra}-228$ & $015262-20-1$ & $2.46 \mathrm{E}-10$ & $9.61 \mathrm{E}-10$ & $0.00 \mathrm{E}+00$ & $2.10 \mathrm{E}+03$ & $8.0 \mathrm{E}-02$ & $9.3 \mathrm{E}-03$ & $5.0 E+01$ & $9.0 \mathrm{E}-04$ & $1.3 \mathrm{E}-03$ \\
\hline $\mathrm{Ra}-228+\mathrm{D}$ & $015262-20-1$ & $2.48 \mathrm{E}-10$ & $9.94 \mathrm{E}-10$ & $3.28 \mathrm{E}-06$ & $2.10 E+03$ & $8.4 \mathrm{E}-02$ & $1.0 \mathrm{E}-02$ & $5.0 \mathrm{E}+01$ & 9.3E-04 & $1.3 E-03$ \\
\hline $\mathrm{Rb}-82$ & $014391-63-0$ & $1.05 \mathrm{E}-14$ & $1.17 \mathrm{E}-14$ & $3.89 \mathrm{E}-06$ & 8.68E-04 & $9.0 \mathrm{E}-01$ & $9.0 \mathrm{E}-02$ & $2.0 \mathrm{E}+03$ & $1.0 \mathrm{E}-02$ & $1.2 \mathrm{E}-02$ \\
\hline $\mathrm{Rb}-86$ & $014932-53-7$ & $7.12 \mathrm{E}-12$ & $4.21 \mathrm{E}-12$ & 3.71E-07 & $1.87 \mathrm{E}+01$ & $9.0 \mathrm{E}-01$ & $9.0 \mathrm{E}-02$ & $2.0 \mathrm{E}+03$ & $1.0 \mathrm{E}-02$ & $1.2 \mathrm{E}-02$ \\
\hline $\mathrm{Rb}-87$ & $013982-13-3$ & $3.68 \mathrm{E}-12$ & $2.26 \mathrm{E}-12$ & $0.00 \mathrm{E}+00$ & $1.73 E+13$ & $9.0 \mathrm{E}-01$ & $9.0 \mathrm{E}-02$ & $2.0 \mathrm{E}+03$ & $1.0 \mathrm{E}-02$ & $1.2 \mathrm{E}-02$ \\
\hline
\end{tabular}

Chemical-specific parameters used in calculating PRGs/radionuclides

Page 11 of 18 
Table 3.6c. (continued)

\begin{tabular}{|c|c|c|c|c|c|c|c|c|c|c|}
\hline Chemical $^{\dagger}$ & CASRN & $\begin{array}{c}\text { Ingestion } \\
\text { SF } \\
(\mathrm{pCi})^{-1}\end{array}$ & $\begin{array}{l}\text { Inhalation } \\
\text { SF } \\
(\mathrm{pCi})^{-1}\end{array}$ & $\begin{array}{c}\text { External } \\
\text { Exposure } \\
\text { SF } \\
\frac{\mathbf{g}}{\mathbf{p C i}-\mathrm{yr}}\end{array}$ & $\begin{array}{l}\text { Half-life } \\
\text { (days) } \\
{\left[T_{R}\right]}\end{array}$ & $\begin{array}{c}\text { Soil-to- } \\
\text { Plant } \\
\text { Uptake } \\
\left(B_{v}\right)\end{array}$ & $\begin{array}{c}\text { Soil-to- } \\
\text { Wet Plant } \\
\text { Uptake } \\
\left(B_{v w}\right)\end{array}$ & $\begin{array}{l}\text { Bioaccum- } \\
\text { ulation } \\
\text { Factor for } \\
\text { Fish } \\
\text { (L/kg) } \\
\text { [BF] }\end{array}$ & 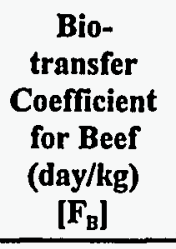 & $\begin{array}{c}\text { Bio- } \\
\text { transfer } \\
\text { Coefficient } \\
\text { for Milk } \\
\text { (day/kg) } \\
{\left[\mathrm{F}_{\mathrm{m}}\right]} \\
\end{array}$ \\
\hline $\mathrm{Rb}-88$ & $014928-36-0$ & $1.46 \mathrm{E}-13$ & $1.36 \mathrm{E}-13$ & $2.68 \mathrm{E}-06$ & $1.24 \mathrm{E}-02$ & $9.0 \mathrm{E}-01$ & $9.0 \mathrm{E}-02$ & $2.0 \mathrm{E}+03$ & $1.0 \mathrm{E}-02$ & $1.2 \mathrm{E}-02$ \\
\hline Rb-89 & $014191-65-2$ & $8.65 \mathrm{E}-14$ & $6.92 \mathrm{E}-14$ & 8.47E-06 & $1.07 \mathrm{E}-02$ & $9.0 \mathrm{E}-01$ & $9.0 \mathrm{E}-02$ & $2.0 \mathrm{E}+03$ & $1.0 \mathrm{E}-02$ & $1.2 \mathrm{E}-02$ \\
\hline $\mathrm{Rh}-103 \mathrm{~m}$ & $007440-16-6$ & $8.19 \mathrm{E}-15$ & $1.28 \mathrm{E}-14$ & $5.85 \mathrm{E}-11$ & $3.90 \mathrm{E}-02$ & $9.0 \mathrm{E}-01$ & $9.0 \mathrm{E}-02$ & $1.0 \mathrm{E}+01$ & $2.0 \mathrm{E}-03$ & $1.0 \mathrm{E}-02$ \\
\hline Rh-105 & 014913-89-4 & 1.93E-12 & $1.22 \mathrm{E}-12$ & 2.49E-07 & $1.48 \mathrm{E}+00$ & $9.0 \mathrm{E}-01$ & $9.0 \mathrm{E}-02$ & $1.0 \mathrm{E}+01$ & $2.0 \mathrm{E}-03$ & $1.0 \mathrm{E}-02$ \\
\hline $\mathrm{Rh}-105 \mathrm{~m}$ & 014913-89-4 & $1.08 \mathrm{E}-15$ & $9.25 \mathrm{E}-16$ & $2.27 \mathrm{E}-08$ & $5.21 \mathrm{E}-04$ & $9.0 \mathrm{E}-01$ & $9.0 \mathrm{E}-02$ & $1.0 \mathrm{E}+01$ & $2.0 \mathrm{E}-03$ & $1.0 \mathrm{E}-02$ \\
\hline $\mathrm{Rh}-106$ & $014234-34-5$ & 3.63E-15 & 4.62E-15 & 7.57E-07 & $3.46 \mathrm{E}-04$ & $9.0 \mathrm{E}-01$ & $9.0 \mathrm{E}-02$ & $1.0 \mathrm{E}+01$ & $2.0 \mathrm{E}-03$ & $1.0 \mathrm{E}-02$ \\
\hline Rn-219 & $014835-02-0$ & & $6.91 \mathrm{E}-14$ & 1.72E-07 & $4.58 \mathrm{E}-05$ & & & & & \\
\hline $\mathrm{Rn}-220$ & $022481-48-7$ & & $1.92 \mathrm{E}-13$ & $1.88 \mathrm{E}-09$ & $6.44 \mathrm{E}-04$ & & & & & \\
\hline $\mathrm{Rn}-222+\mathrm{D}$ & 014859-67-7 & & $7.57 \mathrm{E}-12$ & & $3.82 \mathrm{E}+00$ & $3.1 \mathrm{E}-01$ & $9.7 \mathrm{E}-03$ & $4.1 \mathrm{E}+02$ & $1.1 \mathrm{E}-02$ & $1.5 \mathrm{E}-03$ \\
\hline Ru-103 & 013968-53-1 & 3.32E-12 & $4.59 \mathrm{E}-12$ & $1.70 \mathrm{E}-06$ & $3.94 \mathrm{E}+01$ & $2.0 \mathrm{E}-01$ & $2.4 \mathrm{E}-02$ & $1.0 \mathrm{E}+01$ & $5.1 \mathrm{E}-02$ & 3.3E-06 \\
\hline Ru-105 & 014331-95-4 & $1.15 \mathrm{E}-12$ & 8.02E-13 & $2.88 \mathrm{E}-06$ & $1.85 \mathrm{E}-01$ & $2.0 \mathrm{E}-01$ & $2.4 \mathrm{E}-02$ & $1.0 \mathrm{E}+01$ & $5.1 \mathrm{E}-02$ & 3.3E-06 \\
\hline Ru-106 & 013967-48-1 & $3.45 \mathrm{E}-11$ & $1.15 \mathrm{E}-10$ & $0.00 \mathrm{E}+00$ & $3.68 \mathrm{E}+02$ & $2.0 \mathrm{E}-01$ & $2.4 \mathrm{E}-02$ & $1.0 \mathrm{E}+01$ & $5.1 \mathrm{E}-02$ & 3.3E-06 \\
\hline Ru-106+D & $013967-48-1$ & $3.45 \mathrm{E}-11$ & $1.15 \mathrm{E}-10$ & 7.57E-07 & $3.68 \mathrm{E}+02$ & $1.1 \mathrm{E}+00$ & $1.1 \mathrm{E}-01$ & $2.0 \mathrm{E}+01$ & $5.3 \mathrm{E}-02$ & $1.0 \mathrm{E}-02$ \\
\hline Ru-97 & 015758-35-7 & $5.88 \mathrm{E}-13$ & $4.09 \mathrm{E}-13$ & 4.52E-07 & $2.90 \mathrm{E}+00$ & $2.0 \mathrm{E}-01$ & $2.4 \mathrm{E}-02$ & $1.0 \mathrm{E}+01$ & $5.1 \mathrm{E}-02$ & 3.3E-06 \\
\hline S-35 & $015117-53-0$ & $4.16 \mathrm{E}-13$ & $1.85 \mathrm{E}-13$ & $0.00 \mathrm{E}+00$ & $8.74 \mathrm{E}+01$ & $2.0 \mathrm{E}+00$ & $6.0 \mathrm{E}-01$ & $8.0 \mathrm{E}+02$ & $2.0 \mathrm{E}-01$ & $1.6 \mathrm{E}-02$ \\
\hline $\mathrm{Sb}-122$ & 014374-79-9 & $8.81 \mathrm{E}-12$ & $5.46 \mathrm{E}-12$ & $1.61 \mathrm{E}-06$ & $2.70 \mathrm{E}+00$ & $5.0 \mathrm{E}-02$ & $1.0 \mathrm{E}-02$ & $1.0 \mathrm{E}+02$ & $4.0 \mathrm{E}-05$ & $2.5 \mathrm{E}-05$ \\
\hline$S b-124$ & $014683-10-4$ & $1.07 \mathrm{E}-11$ & $1.32 \mathrm{E}-11$ & 7.35E-06 & $6.02 \mathrm{E}+01$ & $5.0 \mathrm{E}-02$ & $1.0 \mathrm{E}-02$ & $1.0 \mathrm{E}+02$ & 4.0E-05 & $2.5 \mathrm{E}-05$ \\
\hline $\mathrm{Sb}-125$ & 014234-35-6 & 2.97E-12 & $5.20 \mathrm{E}-12$ & $1.34 \mathrm{E}-06$ & $1.01 \mathrm{E}+03$ & $5.0 \mathrm{E}-02$ & $1.0 \mathrm{E}-02$ & $1.0 \mathrm{E}+02$ & $4.0 \mathrm{E}-05$ & $2.5 E-05$ \\
\hline Sb-125+D & 014234-35-6 & $3.54 \mathrm{E}-12$ & $5.85 \mathrm{E}-12$ & $1.34 \mathrm{E}-06$ & $1.01 \mathrm{E}+03$ & $7.1 \mathrm{E}+00$ & $7.1 \mathrm{E}-01$ & $5.0 \mathrm{E}+02$ & 7.0E-03 & $4.8 \mathrm{E}-04$ \\
\hline
\end{tabular}


Table 3.6c. (continued)

\begin{tabular}{|c|c|c|c|c|c|c|c|c|c|c|}
\hline Chemical ${ }^{t}$ & CASRN & $\begin{array}{c}\text { Ingestion } \\
\text { SF } \\
(\mathrm{pCi})^{-1}\end{array}$ & $\begin{array}{c}\text { Inhalation } \\
\text { SF } \\
(\mathrm{pCi})^{-1}\end{array}$ & $\begin{array}{c}\text { External } \\
\text { Exposure } \\
\text { SF } \\
\frac{\mathbf{g}}{\mathrm{pCi}-\mathrm{yr}}\end{array}$ & $\begin{array}{c}\text { Half-life } \\
\text { (days) } \\
{\left[\mathrm{T}_{\mathrm{R}}\right]}\end{array}$ & $\begin{array}{c}\text { Soil-to- } \\
\text { Plant } \\
\text { Uptake } \\
\text { (B) }\end{array}$ & $\begin{array}{c}\text { Soil-to- } \\
\text { Wet Plant } \\
\text { Uptake. } \\
\left(B_{\mathrm{vw}}\right)\end{array}$ & $\begin{array}{l}\text { Bioaccum- } \\
\text { ulation } \\
\text { Factor for } \\
\text { Fish } \\
\text { (L/kg) } \\
\text { [BF] }\end{array}$ & $\begin{array}{c}\text { Bio- } \\
\text { transfer } \\
\text { Coefficient } \\
\text { for Beef } \\
\text { (day/kg) } \\
{\left[F_{\mathrm{B}}\right]}\end{array}$ & $\begin{array}{c}\text { Bio- } \\
\text { transfer } \\
\text { Coefficient } \\
\text { for Milk } \\
\text { (day/kg) } \\
{\left[\mathrm{F}_{\mathrm{m}}\right]}\end{array}$ \\
\hline Sb-126 & $015756-32-8$ & $9.73 E-12$ & 8.41E-12 & 1.03E-05 & $1.24 \mathrm{E}+01$ & $5.0 \mathrm{E}-02$ & $1.0 \mathrm{E}-02$ & $1.0 \mathrm{E}+02$ & $4.0 \mathrm{E}-05$ & $2.5 \mathrm{E}-05$ \\
\hline $\mathrm{Sb}-126 \mathrm{~m}$ & $015756-32-8$ & $7.28 \mathrm{E}-14$ & $6.43 E-14$ & $5.78 \mathrm{E}-06$ & $1.32 \mathrm{E}-02$ & $5.0 \mathrm{E}-02$ & $1.0 \mathrm{E}-02$ & $1.0 \mathrm{E}+02$ & $4.0 \mathrm{E}-05$ & $2.5 \mathrm{E}-05$ \\
\hline Sb-127 & $013968-50-8$ & $8.48 \mathrm{E}-12$ & $6.05 \mathrm{E}-12$ & $2.40 \mathrm{E}-06$ & $3.85 \mathrm{E}+00$ & $5.0 \mathrm{E}-02$ & $1.0 \mathrm{E}-02$ & $1.0 \mathrm{E}+02$ & 4.0E-05 & $2.5 \mathrm{E}-05$ \\
\hline Sb-129 & $014331-88-5$ & $1.86 \mathrm{E}-12$ & $8.60 \mathrm{E}-13$ & $5.56 \mathrm{E}-06$ & $1.83 \mathrm{E}-01$ & $5.0 \mathrm{E}-02$ & $1.0 \mathrm{E}-02$ & $1.0 \mathrm{E}+02$ & 4.0E-05 & $2.5 \mathrm{E}-05$ \\
\hline Sc-46 & $013967-63-0$ & $5.73 \mathrm{E}-12$ & $1.31 \mathrm{E}-11$ & $7.89 \mathrm{E}-06$ & $8.38 \mathrm{E}+01$ & $1.0 \mathrm{E}-02$ & $2.0 \mathrm{E}-03$ & $1.0 \mathrm{E}+02$ & $2.0 \mathrm{E}-03$ & $6.0 \mathrm{E}-05$ \\
\hline Sc-47 & $014391-96-9$ & $2.95 \mathrm{E}-12$ & 2.01E-12 & 2.50E-07 & $3.42 \mathrm{E}+00$ & $1.0 \mathrm{E}-02$ & $2.0 \mathrm{E}-03$ & $1.0 \mathrm{E}+02$ & 2.0E-03 & $6.0 \mathrm{E}-05$ \\
\hline Sc-48 & $014391-86-7$ & $6.65 \mathrm{E}-12$ & $4.20 \mathrm{E}-12$ & $1.31 \mathrm{E}-05$ & $1.82 E+00$ & $1.0 \mathrm{E}-02$ & $2.0 \mathrm{E}-03$ & $1.0 \mathrm{E}+02$ & $2.0 \mathrm{E}-03$ & $6.0 \mathrm{E}-05$ \\
\hline Se-75 & $014265-71-5$ & $6.53 \mathrm{E}-12$ & $4.92 \mathrm{E}-12$ & 8.89E-07 & $1.20 \mathrm{E}+02$ & $5.0 \mathrm{E}-01$ & $1.0 \mathrm{E}-01$ & & $1.0 \mathrm{E}-01$ & $1.0 \mathrm{E}-02$ \\
\hline Si-31 & $014276-49-4$ & $5.04 \mathrm{E}-13$ & $3.29 \mathrm{E}-13$ & $3.45 \mathrm{E}-09$ & $1.09 \mathrm{E}-01$ & $3.5 \mathrm{E}-01$ & $8.8 \mathrm{E}-02$ & & $4.0 \mathrm{E}-05$ & $2.0 \mathrm{E}-05$ \\
\hline Sm-147 & $014392-33-7$ & $2.51 \mathrm{E}-11$ & $6.93 \mathrm{E}-09$ & $0.00 E+00$ & $3.87 \mathrm{E}+13$ & $1.0 \mathrm{E}-02$ & $2.5 \mathrm{E}-03$ & & $5.0 \mathrm{E}-03$ & $2.0 \mathrm{E}-05$ \\
\hline Sm-151 & $015715-94-3$ & $4.60 \mathrm{E}-13$ & 4.63E- 12 & $2.92 \mathrm{E}-13$ & $3.29 \mathrm{E}+04$ & $1.0 \mathrm{E}-02$ & $2.5 \mathrm{E}-03$ & & $5.0 \mathrm{E}-03$ & $2.0 \mathrm{E}-05$ \\
\hline $\mathrm{Sm}-153$ & $015766-00-4$ & $4.02 \mathrm{E}-12$ & $2.18 \mathrm{E}-12$ & $4.65 \mathrm{E}-08$ & $1.95 \mathrm{E}+00$ & $1.0 \mathrm{E}-02$ & $2.5 \mathrm{E}-03$ & & $5.0 \mathrm{E}-03$ & $2.0 \mathrm{E}-05$ \\
\hline Sn-113 & $013966-06-8$ & 3.72E-12 & $6.61 \mathrm{E}-12$ & 2.96E-09 & $1.15 E+02$ & $1.0 \mathrm{E}+00$ & $3.0 \mathrm{E}-01$ & $3.0 \mathrm{E}+03$ & $1.0 \mathrm{E}-02$ & $1.0 \mathrm{E}-03$ \\
\hline $\mathrm{Sn}-121$ & $014683-06-8$ & $1.22 \mathrm{E}-12$ & $6.13 \mathrm{E}-13$ & & $1.13 E+00$ & $1.0 \mathrm{E}+00$ & $3.0 \mathrm{E}-01$ & $3.0 \mathrm{E}+03$ & $1.0 \mathrm{E}-02$ & $1.0 \mathrm{E}-03$ \\
\hline $\mathrm{Sn}-121 \mathrm{~m}$ & $014683-06-8$ & $2.00 \mathrm{E}-12$ & $7.46 \mathrm{E}-12$ & & $2.03 E+04$ & $1.0 \mathrm{E}+00$ & $3.0 \mathrm{E}-01$ & $3.0 \mathrm{E}+03$ & $1.0 \mathrm{E}-02$ & $1.0 \mathrm{E}-03$ \\
\hline $\mathrm{Sn}-125$ & $014683-08-0$ & $1.68 \mathrm{E}-11$ & $1.19 \mathrm{E}-11$ & $1.21 \mathrm{E}-06$ & $9.64 \mathrm{E}+00$ & $1.0 \mathrm{E}+00$ & $3.0 \mathrm{E}-01$ & $3.0 E+03$ & $1.0 \mathrm{E}-02$ & $1.0 \mathrm{E}-03$ \\
\hline Sn-126 & $015832-50-5$ & 2.12E-11 & $4.26 \mathrm{E}-11$ & 3.32E-08 & $3.65 \mathrm{E}+07$ & $1.0 \mathrm{E}+00$ & $3.0 \mathrm{E}-01$ & $3.0 \mathrm{E}+03$ & $1.0 \mathrm{E}-02$ & $1.0 \mathrm{E}-03$ \\
\hline Sr-82 & $014809-50-8$ & $2.58 \mathrm{E}-11$ & 8.87E-12 & $9.00 \mathrm{E}-11$ & $2.50 \mathrm{E}+01$ & $1.1 \mathrm{E}+00$ & 2.1E-01 & $6.0 \mathrm{E}+01$ & $8.0 \mathrm{E}-03$ & $2.8 \mathrm{E}-03$ \\
\hline Sr-85 & $013967-73-2$ & $1.40 \mathrm{E}-12$ & $1.14 \mathrm{E}-12$ & $1.54 \mathrm{E}-06$ & $6.48 \mathrm{E}+01$ & $1.1 \mathrm{E}+00$ & 2.1E-01 & $6.0 \mathrm{E}+01$ & $8.0 \mathrm{E}-03$ & $2.8 \mathrm{E}-03$ \\
\hline
\end{tabular}


Table 3.6c. (continued)

\begin{tabular}{|c|c|c|c|c|c|c|c|c|c|c|}
\hline Chemical $^{\dagger}$ & CASRN & $\begin{array}{l}\text { Ingestion } \\
\text { SF } \\
(\mathrm{pCi})^{-1}\end{array}$ & $\begin{array}{c}\text { Inhalation } \\
\text { SF } \\
(\mathrm{pCi})^{-1}\end{array}$ & $\begin{array}{c}\text { External } \\
\text { Exposure } \\
\text { SF } \\
\frac{\mathrm{g}}{\mathrm{pCi}-\mathrm{yr}}\end{array}$ & $\begin{array}{l}\text { Half-life } \\
\text { (days) } \\
{\left[\mathbf{T}_{\mathbf{n}}\right]}\end{array}$ & $\begin{array}{c}\text { Soil-to- } \\
\text { Plant } \\
\text { Uptake } \\
\left(B_{v}\right)\end{array}$ & $\begin{array}{c}\text { Soil-to- } \\
\text { Wet Plant } \\
\text { Uptake } \\
\left(\mathbf{B}_{\mathrm{vw}}\right)\end{array}$ & $\begin{array}{l}\text { Bioaccum- } \\
\text { ulation } \\
\text { Factor for } \\
\text { Fish } \\
\text { (L/kg) } \\
\text { [BF] }\end{array}$ & $\begin{array}{c}\text { Bio- } \\
\text { transfer } \\
\text { Coefficient } \\
\text { for Beef } \\
(\text { day } / \mathrm{kg}) \\
{\left[\mathrm{F}_{\mathrm{B}}\right]}\end{array}$ & $\begin{array}{c}\text { Bio- } \\
\text { transfer } \\
\text { Coefficient } \\
\text { for Milk } \\
\text { (day/kg) } \\
{\left[F_{m}\right]}\end{array}$ \\
\hline $\mathrm{Sr}-85 \mathrm{~m}$ & 013967-73-2 & $1.80 \mathrm{E}-14$ & 7.13E-15 & $5.24 \mathrm{E}-07$ & 4.70E-02 & $1.1 \mathrm{E}+00$ & 2.1E-01 & $6.0 \mathrm{E}+01$ & 8.0E-03 & $2.8 \mathrm{E}-03$ \\
\hline Sr-89 & $014158-27-1$ & $1.03 \mathrm{E}-11$ & $3.68 \mathrm{E}-12$ & $5.38 \mathrm{E}-10$ & $5.06 \mathrm{E}+01$ & $1.1 \mathrm{E}+00$ & 2.1E-01 & $6.0 \mathrm{E}+01$ & 8.0E-03 & $2.8 \mathrm{E}-03$ \\
\hline Sr-90 & $010098-97-2$ & 4.09E-11 & $5.94 \mathrm{E}-11$ & $0.00 \mathrm{E}+00$ & $1.04 \mathrm{E}+04$ & $1.1 \mathrm{E}+00$ & 2.1E-01 & $6.0 E+01$ & $8.0 \mathrm{E}-03$ & $2.8 \mathrm{E}-03$ \\
\hline Sr-90+D & $010098-97-2$ & $5.59 \mathrm{E}-11$ & $6.93 \mathrm{E}-11$ & $0.00 \mathrm{E}+00$ & $1.04 \mathrm{E}+04$ & $1.1 \mathrm{E}+00$ & 2.1E-01 & $9.0 \mathrm{E}+01$ & $9.0 \mathrm{E}-03$ & $2.8 \mathrm{E}-03$ \\
\hline Sr-91 & $014331-91-0$ & $2.82 \mathrm{E}-12$ & $7.79 \mathrm{E}-13$ & 2.67E-06 & $3.96 \mathrm{E}-01$ & $1.1 \mathrm{E}+00$ & 2.1E-01 & $6.0 \mathrm{E}+01$ & $8.0 \mathrm{E}-03$ & $2.8 \mathrm{E}-03$ \\
\hline Sr-92 & $014928-29-1$ & 2.03E-12 & $4.70 \mathrm{E}-13$ & $5.20 \mathrm{E}-06$ & $1.13 \mathrm{E}-01$ & $1.1 \mathrm{E}+00$ & 2.1E-01 & $6.0 E+01$ & $8.0 \mathrm{E}-03$ & $2.8 \mathrm{E}-03$ \\
\hline Ta-182 & $013982-00-8$ & 7.03E-12 & $1.65 \mathrm{E}-11$ & $4.66 \mathrm{E}-06$ & $1.15 \mathrm{E}+02$ & $1.0 \mathrm{E}-02$ & 2.5E-03 & $1.0 \mathrm{E}+02$ & $6.0 \mathrm{E}-04$ & $3.0 \mathrm{E}-06$ \\
\hline Tb-158 & $015759-55-4$ & $4.20 \mathrm{E}-12$ & $7.04 \mathrm{E}-11$ & & $5.48 \mathrm{E}+04$ & $1.0 \mathrm{E}-02$ & $2.5 \mathrm{E}-03$ & & $4.5 \mathrm{E}-03$ & $2.0 \mathrm{E}-05$ \\
\hline $\mathrm{Tb}-160$ & 013981-29-8 & 7.62E-12 & $1.14 \mathrm{E}-11$ & 4.03E-06 & $7.23 E+01$ & $1.0 \mathrm{E}-02$ & $2.5 \mathrm{E}-03$ & & $4.5 \mathrm{E}-03$ & $2.0 \mathrm{E}-05$ \\
\hline Tc-95 & $014809-56-4$ & $6.81 \mathrm{E}-14$ & $3.38 \mathrm{E}-14$ & 2.72E-06 & 8.33E-01 & $7.6 \mathrm{E}+01$ & $2.1 \mathrm{E}+02$ & $2.0 \mathrm{E}+01$ & $1.0 \mathrm{E}-04$ & $1.4 \mathrm{E}-04$ \\
\hline Tc-95m & $014809-56-4$ & $1.24 \mathrm{E}-12$ & $2.10 \mathrm{E}-12$ & 2.08E-06 & $6.10 \mathrm{E}+01$ & $7.6 \mathrm{E}+01$ & $2.1 \mathrm{E}+02$ & $2.0 \mathrm{E}+01$ & $1.0 \mathrm{E}-04$ & 1.4E-04 \\
\hline Tc-96 & $014808-44-7$ & $2.28 \mathrm{E}-12$ & $1.94 \mathrm{E}-12$ & 9.36E-06 & $4.28 \mathrm{E}+00$ & $7.6 \mathrm{E}+01$ & $2.1 \mathrm{E}+02$ & $2.0 \mathrm{E}+01$ & $1.0 \mathrm{E}-04$ & $1.4 \mathrm{E}-04$ \\
\hline Tc-96m & 014808-44-7 & $2.61 \mathrm{E}-14$ & $2.26 \mathrm{E}-14$ & 7.72E-08 & $3.58 \mathrm{E}-02$ & $7.6 \mathrm{E}+01$ & $2.1 \mathrm{E}+02$ & $2.0 \mathrm{E}+01$ & $1.0 \mathrm{E}-04$ & $1.4 \mathrm{E}-04$ \\
\hline Tc-97 & 015759-35-0 & $1.58 \mathrm{E}-13$ & $3.44 \mathrm{E}-13$ & 2.49E-10 & $9.49 \mathrm{E}+08$ & $7.6 \mathrm{E}+01$ & $2.1 \mathrm{E}+02$ & $2.0 \mathrm{E}+01$ & $1.0 \mathrm{E}-04$ & $1.4 \mathrm{E}-04$ \\
\hline Tc-97m & $015759-35-0$ & $1.20 \mathrm{E}-12$ & $1.96 \mathrm{E}-12$ & 2.67E-10 & $8.90 \mathrm{E}+01$ & $7.6 \mathrm{E}+01$ & $2.1 \mathrm{E}+02$ & $2.0 \mathrm{E}+01$ & $1.0 \mathrm{E}-04$ & $1.4 \mathrm{E}-04$ \\
\hline Tc-99 & $014133-76-7$ & $1.40 \mathrm{E}-12$ & $2.89 \mathrm{E}-12$ & $6.19 \mathrm{E}-13$ & $7.77 \mathrm{E}+07$ & $7.6 \mathrm{E}+01$ & $2.1 \mathrm{E}+02$ & $2.0 \mathrm{E}+01$ & $1.0 \mathrm{E}-04$ & $1.4 \mathrm{E}-04$ \\
\hline Tc-99m & 014133-76-7 & $5.58 \mathrm{E}-14$ & $3.49 \mathrm{E}-14$ & 2.51E-07 & $2.51 \mathrm{E}-01$ & $7.6 \mathrm{E}+01$ & $2.1 \mathrm{E}+02$ & $2.0 \mathrm{E}+01$ & $1.0 \mathrm{E}-04$ & $1.4 \mathrm{E}-04$ \\
\hline Te-125m & $014390-73-9$ & $2.51 \mathrm{E}-12$ & $2.85 \mathrm{E}-12$ & $2.16 \mathrm{E}-09$ & $5.80 \mathrm{E}+01$ & $7.0 \mathrm{E}+00$ & $7.0 \mathrm{E}-01$ & $4.0 \mathrm{E}+02$ & $7.0 \mathrm{E}-03$ & $4.5 \mathrm{E}-04$ \\
\hline Te-127 & $013981-49-2$ & $8.55 \mathrm{E}-13$ & $4.32 \mathrm{E}-13$ & $1.62 \mathrm{E}-08$ & $3.90 \mathrm{E}-01$ & $7.0 \mathrm{E}+00$ & $7.0 \mathrm{E}-01$ & $4.0 \mathrm{E}+02$ & $7.0 \mathrm{E}-03$ & $4.5 \mathrm{E}-04$ \\
\hline
\end{tabular}


Table 3.6c. (continued)

\begin{tabular}{|c|c|c|c|c|c|c|c|c|c|c|}
\hline Chemical $^{t}$ & CASRN & $\begin{array}{c}\text { Ingestion } \\
\text { SF } \\
(\mathrm{pCi})^{-1}\end{array}$ & $\begin{array}{c}\text { Inhalation } \\
\text { SF } \\
(\mathrm{pC})^{-1}\end{array}$ & $\begin{array}{c}\text { External } \\
\text { Exposure } \\
\text { SF } \\
\frac{\mathrm{g}}{\mathrm{pCl}-\mathrm{yr}}\end{array}$ & $\begin{array}{c}\text { Half-life } \\
\text { (days) } \\
{\left[T_{R}\right]}\end{array}$ & $\begin{array}{c}\text { Soil-to- } \\
\text { Plant } \\
\text { Uptake } \\
\text { (B) }\end{array}$ & $\begin{array}{c}\text { Soil-to- } \\
\text { Wet Plant } \\
\text { Uptake } \\
\left(\mathbf{B}_{\text {nw }}\right)\end{array}$ & $\begin{array}{c}\text { Bioaccum- } \\
\text { ulation } \\
\text { Factor for } \\
\text { Fish } \\
\text { (L/kg) } \\
\text { [BF] }\end{array}$ & $\begin{array}{c}\text { Bio- } \\
\text { transfer } \\
\text { Coefficient } \\
\text { for Beef } \\
\text { (day/kg) } \\
{\left[\mathbf{F}_{\mathrm{B}}\right]}\end{array}$ & $\begin{array}{l}\text { Bio- } \\
\text { transfer } \\
\text { Coefficient } \\
\text { for Milk } \\
\text { (day/kg) } \\
{\left[F_{m}\right]}\end{array}$ \\
\hline $\mathrm{Te}-127 \mathrm{~m}$ & $013981-49-2$ & $6.01 \mathrm{E}-12$ & 1.31E-11 & $7.10 \mathrm{E}-10$ & $1.09 \mathrm{E}+02$ & $7.0 \mathrm{E}+00$ & $7.0 \mathrm{E}-01$ & $4.0 \mathrm{E}+02$ & $7.0 \mathrm{E}-03$ & $4.5 \mathrm{E}-04$ \\
\hline Te-129 & $014269-71-7$ & $1.48 \mathrm{E}-13$ & $1.46 \mathrm{E}-13$ & $1.46 E-07$ & $4.83 \mathrm{E}-02$ & $7.0 \mathrm{E}+00$ & $7.0 \mathrm{E}-01$ & $4.0 \mathrm{E}+02$ & $7.0 \mathrm{E}-03$ & $4.5 \mathrm{E}-04$ \\
\hline Te-129m & $014269-71-7$ & $1.17 \mathrm{E}-11$ & $1.33 \mathrm{E}-11$ & $6.92 \mathrm{E}-08$ & $3.36 \mathrm{E}+01$ & $7.0 \mathrm{E}+00$ & $7.0 \mathrm{E}-01$ & $4.0 \mathrm{E}+02$ & $7.0 \mathrm{E}-03$ & $4.5 \mathrm{E}-04$ \\
\hline Te-131 & $014683-12-6$ & $3.90 \mathrm{E}-13$ & $2.48 \mathrm{E}-13$ & $1.35 \mathrm{E}-06$ & $1.74 \mathrm{E}-02$ & $7.0 \mathrm{E}+00$ & $7.0 \mathrm{E}-01$ & $4.0 \mathrm{E}+02$ & $7.0 \mathrm{E}-03$ & 4.5E-04 \\
\hline $\mathrm{Te}-131 \mathrm{~m}$ & $014683-12-6$ & $8.81 \mathrm{E}-12$ & $8.40 \mathrm{E}-12$ & 5.31E-06 & $1.25 E+00$ & $7.0 \mathrm{E}+00$ & $7.0 \mathrm{E}-01$ & $4.0 \mathrm{E}+02$ & $7.0 \mathrm{E}-03$ & $4.5 \mathrm{E}-04$ \\
\hline Te-132 & $014234-28-7$ & $1.22 \mathrm{E}-11$ & 8.38E-12 & 4.31E-07 & $3.26 \mathrm{E}+00$ & $7.0 \mathrm{E}+00$ & $7.0 \mathrm{E}-01$ & $4.0 \mathrm{E}+02$ & $7.0 \mathrm{E}-03$ & $4.5 \mathrm{E}-04$ \\
\hline Th-227 & $015623-47-9$ & $4.04 \mathrm{E}-11$ & 4.31E-09 & $1.74 \mathrm{E}-07$ & $1.87 \mathrm{E}+01$ & $1.1 \mathrm{E}-02$ & $1.4 \mathrm{E}-04$ & $1.0 \mathrm{E}+02$ & 1.0E-04 & $5.0 \mathrm{E}-06$ \\
\hline Th-228 & $014274-82-9$ & $6.29 \mathrm{E}-11$ & $9.45 \mathrm{E}-08$ & $5.28 \mathrm{E}-10$ & $6.97 \mathrm{E}+02$ & 1:1E-02 & $1.4 \mathrm{E}-04$ & $1.0 \mathrm{E}+02$ & $1.0 \mathrm{E}-04$ & $5.0 \mathrm{E}-06$ \\
\hline Th-228+D & $014274-82-9$ & $2.31 \mathrm{E}-10$ & $9.68 \mathrm{E}-08$ & 9.94E-07 & $6.97 \mathrm{E}+02$ & $4.0 \mathrm{E}-01$ & $2.0 \mathrm{E}-02$ & $5.6 \mathrm{E}+02$ & $5.2 \mathrm{E}-02$ & $4.8 \mathrm{E}-03$ \\
\hline Th-229 & $015594-54-4$ & $5.65 \mathrm{E}-11$ & $7.60 \mathrm{E}-08$ & $5.94 \mathrm{E}-08$ & $2.68 \mathrm{E}+06$ & $1.1 \mathrm{E}-02$ & $1.4 \mathrm{E}-04$ & $1.0 \mathrm{E}+02$ & $1.0 \mathrm{E}-04$ & $5.0 \mathrm{E}-06$ \\
\hline Th-229+D & $015594-54-4$ & $3.56 \mathrm{E}-10$ & 8.26E-08 & 5.99E-07 & $2.68 E+06$ & $1.4 \mathrm{E}+00$ & $2.9 \mathrm{E}-01$ & $5.2 \mathrm{E}+02$ & $2.0 \mathrm{E}-02$ & 3.3E-02 \\
\hline Th-230 & $014269-63-7$ & $3.75 \mathrm{E}-11$ & $1.72 \mathrm{E}-08$ & $4.40 \mathrm{E}-11$ & $2.81 \mathrm{E}+07$ & $1.1 \mathrm{E}-02$ & $1.4 \mathrm{E}-04$ & $1.0 \mathrm{E}+02$ & $1.0 \mathrm{E}-04$ & $5.0 \mathrm{E}-06$ \\
\hline Th-231 & $014932-40-2$ & $1.79 \mathrm{E}-12$ & $1.10 \mathrm{E}-12$ & 2.09E-09 & $1.06 \mathrm{E}+00$ & 1.1E-02 & $1.4 \mathrm{E}-04$ & $1.0 \mathrm{E}+02$ & $1.0 \mathrm{E}-04$ & $5.0 \mathrm{E}-06$ \\
\hline Th-232 & $007440-29-1$ & $3.28 \mathrm{E}-11$ & $1.93 \mathrm{E}-08$ & $1.97 \mathrm{E}-11$ & $5.15 E+12$ & $1.1 \mathrm{E}-02$ & $1.4 \mathrm{E}-04$ & $1.0 \mathrm{E}+02$ & $1.0 \mathrm{E}-04$ & $5.0 \mathrm{E}-06$ \\
\hline Th-234 & $015065-10-8$ & $1.93 \mathrm{E}-11$ & $1.90 \mathrm{E}-11$ & $3.50 \mathrm{E}-09$ & $2.41 \mathrm{E}+01$ & $1.1 \mathrm{E}-02$ & $1.4 \mathrm{E}-04$ & $1.0 \mathrm{E}+02$ & $1.0 \mathrm{E}-04$ & $5.0 \mathrm{E}-06$ \\
\hline $\mathrm{Tl}-202$ & $015720-57-7$ & $1.01 \mathrm{E}-12$ & $6.07 \mathrm{E}-13$ & $1.42 \mathrm{E}-06$ & $1.22 \mathrm{E}+01$ & $4.0 \mathrm{E}-03$ & $1.0 \mathrm{E}-03$ & & $4.0 \mathrm{E}-02$ & $2.0 \mathrm{E}-03$ \\
\hline Tl-204 & $013968-51-9$ & 1.97E-12 & $1.15 \mathrm{E}-12$ & $8.72 \mathrm{E}-10$ & $1.38 \mathrm{E}+03$ & $4.0 \mathrm{E}-03$ & $1.0 \mathrm{E}-03$ & & $4.0 \mathrm{E}-02$ & $2.0 \mathrm{E}-03$ \\
\hline Tl-208 & 014913-50-9 & $1.75 \mathrm{E}-14$ & $1.36 \mathrm{E}-14$ & $1.45 \mathrm{E}-05$ & $2.12 \mathrm{E}-03$ & $4.0 \mathrm{E}-03$ & $1.0 \mathrm{E}-03$ & & $4.0 \mathrm{E}-02$ & $2.0 \mathrm{E}-03$ \\
\hline Tl-209 & 015690-73-0 & $1.40 \mathrm{E}-14$ & $1.12 \mathrm{E}-14$ & $7.83 \mathrm{E}-06$ & $1.53 \mathrm{E}-03$ & $4.0 \mathrm{E}-03$ & $1.0 \mathrm{E}-03$ & & $4.0 \mathrm{E}-02$ & $2.0 \mathrm{E}-03$ \\
\hline
\end{tabular}

Chemical-specific parameters used in calculating PRGs/radionuclides

Page 15 of 18 
Table 3.6c. (continued)

\begin{tabular}{|c|c|c|c|c|c|c|c|c|c|c|}
\hline Chemical $^{\dagger}$ & CASRN & $\begin{array}{c}\text { Ingestion } \\
\text { SF } \\
(\mathrm{pCi})^{-1}\end{array}$ & $\begin{array}{c}\text { Inhalation } \\
\text { SF } \\
(\mathrm{pCi})^{-1}\end{array}$ & $\begin{array}{c}\text { External } \\
\text { Exposure } \\
\text { SF } \\
\frac{\mathbf{g}}{\mathbf{p C i}-\mathrm{yr}}\end{array}$ & $\begin{array}{c}\text { Half-life } \\
\text { (days) } \\
{\left[\mathrm{T}_{\mathrm{R}}\right]}\end{array}$ & $\begin{array}{l}\text { Soil-to- } \\
\text { Plant } \\
\text { Uptake } \\
\left(B_{v}\right)\end{array}$ & $\begin{array}{c}\text { Soil-to- } \\
\text { Wet Plant } \\
\text { Uptake } \\
\left(\mathbf{B}_{\mathrm{vw}}\right)\end{array}$ & $\begin{array}{l}\text { Bioaccum- } \\
\text { ulation } \\
\text { Factor for } \\
\text { Fish } \\
\text { (L/kg) } \\
\text { [BF] }\end{array}$ & $\begin{array}{c}\text { Bio- } \\
\text { transfer } \\
\text { Coefficient } \\
\text { for Beef } \\
(\text { day } / \mathrm{kg}) \\
{\left[\mathrm{F}_{\mathrm{B}}\right]}\end{array}$ & $\begin{array}{c}\text { Bio- } \\
\text { transfer } \\
\text { Coefficient } \\
\text { for Milk } \\
\text { (day/kg) } \\
{\left[\mathrm{F}_{\mathrm{m}}\right]}\end{array}$ \\
\hline $\operatorname{Tm}-170$ & $013981-30-1$ & $7.50 \mathrm{E}-12$ & $1.10 \mathrm{E}-11$ & $3.84 \mathrm{E}-09$ & $1.29 \mathrm{E}+02$ & $1.0 \mathrm{E}-02$ & $2.5 \mathrm{E}-03$ & & $4.5 \mathrm{E}-03$ & $2.0 \mathrm{E}-05$ \\
\hline Tm-171 & $014333-45-0$ & $5.86 \mathrm{E}-13$ & $1.84 \mathrm{E}-12$ & $3.15 \mathrm{E}-10$ & $7.01 E+02$ & $1.0 \mathrm{E}-02$ & $2.5 \mathrm{E}-03$ & & 4.5E-03 & 2.0E-05 \\
\hline U-232 & $014158-29-3$ & $8.12 \mathrm{E}-11$ & $5.29 \mathrm{E}-08$ & 3.42E-11 & $2.63 E+04$ & 2.3E-02 & $6.3 \mathrm{E}-04$ & $1.0 \mathrm{E}+01$ & $3.0 \mathrm{E}-04$ & $4.0 \mathrm{E}-04$ \\
\hline U-233 & 013968-55-3 & $4.48 \mathrm{E}-11$ & $1.41 \mathrm{E}-08$ & $3.52 \mathrm{E}-11$ & $5.80 \mathrm{E}+07$ & 2.3E-02 & $6.3 \mathrm{E}-04$ & $1.0 \mathrm{E}+01$ & $3.0 \mathrm{E}-04$ & $4.0 \mathrm{E}-04$ \\
\hline U-234 & $013966-29-5$ & $4.44 \mathrm{E}-11$ & $1.40 \mathrm{E}-08$ & $2.14 \mathrm{E}-11$ & $8.94 \mathrm{E}+07$ & $2.3 \mathrm{E}-02$ & $6.3 \mathrm{E}-04$ & $1.0 \mathrm{E}+01$ & $3.0 \mathrm{E}-04$ & $4.0 \mathrm{E}-04$ \\
\hline U-235 & $015117-96-1$ & $4.52 \mathrm{E}-11$ & $1.30 \mathrm{E}-08$ & 2.63E-07 & $2.57 \mathrm{E}+11$ & $2.3 \mathrm{E}-02$ & $6.3 \mathrm{E}-04$ & $1.0 \mathrm{E}+01$ & $3.0 \mathrm{E}-04$ & $4.0 \mathrm{E}-04$ \\
\hline $\mathrm{U}-235+\mathrm{D}$ & $015117-96-1$ & $4.70 \mathrm{E}-11$ & $1.30 \mathrm{E}-08$ & 2.65E-07 & $2.57 \mathrm{E}+11$ & $3.4 \mathrm{E}-02$ & 7.7E-04 & $1.1 \mathrm{E}+02$ & $4.0 \mathrm{E}-04$ & 4.1E-04 \\
\hline U-236 & $013982-70-2$ & $4.21 \mathrm{E}-11$ & $1.32 \mathrm{E}-08$ & $1.72 \mathrm{E}-11$ & $8.54 E+09$ & 2.3E-02 & $6.3 \mathrm{E}-04$ & $1.0 \mathrm{E}+01$ & $3.0 \mathrm{E}-04$ & $4.0 \mathrm{E}-04$ \\
\hline U-237 & $014269-75-1$ & $3.98 \mathrm{E}-12$ & $3.12 \mathrm{E}-12$ & $1.37 \mathrm{E}-07$ & $6.75 \mathrm{E}+00$ & $2.3 \mathrm{E}-02$ & $6.3 \mathrm{E}-04$ & $1.0 \mathrm{E}+01$ & $3.0 \mathrm{E}-04$ & $4.0 \mathrm{E}-04$ \\
\hline U-238 & $007440-61-1$ & $4.27 \mathrm{E}-11$ & $1.24 \mathrm{E}-08$ & $1.50 \mathrm{E}-11$ & $1.63 E+12$ & 2.3E-02 & $6.3 \mathrm{E}-04$ & $1.0 \mathrm{E}+01$ & $3.0 \mathrm{E}-04$ & $4.0 \mathrm{E}-04$ \\
\hline U-238+D & $007440-61-1$ & $6.20 \mathrm{E}-11$ & $1.24 \mathrm{E}-08$ & $5.25 \mathrm{E}-08$ & $1.63 E+12$ & $3.9 \mathrm{E}-02$ & $2.0 \mathrm{E}-03$ & $1.3 \mathrm{E}+02$ & $4.2 \mathrm{E}-04$ & $4.2 \mathrm{E}-04$ \\
\hline U-240 & $015687-53-3$ & $5.47 \mathrm{E}-12$ & $3.35 \mathrm{E}-12$ & $1.09 \mathrm{E}-10$ & $5.88 \mathrm{E}-01$ & 2.3E-02 & $6.3 \mathrm{E}-04$ & $1.0 \mathrm{E}+01$ & $3.0 \mathrm{E}-04$ & $4.0 \mathrm{E}-04$ \\
\hline V-48 & $014331-97-6$ & $7.56 \mathrm{E}-12$ & $6.84 \mathrm{E}-12$ & $1.12 \mathrm{E}-05$ & $1.60 \mathrm{E}+01$ & $5.5 \mathrm{E}-03$ & $1.4 \mathrm{E}-03$ & $1.0 \mathrm{E}+01$ & $2.5 \mathrm{E}-03$ & $2.0 \mathrm{E}-05$ \\
\hline W-181 & $015749-46-9$ & 2.72E-13 & $8.02 \mathrm{E}-14$ & $2.11 \mathrm{E}-08$ & $1.21 \mathrm{E}+02$ & $3.0 \mathrm{E}+00$ & $1.0 \mathrm{E}-02$ & $1.0 \mathrm{E}+01$ & $4.0 \mathrm{E}-02$ & $3.0 \mathrm{E}-04$ \\
\hline W-185 & $014932-41-3$ & $2.04 \mathrm{E}-12$ & $4.26 \mathrm{E}-13$ & $5.03 \mathrm{E}-11$ & $7.51 \mathrm{E}+01$ & $3.0 \mathrm{E}+00$ & $1.0 \mathrm{E}-02$ & $1.0 \mathrm{E}+01$ & $4.0 \mathrm{E}-02$ & $3.0 \mathrm{E}-04$ \\
\hline W-187 & $014983-48-3$ & $2.46 \mathrm{E}-12$ & $5.29 \mathrm{E}-13$ & $1.63 \mathrm{E}-06$ & $9.92 \mathrm{E}-01$ & $3.0 \mathrm{E}+00$ & $1.0 \mathrm{E}-02$ & $1.0 \mathrm{E}+01$ & $4.0 \mathrm{E}-02$ & 3.0E-04 \\
\hline $\mathrm{Xe}-122$ & $015151-09-4$ & & $3.08 \mathrm{E}-15$ & & $8.38 \mathrm{E}-01$ & $0.0 \mathrm{E}+00$ & $0.0 \mathrm{E}+00$ & & $0.0 \mathrm{E}+00$ & $0.0 \mathrm{E}+00$ \\
\hline $\mathrm{Xe}-123$ & $015700-10-4$ & & $8.92 \mathrm{E}-16$ & & $8.92 \mathrm{E}-02$ & $0.0 \mathrm{E}+00$ & $0.0 \mathrm{E}+00$ & & $0.0 \mathrm{E}+00$ & $0.0 \mathrm{E}+00$ \\
\hline $\mathrm{Xe}-125$ & $013994-18-8$ & & $1.20 \mathrm{E}-15$ & & $7.00 \mathrm{E}-01$ & $0.0 \mathrm{E}+00$ & $0.0 \mathrm{E}+00$ & & $0.0 \mathrm{E}+00$ & $0.0 \mathrm{E}+00$ \\
\hline
\end{tabular}


Table 3.6c. (continued)

\begin{tabular}{|c|c|c|c|c|c|c|c|c|c|c|}
\hline Chemical $^{t}$ & CASRN & $\begin{array}{c}\text { Ingestion } \\
\text { SF } \\
{(\text { pCi })^{-1}}^{-1}\end{array}$ & $\begin{array}{c}\text { Inhalation } \\
\text { SF } \\
(\mathrm{pCi})^{-1}\end{array}$ & $\begin{array}{c}\text { External } \\
\text { Exposure } \\
\text { SF } \\
\frac{\mathrm{g}}{\mathrm{pC} \mathbf{-}-\mathrm{yr}}\end{array}$ & $\begin{array}{c}\text { Half-life } \\
\text { (days) } \\
{\left[T_{R}\right\rfloor}\end{array}$ & $\begin{array}{c}\text { Soil-to- } \\
\text { Plant } \\
\text { Uptake } \\
\text { (B, })\end{array}$ & $\begin{array}{c}\text { Soil-to- } \\
\text { Wet Plant } \\
\text { Uptake } \\
\left(B_{\mathrm{vw}}\right)\end{array}$ & $\begin{array}{l}\text { Bioaccum- } \\
\text { ulation } \\
\text { Factor for } \\
\text { Fish } \\
(\mathrm{L} / \mathrm{kg}) \\
{[\mathrm{BF}]}\end{array}$ & $\begin{array}{c}\text { Bio- } \\
\text { transfer } \\
\text { Coefficient } \\
\text { for Beef } \\
\text { (day/kg) } \\
{\left[\mathrm{F}_{\mathrm{B}}\right]}\end{array}$ & $\begin{array}{c}\text { Bio- } \\
\text { transfer } \\
\text { Coefficient } \\
\text { for Milk } \\
\text { (day/kg) } \\
{\left[\mathrm{F}_{\mathrm{m}}\right]}\end{array}$ \\
\hline Xe-127 & 013994-19-9 & & $4.09 \mathrm{E}-16$ & & $3.64 E+01$ & $0.0 \mathrm{E}+00$ & $0.0 \mathrm{E}+00$ & & $0.0 \mathrm{E}+00$ & $0.0 \mathrm{E}+00$ \\
\hline $\mathrm{Xe}-129 \mathrm{~m}$ & $013965-99-6$ & & $5.74 \mathrm{E}-16$ & & $8.89 \mathrm{E}+00$ & $0.0 \mathrm{E}+00$ & $0.0 \mathrm{E}+00$ & & $0.0 \mathrm{E}+00$ & $0.0 \mathrm{E}+00$ \\
\hline $\mathrm{Xe}-131 \mathrm{~m}$ & $014683-11-5$ & & 4.13E-16 & & $1.18 \mathrm{E}+01$ & $0.0 \mathrm{E}+00$ & $0.0 \mathrm{E}+00$ & & $0.0 \mathrm{E}+00$ & $0.0 \mathrm{E}+00$ \\
\hline Xe-133 & $014932-42-4$ & & $4.14 \mathrm{E}-16$ & & $5.25 E+00$ & $0.0 \mathrm{E}+00$ & $0.0 \mathrm{E}+00$ & & $0.0 \mathrm{E}+00$ & $0.0 \mathrm{E}+00$ \\
\hline $\mathrm{Xe}-133 \mathrm{~m}$ & $014932-42-4$ & & $5.12 \mathrm{E}-16$ & & $2.19 \mathrm{E}+00$ & $0.0 \mathrm{E}+00$ & $0.0 \mathrm{E}+00$ & & $0.0 \mathrm{E}+00$ & $0.0 \mathrm{E}+00$ \\
\hline $\mathrm{Xe}-135$ & $014995-62-1$ & & $7.45 \mathrm{E}-16$ & & $3.80 \mathrm{E}-01$ & $0.0 E+00$ & $0.0 \mathrm{E}+00$ & & $0.0 \mathrm{E}+00$ & $0.0 \mathrm{E}+00$ \\
\hline $\mathrm{Xe}-135 \mathrm{~m}$ & 014995-62-1 & & $1.88 \mathrm{E}-16$ & & 1.07E-02 & $0.0 \mathrm{E}+00$ & $0.0 \mathrm{E}+00$ & & $0.0 \mathrm{E}+00$ & $0.0 \mathrm{E}+00$ \\
\hline Xe-137 & $014835-21-3$ & & $1.39 \mathrm{E}-15$ & & $2.66 \mathrm{E}-03$ & $0.0 \mathrm{E}+00$ & $0.0 \mathrm{E}+00$ & & $0.0 \mathrm{E}+00$ & $0.0 \mathrm{E}+00$ \\
\hline $\mathrm{Xe}-138$ & $015751-81-2$ & & $2.06 \mathrm{E}-15$ & & $9.79 \mathrm{E}-03$ & $0.0 \mathrm{E}+00$ & $0.0 \mathrm{E}+00$ & & $0.0 \mathrm{E}+00$ & $0.0 \mathrm{E}+00$ \\
\hline Y-90 & $010098-91-6$ & $1.50 \mathrm{E}-11$ & $9.90 \mathrm{E}-12$ & $0.00 \mathrm{E}+00$ & $2.67 \mathrm{E}+00$ & $1.0 \mathrm{E}-02$ & $1.0 \mathrm{E}-03$ & $3.0 \mathrm{E}+01$ & $1.0 \mathrm{E}-03$ & $2.0 \mathrm{E}-05$ \\
\hline$Y-91$ & $014234-24-3$ & $1.35 \mathrm{E}-11$ & $1.85 \mathrm{E}-11$ & 1.41E-08 & $5.85 \mathrm{E}+01$ & $1.0 \mathrm{E}-02$ & $1.0 \mathrm{E}-03$ & $3.0 \mathrm{E}+01$ & $1.0 \mathrm{E}-03$ & $2.0 \mathrm{E}-05$ \\
\hline$Y-91 m$ & $014234-24-3$ & $3.69 \mathrm{E}-14$ & $2.99 \mathrm{E}-14$ & $1.90 \mathrm{E}-06$ & $3.45 \mathrm{E}-02$ & $1.0 \mathrm{E}-02$ & $1.0 \mathrm{E}-03$ & $3.0 \mathrm{E}+01$ & $1.0 \mathrm{E}-03$ & $2.0 \mathrm{E}-05$ \\
\hline Y-92 & $015751-59-4$ & $1.95 \mathrm{E}-12$ & $1.61 \mathrm{E}-12$ & $9.80 \mathrm{E}-07$ & $1.48 \mathrm{E}-01$ & $1.0 \mathrm{E}-02$ & $1.0 \mathrm{E}-03$ & $3.0 \mathrm{E}+01$ & $1.0 \mathrm{E}-03$ & $2.0 \mathrm{E}-05$ \\
\hline$Y-93$ & $014981-70-5$ & $5.74 \mathrm{E}-12$ & $3.48 \mathrm{E}-12$ & $3.41 \mathrm{E}-07$ & $4.21 \mathrm{E}-01$ & $1.0 \mathrm{E}-02$ & $1.0 \mathrm{E}-03$ & $3.0 \mathrm{E}+01$ & $1.0 \mathrm{E}-03$ & $2.0 \mathrm{E}-05$ \\
\hline $\mathrm{Zn}-65$ & $013982-39-3$ & $9.93 \mathrm{E}-12$ & $9.98 \mathrm{E}-12$ & 2.27E-06 & $2.44 E+02$ & $9.9 \mathrm{E}-01$ & $2.6 \mathrm{E}-01$ & $1.0 \mathrm{E}+03$ & $1.0 \mathrm{E}-01$ & $1.0 \mathrm{E}-02$ \\
\hline $\mathrm{Zn}-69$ & $013982-23-5$ & $6.19 \mathrm{E}-14$ & $1.04 \mathrm{E}-13$ & $2.03 \mathrm{E}-11$ & $3.86 \mathrm{E}-02$ & 9.9E-01 & $2.6 \mathrm{E}-01$ & $1.0 \mathrm{E}+03$ & $1.0 \mathrm{E}-01$ & $1.0 \mathrm{E}-02$ \\
\hline $\mathrm{Zn}-69 \mathrm{~m}$ & $013982-23-5$ & $1.52 \mathrm{E}-12$ & $1.17 \mathrm{E}-12$ & $1.43 \mathrm{E}-06$ & $5.75 \mathrm{E}-01$ & 9.9E-01 & $2.6 \mathrm{E}-01$ & $1.0 \mathrm{E}+03$ & $1.0 \mathrm{E}-01$ & $1.0 \mathrm{E}-02$ \\
\hline $\mathrm{Zr}-93$ & $015751-77-6$ & $5.21 \mathrm{E}-13$ & $5.26 \mathrm{E}-12$ & $0.00 \mathrm{E}+00$ & $5.58 \mathrm{E}+08$ & $1.0 \mathrm{E}-03$ & $1.0 \mathrm{E}-04$ & $3.0 \mathrm{E}+02$ & $1.0 \mathrm{E}-06$ & 5.5E-07 \\
\hline $\mathrm{Zr}-95$ & $013967-71-0$ & $3.92 \mathrm{E}-12$ & $6.48 \mathrm{E}-12$ & $2.81 \mathrm{E}-06$ & $6.40 \mathrm{E}+01$ & $1.0 \mathrm{E}-03$ & $1.0 \mathrm{E}-04$ & $3.0 \mathrm{E}+02$ & $1.0 \mathrm{E}-06$ & 5.5E-07 \\
\hline
\end{tabular}

Chemical-specific parameters used in calculating PRGs/radionuclides

Page 17 of 18 
Table 3.6c. (continued)

\begin{tabular}{|c|c|c|c|c|c|c|c|c|c|c|}
\hline Chemical $^{\dagger}$ & CASRN & $\begin{array}{c}\text { Ingestion } \\
\text { SF } \\
(\mathrm{pCi})^{-t}\end{array}$ & $\begin{array}{c}\text { Inhalation } \\
\text { SF } \\
(\mathbf{p C i})^{-1}\end{array}$ & $\begin{array}{c}\text { External } \\
\text { Exposure } \\
\text { SF } \\
\frac{g}{\text { pCi-yr }}\end{array}$ & $\begin{array}{c}\text { Half-life } \\
\text { (days) } \\
{\left[T_{R}\right]}\end{array}$ & $\begin{array}{c}\text { Soil-to- } \\
\text { Plant } \\
\text { Uptake } \\
\left(B_{v}\right)\end{array}$ & $\begin{array}{c}\text { Soil-to- } \\
\text { Wet Plant } \\
\text { Uptake } \\
\left(B_{v w}\right)\end{array}$ & $\begin{array}{l}\text { Bioaccum- } \\
\text { ulation } \\
\text { Factor for } \\
\text { Fish } \\
\text { (L/kg) } \\
\text { [BF] }\end{array}$ & $\begin{array}{c}\text { Bio- } \\
\text { transfer } \\
\text { Coefficient } \\
\text { for Beef } \\
(\text { day } / \mathrm{kg}) \\
{\left[\mathrm{F}_{\mathrm{B}}\right]}\end{array}$ & $\begin{array}{c}\text { Bio- } \\
\text { transfer } \\
\text { Coefficient } \\
\text { for Milk } \\
\text { (day/kg) } \\
{\left[F_{\mathrm{m}}\right]}\end{array}$ \\
\hline Zr-97 & 014928-30-4 & $1.04 \mathrm{E}-11$ & 4.73E-12 & $6.85 \mathrm{E}-07$ & $7.04 \mathrm{E}-01$ & $1.0 \mathrm{E}-03$ & $1.0 \mathrm{E}-04$ & $3.0 \mathrm{E}+02$ & $1.0 \mathrm{E}-06$ & $5.5 \mathrm{E}-07$ \\
\hline
\end{tabular}

Note:

$\dagger \quad$ Isotopes designated with an " $\mathrm{m}$ " indicate that the isotope is metastable; isotopes designated with a " $+\mathrm{D}$ " indicate that the radionuclide slope factors include contributions from daughter products. 


\section{DISTRIBUTION}

1. L. V. Asplund

2. L. D. Bloom

3. R. R. Bonczek

4. M. Clauberg

5. S. R. Conder

6. K. M. Golden

7. S. B. Kerr

8-10. S. B. Kerr (U)

11. D. M. Matteo (U)

12. C. W. McGinn

13. J. H. Moyer

14. B. D. Nourse

15-16. P. T. Owen

17. S. T. Purucker

18. A. S. Quist

19. S. E. Stinnette

20. S. G. Thornton (U)

21. D. C. White

22. Central Research Library

23. ER Document Management Center-RC

24. ER Document Management Center (U)

25. A. Temeshy, Bechtel National Inc., 151 Lafayette Dr., Oak Ridge, TN 37831-0350

26. M. Leslie, CDM Federal Programs Corporation, 800 Oak Ridge Turnpike, Suite 500, Oak Ridge, TN 37830

27. D. Gonzalez, Jacobs Engineering, 100 West 3rd St., Piketon, OH 45611 (614) 9478847

28. S. Pack, Science Applications International Corporation, P.0. Box 2501, 301, Laboratory Rd., Oak Ridge, TN 37831

29. T. Perry, DOE Oak Ridge Operations Office, P.O. Box 2001, Oak Ridge, TN 37831-8541 